

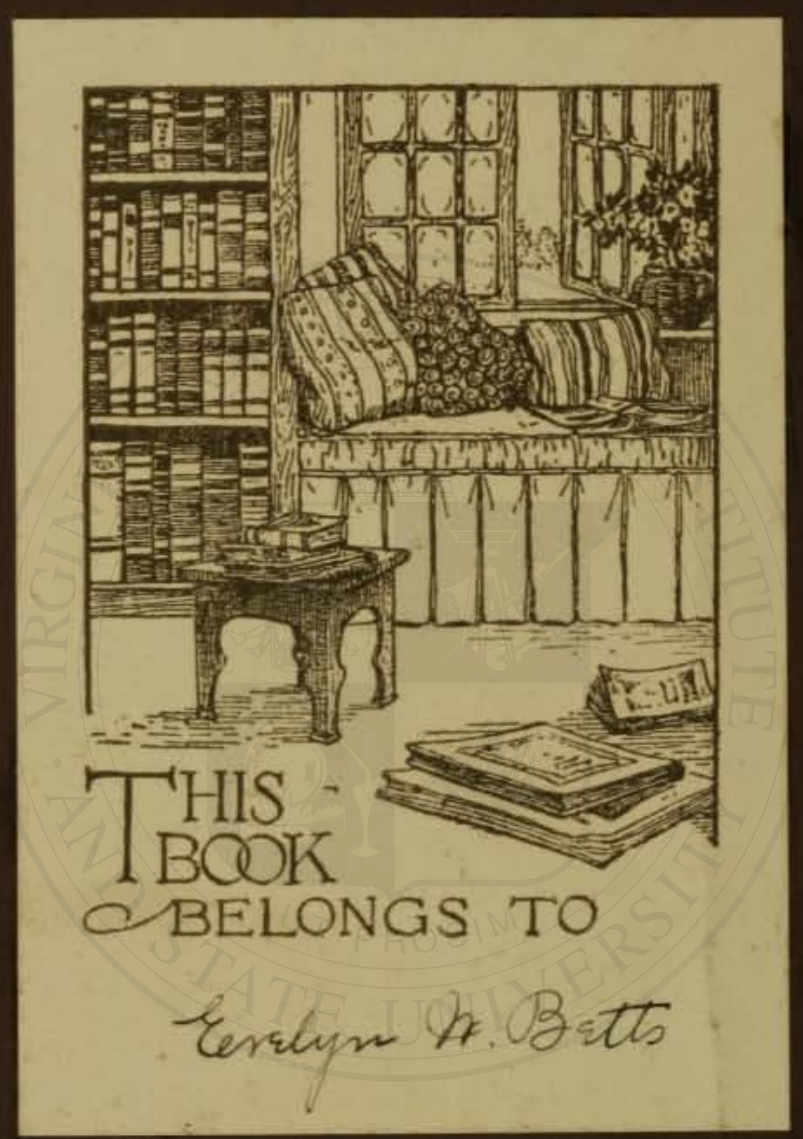





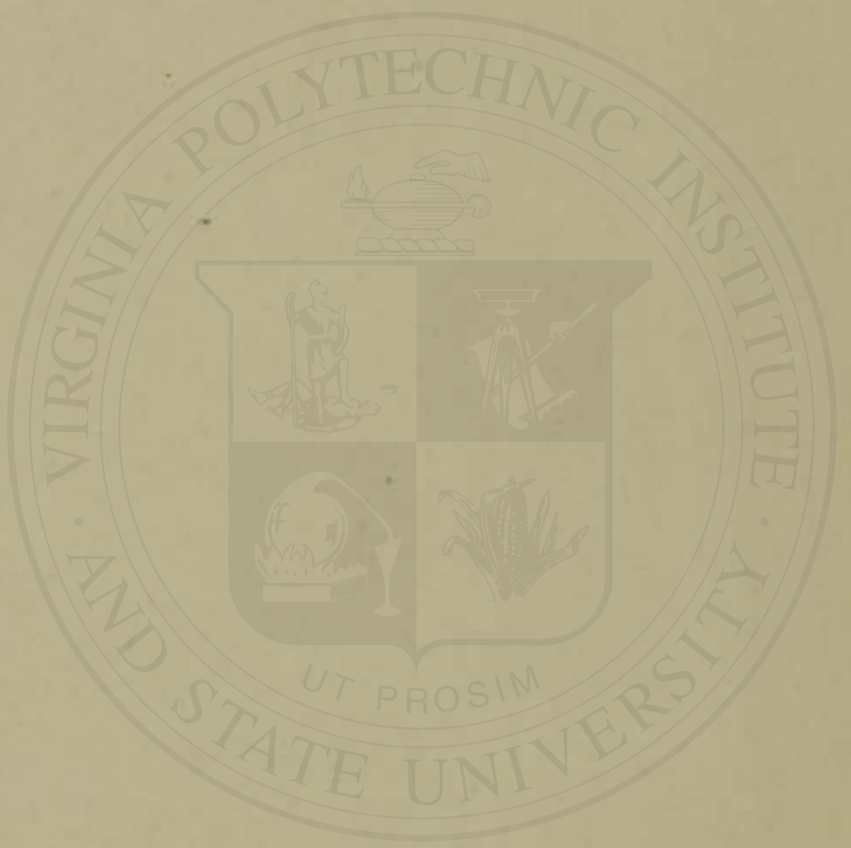


chemiton

$\$ 22^{\circ 0}$

Pood + A T T - 4

wt ptd 1904, thes corpy hus sererae cerrer spotsa lis $\$$ on olisty ele the ed newised 6 ha

$$
\text { tevelyn at Betto }
$$

foly 1914

SCANNED AT VIRGINIA POLYTECHNIC INSTITUTE AND STATE UNHAERSTY - 


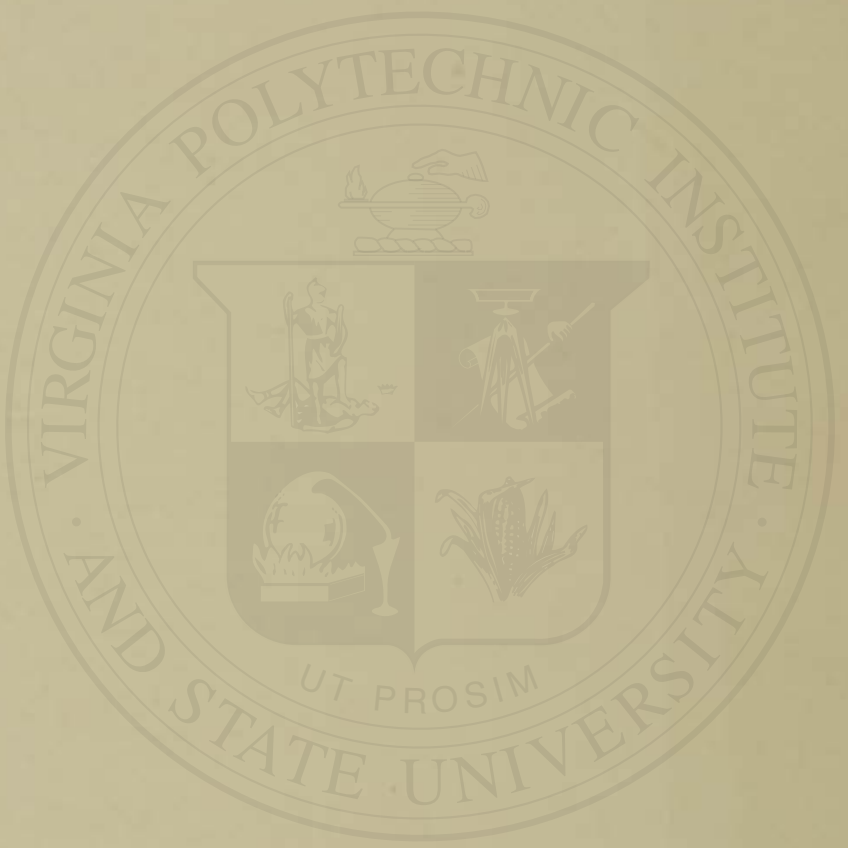




\title{
FOOD INSPECTION AND ANALYSIS.
}

\author{
FOR THE USE OF PUBLIC ANALYSTS, HEALTH \\ OFFICERS, SANITARY CHEMISTS, \\ AND FOOD ECONOMISTS.
}

BX

ALBERT E. LEACH, S.B.,

Late Chief of the Denver Food and Drug Inspection Laboratory, Bureau of Chemistry, U.S. Department of A griculture; Late Chief A nalyst of the Massachusetts Stale Board of Health.

REVISED AND ENLARGED BY

ANDREW L. WINTON, PH.D.,

Chief of the Chicago Food and Drug Inspection Laboratory, Bureau of Chemistry, U.S. Department of Agriculture.

THIRD EDITION, FIRST THOUSAND.

TOTAL ISSUE, FIVE THOUSAND.

NEW YORK:

JOHN WILEY \& SONS.

LONDON: CHAPMAN \& HALL, LIMTTED.

I9I3. 


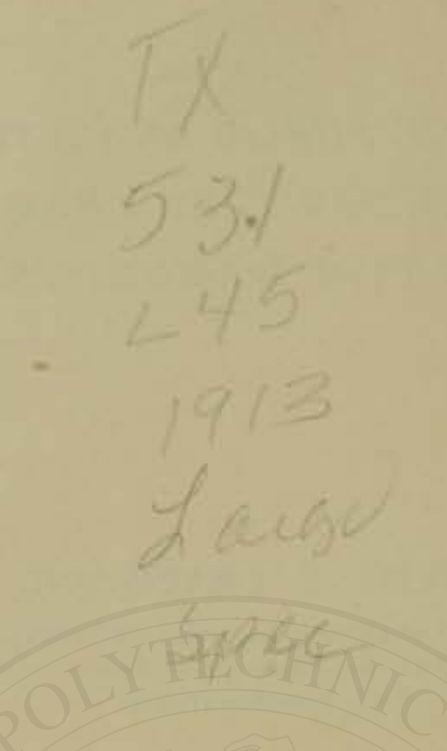

CopYright, 1904, 1909. BY

ALBERT E. LEACH.

First Edition Entered at Stationers' Hall."

Copyrigut, r9r3.

BY

MRS, MARTHA T, LEACH.

1266

\section{DUNGANNON HIGH SCHOOL}

THE SCIENTIFIC PRESE

nOEETT DMUMMOND AND COMRAMT BROOKL $Y$ N, $N, r$. 
Affectionately Dedicated to the Memory of Charles jomeroy Woresster,

former Analyst of the Massachusetts State Board of Health, WHOSE LOVABLE PERSONALITY AND STERLING INTEGRITY WERE

A CONSTANT INSPIRATION DURING MANY YEARS

OF CLOSE COMPANIONSHIP TO

THE AUTHOR. 


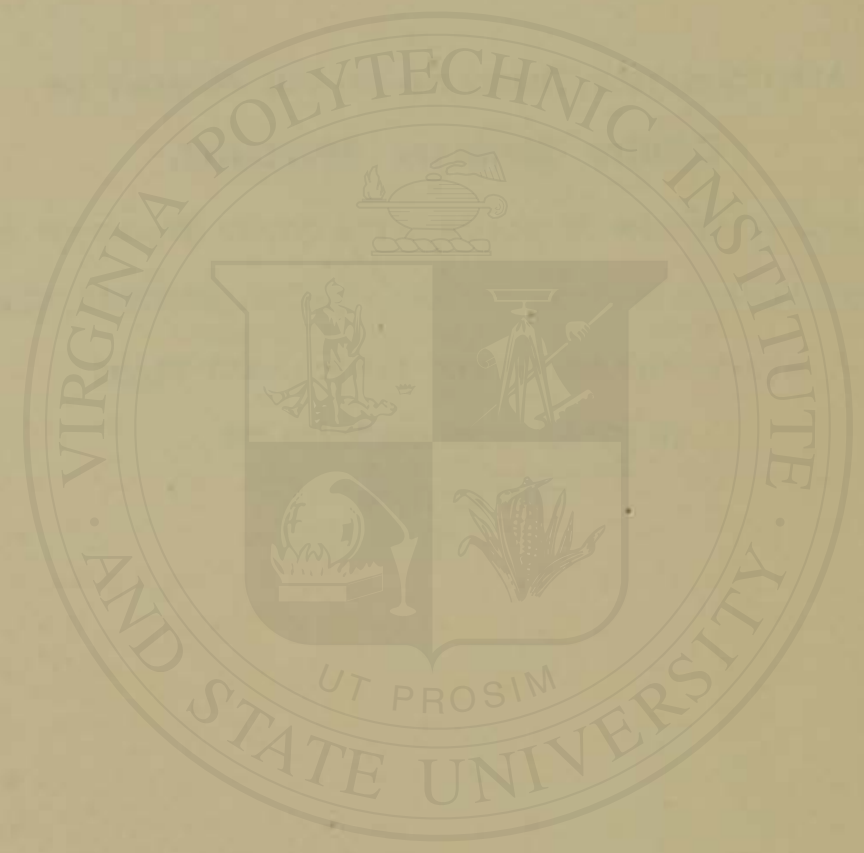




\section{PREFACE TO*THIRD EDITION.}

THE period since the appearance of the second edition has been, in America, one of steady progress in food science as compared with the period of special activity, stimulated by federal legislation, immediately preceding and the pioneer period, in which the author was a prominent figure, that led to the publication of the first edition.

Without changing the general plan of the work, which ought ever to remain as a monument to the author's remarkable grasp of the subject, new matter equivalent to about 80 pages, not including some 40 pages changed in the last thousand of the second edition, and I2 new cuts, have been added. The size of the work, however, has been increased but 47 pages, as much antiquated matter has been replaced by new, thus performing a double service to the reader.

Among the new features are improved general methods and apparatus for the determination of moisture, ash, and arsenic, modern apparatus for the Babcock test, processes for the detection of foreign fat in dairy products, methods for the determination of ammonia and acidity in meat, and of sugars in cereal products, correction of Munson and Walker's sugar table, new methods for vinegar analysis (including glycerine determination), schemes for the separation of food colors, a subchapter on formic acid (recently introduced as a preservative), methods for the analysis of lemon and orange oils, a summary of analyses of authentic samples of vanilla extract, and a complete revision of the final chapter on fruit and vegetable products with new sections on tomato ketchup, dried fruits, preserves (including maraschino cherries), fruit juices, and non-alcoholic carbonated beverages. In the final chapter are included descriptions of recent methods for the determination of tin, vegetable acids, and habitforming drugs, and for the detection of saponin, also microscopical methods for the detection of spoilage.

The text of the Federal Pure Food Law, as amended during the present year, and of the Meat Inspection Law, are added for ready reference as an Appendix. 
The substantial work of Dr. T. B. Osborne in the subchapter on proteins and of Dr. W. D. Bigelow in the chapter on meats, both introduced in the second edition, appear unchanged in the present edition, and grateful acknowledgment, previously expressed by the author, is here repeated.

The revision has been carried out with the generous assistance of Dr. Kate Barber Winton and of a number of chemists whose methods, duly credited, appear in the text.

CHicago, ILI., November, I912.

A. L. W. 


\section{PREFACE TO FIRST EDITION.}

In the preparation of the present work, the requirements of the public analyst are mainly kept in view, as well as of such officials as naturally cooperate with him in carrying out the provisions of the laws dealing with the suppression of food adulteration in states and municipalities. To this end special prominence is given to the nature and extent of adulteration in the various foods, to methods of analysis for the detection of adulterants, and to some extent also to the machinery of inspection.

While the analyst may not in all cases have directly to deal with the minutice of food inspection, his work is so closely allied therewith that this branch of the subject is of vital interest and importance to him. Indeed, in many smaller cities one official often has charge of the entire work, combining the duties of both inspector and analyst.

Endeavor has been made, furthermore, to deal with the general composition of foods, and to give such analytical processes as are likely to be needed by the sanitary chemist, or by the student who wishes to determine the proximate components of food materials.

It has been thought best to include brief synopses of processes of manufacture or preparation of certain foods and food materials, in cases where impurities might be suggested incidental to their preparation.

In view of the fact that Massachusetts was the pioneer state to adopt, over twenty years ago, a practical system of food and drug inspection, and for many years was the only state to enjoy such a system, no apology is perhaps needed for more frequent mention of Massachusetts methods and customs than those of many other states, in which the food laws are now being enforced with equal zeal and efficiency.

Considerable attention has been paid in the following pages to the use of the microscope in food analysis. Of the figures in the text illus- 
trating the microscopical structure of powdered tea, coffee, cocoa, and the spices, fifteen have been reproduced from the admirable drawings of Dr. Josef Moeller, of the University of Graz, Austria. Acknowledgment is gratefully given Dr. Moeller for his kind consent to their use.

The photomicrographs in half-tone, forming the set of plates at the end of the volume, were all made in the author's laboratory, and may be divided into three classes: ist, illustrations of powdered pure foods and food products, as well as of powdered adulterants; $2 \mathrm{~d}$, types of adulterated foods, chosen from samples collected from time to time in the routine course of inspection; and $3 \mathrm{~d}$, photographs of permanently mounted sections of foods and adulterants.

While recent works covering the whole field of general food analysis are comparatively few, the number of treatises, monographs, government bulletins, and articles scattered through the journals, dealing with special subjects relative to food and its inspection, is surprisingly large, and from a painstaking review of these much information has been culled, for which it has been the author's intention at all times to give credit.

Special mention should here be made of the valuable publications of the U. S. Department of Agriculture, both the bulletins issued from Washington, and those from the various experiment stations, an everincreasing number of which are becoming engaged in human food work. The author has freely drawn from these sources, and especially from the data and material furnished by his coworkers in the recent and still pending labor of preparing food methods for the Association of Official Agricultural Chemists, and he wishes to extend his thanks to all of them for their assistance. Appreciation is also expressed for the care and discrimination shown by Mr. L. L. Poates in the preparation of the cuts. Thanks are especially due to Mr. Hermann C. Lythgoe, Assistant Analyst of the Massachusetts State Board of Health, for his invaluable cooperation, and to Dr. Thomas M. Drown for helpful hints and suggestions.

Bostos, Mass., July I, rgo4- 


\section{TABLE OF CONTENTS.}

\section{CHAPTER I.}

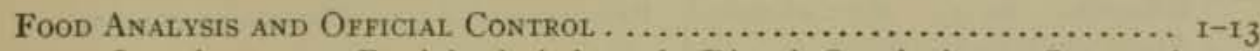

Introductory, I. Food Analysis from the Dietetic Standpoint, 2. Systematic Food Inspection; Functions of the State Analyst; Standards of Purity; Nature of Analytical Methods, 3-5. Adulteration of Food, 5. Misbranding, 6. A Typical System of Food Inspection, 6-9. Practical Enforcement of the Food Laws; Publication; Notification; Prosecution, 10.

References on Food Inspection and Official Control, 11 .

\section{CHAPTER II.}

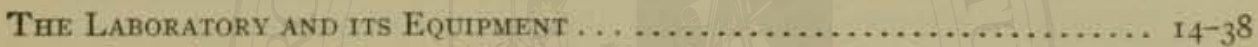

Location, 14. Floor; Lighting; Benches, 15. Hoods, 16. Sinks and Drains, 17. Steam and Electricity; Suction and Blast, 19. Apparatus, 2025. Reagents, 26-35. Equivalents of Standard Solutions; 36-37. Indicators, $3^{8}$.

References on Laboratory Equipment, Reagents, etc., 38 .

\section{CHAPTER III.}

Food, its Functions, Proximate Components, and Nutritive Value . . . . . 39-52

Nature and General Composition of Food; Fats, 39. Protein, and Classification of Nitrogenous Bodies, 40. Proteins, their Subdivisions, Occurrence, and Characterstic Tests, 40-45. Amino Acids, etc., 45. Alkaloids; Nitrates; Ammonia; Lecithin; Carbohydrates and their Classification, 46. Organic Acids; Mineral or Inorganic Materials; Fuel Value of Food; Bomb Calorimeter, $47-48$.

References on Dietetics and Economy of Food, 49.

\section{CHAPTER IV.}

General Analytical Methods

Expression of Results, 53-54. Preparation of Sample, 55. Specific Gravity; Methods and Apparatus, 55-60. Determination of Moisture, 6r. Determination of Ash, $62-63$. Continuous Extraction with Volatile Solvents, $63_{3}-68$. Separation with Immiscible Solvents, 68. Determination of Nitrogen, 69-73. 
Determination of Free Ammonia; Determination of Amido Nitrogen, 74. Determination of Carbohydrates, 74. Poisoned Foods, 74. Detection and Determination of Arsenic, 74-77. Colorometric Analysis, 77. Tintometer, 78 .

References on General Food Analysis, 79.

\section{CHAPTER V.}

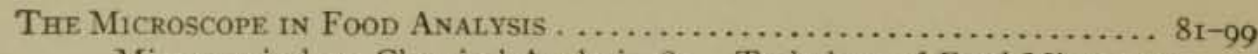

Microscopical vs. Chemical Analysis, 81. Technique of Food Microscopy, 82. Apparatus and Accessories, 82-84. Preparation of Vegetable Foods for Microscopical Examination, 85. Miscroscopical Diagnosis, 86. Vegetable Tissues and Cell Contents, under the Microscope, 87-90. Microscopical Reagents, 90-93. Microchemical Reactions, 90-93. Photomicrography; Appurtenances and Methods, 93-98.

References on the Microscope in Food Analysis, 98.

\section{CHAPTER VI.}

\section{THE REFRACTOMETER .}

Butyro-refractometer, гог. Refractometer Heater, ro2. Manipulation, 102-104. Equivalents of Refractive Indices and Butyro-refractometer Readings, 105-106. Temperature Correction, 107. Abbé Refractometer, 108. Construction; Manipulation, 109-1II. Immersion Refractometer, III-1 I2. Manipulation, $\mathrm{II}_{3}-\mathrm{Ir} 5$. Equivalents of Refractive Indices and Immersion Refractometer Readings, r16-119. Strength of Solutions by Refractometer 120. Temperature Corrections, I2I.

References on the Refractometer, I22.

\section{CHAPTER VII.}

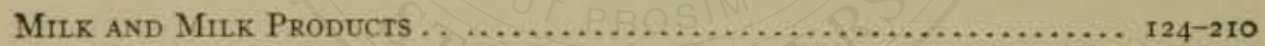

Composition and Characteristics of Milk, 124. Milk Sugar; Milk Proteins, and other Nitrogenous Bodies, 125. Milk Fat; Citric Acid; Composition of the Ash, 126-127. Fore Milk and Strippings, 128. Colostrum; Frozen Milk; Fermentations of Milk, 129. Analysis of Milk, $1_{3}$. Specific Gravity, $1_{3} \mathrm{I}^{-1} 33$. Total Solids, 132. Ash, I34. Fat, by Extraction, by Centrifugal, and by Refractometric Methods, $\mathrm{I}_{34}-\mathrm{I} 44$. Proteins; Casein, 145. Albumin; Other Nitrogenous Bodies, 146. Milk Sugar, by Optical Methods, 147-149, by Fehling's Solution, I49-15I. Relation between the Various Milk Constituents; Calculation by Formulæ, 151-153. Acidity, 153. Boiled Milk, 155. Modified Milk and its Preparation, 155-157. Prepared Milk Foods, Milk Powders, and Artificial Albuminous Foods, 157-159. Koumis, 158. Kephir, 159.

Milk Adulteration and Inspection; Milk Standards, 159-16r. Forms of Adulteration, and Variation in Standard, $161-162$. Rapid Approximate Methods of Examination, $\mathrm{I}_{3}-\mathrm{r} 6_{4}$. Examination of Milk Serum; Constants, 164-168. Systematic Routine Examination, 168. Analytical Methods for Solids, Fat, and Ash, $170-173$. Added Foreign Ingredients, 173. Coloring Matters and their Detection, $174-177$. Preservatives, their Relative Efficiency 
and their Detection, $177^{-185}$. Added Cane Sugar, and Starch, 185 . Added Condensed Milk; Analysis of Sour Milk, 186,

Condensed Milk; Composition, Standards, Adulteration, r86-r88. Methods of Analysis, 188-191. Calculation of Fat in Original Milk, 102.

Cream; Composition, Standards, Adulterants, Foreign Fat, 193-195. Analytical Methods, $1955^{-198}$.

Ice Cream: Standard, Fillers, 198-199. Analytical Methods, 199-20I.

Cheese; Composition, Varieties, 202. Standards; Adulteration, 203-204. Analytical Methods, 204, 205. Separation and Determination of Nitrogenous Bodies, 206, 207. Lactic Acid; Milk Sugar; Foreign Fat, 207.

References on Milk and its Products, 208.

\section{CHAPTER VIII.}

\section{Firsh Foods}

Meat; Structure and Composition. 211. Proximate Components of the Common Meats, 212-217. Meat Inspection, 217. Standards, 218. Meat Preservatives, 218. Curing, 219. Use of Antiseptics; Effect of Cooking, 220. Canned Meats, 221. Sausages, 223-224. Analytical Methods, 225. Fats of Meats, 226-227. Classification, Separation, and Determination of Nitrogenous Bodies, 226-231. Determination of Gelatin, 231. Determination of Nitrates, 232. Preservatives and their Detection, 232. Starch in Sausages, 233. Horseflesh in Sausages, and its Detection, 234-238. Muscle Sugar, 238. Coloring Matters and their Detection, $23^{8-239}$. Detection of Frozen Meat, 239 .

Meat Extracts; Character and Standards, 240-24I. Composition, 242-244. Meat Juices, 245. Miscellaneous Meat Preparations, 246. Methods of Analysis, 246-249. Separation of Nitrogenous Compounds, 249-253. Acidity, 253. Preservatives; Glycerol, 254.

Fish; Structure, Composition, and Methods of Analysis, 254-255. Crustaceans and Mollusks, 256. Floating of Shellfish; Preservatives in Fish and Oysters; Colors, 257.

Concentrated Foods for Armies and Campers, 257.

References on Flesh Foods, 258.

\section{CHAPTER IX.}

Nature and Composition, 26r. The Egg White and its Nitrogenous Compounds, 262. Preparation of Albumin; The Egg Yolk and its Composition, 263. Composition of the Ash, 264 . Analytical Methods; Determination of Lecithin, 265. Preservation of Eggs, 266. Cold Storage Eggs, 267. Physical Methods of Examination, 267. Opened Eggs; Desiccated Eggs, 268. Egg Substitutes, 269. Custard Powders, 270.

References on Eggs, 270. 


\section{CHAPTER X.} of Proximate Analysis, 276-279. Carbohydrates of Cereals, 279. Starch; Detection, Varieties, Classification, Microscopical Examination, 279-283. Starch Determination, by Direct Acid Conversion and by Diastase Methods, 283-284. Sugars, $284-285$. Cellulose; Crude Fiber, 285. Pentosans and their Determination, 285-294. Separation and Determination of the Carbohydrates of Cereals, 295-296. Proteins of Cereais and Vegetables; Separation and Methods of Analysis, 206-208. Proteins of Wheat, their Separation and Determination, 298-300. Proteins of Other Cereals and Vegetables, 300-30r. Ash of Cereals and Vegetables; Scheme for Ash Analysis, 301-305. Sulphur; Chlorine, 305. Microscopy of Cereal Products, 306-31r.

Flour; Milling, 311. Composition, 312. Damaged Flour; Ergot, $3^{1} 3$. Adulteration, 314. Alum; Bleaching, 315. Inspection and Analysis; Fineness, 3r6. Color, Absorption, and Dough Tests, 317. Expansion of Dough, 318. Baking Tests, $3^{17}-3$ I9. Proximate Constituents; Ash, 319. Gluten; Protein; Acidity, 320. Detection of Bleaching; Nitrites, 321. Bamihl Gluten Test, 322.

Bread; Composition; Varieties, 323-325. Methods of Examination, 325326. Adulteration of Bread; Alum, 326. Cake, 327 .

Leavening Materials; Yeast, 327 . Compressed Yeast; Dry Yeast, 328. Composition and Microscopical Examination, 329. Yeast Testing; Available Carbon Dioxide, 330. Starch in Compressed Yeast, 33r.

Chemical Leavening Materials; Baking Powders, their Classification and Composition, 332-334. Adulteration, 334. Cream of Tartar and its Adulteration, 335. Analysis of Baking Chemicals, 336. Carbon Dioxide, 336-339. Tartaric Acid, 339-343. Starch, 343. Aluminum Salts, 344. Lime; Potash; Soda, 345. Phosphoric Acid; Sulphuric Aeid; Ammonia; Arsenic, 346.

Semolina, Macaroni, and Edible Pastes; Noodles, 347-348. Adulteration; Analytical Methods; Lecithin-Phosphoric Acid, 349. Colors, 349-352. Shredded Wheat, 352 .

Prepared Cereal Breakfast Foods; Nature and Composition, 352-354. Analytical Methods, 354 .

Infants' and Invalids' Foods, 354. Classification, 355. Composition, 356.

Diabetic Foods, 357-358. Analytical Methods, 359-360.

References on Cereals, Vegetables, etc., 361.

References on Leavening Materials, 364 .

\section{CHAPTER XI.}

TEA, COFFEE, ANd CoCOA....................................... 365-407

Tea; Varieties, Method of Manufacture, Compositions, $365-368$. Analytical Methods, 368. Extract; Tannin, 370-372. Thein, or Caffeine, 372-374. Adulteration and Detection of Adulterants; Facing..374. Spent Leaves, 375. Foreign Leaves; Stems and Fragments, 376. Added Astringents; Tea Tablets, 377 . Microscopical Structure, 378 .

Coffee; Nature, Composition, Effect of Roasting, 379-38r. Substitutes and Adulterants, 382 . Analytical Methods; Caffetanic Acid. $38_{2}-38_{3}$. 
Caffeine, 384 . Adulteration; Imitation Coffee; Coloring, 384. Glazing; Methods, 385. Microscopical Examination, 386. Chicory; its Microscopical Structure, $3^{86-388}$. Composition of Chicory, and its Determination in Coffee, 380. Date Stones; Hygienic Coffee; Substitutes, 390-392.

Cocoa and Cocoa Products; Composition, Methods of Manufacture, 392395. Theobromine and Nitrogenoús Substances, 396. Milk Chocolate; Compounds, 397. Analytical Methods, 398. Starch; Sucrose; Lactose, 399. Theobromine and Caffeine, $400-40 \mathrm{r}$. Adulteration, and Standards of Purity, 402. Addition of Alkali, Microscopical Structure, 403-404. Cocoa Shells; Added Starch, Sugar, Fat and Colors, 405.

References on Tea, Coffee, and Cocoa, 406.

\section{CHAPTER XII.}

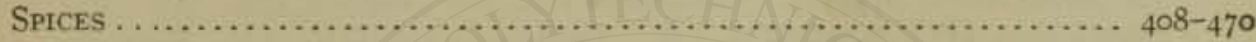

Methods of Proximate Analysis Common to all the Spices, 408. Moisture; Ash; Ether, and Alcohol Extract; Nitrogen; Starch; Crude Fiber; Volatile Oils, 409-4II. Microscopical Examination, 4I2. Spice Adulterants, $4 \mathrm{I}_{2}-4 \mathrm{I} 3$.

Cloves; Composition, 4I2-4I5. Tannin, 4I5. Microscopical Examination, 416. Clove Stems, 4I7. Adulteration and Standard of Purity; Exhausted Cloves, $4 \mathrm{r} 8$. Cocoanut Shells, $4 \mathrm{rg}$.

Allspice; Composition, 420. Tannin Equivalent, 42r. Microscopical Structure, 422-423. Adulteration and Standard of Purity, 424.

Cassia and Cinnamon; Composition, 424-425. Microscopical Structure, 426-427. Adulterants; Standard, 428. Foreign Bark, 428.

Pepper; Composition, 428-432, Nitrogen Determination, 432. Piperin, 433. Microscopical Examination, 433-434. Adulteration and Standards, 435. Pepper Shells and Dust, 435. Olive Stones, 436. Buckwheat, 437. Long Pepper, 438 .

Red Pepper; (Cayenne, Paprika, etc.). Nature; Varieties: Composition, 439-44I; Microscopical Structure, 441-443. Adulteration, 443-445. Added Oil in Paprika, 445.

Ginger; Composition, 445-446. Exhausted Ginger, and its Detection, 447448. Microscopical Structure, 449. Adulteration and Standard, 450.

Turmeric; Composition, 450. Microscopical Structure, 451. Detection, 443 .

Mustard; Composition, Preparation, 453-456. Mustard Oil Determination, 457. Microscopical Structure, 458. Adulteration and Standards; Charlock, 450-460. Coloring Matter, 460. Prepared Mustard; Composition, Adulteration, 460. Analytical Methods, 461 .

Nutmeg and Mace; Composition of Nutmeg, 462-463. Microscopical Structure of Nutmeg; Adulteration; Standard of Purity, 464. Composition of Mace, 465. Microscopical Structure; Adulteration; Standard, 466. Bombay or Wild Mace and its Detection, 467. Macassar Mace, 468.

References on Spices, 468. 
Edible Oils and Fats

Nature and Properties, 471 . Fatty Acids, $471-472$. Saponification, 472 . Analysis; Rancidity; Judgment as to Purity; Filtering, Weighing, and Measuring Fats, 473. Specific Gravity, 474-476. Viscosity, 477. Meltingpoint, 480. Reichert-Meissl Process for Volatile Fatty Acids, $481-482$. Polenske Number, 483 . Soluble and Insoluble Fatty Acids, 484-486. Saponification Number, 486. Iodine Absorption Number; Hübl's Method, 487-49o. Hanus's Method, 491. Wijs's Method, 492. Bromine Apsorption Number, 492-493. Thermal Tests, 493. Maumené Test, 494. Bromination Test,494497. The Acetyl Value, 497-498. The Valenta and Elaidin Tests, 499. Free Fatty Acids, 500. Titer Test, 500-50r. Unsaponifiable Matter, $50 \mathrm{r}$. Cholesterol and Phytosterol, 502. Separation and Crystallization, 503-506. Bömer's Phytosterol Acetate Test, 507. Constants of Edible Oils and Fats, 508-509. Parraffin; Microscopical Examinaltion of Oils and Fats, $5 \mathrm{ro}$. Olive Oil, 511. Composition and Adulteration, 512. Standards, 513. Tests for Adulteration, 513-515. Cottonseed Oil, 516. Bechi's Test, 517. Halphen's Test, $5^{18}$. Sesame Oil, 518 . Adulterants and Tests, 519. Rape Oil, 520. Tests, 521. Corn Oil, 521. Sitosterol, 522. Peanut Oil, 522. Adulterants; Renard's Method, 523. Bellier's Method, 524. Mustard Oil, 525. Poppyseed Oil, 526. Sunflower Oil, 526. Rosin Oil, 527. Cocoanut Oil, 528. Cocoa Butter; Tallow, 529 .

Butter, 529. Composition, 530. Effects of Feeding, 531. Analytical Methods, 531. Water, 531-533. Fat, 533. Ash; Casein; Milk Sugar; Lactic Acid; Salt, 534. Standard Butter Fat, 535. Adulteration, 535. Colors, 535-537. Preservatives, 538-539. Renovated or Process Butter, 540. Oleomargarine; Manufacture, 54I. Coloring; Detection of Palm Oil, 542. Adulterants; Healthfulness, 543. Distinction from Butter, 544. Distinguishing Tests for Butter, Process Butter, and Oleomargarine, 546. Butyrorefractometer, 546-548. Reichert-Meissl Number; Specific Gravity; Foam Test, 549. Milk Test, 550. Curd Tests, 551. Microscopical Examination, 552-553. Foreign Oils, 554 .

Lard, 554. Composition; Lard Oil, 555. Compound Lard; Standards; Adulteration, 556. Foreign Oils, 557. Microscopical Examination, 557-558. Analysis of Lard and Lard Substitutes, 559. Effects of Feeding, 560.

References on Edible Oils and Fats, 561. References on Butter, 562. References on Lard, $5_{6}{ }_{3}$.

\section{CHAPTER XIV.}

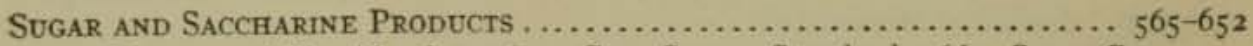

Nature and Classification, 565. Cane Sugar; Standard, 566. Sugar Cane; Manufacture of Cane Sugar, 567. Composition of Cane Sugar Products, 568. Sugar Beet; Manufacture of Beet Sugar, 569. Refining Sugar; Maple Products, 750. Compositions, Standards, and Adulteration of Maple Products, $571-572$. Sorghum, 573. Grape Sugar, 573. Levulose; Malt Sugar, 574. Dextrin; Commercial Glucose, 575. Standards and Healthfulness of Glucose, 576. Milk Sugar; Raffinose, 577 . 
The Polariscope and Saccharimetry, $578-58_{3}$. Comparison of Scales and Normal Weights, 583 . Specific Rotary Power; Birotation, 584 .

Analysis of Cane Sugar and its Products; Tests for Sucrose, 585. Moisture; Ash; Non-sugars; Sucrose Determination by Polariscope, $586-587$. Inversion; Clerget's Formula, 588. Detection and Determination of Invert Sugar, 589 . Ultramarine in Sugar; Copper Reduction, 590. Volumetric Fehling Process, 591-592. Gravimetric Fehling Methods, 593. Defren-O'Sullivan Method, 594-597. Munson and Walker Method, 598-607. Allihn Method; Electrolytic Apparatus 608-6I2. Sucrose Determination by Fehling Solution, 6 I 2.

Analysis of Molasses and Syrups, 6r3. Solids; Ash; Polarization, 6ra620. Double Dilution Method of Polarizing; Raffinose Determination, 620. Adulteration of Molasses and Standards, 621. Glucose Determination, 62I624. Ashing Saccharine Products, 624. Tin Determination, 625.

Separation and Determination of Various Sugars, 625-626.

Analysis of Maple Products, 627. Moisture; Ash; Malic Acid Value, 627.

Lead Number, 628. Hortvet Number, 628-630. Sy's Method, $6_{3}$.

Analysis of Glucose; Polarization Formulx, 630-631. Dextrin; Ash; Sulphurous Acid, 632. Arsenic, 633.

Honey; European, 633. Canadian; American; Hawaiian, 634-635. Adulteration, $6_{3} 6-638$.

Analysis of Honey; Moisture; Ash; Polarization, 639. Reducing Sugars; Levulose; Dextrose; Sucrose; Dextrin, 640. Acids; Glucose, 641. Invert Sugar; Distinction of Honeydew from Glucose, 642 .

Confectionery; Standard; Adulteration; Colors, 645. Analysis of Confectionery; Mineral Adulterants, 646. Ether Extract; Paraffine, 647 . Starch; Polarization, 648. Alcohol; Colors; Arsenic, 649.

References on Sugars, 650.

\section{CHAPTER XV.}

ALCOHOLIC BEVERAGES $653-758$

Alcoholic Fermentation, 653. Alcoholic Liquors and State Control, 654. Liquor Inspection, $655^{-656}$. Analytical Methods common to all Liquors; Specific Gravity, 657. Detection and Determination of Alcohol, 657-66o. Alcohol Tables, 66r-674. The Ebulioscope, 675-676. Extract; Ash; Artificial Sweeteners, 677 .

Fermented Liquors; Cider, 678. Manufacture and Composition, 678-681. Adulteration, 682. Perry, 683. Wine, 684. Classification of Wines, 685 . Composition and Varieties, 686-689. Standards, 689-69r. Adulteration, 691-695. Analytical Methods for Wine; Extract; Acidity, 696. Extract Table, 697-699. Tartaric Acid, 7or. Malic Acid, 702. Sugars; Glycerin, 703. Tannin, 704. Foreign Colors, 704-706.

Malt Liquors; Beer, 707. Varieties of Beer and Ale, 708. Composition, 709. Malt and Hop Substitutes, 710. Adulteration and Standards, 711. Malted vs. Non-malted Liquors, 712. Preservatives; Arsenic, 713. Temperance Beers, 714. Analytical Methods, 714. Alcohol, 715. Extract, 715722. Original Gravity, 722-724. Sugars; Dextrin; Glycerine; Acids, 724. Protein; Phosphoric Acid, 725. Carbon Dioxide, 726. Bitter Principles, 726727. Arsenic, 728. Malt Extract, 729. 
Distilled Liquors; Standards for Spirits, 730. Fusel Oil, 73r. Whiskey, $73 \mathrm{r}$. Manufacture, 73I-732. Standards, 733-734. Composition, 734-737. Adulteration, 738. Brandy; Manufacture; Composition, 739. Standards, 740. Adulteration, 741. Rum; Composition, 742. Standards, 742-743. Gin; Composition, 744. Analytical Methods, for Distilled Liquors; Extract; Acids; Esters; Aldehydes, 745. Furfural, 746. Fusel Oil, 746-749. Methyl Alcohol, 749-752. Caramel, 752-753. Opalescence Test, 753 .

Liqueurs and Cordials, 754. Analysis of Liqueurs, 755 .

References on Alcoholic Beverages; on Beer, 756.

References on Cider and Wine, 757; on Distilled Liquors, 758.

\section{CHAPTER XVI.}

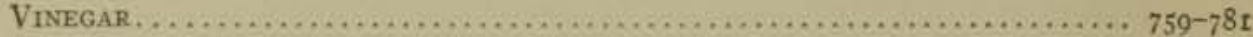

Acetic Fermentation; Varieties of Vinegar, 759. Manufacture and Composition, 760-761. Cider Vinegar, 760. Wine Vinegar, 76r. Malt Vinegar, 762. Spirit, Glucose, and Molasses Vinegars, 763. Wood Vinegar, 764. Analytical Methods; Density; Extract; Ash; Phosphoric Acid, 764. Nitrogen; Acidity, 765. Alcohol; Mineral Acids, 766. Malic Acid, 767. Lead Precipitate, 768. Potassium Tartrate; Sugars, 760. Pentosans, 770. Glycerine, 770-772.

Adulteration of Vinegar; Standards, 772-773. Artificial Cider Vinegar, 774 . Character of Residue and Ash, 774-775. Character of Sugars, 776. Tests, 777 . Composition of Artificial Cider Vinegars, 778. Detection of Adulterants, and Mineral Impurities, 779-780.

References on Vinegar, 780 .

\section{CHAPTER XVII.}

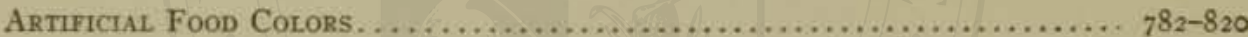

Extent of Use; Objectionable Features, 782. Toxic Effects, 783. Harmful Mineral Colors, 784. Harmful Organic Colors, 785. Harmless Mineral Colors; Harmless Organic Colors, 786-788. Use of Colors in Confectionery, 788.

Vegetable Colors, 789-79r. Special Tests; Orchil; Logwood; Turmeric, $79 \mathrm{r}$. Caramel; Indigo, 792. Cochineal, 792.

Mineral Pigments; Prussian Blue, 792. Ultramarine; Lead Chromate, 793.

Coal-tar Colors, 793. Allowed Colors, 794. Detection in Food; Basic and Acid Dyes; Wool Dyeing, 795. Double Dyeing Method, 796. Vegetable Colors on Wool; Extraction of Colors by Immiscible Solvents, 797. Separation with Ether, 798. Special Tests, 799. Classification and Identification of Coal-tar Dyes; Rota's Scheme, 799-804. Direct Identification of Colors, 805 . Table of Reactions for Colors on the Fiber, 806-813. Reagents, $81_{4}$. Separation and Identification of Allowed and Acid Colors, 814 . Price's Scheme, 815 . Mathewson's Tables, 816-818. Analysis of Colors, 818 . Solubility Tables, 818 .

References on Colors, 8ig.

\section{CHAPTER XVIII.}

Food Preservatives......................................... $821-849$

Preservation of Food, 821. Regulation of Antiseptics, 822. Commercial Food Preservatives, 823. Formaldehyde, 824. Determination in Preservatives, 825. Detection in Food, 826. Determination, 827. Boric Acid; Determination in 
Preservatives, 827 . Detection in Foods, 828 . Determination, $829-830$. Salicylic Acid;. Detection, $8_{31}$. Determination, $8_{32}$. Benzoic Acid, $8_{33}$. Detection, $8_{34}-$ 835. Determination, $835-839$. Sulphurous Acid, 839 . Detection; Determination, 840. Formic Acid; Detection, 841. Determination, 842. Fluorides, Fluosilicates, Fluoborates, $843-844$. Beta-Napthhol; Detection, 845. Asaprol or Abrastol, 845. Detection, 846 .

References on Preservatives and their Use in Food, 846.

\section{CHAPTER XIX.}

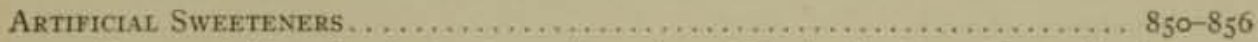

Extent of Use; Saccharin, 850 . Detection of Saccharin, $85 \mathrm{x}$. Determination, 852 .

Dulcin; Detection, 853 . Determination of Dulcin, 854 . Glucin, 855 .

References on Artificial Sweeteners, 855 .

\section{CHAPTER XX.}

Flavoring Extracts and their SUbStITUtes. . . . . . . . . . . . . . . . $857-899$

Vanilla Extract, 857. Vanilla Bean, 857. Composition, 858. Vanillin; Exhausted Vanilla Bean, 850. Composition of Vanilla Extract, 859-86r. Tonka Bean, 860. Coumarin; Standards; Adulteration of Vanilla Extract, 862. Artificial Extracts, 863. Detection of Artificial Extracts, 864. Determination of Vanillin and Coumarin, 865-867. Tests for Coumarin; Vanillin and Coumarin under the Microscope; Normal Lead Number, 867. Acetanilide, 868. Glycerin; Alcohol; Caramel, 860. Limits; Colors, 870.

Lemon Extract, 870. Standards, 870 . Adulteration, 871. Analytical Methods; Determination of Lemon Oil, 872-875. Alcohol; Total Aldehydes, 875 . Citral, 877. Methyl Alcohol; Colors, 878. Solids; Ash; Glycerin; Examination of Lemon Oil, 879. Constants of Lemon and other Oils, 880. Citral, Citronellal, and other Adulterants, 881. Lemon Oil; Analytical Methods; Density; Refraction; Rotation; Citral, 882. Aldehydes; Physical Constants; Pinene; Alcohol, $88_{3}$.

Orange Extract, 884. Almond Extract, 884. Benzaldehyde; Standard, 885. Adulteration; Analytical Methods; Determination of Benzaldehyde, 886. Nitrobenzol, 887. Distinction and Separation from Benzaldehyde; Artificial Benzaldehyde; Alcohol; Hydrocyanic Acid, 888. Wintergreen Extract; Standards, 889. Adulteration; Determination of Wintergreen Oil; Peppermint Extract; Peppermint Oil, 89o. Standards; Analytical Methods; Spearmint Extract, 89r. Spice Extracts; Standards, 892. Analytical Methods, 893-894. Rose Extract; Standards; Determination of Rose Oil, 895. Imitation Fruit Flavors, 895-897. Determination of Esters, 898 .

References on Flavoring Extracts, 898.

\section{CHAPTER XXI}

Vegetable and Frutt Products . . . . . . . . . . . . . . . . . . . . . . . $900-964$

Canned Vegetables and Fruits; Method of Canning, 900-90r. Composition, 902. Decomposition and Detection of Spoiled Cans, 902. Gases from Spoiled 
Cans, 903. Metallic Impurities, 904. Action of Fruit Acids on Tin Plate, 905908. Salts of Lead, 908. Salts of Zine and Copper, 909-910. Salts of Nickel; Toxic Effects of Metallic Salts, 911. Preservatives; Soaked Goods, 912. Analytical Methods; Proximate Analysis; Lead in Tin Alloy, 9r3. Tin, Copper, Lead, Zinc, and Nickel, 914-919.

Ketchup, 919. Standards; Process of Manufacture, 9r9. Composition; Decayed Material; Refuse, 920. Foreign Pulp; Preservatives; Colors; Analytical Methods; Solids, 921. Sand; Sugars, 922. Citric and Lactic Acids, 923. Microscopic Examination, 924-925.

Pickles, 925-926. Adulteration, 926. Horseradish, 927.

Preserves, 927. Fruit Butter; Mince Meat, 927-928. Pie Filling; Maraschino Cherries, 928-920.

Jams and Jellies, 930. Composition; Adulteration, 93I-934. Compounds; Imitations, 936. Analytical Methods; Solids, 936. Ash; Acidity; Protein, 937. Sugars, 938-940. Glucose; Dextrin, 940. Alcohol Precipitate; Organic Acids, 941. Citric Acid; Colors; Preservatives; Sweeteners, 942. Starch; Gelatin; Agar-agar; Apple Pulp, 943. Fruit Tissues, 944.

Dried Fruits, 944. Lye Treatment; Sulphuring; Moisture; Spoilage, 945. Zinc; Analytical Methods, 946.

Fruit Juices, 946-947. Grape Juice, 947. Sweet Cider; Lime Juice, 948. Analytical Methods; Acidity; Tartaric and Malic Acids, 949-950. Citric Acid, 951. Fruit Syrups, 952.

Non-Alcoholic Carbonated Beverages, 952 . Soda Water, 952-953. Syrups, 953. Bottled Beverages; Sweeteners, 954. Acids; Preservatives; Colors; Foam Producers; Habit-forming Drugs, 955. Analytical Methods; Solids; Ash; Acids; Sugars; Flavors; Colors; Preservatives; Sweeteners; Alcohol, 956. Saponin, 956-958. Caffein; Cocaine, 958-96r.

References on Vegetable and Fruit Products, 96r.

\section{APPENDIX.}

The Food and Drugs Act, 965 . The Meat Inspection Law, 969. 


\section{PLATES I-XL.}

Photomicrocraphs of Pure and Adulterated Foods and of Adulterants.

Cereals: Barley, I. Buckwheat, II, III. Corn, III, IV. Oat, IV, V. Rice, V, VI. Rye, VI, VII. Wheat, VIII.

Legumes: Bean, IX. Lentil, IX, X. Pea, X, XI.

Miscellaneous Starches: Potato; Arrowroot; Tapioca, XII. Turmeric; Sago, XIII. Coffee, XIV, XV. Chicory, XV, XVI, Cocoa, XVI, XVII. Tea, XVIII.

Spices: Allspice, XVIII, XIX. Cassia, Cinnamon, XX-XXII. Cayenne, XXIIXXIV. Cloves; Clove Stems, XXIV-XXVII. Ginger, XXVII-XXIX. Mace, XXIX. Nutmeg, XXX. Mustard, XXXI-XXXIII. Pepper, XXXIII-XXXVI.

Spice Adulterants: Olive Stones; Cocoanut Shells, XXXVI. Elm Bark; Sawdust; Pine Wood, XXXVII.

Edible Fats: Pure Butter; Renovated Butter; Oleomargarine, XXXVIII. Lard Stearin, XXXIX. Beef Stearin, XL. 


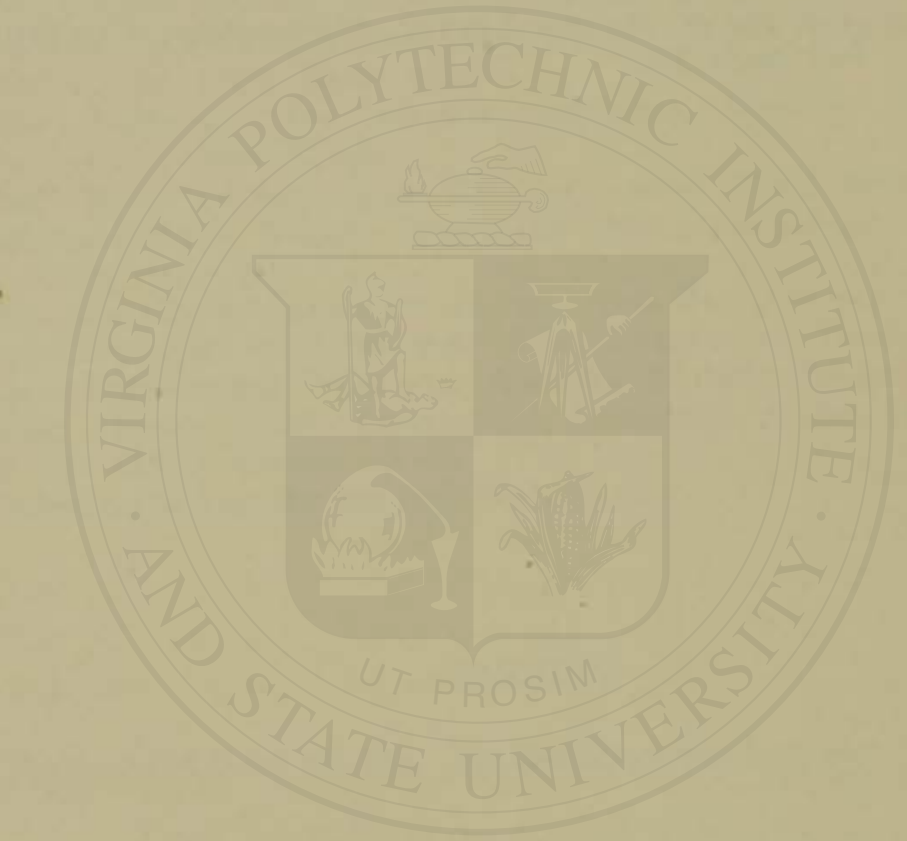




\section{FOOD INSPECTION AND ANALYSIS.}

\section{CHAPTER I.}

FOOD ANALYSIS AND OFFICIAL CONTROL.

\section{INTRODUCTORY.}

THE general subject of food analysis, in so far as the public health is concerned, is to be considered from two somewhat different standpoints: first, from the outlook of the government, state, or municipal analyst, whose mission it is to ascertain whether or not the food may properly be considered pure or free from adulteration; and second, from the point of view of the food economist, whose aim is to determine its actual composition and nutritive value. The one protects against fraud and injury, the other furnishes data for the arrangement of dietaries and for an intelligent conception of the role which the various nutrients play in the metabolism of matter and energy in the body. The two fields are as a rule distinct each from the other, often involving, in the examination of the food, different methods of procedure.

Official Control of Food.-Inview of the importance of the consideration of food with reference to its purity, an ever-increasing number of states have realized the necessity of protecting their citizens from the unscrupulous manufacturers who in various lines are seeking to produce cheaper or inferior articles of food in close imitation of pure goods. Many of the states have laws in accordance with which the sale of such impure or adulterated foods is made a criminal offense, and some, but not all of these, are provided with public analysts and other officers to enforce these laws and punish the offenders. Numerous communities are awake to the importance of municipal control of such commonly used articles of food as milk, butter, and vinegar, and in many cases have machinery of their own for regulating the sale of these foods. 
Since January $\mathrm{I}, \mathrm{I} 90_{7}$, the federal government has been actively engaged in the enforcement of the national food law of June 30 , I9o6, through the Bureau of Chemistry of the U. S. Department of Agriculture. In addition to the central laboratories of this Bureau at Washington, upwards of 20 branch laboratories have been established in the principal cities of the United States to enforce the provisions of the national law which regulates interstate commerce in foods, as well as their manufacture and sale in the territories and the District of Columbia, and their importation from foreign countries.

Food Analysis from the Dietetic Standpoint.-The study of the principles of dietetics has been given increased attention during the last decade in the curricula of many of the technical schools and colleges. Much has been accomplished by certain of the state experiment stations working as a rule in connection with the United States Department of Agriculture along this line. Investigations of this character are especially valuable, and are indeed rendered necessary by the general tendency of the modern physician to regard the hygienic treatment of disease, especially with reference to the matter of diet, as often of far greater importance than the mere administering of drugs.

The food economist studies the varying conditions of age, sex, occupation, environment, and health among his fellow men, with a view to showing what foods are best adapted to supply the special requirements of various classes. The quantity and proportion of protein, fat and carbohydrates, or of fuel value best suited for the daily consumption of a given class or individual having been determined, dietaries are made up from various food materials to supply the need with reference as far as possible to the taste and means of the consumer.

Experiments are made on families, clubs, or individuals, representing various typical conditions of life, and extending over a given period, during which records are kept of the available food materials on hand and received during the term of the experiment, as well as of those remaining at the end. In the case of individuals, additional records may be kept of the amount and composition of the urine and feces. From such data the physiological chemist calculates the amount of nutrients utilized, and studies the metabolism of material in the human body.

$\mathrm{Up}_{\mathrm{p}}$ to this point no very extensive apparatus is required, but if in addition the income and outgo of heat and energy are to be studied, which are important to a complete investigation of the economy of food in the body, the student will require a respiration calorimeter and its appurte- 
nances. The calorimeter is so constructed that an individual may be confined therein for a term of days under close observation and with carefully regulated conditions. Such an equipment involves a large expenditure and is to be found in but few laboratories.

It is not the purpose of the present work to go beyond the strictly chemical or physical processes involved in making the analyses by which the proximate components of the foods are determined. For more complete information in the field of dietary studies and the metabolism of matter and energy in the body, the student is referred especially to the investigations of Atwater and his coworkers, as published in the annual reports of the Storrs Experiment Station at Middletown, and in the bulietins of the U. S. Department of Agriculture, Office of Experiment Stations, a list of which is given at the end of Chapter III.

Commercial Food Analysis.-The proper preparation of food products has long ceased to be carried on by the hap-hazard rule-of-thumb methods that formerly prevailed. Now in the manufacture of many prepared foods and condiments, especially on a large scale, it has become a necessity to use scientific processes, rendered possible only by the employment of skilled chemists. In fact it is coming to be more and more common for food manufacturers to establish chemical laboratories in connection with their works, in the interests both of economy and of improved production.

Frequently disputed points arise in the enforcement of the food laws that render the services of the private food analyst of great importance both to manufacturer and dealer. Thus a wide field is open to the analyst of foods outside the domain of the government or state laboratory, either in connection with the large food manufacturing plants directly, or in private laboratories for experimental research, or for analytical control work.

SYSTEMATIC FOOD INSPECTION.

Functions of the Official Analyst.-The public analyst is employed by city, state, or government to pass judgment on various articles of food taken from the open market by purchase or seizure, either by himself or by duly authorized collectors employed for the purpose. The sole object of his examination is to ascertain whether or not such articles of food conform to certain standards of purity fixed in some cases by special law, and in others by common usage or acceptance. Such a public analyst need not concern himself with the dietetic value of the food or whether it is of high or low grade. It is for him to determine simply whether it is genuine or 
adulterated within the meaning of the law, and, if adulterated, how and to what extent. Aside from his skill as a chemist, it is often necessary for him to possess other no less important qualifications, chief among which are his ability to testify clearly and concisely in the courts, and to meet at any time the most rigid kind of cross-examination, it being of the utmost importance that he understand thoroughly the nature of evidence.

Standards of Purity for Food Products.*-Under an act of Congress approved March 3, I903, standards of purity for certain articles of food have been established as official standards for the United States by the Secretary of Agriculture. The earlier of these standards were formulated under the Secretary's direction by a committee of the Association of Official Agricultural Chemists. Later, however, a joint committee of that association and of the Association of State and National Food and Dairy Departments has had charge of this work. Standards have been and are being thus adopted, covering the entire range of food products.

1. Nature of the Analytical Methods Employed.-Usually but a small percentage of the samples submitted for examination are actually adulterated. The analyst should, therefore, adopt for economy in time the quickest possible reliable processes for separating the pure from the impure, so that most of his attention may be devoted to the latter. The nature of the processes by which this is done varies with the foods. Experience soon enables one to judge much by even the characteristics of taste, appearance, and odor, though such superficial indications should be used with discretion. Orie or two simple chemical or physical tests may often suffice to establish beyond a doubt the purity of the sample, after which no further attention need be paid to it.

A sample failing to conform to the tests of a genuine food must be carefully examined in detail for impurities or adulterants. While in most cases usage or experience suggests the forms of adulteration peculiar to various foods, the analyst should be on the alert to meet new conditions constantly arising. His methods are largely qualitative, since technically he need only show in most cases the mere presence of a forbidden ingredient, though for the analyst's own satisfaction he had best determine the amount, at least approximately.

In reporting approximate quantitative results in court, especially when they are calculated from assumed or variable factors, or when they are the result of judgment based on the appearance of the food under

*U. S. Dept. of Agric., Off. of Sec., Circ. rg. 
the microscope, the analyst should always be conservative in his figures by expressing the lowest or minimum amount of the adulterant, so as to give the defendant the benefit of any doubt. When exact standards are fixed by law, as in the case of total solids or fat in milk, for example, there is of course great necessity for preciseness in quantitative work.

A full analysis of an adulterated food beyond establishing the nature and amount of the adulteration is entirely unnecessary, and in most instances adds nothing to the strength of a contested case, as twenty years' experience in the enforcement of the food laws in Massachusetts has shown.

The responsibility resting upon the analyst is not to be lightly considered, when it is realized that his judgment and findings constitute the basis on which court complaints are made, and the payment of a fine or even the imprisonment of the defendant may be the result of his report. Therefore he should be sure of his ground, knowing that his results are open to question by the defendant. Where court procedure is apt to be involved, a safe rule is for the analyst to consider himself the hardest person to convince that his tests are unquestionable, making every possible confirmatory test to strengthen his position and consulting all available authorities before expressing his opinion; and finally, after being fully convinced that a sample is adulterated, and having so alleged, let him adhere to his statements and not waver in spite of the most rigid crossexamination to which he may be subjected.

While each state or municipality has its own pecuiiar code of regulations and restrictions concerning the duties of the analyst and other officials, these rules are in the main very similar. For instance, it is usually necessary, excepting in the case of such a perishable food as milk, for the analyst to reserve a portion of a sample before beginning the analysis, which sample, in the event of proving to be adulterated, shall be sealed, so that in case a complaint is made against the vendor, the sealed sample may, on application, be delivered to the defendant or his attorney.

Adulteration of Food.-Except in special cases a food in general is deemed to be adulterated if anything has been mixed with it to reduce or lower its quality or strength; or if anything inferior or cheaper has been substituted wholly or in part therefor; or if any valuable constituent has been abstracted wholly or in part from it; or if it consists wholly or in part of a diseased, decomposed, or putrid animal or vegetable substance; or if by coloring, coating, or otherwise it is made to appear of greater value than it really is; or if it contains any added poisonous 
ingredient. These provisions briefly expressed are typical of the general food laws adopted by most states and by the government, though the verbiage may differ. Laws covering compound foods and special foods vary widely with the locality. As to the character of adulteration, nine out of ten adulterated foods are so classed by reason of the addition of cheaper though harmless ingredients added for commercial profit, rather than by the addition of actually poisonous or injurious substances, though occasional instances of the latter are found.

Authentic instances of actual danger to health from the presence of injurious ingredients are extremely rare, so that the question of food adulteration should logically be met largely on the ground of its fraudulent character. Indeed the commoner forms of adulteration are restricted to a comparatively small number of food products, the most staple articles of our food supply, such as sugar and the cereals, eggs, fresh meat, fresh vegetables and fruit being rarely subject to adulteration.

Misbranding.- Under the federal food law and the laws of many of the states misbranding constitutes an offense as well as adulteration. By misbranding is meant any untrue or deceptive statement or design on the label of a food package, either regarding the nature of the contents, or of the place of manufacture or name of manufacturer. One of the commonest forms of misbranding consists in the incorrect statement of weight or measure. Extravagant and untrue claims as to nutritive value have hitherto constituted a frequent form of misbranding.

A Typical System of Food Inspection.-The efficiency of a system of public food inspection is greatly enhanced if the business part of the work, including the bookkeeping and attending to the outside public, be done wholly through some person other than the analyst, as, for example, a health officer, to whom the collectors of samples and the analyst may report independently as to the results of their work, and whose duty it is to determine what shall be done in cases of adulteration. In this way the analyst knows nothing of the data of collection nor the name of the person from whom the sample was purchased, so that he can truthfully state in court that his analysis was unbiased.

Suppose, for example, that three collectors are employed to purchase samples of food for analysis, their duties being to visit at irregular intervals different portions of a state or municipality. Each collector keeps a book in which he enters all data as to the collection of the sample, includ- 


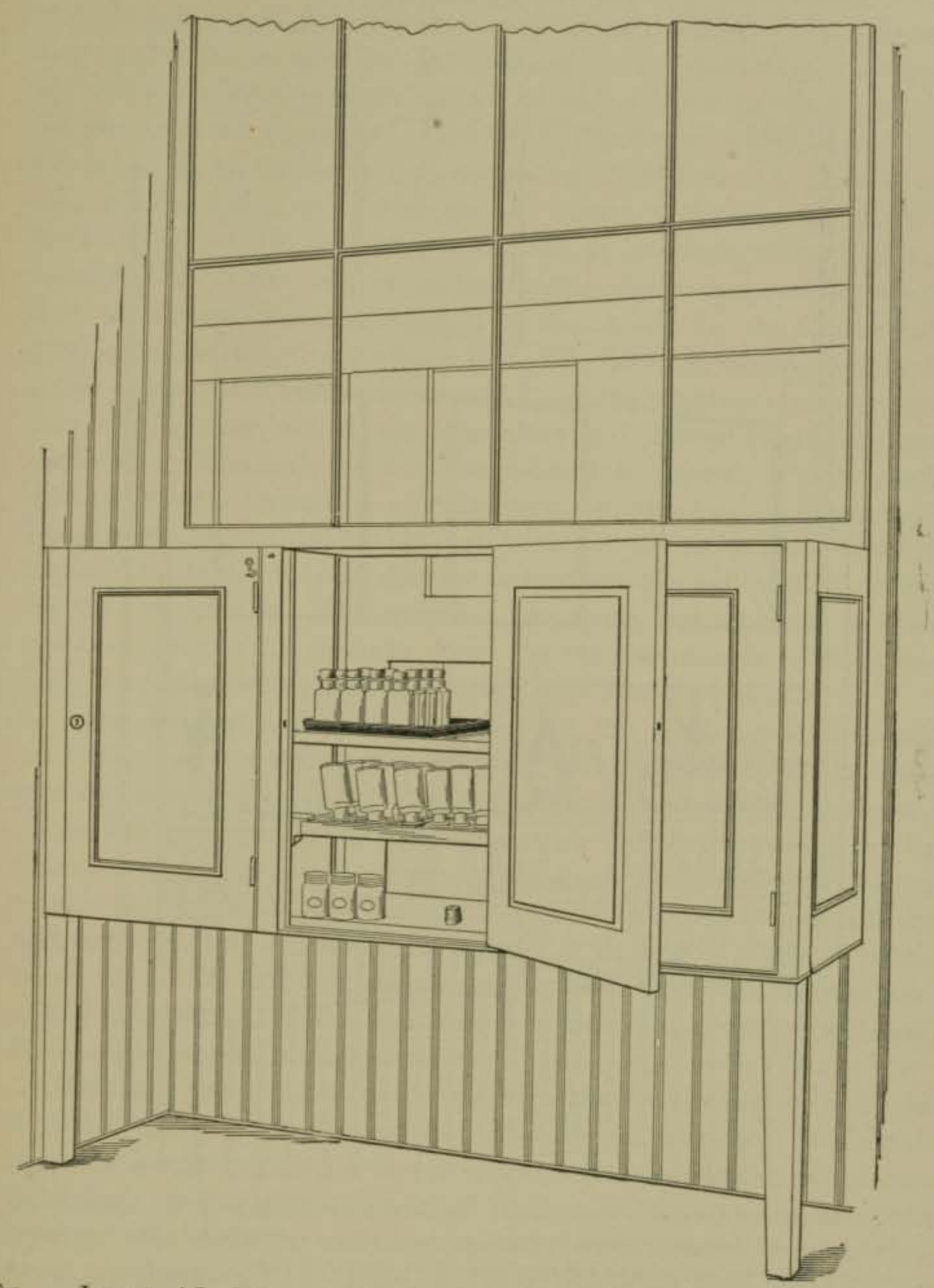

Frg, 1,-Inspectors' Lockers. Insuring safe legal delivery of samples collected by tareo inspectors. Each locker has a door in the rear accessible, from an anteroom, to the in. spector holding key to that locker only. 


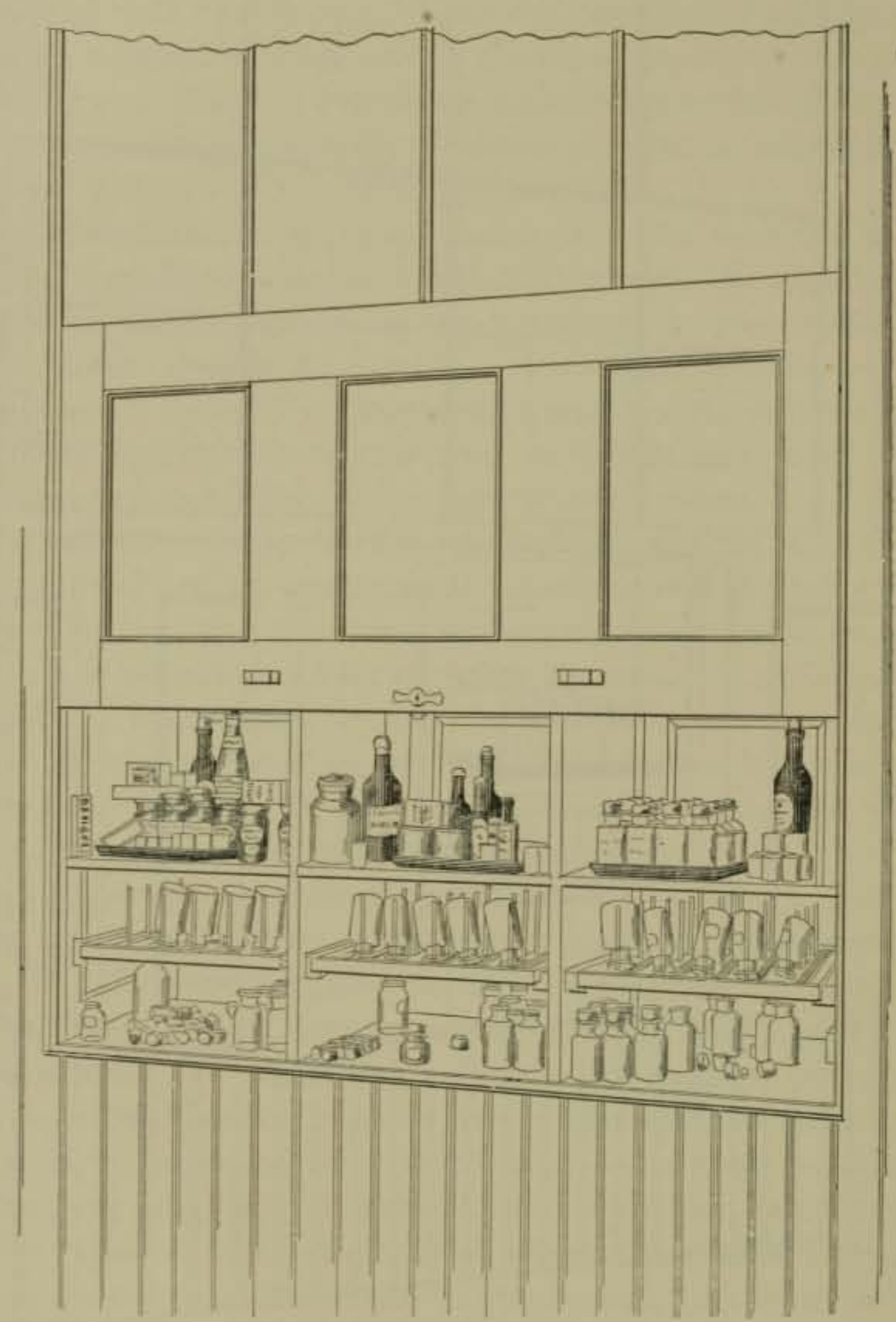

Fis. 2,-Inspectors' Lockers. Front View. The lockers are accessible to the analyst in the laboratory by a single sliding-sash front, provided with a spring lock. The removable sliding-racks are convenient for returning clean sample bottles. 
ing the name of the vendor, assigning a number to each sample, which number is the only distinguishing mark for the analyst. One collector may use for this purpose the odd numbers in succession from I to 9999, the second the even numbers from 2 to 10,000 , while the third may use the numbers from 10,000 up. Each of the two former would begin with a lettered series, as, for instance, $\mathrm{A}$, numbering his samples $\mathrm{IA},{ }_{3} \mathrm{~A}, 5 \mathrm{~A}, 7 \mathrm{~A}$, etc., or $2 \mathrm{~A}, 4 \mathrm{~A}, 6 \mathrm{~A}$, etc., till he reached 10,000 , then beginning on series $\mathrm{B}$ and so on. If the analyst is to be kept in ignorance of the brand or manufacturer in the case of package goods, the collector must remove from the original package sufficient of the sample for the needs of the analyst, and deliver it to the latter in a plain package, bearing simply the name under which the article was sold and the number. Such precautions are, however, not always practicable and depend largely on local regulations. The analyst reports the result of the analysis of each sample with the number thereof on a library card, with appropriate blanks both for data of analysis and for data of collection, the latter to be filled by the collector from his book after the analyst has handed in the card with the data of analysis. This system of recording and reporting analyses has been successfully used for years by the Department of Food and Drug Inspection of the Massachusetts State Board of Health.

Legal Precautions.-The laboratory of the public analyst should preferably be provided with a locker for each collector, to which access may be had only by that collector and the analyst, so that in the absence of the latter, or when circumstances are such that the samples cannot be delivered to him personally, there may be such safeguards with respect to lock and key as to leave no question in the courts as to safe delivery and freedom from accidental tampering. With such a system it is unnecessary for the collector to place under seal the various samples submitted for analysis. Unless such lockers or their equivalent are employed, it is best to carefully seal all samples.

Such a system of lockers for use with three collectors is shown in Figs. $I$ and 2. The same careful attention should afterwards be given to keep the specimens in a secure place both before and during the process of analysis, and to label with care all precipitates, filtrates, and solutions having ir do with the samples, especially when several processes are being simultaneously conducted, in order that there may be no doubt whatever as to their identity. The importance of precautions of this kind in connection with court work can hardly be too strongly emphasized. 
Practical Enforcement of the Food Law.-In the case of foods actually found adulterated, there are three practical methods of suppressing their further sale, viz., by publication, by notification, and by prosecution. These may be separately employed or used in connection with each other, according to the powers conferred by law on the commission, board, or official having in charge the enforcement of the law, and according to the discretion of such official.

Publication.-Under the laws of some states, the only means of protecting the people lies in publishing lists of adulterated foods with their brands and manufacturers' names and addresses in periodical bulletins or reports. Sometimes it is considered best to publish for the information of the public lists of unadulterated brands as well, and, again, it is held that only the offenders should thus be advertised.

Such publication, by keeping the trade informed of the blacklisted brands and manufacturers, certainly has a decidedly beneficial effect in reducing adulteration, and involves less trouble and expense than any other method. It is obviously an advantage, however, in addition to this to be able in certain extreme cases to use more stringent methods when necessary.

Notification and Prosecution.-The adulteration of food is best held in check in localities where under the law cases may be brought in court and are occasionally so brought. The mere power to prosecute is in itself a safeguard, even though that power is not frequently exercised. Under a conservative enforcement of the law, actual prosecution should be made as a last resort. Neither the number of court cases brought by a food commission nor the large ratio of court cases to samples found adulterated are criteria of its good work. Except in extreme cases, it is frequently found far more effective to notify a violator of the law, especially if it is a first offense, giving warning that subsequent infraction will be followed by prosecution. Such a notification frequently serves to stop all further trouble at once and with the minimum of expense. Instances are frequent in Massachusetts where, by such simple notification, widely distributed brands of adulterated foods have been immediately withdrawn from sale.

Massachusetts was the first of all the states to enact pure-food legislation, and since the year $\mathrm{I} 88_{3}$ has had a well-established system of inspection, prosecuting cases under its laws through the Food and Drug Department of the State Board of Health. Cases are brought in court with practically no expense for legal services. Complaints are entered by 
the collector, or, as he is termed, inspector, who makes complaint not in his official capacity, but as a citizen who under the law has been sold a food found to be adulterated, and who is entitled to conduct his own case, which he does with the aid of the analyst and such cther witnesses as he may see fit to employ. Experience is readily acquired by the inspector in conducting such cases in the lower police or municipal courts, where they are first tried, and years ago the services of legal counsel in Massa chusetts were dispensed with as superfluous.*

Statistics in the annual reports of the Massachusetts Board show witb what uniform success these trials have been conducted. While more often settled in the lower courts, occasional appeal cases are carried tc the superior courts, where the services of the regular district attorney are of course availed of in prosecuting the case.

Such a system as the above, while admirable for a state or city after long experience in the enforcement of food laws in the courts, is obviousiy impracticable with newly established systems of state food inspection.

\section{REFERENCES ON FOOD INSPECTION AND OFFICIAL CONTROL.}

Aввот, S. W. Food and Drug Inspection. Article in Reference Handbook of the Medical Sciences, Vol. 3, pp. 162-180. N. Y., 1902.

Andrews, O. W. Public Health Laboratory Work and Food Inspection. London, I 901 .

Bigelow, W. D. Foods and Food Control. U. S. Dept. of Agric., Bur. of Chem., Bul. 69 , rev.

- Food Legislation for the Year Ending June 30, 1907: Ibid., Bul. 112.

- Pure Food Laws of European Countries. U. S. Dept. of Agric., Bur. of Chem., Bul. 6r.

BuchkA, K. von. Die Nahrungsmittelgesetzgebung im Deutschen Reiche. Berlin, IgOI.

Chapin, C. V. Municipal Sanitation in the United States. Providence.

KENWOOD, H. R. Public Health Laboratory Work. London, I904.

LEACH, A. E. Character and Extent of Food and Drug Adulteration in Massachusetts and the System of Inspection of the State Board of Health. Tech. Quarterly, March, rgoo.

Moor, C. D. Suggested Standards of Purity for Foods and Drugs. London, Igo2.

NEufELDT, C. A. Der Nahrungsmittelchemiker als Sachverständiger. Berlin, r907.

* Where such a practice is in vogue an intelligent inspector must of course be chosen with reference to his ability to do this court work. The food laws are few and simple, as are also the court decisions rendered under them, so that it is no great task for the inspector to become much more familiar with them than the average general lawyer whom he meets I in court and who not infrequently consults the inspector for information regarding these laws. 
Polin Et Labit. Examen des Aliments suspects. Paris, 1892 .

Tucker, W. G. Food Adulteration: Its Nature and Extent and How to Deal with It. Med. Rev. of Rev's, Oct., 1903 .

VACHER, F. The Food Inspector's Handbook. London, I893.

Wedderburn, A. J. Reports on Extent and Character of Food and Drug Adulteration in the United States. U. S. Dept. of Agriculture, Div. of Chem., Bulletins 25, 32 , and 41 .

WreEy, H. W. Foods and Their Adulteration. Philadelphia, r9o7.

WÜZBURG, A. Die Nahrungsmittelgesetzgebung im Deutschen Reiche. Leipzig, I903.

The British Food Journal, London, 1899 et seq.

Food and Sanitation, London, 1892-1900 (discontinued August, 1900).

Journal of the Sanitary Institute, 1892 et seg.

Revue International des Falsifications, Amsterdam, 1888 et seq.

The American Food Journal, Chicago, rgo6 et seq.

The Food Law Bulletin, Chicago, 1907 et seq.

Biennial Reports of the Idaho Dairy, Food and Oil Commission, 1903 et seq.

Veröffentlichungen des kaiserlichen Gesundheitsamtes. Berlin, 1877 et seq.

Arbeiten aus dem kaiseslichen Gesundheitsamtes. Berlin, 1886 et seq.

Reports of the Local Government Board of England, 1877 et seq.

Reports of the Paris Municipal Laboratory, 1882 and 1885 .

Reports of the Canton Chemists of Switzerland, I8go et seq.

Annual Reports of the Massachusetts State Board of Health, $188_{3}$ et seq.

Monthly Bulletins of the Massachusetts State Board of Health.

Annual Reports of the Conn. Agric. Exp. Station on Food Products, 1896 et seq.

Annual Reports of the Ohio Dairy and Food Commissioner, 1890 et seq.

Annual Reports of the New Jersey Dairy Commissioner, 1886 et seq.

Annual Reports of New Jersey Laboratory of Hygiene, Chemical Dept., 1903 et seq.

Annual Reports of the Michigan Dairy and Food Department, 1893 et seq.

Monthly Bulletins of the Michigan Dairy and Food Department, Aug., 1895 et seq.

Biennial Reports of the Minnesota Dairy and Food Commissioner.

Annual Reports of the Wisconsin Dairy and Food Commissioner, 1890 et seq.

Annual Reports of the Penn. Board of Agriculeure, r894 et seq.

Annual Reports of the Illinois State Food Commissioner, I899 et seq.

Biennial Reports of the New Hampshire State Board of Health, 1902 et seq.

Quarterly Bulletins of the New Hampshire State Board of Health, I902 et seq.

Annual Reports of the North Carolina State Board of Agriculture on Food Products, rgoo et seq.

Bulletins of the North Dakota Experiment Station.

Annual Reports and Monthly Bulletin of the Indiana State Board of Health, rgo5 et seq.

Official Inspections of the Maine Agricultural Experiment Station. 
Annual Reports of the Wyoming State Dairy Food and Oil Commission, 1904 et seq. Quarterly Bulletins of the Vermont State Board of Health.

Annual Reports of the South Dakota Food and Dairy Commission, rgor et seq.

Proceedings and Methods of Analysis of the Association of Official Agricultural Chemists, published as bulletins of the U. S. Department of Agriculture, Bureau of Chemistry.

Proceedings of the National Association of State Dairy and Food Departments, I902 et seq. 


\section{CHAPTER II.}

\section{THE LABORATORY AND ITS EQUIPMENT.}

Location.-The selection of a location for a food laboratory cannot always be made solely with reference to its needs and its convenience, but it is more often subject to economic conditions beyond the analyst's control. Under very best conditions, such a laboratory should be situated in a building designed from the start exclusively for chemical or biological and chemical work. Almost any well-lighted rooms in such a building can be readily adapted for the purpose. When, however, as is frequently the case, rooms for such a laboratory are provided in municipal, government, or office buildings, in which for the most part clerical work is done, the problem of adequately utilizing such rooms so that they may not at the same time prove offensive to or interfere with the comfort of other occupants of the building is sometimes difficult. It is obvious that basement rooms in such a building, as far as ventilation is concerned, are less readily adapted for the requirements in hand than are those of the top floor, though, if the light is good and there are abundant and well-arranged ventilating-shafts, such rooms may be made to serve every purpose. In the basement one may most easily obtain water, gas, and steam, and dispose of wastes without annoyance to one's neighbors. When, however, it is possible to do so, rooms on the top floor of an office building should be utilized for a food laboratory, for in such rooms the problems of lighting, heating and ventilating are comparatively simple and may usually be solved without regard to other occupants. In such a case ample provision must be made, preferably through shafts which are readily accessible for water-, gas-, steam-, and soil-pipes passing down below.

The actual equipment of the food laboratory depends of course largely on its particular purpose; and while it is manifestly impossible to do otherwise than leave the details to the individual taste and needs of the analyst, 
modified by the means at his disposal, a few general suggestions regarding important essentials may prove helpful. These imply a fairly liberal though not extravagant outlay, with a view to saving both time and energy by convenient surroundings well adapted to the work in hand. At the same time equally satisfactory work is possible under simpler conditions than those described.

Floor.-The best material for the floor of the working laboratory is asphalt. Such a floor is firm but elastic, is readily washed by direct application of running water, if necessary, and resists well the action of ordinary reagents. An occasional thin coating of shellac with lampblack applied with a brush gives the asphalt floor a smooth, hard surface and may be applied locally to cover spots and blemishes.

Lighting.-The lighting of the rooms, if on the top floor, is best effected by both wall windows and skylights. North windows furnish the best light for the microscope; the skylight, when available, is the ideal light for the balance and for general laboratory work.

Ventilation by forced draft is a great convenience. For this purpose an exhaust-fan driven by an electric motor and controlled in speed by a fractional rheostat is admirable. Such a fan had best be located in a small closed compartment or closet near the centre of the series of rooms designed to be ventilated by it, and this closet should have directly over the fan an outlet-shaft passing through the roof of the building. With such a system, a series of branching air-ducts should radiate from the fan closet, conveniently arranged either above or along the ceiling and communicating with the various hoods, closets, and rooms near the top.

Benches.-The working benches should have wooden or glazed tile tops. White glazed tile, if properly laid, furnish a very clean, sanitary, and resistant surface, besides being often convenient for color tests. If laid on a plank surface, cement should not be applied directly, as it swells the wood before drying out and results in a loose and often uneven surface. Cement may be avoided altogether and the tiles after first soaking in oil may be laid in putty directly on the wood. Tiles may be laid in cement by first covering the plank surface with cheap tin plate, overlapping the edges and securing by tacks. This prevents swelling of the wood. The tin may be covered to advantage with cheap paint. The tiles may then be embedded in a layer of cement spread over the tin surface.

Soft encaustic glazed tiles commonly used for wall finish are not as 
effective as hard floor tiles, since the former crackle and lose color when subjected to heat. If the hard floor tiles can be specially glazed, they make by far the most satisfactory and enduring surface.

When wooden bench tops are used they may be treated to advantage by staining with the following solutions:

Solution I. 100 grams of anilin hydrochloride, 40 grams of ammonium chloride, 650 grams of water.

Solution 2. 100 grams of copper sulphate, 50 grams of potassium chlorate, 615 grams of water.

Apply solution I thoroughly to the bare wood and allow it to dry; then apply 2 and dry. Repeat these applications several times. Wash with plenty of hot soap solution, let dry and rub well with vaseline. It is claimed that wood so treated is rendered fire-proof and is not acted on by acids and alkalies. When the finish begins to wear, an application of hot soap solution or vaseline will bring back the deep black color.

The benches should naturally be located with reference to best light from skylights or windows. Gas and water outlets, sinks and waste-pipes should be conveniently arranged with reference to the working benches, as well as suitable provisions for air-blast and exhaust, while in the space beneath the benches such drawers, cupboards, and receptacles as are required should be provided. A clear bench width of 24 inches is ample for most work; if wider there is a temptation to allow apparatus to accumulate at the back. At the back of the bench and within easy reach, a raised narrow shelf should be provided to be used exclusively for common desk reagents. This again should not be so wide as to allow the accumulation of useless bottles. A narrow raised guard or beading at the edge of the reagent shelf prevents the bottles from accidently slipping off.

Hoods.-Closed hoods with sliding sash fronts are almost indispensable. These hoods should be directly connected with the ventilating shafts or pipes, or with the air-ducts that radiate from the exhaust-fan closet, when such a system is provided. Gas outlets inside the hoods are necessary.

When there is a good draft, either natural or forced, a hooded top over the working bench, such as that shown in Fig. 3 , is quite as efficient as a closed hood for most purposes. This is best made of galvanized iron, painted on the outside and treated on the inside with a preparation of graphite ground in oil. Here are best carried out all the processes involving the giving off of fumes and gases, which, if the ventilation is efficient, should pass directly up the flues and not come out in the room. 
Sinks and Drains.-The sinks should preferably be of iron or porcelain. If iron, they should at frequent intervals be treated with a coat of

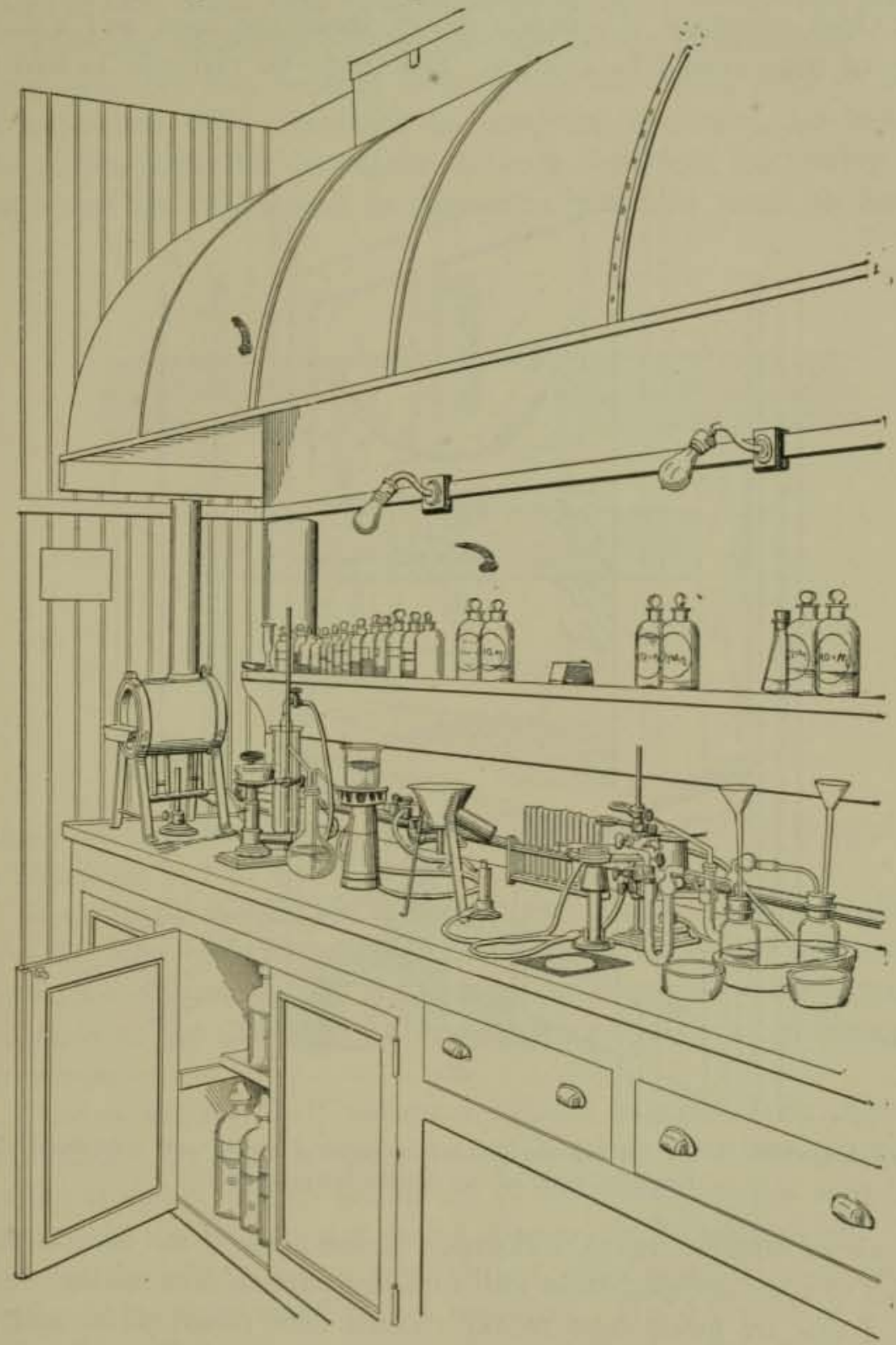

Fig. 3.-Hooded Top of Galvanized Iron over Working-bench, Connected with Ventilating Air-ducts.

asphalt varnish. A great convenience is a hooded sink (Fig. 4) in which foul-smelling bottles, or vessels giving off noxious or offensive fumes 
or gases, may be rinsed under the tap while completely closed in. Openwork rubber mats at the bottom of the sinks help to insure against breakage. Open plumbing of simplest design should be used, and a multiplicity of traps should be avoided. Sinks may be variously located for

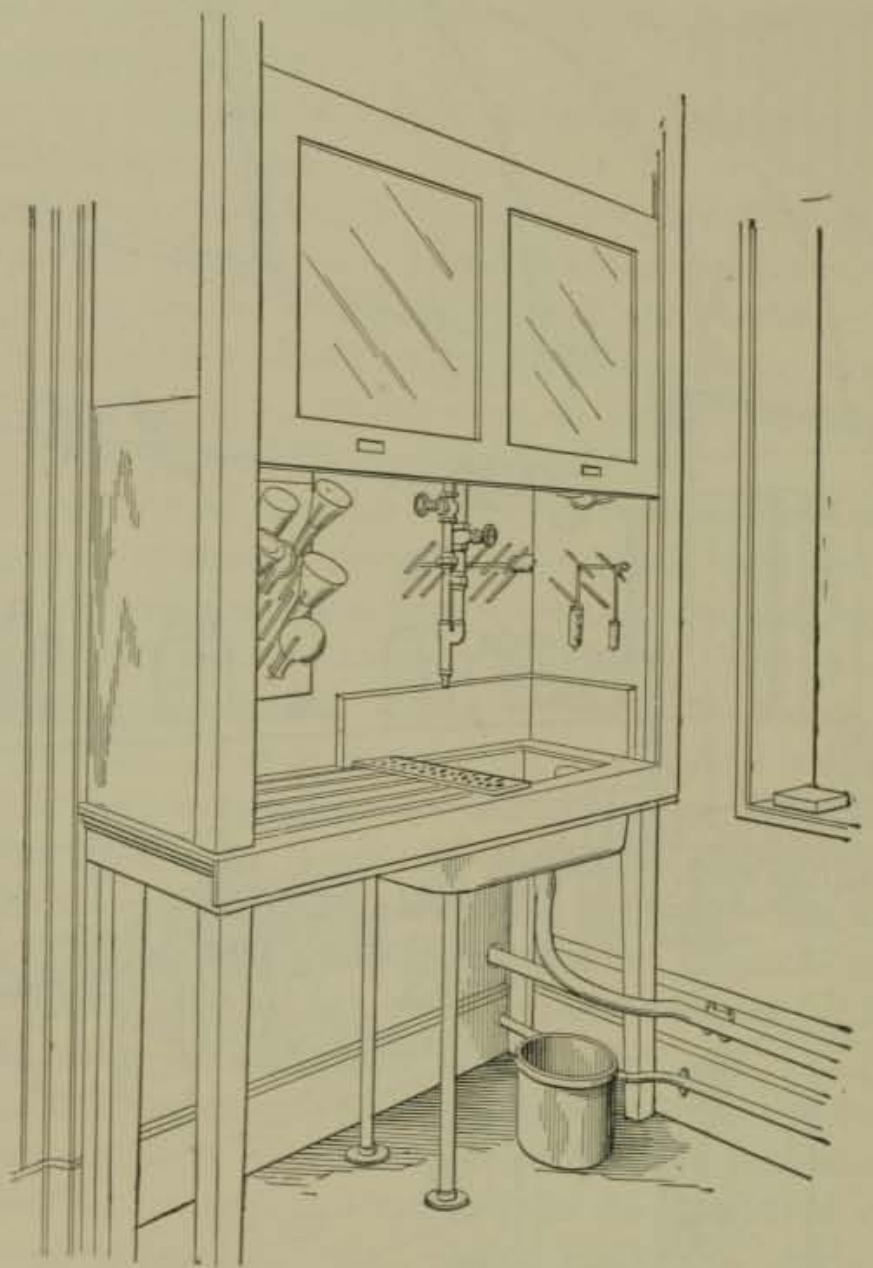

Fro. 4.-A Hooded Sink. An injector-like arrangement of steam and cold-water pipes furnishes water of any desired temperature.

convenience without regard to situation of soil-pipes, if the floor is thick enough to allow an open drain with sufficient pitch to flow readily. Such open drains are much more readily cleaned than closed pipes, and are best constructed by splitting a lead pipe and laying it in an iron box which is sunk into the floor. The edges of the lead pipe are rounded over those of the box as in Fig. 5, filling the joints with hydraulic cement, and the top of the drain is covered by a series of readily removable iron plates 
flush with the top of the floor. Waste-pipes from sinks, still-condensers, refrigerators, and various forms of apparatus involving flowing water may be led into this drain, holes being drilled in the iron cover for their insertion.

Steam and Electricity. - These are useful but not indispensable. Steam, when available, may be used to advantage for boiling ether or benzine in connection with continuous fat-extraction apparatus, for furnishing the motive power for driving the Babcock centrifuge, for heating waterbaths and hot closets, and, in connection with cold water, to furnish a

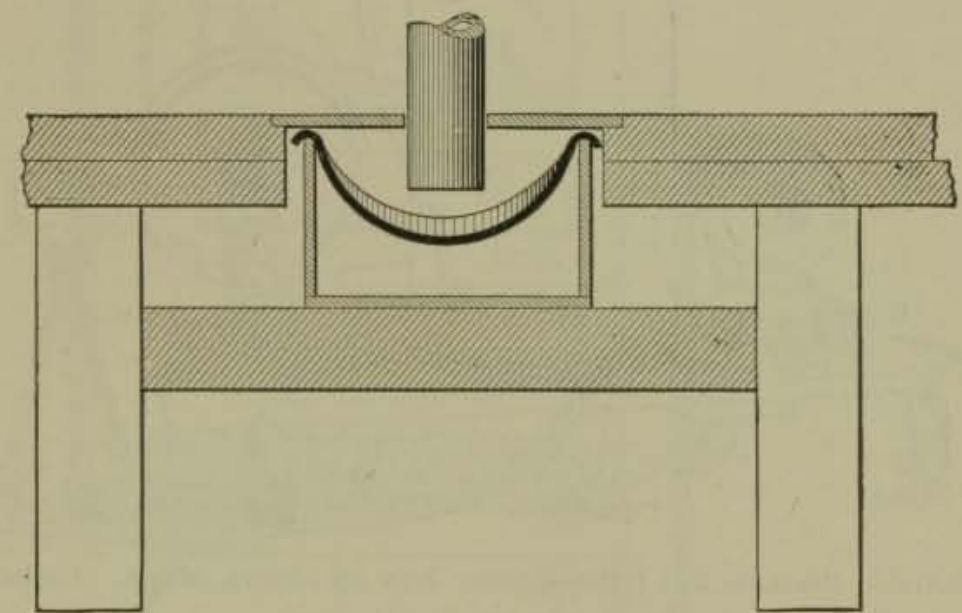

Fig. 5.-Section of Open Drain-pipe in Floor.

supply of hot water when wanted at the sink. The latter application is illustrated in Fig. 4.

If electricity is used for lighting, it may also be applied in a variety of useful ways in the laboratory, as, for instance, for heating coils or electric stoves, for electrolysis, and for running small motors, which in turn may be employed for driving centrifuges, shaking apparatus, ventilating-fans, air-pumps, etc.

Suction and Blast.-If the water-pressure is ample, both air-pressure and exhaust for blast-lamps, vacuum filtration, and other purposes are readily available through the agency of the various devices used in connection with the flow of water, as, for instance, the Richards pump. When, however, the water pressure is insufficient, other means must be employed for furnishing these much-needed requisites. Fig. 6 illustrates a simple and almost noiseless pressure and exhaust pump run by a $\frac{1}{8}$-H.P. electric motor, which with the pressure-equalizing tank and the appropriate connections are mounted on a light wheel truck, and readily movable to any part of the laboratory. By simply screwing the plug into an 
electric-light outlet, either suction or blast may be had at will, depending on the position of a knife-edge switch which determines the direction of the current. By means of a fractional rheostat the speed may be varied and the pressure thus controlled.

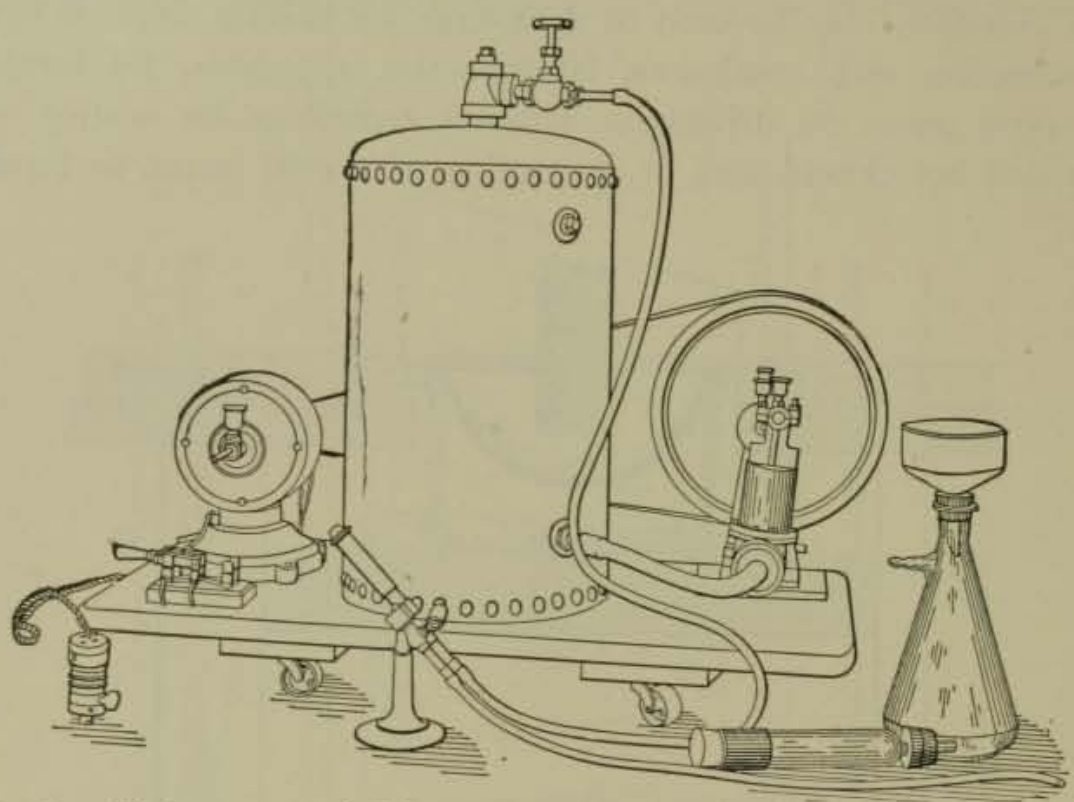

Fic. 6.-Portable Pressure- and Exhaust-pump Run by Electric Motor. Useful for blastlamps, vacuum filtration, etc.

\section{APPARATUS.}

The laboratory is of course to be supplied with the usual assortment of test-tubes, flasks, beakers, evaporating and other dishes of porcelain, platinum and glass, funnels, casseroles, crucibles, mortars, burettes, pipettes, graduates, rubber and glass tubing, lamps, ring-stands and various supports, clamps and holders, the nature, number, and sizes of which are determined by individual requirements. Special forms of apparatus peculiar to certain processes of analysis or to the examination of special foods will be described in their appropriate connection. The following apparatus of a general nature may be regarded as indispensable for the proper fitting out of the food laboratory:

Balances.-These should include ( $\mathrm{I}$ ) an open pan balance for coarse weighing, having a capacity up to I kilogram and sensitive to o.I gram, with a set of weights; and (2) an analytical balance, enclosed in a case, sensitive to $.000 \mathrm{I}$ gram under a load of 100 grams, with an accurate set of non-corrosive weights. The short-beam analytical balance is prefer- 
able for quick work, and as constructed by the best modern makers leaves nothing to be desired.

The Water-bath.-This is such an important accessory to the food analyst that it should, if possible, be specially designed to meet his require-

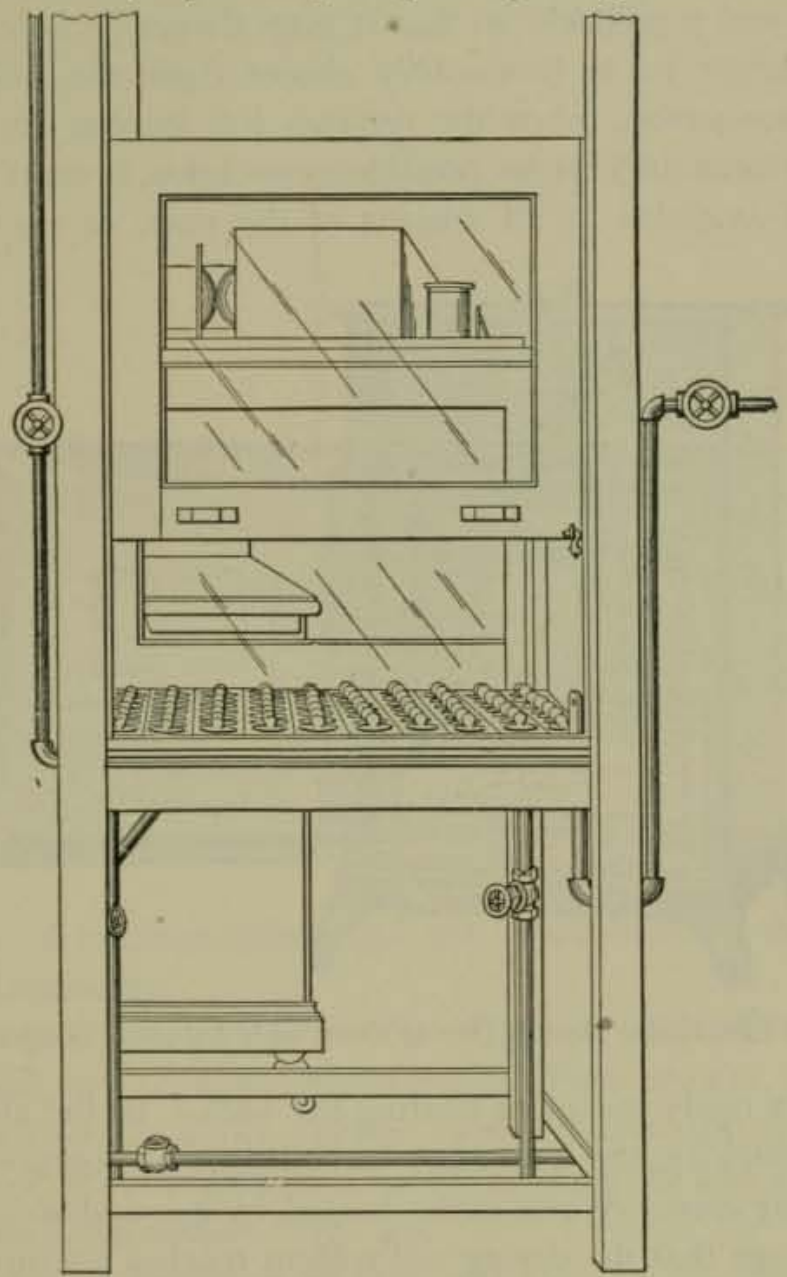

Fic. 7.-Water-bath, Enclosed in Hood, with Sliding-sash Front.

ments, though the ordinary copper baths, supported on legs and designed to be heated by gas-burners, as kept in regular stock by the dealers, will sometimes serve the purpose. For nearly all moisture determinations the platinum dishes described on page 133 and the somewhat larger wine-shells of $100 \mathrm{cc}$. capacity are most used, and for this purpose the top of the bath should have plenty of openings of the right size for these. A very economical construction of bath admirably adapted for the food analyst's use is shown in Fig. 7, being the form employed by the writer. 
The size and number of openings are determined by the number of samples to be simultaneously analyzed. A steam coil within the body of the bath serves to boil the water. Fig. 7 also shows the hood for carrying off the steam and fumes, the sliding front of which is furnished with a hasp and a padlock, so that it may always be kept locked by the analyst whenever he is temporarily absent from the laboratory. This is a useful precaution, when the residues left thereon are from samples which are to form subjects for possible prosecution in court later.

Steam, if available at all seasons of the year, or electric immersion

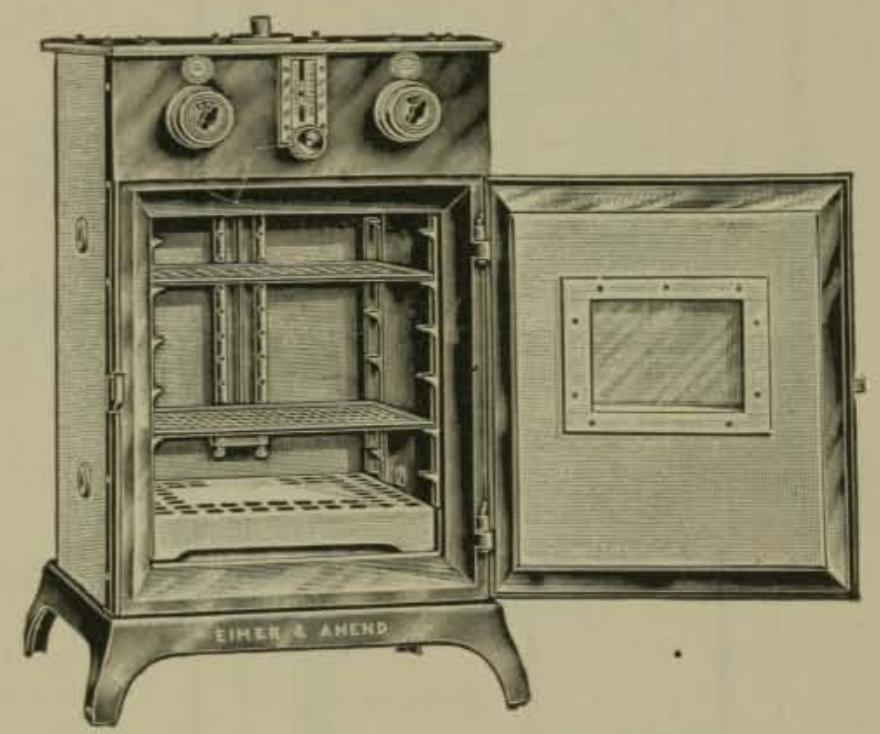

Frg. 8.-Freas Electrically Heated Drying Oven with Accurate Temperature Control.

coils furnish a ready means of heating the bath. In the absence of both steam and electricity, the bath must be boiled by gas burners.

The Drying-oven.-Water ovens heated by gas and steam ovens have the disadvantage that the drying cell seldom reaches a temperature above $98^{\circ} \mathrm{C}$. The electric oven shown in Fig. 8 obviates this difficulty, the regulator permitting of adjustment so that full $100^{\circ}$, as well as any desired temperature, can be attained. Fig. 9 shows an asbestos-covered, jacketed air-oven, heated by a gas burner, with an efficient form of gaspressure regulator.

The Water-still-An efficient still should be provided, capable of supplying the laboratory with an ample quantity of pure water for analytical purposes. Fig, Io illustrates a compact form of still, which is particularly economical in view of the fact that a single stream of inflowing cold 
water first serves to cool the condenser, and, rising, becomes vaporized in the boiler directly connected with the condenser at the top. This apparatus is capable of distilling six gallons of water in twelve hours.

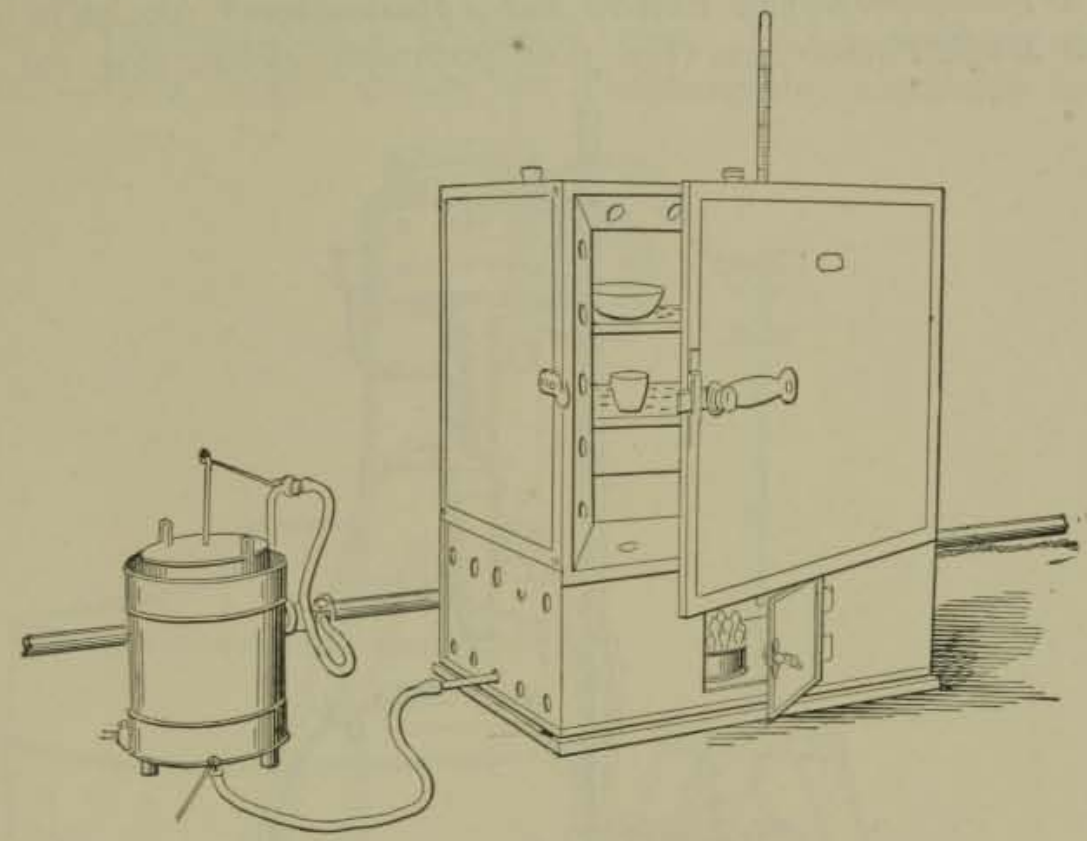

Fig. 9.-Asbestos-covered Air-oven, with Gas-pressure Regulator.

The list of indispensable requisites in addition to the above should include the following:

Continuous Extraction Apparatus (Fig. 20, 21, and 22).

Apparatus for Nitrogen Determination (Figs. 26, 27a, and 27b).

A pparatus for Distilling Various Food Products (pp. 71 and 660).

A Babcock or other Milk-fat Centrifuge (Figs. II and 45).

A Butyro Refractometer (Fig. 38).

An Immersion Refrastometer (Fig. 42).

A Microscope and its Appurtenances (Chapter V).

A Polariscope and its Accessories (Figs. 102, 103, and 104).

A pparatus for Specific Gravity Determination (Figs. 14, I5, I6, and I7). Apparatus for the Determination of Carbon Dioxide (Fig. 71 ).

Apparatus for the Determination of Melting-points (Fig. 93).

Marsh Arsenic A pparatus (Fig. 28).

Electrolytic Apparatus (Fig. IIo).

Separatory Funnels (Figs. 24 and 25).

Following is a list of apparatus and appliances which, while not indispensable, are convenient and at times desirable: 
A Spectroscope, either of the direct-vision variety for the pocket, or the Kirschoff \& Bunsen style on a stand.

Spectroscope Cells, parallel-sided, for observation of absorption spectra. A Photomicrographic Camera and Appurtenances* (pp. 96 to 98). A Muffle Furnace, gas (Fig. 3) or, preferably, electric (Fig. I9).

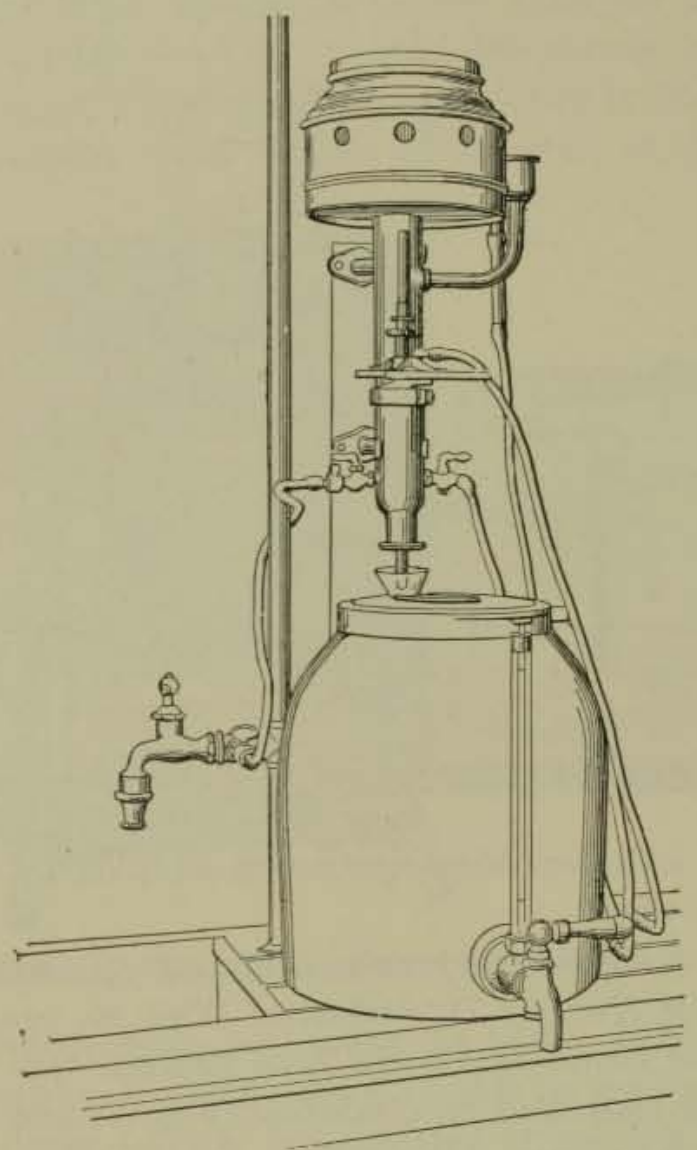

FiG. 10.-A Convenient Laboratory Water-still with Earthenware Receptacle, Provided with Faucet and Glass Gauge.

An Incinerator for a Large Number of Residues (Fig. 52).

An Ebullioscope (Fig. I13).

An Assay Balance, for weighing arsenic mirrors to $0.0 \mathrm{r} \mathrm{mg}$.

An Abbé Refractometer (Fig. 39).

A Schreiner Colorimeter (Fig. 30).

A Lovibond Tintometer (p. 78).

* A photographic dark room is also necessary if photomicrographic work is to be done. 
A Universal Centrifuge.-This convenient apparatus merits a separate brief description, being useful for a wide variety of purposes, such as breaking up ether- and other emulsions, quickly settling out precipitates, and roughly estimating chlorides, sulphates, phosphates, etc., by the volume of the precipitate in graduated tubes. Various-sized aluminum frames, carrying hinged shields, are interchangeably adjustable to the

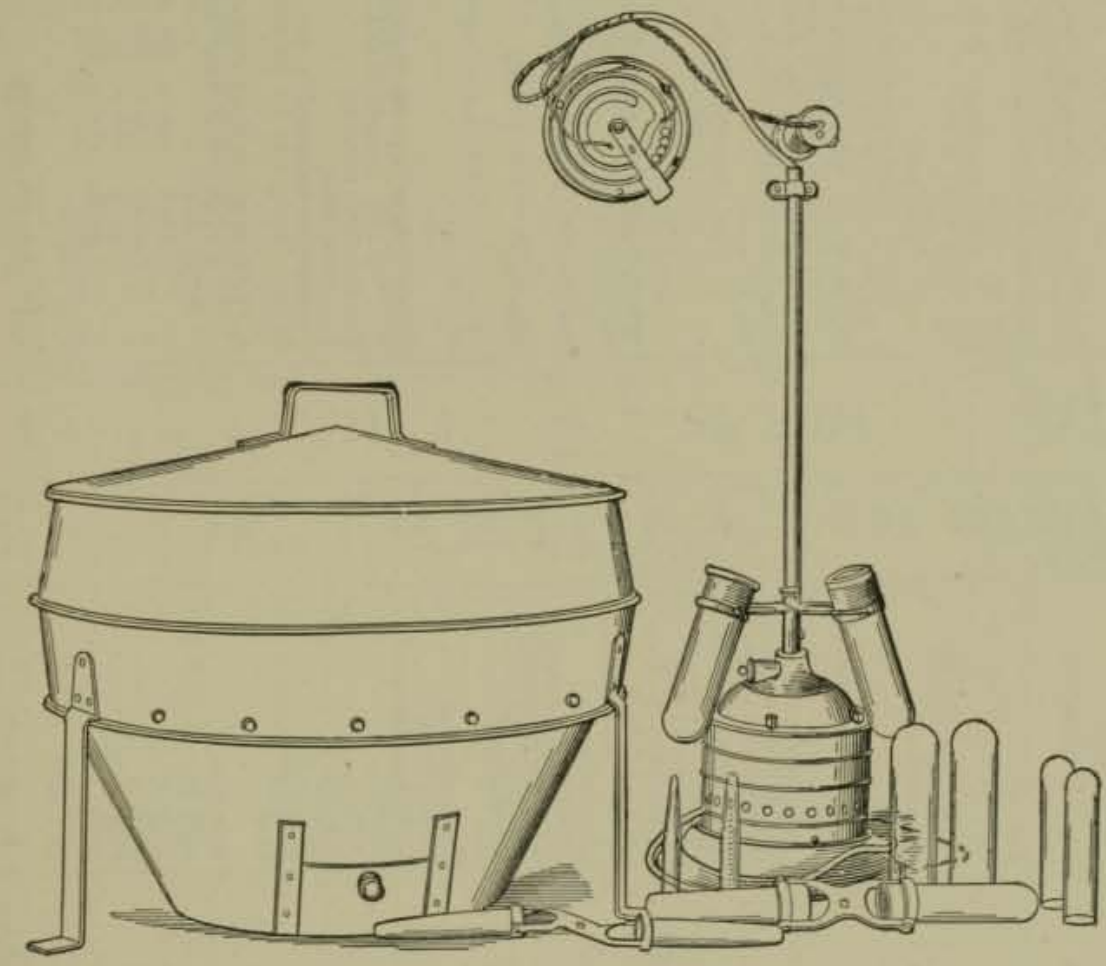

Frg. I1,-The Universal Centrifuge, Driven by an electric motor.

spindle of a vertical electric motor.* The smallest frame has shields adapted to hold two graduated glass tubes of $\mathrm{I}_{5} \mathrm{cc}$. capacity (see Fig. II). This is for the quantitative estimation of small precipitates and the quick settling of sediments. A medium-sized and large frame carry tubes of $80 \mathrm{cc}$. and $\mathrm{I} 20 \mathrm{cc}$. capacity respectively. A frame is also provided with shields adapted for various-sized beakers to be used in settling precipitates. The various types of centrifuges used for the Babcock test (p. I37) may also be used for general work.

* In the absence of electricity a water-motor may be used. 


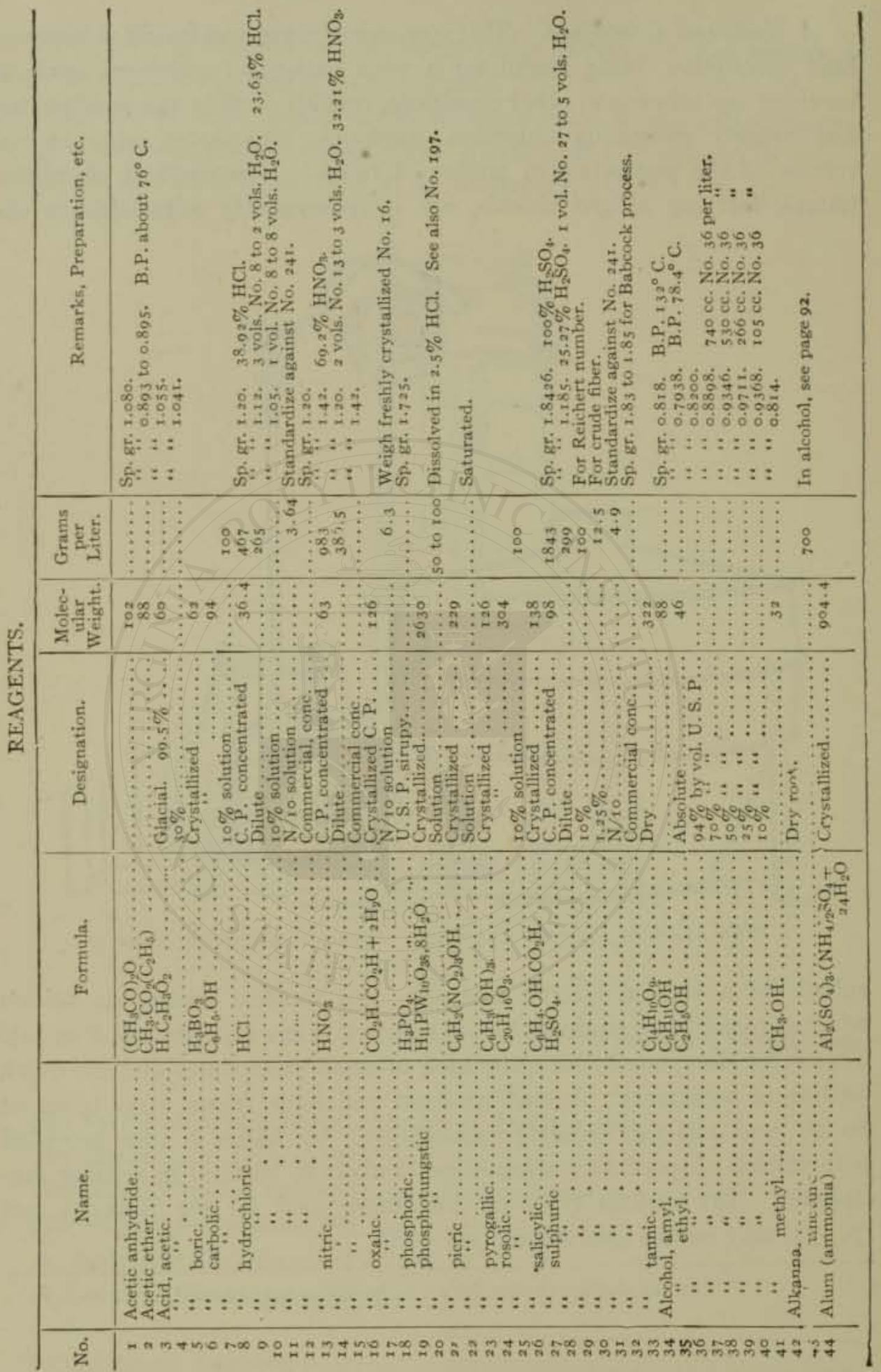




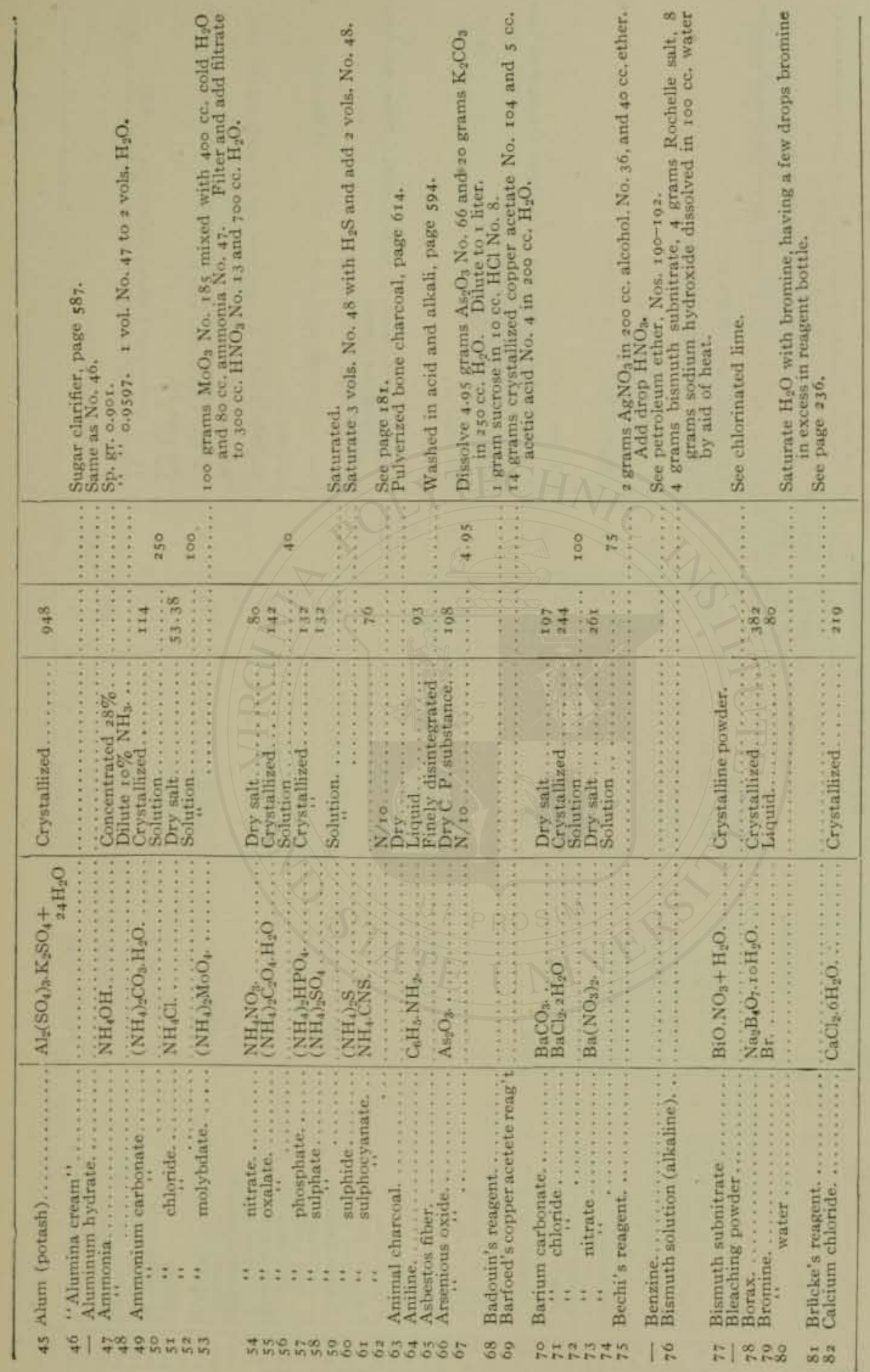




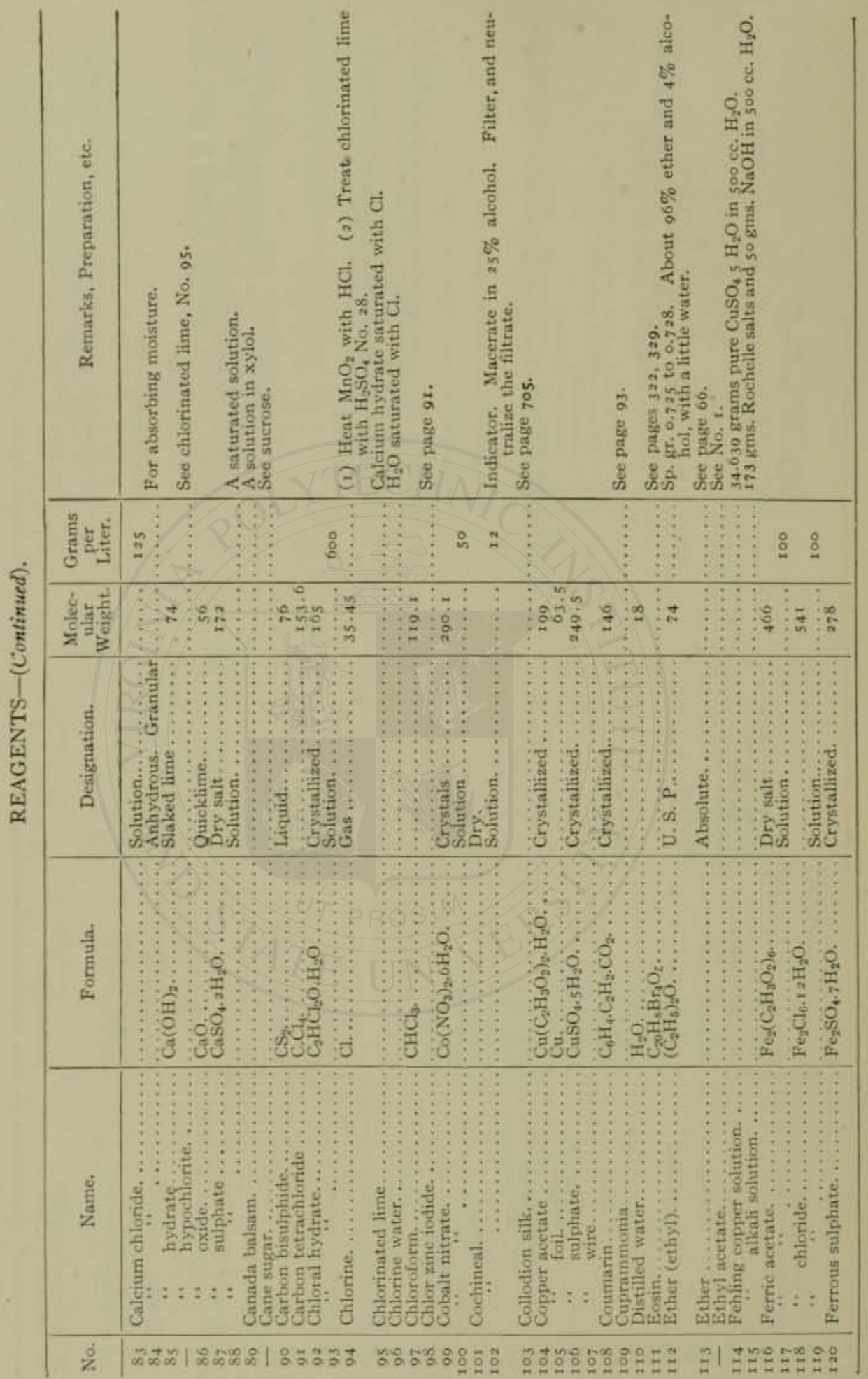




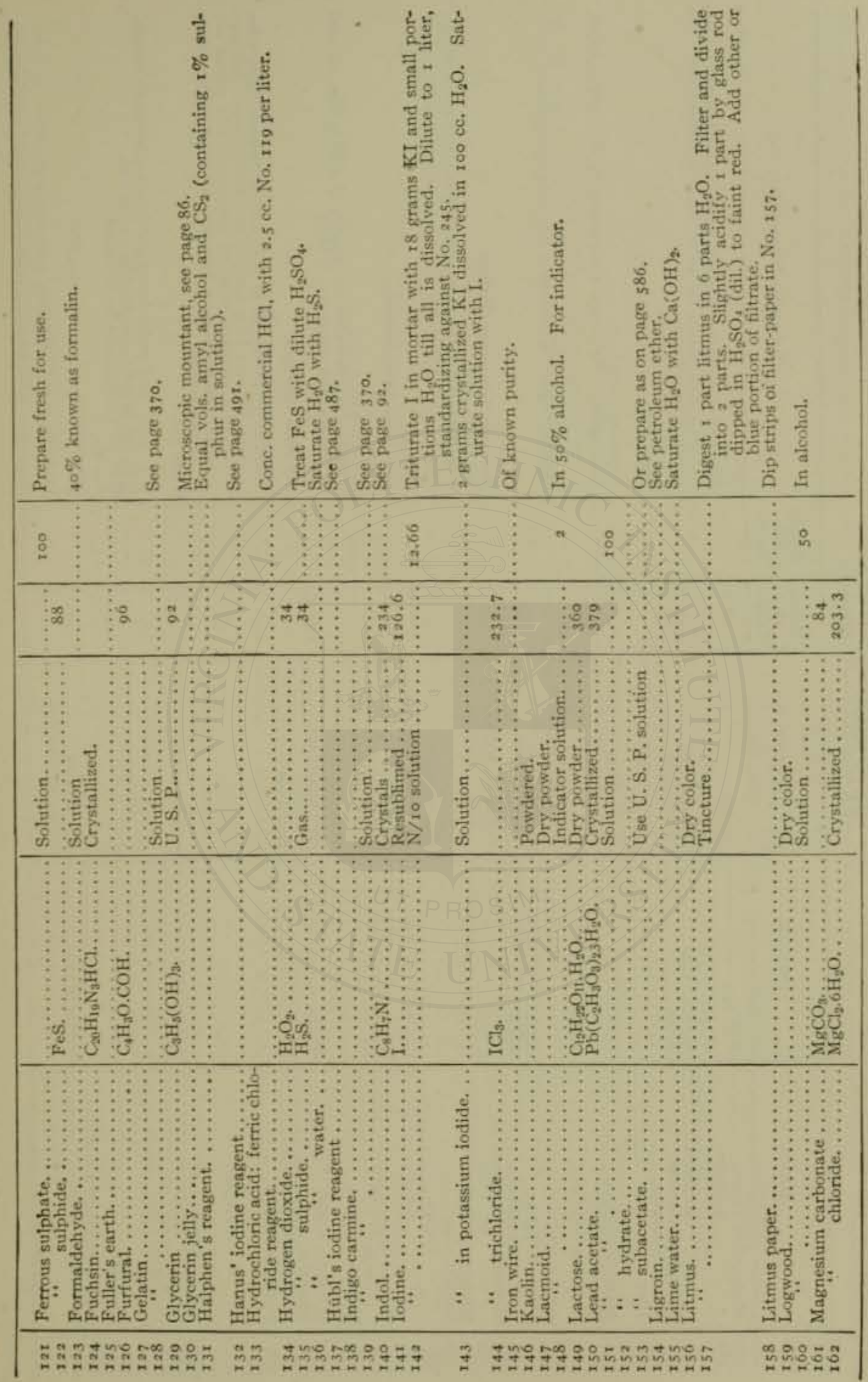




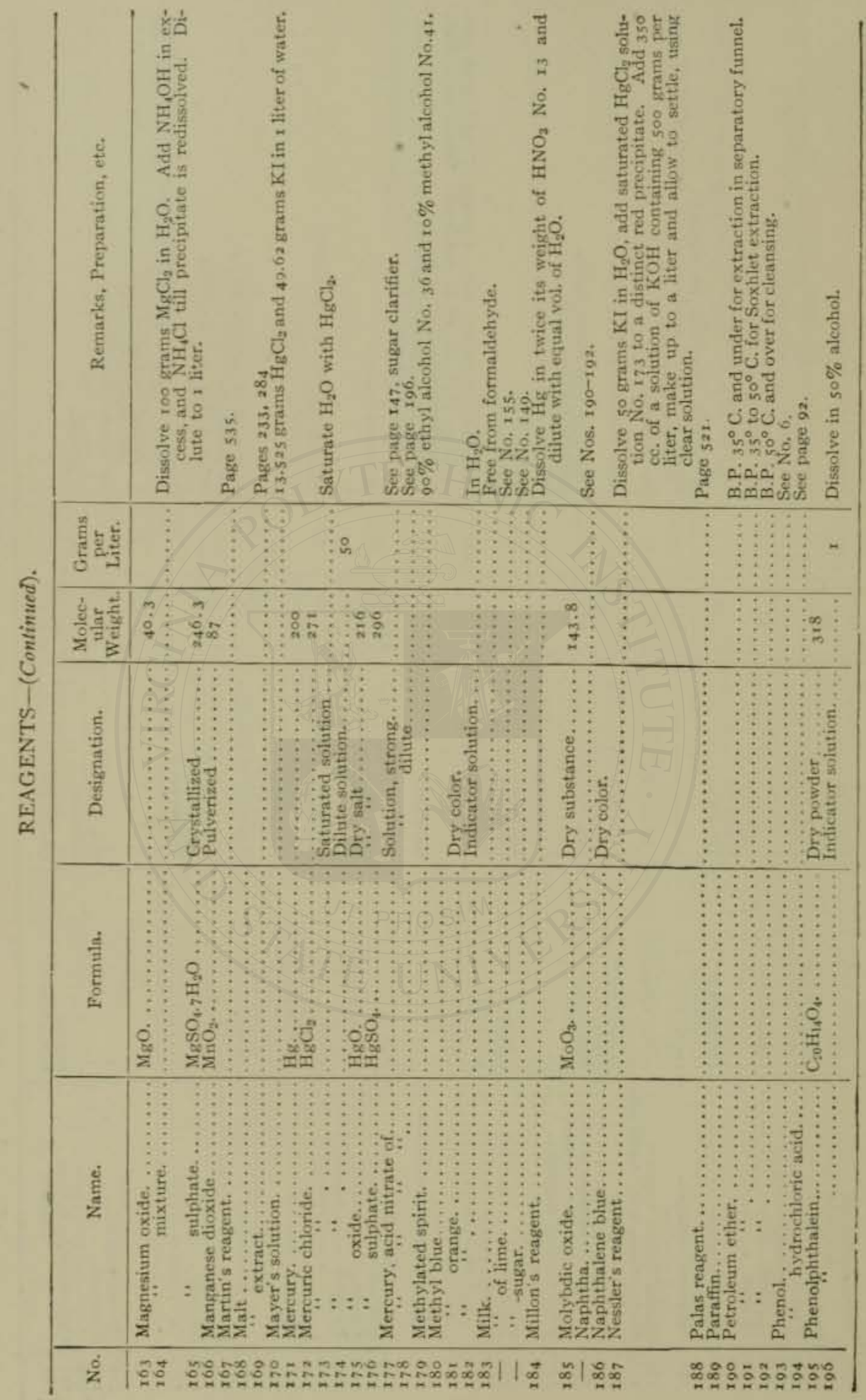




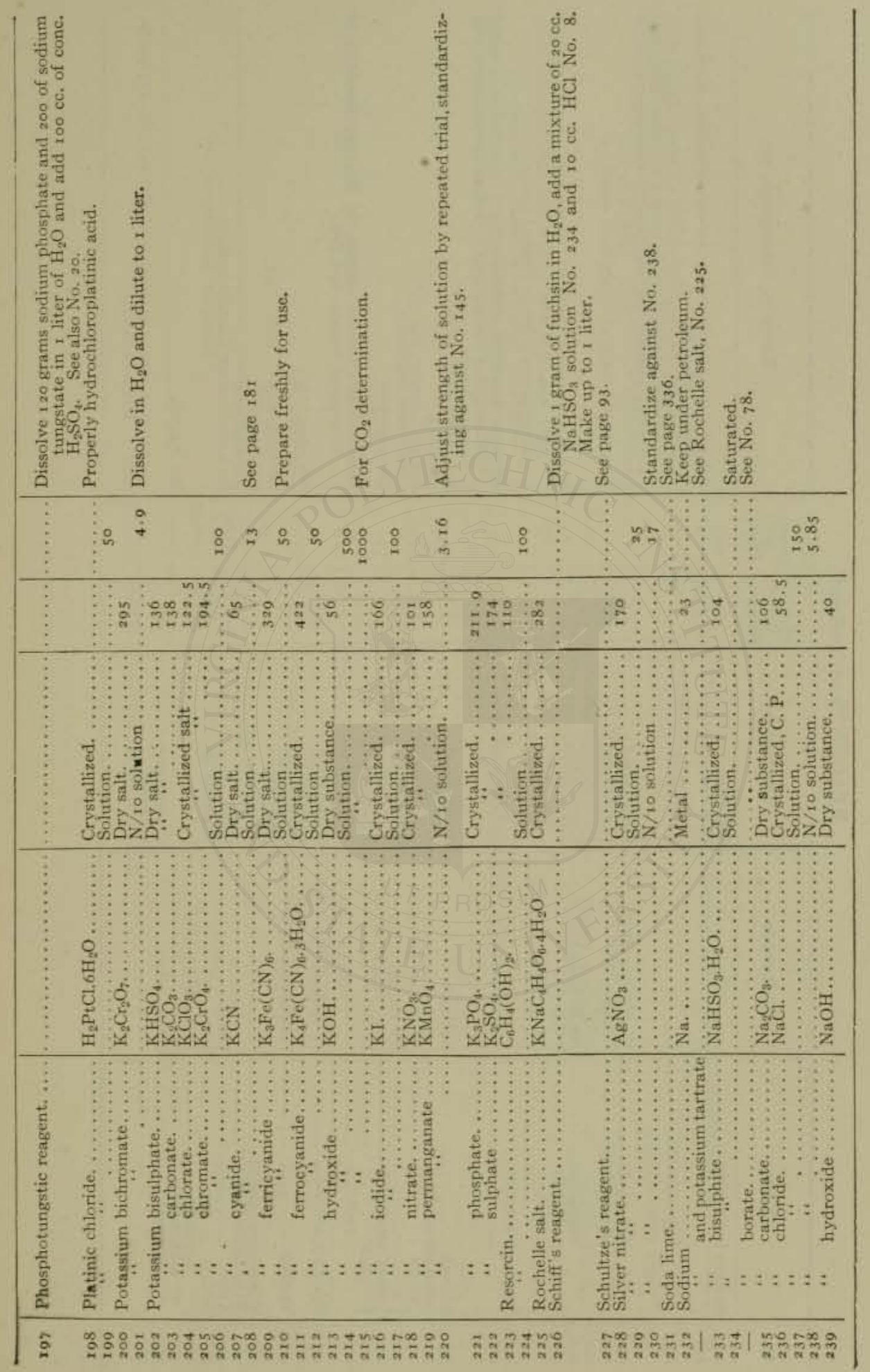




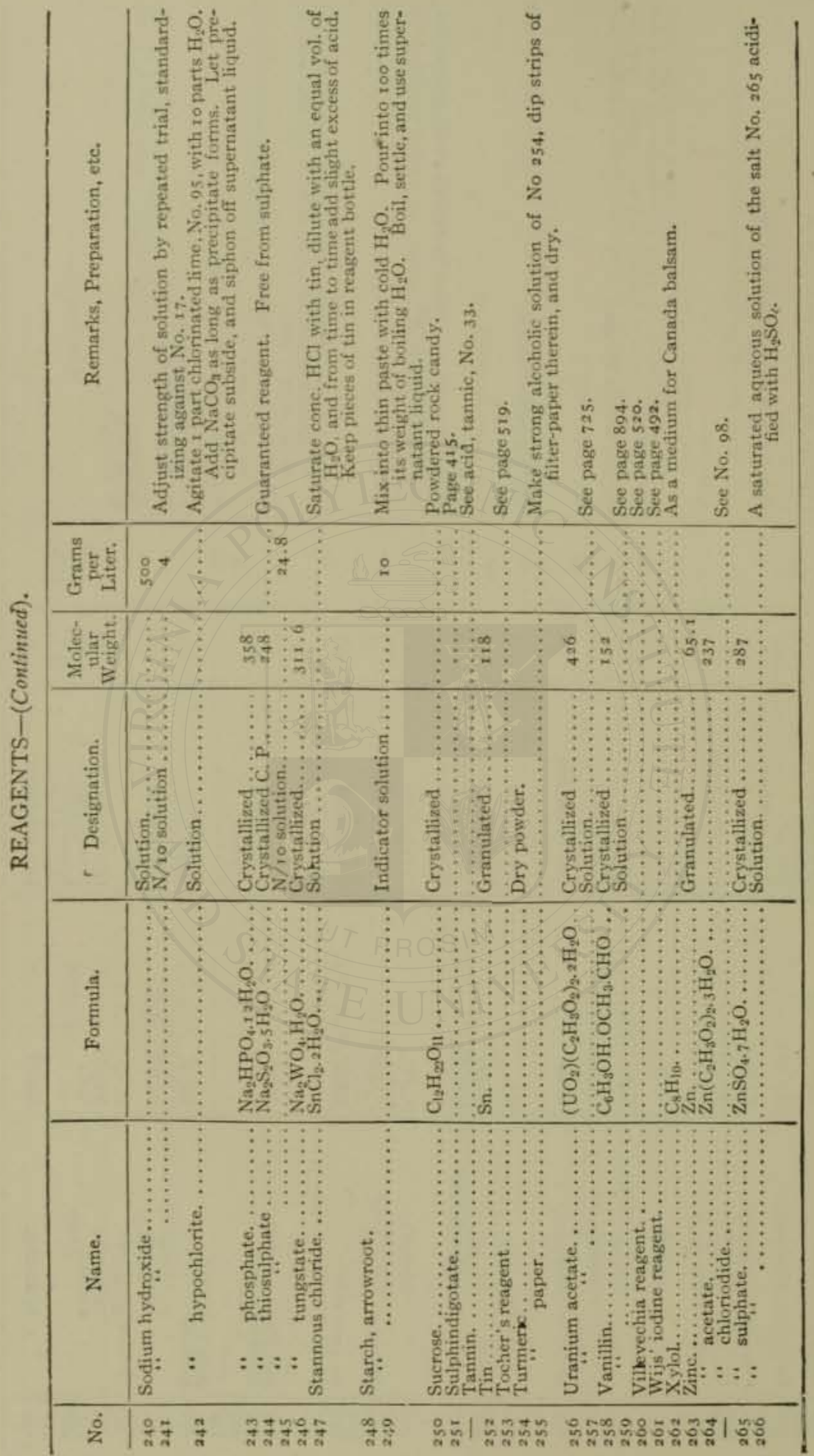


THE LABORATORY AND ITS EQUIPMENT.

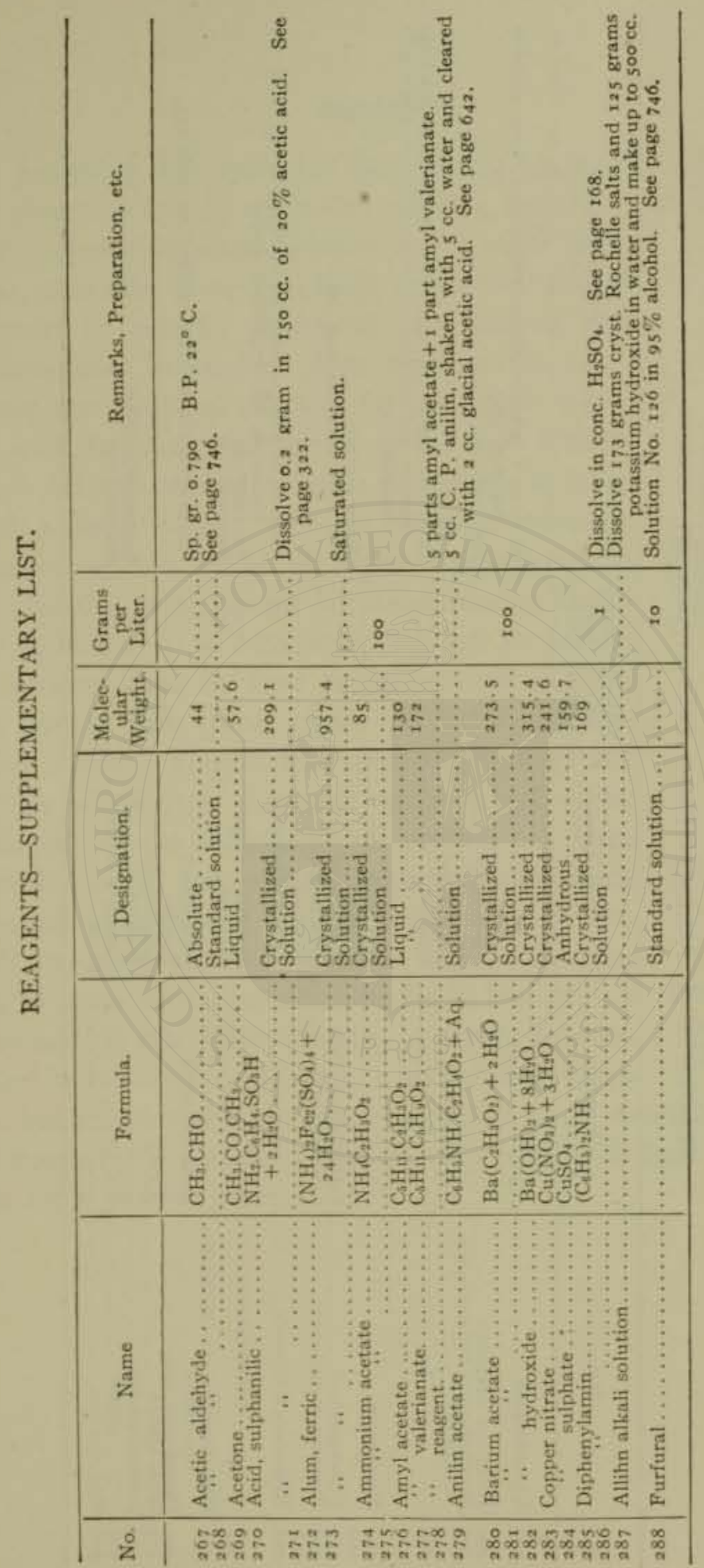


FOOD INSPECTION AND ANALYSIS.

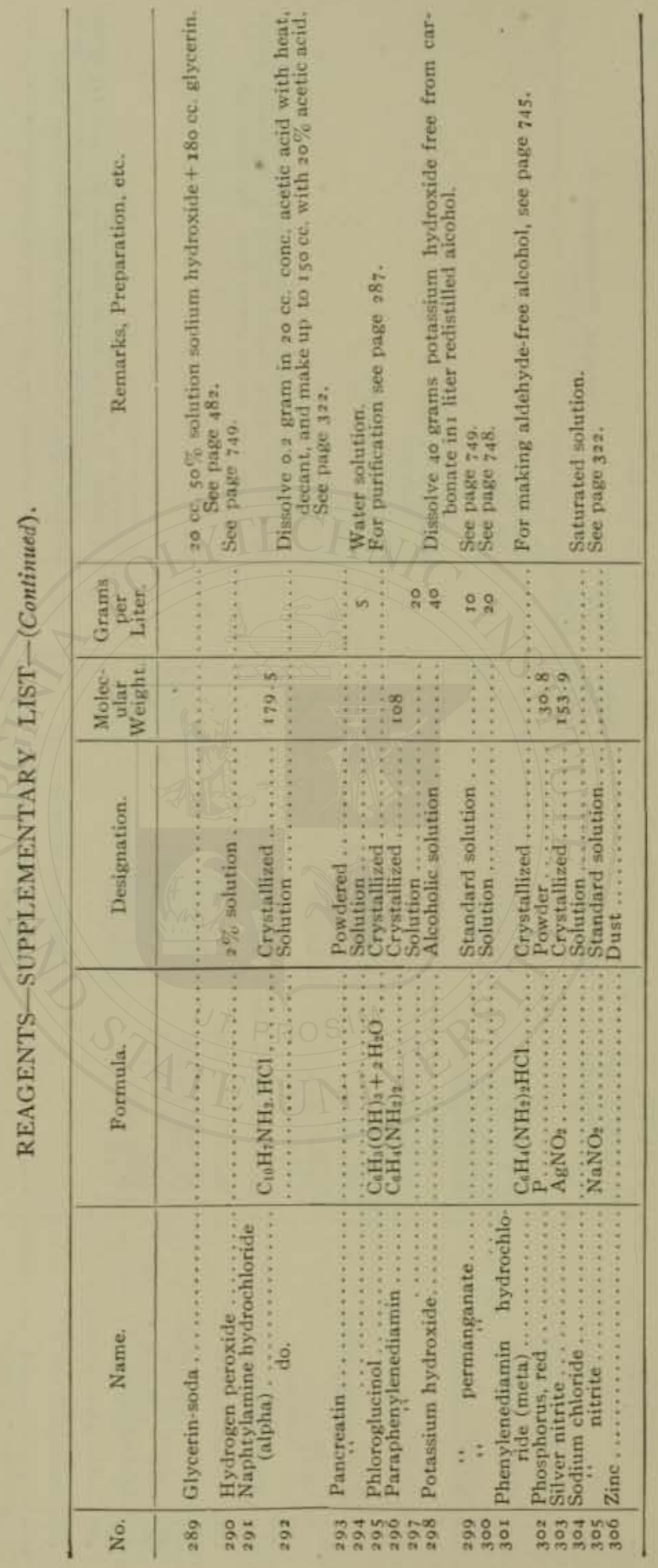




\section{REAGENTS.}

The foregoing list includes the general reagents used in carrying out the processes treated of in this volume, together with their strength, mode of preparation when necessary, and other data. Reagents, especially those constantly employed, should be assigned to regular places on the shelves, and should invariably be kept in place when not in use.

Among the standard solutions for volumetric work, none is more frequently of service in the food laboratory than a tenth-normal solution of sodium hydroxide, and a large supply of this reagent, carefully standardized, should be at all times conveniently at hand. Besides being useful for standardizing tenthnormal solutions, it is constantly needed for determining various acids in food products, such as milk, vinegar, butter, lime juice, cream of tartar, liquors, and many others. Time is well spent in carefully adjusting this solution to its exact tenth-normal value, thus simplifying the calculation of results. A large stock bottle (say of two gallons capacity) containing the standard tenth-normal sodium hydroxide, is conveniently mounted with a side-tube burette in connection, in some such manner as shown in Fig. I2. A small connecting side bottle contains a strong solution of sodium hydroxide (reagent No. 240) through which

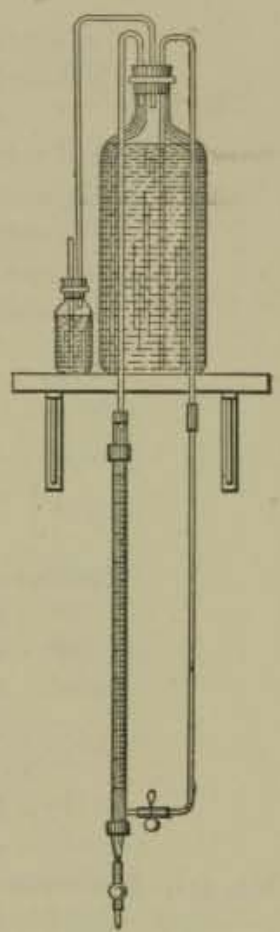

Fig. 12,-Stock Bottle of Tenth-normal Alkali. the air that enters the large bottle is passed, thus depriving it of $\mathrm{CO}_{2}$. In this manner the standard solution may readily be kept of unvarying strength for a year or more. 


\section{EQUIVALENTS OF STANDARD SOLUTIONS.}

Na. 3r. Dectnormal. Sulphuric Acid. One cc, is equivalent to

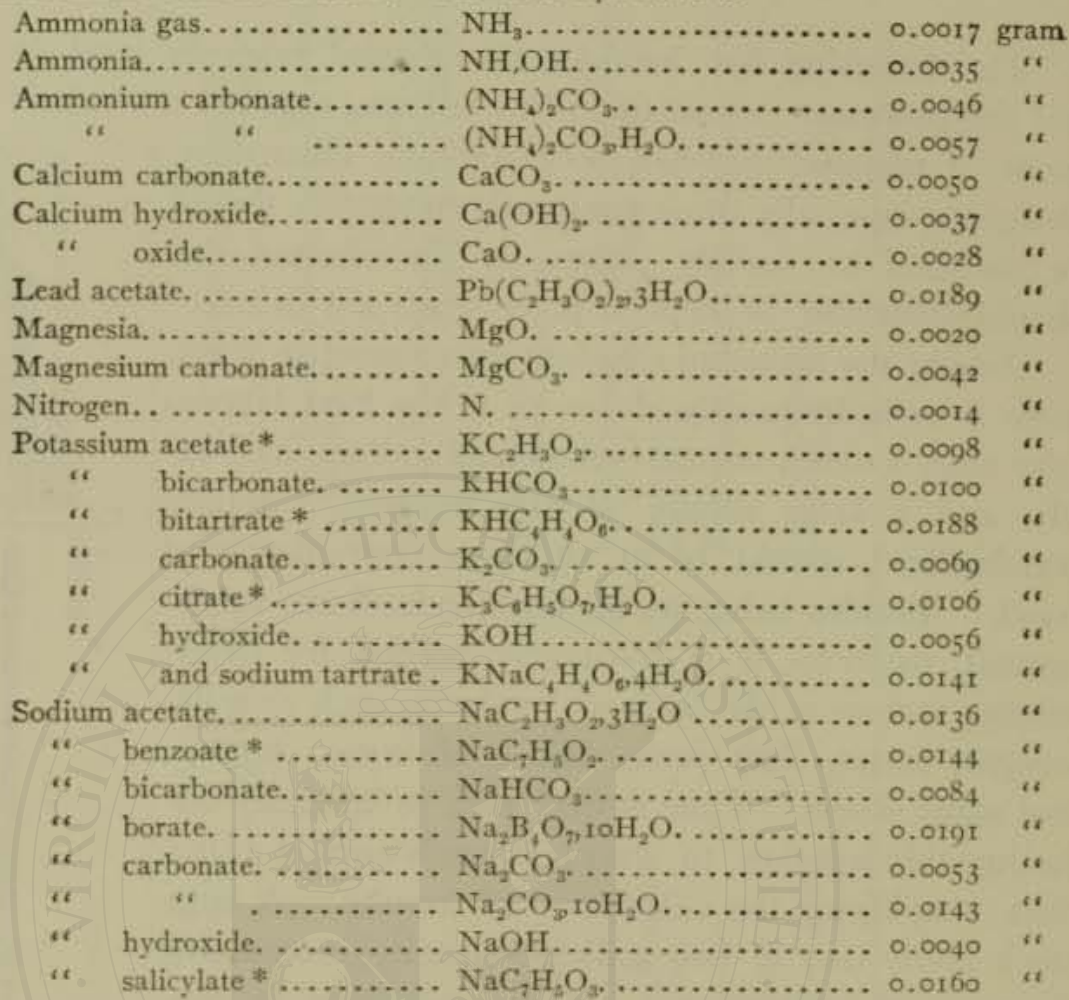

No. 24r. Decrnoparar. Sodrum Hydroxide Sorution. One ce. is equivalent to

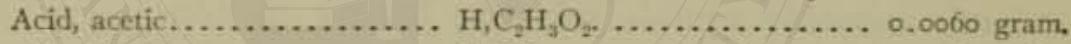

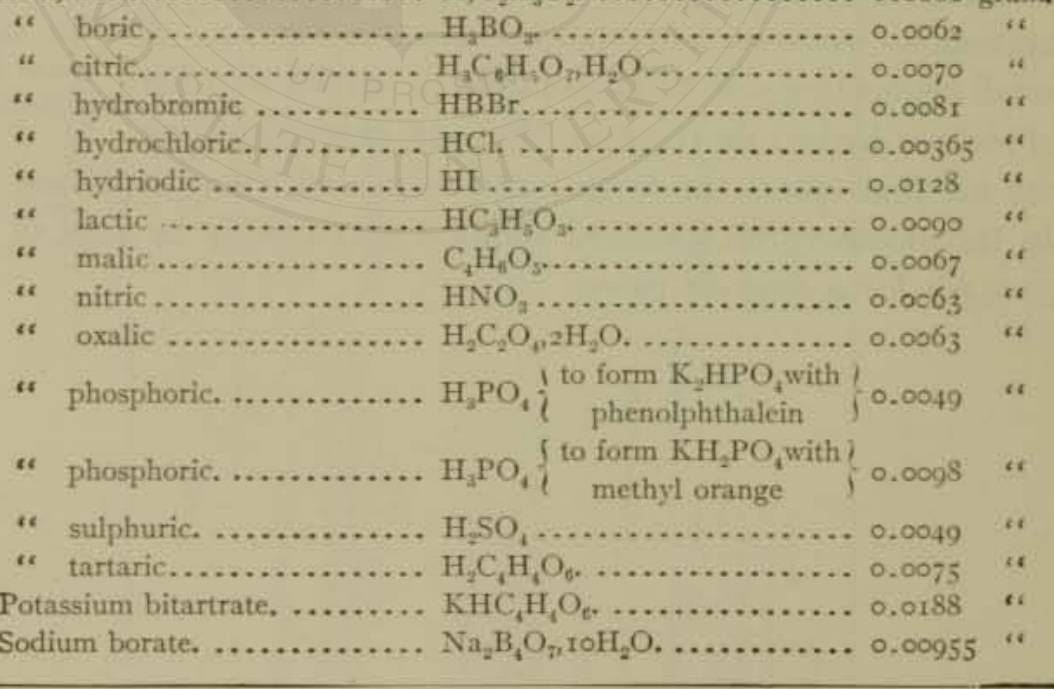

* To be ignited. 
No. I42. Decinorsal Iodine Solution. One cc. is equivalent to
Arsenious oxide.
$\mathrm{As}_{2} \mathrm{O}_{3}$.
0.00495 gram.
Potassium sulphite.
$\mathrm{K}_{2} \mathrm{SO}_{y} \mathrm{H}_{2} \mathrm{O} \ldots \ldots \ldots \ldots \ldots . . .0097$
Sodium bisulphite.

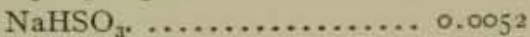
“ sulphite,
$\mathrm{Na}_{2} \mathrm{SO}_{3} 7 \mathrm{H}_{2} \mathrm{O} \ldots \ldots \ldots \ldots \ldots . . .0 .0126$
" thiosulphate.
$\mathrm{Na}_{2} \mathrm{~S}_{2} \mathrm{O}_{3}{ }_{5} \mathrm{H}_{2} \mathrm{O} \ldots \ldots \ldots \ldots \ldots . .0 .0248$
Sulphur dioxide.

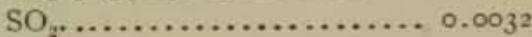
Sulphurous acid............. $\mathrm{H}_{2} \mathrm{SO}_{3} \ldots \ldots \ldots \ldots \ldots \ldots . .004 t$

\section{“}
“
46
6
66
No. 245. Decinormal Sodium Thiosulphate Solution. One cc, is equivalent to
Bromine.................. Br................... 0.0080 gram.
Chlorine....................................... 0.00355 "
Iodine .................... I. .................... 0.01266 "

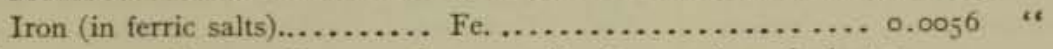

No. 23 . Decinormal Silver Nitrate Solution,* One cc. is equivalent to

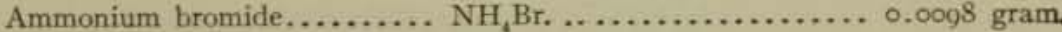

" chloride. ......... $\mathrm{NH}_{4} \mathrm{Cl} \ldots \ldots \ldots \ldots \ldots \ldots \ldots . . .2 .00535$

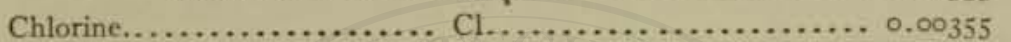

Cyanogen ................. (CN $)_{2} \ldots \ldots \ldots \ldots \ldots \ldots, 0.0052$

Hydrocyanic acid ............ HCN with indicator ........ 0.0027 "

" " .......... HCN $\left\{\begin{array}{c}\text { to formation of precip- } \\ \text { itate }\end{array} 0.0054\right.$ "

Hydrobromic acid. .......... HBr. .................. 0.0080 "

Potassium bromide. ......... KBr, .................. 0.0119 “"

" chloride,.......... KCl. .................. 0.00745 "

" cyanide. .......... KCN with indicator ........ 0.0065 "

" $\quad$. ........... KCN $\left\{\begin{array}{r}\text { to formation of precip- } \\ \text { itate.............. } 0.013^{\circ}\end{array}\right.$

Sodium bromide............ NaBr. ................. 0.0103 "

" chloride. ............ NaCl ................. 0.00585 ",

No. 201. Decinoramal Potassium Bichromate Solution. $f$ One cc. is equivalent to

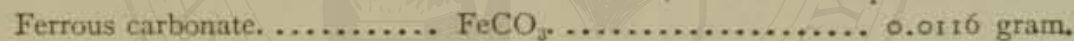

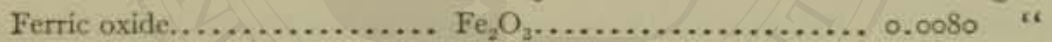

Ferrous oxide. ............. FeO ................. 0.0072 ",

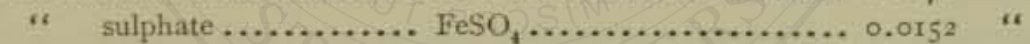

" " $" \ldots \ldots \ldots \ldots, \mathrm{FeSO}_{4}, 7 \mathrm{H}_{2} \mathrm{O} \ldots \ldots \ldots \ldots \ldots \ldots .0 .0278$ "

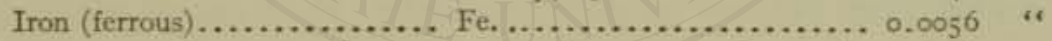

No. 220. Decrnormal Potassium Permanganate Solumon. One cc, is equivalent to

Oxalic acid. ............... $\mathrm{H}_{2} \mathrm{C}_{2} \mathrm{O}_{4}, 2 \mathrm{H}_{2} \mathrm{O} \ldots \ldots \ldots \ldots . . .0063$ gram, and to same weights for iron salts as given under $\mathrm{N} / 10 \mathrm{~K}_{2} \mathrm{Cr}_{2} \mathrm{O}_{7}$.

- Use potassium chromate solution as an indicator, or add till precipitate appears.

+ Use a freshly prepared solution of potassium ferricyanide as an indicator, applying a drop of titrated solution to a drop of indicator ua a white surface. 
The following table from Talbot* shows the reactions of the common indicators used in acidimetry:

\begin{tabular}{|c|c|c|c|c|c|c|}
\hline Indicator. & $\begin{array}{l}\text { Reaction with } \\
\text { Acids. }\end{array}$ & $\begin{array}{l}\text { Reactiop } \\
\text { with" } \\
\text { Alkaties. }\end{array}$ & $\begin{array}{c}\text { Use with } \\
\text { Carbonic } \\
\text { Acid in Cold } \\
\text { Solutiun. }\end{array}$ & $\begin{array}{l}\text { Use with } \\
\text { Carbonic } \\
\text { Accid in Hot } \\
\text { Solution. }\end{array}$ & $\begin{array}{l}\text { Use with } \\
\text { Ammonium } \\
\text { Salts. }\end{array}$ & $\begin{array}{l}\text { Use with } \\
\text { Organic Acid. }\end{array}$ \\
\hline $\begin{array}{l}\text { Litmus. .......... } \\
\text { Methyl orange. . } \\
\text { Phenolphthalein. } \\
\text { Lacmoid. ....... } \\
\text { Cochineal } \\
\text { Rosolic acid ........ } \\
\text { Alizarine. ....... }\end{array}$ & $\begin{array}{c}\text { Red } \\
\text { Pink } \\
\text { Colorless } \\
\text { Purple-red } \\
\text { Purple-red } \\
\text { Yellow } \\
\text { Yellow }\end{array}$ & $\begin{array}{c}\text { Blue } \\
\text { Yellow } \\
\text { Pink } \\
\text { Blue } \\
\text { Blue } \\
\text { Pink } \\
\text { Red }\end{array}$ & $\begin{array}{c}\text { Unreliable } \\
\text { Reliable } \\
\text { Unreliable } \\
\text { Unreliable } \\
\text { Reliable } \\
\text { Unreliable } \\
\text { Unreliable }\end{array}$ & $\begin{array}{c}\text { Reliable } \\
\text { Unreliable } \\
\text { Reliable } \\
\text { Reliable } \\
\text { Reliable } \\
\text { Reliable } \\
\text { Reliable }\end{array}$ & $\begin{array}{c}\text { Reliable } \\
\text { Reliable } \\
\text { Unreliable } \\
\text { Reliable } \\
\text { Reliable } \\
\text { Unreliable } \\
\text { Reliable }\end{array}$ & $\begin{array}{c}\text { Reliabis } \\
\text { Unreliable } \\
\text { Reliable } \\
\text { Unreliable (?) } \\
\text { Unreliable } \\
\text { Unreliable† } \\
\text { Reliable }\end{array}$ \\
\hline
\end{tabular}

* Talbot, Quantitative Analysis, page 75

+ Reliable with oxalic acid.

\section{REFERENCES ON LABORATORY EQUIPMENT, REAGENTS, ETC.}

ADRLANCE, J. S. Laboratory Calculations. New York, 1897.

Arkinson, E. Suggestions for the Establishment of Food Laboratories. U. S. Dept. of Agric., Off. of Exp, Sta., Bul. 17.

Conv, A. J. Tests and Reagents, Chemical and Microscopical, known by their Authors'

Names. New York, $\mathrm{IgO}_{3}$.

K anwood, H. R. Public Health Laboratory Work. The Hygienic Laboratory. Phila. delphia, 1893 .

E RaUCH, C. Testing of Chemical Reagents for Purity. London, 1903 .

I. UNGE, G., and HurTer, F. Alkali-maker's Handbook. London, 189r.

MAARHOFER, J. Instrumente und Apparate zur Nahrungsmittel Untersuchung. Leipzig, 1894 .

Miercks 1907 Index. Merck \& Co., New York.

SCHNEIDER, A. Reagents and Reactions known by the Names of their Authors. 1897 . Sirrron, F. Volumetric Analysis. 8th Ed, Philadelphia, r900.

' zORPE, T. E. Dictionary of Applied Chemistry, London, I9I2.

\section{DUNGANNON HIGH SCHOOL / 266}




\section{CHAPTER III.}

FOOD, ITS FUNCTIONS, PROXIMATE COMPONENTS, AND NUTRITIVE VALUE.

Nature and General Composition.-Food is that which, when eaten, serves by digestion and absorption to support the functions and powers of the body, by building up the material necessary for its growth and by supplying its wastes. The raw materials that constitute our foodsupply are not all available for nourishment, but often contain a proportion of inedible or refuse matter, which it is customary to remove before eating, such as the bones of fish and meat, the shells of clams and oysters, eggshells, the bran of cereals, and the skins, stones, and seeds of fruits and vegetables. The proximate components which make up the edible portion of food include in general water, fat, various nitrogenous bodies consisting chiefly of proteins, carbohydrates, organic acids, and mineral matter. Of these water is hardly to be considered as a nutrient, though it plays an important part in nearly all foods as a diluent and solvent. The fats, proteins, and carbohydrates all contribute in varying degree to the supply of fuel for the production of heat and energy. Besides this universal function, the fats and the carbohydrates serve especially to furnish fatty tissue in the body, while the proteins are the chief source of muscular tissue.

Liebig's classification of foods into nitrogenous, or flesh formers, and non-nitrogeneous, or heat generators, is now no longer accepted as strictly logical, in view of the well-known fact that the nitrogenous materials, besides building up the body, aid in supplying the wastes and yielding energy, and may even be converted into fats or carbohydrates, while the non-nitrogenous aid in furnishing tissue growth in addition to serving as fuel.

THE FAT OF Food,-Fats are the glycerides of the fatty acids, the characteristics of the various edible fats and oils being treated of under

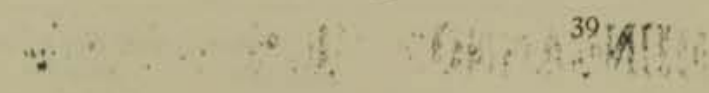


their appropriate headings elsewhere. Fat in human food is supplied by milk and its products, by the adipose tissue of meat, and in slight extent by the oil of cereals and by the edible table oils. The term "ether extract" is sometimes used in stating the results of the analysis of foods and this includes other substances than fat which when present are extracted by ether, such as chlorophyl and other coloring matters, lecithin, alkaloids, etc.

NITROGENOUS COMPOUNDS AND THEIR CLASSIFICATION.-These substances may for convenience be grouped as follows:

A Proteins, B Amino-acids and Amides, C Alkaloids, D Nitrates, E Ammonia, and F Lecithin.

A. PRoteins.-This term includes a large number of nitrogenous bodies consisting, according to our present knowledge, essentially of combinations of $\alpha$-amino-acids and their derivatives. Proteins in one form or another exist in nearly all natural foods both animal and vegetable. The terms "proteids" or "albuminoids" were formerly used generically as synonymous with "protein" to include all nitrogenous bodies of this group, but recently a joint committee on protein nomenclature of the American Physiological Society and the American Society of Biological Chemists recommended that the word "proteid" be abandoned; that "protein " be used to designate the entire group; and that the word "albuminoids" be restricted to a subgroup of proteins. A committee of the Physiological Society of England also made the same recommendation as to the use of the term protein. The classification and most of the definitions here given are those adopted by the American committee.*

Proteins available for food are supplied chiefly by the flesh of meat and fish, by milk, cheese, and eggs, and in the vegetable kingdom by seeds, nuts, and vegetables, especially the legumes. The proportion of crude protein, often designated merely as "protein," is commonly estimated by multiplying by 6.25 the percentage of nitrogen found in the material analyzed. This is done on the assumption that all of the nitrogen present in the substance belongs to protein containing 16 per cent of nitrogen.

There is no marked distinction in chemical constitution between animal and vegetable proteins, although some of the types have as yet been found only in one or the other kingdom. All proteins are insoluble in pure alcohol or in ether. A few are soluble in water but most are not. Nearly all are soluble in very dilute acids or alkalies, while all are decomposed by boiling with concentrated mineral acids or concentrated caustic alkalies. All proteins are lævo-rotary with polarized light.

*Am. Jour. Phys., 21, 1908, p. xxvii. 
Qualitative Test for Proteins.-Xanthoproteic Reaction.-Concentrated nitric acid added to a solution of a protein may or may not form a precipitate. It, however, produces a yellow coloration on boiling. Addition of ammonia in excess turns the precipitate or liquid yellow or orange.

Millon's Reaction,-Millon's reagent No. 184 , page 30 , when added to a protein solution produces a white precipitate, which becomes brick-red on heating. Sodium chloride prevents the reaction. Various organic substances are precipitated by Millon's reagent, but these precipitates do not turn red on heating.

Biuret Reaction.--If a solution of a protein in dilute sulphuric acid be made alkaline with potassium or sodium iydroxide and very dilute copper sulphate be added, a reddish to violet coloration is produced, similar to that formed if biuret* be treated in the same way, hence the name. An excess of copper sulphate should be avoided lest its color obscure that of the reaction.

In solutions which are strongly colored this reaction is of little use unless modified as follows: A considerable quantity of the dilute copper sulphate solution is added to the solution made alkaline with a large excess of potassium hydroxide, and then solid potassium hydroxide is dissolved to almost complete saturation in the solution. The mixture is then shaken with about one half its volume of strong alcohol. On standing the alcohol separates as a clear layer of a violet or crimson color if proteins are present.

I. THE SIMPLE PROTEINS.-Protein substances which yield only $\alpha$ amino acids or their derivatives on hydrolysis.

Although no means are at present available whereby the chemical individuality of any protein can be established, a number of simple proteins have been isolated from animal and vegetable tissues which have been so well characterized by constancy of ultimate composition and uniformity of physical properties that they may be treated as chemical individuals until further knowledge makes it possible to characterize them more definitely.

(a) Albumins.-Simple proteins soluble in pure water and coagulable by heat.

Examples.-Seralbumin of blood and other animal fluids; lactalbumin of milk; leucosin of the seeds of wheat, rye, and barley; legumelin of leguminous seeds.

* Biuret is the substance formed by heating urea to $160^{\circ}$ according to the following reaction:

$$
\underset{\text { Urea }}{\mathrm{CON}_{3} \mathrm{H}_{4}}=\underset{\text { Biuret }}{\mathrm{C}_{2} \mathrm{O}_{2} \mathrm{~N}_{3} \mathrm{H}_{5}}+\underset{\text { Ammonia }}{\mathrm{NH}_{3}}
$$


Coagulation.-Animal albumins usually coagulate at about $75^{\circ}$; vegetable albumins at about $65^{\circ}$.

Miscellaneous Reactions. - Very dilute acids precipitate albumins with the aid of heat. Nitrate of mercury (in dilute nitric acid) precipitates albumins from their solutions; also Mayer's solution acidified with acetic acid. They are precipitated by saturation with ammonium sulphate.

These reactions are not, however, characteristic of the group.

(b) Globulins. - Simple proteins insoluble in pure water, but soluble in neutral solutions of salts of strong bases with strong acids.

Examples.-Myosin of muscle substance; legumin of leguminous seeds; amandin of almonds.

Qualitative Tests.-Globulins are precipitated from their solution by dialysis or dilution. Albumins are not thus precipitated.

(c) Glutelins.-Simple proteins insoluble in all neutral solvents, but readily soluble in very dilute acids and alkalies.

Examples.-Glutenin of wheat is the only well defined protein of this group.

(d) Prolamins.-Simple proteins soluble in relatively strong alcohol (70-80 per cent), but insoluble in water, absolute aicohol, and other neutral solvents.

Examples.-Gliadin of wheat; zein of maize; hordein of barley. Found as yet only in the seeds of cereals.

The use of appropriate prefixes will suffice to indicate the origin of compounds of sub-classes a, b, c, and d, as for example: ovoglobulin, myalbumin, etc.

(e) Albuminoids.-Simple proteins which possess essentially the same chemical structure as the other proteins, but are characterized by great insolubility in all neutral solvents.

Examples.-Keratins of hair, nails, hoofs, horn, feathers, etc.; elastin of connective tissues; collagen of connective tissues and cartilage; fibroin and sericin of raw silk. No albuminoids have yet been discovered in plants.

Gelatin is usually regarded as an albuminoid but does not come strictly within the requirements of the above definition. It is an artificial derivative of collagen and is formed from it by boiling with water or subjecting to steam under pressure. It is prepared from bones and other animal parts, and is insoluble in cold, but soluble in hot water. When the hot water solution containing one per cent or more of gelatin cools, it forms a jelly. By prolonged boiling the gelatinizing power is lost. Aqueous solutions are strongly lævo-rotary. 
Gelatin in common with most proteins is precipitated from its solution by mercuric chloride, picric acid, and tannic acid. It is readily distinguished from soluble proteins, in that it is not precipitated from its solution by lead acetate, nor by most of the metallic salts that throw down proteins.

(f) Histones.- Soluble in water and insoluble in very dilute ammonia, and, in the absence of ammonium salts, insoluble even in an excess of ammonia; yield precipitates with solutions of other porteins, and a coagulum on heating, which is easily soluble in very dilute acids. On hydrolysis they yield a large number of amino-acids, among which the basic ones predominate.

Examples.-Thymus histone. Not found in plants.

(g) Protamins. - Simpler polypeptides than the proteins included in the preceding groups. They are soluble in water, uncoagulable by heat, have the property of precipitating aqueous solutions of other proteins, possess strong basic properties, and form stable salts with strong mineral acids. They yield comparatively few amino-acids, among which the basic amino-acids greatly predominate.

Examples.-Salmin, clupein, and other protamins of fish spermatozoa. Not found in plants.

II. Conjugated PRoteins. - Substances which contain the protein molecule united to some other molecule or molecules otherwise than as a salt.

(a) Nucleoproteins.-Compounds of one or more protein molecules with nucleic acid.

Examples.-The nucleins formed by pepsin digestion.

(b) Glycoproteins.-Compounds of the protein molecule with a substance or substances containing a carbohydrate group other than a nucleic acid.

Examples.-Mucins; ovomucoid; ovalbumin.

(c) Phosphoproteins.-Compounds of the protein moecule with some yet undefined phosphorus-containing substance other than a nucleic acid or lecithins.

Examples,-Casein of milk; vitellin of egg yolk.

(d) Haemoglobins. - Compounds of the protein molecule with haematin or some similar substance.

Example.-Oxyhaemoglobin of red blood corpuscles.

(e) Lecithoproteins.-Compounds of the protein molecule with lecithins, (lecithans, phosphatides).

Examples.-Lecithalbumin; lecithin-nucleovitellin. 


\section{DERIVED PROTEINS.}

i. Primary Protein Derivatives.-Derivatives of the protein molecule, apparently formed through hydrolytic changes which involve only slight altterations of the molecule.

(a) Proteans.-Insoluble products which apparently result from the incipient action of water, very dilute acids or enzymes.

Examples.-Edestan; blood fibrin; insoluble myosin.

(b) Metaproteins. - Products of the further action of acids or alkalies, whereby the molecule is so far altered as to form products soluble in very weak acids and alkalies, but insoluble in neutral fluids.

Examples.-Acid albumin; alkali albumin.

This group will thus include the familiar "acid proteins" and "alkali proteins," not the salts of proteins with acids.

(c) Coagulated Proteins.-Insoluble products which result from (I) the action of heat on their solutions, or (2) the action of alcohol on the protein.

Examples.-Albumin coagulated by heat or alcohol.

2. Secondary Protein Derivatives. Products of the further hydrolytic cleavage of the protein molecule.

(a) Proteoses.- Soluble in water, uncoagulated by heat, and precipitated by saturating their solutions with ammonium or zinc sulphate.

As thus defined this term does not strictly cover all the protein derivatives commonly called proteoses, e.g. heteroproteose and dysproteose.

Subdivision of the Proteoses.-According to the proteins from which they are derived the proteoses may be designated albumose, from albumin, globulose, from globulin, vitellose, from vitellin, caseose, from casein, etc.

Proteoses are subdivided into proto-proteose, soluble in water (both cold and hot) or in dilute salt solutions, but precipitated by saturation with salt; hetero-proteose, insoluble in water, and deutero-proteose, soluble in water, but not precipitated by saturation with salt.

Vegetable proteoses are sometimes called phyt-albumoses.

Qualitative Tests.-Besides responding to the biuret reaction (p. 4I) proteoses are precipitated by nitric acid, the precipitate being soluble on heating, but reappearing on cooling.

Proto-proteose is precipitated from its solution by mercuric chloride and copper sulphate; hetero-proteose is precipitated by mercuric chloride, but not by copper sulphate.

(b) Peptones.-Soluble in water, uncoagulated by heat, and not precipitated by saturating their solutions with ammonium sulphate. 
Qualitative Tests.-Besides giving the biuret reaction, peptones are precipitated from their solution by tannic acid,picric acid, phosphomolybdic acid, and by sodium phosphotungstate acidified by acetic, phosphoric, or sulphuric acid.

Peptones are the only soluble proteins not precipitated by saturation with ammonium sulphate. The following table, showing the reaction of proteoses and peptones, is due to Halliburton:*

\begin{tabular}{|c|c|c|c|c|c|c|c|}
\hline $\begin{array}{l}\text { Variety } \\
\text { of } \\
\text { Protein. }\end{array}$ & $\begin{array}{c}\text { Hot and } \\
\text { Cold Water. }\end{array}$ & $\begin{array}{l}\text { Hot and } \\
\text { Cold Saline } \\
\text { Solutions, } \\
\text { e.g. ro\% } \\
\text { NaCl. }\end{array}$ & $\begin{array}{l}\text { Satura- } \\
\text { tion with } \\
\mathrm{NaCl} \text { or } \\
\mathrm{MgSO}_{4} \text {. }\end{array}$ & $\begin{array}{c}\text { Satura- } \\
\text { tion with } \\
\left(\mathrm{NH}_{4}\right)_{2} \mathrm{SO}_{4}\end{array}$ & Nitric Acid. & $\begin{array}{c}\text { Copper } \\
\text { Sulphate. }\end{array}$ & $\begin{array}{l}\text { Copper } \\
\text { Sulphate } \\
\text { and Caustic } \\
\text { Potash. }\end{array}$ \\
\hline $\begin{array}{l}\text { Proto- } \\
\text { albumose }\end{array}$ & Soluble & Soluble & $\begin{array}{l}\text { Precipi- } \\
\text { tated }\end{array}$ & $\begin{array}{l}\text { Precipi- } \\
\text { tated }\end{array}$ & $\begin{array}{l}\text { Precipit a ted } \\
\text { in cold; pre- } \\
\text { cipitate dis- } \\
\text { solves with } \\
\text { heat and re- } \\
\text { appears on } \\
\text { cooling }\end{array}$ & $\begin{array}{l}\text { Precipi- } \\
\text { tated }\end{array}$ & $\begin{array}{l}\text { Rose-red } \\
\text { color (biu- } \\
\text { ret reac- } \\
\text { tion) }\end{array}$ \\
\hline $\begin{array}{l}\text { Hetero- } \\
\text { albumose }\end{array}$ & $\begin{array}{c}\text { Insoluble; i.e. } \\
\text { precipitated } \\
\text { by dialysis } \\
\text { from saline } \\
\text { solutions }\end{array}$ & $\begin{array}{l}\text { Soluble: part- } \\
\text { ly precipita- } \\
\text { ted, but not } \\
\text { co ag ulated } \\
\text { on heating } \\
\text { to } 65^{\circ} \mathrm{C} \text {. }\end{array}$ & $\begin{array}{l}\text { Precipi- } \\
\text { tated }\end{array}$ & $\begin{array}{l}\text { Precipi- } \\
\text { tated }\end{array}$ & Ditto & $\begin{array}{c}\text { Precipi- } \\
\text { tated }\end{array}$ & Ditto \\
\hline $\begin{array}{l}\text { Deutero- } \\
\text { albumose }\end{array}$ & Soluble & Soluble & $\begin{array}{l}\text { Not pre- } \\
\text { cipitated }\end{array}$ & $\begin{array}{l}\text { Precipi- } \\
\text { tated }\end{array}$ & $\begin{array}{l}\text { This reaction } \\
\text { occurs only } \\
\text { in presence } \\
\text { of excess of } \\
\text { salt }\end{array}$ & $\begin{array}{l}\text { Not pre- } \\
\text { cipitated }\end{array}$ & Ditto \\
\hline Peptone & Soluble & Soluble & $\begin{array}{l}\text { Not pre- } \\
\text { cipitated }\end{array}$ & $\begin{array}{l}\text { Not pre- } \\
\text { cipitated }\end{array}$ & $\begin{array}{l}\text { Not pre- } \\
\text { cipitated }\end{array}$ & $\begin{array}{l}\text { Not pre- } \\
\text { cipitated }\end{array}$ & Ditto \\
\hline
\end{tabular}

(c) Peptides.-Definitely characterized combinations of two or more amino-acids, the carboxyl group of one being united with the amino group of the other, with the elimination of a molecule of water.

The peptones are undoubtedly peptides or mixtures of peptides, the latter term being at present used to designate those of definite structure.

B. AMINO-ACIDS, AMIDES, AND ALLIED PRODUCTS. - Under this head are included products derived from acids or bases, the radicles of which replace one or more of the hydrogen atoms in ammonia. The most common bodies of this class occuring in food products are:

(I) Cholin $\left(\mathrm{C}_{5} \mathrm{H}_{15} \mathrm{NO}_{2}\right)$, found in the muscular tissue of cattle and in yolk of eggs, also in certain fungi.

(2) Betaine $\left(\mathrm{C}_{5} \mathrm{H}_{11} \mathrm{NO}_{2}\right)$, found in certain mollusks, as, for instance, the mussel, in putrefying fish, and (in the vegetable kingdom) in beets and hops. It is formed by the oxidation of cholin.

(3) Asparagin $\left(\mathrm{C}_{4} \mathrm{H}_{8} \mathrm{~N}_{2} \mathrm{O}_{3}\right)$, found in the shoots of asparagus, lettuce, peas and beans, and in the root of the marshmallow. It may be crystal-

* Chemical Physiology and Pathology, page 13I. 
lized out from the expressed juice of the asparagus shoots by evaporation, after having removed the albumin by coagulation (by boiling) and by filtration.*

Asparagin when heated with alkalies forms ammonia, and with acids forms ammonium salts. Freshly prepared copper hydroxide is dissolved by an aqueous solution of asparagin by the aid of heat. If sections of vegetable tissues containing asparagin are placed in alcohol, crystais of asparagin are formed in such a manner as to be detected under the microscope. $\dagger$

Closely allied to the amides are the flesh bases of meat, chief among which are creatin $\left(\mathrm{C}_{4} \mathrm{H}_{4} \mathrm{~N}_{3} \mathrm{O}_{2}\right)$, creatinin $\left(\mathrm{C}_{4} \mathrm{H}_{7} \mathrm{~N}_{3} \mathrm{O}\right)$, derived from creatin by the action of mineral acids and existing in some fish, carnin $\left(\mathrm{C}_{7} \mathrm{H}_{8} \mathrm{~N}_{4} \mathrm{O}_{3}\right)$, and xanthin $\left(\mathrm{C}_{5} \mathrm{H}_{4} \mathrm{~N}_{4} \mathrm{O}_{2}\right)$.

C. ALKALOIDAL NitROgeN. - Alkaloids do not naturally occur in foods, with the exception of tea, coffee, and kola-nuts, which contain caffeine, and cocoa, which contains theobromine.

D. NITROGEN AS NitRATES, - Foods in their natural condition rarely contain nitrates. Meats cured with saltpetre furnish the most common instance of nitrates in food. Nitrates are tested for by extracting the sample with water, and treating the extract with ferrous sulphate and sulphuric acid in the usual manner.

E. NITROGEN AS AMMONIA. - Ammonia occurs very sparingly in food, unless the latter has undergone some form of decomposition. In ripened cheese and in sour milk one sometimes finds it in minute quantities. Its presence is tested for by distilling the finely divided sample in water free from ammonia, and testing the distillate with Nessler's reagent.

F. LEcithin.-This substance $\left(\mathrm{C}_{44} \mathrm{H}_{00} \mathrm{NPO}_{8}\right)$ is a phosphorized fat, and forms a part of the cell material in certain animal and vegetable foods. It is found in considerable quantity in the yolk of egg, and, in traces, in cereals, peas, and beans. It is a yellowish-white solid, soluble in ether and alcohol. Treated with water it swells up, forming an opalescent solution or emulsion, from which it is precipitated by salts of the alkali metals.

THE CARBOHYdRATES AND tHEIR Classification.-The carbohydrates supplied by food are milk sugar and the various sugars, starches, and gums from plant juices, cereals, fruits, and vegetables. Carbohydrates are generally understood as being compounds of carbon, hydrogen, and oxygen, the last two elements being present in the proportion in

* Zeits. fur analytische Chemie, 22, page 325 .

$\dagger$ Wiley, Principles of Agric'l Analysis, Vol. III. p. 427 . 
which they occur in water. They are divided into three main classes, as follows:

A. The Glucose Group, or Monosaccharids $\left(\mathrm{C}_{6} \mathrm{H}_{12} \mathrm{O}_{6}\right)$, including dextrose, levulose, and galactose.

B. The Cane Sugar Group, or Disaccharids $\left(\mathrm{C}_{12} \mathrm{H}_{22} \mathrm{O}_{11}\right)$, including cane sugar, milk sugar, and maltose.

C. The Cellulose Group $\left(\mathrm{C}_{6} \mathrm{H}_{10} \mathrm{O}_{5}\right)$, including starch, cellulose, dextrin, gums, etc.

Closely allied to the carbohydrates, if not actually belonging to them, are inosite $\left(\mathrm{C}_{6} \mathrm{H}_{12} \mathrm{O}_{6}\right)$, occurring in muscular tissue, and pectose, found in green fruits and vegetables.

THE ORGANIC ACIDS.-These acids are minor though important constituents of foods. From their conversion into carbonates within the body, they are useful in furnishing the proper degree of alkalinity to the blood and to the various other fluids, besides being of particular value as appetizers. They exist in foods both in the free state and as salts. Acetic acid is supplied by vinegar; lactic acid by milk, fresh meat, and beer; citric, malic, and tartaric acids by the fruits.

MINERAL OR INORGANIC MATERIALS. - These substances occur in food in the form of chlorides, phosphates, and sulphates of sodium, potassium, calcium, magnesium, and iron, and are furnished by common salt, as well as by nearly all animal and vegetable foods. The inorganic salts are necessary to supply material for the teeth and bones, besides having an important place in the blood and in the cellular structure of the entire body.

FUEL VALUE OF FOOD.-In order to express the capacity of foods for yielding heat or energy to the body, the term fuel value is commonly used. By the fuel value of a food material is meant the amount of heat expressed in calories equivalent to the energy which we assume the body could obtain from a given weight of that food material, if all of its nutritents were thoroughly digested, a calorie being the amount of heat required to raise a kilogram of water $\mathrm{I}^{\circ} \mathrm{C}$. This definition applies to what is known as the large calorie, which is one thousand times as large as the small calorie. Large calories are meant wherever the term occurs in this volume. The fuel value, or, as it is sometimes called, "heat of combustion," may be determined experimentally with a calorimeter, or may be calculated by means of factors based on the result of many experiments showing the mean values for protein, fats, and carbohydrates.

The Bomb Calorimeter.*-This apparatus in its most approved form,

* U. S. Dept. of Agric., Off. of Exp. Sta., Bul, 21, pp. 120-126. 
Fig. ${ }^{3} 3$, consists of a water-tight, cylindrical, platinum lined, sieel bomb, adapted to hold in a capsule the substance whose heat is to be determined, and containing also oxygen under pressure. This bomb is immersed in water contained in a metal cylinder, which is in turn placed inside of concentric cylinders containing alternately air and water. The heat for igniting the substance is supplied by the electric current passing through wires to the interior of the bomb and acting upon a cleverly devised mechanism therein. The heat developed by the ignition is measured by

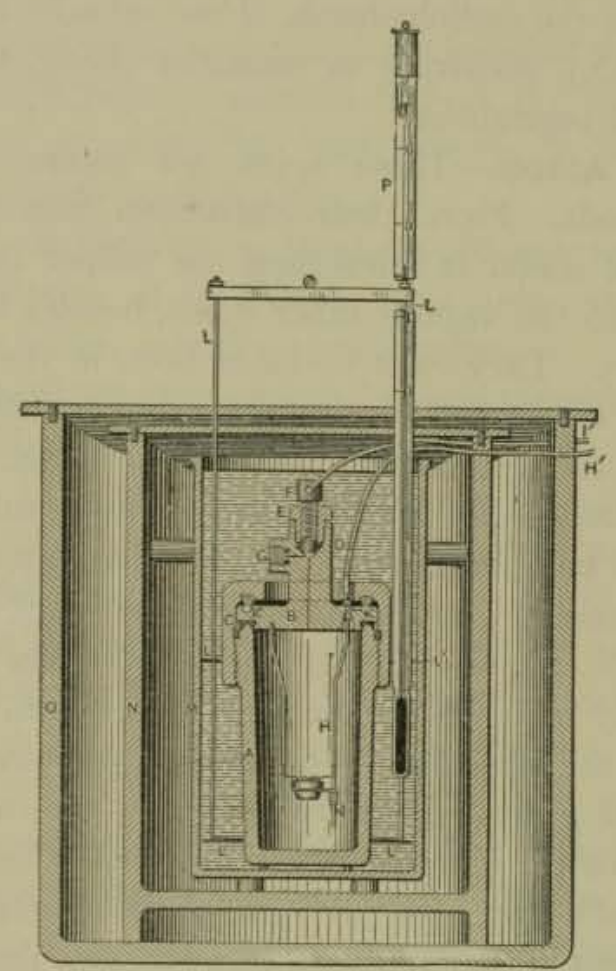

Frg. 13.-Bomb Calorimeter of Hempel and Atwater

the rise in temperature of the water surrounding the bomb, as indicated by a very delicate thermometer graduated to hundredths of a degree, certain corrections being made, as, for instance, for the heat absorbed by the metal of the apparatus. A mechanical stirrer serves to equalize the temperature of the water surrounding the bomb.

Calculation of Fuel Value.-By reason of its great expense the calorimeter is beyond the reach of many laboratories, and on this account the expression of fuel values by calculation is the most common method employed. For this the factors of Rubner are generally used, in accordance with which the amount of energy in one gram of each of the three principal 
classes of nutrients are, for carbohydrates 4.I, for protein 4.I, and for fats 9.3. Expressed in pounds, each pound of carbohydrate or protein has a fuel value of 1860 calories, while each pound of fat has a fuel value of 4220 calories.

\section{REFERENCES ON DIETETICS AND ECONOMY OF FOOD.}

Albu, A., u. Neuberg, C. Mineralstoffwechsel. Berlin, Igo6.

Armssy, H. P. The Principles of Animal Nutrition. New York, 1903.

Atwater, W. O. Dietaries in Public Institutions. Yearbook of U. S. Dept. of Agric., I90r, page 393 .

— Food and Diet. Yearbook of U. S. Dept. of Agric., r894, page 357.

- Principles of Nutrition and Nutritive Value of Food. Farmer's Bulletin, 142.

Bellows, A. J. The Philosophy of Eating. Boston, 1867.

Burnet, R. W. Foods and Dietaries. Phil., 1893 .

Bryant, A. P. Some Results of Dietary Studies in the United States. Yearbook of U. S. Dept. of Agric., 1898 , page 439 .

Chitrenden, R. H. The Nutrition of Man. New York, 1907.

Physiological Economy in Nutrition. New York, 1904.

Halurburton, W. D. Text-book of Chemical Physiology and Pathology. London, I89r.

Hammarsten, O. A Text-book of Physiological Chemistry. New York, rgr2.

Hutchinson, Robt. Food and the Principles of Dietetics. New York, Igor.

Jaffa, M. E. The Study of Human Foods and Practical Dietetics. Cal. Exp. Sta. Bul. 1 ro.

KNIGHT, J. Food and its Functions. London, I895.

Lusk, G. The Science of Nutrition. Philadelphia and London, I9ro.

Neumerster, A. Lehrbuch der physiologische Chemie. 1897.

Pavy, F. W. A Treatise on Food and Dietetics. London, 1874 .

Richards, E. H. The Cost of Living as Modified by Sanitary Science. New York, I900.

The Cost of Food: A Study in Dietaries. New York, rgor.

RUBner, M. Die Gesetze des Energieverbrauchs bei Ernährung. Leipzig, Igo2.

Sherman, H. C. Chemistry of Food and Nutrition. New York, rgrr.

Strommer, F. Die Ernährung des Menschen.

Thompson, W. G. Practical Dietetics. New York, 1895.

Townshend, S. H. The Relation of Food to Health. St. Louis, 1897.

TRUE, A. C., and MILNER, R. D. Development of Nutrition Investigations of the Dept. of Agric. Yearbook of U. S. Dept. of Agric., 1899, page 403 .

Storrs Exp. Station Annual Reports, 1888 et seq.

Dietetic and Hygienic Gazetre.

HyGIENISCHE RUNDSCHAU.

Revue de la Soc. Scientifieque d'Hygifne Altmentaire, 1904 et seq.

Zeitschrift für Physiologische Chemie, 1877 et seq. 
Also the following bulletins of the Office of Experiment Stations, U. S. Department of Agriculture.

Bul. 2I. Methods and Results of Investigations on the Chemistry and Economy of Food, By W. O. Atwater. Pp. 222.

Bul. 28. (Revised edition.) The Chemical Composition of American Food Materials,

By W. O. Atwater and A. P. Bryant. Pp. 87 .

Bul. 29. Dietary Studies at the University of Tennessee in 1895 . By C. E. Wait, with comments by W. O. Atwater and C. D. Woods. Pp. 45 .

Bul. 3r. Dietary Studies at the University of Missouri in 1895 , and Data Relating to Bread and Meat Consumption in Missouri. By H. B. Gibson, S. Calvert, and D. W. May, with comments by W. O. Atwater and C, D. Woods. Pp. 24.

Bul. 32. Dietary Studies at Purdue University, Lafayette, Ind., in 1895 . By W. E. Stone, with comments by W. O. Atwater and C. D. Woods. Pp. 28.

Bul. 35. Food and Nutrition Investigations in New Jersey in 1895 and 1896 . By E. B. Voorhees. Pp. 40.

Bul. 37. Dietary Studies at the Maine State College in 1895 . By W. H. Jordan. Pp. 57 .

Bul. $3^{8}$. Dietary Studies with Reference to the Food of the Negro in Alabama in 1895 and 1896. Conducted with the cooperation of the Tuskegee Normal and Industrial Institute and the Agricultural and Mechanical College of Alabama. Reported by W. O. Atwater and C. D. Woods. Pp. 69.

Bul. 40. Dietary Studies in New Mexico in 1895 . By A. Goss. Pp. 23.

Bul. 44. Report of Preliminary Investigations on the Metabolism of Nitrogen and Carbon in the Human Organism with a Respiration Calorimeter of Special Construction. By W. O. Atwater, C. D. Woods, and F. G. Benedict. Pp. 64.

Bul. 45. A Digest of Metabolism Experiments in which the Balance of Income and Outgo was Determined. By W. O. Atwater and C. F. Langworthy. Pp. 434.

Bul. 46. Dietary Studies in New York City in 1895 and 1896 . By W. O. Atwater and C. D. Woods. Pp. II 7 .

Bul. 52. Nutrition Investigations in Pittsburg, $\mathrm{Pa}$., $1894^{-1896 .}$ By Isabel Bevier. Pp. 48.

Bul. 53. Nutrition Investigations at the University of Tennessee in 1896 and 1897 . By C. E. Wait. Pp. 46.

Bul. 54. Nutrition Investigations in New Mexico in r897. By A. Goss. Pp. 20.

Bul. 55. Dietary Studies in Chicago in $r 895$ and $r 896$. Conducted with the cooperation of Jane Addams and Caroline L. Hunt, of Hull House. Reported by W. O. Atwater and A. P. Bryant. Pp. 76.

Bul. 56. History and Present Status of Instruction in Cooking in the Public Schools of New York City. Reported by Mrs. Louise E. Hogan, with an introduction by A. C. True, Ph.D. Pp. 70.

Bul. 63. Description of a New Respiration Calorimeter and Experiments on the Conservation of Energy in the Human Dody. By W. O. Atwater and E. B. Rosa. Pp. 94.

Bul. 68. A Description of Some Chinese Vegetable Food Materials and their Nutritive and Economic Value. By W. C. Blasdale. Pp. $4^{8}$. 
Bul. 69. Experiments on the Metabolism of Matter and Energy in the Human Body. By W. O. Atwater and F. G. Benedict, with the cooperation of A. W. Smith and A. P. Bryant. Pp. II2.

Bul. $7 \mathrm{r}$. Dietary Studies of Negroes in Eastern Virginia in 1897 and 1898 . By H. B. Frissell and Isabel Bevier. Pp. 45 .

Bul. 75. Dietary Studies of University Boat Crews. By W. O. Atwater and A. P. Bryant. Pp. 72.

Bul. 84. Nutrition Investigations at the California Agricultural Experiment Station, 1896-1898. By M. E. Jaffa. Pp. 39.

Bul. 85. A Report of Investigations on the Digestibility and Nutritive Value of Bread. By C. D. Woods and L. H. Nierrill. P. 5 x.

Bul. 89. Experiments on the Effect of Muscular Work upon the Digestibility of Food and the Metabolism of Nitrogen. Conducted at the University of Tennessee, $1897-1899$. By C. E. Wait. Pp. 77 .

Bul. 9r. Nutrition Investigations at the University of Illinois, North Dakota Agricultural College, and Lake Erie College, Ohio, 1896-1900. By H. S. Grindley and J. L. Sammis, E. F. Ladd, and Isabel Bevier and Elizabeth C. Sprague. Pp. 42.

Bul. 98. The Effect of Severe and Prolonged Muscular Work on Food Consumption, Digestion, and Metabolism, by W. O. Atwater and H. C. Sherman, and the Mechanical Work and Efficiency of Bicyclers, by R. C. Carpenter. Pp. 67 .

Bul. 107. Nutrition Investigations among Fruitarians and Chinese at the California Agricultural Experiment Station, 1899-19or. By M. E. Jaffa. Pp. 43.

Bul. rog. Experiments on the Metabolism of Matter and Energy in the Human Body, 1898-1900. By W. O. Atwater and F. G. Benedict, with the cooperation of A. P. Bryant, A. W. Smith, and J. F. Snell. Pp. I47.

Bul. 116. Dietary Studies in New York City in 1896 and 1897 . By W. O. Atwater and A. P. Bryant. Pp. 83 .

Bul. 117. Experiments on the Effect of Muscular Work upon the Digestibility of Food and the Metabolism of Nitrogen. Conducted at the University of Tennessee, 1899-1900. By C. E. Wait. Pp. 43 .

Bul. I21. Experiments on the Metabolism of Nitrogen, Sulphur, and Phosphorus in the Human Organism. By H. C. Sherman. Pp. 47.

Bul. I26. Studies on the Digestibility and Nutritive Value of Bread at the University of Minnesota in 1900-1902. By Harry Snyder. Pp. 52.

Bul. 129. Dietary Studies in Boston and Springfield, Mass., Philadelphia, Pa., and Chicago, III. By Lydia Southard, Ellen H. Richards, Susannah Usher, Bertha M. Terrill, and Amelia Shapleigh. Pp, ro3.

Bul. 132. Further Investigations Among Fruitarians at the California Agricultural Experiment Station. By M. E. Jaffa. Pp. 8r.

Bul. 136. Experiments on the Metabolism of Matter and Energy in the Human Body, 1900-1902. By W. O. Atwater and F. G. Benedict. Pp. 357.

Bul. I43. Studies on the Digestibility and Nutritive Value of Bread at the Maine Agricultural Experiment Station, 1899-1903. By C. D. Woods and L. H. Merrill. Pp. 77 . 
Bul. 149. Studies of the Food of Maine Lumbermen. By C. D. Woods and E. R. Mansfield. Pp. 60.

Bul. 150. Dietary Studies at the Government Hospital for the Insane, Washington, D. C. By H. A. Pratt and R. D. Milner. Pp. 170.

Bul. 152. Dietary Studies with Harvard University Students. By E. Mallinckrodt, jr. Pp. 63 .

Bul. 156. Studies on the Digestibility and Nutritive Value of Bread and Macaroni at the University of Minnesota, 1903-1905. By Harry Snyder. Pp. 80.

Bul. 159. A Digest of Japanese Investigations on the Nutrition of Man. By K. Oshima. Pp. 224.

Bul. I62. Studies on the Influence of Cooking upon the Nutritive Value of Meats at the University of Illinois, $1903^{-1904}$. By H. S. Grindley and A. D. Emmett. Pp. 230.

Bul. 175 . Experiments on the Metabolism of Matter and Energy in the Human Body. I903-1904. By F. G. Benedict and R. D. Milner. Pp. 335.

Bul. 185. Iron in Food and tts Functions in Nutrition. By H. C. Sherman. Pp. 80.

Bul. 187 . Studies of the Digestibility and Nutritive Value of Legumes at the University of Tennessee, 190I-I905. By C. E. Wait. Pp. 55.

Bul, 193. Studies of the Effect of Different Methods of Cooking upon the Thoroughness and Ease of Digestion of Meat at the University of Illinois. By H. S. Grindley. Pp. 100.

Bul. 208. The Influence of Muscular and Mental Work upon Metabolism and the Efficiency of the Human Body as a Machine. By F. G. Benedict. 


\section{CHAPTER IV.}

\section{GENERAL ANALYTICAL METHODS.}

Extent of a Proximate Chemical Analysis.-For purposes of studying the proximate composition of food for its dietetic value, it is nearly always necessary to make determinations of moisture, ash, fat, total nitrogen, and carbohydrates (when present), as well as of the fuel value. In some cases it may be desirable to proceed further, to make an analysis of the ash, for instance, to separate, at least into classes, the various nitrogenous bodies, especially in flesh foods, and perhaps to subdivide the starch, sugar, gums, and cellulose or crude fiber that make up the carbohydrates in the case of cereals.

An analysis is considered complete whenever the purpose for which the examination has been made has been accomplished, and on that purpose depends solely the extent to which the various compounds present shall be subdivided and determined. Such a subdivision may be extended almost indefinitely. For example, a milk analysis may consist simply in the determination of the total soilids and (by difference) the water. Again, it may be desirable to divide the milk solids into fat and solids not fat, and in some cases to carry the subdivision still farther and separate the solids not fat into casein, albumin, milk sugar, and ash.

Determinations of one or more of the proximate components natural to food are frequently of great service in proving their purity or freedom from adulteration. For the latter purpose, especially in such foods as milk, vinegar, oils, and fats, the determination of specific gravity is also an important factor. Special methods of a peculiar nature are often necessary in the examination of particular foods, and such methods will be treated subsequently under the appropriate headings. In the present chapter only such general methods as are applicable to a large variety of cases will be discussed.

Expression of Results of a Proximate Analysis.-However complete the division of the various proximate compounds or classes of compounds 
which the analyst sees fit to make, the results of his various determinations in a proximate analysis are expected to aggregate about $100 \%$. If every determination be directly made, the result will rarely be exactly 100 , but the precision of the work is apt to be judged by its approach to 100 .

It is often the custom to determine certain compounds or classes of compounds by difference. Thus in cereals moisture, proteins, fat, crude fiber and ash may be determined by the regular analytical methods, and by subtracting their sum from roo the difference may be expressed as "nitrogen-free extract" or carbohydrates. It has long been customary in food analysis to calculate the protein by multiplying the total nitrogen by the factor 6.25 , and on this basis analyses of thousands of animal and vegetable foods have been made. While the figure thus obtained is an arbitrary one, being at best but a rough approximation of the amount of protein present, yet for many reasons there is much to commend this practice of reporting results. In the first place, in most cases it actually does approach the truth. Again, the nitıogenous ingredients of many foods are so numerous and varied, that for the every-day study of dietaries and food values it would be well-nigh impossible with our present knowledge to subdivide these compounds with any degree of accuracy, and especially with uniformity between different chemists, to say nothing of the time involved.

From the fact that so many valuable analyses have already been expressed on the basis of $\mathrm{N} \times 6.25$ for protein, the advantage of comparison with the results thus recorded would seem to be in itself a good reason for continuing the practice, especially until a factor that gives better average results can be adopted. By recording the actual nitrogen found as well as the "protein," old results may at any time be recalculated under new conditions, if found desirable.

In flesh foods, when carbohydrates are known to be absent, the total protein may conveniently be determined by difference. Rather more progress has been made in the separation of the nitrogenous compounds of meats than of the vegetables and cereals, though the methods are by no means accurate or uniform.

Most of the recorded analyses of vegetable foods express the carbohydrates as a whole without attempting to subdivide them, at least further than possibly to express the crude fiber or cellulose separately. A much more intelligible idea of the dietetic value of these foods would be gained by a further separation into starch and sugars. 
Preparation of the Sample.-It is at the outset of the utmost 1-apportance in all cases that a strictly representative portion of the food to be examined should be submitted to analysis. All refuse matter, such as bones, shells, bran, skins, etc., are removed as completely as possible from the edible portion and discarded.

If the composition of the entire mass cannot be made homogeneous throughout, it may be best to select from various portions in making up the sample for analysis, in order to represent as fair an average of the whole as possible.

Finally the sample, if solid or semi-solid, should be divided as finely as possible, by chopping, shredding, pulping, grinding, or pulverizing according to its nature and consistency.

For disintegrating such substances as vegetables and meats for analysis, the common household rotary chopping-machine is admirably adapted. For pulverizing cereals, tea, coffee, whole spices, and the like, the mortar and pestle may be used, or a rotary disk mill or spice-grinder.

Specific Gravity or Density of Liquids.-Where formerly it was customary to compare the density of liquids with that of water at $4^{\circ} \mathrm{C}$. (its maximum density) it is now more common to refer to water at $15.5^{\circ} \mathrm{C}$. or $20^{\circ} \mathrm{C}$., making the determination at that temperature. A common form of expressing the temperature of the determination and the temperature of the standard volume of water with which that of the substance is to be compared, is the employment of a fraction, the numerator of which expresses the temperature of the determination and the denominator that of the standard volume of water, as $\frac{15 \cdot 5^{\circ}}{4^{\circ}}, \frac{15 \cdot 5^{\circ}}{15.5^{\circ}}, \frac{100^{\circ}}{15.5^{\circ}}, \frac{4^{\circ}}{4^{\circ}} \mathrm{C} . *$ When extreme accuracy in the determination of density is required, the pycnometer or Sprengel tube should be employed.

The Hydrometer.-This instrument furnishes the most convenient and ready means of determining the density of liquids where extreme nicety is not required. If well made and carefully adjusted, the hydrometer may be depended on to three decimal places, but before relying on its accuracy, it should be tested by comparison with a standard instrument, or with the pycnometer.

The liquid whose density is to be determined is contained in a jar whose inner diameter should be at least $\frac{3}{8}$ " larger than that of the spindle-

* Unless otherwise stated, all specific gravities in this volume are assumed to be expressed on the basis of $\frac{15.5^{\circ}}{15.5^{\circ}}$ 
bulb, and the temperature of the liquid should be exactly $15.5^{\circ}$ when the reading is taken.

For best results for use with liquids of varying densities, the laboratory should be furnished with a set of finely graduated hydrometers, each limited to a restricted part of the scale, together with a universal hydrometer coarsely graduated, covering the entire range, to show by preliminary test which of the special instruments should be used.

A convenient set of seven such hydrometers are graduated as follows: $0.700-0.850,0.850-1.000,1.000-1.200,1.200-1.400,1.400-1.600,1.600-$ $1.800,1.800-2.000$, while the universal hydrometer has a scale extending from 0.700 to 2.000 . Another less delicate set of three only has one for liquids lighter than water and two for heavier liquids. Some instruments have thermometers in the stem. Others require a separate thermometer.

The Westphal Balance (Fig. I4).-This instrument consists of a scale-beam fulcrumed upon a bracket, which in turn is upheld by a supporting pillar. The scale-beam is graduated into ten equal divisions. From a hook on the outer end of the beam hangs a glass plummet previded with a delicate thermometer, the beam being so adjusted that when the dry plummet hangs in the air, the beam is in exact equilibrium, i.e., perfectly horizontal as shown by the indicator on its inner end. If the large rider be placed on the same hook as the plummet and the latter immersed in distilled water of the standard temperature at which the determinations are to be made (say $15.5^{\circ} \mathrm{C}$.), the scale-beam should again be in equilibrium if the instrument is accurately adjusted As commonly made, the weight of the plummet including the platinum wire to which it is attached amounts to 15 grams, and the displacement of its volume to 5 grams of distilled water at $15.5^{\circ} \mathrm{C}$., the normal temperature on which the determinations are based. Thus the unit (or largest) rider should weigh 5 grams, while the others weigh $0.5,0.05$, and 0.005 gram respectively.

If, instead of distilled water, the plummet be immersed in the liquid whose density is to be determined, the position of the riders on the scalebeam, when so placed as to bring the same into equilibrium, and when read in the order of their relative size (beginning at the largest), indicates directly the specific gravity to the fourth decimal place.

If the liquid is lighter than water, the large rider is first placed in the notch where it comes closest to restoring the equilibrium of the beam, but with the plummet still underbalanced. The rider next in size is then applied in a similar manner, and, unless equilibrium is exactly re- 
stored, the third and the fourth riders successively. If it happens that two riders should occupy the same position on the beam, the smaller is suspended from the larger.

If the liquid is heavier than water, the method employed is the same except that one of the largest or unit riders is in this case always hung from the hook which supports the plummet, while the others cross the

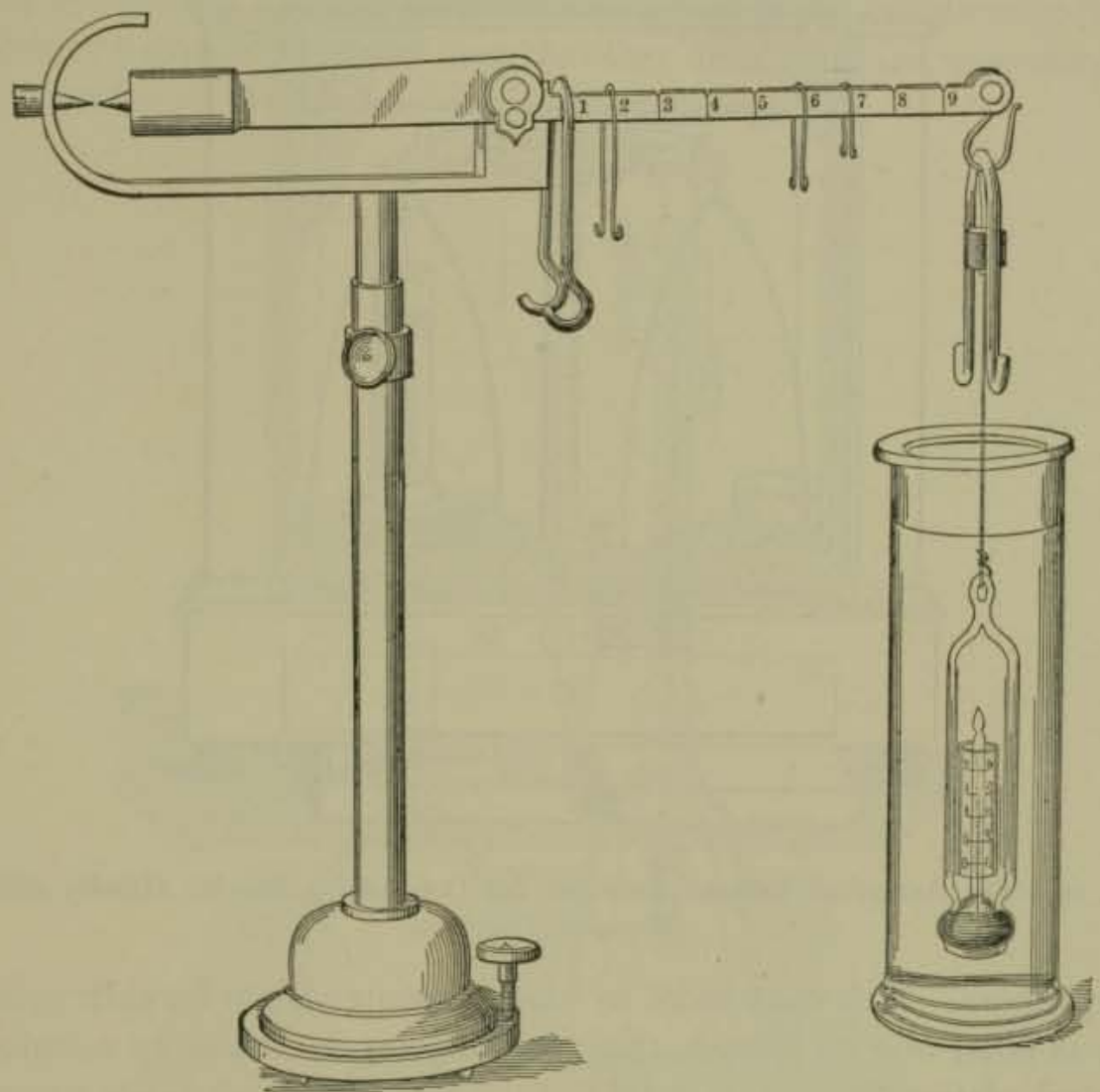

Fic. 14.-The Westphal Balance.

beam at the proper points. If carefully made and adjusted, the Westphal balance is capable of considerable accuracy.

A delicate analytical balance can be used in place of the less carefully adjusted Westphal instrument, by hanging the Westphal plummet from one of the scale-hooks of the same, and employing a fixed support for the glass jar that holds the liquid in which the plummet is to be immersed. The support is so arranged that the scale-pan below it can move freely without coming in contact with it. This arrangement is shown in Fig. 15.

The Pycnometer, or Specific-gravity Botlle. - Fig. I6 shows the two 
forms of pycnometer commonly made. The plain form has a groundglass stopper with a capillary passage through it, the other has a fine thermometer connected with the stopper and a capillary side tube provided with a ground hollow cap. Both are made in different sizes to hold respectively $10,25,50$, and 100 grams of distilled water at the standard

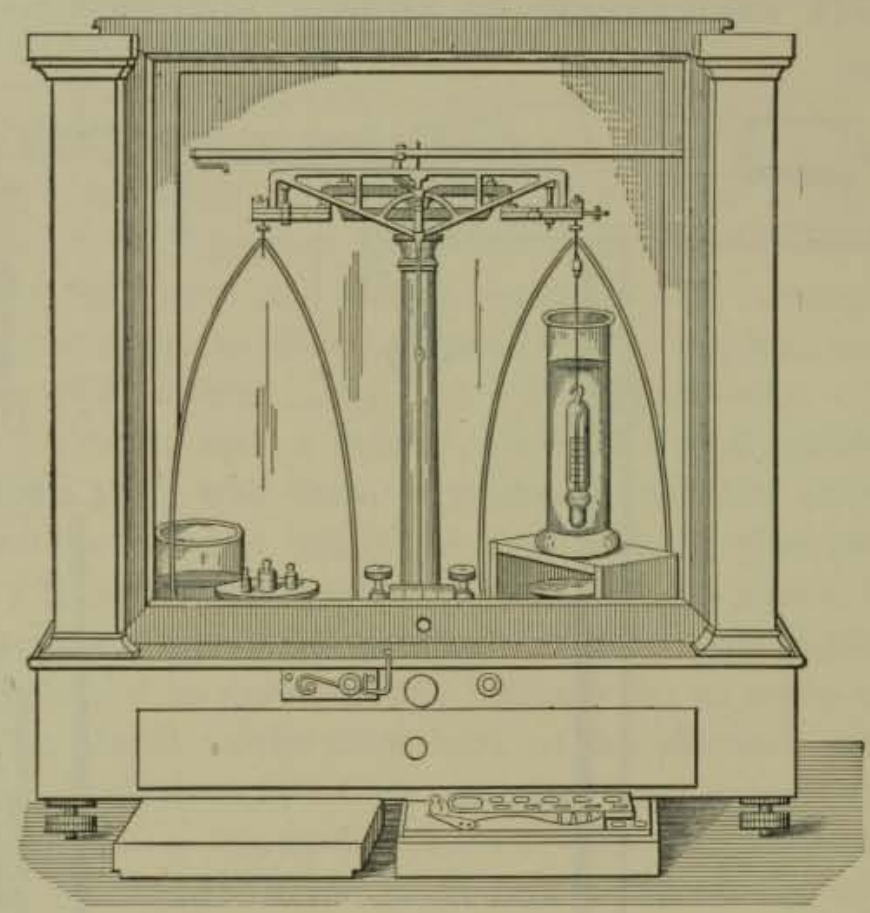

FrG. 15.-The Analytical Balance Arranged for Determining Specific Gravity with the Westphal Plummet.

temperature. It is convenient to have a counterweight for each pycnometer as fitted with its stopper, thus avoiding much trouble in calculation. The calculation of results is simplified also if the pycnometers are accurately constructed to contain exactly the weight of distilled water which they purport to contain at the standard temperature, but it is rather difficult to procure such instruments, especially of the form furnished with the thermometer. Most instruments hold approximately the amount specified, the exact net weight of distilled water which they hold at standard temperature being found by careful test and kept on record. In determining the density of a liquid, the pycnometer is carefully filled with it at a temperature below the standard, the stopper carefully inserted, and the bottle wiped dry. Care should be taken that the liquid completely fills the bottle and is free from air-bubbles. The net weight of the liquid is then taken 
on the balance, when the temperature has reached the standard (say $15.5^{\circ}$ C.), being careful to wipe off the excess of liquid that exudes from the capillary due to expansion. The net weight of the liquid is divided by that of the same volume of distilled water, previously ascertained under the same conditions at the same temperature; the result being the density of the liquid.

The pycnometer with thermometer attachment is obviously susceptible of a greater degree of accuracy than the other form, since the temperature of the liquid, even though $15.5^{\circ} \mathrm{C}$. at the start, soon rises.

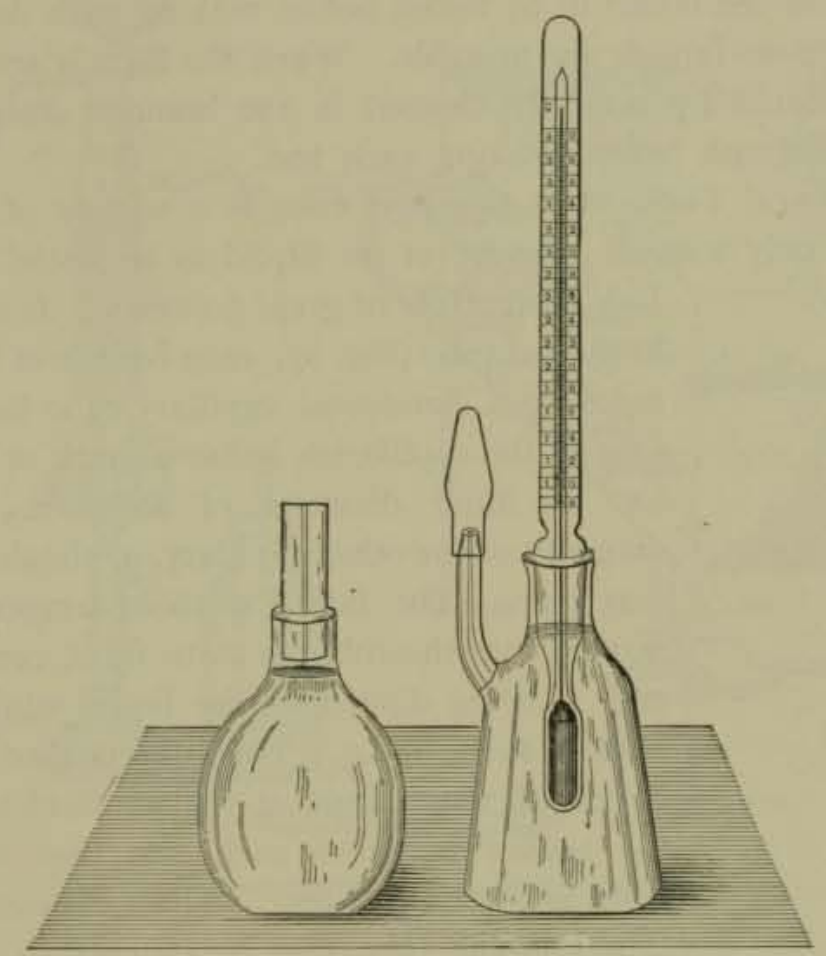

Fig. 16.-Types of Pycnometer.

The writer prefers to use the pycnometer provided with the thermometer, but without the hollow cap that covers the capillary side tube, unless liquids like strong acids are to be operated on, that might otherwise injure the balance. By keeping the liquid to be tested for some time in a refrigerator, it acquires a temperature of from 10 to $\mathrm{I} 2^{\circ} \mathrm{C}$. It is then transferred in the regular manner to the pycnometer and the thermometer-stopper inserted (but not the hollow cap) and the bottle wiped dry. There is ample time to adjust the balance-weights with extreme care while the temperature of the liquid is rising, leisurely wiping off 
at intervals with a soft towel the excess that exudes from the capillary tube, the final weight of the dry bottle and contents being made" at the exact temperature of $15.5^{\circ} \mathrm{C}$.

In taking the tare or adjusting the counterweight of a specific-gravity bottie, the latter should be perfectly clean and dry. It had best be rinsed first with water, then with alcohol, and finally with ether, all traces of the latter being removed by a current of dry air, or otherwise, before weighing.

In making successive determinations of density of a number of different liquids with the same pycnometer, it is sufficient to rinse the bottle once with a little of the liquid to be tested before making each determination, when the various liquids are miscible. When the liquids are immiscible, the bottle should be carefully cleaned in the manner described in the previous paragraph before making each test.

The Sprengel Tube.-The Sprengel tube is a variety of pycnometer useful when only a small quantity of the liquid to be tested is available.

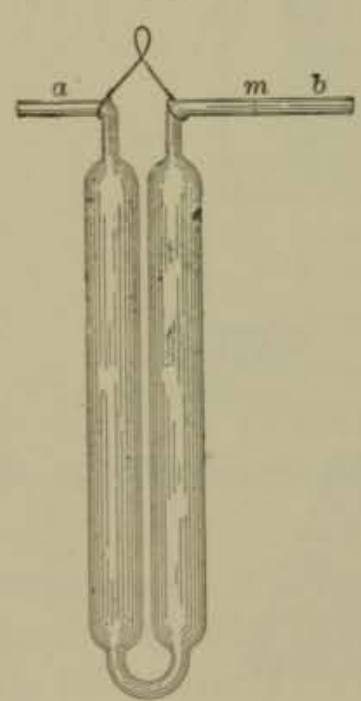

It is susceptible of great accuracy. It consists of a U-shaped tube (Fig. 17), each branch of which terminates in a horizontal capillary tube bent outward. One of the capillaries, $b$, has a mark $m$ thereon and has an inner diameter of about $0.5 \mathrm{~mm}$. The diameter of the other capillary, $a$, should not exceed $0.25 \mathrm{~mm}$. The liquid at room temperature is aspirated into the tube so as to fill it completely, the end $b$ being dipped in the liquid while suction is applied at the end $a$. The tube is then placed in a beaker of water kept at the standard temperature, the beaker being of such size that the capillary ends rest on the edge. The temperature of the liquid in the tube may be assumed to be constant 7r. ${ }^{17}$. -Sprengel Tube when there is no further movement due to contracfor Determining Spe-tion in the larger capillary end, $b$. The meniscus of cific Gravity.

the liquid, when cooled, should not be inside the mark $m$, and is brought exactly to the mark by applying a piece of bibulous paper to the other end, $a$. If a drop or two of liquid has to be added, this may be done by applying to the end $a$ a glass rod dipped in the liquid. When exactly adjusted, the whole is wiped dry and quickly weighed, hung from the arm of the analytical balance. To avoid evaporation by contact with the air, the ends of the capillaries are sometimes ground to receive hollow glass caps not shown in the figure. 
Determination of Moisture.-This is usually calculated from the loss in weight at the temperature of boiling water. Platinum dishes (Fig. 5I) are well adapted for the drying as the residue can be ignited for the determination of ash. If only the moisture is desired, dishes of other metals or glass weighing bottles may be used. Caps for widemouthed bottles made of tinned lead are convenient and can be thrown

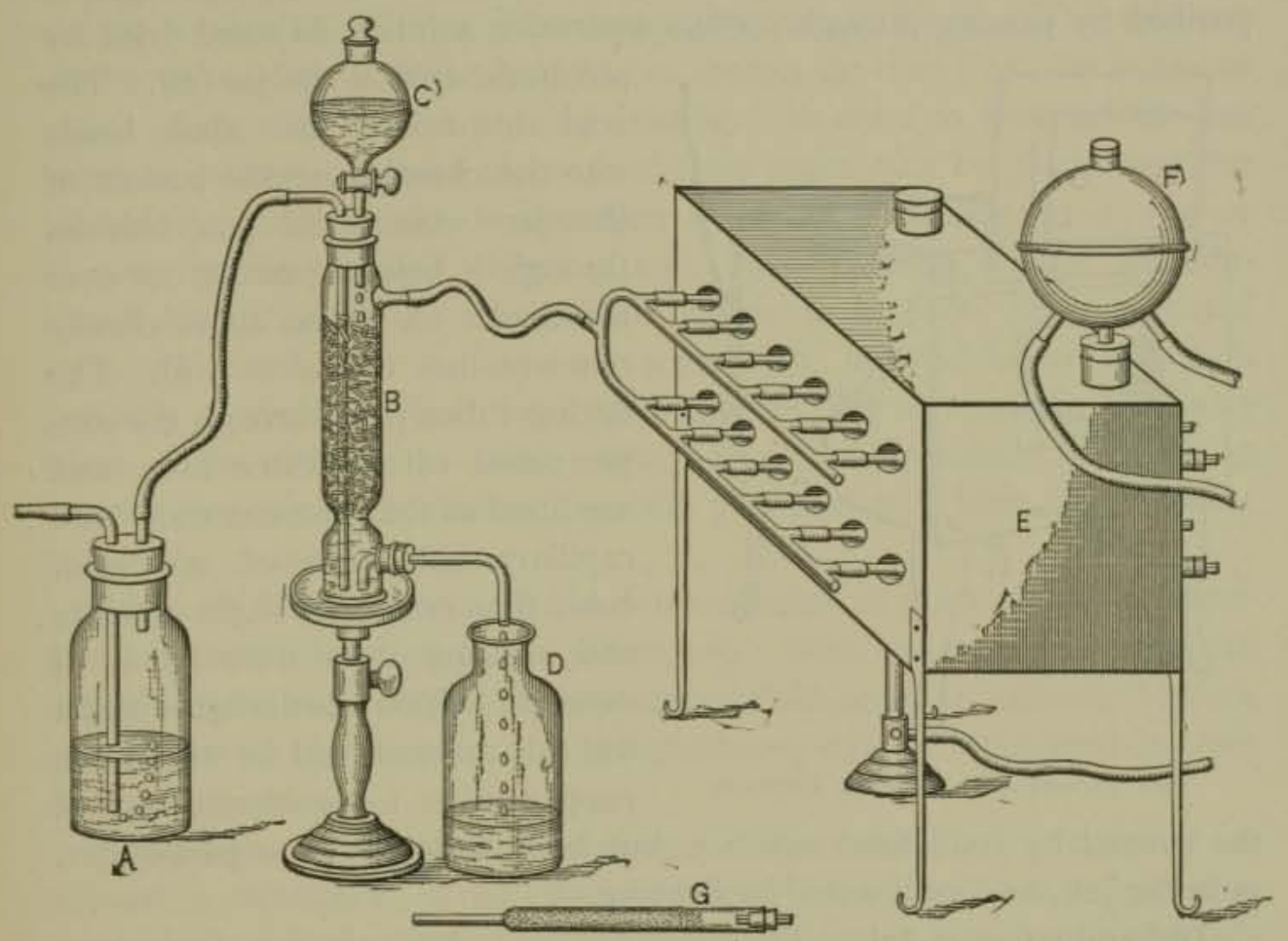

Fig, 18,-Apparatus for Drying in Hydrogen.]

away after using. Viscous substances are best spread over asbestos or sand to hasten the drying.

Some materials must be heated above $100^{\circ} \mathrm{C}$, while certain saccharine products are dried at $70^{\circ} \mathrm{C}$. in vacuo to avoid decomposition. If alcohol, acetic acid, essential oils, or other volatile substances are present the loss includes these as well as moisture. As the water or steam oven seldom attains a temperature above $98^{\circ}$, the loss sustained in these is, strictly speaking, at the "temperature of boiling water." Figs. 8 and 9 show electric and gas ovens for heating at full $100^{\circ}$. Benedict has shown that certain materials can best be dried at room-temperature over sulphuric 
acid in vacuo. Trowbridge * has shortened this process in the case of meat, by gently agitating the desiccator during the drying.

Drying in Hydrogen.-Fig. I8 shows the apparatus devised by Winton $\dagger$ for drying cereal products, cattle foods, etc. (p. 276). The material is weighed out on a watch glass and transferred to the drying tube $(G)$, wisps of cotton, too small to contain an appreciable amount of moisture, being used at both ends to prevent mechanical loss. The hydrogen is purified by passing through sodium hydroxide solution $(A)$ and dried by

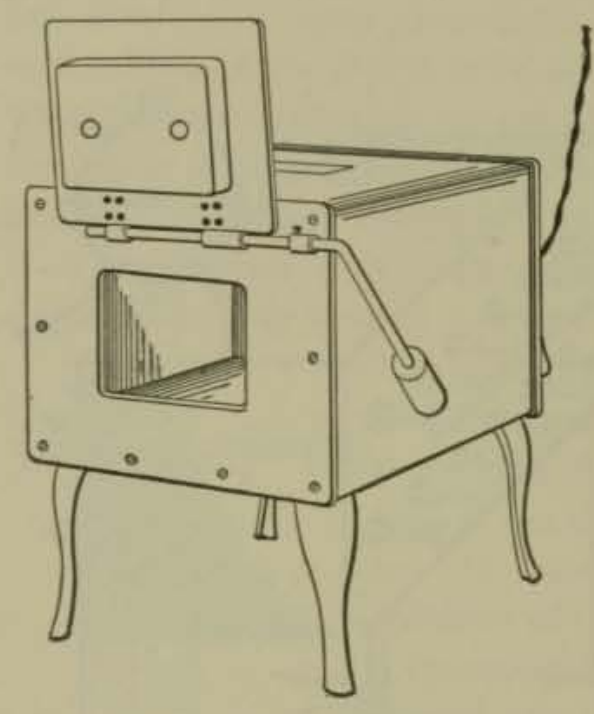

FIG. 19.-Hoskins Electric Furnace. sulphuric acid in the jar $(B)$. The acid drops over the glass beads into the chamber at the bottom of the jar where the gas bubbles through it before passing out over the beads. A siphon automatically removes the excess of acid. The drying tubes pass through the copper tubes of the water oven and are fitted at the posterior ends with capillary exit tubes of $0.5 \mathrm{~mm}$. bore, thus creating a slight pressure and insuring even distribution of current. When the drying is begun the exit tubes should be within the copper tubes to avoid stoppage of the current by condensed moisture, but later they should be pushed out, as in the cut, and each tested by lighting.

Determination of Ash.-The residue from the determination of moisture or else a new portion, is burned at a very faint red heat until white or gray, cooled in a desiccator and weighed. A flat-bottomed platinum dish is most convenient for the purpose. Platinum, however, is attacked by free chlorine, bromine, and iodine, sulphur and phosphorus, sulphates and phosphates with reducing agents, all sulphides, sodium or potassium hydroxide, nitrate and cyanide, metals, and metallic compounds reduced in fusion, such as lead, tin, zinc, bismuth, mercury, arsenic, and antimony. In such cases porcelain must be used.

The degree of heat employed in ashing should be the lowest possible to

* A. O. A. C. Proc, 1908, U. S. Dept. of Agric., Bur. of Chem., Bul. 122, p. 219.

† Conn. Agr. Exp. Sta., Rep. 1889, p. 187. 
insure complete oxidation of the carbon, so as to avoid driving off certain volatile salts that are sometimes present and that would be lost if the heat were too high. At a bright red heat potassium and sodium chloride are slowly volatilized, and calcium carbonate is converted into oxide; furthermore alkali phosphates fuse about*particles of carbon, protecting them from oxidation. To avoid overheating it is recommended not to allow the flame to impinge directly against the dish, but to carry out the burning on a piecce of asbestos paper supported on a triangle. The asbestos also serves to distribute the heat and to protect the dish from the injurious action of the direct flame on long heating. In order to burn off the last traces of carbon, a second piece of asbestos paper may be placed over the top of the dish, or the incineration may be completed in a gas or electric muffle furnace (Figs, 3 and 19). Heating should be continued till the carbon is all oxidized, which is in most cases indicated by a white ash. It is, however, sometimes impossible to obtain a perfectly white ash, but the appearance of the ash usually indicates when all the carbon has been burnt off. It is sometimes necessary to stir the contents of the dish with a stiff platinum wire from time to time during the ignition.

Methods for the detection and determination of the various ash ingredients are considered on pages 301 to 305 . Such cases as are peculiar to certain foods, like the metallic impurities that occur in canned, bottled, and preserved foods under certain conditions, will be considered in their appropriate place.

Extraction with Volatile Solvents. - Whenever it is necessary to exhaust a substance of its ether-soluble or alcohol-soluble ingredients, some form of continuous extraction apparatus is employed with advantage.

Preliminary Drying.-In the case of cereal, legume, and oil-seed products, meats, etc., the portion of the material dried in hydrogen, in vacuo, or in contact with air in an ordinary oven, for the determination of moisture, may be used for extraction. If volatile oil is present, as in spices, the drying must be performed at room temperature in a desiccator.

Milk and other liquids are absorbed in a roll of bibulous paper, in asbestos, or in sand, previous to drying (page I34). The evaporation may be carried on in shells of thin glass (Hoffmeister Schälchen) which are finally broken previous to extraction, or in tinned lead bottle caps which may be crumpled up and inserted in the extractor. 
The Soxhlet Extractor.-This apparatus is shown in Fig. 20. The substance to be extracted is subjected to successive treatment with freshly

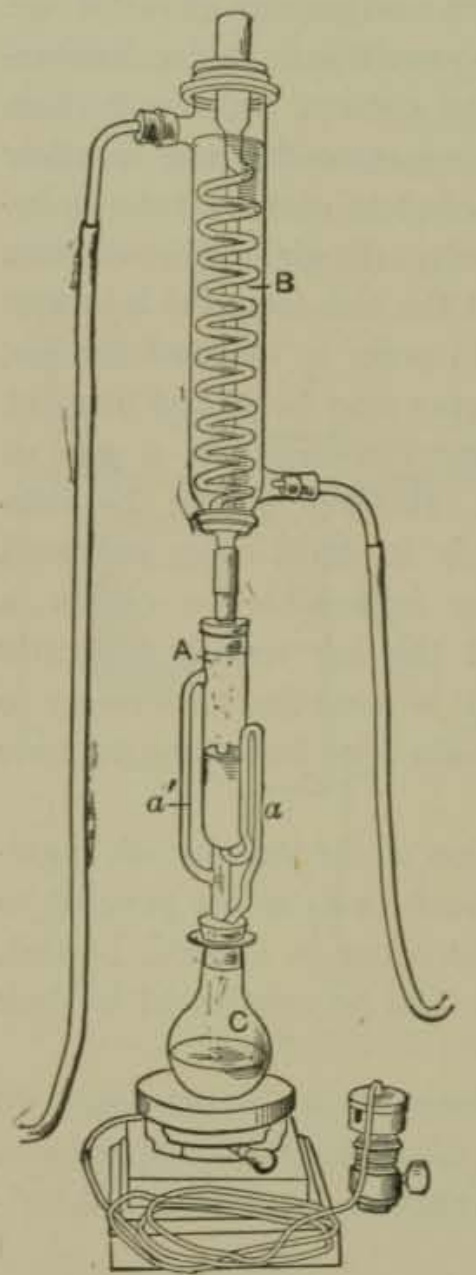

FIG. 20,-The Soxhlet Extractor with Electric Heater. distilled portions of the solvent in the tube $A$. Dry powders are contained in extraction thimbles of filter paper or in filters folded over the end of a flat-bottomed cylinder so as to form a cartridge; liquids, such as milk, previously dried in a paper coil or in a wad of asbestos, are extracted without a filter. The vapor from the solvent, boiling in the flask $C$, passes up through the side tube $a^{\prime}$ into the condenser $B$, where it is liquefied and falls drop by drop on the substance.

When the level of the solvent in the tube $A$ reaches the top of the siphon the liquid drains off into the tared flask $C$, carrying with it whatever it dissolves. The operation is automatically repeated, the substance being successively extracted with freshly distilled portions of the solvent, which leaves behind in the flask $C$ the material in solution.

The heater employed should be a hot plate heated by steam, or, as shown in Fig. 20 , an electric stove, which may be provided with a fractional rheostat for varying the amount of heat. If neither of these is available the extraction flask may be rested on a piece of asbestos paper supported by a lamp stand, the heat being supplied by an ordinary Bunsen burner.

The degree of ebullition is so regulated as to allow the solvent to saturate the sample and siphon over into the flask $C$ from six to twelve times an hour, the extraction being continued from two to six hours, or until all the ether-soluble material has been removed. Care should be taken also that the rate of boiling and the rate of condensation are so regulated that no appreciable loss of reagent occurs during the extraction. A strong smell of ether perceptible a $i$ the top of the condenser indicates a loss. The solvent is recovered at the end of the extraction by disconnecting the weighing flask at a time when nearly all of the solvent is in the part $A$ and before 
it is ready to siphon over. The weighing-flask is then freed from all traces of the solvent by drying first on the water-bath and then in the oven, after which it is cooled in the desiccator and weighed, the difference between this and the first weighing representing the weight of the fat or ether extract.

The Johnson Extractor.-This form of apparatus (Figs. 21 and 22) has the advantage of the Soxhlet extractor in that it is simpler and employs a much smaller amount of ether. The substance is contained in the inner tube of the extractor (Fig. $2 \mathrm{I}$ ), which is closed at the lower end by one thickness each of filter paper and cheese cloth, held tightly in place by nieans of a linen thread wrapped several times about the tube in the constriction and tied in a fast knot. This innner sube properly prepared can be used over and over for extractions. The outer tube, also shown in Fig. $2 \mathrm{I}$, is of such a size that the inner tube fits loosely within it. A slight bulge on one side prevents trapping by means of the condensed solvent. The extraction flask is preferably of only 30 to $35 \mathrm{cc}$. capacity. It is attached to the extractor, as is also the extractor to the condenser tube, by means of a carefully bored cork stopper. For ordinary determinations of ether extract the outer tube should have an inside diameter of $26 \mathrm{~mm}$. and the inner tube an outside diameter of $22 \mathrm{~mm}$, only 8 to $10 \mathrm{cc}$. of the solvent being required. If, however, large amounts of material Fig. 21. - Johnson Extrac( 25 to 50 grams) are to be extracted, the diameters

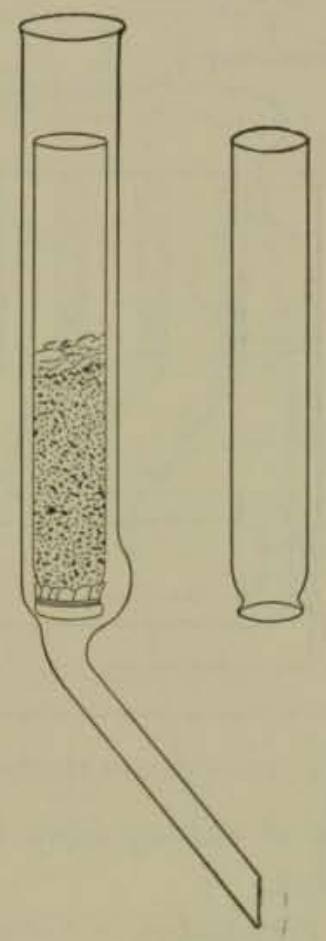
may be made $32 \mathrm{~mm}$. and $28 \mathrm{~mm}$. respectively and a larger amount of solvent employed.

Where only a few extractions are made, the heating can be performed over (but not on) a metal plate heated by a Bunsen burner, and the condensation effected by an ordinary Liebig condenser. If, however, a considerable number of extractions are carried out, the set apparatus shown in Fig. 22 will be found convenient and also economical of space. It may be attached to the wall or placed at the back of a working desk. The heating, as shown in the cut, is effected by means of two steam pipes, but some form of electric heater answers equally well. The case with glazed door prevents the radiation of heat. At the top is shown the multiple condenser consisting 
of a copper tank with block tin tubes. Water is introduced at the left and carried off at the right.

The solvent is best poured through the material, thus obviating in large degree the crawling of the extract. The door should be opened several times during the extraction and kept open for a few minutes for the pur-

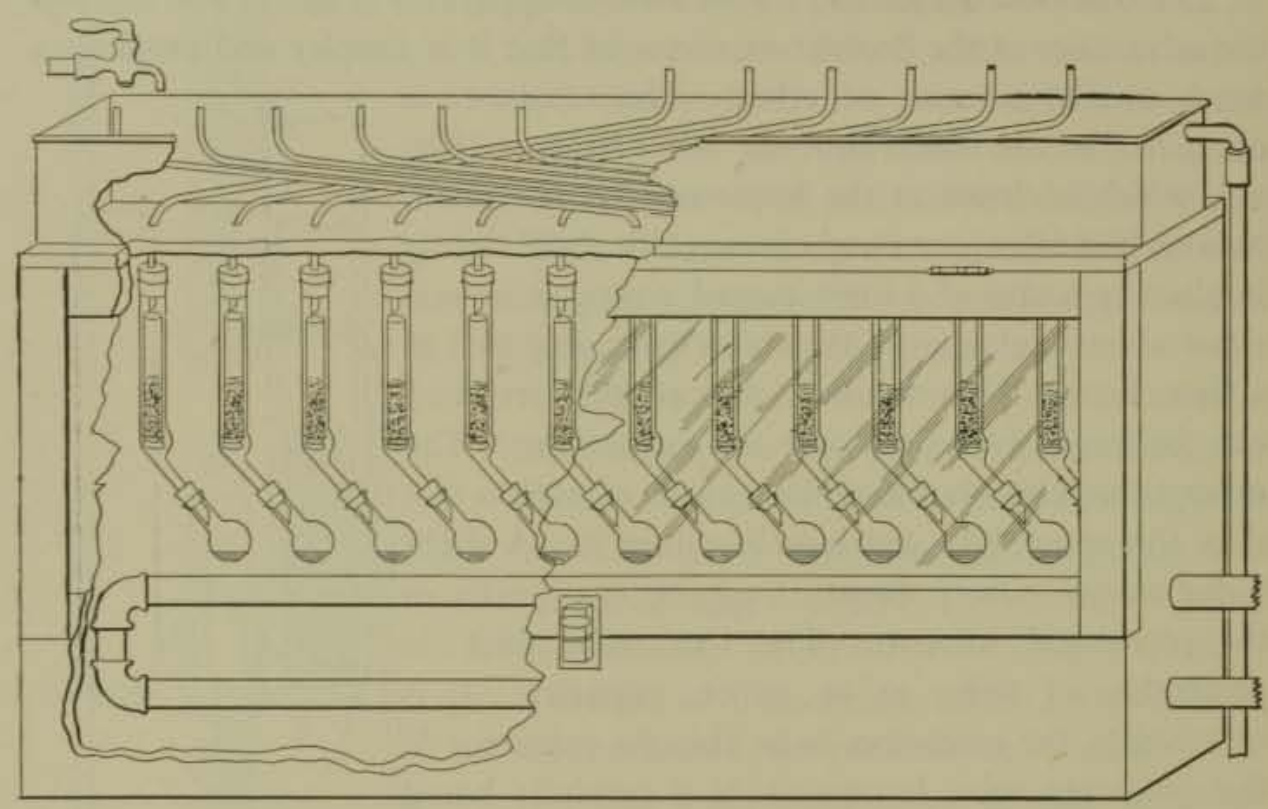

Fic. 22.-Johnson Multiple Extraction Apparatus with Heating Closet and Condenser.

pose of rinsing down the sides of the tubes by means of the condensed vapors.

Preparation of Solvents. - In taking the so-called ether extract, sometimes reckoned as fat, the solvent employed is either ethyl ether or the cheaper petroleum ether. Whichever reagent is employed, certain precautions are necessary for the purity of the reagent. If ethyl ether is used, it should be entirely freed from moisture and alcohol by first shaking with water to remove the larger portion of the alcohol, allowing it to stand for some time over dry calcium chloride, and then distilling over metallic sodium. The ether thus prepared should be kept till used with sodium in the container, the latter being somewhat loosely corked, to allow escape of the hydrogen formed.

Petroleum ether is variously termed benzine, naphtha, or gasoline. It should be low-boiling, preferably between $35^{\circ}$ and $50^{\circ}$, and it is always best to redistil it before using, in order to be sure it is free from residue. As to the choice of the two reagents for use in fat extraction, it may be said that ethyl ether is the solvent most used, but if a large number of determinations are to be made, the lower cost of petroleum ether is to 
GENERAL ANALYTICAL METHODS.

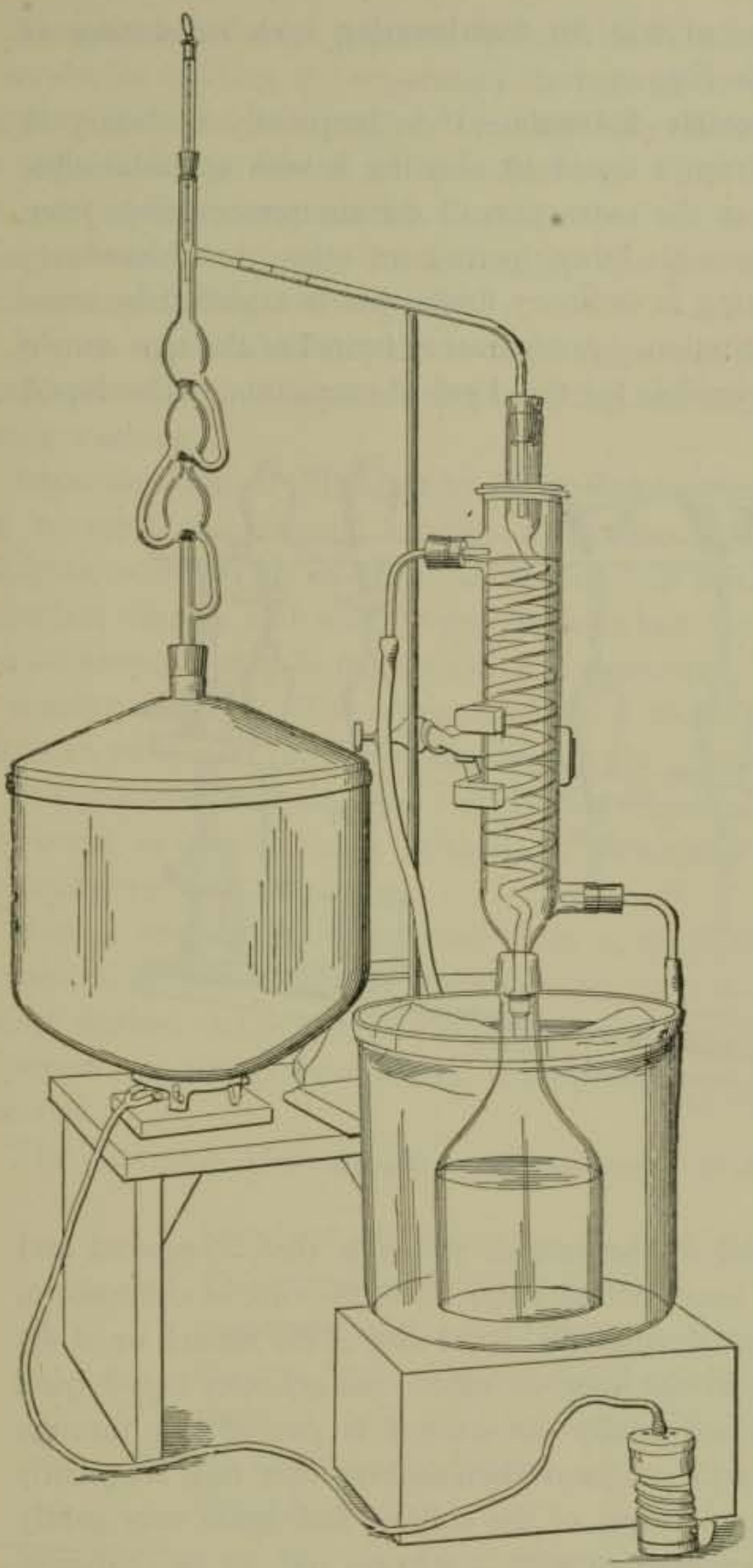

Frg. 23.-Fractionating-still, Arranged for Petroleum Ether.

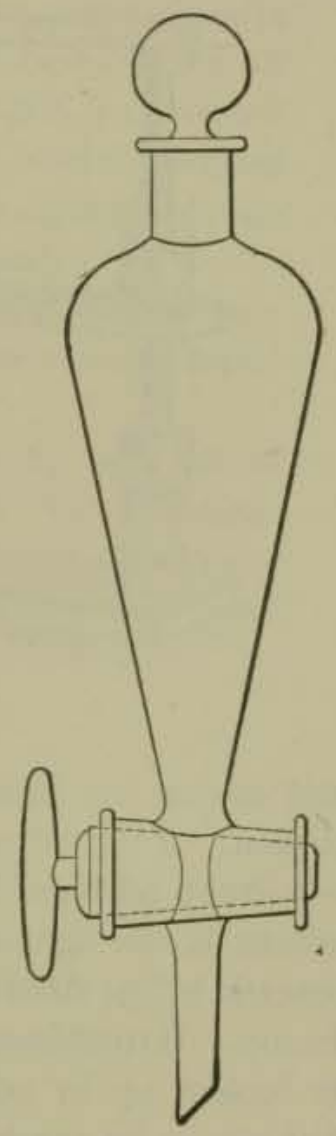

FiG. 24.-A Convenient Form of Separatory Funnel. 
be considered. A convenient still for fractionating such substances as petroleum ether is shown in Fig. 23.

Extraction with Immissible Solvents.- It is frequently necessary to dissolve out a substance from a liqu:d by shaking it with an immiscible solvent, as, for example, in the extraction of certain preservatives from aqueous or acid solutions with ether, petroleum ether, or chloroform. This can be done by shaking in ordinary flasks, but is attended by some difficulty and loss on decantation. A separatory funnel of the type shown in Fig. 24 is almost indispensible for this kind of extraction. The liquid

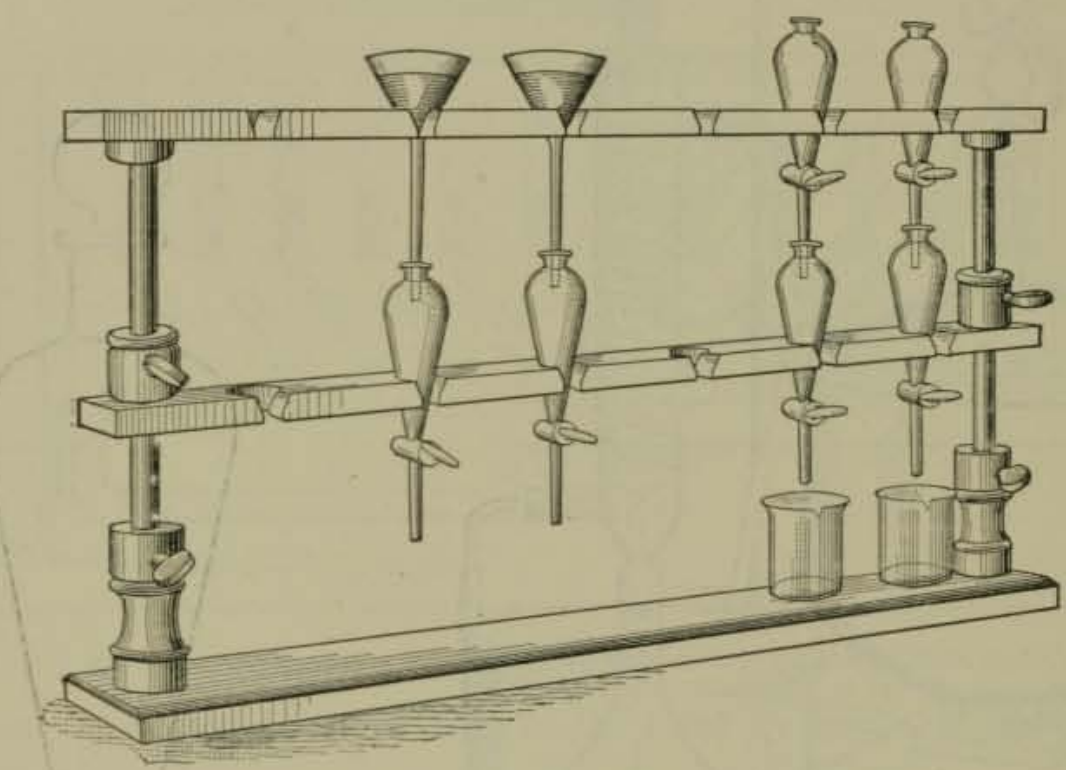

Fig. 25.-Separatory Funnel Support.

and solvent are transferred to the funnel, which is then stoppered and shaken. If the solvent is heavier than water, as in the case of chloroform, it is drawn off from beneath through the outlet-tube of the funnel, or, if the solvent is the lighter, as in the case of ether, the aqueous liquid lying beneath is first drawn off and finally the solvent is poured out through the top. If troublesome emulsions form when shaken, they may frequently be broken up by adding an excess of the solvent and again very gently shaking, or by careful manipulation with a stirring rod, or by centrifuging. If the solvent is ether, and an obstinate emulsion forms, it may frequently be broken by the addition of chloroform. Such a mixture of ether and chloroform sinks to the bottom and may be drawn off as in the case of chloroform alone. 
A separatory funnel support, devised by Winton, is shown in Fig. 25. It serves for holding the separatory funnels while drawing from one into another, and also as a support for ordinary funnels. The two shelves are adjustable by means of thumbscrews. The holes in these shelves are somewhat wider than the slots, so that the separatory funnels after being introduced through the latter drop into position and are held firmly while manipulating the stop-cock.

Winton attaches all stop-cocks and stoppers to the funnel by means of small brass chains, thus preventing breaking and interchange of these parts during washing.

Determination of Nitrogen by Moist Combustion.-In thus determining nitrogen, the organic matter is first decomposed by digestion with sulphuric acid and an oxidizer, the carbon and hydrogen being driven off as carbon dioxide and water respectively, while the nitrogen is converted into an ammonium salt, from which free ammonia $\left(\mathrm{NH}_{3}\right)$ is later liberated by making alkaline. The ammonia is then distilled into an acid solution of known value and calculated by titrating the excess of acid.

In the Kjeldahl process the oxidation is effected by means of a mercury compound, in the Gunning method, by potassium sulphate which forms the bisulphate with the acid.

Neither method in its simplest form is applicable in the presence of nitrates; if these are present, a modification must be used. The GunningArnold method (page 432) is employed for the determination of nitrogen in pepper, as the piperin is not completely decomposed by the usual processes.

The Gunning Method.-Reagents:

\author{
Standard alkali solution, $\mathrm{N} /$ io $\mathrm{NaOH} . *$ \\ Pulverized potassium sulphate. \\ Sulphuric acid, concentrated. \\ Sodium hydroxide, saturated solution. \\ Standard acid solution, $\mathrm{N} / 10 \mathrm{H}_{2} \mathrm{SO}_{4}$ or $\mathrm{HCl}$.* \\ An indicator, cochineal. \\ Granulated zinc.
}

\footnotetext{
* Winton employs standard acid of such a strength that $\mathrm{I} \mathrm{cc}$. is equivalent to $\mathrm{r} \%$ of nitrogen, working on a gram of material, and titrates back with standard alkali two and one-half times weaker than the acid. In order to insure accurate readings, burettes of narrow bore $(\mathrm{I} \mathrm{cc},=2.6 \mathrm{~cm}$.) are employed. The alkali burette is so graduated that a reading of $\mathrm{I}$ corresponds to $2.5 \mathrm{cc}$, thus allowing for the greater dilution. The advantage of this system is that the per cent of nitrogen is obtained by simply subtracting the alkali reading from the number of $\mathrm{cc}$. of acid employed.
} 
The digestion and distillation are preferably carried out in the same flask, which should be pear-shaped with flat or round bottom and made of moderately thick Jena glass. A convenient size has the following dimensions: length $29 \mathrm{~cm}$., maximum diameter $10 \mathrm{~cm}$., tapering gradually to a long neck, which near the end is $28 \mathrm{~mm}$. in diameter with a flaring edge. Its capacity is about $55^{\circ} \mathrm{cc}$.

If desired, the digestion may be conducted in a smaller hard-glass flask of about $25^{\circ} \mathrm{cc}$. capacity and of the same shape as the above, and the distillation in an ordinary round-bottomed flask of $500 \mathrm{cc}$. capacity.

Introduce from 0.5 to 3.5 grams of the sample into the digestion-flask with Io grams of potassium sulphate and from $\mathrm{I}_{5}$ to $25 \mathrm{cc}$. of concentrated sulphuric acid. The flask is inclined over the flame and heated gently for a few minutes below the boiling-point of the acid till the frothing has ceased, after which the heat is gradually increased till the acid boils, and the boiling is continued till the contents have become practically colorless or at least of a pale straw color. Wire gauze may be interposed between the flask and flame, but a triangle or a similiar supfort is to be preferred.

The contents of the flask are then cooled, and, if the digestion has been conducted in the larger flask suitable also for distilling, as above recommended, $300 \mathrm{cc}$. of water are added and sufficient strong sodium hydroxide to make the contents strongly alkaline, using phenolphthalein as an indicator. If a separate flask is used for the distillation, the contents of the digestion-flask are transferred thereto with the water and the alkali added. A few pieces of granulated zinc should also be introduced, which by the evolution of gas prevents bumping and the sucking back of the distillate. The flask is then well shaken and connected with the condenser, the bottom of which is provided with an adapter, dipping below the surface of the standard hydrochloric or sulphuric acid, a measured quantity of which is contained in the receiving-flask. The distillation is then continued till all the ammonia has passed over into the acid, this part of the operation requiring from forty-five minutes to an hour and a half. As a rule the first $250 \mathrm{cc}$. of the distillate will contain all the ammonia.

The excess of acid in the receiving-flask is then titrated with standard alkali, and the amount of nitrogen absorbed as ammonia is calculated. The reagents, unless known to be absolutely pure and free from nitrates and 
ammonium salts, should be tested by conducting a blank experiment with sugar, by means of which any nitrates present are reduced. Any nitrogen due to impurities should be corrected for.

In purchasing sulphuric acid for nitrogen determination it is important to specify that it be "nitrogen-free" as the so-called chemically pure acid often contains a considerable amount of nitrogen.

Modification of Gunning Method to include Nitrogen of Nitrates.-In addition to the reagents used in the simpler Gunning method, sodium thiosulphate and salicylic acid are required.

A mixture of salicylic and sulphuric acids is made in the proportion of $3 \circ \mathrm{cc}$. of concentrated sulphuric to $\mathrm{I}$ gram of salicylic. From 30 to $35 \mathrm{cc}$. of

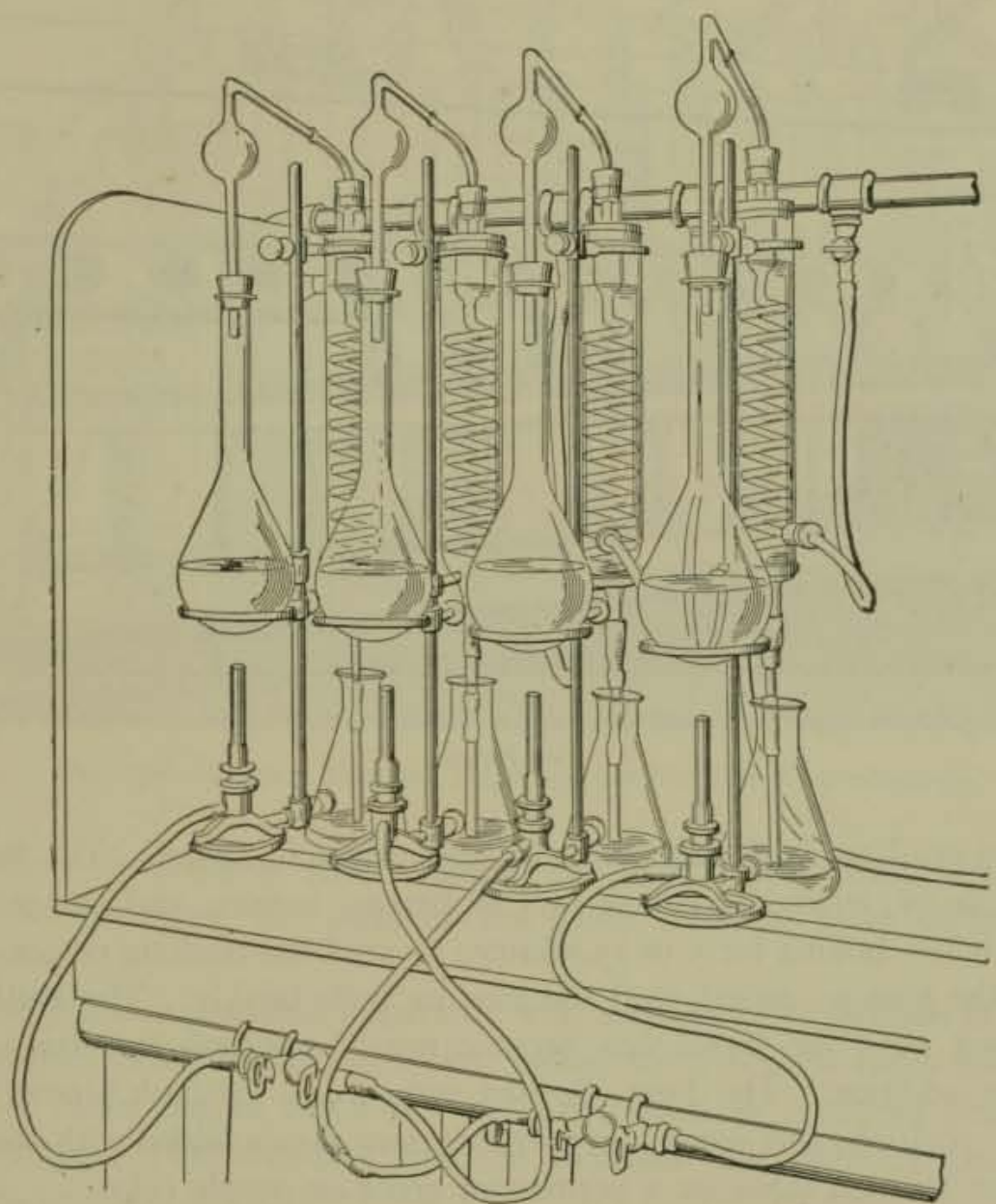

Frg. 26. -Bank of Stills for Nitrogen Determination by Gunning Process.

the mixture are added to the 0.5 to 3.5 grams of the substance in the digestion-flask, the flask is well shaken andallowed to stand a few minutes, 
occasionally shaking. Then 5 grams of sodium thiosulphate are added, and to grams of potassium sulphate, after which the heat is applied, at first very gently and afterwards increasing slowly till the frothing has ceased. The heating is then continued till the contents have been boiled practically colorless. From this point on, proceed as in the Gunning method.

The Kieldahl Method.--One gram of the air dry substance, or a proportionately larger amount of a moist or liquid substance, and 0.7 gram of mercuric oxide (or an equivalent amount of metallic mercury) are placed

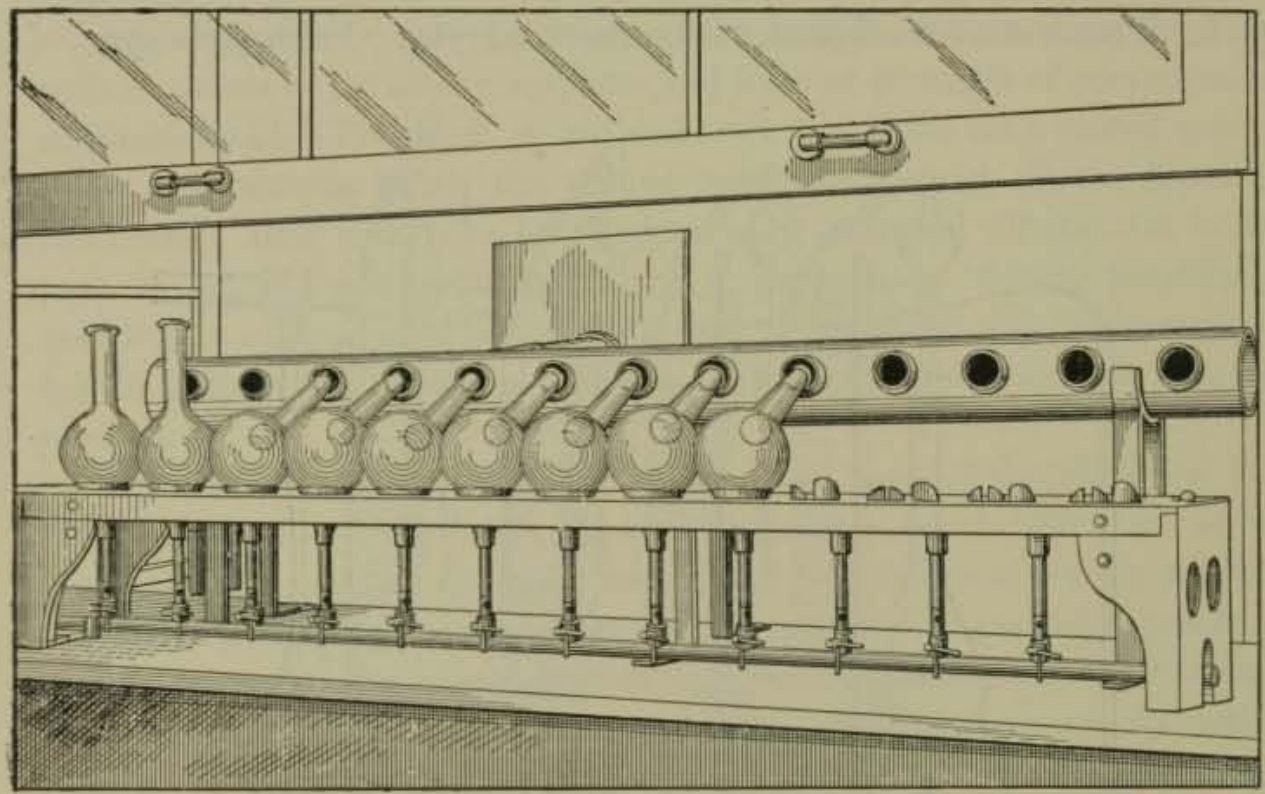

Frg. 27a.- Johnson Digestion Siand for Nitrogen Determination with Lead Y ipe L. C. rry.. of off Fumes.

in a $55^{\circ} \mathrm{cc}$. Jena flask and $20 \mathrm{cc}$. of sulphuric acid added. The flask is placed in an inclined position over a Bunsen burner. and the mixture heated below boiling for 5 to 15 minutes or until the frothing ceases, after which the heat is raised until the mixture boils briskly. The boiling is continued until the liquid has become nearly colorless and for a half hour in addition. The lamp is then turned out, the flask placed in an upright position, and potassium permanganate slowly added with shaking until the solution takes on a permanent green or purple color.

After cooling, $250 \mathrm{cc}$. of water are added, then $25 \mathrm{cc}$. of potassium sulphide solution ( 40 grams of the commercial salt in I liter of water), 
sufficient saturated sodium hydroxide solution to render the solution alkaline, and finally a few grains of granulated zinc, shaking the flask after each addition. Without delay connect with the distillation apparatus, and proceed as in the Gunning method.

Apparatus for Nitrogen Determination.-A bank of stills used by the author in nitrogen determination and in other processes is shown in Fig. 26.

The digestion apparatus shown in Fig. $27 a$ is that devised by Johnson, Winton, and Boltwood. The stand is of cast iron, with holes provided

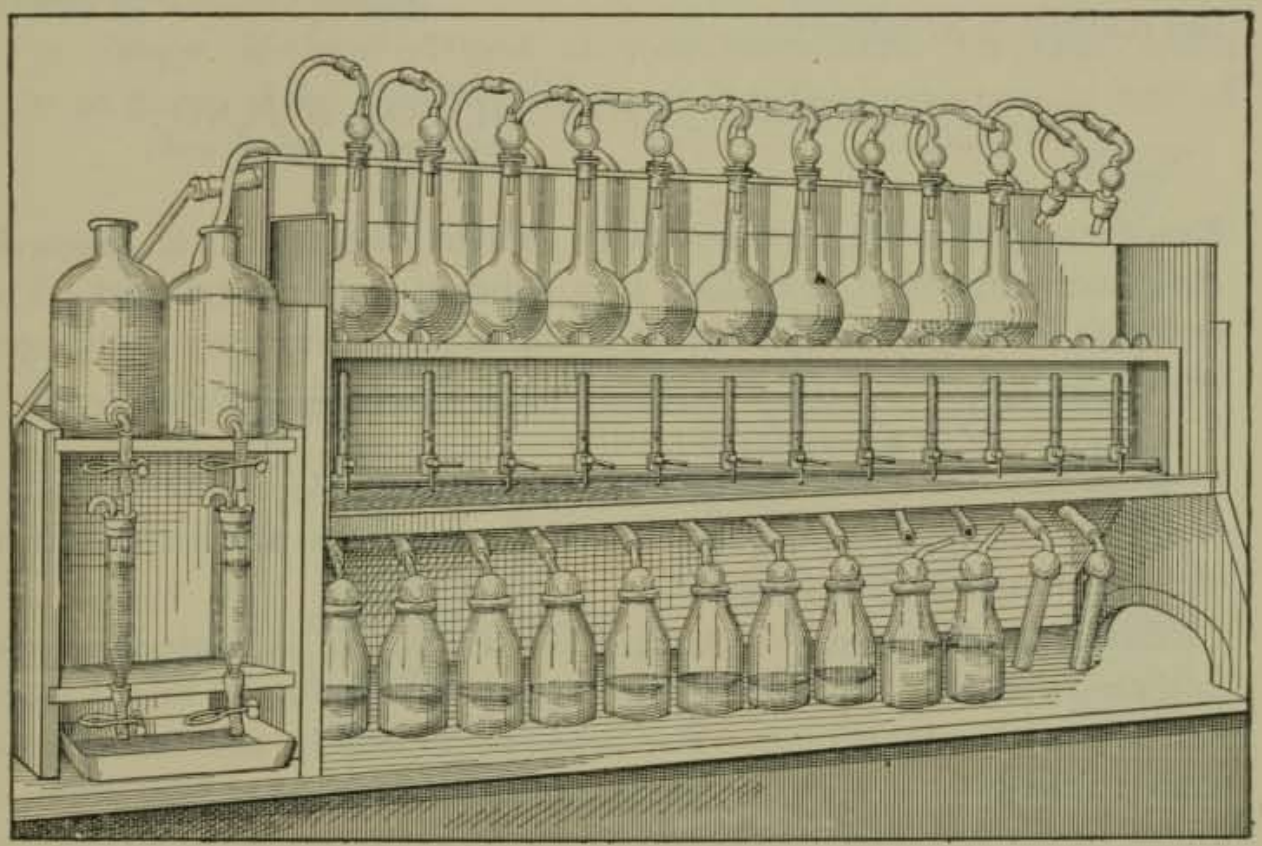

Fí3. 27b,-Johnson Distilling Apparatus for Nitrogen Determination.

with three projections that support the flask. The lead pipe with holes for receiving the ends of the flasks serves to carry off the acid fumes.

The Johnson distilling apparatus with accessories by Winton is shown in Fig. $27 b$. The distillation tubes, except for the glass traps and bulb receiver tubes, are of block tin, and are cooled in a copper tank filled with water. The receivers for the distillate are ordinary pint milk bottles.

At the left are two bottles with suspended tubes for measuring the potassium sulphide and sodium hydroxide solutions. 
Determination of Ammonia.-A weighed quantity of the finely divided sample, treated with ammonia-free water and made alkaline with magnesium oxide free from carbonate, is distilled into a measured quantity of standard acid (tenth-normal hydrochloric or sulphuric acid).and the amount of ammonia determined by titration.

Determination of Amido-nitrogen.*-In the absence of ammonia, or after the removal of the ammonia as described in the preceding section, the sample is boiled for an hour with $5 \%$ hydrochloric or sulphuric acid, which converts the amido-compounds into ammonium salts (chloride or sulphate). Assuming asparagin to be the amido-compound acted upon, the reaction is as follows:

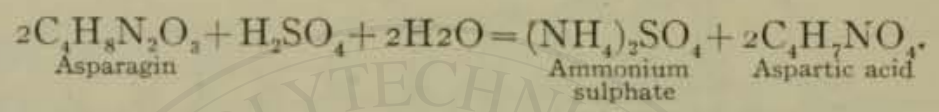

Exactly neutralize the free acid with sodium carbonate, add magnesia (free from carbonate), and distil into standard tenth-normal acid. The ammonia is determined by titration in the usual manner, and its nitrogen represents half of the nitrogen contained in the amido-compound, which it is customary to calculate as asparagin.

Determination of the Various Carbohydrates.- Under title of "Cereals" in Chapter $\mathrm{X}$ are given in detail methods for separation and determination of sugar, dextrin, crude fiber, etc.

Detection of Poisons.-Metallic impurities present in foods incidental to their preparation, or as adulterants, are considered under title of foods liable to such adulteration. The detection of highly toxic substances, such as arsenic, corrosive sublimate, and alkaloids, added with criminal intent, comes within the province of the medico-legal chemist or toxicologist and is beyond the scope of this work. The methods involved are fully described in the treatises of Autenrieth and Blyth (see p. 79), only those for arsenic, which occurs also as an accidental impurity, being here considered.

Detection and Determination of Arsenic.-Methods of Solution.Syrups, baking powders and other materials soluble in water or acid do not need preliminary treatment. Beer is treated as described on page 728. Other methods of solution are as follows:

I. Johnson-Chittenden-Gautier Method. $\uparrow$ - This method is suitable for meat, vegetables, and the like, the proportion of acids used being

- Wiley, Agricultural Analysis, Vol. III. p. 424.

$\dagger$ Am. Chem. Jour., 2, p. 250. 
varied to suit conditions. Heat at $150^{\circ}-160^{\circ} \mathrm{C}$., in a porcelain dish, 100 grams of the finely divided material with $23 \mathrm{cc}$. of pure concentrated nitric acid, stirring occasionally. When the mixture assumes a deep orange color, remove from the heat, add $3 \mathrm{cc}$. of pure concentrated sulphuric acid, and stir while nitrous fumes are given off. Heat to $180^{\circ}$ and add while hot, drop by drop, with stirring, $8 \mathrm{cc}$. of nitric acid, then heat at $200^{\circ}$ till sulphuric fumes come off and a dry charred mass remains. Pulverize the mass, exhaust with hot water, filter, evaporate to small volume, take up in cold $20 \%$ sulphuric acid and treat by the modified Marsh or Gutzeit method.

2. Sanger Method.*-Digest at room-temperature for some hours 5 to 20 grams of the material in a casserole with about an equal bulk of

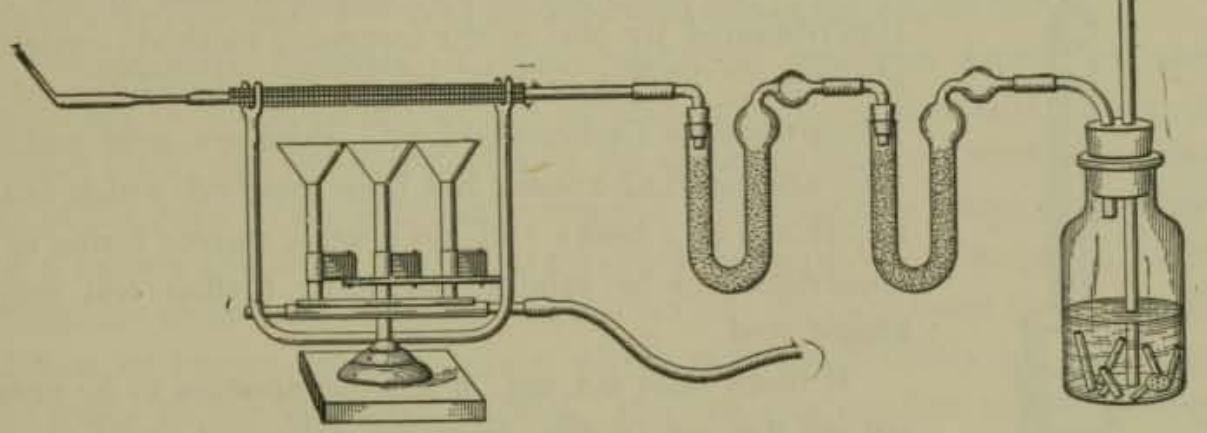

Fic. 28.-Marsh Apparatus for Arsenic.

concentrated nitric acid, add $20 \mathrm{cc}$. of concentrated sulphuric acid and digest further at a gentle heat until the mixture begins to char. Add about 2 cc. of nitric acid and heat until sulphuric fumes appear, repeating the addition of acid and heating until oxidation appears to be practically complete. Remove all nitric acid by dilution and evaporation to the fuming stage, then dilute with 4 volumes of water. At this point about twice the bulk of saturated sulphurous acid solution may be added and the evaporation repeated, thus reducing to the arsenious condition, but this is not usually necessary.

Methods of Determination.-I, Marsh Test.-The apparatus (Fig, 28) consists of a generating flask with funnel tube, a U-tube containing cotton

*Proc. Am. Acad. Arts, Sci, 26, 189r, p. 24. 
moistened with $10 \%$ lead acetate solution (to remove hydrogen sulphide), a calcium chloride drying tube, and a hard glass tube of $6 \mathrm{~mm}$. bore,

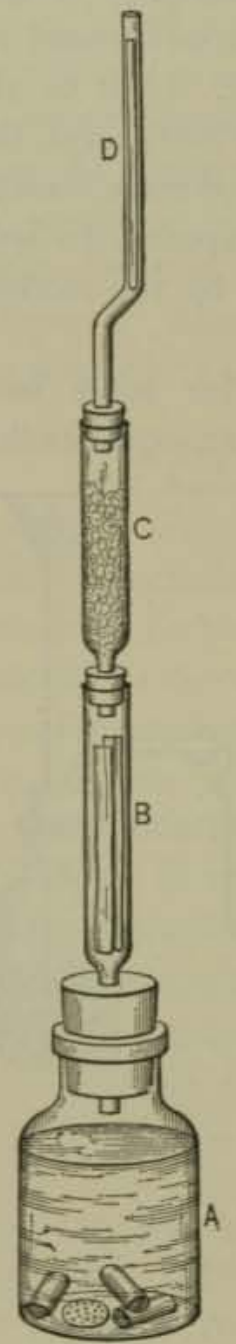

Frc. 29,-Bishop Apparatus for Arsenic. drawn down near the end to a uniform constriction about $4 \mathrm{~cm}$. long and $\mathrm{I} \mathrm{mm}$. inside diameter and also at the very end to a narrow exit tube. The tube is supported over a three-burner furnace the part in contact with the flame being wrapped with wire gauze.

Introduce into the generating flask from 20 to 30 grams of arsenic-free stick zinc and a perforated platinum disk to form an electric couple. Stopper and add through the funnel tube $20 \%$ sulphuric acid sufficient to start the reaction and drive out all air. When danger of explosion is over heat the tube to bright redness. After running the current long enough to prove the absence of arsenic in the reagents add slowly from the funnel tube the solution of the material in $20 \%$ sulphuric acid or the solution obtained by one of the foregoing methods containing about $20 \%$ of that acid, keeping a steady evolution of gas. When the flow slackens add $30 \%$ sulphuric acid and later $40 \%$ acid until all arsenic has been expelled, which usually requires 2 to 3 hours. If no arsenic mirror forms in the constriction of the tube in one hour, further test may be abandoned.

If more than $0.1 \mathrm{mg}$. of arsenic appears to be present cut off the constriction from the tube and weigh it on an assay balance; then dissolve the arsenic in a solution of sodium hypochlorite. (antimony being insoluble), wash with water and then with alcohol, dry, cool, and weigh. The loss is arsenic.

If the amount of arsenic is very small Sanger compares the mirror with a series of standard mirrors prepared in the same apparatus using quantities of a standard solution containing from 0.005 to $0.05 \mathrm{mg}$. of $\mathrm{As}_{2} \mathrm{O}_{3}$. To prepare the standard solution I gram of pure $\mathrm{As}_{2} \mathrm{O}_{3}$ is dissolved in arsenic-free sodium hydroxide, acidified with sulphuric acid, made up to one liter and ro cc. of this stock solution further diluted to $\mathrm{I}$ liter; $\mathrm{I}$ cc. $=0.0 \mathrm{Img}$. $\mathrm{As}_{2} \mathrm{O}_{3}$.

2. Sanger-Black-Gutzeit Method.*-The apparatus (Fig. 29), devised by Bishop, consists of a $30 \mathrm{cc}$. salt-mouth bottle provided with three upright

- Jour. Soc. Chem. Ind., 26, 1907, p. 1115. 
tubes one above the other. The lower tube is $7 \mathrm{~cm}$. long, $1 \mathrm{~cm}$. in bore, and contains strips of filter-paper previously soaked in $5 \%$ lead acetate solution and dried. The middle tube is of the same size as the lower but shorter. It is loosely filled with cotton moistened with $I \%$ lead acetate solution. The upper tube has a uniform bore, of $2.5 \mathrm{~mm}$. and is bent twice so that the upper end is vertical. In this tube is placed a strip of cold-pressed drawing paper $2 \mathrm{~mm}$. wide which has been soaked in $5 \%$ alcoholic murcuric chloride (or bromide) and dried.

Place in the evolution bottle to grams of stick zinc, a few crystals of stannous chloride, a perforated platinum disk and from 2 to 5 grams of the material or else the extract of the charred or digested material prepared as described in the foregoing sections, containing about $20 \%$ of sulphuric acid. Add enough $20 \%(I: 4)$ sulphuric acid to nearly fill the bottle, attach the three tubes and allow to react for 45 minutes. Compare the color on the sensitized strip with that of standard strips obtained with from 0.005 to $0.05 \mathrm{mg}$. of $\mathrm{As}_{2} \mathrm{O}_{3}$ in the same apparatus, using measured quantities of the standard solution described under the Marsh test.

Colorometric Analysis.-Certain analytical processes depend on the formation of a compound of the substance to be determined having a definite color, and the calculation of the quantity present from the intensity of the color of the solution, compared with that of a solution containing a known amount. The comparisons may be made in a special form of cylinder or in a colorimeter. The latter has the advantage that a single solution of known strength serves within reasonable limits for matching any shade in the unknown soiution, and for any number of determinations, the desired depth of the color being secured by varying the length of the column.

Schreiner's Colorimeter.*-This apparatus, shown in Fig. 30 , consists of two graduated tubes $(B)$, containing the standard and unknown colorimetric solutions, the height of the column of liquid in both tubes being changed by two immersion tubes $(A)$, which remain stationary while the graduated tubes are raised or lowered in the clamps $(C)$. The mirror $D$ reflects the light through the tubes, and the mirror $E$ reflects it again to the eye of the operator at $F$.

In making the comparisons, the tube containing the solution of either known or unknown strength is set at a definite point, and the other tube is raised or lowered until the colors match. If $R$ is the reading of the standard solution of the strength $S$, and $r$ the reading of the colorometric solution of unknown strength $s$, then $s=\frac{R}{r} S$.

- Jour. Am. Chem. Soc. 27, 1905, p, 1192. 
If desired, standard slides of colored glass, such as accompany the Lovibond tintometer, may be used at $G$ for matching the solution of un-

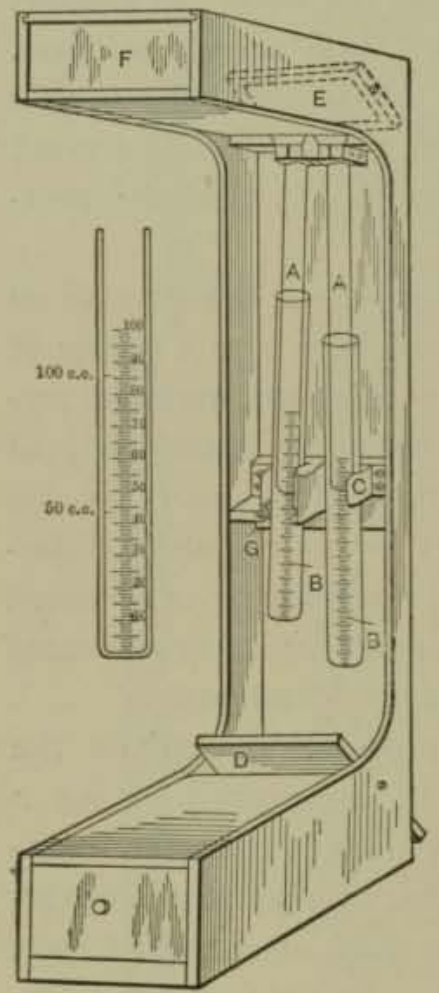

FIG. 30.-Schreiner's Colorimeter with a Tube showing Graduation.

known strength, the value of these slides being determined by comparison with a standard solution.

The Lovibond Tintometer may be used for colorometric chemical analysis, but is not so well suited for this purpose as the Schreiner colorimeter. It is especially designed for determining the color value of liquid and solid technical products, such as beer, wine, oil, flour, paper, etc.

The instrument itself is of simple construction, consisting of an elongated box with an eyepiece at one end and two rectangular openings at the other, one for the solution or substance to be examined, the other for the standard glass slides used for matching the color. Light is reflected through the openings by means of a square piece of opal glass mounted on a jointed standard. Liquids are examined in rectangular cells with glass sides by transmitted light, while powders are pressed into a form and examined by reflected light.

The standard slides used in general work are red, yellow, and blue in even graduation from . 006 to 20 tint units which can be combined so as to produce any desired tint or shade of any color. The results are expressed in terms of standard dominant colors (red, yellow, and blue), subordinate colors (orange, green, and violet) obtained by combining equal values of two dominant colors, and neutral tint (black) obtained by combining equal values of the three dominant colors.

Thus

$$
\begin{aligned}
0.6 R+5.6 Y & =0.6 O+5.0 Y \\
0.08 R+\mathrm{I} .5 Y+0.2 B & =0.08 N+0.12 G+\mathrm{I} .3 Y \\
\mathrm{I} .2 R+\mathrm{I} .0 B & =\mathrm{I} .0 \mathrm{~V}+0.2 R
\end{aligned}
$$

in which $R=$ red, $Y=$ yellow, $B=$ blue, $O=$ orange, $G=$ green, $V=$ violet, $N=$ neutral tint or black. 
Special slides may be obtained for the examination of any desired product. For example, slides of brown shades are furnished for beer, of yellow shades for oils, and so on.

\section{REFERENCES TO GENERAL FOOD ANALYSIS.}

Alt.en, A. H. Commercial Organic Analysis. Philadelphia, I909.

Autenrieth, W. The Detection of Poisons and Strong Drugs. Trans, by W. H. Warren. Philadelphia, 1905.

BaLland, A. Les Aliments. Paris, 1907.

Battershall, J. P. Food Adulteration and its Detection. New York, I887.

BELL, JAS. The Analysis and Adulteration of Foods, Pts. I and II. London, I88I.

Blyth, A. W. and M. W. Foods, their Composition and Analysis. New York, I903.

- Poisons, their Effects and Detection. London, rgo6.

BöнмЕR, C. Die Kraftfuttermittel, ihre Rohstoffe, Herstellung, Zusammensetzung, etc. Berlin, 1903 .

Bretead, P. Guide Pratique des Falsifications et Alterations des Substances alimentaires. Paris, 1907 .

Bujard, A., and BaIer, E. Hilfsbuch für Nahrungsmittel Chemiker. Berlin, I894.

Burcker, E. Traité des Falsifications et Altérations des Substances alimentaires et des Boissons. Paris, 1892.

Clark, E., and Woodman, A. G. The Estimation of Minute Amounts of Arsenic. U. S. Dept. of Agric., Bur. of Chem., Circ. 99.

Ephraim, J. Originalarbeiten über Analyse der Nahrungsmittel. Leipzig, I894.

GIRARD, C. Analyse der Matières alimentaires et Recherche des leurs Falsifications. Paris, 1904.

Hanausek, T. F. Die Nahrungs- und Genussmittel aus dem Pflanzenreiche, 1884.

Hassal, A. H. Food, its Adulterations and the Methods for their Detection. London, I 874 .

KöNIG, J. Chemische Zusammensetzung der menschlichen Nahrungs- und Genussmittel. Berlin, 1903.

- Die Untersuchung landwirtschaftlich und gewerblich wichtiger Stoffe. Berlin, 1906.

LEACH, A. E. Food: Methods of Inspection and Analysis. Article in Reference Handbook of the Medical Sciences, Vol. 3, pages $180-188_{3}$.

Leffyann, H., and Beam, W. Select Methods of Food Analysis. Philadelphia, r905.

Mansfeld, M. Die Untersuchung der Nahrungs- und Genussmittel. Leipzig, 1905.

Neufei.D, C. A. Der Nahrungsmittelchemiker als Sachverständiger. Berlin, 1007.

Polin et Labit. Examen des Aliments suspects. Paris, 1892 .

Richards, E. H., and Woodman, A. G. Air, Water, and Food. New York, rgoo.

Röttger, H. Kurzes Lehrbuch der Nahrungsmittel Chemie. Leipzig, 1903.

RupP, G. Die Untersuchung von Nahrungsmitteln, Genussmitteln und Gebrauchsgegenständen. 1900 . 
Tном⿰, H., und GrLG, E. Einführung in die praktische Nahrungsmittel-Chemie. Leipzig, 1899 .

Vuliers, A., et Coltin, E. Traité des Alterations et Falsifications des Substances alimentaires. Paris, 1000 .

Wiesner, J. Die Rohstoffe des Pflanzenreiches. Leipzig, I9oo.

Wriey, H. W. Principles and Practice of Agricultural Analysis. Vol. III. Agricultural Products. Chem. Pub. Co., Easton, Pa., roo6.

The Analyst. London, 1877 et seq.

Revue International des Falsifications. Amsterdam, 1888 et seq.

Vierteljahresschrift der Chemie der Nahrungs- und Genussmittels. Berlin, $\mathrm{I}_{8} 8_{4}$ et seq. (Discontinued 1897. )

Zeitschrift für Untersuchung der Nahrungs- und Genussmittel. r 898 et seq.

Vereinbarungen zur Untersuchung und Beurtheilung von Nahrungs- und Genussmitteln. Berlin, 1897 .

Also the following bulletins of the Bureau of Chemistry, U. S. Deptartment of Agriculture:

Bulletin $1_{3}$, Parts $1-10$. Food and Food Adulterants. $1887-1902$.

Bulletin 46 . Methods of Analysis adopted by the A. O. A. C. 1899.

Bulletin 65. Provisional Methods for the Analysis of Foods, adopted by the A. O.A. C. Nov. 14-16, 1901. 1902.

Bulletin 107, rev. Official and Provisional Methods of Analysis. A. O. A. C. Igo8. 


\section{CHAPTER V.}

\section{THE MICROSCOPE IN FOOD ANALYSIS.}

Microscopical vs. Chemical Analysis.-A very important means of identification of adulterants in many classes of food products is furnished by the microscope, which in many cases affords more actual information as to the purity of food than can be obtained by a chemical analysis. This is especially true of coffee, cocoa, and the spices, wherein the microscope serves to reveal not only the nature of the adulterants, but also not infrequently the approximate amount of foreign matter present. In the case of the cereal and leguminous products so commonly employed as adulterants, a microscopical examination is of paramount impertance.

The chemical constants of many of the adulterants of coffee and the spices do not always differ sufficiently from those of the pure foods in which they appear to be distinguished therefrom with accuracy and confidence by a chemical analysis alone. On the other hand, one who is familiar with the appearance under the microscope of the pure foods and of the starches and various ground substances used as adulterants, can, with certainty, identify very minute quantities of these materials, when present, with the same ease that one can recognize megascopically the most familiar objects about him.

A chemical test may, for example, indicate the presence of starch, but it cannot reveal the particular kind of starch. The microscope will at once show whether the starch present is wheat or corn or potato or arrowroot, since these starches differ almost as much in microscopical appearance as do the physical characteristics of the grains or tubers from which they are obtained. Again, by a chemical analysis an abnormal amount of crude fiber may show the presence of a woody adulterant, but only the microscope will enable one to decide whether the impurity consists of sawdust or ground cocoanut shells. Not only in such instances as these is the microscopical examination of greater importance 
than a chemical analysis in establishing the purity of the food, but it is at the same time a much quicker guide.

The Technique of Food Microscopy.-The recognition of adulterants by the microscope requires some experience but no more than may be acquired by a chemist who will give the subject serious attention. In the examination of cocoa, coffee, tea, and the spices for adulteration, a careful study of the powdered substance in temporary water mounting will in most cases prove sufficient to familiarize the food analyst with their characteristics under the microscope, and it is not absolutely necessary for him to familiarize himself with the details of section cutting, dissecting, or permanent mounting unless he so desires. The treatment in detail of these latter branches of vegetable histology is beyond the scope of the present work. For full information along these lines the reader is referred especially to such works as those of Behrens*, Zimmerman, $\dagger$ and Chamberlain $\neq$ together with the list of references on page 98 .

Standards for Comparison.-For standards the analyst should provide himself with as complete a set as possible of the various materials to be examined, taking care that their absolute purity is established. Whereever possible, he should grind the sample himself from carefully selected whole goods. These, together with samples of the starches and other adulterants, all of known purity, should be contained in small vials carefully stoppered and plainly labeled, arranged alphabetically or in some equally convenient manner in the desk or table on which the microscope is commonly used. The adulterants included in this set of standards should be not only those which experience has shown most liable to be employed, but any which, by reason of their character, might in the analyst's opinion be used under certain conditions.

\section{APPARATUS.}

The Microscope-stand.-An expensive or complicated stand is unnecessary. The prime requisites for good work in a microscope-stand are firmness or rigidity, and accuracy in centering. An inexpensive stand possessing these features can be used for the best work, providing the optical parts are satisfactory. It is well, if economy must be practiced, to purchase a simple stand provided with the society screw, and let the larger portion of the allowance go for a high grade of lenses, since many of the attachments inherent in a high-priced stand, though often of convenience, may well be dispensed with.

* Guide tó the Microscope in Botany. $\dagger$ Botanical Microtechnique. $\ddagger$ Methods in Plant Histology. 
A stand of the so-called continental type (having the horseshoe base) is preferable. A square stage is rather more convenient than the circular form, and the jointed pillar possesses advantages over the rigid variety in ease of manipulation that are certainly worth considering.

The smooth working of both the coarse and fine adjustments should not be lost sight of. If the microscope is to be used exclusively for food work, a substage condenser is unnecessary, hence the construction of the

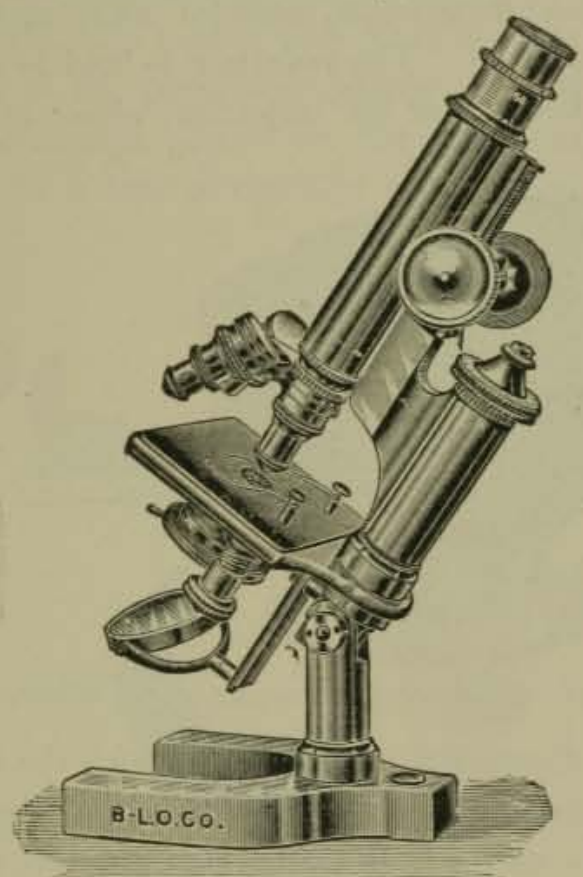

Frc. 3r.-Continental Type of Microscope.

substage may be very simple, unless bacteriological work is to be done as well.

A nose-piece, while not indispensable, is a great convenience for the quick transfer of objectives. A double nose-piece carrying two objectives is ample for routine food work.

The Optical Parts are by far the most important, and should be of superior quality, though not necessarily of the most expensive makers. The food analyst should have at least two objectives, one for high- and one for low-power work, and preferably two oculars.

For the routine examination of powdered food substances the writer prefers a $\frac{1}{6}$-inch objective, used with a medium ocular, the combination giving a magnification of from 240 to 330 diameters, according to the ocular employed. For a low-power objective the $\frac{3}{4}$-inch is a conven- 
ient size. It is useful as a finder preliminary to examination with the higher power, and, in connection with a low-power eyepiece, is well adapted for the examination of butter and lard, and for use with the polariscope.

An eyepiece micrometer mounted in an one inch ocular is indispensable for measuring starch grains and other elements. It is calibrated by means of a stage micrometer.

The Micro-polariscope.-This accessory is useful in the identification of starches and other ingredients, and for ascertaining whether or not fats have been crystallized. The polarizer is held below the stage, while the analyzer is applied above the objective, either in the tube or above the ocular.
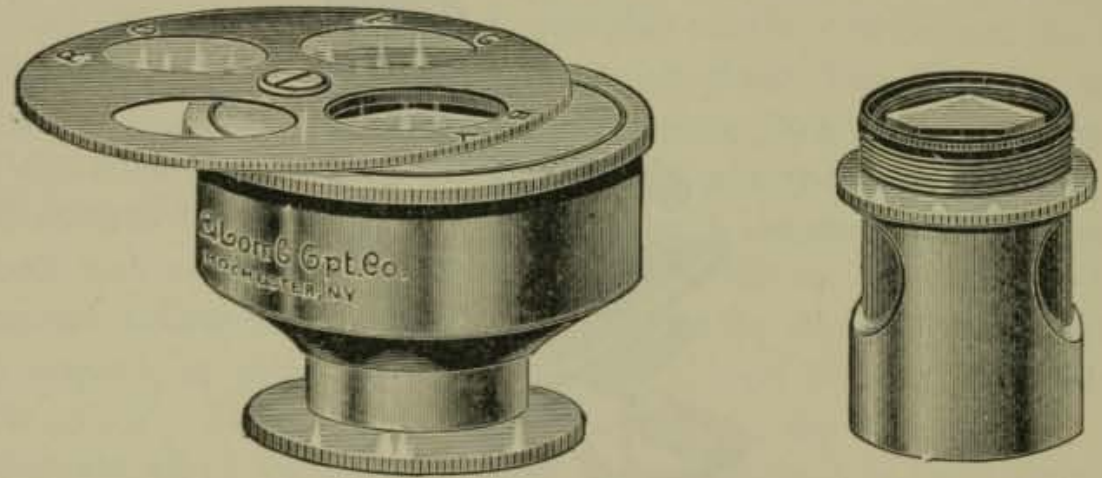

Fro. 32,-Polarizer and Analyzer for the Microscope.

A common form of construction is one in which the substage is adapted to carry interchangeably the diaphragm tube and the polarizer. If the polariscope is much used, it becomes desirable to provide means for quickly changing the polarizer and diaphragm tube below the stage, and for moving the analyzer in and out of place above the objective. Winton* has devised a microscope-stand with this in view, especially adapted to the needs of the food analyst.

If the polariscope is to be used often, it is convenient to have within easy access two stands, one with the polariscope mounted in place in connection with low-power glasses ready for use, and the other stand rovided with the ordinary high- and low-power objectives only.

Microscope Accessories include of necessity a large number of slides and cover glasses. The latter should be No. 2 thickness, $\frac{3}{4}$ inch, either round or square.

One or more dissecting-needles in holders and a small hand magnifying-glass should also be provided.

* Journal App. Microscopy, 2, p. $55^{\circ}$. 
Other useful accessories are a mechanical stage, a pair of fine tweezers, knives, scissors, and, if sections are to be cut, a plano-concave razor.

\section{MICROTECHNIQUE.}

Preparation of Vegetable Food Products for Microscopical Examination.-The ground spices and cocoas of commerce are usually of the requisite fineness for direct examination without further treatment. Coffee, chocolate, starches, and similar products should be ground in a mortar fine enough to pass through a sieve with from 60 to 80 meshes to the inch.

A small portion of the powdered sample is taken up on the tip of a clean, dry knife-blade, and placed on the microscope-slide. By means of a medicine-dropper a drop of distilled water is applied, and the wetted

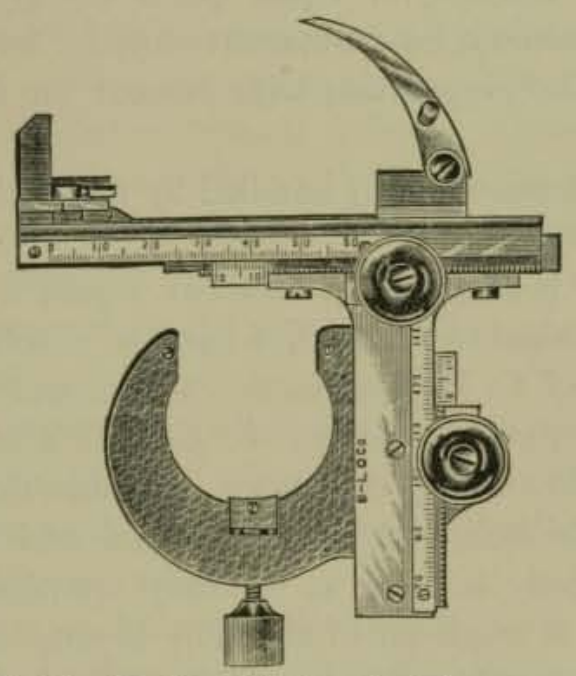

Frg. 33.-Mechanical Stage for Microscope.

powder is then rubbed out under the cover-glass between the thumb and finger to the proper fineness.

The water-mounted slide thus prepared, while useful only for temporary purposes, has proved to be best adapted to the analyst's requirements for routine microscopical examination of powdered food products for adulteration, partly because water is the best medium in most cases for showing up the structural characteristics of these substances and their adulterants, and partly because it serves best for the "rubbing out" process between thumb and finger under the cover-glass, whereby the sample is brought to the requisite degree of fineness.

Experience will soon show how far this rubbing out should be carried for the best effects. Gentle pressure should be applied, care being taken not to break the cover-glass, especially if the sample contain anything of a gritty nature. The rubbing should be continued till the coarser par- 
ticles and overlying masses are separated and distributed uniformly, but if too long persisted in, the forms of the tissues, starch grains, and other characteristic portions will be partially destroyed and of too fragmentary a nature to be readily recognizable.

Canada Balsam in $X y l o l$ is a useful mountant for the examination of starches with polarized light. In this medium, under ordinary illumination, the starches are not plainly visible, since the refractive index of the two are nearly identical, but with crossed nicols the starch grains stand out clearly and distinctly in a dark background. If the material is not perfectly dry it should be soaked in absolute alcohol and then in chloroform or xylol until dehydrated.

Glycerin. - A mixture of equal parts of glycerin and water is perhaps the best medium for permanent mounts, but considerable skill is required to finish the preparation with cement on the edge of the coverglass.

Glycerin jelly is more readily handled by the beginner since no cement is required.

Glycerin Jelly* is prepared as follows: I part by weight of the finest French gelatin is soaked two hours in 6 parts of distilled water, after which 7 parts by weight of C. P. glycerin are added, and to each 100 parts of the mixture add I part of concentrated carbolic acid. Heat the mixture while stirring till flocculency disappears and filter through asbestos while warm, the asbestos being previously washed and put into the funnel while wet. The jelly is solid at ordinary temperatures, and must be warmed to melt. A small bit of this jelly is removed from the mass by a knife-blade and placed on the clean-slide, which is held over a gas flame till the jelly is melted. The powdered specimen being then shaken into the molten drop, the cover-glass is gently placed upon it (being brought down obliquely to avoid formation of air-bubbles) and pressed down in place.

Microscopical Diagnosis.-It is never safe to pass judgment on a spice or other food by the microscopical examination of a single portion. Several slides should be prepared with bits of the powder taken from different parts of the mass, before the character and extent of the adulteration can be safely determined. Care should be taken that the slide, the knife-blade, the water, and the medicine-dropper be perfectly clean and free from contamination with previous specimens.

It should be borne in mind that at best a composite powdered sample

* Botan. Centralbl., Bd. r, p. 25. 
is but a mechanical mixture of various tissues, and that no two portions will show exactly the same composition.

Characteristic Features of Vegetable Foods under the Microscope.The structural features of a powdered spice, examined microscopically, will be found to differ considerably in appearance from those of a thin, carefully mounted section of the same spice. Instead of the beautiful arrangement of cells and cell contents with the perfect order of various parts as seen in the mounted section, one finds in the powdered sample under the microscope what often appears to be a most confusing mass of fragments of various tissues. For this reason the most striking characteristics seem to vary with different observers, and it is a well-known fact that microscopists differ widely as to conceptions of size, shape, and ordinary appearance, even in the case of certain of the well-known starch grains. It is on this account that, irrespective of the description of others, the analyst should familiarize himself with the microscopical appearance of the foods with which he is dealing, as well as of their adulterants, forming his own standards as to what constitute the recognizable features, from specimens prepared by himself.

In the large variety of ground berries, buds, tubers, barks, etc, from which the spices and condiments are prepared, as well as in the grains, legumes, shells, fruit stones, and other materials forming the most familiar adulterants, the kinds of plant tissues and cell contents which, under the microscope, serve as distinguishing marks or guides for identification are comparatively few in number.

The most common of these varieties of cell tissue and of cell contents to be met with by the food microscopist in his work are briefly the following:

Parenchyma.-This is most abundant and widely distributed, forming as it does the thin-walled, cellular tissue of nearly all vegetable food substances. The walls of parenchyma cells are, as a rule, colorless and transparent. The forms of the cells are varied and are often sufficiently characteristic in themselves to identify the substance under examination.

Sclerenchyma, or stone cells, are the thick-walled woody cells forming the hard part of nut shells, fruit stones, and seed coverings, occurring also in some fruits and barks. These cells are more often colored and of various shapes but almost always irregular, sometimes elongated, as in cocoanut shells and olive stones, occasionally nearly rectangular, as in pepper shells, and sometimes polygonal or nearly circular.

In appearance the sclerenchyma cell commonly has a more or less 
deep, central or axial cavity, from which small fissures extend through the thick walls. See Fig. 35 .

Variously shaped sclerenchyma cells are found in allspice, cassia,

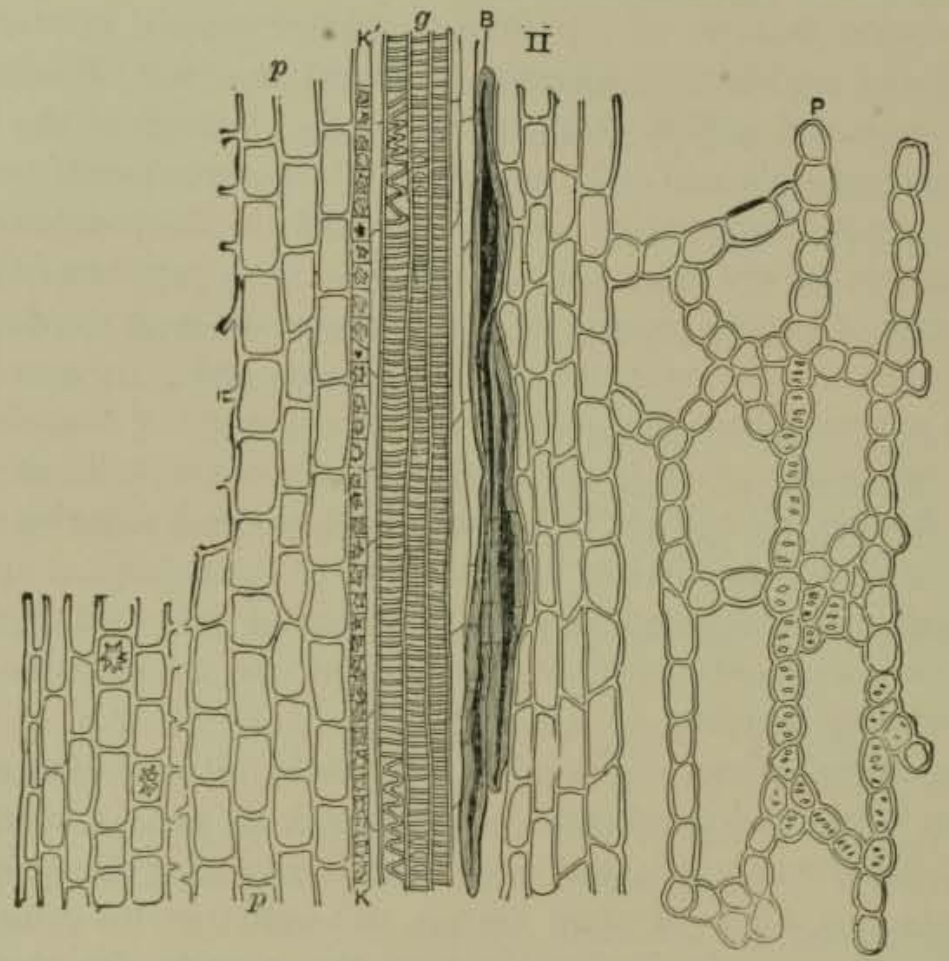

Frg, 34.-Typical Forms of Various Cell Tissues. Longitudinal section through a clove, showing: $P p$, two forms of parenchyma; $B$, bast fibers; $g$, vascular and sieve tissue; $K K^{\prime}$, cells with calcium oxalate crystals. (After Vogl.)

pepper, clove stems, nut shells, etc. Stone cells are optically active to polarized light, and between crossed niccls are very conspicuous by their bright appearance.

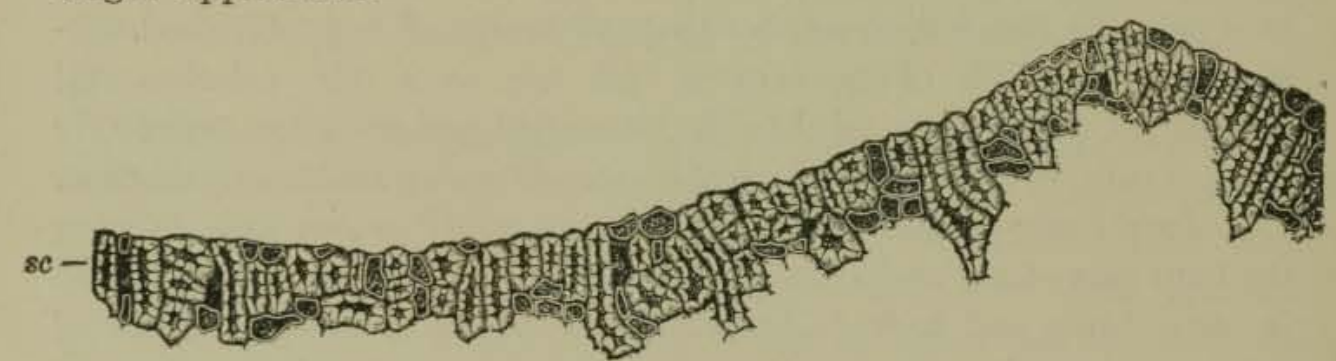

Fig. 35.-Sclerenchyma, or Stone-cell Tissue. A cross-section through the stone-cell layer of the fruit shell of black pepper. (After Vogl.)

Fibro-vascular Bundles are composed of three parts: the bast fibers, or mechanical elements, the phloem, and the xylem. 
Bast Fibers are elongated, pointed sclerenchyma cells, of which flax fibers are examples.

Sieve Tubes, the characteristic elements of the phloem, are thinwalled tubes with perforated partitions known as sieve plates.

Vessels or Ducts occur in the xylem. They are designated as spiral, annular, reticulated, or pitted, according to the nature of the walls.

Corky Tissue, or Suberin, constitutes the thin-walled, spongy cells forming the protective, outer dead layers of the bark. This is found in cassia, and in the barks used as adulterants. Suberin is tested for by potassium hydroxide (p. 93).

Starch wherever it occurs furnishes the most characteristic feature of the cell contents, and, as a rule, will at once indicate under the microsccpe, by the shape and grouping of its granules, the particular substance of which it forms a part. It is very abundantly distributed throughout the vegetable kingdom and occurs in a wide variety of forms. It is particularly conspicuous when viewed by polarized light. Between crossed nicols such starches as corn, potato, and arrowroot show out brightly from a dark background with dark crosses, the bars of which intersect at the hilum of each granule. When a selenite

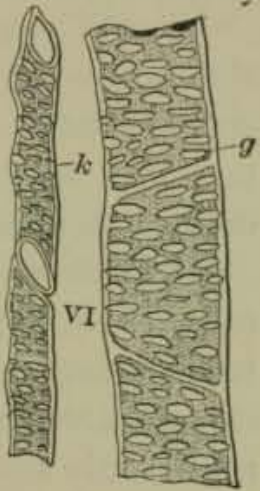

Frg. 36.-Reticulated Ducts of Chicplate is introduced above the polarizer, a beautiful play of colors is seen with various starches, a phenomenon which Blyth applies as a means of identification and classification, but which more modern microscopists regard as of minor importance to distinguishing the various starches morphologically. Starch is found naturally in the cereals, legumes, and many vegetables, in cassia, allspice, nutmeg, pepper, ginger, cocoa, and turmeric. The cereal and leguminous starches from their inertness and cheapness constitute the most common adulterants of the spices and of powdered foods in general. Starch grains are found in the cells of the parenchyma and in other cellular tissues. Iodine is the special reagent (p. 9r).

Gums and Resins occur in characteristic forms among the cell contents of some of the spices. As an example, the portwine-colored lumps of gum in allspice furnish one of the most ready means of recognizing that spice microscopically. Resin is tested for microchemically with alkanna tincture (p. 92). 
Aleurone, or Protein Grains, occur in some of the spices, but are not especially characteristic. They somewhat resemble small starch grains. Most varieties of protein grains are soluble in water, but some are insoluble. The soluble varieties, which are not apparent in water-mounted specimens, must be examined in absolute alcohol, glycerin, or oil. In leguminous seeds aleurone occurs closely intermingled with starch in the same cells, while in the cereals it occupies the whole cell.

Protein grains are tested for under the microscope by iodine in potassium iodide, which turns them brown or yellowish brown, and by Millon's reagent, which colors them brick red.

Plant Crystals are not uncommon in the class of substances which the food analyst examines. Among the common forms are the piperin crystals found in pepper. Calcium oxalate occurs in many vegetable products as prismatic crystals, crystal aggregates, or needle-shaped crystals (raphides).

Crystals of calcium carbonate are sometimes met with also, as, for example, in hops. Calcium oxalate crystals are insoluble in acetic acid, while being readily soluble in dilute hydrochloric. Calcium carbonate crystals are soluble with effervescence in both acids. The acid reagents are directly applied to the sample in water-mount under the cover-glass, and the reaction observed through the microscope.

Fat Globules are of common occurrence in many foods and appear of various sizes, sometimes large and conspicuous, and again almost lost sight of because of their minuteness. They are sometimes colorless, as in mace, and sometimes deeply tinted, as in cayenne. Alkanna tincture is used as a reagent for fat (p. $9^{2}$ ).

Other Cell Contents of less importance, but which may be identified by the microscope with reagents, are tannic acid (tested for by chloriodide of zinc and ierric acetate (Pp. 9 I and $9^{2}$ ), and various essential oils, for the detection of which alkanna tincture is employed.

\section{REAGENTS IN FOOD MICROSCOPY.}

Unless a more extended microscopical investigation of vegetable food substances is contemplated than is involved in the mere identification of adulterants, the analyst will have little need for reagents, * but will depend almost entirely on the form and appearance of the various tissues or tissue fragments, as well as on the abundance, shape, and distribution of such distinctive cell contents as the starches, fat globules, or crystals.

* One reagent that is really necessary on the microscope-table, and will very often be required is iodine in potassium iodide. 
Analytical reagentsare applied to the water-mounted sample by means of a glass rod or pipette, with which a drop of the reagent is deposited on the sample upon the slide, having previously removed the cover, which is afterwards replaced. Or, without removing the cover-glass, a drop of the reagent is placed in contact with one side of it on the slide. Along the opposite side of the cover is then placed a piece of filterpaper. The latter withdraws by capillary attraction a portion of the water from under the cover-glass, and this is replaced by the reagent, which thus intermingles with the particles of the substance.

Following is a brief list of the commoner microchemical reagents, together with their method of preparation and chief uses. For fuller details in this branch of the subject the reader is referred to Poulsen's Botanical Microchemistry, translated by Trelease, and Zimmerman's Botanical Microtechnique.

A. Analytical Reagents.-Iodine in Polassium Iodide.-Two grams of crystallized potassium iodide are first dissolved in $100 \mathrm{cc}$. of distilled water and the solution is saturated with iodine.

This reagent is indispensable for the identification of starch, especially when the latter is present in minute quantities. Starch granules when moistened with water are turned blue by iodine, the reaction being exceedingly delicate under the microscope, even when the starch granules are very minute and insignificant without the reagent.

Iodine in connection with sulphuric acid is also useful in distinguishing pure cellulose from its various modifications, such as lignin and suberin. For this purpose the water-mounted sample is first permeated with the iodine reagent, after which concentrated sulphuric acid is applied, with the result that all pure cellulose is turned a deep-blue color, while the modified forms of cellulose are colored yellow or brown. The cellulose is first converted by the sulphuric acid into a carbohydrate isomeric with starch, known as amyloid.

Protein grains are colored brown or yellow brown by the action of iodine.

Chloriodide of Zinc.-Pure zinc is dissolved in concentrated hydrochloric acid to saturation, and an excess of zinc added. The solution is then evaporated to about the consistency of concentrated sulphuric acid, after which it is first saturated with potassium iodide, and finally with iodine.

This reagent may be used instead of sulphuric acid and iodine for the 
detection of cellulose, since the zinc chloride converts the cellulose into amyloid, which the reagent colors blue.

Chloriodide of zinc is useful for detecting tannic acid in cell contents. For this purpose the above reagent is much diluted by the addition of a $20 \%$ solution of potassium iodide. In this diluted form, when applied to the sample, a reddish or violet coloration is imparted to cell contents having tannin.

Phenol-hydrochloric Acid is prepared by saturating concentrated hydrochloric acid with the purest crystallized carbolic acid. Wood fiber, or lignin, when treated with a drop of this reagent under the cover-glass, and exposed for half a minute to the direct sunlight, will be colored an intense green, which quickly fades.

Indol.-Several crystals of indol are freshly dissolved in warm water. Lignified cell walls assume a deep-red color, when the specimen to be examined is treated first with a drop of the indol reagent, and afterwards washed with dilute sulphuric acid, $\mathrm{I}: 4$.

Millon's Reagent.-This is prepared by dissolving metallic mercury in its weight of concentrated nitric acid, and diluting with an equal volume of water. This reagent, which should be freshly prepared, 'is of use in testing for protein compounds, which turn brick red when treated with it, especially on gently warming the slide.

Tincture of Alkanna.-A $; 0$ or $80 \%$ alcoholic extract of alkanna root, when kept in contact with resins, fixed oils, fats, or essential oils for a short time, stains these cell contents a lively red. The staining is hastened by the aid of heat. Essential oils and resins are soluble in strong alcohol, while fixed oils and fats are insoluble, hence the distinction between these classes of cell contents may be made by the application of alcohol to the alkanna-stained specimen.

Ferric Chloride, Ferric Acetate, or Ferric Sul phate, used in dilute aqueous solution, are all applicable as reagents for tannic acid, which, when present in appreciable amount, will be colored green or blue by either of these reagents.

B. Clarifying Reagents.-Many of the harder cellular tissues are too opaque for careful examination, and may be rendered transparent by clarifying or bleaching. A portion of the powdered sample is either treated with a drop of the reagent under the cover-glass or is allowed to soak for hours or even days in the reagent, using a drop of the same reagent as a medium for examination on the object-glass, instead of water. The clarifying reagents most commonly used are the following: 
Chloral Hydrale.-A $60 \%$ solution.

Ammonia. - Concentrated, or $28 \%$ ammonia is commonly used.

Polassium Hydroxide, used in various degrees of concentration, often in dilute solution, say $5 \%$. This reagent, added to a water mount, causes swelling of the cell wall, and dissolves intercellular substances and protein. It bleaches most of the coloring matters, destroys the starch, and forms soluble soaps with the fats. Potassium hydroxide is also used in testing for suberin, which is extracted from corky tissue on boiling with the reagent, and appears as yellow drops.

Schultze's Macerating Reagent (concentrated nitric acid and chlorate of potassium) is best used by placing the powder or bit of tissue to be treated in a test-tube with an equal volume of potassium chlorate crystals, adding about 2 cc. of concentrated nitric acid, and warming the tube till bubbles are evolved freely, or until the necessary separation of cells is effected. The sample is then removed and washed with water.

By this treatment, bast and wood fibers as well as stone cells are readily separated from other tissues.

Cuprammonia (Schweitzer's Reagent).-This is prepared by adding slowly a solution of copper sulphate to an aqueous solution of sodium hydroxide, forming a precipitate of cupric hydroxide, which is separated by filtration, washed, and dissolved in concentrated ammonia. It should be freshly prepared, and is never fit for use unless it is capable of immediately dissolving cotton. Indeed its chief use is as a test for cellulose, which it readily dissolves. In observing this reaction under the microscope, the powdered specimen under the cover-glass should be only slightly damp before a drop of the fresh reagent is applied. The cell walls are seen to swell up and gradually become more and more indistinct, till they finally disappear.

Cuprammonia is also used as a test for pectose, which occurs in many cell walls, often intermixed with cellulose. When treated with this reagent, cellular tissue containing pectose is acted upon in such a manner that a delicate framework of cupric pectate is sometimes left behind, after the dissolution of the cellulose with which it is mingled.*

\section{PHOTOMICROGRAPHY.}

The photomicrograph serves as a simple means of keeping permanent records of unusual forms of adulteration encountered in the course of routine examination. Besides this, the photomicrograph has at times proved its usefulness as a means of evidence in court, showing as it does with faithfulness the presence of a contested adulterant. It is true

* Poulsen, Botanical Micro-chemistry, p. 15. 
that from an artistic standpoint the photomicrograph of a powdered sample is often disappointing, due to the fact that ordinarily much of the field is out of focus, unless a very simple homogeneous subject is photographed, as, for instance, starch. As compared with the carefully prepared drawing of a section, which is usually idealized, the photomicrograph is in a sense the more truthful representation.

SUMMARY OF MICROCHEMICAL REACTIONS FOR IDENTIFYING

CELLULAR TISSUE AND CELL CONTENTS. BASED ON BEHRENS',*

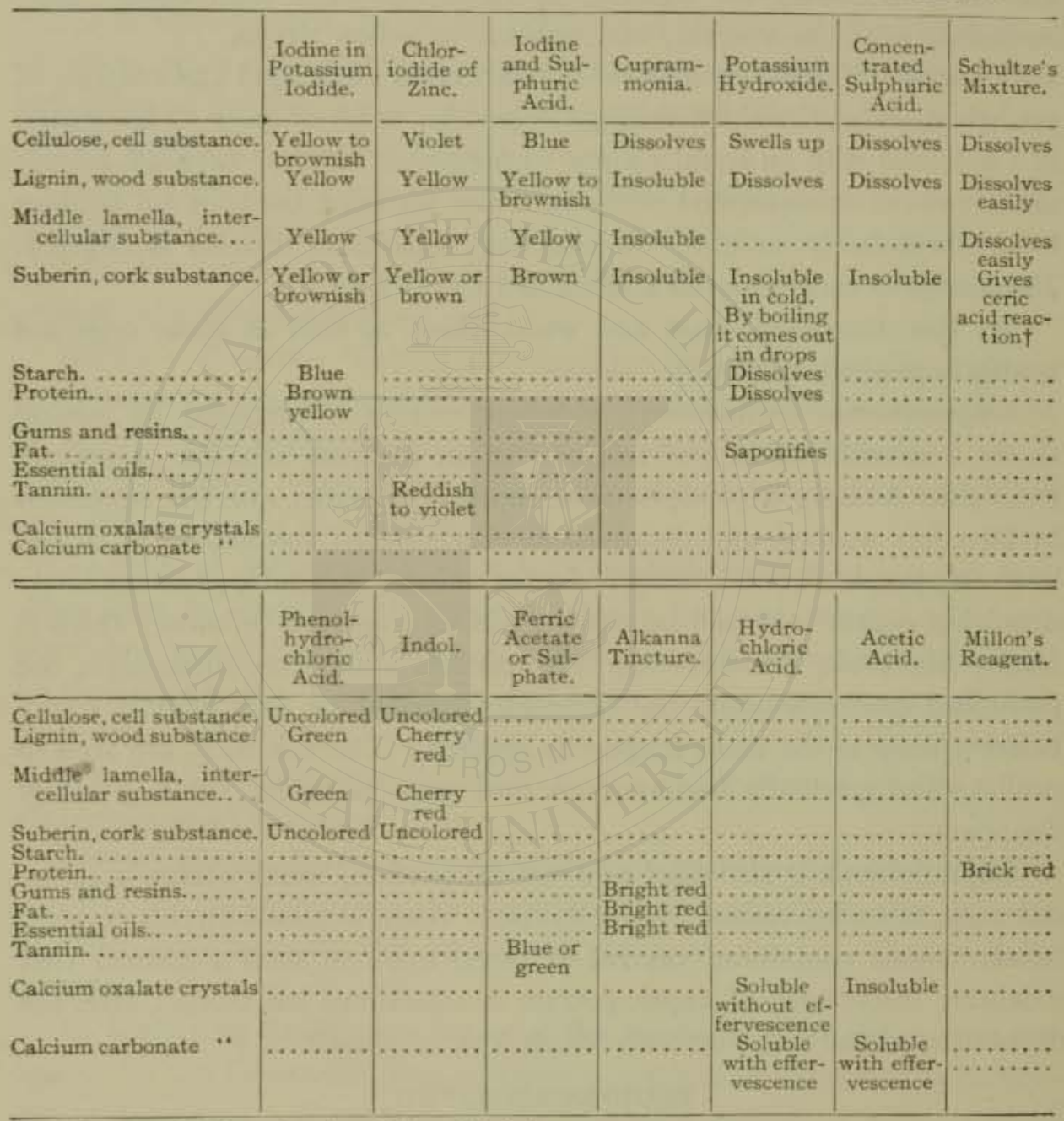

* Microscopical Investigation of Vegetable Substances, page 356.

+ When treated with the reagent, suberin forms masses of ceric acid, saluble in ether, alcohol, or chlaroform.

While the analyst examines microscopically the ordinary powdered spice, for example, he constantly moves with his hand the fine adjustmentscrew, bringing into focus different parts of the field successively. This 
he does unconsciously, so that he does not realize how far from flat the field actually is till he undertakes to photograph it, when, as a rule, only a small portion is in good focus. It is therefore impossible in one photograph to show successfully many varied forms of tissue or cell contents in the powder, but separate photographs shouid be made as far as possible with only a little in each. Thus, for example, with a composite subject like powdered cassia bark, it would be very difficult to show starch, stone cells, and bast fibers in one field, all in equally good focus, and, for the best results only, one, or at most two, such varied groups of elements should be shown in one picture.

Appurtenances and Methods of Procedure.-The temporary method of water-mounting employed by the analyst in routine examination presents many difficulties from a photographic point of view. The vibrating motion of the particles is very annoying, and some skill is required in using just the right amount of water, in avoiding air-bubbles, in waiting the requisite amount of time before exposing the plate for the vibratory motion to cease, and, on the other hand, avoiding too long delay, which would result in the evaporation of the water, and the consequent breaking up of the field. In the writer's experience, however, in spite of these difficulties, the water-mounting gives decidedly the clearest results, and, with patience on the part of the operator, it is in many ways the most desirable method of mounting for photographic purposes. It is in fact the method employed in making most of the photomicrographs of powdered specimens that appear in the plates at the end of this volume, though a few were mounted in glycerin jelly, and the starches for the polarized-light pictures in Canada balsam. The sections of tissues shown in the plates were mounted some in glycerin and others in glycerin jelly.

Experience has shown that two degrees of magnification well calculated to bring out the chief characteristics of the spices and their adulterants in a photomicrograph are 125 and 250 diameters. The starches, which are the most common of any one class of adulterants, vary very widely in the size of their granules. With these the larger magnification of $25^{\circ}$ has been found satisfactory. while the general appearance of the composite ground-spice itself under the microscope, as well as that of such adulterants as ground bark, sawdust, chicory, pea hulls, and the like, is best shown with the lower power of $125 . *$

* The degrees of magnification adopted in the originals of most of the photomicrographs illustrated in the accompanying plates are accordingly 125 and 250 , but in the process of lithographing, the photographs were slightly reduced, so that the actual scales in the reproduction are 110 and 220 respectively. 
The object, mounted in the manner above described, is best examined when held in a mechanical stage, furnished with micrometer adjustments, in such a manner that a typical field may be selected and held in place long enough to photograph.

The Camera.-This need not of necessity be complicated, but may consist simply of a horizontal wooden base on which the microscope

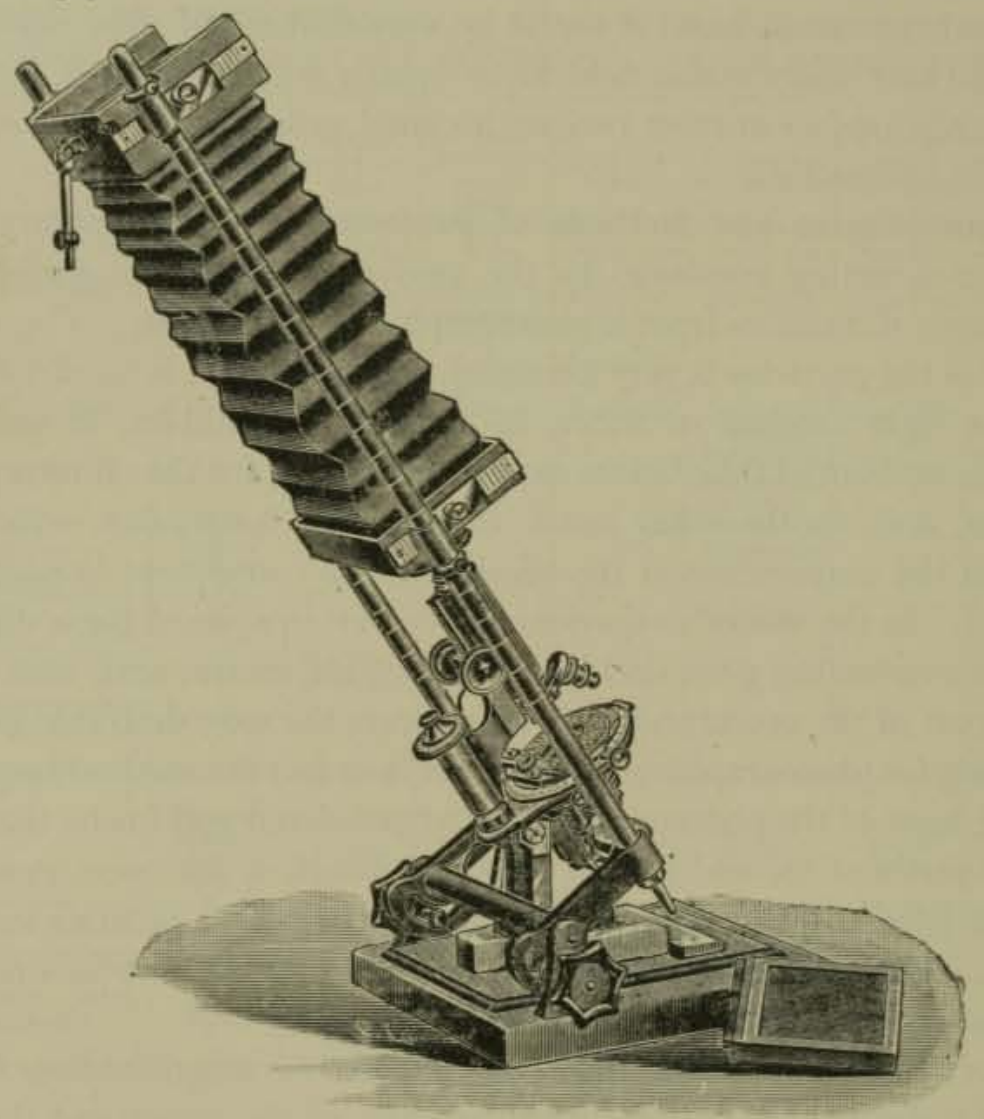

Fig 37a.-A Convenient Photomicrographic Camera.

rests, and an upright board firmly secured to the base, carrying a frame for an interchangeable ground glass and plate-holder, with a rubber gauze skirt hanging from the frame, adapted to be gathered and tied about the top of the microscope-tube. Means are further provided, as by a slotted guide and screw, for adjusting the frame at any desired height on the upright board.*

A more convenient form of apparatus now employed by the writer is that shown in Figs. $37 a$ and $37 b$.

* Such a contrivance as this was employed in making some of the accompanying photomicrographs. 
The base is a solid iron plate upon which the microscope rests (any microscope may be used with this camera), and above which the camera bellows is supported on two solid steel rods. The bellows is supported at either end on wooden frames.

The ground glass is provided with a central transparent area, formed by cementing a cover-glass upon the ground glass, and permits the accurate focusing of the most delicate detail by means of a hand magnifying-glass. The vertical rods supporting the bellows are attached to metal arms, immovably fixed to a horizontal axis, thus permitting the camera to be tilted

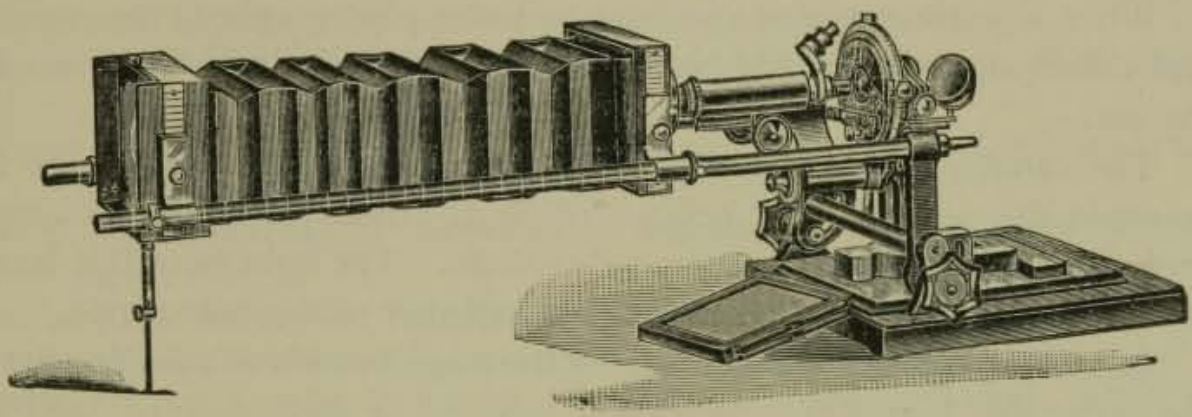

Fig $37 b,-$ Photomicrographic Camera in Horizontal Position

to any angle from vertical to horizontal. It is fixed at the desired angle by means of heavy hand-clamps.

In use the camera is placed in a vertical position and the microscope adjusted on the base so that its tube will coincide with the opening in the front of the camera. The connection between microscope and camera is made light-tight by means of a double chamber, which permits considerable vertical motion of the tube of the microscope without readjustment. A jointed telescoping rod is attached to the upper end of the camera to act as a support, giving perfect steadiness when in a horizontal position, and folding down parallel with the bellows so as to be out of the way when in any other position.

Amplification.-The vertical rods are graduated in inches for determining the amount of amplification, and to show when the ground glass is at right angles to the optical axis. The following simple rule for determining the amount of amplification will give sufficiently accurate results. When photographing without the eyepiece, divide the distance of the ground glass from the stage of the microscope in inches, by the focal length in inches of the objective used. When photographing with the eyepiece, proceed as above and multiply the result by the quotient obtained by dividing to by the focus in inches of the eyepiece used. 
Adjustment and Manipulation.-The microscope can be placed in any position desired, and the camera adjusted to it. The bellows can then be raised and the microscope used as though no camera were present. When an object is to be photographed, the bellows may be slid into position without in any way disturbing the arrangement of light or object, the final focusing on the ground glass being effected quickly by means of the fine adjustment-screw of the microscope. The exposure having been made, observation through the microscope may be continued without interruption by simply raising the bellows again.

When a water-mounted specimen is to be photographed, the camera and microscope tube should be vertical instead of inclined as shown in the cut.

The camera is best kept in a dark room where the exposures are to be made, the source of light being a 16 - or 32-candle-power electric lamp, preferably provided with a ground-glass bulb. The light from this lamp is first carefully centered by moving the reflector of the microscope.

In making pictures, for instance, of the magnification of 250 diameters, the objective, having an equivalent focus of $\frac{1}{6}$ inch, may be used in combination with the one-inch ocular, with the ordinary tube length of microscope. For a lower power, such as 125 diameters, the same objective is employed, but the eyepiece is left out, it being found necessary in this case to remove the upper tube of the microscope, which ordinarily carries the eyepiece, as otherwise the size of the field to be photographed would be restricted. In each case a diaphragm is used in the microscope stage, having an opening of about the same size as that of the front lens of the objective. By means of a stage micrometer scale, the proper position of the camera back is previously determined to give the required magnification.

\section{REFERENCES ON THE MICROSCOPE IN FOOD ANALYSIS.}

Alltmann. Die Elementarorganismen und ihre Beziehungen zu den Zellen. Leipzig, I89o.

BeHrens, J. W. Guide to the Microscope in Botany. Translated by Hervey. Boston, I885.

Bergen, J. Elements of Botany. Gross and Microscopic Structure. Vegetable Histology.

Bessey, C. E. The Essentials of Botany.

Botany for High Schools and Colleges. New York, 1880.

Bonsfiet. D, E. C. Guide to Photomicrography. London.

Chambertan, C. J. Vegetable Tissues.

Methods in Plant Histology. Chicago, 1905. 
ClARK, C. H. Practical Methods in Microscopy, rgoo.

Dammar, O. Illustrirtes Lexicon der Verfälschungen und Verunreinigungen der Nahrungs- und Genussmittel. Leipzig, 1886.

Detmer, W. Das pflanzenphysiologische Praktikum. Jena, r885.

DIETSCH, O. Die wichtigsten Nahrungsmittel und Getränke, deren Verunreinigungen und Verfälschungen. Zurich, 1884 .

GAGE, S. H. The Microscope and Microscopical Methods. Ithaca, 1908.

Greenish, H. G. The Microscopical Examination of Foods and Drugs. Philadelphia, I9I I.

Hanausek, T. F. The Microscopy of Technical Products. Translated by A. L. Winton and Kate G. Barber. New York, 1907.

HAUSHOFER, K. Mikroskopische Reaktionen. Braunschweig, I885. $_{5}$.

HEGLER. Histochemische Untersuchungen verholtzer Zellmembranen. Flora, 189o, page $3^{1 .}$

Hoffmeister, T. Die Rohfaser und einige Formen der Cellulose. Landwirtschaftl. Jahrbücher, 1888, page 239 .

Kосн, L. Mikrotechnische Mittheilungen. Pringsheim's Jahrbücher, Bd. XXIV, page $\mathrm{I}, \mathrm{I} 892$.

Kraemer, H. Botany and Pharmacognosy. Philadelphia, I9ro.

Kraus, G. Zur Kentniss der Chlorophyllfarbstoffe. Stuttgart, I872.

LANGe, G. Zur Kentniss des Lignins. Zeits. für physiologische Chemie. Bd. XIV, page 15.

LEAch, A. E. Microscopical Examination of Foods for Adulteration. An. Rep. Mass. State Board of Health, 1900, p. 679.

LeE, A. B. The Microtomist's Vade Mecum. I893.

MACÉ, E. Les Substances Alimentaire Etudiés au Microscope. Paris, I8gr.

Moeltfr, J. Mikroskopie der Nahrungs- und Genussmittel aus dem Pflanzenreiche. Berlin, 1905.

- Pharmacognostischer Atlas. Berlin, 1892 .

Molisch. Grundriss einer Histochemie der pflanzlichen Genussmittel. Jena, I89r.

NevHauss, R. Lehrbuch der Mikrophotographie. Braunschweig, 1890 .

Poulsen, V. A. Botanical Microchemistry, translated by Trelease. Boston, r 886.

Pringle, A. Practical Photomicrography. New York, 1890.

SCHIMPER, A. F. W. Mikroskopischen Untersuchungen der Nahrungs- und Genussmittel. Jena, rgoo.

Strassburger, E. Manual of Vegetable Histology, translated by Hervey. $\quad$ i 887.

Thomas and DUdLey. A Laboratory Manual of Plant Histology.

Tschirch, A., und Oesterle, O. Anatomischer Atlas der Pharmakognosie und Nahrungsmittelkunde. Leipzig, rooo.

VoGt,A.E. Die wichtigsten vegetabilischen Nahrungs- und Genussmittel. Berlin, 1899-

Winstow, C. E. A. Elements of Applied Microscopy. New York, 1905.

Winton, A. L. The Microscopy of Vegetable Foods. New York, 1906.

Wormley, T. G. The Microchemistry of Poisons. Philadelphia, 1885.

Zrmmerman, A. Botanical Microtechnique. New York, 1893.

- Die Morphologie und Physiologie der Pflanzenzelle. Breslau, I887.

Beiträge zur Morphologie und Physiologie der Pflanzenzelle. Tübingen, r8go. 


\section{CHAPTER VI.}

\section{THE REFRACTOMETER.}

THE refractive index ranks in importance with the specific gravity as a means of determining the identity and purity of various food products, as well as of estimating the percentage of valuable constituents. Various forms of refractometer are used in food analysis.

The Abbé refractometer is of general application in determining the refractive index of fats, fatty oils, waxes, and essential oils, in estimating the solids in saccharine substances, and in other analytical operations. It employs but a few drops of the material, and reads the refractive index directly, using ordinary white light.

The immersion refractometer, an instrument of recent introduction, is suited for the examination of milk serum to detect added water therein, the detection and determination of methyl alcohol in ethyl alcohol, and the standardization of a wide variety of solutions. The instrument is immersed directly in the liquid to be examined, the degree of refraction being indicated on an arbitrary scale.

The Pulfrich is used with the sodium light, and requires a larger amount of material than the Abbé, the liquid being held in a cylinder above the prism. The scale reading is in angular degrees, from which the index of refraction is calculated by a formula or from a table. The instrument is provided with a temperature-controlling apparatus.

In the Amagat and Jean or oleo-refractometer, an outer and an inner cylinder are respectively filled with an oil of known value or purity, and with the oil to be examined. By the comparative displacement to the right or left of a beam of white light passing through both cylinders, the displacement being read in degrees on an arbitrary scale, the refraction of an oil may be measured. Two oils may thus be readily compared under the same conditions, one of known purity, for example, with a doubtful sample of the same kind.

The butyro-refractometer and the Wollny milk fat refractometer (p. I39) are, as their names imply, instruments primarily intended for more restricted fields of work than the foregoing. They involve the same principle as the Abbé, but are simpler in construction and have arbitrary scales.

Unless such widely varying substances as the waxes and the essential oils are to be studied, the Zeiss butyro-refractometer, though primarily 
intended for the examination of butter and lard, answers most of the purposes of the Abbé instrument with the advantage of greater simplicity, being equally well adapted for examining all the common edible oils and fats, as well as other materials.

\section{THE ZEISS BUTYRO-REFRACTOMETER.}

This instrument (shown in Fig. $3^{8}$ ) is so constructed that the degree of refraction of a beam of light, which passes obliquely through a thin

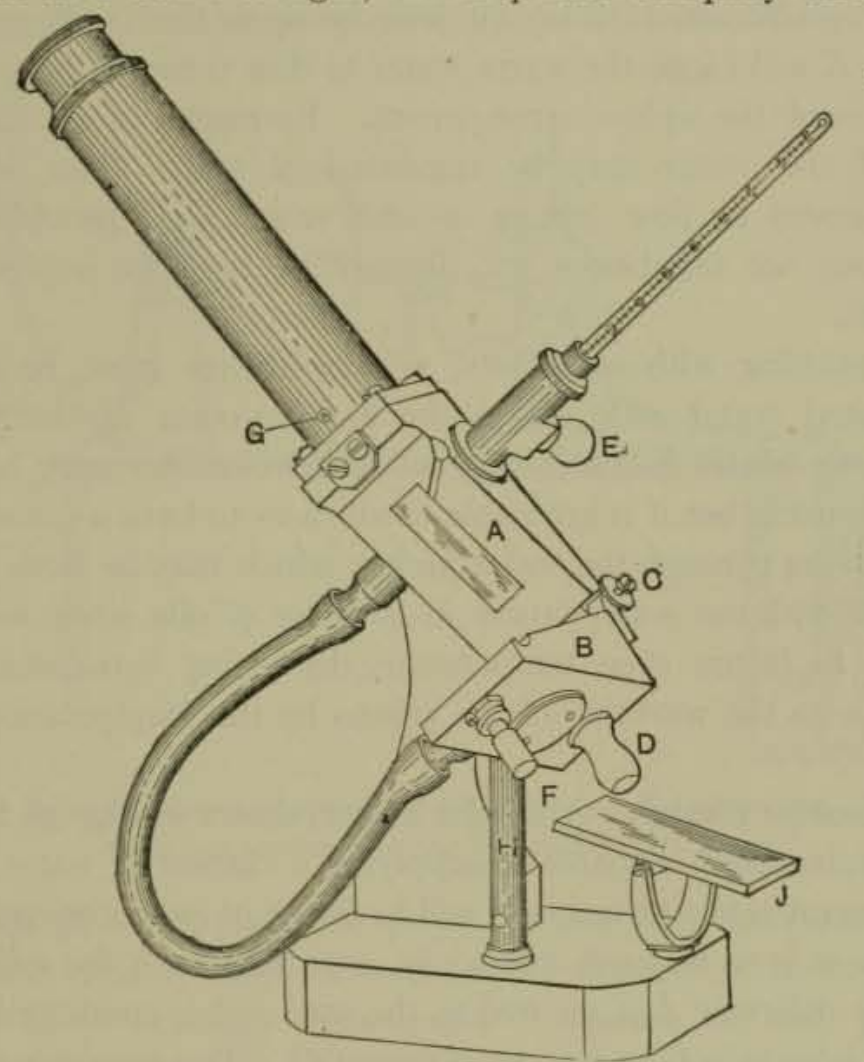

FIG. 38.-The Zeiss Butyro-refractometer.

film of the fat, is read on an arbitrary scale of sufficient extent to cover the widest limits of deviation possible for butter fat and oleomargarine under ordinary temperatures.

The graduation is in divisions from I to 100 , covering a variation in refractive indices of from I.4220 to $1.4895 . A$ and $B$ are the two hinged hollow portions of the prism casing of the instrument, so arranged that when closed together the melted fat is held in a film of sufficient thickness between the two opposed transparent prism surfaces, the beam of light, either diffused daylight or lamplight, being reflected through it by means of the mirror $J$. The transparent scale is within the telescope tube at the height indicated by $G$. 
The refractometer is connected to any kind of heating arrangement, which admits of warm water being transmitted through the prism casing, in at $D$ and out at $E$. A simple arrangement, which suffices for all ordinary cases, may expeditiously be improvised in the following manner: Fill a vessel of say 2 gallons capacity with water of $40^{\circ}$ to $50^{\circ} \mathrm{C}$. Into this vessel dip the free end of an india-rubber tube slipped over the nozzle $D$ and let the vessel be placed at a height of about one-half or one yard above the refactometer. Then it will be seen that suction at a tube attached to $E$ will cause the warm water to flow through the prism casing by the action of the siphon arrangement. By means of a pinch clip the velocity of the water may be regulated at will. The waste water may be allowed to flow into a second vessel and, provided its temperature does not fall below $30^{\circ}$, it may be used for replenishing the upper vessel.

When working with solid fats, a temperature must be maintained by the heated water well above the melting-point of the fat. With liquid oils no heater is necessary, as determinations may be made at room temperature, but it is advisable in all cases to have a constant stream of water passing through the water jacket, which may be done by directly connecting it with the water faucet in the case of oils, since, without such precautions to insure even temperature, disturbing variations are liable to occur, due to the warming of the prisms by the manipulation of cleaning, etc.

Refractometer Heater.-A regular heater, shown in Fig. 39, is furnished by the manufacturers, capable of supplying a current of water of approximately constant temperature, and will be found of great convenience when the instrument is to be used constantly, especially with the solid fats.

A supply reservoir $A$ is secured to the wall and is connected by means of a rubber inlet tube $G$ to the water faucet $C$. The reservoir is provided with a waste overflow pipe and with an outlet tube $D$, the flow through the latter being regulated by the cock $H$. The tube $D$ leads to the spiral heater $H S$, which is heated by a Bunsen burner. From the heater the tube $E$ conducts the warm water through the refractometer, from which it flows through the tube $F$, either directly into the sink, or into the intermediate vessel $B$. The temperature of the water is regulated by adjusting the cock $H$, and the height of the flame of the Bunsen burner.

Manipulation of the Butyro-refractometer.-The prism casing is first opened by giving about half a turn to the right to the pin $F$, Fig. $3^{8}$, until it meets with a stop. Then simply turn the half $B$ of the prism 
casing aside. Pillar $H$ holds $B$ in the position shown in Fig. $3^{8}$. The prism and metallic surfaces must now be cleaned with the greatest care, the best means for this purpose being soft linen, mosstened with a little alcohol or benzine.

If the sample to be examined is a solid fat, maintain the temperature above the melting-point, and apply by a glass rod a drop or two of the clear melted fat (filtered if turbid) to the surface of the prism contained in the casing $B$. For this purpose the apparatus should be raised with

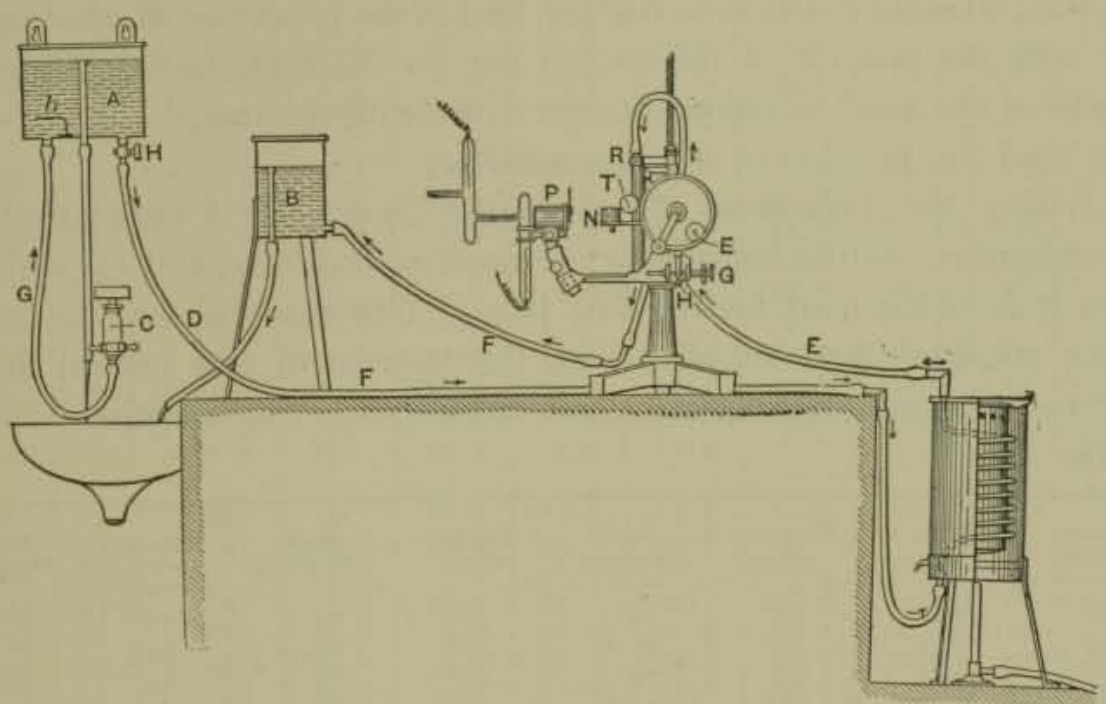

Fic. 39.-The Zeiss Heating Apparatus for all Forms of Refractometer. Shown in the cut in connection with the Pulfrich refractometer.

the left hand so as to place the prism surface in a horizontal position. A liquid oil is directly applied in the same manner without preliminary precautions as to heating. Now press $B$ against $A$, and place $F$ by turning it in the opposite direction, in its original position; thereby $B$ is prevented from falling back, and both prism surfaces are kept in close contact. Place the instrument again upon its sole plate.

While looking into the telescope, give the mirror $J$ such a position as to render the critical line, which separates the bright left part of the field from the dark right part, distinctly visible. It may also be necessary to move or turn the instrument about a little. First it will be necessary to ascertain whether the space between the prism surfaces be uniformly filled with oil or fat, failing which the critical line will not be distinct. For this purpose examine the rectangular image of the prism surface formed about I $\mathrm{cm}$. above the ocular with a hand magnifier or with the 
naked eye, placing the latter at its proper distance from the ocular. Finally adjust the movable front part of the ocular so that the scale becomes clearly visible.

By allowing a current of water of constant temperature to flow through the apparatus some time previous to the taking of the reading, the at first somewhat hazy critical line approaches in a short time, generally after a minute, a fixed position, and quickly attains its greatest distinctness. When this point has been reached, note the appearance of the critical line (i.e., whether colorless or colored, and in the latter case of what color); also note the position of the critical line on the centesimal scale, which admits of the tenth divisions being conveniently estimated; at the same time read the position of the thermometer.

Testing the Adjustment of the Ocular Scale.-It is imperative that the adjustment of the instrument be tested periodically, and in particular when it is being used for the first time. This may be done by means of the standard fluid supplied with the instrument, the critical line of which is approximately colorless, and must occupy the following positions in the scale.

\begin{tabular}{|c|c|c|c|c|c|c|c|}
\hline $\begin{array}{l}\text { Temper- } \\
\text { ature. }\end{array}$ & $\begin{array}{c}\text { Scale } \\
\text { Division. }\end{array}$ & $\begin{array}{l}\text { Temper- } \\
\text { ature. }\end{array}$ & $\begin{array}{c}\text { Scale } \\
\text { Division. }\end{array}$ & $\begin{array}{l}\text { Temper- } \\
\text { ature. }\end{array}$ & $\begin{array}{c}\text { Scale } \\
\text { Division. }\end{array}$ & $\begin{array}{l}\text { Temper- } \\
\text { ature. }\end{array}$ & $\begin{array}{c}\text { Scale } \\
\text { Division. }\end{array}$ \\
\hline $30^{\circ}$ & $68 . \mathrm{I}$ & $25^{\circ}$ & $7^{1} \cdot 2$ & $20^{\circ}$ & $74 \cdot 3$ & $15^{\circ}$ & $77 \cdot 3$ \\
\hline $29^{\circ}$ & 68.7 & $24^{\circ}$ & 71.8 & $\mathrm{Ig}^{\circ}$ & 74.9 & $14^{\circ}$ & 77.9 \\
\hline $28^{\circ}$ & $69 \cdot 3$ & $23^{\circ}$ & 72.4 & $18^{\circ}$ & $75 \cdot 5$ & $13^{\circ}$ & 78.6 \\
\hline $27^{\circ}$ & 70.0 & $22^{\circ}$ & 73.0 & $17^{\circ}$ & 76.1 & $12^{\circ}$ & 79.2 \\
\hline $26^{\circ}$ & 70.6 & $21^{\circ}$ & 73.6 & $16^{\circ}$ & 76.7 & $11^{\circ}$ & 79.8 \\
\hline $25^{2}$ & 71.2 & $20^{\circ}$ & $74 \cdot 3$ & $15^{\circ}$ & $77 \cdot 3$ & $10^{\circ}$ & 80.4 \\
\hline
\end{tabular}

The fractional parts of a degree can accordingly easily be brought into calculation $(0.1=0.06$ scale div.). Deviations of $I$ to 2 decimals of the scale divisions are of no consequence, and are in most cases due to inexact determinations of temperature. Should, however, careful tests result in the discovery of greater deviations, readjustment of the scale will be necessary, which may be effected by means of a watch-key supplied with the instrument, in accordance with the values given in the above table. The watch-key is inserted at $G$ in Fig. $3^{8}$, and by its means the position of the objective, and therefore that of the critical line with respect to the scale may be altered.

Transformation of Scale Divisions into Indices of Refraction.-The following table, adapted from that of Pulfrich, enables one to convert scale readings on the butyro-refractometer into indices of refraction, $n_{D}$, and vice versa: 
EQUIVALENTS OF INDICES OF REFRACTION AND BUTYRO-REFRACTOMETER READINGS.

\begin{tabular}{|c|c|c|c|c|c|c|c|c|c|c|}
\hline \multirow{2}{*}{$\begin{array}{c}\text { Refrac- } \\
\text { tive } \\
\text { Index. } \\
{ }^{n} D .\end{array}$} & \multicolumn{10}{|c|}{ Fourth Decimal of $n_{D}$. } \\
\hline & 0 & 1 & 2 & 3 & 4 & 5 & 6 & 7 & 8 & 9 \\
\hline & \multicolumn{10}{|c|}{ SCALE READINGS. } \\
\hline 1.422 & 0.0 & $0 . \mathrm{I}$ & 0.2 & 0.4 & 0.5 & 0.6 & 0.7 & 0.9 & 1.0 & I. $\mathrm{I}$ \\
\hline 1.423 & I. 2 & 1.4 & I. 5 & 1.6 & 1.7 & 1.9 & 2.0 & 2.1 & 2.2 & 2.4 \\
\hline I. 424 & 2.5 & 2.6 & 2.7 & 2.8 & 3.0 & 3.1 & 3.2 & $3 \cdot 3$ & 3.5 & 3.6 \\
\hline I. 425 & $3 \cdot 7$ & 3.8 & 4.0 & $4 \cdot 1$ & $4 \cdot 2$ & $4 \cdot 3$ & 4.5 & 4.6 & $4 \cdot 7$ & 4.8 \\
\hline 1.426 & 5.0 & 5.1 & 5.2 & $5 \cdot 4$ & 5.5 & 5.6 & $5 \cdot 7$ & 5.9 & 6.0 & 6. 1 \\
\hline 1.427 & 6.2 & 6.4 & 6.5 & 6.6 & 6.8 & 6.9 & 7.0 & $7 \cdot 1$ & 7.2 & $7 \cdot 4$ \\
\hline I. 428 & $7 \cdot 5$ & 7.6 & $7 \cdot 7$ & 7.9 & 8.0 & 8.1 & 8.2 & 8.4 & 8.5 & 8.6 \\
\hline I. 429 & 8.7 & 8.9 & 9.0 & 9.1 & 9.2 & $9 \cdot 4$ & $9 \cdot 5$ & 9.6 & 9.8 & 9.9 \\
\hline I. 430 & 10.0 & I0. I & 10.3 & 10.4 & 10.5 & 10. 6 & I0. 7 & 10.9 & II.O & II. I \\
\hline I. $43 \mathrm{I}$ & II. 3 & II. 4 & II. 5 & II. 6 & 11.8 & 11.9 & 12.0 & 12.2 & 12.3 & 12.4 \\
\hline I. $43^{2}$ & 12.5 & 12.7 & 12.8 & 12.9 & 13.0 & I 3.2 & 13.3 & 13.5 & 1 3.6 & 13.7 \\
\hline I. 433 & 13.8 & 14.0 & 14.1 & 14.2 & 14.4 & 14.5 & I 4.6 & 14.7 & 14.9 & 15.0 \\
\hline I. 434 & 15.1 & 15.3 & I5.4 & 15.5 & 15.6 & 15.8 & 15.9 & 16.0 & 16.2 & 16.3 \\
\hline I. 435 & 76.4 & x6.6 & 16.7 & 16.8 & 17.0 & $\mathrm{I} 7 \cdot \mathrm{I}$ & 17.2 & 17.4 & $17 \cdot 5$ & 17.6 \\
\hline I. $43^{6}$ & 17.8 & 17.9 & 18.0 & 18.2 & 18.3 & 18.4 & 18.5 & 18,7 & 18.8 & 18.9 \\
\hline I. 437 & 19.1 & 19.2 & 19.3 & 19.5 & 19.6 & 19.7 & 19.8 & 20.0 & 20.1 & 20.3 \\
\hline I. $43^{8}$ & 20.4 & 20.5 & 20.6 & 20.8 & 20.9 & 21.1 & 21.2 & $2 \mathrm{r} \cdot 3$ & 21.4 & 21.6 \\
\hline 1. 439 & 21.7 & 21.8 & 22.0 & $22 . \mathrm{I}$ & 22.2 & 22.4 & 22.5 & 22.6 & 22.7 & 22.9 \\
\hline I. 440 & 23.0 & 23.2 & $23 \cdot 3$ & 23.4 & 23.5 & 23.7 & 23.8 & 23.9 & $24 . I$ & 24.2 \\
\hline I. $44 \mathrm{I}$ & $24 \cdot 3$ & 24.5 & 24.6 & 24.7 & 24.8 & 25.0 & $25 \cdot \mathrm{I}$ & 25.2 & 25.4 & 25.5 \\
\hline I. 442 & 25.6 & 25.8 & 25.9 & $26 \cdot x$ & 26.2 & 26.3 & 26.5 & 26.6 & 26.7 & 26.9 \\
\hline I. 443 & 27.0 & $27 \cdot 1$ & $27 \cdot 3$ & $27 \cdot 4$ & 27.5 & $27 \cdot 7$ & 27.8 & 27.9 & $28 . \mathrm{I}$ & 28.2 \\
\hline I. 444 & 28.3 & 28.5 & 28.6 & 28.7 & 28.9 & 29.0 & 29.2 & $29 \cdot 3$ & 29.4 & 29.6 \\
\hline I. 445 & 29.7 & 29.9 & 30.0 & 30.1 & 30.3 & 30.4 & 30.6 & 30.7 & 30.8 & 30.9 \\
\hline I. 446 & $3 \mathrm{I}=\mathrm{I}$ & $3 x \cdot 2$ & 31.4 & $3 \mathrm{t}-5$ & 31.6 & 31.8 & $3 \mathrm{I}-9$ & $3^{2} \cdot 1$ & $3^{2} .2$ & 32.3 \\
\hline I. 147 & $32 \cdot 5$ & 32.6 & $7^{2} .8$ & $3^{2} \cdot 9$ & 33.0 & 33.2 & $33 \cdot 3$ & 33.5 & 33.6 & 33.7 \\
\hline 1. 448 & 33.9 & 34.0 & $3+\cdot 2$ & $34 \cdot 3$ & $34 \cdot 4$ & 34.6 & $34-7$ & 34.9 & 35.0 & $35 \cdot \mathrm{r}$ \\
\hline I. 449 & $35 \cdot 3$ & $35 \cdot 4$ & 35.6 & $35 \cdot 7$ & 35.8 & 36.0 & $36 . \mathrm{r}$ & $3^{6} \cdot 3$ & $3^{6.4}$ & $3^{6} \cdot 5$ \\
\hline I. 450 & $3^{6} \cdot 7$ & 36.8 & $37 \cdot 0$ & $37 \cdot I$ & $37 \cdot 2$ & $37 \cdot 4$ & $37 \cdot 5$ & $37 \cdot 7$ & 37.8 & 37.9 \\
\hline $\mathrm{I} .45 \mathrm{I}$ & $3^{8} \cdot 1$ & $3^{8.2}$ & $3^{8} \cdot 3$ & $3^{8} .5$ & $3^{8} .6$ & $3^{8} .7$ & 38.9 & 39.0 & 39.2 & $39 \cdot 3$ \\
\hline I. $45^{2}$ & 39.5 & 39.6 & 39.7 & 39.9 & 40.0 & 40.1 & 40.3 & 40.4 & 40.6 & 40.7 \\
\hline I. 453 & 40.9 & 41.0 & $4 \mathrm{~T} . \mathrm{T}$ & $41 \cdot 3$ & $4 \mathrm{~T} .4$ & $4 \mathrm{I} \cdot 5$ & $41 \cdot 7$ & 41.8 & 42.0 & $42 . \mathrm{I}$ \\
\hline I. 454 & $42 \cdot 3$ & 42.4 & 42.5 & 42.7 & 42.8 & 43.0 & $43 \cdot 1$ & $43 \cdot 3$ & 43.4 & 43.6 \\
\hline I. 455 & 43.7 & 43.9 & 44.0 & 44.2 & $44 \cdot 3$ & 44.4 & 44.6 & 44.7 & 44.9 & 45.0 \\
\hline I. $45^{6}$ & $45 \cdot 2$ & 45.3 & $45 \cdot 5$ & 45.6 & $45 \cdot 7$ & 45.9 & 46.0 & 46.2 & 46.3 & 46.4 \\
\hline 1. 457 & 46.6 & 46.7 & 46.9 & 47.0 & $47 \cdot 2$ & $47 \cdot 3$ & $47 \cdot 5$ & 47.6 & 47.7 & 47.9 \\
\hline I. $45^{8}$ & 48.0 & 48.2 & 48.3 & 48.5 & 48.6 & 48.8 & 48.9 & $49 . \mathrm{I}$ & 49.2 & 494 \\
\hline I. 459 & $49 \cdot 5$ & $49 \cdot 7$ & 49.8 & 50.0 & 50.1 & 50.2 & 50.4 & 50.5 & 50.7 & 50.8 \\
\hline I. 460 & 51.0 & $5 I .1$ & 5 T. 3 & 51.4 & $5 x .6$ & $5 \mathrm{r} \cdot 7$ & 51.9 & 52.0 & 52.2 & $5^{2} \cdot 3$ \\
\hline I. $46 \mathrm{r}$ & $5^{2} \cdot 5$ & $5^{2} \cdot 7$ & 52.8 & 53.0 & $53 . \mathrm{I}$ & 53.3 & 53.4 & 53.6 & 53.7 & 53.9 \\
\hline I. 462 & 54.0 & $54 \cdot 2$ & $54 \cdot 3$ & $54 \cdot 5$ & 54.6 & 54.8 & 55.0 & 55.1 & 55.3 & 55.4 \\
\hline I. $46_{3}$ & 55.6 & $55 \cdot 7$ & 55.9 & 56.0 & 56.2 & 56.3 & 56.5 & 56.6 & 56.8 & 56.9 \\
\hline I. 464 & 57.1 & $57 \cdot 3$ & $57 \cdot 4$ & 57.6 & 57.7 & 57.9 & $5^{8} .0$ & $5^{8.2}$ & $5^{8} \cdot 3$ & 58.5 \\
\hline I. 465 & $5^{8} .6$ & $5^{8} .8$ & 58.9 & 59.1 & 59.2 & 59.4 & 59.5 & $59 \cdot 7$ & 59.8 & 60.0 \\
\hline I. 466 & 60.2 & 60.3 & 60.5 & 60.6 & 60.8 & 60.9 & $6 \mathrm{r} . \mathrm{I}$ & 61.2 & $6 \mathrm{r} \cdot 4$ & $6 r .5$ \\
\hline ร. 467 & $61 \cdot 7$ & 61.8 & 62.0 & 62.2 & $62 \cdot 3$ & 62.5 & 62.6 & 62.8 & 62.9 & $63 \cdot r$ \\
\hline 1. 468 & 63.2 & 63.4 & $63-5$ & 63.7 & 63.8 & 64.0 & 64.2 & $64 \cdot 3$ & $64 \cdot 5$ & 64.7 \\
\hline I. 469 & 64.8 & $6_{5} .0$ & 65.1 & 65.3 & $65 \cdot 4$ & 65.6 & $65 \cdot 7$ & 65.9 & 66.1 & 66.2 \\
\hline
\end{tabular}


EQUIVALENTS OF INDICES OF REFRACTION AND BUTYRO-REFRAC TOMETER READINGS-(Continued).

\begin{tabular}{|c|c|c|c|c|c|c|c|c|c|c|}
\hline \multirow{2}{*}{$\begin{array}{c}\text { Rofrac- } \\
\text { tive } \\
\text { Index. } \\
n_{D .}\end{array}$} & \multicolumn{10}{|c|}{ Fourth Decimal of $n_{D}$. } \\
\hline & 0 & 1 & 2 & 3 & 4 & 5 & 6 & 7 & 8 & 9 \\
\hline & \multicolumn{10}{|c|}{ SCALE READINGS. } \\
\hline 1. $47^{\circ}$ & 66.4 & 66.5 & 66.7 & 66.8 & 67.0 & 67.2 & $67 \cdot 3$ & 67.5 & $67 \cdot 7$ & 67.8 \\
\hline $1.47 \mathrm{I}$ & 68.0 & 68.1 & 68.3 & 68.4 & 68.6 & 68.7 & 68.9 & 69.1 & 69.2 & 69.4 \\
\hline 1.472 & $69 \cdot 5$ & $69 \cdot 7$ & 69.9 & 70.0 & 70.2 & 70.3 & 70.5 & 70.7 & 70.8 & 71.0 \\
\hline I. 473 & 71.1 & $71 \cdot 3$ & $7^{1} \cdot 4$ & 71.6 & 71.8 & 71.9 & 72.1 & 72.2 & 72.4 & 72.5 \\
\hline I. 474 & 72.7 & 72.9 & 73.0 & 73.2 & $73 \cdot 3$ & $73 \cdot 5$ & $73 \cdot 7$ & 73.8 & 74.0 & $74 . \mathrm{r}$ \\
\hline I. 475 & $74 \cdot 3$ & $74 \cdot 5$ & 74.6 & $7+.8$ & 75.0 & $75 \cdot \mathrm{I}$ & $75 \cdot 3$ & $75-5$ & 75.6 & 75.8 \\
\hline 1.476 & 76.0 & 76.1 & $76 \cdot 3$ & 76.5 & 76.7 & 76.8 & 77.0 & $77 \cdot 2$ & $77 \cdot 3$ & $77-5$ \\
\hline 1.477 & 77.7 & 77.9 & 78.1 & 78.2 & 78.4 & 78.6 & $7^{8} .7$ & 78.9 & $79 \cdot \mathrm{r}$ & 79.2 \\
\hline $1.47^{8}$ & $79 \cdot 4$ & 79.6 & 79.8 & So.o & 80.1 & 80.3 & 80.5 & 80.6 & So. 8 & 81.0 \\
\hline 1. 479 & 81.2 & $81 \cdot 3$ & 81.5 & 81.7 & 81.9 & 82.0 & 82.2 & 82.4 & 82.5 & 82.7 \\
\hline 1.480 & 82.9 & 83.1 & 83.2 & 83.4 & 83.6 & 83.8 & 83.9 & 84.1 & $84 \cdot 3$ & 84.5 \\
\hline I. $48 \mathrm{t}$ & 84.6 & 84.8 & 85.0 & 85.2 & $85 \cdot 3$ & 85.5 & 85.7 & 85.9 & 86.0 & 86.2 \\
\hline 1. 482 & 86.4 & 86.6 & 86.7 & 86.9 & 87.1 & $87 \cdot 3$ & 87.5 & 87.6 & 87.8 & 88.0 \\
\hline I. 483 & 88.2 & 88.3 & 88.5 & 88.7 & 88.9 & 89.1 & 89.2 & 89.4 & 89.6 & S9.8 \\
\hline I. 484 & 90.0 & 90.2 & 90.3 & 90.5 & 90.7 & 90.9 & $91 . I$ & $9 \pi .2$ & $9 \pi .4$ & 91.6 \\
\hline I. 485 & 91.8 & 92.0 & 92.1 & 92.3 & 92.5 & $9^{2.7}$ & $92-9$ & 93.0 & 93.2 & 93.4 \\
\hline 1.486 & 93.6 & 93.8 & 94.0 & 94.1 & $94 \cdot 3$ & $94 \cdot 5$ & 94.7 & 94.8 & & 95.2 \\
\hline 1.487 & 95.4 & 95.6 & 95.8 & 96.0 & $96 . \mathrm{x}$ & 96.3 & 96.6 & $9^{6.7}$ & 96.9 & 97.0 \\
\hline 1.488 & 97,2 & 97.4 & 97,6 & 97.8 & 98.0 & $98 . \mathrm{I}$ & $98 \cdot 3$ & $9^{8} \cdot 5$ & $9^{8} \cdot 7$ & $9^{8.9}$ \\
\hline 1.489 & 99.1 & 99.2 & 99.4 & 99.6 & 99.8 & 100.0 & & & & \\
\hline
\end{tabular}

The Critical Line.-It should be remembered that the instrument is primarily intended for use with butter, and that the prisms are so constructed that the critical line of pure butter is colorless, while various other fats and oils, notably oleomargarine, which have greater dispersive powers than natural butter, show a colored critical line. When too great dispersion is apparent to render possible an accurate reading, or when the critical line presents very broad fringes, as with linseed oil, poppyseed oil, turpentine, etc., use a sodium light, obtained by the application of table salt to the Bunsen gas flame, or the diffused daylight may be reflected in the mirror through a flat bottle filled with a dilute solution of potassium bichromate, to give a yellow light.

The advantages of the refractometer for examination of fats and oils consist in the convenience with which very accurate determinations of the refractive index may be made at any temperature between $10^{\circ}$ and $50^{\circ} \mathrm{C}$., inclusive of thermal variations of refractive powers, and also in the possibility which it affords of distinguishing substances by their different dispersive powers, rendered visible by the different coloring of the critical line, a red-colored critical line being indicative of a relatively low dispersive power, a blue line of relatively high dispersion. 


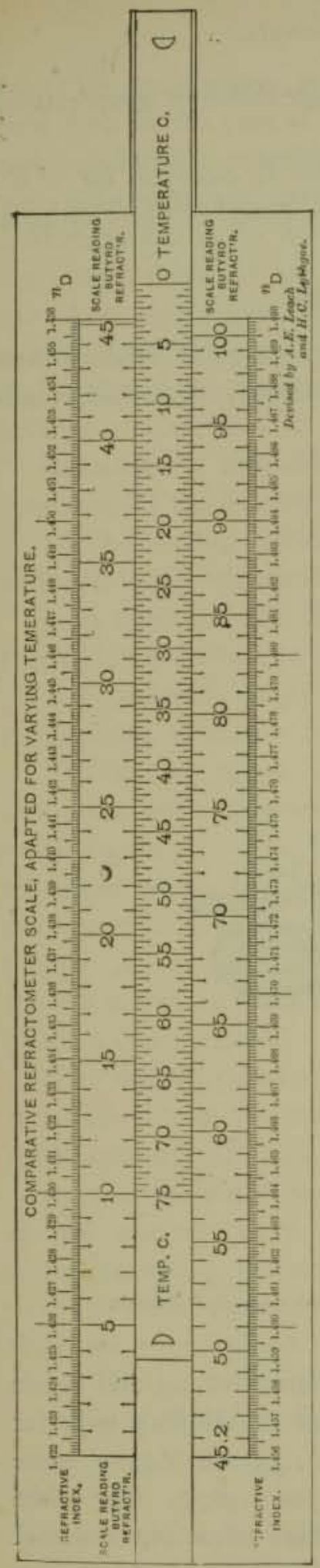

Fig. 40.-Comparative $\mathrm{Re}$ fractometer Scale.

Variation of Reading with the Temperature.No definite temperature has been adopted as a standard for readings of this instrument, but it is easy to reduce readings at any temperature to terms of any other temperature for purposes of comparison. While the change in index of refraction for $\mathrm{I}^{\circ} \mathrm{C}$. is the same whatever the temperature, as Tolman and Munson have pointed out,* the change in scale reading per $\mathrm{I}^{\circ} \mathrm{C}$. decreases as the temperature increases, and varies slightly with different oils. For correcting reading $R^{\prime}$ at a temperature $T^{\prime}$ to a reading $R$ at temperature $T$, their formula is $R=R^{\prime}-X(T-$ $\left.T^{\prime}\right), X$ being the change in scale reading due to change of $\mathrm{I}^{\circ} \mathrm{C}$. in temperature.

For butter, oleomargarine, beef tallow, lard, and other fats reading from $40^{\circ}$ to $50^{\circ}$ or thereabouts on the scale, $X=0.55$. For oils readin between $60^{\circ}$ and $70^{\circ}$, like olive, mustard, rapeseed, cottonseed, peanut, etc., $X=0.58$, and for oils read ing between $70^{\circ}$ and $80^{\circ}$, like corn oil, $X=0.62$.

The slide rul $†$ shown in Fig. 40, for use with the refractometer, has been jointly devised by $\mathrm{H}$, C. Lythgoe and the writer, to render unnecessary the use of tables or formulas. The extreme upper and lower scale divisions indicate indices of refraction, and adjacent to these are the scale divisions indicating readings on the butyrorefractometer. By comparison, therefore, the values of either the Abbé or the butyro scale may be readily ascertained in terms of the other.

The sliding scale, expressing temperature readings in degrees centigrade, is intended to be used in connection with the adjacent scale of butyro-refractometer readings, to readily express the butyro-scale reading of any fat or oil taken at a given temperature, in terms of that at any other temperature. This is frequently convenient London.

* Jour. Am. Chem. Soc., XXIV, p. 755.

rs. Baird and Tatlock, Ltd., 14 Cross Street, Hatton Garden, 
in comparing the work of various observers, where different temperatures have been employed.

The correction for change in $n_{D}$ on the scale is 0.000365 for $I^{\circ} \mathrm{C}$., being based on the experimental work of Tolman, Long, Proctor, Lythgoe, and the author.

\section{THE ABBÉ REFRACTOMETER.}

This instrument, Fig. $4 \mathrm{r}$, has a much wider range in reading than either the butyro or the Wollny instruments already described, read-

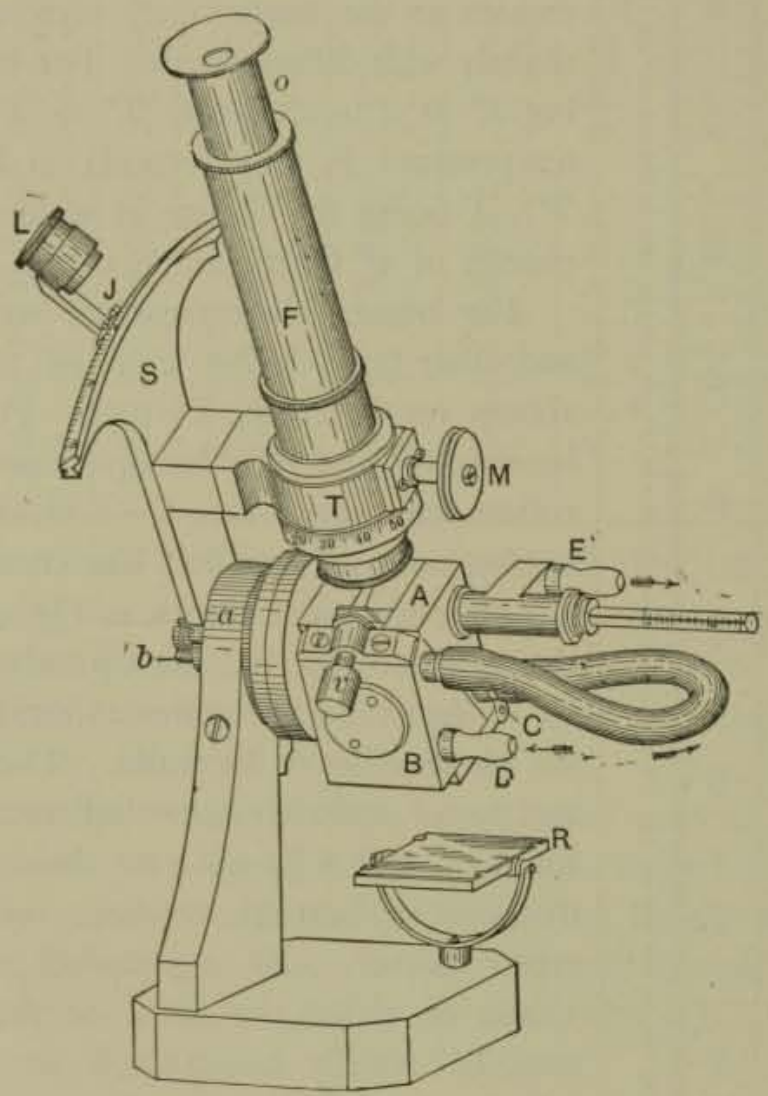

Fig. 41.-The Abbé Refractometer with Temperature-controlled Prisms.

ing as it does to the fourth decimal between the limits of $\mathrm{I} .3$ and $\mathrm{I} .7$ in indices of refraction. The equivalent readings of the Wollny milk fat refractometer, in indices of refraction, range from $\mathrm{I} .3332$ to $\mathrm{I} .422 \mathrm{O}$, while those of the butyro instrument run from 1.4220 to 1.4895 . The Abbé instrument is thus necessary for use with the high-refracting essential 
oils. Its construction is such that the prisms can withstand a higher heat than in the case of the butyro-refractometer, and it is hence better adapted for the examination of samples having a high melting-point, such as beeswax and paraffin. An advantage of the Abbé over the butyro instrument lies in the fact that the wide dispersion, inevitable when reading many substances on the butyro, may be entirely compensated for with the Abbé, and a clear sharp line be obtained. The construction of the prisms in relation to the heating jacket is similar in both instruments, and a film of the substance to be examined is held in the same manner between the surfaces of the prisms.

Construction and Manipulation.-The Abbé refractometer is mainly composed of the following parts (see Fig. 4I):

I. The double Abbé prism $A B$, which contains the fluid and can be rotated on a horizontal axis by means of an alidade.

2. A telescope $O F$ for observing the border-line of the total reflection which is formed in the prism.

3. A sector $S$, rigidly connected with the telescope, on which divisions representing refractive indices are engraved.

The double prism ( $A B$, Fig. $4 \mathrm{r})$ consists of two similar prisms of flint-glass, each cemented into a metal mount and having a refractive index $n_{D}=1.75$. The former of the two prisms, that farthest from the telescope, which can be folded up or removed, serves solely for the purpose of illumination, while the border-line is formed in the second flint prism. A few drops of the fluid to be investigated is deposited between the two adjoining inner faces of the prisms in the form of a thin stratum, about $0.15 \mathrm{~mm}$. thick.

The double prism is opened and closed by means of a screw-head $v$, which acts in the manner of a bayonet catch. In order to apply a small quantity of fluid to the prisms without opening the casing, the screw $v$ is slackened and a few drops of fluid poured into the funnelshaped aperture of a narrow passage, not seen in the figure. On again tightening the screw, the fluid is distributed by capillary action over the entire space between the two prisms. This arrangement facilitates the investigation of rapidly evaporating fluids, such as ether solutions. In the case of viscous fluids (resins, etc.), a drop of moderate size is applied with a glass rod to the dull prism surface, the double prism being opened for the purpose. The prisms are then closed again, and before the measurement is proceeded with, the refractometer is left standing for a few minutes in order to compensate for any cooling or heating of the prisms which might occur while they were separated. 
The arrangement for controlling the temperature of the prisms of the Abbé refractometer is essentially after Dr. R. Wollny's plan of enclosing the prisms in a metal casing with double walls, through which water of a given temperature is circulated.

The border-line is brought within the field of the telescope $O F$ by rotating the double prism by means of the alidade in the following manner: Holding the sector, the alidade is moved from the initial position at which the index points to $n_{D}=1.3$, in the ascending scale of the refractive indices until the originally entirely illuminated field of view is encroached upon from the direction of its lower half by a dark portion; the line dividing the bright and the dark half of the field then is the "border-line." When daylight or lamplight is being employed, the border-line, owing to the total reflection and the refraction caused by the second prism, assumes at first the appearance of a band of color, which is quite unsuitable for any exact process of adjustment. The conversion of this band of color into a colorless line sharply dividing the bright and dark portions of the field is the work of the compensator, which consists of two similar Amici prisms of direct vision for the $D$-line, and rotated simultaneously, though in opposite directions, round the axis of the telescope by means of the screw-head $M$. The dispersion of the border-line, which appears in the telescope as a band of color, can thus be counteracted by rotating the screw-head $M$ till the equal but opposite dispersions are neutralized, making the line colorless and sharp.

The border-line is now adjusted upon the point of intersection of the crossed lines by slightly inclining the double prism to the telescope by means of the alidade. The position of the pointer on the graduation of the sector is then read by the aid of the magnifier attached to the alidade. The reading supplies the refractive index $n_{D}$ of the substance under investigation without any computation, and with a degree of exactness approaching to within about two units of the fourth decimal. Simultaneously, the reading of the scale on the drum of the compensator ( $T$ in Fig. $4 \mathrm{I}$ ) enables the mean dispersion to be arrived at by means of a special table and a short process of computation.

Influence of Temperature. - As the refractive index of fluids varies with their temperature, it is of importance to know the temperature of the fluid contained in the double prism during the process of measurement.

If, therefore, it is desired to measure a fluid with the highest degree of accuracy attainable with the Abbé refractometer (to within one or 
two units of the fourth decimal of $n_{D}$ ), it is absolutely necessary to bring the fluid, or rather the double prism containing it, to a definite known temperature, and to be able to control this temperature so as to keep it constant to within some tenths of a degree for a period of several hours; hence a refractometer principally required for the investigation of fluids must be provided with heatable prisms.

The type of heater shown in Fig. 39. and described in connection with the butyro-refractometer on page 102, is equally adapted for controlling the temperature of the prisms in the Abbé instrument, the flow of water entering at $D$ and passing out at $E$, Fig. $4 \mathrm{I}$.

\section{THE IMMERSION REFRACTOMETER.}

This form of refractometer is of more recent introduction than the others made by Zeiss, and has many features that especially commend it to the use of the food analyst. The construction of the immersion refractometer is such that, as its name implies, it may be immersed directly in an almost endless variety of solutions, the strength of which, within limits, may be determined by the degree of refraction read upon an arbitrary scale. Thus, for example, the strengths of various acids and of a variety of salt solutions used as reagents in the laboratory, as well as of formaldehyde, of sugars in solution, and of alcohol, are all capable of determination by the use of the immersion refractometer.

Figure 42 shows the form used by the writer. $P$ is a glass prism fixed in the lower end of the tube of the instrument, while at the top of the tube is the ocular $O c$, and just below this on a level with the vernier screw $Z$ is the scale on which is read the degree of refraction of the liquid in which the prism $P$ is immersed. The tube may be held in the hand and directly dipped in the liquid to be tested, this liquid being contained in a vessel with a translucent bottom, through which the light is reflected. The preferable method of use is, however, that shown in the cut.

$A$ is a metal bath with inlet and outlet tubes, arranged whereby water is kept at a constant level. The water is maintained at a constant temperature by means of a controller of the same type as the refractometer heater shown in Fig. 39. In the bath $A$ are immersed a number of beakers, containing the solutions to be tested. $T$ is a frame on which is hung the refractometer by means of the hook $H$, at just the right height to permit of the immersion of the prism $P$ in the liquid in any of the beakers in the row beneath. Under this row of beakers the bottom of the tank is composed of a strip of ground glass, through which light is reflected by an adjustable pivoted mirror. 
The temperature of the bath is noted by a delicate thermometer immersed therein, capable of reading to tenths of a degree.

Returning to the main refractometer-tube, $R$ is a graduated ring or collar which is connected by a sleeve within the tube with a compound prism near the bottom, the construction being such that by turning the collar $R$ one way or the other the chromatic aberration or dispersion of any liquid may be compensated for, and a clear-cut shadow or critical line projected across the scale. By the graduation on the collar $R$, the degree of
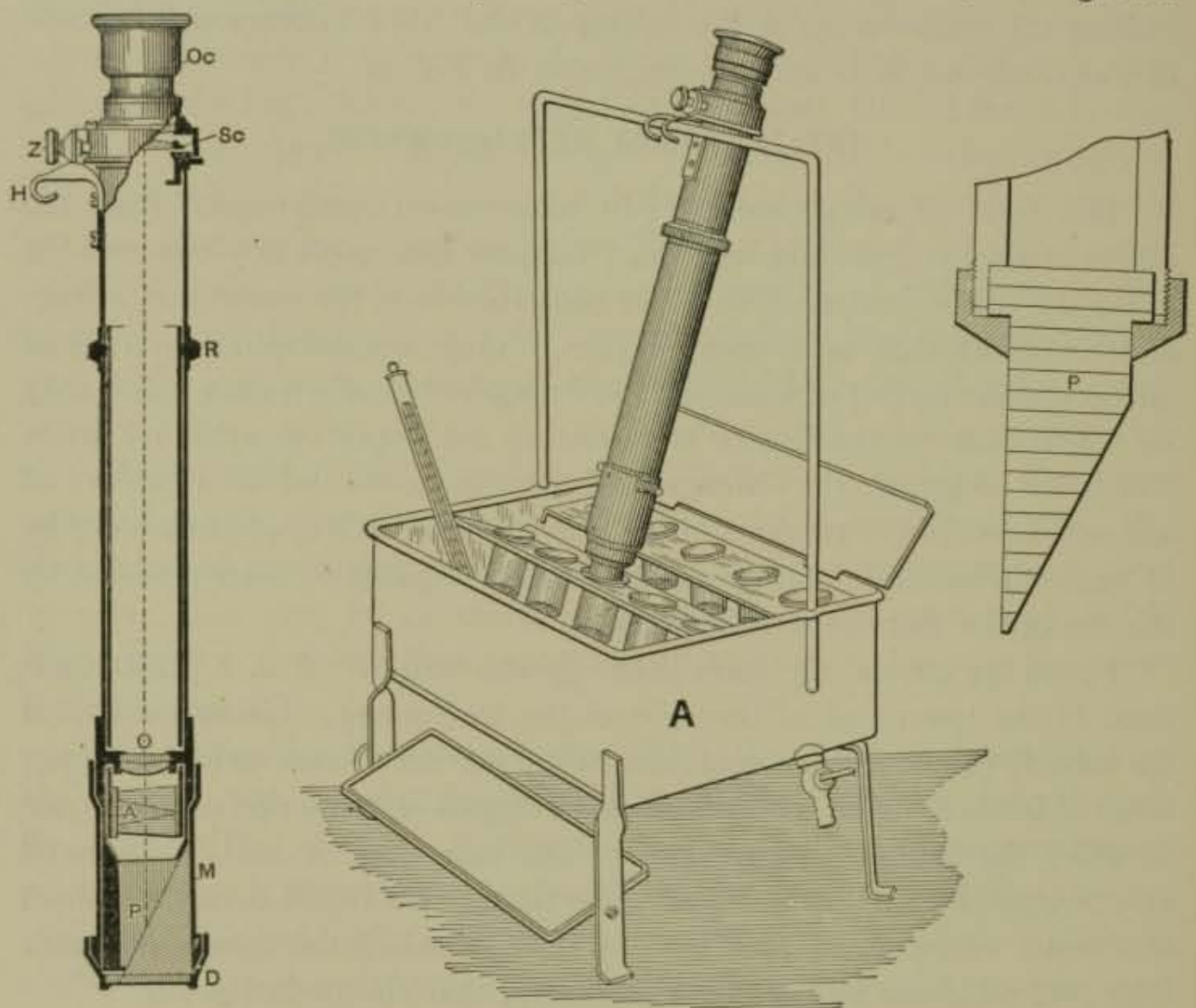

FIG. 42.-The Zeiss Immersion Refractometer.

dispersion may be read. Tenths of a degree on the main scale of the instrument may be read with great accuracy by means of the vernier screw $Z$, graduated along its circumference, the screw being turned in each case till the critical line on the scale coincides with the nearest whole number.

The scale of the instrument reads from -5 to 105 , corresponding to indices of refraction of from 1.32539 to 1.36640 . It should be noted that the index of refraction may be read with a greater degree of accuracy on the immersion refractometer than on the Abbé instrument. 
Manipulation of the Immersion Refractometer.-Before using the instrument for the first time, it is necessary to see that the refractometer is correctly adjusted. For this purpose the bath $A$ is placed with its long side parallel to the window and the mirror turned towards a bright sky, the bath is half filled vith tap-water, and a beaker filled with distilled water is then placed in one of the five holes in the front row immediately above the mirror. Finally, the refractometer is hung by its hook $H$ upon the wire frame, the prism being totally submerged in the water contained in the beaker.

The whole apparatus is now allowed to stand for ten minutes, or until the distilled water has acquired the exact temperature of the bath, and the ocular is focussed upon the divisions of the scale by turning the milled zone of the ocular shell until the lines and numbers are seen quite distinctly, the mirror being adjusted so that the light of the bright sky is seen directly through the beaker. The upper part of the field from -5 to about 15 appears bright, and it is separated from the lower dark part by a sharp line of demarcation, if the index on the ring of the compensator stands at 5 .

SCALE READING AND INDEX OF REFRACTION OF DISTILLED WATER AT $10-30^{\circ} \mathrm{C}$, ACCORDING TO WAGNER.

\begin{tabular}{|c|c|c|c|c|c|c|c|}
\hline $\begin{array}{l}\text { Temper- } \\
\text { ature C. }\end{array}$ & $\begin{array}{c}\text { Scale } \\
\text { Reading. }\end{array}$ & $\begin{array}{l}\text { Index of } \\
\text { Refraction, } n_{D} \text {. }\end{array}$ & $\begin{array}{c}n_{D} \text { Differ- } \\
\text { ence for } \\
x^{\circ} \mathrm{C} \text {. }\end{array}$ & $\begin{array}{l}\text { Temper- } \\
\text { ature C. }\end{array}$ & $\begin{array}{l}\text { Scale } \\
\text { Reading. }\end{array}$ & $\begin{array}{c}\text { Index of } \\
\text { Refraction, } n_{D}\end{array}$ & $\begin{array}{c}n_{D} \text { Differ- } \\
\text { ence for } \\
x^{\circ} \mathrm{C} \text {. }\end{array}$ \\
\hline 30 & II .8 & 1. 33196 & $\ldots$ & 19 & 14.7 & I. 333075 & 8.5 \\
\hline 29 & $I 2 . I$ & I. 33208 & 12.0 & I8 & 14.9 & 1. 33316 & 8.5 \\
\hline 28 & $\mathrm{I} 2.4$ & 1. 332195 & 11.5 & $17 \cdot 5$ & 15.0 & I. 33320 & 438.0 \\
\hline 27 & 12.7 & I. $33^{2} 31$ & II. 5 & 170 & 15.1 & 1. 33324 & $4 \int^{0.0}$ \\
\hline 26 & 13.0 & I. 33242 & 11.0 & 16 & $15 \cdot 3$ & 1. 333315 & $7 \cdot 5$ \\
\hline 25 & ${ }^{1} 3.25$ & 1. $33^{2525}$ & 10.5 & 15 & 15.5 & I. 33339 & $7 \cdot 5$ \\
\hline 24 & 13.5 & 1. 332625 & 10.0 & 14 & 15.7 & I. 33346 & 7.0 \\
\hline 23 & 13.75 & I. 33272 . & 9.5 & 13 & 15.85 & I. 333525 & 6.5 \\
\hline 22 & 14.0 & I. $3328 \mathrm{r}$ & 9.0 & 12 & 16.0 & 1. 33359 & 6.5 \\
\hline 21 & 14.25 & I. 33290 & 9.0 & II & 16.15 & I. 33365 & 6.0 \\
\hline 20 & 14.5 & 1. 33299 & 9.0 & IO & I6. 3 & I. 333705 & $5 \cdot 5$ \\
\hline
\end{tabular}

The reading is taken and the temperature of the distilled water noted. Reference to the above table will show if the refractometer is correctly adjusted. Should the average of several careful readings at a given temperature deviate from that contained in the table, the following should be resorted to:

Readjustment of the Scale.-The ocular end of the refractometer hanging on the wire frame is grasped from behind with the thumb and forefinger of the left hand, the micrometer drum set to ro, and the steel 
spike, housed in the case of the apparatus, inserted into one of the holes of the nickeled cross-holed screw lying on the inner side of the micrometer drum. The spike is then turned anti-clockwise, as seen from the rear, whereupon the nickeled milled nut, which governs the micrometer, becomes loosened. The temperature of the distilled water in the beaker is taken once more to see that it has remained constant, and then the table (page $\mathrm{I}_{3}$ ) is consulted to find the "adjusting number" properly belonging to the temperature indicated. By turning the spike, the borderline is brought exactly upon the integer scale division appertaining to the adjusting number, and the loose micrometer drum is turned so that the index accords with the decimal portion of the adjusting number. The drum is now held firmly with the thumb and forefinger of the left hand, while the nut is screwed up tight again by the right hand, taking care, however, that the drum does not wander off the index. Finally, the new adjustment is tested by repeated readings.

Regulating the Temperature.-In many cases it suffices to allow water at the temperature of the room to flow slowly from a tank suspended high upon the wall through the bath. Should it be required, however, to maintain a given temperature (say $20^{\circ} \mathrm{C}$.) for hours together constant to a tenth of a degree, which is frequently desirable if not actually necessary, a more elaborate temperature-regulating device should be employed. In cold weather, or when the tap-water has a lower temperature than that desired, a refractometer heater of the type shown in Fig. 39, and described on page 102, is convenient.

When, as in the summer, the tap-water temperature is higher than that desired for the refractometer bath, there are various ways of successfully controlling the temperature at a lower degree. An ice-water tank placed above the level of the bath may be employed, the flow from which through the bath is carefully controlled by a pinch-cock or otherwise, or is allowed to mingle, under careful regulation before entering the bath, with the water from the tap direct or with that from the heater.

Investigation of Solutions in Beakers in Bulk.-The first ten solutions are poured into beakers until two-thirds full, and the latter are immersed and brought to the temperature of the bath $A$. When the first five solutions have been measured, they are taken out of the water-bath and the second series of five beakers inserted in their place, bringing at the same time a third series into the water-bath. The second series are measured and so on. Small gummed labels on the outside prove quite satisfactory for numbering the beakers. It is absolutely necessary to 
compare the temperature of the solutions in the beakers with the waterbath from time to time.

After each immersion, the prism should be wiped dry with a clean, soft piece of old linen.

Investigations of Solutions Excludèd from Air.-Quickly evaporating liquids, for instance ether solutions, should be treated individually by means of the metal beaker adapted to fit the prism end of the refractometer. To fill the beaker, the refractometer is held in the left hand with the prism pointing upwards, and the metal beaker ( $M$, Fig. 42) is set and securely fastened by means of the bayonet joint. It is now filled quite full and the cover $D$ carefully fitted and locked.

The solution is now enclosed, air and water tight. The refractometer as before is hung upon the wire frame of the bath, with the metal beaker submerged in the bath.

It is expedient to place the solutions before investigation in closed flasks in the nine unoccupied holes in the bath.

After the measurement, the refractometer is held in the left hand with the prism pointing downwards, and the beaker together with its cover detached by giving a slight turn with the right hand. The solution can be used for other purposes, since it has undergone no change in constitution. Finally, the cover is detached from the beaker, and cover, beaker, and prism cleaned by simple means, and the refractometer made ready for the reception of the next solution, as above.

Investigations of Small Quantities of Solutions with the Auxiliary Prism.-When the solution does not occur in sufficiently large quantities for investigation in the glass beaker, or when the solution is too deeply colored, as in dark beers, molasses, etc., the auxiliary prism is used. As described under "Solutions Excluded from Air," the metal beaker without cover is fitted on the refractometer. The auxiliary prism is held horizontally, and, a few drops of the solution having been applied to the hypothenuse face, the prism is inserted into the metal beaker, held conveniently for the purpose, with its hypothenuse face laid upon the polished elliptical face of the refractometer prism, and then locked in by the cover. If an insufficient quantity of the solution has been taken, the margins of the out-spread drops lying between the two prisms can be recognized by looking through the window of the cover on which abuts the square polished end of the auxiliary prism. It is strongly recommended, wherever possible, to apply a sufficiency of the solution, so that the space between the prisms is completely filled, otherwise a loss in brilliancy occurs, and, under certain circumstances, an unavoidable 
TABLE OF INDICES OF REFRACTION, $n_{D^{*}}$.

(Compared with Scale Readings of Zeiss Immersion Refractometer, according to Wagner.)

\begin{tabular}{|c|c|c|c|c|c|c|c|c|c|}
\hline $\begin{array}{c}\text { Scale } \\
\text { Read- } \\
\text { ing. }\end{array}$ & ${ }^{n} D^{*}$ & $\begin{array}{c}\text { Seale } \\
\text { Read- } \\
\text { ing. }\end{array}$ & $n_{D}$ & $\begin{array}{l}\text { Scale } \\
\text { Read- } \\
\text { ing. }\end{array}$ & $n_{D^{*}}$ & $\begin{array}{c}\text { Scale } \\
\text { Reaad- } \\
\text { ing. }\end{array}$ & ${ }^{n} D$ & $\begin{array}{l}\text { Scale } \\
\text { Read- } \\
\text { ing. }\end{array}$ & ${ }^{n} D^{*}$ \\
\hline 0.0 & I. 327360 & 5.0 & I. 329320 & 10.0 & 1. 331260 & 15.0 & $\mathrm{t}-333200$ & 20.0 & I. 335 I 68 \\
\hline 0.1 & I. 327399 & $5 \cdot \mathrm{I}$ & I. $32935^{\circ}$ & 10.1 & I. 331299 & 15.1 & I. $33323^{8}$ & $20 . \mathrm{I}$ & I. $335+68$ \\
\hline 2 & $43^{8}$ & 2 & $39^{8}$ & 2 & 388 & 2 & 276 & 2 & 206 \\
\hline 3 & 477 & 3 & 437 & 3 & 377 & 3 & $3 \mathrm{I} 4$ & 3 & 244 \\
\hline 4 & 516 & 4 & 476 & 4 & 416 & 4 & 352 & 4 & 282 \\
\hline 5 & 555 & 5 & 515 & 5 & 455 & 5 & 390 & 5 & 320 \\
\hline 6 & 594 & 6 & 554 & 6 & 494 & 6 & 428 & 6 & 358 \\
\hline 7 & 633 & 7 & 593 & 7 & 533 & 7 & 466 & 7 & 396 \\
\hline 8 & 672 & 8 & 632 & 8 & $57^{2}$ & 8 & 504 & 8 & 434 \\
\hline 9 & 711 & 9 & 671 & 9 & 611 & 9 & 542 & 9 & 472 \\
\hline 1.0 & $75^{\circ}$ & 6.0 & 710 & II.O & 650 & 16.0 & $5^{80}$ & 21.0 & 510 \\
\hline I. I & t. $3277^{89}$ & 6.1 & I. 329749 & $11, I$ & I. 33 r689 & $16 . \mathrm{I}$ & T. 333619 & $2 \mathrm{I} . \mathrm{I}$ & I. 335549 \\
\hline 2 & 828 & 2 & 788 & 2 & 728 & 2 & 658 & 2 & $5^{88}$ \\
\hline 3 & 867 & 3 & $\begin{array}{l}827 \\
866\end{array}$ & 3 & $7^{67}$ & 3 & 697 & 3 & 627 \\
\hline 4 & 906 & 4 & 866 & 4 & 806 & 4 & $73^{6}$ & 4 & 666 \\
\hline 5 & & $\begin{array}{l}5 \\
6\end{array}$ & 905 & 5 & 845 & 5 & 775 & 5 & 705 \\
\hline 6 & $\begin{array}{r}984 \\
\text { T. } 328023\end{array}$ & 6 & $\begin{array}{l}944 \\
982\end{array}$ & 6 & 884 & 6 & $\begin{array}{l}814 \\
833\end{array}$ & 6 & 744 \\
\hline $\begin{array}{l}7 \\
8\end{array}$ & $\begin{array}{r}\text { t. } 328023 \\
062\end{array}$ & 8 & $\begin{array}{r}982 \\
\text { I. } 330022\end{array}$ & $\begin{array}{l}7 \\
8\end{array}$ & $\begin{array}{r}932 \\
962 \\
\end{array}$ & $\begin{array}{l}7 \\
8\end{array}$ & $\begin{array}{l}833 \\
892\end{array}$ & $\begin{array}{l}7 \\
8\end{array}$ & $\begin{array}{l}783 \\
822\end{array}$ \\
\hline 9 & IOI & 9 & $06 \mathrm{r}$ & 9 & I. $33200 \mathrm{I}$ & 9 & 931 & 9 & 861 \\
\hline 2.0 & 140 & 7.0 & 100 & 12.0 & 040 & 17.0 & 970 & 22.0 & 900 \\
\hline 2. $\pi$ & r. 328180 & $7 \cdot I$ & 1. 330139 & 12.1 & I. 332078 & $17 \cdot 1$ & 1. 334008 & $22 . \mathrm{I}$ & I. $33593^{8}$ \\
\hline 2 & 220 & 2 & 178 & 2 & II 6 & 2 & 046 & 2 & 976 \\
\hline 3 & 657 & 3 & 217 & 3 & 154 & 3 & 084 & 3 & I. 336014 \\
\hline 4 & 300 & 4 & $25^{6}$ & 4 & 192 & 4 & 122 & 4 & 052 \\
\hline 5 & 340 & 5 & 295 & 5 & 230 & 5 & 160 & 5 & 090 \\
\hline 6 & 380 & 6 & 334 & 6 & 268 & 6 & I 98 & 6 & 128 \\
\hline 7 & 420 & 7 & 373 & 7 & 304 & 7 & $23^{6}$ & 7 & 166 \\
\hline 8 & 460 & 8 & 412 & 8 & 344 & 8 & 274 & 8 & 204 \\
\hline 9 & 500 & 9 & $45 \mathrm{r}$ & 9 & $3^{82}$ & 9 & 312 & 9 & 242 \\
\hline 3.0 & 540 & 8.0 & 490 & 13.0 & 420 & 18.0 & $35^{\circ}$ & 23.0 & 280 \\
\hline 3.1 & I. 328579 & 8. $\mathrm{T}$ & I. 330528 & 13.1 & ז. 332459 & 18.1 & t. $3343^{8 g}$ & $23, I$ & I. $33^{6} 3^{10}$ \\
\hline 2 & 618 & 2 & 566 & 2 & 498 & 2 & 428 & 2 & $35^{8}$ \\
\hline 3 & 657 & 3 & 604 & 3 & 537 & 3 & 467 & 3 & 397 \\
\hline 4 & 696 & 4 & 642 & 4 & 576 & 4 & 506 & 4 & 436 \\
\hline 5 & 735 & 5 & 680 & 5 & 615 & 5 & 545 & 5 & 475 \\
\hline 6 & 774 & 6 & $\begin{array}{l}718 \\
--6\end{array}$ & 6 & 654 & 6 & 584 & 6 & $5^{14}$ \\
\hline 7 & 813 & 7 & $75^{6}$ & 7 & 693 & 7 & 623 & 7 & 553 \\
\hline 8 & 852 & 8 & 794 & 8 & $73^{2}$ & 8 & 662 & 8 & 592 \\
\hline 9 & 891 & 9 & 832 & 9 & 771 & 9 & 701 & 9 & 6,31 \\
\hline 4.0 & $93^{\circ}$ & 9.0 & 870 & 14.0 & 810 & 19.0 & 740 & 24.0 & 670 \\
\hline $4 \cdot \mathrm{r}$ & 1. 328960 & 9.1 & $\mathrm{r}+330909$ & I4. I & I. 332849 & 19.1 & I +334770 & 24.1 & เ. $33^{6} 708$ \\
\hline 2 & I. 329008 & 2 & 948 & 2 & 888 & 2 & $8 \pi 8$ & 2 & $74^{6}$ \\
\hline 3 & $\begin{array}{l}047 \\
0\end{array}$ & 3 & $9^{87}$ & 3 & 927 & 3 & 857 & 3 & $7^{84}$ \\
\hline 4 & & 4 & t. 331026 & 4 & 966 & 4 & 896 & 4 & 822 \\
\hline 5 & 125 & 5 & 104 & 5 & I. 333005 & 5 & 935 & 5 & 860 \\
\hline 6 & 164 & 6 & 104 & 6 & 044 & 6 & 974 & 6 & 898 \\
\hline 7 & 203 & 7 & 143 & 7 & $08_{3}$ & 7 & t. 335013 & 7 & 936 \\
\hline 8 & 242 & 8 & $\mathrm{I} 8 \mathrm{z}$ & 8 & 122 & 8 & 052 & 8 & 974 \\
\hline 9 & $28 \mathrm{I}$ & 9 & 221 & 9 & 161 & 9 & 091 & 9 & 1. 337012 \\
\hline 5.0 & 320 & 10.0 & 260 & 15.0 & 200 & 20,0 & 130 & 25.0 & 050 \\
\hline
\end{tabular}


THE REFRACTOMETER.

TABLE OF INDICES OF REFRACTION, $n_{D}$-(Continued).

\begin{tabular}{|c|c|c|c|c|c|c|c|c|c|}
\hline $\begin{array}{c}\text { Scale } \\
\text { Read- } \\
\text { ing. }\end{array}$ & ${ }^{n} D^{*}$ & $\begin{array}{c}\text { Scale } \\
\text { Read- } \\
\text { ing. }\end{array}$ & $n_{D}$. & $\begin{array}{l}\text { Scale } \\
\text { Read- } \\
\text { ing. }\end{array}$ & ${ }^{n} D^{*}$ & $\begin{array}{l}\text { Scale } \\
\text { Read- } \\
\text { ing. }\end{array}$ & ${ }^{n} D^{*}$ & $\begin{array}{l}\text { Scale } \\
\text { Read- } \\
\text { ing. }\end{array}$ & $n D$. \\
\hline 25.0 & 1. 337050 & 30.0 & t. $33^{8960}$ & 35.0 & I. 340860 & 40.0 & $\mathrm{x} \cdot 34275^{\circ}$ & 45.0 & I. 344630 \\
\hline 25.1 & I. 337088 & 30.1 & I. $33^{8998}$ & $35 \cdot I$ & т. 340898 & $40 . I$ & I. 342788 & $45 \cdot I$ & I. 344667 \\
\hline 2 & 126 & 2 & I. 339036 & 2 & 9.36 & 2 & 826 & 2 & 704 \\
\hline 3 & 164 & 3 & 074 & 3 & 974 & 3 & 864 & 3 & 741 \\
\hline 4 & 202 & 4 & 112 & 4 & I. 341012 & 4 & 902 & 4 & 778 \\
\hline 5 & 240 & 5 & 150 & 5 & $05 \circ$ & 5 & 940 & 5 & 818 \\
\hline 6 & 278 & 6 & IS8 & 6 & 088 & 6 & 978 & 6 & 852 \\
\hline 7 & 316 & 7 & 226 & 7 & 126 & 7 & I. 343016 & 7 & 889 \\
\hline 8 & 354 & 8 & 264 & 8 & 164 & 8 & 054 & 8 & 926 \\
\hline 9 & 392 & 9 & 302 & 9 & 202 & 9 & 092 & 9 & 963 \\
\hline 26.0 & $43^{\circ}$ & 31.0 & 340 & 36.0 & 240 & $4 \mathrm{I} \cdot 0$ & 130 & 46.0 & I. 345000 \\
\hline 26.1 & t. 337468 & $3 I, I$ & I. $33937^{8}$ & $3^{6.1}$ & I. 341278 & $4 \mathrm{I} \cdot \mathrm{I}$ & เ. 343167 & $4^{6 .} \mathrm{I}$ & I. 345037 \\
\hline 2 & 506 & 2 & 416 & 2 & 316 & 2 & 204 & 2 & 074 \\
\hline 3 & 544 & 3 & 454 & 3 & 354 & 3 & 241 & 3 & III \\
\hline 4 & $5^{82}$ & 4 & 492 & 4 & $39^{2}$ & 4 & $27^{8}$ & 4 & ${ }^{1} 48$ \\
\hline 5 & 620 & 5 & 530 & 5 & $43 \circ$ & 5 & 315 & 5 & 185 \\
\hline 6 & 658 & 6 & 568 & 6 & 468 & 6 & $35^{2}$ & 6 & 222 \\
\hline 7 & 696 & 7 & 606 & 7 & 506 & 7 & 389 & 7 & 259 \\
\hline 8 & 734 & 8 & 644 & 8 & 544 & 8 & 426 & 8 & 296 \\
\hline 9 & 772 & 9 & 682 & 9 & $5^{82}$ & 9 & 463 & 9 & 333 \\
\hline 27.0 & $8 \mathrm{ro}$ & 32.0 & 720 & 37.0 & 620 & 42.0 & 500 & 47.0 & $37 \circ$ \\
\hline $27 \cdot I$ & I. $337^{8} 49$ & 32.1 & I. $33975^{8}$ & $37 \cdot 1$ & I. 341657 & 42.1 & I. $34353^{8}$ & $47 \cdot 1$ & I. 345408 \\
\hline 2 & 888 & 2 & 796 & 2 & 694 & 2 & $57^{6}$ & 2 & $44^{6}$ \\
\hline 3 & 927 & 3 & 834 & 3 & 731 & 3 & 614 & 3 & 484 \\
\hline 4 & 966 & 4 & 872 & 4 & $7^{68}$ & 4 & 652 & 4 & 522 \\
\hline 5 & ז. $33^{8005}$ & 5 & 910 & 5 & 805 & 5 & 690 & 5 & 560 \\
\hline 6 & 044 & 6 & 948 & 6 & 842 & 6 & 728 & 6 & 598 \\
\hline 7 & $08_{3}$ & 7 & 986 & 7 & 879 & 7 & 766 & 7 & 636 \\
\hline 8 & 122 & 8 & I. 340024 & 8 & 916 & 8 & 804 & 8 & 674 \\
\hline 9 & 161 & 9 & 062 & 9 & 953 & 9 & 842 & 9 & 712 \\
\hline 28.0 & 200 & 33.0 & 100 & $3^{8} .0$ & 990 & 43.0 & 880 & 48.0 & $75^{\circ}$ \\
\hline 28.1 & ז. $33^{8238}$ & 33.1 & I. 340 r $_{3} 8$ & $3^{8 . I}$ & I. 342028 & 43.1 & I. 343918 & 48.1 & I. 345787 \\
\hline 2 & $27^{6}$ & 2 & I 76 & 2 & 066 & 3 & $\begin{array}{r}956 \\
\end{array}$ & 2 & 824 \\
\hline 3 & $3 \mathrm{t} 4$ & 3 & 214 & 3 & 104 & 3 & 994 & 3 & $86 \mathrm{i}$ \\
\hline 4 & $35^{2}$ & 4 & 252 & 4 & $\mathrm{I}_{42}$ & 4 & I. $34403^{2}$ & 4 & 898 \\
\hline 5 & 390 & 5 & 290 & 5 & 180 & 5 & 070 & 5 & 935 \\
\hline 6 & 428 & 6 & 328 & 6 & 218 & 6 & 108 & 6 & 972 \\
\hline$?$ & 466 & 7 & $3^{66}$ & 7 & 256 & 7 & $I_{4} 6$ & 7 & I. 346009 \\
\hline 8 & 504 & 8 & 404 & 8 & 294 & 8 & 184 & 8 & 046 \\
\hline & 542 & 9 & 442 & 9 & $33^{2}$ & 9 & 222 & 9 & $08_{3}$ \\
\hline 29.0 & $5^{80}$ & 34.0 & 480 & 39.0 & $37^{\circ}$ & 44.0 & 260 & 49.0 & 120 \\
\hline 29.1 & I. $33^{86} 8$ & 34.1 & I. 340518 & $39 \cdot I$ & I. 342408 & 84.1 & t. 344297 & $49 \cdot \mathrm{I}$ & I. $34^{6} \times 5^{8}$ \\
\hline 2 & 656 & 2 & $55^{6}$ & 2 & $44^{6}$ & 2 & 334 & 2 & 196 \\
\hline 3 & 694 & 3 & 594 & 3 & 484 & 3 & 371 & 3 & 234 \\
\hline 4 & 732 & 4 & 632 & 4 & 522 & 4 & 408 & 4 & 272 \\
\hline o & $\begin{array}{l}770 \\
808\end{array}$ & $\begin{array}{l}5 \\
6\end{array}$ & $\begin{array}{l}670 \\
708\end{array}$ & $\begin{array}{l}5 \\
6\end{array}$ & $\begin{array}{l}560 \\
508\end{array}$ & $\begin{array}{l}5 \\
6\end{array}$ & $\begin{array}{l}445 \\
482\end{array}$ & $\begin{array}{l}5 \\
6\end{array}$ & 310 \\
\hline 7 & 846 & 7 & $\begin{array}{l}708 \\
746\end{array}$ & $\begin{array}{l}0 \\
7\end{array}$ & 636 & $\begin{array}{l}0 \\
7\end{array}$ & $\begin{array}{l}482 \\
519\end{array}$ & $\begin{array}{l}0 \\
7\end{array}$ & $\begin{array}{l}348 \\
386\end{array}$ \\
\hline 8 & 884 & 8 & 784 & 8 & 674 & 8 & $55^{6}$ & 8 & 424 \\
\hline 9 & 922 & 9 & 822 & 9 & $7^{12}$ & 9 & 593 & 9 & 462 \\
\hline 30.0 & 960 & 35.0 & 860 & 40.0 & 750 & 45.0 & 630 & 50.0 & 500 \\
\hline
\end{tabular}


TABLE OF INDICES OF REFRACTION, $n_{D}$-(Continued).

\begin{tabular}{|c|c|c|c|c|c|c|c|c|c|}
\hline $\begin{array}{l}\text { Scale } \\
\text { Read- } \\
\text { ing. }\end{array}$ & $" D$. & $\begin{array}{c}\text { Scale } \\
\text { Read- } \\
\text { ing. }\end{array}$ & $n_{D}$. & $\begin{array}{c}\text { Scale } \\
\text { Read- } \\
\text { ing. }\end{array}$ & ${ }^{n} D$ & $\begin{array}{l}\text { Scale } \\
\text { Read- } \\
\text { ing. }\end{array}$ & ${ }^{n} D$ & $\begin{array}{l}\text { Seale } \\
\text { Read- } \\
\text { ing. }\end{array}$ & ${ }^{n} D$ \\
\hline 50.0 & I. 346500 & 55.0 & I. 348360 & 60.0 & I. 350210 & 65,0 & 1.352050 & 70.0 & r. $353^{880}$ \\
\hline 50.1 & I. 346537 & 55.1 & I. $34^{8} 397$ & 60.1 & I. 350247 & 65.1 & 1. 352087 & 70.1 & I. 353917 \\
\hline 2 & 574 & 2 & 434 & 2 & 284 & 2 & 124 & 2 & 954 \\
\hline 3 & 611 & 3 & 471 & 3 & $3^{21}$ & 3 & $16 \mathrm{I}$ & 3 & $99 \mathrm{I}$ \\
\hline 4 & 648 & 4 & 508 & 4 & $35^{8}$ & 4 & 198 & 4 & I. 354028 \\
\hline 5 & 685 & 5 & 545 & 5 & 395 & 5 & 235 & 5 & 065 \\
\hline 6 & 722 & 6 & $5^{82}$ & 6 & $43^{2}$ & 6 & 272 & 6 & 102 \\
\hline 7 & 759 & 7 & 619 & 7 & 469 & 7 & 309 & 7 & 139 \\
\hline & 796 & 8 & 656 & 8 & 506 & 8 & 346 & 8 & 176 \\
\hline 9 & 833 & 9 & 693 & 9 & 543 & 9 & $3_{3}^{8}$ & 9 & $2 \mathrm{I}_{3}$ \\
\hline 51.0 & 870 & 56.0 & $73^{\circ}$ & 61.0 & 580 & 66.0 & 420 & 71.0 & 250 \\
\hline $51 \cdot 1$ & I. 346907 & $56 . \mathrm{I}$ & r. 348767 & 61.1 & I. 350617 & 66.1 & I. $35^{2457}$ & $7 \mathrm{I} \cdot \mathrm{I}$ & I. 354286 \\
\hline 2 & 944 & 2 & 804 & 2 & $\quad 654$ & 2 & 494 & 2 & 322 \\
\hline 3 & $9^{8} \mathrm{r}$ & 3 & $84 \mathrm{r}$ & 3 & 691 & 3 & 531 & 3 & $35^{8}$ \\
\hline 4 & 1. 347018 & 4 & 878 & 4 & 728 & 4 & 568 & 4 & 394 \\
\hline 5 & 055 & 5 & 915 & 5 & 765 & 5 & 605 & 5 & $43^{\circ}$ \\
\hline 6 & 092 & 6 & 952 & 6 & 802 & 6 & 642 & 6 & 466 \\
\hline 7 & 129 & 7 & $9^{89}$ & 7 & 839 & 7 & 679 & 7 & 502 \\
\hline 8 & 166 & 8 & r. 349026 & 8 & 876 & 8 & 716 & 8 & $53^{8}$ \\
\hline 9 & 203 & 9 & 063 & 9 & $9 \times 3$ & 9 & 753 & 9 & 574 \\
\hline 52.0 & 240 & 57.0 & 100 & 62.0 & $95^{\circ}$ & 67.0 & 790 & 72.0 & 610 \\
\hline $5^{2} \cdot \mathrm{I}$ & I. 347277 & $57 \cdot \mathrm{I}$ & r. 349137 & 62.1 & I. 350987 & 67.1 & I. 352827 & 72.1 & x. 354646 \\
\hline 2 & $3 \mathrm{I} 4$ & 2 & 174 & 2 & I. 351024 & 2 & 864 & 2 & 682 \\
\hline 3 & $35 \mathrm{I}$ & 3 & $2 \mathrm{II}$ & 3 & 061 & 3 & 901 & 3 & 718 \\
\hline 4 & 388 & 4 & 248 & 4 & 098 & 4 & $93^{8}$ & 4 & 754 \\
\hline 5 & 425 & 5 & 285 & 5 & 135 & 5 & 975 & 5 & 790 \\
\hline 6 & 462 & 6 & 312 & 6 & 172 & 6 & I. 353012 & 6 & 826 \\
\hline 7 & 499 & 7 & 359 & 7 & 209 & 7 & 049 & 7 & 862 \\
\hline 8 & 536 & 8 & 396 & 8 & 246 & 8 & 086 & 8 & 898 \\
\hline 9 & 573 & 9 & 433 & 9 & $28_{3}$ & 9 & 123 & 9 & 934 \\
\hline 53.0 & 610 & 58.0 & $47^{\circ}$ & 63.0 & 320 & 68.0 & 160 & 73.0 & 970 \\
\hline 53.1 & I. 347647 & $5^{8} .1$ & I. 349507 & 63.1 & I. 351357 & 68.1 & I. 353 I96 & $73 \cdot I$ & I. 355006 \\
\hline 2 & 684 & 2 & 544 & 2 & 394 & 2 & 232 & 2 & 042 \\
\hline 3 & 721 & 3 & $5^{8 I}$ & 3 & $43 \mathrm{I}$ & 3 & 268 & 3 & $07^{8}$ \\
\hline 4 & $75^{8}$ & 4 & 618 & 4 & 468 & 4 & 304 & 4 & $\mathrm{II}_{4}$ \\
\hline 5 & 795 & 5 & 655 & 5 & 505 & 5 & 340 & 5 & I50 \\
\hline 6 & 832 & 6 & 692 & 6 & 542 & 6 & $37^{6}$. & 6 & I86 \\
\hline 7 & 869 & 7 & 729 & 7 & 579 & 7 & 412 & 7 & 222 \\
\hline 8 & 906 & 8 & 766 & 8 & 616 & 8 & 448 & 8 & $25^{8}$ \\
\hline 9 & 943 & $\begin{array}{r}9 \\
-0\end{array}$ & 803 & 9 & 653 & 9 & $4^{8} 4$ & 9 & 294 \\
\hline 54.0 & 980 & 59.0 & 840 & 64.0 & 690 & 69.0 & $5^{20}$ & 74.0 & $33^{\circ}$ \\
\hline $54 \cdot \mathrm{I}$ & I. 3480 r 8 & $59 . \mathrm{I}$ & I. 349877 & 64.1 & I. 35 I 726 & $69 \cdot \mathrm{r}$ & t. $35355^{6}$ & $74 \cdot \mathrm{I}$ & I. 355366 \\
\hline 2 & 056 & 2 & 914 & 2 & 762 & 2 & 592 & 2 & 402 \\
\hline 3 & C94 & 3 & $95 \mathrm{I}$ & 3 & $79^{8}$ & 3 & 628 & 3 & $43^{8}$ \\
\hline 4 & 132 & 4 & 988 & 4 & $8_{34}$ & 4 & 664 & 4 & 474 \\
\hline 5 & 170 & 5 & I. 350025 & 5 & 870 & 5 & 700 & 5 & 510 \\
\hline 6 & 208 & 6 & 062 & 6 & 906 & 6 & 736 & 6 & $54^{6}$ \\
\hline 7 & 246 & 7 & 099 & 7 & 942 & 7 & $\begin{array}{l}772 \\
808\end{array}$ & 7 & $\begin{array}{l}582 \\
6,8\end{array}$ \\
\hline 8 & 284 & 8 & 136 & 8 & $\begin{array}{r}97^{8} \\
\end{array}$ & $\begin{array}{l}8 \\
0\end{array}$ & $\begin{array}{l}808 \\
844\end{array}$ & $\begin{array}{l}8 \\
9\end{array}$ & $\begin{array}{l}018 \\
659\end{array}$ \\
\hline $\begin{array}{r}9 \\
55.0\end{array}$ & 322 & $\begin{array}{r}9 \\
60.0\end{array}$ & I 73 & 6.9 & $\begin{array}{r}1.352014 \\
050\end{array}$ & $\begin{array}{r}9 \\
70.0\end{array}$ & 880 & 75.0 & $\begin{array}{l}059 \\
690\end{array}$ \\
\hline 55.0 & 360 & 60.0 & 210 & 05.0 & $\mathrm{c5}^{\circ}$ & & & & \\
\hline
\end{tabular}


THE REFRACTGMETER.

TABLE OF INDICES OF REFRACTION, $n_{D}-$ (Continued).

\begin{tabular}{|c|c|c|c|c|c|c|c|c|c|}
\hline $\begin{array}{c}\text { Seale } \\
\text { Read- } \\
\text { ing. }\end{array}$ & $n_{D}$ & $\begin{array}{c}\text { Scale } \\
\text { Read- } \\
\text { ing. }\end{array}$ & ${ }^{n} D$. & $\begin{array}{l}\text { Scale } \\
\text { Read- } \\
\text { ing. }\end{array}$ & ${ }^{n} D$ & $\begin{array}{l}\text { Scale } \\
\text { Read- } \\
\text { ing. }\end{array}$ & $n_{D}$ & $\begin{array}{l}\text { Scale } \\
\text { Read- } \\
\text { ing. }\end{array}$ & $n_{0}$ \\
\hline 75.0 & I. 355690 & 80.0 & 1. 357500 & 85.0 & I. 359300 & 90.0 & 1. 361090 & 95.0 & I. 362870 \\
\hline 75.1 & I. 355727 & So. I & x. $35753^{6}$ & 85.1 & I. 359336 & 90.1 & 1. $3^{6+126}$ & 95.1 & I. 362006 \\
\hline 2 & 764 & 2 & 572 & 2 & 372 & 2 & 162 & 2 & 942 \\
\hline 3 & 8 or & 3 & 608 & 3 & 408 & 3 & 198 & 3 & 978 \\
\hline 4 & $8_{3} 8$ & 4 & 644 & 4 & 444 & 4 & 234 & 4 & I. 363014 \\
\hline 5 & 875 & 5 & 680 & 5 & 480 & 5 & 270 & 5 & 050 \\
\hline 6 & 912 & 6 & 716 & 6 & 516 & 6 & 306 & 6 & 086 \\
\hline $\begin{array}{l}7 \\
8\end{array}$ & $\begin{array}{l}949 \\
986\end{array}$ & $\begin{array}{l}7 \\
8\end{array}$ & $\begin{array}{l}752 \\
788\end{array}$ & $\begin{array}{l}7 \\
8\end{array}$ & $\begin{array}{l}552 \\
588\end{array}$ & $\frac{7}{8}$ & $\begin{array}{l}312 \\
378\end{array}$ & 8 & $\begin{array}{l}122 \\
x=8\end{array}$ \\
\hline $\begin{array}{l}8 \\
9\end{array}$ & I. 356023 & 9 & $8_{24}$ & 9 & 624 & $\begin{array}{l}0 \\
9\end{array}$ & $\begin{array}{l}370 \\
414\end{array}$ & $\begin{array}{l}0 \\
9\end{array}$ & $\begin{array}{l}150 \\
104\end{array}$ \\
\hline 76.0 & $\begin{array}{r}200 \\
0\end{array}$ & 81.0 & 860 & 86.0 & 660 & 91.0 & $45^{\circ}$ & 96.0 & 230 \\
\hline 76.1 & I. 356096 & 81.1 & 1. $357^{896}$ & 86.1 & I. 359606 & 91.1 & 1. 361486 & 96.1 & I. 363256 \\
\hline 2 & 132 & 2 & 9.32 & 2 & 732 & 2 & 522 & 2 & 292 \\
\hline 3 & 168 & 3 & 968 & 3 & 768 & 3 & $55^{8}$ & 3 & 328 \\
\hline 4 & 204 & 4 & I. $35^{8004}$ & 4 & 804 & 4 & 594 & 4 & 364 \\
\hline 5 & 240 & 5 & 040 & 5 & 840 & 5 & 630 & 5 & 400 \\
\hline 6 & 276 & 6 & 076 & 6 & 876 & 6 & 666 & 6 & 436 \\
\hline 7 & $3^{12}$ & 7 & 112 & 7 & 912 & 7 & 702 & 7 & 472 \\
\hline 8 & $34^{8}$ & 8 & 148 & 8 & $94^{8}$ & 8 & $73^{8}$ & 8 & 518 \\
\hline 9 & $3^{8} 4$ & 9 & $18_{4}$ & 9 & $9^{84}$ & 9 & 774 & 9 & 554 \\
\hline 77.0 & 420 & 82.0 & 220 & 87.0 & I. 360020 & 92.0 & 810 & 97.0 & 590 \\
\hline $77 \cdot 1$ & I. 356456 & $82 . x$ & I. $35^{8256}$ & $87 \cdot 1$ & 1. 360056 & 92.1 & I. 361846 & $97 \cdot I$ & I. 363625 \\
\hline 2 & 492 & 2 & 292 & 2 & 092 & 2 & 882 & 2 & 660 \\
\hline 3 & 528 & 3 & 328 & 3 & 128 & 3 & $9^{18}$ & 3 & 695 \\
\hline 4 & 564 & 4 & 364 & 4 & 164 & 4 & 954 & 4 & 730 \\
\hline 5 & 600 & 5 & 400 & 5 & 200 & 5 & 990 & 5 & 765 \\
\hline 6 & $6_{3} 6$ & 6 & $43^{6}$ & 6 & 236 & 6 & I. 362026 & 6 & 800 \\
\hline 7 & 672 & 7 & 472 & 7 & 272 & 7 & 062 & 7 & $8_{35}$ \\
\hline 8 & 708 & 8 & 508 & 8 & 308 & 8 & 098 & 8 & 870 \\
\hline-89 & 744 & 9 & 544 & 89 & 344 & 9 & 134 & 9 & 905 \\
\hline 78.0 & 780 & 83.0 & 580 & 88.0 & 380 & 93.0 & 170 & 98.0 & 940 \\
\hline $7^{8.1}$ & I. 3568 r6 & 83.1 & $1 \cdot 35^{9616}$ & 88.1 & 1. 360416 & $93 . \mathrm{I}$ & I. 362205 & 98.1 & I. 363975 \\
\hline 2 & 852 & 2 & 652 & 2 & 452 & 2 & 1) 240 & 2 & I. 364010 \\
\hline 3 & 888 & 3 & 688 & 3 & 488 & 3 & 275 & 3 & 045 \\
\hline 4 & 924 & 4 & 724 & 4 & 524 & 4 & 310 & 4 & 080 \\
\hline 5 & 960 & 5 & 760 & 5 & 560 & 5 & 345 & 5 & 115 \\
\hline 6 & 996 & 6 & 796 & 6 & 596 & 6 & $3^{80}$ & 6 & 160 \\
\hline 7 & 1.357032 & 7 & $8_{32}$ & 7 & 632 & 7 & 415 & 7 & 195 \\
\hline 8 & 068 & 8 & 858 & 8 & 668 & 8 & 450 & 8 & 230 \\
\hline 9 & 104 & 9 & 904 & 9 & 704 & 9 & 485 & 9 & 265 \\
\hline 79.0 & 140 & 84.0 & 940 & 89.0 & 740 & 94.0 & $5^{20}$ & 99.0 & 290 \\
\hline 79.1 & 1. 357176 & 84.1 & I. $35^{8} 97^{6}$ & 89.1 & I. $3^{60775}$ & 94.1 & I $3^{62555}$ & $99 . \mathrm{I}$ & I. 364325 \\
\hline 2 & 212 & 2 & 1. 359012 & 2 & 810 & 2 & 590 & 2 & 360 \\
\hline 3 & 248 & 3 & $0_{4} 8$ & 3 & 845 & 3 & 625 & 3 & 395 \\
\hline 4 & 284 & 4 & 084 & 4 & 880 & 4 & 660 & 4 & $43^{\circ}$ \\
\hline 5 & 320 & 5 & 120 & 5 & $9^{15}$ & 5 & 695 & 5 & 465 \\
\hline 6 & 356 & 6 & I 56 & 6 & 950 & 6 & 730 & 6 & 500 \\
\hline $\begin{array}{l}7 \\
8\end{array}$ & $\begin{array}{l}392 \\
428\end{array}$ & $\begin{array}{l}7 \\
8\end{array}$ & $\begin{array}{l}192 \\
228\end{array}$ & $\begin{array}{l}7 \\
8\end{array}$ & $\begin{array}{r}985 \\
\text { I. } 361020\end{array}$ & $\begin{array}{l}7 \\
8\end{array}$ & $\begin{array}{l}7^{65} \\
800\end{array}$ & 8 & 535 \\
\hline $\begin{array}{l}0 \\
9\end{array}$ & $\begin{array}{l}420 \\
464\end{array}$ & $\begin{array}{l}0 \\
9\end{array}$ & $\begin{array}{l}228 \\
264\end{array}$ & $\begin{array}{l}0 \\
9\end{array}$ & $\begin{array}{r}\text { I. } 301020 \\
055\end{array}$ & $\begin{array}{l}8 \\
9\end{array}$ & $\begin{array}{l}800 \\
835\end{array}$ & 9 & $\begin{array}{l}57^{\circ} \\
605\end{array}$ \\
\hline 80.0 & 500 & 85.0 & 300 & 90.0 & 090 & 95.0 & 870 & 100.0 & 640 \\
\hline
\end{tabular}


degradation of the sharpness of the border-line. On the other hand, with a sufficient quantity of solution, the border-line is surprisingly sharp.

The refractometer is now suspended on the frame, and the measurement proceeded with as before described. After measurement, the cover is first removed, and the prism allowed to fall into the hollow of the hand, then the beaker is removed to enable the refractometer to be conveniently cleaned.

Strengths of Various Solutions.-The most extensive work on the quantitative determination of the strength of a large number of common aqueous solutions with the immersion refractometer has been done by Wagner, who has published a large number of tables. These tatles show the percentage strength (grams per $100 \mathrm{cc}$. at $17.5^{\circ} \mathrm{C}$.) of a large number of salt solutions and of acids, corresponding to the range of scale readings of the instrument, as well as of cane sugar, dextrose, formalde-

SCALE READINGS ON IMMERSION REFRACTOMETER OF VARIOUS STANDAKD REAGENTS USED IN VOLUMETRIC ANALYSIS.*

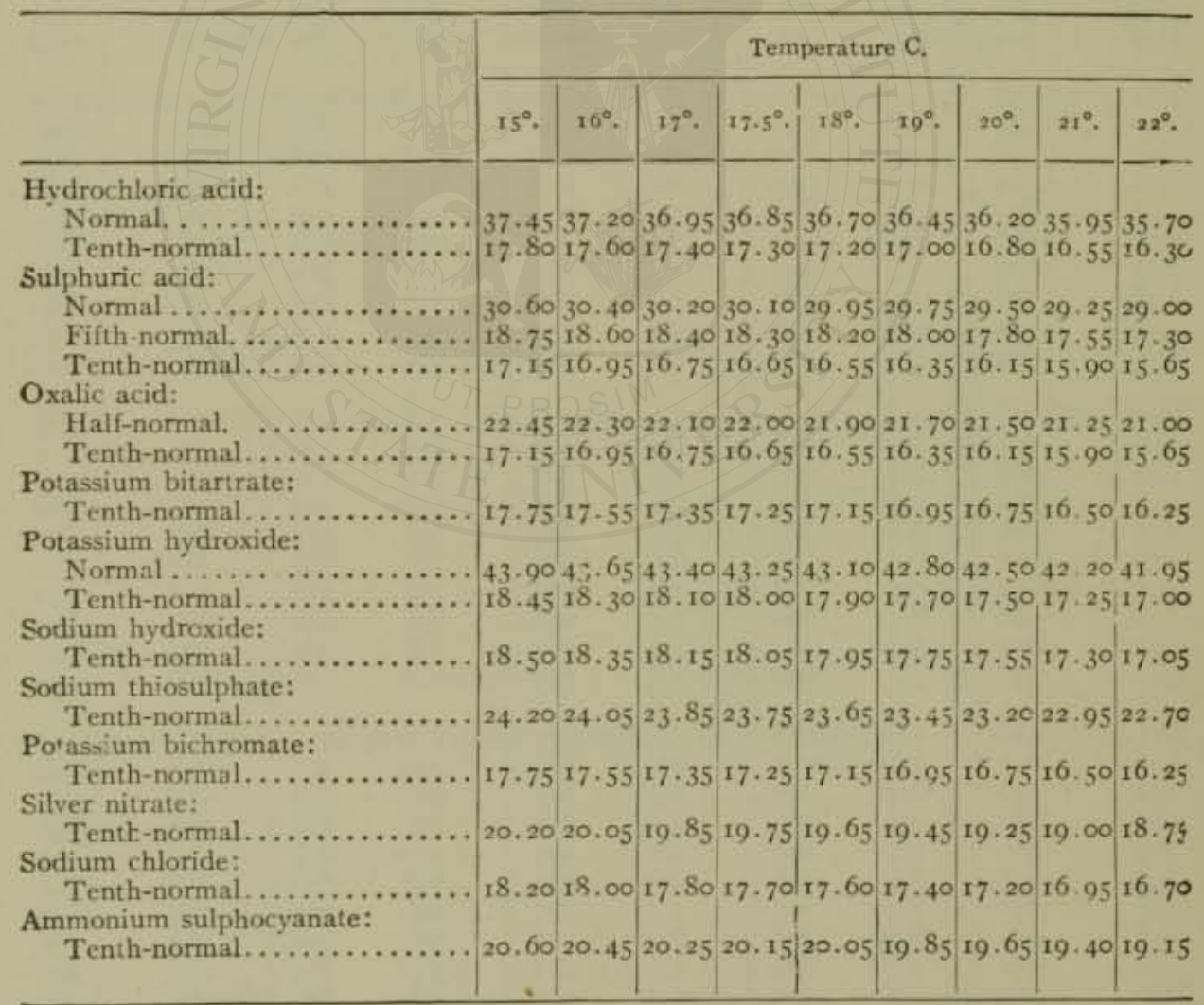

- According to Wagner, all these solutions were made up at $17.5^{\circ} \mathrm{C}$. Readings at different temperatures are given for convenience. 
hyde, alcohol, etc. All these observations have been based on the Mohr liter, at a temperature of $17.5^{\circ}$. More convenient for the American analyst would be tables based on the use of a higher temperature, say $20^{\circ}$, and the analyst is recommended to work out his own standards for comparison, at the temperature best suited to his special locality and convenience. The instrument is especially useful in preparing normal and tenthnormal solutions.

The table on page I20, from Wagner, shows the strength of various common laboratory reagents.

SCALE READINGS AT TFMPERATURES FROM $10-30^{\circ} \mathrm{C}$.

CORRECTED to $17.5^{\circ}$, ACCORDING to Wagner.

\begin{tabular}{|c|c|c|c|c|c|c|c|c|c|c|c|c|c|}
\hline No. & x. & 2. & 3. & 4. & 5. & 6. & 7. & 8. & 9. & 10. & Ir. & $12 \& 13$. & \multirow{3}{*}{ 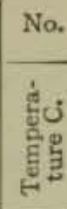 } \\
\hline ț & \multicolumn{12}{|c|}{ Seale Reading at $17.5^{\circ} \mathrm{C}$. } & \\
\hline Eg & 15. & 20. & 25. & 30. & 35. & 40. & 45. & 50. & 60. & 70. & 80. & $00 \& 100$. & \\
\hline $3^{\circ}$ & 3.20 & 3.15 & 3.25 & $3.4 \circ$ & $3 \cdot 55$ & 3.65 & 3.90 & 4.05 & 4.20 & 4.60 & 4.80 & 5.25 & 30 \\
\hline 29 & 2.90 & 2.85 & 2.95 & 3.10 & 3. 25 & $3 \cdot 35$ & .55 & .75 & 3.90 & 4.25 & $4 \cdot 45$ & 4.85 & 29 \\
\hline 28 & 2.60 & 55 & 2.65 & 2.80 & 2.95 & 3.05 & 3.25 & 3.45 & 3.60 & 3.90 & 4. 10 & & 28 \\
\hline 27 & $2 \cdot 30$ & 2.25 & 2.35 & $2.5^{\circ}$ & 2.65 & 2.75 & 2.95 & 3.15 & $3 \cdot 3^{\circ}$ & $3 \cdot 50$ & 3.75 & & 27 \\
\hline 26 & 1.00 & 1. 95 & 2,05 & 2.20 & 2.35 & 2.45 & 2.55 & 2.80 & 2.95 & 3.10 & $3 \cdot 3^{\circ}$ & & 26 \\
\hline 25 & 1. 75 & I. 75 & 1. 80 & I. $99^{\circ}$ & 2.05 & 2.15 & 2.25 & 2.45 & 2.60 & 2.70 & 2.95 & 3.20 & 25 \\
\hline 24 & $\times 50$ & I. 45 & 55 & 1.60 & I. 75 & 1. 85 & .95 & 2.10 & 2.25 & 2.35 & 2,55 & 2.75 & 24 \\
\hline 23 & & & & 1. 35 & & .55 & & & $1.9^{\circ}$ & 2.00 & 2.15 & & 23 \\
\hline 22 & $1, \infty 0$ & $1 . \infty$ & I. 05 & 1. 10 & I.15 & I. 25 & I. 30 & I. 40 & 1.55 & I. 65 & 1. 75 & & 22 \\
\hline 21 & 0.75 & 0.75 & 0.80 & 0.85 & 0.90 & 0.95 & $\mathbf{I . 0 5}$ & 1.05 & 1,20 & 1,25 & I. 35 & I. 45 & $2 \mathrm{I}$ \\
\hline 20 & 0.50 & 0.50 & 0.55 & 0.60 & 0.65 & 0.65 & 0.75 & 0.75 & 0.85 & 0.90 & 0.95 & 1.05 & 20 \\
\hline 19 & & 。 & 30 & 35 & 0.40 & 0.40 & 45 & & 0.45 & 0.55 & 0.55 & 0.60 & 19 \\
\hline 18 & 0.10 & 0.10 & 0.10 & 0.15 & 0.15 & 0.15 & 0.15 & 0.5 & 0.15 & 0.20 & 0.20 & .20 & Is \\
\hline 27.5 & 0.00 & 0.00 & $0.00 \mid$ & 0.00 & 0.00 & 0.00 & 0.00 & 0.00 & 0.00 & 0.00 & 0.00 & 0.00 & ${ }^{17}$. \\
\hline 17 & -0.10 & 10 & 0.10 & 0.10 & 0.10 & 0.10 & .15 & 0.15 & 0.15 & 0.15 & 0.20 & & 17 \\
\hline 16 & $0.3^{\circ}$ & 0.30 & 0.30 & $0.3^{\circ}$ & 0.35 & 0.35 & 0.40 & 0.45 & 0.45 & $0.5^{\circ}$ & 0.55 & 0.55 & 16 \\
\hline I5 & $0.5^{\circ}$ & 0.45 & 0.45 & $0.5^{\circ}$ & 0.60 & 0.60 & 0.65 & 0.75 & 0.75 & 0.80 & 0.85 & 0.90 & I5 \\
\hline $\mathrm{I}_{4}$ & 0.70 & 0.60 & 0.60 & & & & & & .05 & .10 & 1. 25 & & 4 \\
\hline$x_{3}$ & $0.8_{5}$ & 0.75 & 0.75 & $0 . \dot{8}_{5}$ & 1.00 & 1.10 & I. 15 & 1.20 & 1. 35 & $1.40^{\circ}$ & I. 55 & I. & 13 \\
\hline 12 & & & & $\ldots \ldots$ & $\cdots \cdots$ & $\cdots \cdots$ & ..... & $\ldots \ldots$ & ..... & & ...... & $\ldots$ & 12 \\
\hline II & I. 15 & & 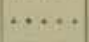 & .. & & $\cdots$ & …. & & & & .. & $\ldots$ & 11 \\
\hline ro & 1.25 & $\cdots \cdots$ & … & $\ldots \ldots$ & . & 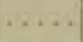 & ..... & .... & & $\cdots$ & $\ldots \ldots$ & $\ldots \ldots$ & . \\
\hline No. & x. & 2. & 3. & 4. & 5. & 6. & 7. & 8. & 9. & ro, & II. & $2 \& 13$. & No. \\
\hline
\end{tabular}




\section{REFERENCES ON THE BUTYRO-REFRACTOMETER.}

Lythgoe, H. C. The Optical Properties of Castor Oil, Cod-liver Oil, Neats-foot Oil, and a few Essential Oils. Jour. Am. Chem. Soc., 27, 1905, p. 887.

Schnemer, C., and Blumfnfit. S, B. Beitrag zur Kenntnis animalischer Fette. Chem. Zeit., 30, 1906, p. 53 .

Sprinkmeyer, H., and Wagner, H. Beiträge zur Kenntnis des Sesamöles. Zeits. Unters. Nahr. Genuss., 10, 1905, p. 347.

Ulzer, F. and Sommer, F. Uber den Nachweis und die Bestimmung des Paraffins in Mischungen mit Ceresin. Chem. Zeit., 30, 1906, p. 142.

\section{REFERENCES ON THE WOLLNY MILK FAT REFRACTOMETER.}

BAIER, E. Untersuchungen über den Nachweis der Wässerung von Milch mit Hilfe des Refraktometers. Ber. d. Nahr. Unters. d. Landw, f. d. Provinz Brandenburg, 1904, p. 14 .

_ Ueber die Zuverlässigkeit der Milchuntersuchungen mit dem Milch-refraktometer von Zeiss-Wollny. Molk. Zeit., 15, 1905, P. $3^{86 .}$

CothereaU, A. Nachweis einer Milchwässerung mittels des Refraktometers. Bull. Sci. Pharm., 7, 1905, p. 68.

Henseval, M., and Mullie, G. La Refractometrie du Lait. Rev, gen. du Lait, 4, 1905, p. 529 .

\section{REFERENCES ON THE ABBE REFRACTOMETER.}

HaRvey, T. F. Temperature Corrections for Use with the Abbé Refractometer, and Refractive Indices of some Fixed and Essential Oils. Jour. Soc. Chem. Ind., 24, 1905, p. 717.

Lithgoe, H. C. The Optical Properties of Castor Oil, Cod-liver Oil, Neats-foot Oil, and a Few Essential Oils. Jour. Am. Chem, Soc., 27, 1905, p. 887.

UTz, Fr. Beiträge zur Untersuchung von Amylalkohol. Allgemeine Chem. Zeit., 6, 1906, p. 106 .

- Die Untersuchung von Spiritus mittels des Refraktometers. Pharm. Nach., I, 1906, p. 74 .

\section{REFERENCES ON THE IMMERSION REFRACTOMETER.}

Ackermann, E. Ueber refraktometrische Bieranalyse. Zeits. f. d. ges. Brauw., 28, I905, p. $44 \mathrm{I}$.

Methode refractometrique rapide d'analyse de la biere a l'aide d'un calculateur automatique. Ann. et Rev. de Chim. Anal., I905, p. I7I.

Ackermann, E, et von Spindler, O. Sur la Determination de l'Extrait de la Bierre. Jour, suisse de Chim. et Pharm, 1903, No. 30 .

Hanus, J., and Chocensky, K. Anwendung des Zeisschen Eintauchrefraktometers in der Nahrungsmittelanalyse. Zeits. Unters. Nahr. Genuss., II, I906, p. $3^{13}$. 
LeAch, A. E., and Lythgoe, H. C. The Detection and Determination of Ethyl and Methyl Alcohols in Mixtures by the Immersion Refractometer. Jour. Am. Chem. Soc., 27, 1905 , p. 964.

- A Comparative Refractometer Scale for Use with Fats and Oils. Jour. Am. Chem. Soc., 26, 1904, p. 1193 .

The Detection of Watered Milk. Ibiç, p. 1195.

Kıonka, H. Ueber natürliche und künstliche Mineralwasser. Balneolog. Zeit., I4, Nos. 34 u. 35 .

Mansfeid, M. Die Verwendbarkeit des Zeiss'schen Eintauchrefractometer bei Nahrungsmittel-Untersuchung. Unters. Anst. östers. Apoth.-Vereins. Ber., 1904-1905, p. ro.

Matrmes, H. Quantitative Bestimmungen wässeriger Lösungen mit dem Zeiss'schen Eintauch-Refraktometer. Zeits. Unters. Nahr. Genuss., 5, 1902, p. 1037.

- Ueber refraktometrische analytische Bestimmungsmethoden. Zeits. anal. Chem., 13,1904, p. 73 .

MонR, M. Die Anwendung des Zeiss'schen Eintauchrefraktometers im Brauereilaboratorium. Wochens. Brau., 22, 1905, p. 616.

MoнR, O. Refraktometrische Extraktbestimmung bei der Malzanalyse. Wochens. $f$. Brau., 23, 1906, P. 136.

Wagner, B. Neue Methoden der quantitativen Bestimmung mit dem Zeiss'schen Eintauchrefraktometer. Zeits, öffentl. Chem., 11, 1905, p. 404.

_ Ueber quantitative Bestimmungen wässeriger Lösungen mit dem Zeiss'-schen Eintauch-Refraktometer. Sondershausen, 1903.

- Tabellen zum Eintauch-Refraktometer. Sondershausen, 1907.

WAGNER, B., and RiNCK, A. Neue Methode der quantitativen Zuckerbestimmung mit dem Zeiss'schen Eintauchrefraktometer. Chem. Zeit., 30, 1906, p. 38.

—Zeits. Chem. Apparat, Berlin, I, 1906, p. 207. 
CHAPTER VII.

MILK AND ITS PRODUCTS.

MILK.

Nature and Composition.-Milk is the secretion of the mammary glands of female mammals for the nourishment of their young. Containing as it does all the requisites for a complete food, i.e., sugar, fat, proteins, and mineral ingredients, combined in appropriate proportion, there is ample reason why it occupies so high a place in the scale of human foods. It is a yellowish-white opaque fluid, denser than water, containing in complete solution the sugar, soluble albumin, and mineral content, and, in less complete solution, the casein, while the fat-globules are held in suspension in the serum, forming an emulsion.

The specific gravity of pure milk ranges from 1.027 to 1.035 .

Milk from various animals has the same general physical properties and the same ingredients, differing, however, in percentage composition. Of all the varieties, the milk of the cow is by far the most important from its universal use, and, unless otherwise qualified, the term milk wherever it occurs in this volume will be understood to mean cow's milk.

Acidity.-When perfectly fresh, milk of carnivorous mammals is, as a rule, acid in reaction, while human milk and that of the herbivora is alkaline. Cow's milk, when freshly drawn, is more often amphoteric in reaction, i.e., it reacts acid with blue and alkaline with red litmus. It soon becomes distinctly acid, and the acidity increases as the milk sugar gradually becomes converted into lactic acid.

Microscopical Appearance.-Under the microscope pure milk shows a conglomeration of various-sized fat globules having a pearly lustre. These globules vary from $0.00 \mathrm{I}$ to $0.0 \mathrm{Im}$. in diameter, averaging about $0.005 \mathrm{~mm}$. When examined under very high powers, it is possible to distinguish bacteria in the milk, the number to be seen depending greatly on the time that has elapsed since the milk was drawn from its source, as well as on the surroundings, the conditions of handling, exposure, etc. 
Color.-The yellow color of milk is imparted to it by the fat globules, and varies greatly in milk from different breeds of cattle, as well as in milk from the same cow at different seasons, being, as a rule, paler during the winter or stall-fed months, and having its greatest intensity soon after the cow is put out to pasture.

Milk Sugar, the carbohydrate of milk, is normally present in amounts varying from 3 to 5 per cent. For the properties of milk sugar see page 577 .

The Proteins of Milk.-Casein constitutes about $80 \%$ of the entire proteins of milk, being present in an average sample to the extent of about $3 \%$. It exists in combination with calcium phosphate, and probably does not form a perfect solution in the milk, but is rather diffused therein in a somewhat colloidal form, being so finely divided, however, as to be incapable of separation by filtration while the milk is fresh.

Pure casein is a white, odorless, and tasteless solid, sparingly soluble in water, and insoluble in ether and alcohol. It is readily soluble in dilute alkalies. Strong acids also dissolve it, but its character is changed. From alkaline solution it is precipitated without change by neutralizing with acid. Its solutions are lævo-rotary.

Lact-albumin is the soluble albumin of milk, existing therein to the extent of about $0.6 \%$ and forming about $15 \%$ or more of the milk proteins. It much resembles the albumin of eggs, being coagulated at $70^{\circ}$ to $75^{\circ} \mathrm{C}$. It is readily soluble in water. Its specific rotary power according to Béchamp is $[\alpha]_{\mathrm{D}}=-67.5$.

Lactoglobulin has been discovered by Emmerling as a constituent in milk, but exists in traces only. According to Babcock, it may be separated from milk whey by carefully neutralizing with sodium hydroxide, and afterwards saturating with magnesium sulphate. It much resembles the globulin of blood serum, being coagulated at $67^{\circ}$ to $76^{\circ} \mathrm{C}$.

Fibrin.-Babcock has discovered in milk very minute traces of a substance anaiogous to the fibrin of blood. This substance, it is claimed, forms a part of the slime found in the separator-bowl of a centrifugal skimmer.

Other Nitrogenous Substances.-Besides the above normal constituents of milk. certain bodies may be formed by proteolytic action during fermentation, such, for example, as caseoses and peptones, formed for the most part by the decomposition of a part of the casein. Galactin is a gelatin-like body of the nature of peptone, occurring in traces in milk. Besides these, minute traces of amido-bodies, such as creatin and urea, are sometimes present, and also ammonia. 


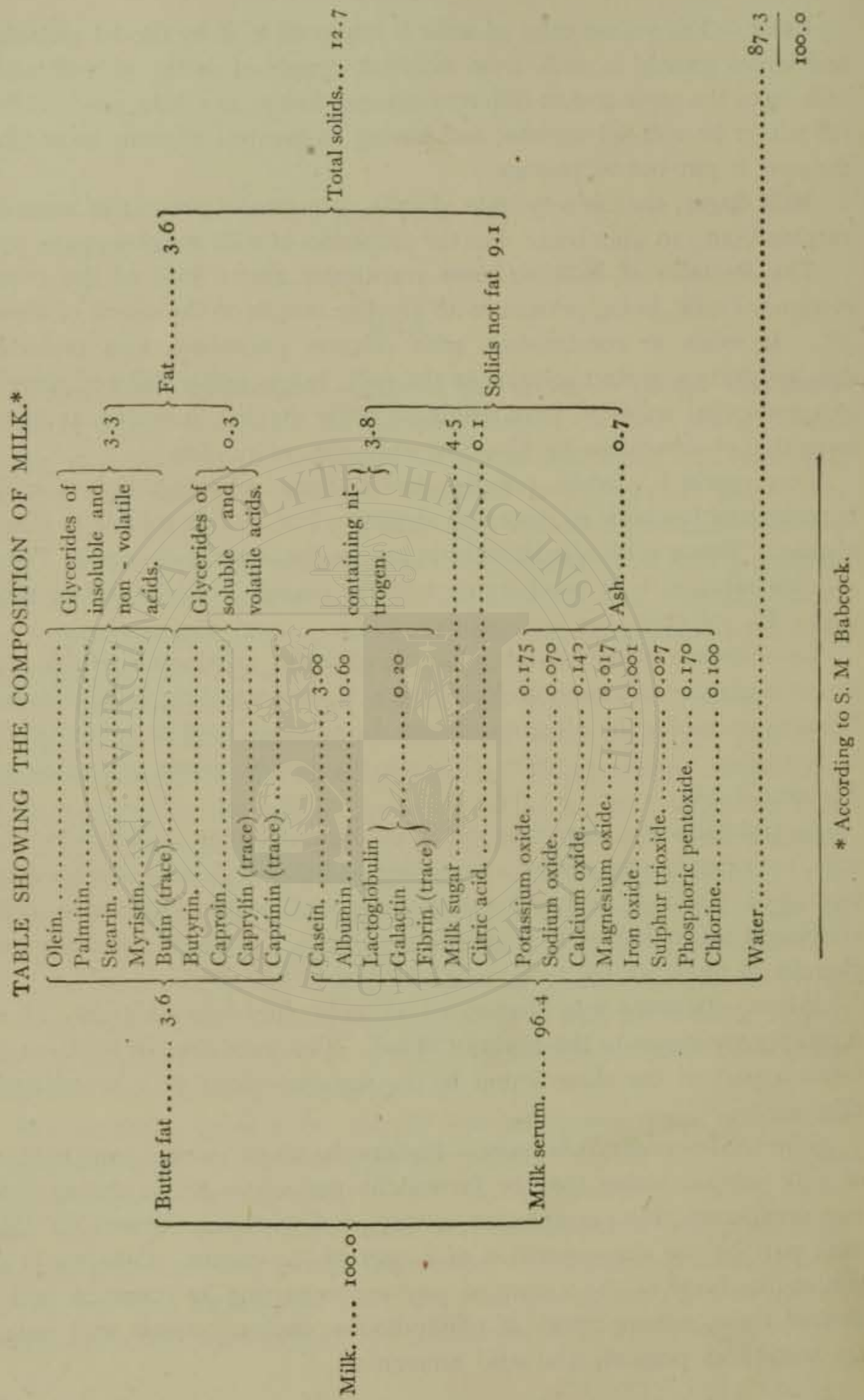


Milk Fat.-Fat forms the most variable constituent of milk, being found in proportions ranging from 2.5 to 7 per cent. For the chemical composition and characteristics of milk fats see Butter (p. 529).

The fat globules are held in suspension in the milk and have long been thought to be surrounded each by a thin nitrogenous membrane, known as Storch's mucoid protein, which becomes broken on churning. This theory, while rendered probable by many of the phenomena connected with the dairy, is by no means universally held at present.

Citric Acid has been found to exist in milk, probably in combination with certain of the mineral constituents, being present to the extent of about $0.1 \%$.

The table on page I 26 arranged by Babcock shows quite clearly the percentage composition of an average cow's milk.

For comparison of milk from different animals the following table * is inserted, showing in most cases minimum, maximum, and mean determinations from a large number of actual analyses:

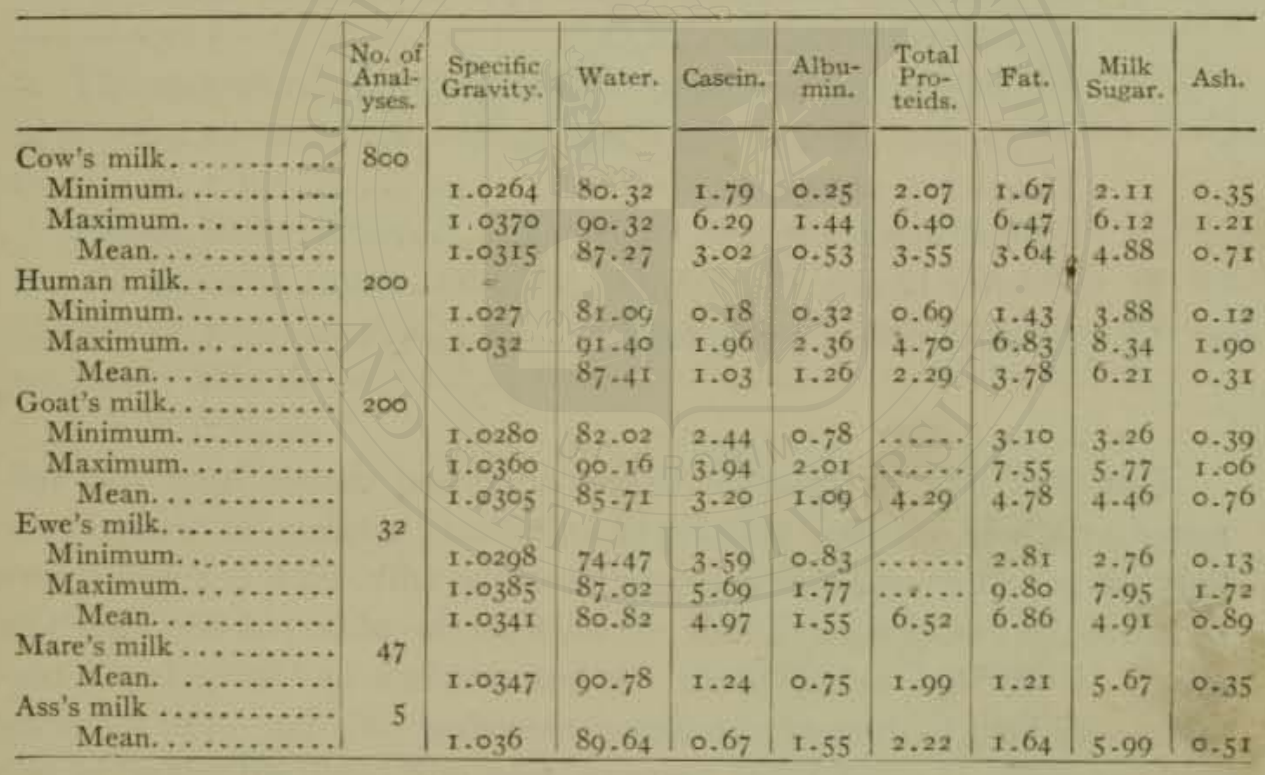

Composition of the Ash of Milk.-The ash of milk does not truly represent the mineral content, since, in the process of incineration, the character of some of the constituents is altered by oxidation and otherwise.

Expressed in parts per 100, the ash of the typical milk sample whose full analysis is given on page 126 would be about as follows:

\footnotetext{
* Compiled from König's Chemie der mens. Nahr, u. Genuss.
} 
Potassium oxide........................ 25.02

Sodium " " $\quad \ldots \ldots \ldots \ldots \ldots \ldots \ldots \ldots \ldots$ 10.01

Calcium " " $\ldots \ldots \ldots \ldots \ldots \ldots \ldots \ldots . .20 .01$

Magnesium "

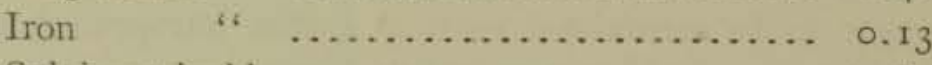

Sulphur trioxide. ........................ 3.84

Phosphoric pentoxide. ................... 24.29

Chlorine.......................... 14.28

100.00

Soldner regards the following as more nearly representing the proportion in which the mineral salts exist in milk:

Sodium chloride, $\mathrm{NaCl} \ldots \ldots \ldots \ldots \ldots \ldots \ldots$ 10.62

Potassium caloride, $\mathrm{KCl} \ldots \ldots \ldots \ldots \ldots \ldots \ldots . \ldots \ldots$

Mono-potassium phosphate, $\mathrm{KH}_{3} \mathrm{PO}_{4} \ldots \ldots \ldots \ldots 12.77$

Di-potassium phosphate, $\mathrm{K}_{2} \mathrm{HPO}_{4} \ldots \ldots \ldots \ldots \ldots \quad 9.22$

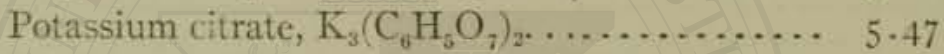

Di-magnesium phosphate, $\mathrm{MgHPO}_{4} \ldots \ldots \ldots \ldots$. $3 \cdot 7 \mathrm{I}$

Magnesium citrate, $\mathrm{Mg}_{3}\left(\mathrm{C}_{6} \mathrm{H}_{5} \mathrm{O}_{7}\right)_{2} \ldots \ldots \ldots \ldots .4 .05$

Di-calcium phosphate, $\mathrm{CaHPO}_{4} \ldots \ldots \ldots \ldots \ldots .7 \cdot{ }^{2}$

Tri-calcium phosphate, $\mathrm{Ca}_{3}\left(\mathrm{PO}_{4}\right)_{2} \ldots \ldots \ldots \ldots . \quad 8.90$

Calcium citrate, $\mathrm{Ca}_{3}\left(\mathrm{C}_{6} \mathrm{H}_{3} \mathrm{O}_{7}\right)_{2} \ldots \ldots \ldots \ldots \ldots \ldots 23.55$

Lime, combined with proteins............... 5.13

100.00

Fore Milk and Strippings.-Unless a portion drawn from the wellmixed or whole complete milking of an animal is taken for analysis, one does not get a fair representative sample of the milk, for it is a well-known fact that the first portion of milk drawn from the udder, termed the "fore milk," is very low in fat, while the last portions or "strippings" contain a very high fat content, sometimes exceeding $10 \%$ fat. The following analyses show the difference between fore milk and strippings in two cases:

\begin{tabular}{|c|c|c|c|}
\hline & $\begin{array}{l}\text { Per Cent } \\
\text { Water. }\end{array}$ & $\begin{array}{l}\text { Per Cent } \\
\text { Solids. }\end{array}$ & $\begin{array}{c}\text { Per Cent } \\
\text { Fat. }\end{array}$ \\
\hline 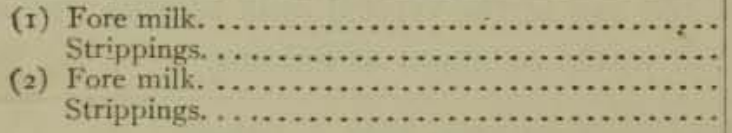 & $\begin{array}{l}88.17 \\
80.82 \\
88.73 \\
80.37\end{array}$ & $\begin{array}{l}11.83 \\
19.18 \\
11.27 \\
19.63\end{array}$ & $\begin{array}{r}1.32 \\
9.63 \\
1.07 \\
10.3^{6}\end{array}$ \\
\hline
\end{tabular}


The per cent of albuminoids, sugar, and ash is nearly the same in both fore milk and strippings.

Colostrum.-The milk given by cows and other mammals for two or three days after the birth of young is termed colostrum, and differs materially in composition from normal milk. It is yellow in color, of an oily consistency, and has a pungent taste. It acts as a purge upon the young. Examined under the microscope, it is found to contain large circular cells larger than fat globules and somewhat similar to blood corpuscles. It is very high in albumin, which seems to be similar to blood albumin. The following analyses were made by Engling, showing the composition of colostrum from a cow eight years old:

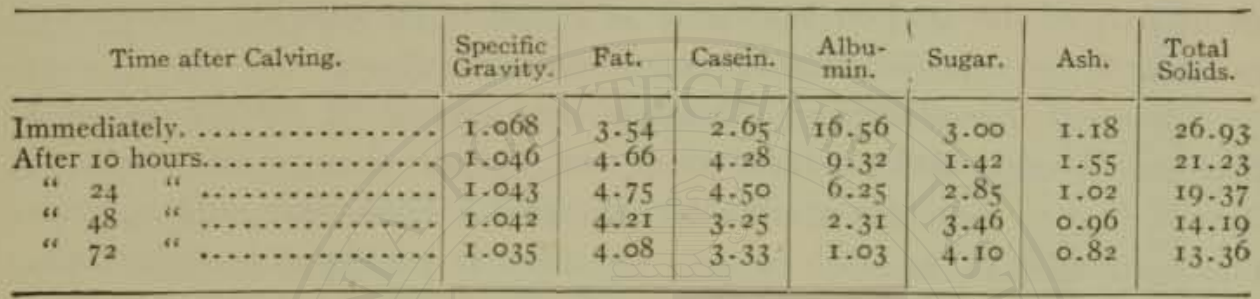

The average of twenty-two analyses of colostrum from different cows by Engling showed total solids $28.3 \mathrm{I}$, fat 3.37 , casein 4.83 , albumin ${ }_{55} .85$, sugar 2.48, ash I. 78 .

Frozen Milk.-Since it is the water in milk that freezes, it follows that in partially frozen milk the unfrozen portion of the milk, or that part which remains still liquid, becomes concentrated by the process of freezing. This is borne out by the following figures of Richmond:*

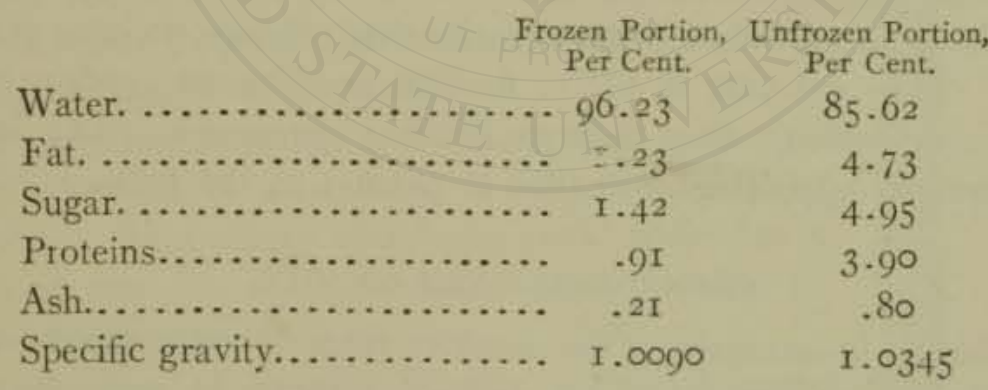

Fermentations of Milk.-These are due to the action of bacteria of various kinds, the most common being the lactic fermentation.

The Souring of Milk is caused by the action of a large number of species of acid-forming bacteria, chief among which is the Bacillus acidi lactici, which multiplies faster than other bacteria in raw milk under

* Analyst, XVIII. p. 53 . 
favorable conditions of temperature. Part of the milk sugar is acted on and transformed, first into dextrose and galactose, the latter sugar subsequently forming lactic acid, as follows:

$$
\begin{aligned}
& \mathrm{C}_{12} \mathrm{H}_{22} \mathrm{O}_{21}, \mathrm{H}_{2} \mathrm{O}=\underset{\text { Lactose }}{\mathrm{C}_{6} \mathrm{H}_{12} \mathrm{O}_{6}}+\underset{\text { Dextrose }}{\mathrm{C}_{6} \mathrm{H}_{12} \mathrm{O}_{6}} \text { Galactose } \\
& \mathrm{C}_{5} \mathrm{H}_{12} \mathrm{O}_{6}={ }_{2} \mathrm{C}_{3} \mathrm{H}_{6} \mathrm{O}_{3} \\
& \text { Galactose Lactic acid }
\end{aligned}
$$

More and more acid is formed until the casein can no longer be held up, curdling ensues, and the casein is precipitated. Finally, after a certain degree of acidity is reached, the ferment is killed and the action stops. Other acids than lactic are also undoubtedly produced, since a small part of the acid in sour milk is found to be volatile. According to Conn* the volatile acids are acetic and formic.

Abnormal Fermentation.-Through the agency of micro-organisms that may develop under certain conditions, various changes are produced in milk that to some extent alter its character. Thus bitter milk is sometimes produced as the result of some organism as yet but little understood.

Occasionally milk is found possessing a peculiarly thick and slimy consistency, whereby it may be drawn out in threads, by dipping a spoon into the milk and withdrawing it therefrom. This is termed ropy milk, and is more often met with in warm weather. It is undoubtedly produced as a result of bacterial action.

Enzyme-forming Bacteria are not uncommonly developed in milk, causing various proteolytic changes, whereby the casein is partially transformed into peptones, caseoses, etc.

Chromogenic Bacteria are the agencies that produce peculiar pigments in milk. Thus red milk is due to Bacillus erythrogenes; yellow milk to Bacillus xynxanthus; blue milk to Bacillus cyanagenes. The latter is quite common, appearing ordinarily in patches in the milk.

\section{CHEMICAL ANALYSIS OF MILK.}

Ordinarily, in ascertaining the nutritive value of milk, one determines its specific gravity, total solids, fat, protein, milk sugar, and ash. Occasionally it is thought desirable to make a distinction in the case of protein between the casein and the albumin. Rarely is it necessary to further subdivide the nitrogenous bodies in milk, unless in connection with a special study of the proteolytic changes which it undergoes.

The total solids, fat, and ash are usually all determined directly, and,

* U. S. Dept. of Agric., Off. of Exp. Stations, Bul. 25, p. 21. 
in the case of the milk sugar and the proteins, a determination of either one may be directly made (whichever is most convenient), the other being calculated by difference.

When foreign ingredients or adulterants are present in milk, special methods are employed to detect them.

Preparation of the Sample.-In procuring a sample for analysis, the greatest care is necessary to insure a homogeneous sample. By far the best method in every case, where possible, is to pour the milk back and forth from one vessel to another (i.e., pour from the original container into an empty vessel and back at least once). Where this is impossible from the size of the container or for any other reason, the milk should be thoroughly mixed with a dipper. A "sampler," of which the Scovell sampling-tube Fig. 43, A) is a convenient form, also aids in securing a representative sample, and is invaluable when it is desirable to secure a definite fraction of the whole for a composite sample.

This instrument consists of a brass or copper tube made in two parts which telescope accurately together as shown in Fig. 43, A, the lower part being closed at the bottom, but provided with three or more lateral slits. The sampler, drawn out to its full length, is carefully inserted in the tank containing the milk and lowered to the bottom, after which the upper part is pressed down over the lower so as to close the slits, and the tube is then lifted out of the tank, containing a fairly representative sample of the milk.

In all operations to which a milk sample is submitted during the process of analysis, it should invariably be poured into a clean empty vessel and back, whenever it has been at rest for an appreciable time, in order to insure a homogeneous mixture.

Determination of Specific Gravity.-This is most readily obtained with the aid of a hydrometer, accurately graduated within the limits of the widest possible variation in the specific gravity of milk. Hydrometers for special use with milk are known as lactometers, and are graduated variously. One of the most convenient forms of this instrument is the Quevenne lactometer, graduated

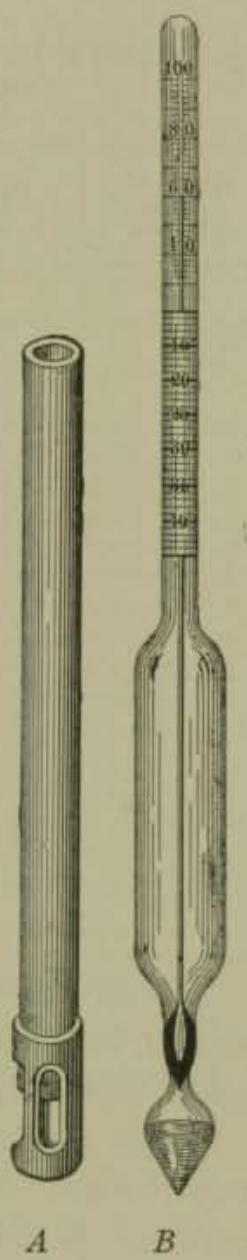

FIG. 43 .

A, Scovell Milksampling Tube. $B$, Quevenne Lactometer. from $15^{\circ}$ to $40^{\circ}$, corresponding to specific gravity 1.015 to 1.040 . This 
instrument, shown in Fig. $43, B$, has a thermometer combined with it, the stem containing a double scale, on the lower part of which the specific gravity is read, while the temperature is read from the upper part.

Another form of instrument is termed the New York Board of Health lactometer, which is not graduated to read the specific gravity directly, but has an arbitrary scale divided into 120 equal parts, the zero being equal to the specific gravity of water, while 100 corresponds to a specific gravity of 1.029. To convert readings on the New York Board of Health scale to Quevenne degrees they must be multiplied by .29.

\section{QUEVENNE LACTOMETER DEGREES CORRESPONDING TO NEW YORK BOARD OF HEALTH LACTOMETER DEGREES.}

\begin{tabular}{|c|c|c|c|c|c|}
\hline $\begin{array}{l}\text { Board of } \\
\text { Health } \\
\text { Degrees. }\end{array}$ & $\begin{array}{l}\text { Quevenne } \\
\text { Scale. }\end{array}$ & $\begin{array}{l}\text { Board of } \\
\text { Health } \\
\text { Degrees. }\end{array}$ & $\begin{array}{l}\text { Quevenne } \\
\text { Scale. }\end{array}$ & $\begin{array}{l}\text { Board of } \\
\text { Health } \\
\text { Degrees. }\end{array}$ & $\begin{array}{l}\text { Quevenne } \\
\text { Scale. }\end{array}$ \\
\hline $\begin{array}{l}60 \\
61 \\
62 \\
63 \\
64 \\
65 \\
66 \\
67 \\
68 \\
69 \\
70 \\
71 \\
72 \\
73 \\
74 \\
75 \\
76 \\
77 \\
78 \\
79 \\
80\end{array}$ & $\begin{array}{l}17.4 \\
17.7 \\
18.0 \\
18.3 \\
18.6 \\
18.8 \\
19.1 \\
19.4 \\
19.7 \\
20.0 \\
20.3 \\
20.6 \\
20.9 \\
21.2 \\
21.5 \\
21.7 \\
22.0 \\
22.3 \\
22.6 \\
22.9 \\
23.2\end{array}$ & $\begin{array}{r}81 \\
82 \\
83 \\
84 \\
85 \\
85 \\
86 \\
87 \\
88 \\
89 \\
90 \\
91 \\
92 \\
93 \\
94 \\
95 \\
96 \\
97 \\
98 \\
99 \\
100\end{array}$ & $\begin{array}{l}23 \cdot 5 \\
23.8 \\
24 \cdot 1 \\
24 \cdot 4 \\
24.6 \\
24 \cdot 9 \\
25 \cdot 2 \\
25 \cdot 5 \\
25.8 \\
26.1 \\
26.4 \\
26.7 \\
27.0 \\
27 \cdot 3 \\
27.6 \\
27.8 \\
28.1 \\
28.4 \\
28.7 \\
29.0\end{array}$ & $\begin{array}{l}101 \\
102 \\
103 \\
104 \\
105 \\
106 \\
107 \\
108 \\
109 \\
110 \\
111 \\
112 \\
113 \\
114 \\
115 \\
116 \\
117 \\
118 \\
119 \\
120\end{array}$ & $\begin{array}{l}29 \cdot 3 \\
29.6 \\
29 \cdot 9 \\
30 \cdot 2 \\
30 \cdot 5 \\
30 \cdot 7 \\
31 \cdot 0 \\
31 \cdot 3 \\
31.6 \\
31 \cdot 9 \\
32.2 \\
32 \cdot 5 \\
32.8 \\
33 \cdot 1 \\
33 \cdot 4 \\
33 \cdot 6 \\
33 \cdot 9 \\
34 \cdot 2 \\
34 \cdot 5 \\
34.8\end{array}$ \\
\hline
\end{tabular}

If extreme accuracy is desired, the Westphal balance or the pycnometer should be used for the determination of specific gravity. For ordinary cases, however, the lactometer, if carefully made, is sufficiently accurate.

With any other form of lactometer than the Quevenne, a separate thermometer is necessary in order to determine the temperature, the common practice being to standardize all such instruments at $60^{\circ} \mathrm{F}$. $\left(15.6^{\circ} \mathrm{C}\right.$.).

Readings at temperatures other than $60^{\circ}$ may be corrected to that temperature by the aid of the table on page $\mathrm{r} 33$.

DETERMINATION OF TOTAL SOLIDS.-Dish Method.-For purposes of milk analysis, platinum dishes are by far the most desirable. These, if made for the purpose, should be of the shape shown in Fig. $5^{\mathrm{I}}$, measur- 
FOR CORRECTING THE SPECIFIC GRAVITY OF MILK ACCORDING TO TEMPERATURE (BY DR. PAUL VIETH).

\begin{tabular}{|c|c|c|c|c|c|c|c|c|c|c|c|c|c|c|c|c|}
\hline \multirow{2}{*}{$\begin{array}{l}\text { Degrees } \\
\text { of } \\
\text { Lactom- } \\
\text { eter. }\end{array}$} & \multicolumn{16}{|c|}{ Degrees of Thermometer (Fahrenheit). } \\
\hline & 45 & 46 & 47 & 48 & 40 & 50 & $5 \mathrm{I}$ & 52 & 53 & 54 & 55 & 56 & 57 & 58 & 59 & 60 \\
\hline $20 \ldots \ldots$ & 19.0 & 19.0 & 19.1 & 19.1 & 19.2 & 19.2 & 19.3 & 19.4 & I9.4 & $19 \cdot 5$ & 19.6 & 19.7 & 19.8 & 19.9 & 19.9 & - \\
\hline $21 \ldots \ldots \ldots$ & 19.9 & 20.0 & 20.0 & 20.1 & 20.2 & 20.2 & 20.3 & $20 \cdot 3$ & 20.4 & 20.5 & 20.6 & 20.7 & 20.8 & 20.9 & 20.9 & 一 \\
\hline $22 \ldots \ldots \ldots$ & 20.9 & 21.0 & 21.0 & $\mid 21 \cdot 1$ & $21 \cdot 2$ & 21.2 & $21 \cdot 3$ & 21.3 & 21.4 & 21.5 & 521.6 & $21 \cdot 7$ & 21.8 & $32 t \cdot 9$ & $2 \mathrm{t} \cdot 9$ & 一 \\
\hline $23 \ldots \ldots \ldots$ & 21.9 & 22.0 & 22.0 & 22.1 & 22.2 & 22.2 & 22.3 & $22 \cdot 3$ & 22.4 & 22.5 & 522.6 & 22.7 & 22.8 & 322.8 & 22.9 & - \\
\hline $24 \ldots \ldots$ & 22.9 & 22.9 & 23.0 & 23.1 & 23.2 & 23.2 & $23 \cdot 3$ & $23 \cdot 3$ & 23.4 & $23 \cdot 5$ & 23.6 & 23.6 & $23-7$ & 23.8 & 23.9 & - \\
\hline $25 \ldots \ldots$. & 23.8 & 23.9 & 24.0 & 24.0 & $24 \cdot 1$ & $24 \cdot I$ & 24.2 & $24 \cdot 3$ & $24 \cdot 4$ & $24 \cdot 5$ & 524.6 & 24.6 & $24 \cdot 7$ & 24.8 & 24.9 & 一 \\
\hline $20 \ldots \ldots$ & 24.8 & 24.9 & 24.9 & 25.0 & 25.1 & 25,1 & 25.2 & 25.2 & $25 \cdot 3$ & 25.4 & 25.5 & 25.6 & $25 \cdot 7$ & 25.8 & 25.9 & - \\
\hline $27 \ldots \ldots$ & 25.8 & 25.9 & $25-9$ & 26.0 & 26.1 & 26.1 & 26.2 & 26.2 & 26.3 & 26.4 & 426.5 & 26.6 & 26.7 & 26.8 & 26.9 & 一 \\
\hline$\cdots \cdots$ & 26.7 & 26.8 & 26.8 & 26.9 & 27.0 & 27.0 & $27 \cdot 1$ & $27 \cdot 2$ & $27 \cdot 3$ & $27 \cdot 4$ & $27 \cdot 5$ & $27 \cdot 6$ & $27 \cdot 7$ & 27.8 & $27 \cdot 9$ & 一 \\
\hline 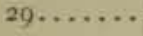 & 27.7 & 27.8 & 27.8 & 27.9 & 28.0 & 28.0 & 28.1 & 28.2 & $28 \cdot 3$ & 28.4 & 428.5 & 28.6 & 28.7 & 28.8 & 28.9 & - \\
\hline $30 \ldots \ldots$ & & 28.7 & 728.7 & 28.8 & 28.9 & 29.0 & $29 . I$ & 199.1 & 29.2 & $29 \cdot 3$ & $329 \cdot 4$ & 29.6 & $529 \cdot 7$ & 29.8 & 29.9 & - \\
\hline $3^{1} \ldots \ldots$ & $29 \cdot 5$ & 29.6 & 629.6 & 29.7 & 29.8 & 29.9 & 30.0 & 30.1 & 30.2 & 30.3 & 30.4 . & $3^{\circ} \cdot 5$ & 30.6 & 30.8 & 30.9 & - \\
\hline $3^{2} \ldots \ldots$ & 30.4 & 30.5 & $530 \cdot 5$ & 30.6 & $\mid 30.7$ & 30.9 & 31.0 & $\mid 31 . x$ & $\left|3^{1} \cdot 2\right|$ & $\mid 3^{x} \cdot 3$ & $33^{1} \cdot 4$. & $3^{x} \cdot 5$ & 31.6 & $31 \cdot 7$ & $|3+.9|$ & 一 \\
\hline $33 \ldots \ldots$ & $3^{\mathrm{I}} \cdot 3$ & $3 x \cdot 4$ & $+31 \cdot 4$ & $3 x-5 \mid$ & 31.6 & 31.8 & $31-9$ & 32.0 & $3^{2} \cdot 1$ & $32 \cdot 3$ & $32 \cdot 4$. & $32 \cdot 5$ & 32.6 & $32 \cdot 7$ & 32.9 & 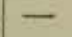 \\
\hline $34 \ldots \ldots$ & 32.2 & $32 \cdot 3$ & $33^{2} \cdot 3$ & $34 \cdot 4$ & $32 \cdot 5$ & $3^{2} \cdot 7$ & 32.9 & 33.0 & 33.1 & 33.2 & $33 \cdot 3$ & $33 \cdot 5$ & 33.6 & $33 \cdot 7$ & 33.9 & - \\
\hline \multirow[t]{2}{*}{$35 \ldots \ldots \ldots$} & 33.0 & $33 \cdot 1$ & 33.2 & $33-4$ & $33 \cdot 5$ & 33.6 & 33.8 & 33.9 & 34.0 & 34.2 & $234 \cdot 3$ & $34 \cdot 5$ & 34.6 & $34-7$ & $34-9$ & - \\
\hline & & $6 x$ & 62 & 63 & 64 & 65 & 66 & 67 & 68 & 60 & 70 & 71 & $7^{2}$ & 73 & 74 & 75 \\
\hline $20 \ldots$. & & 20.12 & 20.2 & 20.22 & 20.3 & $20.4=$ & 20.5 & 20.6 & 20.7 & 20.9 & 21.0 & $2 \mathrm{I} . \mathrm{I}$ & 21.2 & $2 \mathrm{x} \cdot 3$ & $2 x \cdot 5$ & 21.6 \\
\hline $21 \ldots$ & & $2 \mathrm{~T}, \mathrm{I}$ ? & 21.2 & $21-\left.3\right|^{2}$ & $2 \mathrm{I} \cdot 4$ & $21-52$ & 21.6 & 21.7 & 21.8 & 22.0 & 22.12 & 22.2 & $22 \cdot 3$ & 22.4 & 22.5 & 22.6 \\
\hline $22 \ldots \ldots$ & . & 22.1 & 22.2 & $|22 \cdot 3|^{2}$ & 22.4 & $22 \cdot 5$ & 22.6 & 22.7 & 22.8 & 23.0 & $23 . I^{2}$ & 23.2 & $23 \cdot 3$ & 23.4 & $23 \cdot 5$ & $23 \cdot 7$ \\
\hline $23 \ldots \ldots$ & $\cdots$ & $23.1=$ & 23.2 & $23 \cdot 3=$ & $23 \cdot 4$ & $23 \cdot 5$ & 23.6 & $23 \cdot 7$ & 23.8 & 24.0 & 24.12 & 24.2 & $24-3$ & $24 \cdot 4$ & 24.6 & $24 \cdot 7$ \\
\hline $24 \ldots \ldots$ & $*$ & $24 . I 2$ & 24.2 & $24 \cdot 3=$ & $24.4=$ & $24 \cdot 5$ & 24.6 & $24 \cdot 7$ & 24.9 & 25.0 & $|25 \cdot x| 2$ & 25.2 & $25 \cdot 3$ & $25 \cdot 5$ & 25.6 & 25.7 \\
\hline & & 25.1 & 25.2 & $25 \cdot 3=$ & $25 \cdot 4$ & $25 \cdot 5$ & 25.6 & $25 \cdot 7$ & 25.9 & 26.0 & 26.12 & 26.2 & 26.4 & 26.5 & 26.6 & 26.8 \\
\hline $26 \ldots$. & & 26.1 & 26.2 & 26.32 & 26.5 & 26.6 & 26.7 & 26.8 & 27.0 & $e 7 \cdot x$ & 27.22 & $27 \cdot 3$ & $27 \cdot 4$ & $27 \cdot 5$ & $|27-7|$ & 27.8 \\
\hline & & 27.1 & 27 & 27.4 & $27 \cdot 5$ & 27. & $27 \cdot 7$ & 27.8 & 28.0 & $28 . \mathrm{I}$ & 28.22 & 28.3 & 28.4 & 28.6 & 28.7 & 28.9 \\
\hline & & $28.1=$ & 28.3 & $28.4=$ & 28.5 & & 28.7 & 28.8 & 29.0 & 29.1 & 29.22 & 29.4 & 29.5 & 29.7 & 29.8 & 29.9 \\
\hline $29 \ldots \ldots$ & $\cdots$ & $29.1=$ & $29 \cdot 3$ & 29.42 & $29 \cdot 5$ & 29.6 & 29.8 & 29.9 & 30.1 & 30.2 & 30.3 & 30.4 & 30.5 & 30.7 & 30,9 & 31.0 \\
\hline $30 \ldots \ldots$ & $\ldots$ & 30.1 & 30.3 . & 30.43 & 30.5 & 30.7 . & 30.8 & 30.9 & $3 \mathrm{r}, \mathrm{T}$ & $3^{\mathrm{I}} \cdot 2$ & $31 \cdot 3$ & $3^{\mathrm{I}} \cdot 5$ & 31.6 & 31.8 & 31.9 & 32.1 \\
\hline $31 \ldots \ldots$ & $\cdots$ & $3 I, 2$ & $3 \mathrm{I} \cdot 3$ & $3 \mathrm{I} \cdot 4$ & $31 \cdot 5$ & $31 \cdot 7$ & $3^{I} \cdot 7$ & 31.8 & 32.0 & 32.2 & 32.4 & $32 \cdot 5$ & 32.6 & 32.8 & 33.0 & $33 . \mathrm{x}$ \\
\hline $32 \ldots \ldots$. & $\ldots$ & 32.2 & $3^{2} \cdot 3$ & $3^{2} \cdot 5$ & 32.6 & $3^{2} \cdot 7$ & $32 \cdot 9$ & 33.0 & 33.2 & $33 \cdot 3$ & 33.4 & 33.6 & 33.7 & 33.9 & 34.0 & 34.2 \\
\hline $33 \ldots \ldots \ldots$ & $\cdots$ & $33 \cdot 2$ & $33 \cdot 3$ & $33 \cdot 5$ & 33.63 & 33.8 & 33.9 & 34.0 & 34.2 & 34.3 & $34 \cdot 5$ & 34.6 & $34 \cdot 7$ & 34.9 & $35 \cdot x$ & 35. \\
\hline $34 \ldots \ldots$ & . & 34.2 & $34 \cdot 3$ & $34 \cdot 53$ & 34.63 & 34.8 & $34 \cdot 9$ & 35.0 & 35.2 & $35 \cdot 3$ & 35.5 & 35.6 & 35.8 & 36.0 & 36.1 & 36.3 \\
\hline & & 35.2 & $35 \cdot 3$ & $35 \cdot 53$ & 35.63 & 35.8 & $35 \cdot 9$ & $3^{6} \cdot I$ & 36.2 & 36.4 & 36.5 & $3^{6} .7$ & 36.8 & 37.0 & 37.2 & $37 \cdot 3$ \\
\hline
\end{tabular}

ing about $2 \frac{3}{4}$ inches in diameter at the top, and $2 \frac{1}{4}$ inches in diameter at the bottom, having carefully rounded rather than square edges, and being $\frac{1}{2}$ inch deep. The bottom is not perfectly flat, but slightly crowned outward. Such a dish will hold about $35 \mathrm{cc}$.

For purposes of economy it is best to have these dishes spun out with a thick bottom, but with thin sides, not so thin, however, as to be too readily bent.

If platinum dishes cannot be afforded, dishes of porcelain, glass, aluminum, nickel, or even tin may be used, but in all cases should be as thin as practicable.

About $5 \mathrm{cc}$. of the thoroughly mixed sample of milk are carefully transferred by means of a pipette to a tared dish on the scale-pan, and its 
weight accurately determined. The dish with its contents is then transferred to a water-bath, being placed over an opening preferably but little smaller than the diameter of the bottom of the dish, so that as large a surface as possible is in contact with the live steam of the bath. Here it is kept for at least two hours, after which the dish is wiped dry while still hot, transferred to a desiccator, cooled, and weighed.*

Babcock Asbestos Method. $\uparrow-$ Provide a hollow cylinder of perforated sheet metal, $60 \mathrm{~mm}$. long and $20 \mathrm{~mm}$. in diameter, closed $5 \mathrm{~mm}$. from one end by a disk of the same material. The perforations should be about $0.7 \mathrm{~mm}$. in diameter and about $0.7 \mathrm{~mm}$. apart. Fill loosely with from 1.5 to 2.5 grams of freshly ignited, woolly asbestos, free from fine and brittle material, cool in a desiccator, and weigh. Introduce a weighed quantity of milk (between 3 and 5 grams), and dry in a wateroven to constant weight, which is usually reached after four hours' heating.

DETERMINATION OF ASH.-The platinum dish containing the milk residue, obtained in the determination of total solids by the dish method described above, is next placed upon a suitable support above a Bunsen flame (a platinum triangle or a ring stand is convenient for this), and the residue is ignited at a dull-red heat to a perfectly white ash, after which it is cooled and weighed.

DETERMINATION OF FAT. - The Adams Method. - Without doubt the most accurate method of fat determination is by extraction with ether. For this purpose a strip of fat-free filter-paper about $2 \frac{1}{2}$ inches wide and 22 inches long is rolled into a coil and held in place by a wire as shown in Fig. 44.

Schleicher and Schüll furnish fat-free strips especially for this work, but it is very easy to prepare the strips ard extract them with the Soxhlet apparatus.

About 5 grams of milk are run into a beaker with a pipette, and the weight of the beaker and milk are determined. The coil is then introduced into the beaker, holding it by the wire in such a manner that as

* It is a common practice to transfer the milk residue, after a preliminary drying on the water-bath, to an air-oven, kept at a temperature of from $100^{\circ}$ to $105^{\circ}$, where it is dried to a constant weight; but after an experience in analyzing over 30,000 samples of milk, the author is prepared to state that in his opinion the results obtained by the above method of procedure, using the water-bath alone, are more satisfactory. It is impossible to keep a milk residue at a temperature above $100^{\circ}$ for any length of time without its undergoing decomposition, especially as to its sugar content, as is shown by the darkening in color. A milk residue should be nearly pure white, a brownish color showing incipient decomposition. Hence, by continued heating, especially at the temperature of $105^{\circ}$, the residue would continue to lose weight almost indefinitely. If it is thought best to give a final drying in the air-oven, the time should be short and the temperature employed should not in any case exceed $100^{\circ}$.

† A. O. A. C. method, U. S. Dept. of Agric., Bur. of Chem., Bul. I07 (rev.), p. II7. 
much as possible of the milk is absorbed by the paper. It is often possible to take up almost the last drop of the milk. By then weighing the beaker, the amount of milk absorbed by the coil is determined by difference, and the paper coil is hung up and dried, first in the air and then in the oven, at a temperature not exceeding $100^{\circ}$. Another method of charging the paper coil consists in suspending it by the wire and gradually delivering upon it $5 \mathrm{cc}$. of the milk from a pipette, the density of the milk being known.

The coil containing the dried residue is then transferred to the Soxhlet extraction apparatus (see p. 64) and subjected to continuous extraction with anhydrous ether for at least two hours, the receiving-flask being first accurately weighed. The tared flask with its contents is freed from all remaining ether, first on the water-bath and finally in the air-oven. It is then cooled and weighed, the increase in weight representing the fat in the amount of milk absorbed by the coil. If there is any doubt about all the fat having been extracted at first, the process of extraction may be continued till there is no longer a gain in weight of the flask. Experience soon shows the length of time necessary for the complete extraction, which of course depends on the degree of heat employed, and the fre-

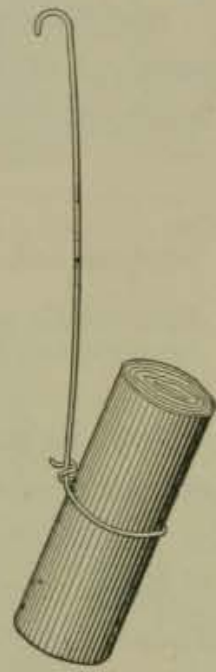

Fig. 44.-The Adams Milkfat Coil. quency with which the extracting-tube overflows. Two hours is ample for most cases, in which the conditions are such that the ether siphons over from the extraction-tube ten times per hour.

Babcock Asbestos Method.*-Extract the residue from the determination of water by the Babcock asbestos method with anhydrous ether in a continuous extraction apparatus, until all the fat is removed, which usually requires two hours. Evaporate the ether, dry the fat in the extraction flask at the temperature of boiling water, and weigh. The fat may also be determined by difference, drying the extracted cylinders at the temperature of boiling water.

Fat Methods Based on Centrifugal Separation. - These methods are the most practicable for commercial work and for use by the public analyst, since they are much more rapid, and, if carefully carried out, practically as accurate as the Adams method. They all depend upon the use of a centrifugal machine, having hinged pockets in which are carried graduated bottles, into each of which a measured quantity of milk is introduced. The milk is then subjected to the action of a suitable reagent, which dissolves the casein and liberates the fat in

* A. O. A. C. method, U. S. Dept. of Agric., Bur. of Chem., Bul. Io7 (rev.), p. IIg. 
a pure state, after which, by whirling at a high speed, the pockets are thrown out horizontally and the milk fat driven into the neck of each bottle, where the amount is directly read.

Various processes of this kind, each having its own special adherents, are in extensive use, among which the best known are the Babcock, the Leffman and Beam, the Gerber, and the Stokes.

A résumé of these processes, showing the reagents employed and other comparative data, is thus tabulated by Allen.*

\begin{tabular}{|c|c|c|c|c|}
\hline , & Babcock. & $\begin{array}{l}\text { Leffman- } \\
\text { Beam. }\end{array}$ & Gerber. & Stokes. \\
\hline 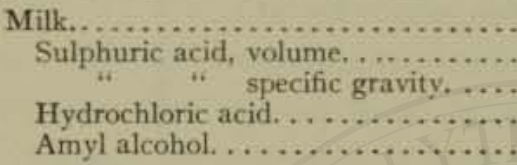 & $\begin{array}{c}17.5 \mathrm{cc} \text {. } \\
17.5 \mathrm{cc} . \\
1.83 \text { to } 1.834 \\
\text { None } \\
\text { None }\end{array}$ & $\begin{array}{l}15 \mathrm{cc} . \\
9 \mathrm{cc} . \\
1.85 \\
1.5 \mathrm{cc} . \\
1.5 \mathrm{cc} .\end{array}$ & $\begin{array}{c}\text { 11 cc. } \\
\text { 10 cc. } \\
\text { I. } 82 \text { to } 1.825 \\
\text { None } \\
1.0 \mathrm{cc} .\end{array}$ & $\begin{array}{l}15 \mathrm{cc} . \\
13 \frac{1}{2} \mathrm{cc} . \\
\mathrm{x} .82 \text { to } \mathrm{x} .83 \\
\text { None } \\
\mathrm{x} .5 \mathrm{cc} .\end{array}$ \\
\hline
\end{tabular}

The Babcock Process, devised originally for the use of creameries and dairymen, is now extensively employed for fat determination in the laboratory.

It has stood the test of over ten years' successful use in the writer's hands. During this time on various occasions results as determined have been compared with those obtained by the Adams process, and the agreement has been as close as could be expected. The following figures show the results of such comparative determinations made in duplicate on three samples of milk, viz., a whole pure milk, ( $\mathrm{I}$ ) and (2); a watered milk, (3) and (4), and a milk centrifugally skimmed, (5) and (6).

\section{COMPARATIVE FAT DETERMINATION BY ADAMS-SOXHLET AND BY} BABCOCK PROCESSES.

\begin{tabular}{|c|c|c|c|}
\hline & & $\begin{array}{l}\text { Per Cent of } \\
\text { Fat by the } \\
\text { Adams-Soxh- } \\
\text { let Process. }\end{array}$ & $\begin{array}{c}\text { Per Cent of } \\
\text { Pat by the } \\
\text { Babcock } \\
\text { Process. }\end{array}$ \\
\hline $\begin{array}{l}\text { A whole milk } \\
\text { A watered milk } \\
\text { A skimmed milk }\end{array}$ & 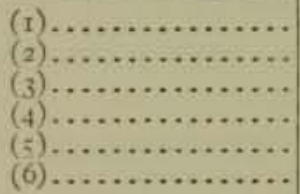 & $\begin{array}{l}4.27 \\
4.28 \\
2.70 \\
2.74 \\
0.16 \\
0.14\end{array}$ & $\begin{array}{l}4 \cdot 30 \\
4.35 \\
2.70 \\
2.80 \\
0.15 \\
0.15\end{array}$ \\
\hline
\end{tabular}

The Centrifuge.-Various styles of centrifuge, carrying from 2 to 40 bottles, are in use for this process.

Two forms of hand machine are shown in Fig. 45, one $(D)$, for two bottles, arranged to screw on the edge of a table, the other for twelve bottles inclosed in a cast-iron case.

\footnotetext{
* Commercial Organic Analysis, 3 Ed., Vol. IV, p. 150.
} 

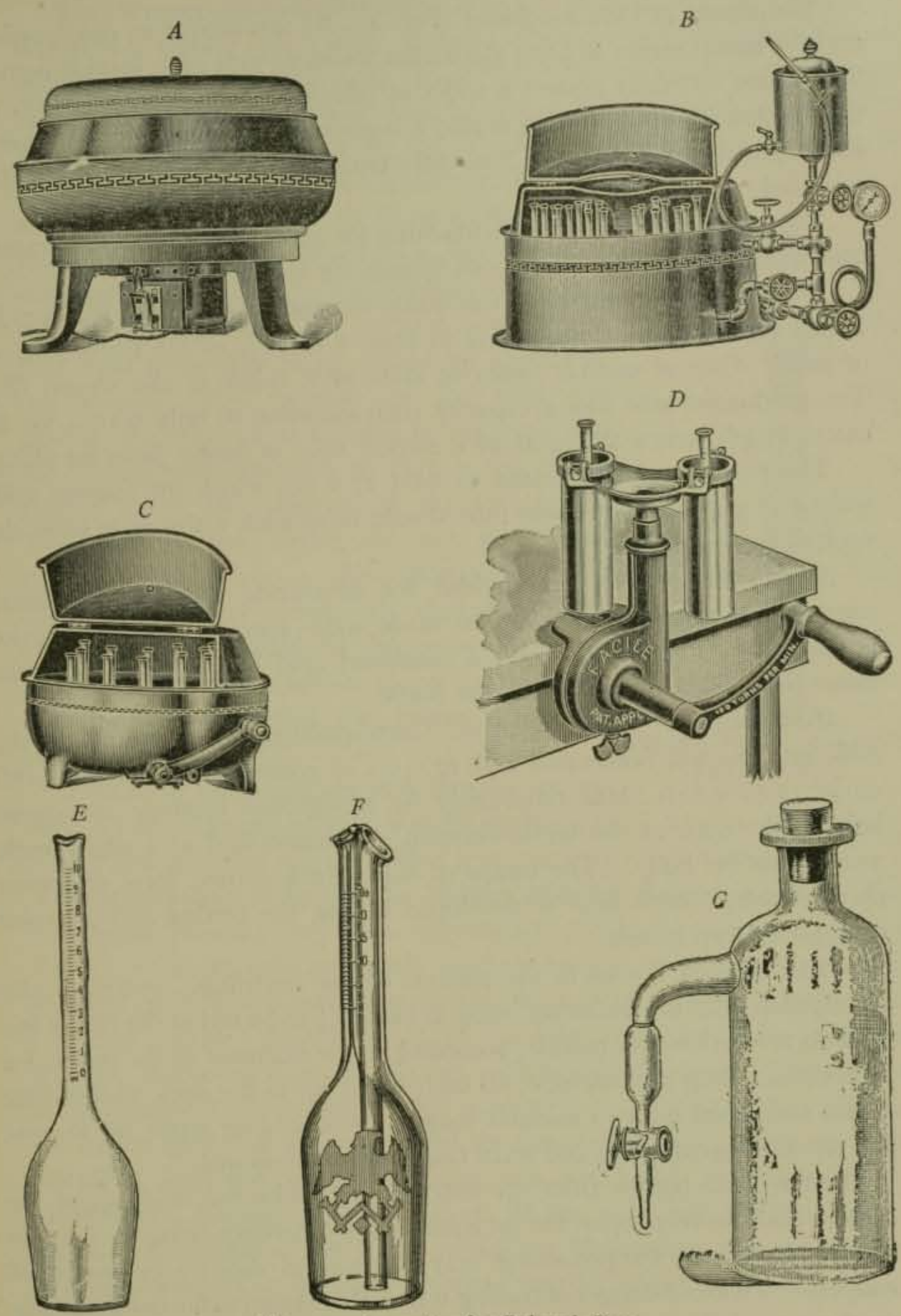

FiG. 45.-Apparatus for Babcock Test.

$A$, Burrell's electric centrifuge; $B$, Burrell's steam turbine centrifuge; $C$ and $D$, Burrell's hand centrifuges; $E$, milk bottle; $F$, Wagner's skim-milk bottle; $G$, Swedish or combined acid bottle. 
The steam turbine machines (Fig. $45, B$ ) are simple in construction and the steam serves to keep the bottles warm as well as to furnish power. The steam impinges against a series of paddles on the outer periphery of the revolving frame, driving it like a horizontal water-wheel. A reverse steam jet, steam gauge, and hot-water tank for filling the bottles are also provided.

Fig. $45, A$ shows an electric machine for 24 to 36 bottles. Laboratory centrifuges are also provided with frames for Babcock bottles.

Glassware.-The ordinary test bottle for milk is shown in Fig. 45, E. It has graduations corresponding to from $\circ$ to $10 \%$ of fat, using $17.6 \mathrm{cc}$. of milk. One of various forms of skim milk bottle is also shown $(F)$. The graduated tube has a capacity corresponding to only $0.25 \%$ for its entire length, hence the need of a second tube of larger bore for filling.

The pipettes are graduated to hold $17.6 \mathrm{cc}$., which for average milk weighs 18 grams. The lower tube should be of such a size as to enter the neck of the test bottle.

A $17.5 \mathrm{cc}$. cylinder is provided for measuring the acid, but where considerable numbers of tests are made some special measuring device is desirable. Fig. $45, G$ shows a combined acid bottle and pipette, the latter being filled by tipping up the bottle.

Manipulation.-Pipette $17.6 \mathrm{cc}$. (corresponding to 18 grams) of the milk into the test bottle and add $17.5 \mathrm{cc}$. of commercial sulphuric acid. (sp.gr. I.82-1.84). Mix thoroughly by a vigorous rotatory movement holding the neck of the bottle between the fingers and at a slight angle away from the body. The lumps of curd which at first form disappear upon shaking; much heat is developed during the mixing and the color changes to deep brown.

Place the test bottles in the pockets of the centrifuge (symmetrically arranged to keep the revolving frame in balance) and whirl at the rate of 800 to Iooo revolutions per minute, according to the diameter of the frame, for 5 minutes. Stop the machine, fill each bottle up to the neck with boiling water and whirl for two minutes longer. Add boiling water up to near the top of the graduation and whirl finally for two minutes.

Remove the bottles from the machine and take the readings of the bottom and the very top of the fat column, the difference being the per cent of fat. If desired, the percentage may be obtained directly by means of calipers. To avoid danger of cooling it is well to immerse the bottles nearly to the top of the neck in water at $60^{\circ} \mathrm{C}$., removing one at a time for reading. 
The Werner-Schmidt Method.-Ten cc. of milk are introduced by means of a pipette into a large test-tube of $50 \mathrm{cc}$. capacity, and ro $\mathrm{cc}$. of concentrated hydrochloric acid are added. The mixture is shaken and heated till the liquid turns a dark brown, either by direct boiling for a minute or two, or by immersing the tube in boiling water for from five to ten minutes. The tube is then cooled by immersion in cold water, and $30 \mathrm{cc}$. of washed ether is added. The tube is closed by a cork provided with tubes similar to a wash-bottle, the larger tube being adapted to slide up and down in the cork, and preferably being urned up slightly at the bottom. The contents of the tube are shaken, the ether layer allowed to separate, and the sliding-tube arranged so that it terminates slightly above the junction of the two layers. The ether is then blown out into a weighed flask. A second and a third portion of ether of Io cc. each are successively shaken with the acid liquid and added to the contents of the weighed flask, from which the ether is subsequently evaporated and the weight of the fat easily obtained.

Instead of measuring the milk into the testing-

Fig. 46.-TheWerner-Schmidt Fat Apparatus. tube, a known weight of milk may be operated on. A sour milk may be readily tested in this way, provided it is previously well mixed.

Determination of Fat by the Wollny Milk-fat Refractometer.*-This instrument presents the same appearance as the butyro-refractometer, Fig. 38 , with an arbitrary scale reading from $\circ$ to 100 , the equivalent readings in indices of refraction of the Wollny instrument varying from I. 3332 to 1.4220 . Exactly $30 \mathrm{cc}$. of the milk to be tested are measured into the stoppered flask $A$, Fig. 47. This may be done by the use of the automatic pipette, which holds exactly $7 \frac{1}{2}$ cc., removing four pipettes full of the milk. $B$ is a numbered tin sampling-tube in which the milk sample is kept for convenience, and into which the automatic pipette readily fits. Having neasured $30 \mathrm{cc}$. of the milk into the flask $A, 12$ drops of a solution of 70 grams potassium bichromate and 3 I2.5 cc. of stronger ammonia in one liter of water may be added as a preservative,

* Milch Zeit., 1900, pp. 50-53. 
if the sample is to be kept for some time before finishing the test. Twelve drops of glacial acetic acid are added to curdle the milk. The flask is then corked and shaken for one to two minutes in a mechanical shaker, after which $3 \mathrm{cc}$. of a standard alkaline solution are added, and the flask corked and shaken for ten minutes in the mechanical shaker, the temperature being kept at $17.5^{\circ} \mathrm{C}$. The standard alkaline solution is prepared

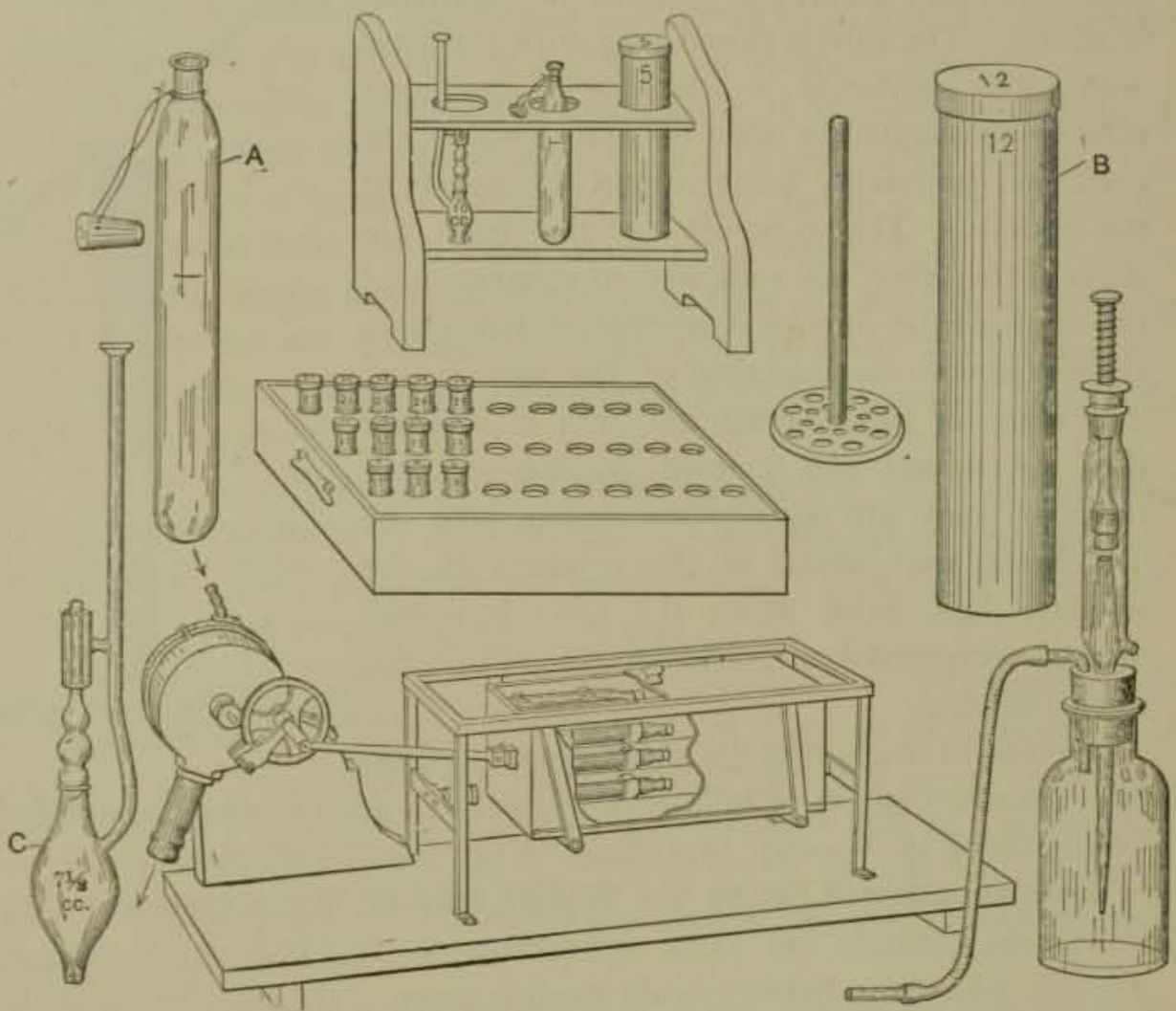

FrG. 47.-Accessories for Carrying Out the Wollny Milk-fat Process.

by dissolving $800 \mathrm{cc}$. of potassium hydroxide in a liter of water, adding $600 \mathrm{cc}$. of glycerin and 200 grams pulverized copper hydrate, the mixture being allowed to stand for several days before using, shaking at intervals. Finally $6 \mathrm{cc}$. of water-saturated ether are added to the mixture in the flask, using for convenience the automatic pipette fitted in the corked bottle as shown. The flask is again shaken for fifteen minutes in the mechanical shaker, and whirled for three minutes in the centrifuge, after which a few drops of the ether solution are transferred to the refractometer, and the reading taken. The percentage of fat is obtained by means of the following table: 
PERCENTAGES OF FAT CORRESPONDING TO SCALE READINGS ON THE WOLLNY REFRACTOMETER.

\begin{tabular}{|c|c|c|c|c|c|c|c|c|c|c|c|}
\hline $\begin{array}{l}\text { Seale } \\
\text { Readd- } \\
\text { ing. }\end{array}$ & $\begin{array}{l}\text { Per } \\
\text { Cent } \\
\text { Fat. }\end{array}$ & $\begin{array}{l}\text { Scale } \\
\text { Read- } \\
\text { ing. }\end{array}$ & $\begin{array}{c}\text { Per } \\
\text { Cent } \\
\text { Fat. }\end{array}$ & $\begin{array}{l}\text { Seale } \\
\text { Read- } \\
\text { ing. }\end{array}$ & $\begin{array}{l}\text { Per } \\
\text { Cent } \\
\text { Fat. }\end{array}$ & $\begin{array}{l}\text { Scale } \\
\text { Read- } \\
\text { ing. }\end{array}$ & $\begin{array}{l}\text { Per } \\
\text { Cent } \\
\text { Fat. }\end{array}$ & $\begin{array}{l}\text { Scale } \\
\text { Read- } \\
\text { ing. }\end{array}$ & $\begin{array}{l}\text { Per } \\
\text { Cent } \\
\text { Fat. }\end{array}$ & $\begin{array}{l}\text { Scale } \\
\text { Reas- } \\
\text { ing. }\end{array}$ & $\begin{array}{l}\text { Per } \\
\text { Cent } \\
\text { Fat. }\end{array}$ \\
\hline 20.0 & & 24.5 & $0.4 \mathrm{I}$ & 29.0 & 0.87 & $33-5$ & 1.34 & $3^{8} .0$ & 1.85 & 42.5 & $2.4 \mathrm{I}$ \\
\hline I & & 6 & 0.42 & I & 0.88 & 6 & 1. 35 & I & 1. 87 & 6 & 2.43 \\
\hline 2 & ... & 7 & 0.43 & 2 & 0.89 & 7 & I. 36 & 2 & I. 88 & 7 & 2.44 \\
\hline 3 & & 8 & 0.44 & 3 & 0.90 & 8 & I. 37 & 3 & r. 89 & 8 & 2.46 \\
\hline 4 & $\ldots$. & 9 & 0.45 & 4 & $0.9 \mathrm{I}$ & 9 & $1.3^{8}$ & 4 & 1.90 & 9 & 2.47 \\
\hline 5 & & 25.0 & 0.46 & 5 & 0.92 & 34.0 & 1. 39 & 5 & 1.91 & 43.0 & 2.49 \\
\hline 6 & 0.00 & I & 0.47 & 6 & 0.93 & I & 1.40 & 6 & I. 92 & I & 2.50 \\
\hline 7 & $0.0 \mathrm{r}$ & 2 & 0.48 & 7 & 0.94 & 2 & 1.42 & 7 & I. 93 & 2 & $2.5 \mathrm{I}$ \\
\hline 8 & 0.02 & 3 & 0.49 & 8 & 0.95 & 3 & 1. 43 & 8 & I. 94 & 3 & 2.52 \\
\hline 9 & 0.03 & 4 & 0.50 & 9 & 0.96 & 4 & I. 44 & 9 & I. 95 & 4 & 2.54 \\
\hline 21.0 & 0.04 & 5 & 0.51 & 30.0 & 0.97 & 5 & 1.45 & 39.0 & 1.96 & 5 & 2.55 \\
\hline I & 0.05 & 6 & 0.52 & I & 0.98 & 6 & I. 46 & I & I. 98 & 6 & 2.56 \\
\hline 2 & 0.06 & 7 & 0.53 & 2 & 0.99 & 7 & 1.47 & 2 & 1.99 & 7 & 2.58 \\
\hline 3 & 0.08 & 8 & 0.54 & 3 & 1.00 & 8 & 1. $4^{8}$ & 3 & 2.00 & 8 & 2.60 \\
\hline 4 & 0.09 & 9 & 0.55 & 4 & 1. or & 9 & 1. 49 & 4 & 2.02 & 9 & $2.6 \mathrm{t}$ \\
\hline 5 & 0.10 & 26.0 & 0.57 & 5 & 1.02 & 35.0 & I. $5^{\circ}$ & 5 & 2.03 & 44.0 & $2.6_{3}$ \\
\hline 6 & 0.11 & I & 0.58 & 6 & $\mathrm{I} .03$ & I & I. $5 \mathrm{I}$ & 6 & 2.04 & I & 2.64 \\
\hline 7 & 0.12 & 2 & 0.59 & 7 & 1.04 & 2 & 1. 52 & 7 & 2.05 & 2 & 2.65 \\
\hline 8 & 0.13 & 3 & 0.60 & 8 & 1.05 & 3 & x. 54 & 8 & 2.07 & 3 & 2.67 \\
\hline 9 & 0.14 & 4 & $0.6 \mathrm{I}$ & 9 & 1. .06 & 4 & 1. 55 & 9 & 2.08 & 4 & 2.68 \\
\hline 22.0 & 0.15 & 5 & 0.62 & 31.0 & 1.07 & 5 & I. 56 & 40.0 & 2.09 & 5 & 2.70 \\
\hline I & 0.16 & 6 & 0.63 & I & 1.08 & 6 & I. 57 & I & 2.10 & 6 & 2.71 \\
\hline 2 & 0.17 & 7 & 0.64 & 2 & 1.09 & 7 & 1.5 & 2 & 2.12 & 7 & 2.72 \\
\hline 3 & 0.18 & 8 & 0.65 & 3 & 1.10 & 8 & I. 59 & 3 & 2.13 & 8 & 2.74 \\
\hline 4 & 0.19 & 9 & 0.66 & 4 & I. II & 9 & 1.60 & 4 & 2.14 & 9 & 2.75 \\
\hline 5 & 0.20 & 27.0 & 0.67 & 5 & 1.12 & 36.0 & $1.6 \mathrm{I}$ & 5 & 2.15 & 45.0 & 2.77 \\
\hline 6 & 0.21 & I & 0.68 & 6 & I. ${ }^{13}$ & $I$ & 1.62 & 6 & 2.16 & I & 2. 78 \\
\hline 7 & 0.22 & 2 & 0.69 & 7 & I. I 4 & 2 & I. & 7 & 2.18 & 2 & 2.79 \\
\hline 8 & 0.23 & 3 & 0.70 & 8 & 1. 15 & 3 & 1.65 & 8 & 2.20 & 3 & 2.80 \\
\hline 9 & 0.24 & 4 & $0.7 \mathrm{r}$ & 9 & 1.16 & 4 & 1.66 & 9 & 2.21 & 4 & 2.82 \\
\hline 23.0 & 0.25 & 5 & 0.72 & 32.0 & 1.17 & 5 & 1.67 & 41.0 & 2.23 & 5 & 2.84 \\
\hline I & 0.26 & 6 & 0.73 & I & 1.18 & 6 & 1. 68 & I & 2.24 & 6 & 2.85 \\
\hline 2 & 0.27 & 7 & 0.74 & 2 & 1.19 & 7 & 1.69 & 2 & 2.25 & 7 & 2.87 \\
\hline 3 & 0.28 & 8 & & 3 & 1.20 & 8 & 1.70 & 3 & 2.26 & 8 & 2.88 \\
\hline 4 & 0.29 & 9 & 0.76 & 4 & 1.22 & 9 & 1. 7 & 4 & 2.27 & 9 & 2.89 \\
\hline 5 & 0.30 & 28.0 & 0.77 & 5 & 1.23 & 37.0 & I. 72 & 5 & 2.28 & 46.0 & 2.90 \\
\hline 6 & $0.3 \mathrm{I}$ & I & 0.78 & 6 & I. 24 & I & 1.7 & 6 & 2.30 & $I$ & 2.92 \\
\hline 7 & 0.32 & 2 & 0.79 & 7 & I. 25 & 2 & I. 7 & 7 & $2 \cdot 3^{2}$ & 2 & 2.93 \\
\hline 8 & 0.33 & 3 & 0.80 & 8 & 1.26 & 3 & & 8 & 2.33 & 3 & 2.94 \\
\hline 9 & 0.34 & 4 & $0.8 \mathrm{I}$ & 9 & 1.27 & 4 & 1. 78 & 9 & 2.34 & 4 & 2.96 \\
\hline 24.0 & 0.36 & 5 & 0.82 & 33.0 & 1.28 & 5 & 1.79 & 42.0 & 2.35 & 5 & 2.98 \\
\hline I & 0.37 & 6 & 0.83 & I & I. 29 & 6 & 1.80 & I & & 6 & 3.00 \\
\hline 2 & $0.3^{8}$ & 7 & 0.84 & 2 & 1.30 & 7 & 1.8 & 2 & 2.3 & 7 & 3.01 \\
\hline 3 & 0.39 & 8 & & 3 & I. $3^{\mathrm{I}}$ & 8 & 1.82 & 3 & 2.39 & 8 & 3.02 \\
\hline 4 & 0.40 & 9 & 0.86 & 4 & I. 32 & & r. 8 & 4 & 2.40 & 9 & 3.03 \\
\hline 5 & 0.41 & 29.0 & 0.87 & 5 & I. 34 & $3^{8} .0$ & 1.85 & 5 & 2.41 & 47.0 & 3.05 \\
\hline
\end{tabular}


PERCENTAGES OF FAT CORRESPONDING TO SCALE READINGS ON THE WOLLNY REFRACTOMETER-(Continued).

\begin{tabular}{|c|c|c|c|c|c|c|c|c|c|c|c|}
\hline $\begin{array}{l}\text { Seale } \\
\text { Read- } \\
\text { ing. }\end{array}$ & $\begin{array}{l}\text { Per } \\
\text { Cent } \\
\text { Fat. }\end{array}$ & $\begin{array}{l}\text { Seale } \\
\text { Read- } \\
\text { ing. }\end{array}$ & $\begin{array}{l}\text { Per } \\
\text { Cent } \\
\text { Fat. }\end{array}$ & $\begin{array}{l}\text { Seale } \\
\text { Read- } \\
\text { ing. }\end{array}$ & $\begin{array}{c}\text { Per } \\
\text { Cent } \\
\text { Fat. }\end{array}$ & $\begin{array}{l}\text { Scale } \\
\text { Read- } \\
\text { ing. }\end{array}$ & $\begin{array}{l}\text { Per } \\
\text { Cent } \\
\text { Fat. }\end{array}$ & $\begin{array}{l}\text { Scale } \\
\text { Read- } \\
\text { ing. }\end{array}$ & $\begin{array}{l}\text { Per } \\
\text { Cent } \\
\text { Fat. }\end{array}$ & $\begin{array}{l}\text { Seale } \\
\text { Read- } \\
\text { ing. }\end{array}$ & $\begin{array}{l}\text { Per } \\
\text { Cent } \\
\text { Fat. }\end{array}$ \\
\hline 47.0 & 3.05 & $5^{\circ} \cdot .5$ & 3.59 & 54.0 & 4.18 & 57.5 & 4.78 & 61.0 & 5.44 & $64 \cdot 5$ & 6.14 \\
\hline I & 3.06 & 6 & 3.60 & I & 4.20 & 6 & 4.80 & I & 5.46 & 6 & 6.16 \\
\hline 2 & 3.08 & 7 & 3.61 & 2 & 4.22 & 7 & 4.82 & 2 & $5 \cdot 4^{8}$ & 7 & 6.18 \\
\hline 3 & 3.10 & 8 & 3.63 & 3 & 4.23 & 8 & 4.84 & 3 & 5.50 & 8 & 6.20 \\
\hline 4 & 3.12 & 9 & 3.64 & 4 & 4.25 & & 4.86 & 4 & 5.52 & 9 & 6.22 \\
\hline 5 & 3.14 & 51.0 & 3.66 & 5 & 4.26 & $5^{8.0}$ & 4.88 & 5 & $5 \cdot 54$ & 65.0 & 6.24 \\
\hline 6 & 3.15 & I & 3.67 & 6 & 4.28 & I & 4.90 & 6 & $5 \cdot 56$ & I & 6.27 \\
\hline 7 & 3.16 & 2 & 3.68 & 7 & 4.29 & 2 & 4.92 & 7 & $5 \cdot 5^{8}$ & 2 & 6.29 \\
\hline 8 & 3.17 & 3 & 3.70 & 8 & $4 \cdot 3 \mathrm{I}$ & 3 & 4.94 & 8 & 5.60 & 3 & $6 \cdot 31$ \\
\hline & 3.18 & 4 & $3 \cdot 7^{2}$ & 9 & $4-33$ & 4. & 4.95 & 9 & 5.61 & 4 & 6.34 \\
\hline 48.0 & 3.20 & 5 & 3.74 & 55.0 & $4-35$ & 5 & 4.97 & 62.0 & 5.63 & 5 & $6.3^{6}$ \\
\hline 1 & 3.21 & 6 & 3.76 & I & $4 \cdot 37$ & 6 & 4.98 & I & 5.65 & 6 & $6.3^{8}$ \\
\hline 2 & 3.23 & 7 & 3.78 & 2 & $4-3^{8}$ & 7 & 5.00 & 2 & 5.66 & 7 & 6.40 \\
\hline 3 & 3.25 & 8 & 3.80 & 3 & 4.40 & 8 & 5.02 & 3 & 5.68 & 8 & 6.42 \\
\hline 4 & 3.27 & 9 & 3.82 & 4 & $4.4^{2}$ & 9 & 5.04 & 4 & 5.70 & 9 & 6.44 \\
\hline 5 & 3.28 & 52.0 & 3.84 & 5 & $4 \cdot 43$ & 59.0 & 5.06 & 5 & $5 \cdot 7^{2}$ & 66.0 & 6.46 \\
\hline 6 & $3 \cdot 30$ & I & 3.85 & 6 & 4.44 & I & 5.08 & 6 & $5 \cdot 74$ & & \\
\hline 7 & $3 \cdot 32$ & 2 & 3.87 & 7 & 4.46 & 2 & 5.10 & 7 & 5.76 & & \\
\hline 8 & $3 \cdot 33$ & 3 & 3.89 & 8 & $4 \cdot 4^{8}$ & 3 & 5.11 & 8 & $5.7^{8}$ & & \\
\hline 9 & 3. 34 & 4 & 3.90 & $\begin{array}{r}9 \\
-69\end{array}$ & 4.49 & 4 & 5.13 & 9 & 5.80 & & \\
\hline 49.0 & $3 \cdot 3^{6}$ & 5 & 3.92 & $5^{6.0}$ & $4 \cdot 5 I$ & 5 & $5 \cdot 15$ & 63.0 & 5.82 & & \\
\hline I & $3 \cdot 3^{8}$ & 6 & 3.93 & I & $4 \cdot 53$ & 6 & 5.17 & I & 5.84 & & \\
\hline 2 & 3.40 & 7 & 3.95 & 2 & 4.55 & 7 & & 2 & & & \\
\hline 3 & 3.42 & 8 & 3.9 & 3 & 4.57 & 8 & 5.20 & 3 & 5.88 & & \\
\hline 4 & $3-43$ & 9 & 3.99 & 4 & $4 \cdot 59$ & 9 & 5.22 & 4 & $5.9 \circ$ & & \\
\hline 5 & 3.44 & 53.0 & 4.01 & 5 & 4.60 & 60.0 & 5.24 & 5 & $5 \cdot 9^{2}$ & & \\
\hline 6 & 3.45 & I & 4.0 & 6 & $4.6 \mathrm{I}$ & I & 5.26 & 6 & 5.94 & & \\
\hline 7 & 3.46 & 2 & 4.04 & 7 & 4.63 & 2 & 5.28 & 7 & 5.6 & & \\
\hline 8 & 3.48 & 3 & 4.06 & 8 & 4.65 & 3 & $5 \cdot 30$ & 8 & 5.98 & & \\
\hline 9 & $3.5^{\circ}$ & 4 & 4.07 & 9 & 4.67 & 4 & $5 \cdot 32$ & 9 & 6.00 & & \\
\hline $5^{\circ} .0$ & $3.5 \mathrm{I}$ & 5 & 4.09 & 57.0 & 4.69 & 5 & $5 \cdot 34$ & 64.0 & 6.02 & & \\
\hline I & 3.53 & 6 & 4.10 & I & 4.71 & 6 & $5 \cdot 36$ & I & 6.04 & & \\
\hline 2 & 3.55 & 7 & 4.12 & 2 & 4.73 & 7 & $5 \cdot 3^{8}$ & 2 & 6.07 & & \\
\hline 3 & 3.56 & 8 & 4.14 & 3 & 4.75 & 8 & 5.40 & 3 & 6.09 & & \\
\hline 4 & 3.57 & 9 & $4 \cdot 16$ & 4 & $4 \cdot 76$ & 9 & 5.42 & 4 & 6.12 & & \\
\hline 5 & $3 \cdot 59$ & 54.0 & 4.18 & 5 & $4 \cdot 7^{8}$ & 61.0 & $5 \cdot 44$ & 5 & 6.74 & & \\
\hline
\end{tabular}

The following table is of use for those who wish to employ the Wollny method, but have the Abbé refractometer instead of the milk-fat refractometer. 
INDICES OF REFRACTION $\left(n_{D}\right)$ CORRESPONDING TO SCALE READINGS OP THE WOLLNY MILK-FAT REFRACTOMETER.

\begin{tabular}{|c|c|c|c|c|c|c|c|c|c|c|}
\hline \multirow{2}{*}{$\begin{array}{c}\text { Refrac- } \\
\text { tive } \\
\text { Index. } \\
n_{D}\end{array}$} & \multicolumn{3}{|l|}{ - } & \multicolumn{7}{|c|}{ Fourth Decimal of $n_{D^{*}}$} \\
\hline & 0 & 1 & 2 & 3 & $4^{*}$ & 5 & 6 & 7 & 8 & 9 \\
\hline & \multicolumn{9}{|c|}{ Seale Readings. } & \\
\hline I. 333 & & & 0.0 & 0.1 & 0.2 & 0.3 & 0.4 & 0.5 & 0.5 & 0.6 \\
\hline 1.334 & 0.7 & 0.8 & 0.9 & I. 0 & $\mathrm{I}, \mathrm{I}$ & 1.2 & I. 3 & I. 4 & 1.5 & 1. 6 \\
\hline 1.335 & 1.7 & 1.8 & 1.9 & 2.0 & 2.1 & 2.1 & 2.2 & 2.3 & 2.4 & 2.5 \\
\hline 1. $33^{6}$ & 2.8 & 2.7 & 2.8 & 2.9 & 3.0 & 3.1 & 3.2 & $3 \cdot 3$ & 3.4 & 3.5 \\
\hline 1.337 & 3.6 & 3.7 & 3.7 & 3.8 & 3.9 & 4.0 & 4.1 & 4.2 & $4 \cdot 3$ & $4 \cdot 4$ \\
\hline 1. $33^{8}$ & $4 \cdot 5$ & 4.6 & 4.7 & 4.8 & 4.9 & 5.0 & 5.1 & 5.2 & $5 \cdot 3$ & 5.4 \\
\hline 1. 339 & $5 \cdot 5$ & 5.6 & 5.7 & 5.8 & 5.9 & 6.0 & 6.1 & 6.2 & 6.3 & 6.4 \\
\hline 1.340 & 6.5 & 6.6 & 6.7 & 6.8 & 6.9 & 6.9 & & $7 \cdot 1$ & 7.2 & $7 \cdot 3$ \\
\hline $1.34 \mathrm{I}$ & 7.4 & 7.5 & 7.6 & 7.7 & 7.8 & 7.9 & 8.0 & 8.1 & 8.2 & 8.3 \\
\hline I. 342 & 8.4 & 8.5 & 8.6 & 8.7 & 8.8 & 8.9 & 9.0 & $9 . \mathrm{I}$ & 9.2 & $9 \cdot 3$ \\
\hline 1.343 & 9.4 & $9 \cdot 5$ & 9.6 & $9 \cdot 7$ & 9.8 & 9.9 & 10.0 & 10.1 & 10.2 & 10. 3 \\
\hline 1.344 & 10.4 & 10.5 & 10.6 & 10.7 & 10.8 & 10.9 & 11.0 & 11.1 & 11.2 & $\mathrm{II} \cdot 3$ \\
\hline I. 345 & 11.4 & II. 5 & II. 5 & 11.6 & 11.7 & 11.8 & 11.9 & 12.0 & 12.1 & 12.2 \\
\hline 1.346 & 12.3 & 12.4 & 12.5 & 12.6 & 12.7 & 12.8 & 12.9 & 13.0 & 13.1 & 13.2 \\
\hline 1.347 & 13.3 & $\mathrm{I}_{3.4}$ & I 3.5 & ${ }_{13} .6$ & 13.7 & 13.8 & 13.9 & 14.0 & 14.1 & 14.2 \\
\hline 1. 348 & $14 \cdot 3$ & 14.4 & I 4.5 & I 4.6 & 14.7 & 14.8 & 14.9 & 15,0 & 15.1 & 15.2 \\
\hline 1. 349 & $15 \cdot 3$ & 15.4 & 15.5 & 15.6 & 15.7 & 15.8 & 15.9 & 16.0 & 16.1 & 16.2 \\
\hline $1.35^{\circ}$ & 16.3 & 16.4 & 16.5 & 16.6 & 16.7 & 16.8 & 16.9 & 17.0 & 17.1 & 17.2 \\
\hline I. 351 & 17.3 & 17.4 & 17.5 & 17.6 & 17.7 & 17.8 & 17.9 & 18.0 & $\begin{array}{l}18.1 \\
\text { I }\end{array}$ & 18.2 \\
\hline 1. 352 & 18.3 & 18.4 & 18.5 & 18.6 & 18.7 & 18.8 & 18.9 & $19: 0$ & 19.1 & 19.2 \\
\hline I. 353 & 19.3 & 19.4 & 19.5 & 19.6 & 10.7 & 19.8 & 19.9 & 20.0 & 20.1 & 20.2 \\
\hline 1. 354 & 20.3 & 20.4 & 20.5 & 20.6 & 20.7 & 20.8 & 20.9 & 210 & 21.1 & 21.2 \\
\hline I. 355 & 21.3 & 21.4 & $21 \cdot 5$ & 21.6 & $2 \mathrm{x} \cdot 7$ & 21.8 & 21.9 & 22.0 & 22.1 & 22.2 \\
\hline I. 356 & 22.3 & 22.4 & 22.5 & 22.6 & 22.7 & 22.8 & 22.9 & 23.0 & 23.1 & 23.2 \\
\hline I. 357 & 23.3 & 23.4 & 23.5 & 23.6 & 23.7 & 23.8 & 23.9 & 24.0 & $24 . \mathrm{I}$ & 24.2 \\
\hline 1. $35^{8}$ & $24 \cdot 3$ & 24.4 & $24 \cdot 5$ & 24.6 & 24.7 & 24.8 & 24.9 & 25.0 & 25.1 & 25.2 \\
\hline 1. 359 & $25 \cdot 3$ & 25.4 & 25.5 & 25.6 & 25.7 & 25.8 & 25.9 & 26.0 & 26.1 & 26.2 \\
\hline x. 360 & 26.3 & 26.4 & 26.5 & 26.6 & 26.7 & 26.8 & 26.9 & & & \\
\hline 1. $36 \mathrm{I}$ & 27.4 & 27.5 & 27.6 & 27.7 & 27.8 & 27.0 & 28.0 & 28.1 & 28.2 & 28.3 \\
\hline 1. 362 & 28.4 & 28.5 & 28.6 & 28.7 & 28.8 & 28.9 & 29.0 & 29.1 & 29.2 & $29 \cdot 3$ \\
\hline 1. 363 & 29.4 & $29 \cdot 5$ & 29.6 & 20.7 & 29.8 & 20.9 & 30.0 & 30.1 & 30.2 & 30.3 \\
\hline 1.364 & 30.4 & 30.5 & 30.6 & & 30.8 & 31.0 & $3 \mathrm{r}, \mathrm{r}$ & $3 \mathrm{I} .2$ & $3 \mathrm{I} \cdot 3$ & $3 \mathrm{I} \cdot 4$ \\
\hline$x .365$ & $3 \mathrm{~T}-5$ & 31.6 & $3^{\mathrm{I}} \cdot 7$ & $3 x .8$ & 31.9 & $3^{2} .0$ & $3^{2.1}$ & 32.2 & 32.3 & $3^{2.4}$ \\
\hline 1. 366 & 32.5 & $3^{2} \cdot 7$ & 32.8 & 32.9 & 33.0 & 33.1 & 33.2 & $33 \cdot 3$ & 33.4 & $33 \cdot 5$ \\
\hline 1. 367 & 33.6 & $33 \cdot 7$ & 33.8 & 33.9 & 34.0 & 34.2 & $34 \cdot 3$ & 34.4 & 34.5 & 34.6 \\
\hline 1. 368 & $34 \cdot 7$ & 34.8 & 34.9 & 35.0 & 35.1 & $35 \cdot 2$ & $35 \cdot 3$ & 35.4 & $35 \cdot 5$ & 35.6 \\
\hline 1.369 & $35 \cdot 7$ & 35.8 & 36.0 & 36.1 & 36.2 & $3^{6} \cdot 3$ & 36.4 & 36.5 & 36.6 & 36.7 \\
\hline 1.370 & $3^{6.8}$ & 36.9 & 37. & 37. & 37.2 & $37 \cdot 3$ & & 37.6 & & 37.8 \\
\hline I. 371 & 37.9 & 38.0 & & 38.2 & $3^{8} \cdot 3$ & 38.4 & 38.5 & 38.6 & $3^{8.7}$ & 38.8 \\
\hline 1.372 & 38.9 & 39.0 & 39.2 & $39 \cdot 3$ & 39.4 & 39.5 & 39.6 & 39.7 & 39.8 & 39.9 \\
\hline I. 373 & 40.0 & 40.1 & 40.2 & 40.3 & 40.4 & 40,5 & 40.7 & 40.8 & 40.9 & 41.0 \\
\hline 1.374 & $4 \mathrm{I}, \mathrm{I}$ & $4 \mathrm{I} \cdot 2$ & $41 \cdot 3$ & & & 41.6 & 41.8 & 41.9 & 42.0 & $42 . x$ \\
\hline 1.375 & 42.2 & 42.3 & 42.4 & 42.5 & 42.6 & 42.7 & 42.8 & 42.9 & 43.0 & $43 \cdot 1$ \\
\hline 1.376 & 43.2 & $43-3$ & 43.4 & 43.6 & & 43.8 & 43.9 & 44.0 & $44 . x$ & 44.2 \\
\hline 1. 377 & $44 \cdot 3$ & $44 \cdot 4$ & 44.6 & $44 \cdot 7$ & 44.8 & 44.9 & 45.0 & $45 . x$ & 45.2 & 45.3 \\
\hline $1.37^{8}$ & 45.4 & 45.6 & 45.7 & 45.8 & 45.9 & 46.0 & 46.1 & 46.2 & 46.3 & 46.4 \\
\hline x. 379 & 46.6 & 46.7 & 46.8 & 46.9 & 47.0 & 47.1 & $47 \cdot 2$ & $47 \cdot 3$ & $47 \cdot 4$ & 47.6 \\
\hline
\end{tabular}


INDICES OF REFRACTION $\left(n_{D}\right)$ CORRESPONDING TO SCALE READINGS OI THE WOLLNY MILK-FAT REFRACTOMETER-(Continued).

\begin{tabular}{|c|c|c|c|c|c|c|c|c|c|c|}
\hline \multirow{2}{*}{$\begin{array}{l}\text { Refrac- } \\
\text { tive } \\
\text { Index, } \\
n_{D} \text {. }\end{array}$} & \multicolumn{10}{|c|}{ Fourth Decimal of $n_{D}$. } \\
\hline & 0 & 1 & 2 & 3 & 4 & 5 & 6 & 7 & 8 & 9 \\
\hline & \multicolumn{10}{|c|}{ Scale Readings, } \\
\hline 1.380 & 47.7 & 47.8 & 47.9 & 48.0 & 48.1 & 48.2 & $4^{8} \cdot 3$ & 48.4 & 48.6 & 48.7 \\
\hline 1. $3^{81}$ & 48.8 & 48.9 & 49.0 & $49 \cdot 1$ & 49.2 & $49 \cdot 3$ & 49.4 & 49.6 & $49 \cdot 7$ & 49.8 \\
\hline 1. $3^{8} 2$ & 49.9 & 50.0 & $50 . x$ & 50.2 & 50.3 & 50.4 & 50.6 & 50.7 & 50.8 & 50.9 \\
\hline I. $3^{8} 3$ & $5^{1} .0$ & $5 \mathrm{I}, \mathrm{I}$ & $5^{\mathrm{X}} \cdot 2$ & $5^{\mathrm{I}}-3$ & $5^{\mathrm{I}} \cdot 4$ & 51.6 & $5^{1} \cdot 7$ & $5=.8$ & $5^{1} \cdot 9$ & 52.0 \\
\hline x. 384 & 52.1 & 52.2 & $5^{2} \cdot 3$ & $5^{2} \cdot 4$ & 52.6 & $5^{2}-7$ & 52.8 & 52.9 & 53.0 & 53.1 \\
\hline 1.385 & 53.2 & $53-3$ & 53.4 & 53.6 & 53.7 & 53.8 & 53.9 & 54.0 & 54.1 & $54 \cdot 2$ \\
\hline 1.386 & $54 \cdot 3$ & 54.4 & 54.6 & 54.7 & 54.8 & 54.9 & 55.0 & 55.1 & 55.2 & $55 \cdot 3$ \\
\hline $1 \cdot 3^{87}$ & 55.4 & 55.6 & 55.7 & 55.8 & 55.9 & 56.0 & $5^{6.1}$ & $5^{6.2}$ & $5^{6} \cdot 3$ & 56.5 \\
\hline 1. 388 & $5^{6.6}$ & $5^{6} .7$ & 56.8 & 56.9 & $57 \cdot I$ & 57.2 & 57.3 & 57.4 & 57.6 & 57.7 \\
\hline I. 389 & 57.8 & $57 \cdot 9$ & 58.0 & 58.1 & 58.2 & 58.3 & $5^{8} .4$ & $5^{8} .6$ & 58.7 & $5^{8} .8$ \\
\hline I. $39^{\circ}$ & $5^{8} .9$ & 59.0 & $59, \pi$ & 59.2 & 59.4 & $59 \cdot 5$ & 59.6 & 59.8 & 59.9 & 60.0 \\
\hline I. $39 \mathrm{I}$ & 60.1 & 60.2 & $60 \cdot 3$ & 60.4 & 60.6 & 60.7 & 60.8 & 60.9 & 61.0 & 61.1 \\
\hline 1. 392 & $6 \mathrm{r} \cdot 3$ & 61.4 & $61-5$ & $6 x .6$ & $6 \mathrm{r} .8$ & 61.9 & 62.0 & 62.1 & 62.2 & $62 \cdot 3$ \\
\hline 1. 393 & 62.4 & 62.6 & 62.7 & 62.8 & 62.9 & 63.0 & 63.2 & $63 \cdot 3$ & 63.4 & 63.5 \\
\hline I. 394 & 63.6 & 63.8 & 63.9 & 64.0 & $64 \cdot I$ & 64.2 & $64 \cdot 4$ & $64 \cdot 5$ & 64.6 & $64 \cdot 7$ \\
\hline I. 395 & 64.8 & 65.0 & 65.1 & 65.2 & $65 \cdot 3$ & 65.4 & $6_{5} .6$ & 65.7 & 65.8 & 65.9 \\
\hline I. 396 & 66.0 & 66.2 & 66.3 & 66.4 & 66.5 & 66.6 & 66.8 & 66.9 & 67.0 & 67.1 \\
\hline 1. 397 & 67.2 & 67.4 & $67 \cdot 5$ & 67.6 & $67 \cdot 7$ & 67.8 & 67.9 & 68.1 & 68.2 & 68.3 \\
\hline 1. $39^{8}$ & 68.4 & 68.6 & 68.7 & 68.8 & 68.9 & 69.0 & 69.1 & $69 \cdot 3$ & 69.4 & 69.5 \\
\hline 1. 399 & 69.6 & 69.8 & $69 \cdot 9$ & 70.0 & 70.1 & 70.2 & 70.4 & 70.5 & 70.6 & 70.8 \\
\hline 1.400 & 70.9 & 71.0 & 71.1 & 71.2 & $7^{1} \cdot 4$ & 71.5 & 71.6 & 71.8 & 71.9 & 72.0 \\
\hline I. 401 & 72.1 & 72.2 & 72.4 & 72.5 & 72.6 & 72.8 & 72.9 & 73.0 & 73.1 & 73.2 \\
\hline 1.402 & 73.4 & $73-5$ & 73.6 & 73.8 & $73-9$ & 74.0 & 74.1 & 74.2 & 74.4 & $74 \cdot 5$ \\
\hline 1.403 & 74.6 & 74.8 & 74.9 & 75.0 & $75 . \mathrm{I}$ & 75.2 & 75.4 & $75 \cdot 5$ & 75.6 & 75.8 \\
\hline 1.404 & 75.9 & 76.0 & 76,1 & 76.2 & $7^{6.4}$ & 76.5 & 76.6 & 76.8 & 76.9 & $77 \cdot 0$ \\
\hline 1.405 & 77.1 & 77.2 & $77 \cdot 4$ & $77 \cdot 5$ & $77 \cdot 7$ & 77.8 & 77.9 & 78.1 & 70.2 & $7^{8} .3$ \\
\hline 1.406 & 78.5 & $7^{8.6}$ & 78.7 & 78.8 & 79.0 & $79 \cdot 1$ & $79 \cdot 2$ & 79.4 & $79 \cdot 5$ & 79.6 \\
\hline 1.407 & 79.8 & 79.9 & 80.0 & 80.1 & 80.2 & 80.4 & & 80.6 & 80.8 & 80.9 \\
\hline 1.408 & 81.0 & 81.1 & 81.2 & $8 \mathrm{r} \cdot 4$ & 81.5 & $8 \mathrm{r} .6$ & 81.7 & $8 \mathrm{r} .9$ & 82.0 & 82.1 \\
\hline 1.409 & $82 \cdot 3$ & 82.4 & 82.5 & 82.6 & 82.8 & 82.9 & 83.0 & 83.2 & $83 \cdot 3$ & 83.4 \\
\hline 1.410 & $8_{3} .6$ & 83.7 & 83.8 & 84.0 & $84 . \mathrm{I}$ & 84.2 & 84. & $8_{4}$ & 84.6 & 84.8 \\
\hline I. 411 & 84.9 & 85.0 & 85.2 & 85.3 & 85.4 & 85.5 & 85.6 & 85.7 & 85.9 & 86.1 \\
\hline 1.412 & 86.2 & 86.3 & 86.5 & 86.6 & 86.7 & 86. & 87.0 & 87.1 & 87.3 & 87.4 \\
\hline I. $4 x_{3}$ & $87 \cdot 5$ & 87.7 & 87.8 & 87.9 & 88.1 & 88.2 & 88 & 88. & 88.6 & 88.7 \\
\hline 1.414 & 88.9 & 89.0 & $89 \cdot I$ & 89.3 & 89.4 & 89.6 & 89.7 & 89.9 & 90.0 & 90.1 \\
\hline I. 415 & 90.2 & 90.4 & 90.5 & 90.6 & 90.8 & 90.9 & 91.0 & 91.2 & $91 \cdot 3$ & \\
\hline I. 416 & 91.6 & $9^{t}-7$ & 91.9 & 92.0 & 92.1 & 92.3 & $9^{2}-4$ & 92.5 & 92.7 & 92.8 \\
\hline 1.417 & 92.9 & $93 \cdot 1$ & 93.2 & 93.3 & $93 \cdot 5$ & 93.6 & 93.8 & 93.9 & 94.0 & 94.2 \\
\hline $\mathrm{I} .4 \mathrm{I} 8$ & 94.3 & 94.4 & 94.6 & $94-7$ & 94.8 & 95.0 & $95 \cdot 1$ & 95.3 & 95.4 & 95.6 \\
\hline 1.419 & $95 \cdot 7$ & 95.8 & 96.0 & $9^{6.1}$ & $9^{6} \cdot 3$ & 96.4 & 96.6 & $9^{6.7}$ & 96.8 & 97.0 \\
\hline 1.420 & $97 . I$ & $97 \cdot 3$ & 97. & 97.6 & 97. & 97.8 & 98.0 & 98.1 & $9^{8} \cdot 3$ & $9^{8.4}$ \\
\hline I. 421 & $9^{8} \cdot 5$ & 98.7 & 98.8 & 99.0 & 99.1 & $99 \cdot 3$ & 99.4 & $99 \cdot 5$ & 99.7 & 99.9 \\
\hline $1.4^{22}$ & 100. & & & & & & & & & \\
\hline
\end{tabular}


DETERMINATION OF PROTEINS.-For determination of the total nitrogen in milk, $5 \mathrm{cc}$. are measured direct into a Kjeldahl digestion-flask, or a known weight from a weighing-bottle may be used, and the regular Gunning method is employed as described on page 69, proceeding with the digestion at once without evaporation.

The total nitrogen, multiplied by 6.38 , gives the total proteins. By many the old factor of 6.25 is still employed, but in view of the fact that both casein and albumin have been found to contain $15.7 \%$ of nitrogen, there would seem to be the best reasons for employing 6.38 as a factor $\left(\frac{100}{15 \cdot 7}\right)$.

Ritthausen's Method.-Ten grams of milk are measured into a beaker and diluted with water to about $100 \mathrm{cc}$. Five cc. of a solution of copper sulphate (strength of Fehling's copper solution, $34.64 \mathrm{grams} \mathrm{CuSO}_{4}$ in $500 \mathrm{cc}$. of water) are added and the mixture stirred. A solution of sodium hydroxide ( 25 grams to the liter) is added cautiously a little at a time, till the liquid is nearly, but not quite neutral, avoiding an excess of alkali, as this would prevent the complete precipitation of the proteins. Allow the precipitate to settle, and pour off the supernatant liquid through a weighed filter, previously dried at $130^{\circ} \mathrm{C}$. Wash a number of times by decantation, and transfer the precipitate to the filter, being careful to remove the portions adhering to the sides of the beaker with a rubber-tipped rod. Wash thoroughly with water, and drain dry, after which the precipitate is washed with strong alcohol, dried, extracted with ether, preferably in a Soxhlet extractor, and then transferred on the filter to the oven, dried at $130^{\circ} \mathrm{C}$., and weighed. The filter and precipitate are then burnt to an asin in a porcelain crucible, and the weight of the residue subtracted from the first weight gives that of the proteins.

Richmond* recommends modifying this process to the extent of neutralizing the milk, using phenolphthalein as an indicator, before adding the copper sulphate solution, and using only $2.5 \mathrm{cc}$. of the latter.

Determination of Casein.-Official Method of the A. O. A. C.-Ten grams of the milk are placed in a beaker, and made up with water to about $100 \mathrm{cc}$. at $40^{\circ}$ to $42^{\circ} \mathrm{C}$. One and one-half cc. of a $10 \%$ solution (by weight) of acetic acid are added, the mixture stirred, warmed to the above temperature, and allowed to stand for from three to five minutes, till a flocculent precipitate separates, leaving a clear supernatant liquid. Decant

* Dairy Chem., p. ro7. 
upon a filter, wash with cold water two or three times by decantation, and finally transfer the whole of the precipitate to the filter, and, after filtering, wash two or three times. The filtrate should be clear or nearly so. If not, it can generally be made so by repeated filtrations, and the washing done afterwards. The filter containing the washed precipitate is transferred to the Kjeldahl digestion-flask and the nitrogen obtained by the Gunning process. $\mathrm{N} \times 6.38=$ casein.

Determination of Albumin.-Optional Methods of the A. O. A. C.-To the filtrate from the direct determination of casein by the acetic acid method as described in the preceding section, exactly nęutralized with sodium hydroxide, $0.3 \mathrm{cc}$. of a 10\% solution of acetic acid is added, and the mixture is boiled till the albumin is completely precipitated. The precipitate is collected on a filter and washed, the nitrogen being determined in the precipitate, and the factor $6.3^{8}$ used in calculating the albumin therefrom.

Leffman and Beam's Modified Method for Albumin and Casein.-Owing to the tedious processes of washing and filtering incidental to the above methods for determining casein, the following is suggested. Twenty cc. of the milk are mixed with saturated magnesium sulphate solution, and the mixture saturated with the powdered salt. The whole is then washed into a graduate with a little of the saturated solution, and the precipitate allowed to settle, leaving a clear supernatant layer. The volume of the mixture in the graduate is read, and as much as possible of the clear portion is withdrawn by a pipette and filtered.

An aliquot part of the filtrate is then taken, and the albumin is precipitated from it by a solution of tannin, after which the precipitate is washed in a filter and the nitrogen determined therein. $\mathrm{N} \times 6.38=$ albumin.

The casein is calculated by difference between the total proteins and the albumin.

Determination of Nitrogen as Caseoses, Amido-compounds, Peptones, and Ammonia.-Van Slyke* proceeds as follows: The filtrate from the determination of the albumin, as above, is heated to $70^{\circ} \mathrm{C}$., I cc. of $50 \%$ sulphuric acid is first added, and afterwards chemically pure zinc sulphate to saturation. The mixture is allowed to stand at $70^{\circ}$ until the caseoses separate out and settle. Cool, filter, wash with a saturated zinc sulphate solution slightly acidified with sulphuric acid, and determine the nitrogen of the caseoses in the precipitate.

* N. Y. Exp. Station, Bul 215, p. 102. 
For Amido-compounds and Ammonia treat 50 grams of the milk in a 250 -cc. graduated flask with I gram sodium chloride and a $12 \%$ solution of tannin, added drop by drop till no further precipitate is formed. Dilute to the 250 -cc. mark, shake, and filter. Determine the nitrogen in $50 \mathrm{cc}$. of the filtrate, the result being the combined nitrogen of the amido-compounds and ammonia.

Distil with magnesium oxide $100 \mathrm{cc}$. of the filtrate from the tannin salt solution, receiving the distillate in a standardized acid, and titrating in the ustial way for the ammonia.

Calculate the nitrogen of the peptones by subtracting from the total nitrogen that due to all other forms.

Van Slyke has furnished the following unpublished analysis of a sample of milk three months old, kept under antiseptic conditions by chloroform.

\begin{tabular}{|c|c|c|c|}
\hline $\begin{array}{c}\text { Per Cent } \\
\text { Total N. }\end{array}$ & $\begin{array}{c}\text { Per Cent } \\
\text { Sol, Nitrogen. }\end{array}$ & $\begin{array}{c}\text { Per Cent } \\
\text { as Paranuclein, } \\
\text { Caseoses, and } \\
\text { Peptones. }\end{array}$ & $\begin{array}{c}\text { Per Cent } \\
\text { N as Amides. }\end{array}$ \\
\hline $0.56 \mathrm{I}$ & 0.099 & 0.074 & 0.025 \\
\hline
\end{tabular}

DETERMINATION OF MILK SUGAR.-If a polariscope is available, the sugar of milk can most readily and conveniently be determined by optical methods. In the absence of a polariscope, the reducing power of milk sugar on copper salts may be utilized quite accurately in determining the sugar, using either volumetric or gravimetric methods as desired.

Determination by Optical Methods.- T. Reagents.-Acid Nitrate of Mercury.-This solution is prepared by dissolving metallic mercury in twice its weight of nitric acid of specific gravity 1.42 , and adding to the solution an equal volume of water. One cc. of this reagent will be found sufficient to precipitate the proteins and fat completely from 65 grams of milk, but if more is employed the result of the analysis is not affected.

Mercuric Iodide Solution. - 33.2 grams of potassium iodide are mixed with 13.5 grams of mercuric chloride, $20 \mathrm{cc}$. of acetic acid, and $640 \mathrm{cc}$. of water.

Subacetate of Lead Solution, U. S. P. See p. $5^{86}$.

Notes.-For the Laurent polariscope, in which the normal weight for sucrose is I6.19 grams, the corresponding normal weight for lactose is 20.496 , while for the Soleil-Ventzke instrument, in which the su- 
crose normal weight is 26.048 grams, the corresponding lactose normal weight is $32.975 *$ *

It is customary to employ three times the normal weight of milk in the case of the Laurent instrument (viz., 6r.48 grams) and twice the normal weight in the case of the Soleil-Ventzke (viz., 65.95 grams).

As it is more convenient to measure the milk than to weigh it, and as the volume varies with the specific gravity, the following table is useful, showing the quantity to be measured in any case, having first determined the specific gravity.

\begin{tabular}{|c|c|c|}
\hline \multirow{2}{*}{ Specific Gravity. } & \multicolumn{2}{|c|}{ Volume of Milk to be Used. } \\
\cline { 2 - 3 } & $\begin{array}{c}\text { For Polariscopes of } \\
\text { which the Sucrose } \\
\text { Normal Weight is } \\
16.19 \text { Grams. }\end{array}$ & $\begin{array}{c}\text { For Polariscopes of } \\
\text { which the Sucrose } \\
\text { Normal Weight is } \\
26.048 \text { Grams. }\end{array}$ \\
\hline 1.024 & $60.0 \mathrm{cc}$ & $64.4 \mathrm{cc}$. \\
1.026 & $59.9 \mathrm{cc}$. & $64.3 \mathrm{cc}$. \\
1.028 & $59.8 \mathrm{cc}$. & $64.15 \mathrm{cc}$. \\
1.030 & $59.7 \mathrm{cc}$. & $64.0 \mathrm{cc}$. \\
1.032 & $59.6 \mathrm{cc}$. & $63.9 \mathrm{cc}$. \\
1.034 & $59.5 \mathrm{cc}$. & $63.8 \mathrm{cc}$. \\
1.035 & $59.35 \mathrm{cc}$. & $63.7 \mathrm{cc}$. \\
\hline
\end{tabular}

For ordinary work it is sufficiently close to have a pipette graduated to deliver $59.7 \mathrm{cc}$. if the Laurent instrument is used, and $64 \mathrm{cc}$. for the Soleil-Ventzke.

2. Process. - Measure as above, the equivalent of $6 \mathrm{r} .48$ grams of the milk for the Laurent, or 65.95 grams for the Soleil-Ventzke, instrument into a 100 -cc. graduated flask, add, in order to clarify, 2 cc. of acid nitrate of mercury solution, or $30 \mathrm{cc}$. of mercuric iodide solution, or 1o cc. of lead subacetate solution. Shake gently and fill to the mark with water. Then add from a pipette enough water to make up for the volume of the precipitated proteins and fat, insuring $100 \mathrm{cc}$. of sugar solution. If the Laurent instrument is used, the amount added as prescribed by the A. O. A. C. is 2.4 cc., and with the Soleil-Ventzke $2.6 \mathrm{cc}$. The contents of the flask are then shaken and poured upon a dry filter. The filtrate, which should be perfectly clear, is polarized in a $200-\mathrm{mm}$. tube, and the reading, divided by 3 for the Laurent and by 2 for the SoleilVentzke, gives the percentage of lactose directly.

Allowance for the Volume of the Precipitate.-This of course varies

* $[\alpha]_{D}$ for lactose $=52.53,[\alpha]_{D}$ for sucrose $=66.5$. hence for the Laurent instrument $52.53: 66.5:: 16.19: 20.496$,

and for the Soleil-Ventzke instrument $52.53: 66.5:: 26.048: 32.975$. 
with the content in proteins, and fat, and while the above allowance gives in most cases sufficiently close results, it is not exact. Leffman and Beam * advise that the amount of water to be added above roo cc. be calculated in each case from the percentage of proteins and fat previously found by analysis, multiplying the actual weight of the fat in grams in the sample taken by 1.075 , and the weight of proteins. by 0.8 , the sum of the two results being the volume in cubic centimeters occupied by the precipitate.

All the calculations are avoide 1 by employing the double-dilution method, which is to be recommended when very particular results are required.

Wiley and Ewell's Double-dilution Method. $\nmid-$ Two flasks are employed graduated at 100 and $200 \mathrm{cc}$. respectively, into each of which are introduced 65.95 grams of milk, if the Soleil-Ventzke instrument is used (or 61.48 grams in case the Laurent is used) and 4 cc. of the mercuric nitrate solution are added, both flasks being filled to the mark and shaken. The contents are filtered and the polarization is made in each case in a $400-\mathrm{mm}$. tube.

The second reading (that of the more dilute solution) is multiplied by 2 , and the product subtracted from the first reading; the remainder is then multiplied by 2 , and the product subtracted from the first reading (that of the stronger or $100 \mathrm{cc}$. solution). The result is the corrected reading, which, divided by 4 , gives the exact per cent of milk sugar in the sample. This method depends on the fact that within ordinary limits the polarizations of two solutions of the same substance are inversely proportional to their volumes.

DETERMINATION OF MILK SUgAR BY FEHLING'S SOLUTION.-Twentyfive grams of the milk $(24.2 \mathrm{cc}$.) are transferred to a 250 -cc. flask, $0.5 \mathrm{cc}$. of a $30 \%$ solution of acetic acid are added and the contents well shaken. After standing for a few minutes, about $100 \mathrm{cc}$. of boiling water are run in, the contents again shaken, $25 \mathrm{cc}$. of alumina cream are next added, the flask shaken once more, and set aside for at least ten minutes. The supernatant liquid is then poured upon a previously wetted ribbed filter, and finally the whole contents of the flask are brought thereon, and the filtrate and washings made up to $250 \mathrm{cc}$. The filtrate must be perfectly clear. The milk sugar in a solution thus precipitated would ordinarily not exceed $\frac{1}{2}$ of I per cent.

* Milk and Milk Products, p. 38 .

† Wiley's Agricultural Analysis, p. 278; Analyst, 21, 1896, p. 182 . 
Volumetric Fehling Process.-From a burette containing the clear milk sugar solution above prepared, run a measured volume into the boiling Fehling liquor containing $5 \mathrm{cc}$. each of copper and alkali solution till sufficient has been introduced to completely reduce the copper, conducting the operation in the manner described in detail on page 59r,

As 0.067 gram of milk sugar will reduce ro cc. of Fehling solution (see p. 593), it follows that the number of cubic centimeters of sugar containing solution required for making the test (using preferably the average of several determinations) will contain 0.067 gram of milk sugar, from which the percentage is readily computed. Thus if $16 \mathrm{cc}$. of the milk sugar solution are necessary to reduce the copper, then 16 cc. contain 0.067 gram milk sugar.

$250 \mathrm{cc}$. of solution contain 25 grams milk,
I cc. " " "
I6 cc. " "

and I.6 grams milk contain 0.067 gram milk sugar. Therefore the sample contains $\frac{.067 \times 100}{1.6}=4.19 \%$.

Gravimetric Fehling Processes.-O'Sullivan-Defren Method.-Twentyfive cc. of the above milk sugar solution are added to the hot mixture of I $5 \mathrm{cc}$. each of Fehling copper and alkali solutions and $50 \mathrm{cc}$. water, prepared as directed on page 59I. and the test carried out in accordance with the details there described. The weight of the cupric oxide, $\mathrm{CuO}$, as formed, may be roughly calculated to anhydrous milk sugar by multiplying by 0.6024 .

For more accurate results, however, the Defren table, page 595, should be used.

Soxhlet's Method.*-Twenty-five cc. of milk are diluted with $400 \mathrm{cc}$. of water in a half-liter graduated flask and ro cc. of Fehling's copper solution are added. Then $8.8 \mathrm{cc}$. of half-normal sodium hydroxide are run in, or a sufficient quantity to nearly but not quite neutralize, the solution being still slightly acid. The flask is filled to the mark, shaken, and the contents filtered, using a dry filter.

One hundred cc. of the filtrate are added to $50 \mathrm{cc}$. of the mixed Fehling solution, which is boiled briskly in a beaker (using $25 \mathrm{cc}$. each of the copper and alkali solution). After boiling for six minutes, filter rapidly through a Gooch crucible provided with a layer of asbestos as described on page 594, and wash with boiling water till free from alkali. The asbestos

*U.S. Dept. of Agric., Bur. of Chem., Bull. 46 , p. 4 r; Bul. 107 (rev.), p. 119. 
film with the adhering cuprous oxide is washed into a beaker by hot dilute nitric acid, and after complete solution of the copper is assured, it is again filtered and washed with hot water till a clean solution containing all the copper is obtained. Add to cc. of cilute sulphuric acid (containing $200 \mathrm{cc}$. of sulphuric acid, specific gravity r. 84 per liter) and evaporate on the steambath till the copper has largely crystallized, then carefully continue the heating over a hot plate till the nitric acid is driven out, as evidenced by the white fumes of sulphuric. Add 8 or 10 drops nitric acid (specific gravity 1.42 ) and rinse into a very clean tared platinum dish of about 100 cc. capacity, in which the copper is deposited by electrolysis. See page 608 .

The weight of milk sugar is determined from that of copper found, from the table on page $\mathrm{I}_{52}$.

If the apparatus for the determination of the copper by the electrolytic method is not at hand, the cuprous oxide may be weighed directly in the Gooch crucible. In order to facilitate drying, it should be washed successively with ro cc. of alcohol, and ro cc. of ether, after which it is dried thirty minutes in a water-oven at $100^{\circ} \mathrm{C}$., cooled, and weighed. The weight of copper is obtained from the weight of the cuprous oxide by the use of the factor $0.888_{3}$.

Munson and Walker Method.-The milk sugar solution is prepared as in Soxhlet's method. For details as to the copper reduction process see page 598 .

Relation between Specific Gravity, Fat, and Total Solids of Milk.The close relationship existing between these factors has long been known, and many formulæ have been devised, whereby, if two of them are known, the third may be computed with considerable approach to accuracy. The specific gravity and the fat are very readily determined by any dairyman, by the aid of a lactometer and the Babcock apparatus. The total solids are ascertained with more difficulty, since the use of more involved and costly apparatus is necessary, besides considerable technical skill. It is therefore common for producers to calculate the total solids from the fat and specific gravity, using one of the many tables prepared for the purpose, based on some one of the best accepted formula. The total solids can thus be calculated to within two or three tenths of a per cent.

The two most commonly used formulæ for this purpose are those of Hehner and Richmond in England, and Babcock in the United States. Hehner and Richmond's formula is

$$
T=0.25 S+1.2 F+0.14,
$$


SOXHLET'S TABLE FOR THE DETERMINATION OF LACTOSE.*

\begin{tabular}{|c|c|c|c|c|c|c|c|c|c|}
\hline $\begin{array}{c}\text { Milli- } \\
\text { grams } \\
\text { of Cop- } \\
\text { per. }\end{array}$ & $\begin{array}{l}\text { Milli- } \\
\text { grams } \\
\text { of Lau- } \\
\text { tose. }\end{array}$ & $\begin{array}{l}\text { Milli- } \\
\text { grams } \\
\text { of Cop- } \\
\text { per. }\end{array}$ & $\begin{array}{l}\text { Milli- } \\
\text { grams } \\
\text { of Lad- } \\
\text { tose. }\end{array}$ & $\begin{array}{l}\text { Milli- } \\
\text { grams } \\
\text { of Cop- } \\
\text { per. }\end{array}$ & $\begin{array}{l}\text { Milli- } \\
\text { grams } \\
\text { of Lac- } \\
\text { tose. }\end{array}$ & $\begin{array}{c}\text { Milli- } \\
\text { grams } \\
\text { of Cop- } \\
\text { per. }\end{array}$ & $\begin{array}{l}\text { Milli- } \\
\text { grams } \\
\text { of Lac- } \\
\text { tose. }\end{array}$ & $\begin{array}{l}\text { Milli- } \\
\text { grams } \\
\text { of Cop- } \\
\text { per. }\end{array}$ & $\begin{array}{l}\text { Milli- } \\
\text { grams } \\
\text { of Lac- } \\
\text { tose. }\end{array}$ \\
\hline 100 & 71.6 & $16 \mathrm{r}$ & $\operatorname{Ix} 7 \cdot x$ & $22 \mathrm{I}$ & 162.7 & $28 \mathrm{I}$ & $209 \cdot \mathrm{t}$ & $34 \mathrm{~T}$ & 256.5 \\
\hline Ior & $72 \cdot 4$ & I62 & I1 7.9 & 222 & 163.4 & 282 & 209.9 & 342 & 257.4 \\
\hline 102 & $73 . \mathrm{I}$ & 163 & 118.6 & 223 & 164.2 & 283 & 210.7 & 343 & 258.2 \\
\hline 103 & 73.8 & 164 & 119.4 & 224 & 164.9 & 284 & 211.5 & 344 & 259.0 \\
\hline $\mathrm{IO4}$ & $74 \cdot 6$ & 165 & 120.2 & 225 & 165.7 & 285 & $212 \cdot 3$ & 345 & 259.8 \\
\hline IO5 & $75 \cdot 3$ & I 66 & 120.9 & 226 & 166.4 & 286 & $213-I$ & 346 & 260.6 \\
\hline 106 & 76.1 & 167 & 121,7 & 227 & $x 67 \cdot 2$ & 287 & 213.9 & 347 & $26 \mathrm{I} \cdot 4$ \\
\hline 107 & 76.8 & 168 & 122.4 & 228 & 167.9 & 288 & 214.7 & $34^{8}$ & $262 \cdot 3$ \\
\hline 108 & $77 \cdot 6$ & 169 & 123.2 & 229 & 168.6 & 289 & 215.5 & 349 & $263 . \mathrm{r}$ \\
\hline 109 & $7^{8} \cdot 3$ & 170 & 123.9 & 230 & I69. 4 & 290 & $216 \cdot 3$ & $35^{\circ}$ & 263.9 \\
\hline 110 & 79.0 & I 7 I & 124.7 & $23 \mathrm{I}$ & 170.1 & $29 I$ & $217 \cdot 1$ & $35 \mathrm{I}$ & $264 \cdot 7$ \\
\hline III & 79.8 & 172 & 125.5 & $23^{2}$ & 170.9 & 292 & 217.9 & $35^{2}$ & 265.5 \\
\hline 112 & 80.5 & 173 & 126.2 & 233 & 171.6 & 293 & 218.7 & 353 & 266.3 \\
\hline 113 & $8 r-3$ & 174 & 127.0 & 234 & 172.4 & 294 & 219.5 & 354 & 267.2 \\
\hline $\mathrm{II}_{4}$ & 82.0 & 175 & 127.8 & 235 & 173.1 & 295 & 220.3 & 355 & 268.0 \\
\hline II5 & $82 \cdot 7$ & 176 & 128.5 & 236 & I 73.9 & 296 & $22 \mathrm{I} \cdot \mathrm{I}$ & 356 & 268.8 \\
\hline 116 & $8_{3 \cdot 5}$ & 177 & 129.3 & 237 & 174.6 & 297 & 221.9 & 357 & 269.6 \\
\hline 117 & $84 \cdot 2$ & 178 & 130,1 & 238 & 175.4 & 298 & 292.7 & $35^{8}$ & 270.4 \\
\hline 118 & $8_{5} .0$ & 179 & 130.8 & 239 & 176.2 & 299 & $223 \cdot 5$ & 359 & $271 \cdot 2$ \\
\hline II9 & 85.7 & 180 & 131.6 & 240 & 176.9 & 300 & 2244 & $3^{60}$ & 272,1 \\
\hline 120 & 86.4 & $18 \mathrm{I}$ & 132.4 & $2+1$ & $177 \cdot 7$ & $30 \mathrm{I}$ & $225 \cdot 2$ & $36 r$ & 272.9 \\
\hline $12 \mathrm{I}$ & 87.2 & 182 & $133 . \mathrm{I}$ & 242 & 178.5 & 302 & 225.9 & 362 & 273.7 \\
\hline 122 & $87 \cdot 9$ & $18_{3}$ & 133.9 & 243 & $179 \cdot 3$ & $3 \circ 3$ & 226.7 & 363 & 274.5 \\
\hline 123 & 88.7 & 184 & I34.7 & 244 & 180.1 & 304 & $227 \cdot 5$ & 364 & $275 \cdot 3$ \\
\hline 124 & $89 \cdot 4$ & 185 & I 35.4 & 245 & 180.8 & 305 & 228.3 & 365 & 276.2 \\
\hline 125 & 90.1 & 186 & 136.2 & 246 & 181.6 & 306 & $229 \cdot \mathrm{I}$ & 366 & $277 \cdot \mathrm{I}$ \\
\hline 126 & 90.9 & 187 & 137.0 & 247 & $182 \cdot 4$ & 307 & 229.8 & 367 & 277.9 \\
\hline 127 & 91.6 & 188 & $137 \cdot 7$ & 248 & $183 \cdot 2$ & 308 & 230.6 & 368 & 278.8 \\
\hline 128 & 92.4 & 189 & 138,5 & 249 & $\mathrm{I}_{4} .0$ & 309 & $231 \cdot 4$ & 369 & 279.6 \\
\hline 129 & $93 . I$ & I90 & I 39.3 & 250 & 184.8 & 310 & 232.2 & 370 & 280.5 \\
\hline 130 & 93.8 & I9I & 140,0 & 251 & 185.5 & $3 I I$ & 232.9 & $37 \mathrm{I}$ & $28 \mathrm{~T} \cdot 4$ \\
\hline $13 I$ & $94 \cdot 6$ & $19^{2}$ & 140.8 & 252 & 186.3 & 312 & $233 \cdot 7$ & 372 & 282.2 \\
\hline 132 & $95 \cdot 3$ & 193 & $1+1.6$ & 253 & $187 \cdot 1$ & 313 & $234 \cdot 5$ & 373 & $28_{3}-1$ \\
\hline 133 & $96 . I$ & 194 & $142 \cdot 3$ & 254 & 187.9 & $3 \mathrm{r} 4$ & $235 \cdot 3$ & 374 & 283.9 \\
\hline 134 & 96.9 & I05 & $\mathrm{I}+3-\mathrm{I}$ & 255 & 188.7 & 315 & $236 . \mathrm{I}$ & 375 & 284.8 \\
\hline 135 & $97 \cdot 6$ & Ig6 & 143.9 & 256 & 189.4 & 316 & 236.8 & $37^{6}$ & 285.7 \\
\hline I36 & $98 \cdot 3$ & I97. & 144.6 & 257 & 190.2 & 317 & $237 \cdot 6$ & 377 & 286.5 \\
\hline 137 & $99-I$ & 198 & I $45-7$ & 258 & 191.0 & 318 & $23^{8} \cdot 4$ & 378 & $287 \cdot 4$ \\
\hline I $38^{8}$ & 99.8 & I99 & $I+6.2$ & 359 & 191.8 & $3^{19}$ & 239.2 & 379 & 288.2 \\
\hline 139 & 100.5 & 200 & 146.9 & 260 & $\operatorname{xg} 2.5$ & 320 & 240.0 & 380 & $289 . \mathrm{I}$ \\
\hline 140 & 101.3 & 201 & $147 \cdot 7$ & 261 & $193-3$ & 321 & 2.40 .7 & $38 \mathrm{I}$ & $280 \cdot 9$ \\
\hline $\mathrm{I}_{4} \mathrm{I}$ & 102.0 & 202 & 1.48 .5 & 262 & $194 . \mathrm{I}$ & $3^{22}$ & $24 \mathrm{I} \cdot 5$ & 382 & 290.8 \\
\hline 142 & 102.8 & 203 & 149.2 & 263 & 194.9 & $3^{23}$ & 242.3 & $3^{8} 3$ & 291.7 \\
\hline 143 & $10_{3} \cdot 5$ & 204 & 150.0 & 264 & 195.7 & 324 & 243.1 & 384 & 292.5 \\
\hline 144 & $104 \cdot 3$ & 205 & 150.7 & 265 & 196.4 & 325 & 243.9 & $3^{85}$ & 293.4 \\
\hline 145 & $105 \cdot 1$ & 206 & 151.5 & 266 & 197.2 & 326 & $24+.6$ & 386 & $294: 2$ \\
\hline 146 & 105.8 & 207 & 152.2 & 267 & 193.0 & $3^{27}$ & 245.4 & 387 & $295 \cdot I$ \\
\hline 147 & 106.6 & 208 & 153.0 & 268 & $\mathrm{rg} 8.3$ & 328 & 246.2 & 388 & 296.0 \\
\hline 148 & $107 \cdot 3$ & 209 & 153.7 & 269 & 199.5 & 329 & 247.0 & 389 & 296.8 \\
\hline I 49 & IOS. 1 & 210 & 154.5 & 270 & $200 \cdot 3$ & $33^{\circ}$ & $247 \cdot 7$ & 390 & $297 \cdot 7$ \\
\hline $155^{\circ}$ & 108.8 & 211 & 155.2 & $27 \mathrm{I}$ & 201 . I & $33 \mathrm{I}$ & 248.5 & $39 I$ & 208.5 \\
\hline I $5 \mathrm{I}$ & 109.6 & 212 & 156.0 & 272 & 201.9 & $33^{2}$ & 249.2 & 392 & $299 \cdot 4$ \\
\hline$I_{52}$ & IIO. 3 & 213 & 156.7 & 273 & $202 \cdot 7$ & 333 & 250.0 & 393 & $300 \cdot 3$ \\
\hline 153 & $I I I . I$ & 214 & 157.5 & 274 & 203.5 & 334 & 250.8 & 394 & 301.1 \\
\hline 154 & 111.9 & 215. & 158.2 & 275 & $204 \cdot 3$ & 335 & 251.6 & 395 & 302.0 \\
\hline 155 & 112.6 & 216 & 159.0 & 276 & $205 . \mathrm{I}$ & $33^{6}$ & 252.5 & 396 & 302.8 \\
\hline I $5^{6}$ & 113.4 & 217 & I59.7 & 277 & 205.9 & 337 & $253 \cdot 3$ & 397 & 303.7 \\
\hline 157 & $\mathrm{II}_{4} \cdot \mathrm{I}$ & 218 & 160.4 & 278 & 206.7 & $33^{8}$ & $254 . \mathrm{I}$ & 398 & 304.6 \\
\hline $15^{8}$ & 114.9 & 219 & 161.2 & 279 & $207 \cdot 5$ & 339 & 254.9 & 399 & 305.4 \\
\hline $\begin{array}{l}159 \\
160\end{array}$ & 115.6 & 220 & $16 r \cdot 9$ & 280 & 208.3 & 340 & $255 \cdot 7$ & 400 & 306.3 \\
\hline
\end{tabular}


whete $T$ is the per cent of total solids, $S$ the lactometer reading, and $F$ the fat. An ingenious instrument known as Richmond's milk-scale (Fig. $4^{8}$ ) is useful in making the calculation, instead of employing either the formula or a table. This is constructed on the principle of the slide rule, and by its use the specific gravity may be corrected to the proper temperature, and the solids calculated from the fat and specific gravity.

Babcock's formula for solids not fat is as follows:

$$
\text { Solids not fat }=\left(\frac{100 S-F S}{100-1.0753 F S}-\mathrm{I}\right)(100-F) 2.5 \text {, }
$$

$S$ being the specific gravity, and $F$ the percentage of fat. On this formula he has prepared a table* by means of which one may calculate solids not fat agreeing quite closely with results obtained by gravimetric analysis. $\dagger$ The table on page 154 has been recomputed and enlarged from that of Babcock, so as to express results in total solids rather than solids not fat.

Calculation of Proteins. -Van Slyke's $\ddagger$ formula for calculating proteins $(P)$ from the fat $(F)$ is:

$$
P=(F-3) \times 0.4+2.8 \text {. }
$$

Olsen $\S$ has devised the following formula for calculating proteins from total solids $(T S)$ :

$$
P=T S-\frac{T S}{\mathrm{I} .34} \text {. }
$$

Approximately $0.8 \times$ proteins $=$ casein.

The proteins being thus calculated, the sugar may be computed by difference. These calculations, while only approximate, give quite satisfactory results for normal, healthy milk, especially from herds.

Determination of Acidity.-While milk is still fresh, i.e., before it has begun to undergo lactic fermentation, it will show an acid reaction, which is sometimes expressed in terms of lactic acid. In view of the fact that

* U. S, Dept. of Agric,, Div. of Chem., Bul. 47, p. 123; Bul. 107 (rev.) p. 225 .

$\dagger$ For approximate work Babcock has suggested the following simplified formula: Solids not fat $=0.25 G+0.2 F$ and total solids $=0.25 G+$ I. $2 F, G$ being the lactometer reading and $F$ the fat.

$\ddagger$ Jour. Am. Chem. Soc. 30, 1908, p ris 2 .

\& Jour. Ind. and Eng. Chem., I, 1909, p. 253.

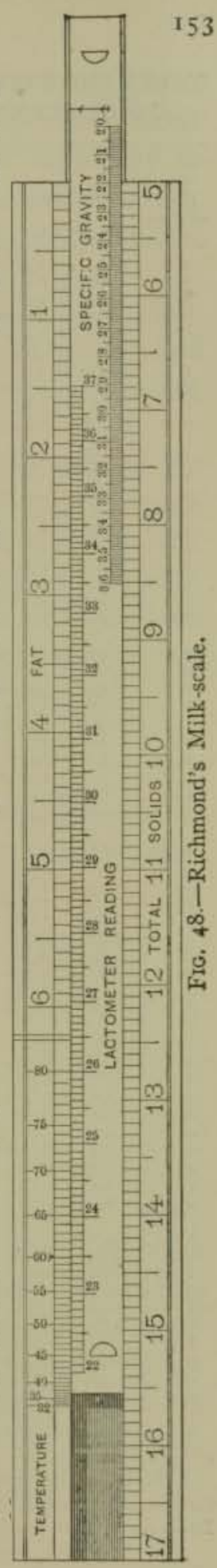


TABLE SHOWING PER CENT OF TOTAL SOLIDS IN MILK CORRESPONDING TO QUEVENNE LACTOMETER READINGS* AND PER CENT OF FAT. $†$

\begin{tabular}{|c|c|c|c|c|c|c|c|c|c|c|c|c|c|c|c|}
\hline \multirow{2}{*}{$\begin{array}{c}\text { Per } \\
\text { Cent } \\
\text { of Fat. }\end{array}$} & \multicolumn{15}{|c|}{ Lactometer Reading at $15.5^{\circ} \mathrm{C}$. } \\
\hline & 22 & 23 & 24 & 25 & & 27 & 28 & 20 & 30 & 31 & 32 & 33 & 34 & 35 & $3^{6}$ \\
\hline 0.0 & .50 & & 6.00 & 6.25 & 6.50 & 6.75 & .00 & 7.25 & $7 \cdot 50$ & 7.75 & 8,00 & 8.25 & 8.50 & 8.75 & \\
\hline & & & & & & 6.87 & & & & $7 \cdot 87$ & & & & & \\
\hline & 5.74 & 5.00 & 6.24 & 6.40 & 6.74 & 6.99 & & & 7.74 & 7.00 & 8.24 & 8.40 & & 3.00 & \\
\hline & & 6.11 & & & & 1 & & & 7,86 & & 8. 36 & $8.6 \mathrm{x}$ & 8.86 & & \\
\hline & 5,0 & 6. & $6.4^{8}$ & .73 & & & .48 & $7-73$ & $7 \cdot 98$ & & 8.48 & 8.73 & 8.00 & & \\
\hline & 6,10 & 6.35 & 5.60 & & 7.10 & & 7.60 & 7.85 & 8. 10 & 8.35 & 8.60 & 8.85 & & & \\
\hline & 6.22 & 6.47 & 6.72 & .97 & $7 \cdot 22$ & $7 \cdot 47$ & $7 \cdot 72$ & 7.97 & 8.22 & 8.47 & 8.72 & 8.97 & 9.22 & 9,47 & \\
\hline .7 & 6.34 & 6.50 & 6.84 & 7.00 & $7 \cdot 34$ & $7 \cdot 59$ & 7.84 & & 8,34 & 8.501 & 8.84 & 9,00 & 9.34 & 9.59 & \\
\hline 8 & 6.46 & $6.7 \mathrm{r}$ & 6.96 & 7 & $7 \cdot 46$ & 7.71 & 7.96 & 8.21 & 46 & 8.71 & 8.96 & & -46 & 9.71 & 9.96 \\
\hline 0.9 & 6.58 & 6.83 & 7.08 & 7.33 & $7 \cdot 58$ & 7.83 & 8,08 & 8,33 & 8.58 & 8.83 & 9.08 & 0.33 & 9.58 & 9.83 & 10. 08 \\
\hline 3.0 & 6.70 & 6.95 & & & 7.70 & 7.95 & 8.20 & 8.45 & .70 & .95 & 9.20 & 9.45 & 9.70 & 9.95 & 10.20 \\
\hline I. 1 & 6.82 & 7.07 & & & 7.82 & 8.07 & -32 & & 8.82 & 9.07 & & & 9.82 & 10.07 & 10.32 \\
\hline 1.2 & 6.94 & $7 \cdot 10$ & 7.4 & 7.69 & $7 \cdot 94$ & 8.70 & 44 & 8.60 & 8.94 & 10 & 44 & 69 & $9-94$ & +9 & 10.44 \\
\hline $1 \cdot 3$ & 7.06 & $7 \cdot 3 x$ & 7.56 & $7.8 \mathrm{t}$ & 8.06 & $8 \cdot 31$ & 8.56 & 8,81 & 26 & 9.31 & 56 & .81 & ro. 06 & & \\
\hline$x \cdot 4$ & 7,18 & $7 \cdot 43$ & 7.6 & 7.9 & 8,78 & 8.43 & 8.68 & 8. & 9.18 & 9.43 & .68 & 031 & 0.18 & .43 & 10.68 \\
\hline .5 & $7 \cdot 3$ & $7 \cdot 55$ & 7.80 & 8.05 & 8.30 & 8,53 & 8.80 & 9. & 0.30 & & 9.80 & 10.05 & 10.30 & .55 & 10.80 \\
\hline 1.6 & $7+42$ & 7.67 & 7,02 & 8.17 & 8.42 & 8.67 & 8.92 & 0. & $9 \cdot 42$ & 9.67 & 9.82 & 10,17 & 10.42 & 10.67 & 10,92 \\
\hline 1.7 & .54 & $7 \cdot 70$ & 8,04 & 8.20 & 8.54 & 8.79 & 0.04 & 9. & $9 \cdot 54$ & 9.79 & 10.04 & 10.20 & 10.54 & 10.79 & it. \\
\hline 1.8 & 7.66 & 7.91 & 8. +6 & $8.4 \mathrm{r}$ & 8.66 & 8.91 & 9.16 & 9.41 & 9.66 & 0.91 & -16 & 10.41 & 10.66 & .91 & it. \\
\hline 1.9 & $7 \cdot 78$ & 8.03 & B. 28 & 8.53 & 8.78 & 9.03 & 9.28 & 9.53 & 9.78 & $r 0.03$ & ro, 28 & 10.55 & 10.78 & 11,04 & 11.20 \\
\hline 2.0 & & 8.15 & 8.40 & 8.65 & 8,00 & & 9.40 & 9.65 & 9.90 & 10.15 & 10.40 & 10.66 & 10.91 & 11,16 & $\mathrm{Ir}, 4 \mathrm{t}$ \\
\hline 2.1 & 8.02 & 8.27 & 8.52 & 8.77 & 0.02 & 9.27 & & 9.77 & 10,02 & 10.27 & 10.52 & 10.78 & 11,03 & $\mathrm{Ir}, 28$ & $1 x .53$ \\
\hline 2.3 & 8.14 & 8.30 & 8.64 & 8.89 & $9 \cdot 14$ & 0.39 & 9.64 & & 10.14 & 10.30 & ro, 64 & 10.90 & 1,15 & $11.4^{\circ}$ & \\
\hline 2 & 8.26 & $8 \cdot 51$ & 8. 76 & $9.0 \mathrm{t}$ & 0,26 & 9.51 & 9.76 & 10. & .26 & 10.51 & 10.76 & 11.02 & 11.27 & 11.52 & 12.77 \\
\hline .4 & $8.3^{8}$ & 8.63 & 8.88 & (3) & 9.38 & 9.63 & 0.88 & 13 & .38 & 10.63 & Io. 88 & $I I . T 4$ & 11.39 & $1 x, 64$ & 1.89 \\
\hline 2 & 8.50 & 8.75 & 0.00 & 25 & 9.50 & 9.75 & 10.00 & .25 & 10.50 & 10.75 & 11.00 & $x x .26$ & 11.51 & 11. 76 & 12.01 \\
\hline 2.6 & 8,60 & 8.87 & 9.12 & 37 & 0.62 & 9.87 & 0,12 & 10.37 & 10.62 & 10.87 & $11 ; 12$ & $|\mathbf{I}, 38|$ & 11.63 & 11.88 & 12.13 \\
\hline 2.7 & 8.74 & 8.99 & .24 & .49 & $9 \cdot 74$ & 9.99 & 10,24 & 10.49 & 10.74 & .99 & 11.24 & $|x .50|$ & $7 x \cdot 75$ & I2.00 & 12.25 \\
\hline 2.8 & 8.86 & $9.1 \mathrm{~T}$ & 9.36 & $9.6 \mathrm{x}$ & 9.86 & 10,11 & 10.36 & 10.61 & 10.86 & 11.11 & 11.37 & $|1 x .62|$ & 11.87 & 12.12 & 12,37 \\
\hline 2.9 & 8,98 & 9.23 & 9.48 & 9.73 & 9.98 & 10.23 & 10.48 & 10.73 & 10.08 & 11.23 & 11.49 & 11.74 & 11.99 & 12.24 & 12.49 \\
\hline 3.0 & 0,10 & .35 & 60 & 85 & 10,10 & 10.35 & 10.60 & 10.85 & & 4.36 & $x+6 x$ & & & 12,36 & \\
\hline 3. & 9.22 & .47 & .72 & 9.97 & 10,22 & 0.47 & .72 & & 11.23 & 11.48 & $1 x-73$ & 11.98 & 12.23 & 12.48 & 12 \\
\hline 3,2 & 34 & .50 & 9.84 & 10,09 & $10,-34$ & 0.59 & 84 & .09 & $11 \cdot 35$ & 11.60 & 12.85 & 12,10 & 12.35 & $t 2.6 x$ & 12.86 \\
\hline 3. & -46 & 9.71 & 9.96 & 0.21 & 10.46 & 10.71 & 20,96 & 11.22 & 11,47 & 11.72 & 11.97 & 12,22 & $12.4^{8}$ & 12.73 & 12.98 \\
\hline 3. & 9.58 & 9.83 & 10,08 & 0.33 & 10.58 & 10.83 & $1 \mathrm{t}, 09$ & $1+.34$ & 11.59 & $\mathrm{II}, 84$ & 12.00 & 12.34 & $x 2.60$ & $\mathrm{r} 2.8 \mathrm{~s}$ & 13.10 \\
\hline 3 & .70 & 9.95 & 10.20 & 0,45 & 10.70 & 10.95 & $\mathrm{IT}=2 \mathrm{I}$ & $x+46$ & $x+7 x$ & 11.96 & 12.21 & 12.46 & 12.72 & 12.97 & 13.22 \\
\hline 3 & 82 & 10.07 & 10,32 & 0.57 & 10,82 & 15,08 & 15.33 & 11.58 & 11.83 & 12,08 & 12,33 & 12,58 & 12.84 & 13.00 & $13 \cdot 34$ \\
\hline 3. & -94 & 0.29 & 10.44 & 9.79 & 10,94 & 11.20 & 11.45 & 11.70 & 11.95 & 12.20 & 12.45 & 12.70 & $x 2.90$ & $t 3,2 \mathrm{r}$ & 13.46 \\
\hline 3.8 & .06 & 10.31 & 10.56 & 10.81 & 11.06 & $|11.32|$ & $x x .57$ & 11,82 & 12.07 & $|12,32|$ & 12.57 & $12,8 z$ & 13,08 & 13,33 & 13.58 \\
\hline 3.9 & 10.18 & 10.43 & 10.68 & 10.93 & 11,18 & 11.44 & 11.60 & 31.94 & $12,+19$ & 12.44 & 12.60 & 12.94 & 13.20 & 13.45 & 13.70 \\
\hline $4 \cdot 0$ & .30 & 0.53 & 10.80 & 1.05 & $I I .30$ & 17.56 & $x x=8 x$ & $r=.06$ & 12,31 & 56 & & & 32 & & \\
\hline 4. & -42 & 10.67 & 10,02 & $x .17$ & 11.42 & 1,68 & 11.93 & .8 & 12.43 & .68 & 12.93 & -18 & $x 3.44$ & 13.69 & \\
\hline $4 \cdot 2$ & 54 & 10.79 & 11,04 & $x+29$ & $1 . x, 54$ & 11.80 & 12.05 & 30 & $\mathrm{r} 2.53$ & 12.80 & 13.05 & $13-31$ & $\mathrm{r} 3.50$ & 13.82 & 14.07 \\
\hline $4 \cdot 3$ & .66 & 10.91 & $1 x+16$ & $\mid 11.41$ & 11.66 & 11.92 & 12,17 & 12.42 & 12.67 & 12.02 & 13.18 & 13.43 & 13.68 & $13-94$ & 14,19 \\
\hline 4. & -78 & 11.03 & $I I=28$ & 12.53 & $7 x-78$ & 12,04 & 12,20 & 12.54 & 12.79 & 13,04 & 13.30 & 13.55 & 13.80 & 14.06 & 14. \\
\hline 4 & 5,00 & 11.15 & 11.40 & $11-65$ & 11.90 & 12,16 & $1=2.41$ & 12.66 & 12.91 & 13.16 & 13.42 & 13.67 & 13.92 & 14.18 & \\
\hline 4 & .02 & 11.23 & 11.52 & $17+78$ & 12.03 & 12.28 & 12.53 & 12,78 & 13.03 & 13.28 & 13.54 & 13. & 14.04 & 14.30 & \\
\hline & . 44 & 11.49 & $1 \pi .65$ & 11,90 & 12.15 & $\mathrm{r} 2.40$ & 12.65 & 12.00 & 13,15 & 13.40 & 13.66 & 13.91 & 14,16 & 14.42 & 2.14 .67 \\
\hline & 11.27 & 11.52 & $7 x, 77$ & 12.02 & 12.27 & 12.52 & 12.77 & $13.02 \mid$ & 13,27 & 13.52 & 13.78 & $\mathrm{~T} 4.03$ & 14.28 & 14.54 & 114.79 \\
\hline $4 \cdot 9$ & 11,39 & 11.64 & 11.89 & 12.14 & 12.30 & $\mathrm{x} 2.64$ & 12.89 & 13,14 & 13.39 & 13.64 & 13.90 & 14,15 & $14.4^{\circ}$ & 14.66 & 14.91 \\
\hline & .51 & 11. 76 & $12,0 x$ & 12.26 & 12,51 & 12.76 & 13.01 & 13.26 & 51 & .76 & 02 & & 14.32 & 14.78 & \\
\hline 5 & 63 & II. 88 & 12,13 & $12 \cdot 38$ & 12.63 & $\mathrm{I} 2.88$ & $x 3.13$ & $13,3^{8}$ & 13.03 & 13.89 & .14 & & 14.64 & 14.90 & \\
\hline 5 & .75 & 12.00 & 12,25 & 12.50 & 12.75 & 13.00 & 13,25 & 13.50 & 13.75 & $14.0 \mathrm{r}$ & 14.26 & 14.51 & $14 \cdot 76$ & 15.02 & 13.27 \\
\hline & .87 & $|x 2, x 2|$ & 12.37 & 12.62 & 12.87 & 13.12 & 13.37 & 13.62 & 13.87 & I 4.13 & 14.38 & & 14.88 & 15.14 & 15.39 \\
\hline & 11.99 & 12.24 & 12.49 & 12.74 & $r 2,99$ & 13,24 & 13,49 & & 14.00 & 14,25 & $x 4.50$ & 14.76 & 15.01 & 15.26 & 15.51 \\
\hline & $\mathrm{I} 2 . \mathrm{II}$ & $|x z-36|$ & 12,61 & 12.86 & 13.11 & 13,36 & 13,61 & 13,86 & 14.12 & 14.37 & 14.62 & 14.88 & 15.13 & 15.38 & \\
\hline & 12,23 & 12.48 & 12.73 & $x=08$ & 13.23 & I 3.48 & 13.73 & 13.99 & 14.24 & 14.49 & 14.75 & I 5.00 & 15.25 & 15.50 & 15.75 \\
\hline 7 & 12.35 & 12,60 & 12.85 & $13 . \mathrm{xO}$ & 13.35 & 13.60 & 13.85 & 1.4 .11 & $14 \cdot 36$ & $\mathrm{r} 4.6 \mathrm{t}$ & 1.4 .87 & 15,12 & 15.37 & 15.62 & 15.87 \\
\hline & $\mathrm{r} 2.47$ & 12,72 & 12.97 & 13.22 & 13.47 & 13.72 & 13.97 & 14.22 & 14.48 & & 14.99 & 15.24 & I5. 49 & 15.74 & $+5,99$ \\
\hline 5.9 & 12,59 & 12.84 & 13.09 & 13.34 & 13.59 & 13.84 & 14.10 & $14 \cdot 35$ & 14.60 & $\mathrm{I} 4.86$ & 15.11 & 15.30 & 15.61 & 15.86 & \\
\hline 6.0 & 12.71 & 12.96 & 13.21 & $13.4^{6}$ & $13.7^{1}$ & 13.96 & 14.22 & $\mathrm{I} 4.47$ & 14.72 & 14.98 & 15.23 & 15.48 & 15.73 & 15.98 & 16.24 \\
\hline
\end{tabular}

* The lactometer reading is expressed in whole numbers for convenience. The true specific gravity corresponding to a given lactometer reading is obtained by writing 1.0 before the lactometer reading. Thus, 1.026 is the specific gravity corresponding to lactometer reading 26 , etc.

†An. Rep. Mass. State Board of Health, rgor, p. 445. (Analyst's Reprint, p. 25.) 
the acidity of "sweet" milk is due partly to the presence of acid phosphates and partly to dissolved carbonic acid in the milk, and not to lactic acid, which is probably absent, a better plan is to express the acidity in terms of the number of cubic centimeters of tenth-normal alkali necessary to neutralize a given quantity of the milk, either 25 or $50 \mathrm{cc}$., using phenolphthalein as an indicator.

If it is desired to calculate the acidity in terms of lactic acid, multiply the number of cubic centimeters of tenth-normal alkali used by 0.897 , and divide by the number of cubic centimeters of milk titrated, the result being the percentage of lactic acid.

Detection of Boiled Milk.-Storch's Method.*-Shake $5 \mathrm{cc}$. of the milk in a test-tube with one drop of a $2 \%$ solution of hydrogen peroxide and two drops of a $2 \%$ solution of paraphenylenediamin. If the milk has not been heated beyond $80^{\circ} \mathrm{C}$., a dark violet color appears at once, but if it has been pasteurized or boiled, no color appears. Siegfeld and Samson $\uparrow$ find that addition of two drops of formalin ( $1: 1$ ) to each 100 cc. of milk previots to boiling causes it to react similar to raw milk.

\section{MODIFIED MILK.}

A comparison of the composition of cow's milk and human milk, as in the following table by Dr. Emmett Holt, $\ddagger$ shows very marked differences.

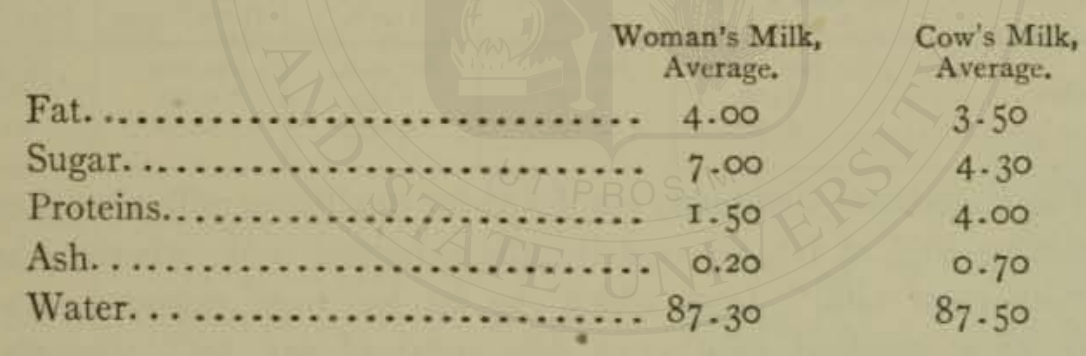

The per cent of fat in the two kinds of milk is nearly the same. There is, however, too little sugar and an excess of proteins and ash in the milk of the cow, assuming human milk as the ideal infant food, so that in basing a diet for infants on the tasis of human milk considerable modification is necessary. Moreover, aside from the actual variation in the amount of ingredients, there are certain inherent differences in the character of the same ingredient, as found in the milk of the cow and in

* Copenhagen Expt. Sta., toth Rep.

‡ "Infancy and Childhood."

$\dagger$ Molk. Ztg., 21, 1907, p. 103. 
human milk. The proteins of cow's milk are, for instance, found to be much more difficult of digestion than those of woman's milk, and the same is probably true of the fat. Aside from the mere statement of a few of these differences, it is obviously beyond the scope of this work to discuss this phase of the subject in detail, reference being made, however, to such books as Dr. T. M. Rotch's "Pediatrics," and "Infancy and Childhood" by Dr. Emmett Holt, for full particulars. So great has been the demand by physicians for "modified milk" for infant feeding, that laboratories for this exclusive purpose have been established in many of the larger cities, in which not only is milk prepared in accordance with certain fixed formulæ supposed to be adapted to average infants of varying age, but milk of any desired composition is prepared, in accordance with special prescriptions of physicians to apply to individual cases.

Methods and Ingredients.-The proteins and the ash in cow's milk are much higher than in human milk, and both are brought to the proper degree of reduction by diluting the milk with water. Milk sugar is ircreased by the addition of lactose, and the fat is increased or diminished by addition of cream or by skimming.

The dilution of cow's milk with a measured amount of water shows the following results on the proteins and ash:

\begin{tabular}{|c|c|c|c|c|c|}
\hline & Cow's Milk. & $\begin{array}{l}\text { Diluted } \\
\text { Once. }\end{array}$ & $\begin{array}{l}\text { Diluted } \\
\text { Twice. }\end{array}$ & $\begin{array}{l}\text { Diluted } \\
\text { Three Times. }\end{array}$ & $\begin{array}{l}\text { Diluted } \\
\text { Four Times. }\end{array}$ \\
\hline $\begin{array}{l}\text { Proteins. } \\
\text { Ash..... }\end{array}$ & $\begin{array}{c}\text { Per cent. } \\
4.00 \\
0.70\end{array}$ & $\begin{array}{c}\text { Per cent. } \\
2.00 \\
0.35\end{array}$ & $\begin{array}{c}\text { Per cent. } \\
\text { ז. } 33 \\
0.23\end{array}$ & $\begin{array}{c}\text { Per cent. } \\
1.00 \\
0.18\end{array}$ & $\begin{array}{c}\text { Per cent. } \\
0.80 \\
0.14\end{array}$ \\
\hline
\end{tabular}

The ingredients commonly employed for modifying milk are (I) cream, containing $16 \%$ of fat, (2) centrifugally skimmed milk, otherwise known as "separator milk" from which the fat has been removed, (3) milk sugar, or a standard solution of "milk sugar of, say, $20 \%$ strength, and (4) lime water. Unusual care should be taken in the selection of the milk supply to insure cleanness, purity, and freshness, as well as in the care of utensils, etc., used in the laboratory, which should in all cases be scrupulously clean. Samples prepared in accordance with a given formula or formulæ are pasteurized in separate bottles, or, if desired, sterilized, and after stoppering with cotton are kept on ice.

Formula.-It is obviously impossible to establish formula universally applicable even to healthy infants, but the following may be regarded as typical formulæ, representing the composition of modified milk to suit the needs of an average growing infant during its first year: 


\begin{tabular}{|c|c|c|c|}
\hline Period. & Fat. & Proteins. & Sugar. \\
\cline { 5 - 5 } Third to fourteenth day....... & Per cent. & Per cent. & Per cent. \\
Second to sixth week......... & 2.5 & 0.6 & 6 \\
Sixth to eleventh week........ & 3 & 0.8 & 6 \\
Eleventh week to fifth month.. & 3.5 & 1.0 & 6 \\
Fifth to ninth month......... & 4 & 1.5 & 7 \\
Ninth to twelfth month....... & 3.5 & 2.5 & 7 \\
& & & 3.5 \\
\hline
\end{tabular}

Milk according to the above formulx can be very simply prepared by the aid of a specially made graduate known as the "Materna" and shown in Fig. 49 .

\section{PREPARED MILK FOODS.}

Milk Powder.-There are numerous brands of desiccated milk or milk powder on the market, sold in bulk and by the can, and largely used by bakers and manufacturers of milk chocolate. Many of these, purporting to contain all the ingredients of milk excepting water, have been found by the author to be pulverized dried skimmed milk. The following are analyses of whole milk, half-skim milk, and skim milk powders:

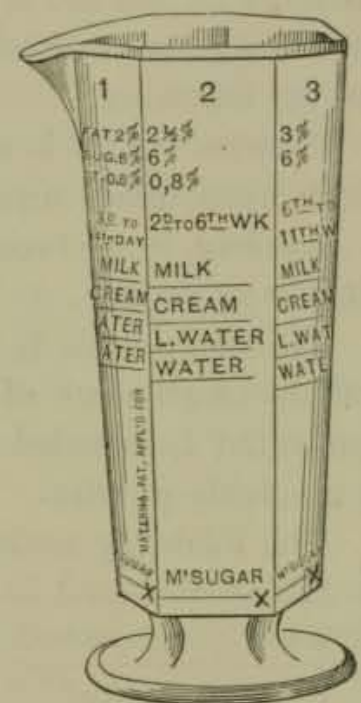

FtG. 49,-The "Materna" Graduate for Modifying Milk.

\begin{tabular}{|c|c|c|c|}
\hline & $\begin{array}{l}\text { Whole Milk, } \\
\text { Powder.* }\end{array}$ & $\begin{array}{l}\text { Half-skim Milk, } \\
\text { Powder.* }\end{array}$ & $\begin{array}{l}\text { Skim Milk, } \\
\text { Powder- } \dagger\end{array}$ \\
\hline Moisture................ & 3.62 & 5.01 & 8.16 \\
\hline Fat...$\ldots \ldots \ldots \ldots \ldots \ldots \ldots$ & 26.75 & 15.26 & I. 73 \\
\hline Proteins $(\mathrm{N} \times 6.25) \ldots \ldots \ldots$ & 32.06 & $3^{8} \cdot 39$ & 33.84 \\
\hline Milk sugar ............... & .31 .90 & 34.67 & $49 \cdot 35$ \\
\hline Ash $\ldots \ldots \ldots \ldots \ldots \ldots \ldots$ & . 5.67 & 6.67 & 6.87 \\
\hline & 100.00 & 100.00 & 99.95 \\
\hline
\end{tabular}

The fat in the skim milk powder corresponds to about $0.16 \%$ fat in the original milk.

Jensen $\ddagger$ states that the casein of dried milk no longer has the power

*C. Huyge, Rev, gén, du Lait, 3, 1904, p. 400.

$\dagger$ Analysis by the author.

$\ddagger$ Molkerei Ztg., Berlin, 15, 1905, p. 565 . 
of swelling when mixed with water. To obviate this difficulty, Hatmaker adds to the milk I to $3 \%$ of sodium bicarbonate, and Elkenberg $2 \%$ of cane-sugar. A Swiss milk powder examined by Jensen contained an excess of sodium and a low acidity, indicating the addition of an alkaline sodium salt.

Artificial Albuminous Foods.-The albumin and casein of milk have furnished the basis of a variety of food preparations, some of which are intended for the use of invalids and people of weak digestion, and others, from their compactness, for travellers and campers. Among these foods are the following:

Nutrose. - This is a caseinate of sodium formed by the action of the alkali upon dried casein. It is soluble in water.

Eucasin is a caseinate of ammonium, a soluble powder somewhat similar to nutrose.

Plasmon.-This is a yellowish powder, prepared by treatment with sodium bicarbonate of the curd precipitated from skimmed milk. The compound is kneaded in an atmosphere of carbon dioxide, and reduced to a soluble powder.

The following analysis of plasmon was made by Woods and Merrill:*

\begin{tabular}{c|c|c|c|c|c}
\hline Water. & Proteids. & Fat. & Carbohydrates. & Ash. & Fuel Value. \\
\cline { 2 - 6 } & 75.0 & 0.2 & 8.9 & 7.4 & 2044 \\
\hline
\end{tabular}

Sanose.-This is also a powder, containing $80 \%$ of pure casein and $20 \%$ of albumose, obtained from the white of egg. The powder possesses a slight taste and an odor suggestive of milk. By briskly stirring the powder with water, an emulsion may be made much resembling milk, but on standing it soon breaks up.

Sanatogen is a grayish-white, tasteless powder, containing $95 \%$ of casein and $5 \%$ sodium glycero-phosphate. When treated with cold water it swells, forming on heating a milk-like emulsion.

Koumis is a stimulating beverage, prepared by allowing milk to undergo alcoholic, lactic, and proteolytic fermentations. The original koumis was made by the Tartar tribes of Asia from mare's milk, which contains more lactose than cow's milk, and apparently lends itself more readily to fermentation. Only a limited amount of koumis is now made from mare's milk, the milk chiefly used for this preparation being that of the cow, treated with yeast and sometimes added sugar. Koumis is a beverage much more commonly used in Europe than in America.

* Maine Exp. Station, Bulletin 178 , p. 101. 
The following analyses were made by Vieth:*

\begin{tabular}{|c|c|c|c|c|c|c|c|c|c|}
\hline & Water. & $\begin{array}{l}\text { Alco- } \\
\text { hol. }\end{array}$ & Fat. & Casein. & $\begin{array}{l}\text { Albu- } \\
\text { min. }\end{array}$ & $\begin{array}{l}\text { Albu- } \\
\text { min- } \\
\text { oses. }\end{array}$ & $\begin{array}{l}\text { Lactic } \\
\text { Acid. }\end{array}$ & Sugar. & Ash. \\
\hline $\begin{array}{l}\text { Mare's milk. } . . \ldots \ldots \ldots \\
\text { Cow's milk. } \\
\text { Skimmed milk.......... }\end{array}$ & $\begin{array}{l}92.07 \\
90.57 \\
92.52\end{array}$ & $\begin{array}{l}2.98 \\
1.04 \\
0.57\end{array}$ & $\begin{array}{l}1 \cdot 30^{*} \\
1 \cdot 3^{8} \\
0.33\end{array}$ & $\begin{array}{l}0.83 \\
1.88 \\
2.03\end{array}$ & $\begin{array}{l}0.24 \\
0.20 \\
0.07\end{array}$ & $\begin{array}{l}0.77 \\
0.77 \\
0.63\end{array}$ & $\begin{array}{l}1.27 \\
1.40 \\
0.56\end{array}$ & $\begin{array}{l}0.23 \\
2.18 \\
2.45\end{array}$ & $\begin{array}{l}0.35 \\
0.58 \\
0.84\end{array}$ \\
\hline
\end{tabular}

Kephir.-This is a fermented milk product similar to koumis, excepting that the fermentation is induced by a fungus known as kephir grains. The proteolytic fermentation is less pronounced in kephir than in koumis. König gives the following table as the mean of twenty-eight analyses:

\begin{tabular}{l|l|l|l|l|l|l|l|l|l|l|l|l}
\hline Water. & $\begin{array}{l}\text { N tro- } \\
\text { gen. }\end{array}$ & Casein. & $\begin{array}{c}\text { Albu- } \\
\text { min. }\end{array}$ & $\begin{array}{c}\text { Acid } \\
\text { Albu- } \\
\text { min. }\end{array}$ & $\begin{array}{c}\text { Hemi- } \\
\text { albumin. }\end{array}$ & $\begin{array}{c}\text { Pep- } \\
\text { tone. }\end{array}$ & Fat. & $\begin{array}{c}\text { Lac- } \\
\text { tose. }\end{array}$ & $\begin{array}{c}\text { Lactic } \\
\text { Acid. }\end{array}$ & $\begin{array}{c}\text { Alco- } \\
\text { hol. }\end{array}$ & Ash. \\
\hline 91.21 & 3.49 & 2.53 & 0.36 & 0.21 & 0.21 & 0.039 & 1.44 & 2.41 & 1.02 & 0.75 & 0.68 \\
\hline
\end{tabular}

ADULTERATION OF MILK.

Systems of Milk Inspection.-A typical method of general food inspection has already been outlined (see pp. 6 and 8), which may easily be modified to include the inspection of milk in connection with other foods, or to provide for a system of milk inspection exclusively. In the examination of such a perishable food as milk, it has not been found practicable for the analyst to reserve for the benefit of the defendant a sealed sample, as in the case of other foods, but experience has shown it had best be made the duty of the collector or inspector to give a sealed sample of milk to the dealer, when the latter requests it at the time of taking the sample. For this purpose the collector is provided with small bottles and sealing pharaphernalia, in addition to the tagged sample bottles or cans in which he collects the milk. The collector should use the same precautions for obtaining a perfectly fair representative sample as does the chemist in making the analysis, i.e., he should carefully pour the milk from the original container into an empty can or vessel and back again, before taking his sample.

Each sample is properly numbered by the collector in presence of the dealer, and the data as to the taking of the sample entered at once under the proper number in the collector's book. If a sealed sample is given,

* Richmond Dairy Chemistry, p. 241 et seq. 
it should bear the same number as the sample reserved for analysis, and a receipt should invariably be required from the dealer, as evidence that his request for a sealed sample has been complied with.

Milk Standards Fixed by Law.-In localities where a systematic form of milk inspection prevails, there is usually in force a statute fixing the legal standard for the total solids, and in many cases for the fat or for the solids exclusive of fat. In some states the statute is so drawn that any deviation from the legal standard constitutes an adulteration in the eye of the law, and hence the offender, who has such milk in his possession with intent to sell, is liable to the same fine as if he actually added water or a foreign substance to the mill.

In other states a distinction is made by the statute between milk that is simply below the legal standard of total solids, and milk containing actually added ingredients (water or otherwise), a much lighter fine being imposed for the former than for the latter offense. Where such a distinction prevails, it often becomes incumbent upon the analyst to show to the satisfaction of the court, in case of milk low in solids, whether or not the milk has been fraudulently watered after being drawn from the cow, it being well understood that cows may give milk below the standard.

Pure milk that is low in solids may owe its deficiency either to poor feeding, or to an inherent tendency on the part of the cow to give milk always of poor quality. Thus the Holstein cow, more than any other breed, is open to the charge of sometimes giving milk below the standard.* That the Holstein cow is a favorite with the producer is by no means strange, from the fact that no other breed can with moderate feeding be made to give so large a quantity of milk.

Wherever there is a statute fixing the standard for milk, it commonly provides also that the addition of any foreign substance whatsoever constitutes an adulteration.

U. S. Standards. $\nmid-$ Slandard milk is the fresh, clean, lacteal secretion obtained by the complete milking of one or more perfectly healthy

* This statement should not be taken as condemning the Holstein, for it is true that cows of this breed often give milk far above the standard. A large number of samples of milk of known purity from Holsteins analyzed by the writer have been found to be of excellent quality. It is a curious fact that among the samples of known purity analyzed by the Massachusetts Board of Health, both the lowest and highest total solids on record came from a Holstein cow; the lowest recorded total solids in a "known purity" milk being $9.9^{6}$ pet cent. (seventh annual report of Massachusetts State Board of Health, Lunacy, and Charity, D. 160 ), and the highest being 17.06 per cent. (twenty-second annual report of the Massa. chusetts State Board of Health, p. 405).

$\dagger$ U. S. Dept. of Agric., Off. of Sec., Cire. r9. 
cows, properly fed and kept, excluding that obtained within fifteen days before and ten days after calving, and contains not less than $8.5 \%$ of solids not fat, nor less than $3.25 \%$ of milk-fat.

Standard Skim-milk is skim-milk containing not less than $9.25 \%$ of milk solids.

FORMS OF ADULTERATION.-Milk is ordinarily adulterated ( 1 ) by watering, (2) by skimming, (3) by both watering and skimming, and (4) by the addition of one or more foreign ingredients.

Watering and Skimming.-The fact that milk is found below the standard of total solids, while more often due to an excess of water, may also be due to a deficiency in fat. In one case the milk is commonly termed watered, and in the other skimmed, using the terms broadly and not necessarily meaning actual and fraudulent tampering with the milk. In a third case, and almost invariably fraudulently, both watering and skimming may be found to have been practiced on the same sample. The analyst judges which of these causes have produced a milk low in solids, by a careful study of the relation between the percentages of total solids, fat, and solids not fat.

If both the total solids and solids not fat are abnormally low, and the proportion of fat to solids not fat about the same as, or higher than, in a normal milk, it is generally safe to assume that the sample has been watered; if both the total solids and the fat are well below the standard, and the solids not fat nearly normal, then the milk has undoubtedly been skimmed; if, in the third place, the total solids and the solids not fat are proportionally reduced below the standard, while the ratio of fat to solids not fat is abnormally small, it is safe to adjudge the milk to be low by reason of both skimming and watering.

Milk of Known Purity.-It is difficult to place the minimum figure for total solids, below which a milk sample may safely be pronounced by the analyst as fraudulently watered after having been drawn from the cow. Nearly nine hundred semples of milk of known purity from various breeds of cow, milked in the presence of an inspector, have been analyzed in the Department of Food and Drug Inspection of the Massachusetts State Board of Health, extending over a period of fifteen years, and among these are many samples from Holstein cows. It is extremely rare that any of these known purity samples have been found with total solids as low as $11 \%$, though there are instances where total solids have run as low as $10 \%$. 
It is safe to assume that in the few cases on record showing less than 10.75\% of total solids, either there was something decidedly abnormal about the health of the cow, or, through some accident, the cow was only partially milked, it being $a^{*}$ well-known fact tnat the last fraction of the milking includes the larger percentage of fat. (See page 128.)

It is therefore nearly always safe to condemn a milk standing below I0.75 as fraudulently watered, if at the same time it has a proportionately high per cent of fat.

The average total solids of 800 samples of milk of known purity analyzed by the Massachusetts Board up to and including the year 1890 amounted to about $13 \frac{1}{1} \%$.

It is rare indeed to find a herd of ten or more well-fed cows of mixed breeds in which the average milk of the herd falls below $12 \frac{1}{2} \%$ of solids.

The milk of forty-seven Holstein cows, examined in 1885 , was found to contain an average of $12.5 \mathrm{I} \%$ of total solids, while the milk of eleven Jerseys examined in the same year averaged $14.02 \%$ of solids. These examples represent the two extremes commonly met. with.

Variation in Standard.-In Massachusetts the law fixes a different standard for total solids in milk during the summer, or pasture-fed season, from that in force during the winter, or stall-fed period. From April to September inclusive the legal standard is $12 \%$ of total solids, of which $9 \%$ are solids not fat, and from October to March inclusive it is $13 \%$, of which $9.3 \%$ are solids not fat. Bearing on the question of difference in normal quality of milk during the two period;, averages were taken of the milks collected by the corps of inspectors of the Massachusetts Board of Health during a month in each period, December and June being selected as most typical, and during these months all the samples were analyzed both for total solids and fat. The samples were taken from stores, milkmen, and producers, and represented as nearly as possible the milk as actually sold to the consumers. In making the averages, all samples of skimmed milk, as well as all samples standing above $17 \%$ of total solids, or under $10.75 \%$, were deducted. The results are summarized as follows: 
QUALITY OF MILK SOLD IN MASSACHUSETTS CITIES AND TOWNS IN WINTER AND SUMMER.

\begin{tabular}{|c|c|c|c|c|c|c|c|c|}
\hline & \multicolumn{8}{|c|}{ December. } \\
\hline & \multirow{2}{*}{$\begin{array}{l}\text { Number } \\
\text { of } \\
\text { Samples. }\end{array}$} & \multicolumn{3}{|c|}{ Total Solids. } & \multicolumn{3}{|c|}{ Fat. } & \multirow{2}{*}{$\begin{array}{l}\text { Solids } \\
\text { not Fat. } \\
\text { Average } \\
\text { Per Cent. }\end{array}$} \\
\hline & & $\begin{array}{l}\text { Highest } \\
\text { Per Cent. }\end{array}$ & $\begin{array}{l}\text { Lowest } \\
\text { Per Cent. }\end{array}$ & $\begin{array}{l}\text { Average } \\
\text { Per Cent. }\end{array}$ & $\begin{array}{l}\text { Highest } \\
\text { Per Cent. }\end{array}$ & $\begin{array}{l}\text { Lowest } \\
\text { Per Cent. }\end{array}$ & $\begin{array}{l}\text { Average } \\
\text { Per Cent. }\end{array}$ & \\
\hline \multirow[t]{4}{*}{$\begin{array}{l}\text { Cities........ } \\
\text { Towns ....... } \\
\text { Summary.... }\end{array}$} & $\begin{array}{r}403 \\
99 \\
502\end{array}$ & $\begin{array}{l}16.86 \\
15 \cdot 48 \\
16.86\end{array}$ & $\begin{array}{l}10.88 \\
12.02 \\
10.88\end{array}$ & $\begin{array}{l}13.21 \\
13 \cdot 44 \\
13 \cdot 32\end{array}$ & $\begin{array}{l}8.50 \\
6.65 \\
8.50\end{array}$ & $\begin{array}{l}2.40 \\
3.50 \\
2.40\end{array}$ & $\begin{array}{l}4 \cdot 37 \\
4 \cdot 4^{8} \\
4 \cdot 4^{2}\end{array}$ & $\begin{array}{l}8.74 \\
8.96 \\
8.85\end{array}$ \\
\hline & \multicolumn{8}{|c|}{ June. } \\
\hline & \multirow{2}{*}{$\begin{array}{c}\text { Number } \\
\text { of } \\
\text { Semples. }\end{array}$} & \multicolumn{3}{|c|}{ Total Solids. } & \multicolumn{3}{|c|}{ Fat. } & \multirow{2}{*}{$\begin{array}{l}\text { Solids } \\
\text { not Fat. } \\
\text { Average } \\
\text { Per Cent. }\end{array}$} \\
\hline & & $\begin{array}{l}\text { Highest } \\
\text { Per Cent. }\end{array}$ & $\begin{array}{l}\text { Lowest } \\
\text { Per Cent. }\end{array}$ & $\begin{array}{l}\text { Average } \\
\text { Per Cent. }\end{array}$ & $\begin{array}{l}\text { Highest. } \\
\text { Per Cent. }\end{array}$ & $\begin{array}{c}\text { Lowest } \\
\text { Per Cent. }\end{array}$ & $\begin{array}{l}\text { Average } \\
\text { Per Cent. }\end{array}$ & \\
\hline $\begin{array}{l}\text { Cities....... } \\
\text { Towns....... } \\
\text { Summary.... }\end{array}$ & $\begin{array}{r}311 \\
76 \\
3^{87}\end{array}$ & $\begin{array}{l}16.90 \\
15.71 \\
16.90\end{array}$ & $\begin{array}{l}10.75 \\
10.99 \\
10.75\end{array}$ & $\begin{array}{l}12.67 \\
12.63 \\
12.65\end{array}$ & $\begin{array}{l}8.80 \\
7.10 \\
8.80\end{array}$ & $\begin{array}{l}2.10 \\
3.00 \\
2.10\end{array}$ & $\begin{array}{l}4.03 \\
4.09 \\
4.06\end{array}$ & $\begin{array}{l}8.54 \\
8.54 \\
8.54\end{array}$ \\
\hline
\end{tabular}

It is interesting to note that the average for total solids of the $88 \mathrm{~g}$ samples examined for both months stands at just $13 \%$, of which $4.24 \%$ is fat and 8.76 is solids not fat.

\section{Rapid Approximate Methods of Determining the Quality of Milk.-} The Lactometer.-A rough idea of the quality of milk can be gained by the use of the lactometer (page $\mathrm{r} 3 \mathrm{I}$ ), but, in view of the fact that a low specific gravity may be the result either of a watered milk or of a milk high in fat, good judgment is necessary in connection with its use. A milk of good standard quality should have a specific gravity between the limits of I.027 and I.033. A watered milk would run below the former and a skimmed milk above the latter figure, though a milk unusually rich in fat would also run low. It should easily be apparent from the taste and appearance of the milk, whether a low specific gravity is due to watering or unusual richness in fat. The fact should also be recognized, that a milk sample may be far below the standard, and still show a specific gravity within the limits of pure milk, by skillfully subjecting the milk to both skimming and watering.

The Lactoscope.-Feser's lactoscope (Fig. 50) gives an approximation to the amount of fat in milk, and its use, especially in connection with the lactometer, is of some value. This instrument consists of a graduated glass barrel, $a$, into the bottom of which is accurately fitted the stopper, bearing 
a white glass cylinder, having black lines thereon. Four cc. of milk are introduced into the barrel by means of a pipette, $c$, and water is added with thorough mixing till the translucence of the mixture is sufficient to allow the black lines to be perceptible through it. The height of the level of milk and water in the barrel $a$ is then read off, the number indicating roughly the percentage of fat in the sample.

As in the case of the lactometer, the purity of a milk sample cannot be positively established by the lactoscope alone. For instance, a watered milk abnormally high in fat would often be found to read within the limits of pure milk, when as a matter of fact its total solids would be below standard. By a careful comparison of the readings of both the lactoscope and lactometer, however, it is rare that a skimmed or watered sample could escape detection.

Thus, if the specific gravity by the lactometer is well within the limits of pure milk, and the fat, as shown by the lactoscope, is above $3 \frac{1}{4}$ per cent., the sample may be safely passed as pure, or as conforming to the standard.

A normal lactometer reading in connection with an abnormally low lactoscope reading shows both watering and skimming, and with an abnormally high lactoscope reading shows a milk high in fat, or a crean. With the lactoscope reading below three, and a low lactometer reading, watering is indicated. A lactometer reading above thirty-three, and a low lactoscope reading, indicate skimming.

Heeren's Pioscope. - This instrument consists of a hard-rubber disk, having in the center a shallow receptacle, the circular rim of which is raised above the level of the disk. Into this receptacle are introduced a few drops of the milk to be tested, and a circular cover-glass containing a number of variously tinted segments is placed over the receptacle, which spreads the milk out into a thin layer, and causes it to assume a tint against the black background that can be matched with one of the colors on the glass, the various tints indicating milks of various grades from the very poorest to rich cream. This test is at best a very rough one.

Examination of the Milk Serum.-Detection of Added Water.-This may often be detected by determining the specific gravity or the degree of refraction of the milk serum, since it has been found that under fixed conditions the composition of the milk serum, or clear "whey," is more constant than that of the milk itself. Hence any considerable amount of watering is manifest from the physical constants of the serum.

In using this method the analyst should carefully work out his own 
standards for comparison, by personal experiment on milk of known composition to which varying amounts of water have been added, using

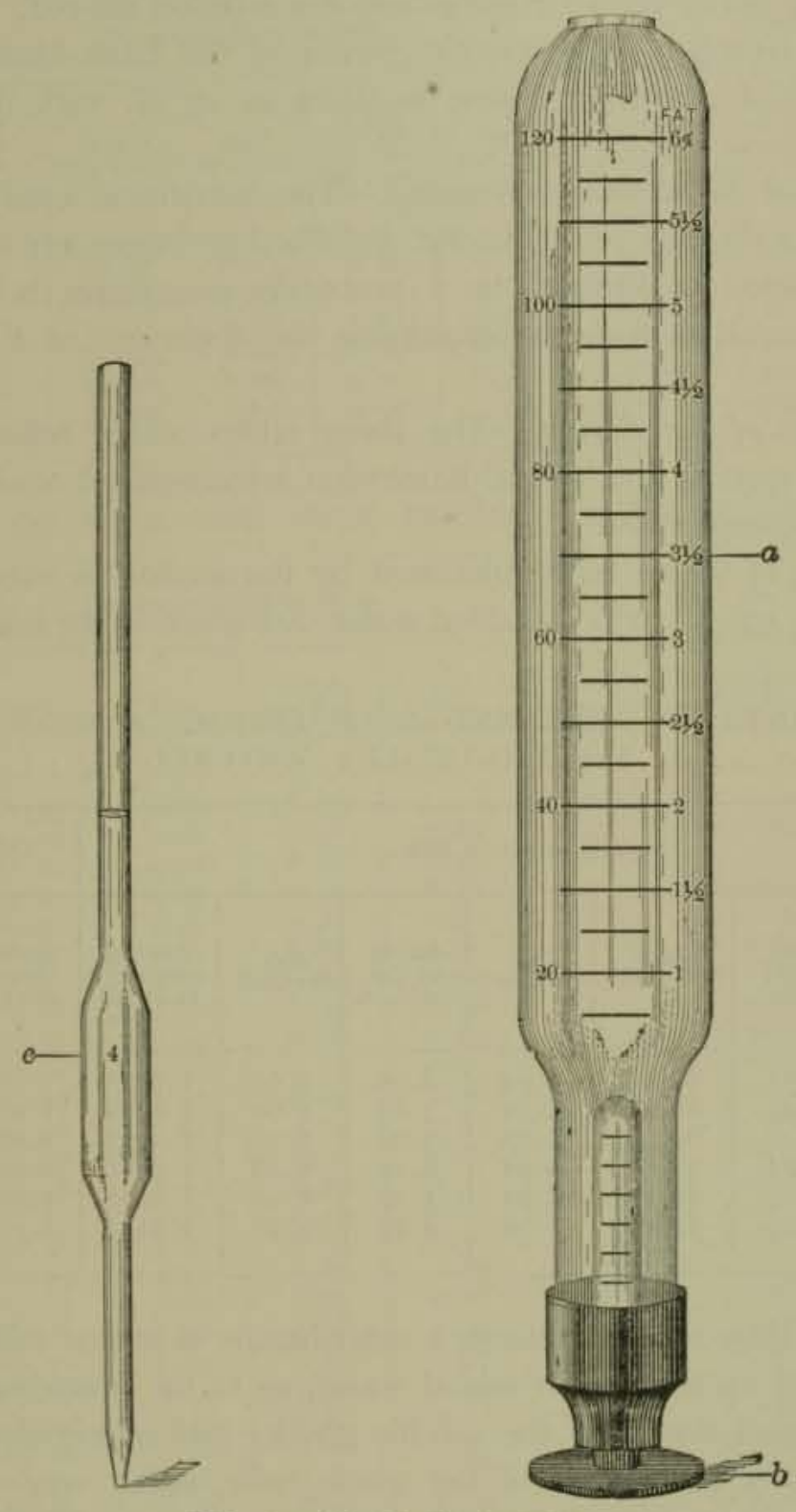

Fig. 50, - Feser's Lactoscope.

the same conditions for obtaining the serum in all cases. Woodman's method* is as follows: To $100 \mathrm{cc}$. of the milk at a temperature of about $20^{\circ} \mathrm{C}$. are added 2 cc. of $25 \%$ acetic acid, specific gravity 1.0350 , in a

* Jour. Am. Chem. Soc., 21, 1899, p. 503. 
beaker, and the beaker, covered with a watch-glass, is heated in a waterbath for 20 minutes at a temperature of $70^{\circ} \mathrm{C}$. After this the beaker is placed in ice water for to minutes and the solution filtered.

Specific Gravity.-The specific gravity of the clear filtrate, obtained by the method described above, is taken at $15^{\circ} \mathrm{C}$. with the Westphal balance.

Immersion Refractometer Reading.- The instrument used is the Zeiss immersion or dipping refractometer described on pages III to I2I. The serum, prepared as directed in a preceding paragraph, is examined in one of the small beakers accompanying the apparatus at a temperature of $20^{\circ} \mathrm{C}$.

Constants of the Serum.-The three tables which follow show the variation of specific gravity and immersion refractometer reading on milk of different composition.

Analyses of whole milk submitted by the author to varying degrees of watering, up to $50 \%$ of added water, are given in the following table:

\section{CONSTANTS OF MILK AND MILK SERUM. A WHOLE MILK SYSTEMATICALLY WATERED.}

\begin{tabular}{|c|c|c|c|c|c|c|c|c|}
\hline \multirow[b]{2}{*}{$\begin{array}{c}\text { Added } \\
\text { Water, } \\
\text { Per Cent. }\end{array}$} & \multicolumn{5}{|c|}{ Determinations on Milk. } & & \multicolumn{2}{|c|}{ On Milk Serum. } \\
\hline & $\begin{array}{c}\text { Total } \\
\text { Solids, } \\
\text { Per Cent. }\end{array}$ & $\begin{array}{l}\text { Water, } \\
\text { Per Cent. }\end{array}$ & $\begin{array}{l}\text { Pat, } \\
\text { Per Cent. }\end{array}$ & $\begin{array}{l}\text { Solids } \\
\text { not Fat, } \\
\text { Per Cent. }\end{array}$ & $\begin{array}{l}\text { Ash, } \\
\text { Per Cent. }\end{array}$ & $\begin{array}{l}\text { Specific } \\
\text { Gravity } \\
\text { at } 15^{\circ} \mathrm{C} \text {. }\end{array}$ & $\begin{array}{l}\text { Specific } \\
\text { Gravity } \\
\text { at } 25^{\circ} \mathrm{C} \text {. }\end{array}$ & $\begin{array}{l}\text { Immersion } \\
\text { Refrac- } \\
\text { tometer } \\
\text { Reading } \\
\text { at } 20^{\circ} \mathrm{C} \text {. }\end{array}$ \\
\hline 0 & 12.65 & $87 \cdot 35$ & 4.00 & 8.65 & 0.65 & $\mathrm{I} .03 \mathrm{I5}$ & I. 0287 & 42.40 \\
\hline 10 & II. 33 & 88.67 & 3.50 & 7.83 & 0.60 & 1.0278 & 1.0260 & 39.75 \\
\hline 20 & 10. 10 & 89.90 & 3.10 & 7.00 & 0.53 & I. 0252 & $\mathrm{I} .0230$ & $3^{6} .90$ \\
\hline 30 & 8.95 & 91.05 & 2.80 & 6.15 & $0.4^{8}$ & 1.0211 & I. 0200 & 34.10 \\
\hline 40 & 7.67 & 92.33 & 2.40 & 5.27 & 0.40 & 1. 0192 & 1.0167 & $31 \cdot 10$ \\
\hline 50 & 6.43 & 93.57 & 2.00 & 4.43 & $0.3^{8}$ & I. Or 54 & I. 0140 & 28.45 \\
\hline
\end{tabular}

The first table on p. 167 shows a centrifugally skimmed milk, systematically watered up to $50 \%$ of added water, as in the preceding table. It will be observed that both the specific gravity and immersion refractometer readings of the serum of the whole milk, agree very closely with those of the skimmed milk in cases having a corresponding amount of added water.

The second table on p. 167 shows analyses of milk selected from a wide range of samples regularly collected and examined in the routine of food inspection by the Massachusetts State Board of Health. 


\section{CONSTANTS OF MILK AND MILK SERUM. A SKIMMED MILK SYSTEMATICALLY WATERED.}

\begin{tabular}{|c|c|c|c|c|c|c|c|c|}
\hline \multicolumn{7}{|c|}{ Determinations on Milk. } & \multicolumn{2}{|c|}{ On Milk Serum. } \\
\hline $\begin{array}{l}\text { Added } \\
\text { Water. } \\
\text { Per Cent. }\end{array}$ & $\begin{array}{c}\text { Total } \\
\text { Solids, } \\
\text { Per Cent. }\end{array}$ & $\begin{array}{l}\text { Water, } \\
\text { Per Cent. }\end{array}$ & $\begin{array}{l}\text { Fat, } \\
\text { Per Cent. }\end{array}$ & $\begin{array}{c}\text { Solids } \\
\text { not Fat, } \\
\text { Per Cent. }\end{array}$ & $\begin{array}{l}\text { Ash, } \\
\text { Per Cent. }\end{array}$ & $\begin{array}{l}\text { Spécific } \\
\text { Gravity } \\
\text { at } 15^{\circ} \mathrm{C} \text {. }\end{array}$ & $\begin{array}{l}\text { Specifie } \\
\text { Gravity } \\
\text { at } x 5^{\circ} \mathrm{C} \text {. }\end{array}$ & $\begin{array}{l}\text { Immersion } \\
\text { Refrac- } \\
\text { tometer } \\
\text { Reading } \\
\text { at } 20^{\circ} \mathrm{C} \text {. }\end{array}$ \\
\hline 0 & 0.05 & 90.05 & 0.03 & 9.02 & 0.64 & I. 0350 & I. 0206 & 42.85 \\
\hline 10 & 8.14 & 91.85 & 0.03 & 8. II & 0.60 & 1.0317 & 1.0260 & 39.60 \\
\hline 20 & 7.27 & 92.73 & 0.02 & 7.25 & 0.56 & I. 0278 & 1.0230 & 36.85 \\
\hline 30 & $6.4 \mathrm{I}$ & 93.59 & 0.02 & 6.39 & $0.4^{8}$ & I. 0247 & I. 0200 & 34.00 \\
\hline 40 & 5.50 & 94.50 & 0.01 & 5.49 & 0.44 & I. 0209 & I. 0170 & $31 \cdot 20$ \\
\hline 50 & $4.6 \mathrm{I}$ & $95 \cdot 39$ & 0.01 & 4.60 & 0.39 & 1.0172 & I. O140 & 28.50 \\
\hline
\end{tabular}

CONSTANTS OF MILK AND MILK SERUM. LABORATORY SAMPLES

\begin{tabular}{|c|c|c|c|c|c|c|c|}
\hline \multicolumn{6}{|c|}{ Determinations on Milk. } & \multicolumn{2}{|c|}{ On Milk Serum. } \\
\hline $\begin{array}{l}\text { Total Solids. } \\
\text { Per Cent. }\end{array}$ & $\begin{array}{l}\text { Water, } \\
\text { Per Cent. }\end{array}$ & $\begin{array}{l}\text { Pat, } \\
\text { Per Cent. }\end{array}$ & $\begin{array}{l}\text { Solids } \\
\text { not Fat, } \\
\text { Per Cent. }\end{array}$ & $\begin{array}{l}\text { Ash, } \\
\text { Per Cent. }\end{array}$ & $\begin{array}{l}\text { Specific } \\
\text { Gravity } \\
\text { at } 15^{\circ} \mathrm{C} \text {. }\end{array}$ & $\begin{array}{l}\text { Specific } \\
\text { Gravity } \\
\text { at } 15^{\circ} \mathrm{C} \text {. }\end{array}$ & $\begin{array}{l}\text { Immersion } \\
\text { Refractom- } \\
\text { eter Read- } \\
\text { ing at } 20^{\circ} \mathrm{C} \text {. }\end{array}$ \\
\hline 16.45 & $83 \cdot 55$ & 8.20 & 8.25 & & I. 0255 & I. 0274 & 40.95 \\
\hline $\mathrm{I}_{5} .9^{\circ}$ & 84.10 & 7.00 & 8.90 & 0.69 & 1.0277 & 1.0285 & 42.00 \\
\hline $14 \cdot 37$ & 85.63 & 5.50 & 8.88 & $0.5^{8}$ & I. $028_{2}$ & I. 0280 & 42.40 \\
\hline 14.17 & 85.83 & 4.85 & $9 \cdot 32$ & 0.62 & I. $03 I_{3}$ & $1.028 \mathrm{I}$ & 44.20 \\
\hline 14.04 & 85.96 & 4.95 & 9.09 & 0.60 & I. 0303 & 1.0274 & 42.70 \\
\hline 13.80 & 86.20 & 5.00 & 8.80 & 0.65 & 1.0302 & I. .0289 & $42 \cdot 75$ \\
\hline 13.59 & 86.41 & $4 \cdot 30$ & 9.29 & 0.64 & 1.0321 & I. 0285 & $44 \cdot 5^{\circ}$ \\
\hline I3. 39 & 86.61 & 4.40 & 8.99 & 0.50 & I. .0324 & 1.0285 & $43 \cdot 70$ \\
\hline 13.28 & 86.72 & 4.40 & 8.88 & 0.60 & 1.0299 & I. 0289 & 42.65 \\
\hline 13.12 & 86.88 & 4.00 & 9.12 & 0.59 & 1.0317 & I. 0280 & 43.75 \\
\hline 13.00 & 87,00 & $4 \cdot 30$ & 8.70 & 0.56 & I. 0310 & I. 0266 & 42.60 \\
\hline 12.90 & 87, ro & 3.85 & 9.05 & $0.6 \mathrm{r}$ & 1.0318 & 1.0289 & 43.40 \\
\hline 12.80 & 87.20 & $4 \cdot 30$ & 8.50 & 0.46 & I. 0304 & 1. 0277 & $42 \cdot 70$ \\
\hline 12.70 & $87 \cdot 30$ & 3.80 & 8.90 & 0.53 & I. 0314 & 1.0280 & 43.10 \\
\hline 12.63 & $87 \cdot 37$ & $3.5^{\circ}$ & 9.13 & 0.65 & I. 0323 & I. 0277 & 43.65 \\
\hline 12.62 & $87 \cdot 3^{8}$ & 4.10 & $8.5^{2}$ & $0.5^{2}$ & 1,0298 & I. 0272 & 42.40 \\
\hline 12.57 & 87.43 & 3.70 & 8.87 & 0.68 & I. 0317 & I. 0278 & $43 \cdot 45$ \\
\hline 12.47 & $87 \cdot 53$ & 3.60 & 8.87 & 0.65 & 1.0303 & 1.0282 & $43 \cdot 15$ \\
\hline $12 \cdot 36$ & 87.64 & 3.20 & $9 \cdot 16$ & 0.55 & 1.0327 & I. 0282 & 43.25 \\
\hline 12.30 & 87.70 & 3.20 & 9.10 & 0.62 & I. 0327 & I. $028_{3}$ & 44.00 \\
\hline 12,16 & 87.84 & $4 \cdot 35$ & $7.8 \mathrm{r}$ & 0.49 & 1.0275 & I. 0265 & $4 I \cdot 10$ \\
\hline 12.00 & 88.00 & 3.40 & 8.60 & 0.62 & I. 0275 & I. 0280 & $41 \cdot 75$ \\
\hline 11.86 & 88.14 & 3.60 & 8.26 & 0.49 & I. 0306 & I. 0266 & 42.40 \\
\hline II. 67 & 88.33 & 3.95 & $7 \cdot 77$ & 0.48 & I. .0265 & I. 0240 & $39 \cdot 30$ \\
\hline II. 60 & 88.40 & 2.75 & 8.85 & 0.65 & 1.0320 & I. 0282 & $43 \cdot 55$ \\
\hline II, 50 & 88.50 & 3.45 & 8.05 & $0.5 \mathrm{I}$ & I. 0290 & I. 0260 & $41 \cdot 40$ \\
\hline II. 40 & 88.60 & 3.10 & 8. 30 & 0.60 & 1. 0297 & I. 0278 & 42.00 \\
\hline II. 25 & 88.75 & 2.80 & 8.45 & $0.5^{8}$ & 1.0280 & I. 0274 & 40.90 \\
\hline 11.07 & 88.93 & 3.00 & 8.07 & 0.62 & 1. 0290 & I. 0270 & 40.75 \\
\hline 10.69 & $89 \cdot 3 \mathrm{I}$ & 2.95 & 7.74 & $\ldots \ldots$ & I. 0288 & I. 0262 & 39.85 \\
\hline 10. 25 & 89.75 & 3.20 & 6.95 & 0.55 & 1.0230 & I. 0223 & 36.40 \\
\hline 8.34 & 91.66 & 2.20 & 6.14 & $0.3^{8}$ & I:0224 & I. 0207 & $34 \cdot 70$ \\
\hline
\end{tabular}


A comparison of the immersion refractometer readings of the serum of milk of varying quality shows at once that the refraction of the serum is a general index to watering. A reading below 40 with the above conditions carefully observed would be suspicious of added water, though 39 might more safely be placed as a limit, below which milk could be declared fraudulently watered. The analyst need not hesitate in testifying to the presence of added water, when the refraction is lower than 39 under the above conditions and the solids not fat below $7.3 \%$.

The tables on page 169 give a summary of refractometric and analytical results of a large number of milk samples from three widely separated localities, namely, Massachusetts, New Jersey, and Great Britain:

Nitrates.-Pure milk, free from contamination with stable filth, contains no nitrates; well water, however, often contains a sufficient amount to enable the detection of a $10 \%$ admixture in milk.

The diphenylamin test, first employed by Soxhlet to detect nitrates in milk, has since been modified by Möslinger,* Richmond, $\dagger$ Hefelmann, $\ddagger$ Reisz, $\S$ Patrick, and others.

Place in a small porcelain crucible one cc. of a solution of o.r gram of diphenylamin in $1000 \mathrm{cc}$. of concentrated sulphuric acid and allow a few drops of the milk serum to flow over the surface. A blue color appearing within to minutes indicates the presence of nitrates. On longer standing, a brown color forms, whether or not nitrates are present.

The delicacy of the test is increased by adding to the reagent a small amount of powdered sodium chloride shortly before using.

SYSTEMATIC EXAMINATION OF MILK FOR ADULTERATION.-If a large number of samples of milk have to be examined daily for adulteration, it may be an advantage to submit all to a preliminary test with the lactoscope and lactometer, excluding from further analysis, as above the standard, such samples as pass certain prescribed limits which experience has proved these tests to be capable of showing to an experienced observer, and submitting the remainder to a chemical analysis. In using such an instrument as the lactoscope for this purpose, the individual element is a most important consideration, and the use of this instrument

\footnotetext{
* Ber. über die 7 Versammlung bayerischer Chemiker. Berlin, 1889 .

$\dagger$ Analyst, 18, 1893. p. 272.

$\ddagger$ Zeits. offentl. Chem., 7, t9or, p. 200.

\$ Pharm. Zeit., 49, 1904, p. 608.

|f See Tillmans: Zeits. Unters. Nahr. Genuss,, 20, 1910, p. 676.
} 


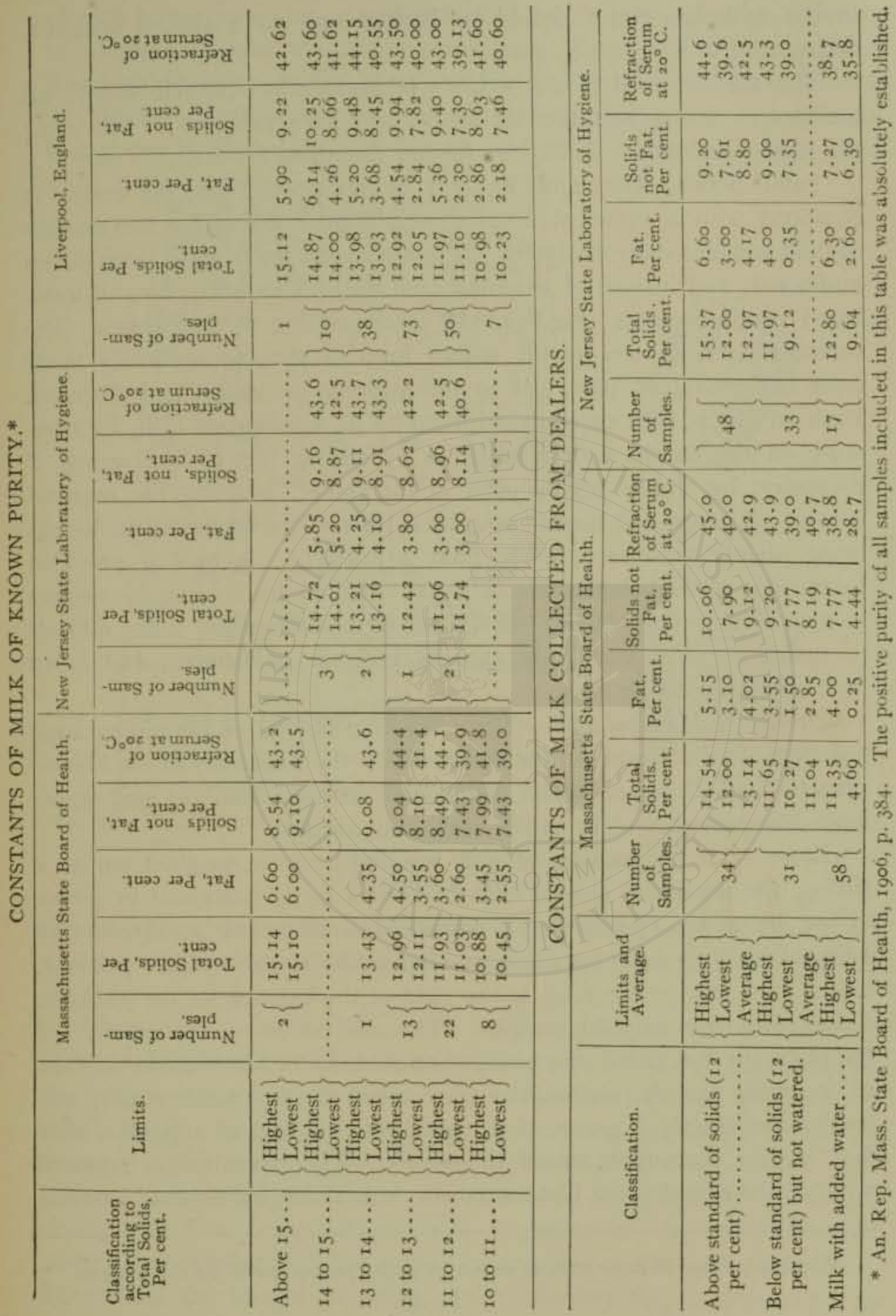


in the milk laboratory should be limited only to a skillful operator, accustomed to interpret its results.

The method used in the writer's laboratory has been to submit all samples to the regular test for solids, and such samples as fall below the legal standard for solids, are further examined for fat.

Total Solids, Ash, and Fat.-It is presupposed that the analyst is equipped with a sufficient number of platinum dishes for the number of milk samples daily analyzed. It is a convenience to have these dishes numbered, and instead of weighing each dish, to have a system of numbered counterweights (Fig. 5I,A) corresponding to the dishes. The counterweights in use by the author for this purpose are easily made from half-inch lead pipe, cut to the appropriate length and flattened. Each weight is then carefully adjusted to its appropriate dish, by trimming off the weight with a knife, or by adding bits of lead scraps, if necessary, by simply prying open in the center, inserting the required amount of scrap, and then closing by a blow of the hammer, the weight being plainly numbered before final adjustment. A rack is provided by the side of the balance-case (Fig. $5^{x}$ ) with slits for holding the weights in their appropriate places. Such a set of counterweights is not difficult to make, requires very little care to keep in adjustment, and is an immense labor-saving device.

Details of Manipulation.-The following method of examining large numbers of milk samples is the one in use in the laboratory of the Massachusetts State Board of Health and is given in some detail, as long experience has proved it to be rapid, easy, and accurate.

From i2 to 20 samples of milk are conveniently weighed out at a sitting, the unopened sample cans or bottles being contained in a tray at the left of the operator on a low stand, another low stand and tray being at his right hand for the cans, after removing the weighed portions, and a third tray on the table at the right of the balance for the platinum dishes with the weighed samples. The analyst enters the number of the platinum dish in his note-book, or on a card,* in line with the number of the milk sample, verifies the correctness of the counterweight, and weighs out exactly 5 grams of the milk with the aid of a pipette, after first having thoroughly mixed the sample. This operation is repeated with all the samples, the platinum dishes containing the weighed amounts

* Specially ruled library cards, as shown on page 172 , are useful for this purpose. 
of each being placed in succession on the tray, which is finally carried to the water-bath and the dishes transferred thereto. The time required for weighing out 12 samples of milk in this manner is about fifteen minutes.

The water-bath is inclosed in a hood, and the sliding front is so arranged that it can be shut down and locked, so that if the analyst has to leave
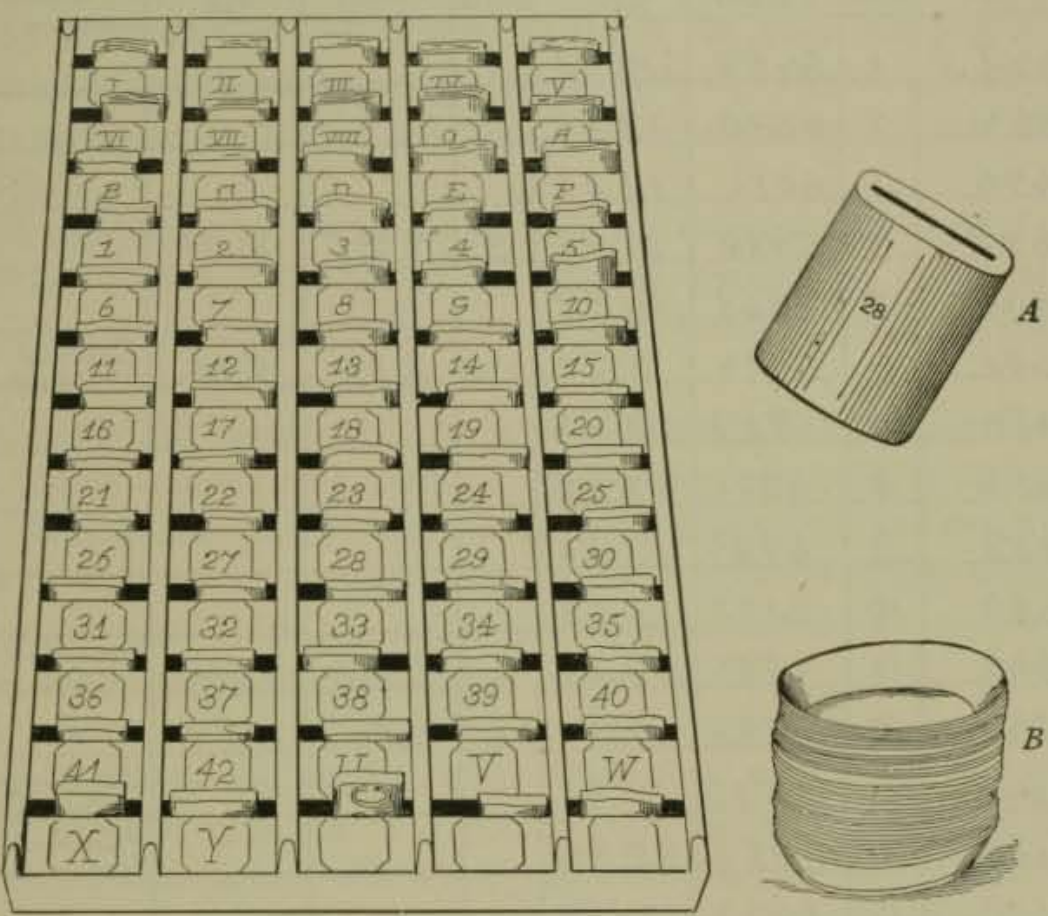

Fig. 51.-Set of Counterweights for Numbered Platinum Dishes, in a Convenient Rack.

A. One of the Counterweights.

B. Platinum Dishes.

the laboratory during the three hours required for the evaporation, he can swear in court that the samples could not be tampered with during his absence (see page $2 \mathrm{I}$ ).

When ready to make the second weighings for the total solids, each dish is taken from contact with the steam, and, while still hot, is wiped dry with a soft towel, till twelve of the dishes are placed on the tray, which is then taken to the balance. Experience has shown that with ordinary rapidity in weighing, twelve of the residues may be thus dealt with at a time without the need of a desiccator, the gathering of moisture during that time being inappreciable, excepting in very damp weather, when a less number of dishes should be removed at a time from the bath. In making the second weighing, and employing the counterweight as 


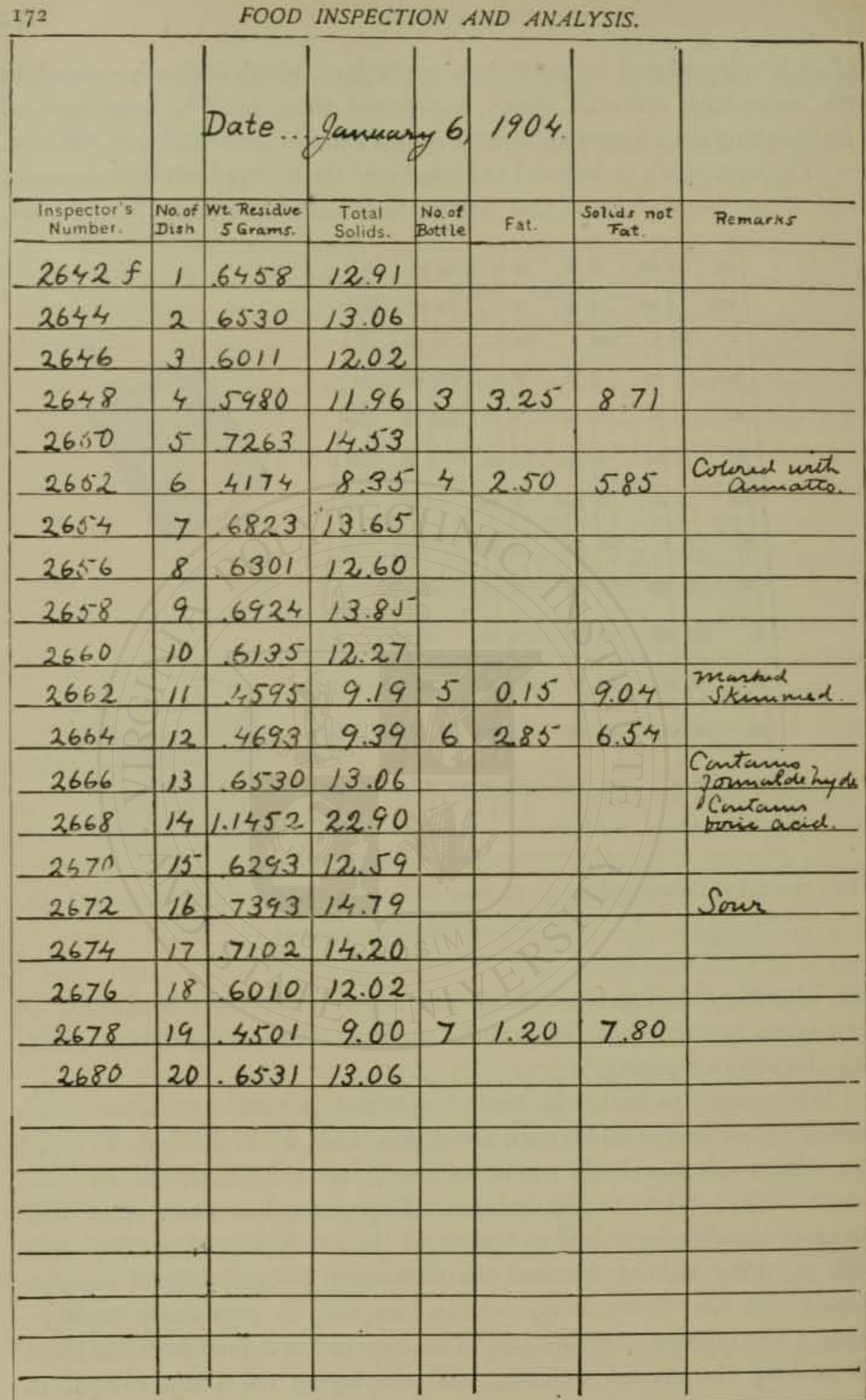

Specimen Card for Analyst's Records of Milk Analyses. To be filed in a cabinet. 
before, the exact net weight of the residue is at once ascertained and entered in the appropriate column in the note-book. Multiplied by 20 it gives at once the percentage of total solids.

It is a great saving of time to weigh out exactly 5 grams as above described. The knack of quickly measuring out the exact amount is easily acquired with practice, the 5-gram weight is the only one required for the operation with the counterweight of the dish, and the laborious figuring of percentage due to using a fraction above or below the 5 grams of milk is avoided.

Such samples as are found to stand below the standard of total solids are further examined for fat by the Babcock process (p. 136), entering the number of the fat bottle in the note-book in the appropnate column, and subsequently the percentage of fat.

Ordinarily the specific gravity is not determined, excepting in some cases of badly watered milk, when, for purposes of a check, it is customary to take the specific gravity, and calculate the solids from the gravity and the fat by Babcock's formula (p. 153), or the Richmond sliding scale, and compare the result with the figure directly determined.

The ash is rarely weighed except in special cases.

The dishes containing the dry residues are easily cleaned by first burning to an ash and cooling, after which they are treated successively with strong nitric acid, which is poured from one to another, the dishes being rinsed thoroughly with water and finally heated to redness.

A convenient device for ashing a large number of residues for purposes of cleaning the platinum dishes and for final heating is the incinerator shown in Fig. 52, made of Russia iron.

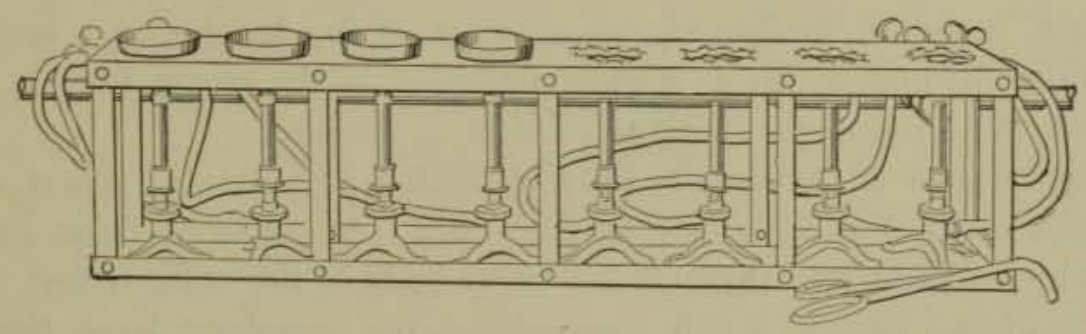

FIG. 52. A Sheet-metal Incinerator, Specially Used for Ashing Milk Residue.

ADDED FOREIGN INGREDIENTS. - Passing over such mythical adulterants as chalk and such rarely used substances as calves' brains, starch, glycerin, sugar, etc., often discussed in manuals on milk, but 
with few authentic instances of their actual occurrence, the commonly found adulterants may be divided into two classes: coloring matters and preservatives.

The coloring matters almost exclusively used are annatto, azo-colors, and caramel. The preservatives commonly met with are formaldehyde, boric acid, borax, and sodium bicarbonate. Rarely salicylic and benzoic acids are found.

Coloring MAtrers.-While it is more often true that an artificially colored milk is also found to be watered, the coloring being added to cover up evidence of the watering, it is not uncommon to find added coloring matter in milk above the standard.*

About $95 \%$ of the milks found colored in Massachusetts show on analysis the fraudulent addition of water.

Statistics of the Massachusetts State Board of Health show that out of 48,000 samples of milk collected throughout the state and analyzed during nine years (from 1894 to 1902 inclusive) 342 samples or $0.7 \%$ were found to contain foreign coloring matter. Of these samples, about $67 \%$ contained annatto, approximately $30 \%$ were found with an azodye, and about $3 \%$ with caramel.

Until comparatively recently annatto was employed almost exclusively for this purpose. Caramel is least desirable of all the above colors from the point of view of the milk-dealer, in that it is difficult to imitate with it the natural color of milk, by reason of the fact that the caramel color has too much of the brown and too little of the yellow in its composition. Annatto, on the other hand, when judiciously used and with the right dilution, gives a very rich, creamy appearance to the milk, even when watered, which accounts for its popularity as a milk adulterant. Of late, however, the use of one or more of the azo-dyes has been on the increase, and so far as a close imitation of the cream color is concerned, these colors are quite as efficient as annatto.

Appearance of Artificially Colored Milk.-The natural yellow color of milk confines itself largely to the cream. An artificial color, on the contrary, is dissipated through the whole body of the milk, so that when the cream has risen in a milk thus colored, the underlying layers, instead of showing the familiar bluish tint of skimmed milk, are still distinctly tinged below the layer of the fat, especially if any considerable quantity of the color has been used. This distinctive appearance is in itself often

\footnotetext{
* In one instance an azo-dye was found by the writer in a milk that contained over $\mathbf{1 7}_{7} \%$ of total solids.
} 
sufficient to direct the attention of the analyst to an artificially colored milk, in the course of handling a large number of samples.

Nature of Annatto.-Annatto, arnatto, or annotto is a reddish-yellow coloring matter, derived from the pulp inclosing the seeds of the Bixa orellana, a shrub indigenous to South America and the West Indies.

A solution of the coloring matter in weak alkali is the form usually employed in milk.

Nature of "Anilin Orange."-Of the coal-tar colors employed for coloring milk, the azo-dyes are best adapted for this purpose and are most used. A few samples of these commercial "milk improvers" have fallen into the hands of the Department of Food and Drug Inspection of the Massachusetts Board of Health, and have proved, on examination, to be mixtures of two or more members of the diazo-compounds of anilin. A mixture of what is known to the trade as "Orange G" and "Fast Yellow" gives a color which is practically identical with one of these preparations, secured from a milk-dealer and formerly used by him.

For purposes of prosecution or otherwise, it is obviously best in our present knowledge of the subject to adopt a generic name stich as "a coal-tar dye" or "anilin orange" * to designate this class of coloring matters in milk, rather than to particularize.

Systematic Examination of Milk for Color.-The general scheme employed by the writer for the examination of milk samples suspected of being colored is as follows: $\dagger$ About $150 \mathrm{cc}$. of the milk are curdled by the aid of heat and acetic acid, preferably in a porcelain casserole over a Bunsen flame. By the aid of a stirring-rod, the curd can nearly always be gathered into one mass, which is much the easiest method of separation, the whey being simply poured off. If, however, the curd is too finely divided in the whey, the separation is effected by straining through a sieve or colander. All of the annatto, or of the coal-tar dye present in the milk treated would be found in the curd, and part of the caramel. The curd, pressed free from adhering liquid, is picked apart, if necessary, and shaken with ether in a corked flask, in which it is allowed to soak for several hours, or until the fat has been extracted, and with it the annatto. If the milk is uncolored, or has been colored with annatto, on pouring off the ether the curd should be left perfectly white. If, on

* The term "anilin orange" has been so commonly applied during the last eight years to any color or mixture of colors of this class in complaints in the Massachusetts courts, as to have acquired a special meaning perfectly well understood.

† Jour. Am. Chem. Soc., 22, 1900, p. 207. 
the other hand, anilin orange or caramel has been used, after pouring off the ether the curd will be colored more or less deeply, depending on the amount of color employed. In other words, of the three colors, annatto, caramel, and anilin orange, the annatto only is extracted by ether. If caramel has been used, the curd will have a brown color at this stage; if anilin orange, the color of the curd will be a more or less bright orange.

Tests for Annatto.- The ether extract, containing the fat and the annatto, if present, is evaporated on the water-bath, the residue is made alkaline with sodium hydroxide, and poured upon a small, wet filter, which will hold back the fat, and, as the filtrate passes through, will allow the annatto, if present, to permeate the pores of the filter. On washing off the fat gently under the water-tap, all the annatto of the milk used for the test will be found to have been concentrated on the filter, giving it an orange color, tolerably permanent and varying in depth with the amount of annatto present. As a confirmatory test for annatto, stannous chloride may afterward be applied to the colored filter, producing the characteristic pink color.

Tests for Caramel.- The fat-freed curd, if colored after the ether has been poured off, is examined further for caramel or anilin orange, by placing a portion of the curd in a test-tube, and shaking vigorously with concentrated hydrochloric acid. If the color is caramel, the acid solution of the colored curd will gradually turn a deep blue on shaking, as would also the white fat-free curd of an uncolored milk, the blue coloration being formed in a very few minutes, if the fat has been thoroughly extracted from the curd; indeed, it seems to be absolutely essential for the prompt formation of the blue color in the acid solution that the curd be free from fat. Gentle heat will hasten the reaction. It should be noted that it is only when the blue coloration of the acid occurs in connection with a colored curd that caramel is to be suspected, and if much caramel be present, the coloration of the acid solution will be a brownish blue. If the above treatment indicates caramel, it would be well to confirm its presence, by testing a separate portion of the milk in the following manner.*

About a gill of the milk is curdled by adding to it as much strong alcohol. The whey is filtered off, and a small quantity of subacetate of lead is added to it. The precipitate thus produced is collected ujon a small filter, which is then dried in a place free from hydrogen sulphide. A pure milk thus treated yields upon the filter-paper a residue which is

\footnotetext{
* See Ninetcenth Annual Report of the Mass. State Board of Health (1887), p. $18_{3}$.
} 
either wholly white, or at most of a pale straw color, while in the presence of caramel, the residue is a more or less dark-brown color, according to the amount of caramel used.

Tests for Coal-tar Dye.-If the milk has been colored with an azo-dye, the colored curd, on applying the strong hydrochloric acid in the test-tube, will immediately turn pink. If a large amount of the anilin dye has been used in the milk, the curd will sometimes show the pink coloration when hydrochloric acid is applied directly to it, before treatment with ether, but the color reaction with the fat-free curd is very delicate and unmistakable.*

Lythgoe $\dagger$ has shown that the amount of anilin orange ordinarily present in a milk for the purposes of coloring can be detected by adding directly to say ro $\mathrm{cc}$. of the sample an equal quantity of strong hydrochloric acid and mixing, whereupon the pink coloration is produced, if the dye is present in more than minute traces. The test is more delicate if carried out in a white porcelain dish. It had best be used as a preliminary test only, and confirmed by a subsequent test on the fat-free curd as above.

\section{SUMMARY OF SCHEME FOR COLOR ANALYSIS.}

Curdle $150 \mathrm{cc}$, milk in casserole with heat and acetic acid. Gather curd in one mass. Pour off whey, or strain, if curd is finely divided. Macerate curd with ether in corked flask. Pour off ether.

\section{Ether Extract.}

Evaporate off ether, treat residue with $\mathrm{NaOH}$ and pour on wetted filter. After the solution has passed through, wash off fat and dry filter, which if colored orange, indicates presence of annatto. (Confirm by $\mathrm{SnCl} \mathrm{C}_{2}$ )

\section{Extracted Curd.}

(1) If Colorless.-Indicates presence of no foreign color other than in ether extract.

(2) If Orange or Brownish.-Indicates presence of anilin orange or caramel. Shake curd in test-tube with concentrated hydrochloric acid.

If solution gradually turns blue, indicative of caramel. (Confirm by testing for caramel in whey of original milk.)
If orange curd immediately turns pink, indicative of anilin orange.

PRESERVATIVES. - In most states and municipalities where pure food laws are in force preservatives in milk are regarded as adulterants

* Occasional samples of milk colored with a coal-tar dye of a different class from those already described have recently been found in Massachusetts. In these cases the color of the separated fat-free curd does not change when treated with hydrochloric acid. The color of the curd is, however, very marked, being deep orange, bordering on the pink.

$\dagger$ Jour. Am. Chem. Soc., 22, 1900, p. 8r 3. 
Their use, however, seems to be on the decrease. Of 6,186 samples of milk examined by the Massachusetts State Board of Health during one year (1899) $7 \mathrm{I}$ samples, or $1.2 \%$, were found to contain a preservative. Of these 55 were found with formaldehyde, $\mathrm{I} 3$ containing boric acid, borax, or a mixture of the two, and 3 contained carbonate of soda.

Comparative tests have been made in the writer's laboratory of the keeping qualities of these commonly used milk preservatives, when present in varying strength, the milk being kept during the experiment at the temperature of the room, which at that season of the year (February) was about $20^{\circ} \mathrm{C}$. $*$ The preservatives were added about five hours after milking. The samples were titrated for acidity each morning, the acidity being expressed by the number of cubic centimeters of decinormal sodium hydroxide necessary to neutralize $5 \mathrm{cc}$. of the milk.

The proportions of preservatives used in this experiment, as shown in the table on page 179 , were intended to cover a wide range, from the weakest that could aid in preserving the milk up to a strength limited only by being perceptible to the taste. The table opposite shows the results.

Formaldehyde, the most commonly used preservative for milk, is sold to the trade under various names, such as "Preservaline," "Freezine," "Iceline," etc., all being dilute aqueous solutions of formaldehyde, containing from 2 to 6 per cent of the gas, being nearly always diluted from the $40 \%$ solution known as formalin. These preparations are usually accompanied by directions, which specify the amount to be used, varying from a tablespoonful of the solution in 5 to Io gallons of the milk. It is commonly used in the strength of I part of the gas in 20,000, and rarely less than I part in 50,000 . The antiseptic power of formaldehyde increases in a marked degree as the strength of the preservative is increased. Milk treated with I part in 10,000 , for instance, according to the table was found to keep sweet $5 \frac{1}{2}$ days. In the strength of I part to 5000 , the milk did not curdle for $10 \frac{1}{2}$ days, while I part of formaldehyde to 2500 parts of milk kept the milk from curdling for 55 days, the acidity up to that time being nearly normal.

Formaldehyde is thus shown to be decidedly the most efficient of all milk preservatives, besides being inexpensive and convenient to use.

Whether the growth of other bacteria than those that produce lactic fermentation is inhibited by formaldehyde in milk is not definitely settled. The claim has been made that pathogenic varieties are destroyed by its use.

* Thirty-first Annual Report Mass. State Board of Health, 1899, p. 611. 


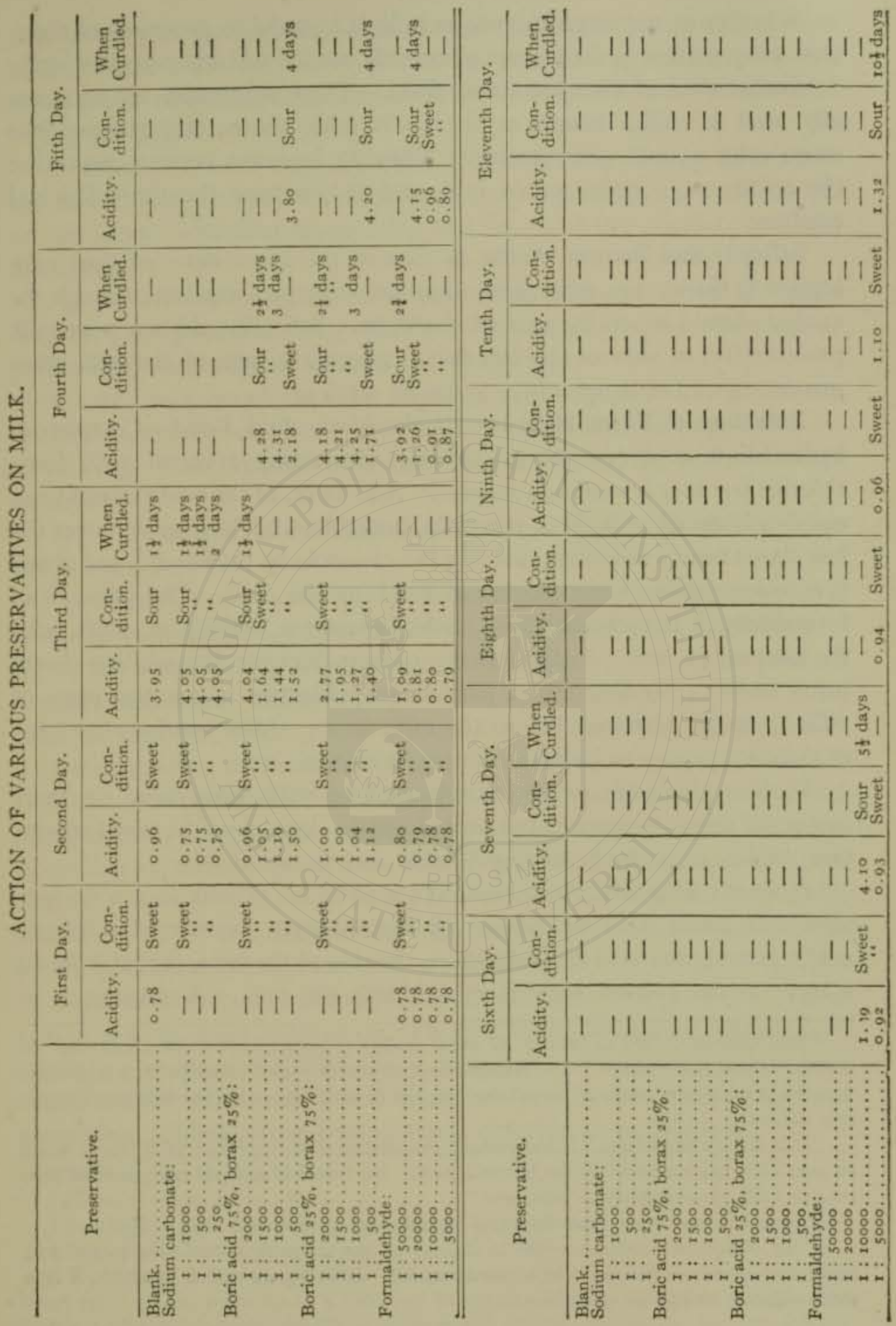


Whether or not formaldehyde in milk is harmful to processes of digestion, when present in the amount commonly used, is still an open question.*

Carbonate and Bicarbonate of Soda.-These substances are occasionally used in milk, though, as the above table shows, they possess little or no value as milk preservatives. They do, however, serve to neutralize the acidity of slightly soured milk and to postpone the time of actual curdling.

Salicylic and Benzoic Acids, in view of the much more efficient antiseptics at hand, are now rarely used as milk preservatives, though the analyst should be on the outlook for them. Salicylic acid is a poor milk preservative, in view of the fact that it affects the taste of the milk, when present in sufficient quantity, to be of service.

Detection of Formaldehyde.-Hydrochloric Acid Test. $\dagger$-Commercial hydrochloric acid (specific gravity 1.2 ) containing $2 \mathrm{cc}$. of $10 \%$ ferric chloride per liter is used as a reagent. Add Io cc. of the acid reagent to an equal volume of milk in a porcelain casserole, and heat slowly over the free flame nearly to boiling, holding the casserole by the handle, and giving it a rotary motion while heating to break up the curd. The presence of formaldehyde is indicated by a violet coloration, varying in depth with the amount present. In the absence of formaldehyde, the solution slowly turns brown. By this test I part of formaldehyde in 250,000 parts of milk is readily detected before the milk sours. After souring, the limit of delicacy proves to be about I part in 50,000.

Various aldehydes, when introduced into milk, give color reactions under the above treatment, but formaldehyde alone gives the violet coloration, which is perfectly distinguishable and unmistakable.

Hehner's Sulphuric Acid Test.-To 5 to ro cc. of milk in a wide testtube add about half the volume of concentrated commercial sulphuric acid, pouring the acid carefully down the side of the tube, so that it forms a layer at the bottom without mixing with the milk. A violet zone at

* Milk-dealers are led to believe, by artful dealers in preservative preparations, that the chemist cannot detect them. The manufacturer of a widely used preservative, a weak solution of formaldehyde, issues an attractive pamphlet in which he makes the following remarkable claims:

"It is not an adulterant. It immediately evaporates, so that no trace of it can be found as soon as it has rendered all the bacteria inert. No chemical analysis can prove its presence in milk, quantitatively or otherwise."

$\dagger$ Annual Report Mass. State Board of Health, 1897 , p. 558 ; also 1899 , p. 699.

$\ddagger$ The coloration produced seems to depend on the presence of iron salts in the acid, hence the use of commercial acid is recommended. If only pure acid is available, a little ferric chloride should be added 
the junction of the two liquids indicates formaldehyde. This test may be combined with the Babcock test for fat, noting whether a violet color forms on addition of the commercial sulphuric acid to the milk in the test bottle.

Confirmatory Tests with Distilled-Milk.-If it is desired to confirm the above tests by further evidence, 100 to $200 \mathrm{cc}$. of the milk sample are subjected to distillation, and the first $20 \mathrm{cc}$. of the distillate are used for testing.

(I) To a few drops of this distillnte in a test-tube add a drop of Schiff's reagent.* In presence of any al Hehyde, a pink coloration will soon be perceptible, deepening in intensity on standing.

(2) Add to $5 \mathrm{cc}$. of the milk distillate a few drops of a $1 \%$ aqueous solution of resorcin or phenol, and proceed as directed on page 826 (preservatives). The crimson color indicates formaldehyde, and not other aldehydes.

(3) Use I or 2 cc. of the milk distillate and apply the phenylhydrazine test, page 826.

(4) A small amount of the distillate from milk (which prior to distilling is acidified slightly with sulphuric acid to fix any free ammonia) is treated with a few drops of Nessler's reagent. $\dagger$ Traces of formaldehyde produce a yellow coloration, while if considerable formaldehyde be present, the color darkens on standing and a grayish precipitate may be formed.

Determination of Formaldehyde in Milk. $\ddagger-$ To $100 \mathrm{cc}$. of milk add I cc. of $\mathrm{I}: 3$ sulphuric acid and subject to distillation in a 500-cc. Kjeldahl nitrogen-flask, using a low circular evaporating burner to avoid frothing. According to Smith, the first $20 \mathrm{cc}$. of the distillate, or one-fifth the original volume, contain very nearly one-third of the total formaldehyde. Collect $20 \mathrm{cc}$. of the distillate and determine the formaldehyde therein by the petassium cyanide method, as follows: $\S$

Treat to cc. of tenth-normal silver nitrate with 6 drops of $50 \%$ nitric acid in a 50-cc. flask, add ro cc. of a solution of potassium cyanide containing 3.I grams of $\mathrm{KCN}$ in $500 \mathrm{cc}$. of water, and make up to the $50-\mathrm{cc}$. mark. Shake, filter, and titrate $25 \mathrm{cc}$. of the filtrate with tenth-normal ammonium sulphocyanate, $\|$ using ferric chloride as an indicator.

* Table of reagents, No. 226 .

† Table of reagents, No. 187 .

$\ddagger$ Smith, Jour. Am. Chem. Soc., 25, 1903, PP. 1032 and 1037 .

\$ Zeits. anal. Chem., 36, pp. 18-24.

\I Theoretically 7.6 grams per liter. On account of the deliquescent nature of the salt weigh out 8 grams, make up to a liter, and titrate against tenth-normal silver nitrate for its exact value, using ferric chloride as an indicator. Sutton, Volumetric Analysis, 8th Ed, p. 155 . 
Acidify another portion of Io cc. of tenth-normal silver nitrate with nitric acid, add ro cc. of the potassium cyanide solution to which the above $20 \mathrm{cc}$. of the formaldehyde distillate has been added. Make up the whole to $50 \mathrm{cc}$., filter and titrate as before $25 \mathrm{cc}$. of the filtrate with tenth-normal ammonium sulphocyanate for the excess of silver.

The amount of potassium cyanide used up by the formaldehyde, in terms of tenth-normal ammonium sulphocyanate, is found by multiplying by two the difference between the two results, and the total formaldehyde is calculated by multiplying by 3 the amount found in the $20 \mathrm{cc}$. of distillate.

The reaction that takes place between the formaldehyde and the potassium cyanide probably results in the formation of an addition product as follows:

$$
\mathrm{CH}_{2} \mathrm{O}+\mathrm{KCN}=\mathrm{KO} \cdot \mathrm{CH}_{2} \mathrm{CN} \text {. }
$$

Detection of Boric Acid.-This is best accomplished by the turmericpaper test applied either directly to the milk or to the ash (page 823). In the former case $10 \mathrm{cc}$. of milk are thoroughly mixed with 6 drops of concentrated hydrochloric acid, after which the tumeric paper is moistened with the mixture and dried.

Determination of Boric Acid.-Use the method of Thompson.* Add Io cc. of a I: I solution of sodium hydroxide to roo cc. of the milk, evaporate to dryness in a platinum dish, and proceed as described on page 829 .

Detection of Carbonate and Bicarbonate of Soda. - The addition of carbonates is manifest by the effervescence caused by treating the milk-ash with acid. Effervescence in the milk-ash is quite perceptible, when as much as $0.05 \%$ of sodium carbonate is present.

Schmidt's method of detecting sodium carbonate or bicarbonate, when present to the extent of $0.1 \%$ or more, is as follows: Ten cc. of milk are mixed with an equal volume of alcohol, and a few drops of a $\mathrm{I} \%$ solution of rosolic acid are added. If carbonate is present, a rosered color will be produced, while pure milk shows a brownish-yellow coloration. The suspected sample thus treated should be compared with a similarly treated sample of pure milk at the same time.

Detection of Benzoic Acid.- Shake $5 \mathrm{cc}$. of hydrochloric acid with $50 \mathrm{cc}$. of the milk in a flask. Then add $150 \mathrm{cc}$. of ether, cork the flask and shake well. Break up the emulsion which forms by the aid of a centrifuge, or, in the absence of a centrifuge, extract the curdled milk by gently shaking with successive portions of ether, avoiding the forma-

* Jour. Soc. Chem. Ind., 12, p. 432 . 
tion of an emulsion. A volume of ether largely in excess over that of the curdled milk has been found to be less apt to emulsionize.* Transfer the ether extract to a separatory funnel, and separate the benzoic acid from the fat by shaking out with dilute ammonia, which takes out the former as ammonium benzoate. Evaporate the ammonia solution in a dish over the water-bath till all free ammonia has disappeared, but before getting to dryness, add a few drops of ferric chloride reagent.

The characteristic flesh-colored precipitate indicates benzoic acid. Care should be taken not to add the ferric chloride till all the ammonia has been driven off, otherwise a precipitate of ferric hydrate is formed.

Detection of Salicylic Acid.-(I) To 50 cc. of the milk add I cc. of acid nitrate of mercury reagent (p. 147), shake and filter. The filtrate, which should be perfectly clear, is then shaken with ether in a separatory funnel, the ether extract evaporated to dryness, and a drop of ferric chloride reagent applied. If salicylic acid be present, a violet color will be produced. In carrying out the test it should be noted that a small portion only of the salicylic acid is in the filtered whey, the larger part being left in the curd. The color test is, however, so delicate as to show its presence, when an appreciable amount is used.

(2) Proceed exactly as directed for benzoic acid (p. 182). On applying the ferric chloride to the final solution, after evaporation of the ammonia, a violet color shows the presence of salicylic acid.

Routine Inspection of Milk for Preservatives.-It was the writer's custom in Massachusetts to examine all the samples of milk collected during the months of June, July, August, and September for the commonly used preservatives, in addition to the regular analysis for total solids and fat. The number of samples thus examined amounted to upwards of 500 per month, varying from 10 to 60 per day. The results of such an examination during four years are thus shown: $\dagger$

PRESFRVATIVES IN MILK.

\begin{tabular}{|c|c|c|c|c|c|c|c|}
\hline Year. & $\begin{array}{c}\text { Samples } \\
\text { Examined. }\end{array}$ & $\begin{array}{l}\text { Number } \\
\text { containing } \\
\text { Form-- } \\
\text { aldehyde. }\end{array}$ & $\begin{array}{l}\text { Per Cent } \\
\text { containing } \\
\text { Form- } \\
\text { aldehyde. }\end{array}$ & $\begin{array}{l}\text { Number } \\
\text { containing } \\
\text { Boric } \\
\text { Acid. }\end{array}$ & $\begin{array}{c}\text { Per Cent } \\
\text { containing } \\
\text { Boric } \\
\text { Acid. }\end{array}$ & $\begin{array}{l}\text { Number } \\
\text { containing } \\
\text { Carbonate. }\end{array}$ & $\begin{array}{l}\text { Total } \\
\text { containing } \\
\text { Preserva- } \\
\text { tive. }\end{array}$ \\
\hline $1898 \ldots \ldots \ldots$ & 1046 & 26 & 2.5 & II & 1.0 & 4 & $4 \mathrm{I}$ \\
\hline I899......... & 2105 & 55 & 2.6 & 13 & 0.6 & 3 & $7 x$ \\
\hline $1900 \ldots \ldots \ldots$ & 2018 & 61 & 3.0 & 6 & 0.3 & - & 67 \\
\hline rgor.......... & 2154 & 42 & I. 9 & 12 & 0.5 & - & 54 \\
\hline rgo2 ........... & 1934 & 29 & I. 5 & I4 & 0.7 & - & 43 \\
\hline Totals. .. & 9257 & $2 I_{3}$ & 2.3 & 56 & 0.6 & 7 & 376 \\
\hline
\end{tabular}

* When this process is used the ether may readily be recovered by distillation.

$\dagger$ An. Rep. Mass, State Board of Health, 1902, p. 474; Analyst's Reprint, p. 22 
Such a system by no means involves a large amount of time or labor, and is really essential before passing judgment upon the purity of the milk, since, unlike added color, there is nothing in the physical appearance of the milk to suggest the presence of preservatives, nor are they rendered apparent by the taste, if skilfully used.

The methods employed are carried out as follows:*

(I) Formaldehyde.-After having been examined for total solids and fat, the milk samples are arranged in order in their original containers, and about ro cc. of each sample are poured into a casserole and tested in succession by means of the hydrochloric acid and ferric chloride test (p. 180). A large stock bottle, which may be fitted with a siphon if desired, is kept on hand containing the hydrochloric acid reagent. Less than one minute is required in making the formaldehyde test for each sample.

2. Carbonate and Boric Acid.-These tests have been so simplified as to be, as it were, a sicie issue in the process of cleaning the platinum dishes used for the determination of total solids. The various residues from the total solids are burnt to an ash in the original numbered dishes in succession, these dishes, after incineration, being arranged side by side on a flat tray. By means of a pipette, one or two drops of dilute hydrochloric acid are introduced into each dish in succession, noting at the time any effervescence that may ensue, which is in itself an indication of sodium carbonate. After every milk ash has been acidulated, a few cubic centimeters of water are added to each dish by means of a washbottle, the dissolving of the ash being hastened by giving a rotary motion to the tray containing the dishes. A strip of turmeric-paper is then allowed to soak for a minute or so in each dish, after which it is withdrawn from contact with the solution and allowed to adhere to the side of the dish above the liquid, where it remains until dry. If the paper when dry is of a deep cherry-red color, turning a dark olive when treated with dilute aikali, the presence of boric acid is assured. These methods are, of course, preliminary tests for quickly singling out the preserved samples. Such confirmatory tests as are desired may in all cases be employed.

Another method of drying the strips outside the dishes is as follows:

In a part of the laboratory free from dust, two long sections of glass rod or tubing are placed in parallel lines over a strip of filter-paper,

* Leach, Analyst, XXVI, p. 289 . An. Rep. Mass. State Board of Health, 1901, p. 447 Food and Drug Reprint, p. 27 . 
with numbers marked on the paper at close intervals corresponding to the numbers of the platinum dishes. The strips of turmeric-paper, after soaking, are removed from the dishes and placed across the glass tubes, over the numbers corresponding to those of the dishes from which they were taken. Here they are allowed to stand till dry, being kept in position by a third section of tube or rod placed over them. When dry, the color of the turmeric strips will indicate whether or not boric acid is present, and also the position will show in what sample to look for it.

VARIOUS ADULTERANTS. - Cane Sugar is said to be used to increase the total solids of milk, but if present to any marked degree, it could hardly fail of detection by reason of the sweet taste imparted to the milk. Cane sugar in milk may be detected * by boiling 5 to ro $\mathrm{cc}$. of the sample with about o.I gram of resorcin and a few drops of hydrochloric acid for a few minutes. In the presenee of cane sugar, a rose-red color is produced.

According to Richmond, cane sugar may be estimated by first ascertaining the total polarization of the sample as in the estimation of milk sugar (p. 147). The milk sugar is then determined by Fehling's solution (pp. 149 to 150 ) either volumetrically or gravimetrically. The difference between the anhydrous milk sugar found by the latter, or Fehling method, and that calculated by dividing the polarization by 1.217 will give the percentage of cane sugar present.

Cotton's $\dagger$ method of detecting cane sugar, when present to the extent of $0.1 \%$, consists in mixing in a test-tube Io cc. of the suspected milk with 0.5 gram of powdered ammonium molybdate, and adding to the mixture Io cc. of dilute hydrochloric acid ( $\mathrm{I}$ to Io). Ten cc. of milk of known purity, or Io cc. of a $6 \%$ solution of milk sugar are similarly treated by way of comparison. Both tubes are placed in a water-bath and the temperature gradually raised to $80^{\circ} \mathrm{C}$. If cane sugar is present, an intense blue coloration is produced, while the genuine milk or the solution of milk sugar remains unchanged at the temperature of $80^{\circ}$. If the temperature is raised to the boiling-point, however, the pure milk or milk sugar solution may also turn blue.

Detection of Starch in Milk.-A small quantity of milk is heated in a test-tube to boiling, cooled, and a drop of iodine in potassium iodide added. A blue coloration indicates starch.

\footnotetext{
* Richards and Woodman, Air, Water, and Food, p. 166.

$\dagger$ Abs. Analyst, 1898 , p. 37 .
} 
Condensed Skimmed Milk as an Adulterant.-The use of condensed unsweetened skimmed milk to raise the solids of a skimmed or watered milk above the standard has been noted in Massachusetts. This sophistication is rendered apparent by the abnormally high solids not fat of the sample, which in some instances have exceeded $11 \%$. A solid not fat in excess of $10 \%$ is suspicious of this form of adulteration. By fixing a legal standard for both fat and solids not fat, such tampering with milk may readily be checked.

Analysis of Sour Milk.-It occasionally becomes necessary for the analyst to deal with samples of sour milk, especially in the summer-time, when the milk has been brought from a long distance. While the process of lactic fermentation results in the formation of traces of volatile acids, unless the sample has become so badly curdled as to render an even homogeneous mixture of the various parts impossible, a fair determination of the solids and fat can readily be made. Experience has proved that, excepting in instances of milk so badly soured as to have become actually putrid, the analysis of sour milk, if carefully made, should not differ materially from that of the same milk before souring.

Care must be taken to secure an even emulsion of the curd and whey. This may sometimes be accomplished by repeatedly pouring the sample back and forth from one container to another. Again, it is sometimes necessary to use an egg-beater of the spiral wire pattern, which preferably should easily fit the can or milk-container. Unless a fine, even emulsion can be secured, it is impossible to make a satisfactory analysis of sour milk. With such an emulsion results can be relied on.

In measuring portions of the thoroughly mixed sample of sour milk for analysis, a pipette should be used having a large opening.*

\section{CONDENSED MILK.}

Canned condensed milk has become a very important article of food, its use having increased considerably during the last few years. The universally accepted meaning of the term "condensed milk" in this country is milk both condensed and preserved with cane sugar, being what is commonly known in England as "preserved milk." The unsweetened variety is more often termed "evaporated cream" and sold as such. It is, however,

* A pipette open to the full size of the tube is convenient for this work. 
as found on the market usually nothing better than condensed ordinary milk, having no added sugar, and has generally no resemblance in composition to cream other than in consistency.

Condensed milk is usually prepared by boiling milk in vacuum-pans under diminished pressure to the proper degree of concentration. Upwards of $35^{\circ}$ samples of sweetened condensed milk have been analyzed in full in the laboratory of the Massachusetts State Board of Health in the course of eight years, representing no less than Iro brands, together with about 30 samples (representing 8 brands) of the unsweetened variety.

In view of the fact that a considerable number of the condensed-milk samples are shown by their analysis to have been produced from skimmed milk, the fat content in the samples analyzed varying from a mere trace to $12 \%$, it is obvious that the typical composition of condensed milk could not fairly be shown by giving maximum, minimum, and mean results from the entire tabulated series, nor would it be possible to draw a hardand-fast line excluding certain samples known to be adulterated in making up the averages. It has therefore been thought best to select a few typical brands and give their analyses in full.

COMPOSITION OF SWEETENED CONDENSED MILK.

\begin{tabular}{|c|c|c|c|c|c|c|c|c|c|}
\hline Points to be Noted. & $\begin{array}{l}\text { Total } \\
\text { Solids, } \\
\text { Per } \\
\text { Cent. }\end{array}$ & $\begin{array}{l}\text { Water } \\
\text { Per } \\
\text { Cent. }\end{array}$ & $\begin{array}{l}\text { Millk } \\
\text { Solids, } \\
\text { Per } \\
\text { Cent. }\end{array}$ & $\begin{array}{l}\text { Cane } \\
\text { Sugar, } \\
\text { Per } \\
\text { Cent. }\end{array}$ & $\begin{array}{c}\text { Milk } \\
\text { Sugar. } \\
\text { Per } \\
\text { Cent. }\end{array}$ & $\begin{array}{c}\text { Pro- } \\
\text { teins, } \\
\text { Per } \\
\text { Cent. }\end{array}$ & $\begin{array}{l}\text { Fat, } \\
\text { Per } \\
\text { Cent. }\end{array}$ & $\begin{array}{l}\text { Ash. } \\
\text { Per } \\
\text { Cent. }\end{array}$ & $\begin{array}{l}\text { Fat in } \\
\text { Origi- } \\
\text { nal } \\
\text { Milk, } \\
\text { Per } \\
\text { Cent. }\end{array}$ \\
\hline $\begin{array}{l}\text { High in fat, much added } \\
\text { sugar. }\end{array}$ & $70 \times 17$ & 20.83 & & & & & & & \\
\hline $\begin{array}{l}\text { High fat, low milk sugar. } \\
\text { Low fat, high milk sugar; }\end{array}$ & 68.70 & 32,30 & $\begin{array}{l}31.32 \\
30,27\end{array}$ & $\begin{array}{l}47.05 \\
38.43\end{array}$ & $\begin{array}{l}9.57 \\
6.38\end{array}$ & $\begin{array}{r}7.05 \\
10.70\end{array}$ & $\begin{array}{l}12.00 \\
15.46\end{array}$ & $\begin{array}{l}1.80 \\
1.73\end{array}$ & $\begin{array}{l}4.00 \\
5.63\end{array}$ \\
\hline $\begin{array}{l}\text { low proteins. } \\
\text { Normal con stituents }\end{array}$ & 69.30 & 30.70 & 31.83 & $37 \cdot 47$ & 16.75 & 6.34 & $7 \cdot 20$ & x. 54 & 2.77 \\
\hline $\begin{array}{l}\text { throughout*... } \\
\text { Condensed from skimmed }\end{array}$ & 74.29 & $25 \cdot 71$ & $32 \cdot 37$ & $41 \cdot 92$ & 11.97 & $8.4^{6}$ & 10.65 & 1.29 & 4.56 \\
\hline $\begin{array}{l}\text { milk. } \\
\text { Condensed from centrifu- }\end{array}$ & 69.30 & 30,70 & 29.15 & 40,15 & $7 x .89$ & 12.15 & 3.06 & 2,05 & 1.11 \\
\hline gally skimmed milk..... & 69.06 & 30.94 & 25.88 & $43 \cdot 18$ & 11.55 & 11.78 & 0.09 & 2.46 & Trace \\
\hline
\end{tabular}

COMPOSITION OF UNSWEETENED CONDENSED MILK.

\begin{tabular}{|c|c|c|c|c|c|c|c|c|}
\hline Points to be Noted. & $\begin{array}{l}\text { Total } \\
\text { Solids, } \\
\text { Per } \\
\text { Cent. }\end{array}$ & $\begin{array}{l}\text { Water, } \\
\text { Per } \\
\text { Cent. }\end{array}$ & $\begin{array}{l}\text { Milk } \\
\text { Sugar, } \\
\text { Per } \\
\text { Cent. }\end{array}$ & $\begin{array}{l}\text { Pro- } \\
\text { teins. } \\
\text { Per } \\
\text { Cent. }\end{array}$ & $\begin{array}{l}\text { Pat, } \\
\text { Per } \\
\text { Cent. }\end{array}$ & $\begin{array}{l}\text { Ash, } \\
\text { Per } \\
\text { Cent. }\end{array}$ & $\begin{array}{c}\text { Fat in } \\
\text { Original } \\
\text { Milk. } \\
\text { Per } \\
\text { Cent. }\end{array}$ & $\begin{array}{l}\text { No. of } \\
\text { Times } \\
\text { Con- } \\
\text { densed. }\end{array}$ \\
\hline 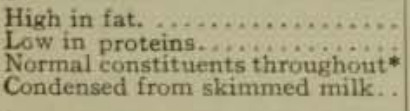 & $\begin{array}{l}36,00 \\
31.25 \\
28.16 \\
35.17\end{array}$ & $\begin{array}{l}64 \cdot 00 \\
86 \cdot 75 \\
69 \cdot 24 \\
64 \cdot 83\end{array}$ & $\begin{array}{r}10.65 \\
13.40 \\
9.85 \\
13.90\end{array}$ & $\begin{array}{r}11.63 \\
7.02 \\
8.66 \\
15.37\end{array}$ & $\begin{array}{r}12.00 \\
9.60 \\
8.10 \\
4.20\end{array}$ & $\begin{array}{l}1.72 \\
1.23 \\
1.55 \\
1.70\end{array}$ & $\begin{array}{l}4.6 \mathrm{r} \\
4.18 \\
3.68 \\
1.28\end{array}$ & $\begin{array}{l}2,6 \\
2.3 \\
2,2 \\
3 \cdot 3\end{array}$ \\
\hline
\end{tabular}

* Can be taken as being very near the a verage for all constituents in honest condensed mill of fair quality. 
In the case of sweetened condensed milk it will be observed that the proteins as a rule run considerably lower than the sugar, whereas in ordinary cow's milk the percentage of proteins and milk sugar are more nearly alike. In making the above analyses all the reducing sugar was reckoned as milk sugar, whereas it is possible that a small amount of the cane sugar is inverted in the process of manufacture, and thus increases the amount of reducing sugar.

U. S. Standards.*-Standard Condensed Milk and Standard Sweetened Condensed Milk are condensed milk and sweetened condensed milk respectively, containing not less than $28 \%$ of milk solids, of which not less than $27.5 \%$ is milk fat. Standard condensed skim-milk is skimmilk from which a considerable portion of water has been evaporated.

The standard for evaporated or condensed milk (unsweetened) has been amended by the Board of Food and Drug Inspection as follows: $\dagger$

(I) It should be prepared by evaporating the fresh, pure, whole milk of healthy cows, obtained by complete milking and excluding all milkings within I5 days before calving and 7 days after calving, provided at the end of this 7 -day period the animals are in a perfectly normal condition.

(2) It should contain such percentages of total solids and of fat that the sum of the two shall be not less than 34.3 and the percentage of fat shall be not less than $7.8 \%$. This allows a small reduction in total solids with increasing richness of the milk in fat.

(3) It should contain no added butter or butter oil incorporated either with whole milk or skimmed milk or with the evaporated milk at any stage of manufacture.

\section{ANALYSIS OF CONDENSED MHLK.}

Preparation of the Sample.-Mix the sample thoroughly, best by transferring the entire contents of the can to a large evaporating-dish, and working it thoroughly with a pestle till homogeneous throughout. Weigh 40 grams of the mixed sample, preferably in a tared weighing-tray for sugar analysis, transfer by washing to a graduated roo-cc. sugarflask and make up to the mark with water.

Total Solids.-Dilute an aliquot part of the mixed solution further with an equal amount of water, and pipette $5 \mathrm{cc}$. of the diluted mixture, corresponding to I gram of the condensed milk, into a tared platinum dish, such as is used for ordinary milk, and rinse the pipette into the

* U. S. Dept. of Agric., Off. of Sec., Circ. I9.

$\dagger$ Food Inspection Decision, $13 \mathrm{r}$. 
dish by means of a wash-bottle. Evaporate, dry at the terrperature of boiling water and weigh as in the case of milk (p. 132).

The character of the residue should be noted. It should not, excepting in the case of a skimmed milk, be caked down hard and glossy on the bottom of the dish, but, if the operation is properly carried out, should have a well-separated fat layer at the top, and the residue should resemble in appearance that from ordinary milk. This result is accomplished by the extreme dilution of the sample.

Ash.-Burn carefully the residue from the total solids as above obtained, cool, and weigh as in the case of ordinary milk (p. I34).

When the total solids-are not to be determined, as in cases where the quality of the milk used in preparation of the sample is decided by the fat and ash alone (see p. 192), $12.5 \mathrm{cc}$. of the above $40 \%$ solution, corresponding to 5 grams of the sample, may be evaporated and burned directly.

Fat.-The Author's Method.*-Fifteen cc. of the $40 \%$ solution prepared as above described, corresponding to 6 grams of the original condensed milk, are measured into an ordinary test-bottle of the Babcock centrifuge. This is filled nearly to the neck with water, and $4 \mathrm{cc}$. of a solution of copper sulphate of the strength of Fehling's copper solution are added. The contents are thoroughly shaken, and the precipitated proteins, carrying with them the fat are rapidly separated out by whirling the fat bottle in the centrifuge, preferably without heating. The writer prefers an electric centrifuge of the Robinson type (p. ${ }^{3} 37$ ) for this purpose, as the heat of the steam-driven machine cakes the precipitate down, so that it is harder to wash. If desired, the precipitate may be allowed to settle out of itself, which it does more quickly in the cold.

The supernatant liquid containing the sugar is drawn off by means of a pipette of large capacity, having a stem sufficiently small to pass easily into the neck of the milk-bottle, a small wisp of absorbent cotton being first twisted over the bottom of the pipette to serve as a filter. On withdrawing the pipette with the sugar solution, the cotton is wiped off into the bottle by rubbing against the inner side.

The precipitated proteins and fat are given two additional washings, as above, by shaking thoroughly with water introduced nearly to the neck of the bottle, separating out in each case by centrifuge or by settling, and finally removing the washings with the pipette, two of such extra washings being found nearly always sufficient to remove all the sugar. If the precipitate is caked down hard after treatment with the centrifuge,

\footnotetext{
* 28 th An. Rep. Mass. State Board of Health, 1896, p 630, and Jour. Am. Chem. Soc., 22 , $19 \circ 0$, p. $5^{89}$.
} 
it may be necessary to employ a stiff platinum wire as a stirrer to aid in mixing with the wash-water. Finally, the volume of 17.6 is completed by addition of water, $17.5 \mathrm{cc}$. of sulphuric acid are added, and the test continued, as in the ordinary Babcock process (p. I36), multiplying the reading obtained by three.

For unsweetened condensed milk, these precautions are, of course, unnecessary.

Roese-Gottlieb Method.-This method (p. I99) is recommended when the accuracy of a gravimetric process is desired.

Proteins.-Calculation from the Nitrogen.-Determine nitrogen by the Kjeldahl or Gunning method in 5 cc. of the $40 \%$ solution, corresponding to 2 grams of the condensed milk, and multiply the result by 6.38 .

Precipitation Method.-Dilute $5 \mathrm{cc}$. of the $40 \%$ solution further to about $40 \mathrm{cc}$. and add sufficient Fehling copper solution, drop by drop, to precipitate the proteins, avoiding a large excess. As a rule, $0.6 \mathrm{cc}$. of copper solution is ample for this. Nearly neutralize with sodium hydroxide, leaving the solution still slightly acid. An excess of alkali tends to dissolve the casein and cause turbidity in the filtrate. Pass through a weighed filter-paper, wash, dry in an air-oven at $100^{\circ} \mathrm{C}$, and weigh. The filter with the dry precipitate is then carefully burnt in a porcelain crucible, and the difference between the weight of the dry precipitate and the weight of the ash is the weight of the proteins and fat. Expressing this in percentage, and deducting from it the per cent of fat previously obtained, the result is the per cent of proteins.

Milk Sugar.-Volumetric Process.-The filtrate and the washings from the preceding operation are made up to $100 \mathrm{cc}$. with water, and the amount of reducing sugar, obtained volumetrically by Fehling's solution, is reckoned as milk sugar. The titration is conducted in the manner described on p. 59 I.

Assuming the solution to be exactly of the strength above described, the milk sugar is calculated as follows: $\frac{100 \times 0.067}{S \times 0.02}=L$, where $L$ is the per cent of lactose or milk sugar, and $S$ the number of cc. of milk solution, prepared as above required to reduce ro cc. of Fehling's solution. Calculation may be avoided by the use of the following table, which may be employed when the above details are minutely carried out: 
PER CENT MILK SUGAR CORRESPONDING TO NUMBER OF CUBIC CENTIMETERS USED.

Strength of solution 2 grams in $100 \mathrm{cc}$.

\begin{tabular}{|c|c|c|c|c|c|c|c|}
\hline $\mathrm{Cu} . \mathrm{Cm}$. & Per Cent. & $\mathrm{Ct} . \mathrm{Cm}$. & Per Cent. & , $\mathrm{Cus} . \mathrm{Cm}$ & Per Cent. & $\mathrm{Cu} . \mathrm{Cm}$. & Per Cent. \\
\hline 18.0 & $18.6 \mathrm{r}$ & 25.0 & 13.40 & 32.0 & 10.47 & 39.0 & 8.59 \\
\hline 18.5 & 18.10 & 25.5 & 13.14 & 32.5 & 10.31 & 39.5 & 8.49 \\
\hline 19.0 & 17.63 & 26.0 & 12.89 & 33.0 & 10.15 & 40.0 & 8.37 \\
\hline 19.5 & 17.18 & 26.5 & 12.64 & $33 \cdot 5$ & 10.00 & 40.5 & 8.27 \\
\hline$=0.0$ & 16.75 & 27.0 & 12.41 & 34.0 & 9.85 & 41.0 & 8.17 \\
\hline 20.5 & 16.34 & 27.5 & $12,+8$ & 34.5 & 9.71 & $4 x-5$ & 8.07 \\
\hline 21.0 & 15.95 & 28.0 & 11.97 & 35.0 & $9 \cdot 57$ & 42.0 & 7.98 \\
\hline 21.5 & $15.5^{8}$ & 28.5 & $11: 75$ & $35 \cdot 5$ & 9.43 & 42.5 & 7.88 \\
\hline 22.0 & 15.22 & 29.0 & I1. 55 & 36.0 & $9 \cdot 30$ & 43.0 & $7 \cdot 7^{8}$ \\
\hline 22.5 & 14.89 & $29 \cdot 5$ & 11.35 & $3^{6} .5$ & 9.17 & $43 \cdot 5$ & $7 \cdot 70$ \\
\hline 23.0 & $14 \cdot 5^{6}$ & 30.0 & 11. 16 & 37.0 & 9.05 & 44.0 & $7.6 \mathrm{r}$ \\
\hline 23.5 & 14.25 & 30.5 & 10.89 & $37 \cdot 5$ & 8.93 & $44 \cdot 5$ & $7 \cdot 53$ \\
\hline 24.0 & 13.95 & 31.0 & 10.80 & $3^{8} .0$ & $8.8 \mathrm{I}$ & & \\
\hline $24 \cdot 5$ & 13.67 & $3^{1} \cdot 5$ & 10.63 & $3^{8} .5$ & 8.70 & & \\
\hline
\end{tabular}

Gravimetric Methods.-Lactose may be determined in the $40 \%$ solution of the condensed milk by the O'Sullivan-Defren method (page I50), the Soxhlet method (page I50), or the Munson and Walker method (page ${ }_{15} \mathrm{I}$ ), the solution being treated exactly as if it were milk.

Cane Sugar.-This is obtained by difference, deducting the milk solids (the sum of the milk sugar, proteins, fat, and ash) from the total solids first obtained.

Detection of Foreign Fats and Oils.-The invention of the homogenizer has led to the preparation of emulsions of oleo, cotton seed and other oils, as well as of butter fat, with skim milk and milk in imitation of whole milk and cream and the use of such homogenized products in condensed milk and ice cream. This substitution is detected by the separation and examination of the fat as follows:

Paul's Method.*-Dilute Ioo grams of the material with $300 \mathrm{cc}$. of water, heat to boiling and add slowly, while boiling, $25 \mathrm{cc}$. of Fehling's copper sulphate solution (p. 28).

Filter through a filter paper on a Büchner funnel, wash three times with hot water and allow to suck dry. Remove filter and precipitate from the funnel, break into small pieces, dry over night at room temperature and grind with about 25 grams of anhydrous copper sulphate.

Place a layer of anhydrous copper sulphate in the bottom of the inner tube of a Johnson extractor (p. 65), then add the powdered mixture and place a loose plug of cotton on the top. Connect the extractor with a flask, pour $50 \mathrm{cc}$. of ether through the mixture and proceed as usual with the extraction. 
Dry the fat as quickly as possible and weigh. Determine the refractive index, volatile fatty acids and such other constants as seem desirable (Chapter XIII).

Calculation of Fat in Original Milk.-The "fat in the original milk," as expressed in the tables on page 187 , was calculated by assuming a percentage of solids not fat of 9.3 in the original milk, this being the standard fixed by the Massachusetts law. Calculate first the fat and the milk solids to the basis of the cane-sugar-free sample. This is done by dividing the per cent of each as found in the sample by roo less the percentage of cane sugar, and multiplying the result by roo. Ascertain the difference between the milk solids and the fat thus obtained in the canesugar-free sample, and divide this percentage of milk solids not fat by 9.3. The result is the "number of times condensed" (if cane sugar were not present as a diluent).

The per cent of fat in the canc-sugar-free sample, divided by the number of times condensed, as above obtained, gives the percentage of fat in the original milk.

The above calculation from the solids not fat of the factor designated as "the number of times condensed," necessitates determinations of fat, ash, proteins, and milk sugar, in fact, a complete analysis of the sample.

A simpler method of calculating the "number of times condensed," involving determinations of fat and ash only in the sample, consists in dividing the per cent of ash found in the condensed milk by 0.7 , this figure being the assumed ash of normal, standard milk. Then, by dividing the fat in the sample by the "number of times condensed " as last calculated, the result is the fat in the original milk. If this is found to be well below $3 \%$, there is reason to suspect that skimmed milk was used in its preparation.

The "fat in the original milk" as thus calculated is, of course, an arbitrary factor and is useful only in deciding whether or not skimmed milk has been used in preparing the sample. By assuming the above very reasonable figures for the solids not fat, or for the ash of natural milk (according to which method is used for calculation), it is readily seen that the highest result is obtained for the "fat in the original milk" and hence the benefit of the doubt as to the use of skimmed milk is given to the manufacturer.

Bigelow and McElroy's Polarimetric Method for Cane Sugar.* 26.048 grams of the mixed sample of condensed milk are transferred to a Ioo-cc.

${ }^{*}$ Jour. Am. Chem. Soc., 15, p. 668. 
graduated sugar-flask and dissolved in water, which is boiled to make sure of normal rotation. The solution is then clarified by the addition of an acetic acid solution of mercuric iodide * and, if necessary, alumina cream, the volume is made up to roo cc., shaken, and filtered through a dry filter. Rejecting the first part, of the filtrate, a further portion is polarized. For inversion, another sample of 26.048 grams is weighed out as before and dissolved, but before clarifying, is heated to $55^{\circ} \mathrm{C}$. and treated with half a cake of compressed yeast, the heating with the yeast being continued at $55^{\circ}$ for five hours. The clarifying solution is added before cooling, and, after cooling, making up to 100 cc., and filtering as before, the invert reading is obtained with the polariscope. By this process of yeast inversion the cane sugar only is inverted, the lactose remaining unchanged. It is best to work with several samples and use the mean of the readings both for direct and invert figures. It is also best to use the double dilution method (p. ${ }^{149)}$ to compensate for the volume of the precipitated fat and proteins.

The per cent of cane sugar is calculated by the formula of Clerget,

$$
S=\frac{a-b}{142.66-\frac{t}{2}},
$$

$S$ being the per cent of cane sugar, $a$ the direct reading,

$b$ the invert reading and $t$ the temperature at which the observation is made.

The above process presupposes the absence of invert sugar in the sample, a supposition which Wiley claims it is fair as a rule to assume.

\section{CREAM.}

Composition.-Cream varies in composition according to the method by which it is obtained, i.e., whether (I) by allowing it to separate from the milk set in shallow pans, whence it is removed by hand-skimming, (2) by setting in deep vessels surrounded by cold water (as for example in the "Cooley" creamer) the skimmed milk being commonly drawn off from below, or (3) by the centrifugal separator. Most of the heavy cream found in the market at the present time is the product of the third or separator process. Analyses of different kinds of cream follow:

- Prepared by dissolving 53 grams of potassium iodide, 22 'grams mercuric chloride, and $32 \mathrm{cc}$. of strong acetic acid in water and making up to $\mathrm{I}$ liter. 
COMPOSITION OF CREAM.

\begin{tabular}{|c|c|c|c|c|c|c|c|c|c|c|}
\hline Chanacter of Cream. & 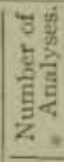 & 㟒 & & है & 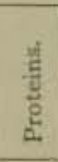 & हैं & $\begin{array}{l}\text { 总 } \\
\text { क् }\end{array}$ & 要 & 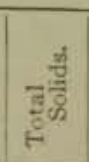 & 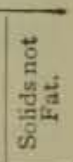 \\
\hline $\begin{array}{l}\text { By natural separation. ....... } \\
\text { By centrifugal separator, }\end{array}$ & 46 & & & & & 22.66 & 4.23 & 0.53 & & \\
\hline "Leavy" cream. ........ & I8 & $\begin{array}{l}\text { Leach } \\
\text { Leach }\end{array}$ & $\begin{array}{l}\text { Maximum } \\
\text { Minimum } \\
\text { Mean } \\
\text { Maximum } \\
\text { Minimum } \\
\text { Mean }\end{array}$ & \begin{tabular}{|l|}
54.80 \\
46.76 \\
51.68 \\
83.29 \\
70.50 \\
77.89
\end{tabular} & & $\begin{array}{r}46.40 \\
38.10 \\
42.02 \\
21.60 \\
8.60 \\
13.86\end{array}$ & & & \begin{tabular}{|l|}
53.24 \\
45.20 \\
48.32 \\
29.50 \\
10.71 \\
22.11
\end{tabular} & $\begin{array}{l}8.50 \\
4.20 \\
6.30 \\
9.30 \\
7.22 \\
8.25\end{array}$ \\
\hline
\end{tabular}

U. S. Standards.*-Standard Cream is cream containing not less than 18\% of milk fat. Standard Evaporated Cream is cream from which a considerable portion of water has been evaporated.

Adulteration of Cream.-In some localities fat standards are fixed for cream both "heavy " and "light," those falling below such standards being deemed adulterated.

Foreign Fats.-Oleo oil, possibly other fats, "homogenized" or emulsified with milk or skim milk, is now being substituted for true cream. A product known as "Syntho" belongs in this class but is sold by its manufacturers under its true name.

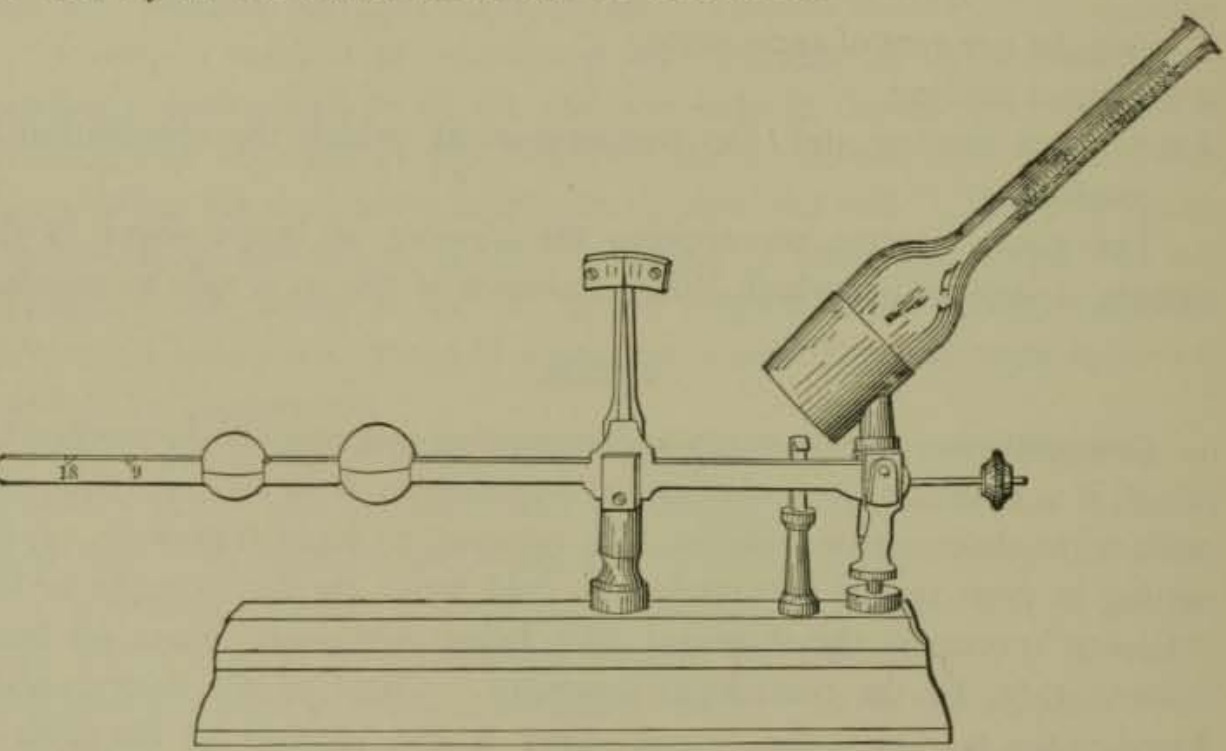

Fic. 53.-A Babcock Cream-test Scale,

Preservatives,-The same preservatives are employed in cream as in milk.

* U. S. Dept, of Agric., Off, of Sec, Circ, Ig. 
Gelatin.-The author has detected this substance in cream sold in Massachusetts. It serves as a thickener and is sometimes sold in powder form mixed with boric acid.

Sucrate of Lime in Milk and Cream.-Pasteurizing reduces the consistency of cream so that its apparent richness and its value for certain culinary preparations is impaired. Babcock and Russell * have shown that sucrate of lime ("viscogen") may be used to thicken such cream, but insist that the treated product be sold under a distinctive name, such as "visco-cream" or "pasteurized visco-cream."

To prepare "viscogen" dissolve $2 \frac{1}{2}$ parts by weight of cane-sugar in 5 parts of water, add, after straining, I part of quicklime slaked in 3 parts of water; shake, allow to settle, siphon off the supernatant liquid, and bottle. For thickening cream use two-thirds of the amount required to neutralize its acidity. It will also thicken milk and condensed milk.

\section{ANALYSIS OF CREAM.}

Total solids, ash, sugar, proteins, and fat (gravimetric) are determined by the methods used in milk analysis (pp. I30-155).

Determination of Fat.-Babcock Process.-Owing to the viscosity of cream and its variation in density strictly accurate results can be secured only by weighing the sample. Fig. 53 shows a cream scale provided with a sliding poise for balancing the bottle and a second for weighing the cream. If a large number of tests are to be made, a balance for weighing several samples on each pan or the Wisconsin hydrostatic cream balance will be found convenient. $\dagger$ The latter, devised by Babcock and Farrington, $\ddagger$ is constructed on the principle of the lactometer. It is provided with a pan on the top of the stem for holding the test bottle and weights.

Two forms of test bottles are shown in Fig. 54. Others with graduations up to $50 \%$ are also obtainable.

The process is as follows: Weigh 9 or 18 grams of the well-mixed sample into the tared test bottle, using a pipette with a wide delivery tube. If 9 grams are used dilute with $9 \mathrm{cc}$. of water. Add $17.5 \mathrm{cc}$. of sulphuric acid of proper strength and proceed as in the case of milk (p. ${ }_{3} 8$ ).

The error due to the curved meniscus of the fat column in the test

\footnotetext{
* Wisconsin Exp. Station, Bull. 54.

$\dagger$ Farrington and Woll, Testing Milk and its Products, zoth ed., Pp. $81-83$. \pm Wisconsin Exp. Station Bul, 195 .
} 
bottle may be overcome by adding a few drops of fat-saturated alcohol (Babcock and Farrington*) or of glymol (Hunzikert).

To prepare fat-saturated alcohol place a teaspoonful of butter in a bottle with $200 \mathrm{cc}$. of denatured or wood alcohol, warm slightly and

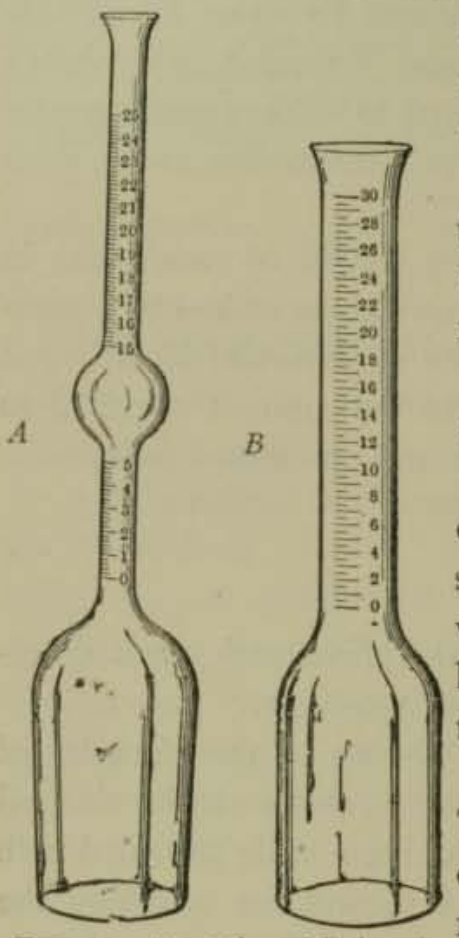

Fig. 54-Varieties of Babcock Test Bottle for Cream. shake until saturated. Coloring matter may be added to further facilitate the reading. Glymol may be colored with alkanet root.

Detection of Foreign Fats.-Determine the refractive index and the volatile fatty. acids of the fat obtained by the Babcock method.

Detection of $\rfloor$ Preservatives.-See pp. I8o$18_{3}$.

In testing for formaldehyde, using ferric chloride and hydrochloric acid, the sample should be diluted with an equal volume of water, heated with the reagents in a casserole but finally poured into a test tube to observe the color.

Detection of Gelatin.-Stokes Method. $\ddagger$ The reagents are as follows: (I) Acid nitrate of mercury, prepared by dissolving metallic mercury in twice its weight of concentrated nitric acid (sp. gr. 1.42) and diluting with $A$, Bartlett Bottle; $B$, Winton twenty-five times its bulk of water, and (2) a Bottle. saturated aqueous solution of picric acid.

To about to cc. of the cream add the same amount of the acid nitrate of mercury solution and $20 \mathrm{cc}$. of cold water. Shake the mixture vigorously and allow to rest for five minutes, after which filter. If much gelatin is present, the filtrate will not be clear, but opalescent. To the whole or a part of the filtrate add a few drops of the picric acid solution. If gelatin be present in any considerable amount, a yellow precipitate is formed. Avoid an excess of acid nitrate of mercury, as this would cause a precipitate with picric acid.

If gelatin is present in small amount only, a cloudiness is produced, best spen against a dark background. The reaction is delicate to I part of gelatin in 10,000 parts of milk or cream.

* Wisconsin Exp. Station, Bull. r95, P. 6.

Purdue, Ind. Exp. Station, Bul. I45, XV, p. 593. ‡Analyst, 22, P. 320 . 
Datection of Sucrate of Lime.-This is indicated by the presence of sucrose, in connection with an abnormally high alkalinity of ash and excessive calcium oxide. The tests are as follows:

Lythgoe's Modification of Baier and Neuman's Test for Detecting Sucrose.*-To $25 \mathrm{cc}$. of milk or cream, add ro cc. of a $5 \%$ solution of uranium acetate, shake well, allow to stand for 5 minutes, and filter. To to cc. of the clear filtrate, (in the case of cream use the total filtrate, which will be less than Io cc.) add a mixture of $2 \mathrm{cc}$. saturated ammonium molybdate and $8 \mathrm{cc}$. dilute hydrochloric acid (I part $25 \%$ acid and 7 parts water), and heat in a water-bath at $80^{\circ} \mathrm{C}$. for 5 minutes. If the sample contains sugar, the solution will be of a Prussian blue color, which should be compared in a colorimeter with standard Prussian blue solution, prepared by adding a few drops of potassium ferrocyanide to a solution of $\mathrm{I}$ cc. of $\mathrm{r} \%$ ferric chloride in $20 \mathrm{cc}$. of water.

Occasional samples of pure milk will give a pale blue color, but this can be entirely removed by filtration, the filtrate being green, while the color due to sugar will pass through the filter, giving the usual blue solution. This color, due to a reduction of molybdic acid, is also produced by levulose and dextrose. Solutions of I gram of lactose, levulose, dextrose, and sucrose in $35 \mathrm{cc}$. of water heated with molybdenum reagent for 5 minutes reacted as follows: lactose no color, levulose a heavy blue, sucrose a weaker blue, and dextrose the weakest blue, the intensity of the last three being as $10: 3: \mathrm{r}$.

Stannous chloride, ferrous sulphate, and hydrogen sulphide give this blue color in the cold, but it disappears on heating except in case the stannous or ferrous salt is present to the extent of at least $1 \%$ (calculated as the metal) which amount would coagulate the cream and impart a very disagreeable taste.

As a confirmatory test for sugar, the resorcine test may be applied to the serum prepared with uranium as described above. This test is given by sucrose and levulose, but not by dextrose or lactose.

Determination of Alkalinity of Ash and Calcium Oxide.-Weigh 25 grams of cream into a platinum dish, place in an oven at about $125-150^{\circ} \mathrm{C}$. over night, and burn to an ash in a muffle at a low-red heat. Dissolve the ash in $20 \mathrm{cc}$. N/ ro sulphuric acid, boil to expel

*Zeits. Unters. Nahr. Genussm., I6, 1908, p. 51 
the carbon dioxide, and titrate back with $\mathrm{N} /$ ro sodium hydroxide, using phenolphthalein as the indicator. Express results as cc. N/Io acid required to neutralize the ash of 100 grams of cream.

Make the final solution of the above determination acid with acetic acid, heat to boiling, add I gram of sodium acetate, and to the clear solution add an excess of ammonium oxalate, boil for a few minutes, filter, and wash with water. Dissolve the, calcium oxalate in hot dilute sulphuric acid, and titrate hot with $\mathrm{N} /$ io potassium permanganate. The number of cubic centimeters of $\mathrm{N} / \mathrm{Io}$ permanganate, multiplied by $0.0112(4 \times 0.0028)$, gives the percentage of $\mathrm{CaO}$ in the sample.

Cream samples treated with calcium sucrate, having a fat content from 26 to $33 \%$, show as a rule an alkalinity of ash of from $I_{4}$ to $I 8$, and a $\mathrm{CaO}$ content of from 0.15 to $0.175 \%$ while the same untreated show in general alkalinity of ash not exceeding 12.5 and a $\mathrm{CaO}$ content not exceeding 0.135 . With higher fat contents both constants drop. For example, a cream of $45 \%$ fat containing calcium sucrate had an alkalinity of ash of 10.2 and a $\mathrm{CaO}$ content of $0.12 \%$. Cream of about $45 \%$ fat untreated had an ash alkalinity of 6.5 and a $\mathrm{CaO}$ content of $0.103 \%$.

\section{ICE CREAM.}

For many years a wide variety of iced foods have been made and sold under the general name of ice cream, many of which are largely composed of ingredients other than milk or cream. In the study and classification of foods of such a miscellaneous nature as ice cream, in its popularly accepted meaning, it is not always easy to satisfactorily define and fix standards. Whether, for example, the product should consist exclusively of frozen cream, sugar and flavoring, or whether eggs and other materials should be allowed under the unqualified name of ice cream, is a subject of some controversy.

Properly speaking, many mixtures sold under the name should be otherwise designated, as, for example, "frozen custard," to specify more aptly their nature and composition. The following standards show the attitude of the government in this regard:

U. S. Standards.*-Ice cream is a frozen product made from cream and sugar with or without a natural flavoring, and contains not less than I $4 \%$ of milk fat.

* U. S. Dept. of Agric., Office of Secretary, Circ. I9. 
Fruit ice cream is a frozen product made from cream, sugar, and sound, clean, mature fruits, and contains not less than $12 \%$ of milk fat.

$N u t$ ice cream is a frozen product made from cream, sugar, and sound, non-rancid nuts, and contains not less than ${ }_{12} \%$ of milk fat.

Fillers or Stiffeners.-In the manufacture of commercial "ice cream" substances are frequently added to cause the product to hold stiff and keep its consistency for many hours after freezing. The thickeners or fillers most commonly thus used are starch, gelatin, and gums such as gum tragacanth. Agar-agar and commercial casein are also said to be employed for this purpose.

Preparations are on the market sold for thickening ice cream, consisting, as a rule, of one or more of the above-named substances.

Homogenized Products. - Unsalted butter emulsified with milk or skim milk is now extensively substituted for true cream in the manufacture of so-called ice cream. Oleo oil and cotton seed oil are also used in such emulsions. None of these emulsions are allowable in the product sold as ice cream.

Ice Cream Cones.-These are cornucopias made of a kind of dry crust used to serve ice cream without a spoon, the cones as well as the ice cream being eaten from the hand. In addition to flour, sugar, and eggs or gelatin, which are proper constitutents, they frequently contain saccharin, artificial color and borax, the latter being used to prevent sticking to the mold during baking.

\section{ANALYSIS OF ICE CREAM.}

Fat.-Roese-Gottlieb Method.*-Prepare a $40 \%$ water solution, as described for condensed milk (p. I88). Of this solution measure Io cc. into a Röhrig tube $\dagger$ (Fig. 55), or a glass cylinder $2 \mathrm{~cm}$. in diameter and $40 \mathrm{~cm}$. high, to which a narrow siphon can be fitted; dilute with $0.5 \mathrm{cc}$. of water, add I.25 cc. of concentrated ammonium hydroxide ( $2 \mathrm{cc}$. if the sample is sour) and mix thoroughly. Add ro cc. of $95 \%$ alcohol and shake well. Then add $25 \mathrm{cc}$. of washed ethyl ether, shake vigorously for half a minute, add $25 \mathrm{cc}$. of petroleum ether (p. 66), preferably redistilled below $60^{\circ} \mathrm{C}$., and shake again for half a minute. Let stand

* Roese, Zeits. Angew. Chem., 1889, p. Ioo; Gottlieb, Landw. Versuchs-Stat., 40, 1892, p. I; Patrick, U. S. Dept. of Agric., Bur. of Chem., Circ. 66; A. O. A. C. Method.

† Zeits. Unters. Nahr. Genussm., 9, 1905, p. 531 . 
twenty minutes or until the upper liquid is clear and its lower level constant.

Draw off as much as possible of the ethereal liquid-usually 0.5 to $0.8 \mathrm{cc}$. is left-through a diminutive filter into a weighed flask. Extract

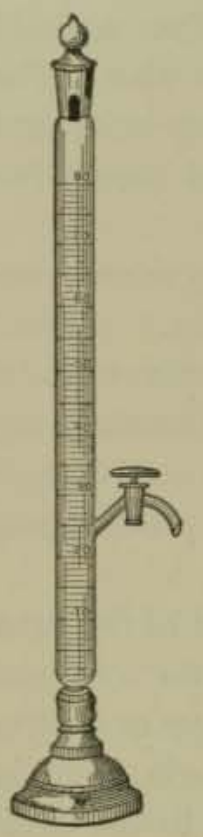

FiG. 55.-Röhrig tube for RoeseGottlieb Method. the liquid remaining in the tube in the same manner as before except that only I5 cc. each of the ethers are used, draw off through the same paper into the flask and wash with a few cc. of the mixed ethers (I:I). Evaporate the drawn off and filtered liquid slowly and dry in a boiling-water oven, one hour at a time, to constant weight. The ether used must be tested for residue upon evaporation and a correction introduced if necessary.

The dried and weighed fats should be dissolved in a little petroleum ether; if a residue be found (due to a trace of the aqueous liquid which may have passed the filter) it must be washed in the flask, dried, and its weight deducted from that of the crude fat.

This method is also applicable to condensed milk, cream, milk, skim milk, buttermilk, and whey. With substances of low fat content the second extraction may be omitted, the weight of the fat being increased to correspond to the entire volume of ethereal liquid measured in the tube.

Detection of Foreign Fats and Oils.-Separate and examine the fat as described on page I9I.

Detection of Thickeners. - Patrick's Method.*-Add $25 \mathrm{cc}$. of water to $50 \mathrm{cc}$. of the sample, and boil till any thickener present is dissolved. Add $2 \mathrm{cc}$. of a $10 \%$ solution of acetic acid, heat to boiling, add 3 heaping teaspoonfuls of kieselguhr, and after shaking pass at once chrough a plaited filter. To $3 \mathrm{cc}$. of the clear filtrate add $12 \mathrm{cc}$. of $95 \%$ alcohol and mix thoroughly, thus precipitating the milk proteins not already removed, and also the gums and some of the gelatin, if much is present. Add $3 \mathrm{cc}$. of a mixture of $95 \mathrm{cc}$. of $95 \%$ alcohol and $5 \mathrm{cc}$. of concentrated hydrochloric acid. This acidified alcohol dissolves completely the milk proteins, and, if a clear solution then remains, no gums or vegetable jellies have been used as thickeners. Turbidity does not, however,

* U. S. Dept. of Agric., Bur. of Chem., Bul. rr6, p. 26. 
necessarily indicate presence of a thickener, as it may be caused by a large amount of eggs, or by the souring of the ice cream. Dilute the mixture, if turbid, by adding 3 cc. of water. Any precipitate due to gelatin or eggs will be dissolved at this dilution, but not that due to vegetable gums. If gum tragacanth be present, the precipitate will be stringy and cohesive, especially after shaking, while agar-agar or other vegetable thickeners will cause a fine flocculent precipitate.

Souring of the ice cream sometimes produces a turbidity or precipitate under the above conditions, which is not always dissolved after diluting with water. Formation of such a precipitate (due to sourness) may, however, apparently be prevented by the previous addition of formaldehyde to the sample.

Howard's Test for Gums.-Precipitate 10 cc. of the melted sample with acetone, and wash with 2 or 3 portions of dilute alcohol, using the centrifuge. Boil the washed residue with 6 to $8 \mathrm{cc}$. of water and I cc. of $10 \%$ sodium hydroxide solution for half a minute. Cool, let stand a few minutes, filter, and heat the filtrate to boiling. Add one and one-half volumes of warm alcohol and shake. If agar-agar or gum tragacanth be present, a flocculent precipitate will immediately separate. Disregard a mere turbidity. To prove the absence of any considerable quantity of milk proteins in the precipitate, dissolve in cold water and saturate the solution with ammonium sulphate.

Gelatin.-Use the method of Stokes (p. I96) on Io to $\mathrm{I}_{5} \mathrm{cc}$. of the sample, disregarding a faint cloudiness at the end.

Starch is detected by the usual iodine test.

Detection of Preservatives.-Formaldehyde and boric acid are tested for as in milk.

Detection of Colors.-See Chapter XVII. The colors used are not merely yellows and oranges such as are added to milk, but include also reds, greens, and even blues, coal-tar dyes being most commonly employed.

\section{BUTTER.}

The value of butter as a food depends almost entirely on its fat content, although minute quantities of protein and milk sugar are also included in its composition.

Hence butter is more logically treated in detail under the heading of fats, page 529 . 


\section{CHEESE.}

Nature and Composition.-Cheese consists principally of the curd and fat removed in a mass from milk, which has been curdled by the natural souring of the milk, or by the action of rennet. The separated mass of curd and fat, after being compressed, is allowed to undergo certain changes, which constitute the ripening or curing, due to the action of micro-organisms and enzymes. Sometimes cream is used as the source of cheese and sometimes skimmed milk. During the ripening process, which requires from a few weeks to several months, the characteristic flavor is developed by the changes which the proteins undergo, and the digestibility of the cheese is improved. The nature of the proteolytic changes that take place during ripening are very little understood, but a variety of complex nitrogenous products are formed, which Van Slyke divides as follows: paracasein, unsaturated paracasein lactate, paranuclein, caseoses (albumoses), peptones, amides, and ammonia. Besides nitrogenous bodies and fat, which are its chief constituents, cheese contains notable quantities of water, milk sugar, lactic acid, and mineral matter.

In some kinds of cheese salt and coloring matter are added.

Varieties.-Well-known cheeses of commerce are often named from districts, towns, or localities where they originated or are still made. They may be classified as cream, whole-milk, or skimmed-milk cheese, according to the quality of the product from which they are made, or again as hard, medium, or soft, according to whether (I) they are pressed, or (2) allowed to drain for days and sometimes weeks without pressure to a firm consistency, or (3) are made in the space of a few hours, being quickly drained on a sieve by hand pressure.

Cheddar Cheese, which is the common cheese of the United States (though originally made some $25^{\circ}$ years ago in England and still made there), is a type of the hard cheese. Stilton, an English, and Gruyère, a Swiss cheese, belong to the medium class, and soft cheeses are represented by Brie and Neufchatel, both French cream cheeses. Other well-known varieties are Edam, a round, mild, long-keeping Dutch cheese, Camembert, a rich cream cheese, and Roquejort, made originally from ewe's milk in the French town of that name, and ripened in caves in the mountains. It is flavored by a peculiar mold. 
The following table, compiled by Woll,* shows the average composition of various cheeses of commerce, both foreign and domestic:

\begin{tabular}{|c|c|c|c|c|c|}
\hline & Water. & Casein. & Fat. & Sugar. & Ash. \\
\hline & Per cent. & Per cent. & Per cent: & Per cent. & Per cent. \\
\hline Cheddar........................... & $34 \cdot 3^{8}$ & $26.3^{8}$ & $32.7 \mathrm{I}$ & 2.95 & $3 \cdot 5^{8}$ \\
\hline Cheshire. ........................ & $3^{2}-59$ & $32 \cdot 5 \mathrm{I}$ & 26.06 & $4 \cdot 53$ & $4 \cdot 3 x$ \\
\hline 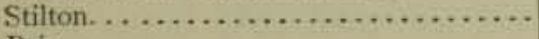 & 30.35 & 28.85 & $35 \cdot 39$ & 1.59 & 3.83 \\
\hline 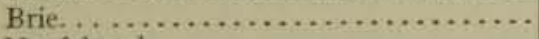 & $50 \cdot 35$ & 17.18 & 25.12 & $1.9+$ & $5 \cdot 41$ \\
\hline 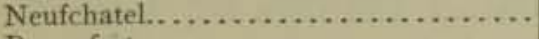 & $44 \cdot 47$ & 14.60 & $33-70$ & 4.24 & 2.99 \\
\hline 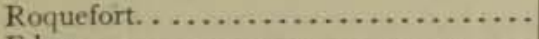 & 31.20 & 27.63 & 33.16 & 2.00 & 6.01 \\
\hline 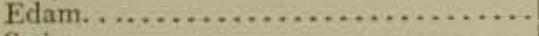 & $3^{6.28}$ & 24.06 & 30.26 & 4.60 & $4 \cdot 90$ \\
\hline Swiss. . . . . . . . . . . . . . . . & 35.80 & 24.44 & $37 \cdot 40$ & $\longrightarrow$ & $2 \cdot 36$ \\
\hline Full cream, mean of ${ }^{4} 43$ analyses...... & $3^{8} .60$ & $25 \cdot 35$ & 30.25 & 2.03 & 4.07 \\
\hline
\end{tabular}

Van Slyke has furnished the following analysis of the nitrogen compounds in a sample of normal American Cheddar cheese six months old and cured at $60^{\circ} \mathrm{F}$.:

\begin{tabular}{c|c|c|c|c|c|c|c}
\hline $\begin{array}{c}\text { Per Cent } \\
\text { N in } \\
\text { Cheese. }\end{array}$ & $\begin{array}{c}\text { Per Cent } \\
\text { Water- } \\
\text { soluble N. }\end{array}$ & $\begin{array}{c}\text { Per Cent } \\
\text { Nas } \\
\text { Paracasein } \\
\text { Mono- } \\
\text { lactate. }\end{array}$ & $\begin{array}{c}\text { Per Cent } \\
\text { N as Para- } \\
\text { nuclein. }\end{array}$ & $\begin{array}{c}\text { Per Cent } \\
\text { N as } \\
\text { Caseoses. }\end{array}$ & $\begin{array}{c}\text { Per Cent } \\
\text { Nas } \\
\text { Peptones. }\end{array}$ & $\begin{array}{c}\text { Per Cent } \\
\text { N as } \\
\text { Amides. }\end{array}$ & $\begin{array}{c}\text { Per Cent } \\
\text { Nas as } \\
\text { Ammonia. }\end{array}$ \\
\hline 3.80. & 1.46 & 0.94 & 0.14 & 0.22 & 0.18 & 0.79 & 0.13 \\
\hline
\end{tabular}

U. S. Standards.t-Cheese is the sound, solid, and ripened product made from milk or cream by coagulating the casein thereof with rennet or lactic acid, with or without the addition of ripening ferments and seasoning, and contains, in the water-free substance, not less than $50 \%$ of milk fat. By act of Congress, approved June 6, 1896 , cheese may also contain added coloring matter.

Skim-milk Cheese is defined the same as cheese except that it is made from skim milk, and no minimum percentage of fat in the waterfree substance is specified.

Adulteration.-Cheese is commonly adulterated in two ways: first, by the partial or total substitution for the milk fat of a foreign fat, as oleomargarine or lard, and, second, by using skimmed milk as a material for its manufacture.

In many localities a standard percentage for fat in cheese is fixed by law, as in the case of the U. S. standard noted above, all samples falling below that standard, unless sold as skim-milk cheese, being deemed adulterated.

* Dairy Calendar, p. 223.

† U. S. Dept, of Agric., Off. of Sec., Circ. Ig. 
Some states have specific standards for varying grades of cheese, classified as to their fat content. Thus under the Pennsylvania law* cheese is divided into five grades, as follows:

Full-cream cheese mușt contain not less than $32 \%$ butter fat.

Three-fourths cream cheese must contain not less than $24 \%$ butter fat.

One-half cream cheese must contain not less than $16 \%$ butter fat.

One-fourth cream cheese must contain not less than $8 \%$ butter fat.

All cheese having less than $8 \%$ fat must be branded "Skimmed Cheese."

The term "filled cheese" is commonly applied to a product in which a foreign fat, as oleo oil or lard, has been used. Filled cheese is more commonly found in localities where a carefully enforced fat-standard law prevails, but, in the absence of a standard for fat in cheese, the manufacturer can cheapen his product much more readily and conveniently by selling a skim-milk cheese in place of the whole-milk article, though not without producing a sensibly inferior product.

\section{METHODS OF ANALYSIS.}

Obtaining a Representative Sample.-Method of the A. O. A.C.†-By means of a cheese-trier remove, if possible, three cylindrical plugs from the cheese perpendicular to the surface and in length equal to about half the thickness of the cheese, one at the centre, one near the circumference, and one midway between the two. About one inch in length is cut off from each plug from the end having the rind, and this is discarded. The remaining portions of the plugs are then finely divided and mixed as intimately as possible.

In place of the plugs a narrow, wedge-shaped segment may be cut from the cheese, reaching from the circumference to the center, the portions near the rind being removed, and the remainder of the piece being finely divided and mixed as before. Analyses should immediately be begun after obtaining the sample.

Determination of Water.-Two or three grams of the sample are carefully weighed in a tared platinum dish, and dried to constant weight in an oven at $100^{\circ} \mathrm{C}$. The loss of weight is reckoned as water. $\ddagger$

* Penn. Laws, 1901, Act. 95, p. 128.

† U. S. Dept. of Agric., Bur. of Chem., Bul. 46, p. 55 .

$\mp$ Previously ignited sand or asbestos is recommended by some as an absorbent to be placed in the dish, but the writer gets better results in most cases directly as above. 
Determination of Ash.-Ignite the residue from the moisture determination at a low, red heat, cool in a desiccator, and weigh.

Determination of Fat.-Lythgoe's Modified Babcock Method.-Weigh accurately about 6 grams of the sample in a tared beaker. Add ro cc. of boiling water, and stir with a rod till the cheese softens and an even emulsion is formed, preferably adding a few drops of strong ammonia to aid in the softening and emulsionizing, and keeping the beaker in hot water till the emulsion is tolerably complete and free from lumps.

If the sample is a full-cream chee:e, which is usually evident from its taste and appearance, a Babcock cream-bottle is employed. The contents of the beaker, after cooling, are transferred to the test-bottle as follows: Add to the beaker about half of the $17.6 \mathrm{cc}$. of sulphuric acid regularly used for the test, stir with the rod and pour carefully into the bottle, using the remainder of the acid in two portions for washing out the beaker. Finally proceed as in the regular Babcock test for milk. Multiply the fat reading by 18 and divide by the weight of the sample taken to obtain the per cent of fat.

Short's Method:*-Grind to a uniform powder 2 to 5 grams of the sample, and about twice its weight of anhydrous copper sulphate. Place a layer of anhydrous copper sulphate about $2 \mathrm{~cm}$. thick on the bottom of the inner tube of a Johnson or Knorr extractor, add the ground mixture, and rinse the mortar first with a little anhydrous copper sulphate and finally with ether. Extract for I 6 hours, evaporate the ether from the extraction-flask, and dry the fat in a boiling-water oven to constant weight.

Werner-Schmidt Method.-Boil 2 to 3 grams of the sample in the Werner-Schmidt tube (p. 139) with $5 \mathrm{cc}$. of water and Io cc. of concentrated hydrochloric acid till, with constant shaking, all but the fat is dissolved. Cool, add $25 \mathrm{cc}$. of ether, and shake the tube well. Draw off as much as possible of the ether, after separation, in the usual manner, and extract with four or five additional portions of the solvent.

Distil off the ether from the combined extractions, and weigh the fat.

Determination of Protein.-From I to 2 grams of the cheese are treated by the Gunning or Kjeldahl method, adding after partial digestion a piece of copper sulphate the size of a pea to aid in the conversion. $\dagger \mathrm{N} \times 6.25=$ protein.

* U. S. Dept. of Agric., Div. of Chem., Bu. 35, pp. 15, 17, 225.

$\dagger$ Van Slyke, N. Y. Exp. Station, Bulletin $2 x_{5}$. 
Separation and Determination of Nitrogen Compounds.-Methods of Van Slyke,*-Twenty-five grams of the sample are mixed in a porcelain mortar with an equal volume of clear quartz sand. Transfer the mixture to a $45^{\circ}-\mathrm{cc}$. Erlenmeyer flask, add about roo cc. of water at $50^{\circ} \mathrm{C}$., and keep the temperature at $50^{\circ}$ to $55^{\circ} \mathrm{C}$. for half an hour with frequent shaking. Decant the liquid through an absorbent-cotton filter into a 500 -cc. graduated flask. Treat the residue with repeated portions of Ioo cc. each of water, heating, shaking, and decanting as above till the filtrate, or water extract, at room temperature amounts to just $500 \mathrm{cc}$. exclusive of the fat floating on top, and use aliquot parts of this water extract for the various determinations.

Water-soluble Nitrogen.-Determine the nitrogen by the Gunning method in $50 \mathrm{cc}$. of the above water extract, corresponding to 2.5 grams of cheese.

Nitrogen as Paranuclein.-Add $5 \mathrm{cc}$. of a $\mathrm{r} \%$ solution of hydrochloric acid to $100 \mathrm{cc}$. of the above water extract (corresponding to 5 grams of cheese), and keep the temperature at $50^{\circ}$ to $55^{\circ}$ till the separation is complete, as shown by a clear supernatant liquid. Filter, wash the precipitate with water, and determine the nitrogen therein by the Gunning method.

Nitrogen as Coagulable Protein.-Neutralize the filtrate from the preceding determination with dilute potassium hydroxide, and heat at the temperature of boiling water till the coagulum, $\dagger$ if any, settles completely. Filter, wash the precipitate, and determine the nitrogen therein.

Nitrogen as Caseoses. - Treat the filtrate from the preceding with I cc. of $50 \%$ sulphuric acid saturated with C. P. zinc sulphate, and warm to about $70^{\circ} \mathrm{C}$. till the caseoses settle out completely. Cool, filter, wash with a saturated solution of zinc sulphate acidified with sulphuric acid. and determine the nitrogen in the precipitate.

Nitrogen as Amides and Peptones.-Place roo cc. of the water extract of cheese in a $250-\mathrm{cc}$. graduated flask, add I gram of sodium chloride and a solution containing $12 \%$ of tannin, till the addition of a drop to the clear supernatant liquid does not further precipitate. Dilute to the 250-cc. mark, shake, pour upon a dry filter, and determine the nitrogen in $50 \mathrm{cc}$. of the filtrate, which gives the amount of nitrogen in the amido-acid and ammonia compounds. Deduct from this the amount of

* Van Slyke, N. Y. Exp. Station, Bulletin 2 r5.

$\dagger$ According to Van Slyke a precipitate at this point is rare in cheese. 
nitrogen as ammonia separately determined, and the difference is the amido-nitrogen.

Nitrogen as peptones is obtained by subtracting the sum of the amounts of nitrogen as paranuclein, coagulable proteins, caseoses, amido-bodies, and ammonia from the total nitrogen in the water extract.

Nitrogen as Ammonia.-Distil roo cc. of the filtrate from the above tannin-salt precipitation into standardized acid, and titrate in the usual manner.

Nitrogen as Paracasein Lactate.-Treat the residue insoluble in water in obtaining the water extract, with several portions of a $5 \%$ solution of sodium chloride, to form a 500-cc. salt extract of the same, in an analogous manner to that employed in preparing the water extract. Determine the nitrogen in an aliquot part of this salt extract.

Determination of Lactic Acid.*-Add water to to grams of the cheese sample at $40^{\circ} \mathrm{C}$. till the volume equals $105 \mathrm{cc}$. Shake and filter. Titrate $25 \mathrm{cc}$. of the filtrate (equivalent to 2.5 grams of cheese) with tenth-normal sodium hydroxide, using phenolphthalein as an indicator.

Each cubic centimeter of decinormal alkali is equivalent to 0.009 gram lactic acid.

Determination of Milk Sugar.-Boil 25 grams of finely divided cheese with two successive portions of about $100 \mathrm{cc}$. each of water, decant through a filter, and finally transfer the residue upon the filter and wash with hot water. Make up the entire aqueous extract thus obtained, when cold, to $250 \mathrm{cc}$, and determine the milk sugar by either Fehling method.

Detection of Foreign Fat.-The cheese fat, separated in the manner described below, is subjected to the various processes detailed under butter, in precisely the same way, the fat of cheese being identical with that of butter. The most ready means for judging its purity consists in determining the refraction with the butyro-refractometer, and the Reichert number.

Separation of the Fat for Examination.-Place a quantity, say 25 grams, of the finely divided sample in a large Erlenmeyer flask, add about $100 \mathrm{cc}$. of petroleum ether, cork the flask and allow it to stand for several hours with frequent shaking. Decant the petroleum ether through a filter, evaporate off the solvent by the aid of heat, and the residue will be found to consist of nearly pure fat.

Or, wrap a sufficient portion of the finely divided sample in a muslin

* U. S. Dept. of Agric., Bureau of Chem., Bul. $4^{6}$, p. 56. 
cloth, place this in a dish, and heat on the water-bath. The fat which runs out is afterward filtered and dried at $100^{\circ}$.

Sufficient cheese fat may usually be obtained for the refractometer reading from the neck of, the test-bottle, after completing the Babcock test, and, usually (except in the case of skimmed-milk cheese), for the Reichert number.

Detection of Skimmed-milk Cheese.-In a cream cheese the fat should greatly exceed the protein; in a whole-milk cheese the per cent of fat should at least equal that of the protein, and is generally in excess. If the fat is considerably less than the protein, the cheese has undoubtedly been made from skimmed milk. The following analyses, made in the writer's laboratory, illustrate these grades:

\begin{tabular}{|c|c|c|c|c|}
\hline Varieties of Cheese & Water. & Fat. & Protein * & Ash. \\
\hline $\begin{array}{l}\text { Full cream (soft) } \ldots \ldots \ldots \ldots \ldots \ldots \\
\text { Whole milk (hard) } \ldots \ldots \ldots \ldots \ldots \ldots \\
\text { Whole milk (soft) } \ldots \ldots \ldots \ldots \ldots \ldots \\
\text { Skimmed milk (soft) } \ldots \ldots \ldots \ldots \ldots \\
\text { Centrifugally skimmed milk (soft).. }\end{array}$ & $\begin{array}{l}37.63 \\
21.89 \\
55.95 \\
62.17 \\
72.80\end{array}$ & $\begin{array}{r}47.40 \\
38.00 \\
24.00 \\
15.20 \\
2.00\end{array}$ & $\begin{array}{l}13 \cdot 70 \\
37 \cdot 71 \\
16 \cdot 49 \\
21 \cdot 36 \\
23 \cdot 52\end{array}$ & $\begin{array}{l}1.27 \\
2.40 \\
3.56 \\
1.27 \\
\text { I. } 68\end{array}$ \\
\hline
\end{tabular}

* By difference.

\section{REFERENCES ON MILK AND ITS PRODUCTS.*}

Arkman. Milk, its Nature and Composition. London, 1899.

Barer, E., und Neunann, P. Ueber den Nachweis und die Beurteilung von Zuckerkalkzusatz zu Milch und Rahm. Zeits, Unters. Nahr. Genussm., 16, 1908, p. 51. Conn, H. W. Milk Fermentations. U. S. Dept. of Agric., Off. of Exp. Station, Bul. 9 .

Dairy Bacteriology. U. S. Dept. of Agric., Off. of Exp. Station, Bul. 25.

Conn, H. W., and Esten. The Ripening of Cream. Storrs Exp. Station, Annual Report, 1900.

Doane, C. F., and Lawson, H. W. Varieties of Cheese. U. S. Dept. of Agric., Bur. of An. Ind., Bul. I46, I9rז.

Euls and Kenrick. Milk and Milk Adulteration. Canada. Int. Rev. Dept., Buls. 21,28 .

Farrington, E. H., and Woin, F. W. Testing Milk and its Products. Madison, Igr2. Fleischarann, W. Lehrbuch der Milchwirthschaft. Bremen, 1893.

FreAr, W. American Milk and Milk Standards. Assn. State and Nat. Dairy and Food Depts., Proc., 19o6, p. 172.

Frerichs, K. Ueber den Nachweis von Zuckerkalk in Milch und Rahm. Zeits. Unters, Nahr. Genussm., 16, 1908, p. 682.

Gerber, N. Die Praktische Milch-Pruefung.

\footnotetext{
* For references en Butter, see p. 562 .
} 
Grotenfelt, G. The Principles of Modern Dairy Practice. New York.

HERz, F. J. Untersuchung der Kuhmilch. Berlin, I88g.

Howard, C. D. The Analysis of Ice Cream. Jour. Am. Chem. Soc., 29, 1907, p. 1622.

Husson, C. Le Lait. Paris, 1878 .

KIRCHNER, W. Handbuch der Milchwirthschaft. Berlin, $189 \mathrm{r}$.

LADD, E. F. Proteids of Cream. Jour. Am. Chem. Soc., 20, 1898, p. 858.

LeACH, A. E., and LythgoE, H. C. The Detection of Watered Milk. Jour. Am. Chem. Soc., 1904, 26, p. 1195.

Le Clere, J. A. Dairy Products. U. S. Dept. of Agric., Bureau of Chemistry, Bul. 65 , p. 35 .

Leffman, H., and Beam, W. Analysis of Milk and Milk Products. Philadelphia, 1893 -

Lehmann, J., und Hempet, W. Die Milchuntersuchungen. Bonn, 1894.

Macfartane, T. Milk and Milk Adulteration. Canada Inl. Rev. Dept., Buls. I, 2,9, II, $17,32,43,6 \mathrm{r}, 74,80$.

C Cheese. Canada Inl. Rev. Dept., Bul, 6.

- Butter. Canada Inl. Rev. Dept., Bul. I6.

Matthes, H., u. Muller, F. Ueber die Untersuchung des Milchserums mit dem Zeiss'schen Eintauchrefraktometer. Zeits. für öffentl. Chem., I9०3, p. 173.

McGr.t, A. Condensed Milk. Canada Inl. Rev. Dept., Buls. 54, 69.

Otro, A. Die Milch und ihre Produkte. Berlin, 1892.

Pearmain, T. H., and Moor, C. G. The Analysis of Food and Drugs. Part I. Milk and Milk Proteids. London, 1897 .

Pearson, R. A. National and State Dairy Laws. U. S. Dept. of Agric., Bureau of An. Ind. Bul. 26, 1900.

Richmond, H. D. Dairy Chemistry. London, I889.

Russel., H. L. Dairy Bacteriology. Madison, 1899 .

SCherer, R., trans. by SAlter, C. Casein: Its Preparation and Technical Utilization. London, 1906.

Schol.. Die Milch.

SChrodt, M. Anleitung zur Prüfung der Milch u. s. w. Bremen, I892.

Sherman, H. C. Seasonal Variations in the Composition of Cow's Milk. Jour. Am. Chem. Soc, 28, 1906, p. I719.

- On the Composition of Cow's Milk. Jour. Am. Chem. Soc., 25, I903, p. I32.

SNYDER, H. The Chemistry of Dairying. Easton, 1897 .

Stutzer, A. Die chem. Untersuchung der Käse. Zeits. f. anal. Chem., I886, p. 493 -

Swithinbank, H. Bacter ology of Milk. Ig०3.

Tourснот, A. L. Milk and Milk Adulteration. Canada Int. Rev. Bul. 53.

Van Freudenreich, E. Die Bakteriologie in der Milchwirthschaft. Basel, r893.

VAN SLyKE. Modern Mcthods of Testing Milk and Milk Products. New York, 1907.

Woo-man, A. G. On the Determination of Added Water in Milk. Jour. Am. Chem. Soc., 21, 1899, p. 503 .

WaNkLYN, J. A. Milk Analysis. London.

Wergmann, H. Die Methoden der Milchcons rvirung. Bremen, I893.

Whitaker, G. M. The Milk Supply of Boston and other New England Cities.

U. S.

Dept. of Agric., Bur. of An. Ind. Bul. 26, 1900. 
Alabama Exp. Sta. Bul. 97. Dairy and Milk Inspection.

Annual Reports of Inspector of Milk and Vinegar, Boston, Mass.

" " " " " " Cambridge, Mass.

Arkansas Exp. Sta. Bul. 45. Milk, its Decomposition and Preservation.

Dairy Products. U. S. Dept. of Agric., Div. of Chem., Bulletin 13, part I, 1887 .

Die Milchzeitung. Bremen, 1872 et seq.

Farmers' Bulletin, No. 2. Bacteria in Milk.

$\begin{array}{llrl}" & \text { " } & \text { 20. } & \text { Milk Fermentation and its Relations to Dairying. } \\ " & 4 & 29 . & \text { Souring of Milk. } \\ " & 4 & 42 . & \text { Facts about Milk. } \\ " & 4 & 74 . & \text { Milk as Food. } \\ \text { " } & \text { " } & 166 . & \text { Cheese-making on the Farm. }\end{array}$

Kansas Exp. Sta. Bul. 88, Keeping Milk in Summer.

Maine Exp. Sta. Bul, 23 (New Series). Cream Preservation.

Massachusetts State Board of Health Reports, $188_{3}$ et seq.

Michigan Exp. Sta, Bul. 140. Ropiness in Milk.

Minnesota Exp. Sta. Bul. 74. Milk and Cheese, Digestibility and Food Value.

New York (Geneva) Exp. Sta. Bul. 70. Reasons for Changing Milk Standards.

" " " " "215. Estimation of Proteolytic Compounds in Cheese and Milk.

" " (Ithaca) " " " 165. Ropiness in Milk.

" " " " " " 195. " " "

North Carolina Exp. Sta, Bul. Ir3. Testing of Milk.

Oklahoma Exp. Sta. Bul. 2I. A New Milk Test.

West Virginia Exp. Sta. Bul. 58. Effect of Pressure in the Preservation of Milk.

Wisconsin Exp. Sta. Bul. 48. Conn. Culture B. 4r, in Butter-making.

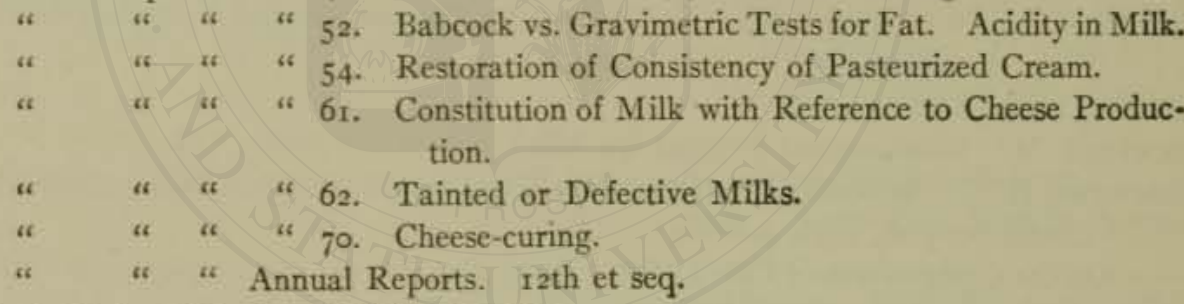

Zeitschrift der Fleisch und Milch Hygiene. 


\section{CHAPTER VIII.}

\section{FLESH FOODS.}

MEAT.

General Structure and Composition.-Meat is structurally made up of muscle fibers, held together by connective tissue, through which fat cells are usually more or less abundantly distributed. Each muscle fiber has a sheath or covering known as sarcolemma, formed of an albuminoid substance similar to elastin, and within the fibers are contained the meat juices, which are solutions in water of proteins, non-protein-nitrogenous extractives, and salts. The substance of the connective tissue is made up largely of the albuminoids elastin (insoluble) and collagen, the latter being convertible by boiling with water or treatment with acids into gelatin. The proteins of the meat juices consist chiefly of the globulin myosin (by far the most abundant), muscle albumin, and the muscle pigment hamoglobin, or a substance closely analogous thereto.

In the living muscle there are no peptones, but the ferment pepsin is present. After death, by the action of the pepsin in presence of lactic acid, a portion of the normal proteins of the muscle undergoes, as it were, digestion, so that in meat both peptones and proteoses* are found.

The non-protein-nitrogenous extractives are mainly creatin, xanthin, hypoxanthin, and carnin, which, from their basic character, are known as flesh bases.

The approximate proportions in which the chief constituents are present in meat is thus shown by König:

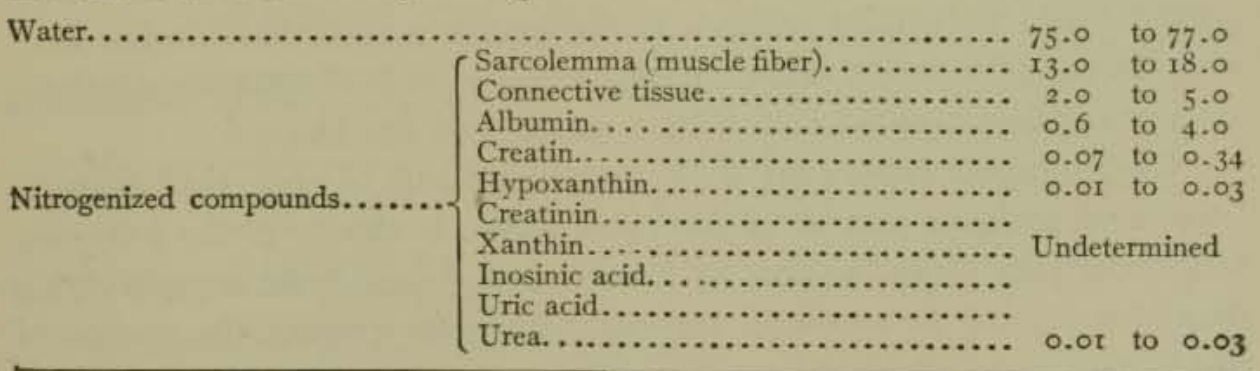

* A proteose or albumose known as myoalbumose normally exists in the live muscle. 


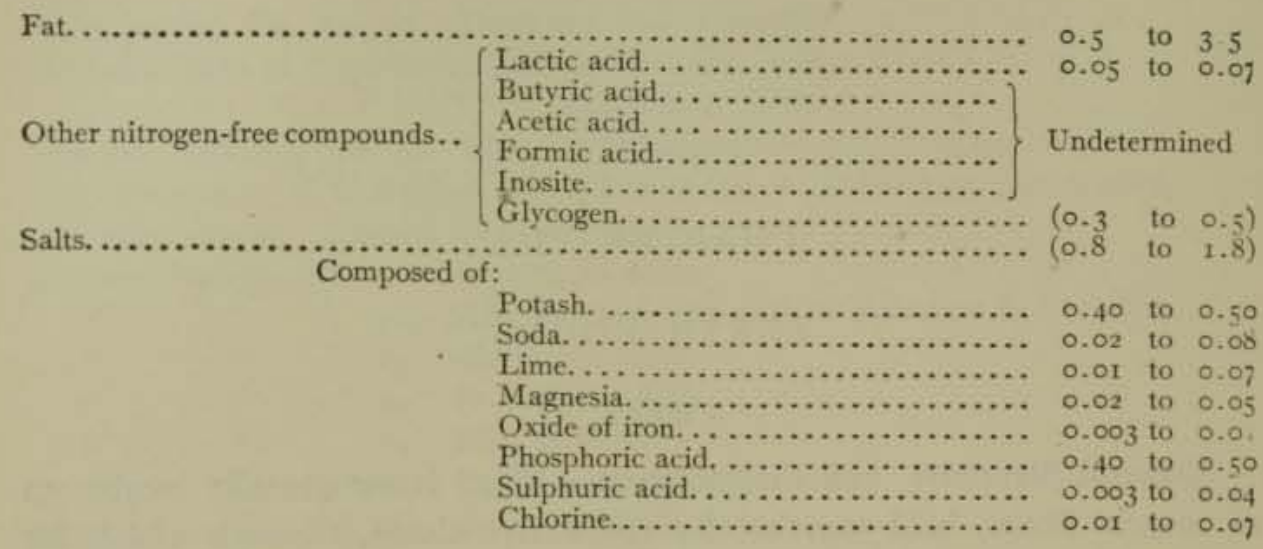

Nitrogen compounds constitute by far the most abundant and important portion of the substance of lean meat. Carbohydrates are almost entirely lacking, the small amount of glycogen and muscle sugar togethe1 constituting rarely more than I per cent.

Glycogen $\left(\mathrm{C}_{6} \mathrm{H}_{10} \mathrm{O}_{5}\right)$, sometimes called animal starch, is a white, amorphous, tasteless, and odorless substance, when pure, much resembling starch. It is soluble in water, forming an opalescent solution, and is insoluble in ether and alcohol. With iodine a port-wine color is produced, which disappears on heating and reappears on cooling. Glycogen is strongly dextro-rotary. It is converted to dextrose by boiling with dilute mineral acid.

Muscle Sugar is either entirely absent in the living muscle, or exists in traces only. After death it is formed presumably from the glycogen, and exists in a very minute quantity, probably as dextrose.

Inosite $\left(\mathrm{C}_{6} \mathrm{H}_{12} \mathrm{O}_{6}+\mathrm{H}_{2} \mathrm{O}\right)$ is found in traces in the muscular substance of the heart, liver, kidneys, and testicles.

Proximate Constituents of the Commoner Meats.-The chief characteristics of the flesh of various animals are in the main very similar, whatever the nature of the animal. So true is this, indeed, that it is extremely difficult from a chemical analysis to distinguish a particular kind of flesh when mixed with that of other animals in finely divided meat preparations, such as sausages, potted and deviled meats, and the like.

The average composition of the commoner cuts of beef, veal, muttor, lamb, and pork, as well as of fowl and game, is shown in the followirg tables, compiled from the work of Atwater and Bryant,* the accompanying diagrams serving to locate, in the case of ordinary meats, the portion of the animal from which the meat is taken.

* U. S. Dept, of Agric,, Off, of Exp. Stations, Bul. 28 (Revised Ed.). 


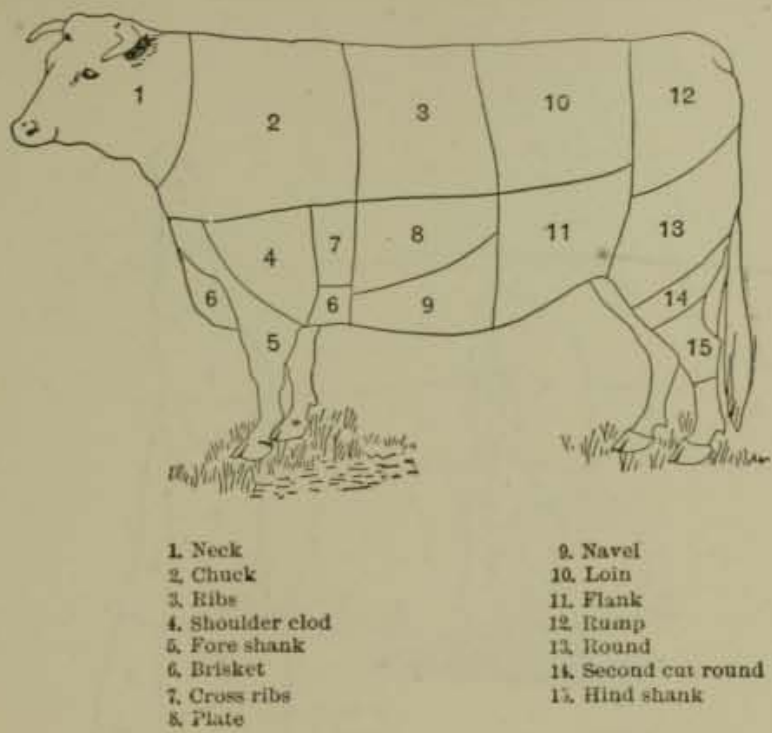

Frg. 56.-Diagram Showing Cuts of Beef.

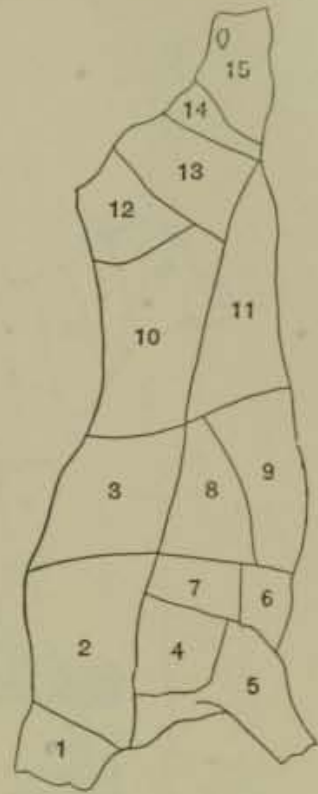

COMPOSITION OF BEEF.

\begin{tabular}{|c|c|c|c|c|c|c|c|c|c|c|}
\hline & & & & & & Pro & tein. & & & \\
\hline & $\mathrm{Cu}$ & it. & $\begin{array}{l}\text { ber of } \\
\text { Anal- }\end{array}$ & Refuse. & Water. & & & Fat. & Ash. & $\begin{array}{l}\text { per } \\
\text { Pound. }\end{array}$ \\
\hline Chuck: & Lean- & $\begin{array}{l}\text { edible portion. . } \\
\text { as purchased. .. }\end{array}$ & 2 & & $71 \cdot 3$ & 20.2 & $19 \cdot 5$ & $\begin{array}{l}8.2 \\
6.6\end{array}$ & $\begin{array}{l}1.0 \\
0.8\end{array}$ & 720 \\
\hline & Medium- & -edible portion. & $\begin{array}{l}2 \\
4\end{array}$ & $\begin{array}{c}\mathrm{I} 9 \cdot 5 \\
\ldots \ldots\end{array}$ & $\begin{array}{l}57 \cdot 4 \\
68 \cdot 3\end{array}$ & $\begin{array}{l}16.3 \\
19.6\end{array}$ & $\begin{array}{l}15.7 \\
18.9\end{array}$ & $\begin{array}{r}0.0 \\
11.9\end{array}$ & $\begin{array}{l}0.8 \\
0.0\end{array}$ & $\begin{array}{l}580 \\
865\end{array}$ \\
\hline & & as purchased... & 4 & 15.2 & $57-9$ & 16.6 & $\begin{array}{l}10.9 \\
16.0\end{array}$ & $10 . I$ & 0.8 & $\begin{array}{l}865 \\
735\end{array}$ \\
\hline & Fat- & edible portion. . & 4 & $\ldots \ldots$ & $62 \cdot 3$ & I8. 5 & 18.0 & I8.8 & 0.9 & $\begin{array}{r}135 \\
1135\end{array}$ \\
\hline & Jean- & & 3 & I $4 \cdot 7$ & $53 \cdot 3$ & 15.9 & 15.4 & 15.9 & 0.7 & 965 \\
\hline Ribs: & Lean- & edible porti & 6 & $\cdots \cdots$ & 66.0 & 16.5 & 16.9 & 9.8 & 0.8 & 790 \\
\hline & Medium- & $\begin{array}{l}\text { as purchased... } \\
\text {-edible portion. }\end{array}$ & 0 & 22.6 & 52.6 & 15.2 & 14.8 & $9 \cdot 3$ & 0.7 & 675 \\
\hline & 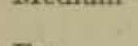 & $\begin{array}{l}\text { portuon... } \\
\text { rchased... }\end{array}$ & $\begin{array}{l}15 \\
15\end{array}$ & 20.8 & $\begin{array}{l}55 \cdot 5 \\
43.8\end{array}$ & $17 \cdot 5$ & 17.0 & 26.6 & 0.9 & $145^{\circ}$ \\
\hline & Fat- & edible portion.. & $\begin{array}{r}15 \\
9\end{array}$ & $\begin{array}{l}20.0 \\
\ldots \ldots .\end{array}$ & 48.5 & $\begin{array}{l}13.9 \\
15.0\end{array}$ & I 3.5 & 21.2 & 0.7 & 1155 \\
\hline & & as purchased... & 8 & 16.8 & 39.6 & $\begin{array}{l}15.0 \\
12.7\end{array}$ & $\begin{array}{l}15.2 \\
12.4\end{array}$ & $\begin{array}{l}35.6 \\
30.6\end{array}$ & $\begin{array}{l}0.7 \\
0.6\end{array}$ & $\begin{array}{l}1780 \\
1525\end{array}$ \\
\hline Loin: & Lean- & cdible portion.. & 12 & $\cdots \cdots$ & 67.0 & 19.7 & $19 \cdot 3$ & $12 \cdot 7$ & 1.0 & 900 \\
\hline & Medium- & as purch & II & 13.1 & $5^{8.2}$ & $17 \cdot x$ & 16.7 & II. I & 0.9 & 785 \\
\hline & & $\begin{array}{l}\text { edible portson } \\
\text { as purchased. }\end{array}$ & $\begin{array}{l}32 \\
32\end{array}$ & 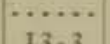 & 60.6 & 18.5 & $\begin{array}{l}18.2 \\
15.8\end{array}$ & 20.2 & 1.0 & $1+90$ \\
\hline & Fat- & edible portion.. & 6 & $\begin{array}{l}13 \cdot 3 \\
\cdots \cdots\end{array}$ & & 17.5 & $\begin{array}{l}15.8 \\
16.8\end{array}$ & $\begin{array}{l}17.5 \\
27.6\end{array}$ & $\begin{array}{l}0.9 \\
0.9\end{array}$ & $\begin{array}{l}1040 \\
1400\end{array}$ \\
\hline Rump: & Jean- & as purchase & 6 & 10.2 & $49 \cdot 2$ & 15.7 & 15.0 & 24.8 & 0.8 & $\begin{array}{l}1490 \\
1305\end{array}$ \\
\hline ixmp: & Lean- & $\begin{array}{l}\text { edible portion.. } \\
\text { as purchased. }\end{array}$ & 4 & $\cdots \ldots$ & $65 \cdot 7$ & 20.9 & I9. 6 & I 3.7 & I.O & 965 \\
\hline & Medium- & $\begin{array}{l}\text { as purchased... } \\
\text {-edible portion... }\end{array}$ & $\begin{array}{r}3 \\
10\end{array}$ & 14.0 & 56.6 & 19.1 & $17 \cdot 5$ & 11.0 & 0.9 & 820 \\
\hline & & as purchased... & $\begin{array}{l}10 \\
10\end{array}$ & $\cdots \cdots$ & 56.7 & 17.4 & 16.9 & $25 \cdot 5$ & 0.9 & $I_{4} 00$ \\
\hline & Fat- & edible portion.. & 5 & $\begin{array}{l}20.7 \\
\cdots \cdots\end{array}$ & $\begin{array}{l}45.0 \\
47.1\end{array}$ & $\begin{array}{l}13.8 \\
16.8\end{array}$ & $\begin{array}{l}13.4 \\
16.4\end{array}$ & $\begin{array}{l}20.2 \\
35.7\end{array}$ & $\begin{array}{l}0.7 \\
0.8\end{array}$ & $\begin{array}{l}1110 \\
1820\end{array}$ \\
\hline Round: & Jean & as purchased... & 5 & 23.0 & $3^{6.2}$ & 12.9 & 12.6 & 27.6 & 0.6 & 1405 \\
\hline Noma: & (t) & $\begin{array}{l}\text { edible porti } \\
\text { as purchase }\end{array}$ & $3 \mathrm{I}$ & $\ldots \ldots$ & 70.0 & 21.3 & 21.0 & $7 \cdot 9$ & $1-1$ & 730 \\
\hline & Medium- & $\begin{array}{l}\text { as purchased.. } \\
\text {-edible portion. }\end{array}$ & $\begin{array}{l}29 \\
18\end{array}$ & 8.1 & $64 \cdot 4$ & $19-5$ & 19.2 & $7 \cdot 3$ & 1.0 & 670 \\
\hline & & as purchased. & I 4 & $\cdots \cdots$ & $65 \cdot 5$ & 20.3 & 19.8 & ${ }^{1} 3.6$ & 1.1 & 950 \\
\hline & Fat- & edible & 5 & $\begin{array}{c}7.2 \\
\cdots \cdots\end{array}$ & 60.4 & $\begin{array}{l}19.0 \\
10.5\end{array}$ & $\begin{array}{l}10.3 \\
19.1\end{array}$ & $\begin{array}{l}12.8 \\
19.5\end{array}$ & $\begin{array}{l}1.0 \\
1.0\end{array}$ & $\begin{array}{l}895 \\
1185\end{array}$ \\
\hline & & as purchased. & 3 & 12,0 & 54.0 & $17 \cdot 5$ & $17 \cdot 1$ & 16.1 & 0.8 & 1005 \\
\hline
\end{tabular}



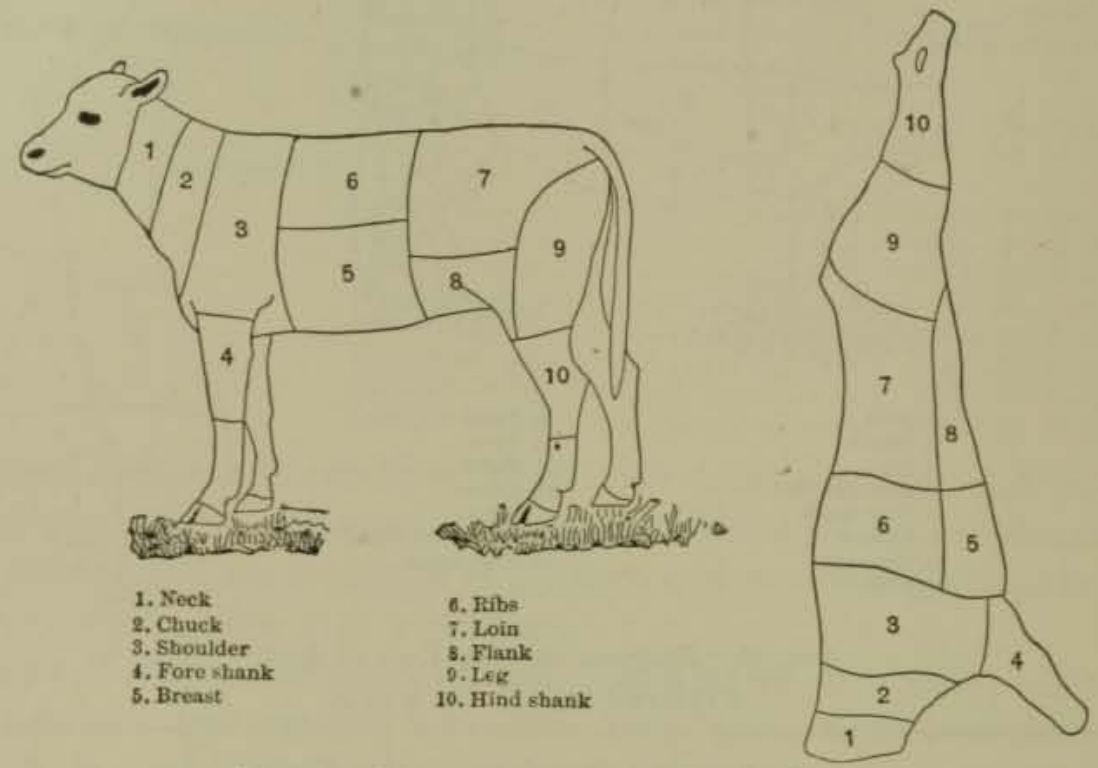

FIG. 57,-Diagram Showing Cuts of Veal.

COMPOSITION OF VEAL.

\begin{tabular}{|c|c|c|c|c|c|c|c|c|c|}
\hline & \multirow[b]{2}{*}{ Cut. } & \multirow{2}{*}{$\begin{array}{c}\text { Num- } \\
\text { ber of } \\
\text { Anal- } \\
\text { yses, }\end{array}$} & \multirow{2}{*}{ Refuse. } & \multirow[b]{2}{*}{ Water. } & \multicolumn{2}{|c|}{ Protein, } & \multirow[b]{2}{*}{ Fat. } & \multirow[b]{2}{*}{ Ash. } & \multirow{2}{*}{$\begin{array}{l}\text { Fuel } \\
\text { Value } \\
\text { per } \\
\text { Pound } \\
\text { Cals. }\end{array}$} \\
\hline & & & & & $\begin{array}{c}\mathrm{N} \times \\
6.25\end{array}$ & $\begin{array}{c}\text { By } \\
\text { Differ- } \\
\text { ence. }\end{array}$ & & & \\
\hline \multirow[t]{4}{*}{ Chuck: } & Lean- edible portion.. & I & ...... & 76.3 & $\ldots \ldots$ & 20.6 & I. 9 & 1.2 & 465 \\
\hline & as purchased... & I & 19.0 & $6 \mathrm{r} .8$ & $\ldots \ldots$ & 16.7 & 1.6 & 0.9 & 380 \\
\hline & Medium-edible portion. & 6 & $\ldots \ldots$ & $73 \cdot 3$ & 19.7 & 19.2 & 6.5 & 1.0 & 640 \\
\hline & as purchased... & 6 & 18.9 & $59 \cdot 5$ & 16.0 & 15.6 & $5 \cdot 2$ & 0.8 & $5^{15}$ \\
\hline \multirow[t]{4}{*}{ Ribs: } & Medium-edible portion.. & 9 & $\ldots \ldots$ & $72 \cdot 7$ & 20.7 & 20.1 & 6.1 & I. 1 & 640 \\
\hline & as purchas & 9 & $25 \cdot 3$ & $54 \cdot 3$ & $15 \cdot 5$ & 15.0 & 4.6 & 0.8 & 480 \\
\hline & edible & 3 & ...... & 60.9 & 18.7 & 18.8 & 19.3 & I. 0 & 1160 \\
\hline & as purchased... & 3 & $24 \cdot 3$ & 46.2 & 14.2 & 14.2 & $14 \cdot 5$ & 0.8 & 875 \\
\hline \multirow[t]{5}{*}{ Loin: } & edible portion.. & 5 & ...... & $73 \cdot 3$ & 20.4 & 19.9 & 5.6 & 1.2 & 615 \\
\hline & as purchased... & 5 & 22.0 & $57 \cdot 1$ & 15.9 & 15.6 & $4-4$ & 0.9 & 480 \\
\hline & Medium-edible portion.. & 6 & $\cdots \ldots$ & 69.0 & 19.9 & 19.2 & 10.8 & I 0 & 825 \\
\hline & as purchased... & 6 & 16.5 & $57 \cdot 6$ & 16.6 & 16.0 & 9.0 & 0.9 & 690 \\
\hline & edible portion.. & 2 & $\cdots \cdots$ & 61.6 & 18.7 & 18.5 & 18.9 & 1.0 & 1145 \\
\hline \multirow{5}{*}{ Leg: } & as purchased... & 2 & $\mathrm{I} 8.3$ & 50.4 & $x_{5} \cdot 3$ & 15.1 & I5.4 & 0.8 & 935 \\
\hline & edible portion.. & 9 & $\ldots \ldots$ & $73 \cdot 5$ & $21 \cdot 3$ & 21.2 & $4 \cdot I$ & 1.2 & $57^{\circ}$ \\
\hline & Modium & 9 & 9.1 & 66.8 & 19.4 & 19.3 & 3.7 & I. I & $5^{20}$ \\
\hline & $\begin{array}{r}\text { Medium-edible portion.. } \\
\text { as purchased. }\end{array}$ & 10 & $\because \cdots$ & 70.0 & 20.2 & 19.8 & 9.0 & 1.2 & 755 \\
\hline & & $\mathbf{9}$ & & 00.1 & 15.5 & 10.9 & $7 \cdot 9$ & 0.9 & 020 \\
\hline
\end{tabular}



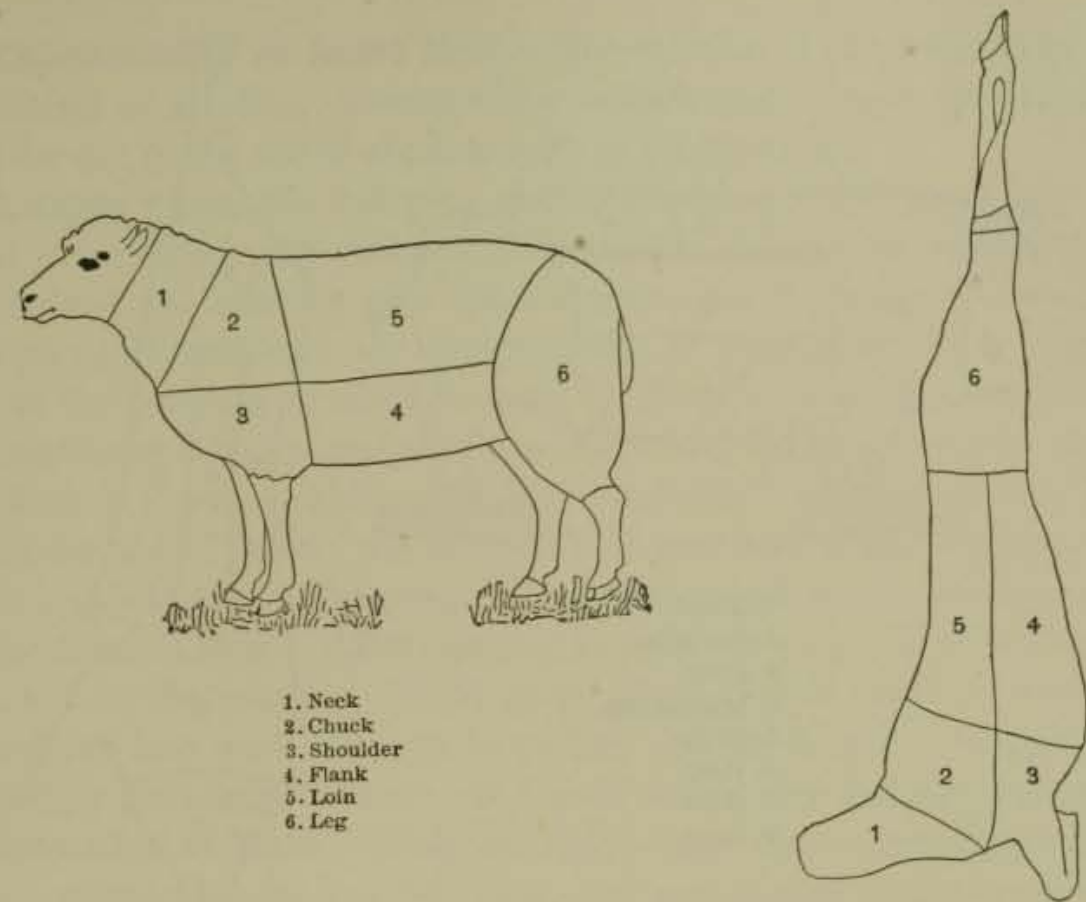

Fic. 58.-Diagram Showing Cuts of Mutton.

COMPOSITION OF MUTTON AND LAMB.

\begin{tabular}{|c|c|c|c|c|c|c|c|c|c|}
\hline \multirow{2}{*}{\multicolumn{2}{|c|}{ Cut. }} & \multirow{2}{*}{$\begin{array}{l}\text { Num- } \\
\text { ber of } \\
\text { Anal- } \\
\text { yses. }\end{array}$} & \multirow{2}{*}{ Refuse, } & \multirow[b]{2}{*}{ Water. } & \multicolumn{2}{|c|}{ Protein. } & \multirow[b]{2}{*}{ Fat. } & \multirow[b]{2}{*}{ Ash. } & \multirow{2}{*}{$\begin{array}{c}\text { Fuel } \\
\text { Value } \\
\text { per } \\
\text { Pound. } \\
\text { Cals. }\end{array}$} \\
\hline & & & & & & & & & \\
\hline & Mutron. & & & & & & & & \\
\hline \multirow[t]{6}{*}{ Chuck: } & Lean- edible portion.. & I & ...... & $64 \cdot 7$ & 17.8 & I8. I & 16.3 & 0.9 & 1020 \\
\hline & as purchased... & 1 & $19 \cdot 5$ & 52.1 & $14 \cdot 3$ & 14.5 & I3.I & 0.8 & 820 \\
\hline & Medium-edible portion.. & 6 & $\ldots \ldots$ & 50.9 & $15 . \mathrm{I}$ & 14.6 & 33.6 & 0.9 & 1700 \\
\hline & as purchase & 6 & $2 \mathrm{r} \cdot 3$ & 39.9 & 11.9 & II. 5 & 26.7 & 0.6 & $135^{\circ}$ \\
\hline & edible portion.. & 2 & $\ldots \ldots$ & 40.6 & 13.9 & 13.7 & $44 \cdot 9$ & 0.8 & 2155 \\
\hline & as purchased... & 2 & 16.5 & 33.8 & 11.6 & 11.5 & $37 \cdot 5$ & 0.7 & 1800 \\
\hline \multirow[t]{4}{*}{ Loin: } & Medium-edible portion.. & I3 & $\ldots \ldots$ & 50.2 & 16.0 & 15.9 & 33.1 & $0 . \dot{8}$ & I695 \\
\hline & as purchased... & 12 & 16.0 & 42.0 & I 3.5 & 13.0 & 28.3 & 0.7 & 1445 \\
\hline & edible portion.. & 3 & $\cdots \cdots$ & $43 \cdot 3$ & 14.7 & 14.2 & $41 \cdot 7$ & 0.8 & 2035 \\
\hline & as purchased... & 3 & 11.7 & $3^{8} \cdot 3$ & 13.0 & 12.5 & 36.8 & 0.7 & 1795 \\
\hline \multirow[t]{2}{*}{ Flank: } & Medium-edible portion.. & 8 & $\cdots \cdots$ & 46.2 & 15.2 & 14.8 & $3^{8} \cdot 3$ & 0.7 & I900 \\
\hline & as purchased... & 2 & 9.9 & 39.0 & $\mathrm{I}_{3} .8$ & I 3.6 & 36.9 & 0.6 & $18 \mathrm{I}_{5}$ \\
\hline \multirow[t]{4}{*}{ Leg: } & edible portion.. & 3 & $\cdots \cdots$ & $67 \cdot 4$ & 19.8 & 19.1 & 12.4 & 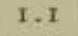 & 890 \\
\hline & as purchased... & 3 & I 6.8 & 56.1 & 16.5 & $1_{5} .9$ & 10.3 & 0.9 & 740 \\
\hline & Medium-edible portion.. & II & $\ldots \ldots$ & 62.8 & 18.5 & 18.2 & 18.0 & 1.0 & 1105 \\
\hline & $\begin{array}{l}\text { as purchased... } \\
\text { LAMB. }\end{array}$ & II & 18.4 & 51.2 & 15.1 & $14 \cdot 9$ & $14 \cdot 7$ & 0.8 & 900 \\
\hline \multirow[t]{2}{*}{ Chuck: } & edible portion.. & I & & $5^{6.2}$ & I9.I & 19.2 & 23.6 & $1 \cdot 0$ & 1350 \\
\hline & as purchased... & I & 19.1 & $45 \cdot 5$ & $15 \cdot 4$ & 15.5 & 19.1 & 0.8 & 1090 \\
\hline \multirow[t]{3}{*}{ Leg: } & Medium-edible portion.. & 2 & $\cdots \cdots$ & 63.9 & 19.2 & 18.5 & I6.5 & I. I & 1055 \\
\hline & as purchased. & 2 & $17 \cdot 4$ & $5^{2} \cdot 9$ & 15.9 & $15 \cdot 2$ & 13.6 & 0.9 & 870 \\
\hline & edible portion & I & $\ldots \ldots$ & 54.6 & I8. 3 & $17 \cdot I$ & $27 \cdot 4$ & 0.9 & 1495 \\
\hline \multirow{3}{*}{ Loin: } & & I & 13.4 & $47 \cdot 3$ & 15.8 & 14.8 & 23.7 & 0.8 & 1295 \\
\hline & edib & 4 & $\cdots \cdots$ & 53.1 & 18.7 & 17.6 & 28.3 & I. O & 1540 \\
\hline & as purchased. & 4 & 14.8 & $45 \cdot 3$ & 16.0 & 15.0 & $24 \cdot 1$ & 0.8 & $\mathrm{I}_{3} \mathrm{I}_{5}$ \\
\hline
\end{tabular}




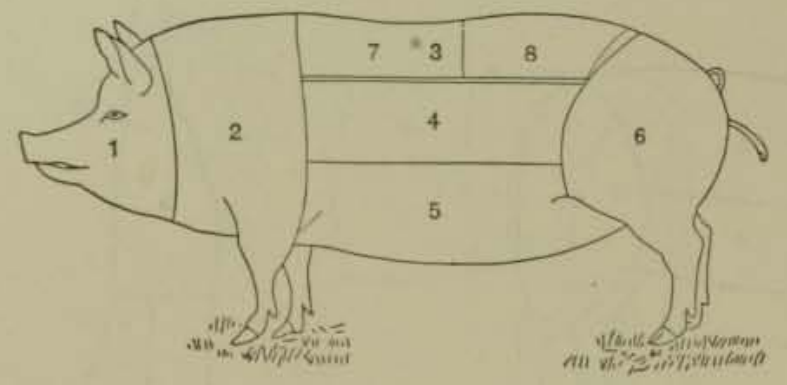
1. Head.
2 Shoulder.
3. Back.
4. Middle cut.
5. Belly.
6. Ham.
7. Ribs.
8. Loin.

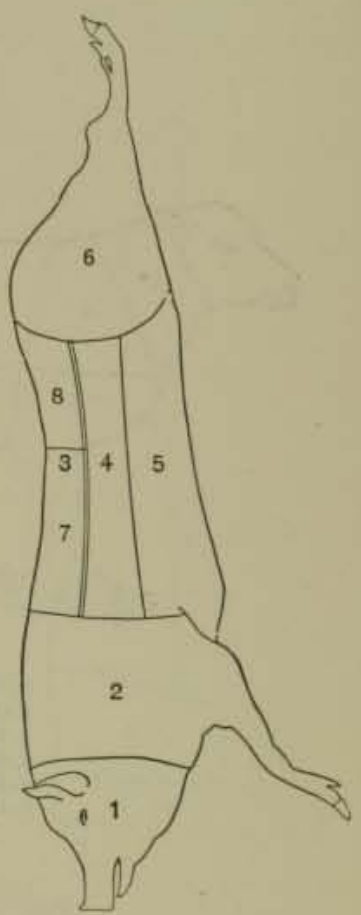

Fic. 59--Diagram Showing Cuts of Pork.

COMPOSITION OF PORK, POULTRY, AND GAME.

\begin{tabular}{|c|c|c|c|c|c|c|c|c|c|c|}
\hline \multirow[t]{2}{*}{ t } & \multirow{2}{*}{\multicolumn{2}{|c|}{ Cut. }} & \multirow{2}{*}{$\begin{array}{l}\text { Num- } \\
\text { ber of } \\
\text { Anal- } \\
\text { yses. }\end{array}$} & \multirow{2}{*}{ Refure. } & \multirow[b]{2}{*}{ Water. } & \multicolumn{2}{|c|}{ Protein. } & \multirow[b]{2}{*}{ Fat. } & \multirow[b]{2}{*}{ Ash. } & \multirow{2}{*}{$\begin{array}{l}\text { Fuel } \\
\text { Value } \\
\text { per } \\
\text { Pound } \\
\text { Cals. }\end{array}$} \\
\hline & & & & & & $\begin{array}{l}\mathrm{N} \times \\
6.25\end{array}$ & $\begin{array}{c}\text { By } \\
\text { Differ- } \\
\text { ence. }\end{array}$ & & & \\
\hline \multicolumn{2}{|c|}{ Shouider: } & Pork. & 19 & $\cdots \cdots$ & $5 \mathrm{x} \cdot 2$ & $13 \cdot 3$ & I 3.8 & $34 \cdot 2$ & 0.8 & 1690 \\
\hline \multirow[t]{4}{*}{ Loin: } & Lean- & $\begin{array}{l}\text { as purchased... } \\
\text { edible portion.. }\end{array}$ & $\begin{array}{r}19 \\
1\end{array}$ & $\begin{array}{l}12 \cdot 4 \\
\cdots \cdots\end{array}$ & $\begin{array}{l}44 \cdot 9 \\
60 \cdot 3\end{array}$ & $\begin{array}{l}12.0 \\
20.3\end{array}$ & $\begin{array}{l}12.2 \\
19.7\end{array}$ & $\begin{array}{l}29.8 \\
19.0\end{array}$ & $\begin{array}{l}0.7 \\
1.0\end{array}$ & $\begin{array}{l}14 \mathrm{SO} \\
1180\end{array}$ \\
\hline & & as purchased... & 1 & 23.5 & 46.1 & 15.5 & $\mathrm{I} 5 . \mathrm{I}$ & 14.5 & 0.8 & 900 \\
\hline & Fat- & edible portion.. & 4 & $\ldots \ldots$ & $4 \mathrm{~T} .8$ & 14.5 & $\mathrm{I} 3 \cdot \mathrm{I}$ & $44 \cdot 4$ & 0.7 & 2145 \\
\hline & & as purchased... & 4 & 16.5 & 34.8 & 11.9 & 10.9 & $37 \cdot 2$ & 0.6 & 1790 \\
\hline \multirow{3}{*}{ Ham: } & Lean- & $\begin{array}{l}\text { edible portion.. } \\
\text { as purchased... }\end{array}$ & 2 & $\cdots$ & $\begin{array}{l}60.0 \\
50.4\end{array}$ & $\begin{array}{l}25.0 \\
24.8\end{array}$ & $=4 \cdot 3$ & 14.4 & 1.3 & 1075 \\
\hline & Fat- & edible portion.. & 5 & $\begin{array}{c}0.9 \\
\ldots . .\end{array}$ & $\begin{array}{l}59.4 \\
38.7\end{array}$ & $\begin{array}{l}2.4 .0 \\
12.4\end{array}$ & 10.6 & 50.0 & 0.1 & 2345 \\
\hline & & as purchased... & 5 & 13.2 & 33.6 & 10.7 & 9.2 & $43 \cdot 5$ & 0.5 & 2035 \\
\hline \multirow{2}{*}{\multicolumn{2}{|c|}{ Chicken: }} & AND GAME. & & & & & & & & \\
\hline & & edible portion.. & 3 & $\cdots \cdots$ & 74.8 & $21 \cdot 5$ & 21.6 & 2.5 & I, 1 & 505 \\
\hline \multirow{2}{*}{\multicolumn{2}{|c|}{ Fowl: }} & edible portion.. & $\begin{array}{r}3 \\
26\end{array}$ & $\begin{array}{c}41.0 \\
\ldots \ldots\end{array}$ & $\begin{array}{l}43.7 \\
63.7\end{array}$ & 12.8 & $\begin{array}{l}12.0 \\
19.0\end{array}$ & $\begin{array}{r}1.4 \\
16.3\end{array}$ & $\begin{array}{l}0.7 \\
1.0\end{array}$ & $\begin{array}{r}295 \\
1045\end{array}$ \\
\hline & & as purchased... & 26 & 25.9 & $47 \cdot 1$ & 13.7 & 14.0 & 12.3 & 0.7 & 775 \\
\hline \multirow{2}{*}{\multicolumn{2}{|c|}{ Goose: }} & edible portion.. & I & $\ldots \ldots$ & 46.7 & 16.3 & 16.3 & 36.2 & 0.8 & $18_{3} \mathrm{O}$ \\
\hline & & as purchased... & I & 17.6 & $3^{8.5}$ & 13.4 & 13.4 & 29.8 & 0.7 & 1505 \\
\hline \multirow{2}{*}{\multicolumn{2}{|c|}{ Turkey: }} & edible portion.. & 3 & $\ldots \ldots$ & $55 \cdot 5$ & 21.1 & 20.6 & 22.9 & 1.0 & 1360 \\
\hline & & as purchased... & 3 & 22.7 & $42 \cdot 4$ & 16.1 & $15 \cdot 7$ & 18.4 & 0.8 & 1075 \\
\hline \multicolumn{2}{|l|}{ Quail: } & as purchased. & I & ....... & 60.9 & 21.8 & ...... & 8.0 & I. 7 & 775 \\
\hline
\end{tabular}


Characteristics of Sound Meat.-The reaction of meat should be acid. If neutral or alkaline, decomposition is indicated, except that alkalinity may be due to the use of alkaline salts as preservatives.

Letheby* gives the following characteristics of sound, fresh meat. In color it is neither pale pink nor deep'purple, the former indicating that the animal was affected with some disease, and the latter that it died a natural death, and was not slaughtered. In appearance it is marbled, due to the presence of small veins of fat distributed among the muscles. In consistency it is firm and elastic to the touch, and should hardly moisten the finger; a wet, sodden, or flabby consistency with a jelly-like fat is indicative of bad meat. As to odor, it is practically free; whatever odor there is should not be disagreeable; a sickly or cadaverous smell is indicative of diseased meat. After standing for a day or so, it should not become wet, but on the contrary should grow drier. When dried at $100^{\circ} \mathrm{C}$. it should not lose more than 70 to 74 per cent in weight; unsound meat frequently loses $80 \%$ or more. It should shrink very little in cooling.

Inspection of Meat.-While carefully drawn laws exist almost everywhere relating to the sale of meat, and government inspectors are appointed to carry out the requirements of the laws, yet in this country there is undoubtedly some meat unfit for food on the market, owing to the small number of inspectors, and the consequent comparative safety with which unscrupulous dealers may sell meats forbidden by law and escape detection. The inspection of meats and fish under municipal ordinances is not always carried out as thoroughly as might be desired.

Unwholesomeness of Meat may be due to a diseased condition of the animal while alive, or to poisonous or injurious toxins developed by the action of bacteria after death. In the first case, the diseased conditions may be due to temporary causes only, or to the presence of animal parasites, such as trichinæ in pork, or as the result of pathogenic bacteria, causing such serious diseases as tuberculosis, anthrax, glanders, etc. It thus requires much skill and judgment on the part of the meat-inspector to correctly pass upon the suitability for food of the various meats as they appear on the market. Coplin and Bevan $\dagger$ give in detail useful data regarding the inspection of meat, as well as of the animal before slaughtering, showing the requisite size, weight, age, conditions of health etc., that should obtain. The detailed physical and microscopical examination involved in such inspection is, however, rarely germane to the work of the public food analyst, and will not be treated of in this manual.

*Lectures on Food, p. 2 ro.

$\dagger$ Practical Hygiene, pp. 130-157. 
It is also beyond the scope of the present work to treat of the harmful toxins developed by bacterial action in meat and fish, causing what is known as ptomaine poisoning. The work of detecting and isolating such poisons comes within the province of the bacteriologist and biologist, rather than that of the chemist, involving many experiments upon guinea-pigs, rabbits, or other animals not usually found in the chemist's laboratory. It has furthermore been recently shown by Vaughn and Novy * that even when these toxins are present in foods in sufficient quantity to produce serious results, very considerable amounts of the food must be taken in order to isolate them by chemical means, more, in fact, than is usually available for analysis.

For the general inspection of meats for animal parasites, poisonous toxins, etc., the reader is referred to such works as those of Vaughn and Novy, Fischöder, Walley, Andrews, Cobbold, and Salmon as cited in the references on pages $25^{8}$ to 260 .

U. S. Standards. $†-$ Standard Meat is any sound, dressed, and properly prepared edible part of animals in good health at the time of slaughter. The term "animals" as herein used includes not only mammals, but fish, fowl, crustaceans, mollusks, and all other animals used as food.

Standard Fresh Meat is meat from animals recently slaughtered, or preserved only by refrigeration.

Standard Salted, Pickled, and Smoked Meats are unmixed meats preserved by salt, sugar, vinegar, spices, or smoke, singly or in combination, whether in bulk or in packages.

Standard Manufactured Meats are meats not included in the above divisions, whether simple or mixed, whole or comminuted, with or without the addition of salt, sugar, vinegar, spices, smoke, oils, or rendered fat, if they bear names descriptive of their composition, and when bearing such descriptive names, if force or flavoring meats are used, the kind and quantity thereof are made known.

Preservation of Meat.-Raw meat soon begins to decompose, unless precautions are taken to destroy, or at least check the growth of putrefying bacteria. From earliest times the subjection of meat to extreme cold has been practiced in order to enhance its keeping qualities. Bacterial growth is inhibited to a greater or less extent by refrigeration, by subjecting the meat to the various processes of curing, by the use of high temperatures and the exclusion of air as in canning, and by the employment of antiseptics.

\footnotetext{
* Cellular Toxines.
} † U. S. Dept. of Agric., Off. of Sec., Circ. No. 19. 
Refrigeration may consist (I) in actually freezing the meat, in which condition it may be kept without decomposition almost indefinitely, until finally thawed for use, or (2) in keeping the meat at or near the temperature of freezing without actually congealing it, as is done by the use of the ordinary refrigerator. The second method, while much less efficacious than the first, serves to prevent decomposition for a considerable time and is preferred for beef, mutton, and pork. The lower temperatures are employed with poultry and game.

Curing consists in subjecting the meat to various processes of drying, smoking, pickling, corning, etc., or to a combination of these processes. In simple drying, the meat is subjected to the heat of the sun or to artificial heat. In smoking, which is commonly practiced on beef and ham, the meat, which may or may not be first salted or otherwise treated, is exposed to the smoke of the burning beech or hickory wood, thus becoming impregnated with the antiseptic properties of the creasote and pyroligenous acid, at the same time being dried by the heat. Treatment with crude pyroligenous acid, instead of smoking, is also commonly practiced. In some cases best results are obtained by a slow smoking at a comparatively low temperature, while in others quick, hot smoking is found most efficacious. The character of the meat is decidedly changed by smoking, and, according to Utescher, smoked meat is always alkaline in reaction. In pickling, the meat may be treated with dry salt and subjected to pressure, so that the meat juice forms the liquid for the brine, in which it is allowed to remain for some time; or, as in the ordinary process of corning, the beef is soaked for some days in a strong solution of salt to which a little saltpetre $\left(\mathrm{KNO}_{3}\right)$ has been added. In the process of pickling, the salts from the brine slowly diffuse into the interior of the meat by osmosis, a part of the soluble albumin passing out into the brine. The effect of the saltpetre is to preserve the natural red color of the meat, which by the action of salt alone becomes destroyed, or at least impaired.

Bacon and ham are frequently cured by pickling in brine containing salt, saltpetre, and cane sugar, and sometimes also such antiseptics as boric acid and calcium bisulphite.

The curing of bacon is sometimes effected by injecting the pickling fluid into the tissues with a " pickle-pump," capable of exerting a pressure of $40 \mathrm{lbs}$. to the square inch, and provided with a hollow, perforated needi-nozzle, which penetrates the flesh. After pickling, the bacon or ham 
may be simply dried, or, if desired, smoked. Oak sawdust is frequently burned for the smoking of ham.

The Use of Antiseptics in Meat.-Most of what might be termed the modern preservatives are to be looked for in one or another of the various meat preparations, though some are better adapted than others for use in particular cases, as will be seen by reference to the composition of typical commercial preservative mixtures given on page 823 .

Borax and boric acid, usually in mixture, have been used more commonly than any other preservatives for the preservation of meat. Like salt, they are used commonly in surface application, in the case of large cuts of meat, or by mixing, in the case of sausage meat. A more recent method of application consists in impregnating the tissue of the meat with a solution of the boric mixture, by means of the above-described pickle-pump. The use of boric acid and its compounds, however, is not permitted under the regulations of the Federal meat inspection law of the United States and Germany.

Sulphurous Acid.-As much as I \% of a solution of sulphurous acid may be added to meat without being apparent to the taste or smell. Mitchell quotes Fischer as having found that $50 \%$ of the preserved meat products (sausages, etc.) sold in Breslau in 1895 contained sulphites, varying in amount from 0.01 to 0.34 per cent of sulphur dioxide. Calcium bisulphite is a salt commonly employed. In Hamburg steak it serves partly as a preservative, but chiefly as a deodorizer and a restorer of the bright red color of fresh meat.

Salicylic Acid is not of such common occurrence in meat products as the other antiseptics mentioned. The writer has found it in prepared mince-meat.

Among other preservative substances sometimes used with meat are solutions containing phosphoric acid and aluminum salts.

The toxic effects of these and other antiseptic chemicals in meats, and the most practical means of controlling their use are questions in controversy, presenting no new phases that have not been elsewhere discussed in treating of the general question of preservatives in food. Methods of detecting preservatives in meats are given elsewhere.

Effect of Cooking on Meat.-The general result of cooking is to render the meat less tough, to develop an agreeable flavor, and to coagulate more or less of the proteins. When subjected to moist heat, such as boiling and steaming, some of the soluble materials are dissolved, so that when the liquor in which the meat is boiled is thrown away, some of the 
valuable substances are lost. This is especially true when meat is placed in cold water which is afterwards brought to boiling, a method to be recommended when the liquor with the dissolved extractives is to be used for broth. When the meat to be boiled is placed at once in boiling water, there is less loss of soluble matter by reason of the formation of a more or less impenetrable costing on the outside, by the coagulation of the proteins. Meat that is boiled becomes softer, owing to a partial dissolving of the gelatin formed. In the dry cooking of meat, as by broiling or roasting, there is usually a hardening of the tissues, and a driving out of some of the meat juices, which are, however, often recovered in the form of gravies.

Canning of Meat.-By far the most effective method of preserving meat and meat preparations of all kinds for long periods of time, consists in the application of the principle of sterilizing by heat, and sealing in air-tight cans. The process of canning cooked meat and its products does not differ materially from that employed in the similar preparation of vegetables. (See Chapter XXI.) Previous to canning, the meats are usually cooked by boiling, during which process the changes described in the preceding paragraph take place.

The practice of misbranding chopped meat with respect to variety has been very prevalent in the past, and many varieties of so-called potted and devilled meats and game have frequently consisted wholly or in large part of a cheaper variety than that specified on the label. This practice has been largely corrected in this country, owing to the enforcement of the regulations of the Federal meat inspection law.

It is largely among the canned meats and prepared meat products that instances of adulteration are to be found, since the fresh meats in whole cuts are rarely subject to adulteration.

Preservatives are sometimes added to canned meats, especially in the case of dried and smoked beef, ham and bacon, and in the potted and devilled mixtures. Boric acid, benzoic acid, and sulphites have been found in these preparations.

It is believed, however, that this practice has been largely discontinued, owing to the enforcement of the Federal regulations mentioned above.

Composition of Canned Meats.-The following table, compiled from results published by Bigelow and others,* shows the composition of various of the most common canned and preserved meats and meat

*U. S. Dept. of Agric,, Bur. of Chem., Bulletin 13, part 10. 


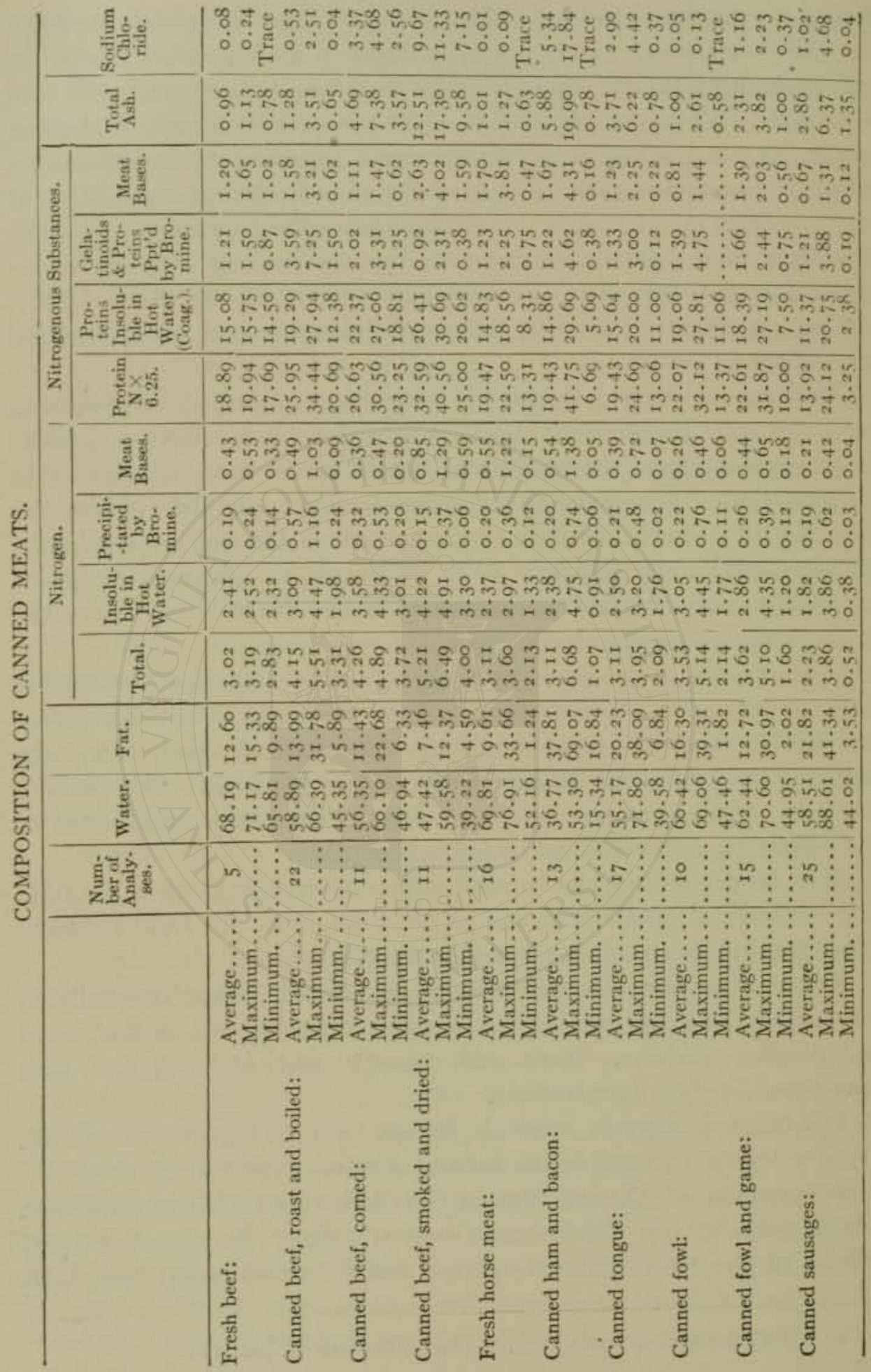


products, and in one or two instances fresh meat has been included for comparison.

Sausages. - Nature and Composition.-Sausages are made from finely chopped meat, highly seasoned with various spices, and, as usually sold, stuffed into casings made of the cleaned and prepared intestine-skin of cattle, sheep, or hogs. The meat most commonly used is pork. Sausages are frequently home-made, especially in farm communities, the chopped and seasoned meat being stuffed in cloth bags instead of casings. Any and all kinds of meat are used in sausages, and much that is undesirable and even unwholesome, is undoubtedly most readily used up in this product. There is little doubt that horse meat occasionally gets into the hands of the marketmen to be worked up in the form of sausages mixed with other meat. The condition in respect to these matters has been greatly improved, however, by the increased vigilance of State and Federal authorities.s. Sausages are sometimes artificially colored, and in some cases contain so-called "fillers" in the nature of dried bread, corn meal, potato starch, crackers, waste biscuit, boiled rice, etc.

\section{CHEMICAL COMPOSITION OF SAUSAGES.*}

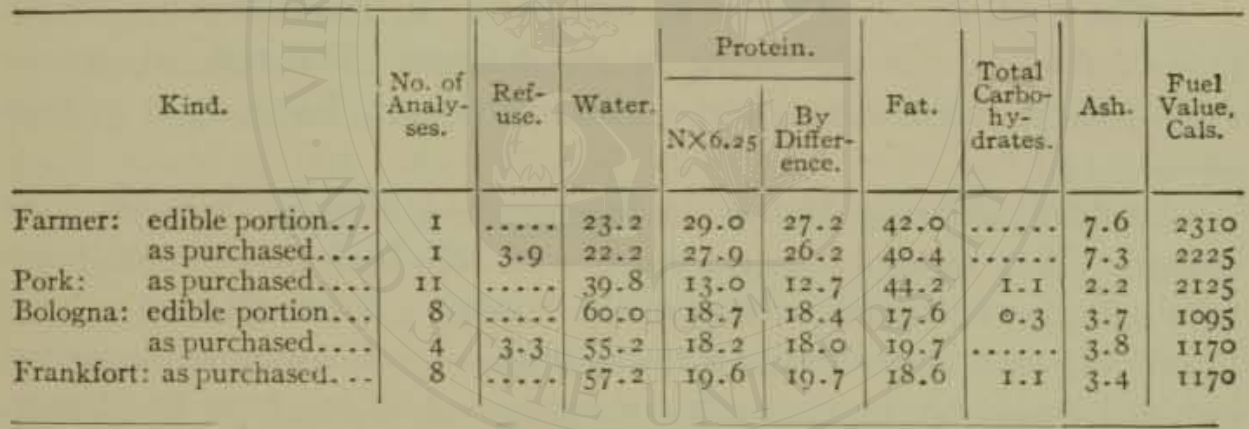

* U. S. Dept of Agric., Off. of Exp. Stations, Bul, 28 (Revised Ed.).

\section{Adulteration of Sausages with Starchy Materials and Water.-} Robison, who has made a special study of these forms of adulteration at the Michigan Dairy and Food Department, states as follows:* "Lean meat carefully chopped has an enormous combining power and can be made to take up a great quantity of water. Frankfurts, bologna, and pork sausage have been found to be adulterated with from 0.5 to $5 \%$ of starch, indicating an addition of approximately I to $10 \%$ of so-called cereal (chiefly corn flour), and from 5 to $40 \%$ of water in addition to

* Personal communication. 
that contained in the meats when in their fresh condition. The main excuse for the use of water is that it renders the meat of such a consistency that it may be easily stuffed into thin cases, such as are usually used for sausages that are eaten without removing the casing. As a matter of fact, this addition is not necessary where fresh meats are used, nor with those cuts of meat which the American public is in the habit of using in the manufacture of sausages in the home. Without doubt, in sausages composed of ox hearts, ears, snouts, lips, etc., in considerable quantities, the addition of water may facilitate the stuffing into thin casings.

"Starch hastens and increases the absorbing or combining power of lean meat. In many instances where inferior products, such as ears, etc., are used, virtually it is the only absorbing agent present in the product. It then serves a two-fold purpose, first, giving an absorbing power to meat which it has not, or inflating the absorbing power of a meat which naturally is deficient in this respect, and second, acting as a skeleton or framework, thereby disguising shrinkage during the process of cooking. Generally, added water and cereal are evidences of inferiority, and they are by no means infrequently added with the very purpose of concealing such inferiority.

"The evidence of adulteration with water is the discrepancy in the ratio of the water to the protein in the sausage. This ratio in sausage made from the fresh carcass varies from $3: 1$ to $3.6: 1$, being on an average about $3 \cdot 35$ :I."

Artificial Coloring Matter in Sausages.-Owing to the rapid color changes which freshly chopped meat, especially beef and mutton, naturally undergo, it is a common practice to employ powdered niter or saltpeter. Treated in this manner, meat remains pink, owing to the action on the hæmoglobin of the oxides of nitrogen resulting from the nitrate. As much as 4 ounces of niter to roo lbs. of meat is sometimes used. A larger quantity would result in a shriveled appearance. The use of artificial colors has been common in the past, in order to permanently dye the flesh a bright red, similar to the tint which the oxy-hæmoglobin naturally imparts to the beef when fresh. A variety of colors have been employed for this purpose, such as red ocher, coal-tar dyes, cochineal, etc. They were sometimes used in admixture with preservatives. Their use has been largely discontinued in this country, owing to the enforcement of the regulations under the Federal meat inspeciton law. 


\section{ANALYTICAL METHODS.}

In analyzing meats and meat products due regard must be paid to their perishable nature, and, for this reason, immediately after their receipt by the analyst the various determinations should be promptly begun and rapidly carried out. If delays are absolutely necessary, the samples, as well as some of the solutions, especially during the earlier course of the analysis, should be kept on ice to prevent decomposition. Even at low temperatures, however, both bacterial and enzymic decomposition occur, and the nature of the proteins is slowly changed. Refuse material, such as bones, skin, gristle, tendons, etc., are separated as completely as possible by means of a knife from the edible portion, and the latter, cut first into small pieces, is passed repeatedly through a sausagemachine or ordinary household meat-chopper, in order to reduce to a homogeneous, finely divided mass.

Determination of Water. - From I to 3 grams of the finely divided material are weighed in a tared platinum dish, and dried to minimum weight at a temperature of $100^{\circ} \mathrm{C}$. in an air-oven. A slight oxidation of the fat may introduce a trifling error, but, excepting for the most exact work, where the drying should be accomplished in an atmosphere of hydrogen, or in vacuo, the above method is sufficiently close.

Determination of Water in Sausages.-Robison's Method.-A large sample ( 100 to 500 grams) is put through a food-chopper, weighed on a large porcelain plate, and allowed to dry at 70 to $90^{\circ} \mathrm{C}$. A steam radiator may be conveniently used for this purpose. After drying to to I2 hours, or over night, it is reweighed and finely ground in a small laboratory mill. If the sample is quite fat, the preliminary drying of the chopped meat may be carried out conveniently on a sieve, which will permit the fat to drain through onto a plate below, thereby making more simple and accurate the sampling and mixing. The fat thus removed should be separately weighed and dried. If the sample is quite lean, the final drying of 2 to 5 grams of the air-dried sample may be made at $100^{\circ}$ C. in an ordinary water or electric oven. If it is quite fat, it is best to conduct this drying in a current of hydrogen.

Determination of Ash. - Incinerate the residue from the total solids in the original dish at a low red heat. It is usually advantageous, especially in the case of salt meat, to exhaust the charred sample with water, collect the insoluble residue on a filter and ignite. The filtrate is then added, evaporated to dryness, and the whole heated to low redness and weighed. A perfectly white ash is difficult to obtain. 
Determination of Fat.-Extraction Method.-Dry 2 grams of the sample at $100^{\circ}$ and extract with anhydrous ether for sixteen hours as in the case of cereal products (p. 277). More complete extraction is obtained by grinding the residue in a mortar and repeating the process and still more complete by digestion with pepsin and intermittent treatment with the fat solvent but this latter is both tedious and open to other errors.

Kita's Centrifugal Method.*-Treat 2.5 grams of meat in a Babcock milk flask with $8 \mathrm{cc}$. of $\mathrm{r}: \mathrm{I}_{2} \mathrm{SO}_{4}$ (or 5 grams with ${ }_{7} \mathrm{cc}$.) and heat in a water-bath to $60-70^{\circ}$ with occasional agitation till the proteins dissolve. Add I cc. of amyl alcohol and sufficient dilute $\mathrm{H}_{2} \mathrm{SO}_{4}$ to bring the layer of fat within the neck. Whirl in a centrifuge for from 3 to 5 minutes and read the amount of fat on the scale. Amyl alcohol is usually necessary for complete separation and a clear fat layer.

Examination of Fat. - Shake a large portion of the original finely divided sample in a corked flask with petroleum ether boiling below $60^{\circ}$ C., and digest for some hours. Pour off the solvent, remove most of the petroleum ether by distillation, and the last traces by allowing to stand in a vacuum desiccator over freshly ignited calcium chloride. Determine the usual constants as described in Chapter XIII. In minced preparations these constants furnish a possible clue to the variety of meat used.

Determination of Acidity of Fat.-Pennington and Hepburn Method. $\dagger$ Weigh to grams of the fat, mechanically separated and ground in a meat chopper, directly into a $25^{\circ} \mathrm{cc}$. Erlenmeyer flask, add $50 \mathrm{cc}$. of neutral alcohol, and phenolphthalein as indicator, and bring to a brisk boil. The hot alcohol dissolves the fat. Titrate immediately with tenth normal sodium hydroxide, shaking vigorously, until a pink color appears, which persists for one-quarter of a minute. Calculate the acid value from the amount of sodium hydroxide used, or the free oleic acid by multiplying the acid value by 0.503 .

Determination of Total Nitrogenous Substance.-Determine nitrogen in 2 grams of the sample by the Gunning or Kjeldahl method (p. 69) and multiply by 6.25 .

Although nitrogenous substances other than proteins are present and the factors for the individual proteins vary, this result is a fairly close approximation to the total nitrogenous substance present. The factors for meat bases are give on page 252 .

Determination of Ammoniacal Nitrogen.-Folin Method modified by Pennington and Greenlee. $\ddagger-T h e$ ammonia, set free by sodium carbonate,

* Arch. f. Hyg. 51, p. 165. † Jour. Amer. Chem. Soc., 32, 1910, p. 568. . † Ibid, p. 561. 


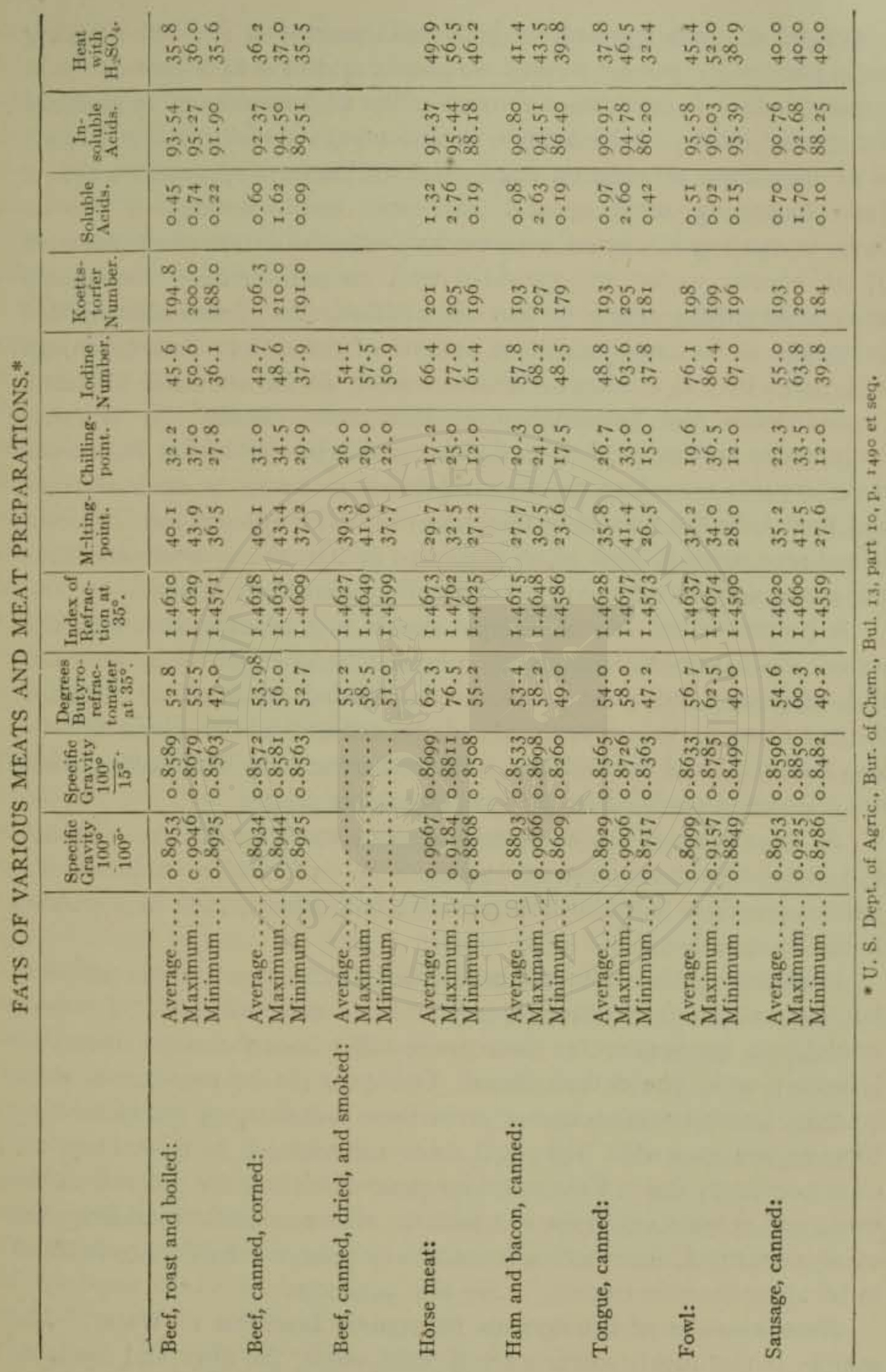


is evolved at room temperature in a rapid current of air. The ingoing air is purified by passing through sulphuric acid in a flask provided with a safety bulb. It next passes through a liter flask containing 25 grams of the ground meat, I gram sodium carbonate, $250 \mathrm{cc}$. of water, and $25 \mathrm{cc}$. of alcohol, then through an empty flask to intercept spray into a $250 \mathrm{cc}$. flask containing tenth normal acid and finally through a roo cc. flask, to catch acid carried over mechanically, to an air pump operated by an electric motor and provided with an anemometer. One pump and one air purifier suffices for four series of flasks, the current being divided by means of four-way tubes. A volume of 8000 cu.ft. passed through each series in 3 to 6 hours suffices to remove all the ammonia liberated.

Separation and Examination of Nitrogenous Bodies. It is rarely necessary to go further than to divide the nitrogenous bodies into several main groups, according to their solubility in water or other solvents, and their behavior toward certain reagents. The nitrogen may be determined separately in each of these groups and by the approximate factor the corresponding substance or class of substances ascertained.

A portion of the fat-free sample should first be exhausted with cold water, which removes the soluble proteins (soluble globulins, proteoses, and peptones) and meat bases, leaving behind the insoluble globulins, the sarcolemma, the albuminoids of the connective tissue (elastin, etc., also insoluble) and the collagen. By next exhausting with boiling water the collagen is removed in the form of soluble gelatin.

By treatment of the combined aqueous extract with zinc sulphate, and with sodium chloride and tannic acid, as hereafter explained, the soluble proteins, including the peptones and gelatin, may be separated from the meat bases.

In obtaining the results from which the table on page 222 was compiled, but three divisions of nitrogenous substances were made, viz., (I) those insoluble in hot water; (2) those precipitated from the water extract by bromine; and (3) the flesh bases. Owing to the incompleteness of the bromine precipitate, the figures given there for nitrogen precipitated by bromine are somewhat high, and those for nitrogen as meat bases are correspondingly low. This fact was observed during the progress of the work, and pointed out in the text with the statement that "considering the small amount of these bodies contained in meat, the results are believed to be approximately correct." See also page 250 .

Determination of Nitrogenous Substances Insoluble in Water.-The sample is thoroughly extracted with cold water, the filter and insoluble material transferred to a flask, and nitrogen determined by the Gunning 
or Kjeidahl method. The insoluble nitrogen thus obtained is multiplied by 6.25 to obtain insoluble proteins. It is obvious that the insoluble nitrogen may be obtained by difference, the cold water extract being diluted to definite volume, the nitrogen determined in an aliquot portion, and calculated to percentage of soluble nitrogen in the weight of original sample corresponding to the aliquot portion taken. The figure thus obtained, deducted from the percentage of total nitrogen, gives the percentage of insoluble nitrogen.

Trowbridge and Grindley* digest the sample (thoroughly ground in a meat chopper) for one hour in ice water, in the proportion of 1000 grams of meat to $1500 \mathrm{cc}$. of water. The resulting solution is filtered through cheese cloth, the process being assisted by squeezing the cloth and its contents with the hand. The residue is divided into smaller portions, placed in beakers, and washed in series, using fresh water with No. I only, and filtering through cheese cloth from one beaker to another until the last filtrate is colorless, neutral to phenolphthalein, and gives no reaction for proteins by the biuret test. The mixed filtrates and washings filter through paper readily, and give a clear red filtrate.

Pennington $\dagger$ proceeds as follows with the meat of chickens:

A portion of the finely divided red or white meat, weighing 60 grams, is put into a tall, slender bottle of $500 \mathrm{cc}$. capacity, constructed to fit a centrifuge capable of carrying I liter of material; $300 \mathrm{cc}$. of water are added, and the flask gently shaken for I 5 minutes. The movement is merely sufficient to keep the particles of meat in motion and the composition of the extract homogeneous. Forcible shaking causes an emulsion to form, as does the very fine grinding of the tissue. After shaking for the required length of time, the flask is rotated in an electric centrifuge for 20 minutes, which causes the heavier particles to settle in a compact mass, and permits the decantation of the supernatant liquid, which is then filtered through paper. The extraction, as outlined, is repeated with portions of $300 \mathrm{cc}$. of water until the filtrate is practically protein free, as indicated by the biuret reaction. The attainment of this result requires ordinarily a volume of 1500 to $2500 \mathrm{cc}$. To guard against bacterial decomposition, thymol is added both to the flesh and to the extract, and to inhibit, so far as possible, the action of the naturally occuring enzymes of the meat, the solution and the meat itself are kept cold, ice being used when necessary.

* Jour. Am. Chem. Soc., 28, 1906, p. 472.

†U. S. Dept. of Agric, Bur. of Chem., Bul. 115 , p. 64 . 
The extraction of the white meat is a much simpler operation than the extraction of the dark meat. The latter does not settle as compactly in centrifuging, filters more slowly, and persists in showing a distinct biuret reaction for a considerable time after the white meat is free of water-soluble proteins. In fact, certain fowls, more especially those which have been in cold storage for long periods of time, never show a dark meat entirely free from water-soluble nitrogen. In such cases, the question of the error due to long manipulation and enzyme action, involving a rise in the actual quantity, has to be considered. It has been found by experiment that after long extraction of such tissue, a point is reached when a very faint biuret reaction, which does not apparently diminish, persists indefinitely. Such extractions are halted after about 26 hours, it being believed that a greater error would result in the gain of what has been originally insoluble material, than the loss of the preformed water-soluble nitrogen. The total extract of the muscle is made up to a definite volume, and neutralized to litmus paper with tenth-normal sodium hydroxide.

Cook weighs 200 grams in a 450 Erlenmeyer flask, adds $25^{\circ} \mathrm{cc}$. of water, and shakes for three hours in a shaking machine. The material is then filtered by means of linen bags, and extracted with water repeatedly by vigorous manipulation with the hands in successive portions of water, pressing out after each extraction until negative biuret reaction is obtained. The operation ordinarily requires from 2200 to $2500 \mathrm{cc}$. of water. A small quantity of phenol and thymol are added as preservatives.

Weber* applied Cook's method at room temperature and with ice water to samples of fresh and storage meat, as well as to samples which he had kept for varying lengths of time in the laboratory. He obtained a larger amount of soluble proteins when working at room temperature. No opinion was expressed as to whether this was due to the greater extracting power of water at room temperature, or to greater enzymic action during the period of extraction.

Determination of Collagen.-The insoluble proteins obtained as directed above are transferred to a beaker, water added, and heated to boiling for some minutes. They are then separated by filtration and washed with boiling water. The nitrogen of the residue insoluble in boiling water is deducted from the nitrogen insoluble in cold water, and multiplied by 5.55 for the per cent of collagen. This method is of doubt-

*U. S. Dept. of Agric, Bur. of Chem., Bul. 122, p. 42. 
ful value, owing to the difficulty of converting the collagen to soluble form on the one hand, and to the tendency to decompose a portion of the protein on the other.

Determination of Coagulable Proteins.-The entire filtrate, or an aliquot portion thereof, from the defermination of nitrogenous bodies insoluble in water is heated sufficiently to coagulate the coagulable proteins, filtered, the insoluble material washed with hot water, and the filter and contents transferred to a Kjeldahl flask and nitrogen determined by the Gunning method. The per cent of nitrogen multiplied by 6.25 gives the per cent of coagulable proteins.

The amount of heating necessary to obtain maximum coagulation varies with different materials. The Association of Official Agricultural Chemists directs that the solution be almost neutralized, but left still faintly acid, and boiled until the globulins are coagulated.*

Pennington, $\uparrow$ working with chickens, evaporates $350 \mathrm{cc}$. to a volume of about $100 \mathrm{cc}$. before filtering. Grindley and Emmettł employ $200 \mathrm{cc}$. of the solution, add alkali till neutral to litmus paper, and evaporate to $50 \mathrm{cc}$. In a later article, Trowbridge and Grindley $\$$ report maximum results from the cold water extract of fresh beef by neutralizing one-fourth of the acidity to phenolphthalein before coagulation.

Determination of Proteoses, Peptones, and Meat Bases.-The filtrate from coagulated proteins, having been diluted by wash water, is concentrated by evaporation and made up to roo cc. Proteoses are then determined by Bömer's method (page 250 ), and meat bases by Sjerning's method, as modified by Bigelow and Cook (page 252). Peptones are determined by difference - the sum of the nitrogen occurring in insoluble nitrogenous bodies, coagulated proteins, meat bases and ammonia being deducted from the total nitrogen.

Determination of Gelatin.-Modified Stutzer's Method.|-A weighed portion of the sample, say to grams, is thoroughly extracted by boiling water, the extract transferred to a porcelain dish containing about 20 grams of previously ignited sand, and evaporated to dryness. The residue is then stirred with four successive portions of absolute alcohol,

*U. S. Dept. of Agric., Bur, of Chem., Bul. 107 (rev.), p. ro8.

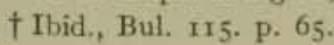

$\ddagger$ Jour. Am. Chem. Soc., 27,1905, p. 665.

\$ Ibid, 28,1906 , p. 494.

II U. S. Dept. of Agric., Bul. 13, part 10, p. ז397. 
using about $50 \mathrm{cc}$, each time and pouring it off through a filter consisting of a layer of asbestos fiber on a perforated porcelain plate within a funnel. This funnel is surrounded by chopped ice, and is so arranged that gentle suction may be used to hasten the filtration. The residue is then repeatedly stirred with successive portions of about $100 \mathrm{cc}$. each of a mixture containing $100 \mathrm{cc}$. of $95 \%$ alcohol, 300 grams of ice, and 600 grams of cold water, the portions being passed through the asbestos filter, and the washing being continued till the solution is colorless as it comes from the filter, keeping the temperature always below $5^{\circ}$. The asbestos is then transferred to a beaker with the washed residue, and the whole thoroughly extracted with boiling water. The hot-water extract is evaporated to small volume, and washed into a Kjeldahl flask, in which it is then evaporated to dryness, and the nitrogen determined by the Gunning method: $\mathrm{N} \times 5.55=$ gelatin.

Detection of Nitrates. - A small portion of the finely divided material is treated in a porcelain dish or on a tile with a little $1 \%$ solution of diphenylamine in concentrated sulphuric acid. Presence of a blue color indicates nitrate.

Detection of Preservatives.-Meats may be systematically tested for preservatives in the same manner as canned goods. The preservatives most commonly used in meat and meat preparations are tested for as follows:

Detection of Sulphurous Acid.-Proceed as directed on page 840.

Traces should be ignored, as slight reactions for sulphurous acid are obtained with meats that have not been chemically preserved. This is probably due to the decomposition of a portion of the proteins. According to Mentzel,* 4 milligrams per 100 grams may be due to this cause.

Detection of Boric Acid. - A portion of the finely divided meat, mechanically freed from the fat as far as possible, is warmed with water acidified with hydrochloric acid, and turmeric-paper is soaked in the extract. The rose-red color of the turmeric-paper after drying (turned blue by weak alkali) is indicative of boric acid.

A more delicate method of procedure consists in burning to an ash a portion of the meat, after treatment with lime water, and testing with turmeric tincture a solution of the ash slightly acidified with hydrochloric acid.

*Zeits. Unters. Nahr. Genuss., 11, 1906, p. 320. 
Detection of Salicylic Acid.-The sample, mechanically freed from fat, is slightly acidified and shaken out with ether. The ether extract evaporated to dryness is tested with a drop of a solution of ferric chloride. A deep-violet coloration indicates salicylic acid.

Starch in Sausages, Meat-balls, etc.-Detection.-The addition of cracker or bread crumbs is best indicated by the presence of considerable starch, which is readily recognized by the iodine test, applied by boiling up a portion of the sample with water, cooling and adding a drop of iodine reagent to the liquid. The characteristic blue color is produced, if starch be present in notable quantity. Traces of starch may be due to the pepper and spices used in seasoning the sausage. A small admixture of starch is rendered apparent when a thin section of the sausage is treated with a drop of iodine reagent and viewed under the microscope. A microscopical examination will sometimes reveal the character of the starch, whether it is from cereals or from pepper, but in some preparations the starch is thoroughly cooked and its structure destroyed.

Estimation of Starch.-The regular acid conversion process, p. 283 , may be applied, but more accurate results are obtained by the method of inversion with malt extract. Medicus and Schwab* prepare the malt extract for this purpose by digesting 5 grams of ground malt with $50 \mathrm{cc}$. of water for one and one-half hours at $20^{\circ}$ to $30^{\circ} \mathrm{C}$. In making the starch estimation, they digest for two hours at a temperature of from $40^{\circ}$ to $50^{\circ}$ C. 20 grams of the sausage mixed with $20 \mathrm{cc}$. of the malt extract, and afterwards for eighteen hours at room temperature. After filtering and washing, the filtrate is boiled to coagulate the albumin and again filtered. The second filtrate is then made up to $200 \mathrm{cc}$., $20 \mathrm{cc}$. of $25 \%$ hydrochloric acid (specific gravity I.I25) are added, and the starch determined in the regular manner.

Mayrhofer's Method. $\dagger$-This is considered the simplest and most reliable method of estimating the starch in such substances as sausages. From 60 to 80 grams of the sample are heated on the water-bath with an $8 \%$ solution of alcoholic potassium hydroxide, which, in the case of pure sausages, dissolves nearly everything except a little cellulose. To prevent gelatinization, warm alcohol is added to dilute the solution, which is then filtered through paper or asbestos. The starch is con-

* Berichte d, chem. Gesell., XII, p. 1285 .

$\dagger$ Zeits. Nahr, Untersuch., I896, p. 33r; Abs. Analyst, I897, p. Ir. 
tained in the insoluble residue, which is washed with alcohol till the washings are no longer alkaline, after which it is treated with an aqueous solution of potassium hydroxide, and the starch solution made up to a definite volume. To an aliquot part of the solution $95 \%$ alcohol is added, whereupon the starch comes down as a flocculent precipitate. This is collected on a weighed filter, washed with alcohol and ether, dried, and weighed. The filter with its contents is then burnt to an ash, the amount of which is deducted.

In order to avoid the ash determination, the starch may be precipitated from a weak acetic acid solution instead of from an alkaline solution, the potassium acetate formed being soluble in the alcohol, and nothing but pure starch is precipitated.

Characteristics of Horse Flesh.-Although certain authorities have found distinguishing characteristics in color, consistency, odor, etc., between horse flesh on the one hand, and beef and pork on the other, it is extremely difficult, by its physical properties, to detect horse flesh when mixed with other meat, especially when the mixture is chopped. Horse flesh has a much coarser texture and is darker in color than beef. The muscle fibers are, as a rule, shorter in horse flesh. On treating horse flesh with formaldehyde, Ehrlich* has found that a very characteristic odor is developed within forty-eight hours, suggestive of roasted goose flesh.

Certain of the constants of the fat of horse meat differ from those of beef and pork, notably the iodine value and the refractometer readings. These constants are compared as follows:

\begin{tabular}{|c|c|c|}
\hline & Iodine Value, & $\begin{array}{l}\text { Butyro-refractom- } \\
\text { eter Readings. } \\
\text { Temperature } 40^{\circ} \text {. }\end{array}$ \\
\hline $\begin{array}{l}\text { Horse fat } \ldots \ldots \ldots \ldots \ldots \ldots \ldots \ldots \\
\text { Beef fat } \ldots \ldots \ldots \ldots \ldots \ldots \ldots \ldots \\
\text { Hog fat } \ldots \ldots \ldots \ldots \ldots \ldots \ldots\end{array}$ & $\begin{array}{l}71-86 \\
3^{8}-46 \\
50-70\end{array}$ & $\begin{array}{l}53 \cdot 7 \\
49 \cdot 0 \\
48.6-51.2\end{array}$ \\
\hline
\end{tabular}

The fact that glycogen usually exists to a much larger extent in horseflesh than in other meat, renders it possible in some cases to detect horseflesh, when present in the mixture.

The following table prepared by Bujard shows the relative amount of glycogen in various kinds of meat and sausages:

* Zeit. Fleisch u. Milch Hyg., 1895, p. 232. 


\begin{tabular}{|c|c|c|c|c|c|}
\hline & \multirow{2}{*}{ Water. } & \multicolumn{2}{|c|}{ Glycogen Direct. } & \multicolumn{2}{|c|}{$\begin{array}{l}\text { Glycogen in Dried } \\
\text { Substance. }\end{array}$} \\
\hline & & $\begin{array}{l}\text { Niebel } \\
\text { Method. }\end{array}$ & $\begin{array}{l}\text { Mayrhofer } \\
\text { Method. }\end{array}$ & Niebel. & Mayrhofer. \\
\hline 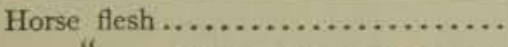 & 74.44 & 0.440 & 0.445 & I. 721 & $1.74 \mathrm{I}$ \\
\hline$" \quad \quad \ldots \ldots \ldots \ldots \ldots \ldots \ldots$ & 74.87 & 0.600 & 0.520 & $2 \cdot 388$ & 2.069 \\
\hline$\ldots \ldots \ldots \ldots \ldots \ldots$ & 76.17 & 1.827 & I. 727 & 7.667 & $7 \cdot 247$ \\
\hline $\ldots \ldots \ldots \ldots \ldots \ldots \ldots$ & 76.00 & 0.592 & 0.610 & 2.466 & 2.542 \\
\hline Red sausage (K nackwurst) ......... & 69.26 & $\ldots \ldots$ & $0.03^{8}$ & $\ldots \ldots$ & 0.124 \\
\hline Pork sausage. .................. & 67.25 & $\cdots$. & 0.24 & $\ldots \ldots$ & 0.733 \\
\hline Veal. $\ldots \ldots \ldots \ldots \ldots \ldots \ldots \ldots \ldots \ldots \ldots$ & 74.6 & $\ldots$. & 0.086 & $\ldots \ldots$ & 0.342 \\
\hline Pork. $\ldots \ldots \ldots \ldots \ldots \ldots \ldots \ldots \ldots \ldots \ldots$ & 75.0 & $\ldots \ldots$ & 0.186 & $\cdots \cdots$ & 0.744 \\
\hline
\end{tabular}

In beef Bujard found 0.74 and 0.073 per cent of glycogen calculated in terms of dried substance, and, in sausages made exclusively from horse meat, amounts of glycogen ranging from 0.05 to 5.34 , the sample in the latter case being made from the liver. It was formerly thought possible to detect as small an amount as $5 \%$ of horse flesh in mixture, but later investigation showed that after the death of the animal, glycogen, though present at first in considerable quantity, decomposes more or less rapidly, going over into muscle sugar (dextrose). Hence, while the presence of much glycogen is suspicious, its absence is by no means proof that horse flesh was not used.

Niebel did not consider the failure of the glycogen test as sufficiently conclusive to establish the absence of horse flesh, on account of the tendency toward decomposition of the glycogen. In the absence of starch, he regards the presence of more than $1 \%$ of dextrose in the fat-free meat, after conversion of the carbohydrates, to be proof of the presence of horseflesh.

Detection of Glycogen.-From the well-known color reaction produced by iodine on glycogen, horse flesh can often be detected, when present in sausages, unless obscured by the presence of starch or dextrin.

Brautigam and Edelmann* proceed as follows: 50 grams of the finely divided meat are boiled with $200 \mathrm{cc}$. of water for an hour, and, after cooling, dilute nitric acid is added to the broth to precipitate the proteins and to decolorize. The broth is then filtered, and a portion of the filtrate is treated in a test-tube with a freshly prepared, saturated, aqueous solution

\footnotetext{
* Pharm. Central., 1898, p. 557 .
} 
of iodine, or, better, with a mixture of 2 parts iodine to 4 parts potassium iodide and 100 parts water, the reagent being carefully added so as not to mix with the broth, but form a layer above it. If glycogen be present in considerable amount, a wine-colored ring is observable at the junction of the two layers. On" heating the test-tube, the coloration disappears if due to glycogen, but it reappears on cooling. This reaction was found to occur with horse flesh and not with beef, mutton, veal, or pork.*

If the color is not clearly apparent, the chopped meat is heated on the water-bath with a solution of potassium hydroxide (using an amount of potassium hydroxide equivalent to $3 \%$ of the weight of the flesh) till the fiber is decomposed, after which the broth is concentrated to half its volume, treated with nitric acid to precipitate the proteins, filtered, and treated with the iodine solution as previously.

Determination of Glycogen.†-Niebel's Modification of Brücke's Method.-This method is applicable only in the absence of dextrose and dextrin. If therefore the presence and character of the starch indicates the presence to a considerable extent of cracker crumbs or other cereal "ffiller," the method is not accurate.

A weighed portion of the flesh is heated on the water-bath with 3 to 4 per cent of potassium hydroxide and four volumes of water for six hours. Evaporate the broth to half its original bulk, and add, after cooling, a solution of mercuric iodide in potassium iodide, $\ddagger$ which precipitates the protein. Filter, and to the clear filtrate add $2 \frac{1}{2}$ times its volume of $95 \%$ alcohol, collect the precipitated glycogen on a filter, wash first with $60 \%$ alcohol, then with $95 \%$ alcohol, then with absolute alcohol, then with ether, and finally with absolute alcohol. Dry at $115^{\circ} \mathrm{C}$. and weigh.

Landwehr's Method.-Applicable in presence of dextrose. The broth prepared as in Niebel's method is freed from protein by the addition of zinc acetate. Filter, wash, and heat the entire filtrate on the waterbath with sufficient of a concentrated solution of ferric chloride, afterwards precipitating the iron with a few drops of a saturated solution of socium hydroxide. Filter, wash the precipitate with hot water, and dis-

* The reaction was found to occur also with the flesh of the human foetus and with the foetus of animals; also with mule meat, but not with the flesh of the dog or cat.

† Jahresb. Nahr. Genuss, $189 \mathrm{r}$, p. 38 .

$\ddagger$ The reagent known as Brucke's reagent is prepared by precipitating a solution of mercuric chloride with potassium iodide, washing the precipitated mercuric iodide till free from chloride, and afterwards saturating, while boiling, a 10\% potassium iodide solution with the mercuric iodide. 
solve in strong acetic acid. Add to the solution, after cooling, sufficient hydrochloric acid to produce a yellow color, then pour into $2 \frac{1}{2}$ volumes of alcohol, and proceed as in the preceding paragraph.

Mayrhofer's Method,* on which the results in the table on page 235 are based, is as follows: Dissolve a weighed portion of the flesh in an aqueous solution of potassium hydroxide, precipitate the proteins by hydrochloric acid and Nessler's reagent, filter, and treat the clear filtrate with alcohol, which precipitates the glycogen. This is collected on a tared filter and washed, first with dilute alcohol, and finally with ether, dried at $110^{\circ} \mathrm{C}$., and weighed.

Pfluger and Nerking's Method.†-Of the finely divided sample 50 grams are heated on the water-bath with $200 \mathrm{cc}$. of $2 \%$ potassium hydroxide till the solution is practically complete. After cooling, the solution is made up to $200 \mathrm{cc}$. with water, shaken, and filtered. To $100 \mathrm{cc}$. of the filtrate, Io grams of potassium iodide and I gram potassium hydroxide are added, and the solution stirrred till clear, after which $50 \mathrm{cc}$. of $95 \%$ alcohol are added and the mixture allowed to stand over night. This precipitates the glycogen. Filter, and wash the precipitate with a solution made up of $\mathrm{I} \mathrm{cc}$. $70 \%$ potassium hydroxide, to grams potassium iodide, Ioo cc. water, and $50 \mathrm{cc} .95 \%$ alcohol. After further washing the glycogen with 2 parts strong alcohol and I part water, dissolve in water and by means of Brücke's mercuric-iodide-in-potassium-iodide reagent (see footnote, p. 236) remove any remaining nitrogenous substances. Filter if turbid, and to the solution add common salt (about 2 milligrams per $100 \mathrm{cc}$. of solution), and reprecipitate the glycogen by adding 2 volumes of $95 \%$ alcohol. Filter, wash first with $95 \%$ alcohol containing a little common salt, then with absolute alcohol, and lastly with ether. Dry and weigh.

Bigelow suggests that the glycogen as above obtained be converted by acid hydrolysis to dextrose, which is determined in the regular manner. Dextrose $\times .9=$ glycogen.

Identification of Raw Horse Flesh by the Blood Serum Test. $\ddagger$-This test depends upon the recent development of the principle that when a rabbit has been inoculated with the blood of a particular animal, as for instance that of the horse, the serum of the rabbit's blood will react with

* Forsch. Ber., I897, IV, 47 .

$\dagger$ Arch. ges. Physiol., $1899,76,531-542 ;$ Bul. 65, Bur. of Chem., p. 13. Recommended for Provis. Adoption by the A. O. A. C.

$\ddagger$ Schitse, A., Ueber weitere Anwendungen der Präcipitine. (Deuts, med. Wochs., 1902, No. 45, p. 804.)

Wassermann, A., u. Schütze, A., Ueber die Entwickelung der biologischen Methode 
the blood of the horse and with that of no other animal. To prepare the blood serum for a reagent, inject a rabbit with io cc. of defibrinated horse's blood every day for five to six days, either subcutaneously or intravenously. The blood afterwards taken from the rabbit is clotted, and the filtered serum is used in making the test, or, if the reagent is to be kept for some time, the rabbit's blood serum is dried and an aqueous solution used for the reagent.

If the clear expressed juice from the suspected flesh, filtered if necessary, be treated with a few drops of the rabbit's blood reagent, prepared as above, a cloudy precipitate will be produced in the case of horse flesh.

By inoculating different rabbits in like manner with the blood of various animals, the flesh of the corresponding animals may be recognized from the reaction of the blood serum of the rabbit with its juices. Only raw flesh responds to the test, as heating destroys the virtue of the reagent.

Determination of Muscle Sugar (Dextrose).-Boil a weighed quantity of the finely divided sample, say 50 grams, with water, add an excess of normal lead acetate solution, and make up with water to a given volume, say $250 \mathrm{cc}$. Filter, and to an aliquot part of the filtrate add enough of a saturated solution of sodium sulphate to precipitate the lead. Again filter, make up to a given volume, and determine the dextrose in a measured part of the solution by either of the regular methods.

Detection of Coloring Matter.-Red Ocher is indicated by an excessive amount of iron in the ash.

Cochineal is most readily tested for by the method of Klinger and Bujard.* The sausage, finely divided, is heated with two volumes of a mixture of equal parts of glycerin and water for several hours on the water-bath, the mixture being slightly acidified. The yellow solution is passed through a wet filter, and the coloring matter, if present, is precipitated as a lake by adding alum and ammonia, the precipitate is filtered off and washed, after which it is dissolved in a small amount of tartaric acid, and the concentrated solution, contained in a test-tube, is examined zur Unterscheidung von menschlichem und tierischem Eiweissmittels Präcipitine. (Ibid., 1902, No. 27 , p. 483 .)

Wassermann, A., Ueber Agglutinine und Prïcipitine. (Zeits. f. Hyg., etc., Bd. 42, 1903,2, p. 267.)

Uhlenhuth, Die Unterscheidung des Fleisches verschiedener Tiere mit Hilfe spezifische Sera und die praktische Anwendung der Methode in der Fleischbeschau. (Deuts. Mfed. Wochs., 1901, No. 45 , p. 780 .)

Miessner, $H$., $u$. Herbst, Die Serum agglutination und ihre Bedeutung für die Fleis huntersuchung. (Arch. f. wissensch. u. prakt. Tierheilk., 1902, Heft 3-4, p. 359.)

*Zeit. angew. Chem., r89r, p. 515. 
through the spectroscope for the characteristic absorption-bands of carmine lake, lying between $b$ and $D$.

Spaeth* has shown that both carmine (cochineal) and anilin red, which are the dyes most commonly used for coloring sausages, can be most readily extracted therefrom by warming the finely divided material a short time on the water-bath with a $5 \%$ solution of sodium salicylate.

Vegetable and Coal-tar Colors.-Various solvents are suggested for the removal of these dyes from sausage meat. Allen $\dagger$ recommends extraction with methylated spirit (a mixture of ethyl alcohol with $10 \%$ methyl); Bigelow $\ddagger$ recommends heating with a mixture of $50 \%$ glycerin slightly acidified; A. S. Mitchell uses alcohol acidified with hydrochloric acid; Spaeth a $5 \%$ solution of salicylate of soda. Other solvents applicable in some cases are dilute ammonia and amyl alcohol. The solvent, after filtering, is evaporated to small volume, acidified with hydrochloric acid, and white wool is boiled in it. If the wool is distinctly dyed, a coal-tar color is undoubtedly present, and this can often be identified by methods given in Chapter XVII. According to Marpmann, pure normal flesh containing natural color only is completely decolorized by macerating for two hours in $50 \%$ alcohol, while artificially colored meat remains colored after this treatment.

Marpmann's Microscopical Method.\$-Moisten a thin section of the sausage with $50 \%$ alcohol, and examine under the microscope. Some colors are readily apparent without further treatment. If only traces of color are present, clarify the substance by treatment with xylol, which is removed by the use of carbon tetrachloride. The mass rendered transparent by this treatment is then immersed in cedar oil and examined, the coloring matters, if present, being especially apparent. If the color used is fuchsin (magenta), carmine, logwood, or orchil, the substance of the cell will appear stained. If acid coal-tar dyes are used, the liquid contents of the cell will show the color.

Detection of Frozen Meat.-Maljean \| detects frozen meat by the aid of a microscope. A drop of the blood or meat juice is pressed out upon a slide and immediately examined before it solidifies. Fresh meat juice contains many red blood corpuscles, floating in a clear colorless serum, and readily apparent. In blood from frozen meat, the red cor-

* Pharm. Central., 1897,38 , p. 884 .

† Commercial Organic Analysis, Vol. IV, p. 294.

‡ U. S. Dept. of Agric., Bureau of Chemistry, Bul. 65, p. 16.

$\$$ Zeits, angewand. Mikrosk, 1895, p. 12 .

II Jour. Pharm. et Chem, 1892, XXV, p. 348 . 
puscles are nearly always completely dissolved in the serum, due to freez. ing, or, if not dissolved, are much distorted and entirely decolorized, the liquid portion being darker than usual.

Megascopically, the fresh meat juice is more abundant than that of frozen meat, and its color is deeper. According to C. A. Mitchell, if a small piece of meat once frozen be shaken in a test-tube with water, color is imparted to the water much more quickly than with fresh meat, and the color is deeper.

\section{MEAT EXTRACTS.}

Character and Composition.-Numerous preparations sold under the name of meat extracts have been on the market for many years. At the beginning of the nineteenth century the value of such extracts was known, but Liebig was the first some fifty years later to produce a commercial extract of meat. Liebig's preparation, as originally made, consisted of a cold-water extract of chopped lean meat, strained free from fiber, heated, filtered, and evaporated, thus containing little of any gelatin or proteins. Later, however, Liebig advocated the use of warm and even boiling water for extraction, by which method of preparation the amount of gelatin was greatly increased. He, however, condemned the use of salt.

The best modern meat extracts consist for the most part of such portions of the meat, previously freed from bone and superfluous fat, as are soluble in water the temperature of which does not exceed $75^{\circ} \mathrm{C}$. The widest latitude, however, prevails as to the temperature employed for the extraction, hence the character of the various products is somewhat varied. It is not an uncommon practice to submit the meat to actual boiling with water, in which case the amount of gelatin will be considerable. In an extract prepared by warm water, one finds very little gelatin, more or less albumin, albumoses, and peptones, and practically all the meat bases, phosphates, and chlorides present in the meat; also minute quantities of lactic acid, inosite, and possibly glycogen. By far the most important of these substances from the physiological standpoint are the meat bases-creatin, creatinin, xanthin, sarkin, etc. To the predominance of these amido-bodies is undoubtedly due the wellknown stimulating effect of meat extracts. Indeed, a properly prepared extract has very little actual food value, but is rather to be regarded as a condiment or as a stimulant, acting on the nervous system in a somewhat analogous manner to tea and coffee.

Commercial meat extracts differ much in consistency according to 
the extent to which evaporation is carried, varying from the thin fluid through the pasty form to the semi-solid. Some preparations have added thereto finely ground dried beef or beef meal.

Owing partly to unfounded claims of manufacturers, meat extracts are commonly confused with meat juices, and products belonging to the former class are sold for the latter. Considering the widely different nature of meat extracts and meat juices, such confusion is a serious matter. Meat extract is employed as a stimulant in the form of beef tea or as a flavor for soups. Meat juices, on the other hand, are employed in the sickroom as a readily available form of food.

Standards.-The following standards for products of this class have been adopted by the Association of Official Agricultural Chemists and the Association of State and National Dairy and Food Departments:

I. Meat extract is the product obtained by extracting fresh meat with boiling water, and concentrating the liquid portion by evaporation after the removal of fat, and contains not less than $75 \%$ of total solids, of which not over $27 \%$ is ash, and not over $12 \%$ is sodium chloride (calculated from the total chlorine present), not over $0.6 \%$ is fat, and not less than $8 \%$ is nitrogen. The nitrogenous compounds contain not less than $40 \%$ of meat bases, and not less than $10 \%$ of creatin and creatinin.

2. Fluid meat extract is identical with meat extract, except that it is concentrated to a lower degree, and contains not more than 75 , and not less than $50 \%$ of total solids.

3. Bone extract is the product obtained by extracting fresh trimmed bones with boiling water and concentrating the liquid portion by evaporation after removal of fat, and contains not less than $75 \%$ of total solids.

4. Fluid bone extract is identical with bone extract, except that it is concentrated to a lower degree and contains not more than 75 and not less than $50 \%$ of total solids.

5. Meat juice is the fluid portion of muscle fiber, obtained by pressure or otherwise, and may be concentrated by evaporation at a temperature below the coagulating point of the soluble proteins. The solids contain not more than $15 \%$ of ash, not more than $2.5 \%$ of sodium chloride (calculated from the total chlorine present), not more than 4 nor less than $2 \%$ of phosphoric acid $\left(\mathrm{P}_{2} \mathrm{O}_{5}\right)$, and not less than ${ }_{12} \%$ of nitrogen. The nitrogenous bodies contain not less than $35 \%$ of coagulable proteins, and not more than $40 \%$ of meat bases.

6. Peptones are products prepared by the digestion of protein material 


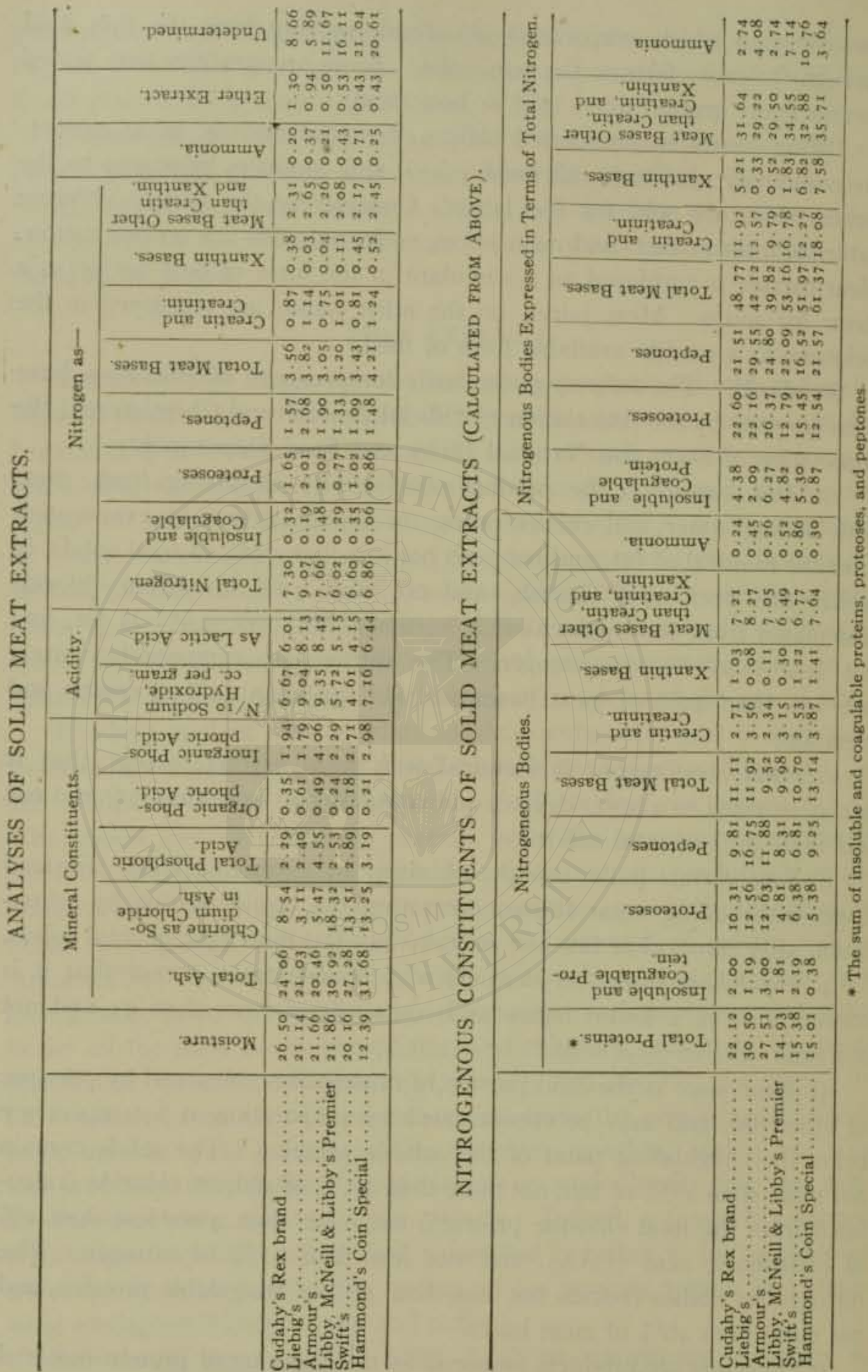



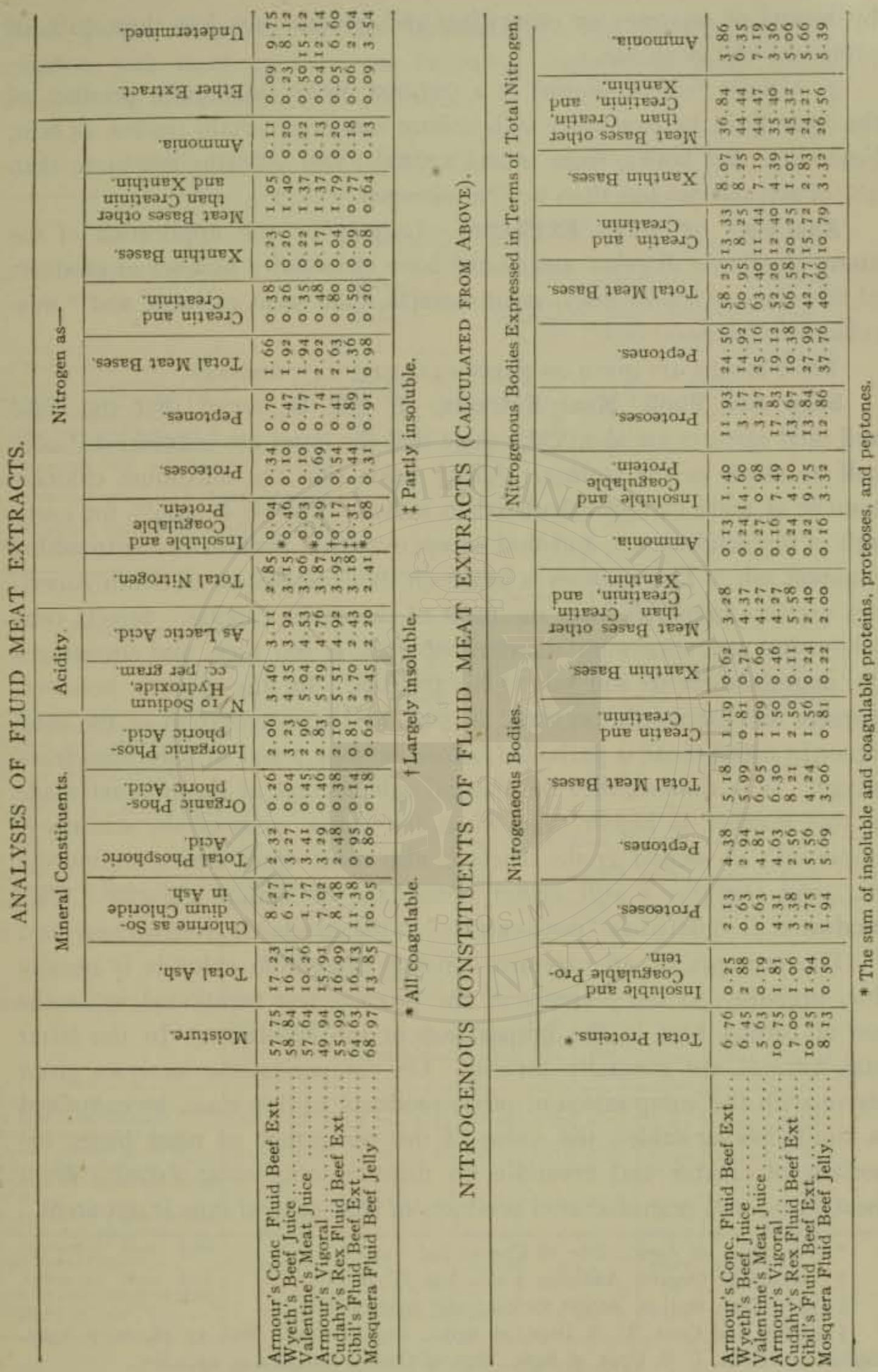
by means of enzymes or otherwise, and contain not less than $90 \%$ of proteoses and peptones.

7. Gelatin (edible gelatin) is a purified, dried, inodorous product of the hydrolysis, by treatment with boiling water, of certain tissues, as skin, ligaments, and bones, from "sound animals, and contains not more than $2 \%$ of ash and not less than $15 \%$ of nitrogen.

ANALYSES OF MEAT EXTRACTS.-Largely by the application of the above standards, Bigelow and Cook* have classified a number of products of this class as solid (pasty) meat extracts, fluid meat extracts, and " miscellaneous preparations."

Their results are given on pages $242,243,247$, and 248 .

Solid and Fluid Meat Extracts. - It will be noted that the solid and fluid extracts are identical, except that the latter are concentrated only half as much as the former. Allent holds that the maximum chlorine content of meat extract calculated to sodium chloride is $0.06 \%$ for every unit of dry solid matter, and that excess over that amount is due to added common salt. This opinion is based on the composition of South American extracts prepared from the meat of the entire carcass. Street $\ddagger$ considers that the maximum standard of $12 \%$ is too high, and encourages the manufacturer to add salt to his product. In this country, however, extracts are commonly prepared in part by the evaporation of the soup liquor in which meat is parboiled before canning, $\S$ and in part from trimmings. It is claimed that the natural salt content of the product made in this manner is higher than when the entire meat of the carcass is employed. A second grade article is also made from bones, trimmings, etc., and contains a still higher percentage of sodium chloride. This product is designated as "bone extract" in the standards given on page $24 \mathrm{~T}$.

The presence of an excessive amount of sodium chloride is usually due, probably, to the presence of the product last mentioned, or to the use of corned beef in the preparation of the substance. In the latter case nitrates are generally present. On comparing the analyses given above with the composition of other products of this class, as contained in the following tables, the value of the percentage of meat bases, especially of creatin and creatinin, in distinguishing meat extracts from meat juices and manufactured products of that general type is apparent.

* U. S. Dept. of Agric., Bur. of Chem., Bul. r14.

† Commercial Organic Analysis, 3 Ed, Vol. IV, p. 307.

$\ddagger$ Conn. Expt. Station, Report for 1907 and 1908, p. 622 .

$\$$ Bigelow and Cook, U. S. Dept. of Agric., Bur. of Chem. Bul. ז3, pt. 10, p. ${ }_{13} 89$. Bigelow and Cook, U. S. Dept. of Agric., Bur, of Chem., Bul. 114, p. 13. 
Meat Juices Prepared in the Laboratory.-For the purpose of comparison with meat extracts, the following analyses of meat juices prepared in the laboratory are of interest.

MEAT JUICES PREPARED ,IN LABORATORY**

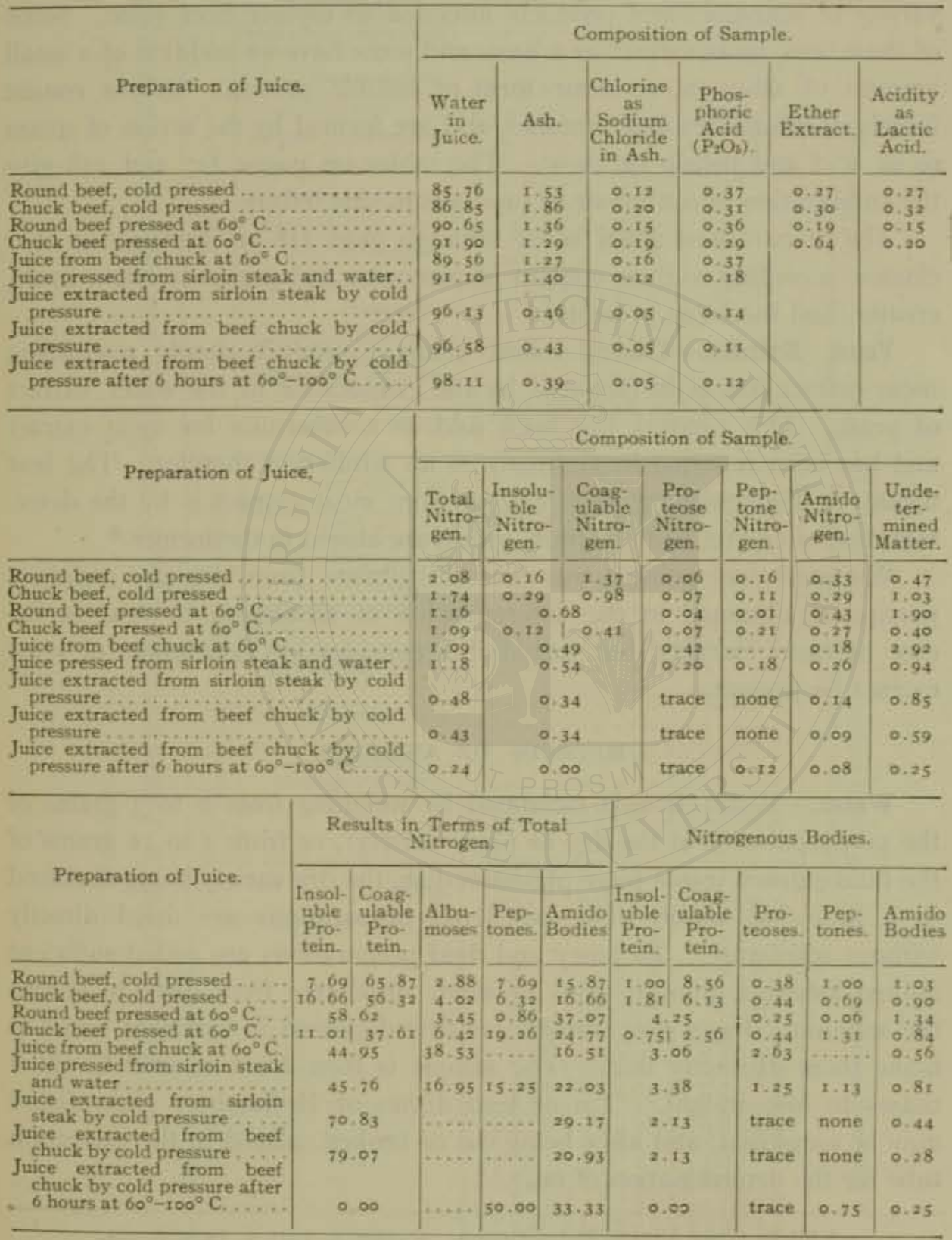

* Bigelow and Cook, U. S. Dept. of Agric., Bul. 1r4, p. rg. 
The composition of these products is widely different from that of the so-called meat juices of commerce, as given in the table on page 247 . It appears to be impracticable to so preserve a true meat juice that it can become an article of commerce.

Miscellaneous Meat Preparations.-There is on the market a wide variety of manufactured products intended to replace beef juice. Some of these have meat extract as a base, and some have an addition of a small amount of albumin, or some form of soluble protein. Others consist largely of albumoses and peptones, and are formed by the action of steam or of acid and pepsin on meat. The tables on pages 247 and 248 give the composition of a number of products of this nature.

The preparations given in the table on page 248 are arranged in four classes, according to their content of proteoses and peptones, meat bases, creatin, and insoluble proteins.

Yeast Extract.-During recent years a product closely resembling meat extract has been prepared by the evaporation of the water extract of yeast. This product has been sold as a substitute for meat extract and has been reported in Germany as an adulterant therefor. The best means of distinguishing yeast extract from meat extract is by the determination of creatin and creatinin, which are absent in the former.*

Wintgen $\dagger$ has pointed out that the filtrate from the zinc sulphate precipitate obtained in the determination of albumoses is clear in the case of meat extracts, but turbid if a considerable percentage of yeast extract be present.

\section{METHODS OF ANALYSIS.}

Water.-Water is best estimated by weighing from 2 to 3 grams of the preparation (if of the dry or pasty variety), or from 5 to 10 grams of the fluid extract, into a large platinum dish, the dry variety being dissolved in a little hot water. The powdered preparations are dried directly without admixture. To pasty and fluid preparations are added sufficient ignited asbestos, pumice stone or sand, sifted free from dust, to absorb the solution. Pasty preparations are first dissolved in sufficient water to make them distinctly fluid. The sample is then dried at $100^{\circ} \mathrm{C}$. till it ceases to lose weight. Tin or lead dishes or Hoffimeister glass dishes may be employed, and after being cut or broken, placed in the extraction tube for the determination of fat.

* Micko, Zeits. Unters. Nahr. Genuss., 5, 1902, p. 193; 6, 1903, p. 781 .

t Arch. Pharm., 242, 1904, p. 537. 


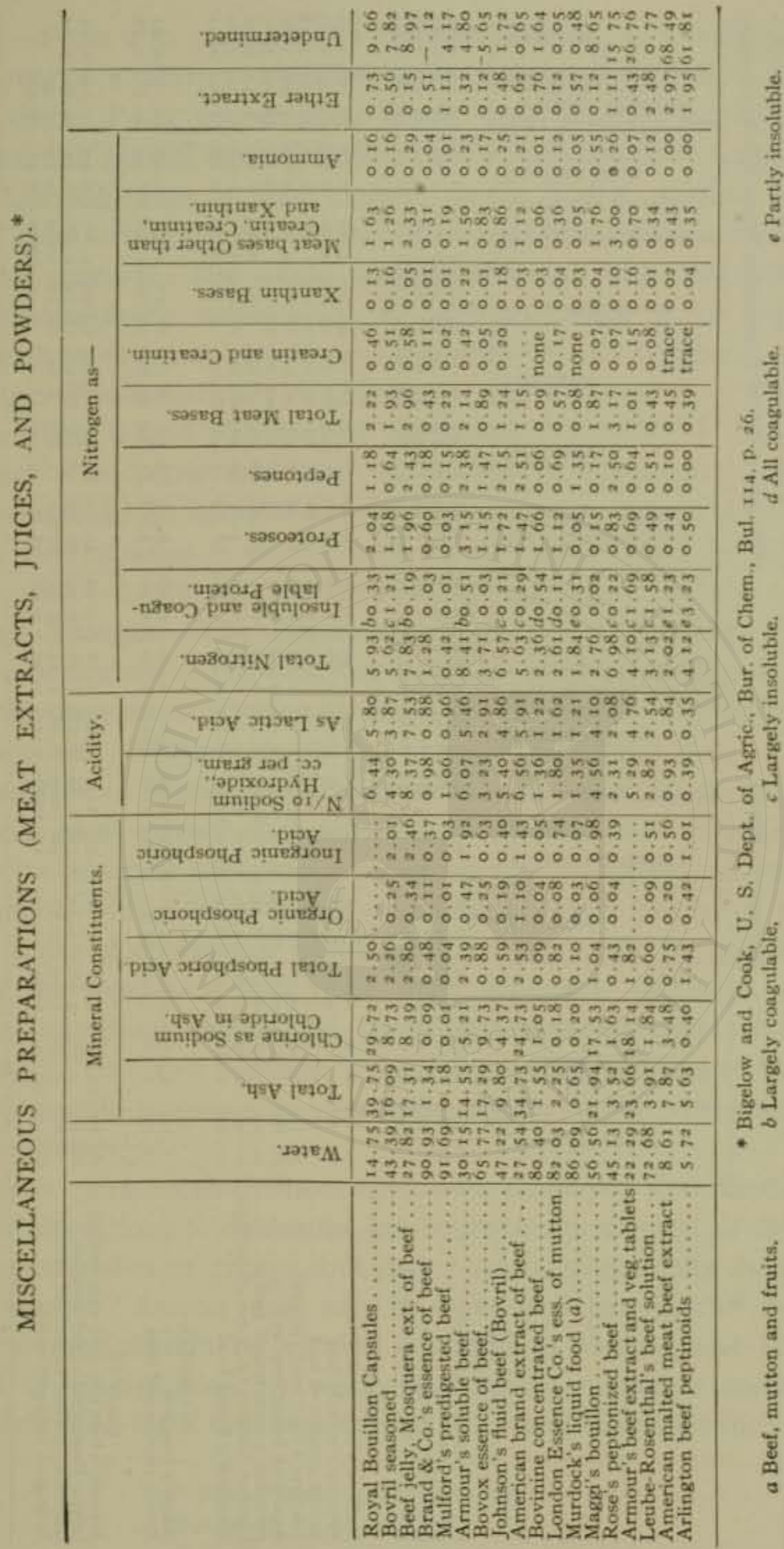




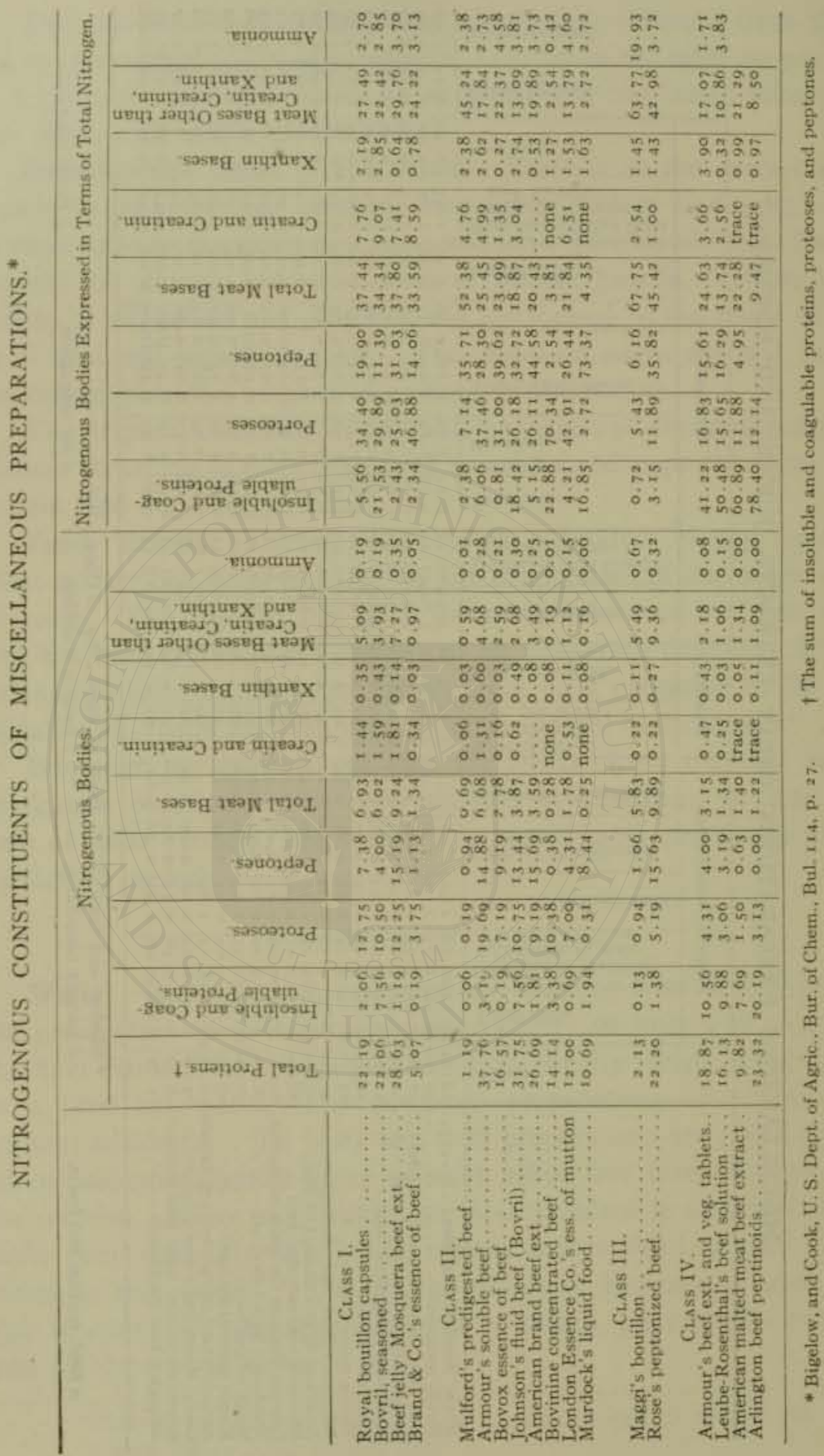


Ash. - From 2 to 3 grams of a fluid preparation, or a correspondingly less amount of a pasty preparation, are evaporated to dryness in a flatbottom dish. Pasty preparations should first be dissolved in water, in order that the sample may distribute itself evenly over the bottom of the dish. The substance is then charred at the lowest possible heat, the charred mass exhausted with water, the insoluble residue collected on a filter and washed. The filtrate and residue are then returned to the dish and completely incinerated, the soluble portion of the ash added, evaporated to dryness, heated to a low redness and weighed. Chlorine is determined volumetrically or gravimetrically in the solution of the ash.

Fat.-This is best obtained by extracting a portion of the air-dried substance with petroleum ether in a Soxhlet apparatus. Petroleum ether extracts the fat only, while ether extracts other substances as well.

The determination is usually made in the residue from the determination of water. A properly prepared extract has very little fat.

Total Nitrogen.-The extract should be tested for nitrates, and the proper modification of the Gunning method should be employed, depending on the presence or absence of nitrates. Use from $I$ to 5 grams for the determination. Nitrates should be properly accounted for.

Separation of Nitrogenous Compounds.-To correctly gauge the food value of a meat extract, it is essential to separate and estimate at least roughly its principal nitrogenous components. To attempt to make such a separation with a high degree of accuracy would involve a long and tedious series of operations, which in most cases would be impracticable. Usually the separation into three main groups is sufficient, insoluble proteins, tannin-salt precipitate (proteoses, peptones and gelatin) and meat bases. At times, however, it may become necessary, or at least desirable for specific purposes, to determine certain of the nitrogenous compounds separately.

Various quick methods have often been employed in connection with technical operations to determine the approximate amount of the several nitrogenous bodies or groups, but they have been generally discarded as untrustworthy. Among these may be mentioned Bruylant's* method of fractional precipitation by varying strengths of alcohol, and Hehner's $\dagger$ method of precipitation by methylated spirits. Another method that was widely used for a time was that of Allen and Searle, $\ddagger$ which is based

* Jour. Pharm. et Chem., 5,1897 , p. 515 .

$\uparrow$ Analyst, 10, p. 221 .

‡ Analyst, 22, 1897, p. 259. 
on the belief that proteoses and peptones were completely precipitated from aqueous solution by saturating with bromine, after acidifying with hydrochloric acid. The experimental evidence on which this method was based consisted of the precipitation of proteins from the filtrate from the zinc sulphate precipitate, diluted with an equal volume of water. From the results so obtained, it appeared that peptones and proteins of larger molecule were completely precipitated by bromine in a half saturated solution of zinc sulphate, and it was assumed that precipitation from aqueous solution would be equally complete. Owing to a lack of methods by which peptones could be completely precipitated, this method has been widely used. The use of the method now appears to have been largely discontinued, as it has been repeatedly found to be unreliable.*

Complete Separation of Nitrogen Compounds would involve a discrimination between meat fiber and insoluble protein, coagulable proteins, acid albumin (syptonin), albumoses, peptones, meat bases, gelatin and ammonia.

(I) Insoluble Proteins.-About 5 grams of the extract of the dry, or 20 to 25 grams of the fluid variety are exhausted with 200 to $250 \mathrm{cc}$. water at about $20^{\circ} \mathrm{C}$., and the residue collected on a tared filter. It is often difficult to filter such an extract in the ordinary way, and the use of the centrifuge is helpful, passing the clear supernatant liquid through the filter, and finally washing the residue thereon. The residue is washed, dried at $100^{\circ}$, and weighed, or the nitrogen may be determined by the Gunning method. The sample may also be placed in a graduated flask, digested in a considerable amount of cold water for several hours with frequent shaking, and the nitrogen determined in an aliquot part of the filtrate. This deducted from total nitrogen gives the nitrogen of insoluble proteins. $\mathrm{N} \times 6.25=$ total insoluble matter, which includes, besides the meat fiber, the insoluble proteins.

(2) Coagulable Proteins. - The filtrate from (I) is neutralized exactly to litmus, and dilute acetic acid added till acidity is just apparent. It is then boiled for some minutes to make insoluble the coagulable proteins, which are collected upon a filter (using to advantage a centrifuge as in the preceding paragraph). Determine the nitrogen in the washed residue, using the factor 6.25 for coagulable protein.

(3) Albumoses or Proteoses. $\dagger$-An aliquot part of the filtrate from (2)

* Bigelow, U. S. Dept. of Agric., Div. of Chem., Bul. r3, pt. ro, p. 1396; Bul. 81, p. ro6. Sjerning, Zeits, anal. Chem., 39, rgoo, p. 545. Fraps and Bizzell, Jour. Am. Chem. Soc., 22, 1900, p. 709. Van Slyke, Chem. News, 88, 1903, p. 92.

† Bömer, Zeit, anal. Chem. 5, 1895, p. 562. 
is saturated with zinc sulphate, adding the powdered salt as long as it continues to dissolve with stirring and shaking. Proteoses and any traces of gelatin or insoluble proteins that have escaped removal are precipitated, but not the peptones or meat bases. Filter, wash, and determine the nitrogen in the residue, using the factor 6.25 for the proteoses.

(4) Peptones.-Sjerning's Tannin-salt Method, modified by Bigelow and Cook.*-An aliquot part of the filtrate from (2), concentrated by evaporation to $20 \mathrm{cc}$. or less, in case it is necessary to take more than 20 cc., is transferred to a roo-cc. flask.

Then $50 \mathrm{cc}$. of a solution containing 30 grams of sodium chloride per Ioo cc, are added, and the flask agitated to insure the thorough mixing of its contents and the solution of the sample. The flask is now placed in the ice box at approximately $12^{\circ} \mathrm{C}$. After the solution has reached the ice box temperature (this requires an hour usually), $30 \mathrm{cc}$. of a $24 \%$ solution of tannin (which must be at ice box temperature) are added. The total volume is now $100 \mathrm{cc}$. The contents of the flask are thoroughly mixed, and the flask returned to the ice box, where it remains over night. In the morning the solution is filtered at ice box temperature into a $50 \mathrm{cc}$. graduated flask. The nitrogen is determined in this filtrate, and also in an aliquot portion of the filtrate from a blank, in which the reagents alone are employed. The nitrogen found in the $50 \mathrm{cc}$. portion, multiplied by two (after correction for the nitrogen in the blank), gives the total nitrogen in the filtrate, and is calculated to per cent of nitrogen on the sample employed. This includes the nitrogen present as ammonia, and all of the nitrogen of the meat bases, except that portion of the creatin precipitated by the tannin-salt reagent. The figure thus obtained is added to the per cent of nitrogen as determined in (I), (2), and (3). This sum, deducted from the total nitrogen, is ordinarily reported as the per cent of nitrogen existing as peptones, and is multiplied by 6.25 for the per cent of peptones.

It is probable that the substances so reported are not true peptones, since the filtrate from (3) commonly gives no biuret reaction. They probably consist largely of peptoids, formed by the action of the hot solution on gelatin and polypeptides.

Bigelow and Cook find that the tannin-salt precipitate is not contaminated with other meat bases than creatin. They believe that about one-quarter of the creatin is found in this precipitate. Accordingly,

* Jour. Am. Chem. Soc., 28, 1906, p. 1496. 
they suggest that the percentage of creatin be determined before and after precipitation with tannin-salt reagent, and correction made by the results so obtained.

Street believes this correction to be impracticable. He finds that it is very difficult, if not impossible, to remove tannin completely from the filtrate, and that the slightest trace of tannin prevents the color reaction for creatin.

(5) Meat bases.-The per cent of nitrogen found in the filtrate from the tannin-salt precipitate in (4), after deducting the per cent of nitrogen found as ammonia in (6), is multiplied by 3.12 to obtain the per cent of meat bases.

(6) Ammonia,-From 5 to Io grams of the original sample are dissolved in a convenient volume of water, and distilled after the addition of powdered magnesia. The distillate is titrated, and its alkalinity reported as per cent of $\mathrm{NH}_{3}$. The corresponding percentage of nitrogen is also calculated, as it is necessary for the calculation of meat bases in (5).

Determination of Creatin and Creatinin.*-This determination may be made in an aliquot of the filtrate from the insoluble and coagulable protein determination. $\dagger$ The aliquot must contain sufficient total creatinin, after dehydration of the creatin to creatinin, to give a reading not far from $8^{\circ}$ on the scale of the Dubosc colorimeter, after applying the colorimetric method as outlined by Folin $\ddagger$ for the estimation of creatinin in the urine. Heat this aliquot with $5 \mathrm{cc}$. of half-normal hydrochloric acid for three and a half hours on a steam bath under a reflux condenser. Neutralize the hydrochloric acid by the addition of $5 \mathrm{cc}$. of half-normal sodium hydroxide, then add I 5 cc. of a saturated picric acid solution, and $5 \mathrm{cc}$. of $10 \%$ sodium hydroxid. Shake the solution, and allow it to stand for five minutes; make up to 500 cc., and compare the color with a half-normal solution of potassium bichromate in the Dubosc colorimeter. The half-normal bichromate solution when the scale is set at $8^{\circ}$ corresponds to $10 \mathrm{mg}$. of creatinin, and from this figure the amount of creatinin in the aliquot is readily calculated.

Hehner $\$$ criticises this method as applied to meat extracts. He believes that more complete results may be obtained by using $25 \mathrm{cc}$. of a $1.0 \mathrm{r} \%$ of picric acid with "a quite small amount of alkali." He considers the

* Bigelow and Cook, Jour. Am. Chem. Soc., 28, 1906, p. 1497.

t Aliquot should represent approximately 0.2 gram of a first class solid beef extract.

$\ddagger$ Zeits. physiol. Chem., $4 \mathrm{I}$, Ig04, p. 223 .

$\$$ Pharm. Jour., 78,1907, p. $68_{3}$. 
precipitate somewhat soluble in excess of alkali. Emmett and Grindley* have made an exhaustive study of the method as applied to meats, meat extracts, and urines. They find that ${ }_{15} \mathrm{cc}$. of $1.2 \%$ picric acid should be employed for the original creatinin determinations, and $30 \mathrm{cc}$. for the dehydrated creatinin. They also recommend $5 \mathrm{cc}$. of alkali for the original creatinin, and $10 \mathrm{cc}$. for the dehydrated creatinin, though an additional $5 \mathrm{cc}$. does not give lower results.

Determination of Xanthin Bases.-In addition to creatin and creatinin, a true meat extract or meat juice should contain small amounts of xanthin bases, including xanthin, hypo-xanthin, guanin, and adenin. These bodies are derived from the nuclei of the cells, and, consequently, in an extract that is prepared from fresh, unaltered beef a certain amount of these bodies should be obtained, together with the salts and other extractive matter. The determination of the xanthin bases is, therefore, of value in determining the origin of an alleged extract of meat.

The xanthin base figures in the tables show a variety of results, which is explained by the fact that in the preparation of the extract under certain conditions of heat and pressure some of these bodies are destroyed. The following method was employed for their determination:

Schittenhelm's Method modified by Cook. $\dagger$ - Use an amount of the standard solution equivalent to 5 grams of the original extract. Place in a large evaporating dish, and add $500 \mathrm{cc}$. of $\mathrm{r} \%$ sulphuric acid. Evaporate to $\mathrm{Ios} \mathrm{cc}$. within 4 to 5 hours. Cool, and neutralize with sodium hydroxide. Add ro cc. of $15 \%$ sodium bisulphate, and $15 \mathrm{cc}$. of $20 \%$ copper sulphate; allow this to stand over night, filter, and wash. The precipitate suspended in water is treated with sodium sulphide, and warmed on the steam bath. Add acetic acid to acidify, and filter hot. To the filtrate add to cc. of 10\% hydrochloric acid, and evaporate to a volume of about 1o cc. Filter, make ammoniacal, and add ammoniacal silver nitrate of $3 \%$ strength. After standing several hours, the solution is filtered and the precipitate washed with distilled water until no longer alkaline. The nitrogen in the precipitate is that of the xanthin bases.

Determination of Gelatin.-This is accomplished by the modified Stutzer method as given on page 231 .

Determination of Acidity. $\ddagger$-In the average solid or pasty extract the lactic acid conteni varies from 4 to 8 per cent, and, as a rule, the extract showing the highest phosphoric acid content likewise shows the highest

* Jour. Biol. Chem., 3, 1907, p. 491.

† U. S. Dept. of Agric., Bur. of Chem., Bul, 114 , p. $4 \mathrm{I}$.

‡ Ibid., p. 39 . 
acidity. This is undoubtedly due to the fact that some of the phosphoric acid is in the form of di-hydrogen or acid phosphate, although the character of the acidity has not been definitely determined.

The method employed for determining acidity consisted in adding tenth-normal sodium hydroxide to a dilute solution of the meat extract in water, until a drop removed by means of a small capillary tube and tested on a piece of litmus paper gives a neutral reaction. The results are expressed in cubic centimeters of tenth-normal sodium hydroxide, also as per cent of lactic acid present. The acidity is commonly expressed as per cent of lactic acid, though it is probably due in large part to acid potassium phosphates. Lactic acid is the chief organic acid, though succinic acid is also present in notable amount.*

Detection of Preservatives in Meat Extracts.-Boric acid is sometimes used as a preservative in these preparations, and is tested for by the usual methods (Chapter XVIII).

Determination of Glycerin.-This substance is sometimes used as a preservative for fluid preparations. Perhaps the most satisfactory method that has been suggested for its determination is that of Bigelow and Cook. $\dagger$ The dried residue is extracted with acetone, the meat bases removed by precipitation with silver nitrate, followed by phosphotungstic aoid. The glycerin is determined in the filtrate by Hehner's method. $\ddagger$

\section{FISH.}

Structure and Composition.-Fish resembles meat both structurally and in the nature of its constituents, but differs from it in a marked degree in the relative proportions of its various components. Thus, there is considerably more refuse matter such as skin and bones in fish than in meat, and in the edible portion of fish the amount of water is much greater. Comparing the nitrogenous components of each, we find in fish more of the gelatin-yielding matter (collagen) and less of the extractives than in meat. There is much less hæmoglobin or allied coloring substance in the flesh and blood of fish than in meat, which accounts for the white color usually characteristic of the former. Certain fish, however, like the salmon, probably owe their distinctive color to a pigment belonging to the lipochrome $\S$ class. The mineral content of fish, as a rule, exceeds that of meat and contains more phosphates. The various edible fishes differ less among themselves in composition than do the meats. According to Chapman the average composition of fish is as follows, in parts per rooo:

*Arb. kais. Gesundheitsamt, t9o6, vol. 24.

$\dagger$ U. S. Dept. of Agric., Bur, of Chem., Bul. 114, p. 42.

$\ddagger$ Jour. Soc. Chem. Ind., 8, 1889 , p. 4 .

$\$$ A series of fatty animal pigments. 


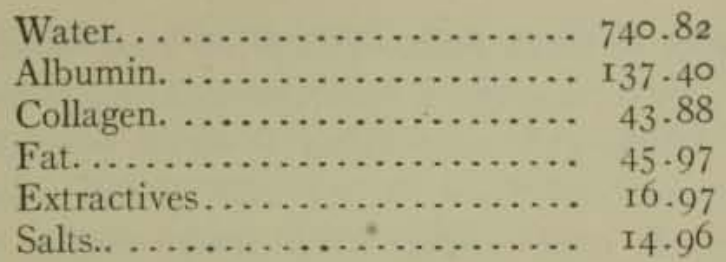

Hutchison classifies fish as follows with reference to their content of fat: Lean.-Fish having less than $2 \%$ fat, such as cod and haddock.

Medium. - Fish having 2 to $5 \%$ fat, such as halibut and mackerel.

Fat.-Fish having more than $5 \%$ of fat, such as eel, $18 \%$; salmon, $12 \%$; turbot, ${ }_{12} \%$, and herring, $8 \%$.

According to Atwater and Bryant * the composition of different varieties of fish is as follows:

COMPOSITION OF FISH.

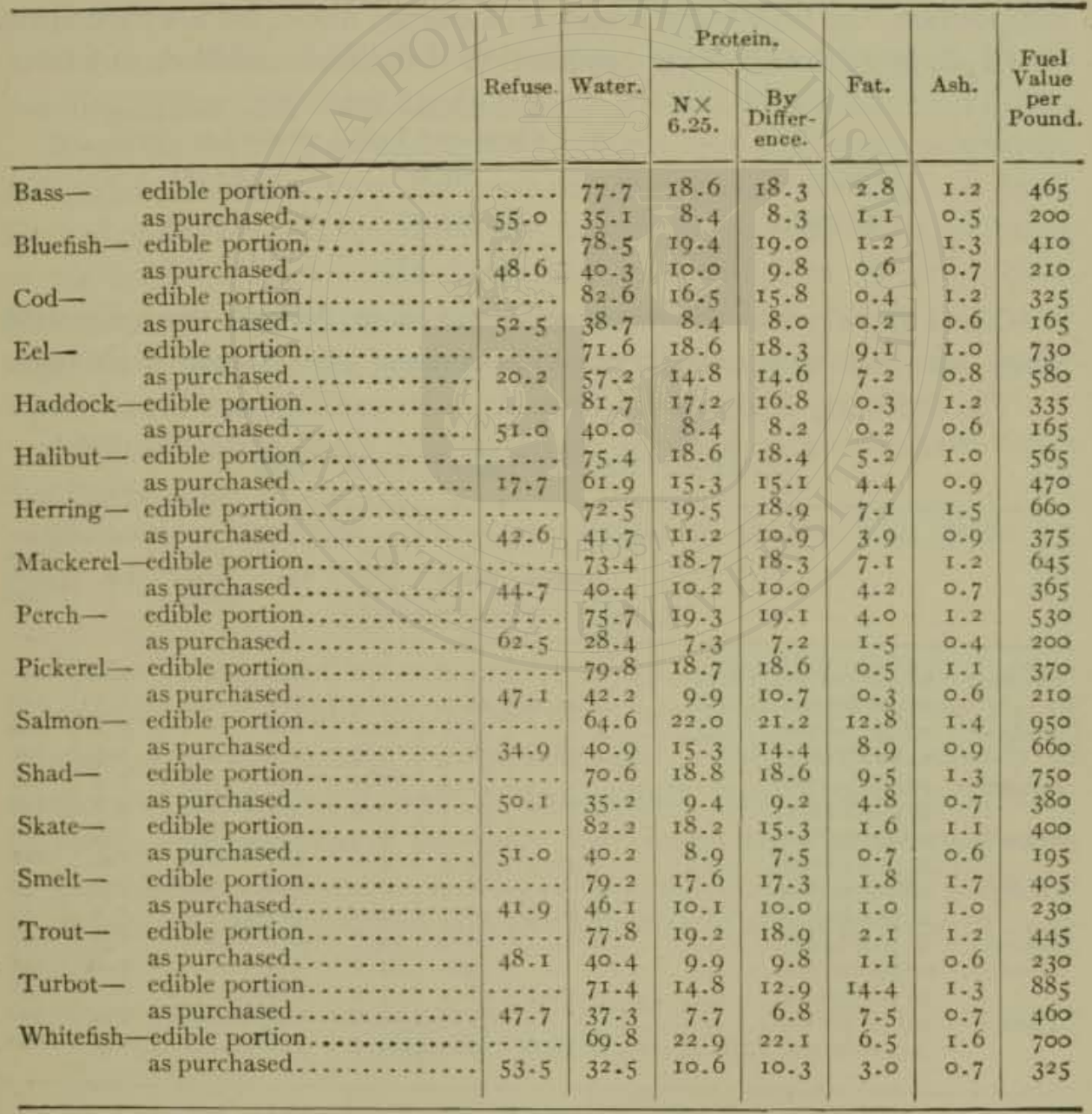

* U. S. Dept. of Agric., Off. of Exp. Sta,, Bul. 28 , p. 47 et seq. 
Characteristics of Fresh Fish.-Fish of all kinds should be eaten when perfectly fresh, as it undergoes decomposition much sooner than mea: when killed. While with meat aging is often beneficial to bring out requisite tenderness and flavor, in the case of fish deterioration begins almost immediately after death. Even though certain varieties of fish may be kept firm and wholesome for some days on ice, the flavor is distinctly impaired by long keeping. Fish that is not perfectly firm to the touch, or that has abnormally dry scales, or that shows blubber at the gills, or that possesses the marked odor that accompanies incipient decomposition, should not be used as food.

Crustaceans and Mollusks.-These differ from the meats and common fish by reason of the presence in considerable proportion of the carbohydrate glycogen contained in the liver. The lobster and crab are nearly alike in composition, the flesh being made up of coarse, dense, thick-walled fibers.

Payen gives the following composition of the flesh and body of lobster:

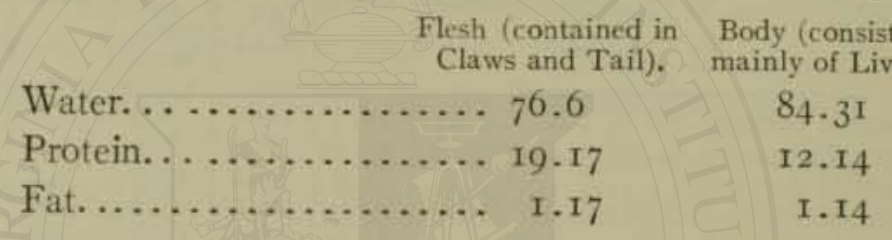

Clams and Oysters are low in solid nutriment, and are more digestible when eaten raw than cooked. Oysters contain $3 \%$ or more of glycogen.

The following analyses are from Atwater and Bryant:*

COMPOSITION OF SHELL FISH, ETC.

\begin{tabular}{|c|c|c|c|c|c|c|c|c|}
\hline$=$ & & Refuse. & Water. & $\begin{array}{l}\text { Pro- } \\
\text { tein. } \\
\text { N } \\
6.25 .\end{array}$ & Fat. & $\begin{array}{l}\text { Car- } \\
\text { bohy- } \\
\text { drates. }\end{array}$ & Ash. & $\begin{array}{c}\text { Fuel } \\
\text { Value } \\
\text { per } \\
\text { Pound. } \\
\text { Cals. }\end{array}$ \\
\hline \multirow[t]{2}{*}{ Clams- } & edible portion. & & 85.8 & 8.6 & 1.0 & 2.0 & 2.6 & ${ }^{\circ} 24^{\circ}$ \\
\hline & as purc & $41 \cdot 9$ & $49 \cdot 9$ & $5 \cdot 0$ & 0.6 & I. 1 & I. 5 & 140 \\
\hline \multirow{2}{*}{ Crabs- } & edible & & $77 \cdot 1$ & 16.6 & 2.0 & 1.2 & 3.1 & 415 \\
\hline & as purchased.. & 52.4 & $3^{6.7}$ & $7 \cdot 9$ & 0.9 & 0.6 & I. 5 & 195 \\
\hline \multirow[t]{2}{*}{ Lobster- } & edible portion. & & 79.2 & 16.4 & 1.8 & 0.4 & 2.2 & 390 \\
\hline & $\begin{array}{l}\text { as purchased.. } \\
\text { edible portion. }\end{array}$ & 61.7 & 30.7 & $5 \cdot 9$ & 0.7 & 0.2 & 0.8 & 140 \\
\hline Mussels- & as purchased... & 46.7 & $\begin{array}{l}84.2 \\
44 \cdot 9\end{array}$ & $\begin{array}{l}0.7 \\
4.6\end{array}$ & $\begin{array}{l}1.1 \\
0.6\end{array}$ & $\begin{array}{l}4.1 \\
2.2\end{array}$ & $\begin{array}{l}1.9 \\
1.0\end{array}$ & $\begin{array}{l}285 \\
150\end{array}$ \\
\hline \multirow[t]{2}{*}{ Oysters - } & edible portion. & & 86.9 & 6.2 & I. 2 & $3 \cdot 7$ & 2.0 & 235 \\
\hline & as purchased & $8 \mathrm{r} .4$ & 16.1 & 1.2 & 0.2 & 0.7 & 0.4 & 45 \\
\hline Scallops- & as purchas & & 80.3 & 14.8 & 0.1 & $3 \cdot 4$ & 1. 4 & 345 \\
\hline \multirow{2}{*}{ Terrapin- } & edible portic & & $74 \cdot 5$ & 21.2 & $3 \cdot 5$ & ........ & 1.0 & 545 \\
\hline & as purchased. & $75 \cdot 4$ & 18.3 & $5 \cdot 2$ & 0.9 & ....... & 0.2 & 135 \\
\hline \multirow[t]{2}{*}{ Turtle- } & $\begin{array}{l}\text { edible portion. } \\
\text { as purchased. }\end{array}$ & & 79.8 & 19.8 & 0.5 & $\ldots$ & 1.2 & $39^{\circ}$ \\
\hline & & 70.0 & 19.2 & 4.7 & 0.1 & & 0.3 & $9^{\circ}$ \\
\hline
\end{tabular}

* U. S. Dept. of Agric., Off. of Exp. Sta., Bul. 28, pp. 52 and 53. 
Floating of Shellfish.-Oysters and other shellfish, either in the shell or, more commonly, after shucking, are often subjected to "floating" or "drinking" in fresh or brackish water or else shipped in direct contact with lumps of ice. Both practices cause the shellfish to greatly increase in size, owing to the absorption of an undue amount of water, and if not labelled "floated" the product is adulterated under the Federal law and the laws of certain States.

It is however, not regarded as improper to drink oysters in water of a saline content equal to that in which they will grow to maturity or to wash the shucked oysters in unpolluted, cold or iced water for the minimum time required for cleaning and chilling. After washing they should be drained and packed for shipment in tight receptacles surrounded by ice but protected from the absorption of the water resulting from the melting of the ice.

Often shellfish is polluted by growing or floating in impure water, handling under insanitary conditions, or packing in unclean receptacles.

Preservatives in Fish and Oysters.-Boric acid and borax in mixture and sodium benzoate form the most common preservatives of salt dried fish and of oysters. In the case of salt codfish, the preservative is sprinkled on the surface. Such surface application in some States, as for example Massachusetts, is allowed by law. In opened oysters sold in casks and kegs, boric mixture has been used commonly in solution in the oyster liquor, but is now infrequent.

Artificial Colors of the coal-tar group are used to give smoked fish a rich brown color.

Methods of Analysis. - These are similar to the methods given for meat.

\section{CONCENTRATED FOODS.}

Under the name of "condensed" or "concentrated foods" or "emergency rations" a number of canned preparations are sold for the use of campers, travelers, armies in the field, etc. These consist usually of mixtures of dried ground meats and vegetables, pressed together in compact form, and preserved in tin cans. The claims made for the food value of these preparations are, as a rule, extravagant and erroneous, as shown by Woods and Merrill,* who give the following analyses of some of these foods:

* Maine Exp. Sta., Bul. 75, p. 103. 


\begin{tabular}{|c|c|c|c|c|c|c|c|}
\hline & \multirow{2}{*}{$\begin{array}{c}\text { Net } \\
\text { Weight } \\
\text { Con- } \\
\text { tents. }\end{array}$} & \multicolumn{5}{|c|}{ Weight of Materials in Package. } & \multirow{2}{*}{$\begin{array}{c}\text { Total } \\
\text { Fuel } \\
\text { Value. } \\
\text { Cals. }\end{array}$} \\
\hline & & Water. & $\begin{array}{l}\text { Pro- } \\
\text { teins. }\end{array}$ & Fat. & $\begin{array}{c}\text { Carbo- } \\
\text { hy- } \\
\text { drates. }\end{array}$ & Ash. & \\
\hline & Grams. & Grams. & Grams. & Grams. & Grams. & Grams. & \\
\hline Ration cartridge, pea, beef, etc. ........ & 241 & $34 \cdot 2$ & 52.9 & 42.0 & 98.0 & 13.9 & $107 \mathrm{I}$ \\
\hline Blue ration campaigning food, $a \ldots \ldots \ldots$ & $\begin{array}{r}169 \\
78\end{array}$ & 76.1 & $37 \cdot 5$ & 9.0 & $37 \cdot 9$ & 8.5 & 432 \\
\hline Red ration campaigning food, $a \ldots \ldots \ldots$ & $\begin{array}{r}78 \\
122\end{array}$ & $\begin{array}{r}10 \\
33.8\end{array}$ & $\begin{array}{r}5.6 \\
26.2\end{array}$ & $\begin{array}{l}23.1 \\
18.5\end{array}$ & $\begin{array}{l}40.9 \\
37.8\end{array}$ & $\begin{array}{l}1.4 \\
5.7\end{array}$ & 436 \\
\hline 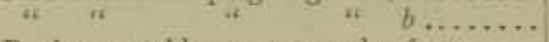 & 77 & 1.2 & 5.0 & 23.0 & 46.6 & $\begin{array}{l}5 \cdot 7 \\
1.2\end{array}$ & $\begin{array}{l}496 \\
424\end{array}$ \\
\hline Ration cartridge, potatoes, beef, etc. ... & 283 & 117.9 & 62.3 & 12.6 & 76.4 & 13.8 & 772 \\
\hline Emer gency ration, $a . \ldots \ldots \ldots \ldots \ldots$ & 120 & 14.2 & 56.1 & 29.6 & II. 9 & 7.8 & 617 \\
\hline$\| \quad \forall \quad b \ldots \ldots \ldots \ldots \ldots$ & 113 & I. 9 & 8.2 & $32 \cdot 7$ & 68.0 & 2.2 & 622 \\
\hline Emergency ration, $a \ldots \ldots \ldots \ldots \ldots \ldots$ & 121 & $4 \cdot 5$ & $7 \mathrm{I} . \mathrm{S}$ & 32.6 & 6.7 & $5 \cdot 4$ & 776 \\
\hline 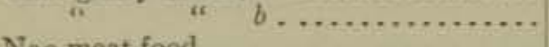 & 127 & $5 \cdot 7$ & 8. 3 & $15 \cdot 3$ & 94.8 & 2.9 & 588 \\
\hline 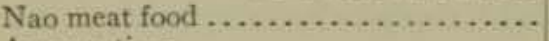 & 437 & $23^{\mathrm{r}} \cdot 3$ & 56.9 & 90.1 & 46.2 & 12.5 & I 328 \\
\hline 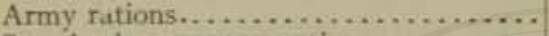 & $66 t$ & 420.2 & 101.2 & $84 \cdot 3$ & 47.9 & $7 \cdot 4$ & 1542 \\
\hline Standard emergency ration. ........... & 418 & 23.6 & 129.6 & $90 \cdot 5$ & 160,3 & $I_{4} .0$ & 2198 \\
\hline$"$ " $"$ " $\quad$ u $\quad$ a...... & 270 & 17.0 & 50.6 & 54.8 & 137.0 & 10.6 & 1402 \\
\hline a $b \ldots \ldots \ldots$. & 49 & 0.5 & 3.2 & 10.5 & 34.0 & 0.8 & 254 \\
\hline$\ldots \ldots \ldots \ldots \ldots \ldots \ldots$ & 423 & 30.7 & $75 \cdot \mathrm{I}$ & $167 \cdot 3$ & 119.8 & $30 . \mathrm{I}$ & 2430 \\
\hline Tanty emergency ration............... & 475 & $313 \cdot 5$ & 60.2 & 48.6 & 41.9 & 10.8 & 1482 \\
\hline F-A Food Company's stew........... & 9647 & 638.0 & 149.2 & $1 \mathrm{r} 4 \cdot 5$ & $52-5$ & 9.8 & 2460 \\
\hline
\end{tabular}

\section{REFERENCES ON FLESH FOODS.}

ACKerman, D., und Kutscher, F. Ueber Krabben-Extrakt. Zeits. Unters. Nahr. Genuss., 13,1907 , pp. 180,610 , and $61_{3}$.

ANDrEws, O. W. Flesh Foods. London, Igoo.

Arnold, C., und Mentzer, C. Zur Untersuchung von Fleischextrakt und Hefeextrakt. Pharm. Ztg., 49, 1904, p. 176.

Association of Official Agricultural Chemists. Methods for the Analysis of Meat and Meat Products. U. S. Dept. of Agric., Bur. of Chem., Bul. I07 (rev.), p. I06.

Barliet, L. Traité de l'inspection des viandes.

Balland, A. The Composition of Fish, Crustaceans and Molluscs. Compt. rend, 126,1898, p. 1721 .

Barschali, H., und BAUR, E. Beiträge zur Kenntnis des Fleischextraktes. Arb. Kaiserl. Gesundheitsamte, 24, 1906, p. 552.

Bigelow, W. D. Preserved Meats. U. S. Dept. of Agric., Bur. of Chem., Bul. 13, Part X.

- Floating of Oysters. A. O. A. C. Proc., 1908, U. S. Dept. of Agric., Bur. of Chem., Bul. 122, p. 215 .

Report on Separation of Meat Proteids. Proceedings of the A. O. A. C., Ig03, 1904, 1905. U. S. Dept. of Agric., Bur. of Chem., Bul. 8I, p. ro4; Bul. go, p. 126 ; Bul. 99 , p. 172 .

Brgelow, W. D., and Cook, F. C. The Separation of Proteoses and Peptones from the Simpler Amino Bodies. Jour. Am. Chem. Soc., 28, 1906, p. I 485.

- Meat Extracts and Similar Preparations. U. S. Dept. of Agric., Bur. of Chem., Bul. II4. 
Boos, W. Chemical Examination of Drawn and Undrawn Poultry Kept in Cold Storage. Mass. State Board of Health, 39th An. Rep, 1907.

Cook, F. C. Report on the Separation of Meat Proteids. Proceedings of the A. O. A. C., 1906, r907. U. S. Dept. of Agric., Bur. of Chem., Bul. 105, p. 9r; Bul. II6, p. 44 .

Davies, H. E. Searl's Test for Yeast Extract. Pharm. Jour., 72, 1904, p. 86.

Dougl.As's Encyclopædia for Bacon Curers, Meat Inspectors, Local Authority Officers, etc. Wm. Douglas \& Sons, London.

Duff, JAs. C. Manufacture of Sausages. New York, 1899.

FIEHE, J. Ueber den Nachweis von Pferdefleisch in Fleisch- und Wurstwaren mittels der Präcipitatreaktion. Zeits. Nahr. Unters. Genuss., 13, 1907, p. 744.

Fischoner, F. Leitfaden der Praktischen Fleischbeshau. Berlin, I899.

Gautier, A. Les Toxines. Paris, 1896.

Grindery, H. S. Losses in Cooking Meat. U. S. Dept. of Agric., Office of Exp. Sta., Bul. ro2.

- The Nitrogenous Constituents of Flesh. J. Am. Chem. Soc., 26, 1904, p. 1086.

GrIndLEY, H. S., and EammtT, A. D. The Chemistry of Flesh. Improved Methods for the Analysis of Animal Substances. Jour. Am. Chem. Soc., 27, 1905, p. 658 .

- The Chemistry of Flesh. A Study of the Phosphorus Centent of Flesh. Jour. Am. Chem. Soc, 28 , 1906, p. 25.

- A Preliminary Study of the Effect of Cold Storage upon Beef and Poultry Jour. Ind. and Eng. Chem., I, I909, P. $4 \mathrm{I}_{3}$.

Grindley, H. S., and Mojonnier, T. The Artificial Method for Determining the Ease and Rapidity of the Digestion of Meats. Studies of the University of Illinois, 1, No. 5,1903 , p. 185 .

Grindley, H. S., and Trowbridge, P. F. The Chemistry of Flesh. A Study of the Proteids of Beef Flesh. Jour. Am. Chem. Soc., 28, 1906, pp. 469-505.

GrindeEY, H. S., and Woods, H. S. The Chemistry of Flesh. Methods for the Determination of Creatinin and Creatin in Meats and their Products. J. Biol. Chem., 2, 1907, p. 309 .

Hast, L. D., and Emmetr, A. D. Relative Economy Composition and Nutritive Value of the Various Cuts of Beef. III. Exp. Sta. Bul. ${ }_{5} 8$.

Kickton, A, und MURDFIELD, R. Ueber den praktischen Wert der Glykogenbestimmung zum Nachweis von Pferdefleisch. Zeits. Unters. Nahr. Genuss., I4, 1907 , p. 501 .

KrтA, T. Ueber die Fettbestimmung im Fleisch und Fleischwaren mittels des Gerberschen Azid-Butyrimeters. Arch. f. Hyg., 51, 1904, p. 165.

Langworthy, C. F. Fish as Food. U. S. Dept. of Agric., Farmer's Bul. 85.

Lebben, S. Preservation and Coloring of Meat Produce. Berlin, Igor.

Leuckart. Human Parasites.

McGill, A. Commercial Beef Extracts. Canada Inland Rev. Dept, Bul. 63 .

Maldet, J. W. Physiological Effect of Creatine and Creatinine, and their Value as Nutrients, U. S. Dept. of Agric., Off. of Exp. Sta., Bul. 66.

Mrско, K. Vergleichende Untersuchung Fleischextrakten und deren Ersatzmitteln. Zeits. Unters. Nahr. Genuss., 5, 1902, p. 193.

- Untersuchung von Fleisch-, Hefen- und anderen Extrakten auf Xanthinkörper. Ibid., 6, 1903, p. $78 \mathrm{r} ; 7$, 1904, p. $257 ; 8$, 1904, p. 225.

- Hydrolyse des Fleischextraktes. Ibid., Io, 1905 , p. 393 ; $\mathrm{II}_{3} \mathrm{x} 906$, p. 705. 
Micko, K. Hydrolyse der Albumosen des Fleischextraktes. Zeits. Unters. Nahr. Genuss., 14, 1907, p. 253.

- Ueber die Untersuchung von Fleischsāften. Zeits. Unters. Nahr. Genuss,, 20, 1910, p. 537.

Missouri Exp. Station, Bul. 25. ,Composition of Flesh of Cattle.

Mrtchele, C. A. Flesh Foods. London, rgoo.

Ostertag. Handbuch der Fleischbeshau.

Pennington, M. E. Changes Taking Place in Chickens in Cold Storage. U. S. Dept. of Agric., Yearbook 1907 , p. 197.

- A Chemical, Bacteriological, and Histological Study of Cold-stored Poultry. Proc, Ist Internat. Congress of Refrig. Industries, 2, 1909, p. 216.

- Studies of Poultry from the Farm to the Consumer. U. S. Dept. of Agric., Bur. of Chem., Cir. 64.

Pennington, M. E., and Greenlfe, A. D. An Application of the Folin Method to the Determination of the Ammoniacal Nitrogen in Meat. Jour. Am. Chem. Soc., 32, 1910, p. 561 .

Pennington, M. E., and Hepburn, J. S. The Determination of the Acid Value of Crude Fat and Its Application in the Detection of Aged Food. Ibid., p. 568.

Richardson, W. D., and Scherubel, E. The Deterioration and Commercial Preservation of Flesh Foods. Jour. Am. Chem. Soc., 30, 1908, p. 1515.

SAlmon, D. E. Inspection of Meats for Animal Parasites. U. S. Dept. of Agric., Bureau of An. Ind., Bul. Ig.

Schmid-Musherm. Handbuch der Fleischkunde. Leipsic, 1884.

SeARt, A. Yeast Extract and Its Detection. Pharm. Jour., 71,1903 , pp. 516 and $704 ; 72,1904$, p. 86.

Street, J. P. Meat Extracts and Meat Preparations. Conn. Agl. Exp. Sta., Rep. I908, Pt. 9, p. 606 .

Trownridge, P. F. Report on the Separation of Meat Proteids. Proceedings of the A. O. A. C., 1908. U. S. Dept. of Agric., Bur. of Chem., Bul. 122, p. 61.

U. S. Food Inspection Decisions: No. 110 . Shellfish. No. 121. The Floating of Shellfish.

Vaughan, V. C., and Novy, F. G. Cellular Toxines.

Walley, Thos. A Practical Guide to Meat Inspection.

We.Ber, F. C. Report on Meat and Fish. Proceedings of the A. O. A. C., 1908. U. S. Dept. of Agric, Bur. of Chem., Bul. 122, p. 42.

WriEx, H. W. Separation of Flesh Bases from Proteids by Bromine. U. S. Dept. of Agric., Div, of Chem., Bul. 54.

Chemical Composition of the Carcasses of Pigs. U. S. Dept. of Agric., Bur. of Chem., Bul. 53 .

WrLey, H. W., and others. A preliminary Study of the Effects of Cold Storage on Eggs, Quail and Chickens. U. S. Dept. of Agric., Bur. of Chem., Bul. $1_{5}$.

Wintgen, M. Ueber den Nachweis von Hefeextrakt in Fleischextrakt. Arch. Pharm., 242, 1904, p. 537.

Woons, C. D. Meats, Composition and Cooking. U. S. Dept. of Agric., Farmer's Bul. 34 .

Zeitschrift für Fleisch und Milch Hygiene, 189r et seq. 


\section{CHAPTER IX.}

\section{EGGS.}

Nature and Composition.-Though eggs of various birds are used to some extent as food, it is the egg of the hen that is in universal use for this purpose, and therefore the one which is here for the most part discussed, bearing in mind that the structure and composition of all varieties of birds' eggs are closely analogous.

Fig. 60 shows the longitudinal section of a hen's egg.

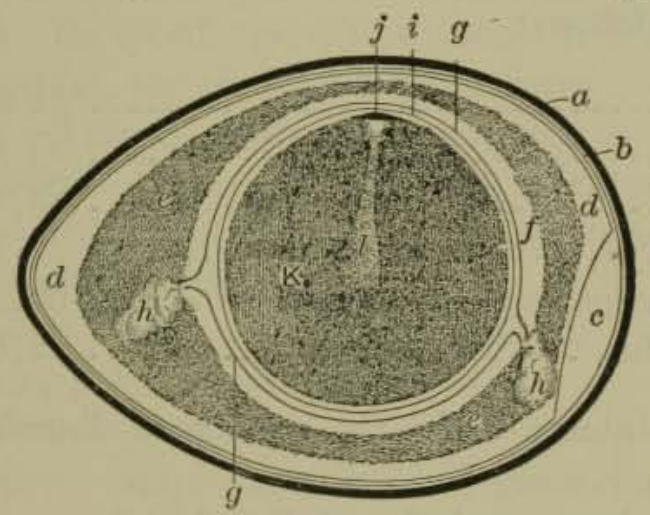

Fro. 60.-Longitudinal Section of a Hen's Egg. $a$, Shell; $b$, Double Membrane of Shell; c, Air-chamher; $d$, Outer, or Fluid Albuminous Layer; $e$, Thick, Middle Albumincus Layer; $f$, Inner Albuminous Layer; $g$, Membrane of the Chalaza; $h h$, the Chalaza; $i$, Vitelline Membrane; $j$, Germ; $k$, Yolk; $l$, Latebra. (After Macé.)

The average weight of a hen's egg is 60 grams, of which the sheli weighs about 6 , the white 36 , and the yolk 18 . Roughly it contains $70 \%$ of water, $12 \%$ of albumin, and $12 \%$ of fat.

The shell, according to König, has the following composition:

Calcium carbonate. . . . . . . . .

Magnesium carbonate....................... o- $2 \%$

Calcium and magnesium phosphate................ $0.5^{-} 5 \%$

Organic substances.......................... $2.0-5 \%$ 
The mean percentage composition of the eggs of the hen, duck, and plover are, according to König, as follows:

\begin{tabular}{|c|c|c|c|c|c|c|c|}
\hline & \multirow{2}{*}{$\begin{array}{l}\text { Water. } \\
\text { Per Cent. }\end{array}$} & \multirow{2}{*}{$\begin{array}{l}\text { Proteins. } \\
\text { Per Cent. }\end{array}$} & \multirow{2}{*}{$\begin{array}{l}\text { Fat. } \\
\text { Per Cent. }\end{array}$} & \multirow{2}{*}{$\begin{array}{l}\text { Nitrogen- } \\
\text { free Sab- } \\
\text { stanice } \\
\text { Per Cent. }\end{array}$} & \multirow{2}{*}{$\begin{array}{c}\text { Salts } \\
\text { Per Cent. }\end{array}$} & \multicolumn{2}{|c|}{$\begin{array}{c}\text { In the Dry Sub- } \\
\text { stance. }\end{array}$} \\
\hline & & & & & & $\begin{array}{l}\text { Nitrogen } \\
\text { Per Cent. }\end{array}$ & $\begin{array}{c}\text { Pat } \\
\text { Per Cent. }\end{array}$ \\
\hline Hen's egg. . . . . . . . . . & 73.67 & 12.55 & 12.11 & 0.55 & 1. 12 & 7.66 & 45.99 \\
\hline Duck's egg. . . . . . . . . . . & 71.11 & 12.24 & $15-49$ & $\ldots$ & I. 16 & 6.78 & 53.62 \\
\hline Plover's egg,....... & 74.43 & 10.75 & 11.66 & 2.18 & 0.98 & 6.75 & 45.78 \\
\hline White of hen's egg..... & 85.75 & 12.67 & 0.25 & $\cdots$ & 0.59 & 14.25 & 1.78 \\
\hline Yolk " " " & 50.79 & 16.24 & 31.75 & 0.13 & 1.09 & $5 \cdot 30$ & $64 \cdot 43$ \\
\hline
\end{tabular}

The Egg-white.-The white of egg has a specific gravity of 1.045 , and its reaction is always alkaline. It is a transparent, albuminous fluid inclosed in a framework of thin membrane. The fibrous portion of the membrane is insoluble in water and in dilute acetic acid.

The composition of the fluid substance of the white of egg, according to Lehmann, is as follows:

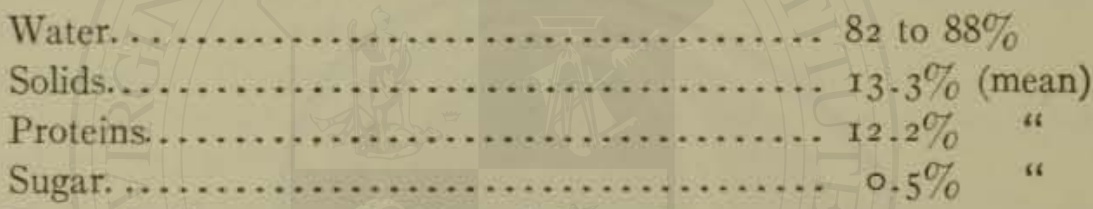

Fats, alkaline soaps, lecithin, cholesterin. ...... traces

Inorganic residue.

$0.66 \%$

The protein substance is for the most part albumin, with a small amount of globulin.

According to Osborne and Campbell* the nitrogen compounds of the white of egg are four in number, which they name ovalbumin, ovomucin, conalbumin, and ovomucoid. No sharp and distinct separation of these bodies has yet been made.

Ovalbumin (albumin) is the chief constituent, and forms by far the largest portion of the protein of the egg-white. In $2.5 \%$ solution in water, ovalbumin starts to coagulate at $60^{\circ}$, and yields a dense coagulum at $64^{\circ}$. Stronger solutions require a somewhat higher temperature for coagulation.

Ovomucin is a globulin-like substance, precipitated from egg-white by dilution with water. It is partly soluble in strong sodium chloride solution. When dried and washed with alcohol, it is a light white powder.

Conalbumin bears a close resemblance to ovalbumin, but coagulates

* Jour. Am. Chem Soc, 22 (1900), P. 422. 
in dilute salt solution at a lower temperature (below $60^{\circ}$ ), and the coagulum is more flocculent than that of ovalbumin.

Ovomucoid is not coagulable by heat, and may thus be separated (imperfectly) by filtering out all the coagulable proteins.

The last two compounds exist in very small amounts only.

Preparation of Albumin.*-By beating up the white of egg in water, the salts and the albumin are dissolved, while the fibrous portion is insoluble and is removed by filtration. The filtrate is then treated with a slight excess of basic lead acetate, the precipitate decomposed by treatment with carbon dioxide, and the lead removed by hydrogen sulphide. The solution is warmed cautiously to $60^{\circ} \mathrm{C}$, , thus beginning to coagulate the albumin, a small part of which, coming down in a flaky form, carries with it the lead sulphide. On filtering or pouring off the supernatant liquid after cooling, one obtains a colorless solution of the albumin, which is evaporated to dryness below $40^{\circ}$. The albumin is obtained in the form of transparent yellowish, horny scales, which may be pulverized in a mortar, if desired. Its specific gravity is 1.262. It is tasteless, odorless, and neutral in reaction, and slowly soluble in water.

The Egg-yolk.-This is much more complex in composition than the white. Halliburton thus enumerates the constituents of the yolk:

(a) Proteins.- Vitellin, the chief one, a globulin resembling myosin.

Albumin, in small quantities.

Nuclein, combined chiefly with the iron present.

(b) Fats.-Olein, palmitin, and stearin.

A yellow lipochrome or lutein.

(c) Carbohydrates.-Grape sugar in small quantities.

(d) Other Organic Constituents. - Lecithin, a phosphorized nitrogenous body allied both to the fats and to the proteins.

Cerebrin.

Cholesterin.

(e) Inorganic Salts, the most abundant of which is potassium chloride. Gobley gives the following composition to the egg-yolk:

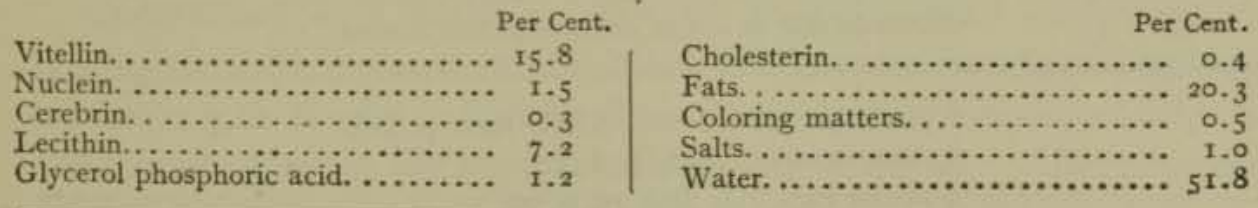

* Allen, Com. Org. Anal., 3 Ed., Vol. IV, p. 42. 
Osborne and Campbell,* as the result of long and careful experiments, consider the protein of egg-yolk to be largely if not wholly a lecithin compound, having properties of a globulin, and soluble in sodium chloride solution.

The fat of the egg yolk, which is used in ointments, has the following characteristics according to Spaeth: $\dagger$

Specific gravity at $100^{\circ} \mathrm{C} \ldots \ldots \ldots \ldots \ldots \ldots \ldots \ldots \ldots \ldots \ldots \ldots . .88 \mathrm{r}$

Iodine number. ............................ 68.48

Reichert-Meissl value. ...................... 0.66

Refractive index at $25^{\circ} \mathrm{C}$. (on butyro-refractometer scale) 68.5

Melting-points of fatty acids..................

Iodine number of fatty acids. . . . . . . . . . . .

The mineral content of the egg is thus shown by König:

COMPOSITION OF THE ASH OF EGGS.

\begin{tabular}{|c|c|c|c|c|c|c|c|c|c|c|c|}
\hline & & $\begin{array}{l}\text { Ash of } \\
\text { the Dry } \\
\text { Sub- } \\
\text { stance. }\end{array}$ & Potash. & Soda. & Lime. & $\begin{array}{l}\text { Mar. } \\
\text { nesia. }\end{array}$ & $\begin{array}{l}\text { Tron } \\
\text { Oxide. }\end{array}$ & $\begin{array}{l}\text { Phos. } \\
\text { Phoric } \\
\text { Aeidi. }\end{array}$ & $\begin{array}{c}\text { Sul- } \\
\text { phuric } \\
\text { Acid. }\end{array}$ & Silica. & $\begin{array}{l}\text { Chlo- } \\
\text { rine. }\end{array}$ \\
\hline Hen's egg: & $\begin{array}{l}\text { entire.. } \\
\text { white.. } \\
\text { yolk... }\end{array}$ & $\begin{array}{l}3.48 \\
4.6 \mathrm{I} \\
2.9 \mathrm{I}\end{array}$ & $\begin{array}{r}17.37 \\
31.41 \\
9.29\end{array}$ & $\begin{array}{r}22.87 \\
31.57 \\
5.87\end{array}$ & $\begin{array}{r}10.91 \\
2.78 \\
13.04\end{array}$ & $\begin{array}{l}1.14 \\
2.79 \\
2.13\end{array}$ & $\begin{array}{l}0.39 \\
0.57 \\
1.65\end{array}$ & $\begin{array}{r}37.62 \\
4.41 \\
65.46\end{array}$ & $\begin{array}{c}0.32 \\
2.12 \\
\cdots \cdots\end{array}$ & $\begin{array}{l}0.31 \\
1.06 \\
0.86\end{array}$ & $\begin{array}{r}8.08 \\
28.82 \\
1.95\end{array}$ \\
\hline
\end{tabular}

The following analyses of eggs were made by Wood and Merrill: $\ddagger$ AVERAGE WEIGHTS OF EGGS AND PARTS AS PREPARED FOR ANALYSIS.

\begin{tabular}{|c|c|c|c|c|c|c|c|c|}
\hline & \multirow{2}{*}{$\begin{array}{c}\text { Weight } \\
\text { as } \\
\text { Received. }\end{array}$} & \multicolumn{4}{|c|}{ Weight Boiled. } & \multirow{2}{*}{$\begin{array}{c}\text { Shell } \\
\text { (Refuse). }\end{array}$} & \multirow{2}{*}{ White. } & \multirow{2}{*}{ Yolk. } \\
\hline & & $\begin{array}{c}\text { Shell } \\
\text { (Refuse). }\end{array}$ & White. & Yolk. & Total. & & & \\
\hline $\begin{array}{l}\text { Turkey........ } \\
\text { Goose......... } \\
\text { Duck. ....... } \\
\text { Guinea fowl... }\end{array}$ & $\begin{array}{l}\text { Grams. } \\
\text { I05.5 } \\
190.4 \\
706 \\
40.2\end{array}$ & $\begin{array}{c}\text { Grams. } \\
11.7 \\
24.1 \\
7.2 \\
5.6\end{array}$ & $\begin{array}{l}\text { Grams. } \\
60.1 \\
98.5 \\
36.5 \\
20.9\end{array}$ & $\begin{array}{l}\text { Grams. } \\
30.9 \\
64.8 \\
24.4 \\
12.5\end{array}$ & $\begin{array}{l}\text { Grams. } \\
102.7 \\
187.4 \\
68.1 \\
39.0\end{array}$ & $\begin{array}{c}\text { Per Cent. } \\
\text { II. } 4 \\
\text { I2.5 } \\
\text { I0.6 } \\
\text { I } 4.4\end{array}$ & $\begin{array}{c}\text { Per Cent. } \\
56.5 \\
52.6 \\
53.6 \\
53.6\end{array}$ & $\begin{array}{c}\text { Per Cent: } \\
30.1 \\
34.6 \\
35.8 \\
32.0\end{array}$ \\
\hline
\end{tabular}

IShrinkage due to loss in preparation and cooking.

* Jour. Am. Chem. Soc., XXII, 1900, p. 413.

$\dagger$ Abst. Analyst, 1896 , p. 233.

$\ddagger$ Maine Exp. Sta, Bul. 75, p. 90 . 
COMPOSITION OF EGGS.

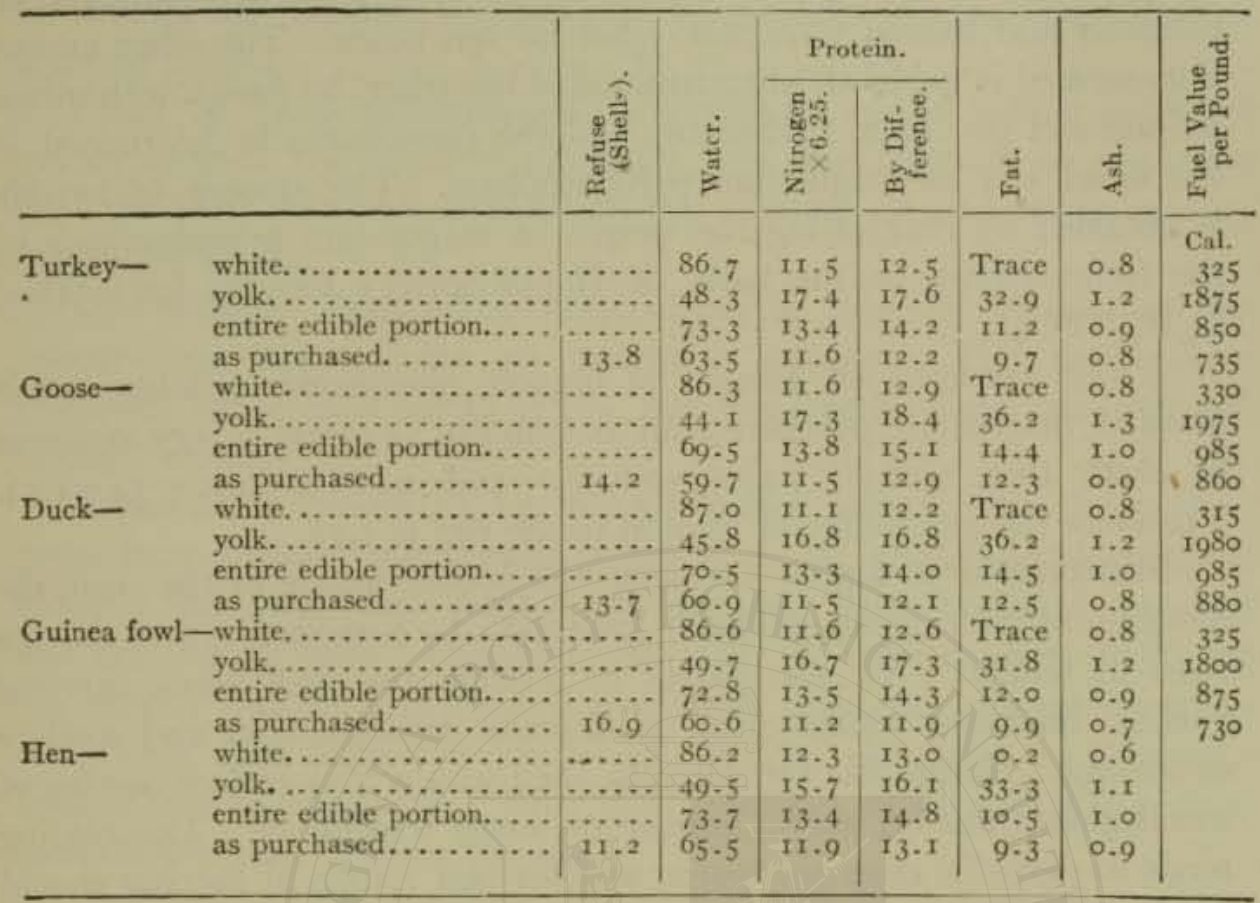

METHODS OF ANALYSIS.

Preparation of the Sample.*-The egg is first weighed as a whole and afterwards boiled hard, cooled, and again weighed. The shell, white, and yolk are then carefully separated and each weighed. After rejecting the shell, the yolk and white are separately reduced by a chopping-knife to the size of wheat grains. These portions are dried partially at a temperature not exceeding $45^{\circ}$, weighed, and afterwards ground to a fine powder in a mortar.

Determinations of water, fat, ash, and total nitrogen are made in practically the same manner as with flesh foods.

Little attention has been paid as yet to the complete separation and determination of the nitrogen compounds in the white and yolk, and it is customary in most cases to express the protein of the whole as $\mathrm{N} \times 6.25$.

Determination of Lecithin.-Wiley's Method. $†-$ The whole egg, excluding the shell, is placed in a flask with a reflux condenser, and boiled for six hours with absolute alcohol. The alcohol is then evaporated off, and the residue treated in like manner for ten hours with ether. After evaporat-

* Woods and Merrill, Maine Exp. Sta., Bul. 75, p. 92.

† Principles and Practice of Agricultural Analysis, Vol. III, p. 43 I. 
ing off the ether, the dry residue is rubbed to a fine powder, placed in an extractor and treated with pure ether for ten hours. The ether extract thus secured is oxidized, after removal of the ether, by fusion with mixed sodium and potassium carbonates, and the phosphorus is determined in the usual way as magnesium pyrophosphate. The amount of lecithin is obtained by multiplying the weight of magnesium pyrophosphate by the factor 7.2703 , on the basis of Hoppe-Seyler's formula for lecithin: $\mathrm{C}_{44} \mathrm{H}_{90} \mathrm{NPO}_{9}$.

If, for example, an amount of organic phosphorus yielding 0.0848 gram of magnesium pyrophosphate is found in 54 grams of egg exclusive of shell, then $0.0848 \times 7.2703=0.61652$ and $0.61652 \times 100 \div 54=1.14$. Therefore the percentage of lecithin in the egg is r.I4.

Preservation of Eggs. - Owing to the porous nature of the shell, the moisture of the contents gradually grows less by evaporation, and the egg loses in weight. Air also passes in through the shell pores, carrying various microbes, which result in ultimate decomposition and spoiling of the egg. Nature has provided the shell with a thin surface coating of mucilaginous matter, which, however, is easily washed off. This coating tends to partially close the pores, and for best results in keeping should not be removed by washing.

Eggs are commonly preserved by protecting them as far as possible from the air. This is accomplished in a variety of ways, the most common being to pack the eggs in salt or bran, so that the packing medium fills up the interstices between the eggs. Eggs thus packed will keep considerably longer then when exposed to the air. A solution of salt is sometimes employed, and also lime water, the eggs being simply packed in the solution. The use of lime water is, however, open to the serious objection that a disagreeable odor and taste are imparted to the eggs.

Eggs are sometimes coated with gelatin, vaseline, wax, or gum, so as to cover them with an impervious layer, either by dipping them in the coating medium, or by varnishing or otherwise applying the substance to the egg shell. By far the most efficacious egg coating has been shown by experiments in the North Dakota Experiment Station,* and also in Germany, to be sodium and potassium silicate, or water glass. The fresh eggs, preferably unwashed, are packed in a jar, and a $10 \%$ solution of water glass is poured over them. According to the North Dakota experiments, at the end of three and a half months, eggs packed in this manner the first of August appeared to be perfectly fresh.

\footnotetext{
* Farmer's Bul, 103 , U. S. Dept. of Agric., p. 18.
} 
One drawback to this method is that eggs so treated break more easily on boiling, but this may be prevented by carefully piercing the shell with a strong needle.

Cadet de Vanx has proposed immersing the egg in boiling water for twenty seconds, the result being that a very thin layer of the egg-white next the shell becomes coagulated, thus forming an impervious coating inside the shell.

Cold-storage Eggs.-The preservation of eggs by storage at low temperatures has become an enormous industry. The temperature employed varies from $24^{\circ}$ to $40^{\circ} \mathrm{C}$, and the length of storage from one to eight months.

Experiments conducted by Wiley,* under authorization from Congress, have brought out certain points as to the physical and chemical changes that take place during cold storage. After breaking the shell and keeping at room temperature one day, the odor of eggs stored for 3.5 months was different from that of fresh eggs, but was not disagreeble. This odor increased on longer storage, and after 12.6 months became very characteristic. After 16.6 months, a musty odor was noticed immediately after opening the egg.

Chemical analysis by Cook showed that eggs in storage for one year lost $10 \%$ of the total weight, due to evaporation of water from the whites. Storage also caused a lowering of the amount of coagulable protein and of lecithin phosphorus, but an increase in lower nitrogen bodies, proteoses, and peptones. The acid reaction of yolks diminished during storage.

Microscopical examination by Howard and Read brought out the interesting fact that small rosette crystals of an unidentified substance appeared in the yolk after storage for I2 months or longer, and this observation has since been utilized in the examination of suspected samples.

Physical Examination of Eggs. - Various physical tests have been prescribed for ascertaining the approximate age of an egg. Thus, according to Delarne, if the egg, when placed in a $10 \%$ salt solution, sinks to the bottom, it may be considered perfectly fresh; if it remains immersed in the liquid, it is to be considered at least three days old; and if it rises to the surface and floats thereon it is more than five days old. This test

*U. S. Dept. of Agric., Bureau of Chem., Bul. 115. 
is a very rough one, and is useful only for eggs that have been kept in the air. Preserved eggs cannot be gauged by this means.

The best method of examining eggs for freshness is "candling," consisting in placing the egg between a bright light and the eye. If the egg is fresh, it will show a uniform rose-colored tint, without dark spots, the air-chamber being small and occupying about one-twentieth the capacity of the egg. If the egg is not fresh, it will appear more or less cloudy, being darker as the egg grows older, becoming in extreme cases opaque. At the same time the air-chamber grows larger as the age increases. So-called "spots" are eggs which show on candling black patches due to fungi.

Opened Eggs.-In the handling of eggs many become cracked or otherwise injured to an extent which renders them unfit for transportation. These are either sold to bakers for immediate use, or else opened and kept from spoiling by freezing, the addition of preservatives, or drying. The portions of "spot eggs" that do not show evidence of damage are also treated by one of these methods. Eggs which, because of their offensive taste, are unfit for food, are used in the tanning industry.

Preservatives commonly employed in opened eggs are boric acid and formaldehyde. The latter is especially effective as an egg preservative. If a small quantity be added and stirred into opened eggs that have become absolutely putrid, the result is astonishing. The product is completely deodorized, and exhibits the outward appearance at least of fresh eggs.

Formaldehyde, if present, may readily be detected by heating some of the egg directly with the hydrochloric-acid ferric-chloride reagent used in testing milk for formaldehyde, carrying out the process exactly as in the case of milk.

Desiccated Egg.-It is possible to evaporate to dryness the contents of the egg to form a powder, the keeping qualities of which far exceed that of ordinary eggs, while it forms a concentrated food which lends itself much more readily to transportation than does the fresh egg in the shell. Several brands of desiccated egg are on the market, which from their analyses are undoubtedly genuine. The following are analyses of two of them, one (A) made by the Bureau of Chemistry, the other (B) by the Massachusetts State Board of Health: 
A. B.

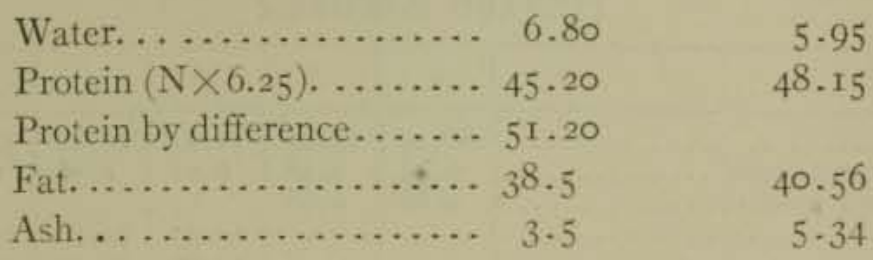

Egg Substitutes.-There have been many preparations in powdered form sold under this name, nearly all claiming to contain all the ingredients of eggs, but most of them falling far short of these claims. Some of them, as for instance those made from desiccated skimmed milk, do contain nitrogenous matter, but as a rule little if any fat.

Two samples of "egg substitute" sold in Massachusetts were analyzed with the following results:*

A.

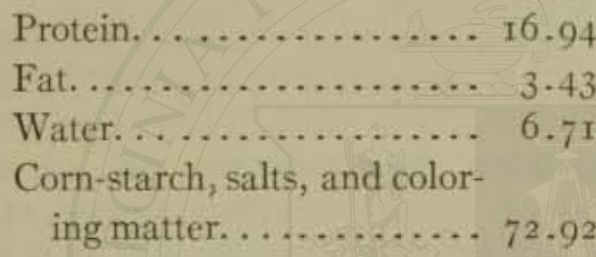

B. 18.72 3.40 7.01 70.87

A ten-cent package of sample A, weighing about 2 ounces, was alleged to be equivalent to $\mathrm{I} 2$ eggs. Starch furnished the chief ingredient in both samples.

One of the most flagrant examples of fraud in this connection was a product sold under the name "N'egg," advertised to contain the nutritive equivalent of the whites and yolks of a dozen eggs, "their composition being based on careful scientific analysis of natural eggs." It was put up in two small boxes, one containing a white and the other a yellow dry powder. Both were entirely devoid of nitrogen, and consisted of nearly pure tapioca starch with a little common salt, the color of the "yolk" being due to Victoria yellow.

Some egg substitutes are sold under the name of "custard powders," and are alleged to take the place of eggs in cooking. These are variously made up of mixtures of skim-milk powder, coloring matter, and baking powder ingredients as shown from the following analyses: $\dagger$

* An. Rep. Mass. State Board of Health, 1895, p. 675

$\dagger$ Food and Sanitation, Nov, 25, 1893 . 
CUSTARD POWDERS.

\begin{tabular}{|c|c|c|c|c|c|c|}
\hline & 1 & 2 & 3 & 4 & 5 & 6 \\
\hline $\begin{array}{l}\text { Starch } \\
\text { Albuminous compounds } \ldots \ldots \ldots \ldots \ldots \ldots \\
\text { Soluble coloring matter } \ldots \ldots \ldots \ldots \ldots\end{array}$ & $\begin{array}{r}86.25 \\
0.59 \\
0.88\end{array}$ & $\begin{array}{r}84.45 \\
0.58 \\
0.98\end{array}$ & $\begin{array}{r}5 \mathrm{I} .03 \\
6.01\end{array}$ & $\begin{array}{r}26.38 \\
2.96\end{array}$ & $\begin{array}{r}52.32 \\
6.00\end{array}$ & $\begin{array}{r}53.82 \\
5.06\end{array}$ \\
\hline Baking soda $\ldots \ldots \ldots \ldots \ldots \ldots \ldots \ldots$ & $\cdots \cdots$ & $\cdots \cdots$ & 15.33 & 50.70 & 22.11 & 26.71 \\
\hline $\begin{array}{l}\text { Tartaric acid } \ldots \ldots \ldots \\
\text { Phosphates. } . \ldots \ldots \ldots \\
\end{array}$ & $\cdots \cdots$ & $\ldots \ldots$ & $\begin{array}{r}13.69 \\
0.24\end{array}$ & 10.33 & $\mathrm{II} .37$ & 6.19 \\
\hline $\begin{array}{l}\text { Carbonates of lime and magnesia } \ldots \ldots \ldots \\
\text { Chlorides and sulphates } \ldots \ldots \ldots \ldots \ldots\end{array}$ & $\cdots \ldots$ & $\ldots \ldots$ & 2.70 & & & \\
\hline $\begin{array}{l}\text { Water. } \\
\text { Ash. } \ldots \ldots \ldots \ldots \ldots \\
\end{array}$ & $\begin{array}{r}\text { Ir. } 83 \\
0.45\end{array}$ & $\begin{array}{r}13.69 \\
0.3^{8}\end{array}$ & 11.00 & 9.63 & 8.20 & 8.22 \\
\hline
\end{tabular}

\section{REFERENCES ON EGGS.}

Borchmann, K. Amtliche Kontrolle des Marktverkehrs mit Eiern. Zeits. Fleisch, u. Milchhyg., 17, 1906, pp. 3, 51, 97, 132 .

LANGWORthy, C. F. Eggs and their Uses as Food. Farmer's Bul. r28.

Osborne, T. B., and CAMpbell, G. F. Proteids of the Egg Yolk. Jour. Am. Chem. Soc., 22, 1900, p. 413 .

— Protein Constituents of Egg White. Jour. Am. Chem. Soc., 22, I900, p. 422.

Prall, F. Ueber Eier-Konservierung. Zeits. Unters. Nahr. Genuss., I4, I907, p. 445. SNyder, H. Digestibility of Potatoes and Eggs. Exp. Sta. Bul. 43, p. 20.

Wrlex, H. W. A Preliminary Study of the Effects of Cold Storage on Eggs, Quail, and Chickens. U. S. Dept. of Agric, Bur. of Chem., Bul. 115.

Farmer's Bul. 87. Food Value of Eggs, p. 24.

" "103. Preserving Eggs. 


\section{CHAPTER X. \\ CEREALS AND THEIR PRODUCTS, LEGUMES, VEGETABLES, AND FRUITS.}

THE chief points of difference in composition between the animal foods already treated of, and those of the vegetable kingdom, are apparent in the relative amounts of proteins and carbohydrates. The proteins present in the cereals and vegetables differ materially both in character and amount from those in the flesh foods, being as a rule present to a much greater extent in the meats than in the grains and vegetables. The leguminous foods, such as peas, beans, and lentils, are, however somewhat exceptional in this respect, being comparatively high in nitrogenous content.

The carbohydrates, which in the flesh foods are almost entirely lacking, and in milk make up about one-third of the solid matter, form the most important and abundant class of constituents in the vegetable foods.

The composition of the principal cereal grains is tabulated as follows by Villier and Collin:

\begin{tabular}{|c|c|c|c|c|c|c|c|c|}
\hline & Wheat. & Barley. & Rye. & Oats. & Rice. & Corn. & Millet. & $\begin{array}{l}\text { Buck- } \\
\text { wheat. }\end{array}$ \\
\hline 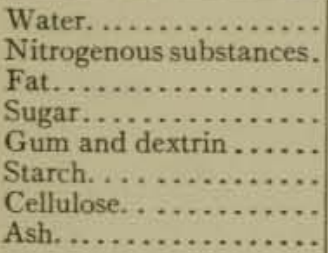 & $\begin{array}{r}13.65 \\
12.35 \\
1.75 \\
1.45 \\
2.38 \\
64.08 \\
2.53 \\
1.81\end{array}$ & $\begin{array}{r}13.77 \\
11.14 \\
2.16 \\
1.56 \\
1.70 \\
61.67 \\
5.31 \\
2.69\end{array}$ & $\begin{array}{r}15.06 \\
11.52 \\
1.79 \\
0.95 \\
4.86 \\
62.00 \\
2.01 \\
1.81\end{array}$ & $\begin{array}{r}12.37 \\
10.41 \\
5.32 \\
1.91 \\
1.79 \\
54.08 \\
11.19 \\
3.02\end{array}$ & $\begin{array}{r}13.11 \\
7.85 \\
0.88 \\
16.52 \\
0.63 \\
1.01\end{array}$ & $\begin{array}{r}13.12 \\
9.85 \\
4.62 \\
3.46 \\
3.38 \\
62.57 \\
2.49 \\
1.51\end{array}$ & $\begin{array}{r}11.66 \\
9.25 \\
3.50 \\
65.95\end{array}$ & $\begin{array}{r}12.93 \\
10.30 \\
2.8 \mathrm{I} \\
55.8 \mathrm{I} \\
16.43 \\
2.72\end{array}$ \\
\hline
\end{tabular}

The following results of the analyses of cereal grains are summarized from the work of the Division of Chemistry, United States Department of Agriculture:*

\section{* Bulletin ${ }^{2} 3$, part 9.}


CEREAL GRAINS.

\begin{tabular}{|c|c|c|c|c|c|c|c|c|c|c|}
\hline & $\begin{array}{l}\text { Num- } \\
\text { ber of } \\
\text { Analy- } \\
\text { ses. }\end{array}$ & $\begin{array}{l}\text { Weight } \\
\text { of } 100 \\
\text { Ker- } \\
\text { nels. } \\
\text { Grams. }\end{array}$ & $\begin{array}{l}\text { Moist- } \\
\text { ure. }\end{array}$ & $\begin{array}{l}\text { Pro- } \\
\text { teins. }\end{array}$ & $\begin{array}{l}\text { Ether } \\
\text { Ex- } \\
\text { tract. }\end{array}$ & $\begin{array}{l}\text { Crude } \\
\text { Fiber. }\end{array}$ & Ash. & 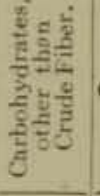 & $\begin{array}{c}\text { Wet } \\
\text { Gluten. }\end{array}$ & $\begin{array}{l}\text { Dry } \\
\text { Gluten. }\end{array}$ \\
\hline $\begin{array}{l}\text { Bariey: } \\
\text { Mean......... }\end{array}$ & $\begin{array}{c}14 \\
\ldots \ldots\end{array}$ & $4 \cdot 533$ & 6.47 & II. 52 & 2.67 & $3.8 \mathrm{I}$ & 2.87 & $7^{2} .66$ & & \\
\hline Buckwheat: & Io & & & & & & & & & \\
\hline $\begin{array}{l}\text { Mean .......... } \\
\text { Corn, domestic: }\end{array}$ & $\ldots \ldots$ & 3.062 & $12-31$ & 10.86 & 2.06 & 10. 57 & I. 85 & $63 \cdot 34$ & & \\
\hline Maximum..... & $\ldots \ldots$ & 48.312 & 12.32 & 11.55 & 5.06 & 2.00 & I. 55 & 75.07 & & \\
\hline Minimum ..... & $\ldots \ldots$ & 10.608 & $9 \cdot 5^{8}$ & 8.58 & 2.94 & 1.00 & 1.19 & 68.97 & & \\
\hline $\begin{array}{l}\text { Mean ........... } \\
\text { Oats, domestic: }\end{array}$ & $\ldots \ldots$ & $3^{8} .979$ & 10.93 & 9.88 & 4.17 & $1 \cdot 71$ & $1 \cdot 3^{6}$ & 71.95 & & \\
\hline Maximum.... & $\ldots \ldots$ & $3.89 t$ & 13.02 & 15.05 & 6.14 & 16.65 & $4 \cdot 37$ & 61.44 & & \\
\hline Minimum ..... & $\ldots \ldots$ & 2.038 & 7.87 & 9.10 & 0.93 & 8.57 & .47 & 53,70 & & \\
\hline Mean......... & ...... & 2.918 & 10.06 & 12.15 & $4-33$ & 12.07 & 3.46 & 58.75 & & \\
\hline $\begin{array}{l}\text { Rice: } \\
\text { Unhuiled... }\end{array}$ & 4 & $2-020$ & 10.28 & & 1.65 & 10.42 & 4.00 & 65.60 & & \\
\hline Unpolished .... & 6 & 2.466 & II. 88 & 8.02 & 1.96 & 0.93 & 1.15 & 76.05 & & \\
\hline Polished ${ }^{*} . .$. . & I4 & 2.132 & 12.34 & $7 \cdot 18$ & 0.26 & $0.4^{\circ}$ & 0.46 & $79 \cdot 3^{6}$ & & \\
\hline $\begin{array}{l}\text { Rye, domestic: } \\
\text { Maximum..... }\end{array}$ & & 4.201 & 11.45 & 18.00 & 2.30 & 2.50 & $2.4 \mathrm{I}$ & & & \\
\hline Minimum ..... & & 1.932 & 9.54 & 8.40 & $\begin{array}{l}2.30 \\
1.16\end{array}$ & 1.65 & $3 .-7$ & $63.6 \mathrm{r}$ & & \\
\hline Mean.......... & $\ldots \ldots$ & 2.493 & 10.62 & 12.43 & 1.65 & 2.09 & 1.92 & $71 \cdot 37$ & & \\
\hline Wheat, domestic: & & & & & & & & & & \\
\hline Maximum..... & $\ldots \ldots$ & 6.190 & $1+\cdot 53$ & 17.15 & 2.50 & $3 \cdot 72$ & $2 \cdot 35$ & 76.05 & 39.05 & 14.65 \\
\hline $\begin{array}{l}\text { Minimum. .... } \\
\text { Mean......... }\end{array}$ & $\ldots \ldots$ & 2,125 & $7-11$ & 8.58 & 0.28 & 1.70 & $1 \cdot 4^{\circ}$ & 66.67 & $\mathrm{r} 2 \cdot 33$ & $4-70$ \\
\hline $\begin{array}{l}\text { Mean . . . . . } \\
\text { Wheat, foreign: }\end{array}$ & $\ldots$ & 3.866 & 10.62 & 12.23 & 1.77 & $2 \cdot 3^{6}$ & 1.82 & 71.18 & 26.46 & 10.31 \\
\hline Maximum..... & $\ldots \ldots$ & $5 \cdot 723$ & 12.97 & 14.52 & 2.26 & 2.89 & 2.04 & 76.14 & 32.57 & 12.33 \\
\hline Minimum. .... & $\ldots \ldots$ & 2.250 & 8.52 & 8.58 & 0.73 & I. 87 & I. 67 & 67.01 & 18.72 & 7.00 \\
\hline Mean......... & $\ldots \ldots$ & 4.076 & 11.47 & 12.08 & 1.78 & 2.28 & 1. 73 & 70.66 & $25 \cdot 3^{6}$ & 9.82 \\
\hline
\end{tabular}

* Polished rice in the United States is commonly coated with gluccse and tale, ostensibly as a protection against dust and the ravages of insects. Such ccating is aloved if declared on the label and directions for its removal are also given.

Balland $\dagger$ gives the following percentage composition of beans, lentils, and peas:

\begin{tabular}{|c|c|c|c|c|c|c|}
\hline & \multicolumn{2}{|c|}{ Beans. } & \multicolumn{2}{|c|}{ Lentils. } & \multicolumn{2}{|c|}{ Peas. } \\
\hline & Min. & Max: & Min. & Max. & Min. & Max. \\
\hline 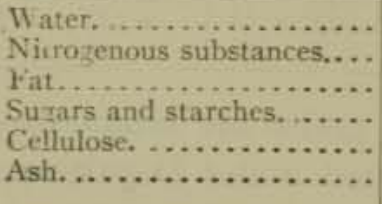 & $\begin{array}{r}10.10 \\
13.81 \\
0.98 \\
52.91 \\
2.46 \\
2.38\end{array}$ & $\begin{array}{r}20.40 \\
25.46 \\
2.46 \\
60.98 \\
4.62 \\
4.20\end{array}$ & $\begin{array}{r}11.70 \\
20.42 \\
0.5^{8} \\
56.07 \\
2.96 \\
1.99\end{array}$ & $\begin{array}{r}13.50 \\
24.24 \\
1.45 \\
62.45 \\
3.56 \\
2.66\end{array}$ & $\begin{array}{r}10.60 \\
18.88 \\
1.22 \\
56.21 \\
2.90 \\
2.26\end{array}$ & $\begin{array}{r}14.20 \\
22.48 \\
1.40 \\
61.10 \\
5.52 \\
3.50\end{array}$ \\
\hline
\end{tabular}

†Jour. Pharm. Chem., 1897, pp. 196, 197. 
The composition of potatoes, according to Balland,* is as follows:

\begin{tabular}{|c|c|c|c|c|c|c|}
\hline & Water. & $\begin{array}{l}\text { Nitroge- } \\
\text { nous } \\
\text { Sub- } \\
\text { stances. }\end{array}$ & Fat. & $\begin{array}{l}\text { Sugar } \\
\text { and } \\
\text { Starch. }\end{array}$ & Cellulose. & Ash. \\
\hline $\begin{array}{ll}\text { Normal state- } & \text { minimum ... } \\
\text { Dried- } & \text { minimum. } \\
\text { maximum. } & \end{array}$ & $\begin{array}{c}66.10 \\
80.60 \\
\cdots \cdots \\
\cdots \cdots\end{array}$ & $\begin{array}{r}1.43 \\
2.81 \\
5.98 \\
13.24\end{array}$ & $\begin{array}{l}0.04 \\
0.14 \\
0.18 \\
0.56\end{array}$ & $\begin{array}{l}15 \cdot 58 \\
29.85 \\
80.28 \\
89.78\end{array}$ & $\begin{array}{l}0.37 \\
0.68 \\
1.40 \\
3.06\end{array}$ & $\begin{array}{l}0.44 \\
1.18 \\
1.66 \\
4.3^{8}\end{array}$ \\
\hline
\end{tabular}

The composition of the common vegetables, fruits, and berries is thus given by Atwater and Bryant. $\dagger$

VEGETABLES.

\begin{tabular}{|c|c|c|c|c|c|c|c|c|c|c|}
\hline & & 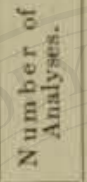 & $\frac{8}{3}$ & $\frac{\mathrm{b}}{\mathrm{a}}$ & $\frac{\text { है }}{\frac{3}{2}}$ & है & 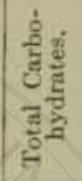 & 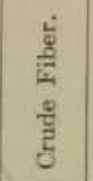 & $\frac{\dot{1}}{2}$ & 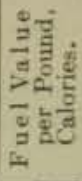 \\
\hline Asparagus - & as purchased.. & 3 & ..... & 94.0 & I. 8 & 0.2 & $3 \cdot 3$ & .8 & .7 & 105 \\
\hline Beans, dried - & as purchased.. & II & $\ldots \ldots$ & 12.6 & 22.5 & 1.8 & 59.6 & $4 \cdot 4$ & $3 \cdot 5$ & 1605 \\
\hline \multirow[t]{2}{*}{ Beans, fresh Lima- } & - edible portion.. & $\mathbf{I}$ & $\ldots .$. & 68.5 & $7 \cdot 1$ & -7 & 22.0 & 1.7 & 1.7 & $57^{\circ}$ \\
\hline & as purchased... & $\ldots$. & 55.0 & 30.8 & 3.2 & -3 & 9.9 & .8 & .8 & 255 \\
\hline \multirow[t]{2}{*}{ Beets, fresh- } & edible portion.. & 24 & 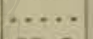 & $87 \cdot 5$ & 1.6 & . 1 & $9 \cdot 7$ & .9 & I. I & 215 \\
\hline & as purchased... & & 20.0 & 70.0 & I. 3 & .1 & $7 \cdot 7$ & $\cdots \cdots$ & .9 & 170 \\
\hline \multirow[t]{2}{*}{ Cabbage - } & edible portion.. & 16 & $\ldots \ldots$ & $9 \mathrm{I} \cdot 5$ & 1.6 & $\cdot 3$ & 5.6 & I. I & I.O & I 45 \\
\hline & as purchased... & $\cdots$ & 15.0 & $77 \cdot 7$ & 1.4 & .2 & 4.8 & $\cdots \cdots$ & .9 & 125 \\
\hline \multirow{2}{*}{ Carrot, fresh- } & edible portion.. & 18 & $\cdots \cdots$ & 88.2 & I. I & .4 & $9 \cdot 3$ & I.I & 1.0 & 210 \\
\hline & as purchased... & $\cdots \cdots$ & 20.0 & 70.6 & .9 & .2 & $7-4$ & $\cdots \cdot$ & .9 & 160 \\
\hline Celery- & $\begin{array}{l}\text { edible portion.. } \\
\text { as purchased... }\end{array}$ & $\begin{array}{r}5 \\
\ldots .\end{array}$ & W... & $\begin{array}{l}94 \cdot 5 \\
75.6\end{array}$ & $\begin{array}{r}\mathrm{I} . \mathrm{I} \\
.0\end{array}$ & $\begin{array}{l}-I \\
-I\end{array}$ & $\begin{array}{l}3 \cdot 3 \\
2.6\end{array}$ & $\cdots \cdots$ & $\begin{array}{r}1.0 \\
.8\end{array}$ & $\begin{array}{l}85 \\
70\end{array}$ \\
\hline Cauliflower- & as purchased... & 2 & $\ldots \ldots$ & 92.3 & 1.8 & .5 & $4 \cdot 7$ & 1.0 & .7 & 140 \\
\hline \multirow[t]{2}{*}{ Cucumber- } & edible portion.. & 4 & $\ldots$. & 95.4 & .8 & .2 & 3.1 & $\ldots \ldots$ & .5 & So \\
\hline & as purchased. & 8 & 15.0 & $81-1$ & .7 & .2 & 2.6 & $\cdots \cdots$ & .4 & 70 \\
\hline \multirow[t]{2}{*}{ Lettuce- } & edible portion... & 8 & $\ldots \ldots$ & 94.7 & I. 2 & $\cdot 3$ & 2.9 & $\cdot 7$ & .9 & 90 \\
\hline & as purchase & $\cdots$ & 15.0 & $80 \cdot-5$ & 1.0 & .2 & 2.5 & $\cdots \cdots$ & .8 & 75 \\
\hline Mushrooms- & as purchased. & II & $\ldots \ldots$ & 88.1 & $3 \cdot 5$ & .4 & 6.8 & .8 & I. 2 & 210 \\
\hline \multirow[t]{2}{*}{ Onion, fresh- } & edible portion.. & 15 & $\ldots \ldots$ & 87.6 & I. 6 & -3 & $9 \cdot 9$ & .8 & .6 & 225 \\
\hline & as purchase & $\ldots \ldots$ & 10.0 & $7^{8} .9$ & I. 4 & -3 & 8.9 & $\cdots \cdots$ & -5 & 205 \\
\hline \multirow[t]{2}{*}{ Parsnip- } & edible portion.. & 3 & $\ldots \ldots$ & 83.0 & I. 6 & $\cdot 5$ & 13.5 & 2.5 & I. 4 & 300 \\
\hline & as purchase & $\cdots \cdots$ & 20.0 & 66.4 & I. 3 & .4 & 10.8 & $\cdots \ldots$ & I. I & 240 \\
\hline \multirow[t]{2}{*}{ Pumpkin- } & edible portion.. & 3 & $\cdots$ & 93.1 & 1.0 & - 1 & $5-2$ & 1.2 & .6 & 120 \\
\hline & as purchased... & $\cdots \cdots$ & 50.0 & $46 \cdot 5$ & -5 & -1 & 2.6 & $\cdots \cdots$ & -3 & 60 \\
\hline \multirow[t]{2}{*}{ Radish- } & edible portion.. & 4 & $\cdots$ & 91.8 & I. 3 & -3 & 8.3 & $\cdot 7$ & 1.0 & 135 \\
\hline & as purchased... & $\cdots \cdots$ & 30.0 & $64 \cdot 3$ & .9 & . I & 5.8 & $\cdot 7$ & -7 & 95 \\
\hline \multirow[t]{2}{*}{ Rhubarb- } & cdible portion.. & 2 & $\because \ldots$ & $94-4$ & .6 & -7 & 3.6 & I. I & -7 & 105 \\
\hline & as purchased... & $\cdots$ & 40.0 & 56.6 & -4 & .4 & 2.2 & $\cdots \cdots$ & -4 & 65 \\
\hline \multirow[t]{2}{*}{ Sqquash - } & edible portion.. & 10 & $\ldots$. & 88.3 & 1.4 & $\cdot 5$ & 9.0 & .8 & .8 & 215 \\
\hline & as purchased... & $\cdots \cdots$ & 50.0 & 44.2 & $\cdot 7$ & .2 & $4 \cdot 5$ & $\cdots \cdots$ & -4 & 105 \\
\hline \multirow{3}{*}{$\begin{array}{l}\text { Tomato, fresh- } \\
\text { Turnip- }\end{array}$} & as purchased... & 27 & 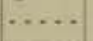 & $94 \cdot 3$ & .9 & $\cdot 4$ & 3.9 & .6 & $\cdot 5$ & 105 \\
\hline & edible portion.. & 19 & $\ldots .$. & 89.6 & 1. 3 & .2 & 8.1 & I. 3 & .8 & 185 \\
\hline & as purchased... & & 30.0 & $62 \cdot 7$ & -9 & $-\mathrm{T}$ & $5 \cdot 7$ & $\cdots \cdots$ & .6 & 125 \\
\hline
\end{tabular}

* Jour. Pharm. Chem., 1897, pp. 298-300.

$\uparrow$ Bul. 28, Office of Exp. Station U, S. Dept. of Agriculture. 
FRUITS.

\begin{tabular}{|c|c|c|c|c|c|c|c|c|c|c|}
\hline & & 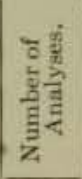 & 逍 & है & 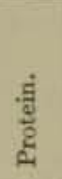 & मैं & 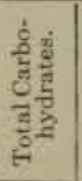 & है & दूँ & 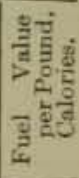 \\
\hline \multirow[t]{2}{*}{ Appies - } & edible portion .... & 29 & & 84.6 & -4 & $\cdot 5$ & 14.2 & I. 2 & $\cdot 3$ & 200 \\
\hline & $\cdots \cdots$ & & 25.0 & $63 \cdot 3$ & -3 & $\cdot 3$ & 10.8 & $\cdots \cdots$ & $\cdot 3$ & 220 \\
\hline Apricots - & $\cdots \cdots$ & II & $\cdots \cdots$ & 85.0 & I. I & $\cdots \cdots$ & $13 \cdot 4$ & $\cdots \cdots$ & $\cdot 5$ & 270 \\
\hline \multirow[t]{2}{*}{ Bananas- } & $\begin{array}{l}\text { as purchared...... } \\
\text { edible portion..... }\end{array}$ & $\cdots$ & $\begin{array}{r}6.0 \\
\ldots \ldots\end{array}$ & $\begin{array}{l}79 \cdot 9 \\
75.3\end{array}$ & $\begin{array}{l}1.0 \\
1.3\end{array}$ & & $\begin{array}{l}12.6 \\
22.0\end{array}$ & 1.6 & $\begin{array}{r}.5 \\
.8\end{array}$ & $\begin{array}{l}255 \\
460\end{array}$ \\
\hline & as purchased...... & & 35.0 & 48.9 & .8 & $\cdot 4$ & $\mathrm{I} 4 \cdot 3$ & $\ldots \ldots$ & .6 & 300 \\
\hline $\begin{array}{l}\text { Blackberries - } \\
\text { Cherries - }\end{array}$ & as purchased.... . & 9 & $\cdots \cdots$ & 86.3 & I. 3 & 1.0 & 10.9 & 2.5 & $\cdot 5$ & 270 \\
\hline Cherries - & $\begin{array}{l}\text { edible portion...... } \\
\text { as purchased...... }\end{array}$ & 16 & $\cdots \cdots$ & $\begin{array}{l}80.9 \\
76.8\end{array}$ & 1.0 & $\begin{array}{l}.8 \\
8\end{array}$ & 16.7 & .2 & .6 & $3^{6} 5$ \\
\hline Cranberries - & as purchased...... & 3 & & 88.9 & .9 & .6 & $\begin{array}{r}15.9 \\
9.9\end{array}$ & $\begin{array}{ll}\cdots \cdot 5 \\
1.5\end{array}$ & .0 & $\begin{array}{l}345 \\
215\end{array}$ \\
\hline Currants- & as purchased...... & I & & 85.0 & I. 5 & $\ldots \ldots$ & 12.8 & $\ldots \ldots$ & .7 & 265 \\
\hline Figs, fresh- & as purchased...... & 28 & $\cdots$ & $79-1$ & I. 5 & $\cdots \cdots$ & I8.8 & - & .6 & 380 \\
\hline Grapes - & $\begin{array}{l}\text { edible portion..... } \\
\text { as purchased...... }\end{array}$ & 5 & ?... & $\begin{array}{l}77.4 \\
58.0\end{array}$ & $\begin{array}{l}\text { I. } 3 \\
\text { I. } 0\end{array}$ & $\begin{array}{l}1.6 \\
1.2\end{array}$ & $\begin{array}{l}19.2 \\
14.4\end{array}$ & $\begin{array}{c}4 \cdot 3 \\
\cdots \cdots\end{array}$ & $\begin{array}{l}.5 \\
.4\end{array}$ & $\begin{array}{l}45^{\circ} \\
335\end{array}$ \\
\hline \multirow{2}{*}{$\begin{array}{l}\text { Huckleberries - } \\
\text { Lemons - }\end{array}$} & edible portion..... & I & $\ldots$. & $81 \cdot 9$ & .6 & .6 & 16.6 & $\cdots \cdots$ & $\cdot 4$ & $\begin{array}{l}335 \\
345\end{array}$ \\
\hline & $\begin{array}{l}\text { edible portion..... } \\
\text { as purchased..... }\end{array}$ & 4 & & $\begin{array}{l}89 \cdot-3 \\
6 ?-2\end{array}$ & 1.0 & $\cdot ?$ & 8.5 & $I \cdot I$ & $\cdot 5$ & 205 \\
\hline Muskmelons- & edible portio & I & 30.0 & $89 \cdot 5$ & .6 & -5 & $\begin{array}{l}5.9 \\
9 \cdot 3\end{array}$ & 2.1 & $\begin{array}{l}.4 \\
.6\end{array}$ & $\begin{array}{l}\text { I } 45 \\
185\end{array}$ \\
\hline \multirow[t]{2}{*}{ Oranges- } & $\begin{array}{l}\text { as purchase } \\
\text { edible port }\end{array}$ & $\cdots_{23}$ & 50.0 & $\begin{array}{l}44.8 \\
86.9\end{array}$ & .3 & $\cdots$ & $\begin{array}{r}4.6 \\
11.6\end{array}$ & $\cdots \cdots$ & $\cdot 3$ & $\begin{array}{r}90 \\
240\end{array}$ \\
\hline & as purchased...... & ... & 27.0 & 63.4 & .6 & .1 & 8.5 & $\ldots$. & .4 & 170 \\
\hline Pears- & $\begin{array}{l}\text { edible portion..... } \\
\text { as purchased.... }\end{array}$ & 2 & & $84 \cdot 4$ & .6 & .5 & $14 \cdot x$ & 2.7 & .4 & 295 \\
\hline Pineapple- & edible portion...... & $\dot{I}$ & 10.0 & $\begin{array}{l}70.0 \\
80.3\end{array}$ & $\cdot 5$ & $\cdot 4$ & 12,7 & $\cdots \cdots$ & $\cdot 4$ & 260 \\
\hline \multirow[t]{2}{*}{ Plums- } & edible portion..... & 3 & & $78 .-4$ & $\begin{array}{r}.4 \\
1.0\end{array}$ & $\begin{array}{c}\cdot 3 \\
\cdots\end{array}$ & $\begin{array}{r}9.7 \\
20.1\end{array}$ & $\begin{array}{c}\cdot 4 \\
\ldots \cdots\end{array}$ & $\cdot 3$ & $\begin{array}{l}200 \\
395\end{array}$ \\
\hline & as purchased....... & $\cdots$ & 5.0 & $74 \cdot 5$ & .9 & $\ldots$. & I9. I & $\cdots \cdots$ & .5 & $37^{\circ}$ \\
\hline Prunes- & $\begin{array}{l}\text { edible portion..... } \\
\text { as purchased...... }\end{array}$ & 24 & $\ldots$ & 79.6 & .9 & $\cdots \cdots$ & 18.9 & $\cdots \cdots$ & .6 & $37^{\circ}$ \\
\hline Raspberries- & irchased. & T. & & $\begin{array}{l}75.0 \\
8=8\end{array}$ & .7 & $\cdots \cdots$ & $17 \cdot 4$ & ..... & $\cdot 5$ & 335 \\
\hline \multirow[t]{2}{*}{ Strawberries- } & edible portion..... & 22 & & 90.4 & 1.0 & .6 & 7.4 & I. 4 & .6 & $\begin{array}{l}255 \\
180\end{array}$ \\
\hline & as purchased...... & & $5 \cdot 0$ & 85.9 & .9 & .6 & 7.0 & $\cdots \cdots$ & .6 & 175 \\
\hline \multirow{2}{*}{ Watermelon- } & jrtion..... & 2 & & $9^{2} \cdot 4$ & .4 & .2 & 0.7 & $\cdots \cdots$ & -3 & 140 \\
\hline & & & & & .2 & -1 & $2 \cdot 7$ & & $\cdot 1$ & 60 \\
\hline
\end{tabular}

The following analyses of apples made by Browne* are of interest. The first four analyses show the changes that occur in the composition of a Baldwin apple at different stages of its growth. Below these is given the average of the analysis of 160 samples, representing 27 varieties of apples.

\section{COMPOSITION OF A BALDWIN APPLE AT DIFFERENT PERIODS.}

\begin{tabular}{|c|c|c|c|c|c|c|c|c|c|c|}
\hline Condition. & Water. & Solids. & $\begin{array}{l}\text { Invert } \\
\text { Sugar. }\end{array}$ & $\begin{array}{l}\text { Su- } \\
\text { crose. }\end{array}$ & $\begin{array}{l}\text { Total } \\
\text { Sugar. }\end{array}$ & $\begin{array}{l}\text { Total } \\
\text { Sugar } \\
\text { atter In- } \\
\text { version. }\end{array}$ & Starch. & $\begin{array}{l}\text { Free } \\
\text { Malic } \\
\text { Acid. }\end{array}$ & Ash. & $\begin{array}{l}\text { Sugar } \\
\text { Co- } \\
\text { efficient. }\end{array}$ \\
\hline $\begin{array}{l}\text { Very green.. } \\
\text { Green..... }\end{array}$ & $\begin{array}{l}81.53 \\
70.81\end{array}$ & $\begin{array}{l}18.47 \\
20.10\end{array}$ & $\begin{array}{l}6.40 \\
6.46\end{array}$ & $\begin{array}{l}1.63 \\
4.05\end{array}$ & $\begin{array}{r}8.03 \\
10.51\end{array}$ & $\begin{array}{r}8.11 \\
10.72\end{array}$ & $\begin{array}{l}4.14 \\
3.67\end{array}$ & $\begin{array}{l}1.14 \\
\ldots .\end{array}$ & $\begin{array}{l}0.27 \\
\ldots .\end{array}$ & $\begin{array}{l}47 \cdot 16 \\
53 \cdot 10\end{array}$ \\
\hline Ripe....... & 80.36 & 19.64 & $7 \cdot 70$ & $6.8 \mathrm{I}$ & $14.5 \mathrm{I}$ & 14.87 & 0.17 & 0.65 & 0.27 & 75.71 \\
\hline Over-ripe.... & 80.30 & 19.70 & $8.8 \mathrm{I}$ & 5.26 & 14.07 & $14 \cdot 35$ & $\ldots$. & 0.48 & 0.28 & 72.84 \\
\hline
\end{tabular}

* Penn. Dept. of Agriculture, Bulletin $5^{8}$. 
AVERAGE COMPOSITION OF 27 VARIETIES OF APPLES.

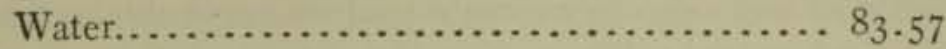

Solids........................... 16.43

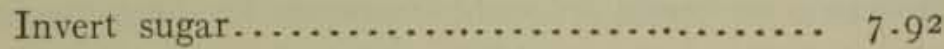

Sucrose........................ 3.99

Total sugar . . . . . . . . . . .

Total sugar after inversion............. I2.12

Free malic acid.................... 0.6 I

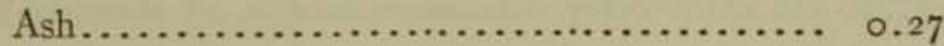

Sugar coefficient................. 73.76

The composition of the commoner nuts is shown in the following table:*

NUTS.

\begin{tabular}{|c|c|c|c|c|c|c|c|c|c|c|}
\hline & & 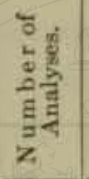 & 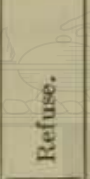 & 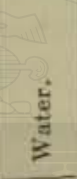 & 递 & 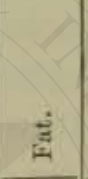 & 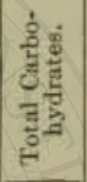 & 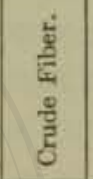 & 莫 & 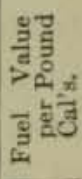 \\
\hline Almonds - & & $\begin{array}{c}\text { II } \\
\ldots \ldots\end{array}$ & & $\begin{array}{l}4.8 \\
2.7\end{array}$ & $\begin{array}{l}20.0 \\
11.5\end{array}$ & $\begin{array}{l}54 \cdot 9 \\
30.2\end{array}$ & $\begin{array}{r}17.3 \\
9.5\end{array}$ & $\begin{array}{c}2.0 \\
\cdots\end{array}$ & $\begin{array}{l}2.0 \\
1.1\end{array}$ & 303 \\
\hline Beechnuts- & & $\begin{array}{c}\mathrm{I} \\
\ldots \ldots\end{array}$ & 40.8 & & $\begin{array}{l}21.9 \\
13.0\end{array}$ & $\begin{array}{l}57 \cdot 4 \\
34.0\end{array}$ & $\begin{array}{r}13.2 \\
7.8\end{array}$ & $\begin{array}{c}\ldots \ldots \\
\ldots \ldots\end{array}$ & $\begin{array}{l}3 \cdot 5 \\
2 \cdot 1\end{array}$ & \\
\hline Brazil-nuts- & & $\begin{array}{l}\mathrm{I} \\
\ldots .\end{array}$ & 49.6 & $\begin{array}{l}5 \cdot 3 \\
2.6\end{array}$ & $\begin{array}{r}7.0 \\
8.6\end{array}$ & $\begin{array}{l}66.8 \\
33-7\end{array}$ & $\begin{array}{l}7 \cdot 0 \\
3 \cdot 5\end{array}$ & $\begin{array}{l}\cdots \\
\cdots \cdots \\
\cdots\end{array}$ & $\begin{array}{l}2.1 \\
3.9 \\
2.0\end{array}$ & $\begin{array}{l}326 \\
165\end{array}$ \\
\hline Butternuts- & & $\begin{array}{l}\mathrm{I} \\
\ldots\end{array}$ & 86.4 & $\begin{array}{r}4 \cdot 4 \\
.6\end{array}$ & $\begin{array}{r}27.9 \\
3.8\end{array}$ & $\begin{array}{r}61.2 \\
8.3\end{array}$ & 35 & $\cdots \cdots$ & $\begin{array}{r}2.9 \\
.4\end{array}$ & $\begin{array}{r}316 \\
43\end{array}$ \\
\hline Chestnuts, fresh - & $\begin{array}{l}\text { portion. } \\
\text { hased.. }\end{array}$ & 9 & $\begin{array}{c}\ldots .0 \\
16.0\end{array}$ & $\begin{array}{l}45.0 \\
37.8\end{array}$ & $\begin{array}{l}6.2 \\
5.2\end{array}$ & $\begin{array}{l}5 \cdot 4 \\
4 \cdot 5\end{array}$ & $\begin{array}{l}42 . \mathrm{t} \\
35 \cdot 4\end{array}$ & $\begin{array}{c}1.8 \\
\ldots . .\end{array}$ & $\begin{array}{l}\text { I. } 3 \\
\text { I. } 1\end{array}$ & $\begin{array}{r}112 \\
94\end{array}$ \\
\hline Cocoanuts- & & I & $\ddot{4} . \ddot{8}$ & $\begin{array}{r}14.1 \\
7.2\end{array}$ & $\begin{array}{l}5.7 \\
2.9\end{array}$ & $\begin{array}{l}50.6 \\
25.9\end{array}$ & $\begin{array}{l}27.9 \\
14.3\end{array}$ & $\begin{array}{l}\cdots \cdots \\
\cdots \cdots \\
\cdots\end{array}$ & $\begin{array}{r}\text { I. } 7 \\
.9\end{array}$ & $\begin{array}{l}276 \\
141\end{array}$ \\
\hline Filberts- & & $\begin{array}{c}\mathrm{I} \\
\cdots\end{array}$ & $\begin{array}{l}\cdots . . \\
52.1\end{array}$ & $\begin{array}{l}3.7 \\
1.8\end{array}$ & $\begin{array}{r}15.6 \\
7.5\end{array}$ & $\begin{array}{l}65 \cdot 3 \\
3 \mathrm{I} \cdot 3\end{array}$ & $\begin{array}{r}13.0 \\
6.2\end{array}$ & ...... & $\begin{array}{l}2.4 \\
\text { I. I }\end{array}$ & $\begin{array}{l}329 \\
157\end{array}$ \\
\hline Hickory-nuts- & & $\begin{array}{l}\text { I } \\
\ldots\end{array}$ & 62.2 & $\begin{array}{l}3 \cdot 7 \\
1-4\end{array}$ & $\begin{array}{r}15.4 \\
5.8\end{array}$ & $\begin{array}{l}67 \cdot 4 \\
25 \cdot 5\end{array}$ & $\begin{array}{r}\text { II. } 4 \\
4.3\end{array}$ & $\cdots \cdots$ & $\begin{array}{r}2.1 \\
.8\end{array}$ & $\begin{array}{l}334 \\
126\end{array}$ \\
\hline Peanuts- & & $\begin{array}{c}4 \\
\cdots \\
\cdots\end{array}$ & $24 \cdot 5$ & $\begin{array}{l}9.2 \\
6.9\end{array}$ & $\begin{array}{l}25.8 \\
19.5\end{array}$ & $\begin{array}{l}38.6 \\
29.1\end{array}$ & $\begin{array}{l}24.4 \\
18.5\end{array}$ & $\begin{array}{r}2 \cdot 5 \\
\cdots \cdots\end{array}$ & $\begin{array}{l}2.0 \\
1.5\end{array}$ & $\begin{array}{l}256 \\
193\end{array}$ \\
\hline Pecans- & & $\begin{array}{c}I \\
\cdots\end{array}$ & $\begin{array}{c}\cdots 3.2 \\
53.2\end{array}$ & $\begin{array}{l}3.0 \\
\mathrm{I} .4\end{array}$ & $\begin{array}{r}11.0 \\
5.2\end{array}$ & $\begin{array}{l}71-2 \\
33-3\end{array}$ & $\begin{array}{r}13.3 \\
6.2\end{array}$ & $\begin{array}{l}\ldots \ldots \\
\cdots \ldots \\
\cdots\end{array}$ & $\begin{array}{r}1.5 \\
-7\end{array}$ & $\begin{array}{l}345 \\
162\end{array}$ \\
\hline $\begin{array}{l}\text { Pistachios- } \\
\text { Walnuts, Calif'nia- }\end{array}$ & $\begin{array}{l}\text { edible portion. } \\
\text {-edible portion. } \\
\text { as purchased.. }\end{array}$ & I & $\begin{array}{l}\cdots \cdots \\
\cdots \cdots \\
73 \cdot 1\end{array}$ & $\begin{array}{r}4 \cdot 2 \\
2 \cdot 5 \\
-7\end{array}$ & $\begin{array}{r}22.3 \\
18.4 \\
4 \cdot 9\end{array}$ & $\begin{array}{l}54 \cdot 0 \\
64 \cdot 4 \\
17 \cdot 3\end{array}$ & $\begin{array}{r}16.3 \\
13.0 \\
3.5\end{array}$ & $\begin{array}{c}\cdots \\
1.4 \\
\cdots\end{array}$ & $\begin{array}{r}3.2 \\
1.7 \\
.5\end{array}$ & $\begin{array}{r}299 \\
330 \\
88\end{array}$ \\
\hline
\end{tabular}

Vegetables and Fruits furnish a large and most important portion of our food supply, but are naturally not included in their fresh state among the foods examined by the public analyst for adulteration, hence

* U. S. Dept. of Agric., Off. of Exp. Station, Bul. 28 . 
but little space need be given them beyond a résumé of their composition, and an outline of methods of proximate analysis applicable to their examination for food values. When, however, these products undergo the various processes incidental to their treatment for long keeping, such as preserving, canning, drying, pickling, and mixing with other ingredients, it is then that many varieties of fraudulent adulteration are practiced. Vegetable foods thus prepared form the subject of a separate chapter. Besides the proximate components that commonly occur in vegetable products, there are three other substances worthy of mention found in vegetables and fruits, viz., inosite, pectose, and inulin.

Inosite, $\mathrm{C}_{6} \mathrm{H}_{12} \mathrm{O}_{6},{ }_{2} \mathrm{H}_{2} \mathrm{O}$, is not a carbohydrate, but, according to $\mathrm{Ham}$ mersten, is an aromatic compound. Besides occurring in unripe fruits, it is found in green asparagus and beans.

Pectose is a substance the exact nature of which has not been fully determined; though it is thought to be a carbohydrate. It gives to unripe fruits and vegetables their peculiar hardness, and furnishes the basis for their gelatinous constituents. When the vegetable or fruit ripens, the insoluble pectose is then transformed by the action of acids and possibly of ferments into pectin, a vegetable jelly, which gives to fruit juice the property of gelatinizing when boiled.

Inulin, $\left(\mathrm{C}_{6} \mathrm{H}_{10} \mathrm{O}_{5}\right)_{n}$, is a starch-like substance, occurring in the roots of chicory and dandelion, and in the tubers of the artichoke. It is a white, starch-like powder, slightly soluble in cold, and readily soluble in hot water, and converted into levulose by boiling with water, or by the action of acids.

\section{METHODS OF PROXIMATE ANALYSIS.}

Preparation of the Sample.-Cereals and other dry products should be ground in a hand mill or iron mortar so as to pass a sieve with round holes I mm. in diameter. Green vegetables, roots, fruits, etc., may be reduced to a pulp in a food chopper.

The following methods, with the exception of the Brown and Duvel method, are those of the Association of Official Agricultural Chemists for the analysis of foods and feeding stuffs.*

Moisture.-Dry a quantity of the substance, representing about 2 grams of the dry material, to constant weight (about five hours) at the temperature of boiling water, in a current of dry hydrogen or in vacuo. The apparatus described on page 62 may be used.

* U. S. Dept. of Agric., Bur. of Chem., Bul. ro7 (rev.). 
Ash.-Burn 2 grams of the substance in a platinum dish at the lowest possible red heat, as described on pages 62 and $5_{3}$. If a white or lightgray ash cannot be obtained in this manner, exhaust the charred mass with water, collect the insoluble residue on a filter, burn, add this ash to the residue from the evaporation of the aqueous extract, and heat the whole at low redness until white or nearly so.

Ether Extract (Fat, etc.).-Extract the residue from the determination of moisture for sixteen hours with anhydrous alcohol-free ether in a continuous extractor (p. 63) and iry the extract to constant weight in a water-oven. The ether extract may also be determined indirectly from the difference in weight of the dried substance before and after extraction.

Protein.-Determine the total nitrogen by the Gunning or Kjeldahl method, using I gram of the substance. Calculate the protein by multiplying the total nitrogen by the appropriate factor, which varies with the different cereals as follows: wheat, 5.70 ; rye, 5.62 ; oats, $6.3 \mathrm{I}$; corn, 6.39; and barley, 5.82. Ordinarily the conventional factor 6.25 is employed.

Crude Fiber (Cellulose, Lignin, etc.).-Transfer the residue, after extraction for the determination of the ether extract, to a 500-cc. Erlenmeyer flask, with a mark showing $200 \mathrm{cc}$., add boiling $1.25 \%$ sulphuric acid to the mark, heat at once to boiling, and boil gently for thirty minutes, shaking cautiously from time to time to prevent the material from crawling up on the sides of the flask. Filter through paper, and wash once with boiling water. Rinse the substance back into the same flask with $200 \mathrm{cc}$. of a boiling $1.25 \%$ solution of sodium hydroxide, free, or nearly so, from sodium carbonate, boil at once, and continue the boiling for thirty minutes in the same manner as directed above for the treatment with acid. Filter on a tared filter-paper or Gooch crucible, and wash with boiling water till the washings are neutral. Dry at $110^{\circ}$ and weigh, after which incinerate completely and correct for ash.

If a tared filter is used, it should be previously dried at $110^{\circ}$ for one hour in a glass-stoppered weighing bottle, cooled for fifteen minutes and weighed. After collecting the fiber on the filter, it is well to wash successively with alcohol and ether to facilitate drying. The filter should not be pushed down into the weighing bottle until the fiber is dry to the touch after which two hours' drying at $110^{\circ}$ will be sufficient. A blank should be made to ascertain the loss sustained by the filter on treatment with alkali and the necessary correction intro- 
duced. This error and others can be avoided by filtering on a Gooch crucible, but with many materials this cannot be used because of clogging. The acid and alkali solutions must be exactly $1.25 \%$ as determined by titration.

Nitrogen-free Extract (Starch, Sugar, Gums, etc.).--Subtract the sum of the moisture, ash, ether extract, protein, and crude fiber, from 100.

Determination of Moisture in Grain, Legumes, Oil Seeds, etc.Brown and Duvel Method.*-This method is especially useful in guarding

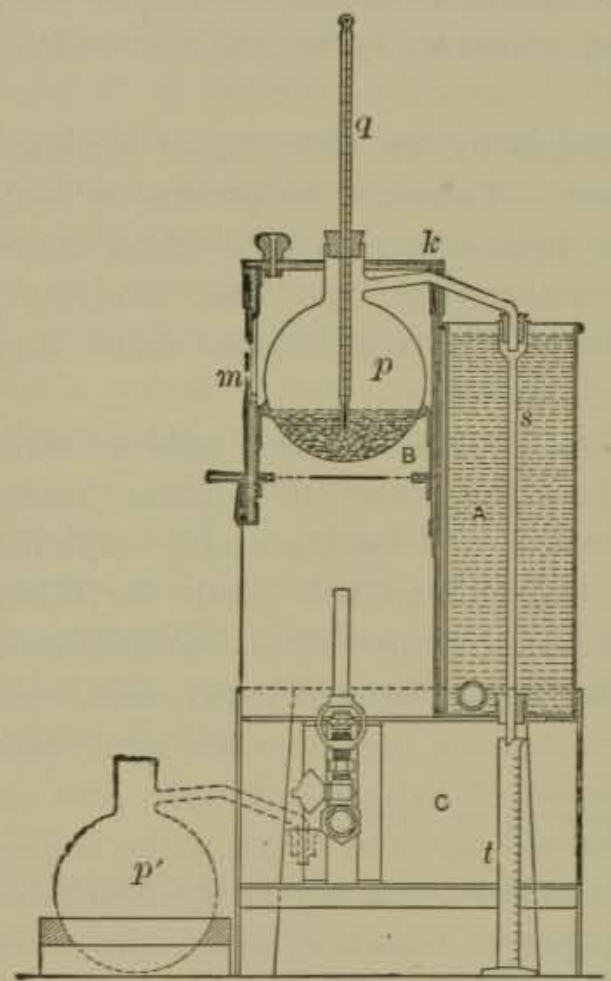

Fig. 6r-Brown and Duvel Apparatus for Determination of Moisture in Grain. End View. against an excessive amount of moisture in grain, which not only adds weight but also causes deterioration through the growth of bacteria and moulds.

The apparatus (Fig. 6r) consists of a condenser-tank $(A)$ and an evaporating-chamber $(B)$ with a cover $(k)$ and a mica window $(m)$, the whole supported on a stand $(C)$. It is arranged for conducting six distillations at the same time.

The distilling-flask is shown at the left $\left(p^{\prime}\right)$ in the wooden rack used only during filling and at the right $(p)$ in position for distillation.

Weigh into the distilling-flask Ioo grams (corn, barley, wheat, rye, unhulled rice, kafir, flaxseed, soy bean) or 50 grams (oats, cottonseed) of the whole grain and add $150 \mathrm{cc}$. of hydrocarbon engine oil with a flash-point in an open cup of $200^{\circ}-$ $205^{\circ} \mathrm{C}$. Close the neck of the flask with a rubber stopper carrying a thermometer $(q)$, the bulb of which extends well into the mixture of oil and corn; connect the side tube by means of another cork with the condenser-tube $(s)$, and heat with the Bunsen burner, so as to bring (in twenty minutes) to the

* U. S. Dept. of Agric,, Bur, of Plant. Ind., Bul. 99, and Circular 72. 
proper temperature, which for corn, barley, rice, kafir, and cottonseed is $190^{\circ}$, for wheat $180^{\circ}$, for rye and flaxseed $175^{\circ}$, for soy bean $170^{\circ}$, and for oats $195^{\circ} \mathrm{C}$. When the desired temperature is reached turn off the flame, and allow to stand until the moisture ceases to drop from the condenser-tube into the graduate $(t)$.. The number of cc. in the graduate represents the amount of moisture in the grain.

The results agree closely with those by drying to constant weight in a water-oven at $100^{\circ}$.

After the determination is finished empty the contents of the flask on a suitable strainer, thus recovering the oil for further use.

\section{CARBOHYDRATES OF CEREALS AND VEGETABLES.}

Classification.-As a rule the same carbohydrates are found in all cereals, being present, however, in varying proportions. By far the greater part of the carbohydrate content of cereals is starch, the other carbohydrates being comparatively small in amount, so that in rough work it is sometimes customary, though incorrect, to assume the entire amount of so-called "nitrogen-free extract" or carbohydrates (as determined by difference) to be starch.

The carbohydrates occurring in cereals may be classified as follows:

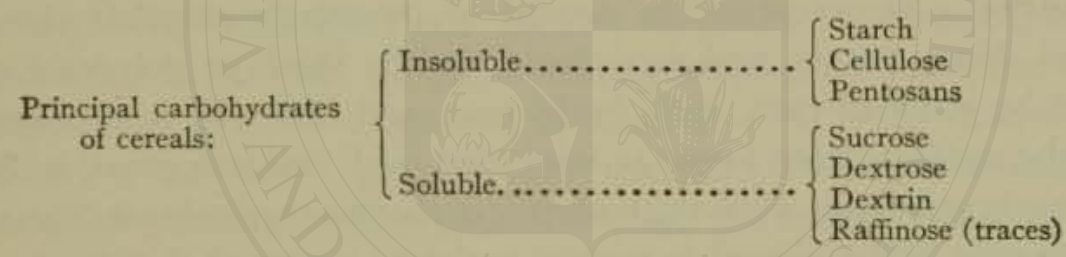

StARCH $\left(\mathrm{C}_{5} \mathrm{H}_{10} \mathrm{O}_{5}\right)_{n}$.-Pure starch is a glistening, white, granular powder having a peculiar feeling when rubbed between the thumb and finger. It is a very hygroscopic, commercial starch containing about $18 \%$ of moisture. Starch is very widely distributed in the vegetable kingdom, occurring in almost every plant at some stage in its growth.

Starch is insoluble in cold water, alcohol, and ether; it is soluble in hot water, though not without change. By boiling with dilute acids, starch is first converted by hydrolysis into a mixture of dextrin and maltose, and finally by prolonged boiling into dextrose. Malt extract also hydrolizes starch in solution.

Detection.--Starch is best detected, when present to any appreciable extent in any mixture, by boiling a portion of the sample in water, cooling, and applying a solution of iodine. A characteristic blue color is pro. duced if starch is present. Very small amounts of starch are best iden. 
tified in powdered mixtures by applying a drop of a solution of iodine to the dry powder on a microscope slide, or, better, to the powder previously rubbed out with water on a slide under a cover-glass; the starch granules, if present, will be colored intensely blue by the iodine, and are at once rendered apparent when viewed through the microscope.

Though the cereal and vegetable starches, whatever their origin, are identical chemically, the various starch granules have certain characteristics, when viewed under the microscope, that render their identifcation easy in most cases. A knowledge of the microscopical appearance of the common vegetable starches is of the utmost importance to the public analyst, who frequently finds them as adulterants of various foods, such as coffee, cocoa, spices, etc. For microscopical examination, powdered samples should be ground fine enough to pass through a 60 or 80 mesh sieve.

Classification. - As seen under the microscope the starch granules of various grains and vegetables differ in form, size, and often in their manner of grouping. Thus, at the outset, the common starches may be divided as to the microscopical form of their granules into three classes, viz., lenticular, irregularly oval, and polygonal. To the first class, in which the starch granule has in general the circular disk form, belong rye, wheat, and barley. Representing the second or irregularly elliptical class are the pea, bean, potato, and arrowroot. In the third, or polygonal class, should be included corn, oats, buckwheat, and rice. In thus characterizing the distinguishing forms as lenticular, oval, and polygonal, it should be borne in mind that while the tendency of the most typical starch granules in each class, when viewed in normal position, is toward the circular, the oval, or the polygonal as the case may be, it is not by any means true that all or even most of the granules in any one instance perfectly conform to one of these shapes throughout. Thus, lenticular wheat granules, when viewed edgewise, will appear elliptical, and are often distorted in shape, especially when roasted; and polygonal buckwheat granules may in many instances have such obtuse angles as to appear circular. It is the general trend of all the starches toward one or another of these shapes that suggests the classification.

The identification of the various starches morphologically is indeed the most natural and ready method. Not only the character of the starch, but a'so its approximate amount, when present in mixtures, can in many instances be ascertained by a careful examination with the microscope. The analyst should be provided with samples of starches of known 
purity conveniently at hand, and in all doubtful cases these should be referred to for comparison.

Wheat Starch (Fig. ${ }^{5} 5_{2}, \mathrm{Pl}$. VIII).--This starch is frequently present in adulterated pepper, mustard, ginger, cocoa, coffee, and other foods. Its granules occur for the most part in two sizes, of which the larger are lenticulars, varying from $0.021 \mathrm{~mm}$. to $0.04 \mathrm{Imm}$, or rarely 0.050 $\mathrm{mm}$, in diameter, while the smaller are rounded or polygonal, averaging about $0.005 \mathrm{~mm}$. in diameter. The smaller granules are grouped irregularly in and around the larger, there being six to ten of the former to one of the latter. The larger granules are, however, the most distinctly characteristic, and are usually readily recognized in a mixture, not only by their shape, but by reason of the concentric rings with which they are provided, and which are generally but not always apparent.

Barley Starch (Fig. 124, Pl. I).-This much resembles wheat, in that it has two sizes of granules, but both sizes are respectively smaller than those of wheat, though present in about the same proportion. The larger lenticular disk-like granules vary from $0.013 \mathrm{~mm}$. to $0.035 \mathrm{~mm}$. in diameter, while the smaller average $0.003 \mathrm{~mm}$. The concentric rings are less apparent in the barley than in the wheat.

Rye Starch (Fig. 148, Pl. VII) has also two sizes of granules, but the larger vary from $0.025 \mathrm{~mm}$. to over $0.05 \mathrm{~mm}$. in diameter, and are considerably larger than the corresponding wheat granules. The smaller granules average about $0.004 \mathrm{~mm}$. in diameter. As in the case of wheat and barley, the larger granules are lenticular, while the smaller are rounded or polygonal. The concentric rings are usually indistinct in the large granules, and many of these show cross-shaped rifts in the center.

Corn Starch (Fig. 133, Pl. IV).-This starch is a common adulterant of spices, cocoa, and other foods. It is placed in a series of four cereal starches whose granules are polygonal, and all of which show more or less tendency to arrange themselves in close contact side by side in masses suggestive of a tessellated or mosaic floor. Arranged in order of the size of their grains, these starches are: Corn, buckwheat, oats, and rice. Corn starch granules tend toward the hexagonal in shape, varying from $0.007 \mathrm{~mm}$. to $0.035 \mathrm{~mm}$. in diameter, and having very marked rifted hila. They are most readily recognized in any mixture, and from their size are readily distinguishable from the other polygonal starches, which never reach $0.017 \mathrm{~mm}$. in diameter.

Buckwheat Starch (Fig. I28, Pl. II, and Fig. I29, Pl. III).-This is a very common adulterant of many spices, especially pepper, which, as 
shown in Fig. 256, Pl. XXXIV, it much resembles in the manner in which its masses of granules group themselves, conforming to the shape of the cells. The individual granules are commonly $0.006 \mathrm{~mm}$. to 0.012 $\mathrm{mm}$. in diameter. Curious rod-shaped aggregates of two to four individuals are of frequent occurrence.

Oat Starch (Fig. 139, Pl. V).-The granules of this starch vary from $0.002 \mathrm{~mm}$. to $0.012 \mathrm{~mm}$. in diameter, and are polygonal, or less often rounded or spindle-shaped in form. They have no rings or hila, and arrange themselves in rounded aggregates of from two to many granules that at first sight might be mistaken for large grains; careful examination, however, shows the dividing lines.

Rice Starch (Fig. I43, PI. VI).-The granules of rice starch resemble closely those of oats both in form and size, but spindle-shaped forms are not present. As in the case of oats, the granules are often united to form rounded aggregates.

Starches of the Pea and Bean.-The starches of these legumes much resemble each other, and are with difficultly distinguished one from the other (see Fig. 164, Pl. XI, and Fig. 154, Pl. IX). The granules are more nearly oval than most other starches, and have both concentric rings and elongated hila. The granules of the pea show a less distinct hilum than those of the bean, and some of them are irregularly swollen. Both peas and beans roasted are commonly used as adulterants of coffee.

Arrowroot.-There are many varieties of arrowroot, including Jamaica, Bermuda, East Indian, Australian, and others, all having certain variations in form and size, but resembling each other in a general way. Fig. ${ }^{6} 67, \mathrm{Pl}$. XII, shows the Bermuda arrowroot, the granules of which are somewhat egg-shaped, being usually smaller at one end than the other, and having rifted hila near the small end.

Potato Starch (Fig. 165, Pl. XII).--This starch has large, irregularly oval granules, with very apparent hila situated eccentrically near one end, and with rings around the hilum. The granules are about $0.07 \mathrm{~mm}$. in large diameter. Fig. 134, Pl. IV, and Fig. I66, Pl. XII, show corn and potato starch when viewed with polarized light with crossed Nicol prisms, the specimens being mounted in Canada balsam.

Tapioca Starch.-The granules of this starch, as shown in Fig. 168, Pl. XII, are more uniform in size throughout than those already described, averaging about $0.018 \mathrm{~mm}$. in diameter, and being quite smoothly circular, without concentric rings, but having a distinctly dotted hilum in 
the center. Many of the grains are cup-shaped, as if a segment of the circle had been removed.

Sago Starch (Fig. 172, Pl. XIII.) - The granules of sago starch vary much in size, and might be called irregularly ellipsoidal in shape with one or more truncated surfaces. Some of them have indistinct concentric rings, and in some, but not all, a hilum is apparent, usually near one end of the granule.

Microscopical Appearances of Starches with Polarized Light.-With polarized light starch granules show dark crosses, the point of intersection being at the hilum (Fig. I66, Pl. XII). These crosses vary in distinctness with the variety. Certain of the starches show a play of colors with polarized light and a selenite plate, especially those whose granules have some sort of hilum. This is particularly striking in such starches as corn, tapioca, potato, and arrowroot. Blyth has made the phenomenon a means of classification of the starches, but the writer considers their appearance with ordinary light sufficient for identification. Canada balsam is the best mountant for examination in polarized light.

Estimation of Starch.-Direct Acid Conversion.-By this method the hemicellulose, if present, or such of the carbohydrates as are capable of being converted to sugar, are reckoned in with the starch. Where little or none of the insoluble carbohydrates other than starch are present, as for instance in the case of commercial starches, this method is sufficiently accurate.

Exhaust 3 grams of the finely divided substance on a fine but rapidly acting filter with ether by washing with 5 successive portions of $10 \mathrm{cc}$. each, and wash the residue first with $150 \mathrm{cc}$. of $10 \%$ alcohol and then with a little strong alcohol. Transfer by washing to a flask with $200 \mathrm{cc}$. of water and $20 \mathrm{cc}$. of hydrochloric acid (specific gravity I.I25), connect with a reflux condenser, and heat the flask in boiling water for $2 \frac{1}{2}$ hours. $\mathrm{Cool}$, and carefully neutralize with sodium hydroxide, clarifying if necessary with alumina cream. Mix well, make up the volume to $500 \mathrm{cc}$., filter, and determine the dextrose in an aliquot part of the filtrate by any of the methods for dextrose. Convert dextrose to starch by the factor 0.9 .

Diastase Method.-By this method the hemicellulose is not converted, only the starch being acted upon. Hence for exact work in the presence of other insoluble carbohydrates this method is to be recommended. Under the action of diastase, starch is first converted into 
maltose and dextrin, and finally into dextrose, in somewhat the following manner:

$$
\begin{aligned}
& \underset{\text { Starch }}{\mathrm{I}_{2} \mathrm{C}_{6} \mathrm{H}_{10}} \mathrm{O}_{5}+{ }_{4} \mathrm{H}_{2} \mathrm{O}=\underset{4}{4 \mathrm{C}_{13} \mathrm{H}_{22} \mathrm{O}_{11}}+\underset{2}{\mathrm{C}_{12} \mathrm{H}_{27} \mathrm{O}_{10}} \\
& \underset{\text { Maltose }}{\mathrm{C}_{12} \mathrm{H}_{22} \mathrm{O}_{14}}+\mathrm{H}_{2} \mathrm{O}=\underset{2}{\mathrm{C}_{6} \mathrm{H}_{12} \mathrm{O}_{6}} \quad \underset{\text { Dextrose }}{\mathrm{C}_{17} \mathrm{H}_{20} \mathrm{O}_{10}}+2 \mathrm{H}_{2} \mathrm{O}=\underset{\text { Dextrin }}{\mathrm{C}_{6} \mathrm{H}_{12} \mathrm{O}_{6}}
\end{aligned}
$$

Exhaust 3 grams of the material, ground to an impalpable powder, with ether and alcohol as in the acid conversion method, wash the residue into a beaker with $50 \mathrm{cc}$. of $\mathrm{w}^{-}$ter and immerse in a boiling water-bath. Keep in the bath for I $5_{5}$ minutes or until completely gelatinized, stirring constantly, and cool to $55^{\circ} \mathrm{C}$. Add $20 \mathrm{cc}$. of malt extract and digest at $55^{\circ} \mathrm{C}$. one hour. Heat again to boiling, boil for ${ }_{15}$ minutes, replacing the water lost by evaporation, $\operatorname{cool}$ to $55^{\circ} \mathrm{C}$, and digest as before with $20 \mathrm{cc}$. of malt extract for one hour or until the residue treated with iodine solution shows no starch under the microscope. Cool, make up to 250 cc., filter, pipette $200 \mathrm{cc}$. into a flask, add $20 \mathrm{cc}$. of hydrochloric acid (specific gravity 1.125) and proceed as in the acid conversion method. Correct for the copper-reducing power of trie malt extract as below.

Preparation of Malt Extract.-Digest at room temperature to grams of freshly pulverized malt with $200 \mathrm{cc}$. of water for 2 to 3 hours with occasional shaking and filter. Determine the amount of dextrose in a given volume of the extract after heating with acid, etc., as in the actual analysis and make the proper correction.

Use of "Animal Diastase."-Pancreatin and similar powdered preparations, such as "vera diastase " and "panase," obtained from the pancreas of cattle and hogs, are preferable to diastase as starch-converting reagents, since, as a rule, they have no copper-reducing power, thus obviating a correction.

Use instead of the malt extract the same amount, viz., $20 \mathrm{cc}$, , of a $0.5 \%$ aqueous solution of powdered U. S. P. pancreatin in starch determinations as above described.

Determination of Sugars in Grain and Cereal Products.-Method of Bryan, Given and Straughn.*-Place 12 grams of the material in a $300-$ cc. graduated flask (if acid add $\mathrm{I}-3$ grams of precipitated calcium carbonate), add $150 \mathrm{cc}$. of $50 \%$ (by vol.) alcohol (carefully neutralized), mix and boil on a steam bath under a reflux condenser for one hour. Cool, and if desired allow to stand overnight. Make up to volume with neutral $95 \%$ alcohol, mix, allow to settle, pipette $200 \mathrm{cc}$. in to a beaker, and evaporate

*U. S. Dept. of Agric. Bur, of Chem., Circ. 7r. 
on the steam bath to $20-30 \mathrm{cc}$. Transfer to a $100 \mathrm{cc}$. graduated flask with water, add enough saturated normal lead acetate solution to produce a flocculent precipitate, and allow to stand 15 minutes or if desired overnight. Make up to the mark with water and pass through a folded filter, saving all the filtrate. Precipitate the lead, with anhydrous sodium carbonate, allow to stand 15 minutes and pour onto an ashless filter. Dilute $25 \mathrm{cc}$. of this clear filtrate with $25 \mathrm{cc}$. of water and determine reducing sugars by the Munson and Walker method (p. 598).

In a $100 \mathrm{cc}$. graduated flask place $50 \mathrm{cc}$. of the same filtrate, neutralize to litmus paper with acetic acid, add $5 \mathrm{cc}$. of concentrated hydrochloric acid, and let stand overnight (or if desired 48 hours) for inversion. Neutralize in a large beaker with anhydrous sodium carbonate; return to the $100 \mathrm{cc}$. flask and make up to the mark. Filter and determine total sugars as invert in $50 \mathrm{cc}$. of the filtrate by the Munson and Walker method.

Multiply the difference between the percentages of invert sugar before and after inversion by 0.95 , thus obtaining the per cent of sucrose. Correct the percentages of both sucrose and reducing sugars for the volume of the alcohol precipitate by multiplying by 0.97 .

Cellulose forms the framework of all vegetable organisms, being next to water, the most abundant substance in the vegetable kingdom. Pure cellulose is white, translucent, and of fibrous or silky texture. It is insoluble in water, alcohol, and ether, but dissolves readily in an ammoniacal solution of cupric hydroxide known as Schweitzer's Reagent or "cuprammonia," (p. 93). Cellulose turns violet when treated with chloriodide of zinc, and blue when treated with sulphuric acid and iodine in potassium iodide (p. 9r).

The "crude fiber," as determined in foods, being the portion that resists the action of hot dilute acid and alkali, is composed largely of cellulose.

The Pentosans are amorphous, insoluble in water, but soluble in dilute alkali, and are converted by boiling with dilute acids into so-called pentose sugars, the best known of which are xylose and arabinose, corresponding to the pentosans xylan and araban respectively. Hemicellulose is the more appropriate generic term for the insoluble carbohydrates capable of hydrolysis by acids to sugars, inasmuch as there are insoluble bodie besides the pentosans that may thus be converted into sugar, such as the hexosans, hydrolyzed by acid to hexose sugars, mannose; galactose, etc. Since the greater portion of these insoluble hydroliz- 
able carbohydrates are pentosans, it is simpler to calculate them all as such.

Determination of Pentosans.-Pentosans are determined either by hydrolyzing to reducing sugar, and estimating the latter as described on page 296 (Stone's method) or by calculation from the furfural* yielded on distillation with hydrochloric acid, as carried out in the provisional method of the A. O. A. C. $†$ as follows:

Place 3 grams of the material in a flask together with $100 \mathrm{cc}$. of $12 \%$ hydrochloric acid (specific gravity 1.06) and several pieces of recently heated pumice stone, connect with a condenser and heat on a wire gauze, rather gently at first, using a gauze top to distribute the flame so as to distil over $30 \mathrm{cc}$. in about ten minutes and passing the distillate through a small filter. Replace the $30 \mathrm{cc}$. driven over with a like amount of the I $2 \%$ acid added through a separatory funnel in such a manner as to wash down the particles on the sides of the flask and continue the process until the distillate amounts to $360 \mathrm{cc}$. To the distillate add gradually a quantity of phloroglucinol (free from diresorcin) dissolved in $12 \%$ hydrochloric acid, about double that of the furfural expected. The solution first turns yellow, then green, and soon an amorphus greenish precipitate appears; which rapidly grows darker, finally becoming almost black. Make the solution up to $400 \mathrm{cc}$. with $12 \%$ hydrochloric acid and allow to stand overnight.

Filter the amorphous black precipitate on a Gooch crucible, wash with ${ }_{50} \mathrm{cc}$. of water, keeping the precipitate covered with the liquid until the last portion has run through, dry for four hours at the temperature of boiling water, cool in a weighing bottle and weigh. Calculate by Kröber's formulæ as follows:

(a) For weight of phloroglucide " $a$ " under 0.03 gram:

$$
\begin{aligned}
& \text { Furfural }=\left(a+0.005^{2}\right) \times 0.5170 . \\
& \text { Pentoses }=\left(a+0.005^{2}\right) \times 1.0170 . \\
& \text { Pentosans }=\left(a+0.005^{2}\right) \times 0.8949 .
\end{aligned}
$$

* Furfural or furfuraldehyde $\left(\mathrm{C}_{6} \mathrm{H}_{4} \mathrm{O}_{2}\right)$ is the aldehyde of pyromucic acid. It is a colorless liquid, having an odor suggestive of cassia. Its boiling-point is $162^{\circ}$ and its specific gravity r.r64. It is sparingly soluble in water and readily soluble in alcohol. Nearly half the tissue of ordinary bran, exclusive of proteins and starch, yields furfural on distillation with acid. I

† U. S. Dept. of Agric., Bur. of Chem., Bul. ro7 (rev.), p. 54. 
(b) For weight of phloroglucide " $a$ " over 0.300 gram.

$$
\begin{aligned}
& \text { Furfural }=\left(a+0.005^{2}\right) \times 0.5180 . \\
& \text { Pentoses }=\left(a+0.005^{2}\right) \times 1.0026 . \\
& \text { Pentosans }=\left(a+0.005^{2}\right) \times 0.8824
\end{aligned}
$$

For weight of phloroglucide " $a$ " from 0.03 to 0.300 gram use Kröber's table (pp. 288-294) to calculate the weight of pentoses (arabinose, xylose), and pentosans (araban, xylan).

The reactions that take place are thought to be somewhat as follows:

$$
\begin{aligned}
& \underset{\text { Pentosan }}{\mathrm{C}_{5} \mathrm{H}_{8} \mathrm{O}_{4}}+\mathrm{H}_{2} \mathrm{O}=\underset{\text { Pentose }}{\mathrm{C}_{5} \mathrm{H}_{10} \mathrm{O}_{5}} \text {. } \\
& \underset{\text { Pentose }}{\mathrm{C}_{5} \mathrm{H}_{10} \mathrm{O}_{5}}=\underset{\text { Furfural }}{\mathrm{C}_{5} \mathrm{H}_{4} \mathrm{O}_{2}}+{ }_{3} \mathrm{H}_{2} \mathrm{O} \text {. }
\end{aligned}
$$

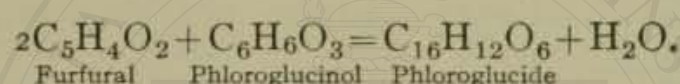

The theoretical yield of phloroglucide should be 2.22 parts to one of furfural, but in practice this is never obtained. The varying factors for calculation as above given are based on experiment.

The phloroglucinol used should be free from diresorcin. To test for the latter, dissolve the reagent in acetic anhydride, heat nearly to boiling, and add a few drops of concentrated sulphuric acid. If more than a faint violet color is produced, the phloroglucinol should be purified as follows:

Heat in a beaker about $300 \mathrm{cc}$. of hydrochloric acid (sp. gr. I.06) and II grams of commercial phloroglucinol, added in small quantities at a time, stirring constantly until it has almost dissolved. Some impurities may resist solution, but it is unnecessary to dissolve them. Pour the hot solution into a sufficient quantity of the same hydrochloric acid (cold) to make the volume $1500 \mathrm{cc}$. Allow it to stand at least overnight-better several days-to allow the diresorcin to crystallize out, and filter immediately before using. The solution may turn yellow, but this does not interfere with its usefulness. In using it, add the volume containing the required amount to the distillate. 
KROBER'S TABLE FOR DETERMINATION OF PENTOSES AND PENTOSANS FROM PHLOROGLUCID.

\begin{tabular}{|c|c|c|c|c|c|c|c|}
\hline Phloroglucia & Furfural. & Arabinose: & Araban. & Xylose. & Xylan. & Pentose. & $\begin{array}{c}8 \\
\text { Pentosan } \\
.\end{array}$ \\
\hline $0.03^{\circ}$ & 0.0182 & 0.0391 & 0.0344 & 0.0324 & 0.0285 & $0.035^{8}$ & 0.0315 \\
\hline .031 & .0188 & .0402 & .0354 & .0333 & .0293 & .0368 & .0324 \\
\hline-032 & .0193 & .0413 & .0363 & .0342 & .0301 & $.037^{8}$ & .0333 \\
\hline-033 & .0198 & .0424 & .0373 & .0352 & .0309 & .0388 & .0341 \\
\hline-034 & .0203 & .0435 & .0383 & .0361 & .0317 & .0398 & $.035^{\circ}$ \\
\hline-035 & .0209 & .0446 & .0393 & $.037^{\circ}$ & .0326 & .0408 & .0359 \\
\hline-036 & .0214 & .0457 & .0402 & .0379 & .0334 & .0418 & .0368 \\
\hline .037 & .0219 & .0468 & .0412 & .0388 & .0342 & .0428 & .0377 \\
\hline .038 & .0224 & .0479 & .0422 & .0398 & .0350 & .0439 & .0386 \\
\hline .039 & .0229 & .0490 & .0431 & .0407 & $.035^{8}$ & .0449 & .0395 \\
\hline .040 & .0235 & .0501 & .0441 & .0416 & .0366 & .0459 & .0404 \\
\hline .041 & .0240 & .0512 & .0451 & .0425 & .0374 & .0469 & .0413 \\
\hline .042 & .0245 & .0523 & .0460 & .0434 & .0382 & .0479 & .0422 \\
\hline .043 & .0250 & .0534 & $.047^{\circ}$ & .0443 & .0390 & .0489 & $.043^{\mathrm{I}}$ \\
\hline .044 & .0255 & .0545 & .0480 & $.045^{2}$ & .0398 & .0499 & .0440 \\
\hline-045 & .0260 & $.055^{6}$ & .0490 & .0462 & .0406 & .0509 & .0448 \\
\hline-046 & .0266 & .0567 & .0499 & .0471 & .0414 & .0519 & .0457 \\
\hline .047 & .0271 & .0578 & .0509 & .0480 & .0422 & .0529 & .0466 \\
\hline .048 & $.027^{6}$ & .0589 & .0519 & .0489 & .0430 & .0539 & .0475 \\
\hline-049 & .0281 & .0600 & .0528 & .0498 & $.043^{8}$ & .0549 & .0484 \\
\hline .050 & .0286 & $.06 \mathrm{rI}$ & $.053^{8}$ & .0507 & .0446 & .0559 & .0492 \\
\hline $.05 x$ & .0292 & .0622 & $.054^{8}$ & .0516 & .0454 & .0569 & .0501 \\
\hline .052 & .0297 & .0633 & .0557 & .0525 & .0462 & .0579 & .0510 \\
\hline .053 & .0302 & .0644 & .0567 & .0534 & .0470 & .0589 & .0519 \\
\hline .054 & .0307 & .0655 & $.057^{6}$ & .0543 & .0478 & .0599 & .0528 \\
\hline .055 & $.03^{12}$ & .0666 & .0586 & .0553 & .0486 & .0610 & .0537 \\
\hline .056 & .0318 & .0677 & .0596 & .0562 & .0494 & .0620 & .0546 \\
\hline .057 & .0323 & .0688 & .0605 & $.057 \mathrm{I}$ & .0502 & .0630 & .0555 \\
\hline .058 & .0328 & .0699 & .0615 & .0580 & .0510 & .0640 & .0564 \\
\hline .059 & .0333 & .0710 & .0624 & $.05^{89}$ & .0518 & $.065^{\circ}$ & .0573 \\
\hline .060 & $.033^{8}$ & .0721 & .0634 & .0598 & .0526 & .0660 & $.05^{8 \mathrm{r}}$ \\
\hline $.06 r$ & .0344 & .0732 & .0644 & .0607 & .0534 & .0670 & .0590 \\
\hline .062 & .0349 & .0743 & .0653 & .0616 & .0542 & .0680 & .0599 \\
\hline .063 & .0354 & .0754 & .0663 & .0626 & $.055^{\circ}$ & .0690 & .0608 \\
\hline .064 & .0359 & .0765 & .0673 & .0635 & $.055^{8}$ & .0700 & .0617 \\
\hline .065 & .0364 & $.077^{6}$ & .0683 & .0644 & .0567 & .0710 & .0625 \\
\hline .066 & .0370 & .0787 & .0692 & .0653 & .0575 & .0720 & .0634 \\
\hline .067 & .0375 & .0798 & .0702 & .0662 & .0583 & $.073^{\circ}$ & .0643 \\
\hline .068 & .0380 & .0809 & .0712 & .0672 & .0591 & $.074 \mathrm{I}$ & $.065^{2}$ \\
\hline .069 & .0385 & .0820 & .0721 & .0681 & .0599 & .0751 & $.066 \mathrm{I}$ \\
\hline
\end{tabular}


KRÖBER'S TABLE FOR DETERMINATION OF PENTOSES AND PENTOSANS FROM PHLOROGLUCID-Continued.

\begin{tabular}{|c|c|c|c|c|c|c|c|}
\hline Phloroglucid & Furfural. & Arabinose. & Araban. & xylose. & xylan. & Pentose. & $\begin{array}{c}8 \\
\text { Pentosan }\end{array}$ \\
\hline 0.070 & $0.039^{\circ}$ & $0.088 \mathrm{I}$ & $0.073 \mathrm{I}$ & 0.0690 & 0.0607 & 0.0761 & 0.0670 \\
\hline .071 & $.039^{6}$ & .0842 & .0741 & .0699 & .0615 & .0771 & .0679 \\
\hline .072 & .0401 & .0853 & .0750 & .0708 & .0623 & $.078 x$ & .0688 \\
\hline .073 & .0406 & .0864 & .0760 & .0717 & $.063 x$ & .0791 & .0697 \\
\hline .074 & $.04 \pi I$ & .0875 & .0770 & .0726 & .0639 & .0801 & .0706 \\
\hline .075 & .0416 & .0886 & .0780 & $.073^{6}$ & .0647 & .0811 & .0714 \\
\hline $.07^{6}$ & .0422 & .0897 & .0789 & .0745 & .0655 & .0821 & .0722 \\
\hline .077 & .0427 & .0908 & .0799 & .0754 & .0663 & .0831 & $.073 \mathrm{I}$ \\
\hline .078 & $.043^{2}$ & .0919 & .0809 & .0763 & .0671 & .0841 & .0740 \\
\hline .079 & .0437 & .0930 & .0818 & $.077^{2}$ & .0679 & $.085 x$ & .0749 \\
\hline .080 & .0442 & .0941 & .0828 & $.078 \mathrm{r}$ & .0687 & $.086 \mathrm{x}$ & $.075^{8}$ \\
\hline $.08 \mathrm{I}$ & .0448 & $.095^{2}$ & $.083_{3} 8$ & .0790 & .0695 & .0871 & .0767 \\
\hline .082 & .0453 & $.096_{3}$ & .0847 & .0799 & .0703 & $.088 x$ & .0776 \\
\hline .083 & $.045^{8}$ & .0974 & .0857 & .0808 & .0711 & .0891 & .0785 \\
\hline .084 & .0463 & .0985 & .0867 & .0817 & .0719 & $.090 x$ & .0794 \\
\hline .085 & .0468 & .0996 & .0877 & .0827 & .0727 & .0912 & .0803 \\
\hline .086 & .0474 & .1007 & .0886 & .0836 & .0735 & .0922 & .0812 \\
\hline .087 & .0479 & . 1018 & .0896 & .0845 & .0743 & .0932 & .0821 \\
\hline .088 & .0484 & .1029 & .0906 & .0854 & .0751 & .0942 & $.08_{3} 0$ \\
\hline .089 & .0489 & .1040 & .0915 & .0863 & .0759 & .0952 & $.083^{8}$ \\
\hline .090 & .0494 &. $\log x$ & .0925 & .0872 & .0767 & .0962 & .0847 \\
\hline $.09 x$ & .0499 & .1062 & .0935 & $.088 \mathrm{I}$ & .0775 & .0972 & .0856 \\
\hline .092 & .0505 & .1073 & .0944 & .0890 & .0783 & .0982 & .0865 \\
\hline .093 & .0510 & .1084 & .0954 & .0900 & .0791 & .0992 & .0874 \\
\hline .094 & .0515 & .1095 & .0064 & .0909 & .0800 & .1002 & $.088_{3}$ \\
\hline .095 & .0520 & .1106 & .0974 & .0928 & .0808 & .1012 & $.089 r$ \\
\hline .096 & .0525 & III & $.098_{3}$ & .0927 & $.08 \times 6$ & .1022 & .0899 \\
\hline .097 & .0531 & .1128 & .0993 & $.093^{6}$ & .0824 & .1032 & .0908 \\
\hline .098 & $.053^{6}$ & . 1139 & .1003 & .0946 & .0832 & .1043 & .0917 \\
\hline .099 & .0541 & $.115^{\circ}$ & .1012 & .0955 & .0840 & .1053 & .0926 \\
\hline .100 & .0546 & .1161 & .1022 & .0964 & .0848 & .1063 & .0935 \\
\hline .101 & $.055 \mathrm{I}$ & $.1 x 7 t$ & .1032 & .0973 & $.085_{5}^{6}$ & .1073 & .0944 \\
\hline .102 & .0557 & .1182 & .1041 & .0982 & .0864 & $-\mathrm{TOS}_{3}$ & .0953 \\
\hline .103 & .0562 & .1193 & $.105 \mathrm{I}$ & .0991 & .0872 & .1093 & $.096 z$ \\
\hline .104 & .0567 & . 1204 & .1060 & .1000 & .0880 & .1103 & $.097 \mathrm{~K}$ \\
\hline .105 & $.057^{2}$ & .1215 & .1070 & . .010 & .0888 &. $\mathrm{IrI}_{3}$ & .0976 \\
\hline .106 & .0577 & .1226 & .1080 & .rorg & .0896 & .1123 & .0988 \\
\hline .107 & .0582 & .1237 & .1089 & .1028 & .0904 & .1133 & .0997 \\
\hline .108 & .0588 & .1248 & .1099 & .1037 & .0912 &. $\mathrm{Ir}_{43}$ & .1006 \\
\hline .109 & .0593 & .1259 & .1108 & .1046 & .0920 & .1153 & rors \\
\hline
\end{tabular}


KRÖBER'S TABLE FOR DETERMINATION OF PENTOSES AND PENTOSANS FROM PHLOROGLUCID-(Continued).

\begin{tabular}{|c|c|c|c|c|c|c|c|}
\hline Phlorogitucid & Furfural. & Arabinose. & Araban. & Xylose. & Xylan. & Pentose. & $\begin{array}{c}8 \\
\text { Pentosan. }\end{array}$ \\
\hline 0.110 & 0.0598 & $0.127^{\circ}$ & 0.1118 & 0.1055 & 0.0928 & $0.116_{3}$ & 0.1023 \\
\hline . III & .0603 & .1281 & .1128 & .1064 & $.093^{6}$ & .1173 & .1032 \\
\hline .112 & .0608 & .1292 & .1137 & .1073 & .0944 & $.118_{3}$ & $.104 \pi$ \\
\hline .113 & .0614 & .1303 & .1147 & .1082 & $.095^{2}$ & .1193 & .1050 \\
\hline .114 & .0619 & .1314 & -1156 & .1091 & .0960 & .1203 & .1059 \\
\hline .115 & .0624 & .1325 & .1166 & .11001 & .0968 & .1213 & .1067 \\
\hline .116 & .0629 & $.133^{6}$ & .1176 & .1110 & $.097^{6}$ & .1223 & .1076 \\
\hline .117 & .0634 & .1347 & .1185 & .11109 & .0984 & .1233 & .1085 \\
\hline .118 & .0640 & . $135^{8}$ & .1195 & .1128 & .0992 & .1243 & .1094 \\
\hline .119 & .0645 & .1369 & .1204 & .1137 & .1000 & .1253 &. $\mathrm{HO}_{3}$ \\
\hline .120 & .0650 & .1380 & .1214 & .1146 & .1008 & .1263 & . IIII \\
\hline .121 & .0655 & .1391 & .1224 & .1155 & 1016 & .1273 & .1120 \\
\hline .122 & .0660 & .1402 & .1233 & .1164 & .1024 & .1283 & .1129 \\
\hline .123 & .0665 & .1413 & .1243 & .1173 & .1032 & .1293 & $.113^{8}$ \\
\hline .124 & .0671 & .1424 & .1253 & .1182 & .1040 & $.13 \circ 3$ & .1147 \\
\hline .125 & $.067^{6}$ & .1435 & .1263 & -1192 & .1049 & .1314 & II 56 \\
\hline .126 & $.068 \mathrm{I}$ & $.144^{6}$ & .1272 & - 1201 & .1057 & .1324 & .1165 \\
\hline .127 & .0686 & .1457 & .1282 & .1210 & .1065 & . 1334 & .1174 \\
\hline .128 & .0691 & .1468 & 1292 & .1219 & 1073 & .1044 & $.118_{3}$ \\
\hline .129 & .0697 & .1479 & .1301 & .1228 & .1081 & .1354 & .1192 \\
\hline .130 & .0702 & .1490 & - 13 II & .1237 & .1089 & .1364 & .1201 \\
\hline .131 & .0707 & .1501 & .1321 & .1246 & .1097 & .1374 & .1210 \\
\hline .132 & .0712 & .1512 & $.133^{\circ}$ & .1255 & .1105 & .1384 & .1219 \\
\hline .133 & .0717 & .0523 & $.1344^{\circ}$ & .1264 & $\mathrm{HII}_{3}$ & .1394 & .1227 \\
\hline .134 & .0723 & .1534 & $.135^{\circ}$ & .1273 & .1121 & .1404 & .1236 \\
\hline .135 & .0728 & . I545 & .1360 & $.128_{3}$ & . II 29 & .1414 & .1244 \\
\hline $.13^{6}$ & .0733 & $.155^{6}$ & $.13^{69}$ & .1292 & .1137 & .3424 & .1253 \\
\hline .137 & $.073^{8}$ & $.15^{67}$ & .1379 & .1301 & .1145 & .1434 & .1262 \\
\hline .138 & .0743 & .1578 & .1389 & .1310 & .1153 & . I 444 & .1271 \\
\hline .139 & .0748 & .1589 & -1 $39^{8}$ & .1319 & .1161 & .1454 & .1280 \\
\hline .140 & .0754 & .1600 & .1408 & .1328 & .1169 & .1464 & .1288 \\
\hline .141 & .0759 & 1611 & .1418 & .1337 & .1177 & - 4474 & .1297 \\
\hline .142 & .0764 & .1622 & .1427 & .1346 & .1185 & .1484 & .1306 \\
\hline .143 & .0769 & .1633 & .1437 & .1355 & .1193 & .1494 & .1315 \\
\hline .144 & .0774 & .1644 & .1447 & .1364 & .1201 & .1504 & .1324 \\
\hline .145 & .0780 & .1655 & .1457 & .1374 & .1209 &.$\times 515$ & . 1333 \\
\hline .146 & .0785 & .1666 & .1466 & $.13^{8} 3$ & .1217 & .1525 & .1342 \\
\hline .147 & .0790 & .1677 & . $47^{6}$ & .1392 & .1225 & -1535 & .1351 \\
\hline .148 & .0795 & .1688 &. $\mathrm{x} 486$ & .1401 & -1233 & .1545 & .1360 \\
\hline .149 & .0800 & x699 & I 495 & .1410 & .1241 & .r555 & .1369 \\
\hline
\end{tabular}


KRÖBER'S TABLE FOR DETERMINATION OF PENTOSES AND PENTOSANS FROM PHLOROGLUCID-(Continued).

\begin{tabular}{|c|c|c|c|c|c|c|c|}
\hline Phloroglucid & Furfural. & Arabinose. & Araban. & Xylose. & $\begin{array}{c}6 \\
\text { xylan. }\end{array}$ & Pentose. & $\begin{array}{c}8 \\
\text { Pentosan. }\end{array}$ \\
\hline $0.15^{\circ}$ & 0.0805 & 0.1710 & 0,1505 & 0.1419 & 0.1249 & 0.1565 & 0.1377 \\
\hline .151 & .0811 & .1721 & .1515 & .1428 & .1257 & .1575 & .1386 \\
\hline .152 & .0816 & .1732 & .1524 & .1437 & .1265 & . 1585 & .1395 \\
\hline .153 & .0821 & . 1743 & .1534 & $\therefore \quad 1446$ & .1273 & . 595 &.$\times 404$ \\
\hline .154 & .0826 & .1754 & .1544 & .1455 & .1281 & .1605 & .1413 \\
\hline .155 & .0831 & .1765 & . 1554 & .1465 & .1289 & .1615 & $.142 \pi$ \\
\hline $\begin{array}{r}.156 \\
.150\end{array}$ & .0837 & .1776 & .1563 & .1474 & .1297 & .1625 & $.143^{\circ}$ \\
\hline .157 & .0842 & .1787 & .1573 & .1483 & -1305 & .1635 & . 1439 \\
\hline .158 & .0847 & -1798 & .1583 & .1492 & .1313 & .1645 & .1448 \\
\hline .159 & .0852 & .1809 & .1592 & .1501 & .1321 & .1655 & .1457 \\
\hline .160 & .0857 & .1820 & .1602 & . 1510 & .1329 & .1665 & .1465 \\
\hline .161 & .0863 & .1831 & .1612 & .1519 & .1337 & .1675 & .1474 \\
\hline .162 & .0868 & .1842 & .1621 & .1528 & r 345 & .1685 & .1483 \\
\hline .163 & .0873 & .1853 & .1631 & I 537 & .1353 & .1695 & $.149^{2}$ \\
\hline .164 & .0878 & .1864 & .1640 & .1546 & ${ }^{-1} 3^{61}$ & .1705 & .1501 \\
\hline .165 & .0883 & .1875 & . $165^{\circ}$ & $.155^{6}$ & .1369 & .1716 & .1510 \\
\hline .166 & .0888 & .1886 & .1660 & .1565 & .1377 & .1726 & .1519 \\
\hline .167 & .0894 & . 1897 & . 1669 & . 5574 & -1385 & .1736 & .1528 \\
\hline .168 & .0899 & .1908 & .1679 & $.15^{8} 3$ & .1393 & .1746 &.+537 \\
\hline .169 & .0904 & rorg & . 6888 & .1592 & .1401 & .1756 & .1546 \\
\hline-170 & .0909 & 1930 & . 1698 & .1601 & .1409 & 1766 & -1554 \\
\hline $.17 x$ & $.09^{14}$ & I 941 & .1708 & 1610 & .1417 & .1776 & $\begin{array}{r}.1563 \\
.\end{array}$ \\
\hline .172 & .0920 & $.195^{2}$ & .1757 & .1619 & .1425 & .1786 & $.157^{2}$ \\
\hline .173 & .0925 & .1963 & .1727 & .1628 & .1433 & -1796 & $.158 \mathrm{r}$ \\
\hline .174 & $.093^{\circ}$ & .1974 & .7736 & .1637 & .1441 & .1806 & .1590 \\
\hline .175 & .0935 & .1985 & .1746 & .1647 & .1449 & .1816 & .1598 \\
\hline .176 & .0940 & .19g6 & $.175^{6}$ & .1656 & .1457 & .1826 & .1607 \\
\hline .177 & .0946 & .2007 & .1765 & .1665 & .1465 & .1836 & .1616 \\
\hline .178 & .0951 & .2018 & .1775 & . 1674 & .1473 & .1846 & .1625 \\
\hline .179 & $.095^{6}$ & .2029 & .1784 & .1683 & .1481 & .1856 & .1634 \\
\hline .180 & $.096 \mathrm{t}$ & .2039 & .1794 & 1692 & .1489 & .1866 & .1642 \\
\hline $.18 \mathrm{I}$ & .0966 & $.205^{\circ}$ & .1804 & I 7 O & . 4497 & 1876 & .1651 \\
\hline .182 & .0971 & .2061 & $.181_{3}$ & .1710 & .1505 & .1886 & .1660 \\
\hline .183 & .0977 & .2072 & .1823 & .1719 &. $\mathrm{I}_{5} \mathrm{I}_{3}$ & .1896 & .1669 \\
\hline .184 & .0982 & .2082 & $.188^{2}$ & .1728 & .1521 & .1906 & .1678 \\
\hline .185 & .0987 & .2093 & .1842 & $.173^{8}$ & .1529 & .1916 & .1686 \\
\hline .186 & .0992 & .2104 & .1851 & $\begin{array}{l}-1747 \\
\end{array}$ & $\begin{array}{r}\times 537 \\
.\end{array}$ & .1926 & $\begin{array}{l}. \\
.\end{array} 695$ \\
\hline .187 & .0997 & .2115 & .1861 & .1756 & .1545 & .1936 & .1704 \\
\hline .188 &. $\mathrm{IOO}_{3}$ & .2126 & .1870 & .1765 & . 5553 & .1946 & .1712 \\
\hline .189 & .1008 & $.213^{6}$ & .1880 & $\times 774$ & .1561 & .1955 & .1721 \\
\hline
\end{tabular}




\section{KRÖBER'S TABLE FOR DETERMINATION OF PENTOSES AND PENTOSANS} FROM PHLOROGLUCID-(Continued).

\begin{tabular}{|c|c|c|c|c|c|c|c|}
\hline$\stackrel{\text { I }}{\text { Phlorglucia }}$ & Purfural. & Arabinose. & Araban. & Xylose. & $x^{6}$ ylan. & Pentose. & $\begin{array}{c}8 \\
\text { Pentosan. }\end{array}$ \\
\hline 0.190 & $0.101_{3}$ & 0.2147 & 0.1889 & 0.1783 & 0.1569 & $0.19^{65}$ & 0.1729 \\
\hline $.19 \mathrm{r}$ & .1018 & .2158 & .1899 & .1792 & .1577 & .1975 & $.173^{8}$ \\
\hline .192 & .1023 & .2168 & .1908 & .1801 & .1585 & .1985 & .1747 \\
\hline .193 & .1028 & .2179 & .1918 & . 1810 & -1593 & .1995 & .1756 \\
\hline .194 & .1034 & .2190 & .1927 & .1819 & .1601 & .2005 & .1764 \\
\hline .195 & .1039 & .2201 & .1937 & .1829 & . I609 & .2015 & . 1773 \\
\hline $.19^{6}$ & .1044 & .2212 & -1946 & $.188_{3}^{8}$ & .1617 & .2025 & .1782 \\
\hline .197 & .1049 & .2222 & $.195^{6}$ & .1847 &.$x 625$ & .2035 & I791 \\
\hline$x 98$ & .1054 & .2233 & .1965 & .1856 & .1633 & .2045 & .1800 \\
\hline .199 & .1059 & .2244 & 1975 & .1865 & $.164 \pi$ & .2055 & .1808 \\
\hline .200 & .1065 & .2255 & .1984 & .1874 & .1649 & .2065 & .1817 \\
\hline .201 & .1070 & .2266 & .1994 & $.188_{3}$ & .1657 & .2075 & .1826 \\
\hline .202 & .1075 & .2276 & .2003 & . 1892 & .1665 & .2085 & .1835 \\
\hline .203 & .1080 & .2287 & .2013 & I I90I & .1673 & .2095 & .1844 \\
\hline .204 & .1085 & .2298 & .2022 & .1910 & .1681 & .2105 & .1853 \\
\hline .205 & . 1090 & .2309 & .2032 & I 1920 & .1689 & .2115 & $.186 r$ \\
\hline .206 & .1096 & .2320 & $.204 \mathrm{I}$ & .1929 & .1697 & .2125 & .1869 \\
\hline .207 & itror & $.233^{\circ}$ & .2051 & $.193^{8}$ & .1705 & .2134 & .1878 \\
\hline .208 & .1106 & .2341 & .2060 & .1947 & .1713 & .2144 & .1887 \\
\hline .209 & .1111 & .2352 & .2069 & .1956 & .1721 & .2154 & .1896 \\
\hline .210 & .1116 & .2363 & .2079 & $.19^{65}$ & .1729 & .2164 & . 1904 \\
\hline .211 & .1121 & .2374 & .2089 &.+975 & .1737 & .2174 & .1913 \\
\hline .212 & .1127 & .2384 & .2098 & .1984 & .1745 & .2184 & .1922 \\
\hline .213 & .1132 & .2395 & .2108 & .1993 & .1753 & .2194 & .1931 \\
\hline .214 & .1137 & .2406 & .2117 & .2002 & $.176 \mathrm{r}$ & .2204 & .1940 \\
\hline .215 & .1142 & .2417 & .2127 & .2011 & .1770 & .2214 & .1948 \\
\hline .216 & .1147 & .2428 & $.213^{6}$ & .2020 & .1778 & .2224 & .1957 \\
\hline .217 & .1152 & $.243^{8}$ & .2146 & .2029 & .1786 & .2234 & . 1966 \\
\hline .218 & .1158 & .2449 & .2155 & $.203^{8}$ & .1794 & .2244 & .1974 \\
\hline .219 & .1163 & .2460 & .2165 & .2047 & .1802 & .2254 & .1983 \\
\hline .220 & .1168 & .2471 & .2174 & .2057 & .1810 & .2264 & .1992 \\
\hline .221 & .1173 & .2482 & .2184 & .2066 & .1818 & .2274 & .2001 \\
\hline .222 & 1178 & .2492 & .2193 & .2075 & .1826 & .2284 & .2010 \\
\hline .223 & .1183 & .2503 & .2203 & .2084 & .1834 & .2294 & .2019 \\
\hline .224 & .1189 & $.25^{14}$ & .2212 & .2093 & .1842 & .2304 & .2028 \\
\hline .225 & .1194 & .2525 & .2222 & .2102 & $.185^{\circ}$ & .2314 & .2037 \\
\hline .226 & .1199 & $.253^{6}$ & $.223^{2}$ & .2111 & $.185^{8}$ & .2324 & $.204^{6}$ \\
\hline .227 & .1204 & .2546 & $.224 \mathrm{I}$ & .2121 & .1866 & .2334 & .2054 \\
\hline .228 & .1209 & .2557 & $.225 \mathrm{I}$ & $.213^{\circ}$ & .1874 & .2344 & .2063 \\
\hline .229 & .1214 & .2568 & .2260 & .2139 & .1882 & .2354 & $.207^{2}$ \\
\hline
\end{tabular}


CEREALS, LEGUMES, VEGETABLES, AND FRUITS.

KRÖBER'S TABLE FOR DETERMINATION OF PENTOSES AND PENTOSANS FROM PHLOROGLUCID-(Continued).

\begin{tabular}{|c|c|c|c|c|c|c|c|}
\hline Phloroglucid & Purfural. & Arabinose. & Araban. & Xylose. & $\begin{array}{c}6 \\
\mathrm{X} y \text { lan. }\end{array}$ & Pentose. & $\begin{array}{c}8 \\
\text { Pentosan. }\end{array}$ \\
\hline 0.230 & 0.1220 & 0.2579 & 0.2270 & 0.2148 & 0.1890 & 0.2364 & $0.208 \mathrm{r}$ \\
\hline .231 & .1225 & .2590 & .2280 & .2157 & .1898 & .2374 & .2089 \\
\hline .232 & $.123^{\circ}$ & .2600 & .2289 & .2166 & .1906 & .2383 & .2097 \\
\hline .233 & .1235 & .2611 & .2299 & .2175 & .1914 & .2393 & .2106 \\
\hline .234 & .1240 & .2622 & .2308 & .2184 & .1922 & .2403 & .2115 \\
\hline .235 & . 245 & .2633 & .2318 & .2193 & .1930 & .2413 & .2124 \\
\hline .236 & .1251 & .2644 & .2327 & .2202 & .1938 & .2423 & .2132 \\
\hline .237 & .1256 & .2654 & .2337 & .2211 & .1946 & .2433 & $.214 \mathrm{I}$ \\
\hline .238 & .1261 & .2665 & .2346 & .2220 & .1954 & .2443 & .2150 \\
\hline .239 & .1266 & .2676 & $.235^{6}$ & .2229 & .1962 & .2453 & .2159 \\
\hline .240 & .1271 & .2687 & .2365 & .2239 & .1970 & .2463 & .2168 \\
\hline $.24 I$ & .1276 & .2698 & .2375 & .2248 & .1978 & .2473 & .2176 \\
\hline .242 & $.128 \mathrm{I}$ & .2708 & .2384 & .2257 & . 1986 & .2483 & .2185 \\
\hline .243 & .1287 & .2719 & .2394 & .2266 & . I994 & .2493 & .2194 \\
\hline .244 & .1292 & .2730 & .2403 & .2275 & .2002 & .2503 & .2203 \\
\hline .245 & .1297 & .2741 & .2413 & .2284 & .2010 & .2513 & .2212 \\
\hline .246 & .1302 & $.275^{2}$ & .2422 & .2293 & .2018 & .2523 & .2220 \\
\hline .247 & .1307 & .2762 & .2432 & .2302 & .2026 & .2533 & .2229 \\
\hline .248 & .1312 & .2773 & .2441 & .2311 & .2034 & .2543 & .2238 \\
\hline .249 & $.13+8$ & .2784 & .2451 & .2320 & .2042 & .2553 & .2247 \\
\hline .250 & .1323 & .2795 & .2460 & .2330 & .2050 & .2563 & .2256 \\
\hline $.25 \mathrm{I}$ & $\mathrm{r}_{3} 28$ & .2806 & .2470 & .2339 & .2058 & .2573 & .2264 \\
\hline .252 & .1333 & .2816 & .2479 & $.234^{8}$ & .2066 & .2582 & .2272 \\
\hline .253 & $.133^{8}$ & .2827 & .2489 & .2357 & .2074 & .2592 & $.228 \mathrm{I}$ \\
\hline .254 & .1343 & $.283^{8}$ & .2498 & .2366 &, 2082 & .2602 & .2290 \\
\hline .255 & . 1349 & .2849 & .2508 & .2375 & .2090 & .2612 & .2299 \\
\hline .256 & .1354 & .2860 & .2517 & .2384 & .2098 & .2622 & .2307 \\
\hline .257 & . I359 & .2870 & .2526 & .2393 & .2106 & $.26_{32}$ & .2316 \\
\hline .258 & .1364 & $.288 \mathrm{t}$ & $.253^{6}$ & .2402 & $.2 \mathrm{Ir} 4$ & .2642 & .2325 \\
\hline .259 & .1369 & .2892 & .2545 & $.24 \mathrm{II}$ & .2122 & .2652 & .2334 \\
\hline .260 & .1374 & .2903 & .2555 & .2420 & .2130 & .2662 & .2343 \\
\hline $.26 \mathrm{r}$ & -1380 & .2914 & .2565 & .2429 & $.213^{8}$ & .2672 & $.235 \mathrm{I}$ \\
\hline .262 & .1385 & .2924 & .2574 & $.243^{8}$ & $.2 r_{4} 6$ & $.268 \mathrm{I}$ & .2359 \\
\hline .263 & .1390 & .2935 & .2584 & .2447 & .2154 & .2691 & .2368 \\
\hline .264 & .1395 & .2946 & .2593 & $.245^{6}$ & .2162 & .2701 & .2377 \\
\hline .265 & .1400 & .2957 & .2603 & .2465 & .2170 & .27111 & .2385 \\
\hline .266 & .1405 & .2968 & $.26 \mathrm{r} 2$ & .2474 & .2178 & .2721 & .2394 \\
\hline .267 & .1411 & .2978 & .2622 & .2483 & .2186 & .2731 & .2403 \\
\hline .268 & $.14+16$ & .2989 & .2631 & .2492 & .2194 & .2741 & .2412 \\
\hline .269 & $.142 \mathrm{I}$ & .3000 & $.264 \mathrm{I}$ & .2502 & .2202 & $.275^{1}$ & .2421 \\
\hline
\end{tabular}


KRÖBER'S TABLE FOR DETERMINATION OF PENTOSES AND PENTOSANS FROM PHLOROGLUCID-(Concluded).

\begin{tabular}{|c|c|c|c|c|c|c|c|}
\hline Phloroglucid & Furfiral. & Arabinose. & Araban. & $\mathrm{xy}$ lose. & Xylan. & Pentose. & $\begin{array}{c}8 \\
\text { Pentosan. }\end{array}$ \\
\hline 0.270 & 0.1426 & 0.3011 & $0.265^{\circ}$ & 0.2511 & 0.2210 & 0.2761 & 0.2429 \\
\hline .271 & .1431 & .3022 & .2660 & .2520 & .2218 & $.277 x$ & .2438 \\
\hline .272 & $.143^{6}$ & $\cdot 3^{\circ} 3^{2}$ & .2669 & .2529 & .2226 & .2781 & .2447 \\
\hline .273 & .1412 & .3043 & .2679 & $.253^{8}$ & .2234 & .2791 & $.245^{6}$ \\
\hline .274 & .1447 & .3054 & .2688 & .2547 & .2242 & .2801 & .2465 \\
\hline .275 & $.145^{2}$ & .3065 & .2698 & $.255^{6}$ & .2250 & $.28 \mathrm{II}$ & .2473 \\
\hline .276 & .1457 & $\cdot 307^{6}$ & .2707 & .2565 & .2258 & .2821 & .2482 \\
\hline .277 & .1462 & -3086 & .2717 & .2574 & .2266 & .2830 & .2490 \\
\hline .278 & .1467 & .3097 & .2726 & .2583 & .2274 & .2840 & .2499 \\
\hline .279 & .1473 & .3108 & $.273^{6}$ & .2592 & .2282 & $.285^{\circ}$ & .2508 \\
\hline .280 & .1478 & .3119 & .2745 & .2602 & .2290 & $.286 \mathrm{I}$ & $.25 \times 7$ \\
\hline $.28 \mathrm{I}$ & .1483 & $.313^{\circ}$ & .2755 & .2611 & .2298 & .2871 & .2526 \\
\hline .282 & .1488 & .3140 & .2764 & .2620 & .2306 & .2880 & .2534 \\
\hline .283 & .1493 & $.315 I$ & .2774 & .2629 & $.23^{14}$ & .2890 & .2543 \\
\hline .284 & .1498 & .3162 & .2783 & .2638 & .2322 & .2900 & $.255^{2}$ \\
\hline .285 & .1504 & $\cdot 3^{1} 73$ & .2793 & .2647 & $.233^{\circ}$ & .2910 & $.256 \mathrm{r}$ \\
\hline .286 & .1509 & .3184 & .2802 & $.265^{6}$ & $.233^{8}$ & .2920 & .2570 \\
\hline .287 & .1514 & .3194 & .2812 & .2665 & .2346 & $.293^{\circ}$ & $.257^{8}$ \\
\hline .288 & .1519 & .3205 & .2821 & .2674 & .2354 & .2940 & .2587 \\
\hline .289 & .1524 & .3216 & .2831 & .2683 & .2362 & $.295^{\circ}$ & .2596 \\
\hline .290 & .1529 & .3227 & $.284^{\circ}$ & .2693 & .2370 & .2960 & .2605 \\
\hline $.29 \mathrm{I}$ & .1535 & $.3^{2} 3^{8}$ & .2850 & .2702 & $.237^{8}$ & .2970 & .2614 \\
\hline .292 & $.154^{\circ}$ & -3248 & .2859 & .2711 & .2386 & .2980 & .2622 \\
\hline .293 & .1545 & .3259 & .2868 & .2720 & .2394 & .2990 & $.263_{3}$ \\
\hline .294 & . $155^{\circ}$ & .3270 & .2878 & .2729 & .2402 & .3000 & .2640 \\
\hline .295 & . 5555 & .3281 & .2887 & $.273^{8}$ & .2410 & .3010 & .2649 \\
\hline .296 & .1560 & $\cdot 3^{292}$ & .2897 & .2747 & .2418 & .3020 & $.265^{8}$ \\
\hline .297 & .1566 & .3302 & .2906 & $.275^{6}$ & .2426 & $.303^{\circ}$ & .2666 \\
\hline .298 & .1571 & $.33^{13}$ & .2916 & .2765 & .2434 & .3040 & .2675 \\
\hline .299 & $.157^{6}$ & .3324 & .2925 & .2774 & $.244^{2}$ & .3050 & .2684 \\
\hline .300 & . $158 x$ & $\cdot 3335$ & .2935 & .2784 & $.245^{\circ}$ & .3060 & .2693 \\
\hline
\end{tabular}


SEPARATION AND DETERMINATION OF THE VARIOUS CARBOHYDRATES OF CEREALS, ETC. STONE'S METHOD.

Stone has thus tabulated the results of a series of analyses of various samples of wheat, flour, corn, and bread, in which he has separated the principal carbohydrates.*

PERCENTAGES OF VARIOUS CARBOHYDRATES IN CERTAIN FOODSTUFFS.

\begin{tabular}{|c|c|c|c|c|c|c|}
\hline & Sucrose. & $\begin{array}{l}\text { Invert } \\
\text { Sugar. }\end{array}$ & Dextrin. & $\begin{array}{l}\text { Soluble } \\
\text { Starch. }\end{array}$ & $\begin{array}{l}\text { Pento- } \\
\text { sans. }\end{array}$ & $\begin{array}{l}\text { Crude } \\
\text { Fiber. }\end{array}$ \\
\hline 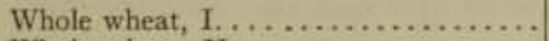 & 0.52 & 0.08 & 0.27 & 0.00 & $4 \cdot 54$ & 2.68 \\
\hline Whole wheat, II. .................. & 0.72 & 0.00 & 0.41 & 0.00 & $4 \cdot 37$ & 2. 51 \\
\hline Wheat flour, I. ...... & 0.18 & 0,00 & 0.90 & 0.00 & 0.00 & 0.25 \\
\hline Wheat flour, II... . & 0.20 & 0.00 & 1,06 & 0.00 & 0.00 & 0.25 \\
\hline Corn. ............. & 9.27 & 0.00 & 0.32 & 0.00 & 5.14 & 1. 99 \\
\hline Sugar-beet. . ........ & 8. $3^{8}$ & 0.07 & 0.35 & 0.00 & 4.89 & 1.00 \\
\hline Bread (wheat, I) .... & 0.05 & 0.32 & 0.68 & 1. 37 & $4 \cdot 16$ & 2.70 \\
\hline Bread (wheat, II) . . . . . . . . . & 0.06 & 0.37 & 0.23 & $2 \cdot 36$ & $4 \cdot 34$ & 2.02 \\
\hline Bread (flour, I) . . ............ & 0.01 & 0.10 & 0.27 & 1.99 & 0.00 & 0.34 \\
\hline Bread (flour, II) . . . . . . . . . . . & 0.15 & $0.3^{8}$ & $0.9 \mathrm{r}$ & 3. 74 & 0.00 & 0.17 \\
\hline 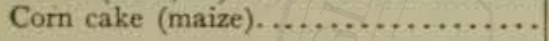 & 0.16 & 0.19 & 0.00 & 2.80 & $3 \cdot 54$ & 2.22 \\
\hline
\end{tabular}

Determination of Cane Sugar.-10 grams of the finely ground material are extracted by boiling under a reflux condenser with $500 \mathrm{cc}$. of $95 \%$ alcohol for three hours, the alcoholic extract is filtered, evaporated nearly to dryness, and then taken up with a small amount of water, to separate the sugar from the oils and waxes dissolved by the alcohol. This aqueous solution is invariably dextro-rotary, and seldom contains any reducing sugar. If the latter is present, it is determined in an aliquot part of the aqueous solution with Fehling's solution, the result being calculated to dextrose. The remainder of the aqueous sugar solution, or the whole of it, if, as is almost always the case, dextrose is absent, is then inverted by heating with hydrochloric acid in the usual manner (page $5^{88}$ ) and the sugar is estimated with Fehling's solution, calculating the result to sucrose (page 6 $6 \mathrm{I}_{2}$ ).

Determination of Dextrin.-Digest the residue from the above alcoholic extraction from eighteen to twenty-four hours with $500 \mathrm{cc}$. of cold distilled water, shaking frequently. On filtering, a clear solution is ob-

* Jour. Am. Chem. Soc., 19, r897, p. 183, and U. S. Dept. of Agric,, Off. of Exp. Sta., Bul. 34. The percentages of normal starch found by Stone are obviously erroneous, and are for this reason excluded from the table as here given. 
tained, which should be tested with iodine for soluble starch. If the latter is not found (which is nearly always the case), the solution is concentrated to a small volume, avoiding a temperature higher than $80^{\circ}$ to $90^{\circ}$, and this is boiled under a reflux condenser for two hours with onetenth its volume of hydrochloric acid (specific gravity I.125). Determine the dextrose by Fehling's solution and calculate to dextrin by the factor o.9. Or, instead of submitting the concentrated aqueous extract to hydrolysis as above, the dextrin may be roughly determined gravimetrically therein by treating with several volumes of strong alcohol until no further precipitation is produced. The flocculent precipitate thus obtained is collected, dried, and weighed.

Determination of Starch.-Dry in an oven the residue from the preceding treatment and determine its quantitative relation to the original sample; 2 grams are then accurately weighed and subjected to the diastase method of starch determination (page $28_{3}$ ).

Determination of Pentosans and Hemicelluloses.-The washed residue, left after filtering off the starch-containing solution from the process of heating with malt extract in the preceding starch determination, is boiled for an hour with $100 \mathrm{cc}$. of $1 \%$ hydrochloric acid, which converts all the pentosans into sugar. Filter, and wash the residue thoroughly, make up the solution to $200 \mathrm{cc}$., and determine the sugar with Fehling's solution, calculating the results for xylan, assuming that the chief sugar formed is xylose. The reducing power of xylose is assumed to be $4.6 \mathrm{I}$ milligrams for each cubic centimeter of Fehling's solution. If the volumetric Fehling method is used, Io cc. of Fehling's solution are thus equivalent to 0.046 gram xylose. Xylose $\times 0.88=x y l a n$.

Crude Fiber (Cellulose, etc.).-The residue from the last dilute acid hydrolysis is boiled with $200 \mathrm{cc}$. of $\mathrm{I} .25 \%$ solution of sodium hydroxide for half an hour, filtered, dried, and weighed. It is then ignited, and the weight of the ash deducted from the first weight.

\section{PROTEINS OF CEREALS AND VEGETABLES.}

Different cereal and vegetable foods present considerable variations in the character and extent of their protein constituents, and by no means all of the common vegetable foods have been studied in detail.

Osborne, in connection with Voorhees and Chittenden, has made a careful study of the proteins of many of the cereals, of potatoes, and of peas. A brief outline only will be given in what follows of methods 
for separation of the vegetable proteins. For fuller details the reader is referred to the work of Osborne et al. in the American Chemical Journal, Vols. - ${ }_{14}$, and ${ }_{15}$, and to the Journal of the American Chemical Society, Vols. $17,18,19$, and 20 .

Proteins Soluble in Water and Dilute Salt Solution.-By the action of various solvents it is possible to separate the different classes of proteins for examination or analysis. Thus water at first applied extracts certain of the soluble proteins, as does a weak salt solution. Osborne and Voorhees recommend the use of a $10 \%$ solution of sodium chloride as the first solvent to apply for separating vegetable proteins, shaking the finely ground material with twice its weight of the salt solution. The salt solution, after filtering, is then subjected to dialysis, the protein matter thus separated out being a globulin, while that not precipitated on dialysis is assumed as the protein matter of the substance soluble in water. Two albumins and a proteose are found in wheat to be thus soluble in water.

If the proteins soluble in salt solution are to have their total nitrogen determined, they are completely precipitated from the solution by saturating with zine or ammonium sulphate.

There are thus two classes of proteins soluble in $10 \%$ salt solution: (a) globulins, insoluble in water alone, and $(b)$ albumins and proteoses, which are soluble in water.

Separation of Albumins, Proteoses, and Globulins.-Starting with the aqueous solution containing the albumins and proteoses, if present, the former are best separated according to Osborne and Vorhees by fractional coagulation, effected by heating at different temperatures, those that precipitate out at a temperature under $65^{\circ}$ being first filtered out, and the filtrate submitted to a higher temperature not exceeding $85^{\circ}$. The two portions thus separated may be collected in filters, and their nitrogen separately determined.

The proteose may be precipitated from the filtrate by saturating with ground salt, or by adding, first salt to the extent of $20 \%$, and finally acetic acid.

The globulins, precipitated in the original 10\% salt solution by the process of dialysis as described, may themselves be separated by employing salt solution of varying strength as solvents.*

Proteins Soluble in Dilute Alcohol, but Insoluble in Water.-The residue from the treatment with $10 \%$ sodium chloride is digested with $75 \%$ alcohol at about $46^{\circ} \mathrm{C}$. for some time and filtered. The residue is further

* Am. Chem. Jour., ${ }^{3} 3$, p. 464 . 
digested at about $60^{\circ}$ with $75 \%$ alcohol three separate times. The evaporated filtrates contain the alcohol-soluble proteins. In this class are the hordein of barley, the gliadin of wheat and rye, and the zein of corn.

Proteins Insoluble in Water, Salt Solution, and Dilute Alcohol.-It is customary to determine the nitrogen in the final residue without further attempt to separate the remaining protein matter. It is, however, possible to further extract with alkaline and acid solvents, if desired, which process, however, changes the nature of the proteins from that in which they originally exist in the substance.

Character and Amount of Proteins in Wheat.*-The proteins of wheat, according to Osborne, are five in number, as follows:

\begin{tabular}{|c|c|c|}
\hline & & $\begin{array}{c}\text { Amount Present, } \\
\text { Per Cent. }\end{array}$ \\
\hline Soluble in water: & $\begin{array}{l}\text { Albumin (leucosin) } \\
\text { Proteose }\end{array}$ & $\begin{array}{l}0.3 \text { to } 0.4 \\
\cdots 0.3\end{array}$ \\
\hline Soluble in ro per cent $\mathrm{NaCl}$ : & Globulin (edestin) & 0.6 to 0.7 \\
\hline Soluble in dilute alcohol: & Gliadin........... & $\ldots 4.25$ \\
\hline Insoluble in above: & Glutenin.......... & $\cdots 4,00$ to 4.5 \\
\hline
\end{tabular}

The term gluten is applied to the protein content of wheat flour insoluble in water, the value of flour for baking bread depending on the amount present. Gluten contains the two definite proteins, gliadin and glutenin. Crude gluten, as obtained by washing the dough in the analytical process (page 320), is a complex mixture of many bodies, containing, besides the two proteins above named, small quantities of cellulose, mineral matter, lecithin, and starch.

Separation and Determination of Wheat Proteins.-Teller's Method. $†-$ Non-gluten Nitrogen.-Two grams of the finely divided sample are mixed with about $\mathrm{I} 5 \mathrm{cc}$. of $\mathrm{I} \%$ sa!t solution in a 250 -cc. flask. The flask is shaken at intervals of ten minutes during one hour, after which it is filled to the mark with the salt solution and al'owed to stand two hours. The supernatant liquid is then filtered through a dry filter into a dry flask, leaving most of the solid material in the flask, passing the first part through twice, if necessary, for a clear filtrate. With a pipette, exactly $50 \mathrm{cc}$. of clear filtrate are run into a 500 -cc. Kjeldahl digestion-flask, $20 \mathrm{cc}$. of the usual reagent sulphuric acid for the Gunning process (p. 69 ) are added, and the contents of the flask brought to a gentle boil. After the water has

\footnotetext{
* Am. Chem. Jour. XV, 392-47r; XVI, 524 .

†'Ark. Exp. Sta. Bul. 42, p. 96 .
} 
been driven off and the acid has stopped foaming, the potassium sulphate is added and the digestion completed. From the per cent of nitrogen thus obtained $0.27 \%$ is deducted, this figure corresponding to the amount of gliadin soluble in $1 \%$ salt solution under the above conditions. The remainder is the percentage of non-gluten nitrogen.

Gluten Nitrogen.-This is obtained by difference between the total nitrogen and the non-gluten nitrogen as above obtained, or by deducting the combined nitrogen of the edestin, leucosin, and the amido-nitrogen from the total nitrogen.

Edestin and Leucosin.-Edestin is a globulin belonging to the vegetable vitellins, and is precipitated from salt solutions by dilution, or by saturation with magnesium or ammonium sulphate, but not by saturating with sodium chloride. It is not coagulated below $100^{\circ} \mathrm{C}$., but is partly precipitated by boiling. Leucosin is an albumin, coagulating at $52^{\circ}$, but precipitates from salt solution by saturating with sodium chloride or magnesium sulphate.

To $50 \mathrm{cc}$. of the clear salt extract, obtained as described under nongluten nitrogen, $250 \mathrm{cc}$. of pure $94 \%$ alcohol are added in a Kjeldahl 500 -cc. digestion-flask, the contents thoroughly mixed, and allowed to stand over night. The precipitate is collected in a ro-cm. filter, which is returned to the flask and the nitrogen determined. This represents the nitrogen of the combined edestin and leucosin. These proteins may, however, be separated by coagulating the leucosin at $60^{\circ}$, and precipitating the edestin by adding alcohol to $50 \mathrm{cc}$. of the clear filtrate, determining the nitrogen separately in each precipitate.

Amido-nitrogen.-Allantoin, asparagin, cholin, and betaine are nitrogenous bases present in wheat.

Ten cc. of a $10 \%$ solution of pure phosphotungstic acid are added to $100 \mathrm{cc}$. of the clear salt extract as above obtained, thus precipitating all the proteins, which are allowed to settle preferably over night. Filter, and determine the nitrogen in the clear filtrate. The filtrate should be tested with a little of the phosphotungstic acid reagent to make sure that all the proteins have been separated. In some cases, as in bran for instance, more than to cc. of the reagent are necessary.

Gliadin is dissolved most readily from flour by hot dilute alcohol, but is entirely insoluble in absolute alcohol. One gram of the material is extracted with $100 \mathrm{cc}$. of hot $75 \%$ alcohol, by shaking the mixture thoroughly in a flask, and heating for an hour at a temperature just below 
the boiling-point of alcohol, with occasional shaking. After standing for an hour, the hot liquid is decanted upon a $10-\mathrm{cm}$. filter, and $25 \mathrm{cc}$. of the hot alcohol are added to the residue and shaken, after which the residue is again allowed to settle, and the liquid decanted. This is repeated six times. The remainder of the alcohol is then driven off by evaporation, and the nitrogen determined in the residue. The difference between the total nitrogen and the nitrogen thus obtained, gives the nitrogen of the alcoholic extract, which includes the amides. Subtracting the latter, or amido-nitrogen, the remainder is the gliadin nitrogen.

Glutenin Nitrogen.-This is the difference between the gluten nitrogen and the gliadin nitrogen.

The factor by which the nitrogen should be multiplied in determining the various proteins, according to Osborne and Voorhees, is 5.7 for wheat.

Proteins of the Common Cereals and Vegetables.-Osborne and his coworkers have made a detailed study of the protein constituents not only of wheat as above outlined, but of other common grains and vegetables, and the results of these investigations may be thus briefly sum. marized:

Proteins of rye: *

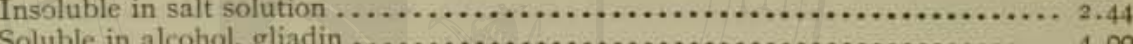

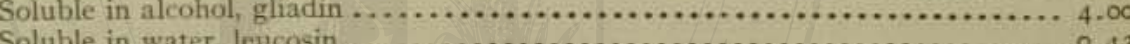

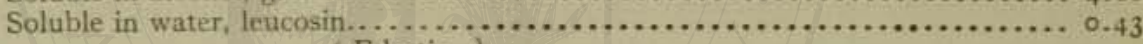

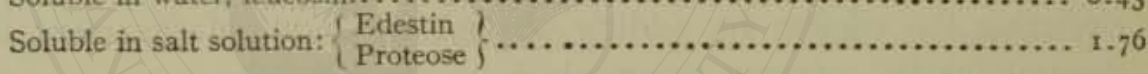

Proteins of barley: $\dagger$

Soluble in water: $\left\{\begin{array}{l}\text { Leucosin } \\ \text { Proteose }\end{array}\right\}$

Per Cent.

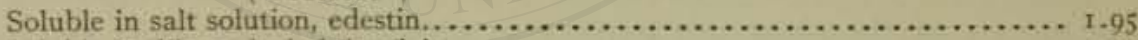

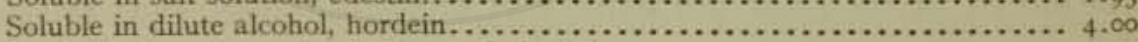

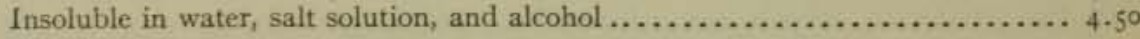
Proteins of corn:

Soluble in water: Proteose. ................................. 0.06

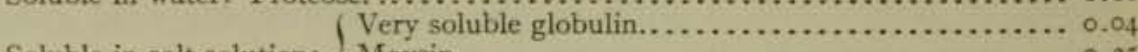

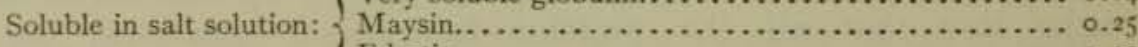

(Edestin............................... 1.10

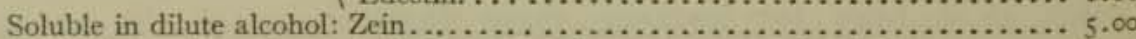

Insoluble in above, but soluble in two-tenths per cent potash solucion......... 3.15 Protein of pea: $\S$

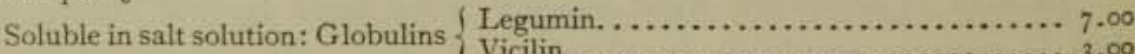

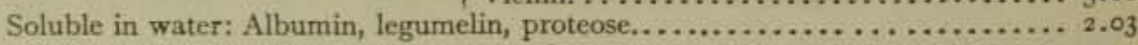

* Jour. Am. Chem. Soc, 17, page 429. † Ibid, 17, P, 539.

† Ibid., 19, p. 525. \$Ibid., 18, p. $583 ; 20$, Pp. 348 and 410 . 
Proteins of Potato.*-Almost the whole protein content of the potato consists of a globulin to which Osborne has applied the name "tuberin." Proteose is also present in very small amount.

\section{MINERAL CONSTITUENTS OF CEREALS AND VEGETABLES.}

The food analyst often finds the determination of one or more of the mineral constituents of a food product of value as a means of detecting adulteration, since the addition of foreign material may alter materially the composition of the ash.

The tablet on page 302 shows the composition of the pure ash of common cereals.

Scheme for Complete Ash Analysis. - The following scheme in essential details was suggested by the late Prof. S. L. Penfield of Yale University, and has been in use for over twenty years at the Connecticut Agricultural Experiment Station.

Preparation of Ash.-The amount of material which should be reduced to ash depends on the percentage of total ash present and the amount of material available. Usually 100 grams is a suitable amount; if, however, the material (e.g., tobacco) is rich in ash, 50 grams is sufficient, while if it contains but a small amount of ash, 200 grams or even more may be required. About 5 gram : of ash is a liberal amount for a complete analysis, but in case of necessity I gram will suffice if care is taken to so adapt the scheme as to make as many determinations as possible on one weighed portion.

The ashing is carried on in a platinum dish heated below redness by a Bunsen burner. In order to distribute the heat and prevent overheating, a piece of asbestos paper is introduced between the dish and the flame. The material first chars, then begins to glow just below the surface, and the combustion gradually extends downward until it reaches the bottom of the dish. Then, and not until then, the unburned carbon on the surface should be stirred in with the ash to facilitate burning. Care should be taken not to heat higher than dull redness, thus avoiding the loss of alkali chlorides and the fusion of alkali phosphates about the particles of carbon. A muffle furnace may be used to complete the burning.

Substances rich in starch or sugar are most difficult of combustion,

* Jour. Am. Chem. Soc., 18, 1896, p. 575 .

† U. S. Dept. of Agric., Bur, of Chem., Bul, r3, part 9, p. 1212. 
COMPOSITION OF ASH OF CEREALS.

\begin{tabular}{|c|c|c|c|c|c|c|c|c|c|}
\hline & $\mathrm{K}_{0} \mathrm{O}$. & $\mathrm{Na}_{2} \mathrm{O}$, & $\mathrm{CaO}$. & MgO. & $\mathrm{Fe}_{2} \mathrm{O}_{3}$. & $\mathrm{P}_{8} \mathrm{O}_{5}$ & $\mathrm{SO}_{2}$. & Cl. & $\mathrm{SiO}_{2}$. \\
\hline & & $9 \cdot 55$ & & 13.24 & & 46.87 & $0.0 \mathrm{I}$ & 0.00 & \\
\hline & 27.60 & 4.64 & $5 \cdot 56$ & II -73 & 5.23 & $4 \mathrm{r} .8 \mathrm{I}$ & 0.52 & $0.5^{8}$ & 2.45 \\
\hline & $24 \cdot 15$ & 6.42 & 2.44 & 8.23 & 0.33 & $35 \cdot 47$ & 0.22 & 0.56 & 22.30 \\
\hline Oat: & I5.9I & $4 \cdot 3^{8}$ & 4.09 & 7.18 & 0.20 & $24 \cdot 34$ & 0.48 & 1.02 & 42.64 \\
\hline Cor & 33.92 & $7 \cdot 72$ & 3.18 & 17.99 & 0.5 & 35. & 0.44 & 0.00 & I. \\
\hline & 20.84 & 13.98 & $4 \cdot 48$ & 9.60 & 0.89 & $43.2 \mathrm{I}$ & 0.24 & 0.80 & 6.1 \\
\hline Buckwheat (U. & $35 \cdot 15$ & 2.26 & 6.62 & 20.55 & I. 68 & 24.09 & 3.59 & 0.67 & $5 \cdot 54$ \\
\hline
\end{tabular}

Teller* obtained the following results of ash analyses of flour, bran, and wheat:

ASH OF WHEAT PRODUCTS.

\begin{tabular}{|c|c|c|c|c|c|}
\hline & $\begin{array}{l}\text { Patent } \\
\text { Flour. }\end{array}$ & $\begin{array}{l}\text { Straight } \\
\text { Flour. }\end{array}$ & $\begin{array}{l}\text { Low } \\
\text { Grade. }\end{array}$ & Bran. & Wheat. \\
\hline Silica...... & $2 \cdot 33$ & 1.28 & .50 & .97 & 1.04 \\
\hline Alumina....... & .41 & .15 & .12 & .07 & .II \\
\hline Ferric oxide...... & .47 & .26 & .25 & .27 & .27 \\
\hline Potash. ......... & $3^{8} \cdot 5^{\circ}$ & $3^{6} \cdot 3^{1}$ & 32.27 & 28.19 & $29.7^{\circ}$ \\
\hline Soda. ............ & 0.00 & 0.00 & 0.00 & 0.00 & 0.00 \\
\hline Lime ...... & $5 \cdot 59$ & 5.65 & $4 \cdot 51$ & 2.50 & 3.10 \\
\hline Magnesia .......... & $4 \cdot 39$ & 6.44 & $9 \cdot 33$ & 14.76 & $\mathrm{I}_{3.23}$ \\
\hline Phosphoric acid. ..... & 48.05 & $49 \cdot 3^{2}$ & 53.10 & $5^{2} .18$ & $5^{2} \cdot 14$ \\
\hline Sulphur trioxide....... & .16 & $.5^{2}$ & .00 & .10 & .22 \\
\hline Chlorine.......... & ....... & …... & …... & .01 & .01 \\
\hline Zinc oxide........ & ....... & .04 & 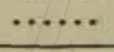 & .27 & .24 \\
\hline Sum........ & 99.90 & 99.97 & 100.08 & 99.95 & 100.06 \\
\hline Per cent of total ash. & $\cdot 31$ & .40 & .70 & $\cdots \cdots$ & 1. 62 \\
\hline
\end{tabular}

König gives the following analyses of the ash of various leguminous and other vegetables:

\begin{tabular}{|c|c|c|c|c|c|c|c|c|c|c|c|}
\hline & 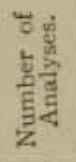 & 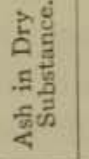 & $\begin{array}{l}\frac{1}{3} \\
\frac{3}{0} \\
\text { م }\end{array}$ & $\begin{array}{l}\text { 迆 } \\
\text { के }\end{array}$ & 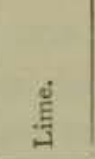 & 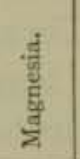 & 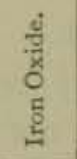 & $\begin{array}{l}\text { हूँ } \\
\text { 递 } \\
\text { हैं }\end{array}$ & 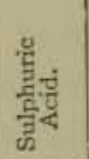 & 总 & $\frac{\stackrel{\text { ही }}{\text { है }}}{\text { है }}$ \\
\hline & 15 & $3 \cdot 57$ & 42.49 & I. 34 & $4 \cdot 73$ & 7.08 & & $3^{8} \cdot 74$ & 2.53 & 0.73 & \\
\hline & 29 & 2.73 & $4 \mathrm{I} \cdot 79$ & 0.96 & $4-99$ & 7.96 & 0.86 & $3^{6.43}$ & 3.49 & 0.86 & \\
\hline & 53 & $3 \cdot 77$ & 60.37 & 2.62 & 2.57 & 4.69 & I. 18 & $17 \cdot 3$ & 6.49 & 2.13 & \\
\hline Beets & 15 & 6.44 & 54.02 & 15.90 & 4.12 & $4 \cdot 54$ & 0.82 & 8.45 & 3.17 & $2 \cdot 3^{8}$ & \\
\hline & II & $5 \cdot 5^{8}$ & $35.2 \mathrm{I}$ & 22.07 & II. 42 & 4.73 & 1.03 & 12.46 & $6.7^{2}$ & 2.47 & \\
\hline Turnips....... & $3^{2}$ & 8.01 & $45 \cdot 40$ & 9.84 & 10.60 & 3.69 & $0.8 \mathrm{I}$ & 12.71 & II. 19 & I. 87 & 5.01 \\
\hline
\end{tabular}

* Ark. Exp. Sta. Bul. 42. 
as the charcoal forms a hard mass, while substances rich in fibrous or woody matter burn quite readily without losing their powdered condition. A certain amount of unburned carbon is no disadvantage, as it is determined in the course of the analysis.

Finally cool the ash, grind to a powder, mix without loss, and weigh, thus determining the percentage of crude ash.

Determination of Water.-Heat I gram of the ash in a platinum crucible well below redness to constant weight.

Determination of Carbonic Acid.-Determine carbonic acid as described on p. 336 using the portion dried for the determination of water.

Determination of Charcoal and Sand.-Weigh I gram of the ash, or transfer the solution and residue from the determination of carbonic acid, into a beaker, add $25 \mathrm{cc}$. of water and $25 \mathrm{cc}$. of ro per cent hydrochloric acid, and boil gently for to minutes. Filter on a Gooch crucible, and wash thoroughly with hot water. Reserve the filtrate for determination of silica, iron oxide, alumina, lime, and magnesia. Wash the residue on the crucible once with alcohol and once with ether, and dry to constant weight at $100^{\circ} \mathrm{C}$. Ignite and weigh again. The loss on ignition is the charcoal, the residue is sand.

Determination of Silica, Iron Oxide, Alumina, Lime and Magnesia.Evaporate to dryness in a platinum dish the filtrate from the determination of charcoal and sand, heat for some hours on the water bath, and dry at $130^{\circ} \mathrm{C}$. until all hydrochloric acid is removed. Moisten the residue thoroughly with concentrated hydrochloric acid, add hot water, stir, and decant the solution on an ashless filter. Treat the residue again with acid and hot water, and repeat the treatment until nothing but silica remains undissolved. Finally collect the silica on the paper, wash with hot water, ignite in a platinum crucible, and weigh.

To the filtrate add ammonia until a precipitate forms which remains on stirring, and then add sufficient hydrochloric acid to just dissolve the precipitate. Heat to $50^{\circ} \mathrm{C}$. and add an excess of ammonium acetate solution and $4 \mathrm{cc}$. of 80 per cent acetic acid. Digest at $50^{\circ} \mathrm{C}$. until the mixed phosphates of iron and alumina have settled, filter, wash with hot water, ignite in a platinum crucible, and weigh. As the precipitate is usually slight and consists almost entirely of iron phosphate, the iron oxide may be calculated with reasonable accuracy using the factor 0.53 . If, however, greater accuracy is desired fuse the weighed precipitate with to parts of sodium carbonate, dissolve in dilute sul- 
phuric acid, reduce with hydrogen sulphide, determine iron by the volumetric permanganate method, and in the same solution determine phosphoric acid by the molybdic method. The alumina is obtained by difference, subtracting the sum of the werghts of the oxide of iron and phosphoric acid from the total weight of the precipitate.

To the filtrate from the mixed phosphates add an excess of ammonium oxalate, allow to stand in a warm place over night, filter, ignite the precipitate in a platinum crucible over a Bunsen burner, and finally to constant weight over a blast lamp, thus obtaining the calcium oxide.

Precipitate the magnesia in the filtrate from the lime by adding ammonia to alkaline reaction, then an excess of sodium phosphate solution with constant stirring, and finally sufficient concentrated ammonia to form one-tenth the final volume. Let stand over night, collect the magnesium ammonium phosphate on a Gooch crucible, ignite to magnesium pyrophosphate, and weigh.

Determination of Sulphuric Acid, Potash, and Soda.-Boil I gram of the ash with dilute hydrochloric acid, and remove charcoal, sand, and silica, as described in the preceding section. Evaporate nearly to dryness to remove the excess of acid. Dilute to $100 \mathrm{cc}$., heat to boiling, and add barium chloride solution drop by drop until the sulphuric acid is precipitated. Allow to stand over night, filter, ignite, and weigh as $\mathrm{BaSO}_{4}$.

Heat the filtrate to boiling, add enough barium hydroxide to make the solution strongly alkaline, filter, and proceed with the determination of potash and soda, as described on p. 345 .

Determination of Phosphoric Acid.-Dissolve 0.5 gram of the ash in hydrochloric acid, filter, and wash. Neutralize with ammonia, clear with nitric acid, and proceed as described on p. 346 .

Determination of Chlorine.-Dissolve I gram of the ash in cold, very dilute nitric acid, filter, and wash. To the filtrate add an excess of silver nitrate, and heat nearly to boiling with constant stirring. Filter on a Gooch crucible, wash with hot water, dry the precipitate at a low heat, and heat cautiously at dull redness until the silver chloride has partially melted.

If desired the chlorine may be determined volumetrically by Volhardt's method, as follows: To the nitric acid solution add a known volume of decinormal silver nitrate solution sufficient to precipitate the chlorine, and $5 \mathrm{cc}$. of saturated solution of ferric alum. Titrate with decinormal ammonium thiocyanate solution until a permanent brown 
color is formed. Subtract the volume required from the volume of decinormal silver nitrate added, and calculate the chlorine.

Determination of Sulphur in Vegetable Materials.*-Place from I. 5 to 2.5 grams of material in a nickel crucible of about $100 \mathrm{cc}$. capacity, and moisten with approximately $2 \mathrm{cc}$. of water. Mix thoroughly, using a nickel or platinum rod. Add 5 grams of pure anhydrous sodium carbonate, and mix. Add pure sodium peroxide, small amounts (approxmately 0.50 gram) at a time, thoroughly mixing the charge after each addition. Continue adding the peroxide until the mixture becomes nearly dry and quite granular, requiring usually about 5 grams of peroxide. Place the crucible over a low alcohol flame (or other flame free from sulphur), and carefully heat with occasional stirring until contents are fused. (Should the material ignite the determination is worthless.) After fusion, remove the crucible, allow to cool somewhat, and cover the hardened mass with peroxide to a depth of about $0.5 \mathrm{~cm}$. Heat gradually, and finally with full flame until complete fusion takes place, rotating the crucible from time to time in order to bring any particles adhering to the sides into contact with the oxidizing material. Allow to remain over the lamp for ten minutes after fusion is complete. Cool somewhat. Place warm crucible and contents in a $600 \mathrm{cc}$. beaker, and carefully add about roo cc. of water. After violent action has ceased, wash material out of crucible, make slightly acid with hydrochloric acid (adding small portions at a time), transfer to a $500 \mathrm{cc}$. flask, cool, and make to volume. Filter, and take a $200 \mathrm{cc}$. aliquot for determination of sulphates by precipitating with barium chloride in the usual manner.

Determination of Chlorine in Vegetable Substances.* - Impregnate 5 grams of substance in a platinum dish with $20 \mathrm{cc}$. of a 5 per cent solution of sodium carbonate, evaporate to dryness, and ignite as thoroughly as possible. Extract the residue with hot water, filter, and wash. Return to the platinum dish, ignite to an ash, dissolve in nitric acid, and determine chlorine by the Volhard method (p. 3०4).

Detection of Sulphuring in Grain.-Carroll + has shown that the following method distinguishes sharply barley, oats and other grains in their natural condition from those bleached by sulphurous acid.

Introduce into a $500 \mathrm{cc}$. flask, provided with a desulphurized perforated stopper and a double-bent delivery tube, to grams of mossy zinc, a few drops of ferric chloride solution, Ico grams of the grain and enough $8 \%$

*A. O. A. C. Method, U. S. Dept, of Agric., Bur. of Chem., Bul, 107 (rev.), pp. $23,24$.

† U. S. Dept. of Agric., Bur. of Plant Ind., Circ. 40. 
hycrochloric acid to cover the grain. Place a loose plug of cotton in the neck, attach the stopper, and run the delivery tube into a test-tube containing to cc. of $2 \%$ lead acetate solution. If a brownish black precipitate of lead sulphide forms, the grain has been sulphured. Particles of dust or zinc, occasionally carried over mechanically, are distinguished from lead sulphide by their insolubility in $10 \%$ ferric chloride solution.

\section{MICROSCOPY OF CEREAL PRODUCTS.}

The histology of the cereals is more fully considered in the works on food microscopy, the brief descriptions here given should, however, enable the food chemist to identify the commoner products. The tissues of the various cereals are quite distinctive, serving usually to determine the particular grain from which a given product is made. In the case of flour the tissues are largely removed in milling, the fragments remaining being small and few in number. Such products are identified either by the character of the starch grains-as in the detection of wheat or corn flour in buckwheat flour-or else, if the starch is not sufficiently characteristic-as in the detection of wheat flour in rye flour-by examining the tissues from a considerable amount of the material.

The most convenient method of accumulating the tissues from flour is to mix thoroughly 2 grams of the material with $200 \mathrm{cc}$. of water and $3 \mathrm{cc}$. of sulphuric acid, bring to a boil, allow to settle, and carefully decant off the liquid from the deposit of tissues. The tissues are mounted for examination in very dilute sodium hydroxide solution

Wheat Products.-Fig. 62 and PI. VIII show the principal elements of the wheat kernel.

The outer layer or epicarp (Fig. 62,epi $i^{1}$ and $e p i^{2}$ ) consists of beaded cells, which on the body of the kernel are elongated, but at the end are polygonal. From this layer at the end of the kernel arise the hairs (Fig. $62, t$, Pl. VIII, Fig. 15I) which form a beard clearly visible under a lens. Some of these hairs become detached in milling, and pass endwise through the bolts, hence their presence in even the highest grade of flour. The second layer or hypoderm (hy) resembles the first, while the third, although likewise made up of beaded cells, is strikingly different and forms the most characteristic tissue of the grain. These cells (Fig. 62, tr, PI. VIII, Fig. 150) being transversely extended are known as "cross cells," and are further distinguished from the outer layers by their arrangement side by side in rows. The cells of the intermediate laver 
(Fig. 62, in) and the tube cells $\left(t u^{1}\right.$ and $\left.t u^{2}\right)$, although of striking appearance, are not of as frequent occurrence as the other layers. The crossing layers of the seed coat or spermoderm ( $i$ and $o$ ), are often met with, and are characterized by the thin walls of the cells and their brownish color.

The perisperm $(P)$, consisting of colorless cells, is seldom seen, except after special preparation, while the next layer, made up of aleurone cells

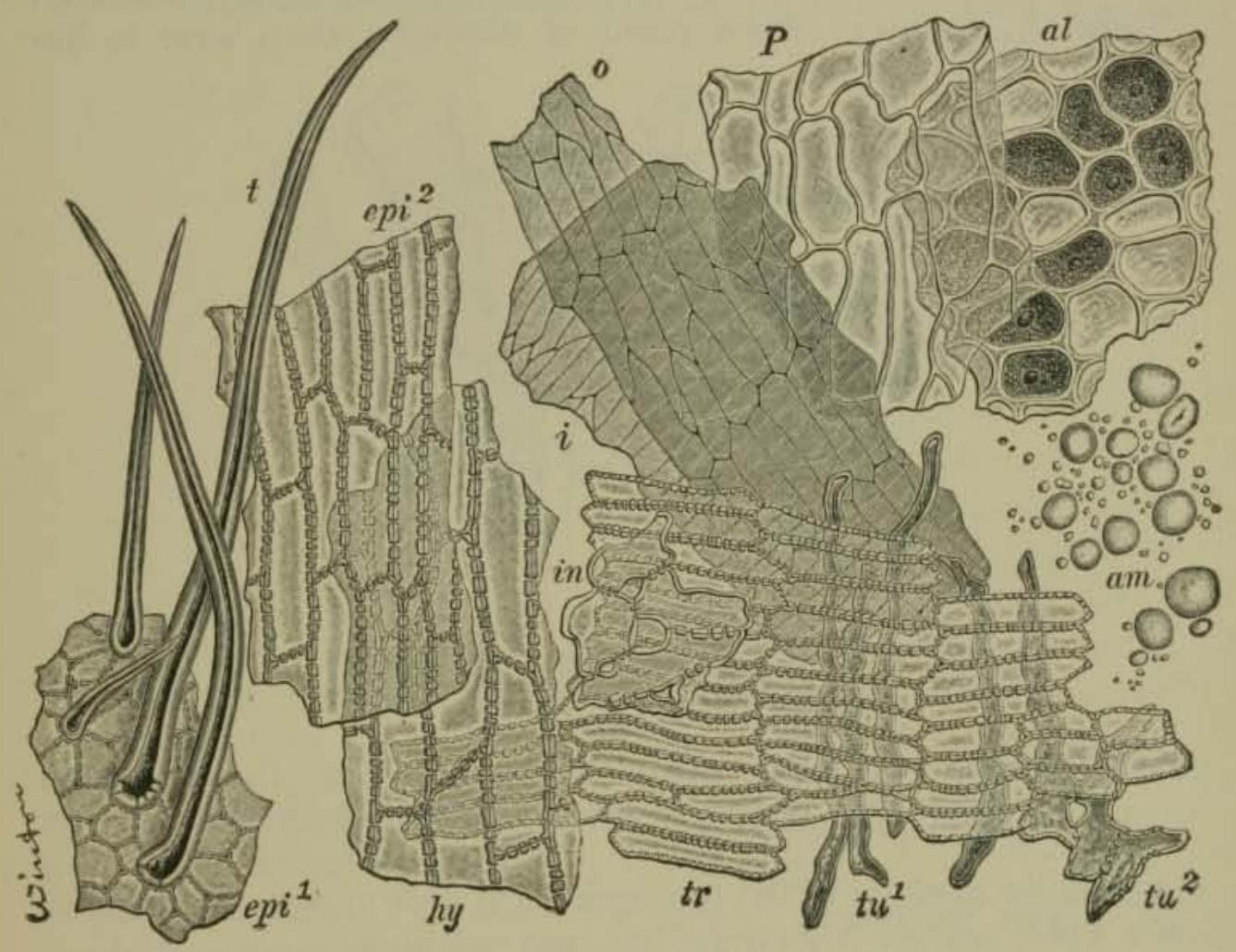

Fic. 62.-Wheat. Elements in Surface View. $X_{160}$. (Wrvion.)

epi $i^{1}$ epicarp at end of grain, with $t$ hairs; epiz epicarp on body of grain; hy hypoderm (first layer of mesocarp); in intermediate cells; $t r$ cross cells; $t u^{2}$ typical tube cells; $t u$ tube cells passing into spongy parenchyma; $o$ outer layer of spermoderm; $i$ inner layer of spermoderm; $P$ perisperm; al aleurone cells; am starch grains.

(Fig. 62, al; Pl. VIII, Fig. I5o), is the most conspicuous of the kernel. This layer is not, however, characteristic of wheat, as it is found in all cereal grains and in buckwheat. The aleurone cells do not contain, as was formerly supposed, the gluten of the grain; this occurs with the starch in the thin-walled cells within the aleurone layer.

The starch gramules (Fig. 62, am; Pl. VIII, Fig. 152) are described 
on page $28 \mathrm{r}$. The starch cells and the aleurone cells together form the endosperm.

The germ, situated at one side of the lower end of the kernel, is made up of very small cells containing fat and protein, but no starch.

Rye Products. - The structure of rye (Fig. 63; Pl. VII) resembles closely that of wheat. The number and general characters of the cell layers are the same in both, and the starch granules are very much alike. There are, however, certain points of difference which serve to dis-

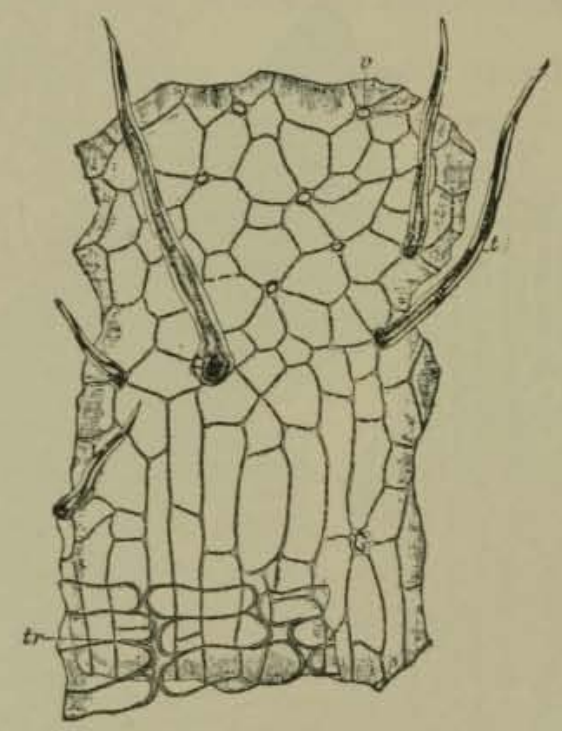

Frc. 53.-Rye, Outer Bran Layers in Surface View. Epicarp consists of porous cells with $t$ hairs, and $v$ hair scars; $t r$ cross cells, Xío. (Moflle.

tinguish the products of the two cereals, and even to detect the presence of a wheat product in a rye product, and vice versa:

First. The breadth of the cavities of wheat hairs is usually less than the thickness of the walls, whereas in rye hairs the reverse is often true (Figs. 62 and $63, t$ ).

Second. The cross cells of wheat have rather thick, distinctly beaded side walls, and thin, pointed end walls; the cross cells of rye have rather thin, indistinctly beaded side walls, and usually swollen, rounded end walls (Figs. $6_{2}$ and 63 , tr; Figs. $1_{50}$ and 146 ).

Third. The large starch granules of wheat seldom reach $0.050 \mathrm{~mm}$. in diameter, while those of rye frequently exceed that limit. Radiating clefts often occur in the starch granules of rye (Pl. VII, Fig. 148).

Fourth. Wheat flour yields a considerable amount of gluten when 
treated according to Bamihl's test (page 322); rye flour yields none or only a trace.

Barley Products.-The common varieties of barley are "chaffy," that is, the grain after threshing is still closely invested by the chaff (Pl. I, Fig. 123). The grain within the chaff is analogous in structure to wheat and rye, but differs from these in that the cross cells are not beaded and form a double layer (Fig. 64, tr), and the starch granules

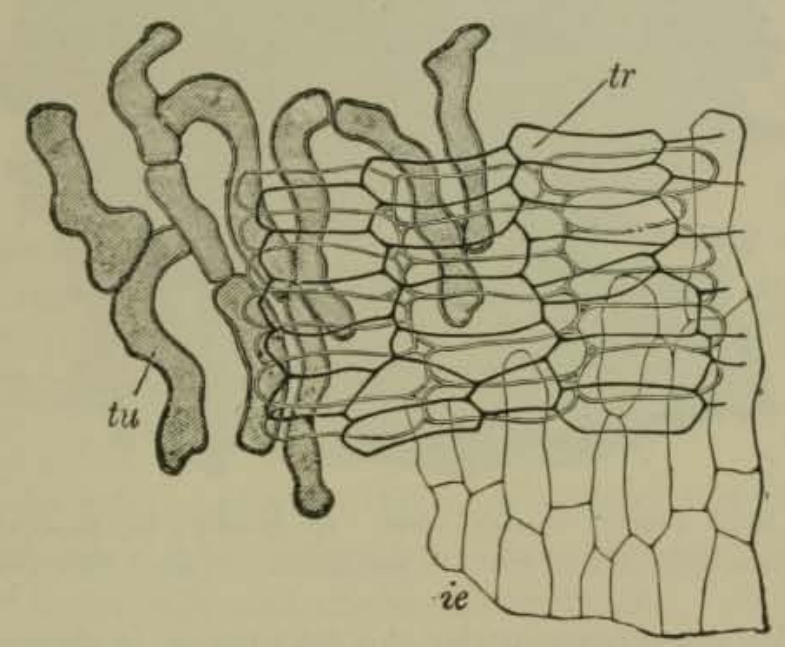

Fic, 64,-Barley. Surface view of $t r$ double layer of cross-cell; $t u$ tube cells; ie seed coat. $\times$ 300. (MOELLER.)

seldom exceed $0.035 \mathrm{~mm}$. in diameter (Pl. I, Fig. I24). The starch is more fully described on page $2 S \mathrm{~s}$.

Corn Products.-The most characteristic element of corn is the starch (page $28 \mathrm{r} ; \mathrm{Pl}$. IV). Polygonal starch granules 0.017 to 0.030 $\mathrm{mm}$. in diameter occur in no other vegetable product of economic importance, excepting the seeds of Kaffir corn and other grains belonging to the genus Sorghum, which are used chiefly for cattle or poultry foods.

Oat Products.-The oat kernel resembles barley in appearance, but is not ribbed. In the preparation of oat meal and other breakfast foods, the chaff (Pl. IV, Fig. 135; Pl. V, Fig. I37) is removed and utilized as a cattle food. The elements of the grain of chief value in identification are the hairs and the starch granules. The hairs (PI. V, Fig. $\left.{ }_{13} 8\right)$ are much longer than those of wheat, rye, and barley, often reaching I mm. They taper toward both ends, so that when detached they often appear to be pointed at the base as well as at the apex. The 
starch granules are small, of the polygonal type, and often occur in eggshaped aggregates (page $282 ; \mathrm{Pl}$. V, Fig. I39).

Rice Products. - The chaff which envelopes this grain is rough and silicious, and after removal from the inner kernel is not suited even for

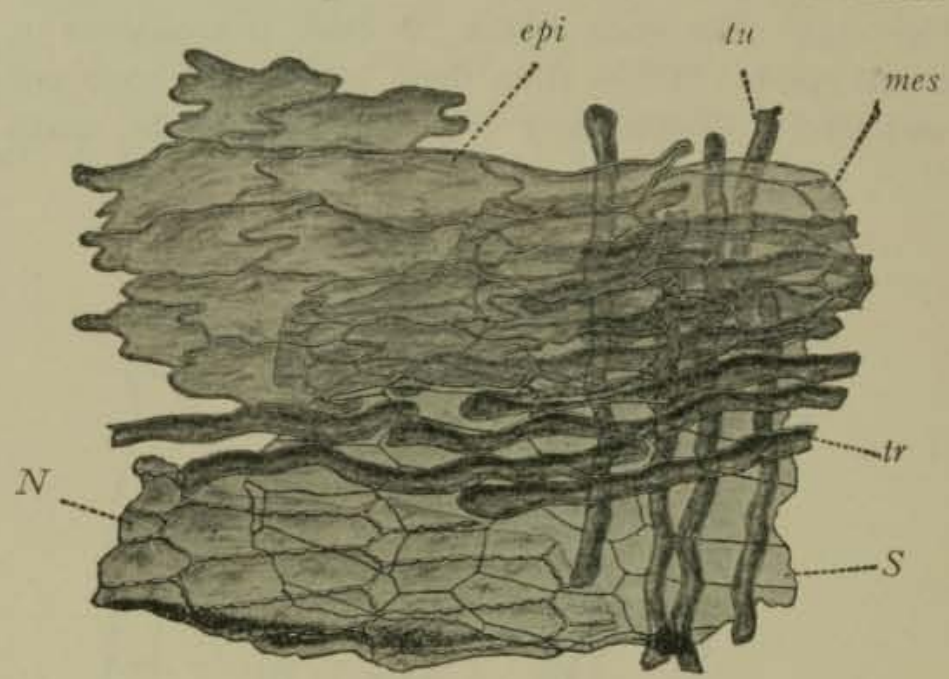

Fic. 65.-Rice. Bran coats in surface view. epi epicarp; mes mesocarp; tr cross cells; $t u$ tube cells; $S$ seed coat; $N$ perisperm. $\times 300$. (Winton.)

cattle food. Its appearance under the microscope is shown in Plate VI, Fig. I42. The thin skin of the kernel proper is largely but not entirely

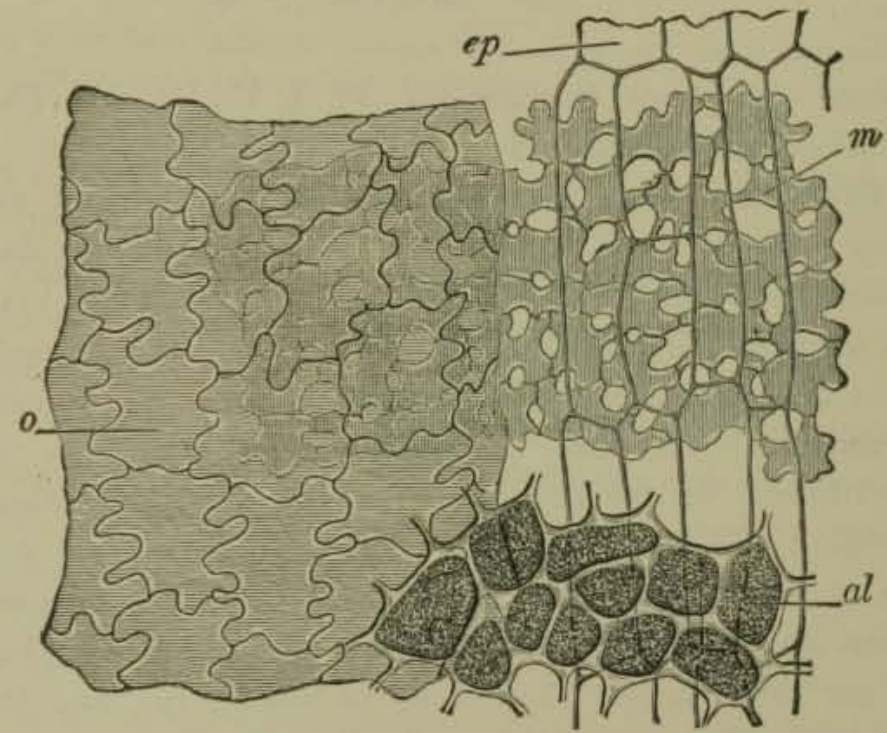

Fig. 66,-Buckwheat. Bran coats in surface view. Seed coat consists of $o$ outer epidermis, $m$ spongy parenchyma, and $e p$ inner epidermis; al aleurone cell. $\times 300$. (MoktLer.) 
removed in the preparation of rice for the market. The elements of this skin are shown in Fig. 65, the outer layer (epi) being the most characteristic. Unlike wheat, rye, and barley, it has no beard. Rice starch (Pl. VI, Fig. I43) is hardly distinguishable from oat starch. It is described on page 282 .

Buckwheat Products.-In the preparation of buckwheat flour the black outer hulls and the inner skin or bran are largely, but not completely, removed. The bran elements are characteristic constituents of the flour, and are rendered especially distinct by adding a drop of dilute potassium hydroxide solution to a water mount (Fig. 66). The cells with wavy walls $(o)$ and the spongy parenchyma $(m)$ are especially noticeable. The starch of buckwheat resembles that of oats, but the individual granules are somewhat larger and occur in rod-shaped, not egg-shaped, aggregates (page 28r; Pl. II, Fig. I28). Masses of starch granules (Pl. III, Fig. 129) conforming to the shape of the cells, occur in abundance in the flour.

\section{FLOUR.}

Flour is the term applied to the finely ground and bolted substance of wheat and other grains, though, unless otherwise qualified, by the term "flour" is generally undertsood that of wheat.

Graham flour is an unbolted meal prepared from the whole wheat kernel.

Process of Milling.-The crude milling process which prevailed until the last quarter of the nineteenth century consisted in grinding the wheat between millstones and bolting to remove the bran and shorts. In the modern or Hungarian process the wheat is first crushed between corrugated rollers, then by sifting separated into middlings, break flour, and bran. The middlings, consisting of the hard glutinous portions of the grain in granular form, are gradually reduced to fine flour between smooth rollers and freed from impurities by means of a series of bolts. A number of grades of flour are thus produced, the streams of which are so combined with each other and the break flour as to form the finished products.

The Grades of Flour commonly made are (1) patent, forming $85 \%$ or less of the flour output; (2) clear or bakers', an intermediate grade inferior to the patent in color and rising properties; and (3) low grade or "red dog," about $5 \%$, suitable only for cattle food. Some mills make two or more grades of patent and clear. On the other hand, it is a frequent practice to combine all the flour streams other than of low grade to form a straight. 
The By-products are (1) tran, the outer coatings of the grain in flakes, (2) shorts, the finer offal containing both starchy matter and bran elements, and (3) germ, rich in oil, often run in with the bran.

Composition of Wheat Flour and By-products. - The following analyses by Clifford Richardson * represent the products of the same milling:

\begin{tabular}{|c|c|c|c|c|c|c|c|c|c|}
\hline & $\begin{array}{l}\text { Mois- } \\
\text { ture. }\end{array}$ & $\begin{array}{l}\text { Protein } \\
\mathrm{N} \times 5.7\end{array}$ & $\begin{array}{l}\text { Moist } \\
\text { Gluten. }\end{array}$ & $\begin{array}{c}\text { Dry } \\
\text { Gluten }\end{array}$ & $\begin{array}{l}\text { Ether } \\
\text { Ex- } \\
\text { tract } \\
\text { (Fat). }\end{array}$ & $\begin{array}{l}\text { Nitro- } \\
\text { gen-free } \\
\text { Extract } \\
\text { (Starch, } \\
\text { ete). }\end{array}$ & $\begin{array}{l}\text { Crude } \\
\text { Fiber. }\end{array}$ & Ash. & $\begin{array}{l}\text { Phos- } \\
\text { phoric } \\
\text { Acid. }\end{array}$ \\
\hline 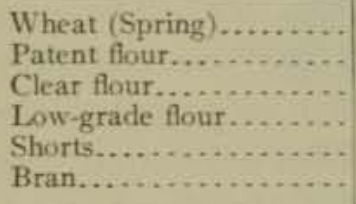 & $\begin{array}{r}9.07 \\
11.48 \\
12.18 \\
12.01 \\
10.94 \\
10.91\end{array}$ & $\begin{array}{l}12.93 \\
11.81 \\
13.57 \\
16.37 \\
15.32 \\
14.84\end{array}$ & $\begin{array}{l}36.14 \\
51.51 \\
10.01 \\
\cdots \\
\cdots\end{array}$ & $\begin{array}{c}10.85 \\
16.97 \\
4.26 \\
\cdots \cdots\end{array}$ & $\begin{array}{l}2.74 \\
1.45 \\
2.00 \\
3.86 \\
4.67 \\
5.03\end{array}$ & $\begin{array}{l}71 \cdot 77 \\
74.69 \\
71.30 \\
64.84 \\
61.76 \\
57.65\end{array}$ & $\begin{array}{l}1.70 \\
0.18 \\
0.33 \\
0.93 \\
3.90 \\
5.98\end{array}$ & $\begin{array}{l}1.79 \\
0.39 \\
0.62 \\
1.99 \\
3.41 \\
5.59\end{array}$ & $\begin{array}{l}0.82 \\
0.18 \\
0.31 \\
1.16 \\
1.62 \\
2.78\end{array}$ \\
\hline
\end{tabular}

From the above it appears that the fat, fiber, ash, and phosphoric acid increase through the series, being least in the patent flour and greatest in the bran, while the nitrogen-free extract decreases. Considering only the flour, the protein is least in the patent and greatest in the low grade, but the gluten, although greater in the clear than in the patent, drops down sharply in the low grade.

The $a s h$ of flour is a valuable index of grade. In a true patent it should not exceed $0.45 \%$. As pointed out by Snyder, the acidity is aiso a guide, although this increases in all grades on aging.

Hard Wheats, such as the Spring varieties of the Northwest and Turkey red Winter wheat of Kansas, yield a "strong" flour rich in protein and gluten, the latter being of good tenacity, while Soft Wheats, such as are grown in States adjoining the Ohio river, yield a white starchy flour, the gluten being smaller in amount and lacking in tenacity.

Analyses of typical hard and soft wheat flour freshly ground, are given in the table on top of page $3{ }^{1} 3$. The percentages are of the total flour, excluding the low grade. Color values are given on page 3 I6.

Color of Flour.- The time-honored test for grade is by the color. A patent is practically free from bran specks while a clear contains such specks in noticeable amount. Both grades have a yellowish tint due to the color associated with the fat, which is more or less pronounced,

* U. S. Dept. of Agric., Bul. 4, 1884, p. 38 .

+ Minn. Agric. Exp. Sta., Bul. 85 . 


\begin{tabular}{|c|c|c|c|c|c|c|c|c|}
\hline & \multicolumn{2}{|c|}{$\begin{array}{l}\text { Mirnesota. } \\
\text { Hard Spring. }\end{array}$} & \multicolumn{2}{|c|}{$\begin{array}{l}\text { Nebraska: } \\
\text { Hard Winter. }\end{array}$} & \multicolumn{2}{|c|}{$\begin{array}{l}\text { Michigan. } \\
\text { Soft Winter }\end{array}$} & \multicolumn{2}{|c|}{$\begin{array}{l}\text { Missouri. } \\
\text { Soft Winter. }\end{array}$} \\
\hline & $\begin{array}{c}78 \% \\
\text { Patent. }\end{array}$ & Clear. & $\begin{array}{c}80 \% \\
\text { Patent. }\end{array}$ & $\begin{array}{l}20 \% \\
\text { Clear. }\end{array}$ & $\begin{array}{l}80 \% \\
\text { Patent. }\end{array}$ & $\begin{array}{l}20 \% \\
\text { Clear. }\end{array}$ & $\begin{array}{l}40 \% \\
\text { Patent. }\end{array}$ & $\begin{array}{l}60 \% \\
\text { Clear. }\end{array}$ \\
\hline Moisture,............. & I3. 74 & 13.26 & $\$ 3 \cdot 33$ & 12.85 & 13.22 & 12.62 & 12.27 & 12.02 \\
\hline Ash......... & 0.44 & 0.85 & 0.39 & 0.67 & 0.42 & 0.89 & 0.39 & 0.50 \\
\hline Crude fiber........... & 0.06 & 0.26 & 0.18 & 0.24 & 0.19 & 0.27 & 0.34 & $0.3^{8}$ \\
\hline Protein $(\mathrm{N} \times 5.7) \ldots \ldots$ & 10.60 & II. 74 & 11.09 & Ir. 86 & 8.66 & 12.26 & 9.01 & 10.72 \\
\hline Alcohol sol. protein .... & 5.84 & 6.21 & $5 \cdot 79$ & 6.55 & $5 \cdot 24$ & $5 \cdot 53$ & 5.04 & 6.21 \\
\hline Salt sol, protein...... & 1.62 & 2.19 & 1.48 & 2.02 & I. 45 & 2.19 & x. 25 & 1.43 \\
\hline Moist gluten......... & $3^{6.93}$ & 38.76 & 30.48 & 42.50 & 20.23 & $3 \mathrm{I} .24$ & 17.90 & 33.21 \\
\hline Dry gluton............. & 12.48 & 13.41 & 9.85 & 13.08 & 6.97 & 10. 55 & 5.90 & 10.22 \\
\hline Nitrogen-free extract... & 74.07 & 71.91 & $75 \cdot 16$ & 73.06 & 76.40 & 72.19 & $77-12$ & $75 \cdot 23$ \\
\hline 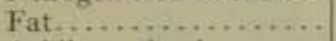 & I. 09 & I. 98 & 0.85 & I. 32 & I.II & I. 77 & 0.87 & 1.15 \\
\hline Acidity as lactic....... & 0.108 & 0.230 & 0.081 & 0.158 & 0.110 & 0.250 & 0.063 & 0.095 \\
\hline
\end{tabular}

according to the kind of wheat, but is not proportional to the per cent of fot. This is measured by the gasoline color value (pp. 316 and $3^{2}$ ).

Composition of Various Flours.-The following analyses are from Bulletin $1_{3}$, Part 9, of the Bureau of Chemistry:

\begin{tabular}{|c|c|c|c|c|c|c|c|c|}
\hline & $\begin{array}{l}\text { No, of } \\
\text { Analyses. }\end{array}$ & Moisture. & $\begin{array}{l}\text { Protein } \\
\mathrm{N} \times 6,25 .\end{array}$ & $\begin{array}{l}\text { Ether } \\
\text { Extract } \\
\text { (Fat). }\end{array}$ & $\begin{array}{l}\text { Nitrogen- } \\
\text { free Ex- } \\
\text { tract } \\
\text { (Starch. } \\
\text { etc.). }\end{array}$ & $\begin{array}{l}\text { Crudie } \\
\text { Fiber. }\end{array}$ & Ash. & $\begin{array}{l}\text { Caleu- } \\
\text { hited } \\
\text { Calories } \\
\text { of Com- } \\
\text { bustion. }\end{array}$ \\
\hline $\begin{array}{l}\text { Corn flour...... } \\
\text { Rye flour....... } \\
\text { Barley flour..... } \\
\text { Buckwheat flour }\end{array}$ & $\begin{array}{l}3 \\
1 \\
1 \\
1\end{array}$ & $\begin{array}{l}12.57 \\
11.41 \\
10.92 \\
11.89\end{array}$ & $\begin{array}{r}7.13 \\
13.56 \\
7.50 \\
8.75\end{array}$ & $\begin{array}{l}1.33 \\
1.97 \\
0.89 \\
1.58\end{array}$ & $\begin{array}{l}78.36 \\
73 \cdot 37 \\
80.50 \\
75 \cdot 41\end{array}$ & $\begin{array}{l}0.87 \\
1.86 \\
0.67 \\
0.52\end{array}$ & $\begin{array}{l}0.61 \\
1.55 \\
0.86 \\
1.85\end{array}$ & 38.37 \\
\hline
\end{tabular}

Damaged Flour.-Grain is often damaged by the growth of smuts, rusts, and ergot. Both grain and flour are also liable to attacks of molds, yeasts, algae, and bacteria.

Various insects and other forms of animal life frequently infest grain or flour. Among these are weevils and various other beetles, flour moths, mites, and the wheat worm, a nematode related to trichina.

Grain may also be damaged by sprouting, the diastate thus formed partially dissolving the starch granules with the formation of fissures and branching channels, readily seen under the microscope. Flour thus damaged is high in cold-water extract (p. 320).

Ergot.-Ergot, a fungous growth containing a poisonous alkaloid, sometimes develops in rye and, less often, in wheat. Under the microscope it appears as a fine network of mostly colorless parenchyma cells, containing globules of fat (Fig. 67). Some of the cells are circular, others considerably elongated, and some contain a deep-brown coloring 
matter, which, with ammonia, becomes violet-red, changing to red with acid. Occasionally the cell walls appear of a dark color. If flour containing ergot be treated with a very dilute solution of anilin violet, the stain will be practically absorbed by the damaged particles of the grain, and resisted by the normal granules. A hot, alcoholic extract of flour containing ergot is colored red when treated with dilute sulphuric acid.

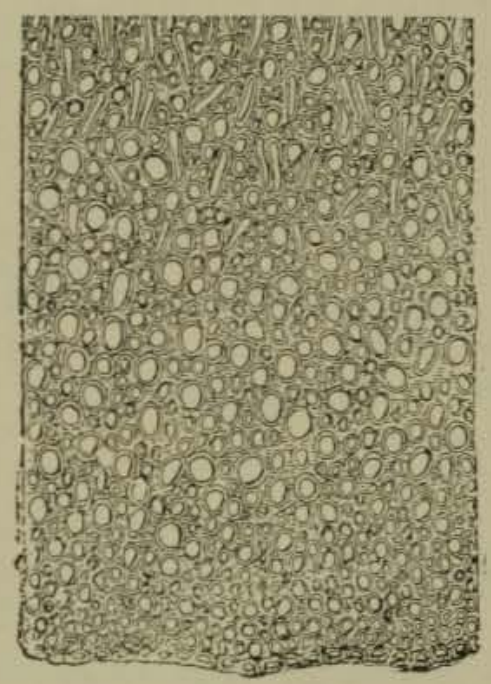

A

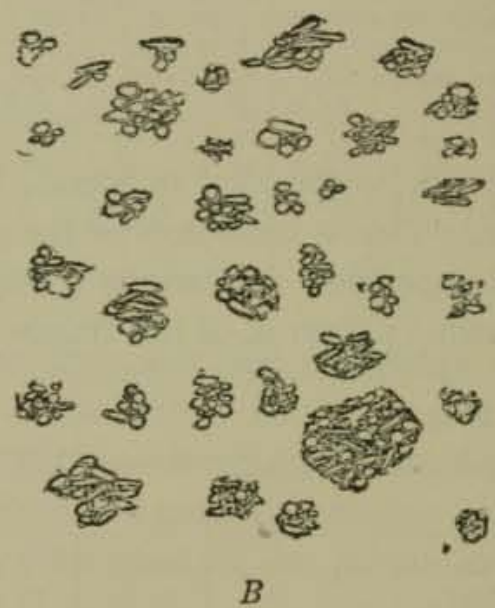

Fig. $67 .-A$, Transverse Section of the Ergot of Wheat under the Microscope; $B$ Powdered Wheat Ergot. (After Villiers and Collin.)

Adulteration of Flour. - Besides the substitution of cheaper or inferior grades for those of higher quality, the fraudulent admixture of corn flour to wheat flour was at one time extensively practiced. This adulterant is best detected by the microscope (p. 28r).

Rye flour is often adulterated with cheap grades of wheat flour or middlings. These admixtures are detected by the Bamihl test (p. 322) and by microscopic examination of the residue after boiling with dilute acid (p. 306), noting especially the cross cells.

Much of the so-called buckwheat flour consists of mixtures containing wheat or corn flour, or both. Rice flour is also used in pancake flours, although probably not to cheapen the product. Self-raising pancake flours are usually mixtures of two or more flours with leavening material. The microscopic characteristics of the starch grains and tissues, serve to identify the different flours present in such mixtures. 
Finely ground mineral adulterants are said to have been used in flours, but no authentic instance of this kind has come to the writer's knowledge. Any considerable admixture of such a nature would be manifest in the increased ash.

Alum in Flour.-Alum was formerly used in Europe, both by miller and baker, to improve the appearance of inferior or slightly damaged flour, but now is rarely if ever employed, and the presence of notable quantities of aluminium compounds in flour or bread is usually due to alum baking powder.

Detection.-Mix to grams of the sample with Io cc. of water and stir in I cc. of logwood tincture ( 5 grams logwood digested in roo cc. alcohol) and I cc. of a saturated solution of ammonium carbonate. If the sample is pure, the color will be a faint brown or pink, but if alum is present, a distinct lavender-blue color is produced, which should remain after heating for two hours in the water-oven.

Alum may also be detected by the ammonium chloride method, described on page $3+4$.

Bleaching of Flour.-In 1908 about $80 \%$ of the flour produced in the United States was bleached by nitrogen peroxide, but as a result of the enforcement of the federal law the practice has been largely discontinued. The gas is generated by electrical, chemical, or electro-chemical means, and is diluted with air before treatment of the flour. In the Alsop process, which is most commonly employed, it is formed by a flaming discharge of electricity, which causes the nitrogen and oxygen of the air to combine.

Nitrogen peroxide destroys almost immediately the yellow color which is associated with the fat of the flour, thus increasing the whiteness of the product. It also forms with the moisture of the flour nitrous and nitric acids, the former (free or combined) being easy of detection. A considerable part of the nitrous nitrogen remains in yeast bread after baking and nearly all of it in soda biscuit. Bleaching also diminishes the iodine number of the fat, affects the quality of the gluten, and injures the flavor of the bread.

Aging versus Bleaching. - Storage under proper conditions slowly whitens flour, improves its baking properties, increases its organic acidity, diminishes its water-content and brings about other changes not well understood. Bleaching immediately whitens flour but does not improve its baking properties, increase its organic acidity nor appreciably affect its water-content. It does, however, introduce nitrous 
and nitric acids. Often 2 parts of nitrous nitrogen per million are recoverable and sometimes 6 or 7 parts, but this gradually disappears so that after some months hardly a trace remains.

The extent to which typical flours are whitened by aging and by bleaching so as to contain 2 parts of nitrous nitrogen per million is apparent from the gasoline color values in the following table by Winton:

\begin{tabular}{|c|c|c|c|c|c|c|c|c|}
\hline & \multicolumn{2}{|c|}{$\begin{array}{l}\text { Minnesota, } \\
\text { Harn Spring. }\end{array}$} & \multicolumn{2}{|c|}{$\begin{array}{l}\text { Nobraska, } \\
\text { Hard Winte- }\end{array}$} & \multicolumn{2}{|c|}{$\begin{array}{l}\text { Michigan, } \\
\text { Soft Whater. }\end{array}$} & \multicolumn{2}{|c|}{$\begin{array}{l}\text { Missouri, } \\
\text { Soft Winter. }\end{array}$} \\
\hline & Patent. & Clear. & $\begin{array}{l}\text { 8o\% } \\
\text { Patent: }\end{array}$ & $\begin{array}{l}20 \% \\
\text { Clear. }\end{array}$ & $\begin{array}{l}80 \% \\
\text { Patent: }\end{array}$ & $\begin{array}{l}20 \% \\
\text { Clear. }\end{array}$ & Paterit. & $\begin{array}{l}60 \% \\
\text { Clear. }\end{array}$ \\
\hline \multicolumn{9}{|l|}{$\begin{array}{l}\text { Gasoline color value of } \\
\text { Unisleached: }\end{array}$} \\
\hline New............. & 2.00 & 2.00 & 2.63 & 2.50 & $1 . \div 3$ & $1.6 \mathrm{t}$ & 1.47 & 2.60 \\
\hline Aged to week & 1. 78 & 1.83 & 2.12 & 3.17 & 1.20 & 1.49 & 1.22 & 1. 33 \\
\hline Aged $20 \quad \ldots$ & 1.20 & 1.34 & 1. 36 & 1.68 & 0.80 & 1.20 & 0.68 & 0.88 \\
\hline Aged $30 "$ & 0.72 & 0.88 & 0.70 & 0.82 & 0.56 & 0.72 & 0.48 & 0.52 \\
\hline New........... & 0.60 & 0.66 & 0.80 & 0.80 & 0.40 & 0.50 & 0.32 & 0.40 \\
\hline Aged to weeks, & 0.44 & 0.54 & 0.46 & 0.48 & 0.06 & $0.3 \$$ & 0.22 & 0.26 \\
\hline Aged $20 \quad \%$ & 0.30 & 0,50 & 0.34 & 0.40 & 0.20 & 0.36 & 0.18 & 0.24 \\
\hline Aged 30 & 0.30 & 0.50 & 0.24 & 0.36 & 0.18 & 0.40 & 0.14 & 0.16 \\
\hline
\end{tabular}

\section{INSPECTION AND ANALYSIS OF FLOUR.}

In some of the larger cities, authorized inspectors are appointed by boards of trade to pass upon the quality of flour. To such inspectors dealers submit samples, which are gauged as to color, soundness, weight, etc., comparing them usually with a series of graded samples, and stamping or branding them officially with the date as well as the grade. Market quotations also are based on the standard terms adopted. The names of the various grades differ with the locality. In St. Louis, the following names are adopted in order of their quality, viz., Patent, Extra Fancy, Fancy, Choice, and Family.

The grade or quality of flour is determined largely by its color, fineness, odor, absorption, and dough-making properties. Baking tests are also relied on to a considerable extent by millers and buyers.

Of the chemical methods those for ash, protein, gluten, acidity, fat, and fiber are of chief importance.

Fineness.-The granulation is determined by rubbing the flour between the thumb and fingers. A gritty flour is one that feels rough and granular, due to aggregates of cells with contents intact. Smooth flour, on the other hand, feels soft and slippery. It is so finely ground that the cells are isolated and often ruptured, thus liberating the contents. 
Pelsar Color-test.-Place IO-I5 grams of the flour on a rectangular glass plate, about $12 \mathrm{~cm}$. long and $8 \mathrm{~cm}$. wide, and pack on one side in a straight line by means of a flour trier. Treat the same amount of the standard flour used for comparison in the same manner, so that the straight edges of the two flours are adjacent. Carefully move one of the portions so as to be in contact with the other, and "slick" both with one stroke of the trier, in such a manner that the thickness of the layer diminishes from about $0.5 \mathrm{~cm}$. on the middle of the plate to a thin film at the edge, and the line of demarcation between the two flours is distinct. Cut off the edges of the layer with the trier, so as to form a rectangle, and compare the color of the two flours. The difference in color becomes more apparent after carefully immersing the plate with the flour in water, and still more apparent after drying.

Gasoline Color Value. - Winton Method.-Place 20 grams of the flour in a wide-mouthed glass-stoppered bottle of about I20 cc. capacity and add roo cc. of colorless gasoline. Stopper tightly and shake vigorously for five minutes. After standing sixteen hours, shake again for a few seconds until the flour has been loosened from the bottom of the the bottle and thoroughly mixed with the gasoline, then filter immediately through a dry II-cm. paper, previously fitted to the funnel with water and thoroughly dried, into a flasi, keeping the funnel covered with a watch-glass to prevent evaporation. In order to secure a clear filtrate, a certain quantity of the flour should be allowed to pass over on to the paper and the first portion of the filtrate passed through a second time.

Determine the color value of the clear gasoline solution in a Schreiner colorimeter, using for comparison a $0.005 \%$ water solution of potassium chromate. This solution corresponds to a gasoline number of 1.0 and may be prepared by making to cc. of a $0.5 \%$ solution up to one liter. The colorimeter tube containing the gasoline solution should first be adjusted so as to read $50 \mathrm{~mm}$., then the tube containing the standard chromate solution raised or lowered until the shades in both tubes match. The reading of the chromate solution, divided by the reading of the gasc ine solution gives the gasoline color value.

Absorption and Dough Test.-Stir 30 grams of the flour in a heavy coffee cup with $15 \mathrm{cc}$. of water by means of a spatula until a smooth ball of dough has been formed. If after standing two minutes the amount of water proves insufficient to thoroughly dough up the flour, repeat the operation, using ${ }^{1} 5.5 \mathrm{cc}$. of water, and, if necessary, continue to repeat until the quantity is found that will yield a stiff, but thoroughly 
elastic dough. From the results of this test, calculate the absorption of Tooo grams of flour in terms of cc. of water.

The physical characters of the dough, such as color and elasticity, furnish valuable indications of the quality or grade of the flour.

Expansion of Dough.-Rub to a smooth paste 3.5 grams of granulated sugar, I.2 grams of salt, and 3 grams of compressed yeast, and thoroughly mix with $60 \mathrm{cc}$. of water at $35^{\circ} \mathrm{C}$. Warm roo grams of the flour in a shallow pan to $35^{\circ} \mathrm{C}$., add to it the yeast mixture, mix with a spatula, and knead with the fingers until a smooth ball of dough has been formed. Drop the dough into a graduated, 500-cc. cylinder, pat down so as to force out the air, and note the volume of the mass. Place in a raising closet kept at $35^{\circ} \mathrm{C}$. Read the volume at the end of the first hour and every half hour thereafter until the maximum is reached.

Baking Tests.*-Koelner or Straight Dough Method.-This process yields a close-grained loaf of even texture, and serves well to determine the flavor and relative size of the loaf.

Place 220 grams of the flour, previously warmed in a shallow pan, in a raising closet kept at $35^{\circ} \mathrm{C}$., in a Koelner dough kneader, which has previously been warmed to $35^{\circ} \mathrm{C}$. by means of water placed in the special compartment for this purpose. To the flour add $\mathrm{I} 2 \mathrm{grams}$ of sugar, 5 grams of salt, and 10 grams of compressed yeast, rubbed smooth and thoroughly mixed in a cup with roo cc. of water at $35^{\circ} \mathrm{C}$. Rinse the cup with sufficient water to make the total quantity required, as calculated from the absorption test. This amount is usually about $87 \mathrm{cc}$.

Adjust the blades of the kneader for mixing, and turn the crank at the rate of 90 revolutions per minute for to minutes. Adjust the blades for kneading, add 120 grams of flour, previously warmed to $35^{\circ} \mathrm{C}$., and turn the crank for ten minutes at the rate of 60 revolutions per minute. Remove the dough immediately to a warmed plate, cut into two equal parts, mould the two separately, and place end to end in a warmed, greased, and tared baking tin measuring $27 \times 6.3 \mathrm{~cm}$. at the top, $25.4 \times 5$ $\mathrm{cm}$. at the bottom, and $8.8 \mathrm{~cm}$. deep-all inside measurements.

Weigh the tin with dough, place a tin gauge across the top, and set the whole in the raising closet. After the dough has risen to the gauge, place the tin in a suitable oven heated to $200^{\circ} \mathrm{C}$., and bake at 200 to $205^{\circ}$ until 30 grams of water have been removed, which usually requires from 30 to 35 minutes. Break the loaf in two, and note the odor when hot and again when cold, also the flavor when cold.

* Descriptions by Miss H. L. Wessling, Chicago Laboratory, Bur, of Chemistry. 
When thoroughly cool, determine the volume of the loaf as follows: cover the bottom of a box $7.6 \times 12.7 \times 28 \mathrm{~cm}$,, inside measurements, with flaxseed, place the loaf in the box, pour flaxseed without jarring into the box until filled, and strike off the surplus seed by means of a straight edge. Remove the seed from the box, weigh, and divide the weight by the weight of $\mathrm{I} \mathrm{cc}$. of the seed, as calculated from an actual weighing of the seed required to fill the box. Subtract this figure from the cubic contents of the box in cc., thus obtaining the volume of the loaf.

Long Fermentation Method.-This method, used in some of the large mills in the northwest, differs from the Koelner method in that (I) a sponge is set, (2) the dough is kneaded twice, and (3) the dough is finally expanded to the limit. The bread is coarse in texture, but serves well to test the strength and flavor.

To 255 grams of warmed flour contained in a jar or earthenware crock, add 3.5 grams of salt and 8.6 grams of compressed yeast, mixed thoroughly with $170 \mathrm{cc}$. of water at $35^{\circ} \mathrm{C}$. Stir together until a soft sponge is formed, and keep in the raising closet, at $35^{\circ} \mathrm{C}$., until the volume has been doubled, then mix with 85 grams of warmed flour, 12 grams of sugar, 6 grams of lard, and the remainder of the water, the total quantity for 340 grams of flour being calculated from the absorption test. Knead steadily for six minutes, transfer the dough to the jar or crock, and set it in the raising closet until it has again doubled its volume.

Remove the dough to a warmed plate, knead lightly in the hands for a minute or two, then place in a warmed and greased standard baking tin, $16.8 \times 8.8 \mathrm{~cm}$. across the top, $14.9 \times 6.9 \mathrm{~cm}$, across the bottom, and $13.9 \mathrm{~cm}$. deep, all inside measurements, with extensions of the metal at the top of the two sides. Prick the dough about a dozen times with a fine-pointed wire, and raise again in the closet, until the bubbles of gas just begin to break and form larger ones. This is a matter of judgment and can be learned only by experience. The dough must not be raised to its limit beforehand, but must be put in the oven at such a stage that with the additional rising in the oven it will have attained the maximum volume. Bake from 30 to 35 minutes, raising the temperature gradually from $180^{\circ} \mathrm{C}$. at the beginning to $210^{\circ} \mathrm{C}$. at the end.

Determination of Moisture, Protein, Crude Fiber and Fat. Employ the methods described on pages 277 to 279 . The crude fiber should be collected and weighed on a Gooch crucible.

Determination of Ash.-Char 5 grams of the flour in a flat-bottomed platinum dish heated on a piece of thin asbestos board over a Bunsen burner. 
Complete the burning at dull redness, preferably in a muffle furnace. If the ash is black or dark gray add a few drops of nitric acid, evaporate to dryness on a water-bath and again heat at dull redness, repeating the treatment if necessary.

Determination of Moist "and Dry Gluten.*-Place 25 grams of the flour in a coffee cup, add ${ }_{15} \mathrm{cc}$. of water at a temperature not to exceed $15^{\circ}$, and work the mass into a ball with a spatula, taking care that none of it adheres to the dish. Allow the dough to stand one hour, then knead in a stream of cold water over a piece of bolting cloth held in place by two embroidery hoops, until the starch and soluble matters are removed. Place the gluten thus obtained in cold water, and allow to remain for one hour, after which press as dry as possible between the hands, roll into a ball, place in a tared flat-bottomed dish, and weigh as moist gluten. Spread the gluten out in the dish, dry for 24 hours in a boiling water-oven, and weigh again, thus obtaining the dry gluten.

Determination of Alcohol-soluble Protein (Crude Gliadin.)Chamberlain Method.*-Digest 5 grams of the sample with $250 \mathrm{cc}$. of $70 \%$ (by vol.) alcohol for 24 hours, shaking every half hour during the first 8 hours. Filter through a dry paper, determine nitrogen in $100 \mathrm{cc}$. of the filtrate and multiply the result by 5.7 .

The amount of alcohol-soluble protein may also be expressed in terms of percentage of the total protein. This percentage is known by some authors as the "gliadin ratio."

Determination of Salt-soluble Protein.-Chamberlain Method.*Digest to grams of the flour with $250 \mathrm{cc}$. of $5 \%$ potassium sulphate solution, as described under Alcohol-soluble Protein. Determine nitrogen in $50 \mathrm{cc}$. of the filtrate, and multiply the result by 5.7 .

Determination of Acidity of Flour.-Titrate $100 \mathrm{cc}$. of the solution prepared as described for the determination of nitrites (p. 32I), with tenth-normal sodium hydroxide solution, using phenolphthalein as indicator. If the distilled water used contains an appreciable amount of carbon dioxide, it should previously be boiled in a Jena flask until neutral but not long enough to dissolve alkali from the glass. Two hundred cc. of the boiled water should remain colorless on addition of phenolphthalein, but should take on a distinct pink color when mixed with a single drop of tenth-normal alkali.

Determination of Cold-water Extract.-Wanklyn Method.-Mix

*U. S. Dept, of Agric, Bur. of Chem., Bul. 81, p. r18. 
Ioo grams with distilled water in a graduated liter flask, shake frequently during six or eight hours and allow to stand over night. Decant on a filter, rejecting the first portions that run through, and evaporate $50 \mathrm{cc}$. of the clear filtrate to dryness in a tared metal dish on a waterbath. The weight of the dried residue multiplied by 20 gives the cold-water extract which, according to Wanklyn, should not exceed $5 \%$.

Determination of Iodine Number of the Fat.-Dry over sulphuric acid for three days sufficient flour to yield 0.2 to 0.25 gram of fat and extract for sixteen hours in a Johnson extractor with $25 \mathrm{cc}$. of absolute

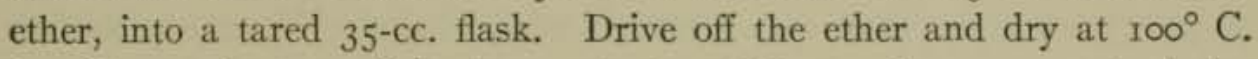
for fifteen-minute periods to constant weight, passing a current of dry hydrogen through the flask. Proceed according to the Hanus method, adding the chloroform and iodine solution directly to the flask, and breaking the flask within a wide-mouthed glass stoppered bottle for the final dilution and titration.

Detection of Bleaching in Flour.-Place on the "slicked" surface of the flour a drop or two of a mixture of equal parts of solutions $(a)$ and $(b)$, described in the following section. If the flour is unbleached and has not been stored under conditions permitting absorption of nitrous acid the liquid, which does not immediately soak into the flour, will remain colorless or nearly so, while if it is bleached it soon takes on a marked pink or crimson color, varying in degree with the extent of bleaching. A positive test should be supplemented by determinations of nitrous nitrogen and gasoline color value.

Determination of Nitrous Nitrogen.-Griess-Ilosvay Method.*-This method, commonly employed for the determination of nitrites in water, is well adapted for the estimation of the extent to which flour has been bleached by nitrogen peroxide or nitrosyl chloride.

I. Reagents.-(a) Sulphanilic Acid Solution.-Dissolve 0.5 gram of sulphanilic acid in $150 \mathrm{cc}$. of $20 \%$ acetic acid.

(b) Alpha-naphtylamine Hydrochloride Solution.-Dissolve 0.2 gram of the salt in $150 \mathrm{cc}$. of $20 \%$ acetic acid with the aid of heat.

(c) Standard Sodium Nitrite Solution.-Dissolve 0.1097 gram of dry C.P. silver nitrite in about $20 \mathrm{cc}$. of hot water, add 0.05 gram of C.P. sodium chloride, shake until the silver chloride flocks and make up to $1000 \mathrm{cc}$. Draw off Io cc. of the clear solution and dilute to

* Bull. chim. [2], 2, p. $3 \times 7$. 
one liter. One cc, of this solution contains $0.000 \mathrm{I} \mathrm{mg.} \mathrm{of} \mathrm{nitrogen} \mathrm{as}$ nitrite.

Suitable silver nitrite is on the market; it may also be prepared as follows: mix a warm concentrated solution of 8 parts of sodium nitrite with a warm concentrated solution of 16 parts of silver nitrate. When cool collect the precipitate on a Buchner funnel and wash with cold water. Dry quickly on a water-bath with as little exposure to light as possible. Long continued drying at $100^{\circ} \mathrm{C}$. causes it to slowly decompose.

2. Determination.-Weigh out 20 grams of the flour into an Erlenmeyer flask, add $200 \mathrm{cc}$. of water free from nitrites, previously heated to $40^{\circ} \mathrm{C}$., close the flask with a rubber stopper, shake vigorously for five minutes, digest one hour at $40^{\circ}$, shaking every ten minutes, and filter on a dry folded filter free from nitrites. As the first portion of the filtrate is usually turbid, it should be returned to the filter and the operation repeated until a clear liquid is secured. Dilute $50 \mathrm{cc}$. of the filtrate and also $50 \mathrm{cc}$. of the standard nitrite solution each with $50 \mathrm{cc}$. of water, add $2 \mathrm{cc}$. each of solutions $(a)$ and $(b)$; shake and allow to stand one hour to bring out the color. Compare the two solutions in a colorimeter (page 77). Divide the height of the column of the standard solution by that of the solution of the sample, thus obtaining the parts of nitrogen as nitrous acid (free or combined) per million of flour.

Bamihl Test for Gluten (Modified by Winton*).-This test serves to detect wheat flour mixed with rye and other flours.

Place a very small quantity of the flour (about 1.5 milligrams) on a microscope slide, add a drop of water containing 0.2 gram of watersoluble eosin in $1000 \mathrm{cc}$., and mix by means of a cover glass, holding the latter at first in such a manner that it is raised slightly above the slide, and taking care that none of the flour escapes from beneath it. Finally allow the cover glass to rest on the slide, and rub it back and forth until the gluten has collected into rolls. The operation should be carried out on a white paper so that the formation of gluten rolls can be noted.

Wheat flour or other flours containing it yields by this treatment a copious amount of gluten, which absorbs the eosin with avidity, taking on a carmine color. Rye and corn flour yield only a trace of gluten, and buckwheat flour no appreciable amount. The preparations are best examined with the naked eye, thus gaining an idea of the amount of

* U. S. Dept. of Agric., Bur. of Chem., Bul. 122, p. 217 . 
gluten present. Under the microscope traces of gluten, such as are formed in rye flour, are so magnified as to be misleading.

In case the flour is coarse, or contains a considerable amount of branelements, as is true of buckwheat flour and low grade wheat flour, the cest should be made after bolting, as the bran particles and coarse lumps interfere with the formation of gluten rolls.

This test should be supplemented by microscopic examination of the untreated flour and also of the tissues, accumulated after boiling with $1 \frac{11}{4} \%$ sulphuric acid as described on page 306 . In the case of rye flour adulterated with wheat flour the difference in the cross cells (pp. 306-308) should be especially noted; these, however, are present in considerable amount only in the cheaper grades of wheat flour.

\section{BREAD.}

Bread is a term broadly applied to any baked mixture of finely divided grain and water, whether or not other ingredients are used. Pilot, or ship bread, crackers, and unleavened bread, consist almost entirely of flour and water with a slight addition of salt.

Similarly, corn bread or corn cake is frequently made exclusively from corn meal and water. In a narrower sense, however, bread is generally understood to mean the raised or leavened product, rendered light and porous by the aid of gas, which is generated either before or during baking. Commonly the gas employed is carbon dioxide, generated either by the fermentative action of yeast on the sugar of the dough, yielding both alcohol and gas, or by the agency of baking chemicals mixed with the dough, whereby an alkaline bicarbonate is decomposed by the action of an acid to produce the gas. Again, the gas may consist wholly or in part of ammonia, yielded by the vaporization during baking of ammo. nium carbonate mixed with the dough; and finally, the expansion dur. ing baking of the air itself confined in the dough may be the leavening agent, as in the case of fuff paste and pie crust.

Wheat flour is of chief value for bread on account of its high content of gluten, in which other cereals are lacking. In the preparation of ordinary white bread, the flour is mixed with water or milk, salt, and yeast, the materials are mingled thoroughly by kneading and allowed to remain for some time in a warm place, during which, by the vinous fermentation induced by the yeast, the mass "rises" or forms a light sponge, due to the action of the gas on the glutinous dough.

Shrrine the subsequent process of baking, which should take place 
a temperature between $230^{\circ}$ and $260^{\circ} \mathrm{C}$., further expansion ensues, much of the water is driven off, and the porous mass sets to form the loaf, the outside of which is converted into a brown crust, due to the caramelizing of the dextrin and sugar into which the starch of the outer layers is converted. Among other changes that take place in the interior or "crumb" during baking are (I) the partial breaking up of the starch grains, which, however, largely retain their identity, though in some degree distorted in shape; (2) somewhat obscure changes in the character of the proteins; and (3) partial oxidation of the oil or fat.

The standard for judging the quality of commercial bread may wel be based on that of the best home-baked family loaf. The well-made loaf should possess an agreeable odor, and a sweet, nutty flavor, entirely free from mustiness. It should be well "raised," with a good crumbling fracture; it should not be tough or soggy on the one hand (due to underraising), nor extremely dry and spongy on the other (indicative of overraising). Over-raising, moreover, produces sourness, due to advanced lactic fermentation.

Composition of Bread.-The following analyses made in the U. S. Bureau of Chemistry of common varieties of bread were summarized from Bulletin $\mathrm{I}_{3}$, part 9, averages of a number of analyses being given in each case:

\begin{tabular}{|c|c|c|c|c|c|}
\hline & $\begin{array}{l}\text { No. of } \\
\text { Analyses. }\end{array}$ & Moisture. & $\begin{array}{l}\text { Protein, } \\
\mathrm{N} \times 6.25 .\end{array}$ & $\begin{array}{l}\text { Protein, } \\
\mathrm{N} \times 5.70 .\end{array}$ & $\begin{array}{c}\text { Ether } \\
\text { Extract. }\end{array}$ \\
\hline 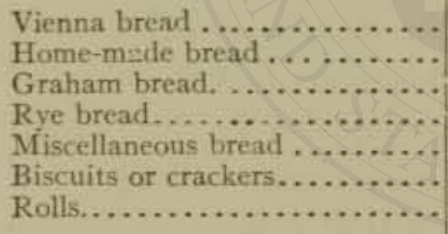 & $\begin{array}{r}10 \\
2 \\
79 \\
7 \\
9 \\
48 \\
11\end{array}$ & $\begin{array}{r}38.71 \\
33.02 \\
34.80 \\
33.42 \\
34.4 \mathrm{t} \\
7.13 \\
27.98\end{array}$ & $\begin{array}{r}8.87 \\
7.94 \\
8.93 \\
8.63 \\
7.60 \\
10.34 \\
8.20\end{array}$ & $\begin{array}{l}8.09 \\
7.24 \\
8.15 \\
7.88 \\
6.93 \\
9.43 \\
7.48\end{array}$ & $\begin{array}{l}1.06 \\
1.95 \\
2.03 \\
0.66 \\
1.48 \\
8.67 \\
3.41\end{array}$ \\
\hline$=$ & $\begin{array}{l}\text { Crude } \\
\text { Fiber. }\end{array}$ & Salt. & Ash. & $\begin{array}{l}\text { Carbohy- } \\
\text { drates, } \\
\text { Excluding } \\
\text { Fiber. }\end{array}$ & $\begin{array}{c}\text { Calculated } \\
\text { Calories of } \\
\text { Combus- } \\
\text { tion. }\end{array}$ \\
\hline 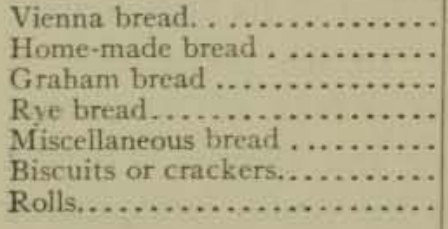 & $\begin{array}{l}0.62 \\
0.24 \\
1.13 \\
0.62 \\
0.30 \\
0.47 \\
0.60\end{array}$ & $\begin{array}{l}0.57 \\
0.56 \\
0.69 \\
1.00 \\
0.49 \\
0.99 \\
0.69\end{array}$ & $\begin{array}{l}\text { I. } 19 \\
\text { I. } 05 \\
\text { I. } 59 \\
\text { I. } 84 \\
\text { I. } 00 \\
\text { I. } 57 \\
\text { I. } 31\end{array}$ & $\begin{array}{l}53.72 \\
56.75 \\
53.40 \\
56.21 \\
56.18 \\
73.17 \\
59.82\end{array}$ & $\begin{array}{l}4435 \\
4467 \\
4473 \\
4338 \\
4429 \\
4755 \\
4538\end{array}$ \\
\hline
\end{tabular}

In the examination of bread for its general quality, without regard to its food value, much information may be gained by carefully observing the 
physical characteristics of the loaf, its color, taste, odor, porosity, etc. In addition to such data, determination of moisture, ash, and acidity will usually suffice to enable the analyst to pass judgment on its wholesomeness. The following summary gives such analytical data on upwards of fifty samples of bread, purchased from cheaper bakeries and stores, and examined in the author's laboratory.

BREAD.

\begin{tabular}{|c|c|c|c|c|c|}
\hline Kind of Bread. & $\begin{array}{l}\text { No. of } \\
\text { Analyses. }\end{array}$ & $\begin{array}{l}\text { Weight of } \\
\text { Loaf in } \\
\text { Grams. }\end{array}$ & $\begin{array}{l}\text { Water, } \\
\text { Per Cent. }\end{array}$ & $\begin{array}{l}\text { Per Cent } \\
\text { Ash in } \\
\text { Terms of } \\
\text { Solids. }\end{array}$ & Acidity,* \\
\hline White .................. & 44 & & & & \\
\hline 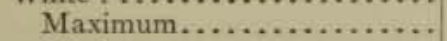 & .. & 653 & 45.20 & I. 83 & 6.2 \\
\hline Minimum.............. & .. & 126 & 33.00 & 0.60 & I. 3 \\
\hline 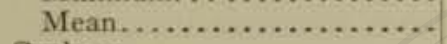 & .. & 430 & $40.7^{2}$ & 0.85 & 2.6 \\
\hline Graham . ................. & 7 & & & & \\
\hline 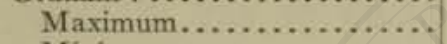 & -. & 500 & $45 \cdot 20$ & I. 55 & $4 \cdot 2$ \\
\hline Minimum .............. & .. & 367 & 40.10 & 0.96 & 2.I \\
\hline Mean.................... & $\cdots$ & 420 & $4^{x} \cdot 5^{\circ}$ & I. 26 & $3 \cdot 5$ \\
\hline Whole wheat .............. & 1 & 507 & 45.10 & $x .20$ & \\
\hline Diabetic .................. & I & 445 & 47.00 & 2.20 & \\
\hline Muffins .................. & I & 194 & 48.20 & I. I5 & I. 7 \\
\hline 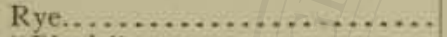 & I & I $29 \mathrm{I}$ & $47 \cdot 15$ & 2.13 & 10.0 \\
\hline "Black" $" \ldots \ldots \ldots \ldots \ldots \ldots$ & I & $55^{\circ}$ & 47.00 & 2.20 & \\
\hline German with seeds .......... & I & 417 & $42 \cdot 30$ & 0.95 & \\
\hline Brown..$\ldots \ldots \ldots \ldots \ldots \ldots \ldots$ & I & 500 & 48.10 & 3.50 & \\
\hline "Knackerbrod" ............ & I & Iro & 8.00 & I. 94 & \\
\hline
\end{tabular}

* Cubic centimeters of tenth-normal soda required to neutralize ro grams of the fresh bread.

Water in Bread.- The amount of water is of considerable importance, and, in the best bread, varies from 33 to 40 per cent. A larger content of water than $40 \%$ should be considered objectionable in a white bread, both on the ground of acting as a make weight, and because a large excess of moisture tends to cause the growth of mold.

Acidity of Bread.-The degree of sourness of a sample of bread is one of the most important indications as to its quality, and is most readily obtained by rubbing up in water, by means of a pestle, to grams of the "crumb," and titrating with tenth-normal alkali, using phenolphthalein as an indicator. To neutralize the acidity of ro grams of the normally sweet loaf, an average of $2 \mathrm{cc}$. of the standard alkali solution is required, corresponding to 0.72 gram of lactic acid per loaf of an average weight of 400 grams. The loaf exhibiting the maximum sourness or acidity in the above table required ro cc. of standard alkali per to grams of bread, corresponding to Ir.6I grams lactic acid in the loaf of $\mathrm{I}, 29 \mathrm{I}$ grams. 
Fat in Bread.-It is well known that the results of fat or ether extract as obtained by the ordinary method and expressed in most bread analyses are too low, being considerably less than the combined fat of the materials entering into its composition. This is probably due to the fact that during baking the fat particles are incrusted with insoluble matter, which protects thein from the subsequent action of the ether. It is further claimed by some that the partial oxidation of the fat during baking has something to do with the low results. No perfectly satisfactory improvement over the regular ether method for fat extraction in bread has been discovered, and therefore this method, as described elsewhere, is recommended.

Adulteration of Bread.-The fraudulent addition of inert foreign ingredients to bread is almost never practiced, and is mainly of historic interest. Gypsum, chalk, bone ash, and various other minerals have been mentioned as possible adulterants, but the amount of any of these materials necessary to add for purposes of profit could scarcely be present without very apparent injury to the quality of the bread. Their presence in any considerable degree would be apparent in the abnormally high ash content of the bread.

The employment of alum to "improve" inferior or unsound flour has already been referred to, and, for the same purpose, sulphate of copper in small quantities is also said to have been used, enabling the making of bread of fairly good appearance from flour that was distinctly damaged.

Alum in Bread * is tested for by a modification of the logwood process described on page $3^{1} 5$ as follows: $5 \mathrm{cc}$. of the logwocd tincture and $5 \mathrm{cc}$. of the saturated ammonium carbonate solution are diluted to $100 \mathrm{cc}$, and the freshly prepared mixture poured over about Io grams of the bread crumbs in a porcelain evaporating-dish. After standing a few minutes, as much as possible of the liquid is drained off, the bread is slightly washed by one treatment with water, and dried in the wateroven. In presence of alum, a dark-blue color is given to the bread, which becomes deeper on drying. The color is proportional to the amount of alum present. If the sample is free from alum, the color varies from red to light brown. The reagent solution must be freshly prepared. This test is not perfectly reliable in the case of very old or sour breads, which have been known to give the color test with logwood in the absence of alum.

* Jago on Bread, p. 634. 
Copper Salts in Bread are detected in the ash by the same method as that used for canned goods (p. 9r8).

Cake and Similar Preparations.-These differ from bread chiefly by the addition of considerable sugar, butter, spices, and other flavoring materials. In gingerbread, molasses is used as an important ingredient besides ginger. The adulterants of molasses, such as glucose, salts of tin, etc., would thus sometimes occur in gingerbread. In fact stannous chloride has been found in ginger cakes.*

The following analyses of a few typical varieties of cakes are selected from Bulletin ${ }^{2} 3$ of the Bureau of Chemistry:

\begin{tabular}{|c|c|c|c|c|c|}
\hline & Moisture. & $\begin{array}{l}\text { Proteins, } \\
\text { N } \times 6.25 \text {. }\end{array}$ & $\begin{array}{l}\text { Proteins, } \\
\text { N } \times 5.7 \circ \text {, }\end{array}$ & $\begin{array}{c}\text { Ether } \\
\text { Extract. }\end{array}$ & $\begin{array}{l}\text { Crude } \\
\text { Fiber. }\end{array}$ \\
\hline 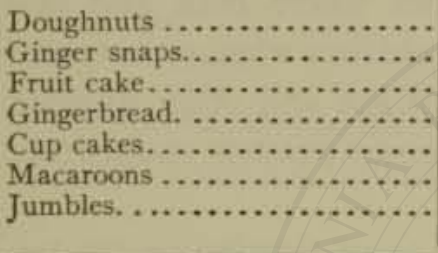 & $\begin{array}{r}21.61 \\
4.86 \\
24.47 \\
21.49 \\
14.81 \\
8.06 \\
13.34\end{array}$ & $\begin{array}{l}6.73 \\
6.06 \\
4.56 \\
6.25 \\
5.24 \\
6.67 \\
7.62\end{array}$ & $\begin{array}{l}6.14 \\
5.53 \\
4.16 \\
5.70 \\
4.78 \\
6.08 \\
6.95\end{array}$ & $\begin{array}{r}19 \cdot 33 \\
15 \cdot 44 \\
12 \cdot 35 \\
8.42 \\
15 \cdot 56 \\
12.97 \\
14.79\end{array}$ & $\begin{array}{l}0.60 \\
0.79 \\
0.90 \\
0.27 \\
1.41 \\
1.04\end{array}$ \\
\hline & Ash. & Salt. & Sugar. & $\begin{array}{l}\text { Carbohy- } \\
\text { drates } \\
\text { other than } \\
\text { Fiber and } \\
\text { Sugar. }\end{array}$ & $\begin{array}{l}\text { Calculated } \\
\text { Calories. }\end{array}$ \\
\hline 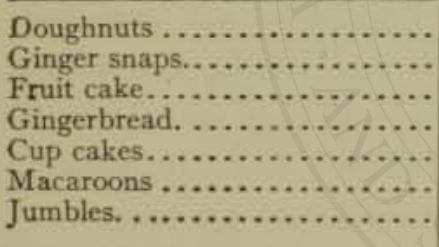 & $\begin{array}{l}0.40 \\
1.82 \\
1.55 \\
1.21 \\
0.82 \\
0.97\end{array}$ & $\begin{array}{l}0.03 \\
0.47 \\
0.28 \\
0.34 \\
0.07 \\
0.39\end{array}$ & $\begin{array}{r}\mathrm{r} .28 \\
28.66 \\
\\
9.48 \\
32.48 \\
58.77 \\
16.60\end{array}$ & $\begin{array}{l}50.64 \\
24.90 \\
52.46 \\
30.89 \\
10.89 \\
46.31\end{array}$ & $\begin{array}{l}5529 \\
4971 \\
\\
4757 \\
5073 \\
4835 \\
5133\end{array}$ \\
\hline
\end{tabular}

YEAST.

The yeast plant is a fungus of the genus Saccharomyces, widely distributed through the vegetable kingdom and in the air. It is capable of rapid growth by the multiplication of its cells when present in a favorable medium, such as malt wort, and with propitious conditions of temperature, moisture, etc. Under such conditions, it forms a yellowish, viscous, frothy substance, the chief value of which, in the liquor industry, is the production of alcohol, while for bread-making, as a result of the same kind of fermentation, the end desired is the leavening of the doughy mass by the carbon dioxide liberated.

* See U. S. Dept. of Agric., Bur. of Chem., Bul. 13, p. ${ }_{1369}$ 
A vigorous, pure yeast which will "raise" quickly is a great preventive against sour bread, for not only is it comparatively free from the germs and products of lactic acid fermentation, but by doing its work quickly it enables the baker to check the fermentation or raising process before the lactic acid or sour decomposition is far advanced.

Yeast most commonly used in bread-making is of the so-called "compressed" variety. The use of compressed yeast is almost universal for domestic purposes, and is more or less common in bakeries. A small amount of brewers' yeast in liquid form from beer wort is used, especially in the immediate neighborhood of breweries, and dry yeasts are used to some extent in localities so remote that fresh compressed yeast cannot readily be obtained.

Compressed Yeast is a product of distilleries where malt and raw grain are fermented for spirits. Most of it comes from whisky wort, and some from the worts used in the manufacture of gin and other distilled liquors. Little if any of the commercial compressed yeast is made from beer wort yeast.

In the manufacture of compressed yeast, the yeast floating on the top of the wort is separated by skimming, while that settling to the bottom is removed by running the wort into shallow settling trays. Top yeast is considered more desirable than bottom yeast for bread-making. The separated yeast is washed in cold water, and impurities are removed, either by sieving through silk or wire sieves, or by fractional precipitation while washing. The yeast, with or without the addition of starch, is finally pressed in bags in hydraulic presses, after which it is cut into cakes, packed in tin-foil, and kept in cold storage till distributed for use.

Such yeast should be used when fresh, as it readily decomposes and soon becomes stale. When fresh, it should have a creamy, white color, uniform throughout, and should possess a fine, even texture; it should be moist without being slimy. It should quickly melt in the mouth without an acid taste. Its odor is characteristic, and should be somewhat suggestive of the apple. It should never be "cheesy," such an odor indicating incipient decomposition, as does a dark or streaked color.

Dry Yeast is prepared by mixing fresh yeast with starch or meal, molding into a stiff dough, and drying, either in the sun or at a moderate temperature under reduced pressure. Such yeast, when dry, is cut into cakes and put in packages. It will keep almost indefinitely. During the drying process, many of the yeast cells are rendered torpid and temporarily inert, and for this reason the dried yeast does not act so promptly 
in leavening as does compressed or brewers' yeast, but when once it begins to act it is quite as efficacious.

Composition of Yeast.-The following is the result of the analysis of under-fermentation yeast, after drying, by Nägele and Loew:

Cellulose and mucilage $\ldots \ldots \ldots \ldots \ldots \ldots \ldots, 37$

Albuminoids (mycroprotein, etc.)......... $3^{6}$

" soluble in alcohol............. 9

Peptones (precipitable by subacetate of lead) ... $\quad 2$

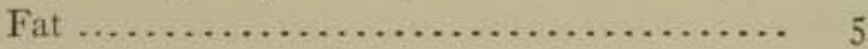

Extractive matters (leucin, glycerin, etc.) $\ldots \ldots .4$

Ash $\ldots \ldots \ldots \ldots \ldots \ldots \ldots \ldots \ldots \ldots \ldots \ldots \ldots \ldots \ldots, 7$

I00

Lintner gives the following average analyses of the ash of three samples of yeast, analyzed by him:

Silica............................. ${ }^{1} \cdot 34$

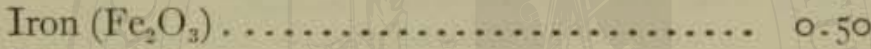

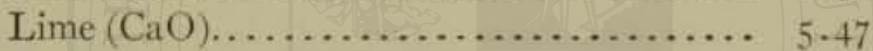

Sulphuric anhydride $\left(\mathrm{SO}_{3}\right) \ldots \ldots \ldots \ldots \ldots \ldots, 0.5^{6}$

Magnesia (MgO) ..................... 6.12

Phosphoric anhydride $\left(\mathrm{P}_{2} \mathrm{O}_{5}\right) \ldots \ldots \ldots \ldots \ldots .50 .60$

Potash $\left(\mathrm{K}_{2} \mathrm{O}\right)$ and a little soda............ 33.49

98.08

Matthews and Scott give the following as the ash composition of yeast:

Potassium phosphate................. 78.5

Magnesium phosphate................ I3.3

Calcium phosphate................ 6.8

Silica, alumina, etc................. I.

100.0

Microscopical Examination of Yeast.-Mix a bit of the yeast in water on the glass slip till a milky fluid is formed, and stir in a drop of a very weak anilin dye solution, such as methyl violet, eosin, or fuch- 
sin.* Put on the cover-glass, and examine under the microscope. Living, active cells resist the stain, if the latter is dilute enough, and appear colorless or nearly so, while the decayed and lifeless cells are stained, and can easily be distinguished by their color. Yeast cells are circular or oval in shape, and vary from 0.007 to $0.009 \mathrm{~mm}$. in diameter. They are sometimes isolated, and sometimes grouped in colonies; each cell has an outer, mucilaginous coating or envelope. The interior, granular mass or substance of the cell is the protoplasm, and within the protoplasm are frequently seen one or more circular empty spaces known as vacuoles.

Yeast cells multiply by the process of budding. The decadence of yeast cells is marked by the increased size of vacuole, and by the thicken. ing of the cell wall.

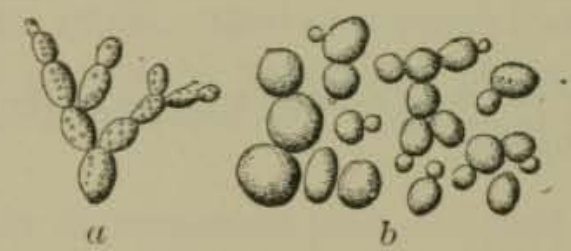

Fic. 68.-Sprouting Yeast-cells (Saccharomycas cerevisia). ( $a$, after Lürssen; $b$, after Hansen.)

Yeast-testing.-Avnilable Carbon Dioxide.-The value of yeast in bread-making depends on the amount of carbon dioxide which it is capable of generating under given circumstances, hence the available carbon dioxide is the chief factor in gauging a yeast. There are various methods of determination, (I) either by measuring the volume of gas set free by the action of a weighed quantity of yeast in a sugar solution of known strength, kept for a fixed time at a fixed temperature (say $30^{\circ}$ ), or (2) by conducting the gas from such a fermenting solution through a weighed absorption bulb, containing potassium hydroxide and noting the increase in weight, or (3) by the more convenient method of Meissl as follows:

A mixture is made of 400 grams pure, concentrated sugar, 25 grams ammonium phosphate, and 25 grams potassium phosphate. A small, wide-mouthed flask of about roo cc. capacity is fitted with a doubly perforated rubber stopper, having two tubes as shown, one of which is bent and passes nearly to the bottom of the flask, being fitted at the outer end with a rubber tube and glass plug, while the other is connected with a small calcium chloride tube. Measure $50 \mathrm{cc}$. of distilled water into

* I gram crystallized fuchsin in I6o cc. water having I cc. alcohol. 
this flask, and dissolve 4.5 grams of the above sugar phosphate mixture. Finally add I gram of the yeast to be tested, stir it well till there are no lumps, and cork the flask. Carefully weigh on a delicate balance the flask with its contents, and immerse in a water-bath at $30^{\circ} \mathrm{C}$., keeping it at that temperature for six hours. At the end of this time, remove the flask from the bath, and immediately immerse in cold water to cool the contents. Remove the rubber tube with the glass plug, and by suction draw out the remaining carbon dioxide. Replace the plug, and having carefully wiped off the flask, again weigh. The loss in weight is due to carbon dioxide set free by the fermentation of the yeast.

Starch in Compressed Yeast.-Potato, corn, or tapioca starch has long been added to yeast before pressing, on the ground that the starch acts as a drier, producing a much cleaner product, and one that can be more readily and intimately mingled with the materials of the bread, besides enhancing the keeping qualities of the yeast, especially in

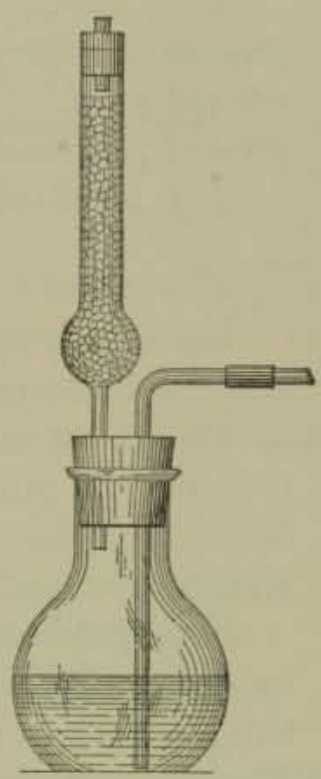

Fig. fin.-Apparatus for Determining Leavening Power of Yeast. warm weather. The quantities used vary from about $5 \%$ up to over $50 \%$. Undoubtedly the larger amounts are added as a make weight. Some manufacturers use no starch whatever.

The question has frequently been raised whether, with improved methods of manufacture, whereby yeast can be produced comparatively free from slime, and thus capable of pressure without the admixture of starch, the use of the latter should not be considered as an adulterant.

Briant claims that the admixture of starch up to $5 \%$ increases rather than decreases the actual content of yeast, in that the starch abstracts moisture from the yeast cells themselves, the proportion of water being much smaller, and that of the yeast larger in the starch-mixed substance. T. J. Bryan,* on the other hand, finds that the addition of starch to yeast reduces the carbon dioxide value, and that the percentage reduction is greater than the percentage of starch present. His experiments further indicate that the keeping qualities of starch yeast is not greater, but actually less than that of pure yeast.

* A. O. A. C. Proc, 1907 , U. S, Dept. of Agric., Bur. of Chem., Bul. 116, p. 25. 
U. S. Rulings.*-r. The term "compressed yeast," without qualification, means distillers' yeast without admixture of starch.

2. If starch and distillers' yeast be mixed and compressed such product is misbranded if labeled or sold simply under the name "compressed yeast." Such a mixture or compound should be labeled "compressed yeast and starch."

3. It is unlawful to sell decomposed yeast under any label.

\section{CHEMICAL LEAVENING MATERIALS.}

Under this heading are included the various ingredients that enter into the mixtures commonly known as "baking powders" which have no food value in themselves, but are, strictly speaking, instruments or tools that by purely chemical reactions bring about, under certain conditions, the comparatively quick liberation of gas and the consequent aeration of biscuit, bread, and cake.

Baking Powders and their Classification.-Formerly the housewife was accustomed to measure out in proper proportion a mixture of sour milk, or cream of tartar, with saleratus to produce quick aeration of bread. The modern baking powder is a natural outgrowth of the former practice, and has almost wholly displaced it, producing, as it does, a mixture ready for immediate use of an acid and an alkaline constituent in proper proportion for chemical combination to form the gas. A third ingredient is, however, generally considered as necessary to check deterioration, viz., a dry, inert material, which by absorbing moisture prevents the premature chemical action between the reagents. Starch is nearly always used for this purpose, though sugar of milk has a limited use. The alkaline principle of nearly all baking powders is bicarbonate of soda, or saleratus. Baking powders are divided naturally into three main classes, with reference to the acid principle:

(I) Tartrate Powders, wherein the acid principle is (a) bitartrate of potassium or $(b)$ tartaric acid, typified by the following reactions:

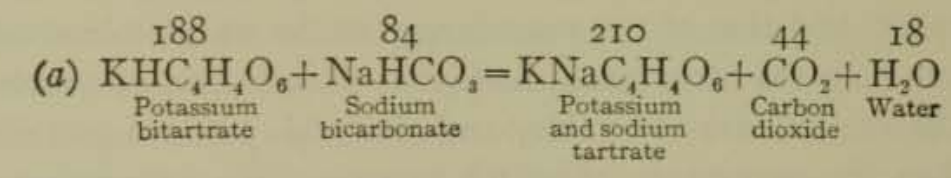

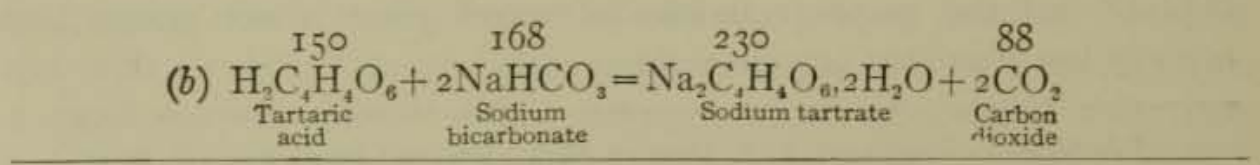

* Food Inspection Decision, No. 111, Jan. 7, 1910. 
(2) Phosphate Powders, in which calcium acid phosphate is the acid principle:

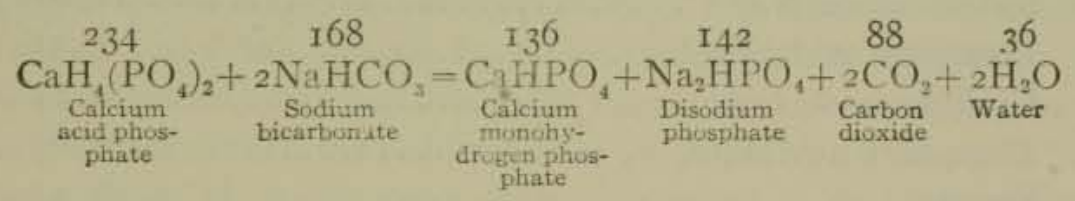

(3) "Alum Powders," wherein the acidity is due wholly or in part to sulphate of aluminum as it occurs in potash or ammonia alum, or in the double sulphates of aluminum and sodium.*

Assuming burnt potash alum as the substance used, the reaction would be as follows:

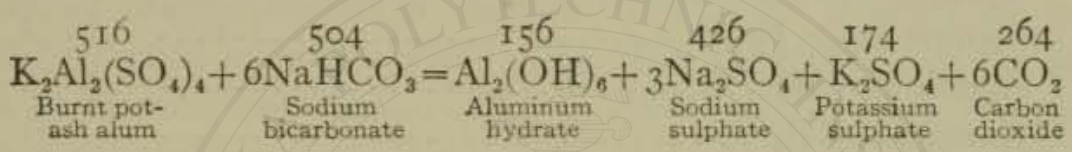

Naturally many baking powders of complex composition are met with, embodying various mixtures of the above classes.

Composition of Various Baking Powders.-Following are analyses of typical baking powders of the above classes: $\dagger$

I. Cream of Tartar Baking Powder:

Total carbon dioxide, $\mathrm{CO}_{2} \ldots \ldots \ldots \ldots \ldots \ldots \ldots . . \ldots \ldots$

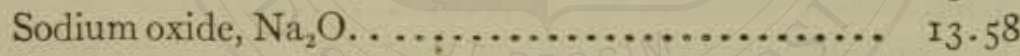

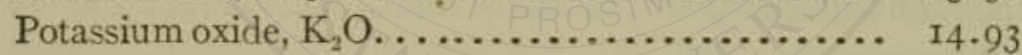

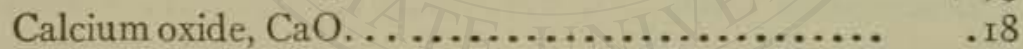

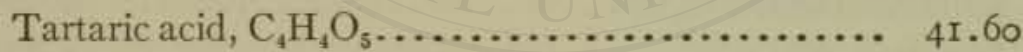

Sulphuric acid, $\mathrm{SO}_{3} \ldots \ldots \ldots \ldots \ldots \ldots \ldots \ldots \ldots$. Io

Starch............................. $7 \cdot 4^{2}$

\begin{tabular}{ll} 
Water of combination and association by difference... $\quad 8.98$ \\
\cline { 2 - 2 }
\end{tabular}

Available carbon dioxide $12.58 \%$.

100.00

* It is probable that very little ammonia or potash alum is actually used at present in this class of powders. A product largely used is known in the trade as S. A. S. (sodium aluminum sulphate) and is a calcined double sulphate of aluminum and sodium.

$\dagger$ Div, of Chem., Bul. r3, part 5, pp, 600, 604, and 606 . 
2. Phosphate Baking Powder:

Total carbon dioxide, $\mathrm{CO}_{2} \ldots \ldots \ldots \ldots \ldots \ldots \ldots . \quad{ }_{3} 3.47$

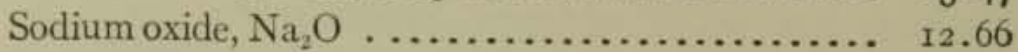

Potassium oxide, $\mathrm{K}_{2} \mathrm{O} \ldots \ldots \ldots \ldots \ldots \ldots \ldots \ldots \ldots . . . .3 \mathrm{I}$

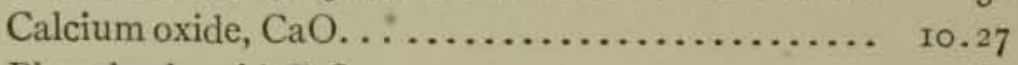

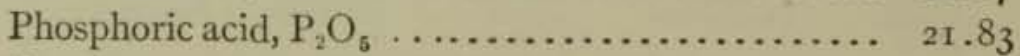

Starch. ............................. $26.4 \mathrm{I}$

Water of combination and association by difference. .. $\quad 15.05$

100.00

Available carbon dioxide $12.86 \%$.

3. Alum Baking Powder:

Total carbon dioxide, $\mathrm{CO}_{2} \ldots \ldots \ldots \ldots \ldots \ldots \ldots . \quad 9 \cdot 45$

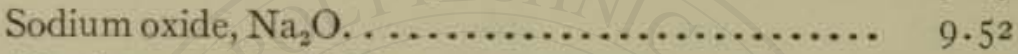

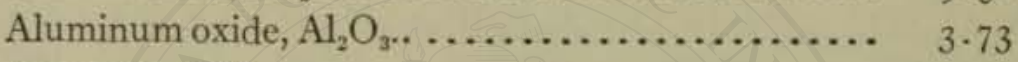

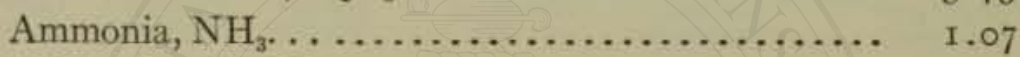

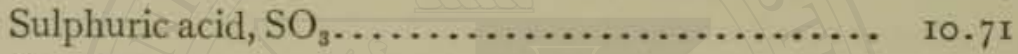

Starch. ............................ 43.25

$\begin{array}{lll}\text { Water of combination and association by difference } . & 22.27\end{array}$

Available carbon dioxide $8.10 \%$.

Mixed Powders:

Total carbon dioxide, $\mathrm{CO}_{2} \ldots \ldots \ldots \ldots \ldots \ldots \ldots . \quad$ Io. 68

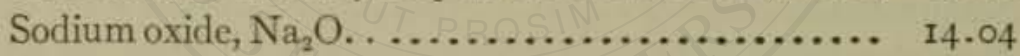

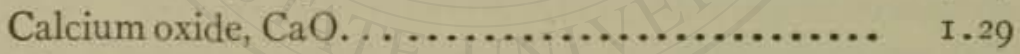

Aluminum oxide, $\mathrm{Al}_{2} \mathrm{O}_{3} \ldots \ldots \ldots \ldots \ldots \ldots \ldots \ldots . \quad 4.59$

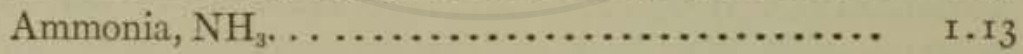

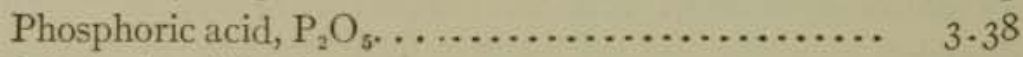

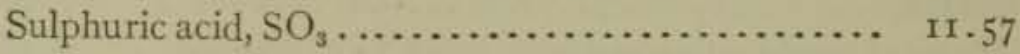

Starch. ................................ $4^{22.93}$

$\begin{array}{lll}\text { Water of combination and association by difference } . . & \text { 10.39 }\end{array}$

100.00

Available carbon dioxide $10.37 \%$.

The Adulteration of Baking Powder.-No substance that comes within the domain of food inspection is the subject of so much controversy 
as baking powder. Unless a specific law forbids the use of a particular ingredient or class of ingredients, or in some manner regulates the labelling of the package, no baking powder of any kind can be considered adulterated under the general food law, unless it can be proved to be injurious to health, or unless it contain inert and useless mineral matter.

As a matter of fact, the residue left in the bread by all classes of baking powder consists of one or more drugs recognized in the Pharmacopœia, all of which in large quantity exercise well-marked toxic effects on the human system. Artificial digestion experiments, and physiological tests on the lower animals, using excessive doses of any of the above drugs, do not show the effect of the every-day use of baking powder in bread on the human system, and only a systematic examination of the effect of such use on large numbers of people can prove conclusively whether or not any one class of baking powders is harmful, and hence whether or not it should be classed as adulterated. Aside from the question of the harmfulness of the acid ingredients, which is the subject of much controveisy among rival manufacturers, there can be no doubt that such inert mineral substances as calcium sulphate, terra alba, or clay, which are entirely useless, and lower the strength of the powder, are to be considered in the light of adulterants.

Traces of arsenic derived from the raw materials used in manufacture often occur in both alum and phosphate powders while lead is an accidental impurity of tartrate powders.

Cream of Tartar.-Its Nature and Adulteration.-Cream of Tartar, or potassium bitartrate $\left(\mathrm{KH}_{5} \mathrm{C}_{4} \mathrm{O}_{6}\right)$, is the purified product obtained by the recrystallization of the crude argols or lees deposited in the interior of wine casks. It is usually guaranteed $99 \%$ pure.

The lees, or argols, consist chiefly of crude potassium bitartrate, which is present in the juice of the grape, but is insoluble in the alcohol formed in the fermentation, and is hence deposited. If, for the clarification of the wine, such substances as gypsum or plaster of Paris are used, tartrate of calcium will be found mixed with the bitartrate of potassium in the lees and also, if not eliminated, in the cream of tartar.

Other common adulterants of cream of tartar are calcium acid phosphate, gypsum or plaster of Paris, starch, and alum.

Small amounts of lead from the tanks in which the cream of tartar is crystallized constitutes a common impurity.

Potassium bitartrate is insoluble in alcohol, sparingly soluble in cold, and readily soluble in hot water. 
CHEMICAL ANALYSIS OF BAKING CHEMICALS AND BAKING POWDERS.

Cream of Tartar.-The degree of purity of commercial cream of tartar is best determined by weighing out exactly 0.188 gram of the sample, dissolving in hot water, and titrating with tenth-normal sodium hydroxide, using phenolphthalein as an indicator. If the article is pure, exactly $10 \mathrm{cc}$, of the standard alkali will be required for the titration. All the above-named adulterants, with the exception of alum, are either insoluble, or sparingly soluble in hot water, and will indicate the impurity of the sample even before titration. If the adulterant be alum, the sample would go into solution in the water, but the alum would be precipitated by the sodium hydroxide, the precipitate being, however, soluble in an excess of the alkali.

Sodium Bicarbonate on account of its cheapness is rarely adulterated, save by the occasional presence of common salt, an impurity incidental to its manufacture. The degree of purity of sodium bicarbonate is best ascertained by titration with standard acid, each cubic centimeter of tenthnormal acid being equivalent to 0.0084 gram of sodium bicarbonate.

Determination of Total Carbon Dioxide.-Reagents.-Calcium Chloride.-This can be obtained in granulated form in pellets of abcut the size of peas, specially prepared for moisture absorption.

Soda Lime.*-To a kilogram of commercial sodium hydroxide, 500 to $600 \mathrm{cc}$. of water are added, and the mixture heated in an iron kettle to form a thin paste. While still hot, a kilogram of coarsely powdered quicklime is added, stirring with an iron rod. The lime is slaked, and the whole mass heats and steams up. No outside heat is necessary at this stage, but the mass is stirred and the lumps broken up. As soon as cool, place the product in wide-mouthed bottles, and seal with paraffin wax. The product should be slightly moist to give the best results.

Hydrochloric Acid.-Specific gravity I.I.

Sulphuric Acid.-Specific gravity 1.85 .

Potassium Hydroxide Solution.-Specific gravity 1.55 .

Two varieties of apparatus are in use for the d termina ion of carbon dioxide. In one form the amount of carbon dioxide is obtained by difference in weight of the apparatus, before and after elimination of the gas. In the other, the gas driven out of a given weight of the samp'e is absorbed, and its amount calculated from the increase in weight of the

* Benedict and Tower, Jour. Am. Chem. Soc., Vol. XXI, F. 396. 
absorbent. Types of these varieties are the Geissler and the Knorr apparatus

The Geissler Apparatus.-This consists of a flask $A$, having a ground neck $a$, and a flaring funnel-top $A^{\prime} . \quad B$ is an elongated bulb, closed at the top by the hollow stopper $K$, and terminating below in the hollow stem $B^{\prime}$, which is accurately ground at $b$ to fit the neck $a$. Fused into the bulb $B$ is the tube $C$, and within this is the small tube $D$, open at the top and communicating directly with the hollow stem $B^{\prime}$. $g g$ are openings between $B$ and $C$.

$E$ is a fine glass tube, passing from the bottom of the hollow stem $B^{\prime}$ and to the height of a small protuberance $e$ in the bottom of the funnel $A^{\prime}$, the construction being such that by turning the bulb and stem $B B^{\prime}$ in the neck $a$ of the flask $A$ the tube $E$ may be opened or closed at the top. $H$ is a side tube in the flask. $A$, closed by the ground stopper $h$.

The bulb $B$ and the tube $C$ are filled with strong sulphuric acid nearly to the top of the tube $D$, by passing through the neck at the top, which is then closed by the stopper $K$.

About 0.5 gram of the dried sodium bicarbonate, or I gram of the baking powder, is introduced into the flask $A$ through the neck $a$ from a weighing-tube or otherwise, so that its exact weight is known. The stem $B^{\prime}$ is then inserted, and the funnel-top $A^{\prime}$ is nearly filled with the hydrochloric acid, the tube $e$ being closed.

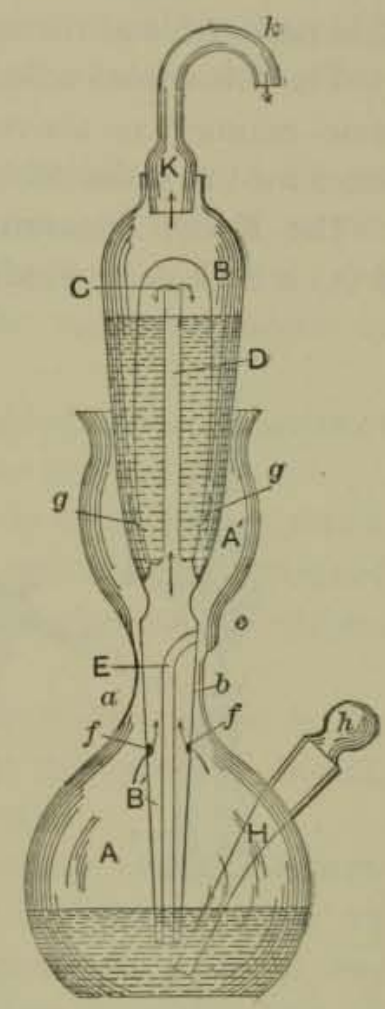

Fic. 7\%.-Geissler's $\mathrm{CO}_{2} \mathrm{Ap}$ paratus or Alkalimeter.

The entire apparatus is then weighed, after which the stem is turned to bring the protuberance $e$ nearly opposite the tube $E$, uncovering it enough to allow the acid to pass slowly down the tube into the flask and upon the powder in the bottom of the flask. The carbon dioxide evolved passes through the opening $f$ into the hollow stem $B^{\prime}$, thence up through he tube $D$, and down and up (as indicated by the arrows) through the sulphuric acid which absorbs the moisture. Finally the gas passes out through the tube $K$.

After the evolution of the gas has continued for two or three minutes, gentle heat is applied to the flask from a gas flame, and the solution is 
brought to boiling, which is continued for a few minutes, during the latter portion of which the stopper $h$ is removed, and the tubulure connected by rubber tubing with a system of two $U$ tubes, one containing soda lime, and the other calcium chloride. The tube $k$ is then connected with the aspirator, and a current of "dried air is passed through the apparatus at the rate of about two bubbles per second, long enough to displace all the carbon dioxide. The rubber tubes are then disconnected, the stopper $K$ is replaced, and the apparatus cooled to room temperature and weighed.

The available carbon dioxide in baking powder is determined in the same manner as above, by simply substituting freshly boiled, distilled water for the hydrochloric acid in the funnel-top $A^{\prime}$.

The Knorr Apparatus (Modified). -The apparatus (Fig. 7I) consists of (I) a flask, into which is introduced an accurately weighed amount of

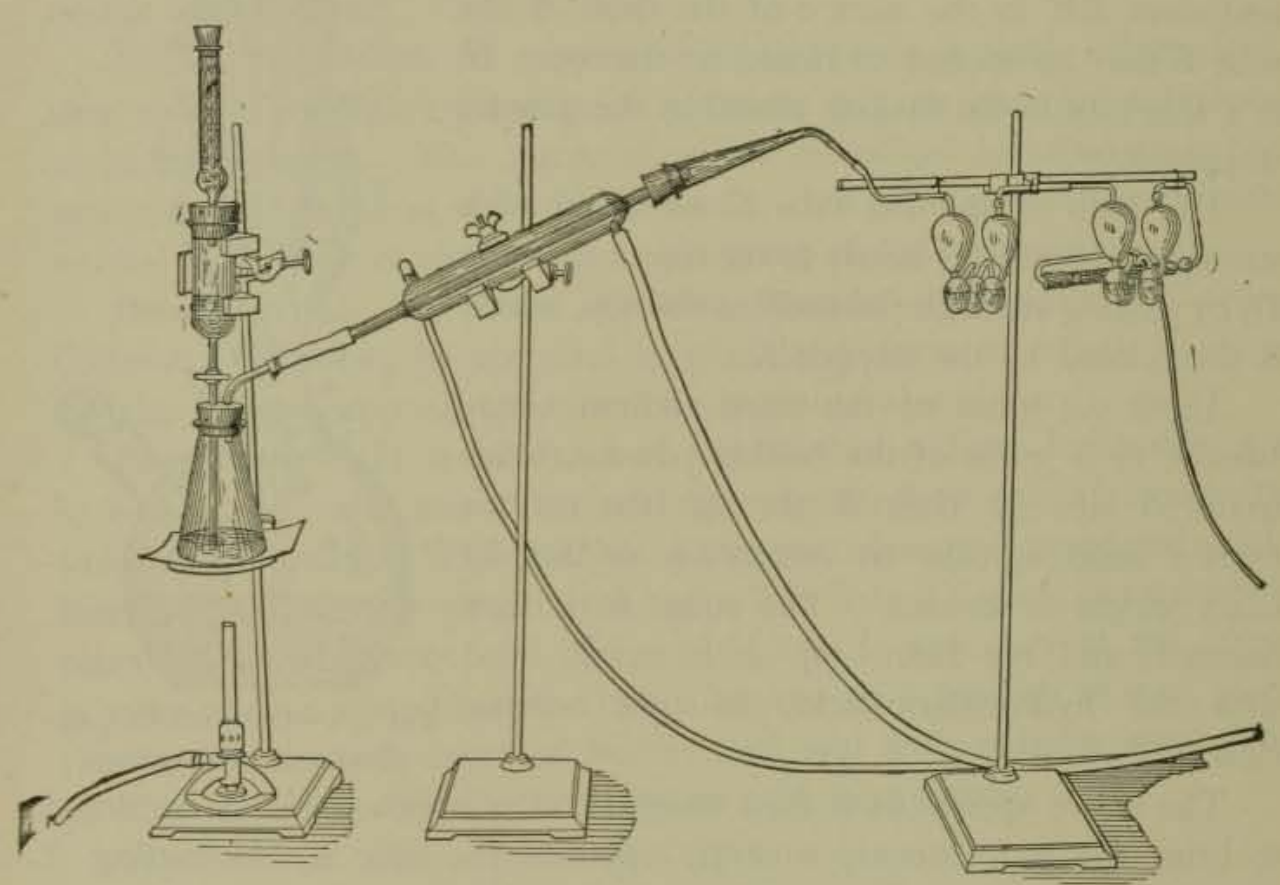

Fic. 71.-Modified Knorr Apparatus for Determining Carbon Dioxide.

the dry samp'e ( 0.5 to I gram of sedium bicarbonate or I to 2 grams of baking powder); (2) a funnel, the tube of which, provided with a stopcock enters the stopper of the flask; (3) a soda lime tube, entering a stopper at the top of the funnel; (4) a Liebig condenser, connecting with a tube passing through the stopper of the flask; (5) a Geissler bulb, filled with the sulphuric acid; (6) a potash absorption-bulb, and (7) a calcium 
chloride tube, which may if desired be replaced by a second sulphuric acid bulb. The potash absorption apparatus is accurately weighed before being connected up, and the funnel is nearly filled with the hydrochloric acid reagent, after which the soda lime tube is attached. The calcium chloride tube is connected by a rubber tube with the aspirator, and a current of cold water is allowed to run through the outer Liebig condenser-tube.

The stop-cock in the funnel-tube is first opened to allow the acid to slowly run into the flask, the flow being regulated to insure slow evolution of the gas.

The aspirator is then turned on so that about two bubbles of air per second pass through the apparatus, and gentle heat is applied to the flask by the gas flame, the solution within being brought to boiling, and the boiling continued for several minutes after the vapor has begun to gather in the condenser.

Prolonged boiling of the solution should be avoided, and in a series of tests the time of boiling should be precisely the same in all cases.

After removing the flame, the flask is allowed to cool, the aspiration being continued. The absorption-tube is then removed and weighed at room temperature, the increase in weight being due to the carbon dioxide.

The Available Carbonic Acid in Baking Powder is determined in the same manner as the total carbon dioxide, except that recently boiled, distilled water is substituted for the hydrochloric acid.

Detection of Tartaric Acid.*-It is often desirable to test a "compound " cream of tartar, or a "cream of tartar substitute," or an adulterated sample made up largely of foreign ingredients, to see if any tartaric acid, free or combined, be present. The following test is applicable in presence of phosphates:

If the substance to be tested is found to be free from starch, mix a little of the dry powder in a test-tube with a bit of dry resorcin, add a few drops of concentrated sulphuric acid, and heat slowly. A rose-red color indicates tartaric acid or a tartrate, the ccior being discharged on dilution with water.

In case of baking powder, or a cream of tartar substitute containing starch, shake repeatedly from 3 to 5 grams of the sample with about

*Wolff, Rev. Chim. Analyt. et appr. 4 (1899), p. 263r. I 
$25^{\circ} \mathrm{cc}$. of cold water in a large flask, allowing the insoluble portion to subside.

Decant the solution through a filter, and evaporate the filtrate to dryness, after which test the dried residue or a portion thereof with resorcin and sulphuric acid as above described.

Determination of Total Tartaric Acid.-Modified Heidenhain Method:*-Applicable only in the absence of phosphates and salts of aluminum and calcium.

Into a shallow porcelain dish, 6 inches in diameter, weigh out 2 grams of the material and sufficient potassium carbonate to combine with all tartaric acid not in the form of potassium bitartrate. Mix thoroughly with ${ }_{5} \mathrm{cc}$. of cold water, and add $5 \mathrm{cc}$. of $99 \%$ acetic acid. Stir for half a minute with a glass rod bent near the end. Add roo cc. of $95 \%$ alcohol, stir violently for five minutes, and allow to settle at least thirty minutes. Filter on a Gooch crucible with a thin layer of paper pulp, and wash with $95 \%$ alcohol until $2 \mathrm{cc}$. of the filtrate do not change the color of litmus tincture diluted with water. Place the precipitate in a small casserole, dissolve in $50 \mathrm{cc}$. of hot water, and add standard fifth-normal potassium hydroxide solution, leaving it still strongly acid. Boil for one minute. Finish the titration, using phenolphthalein as indicator, and correct the reading by adding $0.2 \mathrm{cc}$. One cc. of fifth-normal potassium hydroxide solution is equivalent to 0.026406 gram tartaric anhydride $\left(\mathrm{C}_{4} \mathrm{H}_{4} \mathrm{O}_{5}\right)$, $0.0300 \mathrm{I}$ gram tartaric acid $\left(\mathrm{H}_{2} \mathrm{C}_{4} \mathrm{H}_{4} \mathrm{O}_{6}\right)$, and 0.03763 gram potassium bitartrate $\left(\mathrm{KHC}_{4} \mathrm{H}_{4} \mathrm{O}_{6}\right)$.

The standard of the potassium hydroxide solution should be fixed by pure dry potassium bitartrate.

The accuracy of this method is indicated by the agreement of the percentages of potassium bitartrate in cream of tartar powders containing no free tartaric acid, obtained by calculation from the tartaric acid, with those obtained by calculation from the potassium oxide.

in presence of phosphates or of aluminum and calcium salts, the only satisfactory method of arriving at the amount of tartaric acid present is by difference, having determined or calculated the other ingredients.

Kenrick's Polariscopic Methods.-Method I. (Applicable to Cream of Tartar).- The method is based on the fact that in the presence of excess of ammonia, the rotation of the solution is proportional to the

* Provisional methods of the A. O. A. C., Bur. of Chem., Bul. 65, p. r04; Bul. 107 (rev.), p. 175 . 
concentration of the tartaric acid, and is independent of the other bases and acids present.

(a) The Substance is Completely Soluble in Dilute Ammonia.-A weighed quantity of the material containing not more than I gram tartaric acid is placed in a $25 \mathrm{cc}$. measuring flask, moistened with 3 or $4 \mathrm{cc}$. of water, and concentrated ammonia (sp. gr, o.880) added in quantity sufficient to neutralize all acids that may be present, and leave about I cc. in excess. The actual amount of the excess is not of importance, but a greater quantity than I cc. of free ammonia should be avoided. The solution is then made up to $25 \mathrm{cc}$. with water, filtered, if necessary, through a dry filter, and measured in a $20 \mathrm{~cm}$. tube in the polarimeter.

The amount of tartaric acid $\left(\mathrm{C}_{4} \mathrm{H}_{6} \mathrm{O}_{6}\right)$ in grams $(y)$ in the material taken is given by the formula:

$$
y=0.005^{\operatorname{Ig} x}
$$

where $x$ is the rotation in minutes.

(b) The Substance is not Completely Soluble in Dilute Ammonia.-In this case calciun tartrate is probably present, and may be determined as follows: Treat I gram of the substance (or an amount containing not more than I gram of tartaric acid) in a small beaker with ${ }^{15} \mathrm{cc}$. of water, and to drops of concentrated hydrochloric acid. Heat gently till both the potassium and calcium tartrates have passed into solution, and then, while still hot, add $2 \mathrm{cc}$. of concentrated ammonia (or enough to produce an ammoniacal smelling liquid), and about 0. I gram of sodium phosphate dissolved in a little water. Transfer to a $25-\mathrm{cc}$. measuring flask, cool, make up to the mark with water, filter through a dry filter, and polarize the filtrate in a $20-\mathrm{cm}$. tube. The tartaric acid is calculated from the formula given under $(a)$.

The precipitation of the calcium by means of sodium phosphate is not absolutely necessary, but when this is not done, in cases where the proportion of calcium in the sample is high, there is a great tendency for the calcium tartrate to crystallize out from the ammoniacal solution before the reading is made.

The tartaric acid present as bitartrate of potash may be determined by proceeding as in $(a)$, the calcium tartrate being practically insoluble in cold ammonia solution.

The tartaric acid present as calcium tartrate is given, with sufficient accuracy for most purposes, by the difference between the results of $(a)$ and $(b)$. If more accurate results are required, the residue insoluble in 
ammonia in (a) may be dissolved in a little hydrochloric acid and treated as above with sodium phosphate and ammonia.

Method 2, (Applicable to Baking Powder and Cream of Tartar mixed with Substitutes).-Direct readings of rotation in ammoniacal solution are inadmissible in analyses of the substances of this class, on account of the influence of iron and aluminum on the rotation of tartaric acid, and on account of the small but unknown rotation of the trace of inverted starch.

Accurate determinations, however, may be made in the presence of excess of ammonium molybdate in neutral solution. The latter substance has the property of greatly increasing the rotation of tartaric acid, so that by its use the small rotation of the inverted starch is made insignificant. It is to be noted, however, that this increased rotation is very sensitive to the presence of alkali and acid, and is, moreover, modified by phosphates. It is therefore necessary, in the first place, to remove the phosphoric acid, and, secondly, to bring the solution to a definite state of neutrality. Both these results are attained by the following procedure, the details of which must be carefully adhered to:

(a) Reagents.-The following solutions must be prepared, but need not be made up very accurately:

Molybdate solution: 44 grams ammonium heptamolybdate in $250 \mathrm{cc}$.

Citric acid solution: 50 grams citric acid in $500 \mathrm{cc}$.

Magnesium sulphate solution: 60 grams $\mathrm{MgSO}_{4} \cdot 7 \mathrm{H}_{2} \mathrm{O}$ in $500 \mathrm{cc}$.

Ammonia solution: $80 \mathrm{cc}$. concentrated ammonia (sp. gr. 0.880 ) in $500 \mathrm{cc}$.

Hydrochloric acid: $60 \mathrm{cc}$. concentrated hydrochloric acid in $500 \mathrm{cc}$. Methyl orange solution:

(b) Process.-An amount of material containing not more than 0.2 gram tatraric acid, not more than 0.3 gram alum, and not more than 0.3 gram calcium superphosphate, is accurately weighed, and placed in a dry flask. To this, $5 \mathrm{cc}$. of citric acid and ro cc. of molybdate solution are added, and allowed to react with the substance for 10 or 15 minutes (with an occasional shake). Next, $5 \mathrm{cc}$. of magnesium sulphate solution are added, and $\mathrm{I}_{5} \mathrm{cc}$. of ammonia solution stirred in. After a few minutes (not more than one hour), the solution is filtered through a dry filter, a slight turbidity of the filtrate being disregarded. To $20 \mathrm{cc}$. of the filtrate are then added a few drops of methyl orange and hydrochloric acid, from a burette, till the pink color appears ( 2 or 3 drops too much or too little are of no consequence). Finally, ro cc. more of the molybdate 
filter, wash with water, and transfer the starch by a wash-bottle from the filter-paper back into the original flask, using $200 \mathrm{cc}$. of water.

If the sample be free from lime, weigh 5 grams directly into the $500-\mathrm{cc}$. flask with $200 \mathrm{cc}$. of water. In either case add $20 \mathrm{cc}$. of hydrochloric acid (specific gravity 1.125) and heat the flask in boiling water for $2 \frac{1}{3}$ hours, the flask being provided with a reflux condenser. Determine the dextrose, and from this the starch in the regular manner.

Detection of Aluminum Salts.*-(a) In Baking Powder.-Applicable in presence of phosphates. Burn to an ash about 2 grams of the sample in a platinum dish. Extract with boiling water and filter. Add to the filtrate sufficient ammonium chloride solution to produce a distinct odor of ammonia. A flocculent precipitate indicates aluminum.

In igniting, as above directed, sodium aluminate results from the more or less complete fusion. The reaction which occurs may be represented as follows:

$$
\underset{\substack{\text { Sodiur. } \\ \text { aluminate }}}{\mathrm{Na}_{2} \mathrm{Al}_{2} \mathrm{O}_{4}}+\underset{\substack{\text { Ammonium } \\ \text { chloride }}}{2 \mathrm{NH}_{4} \mathrm{Cl}}+\underset{4}{4 \mathrm{H}_{2} \mathrm{O}}=\underset{\substack{\text { Aluminum } \\ \text { hydroxide }}}{\mathrm{Al}_{2}(\mathrm{OH})_{6}}+\underset{\text { Ammonia }}{2 \mathrm{NH}_{4} \mathrm{OH}}+\underset{\text { Salt }}{2 \mathrm{NaCl}}
$$

If any phosphate of lime be present, it will be insoluble in the solution of the ash. If phosphate of sodium or potassium be present, it will go into solution, but will only precipitate out when an aluminum salt is also present on the addition of the ammonium chloride reagent.

(b) In Cream of Tartar.-Mix about I gram of the sample with an equal quantity of sodium carbonate, burn to an ash, and proceed as in the case of baking powder (a).

Determination of Alumina.-The above qualitative method with ammonium chloride may be made quantitative in presence of phosphates as follows: After carrying out the qualitative method as above directed, filter off the final precipitate, dissolve it in nitric acid, and test it for phosphate with ammonium molybdate. If phosphates are found absent, proceed as before with a weighed amount of the sample and wash, ignite, and weigh the residue as $\mathrm{Al}_{2} \mathrm{O}_{3}$.

If phosphate is found present in the ammonium chloride precipitate, proceed as before, igniting and weighing the total residue. Then determine the $\mathrm{P}_{2} \mathrm{O}_{5}$ in the latter and subtract from the total. The difference will be the $\mathrm{Al}_{2} \mathrm{O}_{3}$.

* Leach, 3 Ist An. Rep. Mass. State Board of Health, 1899 , p. $6{ }_{3} 8$. 
Determination of Lime. -5 grams of the sample are treated in a $500 \mathrm{cc}$. graduated flask with $50 \mathrm{cc}$. of water and $25 \mathrm{cc}$. of concentrated hydrochloric acid. Add water to the mark, shake, and allow the starch to settle. Decant through a dry filter, and to $50 \mathrm{cc}$. of the filtrate add ammonia nearly to neutralization, an excess of ammonium acetate solution, and $4 \mathrm{cc}$. of $80 \%$ acetic acid, and heat at $50^{\circ} \mathrm{C}$. Filter if necessary, and precipitate the lime with an excess of ammonium oxalate. Filter, wash, and ignite over a blast-lamp. Weigh as $\mathrm{CaO}$.

Determination of Potash and Soda.*-Weigh out 5 grams into a platinum dish, and incinerate in a muffle at a low heat. The charred mass is well rubbed up in a mortar, then boiled fifteen minutes with about $200 \mathrm{cc}$. of water, to which has been added a little hydrochloric acid. The whole is transferred to a $500-\mathrm{cc}$. flask, and, after cooling, made up to the mark and filtered. Of the filtered liquid too cc., representing I gram of the sample, are measured out, heated to boiling, and a slight excess of barium chloride solution added; then without filtering barium hydroxide is added in slight excess, the precipitate filtered off, and washed. To the filtrate is added a little ammonium hydroxide, and ammonium carbonate solution until the barium is precipitated. This precipitate is filtered and washed, the filtrate evaporated to dryness, and carefully ignited below redness until all volatile matter is driven off. The residue is dissolved in a few cc. of water, and a few drops of ammonium carbonate solution added. The precipitate, if any, is removed by filtering and washing, and the filtrate evaporated in a small tared platinum dish, ignited below redness, and weighed. This gives the weight of the mixed chlorides. The residue is taken up with hot water, from 5 to $10 \mathrm{cc}$. of a $10 \%$ solution of platinic chloride added, and the whole evaporated to a sirupy consistency on the waterbath; it is then treated with $80 \%$ alcohol, the precipitate washed with $80 \%$ alcohol by decantation, transferred to a Gooch crucible, dried at $100^{\circ} \mathrm{C}$., and weighed. The weight of the precipitate, multiplied by 0.19308 , gives the weight of $\mathrm{K}_{2} \mathrm{O}$, and by 0.3056 the equivalent amount of $\mathrm{KCl}$. The weight of $\mathrm{KCl}$ found is subtracted from the weight of the mixed chloride, the remainder being $\mathrm{NaCl}$, which, multiplied by 0.5300 gives the weight of $\mathrm{Na}_{2} \mathrm{O}$ in the sample.

* U. S. Dept, of Agric., Div, of Chem., Bul. 13, part 5, p. 593. 
Determination of Phosphoric Acid.-Method of the A. O. A. C.*Mix 5 grams of the material with $10 \mathrm{cc}$, of magnesium nitrate solution, $\dagger$ dry, ignite, and dissolve in hydrochloric acid. Take an aliquot part of the solution prepared above, corresponding to 0.25 gram, 0.50 gram, or I gram, neutralize with ammonia, and clear with a few drops of nitric acid. In case hydrochloric or sulphuric acid has been used as solvent, add about 15 grams of dry ammonium nitrate, or a solution containing that amount. To the hot solution add $50 \mathrm{cc}$. of molybdic solution‡ for every decigram of $\mathrm{P}_{2} \mathrm{O}_{5}$ that is present. Digest at about $65^{\circ}$ for an hour, filter, and wash with cold water, or preferably ammonium nitrate solution. Test the filtrate for phosphoric acid by renewed digestion and addition of more molybdic solution. Dissolve the precipitate on the filter with ammonia and hot water and wash into a beaker to a bulk of not more than $100 \mathrm{cc}$. Nearly neutralize with hydrochloric acid, cool, and add magnesia mixture from a burette; add slowly (about I drop per second), stirring vigorously. After fifteen minutes add $30 \mathrm{cc}$. of ammonia solution of density 0.96 . Let stand for some time; two hours is usually enough. Filter, wash with $2.5 \% \mathrm{NH}_{3}$ until practically free from chlorides, ignite to whiteness or to a grayish white, and weigh.

Determination of Sulphuric Acid.-Provisional Method A. O. A. C.\|Boil 5 grams of the powder gently for one and one-half hours with a mixture of $300 \mathrm{cc}$. of water and $15 \mathrm{cc}$. of concentrated hydrochloric acid. Dilute to 500 cc., draw off an aliquot portion of 100 cc., dilute considerably, precipitate with barium chloride, filter through a Gooch crucible, ignite, and weigh. Direct solution of the material without burning the organic matter was proposed by Crampton. 9

Determination of Ammonia (present in the form of ammonia alum or ammonium carbonate). Mix 5 grams of the sample with $200 \mathrm{cc}$. of water, and add an excess of sodium hydroxide. Distil into standard acid, and determine the ammonia by titration.

Detection and Determination of Arsenic.-Proceed according to tha Marsh or Sanger-Black-Gutzeit method without preliminary treatment (page 75).

* U. S. Dept. of Agric., Div, of Chem., Bul. 46, p. I2; Bul. 107 (rev.), p. 4.

$\dagger$ Prepared as follows: Dissolve 80 grams calcined magnesia in nitric acid, avoiding an excess of acid, then add a little calcined magnesia in excess, boil, filter from the excess of magnesia, ferric oxide, etc., and dilute with water to $500 \mathrm{cc}$.

$\ddagger$ Reagent No. 53 .

$\$$ Prepared by dissolving 100 grams of ammonium nitrate, Reagent No. 54 , in I liter of water.

\U. S. Dept. of Agric., Bur. of Chem., Bul. 65, p. I07; Bul. 107 (rev.), p. 178.

I U. S. Dept. of Agric., Div. of Chem., Bul. 13, part 5, p. 596. 


\section{SEMOLINA, MACARONI, AND EDIBLE PASTES.}

Semolina is the ccarse meal ground from certain varieties of hard or "durum" wheats, grown originally in Italy, Sicily, and Russia, but at present in France and certain parts of the United States and Canada. This hard wheat is high in gluten, and especially adapted for the preparation of macaroni and the various pastes. A peculiar process is employed in preparing the wheat, whereby the husk is removed by wetting, heating, grinding, and sifting, the resulting meal or semolina, being in the form of small, round, glazed granules.

Italian Pastes. - Semolina furnishes the basis of the Italian edible pastes, being mixed with warm water, kneaded, and molded into various forms, either by pressure through holes in an iron plate, or otherwise, and finally dried. In parts of Italy juices of carrots, onions, and other vegetables are said to be mingled with the paste, but for local consumption only. Saffron is sometimes added to pastes for the purpose, so it is claimed, of imparting a spicy flavor, although the quantity used is often so small as to be apparent only to the eye, thus indicating that the real object of its addition is to impart a color in imitation of an egg paste.

Macaroni is the larger of the slender-tube or pipe-shaped products; vermicelli is the worm-shaped variety, produced when the holes in the plate are very small; spaghetti is the term applied to the cord-like paste intermediate in size between the others. A variety of Italian pastes or pâtês is made by rolling the kneaded semolina into thin sheets, and cutting out in shapes of animals, letters of the alphabet, etc.

The composition of some of these products is as follows:

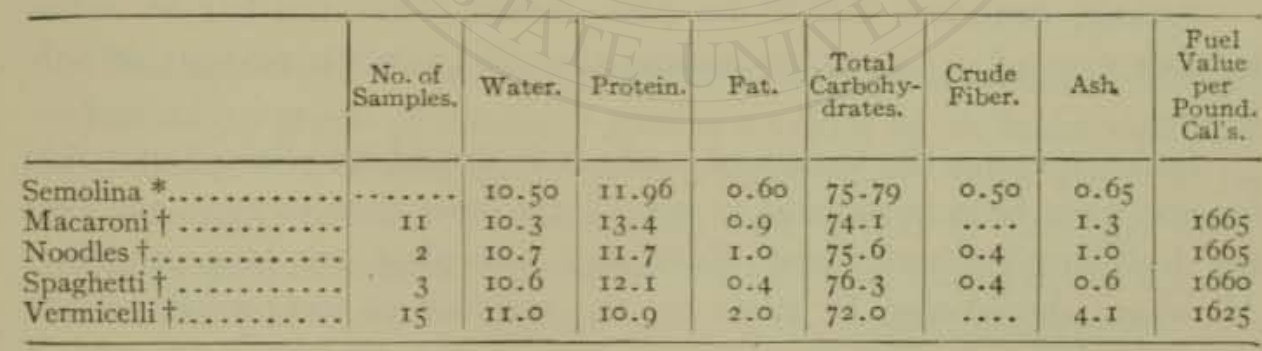

Balland.

$\uparrow$ Atwater and Bryant.

Noodles are a strap-shaped form of paste made in German households as well as in factories. True, or egg-noodles, contain a certain percentage of eggs, while water-noodles are practically the same in composition as Italian pastes. The difference in composition between water- 
noodles and noodles made with different numbers of eggs or egg yolks per German pound of flour, is shown by the analyses of Juckenack and Pasternack* given in the following table: $\dagger$

\begin{tabular}{|c|c|c|c|c|c|c|c|c|c|c|c|}
\hline \multirow{2}{*}{ 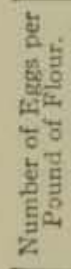 } & \multicolumn{5}{|c|}{ Composition of the Dry Matter. } & \multirow{2}{*}{ 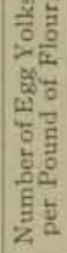 } & \multicolumn{5}{|c|}{ Composition of the Dry Matter. } \\
\hline & Ash. & $\begin{array}{l}\text { Total } \\
\text { Phos: } \\
\text { phoric } \\
\text { Acid. }\end{array}$ & $\begin{array}{l}\text { Lecithin } \\
\text { Phos- } \\
\text { pharic } \\
\text { Acid. }\end{array}$ & $\begin{array}{l}\text { Ether } \\
\text { Extract }\end{array}$ & $\begin{array}{l}\text { Protein } \\
\mathrm{N} \times 0_{\frac{1}{2}}\end{array}$ & & Ash. & $\begin{array}{l}\text { Total } \\
\text { Phos.- } \\
\text { phoric } \\
\text { Acid. }\end{array}$ & $\begin{array}{l}\text { Lecithin } \\
\text { Phos- } \\
\text { phoric } \\
\text { Acid. }\end{array}$ & $\begin{array}{l}\text { Ether } \\
\text { Extract }\end{array}$ & $\begin{array}{l}\text { Protein } \\
N \times 6 \frac{1}{2}\end{array}$ \\
\hline$\circ$ & $\begin{array}{c}\% \\
0.460\end{array}$ & $\begin{array}{c}\% \\
0.2300\end{array}$ & $\begin{array}{c}\% \\
0.0225\end{array}$ & $\begin{array}{c}\% \\
0.66\end{array}$ & $\begin{array}{c}\% \\
12.00\end{array}$ & 0 & $\begin{array}{c}\% \\
0.460\end{array}$ & $\begin{array}{c}\% \\
0.2300\end{array}$ & $\begin{array}{c}\% \\
0.0225\end{array}$ & $\begin{array}{c}\% \\
0.66\end{array}$ & $\%$ \\
\hline I & $0.5^{65}$ & $0.27 \times 6$ & 0.0513 & I. 56 & 12.99 & I & 0.488 & 0.2720 & 0.0518 & I. 57 & \\
\hline 2 & 0.664 & 0.3110 & 0.0786 & 2.42 & 13.92 & 2 & 0.516 & 0.3127 & 0.0801 & 2.47 & \\
\hline 3 & $0.75^{8}$ & $0.34^{82}$ & 0, ro 44 & 3.24 & $\begin{array}{c}14.81 \\
*\end{array}$ & 3 & 0.542 & 0. 3520 & $\begin{array}{c}0.1075 \\
*\end{array}$ & $3 \cdot 33$ & ${ }^{13} .07$ \\
\hline 12 & I. 426 & $0.6 \times 23$ & 0.2875 & 7.94 & 21.09 & 12 & 0.745 & 0.6533 & 0.3171 & 8.64 & $15 \boldsymbol{\jmath}^{\mathrm{I}}$ \\
\hline
\end{tabular}

From these results it appears that the percentages of ash, total phosphoric acid, and protein are appreciably increased by the addition of each egg or egg yolk, while the percentages of lecithin-phosphoric acid and ether extract are more than doubled by the addition of the first egg, and are increased in corresponding proportion by the addition of two or more eggs.

The German Association of Food Chemists require that commercial egg-noodles contain at least $0.045 \%$ of lecithin-phosphoric acid, and $2.00 \%$ of ether extract, corresponding to the minimum in noodles with two eggs per half kilogram of flour.

Spaeth $\ddagger$ considers that if the ether extract of noodles has an iodine number over 98 , it is safe to assume that they contain no eggs or only traces.

In interpreting the results of analysis it should be remembered that fat may have been introduced in some form other than in eggs, and that the lecithin-phosphoric acid diminishes somewhat on long standing. Allowance should also be made for the variation in composition of the eggs and flour.

Of 22 brands of American noodles examined by Winton and Bailey $\S$ only 5 appeared to be made with eggs; the lecithin-phosphoric acid in

*Zeits. Unters. Nahr. Genuss., 3, 1900, p. I3; 8, I904, p. 94.

$\dagger$ The German pound is approximately 468 grams; the avoirdupois pound is 454 grams.

$\ddagger$ Forsch. über Lebensm., 3, 1896 , p. 49.

\$ Jour. Am. Chem. Soc. 1905,37 , p. 137 ; Rep. Conn. Exp. Sta., r904, p. 138 . 
these ranged from 0.036 to 0.058 , and the ether extract from 1.83 to 2.33 per cent, while in the other samples the lecithin-phosphoric ranged from 0.015 to 0.032 and the ether extract from 0.28 to 2.50 per cent.

Adulteration of Pastes.-Rice, corn, and potato flours have been used in the preparation of the cheaper varieties of semolina, but rarely in this country. A more common form of adulteration is the substitution of water-noodles for egg-noodles, artificial colors being used to carry out the deception. Substitutions of this kind are detected by determinations of lecithin-phosphoric acid and ether extract, supplemented by tests for artificial colors.

Shredded Wheat is a whole-wheat preparation, put out in the form of light biscuits built up of fine porous threads, not unlike those of vermicelli. The wheat, softened by boiling, is shredded by passing through a peculiar machine, after which the biscuits are made by lightly putting together the threads and by final baking. The comparative composition of shredded wheat and of typical whole wheat is thus shown by Wiley:*

\begin{tabular}{|c|c|c|}
\hline Constituents. & $\begin{array}{l}\text { Shredded } \\
\text { Biscuit. } \\
\text { Per Cent. }\end{array}$ & $\begin{array}{l}\text { Typical } \\
\text { Wheat. } \\
\text { Per Cent. }\end{array}$ \\
\hline Moisture. & 10.57 & 10.60 \\
\hline Proteins.... & 12.06 & 12.25 \\
\hline Ether extract . & 1.03 & I. 75 \\
\hline Ash ........ & 2.65 & I. 75 \\
\hline Crude fiber. . ..................... & $2 \cdot 5^{8}$ & 2.40 \\
\hline Carbohydrates other than fiber. & 71.11 & 71.25 \\
\hline
\end{tabular}

ANALYSIS OF PASTES.

Determination of Lecithin-phosphoric Acid.-Juckenack's Method. $\dagger$ -Extract 30 grams of the finely ground material for to hours with absolute alcohol in a Soxhlet extractor at a temperature, inside the extractor, not below $55^{\circ}-60^{\circ} \mathrm{C}$. The extraction flask should be provided with a small quantity of pumice stone to prevent bumping during the boiling, and the extractor enclosed by asbestos paper, if the desired temperature is not readily maintained. After the extraction is completed, add $5 \mathrm{cc}$. of alcoholic solution of potash (prepared by dissolving 40 grams of phosphorusfree caustic potash in $1000 \mathrm{cc}$. alcohol), and distil off all the alcohol. Transfer the residue to a platinum dish by means of hot water, evaporate to dryness on a water bath, and char over asbestos. Treat the charred

* U. S. Dept. of Agric,, Bur. of Chem., Bul, 13, p. 1337.

$\dagger$ Zeits. Unters, Nahr. Genuss., 3, 1900, p. 13 . 
solution are added to the pink solution, which now becomes colorless or pale yellow, and water is added to make up the volume to $50 \mathrm{cc}$. This solution, after filtering if necessary, is polarized in a $20-\mathrm{cm}$. tube.

The amount of tartaric acid in grams $(y)$ in the weight of substance originally taken is given by the following formula, in which $x$ is the rotation in minutes:

$$
y=0.001086 x+0.001601 \sqrt{ } x .
$$

But if the rotation is not less than $40^{\prime}$, the simpler formula,

$$
y=0.0075+0.001168 x
$$

may be employed.

The following table gives the tartaric acid in grams for every 10 minutes

\begin{tabular}{|c|c|c|c|}
\hline Rotation in Minutes. & $\begin{array}{c}\text { Grams } \\
\text { Tartaric } \\
\text { Acid. }\end{array}$ & Rotation in Minutes. & $\begin{array}{l}\text { Grams } \\
\text { Tartaric } \\
\text { Acid. }\end{array}$ \\
\hline 10. & 0.016 & $90 \ldots . .$. & 0.1130 \\
\hline 20. & 0.029 & $100 \ldots \ldots$ & 0.1246 \\
\hline ........ & 0.0415 & $110 \ldots \ldots \ldots .$. & 0.1365 \\
\hline $40 \ldots \ldots \ldots$ & 0.0535 & $120 \ldots \ldots \ldots \ldots$ & 0.1479 \\
\hline$\ldots \ldots$ & 0.0657 & $1_{30} \ldots \ldots \ldots \ldots$ & 0.1595 \\
\hline$\ldots \ldots$ & 0.0776 & $\tau_{40} \ldots \ldots \ldots \ldots$ & 0.1710 \\
\hline$\ldots \ldots$ & 0.0895 & $150 \ldots \ldots \ldots$ & 0.1825 \\
\hline $80 \ldots$ & 0.1013 & & \\
\hline
\end{tabular}
rotation:

Determination of Starch.-McGill's Method* (Modified).-Digest I gram of the sample with $150 \mathrm{cc}$. of a cold $3 \%$ solution of hydrochloric acid during twenty-four hours, with occasional shaking. Filter through a tared Gooch crucible, wash first with water until neutral, then once with alcohol, and finally with ether. Dry at $110^{\circ} \mathrm{C}$. for four hours, cool, and weigh. Burn off the starch, and again weigh. The difference in the two weights indicates the weight of the starch. The purity of the starch is insured by examination with the microscope.

Acid Conversion Method. $\uparrow$-If the sample contains lime, mix 5 grams in a $500-\mathrm{cc}$. flask with $200 \mathrm{cc}$. of $3 \%$ hydrochloric acid, and let the mixture stand an hour with frequent shaking. Filter through a wetted II $\mathrm{cm}$.

* Canada Inland Rev. Bul. 68, p. 33 .

† U. S. Dept. of Agric., Bur. of Chem., Bul. 65, p. ro5; Bul. 107 rev., p. 176. 
mass with dilute nitric acid, filter, and wash with water. Return the residue with the paper to the platinum dish, and burn to a white ash. Treat again with nitric acid, filter and wash, uniting the filtrates. Determine phosphoric acid by the usual method.

Detection of Artificial Colors in Pastes. - The following colors have been used in noodles and other pastes: turmeric, saffron, annatto, naphthol yellow (Martius yellow), naphthol yellow S, picric acid, aurantia, Victoria yellow, tartrazine, metanil yellow, azo yellow, gold yellow, and quinoline yellow. Of these naphthol yellow, picric acid, metanil yellow, and Victoria yellow are injurious to health, and their use is illegal in European countries as well as in the United States. Fortunately, they are rarely found in the products now on the market.

The detection of artificial colors is complicated by the presence of the natural coloring matter of the flour and the lutein of eggs. These are conveniently extracted by ether, which does not remove the artificial colors, although most of them unmixed dissolve freely in this solvent.

Juckenack's Method,*-Thoroughly shake two portions of the finely ground material, each of about 10 grams, in test tubes with I5 cc. of ether and $\mathrm{I}_{5} \mathrm{cc}$, of $70 \%$ alcohol respectively, and allow to stand $\mathrm{I}_{2}$ hours.

(a) If the ether remains uncolored or only slightly tinted and the material below it remains yellow, while the alcohol is distinctly colored and the material is decolorized, a foreign dye is indicated.

(b) If both ether and alcohol are colored, either (I) lutein (egg color) alone, or (2) this with a foreign dye is present.

I. Treat a portion of the ether solution with dilute nitrous acid, according to Weyl. If the ether is not completely decolorized, a foreign dye is present.

2. If the deposit of material below the alcohol is decolorized, while that below the ether is colored, tests should be made for foreign dyes as follows: Shake the portion previously treated with ether with three or more fresh portions of the same solvent, until no more color is extracted, and then shake the residue with $70 \%$ alcohol and allow to stand $I_{2}$ hours. After filtering, concentrate the solution slightly, acidify with hydrochloric acid, boil with sensitized wool, and identify the color in the usual manner (page 8or).

Schlegel's Method. $\dagger-E x t r a c t ~ 100$ grams of the finely powdered material with ether in a continuous extraction apparatus, and shake the residue

* Zeits. Unters. Nahr. Genuss., 3, 1900, p. x.

† Untersuchungsanstalt, Nürnberg, Ber., 1906, p. 24. 
at frequent intervals for half a day with a mixture of $-\mathrm{r} 4 \mathrm{o} \mathrm{cc}$. of alcohol, $5 \mathrm{cc}$. of ammonia, and $\mathrm{IO}_{5} \mathrm{cc}$. water. Filter, evaporate to remove alcohol and ammonia, acidify slightly with hydrochloric acid, and again filter. Boil the filtrate with sensitized wool, and identify the color on the dyed fiber by the usual tests (page 801 ).*

Fresenius Method.*-Extract 20 to 40 grams of the powdered material with ether in a continuous extraction apparatus. Dry the residue to remove ether, shake for $\mathrm{I}_{5}$ minutes with $\mathrm{I} 20 \mathrm{cc}$. of $60 \%$ acetone, and allow to stand 12 to 24 hours. Filter, evaporate until the acetone is removed, and divide into two portions, a larger and a smaller. To the larger portion add sufficient acetic acid to dissolve flocks, and boil with sensitized wool. Remove natural coloring matter from the wool by boiling in dilute acetic acid. If after this treatment the wool is dyed, the presence of a foreign color is indicated, which may be identified by the usual tests.

To the smaller portion of the aqueous solution, obtained after removal of the acetone as above described, add an equal volume of alcohol, heat to dissolve flocks, divide into four portions, and apply special tests to three of these, reserving the fourth for comparison. The natural color of the flour is decolorized by hydrochloric acid, intensified by ammonia, but not affected by stannous chloride, even on heating. Saffron reacts in a similar manner, but is only slightly bleached by the acid, and is not affected by the other two reagents.

Piutti and Bentivoglio Method. $\dagger-$ This method is especially designed to detect the four colors forbidden by Italian law, and to distinguish these from naphthol yellow $\mathrm{S}$.

Add 50 grams of the paste to $500 \mathrm{cc}$. of boiling water, made alkaline with $2 \mathrm{cc}$. of concentrated ammonia water, add 60 to $70 \mathrm{cc}$. of alcohol, and continue the boiling 40 minutes. After filtering, acidify the liquid with 2 to $3 \mathrm{cc}$. of dilute hydrochloric acid and boil with 5 to 6 strands of sensitized wool, each strand weighing about 0.5 gram. Wash the wool, dissolve the color in dilute ammonia, and repeat the dyeing. After dissolving a second time in ammonia, evaporate the solution of the dye to dryness, avoiding as far as possible the formation of a skin, and take up the residue in water. If a skin has formed, filter and test the insoluble matter for metanil yellow with dilute hydrochloric acid, and for picric acid with ammonium sulphide.

*Zeits. Unters. Nahr. Genuss., 13, 1907, p. 132.

$\dagger$ Gaz, chim. Ital. 36 , II, 1806 , p. 385 . 
To I cc. of the filtrate add stannous chloride solution and a little sodium hydroxide, or preferably sodium ethylate. If no red color forms, nitro-colors are absent; if, also, in another portion dilute hydrochloric acid produces no violet color, thus showing the absence of metanil yellow, no further test is necessary. "In the presence of these colors, acidify the remainder of the solution with acetic acid, shake violently with carbon tetrachloride, and identify the color according to the following scheme:

A. Color dissolves in carbon tetrachloride to colorless solution. Extract with very dilute ammonia, concentrate and divide into two parts.

I. Acidify with hydrochloric acid, and add I to 2 drops of stannous chloride and ammonia in excess. A rose colored solution and precipitate form...................................

2. Acidify slightly with hydrochloric acid, add a little zinc dust and stir. Solution becomes rose-violet. ................ Victoria yellow.

$B$. Color is insoluble in carbon tetrachloride. Evaporate to dryness on water-bath, take up in water and divide into three parts.

I. Hydrochloric acid produces a violet coloration...... Metanil yellow.

2. Ammonium sulphide produces a red brown coloration.

Picric acid.

3. Stir on a water-bath with zinc dust and ammonia, filter, treat with zinc dust and hydrochloric acid and again filter. (a) Potassium hydroxide produces a yellow coloration, and $(b)$ ferric chloride an orange coloration. Naphthol yellow $S$.

Schmitz-Dumont Test for Tropeolins.*-Moisten a small portion of the material with a few drops of dilute hydrochloric acid. The formation of a reddish or bluish color shows the presence of an azo color or some other coal-tar color.

Test for Turmeric.- Extract the color from the ground material by alcohol and identify by the boric acid test (page 79r).

\section{PREPARED CEREAL BREAKFAST FOODS.}

The large number and variety of these preparations now on the market testify to the fact that the breakfast cereal forms a most important, as well as considerable, portion of our food supply. These foods are generally prepared from wheat, oats, and corn, and are, as a rule, remarkably pure and free from adulteration, though the food value of different varieties

* Zeits. offent. Chem., 8, I902, p. 424. 
is often grossly misstated by their manufacturers. Formerly the breakfast food consisted entirely of the coarsely ground, generally decorticated, raw cereal grain, and required a long period of cooking to prepare it for use. At present many of the oat products, and to some extent also those of corn, rice, and wheat, are subjected to a more or less preliminary cooking and drying, whereby they are capable of being prepared for use in a much shorter time, and their keeping qualities are enhanced. The so-called rolled oats are prepared by softening the grains through steaming, after which they are crushed between rollers and afterwards dried. The steaming process is a typical one for various other cereals, though in some cases the heating consists in baking or kiln drying.

The effect of the preliminary cooking on the finished product varies somewhat according to whether dry or moist heat has been applied, and is chiefly noticeable in the altered character of the carbohydrates. In all cases the starch is rendered more soluble, whether by the conversion of a portion into dextrin and dextrose, or by a simple breaking down of the starch grains, as in the case of bread in baking.

In spite of the seemingly endless variety of the package cereals, they divide themselves as a matter of fact into a very few well-defined classes, the members of which differ but little from each other except in name.

First there are the raw cereal grains of the oat, wheat, and corn, prepared by simple crushing to various degrees of fineness, after decorticating; next comes the classes of partially cooked preparations of each of these grains, appearing in various forms of "flakes," "granules," "grits," etc., and again a class known as malted cereals, in which the moist, ground grain is mixed with malted barley, and, by controlling the temperature, a portion of the starch is converted to maltose and dextrin, after which the mixture is crushed between hot rollers and dried.

In the preparation of most of the corn breakfast products, such as samp and hominy, it is customary to remove the germ, which contains the oil and fat, lest the tendency of the latter to become rancid should result in the deterioration of the food. In wheat foods the germ is less often removed, and rarely, if ever, in oat preparations. The amount of fat found in the prepared cereal food as compared with that in the whole grain is of interest in this connection.

Composition of Some of the Common Breakfast Cereals.-The following analyses will serve to typify the various classes of these preparations as they appear on the market: 


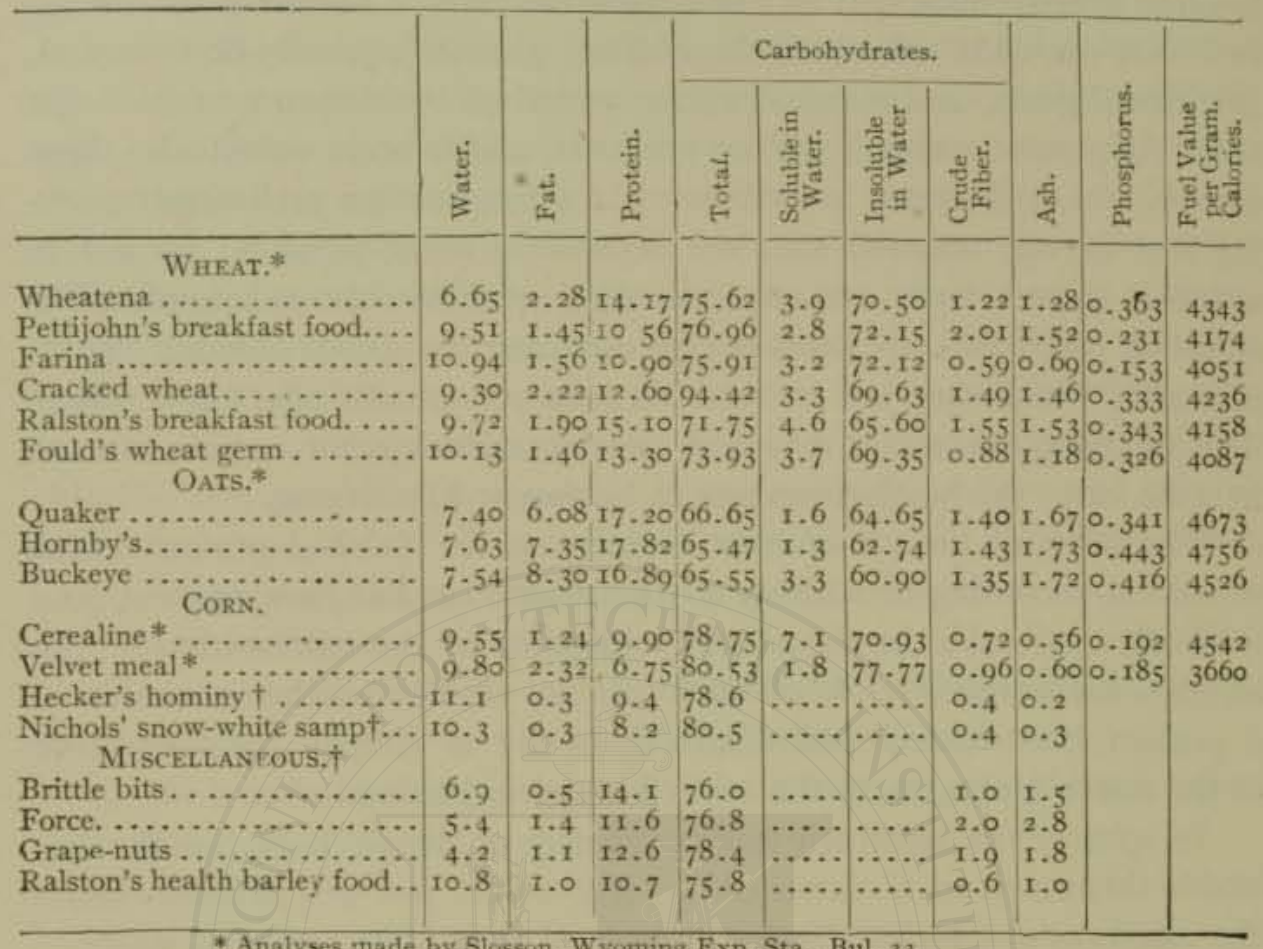

* Analyses made by Stosson, Wyoming Exp. Sta., Bul. 33

† Analyses made by Merrill and Mansfield, Maine Exp. Sta., Bul, 84.

The methods of analysis employed for these preparations are the same as for ordinary cereals (p. 277), the sample being ground fine enough to pass through a $\mathrm{I}-\mathrm{mm}$. sieve.

\section{PREPARED FOODS FOR INFANTS AND INVALIDS.}

In dealing with the composition and analysis of this class of proprietary foods more than ordinary care is necessary, in view of the fact that one or another of these preparations are frequently prescribed for the exclusive diet of those whose very life may depend on the character and suitability of the food to the case in hand. Many of these foods do, as a matter of fact, honestly fulfil the claims of their manufacturers, but others fall far short of so doing, so that it is hardly safe to use them unless some intelligent idea of their composition can be gained. It is not, as a rule, within the province of the analyst to furnish an opinion regarding the adaptability of a certain food to the requirements of an infant or invalid, but rather to provide the necessary data whereon such an opinion may be intelligently based. 
A simple statement of moisture, fat, protein, carbohydrates (by difference), and ash, which in the case of ordinary foods would often be sufficient, would be obviously inadequate in expressing the analysis of an infant food, since it is of much more vital importance than in other foods to know the solubility of the food itself, and, to as great an extent as possible, the character of the carbohydrates.

The chief ingredients of many of these preparations are wheat, or mixed cereals high in starch. Many of the foods are, according to the directions, to be used practically without cooking, but by simply mixing with milk or water, and, in some cases, bringing to the boiling-point. Hence the degree of conversion which the raw starch has undergone in the process of manufacture of the food should, if possible, be ascertained as a prime factor in judging of its character and adaptability to the needs of the young child and of the sick. Incidentally it should be said that few if any of the infant foods, even those whose high character has long been established by continued trial, conform very closely to the composition of woman's milk, which was long accepted as the true standard on which to base their efficiency. Hence it is no easy task to pass judgment on a particular food from its chemical composition alone without trial, nor is it right to unqualifiedly condemn in all cases food high in insoluble carbohydrates, since there are undoubtedly many instances in which such foods are successfully used.

Classification and Preparation of Infants' Foods.-These foods may for convenience be divided into two main classes, viz., farinaceous foods, or those which are prepared wholly or chiefly from one or more cereal grains, and lactated foods, or those in which cow's milk forms the basis, but which may contain in addition thereto various other substances, such as cereals, sugars, etc.

The farinaceous foods, which are usually directed to be mixed with milk before using, may be further subdivided into $(a)$ those that consist chiefly of unconverted starch, $(b)$ those whose starch has been nearly all hydrolyzed to soluble form in the process of manufacture, and $(c)$ those which contain much unconverted starch, but in addition thereto diastase or some other ferment, which, when the food is mixed with warm water or milk, is supposed to convert all the starch to soluble form.

The unconverted starch foods are nearly all made up of baked dry flour, chiefly that of wheat, but sometimes a mixture of cereals (as oats, barley, and wheat) and sometimes oats or barley alone. The baking 
breaks down to some extent the starch grains, as in the case of bread or crackers, but does not actually convert much of it to sugar.

The soluble farinaceous foods are usually prepared somewhat as follows: A mixture of ground wheat and barley malt (with sometimes a little wheat bran) is mixed with water to form a paste, and a little bicarbonate of potash added. The mixture is heated at $65^{\circ} \mathrm{C}$. for sufficient time to convert the starch, after which it is exhausted with warm water, the extract being strained, and the filtrate evaporated to dryness to form the food. The sugars of such foods consist largely of maltose mixed with dextrin.

The farinaceous foods, which depend for the conversion of their starch on the method of cooking or heating before serving, are usually mixtures of wheat or other cereal flour with malt or pancreatic extract.

The milk foods are variously prepared, either by the simple desiccation of cow's milk (usually previously skimmed) or, when whole milk is used, by mingling the desiccated milk with sugars or baked cereal flour. Sometimes desiccated milk is used in mixture with a dried extract of malted cereals. In fact all sorts of mixtures are found on the market, involving, however, in nearly all cases, one modification or another of the above general processes of preparation.

Composition.-Few complete analyses of these classes of foods have recently been made. Among the best are those of McGill,* from whose work the following figures have been selected, illustrating typical examples of foods on the market:

\begin{tabular}{|c|c|c|c|c|c|c|c|c|}
\hline & है & $\frac{\stackrel{d}{g}}{\frac{d}{0}}$ & 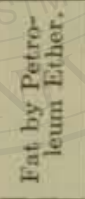 & $\frac{\frac{\frac{1}{3}}{3}}{\frac{3}{3}}$ & 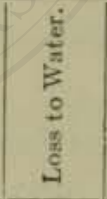 & 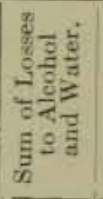 & 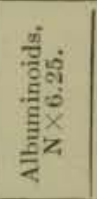 & $\frac{1}{4}$ \\
\hline \multicolumn{9}{|l|}{ Farinaceous foods: } \\
\hline Imperial granum. . .......... & 5 & 6.04 & 0.72 & . & 3.94 & 3.94 & 13.77 & 0.49 \\
\hline Ridge's food. ............... & 9 & 8.12 & $0.4^{8}$ & 0.34 & 4.67 & 5.02 & 13.83 & 0.53 \\
\hline Mother's food. .......... & 2 & $9 \cdot 99$ & 0.13 & & $\cdots \cdots$ & 8.83 & 8.60 & 2.08 \\
\hline Robinson's barley. ....... & 7 & 9.41 & $0.4 \mathrm{I}$ & 0.65 & 2.26 & $2.9 \mathrm{I}$ & $7 \cdot 4^{6}$ & 0.94 \\
\hline \multicolumn{9}{|l|}{ Mixed foods: } \\
\hline Horlick's malted milk........ & 9 & 2.55 & $\pi \cdot 4 \mathrm{I}$ & $\cdots \cdots$ & $\ldots \ldots$ & 63.87 & I 4.00 & $3 \cdot 57$ \\
\hline Lactated food.............. & 12 & $5-77$ & 0.48 & 28.24 & $4 \cdot 27$ & 32.90 & 10.01 & 2.57 \\
\hline Mellin's food. . ............. & 8 & $4 \cdot 72$ & 0.30 & $\ldots \ldots$ & $\ldots \ldots$ & 82.0 & 10.10 & 3.50 \\
\hline Nestle's milk food. . . . . . . . . & 9 & 2.18 & $4 \cdot 45$ & $39 \cdot 54$ & $4 \cdot 30$ & 43.84 & 10.72 & 1.60 \\
\hline Reid \& Carnrick's baby food .. & 2 & 5.69 & 2.18 & & ....... & $3^{8} .21$ & 16.60 & $2.7^{8}$ \\
\hline
\end{tabular}

* Canadian Dept. of Inland Rev., Bul. 59. 


\begin{tabular}{|c|c|c|c|c|c|}
\hline & $\begin{array}{l}\text { Stareh, } \\
\text { Fiber. } \\
\text { etc. by } \\
\text { Differ- } \\
\text { ence. }\end{array}$ & Maltose. & Lactose. & $\begin{array}{l}\text { Cane } \\
\text { Sugar. }\end{array}$ & Remarks. \\
\hline 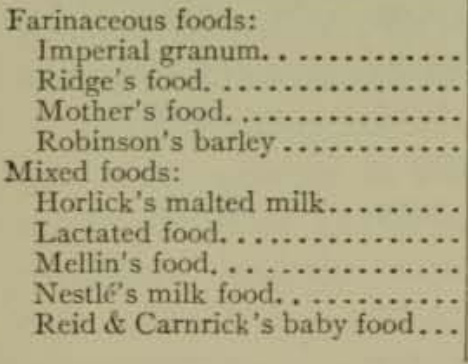 & $\begin{array}{l}76.60 \\
72.01 \\
60.24 \\
78.66 \\
15.68 \\
47 \cdot 72 \\
\cdots \cdots \cdots \\
35 \cdot 34 \\
34 \cdot 54\end{array}$ & 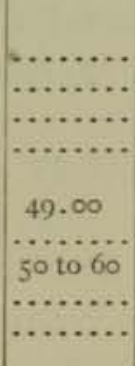 & $\begin{array}{c}\ldots \ldots \\
\ldots \ldots \ldots \\
\ldots \ldots \ldots \\
\ldots \ldots \ldots \\
30.00 \\
\ldots \ldots \\
8.96 \\
30.00\end{array}$ & $\begin{array}{c}\ldots \ldots \\
\ldots \ldots \\
3.00 \\
\cdots \ldots \\
8.00 \\
\text { Trace } \\
\cdots \ldots \\
36.34 \\
8 \text { to } 9\end{array}$ & $\begin{array}{l}\text { Wheat starch } \\
\text { Wheat starch } \\
\text { Corn and wheat starch } \\
\text { Barley starch }\end{array}$ \\
\hline
\end{tabular}

Diabetic Foods..-Gluten flour and similar preparations are primarily intended for the use of diabetics, from whose dietary carbohydrates must be excluded.

The following analyses of commercial gluten preparations were made by Woods and Merrill.*

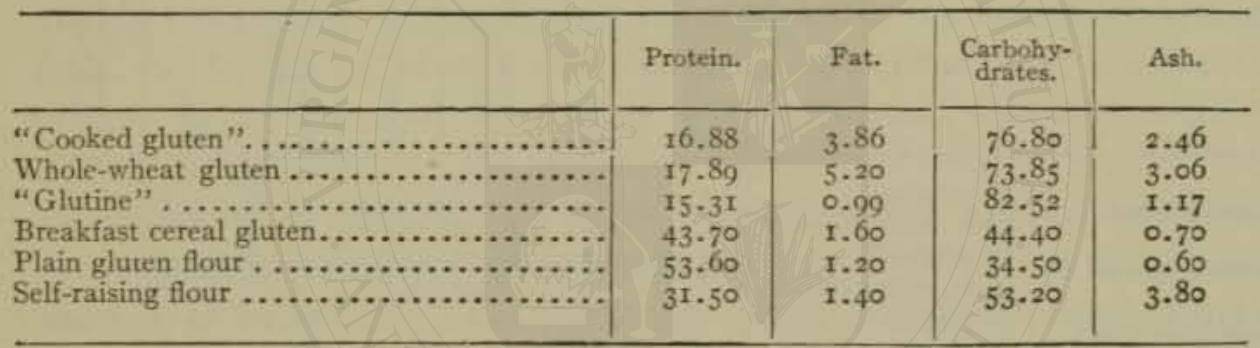

Many brands of gluten flour are put on the market by dealers in socalled "health foods," and in many cases are represented to be practically free from starch. Thirteen samples of gluten flour were analyzed by the author in 1899 , varying in price from II to 50 cents per pound. Of these, 3 , the product of one manufacturer, contained less than $1 \%$ of starch, 3 contained from to to 20 per cent, while 7 contained from $5^{6}$ to 70 per cent of starch, the substance which, of all others, the diabetic patient tries to avoid. Some of these preparations were little better than wholewheat flour. An analysis of one of them, known as "Pure Vegetable Gluten," and sold for 50 cents per pound, and of two similar diabetic flours reported by Winton follow: 


\begin{tabular}{|c|c|c|c|}
\hline Moisture. . . . . . . & $\begin{array}{l}\text { ure Vegetable } \\
\text { Gluten" } \\
\text { I0. } 78\end{array}$ & $\begin{array}{l}\text { "Diabetic } \\
\text { Food." } \\
\text { I2.67 }\end{array}$ & $\begin{array}{l}\text { "Diabetic } \\
\text { Flour." } \\
9.26\end{array}$ \\
\hline Ash............... & 2.20 & 0.43 & I. 30 \\
\hline 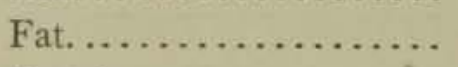 & $3 \cdot 25$ & 0.90 & $2.2 I$ \\
\hline Protein. . . . . . . . . & $r_{4} .25$ & II. 37 & 14.25 \\
\hline Crude Fiber. ............ & $\ldots$ & 0.25 & I.03 \\
\hline Sugars. . . . . . . . . . . . & I.70। & & \\
\hline $\begin{array}{l}\text { Dextrin } \ldots \ldots \ldots \ldots \ldots \ldots \\
\text { Starch. ............. }\end{array}$ & $\left.\begin{array}{r}2.55 \\
56.55\end{array}\right\}$ & $7 \mathrm{I} \cdot 5 \mathrm{I}$ & 66.63 \\
\hline Undetermined......... & $8.7^{2}$ & 2.87 & $5 \cdot 3^{2}$ \\
\hline
\end{tabular}

Winton has reported the following analyses of flours and meals well suited for the preparation of diabetic biscuit, and of the biscuit made from two of these by a cook in the family of a diabetic patient:

\begin{tabular}{|c|c|c|c|c|c|c|c|c|}
\hline & & 递 & $\frac{d}{4}$ & 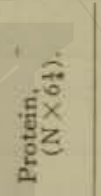 & $\frac{\stackrel{f}{5}}{\stackrel{5}{E}}$ & 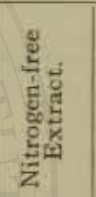 & 迹 & 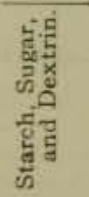 \\
\hline Gluten flour & & 10.12 & $\begin{array}{l}0.22 \\
0.24\end{array}$ & $\begin{array}{l}85 \cdot 38 \\
05.00\end{array}$ & $\begin{array}{l}0.03 \\
0.03\end{array}$ & $\begin{array}{l}3.69 \\
4.11\end{array}$ & $\begin{array}{l}0.56 \\
0.62\end{array}$ & $\begin{array}{l}4.46 \\
4.06\end{array}$ \\
\hline Gluten biscuit. & & $25 \cdot 5^{8}$ & $\begin{array}{l}0.24 \\
2.35 \\
3.16\end{array}$ & $\begin{array}{l}95.00 \\
50.91 \\
68.41\end{array}$ & $\begin{array}{l}0.03 \\
0.64 \\
0.86\end{array}$ & $\begin{array}{l}4.11 \\
3.18 \\
4.27\end{array}$ & $\begin{array}{r}0.02 \\
17.34 \\
23.30\end{array}$ & \\
\hline Soja bean meal.. & $\begin{array}{l}\text { In original......... } \\
\text { Calc, water-free.. }\end{array}$ & $7 \cdot 75$ & $\begin{array}{l}3.10 \\
4 \cdot 38 \\
4.75\end{array}$ & $\begin{array}{l}08.41 \\
39.87 \\
43.22\end{array}$ & $\begin{array}{l}0.80 \\
3.85 \\
4.17\end{array}$ & $\begin{array}{r}4.27 \\
25.09 \\
27.20\end{array}$ & $\begin{array}{l}23.30 \\
19.06 \\
20.66\end{array}$ & $\begin{array}{l}8.95 \\
9.70\end{array}$ \\
\hline Soja bean biscuit & $\begin{array}{l}\text { In original........ } \\
\text { Calc. water-free. }\end{array}$ & 27.66 & $\begin{array}{l}4.75 \\
5 \cdot 33 \\
7.37\end{array}$ & $\begin{array}{l}43.71 \\
16.71 \\
23.10\end{array}$ & $\begin{array}{l}1.17 \\
1.55 \\
2.14\end{array}$ & $\begin{array}{l}12.84 \\
17.75\end{array}$ & $\begin{array}{l}35.91 \\
49.64\end{array}$ & $\ldots \ldots$ \\
\hline Casoid flour & $\begin{array}{l}\text { In original........ } \\
\text { Calc. water-free. }\end{array}$ & 10.01 & $\begin{array}{l}2.51 \\
2.46 \\
2.73\end{array}$ & $\begin{array}{l}85.56 \\
95.08\end{array}$ & & & $\begin{array}{l}0.4 \\
0.50 \\
0.56\end{array}$ & $\begin{array}{l}\text { none } \\
\text { none }\end{array}$ \\
\hline Almond meal . & $\begin{array}{l}\text { In original........ } \\
\text { Calc. water-free. }\end{array}$ & $\begin{array}{l}8.51 \\
\ldots .\end{array}$ & $\begin{array}{l}6.42 \\
7.02\end{array}$ & $\begin{array}{l}50.62 \\
55.32\end{array}$ & $\begin{array}{l}2.86 \\
3.12\end{array}$ & $\begin{array}{l}15.96 \\
17.45\end{array}$ & $\begin{array}{l}15.63 \\
17.09\end{array}$ & $\begin{array}{l}7.18 \\
7.85\end{array}$ \\
\hline
\end{tabular}

In the analysis of diabetic foods, the determination of starch, sugar and dextrin together is of greater value than of starch alone, since all three classes of carbohydrates are about equally injurious to diabetics, the starch and dextrins being converted into sugars by the digestive fluids. The nitrogen-free extract of cereal preparations corresponds closely with the sum of the starch, sugar and dextrin, but in the case of soja bean meal, almond meal and other products of legumes and oil seeds, as well as vegetables, it is considerably greater, as it includes pentosans and other substances. 


\section{METHODS OF ANALYSIS.}

The sample is prepared for analysis by grinding it sufficiently fine in a mortar or mill to pass through a $\mathrm{I}-\mathrm{mm}$. sieve. Moisture, fat, ash, and nitrogen are determined as in the régular methods for cereals (pp. 276-278).

In determining loss of weight due to solubility of the sample in alcohol and water, proceed as follows:* The fat-free residue left in the Soxhlet apparatus, after extraction with ether or petroleum ether, is subjected to further extraction with $95 \%$ alcohol, till all soluble matter has been extracted. If 5 grams of the sample were originally taken for the fat extraction, this operation would require about five hours. Evaporate the alcoholic extract to dryness, and weigh the residue as in the case of the ether extract. Dry the residue left in the Soxhlet from the alcoholic extract, or a portion thereof, in a platinum dish over the water-bath, cool, and weigh. Transfer to a Gooch crucible, provided with asbestos and previously tared, a portion, the relation of which to the original weight taken is calculated from the moisture, ether, and alcohol extracts as previously determined. Pass through the contents in the Gooch by suction from 200 to $300 \mathrm{cc}$. of cold water at room temperature, dry the Gooch and its contents at $100^{\circ}$ to constant weight, cool and weigh, thus determining the solubility of the sample in water.

According to McGill, five hours' extraction with alcohol under the above conditions removes all cane sugar, but probably not all the lactose, maltose, and dextrose, if a considerable quantity of these sugars is present. Water dissolves the dextrin and gum and such of the sugar as escapes solution in the alcohol, hence the sum of the alcohol and water extract is of value. In the calculation of the starch, fiber, etc., by difference, it should be borne in mind that the result is only approximate, by reason of the fact that the small amount of soluble albuminoids (which McGill states never exceeds $2 \frac{1}{2} \%$ ) are reckoned in, hence a small error is introduced, which could be corrected, if considered worth while, by determining the amount of soluble albuminoids.

Separation of the Carbohydrates can be effected by Stone's method (pp. 295, 296), but a very satisfactory idea of the solubility of these foods, which is of chief importance, can be gained by the much simpler modified method of McGill, as described in the preceding paragraphs.

* McGill, Canada Inland Rev. Dept., Bull. $5^{8}$. 
Starch, Sugar, and Dextrin are determined together in diabetic preparations by the diastase method (p. $28_{3}$ ) omitting the preliminary washing with dilute alcohol.

Cold-water Extract.-The equivalent of ro grams of the moisture-free substance, finely ground, is weighed in a tared flask, and water added in several portions with gentle shaking till the contents of the flask weigh I 10 grams. The flask is then corked and vigorously shaken at intervals during six or eight hours and allowed to stand over night. The supernatant liquid is then decanted into the large tubes of a centrifuge, and whirled till the sediment settles out. The comparatively clear liquid may then be readily filtered. $20 \mathrm{cc}$. of the filtrate, corresponding to 2 grams of the original sample, are then transferred to a tared dish, evaporated to dryness, and dried to constant weight, as in the determination of the total solids.

Additional information may be gained from the specific gravity of the $10 \%$ solution of the cold-water extract, best obtained by means of a pycnometer.

Reducing Sugars are determined in an aliquot part of the above $10 \%$ solution, diluted to proper strength.

Effects of Subsequent Heating.-It is hardly fair in the case of those farinaceous foods which, according to directions, are to be subsequently subjected to heating, or boiling with water or milk, to condemn them as containing much insoluble matter, without comparing the figures expressing results of the analyses of the raw foods, calculated to the water-free basis, with those obtained on analyzing the food after boiling or otherwise cooking with pure distillec water, for a length of time specified in the directions, and afterwards drying. It is possible that the presence in the food of diastase, or other ferment, may be depended on to hydrolyze a whole or a portion of the starch, and only by such comparison will this be shown.

Microscopical Examination of the food is of value in determining its general character, showing especially whether or not starch is present in its original form, or has been converted in whole or in part. The particular varieties of cereal grain employed are generally evident, as well as the presence and proportion of the different tissues of the grain. 
REFERENCES ON CEREALS, VEGETABLES, LEGUMES, FRUITS, ETC.

Alway, F. J. The Effects of Bleaching upon the Quality of Wheat Flour. Neb. Exp. Sta. Bul. 102.

Atwater, Heien W. Bread and the Principles of Bread Making. Farmers' Bul. ir 2. - Beans, Peas and other Legumes as Food. Farmers' Bul, 12I.

Avery, S. A Contribution to the Chemistry of Bleached Flour. J. Amer. Chem. Soc., 29, 1907 , p. 571 .

Batland. Recherches sur les Blés, les Farines et le Pain. Paris, 1894.

Batrard. The Bleaching of Flour. Compt. rend, I39, I904, p. 822.

Bigelow, Munson, Tolman, and Howard. Fruits and Fruit Products. Bur. of Chem., Bul. 66.

Blasdale, W. C. Some Chinese Vegetable Food Materials. Exp. Sta. Bul. 68.

Blythine, A., u. Wrampelmeyer, E. Breiträge zur Untersuchung und Beurtheilung der Eierteigwaaren. Zeits. Unters. Nahr. Genuss., 4, Igor, p. I45.

BRAHM, K. Ueber den Einfluss von Ozon auf die Backfähigkeit von Weizenmehl. Zeits. Unters. Nahr. Genuss., 8, 1904, p. 669.

Chambert.ain, J. S. Investigations on the Properties of Wheat Proteins. Jour. Am. Chem. Soc., 28, 1906, p. 1657 .

- The Feeding Value of Cereals. U. S. Dept. of Agric, Bur. of Chem., Bul. r20.

Chittenden, R. H., and Osborne, T. B. Proteids of the Corn Kernel. Am. Chem. Jour., XIII, No. 7 , and XIV, No. I.

Cochran, C. B. The Estimation of Fat in Infant and Invalid Foods. Jour. Am. Chem. Soc., 27, 1905, p. 906.

Fleurent, E. The Bleaching of Flour. Compt. rend., 142, 1906, p. 180.

GaLt, H. The Microscopy of the Starches. London, Igoo.

Goodfellow, J. The Dietetic Value of Bread. London, I892.

Griffiths, W. The Principal Starches Used as Food. Cirencester, I892.

Guess, H. A. The Gluten Constituents of Wheat and Flour and their Relation to Bread-Making Qualities. Jour. Am. Chem. Soc., 22, rgoo, p. 263.

Hanausek, T. F. Die Nahrungs- und Genussmittel aus dem Pflanzenreiche. Kassel, 1884 .

- Microscopy of Technical Products (Trans. by Winton, A. L., and Barber, Kate G.). New York, 1907. Wheat and Flour, p. 334 et seq.

Harz, C. O. Landw. Samenkunde. Berlin, I885.

JAGO, W., and JAGO, W. C. The Technology of Breadmaking. London, I9Ir.

JuCKENACK, A. Ueber die Untersuchung und Beurtheilung der Teigwaren des Handels. Zeits. Unters. Nahr. Genuss., 3, I900, p. I.

Juckenack, A., u. Pasternack, R. Die Beurteilung des Gehaltes der Eierteigwaren und eigelbhaltigen Nahrungsmittel an Eimasse. Zeits. Unters. Nahr. Genuss., 8 , 1904 , p. 94.

Juckenack, A., u. Sendtner, R: Neure Beiträge zur Beurtheilung und Untersuchung der Teigwaaren des Handels. Zeits. Unters. Nahr. Genuss., 5, I902, p. 997 Körnicke, F., u. Werner, H. Handbuch des Getreidebaues. Berlin, I885. 
Krazarer, H. An Examination of Commercial Flour. Jour. Am. Chem. Soc., 2r, 1899, p. $65^{\circ}$.

KRUG, W. H. Analytical Methods for Carbohydrates as Applied to Foods, etc. Jour.

Frank. Inst., I902 (CLIV), pp. 349-40I.

LADD, E. F., and BAssetr, H. P. , Bleaching of Flour. N. Dak. Exp. Sta., Rep. rgo8, p. 133; Jour. Biolog. Chem., 6, 1909, p. 75.

LADD, E. F., and Stallings, R. E. Bleaching of Flour. N. Dak. Exp. Sta., Bul. 72.

LADD, E. F., and White, H. L. The Effects of Bleached Flour on Rabbits. N. Dak. Agr. Exp. Sta., Rep roog, p. 145.

Lawes and Gilbert. Composition of Wheat Grain, its Products in the Mill, and Bread.

McGrt, A. Infants' and Invalids' Foods. Canada Inl. Rev. Dept., Bul. 59.

Cereal Breakfast Foods. Canada Inl. Rev. Dept., Bul. 84 .

Maurizio, A. Getreide, Mehl und Brot. Berlin, 1903.

Moeller, J. Mikroskopie der Nahrungs- und Genussmittel aus dem Pflanzenreiche. Berlin, 1886. 2 Aufl. 1905.

Mor.ssch, H. Grundriss einer Histochemie der pflanzlichen Genussmittel. Jena, 189r.

Munson, L. S., and Torman, L. M. Fruits and Fruit Products. Bur. of Chem., Bul. 65, Part XIII, p. 74.

Norton, F. A. Crude Gluten. Jour. Am. Chem. Soc., 28, rgo6, p. 8.

Osborne, T. B. Crystallized Vegetable Proteids. Am. Chem. Jour., XIV, No. 3.

- Oat Proteids. Conn. Exp. Station, An. Reps., 189o, p. 115; 1891, p. 124.

The Proteose of Wheat. Am. Chem. Jour., XIX, No. 3 .

- Proteids of Rye. Jour. Am. Chem. Soc., 17, p. 429.

— Proteids of Barley. Jour. Am. Chem. Soc., 17, p. 539.

- Amounts and Properties of the Proteids of Maize. Jour. Am. Chem. Soc., r9, p. 525 .

Osborne, T. B., and Campbelt, G. F. Proteids of the Potato. Jour. Am. Chem. Soc., 18 , p. 575 .

- Proteids of the Pea. Jour. Am. Chem. Soc., 18, p. 583 , and 20, p. 348 .

Osborne, T. B., and Voorress, C. L. Proteids of Wheat. Jour. Am. Chem. Soc. I6, p. 524 .

- Proteids of the Wheat Kernel. Am. Chem. Jour., XV, No. 6.

Rolfe, Geo. W., and Germanos, H. W. Notes on the Hydrolysis of Starch by Acids. Jour. Am. Soc., 25, 1903 , p. 1003.

Rolfe, Geo. W., and HadDock, IsaAc T. The Presence of Maltose in Acid-Hydrolized Starch Products. Jour. Am. Chem. Soc., 25, 1903, p. 1015.

Schimper, A. F. W. Anleitung z. mikrosk. Unters. der Nahrungs- und Genussmittel. Jena, $\mathbf{1} 886$.

Shepard, J. H. Nitrous Acid as an Antiseptic. Am. Food Jour., 3, 1908, p. 21.

Sherman, H. C. Carbohydrates of Wheat. Jour. Am. Chem. Soc., 19, 1897, p. 29 r.

Skinner, R. P. Manufacture of Semolina and Macaroni. Bur. of Plant. Ind., Bul. 20.

SNyder, H. Digestibility and Nutritive Value of Bread. Exp. Sta. Bul. 126.

- Testing Wheat Flour for Commercial Purposes. Jour. Am. Chem. Soc., 27, 1905 , p. 1068.

Wheat and Flour Investigations. Minn. Agric. Exp. Sta., Bul. 85, 1904. 
Snyder, H. The Proteids of Wheat Flour. Minn. Agric. Exp. Sta. Bul. 63, p. 5 I9. Report on the Bleaching of Flour. Dec. 28, 1906.

Snyder, Frisby, and Bryant. Losses in Boiling Vegetables. Exp. Sta. Bul. 43

Snymer, H., and Voorhers, M. A. Studies on Bread and Bread-Making. Exp. Sta. Bul. 67 .

Stone, W. E. Carbohydrates of Wheat, Maize, Flour, and Bread. Action of Enzymes on Starches. Exp. Sta. Bul. 24.

The Quantitative Determination of Carbohydrates in Food Stuffs. Jour. Am. Soc., 19, 1897, p. 347 .

Thatcher, R. W. A Comparison of Various Methods of Estimating the Baking Qualities of Flour. Jour. Am. Chem. Soc., 29, 1907, p. 910.

Wheat and Flour Investigations. Washington Agric. Exp. Sta. Bul. 84.

Tschirch, A., und Oesterle, O. Anatomischer Atlas der Pharmakognosie und Nahrungsmittelkunde. Leipzig, 1893 .

VogL, A. E. Verfälschungen und Verunreinigungen des Mehles und deren Nachweisung. Wien, 1880 .

Die wichtigsten vegetabilischen Nahrungs- $u$. Genussmittel. Wien u. Leipzig, $r 899$.

WANklyn, J. A., and Cooper, W. J. Bread Analysis. London, 1886.

Wriex, H. W. Sweet Casava. Div. of Chem., Bul. 44.

Analysis of Cereals Collected at the. World's Columbian Exposition. Div. of Chem., Bul. 45 .

- Composition of Maize. Div. of Chem., Bul. 50.

Wruex, H. W., et al. Cereals and Cereal Products. Div, of Chem., Bul. 13, Part IX. Winton, A. L. The Microscopy of Vegetable Foods. New York, I906.

- Diabetic Foods. An. Rep. Conn. Exp. Sta. 1906, p. 153.

- A Modification of the Bamihl Test for Detecting Wheat Flour in Rye Flour.

A. O. A. C. Proc., 1908, U. S. Dept. of Agric., Bur. of Chem., Bul. r22, p. 217.

The Color of Flour and a Method of Determining the Gasoline Color Value.

A. O. A. C. Proc, 1910.

Winton, A. L., and Bamlex, E. M. On the Composition of American Noodles and Methods for the Analysis of Noodles. An. Rep. Conn. Exp. Sta., 1904, p. 138; Jour. Am. Chem. Soc., 37, 1905, p. 137 .

Winton, A. L., and Ogden, A. W. Macaroni, Sphaghetti, Vermicelli, and Noodles. An. Rep. Conn. Exp. Sta., r9or, p. I96.

Wrinton, A. L., and Shanley, E. J. Simple Tests for Detecting Bleaching in Flour. A. O. A. C. Proc., 1908, p. 216.

Woods and Merrm.t. Digestibility and Nutritive Value of Bread. Exp. Sta. Bul. 85.

Arkansas Exp. Station Bul. 42. Wheat and its Mill Products.

California " " " $" 93$. Oranges and Lemons.

" " " "

" " " " 102 . Figs.

" " " An. Reports, 1892 et seq.

Maine Exp. Station Bul. 54. Nuts as Food.

" " " " 55 . Cereal Breakfast Foods.

" " " "75. Analyses of Self-raising Flours, Pea Flours, Gluten Foods, Chestnuts, Malted Nuts. 
Maine Exp. Station Bul. 84. Cereal Breakfast Foods.

Minnesota Exp. Station Bul. 74. Digestibility and Food Value of Beans.

Penn. Dept. of Agriculture Bul. ro. Special Report on Prepared Foods for Infants and Invalids.

Wyoming Exp. Sta. Bul. 33. Composition of Prepared Cereal Foods.

Great Britain. Local Govt. Board of Public Health, Report on Bleaching of Flour and "Improvers.", n. ser, rorr, No. 49. Hamil, J. M., pp. 1-33. MonierWilliams, G. W., pp. 34-65.

U. S. Dept. of Agric., Notices of Judgment, Nos. 382 and 722. New Orleans and Kansas City Bleached Flour Trials.

\section{REFERENCES ON LEAVENING MATERIALS.}

Bryan, T. J. The Carbon Dioxide Value of Pure Compressed Yeast and Starch Compounds. A. O. A. C. Proc, r907, U. S. Dept. of Agric., Bur. of Chem., Bul. I16, page 25 .

Catum, Chas, A. Baking Powders, with Special Reference to Phosphate Powders. Providence, 1899 .

Crantrton, C. A. Baking Powders. Div. of Chem., Bul. 13, Pt. 5.

GrFen, J. R. Die Enzyme. Berlin.

- The Soluble Ferments and Fermentation. rgor.

Hansen, E. C. Practical Studies in Fermentation. London, 1896.

Jorgensen, A. Die Mikro-organismen der Gärungsindustrie. Berlin.

- Micro-organisms and Fermentation. London, 1900.

Kenrick, G. B., and F. B. The Application of Polarimetry to the Estimation of Tartaric Acid in Commercial Products. Jour. Am. Chem. Soc., 24, 1902, page 928 .

KLOCKFR, A. Fermentation Organisms. 1903.

LiNINER, P. Mikroskopische Betriebskontrolle in den Gärungsgewerben. Berlin, I895.

McGrı., A. Baking Powders. Canada Inl. Rev. Dept., Buls. 10, 26, 68.

- Cream of Tartar. Canada Inl. Rev. Dept., Buls. 12, 26, 71 .

Matrhews, Chas. G. Manual of Alcoholic Fermentation. London, rgor.

Oppenhermer, C. Trans. by Mitchell, C. A. Ferments and their Action. London, IgOr.

Plummer, R. H. A. Chemical Changes and Products Resulting from Fermentation. London, 1903 .

Winton, A. L. Baking Powders. Bur. of Chem. Bul. 65, Part XV. Bul. ro7, Part XXVI.

Conn. Exp. Sta. An. Rep., I900, page I5.

Michigan Dairy and Food Commission, Bul. 2, page 12; Bul. 3, page 7 .

Penn. Board of Agriculture An. Report, 1897, page 166.

North Carolina Exp. Sta., Bul. 155. 


\section{CHAPTER XI.}

TEA, COFFEE, AND COCOA.

\section{TEA.}

Nature and Classification.-Tea consists of the prepared leaves or leaf buds of Camellia Thea also known as Thea chinensis.

The best teas are made from young leaves only, the Chinese teas being classified with reference to the age and position of the leaf on the young shoot. Thus, the very choicest Chinese tea, rarely found outside of China, is prepared from the youngest or end leaves of the shoot, which are scarcely more than buds, and form the tea known as pekoe tip, or flowery pekoe. The next leaves are the orange pekoe and pekoe, which produce a very high grade of tea, while next in order as to age, size, and grade of leaf are the souchong Ist and $2 d$, and the congou, producing teas called by the same names.

More than $50 \%$ of the tea consumed in the United States comes from China, and over $40 \%$ from Japan, the remainder being derived largely from India, Ceylon, and other East Indian ports.

In the manufacture of, tea the fresh leaves, which are nearly $80 \%$ water, are rolled, withered by exposure to light, heat, and air, and finally dried or "fired" by treatment with artificial heat over charcoal fires, or in properly constructed furnaces.

Teas are divided into two groups, black and green, which differ from each other, not as formerly supposed in being derived from different plants, but in their process of manufacture, the same species of plant furnishing both varieties. Genuine green tea is prepared by first steaming and afterwards drying the leaves while still fresh, thus retaining the bright color. Black tea is allowed to undergo oxidation or fermentation by exposure to the sun, which gradually turns the leaves black. Less tannin is present in black tea than in green. 
Composition of Tea.-König gives the following composition of fully developed tea leaves, being the mean of 50 to 70 analyses:

\begin{tabular}{|c|c|c|c|c|c|c|c|c|c|}
\hline Water. & $\begin{array}{l}\text { Nitroge- } \\
\text { nous Sub- } \\
\text { stances. }\end{array}$ & Theine. & $\begin{array}{c}\text { Essential } \\
\text { Oil. }\end{array}$ & $\begin{array}{l}\text { Fat, Chil- } \\
\text { rophyl, } \\
\text { and Wax. }\end{array}$ & $\begin{array}{l}\text { Gum, } \\
\text { Dextrin, } \\
\text { etc. }\end{array}$ & Tannin. & $\begin{array}{l}\text { Pectin, } \\
\text { etc. }\end{array}$ & $\begin{array}{l}\text { Crude } \\
\text { Fiber. }\end{array}$ & Ash. \\
\hline $9 \cdot 5^{I}$ & $24 \cdot 5^{\circ}$ & $3 \cdot 5^{8}$ & 0.68 & 6.39 & 6.44 & ${ }_{15} .65$ & 16.02 & II. $5^{8}$ & 5.65 \\
\hline
\end{tabular}

Though the nitrogenous substances of tea predominate in amount over any other class of constituents, yet, with the exception of theine or

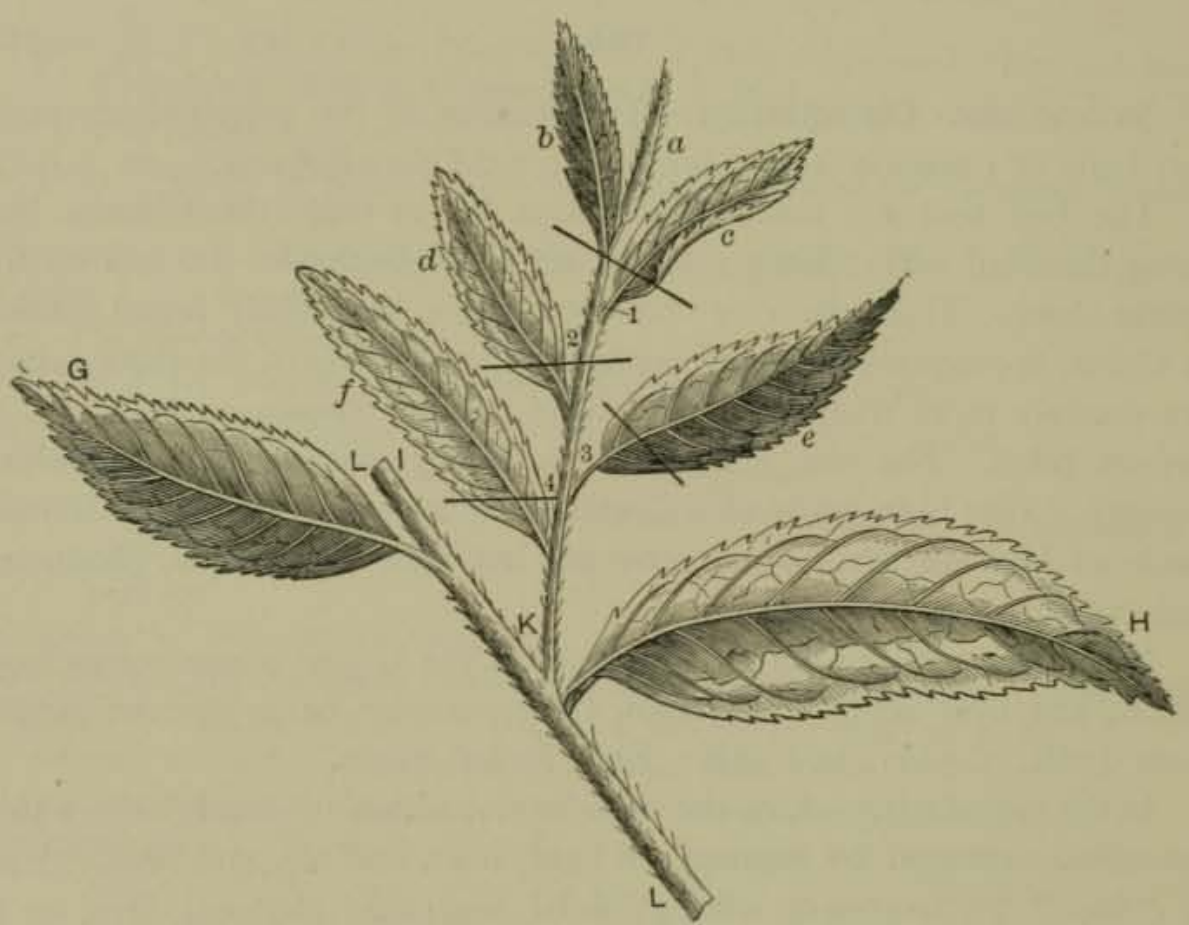

Frg. 72.- $a$, Flowery Pekoe; $b$, Orange Pekoe; $c$, Pekoe; $d$, Souchong, Ist; $e$, Souchong, $2 \mathrm{~d}$; f, Congou; $a, b$ (when mixed together), Pekoe; $a, b, c, d, e$ (when mixed together), Pekoe Souchong. If there be another leaf below $f$, it is termed Bohea. At base of leaves are buds $1,2,3,4$, from which new shoots spring. (After Money.)

caffeine, they have been little studied. Theine, tannin, and essential oil give to the infusion of tea its chief characteristics.

Zollinski* gives the following summarized results of analyses of a number of the cheaper grades of Chinese black tea:

* Zeits. anal. Chem., $1898,37,365$. 


\begin{tabular}{|c|c|c|c|c|c|c|c|c|}
\hline & Water. & $\begin{array}{c}\text { Total } \\
\text { Nitrogen. }\end{array}$ & $\begin{array}{l}\text { Albumin- } \\
\text { oid and } \\
\text { Amido- } \\
\text { nitrogen. }\end{array}$ & $\begin{array}{l}\text { Protein, } \\
\mathrm{N} \times 6.25\end{array}$ & Theine. & Ash. & $\begin{array}{l}\text { Soluble } \\
\text { Ash. }\end{array}$ & $\begin{array}{l}\text { Insoluble } \\
\text { Ash. }\end{array}$ \\
\hline Maximum.... & II. 57 & 4.12 & $3 \cdot 7^{8}$ & $23.8_{3}$ & 2.06 & $6.7^{8}$ & 31.17 & 61.03 \\
\hline Minimum .... & 9.96 & $3 \cdot 7^{6}$ & $3 \cdot 37$ & 21.06 & 1.14 & $4 \cdot 79$ & 28.13 & $57 \cdot 74$ \\
\hline Average....... & $10.5^{8}$ & $3 \cdot 93$ & $3 \cdot 5^{2}$ & 22.01 & I. 55 & $5 \cdot 94$ & 29.67 & $59 \cdot 75$ \\
\hline
\end{tabular}

A very complete series of analyses of tea was made by Joseph F. Geissler in $1884, *$ from which the following summaries are taken:

\begin{tabular}{|c|c|c|c|c|c|c|c|c|c|c|c|c|}
\hline & & 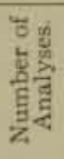 & $\frac{d}{\frac{d}{2}}$ & 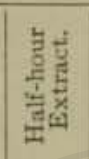 & 竎造 & 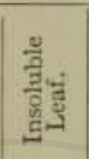 & 嶽 & $\begin{array}{l}\text { E } \\
\text { E }\end{array}$ & 䗒 & हैं & 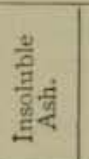 & 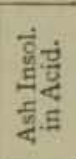 \\
\hline \multirow[t]{2}{*}{ Indian: } & & 6 & & 39.66 & 45.64 & 53.07 & 18.86 & $3 \cdot 3$ & $5 \cdot 79$ & 3.68 & 2.22 & \\
\hline & & : & & & & & & & & & $\begin{array}{l}1.93 \\
2.12\end{array}$ & \\
\hline \multirow[t]{3}{*}{ Oolong: } & Maxi & 13 & 6,88 & 44.02 & 48.87 & 53.15 & 20.07 & 3.50 & 6.11 & 3.71 & 3.17 & \\
\hline & & $\cdots$ & 5.09 & 34. & 40.6 & 44.8 & 11.93 & 1. 15 & $5 \cdot 44$ & 2.60 & I. 84 & \\
\hline & & & 5.89 & 37.88 & $43 \cdot 32$ & 50.7 & 16.38 & $2 \cdot 32$ & $5.8 \mathrm{I}$ & 3.2 & 2.68 & \\
\hline \multirow{2}{*}{ Congou: } & & 11 & $\begin{array}{l}9.15 \\
7.65\end{array}$ & $\begin{array}{l}32.14 \\
23.48\end{array}$ & $\begin{array}{l}37.06 \\
27.48\end{array}$ & $\begin{array}{l}3.85 \\
54.5\end{array}$ & $\begin{array}{r}13.89 \\
8.44\end{array}$ & $\begin{array}{l}2.87 \\
\text { I. } 70\end{array}$ & $\begin{array}{l}0.48 \\
5.52\end{array}$ & $\begin{array}{l}3.52 \\
2.28\end{array}$ & $\begin{array}{l}3.86 \\
1.00\end{array}$ & I. 3 \\
\hline & & & 8.37 & 28 & & 57.2 & II. 54 & 2.37 & $5-75$ & 3.06 & 2.68 & \\
\hline
\end{tabular}

Kenrick $\uparrow$ gives the following averages of a series of analyses of tea made by him in 1891 :

\begin{tabular}{|c|c|c|c|c|c|c|c|c|c|}
\hline & \multirow{2}{*}{ 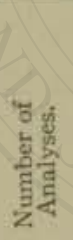 } & \multicolumn{3}{|c|}{$\begin{array}{l}\text { Substances Extracted } \\
\text { by Io Minutes' } \\
\text { Infusion. }\end{array}$} & \multirow[b]{2}{*}{$\frac{\frac{\pi}{2}}{\frac{\pi}{2}}$} & \multirow{2}{*}{$\begin{array}{l}\frac{j}{3} \\
\frac{0}{3} \\
\frac{a}{3} \\
\text { on }\end{array}$} & \multirow{2}{*}{ 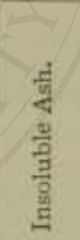 } & \multirow[b]{2}{*}{ 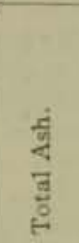 } & \multirow{2}{*}{ 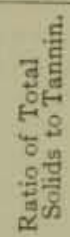 } \\
\hline & & 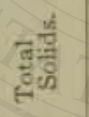 & 总 & $\frac{\stackrel{d}{E}}{\stackrel{E}{E}}$ & & & & & \\
\hline Congou tea. & 10 & $23 \cdot 37$ & 5.18 & 2.65 & 7.60 & 3.55 & 2.28 & 5.83 & \\
\hline Assam tea & 3 & 38.53 & & $2.9^{8}$ & 5.75 & 3.69 & 2.16 & 5.84 & $3.8 \mathrm{x}$ \\
\hline Ceylon tea ....... & 2 & 27.45 & 7.85 & 2.68 & 6.31 & $3 \cdot 34$ & 1.88 & 5.22 & 3.50 \\
\hline Unclassed black.. & 13 & $23 \cdot 7^{6}$ & $5.40^{\circ}$ & 2.82 & 6.54 & 3.53 & 2.37 & 5.90 & $4 \cdot 40$ \\
\hline & 18 & 30.07 & $9 \cdot 3^{8}$ & 2.45 & 4.00 & 3.62 & 2.73 & 6.35 & 3.20 \\
\hline Gunpowder & 2 & 28.55 & 8.00 & 2.39 & $4 \cdot 7^{2}$ & $3 \cdot 3^{6}$ & 3.70 & 7.06 & $3-57$ \\
\hline Young Hyso & 5 & 34.22 & 10.98 & $2.5^{2}$ & $5 \cdot 4^{\circ}$ & $3.8_{3}$ & $2 . \mathrm{IC}$ & 5.93 & 3.12 \\
\hline
\end{tabular}

The ash of many genuine teas has been examined by Battershal $\ddagger$ with the following results:

* Am. Grocer, Oct. 23, 1884 .

$\dagger$ Canada Inland Rev. Dep. Bul. 24.

$\ddagger$ Food Adulteration and its Detection. 


\begin{tabular}{|c|c|c|c|}
\hline & $\begin{array}{l}\text { Oolong. } \\
\text { Average of so } \\
\text { Samples. }\end{array}$ & Japan. & $\begin{array}{c}\text { Spent } \\
\text { Black Tea. }\end{array}$ \\
\hline 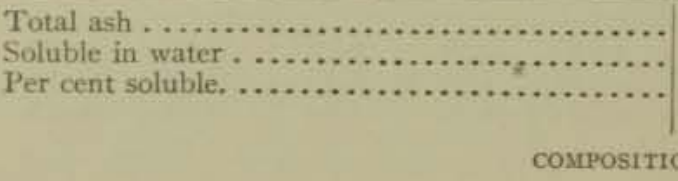 & $\begin{array}{r}6.04 \\
3.44 \\
57.00\end{array}$ & $\begin{array}{r}5.55 \\
3.60 \\
64.55\end{array}$ & $\begin{array}{r}2.52 \\
0.28 \\
11.11\end{array}$ \\
\hline 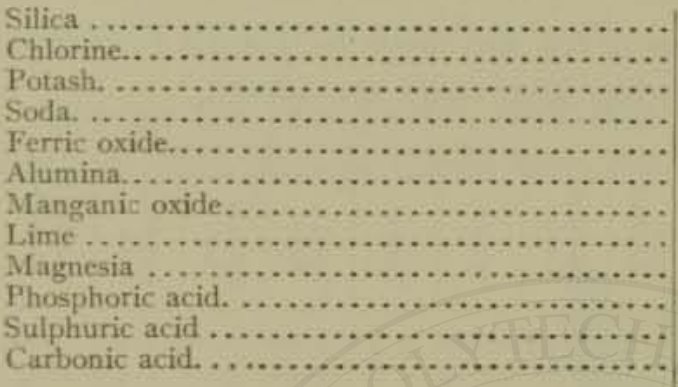 & $\begin{array}{r}11.30 \\
1.53 \\
37.46 \\
1.40 \\
1.80 \\
5.13 \\
2.10 \\
9.43 \\
8.00 \\
12.27 \\
4.18 \\
5.40\end{array}$ & $\begin{array}{r}9.30 \\
1.60 \\
41.63 \\
1.12 \\
1.12 \\
4.26 \\
1.30 \\
8.18 \\
5.33 \\
16.62 \\
3.64 \\
5.90\end{array}$ & $\begin{array}{r}27.75 \\
0.79 \\
16.00 \\
19.66 \\
11.20 \\
15.80 \\
1.10 \\
6.70\end{array}$ \\
\hline & 100.00 & 100.00 & 99.00 \\
\hline
\end{tabular}

Kozai * gives the following as the results of analyses made by him of Japanese teas:

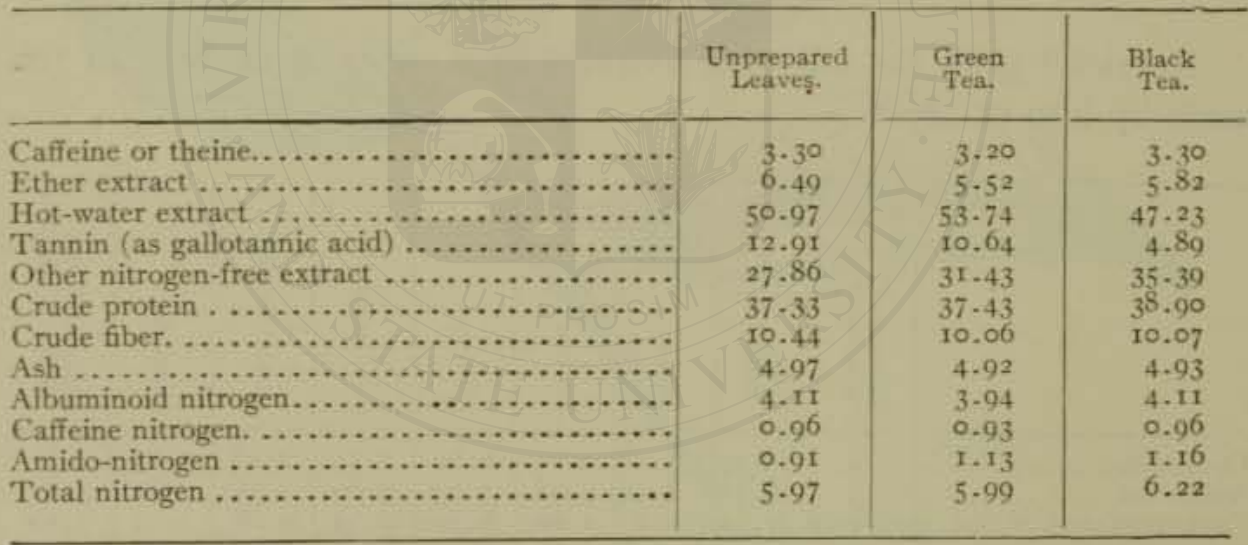

\section{PROXIMATE COMPONENTS AND ANALYTICAL METHODS.}

Preparation of Sample.-Grind the material so as to pass a sieve with holes $0.5 \mathrm{~mm}$. in diameter.

Moisture, Ether Extract, and Crude Fiber are determined in the same weighed portion of 2 grams, following the methods described under cereals (p. 276$)$.

* Bul. 7, Imperial College of Agriculture, Japan. 
Protein.-Determine total nitrogen by the Kjeldahl or Gunning method; from this subtract the nitrogen due to caffein (obtain by dividing by 3.464 ) and multiply the difference by 6.25 .

Total Ash.-Burn 2 grams of the material to a white ash in a platinum dish at a faint red heat: The total ash of pure tea should not be less than 4 nor more than $7 \%$.

Soluble and Insoluble Ash.*-The total ash, as obtained above, is transferred to a beaker with hot water and boiled for some time, after which it is poured upon a filter and the residue washed with hot water. The residue is then dried, ignited at a faint red heat in a platinum dish, cooled, and weighed, thus giving the amount of insoluble ash. The soluble ash is calculated by difference from the total and insoluble ash.

Ash Insoluble in Acid.*-The portion of the ash insoluble in water is washed upon a tared filter with hot $10 \%$ hydrochloric acid and further washed with the latter reagent till the acid-soluble matter is dissolved out, after which it is washed with water, dried, and weighed.

Alkalinity of Ash.*-This is expressed in terms of cc. of tenthnormal acid required for the ash of I gram of sample.

Soluble Ash.-Cool the filtrate from the determination of insoluble ash, as described above, and titrate with tenth-normal hydrochloric acid, using methyl orange as an indicator.

Insoluble Ash. - Add excess of tenth-normal hydrochloric acid (usually Io to $15 \mathrm{cc}$.) to the ignited insoluble ash as obtained above in the platinum dish, heat to the point of boiling over an asbestos plate, cool, and titrate excess of hydrochloric acid with tenth-normal sodium hydroxide, using methyl orange as an indicator.

Essential Oils.-Distil too grams of the tea with $800 \mathrm{cc}$. of water, and shake out the distillate with several portions of ether. The residue from the combined ether extracts contains the volatile oil.

Insoluble Leaf. $\dagger$-Boil 2 grams of the tea in a $500-\mathrm{cc}$. Erlenmeyer flask over a low flame with $200 \mathrm{cc}$. of water, replacing from time to time by addition of hot water the loss from evaporation. Filter through a tared filter, and wash the residue until the filtrate measures $500 \mathrm{cc}$, stirring the contents of the filter throughout the process to facilitate filtering. Reserve filtrate for determination of tannin and theine. Dry the filter and residue until dry to the touch, transfer to the weighing bottle, and dry to constant weight at $100^{\circ} \mathrm{C}$. If the amount of insoluble

* A. O. A. C. Method, U. S. Dept. of Agric., Bur. of Chem., Bul. ro7 (rev.), p. 69.

† Doolittle and Woodruff, A. O. A. C. Proc. 1906, U. S. Dept. of Agric., Bur. of Chem., Bul. 105, p. 48. Winton, Ogden and Mitchell, Conn. Exp. Sta. An. Rep., 1898, p. ${ }_{32}$. 
leaf is above $60 \%$, the presence of spent or exhausted leaves may be suspected.

Extract.-By this term is meant the total amount of water-soluble matter in tea, including such compounds as tannin, caffeine, albuminous matter, dextrin, gum, certain parts of the ash, etc.

The value of a tea from a food standpoint depends obviously upon the character and amount of the extract, rather than on the composition of the dry tea. The relative composition of the extract and of the insoluble leaf, as found by Eder, is given in the following table.

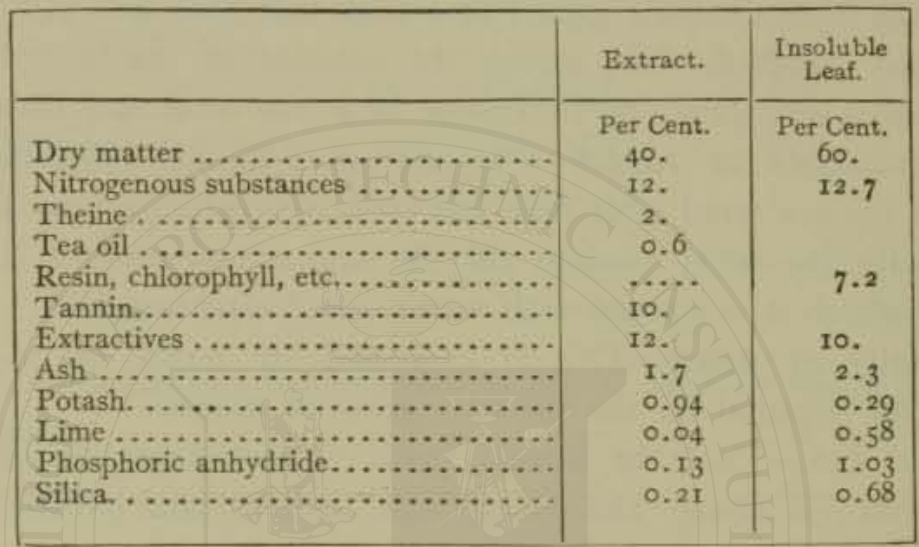

Determination.-The sum of the percentages of insoluble leaf and moisture subtracted from too gives the percentage of extract.

Tannin.-Proctor's Modification of Lowenthal's Method.*

Reagents: (I) Potassium permanganate solution containing about I.33 grams per liter.

(2) Tenth-normal oxalic acid solution ( 6.3 grams per liter).

(3) Indigo carmine solution, containing 6 grams indigo carmine (free from indigo blue) and $50 \mathrm{cc}$. concentrated sulphuric acid per liter.

(4) Gelatin solution, prepared by soaking 25 grams gelatin for an hour in a saturated sodium chloride solution, heating till the gelatin is dissolved, and making up to a liter after cooling.

(5) Mixture of $975 \mathrm{cc}$. saturated sodium chloride solution and 25 cc. concentrated sulphuric acid.

(6) Powdered kaolin.

Obtain the value of the potassium permanganate solution in terms of the tenth-normal oxalic acid solution.

* U. S. Dept. of Agric., Div. of Chem., Bul. 13, part 7, p. 890; Bul. ro7 (rev.), p. 150 . 
Boil 5 grams of the powdered tea for half an hour with $400 \mathrm{cc}$. of water, cool, transfer to a graduated 500-cc. flask, and make up to the mark. To ro cc. of the infusion (filtered if not clear) add $25 \mathrm{cc}$. of the indigo carmine solution and about $750 \mathrm{cc}$. of water. Then add from a burette the potassium permanganate solution, a little at a time while stirring, till the color becomes light green, then cautiously drop by drop till the color changes to bright yellow,* or further to a faint pink at the rim. The volume in cubic centimeters of permanganate furnishes value $a$ of the formula.

Mix $100 \mathrm{cc}$. of the clear infusion of tea with $50 \mathrm{cc}$. of gelatin solution, Ioo cc. of salt acid solution, and to grams of kaolin, and shake several minutes in a corked flask. After settling, decant first the clear supernatant liquid through a filter, and finally bring the precipitate upon the filter. Mix $25 \mathrm{cc}$. of the filtrate (corresponding to ro $\mathrm{cc}$. of the original infusion) with $25 \mathrm{cc}$. of the indigo carmine solution, and about $75^{\circ} \mathrm{cc}$. of water, and titrate with permanganate as before. The volume in cubic centimeters of permanganate used gives value $b$.

$a=$ quantity of permanganate solution required to oxidize all oxidizable substances present.

$b=$ quantity of permanganate solution required to oxidize substances other than tannin.

$\therefore a-b=c$, permanganate required for the tannin. Assuming that $0.04 \mathrm{r} 57$ gram tannin (gallotannic acid) is equivalent to 0.063 gram oxalic acid, the tannin in the tea is readily calculated.

As recommended by Doolittle and Woodruff $\dagger$ the determination may be conveniently made on aliquot portions of the solution obtained in the determination of insoluble leaf.

Method of Fletcher and Allen. $\ddagger$-This method depends upon the precipitation of the tannin and other astringent matters in tea infusion by lead acetate, the point of complete precipitation being indicated by an ammoniacal solution of potassium ferricyanide.

Five grains of neutral lead acetate are dissolved in water, made up to I liter, and after standing the solution is filtered.

As an indicator, 0.05 gram of pure potassium ferricyanide is dissolved in $5 \circ \mathrm{cc}$. of water, and an equal volume of concentrated ammonia-

\footnotetext{
* Various shades of color may be produced, but the same shade should obviously be adopted as an end-point by the operator as when standardizing.

† A. O. A. C. Proc. 1906, U. S. Dept. of Agric., Bur. of Chem., Bul. 105, p. 49.

$\ddagger$ Chem. News, XXIX, 169,189 .
} 
water is added. This indicator produces a red coloration with tannin, gallic acid, or gallotannic acid in solution, being so sensitive that a drop of the indicator will detect I part of tannin in 10,000 parts of water.

Three separate quantities of $10 \mathrm{cc}$. each of the standard lead acetate solution, as above prepared, are measured into as many beakers, and each diluted to $100 \mathrm{cc}$. with boiling water. Two grams of powdered tea are boiled in $250 \mathrm{cc}$. of water, and varying quantities of this decoction are measured from a burette or pipette into the beakers containing the lead solution, the first beaker receiving, say, ${ }^{2} 2 \mathrm{cc}$., the second $15 \mathrm{cc}$., and the third 18 cc., in the case of black tea, and, with green tea, 8, I0, and 12 cc., respectively.

About I cc. of each of these trial quantities is removed from the various beakers by means of a pipette, passed through small filters, and tested with the ammoniacal ferricyanide indicator, the diops of filtered solution being allowed to fall directly on spots of the indicator, previously placed on a white slab or plate.

It is thus easy to ascertain the approximate amount of tea solution which it is necessary to add to produce a pink coloration with the indicator, so that by repeated tests, nearly the right amount may be added at once. If no coloration in a given case is produced when a drop of the filtrate from the solution in the beaker is allowed to fall on the drop of indicator solution, a little more of the tea decoction is added, and this process is repeated until the pink color is apparent.

It should be noted how much of the tea decoction is necessary to add to roo $\mathrm{cc}$. of pure water, that a drop of the solution may produce the pink coloration with the ferricyanide, and this a mount should be subtracted from the amount of decoction found necessary to add to the known lead solution in the beaker. It was found by repeated experiment that $10 \mathrm{cc}$. of lead solution would precipitate 0.0 r gram of pure gallotannic acid; hence, carrying out the process exactly as above described, 125 divided by the number of cubic centimeters of tea decoction required gives the percentage of tannin in the sample.*

Theine or Caffeine $\left(\mathrm{C}_{8} \mathrm{H}_{10} \mathrm{~N}_{4} \mathrm{O}_{2}\right)$. - This alkaloid when pure exists in white silky needles. It is odorless and sparingly soluble in cold water,

\footnotetext{
* This process estimates the total astringent matter, all of which is counted in as tannin.
} 
but more so in hot. It is less soluble in alcohol, and almost insoluble in ether. It readily dissolves in chloroform. It is present in tea, coffee, and kola. Graf* has shown that the amount of caffeine present in tea is in most cases proportional to the commercial value and quality.

Detection.-Caffeine may be detected, if present in a suspected residue, by the so-called "murexid test," which is made with the material in a solid state, or with the residue from the evaporation of a liquid. A small quantity of the solid or powdered material is heated in a white porcelain dish and covered with a few drops of strong hydrochloric acid, after which a fragment of potassium chlorate is immediately added. The mixture is then evaporated to complete dryness on the water-bath, whereupon, if caffeine is present, a reddish-yellow or pink color is produced. After cooling, the residue is treated with a very little ammonia water applied on the point of a stirring-rod. In the presence of caffeine, a purple color (that of murexoin) is produced on application of the ammonia.

Determination of Theine or Caffeine.-Dvorkovitsch Method. $†-$ Digest 10 grams of the powdered tea with $200 \mathrm{cc}$. of boiling water for 5 minutes and decant the solution; repeat the treatment twice, and boil the residue with $200 \mathrm{cc}$. of water. Make up the combined solutions to I000 cc. and extract a portion with petroleum ether to remove fat, etc. To $600 \mathrm{cc}$. of the fat-free solution (equivalent to 6 grams of tea) add roo cc. of $4 \%$ barium hydroxide solution, mix and filter. To $583 \mathrm{cc}$. of the filtrate (equivalent to 5 grams of tea) add roo cc. of a $20 \%$ solution of sodium chloride, and extract three times with chloroform. Distil the greater part of the chloroform from the combined extracts, place the residue in a tared dish, evaporate the remainder of the chloroform, dry at $100^{\circ} \mathrm{C}$., and weigh. The caffeine is usually of sufficient purity to render a nitrogen determination unnecessary.

Doolittle and Woodruff $\ddagger$ proceed as follows: Extract in a separating funnel with petroleum ether $225 \mathrm{cc}$. of the filtrate from the determination of insoluble leaf (p. 369 ) made up to $500 \mathrm{cc}$. To the fat-free portion add $5 \circ \mathrm{cc}$. of a $4 \%$ barium hydroxide solution, shake well,

Forsch, Ber., 4, 1897, pp. 88, 89 .

† Ber. d. chem. Ges., 24, 1891, p. 1945; U. S. Dept, of Agric., Bur. of Chem., Bul, 107 (rev.), p. 150.

$\ddagger$ loc. cil. 
and filter. To the filtrate add $50 \mathrm{cc}$. of a $20 \%$ sodium chloride solution and proceed as above described.

Modification of Stahlschmidt's Method.-Six grams of finely powdered tea are boiled in a flask with several successive portions of water for ten minutes each, and the combined aqueous extracts thus obtained are made up to $600 \mathrm{cc}$. with water. Four grams of powdered lead acetate are added to the decoction, which is then boiled for ten minutes, using a reflux condenser, or making up the loss by occasional addition of water. The solution is then poured upon a dry filter, and $500 \mathrm{cc}$. of the filtrate, corresponding to 5 grams of the tea, are evaporated to about $50 \mathrm{cc}$. and enough sodium phosphate added to precipitate the remaining lead. The solution is then filtered, and the precipitate thoroughly washed, the filtrate and washings being evaporated to about $40 \mathrm{cc}$. Finally, the solution thus concentrated is extracted with chloroform in a separatory funnel for at least four times, and the chloroform extract evaporated to dryness, leaving the caffeine, which is dried to constant weight at $75^{\circ}$ and weighed.

\section{ADULTERATION OF TEA.}

Facing.-The most common form of tea adulteration, if such it may be called, is the practice of "facing" the dried leaves, or treating them with certain pigments and coloring materials to impart a bright color or gloss to the tea, thus causing an inferior grade to appear of better quality than it really is. This practice is more often applied to green tea. The materials for facing include such substances as Prussian blue, indigo, plumbago, and turmeric, often accompanied by such minerals as soapstone, gypsum, etc. Only a small amount of foreign material is actually added to the tea, but the adulteration consists in the deceptive appearance imparted thereto.

Battershal has examined various samples of the preparations used in Japan for facing tea. He found in one case the following composition: Soapstone, $47.5 \%$; gypsum, $47.5 \%$; Prussian blue, $5 \%$. Another sample consisted of soapstone, $75 \%$; indigo, $25 \%$. A third was composed of soapstone, $60 \%$, and indigo, $40 \%$. In applying the facing to the tea, the latter is first heated in an iron pan over the fire, the facing mixture is then added while still hot, and the whole is stirred briskly till the desired color is imparted. The Chinese and Japanese do not face the tea which they themselves consume, but only that intended for export trade. 
Detection of Facing.-The most delicate test for facing is to examine under the microscope, or lens, the dust obtained by sifting the leaves or the sediment obtained after shaking them with water. Plumbago appears glossy black, soapstone gray, gypsum white, Prussian blue, ultramarine and indigo shades of blue, and turmeric yellow. Prussian blue is decolorized by sodium hydroxide solution. Ultramarine is not affected by alkali but is decolorized by hydrochloric acid. Indigo is not decolorized by either reagent.

Read * rubs the siftings with a spatula on sheets of white and black paper and removes the loose dust. The colors after this treatment are recognized under the lens as streaks on the paper. West $\dagger$ detects Prussian blue by the blue spots formed by sprinkling the ground tea on filter paper moistened with oxalic acid solution and drying.

Prussian blue if present in considerable amount may be detected in the sediment, as above obtained, by the blue precipitate which forms after dissolving in hot alkali, filtering, acidifying with hydrochloric acid, and then adding a drop of ferric chloride. If the residue on the paper after treatment with hot alkali, on removal to a porcelain dish and treatment with concentrated sulphuric acid, yields hydrogen sulphide (recognized by its odor or by the blackening of lead acetate paper) ultramarine is indicated.

Such minerals as gypsum and soapstone are readily separated as a sediment by shaking the leaves in water, and the sediment is examined by the appropriate qualitative methods for these substances.

Spent or Exhausted Leaves.-These consist of leaves of tea that have been previously steeped or infused, and afterwards rerolled and dried. Such leaves are sometimes mixed with tea as an adulterant. Any considerable admixture of spent leaves is evident, both by the extremely low ash, and the abnormally small proportion of water-soluble ash in the sample. It is rare that the total ash of genuine tea is under $5 \%$, while the soluble ash is seldom less than $3 \%$.

The ash of spent tea leaves sometimes runs as low as $2.5 \%$, of which generally not more than 0.3 to 0.8 per cent is soluble. Spent leaves are also naturally low in tannin and in extract.

If the extract is much below $32 \%$, spent leaves may be suspected. Allen determines the per cent of spent leaves by subtracting the per cent of extract from 32 , multiplying by 100 and dividing by 30 .

*U. S. Treasury Decision, No. 32322 .

$\dagger$ Jour. Ind. Eng. Chem., 4, 1912, p. 528 . 
The use of spent or exhausted leaves as an adulterant is very rare at present, though formerly of common occurrence.

Foreign Leaves as a Substitute for Tea.-This sophistication is not common, but the detection of leaves other than tea is readily accomplished by a careful examination of the shape and character of the leaves. For this purpose the dried leaves are opened out by soaking a short time in hot water, after which they are spread upon a glass plate, and examined by the aid of a magnifying-glass.

The genuine tea leaf (Fig. 73) is very characteristic, and is readily distinguished from other leaves. It is oval or lanceolate, 5 to $8 \mathrm{~cm}$. long

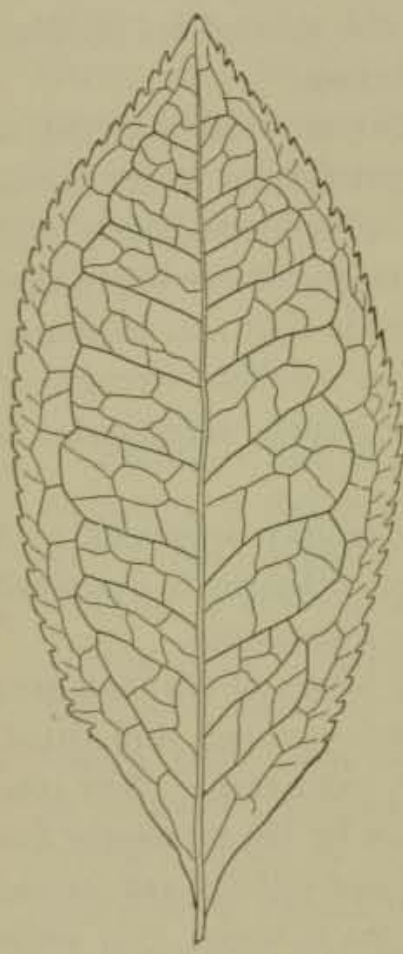

Fig, 73. - The Leaf of Genuine Tea. and 2 to $3 \mathrm{~cm}$. wide. It is short-stemmed, somewhat thick and fleshy, attenuated at the bottom and usually pointed at the top. At a certain height from the base, from a third to a quarter up, the smooth or wavy border becomes peculiarly, though not deeply, serrated in a regular manner, the serrations, which are hook-shaped, continuing to the tip of the leaf. Mature leaves always show these serrations, but they are somewhat obscure in young leaf buds. The latter, however, are rarely found in this country. The veins extend outward from the central rib nearly parallel to each other, but before reaching the border, each bends upward to form a loop with the one above.

Foreign leaves, said to be used as adulterants, are those of the willow, poplar, elder, birch, elm, and rose, but the writer has never found any of these in tea. All of them differ materially from the genuine tea leaf, and if foreign leaves are apparent in a sample under examination, they should be compared with various leaves collected by the analyst for the purpose.

Stems and Fragments.-These, as well as "tea dust," are apparent by an examination of the leaves, opened out in hot water as explained above. The ash of tea stems and dust is abnormally high. The term "lie tea" is applied to an imitation of tea, consisting of fragments, stems, and tea dust, mixed with foreign leaves, mineral matter, gum, etc. 
The ash of such "tea" has been found as high as $50 \%$. Such imitations are now almost unknown. Make-weight substances, such as brickdust, iron salts, metallic iron, sand, etc., have been found in tea. If present, they are to be found in the sediment, obtained on shaking out the tea in water.

Added Astringents. - Catechu is sometimes said to be added to tea to give it increased astringency, especially to such tea as has been adulterated by the addition of exhausted tea. Hagar's method for detecting catechu is as follows:

A hot-water extract of the tea (I to IOO) is boiled with an excess of litharge and filtered. To a part of the filtrate, which should be perfectly clear, nitrate of silver is added. If catechu be present, a yellow flocculcnt precipitate, rapidly becoming dark-colored, is formed. Pure tea treated in like manner gives a gray precipitate.

Spencer * adds, instead of silver nitrate, a drop of ferric chloride to the clear filtrate. With catechu a green precipitate is formed.

As a matter of fact the worst forms of tea adulteration, such as the actual substitution of foreign leaves, once so commonly practiced, are now extremely rare in this country and have been for some years, by reason of the careful system of government inspection in force at the various ports of entry. The greater portion of the tea on our market to-day is genuine, but fraud is practiced to c considerable extent by the substitution of inferior grades for those of good quality. This form of deception is in many cases beyond the power of the analyst to detect, and properly comes within the realm of the professional tea-taster.

Tea Tablets.-Finely ground tea of varying quality is sometimes pressed into tablets, to be used by travelers and campers for preparing a beverage, by simply dissolving in hot water.

The composition of one of these preparations sold under the name of Samovar Tea Tablets, analyzed by the Mass. State Board of Health, is as follows:

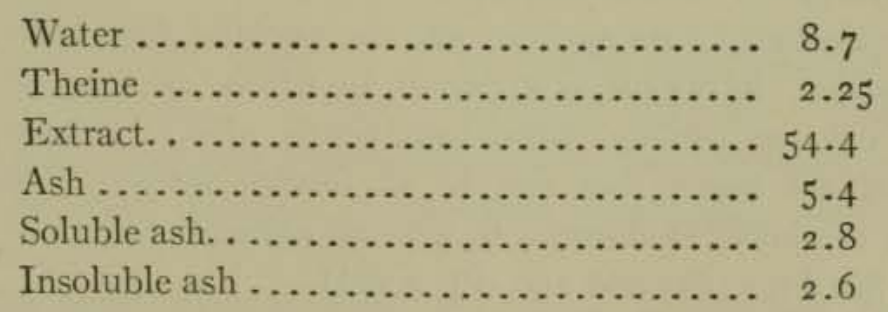

* U. S. Dept. of Agric., Div. of Chem., Bul. 13, p. $88_{5}$. 
Microscopicar Structure of Tea.-The powdered tea may be examined directly in water-mount. Schimper recommends treating the powdered tea with chloral hydrate or potash lye, to render it more transparent.

By far the most characteristic element is the peculiarly shaped sclerenchyma, or stone cell, st, Fig. 74, entirely unlike anything to be found in other leaves. These cells are very irregular in form, being sometimes star-shaped, sometimes branched, almost always with deeply wrinkled sides,

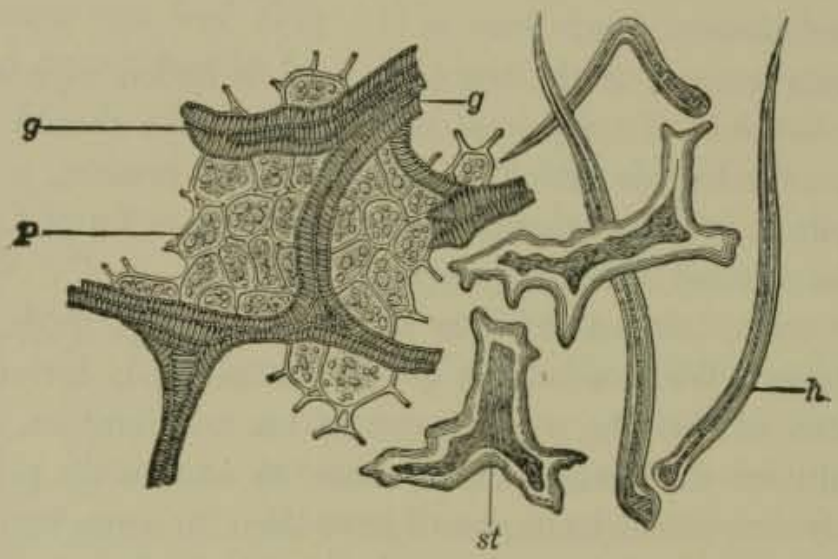

FrG. 74.-Powdered Tea under the Microscope. $X_{1} 60 . g$, end of leaf nerve; $p$, chlorophyll parenchyma; st, stone cells; $h$, hairs. The tissues were warmed in potash to render transparent. (After Moeller.)

and often with sharp points. In most foreign leaves such sclerenchyma cells are lacking, but they are abundant in all genuine tea leaves, excepting rarely in the very young leaves, where they are sometimes not fully developed. They are especially numerous in the main vein and in the stem. They may be seen to best advantage in a section of the stem, or midrib, made parallel to the surface of the leaf. To make such a section, soak the leaf first in water, and afterwards dry in alcohol. The interior of the leaf is composed chiefly of ground tissue, having rounded cells full of chlorophyll grains and the fibro-vascular bundles of the veins.

Other important characteristics are the peculiar hair growth on the under epidermis, $B$, which is apparent in nearly all teas, also crystal rosettes of calcium oxalate, which are nearly always present, even in fragments of tea leaves, but not in all foreign leaves. The peculiar structure of the lower epidermis, $B$, with its numerous stomata is also to be noted. See Figs. 189 and 190, Pl. XVIII. 


\section{COFFEE.}

Nature of Coffee.-Coffee is the seed of the Coffea arabica, a tree which, when under cultivation, is not allowed to exceed twelve feet in height, but when wild sometimes reaches a height of twenty feet. It is indigenous in Southern Abyssinia, and was cultivated in Arabia in the sixteenth century, and in the East Indies in the seventeenth, afterward being introduced into the West Indies and South America. The coffeebeans are usually inclosed in pairs in the berry, being plano-convex with their flat sides together but in "péa berry " coffee only a single, rounded bean is present.

When the ripe fruit is gathered, it is first dried and then freed from the hulls, usually by machinery, or, in the West Indies, the green berries are "pulped" or "hulled" under water by a peculiar macerating machine. The raw beans are roasted, and afterwards ground for preparing the infusion.

Brazil furnishes more than half the world's supply of coffee, and nearly $75 \%$ of that consumed in the United States.

Composition of Coffee.-Most of the coffee in the retail market is roasted, being sold either in the whole bean or ground. Comparatively little raw coffee is sold at retail.

The constituents of raw coffee, besides water, are, in the order of their comparative amounts, cellulose or crude fiber, fat, protein, caffetannic acid, sugar, caffein, gum, dextrin, and ash. The effect of roasting coffee, besides driving off most of the water, is to caramelize a large part of the sugar, to make the bean less tough and more brittle, and, most important of all, to develop an empyreumatic, oily substance, known as caffeol $\left(\mathrm{C}_{8} \mathrm{H}_{10} \mathrm{O}_{2}\right)$, to which the characteristic flavor and aroma of coffee are largely due. Caffeol may be obtained by distilling an infusion of roasted coffee, and extracting the distillate with ether. It is a brown oil, almost insoluble in water.

According to Génin, there are in raw coffee small amounts of two essential oils, one soluble in water, the other insoluble. During the roasting, these are partially transformed into the substance caffeol.

The fat in coffee forms a considerable constituent, amounting in some cases to $15 \%$.

Caffetannic Acid $\left(\mathrm{C}_{15} \mathrm{H}_{18} \mathrm{O}_{8}\right)$ is, when pure, a colorless, crystalline compound. It exists in coffee either as a salt of calcium or magnesium, or, according to Payen, as a caffetannate of potassium and caffeine. 
The following summary of analyses of coffee of various kinds made by König show in general its composition:

\begin{tabular}{|c|c|c|c|c|}
\hline & \multicolumn{2}{|c|}{ Raw Coffee. } & \multicolumn{2}{|c|}{ Roasted Coffee. } \\
\hline & Mínimum. & Maximum. & Minimum. & Maximum. \\
\hline 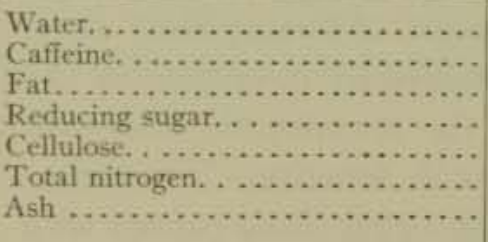 & $\begin{array}{r}8.0 \\
0.8 \\
11.4 \\
5.8 \\
16.6 \\
1.1 \\
3.5\end{array}$ & $\begin{array}{r}12.0 \\
1.8 \\
14.2 \\
7.8 \\
42.3 \\
2.2 \\
4.0\end{array}$ & $\begin{array}{r}0.4 \\
0.8 \\
10.5 \\
0.0 \\
26.3 \\
1.3 \\
4.0\end{array}$ & $\begin{array}{r}4.0 \\
1.8 \\
16.5 \\
1.1 \\
51.0 \\
2.7 \\
5.0\end{array}$ \\
\hline
\end{tabular}

The change in composition that takes place in roasting coffee is well shown by the following figures, which give the mean of analyses by König of four samples of coffee before and after roasting:

\begin{tabular}{|c|c|c|}
\hline & Raw Coffee. & Roasted Coffee. \\
\hline 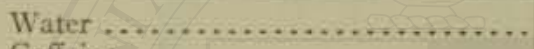 & 11.23 & I. 15 \\
\hline Caffeine..$\ldots \ldots \ldots \ldots \ldots \ldots \ldots \ldots$ & 1.21 & 1.24 \\
\hline 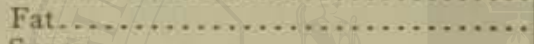 & 12.27 & $\mathrm{I} 4 \cdot 48$ \\
\hline$\ldots \ldots \ldots \ldots \ldots \ldots \ldots$ & 8.55 & 0.66 \\
\hline 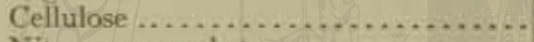 & 18.17 & 10.89 \\
\hline Nitrogenous substances ........... & 12.07 & 13.98 \\
\hline Other non-nitrogenous matter........ & $32.5^{8}$ & 45.09 \\
\hline 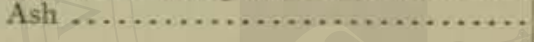 & 3.92 & 4.75 \\
\hline
\end{tabular}

[COMPOSITION OF THE ASH OF COFFEE, *

\begin{tabular}{|c|c|c|c|c|}
\hline Constituents. & Mocha. & Maracaibo. & Java. & Rio. \\
\hline 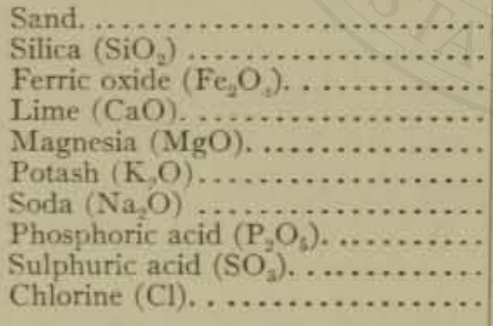 & $\begin{array}{r}1.44 \\
0.88 \\
0.89 \\
7.18 \\
10.68 \\
59.84 \\
0.48 \\
12.93 \\
4.43 \\
1.25\end{array}$ & $\begin{array}{r}0.72 \\
0.88 \\
0.89 \\
5.06 \\
11.30 \\
61.82 \\
0.44 \\
13.20 \\
5.10 \\
0.59\end{array}$ & $\begin{array}{r}0.74 \\
0.91 \\
1.16 \\
4.84 \\
11.35 \\
62.08 \\
1 \ldots \\
14.09 \\
4.10 \\
0.73\end{array}$ & $\begin{array}{r}1.34 \\
0.69 \\
1.77 \\
4.94 \\
10.60 \\
63.60 \\
0.17 \\
11.53 \\
4.88 \\
0.48\end{array}$ \\
\hline & 100.00 & 100.00 & 100.00 & 100.00 \\
\hline
\end{tabular}

The following are analyses of common varieties of roasted coffee, also of coffee substitutes and adulterated coffee made by Lythgoe: $\dagger$

* U. S. Dept. of Agric., Div. of Chem,, Bul. 13, p. 904 .

$\dagger$ An. Rep. Mass. State Board of Health, 1904, p. 3\%0, U, S. Dept. of Agric., Bur. of Chem., Bul. 9o, pp. 43-45. 
COMPOSITION OF ROASTED COFFEE.

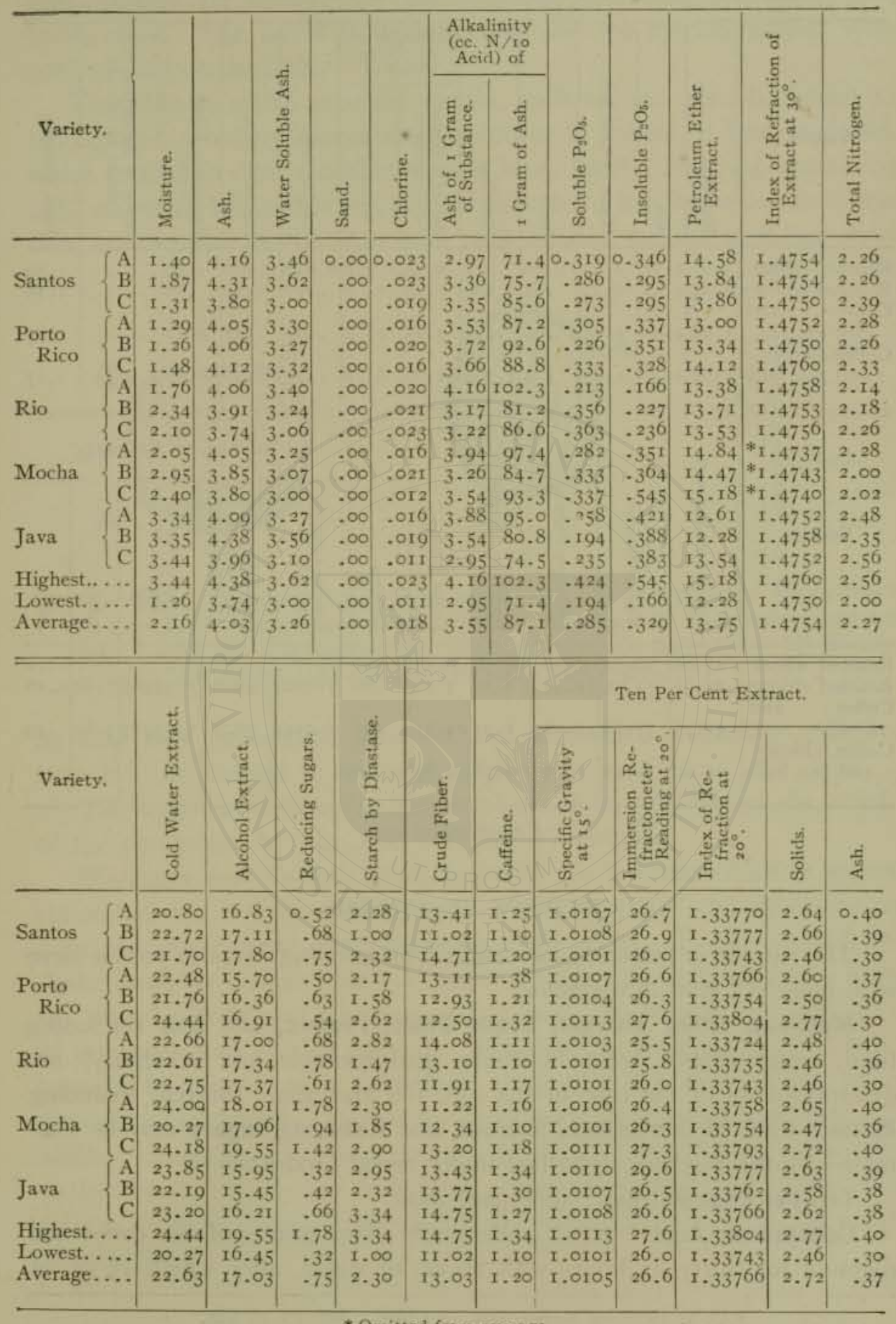

* Omitted from average. 
COMPOSITION OF COFFEE SUBSTITUTES AND OF ADULTERATED COFFEE.

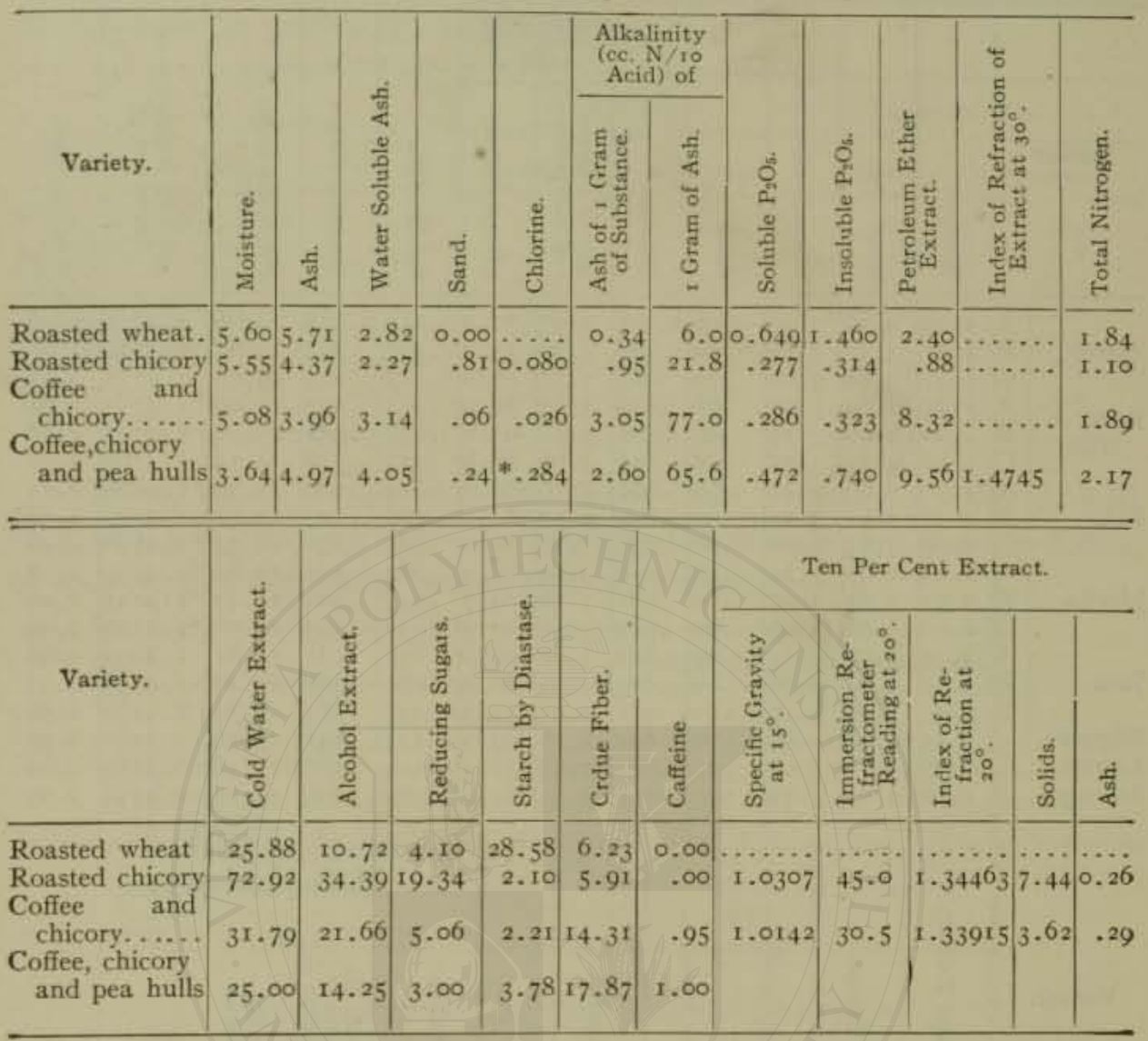

* Admixture of salt.

\section{METHODS OF ANALYSIS.}

The sample is prepared for analysis by grinding so as to pass a sieve with holes $0.5 \mathrm{~mm}$. in diameter.

Moisture, Ether Extract, Crude Fiber, Protein, and Ash (including total, water-soluble, water-insoluble, acid-insoluble and alkalinity) are determined as in the case of tea (pp. 368 and 369 ). Starch, Reducing Matters by Acid Conversion, Sucrose, and Reducing Sugars may be estimated by the methods described under cereal products.

Ten Per Cent Extract. (See page 389 .)

Caffetannic Acid.-Krug's Method.*-Two grams of the coffee are digested for thirty-six hours with - Io cc. of water, after which $25 \mathrm{cc}$. of

* U. S. Dept. of Agric., Div. of Chem., Bul. r3, p. 908. 
$90 \%$ alcohol are added, and the digestion continued for twenty-four hours more. The liquid is then filtered, and the residue washed with $90 \%$ alcohol on the filter.

The filtrate, which contains tannin, caffeine, fat, etc., is heated to boiling and a boiling concentrated solution of acetate of lead is added, which precipitates out a caffetannate of lead, $\mathrm{Pb}_{3}\left(\mathrm{C}_{15} \mathrm{H}_{15} \mathrm{O}_{8}\right)_{2}$, containing $49 \%$ of lead. When this has become flocculent, it is separated by filtration, and washed on the filter with $90 \%$ alcohol, till the washings show

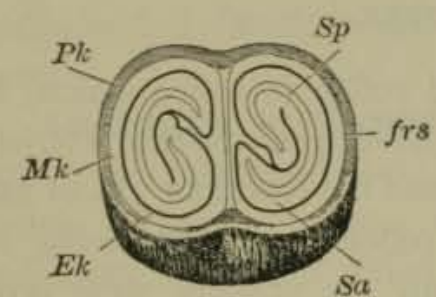

I.

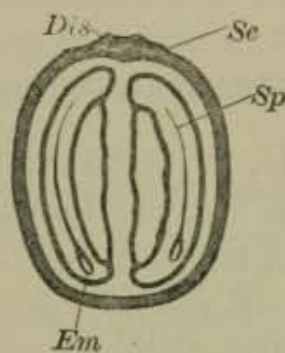

II.

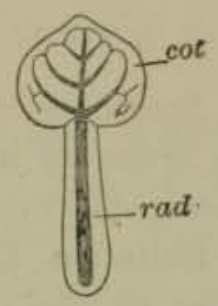

III.

Fic. 75.-Coffee. I. cross-section of berry, natural size. $P k$ outer pericarp; $M k$ endocarp; Ek spermoderm; $S a$ hard endosperm; $S p$ soft endosperm. II. Longitudinal section of berry, natural size; $D$ is bordered dis:; Se remains of sepals; $E m$ embryo. III. Embryo enlarged; col cotyledon; rad radicle. (TSCHIRCH AND OESTERLE.)

no lead with ammonium sulphide, and afterwards with ether, till free from fat. It is dried at $100^{\circ}$ and weighed.

The weight of caffetannic acid is obtained by multiplying the weight of the precipitate by $65^{2}$, and dividing by $126_{3} .6_{3}$.

Woodman and Taylor's Modification.*-To 2 grams of finely ground coffee (passing $0.5 \mathrm{~mm}$. sieve), add $10 \mathrm{cc}$. of water, and shake for an hour in a mechanical shaking device. Add $25 \mathrm{cc}$. of $90 \%$ alcohol and shake again for half an hour. Filter and wash with $90 \%$ alcohol. Bring the united filtrate and washings, about $50 \mathrm{cc}$., to boiling, and add $6 \mathrm{cc}$. of saturated lead acetate solution. Separate the precipitated lead caffetannate by means of a centrifuge, decanting the supernatant liquid through a tared filter. Repeat the centrifugal treatment twice with $90 \%$ alcohol, decanting each time through the filter. Transfer the precipitate to the filter, and wash free from lead. Wash with ether, dry at $100^{\circ}$, and weigh. The weight of the precipitate multiplied by $0.5^{1} 6$ gives the weight of caffetannic acid.

* A. O. A. C. Proc. 1908, U. S. Dept. of Agric., Bur. of Chem., Bul, 122, p. 82. 
Caffeine.-Görter Method.*-Moisten Ir grams of the finely powdered coffee with 3 cc. of water, allow to stand for half an hour, and extract for three hours in a Soxhlet or Johnson extractor with chloroform. Evaporate the extract, treat the residue of fat and caffeine with hot water, filter through a cotton plug, and wash with hot water. Make up the filtrate and washings to $55 \mathrm{cc}$., pipette off $50 \mathrm{cc}$, and extract four times in a separatory funnel with chloroform. Evaporate this chloroform extract in a tared flask, dry the caffeine at $100^{\circ} \mathrm{C}$., and weigh.

Calculate the caffeine also from the nitrogen content.

\section{ADULTERATION OF COFFEE.}

According to the U. S. Standard roasted coffee is coffee which, by the action of heat, has become brown and developed its characteristic aroma, and contains not less than $10 \%$ of fat and not less than $3 \%$ of ash.

Imitation Coffee.-Formerly, artificial coffee-beans containing no coffee whatever, but cleverly molded to imitate the original, were occasionally to be found, mixed with genuine, whole coffee. $\dagger$

"Coffee pellets" are occasionally sold in bulk to dealers as an adulterant of whole coffee. These do not closely resemble the real berries in appearance, but are approximately of the same size, and are not apparent to the purchaser when the whole coffee is ground at the time of purchase. A sample of these "pellets" examined recently was found to consist of roasted wheat mash, colored with red ocher.

Coloring Coffee Beans.-The practice of treating raw coffee beans in a manner somewhat analogous to the facing of tea leaves has been sometimes practiced, with a view to giving to cheaper or inferior grades the appearance of high-priced coffee. For this purpose various pigments have been employed, such as yellow ocher, chrome yellow, burnt umber, venetian red, Scheele's green, iron oxide, tumeric, indigo, Prussian blue, etc., the coffee beans being first moistened with water containing a little gum, and shaken with the pigment. As a rule such pigments, especially when inorganic, are best sought for either in the ash, or in the sediment obtained by shaking the coffee beans in cold water, using the

*Annalen, 358, 1908 , p. 327 . U. S. Dept. of Agric., Bur. of Chem., Bul. ${ }^{132}$, p. 135 . A. O. A. C. method.

t A sample of such imitation whole coffee in the possession of the writer consists almost entirely of roasted wheat molded into beans with difficulty to be distinguished in appearance from those of genuine coffee, so closely do they resemble the original, even to the cleft in the sides. The chaff in the cleft is, however, lacking. 
ordinary qualitative chemical methods. Organic coloring matters can be best extracted with alcohol. Prussian blue and indigo are tested for as in the case of tea leaves (p. 375).

Glazing.-This is a more recent form of treatment of the whole bean, which consists in coating the beans by dipping in egg or sugar, or a mixture of the two, sometimes using various gums. Such glazing is alleged to improve the keeping qualities of the coffee, as well as to aid in clarifying the infusion, and if this is the sole purpose, the practice cannot be condemned as a form of adulteration. If, however, it is done to give inferior varieties of coffee a better appearance, in order to deceive the consumer, it clearly constitutes adulteration within the meaning of the law.

Adulterants of Ground Coffee.-Of the adulterants used in ground coffee the following have been found in Massachusetts: Roasted peas, beans, wheat, rye, oats, chicory, brown bread, pilot bread, charcoal, red slate, bark, and dried pellets, the latter consisting of ground peas, pea hulls, and cereals, held together with molasses.

Methods of Detecting Adulterants.-These methods are, as a rule, physical rather than chemical. A rough test of the genuineness of ground coffee consists in shaking some of the sample in cold water. Pure coffee, under these conditions, usually floats on the surface, while the ordinary adulterants, such as cereals, chicory, mineral ingredients, etc., sink, the grains of chicory coloring the water a brownish-red as they subside.

Macfarlane recommends the use of a saturated solution of common salt, in which a portion of the suspected sample, divided in small grains, is shaken in a test-tube. If the liquid is colored pale amber, while all or nearly all the material floats, the coffee is pure. Any considerable sediment at the bottom of the tube, accompanied by a dark-yellow to brown color imparted to the liquid, indicates adulteration by roasted cereals, or chicory, or both.

A careful examination of the coarsely crushed grains of a ground sample with the naked eye will often serve to detect, and in some cases identify, certain adulterants, such as chicory and ground peas or beans. A magnifying-glass will aid in such an examination, and the observer can often separate the various ingredients of a coffee mixture, first spreading a small portion of the sample on a sheet of white paper. The chicory grains are apparent from their dark and somewhat gummy appearance, and can usually be recognized by crushing them between the teeth. Their soft consistency and sweetish bitter taste are very distinctive. The dull 
outer surface of the crushed coffee grains is in marked contrast to the polished appearance of the surface of the broken peas or beans, often to be found as adulterants, while fragments of broken cereal grains are readily distinguished from coffee with a low-power magnifier, though perhaps not easily identified by the eye alone.

Determination of Added Starch.-Starch is determined in the finely powdered sample as directed on page $28_{3}$.

Microscopical Examination of Coffee.-By far the best means of detecting adulteration is furnished by the microscope. The individual grains of coarsely ground coffee and adulterants, separated by the cold water test or by picking over the mixture, are identified by microscopic examination either after sectioning with a razor or crushing to a powder. In addition, examination is made of a small portion of the sample pulverized in a mortar to a degree fine enough to allow the cover-glass to lie flat on the wetted powder, yet not so fine that it ceases to feel granular when rubbed between the fingers. The writer finds it sufficient to examine this powder in water without further treatment, although Schimper recommends maceration for twenty-four hours with ammonia, in order to render the tissues more transparent, using this reagent also as a mountant.

In general the interior of the coffee tissue or endosperm consists of polygonal cells with highly characteristic, knotty, thickened walls, which are best seen in razor sections, Fig. 76,2 . These cells contain brilliant, colorless, spherical oil drops, and also proteins.

The seed coat is also very characteristic, showing in the powder as occasional delicate silver-like patches, with peculiar, spindle-shaped, thick-sided cells, some of which are loosened from the tissue.

Plates XIV and XV illustrate photomicrographs of pure and adulterated coffee. Fig. 174 shows genuine coffee, with its loose mesh of irregularly polygonal cells, thick-walled, and inclosing oil drops with amorphous material. It is not to be expected that every pulverized sample of genuine coffee, mounted as above, will show in every microscopic field the even, continuous structure that Fig. 174 illustrates, but careful examination will show in nearly every field fragments, and more or less disjointed portions of the polygonal cells, grouped in the form so characteristic of coffee. See Fig. 176 .

Chicory under the Microscope.-Fig. 77, after Moeller, shows structural features of chicory, The most striking elements are the fine, thickwalled, long-celled, parenchyma of the bark $r p$ and $b p$ with its delicate 


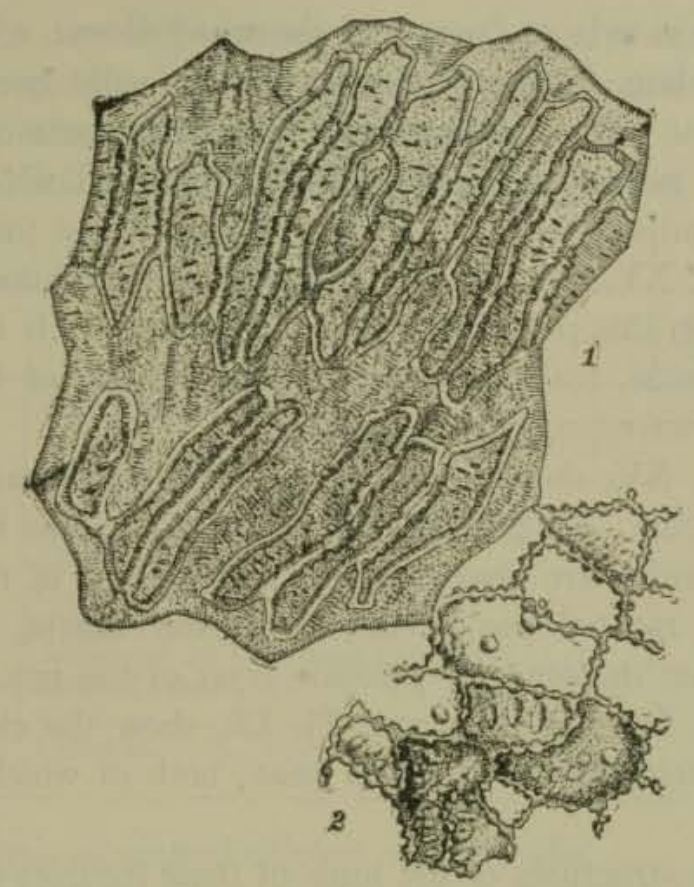

FIG. 75,--Powdered Coffee under the Microscope. $X_{125}$. (After Moeller.) I, seed coat (surface). 2, endosperm parenchyma.
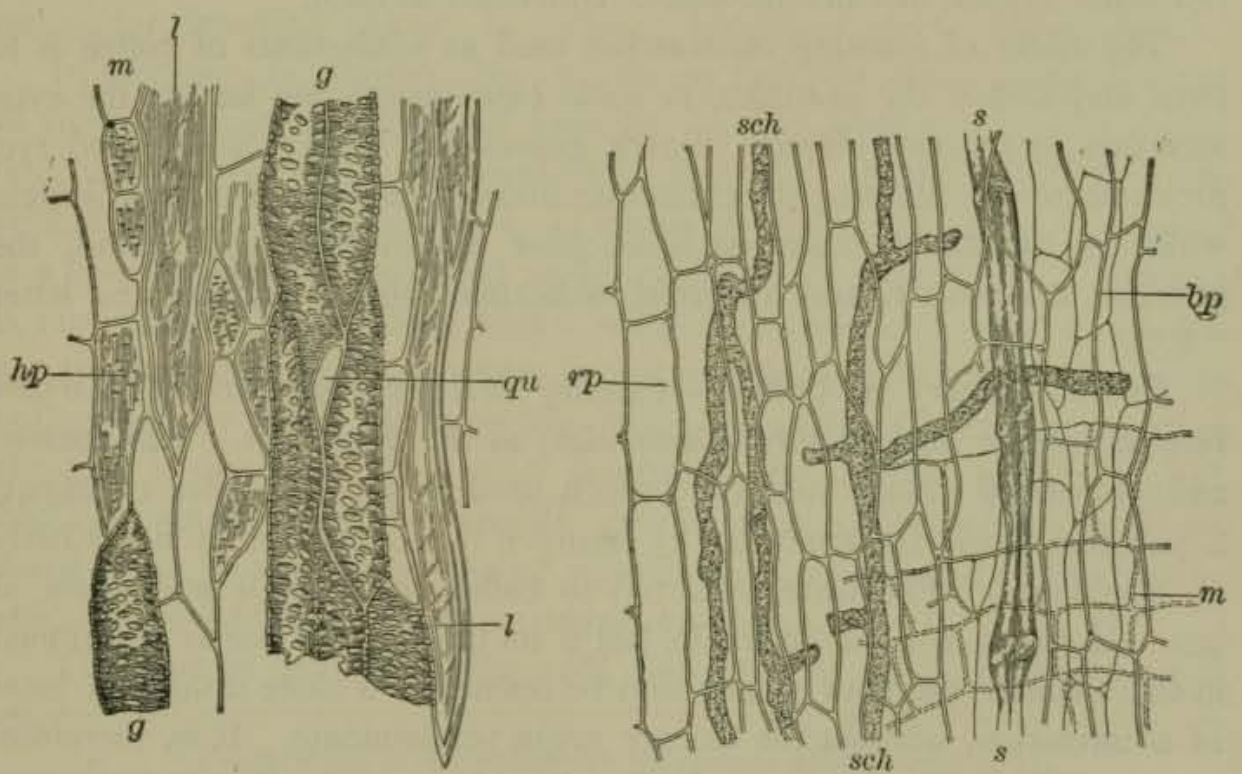

Fig. 77.-Chicory Root in Tangential and Radial Sections. XI60. $g$, reticulated ducts with perforations $q u ; h p$, wood parenchyma; $l$, wood fibers; $r p$, bark parenchyma; $s c h$, milk ducts; $b p$, bast parenchyma; $m$, medullary rays. (After Moeller.) 
tracery, and the vessels or ducts $g$ of the wood fibers. These ducts are tubular, resembling jointed cylinders, often with overlapping joints. Less distinct, but very characteristic of certain roots of the composite family, are the narrower branching milk ducts sch which do not exist in beets and turnips, which are sometimes substituted for chicory.

Fig. ${ }_{178}, \mathrm{Pl} . \mathrm{XV}$, is a photomicrograph of an adulterated sample of coffee, showing in this particular field chicory alone. It is a mass of confused cellular tissue, traversed by two broad bands of the vessels, with their striking, transverse, dotted markings.

Fig. ${ }^{177}, \mathrm{Pl}$. XV, shows a sample of coffee adulterated with roasted peas and pea hulls. No genuine coffee appears in this field. The chief masses in the center are characteristic aggregations of the round starch granules of the roasted pea. The rectangular billets, like bunches of matches, are from the outer or palisade layer of the pea.

Fig. I64, Pl. XI, and Fig. I54, Pl. IX, show the close resemblance between the starches of the pea and bean, both of which are commonly used in coffee.

The palisade structures of the hulls of these legumes also bear a close resemblance, but the cells of the next layer in the pea are hour-glass shaped, while in the bean they are not remarkable for their shape, but for the single crystal of calcium oxalate contained in each.

The effect of roasting on starches used as adulterants of coffee is to twist and distort the granules, in some cases destroying largely the even structure of the raw starch. Starch granules of wheat, barley, and rye, for example, are almost perfect circular disks in the case of the raw starch, while in roasted products, such as pilot biscuit and stale bread, the granules are twisted and distorted, sometimes almost forming the letter "S."

Use of Chicory in Coffee.-Chicory is a perennial herb (Cicleorium intybus) of the same family (Compositce) as the dandelion. The roasted and pulverized chicory root is so much used in ground coffee to impart a peculiar flavor thereto, that by many it is considered as not structly an adulterant. The taste imparted to coffee by a small admixture of pure chicory is to some desirable, but if its unrestricted use is sanctioned in this manner, the door would soon be opened to a more unlimited form of adulteration, wherein the chicory might predominate. It is, therefore, best to regard chicory as an adulterant, and to require the package containing a mixture of coffee and chicory, if sold legally, to have plainly printed thereon the percentage of chicory in the mixture. 
Chicory, when roasted, consists of gum, partly caramelized sugar, and insoluble vegetable tissue. Common adulterants of chicory are dried beets and other roots, also cereal matter.

Villiers and Collin* give the following analyses of two samples of chicory:

\begin{tabular}{|c|c|c|c|}
\hline & & $\begin{array}{l}\text { In Large } \\
\text { Granules. }\end{array}$ & In Powder. \\
\hline \multirow{6}{*}{ Soluble in water: } & Water (loss at $100^{\circ}$ to $\left.103^{\circ}\right) \ldots \ldots \ldots \ldots \ldots$ & 16.28 & 16.96 \\
\hline & Weight of total matter soluble in water. .... & $57 \cdot 9^{6}$ & 56.90 \\
\hline & Reducing sugar $\ldots \ldots \ldots \ldots \ldots \ldots \ldots \ldots \ldots \ldots$ & 26.12 & 23.79 \\
\hline & Dextrin, gum, inulin. ................... & 9.63 & $9 \cdot 3 \mathrm{I}$ \\
\hline & 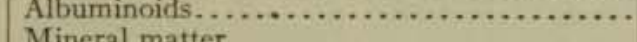 & 3.23 & 3.66 \\
\hline & Coloring matter. $\ldots \ldots \ldots \ldots \ldots \ldots \ldots \ldots \ldots \ldots$ & $\begin{array}{r}2.5^{8} \\
16.40\end{array}$ & $\begin{array}{r}2.55 \\
-50\end{array}$ \\
\hline \multirow{5}{*}{ Insoluble in water: } & 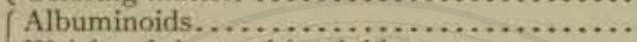 & 3.15 & 2.98 \\
\hline & Weight of the total insoluble matter. ......... & 25.76 & 26.14 \\
\hline & Mineral matter. $\ldots \ldots \ldots \ldots \ldots \ldots \ldots \ldots \ldots$ & $4.5^{8}$ & 5.87 \\
\hline & Fat $\ldots \ldots \ldots \ldots \ldots \ldots \ldots \ldots \ldots \ldots \ldots \ldots \ldots$ & $5 \cdot 7 \mathrm{I}$ & 3.92 \\
\hline & Cellulose, ............... & 12.32 & 13.37 \\
\hline
\end{tabular}

See also analysis of roasted chicory on page 382 .

Detection and Estimation of Chicory.-Various chemical tests for detection of chicory in coffee infusions have been suggested, depending on color reactions, $\uparrow$ but these are, as a rule, unreliable. By far the best means for detecting chicory in coffee is furnished by the microscope.

In mixtures containing coffee and chicory only, the approximate amount of the latter can be calculated from the specific gravity of a $10 \%$ decoction, using conveniently the method of McGill. $\ddagger$ A quantity of the pulverized sample, corresponding to 10 grams of the dry substance, is weighed in a counterbalanced flask, and water added till the weight of the contents is rio grams. Fit the flask with a reflux condenser, and after so regulating the heat that boiling begins in ten to fifteen minutes, continue the boiling for an hour. Remove the flame, and after fifteen minutes pass through a dry filter, cool, and determine the specific gravity at $15^{\circ}$. McGill found the average specific gravity of a $10 \%$ decoction as above carried out to be, in the case of pure coffee, 1.00986 and in the case of chicory 1.02821 , the difference being 0.01835 .

The specific gravity of the $10 \%$ decoction of the suspected sample

\footnotetext{
* Falsifications et Alterations des Substances A limentaires, p. 234. † See Allen's Commercial Org. Analysis, 4 Ed., Vol. VI, pp. 671, 672. $\ddagger$ Trans. Royal Soc. of Canada, 1887 .
} 
at $15^{\circ}$ being $d$, the per cent of chicory, $c$, can be calculated roughly by the formula

$$
c=100-\frac{(\mathrm{I} .0282 \mathrm{I}-d) \mathrm{ro0}}{0.01835}
$$

This method is of course inapplicable when other substances than chicory are present.

Date Stones, roasted and ground, have been used to some extent as a coffee adulterant. Fig. 78 shows the structural features of date stones

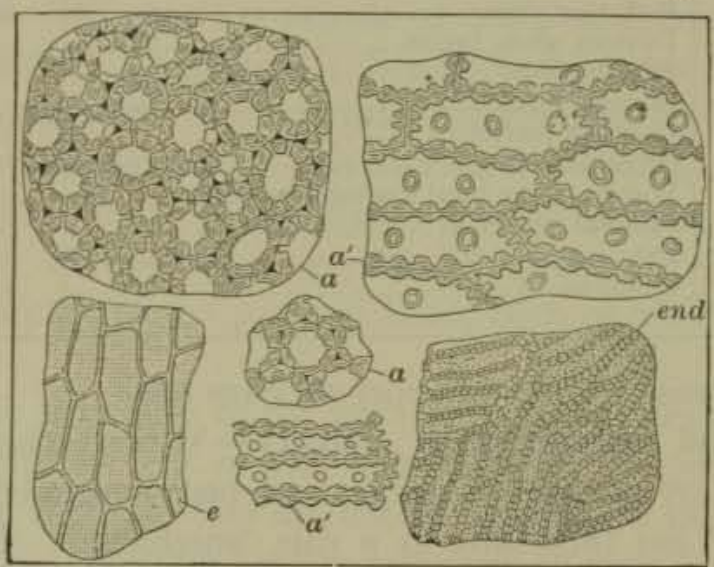

Frc. 78.-Powdered Date Stones under the Microscope. end, endocarp; $e$, episperm; $a$, albumen in cross-section; $a^{\prime}$, albumen in longitudinal section. (After Villiers and Collin.)

under the microscope. End represents a fragment of endocarp with its elongated, thick-walled cells, peculiarly arranged as shown, adjacent cells often lying with axes at right angles to each other. The more evenly formed episperm cells, $e$, are thin-walled and of a brown color. The albumen, $a$, is made up of very thick-walled, somewhat regularly arranged cells, indented from within with deep channels. Date stones are readily distinguished from coffee by these features.

Hygienic Coffee.-Various processes have been devised for removing the caffeine from coffee. One of these, patented in Germany, has recently come into extensive use, as the flavor of the beverage is not greatly injured by the treatment. In following out this process the whole beans are first exhausted with water in a vacuum, and the infusion extracted with a suitable solvent for caffeine. The exhausted beans are then impregnated with the decaffeinated infusion and dried in a vacuum. This treatment, 
as shown by the investigations of Lendrich and Murdfield,* does not completely remove all the caffeine, the quantity remaining being from 0.14 to $0.26 \%$, or about one-sixth of that in the untreated coffee. Further effects of the treatment are a decrease in the water extract and an increase in the fat. The following are the average of analyses, made by these authors, of caffeine-free and untreated coffee:

\begin{tabular}{|c|c|c|c|c|c|c|c|c|c|}
\hline & \multirow[b]{2}{*}{ 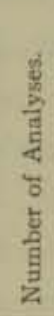 } & \multirow[b]{2}{*}{$\frac{\frac{j}{2}}{\frac{5}{3}}$} & \multicolumn{7}{|c|}{ Analysis of the Dry Substance. } \\
\hline & & & $\frac{1}{4}$ & 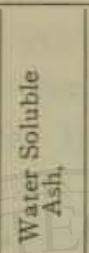 & 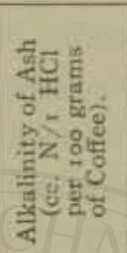 & 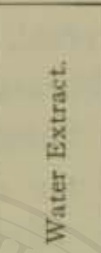 & 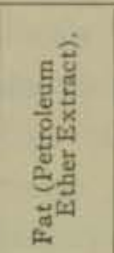 & 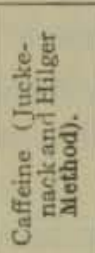 & 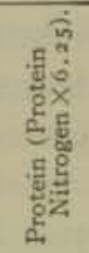 \\
\hline $\begin{array}{l}\text { "Caffeine-free Coffee". .. } \\
\text { Untreated coffee. ...... }\end{array}$ & $\begin{array}{r}14 \\
9\end{array}$ & $\begin{array}{l}\% \\
2.13 \\
1.46\end{array}$ & $\begin{array}{l}\% \\
4.23 \\
4.71\end{array}$ & $\begin{array}{c}\% \\
3.22 \\
3.77\end{array}$ & $\begin{array}{l}47.72 \\
5^{6} \cdot 43\end{array}$ & $\begin{array}{c}\% \\
21.30 \\
26.17\end{array}$ & $\begin{array}{c}\% \\
17.13 \\
15.73\end{array}$ & $\begin{array}{c}\% \\
0.22 \\
1.19\end{array}$ & $\begin{array}{l}\% \\
11.83 \\
11.75\end{array}$ \\
\hline
\end{tabular}

Several brands of coffee, advertised to be free from tannin and in some cases also from caffeine, have been placed on the market in the United States. Some of these consist merely of ground coffee from which the chaff (which is represented to contain not only the tannin but also most of the caffeine) has been removed by mechancial means. The absurdity of the claims of the manufacturers is shown by the following analyses made in New Hampshire by C. D. Howard. $\dagger$

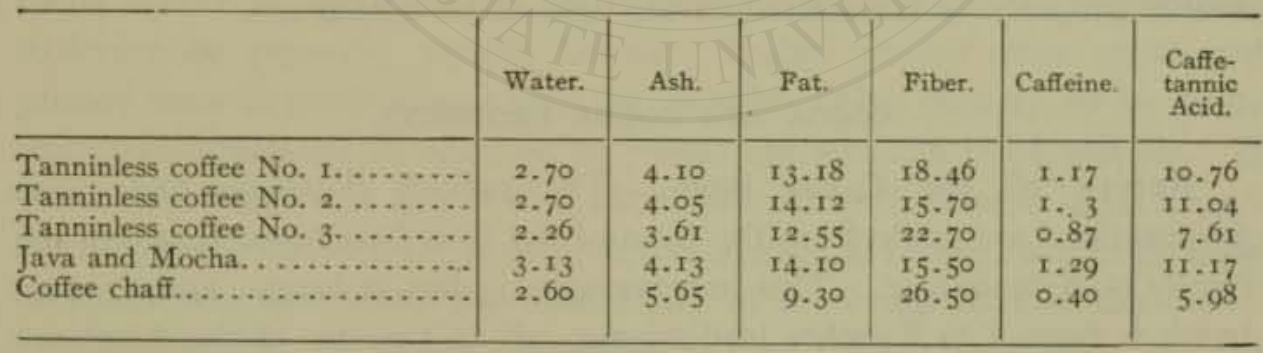

The following analyses made at the Connecticut Station by E. J. Shanley $\ddagger$ corroborate those of Howard:

* Zeits. Unters. Nahr. Genuss., 15, 1908, p. 705.

† A. O. A. C. Proc, rgo6, U. S. Dept. of Agric., Bur. of Chem., Bul. ro5, p. 4 I.

$\ddagger$ An. Rep. Conn. Exp. Sta., 1907, p. 141. 


\begin{tabular}{|c|c|c|c|}
\hline & $\begin{array}{l}\text { Caffetannic } \\
\text { Acid in the } \\
\text { Coffee. }\end{array}$ & $\begin{array}{l}\text { Caffetannic } \\
\text { Acid in the } \\
\text { Chaft. }\end{array}$ & $\begin{array}{c}\text { Per Cent of } \\
\text { Chaft in the } \\
\text { Coffee. }\end{array}$ \\
\hline 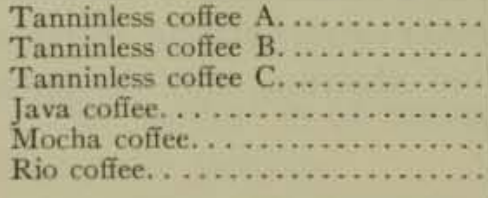 & $\begin{array}{l}9.89 \\
9 \cdot 45 \\
9 \cdot 96 \\
9 \cdot 51 \\
9 \cdot 96 \\
9 \cdot 47\end{array}$ & $\begin{array}{l}5.46 \\
7.55 \\
6.79\end{array}$ & $\begin{array}{l}1.80 \\
2.38 \\
1.77\end{array}$ \\
\hline
\end{tabular}

Coffee Substitutes. - A large number of preparations sold as " coffee substitutes" or "cereal coffee" are now on the market in the United States, most of which are composed, as alleged on the labels, of cereals, ground peas, etc. Some contain roasted wheat, malt or some other cereal ai:ne, others are mixtures of cereals or cereal products and peas, and a few contain chicory. Some of these preparations have labels calling attention to the evil effect's of coffee, and one of the latter class, extensively advertised, and purporting to contain nothing but the entire wheat kernel roasted and ground, was found to contain peas, and about $30 \%$ of that "most harmful ingredient" coffee itself. Various substitutes are also made from dried fruits such as figs, prunes and bananas.

In addition to the materials named the following have been used in Europe: beans, lupine seeds, cassia seeds, astragalus seeds, Parkia seeds, chick peas, soja beans, dried pears, carob bean pods, date stones, ivory nuts, acorns, grape seeds, fruit of the wax palm, cola nuts, false flaxseed, dandelion roots, beets, turnips and carrots.*

As in the case of coffee the analyst must depend chiefly on the microscope in identifying the constituents of coffee substitutes. Coffee itself should properly be considered in the light of an adulterant.

\section{COCOA AND COCOA PRODUCTS.}

Nature of the Cocoa Bean.-The various chocolate and cocoa preparations are made from the bean of the tree Theobroma cacas, of the family of Bytmeriacee. This tree averages $I_{3}$ feet in height, and its main trunk is from 5 to 8 inches in diameter. It is a native of the American iropics, being especially abundant and growing under best conditions in Mexico, Central America, Brazil, and the West Indies.

The cocoa beans of commerce are derived chiefly from Ariba, Bahia, Caracas, Cayenne, Ceylon, Guatemala, Haiti, Java, Machala, Mara-

*Winton's Microscopy of Foods, p. 435. 
caibo, St. Domingo, Surinam, and Trinidad. Besides these, the Seychelles and Martinique furnish a small amount.

The plant seeds, or beans, grow in pods, varying in length from 23 to $30 \mathrm{~cm}$., and are from 1o to $15 \mathrm{~cm}$. in diameter. The beans, which are about the size of almonds, are closely packed together in the pod. Their color when fresh is white, but they turn brown on drying.

The gathered pods are first cut open, and the seeds removed to undergo the process of "sweating" or fermenting, which is carried out either in boxes or in holes made in the ground. This process requires great care and attention, as upon it depends largely the flavor of the seed. The sweating operation usually takes two days, after which the seeds are dried in the sun till they assume their characteristic warm red color, and in this form are shipped into our markets.

Manufacture of Chocolate and Cocoa.-For the production of chocolate and cocoa the beans are cleaned and carefully roasted, during which process the flavor is more carefully developed, and the thin, paperlike shell which surrounds the seed is loosened, and is very readily removed. The roasted seeds are crushed, and the shells, which are separated by winnowing, form a low-priced product, from which an infusion may be made, having a taste and flavor much resembling chocolate.

The crushed fragments of the kernel or seed proper are called cocoa $n i b s$, and for the preparation of chocolate they are finely ground into a paste and run into molds, either directly, or after being mixed with sugar and vanilla extract or spices, according to whether plain or sweet chocolate is the end product.

For making cocoa, however, a portion of the oil or fat known as the cocoa butter is first removed, by subjecting the ground seed fragments to hydraulic pressure, usually between heated plates; after which the pressed mass is reduced to a very fine powder, either directly, or by treatment with ammonia or alkalies, to render the product more soluble. It is held that the large amount of fat contained in the cocoa seeds (varying from 40 to 54 per cent) is difficult of digestion to many, such as invalids and children, and hence the desirability of removing part of the fat.

Composition of Cocoa Products. - The chief constituents of the raw cocoa bean, named in the order of their relative amount, are fat, protein, starch, water, crude fiber, ash, theobromine, gum, and tannin. During the roasting there is reason to believe a volatile substance is developed much in the nature of an essential oil, which gives to the 
product its peculiar flavor, and is somewhat analogous to the caffeol of coffee.

Tannin, the astringent principle of cocoa, exists as such in the raw bean, but rapidly becomes oxidized to form cocoa red, to which the color of cocoa is due. shells:

Weigmann gives the following results of analyses of cocoa nibs and COMPOSITION OF COCOA NIBS.

\begin{tabular}{|c|c|c|c|c|c|c|c|c|c|}
\hline Commercial Varieties. & 递 & 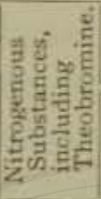 & है & 宩 & 递 & 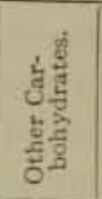 & $\begin{array}{l}\text { हु } \\
\text { Jु }\end{array}$ & प्रें & हु \\
\hline 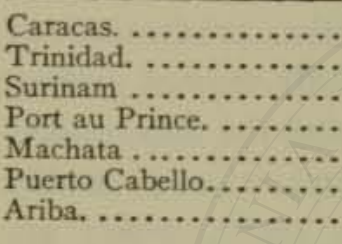 & $\begin{array}{l}7 \cdot 77 \\
7.87 \\
7 \cdot 53 \\
7.77 \\
8.17 \\
8.08 \\
8.27\end{array}$ & $\begin{array}{l}14.13 \\
14.06 \\
13.69 \\
14.56 \\
14.06 \\
13.50 \\
15.37\end{array}$ & $\begin{array}{r}1.48 \\
1.31 \\
1.66 \\
\cdots \cdots \\
\cdots \cdots \\
1.51 \\
\cdots \cdots\end{array}$ & $\begin{array}{l}45 \cdot 54 \\
44 \cdot 62 \\
44.74 \\
46.35 \\
45 \cdot 93 \\
46.61 \\
45 \cdot 15\end{array}$ & $\begin{array}{r}19.40 \\
25.30 \\
26.45 \\
5.97 \\
5.69 \\
22.9 \\
5.83\end{array}$ & $\begin{array}{l}15.53 \\
17.50\end{array}$ & $\begin{array}{l}6.19 \\
4 \cdot 55 \\
4 \cdot 30 \\
5 \cdot 19 \\
4 \cdot 36 \\
4 \cdot 43 \\
4 \cdot 48\end{array}$ & $\begin{array}{l}4.91 \\
3.48 \\
3.16 \\
4.15 \\
4.09 \\
4.28 \\
3.88\end{array}$ & $\begin{array}{l}2.06 \\
0.10 \\
0.13 \\
1.48 \\
0.22 \\
0.18 \\
0.14\end{array}$ \\
\hline
\end{tabular}

COMPOSITION OF COCOA SHELLS.

\begin{tabular}{|c|c|c|c|c|c|c|c|c|c|}
\hline Commercial Varieties. & 部 & 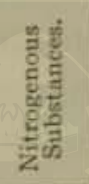 & 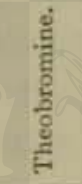 & लैं & 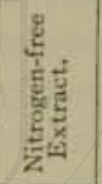 & 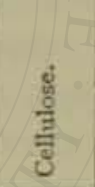 & $\frac{\dot{1}}{4}$ & 鴶 & 良 \\
\hline $\begin{array}{l}\text { Tunidad. ......... } \\
\text { Suerto Cabello. }\end{array}$ & $\begin{array}{l}12.49 \\
14.64 \\
13.93 \\
14.89\end{array}$ & $\begin{array}{l}13.18 \\
14.62 \\
16.25 \\
16.18\end{array}$ & $\begin{array}{l}0.5^{8} \\
0.74 \\
0.78 \\
0.75\end{array}$ & $\begin{array}{l}2.3^{8} \\
3.45 \\
2.54 \\
2.01\end{array}$ & $\begin{array}{l}40 \cdot 30 \\
44 \cdot 89 \\
42 \cdot 47 \\
43 \cdot 32\end{array}$ & $\begin{array}{l}16.33 \\
15.79 \\
17.04 \\
15.25\end{array}$ & $\begin{array}{l}9.06 \\
6.19 \\
6.63 \\
8.08\end{array}$ & $\begin{array}{l}6.26 \\
0.42 \\
0.85 \\
0.27\end{array}$ & $\begin{array}{l}2.11 \\
2.34 \\
2.60 \\
2.59\end{array}$ \\
\hline
\end{tabular}

The following are the summarized results of the analyses of seventeen varieties of cocoa seeds and shells, made by Winton, Silverman, and Bailey.*

* An. Rep. Conn. Agric. Exp, Sta., 1902, p. 270. 


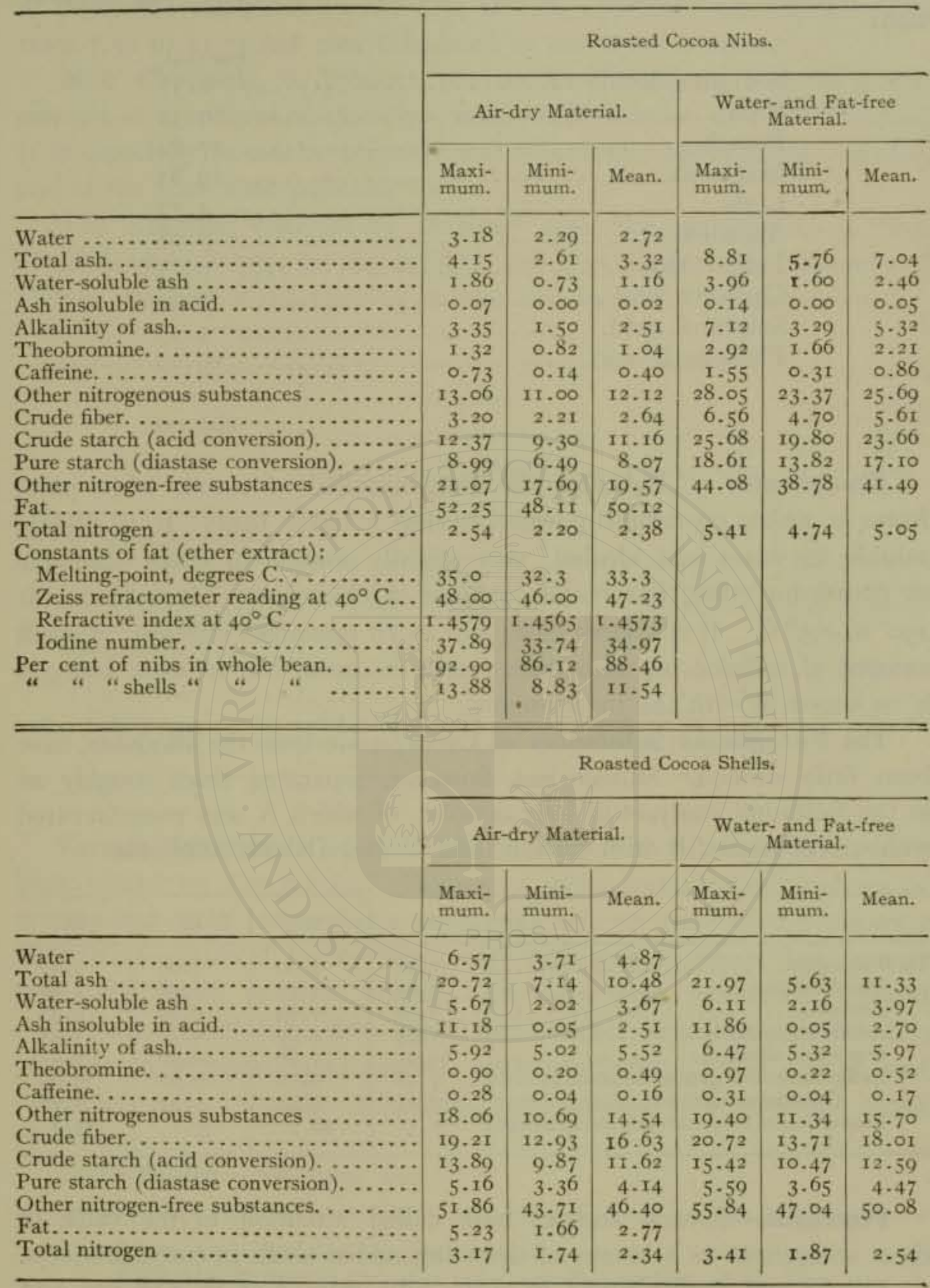



tion:

According to Bell * the ash of cocoa nibs has the following composi-

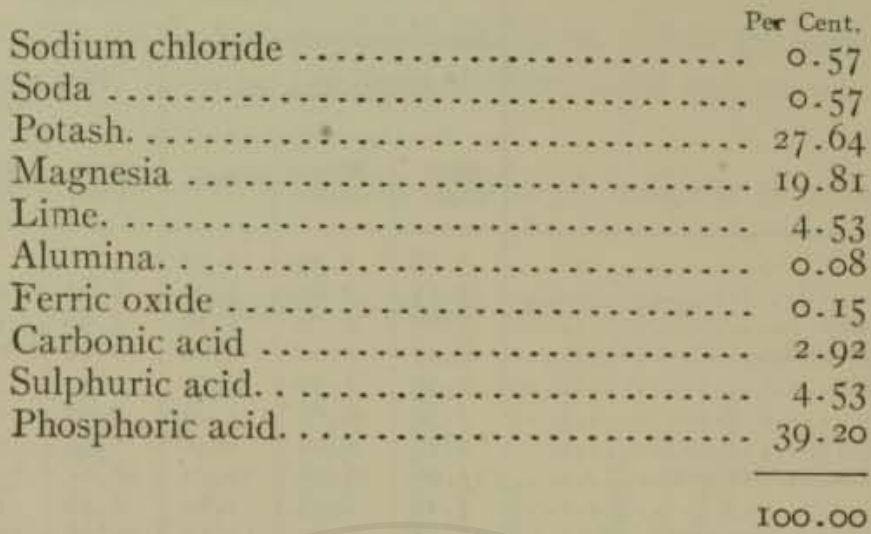

Theobromine $\left(\mathrm{C}_{7} \mathrm{H}_{8} \mathrm{~N}_{4} \mathrm{O}_{2}\right)$, the chief alkaloid of cocoa, when pure, forms a white, crystalline powder, having a bitter taste. It is slightly soluble in water and alcohol, very slightly soluble in ether, insoluble in petroleum ether, but readily soluble in chloroform. It sublimes at $290^{\circ}$ to $295^{\circ} \mathrm{C}$. It is a weak base, and much resembles caffeine. A small amount of caffeine has also been found in cocoa, but in most analyses is reckoned in with the theobromine.

The Nitrogenous Substances of Cocoa, aside from the alkaloids, have been little studied. Stutzer has, however, separated them roughly as in the following analyses of four samples, of which A was manufactured without chemicals, B with potash, and C and D with ammonia:

\begin{tabular}{|c|c|c|c|c|}
\hline & A. & B. & c. & D. \\
\hline Total nitrogen ... & 3.68 & $3 \cdot 3^{\circ}$ & 3.95 & $3 \cdot 57$ \\
\hline Theobromine & 1,92 & 1.73 & 1.98 & i. 80 \\
\hline Ammonia............ & 0.06 & 0.03 & 0.46 & 0.33 \\
\hline Amido compounds. .. & 1.43 & I. 25 & $0.3 \mathrm{I}$ & 1.31 \\
\hline Digestible albumin.................. & 10.25 & 7.68 & 10.50 & 7.81 \\
\hline Indigestible nitrogenous substances ..... & 7.18 & 9.19 & 7.68 & 8.00 \\
\hline Containing nitrogen..$\ldots \ldots \ldots \ldots$ & 1.15 & 1. 47 & 1.23 & 1. 28 \\
\hline Proportion of total nitrogen indigestible. & $3 \mathrm{t} \cdot 2$ & $44 \cdot 5$ & $3 \mathrm{I} \cdot 2$ & 35.8 \\
\hline
\end{tabular}

Pentosans.-Several authors have called attention to the value of these substances as a means of detecting added shells in cocoa products.

Lührig and Segin† found in cocoa nibs from $2.5^{\mathrm{I}}$ to $4.5^{8}$ per cent

* Analysis and Adulteration of Foods.

† Zeits. Unters. Nahr. Genuss., 12, 1906, p. r61. 
of pentosans calculated to the dry, fat-free substance, and in the shells from 7.59 to II.23 per cent calculated to the dry substance.

Milk Chocolate, a product of comparatively recent introduction, consists of a mixture of chocolate, sugar, milk powder, and cocoa butter. It is especially prized by travelers and others who desire a concentrated, and at the same time palatable food.

The following analyses by Dubois* show the composition of three of the leading brands on the market, and also illustrate the accuracy of Dubois' method of determining sucrose and lactose given on page 399 .

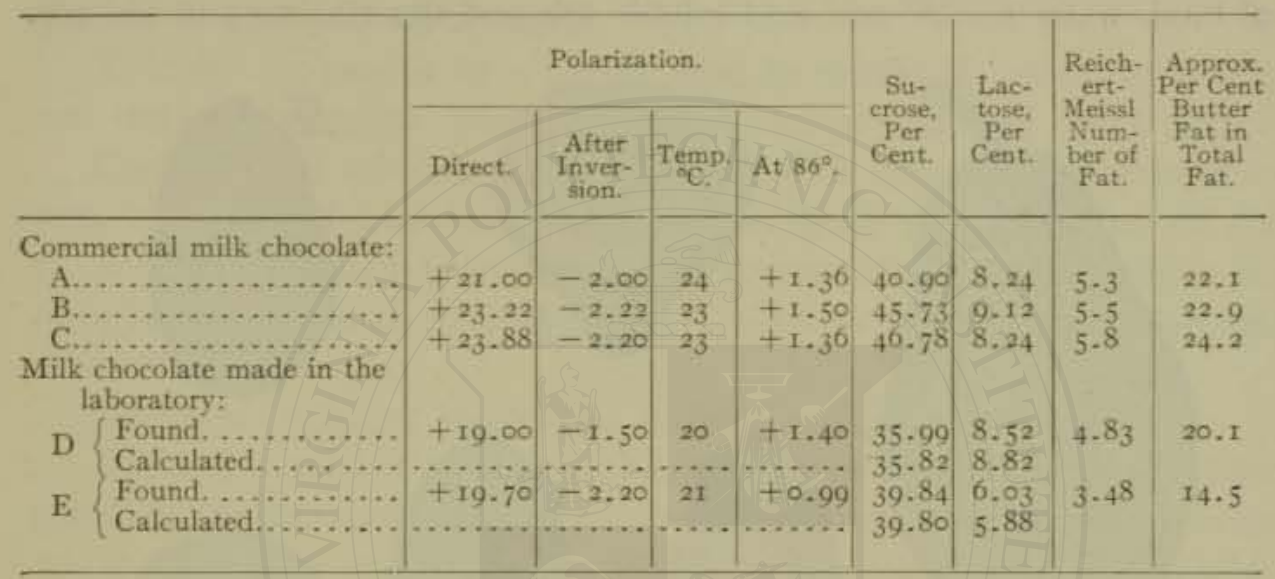

Various Compounds of chocolate or cocoa with other materials have been placed on the market. Zipperer $\dagger$ gives formulas or analyses of seventy-four such preparations, containing one or more of the following ingredients: oatmeal, barley meal, malt, malt extract, wheat flour, potato flour, rice, peas, peanuts, acorns, cola nuts, sago, arrowroot, Iceland moss, gum Arabic, salep, dried meat, meat extract, peptones, milk powder, plasmon (a preparation of casein), eggs, saccharin, vanilla, spices, and inorganic salts. Certain medicinal preparations also contain cocoa products.

Cocoa Butter.-See page 529 .

* Jour. Am. Chem. Soc., 29, 1907, p. $55^{6}$.

$\dagger$ The Matufacture of Chocolate and Cacao Preparations, ad ed., Igo2. 


\section{METHODS OF ANALYSES.}

Preparation of the Sample.-Cocoa is usually in a fine powder, and needs merely to be put through a sieve, to break up lumps, and mixed. Chocolate should be grated or shaved so as to permit mixing. It can not be ground, as the heat of grinding reduces it to a paste.

Moisture.-Dry two grams of the material to constant weight at $100^{\circ}$ C. in a current of dry hydrogen. Somewhat lower results are obtained by drying in a dish in air.

Ash.-Proceed as described under tea (page 369 ) in the determination of total. water-soluble and acid-soluble ash, and the alkalinity of the ash.
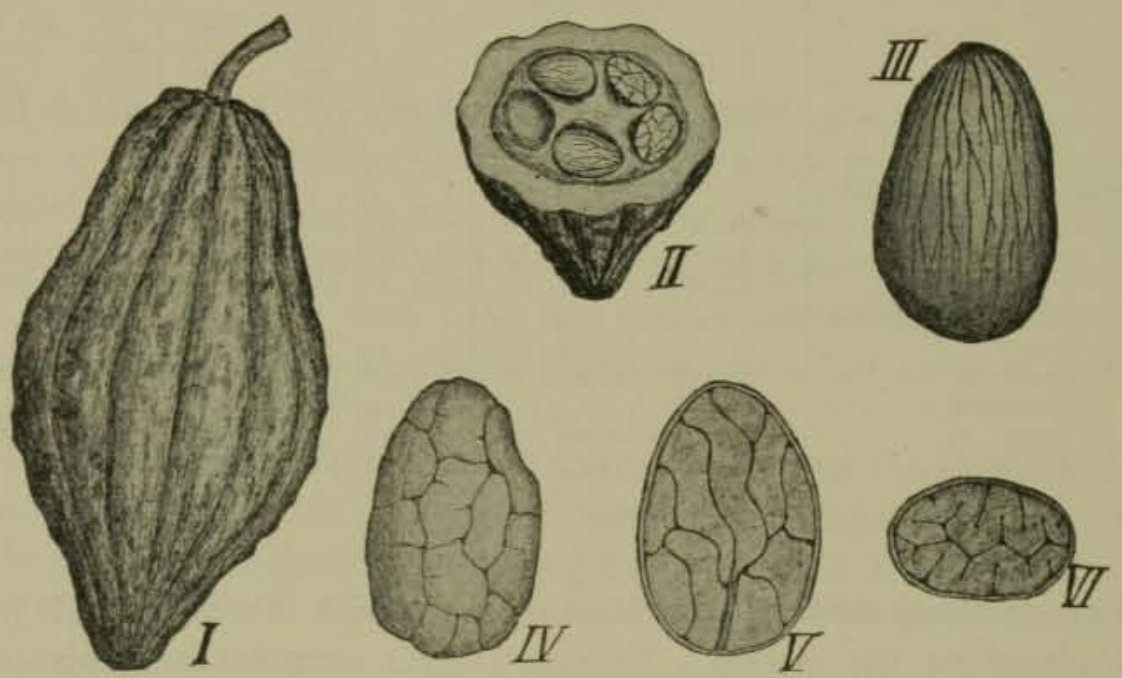

FIG. 79.-Cocoa. I entire fruit, $\times \frac{1}{4} ; I I$ fruit in cross-section; III seed (cocoa bean) natural size: $I V$ seed deprived of seed coat; $V$ seed in longitudinal section, showing radicle (germ); VI seed in cross-section. (Winton.)

Protein.-Determine total 'nitrogen by the Kjeldahl or Gunning method. From the percentage of total nitrogen subtract the nitrogen of the theobromin and caffeine, obtained by multiplying the percentages found by $0.3 \mathrm{II}$ and 0.289 respectively, and multiply the remainder by 6.25 .

Fat (Ether Extract).- Extract two grams of the material in a continuous extractor until no more fat is removed. Grind the residue and repeat the extraction. Dry the combined extract at $100^{\circ} \mathrm{C}$. and weigh.

Constants of Fat.- See chapter on Edible Oils and Fats.

Crude Fiber.-Proceed as in the analysis of cereal products (page 277), using the residue from the ether extraction. 
Reducing Matters by Acid Conversion (Crude Starch).*-Weigh four grams of the material into a small Wedgewood mortar, add $25 \mathrm{cc}$. of ether, and grind with a pestle. After the coarser material has settled out, decant off the ether with the fine suspended matter on a II crn. paper. Repeat this treatment until no more coarse material remains. After the ether has evaporated, transfer the fat-free residue from the filter to the mortar by means of a jet of cold water, and rub to an even paste. Filter the liquid on the paper previously employed. Repeat the process of transferring from the filter to the mortar, grinding, and filtering, until all sugar is removed. In the case of sweetened cocoa products, at least $500 \mathrm{cc}$. of water should be used.

Transfer the residue to a $500-\mathrm{cc}$. flask by means of $200 \mathrm{cc}$. of water, and convert the starch into dextrose by Sachsse's method (page $2_{3}$ ).

Cool the acid solution, nearly neutralize with sodium hydroxide solution, add $5 \mathrm{cc}$. of lead sub-acetate solution (page 586 ), make up to $25^{\circ}$ cc. and filter through a dry filter. To Ioo cc. of the filtrate, add I cc. of $60 \%$ sulphuric acid, shake thoroughly, allow to settle, and filter through a dry filter.

Determine reducing matters by Allihn's method (page 608).

Dubois, $\dagger$ instead of treating with ether as above described, shakes four grams of the unsweetened product or eight grams of the sweetened with roo cc. of gasoline, and whirls in a centrifuge to separate from the insoluble matter. After decanting off the gasoline layer, sweetened products are treated in like manner with two portions of $100 \mathrm{cc}$. of water to remove the bulk of the sugar, and finally washed on the paper.

Starch.-Diastase Method.-Remove the fat and sugar from four grams of the material by treatment with ether and water, as described in the preceeding section, and determine starch in the residue by the diastase method (page 283).

Pentosans. See page 285 .

Determination of Sucrose and Lactose.-Dubois Method. $\ddagger$-Place 26 grams of the material in an 8-ounce nursing bottle, add about roo cc. petroleum ether and shake for five minutes. Whirl in a centrifuge until the solvent is clear, draw off the same by suction and repeat the treatment with petroleum ether. Keep the bottle containing the defatted residue in a warm place until the petroleum ether is practically expelled.

* Winton, Silverman and Bailey, An. Rep. Conn. Exp. Sta., I902, p. 275.

$\dagger$ A. O. A. C. Proc. 1908, U. S. Dept. of Agric., Bur. of Chem., Bul. 122, p. 214.

‡A. O. A. C. Method, U. S. Dept. of Agric., Bur. of Chem., Circ. 66, p. 15. 
Add 100 cc. water and shake until all the chocolate is loosened from the sides and bottom of the bottle and continue the shaking for three minutes longer. Add ro cc. of lead subacetate solution (p. 586), mix thoroughly and filter through a folded filter. Make the direct polariscopic reading (a) in a $200-\mathrm{mm}$. tube, then precipitate the excess of lead by dry potassium oxalate. Invert by one of the methods given on page 588 , polarize, and multiply the invert reading by 2 to correct for dilution (b). Calculate the approximate percentages of sucrose $(S)$ and lactose $(L)$ by the following formulas:

$$
S=\frac{(a-b) \times \mathrm{IIO}}{142.66-\frac{t}{2}} \quad L=\frac{(a \times \mathrm{I} .10)-S}{0.79} .
$$

From the sum of $S$ and $L$ calculate the approximate number of grams of total sugar $G$ present in the 26 grams of sample taken and determine the factor $X$ thus:

$$
X=110+(G \times 0.62),
$$

in which 0.62 is the volume in cc. displaced by I gram of sugar in water solution. Applying this correction,

True per cent sucrose $=\frac{S X}{110} . \quad$ True per cent lactose $=\frac{L X}{110^{\circ}}$.

The following method of solution may be substituted for that given above:

Transfer 26 grams to a flask, add $100 \mathrm{cc}$. water, cork, and heat in steam-bath for twenty minutes, releasing the pressure occasionally during the first five minutes. Twice during the twenty minutes shake thoroughly so as to emulsify completely. Finally cool to room temperature, add $10 \mathrm{cc}$. lead subacetate solution, mix, and filter.

Theobromine and Caffeine (Decker-Kunze Method).*-Boil Io grams of the powdered material and 5 grams of calcined magnesia for 30 minutes with $300 \mathrm{cc}$. of water. Filter by the aid of suction on a Buchner funnel, using a round disk of filter paper. Transfer the material and paper to the same flask used for the first boiling, add I $50 \mathrm{cc}$. of water,

\footnotetext{
* Schweiz. Wchschr. Phar., 40, 1902, pp. 527, 541, 553; Abstract Chem. Centr., 74, 1903, p. 62 ; An. Rep. Conn. Exp. Sta., 1902, p. 274 .
} 
and boil $\mathrm{I}_{5}$ minutes. Filter as before, and repeat the operation of boiling with $150 \mathrm{cc}$. of water and filtering. Wash once or twice with hot water. Evaporate the united filtrates (with quartz sand if sugar be present): to complete dryness in a thin glass dish of about $300 \mathrm{cc}$. capacity.*

Grind to a coarse powder in a mortar provided with a suitable cover to prevent loss by flying. Transfer to the inner tube of a continuous fat extractor, and dry thoroughly in a water oven. Extract with chloroform for 8 hours, or until the theobromine and caffeine are completely removed, into a weighed flask. It is important that the material be thoroughly dry, that an extractor be used that permits of a hot extraction, and that a considerable volume of chloroform passes through the material. Distil off the chloroform, and dry at $100^{\circ} \mathrm{C}$. to constant weight.

If the material be pure chocolate or cocoa, the extract thus obtained is practically pure theobromine and caffeine, but if the material is cocoa shells or a cocoa product mixed with a large amount of shells, the extract may be brown in color, due to the presence of considerable amounts of impurities.

In either case, separate the caffeine by treating the extract in the flask at the room temperature for some hours with $50 \mathrm{cc}$. of pure benzol. Filter through a small paper into a tared dish, evaporate to dryness, and dry to constant weight at $100^{\circ} \mathrm{C}$., thus obtaining the amount of caffeine.

Determine theobromine by Kunze's $\dagger$ method, as follows:

Add to the residue and paper $150 \mathrm{cc}$. of water, enough ammonia water to make the liquid slightly alkaline, and an excess of decinormal silver nitrate solution. Boil to half the original volume, add $75 \mathrm{cc}$. of water, and repeat the boiling. The solution should be perfectly neutral. If it contains the slightest amount of free ammonia, add water and boil until it is completely removed.

Filter from the insoluble silver theobromine compound, and wash with hot water. In the filtrate determine the excess of silver nitrate by Volhard's $\ddagger$ method as follows:

Add $5 \mathrm{cc}$. of cold saturated solution of ferric ammonium sulphate (ferric-ammonium alum), and enough boiled nitric acid to bleach the liquid. Titrate with decinormal ammonium sulphocyanide solution until a permanent red color appears.

*A "Hoffmeister Schälchen" may be used, or dishes may be made from broken flasks by making a scratch with a diamond and leading a crack from this scratch about the flask by means of a glowing springcoal.

$\dagger$ Ztschr $\mathrm{f}$, anal. Chem., 33, 1894, p. 1 .

[ $\ddagger$ Ibid., 13,1874 , p. 171 . 
One cc. of decinormal $\mathrm{AgNO}_{3}$ solution is equivalent to 0.01802 gram of theobromine. If the mixed alkaloids were colorless, the theobromine obtained by subtracting the weight of caffeine from the weight of the mixed alkaloids will usually agree closely with that obtained by silver titration.

\section{ADULTERATION OF COCOA PRODUCTS AND STANDARDS OF PURITY.}

The following are the U. S. standards** Standard chocolate should contain not more than $3 \%$ of ash insoluble in water, $3.5 \%$ of crude fiber, and $9 \%$ of starch, nor less than $45 \%$ of cocoa fat.

Standard sweet chocolate and standard chocolate coating are plain chocolate mixed with sugar (sucrose), with or without the addition of cocoa butter, spices, or other flavoring material, containing in the sugarand fat-free residue no higher percentage of either ash, fiber, or starch than is found in the sugar- and fat-free residue of plain chocolate.

Standard cocoa should contain percentages of ash, crude fiber, and starch corresponding to those of plain chocolate, after correcting for fat removed.

Standard sweet cocoa is cocoa mixed with sugar (sucrose) containing not more than $60 \%$ of sugar, and in the sugar- and fat-free residue no higher percentage of either ash, crude fiber, or starch than is found in the sugar- and fat-free residue of plain chocolate.

The removal of fat, or the addition of sugar beyond the above prescribed limits, or the addition of foreign fats, foreign starches, or other foreign substances, constitutes adulteration, unless plainly stated on the label.

The most common adulterants of cocoa are sugar and various starches, especially those of wheat, corn, and arrowroot. Starch is sometimes added for the alleged purpose of diluting the cocoa fat, instead of removing the latter by pressure, thus, it is claimed, rendering the cocoa more digestible and more nutritious. Unless its presence is announced on the label of the package, starch should be considered as an adulterant. Cocoa shells are also commonly employed as a substitute for, or an adulterant of, cocoa. Other foreign substances found in cocoa are sand and ground wood fiber of various kinds. Iron oxide is occasionally used as a coloring matter, especially in cheap varieties.

* U. S. Dept. of Agric., Off. of Sec., Circ. 19. 
Such adulterants as the starches and cocoa shells are best detected by the microscope. The presence of any considerable admixture of sugar is made apparent by the taste. Mineral adulterants are sought for in the ash.

Addition of Alkali.-The amount of water-soluble matter in cocoa is very small (about 20 to 25 per cent), and in preparing the beverage, the desideratum aimed at is to produce as perfect an emulsion as possible. The legitimate means of accomplishing this is by pulverizing the cocoa very fine, so that particles remain in even suspension and form a smooth paste. Another means sometimes resorted to for producing a so-called "soluble cocoa" is to add alkali in its manufacture, the effect being to act upon a part of the fat, and produce a more perfect emulsion with less separation of oil particles. Such treatment with alkali is regarded with disfavor, even if not considered as a form of adulteration. Cocoa thus treated is generally darker in color than the pure article.

The use of alkali is usually rendered apparent by the abnormally high ash, and by the increased alkalinity of the ash, the latter constant being expressed in terms of the number of cubic centimeters of decinormal acid necessary to neutralize the ash of I gram of the sample. In pure, untreated cocoa, the ash rarely exceeds $5.5 \%$, and the alkalinity of the ash is generally not more than 3.75 . In cocoa treated with alkali, the ash sometimes reaches $8.5 \%$, with the alkalinity running as high as 6 or even 8 .

Microscopical Structure of Cocoa.-Fig. 8o shows elements of the powdered cocoa bean, both of the shell and of the kernel. The powder of the latter should constitute pure cocoa, with occasional fragments only of the shell. The irregular lobes constituting the kernel are each inclosed in a membrane made up of angular cells, filled with granular matter. (4), (5), and (6) show elements of the powdered cotyledons, or seed kernels. The polygonal tissue of the cotyledon is shown in crosssection at (4). In the powder one finds also dark granular matter, bits of débris, and fragments, with masses of yellow, reddish-brown, and sometimes violet coloring matter, together with numerous starch granules and aleurone grains.

The starch granules are nearly circular, with rather indistinct central nuclei, and range in size from 0.0024 to $0.0127 \mathrm{~mm}$., averaging about $0.007 \mathrm{~mm}$. They are more often found in single detached grains, but sometimes in groups of two or three. Occasional spiral ducts, $s p$, are seen, but these are not abundant in the pure cocoa. 
The masses of color pigment are shown up with striking clearness, according to Schimper, by applying a drop of sulphuric acid to the edge of the cover-glass and allowing it to penetrate the tissue. The bits of coloring matter are for a short time colored a brilliant red, which, however, soon fades. Ferric chloride colors them indigo blue.

Schimper recommends mounting the powder in a drop of chloral hydrate, which soon renders most of the tissues transparent. It is sometimes necessary to allow the chloral to act on the powder in a closed

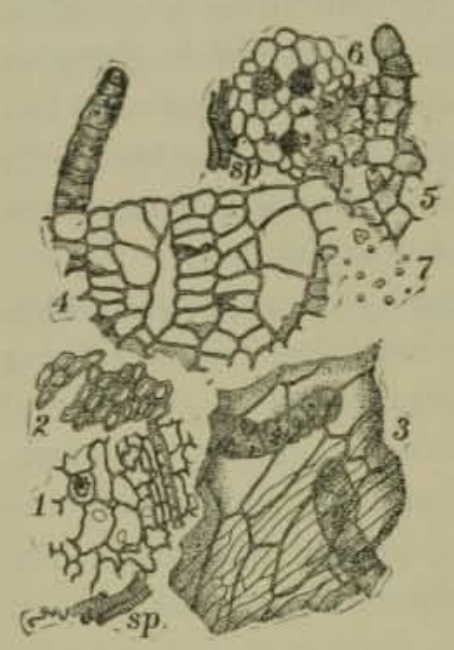

$A$

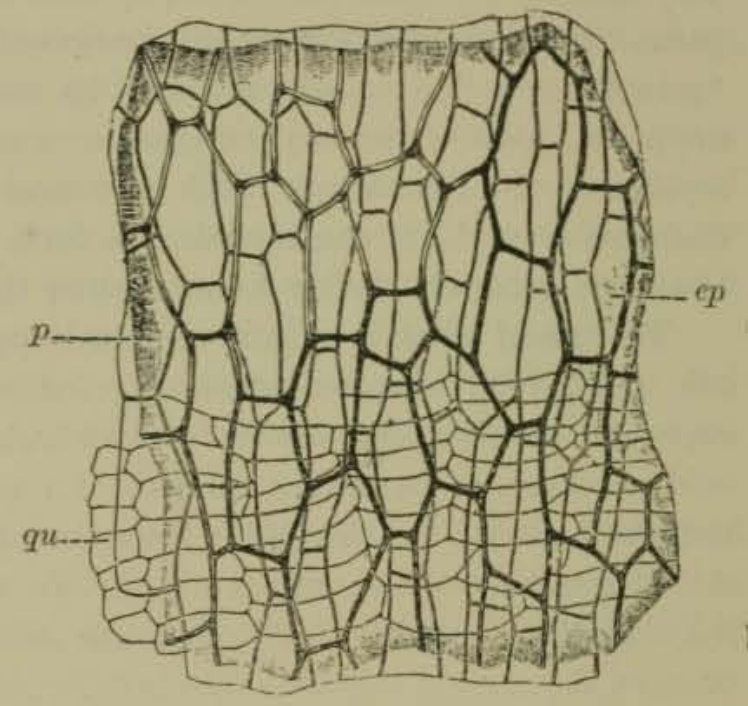

$B$

Fig. 80.-Cocoa under the Microscope.

A. Powdered Cocoa under the Microscope. X125. (After Moeller.) I, cross-section through shell parenchyma; 2 , thick-walled cells; 3 , epidermis of shell (surface section); 4, cross-section of cotyledon tissue; 5,6 , cotyledon parenchyma; 7 , starch.

$B$. Cocoa Shell in Surface Section. X160. ep, epicarp; $p$, parenchyma of the fruit; $q u$, layer of transverse cells. (After Moeller.)

vessel for twenty-four hours, before all the elements, of pure cocoa are rendered transparent. If after that time opaque masses are still found, these are due to foreign material.

Ammonia may be used instead of chloral with even better results, but this reagent requires longer treatment, soaking for several days or a week being sometimes necessary.

Fig. $185, \mathrm{Pl}$. XVII, shows the microscopical appearance of genuine powdered cocoa with its variously sized starch grains and the débris of the ground cotyledons. Fig. 186 shows cocoa adulterated with arrowroot. 
Cocoa Shells.-A cross-section of the shell parenchyma and the stonecell layer, also some of the numerous spiral ducts, all characteristic of the ground shell, are shown at I, Fig. 8o.

The thick-walled stone-cells are shown in surface view at 2, and the spongy, outer seed-skin, composed of two layers, with elongated cells running crosswise to each other in striated fashion, and with the underlying hairs or so-called "Mitscherlich bodies," is shown at 3 . The presence of an abnormally large number of yellow and brown frag. ments in the water-mounted cocoa specimen, even under small magnification, arouses suspicion of the presence of shells, the most distinctive elements of which are the spongy tissue, the stone cells, and the abundant spiral ducts, the latter being scarce in pure cocoa powder.

Cocoa shells are indicated on chemical analysis by the abuormally high ash, crude fiber and pentosans.

Added Starch.-This can only be approximately determined by a careful examination with the microscope. Long experience will enable the analyst to familiarize himself with the appearance and abundance of starch grains of various kinds in a series of fields, so that he can roughly estimate the amount of each starch present in the mixture, by careful comparison with mixtures of known percentage composition.

If the amount of starchy adulterant is considerable, evidence may be secured by determinations of starch by the diastase method and reducing matters by acid conversion.

Added Sugar.-Any appreciable amount of added cane sugar is shown by the sweet taste. The amount of cane sugar may be determined by means of the polariscope, as described on page 399 .

An abnormally low ash is indicative of the addition of starch or sugar or both.

Foreign Fat.-Certain manufacturers have found it profitable to remove a portion of the cocoa butter from chocolate and substitute for it a cheaper fat, such as cocoanut oil, tallow or even paraffine. Such adulteration is detected by determination of the physical and chemical constants of the fat obtained by extraction with ether.

Dyes and Pigments, such as Bismark brown and Venetian red, have been employed to hide the presence of diluents. They are detected by dyeing tests, and by examination of the ash. 


\section{REFERENCES ON TEA, COFFEE, AND COCOA.}

BAKer, W., \& Co. The Chocolate Plant and Its Products. Boston, r8gr.

Bertrand, G. Coffees without Caffein. Compt. rend., I4r, 1905, p. 209.

BeythIEN, A., BoHRIscH, P., and,DeItER, J. Beiträge zur Chemischen Untersuchung des Thees. Zeits. Unters, Nahr. Genuss., 3, rgoo, p. 145.

Crayton, E. G. Roasted Beetroot. Analyst, 29, 1904, p. 279.

Crole, D. Tea: a Textbook of Tea Planting and Manufacture. London, 1897 .

v. CZADEk, O. Beitrag zur Beurteilung von Feigenkaffee. Z land. Versuchs. Oester. 5 , r902, p. 761 .

Davies, S. H., and McLellan, B. G. Amount of Cocoa Butter contained in the Cocoa Bean. Jour. Soc. Chem. Ind., 23, 1904, p. 480.

DECkER, J. Zur Kenntnis der Kakaoschalen. Pharm. Cent., 46, 1905, p. 863.

DUсні́сек, F. Beiträge zur Kenntnis der chemischen Zusammensetzung des Kaffees und der Kaffee-Ersatzstoffe, Zeits. Unters, Nahr, Genuss., 8, 1904, p. I39.

Dusors, W. L. Determination of Lactose and Butter Fat in Milk Chocolate. Jour. Am. Chem. Soc, 29 , 1907, p. 556.

Dyer, B. Chicory, and Variations in its Composition. Analyst, 23, 1898, p. 226.

Ewent, E. E. The Carbohydrates of the Coffee Bean. Am. Chem. Jour., I4, I892, p. 373 .

Filsinger. Zur Untersuchung und Begutactung der Kakaofabrikate. Z. öffent. Chem., 9, 1903, p. 6.

Génin, V. Café, Chicorée, Thé, Maté, Coca et Cacao. Analyse des Matieres Alimentaires. Girard et Dupré. Paris, 1894.

Hanausek, T. F. Ueber die Harzglasur des Kaffees. Zeits. Unters. Nahr. Genuss, 2, 1899, p. 275 .

Hanus, J. Zur Fettbestimmung in Kakao nach dem Gottlieb-Röse'schen Verfahren. Zeits, Unters. Nahr. Genuss,, II, 1906, p. 738.

Hehner, O, and Skertchly, W. P. Estimation of Pentosans and its Application to the Analysis of Foods. Analyst, 24, 1899, p. 178.

JAEGER, R., and Unger, E. Beitrag zur Kenntnis der Kakaoschalen. Zeits. Unters. Nahr. Genuss., 10, I905, p. 76 I; Ber. d. Chem. Ges., 35, 1902, p. 4440; 36, 1903, p. 1222.

Kat7, J. Der Koffeingehalt des als Getränk benutzten Kaffeeaufgusses. Arch. Pharm., 242, 1904, p. 42.

Kenrick, A. Tea. Canada Inland Rev, Dept, Bul. 24.

Kirschner, A. Die Bestimmung des Fettes in Kakao. Zeits. Unters. Nahr. Genuss., II, I906, P. $45^{\circ}$.

Kornig, J. Chemie der menschlichen Nahrungs- und Genussmittel. Vierte Aufl. Berlin, 1903 .

Kunze, W. E. Quantitative Separation and Estimation of the Alkaloids of Pure Coffee. Analyst, 19, p. 194.

LAxA, O. Ueber Milch-Schokoladen. Zeits. Unters. Nahr. Genuss., 7, 1904, p. 491. Lemmann, K. Die Fabrikation des Surrogatkaffees und des Tafalsenfes. Vienna, I893.

Lendrich, K., und MURdfield, R. "Coffein-freier Kaffee." Zeitr. Unters. Nahr. Genuss., 15, 1908, p. 705. 
Lodge, J. L. Coffee: History, Growth, and Cultivation; its Preparation and Effect on the System. London, 1894 .

Lunwig, W. Die Bestimmung der Rohfaser in Kakao. Zeits. Unters. Nahr. Genuss., 12, 1906, p. 153 .

LÜHRIG, H. Zur Kenntnis der Kakaoschalen. Zeits. Unters. Nahr. Genuss,, 9, 1905 , p. 263.

LÜHRIG, H., and SegrN, A. Pentosangehalt der Kakaobohnen und seine Verwertung zum Schalennachweis im Kakaopulver. Zeits. Unters. Nahr. Genuss,, 12, 1906, p. 16r.

Macfarlane, T. Coffee. Canada Inl. Rev. Dept. Buls. 3, 29, 3 t.

McGitl, A. Cocoa and Chocolate. Canada Inl. Rev. Dept. Bul. 72.

Matrhes, H., and MÚmer, F. Beiträge zur Kenntnis des Kakaos. Zeits. Unters. Nahr. Genuss., 12, 1906, p. 88 .

- Die Bestimmung der Rohfaser in Kakaowaren. Ibid., p. 159.

Mrchaelis, A. Der Kaffee als Genuss- und Heilmittel nach seinen botanischen, chemischen, dietetischen und medicinischen Eigenschaften.

ORTH, E. Beitrag zur Untersuchung und Beurteilung kandierter Kaffees. Zeits. Unters. Nahr. Genuss., 9, 1905, p. 137.

Pearmain, T. H., and Moor, C. G. On the Adulteration of Coffee. Analyst, 20, 1895, p. 176.

Suethane, A. Composition of Some Samples of Pure Coffee. Analyst, 7, 1882, p. 73.

Spencer, G. L. Tea, Coffee, and Cocoa Preparations. Div. of Chem., Bul. I3, Part VII, 1892 .

Sternmann, A. Ueber die Bestimmung des Zuckers in Schokolade. Schw, Woch. Chem. Pharm., 40, 1902 , p. $581 ; 4 x, 1903$, p. 65.

Trпцich, H. Die Kaffeesurrogate, ihre Zusammensetzung und Untersuchung. Munich, 1889.

Wanklyn, J. A. Tea, Coffee, and Cocoa. London, $\mathrm{rS8}_{3}$.

Wetmans, P. Zur Prufung von Schokolade auf den Gehalt an Zucker. Z. öffent. Chem., 9, 1903, Pp. 93 and 115.

Kakao und Schokolade. Ibid, p, 206.

Wigner, G. W. Nitrogenous Constituents of Cocoa. Analyst, 4, I879, p, 8.

Winton, A. L., Silverman, M., and Batley, E. M. Cocoa. An. Rept. Conn. Exp. Sta., 1902, p. 248. Chocolate. Ibid,, 1903, p. 123.

Worff, J. Ueber die Zusammensetzung und die Untersuchung der Cichorienwurıel. Zeits. Unters. Nahr. Genuss., 3, 1900, p. 593.

YAPple, F. Analyses of Cocoa. Amer. Jour. Pharm., 67, 1895, p. 318.

ZIPPERER. The Manufacture of Chocolate and Other Cacao Preparations. 2d ed. Berlin, 1902.

Conn. Exp. Sta. Annual Reports, 1896 et seq.

Maine Exp. Sta. Bul. 65. Analysis of Coffee Substitutes.

Massachusetts State Board of Health Reports, i882 et seq.

N. H. Sanitary Bul., Jan., I906, p. I68.

North Carolina Exp. Sta. Bul. I54. Adulteration of Coffee and Tea.

Penn. Dept. of Agric. An. Rept., I897, p. 178. Substitutes for Coffee.

$\begin{array}{llll}" \text { " } & \text { " } & \text { " } & \text { 1898, pp. } 75 \text { and } 548 \text {. Coffee and its Adulterations. }\end{array}$




\section{CHAPTER XII.}

\section{SPICES.}

THESE aromatic vegetable substances are classed as condiments, and depend for their use on the pungency which they possess in giving flavor or relish to food. As such seasoning or zest-giving substances, they are of considerable importance dietetically, but from the fact that they are used in comparatively insignificant amount, the determination of their chemical ccmposition or actual value as nutrients per se is of little importance to the food economist.

Spices are, however, of chief interest to the public analyst, because of all food materials they constitute from their nature a class more susceptible than others to fraudulent adulteration of the most skilled variety.

In many cases not only the megascopic appearance and taste of the skillfully adulterated article are made to counterfeit the genuine spice, but even the microscopical appearance is intended to deceive, since it is the microscope that is most useful in the detection of adulteration, and in many cases in the determination of the approximate amount of the adulterants.

Indeed it is very rare that the microscope will fail to detect the presence of any foreign substance in spice, and hence its use is indispensable in the study of this class of foods by the analyst. Chemical methods, as a rule, while of secondary importance, are, however, very helpful, both as confirmatory of the microscopical research, and in some cases showing instances of adulteration not readily apparent with the microscope, such, for example, as in the case of exhausted spices, or those deprived of a whole or a part of their volatile oil. Sophistication of this kind is best shown by the ether extract.

General Methods of Proximate Analysis.-The following methods common to all the spices are for the most part those adopted provisionally by the A. O. A. C.* Methods peculiar to special spices will be treated

* U. S. Dept. of Agric., Bur, of Chem., Bul. 65 and Bul. 107 (rev.). 
under the discussion of the spice in question. For these determinations the spices should be powdered fine enough to pass through a 6o-mesh sieve.

Determination of Moisture.-Richardson's Method.*-Two grams of the sample are weighed in a tared platinum dish and dried in an air-oven at $110^{\circ}$ to a constant weight, which generally requires about twelve hours. The loss in weight includes the moisture and the volatile oil. The latter is determined from the ether extract, as described on page $4 \mathrm{ro}$, and deducted from the total loss to obtain the moisture.

McGill $\dagger$ determines the moisture by exposure of a weighed portion of the sample in vacuo over perfectly colorless sulphuric acid. The spice gives up its moisture before the volatile oil comes off, and any appreciable amount of the volatile oil, when absorbed by the acid, causes the latter to be discolored, so that by carefully observing the beginning of the discoloration, and removing the sample, the loss due to moisture may be obtained by weighing at the proper stage. The abstraction of the moisture in this manner requires about twenty-four hours.

Determination of Ash.-Two grams of the spice are burned in a platinum dish heated to faint redness on a piece of asbestos paper by means of a Bunsen burner. The burning is best finished in a muffle furnace. If the ash contains an appreciable amount of carbon, it is exhausted on a filter with hot water, and the filter with the residue is burnt in the dish previously used. After adding the aqueous extract and a few drops of ammonium carbonate solution, the whole is evaporated to dryness and ignited at a faint red heat.

The Water-soluble Ash $\ddagger$ is found by boiling the total ash as above obtained with $5 \circ \mathrm{cc}$. of water, and filtering on a tared Gooch crucible, the insoluble residue being washed with hot water, dried, ignited, and weighed. The insoluble ash, subtracted from the total, leaves the watersoluble ash.

Sand.-This is assumed to be the percentage of ash insoluble in hydrochloric acid. The ash from 2 grams of the substance, obtained as above described, is boiled with $25 \mathrm{cc}$. of 10\% hydrochloric acid (spezific gravity 1.050 ) for five minutes, the insoluble residue is collected on a tared Gooch crucible, thoroughly washed with hot water, and finally dried and weighed.

* U. S. Dept. of Agric., Div. of Chem., Bul. 13, pt. 2, p. 165.

† Canada Dept. of Inland Rev, Bul. 73, p. 9.

$\ddagger$ U. S. Dept. of Agric., Bur. of Chem., Bul. 65, p. 55; Bul. 107 (rev.), p. 162. 
Lime is determined from the ash as directed on page 303 , having first separated the iron and phosphates.

The sulphuric acid due to calcium sulphate (added as an adulterant) is determined by precipitation with barium chloride of a very weak hydrochloric acid solution of the "ash, the separated barium sulphate being washed, dried, ignited, and weighed.

Ether Extract.-Total, Volatile, and Non-volatile*-Two grams of the air-dry, powdered substance are placed in some form of continuous extraction apparatus, such as Soxhlet's or Johnson's (pp. 64 and 65), and are subjected to extraction for sixteen hours with anhydrous, alcoholfree ether. $\dagger$ The ether solution is then transferred to a tared evaporatingdish, and allowed to evaporate spontaneously at the temperature of the room. After the disappearance of the ether, the evaporating-dish is placed in a desiccator over concentrated sulphuric acid and left over night, or for at least twelve hours, after which it is weighed, the residue in the dish being regarded as the total ether extract.

The dish and its contents are then subjected to a heat of about $100^{\circ} \mathrm{C}$. for several hours, taking a long time to bring the temperature up to that point so as to avoid oxidation of the oil. Finally heat at $110^{\circ} \mathrm{C}$. till the weight is constant. The final residue is the non-volatile, and the loss in weight the volatile ether extract.

Alcohol Extract.-Method of Winton, Ogden, and Mitchell. $\neq-T w o$ grams of the powdered sample are placed in a roo-cc. graduated flask, which is filled to the mark with $95 \%$ alcohol. The flask is stoppered and shaken at half-hour intervals during eight hours, after which it is allowed to stand for sixteen additional hours without shaking, and the contents poured upon a dry filter. Of the filtrate, $50 \mathrm{cc}$. are evaporated to dryness in a tared platinum dish on the water-bath, and heated at $110^{\circ} \mathrm{C}$. in an air-oven to constant weight. This method, while only approximate, is so much simpler than the tedious operation of continuous extraction, considering the long time required, that it is regarded as preferable for ordinary work, and, unless great care is taken, is nearly as accurate.

Determination of Nitrogen.-This, in spices other than pepper, is best done by means of the Gunning or Kjeldahl method (p. 60).

* Richardson, U. S. Dept. of Agric., Div, of Chem., Bul, 13, p. 165.

† Petroleum ether may be used, yielding results which differ but slightly from those obtained with ethyl ether. As the latter has been used in the analyses of a large number of samples of spices, if these analyses are to be taken for standards of comparison it is evident that the same solvent should be used.

$\ddagger$ U. S. Dept. of Agric., Bur. of Chem., Bul. 65, p. 56; Bul. 107 (rev.), p. $166_{3}$. 
Determination of Starch.-In spices like white pepper, ginger, and nutmeg that normally contain a high content of starch and very little other copper-reducing matter, the direct acid conversion process of starch determination is satisfactory.

In spices normally free from, starch, such as cloves, mustard, and cayenne, where a starch determination indicates the amount of a foreign starch present as an adulterant, it is safer to use the diastase process.

Four grams of the powdered sample are extracted on a filter-paper (fine enough to retain all starch particles) first with five successive portions of ro cc. of ether, then with $150 \mathrm{cc}$. of $10 \%$ alcohol. Owing to difficulty of filtering in the case of cassia and cinnamon, Winton recommends that all washing in the determination of starch in these substances be omitted. The residue is washed from the filter-paper by means of a stream of water into a 500-cc. flask, if the direct acid conversion method is used, using $200 \mathrm{cc}$. of water; $20 \mathrm{cc}$. of hydrochloric acid (specific gravity I.I25) are added, and the method from this point on followed, as detailed on page $28_{3}$.

If the starch is to be determined by the diastase method, wash the residue from the filter-paper into a beaker with $100 \mathrm{cc}$. of water, and proceed as on page $28_{3}$.

Determine the dextrose in either case by the Defren or Allihn method, or volumetrically, and convert dextrose to starch by the factor o.9.

Determination of Crude Fiber. - Two grams of the substance are extracted with ordinary ether (or the residue left from the determination of the ether extract may be taken) and subjected to the regular method for determining crude fiber, by boiling successively with acid and alkali (page 277).

McGill recommends the use of the centrifuge in separating the crude fiber, after boiling with the alkaline solution.

Determination of Volatile Oil.-Method of Girard and Dupré.*The spice is mixed with water and subjected to distillation, receiving the distillate in a graduated cylinder. The volume occupied by the essential oil (which is immiscible with water) can be thus rearl off and its content roughly determined. If the volatile oil is slightly soluble in water, separate out the water layer, having first read the volume of the oil layer, and extract the aqueous solution with petroleum ether. Evaporate the petroleum ether extract to dryness at room temperature

* Analyse des Matieres Alimentaires, and ed., p. 787 . 
in a tared dish, and add the volume due to the weight of the residue to the volume read off in the graduate.

Microscopical Examination of Powdered Spices. - As a rule few micrcscopical reagents are necessary in the routine examination of powdered spices for adulteration, unless a more careful study of the structure than is necessary to prove the presence of adulterants is desirable. The simple water-mounted specimen is usually sufficient to show the purity or otherwise of the sample. If in doub: as to the presence of starch in small quantities, iodine in potassium iodide should be applied to the specimen, well rubbed out under the cover-glass.

The tissues may be cleared by adding to the water mount a small drop of $5 \%$ sodium hydroxide, or by soaking a portion of the spice for a day in chloral hydrate solution. A valuable means of clearing dense tissues is to boil about 2 grams of the material successively with dilute acid and alkali as in the crude fiber process (p. 277), decanting (not filtering) the solution after each boiling.

The presence of occasional traces of a foreign substance, when viewed under the microscope, is hardly sufficient to condemn the sample as adulterated, since such traces are apt to be accidental.

Composition of Miscellaneous Spice Adulterants. - The chemical analyses of various spice adulterants commonly met with are given on page 413 .

\section{CLOVES.}

Nature and Composition.-Cloves are the dried, undeveloped flowers of the clove tree (Caryophyllus aromaticus or Eugena caryophyllata), which belongs to the myrtle family (Myrtacea). The tree is an evergreen, from twenty to forty feet in height, cultivated extensively in Brazil, Ceylon, India, Mauritius, the West Indies, and Zanzibar. Its leaves are from 7.5 to $I_{3} \mathrm{~mm}$. long, and its flowers, of a purplish color, grow in clusters. The green buds in the process of growth change to a reddish color, at which stage they are removed from the tree, spread out in the sun, and allowed to dry, the color changing to a deep brown. Each whole clove consists of a hard, cylindrical calyx tube, having at the top four branching sepals, surrounding a ball-shaped casing, which consists of the tightly overlapping petals, and within which are the stamens and pistil of the flower. In taste the clove possesses a strong and peculiar pungency. One of its most valuable ingredients is the volatile clove oil. This is composed largely of eugenol $\left(\mathrm{C}_{10} \mathrm{H}_{12} \mathrm{O}_{2}\right)$, which forms 70 to 
COMPOSITION OF SPICE ADULTERANTS.

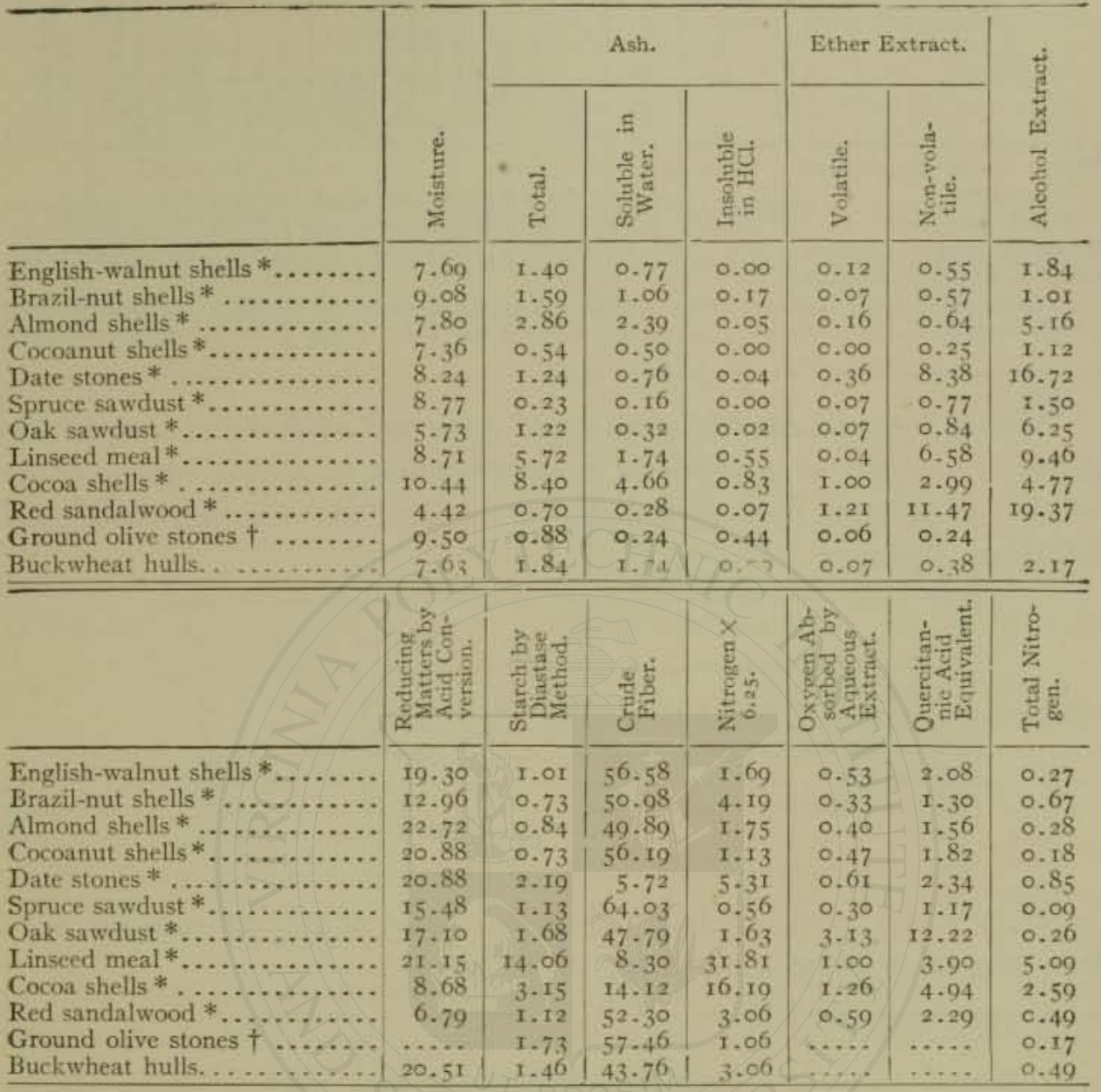

75 per cent of the oil, and a sesquiterpene known as caryophyllene. There are also in cloves a notable amount of fixed oil and resin, and also a peculiar form of tannin.

Very few complete analyses of cloves are on record. Richardson $\ddagger$ seems to have been the earliest worker in the field to give anything at all satisfactory in the way of a number of determinations of value.

The following are maximum and minimum figures from the tabulated results of Richardson's analyses:

*Winton, Ogden, and Mitchell, Conn. Exp. Sta. An. Rep., r\&98, p. 2 ro.

$\dagger$ Doolittle, Mich. Dairy and Food Dept. Bul. 94, 1903, p. 12.

$\ddagger$ U. S. Dept. of Agric., Div. of Chem., Bul. 13. 


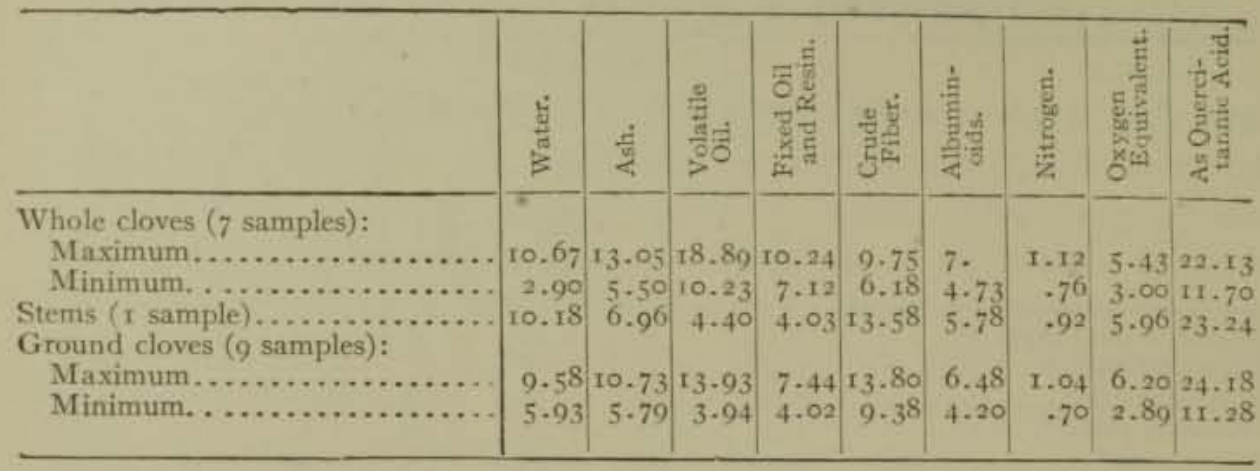

McGill * gives tables of analyses of pure and adulterated samples of cloves. Analyses of upwards of twenty samples of genuine cloves, both whole and ground, from these tables show the following maximum and minimum figures:

\begin{tabular}{|c|c|c|}
\hline & Maximum. & Minimum. \\
\hline Moisture. ............. & II. 80 & 5.05 \\
\hline 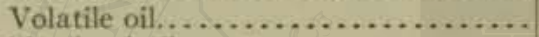 & 19.63 & 9.24 \\
\hline Total volatile matter............... & 30.68 & 16.25 \\
\hline Fixed oil...$\ldots \ldots \ldots \ldots \ldots \ldots \ldots$ & 10.23 & 0.94 \\
\hline Total extraction.................. & $31 \cdot 40$ & 22.23 \\
\hline 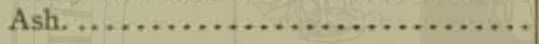 & 7.00 & 5.03 \\
\hline
\end{tabular}

McGill also made analyses of whole cloves of several varieties, the following table being a summary of his results:

\begin{tabular}{|c|c|c|c|c|c|c|c|}
\hline & & $\begin{array}{l}\text { No of } \\
\text { Analyses. }\end{array}$ & Moisture. & $\begin{array}{l}\text { Total } \\
\text { Volatile } \\
\text { Matter. }\end{array}$ & $\begin{array}{l}\text { Yoliatile } \\
\text { Oil. }\end{array}$ & $\begin{array}{l}\text { Total } \\
\text { Extract- } \\
\text { ive } \\
\text { Matter. }\end{array}$ & $\begin{array}{l}\text { Fixed } \\
\text { Oil. }\end{array}$ \\
\hline \multirow[t]{3}{*}{ Penang cloves: } & Maximum..... & 8 & $7 \cdot 4$ & $24 \cdot 3$ & 17.2 & 28.2 & 12.0 \\
\hline & Minimum. .... & $\ldots$ & 5.0 & 20.7 & 14.8 & $24 \cdot 4$ & 9.5 \\
\hline & Mean......... & $\ldots$ & 6.2 & 22.4 & 16.2 & 27.0 & 10.8 \\
\hline \multirow[t]{3}{*}{ Amboyna cloves: } & Maximum..... & 8 & 6.7 & 25.9 & 10.2 & 29.2 & 10.0 \\
\hline & Minimum..... & $\ldots$ & $5 \cdot 5$ & $23 \cdot 5$ & 18.0 & 26.5 & 8.2 \\
\hline & Mean......... & $\ldots$ & 6.1 & 24.6 & 18.5 & $27 \cdot 5$ & 9.0 \\
\hline \multirow[t]{3}{*}{ Zanzibar cloves: } & Maximum.... & 13 & 6.7 & 23.6 & 18.3 & 28.1 & 10.7 \\
\hline & Minimum. ... & $\ldots$ & $4 \cdot \mathrm{I}$ & 18.6 & 12.1 & $2 \mathrm{I} \cdot 3$ & 8.0 \\
\hline & Mean........ & $\ldots$ & $5 \cdot 7$ & $21 \cdot 7$ & 16.0 & $25 \cdot 5$ & 9.6 \\
\hline
\end{tabular}

Maximum and minimum figures of thirteen samples of unadulterated cloves, as purchased from retail dealers in Connecticut and analyzed by Winton and Mitchell, $\uparrow$ are as follows:

* Canada Inland Rev. Dept. Bul. 73 .

† Conn. Exp. Sta. Rep., 1898 , pp. 176-177 


\begin{tabular}{|c|c|c|}
\hline & Maximum. & Minimum. \\
\hline 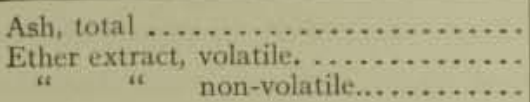 & $\begin{array}{r}7.92 \\
18.25 \\
7.19\end{array}$ & $\begin{array}{r}5.99 \\
11.03 \\
4.87\end{array}$ \\
\hline
\end{tabular}

Winton, Ogden, and Michell * give more complete analyses of eight samples of whole cloves of known purity, representing Penang, Amboyna, , and Zanzibar varieties, and two samples of clove stems, as follows:

\begin{tabular}{|c|c|c|c|c|c|c|c|}
\hline & \multirow{2}{*}{ Moisture. } & \multicolumn{3}{|c|}{ Ash. } & \multicolumn{2}{|c|}{ Ether Extract. } & \multirow{2}{*}{$\begin{array}{l}\text { Alcohol } \\
\text { Extract. }\end{array}$} \\
\hline & & Total. & $\begin{array}{l}\text { Soluble in } \\
\text { Water. }\end{array}$ & $\begin{array}{l}\text { Insoluble } \\
\text { in } \mathrm{HCl} \text {. }\end{array}$ & Volatile. & $\begin{array}{c}\text { Non- } \\
\text { volatile. }\end{array}$ & \\
\hline \multirow[t]{2}{*}{$\begin{array}{l}\text { Maximum } . . . \ldots \ldots \ldots \\
\text { Minimum. . . . . . . . } \\
\text { Mean............... } \\
\text { Clove stems, mean. . }\end{array}$} & $\begin{array}{l}8.26 \\
7.03 \\
7.8 t \\
8.74\end{array}$ & $\begin{array}{l}6.22 \\
5.28 \\
5.92 \\
7.99\end{array}$ & $\begin{array}{l}3 \cdot 75 \\
3 \cdot 25 \\
3 \cdot 58 \\
4 \cdot 26\end{array}$ & $\begin{array}{l}0.13 \\
0.00 \\
0.06 \\
0.60\end{array}$ & $\begin{array}{r}20.53 \\
17.82 \\
19.18 \\
5.00\end{array}$ & $\begin{array}{l}6.67 \\
6.24 \\
6.49 \\
3.83\end{array}$ & $\begin{array}{r}15.58 \\
13.99 \\
14.87 \\
6.79\end{array}$ \\
\hline & $\begin{array}{l}\text { Reducing } \\
\text { Matters } \\
\text { by Acid } \\
\text { Conver- } \\
\text { sion, as } \\
\text { Starch. }\end{array}$ & $\begin{array}{c}\text { Starch by } \\
\text { Diastiase } \\
\text { Method. }\end{array}$ & $\begin{array}{l}\text { Crude } \\
\text { Fiber. }\end{array}$ & $\begin{array}{c}\text { Nitrogen, } \\
\times 6.25 \text {. }\end{array}$ & $\begin{array}{l}\text { Oxygen } \\
\text { Absorbed } \\
\text { by Aque- } \\
\text { ous Ex- } \\
\text { tract. }\end{array}$ & $\begin{array}{l}\text { Querci- } \\
\text { tannic } \\
\text { Acid. }\end{array}$ & $\begin{array}{c}\text { Total } \\
\text { Nitrogen. }\end{array}$ \\
\hline $\begin{array}{l}\text { Maximum........... } \\
\text { Minimum. ............ } \\
\text { Mean............... } \\
\text { Clove-stems, mean ..... }\end{array}$ & $\begin{array}{r}9.63 \\
8.19 \\
8.99 \\
14.13\end{array}$ & $\begin{array}{l}3.15 \\
2.08 \\
2.74 \\
2.17\end{array}$ & $\begin{array}{r}9.02 \\
7.06 \\
8.10 \\
18.71\end{array}$ & $\begin{array}{l}7.06 \\
5.88 \\
6.18 \\
5.88\end{array}$ & $\begin{array}{l}2.63 \\
2.08 \\
2.33 \\
2.40\end{array}$ & $\begin{array}{l}20.54 \\
16.25 \\
18.19 \\
18.79\end{array}$ & $\begin{array}{l}1.13 \\
0.94 \\
0.99 \\
0.94\end{array}$ \\
\hline
\end{tabular}

The Tannin Equivalent in Cloves.-The amount of tannin in cloves was shown by Ellis to be so constant as to be of valuable assistance as a guide to their purity. The actual determination of tannin is, however, a long and difficult proceeding, and Richardson $\dagger$ has pointed out that it is not necessary, but that simply using the first part of the Lowenthal tannin process, and noting the "oxygen absorbed" as expressed by the oxidizing power of permanganate of potash on the material after extraction with ether, is quite as useful as determining the tannin, and is in effect proportional to the tannin present. The result is sometimes expressed as in Richardson's figures above, as the oxygen equivalent, or as quercitannic acid.

Determination of Tannin Equivalent.\$-Reagents: Indigo Solution.Six grams of the indigo salt $\delta$ are dissolved in $500 \mathrm{cc}$. of water by heat-

* Conn. Exp. Sta. Rep., 1898 , pp. 206, 207.

$\dagger$ U. S. Dept. of Agric., Div. of Chem., Bul. 13, p. 167 .

$\ddagger$ U. S. Dept. of Agric., Bur. of Chem., Bul. 65, p. 60; Bul, 107 rev., p. 164 .

\$ The quality of the indigo used is of great importance since with inferior brands it is 
ing. After cooling, $50 \mathrm{cc}$. of concentrated sulphuric acid are added, the solution made up to a liter and filtered.

Standard Permanganate Solution.-Dissolve I.333 grams of pure potassium permanganate in a liter of water. This should be standardized by titrating against ro cc. of tenth-normal oxalic acid $(6.3$ grams pure crystallized oxalic acid in $1,000 \mathrm{cc}$.), diluted to $500 \mathrm{cc}$. with water, heated to $60^{\circ} \mathrm{C}$., and mixed with $20 \mathrm{cc}$. of dilute sulphuric acid ( $1: 3$ by volume). The permanganate solution is added slowly, stirring constantly, till a pink color appears.

Two grams of the material are extracted for twenty hours with pure anhydrous ether. The residue is boiled for two hours with $300 \mathrm{cc}$. of water, cooled, made up to $500 \mathrm{cc}$, and filtered.

Twenty-five cc. of the filtrate are pipetted into a I200-cc. flask, $750 \mathrm{cc}$. of distilled water are added and $20 \mathrm{cc}$. of indigo solution.

The standard permanganate solution is then run in from a burette a drop at a time with constant shaking, until a bright golden yellow color appears, which indicates the end-point. Note the number of cubic centimeters required, represented by $(a)$.

In a similar manner determine the number of cubic centimeters of standard permanganate solution consumed by $20 \mathrm{cc}$. of the indigo solution alone, represented by $(b)$, and subtract this from $(a)$.

The oxygen equivalent, or, as it is sometimes called, the "oxygen absorbed," is calculated from the equivalent in tenth-normal oxalic acid of the number of cubic centimeters of standard permanganate represented by $a-b$. $10 \mathrm{cc}$. of tenth-normal oxalic acid are equivalent to 0.008 gram of oxygen absorbed, or 0.0623 gram of quercitannic acid.

Microscopical Examination of Cloves.-Unless the finely powdered, water-mounted sample is well rubbed out under the cover-glass, many of the masses of cellular tissue will be too dense to recognize. With a little care, however, it is possible to make a very satisfactory water mount, though by scaking for twenty-four hours in chloral hydrate solution the more opaque masses are rendered very translucent.

Fig. 81, from Moeller, shows some of the characteristics of pnwdered cloves. The outer skin of the calyx tube is shown at (I) with its polygonal cells and large oil spaces showing through them; (2) shows the epidermis of the outer part of the lobes or wings of the calyx, with stomata

impossible to get a sharp end-point. The indigo solution should be made from the very best variety of sulphindigotate, which may be obtained from Grueber \& Co., of Leipzig, or Gehe \& Co., of Dresden, under the name of carminium caruleum. 
surrounded by irregularly shaped cells; (3) represents the epidermis of the petals, with crystals of calcium oxalate; a cross-section of the epidermis of the calyx is shown at (4); (5) shows the parenchyma, with calcium oxalate crystals and with one of the slender spiral ducts; (6) and (7) represent in cross-section and longitudinal section respectively the parenchyma of the middle layers of the ovary, one of the rounded, triangular pollen grains being shown at (12).

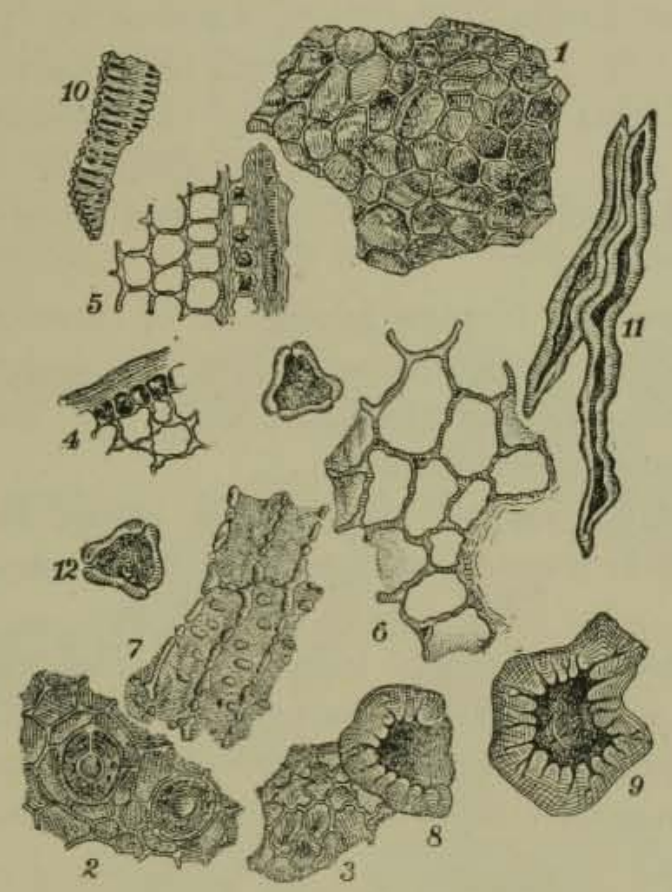

Fig. 81,-Powdered Cloves under the Microscope. X125. (After Moeller.)

Characteristics of clove stems, which are frequently used as adulterants of cloves, are found in (8), (9), (IO), and (II). Stone cells of the outer skin and the inner portion of the clove stem are shown at (8) and (9) respectively; (10) shows one of the vascular ducts, and (II) two of the bast fibers. Both the vascular ducts and the stone cells are very characteristic of clove stems. Pure cloves have no stone cells and comparatively few bast fibers. Stem under the microscope show a large number of bast fibers and frequent stone cells, the latter being of a distinctly yellow color.

A plain water-mounted slide rarely shows all the structural details depicted in Fig. 8r, but is nearly always sufficiently characteristic to 
prove the purity of the sample. Fig. 220, Pl. XXV, shows the actual appearance of powdered cloves, mounted in water and examined under a magnification of 130. The general appearance of the cellular tissue is that of a loose, spongy mass filled with brown, granular material. Throughout the masses of tissue are to be seen small oil globules.

Cloves have no starch whatever. Aside from the stems, cloves are sometines adulterated with clove fruit or "mother cloves," which have a small amount of a sago-like starch, and also contain some stone cells.

Adulteration of Cloves.-The U. S. standard for pure cloves is as follows: Volatile ether extract not less than $10 \%$; quercitannic acid, calculated from the total oxygen absorbed by the aqueous extract, should not be less than $12 \%$; total ash should not exceed $8 \%$; ash insoluble in hydrochloric acid should not exceed $0.5 \%$, and crude fiber should not be more than $10 \%$.

Clove Stems are verry frequent adulterants of cloves and possess some slight pungency. They are commonly identified under the microscope by the large number of bast fibers and stone cells, and should not be found in pure cloves in excess of $5 \%$.

Allspice, being considerably cheaper than cloves, is sometimes used as an adulterant. It is readily recognized by the characteristics described on page 422 .

Other Adulterants commonly found are cereal starches (especially corn and wheat) and ginger (for the most part "exhausted"). Besides the above, pea starch, rice, turmeric, charcoal, sand, pepper, ground fruit stones, and sawdust have been found in samples of cloves examined in Massachusetts.

Exhausted Cloves, both whole and in powdered form, are not infrequently found on the market. These have been deprived of a portion of the volatile oil, and are much less pungent than the pure article, so that the difference in taste between the two varieties is quite marked. It is, however, rare that powdered cloves are sold consisting entirely of the exhausted variety, the more common practice being to mix from Io to 25 per cent of exhausted cloves with the pure powder, so that the sophistication is less apparent.

A determination of the volatile oil is the only reliable means of showing whether or not the material has been wholly or in part exhausted, though Villier and Collin claim that under the microscope an exhausted sample of cloves shows the oil glands to be nearly empty, or to inclose much smaller droplets of oil than the pure variety. 
With the exception of exhausted cloves, the presence of nearly every foreign ingredient is best and most quickly shown by the use of the microscope, though much information as to the purity of the sample can be gained by the ether extract, the percentage of ash, and of crude fiber.*

Cocoanut Shells.-Figs. 226 and $227, \mathrm{Pl}$. XXVII, show samples of cloves adulterated with ground cocoanut shells. The long, spindle-shaped, yellowbrown and deeply furrowed stone cells of the adulterant with their thick walls and central branching pores are unmistakable. The dark-brown contents of the cells turn reddish brown when treated with potassium hydroxide. The anatomy of the cocoanut, including the shell, has been carefully studied by Winton. $\dagger$

Fig. 82, after Winton, shows elements of powdered cocoanut shell under the microscope. st are the dark, elongated, yellow, porous stone

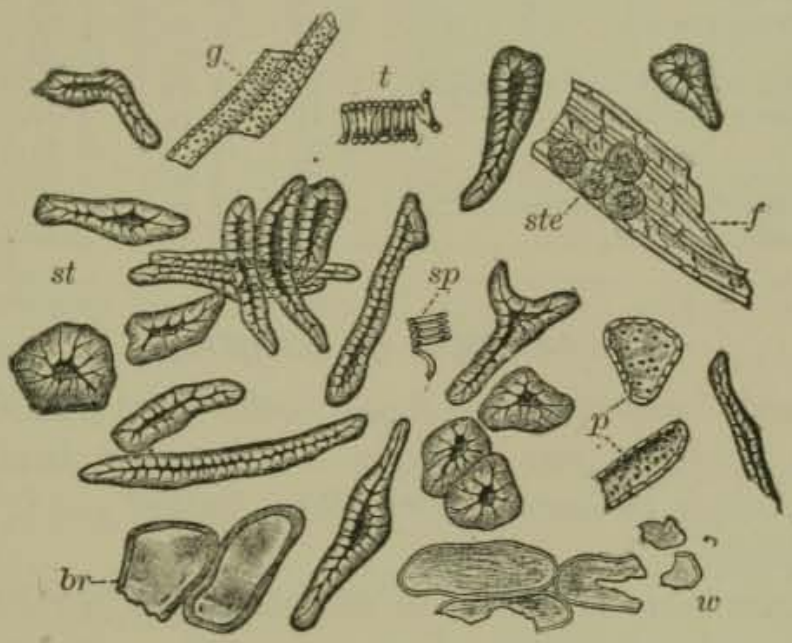

Fig. 82.-Cocoanut-shell Powder. st, dark-yellow stone cells with brown contents; $b$, reticulated trachea; $s p$, spiral trachea; $g$, pitted trachea; $w$, colorless, and $b r$, brown, parenchyma of mesocarp; f, bast fibres, with stegmata (ste). Xío. (After Winton.)

cells with their brown contents, these stone cells being the most distinctive characteristic of the ground shells. $t, s p$, and $g$ are the various forms of trachea; $w$ and $b r$ are respectively colorless and brown parenchyma of the mesocarp or outer coat, portions of which always adhere to the nutshell and are ground with it.

* Note especially the sharp distinction between these values in the case of pure cloves and of clove stems in Richardson's table.

† The Anatomy of the Fruit of the Cocoanut. Conn. Exp. Sta. Rep., I9or, p. 208. 
Fig. 264, Pl. XXXVI, shows a photomicrograph of powdered cocoanut shells, mounted in gelatin. The long, spindle-shaped stone cells are especially apparent,

Ground cocoanut shells have been used in various spices besides cloves, especially allspice and pepper. In the following tabulated results of analyses by Winton, Ogden, and Mitchell * are shown the wide deviation between the chemical constants of cocoanut shells and several of the spices in which they appear as adulterants.

\begin{tabular}{|c|c|c|c|c|c|}
\hline & $\begin{array}{c}\text { Black } \\
\text { Pepper. }\end{array}$ & Cloves. & Allspice. & Nutmeg. & $\begin{array}{l}\text { Cocoanut } \\
\text { Shells. }\end{array}$ \\
\hline 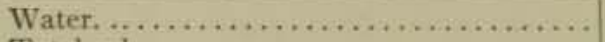 & II. 96 & $7.8 \mathrm{I}$ & $9 \cdot 7^{8}$ & 3.63 & $7 \cdot 36$ \\
\hline Total ash. ................ & $4 \cdot 7^{6}$ & 5.92 & $4 \cdot 47$ & 2.28 & 0.54 \\
\hline Ash soluble in water. . . . . . . . . . . & 2.54 & $3 \cdot 5^{8}$ & 2.47 & 0.86 & 0.50 \\
\hline Ash insoluble in hydrochloric acid. ....... & 0.47 & 0.06 & 0.03 & 0.00 & 0.00 \\
\hline Volatile ether extract. ................. & 1.14 & 19.18 & 4.05 & 3.02 & 0.00 \\
\hline Non-volatile ether extract. ............. & 8.42 & 6.49 & 5.84 & $3^{6.70}$ & 0.25 \\
\hline 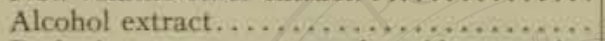 & 9.62 & 14.87 & II. 79 & 10.77 & 1,12 \\
\hline Reducing matters, as starch, acid conversion & $3^{8}, 63$ & 8.99 & 18.03 & $25 \cdot 56$ & 20.88 \\
\hline Starch by diastase method. .............. & 34.15 & 2. 74 & 3.04 & $23 \cdot 72$ & 0.73 \\
\hline 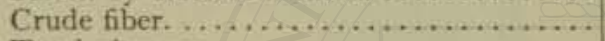 & 13.06 & 8.10 & 22.39 & $2.5 \mathrm{I}$ & 56.19 \\
\hline Total nitrogen. . . . . . . . . . ......... & 2.26 & 0.99 & 0.92 & 1.08 & 0.18 \\
\hline Oxygen absorbed by aqueous extract. ..... & $\ldots \ldots$ & 2. 33 & I. 24 & $\ldots$. & 0.23 \\
\hline Quercitannic acid equivalent............ & $\ldots$. & 18. I9 & 9.71 & $\ldots \ldots$ & 1.83 \\
\hline
\end{tabular}

\section{ALLSPICE, OR PIMENTO.}

Nature and Composition.-Allspice is the dried fruit of the Eugenia pimenta, an evergreen tree belonging to the same family (Myrtacea) as the clove. It is indigenous to the West Indies, and is especially cultivated in Jamaica.

The allspice berry is grayish or reddish brown in color, and is hard and globular, measuring from 4 to $8 \mathrm{~mm}$. in diameter, being surmounted by a short style. This is imbedded in a depression, and around it are the four lobes of the calyx, or the scars left by them after they have fallen off. The berry has a wrinkled, ligneous pericarp, with many small excrescences filled with essential oil. The pericarp is easily broken between the fingers, showing the berry to be formed of two cells with a single, brown, kidney-shaped seed in each, covered with a thin, outer coating, inclosing an embryo rolled up in a spiral.

The berries are gathered when they have attained their largest size, but before becoming fully ripe. If allowed to mature beyond this stage, some of the aroma is lost.

* Conn. Ag. Exp. Sta. Rep., rgor, p. 225. 
Though considerably less pungent than other spices, allspice possesses an aroma not unlike cloves and cassia. In chemical composition it most resembles cloves, containing both volatile oil and tannin; but, unlike cloves, it contains much starch, the starch being contained in the seeds. The volatile oil of allspice is very similar to clove oil. It is slightly lævorotary, and is composed of eugenol and a sesquiterpene not determined. It is present in allspice to the extent of 3 to 4.5 per cent. The boilingpoint of the oil is $255^{\circ} \mathrm{C}$.

Authoritative full analyses of allspice are even more meager than of cloves. Analyses of one sample of whole allspice and five samples of the ground spice, made by Richardson,* are thus summarized:

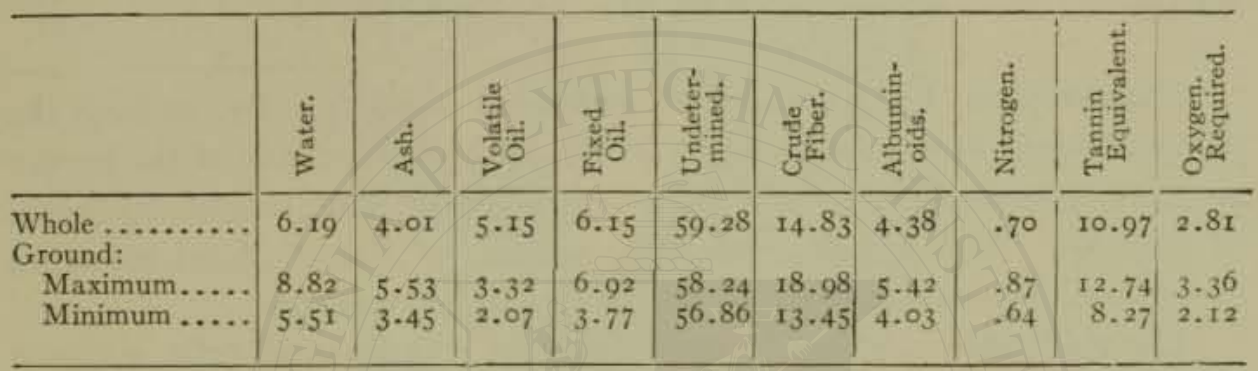

Seventeen samples of unadulterated allspice, as sold on the Connecticut market, were analyzed by Winton and Mitchell, $\dagger$ with maximum and minimum results as follows:

\begin{tabular}{|l|c|c|}
\hline \multicolumn{1}{|c|}{ Ash. } & Maximum. & Minimum. \\
\hline Total. ....................... & $7.5 \mathrm{I}$ & 4.34 \\
Insoluble in hydrochloric acid (sand)... & .95 & .40 \\
Ether extract, volatile............. & 3.50 & 1.34 \\
Ether extract, non-volatile ............ & 6.22 & $3 \cdot 78$ \\
\hline
\end{tabular}

Three samples of pure whole allspice were more fully analyzed by Winton, Mitchell, and Ogden with the results given on page $422 .+$

The Tannin Equivalent in Allspice.-Tannin is present in allspice, though to a less extent than in cloves. The exact amount present is rarely determined, but rather the "oxygen equivalent," or quercitannic acid, as explained on page $4 \mathrm{I}_{5}$, the determination being carried out as there detailed.

* U. S. Dept. of Agric., Div. of Chem., Bul. I3, p. 229.

$\dagger$ An. Rep. Conn. Exp. Sta., 1898 , pp. 178 , 179 .

† Ibid., pp. 208, 209 . 


\begin{tabular}{|c|c|c|c|c|c|c|c|}
\hline & \multirow{2}{*}{ Moisture. } & \multicolumn{3}{|c|}{ Ash. } & \multicolumn{2}{|c|}{ Ether Extract. } & \multirow{2}{*}{$\begin{array}{l}\text { Alcohol } \\
\text { Extract. }\end{array}$} \\
\hline & & Total. & $\begin{array}{l}\text { Soluble } \\
\text { in Water. }\end{array}$ & $\begin{array}{l}\text { Insoluble } \\
\text { in } \mathrm{HCl}\end{array}$ & Volatile. & $\begin{array}{l}\text { Non- } \\
\text { volatile. }\end{array}$ & \\
\hline \multirow[t]{2}{*}{ 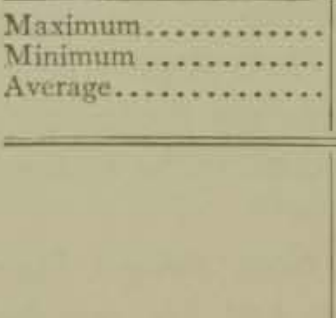 } & $\begin{array}{r}10.14 \\
9.45 \\
9.78\end{array}$ & $\begin{array}{l}4 \cdot 76 \\
4 \cdot 15 \\
4 \cdot 47\end{array}$ & $\begin{array}{l}2.69 \\
2.29 \\
2.47\end{array}$ & $\begin{array}{l}0.06 \\
0.00 \\
0.03\end{array}$ & $\begin{array}{l}5.21 \\
3 \cdot 38 \\
4.05\end{array}$ & $\begin{array}{l}7 \cdot 72 \\
4 \cdot 35 \\
5 \cdot 84\end{array}$ & $\begin{array}{r}r .27 \\
7.39 \\
\text { II. } 79\end{array}$ \\
\hline & $\begin{array}{l}\text { Reducing } \\
\text { Matters } \\
\text { by Acid. } \\
\text { Conver- } \\
\text { sion, as } \\
\text { Starch. }\end{array}$ & $\begin{array}{c}\text { Starch } \\
\text { by } \\
\text { Diastase. }\end{array}$ & $\begin{array}{l}\text { Crude } \\
\text { Fiber. }\end{array}$ & $\begin{array}{l}\text { Nitrogen, } \\
\times 6.25 \text {. }\end{array}$ & $\begin{array}{l}\text { Oxygen } \\
\text { Absorbed } \\
\text { by Aque- } \\
\text { ous Ex- } \\
\text { tract. }\end{array}$ & $\begin{array}{l}\text { Querci- } \\
\text { tannic } \\
\text { Acid. }\end{array}$ & $\begin{array}{c}\text { Total } \\
\text { Nitrogen. }\end{array}$ \\
\hline $\begin{array}{l}\text { Maximum } \ldots \ldots \ldots \ldots \ldots \\
\text { Minimum } \ldots \ldots \ldots \ldots \ldots \ldots \\
\text { Average............ }\end{array}$ & $\begin{array}{l}20.65 \\
16.56 \\
18.03\end{array}$ & $\begin{array}{l}3 \cdot 76 \\
1.82 \\
3 \cdot 04\end{array}$ & $\begin{array}{l}23 \cdot 98 \\
20.46 \\
22 \cdot 39\end{array}$ & $\begin{array}{l}6.37 \\
5.19 \\
5 \cdot 75\end{array}$ & $\begin{array}{l}1.59 \\
1.03 \\
1.24\end{array}$ & $\begin{array}{r}12.48 \\
8.06 \\
9 \cdot 71\end{array}$ & $\begin{array}{l}1.02 \\
0.83 \\
0.92\end{array}$ \\
\hline
\end{tabular}

Microscopical Examination of Powdered Allspice.-By soaking the powder twenty-four hours or more in chloral hydrate, many of the harder portions are rendered much more transparent than would otherwise be possible. Fig. $8_{3}$, after Moeller, shows the microscopical structure of various elements that go to make up allspice powder.

The epidermis, or outer layer of the berry with its small cells, is shown in cross-section at ( $\mathrm{I} a$ ) and in surface view at (2). Just beneath the outer coat are the large oil spaces $(\mathrm{I} b)$ and still further below the stonecells (IC). The fruit parenchyma (3) has vascular tissues running through it. (4) and (5) are the inner epidermis and stone cells of the dividing partitions between the seeds. Small hairs connected with the outer epidermis are shown at (6). (7) and (8) show in cross-section a portion of the seed-shell and inclosed seed or embryo, with the starch $(8 a)$ and the colored lumps of gum or resin $(8 b)$ of a port-wine color. These colored cells exist in the seed coating, and, although only one is here shown, constitute a very important and striking characteristic of allspice. (9) represents the spongy parenchyma of the seed shell, and (ro) shows its epidermis. In the parenchyma of the fruit and of the partitions between the cells are seen, but not always plainly, minute crystals of calcium oxalate (see (4) and (5)).

These details so closely drawn by Moeller are idealized, but serve well to indicate what should be looked for. In practice the watermounted specimen shows all the characteristics necessary to identify pure allspice, and most if not all its adulterants. In fact pimento is one of the easiest spices to identify under the microscope, by reason of its striking characteristics. 
Three distinctive features are especially typical, viz.: First, the starch grains, which are very uniform in size, measuring about $0.008 \mathrm{~mm}$. in diameter, being nearly circular as a rule, and often arranged in groups not unlike masses of buckwheat starch. Ordinarily these masses contain fewer granules than do those of buckwheat. The granules are

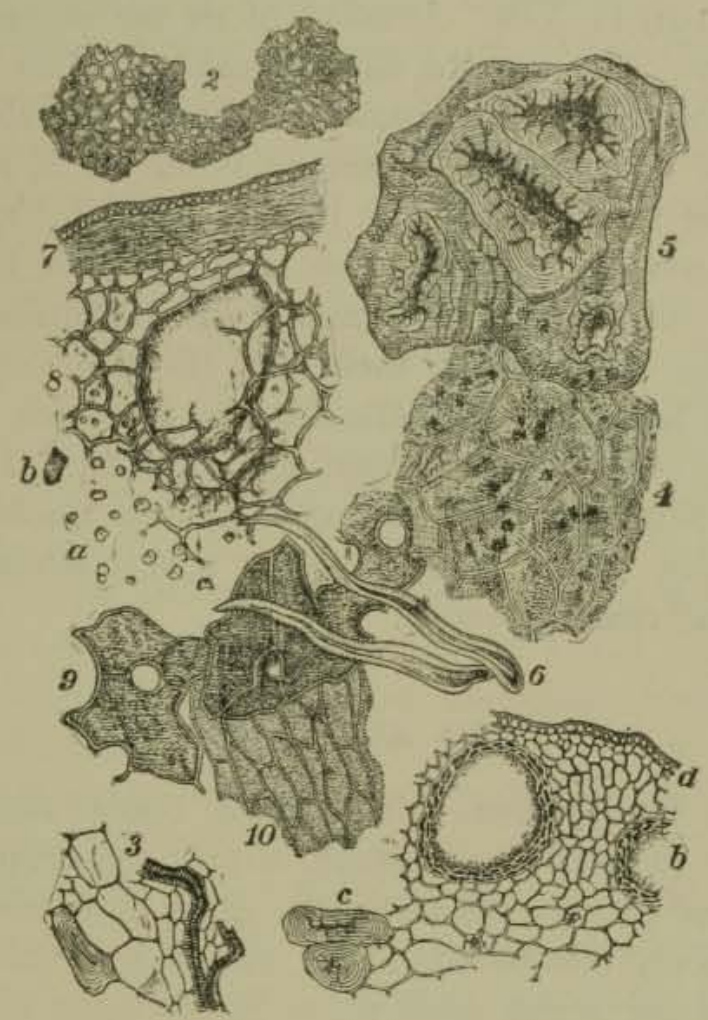

Frg. 83.-Powdered Allspice under the Microscope. Xr25. (After Moeller.)

smaller and more inclined to the circular than to the polygonal form, while in many cases they have distinct central hila. The starch grains are very numerous and are found in nearly every field. See Fig. I95, Pl. XIX.

A second distinctive feature of allspice is the stone cells, of which there are many. These are more often colorless, and in most cases very large and plainly marked. They are sometimes seen singly and at other times grouped together. Frequently they are attached to pieces of brown parenchyma. 
The third and most characteristic feature of allspice powder under the microscope is the striking appearance of the lumps of gum or resin, which are of a more or less deep port-wine or amber color and are contained in the middle layers of the seed coat. These cells are very striking, occurring sometimes in isolated bits, and in other cases in aggregations of from 2 to 4 or even 6 to 8 cells. These resinous lumps appear plainly in Fig. 194, Pl. XIX. Droplets of oil are occasionally seen, but noc in profusion. As a rule the oil is forced out of its large containing cells and into the surrounding tissue by the process of drying.

Adulteration of Allspice.-According to the U. S. standard for allspice, quercitannic acid should not be less than $8 \%$, total ash not more than $6 \%$, ash insoluble in hydrochloric acid not more than $0.5 \%$, crude fiber not more than $25 \%$. The most common adulterants found in powdered allspice are cocoanut shells and the cereal starches. Besides these the writer has found in Massachusetts, peas, pea hulls, exhausted ginger, cayenne, olive stones, pepper, and turmeric. To this list may be added clove stems, which are on record as a not uncommon adulterant in some localities. All of these are to be readily recognized by a careful microscopical examination.

\section{CASSIA AND CINNAMON.}

Nature and Composition. - The terms cassia and cinnamon are interchangeable in commerce, though, strictly speaking, they represent two separate and distinct species of the genus Cinnamomum, belonging to the laurel family (Lauracece). True cinnamon is the bark of Cinnamomum zeylanicum, a tree from 20 to 30 feet high, having horizontal or drooping branches, and native to the island of Ceylon, but cultivated also in some parts of tropical Asia, in Sumatra, and in Java. The entire yield of pure Ceylon cinnamon is extremely small, and but little of it is found in this country. It is the very thin, inner bark of the tree, and is of a pale, yellowish-brown color, being found on the market in long, cylindrical, quill-like rolls or pieces, the smaller rolls being inclosed in the larger. The outer surface is marked by round dark spots, corresponding to points of insertion of the leaves, and it is also furrowed lengthwise by somewhat wavy, light-colored lines. The inner surface of the bark is darker colored, and has no lines. In thickness the bark varies from I. 5 to $3 \mathrm{~mm}$. Both the inner and outer coatings of the bark of Ceylon cinnamon are usually removed in the process of preparation, so 
that it is of a much cleaner and more even texture than the cassia bark, which is thicker and heavier by reason of the outer cork layer usually left on it.

The cheaper and more common cassia is the bark of the Cinnamomum cassia, which comes from China, Indo-China, and India. It is of a darker color than that of cinnamon, of coarser texture, and as a rule about four times as thick. Most varieties of cassia bark are less tightly rolled than cinnamon, and are not arranged one within the other in layers. The outer surface is marked by elliptical spots left by the leaves, and by small, dark-brown, wart-like protuberances. Cassia does not have the wavy, light-colored lines found in the cinnamon. Both cinnamon and cassia barks are very aromatic in taste, somewhat astringent, and slightly sweet.

Cassia buds are the dry flower buds of China cassia, and are found in the market both in whole and in powdered form. Powdered cassia often consists of a mixture of several varieties of bark, while the cheaper grades sometimes contain an admixture of the ground buds.

The best grade of cassia is that from Saigon, a much cheaper, from Batavia, while the cheapest is the China cassia.

The odor of cassia and cinnamon bark is due to the volatile oil, of which from I to 2 per cent is usually found. Cassia and cinnamon oil greatly resemble each other, the principal constituent in either case being cinnamic aldehyde, $\mathrm{C}_{6} \mathrm{H}_{5} \mathrm{CH}$ : CH.CHO. Besides this, one or more esters of acetic acid are present. Both oils are very pungent and intensely sweet.

Starch is present in cassia to the extent of from 16 to 30 per cent. A very small amount of tannin is found, as well as cinnamic acid and mucilaginous matters. Cassia buds are somewhat similar in composition to the bark. They have, however, less starch and crude fiber, and higher contents of volatile oil and nitrogen than the bark.

Richardson* has made analyses of a few samples of pure whole cinnamon and cassia, from which the following are taken:

\begin{tabular}{|c|c|c|c|c|c|c|c|c|}
\hline & $\frac{\vec{s}}{\grave{a}}$ & 进 & 递市 & 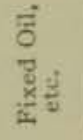 & 郭若 & 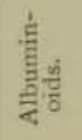 & 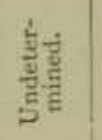 & $\frac{\text { है }}{\mathrm{z}}$ \\
\hline Ceylon cinnamon, $1, \ldots \ldots \ldots \ldots \ldots$ & $\begin{array}{r}5.40 \\
7.43\end{array}$ & $\begin{array}{l}4.55 \\
3.40\end{array}$ & $\begin{array}{l}1.05 \\
.82\end{array}$ & $\begin{array}{l}1.66 \\
1.58\end{array}$ & $\begin{array}{l}33.08 \\
25.63\end{array}$ & $\begin{array}{l}2.9^{8} \\
3.80\end{array}$ & $\begin{array}{r}5 \mathrm{I} \cdot 28 \\
56.84\end{array}$ & .48 \\
\hline Cassia bud $=\ldots \ldots \ldots \ldots \ldots \ldots$ & $\begin{array}{r}7.43 \\
4.79\end{array}$ & $\begin{array}{l}3 \cdot 4^{\circ} \\
5 \cdot 5^{8}\end{array}$ & 3.59 & $\begin{array}{l}1.50 \\
5.21\end{array}$ & $\begin{array}{r}25.63 \\
8.60\end{array}$ & $\begin{array}{l}3.80 \\
7.00\end{array}$ & $\begin{array}{l}50.84 \\
65.23\end{array}$ & $\begin{array}{r}.02 \\
1.12\end{array}$ \\
\hline $\begin{array}{l}\text { Cassia bark }(4 \text { samples }): \\
\text { Maximum } \ldots \ldots \ldots \ldots \ldots \ldots \ldots \ldots \ldots \ldots \ldots \\
\text { Minimum } \ldots \ldots \ldots \ldots \ldots \ldots \ldots\end{array}$ & $\begin{array}{r}17 \cdot 45 \\
9 \cdot 3^{2}\end{array}$ & $\begin{array}{l}8.23 \\
2.48\end{array}$ & $\begin{array}{r}3 \cdot 5 \mathrm{I} \\
\cdot 55\end{array}$ & $\begin{array}{r}2.38 \\
.74\end{array}$ & $\begin{array}{l}26.29 \\
14 \cdot 33\end{array}$ & $\begin{array}{l}4.55 \\
2.63\end{array}$ & $\begin{array}{l}65.33 \\
48.65\end{array}$ & $\begin{array}{l}.73 \\
.42\end{array}$ \\
\hline
\end{tabular}

* U. S. Dept. of Agric., Div. of Chem., Bul. I3, p. 22 I. 
Winton, Ogden, and Mitchell's* results of analyses of whole samples of cinnamon, cassia, and cassia buds are thus summarized:

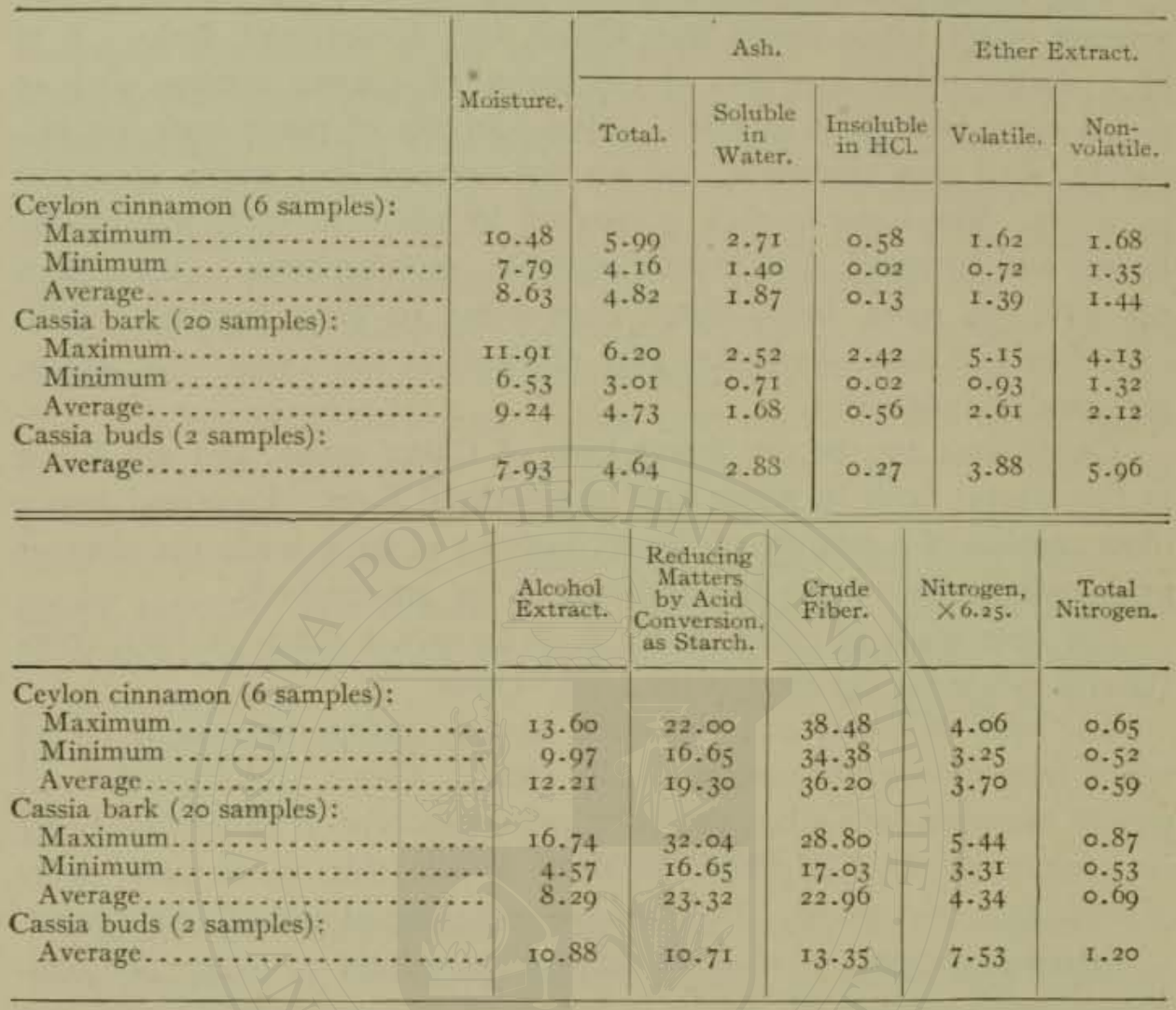

Structure of Powdered Cassia under the Microscope.-Fig. 84, from Moeller, shows various elements of cassia bark as veiwed microscopically. (I) shows in cross-section a portion of the cork and outer layer of the bark rind, with flat cells nearest the surface, having somewhat thick walls and reddish-brown contents, and, farther in, the cells $s$, with mucilaginous material.

The stone cells of the intermediate layer of bark are shown at (2). Here the tendency of the stone cells is to be thicker on one side than on the other, as is plainly shown. (3) represents the structure of the inner layer of the bark, showing bast fibers $b$ cut across, and more of the socalled mucilaginous cells $s$ of large size, which normally contain the ethereal or volatile oil. The starch granules (4) are contained in great abundance in the polygonal cells of the parenchyma of the intermediate

* Twenty-second Annual Report Conn. Exp. Sta., I8g8, pp. 204, 205. 
and inner bark layers. (6) represents a fragment of a bast fiber, which is often shown in cassia powder with connecting parenchyma. The stone-cells of the cork are shown in plan view at (7). Very small, needlelike crystals of oxalate of calcium are occasionally to be seen if looked for carefully. They occur in the parenchyma cells of the inner and intermediate layers of the bark.

The microscopical structure of Ceylon cinnamon much resembles that of cassia. Cassia starch grains measure from 0.0132 to $0.0222 \mathrm{~mm}$.,

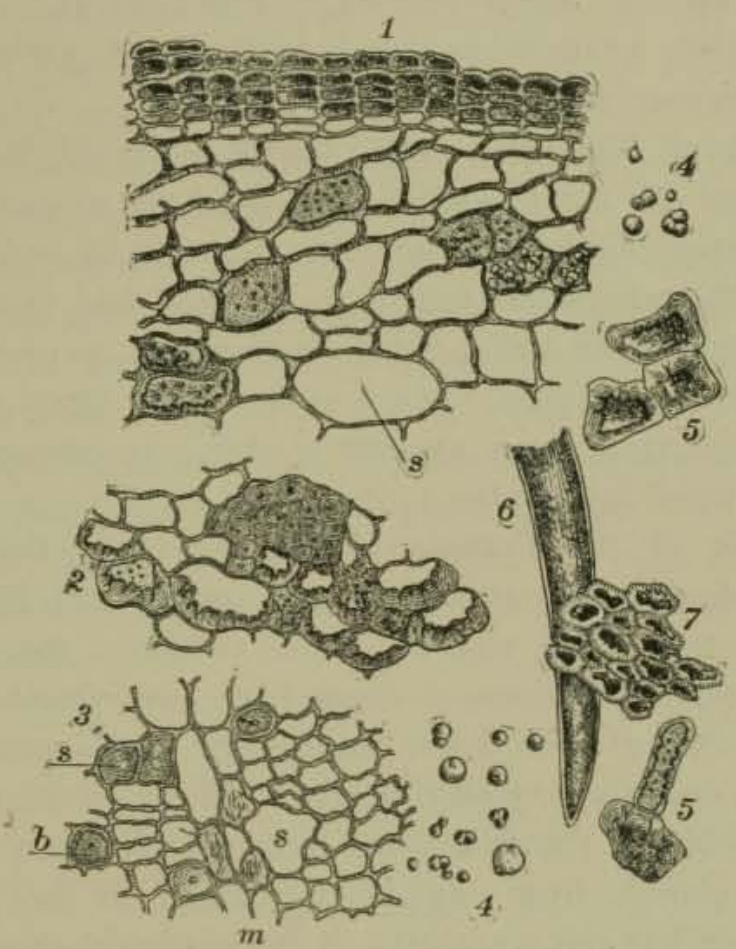

Frg. 84.-Powdered Cassia under the Microscope. X 125 . (After Moeller.)

being considerably larger and more abundant that those of true cinnamon. As a rule the bast fibers of cassia are larger, but shorter, than those of cinnamon, and provided with thicker walls.

Figs. 203 and 204, Pl. XXI, show various phases of pure cassia bark as photographed from water-mounted specimens of the powder. Cassia starch somewhat resembles that of allspice, but it is not as a rule found in masses containing as many granules as does the allspice starch. Very commonly two or three of the starch granules are arranged together in 
such a manner that at first sight they appear to form a single large granule, but on more careful examination are seen to be two- and three-lobed, consisting of several smaller grains. Stone cells, which are very abundant in the powdered cassia, do not happen to be included to any extent in the photographed fields. Cassia stone cells are generally more oblong than those of allspice, and are more often brown in color, while the allspice stone cells are generally colorless.

A distinctive feature of powdered cassia consists in the long, ambercolored wood fibers, some distributed in bundles, and others arranged singly. These are very clearly shown in Figs. 204 and 205.

Yellow patches of cellular tissue with starch grains interspersed among them are very abundant in the powder.

Adulteration of Cinnamon and Cassia.-The U. S. standards are as follows: Total ash not to exceed $8 \%$; sand not to exceed $2 \%$.

The commonest adulterants are cereal products and foreign bark. Besides these, the writer has found, in samples sold in Massachusetts, leguminous starches, pea hulls, nutshells, turmeric, pepper, olive stones, ginger, mustard, and sawdust. Much of the China cassia when imported contains an inexcusably large amount of dirt. In one sample Winton, Ogden, and Mitchell found over $15 \%$ of sand.

Ground Bark of the Common Trees, especially that of the elm, resenbles in physical appearance ground cassia, and is to be looked for as an adulterant. Fig. 265, Pl. XXXVII, shows the appearance of ground elm bark. The fibers of cassia bark have starch granules as a rule interposed among them, while the foreign bark, usually of a much coarser texture, shows no starch connected with its structure.

Fig. 206, Pl. XXH, shows a water-mounted specimen of adulterated cassia powder, chosen from samples purchased in the Massachusetts market. Nothing but the adulterant (a foreign bark) shows in the field. The tissue is loose and considerably coarser than that of cassia bark.

PEPPER.

Nature and Composition.-Pepper is the dried berry of the pepper plant (Piper nigrum), a climbing shrub belonging to the family Piperacea, native to the East Indies, but cultivated in many tropical countries. The height of the pepper plant is from twelve to twenty feet. When the fruit begins to turn red, it is gathered and then dried, by which process it turns black and shrivels up, forming the black peppercorns of commerce. They are spherical single-seeded berries, about $5 \mathrm{~mm}$. in diam- 
eter, covered with a brownish-gray epicarp, and having on the under side the remains of a short stem. At the top of the berry is an indistinct trace of a style, and of a lobed stigma.

Varieties of black pepper are named from the localities in which they are grown or from which they "are shipped, as Singapore, Lampong, Sumatra, Tellichery, Malabar, Acheen, Penang, Alleppi, Trang, Mangalore, etc.

White pepper is obtained by decorticating the fully ripened black peppercorns, or removing the dark skin. This is accomplished by macerating them in water to loosen the skin, which is then removed readily by drying and rubbing between the hands. White whole pepper grains are grayish white, and a trifle larger than the black pepper berries. They are nearly spherical in shape, and have a number of light-colored lines that, like meridians, run from top to bottom. The common varieties are Siam, Singapore and Penang, the latter being coated with lime.

The pungent taste of pepper is due in great part to its essential oil, a hydrocarbon of the formula $\mathrm{C}_{10} \mathrm{H}_{16}$, present in amounts varying from 0.5 to $\mathrm{I} .7$ per cent. Pepper oil contains phellandrene and a terpene.

Other important constituents of pepper are piperidine, and the crystalline base piperin, $\mathrm{C}_{17} \mathrm{H}_{19} \mathrm{NO}_{3}$, insoluble in water, but soluble in ether, and in alcohol. Starch is present in pepper to a large extent.

Burcker gives the following average percentage composition of black and white pepper:

\begin{tabular}{|c|c|c|c|c|c|c|c|c|}
\hline & $\frac{\dot{5}}{4}$ & $\frac{\dot{8}}{\vec{B}}$ & $\frac{\mathrm{g}}{\mathrm{g}}$ & 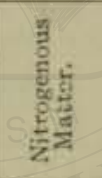 & $\begin{array}{l}\text { हैं } \\
\frac{\text { है }}{\text { है }} \\
\text { है }\end{array}$ & 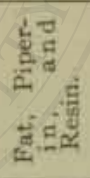 & 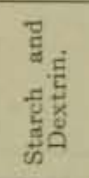 & 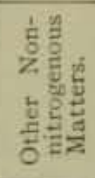 \\
\hline $\begin{array}{l}\text { Black pepper.. } \\
\text { White pepper. }\end{array}$ & $\begin{array}{l}4.57 \\
1.80\end{array}$ & $\begin{array}{r}12.45 \\
6.08\end{array}$ & $\begin{array}{l}12.50 \\
13.56\end{array}$ & $\begin{array}{l}\text { II. } 98 \\
\text { II. } 12\end{array}$ & $\begin{array}{l}1.36 \\
0.94\end{array}$ & $\begin{array}{l}6.85 \\
7.11\end{array}$ & $\begin{array}{l}42.90 \\
56.04\end{array}$ & $\begin{array}{l}7 \cdot 39 \\
3 \cdot 35\end{array}$ \\
\hline
\end{tabular}

Richardson's* analyses of three samples of whole black and two samples of whole white pepper, all pure, are as follows:

\begin{tabular}{|c|c|c|c|c|c|c|c|}
\hline & & Water. & Ash. & $\begin{array}{l}\text { Volatile } \\
\text { Oil. }\end{array}$ & $\begin{array}{l}\text { Piperin } \\
\text { and } \\
\text { Resin. }\end{array}$ & $\begin{array}{l}\text { Alcohol } \\
\text { Extract. }\end{array}$ & $\begin{array}{l}\text { Starch } \\
\text { (Acid Con- } \\
\text { sersion) }\end{array}$ \\
\hline $\begin{array}{l}\text { Black pepper: } \\
\text { White pepper: }\end{array}$ & 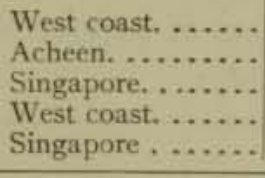 & $\begin{array}{r}8.91 \\
8.29 \\
9.83 \\
9.85 \\
10.60\end{array}$ & $\begin{array}{l}4.04 \\
4.70 \\
3.70 \\
1.41 \\
1.34\end{array}$ & $\begin{array}{r}.70 \\
1.69 \\
1.60 \\
.57 \\
1.26\end{array}$ & $\begin{array}{l}7.29 \\
7.72 \\
7.15 \\
7.24 \\
7.76\end{array}$ & $\begin{array}{l}6.06 \\
5 \cdot 74 \\
\dddot{2.5} \\
2.57\end{array}$ & $\begin{array}{l}36 \cdot 52 \\
37 \cdot 50 \\
37 \cdot 30 \\
40.61 \\
43 \cdot 10\end{array}$ \\
\hline
\end{tabular}

* U. S. Dept. of Agric., Bur. of Chem., Bul. I3, part 2, p. 206. 


\begin{tabular}{|c|c|c|c|c|c|c|}
\hline & - & $\begin{array}{l}\text { Undeter- } \\
\text { mined. }\end{array}$ & $\begin{array}{l}\text { Crude } \\
\text { Fiber. }\end{array}$ & $\begin{array}{l}\text { Albumin- } \\
\text { oids. }\end{array}$ & $\begin{array}{l}\text { Total } \\
\mathrm{N} \times 6.25 .\end{array}$ & Total N. \\
\hline \multirow[t]{2}{*}{ Black pepper: } & West coast. ... & 24.62 & 10.23 & 7.69 & $9.8 \mathrm{I}$ & 1.57 \\
\hline & Acheen. ... & 13.64 & 10.02 & 10. $3^{8}$ & 12.60 & 2.02 \\
\hline \multirow{3}{*}{ White pepper: } & Singapore ........... & 17.66 & 10.02 & 10.00 & 12.08 & 1. 93 \\
\hline & $\begin{array}{l}\text { West coast. ............ } \\
\text { Singapore. }\end{array}$ & $\begin{array}{l}23.28 \\
10.55\end{array}$ & $7 \cdot 73$ & $9 \cdot 3 \mathrm{I}$ & 11.48 & 1.83 \\
\hline & singapore . ......... & 19.55 & 4.20 & 9.62 & 11.90 & 1.90 \\
\hline
\end{tabular}

Richardson gives the following variations in the constituents of pure pepper:

\begin{tabular}{|c|c|c|}
\hline & Black. & White. \\
\hline Water & 8.0 to 11.0 & 8.0 to 11.0 \\
\hline Ash..... & 2.75 to 5.0 & 1.0 to 2.0 \\
\hline Volatile oil....... & .50 to 1.75 & .50 to 1.75 \\
\hline Piperin and resin. & 7.0 to 8.0 & 7.0 to 8.0 \\
\hline Starch .... & 32.0 to 38.0 & 40.0 to 44.0 \\
\hline Crude fiber & 8.0 to 11.0 & 4.11 to 8.0 \\
\hline Albumino & 7.0 to 12.0 & 8.0 to 10.0 \\
\hline
\end{tabular}

McGill's * analyses of six samples of whole black, and five samples of whole white pepper, all genuine, are thus summarized:

\begin{tabular}{|c|c|c|c|c|c|c|c|c|}
\hline & \multirow[b]{2}{*}{$\begin{array}{l}\text { Moisture, } \\
\text { etc., Lost } \\
\text { at } 100^{\circ} \mathrm{C} \text {. }\end{array}$} & \multicolumn{4}{|c|}{ Ash. } & \multirow[b]{2}{*}{$\begin{array}{l}\text { Sand } \\
\text { Expressed } \\
\text { as Per } \\
\text { Cent of } \\
\text { Total } \\
\text { Ash. }\end{array}$} & \multirow[b]{2}{*}{$\begin{array}{l}\text { Alcohol } 1 \text { Extract. } \\
\text {. }\end{array}$} \\
\hline & & & $\begin{array}{l}\text { Soluble } \\
\text { in Hot } \\
\text { Water. }\end{array}$ & $\begin{array}{l}\text { Insoluble } \\
\text { in Water. }\end{array}$ & Total. & $\begin{array}{l}\text { Insolubie } \\
\text { in Hydro- } \\
\text { chloric } \\
\text { Acid. }\end{array}$ & & \\
\hline \multirow[t]{6}{*}{ Black: } & Maximum & 14.10 & 2.64 & 3.06 & 5.16 & 1.08 & 21 & 9.06 \\
\hline & Minimu & 10.62 & 2.07 & 1. 46 & 3.98 & .06 & 2 & 8.28 \\
\hline & Mean & 12.03 & $2.4 \mathrm{I}$ & 2.05 & $4 \cdot 47$ & $0.3^{6}$ & 8 & 8.71 \\
\hline & Maxin & 13.00 & 0.72 & 3.04 & 3.65 & 0.88 & 42 & 8.92 \\
\hline & Minimum & II. 30 & 0.14 & 1. $50^{\circ}$ & 1.64 & 0.26 & 9 & 7.00 \\
\hline & Mean.... & 12.34 & 0.54 & 2.46 & 3.00 & 0.55 & 21 & $7 \cdot 73$ \\
\hline
\end{tabular}

Winton, Ogden, and Mitchell's, and Winton and Bailey's $\dagger$ analyses of whole black pepper and whole white pepper, representing the leading varieties imported into the United States, also of pepper shells and long pepper, are summarized in the following table:

* Canada Inl. Rev. Dept. Bul. 20, 189o.

$\dagger$ An. Rep. Conn. Exp. Sta., 1898, pp. 198-199; 1903, pp. 158-164. 


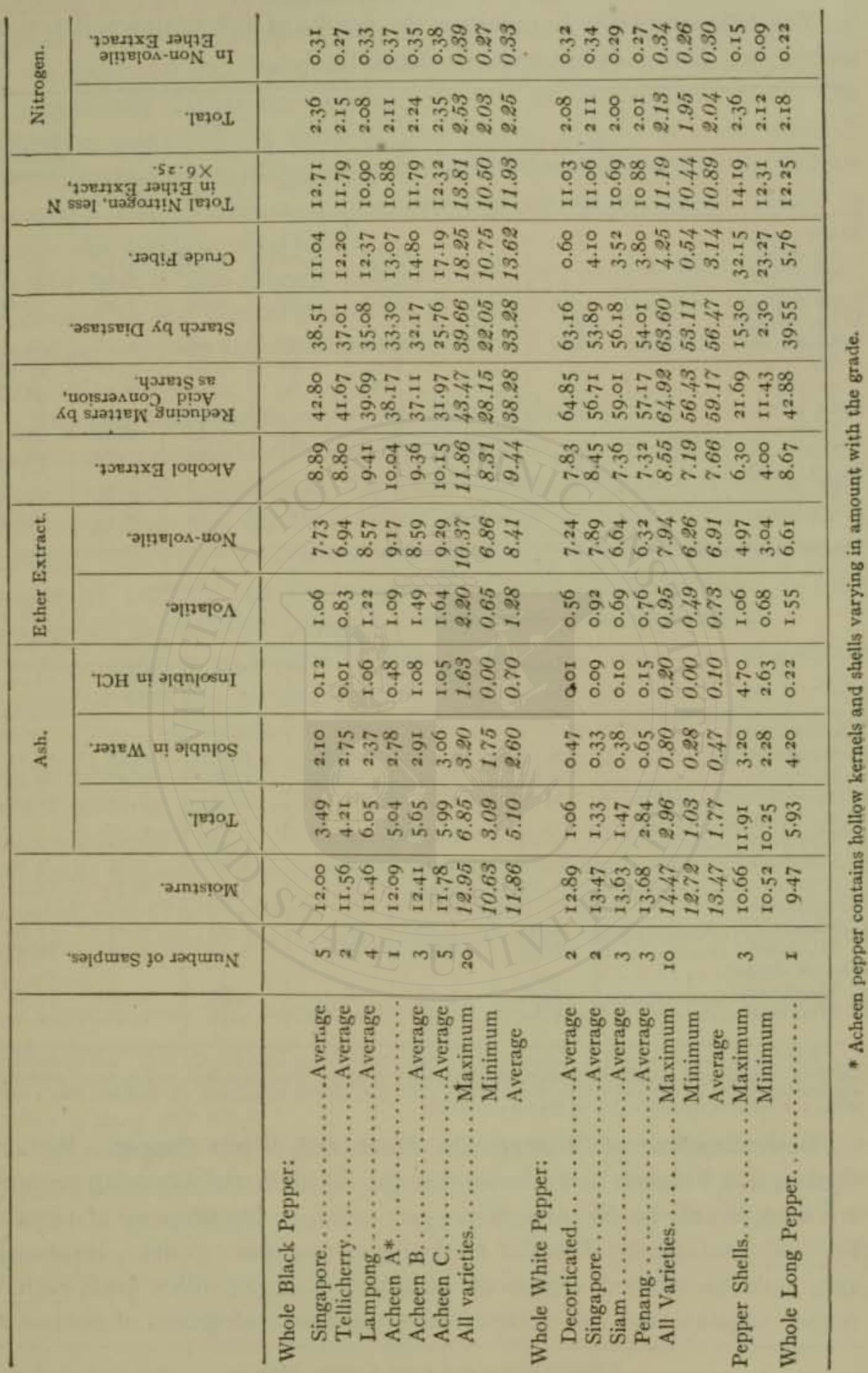


The following table summarizes the results of full analyses of pepper and pepper shells recently made by Doolittle:*

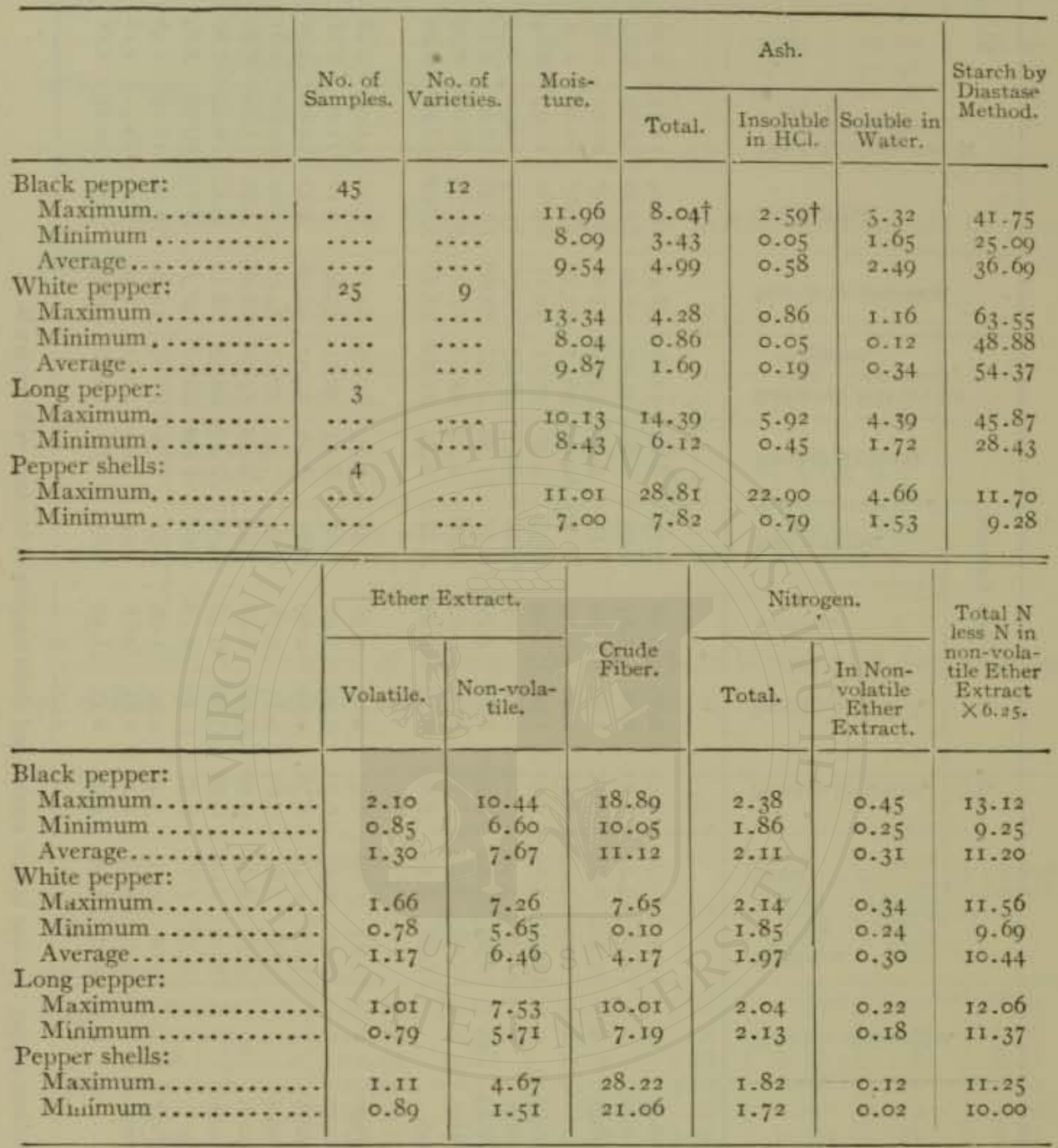

+ Two samples of Acheen $\mathrm{C}$ pepper had a total ash of $8.00 \%$ and $8.04 \%$, with " ash insoluble in HCl" of $2.50 \%$ and $2.40 \%$ respectively. Eliminating these two samples, which were evidentiv abnorually high in sand and dirt, the highest total ash of the remaining 43 samples was $7.00 \%$. while the highest ash insoluble in $\mathrm{HCl}$ was $x .80 \%$.

Determination of Nitrogen in Black and White Pepper.-Winton, Ogden, and Mitchell have shown that the Kjeldahl and Gunning methods are inapplicable in the case of pepper, owing to the presence of piperin, bus that the Gunning-Arnold † method gives accurate results. In accordance with this method, I gram of the sample is mixed with a gram each of copper sulphate and red oxide of mercury, about 16 grams of potassium

* Mich. Dairy and Food Comm. Bul. 94.

† Zeits, anal. Chem., 31, 1892, p. 525 . 
sulphate, and $25 \mathrm{cc}$. of sulphuric acid in a Kjeldahl flask, for both digestion and distillation, of about $600-\mathrm{cc}$. capacity. The heating is conducted in the usual manner, beginning with a gentle heat till the frothing ceases, and gradually increasing the temperature till the mixture boils. The boiling is continued for three or four hours, after which the flask is cooled, and to it are added $300 \mathrm{cc}$. of water, $50 \mathrm{cc}$. of potassium sulphide solution,* and enough of a saturated solution of sodium hydroxide to render the reaction alkaline.

The flask is then connected to the condenser, and the distillation conducted as in the Gunning method (p. 69), using zinc dust to prevent bumping, receiving the distillate into standard acid, and titrating against standard alkali.

Nitrogen Determination in the Ether Extract. $\dagger$-Ten grams of the sample are extracted with absolute ether for twenty hours in a continuous-extraction apparatus, the extract being collected in a tared Kjeldahl extraction- and distillation-flask, the same as used in the preceding section. The ether is then evaporated off, the residue dried to constant weight at $110^{\circ} \mathrm{C}$. and its weight ascertained. The nitrogen is then determined in the ether extract by the Gunning-Arnold method.

Determination of Piperin. - Fifty grams of the sample are thoroughly exhausted with hot alcohol, and the alcohol extract evaporated to dryness. The dry residue is then treated with a solution of potassium hydroxide, and washed upon a filter. The residue is washed several times with the caustic alkali, which dissolves the resinous matters, and afterwards with water. It is then dissolved in alcohol, from which crystals of crude piperin separate on evaporation. These are redissolved in alcohol, and precipitated by the addition of water. The crystalline precipitate is collected on a tared filter, washed with water, dried, and weighed.

Piperin may be roughly estimated by multiplying the nitrogen in the ether extract by the factor 20.36 .

The amount of piperin varies considerably, ranging in black pepper from 4 to 9 per cent.

Microscopical Characteristics of Ground Pepper.-Moeller's representation of powdered black pepper shows what should be looked for under the microscope with the best conditions (Fig. 85). The shell of the peppercorn, a cross-section of which is shown at (t), consists of the

* Forty grams $\mathrm{K}_{2} \mathrm{~S}$ in 1 liter or water.

† Method of Winton, Ogden and Mitchell.

$\ddagger$ Villiers et Collin, Substances Alimentaires, p. $37 x$. 
epidermis, $a$, under which is a thin layer of brown parenchyma, $c$, while below this layer is shown the most characteristic portion of

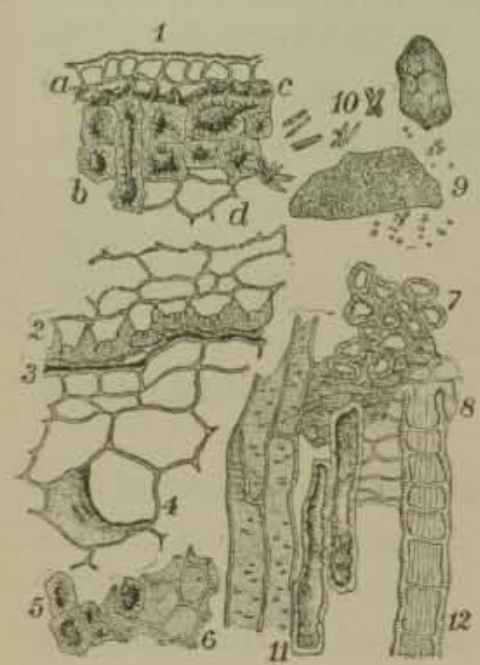

Frg. 85.-Powdered Black Pepper under the Microscope. $\times 125$. (After Moeller,) the pepper shell, viz.: the thickened, colored, stone cells, $b$. These are as a "rule inclined to be rectangular rather than rounded. At $d$ is shown a bit of the colorless parenchyma of the fruit itself.

(2), (3), and (4) show a cross-section of the outer part of the berry, (2) representing the inner stone-cell layer, a single row of horseshoe-like cells, (3) the thin seed coat, and (4) the white perisperm, with its large cells. Here and there through the perisperm certain yellow contents are visible, consisting largely of resinous matter. A dark resin cell is shown at (4). The ethereal oil, starch, and piperin are found in this part of the berry.

(5) shows in surface view the mostly rectangular stone cells of the pepper shell, resting upon the epidermis (6). Groups of stone cells are frequently thus found with portions of the epidermis.

The inner rounded, or cup-shaped cells are shown in plan view at (7) and the seed skin at (8), masses of starch and separate starch granules are shown at (9), and crystals of piperin at (10).

The bast-parenchyma of the pepper stem is shown at (II), pieces of which are commonly found in powdered pepper, and (I2) shows a fragment of one of the many-celled hairs which grow on the stem.

The rounded cup cells (7) are readily distinguished from the more rectangular stone cells (5). The walls of the cup cells are nearly always colorless, and the cells themselves empty.*

A water-mounted specimen of finely ground, black pepper, when viewed microscopically, shows most of the elements above described, at least in fragmentary form, though, in the case of the coarser particles,

* The harder portions of the pepper, especially of the shell, are best examined by soaking for at least twenty-four hours in chloral hydrate, and mounting in this reagent on the slide. 
by no means as clearly as by the use of chloral hydrate. Large polygonal masses of starch appear grouped as photographed in Fig. 256 , Pl. XXXIV, if not rubbed out too fine under the cover-glass. Starch, indeed, is the most conspicuous element of pepper, being distributed more or less evenly throughout the masș. The powder may, however, be so finely reduced by abrasion under the cover-glass as to break up these starch masses wholly or in part, so that the granules may appear in much smaller groups or even singly. Fig. 255 shows such a field under a higher magnification. The individual granules of pepper starch average $0.003 \mathrm{~mm}$. in diameter.

Besides the starch, and next to it the most numerous, one finds in the water-mounted black-pepper specimen many of the dark-yellow, thickwalled stone cells, patches of the colored parenchyma, and epidermis of the shell. Other elements of the perisperm, besides the starch, are seen in fragments, such as bits of resin, small droplets of oil, pieces of stems, and occasionally the needle-shaped crystals of piperin. Some of the rounded, cup-shaped cells are also usually found.

White pepper contains, of course, the same elements, but without the deeply colored stone cells and other characteristics of the shell, which has been removed from it.

Adulteration of Pepper.-The following U. S. standards for pepper have been adopted: For white pepper, non-volatile ether extract should not to be less than $6 \%$; starch should not be less than $50 \%$ by the diastase method; total ash should not be more than $4 \%$; ash insoluble in hydrochloric acid should not exceed $0.5 \%$; crude fiber should not exceed $5 \%$. One hundred parts of the non-yolatile ether extract should contain not less than 4 parts of nitrogen. For black pepper, which should be free from added pepper shells, pepper dust, and other pepper by-products, non-volatile ether extract should not be less than $6 \%$; starch by the diastase method should not be less than $25 \%$; total ash should not exceed $7 \%$; and crude fiber should not exceed $15 \%$. One hundred parts of the non-volatile ether extract should contain not less than 3.25 parts of nitrogen. The adulterants used in ground pepper are many and varied.

Pepper Shells, which have been removed from the white pepper of commerce, are not infrequently ground and added to the cheaper grades of black pepper. When a sample of black pepper is shown by the microscope to contain more shells in proportion to the other elements than could be possible in a ground whole berry, added shells are indicated. 
The analyst should, for comparison, grind in a mortar single berries of various grades, and familiarize himself with the appearance of the ground powder under the microscope, when the maximum amount of shells possible under natural conditions are present, noting especially the apparent number of stone cells of the outer coating. The familiar title of P. D. (pepper dust) originally given to ground pepper shells, stems, and "sweepings " is now applied in the trade not only to almost any cheap and appropriate material for admixture with pepper, but also, in a broader sense, to ground powder suitable as an adulterant for any spice.

The presence of pepper shells is indicated by an excess of ash, sand, and crude fiber, and a deficiency of starch.

Hilger and Bauer, also Hanus and Bien, advocate the determination of pentosans as a means of detecting pepper shells.

Ground Olive-stones constitute one of the most commonly found foreign materials used as an adulterant of pepper. The powder, sometimes called "poivrette," is very like white pepper in appearance, is wholly inert in taste, and thus forms an admirable adulterant. While best detected by their characteristic appearance under the microscope, the presence of ground olive stones may be shown by color tests with certain chemical reagents.

Pabst has adopted for this purpose a test first suggested by Wurster for the detection of wood pulp in paper. The reagent is prepared as follows: In a porcelain capsule to grams of commercial dimethyl anilin are mixed with 20 grams of pure concentrated hydrochloric acid, and at least 100 grams of cracked ice are added. Then, while stirring, a solution of 8 grams of nitrite of soda in $100 \mathrm{cc}$. of water are added little by little, and the mixture allowed to remain for half an hour, after which 30 or $40 \mathrm{cc}$. of hydrochloric acid are added, and 20 grams of tin-foil. The reduction is allowed to go on for half an hour, heating on the waterbath, if necessary. The tin is then precipitated by granulated zinc, the liquid is filtered, and the filtrate neutralized with carbonate of potassium or sodium to the point of forming a precipitate, the precipitate being dissolved by a few drops of acetic acid. Finally the volume is made up with water to 2 liters, adding, before doing so, 3 or $4 \mathrm{cc}$. of a concentrated solution of sodium bisulphite, to prevent oxidation. The reagent thus prepared will keep for several years in a brown, tightly stoppered bottle.

If a pinch of pepper, which contains ground olive stones, be heated gently with a little of the above reagent in a test-tube, the stone cells of the adulterant will be colored a bright red brown, and the colored particles will be seen to settle to the bottom of the tube, after shaking, 
more quickly than the rest of the powder. Or, if the whole is poured from the test-tube into a porcelain dish, the color is more marked. Pure pepper is not colored under this treatment with the reagent.

Jumeau uses for a color reagent 5 grams of iodine in roo cc. of a mixture of equal parts of ether and alcohol. Enough of the finely ground pepper to be examined is placed in a porcelain capsule to cover the bottom of the dish, and sufficient iodine reagent is added to wet the entire mass, carefully avoiding excess. The thick paste is first mixed till homogeneous, and then allowed to dry in the air, after which it is broken up by a pestle, and the powder examined, either under the microscope, or by the naked eye. With pure pepper, a more or less deep-brown coler is produced uniformly through the powder, but if olive stones are preseni, particles of these are colored yellow. With the naked eye as smali an admixture as $2 \%$ of olive stones can thus be detected.

A solution of anilin acetate colors olive stones yellowish browr, while pure pepper appears grayish, or white.

Under the microscope olive stones are readily apparent, since the stone cells differ in size, form, and mode of grouping from those of pepper. Fig. ${ }^{2} 6_{3}$, Pl. XXXVI, is a photograph of a water-mounted specimen of olive stones. They are for the most part entirely devoid of color, being long and narrow. In shape and manner of grouping they much resemble cocoanut shells (p. $4 \mathrm{I} 9$ ), but are distinguished from the latter from their lack of color.

Fig. 26I shows under low magnification a sample of pepper, bought on the market in Massachusetts, highly adulterated with olive stones. A large mass of the stone cells of the adulterant appears in the center of the field. Many of the stone cells are shown arranged end to end, so that what at first sight appear to be single, very long cells are in reality made up of several shorter ones. In ground olive stones one frequently finds, besides the stone cells, bits of the outer tegument of the seed, showing large cells with sinuous, rather thick walls; also bits of parenchyma, crossed frequently by fibro-vascular duct bundles.

Buckwheat Products. - Both the hulls and the middlings have been added to black pepper, and the middlings to white pepper. The starch of buckwheat possesses the added advantage, from the point of view of the spice-grinder, that it somewhat resembles pepper starch in microscopical appearance, not only in the shape of the starch granules, but also in the manner of grouping into masses. Compare Figs. I 28 and I29, Plates II and III, showing buckwheat starch, with Figs. 255 and 256 , PI. XXXIV, respectively, showing pepper starch made under similar 
conditions of magnification, etc. The starch granules and masses are coarser in the case of buckwheat than of pepper.

Fig. 260, Pl. XXXV, shows a photograph of a pepper sample adulterated with buckwheat, masses of both starches appearing in the same field.

Other Adulterants found "in Massachusetts samples of pepper have been wheat and corn products, nutshells, cayenne, charcoal, turmeric, rice, sand, and sawdust. Charred cocoanut shells were at one time extensively used (see pp. 419 and 420 ).

Long Pepper, according to English analysts, has been used to a considerable extent as an adulterant. This is the fruit of the Chavica Roxburghii, a wild plant growing in India on the banks of rivers. The fruit, as its name implies, is long and cylindrical, while of about the same diameter as the spherical true peppercorns. Long pepper contains, as a rule, less than half the amount of piperin that true pepper does, and rather more starch than black pepper. Its taste is much less pungent than that of true pepper.

From its method of growth, long pepper is found with considerable dirt and sand adhering to the outer surface of the dried grains. This is due to the fact that the fruit often trails on the ground, and in gathering it the natives are not particular about removing the adhering soil. The surface of the fruit grains being very rough and irregular, much of the dirt remains dried thereon. The presence of long pepper thus materially increases the ash.

Long pepper possesses a very disagreeable, but peculiar odor, developed more especially when slightly warmed. For this reason, if for no other, it is not an ideal adulterant, since pepper containing it would not be palatable with warm food. At the present time it costs more than black pepper, and is used chiefly in mixed whole spices for pickles.

Brown gives the following analyses of samples of long pepper:

\begin{tabular}{|c|c|c|c|c|c|c|c|c|}
\hline & $\begin{array}{l}\text { Total } \\
\text { Ash. }\end{array}$ & $\begin{array}{l}\text { Sand and } \\
\text { Ash Insol- } \\
\text { uble in } \\
\text { Hydrochlo- } \\
\text { ric Acid. }\end{array}$ & $\begin{array}{c}\text { Starch and } \\
\text { Matters } \\
\text { Converti- } \\
\text { ble into } \\
\text { Sugar. }\end{array}$ & $\begin{array}{l}\text { Albumin- } \\
\text { ous Matter } \\
\text { Soluble in } \\
\text { Alkali. }\end{array}$ & Cellulose. & $\begin{array}{l}\text { Aleoholic } \\
\text { Extract. }\end{array}$ & $\begin{array}{c}\text { Ether } \\
\text { Bxtract. }\end{array}$ & $\begin{array}{c}\text { Total } \\
\text { Nitrogen. }\end{array}$ \\
\hline I & 8.91 & I. 2 & 44.04 & 15.47 & $15 \cdot 7$ & $7 \cdot 7$ & $5 \cdot 5$ & 2.1 \\
\hline 2 & 8.98 & I. I & $49 \cdot 34$ & 17.42 & 10.5 & 7.6 & 4.9 & 2.0 \\
\hline 3 & 9.61 & I. 5 & 44.61 & $15.5 \mathrm{I}$ & 10.37 & 10.5 & 8.6 & $2 \cdot 3$ \\
\hline
\end{tabular}

According to Brown and Heisch, the granules of long pepper starch under the microscope are larger than those of true pepper, and more angular. Stokes, $*$ however, finds no such marked difference in the size

* Analyst, XIII, p. Iog. 
of starch granules and his experience is shared by the writer. When the two specimens (long and true pepper) are viewed side by side in water mounts under the microscope, the average size of the long pepperstarch grains is a trifle larger than those of true pepper, though, unless compared directly, the difference is not readily apparent. Stokes suggests a method of distinguishing the two by polarized light. With crossed Nicols, so that a dark field is given, and with the specimen mounted in glycerin, true pepper starch shows an evenly dark appearance, using E low power, while with long pepper a "ghostly white" image is shown. Long pepper, when present in true pepper powder, may generally be rendered apparent by the development of the characteristic odor on heating. Bits of fluffy fiber from the catkin of the long pepper will always be found in the ground powder, and will be apparent under the magnifying-glass.

Microscopic examination of the crude fiber discloses the highly characteristic, large, beaded cells of the endocarp, also elements of the spindle.

\section{RED PEPPER.}

Nature and Composition.-According to the U. S. Standards red pepper is the red, dried, ripe fruit of any species of Capsicum, a genus of the nightshade family (Solanacee), indigenous to the American tropics, but now cultivated in nearly all warm and temperate countries, and is of two distinct kinds: cayenne pepper or cayenne, the dried ripe fruit of C. frutescens, C. baccatum, or some other small fruited species of Capsicum, and paprika, the dried ripe fruit of $C$. annuum, or some other large-fruited species of the genus, excluding seeds and stems.

Cayenne is characterized by its extreme pungency and the small size of the pods, which seldom exceed $2 \mathrm{~cm}$. in length. The leading commercial varieties are Zanzibar and Japan, the latter being the more brilliant in color.

"Capsicums" or "Bombay Chillies" are low grade peppers of a brown color, with pods 2 to $3 \mathrm{~cm}$. long, which now are said to come from the vicinity of the river Niger in Africa.

Paprika is a variety of $C$. annuum grown in Hungary. The powder is of a deep red color and has a sweetish, mildly pungent flavor.

Pimiento is a large-fruited pepper grown in Spain. The succulent pericarp is much used for stuffing olives while the dried pod is ground as a spice, often being substituted for the more valuable Hungarian varieties. The kitchen garden peppers, of which over thirty varieties are cultivated in the United States, also belong to the species $C$. annuum.

The capsicum plant has solitary flowers, with a five-cleft corolla, and the fruit is of an elongated, conical form. The surface of the fresh fruit 
is smooth and very red, but it loses some of its brilliance in drying, and becomes shriveled. The pericarp is thin and tough, and at its base is a five-lobed calyx, greenish brown in color, terminating in a thick stem. The fruit proper is divided into two or three cells, which are separate and distinct at the lower portion, but which unite and form one at the top. The cells inclose a large number of yellow, wrinkled, kidneyshaped seeds, containing a fleshy endosperm, and a curved embryo.

Red pepper contains a fixed, bland oil, found in both pod and seed, but more abundantly in the latter, considerable resinous and mucilaginous material, a red coloring matter confined to the pod, and the active principle capsicin, a crystalline alkaloid, to which much of the pungency is due. The capsicin is present in both seeds and pod, but is more abundant in the latter, where it is dissolved in the oil.

Capsicin may be isolated, according to Thresh, by extracting powdered cayenne with petroleum ether, mixing the red residue left on evaporating off the solvent with two or three times its weight of oil of almonds, and exhausting the mixture with alcohol. On evaporating the alcohol extract, the capsicin crystallizes out in narrow, thin plates, very soluble in alcohol, but insoluble in water. They volatilize at $100^{\circ}$, and condense in small drops.

The red coloring matter is soluble in ether, petroleum ether, carbon bisulphide, and chloroform, but sparingly soluble in alcohol.

Analyses of Cayenne.-Richardson * gives the following data of analyses of two pure samples of cayenne:

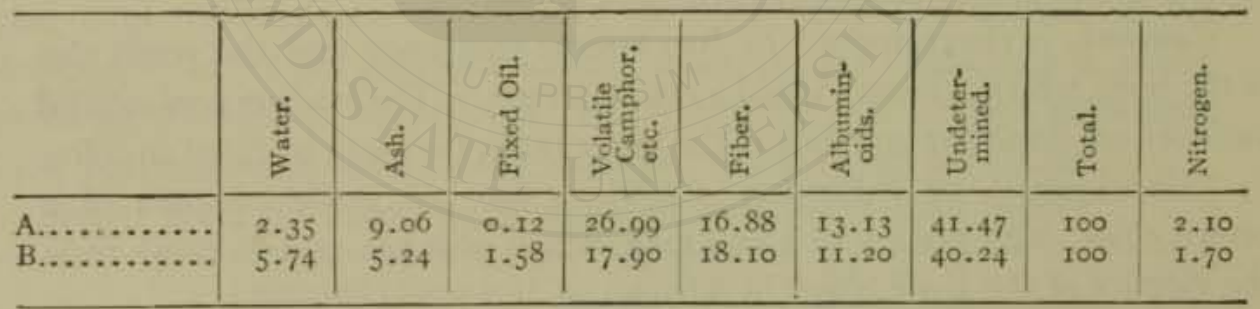

Maximum and minimum data of ash and non-volatile ether extract of fourteen samples of cayenne, sold in sealed packages in Connecticut, and analyzed by Winton and Mitchell are as follows: $\dagger$

\begin{tabular}{|c|c|c|}
\hline & Ash. & $\begin{array}{l}\text { Non-volatile } \\
\text { Ether Extract. }\end{array}$ \\
\hline 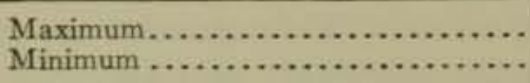 & $\begin{array}{l}7.1 \varepsilon \\
5.89\end{array}$ & $\begin{array}{l}19.14 \\
15.59\end{array}$ \\
\hline
\end{tabular}

* U. S. Dept, of Agric., Div. of Chem., Bul. I3, p. 211.

$\dagger$ An. Rep. Conn. Exp. Sta., 1898 , p. 175. 
Winton, Ogden, and Mitchell * analyzed eight samples of whole chillies, representing three varieties, namely Zanzibar, Japan, and Bombay, the summarized results being as follows:

\begin{tabular}{|c|c|c|c|c|c|c|}
\hline & \multirow{2}{*}{ Moisture. } & \multicolumn{3}{|c|}{ Ash. } & \multicolumn{2}{|c|}{ Ether Extract. } \\
\hline & & Total & $\begin{array}{l}\text { Soluble in } \\
\text { Water. }\end{array}$ & $\begin{array}{l}\text { Insoluble } \\
\text { in } \mathrm{HCl} \text {. }\end{array}$ & Volatile. & $\begin{array}{l}\text { Non-vola- } \\
\text { tile. }\end{array}$ \\
\hline \multirow[t]{2}{*}{$\begin{array}{l}\text { Maximum......... } \\
\text { Minimum .......... } \\
\text { Average........... }\end{array}$} & $\begin{array}{l}7.08 \\
3.67 \\
5.73\end{array}$ & $\begin{array}{l}5.06 \\
5.08 \\
5.43\end{array}$ & $\begin{array}{l}4.93 \\
3.30 \\
3.98\end{array}$ & $\begin{array}{l}0.23 \\
0.05 \\
0.15\end{array}$ & $\begin{array}{l}2.57 \\
0.73 \\
1.35\end{array}$ & $\begin{array}{l}21.81 \\
17.17 \\
20.15\end{array}$ \\
\hline & $\begin{array}{l}\text { Alcohol } \\
\text { Extract. }\end{array}$ & $\begin{array}{l}\text { Reducing } \\
\text { 3fatters as } \\
\text { Starch, } \\
\text { Acid Con- } \\
\text { version. }\end{array}$ & $\begin{array}{l}\text { Starch by } \\
\text { Diastase } \\
\text { Method. }\end{array}$ & $\begin{array}{l}\text { Crude } \\
\text { Fiber. }\end{array}$ & $\begin{array}{l}\text { Nitrogen, } \\
\times 6.25 \text {. }\end{array}$ & $\begin{array}{c}\text { Total } \\
\text { Nitrogen. }\end{array}$ \\
\hline $\begin{array}{l}\text { Maximum } \\
\text { Minimum } \ldots \ldots \ldots \ldots \\
\text { Average } . . . \cdots \cdots \cdots\end{array}$ & $\begin{array}{l}27 \cdot 61 \\
21 \cdot 52 \\
24 \cdot 35\end{array}$ & $\begin{array}{l}9.31 \\
7.15 \\
8.47\end{array}$ & $\begin{array}{l}1.46 \\
0.80 \\
1.01\end{array}$ & $\begin{array}{l}24.91 \\
20.35 \\
22.35\end{array}$ & $\begin{array}{l}14.63 \\
13 \cdot 31 \\
13.67\end{array}$ & $\begin{array}{l}2.34 \\
2.13 \\
2.18\end{array}$ \\
\hline
\end{tabular}

The percentages of "starch by the diastase method" given in the above table represent errors of the process as neither cayenne or paprika contain an appreciable amount of starch.

Analyses of Paprika and Pimiento.-Doolittle and Ogden $†$ have made exhaustive analyses of known samples of Hungarian and Spanish red pepper, including determinations of non-volatile ether extract, and iodine number of this extract, which are of especial value in detecting added oil. A summary of their results is given on page 442 .

Microscopical Structure of Red Pepper.-Fig. 86, from Moeller, shows the appearance under the microscope of various elements of powdered paprika. (I) is a sectional view through the outer portion of the fruit shell or pod, showing the epidermis $a$, and beneath this the collenchyma layer. The inner epidermis is shown at (2), with its cells thick-walled in places, and inclosing brilliant, red oil drops of coloring matter. (3) represents the outer, and $(4)$ and $(5)$ the inner epidermis in surface view. The outer epidermis of cayenne, which is the element of chief value in distinguishing this from paprika, is shown at (6).

A cross-section through the seed shell is shown at (7), $a$ being the epidermis of the seed, $b$ the parenchyma layer directly beneath, and $c$ the tissues of the endosperm. (8) shows in surface view the peculiar seed

* Am. Rep. Conn. Exp. Sta., 1898, pp. 200-201.

$\dagger$ Jour. Am. Chem. Soc., 30, 1908, p. 1481 . 


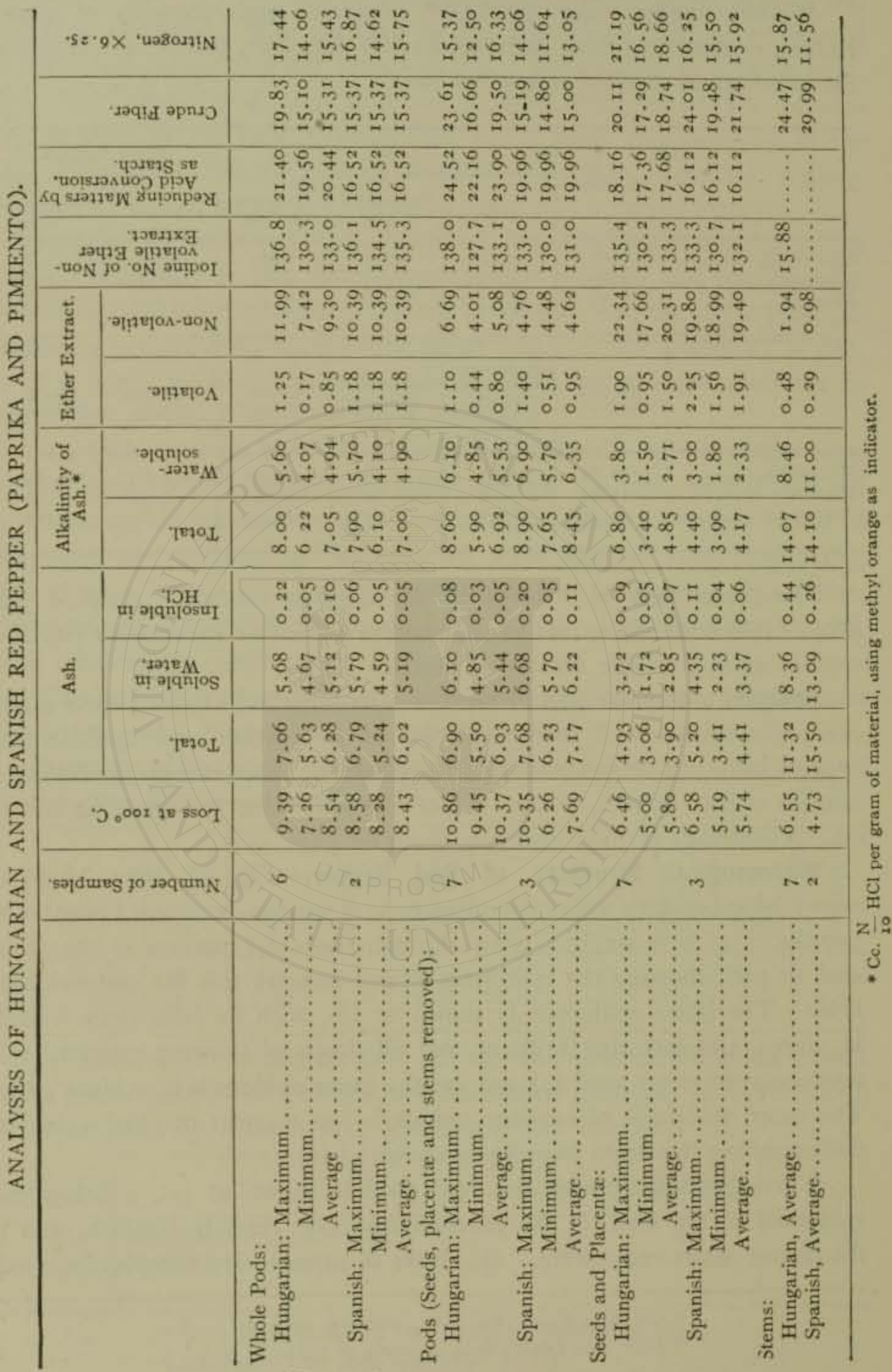


epidermis, the appearance of which Moeller compares with that of intestines. At (9) is shown one of the isolated cells of this epidermis more highly magnified, while (Io) shows the epidermis of the calyx.

Figs. 2 II and 2I2, Pl. XXIII, show photomicrographs of powdered cayenne. In Fig. $2 \mathrm{II}$ is shown a large bit of the outer epidermis of the fruit pod, while in Fig. 212 appears a smaller portion of this same kind of epidermis, and next to this the characteristic skin of the seed shell, with its striking markings suggestive of the convolutions of the intestines. Yellow or yellowish-red droplets of oily coloring matter are distributed through the field. Starch grains are absent.

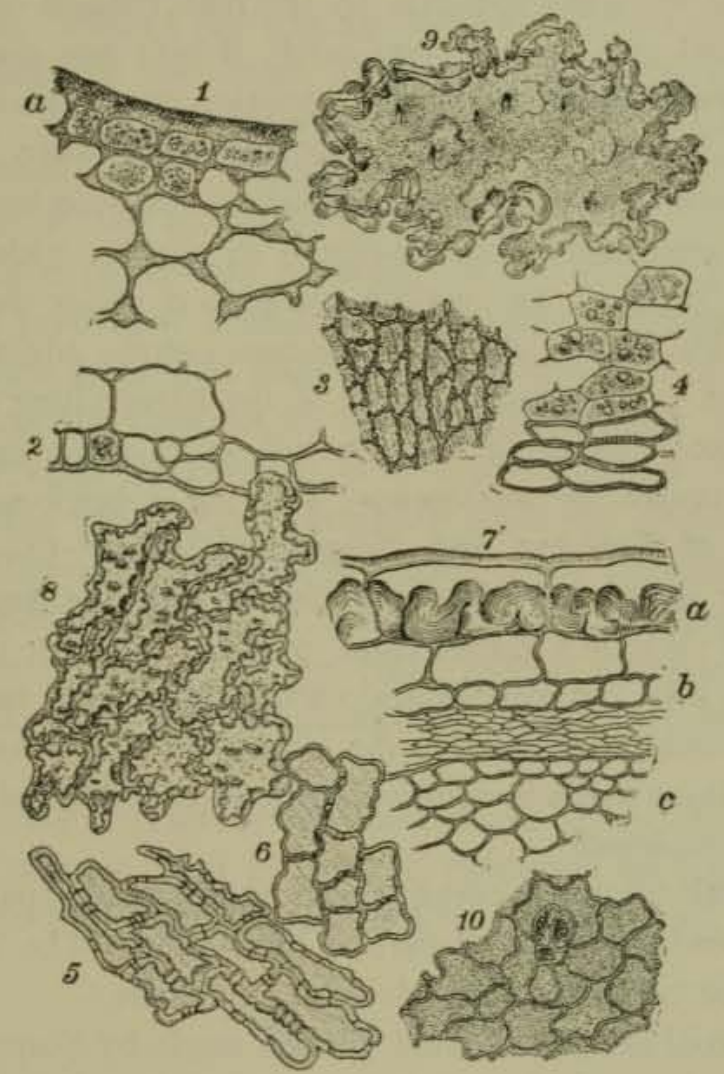

Fig, 86.-Powdered Red Pepper under the Microscope. X125. (After Moeller.)

Adulteration of Red Pepper.-The U.S. standards for cayenne are the following: Non-volatile ether extract should be not less than $15 \%$; total ash should not exceed $6.5 \%$; ash insoluble in hydrochloric acid should not exceed $0.5 \%$; starch by the diastase method should not exceed I. $5 \%$, and crude fiber should not exceed $28 \%$. 
The most common adulterants of cayenne are the starches of the cereal grains, corn and wheat. Ground pilot bread and crackers are especially common. Besides these the writer has found in the routine examination of cayenne samples in Massachusetts, ginger, nutshells, turmeric, rice, gypsum, buckwheat, olive stones, mustard hulls, ground redwood, red ocher, and coal-tar dyes. Fig. $2 I_{3}$, Pl. XXIV, shows a sample adulterated with wheat, corn, and cocoanut shells.

Mineral Adulterants, such as gypsum, and red ocher and other pigments, are all to be looked for in the ash by methods of qualitative analysis. An abnormally high ash is suggestive of adulteration. According to Vedrodi, the ash of genuine cayenne should not exceed 5.96. The presence of red ocher is rendered apparent by the high content of iron.

Salts of lead and mercury are rarely if ever now used for color.

Ground Redwood--Numerous varieties of redwood are commonly used to intensify the color of cayenne, especially when otherwise highly adulterated with colorless materials, such as the starches. The redwood is sometimes used alone, and sometimes in mixture with turmeric. Both redwood and turmeric are readily recognized under the microscope.

Fig. 214, Pl. XXIV, shows a cayenne sample adulterated with corn starch and red sandalwood, a mass of the latter filling the center of the field. The wood fibers of the dyestuff, even when finely ground, are very striking under the microscope, showing a brick-red color.

Detection of Coal-tar and Vegetable Colors.-Oil-soluble coal-tar and vegetable colors may be tested for in cayenne and paprika by an adaptation of Martin's butter-color method, shaking the ether extract of the sample with the alcohol and carbon bisulphide mixture, page 535 . The carbon bisulphide dissolves the oil and natural color, while the overlying alcohol layer holds in solution many of the artificial coloring matters that may be employed.

The natural colors of cayenne and paprika are sparingly soluble in alcohol, but readily soluble in carbon bisulphide. The separated alcohol is examined for colors by methods given elsewhere.

Tests for coal-tar dyes should also be made by Sostegni and Carpentieri's, or Arata's method (p. 796).

Szigeti * treats the suspected sample with water acidified with acetic acid, and boils in this solution a bit of wool, which, if carotin or a coal-tar dye be present, is colored red. If the color is carotin, it will be removed

*Zeits. landw. Versuchs. Oesterreich, 5, 1902, pp. 1208, 1222. 
from the wool by treatment with petroleum ether, or by heating at $100^{\circ}$ C. for some hours, but if a coal-tar dye, it will still remain fixed thereon.

Detection of Olive Oil in Red Pepper.-The color of paprika and pimiento is often intensified by grinding with olive oil. This form of adulteration is detected by determination of the iodine number of the nonvolatile ether extract. The following method elaborated by Seeker has been adopted by the A. O. A. C.:

Dry 5 grams on a watch-glass over sulphuric acid for at least twelve hours. Measure $25^{\circ} \mathrm{cc}$. of anhydrous alcohol-free ether (p. 66) into a graduated flask with the mark near the lower end of the neck, and brush the paprika into it. Place a mark on the neck of the flask at the meniscus, and allow to stand for one hour, shaking at twenty-minute intervals during that time. Bring the meniscus back to the mark either by cooling if the level has risen, or by adding absolute ether if it has fallen, and let settle. Pipette off $100 \mathrm{cc}$. of the supernatant liquid, filter through an II-cm. close-textured paper into a tared, air-dry glass-stoppered $250-\mathrm{cc}$. Erlenmeyer flask previously counterpoised against a similar flask, wash with a little absolute ether, and distil off the solvent until the ether ceases to come over. Lay the flask on its side in a water-oven, heat for thirty minutes, cool the open flask for at least thirty minutes in the air and weigh. Repeat this heating and weighing until the weight is constant to within one milligram, two heatings usually being sufficient, and calculate the per cent of ether extract. If more than $I_{2} \frac{1}{2}$ hours' heating is required to obtain constant weight or if the ether extract becomes colorless it should be rejected, and a new determination started with freshly purified ether.

Dissolve the ether extract in the flask in ro cc. of chloroform, add $30 \mathrm{cc}$. of Hanus solution, and proceed as described on page $49 \mathrm{I}$. The iodine number thus determined should not be less than 125 .

\section{GINGER.}

Nature and Composition.-Ginger as a spice is the ground rootstock of the Zingiber officinale, an annual herb of the family Zingiberacea, growing to a height of from 3 to 4 feet. It is a native of India and China, but is cultivated quite extensively in tropical America, Africa, and Australia.

The root is dug when the plant is a year old, and when the stem has 
withered. If the root, when freshly dug and scalded to prevent sprouting, is dried at once, it forms the so-called black ginger, of which Calcutta and African are the common varieties. When decorticated, the product is known in commerce as white ginger, the chief varieties being Jamaica, Cochin, and Japan. The best variety is Jamaica ginger. The scraped root is sometimes bleached to make it still whiter, or sprinkled with carbonate of lime.

In commerce whole or black ginger appears in "hands" 4 to $10 \mathrm{~cm}$. long, and from to to $15 \mathrm{~mm}$. in diameter. These usually have three or four various-sized, irregular branches, some short and thick, others elongated. The epidermis is gray or yellowish gray in color, more or less wrinkled, and beneath it is a reddish-brown layer. The inner portion of the dried root is white or yellowish. The root is hard, and of a compact, horny structure.

White or decorticated ginger appears in "hands " of smaller diameter than the black, and yields a lighter colored powder on grinding. Preserved ginger root is prepared by boiling the root in water, and curing with sugar or honey. Much of the preserved ginger comes from Canton.

The distinguishing features of ginger are its large content of starch, its volatile oil, and its resinous matter. Inasmuch as the epidermis contains a large amount of pungent resin, it is easy to see how the peeled or decorticated variety is inferior.

Oil of ginger is very aromatic, and of a greenish-yellow color. Its specific gravity ranges from 0.875 to 0.885 . It is slightly soluble in alcohol. Of its composition little is known.

Richardson's analyses in full of five samples of whole ginger-root are as follows:

\begin{tabular}{|c|c|c|c|c|c|c|c|c|c|}
\hline & $\frac{\vec{J}}{\mathrm{~d}}$ & $\frac{\pi}{4}$ & $\frac{0}{30}$ & 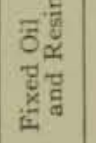 & हुँ & 鄗 & $\begin{array}{l}\frac{1}{2} \\
\frac{1}{4} \cdot \frac{1}{8}\end{array}$ & 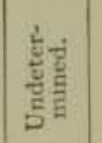 & 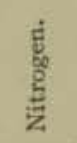 \\
\hline Calcutta . & 9.60 & 7.02 & 2.27 & $4 \cdot 5^{8}$ & $49 \cdot 34$ & 7.45 & $6.3 c$ & 13.44 & I.OI \\
\hline Cochin ........ & $9.4 \mathrm{t}$ & $3 \cdot 39$ & 1.84 & $4.07:$ & $53 \cdot 33$ & 2.05 & 7.00 & 18.91 & 1.12 \\
\hline Unbleached Jamaica.............. & 10.49 & $3-44$ & 2.03 & 2.29 & $50.5^{8}$ & 4.74 & $10.8_{5}$ & $15.5^{8}$ & 1.74 \\
\hline Bleached Jamaica, London. ...... & 11.00 & 4.54 & $\mathrm{I} .89$ & 3.04 & $49 \cdot 34$ & 1.70 & 9.28 & $19.2 x$ & 1.48 \\
\hline " $\quad$ American ... & 10.11 & $5 \cdot 5^{8}$ & 2.54 & 2.69 & 50.67 & 7.65 & $9 \cdot 10$ & 11.66 & I. 46 \\
\hline
\end{tabular}

Summaries of Winton, Ogden, and Mitchell's analyses of eighteen samples of whole ginger, representing the common white and black varieties, as well as of two samples of exhausted ginger, are as follows: 


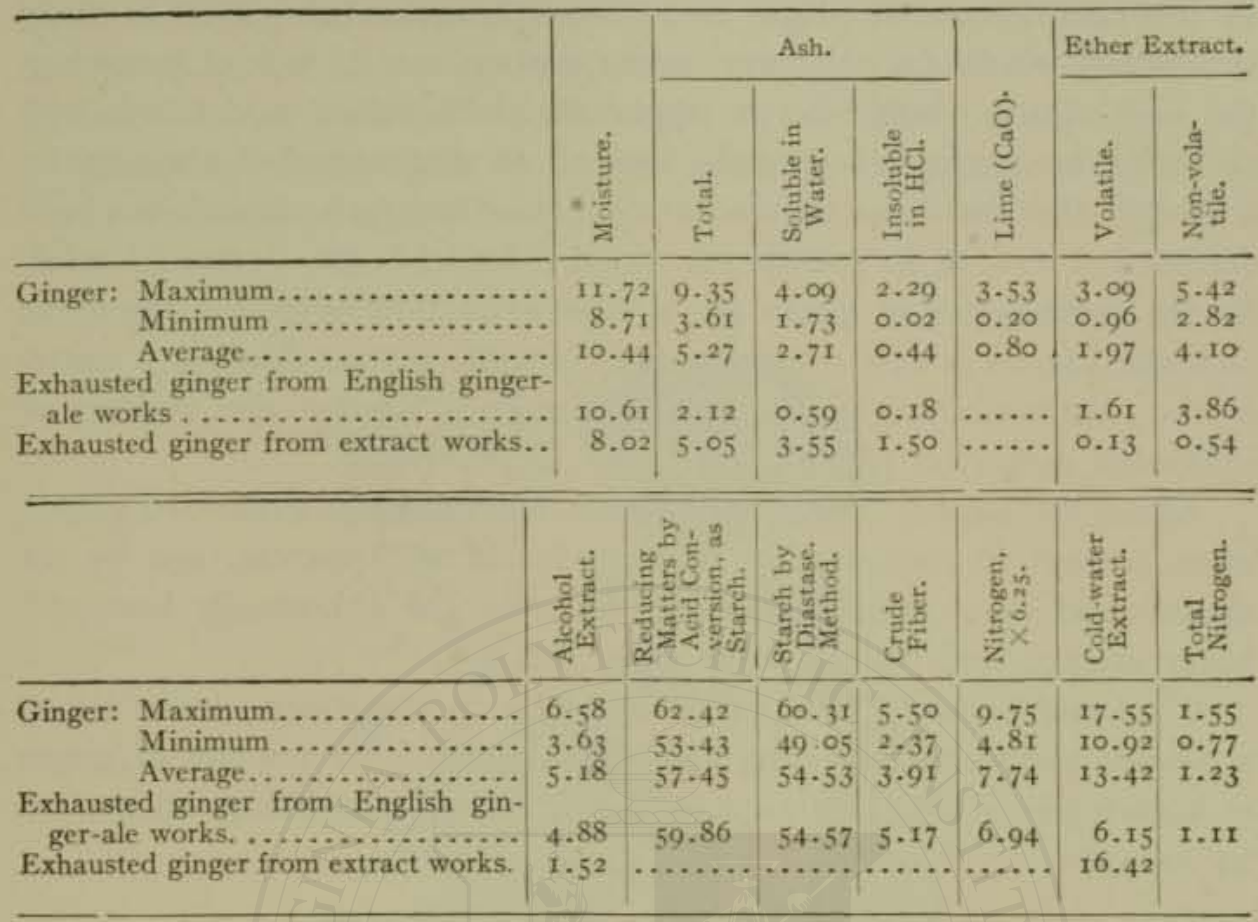

McGill * records the analyses of ninety-eight samples of ground ginger as sold in the Canadian market. Of thirty-two of these, pronounced pure on analyses, the following is a summary:

\begin{tabular}{|c|c|c|c|c|c|c|c|}
\hline & $\begin{array}{l}\text { Moisture } \\
\text { or Loss } \\
\text { on Dry- } \\
\text { ingat } \\
\text { roo․ }\end{array}$ & $\begin{array}{l}\text { Petro- } \\
\text { leum- } \\
\text { ether } \\
\text { Bxtract. }\end{array}$ & $\begin{array}{c}\text { Cold- } \\
\text { waters } \\
\text { Extract. }\end{array}$ & Total. & Soluble. & Insoluble. & $\begin{array}{c}\text { Alkalin- } \\
\text { ity of } \\
\text { Soluble } \\
\text { Ash as } \\
\mathrm{K}_{2} \mathrm{O} \text {. }\end{array}$ \\
\hline $\begin{array}{l}\text { Maximum... } \\
\text { Minimum ... }\end{array}$ & $\begin{array}{r}12.00 \\
9.50\end{array}$ & $\begin{array}{l}6.13 \\
2.78\end{array}$ & $\begin{array}{l}15.4^{8} \\
14.04\end{array}$ & $\begin{array}{l}7.84 \\
3.67\end{array}$ & $\begin{array}{l}3.15 \\
2.28\end{array}$ & $\begin{array}{l}3.99 \\
1.96\end{array}$ & $\begin{array}{r}133 \\
.103\end{array}$ \\
\hline
\end{tabular}

According to Vogl, the proportion of ginger ash varies quite widely according to the kind, but should never exceed $8 \%$.

Exhausted Ginger and Methods of Detection.-There are two kinds of exhausted ginger commercially available for admixture with ground spice, as an adulterant. One is the product left after extraction with strong alcohol in the making of extract of Jamaica ginger, and the other the residue from extraction with either very dilute alcohol, or with water,

* Dept. Inl. Rev. Canada Bul. 48 , pp. ro, 11 . 
in the manufacture of ginger ale. Ground, exhausted ginger is rarely substituted wholly for the pure variety, since, from its lack of pungency, the sophistication would be too apparent. It is rather used to mix with the latter in varying proportions, and as an adulterant of other spices.

Ginger that has been exhausted by extraction with alcohol has been deprived of most of its volatile oil, which is found in the "extract," while for the manufacture of ginger ale, a water extract, or at most a very dilute alcoholic extract is best adapted. Such a water extract does, as a matter of fact, remove much of the valued pungency, so that the residue, or exhausted ginger, is rather inert.

Either the alcohol- or the water-extracted variety of exhausted ginger, when present in considerable amount, would be apparent, one by the alcohol and ether extract, and the other by the abnormally low coldwater extract, and water-soluble ash.

Dyer and Gilbard* first called attention to the water-soluble ash as a reliable means of indicating exhausted ginger. Six samples of ginger of known purity were analyzed by them, their results being summarized as follows:

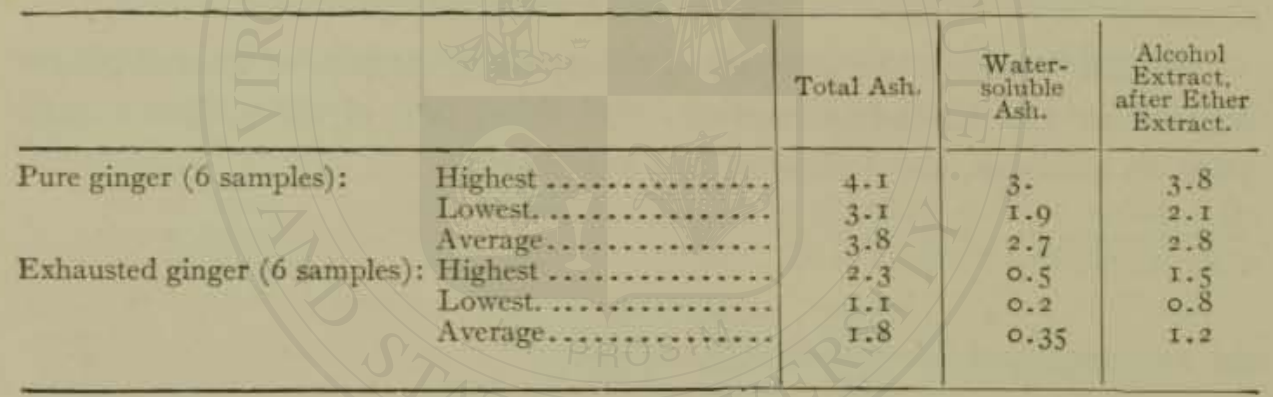

Allen and Moor $\dagger$ pointed out the value of the cold-water extract as a help in detecting exhausted ginger, especially when taken in connection with the soluble ash, showing that the presence of this adulterant is assured, when the soluble ash is as low as $1 \%$, and the cold-water extract is less than $8 \%$.

Determination of Cold-water Extract.-Winton, Ogden, and Mitchell's Method. $\$-F o u r$ grams of the ground sample are placed in a $200-\mathrm{cc}$. graduated flask, and the latter is filled to the mark with water, and shaken at half-hour intervals during eight hours, after which it is allowed to

* Analyst, XVIII (I893), p. I97.

† Analyst, XIX (1894), p. 194.

$\ddagger$ U. S. Dept. of Agric., Bur, of Chem., Bul. 65, p. 59; Bul. 107 (rev.), p. 164. 
stand at rest for sixteen hours in addition. The contents are then filtered, and $50 \mathrm{cc}$. of the filtrate evaporated to dryness in a platinum dish. It is then dried at $100^{\circ}$ to constant weight and weighed.

Microscopical Structure of Ground Ginger.-Fig. 87, from Moeller, shows elements of ginger root, from which the epidermis has not been

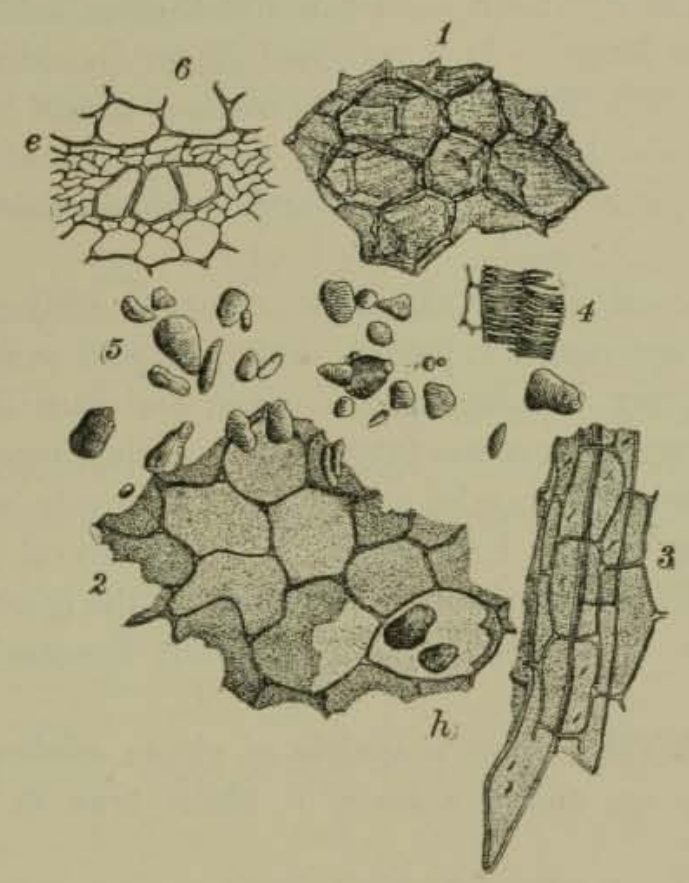

Fic. 87.-Powdered Ginger under the Microscope. X125. (After Moeller.)

removed. A bit of the large-celled cork (or dead protective tissue of the epidermis) is shown in surface view at (I); at (2) is shown in crosssection the parenchyma in which the starch is contained, $h$ being an oilcell; (3) shows the parenchyma in longitudinal section, with bast fibers. Fragments of spiral ducts are shown at (4), and starch grains at (5). is a cross-section in the extreme interior of the root.

The most prominent feature of powdered ginger is the starch grains (5), which Moeller compares in shape to tied sacks.

Fig. 228, Pl. XXVII, is a photomicrograph of pure, ground ginger, mounted in water, showing the starch grains inclosed in the cells of the parenchyma. Fig. 23 $\mathrm{I}$ shows the starch grains alone. The granules of ginger starch are ellipsoidal, and as a rule very clear and transparent, being for the most part entirely devoid of either hilum or concentric rings. 
Occasionally granules are to be found, however, with faint concentric markings, and even with an apparent hilum. The characteristic form of the ginger starch granule is more or less egg-shaped, with a small protuberance near one end. This protuberance serves to readily distinguish the starch granules of ginger from those of wheat, with which ginger is frequently adulterated. While wheat granules are of various sizes, the grains of ginger starch are as a rule much more uniform.

Adulteration of Ginger.-U.S. standard ginger should meet the following requirements: Starch by the diastase method should not be less than $42 \%$; crude fiber should not exceed $8 \%$; total ash should not exceed $8 \%$; lime should not exceed $\mathrm{I} \%$; ash insoluble in hydrochloric acid should not exceed $3 \%$.

Besides exhausted ginger, the most common adulterants found in powdered ginger are turmeric, wheat, corn, rice, and sawdust. Sawdust of soft wood is a not uncommon adulterant, and care should be taken to distinguish between the wood fiber natural to the ginger root, and that of the foreign variety. A careful study should be made of finely ground, soft-wood sawdust, with its long spindle cells and lateral pores, as shown in Fig. 266, Pl. XXXVII, and the wood fiber of the genuine ginger root. A large admixture of sawdust would materially increase the percentage of crude fiber.

Fig. 234, PI. XXIX, shows a sample of ginger adulterated with corn and wheat. Fig. $23^{2}$ shows a mass of wheat bran in an adulterated sample.

Fig. 233 shows ginger adulterated with turmeric.*

\section{TURMERIC.}

Nature and Composition.-Turmeric, while largely used as an adulterant of other spices (especially of ginger and mustard), possesses some value as a condiment in itself, forming, for instance, the chief ingredient of curry powder. $\dagger$ Turmeric (Curcuma longa) belongs to the same family (Zingiberacea) as ginger, having a perennial rootstock, and an annual stem. It is a native of the East Indies and Cochin-China. Its chief ingredients are starch, a volatile oil, a yellow coloring matter (curcumin), cellulose, and gum.

\footnotetext{
* This photomicrograph is very disappointing, in that it fails to show the intense yellow of the central mass of turmeric.

$\dagger$ Curry powder consists of a mixture of turmeric, cayenne, and various pungent spices.
} 
Curcumin $\left(\mathrm{C}_{14} \mathrm{H}_{14} \mathrm{O}_{4}\right)$ is insoluble in cold water, but readily soluble in alcohol. It is extracted from powdered turmeric by boiling the latter with water, filtering, and extracting the residue with boiling alcohol. The alcoholic solution is filtered, evaporated, and the residue extracted with ether. The ether extract contains the curcumin, together with a small amount of volatile oil.

Curcuma oil is an orange-yellow, slightly fluorescent liquid, its specific gravity being 0.942 .

The following analyses of turmeric were made in the writer's laboratory:

\begin{tabular}{|c|c|c|c|c|c|c|c|c|}
\hline Variety. & $\begin{array}{l}\text { Mois- } \\
\text { ture. }\end{array}$ & $\begin{array}{l}\text { Total } \\
\text { Ash. }\end{array}$ & \multicolumn{2}{|c|}{$\begin{array}{c}\text { Ash } \\
\text { Soluble } \\
\text { inWater. }\end{array}$} & $\begin{array}{l}\text { Ash } \\
\text { Insoluble } \\
\text { in HCl. }\end{array}$ & $\begin{array}{c}\text { Total } \\
\text { Nitrogen }\end{array}$ & $\begin{array}{l}\text { Protein, } \\
\mathrm{N} \times 6.25\end{array}$ & $\begin{array}{c}\text { Total } \\
\text { Ether } \\
\text { Extract. }\end{array}$ \\
\hline China. ............. & 9.03 & $6.7^{2}$ & & & 0.1 & I. 73 & 10.81 & 10.86 \\
\hline & 9.08 & 8.5 & & & $\cdots$ & 0.97 & 6.06 & \\
\hline ppi............... & 8.07 & 5.99 & & & .. & I. 56 & $9 \cdot 75$ & 10.66 \\
\hline Average.. & 8.73 & 7.07 & & & $\cdots$ & 1.42 & 8.88 & II. 17 \\
\hline Variety. & $\begin{array}{c}\text { Volatile } \\
\text { Ether } \\
\text { Extract. }\end{array}$ & $\begin{array}{l}\text { Non- } \\
\text { ati } \\
\text { Eth } \\
\text { Extr }\end{array}$ & & $\begin{array}{l}\text { Alec } \\
\text { Ext: }\end{array}$ & $\begin{array}{l}\text { ohol } \\
\text { ract. }\end{array}$ & $\begin{array}{l}\text { Crude } \\
\text { Fiber. }\end{array}$ & $\begin{array}{l}\text { Reducing } \\
\text { Matter by } \\
\text { Acid Con- } \\
\text { version, as } \\
\text { Starch. }\end{array}$ & $\begin{array}{l}\text { Starch by } \\
\text { Diastase } \\
\text { Method. }\end{array}$ \\
\hline hina & 2.01 & 8. & & & .22 & $4 \cdot 45$ & $4^{8} .69$ & 40.05 \\
\hline & & & & & & 5.84 & & \\
\hline . & 3.16 & & & & .37 & 5.83 & $5^{\circ} .44$ & 33.03 \\
\hline Average...... & 3.19 & 7.8 & & & .96 & $5 \cdot 37$ & $49 \cdot 73$ & 34.21 \\
\hline
\end{tabular}

Microscopical Structure of Turmeric.-Moeller's representation of characteristics of powdered turmeric is reproduced in Fig. 88. The epidermis is shown at ( $\mathrm{I}$ ) with one of the numerous, one-celled hairs that grow from it, also the scar left after one of the hairs has been removed; (2) shows in plan view the cork immediately under the epidermis. The tender-celled parenchyma is shown in cross-section at (3), and in longitudinal section at (4). In some of the cells of the parenchyma are found dark-yellow lumps of resin $(h)$, and vascular ducts $(g)$, but by far the most numerous and striking contents of the parenchyma-cells are the brightyellow masses of "paste balls" $(3 a)$ and the starch granules, one of which is shown in (3). See also Plate XIII. The starch grains in the water-mounted powder show under the microscope in masses, usually of a deep-yellow color, unless very finely rubbed out, when they appear for the most part in fragments. 
The whole starch granule appears somewhat in the form of a clamshell, with very distinct markings. When fragments of the starch granules are carefully examined, these distinct markings are so strongly characteristic, even in the smallest pieces commonly found in the powdered sample, as to nearly always serve to identify them. See Fig. I7I, Pl. XIII.

Turmeric as an Adulterant.-Turmeric is a material especially adapted by its deep-yellow color to intensify mustard and ginger, especially when

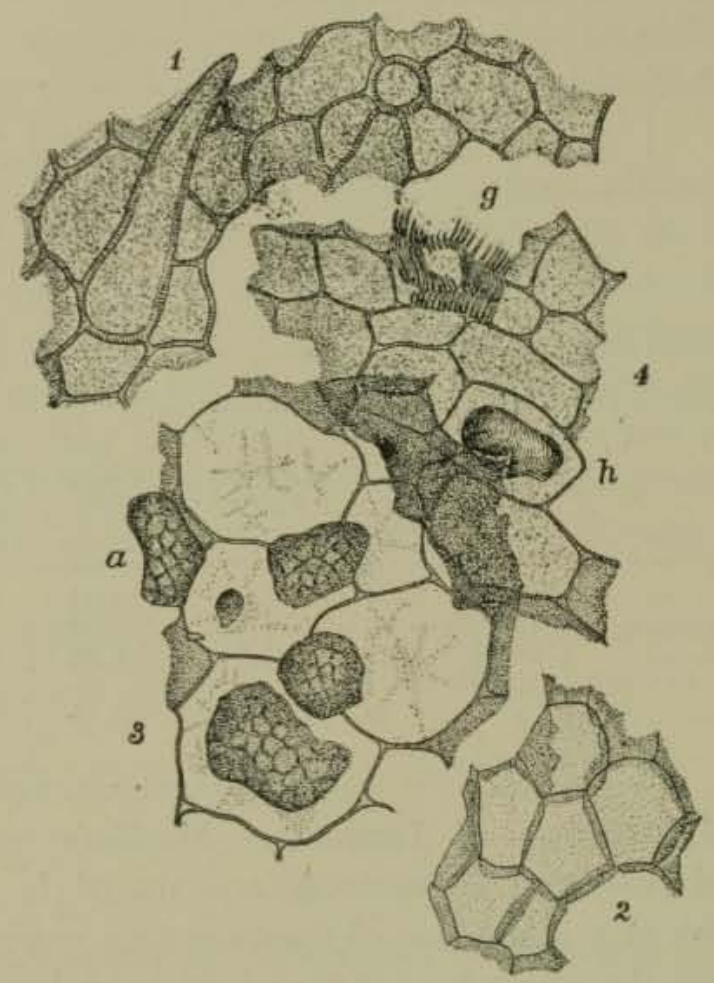

Fig. 88.-Powdered Turmeric under the Microscope. Xi25. (After Moeller.)

these spices are adulterated with the lighter-colored cereal starches, hence it is very commonly found in these spices, both with and without other adulterants.

It is also frequently used in small quantities in adulterated cayenne, mace, and various spices, to counteract the colors of other dyestuffs, such as ground redwood, which in itself would sometimes be too intense if used alone. 
Turmeric, when present to any marked extent in a powdered spice, may be detected chemically, by extracting the material with alcohol, pouring off the latter, and soaking in it a piece of filter-paper. Turmeric, if present, will stain the latter yellow, turning red with alkali, especially apparent after drying. Soak the yellow paper in a solution of borax, acidulated slightly with hydrochloric acid. When dry, a rose-red color will indicate turmeric, turning dark olive when dilute alkali is applied.

\section{MUSTARD.}

Nature and Composition.-Mustard is the seed of the mustard plant, an annual belonging to the family Cruciferce, and to the genus Sinapis, or Brassica, as it is sometimes called. The plant is an herb, native throughout Europe, and cultivated extensively in the United States. It grows to a height of from 3 to 6 feet, having yellow flowers and lyrate leaves.

Two varieties commonly used are Brassica or (Sinapis) alba, white mustard, and Brassica (or Sinapis) nigra, black mustard, the ground spice being as a rule a mixture of the two. In the trade these varieties are known as brown and yellow mustard respectively. The seeds of both varieties are globular, those of the black mustard being small, and of a dark-brown color on the outside and yellow within. White mustard seeds are considerably larger than the black, being pale yellow in color on the outside.

The surface of the black nuustard seeds is reticular, and full of small depressions, while the white variety is much smoother. There are several layers forming the husk of the seed of both varieties, and within the husk is the yellowish-colored kernel or embryo, with two cotyledons.

Both black and white mustard contain from $3 \mathrm{I}$ to $37 \%$ of fixed oil, a soluble ferment known as myrosin, and a sulphocyanate of sinapin. Mustard seeds contain no starch, and very little volatile oil as such. Black mustard seed contains sinigrin, or myronate of potash (not found in the white seed), which, when moistened with water, forms by hydrolysis the volatile oil of black mustard, otherwise known as allyl isothiocyanate, in accordance with the following equation:

$$
\underset{\substack{\text { Potasium } \\ \text { myronate }}}{\mathrm{KC}_{10} \mathrm{H}_{10} \mathrm{NS}_{2} \mathrm{O}_{9}}+\mathrm{H}_{2} \mathrm{O}=\underset{\text { Glucose }}{\mathrm{C}_{6} \mathrm{H}_{12} \mathrm{O}_{6}}+\underset{\substack{\text { Mustard } \\ \text { oil }}}{\mathrm{C}_{3} \mathrm{H}_{5} \mathrm{CNS}}+\underset{\substack{\text { Potassium } \\ \text { bisulphate }}}{\mathrm{KHSO}_{4}} .
$$

Mustard Oil (volatile) is a colorless, or slightly yellow, highly refractive liquid of a very strong odor, and capable of blistering the skin when 
brought in contact with it. It is optically inactive. Its specific gravity varies between 1.016 and 1.030 . It boils between $148^{\circ}$ and $156^{\circ}$. It turns reddish brown by exposure to light. follows:

Volatile oil of black mustard forms thiosinamine with ammonia, as

$$
\mathrm{C}_{3} \mathrm{H}_{5} \mathrm{CNS}+\mathrm{NH}_{3}=\mathrm{CS} \cdot \mathrm{NH}_{2} \cdot \mathrm{NH} \cdot \mathrm{C}_{3} \mathrm{H}_{5} \text {. }
$$

Thiosinamine is soluble in hot water, from which it crystallizes in tufts of monoclinic crystals, having a melting-point of $74^{\circ} \mathrm{C}$. It is precipitated by silver nitrate, mercuric chloride, and Mayer's solution.

White mustard differs from the black in containing a sulphur compound, sinalbin, $\mathrm{C}_{30} \mathrm{H}_{42} \mathrm{~N}_{2} \mathrm{~S}_{2} \mathrm{O}_{15}$. This is a glucoside. Sinalbin by hydrolysis forms an oil of white mustard, in a somewhat similar manner to the potassium myronate of black mustard, and according to the following equation:

$$
\underset{\text { Sinalbin }}{\mathrm{C}_{30} \mathrm{H}_{42} \mathrm{~N}_{2} \mathrm{~S}_{2} \mathrm{O}_{15}}+\mathrm{H}_{2} \mathrm{O}=\underset{\substack{\text { Sinatbin } \\ \text { mustard oil }}}{\mathrm{C}_{7} \mathrm{H}_{2} \mathrm{ONCS}}+\underset{\text { Glucose }}{\mathrm{C}_{6} \mathrm{H}_{12} \mathrm{O}_{6}}+\underset{\substack{\text { Sinapin acid } \\ \text { sulphate }}}{\mathrm{C}_{18} \mathrm{H}_{24} \mathrm{NO}_{5} \mathrm{HSO}_{4}} \text {. }
$$

Sinalbin Mustard Oil cannot be obtained by the distillation of white mustard, being sparingly volatile with steam.

Sinalbin mustard oil somewhat resembles that from black mustard, being quite as pungent, but less strong in odor when cold. It is soluble in dilute alkali.

Fixed oil of mustard is a bland, tasteless, and nearly odorless oil, its specific gravity at $15^{\circ}$ varying between the limits of 0.914 to $0.9 \mathrm{r} 8$. It is said to be used to some extent as an adulterant of table oils, being separated by pressure from the crushed mustard seeds before the latter are ground into "flour." The chief use of mustard oil is in mixture with other oils as an illuminant.

MUSTARD FLOUR.-In the process of preparing the ground spice commonly known as mustard "flour," the seeds are first crushed and separated by winnowing from the hulls, the latter being incapable of the fine grinding necessary to produce a smooth flour. The yellow hulls are, however, found in the cheaper grades ot ground mustard, and both varieties of hull are frequently used in the wet mustard preparations, sold in bottled form. In order to produce an even, dry powder, free from lumps, it is necessary to remove a large portion of the fixed oil, which is indeed of no value in the final product, and this is done by subjecting the crushed material to hydraulic pressure, during which process the 
mustard is molded together into thin, hard plates, called "mustard cake." This is then broken up and reduced to fine powder by pounding.

Richardson's * analyses of whole-seed flour, prepared by himself without the removal of the fixed oil, are as follows:

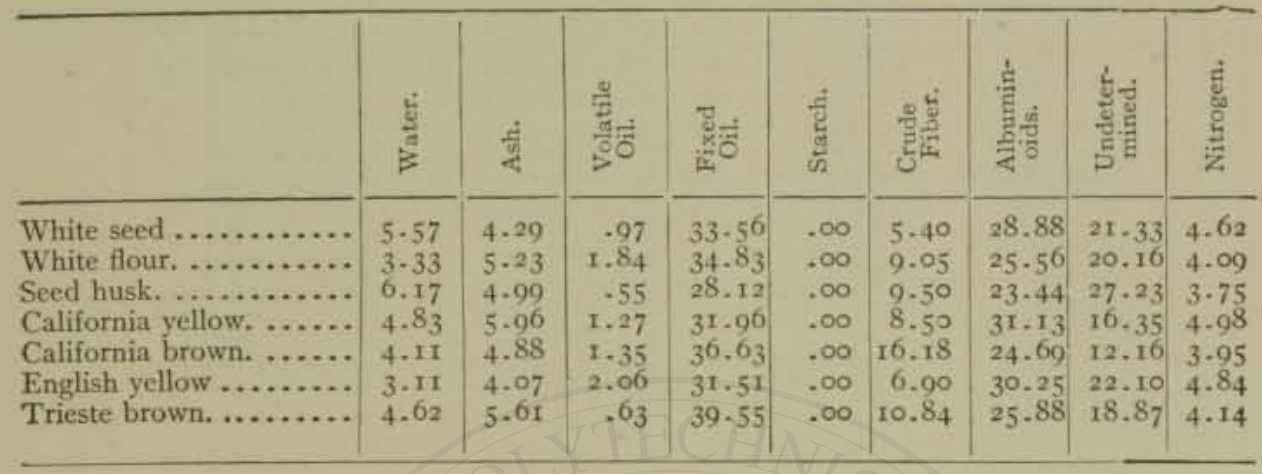

Winton and Mitchell made no full analyses of mustard seed of known purity, but the following is a summary of analyses of 18 samples of commercial mustards, sold in packages in Connecticut, and not found to be adulterated:

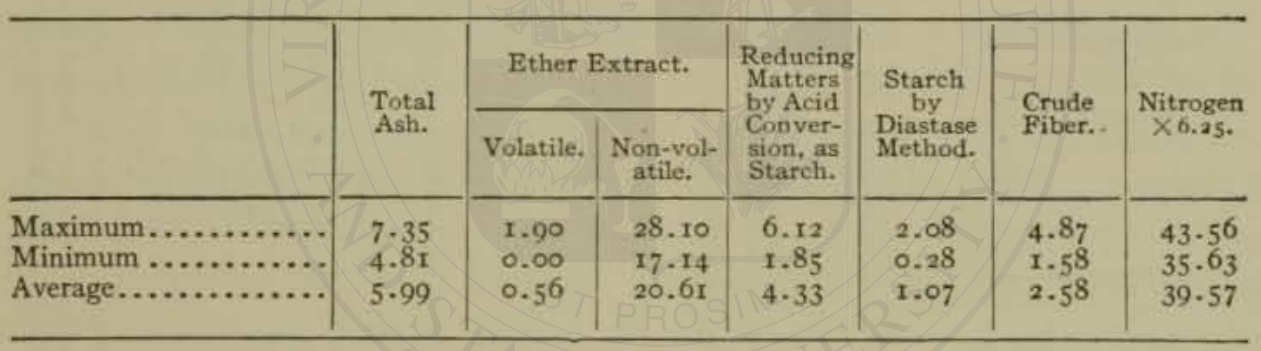

The following analyses of 5 samples of mustard flour, 6 samples of mustard hulls, and 6 samples of whole mustard, were made in the author's laboratory in 1903 :

* U. S. Dept. of Agric., Div, of Chem., Bul. 13, part 2. 


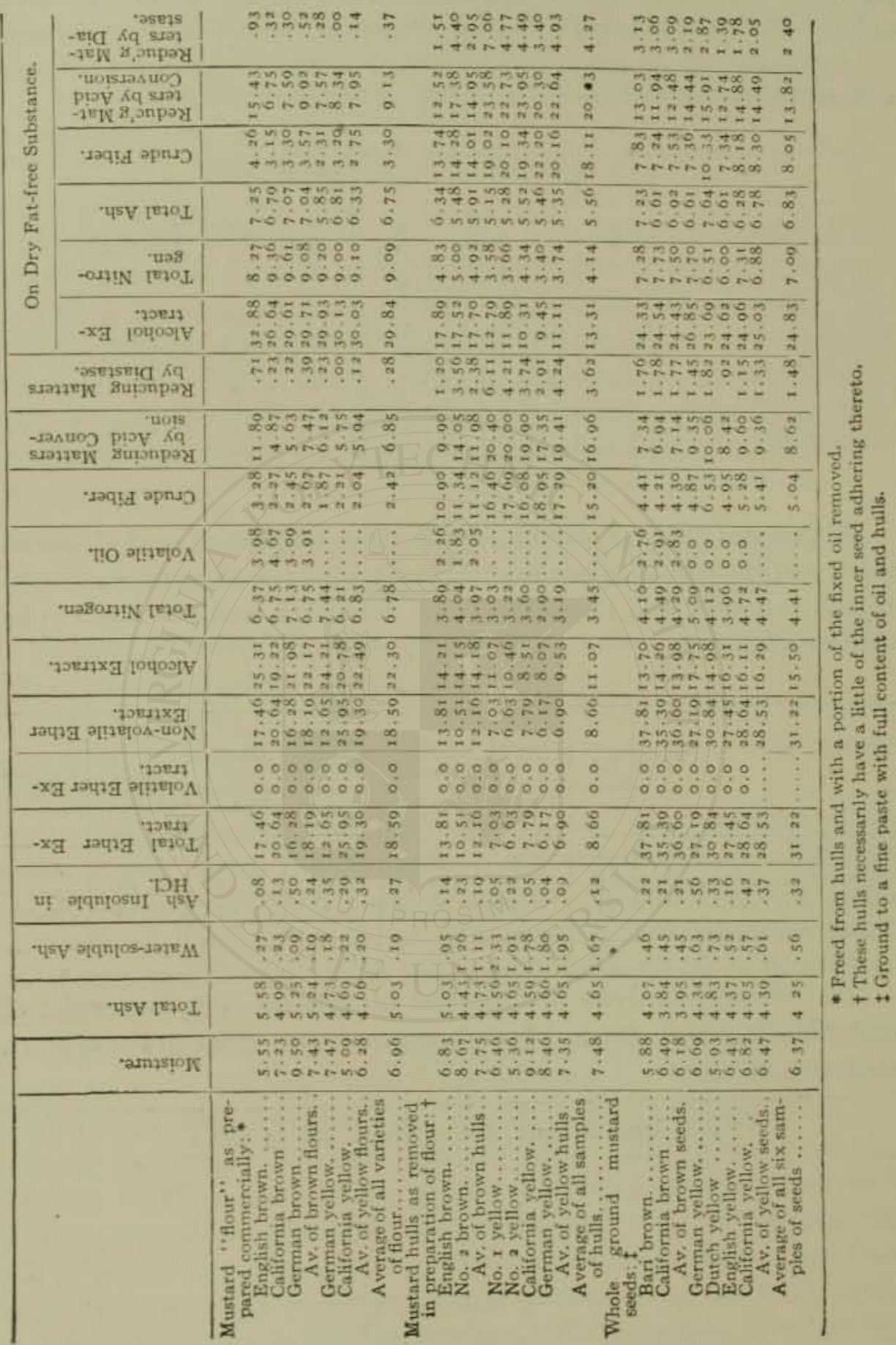


Piesse and Stansell give the following composition of mustard ash:

\begin{tabular}{|c|c|c|c|}
\hline & \multicolumn{2}{|c|}{ White Seeds, } & \multirow{2}{*}{$\begin{array}{l}\text { Brown Seeds. } \\
\text { Cambriage. }\end{array}$} \\
\hline & Yorkshire, & Cambridge. & \\
\hline Potash... & $2 \mathrm{~T} .29$ & t8.88 & $2 \mathrm{I} .4 \mathrm{I}$ \\
\hline Soda... & 0.18 & 0.21 & 0.35 \\
\hline Lime .... & 13.46 & $9 \cdot 34$ & $x_{3} .57$ \\
\hline Magnesii. . & 8.17 & 10. 49 & I0.04 \\
\hline Iron oxide.... & 1.18 & 1.03 & 1.06 \\
\hline Sulphuric acid ............ & 7.06 & 7.16 & $5 \cdot 56$ \\
\hline 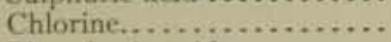 & 0.11 & 0.12 & 0.15 \\
\hline Phosphoric acid, ............ & $3^{2} \cdot 74$ & 35.00 & 37.20 \\
\hline Silica. . . . . . . . $\ldots \ldots \ldots$ & 1.00 & 1,12 & $1.4 \mathrm{I}$ \\
\hline & 1.82 & 1.95 & $x .3^{8}$ \\
\hline \multirow[t]{2}{*}{ Charcoal. .................. } & 12.82 & 15.14 & 7.57 \\
\hline & 99.85 & 100.48 & 99.70 \\
\hline
\end{tabular}

\section{Determination of Myronate of Potassium and Sinapin Sulphocyanate.*} -Extract at least 50 grams of the powdered material with several portions of a mixture of equal parts of water and alcohol, digesting with the aid of heat in a flask with a return-flow condenser. Evaporate the alcoholic extract in a tared dish to dryness, and heat at $105^{\circ}$ to constant weight. After weighing, incinerate the residue at a temperature sufficiently high to transform to the neutral sulphate the potassium bisulphate resulting from the decomposition of the myronate. The weight of myronate of potassium is obtained by multiplying the weight of neutral sulphate (the final ash) by the factor 4.77 . This, deducted from the total weight of the dried alcoholic residue as above, gives that of the sulphocyanate of sinapin.

Determination of Mustard Oil in Mustard Flour.-Roeser's Method. $\dagger$ -Mix 5 grams of the sample with $60 \mathrm{cc}$. of water and $I_{5} \mathrm{cc}$. of $60 \%$ alcohol, and let stand for two hours. Distil into a flask containing to cc. of ammonia, and, after about two-thirds of the solution have been distilled off, mix the ammoniacal distillate with Io cc. of tenth-normal silver nitrate solution, and allow the mixture to stand for twenty-four hours, after which make up with water to $100 \mathrm{cc}$. Filter, and treat $50 \mathrm{cc}$. of the filtrate with $5 \mathrm{cc}$. of tenth-normal potassium cyanide solution. Titrate the excess of cyanide with the tenth-normal silver nitrate, using as an indicator a $5 \%$ solution of potassium iodide, made slightly ammoniacal.

* Girard, Analyse des Matières Alimentaires, p. 8ro.

$\dagger$ Abs. Analyst, XXVII, 1902, p. 197. 
The percentage of mustard oil present is found by multiplying by 2 the number of cubic centimeters of silver nitrate solution taken up by the oil, and multiplying this product by the factor 0.3137 .

Microscopical Characteristics of Powdered Mustard.-The principal features of powdered black mustard are represented in Fig. $89^{\circ}$ The

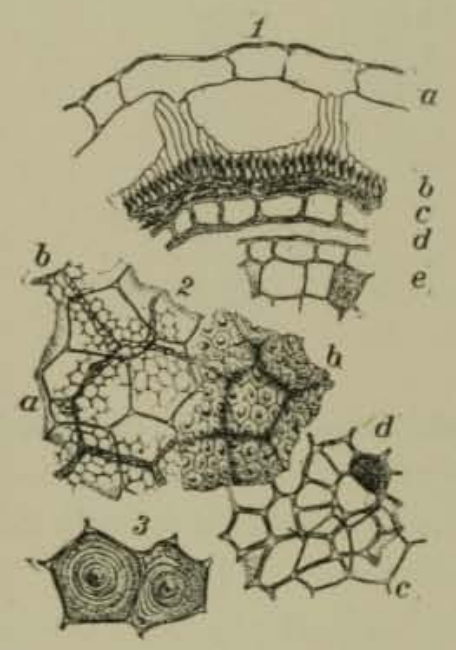

Fig. 89. - Powdered Mustard under the Microscope. $X_{125}$. (After Moeller.) seed shell or hull is shown in cross-section at (I), $a$ being the polygonal-celled epidermis, $b$ a layer of palisade-shaped cells, and $c$ a thin pigment layer, the brown coloring matter of which is colored blue by iron salts; $d$ is the aleurone layer and obscure parenchyma, and $e$ the small-celled tissue of the cotyledons, containing fixed oil and albumen.

(2) shows in surface view the various layers of the seed shell, the letters of reference corresponding to those of (I).

(3) shows in surface view a bit of the extreme outer mucilaginous layer of the seedhull.

Fig. 247, Pl XXXII, shows the appearance in water-mount of pure ground mustard. This is a photomicrograph of the ground hulled seed without the extraction of the oil, and should not be taken as a standard for commercial mustard "flour," from which, as a rule, a large portion of the oil has been removed. The cellular tissue of the mustard shows in the form of granular masses of loose, fine gray texture; the globular bodies are oil drops. Here and there through the field of ordinary ground mustard are to be seen patches of the yellowish layer of the seed skin of the brown mustard, a mass of which is shown in Fig. 248, with dark-brown spots distributed regularly through it. This is the layer shown at (2) b, Fig. 89. The hull of the yellow seed, also common in powdered mustard, is similar in appearance, having darkbrown spots, but with nearly colorless or gray cell walls, instead of yellow.

Patches of the outer hull layer represented by (3) in Fig. 89 are also very common in the commercial mustard flour. Mustard contains no starch. 
Adulteration of Mustard.-U. S. standards for mustard are as follows: Starch, by diastase method, should not exceed $2.5 \%$ and total ash should not exceed $8 \%$.

It is difficult to draw the line between the amount of mustard hulls which may naturally occur in ground mustard, and the excess amount which is sometimes added as an adulterant. Samples in which the patches of hulls predominate in number over the regular cellular tissue of the seed, as seen under the microscope, are undoubtedly adulterated by the fraudulent admixture of ground hulls, that have been separated out from the crushed mustard seeds intended for higher grades. Samples of mustard flour thus adulterated are common.*

In determining starch in mustard, it should be borne in mind that mustard huils have considerable reducing matter by the diastase process.

The most common adulterants of mustard, other than excess of hulls, are wheat, rice, millet, turmeric, charlock and other weed seeds. Yellow, oil-soluble azo-dyes are also employed.

Other adulterants found in Massachusetts have been potato starch, cayenne, corn, and gypsum or "terra alba" (the latter being found in one instance to the extent of $2 \mathrm{r} \%$ ).

Fig. 250, Pl. XXXIII, shows a sample of mustard adulterated with wheat bran. Very little besides the adulterant appears in this field.

The common practice of adulterating mustard with wheat is an outgrowth of the old notion that a certain amount of wheat flour was necessary to prevent lumping.

Charlock or Wild Mustard (Brassica Sinapistruin) grows luxuriantly in the grain fields of the Northwest and the seed is a common impurity of the uncleaned wheat from that region. It is an important constituent of wheat screenings, from which it is separated and placed on the market under such names as "Dakota mustard," "Domestic mustard," etc. This product also contains other weed seeds, notably those of the mustard family, and also a certain amount of broken wheat. Ground charlock or charlock cake and charlock flour are common adulterants

\footnotetext{
* It is claimed by some manufacturers that the hulls thus removed are not used as an adulterant of cheaper mustard flours, in view of the fact that it is difficult or impossible to grind them finely enough, but that they are used up in the manufacture of compound mustard pastes. A sample of ground mustard was recently found by the writer, in which it was noticed that a large number of yellow lumps were distributed through it. These lumps were picked out, transferred to the microscope slide, and crushed and rubbed out under the cover-glass. Examined under the microscope, they were found to consist entirely of a mixture of mustard hulls and turmeric, which would seem to show that hulls were present in this case as an adulterant.
} 
of prepared mustard and mustard flour. These rank-tasting adulterants often contain an appreciable amount of starch derived from the broken wheat and starchy weed seeds. See Fig. 249; Pl. XXXIII.

Charlock is identified by the presence in the palisade cells of a black substance which on heating in various acid reagents (such as chloral hydrate, glycerine, or zinc chloride, acidified with hydrochloric acid, syrupy phosphoric or citric acid), becomes bright carmine. A satisfactory reagent is a solution of 16 grams of chloral hydrate in $10 \mathrm{cc}$. of water and I cc. of concentrated hydrochloric acid. Mount to mg. of the material in a drop of the reagent, heat gently, and examine under a lens.

Detection of Coloring Matter.*-Turmeric is best detected by the microscope (see pp. 45I and 452). OH-soluble coal-tar dyes should be tested for as in the case of cayenne. Nitro colors, such as naphthol yellow (Martius yellow) and naphthol yellow S, are detected by dyeing tests, with subsequent examination of dyed fabric according to the scheme on p. 801 .

PREPARED MUSTARD. - This product consists of a mixture of ground mustard seed or mustard flour with salt, spices, and vinegar. The U. S. standards require that it should contain not more than $24 \%$ of carbohydrates calculated as starch, not more than I2 \% of crude fiber, and not less than $35 \%$ of protein.

Most of the product consumed in the United States is of domestic manufacture, although until the passage of the federal food law it was customary to designate it German or French mustard, or label it in a foreign language.

Composition and Adulteration.-The common admixtures are wheat flour, maize flour, and other starchy matter, mustard hulls, sugar, chemical preservatives, and artificial colors.

Of 28 brands examined in Connecticut in 1905 by Winton and Andrew, $\uparrow$ 13 contained cereal flour (wheat or corn), 4 salicylic acid, and 25

* Recently some very yellow samples of powdered mustard have appeared on the market that are apparently free from foreign color. Their method of manufacture is kept secret. From the fact that they contain nearly, if not quite, the full content of fixed mustard oil that would be present if the oil had not been previously expressed, and for various other reasons, it is probable that the color is due largely to the presence of the fixed oil, which has a deep-yellow color, and which has hitherto been generally removed for purposes of fine pounding and to avoid caking.

In such samples, the oil, previously pressed out, is, after pounding, restored, and with it much of the color. Incidentally in such a process oil-soluble coal-tar dyes may conveniently be dissolved in the mustard oil, in order to intensify the color, and the analyst should be on the outlook for such foreign colors.

$\dagger$ An. Rep. Conn. Exp. Sta., 1905, p. 123 . 
artificial color (turmeric, nitro-color or azo-color). A summary of the analyses of those brands free from cereal flour and those containing it follows:

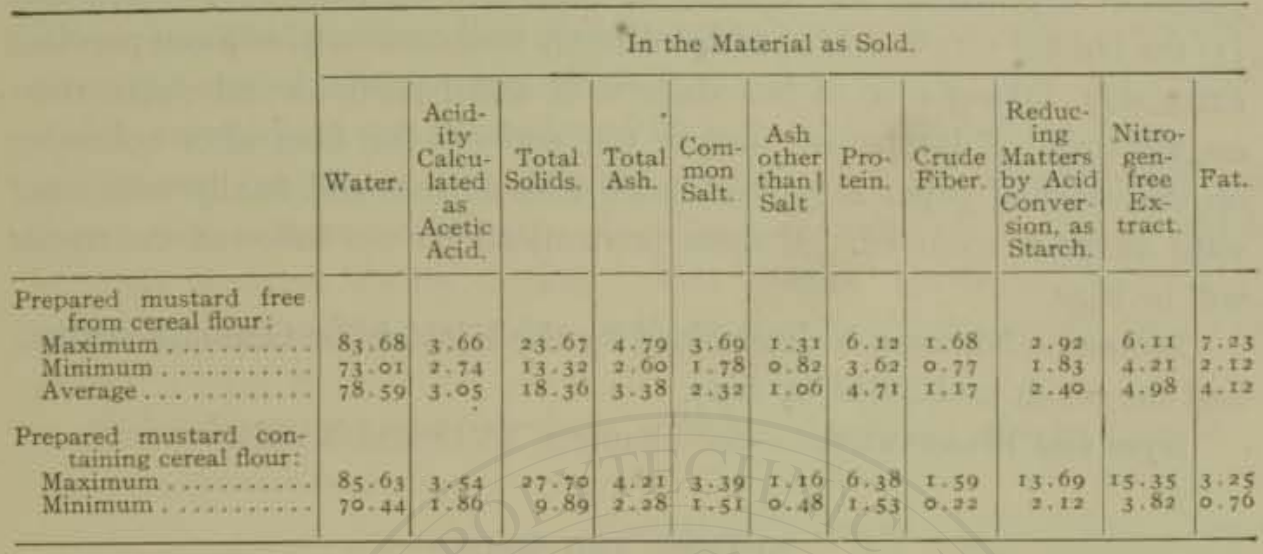

\begin{tabular}{|c|c|c|c|c|c|}
\hline & In & e Dry, $\mathrm{F}_{\mathrm{i}}$ & and $S$ i & - free Mate & rial. \\
\hline & Ash. & Protein. & $\begin{array}{l}\text { Crude } \\
\text { Piber. }\end{array}$ & $\begin{array}{l}\text { Reducing } \\
\text { Matters } \\
\text { by Acid } \\
\text { Conver- } \\
\text { sion, as } \\
\text { Starch. }\end{array}$ & $\begin{array}{l}\text { Nitrogen- } \\
\text { free } \\
\text { Extract. }\end{array}$ \\
\hline 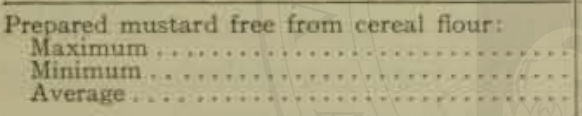 & $\begin{array}{r}10.66 \\
7.35 \\
8.94\end{array}$ & $\begin{array}{l}43-94 \\
32.01 \\
39-44\end{array}$ & $\begin{array}{r}14.12 \\
7 \cdot 77 \\
9 \cdot 89\end{array}$ & $\begin{array}{l}24 \cdot 37 \\
16 \cdot 82 \\
20 \cdot 17\end{array}$ & $\begin{array}{l}44 \cdot 76 \\
34=98 \\
4+73\end{array}$ \\
\hline $\begin{array}{l}\text { Prepared mustard containing cereal flour: } \\
\text { Maximum } \ldots \ldots \ldots \ldots \ldots \ldots \ldots \ldots \ldots \ldots \ldots \ldots \\
\text { Minimum } \ldots \ldots \ldots \ldots \ldots \ldots \ldots \ldots \ldots \\
\end{array}$ & $\begin{array}{r}9.68 \\
4.84\end{array}$ & $\begin{array}{l}33.89 \\
21.37\end{array}$ & $\begin{array}{r}18.44 \\
0.45\end{array}$ & $\begin{array}{l}59.22 \\
24.51\end{array}$ & $\begin{array}{l}66.42 \\
42.79\end{array}$ \\
\hline 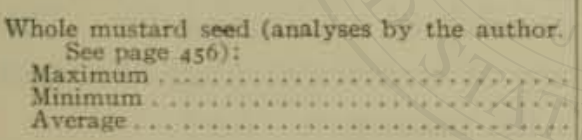 & $\begin{array}{l}7.64 \\
6.28 \\
6.83\end{array}$ & $\begin{array}{l}48.31 \\
37.50 \\
44 \cdot 31\end{array}$ & $\begin{array}{r}10.3 .3 \\
7.24 \\
8.05\end{array}$ & $\begin{array}{l}15.91 \\
17.94 \\
13.82\end{array}$ & $\begin{array}{l}48.55 \\
37.84 \\
40.81\end{array}$ \\
\hline
\end{tabular}

The following methods for the analysis of prepared mustard were used by Winton and Andrew, and afterwards adopted by the Association of Official Agricultural Chemists:

Solids, Ash and Salt are determined in one portion of 5 grams of the thoroughly mixed material, following the usual methods. The salt is calculated from the percentage of chlorine.

Ether Extract.-Ten grams of the material and about 30 grams of sand are placed in a capsule, and dried on a water bath with stirring. The dried residue is ground and extracted with anhydrous ether in the usual manner. 
Reducing Matters by Acid Conversion are determined directly in the material, without previously washing, as described on page $4 \mathrm{Ir}$.

Crude Fiber.-Eight grams of the material (equivalent to about 2 grams of dry matter) are treated as described on page 277 , except that (I) the boiling $1.25 \%$ acid is added directly to the material without previous extraction, taking care to introduce it in small portions and shake thoroughly until all lumps are broken up, and (2) the fiber after collecting on the weighed paper is washed twice with alcohol and finally with ether until all fat is removed. If these precautions are not followed the results will be high.

Protein.-Nitrogen is determined by the Kjeldahl or Gunning method, and the result multiplied by 6.25 .

Dyes and Preservatives.-See chapters XVII and XVIII.

\section{NUTMEG AND MACE.}

Nature and Composition.-Both nutmeg and mace occur in the fruit of several varieties of trees of the genus Myristica, especially of the Myristica fragrans or Myristica moschata, belonging to the family Myristicacece. The nutmeg tree is a native of the Malay archipelago, and grows from 20 to 30 feet high, somewhat resembling the orange tree in appearance. It does not produce flowers till its eighth or ninth year, after which it bears fruit constantly for many years. The fruit is a globular, pendant drupe, about $5 \mathrm{~cm}$. in diameter, of a yellowish-green color, the pericarp of which, when ripe, splits in two, showing within it the kernel, completely surrounded by a fleshy, fibrous aril, or covering of a crimson color. This covering, when dried, furnishes the mace of commerce, while the inner kernel, which is a hard, brown seed, is the nutmeg.

The nutmeg seed or kernel, when gathered, is surrounded by a thick tegument, marked with depressions corresponding to the lobes of the aril or mace, and by a second thin, inner envelope, closely adhering to the seed. The whole seed is dried in the sun for about two months, or by the aid of heat, the tegument becoming separated from the kernel, and, by breaking with a hammer, is readily removed. The kernels are then commonly washed in milk of lime, and again dried, or they are sometimes treated with dry, powdered, air-slaked lime. Liming is alleged to prevent sprouting and ward off the attacks of insects. The so-called brown nutmegs of commerce are those which have not been treated or coated with lime. 
NUTMEGS are spheroidal, sometimes nearly spherical, from 20 to 25 $\mathrm{mm}$. long and 15 to $18 \mathrm{~mm}$. in diameter. The outer surface is somewhat furrowed. A cross-section of the kernel shows the grayish-brown, starchy endosperm, mottled with the dark-brown, resinous veins of the perisperm. These veins on pressure with the finger nail present an oily appearance. Near the end of the nutmeg which is attached to the stem, is a small cavity, in which is the undeveloped embryo with two cotyledons.

Nutmeg contains a considerable amount of fixed oil, a volatile oil, starch, and albuminous matter. Its volatile oil is colorless, and is soluble in three parts of strong alcohol. The specific gravity of nutmeg oil varies between 0.865 and 0.920 , and its specific rotary power $(a)_{D}=14$ to 28 .

Richardson's analyses of three samples of nutmeg are as follows:

\begin{tabular}{|c|c|c|c|c|c|c|c|c|}
\hline & Water. & Ash. & $\begin{array}{l}\text { Volatile } \\
\text { Oil. }\end{array}$ & $\begin{array}{l}\text { Fixed } \\
\text { Oil or } \\
\text { Fat. }\end{array}$ & $\begin{array}{l}\text { Starch, } \\
\text { etc. }\end{array}$ & $\begin{array}{l}\text { Crude } \\
\text { Fiber. }\end{array}$ & $\begin{array}{l}\text { Albu- } \\
\text { minoids. }\end{array}$ & $\begin{array}{l}\text { Nitro- } \\
\text { gen. }\end{array}$ \\
\hline Whole limed. . & 6.08 & 3.27 & 2.84 & $34 \cdot 37$ & 36.98 & 11.30 & 5.16 & .83 \\
\hline Ground limed. & 4.19 & 2.22 & 3.97 & $37 \cdot 3 \circ$ & 40.12 & 6.78 & $5 \cdot 42$ & .87 \\
\hline Ground... & 6.40 & 3.15 & 2.90 & $30.9^{8}$ & 41.77 & $9 \cdot 55$ & 5.25 & .84 \\
\hline
\end{tabular}

König gives the following minimum and maximum composition of nutmeg:

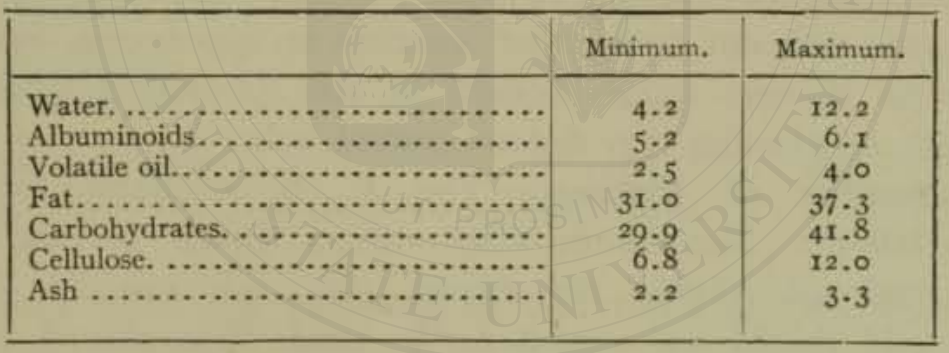

Winton, Ogden, and Mitchell analyzed four samples of nutmeg of known purity, the following being maximum and minimum results:

\begin{tabular}{|c|c|c|c|c|c|c|}
\hline & \multirow{2}{*}{ Moisture. } & \multicolumn{3}{|c|}{ Ash. } & \multicolumn{2}{|c|}{ Ether Extract. } \\
\hline & & Total. & $\begin{array}{l}\text { Soluble in } \\
\text { Water. }\end{array}$ & $\begin{array}{l}\text { Insoluble } \\
\text { in } \mathrm{HCl} \text {. }\end{array}$ & Volatile. & $\begin{array}{c}\text { Non-vola- } \\
\text { tile. }\end{array}$ \\
\hline $\begin{array}{l}\text { Maximum } . . . \cdots \cdots \cdots \cdots \cdots \\
\text { Minimum } . . . \cdots \cdots \cdots \cdots\end{array}$ & $\begin{array}{r}10.83 \\
5.79\end{array}$ & $\begin{array}{l}3.26 \\
2.13\end{array}$ & $\begin{array}{l}1.46 \\
0.82\end{array}$ & $\begin{array}{l}0.01 \\
0.00\end{array}$ & $\begin{array}{l}6.94 \\
2.5^{6}\end{array}$ & $\begin{array}{l}36.94 \\
28.73\end{array}$ \\
\hline
\end{tabular}




\begin{tabular}{|c|c|c|c|c|c|c|}
\hline & $\begin{array}{l}\text { Alcohol } \\
\text { Extract. }\end{array}$ & $\begin{array}{l}\text { Reducing } \\
\text { Matters by } \\
\text { Acid Con- } \\
\text { version, as } \\
\text { Starch. }\end{array}$ & $\begin{array}{l}\text { Starch by } \\
\text { Diastase. }\end{array}$ & $\begin{array}{l}\text { Cruide } \\
\text { Fiber. }\end{array}$ & $\begin{array}{l}\text { Nitrogen } \\
\times 6.25 \text {. }\end{array}$ & $\begin{array}{c}\text { Total } \\
\text { Nitrogen. }\end{array}$ \\
\hline $\begin{array}{l}\text { Maximum............. } \\
\text { Minimum } \ldots \ldots \ldots \ldots \ldots\end{array}$ & $\begin{array}{l}17.3^{8} \\
10.4^{2}\end{array}$ & $\begin{array}{l}25.60 \\
17.19\end{array}$ & $\begin{array}{l}24.20 \\
14.62\end{array}$ & $\begin{array}{l}3 \cdot 72 \\
2 \cdot 38\end{array}$ & $\begin{array}{l}7.00 \\
6.56\end{array}$ & $\begin{array}{l}\text { I. } 12 \\
1.05\end{array}$ \\
\hline
\end{tabular}

Microscopical Structure of Nutmeg. (Fig. 90.)-The thin-walled cells of the parenchyma of the endosperm or albumen are shown at

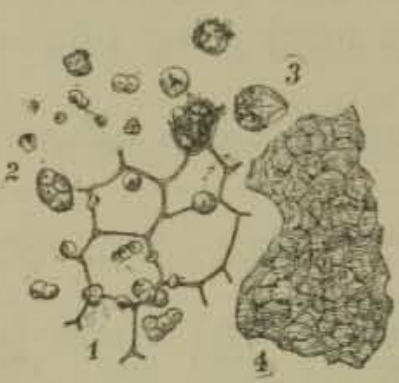

FIG. 90.-Powdered Nutmeg under the Microscope. X125. (After Moeller.) (I), with starch grains. Simple and compound granules of the starch are shown at (2). Aleurone grains appear as shown at (3), and (4) represents in surface view the epidermis, or brown seed coat, with its numerous layers of flat cells. Powdered nutmeg under the microscope in watermount shows most commonly a spongelike, loose meshwork of bruised or broken cellular tissue, with many starch granules, and occasional fragments of the epidermis.

Fig. 240, Pi. XXX, is a photomicrograph of a water-mounted sample of pure nutmeg. The starch granules of nutmeg are different from other starches in appearance, being almost circular as a rule, quite uniform in size (averaging $0.006 \mathrm{~mm}$. in diameter), and having very distinct central hyla.

Adulteration of Nutmeg. - The U. S. standards for nutmegs are as follows: Non-volatile ether extract should be not less than $25 \%$; total ash should not exceed $5 \%$; ash insoluble in hydrochloric acid should not exceed $0.5 \%$; crude fiber should not exceed $10 \%$.

This spice is more often sold in the whole form, since the housewife much prefers to grate the whole nutmeg, rather than to use the ground material. It is hence less liable to adulteration than the other spices, though of late more of the ground nutmeg is being sold in packages. Samples of ground nutmeg have been found in Massachusetts adulterated with wheat and nutshells. Onc sample was found to contain at least $25 \%$ of ground cocoanu shells.

Nutmegs which have become mouldy, or have been eaten out by insects, have been imported for grinding, as sound nutmegs are not readily reduced to a powder. Such a product is obviously unit for consumption. 
An inferior variety is known as the Macassar nufmeg. This lacks much of the agreeable pungency of the better grades.

MACE.-The crimson-colored aril that surrounds the nutmeg kernel within the pericarp, as above described (p. 462), has many narrow, flattened lobes. In the process of drying to form the mace of commerce, it loses its brilliant red color, and turns a yellowish brown. When dried, it is brittle, somewhat translucent, and of a pungent odor. Whole mace appear on the market in the form of flat membranous masses, 3 to $4 \mathrm{~cm}$. long.

It contains no starch as such, but has a modified form of starch known as amylodextrin. This is a carbohydrate, $\mathrm{C}_{30} \mathrm{H}_{n 3} \mathrm{O}_{31}+\mathrm{H}_{2} \mathrm{O}$, which produces with iodine a red coloration. Mace has a large amount of fixed oil, as well as considerable resinous and albuminous matter, and a volatile oil which much resembles that of nutmeg.

The specific gravity of volatile oil of mace is rather higher than that of nutmeg oil. Its specific rotary power, $(\boldsymbol{a})_{D}=10$ to 20 .

König's figures for the composition of mace are as follows:

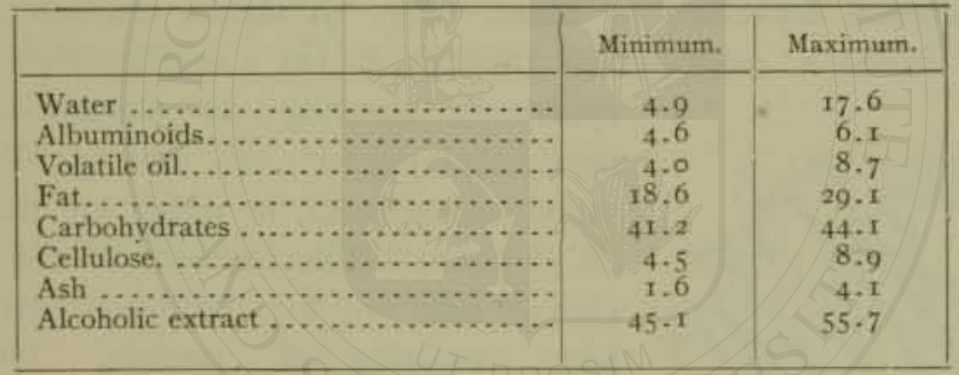

Richardson gives the following as the results of analyses of three samples made by him:

\begin{tabular}{|c|c|c|c|c|c|c|c|c|}
\hline & Water. & Ash. & $\begin{array}{l}\text { Volatile } \\
\text { Oil. }\end{array}$ & Resin. & $\begin{array}{l}\text { Unde- } \\
\text { ter-- } \\
\text { mined. }\end{array}$ & $\begin{array}{l}\text { Crude } \\
\text { Fiber. }\end{array}$ & $\begin{array}{l}\text { Albu- } \\
\text { minoids. }\end{array}$ & $\begin{array}{l}\text { Nitro- } \\
\text { gen. }\end{array}$ \\
\hline $\begin{array}{l}\text { Whole mace ........... } \\
\text { Ground mace. ........ } \\
\text { Ground mace. ........ }\end{array}$ & $\begin{array}{r}5.67 \\
4.86 \\
10.47\end{array}$ & $\begin{array}{l}4.10 \\
2.65 \\
2.20\end{array}$ & $\begin{array}{l}4.04 \\
8.66 \\
8.68\end{array}$ & $\begin{array}{l}27 \cdot 50 \\
29.08 \\
23.33\end{array}$ & $\begin{array}{l}41.17 \\
35 \cdot 50 \\
34.68\end{array}$ & $\begin{array}{l}8.93 \\
4.48 \\
6.88\end{array}$ & $\begin{array}{l}4.55 \\
6.13 \\
5.08\end{array}$ & $\begin{array}{l}.73 \\
.98 \\
.81\end{array}$ \\
\hline
\end{tabular}

Winton, Ogden, and Mitchell's analyses of four samples of pure Banda or Penang mace, as well as of Bombay and Macassar mace, are summarized as follows: 


\begin{tabular}{|c|c|c|c|c|c|c|}
\hline & \multirow{2}{*}{ Moisture. } & \multicolumn{3}{|c|}{ Ash. } & \multicolumn{2}{|c|}{ Ether Bxtract. } \\
\hline & & Total. & $\begin{array}{c}\text { Soluble in } \\
\text { water. }\end{array}$ & $\begin{array}{l}\text { Insolubie } \\
\text { in } \mathrm{HCl}\end{array}$ & Volatile, & $\begin{array}{c}\text { Non-vola- } \\
\text { tile. }\end{array}$ \\
\hline 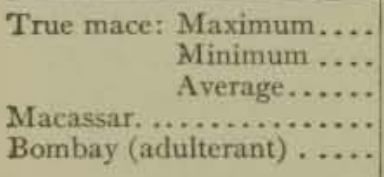 & $\begin{array}{r}12.04^{*} \\
9.7^{*} \\
11.05 \\
4.18 \\
0.32\end{array}$ & $\begin{array}{l}2.54 \\
1.81 \\
2.01 \\
2.01 \\
1.08\end{array}$ & $\begin{array}{l}1.33 \\
1.06 \\
1.13 \\
\text { I. } 11 \\
\text { I. } 37\end{array}$ & $\begin{array}{l}0.21 \\
0.00 \\
0.07 \\
0.03 \\
0.07\end{array}$ & $\begin{array}{l}8.65 \\
6.27 \\
7.58 \\
5.89 \\
4.65\end{array}$ & $\begin{array}{l}23 \cdot 72 \\
21.63 \\
22.48 \\
53 \cdot 54 \\
59 \cdot 81\end{array}$ \\
\hline & $\begin{array}{l}\text { Alcohol } \\
\text { Extract. }\end{array}$ & $\begin{array}{l}\text { Reducing } \\
\text { Matters by } \\
\text { Acid Con- } \\
\text { version, as } \\
\text { Starch. }\end{array}$ & $\begin{array}{l}\text { Starch by } \\
\text { Diastase.* }\end{array}$ & $\begin{array}{l}\text { Crude } \\
\text { Fiber }\end{array}$ & $\begin{array}{l}\text { Nitrogen } \\
\times 6.25 \text {. }\end{array}$ & $\begin{array}{c}\text { Total } \\
\text { Nitrogen. }\end{array}$ \\
\hline 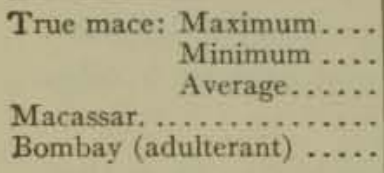 & $\begin{array}{l}24.76 \\
22.07 \\
23.11 \\
32.89 \\
44.27\end{array}$ & $\begin{array}{l}34 \cdot 42 \\
26.77 \\
31.73 \\
10.39 \\
16.20\end{array}$ & $\begin{array}{r}30.43 \\
23.12 \\
27.87 \\
8.78 \\
14.51\end{array}$ & $\begin{array}{l}3.85 \\
2.94 \\
3.20 \\
4 \cdot 57 \\
3.21\end{array}$ & $\begin{array}{l}7.00 \\
6.25 \\
6.47 \\
7.00 \\
5.06\end{array}$ & $\begin{array}{l}1.12 \\
1 . \infty \\
1.03 \\
1.12 \\
0.8 \mathrm{I}\end{array}$ \\
\hline
\end{tabular}

* The figures in this column do not express starch, but amylo-dextrin, which like starch may be determined by the diastase method.

Microscopical Structure of Mace.-Fig. 9I shows characteristics of mace, (I) being a cross-section through it, (2) a surface view of the

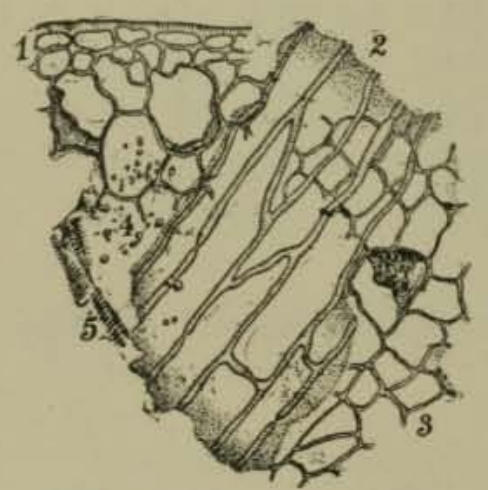
epidermis, showing its elongated, often nearly rectangular cells, and (3) the largecelled parenchyma, in which are numerous oil globules. The contents of the parenchyma cells are for the most part colorless, consisting of protein, fat, and granules of amylodextrin, which are shown at (4). At (5) are shown fragments of vascular tissue.

The water-mounted powder of pure mace shows no highly colored fragments, Frc. 9r.-Powdered Mace under but as a mass, is white or grayish, and the Microscope. $X_{125}$. (After of loose texture. Occasional pale, yelMoeller.)

lowish, lumpy masses appear, and palebrown fragments of the seed coating. The amylodextrin granules (which are colored red-brown by solution of iodine) are very small.

Adulteration of Mace.-U. S. standard mace should contain not less than 20 nor more than $30 \%$ of non-volatile ether extract; nor more than 
${ }_{3} \%$ of total ash; nor more than $0.5 \%$ ash insoluble in hydrochloric acid; nor more than $10 \%$ of crude fiber.

Turmeric and cereal starches are not uncommonly found in mace, but by far the most common adulterant is the so-called false, or wild mace, otherwise known as Bombay *mace.

Bombay Mace (Myristica fatua) is almost entirely devoid of odor or taste, being nearly as inert as so much starch. It is most properly regarded as an adulterant from its lack of pungency, even though in a sense it is a variety of mace.

Its non-volatile ether extract is twice as high as that of Penang mace, and at room temperature the fixed oil of Bombay mace is a thick and viscous fat, while that of Penang and other maces is a thin oil.

The refractive indices of the fixed oils of various species of pure, as well as of Bombay mace, as determined by Lythgoe in the writer's laboratory, are as follows:

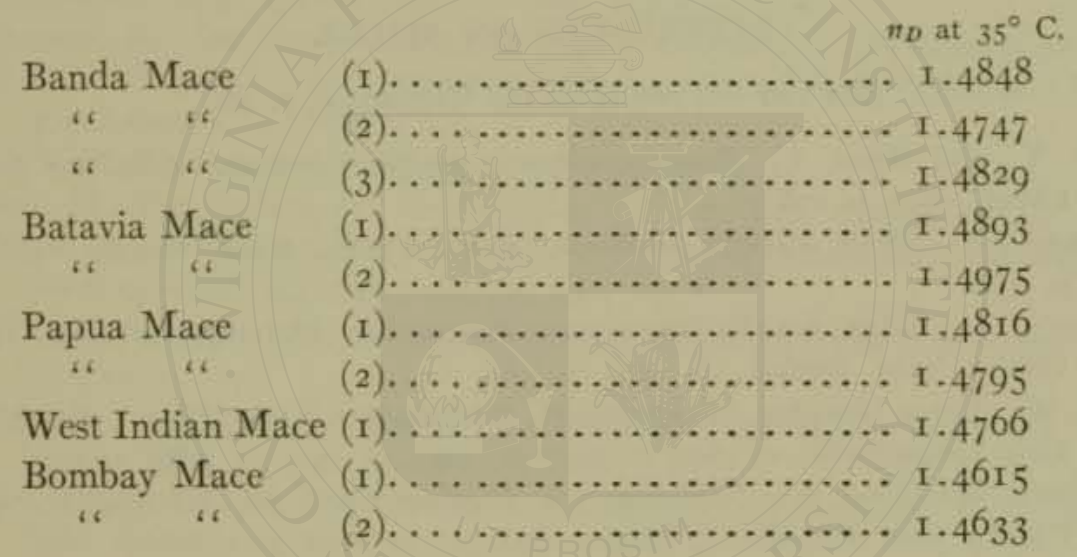

The microscope indicates at once when Bombay mace is present in a sample. The oil glands situated in the outer layers of Bombay mace are strongly colored and contain a reddish resinous substance, while the glands of the more interior layers have balsam-like contents of bright yellow color. Both the red and yellow lumps are visible in water mounts, but a $5 \%$ potassium hydroxide solution colors them a brilliant blood-red, making possible a close percentage estimation of Bombay mace in true mace.

Hefelmann's Test for Bombay Mace* consists in saturating a strip of filter-paper with an alcoholic solution of the mace, and removing the excess of liquid by pressure between filter-paper. On treating with a

* Pharm. Zeit., 1891. 
drop of dilute sodium or potassium hydroxide solution, a red color is produced in presence of the wild mace.

Waage's Test.-One part of the mace is extracted with ten parts of alcohol, and potassium chromate solution is added to the extract. If Bombay mace is present, the solution becomes red, and the precipitate, which is at first yellow, becomes red on standing. True mace gives a yellow solution and precipitate, and the latter does not change greatly on standing.

Turmeric is tested for chemically as on p. $79 \mathrm{r}$.

Macassar Mace is sometimes designated as wild mace, but it is by no means as inert as the Bombay variety, and possesses a wintergreenlike odor. Its taste, while distinctive, is not that of true Penang mace. It is distinctly an inferior article, and its volatile oil content, as shown by the analyses on p. 466 , is considerably below the minimum for true mace.

\section{REFERENCES ON SPICES.}

(See also References on the Microscope, p. 98.)

ARnst, T., and HaRT, F. Zusammensetzung einiger Gewürze. Zeits. für Angew. Chem., 1893 , p. 136 .

Beythien, A. Einige Paprika-Analysen. Zeits. Unters. Nahr. Genuss., 5, 1902, p. 858 .

BoHriscH, P. Ueber den Nachweis einer Künstlichen Färbung des Senfs. Zeits. Unters. Nahr. Genuss., 8, I904, p. 285.

Busse, W. Ueber Gewürze. I. Pfeffer. II. Muskatnüsse. III. Macis. Arbeit. a. d. Kais. Gesundheits., 9, I894, p. 509; II, I895, p. 390; 12, I896, p. 628.

- Notiz, betreffend den Nachweis von Bombay-Macis im Macispulver. Zeits. Unters. Nahr. Genuss., 7 , 1904, p. 590.

Delaite, J. Untersuchung von Senfmehl. Rev. Int, des Falsif., 1897, pp. ro, 37.

DENIS, W. Determination of the Iodine Number of the Non-volatile Ether Extract of Paprika. A. O. A. C. Proc,, 1908. U. S. Dept. of Agric., Bur. of Chem., Bul. 122, p. 213.

Doolittre and Ogden. Composition of Known Samples of Paprika. Jour. Am. Chem., Soc. 30 , 1908, p. 148 r.

DYER u. GriBARD. Unterscheidung zwischen unverfälschtem und extrahirtem Ingwer. Chem. Ztg., 17,1893 , p. $83_{3} 8$.

Fonster, A. Ueber Gewürze. Zeits. f. öffentl. Chem., 4, 1898, p. 626.

GínIN, V. Epices et Aromates. Analyse des Matières Alimentaires. Girard et Dupré, Paris, 1894.

GICHARD, B. Verfälschung von Zimmetrindenpulver. Zeits, f. Nahr. Hyg. Waarenk., 9, 1895 , p. $28 \mathrm{r}$.

GladHILL, J. W. Examination of Commercial Peppers. Am. Jour. Pharm., 76, 1904, p. 7 r. 
HÄRTFL, F. Untersuchung und Beurteilung von gemahlenen schwarzen Pfeffer. Zeits. Unters. Nahr. Genuss., 13, 1907, p. 665.

- u. WmL, R. Untersuchung und Beurteilung von Pfeffer. Zeits. Unters. Nahr. Genuss., 14,1907 , p. 567 .

HANAUSEk, T. F. Zur Charakteristik des Cayenpfeffers. Zeits. für Nahr. Unters. Hyg., 7, 1893, p. 297.

- Verfälschung von Gewürzen. Zeits. für Nahrungsm. Unters. u. Hyg, 8, 1894, 95.

Gewürzfälschungen. Apoth. Ztg., 1894, p. 582 .

Gefälschte und echte Macis. Rev. I-ter. Fals., I, 1887 , p. 23 ,

Hanus, J. Beitrag zur Kenntnis verschiedener Arten von Zimmet. Zeits. Unters. Nahr. Genuss., 7, 1904, p. 669 .

u. Bien, F. Zur Kentniss der Zuckerarten der Gewürze. Zeits. Unters. Nahr. Genuss., 12, 1906, p. 395.

Hartwich, C. Eine Bemerkungen über den Pfeffer. Zeits. Unters. Nahr. Genuss., 12 , 1906 , p. 524 .

Heberrand, A. Die Beurteilung des Pfeffers nach dem Gehalte an Rohfaser und Piperin. Zeits. Unters. Nahr. Genuss, 6, 1903, p. 345.

Hefelmann, R. Zur Untersuchung von Macis. Pharm. Ztg., 36, 1891, p. 122.

Heid, F., u. Hillger, A. Zur chemischen Charakteristik der Bombay Macis. Forsch. über Lebensm., I, 1894, p. 136 .

Jones, E. W. T. Analysis of Ginger. Analyst, 1886, p. 75.

Krasier, H. Zur Prüfung der Gewürznelken. Apoth. Ztg., 1894, p. 870 .

LEACH, A. E. Microscopical Examination of Foods for Adulteration. Mass. State Board of Heatlh An. Rep., I900, p. 679.

Composition and Adulteration of Ground Mustard. Jour. Am. Chem. Soc., 26, 1904, P. 1203 .

LuDwig, W, u. HAUPT, H. Zucker als Natürlicher Bestandteil der Macis. Zeits. Unters. Nahr. Genuss,, 9, 1904, p. 200.

Lührig, H., u. Thaмm, R. Beiträge zur Kenntniss der Gewürze. Zeits. Unters. Nahr. Genuss., II, 1906, p. 129.

Macfart.ane, T. Mustard. Canada Inl. Rev. Dept., Bul. ig.

- Mustard. Canada Inl. Rev. Dept, Bul. 50.

McGru, A. Cloves. Canada Inl. Rev. Dept., Bul. 73 .

- Ground Ginger. Canada Inl. Rev. Dept., Bul. 48.

- Pepper. Canada Inl. Rev. Dept., Bul. 20.

REICH, R. Ingwer und extrahierter Ingwer. Zeits. Unters. Nahr. Genuss., I4, 1907 , p. 549.

Richardson, C. Spices and Condiments. U. S. Dept, of Agric, Div. of Chem., Bul. 13, part 2, 1887 .

RoETrger, H. Die Gewürznelken, ihre Verfälschung und Beurtheilung. Ber. XI. Vers. d. freien Verein bayr. Vertreter d. angew. Chem. in Regensburg, 1892, p. 66 .

Sorstern, P. Banda und Bombay Maces, Pharm. Ztg., 1893, pp. 454, 467.

SPÄTH, E. Neue Verfälschungen von Gewürzen. Forsch. über Lebensm., 3, 18g6, p. 308 . 
SpÄтH, E. Zur mikroskop. Prüfung des Piments. Forsch. über Lebensm., 2, 1895, p. 4 I9.

_ Zur Prüfung und Beurteilung des gemahlenen schwarzen Pfeffers. Zeits. Unters. Nahr. Genuss., 9, I905, p. 576 .

— Vorschläge des Ausschusses zur Abänderung des Abschnittes "Gewürze" der "Vereinbarungen." Zeits. Unters. Nahr. Genuss., I0, 1905, p. I6; I2, I906, p. 12 ,

— Der Nachweis von Zucker in Macis und in Zimt. Zeits. Unters. Nahr. Genuss., II, 1906, p. 447 .

Sprinkmeytr, H., u. Fürstenberg, A. Beiträge zur Kenntnis der Gewürze. Zeits. Unters. Nahr. Genuss., 12, 1906, p. 652.

Vogi, A., and Hanausek, T. F. Untersuchung der Gewürze. Sudd. Apoth. Ztg., I896.

WAage, T. Banda und Bombay Maces. Pharm. Centralbl., 33, 1892, p. 372.

WARBURG, O. Die Muskatnuss, ihre Geschichte, Botanik, Kultur, u. s. w. Leipzig, 1897.

Weigle, T. Untersuchungen über die Zusammensetzung des Pfeffers. Ber. Pharm. Ges., 1893 , p. 210.

Windisch, R. Beiträge zur Kenntnis des Aschengehaltes des Paprika. Zeits, Unters. Nahr. Genuss., I3, I907, p. 389 .

Winton, A. L. Spices, U. S. Dept. of Agric., Bur. of Chem., Bul. 65, 1902.

- Microscopy of Vegetable Foods. New York, Igo6.

Conn. Exp. Sta. An. Rep. 1896 , et seq.

Mass. State Board of Health An. Reports, $188_{3}$, et seq.

U. S. Dept. of Agric., Bur. of Chem., Bul. 107 (rev.), p. 162 


\section{CHAPTER XIII.}

\section{EDIBLE OILS AND FATS.}

Nature and Properties.-The oils and fats are the glycerin salts or glycerides of the fatty acids, the most important, on account of their occurrence in nearly all fats and oils, being the triglycerides of oleic, palmitic, and stearic acids, known as olein, palmitin, and stearin, respectively.

Fats and oils are insoluble in water, and are almost insoluble in cold $95 \%$ alcohol, thonigh they are somewhat soluble in absolute alcohol. They are readily soluble in ether, petroleum ether, chloroform, amyl alcohol, oil of turpentine, and carbon bisulphide.

Following is a list of the fatty acids whose glycerides are found in edible oils and fats, together with their melting- and boiling-points when these have been determined, and the oils and fats in which they occur.

ACIDS OF THE ACETIC SERIES $\mathrm{C}_{n} \mathrm{H}_{2 n} \mathrm{O}_{2} *$

\begin{tabular}{|c|c|c|c|c|}
\hline Name. & Formula. & $\begin{array}{l}\text { Melting- } \\
\text { point. }\end{array}$ & $\begin{array}{l}\text { Boiling- } \\
\text { point. }\end{array}$ & Occurrence in Oils and Pats. \\
\hline Butyrict.. & $\mathrm{C}_{4} \mathrm{H}_{8} \mathrm{O}_{2}$ & $-6.5^{\circ}$ & $162 \cdot 3$ & Butter, cocoa butter. \\
\hline Caproict..... & $\mathrm{C}_{0} \mathrm{H}_{12} \mathrm{O}_{2}^{2}$ & & 200 & Butter, cocoanut oil. \\
\hline Caprylicł...... & $\mathrm{C}_{8} \mathrm{H}_{10} \mathrm{O}_{2}$ & 16.5 & 236 & \\
\hline Caprict. ...... & $\mathrm{C}_{10} \mathrm{H}_{20} \mathrm{O}_{2}$ & $3^{\mathrm{I}} \cdot 3$ & $268-270$ & " " " \\
\hline Lauric. ....... & $\mathrm{C}_{12} \mathrm{H}_{24} \mathrm{O}_{2}$ & 43.6 & $176^{\circ}$ & Cocoanut oil, cocoa butter. \\
\hline Myristic...... & $\mathrm{C}_{14} \mathrm{H}_{28} \mathrm{O}_{2}$ & 53.8 & 196.5 & _. sesame oil. \\
\hline Palmitic..... & $\mathrm{C}_{10} \mathrm{H}_{32} \mathrm{O}_{2}$ & 62.6 & 215 & Nearly all oils and fats. \\
\hline & $\mathrm{C}_{18} \mathrm{H}_{30} \mathrm{O}_{2}$ & $69 \cdot 3$ & 232.5 & $\begin{array}{l}\text { Fats and nearly all oils, except } \\
\text { olive and corn. }\end{array}$ \\
\hline $\begin{array}{l}\text { Arachidic. ..... } \\
\text { Behenic. ..... }\end{array}$ & $\mathrm{C}_{20} \mathrm{H}_{40} \mathrm{O}_{2}$ & & $\cdots$ & Peanut, olive (trace), rape (trace). \\
\hline Lignoceric. ... & $\begin{array}{l}\mathrm{C}_{22} \mathrm{H}_{44} \mathrm{O}_{2} \\
\mathrm{C}_{24} \mathrm{H}_{43} \mathrm{O}_{2}\end{array}$ & $\begin{array}{l}83^{-8} \\
8 \mathrm{r}\end{array}$ & $\cdots$ & $\begin{array}{l}\text { Rape, mustard. } \\
\text { Peanut. }\end{array}$ \\
\hline & & & $\cdots$ & \\
\hline
\end{tabular}

- Lewkowitsch, Oils, Fats, and Waxes, 3 d ed. (r904), pp. $63-7 \mathrm{r}$.

† These four acids are the only ones that can be distilled under ordinary pressure without becoming decomposed. 
ACIDS OF THE OLEIC SERIES $\mathrm{C}_{n} \mathrm{H}_{2 n-2} \mathrm{O}$.

\begin{tabular}{|c|c|c|c|c|}
\hline Name. & Formula. & $\begin{array}{l}\text { Melting- } \\
\text { point. }\end{array}$ & $\begin{array}{l}\text { Boiling- } \\
\text { point. }\end{array}$ & Occurrence in Oils and Pats. \\
\hline $\begin{array}{l}\text { Hypogzeic..... } \\
\text { Oleic. . ...... } \\
\text { Iso-oleic* .... } \\
\text { Rapic. ........ } \\
\text { Erucic. ....... }\end{array}$ & $\begin{array}{l}\mathrm{C}_{10} \mathrm{H}_{30} \mathrm{O}_{2} \\
\mathrm{C}_{15} \mathrm{H}_{34} \mathrm{O}_{2} \\
\mathrm{C}_{15} \mathrm{H}_{34} \mathrm{O}_{2} \\
\mathrm{C}_{18} \mathrm{H}_{34} \mathrm{O}_{2} \\
\mathrm{C}_{22} \mathrm{H}_{42} \mathrm{O}_{2}\end{array}$ & $\begin{array}{l}33^{\circ} \\
14^{\circ} \\
44^{-4}-45^{\circ} \\
\cdots \cdots \\
33-34^{\circ}\end{array}$ & $\begin{array}{l}23^{\circ} \\
23^{2} \cdot 5^{\circ} \\
\cdots \\
\cdots \ddot{*}^{\circ}\end{array}$ & $\begin{array}{l}\text { Peanut. } \\
\text { Nearly all fats and oils. } \\
\text { Rape and mustard. }\end{array}$ \\
\hline
\end{tabular}

ACIDS OF THE LINOLEIC SERIES $\mathrm{C}_{n} \mathrm{H}_{2 n-4} \mathrm{O}_{2}$.

\begin{tabular}{c|c|c|c|c}
\hline Name. & Formula. & $\begin{array}{c}\text { Melting- } \\
\text { point. }\end{array}$ & $\begin{array}{c}\text { Boiling- } \\
\text { point. }\end{array}$ & Occurrence in Oils and Pats. \\
\hline Linoleic...... & $\mathrm{C}_{13} \mathrm{H}_{22} \mathrm{O}_{2}$ & Under $-18^{\circ} \mathrm{C} . \ldots \ldots \ldots \ldots$ & $\begin{array}{c}\text { Olive, cottonseed, peanut, sesame, } \\
\text { cocoa butter, poppy seed, sun- } \\
\text { flower. }\end{array}$ \\
\hline
\end{tabular}

* Solid oleic acid.

Saponification of Fats and Oils.-By this term is meant the decomposition of the glycerides composing the fats or oils, whereby the triatomic alcohol glycerin and the fatty acids are separated. The saponification process is commonly applied in carrying out many determinations of value on fats and oils, such as those of the soluble and insoluble fatty acids, the Reichert value, etc. As commonly carried out, the triglycerides are first split up into glycerin and the soluble soaps of the fatty acids by the action of caustic alkali, usually in solution in alcohol. This part of the process in the case of a given oil, composed, for example, of stearin, olein, and palmitin, is illustrated as follows:

$$
\begin{aligned}
& \text { (I) } \mathrm{C}_{3} \mathrm{H}_{5}\left(\mathrm{C}_{18} \mathrm{H}_{35} \mathrm{O}_{2}\right)_{3}+{ }_{3} \mathrm{KOH}=\mathrm{C}_{3} \mathrm{H}_{5}(\mathrm{OH})_{3}+{ }_{3} \mathrm{~K}\left(\mathrm{C}_{18} \mathrm{H}_{35} \mathrm{O}_{2}\right) \\
& \text { Stearin or } \quad \text { Glycerin Potassium } \\
& \text { triglyceryl } \\
& \text { (2) } \mathrm{C}_{3} \mathrm{H}_{5}\left(\mathrm{C}_{16} \mathrm{H}_{31} \mathrm{O}_{2}\right)_{3}+3 \mathrm{KOH}=\mathrm{C}_{3} \mathrm{H}_{5}(\mathrm{OH})_{3}+{ }_{3} \mathrm{~K}\left(\mathrm{C}_{16} \mathrm{H}_{31} \mathrm{O}_{2}\right) \\
& \begin{array}{ll}
\begin{array}{l}
\text { Palmitin or } \\
\text { triglyceryl }
\end{array} & \text { Potassium } \\
\text { palmitate } &
\end{array}
\end{aligned}
$$

These "soaps," or potassium salts of the fatty acids, are further ciecomposed by the action of sulphuric acid into the free fatty acids and fe' assium sulphate, in the case of potassium stearate, as follows:

$$
\underset{\text { Potassium stearate }}{2 \mathrm{~K}}\left(\mathrm{C}_{18} \mathrm{H}_{35} \mathrm{O}_{2}\right)+\mathrm{H}_{2} \mathrm{SO}_{4}=\mathrm{K}_{2} \mathrm{SO}_{4}+\underset{2}{\mathrm{H}}\left(\mathrm{C}_{18} \mathrm{H}_{35} \mathrm{O}_{2}\right)
$$




\section{ANALYSIS OF EDIBLE OILS AND FATS.}

No class of food products presents more difficulties to the analyst than the fats and oils, in that the various physical and chemical constants by which one derives information as to their nature or purity differ within such wide limits that it is not easy to prescribe absolute standards. Many elements enter in to cause this variation, chief among which are, in vegetable oils, the large number of varieties of fruits or seeds from which each oil is in different localities obtained, as well as the valious grades of each oil with respect to refining. In the animal fats, butter and lard, the kind of food fed to the animal undoubtedly influences the constants of the fat, and in all fats and oils much depends upon their age, and the conditions under which they are kept as to temperature, exposure to moisture, light and air, etc.

Rancidity should not be confounded with acidity, although rancid oils usually are high in acids. Lewkowitsch holds that fatty acids are liberated by the action of moisture in the presence of enzymes. If in addition the oil is exposed to air and light, the fatty acids are acted on causing rancidity, which is detected by taste and smell, although chemically little understood. As a rule rancidity develops more. readily in liquid oils in which olein predominates than in solid fats. To avoid changes samples should be kept in a dark, cool place in tight containers.

Judgment as to Purity of a given oil or fat should not be hastily given. It is sometimes comparatively easy to prove the presence and approximate amount of an adulterant, the various constants all serving to identify it without fail. Again, in some cases it is easy to pronounce the sample adulterated, without being able to definitely state the exact nature of the adulterant. The tests to be employed depend on the particular case in hand. Sometimes the determination of two or three constants will be sufficiently definite.

Again, a large number of tests must be made before one can intelligently form an opinion. It should be borne in mind that skilful manufacturers may adulterate the edible oils and fats with mixtures intended to confuse the chemist, and yield on analysis constants that are entirely misleading.

Much information may usually be gained by carefully noting the color, taste, odor, and appearance of the sample.

Filtering, Measuring, and Weighing of Fats.-These manipulations naturally present some difficulties in the case of solid fats not encountered with liquid oils. 
A steam- or hot-water-jacketed funnel as represented in Fig. $9^{2}$ is convenient for filtering fats, or, in the absence of this contrivance for keeping the fat in a molten condition, a hot funnel may be employed, the filtering being best conducted in a warm closet or oven.

Portions of the fat for the various determinations may be measured off with a pipette while the fat is still hot, but a much better way is to

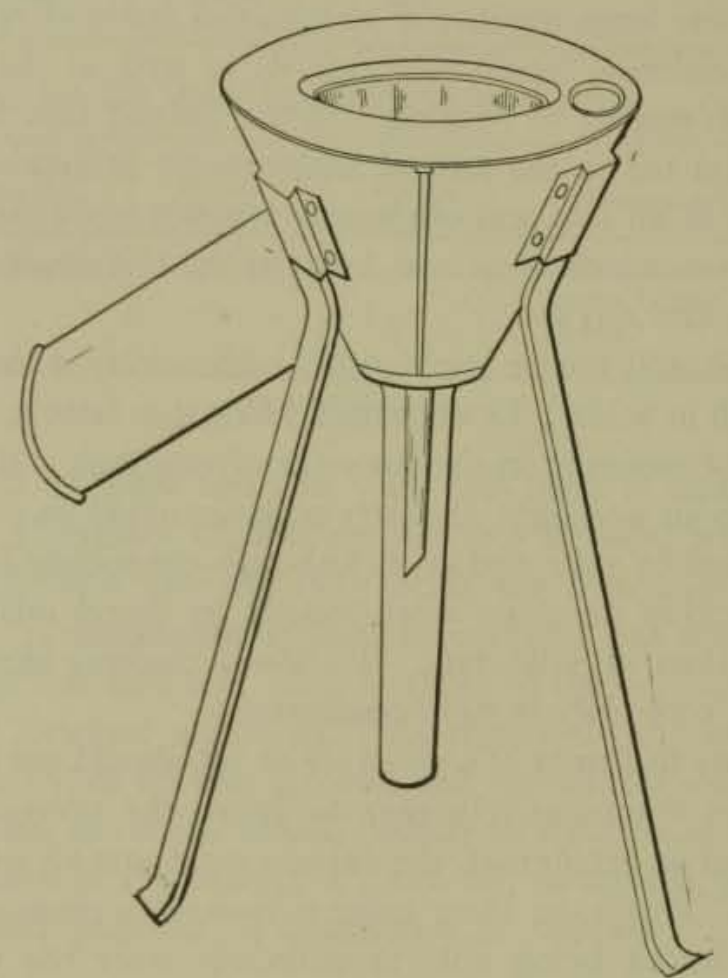

FIG. 92,-Jacketed Funnel for Hot Filtration.

cool the fat (over ice if necessary), and to weigh the desired amounts in the solid state. This can very readily be done by placing a flat platinum or other dish on the scale-pan, covering it with a moderately thick, cut filter-paper somewhat larger in diameter than the dish and designed to lie flat upon it, and taking the tare of both dish and filter. The solidified fat, after mixing with a stirring-rod, is transferred in one or more portions to the middle of the filter, and the exact weighed amount is obtained, after which, by carefully handling the edges of the filter and folding in the latter, the fat with the filter may be transferred to a flask or other receptacle.

Specific Gravity.-The specific gravity of liquid oils is most conveniently taken either at room temperature or at $15.5^{\circ}$, being always 
best referred to the latter. Either the hydrometer, Westphal balance, Sprengel tube, or pycnometer are employed, according to the degree of accuracy required. If taken at any other temperature than $15.5^{\circ}$, say at room temperature, $T$, the specific gravity may be computed at $15.5^{\circ}$ by the formula

$$
G=G^{\prime}+K(T-15 \cdot 5), *
$$

in which $G$ is the specific gravity at $15.5^{\circ}, G^{\prime}$ the specific gravity at $T^{\circ}$, and $K$ a factor varying with the different oils as follows:

FACTORS FOR CALCULATING SPECIFIC GRAVITY.

\begin{tabular}{|c|c|c|}
\hline Oil. & $\begin{array}{l}\text { Correction } \\
\text { for } 1^{\circ} \mathrm{C} \text {. }\end{array}$ & Observer. \\
\hline $\begin{array}{l}\text { Cod-liver oil. } \ldots \ldots \ldots \ldots \ldots \ldots \\
\text { Lard oil. } \ldots \ldots \ldots \ldots \ldots \ldots \ldots \\
\text { Olive oil. } \ldots \ldots \ldots \ldots \ldots \ldots \\
\text { Peanut oil. } \ldots \ldots \ldots \ldots \ldots \ldots \\
\text { Rape oil. } \ldots \ldots \ldots \ldots \ldots \ldots \ldots \\
\text { Sesame oil. } \ldots \ldots \ldots \ldots \ldots \ldots \\
\text { Cottonseed oil. } \ldots \ldots \ldots \ldots \ldots \ldots\end{array}$ & $\begin{array}{r}0.000646 \\
.000658 \\
.000629 \\
.000655 \\
.000620 \\
.000624 \\
.000629\end{array}$ & $\begin{array}{l}\text { A. H. Allen } \\
\text { C. M. Wetherill } \\
\text { C. M. Stillwell } \\
\text { A. H. Allen } \\
\text { " } \\
\text { " }\end{array}$ \\
\hline
\end{tabular}

Unless the most accurate work is necessary, it is sufficient to assume in all cases $K=0.00064$, in which case the formula becomes $G=G^{\prime}+$ $0.00064(T-15.5)$.

In the case of solid fats, it is most convenient to take the specific gravity of the melted fat. This may be done at any temperature above the melting-point by either of the instruments above described, or at the temperature of boiling water by the Westphal balance or pycnometer.

When the pycnometer is used, it is immersed in a water-bath, the temperature of which is well above the melting-point of the fat, say $35^{\circ}$ or $40^{\circ}$. While still immersed nearly to the neck, it is carefully filled with the melted fat and kept in the bath till the fat has acquired the same temperature, usually about fifteen minutes. If the pycnometer is provided with a thermometer stopper, this will serve to indicate the temperature; otherwise a separate thermometer is inserted in the bath. The pycnometer is then removed, cleaned, dried, and cooled to the room temperature, at which it is weighed. The factors employed in the above formula for calculation of specific gravity of solid fats at $15.5^{\circ}$ are as follows:

* Allen, Com. Org. Anal., 4 Ed.; Vol. II, p. 49. 
FACTORS FOR CALCULATING SPECIFIC GRAVITY.

\begin{tabular}{|c|c|}
\hline Fats. & $\begin{array}{l}\text { Correction } \\
\text { for t }{ }^{\circ} \mathrm{C} \text {. }\end{array}$ \\
\hline 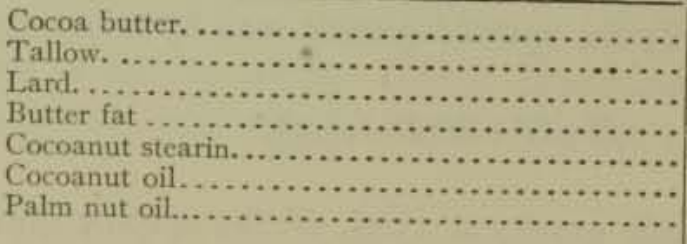 & $\begin{array}{r}0.000717 \\
.000675 \\
.000650 \\
.000617 \\
.000674 \\
.000642 \\
.000657\end{array}$ \\
\hline
\end{tabular}

Either the Westphal balance or the hydrometer may be used directly on the melted fat, carefully recording the temperature and calculating as above.

For making the determination with the Westphal balance at the temperature of boiling water, the melted fat is contained in a vessel immersed in a boiling water-bath, and kept sufficiently long to acquire that temperature, which is carefully noted.

A. O. A. C. Method.*-The pycnometer, being perfectly clean, is first weighed with the stopper, after which it is filled with freshly boiled, hot, distilled water and placed in a bath of boiling water, where it is kept for half an hour, replacing any loss by evaporation in the flask with boiling distilled water. The stopper of the pycnometer, previously heated at $100^{\circ}$, is then inserted, and the flask removed and wiped perfectly dry. It is then allowed to cool nearly to room temperature, and weighed on the balance when the temperature is the same as that of the room.

The flask, being again perfectly clean and dry, is filled while hot with freshly melted and filtered hot fat, free from air-bubbles, and kept for half an hour in a boiling water-bath, after which the stopper, previously heated as before to $100^{\circ}$, is inserted, and the flask taken from the bath and wiped dry. It is then allowed to cool and weighed when the temperature of the room has been reached.

The specific gravity is calculated by dividing the weight of the fat by the weight of the water previously found.

Having once obtained the weight of the flask and the weight of a volume of water contained therein when at boiling temperature, these figures can be constantly used without redetermination, if the flask is cleaned thoroughly each time.

Calculation of Proportions of Two Known Oils in Mixture. $\dagger-$ This may be roughly accomplished from the specific gravity of the mixture and of the oils known to compose it.

*U. S. Dept. of Agric., Bur, of Chem., Bul. 65, p. 21.

t Villiers et Collin, Les Substances Alimentaires, p. 646 . 
Le: $G=$ specific gravity of mixture,

$D$ and $D^{\prime}=$ specific gravity of the two oils, and $X=\%$ oil of specific gravity $D$.

Then $X=\frac{100\left(G-D^{\prime}\right)}{D-D^{\prime}}$.

EDIBLE OILS AND FATS ARRANGED IN ORDER OF SPECIFIC GRAVITY.

\begin{tabular}{|c|c|}
\hline & Specifie Gravity. \\
\hline 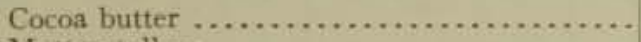 & .976 to .950 \\
\hline 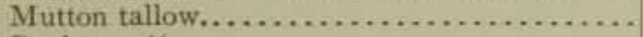 & $.953^{\prime \prime} .937$ \\
\hline Beef $\quad$ " $\quad \ldots \ldots \ldots \ldots \ldots \ldots \ldots \ldots \ldots \ldots \ldots \ldots \ldots \ldots \ldots$ & $.952 \div .943$ \\
\hline 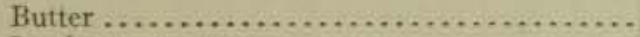 & $.940 \% .926$ \\
\hline 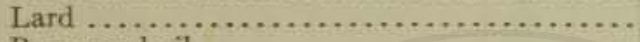 & $-93^{8 *}-934$ \\
\hline 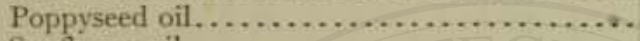 & $.927 \% .924$ \\
\hline 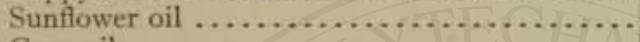 & $.926 \cdots .924$ \\
\hline 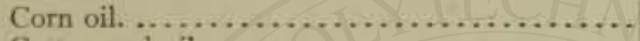 & $.926^{\prime \prime} .921$ \\
\hline 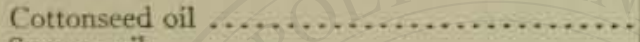 & $.925^{4 .} .922$ \\
\hline 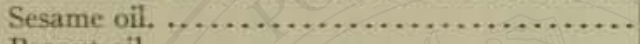 & $.924^{\circ} \times-923$ \\
\hline 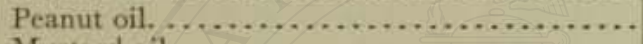 & $.921 \% .917$ \\
\hline 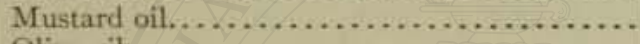 & $.920 \% .916$ \\
\hline Olive oil......................... & $.918 \% .916$ \\
\hline 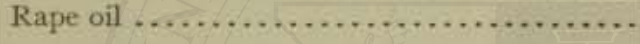 & $.917^{\prime \prime}-913$ \\
\hline
\end{tabular}

The Viscosity, or degree of fluidity in the case of edible oils, is of less importance than in the case of lubricating oils, and gives little insight into the nature or purity of the sample.

Hence a discussion of various viscosimeters and their use will not be included here, but reference is made to Lewkowitsch * for information regarding them.

The Refractive Index, and the reading on the arbitrary scale of the butyro-refractometer, express in two different and interchangeable terms the refraction value, a useful constant of fats and oils and easily determined.

For the routine examination of fats and oils the butyro-refractometer is more convenient than the Abbé refractometer, and the readings obtained by the former instrument are less cumbersome than refractive indices.

These instruments and details with regard to their manipulation are described in Chapter VI.

The readings on the scale of the butyro-refractometer may be readily transformed into refractive indexes and vice versa by table or by means of the Leach and Lythgoe slide rule (page 107). Lythgoe'st table on pp. 478 and 479 is useful as showing readings on the butyro-refractometer of all the edible oils and fats at various temperatures.

* Chemical Analysis of Oils and Fats, 3d ed., 1904, pp. 197-209.

$\dagger$ Tech. Quarterly, 16, 1903, p. 222. 
CALCULATED READINGS ON BUTYRO-REFRACTOMETER OF EDIBLE OILS AND FATS.

\begin{tabular}{|c|c|c|c|c|c|c|c|c|c|}
\hline $\begin{array}{c}\text { Temp. } \\
\text { C. }\end{array}$ & $\begin{array}{c}\text { Cocoanut } \\
\text { Oil. }\end{array}$ & Butter.* & $\begin{array}{c}\text { Bref } \\
\text { Stearin. }\end{array}$ & $\begin{array}{c}\text { Cacao } \\
\text { Butter. }\end{array}$ & $\begin{array}{c}\text { Baef } \\
\text { Tallow. }\end{array}$ & $\begin{array}{c}\text { Lard } \\
\text { Stearin. }\end{array}$ & $\begin{array}{l}\text { Beef } \\
\text { Óleo. }\end{array}$ & Lard. $†$ & $\begin{array}{l}\text { Lard } \\
\text { Oil. }\end{array}$ \\
\hline 45.0 & 31.6 & $41 \cdot 5$ & 41.9 & $43 \cdot 7$ & $44 \cdot 1$ & 44.9 & 45.0 & $4^{8.2}$ & \\
\hline $44 \cdot 5$ & $3 \mathrm{x}-9$ & 41.8 & 42.2 & 44.0 & 44.3 & $45 \cdot I$ & $45 \cdot 3$ & 48.4 & \\
\hline 44.0 & 32.2 & 42.0 & $4^{2} \cdot 4$ & 44.2 & 44.6 & $45 \cdot 5$ & 45.6 & $4^{8.7}$ & \\
\hline $43 \cdot 5$ & $32 \cdot 4$ & $42 \cdot 3$ & $4^{2} .6$ & $44 \cdot 5$ & 44.8 & $45 \cdot 7$ & 45.9 & 49.0 & \\
\hline 43.0 & 32.7 & 42.6 & $4^{2} \cdot 9$ & 44.8 & $45 \cdot \mathrm{I}$ & 46.0 & $46 . I$ & $49 \cdot 3$ & \\
\hline $42 \cdot 5$ & $5^{2} \cdot 9$ & 42.9 & $43 \cdot 2$ & 45.0 & $45 \cdot 4$ & $46 \cdot 3$ & $4^{6.4}$ & 49.6 & \\
\hline 42.0 & 33.2 & $43 . I$ & $43 \cdot 5$ & $45 \cdot 3$ & 45.6 & $46 \cdot 5$ & 46.7 & 49.9 & \\
\hline $4 \mathrm{I} \cdot 5$ & $33 \cdot 5$ & 43.4 & $43 \cdot 7$ & 45.6 & 45.8 & 46.8 & 47.0 & 50.1 & \\
\hline 41.0 & $33-7$ & $43 \cdot 7$ & 44.0 & 45.9 & 46.1 & 47.0 & $47 \cdot 3$ & 50.4 & \\
\hline 40.5 & 34.0 & 43.9 & $44 \cdot 2$ & 46.1 & 46.3 & $47 \cdot 3$ & 47.6 & 50.7 & \\
\hline 40.0 & $34 \cdot 3$ & $44 \cdot 2$ & $44 \cdot 3$ & 46.4 & 46.6 & $47 \cdot 6$ & 47.8 & 51.0 & $5^{1} .6$ \\
\hline $39 \cdot 5$ & $34 \cdot 5$ & 44.5 & & 46.6 & 46.8 & 47.8 & 48.1 & $5 \mathrm{r} \cdot 3$ & 51.8 \\
\hline 39.0 & 34.8 & 44.8 & ........ & 46.8 & $47 \cdot 1$ & 48.1 & 48.4 & 51.6 & 52,1 \\
\hline $3^{8} .5$ & 35.0 & 45.0 & -........ & $47 \cdot 1$ & $47 \cdot 4$ & 48.4 & $4^{8} \cdot 7$ & 51.9 & 52.4 \\
\hline $3^{8} .0$ & $35 \cdot 3$ & 45.3 & ......... & $47 \cdot 4$ & 47.6 & 48.6 & 48.9 & 52.1 & 52.6 \\
\hline $37 \cdot 5$ & $35 \cdot 5$ & 45.6 & $\ldots \ldots \ldots$ & 47.6 & 47.8 & $4^{8} \cdot 9$ & 49.2 & $5^{2} \cdot 4$ & 52.8 \\
\hline 37.0 & 35.8 & 45.9 & $\ldots \ldots \ldots$ & $47 \cdot 9$ & $48 . \mathrm{I}$ & $49 \cdot 2$ & 49.5 & 52.7 & $53 . \mathrm{I}$ \\
\hline 36.5 & 36.1 & 46.1 & ......... & $4^{8.2}$ & $4^{8} \cdot 3$ & $49 \cdot 4$ & 49.8 & 53.0 & 53.4 \\
\hline 36.0 & 36.3 & 46.4 & $\ldots \ldots \ldots$ & $4^{8.5}$ & 48.6 & $49 \cdot 7$ & 50.0 & $53 \cdot 3$ & $53 \cdot 7$ \\
\hline $35 \cdot 5$ & 36.6 & 46.7 & $\ldots \ldots \ldots$ & $4^{8} .7$ & 48.8 & 50.0 & 50.3 & 53.6 & 54.0 \\
\hline 35.0 & $3^{6.9}$ & $47 \cdot 0$ & $\cdots \ldots \ldots$ & 49.0 & 49.1 & 50.2 & 50.6 & 53.9 & 54.2 \\
\hline $34-5$ & $37-1$ & $47 \cdot 2$ & & $\ldots$ & ...... & $\ldots \ldots \ldots$ & 50.9 & $54 \cdot 2$ & 54.5 \\
\hline 34.0 & $37 \cdot 4$ & $47 \cdot 5$ & $\ldots \ldots \ldots$ & $\ldots \ldots$ & $\ldots$. & $\ldots \ldots \ldots$ & $5 x \cdot 2$ & $54 \cdot 4$ & $54 \cdot 7$ \\
\hline $33 \cdot 5$ & 37.6 & 47.8 & $\ldots \ldots \ldots$ & $\ldots \ldots .$. & $\ldots \ldots$ & .......... & $5 \mathrm{t} .5$ & $54 \cdot 7$ & 55.0 \\
\hline 33.0 & $37 \cdot 9$ & 48.1 & $\ldots \ldots$. & $\ldots \ldots \ldots$ & $\ldots .$. & $\cdots, \cdots$ & 51.7 & 55.0 & $55 \cdot 3$ \\
\hline 325 & $3^{8.1}$ & $4^{8} \cdot 3$ & $\ldots \ldots \ldots$ & $\ldots \ldots \ldots$ & $\ldots \ldots$ & $\ldots \ldots$ & 52.0 & $55 \cdot 3$ & 55.6 \\
\hline 32.0 & 38.4 & 48.6 & $\ldots$. & $\ldots \ldots \ldots$ & $\ldots \ldots$ & $\ldots \ldots$ & $52 \cdot 3$ & 55.6 & 55.9 \\
\hline $3^{1} \cdot 5$ & 38.6 & 48.9 & $\ldots . . .$. & $\cdots \cdots$ & , . . . & $\ldots \ldots$ & 52.6 & 55.9 & 56.1 \\
\hline $3^{1} \cdot 0$ & $3^{8.9}$ & $49 \cdot 2$ & $\ldots \ldots \ldots$ & $\ldots \ldots . .$. & $\ldots \ldots$ & $\ldots \ldots \ldots$ & 52.8 & 56.1 & 56.4 \\
\hline 30.5 & $39 \cdot 2$ & $49 \cdot 5$ & $\ldots \ldots$ & $\ldots \ldots \ldots$ & $\cdots \cdots$ & $\cdots \cdots \cdots$ & 53.1 & 56.4 & 56.7 \\
\hline 30.0 & $39 \cdot 5$ & 49.8 & & $\ldots \ldots \ldots$ & $\ldots \ldots$ & $\cdots \cdots \cdots$ & 53.4 & 56.7 & 57.0 \\
\hline 29.5 & $39 \cdot 7$ & 50.0 & $\ldots \ldots \ldots$ & $\cdots \cdots \cdots$ & $\ldots \ldots \ldots$ & $\ldots \ldots \ldots$ & $53 \cdot 7$ & 57.0 & 57.2 \\
\hline 29.0 & 40.0 & $5^{0} \cdot 3$ & $\ldots \ldots \ldots$ & $\ldots \ldots \ldots$ & $\ldots \ldots \ldots$ & $\ldots \ldots \ldots$ & 53.9 & $57 \cdot 3$ & $57 \cdot 5$ \\
\hline 28.5 & 40.3 & 50.5 & ......... & $\ldots \ldots \ldots$ & $\ldots \ldots \ldots$ & $\ldots \ldots \ldots$ & 54.1 & 57.6 & 57.8 \\
\hline 28.0 & 40.5 & 50.8 & ........ & $\ldots \ldots \ldots$ & $\ldots \ldots \ldots$ & $\ldots \ldots \ldots$ & $54 \cdot 4$ & 57.8 & 58.1 \\
\hline $27 \cdot 5$ & 40.8 & $51 \cdot x$ & & $\ldots \ldots \ldots$ & n. $\ldots \ldots$ & $\ldots \ldots \ldots$ & $54 \cdot 7$ & $58 . x$ & $5^{8} .3$ \\
\hline 27.0 & 41.0 & $51 \cdot 4$ & . $\ldots \ldots \ldots$ & $\ldots \ldots \ldots$ & …….. & $\ldots \ldots$ & 55.0 & $5^{8} .4$ & $5^{8.6}$ \\
\hline 26.5 & $4 \mathrm{~T} \cdot 3$ & 51.6 & & $\ldots \ldots \ldots$ & $\ldots \ldots \ldots$ & $\ldots \ldots \ldots$ & 55.2 & 58.7 & 58.9 \\
\hline 26.0 & $4 I \cdot 5$ & 51.9 & ......... & $\ldots \ldots \ldots$ & $\ldots \ldots$ & $\cdots \ldots \ldots$ & $55 \cdot 5$ & 59.0 & 59.2 \\
\hline $25 \cdot 5$ & 41.8 & $5^{2} \cdot 2$ & ......... & $\ldots \ldots \ldots$ & $\ldots \ldots$ & $\ldots \ldots \ldots$ & 65.8 & $59 \cdot 3$ & 59.5 \\
\hline 25.0 & 42.0 & $5^{2} \cdot 5$ & ........ & $\ldots \ldots \ldots$ & & $\ldots \ldots \ldots$ & $66 . \mathrm{I}$ & 59.6 & 59.8 \\
\hline
\end{tabular}

* Butter readings by Zeiss.

† Lard readings by Hefelmann. 
CALCUL.ATED READINGS-(Continued).

\begin{tabular}{|c|c|c|c|c|c|c|c|c|c|c|}
\hline $\begin{array}{l}\text { Temp. } \\
\text { C. }\end{array}$ & $\begin{array}{l}\text { Olive } \\
\text { Oil. }\end{array}$ & $\begin{array}{l}\text { Peanut } \\
\text { Oil. }\end{array}$ & $\begin{array}{l}\text { Cotton- } \\
\text { seed } \\
\text { Oil. }\end{array}$ & $\begin{array}{l}\text { Rape- } \\
\text { sedd } \\
\text { Oil. }\end{array}$ & $\begin{array}{l}\text { Sesame } \\
\text { Oil. }\end{array}$ & $\begin{array}{l}\text { Yellow } \\
\text { Mustard } \\
\text { Oil. }\end{array}$ & $\begin{array}{c}\text { Black } \\
\text { Mnistard } \\
\text { Oil. }\end{array}$ & $\begin{array}{c}\text { Sun- } \\
\text { flower } \\
\text { Oil. }\end{array}$ & $\begin{array}{l}\text { Corn } \\
\text { Oil. }\end{array}$ & $\begin{array}{l}\text { Poppy- } \\
\text { seed } \\
\text { Oil. }\end{array}$ \\
\hline 35.0 & 57.0 & 59.8 & 61.8 & $62 . t$ & 62.3 & $6_{3} .0$ & 64.2 & $64 \cdot 5$ & 65.0 & $C_{5.5}$ \\
\hline $34 \cdot 5$ & 57.2 & 60.0 & 62.1 & & & $63 \cdot 3$ & 64.5 & 64.8 & 65.3 & 5.8 \\
\hline & & & & & & & & 65.1 & 65.6 & 6.1 \\
\hline & 57.7 & & $\begin{array}{l}62.5 \\
62.8\end{array}$ & $\begin{array}{l}83.0 \\
63.3\end{array}$ & $\begin{array}{l}63-1 \\
63-4\end{array}$ & -9 & $\begin{array}{l}65 \cdot 1 \\
65.3\end{array}$ & $\begin{array}{l}65.4 \\
65.4\end{array}$ & $\begin{array}{l}65.9 \\
66.2\end{array}$ & 6.4 \\
\hline $\begin{array}{l}33.0 \\
32.5\end{array}$ & 58.3 & 61.1 & $6_{3} .0$ & 63.6 & 63.7 & 64.4 & 65.6 & 66.0 & 66.5 & 67.0 \\
\hline 32.0 & $5^{8} .5$ & 61.4 & 63.2 & 63.8 & 64.0 & .7 & 9 & 66. & 66.8 & 7.3 \\
\hline $3 \mathrm{I} \cdot 5$ & & & & 64.1 & & & & & 67.1 & 67.6 \\
\hline 31.0 & 59.2 & 62.0 & 6 & $4 \cdot 4$ & 64.6 & $5 \cdot 3$ & 66.5 & 66. & 67.4 & 67.9 \\
\hline 30.5 & $59 \cdot 5$ & 62.2 & 64.2 & $4 \cdot 7$ & 64.9 & 65.6 & 66.8 & 67.2 & $67 \cdot 7$ & 68.2 \\
\hline 30.0 & 59.9 & 62.5 & $64 \cdot 5$ & 65.0 & 65.1 & 65.8 & 67.0 & $67-5$ & 68.0 & 68.5 \\
\hline 29.5 & 60.1 & 62.8 & 64.9 & $65 \cdot 3$ & 65.4 & 6.1 & 67.3 & 47. & 68.2 & 68.7 \\
\hline & & & & & & & 67.6 & & 68.5 & 9.0 \\
\hline 28.5 & 60.6 & 63.3 & 65.3 & .9 & 0 & & 67.9 & 68. & 68.8 & $9 \cdot 3$ \\
\hline 28.0 & 60. & 6 & 5,7 & 66.1 & 5.2 & 6.9 & 68.1 & 68.6 & 69.1 & 69.6 \\
\hline $27 \cdot 5$ & 61.1 & 63.9 & 66.0 & 66.4 & 66.5 & 67.2 & 68.4 & 68.9 & 69.4 & 69.9 \\
\hline 27.0 & $6 \mathrm{x} \cdot 5$ & $64 \cdot 2$ & 5.5 & & 5.8 & $7 \cdot 5$ & 68.7 & $69 \cdot 2$ & 69.7 & 0.2 \\
\hline & 62. & & c & 7.0 & .1 & 7.8 & 69. & 69. & 70.0 & 70.5 \\
\hline 26.0 & & & & & .0 & & 6 & 60 & 70.3 & 0.8 \\
\hline $25 \cdot 5$ & 62.4 & 65.0 & $7 \cdot 5$ & 7.6 & $7 \cdot 7$ & 8.3 & 69.5 & 70.1 & 70.6 & 71.1 \\
\hline 25.0 & 63.0 & $65 \cdot 3$ & 67.9 & 67.8 & 67.9 & 68.6 & 69.8 & 70.4 & 70.9 & $7^{1} \cdot 4$ \\
\hline $24 \cdot 5$ & 6 & & & & & & & & 71.2 & $7^{11} \cdot 7$ \\
\hline 24.0 & 63. & 65.8 & & & & 2 & 70.4 & 71.0 & $71 \cdot 5$ & 72.0 \\
\hline $23 \cdot 5$ & & & & & 8.8 & & & & & \\
\hline 23.0 & 64.2 & 66.4 & 69.1 & 69.0 & 69.1 & $9 \cdot 7$ & 70.9 & 1. 6 & 72.1 & 72.6 \\
\hline 22.5 & $64 \cdot 5$ & 66.6 & $69-4$ & $69 \cdot 3$ & 69.4 & 70.0 & 71.2 & $71 \cdot 9$ & 72.4 & $7^{2} .9$ \\
\hline 22.0 & 64 & & & & & & & & & 73.2 \\
\hline 21.5 & 65. & 6 & & & & & 1.8 & & 73.0 & $3 \cdot 5$ \\
\hline & & & & & & & & & & 3.8 \\
\hline 20.5 & 65. & 67. & 7 & & & 2 & 7 & 73.1 & 73.6 & $74-\mathrm{r}$ \\
\hline 20.0 & 66.0 & 68.0 & 70.9 & 70.8 & 70.8 & 71.4 & 72.6 & 73.4 & 73.9 & 74.4 \\
\hline I9.5 & & & & & & & & & & 74.6 \\
\hline & & & & & & & 73. & & & 4.9 \\
\hline 18. & 66. & 68.8 & & & & & & 74.2 & & 5.2 \\
\hline 18.0 & & & 7 & 72.0 & 72.0 & 2.6 & & & 5.0 & $75 \cdot 5$ \\
\hline 17.5 & 67.5 & $69 \cdot 3$ & 72.4 & $72 \cdot 3$ & $72 \cdot 3$ & 72.9 & 74.1 & 74.8 & $75 \cdot 3$ & 75.8 \\
\hline 17.0 & 6 & 69. & & & & 7. & & 75 & & $76 \cdot x$ \\
\hline & & & & & & & & & & 6.4 \\
\hline 16.0 & & 70. & & & 7 & & $4-9$ & & & $7^{6 \cdot 7}$ \\
\hline I5. & & & & & & & & & 76.5 & 77.0 \\
\hline 15.0 & 68.9 & 70.8 & 73.8 & 73.8 & 73.7 & $74-3$ & $75 \cdot 5$ & $76 \cdot 3$ & 76.8 & 77.3 \\
\hline
\end{tabular}


Melting-point.-A piece of small glass tubing is drawn out to a capillary open at both ends, and this is inserted into a beaker of the fat, melted at a temperature slightly above its fusing-point. A portion of the melted fat being drawn up into the capillary, the latter is removed and the fat allowed to solidify spontaneously. After an interval of not less than twelve hours, the capillary is attached by a rubber band to the stem of a delicate thermometer (preferably capable of being read to tenths of a degree), the portion of solidified fat being opposite the thermometer bulb. A test-tube containing water is held in the neck of a flask in such a manner as to be immersed in water contained in the flask, as shown in Fig. 93, the flask being held on the ring of a stand, with wire gauge interposed between flask and flame. The thermometer with attached capillary is then held immersed in the water of the test-tube and below the

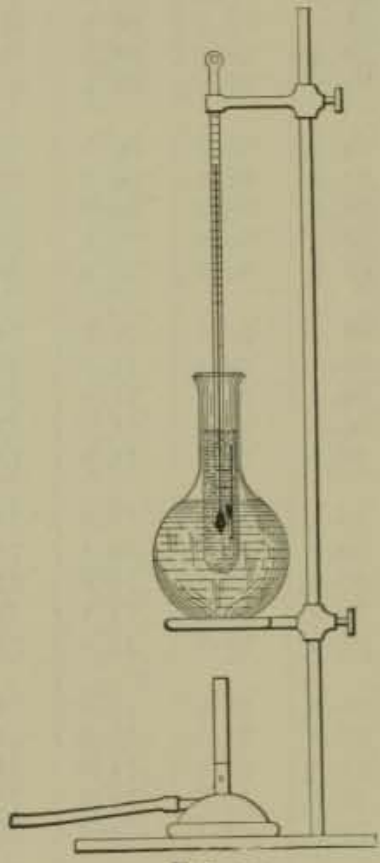

Fig. 93.

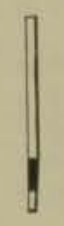

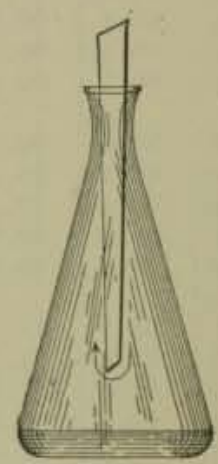

Fig. 94.

Frg. 93,-Apparatus for Determining Melting-point. Capillary tube with enclosed fat shown on the right, enlarged.

FIG. 94.- Reichert Flask with Card Inserted for Quick Evaporation.

level of the water in the flask, as shown. The water in the flask is then heated very gradually, so that the rise of temperature as shown by the thermometer does not exceed $0.5^{\circ} \mathrm{C}$. per minute, the exact temperature at which fusion of the fat occurs being recorded as the melting-point.

The flame is then removed, and the temperature at which the fat solidifies is noted as the solidifying-point. 
The mean of two or three determinations is usually taken as the true melting and solidifying-points.

Reichert-Meissl Process for Volatile Fatty Acids. - This process has undergone various modifications from time to time. Reichert originally used 2.5 grams of fat, but Meissl, who improved the process, used

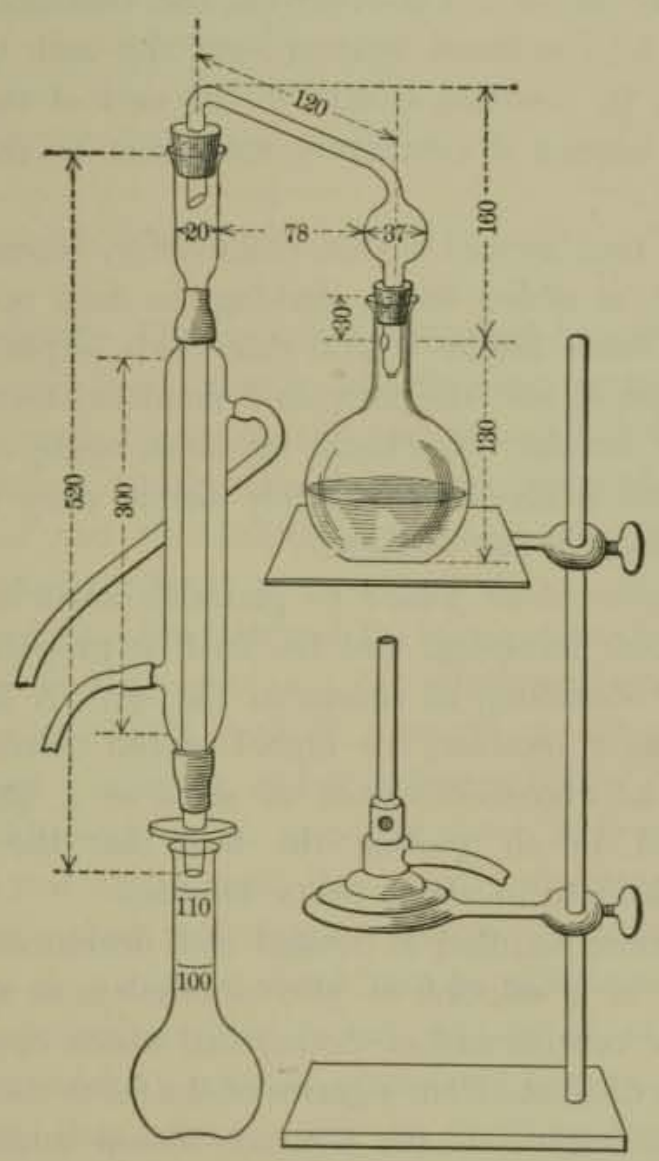

Fig. 95.-Apparatus for Reichert-Meissl and Polenske Distillation.

5 grams, so that the Reichert-Meissl number is now expressed on the basis of 5 grams of fat. The method is conveniently carried out as follows:

Five grams of the fat are transferred to a dry, clean Erlenmeyer flask of about $300 \mathrm{cc}$. capacity, Io cc. of $95 \%$ alcohol are added, and $2 \mathrm{cc}$. of sodium hydroxide solution (prepared by dissolving Ioo grams of sodium hydroxide in $100 \mathrm{cc}$. of water). The flask with its contents is then heated 
on a water-bath with a funnel in the neck, which satisfactorily replaces the return-flow condenser originally prescribed. The heating is continued with occasional shaking till saponification is complete. This stage of the process is indicated by the appearance of the solution, which is then perfectly clear and free from fat globules.

The condenser-funnel being removed, the contents of the flask are next evaporated by continued heating over the bath to dryness. This may be hastened by inserting a card in the neck of the flask, as shown in Fig. 94, thus starting a circulatory movement to the air through the flask.

The dry soap thus formed is then dissolved by warming on the waterbath with 135 cc. of added water, shaking the flask occasionally. After cooling, 5 cc. of dilute sulphuric acid (200 parts sulphuric acid in I liter of water) are added, and the fatty acid emulsion formed is melted by heating the flask on the water-bath, the flask being corked during the heating. The fatty acids are completely melted when they form an oily layer on the surface of the solution.

Scraps of pumice stone joined by platinum wires are next placed in the flask to prevent bumping, and the flask is properly connected with the condenser for distilling, as shown in Fig. 95. A flask graduated at IIO cc. is used as a receiver, the funnel placed therein being provided with a loose tuft of absorbent cotton to serve as a filter. The distillation is conducted by so grading the heat that the receiving flask is filled with the distillate in about thirty minutes.

Finally the entire distillate is titrated with decinormal sodium hydroxide, using $0.5 \mathrm{cc}$. of a solution of phenolphthalein as an indicator. The number of cubic centimeters of decinormal alkali required to neutralize the acidity of the distillate from 5 grams of the fat in the manner described expresses what is known as the Reichert-Meissl number.

Leffmann and Beam's Modification.*-Five grams of the fat placed in the flask are treated with $20 \mathrm{cc}$. of a solution of soda in glycerin $(20 \mathrm{cc}$. of a $50 \%$ solution of sodium hydroxide in $180 \mathrm{cc}$. of glycerin), heating the flask till the contents are completely saponified. The solution becomes perfectly clear, showing complete saponification in about five minutes, after which $135 \mathrm{cc}$. of water are added to the clear soap solution, at first drop by drop to prevent foaming; 5 cc. of the dilute sulphuric acid are then added, and the distillation conducted at once without first melting the fatty acids.

\footnotetext{
* Lefimann and Beam, Select Methods of Food Analysis, p. 146.
} 
EDIBLE OILS AND FATS IN THE ORDER OF THEIR REICHERT-MEISSL. NUMBER.

\begin{tabular}{|c|c|c|c|}
\hline & Lowest. & Highest. & Average. \\
\hline 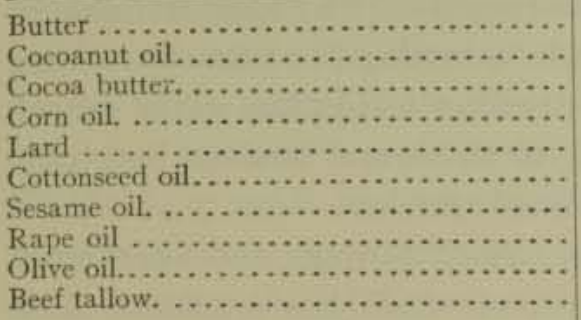 & $\begin{array}{l}24.5 \\
6.65 \\
0.20 \\
1.32 \\
\ldots \\
\cdots \\
0.70 \\
0.58 \\
\ldots \ldots \\
\ldots \ldots\end{array}$ & $\begin{array}{r}32 \\
7.8 \\
0.80 \\
5.0 \\
\ldots \\
\ldots \\
1.20 \\
0.90 \\
\cdots \\
\cdots\end{array}$ & $\begin{array}{l}28.25 \\
7.2 \\
0.5 \\
3.16 \\
1.10 \\
0.95 \\
0.95 \\
0.74 \\
0.60 \\
0.5\end{array}$ \\
\hline
\end{tabular}

Polenske Number.*-This number represents the volatile fatty acids insoluble in water, and is of value in detecting cocoanut oil in butter and other fats. The details of apparatus and manipulation here described should be closely adhered to in order to secure comparable results. Both the Reichert-Meissl and the Polenske number may be determined in one weighed portion of the fat.

Place 5 grams of the clear filtrated fat in a 300 -cc. Jena flask, add 20 grams of glycerine and $2 \mathrm{cc}$. of a $50 \%$ solution of sodium hydroxide. Heat the flask on a wire gauze until the contents are completely saponified, which requires about 5 minutes, and is indicated by the clearing up of the liquid. While still hot add $90 \mathrm{cc}$. of boiled water, at first drop by drop to prevent foaming, and shake until the soap is dissolved. The solution should be completely clear and almost colorless. Rancid or oxidized fats that yield a brown soap solution should not be examined.

To the soap solution, warmed to $50^{\circ}$, add $50 \mathrm{cc}$. of dilute sulphuric acid ( 25 cc.: I liter) and 0.5 gram of granulated pumice stone with grains I $\mathrm{mm}$. in diameter, then connect with the distilling apparatus shown in Fig. 95. Distil over a $0.5 \mathrm{~mm}$. mesh copper gauze, using a Bunsen flame so regulated as to give a distillate of $\mathrm{r}$ ro cc. in $19^{-20}$ minutes, and a stream of water that will cool the distillate to about $20-23^{\circ}$. The room should have a temperature of about $18-22^{\circ}$. As soon as IIo cc. have come over, replace the flask by a 25 -cc. measuring cylinder.

Without mixing the distillate place the flask for Io minutes in water at $15^{\circ}$, so that the $110 \mathrm{cc}$. mark is about $3 \mathrm{~cm}$. below the surface of the water. After the first five minutes, gently move the neck of the flask in

* Polenske, Zeits. Unters. Nahr. Genuss., 7, 1904, p. 274. Fritsche, ibid., p. 193. 
the water so that the fatty acids floating on the surface come in contact with the glass, noting at the end of rominutes the condition of these acids. If the butter is pure, the floating acids are either solid or form a half solid turbid mass, according as the Reichert-Meissl number is high or low; if it is adulterated with $10 \%$ or more of cocoanut oil, they form transparent oil drops. Stopper the I Io-cc. flask, mix by inverting 4 or 5 times, avoiding violent shaking, filter through an 8-cm. dry filter fitted close to the funnel, and titrate roo cc. of the liquid with testh-normal barium hydroxide solution, thus obtaining the Reichert-Meissl number.

After the last drop of distillate has passed through the filter, wash with three $1_{5} \mathrm{cc}$. portions of water, each of which has previously been used to rinse the condenser tube, the measuring cylinder and the rio cc. flask. Then repeat this treatment, using $\mathrm{I}_{5} \mathrm{cc}$. portions of neutral $90 \%$ alcohol. Titrate the united alcoholic washings with tenth-normal barium hydroxide solution, using phenolphtalein as indicator. The number of cc. required is the Polenske number.

The following results illustrate the value of the method:

\begin{tabular}{|c|c|c|}
\hline & $\begin{array}{l}\text { Reichert-MeissI } \\
\text { Number. }\end{array}$ & $\begin{array}{l}\text { Polenske } \\
\text { Number. }\end{array}$ \\
\hline ples of butter (Polenske) & N. $23.3-30.1$ & 1. $5-3.0$ \\
\hline les of cocoanut oil (Polenske) & $6.8-7 \cdot 7$ & $16.8-17.8$ \\
\hline Oleomargarine (Arnold) ... . & 0.5 & 0.53 \\
\hline Lard (Arnold) ........... & 0.35 & 0.5 \\
\hline (Arnold) & 0.55 & $0.5^{6}$ \\
\hline
\end{tabular}

Determination of Soluble and Insoluble Fatty Acids. $-A . O . A . C$. Method.*-Soluble Acids.-Five grams are weighed out and transferred to an Erlenmeyer flask of the same size and in the same manner as that used for the Reichert-Meissl process. $50 \mathrm{cc}$. of alcoholic potash solution are added (40 grams of potassium hydroxide in I liter of $95 \%$ redistilled alcohol) and the flask, provided with a return-flow condenser, is heated on the water-bath till saponification is complete, as evidenced by the clear solution free from fat globules. The alcoholic solution of potash is preferably measured from a pipette, from which it is allowed to drain for a noted interval of time, say thirty seconds.

After complete saponification, the condenser is removed and the alcohol is evaporated by further heating. One or more blanks are pre-

*U. S. Dept. of Agric., Div, of Chem., Bul. 46, p. 47; Bul. 107 (rev.), p. 138. 
pared at the same time, using the same 5o-cc. pipette for measuring, and applying the same time limit for draining the pipette. The blanks are first titrated, after evaporation, with half-normal hydrochloric acid, using phenolphthalein as an indicator. Then add to the flask containing the fatty acids I cc. more of the half-normal acid than is found necessary to neutralize the alkali in the blanks, after which the flask is again heated with a funnel in the neck till the fatty acids have completely separated in a layer on top of the solution. Then cool the flask in ice water till the fatty acids are solidified, after which decant the liquid portion through a filter, previously dried in the oven and weighed, into a liter flask, keeping the solid mass of fatty acids intact. Next add 200 or 300 cc. of hot water to the flask containing the fatty acids, and again melt over the water-bath till they collect as before on top, having again inserted the funnel to act as a condenser, and occasionally shaking the contents of the flask during heating. Cool as before in ice water, after which again decant the liquid from the solid mass through the same filter into the liter flask. Repeat this process of washing, melting, cooling, and decanting three times, receiving all the wash water through the same filter in the same flask. Make up the washings with water to the liter mark, and, after mixing, two portions of $100 \mathrm{cc}$. each are titrated with tenth-normal sodium hydroxide, using phenolphthalein for an indicator. Each reading is multiplied by ten to represent the total volume, and the figure thus obtained represents the number of cubic centimeters of tenthnormal alkali necessary to neutralize the acidity of the soluble fatty acids, together with the excess of half-normal acid used, amounting to I cc. This I cc. of half-normal acid corresponds to $5 \mathrm{cc}$, of tenth-normal alkali, hence $5 \mathrm{cc}$. are to be deducted from the total number of cubic centimeters required for the titration, the corrected figure thus obtained being multiplied by the factor 0.0088 , which gives the weight of soluble fat acids in the 5 grams of the sample, calculated as butyric acid.

Insoluble Acids.-Transfer the fatty acids left in a cake in the flask from the separation of the soluble acids, to a weighed glass evaporating dish, using strong alcohol to wash them out thoroughly. Dry the filter used in the separation, transfer it to an Erlenmeyer flask, and thoroughly wash it with strong alcohol, transferring all the washings to the dish. The alcohol is then evaporated by placing the dish on the water-bath, after which it is dried for two hours in the air-oven at $100^{\circ}$, cooled in the desiccator, and weighed. After once heating for two hours, cooling and weighing, heat again for half an hour, cool, and weigh. If a considerable loss 
in weight is found, heat for an additional half-hour. It is best, however, to avoid too prolonged heating, lest oxidation of the fatty acids should produce an increase in weight.

Hehner's Method.-Transfer the fatty acids left in the original Erlenmeyer flask to the thoroughly wet, tared filter, washing out the flask with hot water, thus bringing all the fatty acids upon the filter, which, if of good quality and thoroughly wet beforehand, will retain them. If, however, oily particles are noticed in the filtrate, they may be solidified by cooling in ice water, and afterwards removed by a glass rod and transferred to the filter. After draining dry, the funnel is immersed in cold water to solidify the fatty acids, and the filter containing them is transferred to a weighed dish, which is dried for two hours in the oven at $100^{\circ}$, cooled in the desiccator, and weighed, subtracting the weight of the dish and filter.

EDIBLE OILS AND FATS ARRANGED IN ORDER OF INSOLUBLE FATTY ACIDS.

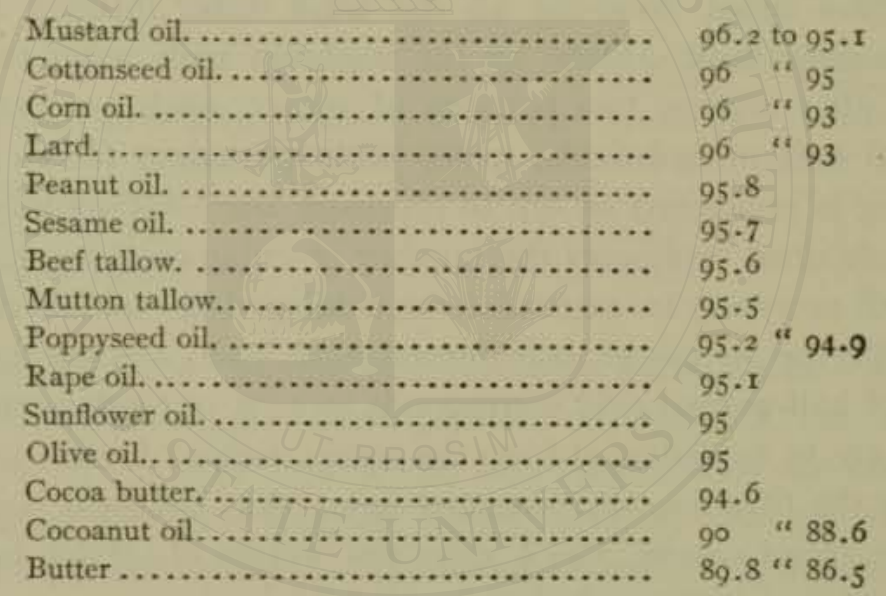

Saponification Number. - Koettstorjer's Method. - By the saponification number is meant the number of milligrams of potassium hydroxide necessary to completely saponify $\mathrm{I}$ gram of the fat. Between $\mathrm{I}$ and 2 grams of the fat are transferred in the usual manner (see p. 474) to an Erlenmeyer flask, and $25 \mathrm{cc}$. of the alcoholic potash solution ( 40 grams of potassium hydroxide free from carbonates in I liter of $95 \%$ alcohol redistilled after standing for some time with potassium hydroxide) are added with a graduated pipette, which is allowed to drain for a noted period of time, say thirty seconds. The determination should preferably 
be made in duplicate. Conduct the saponification as in the case of the soluble fatty acids by heating on the water-bath. After saponification, remove from the bath, cool, and titrate with half-normal hydrochloric acid, using phenolphthalein as an indicator. Titrate also several blanks in which $25 \mathrm{cc}$. of the alcoholic potash solution are measured out with the same pipette as before, and allow to drain for the same amount of time. Subtract the number of cubic centimeters of half-normal acid necessary to neutralize the alkali in the case of the saponified fat from that necessary to neutralize the blank, multiply the result by 28.06 , and divide the product by the number of grams of fat taken.

EDIBLE OILS AND FATS ARRANGED IN ORDER OF THEIR SAPONIFICATION NUMBER.

\begin{tabular}{|c|c|c|c|}
\hline & Minimum. & Maximum. & Mean. \\
\hline 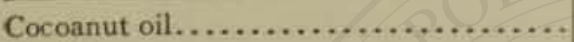 & 246.2 & 268.4 & $257 \cdot 3$ \\
\hline 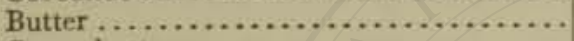 & 225 & 230 & $227 \cdot 5$ \\
\hline 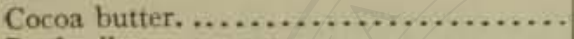 & $19 ?$ & 202 & 197 \\
\hline 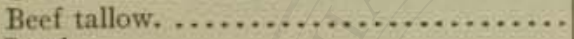 & 193.2 & 200 & I96.6 \\
\hline 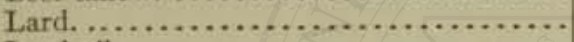 & $195 \cdot 3$ & 196.6 & 196 \\
\hline 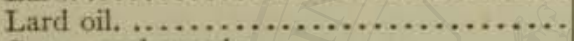 & 195 & 196 & $x 95 \cdot 5$ \\
\hline Cottonseed stearin................. & 194.6 & $195 \cdot \mathrm{I}$ & 194.8 \\
\hline Poppyseed oil ...................... & 190 & 198 & I94 \\
\hline 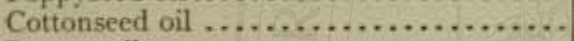 & I9I & 196.6 & 193.8 \\
\hline 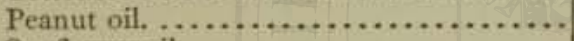 & 190 & 197 & 193.5 \\
\hline Sunflower oil ..................... & 193 & 194 & 193.5 \\
\hline 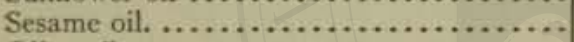 & 187.6 & 192.4 & 192.6 \\
\hline 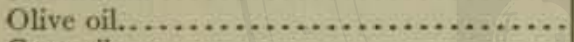 & 185 & 196 & $\mathrm{rg} \cdot 5$ \\
\hline 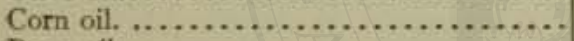 & 188 & 193.4 & 190.7 \\
\hline 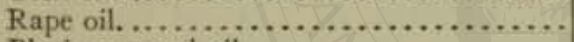 & 170.2 & 179.2 & 174.6 \\
\hline 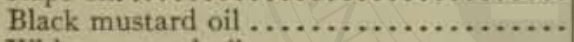 & 174 & 174.6 & $174 \cdot 3$ \\
\hline 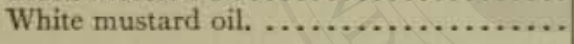 & 170.3 & 174.6 & $172 \cdot 4$ \\
\hline
\end{tabular}

The Iodine Absorption Number.-This determination is based on the well-known property of the unsaturated fatty acids to absorb a fixed amount of iodine under given conditions of time, strength of reagent, etc.

Hübl's Method.*-The following reagents are necessary:

(I) Iodine Solution, made by dissolving 26 grams of pure iodine in $500 \mathrm{cc}$. of $95 \%$ alcohol, and, separately, 30 grams of mercuric chloride in $500 \mathrm{cc}$. of the same strength of alcohol. Filter the latter solution, if necessary, and mix the two together, allowing the mixture to stand at least twelve hours before using.

(2) Decinormal Thiosulphate Solution, made by dissolving 24.6 grams of the freshly powdered, chemically pure salt in water, and making up to I liter.

* A. O. A. C. Method, U. S. Dept. of Agric., Bur. of Chem., Bul. 65, p. 24; Bul. ro7 (rev.), P. 136 . 
(3) Starch paste, prepared by boiling I gram of starch in $200 \mathrm{cc}$. of water for ten minutes, then cooling.

(4) Potassium Iodide Solution, made by dissolving 150 grams of the salt in water, and making up the volume to I liter.

(5) Potassium Bichromate Solution for standardizing the thiosulphate, made by dissolving 3.874 grams of chemically pure potassium bichromate in distilled water, and making up the volume to I liter.

The sodium thiosulphate solution is standardized as follows: $20 \mathrm{cc}$. of the potassium bichromate solution are introduced into a glass-stoppered flask together with ro cc. of potassium iodide and $5 \mathrm{cc}$. of strong hydrochloric acid. Then slowly add from a burette the sodium thiosulphate solution, till the yellow color of the solution has nearly disappeared, after which a little of the starch paste is added, and the titration carefully continued to just the point of disappearance of the blue color. The reaction which takes place is as follows:

$$
\mathrm{K}_{2} \mathrm{Cr}_{2} \mathrm{O}_{7}+{ }_{4} \mathrm{HCl}+6 \mathrm{KI}={ }_{2} \mathrm{CrCl}_{3}+8 \mathrm{KCl}+6 \mathrm{I}+{ }_{7} \mathrm{H}_{2} \mathrm{O} .
$$

The equivalent of I gram of iodine in terms of the thiosulphate solution is found by multiplying the number of cubic centimeters of the latter solution required for the above titration by 5 .

If, for example, $\mathrm{I} 6.4 \mathrm{cc}$. of the thiosulphate solution are required for $20 \mathrm{cc}$. of the bichromate solution, then I gram of iodine is equivalent to $\mathrm{I} 6.4 \times 5=82.0 \mathrm{cc}$. of sodium thiosulphate solution, or $\mathrm{I} \mathrm{cc}$. of the thiosulphate solution $=\frac{1}{82}=0.0122$ gram of iodine. I cc. of exactly decinormal thiosulphate is theoretically equivalent to 0.0127 gram of iodine.

The thiosulphate solution may also be standardized by means of iodine. A short tube closed at one end is tared, together with another tube of such a size as to fit over the first. Into the inner tube are introduced about 0.2 gram of resublimed iodine and the tube heated until the iodine melts, after which it is closed by the second tube and the whole cooled in a desiccator and weighed. The iodine is dissolved in 10 $\mathrm{cc}$. of $10 \%$ potassium iodide solution, the solution diluted with water, and the thiosulphate solution added with constant stirring until only a yellow color remains. Starch paste is then added, and the titration continued until the blue color disappears.

Manipulation.-Place 0.4 to I gram of the solid fat, or from 0.2 to 0.4 gram of oil, in a glass-stoppered flask or bottle of $300 \mathrm{cc}$. capacity. 
In the case of oils, this may conveniently be done by difference, weighing first a small quantity of the oil in a beaker with a short piece of glass tubing to serve as a pipette, transferring a number of drops of the oil from the beaker to the bottle, and again weighing the beaker and contents. The number of drops of oil required for the desired weight is first ascertained experimentally.

The material may also be conveniently and accurately weighed in small, flat bottomed cylinders of glass about to $\mathrm{mm}$. in diameter and 15 $\mathrm{mm}$. high, which may be made by cutting off so-called "shell vials." Fats are introduced while melted, the weight being taken after cooling. The cylinder and fat are transferred together by means of forceps to the glass-stoppered bottle.

Dissolve the oil in ro cc. of chloroform, and after solution has taken place, add $30 \mathrm{cc}$. of the iodine solution, shake, and set in a dark place for three hours, shaking occasionally. The excess of iodine should be at least as much as is absorbed. When ready for the titration, add $20 \mathrm{cc}$. of the potassium iodide solution (the purpose of which is to keep in solution the mercuric iodide formed, which would otherwise precipitate on dilution) and roo cc. of distilled water. Titrate the excess of iodine by the thiosulphate solution, which is slowly added from a burette till the yellow color has nearly disappeared, then add a little starch paste, and finally thiosulphate solution drop by drop until the blue color of the iodized starch is dispelled. Near the end of the reaction the flask should be stoppered and vigorously shaken, in order that all the iodine may be taken up, and sufficient thiosulphate should be added to prevent a reappearance of any blue color in five minutes.

Two blanks are conducted at the same time and in similar flasks or bottles, in exactly the same manner as in the case of the above titration, except that the fat is omitted. This is to get the true value of the iodine solution in terms of the thiosulphate solution.

Suppose, for example, in the case of the blanks, $30 \mathrm{cc}$. of the iodine solution required in one instance, $46.2 \mathrm{cc}$. of sodium thiosulphate solution and in the other $46.4 \mathrm{cc}$. The mean is 46.3 . Suppose $30.7 \mathrm{cc}$. of thiosulphate solution were required for the excess of iodine remaining over and above that absorbed by 0.5 gram of the fat in the above process. Then the thiosulphate equivalent to the iodine absorbed by the fat would be $46.3-30.7=15.6 \mathrm{cc}$., and the per cent of iodine absorbed would be $\frac{15.6 \times 0.0122 \times 100}{0.5}=38.06$. 
EDIBLE OILS AND FATS ARRANGED IN ORDER OF THEIR HÜBL NUMBER.

\begin{tabular}{|c|c|c|c|}
\hline & Lowest. & Highest. & Average. \\
\hline 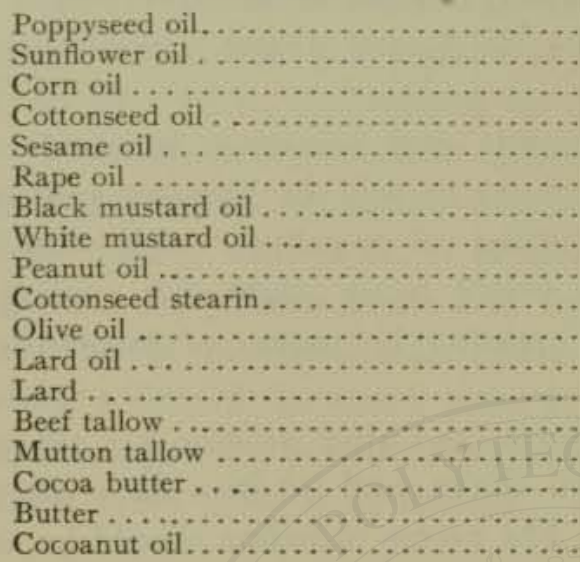 & $\begin{array}{l}132.6 \\
118 \\
111,2 \\
108 \\
103 \\
94 \\
96 \\
92.1 \\
83 \\
88.7 \\
79 \\
56 \\
46 \\
35 \cdot 4 \\
32.7 \\
32 \\
25.7 \\
8\end{array}$ & $\begin{array}{l}143 \cdot 3 \\
133 \cdot 3 \\
130 \\
110 \\
115 \\
105 \\
110 \\
97-7 \\
103 \\
103.8 \\
88 \\
85 \\
70 \\
47.5 \\
46.2 \\
41.7 \\
37.9 \\
9.5\end{array}$ & $\begin{array}{l}138 \\
125.7 \\
120.6 \\
109.5 \\
109 \\
99.5 \\
103 \\
94.9 \\
93 \\
91.2 \\
83.5 \\
70.5 \\
58 \\
41.4 \\
39.5 \\
34.9 \\
33.3 \\
8.7\end{array}$ \\
\hline
\end{tabular}

The Hübl method has long been almost universally used for estimating the per cent of iodine absorbed, but is open to serious objections, chief of which are the tendency of the iodine solution to lose strength, and the length of time required to insure saturation of the oil with the iodine.

Of late two other methods have come into prominence, viz., the Wijs and the Hanus. The reagents in both these methods hold their strength for months without change, and the time required for carrying out the reaction in the case of most of the edible oils and fats is very short.

Of the three methods, that of Hanus has the advantage of greatest simplicity in the composition and preparation of the chief reagent.

Tolman and Munson* have shown that with oils and fats having iodine numbers below 100 , the three methods give practically identical figures, while with oils having high iodine numbers, the Wijs and Hanus methods give higher results than the Hübl, but are doubtless more nearly correct.

The following are comparative results of the three methods:*

* Jour. Am. Chem. Soc., 25 (1903), p, 244. 


\begin{tabular}{|c|c|c|c|c|c|c|}
\hline 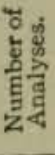 & & 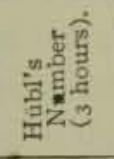 & 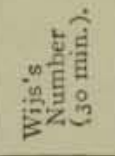 & 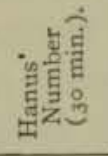 & 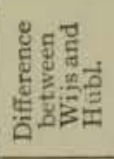 & 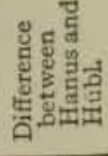 \\
\hline $\mathbf{I}$ & Cocoanut oil.. & 8.93 & 9.05 & 8.60 & +0.12 & -0.33 \\
\hline 2 & Butter- $\quad \begin{array}{l}\text { minimum } \\
\text { maximum.......... }\end{array}$ & $\begin{array}{l}34.8 \\
35 \cdot 3\end{array}$ & $\begin{array}{l}35.9 \\
36.2\end{array}$ & $\begin{array}{l}35 \cdot 4 \\
35 \cdot 3\end{array}$ & $\begin{array}{l}+1.1 \\
+0.9\end{array}$ & $\begin{array}{l}+0.6 \\
+0.0\end{array}$ \\
\hline $\mathbf{I}$ & Oleo oil. ....................... & 42.6 & $43 \cdot 5$ & $43 \cdot 3$ & +0.9 & +0.7 \\
\hline 4 & Oleomargarine - minimum $\ldots . .$. & $52 \cdot 5$ & 52.9 & 52.0 & +0.4 & -0.5 \\
\hline 2 & Lard oil $-\quad \quad \begin{array}{l}\text { maximum. } \\
\text { minimum ........ }\end{array}$ & $\begin{array}{l}66 \cdot 3 \\
69 \cdot 3\end{array}$ & $\begin{array}{l}66.0 \\
70.5\end{array}$ & 69.8 & $\begin{array}{l}-0.3 \\
+1.2\end{array}$ & $\begin{array}{l}-1.5 \\
+0.5\end{array}$ \\
\hline & maximum. ..... & $73 \cdot 7$ & $74 \cdot 5$ & 73.9 & +0.7 & +0.2 \\
\hline $3^{6}$ & $\begin{array}{l}\operatorname{minimum} . \ldots . \\
\text { maximum. } . . .\end{array}$ & $\begin{array}{l}79.2 \\
80.8\end{array}$ & $\begin{array}{l}79.9 \\
91.4\end{array}$ & 80.6 & $\begin{array}{l}+0.7 \\
+\end{array}$ & +1.4 \\
\hline & $\begin{array}{l}\text { maximum. } . . \\
\text { average } . . . .\end{array}$ & 84.0 & $\begin{array}{l}91 \cdot 4 \\
85 \cdot 3\end{array}$ & 84.6 & +1.0 & +0.6 \\
\hline 3 & minimum.... & $94 \cdot 5$ & 95.2 & $94 \cdot I$ & +0.7 & -0.1 \\
\hline 5 & 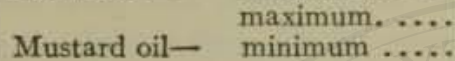 & $107 \cdot 7$ & 109.5 & 107.7 & +1.8 & +0.0 \\
\hline & Mustard oil- $\operatorname{minimum}_{\text {maximum. }} . . .$. & $\begin{array}{r}98.4 \\
113.0\end{array}$ & $\begin{array}{l}104 \cdot 3 \\
118 \cdot 2\end{array}$ & $\begin{array}{l}103.8 \\
116.8\end{array}$ & $\begin{array}{l}+5 \cdot 9 \\
+5 \cdot 2\end{array}$ & $\begin{array}{l}+5.4 \\
+3.8\end{array}$ \\
\hline 2 & minimum ..... & 100.2 & 104.1 & 102.8 & $+3 \cdot 9$ & +2.6 \\
\hline & maximum. ..... & 101.3 & $105 \cdot 7$ & 105.2 & $\begin{array}{l}+4.4 \\
+2.8\end{array}$ & $\begin{array}{l}+3.8 \\
+0.8\end{array}$ \\
\hline $\begin{array}{l}1 \\
3\end{array}$ & 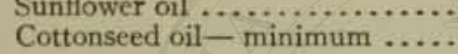 & $\begin{array}{l}106.4 \\
103.8\end{array}$ & $\begin{array}{l}109 \cdot 2 \\
105 \cdot 3\end{array}$ & 105.2 & +1.5 & +1.4 \\
\hline & maximum.... & 106.2 & $107 \cdot 3$ & 107.8 & $+1 . I$ & +1.6 \\
\hline 1 & Sesame oil. ................... & 106.4 & 107.0 & 106.5 & +0.6 & to.I \\
\hline 3 & Corn oil- $\quad$ minimum ..... & 119.0 & 122.2 & II9. 6 & +3.0 & +0.4 \\
\hline & maximum. .... & $123 \cdot 3$ & 129.2 & 126.0 & +5.8 & +2.7 \\
\hline 2 & Poppyseed oil $-\underset{\text { maximum }}{\text { minimum }} . . . .$. & $\begin{array}{l}\text { I33.4 } \\
\text { I34.0 }\end{array}$ & $\begin{array}{l}135 \cdot 2 \\
139 \cdot 1\end{array}$ & $\begin{array}{l}132.9 \\
138.4\end{array}$ & $\begin{array}{l}+1.8 \\
+4.2\end{array}$ & $\begin{array}{l}-0.5 \\
+3.5\end{array}$ \\
\hline
\end{tabular}

Hanus' Method.*-Reagents.-Iodine Solution.-Dissolve I3.2 grams of pure iodine in I liter of pure glacial acetic acid $(99 \%)$, and to the cold solution add $3 \mathrm{cc}$. of bromine, or sufficient to practically double the halogen content when titrated against the thiosulphate solution, but with the iodine slightly in excess.

Decinormal Thiosulphate Solution, Starch Paste, and Potassium Iodide Solution, as in Hübl's method.

Method of Procedure.-Proceed as in Hübl's method, substituting $30 \mathrm{cc}$. of the Hanus iodine reagent for that of Hübl, stirring the solution before adding the water, and, instead of adding $20 \mathrm{cc}$. of the potassium iodide solution, use only to $\mathrm{cc}$. The excess of iodine should be at least $60 \%$ of that added.

*Zeits. Unters. Nahr. Genuss., 4, xgor, p. $9^{1} 3$. Also Hunt, Jour. Soc. Chem. Ind., 2I, 1902, p. 454; U. S. Dept. of Agric., Bur. of Chem., Bul, 107 (rev.), p. 136. 
Only half an hour is required for full saturation of the oil by the iodine in the Hanus method, as against three hours in the Hübl. In case of the non-drying oils and fats, the reaction takes place in from eight to fifteen minutes, though it is best to let the flask set for half an hour at least, in all cases. With oils having an iodine number in excess of 1oo, Tolman and Munson recommend one hour's standing.

On account of the high coefficient of expansion of acetic acid, care should be taken that the temperature is the same when the iodine solution is measured for the blank and for the determination, as otherwise a serious error may be introduced.

Wijs's Method.*-Reagents.-Iodine Solution.-Dissolve I3.2 grams of pure iodine in I liter of pure glacial acetic acid, and pass through the larger portion of this solution a current of carefully washed and dried chlorine gas $\dagger$ until the solution is practically decolorized. Finally add enough of the original solution of iodine in acetic acid to restore the iodine color, so that there is a slight excess of iodine.

Hunt's Modified Iodine Solution.-Dissolve to grams of iodine trichloride in I liter of pure glacial acetic acid, and finally add and dissolve Io. 8 grams of pure iodine.

Other Reagents, as in the Hübl and Hanus methods.

Method of Procedure.-Proceed as in the Hanus method, observing the same precautions, the only difference being in the use of the Wijs iodine reagent.

Wijs recommends the following periods of time for absorption of the iodine in the case of various oils: For non-drying oils and fats, such as peanut and olive oil, $\ddagger$ fifteen minutes; for semi-drying oils, such as cottonseed, rape, sesame, corn, and mustard, thirty minutes; for drying oils, such as sunflower and poppyseed, one hour.

The Bromine Index or Bromine Absorption Number.-The measure of the amount of bromine absorbed by the oils and fats is a useful factor. By the bromine index is understood the weight of bromine which is

* Ber. d. chem. Ges., 31 ( 1898$)$, p. $75^{\circ}$.

† The chorine is conveniently prepared by treatment of bleaching powder with dilute sulphuric acid, using gentle heat, and washing the gas by passing through strong sulphuric acid.

$\ddagger$ For butter, oleo oil, lard oil, and cocoanut oil, fifteen minutes is sufficient. 
absorbed by I gram of a given oil. The bromine index of various oils has been determined as follows:

\begin{tabular}{|c|c|c|}
\hline & Bromine Index. & Observer. \\
\hline 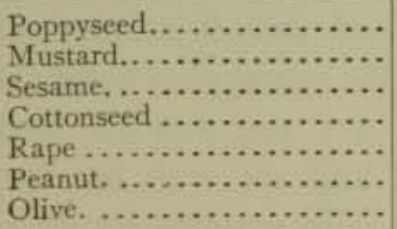 & $\begin{array}{c}0.835 \\
0.763 \\
0.695 \\
0.645 \\
0.632 \\
0.530 \\
0.500 \text { to } 0.544\end{array}$ & $\begin{array}{l}\text { Levallois } \\
\text { Girard } \\
\text { Levallois } \\
\quad \text { Girard } \\
\text { Levallois } \\
\text { " }\end{array}$ \\
\hline
\end{tabular}

Method of Levallois.-Five grams of the oil are saponified with alcoholic potash in a 50-cc. graduated flask by the aid of a gentle heat. At the end of the saponification and after cooling, the flask is filled to the mark with alcohol, and, after shaking, $5 \mathrm{cc}$. are removed by means of a pipette and transferred to a flask. A slight excess of hydrochloric acid is added to set free the fatty acids, and from a burette a standardized solution of bromine water is run in till with constant shaking a permanent yellow color persists. The bromine is previously standardized with potassium iodide and sodium thiosulphate. The weight of bromine fixed by I gram of the fat is then calculated.

Mill's Method.-Modified.†-Dissolve o.I gram of the filtered and dried fat in $50 \mathrm{cc}$. of carbon tetrachloride or chloroform in a Ioo-cc. stoppered bottle. From a burette a standard solution of bromine in carbon tetrachloride, approximately tenth-normal ( 8 grams to a liter), is slowly added to the oil solution till, after fifteen minutes, a permanent coloration remains. The amount of bromine absorbed is calculated by comparing with the color similarly produced in a blank experiment, or an excess of bromine solution may be run in and the solution titrated back with a standard solution of thiosulphate, using potassium iodide and starch.

Thermal Tests.-The rise in temperature produced by the action of certain reagents on various oils and fats, when applied in a definite manner, has been found to be of considerable value, especially in the case of sulphuric acid and of bromine.

* Villiers et Collin, Les Substances Alimentaires, p, 680 .

$\dagger$ Jour. Soc. Chem. Ind., 2, p. $435 ; 3$, p. 366 . 
The Maumené Test,* or thermal reaction with sulphuric acid, is most readily carried out in a beaker of say $150 \mathrm{cc}$. capacity, which is set into a larger beaker or vessel of any kind, the space between the two being packed with felt or cotton waste. The inner beaker is removed, and into it is weighed 50 grams of the oil. It is then replaced and the packing adjusted, if necessary, after which the temperature of the oil is noted with a thermometer. From a burette containing the strongest sulphuric acid of the same temperature as the oil, Io cc. are run into the beaker, at the same time stirring the mixture of acid and oil with the thermometer. The temperature rises somewhat rapidly, and remains for an appreciable time at its maximum point, which should be noted. The difference in degrees centigrade between the initial temperature of the oil and the maximum temperature of the mixture expresses the Maumené number.

With certain oils, as cottonseed, considerable frothing ensues when concentrated acid is employed, making an accurate determination of the Maumené number somewhat difficult. In this case it is better to employ a somewhat weaker acid, and to express results in terms of what is called the "specific temperature reaction." This is the result obtained by dividing the rise of temperature in the case of the oil by the rise of temperature in the case of water, using the same strength of acid, and multiplying the quotient by roo. Indeed, it is of importance in all cases to compare results on oils with those obtained by carrying out the same test on water.

Bromination Test.-This test depends upon the avidity with which the oils and fats absorb bromine, the rise in temperature caused by the reaction being measured in this case rather than the actual amount of bromine absorbed, as in the case of the iodine absorption. Indeed, there is such a close relation between the iodine number and the heat of bromination, that when one is determined the other may be calculated quite closely by multiplying by a factor. In view of the fact that the heat of bromination is much more readily determined than the iodine number, it is often convenient to calculate the latter from the former, the result in the case of the edible oils and fats being quite sure to fall within the limits of variation of the iodine number of different oils of the same class. The bromination test was devised by Hehner and Mitchell, $\uparrow$ who employed a vacuum jacketed tube for a calorimeter in which to make the test. Various modifications have been suggested both in the

* Maumené, Compt. Rend., XXXV (1852), p. $57^{2}$.

† Analyst, XX (1895), p. 146. 
apparatus employed and in the manner of diluting the oil and applying the reagent. The calorimeter employed by Gill and Hatch,* Fig. 96, is conveniently made and is very satisfactory. It consists of a long, narrow, flat-bottomed tube, held by a cork in a small beaker, in such a manner that it is surrounded by an air jacket. The small beaker is set into one of larger size, the space between the two being packed with cotton waste. Five grams of the oil or fat are dissolved in $25 \mathrm{cc}$.

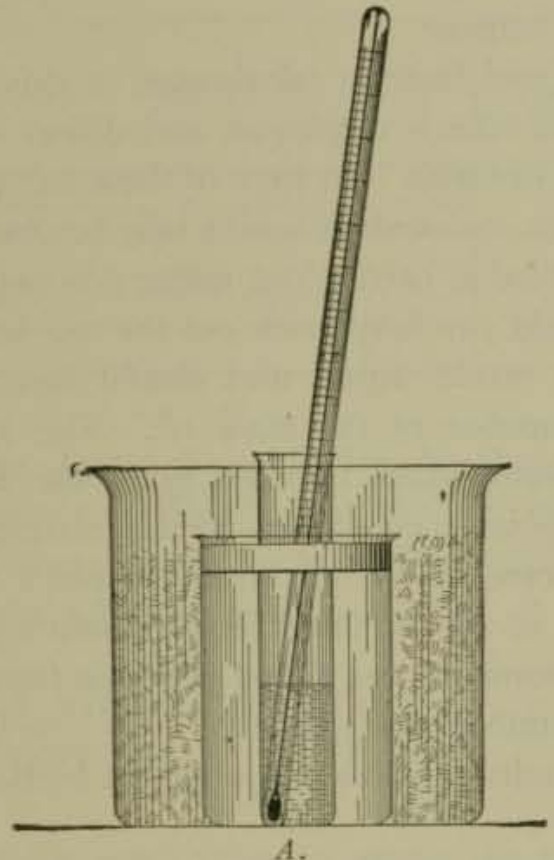

Fig. 96.

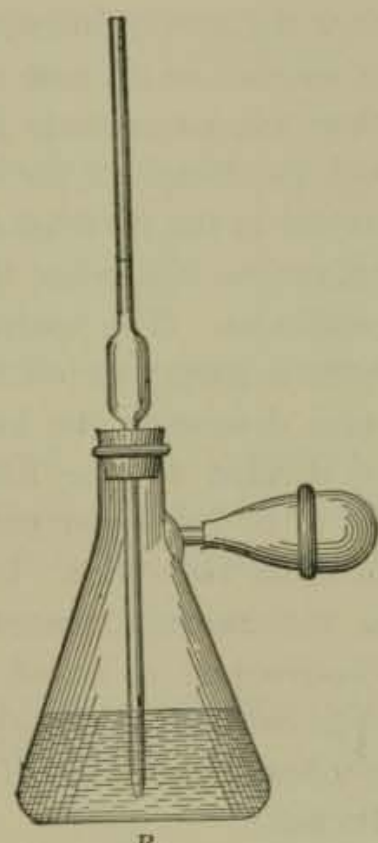

B.

A. Gill and Hatch's Calorimeter for the Bromination Test with Oils.

B. Wiley's Pipette for Measuring Bromine in Chloroform.

of chloroform or carbon tetrachloride, and $5 \mathrm{cc}$. of this solution (containing I gram of the oil) are transferred by a pipette to the inner tube of the above calorimeter, being careful not to let it flow down the sides of the tube. The temperature of the oil is then taken by a thermometer graduated to $0.2^{\circ}$. The bromine reagent, which should be freshly prepared, is made up by measuring from a burette one part by volume of bromine into four parts of chloroform or carbon tetrachloride. The reagent is transferred to a measuring-flask devised by Wiley, $\uparrow$ consisting of a side-necked filter-flask provided with a per-

* Jour. Am. Chem. Soc., XXI (1899), p. 27. Gill, Oil Analysis, p. 50. † Jour. Am. Chem. Soc., XVIII (1896), p. $37^{8}$. 
forated rubber stopper into which the stem of a 5 -cc. pipette is fitted, Fig. 96. A bulb on the side-neck serves to fill the pipette. This pipette, filled to the mark with the bromine reagent (which should be at the same temperature as the oil solution in the calorimeter), is first covered by the finger and removed, and its contents of $5 \mathrm{cc}$. allowed to flow down the sides of the inner tube of the calorimeter and mingle with the oil without stirring. The rise in temperature is very quick, and the highest point is noted. The difference between the highest and the initial temperature constitutes the heat-of-bromination number.

This number, in the case of Gill and Hatch's calorimeter, is somewhat lower than when a vacuum jacketed tube is employed, and differs somewhat with the diluent of the oil and bromine. In spite of these variations and that due to the personal equation, concordant results may be obtained with the various oils, when the method is carried out under precisely the same conditions. The analyst should carefully work out the test several times with a particular oil till the results agree, and should then with equal care determine the iodine number of the same oil. The iodine number, divided by the heat-of-bromination number, gives the factor which is to be employed under the same conditions for calculating one constant from the other. In the case of Hehner and Mitchell's work with the vacuum tube, measuring I cc. of undiluted bromine into I gram of oil dissolved in Io cc. of chloroform, it was found that the factor to be used in calculating the iodine number was 5.5 .

The following are some of the results on edible oils obtained by Hehner and Mitchell:

\begin{tabular}{|c|c|c|c|}
\hline Oil. & $\begin{array}{c}\text { Heat of } \\
\text { Bromination. }\end{array}$ & $\begin{array}{l}\text { Iodine } \\
\text { Number. }\end{array}$ & $\begin{array}{l}\text { Calculated } \\
\text { Iodine Number. }\end{array}$ \\
\hline 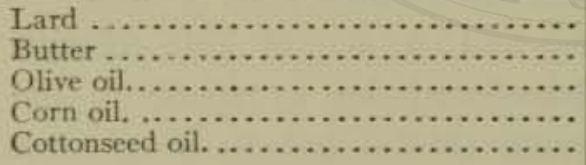 & $\begin{array}{l}10.6 \\
6.6 \\
15 \\
21.5 \\
19.4\end{array}$ & $\begin{array}{r}57 \cdot 15 \\
37.07 \\
80.76 \\
122 \\
107.13\end{array}$ & $\begin{array}{r}58.3 \\
36.3 \\
82.5 \\
118.2 \\
106.7\end{array}$ \\
\hline
\end{tabular}

As in the case of the Maumené test with sulphuric acid (wherein the rise in temperature of sulphuric acid and water is taken as a standard), it is convenient to employ some standard for the bromination test, whereby varying results due to difference in apparatus, etc., may be compared.

In this case Gill and Hatch found that sublimed camphor may be prepared sufficiently pure to be used for such a standard. Applying the bromination test with their calorimeter, as above described, to $5 \mathrm{cc}$. of a 
solution of $7 \frac{1}{2}$ grams of camphor in $25 \mathrm{cc}$. of carbon tetrachloride, an average rise in temperature of $4.2^{\circ}$ was obtained, and the specific temperature reaction is calculated for each oil by dividing the heat of bromination by this number. Furthermore, by dividing the iodine number of several oils by this specific temperature reaction, the factor to be employed for the calculation of the iodine number was found to be 17.18 , as in the following cases:*

\begin{tabular}{|c|c|c|c|}
\hline \multirow{2}{*}{ Oil. } & \multirow{2}{*}{$\begin{array}{l}\text { Specific Tem- } \\
\text { perature } \\
\text { Reaction. }\end{array}$} & \multicolumn{2}{|c|}{ Iodine Number. } \\
\hline & & Caleulated. & Found. \\
\hline 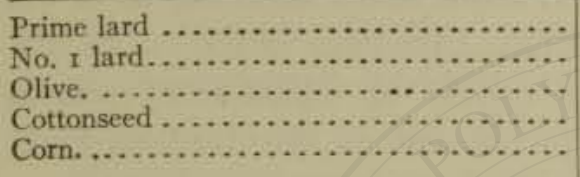 & $\begin{array}{l}3.705 \\
4.096 \\
4.762 \\
5.667 \\
6.381\end{array}$ & $\begin{array}{r}63.8 \\
70 \cdot 3 \\
81.8 \\
97 \cdot 3 \\
109 \cdot 5\end{array}$ & $\begin{array}{r}63.8 \\
73.9 \\
82.0 \\
103.0 \\
107.8\end{array}$ \\
\hline
\end{tabular}

The Acetyl Value. - On heating fats with acetic anhydride they become "acetylated"; i.e., the hydrogen atom of their alcoholic hydroxyl group is exchanged for the acetic acid radicle, in accordance, for example, with the following reaction:

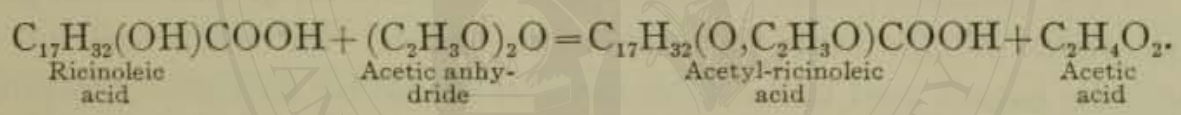

By the actyl value is meant the number of milligrams of potassium hydroxide necessary to neutralize the acetic acid formed by the saponification of I gram of the acetylated fat.

Lewkowitsch's method of procedure is as follows: to grams of the oil are boiled with an equal volume of acetic anhydride for two hours in a flask with a return-flow condenser, and the mixture is then transferred to a large beaker containing $500 \mathrm{cc}$. of water, and boiled for half an hour. To prevent bumping, a current of carbon dioxide is slowly passed through it during the boiling, introduced through a finely drawn, bent glass tube reaching nearly to the bottom of the beaker. The mixture on standing separates into two layers, of which the lower, or aqueous layer, is siphoned off, and the oily layer boiled with fresh portions of 
water, which are in turn siphoned off, the operation being repeated till the wash water tests free from acid by litmus paper.

The acetylated fat is then separated from the water by drying at $100^{\circ}$ in an oven.

From 2 to 4 grams of the acetylated fat is weighed into a flask, and saponified with alcoholic potash in precisely the same manner as for the determination of the saponification number. Evaporate the alcohol and dissolve the soap in water. One of two methods may be carried out for freeing the acetic acid for titration, one by distillation and the other by filtration.

For the former or distillation process, acidify the aqueous solution of the soap with $\mathrm{I}$ : Io sulphuric acid, and distill in the same way as in the Reichert process, excepting that in this case from 600 to $700 \mathrm{cc}$. of distillate must be obtained, so that water should be added from time to time through a stoppered funnel fixed in the cork of the distilling-flask. The distillate should be received in a funnel with a loose cotton plug, so as to filter it free from insoluble acids mechanically carried over. The filtrate is titrated with tenth-normal sodium hydroxide, using phenolphthalein as an indicator. The number of cubic centimeters of alkali used is multiplied by $5.6 \mathrm{I}$, and the product divided by the number of grams of acetylated fat taken. The result is the acetyl value.

If the filtration process is used (which is more rapid and should give concordant results with the distillation process), the exact amount of alcoholic potash used in the saponification should be accurately measured in carrying out the former part of the test, and the exact number of cubic centimeters of standard acid corresponding to the amount of alkali employed should be added to the aqueous soap solution. The mixture should be gently warmed, and the fatty acids will gather in a layer at the top. These are filtered off and washed, till free from acid, with boiling water. The filtrate is titrated with tenth-normal sodium hydroxide, and the acetyl value calculated as in the distillation process.

EDIBLE OILS ARRANGED IN ORDER OF ACETYL VALUE.

Cottonseed oil .......................... Average.

Cottonseed oil .................... 18.0

Rape oil . .................... I4.7

Poppyseed oil .................. I3.I

Sesame oil...................... 11.5

Olive oil . . . . . . . . . 6

Peanut oil...................... $3 \cdot 4$ 
The Valenta Test.-This depends upon the solubility of the oil in glacial acetic acid. Pour from 3 to $5 \mathrm{cc}$. of the oil into a test-tube, and add an equal volume of glacial acetic acid (specific gravity 1.0562 ). Place a thermometer in the tube and warm gently till the oil goes into solution. Then allow the mixture to cool, and observe the temperature at which the solution begins to appear turbid.

Castor oil and oil of the olive kernel are soluble in glacial acetic acid at ordinary temperatures, while rape and mustard seed oils are insoluble even in the boiling acid.

Elaïdin Test.-This is based on the conversion by nitrous oxide of liquid olein into the solid elaïdin, a crystalline compound isomeric with olein, while other common glycerides remain liquid under treatment with this reagent. By the consistency of the final product, when subjected under certain conditions to the action of nitrous oxide, some idea as to the character of the oil may be gained.

Manipulation.-To carry out the test according to Pontet (modified), weigh 5 grams of the oil into a beaker, add 7 grams of nitric acid (specific gravity 1.34 ) and about 0.5 gram of copper wire. Place the beaker in water at $15^{\circ}$ and stir thoroughly with a glass rod in such a manner as to make an intimate mixture of the oil and the evolved nitrous oxide gas. After the wire has been dissolved, add another piece of about the same size and again stir vigorously. Set aside for about two hours, at the end of which, in the case of pure olive, almond, peanut, or lard oil, it will have been changed into a solid white mass.

Nearly all the seed oils, especially cottonseed and mustard, are turned into a pasty or buttery mass.

Another modification of Pontet's test consists in mixing Io grams of the oil, 5 grams of nitric acid (specific gravity I. $3^{8}$ ), and I gram of mercury in a test-tube, shaking for three minutes and allowing to stand twenty minutes, when it is again shaken. follows:

The behavior of various oils after that time on further standing is as

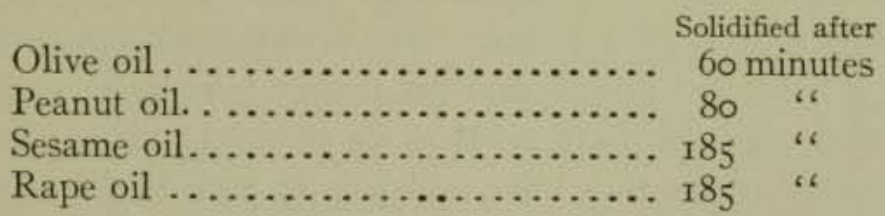

Free Fatty Acids.*-Weigh 20 grams of the oil or fat into a $150-c c$. Erlenmeyer flask, and add $50 \mathrm{cc}$. of $95 \%$ alcohol, which has previously

* Allen, Com, Org. Anal., 4 Ed., Vol. II, p. 9. 
been carefully neutralized with a weak solution of sodium hydroxide, using phenolphthalein as an indicator. Warm the mixture to about $60^{\circ}$, and add carefully from a burette tenth-normal sodium hydroxide (using the above indicator) till a pink color is produced, shaking thoroughly during the titration.

The result may be reported in terms of percentage of oleic acid (each cubic centimeter of tenth-normal alkali is equivalent to 0.0282 gram of oleic acid) or as the "acid number," by which is meant the number of cubic centimeters of tenth-normal alkali necessary to saturate the free acid in I gram of the fat or oil.

Constants of the Free Fatty Acids.-Often much information as to the character of an oil or fat may be obtained by determining such constants of its fatty acids as the melting-and solidifying-point, the iodine number, etc.

To prepare the fatty acids for examination, saponify a quantity of the oil or fat with alcoholic potash, evaporate the alcohol, and dissolve the soap in hot water. Decompose the soap by the addition of an excess of hydrochloric or sulphuric acid, continuing the heating till the fatty acids rise in a layer to the top of the liquid, from which they may be removed. The melting-point, iodine number, etc., are determined as with the oil or fat itself.

Solidifying-point of the Fatty Acids, or Titer Test.-Modified Wolfbauer Method.*-Saponify 75 grams of fat in a metal dish with $60 \mathrm{cc}$. of $30 \%$ sodium hydroxide $\left(36^{\circ}\right.$ Baumé) and 75 cc. of $95 \%$ by volume alcohol or $120 \mathrm{cc}$. of water. Boil to dryness, with constant stirring to prevent scorching, over a very low flame, or over an iron or asbestos plate. Dissolve the dry soap in a liter of boiling water, and if alcohol has been used, boil for forty minutes in order to remove it, adding sufficient water to replace that lost in boiling. Add $100 \mathrm{cc}$. of $30 \%$ sulphuric acid $\left(25^{\circ}\right.$ Baumé) to free the fatty acids, and boil until they form a clear, transparent layer. Wash with boiling water until free from sulphuric acid, collect in a small beaker, and place on the steam bath until the water has settled and the fatty acids are clear; then decant them into a dry beaker, filter, using a hot-water funnel, and dry twenty minutes at $100^{\circ} \mathrm{C}$.

When dried, cool the fatty acids to 15 or $20^{\circ} \mathrm{C}$. above the expected titer, and transfer to the titer tube, which is $25 \mathrm{~mm}$. in diameter and 100 $\mathrm{mm}$. in length ( $\mathrm{I}$ by 4 inches), and made of glass about $\mathrm{I} \mathrm{mm}$. in thickness. Place in a 16-ounce saltmouth bottle of clear glass, about $70 \mathrm{~mm}$. in diameter and $150 \mathrm{~mm}$. high ( 2.8 by 6 inches), fitted with a cork, which is perforated so as to hold the tube rigidly when in position. Suspend the

* A. O. A. C. Method, U. S. Dept. of Agric., Bur. of Chem. Bul, ro7, p. 135 . 
thermometer, graduated to $0.10^{\circ} \mathrm{C}$., so that it can be used as a stirrer, and stir the mass slowly until the mercury remains stationary for thirty seconds. Then allow the thermometer to hang quietly, with the bulb in the center of the mass, and observe the rise of the mercury. The highest point to which it rises is recorded as the titer of the fatty acids.

Test the fatty acids for complete saponification as follows:

Place $3 \mathrm{cc}$. in a test tube and add $\mathrm{I}_{5} \mathrm{cc}$. of alcohol ( $95 \%$ by volume). Bring the mixture to a boil and add an equal volume of ammonium hydroxide ( $0.96 \mathrm{sp}$. gr.). A clear solution should result, turbidity indicating unsaponified fat. The titer must be made at about $20^{\circ} \mathrm{C}$. for all fats having a titer above $30^{\circ} \mathrm{C}$. and at $10^{\circ} \mathrm{C}$. below the titer for all other fats.

The thermometer must be graduated in tenth degrees from $10^{\circ}$ to $60^{\circ}$, with a zero mark, and have an auxiliary reservoir at the upper end, also one between the zero mark and the $10^{\circ}$ mark. The cavity in the capillary tube between the zero mark and the $10^{\circ}$ mark must be at least $\mathrm{I} \mathrm{cm}$. below the $10^{\circ}$ mark, the $10^{\circ}$ mark to be about 3 or $4 \mathrm{~cm}$. above the bulb, the length of the thermometer being about $1_{5}$ inches over all. The thermometer is annealed for 75 hours at $45^{\circ} \mathrm{C}$., and the bulb is of Jena normal $16^{\mathrm{II}}$ glass, moderately thin, so that the thermometer will be quick acting. The bulb is about $3 \mathrm{~cm}$. long and $6 \mathrm{~mm}$. in diameter. The stem of the thermometer is $6 \mathrm{~mm}$. in diameter and made of the best thermometer tubing, with scale etched on the stem, the graduation to be clear cut and distinct, but quite fine.*

Unsaponifiable Matter.-As will be seen by reference to the table on page 509, the unsaponifiable matter in pure edible oils and fats is comparatively insignificant in amount, consisting largely of cholesterol or phytosterol. A high content of unsaponifiable matter is indicative of adulteration, pointing to the presence of mineral or coal-tar oils, or to paraffin.

Determination of Unsaponifiable Matter. $\dagger$-Weigh 7 to ro grams of the fat or oil in a $250-\mathrm{cc}$. flask, and saponify by boiling with $25 \mathrm{cc}$. of alcoholic potassium hydroxide and $25 \mathrm{cc}$. of alcohol under a returnflow condenser. After saponification, add 30 to $40 \mathrm{cc}$. of water, and bring to the boiling-point. Cool and transfer the contents from the flask to a separatory funnel, washing out the flask first with a small amount

* Tolman, U. S. Dept. of Agric, Bur. of Chem., Bul. 90, p. 75.

† Hönig and Spitz, Jour. Soc. Chem. Ind., 189r, p. 1039. 
of $50 \%$ alcohol, and finally with $50 \mathrm{cc}$. of petroleum ether (B.P. $40^{\circ}-70^{\circ}$ ), adding both washings to the separatory funnel. Shake the latter thoroughly, but avoid if possible forming an emulsion. If the latter persists in forming, add a volume of water equal to that of the soap solution, which will sometimes break it up. After separation of the petroleum ether layer, draw off the underlying soap solution into a beaker, and wash the petroleum ether two or three times with $50 \%$ alcohol, which is drawn off and added to the soap solution. The petroleum ether is then run into a tared Erlenmeyer flask, and the soap solution extracted twice more with fresh portions of petroleum ether, washing the ether each time with $50 \%$ alcohol as before and then transferring the ether to the tared flask. The petroleum ether is then removed by placing the flask on the water-bath, bumping being prevented by means of a spirai of platinum wire weighed with the flask. Finally remove all traces of remaining ether by blowing hot air through the flask, or, in the absence of mineral oils (some of which are volatile), dry in the water-oven to constant weight, cool in a desiccator, and weigh.

Cholesterol and Phytosterol.-These are monatomic alcohols, and combine with the fatty acids forming esters. Both respond to the same reactions, and are separated by the same process from the oils and fats in which they occur. Phytosterol was long thought to be the same as cholesterol, and some confusion seems to have arisen from the fact that early writers purport to have found cholesterol in vegetable oils, when in reality the substance was phytosterol. The latter was first distinguished from cholesterol by Hesse, who named it.

Cholesterol $\left(\mathrm{C}_{26} \mathrm{H}_{44} \mathrm{O}\right)$ crystallizes in white, nacreous, monoclinic laminx, having a melting-point of $145^{\circ}$ and specific gravity 1.067. Its reaction is neutral, it is devoid of taste or smell, insoluble in water, sparingly soluble in cold, but readily soluble in boiling alcohol, and soluble in ether, chloroform, methyl alcohol, benzene, and oil of turpentine. It sublimes unchanged at $200^{\circ}$, but at higher temperatures decomposes.

Commercial cholesterol is obtained from wool oil and is known as lanolin, being used largely in medicine as a basis for ointment.

Cholesterol occurs also in the yolk of eggs, in many animal secretions, and in most animal oils and fats.

It separates in laminated, transparent crystals from a mixture of 2 volumes alcohol and I volume ether, and in the form of anhydrous needles from chloroform.

Phytosterol $\left(\mathrm{C}_{26} \mathrm{H}_{44} \mathrm{O}, \mathrm{H}_{2} \mathrm{O}\right)$ is most abundantly found in the legu- 
minous seeds, and is prepared commercially from these, especially from peas and lentils. It is a constituent of most vegetable oils.

It crystallizes in slender, glittering plates from chloroform, ether, and petroleum ether, and from alcohol in tufts of needles. In solubility it much resembles cholesterol, but its melting-point from $I_{3} 2^{\circ}$ to $I_{3} 4^{\circ}$ is lower.

Determination of Cholesterol and Phytosterol.-Method of Forster and Reichmann.*-50 grams of the oil or fat are boiled for five minutes in a flask connected with a reflux condenser with two successive portions of $75 \mathrm{cc}$. of $95 \%$ alcohol, and in each case the alcoholic solution is separated by means of a separatory funnel. The combined alcoholic solutions are then boiled in a flask provided with a funnel in the neck, till onefourth of the alcohol is evaporated, and then poured into an evaporating dish and brought to dryness. The residue is then extracted with ether, and the ether solution is evaporated to dryness, taken up again with ether, filtered, evaporated once more, and dissolved in hot $95 \%$ alcohol, from which it is allowed to crystallize. Cholesterol or phytosterol will crystallize out under these conditions, and may be weighed.

Distinguishing between Cholesterol and Phytosterol.-It is sometimes of importance to determine which of these substances is present in an oil, or whether indeed both occur. Confirmatory proof as to the presence of vegetable in animal oils may, for instance, be established by showing whether the unsaponifiable residue in the sample contains cholesterol or phytosterol or both. Hehner $\dagger$ has made use of this test in determining the presence of cottonseed oil in lard.

The most ready means of distinguishing between cholesterol and phytosterol is furnished by the marked difference between the form of the crystals, the manner of crystallization of the two substances, and the melting points of the acetates.

\section{Separation and Crystallization of Cholesterol and Phytosterol.-} Bömer's Method. $\ddagger$-Saponify 100 grams of the fat by heating in a liter Erlenmeyer flask on a boiling water bath with $200 \mathrm{cc}$. of alcoholic potash sslution (200 grams of potassium hydroxide $+\mathrm{I}$ liter of alcohol). The flask should be provided with a perforated rubber stopper, through which passes a glass tube $700 \mathrm{~cm}$. long, which serves as a reflux condenser. During the first part of the heating shake often and vigorously until the solution is clear, after which continue the heating one-half to one hour longer with occasional shaking.

* Analyst, 22, 1897, p. I3I.

† Ibid., 13,1888 , p. 165 .

† Zeits. Unters. Nahr. Genuss., 1, 1898, p. 31 . I 
While still warm, transfer to a separatory funnel of about I.5 liters capacity, rinsing the flask with $400 \mathrm{cc}$. of water. When cool, add $500 \mathrm{cc}$. of ether, shake vigorously for one-half to one minute, opening the cock repeatedly, and allow to stand for two to three minutes until the liquids separate. Remove the ether solution to a flask, and distil off the ether, using a few pieces of pumice stone to prevent bumping. Shake the soap solution two to three more times in the same manner with 200 to $250 \mathrm{cc}$. of ether, add the ether solution after each shaking to the residue in the distilling flask, and distil off the ether.

Usually a small amount of alcohol remains in the flask after removal of the ether, which may be removed by heating on a boiling water bath in a blast of air. To saponify any remaining fat, add $20 \mathrm{cc}$. of the alcoholic potash solution, and heat for five to ten minutes as before. Transfer to a small separatory funnel, rinse with $40 \mathrm{cc}$, of water, cool and shake with $150-200 \mathrm{cc}$. of ether from one-half to one minute, allow to stand two to three minutes, and draw off the lower layer. Wash the ether solution three times with $10-20 \mathrm{cc}$. of water, filter, to remove drops of water, into a small beaker, and remove the ether by cautious evaporation on the water bath, thus obtaining the crude cholesterol or phytosterol.

The unsaponifiable residue, which may be weighed after drying, in the case of animal fats shows beautiful radiating crystals, and consists largely of cholesterol, while in the case of vegetable fats it consists largely of phytosterol. Dissolve the residue in $4-20 \mathrm{cc}$. of absolute alcohol with the aid of heat, and allow to crystallize slowly in a shallow dish.

The crystallization in the case of cholesterol alone begins from the margin of the liquid and gradually extends inward toward the center, forming a uniformly bright, thin, colorless film over the whole surface. This film is best removed with a knife or spatula and pressed between filter-paper. The film will be seen, even megascopically, to be composed of large, glossy plates with a silk-like luster. After the removal of the first film a second will form similar to the first, but composed as a rule of smaller crystals. These are removed in like manner, dried between filters, and added to the first in a glass. After the second crop, the mother liquid is thrown away. The crystals are then redissolved in absolute alcohol, and again allowed to separate out, being repeatedly recrystallized till the melting-point is constant. In lard and most fats the crystals were found pure by Bömer after the second crystallization.

Phytosterol is crystallized with greater difficulty, especially when derived from seed oils, on account of the presence of pigments and other 
foreign matter. The first procedure is the same as above described for cholesterol, the crystals being allowed to separate slowly out of a solution in absolute alcohol. Unlike cholesterol, no film is formed on the surface, but needles (sometimes I cm. in length) are gradually eliminated, beginning at the margin and extending inward mostly at the bottom. In concentrated solutions, fine needles would be uniformly deposited through the liquid. These are best separated from the mother liquid by filtration, as they are not easily taken out with a knife. They may be washed on the filter with small amounts of absolute alcohol for microscopical examination, or repeatedly recrystallized, as in the case of cholesterol, till the melting-point is constant.

I. Cholesterol Crystals. - When crystallized separately under above conditions, cholesterol crystals viewed under the microscope show generally rhomboidal forms of plates, as in Fig. 97 , but sometimes with a reenter-
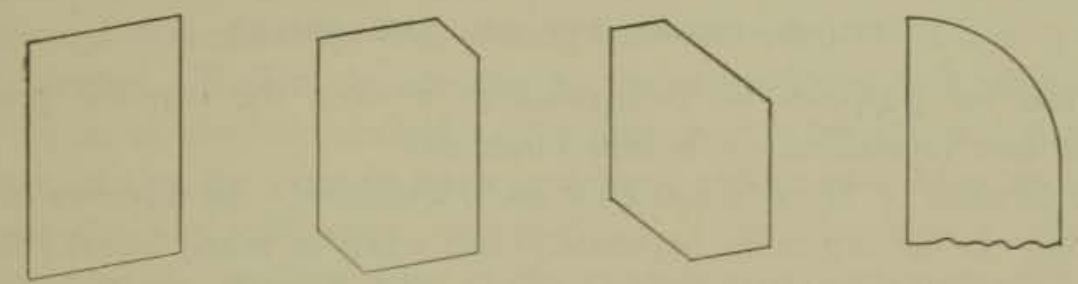

Fig. 97.-Cholesterol Crystals under the Microscope. (After Bömer.)

ing angle. The plates are often grown together in masses. The most characteristic forms are found from the first crystallization or from the first film removed. Sometimes quadrilateral crystals predominate ameng the plates, often also the other shapes shown are found most numerous.

2. Phytosterol Crystals,-Pure phytosterol crystallizes in needles or narrow plates, arranged commonly in star form or in bunches. The most common forms are shown in Fig. 98, best conditions as to shape of crystals being obtained from slow crystallization, in which case the needles are finer and more regular.

The crystals are commonly in the form of long, narrow plates, thin and slender, often pointed at both ends. Sometimes the points are lacking, or the ends are beveled. The more frequently they are recrystallized, the larger and more varied are the crystal forms. The broad, hexagonal and quadrilateral plates shown are products of re- 
crystallization; the shorter forms are rarely met with. Sometimes various forms are found side by side in the same crystallization.

Phytosterol crystals, from a second or third recrystallization, sometimes grow together in bunches resembling at first glance to the naked eye the cholesterol masses. They never do this in the first crystallization, whereas in the case of cholesterol the growing together in masses is very characteristic of the first crystallization.
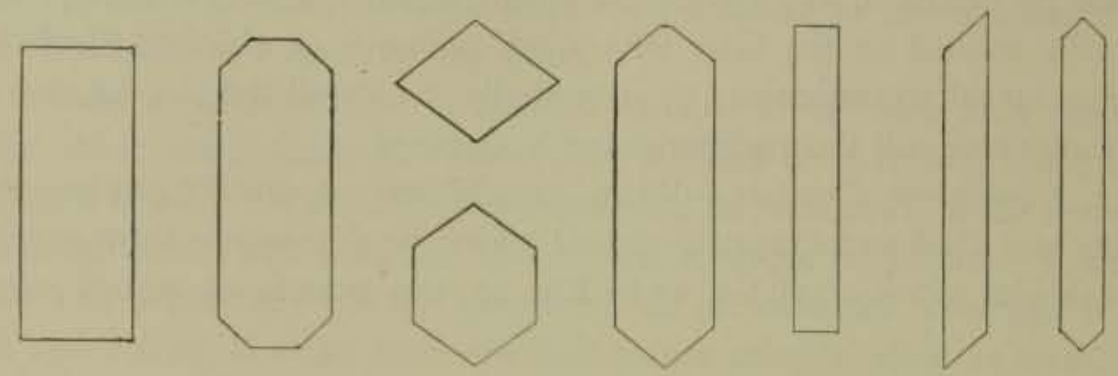

Fic. 98.-Phytosterol Crystals. (After Bömer.)

Thus for purposes of distinguishing between the two the product of the first crystallization is best observed.

3. Crystals of Mixed Cholesterol and Phytosterol.-In mixtures of the two they do not crystallize separately, but when in nearly equal proportion, or with phytosterol predominating, the crystals much resemble phytosterol. Even when cholesterol predominates to the extent of 20 parts to I of phytosterol, the mode of crystallization leans most toward that of phytosterol, though the needles are of different shape. Such a mixture, for instance, does not form in a film like cholesterol, but, like phytosterol, comes out in needle-like bunches. The needles, however, are more often like those shown in Fig. 99 when viewed under the micro-
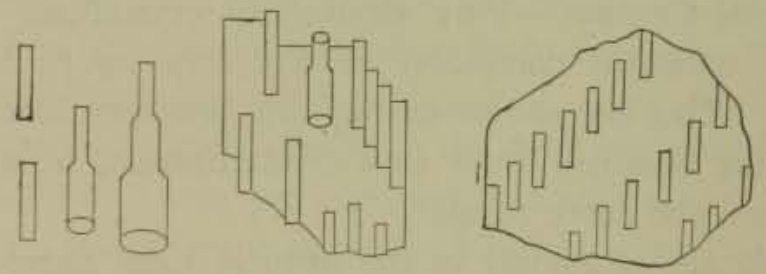

Frg. 99.-Characteristic Forms of Crystallization of Mixed Cholesterol and Phytosterol (After Bōmer.)

scope, showing needles for the most part squarely cut off at the ends, and sometimes placed end to end, and of varying diameter, giving the appearance of a spy-glass. When cholesterol predominates over phytosterol $5 \circ$ to $I$, the plates resemble those of cholesterol. 
Bömer's Phytosterol Acetate Test for Vegetable Fats.*-Dissolve the crude cholesterol or phytosterol, or the mixture of the two, obtained by Bömer's method, as described on page $50_{3}$, in the smallest possible amount of absolute alcohol, and allow to crystallize. Examine under the microscope the first crystals that separate, comparing with the cuts and descriptions given in the preceding section. Remove the alcohol completely by evaporation on the water bath, add 2 to $3 \mathrm{cc}$. of acetic anhydride, cover with a watch glass, and boil for one-fourth minute on a wire gauze; then remove the watch glass, and evaporate the excess of acetic anhydride on the water bath. Heat the residue with sufficient absolute alcohol to dissolve the esters, and add enough more to prevent immediate crystallization on cooling. Cover until the room temperature is reached and allow to crystallize.

After one-half to one-third of the liquid has evaporated and the greater part of the esters have crystallized, transfer the crystals to a small filter by the aid of a small spatula, rinsing with two portions of 2 to $3 \mathrm{cc}$. of $95 \%$ alcohol. Return the crystals to the crystallizing dish, dissolve in 5 to ro cc. of absolute alcohol, and again allow to crystallize. After the greater part of the crystals have separated, collect on a filter as before. Repeat the recrystallization several times ( 5 to 6 is usually sufficient), determining the melting point of the crystals after each recrystallization beginning with the third.

If after the last crystallization the corrected melting point of the crystals is above $116^{\circ}$, the presence of a vegetable fat or oil is indicated, if it is II $7^{\circ}$ or higher the proof may be regarded positive.

The standard thermometer used should be graduated to tenths of a degree. Correct the reading by the following formula:

$$
S=T+0.000154 n(T-t)
$$

in which $S=$ the corrected melting point, $T=$ the observed melting point, $n=$ the length of the mercury column above the surface of the liquid, expressed in degrees, and $t=$ the temperature of the air about the mercury column as determined by a second thermometer.

Bömer states that by this method the analyst can detect in edible animal fats $I$ to 2 per cent of oils rich in phytosterol (cottonseed, peanut, sesame, rape, hemp, poppy, and linseed), and 3 to 5 per cent of oils containing smaller amounts of this constituent (olive, palm, palm kernel, and probably cocoanut). He found the corrected melting point of cholesterol acetate to be $I I 4.3^{\circ}$ to $114.8^{\circ}$ and of phytosterol acetate, $125.6^{\circ}$ to I $37.0^{\circ}$ according to the source.

\footnotetext{
*Zeits. Unters, Nahr. Genuss., 4, rgor, p, 1070.
} 


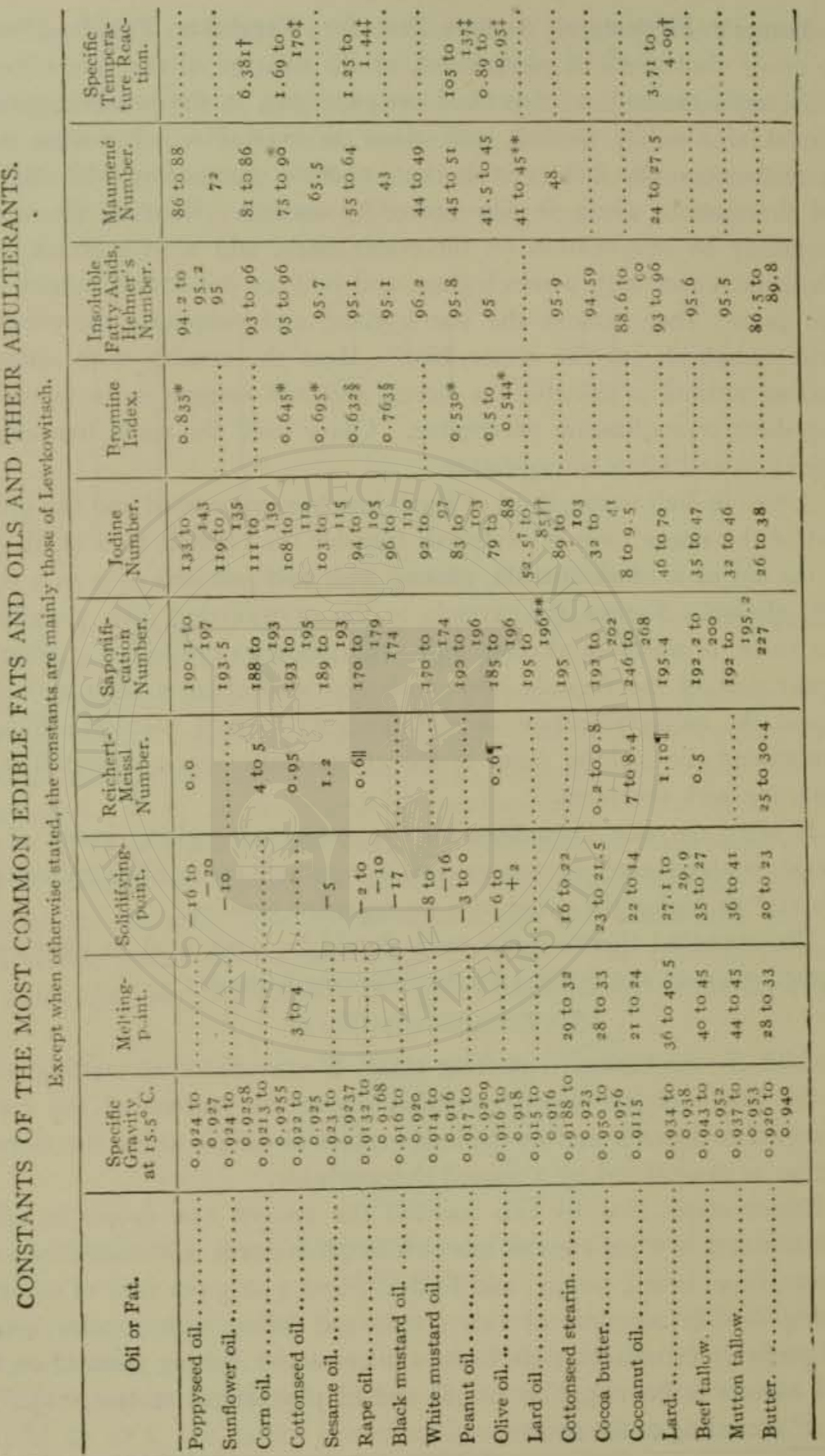




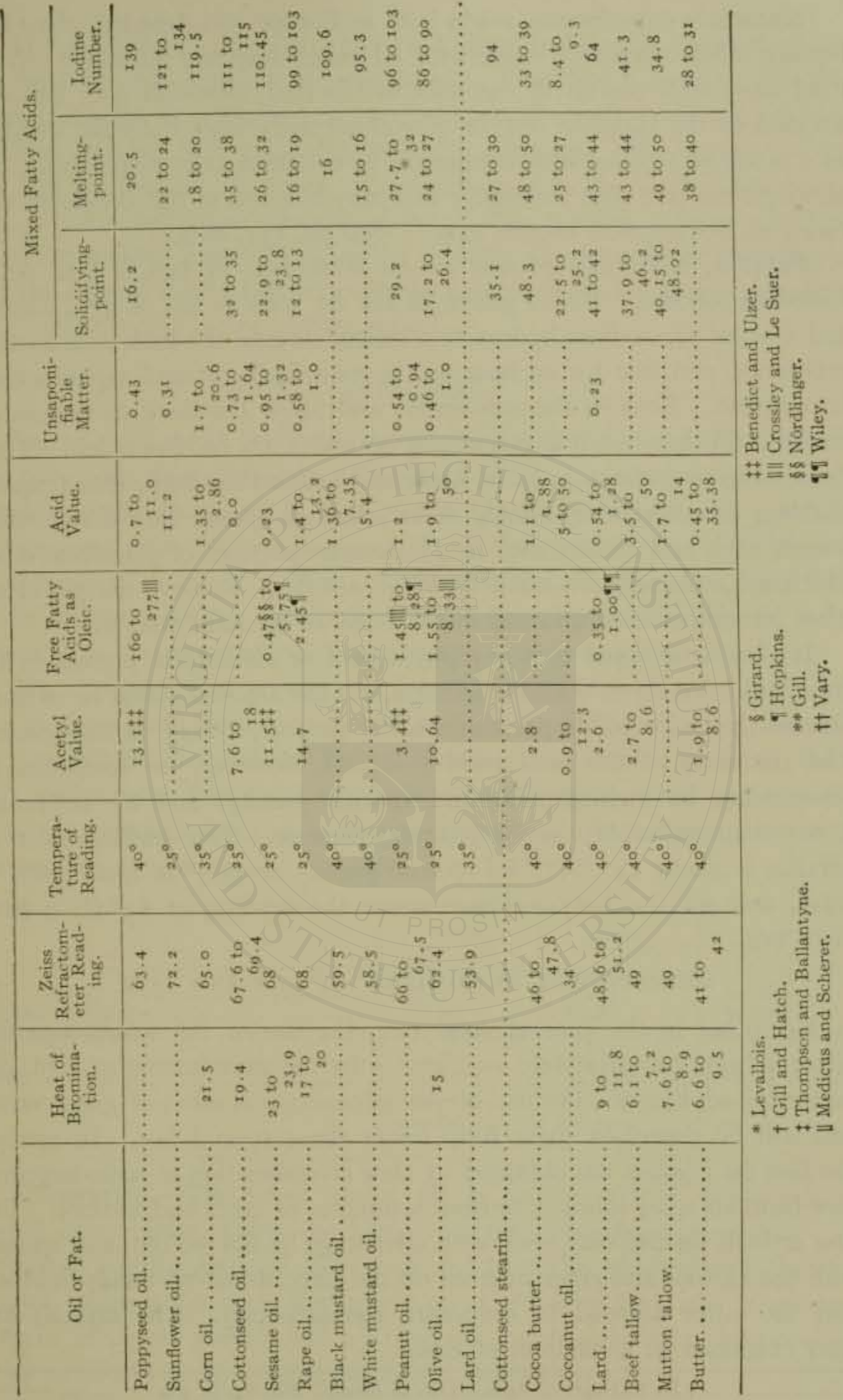


Numerous experiments, made both in Europe and America, show that feeding milch cows and swine with oil cakes does not introduce phytosterol into either the fat of the milk or the lard, although both fats may respond to the Halphen test, or give abnormally high Polenske numbers as a result of feeding with cottonseed or cocoanut cake respectively, and although the lard (not the butter fat) may respond to the Baudouin test, owing to feeding with sesame cake. (See Pp. 531, 560 ).

Paraffin, sometimes present as an adulterant of fats, is best determined as follows: * Boil 2 grams of the fat with io cc. of $95 \%$ alcohol and $2 \mathrm{cc}$. of I : I sodium hydroxide solution, connect the flask with a reflux condenser, and heat for an hour on the water-bath, or until saponification is complete. Remove the condenser, and allow the flask to remain on the bath till the alcohol is evaporated off and a dry residue is left. Treat the residue with about $40 \mathrm{cc}$. of water and heat on the bath, with frequent shaking, till everything soluble is in solution. Wash into a separatory funnel, cool, and extract with four successive portions of petroleum ether, which are collected in a tared flask or capsule. Remove the petroleum ether by evaporation and dry in the oven to constant weight.

It should be noted that any phytosterol or cholesterol present in the fat would come down with the paraffin, but the amount would be so insignificant that with added paraffin actually present, it may be disregarded. The character of the final residue should, however, be confirmed by determining its melting-point and specific gravity, and by subjecting it to examination in the butyro-refractometer. The melting-point of paraffin is about $54.5^{\circ} \mathrm{C}$.; its specific gravity at $15.5^{\circ}$ is from 0.868 to $0.9{ }^{1} 5$, and on the butyro-refractometer the reading at $65^{\circ} \mathrm{C}$. is from II to 14.5 .

\section{MICROSCOPICAL EXAMINATION OF OILS AND FATS.}

Excepting in the case of solid fats, the use of the microscope has hitherto been comparatively restricted. In the examination of lard and butter for adulterants, the use of the microscope is often of great value, and will be described more fully under these special fats. In general the best fat crystals are obtained by slow crystallization at room temperature from an ether solution, or from a mixture of ether and alcohol. The first crystals formed may often with advantage be filtered out, and washed with the alcohol and ether mixture on the filter, dissolved finally in ether, and the latter allowed to evaporate spontaneously. The crystals are then examined in a medium of ether. 
If it be desired to separate the liquid oleins from an oil, so that crystals of the solid fats are left for examination, Gladding* recommends dissolving the fat in a mixture of two volumes of absolute alcohol and one volume of ether in a test-tube, which is stoppered with cotton and set for half an hour in ice water, during which time the more solid stearin and palmitin will have crystallized out. This portion is then separated from the mother liquor by filtration through an alcohol-wet filter-paper, and the crystals finally treated as in the preceding section, being examined in a medium of olive or cottonseed oil.

\section{OLIVE OIL.}

Source.-Olive oil is derived from the fruit of the cultivated thornless olive tree, Olea Europaea sativa, $\boldsymbol{\dagger}$ of which there are a great many varieties, originally grown in Asia Minor, Greece, Palestine, and southern Europe, and now cultivated extensively in California, Peru, and Mexico, as well as in Australia. Most of the olive oil of commerce, especially of the choicest varieties, is supplied by southern France, Spain, and Italy. The tree is an evergreen of slow growth and great longevity.

The ripe olive fruit is purple or purplish black in color; it is round or oval in shape, and from 2.5 to $4 \mathrm{~cm}$. in diameter. The oil is contained in the parenchyma cells of the fruit suspended in a watery fluid. A thick skin incloses the fruit, and within is a kernel, which itself contains oil. The fruit contains from 40 to 60 per cent of oil. According to Brannt, $\ddagger$ the average composition of the olive is as follows:

\begin{tabular}{|c|c|c|c|}
\hline & $\begin{array}{l}\text { Flesh, } \\
\text { Per Cent. }\end{array}$ & $\begin{array}{l}\text { Stone, } \\
\text { Per Cent. }\end{array}$ & $\begin{array}{l}\text { Seed, } \\
\text { Per Cent. }\end{array}$ \\
\hline 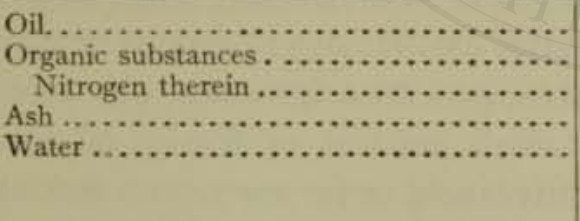 & $\begin{array}{r}56.4 \\
16.70 \\
2.68 \\
24.22 \\
100.00\end{array}$ & $\begin{array}{r}85.75 \\
\quad 2.50 \\
4.16 \\
\begin{array}{c}4.20 \\
100.00\end{array}\end{array}$ & $\begin{array}{r}12.26 \\
79.3^{8} \\
2.16 \\
6.20 \\
100.00\end{array}$ \\
\hline
\end{tabular}

Preparation.-The finest virgin oil is produced from hand-picked, peeled olives, from which the kernels or pits have been removed. A somewhat inferior grade of oil is produced from the whole olive including the pit, while a distinctly low grade oil is obtained from the stones, or kernels, which are ground into a coarse meal and subjected to pressure, or to the action of such solvents as carbon bisulphide.

* Jour. Am. Chem. Soc, 1896,18 , p. 189 .

† As distinguished for the wild thorny species, Europaa sylvestris.

$\ddagger$ Animal and Vegetable Fats and Oils. 
In the process of manufacture the fruit, after first being dried, is reluced to a pulp in a stone or iron mill, and the pulpy mass, contained in baskets or bags, is subjected to pressure in an iron press. The very highest grade of virgin oil is that which runs out from the pulp with little or no pressure. After the first pressing, the pomace is ground, treated with water, and again subjected to pressure. Several pressings in this manner may be carried out, each yielding an oil inferior to that preceding, the lowest grades being used for lubricants and in the manufacture of soap.

Nature and Composition.-The better grades of olive oil, suitable for table and medicinal purposes, possess a pleasant, bland taste, and a distinctive and agreeable odor, unmistakable in character for that of any other oil. The finest virgin oil is pale green in color, due to the presence of chlorophyll, which is closely associated with the oil globules in the cellular tissue of the fruit. Some varieties of olive oil are nearly colorless, while others are a deep golden yellow.

Olive oil contains $28 \%$ of solid glycerides, chiefly palmitin and a very small amount of arachin, and $72 \%$ of liquid glycerides, mainly olein with a little linolein. Stearin is practically absent. Lewkowitch * states that olive oil differs from most vegetable oils in containing cholesterol but not phytosterol. Gill and Tufts $\dagger$ show that olive oil is not thus exceptional, but that the unsaponifiable alcohol is phytosterol and not cholesterol.

Olive oil is very soluble in chloroform, benzol, and carbon bisulphide, but is sparingly soluble in alcohol. Five parts of ether will dissolve 3 parts of the oil.

For customs purposes the United States Government considers a gallon equivalent to 7.56 pounds which is slightly below the truth.

ADULTERANTS.-As a rule the low grade olive oils are most subject to adulteration, by reason of the fact that it hardly pays to destroy or even modify the fine quality and delicacy possessed by a first-class oil, which would inevitably be the result if even a small amount of foreign oil were added. Furthermore, if olive oil be slightly rancid or for any reason lacking in flavor, the admixture of a bland oil tends rather to minimize the fact.

The most common adulterant of olive oil in this country is naturally cottonseed oil, which is often substituted wholly for it. In Europe peanut oil is sometimes used both as an admixture and even as a substiiute, since it possesses in itself a rather pleasant flavor, rendering it especially adapted for use as an adulterant. Other cheap oils used for this purpose are corn, mustard, poppyseed, rape, sesame, and sunflower

* Chem. Anal. of Oils, Fais, and Waxes, ad ed, p. $45^{2}$.

† Jour. Am. Chem. Soc., XXV, r9o3, p. 498. 
oil. The writer has also found in samples of alleged olive oil sold in Massachusetts cocoanut oil* and even fish oil.

Pure Olive Oil of the U. S. Pharmacopœia.-The requirements of the Pharmacopocia are as follows:

Specific gravity, 0.910 to 0.915 at $25^{\circ} \mathrm{C}$. $\left(77^{\circ} \mathrm{F}\right.$.); iodine value not less than 80 nor more than 88 ; saponification value I9I to I95. Very sparingly soluble in alcohol, but readily soluble in ether, chloroform, or carbon disulphide.

When cooled to about $10^{\circ} \mathrm{C}$. $\left(50^{\circ} \mathrm{F}\right.$.), the oil should become somewhat cloudy from the separation of crystalline particles, and at $0^{\circ} \mathrm{C}$. $\left(32^{\circ} \mathrm{F}\right.$.) it should form a whitish, granular mass.

If $2 \mathrm{cc}$. of olive oil be shaken vigorously with an equal volume of nitric acid (sp. gr. 1.37), the oil should retain a light yellow color, not becoming orange or reddish brown, and after standing for six hours, should change into a yellowish-white solid mass and an almost colorless liquid (absence of appreciable quantities of cottonseed oil and other seed oils).

Olive oil should not show the cottonseed oil reaction with the Bechi and Halphen test, p. 5 I8, nor the sesame oil reaction with the Baudouin test, p. 5 I9.

U. S. Standards. Olive oil is the oil obtained from the sound, mature fruit of the cultivated olive tree (Olea europoea L.) and subjected to the usual refining processes; is free from rancidity; has a refractive index $\left(25^{\circ} \mathrm{C}\right.$.) not less than 1.4660 and not exceeding 1.4680 ; and an iodine number not less than 79 and not exceeding 90. Virgin alive oil is olive oil obtained from the first pressing of carefully selected, hand-picked olives

Reaction with Strong Acid.-Pure olive oil, when shaken or stirred with an equal volume of concentrated nitric or sulphuric acid, turns from a pale to a dark-green color in a few minutes. If, under this treatment, a reddish to an orange, or brown coloration is produced, the presence of a foreign vegetable oil (usually a seed oil) is to be suspected.

Bach gives the following table showing the action of strong nitric acid on various oils:

\begin{tabular}{|c|c|c|c|}
\hline Kind of Oit. & $\begin{array}{l}\text { After Agitation } \\
\text { with Nitric Acid. }\end{array}$ & $\begin{array}{l}\text { After Heating for } \\
\text { Five Minutes. }\end{array}$ & $\begin{array}{l}\text { Conisistency after } \\
\text { Standing Twelve to } \\
\text { Eighteen Hours. }\end{array}$ \\
\hline 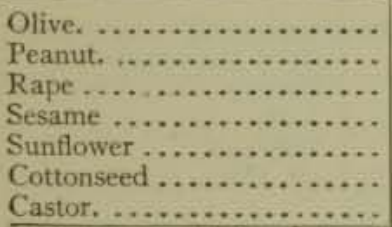 & $\begin{array}{l}\text { Pale green } \\
\text { ". rose } \\
\text { White } \\
\text { Dirty white } \\
\text { Yellowish brown } \\
\text { Pale rose }\end{array}$ & $\begin{array}{l}\text { Orange-yellow } \\
\text { Brownish yellow } \\
\text { Orange-yellow } \\
\text { Brownish yellow } \\
\text { Reddish yellow } \\
\text { Reddish brown } \\
\text { Golden yellow }\end{array}$ & $\begin{array}{l}\text { Solid } \\
\text { "t } \\
\text { Liquid } \\
\text { Buttery } \\
\text { ". }\end{array}$ \\
\hline
\end{tabular}

* A sample of alleged olive oil purchased in a Massachusetts drug store and found to be adulterated with cocoanut oil, had the following constants:

Specific gravity ............... 0.911

Reichert-Meissl number ........... 2.90

Iodine number............... 74.5

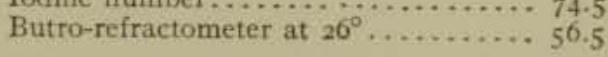


The Zeiss Butyro-refractometer furnishes one of the most useful and easily applied preliminary means of judging the purity of the sample. If the reading is beyond the limits of pure olive oil, it at once indicates adulteration and often points to the particular adulterant. On the other hand, it is not always safe to assume the oil to be pure if the reading is correct, since mixtures of higher and lower refracting foreign oils may be so skillfully prepared as to read well within the limits of the pure oil on the refractometer scale. The refractometer reading of pure cottonseed oil is almost five degrees higher than that of pure olive.

\section{READINGS ON ZEISS REFRACTOMETER OF OLIVE AND COTTONSEED} OILS.*

\begin{tabular}{|c|c|c|c|c|c|}
\hline \multirow{2}{*}{$\begin{array}{l}\text { Temperature } \\
\text { (Centigrade). }\end{array}$} & \multicolumn{2}{|c|}{ Scale Reading. } & \multirow{2}{*}{$\begin{array}{l}\text { Temperature } \\
\text { (Centisrade). }\end{array}$} & \multicolumn{2}{|c|}{ Scale Reading. } \\
\hline & Olive Oil. & Cottanseed Oil. & & Olive Oit. & Cottonseed Oil. \\
\hline $35 \cdot 0$ & 57.0 & 61.8 & $25 \cdot 5$ & 62.4 & $67 \cdot 5$ \\
\hline $34 \cdot 5$ & $57 \cdot 2$ & 62.1 & 25.0 & 63.0 & 67.9 \\
\hline 34.0 & $57 \cdot 4$ & $62 \cdot 3$ & $24 \cdot 5$ & $63 \cdot 3$ & 68.2 \\
\hline $33 \cdot 5$ & $57 \cdot 7$ & 62.5 & 24.0 & 63.6 & 68.5 \\
\hline 33.0 & 58.0 & 62.8 & $23 \cdot 5$ & 63.9 & 68.8 \\
\hline $3^{2} \cdot 5$ & 58.3 & 63.0 & 23.0 & $64 \cdot 2$ & $69 \cdot \mathrm{r}$ \\
\hline 32.0 & $5^{8} \cdot 5$ & 63.2 & 22.5 & 64.5 & 69.4 \\
\hline $3^{I}-5$ & 59.0 & 63.6 & 22.0 & 64.8 & 69.7 \\
\hline 31.0 & 59.2 & 64.0 & $2 \mathrm{I} \cdot 5$ & $65 . \mathrm{I}$ & 70.0 \\
\hline 30.5 & 59.4 & 64.2 & $2 \mathrm{I} .0$ & $65 \cdot 4$ & 70.3 \\
\hline 30.0 & 59.9 & $64 \cdot 5$ & 20.5 & $65 \cdot 7$ & 70.6 \\
\hline $29 \cdot 5$ & 60.1 & 64.9 & 20.0 & 66.0 & 70.9 \\
\hline 29.0 & 60.3 & 65.1 & 19.5 & 66.3 & 71.2 \\
\hline 28.5 & 60.6 & $65 \cdot 3$ & 19.0 & 66.6 & 71.5 \\
\hline 28.0 & 60.9 & $65 \cdot 7$ & 18.5 & 66.9 & 71.8 \\
\hline $27 \cdot 5$ & 61.1 & 66.0 & 18.0 & $67 \cdot 2$ & 72.1 \\
\hline 27.0 & $6 r \cdot 5$ & 66.5 & 17.5 & 67.5 & 72.4 \\
\hline 26.5 & 62.0 & 67.0 & 17.0 & 67.8 & 72.7 \\
\hline 26.0 & 62.2 & $67 \cdot 3$ & 16.5 & 68.1 & 73.0 \\
\hline
\end{tabular}

The Elaidin Test, in the case of pure olive oil, is very distinctive, since it yields by far the hardest elaidin of all the common oils, and solidifies the most quickly.

Archbutt $\dagger$ shows the effect on this test of the mixture with olive oil of various proportions of rape and cottonseed oil, as follows:

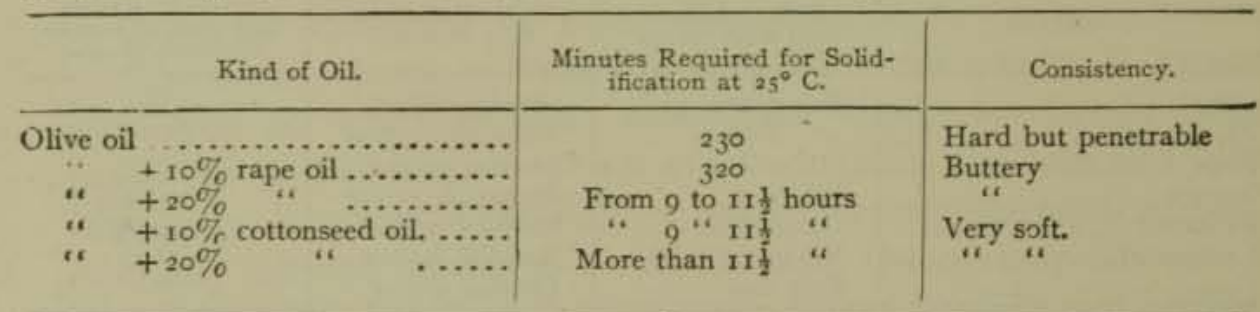

* Ann. Rep. Mass. State Bd. of Health, 1899, p. 647. † Jour. Soc. Chem. Ind., 1897, p. 447. 
Cottonseed Oil as an adulterant is best detected by means of the Halphen or Bechi tests. Its presence in notable quantities increases the specific gravity, refractometer reading, and iodine number very materially. Its high Maumené figure is also distinctive.

Peanut Oil, when present to a considerable extent, betrays its presence by its peculiar bean-like flavor. Most of the constants of peanut oil lie within the limits of olive oil, with the exception of the higher odine number and refractometer reading. A considerable admixture of peanut oil raises the refractometer reading perceptibly over that of pure olive. Its presence is best shown positively by tests for arachidic acid (p. 523), noting that traces of arachin have been reported in pure olive oil, insufficient, however, to interfere with the detection of added peanut oil.

Sesame Oil differs more particularly from olive in its higher specific gravity and iodine and Maumené numbers, and is readily detected by distinctive color tests (p. 5I9).

Rape Oil is characterized by a much lower saponification value and higher iodine number than olive.

Corn Oil differs materially from olive in its exceedingly high iodine number and refractometer reading. Its specific gravity and saponification numbers are also higher.

Lard Oil, when present in considerable quantity, is often rendered apparent by its characteristic odor on warming. Its low refractometer reading and iodine number are also distinctive.

Poppyseed Oil differs most widely from olive oil in its refractometric reading, its high dispersion, and its Maumené number, which in the case of poppyseed is $87^{\circ}$ and of olive about $42^{\circ}$.

Cocoanut $\mathrm{Oil}$ in mixture with olive perceptibly raises the solidifyingpoint. When more than $12 \%$ of cocoanut oil is present, the sample will become solid when placed in ice water.

Fish Oils, when present, are rendered apparent by reason of their strong taste and smell, and by their very high iodine number. Boiling the sample with sodium hydroxide develops a peculiar reddish coloration, when fish oils are present.

Routine Examination of Olive Oil for Adulterants.-First note the smell and taste of the sample, and then take the refractometric reading. An abnormally high refraction indicates adulteration. Then test with strong nitric acid (p. $5^{1} 3$ ). If the refraction is normal, and the color resulting from the acid reaction a pale green, the presumption is that the oil is pure. Test first for cottonseed oil by the Halphen reaction, 
and then in succession try the various color reactions for sesame and rape oils. If alk these are absent, and, by abnormal constants, or by color with nitric acid, there is reason to believe the oil is adulterated, determine carefully such of the constants as are most indicative, by their wide variation from olive, of poppyseed, mustard, and corn oils.

If all these oils are presumably absent, and either a high refractometer reading or a color reaction with nitric acid still indicates adulteration, peanut oil is more than likely to be present, and should be tested for either by Renard's or Bellier's method.

The edible oils and adulterants are arranged in order of their relative price about as follows: Olive oil, peanut oil, lard oil, sesame oil, poppyseed oil, rape oil, corn oil, cottonseed oil.

\section{COTTONSEED OIL.}

Source and Preparation.-This oil, largely used as a table oil and as an adulterant of olive oil, is derived from seeds of the various species of the cotton plant, Gossipium, of which the most common are G. herbaceum, native to Asia, but cultivated extensively in southern Europe and in the United States, G. arboreum, in Asia and Africa, and G. barbadense, in the West Indies. G. religiosum and hirsutum are varieties of G. herbaceum.

The seeds are in reality a by-product in cotton manufacture. In shape they are irregularly oval, measuring from 5 to $8 \mathrm{~mm}$. greatest diameter. The seed skin or pod is covered with the fiber of the cotton.

The seeds are first cleaned and separated from dirt by sifting machines and from the fiber by specially constructed gins, after which they are cut into small pieces, freed from their hulls, crushed between rollers, and afterward submitted to hydraulic pressure in bags to express the oil. which is clarified by filtration or refined. The refining consists in washing the crude oil with sodium hydroxide solution, whereby the impurities are dissolved and thus removed.

Nature and Composition of Seeds and Oil.-The seeds of the cotton plant are rich in oil, containing from to to 29 per cent, according to the variety. Four samples of American cottonseed were found to be composed as shown in table on top of page $5^{1} 7$, according to Brannt.*

Refined cottonseed oil is a pale yellow oil of thick consistency, possessing a bland though pleasant taste and odor. It consists of the glycerides of oleic, stearic, palmitic, and linoleic acids, and evidently also a small content of hydroxyacids, though this has not been investigated as yet.

\footnotetext{
* Vegetable Fats and Oils, p. 223.
} 


\begin{tabular}{|c|c|c|c|c|}
\hline Constituents. & $\begin{array}{c}\text { South } \\
\text { Carolina. }\end{array}$ & $\begin{array}{c}\text { Georgia } \\
\text { I. }\end{array}$ & $\begin{array}{l}\text { Georgia } \\
\text { II. }\end{array}$ & $\begin{array}{l}\text { Georgia } \\
\text { III. }\end{array}$ \\
\hline 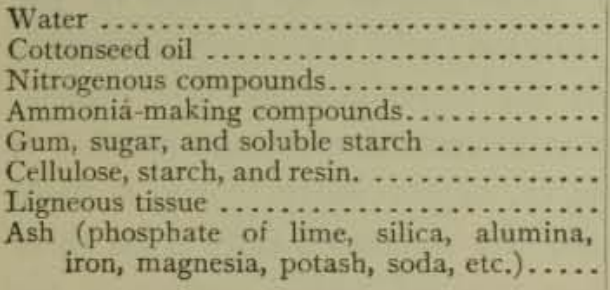 & $\begin{array}{r}9.5 \\
20.1 \\
17.8 \\
2.3 \\
.8 \\
26.2 \\
17.6 \\
5.7\end{array}$ & $\begin{array}{r}10.1 \\
16.2 \\
17.4 \\
2.9 \\
.9 \\
27.4 \\
19.2 \\
5.9\end{array}$ & $\begin{array}{r}9.8 \\
17.1 \\
17.2 \\
3.2 \\
-7 \\
26.1 \\
19.8 \\
6.1\end{array}$ & $\begin{array}{r}8.2 \\
19.6 \\
18.1 \\
3.7 \\
-9 \\
20.7 \\
22.4 \\
6.4\end{array}$ \\
\hline & 100.0 & 100.0 & 100.0 & 100.0 \\
\hline
\end{tabular}

On cooling the oil to a temperature below $12^{\circ} \mathrm{C}$. particles of solid fat will separate. At about $0^{\circ}$ to $-5^{\circ} \mathrm{C}$. the oil solidifies. When the oil is brought in contact with concentrated sulphuric acid, a dark, reddish-brown color is instantly produced.

U. S. Standards.-Cottonseed oil is the oil obtained from the seeds of cotton plants and subjected to the usual refining processes; is free from rancidity, has a refractive index $\left(25^{\circ} \mathrm{C}\right.$.) not less than 1.4700 and not exceeding 1.4725 ; and an iodine number not less than 104 and not exceeding r 10 .

"Winter-yellow" cottonseed oil is expressed cottonseed oil from which a portion of the stearin has been separated by chilling and pressure, and has an iodine number not less than 110 and not exceeding 116.

Cottonseed Stearin.-This product, used as an adulterant of lard as well as a substitute therefor, is obtained as a by-product in the manufacture of winter-yellow cottonseed oil. It is a light yellow fat, resembling butter in consistency.

Bechi's Silver Nitrate Test.-Hehner's Modification.-Two grams of silver nitrate are dissolved in $200 \mathrm{cc}$. of $95 \%$ alcohol free from aldehyde, $40 \mathrm{cc}$. of ether are added, and the reagent made very slightly acid with nitric acid.

In applying the test, a small quantity of the melted fat or oil is mixed in a test-tube with half its volume of the above reagent, and the tube is immersed in boiling water for fifteen minutes. With proper precautions the presence of cottonseed oil is indicated by a more or less strong reduction of the silver, while an oil or fat free from cottonseed oil causes no appreciable reduction.

Certain oils free from cottonseed that have become rancid or decomposed, as well as fats that have been subjected to a high temperature, 
sometimes show a slight reduction with Bechi's test. In cases of doubt it is well to apply the test on the fatty acids as follows:

Milliau's Modification of Bechi's Test.*-Heat 20 grams of the sample with $30 \mathrm{cc}$. of alcoholic potash solution $(20 \%$ potassium hydroxide in $70 \%$ alcohol), shaking at intervals till saponification is complete. Continue the heating for some minutes afterward until the alcohol is driven off, and dissolve the soap in $250 \mathrm{cc}$. of hot water. Add a slight excess of $10 \%$ sulphuric acid, and wash the separated fatty acids three times by decantation with water. Then proceed with a portion of the fatty acids as in Bechi's test.

Halphen's Test.-This is a much more delicate test for cottonseed oil than either of the preceding, as little as $2 \%$ of cottonseed oil being rendered apparent in olive oil. A mixture is made of equal volumes of amyl alcohol and carbon bisulphide in which $1 \%$ of sulphur has been dissolved. From 3 to $5 \mathrm{cc}$. of melted fat are mixed with an equal volume of the above reagent in a test-tube, loosely stoppered with cotton, and heated in a bath of boiling saturated brine for fifteen minutes. If cottonseed oil is present, a deep-red or orange color is produced. In its absence little or no color is developed.

Previous heating of the oil diminishes the delicacy of the Halphen test, and Holde and Pelgry + state that if cottonseed oil has been heated at $250^{\circ}$ for ten minutes, it will fail to respond to the test. Fulmer finds that it is necessary to heat to 265 to $270^{\circ}$ to render it wholly inactive to the test.

Gastaldi finds that it is the pyridin bases in amyl alcohol that render it useful. The test can be made by heating $5 \mathrm{cc}$. oil, $4 \mathrm{cc}$. carbon bisulphide containing $I \%$ of sulphur, and I drop of pyridin for from $I_{5}$ minutes to one hour in a water-bath.

\section{SESAME OIL.}

Sesame or benné oil is pressed from the seeds of Sesamum indicum and $S$. orientale, both of which are now regarded as varieties of the same species, and $S$. radiatum. These plants are native to southern Asia, but now cultivated in nearly all tropical countries. The larger portion of commercial sesame oil is manufactured in England, France, Germany, and Austria.

The seeds are yellow to dark brown, and in some cases black, inclined to the oval in form, the average longest diameter being about $4 \mathrm{~mm}$.

* Moniteur Scientifique, 1888, p. 366 . † Jour. Soc, Chem. Ind., I8, 1899, p. 711.

$\mp$ Chem. Ztg., 35, 191 I, p. 688. 
The seeds are commonly subjected to cold pressure once, and afterwards twice pressed when warm, thus yielding three grades of oil. From 47 to 60 per cent of oil is contained in the seeds.

According to Brannt* the composition of sesame seeds is as follows:

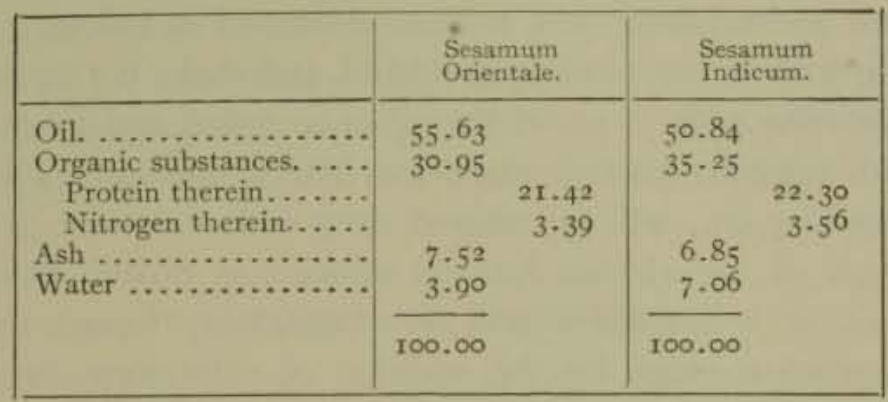

Sesame oil consists of the glycerides of oleic, stearic, palmitic, and myristic acids. It is golden yellow in color, free from odor, and possesses a delicate and characteristic flavor, on account of which the highest grades are by some considered equal to olive oil as a condiment. It is accordingly sold to some extent as an edible oil. It was formerly used as an adulterant of olive oil, but has of late years been largely displaced by cheaper oils for purposes of adulteration. When cooled to $-3^{\circ} \mathrm{C}$., sesame oil congeals to a yellowish-white mass. Concentrated sulphuric acid converts it into a brownish-red jelly.

U. S. Standards. - Refractive index $\left(25^{\circ}\right)$ I.4704 to 1.4717 ; iodine number $\mathrm{IO}_{3}$ to $\mathrm{II} 2$.

Adulterants to be looked for in sesame oil are cottonseed, poppyseed, corn, and rape oils.

Tocher's Test. $†-O n e$ gram of pyrogallic acid is dissolved in ${ }_{15} \mathrm{cc}$. of concentrated hydrochloric acid and mixed with ${ }_{5} \mathrm{cc}$. of the sample in a separatory funnel. After standing for a minute, the aqueous solution is withdrawn and boiled. If sesame oil is present, the solution shows a red coloration by transmitted, and blue by reflected, light.

Baudouin's Test. $\neq-D i s s o l v e$ o.I gram of cane sugar in ro cc. of hydrochloric acid (specific gravity I.2O) in a test-tube, and shake thoroughly with $z 0$ grams of the oil to be tested for one minute. Then allow the mixture to stand. The aqueous solution quickly separates from the oil, and in the presence of $\mathrm{I} \%$ or more of sesame oil will be colored deep red.

Certain pure Tunisian and Algerian olive oils have been found to cause a slight coloration with this test, but of a different shade from sesame. Moreover, if the test is applied to the fatty acids, no coloration in the case of olive oil is produced, while with sesame the color is the same as with the oil.

* Vegetable Fats and Oils, p. $25 \mathrm{r}$.

† Chem. Zeit. Rep., 5, 1891, p. 15.

$\ddagger$ Zeits, angew. Chem., 1892, p. 509 . 
Villavecchia and Fabris Test.*-This test was suggested on account of the fact that the color reaction in the Baudouin test was attributed to the agency of the levulose produced by the inversion of the sugar by hydrochloric acid. As furfurol is the chief product of the reaction between levulose and hydrochloric acid, it was substituted as follows: Dissolve 2 grams of furfurol in $100 \mathrm{cc}$. of $95 \%$ alcohol, and shake $0.1 \mathrm{cc}$. of this solution in a test-tube with ro cc. of the oil to be tested and ro cc. of hydrochloric acid (specific gravity I.20) for half a minute. The aqueous layer, on settling out, will be colored deep red, if sesame is present.

Or o.I cc. of the alcohol furfurol solution is mixed with ro cc. of oil and I cc. of hydrochloric acid in a separatory funnel, shaken well, and the separation aided by the addition of chloroform, which causes the aqueous layer, showing color with sesame oil, to float.

Since furfurol produces with hydrochloric acid alone a violet coloration, it is necessary to use it in dilute solution as above.

\section{RAPE OIL.}

Rape or colza oil is expressed from the seeds of the Brassica or rapeplant, of which there are three principal varities, Brassica napus, B. campestris, and B. rapa, one or another of which are cultivated in nearly every country of Europe, excepting Greece. Large amounts are also grown in India and China. The seeds are small, round grains, from 2 to $2.5 \mathrm{~mm}$. in diameter, yielding from 30 to 45 per cent of oil. The seeds, according to Brannt, $\uparrow$ have the following average composition:

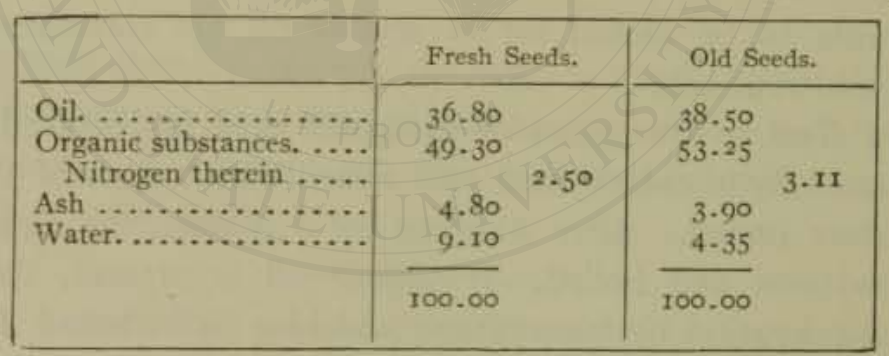

In the process of preparation the seeds are first crushed, and the oil removed by pressing or extraction. The crude oil is of a brownishyellow color, and when fresh is almost free from taste and smell, so that it serves, when cold pressed, as an edible oil, or an adulterant of such oils. It develops a disagreeable and peculiar taste and odor on long standing, due to the presence of certain albuminous and mucilaginous substances which it contains. These may be removed by refining, usually by treatment with sulphuric acid, but the refined oil has an unpleasant taste and odor.

\footnotetext{
* Jour. Soc. Chem. Ind., 1894, pp. 13-69. † Vegetable Fats and Oils, p. 240.
} 
The principal components of rape oil are the glycerides of stearic, oleic, erucic, and rapic acids. The chief adulterants are cottonseed and poppyseed oils.

Palas Test for Rapeseed Oil.*-Mix in the cold $30 \mathrm{cc}$. of a $\mathrm{r} \%$ solution of fuchsin, $20 \mathrm{cc}$. of sodium bisulphite (specific gravity I.3I), $200 \mathrm{cc}$. of water, and $5 \mathrm{cc}$. sulphuric acid. If the sample of oil to be tested be shaken with the reagent, a rose-red coloration is obtained in the presence of rape oil, said to be delicate to the extent of detecting $2 \%$ of the oil in mixtures.

\section{CORN OR MAIZE OIL.}

Corn oil is derived from the seed of the American grain Zea mays, or Indian corn, the constitution of the yellow and white varieties of which is, according to Andés, $\nmid$ as follows:

\begin{tabular}{|c|c|c|c|}
\hline & $\begin{array}{l}\text { Yellow Corn, } \\
\text { Per Cent. }\end{array}$ & \multicolumn{2}{|c|}{$\begin{array}{l}\text { White Corn, } \\
\text { Per Cent. }\end{array}$} \\
\hline $\begin{array}{l}\text { Organic matter .......... } \\
\text { Starch ............. } \\
\text { Albuminoids.......... }\end{array}$ & $\begin{array}{ll}82.93 & \\
& 61.95 \\
& 10.71\end{array}$ & 80.76 & $\begin{array}{r}62.23 \\
9.62\end{array}$ \\
\hline 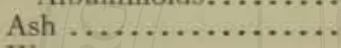 & 1.32 & I. 04 & \\
\hline Water .......... & 9.50 & 10.60 & \\
\hline Oil. .......... & 6.25 & 7.60 & \\
\hline & 100.00 & 100.00 & \\
\hline
\end{tabular}

Nearly all the oil is contained in the germ of the seed, the oil constituting in fact over $20 \%$ of the germ. Corn oil consists chiefly of the glycerides of palmitic and oleic acids. There is some doubt as to the presence of stearin. It is golden yellow in color, and possesses a pleasant odor and taste, resembling in flavor freshly ground grain.

It is prepared by subjecting to hydraulic pressure the germ separated in the manufacture of starch and of glucose, the germs yielding about ${ }_{15} \%$ of pure oil. While most of the oil of commerce is a by-product from starch and glucose factories, a small amount is recovered from the residte of fermentation vats in the manufacture of alcohol. Corn oil is coming to be used more and more as an adulterant of olive oil, and, according to Lewkowitsch, of lard.

It is claimed by Hopkins, $\ddagger$ by Hoppe-Seyler, and others, that corn oil,

* Analyst, XXII, p. 45.

t Vegetable Fats and Oils, p. ז 13 .

$\ddagger$ Jour. Am. Chem. Soc., 1898 , 20, p. 948 . 
unlike most vegetable oils, contains cholesterol. Olive oil was long supposed to be unique as a vegetable oil in containing this substance. Hopkins, on the assumption that cholesterol occurs in corn oil, suggested that a test for corn oil as an adulterant of certain vegetable oils lay in the identification of cholesterol.

Gill and Tufts * claim that, while the alcohol of corn oil is not phytosterol, neither is it cholesterol, but a third substance, known as sitosterol, $\uparrow$ occurring in wheat and rye.

There are no color reactions identifying corn oil as such. Its presence in other oils is indicated only by its influence on the various constants, the iodine number and refractometric reading especially being much higher than those of other edible oils.

\section{PEANUT OIL.}

Peanut or arachis oil is obtained from the seeds of the Arachis hypogaa (peanut, ground nut, or earth nut) cultivated in most tropical countries, notably in South America, China, India, and Japan. The plant is a creeping herb, developing its blossoms in the axes of the leaves. The fruit buds grow down into the earth, where the fruit is ripened, forming the well-known peanuts of commerce, the composition of which, according to Brannt, is as follows:

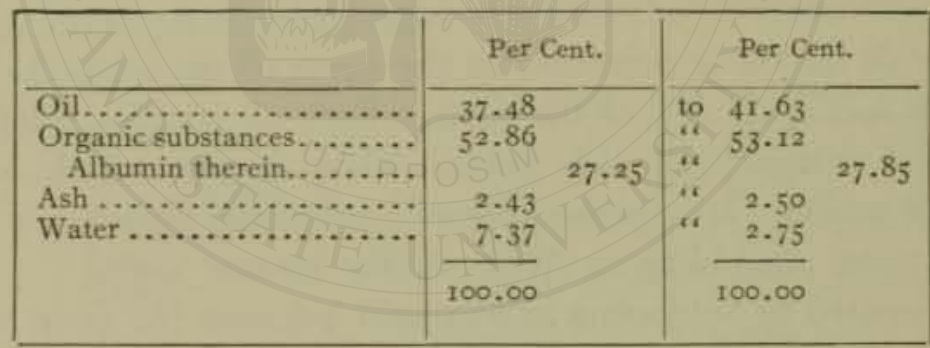

Peanut oil is composed chiefly of the glycerides of oleic, palmitic, hypogæic, and arachidic acids. The oil is extracted by pressure, the first cold-drawn oil being practically colorless, and possessing a pleasant taste suggestive of kidney beans. It is especially adapted for use as a salad or table oil. A second pressure of the moistened residue from the first yields an inferior oil, yellowish in color, also somewhat used for edible purposes, and sometimes commercially called "butterine oil."

U. S. Standards. - Refractive index $\left(25^{\circ}\right)$ I. 4690 to $\mathrm{I} .4707$; iodine number 87 to 100 .

* Jour. Am. Chem. Soc., XXV, 1903. † Burian, Monatsh. Chem., 18, 1897, p. 55 I. 
Adulterants of peantt oil are cottonseed, poppyseed, rape, and sesame oils. Very little pure peanut oil is found in commerce in the United States. It is to be looked for as an adulterant of French and Italian olive oils.

Characteristic Tests.-Peanut oil, when pure or nearly pure, may as a rule be readily identified from other oils. When present in large admixture in other oils it is not difficult to detect, but when only a small amount is present, in olive oil for instance, its detection becomes a more troublesome matter.

This difficulty arises from the fact that the constants of peanut oil are nearly the same as those of olive, with the single exception of the refractometric reading. Furthermore, there is no readily applied color test identifying peanut oil.

All the other common adulterants of olive oil, as cottonseed, sesame, corn, poppyseed, and rape oils, are readily identified, when present in small amounts, either by special color tests, or by reason of the fact that certain of their constants differ very widely from those of olive oil. Much more care and precaution are necessary in dealing with small admixtures of peanut oil than with almost any other adulterant.

The Renard Test* has long been in use for detecting and estimating peanut oil in mixtures. In its original form this test did not give entirely satisfactory results, and earlier led to some erroneous conclusions. In recent years, however, it has been so modified and improved as to be capable of quite positive results when carefully carried out. While arachin is said to occur in minute traces in olive oil, its presence is not sufficiently marked to interfere with the use of the Renard method in detecting any decided admixture of peanut oil.

The following modification of the Renard method, devised by Tolman, $\uparrow$ has been adopted by the A. O. A. C.:

Twenty grams of the oil are saponified in a $250-\mathrm{cc}$. Erlenmeyer flask with $200 \mathrm{cc}$. of alcoholic potassium hydroxide (40 grams potassium hydroxide in I liter of $95 \%$ redistilled alcohol). Neutralize with dilute acetic acid, using phenolphthalein as an indicator, and wash into a 500-cc. flask containing a boiling mixture of $\mathrm{I00}$ cc. water and I20 cc. $20 \%$ solution of lead acetate.

Boil for a minute and cool the contents of the flask by immersing in cold, or, preferably, ice water, whirling the flask occasionally so that

* Comp. Rend., 73,1871 , p. 1330.

† U. S. Dept. of Agric., Bur, of Chem., Bul. 65; also Bul. 77, and Bul. 107 (rev.). 
the soap when cold adheres to the sides of the flask. The water and excess of lead acetate can then be poured out, leaving the soap in the flask. Wash by shaking and decantation, first with cold water and then with $90 \%$ alcohol. Add $200 \mathrm{cc}$. of ether, cork the flask, and allow to stand with occasional shaking till the soap is disintegrated, after which boil on a water-bath under a reflux condenser for five minutes. Cool the soap solution down to a temperature between $15^{\circ}$ and $17^{\circ}$, and allow it to stand for about twelve hours.

Filter and thoroughly wash the precipitate with ether, after which the soap in the filter is washed back into the original flask with a stream of hot water acidulated with hydrochloric acid.

Add an excess of dilute hydrochloric acid, partially fill the flask with hot water, and heat until fatty acids form a clear oily layer. Fill the flask with hot water, allow the fatty acids to harden and separate from the precipitated lead chloride, wash, drain, repeat washing with hot water, and dissolve the fatty acids in roo cc. of boiling $90 \%$ by volume alcohol. Cool to $15^{\circ} \mathrm{C}$, shaking thoroughly to aid crystallization.

From 5 to 10 per cent of peanut oil can be detected by this method, as it effects a complete separation of the soluble acids from the insoluble, which interfere with the crystallization of the arachidic acid. Filter, wash the precipitate twice with 10 cc. of $90 \%$ alcohol, and then with $70 \%$ alcohol. Finally dissolve off the precipitate with boiling absolute alcohol, evaporate to dryness in a tared dish, dry and weigh. To the weight add 0.0025 gram for each $10 \mathrm{cc}$. of $90 \%$ alcohol used in the crystallization and washing, if done at $15^{\circ} \mathrm{C}$., and 0.0045 gram for each $10 \mathrm{cc}$. if done at $20^{\circ}$. The approximate amount of peanut oil is found by multiplying the weight of arachidic acid by 20 .

Arachidic acid crystals thus obtained should be examined microscopically. The melting-point should lie between $71^{\circ}$ and $72^{\circ} \mathrm{C}$.

Methods of J. Bellier.*-Qualitative Test.-Saponify I gram of the oil with $5 \mathrm{cc}$. of an alcoholic potash solution containing 85 grams potassium hydroxide per liter of strong alcohol, conducting the saponification in a small Erlenmeyer flask on the water-bath. After saponification, boil for two minutes, neutralize with dilute acetic acid, using phenolphthalein as an indicator, and cool by setting the flask in water at a temperature of from $17^{\circ}$ to $19^{\circ}$. After a short time, a precipitate nearly always comes down. Then add to the solution $50 \mathrm{cc}$. of $70 \%$ alcohol, containing $\mathrm{I} \%$ by volume of strong hydrochloric acid (specific

* Ann. Chim. Anal., r899, 4, p. 49; Zeits, für untersuch. Nahr., 1899, 2, p. 726. 
gravity 1.20). Cork the flask, shake vigorously, and again cool by setting the flask in the above cooling-bath. In the absence of a precipitate, the oil may be pronounced free from peanut. If $10 \%$ or more of peanut oil is present, a more or less characteristic precipitate forms, and often with less than $10 \%$ a cloudiness in the solution is perceptible after standing between $17^{\circ}$ and $19^{\circ}$ for half an hour. Pure olive oil remains perfectly clear as a rule.

A few varieties of olive oil from Tunis especially high in solid fat acids, as well as cottonseed oil and sesame oil, give similar turbidity on the addition of the $70 \%$ alcohol. To distinguish between these oils and peanut oil, heat the mixture on the water-bath till complete solution takes place, and again cool to $17^{\circ}$ to $19^{\circ}$. In the case of peanut oil the cloudiness or precipitate again occurs to the same extent as before, while in the other cases the solution should remain clear or nearly so.

Quantitative Determination,-Saponify 5 grams of the oil with $25 \mathrm{cc}$. of the above alcoholic potash solution in a $250-c c$. Erlenmeyer flask, neutralize exactly with acetic acid, and cool quickly in water. After standing an hour, pour upon a 9-cc. filter and wash the precipitate with $70 \%$ alcohol containing $18 \%$ by volume of hydrochloric acid, the temperature of the solution being not less than $16^{\circ}$ nor more than $20^{\circ}$. Continue the washing till the wash water no longer shows turbidity when diluted with water.

Dissolve the precipitate in 25 to $30 \mathrm{cc}$. of hot $95 \%$ alcohol, dilute with water until the alcohol is $70 \%$, let stand in water at $20^{\circ}$, filter, wash with $70 \%$ alcohol, dry at $100^{\circ}$, and weigh.

Bellier states that he has recognized with certainty as small an admixture as $2 \%$ of peanut oil by this method.

\section{MUSTARD OIL.}

The fixed oil of mustard is a by-product expressed from the seeds of the black and white mustard (Sinapis nigra and $S$. alba) in the process of preparation of mustard flour as a spice. The seeds contain from 25 to 35 per cent of oil.

Mustard oil somewhat resembles rape in composition, containing glycerides of erucic, behenic, and probably rapic acid.

Black mustard oil is brownish yellow in color, having a mild flavor, and an odor but slightly suggestive of mustard. White mustard oil is golden yellow, and has a somewhat sharp taste. 
Mustard oil is an alleged adulterant of edible oils, though by no means a common one.

\section{* POPPYSEED OIL.}

This oil is obtained from the seeds of the opium poppy (Papaver somniferum), native in the countries east of the Mediterranean, and cultivated extensively for opium and for oil in all parts of Europe, Asiatic Turkey, Persia, Egypt, India, and China. Most of the oil of commerce comes from France and Germany.

There are two chief varieties of poppy, the black ( $P$. nigrum) and the white $(P$. album), the finest oil being produced from the white. The seeds are somewhat flattened in form and kidney-shaped, yielding from 40 to 60 per cent of oil. According to Brannt the seeds have the following composition:

\begin{tabular}{|c|c|c|c|}
\hline \multirow{5}{*}{$\begin{array}{l}\text { Oil. } \\
\text { Organic substances. .... } \\
\text { Protein therein ....... } \\
\text { Ash .................... } \\
\text { Water .............. }\end{array}$} & \multirow{3}{*}{$\begin{array}{l}\begin{array}{l}\text { White Poppy- } \\
\text { seed. }\end{array} \\
\begin{array}{l}55.62 \\
32.11 \quad \\
\text { I } 680\end{array}\end{array}$} & \multicolumn{2}{|c|}{$\begin{array}{l}\text { Black Poppy- } \\
\text { seed. }\end{array}$} \\
\hline & & $51 \cdot 36$ & \\
\hline & & $35 \cdot 14$ & 17.50 \\
\hline & 3.42 & 4.00 & \\
\hline & 8.85 & 9.50 & \\
\hline & 100.00 & 100.00 & \\
\hline
\end{tabular}

The oil is obtained by crushing the seeds and applying pressure. The best grade of cold-drawn oil is pale yellow in color, possessing a pleasant taste when fresh, and being practically free from odor. Lower grades shade into deeper yellow and even reddish color, possessing a strong taste and odor. Poppyseed oil is much used in Europe as a table oil, and does not readily turn rancid. It is composed of the glycerides of stearic, palmitic, and linoleic acids. Poppyseed oil has been used to some extent as an adulterant of olive oil. It is itself not infrequently adulterated with sesame oil.

\section{SUNFLOWER OIL.}

Sunflower oil is derived from the seed kernels of the plant of the same name (Helianthus annuus), originally grown in Mexico, but now cultivated most extensively on a commercial scale in southern Russia. 
According to S. M. Babcock * the composition of sunflower seeds is as follows:

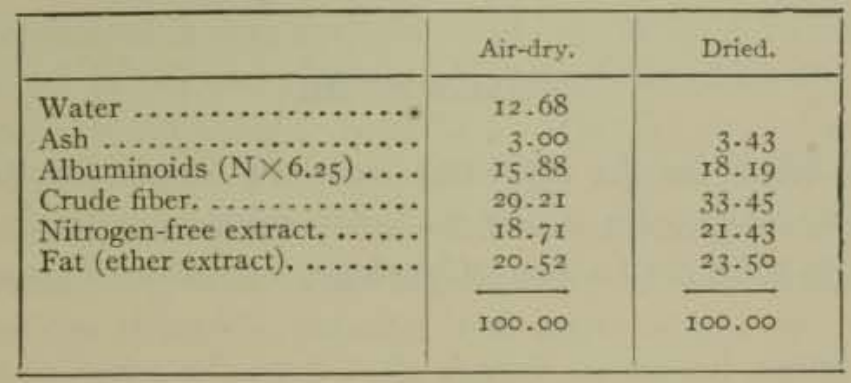

The seeds are long, black, and oval in shape, yielding from 18 to 28 per cent of oil. The liquid fatty acids of sunflower oil consist for the most part of linoleic, but little oleic acid being found.

The seeds are first shelled, then crushed, and finally submitted to pressure both cold and hot.

Sunflower oil is pale yellow in color, has a mild, pleasant taste, and is nearly free from odor. The cold-drawn oil is the variety most used for edible and culinary purposes in Russia, and as an adulterant of olive oil. Its use as an adulterant is, however, limited, and the writer has no knowledge of its having been found in olive oils used in the United States.

\section{ROSIN OIL.}

Rosin oil is prepared by the distillation of common rosin, and is an alleged adulterant of olive oil. It may be detected when present by shaking I to $2 \mathrm{cc}$. of the sample with acetic anhydride while warming. Cool, remove the anhydride by a pipette, and add a drop of sulphuric acid (specific gravity I.53). Rosin oil gives a fugitive-violet color. $\dagger$

Cholesterol also responds to this color reaction.

Renard's Test for Rosin Oil.-Prepare a solution of stannic bromide by allowing dry bromine to fall drop by drop upon tin in a dry, cool flask, and dissolving the product in carbon bisulphide.

Add a drop of this reagent to I cc. of the oil. In presence of rosin oil a violet color will be produced.

Polarization Test for Rosin Oil. $\nmid-$ The oil is dissolved in definite proportion in petroleum ether, and polarized in a $200-\mathrm{mm}$. tube. Rosin

* The Sunflower Plant, its Cultivation, Composition, and Uses. U. S. Dept. of Agric., Div. of Chem., Bul. 60, p. 18 .

† U. S. Dept. of Agric., Bur, of Chem., Bul. 65, p. 32. 
oil polarizes from +30 to +40 on the cane sugar scale, while other oils have a reading between $+\mathrm{I}$ and $-\mathrm{I}$.

\section{- COCOANUT OIL.}

Cocoanut oil is the fat expressed from the kernels of the cocoanut or fruit of the cocoa palm (Cocos nucifera), indigenous to the South Sea Islands and to the East-Indian archipelago, but grown in many tropical countries.

It is sometimes known as "copra oil," from the copra or pulp, which contains from 60 to 70 per cent. of fat. According to Brannt, the composition of the pulp is as follows:

\begin{tabular}{|c|c|c|c|}
\hline & Indian Copra, & African & copra. \\
\hline Oil. ............. & 68.75 & 66.80 & \multirow{4}{*}{10.20} \\
\hline $\begin{array}{l}\text { Organic substances. . ............ } \\
\text { Albuminous substances }\end{array}$ & 23.65 & 25.25 & \\
\hline 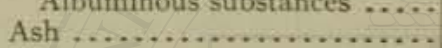 & 9.10 & I. 50 & \\
\hline 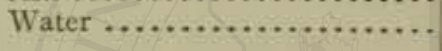 & 6.15 & 6.45 & \\
\hline & 100.00 & 100.00 & \\
\hline
\end{tabular}

In the preparation of the oil the moist copra is separated from the shell, crushed in mortars and subjected to pressure, yielding a milky mass. This is then heated in boilers and the oil removed by skimming. In some localities the pulp is first dried and then pressed.

Cocoanut oil is usually white and possesses a mild taste and pleasant odor. The oil easily becomes rancid but is seldom adulterated. The colddrawn Malabar oil is of greenish color and is used by the natives as an edible oil or substitute for butter. This variety is seldom found in commerce.

The oil contains, besides palmitin and olein, large proportions of myristin and laurin. Unlike the other vegetable oils, it contains also notable quantities of the glycerides of the volatile fatty acids caproic, capric, and caprylic, hence the high saponification value and Reichert number. The most characteristic constant is the Polenske number. The iodine number $(8-9.5)$ is strikingly low, although oil from the rind, according to Richardson, ${ }^{*}$ runs as high as 40 .

According to Andés, crystals of cocoanut oil appear under the microscope as a thick network of long needles.

\footnotetext{
- Jour. Ind. Eng. Chem., 3, 1911, p. 574 .
} 
COCOA (CACAO) BUTTER.

This preparation is not, properly speaking, in itself an edible fat. It is a by-product in the manufacture of cocoa, being removed by pressure from the crushed and ground cocoa nibs. The fat in cocoa beans varies from 36 to 50 per cent. The expressed fat is yellowish white, of a tallowlike consistency, has a pleasant taste and an odor suggestive of chocolate. It keeps a long time without turning rancid. In composition it consists of the glycerides of stearic, palmitic, and lauric acids, with traces of the glycerides of arachidic and butyric acids.

Its demand for pharmaceutical purposes is, however, sufficiently great to render the use of cocoa-butter as an adulterant of food-fats extremely rare. It should be borne in mind as a possible adulterant in examining various oils.

It is subject to adulteration with paraffin, tallow, and cottonseed stearin.

\section{TALLOW.}

The rendered fats of various animals, especially the cow and sheep, constitute what is generally known as tallow. The untreated fatty tissues are more properly known as suet, the tallow being the clear fat separated entirely by heat from the cellular material.

Tallow consists almost entirely of olein, palmitin, and stearin. Mutton tallow is usually, but not always, harder than beef tallow.

Excepting in the manufacture of material for oleomargarine, wherein the heart and caul fats of beef are almost exclusively used, the fats from different parts of the animal are not, as a rule, separated.

Fresh tallow has very little free fatty acid, but when it becomes rancid, the fat contains sometimes as high as $12 \%$ of free acid, reckoned as oleic.

Tallow is of chief interest to the food analyst in connection with its use as an adulterant of lard.

\section{BUTTER.}

Nature and Composition. - Butter is the product obtained by the churning of cream or milk, whereby the fat particles are caused to adhere together into a compact mass, inclosing a certain portion of the casein, the excess of milk serum being subsequently largely removed by washing and mechanical working. 
Butter fat is of extremely complex composition, containing a larger variety of glycerides than any other fat. Besides olein, palmitin, and stearin, the usual glycerides of the insoluble or fixed fatty acids found in most fats, butter contains notable quantities of the glycerides of a number of the volatile fatty acids, chief among which are butyrin, caproin, caprin, and caprylin, to which are due its distinctive taste, and which by exposure to light and air readily become decomposed into their fatty acids-butyric, caproic, capric, and caprylic, respectively. This decomposition in butter causes, or, more properly speaking, accompanies, what is commonly known as "rancidity."

The process of separation of butter fat into its component glycerides is a matter of extreme difficulty, and results obtained by different chemists vary widely. Separation has been attempted by fractional distillation, by methods depending on the difference in chemical affinity of the various acids, and on the difference in solubility of the various lower homologues in water at different temperatures.*

According to Browne, the composition of butter fat is as follows:

\begin{tabular}{|c|c|c|}
\hline Acid. & $\begin{array}{c}\text { Percentage of } \\
\text { Acid. }\end{array}$ & $\begin{array}{l}\text { Percentage of } \\
\text { Triglycerides. }\end{array}$ \\
\hline Dioxystearic. .. & 1.00 & I. 04 \\
\hline Oleic......... & $3^{2} \cdot 50$ & 33.95 \\
\hline Stearic...... & I. 83 & I. 9 I \\
\hline Palmitic..... & $3^{8.6 r}$ & $40.5 \mathrm{I}$ \\
\hline Myristic....... & 9.89 & 10.44 \\
\hline Lauric. ....... & 2.57 & 2.73 \\
\hline Capric. ....... & $0.3^{2}$ & 0.34 \\
\hline Caprylic ..... & 0.49 & 0.53 \\
\hline Caproic. ...... & 2.09 & $2 \cdot 32$ \\
\hline Butyric........ & 5.45 & 6.23 \\
\hline Totals. & $94 \cdot 75$ & 100.00 \\
\hline
\end{tabular}

Upwards of 300 analyses of butter are summarized by König in the following table:

\begin{tabular}{|c|c|c|c|c|c|c|c|}
\hline & $\begin{array}{l}\text { Water. } \\
\text { Per Cent. }\end{array}$ & $\begin{array}{l}\text { Pat, } \\
\text { Per Cent. }\end{array}$ & $\begin{array}{l}\text { Casein, } \\
\text { Per Cent. }\end{array}$ & $\begin{array}{l}\text { Milk, } \\
\text { Per Cent. }\end{array}$ & $\begin{array}{l}\text { Sugar. } \\
\text { Per Cent. }\end{array}$ & $\begin{array}{l}\text { Lactic Acid, } \\
\text { Per Cent. }\end{array}$ & $\begin{array}{l}\text { Salts, } \\
\text { Per Cent. }\end{array}$ \\
\hline $\begin{array}{l}\text { Minimum. .... } \\
\text { Maximum..... } \\
\text { Mean........ }\end{array}$ & $\begin{array}{r}4.15 \\
35.15 \\
13.59\end{array}$ & $\begin{array}{l}69.96 \\
86.15 \\
84.39\end{array}$ & $\begin{array}{l}0.10 \\
4.78 \\
0.74\end{array}$ & $0.5^{\circ}$ & $\begin{array}{l}0.45 \\
\text { I. } 16\end{array}$ & 0.12 & $\begin{array}{r}0.02 \\
15.08 \\
0.66\end{array}$ \\
\hline
\end{tabular}

* Browne, A Contribution to the Chemistry of Butter Fat, Jour. Am. Chem. Soc., 21, r899, p. 807 . 
Effects of Feeding Oil Cakes on the Composition of Butter.-Experiments have shown that the substance which causes cottonseed oil to respond to the Halphen test passes into the milk fat on feeding cows with cottonseed cake, but the substance that gives the Baudouin reaction is never carried into the milk on feeding with sesame cake. A number of investigators have found that feeding with cocoanut cake raises somewhat the Polenske number of the milk fat. There is good evidence, however, that, while the addition of vegetable oils to butter introduces phytosterol, as detected by Bömer's phytosterol acetate test, this substance can not be introduced into the milk fat by feeding. These facts should be borne in mind in the examination of butter for foreign fats.

\section{ANALYSIS OF BUTTER.}

Preparation of the Sample.-A. O.A. C. Method.*-If large quantities of butter are to be sampled, a butter-trier or sampler may be used. The portions thus drawn, about 500 grams, are to be perfectly melted in a closed vessel at as low a temperature as possible, and when melted, the whole is to be shaken violently for some minutes till the mass is homogeneous, and sufficiently solidified to prevent the separation of the water and fat. A portion is then poured into the vessel from which it is to be weighed for analysis, and should nearly or quite fill it. This sample should be kept in a cold place till analyzed.

Water.-A. O. A.C. Method.-About two grams of the sample are weighed in a flat-bottomed platinum dish, such as is used for determining water in milk, and the dish and its contents kept in contact with the live, stream of a water-bath till a constant weight is attained.

Patrick's Rapid Method. $†-T h i s$ method is especially suited for the use of dairymen, inspectors and others not provided with laboratory facilities.

Ten grams of the thoroughly mixed butter are weighed into a $250-\mathrm{cc}$. aluminium beaker, which, together with a glass rod has been previously tared, and boiled over (but not in) the flame of an alcohol lamp provided with a conical asbestos chimney, holding the beaker by means of a wire clamp in a nearly horizontal position to avoid loss from spattering or foaming, and whirling constantly to prevent overheating. The rod serves to break up lumps of curd which form, thus facilitating the drying.

*U. S. Dept. of Agric., Bur, of Chem., Bul. 46, p. 43; Bul, to7 (rev.), p. 123.

† Jour. Am. Chem. Soc., 28, 1906, p. 1611; 29, 1907 , p. 1126. 
The heating should be so conducted as to avoid any considerable dis-

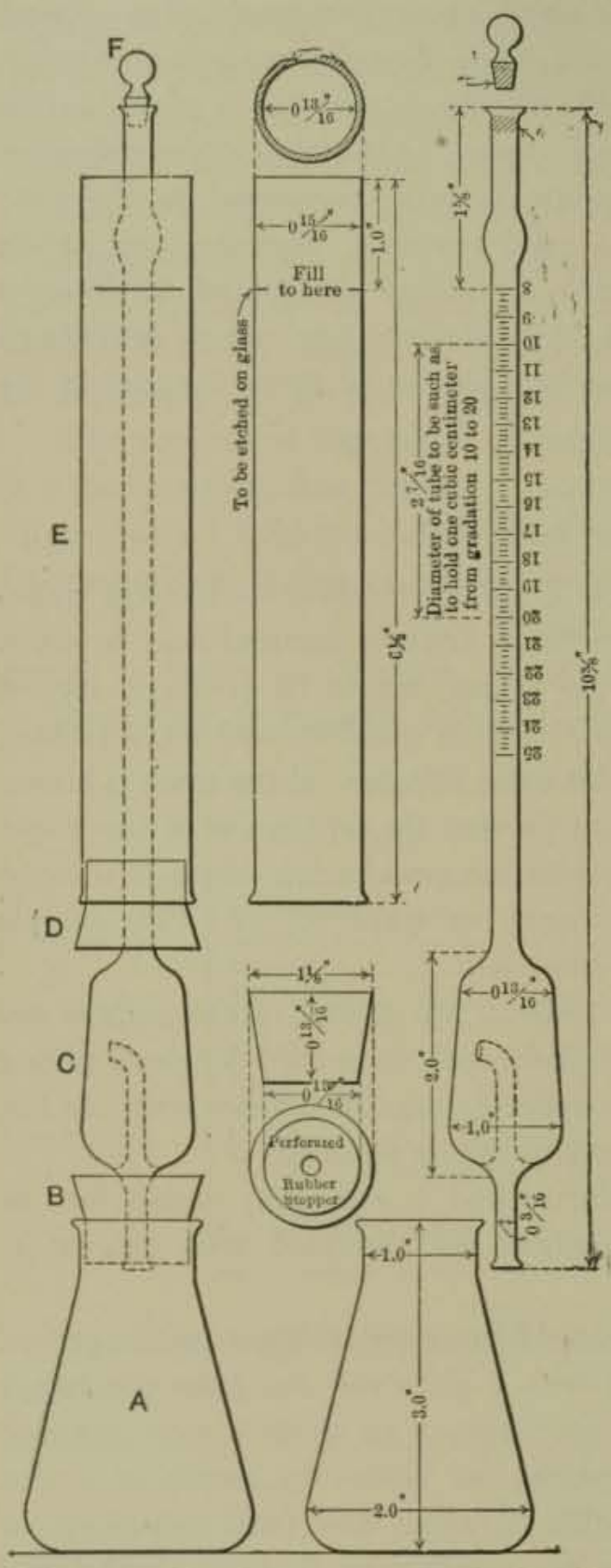

Fig. 100.-Gray's Apparatus for the Rapid Determination of Water in Butter. coloration of the curd. With suitable heating the water may be removed in less than 15 minutes, after which the beaker is cooled in water and weighed. A balance sensitive to to milligrams, such as is used in weighing cream for testing by the Babcock method, is sufficiently accurate for weighing the butter.

Gray's Method.*-1. The Special Apparatus for this method, shown in Fig. Ioo, consists of a flask (A) connected by a close-fitting rubber stopper $(B)$ with a graduated tube $(C)$, and this in turn with a condenser jacket $(E)$ by a rubber stopper $(D)$. The tube $C$ is closed by a glass stopper, the zero mark being the end of the stopper. Each mark of the graduation represents $0.02 \mathrm{cc}$, or, when Io grams of butter are used, $0.2 \%$.

2. Process. -Weigh to grams of the well mixed butter on a piece of parchment paper $\mathrm{I}_{3} \mathrm{~cm}$. square, introduce into the flask, and add $6 \mathrm{cc}$. of a mixture of 5 parts of amyl acetate and I part of amyl valerianate, free from water-soluble impurities. Connect the apparatus as shown in Fig. IOO, fill the condenser jacket with cool water to within $2.5 \mathrm{~cm}$. of the top, and remove the glass stopper $F$. Heat the flask over a Bunsen burner, thus melting the butter and boiling the water. Watch the con-

* U. S. Dept. of Agric., Bur. of Animal Ind, Circ. 100. 
densation of the steam in the graduated part of the tube $C$, and do not allow the steam to get higher than the $15 \%$ mark. In case of continued foaming, allow the mixture to cool, add $2 \mathrm{cc}$. of the amyl reagent, and continue heating. After the water in the sample has boiled out, the temperature rises and the amyl reagent boils, driving the last traces of water and water-vapor from the flask and bottom of the stopper. Some of the amyl reagent is carried into the tube $C$ with the steam, and some is boiled over after the water has been driven off. This amyl reagent in the tube is no disadvantage. When the mixture in the flask becomes a brown color and all the crackling noises in boiling cease, which usually requires 5 to 8 minutes, it is safe to conclude that all water has been driven from the flask.

Disconnect the flask $A$ from the stopper $B$, place the glass stopper $F$ in the tube $C$, giving it a turn to insure its being held firmly; invert the tube $C$, first being sure that the mouth of the small tube inside the bulb is held upwards, pour the water from the condensing jacket $E$, and remove the jacket. When the tube $C$ is inverted, the water and reagent flow into the graduated part of the tube. To separate these and to get the last traces of water down into the graduated part, the tube $C$ is held with the bulb in the palm of the hand, and the stoppered end away from the body, raised to a horizontal position, and swung at arm's length sharply downward to the side. This is repeated a number of times until the dividing line between the water and reagent is very distinct, and no reagent can be seen with the water or vice versa. The tube should then be held a short time with the stoppered end downward, and the amyl reagent in the bulb agitated in order to rinse down any adhering water.

The reading should not be taken until the tube and contents have cooled so little warmth is felt. When 10 grams of butter are used, the percentage is read directly at the lower meniscus.

With butter very low in moisture it may be desirable to use $1_{5}$ grams, and with butter very high, 5 grams.

Fat. - This may be determined either directly or indirectly. For the direct determination, a weighed amount of the sample, from 2 to 3 grams, is first dried at $100^{\circ}$ in sand or asbestos, contained in a thin and fragile round-bottomed evaporating-shell (Hoffmeister's Schälchen). If desired, the moisture may be determined in this connection by loss in weight after drying. The shell is afterwards inclosed in a piece of fat-free filter-paper, and crushed in pieces between the fingers in such a manner as to avoid loss. The pieces are gathered in a mass and folded together 
in the filter-paper to form a packet of a size readily transferable to a Soxhlet extractor, in which the fat is removed in the usual manner and weighed, after drying, in a tared flask.

Or, the fat may be indirectly determined by subtracting the sum of the water, casein, and ash from 100.

Casein.-The residue from the determination of water by the A.O. A. C. method is stirred with petroleum ether until the fat is dissolved, and transferred to a tared Gooch crucible. After thorough washing with petroleum ether, the crucible is dried at $100^{\circ}$, cooled, and weighed, thus obtaining the casein and ash. The loss on ignition at a dull red heat represents the casein.

If desired nitrogen may be determined in the residue after removal of the fat with petroleum ether, and casein calculated from the nitrogen, using the factor 6.37 .

Ash.- The residue left on the Gooch crucible after ignition, obtained as described in the preceding section is the ash. It consists largely of salt, which may be calculated from the percentage of chlorine determined by titration.

Milk Sugar and Lactic Acid compose most of the undetermined matter remaining after deducting from the total solids the sum of the fat, casein, and ash. Determine milk sugar, if desired, in an aqueous extract of the butter by Fehling's solution.

Determination of Salt.-In a tared dish or beaker weigh out about 5 grams of butter, taking a gram or so at a time from different parts of the sample. Add hot water to the weighed part, and after it has melted, the contents of the dish are poured into a separatory funnel, shaken and allowed to stand till the fat collects at the top, after which the underlying aqueous solution is drawn off into an Erlenmeyer flask, leaving the fat in the funnel bulb. Hot water is again added, and from ten to fifteen extractions are made, using about $20 \mathrm{cc}$. of water each time, all the water being collected in the Erlenmeyer flask.

A few drops of a solution of potassium chromate are then added for an indicator, and the sodium chloride volumetrically determined by a standard silver nitrate solution.

Salted butter contains from 0.5 to $6 \%$ of salt.

Examination of Butter Fat.-The butter fat is best obtained free from curd and salt by filtering when hot, the sample being best melted in a beaker on the water-bath. The water, with the curd and salt, will settle to the bottom. The clear fat is then filtered at a temperature not 
exceeding $50^{\circ} \mathrm{C}$., and subjected to such examination as may be desired to determine its purity.

U. S. Standard. Butter Fat has a Reichert-Meissl number not less than 24 and a specific gravity not less than 0.905 at $\frac{40^{\circ}}{40^{\circ}} \mathrm{C}$.

\section{ADULTERATION OF BUTTER.}

The artificial coloring of butter is an art practiced for so many years, and is so far in accord with the popular demand, that it can hardly be considered as an adulteration. The most recent custom of adding preservatives other than salt to butter is, however, very properly considered in most localities as reprehensible, unless the character and amount of the preservative be made clear to the purchaser by a suitable label.

The most common and time-honored sophistication is the substitution in whole or in part of foreign fat, as in the case of oleomargarine, and, more recently, in the fraudulent sale of renovated or process butter for the freshly made article.

U. S. Standard Butter is butter containing not less than $82.5 \%$ of butter fat. By acts of Congress approved August 2, I886, and May 9, IgO2, butter may also contain added coloring matter.

ARTIFICIAL COLORING MATTER IN BUTTER.-Formerly carrot juice and annatto were used almost entirely as butter colors. The carrot furnished to the farmer a ready means of coloring his dairy butter, and its use was lung in vogue for this purpose, before the commercial butter colors were available. Other vegetable colors, such as turmeric, marigold, saffron, and safflower, are said to have been used for this purpose, but, with the possible exception of turmeric, the writer is not aware of authentic cases in which they have been found in recent years. While annatto as a butter cclor is still in use, it is rapidly giving place to various oil-soluble, azo coaltar colors, which are admirably adapted to the purpose. All butter colers are now put on the market in solution in oil, usually cottonseed in this country and sesame in Europe.

Detection.-Martin* devised a general scheme, applicable for the detection of various colors in butter. His reagent consists of a mixture of 2 parts of carbon bisulphide with 15 parts of ethyl or methyl alcohol. $25 \mathrm{cc}$. of this solution are shaken with about 5 grams of the butter to be tested, and, after standing for some minutes, the mixture separates into two layers, of

\footnotetext{
* Analyst, 12, p. 70.
} 
which the lower consists of the fat in solution in the carbon bisulphide, while the upper is the alcohol, which dissolves out and is colored by the artificial dye employed. If saffron is present, the alcoholic extract will be colored green by nitric acid and red by hydrochloric acid and sugar.

Coal-tar dyes, if present, may be fixed on silk or wool by boiling bits of the fiber in the alcoholic extract, diluted with water and acidulated with hydrochloric acid.

Turmeric is to be suspected, if ammonia turns the alcoholic extract brown; marigold, if silver nitrate turns it black, and annatto, if on evaporating the alcoholic solution to dryness and applying to the residue a drop of concentrated sulphuric acid, a greenish-blue coloration is produced.

Turmeric is further tested for in the residue from the alcoholic extract as above obtained, by boiling the residue in a few cubic centimeters of a dilute solution of boric acid (or a solution of borax acidulated with hydrochloric acid), and soaking a strip of filter-paper therein. On drying the paper, if it assumes a cherry-red color, turning dark olive by dilute alkali, the presence of turmeric is assured.

Carrotin (the coloring matter of the carrot root) does not impart its color to the alcohol layer in Martin's test. Moore* has pointed out this exception, and shown that while with carrotin present the alcohol layer in Martin's test remains colorless, as in the case of uncolored butter, that when, however, a drop of very dilute ferric chloride is added, and the test-tube shaken, if carrotin be present, the alcohol will gradually absorb the yellow color from the butter. Care must be taken to avoid an excess of ferric chloride, as very little of this reagent will suffice.

Allen states that a butter color commercially known as "carrotin" consists in reality of I part of annatto in 4 parts of oil.

Detection of Annatto in Butter.-Treat 2 or 3 grams of the melted and filtered fat (freed from salt and water) with warm, dilute sodium hydroxide. After stirring, pour the mixture while warm upon a wet filter, using to advantage a hot funnel. If annatto is present, the filter will absorb the color, so that, when the fat is washed off by a gentle stream of water, the paper will be dyed straw color. It is well to pass the warm alkaline filtrate two or three times through the fat on the filter to insure removal of the color.

If, after drying the filter, the color turns pink on application of a drop of stannous chloride solution, annatto is assured.

* Analyst, $11, \mathrm{P} \quad 163$. 
Detection of Coal-tar Colors in Butter.-Geisler's Method.*-A few drops of the clarified fat are spread out on a porcelain surface and a pinch of fullers' earth added. In the presence of various azo-colors, a pink to violet-red coloration will be produced in a few minutes. Some varieties of fullers' earth react much more readily: with the azo-dyes than do others. In fact some do not respond at all. When once a satisfactory sample of this reagent is obtained, a large stock should be secured of the same variety.

Low's Method. $\dagger$-A small amount of material to be tested is melted in a test-tube, an equal volume of a mixture of I part of concentrated sulphuric acid and 4 parts of glacial acetic acid are added, and the tube is heated nearly to the boiling-point, the contents being thoroughly mixed by shaking; the tubes are set aside, and after the acid solution has settled out it will have been colored wine-red in the presence of azo-color, while with pure butter fat, comparatively no color will be produced.

Doolittle's Method for Azo-colors and Annatto. $\ddagger$-The melted sample is first filtered. Two test-tubes are taken and into each are poured about 2 grams of the filtered fat, which is dissolved in ether. Into one testtube are poured I or 2 cc. of dilute hydrochloric acid, and into the other about the same volume of dilute potassium hydroxide solution. Both tubes are well shaken and allowed to stand. In the presence of azodye, the test-tube to which the acid has been added will show a pink to wine-red coloration, while the potash solution in the other tube will show no color. If annatto has been used, on the other hand, the potash solution will be colored yellow, while no color will be apparent in the acid solution.

Cornelison's Test for Artificial Colors. $\S$-Melt ro grams of the clear, dry fat, and shake well in a separatory funnel with Io to 20 grams of $99.5 \%$ acetic acid. If the materials are too hot, the fat will dissolve, but at about $35^{\circ}$ it separates quickly and almost completely. Draw off the clear acid, and after noting its color, test by adding to one portion of $5 \mathrm{cc}$. a few drops of concentrated nitric acid, and to another portion a few drops of concentrated sulphuric acid.

Natural yellow butter gives by this test a colorless extract, which remains colorless on adding nitric or sulphuric acid. The acid extracts of annatto, curcumin, and carrot are various shades of yellow, both before

\footnotetext{
* Jour, Am. Chem. Soc., 20, 1898, p. 11 ro.

† Ibid., 20, p. 889 .

$\ddagger$ U. S. Dept. of Agric., Bur. of Chem., Bul. 65, p. 152 .

\& Jour. Am. Chem. Soc., 30, 1908, p. 1478.
} 
and after addition of nitric acid, while with sulphuric acid they take on a pink coloration on standing, which in the case of curcumin is very decided. Soudan I and butter yellow give pink extracts, which remain pink on adding the stronger acids, while cerasine orange G, yellow O.B., yellow A.B. and certain other coal-tar dyes give extracts of various shades of yellow, which on treatment with the heavy acids in some cases remain colorless, but in others become pink, while the oil globule which separates remains colorless or takes on a pinkish color according to the dye.

PRESERVATIVES AND THEIR DETECTION.-Fresh or unsalted butter and renovated butter are often found with an added preservative, the one most commonly used for this purpose being the so-called "boric mixture" (borax and boric acid) already discussed under milk adulteration. Salted butter is occasionally, though not so often, found preserved. Other preservatives used in butter are formaldehyde, and salicylic and sulphurous acids. These latter are, however, rarely found.

Boric Acid.-This, if present, is best detected in the aqueous solution that settles to the bottom when butter is melted at the temperature of the boiling water bath, the supernatant fat being decanted off. Richmond* claims to be able to distinguish free boric acid from borax as follows: If on applying turmeric-paper directly to the aqueous liquid the paper turns red, the color being especially evident on drying, free boric acid is indicated. As a confirmatory test the reddened turmericpaper is treated with dilute caustic alkali, whereupon it turns a dark olive. green if boric acid is present.

In the absence of a red color by the above test, or when this color is faint, the aqueous solution is acidified slightly with hydrochloric acid and the turmeric-paper applied as before. If borax be present to an appreciable extent, the red color will now be quite marked, even though not appearing before. In other words, testing with turmeric-paper without acidifying with hydrochloric acid shows, according to Richmond, a slight coloration due to the free acid alone, while the more intense color formed by first acidifying is due to the combined acid or borax.

Determination of Boric Acid.-Ten grams of the butter fat are weighed in a beaker and transferred with hot water to a separatory funnel in which the fat is extracted with Io to 15 portions of hot water as described on page 534. The combined aqueous extract is evaporated to dryness in a platinum dish, the residue made alkaline, and ignited at

* Dairy Chemistry, p. 254. 
a dull red heat. Boil the ash with water, filter, and wash with hot water, keeping the volume of the filtrate under $60 \mathrm{cc}$. Make sure that the soluiion is perfectly neutral to methyl orange by treatment, if necessary, with sulphuric acid and tenth-normal alkali, add $30 \mathrm{cc}$. of glycerin, a few drops of the phenolphthalein indicator, make up to roo cc., and titrate with tenth-normal sodium hydroxide according to Thompson's method (p. 829).

Butter being practically free from phosphates, the preliminary treatment for removing phosphoric acid in Thompson's method may be omitted.

Formaldehyde.-The aqueous solution from which the fat of the butter melted at low temperature has been poured off, is added to some milk previously found free from formaldehyde, and the test for the latter with hydrochloric acid and ferric chloride is tried directly in the milk.

Salicylic Acid.-Detection.-See method No. 2 for detection in milk, page $18_{3}$.

Determnation of Salicylic Acid.-Method of the Paris Municipal Laboratory.-Repeatedly exhaust 20 grams of butter in a separatory funnel with a solution of sodium bicarbonate, thus obtaining soluble sodium salicylate, if salicylic acid be present. Acidulate the aqueous extract with dilute sulphuric acid, and extract with ether. Evaporate the ether, and to the residue add a little mercuric nitrate, forming a precipitate nearly insoluble in water. Filter this off, wash the precipitate with water, and decompose into free salicylic acid with dilute sulphuric acid. Redissolve in ether, evaporate the solvent as before, and dry the residue at a temperature of $80^{\circ}$ to $100^{\circ}$. Extract the residue with petroleum ether, dilute the ethereal liquid with an equal volume of $95 \%$ alcohol, and titrate with tenth-normal alkali, using phenolphthalein as an indicator.

I cc. of tenth-normal alkali $=0.0{ }_{3} 8$ gram salicylic acid.

Sulphurous Acid.-The aqueous liquid, separated from the butter fat, is distilled, and the distillate treated with bromine water and barium chloride. A precipitate on the addition of the latter reagent indicates the presence of sulphurous acid or a sulphite in the butter.

Glucose in Butter.*-Crampton states that glucose has been found by him in butter intended for export to tropical countries, added to pre-

* Jour, Am. Chem. Soc., 20, 1898, p. 201. 
vent decomposition. In one sample made for export to Guadeloupe he found over 10\% of glucose.

For its detection or estimation to grams of the sample are weighed out and transferred to a separatory funnel with hot water, and shaken out with successive portions of hot water. These are combined, and the aqueous extract made up to $25^{\circ} \mathrm{cc}$. The reducing sugar may be determined by Fehling's solution or by polarization, using in the latter case alumina cream as a clarifier. While a slight reduction should be disregarded, any considerable reduction may be undoubtedly ascribed to glucose.

BUTTER "FILLED" WITH WATER.-Various preparations have been placed on the market to aid in incorporating water with butter. So called "black pepsin" has been used for this purpose. By churning the butter with water and a certain amount of the preparation in such a manner as to destroy the grain, it is possible to introduce two or three times the normal amount of water.

\section{RENOVATED OR PROCESS BUTIER.}

This product is also variously termed "boiled," "aerated," and "sterilized" butter. There are various modifications of the process of manufacture, but the object is to melt up and treat rancid butter in such a manner that for a time at least it is sweet. The following manner of treatment is typical, and shows in the main the necessary steps in carrying out the process, though details of manipulation vary in different localities.

The butter is melted in large tanks surrounded with hot water jackets at a temperature varying from $40^{\circ}$ to $45^{\circ} \mathrm{C}$. By this means the curd and brine settle to the bottom, whence they are drawn off, while the lighter particles rise to the top in the form of a froth or scum and are removed by skimming.

The clear butter fat is then, as a rule, removed to other jacketed tanks, and, while still in a molten condition, air is blown through it, which removes the disagreeable odors. The melted fat is then churned with an admixture of milk (more often skimmed) till a perfect emulsion is formed, after which it is rapidly chilled by running into ice cold water, with the result that it becomes granular in form. It is then drained and "ripened" for some hours, after which it is worked free from excess of milk and water, salted, and packed.

Under some state laws this product, to be legally sold, must conform to rules of labeling as strict as those prescribed for oleomargarine. In 
other localities it may be sold with impunity. Not infrequently it is sold as choice creamery butter, and sometimes at the same price.

U. S. Standard Renovated or Process Butter should contain not more than $16 \%$ of water, and at least $82.5 \%$ of butter fat.

\section{OLEOMARGARINE.}

According to the U.S. revenue laws, artificial butter composed wholly or in part of fat other than butter fat must be branded oleomargarine. The name butterine, although used in advertising matter, does not have the sanction of the government. The product is commonly known in England as margarine. As a rule the oleomargarine of commerce is composed of refined oleo oil, usually churned up with neutral lard, milk, and a small amount of pure butter, the whole being salted and sometimes colored to resemble butter. Cottonseed oil and other vegetable oils are also used to some extent.

Oleo oil is prepared from the fat of beef cattle somewhat as follows:* Immediately after the animals are killed the fresh intestinal and caul fat are removed and placed in tanks of water at a temperature of about $80^{\circ} \mathrm{F}$. From this water they are transferred to other tanks of cold water and chilled until all animal heat is removed. The fat is then cut or hashed into small pieces and melted at about $150^{\circ} \mathrm{F}$. in jacketed steam kettles, until the clear oil is separated from the connective tissue.

This oil is then drawn off into vats, which, on account of the appearance of the oil on cooling, are called graining or seeding vats, where it is allowed to stand for twenty-four hours or more at a temperature of about $85^{\circ} \mathrm{F}$. From these vats the semi-solid emulsion of oil and stearin is dipped into cloths, which are folded and placed in a press between sheets of metal and subjected to powerful pressure. By this means the oil is separated from the stearin, and is drawn into casks for export or for manufacture into oleomargarine. Large quantities are annually exported to Holland, where oleomargarine is manufactured, and either sold for consumption in that country, or re-exported to other countries in Europe.

The oleo oil thus expressed is a mixture of olein and palmitin. When first prepared, it is a clear amber-colored fluid, free from odor or fatty taste. It is packed in tierces, and, when opened at ordinary temperature, is a light-yellow solid.

The further process of manufacture of oleomargarine consists in

* Report on Oleomargarine, Its Manufacture and Sale, Igth An. Report, Mass. St. Bd. of Health, 1887 . 
the main of mixing the oleo oil as above obtained with varying proportions of neutral lard, milk, and genuine butter, with or without added coloring matter, and churning the mixture at a temperature above the melting-point of the fats, the neutral lard having previously been cured for at least forty-eight hours in salt brine. Occasionally small quantities of other vegetable oils, as cottonseed, peanut, or sesame, are included in the above mixture. After the churning, the whole mass is cooled by contact with ice water. The chilled mass is drained, and afterwards salted, worked, and given much the same treatment as butter.

The composition of commercial oleomargarine varies between the following limits:

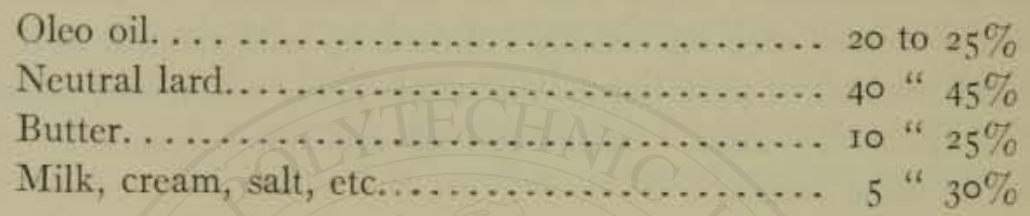

Coloring of Oleomargarine.-The artificial coloring matters employed are the same as in the case of butter, and are similarly tested for.

In many states oleomargarine cannot be legally sold when colored to resemble butter. Under other state laws coloring matter is allowable. The federal law and most state laws prescribe the most rigid rules for marking packages containing oleomargarine, with a view to affording the utmost protection to the producer of butter against the fraudulent substitution therefor.

Crampton and Simon's Tests for Palm Oil.*-So called "butter oils," consisting of cottonseed oil to which has been added 2 to 5 per cent of palm oil are used to color oleomargarine. The following tests serve for the detection of palm oil.

Preparation of Sample.- The sample should be kept in a cool, dark place until tested, as exposure to air and light, or the presence of water, alcohol, ether or similar reagents interfere with the tests. Immediately before testing, the sample is filtered as quickly as possible at a temperature not exceeding $70^{\circ} \mathrm{C}$.

First Method.-Dissolve roo cc. of the fat in $300 \mathrm{cc}$. of petroleum ether, and shake out with $50 \mathrm{cc}$. of $0.5 \%$ potassium hydroxide. Draw off the watery layer, make distinctly acid with hydrochloric acid, and shake out

* Jour. Am. Chem. Soc., 27, 1905, p. 270. 
with to cc. of colorless C. P. carbon tetrachloride. Separate the carbon tetrachloride solution, transfer a portion to a porcelain crucible, add $2 \mathrm{cc}$. of a mixture of one part of colorless, crystallized C. P. phenol and 2 parts of carbon tetrachloride, then 5 drops of hydrobromic acid (sp. gr. 1.19), and mix by gentle agitation.*

The almost immediate development of a bluish-green color is indicative of palm oil.

Second Method.-Shake ro cc. of the melted and filtered fat with an equal volume of colorless C. P. acetic anhydride, add one drop of sulphuric acid (sp. gr. I.53), and shake a few seconds longer. $\dagger$

If palm oil be present, the lower layers on settling out will be found to be colored blue with a tint of green. The color in this as in the preceeding test is transient.

Of the edible oils only sesame and mustard oils give a similar color reaction. Sesame oil, after repeated extractions with alcohol, will not give the blue color, but cottonseed oil containing as little as $1 \%$ of palm oil still responds to the test.

Adulterants of Oleomargarine. - This product is liable to adulteration not only by the use of inferior and unwholesome fat, but by the admixture in some cases of paraffin. $\ddagger$ This sophistication is made manifest, if an appreciable amount of the adulterant has been used, by the high melting-point and the low saponification number, as well as by the low specific gravity. If a clear saponification is impossible under ordinary conditions, paraffin is to be suspected. It may be separated and quantitatively determined as described on p. 5 ro.

Healthfulness of Oleomargarine.- Under the directions of the Massachusetts Board of Health, $\S$ a large number of artificial digestion experiments were made to show the relative nutritive value of butter and oleomargarine, and at the same time the wholesomeness of oleomargarine as a food was carefully investigated. The general conclusions reached were that, when comparing the best grades of both products, there is little if any difference between butter and oleomargarine on grounds of digestibility, while a good oleomargarine is much to be preferred to a

\footnotetext{
* Halphen uses a similar reagent to detect rosin oil in mineral oil. Jour. Soc. Chem. Ind., 21, 1902, p. 1474 .

† The reagents are the same as used in the Liebermann-Storch test for rosin oil.

$\ddagger$ Geissier, Jour. Am. Chem. Soc., 21, 1899, p. 605 .

8 igth An. Report, Mass. State Board of Health, I887, p. 248.
} 
poor butter from a nutritive standpoint. As to its wholesomeness, a large number of experts consulted were unanimous in expressing their favorable opinions of oleomargarine as a healthful article of food.

When sold on its own basis in accordance with the law, it forms an excellent cheap substitute for butter. It is only when fraudulently sold as butter or in violation of the various state and federal laws, that it comes within the province of the health authorities to condemn it, and, unfortunately, by reason of its close resemblance to the dairy product, the temptation to sell it for what it is not is always great.

Distinguishing Oleomargarine from Butter.-The two products, made up as they are of mixtures of the same fats, and differing for the most part only in the percentage composition of these fats, show many properties in common. For instance, the melting-point is so nearly the same for both products as to be of no use as a distinguishing indication. Other physical characteristies, as of taste and smell, are verry similar in both products, except in the hands of the expert. The microscope is of limited value, except in so far as it indicates that the fat has first been melted and afterwards solidified.

From the fact that oleo oil and neutral lard form by far the larger portion of the mixture known as oleomargarine, the glycerides that make up the fat of the latter are chiefly those of the insoluble fatty acids, stearic, oleic, and palmitic. The percentage of volatile fatty acids present in oleomargarine is very small, and the presence of these volatile acids is due entirely to the admixture of butter which it contains. This furnishes the most ready means of distinguishing chemically between the two products, and, as indicated by the Reichert number, is the chief reliance of the analyst for court evidence.

Incidentally, as will be seen by the accompanying table, the refrac-

CONSTANTS OF BUTTER FAT AND OLEOMARGARINE.

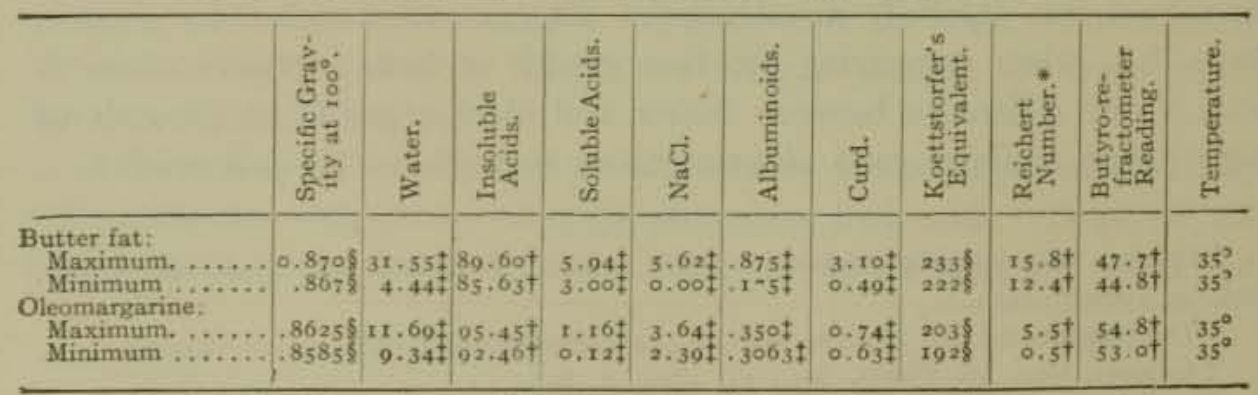

* Number of cubic centimeters $\mathrm{N} / 10$ alkali neutralizing volatile acids in 2.5 grams fat.

+ From analyses made in Mass. State Board of Health laboratory.

From analyses made in laboratory of U.S. Dept, of Agric., Bur. of Chem.

$\$$ From analyses by A. H. Allen. 
tometer reading, the iodine number, the saponification equivalent, and the specific gravity are all useful constants in indicating points of difference between the two fats, it being understood that in oleomargarine, as in butter, the fat for examination is melted and separated by filtration or otherwise from the curd, salt, and other constituents.

The constants for varying mixtures of butter with foreign fat as found by Villiers and Collin* are tabulated below.

Odor and Taste. - It is easy with a little practice to become so accustomed to the odor and taste of oleomargarine, as to be able to pass judgment with considerable confidence by these senses alone, whether a sample in question is oleomargarine or butter. The distinction is rendered more apparent by melting a portion of the sample on the waterbath. If the product is butter, either fresh or renovated, the butyric odor of the melted fat is very characteristic, while the melted oleomargarine not only is lacking in the butyric odor (a negative property), but possesses a distinctive "meaty" smell peculiar to itself, which, while not unpleasant, is unmistakable. The flavor of oleomargarine to one experienced in distinguishing between the two products is very apparent. This flavor, slight though it is, might be compared to that of cooked meat.

\begin{tabular}{|c|c|c|c|c|c|c|c|c|}
\hline & & & & & $\begin{array}{l}\text { Hebner's } \\
\text { Number. }\end{array}$ & $\begin{array}{l}\text { Soluble } \\
\text { Acids. }\end{array}$ & $\begin{array}{l}\text { Koettstorfer's } \\
\text { Equivalent. }\end{array}$ & $\begin{array}{l}\text { Volatile } \\
\text { Acids. }\end{array}$ \\
\hline Pure & utter. & & & & 88 & 5 & 224 & 26 \\
\hline Butte & $05 \%$ & foreig & fat, & $5 \%$ & 88.35 & 4.8 & 222.6 & 24.7 \\
\hline & & 40 & & $10 \%$ & 88.70 & $4 \cdot 5$ & 221.2 & 23.4 \\
\hline " & $85 \%$ & "๘ & 6 & $15 \%$ & 89.05 & $4 \cdot 3$ & 219.8 & 22.2 \\
\hline " & $80 \%$ & $"$ & " & $20 \%$ & 89.40 & $4 \cdot I$ & 218.4 & 20.9 \\
\hline " & $75 \%$ & " & " & $25 \%$ & 89.75 & 3.9 & 217 & 19.6 \\
\hline " & $70 \%$ & " & ". & $30 \%$ & 90.10 & 3.6 & 215.6 & $18 \cdot 3$ \\
\hline " & $65 \%$ & “ & $" a$ & $35 \%$ & 90.45 & 3.4 & 214.8 & 17.1 \\
\hline " & $60 \%$ & "“ & " & $40 \%$ & 90.80 & 3.2 & 212.8 & 15.8 \\
\hline$a$ & $55 \%$ & " & " & $45 \%$ & 91.15 & 3 & 211.4 & 14.5 \\
\hline$"$ & $50 \%$ & " & $"$ & $50 \%$ & 91.50 & 2.7 & 210 & 13.2 \\
\hline 4 & $45 \%$ & " & " & $55 \%$ & $9 \mathrm{r} .85$ & 2.5 & 208.6 & 12 \\
\hline et & $40 \%$ & " & " & $60 \%$ & 92.20 & 2.3 & 207.2 & 10.7 \\
\hline "4 & $35 \%$ & $"$ & $"$ & $65 \%$ & 92.55 & 2.1 & 205.8 & 9.4 \\
\hline$n$ & $30 \%$ & " & " & $70 \%$ & 92.90 & 1.8 & 204.4 & 8.1 \\
\hline " & $25 \%$ & " & " & $75 \%$ & 93.25 & 1. 6 & 203 & 6.9 \\
\hline$\because z$ & $20 \%$ & " & 4 & $80 \%$ & 93.60 & 1.4 & 201.6 & 5.6 \\
\hline “ & & " & $"$ & $85 \%$ & 93.95 & 1.2 & 200.2 & $4 \cdot 3$ \\
\hline " & $10 \%$ & $"$ & " & $90 \% \ldots$. & $94.3^{\circ}$ & 0.9 & 198.8 & 3 \\
\hline “ & $5 \%$ & " & $"$ & $95 \% \ldots$ & 94.65 & 0.7 & 197.4 & 1.8 \\
\hline Forei & $\mathrm{n}$ fat. & 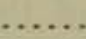 & & & 95 & 0.5 & 196 & 0.5 \\
\hline
\end{tabular}

* Les Substances Alimentaires, p. 731. 
DISTINGUISHING BETWEEN BUTTER, PROCESS BUTTER, AND OLEOMARGARINE.

With the increased occurrence in the market of the commercial product known as "process" butter, especially in localities where its sale is restricted or regulated by law, it becomes incumbent on the analyst to distinguish it from the other products which it resembles.

As a rule, the tests, chiefly physical, that are applied on the edible product as a whole (i.e., without separation of the curd, salt, etc.), such as the foam test, the milk test, the microscopical examination, and the appearance of the melted sample, distinguish broadly between pure fresh butter on the one hand, and oleomargarine on the other. In other words, although there are those skilled in making the above tests who claim to be and doubtless are able to note distinguishing features between oleomargarine and process butter, yet these two products respond alike, though perhaps in varying degrees, to these tests, and are classed together as distinguished from pure butter.

On the other hand, such tests as depend upon the refractometer, the Reichert number, and, infeet, all the so-called chemical constants, which are applied to the separated fat, freed from other substances, will serve to distinguish between oleomargarine and butter, whether "process" butter or otherwise, since the "processing" or "renovating" of butter does not change the character of its fat sufficiently to materially alter these constants.

It is best, therefore, for purposes of routine preliminary separation to submit all samples to the "foam" test and to examine them by the butyro-refractometer.* These tests alone, which are very quickly and readily applied, will rarely fail to separate into the three classes, butter, process butter; and oleomargarine, the products under examination, after which such confirmatory tests as are desired are made on adulterated samples.

The Butyro-refractometer.-This instrument, as its name implies was primarily intended by Zeiss for the examination of butter, and, while its use has been extended for work with other fats and oils, its construction is such as to show particularly a distinction between butter and oleomargarine by the appearance of the critical line of the fat. This mode of differentiation is due to the peculiar construction of the double

\footnotetext{
* Out of the large number of samples of butter and oleomargarine examined on the butyro-refractometer in the author's laboratory during eight years, he has never found a single instance where the instrument failed to show the difference between the two products
} 
prism, which shows differences of dispersive power by different appearances of the critical line. The prisms are so constructed that the critical line of pure butter is colorless, while margarine and artificial butter, which have greater dispersive powers than natural butter, show a blue-colored critical line. But anomalies in the color, both with pure butter and mixtures, are more or less observable, which render it impossible to draw a sharp line between adulterated and genuine butter. The appearance of a blue fringe may, however, be a useful factor in cases of suspected adulteration.

The following particulars respecting the application of the refractometer for analysis of butter are contained in a paper of Dr. R. Wollny of Kiel,* who assisted in the construction of the instrument. The readings of the refractive indices of a large number of butter samples taken at $25^{\circ} \mathrm{C}$. by Dr. Wollny have been directly reduced to scale divisions and yield the following equivalents:

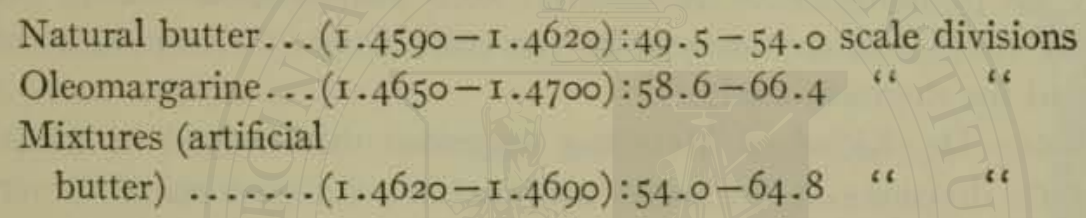

Limit of Scale Reading for Pure Butter.-Whenever in the refractometric examination of butter at a temperature of $25^{\circ} \mathrm{C}$. higher values than 54.0 are found for the critical line, these samples will, according to Wollny, by chemical analysis always be found to be adulterated; but with all samples in which the value for the position of the critical line does not reach 54.0 chemical analysis may be dispensed with, and the samples may be pronounced to be pure butter. Wollny suggests, as a means of removing all chances of adulterated butter escaping detection, that the above limit be placed still lower, and that all samples exhibiting values exceeding 52.5 (at a temperature of $25^{\circ} \mathrm{C}$.) be set aside for chemical analysis.

In calculating the position of the critical line for other temperatures than $25^{\circ} \mathrm{C}$. allow per $1^{\circ} \mathrm{C}$. variation of temperature a mean value of

* Dr, R. Wollny, Schlussbericht tuher die Butteruntersuchungsfrage, Milchwirthschaftlicher Verein, Korrespondenzblatt, No. 39, 1891, p. 15.

Older papers on butter tests by refraction of light will be found in: Mueller, Rep. d. anal. Chemie, 1886 , pp. $346,{ }_{3} 66$. Skalweit, Milchzeitung, 1886,15, p. 462 . Wollny, Ueber die Kunstbutterfrage, Leipzig, 1887 , p. 50. 
0.55 scale division.* The following table, which has been compiled in this manner, shows the values corresponding to various temperatures, each value being the upper limit of scale divisions admissible in pure butter:

\begin{tabular}{|c|c|c|c|c|c|c|c|}
\hline $\begin{array}{l}\text { Temper- } \\
\text { ature. }\end{array}$ & $\begin{array}{c}\text { Scale } \\
\text { Division. }\end{array}$ & $\begin{array}{l}\text { Temper- } \\
\text { ature. }\end{array}$ & $\begin{array}{c}\text { Scale } \\
\text { Division. }\end{array}$ & $\begin{array}{l}\text { Temper- } \\
\text { ature. }\end{array}$ & $\begin{array}{c}\text { Scale } \\
\text { Division. }\end{array}$ & $\begin{array}{l}\text { Temper- } \\
\text { ature. }\end{array}$ & $\begin{array}{c}\text { Scale } \\
\text { Division. }\end{array}$ \\
\hline $45^{\circ}$ & 41.5 & $40^{\circ}$ & 44.2 & $35^{\mathrm{C}}$ & 47.0 & $30^{\circ}$ & 49.8 \\
\hline $44^{\circ}$ & 42.0 & $39^{\circ}$ & 44.8 & $34^{\circ}$ & 47.5 & $29^{\circ}$ & $5^{\circ}, 3$ \\
\hline $43^{\circ}$ & 42.6 & $3^{\circ}$ & $45 \cdot 3$ & $33^{\circ}$ & 48.1 & $28^{\circ}$ & 50.8 \\
\hline $42^{\circ}$ & $43 . x$ & $37^{\circ}$ & 45.9 & $32^{\circ}$ & 48.6 & $27^{\circ}$ & 51.4 \\
\hline $4 x^{\circ}$ & 43.7 & $36^{\circ}$ & $4^{6} .4$ & $3 x^{\circ}$ & 49.2 & $26^{\circ}$ & 51.9 \\
\hline $40^{\circ}$ & $44 \cdot 2$ & $35^{\circ}$ & 47.0 & $30^{\circ}$ & 49.8 & $25^{\circ}$ & 52.5 \\
\hline
\end{tabular}

If, therefore, at any temperature between $45^{\circ}$ and $25^{\circ}$ values be found for the critical line which are less than the values corresponding to the same temperature according to the table, the sample of butter may safely be pronounced to be natural, i.e., unadulterated butter. If the reading shows higher numbers for the critical line, the sample should be reserved for chemical analysis.

Note.-Dr. Eichel of Metz has suggested that instead of comparing the scale divisions at the same temperature, the position of the critical line may be determined at the moment when the butter begins to set. In this case he gives fifty-four as the highest admissible number for the critical line of pure butter.

No sharp distinction is apparent between pure and renovated butter on the refractometer.

Special Thermometer for the Butyro-refractometer.-Instead of employing the ordinary thermometer, as shown in Fig. 36, a special thermometer (Fig. IOI) has been devised for work both with butter and with lard. This instrument has two scales, arranged side by side, one for butter and one for lard, each of which indicates at once the highest allowable reading for the pure fat, corresponding to the temperature at which the observation is made, which, however, need not be noted.

If the scale reading of the instrument, as observed through the telescope, differs materially from the reading of the special thermometer, the fat under examination is undoubtedly adulterated, or, in the case of butter, a higher reading indicates oleomargarine. The special thermometer thus indicates the highest permissible number for pure butter.

* With natural butter this number is, as a rule, somewhat less $(0.53)$, with oleomargarine a little greater $\left(0.5^{6}\right)$. 
The Reichert or Reichert-Meissl Number * is by far the most important single determination in establishing proof of the character of the sample, whether butter or oleomargarine, for evidence in court, and in such cases this determination is indispensable. The result is conclusive, excepting in those rare instances where the admixture of foreign fat is so small as to cause the Reichert number to approximate that of pure butter. In common instances of creamery butter and commercial oleomargarine the Reichert number shows a very marked distinction (see table, p. 544).

It is difficult to fix a minimum figure below which, in doubtful cases, a sample may be pronounced impure by reason of admixture with foreign fat. In general, however, a Reichert number under to would be almost sure to show adulteration, though instances are on record where butter of known purity has shown a Reichert number even lower than this. It is in fact rare that pure butter has a Reichert number under 12.

Stebbins $†$ gives the maximum, minimum, and average of the Reichert number obtained by him on $3^{17} 7$ samples of unadulterated butter, some of which were of low grade, as follows: Maximum, 18.2; Minimum, 11.2; Average, 14.7.

As a rule little difference is apparent between pure and "renovated" samples as regards their Reichert number.

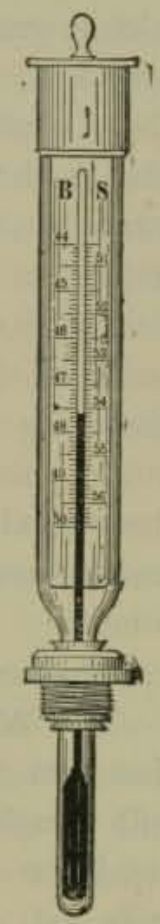

Frg, Ior.-Special Butyro-refractometer Thermometer for Butter and Lard.

Vieth has shown that the Reichert number of butter is generally a trifle lower after it becomes rancid.

Specific Gravity.-Skalweit has shown that the specific gravity of butter and oleomargarine relative to each other varies with the temperature at which it is taken, the difference between the two growing less and less as the temperature increases above $35^{\circ}$. The greatest variation being at $35^{\circ}$, he recommends this temperature as the best at which to make the determination.

The Foam Test, also known as the "boiling" or "spoon" test. $\ddagger$ This, though originally intended as a household test, is in reality one of

* The writer prefers to carry out this process on 2,5 grams of the butter fat, expressing thus the Reichert number, this being practically the half Reichert-Meissl number, which is based on the use of 5 grams.

† Jour. Am, Chem. Soc., 21 , 1899 , p. 939.

$\ddagger$ Farmer's Bulletin, No, I3 $_{3}$. 
the very best laboratory methods of separating pure butter samples from renovated butter and oleomargarine. A small lump of the sample (from 3 to 5 grams) is heated in a large spoon over a Bunsen flame, turned very low, stirring constantly during the heating. Genuine butter, under these conditions, will boil quietly, but with the production of considerable froth or foam, which will often swell up over the sides of the spoon, when, just after boiling, the latter is raised from the flame. Renovated butter or oleomargarine, under this treatment, will bump and sputter noisily like hot grease containing water, but will not foam.* Another point of difference is that on removing the spoon from the flame and observing the character of the curdy particles, in the case of genuine butter these particles of curd will be very small and finely divided in the melted fat, being indeed hardly perceptible, while with oleomargarine and renovated butter, the curd will gather in somewhat large masses or lumps.

The test may be carried out in a test-tube if desired.

The Waterhouse or Milk Test. $†$-This test is based on the assumption that butter fat, which is in itself exclusively the product of milk, will mingle intimately with the milk when added thereto in a melted condition and cooled therein, whereas oleomargarine, being foreign to milk fat, will, under like conditions, refuse to diffuse itself naturally in milk as a medium.

About $50 \mathrm{cc}$. of well-mixed sweet milk are heated nearly to boiling in a beaker, and from 5 to Io grams of the fat sample are added. The mixture is then stirred, preferably with a small wooden stick, until the fat is melted. The beaker is then placed in a dish of ice cold water, and the stirring continued till the fat reaches the solidifying-point, at which period, if the sample is oleomargarine, the fat can readily be collected by the stirrer into one lump or clot, but, if butter, it cannot be so collected, but remains in a granulated condition, distributed through the milk in small particles. It is not necessary to keep up the stirring through the entire term of cooling, but to begin stirring before the fat starts to solidify, which should require from ten to fifteen minutes after the mixture is placed in cold water.

This test, if carefully carried out, shows a marked distinction between butter, whether pure or renovated, and oleomargarine. Under certain conditions, as when the cooling is too rapid, samples of renovated butter.

\footnotetext{
* A very slight foam is sometimes observable with occasional renovated samples, but nothing like the abundant amount produced by the genuine product.

† Parsons, Jour. Am. Chem. Soc., 23, 190r, p. 200.
} 
fat will sometimes show a slight tendency to clot together as in the case of oleomargarine, but to no such extent as the latter.

The author's experience with this test has shown it to be very reliable not only in identifying oleomargarine from butter, but in nearly every case renovated butter can be distinguished from genuine. As a rule, genuine butter fat, even after cooling to the solidifying-point, shows the greatest tendency to emulsionize with the milk when stirred, without adhering to the wooden rod, and is slow to come to the surface when the stirring is stopped. Renovated butter fat, when stirred in the cold milk, almost instantly gathers in a film on the surface of the milk when the stirring is stopped, without emulsionizing. It does not clot together like oleomargarine, but it tends to adhere to the wooden rod.

Patrick * recommends the use of skimmed or partially skimmed milk, and heats to the boiling-point after the fat has been introduced into the hot milk.

Examination of the Curd.-The curd of genuine butter is made up largely of such of the milk proteins as are insoluble in water and hence pass into the cream when separated. These proteins form a gelatinous mass in the butter, readily clotting together when the fat is melted. On the other hand, the curd of process butter, which is, as it were, artificially derived from the entire or skim milk used in its manufacture (in order to replace the natural curd which has been removel in the "purifying" process), differs from the proteins of cream in that it is granular and flaky, consisting chiefly of coagulated casein. Hence the distinction noted as to the appearance of the curd in the foam test.

For the same reason, if beakers containing pure and renovated butter are melted on the water-bath, the curd of the pure sample will settle at once, or in a very few minutes, to the bottom after melting, leaving a comparatively clear supernatant fat. The renovated sample will nearly always fail to settle out clear, even after standing on the water-bath for half an hour or more, but will still be cloudy throughout the mass, due to particles of non-cohesive, floating curd.

In the case of oleomargarine, the curd of which is composed partly of pure butter curd (from cream proteins) and partly of the proteins of the milk with which it is churned, the cloudiness of the fat on melting depends on the relative proportion of milk proteins, and in general is not especially characteristic.

* Farmer's Bulletin, No. r3r. 
Identification of the Source of the Curd.* Half fill a small beaker with the sample and melt on the water-bath. Decant as much as possible of the fat and pour the rest, consisting largely of the water, salt, and curd, upon a wet filter. Acidify the filtrate, which contains the salt and soluble proteins, with acetic acid and boil. If the sample is pure butter, only a slight milkiness is found, indicating absence of albumins, whereas, in the case of process butter, a white, flocculent albuminous precipitate is produced.

Apply to the filtrate also Liebermann's test for albumin; i.e., add strong hydrochloric acid. If a violet coloration is produced, the sample is presumably "process" butter.

Microscopical Examination of Butter. Considerable information may in general be gained by an examination of the sample under ordinary light and with a rather low power, say from 120 to 150 diameters. For examination in this way a bit of the sample on the edge of a knife blade is placed on the glass slide, and simply pressed lightly into a thin film by the cover-glass. A very characteristic difference between genuine and renovated butter is at once seen in the relative opacity of the fields. The fat film, in the case of the fresh, pure butter, is much more transparent than that of the renovated. Again, the curd is so finely divided throughout the mass of genuine butter fat that the field is much more even than that of the renovated, wherein often large and opaque patches of curd are frequently distributed throughout the field.

When a renovated butter sample, mounted as above, is viewed by reflected light, for which purpose the microscope mirror is turned so as not to transmit light through the instrument, one sees a very dark and scarcely perceptible field; but the opaque patches of curd above referred to are strikingly apparent as white masses against a dark background.

With Polarized Light.-It has already been stated that the microscope is useful in showing whether or not a fat has been melted, the crystalline structure of the fat once melted and afterward cooled being rendered apparent, especially when viewed by polarized light. This fact has long been known and put to practical use in the identification microscopically of butter and oleomargarine. $\dagger$

When viewed by polarized light between crossed Nicols under a low magnification, pure butter not previously melted should show no

* Hess and Doolittle, Jour. Am. Chem. Soc., 22, 1900, p. 151.

$\dagger$ Hummel, ibid., 22, p. 327 ; Crampton, loc, cit., supra, p. 703. 
crystalline structure, being uniformly bright throughout, and, if the selenite plate be used, should present an even colored field, entirely devoid of fat crystals. On the other hand, with process butter or oleomargarine, both of which have been melted and subsequently cooled the crystalline structure should be marked, showing with polarized light a more or less mottled appearance, and a play of colors with the selenite.

Various conditions enter in to affect the reliability of the polarized light test. It is nearly always possible in cold weather to observe these distinctions in practice, as above described, in a sharp and striking manner. Figs, 269,270 , and $27 \mathrm{I}$, Pl. XXXVIII, show typical fields of the three products with crossed Nicols and selenite plate. The appearance of pure butter is perfectly blank, while oleomargarine presents a much more mottled appearance than renovated butter. Such well-defined points of variation as are shown in Plate XXXVIII are not always to be seen in practice, even in the hands of an expert. Pure butter sometimes exhibits a somewhat mottled field, due to a slight crystallization at some period of its history. In the summer-time, for instance, when butter melts so easily at ordinary temperature, these distinctions between pure and adulterated samples as shown by polarized light are by no means as satisfactory as in the winter.

Great care should be taken on this account, on the part of the collector of samples as well as the analyst, to keep the sample from melting under ordinary conditions before it is examined.

Hess and Doolittle's Method of Examining the Curd.*-A convenient portion of the sample of suspected butter is melted in a beaker, as much of the fat as possible is decanted off, and the remaining curd, washed free from fat with ether, is poured out on a glass plate and dried. A sample of pure butter is treated in like manner by way of comparison. When examined under a very low magnification of from 3 to 6 diameters, the curd from the pure sample will be seen to be non-granular and amorphous in appearance, while, in the case of renovated butter, the curd will appear very coarse grained and mottled.

Zega's Test for Oleomargarine. + A portion of the filtered fat is poured into a test-tube and kept for two minutes in a boiling water-bath. I cc. of this fat is then measured with a hot pipette into a $50-\mathrm{cc}$, tube containing $20 \mathrm{cc}$. of a mixture of 6 parts ether, 4 parts alcohol, and I part glacial acetic acid. The tube is stoppered, shaken well, and cooled in

* Jour. Am. Chem. Soc., 22, 1900, p. I5 I.

† Chem, Zeit., 1899, 23, 312; Abs. Analyst, 24, p. 206. 
water at $15^{\circ}$ to $18^{\circ} \mathrm{C}$. In the case of pure butter fat, the solution remains clear for some time, a slight deposit being apparent only after standing an hour or more. With oleomargarine, a deposit is evident in a very short time, and in ten minutes a heavy precipitate comes down. With $10 \%$ of oleomargarine in butter, a separation occurs in about fifteen minutes. When a few solid particles have separated out, they are withdrawn and examined under the microscope. With genuine butter, long narrow rods appear, sometimes pointed at the ends, often bent, and grouped as a rule centrally in star-shaped bundles. Oleomargarine presents an appearance of bundles of fine needles, closely packed to form masses frequently resembling sheaves and dumb bells in shape.

Identification of Various Oils and Fats. - Cottonseed oil may be recognized, if present in butter or its substitutes, by the Halphen test, and sesame oil by the Baudouin test. Peanut oil is tested for by the Bellier or Renard test.

Cocoanut oil is sometimes said to be present in butter substitutes. It has a higher Reichert number than most adulterants, and hence a larger admixture of this than of other foreign fats could be used, without lowering the Reichert number of the whole below the allowable limits of pure butter. Its presence would, however, be rendered apparent by the low iodine and refractometer numbers and the high Polenske number.

\section{LARD.}

Nature and Composition.-Lard is the fat of hogs, separated by heat from the scraps or containing tissues. The choicest or highest grade of lard is known as leaf lard, and is derived from the fat which surrounds the kidneys. A comparatively small part of the lard of commerce is, however, strictly speaking, pure leaf lard. Most of it is derived from the whole fat of the animal by rendering, by the aid of steam under pressure, either in open kettle or in closed tanks, the former being used more often for rendering lard on a small scale, and the latter being the most common commercial method.

Next to the leaf, the fat from the hog's back is considered the best in quality, after which is graded, in the order named, the fat from the head, the region of the heart, and the small intestines, the last two grades constituting what is commonly known as "trimmings."

Good lard is white and granular, having the consistency of salve. It has an agreeable, characteristic odor and taste.

The leaf or kidney fat furnishes also the source of the so-called neutral lard, already mentioned as an ingredient of oleomargarine. The leaf, 
being first chilled and finely gro!nd, is placed in the kettle and rendered at a temperature of from $40^{\circ}$ to $50^{\circ} \mathrm{C}$., at which heat only a portion of the lard separates. This portion is, while melted, washed with water containing salt or dilute acid, and forms the neutral lard, a product almost entirely free from odor. The remainder of the leaf is then transferred to the closed tank and subjected for some hours to steam under pressure at a temperature of $230^{\circ}$ to $290^{\circ} \mathrm{F}$., the resulting lard being graded as pure leaf lard.

The composition of the mixed fatty acids of lard is thus calculated by Twitchell:

Linoleic acid.......................... 10.06\%

Oleic acid. . . . . . . . . . . . . . . . . . .

Solid acids (by difference), stearic and palmitic .. $48.55 \%$

Lewkowitsch* gives the following constants for American lards made from fat from different parts of the animal:

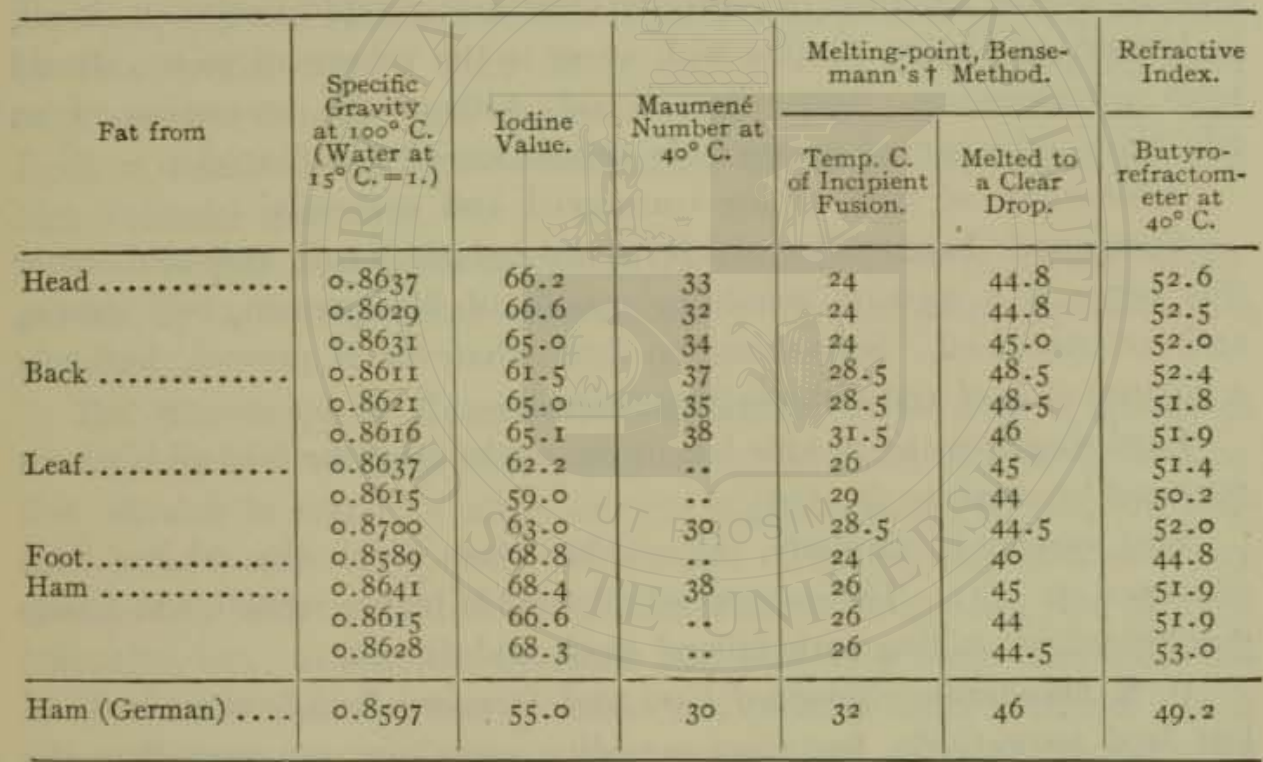

Lard Oil.-This oil is obtained by subjecting lard contained in woolen bags to hydraulic pressure in the cold. The lard oil (chiefly olein) thus expressed constitutes nearly $60 \%$ of the whole, and the residue is known as lard stearin.

Lard oil is a thin fluid, pale yellow in color, and with varying specific

* Oils, Fats, and Waxes, Igo4, p. 78r.

$\dagger$ Bensemann distinguishes between the temperature at which the fat begins to liquefy and that at which it becomes completely transparent. 
gravity, due to varying conditions of pressure and temperature. It has a pleasant, though somewhat bland taste, and is used to some extent as an edible oil. It is used in France as an adulterant of olive oil, and with the Maumené, elaĩdin, and nitric acid tests, it behaves much like olive oil.

According to the U. S. Pharmacopœia, the specific gravity of lard oil should be from 0.910 to 0.920 at $15^{\circ} \mathrm{C}$.

At a temperature a little below $10^{\circ} \mathrm{C}$. it should form a semi-solid white mass.

When it is brought in contact with concentrated sulphuric acid, a dark reddish-brown color should instantly be produced.

Lard oil should not respond to the Bechi test for cottonseed oil.

If $5 \mathrm{cc}$. of the oil, contained in a small flask, be mixed with a solution of 2 grams of potassium hydroxide in $2 \mathrm{cc}$. of water, then $5 \mathrm{cc}$. of alcohol added, and the mixture heated for about five minutes on a water-bath with occasional agitation, a perfectly clear and complete solution should be formed, which, on dilution with water to the volume of $50 \mathrm{cc}$., should form a transparent, light-yellow liquid, without the separation of an oily layer (absence of appreciable quantities of paraffin oils).

Adulterants of lard oil are cottonseed and corn oils.

Compound Lard.-The article so extensively made and sold under this name is a mixture consisting usually of lard stearin, beef stearin, and cottonseed oil. Sometimes no lard whatever is present, but only a mixture of beef and cottonseed stearins.

Lard stearin is the residue left in the cloths after the lard oil has been removed by pressure (p. 555).

Beef stearin is, similarly, the residue from which oleo oil has been expressed (p. 54I). The cottonseed oil used is highly refined, and finally decolorized by mixing with fullers' earth and filtering.

U. S. Standards.-Standard Lard and Standard Leaf Lard are lard and leaf lard respectively, free from rancidity, containing not more than $\mathrm{r} \%$ of substances other than fatty acids, not fat, necessarily incorporated therewith in the process of rendering, and standard leaf lard has an iodine number not greater than 60 .

Adulteration of Lard.-The mixture known as "compound lard" is quite commonly fraudulently sold for pure lard. Indeed, the adulterants of lard usuaily met with ate cottonseed oil or stearin and beef stearin. Other oils said to have been used as adulterants are peanut, sesame, corn, and cocoanut. Formerly water was incorporated into 
the fat to such an extent as to materially cheapen it, but this sophistication is now rare. Moisture is determined as in the case of butter.

The Butyro-refractometer Reading.-The refracting degree of cottonseed oil on the butyro-refractometer is about 8.9 in excess of the standard refraction of lard, while that of beef tallow is about 3.8 less than the standard. If, therefore, the refractometer reading is unusually low, the presence of beef stearin is to be suspected; if unusually high, cottonseed oil should be looked for. A mixture of the two adulterants with pure lard such as is found in "compound lard," may sometimes, though not often, be found to give refractometric readings within the limits of pure lard.

Detection of Foreign Oils.-Cottonseed oil is best detected by the Halphen test. A very slight color reaction should not be taken as proof positive of the admixture of cottonseed oil, since it has been found that the fat of hogs fed on cottonseed meal gives a slight reaction with both the Bechi and the Halphen tests. Sesame and peanut oils are detected by their special tests. Corn oil is indicated by the abnormally high refractometric reading and iodine number, cocoanut oil by the high Reichert number, the high saponification equivalent, and especially the high Polenske number.

Beef stearin is difficult to identify chemically, but is usually distinguished by a microscopical examination of the fat after crystallization as follows:

The Microscopical Examination of Lard.-From 2 to 5 grams of the fat are dissolved in 10 to $20 \mathrm{cc}$. of ether $*$ in a test-tube, and the solution allowed to stand 12 hours or over night at about $20^{\circ} \mathrm{C}$., the testtube being loosely stoppered with cotton. The crystals obtained vary considerably with the condition of heat, amount of solvent, rate of crystallization, etc., so that the operator had best vary these conditions till he is satisfied that the best possible results have been obtained. It is often advantageous to separate the crystals first obtained by filtration from the mother liquor, and to redissolve in ether and recrystallize in a second test-tube. The crystals formed at the bottom of the test-tube are, for the purpose of thus purifying, separated from the mother liquor by filtration through a small filter, and the precipitate washed several times with ether. The washing with ether should not be continued so long that the crystals are perfectly freed from mother liquor and olein, for in this case they are so dry and pulverulent as to require a mountant when on the slide for microscopical examination. The writer prefers

* Some analysts get better results with a mixture of ether and alcohol, 
to have them slightly oleaginous, so that when applied to the slide no mountant need be used. In this case the crystals seem to stand out in wider contrást to the background than when cottonseed oil, the usual medium, is used.

If the crystals are, however, in a pulverulent condition, a drop of alcohol can be used as a mountant, or oil, as preferred. Mounted under a cover-glass they are examined under various powers of the microscope.

Figs. 272 and 273 , PI. XXXIX, show the typical appearance of pure lard stearin from a leaf lard of known purity, and Figs. 276, 277, and 278 illustrate beef stearin. These figures show distinctive crystallization of each form under the best conditions. The lard stearin crystals when thus obtained are flat rhomboidal plates cut off obliquely at one end, and are grouped irregularly, as if thrown carelessly together. The beef stearin crystals, on the other hand, are cylindrical rods or needles, often curved, with sharp ends, and are arranged as shown in fanshaped clusters. Conditions of crystallization are frequently such as not to show the sharp distinctions noted above. Both forms of crystals are at times apt to gather in clusters that at first sight appear somewhat similar, and are often misleading as to their true character. It is found almost invariably that the beef stearin crystals gather in clusters, radiating from a common center or point, often with a peculiar twisted appearance, breaking up into little fans. Lard crystals, it is true, do not always lie flat in irregular groups as shown in Fig. 272, but, as in Fig. 274, form clusters that, unless studied carefully, might at first sight be considered as identical with the fan shapes of the beef stearin already described. It will be seen, however, that if the best possible conditions are attained, the crystals of lard, instead of radiating from a point, are arranged more like feathers or alternate leaves on a branch, each crystal being given forth from another close at hand. Moreover, the lard crystals are themselves straight and not curved, the apparent curve in the appearance of the clusters being, on careful examination, especially under high power, seen to be chiefly due to several of these straight crystals arranged at angles to each other.

Even when the highest powers of the microscope are applied to the beef stearin crystals, they will always appear as cylindrical, sharppointed rods, some straight, others curved; while with the lard crystals they should be capable of showing the thin, flat, oblique-ended structure when examined with higher powers, even when they are arranged in the feathery clusters, the apparently pointed ends of some of the crystals 
being due to the fact that the plates are viewed edgewise. This is apparent in Fig. 275, in which the crystals are magnified to 480 diameters.

According to Belfield, who was one of the earliest to employ the microscope for identification of foreign fat in lard, it is possible to detect welldefined crystals of both lard and beef-stearin in mixtures crystallized out in the above manner from ether. Later investigators, however, find difficulty in getting both kinds of crystals in the final deposit, it being the more common experience that the character of the final crystals from a mixture of the two fats more often tends to one or the other forms of crystallization. Repeated crystallizations may change the character of the crystals and a number of such crytallizations should therefore be made before final judgment is passed.

The Iodine Number (p. 487). - This test is generally prefigured by the refractometer. Cottonseed oil will absorb about $108 \%$ of its weight of iodine, while beef fat will absorb about $37 \%$.

ANALYSES OF SAMPLES ILLUSTRATING TYPES OF LARD, LARD SUBSTITUTES, AND MIXTURES.

\begin{tabular}{|c|c|c|c|c|c|c|c|c|}
\hline & \multirow[b]{2}{*}{ Nitric Acid Test. } & \multirow[b]{2}{*}{ Crystallization. } & \multirow[b]{2}{*}{$\begin{array}{l}\text { Bechi Reac- } \\
\text { tion. }\end{array}$} & \multicolumn{3}{|c|}{$\begin{array}{l}\text { Butyro-refrac- } \\
\text { tometer. }\end{array}$} & \multirow{2}{*}{$\begin{array}{l}\frac{d}{d} \\
\frac{E}{2} \\
\frac{E}{2} \\
\frac{E}{2} \\
\frac{E}{2}\end{array}$} & \multirow[b]{2}{*}{ Conclusion. } \\
\hline & & & & 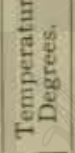 & है & 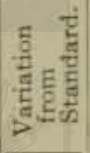 & & \\
\hline A & Slight color..... & Lard stearin & None & $42-5$ & $49 \cdot 7$ & $+0,1$ & $5^{8,1}$ & Lard \\
\hline B & Red.......... & 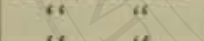 & $"$ & 42 & $5 \circ$ & +0.2 & 59.9 & $\%$ \\
\hline C & Slight color .... & $"$ & 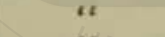 & $41-5$ & $50 . \mathrm{I}$ & +0.0 & 58.7 & “ \\
\hline D & is $\quad 4 \quad \ldots$ & 16 & $\because 6$ & 43 & 50 & +0.6 & 63.7 & “ \\
\hline E & " $"$ " $\quad \ldots$. & $\because$ & 4 & $4 \mathrm{I}-3$ & $5 x$ & +0.8 & 64.6 & $" 6$ \\
\hline $\mathrm{F}$ & " $\quad$ " $\quad \ldots$. & 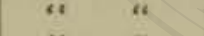 & “ & 42 & 50.5 & +0.7 & 64.8 & " \\
\hline G & " $\quad$ " $\quad \ldots$. & " $\quad \pi$ & c & 42 & 49.7 & -0.1 & $5^{6} .4$ & Leaf lard \\
\hline $\mathrm{H}$ & Very slight color & Beef stearin & " & 50 & 41.2 & -3.8 & $37 \cdot 3$ & Beef tallow \\
\hline$I$ & Deep-brown red & $\begin{array}{l}\text { Few small } \\
\text { bunches }\end{array}$ & Deep color & 42 & $5^{8} \cdot 7$ & +8.9 & 108 & Cottonseed oil \\
\hline $\mathrm{J}$ & Red .......... & Lard stearin & at it & 43 & 50.5 & $+\mathrm{I} \cdot 3$ & $69 \cdot 5$ & $\begin{array}{l}\text { Lard and cotton- } \\
\text { seed oil }\end{array}$ \\
\hline K & Very slight color & $\begin{array}{l}\text { Lard and } \\
\text { beef stearin }\end{array}$ & None & 43 & $48 \cdot 5$ & -0.7 & 55.2 & $\begin{array}{l}\text { Lard and beef } \\
\text { tallow }\end{array}$ \\
\hline L & Deep brown.... & Lard stearin & Deep color & $43 \cdot 5$ & 51 & $+1-1$ & $71-4$ & $\begin{array}{l}\text { Lard and cotton- } \\
\text { seed oil }\end{array}$ \\
\hline M & Red........... & " & "6 & $43 \cdot 7$ & 50.1 & $+\mathrm{I} \cdot 3$ & 66.7 & Ditto \\
\hline $\mathrm{N}$ & " $\quad \ldots \ldots \ldots$ & $\begin{array}{l}\text { Lard and } \\
\text { beef stearin }\end{array}$ & $"$ & $43 \cdot 5$ & $49 \cdot \mathrm{I}$ & +0.3 & $54-7$ & $\begin{array}{l}\text { Lard, beef tallow, } \\
\text { and cottonseed } \\
\text { oil }\end{array}$ \\
\hline
\end{tabular}

Notes on the Above Table.- - It will in general be noted that adulteration of lard with cottonseed oil alone is indicated by an abnormally high 
refractometer number, while the presence of tallow will result in an abnormally low refraction. But both adulterants may be present and a normal refraction result. In such a case the positive detection of one of them, such as the cottonseed oil by the Bechi or Halphen test, will indirectly show the presence of the other (tallow), and this indirect proof will be confirmed by crystallization.

Samples A, B, and $\mathrm{C}$ give reactions corresponding to normal, pure lard. D, E, and F show somewhat high refractometer and iodine numbers, but give no direct reaction for cottonseed oil by the Bechi test. $\mathrm{G}$, although showing low iodine and refractometer numbers, gives no evidence of the presence of tallow by crystallization. In fact, the crystals from this sample proved under all circumstances to be most clearly typical of pure lard, broad and flat plates with obliquely cut ends.

This sample was, in fact, pure leaf lard. It is generally true that a stiff, strictly pure leaf lard, which both by its consistency and by its low iodine and refractometer numbers might suggest the presence of beef fat, shows on crystallization much more definitely characteristic lard stearin than does a whole-hog lard, whose iodine and refractometer numbers are more nearly the normal standard.

In distinction from such leaf lard, a sample which may have a similar consistency and iodine and refractometer numbers, but which is composed of a whole-hog lard of a comparatively high iodine number, together with beef fat, gives unmistakable proof of its adulteration by its crystallization.

Effects of Feeding Hogs on Oil Cakes.-Fulmer,* Emmett and Grindley $\dagger$ and other investigators have found that feeding cottonseed meal to hogs causes the lard from these hogs to give a color with the Halphen test, but Tolman, $\ddagger$ Farnsteiner $\S$ and Polenske\| have shown that the lard does not contain phytosterol when examined by Bömer's phytosterol acetate method.

Lard from hogs fed on sesame cake has been shown to respond to the Baudouin test, but not to the phytosterol acetate test.

* Jour. Am. Chem. Soc., 26, 1904, p. 837 .

$\dagger$ Ibid., 27, 1905, p. 263 .

$\ddagger$ Ibid., p. 589 .

$\$$ Zeits, Unters. Nahr. Genuss., II, I906, p, r.

|| Arb. Kaiserl. Gesundheitsamt., 22, 1905, p. 568. 


\section{REFERENCES ON EDIBLE OILS AND FATS.}

ANDES, L. E. Animal Fats and Oils.

- Vegetable Fats and Oils. London, 1897 .

Arnold, W. Beiträge zur Analyse der Speisefette. Zeits. Unters. Nahr. Genuss., I0, 1905, P. $20 \mathrm{t}$.

-Beiträge zum Ausbau der Chemie der Speisefette. Ibid., r4, 1907, p. 147.

BenEDikt, R. Analyse der Fette und Wachsarten. Berlin, 1892 .

Beauvisage, G. Les Matières Gras. Paris, r8gr.

Bockarry, L. Huiles Comestibles. Analyse des Matières Alimentaires (Girard et Dupré), p. 4or. Paris, 1894 .

Bornmann, G. Die Fetten Oele des Pflanzen und Thierreiches. Weimar, r889.

Brannt, W. T. Animal and Vegetable Fats and Oils. Phila., I888.

Cannizzaro, S., and Fabris, G. Reliability of Tests for Determining the Purity of Olive Oil. Rome, 189 r.

Dunlap, F. L. The Preparation of Aldehyde-Free Ethyl Alcohol for Use in Oil and Fat Analysis. Jour. Am. Chem. Soc, 28, 1906, p. 395.

FARnstriner, K. Vorschläge des Ausschusses zur Abänderung des Abschnittes

"Speisefette und Oele" der Vereinbarungen. Zeits. Unters. Nahr. Genuss., 10, 1905 , p. $5 \mathrm{r}$.

Fischer, $\mathrm{K}$, und Peyan, $\mathrm{H}$. Beiträge zur Kenntnis des Baumwollsamenöles und der Halphen'schen Reaktion. Zeits. Unters. Nahr. Genuss., 9, I905, p. 8I

Giri, A. A Short Handbook of Oil Analysis. Phila., 1900.

Hanus, J, und Stekt, L. Die Aethylesterzahl, eine neue Konstante Zum Nachweise Cocosfettes. Zeits. Unters. Nahr. Genuss., I5, I908, p. 577.

Hehner, O, and Mrtchen, C. A. On the Determination of Stearic Acid in Fats. Analyst, 21, 316.

Hopkrss, E. H. The Oil Chemists' Handbook. New York, rgor.

Hunt, F. W. A Comparison of Methods used to Determine the Iodine Values of Oils. Jour. Soc. Chem. Ind., I902, 454.

Lewkowitsch, J. Chemical Analysis of Oils, Fats, and Waxes. $3 \mathrm{~d}$ ed. London, I904.

Laboratory Companion to Fats and Oils Industries. London, Igor.

Lichtenberg, C. Die Fettwaaren und fetten Oele. Weimar, I880.

LythGoE, H C. Readings on the Zeiss Butyro-refractometer of Edible Oils and Fats. Technology Quarterly, 16, 1903, p. 222.

Macrarlane, T. Olive Oil. Can. Inl. Rev. Dept., Bul. 67.

Mareille, R. Die Bildung der freien Säure und das Ranzigwerden der Olivenöle Seifensieder Ztg., 3r, r904, pp. 630, 656, 67r, $69 \mathrm{r}$.

McPherson, W, and Rutr, W. A. Corn Oil-Its Possibilities as an Adulterant in Lard and its Detection. Jour. Am. Chem. Soc., 29, 1907, p. 921.

Polenske, E. Beiträge zur Untersuchung von Schweineschmalz und Butter. Arb. Kaiserl. Gesundh. Amt., 22, 1905, p. 557.

RaE \& Co. Prima Arborum. The Olive Tree and its Fruit. New York, r887.

SCHÄDler, C. Die Untersuchung der Fette, Oele, Wachsarten, etc. Leipzig, 1889.

- Die Technologie der Fette und Oele des Pflanzen und Thierreichs. Leipzig, 1892. 
Thamann, F. Die Fette und Oele, Leipzig, r88r.

Torman, L. M. Edible Oils and Fats. U. S. Dept. of Agric., Bur."of Chem., Bul. 65, p. 20

- Report of Coopperative Work on the Dalican Titer Test. U. S. Dept.Agric., Bur. Chem., Bul. 81, p. 65 .

- Report on Fats and Oils. Ibid. Bul. 90 , p. 69 .

Tolman, L. M., and Munson, L. S. Use of the Bechi Test in Olive Oils. Jour. Am. Chem., Soc. 24, 1902, 397.

- Refractive Indices of Salad Oils. Jour. Am. Chem. Soc., 24, 1902, 754.

- Iodine Absorption of Oils and Fats. Jour. Am. Chem. Soc., I903, 244.

- Olive Oil and its Substitutes. U. S. Dept. of Agric., Bur. of Chem., Bul. 77. Villon, A. M. Les Corps Gras. Paris, 1890.

Vulte, H. T., and Grbson, H. W. Nature and Properties of Corn Oil. Jour. Am. Chem. Soc., 23, 1901. p. I.

Wright, A. C. Analysis of Oils and Allied Substances. New York, 1903.

Wright, C. R. A. Animal and Vegetable Fixed Oils, Fats, Butters, and Waxes. London, 1804 .

California Exp. Sta. Buls. 104, 123, 129, I37, et al., on California Olives and Olive Oils.

California Exp. Sta. An. Reports, 1892 et seq.

Connecticut Exp. Sta. An. Report, 1897, p. 44.

\section{REFERENCES ON BUTTER.}

Alvord, H. E. Composition and Characteristics of Butter. Penn. Dept of Agric. An. Rep., r898, p. 558 .

Bockarry, L. Beurre, Analyse des Matières Alimentaires (Girard et Dupré), p. $35 \mathrm{r}$. Paris, 1894 .

Brackett, E. G. Healthfulness of Oleomargarine as an Article of Food. Mass. State Board of Health An. Rep., 1887 , p. 248.

Browne, C. A., Jr. A Contribution to the Chemistry of Butter Fat. Jour. Am. Chem. Soc., 21, 1899, pp. 612, $807,975$.

Cochran, C. B. Butter and Butter Adulterants. Jour. Frankl. Inst., I47, 1899, p. 85.

_ Butter and Butter Adulterants. Penn. Dept. of Agric. An. Rep., 1898, p. 616.

Detection of Foreign Fats in Butter. Jour. Am. Chem. Soc., 19, p. 796.

Cornwalt, H. B. Examination of Butter Colors. Chem. News, 55, p. 49.

Crampton, C. A. The Composition of Process or Renovated Butter. Jour. Am. Chem. Soc., 25, 1903 , p. 358 .

Fritzsche, M. Die bisherigen Erfahrungen und Urteile uber die Polenske'sche Zahl und ein Beitrag zur Kenntnis derselben bei holländischer Versandbutter. Zeits. Unters. Nahr. Genuss., 15, 1908, p. 193.

Geissler, J. F. A Delicate Test for Color in Butter. Jour. Am. Chem. Soc,, 20, p. IIO.

Genth, F. A. The Necessity for a Butter Standard. Penn. Dept. of Agric. An. Rep., I897, p. 549 .

Grrard et Brevans. Le Margarin et le Beurre Artificiel. Paris, r889. 
GRAY, C. E. A Rapid Method for the Determination of Water in Butter. U. S. Dept. Agric., Bur. Animal Ind., Circ. No. 100.

Harris, F. W. The Estimation of Cocoanut Oil in Butter Fat. Analyst, 3I, I906, p. 353 .

Hehner, O., and Angel., A. Butter, its Analysis and Adulteration, London, 1877 .

Hess, W. H. and Doolitrie, R E. Methods for the Detection of Process or Renovated Butter. Jour. Am. Chem. Soc., 22, 1900, p. 150.

Hummer, J. A. Examination of Brown and Taylor's Official Method of Identifying Butter. Jour. Am. Chem. Soc.. 22, 1900, pp. 327, 703

LANG, V. Fabrikation von Kunstbutter. Leipzig, 1885 .

Low, A. W. Testing for a Yellow Azo-Color in Fats. Jour. Am. Chem. Soc., 20 I 898 , p. 889 .

Macfarlane. T. Butter. Can. Inl. Rev. Dept., Bul. 16.

Martin, E. W. Detection of Artificial Coloring Matter in Butter, Oleomargarine, etc Analyst, 12, 1887 . p. 70 .

Moore, R. W. A Test for Carrot Color in Butter. Analyst, II, I886, p. I63.

PAtrick G. F. The Rapid Determination of Water in Butter. Jour Am. Chem. Soc., 28 , 1906 , p. $1611 ; 29$, 1907 , p. 1126.

Polenske, E. Eine neue Methode zur Bestimmung des Kokosfettes in der Butter. Zeits. Unters. Nahr. Genuss., 7, 1904, p. 273.

von Ryn, J. J. L. Composition of Dutch Butter. London, r9o2.

DE SCHWEINitz and Emery. Use of the Calorimeter in Detecting Adulterations of Butter. Jour. Am. Chem. Soc., 18,1896 , p. 174 .

SeI., E. Ueber Kunstbutter, ihre Herstellung, etc. Berlin, 1886.

Stebrins, J. H. On the Reichert Figure of Butter.

ZANE, A. J. General Analyse des Beurres. Paris, 1892.

Farmer's Bulletin I2. Nostrums for Increasing the Yield of Butter.

" " 57. Butter Making on the Farm.

" " 92. Pasteurization in Butter Making.

" " I3r. Household Tests for the Detection of Oleomargarine and Renovated Butter.

Minnesota Exp. Sta. Bul. 74. Digestibility and Food Value of Butter.

New York Exp. Sta. (Ithaca) Bul. 118. Butter Increasers.

North Carolina Exp. Sta. Bul. 166. Butter, its Composition, Artificial Imitation, and Adulterants.

Storrs, Conn. Exp. Sta. IIth An. Rep., page 85. Bacteriology in Butter Making.

\section{REFERENCES ON LARD.}

Cochran, C. B. Detection of Foreign Fats in Lard and Butter.

Conroy, M. Lard, its Adulteration with Cottonseed Oil and Detection Thereof. Analyst, $\mathrm{I}_{3}, 203$.

Farnsteiner, K. Kendrich, K, und Buttenberg, P. Zusammensetzung des Fettes von Stark mit ölhaltigen Futtermitteln gefütterten Schweinen. Zeits. Unters. Nahr. Genuss., II, I906, p. I. 
Fulmer, E. Some Notes Concerning Halphen's Test for Cottonseed Oil. Jour. Am. Chem. Soc., 24, I902, p. Ir 49 .

- On Reactions of Lard from Cottonseed Meal-fed Hogs with Halphen's Reagent. Ibid. 26,1904, p. 837 .

Gladoing, T. S. Examination of Lard for Adulteration. Analyst, I4, 1889, p. 32.

- Microscopic Detection of Beef Fat in Lard. Jour. Am. Chem. Soc., 18, p. 189.

Henner, O. On Belfield's Test for Beef Stearin in Lard. Analyst, 27, 1902, p. 247.

KöNig, J., und Schluekebier, J. Ueber den Einfluss des Futterfettes auf das Körperfett bei Schweinen mit besonderer Berücksichtigung des Verbleibs des Phytosterins. Zeits. Unters. Nahr. Genuss., 15, 1908, p. 64 r.

Macfarlane, T. Lard. Canadian Inl. Rev. Dept., Bul. 7 .

Stock, W. F. K. On the Estimation of Beef Fat in Lard. Analyst, I9, I894, p. 2.

Tennile, G. F. Determination of Solid Fats in Compound Lard. Jour. Am. Chem. Soc., 19,1897 , p. 51 .

Tolman, L. M. Examination of Lard from Cottonseed Meal-fed Hogs, by Phytosterol Acetate Method of Bömer. Jour. Am. Chem. Soc., 27, 1905, p. 589.

Wesson, D. Examination of Lard for Impurities. Jour. Am. Chem. Soc., 17, 1895, p. 723 .

WrLey, H. W. Quantitative Estimation of Adulterants in Lard. Analyst, I4, I889, p. 73 .

- Lard and Lard Adulterations. U. S. Dept. of Agric., Div, of Chem., Bul. I3, part 4

Wrrthle, F. Detection of Cottonseed Stearin in Lard. Analyst, 27, 1902, p. 247.

Conn. Exp. Sta. An. Rep., 1896 , p. 128.

Mass. State Board of Health An. Rep., 1895, p. 668. 


\section{CHAPTER XIV.}

\section{SUGAR AND SACCHARINE PRODUCTS.}

Nature and Classification of Sugars.-Of all classes of food materials the sugars from their great solubility are the most readily available, and on this account are very valuable as nutrients. As in the natural processes of digestion the starches and more difficultly digestible of the carbohydrates are converted into sugar and thus rendered assimilable, so by processes quite analogous to those that take place in the alimentary tract, the chemist converts these same carbohydrates into sugar as an end-point for purposes of definite determination.

The sugars are characterized by their sweet taste, their ready solubility in water, their power to rotate the plane of polarized light, and their insolubility in ether and absolute alcohol.

The sugars occurring commonly in food naturally divide themselves into two groups: First, the Saccharoses, or cane sugar group, having the composition $\mathrm{C}_{12} \mathrm{H}_{23} \mathrm{O}_{11}$, of which the most prominent members are sucrose, maltose, and lactose; and, second, the Glucoses, or grape sugar group, expressed by the formula $\mathrm{C}_{6} \mathrm{H}_{12} \mathrm{O}_{6}$, which includes dextrose, levulose and galactose, besides other less common sugars.

The members of both groups are intimately related. Thus by the ordinary process of so-called inversion sucrose, or cane sugar, belonging to group $\mathrm{I}$, is converted by the action of heat and dilute acid into two sugars, dextrose and levulose, which are members of group 2, in accordance with the following reaction:

$$
\underset{\text { Cine sugar }}{\mathrm{C}_{12} \mathrm{H}_{22} \mathrm{O}_{11}}+\mathrm{H}_{2} \mathrm{O}=\underset{\text { Dextrose }}{\mathrm{C}_{6} \mathrm{H}_{12} \mathrm{O}_{8}}+\underset{\text { Levulose }}{\mathrm{C}_{6}} \mathrm{H}_{12} \mathrm{O}_{6} \text {. }
$$

The same formula expresses also the result that takes place when lactose, or milk sugar, is heated with dilute acids, breaking up into dextrose and galactose. 
Occurrence.-Sugars occur in roots, grasses, stems of plants, trunks of trees, leaves, and fruits, usually in the form of cane sugar, or sucrose, and of invert sugar (dextrose and levulose) mixed in varying proportions.

The following table from Buignet* shows the kind and amount of sugars occurring in some of the common fruits:

\begin{tabular}{|c|c|c|c|}
\hline & Cane Sugar. & $\begin{array}{l}\text { Reducing } \\
\text { Sugar. }\end{array}$ & Acid. \\
\hline Apricots $, \ldots \ldots, \cdots, \cdots, \cdots$ & 6.04 & 2.74 & I. 864 \\
\hline Pineapples.$\ldots \ldots \ldots \ldots \ldots$ & II. 33 & I. 98 & .547 \\
\hline English cherries............. &.$\infty 0$ & 10.00 & $.66 t$ \\
\hline Lemons................... & $.4 t$ & 1.06 & 4.706 \\
\hline Figs $\ldots \ldots \ldots \ldots \ldots$ & .00 & II. 55 & .057 \\
\hline Strawberries. ................ & 6.33 & $4 \cdot 9^{8}$ & $.55^{\circ}$ \\
\hline Raspberries.... & 2.01 & 5.22 & 1. 380 \\
\hline Gooseberries .............. & .00 & 6.40 & 1. 574 \\
\hline Oranges,$\ldots \ldots \ldots \ldots \ldots$ & $4 \cdot 22$ & $4 \cdot 3^{6}$ & .448 \\
\hline Peaches (green) ............. & .92 & 1.07 & 3.900 \\
\hline Pears (Madeleine)............ & .36 & 8.42 & .115 \\
\hline Apples....................... & 5.28 & 8.72 & I. 148 \\
\hline Prupes & 2.19 & $5 \cdot 45$ & .633 \\
\hline 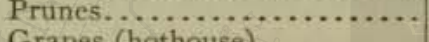 & $5 \cdot 24$ & 2.43 & 1. 288 \\
\hline Grapes (hothouse) ........... & .00 & 17.26 & .345 \\
\hline &.$\infty 0$ & 1.60 & 2.485 \\
\hline
\end{tabular}

CANE SUGAR, OR SUCROSE.

Nature and Occurrence.-This, the most common of all the sugars, is nearly always understood by the unqualified term of sugar. It crystallizes in monoclinic prisms. Its specific gravity is $\mathrm{I} .595$. Its meltingpoint is about $160^{\circ} \mathrm{C}$. Its specific rotary power $[\alpha]_{D}$ in solutions having a concentration of from to to 20 grams in $100 \mathrm{cc}$. is, according to Tollens, $66.48^{\circ}$. Sucrose is extremely soluble in water, which, when cold, will hold in solution twice its weight of the sugar.

Cane sugar is ordinarily derived from four sources-the sugar beet, the sugar cane, the maple tree, and the sorghum plant. The first two sources supply the principal output of commercial cane sugar, about half the sugar on the world's market being furnished, by the sugar beet and the other half by the sugar cane. It should be understood that the product sucrose, or cane sugar, is chemically the same whether derived from either of the above sources and thoroughly refined.

U. S. Standard Sugar is white sugar containing at least $99.5 \%$ of sucrose.

* Ann. Chim. Phys., 59, 233. 
The Sugar Cane (Saccharum officinarum) is cultivated principally in Louisiana and other southern states, in Cuba and the West Indies, and in the Hawaiian Islands. Its growth and cultivation form an industry in nearly all tropical countries.

Allen * has compiled the following table showing the composition of the juice of the sugar cane from different localities:

\begin{tabular}{|c|c|c|c|c|c|}
\hline $\begin{array}{l}\text { Locality and Kind } \\
\text { of Cane. }\end{array}$ & Water. & Sugar. & $\begin{array}{l}\text { Woody } \\
\text { Fiber. }\end{array}$ & Salts. & Authority. \\
\hline 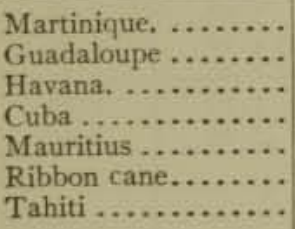 & $\begin{array}{l}72.1 \\
72.0 \\
77.0 \\
65.9 \\
69.0 \\
76.73 \\
76.08\end{array}$ & $\begin{array}{l}18.0 \\
17.8 \\
12.0 \\
17.7 \\
20.0 \\
13.39 \\
14.28\end{array}$ & $\begin{array}{c}9.9 \\
9.8 \\
\text { I1.0 } \\
16.4 \\
10.0 \\
9.07 \\
8.87\end{array}$ & $\begin{array}{l}\ldots \ldots \\
0.4 \\
\cdots \ldots \\
\cdots \cdots \\
1.0 \\
.39 \\
.35\end{array}$ & $\begin{array}{l}\text { Peligot } \\
\text { Dupuy } \\
\text { Casaseca } \\
\text { Casaseca } \\
\text { Icery } \\
\text { Avequin } \\
\text { Avequin }\end{array}$ \\
\hline
\end{tabular}

The composition of raw cane sugar ash according to Monier is as follows:

RAW CANE SUGAR ASH.

Carbonate of calcium. .................. 49.00

" $\quad$ " potassium................... 6.50

Sodium and potassium sulphate............. 16.00

Sodium chloride........................ 9.00

Silica and alumina. . . . . . .

100.00

Manufacture of Cane Sugar.-The process of manufacturing raw sugar from sugar cane is briefly as follows: The juice is first extracted from the canes by crushing in roll mills and is freed from nitrogenous bodies, organic acids, etc., by the process of defecation, which consists in heating to coagulate the albumin, and nearly neutralizing with milk of lime, the impurities being removed as a scum. The juice is then subjected to evaporation and crystallization, the raw, or muscovado sugar, which contains from $8_{7}$ to $9 \mathrm{I}$ per cent of sucrose, being separated from the molasses, which is the mother liquor, by draining or by centrifugal.

Some of the best grade of muscovado, or raw sugar, is used as "brown sugar" without further refining, and much of the molasses is used as a table syrup and for cooking, while the lower grades of molasses are used in the manufacture of rum.

- Com. Org. Anal., 4 Ed., Vol. I, p. 359. 
The following table from Thorpe* shows the average composition of raw and refined sugar:

\begin{tabular}{|c|c|c|c|c|c|}
\hline & - Cane & Glucose ${ }^{1}$. & Water. & $\begin{array}{l}\text { Organic } \\
\text { Matter. }\end{array}$ & Ash. \\
\hline RAW SUGAR. & & & & & \\
\hline Good centrifugal ............. & 96.5 & 0.75 & I. 50 & 0.85 & 0.40 \\
\hline Poor centrifugal............. & 92.0 & 2.50 & 3.00 & I. 75 & 0.75 \\
\hline Good muscovado............ & $9 x \cdot 0$ & 2.25 & 5.80 & I. 10 & 0.65 \\
\hline Poor muscovado ............ & 82.0 & 7.00 & 6.00 & $3 \cdot 50$ & $1.50^{\circ}$ \\
\hline Molasses sugar.............. & 85.0 & 3.00 & 5.00 & 5.00 & 2.00 \\
\hline Jaggary sugar............... & 75.0 & 11.00 & 8.00 & 4.00 & 2.00 \\
\hline Manilla sugar............... & 87.0 & $5 \cdot 50$ & 4.00 & 2.25 & 1.25 \\
\hline Beet sugar, 1st.............. & 95.0 & 0.00 & 2.00 & 1.75 & 1.25 \\
\hline Beet sugar, $2 \mathrm{~d} . . . \ldots \ldots \ldots . .$. & 91.0 & 0.25 & 3.00 & 3.25 & 2.50 \\
\hline REFINED SUGAR. & & & & & \\
\hline Granulated sugar ........... & 99.8 & 0.20 & 0.00 & 0.00 & 0.00 \\
\hline White coffee sugar........... & 91.0 & 2.40 & $5 \cdot 50$ & 0.80 & 0.30 \\
\hline Yellow X C sugar. ........... & 87.0 & $4 \cdot 5^{\circ}$ & 6.00 & 1. 50 & 1.00 \\
\hline Yellow sugar ............... & 82.0 & $7 \cdot 50$ & 6.00 & 2.50 & 2.00 \\
\hline Barrel sugar. .............. & 40.0 & 25.00 & 20.00 & 10.00 & 5.00 \\
\hline
\end{tabular}

i The term "glucose" includes sugars which reduce Fehling's solution, but are not necessarily optically active.

The following minimum and maximum figures are taken from analyses made by Babington $\dagger$ of twenty-two samples of brown sugar and thirtyone samples of molasses.

BROWN SUGAR.

Direct polarization. ................... $8_{4}$ to $8_{7}$

Invert " $\quad \ldots \ldots \ldots \ldots \ldots \ldots \ldots \ldots,-27$ " -29

Sucrose by Clerget.................. 83.5 " $9^{1} \cdot 5$

Reducing sugar................... 3 " 6

Moisture.............................. 3.5 " 6

Ash........................... 0.8 " 3.0

MOLASSES.

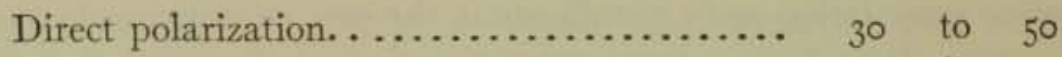

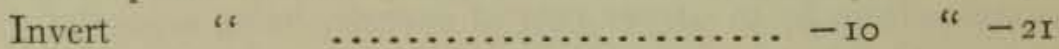

Sucrose by Clerget................... 32 " $3^{2}$

Reducing sugar.................... I3 " 4

Moisture........................ " 29 " 32

Ash............................. 0.5 " 4

* Outlines of Industrial Chem., p. $3^{8} 3$.

† Can. Inl. Rev. Dept. Bul. 25. 
The Sugar Beet (Beta vulgaris) is grown chiefly in France and Germany, and to a lesser extent in Holland and England. The successful growth of the sugar beet in the United States is confined mainly to California, Colorado, Utah, and Nebraska, and the entire output of beet sugar in this country is comparatively small.

According to R. Hoffmann, sugar beets have about the following composition, three types being selected-first, those poor in sugar; second, those having a medium sugar content, and third, those rich in sugar:

COMPOSITION OF THE SUGAR BEET.

\begin{tabular}{|c|c|c|c|}
\hline & First Type: & Second Type. & Third Type. \\
\hline 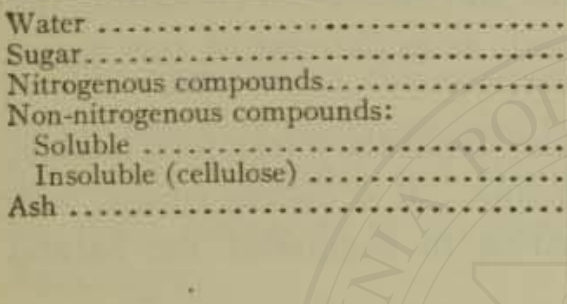 & $\begin{array}{r}89.20 \\
4.00 \\
1.00 \\
4.13 \\
1.01 \\
0.66 \\
100.00\end{array}$ & $\begin{array}{r}83.20 \\
9.42 \\
1.64 \\
3.34 \\
1.50 \\
0.90 \\
100.00\end{array}$ & $\begin{array}{r}75.20 \\
15.00 \\
2.20 \\
\\
4.23 \\
2.07 \\
1.30 \\
100.00\end{array}$ \\
\hline
\end{tabular}

The following is the mean composition of ten samples of California sugar beet: *

Per cent juice extracted............... $6 \mathrm{r} .38$

Specific gravity.................... $\mathrm{r} .062$ to $\mathrm{I} .075$

Per cent of reducing sugar. . .......... $0.9 \mathrm{I}$

Per cent of sucrose. . . . . . . . . . . . $\mathrm{I}_{4} \cdot 3^{8}$

Total solids calculated ............... $16,5^{8}$

Total solids weighed................ 17.20

Per cent of ash. ................... 0.994

The composition of beet sugar ash according to Monier is as follows: RAW BEET SUGAR ASH.

Carbonates of potassium and sodium ............... 82.20

Carbonate of calcium....................... 6.70

Potassium and sodium sulphate and sodium chloride. ... II. IO

100.00

Manufacture of Beet Sugar.-In making raw sugar from sugar beets the latter are first washed and sliced by machinery and the juice extracted

* U. S. Dept. of Agric, Div. of Chem., Bul. 27, p. 202. 
by diffusion or digestion with warm water. The juice is then clarified or defecated in much the same manner as that from the sugar cane, after which it is usually bleached with sulphur dioxide.

The subsequent evaporation and crystallization are carried out usually in vacuum pans, and the sugar separated out by centrifugals.

Beet sugar molasses is unfit for food, due to the presence of nitrogenous bodies, which give it a very unpleasant taste and smell.

Process of Refining.-In refining raw sugar, a syrup is made, which is subjected to centrifuging and further defecation, using lime, clay, liquid blood, calcium acid phosphate, and other substances as clarifiers. The juices are then filtered, first through cloth bags and then through bone char, after which they are evaporated and allowed to crystallize, the resulting granulated sugar being separated, as in the case of raw sugar, by centrifugal machines.

Gramulated Sugar of commerce is without doubt the purest food product on the market, being generally $99.8 \%$ sucrose. It is usually treated with an extremely weak solution of ultramarine to counteract the natural yellow color.

The syrup from which the granulated sugar is separated forms the "golden," or "drip," syrup used on the table. Its typical composition is as follows: Sucrose, $40 \%$; reducing sugars, $25 \%$; water, $20 \%$; organic matter, 10\%; ash, $5 \%$.

The dry sugars, whether white or brown, are rarely subjected to adulteration.

Maple Sap.-The sap of the maple tree, Acer saccharinum, or Acer barbatum, furnishes a sugar considerably prized for its peculiar flavor. The maple sugar industry is largely confined to the northeastern states and to Canada, and the maple sugar season is generally limited to six weeks or two months in the spring.

The following are minimum and maximum figures from the analyses of five samples of maple sap made in Massachusetts:

Specific gravity.................... I.007 to 1.015

Sucrose. .......................... 0.769 " 2.777

Reducing sugar..................... " 0.012

The ash of maple sap varies from 0.04 to 0.1 per cent. Albuminoids are present in amount varying from 0.008 to 0.03 per cent.

Maple Sugar and Syrup are made by simply boiling down the sap to the proper consistency, usually in open pans, and removing the scum 
with great care, since this contains nitrogenous matters that would cause fermentation in the finished product. Pure cane sugar is never commercially produced from the maple sap, since the refining process would deprive it of the flavor which gives to maple sugar the chief value.

McGill gives the following as the "average analyses of six samples of maple syrup of known purity:

\begin{tabular}{|c|c|c|c|c|c|c|c|}
\hline \multirow{2}{*}{$\begin{array}{l}\text { Saccharim- } \\
\text { eter Direct. }\end{array}$} & \multirow{2}{*}{$\begin{array}{l}\text { Saccharim- } \\
\text { eter Invert. }\end{array}$} & \multirow{2}{*}{$\begin{array}{l}\text { Cane Sugar } \\
\text { by Clerget. }\end{array}$} & \multicolumn{2}{|c|}{ By Copper. } & \multirow{2}{*}{ Ash. } & \multirow{2}{*}{ Water. } & \multirow{2}{*}{ Solids. } \\
\hline & & & $\begin{array}{l}\text { Reducing } \\
\text { Sugar. }\end{array}$ & Cane Sugar. & & & \\
\hline+62.2 & -21.2 & 62.4 & .42 & $63 \cdot 3^{6}$ & .53 & $35 \cdot 70$ & $64 \cdot 3^{\circ}$ \\
\hline
\end{tabular}

The variation in the composition of pure maple products is shown by the following table compiled by A. H. Bryan * from analyses published by Hortvet, $\dagger$ Jones, $\ddagger$ and Winton $\S$, and some sixty analyses made at the sugar laboratory of the Bureau of Chemistry, U. S. Department of Agriculture.

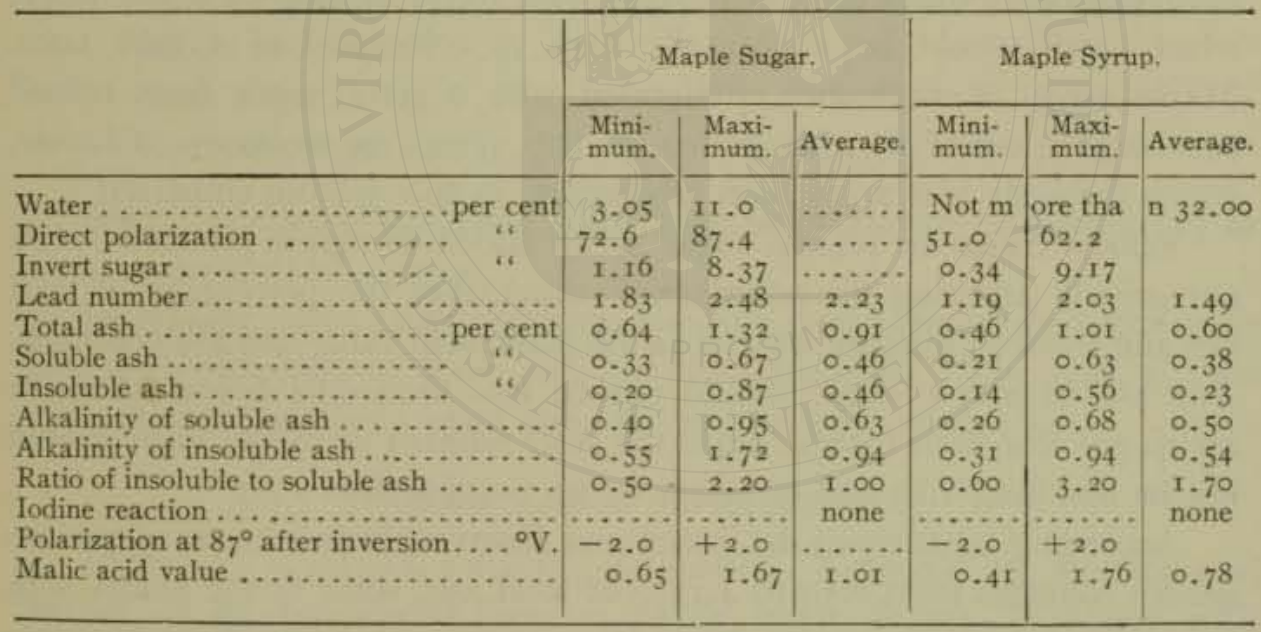

Partial ash analyses of maple products and brown sugar have been made by Jones $\|$ with the following maxima and minima results:

* U. S. Dept. Agric., Bur. of Chem., Circular No, 40, p. ro.

$\dagger$ Jour. Am. Chem. Soc., 26, 1904, p. 1523.

‡ Vt. Agric. Exp. Sta. Rep., 1904, p. 446; 1905, p. 315 .

$\$$ Jour. Am. Chem. Soc., 28, r9o6, p. 1204.

|| Loc. cit., I905, p. 331 . 


\begin{tabular}{|c|c|c|c|c|c|c|c|c|}
\hline & & \multirow{2}{*}{$\begin{array}{l}\text { Number } \\
\text { of } \\
\text { Analysis. }\end{array}$} & \multicolumn{3}{|c|}{ 100 Parts of Ash Contain } & \multicolumn{3}{|c|}{ Ratio of } \\
\hline & & & $\mathrm{CaO}$. & $\mathrm{K}=\mathrm{O}$. & $\mathrm{SO}_{3}$. & $\begin{array}{c}\mathrm{CaO} \text { to } \mathrm{K}=\mathrm{O} \\
\times \text { roo. }\end{array}$ & $\mathrm{CaO}_{\times}$to $\mathrm{SO}_{3}$ & $\begin{array}{l}\mathrm{K} \cdot \mathrm{O} \text { to } \mathrm{SO}_{3} \\
\times \text { too. }\end{array}$ \\
\hline Maple syrup: & \multirow{3}{*}{$\begin{array}{l}\text { Min..... } \\
\text { Max. .. } \\
\text { Min.... } \\
\text { Max.... }\end{array}$} & 6 & $\begin{array}{l}18.03 \\
23.08\end{array}$ & $\begin{array}{l}30.00 \\
38.08\end{array}$ & 0.68 & 150 & $3 \cdot 4$ & 1.9 \\
\hline \multirow{2}{*}{ Maple sugar: } & & \multirow{2}{*}{4} & $\begin{array}{l}23.90 \\
21.03\end{array}$ & $\begin{array}{l}30.90 \\
18.26\end{array}$ & $\begin{array}{l}2.30 \\
1.51\end{array}$ & $\begin{array}{r}181 \\
57\end{array}$ & $\begin{array}{r}12.7 \\
5.2\end{array}$ & 7.2 \\
\hline & & & $3 x .74$ & 32.95 & 2.42 & $\begin{array}{r}57 \\
153\end{array}$ & $\begin{array}{r}5.2 \\
10.4\end{array}$ & $\begin{array}{l}5.1 \\
9.4\end{array}$ \\
\hline \multirow[t]{2}{*}{ Brown sugar: } & Min.... & \multirow[t]{2}{*}{$4^{*}$} & 4.17 & 30.72 & $4 \cdot 5^{8}$ & 257 & 27 & 11 \\
\hline & Max.... & & 21.62 & $55 \cdot 4^{\circ}$ & 17.78 & 949 & ${ }^{x} 57$ & $5^{8}$ \\
\hline
\end{tabular}

* Including one analysis by Hortvet.

U. S. Standards for Maple Products.-Maple Sugar is the solid product resulting from the evaporation of maple sap, and contains in the water-free substance not less than $0.65 \%$ of maple sugar ash.

Maple syrup is syrup made by the evaporation of maple sap or by the solution of maple concrete, and contains not more than $32 \%$ of water and not less than $0.45 \%$ of maple syrup ash.

Adulteration of Maple Sugar and Syrup.-The chief adulterants of maple sugar are brown, or molasses sugar, and white, or refined sugar, the latter being often used in mixture with burnt or inferior maple stock, which itself would be abnormally dark in color and of a rank taste. Maple syrup is commonly adulterated with a syrup made from refined cane sugar, less often with golden or drip syrup, or molasses. Glucose, which formerly was a common adulterant, is now seldom employed.

Refined Sugar or refined sugar syrup added to maple products, while not greatly affecting the polarization, diminishes the percentage of total ash and the lead number, as well as the malic acid value and ash constants. The ash of maple sugar should not be less than $0.64 \%$ and of maple syrup not less than $0.45 \%$, while the lead number of maple sugar should not be less than 1.83 and of maple syrup not less than I.19.

According to analyses by Jones and Hortvet, brown sugar of various grades contains from 0.59 to $4.33 \%$ of total ash, some of the grades with low ash content, or syrups made from them, not being distinguishable from maple sugar or maple syrup respectively by this determination alone; the ratio of insoluble to soluble ash, however, is commonly higher in brown sugar than in maple products. It is frequently possible to identify brown, or molasses sugar, especially when it forms the larger portion of the alleged maple sugar or syrup, by the physical sense of taste. When the perfectly characteristic taste of brown, or molasses sugar, or of "drip syrup," so far predominates over the maple flavor as to be unmistakable, 
especially in cases where the maple flavor is entirely lacking, one need have little hesitation in condemning the product.*

Glucose in maple products is detected by polarization both before and after inversion. A reading of the inverted solution much in excess of 3 degrees Ventzke at $87^{\circ} \mathrm{C}$. furnishes evidence of the presence of this adulterant.

Sorghum (Andropogon sorghum, variety saccharatus) has for many years been grown quite extensively in the southern and western states, and used as a source of syrup, but only in recent years has it been found practicable to produce crystallized cane sugar from it on account of the presence of starch, uncrystallizable sugar, etc.

Much experimental work has been done of late along this line by the U. S. Department of Agriculture. The sorghum plant is as yet, however, a very small factor in the production of cane sugar, though much progress is being made.

The composition of the juice of the sorghum plant is shown by the following results of analyses of eleven varieties made by Hardin: $\dagger$

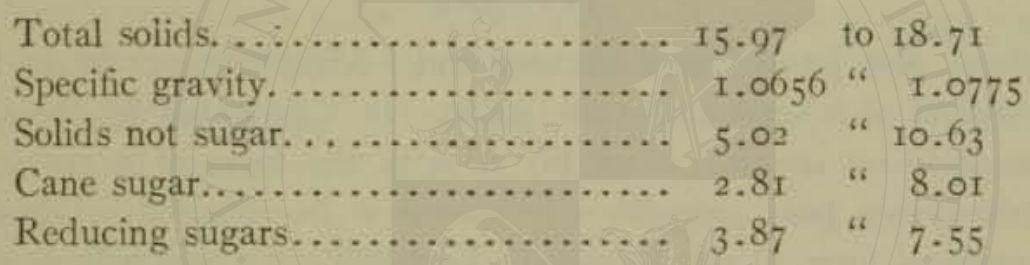

Some varieties of sorghum juice have been known to contain $I_{5}$ or even 17 per cent of sucrose.

In making syrup from sorghum, the ripe canes are crushed, the juice is heated with milk of lime, and the scum removed. The juice is then concentrated usually in open pans to the required consistency.

GRAPE SUGAR, OR DEXTROSE.

Dextrose $\left(\mathrm{C}_{6} \mathrm{H}_{12} \mathrm{O}_{6}+\mathrm{H}_{2} \mathrm{O}\right)$, designated $d$-glucose by Fischer and known in its commercial form as starch sugar, occurs in honey with levulose, and in fruits with both levulose and cane sugar. It is produced by the action of dilute acids or of certain ferments on starch, dextrin, or cane sugar. Grapes contain about $15 \%$ of dextrose. Anhydrous

* The sense of taste, if properly cultivated, and with its limitations recognized, should be entitled to as much consideration as the other senses in forming an opinion. Taste and smell are often very useful factors in detecting adulterants, but should of course be used with discretion.

1 †. S. Dept. of Agric., Div. of Chem., Bul. 37 , p. 75. 
dextrose is soluble in 1.2 parts of cold water. It is soluble in alcohol, but less so than cane sugar. It is much less sweet than cane sugar.

The specific rotary power of dextrose is

$$
[\alpha]_{D}=5^{2} \cdot 3, \quad[\alpha]_{j}=5^{8} .
$$

A normal solution of dextrose on the Soleil-Ventzke scale polarizes at $78.6^{\circ}$. For the commercial preparation of dextrose see p. 576 .

U. S. Standards for Various Sugars.-Standard 70 sugar, or brewers' sugar, is hydrous starch sugar containing not less than $70 \%$ of dextrose, and not more than $0.8 \%$ of ash.

Standard 8o sugar, climax, or acme sugar, is hydrous starch sugar containing not less than $80 \%$ of dextrose, and not more than $\mathrm{r} .5 \%$ of ash.

Standard anhydrous starch sugar is anhydrous starch sugar containing not less than $95 \%$ of dextrose without water of crystallization, and not more than $0.8 \%$ of ash.

The ash of these standard products consists almost entirely of chlorides and sulphates of lime and soda.

\section{LEVULOSE.}

Levulose, also known as $d$-fructose and $l$ - $\hat{-}$-fructose, occurs in foods as the product of inversion of cane sugar. It is prepared by the action of dilute acids on inulin. Normally it is in the form of a syrup, but with extreme care pure anhydrous levulose can be obtained. Diabetene is a commercial form of dry levulose. Levulose is formed with dextrose in the inversion of cane sugar (p. 565), and with dextrose occurs in honey and in many fruits. The specific rotary power of levulose varies with the temperature. At $I 5^{\circ}$ C. $[x]_{D}=-98.8^{\circ}$, decreasing by $0.6385^{\circ}$ for each degree increase in temperature. Its left-handed reading on the Ventzke sugar scale at $15^{\circ} \mathrm{C}$. is equivalent to $148.6^{\circ}$. Levulose is sweeter than dextruse. Its reducing power on Fehling's solution is assumed to be the same as that of dextrose.

\section{MALT SUGAR, OR MALTOSE.}

Maltose $\left(\mathrm{C}_{12} \mathrm{H}_{22} \mathrm{O}_{11}+\mathrm{H}_{2} \mathrm{O}\right)$ is of little importance from the standpoint of the food analyst, excepting as an ingredient of commercial glucose, and as being the sugar produced by the action of ptyaline, the ferment of the saliva on the starch of food in the ordinary process of digestion. When gelatinized starch is subjected to treatment with malt extract at $55^{\circ}$ to $60^{\circ} \mathrm{C}$., it is converted into dextrin and maltose as follows:

$$
\text { Io }_{12} \mathrm{H}_{20} \mathrm{O}_{10}+8 \mathrm{H}_{2} \mathrm{O}={ }_{2} \mathrm{C}_{12} \mathrm{H}_{20} \mathrm{O}_{10}+\underset{\text { Dextrin }}{8 \mathrm{C}_{12} \mathrm{H}_{22} \mathrm{O}_{11}} \text { Maltose }
$$


In its commercial preparation maltose is separated from dextrin by crystallization in alcohol. By the action of weak acids and heat both dextrin and maltose are further converted into dextrose.

Maltose usually crystallizes in minute needles, and its molecule of water is expelled at $110^{\circ} \mathrm{C}$. It is somewhat less soluble in water than dextrose. It is slightly soluble in alcohol, though less than sucrose. Solutions of maltose possess the property of birotation; i.e., when freshly prepared they do not at once assume their true optical activity. The rotation of a freshly prepared solution of maltose increases on standing, requiring several hours to reach its maximum. The specific rotary power, according to O'Sullivan, of anhydrous maltose is $[\alpha]_{D}=I 39.2$, $[\alpha]_{j}=154.5$. For hydrated maltose $[\alpha]_{D}$ would thus be $\mathrm{I}_{32.2}$.

A normal solution of maltose hydrate on the Soleil-Ventzke scale should polarize at $198.8^{\circ}$.

\section{DEXTRIN. COMMERCIAL GLUCOSE.}

DEXTRIN, $\left(\mathrm{C}_{6} \mathrm{H}_{10} \mathrm{O}_{5}\right)_{n}$, possesses more the nature of a gum than of a sugar, and is sometimes called British gum. It is said to occur naturally in the sap of various plants, but this is not definitely assured.

It undoubtedly occurs in beer and in bread crust, and is one of the constituents of commercial glucose. Like starch, it is convertible by hydrolysis with acid into dextrose. By treatment of starch with malt exiract or diastase, starch is converted into dextrin and maltose, these two bodies being separated, in the commercial preparations of dextrin, by repeated treat ment with alcohol.

Dextrin is an uncrystallized, colorless, tasteless body, capable of being pulverized. It is readily soluble in water, slightly soluble in dilute alcohol, but insoluble in alcohol of $60 \%$ or stronger. It is not colored by iodine, and exercises no reducing action on alkaline copper solution. Its specific rotary power is $[\alpha]_{D}=200,[\alpha] j=222$.

Amylodextrin, erythrodextrin and achroodextrin are intermediate products formed in the transformation of starch into dextrose. Amylodextrin is colored purple and erythrodextrin red by iodine solution, while achroodextrin produces no coloration. It is probable that some of these dextrins are not simple substances.

Commercial Glucose, otherwise known as mixing syrup, crystal syrup, and starch, or corn syrup, is a heavy, mildly sweet, colorless, semi-fluid substance, having a gravity of $40^{\circ}$ to $45^{\circ}$ Baumé. It is largely used as an adulterant of maple syrup, molasses, honey, drip syrup, and jellies and jams, and as an ingredient of confectionery.

In France and Germany it is made from potato starch, but in the United 
States mainly from corn starch. The conversion is effected by boiling with dilute sulphuric or hydrochloric acid, after which the acid is neutralized with marble dust, or sodium carbonate respectively, the juice is filtered through bone black, and finally concentrated by evaporation, the degree of conversion and of concentration depending on whether the liquid glucose or the solid dextrose is wanted for the final product. The end product obtained by complete conversion is the dry commercial grape sugar, or dextrose, which is purified by repeated crystallization.

Commercial glucose is a mixture of dextrin, maltose, and dextrose of the following varying composition:

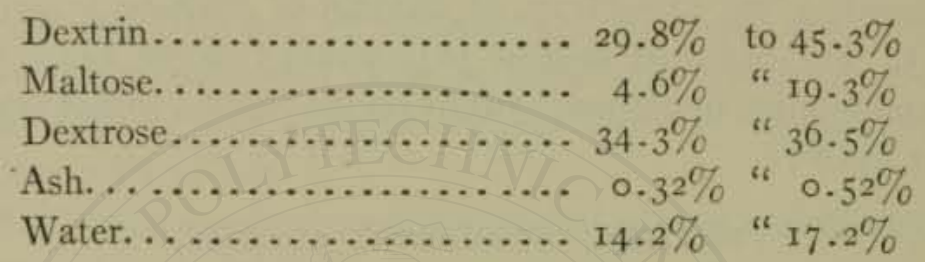

Calcium sulphate is usually found in the ash if sulphuric acid was used for conversion.

Solid commercial grape sugar, or dextrose, has the following com position:
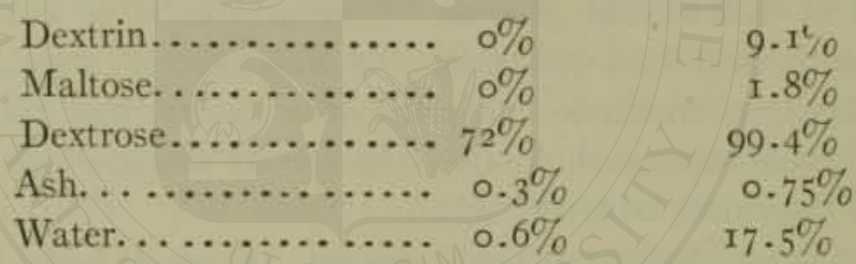

$U$. S. Standard glucose, mixing glucose, or conjectioners' glucose, is colorless glucose, varying in density between $4 \mathrm{I}^{\circ}$ and $45^{\circ}$ Baumé, at a temperature of $100^{\circ} \mathrm{F}$. $\left(37.7^{\circ} \mathrm{C}\right.$. $)$. It conforms in density, within these limits, to the degree Baumé it is claimed to show, and for a density of $4 \mathrm{I}^{\circ}$ Baumé contains not more than $21 \%$ of water, and for a density of $45^{\circ}$ not more than $14 \%$. It contains on a basis of $4 \mathrm{I}^{\circ}$ Baumé not more than $1 \%$ of ash, consisting chiefly of chlorides and sulphates of lime and soda.

Healthfulness of Glucose.-The analyst alleging commercial glucose as an adulterant is frequently asked in court as to its healthfulness, so that the following conclusions of a committee appointed some years ago by the National Academy of Sciences to ascertain among other things whether there is any danger attending the use of this product in food are in point: "First, that the manufacture of sugar from starch is a longestablished industry, scientifically valuable and commercially important; 
second, that the processes which it employs at the present time are unobjectionable in their character and leave the product uncontaminated; third, that the starch sugar thus made and sent into commerce is of exceptional purity and uniformity of composition and contains no injurious substances; and fourth, that though having at best only about twothirds the sweetening power of cane sugar, yet starch sugar is in no way inferior in healthfulness, there being no evidence before the committee that maize starch sugar, either in its normal condition or fermented, has any deleterious effect upon the system, even when taken in large quantities."

MILK SUGAR, OR LACTOSE.

Lactose $\left(\mathrm{C}_{12} \mathrm{H}_{22} \mathrm{O}_{11}+\mathrm{H}_{2} \mathrm{O}\right)$ is prepared commercially from skimmilk by coagulating with rennet and digesting the whey with chalk and aluminum hydroxide. The insoluble matter is filtered out, and the filtrate is concentrated in vacuo to a syrup, which, on standing, yields crystals of lactose. The product is purified by repeated crystallization.

Lactose ordinarily crystallizes in rhombic, hemihedral crystals. Its specific gravity is $\mathbf{1} .5^{25}$. Its water of crystallization is lost by drying at $130^{\circ} \mathrm{C}$. It is soluble in 6 parts of cold water, and in $2 \frac{1}{2}$ or less of boiling water. It is insoluble in absolute alcohol and ether. It has a very slightly sweet taste.

The specific rotary power of milk sugar, after remaining in solution long enough to overcome its birotation, is

$$
[\alpha]_{D}=5^{2} \cdot 5 \text {. }
$$

In the ordinary souring of milk the lactose becomes converted into lactic acid.

On heating lactose with dilute acids it undergoes inversion, forming dextrose and galactose in accordance with the formula given on p. $5^{6}$, illustrating the inversion of cane sugar.

Milk sugar is of considerable importance by reason of the large amount used of late in the preparation of modified milk for infant feeding. .

Grape sugar and cane sugar are to be looked for as adulterants of milk sugar.

The purity of milk sugar is best established by titrating against Fehling's solution, to cc. of which are equivalent to 0.067 gram of lactose.

RAFFINOSE.

Raffinose, $\mathrm{C}_{18} \mathrm{H}_{32} \mathrm{O}_{16} \mathrm{H}_{2} \mathrm{O}$, is a sugar belonging neither to the saccharose nor the glucose group, but to the so-called saccharoid group, the other members of which do not occur in foods. 
Raffinose occurs in beet root molasses to the extent of from 3 to 4 per cent. It is a crystalline, slightly sweet substance, soluble in water and slightly soluble in alcohol. It does not reduce Fehling's solution, but readily undergoes fermentation with bottom yeast. On inversion it splits up into levulose and melibiose $\left(\mathrm{C}_{12} \mathrm{H}_{23} \mathrm{O}_{11}\right)$.

The melting-point of raffinose is $118^{\circ}$ to $119^{\circ} \mathrm{C}$. Its specific rotary power $[\alpha]_{D}=+104.5$ at a temperature of $20^{\circ} \mathrm{C}$.

\section{THE POLARISCOPE AND SACCHARTMETRY.}

A full discussion of the principles of polarized light and even a detailed description of their application to the polariscope will not be given here, but the reader who wishes full information along this line is referred to the various text-books, and especially to those of Tucker, Spencer, and Landolt,* in which various forms of polariscopes are described and their underlying principles discussed.

The Soleil-Ventzke Saccharimeter is the one most commonly used in this country, being adopted as the standard for all United States government work. Fig. 102 shows this instrument, known as the half-shadow

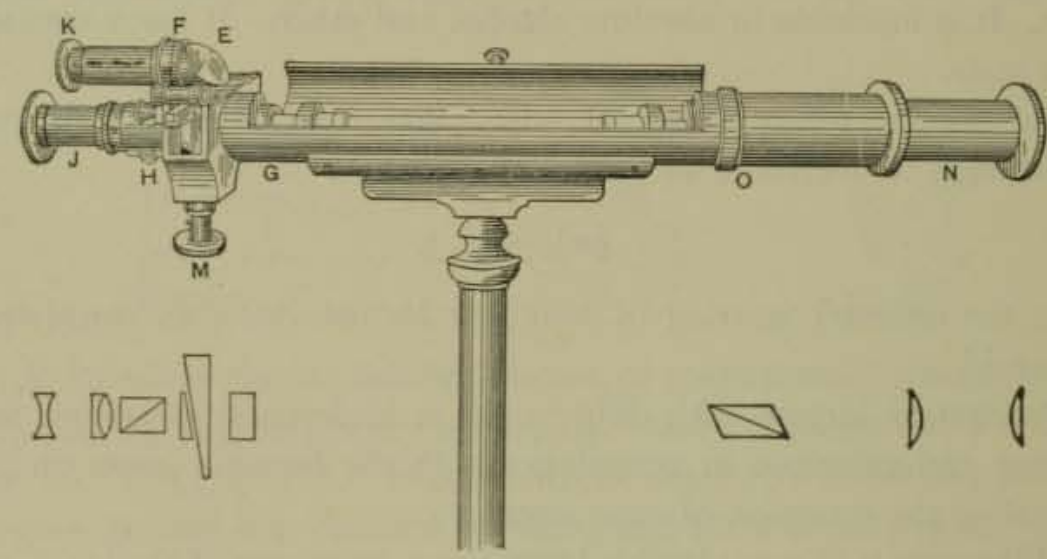

FtG. 102,- Single-wedge Saccharimeter.

apparatus, in its simplest form with a single movable wedge in its com. pensating system.

An excellent light for work with this instrument is that furnished by the Welsbach burner, a convenient form of lamp being shown in Fig. III, in which the burner is inclosed in a sheet-metal chimney of suitable construction. An argand, gas, or kerosene burner may however be used, 
and in a late form of Schmidt and Haensch instrument, Fig. I03, a specially constructed incandescent electric lamp is supplied.

The Single-wedge Saccharimeter.-The following description of the saccharimeter and directions for its use are from the revised regulations of the U. S. Internal Revenue Department. The tube N, Fig. Io2, contains the illuminating system of lenses and is placed next to the lamp; the polarizing prism is at $O$ and the analyzing prism at $H$. The quartz wedge compensating system is contained in the portions of the tube marked $F E G$ and is controlled by the milled head $M$. The tube $J$ carries a small telescope, through which the field of the instrument is viewed, and just above is the reading-tube $K$, which is provided with a mirror and magnifying lens for reading the scale.

The tube containing the sugar solution is shown in position in the trough between the two ends of the instrument. In using the instrument the lamp is placed at a distance of at least $200 \mathrm{~mm}$. from the polarizing end; the observer seats himself at the opposite end in such a manner as to bring his eye in line with the tube $J$. The telescope is moved in or out until the proper focus is secured to give a clearly defined image, when the field of the instrument will appear as a round, luminous disk, divided into halves by a vertical line passing through its center, and darker on one half of the disk than on the other, when the compensating quartz wedge is displaced from the neutral position. If the observer, still looking through the telescope, will now grasp the milled head $M$ and rotate it first one way and then the other, he will find that the appearance of the field changes, and at a certain point the dark half becomes light and the light half dark. By rotating the milled head delicately backward and forward over this point he will be able to find the exact position of the quartz wedge operated by it, in which the field is neutral, or of the same intensity of light on both halves. The three different appearances presented by the field are shown in Fig. Io6, opposite page 582 ,

One of the compensating quartz wedges is fixed and the other is movable, sliding one way or the other according as the milled head is turned, so that for different relative positions of the two wedges a different thickness of quartz is interposed in the path of the polarized ray. By this means the amount of the rotation which the sugar solution or other optically active substance examined exerts upon the light polarized by the prism at $O$ may be, as it were, counteracted by varying the relative position of the wedges. 
With the milled head set at the point which gives the appearance of the middle disk shown in Fig. 106, the eye of the observer is raised to the reading tube $K$, which is adjusted to secure a plain reading of the divisions, and the position of the scale is noted. It will be seen that the scale proper is attached to the quartz "wedge, which is moved by the milled head; and attached to the other quartz wedge is a small scale called a vernier, which is fixed, and which serves for the exact determination of the position of the movable scale with reference to it. On each side of the zero line of the vernier a space corresponding to nine divisions of the movable scale is divided into ten equal parts. By this device the fractional part of a degree indicated by the position of the zero line is ascertained in

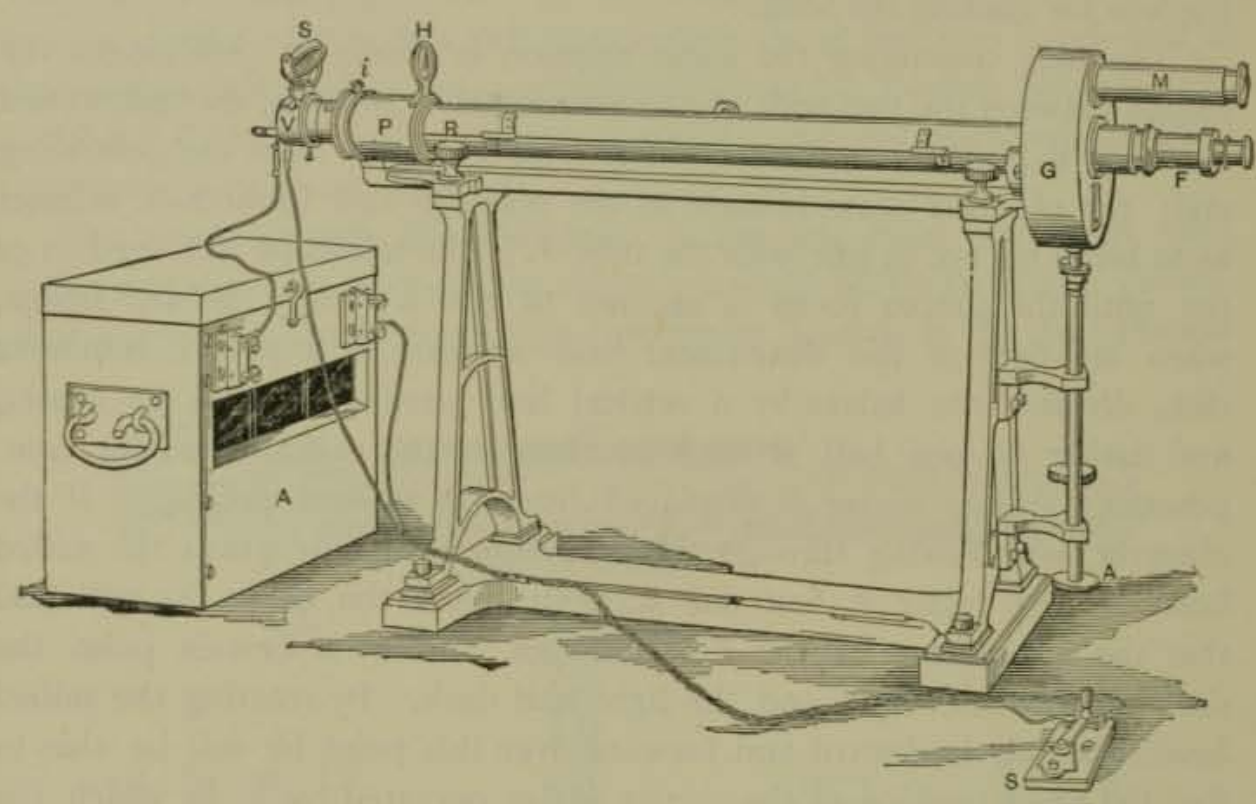

Fic. 103.-Double-wedge Soleil-Ventzke Saccharimeter, mounted on Bock Stand and provided with Incandescent Electric Lamp.

tenths; it is only necessary to count from zero until a line is found which makes a continuous line with one on the movable scale.

With the neutral field, as indicated above, the zero of the movable scale should correspond closely with the zero of the vernier, unless the zero point is out of adjustment.

Adjusting the Instrument.-If the observer desires to secure an exact adjustment of the zero of the scale, or in any case if the latter deviates more than two-tenths of a degree, the zero lines are made to coincide by moving the milled head and securing a neutral field at this point by 
means of the small key which comes with the instrument, and which fits a small nipple on the lefl-hand side of $F$, the fixed quartz wedge of the compensating system. This nipple must not be confounded with a similar nipple on the right-hand side of the analyzing prism $H$, which it fits as well, but which must never be touched, as the adjustment of the instrument would be seriously disturbed by moving it. With the key on the proper nipple it is turned one way or the other until the field is neutral. Unless the deviation of the zero be greater than $0.2^{\circ}$ it will not be necessary to use the key, but only to note the amoun: of the deviation, and for this purpose the observer must not be content with a single setting, but must perform the operation five or six times and take the mean of these different readings. If one or more of the readings show a deviation of more than $0.2^{\circ}$ from the general average they should be rejected as incorrect. Between each observation the eye should bc allowed a moment of rest.

The Scale usually has rio equal divisions on one side of the zero for reading right-handed polarization, and 20 equal divisions on the othe: side for left-handed polarization. The scale is an arbitrary one, based on the plan that a normal aqueous solution of pure cane sugar $(26.048$ grams made up to $100 \mathrm{cc}$.) will read exactly $100^{\circ}$ or divisions to the right of the zero.

The accuracy of various portions of the scale may be verified by quartz control plates of varying thickness, usually mounted in tubes, the correct polariscopic reading of each of which plates has been accurately cetermined, this reading being as a rule marked on the tube. As the sugar value of such a quartz plate varies with the temperature, the temperature at which the particular reading marked thereon applies is usually specified, and in many cases a table giving its exact value at different temperatures from $10^{\circ}$ to $35^{\circ}$ accompanies the plate.

The Double-wedge Saccharimeter is shown in Fig. I04, the arrangement of the optical parts being also shown.

In this instrument the two sets of wedges employed are of opposite optical properties, so that extreme accuracy may be arrived at by making the readings with both, the inaccuracies of one being compensated for by the other. Ordinarily in using this form, one movable wedge, say the one controlled by the right-hand milled screw head, is set at zero, while the reading of the sugar solution or other substance to be polarized is made with the other movable wedge.

The Triple-field Saccharimeter.-The latest form of saccharimeter 


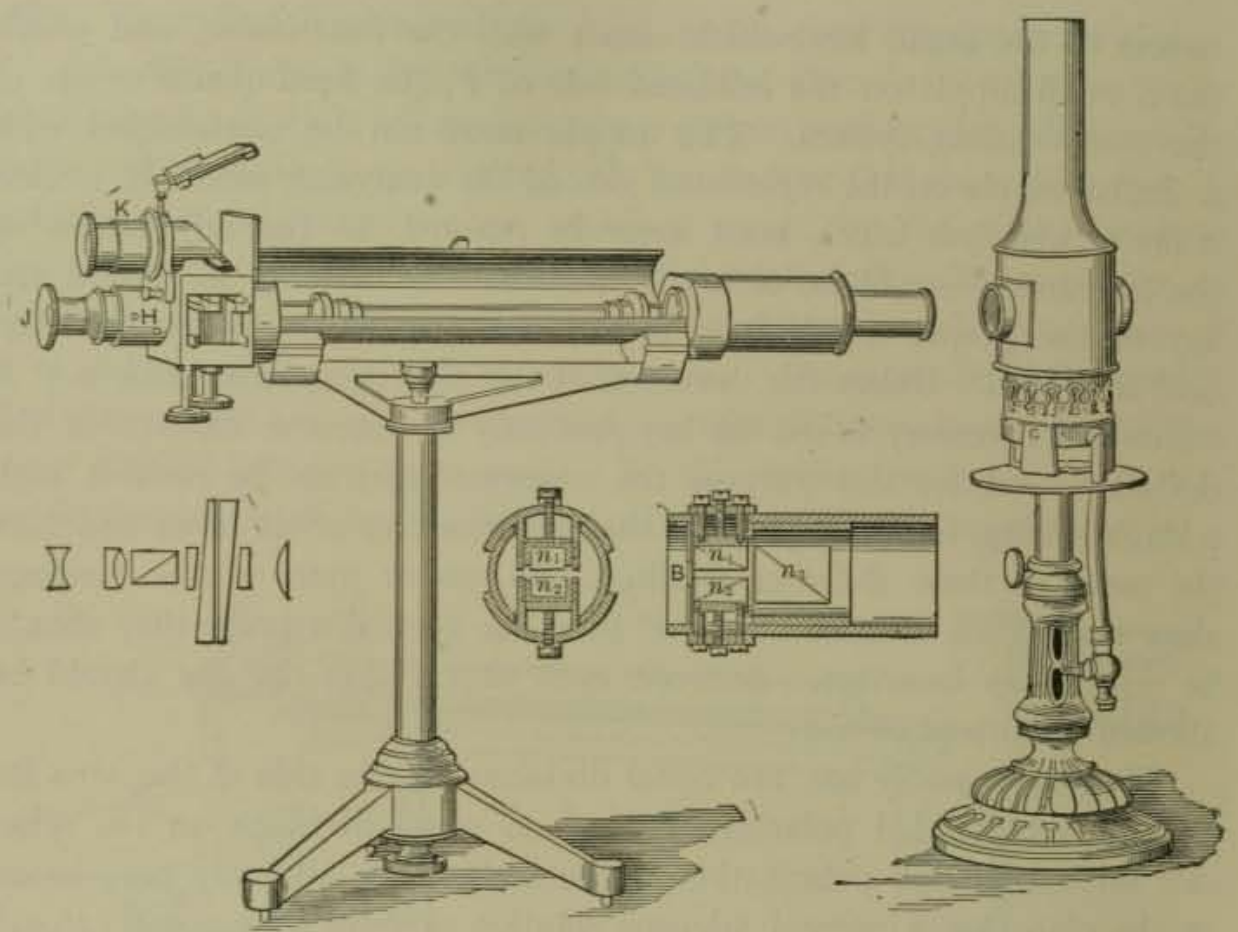

FIG. 104,-Triple-wedge, Triple-field Soleil-Ventzke Saccharimeter.

is the triple-field instrument, the construction of the polarizer being shown in Fig. 105.

In this form the analyzer is the same as in the foregoing instruments, but the polarizer consists of one large and two small Nicol prisms I, II, and III, the construction and arrangement being such that when the compensating wedges are at the neutral point, sections 1,2 , and 3 of the circular field (corresponding respectively to the prisms I, II, and III) are evenly lighted, forming a circular uniformly colored field, while in any other position of the wedges section I is dark while 2 and 3 are light or vice versa. The accompanying diagram, Fig. 106, shows the appearance of the field of this instrument in the three positions of the quartz wedge, viz., at the neutral point and at both sides thereof.

The lamp used for illumination should be separated from the polariscope on account of the influence of its
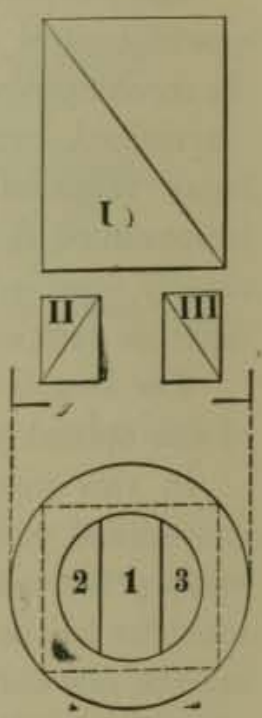

Fig. 105. heat on the readings. This is best accomplished by having the lamp in a separate compartment from the polariscope, so 

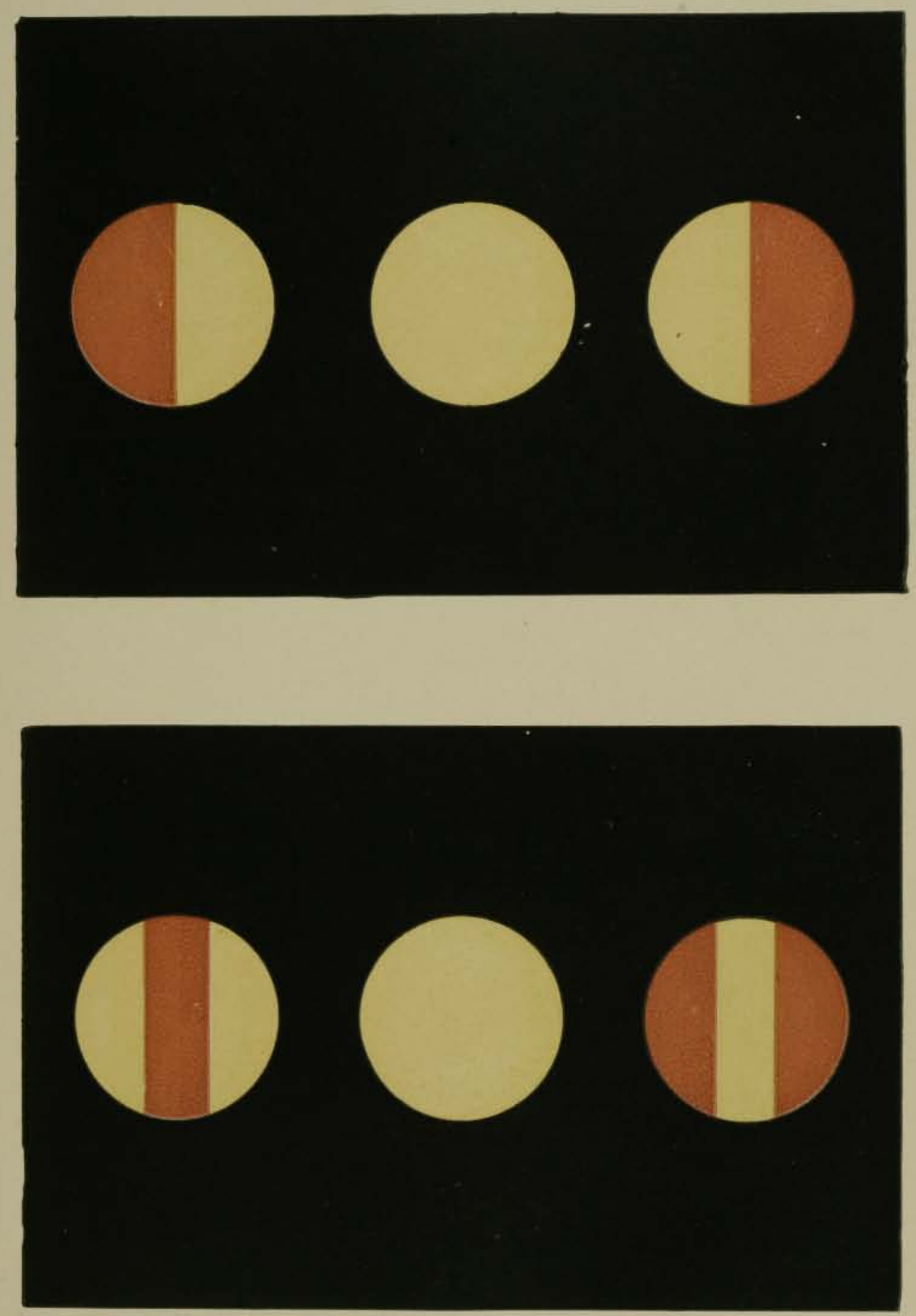

F1G. 106.-Appearance of the Field in the Half-shade (above) and Triple-shade (below) Saccharimeter. 


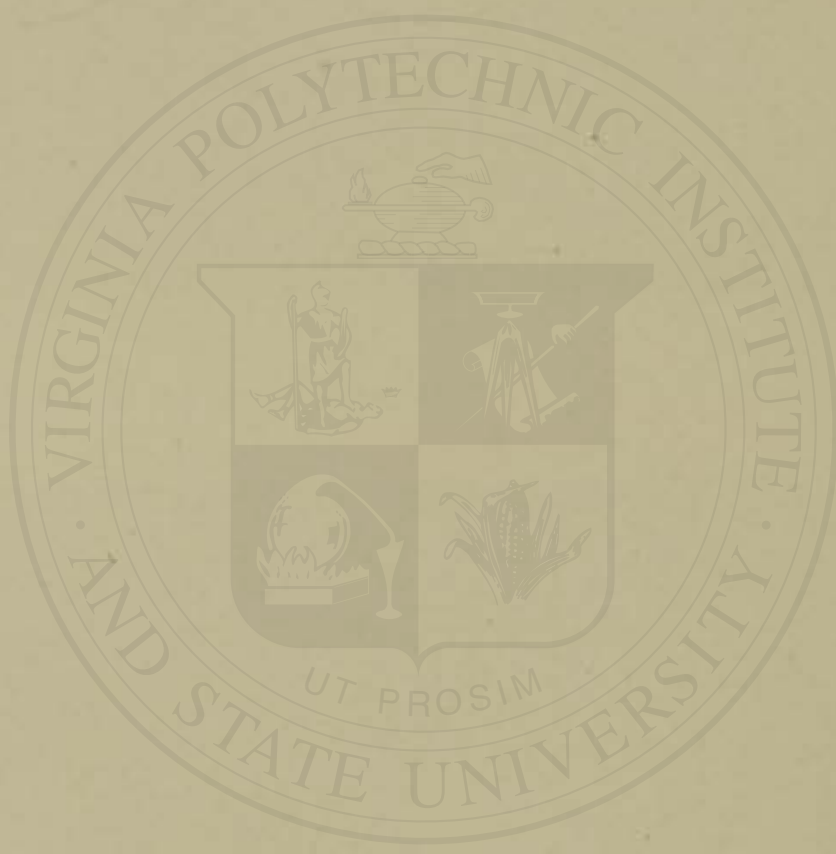


that both are on opposite sides of a partition, an opening in which transmits the light. In any event some kind of screen should be interposed between the two. Best results are obtained if the room in which the observations are made is dark.

Comparisons of Scales of Various Polariscopes.-Besides the SoleilVentzke instrument, there are various other forms of polariscope. Among the best known of these are Laurent's, Wild's, and Duboscq's, all of which are made with scales reading in circular degrees, while in some cases modified forms have scales in which, like the Soleil-Ventzke, percentages of sugar are directly read off. Some instruments are provided with double scales reading both circular degrees and percentages of sugar, and in certain of the Duboscq instruments additional scales for percentages of milk sugar and diabetic sugar are provided.

In the Wild, Duboscq, and Laurent instruments the source of light is the sodium flame, yielding what is termed a monochromatic light. This is produced by fused sodium chloride passing through a Bunsen flame, various mechanical devices being employed for making the light continuous. In the Ventzke instrument, as was stated above, the ordinary light from a bright gas or oil flame is used.

For convenience in conversion of readings on one instrument to their equivalents on other scales, the following factors can be used:

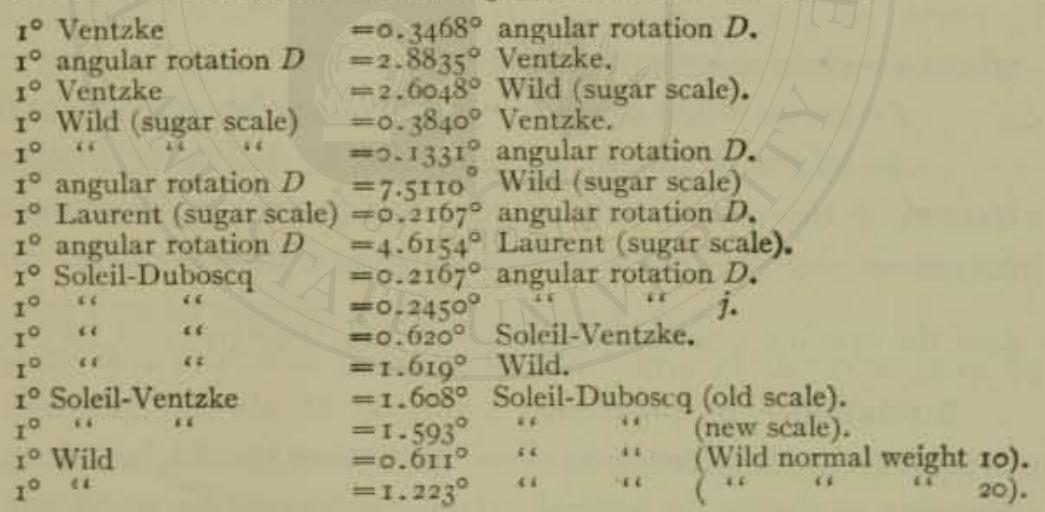

Normal Weights of Sugar for Different Instruments.-The following normal weights (number of grams in $100 \mathrm{cc}$. at $17.5^{\circ} \mathrm{C}$.) are those on which the scales of the various instruments are based: Soleil-Ventzke, 26.048; Soleil-Duboscq 16.29 (formerly I6.I9); Wild, usually, Io or 20; Laurent, I6.29.

The International Commission for Uniform Methods in Sugar Analysis has decided to use for the Ventzke scale 26 grams and make up at $20^{\circ} \mathrm{C}$. to 100 metric cc., which figures are approximately equivalent to 26.048 grams made up to 100 Mohr cc. 
Specific Rotary Power.-This is a theoretical term to express a standard by which the various optically active substances may be compared, and is understood to mean the amount in angular degrees through which the plane of polarization of a ray of light of stated wave length is rotated by I gram of a given substance in aqueous solution of I cc. and forming a column I decimeter in length. The actual rotary power of a solution varies directly with the length of the column traversed by the light, with the concentration of the solution, and with the wave length of light, hence the need of a purely theoretical basis for purposes of comparison.

The specific rotary power is usually expressed as $[\alpha]_{D}$ or $[\alpha]_{j}$, the letters $D$ or $j$ indicating the character of the light. Thus, $D$ indicates the monochromatic light obtained from the sodium flame, named from the $D$ line of Fraunhofer in the yellow portion of the spectrum, while $j$ (from the French jaune) indicates what is known as the transition tint, the rose-purple color produced when ordinary white light passes througi the polarizer and analyzer, placed with their principal sections parallel to each other and with a plate of quartz $3.75 \mathrm{~mm}$. thick interposed between them.*

The specific rotary power is determined as follows:

$$
[\alpha]_{D} \text { or }[\alpha]_{j}=\frac{\operatorname{Ioo} a}{c l},
$$

where $a=$ observed angular rotation,

$c=$ grams of the substance in $100 \mathrm{cc}$. of the solution, and

$l=$ length of the observation-tube in decimeters; or, in cases where, instead of the grams per $100 \mathrm{cc}$, the percentage composition is known (expressed by $p=$ grams of the substance in roo grams of the solvent), and the specific gravity (expressed by $d$ ), then $[\alpha]_{D}$ or $[\alpha]_{j}=\frac{100 a}{p d l}$.

Birotation.-In polarizing solutions of all the common sugars other than sucrose the phenomenon of birotation should be taken into account, whereby a change in optical activity is shown by standing. Thus, solutions of dextrose, levulose, and lactose polarize much higher when freshly prepared than after long standing, requiring in some instances several hours before the lowest or normal figure is reached. Maltose, on the other hand, increases in polarization after standing in solution. By

* Some confusion is caused by the adoption of the characters $D$ and $j$, since both would naturally seem to indicate yellow light. The so-called transition tint above defined is, however, complementary to the mean yellow, or joune moyen, and it is the complementary color and not the yellow itself that is indicated by the character $j$. 
boiling the solution it may be at once brought to its correct reading. 'The desired result may also be accomplished by adding a few drops of ammonia, either treatment being resorted to before the solution is made up to the required volume.

\section{ANALYSIS OF CANE SUGAR AND ITS PRODUCTS.}

Qualitative Tests for Sucrose.-(a) Polariscope Test.-The substance to be tested, if not already in solution, is dissolved in water, and if the solution is not perfectly clear, is clarified by the addition of alumina cream or by subacetate of lead (p. $5^{86)}$ and filtered. An observation tube is filled with the clear solution and the polariscope reading noted. A measured portion of the same solution is then treated with one-tenth its volume of concentrated hydrochloric acid and is subjected to inversion (p. 588), after which the same tube as before is filled with the inverted solution and a second reading obtained, one-tenth of the observed reading being added for the true invert polariscopic reading. If the two readings are virtually the same, sucrose is absent, but, in the presence of sucrose, the second reading will be considerably lower than the first or may even be to the left of the zero.

(b) Test with Nilrate of Cobalt.*-Prepare a $5 \%$ solution of cobaltous nitrate, and a $50 \%$ solution of potassium hydroxide. If the sugar solution to be tested contains dextrin or gums, these should first be removed by treatment with alcohol. $\mathrm{I}_{5} \mathrm{cc}$. of the sugar solution to be tested are mixed with $5 \mathrm{cc}$. of the cobaltous nitrate reagent, and $2 \mathrm{cc}$. of the potassium hydroxide solution are added. Sucrose produces under these conditions a permanent amethyst-blue color, while dextrose gives at first a turquoise-blue passing over into light green. In a mixture of the two sugars the color due to sucrose will predominate.

According to Wiley, I part of sucrose in 9 parts of dextrose may be detected by this test.

ANALYSIS OF CANE SUGAR.-In the case of commercial granulated or loaf sugar the sucrose determination is usually all that is necessary to determine its purity, and the same is true, as a rule, of the powdered white sugars. A fairly complete analysis of raw or brown sugar consists in the determinations of moisture, sucrose, invert sugar, ash, organic non-sugars, and quotient of purity. Care should be taken that the portion subjected to analysis is a fair representation of the whole, and is perfectly homogeneous.

*Wiley, Ag. Anal., p. r8g. 
Determination of Moisture. -2 to 5 grams of the sample are dried in a flat, tared metal dish, to constant weight in vacuo, or in a McGill oven* in a current of air, at about $70^{\circ} \mathrm{C}$., at which temperature levulose is not decomposed. For ordinary purposes sufficiently accurate results may be obtained by the A. O. A. C. method of drying to constant weight at $100^{\circ} \mathrm{C}$. in a water oven.

Determination of the Ash.-The residue from the moisture determination is burned slowly and cautiously over a low flame until frothing has ceased. Afterwards increase the flame and ignite to a white ash at a low, red heat.

In igniting saccharine substances which contain an appreciable amount of cane sugar, the contents of the dish will swell up and froth, unless great care be taken, to such an extent as to flow over the sides of the dish, occasioning loss and inconvenience. Such frothing may be largely held in check by directing the flame at first down from above upon the pasty mass, instead of from under the dish as ordinarily, till all is reduced to a dry char, afterwards continuing the ignition from below in the usual manner.

Organic Non-sugars. - These consist mainly of compounds of organic acids, together with gum, coloring matter, albuminous bodies, etc. They are determined by difference between $100 \%$ and the sum of the sucrose, invert sugar, moisture, and ash.

Quotient of Purity.-By this term is meant the percentage of pure sugar in the dry substance. It is calculated by dividing the per cent of sucrose by the percentage of total solids and multiplying the result by 100 .

Determination of Sucrose by the Polariscope.-Reagents.-Lead Subacetate Solution. + -Boil for half an hour $43^{\circ}$ grams of normal lead acetate, 130 grams of litharge, and $1000 \mathrm{cc}$. of water, allow to cool and settle. Dilute the supernatant liquid to 1.25 specific gravity with recently boiled water.

* A. McGill, Laboratory of Inland Revenue, Ottawa, Canada, has devised a forceddraft water-oven for drying at temperatures between $60^{\circ}$ and $90^{\circ} \mathrm{C}$. The oven is heated by means of ordinary gas-burners, and the temperature is controlled by introducing at the bottom of the oven a blast of air from a blower run by a small water-motor. Before dis- ? charging into the oven, the air-tube enters the water-chamber and is coiled a number of times in order to sufficiently warm the air before it enters the oven. The exit end of the air-tube is covered with a concavo-convex dis's in order to distribute the blast and to prevent harmful currents. By regulating the burners and the flow of air, a fairly constant temperature can be obtained. The bottom of the oven is curved instead of flat, to prevent bumping when the water is boiling; a perforated plate serves as a false bottom.

t U. S. P. lead subacetate, sometimes sold as Goulard's extract, may also be used. 
Anhydrous lead subacetate, first proposed by Horne,* may be substituted for the solution.

Alumina Cream.-Divide a cold, saturated solution of alum into two unequal portions, add to the larger a slight excess of ammonia, then by degrees the remaining portion to faint acid reaction.

Process.-If the Soleil-Ventzke polariscope is to be used, weigh out 26 grams of the sugar, which may conveniently be done in the German-

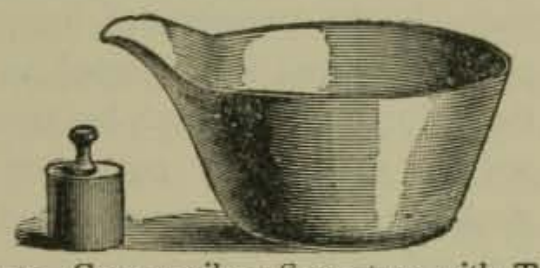

Fig. 107.-German-silver Sugar-tray with Tare.

silver, tared tray especially designed for this purpose (Fig. 107). If any other instrument is employed, weigh out the standard or normal weight for that instrument (see p. $5^{8} 3$ ). Transfer the sugar by washing to a

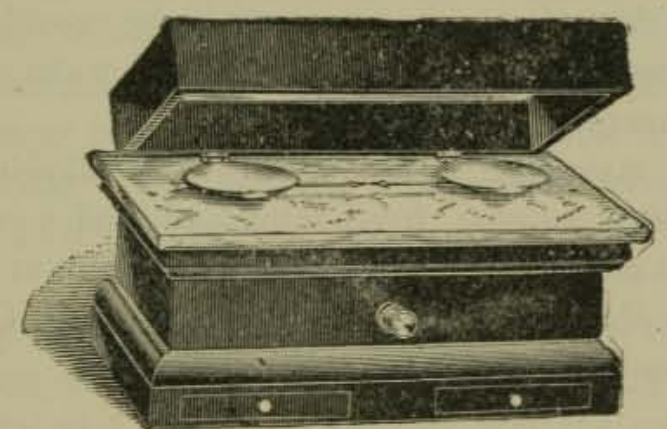

Frc. 108.-A Convenient Sugar-scale.

Ioo-cc. graduated sugar-flask, and if the solution is perfectly clear, as would be the case with a refined sugar, make up to the mark and shake to insure a uniform solution. If the solution is slightly turbid, or more or less opaque or dark-colored, a clarifier must be added before making up to the mark to obtain a clear solution for polarization. The kind and amount of clarifier to be used depends on the nature of the sugar solution and must be learned by experience. If the turbidity is only slight, from 5 to $10 \mathrm{cc}$. of alumina cream alone will often prove sufficient; if more opaque, 1o cc. of lead subacetate solution or a small amount of the dry salt may be used.

* Jour, Am. Chem. Soc., 26, 1904, p. 186. 
For additional details as to clarification see page 6r4. under Molasses.

After adding the clarifier, the flask is filled to the mark with water and shaken, the solution being poured upon a dry filter and the first few cubic centimeters of the filtrate rejected. A $200-\mathrm{mm}$. observationtube is filled with the clear sugar solution and the polarization noted. If sucrose is the only optically active substance present, the direct reading on the polariscope will indicate its percentage.

Process of Inversion.- In the presence of invert or other sugars the normal solution as above prepared is subjected to inversion as follows: Free a portion of the solution from lead by treating with anhydrous sodium carbonate, sodium sulphate or potassium oxalate, filter, place $50 \mathrm{cc}$. in a roo-cc. flask, add $25 \mathrm{cc}$. of water and little by little, while rotating the flask, $5 \mathrm{cc}$. of $38.8 \%$ hydrochloric acid. Heat in a water bath at $70^{\circ} \mathrm{C}$., so that the solution in the flask reaches $67^{\circ}$ to $69^{\circ} \mathrm{C}$. in two and one-half to three minutes. Maintain at $69^{\circ} \mathrm{C}$. during seven to seven and one-half minutes, making a total time of heating of ten minutes. Remove the flask, cool the contents rapidly to $20^{\circ} \mathrm{C}$., and dilute to $100 \mathrm{cc}$. Polarize this solution in a $200-\mathrm{mm}$. tube provided with a lateral branch and a water jacket, passing a current of water around the tube to maintain a temperature of $20^{\circ} \mathrm{C}$.

The inversion may also be accomplished by allowing a mixture of $50 \mathrm{cc}$. of the clarified solution, freed from lead, and $5 \mathrm{cc}$. of the acid to stand for 24 hours at not less than $20^{\circ} \mathrm{C}$. or for to hours at not less than $25^{\circ}$.

The sucrose is obtained by the following formula of Clerget, based on the rotation of cane sugar before and after inversion,

$$
S=\frac{100(a-b)}{142.66-t / 2},
$$

where $S=$ per cent of sucrose, $a=$ direct polarization, $b=$ invert polarization, and $t=$ temperature. Note that if the direct polarization is to the right or positive, and the invert to the left or negative, then $a-b$ would be the sum of the two polarizations.

In many cases where it is almost impossible to obtain a colorless solution for polarization in the $200-\mathrm{mm}$. tube, a $100-\mathrm{mm}$. tube may be employed, and the readings multiplied by 2 , or half the normal weight,* viz., I3 grams, of the sample may be taken and made up to roo cc., the 200-mm. tube employed, and the readings multiplied by 2 .

*Wherever the term "normal weight" occurs hereafter will be meant, unless otherwise noted, the normal weight of sugar for the Soleil-Ventzke polariscope, viz., 26 grams, and by a "normal solution" will be meant 26 grams in too cc, of water at $20^{\circ} \mathrm{C}$. Clerget's formula, as originally worked out by him, was not based on this normal weight, but on 16.35 grams. It is, however, applicable to 26 grams. 
The determination of sucrose by the Clerget formula is applicable to all mixtures of the common sugars excepting those in which lactose, or milk sugar, is present.

Theory of Inversion.-On p. 565 a reaction is given showing that when sucrose is subjected to inversion by the action of dilute acids or of invertase or yeast it splits up into the two sugars dextrose and levulose, forming equal quantities of each. The dextrose is, however, dextrorotary and the levulose lævorotary. Invert sugar is the term applied to the mixture of dextrose and levulose formed by the inversion of sucrose. The specific rotary power of sucrose varies so little with the temperature as to be regarded for practical purposes as constant. At $87^{\circ}$ a solution of invert sugar polarizes at zero. This is due to the fact that the rotary power of levulose, unlike that of sucrose and dextrose, varies with the temperature. At from $87^{\circ}$ to $88^{\circ}$ the left-handed rotation of the levulose balances the right-handed rotation of the dextrose in the invert sugar, hence the zero reading. As the temperature decreases from $87^{\circ}$, the rotary power of the levulose proportionally increases, till at $0^{\circ}$ the normal invert sugar solution would polarize $44^{\circ}$ to the left of the zero. On these facts Clerget's formula (p. ${ }_{588}$ ) is based, assuming that a normal solution of pure cane sugar polarizes at $+100^{\circ}$, while after inversion the reading for $0^{\circ}$ temperature would be $-44^{\circ}$ and would decrease half a degree for each degree in temperature above $0^{\circ}$. Thus at $20^{\circ}$ the invert reading would be -34 .

Detection of Invert Sugar.-Methyl-blue Test.-This test depends on the decolorization of methyl blue by invert sugar. 20 grams of sugar are dissolved in water and made up to $100 \mathrm{cc}$. If the solution is not clear, sufficient subacetate of lead solution is added to clarify before making up to the mark, and the solution is filtered. Add to the filtrate enough 10\% sodium carbonate solution to make alkaline, and filter a second time. Take about $50 \mathrm{cc}$. of the filtrate in a casserole, add 2 drops of a ${ }_{1} \%$ solution of methyl blue, and boil over a free flame, noticing particularly the time the solution begins to boil.

If the color disappears in one minute after boiling, there is present at least $0.01 \%$ of invert sugar. If it is not completely decolorized by three minutes' boiling, no invert sugar is present.

Determination of Invert Sugar in Cane Sugar Products by the Polariscope.-While invert sugar is best determined by Fehling's solution as described elsewhere, it may be approximately estimated by the polari- 
scope, though less satisfactorily. On p. 626 a method is given for the determination of levulose by polariscopic readings at two different temperatures. Since invert sugar is composed of equal parts by weight of dextrose and levulose, the percentage of levelose multiplied by 2 would give that of invert sugar.

Test for Ultramarine in Sugar.*-A large amount of the sugar is. dissolved in water and the coloring matter is allowed to settle out, washing the residue several times by decantation. On treatment with hydrochloric acid, the blue color is discharged if due to ultramarine.

\section{SUGAR DETERMINATION BY COPPER REDUCTION.}

Various convenient methods of determining sugars depend on the readiness with which certain of them, known as reducing sugars, act on copper salts, especially on the tartrate of copper, reducing it to cuprous oxide.

This reducing power is exercised in a definite degree under fixed conditions, so that the amount of reducing sugar present may be accurately determined. Of the common sugars, sucrose is the only one that has no direct reducing action, but on undergoing inversion it is converted into reducing sugars, which are readily determined.

Use of Fehling's Solution.-There are various well-known mixtures of copper sulphate, tartaric acid salts (usually Rochelle salts or cream of tartar), and alkalies, called after chemists who have employed them in the determination of the reducing sugars, each one possessing certain advantages, but none have become so widely adopted as Fehling's solution, the use of which in one form or another is now well-nigh universal.

There are a number of methods by which Fehling's solution is employed for this purpose, both volumetric and gravimetric. The former are simpler and quicker of manipulation, and thus are preferable for commercial work where extreme accuracy is not required. The gravimetric methods are usually considered more delicate and accurate, calling for less skill, but more time in arriving at results, and with less of the "personal element" than the volumetric.

- Some modifications of the. Fehling method, especially as carried out gravimetrically, differ for the various reducing sugars to be determined,

* Leffmann and Beam, Select Methods of Food Analysis, p. 126. 
and others are carried out alike, so far as manipulation is concerned, whether the particular sugar to be determined be dextrose, maltose, or lactose.

While, strictly speaking, the reducing power of dextrose, levulose, and invert sugar are not identical, it is customary in commercial work to regard them as such, and no appreciable error arises in consequence except in extreme cases. Thus the term "reducing sugars" is commonly applied indiscriminately to dextrose, levulose, and invert sugar, the same factor being used in calculating either, in mixtures wherein other reducing sugars, as lactose, maltose, etc., having widely different reducing powers are absent.

Fehling's solution is made up in two separate parts as follows:

A. Fehling's Copper Solution.- 34.639 grams of carefully selected crystals of pure copper sulphate dissolved in water and diluted to exactly $500 \mathrm{cc}$.

B. Fehling's Alkaline Tartrate Solution. - I73 grams Rochelle salts and $5 \circ$ grams sodium hydroxide are dissolved in water and diluted to. exactly $500 \mathrm{cc}$.

The Fehling solution should be standardized by dissolving 0.5 gram of pure anhydrous dextrose in water, and diluting to exactly $100 \mathrm{cc}$. $10 \mathrm{cc}$. of this dextrose solution should exactly reduce the copper in Io cc. of the Fehling ( $5 \mathrm{cc}$. each of solutions $A$ and $B$ ) when conducted according to the volumetric process describe 1 below.

VolUMETRIC FEHLING PROCEss.-For determining dextrose, levulose, or invert sugar in a raw or brown sugar, make a solution of the sugar of such a strength that an accurately weighed amount dissolved in water and made up to $100 \mathrm{cc}$. shall contain not more than $1 \%$ of the reducing sugar, as nearly as can be guessed at with reference to the class of sugar under examination, or from a rough preliminary titration.

Measure accurately into a flask of about $250 \mathrm{cc}$. capacity $5 \mathrm{cc}$. Fehling's copper sulphate solution, $A$, and $5 \mathrm{cc}$. of the alkali solution, $B$. Add about $40 \mathrm{cc}$. of water, mix and boil over a free flame, with copper gauze beneath the flask. While still boiling, add from a pipette or burette a measured quantity of the sugar solution, prepared as above, until the copper after three minutes' boiling is all reduced to cuprous oxide. The end-point is determined in a variety of ways. Practice will soon enable the eye to judge the near approach of the end-point by the changes in color that take place in the solution, which turns from a deep blue, first to green, then to a dull-red tint, and finally to a bright brick-red. The sugarcontaining solution may be added from the burette quite rapidly until 
the solution reaches the dull-red tint, after which care is taken to add a little at a time, keeping account of the total amount added. If the flask be removed from the flame, and the bright, diffused light from a window viewed through the solution with the eye on a level with the surface, a thin film scarcely wider than a line will be observed just below the surface (see Fig. ro9), which is blue so long as some of the copper in the solution remains unreduced. When, however, all the copper has been reduced, this film ceases to be blue and becomes colorless or yellow.

If the film is not at once apparent, it may often be made quite noticeable by simply diluting the solution in the flask with water. At the approach of the end-point the sugar-containing solution should be added a very little at a time. The exact end-point is best arrived at by decanting a few drops of the mixture in the flask through a filter, acidifying the filtrate with acetic acid, and adding a drop of a solution of ferrocyanide of potassium. As long as there is unreduced copper present, a precipitate or brown-red coloration will appear when the ferrocyanide is added. The sugar solution toward the end should be added to the contents in the flask in small installments (say half a cubic centimeter each time), boiling the liquor for at least three minutes after each addition, until no brown-red coloration is pro-

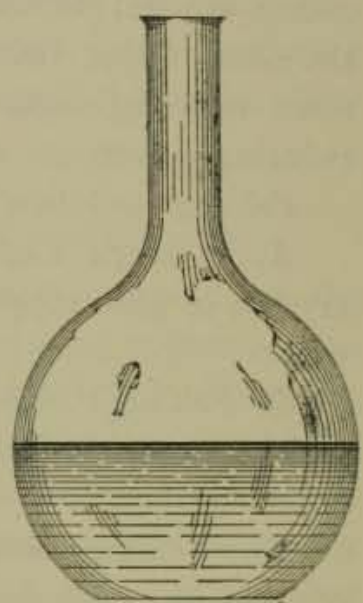

Frg. rog.-Flask and Contents used in Volumetric Fehling Determinations. Showing layer just beneath the surface, the color of which indicates the end-point in adding the sugar-containing liquid. duced by adding the ferrocyanide to a little of the filtered acidified liquid. When the number of cubic centimeters of sugar solution necessary to reduce the copper has thus been determined, a second titration should be made to verify the first, running the entire amount of sugar-containing liquid found necessary in the first case into the second flask.

The equivalents of ro cc. of Fehling's solution in the above volumetric method are, in terms of the common reducing sugars, as follows:

$$
\begin{aligned}
& 0.05 \text { gram of }\left\{\begin{array}{c}
\text { invert sugar, } \\
\text { dextrose, or } \\
\text { levulose }
\end{array}\right\} \text { will reduce ro cc. Fehling's solution. } \\
& 0.0475 \text { gram of }\left\{\begin{array}{c}
\text { cane sugar } \\
\text { after in- } \\
\text { version }
\end{array}\right\} \text { will reduce to cc. Fehling's solution. }
\end{aligned}
$$


0.0807 gram of maltose will reduce to cc. Fehling's solution. 0.067 gram of lactose " " I0 cc. "“ "

Suppose, for example, a sample of brown sugar is to be examined for invert sugar. This class of sugar has usually from 2 to 6 per cent of invert sugar. Hence, if ro grams of the sample are dissolved in 100 cc., the resulting solution will contain not more than $1 \%$ of invert sugar.

Suppose $12.9 \mathrm{cc}$. of this $10 \%$ sugar solution were found by the above process to reduce ro cc. of Fehling's solution.

Io cc. Fehling's solution are equivalent to 0.05 gram invert sugar.

Therefore $12.9 \mathrm{cc}$. of the sugar solution contain 0.05 gram invertsugar.

I0o cc. sugar solution contain to grams sample, and $12.9 \mathrm{cc}$. contain I.29 grams sample, the equivalent of 0.05 gram invert sugar.

Hence per cent invert sugar $=\frac{0.05 \times 100}{1.29}=3.9$.

GRAVIMETRIC FEHLING PROCESSES.-In determining reducing sugars by gravimetric processes, a measured volume of the sugar solution is allowed to act upon a measured volume of hot Fehling's solution for a fixed time, thus forming cuprous oxide. This may be dried and weighed direct, but is more commonly converted either into cupric oxide by ignition, or into metallic copper by reduction with hydrogen or by electrolysis. In any case the sugar is calculated from the weight of the cuprous oxide, the cupric oxide, or the metallic copper (whichever method be used) by the employment of the proper factor, or by the use of tables compiled for the purpose.

Note.-Much difference of opinion exists as to the best and most accurate Fehling gravimetric method to employ. For the determination of dextrose, the Association of Official Agricultural Chemists has given its approval to the Allihn method, wherein the cuprous oxide deposited is further reduced to metallic copper and the dextrose calculated from the copper by Allihn's table.

The author for two reasons prefers the method of O'Sullivan as employed by Defren, with the use of the Defren tables, in accordance with which the reducing sugar is expressed in terms of its equivalence to cupric oxide, first because of its comparative simplicity, involving as it does less processes than the Allihn method (each additional process introducing a possible source of error), and, second, because the same method as carried out is applicable for the determination not only of dextrose, but also of maltose and lactose, Defren having worked out 
tables adapted for them all. Munsen and Walker* have also devised a simple method with accompanying tables, adapted, with a uniform system of procedure, to the determination of the various reducing sugars. In using the tables for dextrose, maltose, and lactose compiled by Allihn, Wein, and Soxhlet, the method employed must in each case be carried out in strict accordance to the minutest details adopted by each of the above authorities, and they are by no means uniform.

The Defren-0'Sullivan Method.†-Mix I5 cc. of Fehling's copper solution, $A$ (p. 59I), with I $_{5} \mathrm{cc}$. of the tartrate solution, $B$, in a quarterliter Erlenmeyer flask, and add $50 \mathrm{cc}$. of distilled water. Place the flask and its contents in a boiling water bath and allow them to remain five minutes. Then run rapidly from a burette into the hot liquor in the flask $25 \mathrm{cc}$. of the sugar solution to be tested (which should contain not more than one-half per cent of reducing sugar). Allow the flask to remain in the boiling water bath just fifteen minutes after the addition of the sugar solution, remove, and with the aid of a vacuum filter the contents rapidly in a platinum or porcelain Gooch crucible containing a layer of prepared asbestos fiber about I $\mathrm{cm}$. thick, the Gooch with the asbestos having been previously ignited, cooled, and weighed. The cuprous oxide precipitate is thoroughly washed with boiling distilled water till the water ceases to be alkaline.

The asbestos used should be of the long-fibered variety, and should be specially prepared as follows: Boil first with nitric acid (specific gravity 1.05 to 1.10 ), washing out the acid with hot water, then boil with a $25 \%$ solution of sodium hydroxide, and finally wash out the alkali with hot water. Keep the asbestos in a wide-mouthed flask or bottle, and transfer it to the Gooch by shaking it up in the water and pouring it quickly into the crucible while under suction.

Dry the Gooch with its contents in the oven, and finally heat to dull redness for fifteen minutes, during which the red cuprous oxide is converted into the black cupric oxide. If a platinum Gooch is used (and this variety is preferred by the writer), it may be heated directly over the low flame of a burner. If the Gooch is of porcelain, considerable care must be taken to avoid cracking the crucible, the heat being increased cautiously and the operation preferably conducted in a radiator or muffle. After oxidation as above, the crucible is transferred to a desiccator, cooled, and quickly weighed. From the milligrams of cupric oxide, calculate the milligrams of dextrose from the following table:

* U. S. Dept. of Agric, Bur, of Chem., Bul. 107 (rev.), p. 241.

† Jour. Am. Chem. Soc., 18, 1896 , p. 749 , and Tech. Quart., 10, 1897, p. 267 . 
DEFREN'S TABLE FOR THE DETERMINATION OF DEXTROSE, MALTOSE, AND LACTOSE.

\begin{tabular}{|c|c|c|c|c|c|c|c|}
\hline $\begin{array}{l}\text { Milligrams } \\
\text { of Cupric } \\
\text { Oxide. }\end{array}$ & $\begin{array}{l}\text { Milligrams } \\
\text { of Dextrose. }\end{array}$ & $\begin{array}{l}\text { Milligrams } \\
\text { of Maltose. }\end{array}$ & $\begin{array}{l}\text { Milligrams } \\
\text { of Lactose. }\end{array}$ & $\begin{array}{c}\text { Milligrams } \\
\text { of Cupric } \\
\text { Oxide. }\end{array}$ & $\begin{array}{l}\text { Milligrams } \\
\text { of Dextrose. }\end{array}$ & $\begin{array}{l}\text { Milligrams } \\
\text { of Maltose. }\end{array}$ & $\begin{array}{l}\text { Mrilligrams } \\
\text { of Lactose. }\end{array}$ \\
\hline 30 & 13.2 & 21.7 & I8.8 & So & 35.4 & 58.1 & 50.5 \\
\hline $3 \mathrm{I}$ & 13.7 & 22.4 & 19.5 & $8 \mathrm{I}$ & 35.9 & 58.9 & $5 \mathrm{~T} . \mathrm{I}$ \\
\hline $3^{2}$ & $\mathrm{I} 4 . \mathrm{I}$ & $23 . I$ & 20.1 & 82 & $3^{6} .3$ & 59.6 & 51.7 \\
\hline 33 & 14.6 & 23.9 & 20.7 & $8_{3}$ & 36.8 & 60.3 & $5^{2} \cdot 4$ \\
\hline 34 & 15.0 & 24.6 & $2 \mathrm{I} \cdot 4$ & 84 & 37.2 & $6 \mathrm{r} . \mathrm{I}$ & 53.0 \\
\hline 35 & I 5.4 & $25 \cdot 3$ & 22.0 & 85 & 37.7 & 6 r. 8 & 53.6 \\
\hline 36 & 15.9 & 26.1 & 22.6 & 86 & $3^{8.1}$ & 62.5 & $54 \cdot 3$ \\
\hline 37 & 16.3 & 26.8 & $23 \cdot 3$ & 87 & $3^{8} .5$ & 63.3 & 54.9 \\
\hline $3^{8}$ & 16.8 & $27 \cdot 5$ & 23.9 & 88 & 39.0 & 64.0 & $55 \cdot 5$ \\
\hline 39 & 17.2 & 28.3 & 24.5 & 89 & $39 \cdot 4$ & $64 \cdot 7$ & 56.2 \\
\hline 40 & 17.6 & 29.0 & 25.2 & 90 & 39.9 & 65.5 & 56.8 \\
\hline $4 \pi$ & 18.1 & 29.7 & 25.8 & 91 & 40.3 & 66.2 & 57.4 \\
\hline 42 & 18.5 & 30.5 & 26.4 & 92 & 40.8 & 66.9 & 58.1 \\
\hline 43 & 19.0 & 31.2 & $27 \cdot 1$ & 93 & $4 \pi \cdot 2$ & $67 \cdot 7$ & $5^{8.7}$ \\
\hline 44 & 19.4 & 31.9 & 27.7 & 94 & $4 x \cdot 7$ & 68.4 & $59 \cdot 3$ \\
\hline 45 & 19.9 & $3^{2} \cdot 7$ & 28.3 & 95 & $42, T$ & 69.1 & 60.0 \\
\hline 46 & 20.3 & 33.4 & 29.0 & 96 & 42.5 & 69.9 & 60.6 \\
\hline 47 & 20.7 & 34.1 & 29.6 & 97 & 43.0 & 70.6 & 61.2 \\
\hline 48 & $21 \cdot 2$ & 34.8 & 30.2 & $9^{8}$ & 43.4 & $71 \cdot 3$ & $6 r \cdot 9$ \\
\hline-49 & 21.6 & $35 \cdot 5$ & 30.8 & 99 & 43.9 & $72 . \mathrm{I}$ & 62.5 \\
\hline 50 & 22.1 & $3^{6.2}$ & $3 \mathrm{~T} \cdot 5$ & 100 & $44 \cdot 4$ & 72.8 & 63.2 \\
\hline $5 \mathrm{I}$ & 22.5 & 37.0 & $32 . I$ & 101 & 44.8 & 73.5 & 63.8 \\
\hline 52 & 23.0 & $37 \cdot 7$ & $3^{2} \cdot 7$ & 102 & 45.3 & 74.3 & $64 \cdot 4$ \\
\hline 53 & 23.4 & $3^{8.4}$ & $33 \cdot 3$ & $\mathrm{rO}_{3}$ & 45.7 & 75.0 & $65 \cdot r$ \\
\hline 54 & 23.8 & 39.2 & 34.0 & 104 & 46.2 & $75 \cdot 7$ & $65 \cdot 7$ \\
\hline 55 & 24.2 & 39.9 & 34.6 & 105 & 46.6 & $7^{6.5}$ & 66.3 \\
\hline $5^{6}$ & $24 \cdot 7$ & 40.5 & 35.2 & 106 & 47.0 & $77 \cdot 2$ & 67.0 \\
\hline 57 & $25 . \mathrm{I}$ & $4 \mathrm{~T} \cdot 3$ & 35.9 & 107 & 47.5 & $77-9$ & 67.6 \\
\hline $5^{8}$ & 25.5 & 42.1 & 36.5 & 108 & 48.0 & 78.7 & 68.2 \\
\hline 59 & 26.0 & 42.8 & $37 \cdot I$ & rog & $4^{8} .4$ & 79.4 & 68.9 \\
\hline 60 & 26.4 & $43 \cdot 5$ & 37.8 & 110 & $4^{8} .9$ & 80.1 & 69.5 \\
\hline 61 & 26.9 & $44 \cdot 3$ & $3^{8}-4$ & 111 & $49 \cdot 3$ & 80.9 & 70.1 \\
\hline 62 & $27 \cdot 3$ & 45.0 & 39.0 & 112 & 49.8 & 81.6 & 70.8 \\
\hline 63 & 27.8 & $45 \cdot 7$ & $39 \cdot 7$ & $\mathrm{II}_{3}$ & 50.2 & $82 \cdot 3$ & $71 \cdot 4$ \\
\hline 64 & 28.2 & 46.5 & 40.3 & 114 & 50.7 & $8_{3}, \mathrm{I}$ & 72.0 \\
\hline 65 & 28.7 & 47.2 & 40.9 & II 5 & $5 \mathrm{I} \cdot \mathrm{I}$ & $8_{3.8}$ & $72 \cdot 7$ \\
\hline 66 & $29 . \mathrm{I}$ & $47 \cdot 9$ & 41.6 & 116 & 5 x. 6 & 84.5 & $73 \cdot 3$ \\
\hline 67 & 29.5 & $4^{8} .6$ & 42.2 & $\mathrm{II} 7$ & 52.0 & 85.2 & 74.0 \\
\hline 68 & 30.0 & $49 \cdot 4$ & 42.8 & II & 52.4 & 85.9 & 74.6 \\
\hline 69 & 30.4 & 50.1 & $43 \cdot 5$ & 119 & $5^{2} \cdot 9$ & 86.6 & $75 \cdot 2$ \\
\hline 70 & 30.9 & 50.8 & $44 \cdot I$ & 120 & $53 \cdot 3$ & 87.4 & $75 \cdot 9$ \\
\hline $7 \mathrm{x}$ & $3 \mathrm{~T} \cdot 3$ & 51.6 & $44 \cdot 7$ & $12 \mathrm{I}$ & 53.8 & 88. I & 76.6 \\
\hline 72 & 3 T. 8 & $5^{2} \cdot 3$ & $45 \cdot 4$ & 122 & 54.2 & 88.9 & 77.2 \\
\hline 73 & $3^{2} \cdot 2$ & 53.0 & 46.0 & 123 & $54 \cdot 7$ & 89.6 & 77.9 \\
\hline 74 & 32.6 & 53.8 & 46.6 & 124 & 55.1 & 90.3 & 78.5 \\
\hline 75 & $33 . \mathrm{I}$ & $54 \cdot 5$ & $47 \cdot 3$ & 125 & 55.6 & $91 . x$ & $79 \cdot x$ \\
\hline $7^{6}$ & $33 \cdot 5$ & 55.2 & 47.9 & 126 & 56.0 & 9 r. 8 & 79.8 \\
\hline 77 & 34.0 & 56.0 & $4^{8} .5$ & 127 & 56.5 & $9^{2} \cdot 5$ & 80.4 \\
\hline $7^{8}$ & $34-4$ & 56.7 & 49.2 & 128 & 56.9 & 93.3 & $8 \mathrm{r} \cdot \mathrm{I}$ \\
\hline 79 & 34.9 & 57.4 & 49.8 & 129 & $57-3$ & 94.0 & $8 x \cdot 7$ \\
\hline
\end{tabular}


DEFREN'S TABLE FOR THE DETERMINATION OF DEXTROSE, MALTOSE, AND LACTOSE-(Continued).

\begin{tabular}{|c|c|c|c|c|c|c|c|}
\hline $\begin{array}{l}\text { Milligrams } \\
\text { of Cupric } \\
\text { Oxide. }\end{array}$ & $\begin{array}{l}\text { Milligrams } \\
\text { of Dextrose. }\end{array}$ & $\begin{array}{l}\text { Milligrams } \\
\text { of Maltose. }\end{array}$ & $\begin{array}{l}\text { Milligrams } \\
\text { of Lactose. }\end{array}$ & $\begin{array}{c}\text { Milligrams } \\
\text { of Cupric } \\
\text { Oxide. }\end{array}$ & $\begin{array}{l}\text { Milligrams } \\
\text { of Dextrose. }\end{array}$ & $\begin{array}{l}\text { Milligrams } \\
\text { of Maltose. }\end{array}$ & $\begin{array}{l}\text { Milligrams } \\
\text { of Lactose. }\end{array}$ \\
\hline 130 & 57.8 & 94.8 & $82 \cdot 4$ & 180 & 80.4 & I $3 \mathrm{I} .8$ & 114.6 \\
\hline $13 I$ & 58.2 & $95 \cdot 5$ & $8_{3} .0$ & $18 \mathrm{I}$ & 80.8 & 132.5 & $\mathrm{II}_{5.2}$ \\
\hline $13^{2}$ & $5^{8.7}$ & 96.2 & $8_{3} .6$ & 182 & $8 x \cdot 3$ & 133.2 & II 5.8 \\
\hline 133 & 59.1 & 97.0 & 84.2 & $18_{3}$ & $8 \mathrm{r} .8$ & 134.0 & 116.5 \\
\hline${ }^{1} 34$ & 59.6 & 97.7 & 84.9 & 184 & 82.2 & 134.7 & 117.1 \\
\hline 135 & 60.0 & 98.4 & 85.5 & 185 & 82.7 & 135.5 & $\mathrm{II}_{7.8}$ \\
\hline 136 & 60.5 & 99.2 & $86 . \mathrm{I}$ & 186 & $8_{3} \cdot 1$ & 136.2 & 118.4 \\
\hline 137 & 60.9 & 99.9 & 86.8 & 187 & 83.5 & I 36.9 & $1+9.1$ \\
\hline $13^{8}$ & $6 \mathrm{r} \cdot 3$ & 100.7 & 87.4 & I 88 & 84.0 & 137.7 & 110.7 \\
\hline 139 & $6 \mathrm{r} .8$ & 101.4 & 88.1 & 189 & 84.4 & $13^{8.4}$ & 120.4 \\
\hline 140 & 62.2 & 102,1 & 88.7 & 190 & $84 \cdot 9$ & 139.1 & 121.0 \\
\hline 141 & $62 \cdot 7$ & 102.8 & 80.3 & 191 & 85.4 & 139.9 & 121.7 \\
\hline 142 & $63 . \mathrm{I}$ & 103.5 & 90.0 & 192 & 85.9 & 140.6 & 122.3 \\
\hline 143 & 63.6 & 104.3 & 90.6 & 193 & 86.3 & 141.4 & 123.0 \\
\hline 144 & 64.0 & 105.0 & $9 \mathrm{I} .3$ & 194 & 86.8 & $\mathrm{I}_{4} 2 . \mathrm{I}$ & 123.6 \\
\hline $\mathrm{T} 45$ & $64 \cdot 5$ & 105.8 & 91.9 & 195 & 87.2 & 142.8 & 124.3 \\
\hline 146 & 64.9 & 106.5 & 92.6 & 196 & $87-7$ & 143.6 & 124.9 \\
\hline 147 & $65-4$ & 107.2 & 93.2 & 107 & 88. I & $144 \cdot 3$ & 125.6 \\
\hline 148 & 65.8 & 108.0 & 93.9 & I 98 & 88.6 & I 45.1 & 126.2 \\
\hline 149 & 66.3 & 108.7 & 94.5 & 199 & 89.0 & 145.8 & 126.9 \\
\hline 150 & 66.8 & 109.5 & 95.2 & 200 & 89.5 & $x_{4} 6.6$ & 127.5 \\
\hline $\mathrm{I}_{5} \mathrm{I}$ & $67 \cdot 3$ & 110.2 & 95.8 & 201 & 89.9 & $147 \cdot 3$ & 128.2 \\
\hline 152 & 67.7 & 111.0 & 96.5 & 202 & 90.4 & $\mathrm{I}_{4} 8 . \mathrm{I}$ & 128.8 \\
\hline 153 & 68.3 & 111.7 & 97.1 & 203 & 90.8 & 148.8 & 129.5 \\
\hline 154 & 68.7 & 112.4 & 97.8 & 204 & $9^{1}-3$ & 149.6 & 130.1 \\
\hline 155 & 69.2 & $1 \times 3.2$ & 98.4 & 205 & 91.7 & I 50.3 & 130.8 \\
\hline 156 & 69.6 & 113.9 & $99 . \mathrm{I}$ & 206 & 92.2 & I5I. I & 131.5 \\
\hline 157 & 70.0 & 114.7 & $99 \cdot 7$ & 207 & 92.6 & 151.8 & 132.1 \\
\hline 158 & 70.5 & 115.4 & 100.4 & 208 & $93, \mathrm{I}$ & $15^{2} .5$ & 132.8 \\
\hline 159 & 70.9 & 116.1 & 101.0 & 209 & 93.5 & 153.3 & 1 33.4 \\
\hline 160 & $71 \cdot 3$ & 116.9 & 101.7 & 210 & 94.0 & 154.1 & $I_{34.1}$ \\
\hline $16 \mathrm{r}$ & 71.8 & 117.6 & $102 \cdot 3$ & $2 \mathrm{II}$ & 94.4 & 154.8 & 134.7 \\
\hline 162 & $72 \cdot 3$ & 118.4 & 103.0 & 212 & 94.9 & 155.6 & 135.4 \\
\hline 163 & 72.7 & 119.1 & 103.6 & 213 & 95.3 & I 56.3 & 136.0 \\
\hline 164 & 73.2 & 119.9 & $104 \cdot 3$ & 214 & 95.8 & 157.1 & 136.7 \\
\hline 165 & 73.6 & 120.6 & 104.9 & 215 & 96.3 & I 57.8 & $137 \cdot 3$ \\
\hline 166 & $74 . I$ & $12 \mathrm{~T} \cdot 4$ & 105.6 & 216 & 96.7 & 158.6 & 138.0 \\
\hline 167 & $74 \cdot 5$ & 122.1 & 106.2 & 217 & $97 \cdot 2$ & 159.3 & 138.6 \\
\hline 168 & 74.9 & 122.9 & 106.9 & 218 & 97.6 & 160.2 & 139.3 \\
\hline 169 & 75.4 & 123.6 & 107.5 & 219 & $9^{8} . \mathrm{I}$ & 160.8 & 139.9 \\
\hline 170 & 75.8 & 124.4 & 108.2 & 220 & 98.6 & $16 x \cdot 5$ & 140.6 \\
\hline 171 & 76.3 & $125 . \mathrm{I}$ & 108.8 & 211 & 99.0 & 162.3 & 141.2 \\
\hline 172 & 76.8 & 125.8 & 109.5 & 222 & $99 \cdot 5$ & 163.0 & 141.9 \\
\hline 173 & $77 \cdot 3$ & 126.6 & 110.1 & 223 & 99.9 & 163.7 & 142.5 \\
\hline 174 & $77 \cdot 7$ & $127 \cdot 3$ & 110.8 & 224 & 100.4 & $164 \cdot 5$ & 143.2 \\
\hline 175 & $78 \cdot 2$ & 128.1 & III. 4 & 225 & 100.9 & 165.3 & $1_{43.8}$ \\
\hline 176 & 78.6 & 128.8 & 112.0 & 226 & $101 \cdot 3$ & 166.0 & 144.5 \\
\hline 177 & 79.1 & 129.5 & 112.6 & 227 & 101.8 & 166.8 & 145.1 \\
\hline 178 & 79.5 & 130.3 & $\mathrm{Ix}_{3.3}$ & 228 & 102.2 & 167.5 & 145.8 \\
\hline I 79 & 80.0 & 131.0 & $113-9$ & 229 & 102.7 & 168.3 & 146.4 \\
\hline
\end{tabular}


DEFREN'S TABLE FOR THE DETERMINATION OF DEXTROSE, MALTOSE, AND LACTOSE-(Concluded).

\begin{tabular}{|c|c|c|c|c|c|c|c|}
\hline $\begin{array}{c}\text { Milligrams } \\
\text { of Cupric } \\
\text { Oxide. }\end{array}$ & $\begin{array}{l}\text { Milligrams } \\
\text { of Dextrose. }\end{array}$ & $\begin{array}{l}\text { Milligrams } \\
\text { of Maltose. }\end{array}$ & $\begin{array}{l}\text { Milligrams } \\
\text { of Lactose. }\end{array}$ & $\begin{array}{c}\text { Milligrams } \\
\text { of Cupric } \\
\text { Oxide. }\end{array}$ & $\begin{array}{l}\text { Milligrams } \\
\text { of Dextrose. }\end{array}$ & $\begin{array}{l}\text { Milligrams } \\
\text { of Maltose. }\end{array}$ & $\begin{array}{l}\text { Milligrams } \\
\text { of Lactose. }\end{array}$ \\
\hline $23^{\circ}$ & 103.1 & 169.1 & $147.0^{\circ}$ & 280 & 126.1 & 206.8 & 179.6 \\
\hline $23 I$ & 103.6 & 169.8 & $147-7$ & $28 \mathrm{I}$ & 126.5 & $207 \cdot 5$ & 180.2 \\
\hline 232 & 104.0 & 170.6 & $\mathrm{I}_{4} 8.3$ & 282 & 127.0 & 208.3 & 180.9 \\
\hline 233 & $104 \cdot 5$ & $171 \cdot 3$ & I 49.0 & 283 & 127.4 & 209.0 & $181 \cdot 5$ \\
\hline 234 & 105.0 & 172.1 & 149.6 & 284 & 127.9 & 209.8 & 182.2 \\
\hline 235 & 105.4 & 172.8 & 150.3 & 285 & 128.3 & 210.5 & 182.9 \\
\hline 236 & 105.9 & 173.6 & 150.9 & 286 & 128.8 & $211 \cdot 3$ & 183.6 \\
\hline 237 & 106.3 & 174.3 & 151.6 & 287 & 129.3 & $2 \mathrm{I} 2 . \mathrm{I}$ & 184.2 \\
\hline 238 & 106.8 & $175 . \mathrm{I}$ & 152.2 & 288 & 129.7 & $2 \mathrm{I}_{2} \cdot 8$ & 184.9 \\
\hline 239 & $107 \cdot 2$ & 175.8 & 152.9 & 289 & 130.2 & 213.6 & 185.6 \\
\hline 240 & $107 \cdot 7$ & 176.6 & 153.5 & 290 & I 30.6 & $214 \cdot 3$ & I 86.2 \\
\hline 241 & 108.1 & $177 \cdot 3$ & 154.2 & 291 & 131.1 & $215 \cdot 1$ & $\mathbf{1 8 6} .9$ \\
\hline 242 & 108.6 & 178.1 & 154.8 & 292 & $131 \cdot 5$ & 215.9 & 187.6 \\
\hline 243 & IO9.0 & 178.8 & 155.5 & 293 & 132.0 & 216.6 & I88. 2 \\
\hline 244 & $109 \cdot 5$ & 179.6 & I56.I & 294 & $132 \cdot 5$ & $217 \cdot 4$ & 188.9 \\
\hline 245 & 100.9 & 180.3 & 156.8 & 295 & 133.0 & 218.2 & 180.5 \\
\hline 246 & 110.4 & $18 \mathrm{r}, \mathrm{I}$ & $157 \cdot 4$ & 296 & 133.4 & 218.9 & 190.2 \\
\hline 247 & 110.9 & I 81.8 & 158.1 & 297 & 133.9 & 219.7 & 190.8 \\
\hline 248 & 111.3 & $18 z .6$ & 158.7 & 298 & $134 \cdot 3$ & 220.4 & $191 \cdot 5$ \\
\hline 249 & $\operatorname{Irr} .0$ & 183.3 & I59.4 & 299 & 134.8 & $221 \cdot 2$ & $192 . I$ \\
\hline 250 & 112.3 & $184 \cdot \mathrm{I}$ & 160.0 & 300 & 135.3 & 221.9 & I92.8. \\
\hline $25 \mathrm{I}$ & $112 \cdot 7$ & 184.8 & 160.7 & 301 & 135.7 & $222 \cdot 7$ & 193.4 \\
\hline 252 & 113.2 & 185.5 & $16 r \cdot 3$ & 302 & 136.2 & $223 \cdot 5$ & $194 . \mathrm{I}$ \\
\hline 253 & II $3-7$ & 186.3 & I 62.0 & 303 & 136.6 & 224.2 & 194.7 \\
\hline 254 & $\mathrm{II}_{4, \mathrm{I}}$ & $187 \cdot \mathbf{I}$ & 162.6 & 304 & 137.1 & 225.0 & I95.3 \\
\hline 255 & $\mathrm{II}_{4} \cdot 6$ & I87.8 & 163.3 & 305 & 137.6 & 225.8 & r96.0 \\
\hline 256 & II5.0 & I88.6 & 163.9 & 306 & 138.0 & $226 \cdot 5$ & 196.6 \\
\hline 257 & II5. 5 & $189 \cdot 3$ & I64.6 & 307 & 138.5 & $227 \cdot 3$ & $197 \cdot 3$ \\
\hline $25^{8}$ & 116.0 & 190.1 & 165.2 & 308 & I 38.9 & 228. I & $197-9$ \\
\hline 259 & 110.4 & 190.8 & 165.9 & 309 & 139.4 & 228.8 & 190.0 \\
\hline 260 & 116.9 & 191.6 & $\mathrm{x} 66.5$ & 310 & $\times 39.9$ & 229.6 & 199.3 \\
\hline 261 & II $7 \cdot 3$ & 192.4 & 167.2 & $3 I I$ & 140.3 & 230.4 & I99.9 \\
\hline 262 & 117.8 & 193.1 & 167.8 & 312 & 140.8 & $23 \mathrm{I} \cdot \mathrm{I}$ & 200.6 \\
\hline 263 & 118.3 & 193.9 & 168.1 & 313 & 141.2 & $231 \cdot 9$ & $201 \cdot 3$ \\
\hline 204 & 118.7 & I94.6 & 169.5 & 314 & $14 \mathrm{I} .7$ & $23^{2} \cdot 7$ & 202.0 \\
\hline 265 & II9.2 & I 95.4 & 160.8 & 315 & 142.2 & $233 \cdot 4$ & 202.6 \\
\hline 266 & 119.6 & 196.1 & 170.4 & $3^{16}$ & 142.6 & $234 \cdot 2$ & $203 \cdot 3$ \\
\hline 267 & 120.1 & 196.9 & 171.1 & 317 & 143.1 & 234.9 & 203.9 \\
\hline 268 & 120.6 & I97.7 & 171.7 & 318 & 143.6 & $235 \cdot 7$ & $204 \cdot 6$ \\
\hline 269 & 121.0 & I98.4 & $172 \cdot 4$ & 319 & 144.0 & 236.5 & $205 \cdot 3$ \\
\hline 270 & $121 \cdot 4$ & 199.2 & 173.0 & 320 & $144 \cdot 5$ & 237.2 & 205.9 \\
\hline 271 & 121.9 & I99.9. & 173.7 & & & & \\
\hline 272 & 122.4 & 200.7 & 174.4 & & & & \\
\hline 273 & 122.8 & $201 \cdot 5$ & 175.0 & & & & \\
\hline 274 & $12 j \cdot \hat{j}$ & $202 \cdot 2$ & I75.7 & & & & \\
\hline 275 & 123.7 & 203.0 & 176.3 & & & & \\
\hline 276 & 124.2 & 203.7 & 177.0 & & & & \\
\hline $\begin{array}{l}277 \\
278\end{array}$ & $\begin{array}{l}124.6 \\
125 . I\end{array}$ & $\begin{array}{l}204 \cdot 5 \\
207 \cdot 2\end{array}$ & $\begin{array}{l}177.6 \\
178.3\end{array}$ & & & & \\
\hline $\begin{array}{l}278 \\
279\end{array}$ & $\begin{array}{l}125.1 \\
\mathrm{~T} 25.6\end{array}$ & $\begin{array}{l}207.2 \\
206.0\end{array}$ & $\begin{array}{l}170.3 \\
178.9\end{array}$ & & & & \\
\hline
\end{tabular}


Munson and Walker Method.*-I. Preparation of Solutions and Asbestos. - Use the copper sulphate solution and alkaline tartate solution as given on page 591. Prepare the asbestos, which should be the amphibole variety, by first digesting with $\mathrm{I}: 3$ hydrochloric acid for tivo or three days. Wash free from acid, and digest for a similar period with soda solution, after which treat for a few hours with hot alkaline copper tartrate solution of the strength employed in sugar determinations. Then wash the asbestos free from alkali, finally digest with nitric acid for several hours, and after washing free from acid, shake with water for use. In preparing the Gooch crucible, load it with a film of asbestos one-fourth inch thick, wash this thoroughly with water to remove fine particles of asbestos; finally wash with alcohol and ether, dry for thirty minutes at $100^{\circ} \mathrm{C}$., cool in a desiccator and weigh. It is best to dissolve the cuprous oxide with nitric acid each time after weighing, and use the same felts over and over again, as they improve with use.

2. Process,-Transfer $25 \mathrm{cc}$. each of the copper and alkaline tartrate solutions to a $400-\mathrm{cc}$. Jena or Non-sol beaker, and add $50 \mathrm{cc}$. of reducing sugar solution, or, if a smaller volume of sugar solution be used, add water to make the final volume $100 \mathrm{cc}$. Heat the beaker upon an asbestos gauze over a Bunsen burner, so regulate the flame that boiling begins in four minutes, and continue the boiling for exactly two minutes. Keep the beaker covered with a watch-glass throughout the entire time of heating. Without diluting, filter the cuprous oxide at once on an asbestos felt in a porcelain Gooch crucible, using suction. Wash the cuprous oxide thoroughly with water at a temperature of about $60^{\circ} \mathrm{C}$., then with Io cc. of alcohol, and finally with ro cc. of ether. Dry for thirty minutes in a water oven at $100^{\circ} \mathrm{C}$., cool in a desiccator and weigh as cuprous oxide.

The number of milligrams of copper reduced by a given amount of reducing sugar differs when sucrose is present and when it is absent. In the tables on pp. 599 to 607 the absence of sucrose is assumed, except in the two columns under invert sugar, where one for mixtures of invert sugar and sucrose ( 0.4 gram of total sugar in $50 \mathrm{cc}$. of solution), and one for invert sugar and sucrose when the $50 \mathrm{cc}$. of solution contains 2 grams of total sugar are given, in addition to the column for invert sugar alone.

* Jour. Am. Chem. Soc., 28, 1906, p. 16 3 ; 29, 1907, p. 541; U. S. Dept. Agric., Bur. of Chem., Bul. 107 (rev.), p. 24I; Circ. 82. 
MUNSON AND WALKER'S TABLE FOR CALCULATING DEXTROSE, INVERT SUGAR, LACTOSE, AND MALTOSE.

[Weights in milligrams.]

\begin{tabular}{|c|c|c|c|c|c|c|c|c|c|c|c|}
\hline \multirow[b]{2}{*}{ 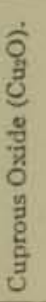 } & \multirow[b]{2}{*}{$\begin{array}{l}3 \\
3 \\
\frac{5}{3} \\
\frac{8}{3}\end{array}$} & \multirow[b]{2}{*}{$\frac{d}{\frac{d}{5}}$} & \multirow[b]{2}{*}{$\begin{array}{l}\frac{5}{5} \\
\text { जै } \\
\text { है } \\
\text { E् }\end{array}$} & \multicolumn{2}{|c|}{$\begin{array}{l}\text { Invert Sugar } \\
\text { and Sucrose. }\end{array}$} & \multicolumn{3}{|c|}{ Lactose. } & \multicolumn{2}{|c|}{ Maltose. } & \multirow[b]{2}{*}{ 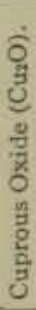 } \\
\hline & & & & 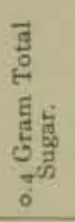 & 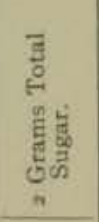 & $\frac{j}{3}$ & 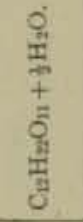 & $\begin{array}{l}0 \\
0 \\
\pm \\
0 \\
0 \\
0 \\
0 \\
0 \\
0\end{array}$ & $\frac{3}{3}$ & $\begin{array}{l}0 \\
\text { II } \\
+ \\
0 \\
0 \\
\text { in } \\
\frac{3}{3}\end{array}$ & \\
\hline 10 & 8.9 & 4.0 & $4 \cdot 5$ & x. 6 & $\cdots \cdots$ & 3.8 & 3.9 & 4.0 & $5 \cdot 9$ & 6.2 & ro \\
\hline II & 9.8 & $4 \cdot 5$ & 5.0 & 2,1 & $\ldots \ldots+\cdots$ & 4.5 & 4.6 & 4. 7 & $6-7$ & 7.0 & 11 \\
\hline 12 & 10.7 & 4.9 & 5. 4 & 2.5 & 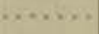 & 5.1 & $5 \cdot 3$ & $5 \cdot 4$ & $7 \cdot 5$ & $7 \cdot 9$ & 12 \\
\hline 13 & $\mathrm{rt} \cdot 3$ & $5 \cdot 3$ & 5.8 & 3.0 & $\cdots \cdots$ & 5.8 & 5.9 & 6.1 & 8.3 & 8.7 & 13 \\
\hline 14 & 12.4 & $5 \cdot 7$ & 6.3 & $3 \cdot 4$ & $\ldots \ldots$. & 6.4 & 6.6 & 6.8 & $9, \mathrm{t}$ & 9.5 & 14 \\
\hline 15 & 53,3 & 6.2 & 6.7 & $3 \cdot 9$ & $\cdots+\cdots$ & $7 \cdot 3$ & $7 \cdot 3$ & $7 \cdot 5$ & 9.9 & 10.4 & 15 \\
\hline 16 & 14.2 & 6.6 & 7.2 & $4 \cdot 3$ & 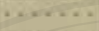 & 7.8 & 8.0 & 8.2 & 10,6 & $11 \cdot 2$ & 16 \\
\hline 17 & $15 . \mathrm{t}$ & 7.0 & 7.6 & 4.8 & CNot & 8,4 & 8.6 & 8.9 & It $\cdot 4$ & 12.0 & 17 \\
\hline 18 & 16,0 & $7 \cdot 5$ & 8,1 & 5.2 & $\because x, 1$, & 9.1 & 9.3 & 9.5 & 12.2 & 12.9 & $x 8$ \\
\hline 19 & 16.9 & $7 \cdot 9$ & 8.5 & 5.7 & 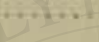 & 9.7 & 10.9 & 10,2 & 13.0 & $23 \cdot 7$ & 19 \\
\hline 20 & 17.8 & $8 \cdot 3$ & 8,9 & 6.1 & $\ldots \ldots$ & 10.4 & 10.7 & 10.9 & $x 3.8$ & 1.4 .6 & 20 \\
\hline 21 & 18.7 & 8.7 & 9.4 & 6.6 & $\ldots \ldots$ & 11.0 & $\mathrm{Ir}, 3$ & TI. 6 & 14.6 & 15.4 & 21 \\
\hline 22 & 19.5 & 9.2 & 9.8 & 7.0 & $\cdots \cdots, n$ & ii. 7 & 12.0 & 12,3 & 15.4 & 16.2 & 22 \\
\hline 23 & 20.4 & 9.6 & 10.3 & $7 \cdot 5$ & $\cdots+\ldots$ & 12,3 & 12.7 & 13,0 & 16.2 & $17+8$ & 23 \\
\hline 24 & 21.3 & 10.0 & 10,7 & 7.9 & $\ldots \ldots$ & 13.0 & 13.4 & 13.7 & 17.0 & 17.9 & 24 \\
\hline 25 & 22.2 & 10.5 & 11,2 & 8.4 & nten. & 13.7 & 14.0 & 14.4 & 17.8 & 18.7 & 25 \\
\hline 26 & $23, x$ & 10.9 & 11,6 & 8.8 & $\therefore \times 2.0$ & 14.3 & t 4.7 & $15, \mathrm{I}$ & 28.6 & 19.6 & 26 \\
\hline 27 & 24,0 & $1 \mathrm{~T} \cdot 3$ & 12.0 & 9.3 & $\cdots+\cdots$ & 15.0 & 15.4 & 15.8 & 19.4 & 20.4 & 27 \\
\hline 28 & $24 \cdot 9$ & $1 \times .8$ & 12.5 & 9.7 & $\cdots+\infty$ & 15,6 & 16.1 & 16.5 & 20.2 & 21.2 & 28 \\
\hline 29 & 25.8 & 12.2 & 72,9 & 10.2 & $\because \ldots$. & 10.3 & 16.7 & 17.1 & 21.0 & 22.1 & 29 \\
\hline 30 & 26.6 & 12.6 & 13.4 & 10.7 & $4 \cdot 3$ & 16.9 & 17.4 & 17.8 & 21.8 & 22.9 & 30 \\
\hline $3 x$ & $27 \cdot 5$ & $13 . \mathrm{T}$ & 13.8 & 11.1 & $4 \cdot 7$ & 17.6 & $18, \mathrm{t}$ & 18.5 & 32.6 & 23.7 & 31 \\
\hline 32 & 28.4 & 13,5 & 14.3 & 11.6 & 5.2 & 18.3 & 18.7 & 19.2 & $23-3$ & 24.6 & 32 \\
\hline 33 & $29 \cdot 3$ & 13.9 & 14.7 & 12.0 & 5.6 & 18.9 & 19.4 & 19.9 & 24.1 & 25.4 & 33 \\
\hline 34 & 30.2 & 14.3 & 15.2 & 12.5 & $6 . t$ & 19.6 & 20.1 & 20.6 & $24 \cdot 9$ & 26.2 & 34 \\
\hline 35 & $3 \pi, 5$ & 14.8 & 13.6 & 12.9 & 6.5 & 20,2 & 20.8 & $2 t \cdot 3$ & 23.7 & $27 . x$ & 35 \\
\hline 36 & 32.0 & 15.2 & $x 6,1$ & 13,4 & 7.0 & $20 \cdot 9$ & 21.4 & 22.0 & 26.5 & 27.9 & 36 \\
\hline 37 & 32.9 & 15.6 & 16.5 & 13.8 & $7 \cdot 4$ & $21 \cdot 5$ & 22.1 & $22 \cdot 7$ & $27 \cdot 3$ & 28.7 & 37 \\
\hline 38 & 33.8 & 16.1 & $x 6.9$ & 14.3 & $7 \cdot 9$ & 22.2 & 22.8 & 23,4 & 28,1 & 29.6 & 38 \\
\hline 39. & 34.6 & 16.5 & 17.4 & 14.7 & 8.4 & 22.8 & 23.5 & 24.1 & 28.9 & 30.4 & 39 \\
\hline 40 & 35.5 & $16: 9$ & 17.8 & 15.2 & 8.8 & $23 \cdot 5$ & $24 . \mathrm{I}$ & 24.8 & 29.7 & $3 x \cdot 3$ & 40 \\
\hline $4 t$ & 36.4 & 17.4 & 18.3 & 15.6 & $9-3$ & 24.2 & 24.8 & $25 \cdot 4$ & 30.5 & 32.1 & $4 \pi$ \\
\hline 42 & $37 \cdot 3$ & 17.8 & 18,7 & 16.1 & 9.7 & 24.8 & 25.5 & 26.1 & 31,3 & 32.9 & 42 \\
\hline 43 & 38.2 & 18.2 & 19.2 & 16.6 & 10.2 & $25 \cdot 5$ & 26.2 & 36.8 & 32.1 & 33.8 & 43 \\
\hline 44 & $39 . x$ & 18.7 & 19.6 & $x 7,0$ & 10,7 & 26.1 & 26.8 & 27.5 & 32.9 & 34.6 & 44 \\
\hline 45 & 40.0 & $19 . \mathrm{t}$ & $20 . \mathrm{I}$ & $17 \cdot 5$ & $I T, r$ & 26.8 & 27.5 & 28.2 & $33 \cdot 7$ & 35.4 & 45 \\
\hline 46 & 40.9 & 19,6 & 20.5 & 17.9 & $\mathrm{xt}, 6$ & 27.4 & 28.2 & 28.9 & $34 \cdot 4$ & 36.3 & 46 \\
\hline 47 & $4 \mathrm{I}, 7$ & $20: 0$ & 21.0 & 18.4 & 12,0 & $28 \cdot \mathrm{I}$ & 28.9 & 29.6 & $35 \cdot 2$ & $37+1$ & 47 \\
\hline 48 & 42.6 & 20.4 & $21 \cdot 4$ & 18.8 & 12.5 & 28.7 & 29.5 & 30.3 & 36.0 & 37.9 & 48 \\
\hline 49 & $43 \cdot 5$ & 20.9 & $2 x \cdot 9$ & 19.3 & $\mathrm{r} 2.9$ & 29.4 & 30,2 & 31.0 & 36.8 & 38.8 & 49 \\
\hline 50 & 44.4 & 21.3 & 22.3 & 19.7 & 13.4 & $30 . \mathrm{I}$ & 30,9 & $31: 7$ & 37.6 & 39.6 & 50 \\
\hline $5 t$ & $45 \cdot 3$ & $2 \mathrm{x}, 7$ & 22.8 & 20,2 & 13.9 & 30.7 & $3 x, 5$ & 32.4 & 38.4 & 40.4 & $5 \mathrm{r}$ \\
\hline 52 & 46.2 & 22.2 & 23.2 & 20.7 & 14.3 & 31,4 & 32.2 & 33.0 & 39.2 & 41,3 & 52 \\
\hline 53 & $47, x$ & 22.6 & 23.7 & $2 I, I$ & 1.4 .8 & 32.1 & 32.9 & 33.7 & 40.0 & 42,1 & 53 \\
\hline 54 & 48.0 & 23.0 & 24.1 & 21,6 & 15.2 & 32.7 & 33.6 & 34.4 & 40.8 & 42.9 & 54 \\
\hline 55 & 48.9 & 23.5 & 24.6 & 22,0 & $x 5,7$ & 33.4 & $34 \cdot 3$ & $35 \cdot 1$ & $4 \pi .6$ & 43.8 & 55 \\
\hline 56 & 49.7 & 23.9 & 25.0 & 22.5 & 16.2 & 34.0 & 34.9 & 35.8 & 42.4 & 44.6 & 56 \\
\hline 57 & 50.6 & $24 \cdot 3$ & 25.5 & 22.9 & 16.6 & 34.7 & 35.6 & 36.5 & 43.2 & $45 \cdot 4$ & 57 \\
\hline 58 & 51.5 & 24.8 & 25.9 & 23.4 & 17,5 & 35.4 & 36.3 & 37.2 & 44.0 & $46 \cdot 3$ & 58 \\
\hline 59 & 52.4 & 25.2 & 26.4 & 23.9 & 17.5 & 36.0 & 37.0 & 37.9 & 44.8 & 47.1 & 59 \\
\hline 60 & 53.3 & 25.6 & 26.8 & $24 \cdot 3$ & 18.0 & 36.7 & 37.6 & 38,6 & 45.6 & 48,0 & 60 \\
\hline $6 x$ & 54.2 & 26,1 & $27 \cdot 3$ & 24.8 & 18.5 & 37.3 & 38.3 & 39.3 & 46.3 & 48.8 & $6 r$ \\
\hline 62 & $55 \times 1$ & 26.5 & 27.7 & 25.2 & 18.9 & 38.0 & 39.0 & 40.0 & $47 \cdot \mathrm{t}$ & 49.6 & 62 \\
\hline 63 & 56.0 & 27.0 & 28.2 & 25.7 & 89.4 & 38.6 & 39.7 & 40.7 & $47 \cdot 9$ & 50.5 & 63 \\
\hline 64 & 56,8 & 27.4 & 28.6 & 26.2 & 19.8 & 39.3 & 40.3 & $4 I, 4$ & 48.7 & $5 \mathrm{I} \cdot 3$ & 64 \\
\hline
\end{tabular}


MUNSON AND WALKER'S TABLE FOR CALCULATING DEXTROSE, INVERT SUGAR, LACTOSE, AND MALTOSE-(Continued).

[Weights in milligrams,]

\begin{tabular}{|c|c|c|c|c|c|c|c|c|c|c|c|}
\hline \multirow[b]{2}{*}{ 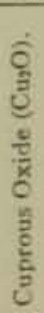 } & \multirow[b]{2}{*}{ 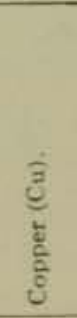 } & \multirow[b]{2}{*}{ 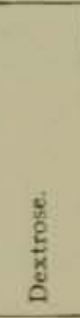 } & \multirow[b]{2}{*}{ 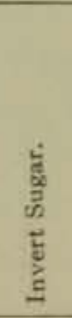 } & \multicolumn{2}{|c|}{$\begin{array}{l}\text { Invert Sugar } \\
\text { and Sucrose. }\end{array}$} & \multicolumn{3}{|c|}{ Lactose. } & \multicolumn{2}{|c|}{ Maltose. } & \multirow[b]{2}{*}{ 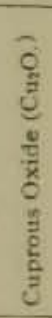 } \\
\hline & & & & 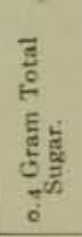 & 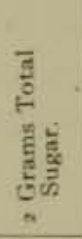 & $\begin{array}{l}\bar{b} \\
\frac{1}{2}\end{array}$ & $\begin{array}{l}0 \\
\pm \\
\pm \\
\pm \\
0 \\
0 \\
\frac{1}{n} \\
y\end{array}$ & $\begin{array}{l}0 \\
\frac{1}{1} \\
+ \\
\frac{1}{5} \\
\frac{1}{2} \\
\frac{E}{E}\end{array}$ & $\begin{array}{l}0 \\
0 \\
\frac{1}{5} \\
\text { v }\end{array}$ & 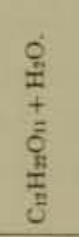 & \\
\hline 65 & $57 \cdot 7$ & 27.8 & 29.1 & 26.6 & 20.3 & 40.0 & 41.0 & 42,1 & 49.5 & 52,1 & 65 \\
\hline 66 & 58.6 & 28.3 & 29.5 & 27.1 & 20.8 & 40.6 & 41.7 & 42.8 & 50.3 & 53.0 & 66 \\
\hline 67 & 59.5 & 28.7 & 30.0 & 27.5 & 21.2 & $4 \mathrm{I}, 3$ & 42.4 & 43.5 & $51 . x$ & 53.8 & 67 \\
\hline 68 & 60,4 & 29.2 & 30.4 & 28.0 & 21.7 & 41.9 & $43 . \mathrm{t}$ & 44.2 & 51.9 & 54.6 & 68 \\
\hline 69 & $61 \cdot 3$ & 29.6 & 30.9 & 28.5 & 22.2 & 42.6 & 43.7 & 44.8 & 52.7 & 55.5 & 69 \\
\hline 70 & 62,2 & 30.0 & 31.3 & 28,9 & 22.6 & $43-3$ & 44.4 & $45 \cdot 5$ & 53.5 & $5^{6} \cdot 3$ & 70 \\
\hline 71 & 63.1 & 30.5 & 31.8 & 29.4 & 23.1 & 43.9 & 45.1 & 46.2 & $54 \cdot 3$ & $57-1$ & 71 \\
\hline 72 & 64.0 & 30.9 & 32.3 & 29.8 & 23.5 & 44.6 & 45.8 & 46.9 & 55.1 & 58.0 & 72 \\
\hline 73 & 64.8 & 31.4 & $32 . ?$ & 30.3 & 24.0 & 45.2 & $4^{6} .4$ & 47.6 & 55.9 & 58.8 & 73 \\
\hline 74 & 65.7 & 31.8 & 33.2 & 30.8 & $24 \cdot 5$ & 45.9 & 47.1 & 48.3 & 56.7 & 59.6 & 74 \\
\hline 75 & 66.6 & 32.2 & 33.6 & 31.2 & 24.9 & $4^{6} 6.6$ & 47,8 & 42.0 & 57.5 & 60.5 & 75 \\
\hline 76 & 67.5 & 32.7 & 34.1 & 31,7 & 25.4 & $47=2$ & 48.5 & 49.7 & 58.2 & $6 x+3$ & 76 \\
\hline 77 & 68.4 & 33.1 & $34-5$ & $32=1$ & 25.9 & 47.9 & 49.1 & 50.4 & 59:0 & 62.1 & 77 \\
\hline 78 & 69.3 & 33.6 & 35.0 & $32-6$ & $26 \cdot 3$ & 48.5 & 49.8 & 51.1 & $\begin{array}{l}59.8 \\
60.6\end{array}$ & 63.0 & 78 \\
\hline 79 & 70.2 & 34.0 & 35.4 & 33.1 & 26.8 & 49.2 & 50.5 & 51.8 & & & 79 \\
\hline 80 & 71.1 & 34.4 & $35: 9$ & 33.5 & $27-3$ & $49-9$ & 51.2 & 52.5 & $6 \mathrm{r} \cdot 4$ & 64.6 & 80 \\
\hline 81 & 71.9 & 34.9 & 36.3 & 34.0 & 27.7 & 50.5 & 51.9 & 53.2 & 62.2 & $65 \cdot 5$ & $8 \mathrm{I}$ \\
\hline 82 & 72.8 & $35 \cdot 3$ & 36.8 & 34.5 & 28.2 & 51.2 & 52.5 & 53.9 & 63.0 & 66.3 & 82 \\
\hline 83 & 73.7 & 35.8 & 37.3 & 34.9 & 28.6 & 51.8 & 53.2 & 54.6 & 63.8 & 67.1 & 83 \\
\hline 84 & 74.6 & $36: 2$ & 37.7 & 35.4 & 29.1 & 52.5 & 53.9 & 55.3 & 64.6 & 68.0 & 84 \\
\hline 85 & 75.5 & 36.7 & 38.2 & 35.8 & 29.6 & 53.1 & 54.6 & $56: 0$ & 65.4 & 68.8 & 85 \\
\hline $80^{\circ}$ & 76.4 & 37.1 & 38.6 & 36.3 & 30,0 & 53.8 & 55.2 & 56.6 & 66.2 & 69.7 & 86 \\
\hline 87 & $77-3$ & 37.5 & 39.5 & 36.8 & 30.5 & 34.5 & 55.9 & 57.3 & 67.0 & 70.5 & 87 \\
\hline 88 & $78=$ & 380 & 39.5 & 37.2 & 31.0 & 55.1 & 56.6 & 58.0 & $\begin{array}{r}67.8 \\
68 .\end{array}$ & $2 x \cdot 3$ & 88 \\
\hline 89 & 79.1 & 38.4 & 40.0 & 37.7 & $31-4$ & 55.8 & $57 \cdot 3$ & 58.7 & 68.5 & 72,2 & 89 \\
\hline 90 & 79.9 & $38 \cdot 9$ & 40,4 & 38.2 & $3 t \cdot 9$ & 56.4 & 58.0 & 59.4 & 69.3 & 73.0 & 90 \\
\hline 91 & 80.8 & $39 \cdot 3$ & 40,9 & 38.6 & 32.4 & 57.1 & 58.6 & 60.1 & 70.1 & 73.8 & $9 x$ \\
\hline 92 & $8 x .7$ & 39.8 & 41.4 & 39.3 & 32.8 & 57.8 & 59.3 & 60.8 & 70.9 & 74,7 & 92 \\
\hline 93 & 82.6 & $40: 2$ & 41.8 & 39.6 & $33-3$ & 58.4 & 60.0 & $6 \mathrm{x}, 5$ & 71.7 & 75,5 & 93 \\
\hline 94 & 83.5 & 40,6 & 42.3 & 40.0 & 33.8 & 59.1 & 60.7 & 62,2 & 72.5 & 76,3 & 94 \\
\hline 95 & 84.4 & $41-1$ & 42.7 & 40.5 & $34 \cdot 2$ & 59.7 & 61,3 & 62.9 & $73-3$ & 77.2 & 95 \\
\hline 96 & 85.3 & $4 \mathrm{~T}, 5$ & 43.2 & 41.0 & 34.7 & 60.4 & 62.0 & 63.6 & & $\begin{array}{l}78.0 \\
78.8\end{array}$ & 96 \\
\hline 97 & 86.2 & 42.0 & 43.7 & $41-4$ & 35.2 & $61 . t$ & 62.7 & $64 \cdot 3$ & $\begin{array}{l}74-9 \\
75,7\end{array}$ & 79.7 & $\begin{array}{l}97 \\
98\end{array}$ \\
\hline 98 & 87.1 & 42.4 & 44.1 & $41-9$ & 35.6 & $61-7$ & 63.4 & $\begin{array}{l}65.0 \\
65 .-\end{array}$ & $\begin{array}{l}75.7 \\
76.5\end{array}$ & 80.5 & $\begin{array}{l}98 \\
99\end{array}$ \\
\hline 99 & 87.9 & $f=.9$ & 44.6 & $42: 3$ & 36.1 & 62.4 & 64.0 & & & & \\
\hline 100 & 88.8 & $43 \cdot 3$ & 45.0 & 42.8 & 36.6 & 63.0 & 64.7 & $66: 4$ & $77 \cdot 3$ & $8 \mathrm{t}, 3$ & 100 \\
\hline tor & $89 . ?$ & 43.8 & $45-5$ & 43.3 & 37.0 & 63.7 & 65.4 & 67.1 & $\begin{array}{r}78 \cdot 1 \\
78.8\end{array}$ & 82.2 & 101 \\
\hline 102 & 90.6 & 44.2 & 46.0 & 43.8 & 37.5 & 64.4 & 66.1 & 67.8 & $\begin{array}{r}78.8 \\
-9.6\end{array}$ & $\begin{array}{l}83.0 \\
83.8\end{array}$ & 102 \\
\hline 103 & 91.5 & 44.7 & 46.4 & 44.2 & 38.0 & 65.0 & 66.7 & 68,5 & $\begin{array}{l}79.0 \\
80: 4\end{array}$ & $\begin{array}{l}83,8 \\
84.7\end{array}$ & $\begin{array}{l}103 \\
104\end{array}$ \\
\hline 104 & 92.4 & 45.1 & 46.9 & 44.7 & 38.5 & 65.7 & 67.4 & 69.1 & & & 104 \\
\hline 105 & 93.3 & 45.3 & $47 \cdot 3$ & 45.2 & 38,9 & 66.4 & 68.1 & 69.8 & 81,2 & 85.5 & 105 \\
\hline 106 & $94-2$ & 46.0 & 47.8 & 45.6 & 39.4 & 67.0 & 68.8 & 70. & 82.0 & 86.3 & 106 \\
\hline 107 & 95.0 & 46.4 & $48 \cdot 3$ & 46.2 & 39.9 & 67.7 & 69.5 & 71.2 & $\begin{array}{l}82.8 \\
83.6\end{array}$ & $\begin{array}{l}87.2 \\
88.0\end{array}$ & $\begin{array}{l}207 \\
108\end{array}$ \\
\hline $\begin{array}{l}108 \\
109\end{array}$ & $\begin{array}{l}95.9 \\
96.8\end{array}$ & $\begin{array}{l}46 \cdot 9 \\
47 \cdot 3\end{array}$ & 48,7 & 46.6 & $40 \cdot 3$ & 68.3 & 70.1 & 71.9 & $\begin{array}{l}83.6 \\
84.4\end{array}$ & 88.8 & $\begin{array}{l}108 \\
109\end{array}$ \\
\hline & & 47.3 & 49,2 & 47.0 & 40.8 & 69.0 & 70.8 & 72,6 & & & \\
\hline $\begin{array}{l}\text { ito } \\
\text { iit }\end{array}$ & $\begin{array}{l}97=7 \\
98.6\end{array}$ & $\begin{array}{l}47,8 \\
48,2\end{array}$ & 49.6 & 47.5 & $4 x-3$ & 69.7 & 21.5 & 73.3 & $\begin{array}{l}85.2 \\
86.0\end{array}$ & $\begin{array}{l}89.7 \\
90.5\end{array}$ & $\begin{array}{l}110 \\
111\end{array}$ \\
\hline $\begin{array}{l}111 \\
112\end{array}$ & $\begin{array}{l}98.6 \\
99.5\end{array}$ & $\begin{array}{l}48.2 \\
48.7\end{array}$ & 50,8 & 48.0 & 41,7 & 70.3 & $72 \cdot 2$ & 74. & 86.8 & 90.5 & 112 \\
\hline$x+3$ & $\begin{array}{r}99.5 \\
100.4\end{array}$ & $\begin{array}{l}48.7 \\
49.7\end{array}$ & $\begin{array}{l}50.6 \\
51.0\end{array}$ & $\begin{array}{l}48.4 \\
48.9\end{array}$ & $\begin{array}{l}42 \cdot 2 \\
42 \cdot 7\end{array}$ & $\begin{array}{l}71.0 \\
71.6\end{array}$ & $\begin{array}{l}72.8 \\
73.5\end{array}$ & $\begin{array}{l}7.4 .7 \\
75.4\end{array}$ & 87.6 & $92 . a$ & 113 \\
\hline 114 & $101-3$ & 49.6 & 51.5 & 49.4 & 43.2 & 72.3 & 74.2 & $76 . \mathrm{x}$ & 88.4 & 93.0 & 114 \\
\hline $1 \times 5$ & 102.2 & 50.0 & $51 \cdot 9$ & 49.8 & 43,6 & 73.0 & 74.9 & 76.8 & 89.2 & 93.9 & Ixs \\
\hline 116 & 103.0 & 50.5 & $5=-4$ & 50.3 & 44.1 & 73.6 & 75.6 & 77.5 & 90.0 & 94.7 & $x+6$ \\
\hline 117 & 103.9 & 50.9 & 52.9 & 50.8 & 44.6 & 74.3 & 76.2 & 78.2 & $90 . ?$ & 95.5 & 1xy \\
\hline 118 & 104.8 & 51.4 & $53 \cdot 3$ & 51.2 & 45.0 & 75.0 & 76.9 & 78.9 & $\begin{array}{l}91.5 \\
92.3\end{array}$ & 96.4 & 123 \\
\hline 119 & 105.7 & 52.8 & 53.8 & 51,7 & 45.5 & 75.6 & 77.6 & 79.6 & 92.3 & & 109 \\
\hline
\end{tabular}


MUNSON AND WALKER'S TABLE FOR CALCULATING DEXTROSE, INVERT SUGAR, LACTOSE, AND MALTOSE-(Contimued).

[Weights in milligrams.]

\begin{tabular}{|c|c|c|c|c|c|c|c|c|c|c|c|}
\hline \multirow{2}{*}{ 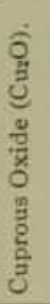 } & \multirow[b]{2}{*}{$\begin{array}{l}\frac{3}{3} \\
\frac{b}{2} \\
\frac{a}{0}\end{array}$} & \multirow[b]{2}{*}{$\frac{8}{x}$} & \multirow[b]{2}{*}{ 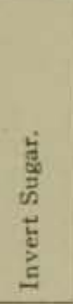 } & \multicolumn{2}{|c|}{$\begin{array}{l}\text { Invert Sugar } \\
\text { and Sucrose. }\end{array}$} & \multicolumn{3}{|c|}{ Lactose. } & \multicolumn{2}{|c|}{ Maltose. } & \multirow{2}{*}{ 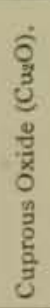 } \\
\hline & & & & 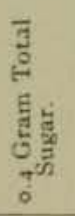 & 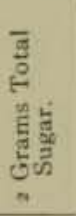 & $\frac{\dot{\bar{n}}}{\mathrm{~b}}$ & $\begin{array}{l}0 \\
\text { I. } \\
\text { I. } \\
+ \\
\text { b } \\
\frac{5}{3} \\
\frac{3}{3}\end{array}$ & 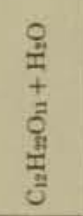 & $\frac{\vec{b}}{3}$ & 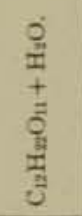 & \\
\hline 120 & 106.6 & $52-3$ & $54 \cdot 3$ & $52: 2$ & 46.0 & $76 \cdot 3$ & $78 \cdot 3$ & 80.3 & 93 - I & 98,0 & 120 \\
\hline 121 & 107.5 & 52.7 & $54 \cdot 7$ & 52,7 & 46.5 & 76.9 & 79.0 & 81.0 & $93-9$ & 98.9 & $12 \mathrm{I}$ \\
\hline 122 & 108.4 & 53.2 & 55.2 & $53 . \mathrm{r}$ & 46.9 & 77.6 & 79.6 & $8 x, 7$ & 947 & 99.7 & 122 \\
\hline 123 & 109.3 & 53.6 & 55.7 & 53,6 & 47.4 & $78 \cdot 3$ & 80.3 & 82.4 & $95 \cdot 5$ & $100-5$ & 123 \\
\hline 124 & tro. 1 & $54-1$ & $56, i$ & $54-1$ & 47.9 & 78.9 & 81,0 & $83 . \mathrm{t}$ & $96-3$ & to: 4 & 124 \\
\hline 125 & $1 \times \mathrm{T}, 0$ & 54.5 & 56.6 & $54 \cdot 5$ & $48: 3$ & 79.6 & 81.7 & 8.3 .8 & $97 \times x$ & 102,2 & 125 \\
\hline 126 & in 9 & 55.0 & 57,0 & 55.0 & 48.8 & 80.3 & 82.4 & 84.5 & $97-9$ & 103.0 & 126 \\
\hline 127 & $1 \times 2.8$ & 55,4 & $57 \cdot 5$ & $55 \cdot 5$ & 49.3 & 80.9 & 83.0 & 85.2 & 98.7 & 103.9 & 127 \\
\hline 128 & $113 \cdot 7$ & 55.9 & 58,0 & 55.9 & 49.8 & 81.6 & $83-7$ & 85.9 & 99.4 & 104.7 & 128 \\
\hline $4=9$ & $1 \times 4.6$ & $5^{6} \cdot 3$ & 58.4 & 56.4 & 50.2 & 82,2 & 84.4 & 86.6 & 100.2 & 105.5 & 129 \\
\hline 130 & 115.5 & 56.8 & 58,9 & 56,9 & 50.7 & 82.9 & $85-1$ & $87 \cdot 3$ & ror 0 & 206.4 & 130 \\
\hline 131 & $1+6.4$ & $57 \cdot 2$ & 59.4 & 57.4 & 51.2 & 83,6 & 85.7 & 88.9 & 101.8 & $107, z$ & 131 \\
\hline 132 & $117 \cdot 3$ & $57 \cdot 7$ & 59.8 & 57.8 & 51.7 & 84.2 & 86.4 & 88.7 & 102,6 & 108.0 & 132 \\
\hline 133 & 118,3 & $\begin{array}{l}58,1 \\
-8\end{array}$ & $60 \cdot 3$ & 58,3 & 52,2 & $84 \cdot 9$ & $87-1$ & 80.4 & 103.4 & 108.9 & 133 \\
\hline 134 & 119.0 & 58.6 & 60,8 & 58.8 & 32.6 & 85.5 & 87.8 & 90.1 & 104.2 & $109-7$ & 134 \\
\hline 135 & 179.9 & 59.0 & $61>2$ & 59.3 & $33 \cdot \mathrm{I}$ & 86.2 & 88.5 & 90.8 & 105.0 & 110.5 & 135 \\
\hline 136 & 120.8 & 59.5 & $61-7$ & 59.7 & 53.6 & 86.9 & 80.1 & 91.5 & $105-8$ & $11 x \cdot 4$ & 136 \\
\hline 137 & 121.7 & 60.0 & 62,2 & 60.2 & 54.0 & $87 \cdot 5$ & 89,8 & 92.1 & $106: 6$ & $112: 2$ & 137 \\
\hline 138 & 122.6 & 60.4 & 62.6 & 60.7 & $54 \times 5$ & 88.2 & 90.5 & 92.8 & $107 \cdot 4$ & 113.0 & r38 \\
\hline 139 & 123.5 & 60.9 & $63 \cdot 1$ & $6 x, 2$ & $35: 0$ & 88.9 & $91 \cdot 2$ & 93.5 & 108.2 & 113.9 & 139 \\
\hline 140 & 1.24 .4 & 61,3 & 63.6 & $6 t .6$ & 55.5 & $89 \cdot 5$ & 91.9 & 9.4 .2 & 1090 & 114.7 & 1.40 \\
\hline 141 & 125.2 & 61.8 & 64.0 & $62-1$ & 55.9 & 90,2 & 92.5 & 94.9 & 109.8 & $1+5.5$ & $14 \mathrm{t}$ \\
\hline 142 & $126 . \mathrm{r}$ & 62.2 & $64 \cdot 5$ & 62,6 & $56 \cdot 4$ & 90,8 & $93-2$ & 95.6 & 110,5 & $x+6.4$ & 142 \\
\hline$x+3$ & $127-0$ & 62,7 & 65.0 & $63 . t$ & 56.9 & 91.5 & 93.9 & 96.3 & 711,3 & 177,2 & 143 \\
\hline 144 & 127.9 & $63+1=$ & 65.4 & 63.5 & 57.4 & 92.2 & 94.6 & 97.0 & $x+2, x$ & 118.0 & 144 \\
\hline 145 & 128.8 & 63.6 & 65,9 & 64,0 & 57.8 & 92.8 & $95-3$ & 97.7 & 112,9 & $1 \times 8.9$ & 145 \\
\hline I 46 & $129 \cdot 7$ & $64: 0$ & 66.4 & $64 \cdot 5$ & 58.3 & 93.5 & 95.9 & 98.4 & $1 \times 3-7$ & 119.7 & 146 \\
\hline 147 & 130.6 & $64 \cdot 5$ & 66.9 & 65.0 & 58.8 & 94.3 & 06.6 & 99.1 & $x y .5$ & 120.5 & 147 \\
\hline 148 & 131,5 & 65.0 & $67 \cdot 3$ & 65.4 & 59.3 & 94.8 & 97.3 & 99.8 & 115.3 & $121-4$ & 148 \\
\hline $\mathrm{I} 49$ & 132.4 & 65.4 & 67.8 & 65.9 & $59: 7$ & 95.5 & 98.0 & 100.5 & $116 . t$ & 122.2 & 149 \\
\hline 150 & $133-2$ & 65,9 & $68 ; 3$ & 66.4 & 60.2 & 96.1 & $98 \cdot 7$ & for, 2 & $1+6,9$ & 123.0 & 150 \\
\hline 151 & 134.1 & 66.3 & 68.7 & $66 \cdot 9$ & $60 \cdot 7$ & 96.8 & 99.3 & Tor, 9 & 117.7 & $123 \cdot 9$ & $15 \mathrm{r}$ \\
\hline 152 & 135.0 & 66.8 & 69.2 & $67 \cdot 3$ & 67.2 & 97.5 & 100.0 & 102,6 & $x+8.5$ & 124.7 & 152 \\
\hline 153 & 135.9 & 67.2 & 69.7 & 67.8 & $6 t \cdot 7$ & 98.1 & 100.7 & 103,3 & 119.3 & 125,5 & 153 \\
\hline t54 & 136.8 & 67.7 & 70.1 & $68 \cdot 3$ & $62 . \mathrm{I}$ & 98.8 & ror .4 & 104,0 & 120.0 & 126.4 & 154 \\
\hline 155 & 137.7 & 68.2 & 70.6 & 68.8 & 62.6 & 99.5 & 102,1 & 104.7 & 120.8 & $127 \cdot 2$ & 155 \\
\hline 156 & 138.6 & 68.6 & $75 \cdot x$ & 69.2 & 63.1 & 100. $\mathrm{r}$ & 102.8 & 105.4 & 121.6 & 128,0 & 156 \\
\hline 157 & 139.5 & $69 . t$ & 72.6 & 69.7 & 63.6 & too. 8 & 103.4 & 106.1 & 122.4 & 128,9 & 157 \\
\hline 158 & $140-3$ & 69.5 & 72.0 & 70.2 & 64,1 & 101.3 & 104.1 & 106.8 & 123.2 & 129.7 & 158 \\
\hline$\times 59$ & $14 x-2$ & 70.0 & $72 \cdot 5$ & $70 \cdot 7$ & $64 \cdot 5$ & $102, I$ & 104.8 & 107.5 & 124.0 & 130.5 & r 59 \\
\hline 160 & $I 42, x$ & 70.4 & 73.0 & $71 \cdot 2$ & 65.0 & 102.8 & ro5.5 & 108.2 & 124.8 & $13 x=4$ & 160 \\
\hline $86 t$ & 143.0 & 70,9 & 73.4 & $71 \cdot 6$ & 65.5 & 103.4 & 106.2 & 108.9 & 125.6 & 132.2 & $16 t$ \\
\hline 162 & $\mathrm{r} 43.9$ & 71.4 & $73 \cdot 9$ & 72,1 & 66.0 & 104.1 & 106.8 & 109.6 & 126.4 & 133.0 & 162 \\
\hline 163 & 144.8 & 71.8 & 74.4 & 72.6 & 66.5 & 104.8 & $107-5$ & 110.3 & 127,2 & $133-9$ & 163 \\
\hline 164 & $1.45-7$ & 72.3 & 74.9 & 73.1 & 66.9 & 105.4 & 108.2 & 111.0 & 128,0 & 134.7 & 164 \\
\hline 165 & I 46.6 & 72.8 & $75 \cdot 3$ & 73.6 & 67.4 & 106. I & 108.9 & $11 x .7$ & 128.8 & 135,5 & 165 \\
\hline 166 & $1.47 \cdot 5$ & $73 \cdot 2$ & 75.8 & 74.0 & 67.9 & 106,8 & $\operatorname{rog} .6$ & $1 \mathrm{r} 2.4$ & 129.6 & 136,4 & 166 \\
\hline 167 & $x+48.3$ & $73 \cdot 7$ & $76 \cdot 3$ & 74.5 & 68.4 & 107.4 & $1+0.3$ & 113.1 & 130.3 & $137 \cdot 2$ & 167 \\
\hline 168 & 149.2 & 74.1 & 76.8 & $75 \%$ & 68.9 & $108 . \frac{7}{2}$ & 110.9 & II3.8 & $13 \mathrm{I}, \mathrm{i}$ & 136.0 & 168 \\
\hline 169 & $150, t$ & 74.6 & 77.2 & 75.5 & 69.3 & ros. 8 & $11 t .6$ & 114.5 & 131.9 & 138.9 & 169 \\
\hline 170 & 151.0 & $75 . t$ & 77.7 & 76.0 & 69.8 & I09. 4 & 112.3 & II5. 2 & 132,7 & 139.7 & 170 \\
\hline 171 & 151.9 & $75 \cdot 5$ & 78.2 & 76.4 & 70.3 & Iro, I & 113.0 & 115.9 & t33.5 & 140.5 & $27 x$ \\
\hline 172 & 152.8 & 76.0 & 78.7 & 76.9 & 70.8 & 110.8 & 113.7 & 116.6 & 134.3 & $14 t-4$ & 172 \\
\hline 173 & 153.7 & $76 \cdot 4$ & 79.1 & 77.4 & $7 x \cdot 3$ & III. 4 & 114.3 & 117.3 & $135 . \mathrm{t}$ & 142,2 & 173 \\
\hline 174 & 154.6 & 76.9 & 79.6 & 77.9 & $71 \cdot 7$ & 112,1 & 115.0 & 118,0 & 135.9 & 143.0 & 174 \\
\hline
\end{tabular}


MUNSON AND WALKER'S TABLE FOR CALCULATING DEXTROSE, INVERT SUGAR, LACTOSE, AND MALTOSE-(Continued).

[Weights in milligrams.]

\begin{tabular}{|c|c|c|c|c|c|c|c|c|c|c|c|}
\hline \multirow[b]{2}{*}{ 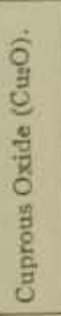 } & \multirow[b]{2}{*}{ 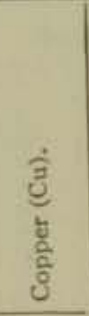 } & \multirow[b]{2}{*}{ 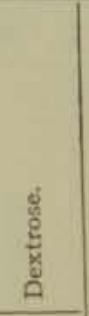 } & \multirow[b]{2}{*}{ 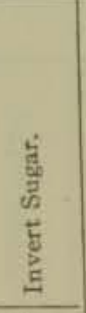 } & \multicolumn{2}{|c|}{$\begin{array}{l}\text { Invert Sugar } \\
\text { and Sucrose. }\end{array}$} & \multicolumn{3}{|c|}{ Lactose. } & \multicolumn{2}{|c|}{ Maltose. } & \multirow[b]{2}{*}{ 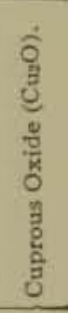 } \\
\hline & & & & 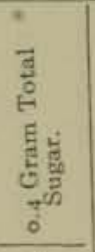 & 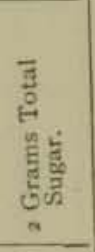 & 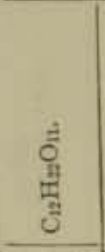 & 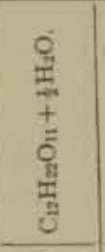 & 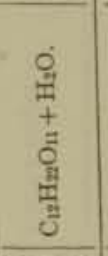 & : & 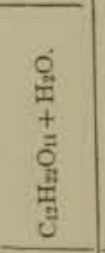 & \\
\hline $\begin{array}{l}175 \\
176 \\
177 \\
178 \\
179\end{array}$ & $\begin{array}{l}155.5 \\
156.3 \\
157.2 \\
158.1 \\
159.0\end{array}$ & $\begin{array}{l}77.4 \\
77.8 \\
78.3 \\
78.8 \\
79.2\end{array}$ & $\begin{array}{l}80.1 \\
80.6 \\
81.0 \\
81.5 \\
82.0\end{array}$ & $\begin{array}{l}78.4 \\
78.8 \\
79.3 \\
79.8 \\
80.3\end{array}$ & $\begin{array}{l}72.2 \\
72.7 \\
73.2 \\
73.7 \\
74.2\end{array}$ & $\begin{array}{l}112.8 \\
113.4 \\
114.1 \\
114.8 \\
115.4\end{array}$ & $\begin{array}{l}115.7 \\
116.4 \\
117.1 \\
117.8 \\
118.4\end{array}$ & $\begin{array}{l}118.7 \\
119.4 \\
120.1 \\
120.8 \\
121.5\end{array}$ & $\begin{array}{l}136.7 \\
137.5 \\
138.3 \\
139.1 \\
139.8\end{array}$ & $\begin{array}{l}143.9 \\
1.4 .7 \\
1.45 .5 \\
x 46.4 \\
1.47 .2\end{array}$ & $\begin{array}{l}175 \\
176 \\
177 \\
178 \\
179\end{array}$ \\
\hline $\begin{array}{l}180 \\
18 x \\
182 \\
183 \\
184\end{array}$ & $\begin{array}{l}159.9 \\
160.8 \\
161.7 \\
162.6 \\
163.4\end{array}$ & $\begin{array}{l}79.7 \\
80.7 \\
80.6 \\
81.1 \\
81.5\end{array}$ & $\begin{array}{l}82.5 \\
82.9 \\
83.4 \\
83.9 \\
84.4\end{array}$ & $\begin{array}{l}80.8 \\
81.3 \\
81.7 \\
82.2 \\
82.7\end{array}$ & $\begin{array}{l}74.6 \\
75.1 \\
75.6 \\
76.1 \\
76.6\end{array}$ & $\begin{array}{l}116.1 \\
116.7 \\
117.4 \\
118.2 \\
118.7\end{array}$ & $\begin{array}{l}119.1 \\
110.8 \\
120.5 \\
121.2 \\
121.8\end{array}$ & $\begin{array}{l}122.2 \\
122.9 \\
123.6 \\
124,3 \\
125.0\end{array}$ & $\begin{array}{l}140.6 \\
141.4 \\
142.2 \\
143.0 \\
143.8\end{array}$ & $\begin{array}{l}148.0 \\
148.9 \\
149.7 \\
150.5 \\
151.4\end{array}$ & $\begin{array}{l}180 \\
181 \\
182 \\
183 \\
184\end{array}$ \\
\hline $\begin{array}{l}x 85 \\
186 \\
187 \\
187 \\
189 \\
189\end{array}$ & $\begin{array}{l}164 \cdot 3 \\
165 \cdot 2 \\
166-2 \\
167.0 \\
167.9\end{array}$ & $\begin{array}{l}82.0 \\
82.5 \\
82.9 \\
83.4 \\
83.9\end{array}$ & $\begin{array}{l}84.9 \\
85.3 \\
85.8 \\
86.3 \\
86.8\end{array}$ & $\begin{array}{l}83.2 \\
83.7 \\
84.2 \\
84.6 \\
85,1\end{array}$ & $\begin{array}{l}77.1 \\
77.6 \\
78.0 \\
78.5 \\
79.0\end{array}$ & $\begin{array}{l}119.4 \\
120.1 \\
120.7 \\
121.4 \\
122.1\end{array}$ & $\begin{array}{l}122.5 \\
123.2 \\
123.9 \\
124.0 \\
125.3\end{array}$ & $\begin{array}{l}125,7 \\
126,4 \\
127,1 \\
127.8 \\
128,5\end{array}$ & $\begin{array}{l}144.6 \\
145.4 \\
146.2 \\
147.0 \\
147.8\end{array}$ & $\begin{array}{l}152.2 \\
153.0 \\
153.9 \\
154.7 \\
155.5\end{array}$ & $\begin{array}{l}185 \\
186 \\
187 \\
188 \\
189\end{array}$ \\
\hline $\begin{array}{l}190 \\
191 \\
191 \\
192 \\
193 \\
194\end{array}$ & $\begin{array}{l}168.8 \\
169.7 \\
170.5 \\
171.4 \\
172.3\end{array}$ & $\begin{array}{l}84.3 \\
84.8 \\
85.3 \\
85.7 \\
86.2\end{array}$ & $\begin{array}{l}87.2 \\
87.7 \\
88.2 \\
88.7 \\
89.2\end{array}$ & $\begin{array}{l}85.6 \\
86.1 \\
86.6 \\
87.5 \\
87.6\end{array}$ & $\begin{array}{l}79 \cdot 5 \\
80.0 \\
80.5 \\
81.0 \\
81.4\end{array}$ & $\begin{array}{l}122,7 \\
123,4 \\
124,1 \\
124,7 \\
125\end{array}$ & $\begin{array}{l}125.9 \\
126.6 \\
127.3 \\
128.0 \\
128.7\end{array}$ & $\begin{array}{l}129.2 \\
129.9 \\
130.6 \\
131.3 \\
132.0\end{array}$ & $\begin{array}{l}148.6 \\
149.3 \\
150.1 \\
150.9 \\
151.7\end{array}$ & $\begin{array}{l}156.4 \\
157.2 \\
158.0 \\
158.9 \\
159.7\end{array}$ & $\begin{array}{l}190 \\
191 \\
192 \\
193 \\
194\end{array}$ \\
\hline $\begin{array}{l}195 \\
196 \\
197 \\
198 \\
199\end{array}$ & $\begin{array}{l}173.2 \\
174.1 \\
175.0 \\
175.9 \\
176.8\end{array}$ & $\begin{array}{l}86.7 \\
87.7 \\
87.6 \\
88.1 \\
88.5\end{array}$ & $\begin{array}{l}89.6 \\
90.1 \\
90.6 \\
91.1 \\
91.6\end{array}$ & $\begin{array}{l}88.0 \\
88.5 \\
89.0 \\
89.5 \\
90.0\end{array}$ & $\begin{array}{l}81.9 \\
82.4 \\
82.9 \\
83.4 \\
83.9\end{array}$ & $\begin{array}{l}126.1 \\
126.7 \\
127.4 \\
128.1 \\
128.7\end{array}$ & $\begin{array}{l}129.4 \\
130.0 \\
130.7 \\
131.4 \\
132.1\end{array}$ & $\begin{array}{l}132.7 \\
133.4 \\
134.1 \\
134.8 \\
135.5\end{array}$ & $\begin{array}{l}152.5 \\
153.3 \\
154.1 \\
154.9 \\
155.7\end{array}$ & $\begin{array}{l}160.5 \\
161.4 \\
162.2 \\
163.0 \\
163.9\end{array}$ & $\begin{array}{l}195 \\
196 \\
197 \\
198 \\
199 \\
199\end{array}$ \\
\hline $\begin{array}{l}200 \\
201 \\
202 \\
203 \\
204 \\
204\end{array}$ & $\begin{array}{l}177.7 \\
178.5 \\
179.4 \\
180.3 \\
181.2\end{array}$ & $\begin{array}{l}89.0 \\
89.5 \\
89.9 \\
90.4 \\
90.9\end{array}$ & $\begin{array}{l}92.0 \\
92.5 \\
93.0 \\
93.5 \\
94.0\end{array}$ & $\begin{array}{l}90.5 \\
91.0 \\
91.4 \\
91.9 \\
92.4\end{array}$ & $\begin{array}{l}84.4 \\
84.8 \\
85.3 \\
85.8 \\
86.3\end{array}$ & $\begin{array}{l}129.4 \\
130.0 \\
130.7 \\
131.4 \\
132.0\end{array}$ & $\begin{array}{l}132.8 \\
133.5 \\
134.1 \\
134.8 \\
135.5\end{array}$ & $\begin{array}{l}136.2 \\
136.9 \\
137.6 \\
138.3 \\
139.0\end{array}$ & $\begin{array}{l}156.5 \\
157.3 \\
158.1 \\
158.8 \\
159.6\end{array}$ & $\begin{array}{l}x 6.7 .7 \\
165.5 \\
166.4 \\
167.2 \\
168.0\end{array}$ & $\begin{array}{l}200 \\
201 \\
202 \\
203 \\
204\end{array}$ \\
\hline $\begin{array}{l}205 \\
206 \\
207 \\
208 \\
209\end{array}$ & $\begin{array}{l}182.1 \\
183.0 \\
183.9 \\
184.8 \\
185.6\end{array}$ & $\begin{array}{l}92.4 \\
91.8 \\
92.3 \\
92.8 \\
93.2\end{array}$ & $\begin{array}{l}94.5 \\
94.9 \\
95.4 \\
95.9 \\
96.4\end{array}$ & $\begin{array}{l}92.9 \\
93.4 \\
93.9 \\
94.4 \\
94.9\end{array}$ & $\begin{array}{l}86.8 \\
87.3 \\
87.8 \\
88.3 \\
88.8\end{array}$ & $\begin{array}{l}132.7 \\
133.4 \\
134.0 \\
134.7 \\
135.4\end{array}$ & $\begin{array}{l}136.2 \\
136.9 \\
137.6 \\
138.3 \\
138.9\end{array}$ & $\begin{array}{l}139.7 \\
140.4 \\
14 \mathrm{x} .1 \\
141.8 \\
142.5\end{array}$ & $\begin{array}{l}160.4 \\
161.2 \\
162.0 \\
162.8 \\
163.6\end{array}$ & $\begin{array}{l}168.9 \\
169.7 \\
170.5 \\
171.4 \\
172.2\end{array}$ & $\begin{array}{l}205 \\
206 \\
207 \\
208 \\
209\end{array}$ \\
\hline $\begin{array}{l}210 \\
211 \\
212 \\
213 \\
213 \\
214\end{array}$ & $\begin{array}{l}186.5 \\
187.4 \\
188.3 \\
189.2 \\
190.1\end{array}$ & $\begin{array}{l}93.7 \\
94.2 \\
94.6 \\
95.1 \\
95.6\end{array}$ & $\begin{array}{l}96.9 \\
97.4 \\
97.8 \\
98.3 \\
98.8\end{array}$ & $\begin{array}{l}95.4 \\
95.8 \\
96.3 \\
96.8 \\
97.3\end{array}$ & $\begin{array}{l}89.2 \\
89.7 \\
90.2 \\
90.7 \\
91.2\end{array}$ & $\begin{array}{l}136.0 \\
136.7 \\
137.4 \\
138.0 \\
138.7\end{array}$ & $\begin{array}{l}139.6 \\
140.3 \\
141.0 \\
141.7 \\
142.4\end{array}$ & $\begin{array}{l}143.2 \\
143.9 \\
144.6 \\
145.3 \\
146.0\end{array}$ & $\begin{array}{l}164.4 \\
165.2 \\
166.0 \\
166.8 \\
167.5\end{array}$ & $\begin{array}{l}173.0 \\
173.8 \\
174.7 \\
175.5 \\
176.4\end{array}$ & $\begin{array}{l}210 \\
211 \\
212 \\
213 \\
214\end{array}$ \\
\hline $\begin{array}{l}215 \\
216 \\
217 \\
218 \\
219\end{array}$ & $\begin{array}{l}191.0 \\
191.9 \\
192.8 \\
193.6 \\
194.5\end{array}$ & $\begin{array}{l}96.1 \\
96.5 \\
97.0 \\
97.5 \\
98.0\end{array}$ & $\begin{array}{r}99.3 \\
99.8 \\
100.3 \\
100.8 \\
101.2\end{array}$ & $\begin{array}{l}97.8 \\
98.3 \\
98.8 \\
99.3 \\
99.8\end{array}$ & $\begin{array}{l}91.7 \\
92.2 \\
92.7 \\
93.2 \\
93.7\end{array}$ & $\begin{array}{l}139.4 \\
140.0 \\
140.7 \\
141-4 \\
142.0\end{array}$ & $\begin{array}{l}\mathrm{r} 43.0 \\
\mathrm{~T} 43.7 \\
\mathrm{I} 44.4 \\
\mathrm{r} 45 . \mathrm{I} \\
\mathrm{T} 45.8\end{array}$ & $\begin{array}{l}146.7 \\
147.4 \\
148.1 \\
148.8 \\
149.5\end{array}$ & $\begin{array}{l}168.3 \\
169.3 \\
169.9 \\
170.7 \\
171.5\end{array}$ & $\begin{array}{l}177.2 \\
178.0 \\
178.9 \\
179.7 \\
180.5\end{array}$ & $\begin{array}{l}215 \\
216 \\
217 \\
218 \\
219\end{array}$ \\
\hline $\begin{array}{l}220 \\
221 \\
222 \\
223 \\
224 \\
224\end{array}$ & $\begin{array}{l}195.4 \\
296.3 \\
197.2 \\
198.1 \\
199.0\end{array}$ & $\begin{array}{r}98.4 \\
98.9 \\
99.4 \\
99.9 \\
100.3\end{array}$ & $\begin{array}{l}101.7 \\
102.2 \\
102.7 \\
103.2 \\
103.7\end{array}$ & $\begin{array}{l}100.3 \\
100.8 \\
101.2 \\
101.2 \\
102.2\end{array}$ & $\begin{array}{l}94.2 \\
94.7 \\
95.1 \\
95.6 \\
96.1\end{array}$ & $\begin{array}{l}142 \cdot 7 \\
143 \cdot 4 \\
144 \cdot 0 \\
144 \cdot 7 \\
145 \cdot 4\end{array}$ & $\begin{array}{l}146.5 \\
147.2 \\
147.8 \\
148.5 \\
149.2\end{array}$ & $\begin{array}{l}150.2 \\
150.9 \\
151.6 \\
152.3 \\
153.0\end{array}$ & $\begin{array}{l}172.3 \\
173.1 \\
173.9 \\
174.7 \\
175.5\end{array}$ & $\begin{array}{l}18 \mathrm{r} \cdot 4 \\
182.2 \\
183.0 \\
183.9 \\
184.7\end{array}$ & $\begin{array}{l}220 \\
221 \\
222 \\
223 \\
224\end{array}$ \\
\hline $\begin{array}{l}225 \\
226 \\
227 \\
228 \\
229\end{array}$ & $\begin{array}{l}199.9 \\
200.7 \\
201.6 \\
202.5 \\
203.4\end{array}$ & $\begin{array}{l}100.8 \\
101.3 \\
101.8 \\
102.2 \\
102.7\end{array}$ & $\begin{array}{l}104.2 \\
104.6 \\
105.1 \\
105.6 \\
106.1\end{array}$ & $\begin{array}{l}102.7 \\
103.2 \\
103.7 \\
104.2 \\
104.7\end{array}$ & $\begin{array}{l}96.6 \\
97.7 \\
97.6 \\
98.1 \\
98.6\end{array}$ & $\begin{array}{l}146.0 \\
146.7 \\
147.4 \\
148.0 \\
148.7\end{array}$ & $\begin{array}{l}149.9 \\
150.6 \\
151.3 \\
152.0 \\
152.6\end{array}$ & $\begin{array}{l}153.7 \\
154.4 \\
155.1 \\
155.8 \\
156.5\end{array}$ & $\begin{array}{r}176.2 \\
177.0 \\
177.8 \\
178.6 \\
179.4\end{array}$ & $\begin{array}{l}185.5 \\
186.4 \\
187.2 \\
188.0 \\
188.8\end{array}$ & $\begin{array}{l}225 \\
226 \\
227 \\
228 \\
229\end{array}$ \\
\hline
\end{tabular}


MUNSON AND WALKER'S TABLE FOR CALCULATING DEXTROSE, INVERT SUGAR, LACTOSE, AND MALTOSE-(Continued).

[Weights in milligrams.]

\begin{tabular}{|c|c|c|c|c|c|c|c|c|c|c|c|}
\hline \multirow{2}{*}{ 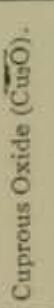 } & \multirow[b]{2}{*}{$\begin{array}{l}\frac{\dot{3}}{0} \\
\frac{0}{0} \\
\frac{0}{0}\end{array}$} & \multirow[b]{2}{*}{$\frac{\hat{E}}{\frac{E}{6}}$} & \multirow[b]{2}{*}{$\begin{array}{l}\frac{b}{2} \\
\frac{a}{d} \\
\frac{2}{2} \\
\frac{5}{3} \\
\frac{5}{5}\end{array}$} & \multicolumn{2}{|c|}{$\begin{array}{l}\text { Invert Sugar } \\
\text { and Sucrose }\end{array}$} & \multicolumn{3}{|c|}{ Lactose. } & \multicolumn{2}{|c|}{ Maltose. } & \multirow{2}{*}{ 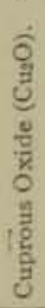 } \\
\hline & & & & 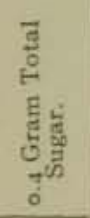 & 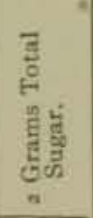 & $\frac{j}{0}$ & $\begin{array}{l}\frac{0}{6} \\
\frac{1}{2} \\
+ \\
+ \\
0 \\
0 \\
\frac{1}{2} \\
\frac{1}{3}\end{array}$ & $\begin{array}{l}0 \\
0 \\
\text { I } \\
+ \\
\overline{0} \\
0 \\
y \\
y \\
0\end{array}$ & $\frac{\frac{3}{5}}{\frac{y}{3}}$ & $\begin{array}{l}\frac{0}{3} \\
\frac{3}{3} \\
\frac{5}{2} \\
\frac{3}{3} \\
\frac{3}{3}\end{array}$ & \\
\hline 230 & $204 \cdot 3$ & 103.2 & 106.6 & 105.2 & $99 \cdot 1$ & I 49.4 & 153.3 & 157.2 & 180,2 & $189 \cdot 7$ & 230 \\
\hline 231 & 205.2 & $103-7$ & $107, I$ & 105.7 & 99.6 & 150.0 & 154.0 & 137.9 & $181=0$ & 190.5 & 231 \\
\hline 232 & 206,1 & 104.3 & 107.6 & 106.2 & 100.1 & 150.7 & 154.7 & 138.6 & $78 \mathrm{r}, 8$ & 191.3 & 232 \\
\hline 233 & 207.0 & 104.6 & $108 \cdot 1$ & 106.7 & 100.6 & 151.4 & 155.4 & I 59.3 & $182-6$ & 192.2 & 233 \\
\hline 234 & 207.9 & 105.2 & 108.6 & $107: 2$ & 101.1 & 152,0 & 156.1 & $160 ; 0$ & 183.4 & 193.0 & 234 \\
\hline 235 & 208.7 & 105.6 & rog $\mathrm{x}$ & $107 \cdot 7$ & 101.6 & 152,7 & 156.7 & 160.7 & $x 8_{4} \cdot 2$ & 193.8 & 235 \\
\hline 236 & 209.6 & 106.0 & 109.5 & 108.2 & 102.1 & 153.4 & 157.4 & 161.4 & $\times 84.9$ & 194.7 & 236 \\
\hline 237 & 210.5 & 106.5 & 110.0 & 108.7 & 102.6 & 154.0 & 158,1 & 162.1 & 185.7 & 195.5 & 237 \\
\hline 238 & $211-4$ & 107.0 & 120.5 & $109-2$ & 103.1 & $154-7$ & r 58.8 & 162,8 & 186.5 & 196.3 & 238 \\
\hline 239 & 212.3 & $107 \cdot 5$ & III. 0 & 109,6 & 103.5 & r 55.4 & 159.5 & 163.5 & 187.3 & 197.2 & 239 \\
\hline 240 & $213 \cdot 2$ & 108.0 & 111.5 & 110.1 & 104.0 & 156.1 & 160.2 & I 6.4 .3 & 188.1 & 198.0 & 240 \\
\hline 241 & $214, I$ & to8.4 & $x+2,0$ & 110,6 & $104 \cdot 5$ & 156.7 & 160.9 & 165.0 & 788.9 & 198.8 & 241 \\
\hline 242 & 215,0 & to8.9 & $1+2.5$ & $111-1$ & 105.0 & 157.4 & 161,5 & 165.7 & 189.7 & 199.7 & 242 \\
\hline 243 & 215.8 & rog. 4 & 113.0 & 111.6 & 105.5 & $158, \mathrm{I}$ & 162.2 & 166.4 & 190.5 & 200.5 & 243 \\
\hline 244 & 216.7 & tog. 9 & $1+3 \cdot 5$ & $x+2 . I$ & 106.0 & 158,7 & 162,9 & 167.1 & $19 t-3$ & $201-3$ & 244 \\
\hline 245 & 217,6 & 110,4 & 124.0 & 112.6 & 106.5 & I 59.4 & 163.6 & 167.8 & 192.2 & 202.2 & 245 \\
\hline 246 & 278.5 & 110.8 & $1+4.5$ & $1+3,1$ & 107,0 & $160, i$ & 164.3 & 168,5 & 192.9 & 203.0 & 246 . \\
\hline 247 & 219.4 & 111.3 & $1 \times 5.0$ & 113.6 & 107,5 & 160.7 & 165.0 & 169.2 & 193.6 & 203.8 & 247 \\
\hline 248 & 220.3 & 211.8 & $\mathrm{I}+5,4$ & 114.1 & 108.0 & 161.4 & 165.7 & 169.9 & 19.4 .4 & $204 \cdot 7$ & 248 \\
\hline 249 & 221.2 & $1+2.3$ & 715.9 & 114,6 & 108.5 & 152.1 & 160.3 & 170,6 & 195.2 & 205.5 & 249 \\
\hline 250 & 222.1 & $1 \times 2,8$ & $1 \pm 6.4$ & I I $5, x$ & 109.0 & 162.7 & 167.0 & $171 \cdot 3$ & 196.0 & 206.3 & 250 \\
\hline 251 & 223,0 & 113.2 & $1 \times 6.9$ & 115.6 & 109.5 & 163.4 & $167 \cdot 7$ & 172.0 & 296.8 & $207 \cdot 2$ & $25 \mathrm{t}$ \\
\hline 252 & 223,8 & 113,7 & 117.4 & 116,1 & 110.0 & 164.1 & 168.4 & 172.7 & 197.6 & 208.0 & 252 \\
\hline 253 & $224 \cdot 7$ & $1 \times 4.2$ & 117,9 & 116.6 & 110.5 & 164.7 & 169,1 & 173.4 & 798.4 & 208.8 & 253 \\
\hline 254 & 225.6 & 114.7 & 118.4 & $127, x$ & 112.0 & 165.4 & 169.8 & 174.1 & 199.2 & 209.7 & 254 \\
\hline 255 & 226.5 & $\operatorname{Ir} 5.2$ & 118.9 & 117.6 & IrI. 5 & $166 \cdot \mathrm{I}$ & 170.5 & 174.8 & 200.0 & 210.5 & 255 \\
\hline 256 & 227.4 & $x+5.7$ & 159.4 & 118,1 & 112.0 & 166.8 & 171,1 & 175.5 & 200.8 & $211 \cdot 3$ & $23^{6}$ \\
\hline 257 & 228.3 & 116.1 & 119.9 & $1+8.6$ & 112.5 & 167.4 & 171.8 & 176.2 & 201.6 & 212.2 & 257 \\
\hline 258 & 229.2 & $\mathrm{x} 16.6$ & 120,4 & II 9.1 & 113.0 & 168.1 & 172.5 & 176.9 & 202,3 & $2 \times 3.0$ & 258 \\
\hline 259 & 230,1 & 117.1 & 120.9 & 119.6 & $1 \times 3.5$ & 168.8 & 173.2 & 177,6 & $203 \cdot \mathrm{I}$ & 213.8 & 259 \\
\hline 260 & 231.0 & $x 7.6$ & $121 \cdot 4$ & 120.1 & $\mathrm{xr} 4,0$ & 169.4 & $\mathrm{I} 73.9$ & $\mathrm{I} 78.3$ & 203.9 & $2 \mathrm{r} 4 \cdot 7$ & 260 \\
\hline 261 & 231.8 & $1 x 8.1$ & 121.9 & 120,6 & 114.5 & 170,1 & 174.6 & 179.0 & 204.7 & 215.5 & $26 \mathrm{r}$ \\
\hline 262 & 232.7 & $x+8.6$ & 122.4 & 121.1 & 115.0 & 170.8 & 175.3 & 179.8 & 205.5 & 216.3 & 262 \\
\hline 263 & 233.6 & 119.0 & 122.9 & 121,6 & xis. 5 & 171.4 & 176.0 & 180.5 & 206.3 & $2 \times 7 \cdot 2$ & 263 \\
\hline 264 & 234.5 & 119.5 & 123.4 & 122.1 & $1 \times 6.0$ & 172.1 & 176.6 & $18 \mathrm{r} .2$ & 207.1 & 218.0 & 264 \\
\hline 265 & $235 \cdot 4$ & 120.0 & 123.9 & 122.6 & 176.5 & 172,8 & 177.3 & 181.9 & $207-9$ & $2 \pm 8.8$ & 265 \\
\hline 266 & $236 \cdot 3$ & 120.5 & $\mathrm{t} 24.4$ & 123,1 & 117,0 & 173.5 & 178.0 & 182.6 & 208.7 & 219.7 & 266 \\
\hline 267 & 237.2 & 121.0 & 124.9 & 123.6 & $1 \times 7,5$ & 174.1 & 178.7 & 183.3 & 209.5 & 220.5 & 267 \\
\hline 268 & 238.1 & 121.5 & 125.4 & 124.1 & 118.0 & 174.8 & 179.4 & 184.0 & 210.3 & 221.3 & 268 \\
\hline 269 & 238.9 & 122.0 & 125.9 & 1.24 .6 & 118.5 & 175.5 & 180.1 & 184.7 & 211,0 & 222.1 & 269 \\
\hline 270 & 239.8 & 122.5 & 126.4 & $t 25 . t$ & $1+9.0$ & $176, \mathrm{t}$ & 180.8 & 185.4 & 217.8 & 223.0 & 270 \\
\hline 271 & 240.7 & 122.9 & 126.9 & 125.6 & 119.5 & 176.8 & 181.5 & 186.1 & 212.6 & 223.8 & 271 \\
\hline 272 & 241.6 & $123 \cdot 4$ & 127.4 & 726.2 & 120.0 & $177 \div 5$ & 182.1 & 186.8 & $2 \times 3 \cdot 4$ & 224.6 & 272 \\
\hline 273 & 2.42 .5 & 123.9 & 127.9 & 126.7 & 120,6 & 178.1 & 182.8 & 187.5 & 214.2 & $225 \cdot 5$ & 273 \\
\hline 274 & 243.4 & $124 \cdot 4$ & 128.4 & 127.2 & $221, t$ & 178.8 & 183.5 & 188,2 & $2 \times 5,0$ & 226.3 & 274 \\
\hline 275 & 244.3 & 124.9 & 128,9 & 127.7 & 121.6 & 179.5 & 184.2 & 188.9 & 215.8 & 227,1 & 275 \\
\hline 276 & 245.2 & $125 \cdot 4$ & $129 \cdot 4$ & 1.28 .2 & 122.1 & I 80.2 & 184.9 & 189.6 & $2 \times 6.6$ & 228.0 & 276 \\
\hline 277 & $246, I$ & 125,9 & 129.9 & 128.7 & 122.6 & 180.8 & 185.6 & 190.3 & $2 \times 7.4$ & 228.8 & 277 \\
\hline 278 & 246.9 & 126.4 & 130.4 & 129.2 & 123.1 & 181,5 & 186.3 & 191,0 & $2+8,2$ & 229.6 & 278 \\
\hline 279 & 247.8 & 126.9 & 130.9 & 129.7 & 123.6 & 182.2 & 187.0 & 191.7 & 218.9 & 230.5 & 279 \\
\hline 280 & 248,7 & $x 27+3$ & 131,4 & 130.2 & $124-1$ & 182.8 & 187.7 & $192: 4$ & $2 \times 9.7$ & 231.3 & 280 \\
\hline $28 \mathrm{t}$ & 249.6 & $x 27.8$ & 135.9 & $130 \cdot 7$ & 124.6 & 183.5 & 188.3 & 193.1 & $220 \cdot 5$ & 232.1 & 281 \\
\hline 282 & 250.5 & 1.28 .3 & 132.4 & 131,2 & $125, \mathrm{t}$ & 184.2 & 189.0 & 193.9 & $221 \cdot 3$ & 233.0 & 282 \\
\hline 283 & 251.4 & 128.8 & 132.9 & 131,7 & 125.6 & 184.8 & 189.7 & 194.6 & 222.1 & 233.8 & 283 \\
\hline 284 & $252 \cdot 3$ & 129.3 & $\mathrm{r} 33.4$ & 132.2 & $126 . \mathrm{I}$ & 185.5 & 190.4 & 195.3 & 222.9 & 234.6 & 284 \\
\hline
\end{tabular}


MUNSON AND WALKER'S TABLE FOR CALCULATING DEXTROSE, INVERT SUGAR, LACTOSE, AND MALTOSE-(Continued).

[Weights in milligrams.]

\begin{tabular}{|c|c|c|c|c|c|c|c|c|c|c|c|}
\hline \multirow{2}{*}{ 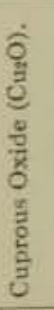 } & \multirow[b]{2}{*}{$\begin{array}{l}\dot{3} \\
\frac{3}{8} \\
\frac{1}{3}\end{array}$} & \multirow[b]{2}{*}{$\frac{\frac{g}{2}}{\frac{g}{x}}$} & \multirow[b]{2}{*}{ 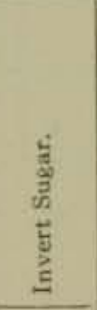 } & \multicolumn{2}{|c|}{$\begin{array}{l}\text { Invert Sugar } \\
\text { and Sucrose. }\end{array}$} & \multicolumn{3}{|c|}{ Lactose. } & \multicolumn{2}{|c|}{ Maltose. } & \multirow[b]{2}{*}{ 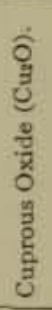 } \\
\hline & & & & 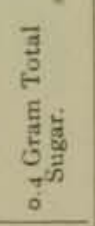 & 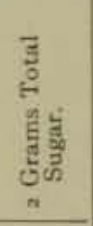 & $\frac{\dot{z}}{0}$ & 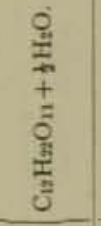 & $\begin{array}{l}0 \\
\text { II } \\
+ \\
\overline{0} \\
0 \\
\text { in } \\
\text { ü }\end{array}$ & $\frac{\frac{3}{3}}{\frac{8}{3}}$ & $\begin{array}{l}0 \\
\text { In } \\
+ \\
5 \\
0 \\
\frac{5}{1} \\
\frac{1}{3} \\
\end{array}$ & \\
\hline 285 & 253.2 & 129.8 & 133.9 & 132.7 & 126.6 & 186.2 & IOI. I & 196.0 & $223-7$ & 235.5 & 285 \\
\hline 286 & 254.0 & 130.3 & 134.4 & 133.2 & 127,1 & 186.9 & 191.8 & 196,7 & $224 \cdot 5$ & 236.3 & 286 \\
\hline 287 & $254-9$ & 130.8 & 134.9 & $533-7$ & 127.6 & 187.5 & 192.5 & 197.4 & $225-3$ & 237,1 & 287 \\
\hline 288 & 255.8 & $I 3 I-3$ & $135 \cdot 4$ & 134.3 & 128.1 & 188.2 & 193.2 & $198-1$ & 226.1 & 238.0 & 288 \\
\hline 289 & 250.7 & 131.8 & 135.9 & 134.8 & 128.6 & 188.9 & 193.8 & 198.8 & 226.9 & 238.8 & 289 \\
\hline 290 & 257.6 & 132.3 & 136,4 & 135.3 & 129.2 & 180.5 & 194.5 & 190.5 & 227.6 & 239.6 & 290 \\
\hline 291 & 258,5 & 132.7 & 136.9 & 135,8 & 129.7 & 190,2 & 195.2 & 200.3 & 228.4 & 240.5 & 291 \\
\hline 292 & $259 \cdot 4$ & 133.2 & 137.4 & 136.3 & 130.2 & $190-9$ & 195.9 & 200.9 & $229 \cdot 2$ & $24 \pi-3$ & 292 \\
\hline 293 & $260 \cdot 3$ & 133.7 & $137-9$ & 136.8 & 130.7 & $101-5$ & 196.6 & 201.6 & 230.0 & 242.1 & 293 \\
\hline 294 & 261.2 & 134.2 & 338.4 & 137,3 & $13 t .2$ & 192.2 & 197.3 & $202 \cdot 3$ & 230.8 & $242: 9$ & 294 \\
\hline 295 & 262.0 & 134,7 & 138.9 & 137.8 & 131,7 & 192.9 & 198.0 & 203.0 & $235 \cdot 6$ & 2.43 .8 & 295 \\
\hline 296 & 262,9 & $135-2$ & 139.4 & 138,3 & 132.2 & 193.6 & 198.7 & $203-7$ & $232 \cdot 4$ & 244.6 & 296 \\
\hline 297 & 263.8 & 135.7 & 140.0 & 138.8 & 132.7 & $194 \cdot 2$ & 199.3 & 204.4 & $233 \cdot 2$ & 245.4 & 297 \\
\hline 298 & 264.7 & 136.2 & 140.5 & 139.4 & 133.2 & 104.9 & 200,0 & 205.1 & 234.0 & 246.3 & 298 \\
\hline 299 & 265.6 & 136.7 & 141.0 & 139.9 & 133,7 & $195=6$ & 200.7 & 205.8 & 234.8 & $247 \cdot 1$ & 299 \\
\hline 300 & 266.5 & 137.2 & $14 t \cdot 5$ & 140.4 & 134.2 & 196.2 & 201.4 & 206.6 & 235.5 & 247.9 & 300 \\
\hline 301 & $267 \cdot 4$ & 137,7 & 142.0 & 140.9 & 134.8 & $106-9$ & 202,1 & 207.3 & 236.3 & 248.8 & 301 \\
\hline 302 & 268.3 & 138.2 & 142,5 & $r 4 x-4$ & 135.3 & 197.6 & 202.8 & 208,0 & $237-\mathrm{t}$ & 249.6 & 302 \\
\hline 303 & 269.1 & 138.7 & 1.43 .0 & 141,9 & 135.8 & 198,3 & 203.5 & $208-?$ & 237.9 & 250.4 & 303 \\
\hline 304 & 270.0 & 139.2 & $1.43 \cdot 5$ & 242.4 & $136-3$ & 198.9 & $204 \cdot 2$ & 209,4 & 238.7 & $251 \cdot 3$ & 304 \\
\hline 305 & 270.9 & 139.7 & 144.0 & 142.9 & 136.8 & 190,6 & 204.9 & 210.1 & 239.5 & 252.1 & 305 \\
\hline 306 & 271.8 & 140.2 & $t+4.5$ & $\mathrm{I} 43.4$ & $137 \cdot 3$ & $200 \cdot 3$ & $205-5$ & $210 \times 8$ & 240.3 & $252-9$ & 306 \\
\hline 307 & 272.7 & 140.7 & 145.0 & 144.0 & 137.8 & 201.0 & $206 \cdot 2$ & 211.5 & $241-1$ & 253.8 & 307 \\
\hline 308 & 273.6 & $1.41,2$ & 1.45 .5 & 1.4 .5 & 138.3 & 201,6 & 206,9 & 212.2 & $24 x-9$ & 254,6 & 308 \\
\hline 309 & 274.5 & 141,7 & $1,46,1$ & 145,0 & 138.8 & $202: 3$ & 207.6 & 212.9 & 242.7 & $255-4$ & 309 \\
\hline 310 & $275 \cdot 4$ & 1.42 .2 & 146.6 & $1,45,5$ & 139.4 & 2030 & 208.3 & 213.7 & $243 \cdot 5$ & 256.3 & 310 \\
\hline $3 t 2$ & 276.3 & 142,7 & 147,1 & 146.0 & 139.9 & 203.6 & 209.0 & 214.4 & $244 \cdot 2$ & $257-1$ & $3 \mathrm{It}$ \\
\hline 312 & 277,1 & 1.43 .2 & 147,6 & 146.5 & I 40.4 & 20.3 .3 & 209.7 & $2+5.1$ & 2.45 .0 & 257.9 & 312 \\
\hline 313 & 278.0 & $7.43-7$ & 148,1 & 147.0 & I 40.9 & 205.0 & 210.4 & 215.8 & 245.8 & 258.8 & 313 \\
\hline $3 t 4$ & 278.9 & 144,2 & 148.6 & 147.6 & 1.41 .4 & 205.7 & $211, x$ & 216.5 & 246.6 & 259.6 & 3 I. 4 \\
\hline 315 & 279.8 & 144.7 & $1+9.1$ & 148,1 & I $4 \mathrm{t}, 9$ & $206-3$ & 211.8 & 217.2 & $247-4$ & $260 \cdot 4$ & 313 \\
\hline 316 & 280.7 & 145.2 & 149.6 & 148.6 & 142.4 & 207.0 & 212.5 & 217.9 & 248.2 & 261.2 & $3 \times 6$ \\
\hline 317 & $28 \pi, 6$ & $\mathrm{r}+5,7$ & 150.1 & 149.1 & 143.0 & 207.7 & 213,1 & 218.6 & 249.0 & $262, \mathrm{I}$ & 317 \\
\hline 318 & 282.5 & 1.46 .2 & $150,-7$ & +49.6 & 143.5 & 2084 & 213.8 & 219.3 & 249.8 & 262.9 & $3 \times 8$ \\
\hline 319 & 283.4 & 146.7 & 151.2 & 150.1 & 144.0 & 209.0 & 214.5 & 220.0 & 250.6 & 263.7 & 319 \\
\hline 320 & 284.2 & $1.47 \cdot 2$ & 155.7 & 150.7 & 144.5 & 209.7 & 215.2 & 220.7 & $25 x-3$ & 264.6 & 320 \\
\hline $32 \mathrm{r}$ & $285-1$ & $147 \cdot 7$ & 152.2 & 151,2 & 145.0 & 210.4 & 215.9 & 221.4 & 252.1 & 265.4 & $32 \mathrm{r}$ \\
\hline 322 & 286.0 & 148.2 & I $52-7$ & $\mathrm{x} 5 \mathrm{t}, 7$ & 145.5 & 211.0 & 216.6 & 222,2 & 252.9 & 266.2 & 322 \\
\hline 323 & 286.9 & 148.7 & 153.2 & 152.2 & 1.46 .0 & 211.7 & $217 \times 3$ & 222,9 & 253.7 & $267 \cdot 1$ & 323 \\
\hline 324 & 287.8 & 149.2 & 153.7 & 152.7 & 146.6 & 212.4 & 218,0 & 223.6 & 254.5 & 267,9 & 324 \\
\hline 325 & 288.7 & 149.7 & 154.3 & 153.2 & 147.1 & $213 . \mathrm{t}$ & 218,7 & $224 \cdot 3$ & $255 \cdot 3$ & 268.7 & 325 \\
\hline 326 & 289.6 & 150.2 & 154.8 & 153.8 & 147.6 & 213.7 & 219.4 & 225.0 & $256, t$ & 269.6 & 326 \\
\hline 327 & $2,90 \cdot 5$ & 150.7 & 155.3 & 154.3 & 148,1 & 21.4 .4 & 720.1 & $225-7$ & $256 \cdot 9$ & 270.4 & 327 \\
\hline 328 & 291.4 & 151.2 & 155.8 & 154.8 & 148.6 & $215 . \mathrm{I}$ & $220 \cdot 7$ & $226 \cdot 4$ & $257 \cdot 7$ & $27 \mathrm{I}-2$ & 328 \\
\hline 329 & 292.2 & 151.7 & $156 \cdot 3$ & $155-3$ & 149.1 & 215.8 & 221.4 & 227.1 & 258.5 & 272,1 & 329 \\
\hline 330 & $293, t$ & 152.2 & 156.8 & 155.8 & 149.7 & 216.4 & $222 . \mathrm{I}$ & $227 \cdot 8$ & $259: 3$ & 222.9 & 330 \\
\hline $33 t$ & 294.0 & 152,7 & $157 \cdot 3$ & 156.4 & 150.2 & 317.1 & 222.8 & $228-5$ & 260,0 & $273-7$ & $33 t$ \\
\hline 332 & 294.9 & 153.2 & 157.9 & 156.9 & 150.7 & 217.8 & 223.5 & 229.2 & 260.8 & 274.6 & 332 \\
\hline 333 & 295,8 & 153.7 & 158.4 & 257.4 & $151-2$ & $2 \times 8.4$ & 224.2 & 230,0 & $26 t-6$ & 275.4 & 333 \\
\hline 334 & 296.7 & 154.2 & 158.9 & 157.9 & 151.7 & 210.1 & 224.9 & 230.7 & $262 \cdot 4$ & 276.2 & 334 \\
\hline 335 & 297.6 & 154.7 & 159.4 & 158.4 & 152,3 & $2 \times 9.8$ & 225.6 & 231.4 & $263 \cdot 2$ & 277.0 & 335 \\
\hline 336 & $298 \cdot 5$ & 755.2 & I 59.9 & 159.0 & 152.8 & 220.5 & 226.3 & $2 \cdot 2 \cdot \mathrm{I}$ & 264.0 & 277.9 & 336 \\
\hline 337 & $299 \cdot 3$ & 155.8 & 160.5 & $159-5$ & 153.3 & 221,1 & 227.0 & 232.8 & 264.8 & 278.7 & 337 \\
\hline $33^{8}$ & $300 \cdot 2$ & 156.3 & $16 \mathrm{r}, 0$ & 160.0 & $\mathrm{t} 53.8$ & 221,8 & $227 \cdot 7$ & 233.5 & 265.6 & 279.5 & 338 \\
\hline 339 & 301.1 & 156.8 & $\mathrm{I} 6 \mathrm{I}, 5$ & $160-5$ & 154.3 & 222.5 & 228,3 & 234.2 & 266.4 & 280.4 & 339 \\
\hline
\end{tabular}


MUNSON AND WALKER'S TABLE FOR CALCULATING DEXTROSE, INVERT SUGAR, LACTOSE, AND MALTOSE-(Continued).

[Weights in milligrams.]

\begin{tabular}{|c|c|c|c|c|c|c|c|c|c|c|c|}
\hline \multirow[b]{2}{*}{ 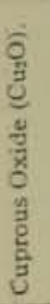 } & \multirow[b]{2}{*}{$\begin{array}{l}\dot{3} \\
\frac{\hat{y}}{\frac{0}{3}}\end{array}$} & \multirow[b]{2}{*}{ 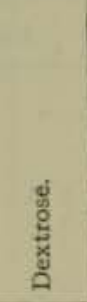 } & \multirow[b]{2}{*}{$\begin{array}{l}\text { हैं } \\
\text { हैं } \\
\text { है } \\
\text { है }\end{array}$} & \multicolumn{2}{|c|}{$\begin{array}{l}\text { Invert Sugar } \\
\text { and Sucrose. }\end{array}$} & \multicolumn{3}{|c|}{ Lactose. } & \multicolumn{2}{|c|}{ Maltose. } & \multirow[b]{2}{*}{ 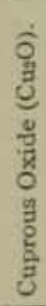 } \\
\hline & & & & 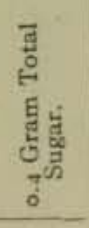 & 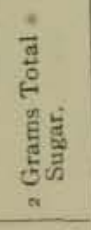 & $\frac{\hat{0}}{\frac{1}{3}}$ & 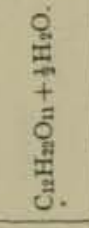 & $\begin{array}{l}0 \\
\frac{1}{1} \\
+ \\
\frac{1}{3} \\
\frac{1}{2} \\
\frac{1}{3}\end{array}$ & $\frac{\frac{1}{3}}{\frac{1}{3}}$ & $\begin{array}{l}0 \\
\pm \\
\pm \\
\pm \\
0 \\
\frac{1}{1} \\
\frac{1}{3}\end{array}$ & \\
\hline 340 & 302.0 & 157.3 & 162,0 & 161.0 & 154.8 & 223,2 & 229.0 & 234.9 & 267.1 & $28 x, 2$ & 340 \\
\hline 341 & $302+9$ & 157.8 & 162,5 & 161.6 & 155.4 & 223.8 & 229.7 & 235.6 & $267 \cdot 9$ & 282.0 & $34 \mathrm{I}$ \\
\hline 3.42 & 303.8 & 158.3 & 163.1 & 162.3 & 155.9 & 224.5 & $230 \cdot 4$ & 236,3 & 268.7 & 282,9 & 342 \\
\hline 343 & 304.7 & 158.8 & 163,6 & 162.6 & 156.4 & 225.2 & $231, t$ & 237.0 & 269.5 & 283.7 & 343 \\
\hline 344 & 305.6 & 159.3 & $104, t$ & 163.1 & 156.9 & $225 \cdot 9$ & $231-8$ & $237 \cdot 8$ & 270.3 & 284.5 & 344 \\
\hline 345 & $306 \cdot 5$ & I 59.8 & 164.6 & 163.7 & 157.5 & 226.5 & 232.5 & 238,5 & $271 \cdot x$ & $285 \cdot 4$ & 345 \\
\hline 346 & $307 \cdot 3$ & 160.3 & 165.1 & 164,2 & I 58.0 & 227.2 & 233.2 & 239.2 & 271.9 & 286.2 & 346 \\
\hline 347 & 308.2 & 160.8 & $165 \cdot 7$ & 164.7 & 158.5 & 227.9 & 233.9 & 239.9 & 272.7 & 287.0 & 347 \\
\hline 348 & $309 \cdot 1$ & 161.4 & 166.2 & 165.2 & 159.0 & 228,5 & 234.6 & 240.6 & $273 \cdot 5$ & $287-9$ & 348 \\
\hline 349 & $3 \times 0.0$ & $16 x, 9$ & $x 66.7$ & 165.7 & 159.5 & 229.2 & 235.3 & 241,3 & 274.3 & 288.7 & 349 \\
\hline 350 & 310.9 & 162,4 & 167.2 & 166.3 & 160.1 & $229-9$ & 235.9 & 242.0 & 275.0 & $289 \cdot 5$ & 350 \\
\hline 351 & $3 x+8$ & 162.9 & 167.7 & 166.8 & 160,6 & 230.6 & 236.6 & 242,7 & 275.8 & 290.4 & 351 \\
\hline 352 & 312,7 & 163.4 & +68.3 & $167 \cdot 3$ & $16 x-1$ & 231,2 & $237 \cdot 3$ & 243.4 & 276.6 & $291 \cdot 2$ & 352 \\
\hline 353 & 313.6 & $\begin{array}{r}63.9 \\
.60\end{array}$ & x 68.8 & 167.8 & 161.6 & 2319 & 238.0 & 244.1 & $277 \cdot 4$ & 292.0 & 353 \\
\hline 354 & 31.4 .4 & $164 \cdot 4$ & $109 \times 3$ & 168.4 & 102.2 & 232.6 & $238 \cdot 7$ & 244.8 & 278.2 & 292,8 & 354 \\
\hline 355 & $3 \times 3.3$ & $164 \cdot 9$ & 169.8 & 168.9 & 162.7 & $233 \cdot 3$ & 239.4 & 245.6 & 279.0 & 293.7 & 355 \\
\hline 356 & 316,2 & $165 \cdot 4$ & $170: 4$ & 169.4 & 163.2 & 233.9 & 240,1 & 246.3 & 279.8 & 294.5 & 356 \\
\hline 357 & $3+7,1$ & 166.0 & 170.9 & 170,0 & 163.7 & 234.6 & 240,8 & 247.0 & 280.6 & 295.3 & 357 \\
\hline 358 & 358.0 & 166.5 & 171,4 & 170.5 & $164 \cdot 3$ & 235.3 & 241.5 & $247 \cdot 7$ & $28 \mathrm{r}-4$ & 296.2 & 358 \\
\hline 359 & 318.9 & 107.0 & 171.9 & $17 t .0$ & 164.8 & 236.0 & 242.2 & 248.4 & 282,2 & 297.0 & 359 \\
\hline 360 & 319.8 & 167.5 & 172.5 & $x 7 x-5$ & 165.3 & 236.7 & 242.9 & $249 . \mathrm{I}$ & 282,9 & 297.8 & 360 \\
\hline 361 & 320.7 & 168.0 & 173.0 & $172, t$ & 165.8 & $237 \cdot 3$ & 243.6 & 249,8 & 283,7 & 298.7 & $36 t$ \\
\hline 362 & 321.6 & 168.5 & 173.5 & 172.6 & I66. 4 & 238.0 & 244.3 & 250.5 & $284 \cdot 5$ & 299.5 & 362 \\
\hline 363 & $322 \cdot 4$ & $\times 69.0$ & 174.0 & I $73 . \mathrm{I}$ & $x: 6.9$ & $238-7$ & 245.0 & 251,2 & 285.3 & 300,3 & 363 \\
\hline 364 & $323 \cdot 3$ & 169.6 & 174.6 & 173.7 & 167.4 & 239.4 & 245.7 & 252,0 & $286, t$ & $301 \cdot 2$ & 364 \\
\hline 365 & $32.4 \cdot 2$ & 170.1 & 175.1 & 174.2 & 167.9 & 240.0 & 246,4 & 252.7 & 286.9 & 302.0 & 365 \\
\hline 366 & 325,1 & 170.6 & 175.6 & 174.7 & 168.5 & 240.7 & 247,0 & 253.4 & 287,7 & 302.8 & 366 \\
\hline 367 & 326.0 & $17 \mathrm{I}, \mathrm{I}$ & 176.1 & 175.2 & 169,0 & 241.4 & 247,7 & 254.1 & 288.5 & 303.6 & 367 \\
\hline 368 & 326,9 & 171.6 & 176,7 & 173.8 & 169.5 & $242 . \mathrm{I}$ & $248 \cdot 4$ & 254.8 & 289.3 & 304.5 & 368 \\
\hline 369 & 327.8 & $172, t$ & 177.2 & 176.3 & 170.0 & $242 \cdot 7$ & 249.1 & 255.3 & 290.0 & 305.3 & 369 \\
\hline 370 & 328.7 & 172.7 & 177.7 & 176.8 & 170.6 & 243.4 & 249.8 & 256.2 & 290.8 & 306.1 & 370 \\
\hline 371 & 329.5 & 173.2 & $178: 3$ & 172,4 & I, $1, \mathrm{I}$ & 244,1 & 250.5 & 236.9 & 291.6 & 307.0 & 371 \\
\hline 372 & $320 \cdot 4$ & 173.7 & 178.8 & 177.9 & 171.6 & 244.8 & $25 x: 2$ & 257.7 & 292.4 & 307.8 & 372 \\
\hline 373 & $33 \div-3$ & 174.2 & 179.3 & $\begin{array}{r}178.4 \\
.78\end{array}$ & $172: 2$ & $245 \cdot 4$ & 251,9 & 258.4 & 293.2 & 308.6 & 373 \\
\hline 374 & 332.2 & 174.7 & 179.8 & 179.0 & 172.7 & $246, \mathrm{I}$ & 252.6 & $259 \cdot \mathrm{t}$ & 294.0 & $309 \cdot 5$ & 374 \\
\hline 375 & $333 . \mathrm{x}$ & $175 \cdot 3$ & 180.4 & 179.5 & 173.2 & 246,8 & 253.3 & 259.8 & 294.8 & 310.3 & 375 \\
\hline 376 & 334.0 & 175.8 & 180.9 & 180.0 & 173.7 & $247 \cdot 5$ & 254.0 & 260,5 & 295.6 & 311,1 & 376 \\
\hline 377 & $334 \cdot 9$ & 176.3 & $18 x+4$ & 180.6 & 174.3 & $2.48,1$ & 254.7 & $26 \mathrm{I} / 2$ & 296,4 & 312.0 & 377 \\
\hline 378 & 335.8 & 176.8 & 182.0 & 181,1 & 174.8 & 248.8 & 255.4 & 261.9 & 297.2 & 312.8 & 378 \\
\hline 379 & 336.7 & $177-3$ & 182.5 & 181.6 & I 75.3 & 249.5 & 256.1 & 262.6 & 297.9 & 313.6 & 379 \\
\hline 380 & $337 \cdot 5$ & 177.9 & 183.0 & 182,1 & 175.9 & 250.2 & 256,8 & 263.4 & 298,7 & 314.5 & 380 \\
\hline $38 \mathrm{r}$ & 338.4 & 178.4 & 183.6 & 182,7 & $1.76,4$ & 250.8 & 257.5 & 264.1 & 299.5 & $315 \cdot 3$ & $38 \mathrm{t}$ \\
\hline 382 & 339.3 & 178.9 & 184.1 & $183 \cdot 2$ & 176.9 & $25 I, 5$ & $258, \mathrm{I}$ & 264.8 & $300 \cdot 3$ & $3 \times 6,1$ & 382 \\
\hline 383 & 340,2 & 179.4 & +84.6 & $183-8$ & 177.5 & 252.2 & 258,8 & 265.5 & 301,1 & 316.9 & 383 \\
\hline 384 & 341,1 & 180.0 & 185.2 & $184-3$ & 178.0 & 252.9 & 259.5 & 266.2 & $30 \times .9$ & 317.8 & 384 \\
\hline 385 & 342,0 & 180.5 & 185,7 & 184,8 & 178.5 & 253,6 & $260 \cdot 2$ & 266.9 & 302.7 & 318.6 & 385 \\
\hline 386 & 342.9 & $18 \pi .0$ & 186.2 & 185.4 & 179.1 & 254.2 & 260.9 & 267.6 & 303.5 & 319.4 & 386 \\
\hline 387 & 343.8 & $18 r .5$ & 186,8 & 185.9 & 179.6 & 254.9 & 261.6 & 268.3 & 304.2 & $320 \cdot 3$ & 387 \\
\hline 388 & 344.6 & 182.0 & 187.3 & 186.4 & 180.1 & 255.6 & 262,3 & 269.0 & 305.0 & $321-1$ & 388 \\
\hline 389 & $345 \cdot 5$ & 182.6 & 187.8 & 787.0 & $\times 80.6$ & 256.3 & 263.0 & 269.8 & 305.8 & 321.9 & 389 \\
\hline 390 & $346-4$ & $x 83.1$ & $+88+4$ & 187,5 & 181.2 & 256,9 & 263,7 & 270.5 & 306.6 & 322.8 & 390 \\
\hline $39 \mathrm{r}$ & $347 \cdot 3$ & $\times 83.6$ & T88.9 & 188.0 & $18 x-7$ & 257.6 & 264.4 & $271-2$ & $307 \cdot 4$ & 323.6 & $39 \mathrm{r}$ \\
\hline 392 & 348,2 & 184,1 & 189.4 & 188.6 & $182 \cdot 3$ & 258.3 & $265 . t$ & 271.9 & 308,2 & $324 \cdot 4$ & 392 \\
\hline 393 & $349 \cdot \mathrm{t}$ & 184.7 & 190.0 & $189 \cdot 1$ & 182.8 & 259.0 & 265.8 & 272.6 & 309.0 & $325 \cdot 2$ & 393 \\
\hline 394 & 350.0 & 185.2 & 190.5 & I 89.7 & 183.3 & 259.6 & 266.5 & 273.3 & 309.8 & 326.1 & 394 \\
\hline
\end{tabular}


MUNSON AND WALKER'S TABLE FOR CALCULATING DEXTROSE, INVERT SUGAR, LACTOSE, AND MALTOSE-(Continued).

[Weights in milligrams.]

\begin{tabular}{|c|c|c|c|c|c|c|c|c|c|c|c|}
\hline \multirow{2}{*}{ 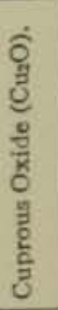 } & \multirow[b]{2}{*}{$\begin{array}{l}\dot{3} \\
\dot{3} \\
\frac{\hat{\alpha}}{0} \\
8\end{array}$} & \multirow[b]{2}{*}{$\begin{array}{l}\frac{g}{2} \\
\text { ğ } \\
\text { x. } \\
\text { a }\end{array}$} & \multirow[b]{2}{*}{$\begin{array}{l}\frac{5}{8} \\
\frac{5}{5} \\
\frac{5}{5} \\
5\end{array}$} & \multicolumn{2}{|c|}{$\begin{array}{l}\text { Invert Sugar } \\
\text { and Sucrose. }\end{array}$} & \multicolumn{3}{|c|}{ Lactose. } & \multicolumn{2}{|c|}{ Maltose. } & \multirow{2}{*}{ 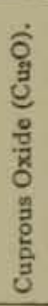 } \\
\hline & & & & 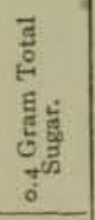 & 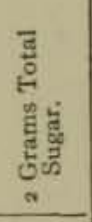 & $\frac{0}{0}$ & 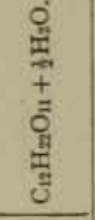 & $\begin{array}{l}0 \\
0 \\
7 \\
+ \\
0 \\
0 \\
\frac{1}{3} \\
\frac{1}{3} \\
0\end{array}$ & 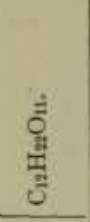 & $\begin{array}{l}0 \\
8 \\
\mathbb{1} \\
+ \\
5 \\
0 \\
\frac{1}{4} \\
\frac{\pi}{0}\end{array}$ & \\
\hline 395 & 350.9 & 185.7 & 191.0 & 190.2 & 183.9 & 260.3 & 267.2 & 274,0 & 310.6 & $326-9$ & 395 \\
\hline 396 & 351.8 & 186.2 & 191.6 & 190.7 & 184.4 & 261,0 & 267,9 & 274.7 & $311=4$ & $327 \cdot 7$ & 396 \\
\hline 397 & 352.6 & 186.8 & 192,1 & 191.3 & $184 \cdot 9$ & 261.7 & 268.6 & $275 \cdot 5$ & 312.1 & 328.6 & 397 \\
\hline 398 & 353.5 & $\begin{array}{l}187,3 \\
87,8\end{array}$ & 192.7 & 191.8 & 185.5 & 262,3 & $269 \cdot 3$ & 276,2 & $312-9$ & 329.4 & 398 \\
\hline 399 & $354 \cdot 4$ & 187.8 & 193.2 & 192,3 & 180.0 & 263,0 & 209.9 & 270.9 & $313 \cdot 7$ & 330.2 & 399. \\
\hline 400 & $335 \cdot 3$ & 188,4 & 193.7 & $192+9$ & 186.5 & 263,7 & 270.6 & 277.6 & $3 \times 4,5$ & 331,1 & 400. \\
\hline 401 & 356.2 & 188.9 & 194.3 & 193.4 & 187.1 & 264.4 & $271-3$ & 278.3 & $3 \times 5-3$ & $331 \cdot 9$ & 401 \\
\hline 402 & $357 \cdot t$ & 189.4 & I 94.8 & 194.0 & 187.6 & 265.0 & 272,0 & 279.0 & 316.1 & $332-7$ & $402=$ \\
\hline 403 & 358.0 & 189.9 & 195.4 & 194.5 & $t 88, t$ & $265 \cdot 7$ & 272.7 & 279.7 & 316.9 & 333.6 & 403 \\
\hline 404 & 358.9 & 190.5 & $195: 9$ & 195,0 & 188.7 & 266,4 & 273.4 & 280.4 & $3 \times 7 \cdot 7$ & $334 \cdot 4$ & 404 \\
\hline 405 & $359 \cdot 7$ & 191.0 & 196.4 & 195.6 & 289.2 & $267 . \mathrm{I}$ & 274.1 & $28 \mathrm{I}, \mathrm{I}$ & $3 \times 8,5$ & 335.2 & 405 \\
\hline 406 & 360.6 & 191.5 & 197.0 & 196.1 & 189.8 & 267.8 & 274.8 & 281.9 & 319.2 & 336.0 & 406 \\
\hline 407 & $361 \cdot 5$ & 192.1 & $197+5$ & 196.7 & 190.3 & 268.4 & 275.5 & $282 \cdot 6$ & 320.0 & 336.9 & 407 \\
\hline 408 & $362 \cdot 4$ & 192.6 & 198.1 & 197.2 & 190.8 & $269 \cdot \mathrm{I}$ & 276,2 & 283.3 & 320.8 & $337 \cdot 7$ & 408 \\
\hline 409 & $363 \cdot 3$ & 193 - 1 & 198.6 & 197.7 & 191.4 & 269.8 & 276.9 & 284,0 & 321.6 & 338.5 & 409 \\
\hline 410 & 364.2 & 193.7 & $199 \cdot 1$ & 198.3 & 191.9 & 270.5 & 277.6 & 284.7 & 322.4 & 339.4 & 410 \\
\hline 411 & 365.1 & 194.2 & 199.7 & 198.8 & 192.5 & $271-2$ & 278.3 & $285 \cdot 4$ & 323.2 & 340.2 & $4 \mathrm{II}$ \\
\hline 412 & 360.0 & 194.7 & 200.2 & 199.4 & 193.0 & 271.8 & 279.0 & 286,2 & 324.0 & 341,0 & 422 \\
\hline 413 & 366.9 & 195,2 & 200.8 & 199.9 & 193.5 & $272: 5$ & 279.7 & 286,9 & 324.8 & 341.9 & 413 \\
\hline 414 & $367 \cdot 7$ & 195.8 & $201: 3$ & 200.5 & $194 \cdot 1$ & 273.2 & 280.4 & 287.6 & 325.6 & 342.7 & 474 \\
\hline 415 & 368.6 & 196.3 & 201,8 & $201 \cdot 0$ & 194.6 & 273.9 & 281,1 & 288.3 & 326.3 & 343.5 & $4 \times 5$ \\
\hline 416 & $369 \cdot 5$ & 196,8 & 202.4 & 201.6 & 195.2 & 274.6 & $28 I, 8$ & 289,0 & $327 \cdot I$ & $344 \cdot 4$ & 416 \\
\hline $\begin{array}{l}417 \\
48\end{array}$ & 370.4 & 797.4 & 202.9 & $202 \cdot 1$ & 195.7 & 275.2 & 282.5 & $289 \cdot 7$ & 327,9 & $345 \times 2$ & 417 \\
\hline 418 & $\begin{array}{l}371 \cdot 3 \\
372 \cdot 2\end{array}$ & 197.9 & 203.5 & 202.6 & $196-2$ & 275.9 & $283 \cdot 2$ & $290 \cdot 4$ & 328.7 & $\begin{array}{l}346,0 \\
346,8\end{array}$ & 418 \\
\hline 419 & 372.2 & 198.4 & 204.0 & 203.2 & t 96.8 & 276.6 & 283.9 & 291.2 & $329 \cdot 5$ & 346.8 & $4: 9$ \\
\hline 420 & 373,1 & 199.0 & 204.6 & $203 \cdot 7$ & 197.3 & 277.3 & 284.6 & $291 \cdot 9$ & 330.3 & $347 \cdot 7$ & 420 \\
\hline 421 & 374.0 & 199.5 & 205,1 & 204.3 & 197.9 & 277.9 & $285 \cdot 3$ & 292.6 & 331.1 & $3.48 \cdot 5$ & 421 \\
\hline 422 & 374.8 & 200,1 & $205 \cdot 7$ & 204.8 & 198.4 & 278.6 & 286.0 & 293.3 & 331.9 & $349 \cdot 3$ & 422 \\
\hline 423 & 375.7 & 200.6 & 206.2 & 205.4 & 198.9 & 279.3 & 286.7 & $294: 0$ & $332 \cdot 7$ & 350.2 & 423 \\
\hline 424 & 376.6 & $20 x, 1$ & $206 \cdot 7$ & 205.9 & $199+5$ & 280.0 & $287 \cdot 4$ & 294.7 & $333 \cdot 4$ & 351.0 & 424 \\
\hline $\begin{array}{l}425 \\
426\end{array}$ & 377.5 & $201 \cdot 7$ & $207 \cdot 3$ & $206 \cdot 5$ & 200.0 & $280-7$ & $288, I$ & $295: 4$ & $334 \cdot 2$ & 351.8 & 425 \\
\hline $\begin{array}{l}426 \\
427\end{array}$ & $\begin{array}{l}378.4 \\
379.3\end{array}$ & 202.2 & 207.8 & 207.0 & 200,6 & $28 \mathrm{t}-3$ & 288.8 & 296,2 & 335.0 & 352,7 & 426 \\
\hline $\begin{array}{l}427 \\
428\end{array}$ & $\begin{array}{l}379 \cdot 3 \\
380 \cdot 2\end{array}$ & 202.8 & 208.4 & 207.6 & 201,1 & 282,0 & $289 \cdot 4$ & 296.9 & 335.8 & $353 \cdot 5$ & 427 \\
\hline $\begin{array}{l}428 \\
429\end{array}$ & $\begin{array}{l}380 \cdot 2 \\
38 \mathrm{r} \cdot \mathrm{t}\end{array}$ & $203 \cdot 3$ & 208.9 & $208-\mathrm{t}$ & $201 \cdot 7$ & $282 \cdot 7$ & $290 \cdot \mathrm{I}$ & 297.6 & 336.6 & $354 \cdot 3$ & 428 \\
\hline 429 & $38 \mathrm{r} \cdot \mathrm{I}$ & 203.8 & $209 \cdot 5$ & $208 \cdot 7$ & $202 \cdot 2$ & $283 \cdot 4$ & 290.8 & 298.3 & $337 \cdot 4$ & $355 \times 1$ & 429 \\
\hline $\begin{array}{l}430 \\
431\end{array}$ & $38 z .0$ & 204,4 & 210.0 & 209.2 & 202.7 & $284-1$ & 291.5 & 299.0 & $33^{8} \cdot 2$ & 356.0 & 430 \\
\hline $\begin{array}{l}431 \\
432\end{array}$ & 382.8 & $204 \cdot 9$ & 210.6 & 209.8 & $203 \cdot 3$ & 284.7 & 292,2 & 299.7 & 339.0 & 356.8 & 431 \\
\hline 432 & 383.7 & $205 \cdot 5$ & 211.1 & 210.3 & 203.8 & $285-4$ & 292.9 & 300,5 & 339.7 & $357 \cdot 6$ & 432 \\
\hline 433 & 384.6 & 206.0 & 211.7 & 210.9 & $204 \cdot 4$ & $286 \cdot i$ & 293.6 & 301,2 & 340,5 & 358.5 & 433 \\
\hline 434 & $385 \cdot 5$ & 206.5 & 212.2 & $2 \mathrm{II} \cdot 4$ & $204 \cdot 9$ & 286.8 & $294 \cdot 3$ & 301.9 & $341+3$ & 359.3 & 434 \\
\hline $\begin{array}{l}435 \\
436\end{array}$ & $386 \cdot 4$ & 207.1 & 212.8 & $212: 0$ & $205 \cdot 5$ & $287 \cdot 5$ & 295.0 & 302.6 & $342, \mathrm{I}$ & 360,1 & 435 \\
\hline 436 & $387 \cdot 3$ & 207,6 & 213.3 & 212.5 & 206.0 & 288,1 & 295.7 & 303.3 & $342 \cdot 9$ & 361,0 & 436 \\
\hline 437 & 388.2 & 208,2 & 213.9 & 213,1 & 206.6 & 288.8 & 296.4 & $304 \cdot 0$ & $343 \cdot 7$ & $36 x \cdot 8$ & 437 \\
\hline 438 & $3^{89} \cdot \mathrm{I}$ & 208.7 & $214 \cdot 4$ & 213.6 & $207 \cdot 1$ & 289.5 & $297 \times 1$ & $304 \cdot 7$ & $344 \cdot 5$ & 362,6 & 438 \\
\hline 439 & 390.0 & $209 \cdot 2$ & 215.0 & $21.4 \cdot 2$ & 207.7 & 290.2 & 297.8 & 305.5 & $345 \cdot 3$ & $363 \cdot 4$ & 439 \\
\hline 440 & 390.8 & 209.8 & 215.5 & 214.7 & 208,2 & 290.9 & 298.5 & 306.2 & 346.1 & $364 \cdot 3$ & 440 \\
\hline $44 \mathrm{I}$ & 391.7 & 210.3 & 216,1 & 215.3 & 208.8 & 291.5 & 299.2 & 306,9 & 346.8 & 365,1 & 441 \\
\hline 442 & 392.6 & 210.9 & 216,6 & 215.8 & $209 \cdot 3$ & 202.2 & $299 \cdot 9$ & 307.6 & 347.6 & 365,9 & 442 \\
\hline 443 & $393 \cdot 5$ & 211,4 & 217.2 & 276.4 & 209.9 & 292.9 & 300,6 & 308.3 & 348.4 & 366.8 & 443 \\
\hline 444 & $394 \cdot 4$ & 212.0 & 217.8 & 216.9 & 210.4 & 293.6 & $301 \cdot 3$ & 309.0 & $349 \cdot 2$ & 567.6 & 444 \\
\hline 445 & $395 \cdot 3$ & $2 \times 2.5$ & $2 \pm 8.3$ & 217.5 & $211: 0$ & 294,2 & 302.0 & 309.7 & 350.0 & 368,4 & 445 \\
\hline 446 & 396.2 & 213,1 & $2 \times 8.9$ & 218.0 & 211.5 & 294.9 & 302.7 & 310.5 & 350.8 & 369.3 & 446 \\
\hline 447 & 397,1 & 213.6 & 219.4 & 218.6 & $2 \mathrm{I} 2, \mathrm{I}$ & 295.6 & $303 \cdot 4$ & $3 I I .2$ & 351.6 & 370.1 & 447 \\
\hline 448 & $397 \cdot 9$ & 2141 & 220,0 & 219.1 & 212.6 & 296.3 & 304.1 & $3 r 1.9$ & 352.4 & 370.9 & $44^{8}$ \\
\hline 449 & 398.8 & 214.7 & 220.5 & 219.7 & 213.2 & 297.0 & 304,8 & 312,8 & 353.2 & 371.7 & 449 \\
\hline
\end{tabular}


MUNSON AND WALKER'S TABLE FOR CALCULATING DEXTROSE, INVERT SUGAR, LACTOSE, AND MALTOSE-(Continued).

[Weights in milligrams,]

\begin{tabular}{|c|c|c|c|c|c|c|c|c|c|c|c|}
\hline \multirow{2}{*}{ 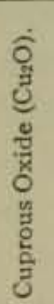 } & \multirow[b]{2}{*}{$\begin{array}{l}\dot{3} \\
\dot{3} \\
\dot{8} \\
\frac{\hat{\theta}}{3}\end{array}$} & \multirow[b]{2}{*}{$\frac{\text { है }}{\frac{5}{6}}$} & \multirow[b]{2}{*}{ 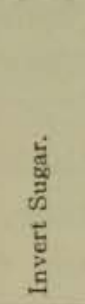 } & \multicolumn{2}{|c|}{$\begin{array}{l}\text { Invert Sugar } \\
\text { and Sucrose. }\end{array}$} & \multicolumn{3}{|c|}{ Lactose. } & \multicolumn{2}{|c|}{ Maltose. } & \multirow{2}{*}{ 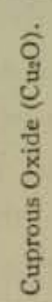 } \\
\hline & & & & 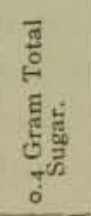 & 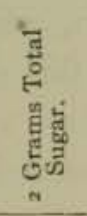 & $\frac{\stackrel{3}{\bar{H}}}{\frac{\mathrm{H}}{\mathrm{I}}}$ & 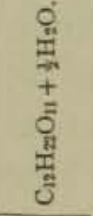 & $\begin{array}{l}0 \\
\vdots \\
+ \\
\vdots \\
0 \\
\frac{1}{1} \\
\frac{1}{3}\end{array}$ & $\frac{\dot{0}}{\frac{5}{5}}$ & 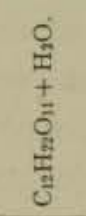 & \\
\hline $45^{\circ}$ & 399.7 & 215.2 & 221,1 & 220.2 & 213.7 & 297,6 & $305 \cdot 5$ & 313.3 & 353.9 & 372,6 & 450 \\
\hline 451 & 400.6 & 215,8 & 221,6 & 220.8 & $214 \cdot 3$ & $298: 3$ & 306,2 & $3 \mathrm{I} 4.0$ & 354.7 & 373.4 & 451 \\
\hline 452 & $40 x \cdot 5$ & 216,3 & 222.2 & 221.4 & 214.8 & 299.0 . & 306.9 & 314.7 & 355.5 & 374.2 & 452 \\
\hline 453 & 402.4 & 216.9 & 222.8 & 221.9 & 215.4 & $299.7^{\circ}$ & 307.6 & 315.5 & 356.3 & $375 \cdot 1$ & 453 \\
\hline 454 & $403-3$ & 217.4 & $223 \cdot 3$ & $222 \cdot 5$ & 215.9 & $300 \cdot 4$ & $308 \cdot 3$ & 316.2 & 357,1 & $375 \cdot 9$ & 454 \\
\hline 455 & $404 \cdot 2$ & 218.0 & $223 \cdot 9$ & 223.0 & 216.5 & 301.1 & 309.0 & 316.9 & 357.9 & 376.7 & 455 \\
\hline 456 & 405.1 & 218.5 & 224.4 & 223.6 & $217=0$ & 301,7 & $309 \cdot 7$ & 317.6 & 358.7 & 377.6 & 456 \\
\hline 457 & 405.9 & 279.1 & 225.0 & $224 \cdot 1$ & 217.6 & 302,4 & 310.4 & 318,3 & 359.5 & 378.4 & 457 \\
\hline 458 & 406.8 & 219.6 & 225.5 & 224.7 & 218,1 & 303.1 & 311,1 & 319.0 & 360.3 & 379.2 & 458 \\
\hline 459 & $407 \cdot 7$ & 220.2 & 220.1 & 225,3 & $2+8.7$ & 303.8 & 311,8 & 319.8 & 361.0 & 380.0 & 459 \\
\hline 460 & 408.6 & 220.7 & 226.7 & 225.8 & 219.2 & 304.5 & 312,5 & 320.5 & 361,8 & 380,9 & 460 \\
\hline $46 r$ & 409.5 & $22 \mathrm{I} \cdot 3$ & 227.2 & 220.4 & 219.8 & 305,1 & 313,2 & 321,2 & 362.6 & $38 x \cdot 7$ & 461 \\
\hline 462 & 410.4 & 221.8 & 227,8 & 226.9 & -20.3 & 305.8 & $313=9$ & 321,9 & $363 \cdot 4$ & 382.5 & 462 \\
\hline 463 & $411 \cdot 3$ & $222+4$ & 228.3 & 227.5 & 2209 & 306.5 & $3+4.0$ & 322,6 & 364.2 & 383.4 & 463 \\
\hline 464 & 412.2 & 222.9 & 228,9 & 228.1 & 221.4 & 307.2 & 315,3 & 323.4 & 365.0 & 384.2 & 464 \\
\hline 465 & 413.0 & $223 \cdot 5$ & 229.5 & 228.6 & 222.0 & $307 \cdot 9$ & 316.0 & 324.1 & 365.8 & 385.0 & 465 \\
\hline 466 & 413.9 & 224.0 & 230.0 & 229.2 & 222.5 & 308.6 & 316.7 & 324.8 & 366.6 & 385.9 & 466 \\
\hline 467 & 414.8 & 224.6 & 230,6 & 229.7 & 223,1 & 309.2 & 317.4 & $325-5$ & $367 \cdot 3$ & 386,7 & 467 \\
\hline 468 & $4 \times 5.7$ & 225,1 & $231 \cdot 2$ & 230.3 & $223 \cdot 7$ & 309.9 & 318 i & 326.2 & 368,1 & 387.5 & 468 \\
\hline 469 & $4+6.6$ & 225.7 & 231.7 & 230.9 & 224.2 & 310.6 & $3+8.8$ & 326.9 & 368.9 & 388.3 & 469 \\
\hline 470 & 417.5 & 226.2 & 232.3 & 231.4 & 224.8 & 311.3 & 319.5 & 327.7 & 369.7 & $389 \cdot 2$ & 470 \\
\hline 471 & $4+8.4$ & 226.8 & 232.8 & 232,0 & 225.3 & 312.0 & 320,2 & 328.4 & 370.5 & 390.0 & 471 \\
\hline 472 & 419.3 & 227.4 & $233 \cdot 4$ & $232 \cdot 5$ & 225.9 & 312.6 & 320.9 & 329.1 & 371,3 & 390.8 & 472 \\
\hline 473 & 420.2 & 227.9 & 234,0 & $233 \cdot \mathrm{I}$ & 226.4 & 313.3 & 321,6 & 329.8 & 372,1 & 391.7 & 473 \\
\hline 474 & 421.0 & 228.5 & 234.5 & 233.7 & 227.0 & 314.0 & 322.3 & 330,5 & 372.9 & 392.5 & 474 \\
\hline 475 & $42 \times \cdot 9$ & 229.0 & $235 \cdot I$ & 234.2 & 227.6 & 314.7 & 323,0 & $331-3$ & 373.7 & $393 \cdot 3$ & 475 \\
\hline 476 & 422.8 & 229.6 & 235,7 & 234.8 & 228,1 & 315.4 & 323.7 & 332.0 & 374.4 & 394.2 & 476 \\
\hline 477 & 423.7 & 230.1 & $236-2$ & 235.4 & 228.7 & 316,1 & $324 \cdot 4$ & 332.7 & 375.2 & 395.0 & 477 \\
\hline 478 & 424.6 & 230.7 & 236.8 & 235.9 & 229.2 & 316.7 & $325, \mathrm{I}$ & 333.4 & 376.0 & 395.8 & 478 \\
\hline 479 & 425.5 & $23 t \cdot 3$ & 237.4 & 236.5 & 229.8 & 317.4 & 325.8 & 334.1 & 376.8 & 396.6 & 479 \\
\hline 480 & $426: 4$ & $23 t, 8$ & 237.9 & 237.1 & 230.3 & $318, t$ & 326.5 & 334.8 & 377.6 & 397.5 & 480 \\
\hline 481 & $427-3$ & 232.4 & 238.5 & 237.6 & $230-9$ & 318.8 & 3272 & 335.6 & 378.4 & 398,3 & 481 \\
\hline 482 & 428.1 & 232.9 & $239-\pi$ & 238.2 & $231-5$ & 319.5 & 327.9 & 336.3 & 379.2 & $399 . \mathrm{I}$ & 482 \\
\hline 483 & 429,0 & 233.5 & 239.6 & 238,8 & 232.0 & 320.1 & 328,6 & 337.0 & 380.0 & 400.0 & 483 \\
\hline 484 & 429.9 & 234.1 & 240.2 & $239 \cdot 3$ & $232-6$ & 320,8 & $329 \cdot 3$ & $337 \cdot 7$ & 380.7 & 400.8 & 484 \\
\hline 485 & 430.8 & 234.6 & 240.8 & 239.9 & 233.2 & 321.5 & 330.0 & 338.4 & $38 x \cdot 5$ & $40 x-6$ & 485 \\
\hline 486 & $431 \cdot 7$ & $235 \cdot 2$ & $241 \cdot 4$ & 240.5 & $233 \cdot 7$ & 322.2 & 30.7 & $339 \cdot \mathrm{I}$ & $382 \cdot 3$ & $402 \cdot 4$ & 486 \\
\hline 487 & 432,6 & 235.7 & 241,9 & $241 \cdot 0$ & $234: 3$ & 322.9 & $33 I-4$ & 339.9 & $383 . x$ & $403 \cdot 3$ & 487 \\
\hline 488 & 433.5 & 266.3 & $2.42 \cdot 5$ & 241.6 & 234.8 & 323,6 & 332,1 & 340.6 & $383 \cdot 9$ & $404 \cdot 1$ & 488 \\
\hline 489 & $434 \cdot 4$ & 236.9 & 243.1 & 2.42 .2 & $235 \cdot 4$ & 324.2 & 332.8 & $34^{1}$ - 3 & $384 \cdot 7$ & $404 \cdot 9$ & 489 \\
\hline 490 & $435 \cdot 3$ & 237.4 & 243.6 & 242.7 & 236.0 & 3249 & 333.5 & 342.0 & $3^{85} \cdot 5$ & 405,8 & 490 \\
\hline
\end{tabular}


Allihn's Method for the Determination of Dextrose.*-The solutions used are those described on page 591 , except that 125 grams of potassium hydroxide are used in place of 50 grams of sodium hydroxide in preparing the alkaline tartrate solution. Place $30 \mathrm{cc}$. of Fehling's copper solution, $30 \mathrm{cc}$. of the alkaline tartrate solution, and $60 \mathrm{cc}$. of water in a beaker and heat to boiling. Add $25 \mathrm{cc}$. of the sugar solution, which must be so prepared as not to contain more than $\mathrm{I} \%$ dextrose, and boil over the flame for two minutes. Filter immediately without diluting through a Gooch srucible containing a layer of asbestos fiber, prepared as described on page 594, and wash thoroughly with hot water, using reduced pressure. Transfer the asbestos fiber and the adhering cuprous oxide by means of a glass rod to a beaker and rinse the crucible with about $30 \mathrm{cc}$. of a boiling mixture of dilute sulphuric and nitric acids containing $6_{5} \mathrm{cc}$. of sulphuric acid (specific gravity 1.84) and 50 cc. of nitric acid (specific gravity I.42) per liter. Heat and agitate till the solution is complete, then filter into a scrupulously clean, tared platinum dish of roo-cc. capacity, taking care to wash out all the copper solution from the filter into the dish. Deposit the copper electrolytically in the platinum dish and weigh. Determine the dextrose from Allihn's table. p. 609.

Or, the metallic copper may be calculated by means of the factor 0.7989 from the cupric oxide obtained as in Defren's method (p. 594) and Allihn's table used.

Or, the cuprous oxide as directly obtained by either Allihn's or Defren's method may be washed with alcohol and ether, dried for twenty minutes at $100^{\circ} \mathrm{C}$., and weighed, its equivalent in dextrose being ascertained from Allihn's table.

Electrolytic Apparatus. - The author has devised the apparatus shown in Fig. I ro for the electrolytic deposition of copper in sugar analysis and for other work of like nature. A, Fig. IIO, is a hard-rubber plate $50 \mathrm{~cm}$. long and $25 \mathrm{~cm}$. wide provided with four insulated metal binding posts, $B$, each carrying at the top a thumb screw by which a coiled platinum wire electrode, $C$, may be attached. In front of each post is a copper plate about $4 \mathrm{~cm}$. square covered with thin platinum foil, $P$, which is bent around the edges of the copper plate and so held in place, the copper plate being screwed to the rubber from beneath. On the square platinumcovered plate is set the platinum evaporating-dish which holds the solution from which the copper is to be deposited, the inside of the dish forming the cathode, while the electrode $C$, dipping below the surface of the solution, forms the anode. In front of each platinum-covered plate

\footnotetext{
* Jour. für praktische Chemie. 22 (1880), p. 46.
} 
ALLIHN'S TABLE FOR THE DETERMINATION OF DEXTROSE.*

\begin{tabular}{|c|c|c|c|c|c|c|c|c|c|c|c|}
\hline $\begin{array}{l}\text { Milli- } \\
\text { grams } \\
\text { of } \\
\text { Cop- } \\
\text { per. }\end{array}$ & $\begin{array}{l}\text { Milli- } \\
\text { grams } \\
\text { of Cu- } \\
\text { prous } \\
\text { Oxide. }\end{array}$ & $\begin{array}{l}\text { Mili- } \\
\text { grams } \\
\text { of } \\
\text { Dex- } \\
\text { trose. }\end{array}$ & $\begin{array}{c}\text { Milli- } \\
\text { grams } \\
\text { of } \\
\text { Cop- } \\
\text { per. }\end{array}$ & $\begin{array}{l}\text { Milli- } \\
\text { grams } \\
\text { of Cu- } \\
\text { prous } \\
\text { Oxide. }\end{array}$ & $\begin{array}{l}\text { Milli- } \\
\text { grams } \\
\text { of } \\
\text { Dex- } \\
\text { trose. }\end{array}$ & $\begin{array}{c}\text { Milli- } \\
\text { grams } \\
\text { of } \\
\text { Cop- } \\
\text { per. }\end{array}$ & $\begin{array}{l}\text { Milli- } \\
\text { grams } \\
\text { of Cu- } \\
\text { prous } \\
\text { Oxide. }\end{array}$ & $\begin{array}{l}\text { Milli- } \\
\text { grams } \\
\text { of } \\
\text { Dex- } \\
\text { trose. }\end{array}$ & $\begin{array}{l}\text { Milli- } \\
\text { grams } \\
\text { of } \\
\text { Cop- } \\
\text { per. }\end{array}$ & $\begin{array}{l}\text { Milli- } \\
\text { grams } \\
\text { of Cu- } \\
\text { prous } \\
\text { Oxide. }\end{array}$ & $\begin{array}{l}\text { Milli- } \\
\text { grams } \\
\text { of } \\
\text { Dex- } \\
\text { truse. }\end{array}$ \\
\hline 11 & 12.4 & 6.6 & 76 & 85,6 & 38.8 & 141 & 158.7 & 71.8 & 206 & 231.9 & 105.8 \\
\hline 12 & 13.5 & $7 . t$ & 77 & 86.7 & 39.3 & 142 & 150.9 & 72.3 & 207 & 233,0 & 106.3 \\
\hline 13 & 14.6 & 7.6 & 78 & 87.8 & 39.8 & 143 & 161.0 & 72.9 & 208 & 234.2 & 106.8 \\
\hline 14 & 15.8 & 81 & 70 & 88.9 & $40 \cdot 3$ & 144 & 162,1 & $73 \cdot 4$ & 209 & $235 \cdot 3$ & 107.4 \\
\hline 15 & $\times 6.9$ & 8.6 & So & $90, x$ & 40.8 & 145 & 163.2 & 73.9 & 210 & 236.4 & 107.9 \\
\hline 16 & 18.0 & 9.0 & $8 \mathrm{I}$ & 01.2 & $4 \pi \cdot 3$ & 146 & I 64.4 & $74 \cdot 4$ & $2 \mathrm{rI}$ & $237 \cdot 6$ & 108.4 \\
\hline 17 & 10.1 & 9.5 & 82 & $92 \cdot 3$ & $4 \pi .8$ & 147 & 265.5 & 74.0 & 212 & 238.7 & 109.0 \\
\hline 18 & 20.3 & 10.0 & 83 & $93 \cdot 4$ & $42 \cdot 3$ & 148 & 166.6 & $75 \cdot 5$ & 213 & 239.8 & 100.5 \\
\hline 19 & 21.4 & ro. 5 & 84 & 94.6 & $42: 8$ & 149 & $167 \times 7$ & 76.0 & 214 & $240 \cdot 9$ & $1 \times 0,0$ \\
\hline 20 & 22.3 & $1 x, 0$ & 85 & $95 \cdot 7$ & $43 \cdot 4$ & 150 & 168.9 & 76.5 & 215 & 242.1 & 110.6 \\
\hline 21 & 23.6 & $\mathrm{Ix} \cdot 5$ & 86 & 96.8 & 43.9 & $x 51$ & 170,0 & 77.0 & 216 & 243,2 & 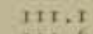 \\
\hline 22 & 24.8 & 12.0 & 87 & 97.9 & $44: 4$ & 152 & 171,1 & $77 \cdot 5$ & $2 i 7$ & 244,3 & 113.6 \\
\hline 23 & 25.9 & 12.5 & 88 & $99, x$ & 44.9 & 153 & 172.3 & 78.1 & 218 & $2.45 \cdot 4$ & $I X 2 . I$ \\
\hline 24 & $27 \cdot 0$ & 13.0 & 89 & 100.2 & $45 \cdot 4$ & 154 & 173.4 & 78.6 & 219 & 246.6 & $1 x 2.7$ \\
\hline 25 & 28,1 & 13.5 & 90 & 101.3 & $45 \cdot 9$ & 155 & 174.5 & 79.1 & 220 & 247.7 & 113.2 \\
\hline 26 & $29 \cdot 3$ & 14.0 & 91 & 102.4 & $4^{6} \cdot 4$ & 156 & $x 75.6$ & 70.6 & 221 & 248.7 & 113.7 \\
\hline 27 & 20.4 & 14.5 & 92 & 103.6 & 46,9 & 157 & 176.8 & 80.1 & 222 & 249.9 & 214.3 \\
\hline 28 & $3 x \cdot 5$ & 15.0 & 93 & 104.7 & $47 \times 4$ & 158 & 177.9 & 80.7 & 223 & $251=0$ & 114.8 \\
\hline 29 & $32 \cdot 7$ & 15.5 & 94 & 105.8 & 47.9 & 159 & 179.0 & $8 t, 2$ & 224 & $25^{2} \cdot 4$ & 115.3 \\
\hline 30 & 33.8 & 16.0 & 95 & 107,0 & $4^{8} \cdot 4$ & 160 & 180,1 & 81.7 & 225 & $253 \cdot 3$ & 175.9 \\
\hline $3 t$ & $34 \cdot 9$ & 16.5 & 06 & $x 08, x$ & 48.9 & $16 t$ & $18 t, 3$ & 82.2 & 226 & 254.4 & 176.4 \\
\hline 32 & 36.0 & 17.0 & 97 & 109.2 & $49-4$ & 162 & 182.4 & 82,7 & 227 & 255.6 & $1 \times 6.9$ \\
\hline 33 & $37 \cdot 2$ & 17.5 & $9^{8}$ & $1 \times 0.3$ & 40.9 & 103. & 183.5 & 83,3 & 228 & 256.7 & 177.4 \\
\hline 34 & 38.3 & 18.0 & 99 & 111.5 & 50.4 & $x 64$ & 184.6 & $83: 8$ & 229 & 257.8 & $1+8,0$ \\
\hline 35 & $30 \cdot 4$ & 18.5 & 100 & 112.6 & 50.9 & 165 & 185.8 & $84 \cdot 3$ & 230 & 258.9 & 118.5 \\
\hline 36 & $40 \cdot 5$ & 18.0 & rot & $x+3.7$ & $5 r-4$ & 166 & 186.0 & 84.8 & 231 & $260, t$ & 319.0 \\
\hline 37 & $41 \cdot 7$ & 10.4 & $x 02$ & $1 \times 4.8$ & $5 x-9$ & 167 & 188.0 & 85.3 & 232 & $26 x-2$ & $x \times 0.6$ \\
\hline 38 & 42.8 & 10.0 & 103 & $x+6.0$ & $52 \cdot 4$ & 168 & $189 \cdot r$ & 85.9 & 233 & $262-3$ & 120,1 \\
\hline 39 & 43.9 & 20.4 & 104 & $x+7, x$ & 52.9 & 160 & $x 90,3$ & 86.4 & 254 & $263: 4$ & 120.7 \\
\hline 40 & 45.0 & 20.9 & 105 & 118.2 & 53.5 & 170 & 191.4 & 86.9 & 235 & $26+6$ & $121: 2$ \\
\hline $4 \pi$ & 46.2 & $25 \cdot 4$ & 106 & 119.3 & 54,0 & 171 & to2, 5 & 87,4 & 236 & 265.7 & 128,7 \\
\hline 42 & $47 \cdot 3$ & $2 x, 9$ & 107 & 120.5 & $54 \cdot 5$ & $x>2$ & 103.6 & 87.0 & 237 & 266.8 & 122.3 \\
\hline 43 & 48.4 & 22.4 & 108 & 121.6 & 55.0 & 173 & 194.8 & 88,5 & 238 & 268,0 & 122.8 \\
\hline 44 & $49 \cdot 5$ & 22.9 & 109 & 122.7 & 55.5 & 174 & 105.9 & 89,0 & 230 & 269.1 & 123.4 \\
\hline 45 & 50.7 & $23 \cdot 4$ & I 10 & 1.23 .8 & 56.0 & 175 & 107.0 & $89 \cdot 5$ & 240 & 270.2 & 123.9 \\
\hline 46 & $5 \pi .8$ & 23.0 & 111 & 125.0 & 56.5 & 176 & $t 98, t$ & 90.0 & 241 & 271.3 & 124.4 \\
\hline 47 & 52.9 & $24 \cdot 4$ & 112 & $126 . t$ & $57: 0$ & 177 & 190.3 & 90.5 & 242 & $272 \cdot 5$ & 125.0 \\
\hline 48 & 54.0 & 24.9 & 113 & 127,2 & 57.5 & 178 & $200 \cdot 4$ & $9 \mathrm{r}+\mathrm{x}$ & 243 & 273.6 & $125 \cdot 5$ \\
\hline 49 & $55: 2$ & $25 \cdot 4$ & 114 & r.28.3 & 58.0 & 170 & $201-5$ & $9 x-6$ & 244 & 274,7 & 126.0 \\
\hline so & 56.3 & 25.9 & 115 & 129.6 & 58.6 & 180 & 202.6 & $92 \cdot x$ & 245 & 275.8 & 126.6 \\
\hline 51 & $57 \cdot 4$ & 26.4 & 116 & 130.6 & 59.1 & 181 & 203.8 & 92,6 & 246 & 277.0 & 127.1 \\
\hline 52 & 58.5 & 26.9 & 117 & $131 \cdot 7$ & 59.6 & 182 & 204.9 & 93.1 & 247 & 278.1 & 127.6 \\
\hline 53 & $59 \cdot 7$ & $27 \cdot 4$ & $1 \times 8$ & 132.8 & $60 \cdot 1$ & 183 & 206,0 & 93.7 & 248 & $279 \cdot 2$ & 128.1 \\
\hline 54 & 60.8 & $27-9$ & 119 & 134.0 & 60.6 & 184 & 207.1 & $04 \cdot 2$ & 249 & $280 \cdot 3$ & 128.7 \\
\hline 55 & $6 x \cdot 9$ & 28.4 & 120 & $135 . \mathrm{r}$ & 61.1 & 135 & 208.3 & $94 \cdot 7$ & 250 & $281-5$ & 139.2 \\
\hline 56 & 63.0 & 28.8 & 121 & 136.2 & $6 r \cdot 6$ & 186 & $200 \cdot 4$ & 95,2 & 251 & 282.6 & $129 \cdot 7$ \\
\hline 57 & $64 \cdot 2$ & $29 \cdot 3$ & 122 & 137.4 & 62.1 & 187 & 210.5 & $95 \cdot 7$ & 252 & $283+7$ & 130.3 \\
\hline 58 & $65 \cdot 3$ & 29.8 & 123 & $x 38.3$ & 62.6 & 188 & 211.7 & $96 \cdot 3$ & 253 & 284.8 & 130.8 \\
\hline 50 & 66.4 & $30 \cdot 3$ & $x 24$ & 139.6 & $63 \cdot r$ & 189 & 212.8 & 96.8 & 254 & 286.0 & $13 x-4$ \\
\hline 60 & 67.6 & 30.8 & 125 & 740.7 & $63 \cdot 7$ & 190 & $2 \times 3.9$ & $97 \cdot 3$ & 255 & $287-1$ & 131.9 \\
\hline $6 x$ & 68.7 & $3 x=3$ & 126 & $x 4 t .9$ & $64 \cdot 2$ & $19 \pi$ & 215.0 & 97.8 & 256 & 288,2 & 132,4 \\
\hline 62 & 60.8 & 31.8 & 127 & $x+3.0$ & $64 \cdot 7$ & 102 & $2 \times 6,2$ & $98 \cdot 4$ & 257 & $289-3$ & 133.0 \\
\hline 63 & 70.9 & 32.3 & 128 & 1.44 .1 & 65.2 & 19.3 & $2 \times 7 \cdot 3$ & 98,9 & 258 & 200.5 & 133,5 \\
\hline 64 & 72.1 & 32.8 & $\times 29$ & 145.2 & $65 \cdot 7$ & 194 & $2 \times 8.4$ & $99 \cdot 4$ & 250 & $20 \pi, 6$ & $134-1$ \\
\hline 65 & 73.2 & $33 \cdot 3$ & 130 & $1 \times 46.4$ & 66.2 & 195 & $2 \times 9.5$ & 100,0 & 260 & 292.7 & $\times 34,6$ \\
\hline 66 & $74 \cdot 3$ & 33.8 & $13 \mathrm{I}$ & $1.47 \cdot 3$ & 66.7 & 106 & 220.7 & 100.5 & 261 & 293.8 & 135.1 \\
\hline 67 & $75 \cdot 4$ & 34.3 & 132 & 148.6 & $67 \cdot 2$ & 197 & $22 x-8$ & 101.0 & 262 & 295.0 & $135 \times 7$ \\
\hline 68 & 76.6 & 34,8 & 133 & 149.7 & $67 \cdot 7$ & 108 & 222.9 & $t 0 t, 5$ & 263 & 206.1 & 136.2 \\
\hline 69 & 77.7 & $35 \cdot 3$ & I 34 & 150.9 & 68.2 & 190 & 224.0 & 102.0 & 264 & $207-2$ & 136,8 \\
\hline 70 & 78.8 & 35.8 & 135 & 152.0 & 68.8 & 200 & 225.2 & 102.6 & 265 & 298.3 & 137.3 \\
\hline $7 \mathrm{r}$ & 70.9 & $36 \cdot 3$ & 136 & $153, x$ & $60 \cdot 3$ & 201 & 226.3 & 103.1 & 266 & $299-5$ & 137,8 \\
\hline 72 & $81, t$ & 36.8 & 137 & 154.2 & 60.8 & 202 & $227 \cdot 4$ & 103.7 & 267 & 300.6 & 138.4 \\
\hline 73 & 82.2 & $37 \cdot 3$ & 138 & 155.4 & $70 \cdot 3$ & 203 & 228.5 & 104,2 & 268 & 301.7 & 138,9 \\
\hline 74 & $83 \cdot 3$ & 37.8 & 130 & 150.5 & 70.8 & 204 & 229.7 & 104.7 & 269 & 302.8 & 130.5 \\
\hline 75 & 84.4 & 38.3 & 140 & 157.6 & 71,3 & 205 & 230.8 & 105.3 & 270 & $304: 0$ & 140,0 \\
\hline
\end{tabular}

* U. S. Dept. of Agric.. Bur. of Chem.. But. 65.p 243 
ALLIHN'S TABLE FOR THE DETERMLNATION OF DEXTROSE-(Continued).

\begin{tabular}{|c|c|c|c|c|c|c|c|c|c|c|c|}
\hline $\begin{array}{c}\text { Milli- } \\
\text { grams } \\
\text { of } \\
\text { Cop- } \\
\text { per. }\end{array}$ & $\begin{array}{l}\text { Milli- } \\
\text { grams } \\
\text { of Cu- } \\
\text { prous } \\
\text { Oxide. }\end{array}$ & $\begin{array}{l}\text { Milli- } \\
\text { grams } \\
\text { of } \\
\text { Dex- } \\
\text { trose. }\end{array}$ & $\begin{array}{l}\text { Milli- } \\
\text { grams } \\
\text { of } \\
\text { Cop- } \\
\text { per. }\end{array}$ & $\begin{array}{l}\text { Milli- } \\
\text { grams } \\
\text { of Cu- } \\
\text { prous } \\
\text { Oxide. }\end{array}$ & $\begin{array}{l}\text { Milli- } \\
\text { grams } \\
\text { of } \\
\text { Dex- } \\
\text { trose. }\end{array}$ & $\begin{array}{l}\text { Milli- } \\
\text { grams } \\
\text { of } \\
\text { Cop- } \\
\text { per. }\end{array}$ & $\begin{array}{l}\text { Milli- } \\
\text { grams } \\
\text { of Cu- } \\
\text { prous } \\
\text { Oxide. }\end{array}$ & $\begin{array}{l}\text { Milli- } \\
\text { grams } \\
\text { of } \\
\text { Dex- } \\
\text { trose. }\end{array}$ & $\begin{array}{l}\text { Milli- } \\
\text { grams } \\
\text { of } \\
\text { Cop- } \\
\text { per. }\end{array}$ & $\begin{array}{l}\text { Milli- } \\
\text { grams } \\
\text { of Cu- } \\
\text { prous } \\
\text { Oxide. }\end{array}$ & $\begin{array}{l}\text { Milli- } \\
\text { grams } \\
\text { of } \\
\text { Dex- } \\
\text { trose. }\end{array}$ \\
\hline 271 & 305.1 & 140,6 & 321 & 361,4 & 168,1 & 378 & $417 \cdot 7$ & 206.3 & $42 \pi$ & 474.0 & $225 . \mathrm{I}$ \\
\hline 272 & 306.2 & 141,1 & 322 & 362,5 & 168.6 & 372 & 418.8 & 106.8 & 422 & 475.6 & 225.7 \\
\hline 273 & $307 \cdot 3$ & $141 \cdot 7$ & 323 & 363,7 & 169.2 & 373 & 420.0 & $197: 4$ & 423 & 476.2 & $226 \cdot 3$ \\
\hline 274 & 308.5 & 142.2 & 324 & 364.8 & 169.7 & 374 & $42 t, r$ & 108,0 & 424 & $477 \cdot 4$ & 226.9 \\
\hline 275 & 309.6 & 142.8 & 325 & $365 \cdot 9$ & 170.3 & 375 & $422-2$ & 198.6 & 425 & 478.5 & 227.5 \\
\hline 276 & 310.7 & 1.43 .3 & 326 & 367,0 & 170.9 & 376 & $423-3$ & $\mathrm{~T} 00 . \mathrm{t}$ & 426 & 479.6 & 228.0 \\
\hline 277 & 311.9 & 143.9 & 327 & 368,2 & $17 x, 4$ & 377 & $424 \cdot 5$ & 199.7 & 427 & 480.7 & 228,6 \\
\hline 278 & 313.0 & $7.4: 4$ & 328 & $300-3$ & 172.0 & 378 & 425.6 & $200 \cdot 3$ & 428 & $48 x-9$ & 229.2 \\
\hline 279 & 314.1 & 145.0 & 320 & 370.4 & 172.5 & 379 & 426.7 & $200 . \overline{8}$ & 429 & $483=0$ & 229.8 \\
\hline $280^{\circ}$ & 315.2 & $\mathrm{I} 45 \cdot 5$ & 330 & $37 t .5$ & 173.1 & 380 & 427.8 & 201.4 & 430 & $484-1$ & 230.4 \\
\hline $28 \mathrm{r}^{-}$ & $3 \times 6.4$ & $\mathrm{t} 46 . \mathrm{t}$ & $33 x$ & 372.7 & 173.7 & 381 & 429.0 & 202.0 & $43 x$ & 485,3 & $23 t+0$ \\
\hline 282 & 317.5 & $\mathrm{x} 46.6$ & 332 & 373.8 & $174 \cdot 2$ & 382 & $430 . t$ & 202.5 & 432 & 486.4 & 231,6 \\
\hline 283 & 318.6 & $147 \cdot 2$ & 333 & 374.9 & 174.8 & 383 & $43 x=2$ & $293, x$ & 433 & 487.5 & 232,2 \\
\hline 284 & 359.7 & $147-7$ & 334 & 376.0 & 175.3 & 384 & 432,3 & 203.7 & 434 & 488.6 & 232,8 \\
\hline 285 & 320.9 & $x+48+3$ & 335 & 377.2 & 175.9 & 385 & 433.5 & $204 \cdot 3$ & 435 & $489 \cdot 7$ & 233.4 \\
\hline 286 & 322.0 & 148.8 & 336 & 378.3 & $\pm 76,5$ & 386 & $434-6$ & 204.8 & 436 & 490.9 & 233.9 \\
\hline 287 & 323.1 & I $49-4$ & 337 & 379.4 & 177.0 & 387 & $435 \cdot 7$ & 205.4 & 437 & 492.0 & 234.5 \\
\hline 288 & $324 \cdot 2$ & 149.9 & 338 & 380.5 & 177.6 & 388 & 436.8 & 206.0 & 438 & $493 . I$ & $235, x$ \\
\hline 280 & $325: 4$ & 150.5 & 330 & 381,7 & $178 . x$ & 380 & 438.0 & 206.5 & 439 & $404 \cdot 3$ & $235 \cdot 7$ \\
\hline 290 & 326.5 & ISI.0 & 340 & 382,8 & 178.7 & $39^{\circ}$ & $439: 1$ & 207.1 & $44^{\circ}$ & $495 \cdot 4$ & 236.3 \\
\hline 291 & $327 \cdot 4$ & t5 $x, 6$ & 341 & $383 \cdot 9$ & $179: 3$ & $39 t$ & 440.2 & $207 \cdot 7$ & $4+1$ & 496.5 & 236.9 \\
\hline 202 & 328.7 & I $52, \mathrm{I}$ & 342 & 385,0 & 179.8 & 302 & $44 x-3$ & $208 \cdot 3$ & 442 & $497 \cdot 6$ & 237.5 \\
\hline 293 & 329.9 & 152.7 & 343 & 386.2 & 180.4 & 303 & $44^{2}-4$ & 208.3 & 443 & 408.8 & 238.1 \\
\hline 294 & $33 t .0$ & 153,2 & 344 & $387 \cdot 3$ & 180,9 & 304 & 443.6 & $209 \cdot 4$ & 444 & $409 \cdot 9$ & 238.7 \\
\hline 295 & 332.7 & 153.8 & 345 & 388,4 & $18 \pi \cdot 5$ & 395 & $444 \cdot 7$ & 210,0 & 445 & $501: 0$ & 239.3 \\
\hline 206 & 333.3 & $15,4.3$ & 346 & $38 \mathrm{~g} \cdot 6$ & 182.1 & 396 & $445 \times 9$ & 210,6 & $44^{6}$ & 502,1 & 239.8 \\
\hline 207 & $334 \cdot 4$ & 154,9 & 347 & $390 \cdot 7$ & 182.6 & 397 & 447.0 & 211.2 & 447 & 503.2 & 240.4 \\
\hline 208 & 335.5 & 155.4 & 348 & $397 \times 8$ & 183.2 & 308 & 448.1 & $2 \times 1,7$ & 448 & 504.4 & 241.0 \\
\hline 290 & 336.6 & 156.0 & 349 & 392,9 & 183.7 & 399 & 440.2 & $212 \cdot 3$ & 449 & 505.5 & 241,6 \\
\hline 300 & 337.8 & 156.3 & 350 & 394,0 & 184.3 & 400 & 450.3 & 212.9 & 450 & 506.6 & 242.2 \\
\hline 301 & 338.9 & 157.1 & $35 \mathrm{t}$ & $305 \cdot 2$ & 184.9 & 401 & $45 x-5$ & $2 \times 3.5$ & $45 \mathrm{I}$ & 507.8 & 242.8 \\
\hline 302 & 340.0 & 157.6 & 352 & $396 \cdot 3$ & 185.4 & 402 & 452,6 & $2 x-1 . x$ & 452 & 508.9 & 243.4 \\
\hline 303 & $34 t, 1$ & 158.2 & 353 & $397+4$ & 186.9 & 403 & 453,7 & 25.4 .6 & 453 & 510.0 & 244.0 \\
\hline 304 & $342 \cdot 3$ & 158,7 & 354 & 398,6 & 186.6 & 404 & 454.8 & 215.2 & 454 & 511,1 & $244 \cdot 6$ \\
\hline 305 & $343 \cdot 4$ & 159.3 & 355 & 399.7 & 287.2 & 405 & 456.0 & 215.8 & 455 & $5 \times 2.3$ & $245 \cdot 2$ \\
\hline 306 & 344.5 & 159.8 & 356 & 400.8 & $187-7$ & 406 & $457 \cdot 1$ & 256.4 & 456 & 513.4 & $245 \cdot 7$ \\
\hline 207 & $345 \cdot 6$ & 160.4 & 357 & $401 \cdot 9$ & 188.3 & 407 & 458.2 & $217=0$ & 457 & 514.5 & $246 \cdot 3$ \\
\hline 368 & 346.8 & 160.9 & 358 & $403 . t$ & 188.9 & 408 & $459 \cdot 4$ & 217.5 & $45^{8}$ & 515.6 & $246 \cdot 9$ \\
\hline 309 & 347.9 & $161+5$ & 359 & $404 \cdot 2$ & $180=4$ & 400 & $460 \cdot 5$ & $278 \cdot 1$ & 459 & 516.8 & 247.5 \\
\hline $3 \times 0$ & 349.0 & 162.0 & 360 & $405 \times 3$ & 190.0 & 410 & $46 \pi, 6$ & 218.7 & 460 & $5 \times 7.9$ & 248,1 \\
\hline 311 & 350,1 & 162.6 & $36 \mathrm{r}$ & 406.4 & 190.6 & 47 & 463,7 & 279.3 & $46 x$ & 510.0 & 248.7 \\
\hline 312 & $35 \mathrm{r} \cdot 3$ & $\mathrm{I} 63 . \mathrm{I}$ & 362 & 407.6 & $19 \mathrm{I}, \mathrm{I}$ & 412 & $4^{63} .8$ & 210.9 & 462 & 520.1 & $249 \cdot 3$ \\
\hline 313 & 352.4 & $163+7$ & 363 & 408,7 & 101,7 & 413 & 465.0 & 220.4 & 463 & $52 t \cdot 3$ & 249.9 \\
\hline 314 & $353 \cdot 5$ & $164+2$ & 364 & 409.8 & $\mathrm{x} 02,3$ & 414 & 466.1 & 221.0 & & & \\
\hline $3 \pm 5$ & 354.0 & 164.8 & 365 & 410.9 & 192.9 & 415 & 467.2 & $221 \cdot 6$ & & & \\
\hline 316 & 355.8 & 165.3 & 366 & $412 . \mathrm{r}$ & 193.4 & 416 & 468.4 & 222,2 & & & \\
\hline $3 \times 7$ & 356.9 & 165,0 & 367 & $413 \cdot 2$ & 104.0 & 417 & 460.5 & 222.8 & & & \\
\hline $3+8$ & 358.0 & r 66.4 & 368 & 41.4 .3 & 104.6 & 418 & 470.6 & 223.3 & & & \\
\hline 329 & $359-1$ & 167.0 & 360 & $4 \times 5.4$ & 195.1 & 410 & 471.8 & 223.9 & & & 1 \\
\hline 320 & $360 \cdot 3$ & 167.5 & 370 & 416.6 & 105.7 & 420 & 472.9 & $224 \cdot 5$ & & & 1 \\
\hline
\end{tabular}

is a switch, $S$, and at either end of the hard-rubber plate is a binding post, $R$, for connection with the electric current. The wiring, which is on the under side of the rubber plate, is best illustrated by the diagram in Fig. Iro.

Four determinations may be carried on simultaneously in four platinum dishes, if desired, the wiring and the switches being so arranged that beginning at one end of the plate either the first dish or the first 

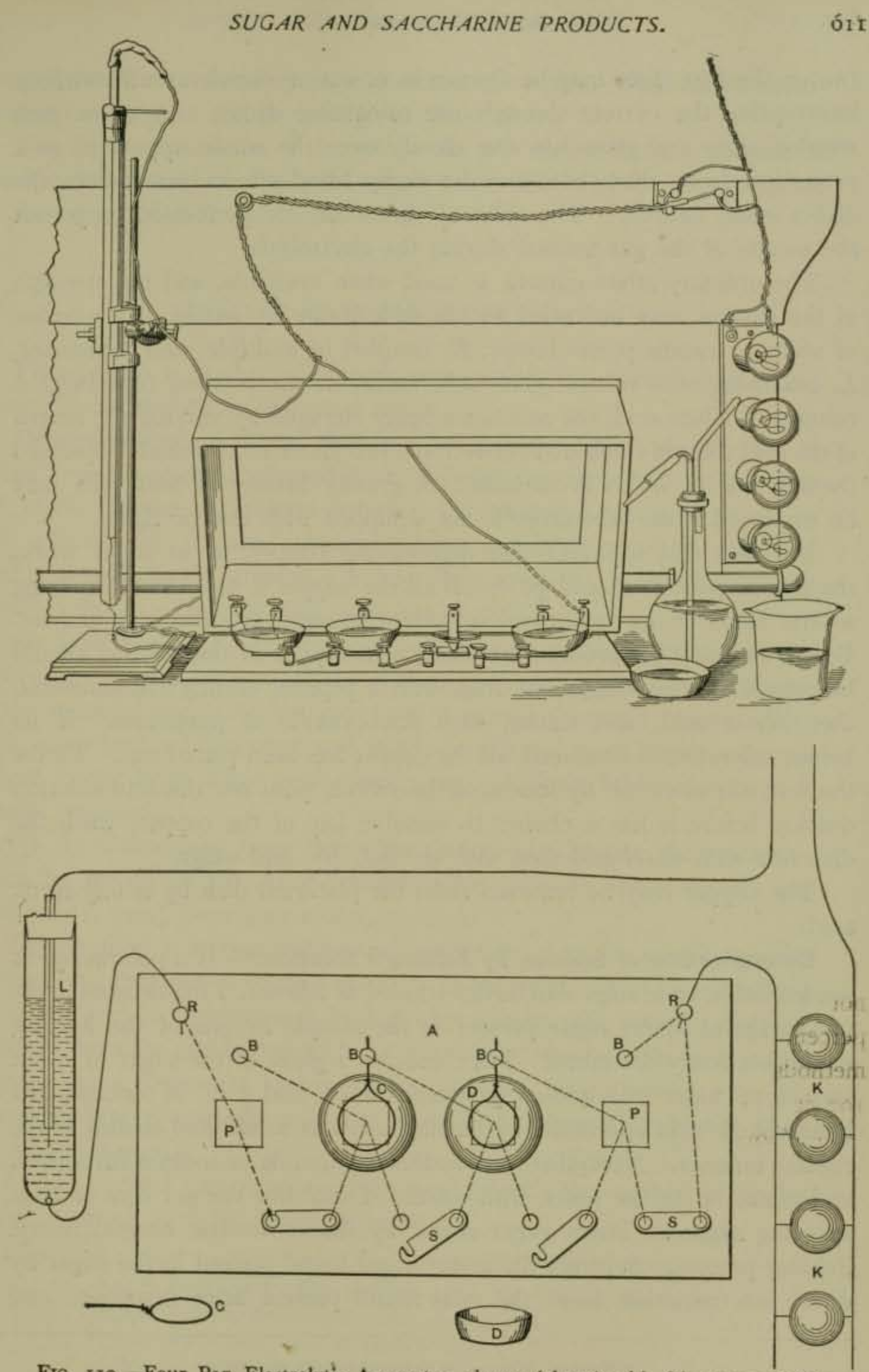

Fic. 1ro.-Four Pan Electrolytic Apparatus, shown (above) with Glass-covered Top Partially Removed, and (below) in Diagram. 
two or the first three may be thrown in or out of circuit at will without interrupting the current through the remaining dishes. A cover with wooden sides and glass top fits closely over the whole apparatus as a protection from dust, but may be easily lifted off to manipulate the dishes when desired. The sides of the cover are perforated to permit the escape of the gas formed during the electrolysis.

The ordinary street current is used when available, and the strength of the current may be varied within wide limits by means of a number of 16 or 32 candle-power lamps, $K$, coupled in multiple, and a rheostat, $L$, consisting of a vertical glass tube sealed at the bottom, containing a column of dilute acid, the resistance being changed by varying the length of the acid column contained between the two platinum terminals immersed therein, one of which is movable. A gravity battery of four cells may be employed if the laboratory is not equipped with electric lights.

In using this apparatus for determining copper, as in sugar work, the plating process should go on till all the copper is deposited, requiring several hours or over night with a current strength of about 0.25 ampere. Before stopping the process, the absence of copper in the solution should be proved by removing a few drops with a pipette, adding first ammonia, then acetic acid, and testing with ferrocyanide of potassium. If no brown coloration is produced, all the copper has been plated out. Throw the dish out of circuit by means of the switch, pour out the acid solution quickly before it has a chance to dissolve any of the copper, wash the dish first with water and then with alcohol, dry, and weigh.

The copper may be removed from the platinum dish by strong nitric acid.

Determination of Sucrose by Fehling's Solution.*-If a polariscope is not available, cane sugar can be determined as follows: First determine the percentage of invert sugar present in the sample by one of the Fehling methods already described. Then dissolve I gram of the sugar in about $100 \mathrm{cc}$. of water in a $500-\mathrm{cc}$. graduated flask, add $3 \mathrm{cc}$. of concentrated hydrochloric acid and invert by heating in water to $68^{\circ}$ and cooling in the regular manner. Neutralize with sodium hydroxide or sodium carbonate, and make up to the mark with water. Determine the per cent of total reducing sugar as invert sugar either by the volumetric or gravimetric Fehling process. Subtract the invert sugar found present in the sugar by direct determination from the total found present after inversion, and

* Tucker, Manual of Sugar Analysis, p. 182. 
the remainder is the invert sugar due to cane sugar. This figure multiplied by 0.95 gives the percentage of cane sugar.

For the determination of sucrose by the gravimetric Fehling process on the inverted sample, multiply the cupric oxide $(\mathrm{CuO})$ by the factor 0.4307 , or the copper $(\mathrm{Cu})$ by the factor 0.5394 .

\section{ANALYSIS OF MOLASSES AND SYRUPS.}

First insure a perfectly homogeneous sample by stirring with a rod to evenly distribute any separated sugar.

Determination of Total Solids.-(I) Asbestos Method.-Weigh 20 grams into a roo-cc. graduated flask, dissolve in water, and make up to the mark. Insure a uniform solution by shaking. Measure ro cc. of this solution into a tared platinum dish containing about 5 grams of freshly ignited, finely divided asbestos fiber, and dry to constant weight at $70^{\circ}$ in vacuo, or in a McGill oven (see p. 586 ).

(2) Sand Method.*-Place about 15 grams of ignited quartz sand and a stirring rod in a flat-bottom metal dish and weigh. Add 2 to 4 grams of the material and sufficient moisture to permit thorough mixing. Dry on a water bath with stirring and finally in a water oven until the loss in weight in one hour is not more than $3 \mathrm{mg}$. At least 8 hours' heating is usually required.

(3) By Calculation from Refractive Index.-Determine the refractive index by means of the Abbé refractometer (p. 108), and calculate the total solids, using Geerligs's tables (p. 6r 5 ).

This method is more accurate and convenient than the specific gravity method and employs a smaller quantity of material. The investigations of Stolle $\dagger$ and of Tolman and Smith $\ddagger$ have shown that sucrose, maltose, dextrose, levulose and lactose all have practically the same refractive index. Dextrin has a somewhat higher refractive index, nevertheless the solids of commercial glucose do not give a reading appreciably higher than the sugars named.

A. H. Bryan, $\S$ has compared this method with the method of drying at $70^{\circ}$ in vacuo, with the following results:

* U. S. Dept. Agric., Bur. of Chem., Bul. 107 (rev.), p. 65.

† Zeits. deutsch. Zucker-Ind., Igor, pp. 335, 469 .

$\ddagger$ Jour. Am. Chem. Soc., 28, 1906, p. 1476 .

\$ Ibid., 30, 1908, p. I 443 . 


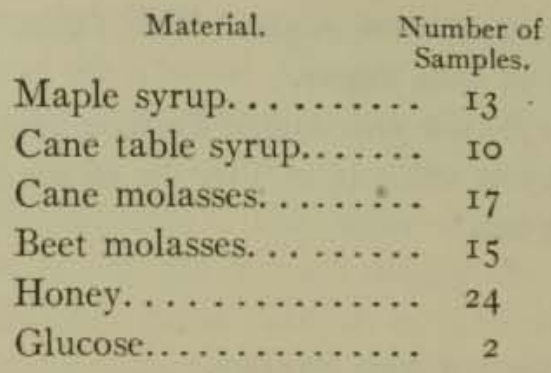

Difference compared with the Gravimetric Method.

Maple syrup......... I3

$-\mathrm{I} .34$ to +0.72

-0.79 " +0.62

-1.53 " +o. 59

$-\mathrm{r} .83$ " -0.07

$-2.5^{2}$ " +0.91

Glucose............. $\quad 2$

$-0.27 "+0.27$

(4) By Calculation from Specific Gravily.-Weigh 25 grams of the sample into a roo-cc. graduated flask, dissolve in water, and make up to the mark. Determine the specific gravity, at $\frac{20^{\circ}}{4^{\circ}} \mathrm{C}$., of the diluted solution by means of a pycnometer or accurate hydrometer. Ascertain from the table on pp. 617 and 618 the percentage by weight of solids (sugar) corresponding to the specific gravity of the diluted solution, and calculate the total solids in the original sample by the formula

Solids in original sample $={ }_{4} D S$,

$D$ being the specific gravity of the diluted solution and $S$ the per cent of solids in the diluted solution.

The solids may also be obtained directly by means of the saccharometer, also known as the Brix spindle. This instrument is a hydrometer graduated so as to show the per cent of sugar when the temperature of the liquid is $20^{\circ} \mathrm{C}$.

If the specific gravity or saccharometer reading is taken at any other temperature than $20^{\circ} \mathrm{C}$. the necessary correction may be found in the table on page 619 .

Determination of Ash.-Weigh from 5 to ro grams of the sample into a tared platinum dish, evaporate to dryness on the water-bath, and proceed as directed for ash of sugar (p. 586).

Polarization and Determination of Sucrose.-Molasses and golden syrup require the application of clarifying reagents before a sufficiently clear solution can be obtained for reading on the polariscope. Even then it is not possible nor is it necessary to get a water-white solution, so that in this class of products greater accuracy can usually be attained by polarizing in a roo-mm. tube (half the standard length) and multiplying the reading by 2 . The clarifier best adapted as a rule for molasses and golden syrup is lead subacetate either in solution (p. 586) or, as first proposed by Horne,* as the anhydrous salt.

* Jour. Am. Chem. Soc., 26, 1904, p. 186. 
GEERLIGS'S TABLE FOR DRY SUBSTANCE IN SUGAR-HOUSE PRODUCTS BY THE ABBE REFRACTOMETER, AT $28^{\circ} \mathrm{C} . *$

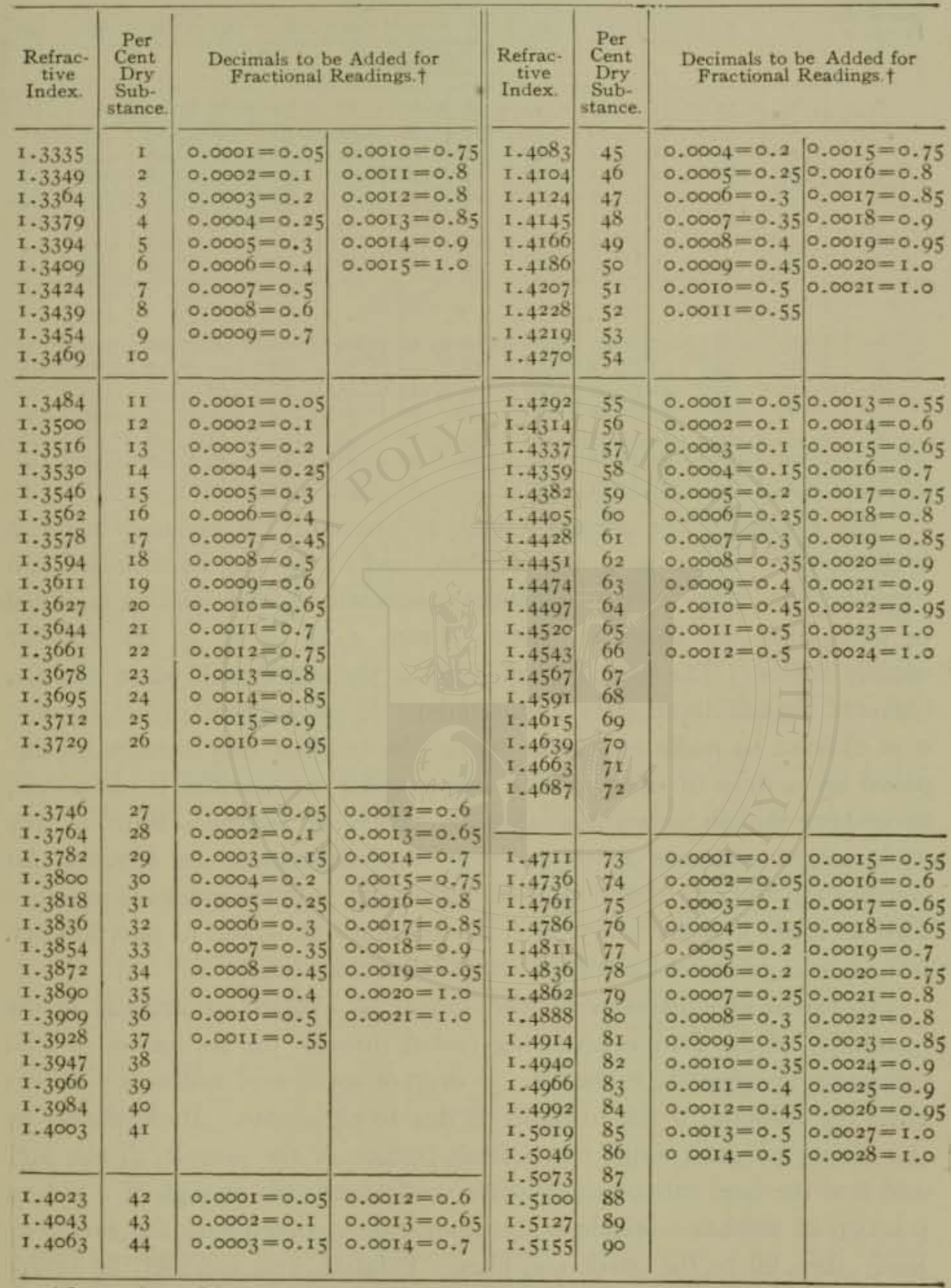

* Intern. Sugar Jour., 10, DP. 69-70.

$\uparrow$ Find in the table the refractive index which is next lower than the reading actually made and note the corresponding whole number for the per cent of dry substance. Subtract the refractive index obtained from the table from the observed reading; the decimal corresponding to this difference, as given in the column so marked, is added to the whole per cent of dry substance as
first obtained. 


\begin{tabular}{|c|c|c|c|c|c|c|c|c|c|c|c|c|c|}
\hline \multirow{3}{*}{$\begin{array}{l}\text { Tempera- } \\
\text { ure of the } \\
\text { Prisms in } \\
{ }^{\circ} \mathrm{C} \text {. }\end{array}$} & \multicolumn{13}{|c|}{ Dry Substance. } \\
\hline & 0 & 5 & 10 & 15 & 20 & 25 & 130 & 40 & 50 & 60 & 70 & 80 & 90 \\
\hline & \multicolumn{13}{|c|}{ Subtract- } \\
\hline 20 & 0.53 & 0.54 & 0.55 & 0.56 & 0.57 & 0.58 & 0.60 & 0.62 & 0.64 & 0.62 & 0.61 & 0.60 & $0.5^{8}$ \\
\hline $2 \mathrm{I}$ & .46 & .47 & .48 & .49 & $.5^{\circ}$ & .51 & .52 & .54 & .56 & .54 & .53 & .52 & .50 \\
\hline 22 & .40 & .41 & .42 & .42 & .43 & .44 & .45 & .47 & .48 & .47 & .46 & .45 & .44 \\
\hline 23 & .33 & $\cdot 33$ & $\cdot 34$ & $\cdot 35$ & -36 & .37 & $-3^{8}$ & $\cdot 39$ & .40 & .39 & $.3^{8}$ & .38 & -38 \\
\hline 24 & .26 & .26 & .27 & .28 & .28 & .29 & -30 & .31 & .32 & $.3 i$ & .31 & .30 & .30 \\
\hline$\angle 5$ & .20 & .20 & .21 & .21 & .22 & .22 & .23 & .23 & .24 & .23 & .23 & .23 & .22 \\
\hline 26 & .12 & .12 & .13 &. $\mathrm{I}_{4}$ & .14 & .15 & .15 & . I6 & .16 & .16 & .15 & .15 & .14 \\
\hline \multirow[t]{2}{*}{27} & .07 & .07 & .07 & .07 & .07 & .07 & .08 & .08 & .08 & .08 & .08 & .08 & .07 \\
\hline & \multicolumn{13}{|c|}{ Add- } \\
\hline 29 & 0.07 & 0.07 & 0.07 & 0.07 & 0.07 & 0.07 & 0.08 & 0.08 & 0.08 & 0.08 & 0.08 & 0.08 & 0.07 \\
\hline 30 & .12 & -12 & .13 & .14 & .14 & .14 & .15 & .15 & .16 & .16 & .16 & .15 & .14 \\
\hline 31 & .20 & .20 & .21 & .21 & .22 & .22 & .23 & .23 & .24 & .23 & .23 & .23 & .22 \\
\hline 32 & .26 & .26 & .27 & -28 & .28 & .29 & -30 & -31 & .32 & -31 & -31 & .30 & .30 \\
\hline 33 & .33 & -33 & -34 & -35 & .36 & -37 & -38 & .39 & .40 & .39 & -38 & -38 & .38 \\
\hline 34 & .40 & $.4 \mathrm{I}$ & .42 & .42 & .43 & .44 & .45 & .47 & .48 & .47 & .46 & .45 & .44 \\
\hline 35 & .46 & .47 & .48 & .49 & .50 & .51 & .52 & .54 & .56 & .54 & .53 & .52 & .50 \\
\hline
\end{tabular}

The Process. - The normal weight, 26 grams, of the molasses or syrup is dissolved in water in a roo-cc. flask, and in the case of molasses and "golden," or "drip" syrup, sufficient subacetate of lead solution is added to precipitate the coloring matter. From 5 to $10 \mathrm{cc}$. of the clarifier usually suffice. The flask is then filled to the mark with water and the contents shaken thoroughly and filtered. If on account of air bubbles it is difficult to make up to the mark, the bubbles may usually be dispelled by a drop of ether. With maple syrup no clarifier is, as a rule, necessary, though sometimes alumina cream is helpful. With a very dark-colored molasses 20 to $30 \mathrm{cc}$. of lead subacetate are required for clarification and in extreme cases (though rarely with the grades of molasses used as food) it is necessary, after the ordinary filtration, to pass through from 5 to 6 grams of powdered, dried bone charcoal.*

An excess of subacetate of lead should be avoided on account of the possibility of the filtrate becoming turbid through the formation of lead carbonate by exposure to the air. A drop of acetic acid will nearly always clear the solution, if the turbidity is due to carbonate. If cloudiness in the filtrate persists, weigh out a fresh portion of the sample, dilute, and add first the lead subacetate solution, and afterwards enough of a strong solution of sodium sulphate or common salt to precipitate the excess of lead; then fill to the mark and filter. Polarize, and conduct the inversion as directed on p. $5^{88}$, using, however, a $100-\mathrm{mm}$. tube, and multi-

* The treatment with bone char should be used only as a list resort, as, on account of slight absorption of sugar, observed readings are from $0.4^{\circ}$ to to $0.5^{\circ}$ too low. 
DENSITY OF SOLUTIONS OF CANE SUGAR AT $\frac{20^{\circ}}{4^{\circ}} \mathrm{C}, *$

\begin{tabular}{|c|c|c|c|c|c|c|c|c|c|c|}
\hline \multirow{2}{*}{ 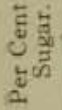 } & \multicolumn{10}{|c|}{ Tenths of Per Cent. } \\
\hline & o & I & 2 & 3 & 4 & 5 & 6 & 7 & 8 & 9 \\
\hline 0 & $0.998 z$ & 0.9986 & 0.9990 & 0.9994 & 0.9998 & 1.0002 & f.,0006 & 1.0010 & 1.0013 & 1.00 \\
\hline$i$ & 1.0021 & 1.0025 & 1.0029 & 1. 0033 & I. 003 ? & 1.0041 & I. .0045 & $1.00+8$ & I. 0052 & 1.0058 \\
\hline 2 & I. 0060 & $x, 0064$ & I. .0068 & 1.0072 & I. 0076 & $x, 0080$ & T. .0084 & x. .0088 & I. $009 \mathrm{I}$ & 1. 0095 \\
\hline 3 & 1.0099 & 1.0103 & 1.0107 & t.otit & 1.0115 & 1.0119 & 1.0123 & 1.0127 & 1.0131 & 1.0135 \\
\hline 4 & 1.0139 & 1.0143 & 1.0147 & 1.0151 & 1.0155 & 1.0159 & 1.0163 & 1.0167 & 1.0171 & 1.0175 \\
\hline 5 & 1.0179 & T. 0183 & t. 0187 & 1.0191 & 1.0195 & 1,0199 & 1.0203 & 1,0207 & 1.0211 & 1.021 \\
\hline 6 & 1,0219 & 1.0223 & 1.0227 & 1.0231 & 1.0235 & 1.0239 & 1.0243 & 1.0247 & 1. 0251 & 1.0255 \\
\hline 7 & 1.0259 & 1.0263 & $x .0267$ & 1,0271 & $x .0276$ & 1.0279 & $x .0283$ & $x .0287$ & $x .0291$ & 1.0295 \\
\hline 8 & 1,0299 & 1.0303 & 1. .0308 & 1,0312 & 1.0316 & 1.0320 & 1.0324 & 1.0328 & 1.0332 & 1.0336 \\
\hline 9 & 1.0340 & 1,0344 & 1.0349 & 1.0353 & 1.0357 & 1.0361 & 1.0365 & 1.0369 & 1.0373 & 1.0377 \\
\hline to & 1.0381 & ז.0386 & t. .0390 & 1,0394 & 1.0398 & 1,0402 & r. 0406 & 1.0410 & t. 0415 & $1.04 !$ \\
\hline II & 1. 0423 & 1.0427 & $\mathrm{t}$ & 1. 04 & & & I. 0448 & $\mathrm{I}, 0$ & & 1.0460 \\
\hline 12 & 1.0465 & I. 0469 & I. .0473 & I. 0477 & $t .048 x$ & t. 0486 & 1.0490 & 1. 0494 & & 1.0502 \\
\hline 13 & I. 0507 & 1.0511 & 1.0515 & $t .0510$ & 1. 0524 & 1.0528 & 1.0532 & 1.0536 & 1.0540 & 1.0545 \\
\hline 14 & I. 0549 & 1.0553 & $x .055^{8}$ & 1.0562 & 1.0506 & 1.0570 & 1.0575 & 1.0579 & $x, 0583$ & 1. 058 ? \\
\hline I5 & 1.0592 & $x .0596$ & $x .0600$ & 1.0605 & 1. .0609 & 1.0613 & 1.0617 & 1.0622 & 1. 0626 & 1.06 \\
\hline 16 & 1.0635 & 1.0639 & $x .0643$ & T. 0648 & & & & & & 1. $.06 ?$ \\
\hline 17 & 1.0678 & 1.0682 & 1.0687 & 1.009r & t.0695 & 1.0700 & 1.0704 & 1.0708 & 1.0713 & 1.07 \\
\hline 18 & 1.0721 & 1.0726 & 1.0730 & 1.0735 & & & & & & 1.076 \\
\hline 19 & 1.0765 & x. 0770 & x. 0774 & 1.0779 & 1.0783 & 1.0787 & 1.0792 & 1.0796 & I. OSor & 1. 0805 \\
\hline 20 & 1.0810 & $t .0814$ & 1,0818 & 1. 0823 & x.0827 & 1.0832 & 1,0836 & $x, 084 x$ & 1. 08.45 & .08 \\
\hline is & & & & & & & & & & I. 089 \\
\hline $2 z$ & I. 0899 & 1.0904 & 1. 0908 & 1.0913 & 1.0917 & 1.0922 & r.0926 & 1.0931 & 100355 & 1.0949 \\
\hline 23 & 1.0944 & 1.0949 & 1,0953 & 1. .0958 & 1.0962 & 1.0967 & 1. .0971 & 1.0976 & 1.0981 & $x .09$ \\
\hline 24 & 1.0990 & I. 0994 & I. 0999 & 1.1003 & r, 1008 & I. 1013 & T. 1017 & x. 1022 & 1. 1026 & 1. 10 \\
\hline 25 & 1. 1036 & & & & & & & 1. 1068 & & \\
\hline 26 & I. 1082 & I & 1.1001 & 1,10 & & & 1. 1110 & & & 1. $\pi x$ \\
\hline 27 & 1.1128 & & & 1. 1 & $1:$ & & & & & $x, 11$ \\
\hline 28 & 1.1175 & 1.1180 & $x, 1185$ & I. 1789 & $t, x+194$ & 1,7199 & $1,1 \geqslant 03$ & I. $x 208$ & 1.1213 & r. $13 \mathrm{r}$ \\
\hline 29 & 1.1222 & $x .1227$ & 1. .1232 & 1. 1237 & $t=124 t$ & I. 1246 & 1.1251 & $x .1256$ & 1.1260 & 1.120 \\
\hline 30 & & & & TI & 1,1289 & & & & & \\
\hline 35 & & & 1. & I & $x-1$ & t. & & & & 1.130 \\
\hline 32 & & & & & & & & & & 1.14 \\
\hline 33 & & & $\mathrm{I}, \mathrm{I}$. & $x=1$. & I. 12 & & & & & I. $\mathrm{I}$ \\
\hline 34 & I. 5463 & 1. 1.468 & 1. 1473 & x. 7478 & f. .483 & 1.1488 & 1.1493 & 1,1498 & 1,1503 & 1.150 \\
\hline 35 & 1.151 & & & I. .152 & & & & & & \\
\hline 36 & & & & & & & & & & 1.1 \\
\hline 37 & & & & & & & & & & \\
\hline 38 & & & & 1. 16 & 168 & & & & & I. 178 \\
\hline 39 & 1.1713 & 1. 1718 & $x+1724$ & 1,1729 & 1.7734 & 1.1739 & 1. 17744 & 1. $\times 749$ & 1.1754 & 1.175 \\
\hline 40 & & & & & & & & & & \\
\hline $4 \mathrm{r}$ & & & & & & & & & & \\
\hline 42 & & & & 1. $1 !$ & 1,18 & 1.18 & 1,18 & I. 1904 & I. T909 & 1.18 \\
\hline 43 & & & & & & & & & & \\
\hline 44 & 1.1972 & 1.1978 & $i+198$ & 1.198 & T. 1994 & 1.1999 & 2.2004 & I. 2009 & I. 2015 & T. 20 \\
\hline 45 & & & & & & & & & & \\
\hline 46 & & & & & & & & & & \\
\hline 47 & & & & & & & & & & 1.21 \\
\hline 48 & & & & & & & & $x .2224$ & I. 2230 & 1.22 \\
\hline 49 & $1,224 \mathrm{t}$ & I. 2246 & 1.2252 & 1,2257 & 1.2263 & 1.2268 & 8.2274 & 1,2279 & I. 2285 & 1,229 \\
\hline 50 & I. 2296 & 1.2301 & $x, 2307$ & 1.2312 & t. 2358 & 1.2323 & ז. 2329 & $x, 2334$ & I, 2340 & 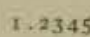 \\
\hline
\end{tabular}

* According to Dr, F. Plato (Kaiserlichen Normal-Eichungs-Kommission, Wiss, Abh., 2, 1900. p. 153). This table is given by the U.S. Burean of Standards (Circular 19. Pp, 12 and 13 ) as the basis for standardizing hydrometers, Indicating per cent of sugar at $20^{\circ}$, known as saccharometers or Brix spindles. The table is also useful in calculating the per cent of sugar from the specific gravity as determined by the pycnometer. Temperature corrections are given on page 619 . 
DENSITY OF SOLUTIONS OF CANE SUGAR AT $\frac{20^{\circ}}{4^{\circ}} \mathrm{C}$.-Continued

\begin{tabular}{|c|c|c|c|c|c|c|c|c|c|c|}
\hline \multirow{2}{*}{ 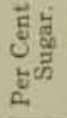 } & \multicolumn{10}{|c|}{ Tenths of Per Cent. } \\
\hline & $\circ$ & 1 & $z$ & 3 & 4 & 5 & 6 & 7 & 8 & 9 \\
\hline in & x. 2296 & 1. 2301 & 1. 2307 & 1.2312 & 1. $23 \times 8$ & $x .2323$ & I. 2329 & $x .2334$ & $x .2340$ & t. 2345 \\
\hline 51 & 1. 2351 & 1.2356 & 5.2362 & 1.2367 & & .2379 & 1,2384 & 1.2390 & $i, 2395$ & 1.2401 \\
\hline 52 & 1.2406 & 1.2412 & 1.2418 & 1.2423 & 1. 2429 & $x .2434$ & 1. 2440 & $1: 2446$ & 1.2451 & 1. 2452 \\
\hline 53 & I. 2462 & x. 2468 & 1.2474 & 1.2479 & I. 2485 & $x .2490$ & $x-2496$ & 1. 2302 & 1,2507 & 1.2513 \\
\hline 54 & 1. 2519 & 1.2524 & 1.2530 & $i, 2536$ & 1. 2541 & 1.2547 & $x, 2553$ & 1.2558 & 1.2564 & 1.2570 \\
\hline 55 & 1.2575 & I. $258 \mathrm{I}$ & r. 2587 & 1.2592 & I, 2598 & 1.2604 & 1.2610 & $1.26 \times 5$ & 1. 2621 & 1. 2627 \\
\hline 56 & 1. 2632 & i. 2638 & 1.2644 & $\begin{array}{r}1.2650 \\
0\end{array}$ & $\begin{array}{r}1.2655 \\
\end{array}$ & 1.2661 & 1.2667 & 1.2673 & 1.2678 & 1.2684 \\
\hline 57 & 1.2690 & 1. 2696 & 1.2701 & 1.2707 & 1.2713 & 1.2719 & $x .2725$ & $x .2730$ & 1.2736 & 1.2742 \\
\hline 58 & I. 2748 & 1.2754 & 1.2759 & $x, 2705$ & $2.277 x$ & & $i, 2783$ & 1.2788 & 8.2794 & 1.2800 \\
\hline 59 & 3. 2806 & 1.2812 & t. .2818 & 1,2823 & t. 2829 & 1.2835 & 1.2841 & 1.2847 & 1.2853 & 1. 2859 \\
\hline 60 & T. 2865 & 1.2870 & x. 2876 & T. $288 z$ & 1.2888 & 1.2894 & $t .2900$ & $t .2906$ & 1.2912 & t. $29+8$ \\
\hline 61 & 3.2924 & 1.2929 & 1.2935 & 1.2941 & & & 1.2959 & 1.2965 & 1.2971 & 1.2977 \\
\hline 62 & 1.2983 & I. 2989 & I. 2995 & $1+300 \mathrm{x}$ & 1.3007 & I. 3013 & 1.3019 & 1.3025 & & I. 3037 \\
\hline 63 & T. 3043 & x. 3049 & I. 3055 & 1. $306 \mathrm{I}$ & 1.3067 & 1. 3073 & 1. 3079 & 1.3085 & 1.3091 & 1. 3097 \\
\hline 64 & 2.3103 & t. 3109 & 1.3115 & 1.3121 & 1.3127 & 1. 3133 & 1.3139 & I. 3145 & 1.3151 & t. 3157 \\
\hline 6 & I. 3163 & x. $3 x 69$ & 1.3175 & 1.3182 & t. 3288 & 1.3194 & 1,3200 & 1. 3206 & 1.3212 & 1. 3218 \\
\hline 66 & 8.3224 & 1.3230 & & 1,3243 & $i-3$ & & & & & 1. 3279 \\
\hline 67 & 1.3286 & 1,3292 & 1.3298 & 1.3304 & I. 3 & 1.3 & 1. 3. & 1,33 & 1.3 & 1.3341 \\
\hline 68 & t. 3347 & $x, 3353$ & I. 3360 & 7.3366 & t. 3372 & 1. 3378 & 1.3384 & & & 1. 3403 \\
\hline 69 & 1.3409 & $1-3416$ & 1.3422 & $x-3428$ & 1.3434 & 1.3440 & I. 3447 & I. 3453 & 1.3459 & $x .3465$ \\
\hline 70 & 1. 3472 & $x=3478$ & I. 3484 & t. $349 t$ & f. 3497 & 1.3503 & $x \cdot 3509$ & 1. 3516 & 1. 3522 & $x, 3528$ \\
\hline 71 & $\begin{array}{r}7.3535 \\
\end{array}$ & i. 35 & & i. 3 & & & & & & \\
\hline & $1.35 \mathrm{c}$ & & & $x .3617$ & & & & & & 3. 3655 \\
\hline 73 & 1.3661 & 1. 3668 & I. 3674 & $t .368 t$ & 1. 3687 & 1.369 & 1.37 & 1.3706 & 1.3713 & 1.3719 \\
\hline 74 & 1.3725 & 1.3732 & 1.3738 & 1.3745 & 1.3751 & 1.3757 & 1.3764 & $x .3770$ & $x .3777$ & $x, 3783$ \\
\hline 75 & $x .3790$ & x. 3796 & t. 3803 & 1. 3809 & x. 3816 & 1.3822 & $1=3829$ & 1. 3835 & & I. 3848 \\
\hline 76 & $x .3854$ & 1,3 & 1.3867 & $x, 3$ & & & & & & \\
\hline 7 & $x .3920$ & 1. 3926 & I. 3933 & I. 39 & 1.39 & 1.3 & & $\begin{array}{l}1.39 \\
\end{array}$ & & \\
\hline 78 & i. 3985 & f. 3992 & 1,3998 & 1,4005 & 1.4011 & 1.401 & 1.4025 & $x .4031$ & & T. 4044 \\
\hline 79 & 1. 4051 & 1.4058 & 1.4064 & $1.407 x$ & 1.4077 & 1. 4084 & I. 4091 & 1. 4097 & $1 ., 4104$ & I. $4 \pi 11$ \\
\hline 8o & 1. 4117 & 1. 4124 & 1. 4130 & & & & & I. 4164 & & 1.4177 \\
\hline $8 I$ & 1.418 & $x, 4$ & & & & 3. 4 ? & & & & \\
\hline 82 & T. 425 & I. 4 & i & 3.4 & 1.427 & 3.428 & & & & 1. -4311 \\
\hline 83 & T. 4318 & $1.43=5$ & 1.4332 & 1. 4338 & 1. 4345 & 1.43 & & 1.43 & 1. 4372 & I. 4379 \\
\hline 84 & 1.4386 & 1. 4393 & 1.4399 & I. 4406 & 1. 4413 & I. 4420 & 1.4427 & 1. 4433 & $1.444^{\circ}$ & 1.4447 \\
\hline 85 & I. 445 & 1. $446 t$ & & & I. 448 & 1.448 & & & & \\
\hline & $x .45$ & 1. 4 & & & & & & & & \\
\hline & $x .45$ & I. 45 & & $1.4 t$ & & & & & & \\
\hline 88 & 1. 4660 & i. .4667 & i. 4674 & 1.4681 & $t=4 t$ & x. 46 & & & & $1 .-4723$ \\
\hline 89 & $\mathrm{I} .4730$ & 1.4737 & 1.4744 & 1.4751 & 1.4758 & $x .4765$ & 1.4772 & 1.4779 & 3.4786 & 1. 4793 \\
\hline 90 & 1.4800 & & & 1. 4821 & 1. 48 & & & & & 1.4863 \\
\hline & 1. 487 & 1.4 & & 1.489 & 1. 480 & & & & & \\
\hline 92 & I. 49 & I. 49 & t. 4 & & 1.496 & & 1. 4 & & & \\
\hline 93 & 1. 5012 & 1. 5019 & & & I. 5040 & & & & & \\
\hline 94 & 1. 5083 & I, 5090 & 1. 5097 & $x .5104$ & I. 5112 & 1. 5119 & 1.5126 & 1. 5133 & 1.5140 & 1. 5147 \\
\hline 9 & & & & & & & & & & 1. 5219 \\
\hline 9 & & 1.5 & & & $x \cdot 5$ & & & & & \\
\hline & & I. 5 & & 1.53 & & $x-3$ & 1. 5 & 1.5 & 5. 5357 & I. 5364 \\
\hline 98 & 1,53 & 1. 5379 & & I. 5393 & I. $540 \mathrm{I}$ & & I. 5 . & I. 5423 & 1. 5430 & 0.5437 \\
\hline 99 & I. 5445 & 1.5452 & 1. 5459 & I. 5467 & I. 5474 & I. $548 \mathrm{t}$ & 3.5489 & 1. 5496 & 1. 5503 & i. $55 \mathrm{HI}$ \\
\hline & I. 5518 & & & & & & & & & \\
\hline
\end{tabular}


TEMPERATURE CORRECTIONS TO SACCHAROMETER READINGS (STANDARD AT $20^{\circ} \mathrm{C}$.).*

\begin{tabular}{|c|c|c|c|c|c|c|c|c|c|c|c|c|c|c|}
\hline \multirow{3}{*}{$\begin{array}{l}\text { Tempera- } \\
\text { ture in } \\
\text { Degrees } \\
\text { Centigrade: }\end{array}$} & \multicolumn{14}{|c|}{ Observed Per Cent of Sugar. } \\
\hline & 0 & 5 & 10 & 15 & 20 & $25^{*}$ & 30 & 35 & 40 & 45 & 50 & 55 & 60 & 70 \\
\hline & \multicolumn{14}{|c|}{ Subtract from Observed Per Cent. } \\
\hline 0 & 0.30 & 0.49 & 0.65 & 0.77 & 0.89 & 0.09 & 1.08 & $t=16$ & $t \cdot 24$ & I. $3 x$ & 2.37 & 1.41 & $x, 44$ & 1.49 \\
\hline 5 & 0.36 & 0.47 & 0.56 & 0.65 & 0.73 & 0.80 & 0.86 & 0.91 & 0.97 & $\mathrm{I}, \mathrm{OI}$ & $x, 05$ & 1.08 & 1,10 & $\mathrm{~T}, \mathrm{~T}, 4$ \\
\hline 10 & 0.32 & 0.38 & 0.43 & 0.48 & 0.52 & 0.57 & 0.60 & 0.64 & 0.67 & 0.70 & 0.72 & 0.74 & 0.75 & 0.77 \\
\hline It & 0.31 & 0.35 & 0.40 & 0.44 & 0.48 & 0.51 & 0.55 & 0,58 & 0.60 & 0.63 & 0.65 & 0.66 & 0.68 & 0.70 \\
\hline 12 & 0.29 & 0.32 & 0.36 & 0.40 & 0.43 & 0.46 & 0.50 & 0.52 & 0.54 & $0,5^{6}$ & 0.58 & 0.59 & 0.60 & 0.62 \\
\hline 13 & 0.26 & 0.29 & 0.32 & 0.35 & 0.38 & 0.41 & 0.44 & 0.46 & 0.48 & 0.49 & 0.51 & 0.52 & 0.53 & $0 / 55$ \\
\hline 14 & 0.24 & 0.26 & 0.29 & $0.3 t$ & 0.36 & 0.36 & 0.38 & 0.40 & 0.41 & 0.42 & 0.44 & 0.45 & 0.46 & 0.47 \\
\hline 15 & 0.20 & 0.22 & 0.24 & 0.26 & 0.28 & $0.3 \mathrm{C}$ & 0.32 & 0.33 & 0.34 & 0.36 & $0.3 t$ & 0.37 & 0.38 & 0.39 \\
\hline 16 & 0,17 & 0.78 & 020 & 0.22 & 0.23 & 0,25 & 0.26 & 0.27 & 0.28 & 0.28 & 0.29 & 0.30 & 0.31 & 0.32 \\
\hline 17 & 0,13 & 0.14 & 0.15 & 0.16 & 0.18 & 0.19 & 0.20 & 0.20 & $0 / 21$ & 0.21 & 0,28 & 0.23 & 0.23 & 0.24 \\
\hline 18 & 0,09 & 0,10 & 0,10 & 0.11 & 0.12 & $\begin{array}{lll}0 & \mathrm{I}_{3}\end{array}$ & 0.13 & o. $\mathrm{r}_{4}$ & $0 . x_{4}$ & 0,14 & $0.1 E$ & 0.15 & 0.15 & 0.16 \\
\hline 19 & 0.05 & 0.05 & 0.05 & 0.06 & 0.06 & 0.06 & 0.07 & 0.07 & 0.07 & 0.07 & $0.0 \varepsilon$ & 0.08 & 0.08 & 0,08 \\
\hline $27 \cdot 5$ & $0, \pi 1$ & $0, t z$ & 0.12 & 0.14 & $0 \times 1=$ & 0.26 & 0,16 & 0.17 & 0,17 & 0.18 & $0.1 \varepsilon$ & 0.19 & 0.19 & 0.20 \\
\hline \multirow[t]{2}{*}{$\left(60^{\frac{15}{5}} ;\right)^{6}$} & 0,18 & $0.2 c$ & 0.22 & 0.24 & $0.2 t$ & 0.28 & 0.29 & $0.3 c$ & 0.30 & $0.3=$ & 0.33 & 0.33 & 0.34 & 0.34 \\
\hline & \multicolumn{14}{|c|}{ Add to Observel Por Cent. } \\
\hline 21 & 0.04 & 0.05 & 0.05 & 0.06 & 0.06 & 0.07 & 0.07 & 0.07 & 0.07 & $0.0 s$ & $c, c k$ & 0.08 & 0.08 & 0.09 \\
\hline 22 & $0+10$ & 0.10 & 0.11 & 0.12 & 0.12 & 0,13 & 0.14 & $0 . I_{4}$ & 0,15 & 0.15 & 0,16 & 0,16 & 0.16 & 0.16 \\
\hline 23 & 0,26 & 0,16 & 0.17 & 0.17 & 0.19 & 0.20 & $0.2 \mathrm{r}$ & 0.21 & 0,22 & 0.23 & 0.2 .4 & 0,24 & 0.24 & 0.24 \\
\hline 24 & 0.21 & 0.22 & 0.23 & 0.24 & 0.26 & 0.27 & 0.28 & 0.29 & 0.30 & 0.31 & 0.32 & 0.32 & 0.32 & 0.32 \\
\hline 25 & 0.27 & 0.28 & 0.30 & 0.31 & 0.32 & 0.34 & 0.35 & 0.36 & 0,38 & 0,38 & 0,39 & 0.39 & 0,40 & 0.39 \\
\hline 26 & 0.33 & 0.34 & $0.3 c$ & 0.37 & 0.40 & 0.40 & 0.42 & 0.44 & 0.46 & 0.47 & 0.47 & 0.48 & 0.48 & 0.48 \\
\hline 27 & 0.45 & 0.41 & 0.42 & 0.44 & 0.46 & 0.48 & 0.50 & 0.52 & 0.5 .4 & $0,5.4$ & 0.58 & 0.56 & 0.56 & 0.56 \\
\hline 28 & $0.4^{6}$ & 0.47 & 0.45 & 0,51 & 0.54 & 0.561 & 0.58 & $0.6 c$ & 0.61 & 0.62 & 0.63 & 0.63 & 0.62 & 0.64 \\
\hline 29 & 0.54 & 0.55 & $0.5 c$ & 0.59 & 0,61 & 0.63 & 0.66 & 0.68 & 0.70 & 0.70 & 0.73 & 0,72 & 0.72 & 0.72 \\
\hline 30 & 0.61 & 0.02 & 0.63 & 0.66 & 0.68 & 0.71 & 0.73 & 0.76 & 0.78 & 0,78 & 0.79 & $0.8 c$ & $0.8 \mathrm{c}$ & $0.3 r$ \\
\hline 35 & 0.99 & $t .0 t$ & $x .02$ & $x .06$ & 1,10 & $x, 13$ & 1. 16 & I. 58 & 1,20 & 1,21 & 1.22 & $x: 22$ & $x \cdot 23$ & 1.22 \\
\hline 40 & 3.42 & t. 45 & I. .47 & 1,51 & 1.54 & 1.57 & 1.60 & 1.62 & 1.64 & 1. 65 & 1.65 & 1.65 & 1.66 & t. 65 \\
\hline 45 & 1.93 & $x-94$ & 1.96 & 2.00 & 2.03 & 2,05 & 2.07 & 2,00 & 2.10 & 2.10 & $2,+1 c$ & 2.10 & 2,10 & 2.08 \\
\hline 50 & 2.46 & $2 \cdot 48$ & 2.50 & 2.53 & 2.56 & $=-57$ & 2.58 & 2.59 & 2,59 & 2,58 & $2.5 \varepsilon$ & 2.57 & 2,56 & 2.52 \\
\hline 55 & 3.05 & 3.07 & 3.09 & 3.12 & $3-12$ & 3.12 & 3.12 & 3.11 & 3.10 & 3.08 & 3.07 & 3.05 & $3.0,3$ & 2.97 \\
\hline 60 & 3.69 & $3-72$ & 3.73 & 3.73 & $3 \cdot 72$ & 3.70 & 3.67 & 3.65 & 3,62 & 3,60 & 3.57 & 3.54 & 3.50 & $3 \cdot 43$ \\
\hline 27.5 & 0.43 & 0.44 & $0.4 c$ & 0.48 & 0.50 & 0.52 & 0.54 & 0.56 & 0.58 & 0.58 & 0.59 & 0.60 & 0.60 & 0.60. \\
\hline
\end{tabular}

*U. S, Dept. of Commerce and Labor, Bur, of Standards, Circular 29, 1909, p. II. This table is calculated using the data on thermal expansion of sugar solutions by Plato (Wiss. Abh. der Kaiserlichen Normal-Eichungs-Kommission, 2, 1900, P. T.40), assuming the instrument to be of Jena $16^{111}$ glass. The table should be used with caution and only for approximate results when the tampersture differs much from the standard temperature or from the temperature of the surrounding air. 
plying the reading by 2 , both direct and invert.* Use Clerget's formula for calculation of the sucrose.

For medium- or light-colored grades of molasses, which yield but a smail precipitate with lead subacetate, the above method of simple polarization, both direct and invert, gives results sufficiently accurate for ordinary work. For dark-colored, or "black-strap" molasses, or wherever extreme accuracy is required, the solution should be first made up to the mark and then clarified by the addition of a slight excess of anhydrous lead subacetate (p. $5^{87}$ ), as proposed by Horne, or else the double dilution method of Wiley should be employed. Both methods make due allowance for the volume of the precipitate.

Doutle Dilution Method. $†$ - Take half the normal weight of the sample and make up the solution to Ioo cc., using the appropriate clarifier. Take the normal weight of the sample and make up a second solution with the clarifier to Ioo cc. Filter and obtain direct polariscopic readings of both solutions. Invert each in the usual manner and obtain the invert reading of the two.

The true direct polarization of the sample is the product of the two direct readings divided by their difference. The true invert polarization is the product of the two invert readings divided by their difference.

Determination of Raffinose in Beet Sugar Molasses.-For the determination of sucrose and raffinose when present in the same solution, use the following formulas of Creydt: $\ddagger$

and

$$
\begin{aligned}
& \text { Sucrose }=\frac{0.5188 a-b}{0.8454} . . . . . \\
& \text { Raffinose }=\frac{a-S}{1.85}, . . . . . . .
\end{aligned}
$$

where $a=$ direct reading, $b=$ reading after inversion, and $S=$ per cent of sucrose.

* The short tube (100 mm.) is preferred for polarizing molasses, not only on account of the more or less deep color of the clarified solution, but also because a molasses sample containing considerable commercial glucose would not read within the scale limits, if the 200 $\mathrm{mm}$. tube were employed.

$\dagger$ Wiley and Elwell, Analyst, 1896,21, p. 184 .

$\ddagger$ U. S. Dept Agric, Bur. of Chem., Bul. 107 (rev.), p. 43 . 
Davoll * recommends for purposes of clarification of the molasses the use of powdered zinc after inversion of the molasses sample according to Clerget's method. He adds I gram of the zinc to the sample after inversion while at the temperature of $69^{\circ} \mathrm{C}$., allowing it to act for three to four minutes at that temperature, after which he cools and filters, with the production of an almost colorless solution.

Determination of Reducing Sugar.-(Estimated as Dextrose.)-Dilute 5 grams of molasses or syrup with water in a 100 -cc. graduated flask, using 2 to $5 \mathrm{cc}$. normal lead acetate. Make up to $100 \mathrm{cc}$., filter, take an aliquot part of the filtrate ( 25 to $50 \mathrm{cc}$.) and make this up to 100 cc., the amount taken being such that, when diluted, the solution will contain not more than $\frac{1}{2} \%$ of dextrose. Since lead acetate has been used to clarify, add to the aliquot part taken and before dilution, enough sodium sulphate to precipitate the excess of lead, then filter and make up to the roo cc. mark.

Determine the reducing sugar in this solution by either volumetric or gravimetric Fehling processes.

U. S. Standard Melasses is molasses containing not more than $25 \%$ of water, nor more than $5 \%$ of ash.

Adulteration of Molasses and Syrups.-A common adulterant of all these products is commercial glucose. From its water-white color and inert sweetness, no less than from its cheapness, it forms an admirable adulterant for dark-colored or low-grade molasses and syrups, counteracting to a great extent by its smoothness the strong and often disagreeable taste of the inferior products with which it is mixed. Thus a grade of molasses too cheap to be ordinarily used for food purposes can be made to assume the appearance, and to some extent the taste, of the higher-priced and light-colored grades, by admixture with commercial glucose.

Tin salts are also used to improve the color of low-grade or dark molasses, and bleaching agents, such as sulphurous acid, are frequently employed. Copper is sometimes found, due to utensils or vessels used in processes of manufacture.

Lead may occur in maple syrup, due to the leaden plugs or spigots through which the sap is sometimes drawn from the trees.

Detection and Determination of Commercial Glucose. $\uparrow-F r o m$ the direct polarization of a normal solution of molasses or syrup the presence

* Jour. Am. Chem, Soc., 25 (1903), p. rorg.

† Leach, ibid., p. 982 . 
or absence of commercial glucose can usually be established. The direct polarization of a normal solution of pure molasses should not be much in excess of $50^{\circ}$ on the Soleil-Ventzke scale, while a pure, dark-colored molasses should polarize well under $40^{\circ}$. Golden syrup and maple syrup read higher than molasses, and a normal solution of pure maple syrup may have a direct polarization as high as $65^{\circ}$, being more often than not above $60^{\circ}$.

An excessively high direct polarization is at once an indication of the presence of commercial glucose, while an invert reading at ordinary room temperature to the right of the zero-point is an almost positive proof of its presence in either of the above products.

The optically active constituents of commercial glucose, viz., dextrin, maltose, and dextrose, are present in such varying amounts, that it is impossible to determine accurately the exact amount of this adulterant in complex saccharine products which themselves contain components common to glucose. Its approximate amount can, however, be very satisfactorily estimated in molasses and syrups by the use of the following formula:

$$
G=\frac{(a-S) 100}{175}, *
$$

where $G=$ per cent of commercial glucose, $a=$ direct polarization, and $S=$ per cent of cane sugar previously obtained from the Clerget formula (p. 588). A large amount of invert sugar present affects the accuracy of this formula. It is especially applicable to maple syrup, wherein the per cent of invert sugar is small, but may be applied also to molasses and golden syrup, wherein the amount of invert sugar is not so large but that results may be obtained as close as could be expected from an empirical formula. $\dagger$

In saccharine products containing considerable invert sugar the invert reading at $87^{\circ} \mathrm{C}$. obtained as directed on page 639 , is divided by

* Leach, U. S. Dept. of Agric., Bur. of Chem., Bul. 65, p. 48.

† This formula is based on the assumption that $42^{\circ} \mathrm{Bé}$. mixing glucose, the grade specially made and used for admixture with molasses, syrups, and honey, has a maximum polarization of $175^{\circ} \mathrm{V}$. It was adopted as a result of investigations made some years ago by the author, but subsequently it appeared that $42^{\circ}$ Bé. mixing glucose po'-rizes lower than formerly. Thus a sample recently examined by the author polarized at $162,1^{\circ} \mathrm{V}$. Pending further investigations it seems best for the present to retain the old formula, for, while it undoubtedly gives low results, especially with higher admixtures of glucose, it approximates the truth more closely than would be expected, perhaps because it tends to compensate for the error due to substances in genuine molasses and honey that polarize to the right after inversion. Furthermore, it has been adopted by the A. O. A. C. To avod misunderstanding, express results in terms of glucose polarizing at that factor. 
the appropriate factor $\left(\mathrm{I}_{3}\right)$ to obtain the percentage of commercial glucose.

While theoretically pure molasses and syrups would be expected to show no rotation when polarized at $87^{\circ} \mathrm{C}$. after inversion, as a matter of fact most samples exhibit a decidedly right-handed reading at that temperature. Occasionally a zero reading is noted, and in rare instances a slight left-handed rotation occurs under the above conditions.

Dextro-rotation is undoubtedly caused by some form of decomposition or fermentation. It may be due to a preponderance of dextrose in the reducing sugars, since levulose is more easily decomposed than dextrose, or it may be caused by the decomposition products formed when the raw juice is being defecated with lime, or again it might result from a special fermentation forming dextran.

The following table shows results by A. H. Bryan* of polarization of samples of Louisiana molasses and syrup of known purity, showing especially the invert readings at $87^{\circ} \mathrm{C}$.:

POLARIZATION OF LOUISIANA MOLASSES AND SYRUP.

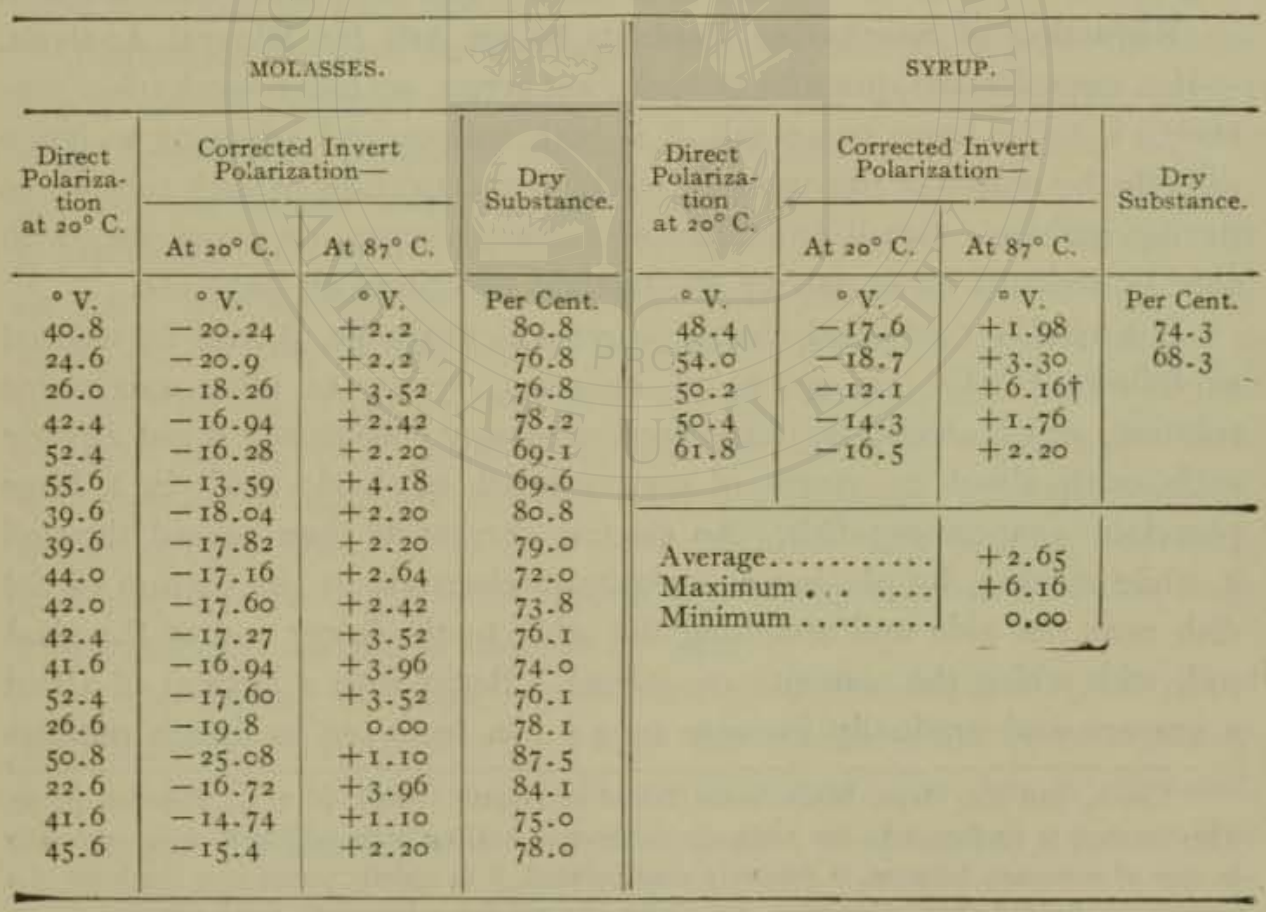

* A. O. A. C. Proc., 1908, U. S. Dept. of Agric., Bur, of Chem., Bul. 122, p. 182. $\dagger$ Sample ropy and badly fermented. 
TYPICAL ANALYSES OF MOLASSES AND SYRUPS ADULTERATED WITH COMMERCIAL GLUCOSE.

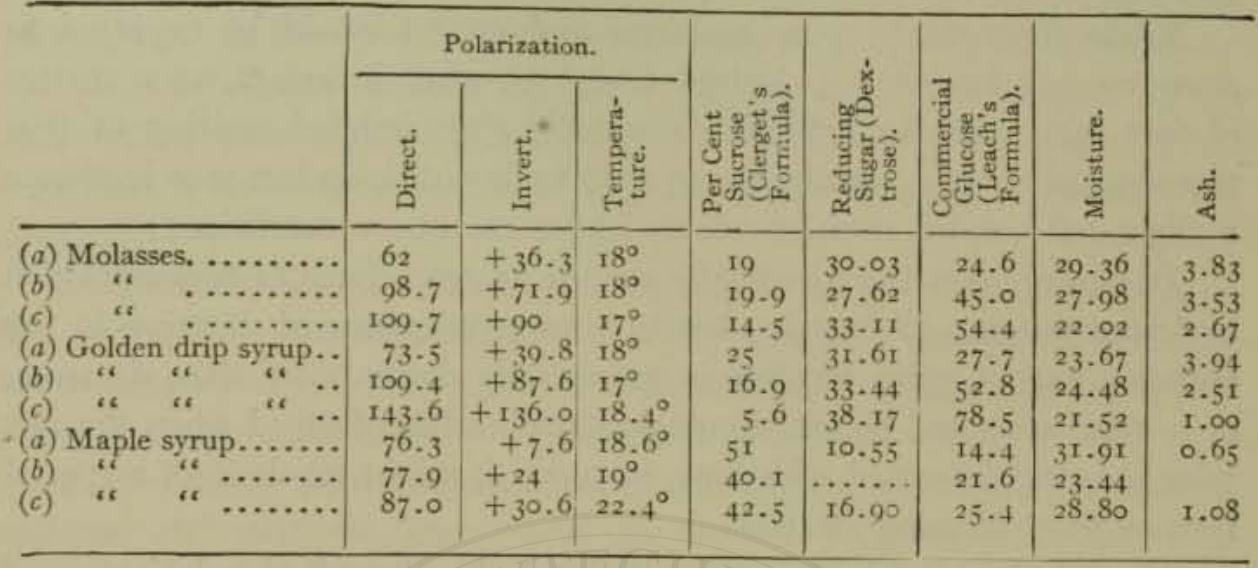

Determination of Dextrin. - According to Beckman's method a weighed amount of the honey or molasses is diluted with an equal volume of water and from ten to twelve times its volume of methyl alcohol is added. The precipitated dextrin is collected in a tared filter and thoroughly washed with methyl alcohol, after which it is dried and weighed.

Reduction of Saccharine Products to an Ash for Mineral Analysis. -If a considerable quantity of molasses, syrup, or other saccharine substance is to be burnt to an ash, it is both tedious and annoying to ignite directly, by reason of the excessive swelling and frothing of such substances during ignition. Small quantities of molasses, syrup, or honey may with care be reduced to an ash by the method described on page 586 .

If a readily controlled electric current is available, it may be utilized as follows: * Mix 100 grams of molasses, syrup, or other saccharine solution, which should be evaporated to syrupy consistency if not already such, with about 35 grams of concentrated sulphuric acid in a large porcelain evaporating-dish. An electric current is then passed through it while stirring, by placing one platinum electrode in the bottom of the dish near one side and attaching the other to the lower end of the glass rod, with which the contents are stirred. Begin with a current of about I ampere and gradually increase to $4 . \dagger$ In from ten to fifteen minutes

* Leach, 32 d An. Rept. Mass. State Board of Health (1900), p. 653. Reprint, p. 37. This method is preferred to the ordinary method of heating with sulphuric acid, especially in case of molasses, because, if properly manipulated, it so quietly comes into the form of a very finely divided char or powder, especially adapted for subsequent quick ignition.

$\uparrow$ Modified from method of Budde and Schou for determining nitrogen electrolytically. Ztschr. anal. Chem., 38 (r899), p. 345. 
the mass is reduced to a fine, dry char, which may then be readily burnt to a white ash in the original dish over a free flame or in a muffle.

Or, Ioo grams of the molasses or syrupy solution to be ashed may be first evaporated to dryness and afterward mixed with from to to $20 \mathrm{cc}$. of concentrated sulphuric acid in a porcelain evaporating-dish, or if the substance to be ashed be a dry sugar or confectionery, 20 grams are mixed with the above amount of acid. Heat is gently applied by means of the gas flame till the swelling and frothing have ceased, which usually requires only a few minutes. The final ignition is then accomplished in the usual manner, nitric acid being added if necessary to completely destroy the organic matter.

Determination of Tin in Molasses.-Fuse the ash from a weighed portion of the sample with sodium hydroxide in a silver crucible, dissolve in water, and acidulate with hydrochloric acid; filter and precipitate the tin from this solution with hydrogen sulphide; wash the precipitate on a filter and dissolve it in an excess of ammonium sulphide. Filter this solution into a tared platinum dish, and deposit the tin directly in the dish by electrolysis, using a current of 0.05 ampere and the apparatus described on page 608 .

Distinction between Invert Sugar, Maltose, and Lactose.*-All these sugars reduce Fehling's solution. Dextrose and levulose (invert sugar) when boiled with Barfoed's copper acetate solution (I4 grams crystallized copper acetate and $5 \mathrm{cc}$. acetic acid in $200 \mathrm{cc}$. water) will form a precipitate of cuprous oxide, while neither maltose nor lactose will do this. The solution, which has thus been tested for invert sugar and found to be free, or the filtrate from the cuprous oxide precipitate, is treated with an excess of basic lead acetate, filtered, and to the filtrate is added an excess of sodium sulphate solution to precipitate the lead. The solution is again filtered and treated with copper sulphate solution, if not already blue. It is then made alkaline with sodium hydroxide and heated to boiling. A red precipitate of cuprous oxide at this stage indicates either lactose or maltose or both.

A solution of the sugar, made strongly ammoniacal, is then mixed with alkaline bismuth solution $\dagger$ and the container is set in a waterbath at $60^{\circ} \mathrm{C}$. Maltose soon reduces the bismuth, but lactose does not.

To test for lactose, add strong nitric acid to the solid sugar residue

\footnotetext{
* Bartley and Mayer, Merck's Report, 12 (1903), p. roo.

$\dagger$ This reagent is prepared as follows: Bismuth subnitrate, 2 grams; Rochelle salt, 4 grams; sodium hydroxide, 8 grams; dissolved in $100 \mathrm{cc}$. of water by the aid of heat.
} 
and warm gently till red fumes come off. Then set the container in hot water and cool gradually. Crystals of mucic acid appear after a time if any appreciable amount of lactose be present.

Determination of Lactose or Maltose.-Either sugar, if in solution free from other reducing sugars, may be determined by the volumetric Fehling methot (p. 59I) or by the Defren method, using the table on page 595 .

For the determination of maltose in commercial glucose, see page 630 .

Estimation of Cane Sugar and Dextrose in Mixtures.-Obtain true direct and invert readings of a normal solution of the mixture. Determine the per cent of sucrose by Clerget's formula (p. 588). This figure represents the right-handed rotation due to sucrose. Subtracting this from the direct polarization, the difference represents the right-handed rotation due to dextrose. The specific rotary power of sucrose is 66.5 and that of dextrose 52.3 .

Calling $d$ the percentage of dextrose and $R^{\prime}$ the right-handed rotation due to dextrose as above obtained, if the Soleil-Ventzke scale is used,

whence

$$
66 \cdot 5: 5^{2} \cdot 3=d: R^{\prime}
$$

$$
d=\frac{66 \cdot 5 R^{\prime}}{5^{2} \cdot 3} .
$$

Determination of Levulose.*-On page $5^{89}$ attention was called to the variation in the rotary power of levulose with the temperature. This variation is constant, and I gram of levulose in roo cc. of water produces a decrease in left-handed reading of $0.0357^{\circ}$ on the cane sugar (Ventzke) scale for each $\mathrm{I}^{\circ} \mathrm{C}$. increase in temperature. Therefore, the weight of levulose present in a given solution can be calculated from the polariscopic readings at two temperatures, using a water-jacketed tube, as described on page 639 .

$$
L=\frac{R-R^{\prime}}{0.0357\left(t-t^{\prime}\right)}
$$

where

$L=$ weight of levulose,

$R=$ reading at higher temperature $t$,

$R^{\prime}=$ reading at lower temperature $t^{\prime}$.

The percentage of levulose present in the solution may readily be calculated as follows:

* Wiley, Agric. Anal., p. 272. 
$L^{\prime}=$ percentage of levulose,

$L=$ weight of levulose in solution,

$W=$ weight of sugar sample made up to roo cc.,

$$
L^{\prime}=\frac{L \times 100}{W} \text {. }
$$

In a normal solution $W=26.048$.

\section{ANALYSIS OF MAPLE PRODUCTS.}

Determination of Moisture.-This is accomplished by direct drying with sand, or by calculation from the specific gravity, or, preferably from the refractive index. See molasses methods, page 613.

Determination of Ash.-Burn 5 grams in a platinum dish by the usual method, observing the precautions given for molasses, page 6r 4 .

Soluble and Insoluble Ash.*-To the platinum dish containing the ash add $40 \mathrm{cc}$. of hot water and boil gently for two minutes. Filter through a small ashless filter, and wash with hot water until the filtrate amounts to $100 \mathrm{cc}$. Return the filter to the dish used for ashing, burn at a low red heat, cool and weigh, thus obtaining the insoluble ash. The soluble ash is obtained by difference, subtracting the weight of insoluble from that of total ash.

Alkalinity of Soluble Ash.†-Allow the filtrate from the above determination to cool, then titrate with tenth-normal hydrochloric acid, using methyl orange as an indicator.

Alkalinity of Insoluble Ash. $\dagger$-Add excess of tenth-normal hydrochloric acid (usually Io to $\mathrm{I}_{5} \mathrm{cc}$.) to the ignited insoluble ash in the platinum dish, heat to the point of boiling over an asbestos plate, allow to cool, and titrate excess of hydrochloric acid with tenth-normal sodium hydroxide, using methyl orange as an indicator.

Express the alkalinity in each case as the number of cubic centimeters of tenth-normal acid used on the ash of I gram of sample.

Polarization.--See page $6 r_{4}$.

Determination of Reducing Sugar.-See page 62I.

Determination of Malic Acid Value.-Modified Leach and Lythgoe Method. $\ddagger$ Weigh 6.7 grams of the sample into a $200 \mathrm{cc}$. beaker, and add

*A. H. Bryan, U. S. Dept. of Agric, Bur. of Chem., Circ. No. 40, p. 6.

† U. S. Dept. of Agric, Bur. of Chem., Bul. 107 (rev.), p. 69.

$\ddagger$ Jour. Am. Chem. Soc., 26, 1904 , pp. 380 and 1536 ; U. S. Dept. of Agric., Bur. of Chem., Bul. to 7 (rev.), p. 74 . 
water to make a volume of $20 \mathrm{cc}$. Add 2 drops of ammonium hydroxide (specific gravity, 0.90), I cc. of a $10 \%$ solution of calcium chloride, and $60 \mathrm{cc}$. of $95 \%$ alcohol. Cover the beaker with a watch glass, heat for one-half hour on a water bath, then turn off the flame and allow the beaker to stand overnight. Filter the material in the beaker through good quality filter paper, wash the precipitate with hot $75 \%$ alcohol until the filtrate measures $100 \mathrm{cc}$., dry and ignite. Add from $\mathrm{I}_{5}$ to $20 \mathrm{cc}$. of tenth-normal hydrochloric acid to the ignited residue, thoroughly dissolve the lime by heating carefully to just below boiling, cool and titrate the excess of acid with tenth-normal sodium hydroxide, using methyl orange as an indicator. One-tenth of the number of cubic centimeters acid neutralized by the ignited residue expresses the malic acid value. Run blank determinations on reagents, using the same amounts, particularly of ammonium hydroxide, as were used in the original determination, and make the necessary correction.

Determination of Lead Number.-Winton Method.*-Weigh 25 grams of the material (or 26 grams if a portion of the filtrate is to be used for polarization) and transfer by means of boiled water into a Ioo-cc. flask. Add $25 \mathrm{cc}$. of standard lead subacetate solution, fill to the mark, shake, allow to stand at least three hours and filter through a dry filter. From the clear filtrate pipette off ro cc., dilute to $50 \mathrm{cc}$., add a moderate excess of sulphuric acid, and $100 \mathrm{cc}$. of $95 \%$ alcohol. Let stand over night, filter on a Gooch crucible, wash with $95 \%$ alcohol, dry at a moderate heat, ignite at low redness for three minutes, taking care to avoid the reducing cone of the flame, cool, and weigh. Calculate the amount of lead in the precipitate, using the factor $0.68_{31}$, subtract this from the amount of lead in $2.5 \mathrm{cc}$. of the standard solution, multiply the remainder by 100 , and divide by 2.5 , thus obtaining the lead number.

The standard lead subacetate is prepared by diluting one part of the ordinary solution (page 586) with four volumes of water, filtering if not clear. It is standardized by a blank determination conducted as above described. The solution deposits a slight precipitate on standing, but this does not usually appreciably affect its strength.

Ross Modification. $\uparrow-$ This process is specially adapted for the examination of mixtures of maple and cane sugar syrups, as the results are proportional to the per cent of maple syrup present, which is not true of the Winton method. The lead numbers of pure maple syrup range

\footnotetext{
* Jour. Am. Chem. Soc., 28, 1906, p. 1204.

† U. S. Dept. of Agric., Bur. of Chem., Circular 53.
} 
from 1.8 to 3.0 , whereas by the Winton method they range from 1.2 to 2.5 .

Transfer 25 grams of the syrup to a roo-cc. flask, using about $25 \mathrm{cc}$. of freshly boiled water, add $10 \mathrm{cc}$. of potassium sulphate solution ( 7 grams. per liter), then $25 \mathrm{cc}$. of lead subacetate solution of the strength employed in the foregoing method. Make up to the mark with boiled water and proceed as in the Winton method.

Run the blank in exactly the same way, substituting 25 grams of pure cane sugar syrup (66 grams of sucrose dissolved in 34 grams of water) for the maple syrup.

Determination of Hortvet Number.*-Apparatus.-(I) A tube, I5.3 $\mathrm{cm}$. in length, consisting of a wide cylindrical portion $3 \mathrm{~cm}$. in diameter, narrowed at the top to a neck $2 \mathrm{~cm}$. in diameter, and at the bottom to a stem graduated in tenths to $5 \mathrm{cc}$.

(2) A holder, made of pine or white wood, of a size adapted to carry the tube in the shield of the centrifuge. The holders and tubes should be arranged in balanced pairs in the centrifuge.

Procedure.-Introduce $5 \mathrm{cc}$. of syrup or 5 grams of sugar into the tube. Add ro cc. of water, and dissolve completely. Next add ro drops of alumina cream, and I.5 cc. of lead subacetate. Shake thoroughly, and allow to stand from forty-five to sixty minutes. Place the tube in its holder in the centrifuge shield, and run six minutes. If, after the end of this time, any material adheres to the sides of the wide part of the tube, loosen with a small wire or-by giving the tube a slight twist, then run the tube six additional minutes, and finally rear the volume of the precipitate in the stem, estimating to $0.01 \mathrm{cc}$.

Run a blank with the above reagents in water, subtracting the blank reading from that of the precipitate. In the case of syrup, reduce to the 5 -gram basis by dividing by the specific gravity of the sample. If the sugar content of the sample is known, the specific gravity can be calculated from the table on page 617 . For pure maple syrup $\mathrm{r} .33$ is very nearly correct.

The centrifuge used by Hortvet had a radius of $18.5 \mathrm{~cm}$. and was run at a speed of 1600 revolutions per minute. The corresponding velocity in $\mathrm{cm}$. per second $(v)$ and revolutions per minute $(R)$ for any given centrifuge with a radius of $r \mathrm{~cm}$. may be calculated by the following formulæ:

$$
v=\sqrt{520,000 r}, \quad R=60 v / 2 \pi r \text {. }
$$

\footnotetext{
* Jour. Am. Chem. Soc., 26, 1904, p. 1532.
} 
Results by Hortvet on pure maple syrups vary from I.2 cc. to about $2.5 \mathrm{cc}$., and on pure maple sugars from $\mathrm{r} .8 \mathrm{cc}$, to $4 \mathrm{cc}$.

Commercial brands of adulterated syrups and sugars give such precipitates as $0.00 \mathrm{cc}$., $0.02 \mathrm{cc} ., 0.05 \mathrm{cc}$., and $0.08 \mathrm{cc}$. Hortvet regards with suspicion a syrup testing lower than I.2 cc., and when the result is below I cc., the sample is positively condemned as being mixed with refined cane sugar. In the case of sugar, a somewhat higher minimum figure is adopted than with syrup. In view of the fact that the speed has much to do with the volume of the precipitate, the analyst should make a series of similar experiments with his own centrifuge, and work out his own standards. Results may be better compared with each other, if calculated on the water-free basis.

In case of doubt, and in fact in all cases at first, it would be well to make confirmatory tests, such as determining the ash and reducing sugar.

Sy's Lead Method.*-In a 25 -cc. graduated cylinder introduce $5 \mathrm{cc}$. of syrup, or 5 grams of sugar which is afterwards dissolved in a little water. Add water to the ${ }_{5} \mathrm{cc}$. mark and $2 \mathrm{cc}$. of lead subacetate solution. Shake thoroughly and allow the mixture to stand twenty hours. Then read the volume of the precipitate, which for pure maple products should be at least $3 \mathrm{cc}$. and is usually over $5 \mathrm{cc}$.

\section{ANALYSIS OF COMMERCIAL GLUCOSE.}

Wileyt has worked out a method for calculating the percentage of dextrin, maltose, and dextrose present in commercial glucose, based on the specific rotary power of these substances and on the reducing power of maltose and dextrose. To apply this method, the operator, if he has a polariscope reading in sugar scale degrees, must ascertain the equivalent readings in angular degrees from the table on page $5^{8}{ }_{3}$, and calculate the specific rotary power in each case from the formula $(\alpha)_{D}=\frac{\text { Ioo } a}{c l}$, page $5^{84}$.

Thus, if he possesses a Schmidt and Haensch instrument, he should multiply the true reading, as obtained on that instrument, with a normal

* Jour. Am. Chem. Soc., 30, rgo8, p. 1430 .

† Chem. News, 46, p. 175; Agric. Anal., 3, pp. 288-29o. 
solution of the given sugar or mixture, by the factor 0.3468 , to convert the reading into circular degrees from which to figure the specific rotary power as above.

The specific rotary power of dextrin is fixcd at 193 , that of maltose ${ }_{13} 8$, and that of dextrose at 53 .

Then if $P=$ total polarization of the mixture in terms of specific rotary power, $d=$ per cent dextrose, $m=$ per cent maltose, and $d^{\prime}=$ per cent dextrin,

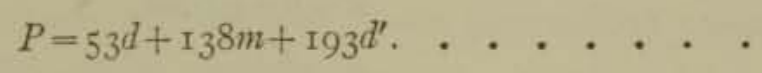

The value of $P$ is obtained from observation and calculation as above described on a known solution of the sample, say to grams in 100 cc. The reducing sugars, maltose and dextrose, are then removed, preferably by oxidation with cyanide of mercury, as follows:*

Prepare the reagent by dissolving 120 grams mercuric cyanide and I20 grams sodium hydroxide in water, mixing the two solutions, and making up to $I, 000 \mathrm{cc}$. Remove any precipitate that may gather by filtration.

Make a solution of ro grams of the glucose sample in roo cc. and take to cc. of this solution in a $50-\mathrm{cc}$. graduated flask. Add sufficient mercuric cyanide solution to have an excess of reagent after the oxidation (from 20 to $25 \mathrm{cc}$.), and boil for three minutes under a hood with a good draft. Cool and neutralize the alkali with concentrated hydrochloric acid, adding the latter till the brown color is discharged. By this method the optical activity of the maltose and dextrose is discharged, while that of the dextrin remains unaffected. From the polariscope reading calculate as above the specific rotary power of the dextrin $\left(P^{\prime}\right)$. Then

$$
P^{\prime}=193 d^{\prime} . \text {. . . . . . . . . }
$$

The reducing power on Fehling's solution of dextrose is to that of maltose as 100 is to 62 . Whence, if $R=$ reducing sugar (reckoned as dextrose) we have

$$
R=\dot{d}+0.62 m
$$

Subtracting equation (2) from equation (I) we have

$$
P-P^{\prime}=53^{d}+{ }_{3} 8 m \text {. }
$$

*Wiley, Agric. Analysis, p. 290. 
Multiplying equation (3) by 53 and subtracting from equation (4),

Therefore

$$
\begin{aligned}
& P-P^{\prime}=53 d+138 m, \\
& 53 R=53 d+32.86 m ; \\
& P-P^{\prime}-53 R=105.14 m .
\end{aligned}
$$

$$
\begin{aligned}
& m=\frac{P-P^{\prime}-53 R}{105.14}, \ldots . . . . . \\
& d=R-0.62 m, \quad . \quad . \quad . \quad . \quad . \quad . \quad(7) \\
& d^{\prime}=\frac{P^{\prime}}{193} . \quad . \quad . \quad . \quad . \quad . . . . .
\end{aligned}
$$

Determination of Dextrin in Commercial Glucose.-One volume of the sample is well shaken with about Io volumes of $90 \%$ alcohol, and the precipitated dextrin is separated by filtration through a tared filter, washed thoroughly with strong alcohol, dried at $100^{\circ}$, and weighed.

Qualitative Tests for Commercial Glucose. - Several confirmatory chemical tests may be employed for commercial glucose, aside from the optical test with the polariscope. Thus a precipitate of dextrin by treatment of the sample with an excess of strong alcohol, in the absence of mineral salts insoluble in alcohol, is strongly indicative of commercial glucose. An excess of sodium chloride in the ash also points strongly to the presence of glucose.

Determination of Ash.-Formerly when sulphuric acid was used for conversion of the starch the ash consisted largely of calcium sulphate, but at present when hydrochloric acid is almost exclusively used the mineral matter is almost entirely common salt, formed by the neutralization of the acid.

Determine ash by burning in a platinum dish at dull redness as in the case of other saccharine products. Qualitative or quantitative tests may be made for chloride, in the latter case calculating the equivalent amount of sodium chloride. If the amount of sodium chloride found does not equal the total ash, sulphates may be looked for.

Determination of Sulphurous Acid. - At the present time glucose usually is free from an appreciable amount of sulphurous acid which formerly was extensively employed for bleaching. It may be determined by distillation, oxidation to sulphuric acid, and precipitation with barium chloride as described on page 840 . 
Detection of Arsenic.-Since the Manchester epidemic of arsenical poisoning, due to the consumption of beer prepared from glucose contaminated through the sulphuric acid with this poison, it is highly important that both the acid used for conversion and the glucose be frequently tested for this contamination.

The tests may be made on 2 to 5 grams of the materials without charring or destruction of the organic matter, by the Marsh test or the SangerBlack-Gutzeit test as described under general methods on pages 74 to 77 .

The English limit of one and one-half parts per million calculated as metallic arsenic should not be exceeded.

\section{HONEY.}

Composition and Occurrence.-Honey is the saccharine product deposited by bees (Apis mellifica and $A$. dorsata) in the cells of honey comb, which the insect forms out of wax secreted by its body. Honey has its source chiefly in the nectaries of flowers, from which the bees abstract it, also in the juices of ripe fruits and the exudations of leaves (honeydew). While in the honey-sac of the bee, the sucrose, which forms the chief constituent of the fruit juice or nectar, becomes for the most part inverted, forming, in the honey, dextrose and levulose. The evaporation to a syrupy consistency is effected in the hive by exposure to a current of air, produced by fanning of the wings of the bees.

The flavor of honey varies considerably, according to its source. Besides water, the sugars, and mineral matters, pollen is usually present, derived from the flowers, also as a rule a small quantity of wax, and nearly always appreciable amounts of various organic acids. Fincke $\dagger$ states real honey may or may not contain formic acid.

European Honey.-Neufeld* gives the following limits for pure honey:

Water. ....................... 8.30 to $33.59 \%$

Protein. ...................... 0.03 " $2.67 \%$

Invert suger. .................. 49.59 " $93.96 \%$

Sucrose.................... 0. 10 " 10.1 $2 \%$

Dextrin....................... 0.99 " $9.70 \%$

Formic acid................... 0.03 " $0.21 \%$

Ash. ....................... $0.02 " 0.68 \%$

* Der Nahrungsmittelchemiker als Sachverständiger., Berlin, r907, p. 275.

$\dagger$ Zeits. Unters. Nahr. Genussm., 23, 1912, p. 255. 
Canadian Honey.-A large number of samples of genuine honey analyzed in 1897 for the Department of Inland Revenue, Canada (Bul. 47), showed the following variations:

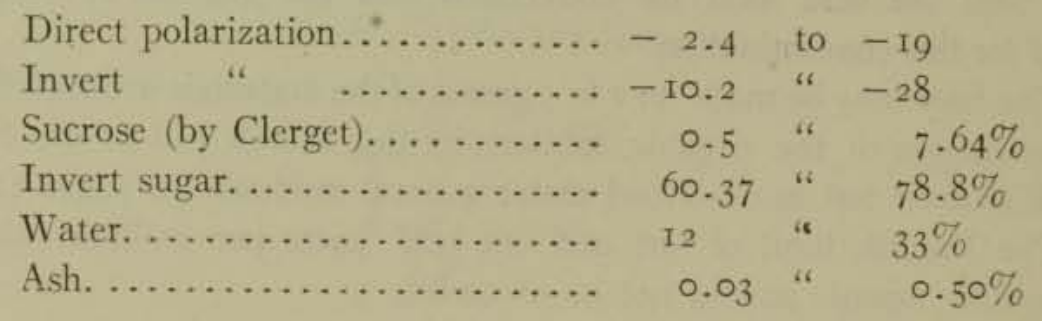

American Honey.-Browne* has examined 97 samples of American and Hawaiian honey, representing the product made from the nectar of numerous flowers as well as honeydew. Maxima and minima of polarizations and analyses of some of the more important kinds, and of all the levorotatory and the dextrorotatory samples are given in the table on page 635 .

As regards the chemical characteristics of honey from different flowers, Browne states that alfalfa honey usually has less dextrin and undetermined matter-the so-called "impurities"-and more sucrose than the other varieties, although the low amount of impurities is, to some extent, characteristic of the honey of the whole family (leguminosæ). The compositæ yield honey with about the average amount of organic non-sugars; the rosaceæ yield a product low in dextrin, but high in undetermined $\mathrm{m}$ t ter. Buckwheat and other polygonaceous honeys contain almost no sucrose, but give tests for tannins. Basswood honey is relatively high in dextrin, and that from poplar, oak, hickory and other trees, all of which contain considerable quantities of honeydew, are rich in both dextrin and ash. Pronounced tannin reactions are obtained in honey gathered from the flowers or plants of the sumac, hop and others rich in tannin. Tupelo, mangrove and sage honeys are distinguished by their high levulose content.

Browne found the average per cent of water in honey from the arid states of Arizona, Nevada, Utah, and Colorado was 15.60, and from the humid states of Minnesota, Wisconsin, Illinois, Missouri and Iowa was I8.88.

Hawaiian Honey.-This is characterized by its high ash and the presence of decided amounts of chlorides in the ash. Van Dinet states

* U, S. Depi. of Agric., Bur. of Chem., Bul, r10 (1908).

† Ibid., p. 52 . 


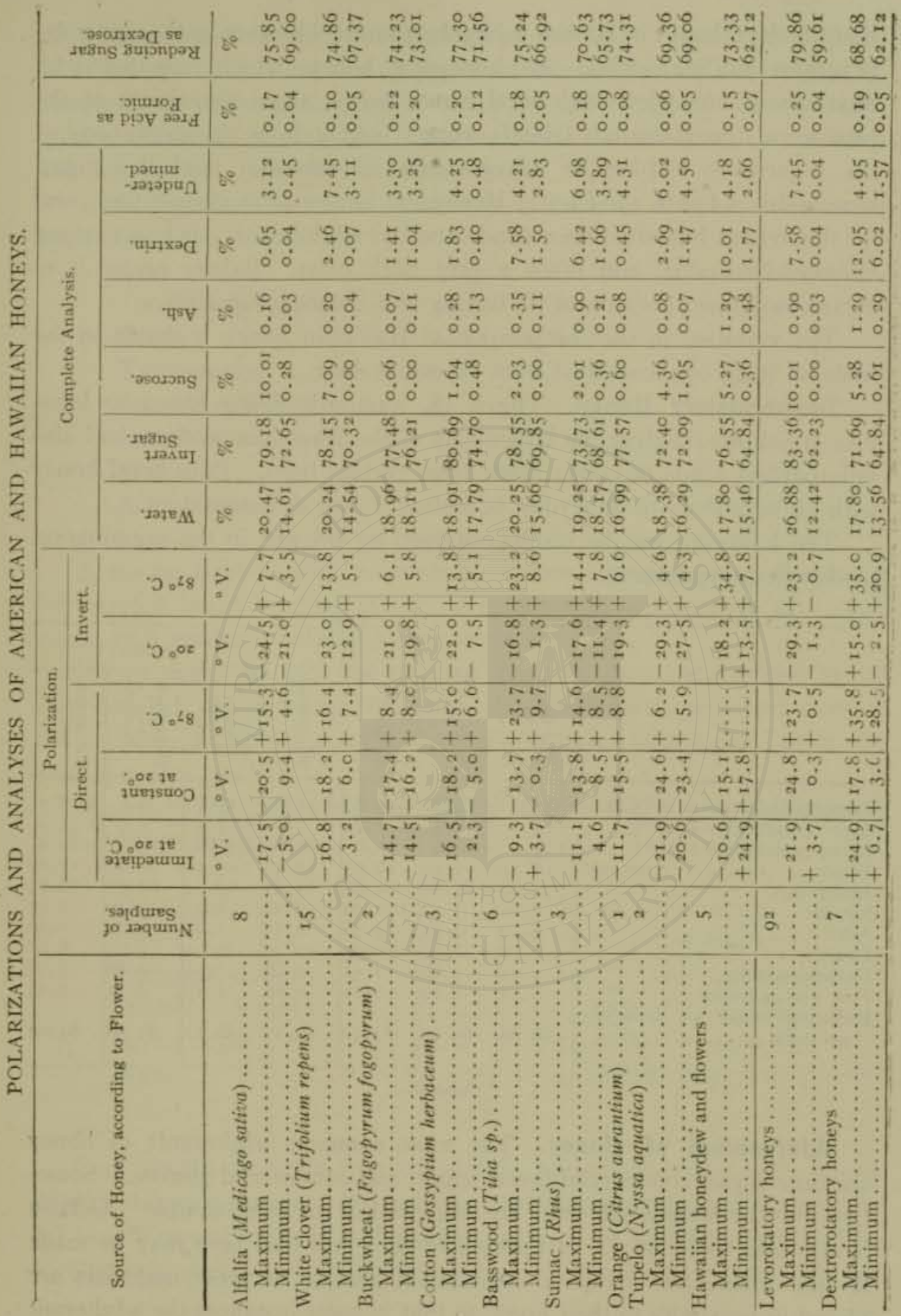


that the floral honey of Hawaii is largely from the blossoms of the algarroba (Prosopis julifera), while the honeydew honey, which, together with mixtures of honeydew and floral honey forms about two-thirds of the product of the Hawaiian Islands, comes largely from the exudations of the sugar-cane leaf-hopper (Perkinsiella saccharicida), and the sugarcane aphis (Aphis sacchari). Honeydew honey is dextrorotatory, and for this reason has often been condemned as adulterated. It has a strong molasses-like odor, and often a very dark color. Bakers prefer it to algarroba honey, because of its baking and boiling properties.

The variation in the composition of Hawaiian honey is shown in the table on page 635 , compiled from Browne's data.

Dextrorotatory Honey.-The U. S. standards define honey as levorotatory, thus excluding the larger part of the Hawaiian product, and also unimportant kinds of honey made from certain trees. Pure floral honey with no admixture of honeydew is seldom if ever dextrorotatory.

The following are the results obtained by Browne in the examination of detrorotatory honeys:

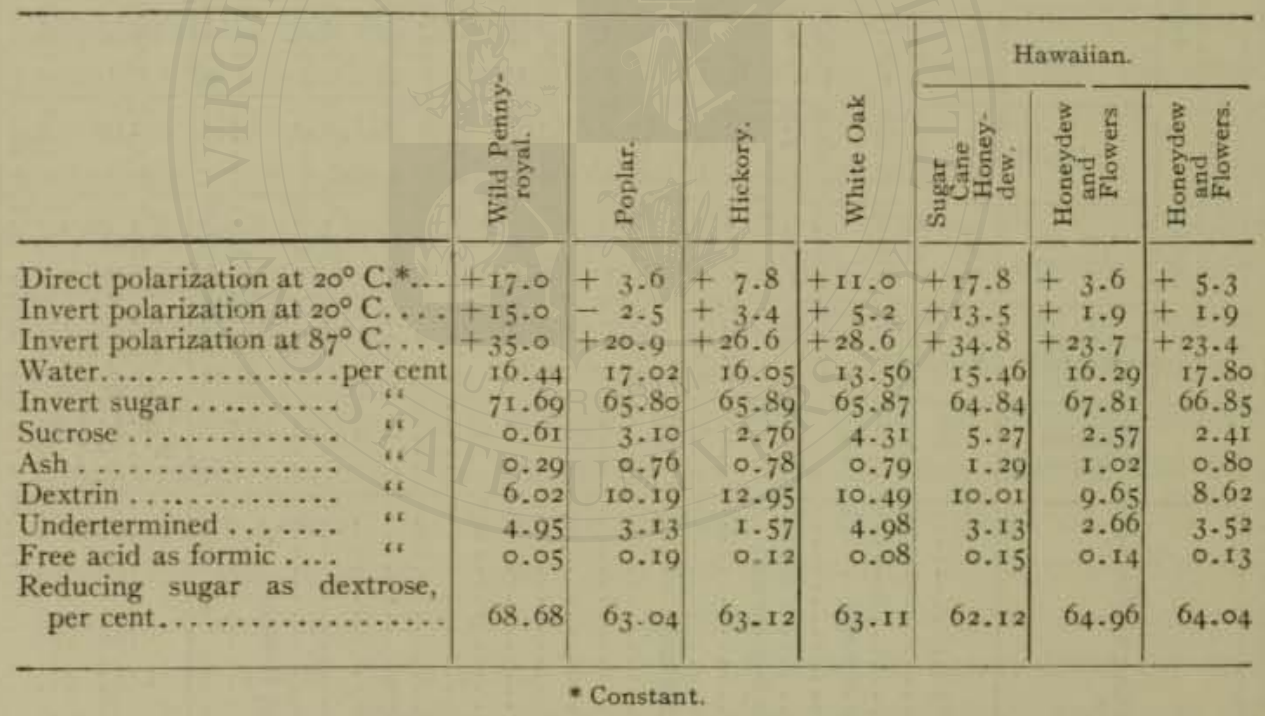

Adulteration of Honey.-The most common adulterants of honey are cane sugar, commercial invert sugar, and commercial glucose. Sometimes two or more adulterants are employed in the same sample. Gelatin is also said to be used. It appears to be a fact that bees may be made to feed upon cane syrup or commercial glucose, if these materials are placed in proximity to their hives, so that in some instances the adulterant 
may be supplied through the medium of the bee. Sophisticated honey is often put up in tumblers or jars containing pieces of honeycomb, so that presence of the comb is by no means proof of its purity. Comb-honey, sold in the frame as sealed by the bees, is never adulterated, except when the bees are fed upon glucose or căne sugar.

Cane Sugar.-The following are typical analyses of honey adulterated with cane sugar:

A. B. C.

\begin{tabular}{|c|c|c|c|}
\hline Direct polarization. . . . & $+34 \cdot 7$ & $+\mathrm{I} 2$ & +1.2 \\
\hline Invert $\quad \| \quad \ldots \ldots$ & -24 & -17.6 & $-2 I \cdot 5$ \\
\hline Temperature. ........ & $14^{\circ}$ & $\mathrm{r} 5^{\circ}$ & $19 \cdot 5^{\circ}$ \\
\hline Sucrose (Clerget) . . . . & $43 \cdot 16 \%$ & $21.8 \%$ & $17.07 \%$ \\
\hline Invert sugar. . . . . . . & $42.48 \%$ & $60.03 \%$ & $67.2 \%$ \\
\hline Water. & $42.42 \%$ & $2 \mathrm{I} .15 \%$ & ${ }^{1} 5.56 \%$ \\
\hline Ash. & $\ldots \ldots \ldots$ & $.11 \%$ & $0.06 \%$ \\
\hline
\end{tabular}

A strong right-handed polarization before inversion, coupled with a left-handed invert reading at $20^{\circ}$, is evidence of adulteration with cane sugar, or a product containing cane sugar.

Honey stored by bees fed on cane sugar is also characterized by its right-handed polarization. Although the bee inverts the larger part of the cane sugar in its body, this inversion is never as complete as in the case of nectar honey.

Glucose.-The following are typical analyses of honey adulterated with commercial glucose:

A.* B. $\mathrm{C}$.

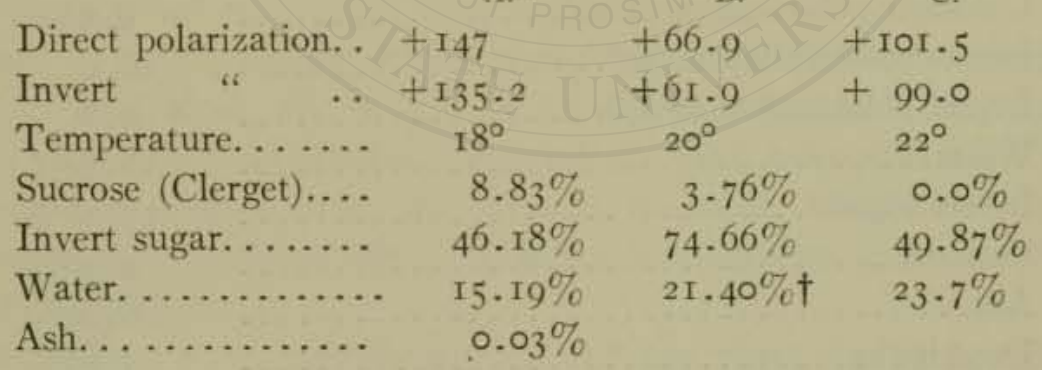

Care should be taken not to confuse honeydew honey with honey adulterated with glucose. Browne gives the following means of distinction: (I) the difference in invert polarization between 20 and $87^{\circ}$, corrected to $77 \%$ invert sugar, (2) Beckman's iodine test (page 64I), and (3) the

* Both commercial glucose and added cane sugar. 
König and Karsch test (page 642). He also finds the quantity and character of the ash, the acidity, and microscopic examination of value.

The following analyses of mixtures of commercial glucose and honey were made by A. H. Bryan.*.

\begin{tabular}{|c|c|c|c|c|c|c|c|c|c|c|}
\hline \multicolumn{2}{|c|}{ Mixture, } & \multirow[b]{2}{*}{$\begin{array}{c}\text { Constant } \\
\text { Direct } \\
\text { Polariza- } \\
\text { tion at } \\
z^{\circ} \mathrm{C} \text {. }\end{array}$} & \multicolumn{2}{|c|}{ Invert Polarization- } & \multirow[b]{2}{*}{$\begin{array}{c}\text { Polariza- } \\
\text { tion } \\
\text { Differ- } \\
\text { ence } \\
\left(87^{\circ}-\right. \\
\left.20^{\circ}\right) .\end{array}$} & \multicolumn{2}{|c|}{ Invert Sugar } & \multicolumn{3}{|c|}{ Calculated Glucose. } \\
\hline Glucose & Honey. & & At $20^{\circ} \mathrm{C}$ & At $87^{\circ} \mathrm{C}$. & & $\begin{array}{c}\text { Before } \\
\text { Inver- } \\
\text { sion. }\end{array}$ & $\begin{array}{c}\text { After } \\
\text { Inver- } \\
\text { sion. }\end{array}$ & $\begin{array}{c}\text { Invert } \\
\text { Polariza- } \\
\text { tion at } \\
87^{\circ}+ \\
t .63 \text {. }\end{array}$ & $\begin{array}{c}\text { Invert } \\
\text { Polariza- } \\
\text { tion at } \\
\left(20^{\circ} \mathrm{C} .+\right. \\
17.5) \div \\
1.93\end{array}$ & $\begin{array}{l}\text { 100- } \\
\text { (Correct- } \\
\text { ed Polar- } \\
\text { ization } \\
\text { Differ- } \\
\text { ence X } \\
\text { roo } \\
26.7 \text { ) }\end{array}$ \\
\hline $\begin{array}{r}\% \\
100\end{array}$ & $\begin{array}{c}\% \\
\cdots \cdots\end{array}$ & $\begin{array}{r}\circ \mathrm{V} . \\
+153.8\end{array}$ & $\begin{array}{c}\circ \mathrm{V} . \\
+153.34\end{array}$ & $\begin{array}{r}\circ \mathrm{V} . \\
+\quad+44.32\end{array}$ & $\begin{array}{l}{ }^{\circ} \mathrm{V} . \\
\cdots \\
\cdots\end{array}$ & $\begin{array}{c}\% \\
30.02\end{array}$ & $\begin{array}{c}\% \\
30.45\end{array}$ & $\begin{array}{c}\% \\
88.5\end{array}$ & $\frac{\%}{88.5}$ & $\%$ \\
\hline 50 & 50 & +67.0 & +65.67 & $+73.8 \mathrm{I}$ & 8.14 & 53.67 & $54 \cdot 5^{c}$ & $45 \cdot 3$ & $43 \cdot .1$ & $5^{6.9}$ \\
\hline 20 & 80 & +15.4 & +13.42 & +33.00 & $19.5^{8}$ & 69.00 & 70.35 & 20.2 & 16.0 & 19.2 \\
\hline 10 & 90 & -2.4 & -4.84 & $+\quad 18.59$ & 23.43 & $74 \cdot 42$ & $74 \cdot 1=$ & 11.4 & 6.6 & 8.8 \\
\hline 5 & 95 & -11.5 & $-14 \cdot 31$ & +11.66 & 25.96 & $75 \cdot 74$ & $77.8 \mathrm{c}$ & $7 \cdot 2$ & 1. 6 & 3.8 \\
\hline 3 & 97 & -14.2 & -16.94 & +9.13 & 26.07 & 76.62 & 78.01 & 5.6 & 0.29 & 3.7 \\
\hline 2 & 98 & -16.0 & -18.70 & 8.14 & 26.84 & 76.64 & $78 \cdot 34$ & 5.0 & 0.00 & 1.2 \\
\hline I & 99 & -18.2 & -20.90 & 6.93 & $27.8_{3}$ & $77 \cdot 20$ & 78.87 & 4.2 & 0.00 & 0.0 \\
\hline$\ldots \ldots$ & 100 & -19.5 & -22.11 & $+\quad 5.94$ & 28.05 & 77.68 & $78 \cdot 9$ & 3.2 & 0.00 & 0.0 \\
\hline
\end{tabular}

Commercial Invert Sugar is the most difficult of detection of all the adulterants. Herzfeld's process $\dagger$ for the manufacture of invert sugar syrups consists in boiling for thirty to forty-five minutes I kilogram of refined sugar in $300 \mathrm{cc}$. of water with I.I gram of tartaric acid. Brown $\ddagger$ gives the following analysis of the product made by this process:

Direct polarization at $20^{\circ} \ldots \ldots \ldots \ldots \ldots \ldots-6.2$

Constant polarization at $20^{\circ} \ldots \ldots \ldots \ldots \ldots . . .6 .5$

Invert polarization at $20^{\circ} \ldots \ldots \ldots \ldots \ldots \ldots-16.9$

Invert polarization at $87^{\circ} \ldots \ldots \ldots \ldots \ldots \ldots+4.8$

Water............................... $16.32 \%$

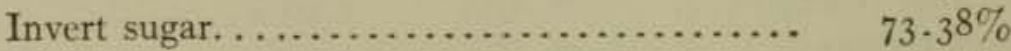

Sucrose. .......................... $4.36 \%$

Ash. ................................. $0.00 \%$

Dextrin.............................. $4.86 \%$

100.00

Acids as formic......................... $0.06 \%$

*A. O. A. C. Proc., 1908, U. S. Dept. of Agric., Bur. of Chem., Bull. 122, p. 18r. $\dagger$ Zeits. ver. d. Zucker-Ind., 3I, p. 1988.

$\ddagger$ loc. cit., page 64 . 
This adulterant is best detected by Browne's test (page 642). Ley's test* has value in confirming the results of Browne's test, but should be used with caution, as American honeys do not react like the European.

Gelatin is indicated if a precipitate occurs in the diluted sample with a solution of tannic acid.

\section{ANALYSIS OF HONEY.}

Preparation of Sample.-In the case of strained honey, stir with a rod till any separated sugars are evenly distributed throughout the mass, or, if the honey has become solidified wholly or in part by crystallization, use a gentle heat on a closed water-bath to restore it to fluid form.

In the case of comb honey, cut with a knife across the top of the comb if sealed, and separate completely from the comb by straining through a 40 -mesh sieve.

Determination of Moisture.t-Weigh 2 grams into a flat-bottom metal dish $2 \frac{1}{2}$ inches in diameter, which, together with Io to 15 grams of fine quartz sand and a short stirring rod, has been previously tared, add 5 to ro cc. of water, stir until the whole has been thoroughly incorporated, and dry to constant weight at 65 to $70^{\circ} \mathrm{C}$. in a vacuum oven. Honeys of high purity usually dry in twelve hours, while those of the honeydew class rich in dextrin and gum require thirty-six hours, or longer.

Determination of Ash.-See page 6r4.

Polarization.-Direct and Invert at $20^{\circ} \mathrm{C}$.- Proceed as directed under molasses (page $6 \mathrm{r}_{4}$ ), except that only alumina cream is used as a clarifier. To destroy birotation add a drop or two of ammonia before making up to the mark. $\ddagger$

Invert at $87^{\circ} \mathrm{C}$.- Invert a half normal portion in the usual manner in a roo-cc. flask, cool, add a few drops of phenolphthalein and enough sodium hydroxide to neutralize; discharge the pink color with a few drops of dilute hydrochloric acid, add from 5 to $10 \mathrm{cc}$. of alumina cream, make up to the mark and filter. Polarize in a $200-\mathrm{mm}$. tube at $87^{\circ}$, and multiply reading by 2 .

Polarization at the temperature of $87^{\circ}$ can most readily be effected by the use of a water-jacketed tube, as shown in Fig. III. An all-metal tube, the interior of which is heavily gold-plated to avoid corrosion by acid, is preferable to one in which the inner tube is glass with a metal

* Pharm. Zeits., 47, 1902, p. 603.

$\dagger$ Browne, U. S. Dept. of Agric., Bur. of Chem., Bul. I10, p. I8.

$\ddagger$ Frühling, Zeits. öffentl. Chemie, 4, r898, p. 4 ro. 
jacket, as in the latter leaky joints are liable to occur, due to uneven expansion. A tubulure is provided in the outer tube for a thermometer, so that the exact temperature may be noted. A tank of boiling water placed on a shelf above the polariscope is connected by rubber tubing with the jacketed tube as it rests in the polariscope, as shown in Fig. III.

Determination of Reducing Sugars.-Determine by Allihn's method (page 608) in an aliquot of $25 \mathrm{cc}$. of a solution obtained by making $10 \mathrm{cc}$. of the solution prepared for polarization up to $250 \mathrm{cc}$. If desired the sugar may be determined by the volumetric Fehling process (page 59I).

The reducing sugars may be calculated as dextrose as obtained from Allihn's table, or as levulose by multiplying the dextrose by r.044.

Determination of Levulose.-Wiley's Method.*-This may be calculated approximately by the following fornula:

$$
l=\frac{100(1.0315 A-a)}{(2.3919) 26}=\frac{100(1.0315 A-a)}{62.19},
$$

in which $l=$ levulose, $a=$ the direct polarization at $20^{\circ}$ of a solution of the normal quantity of honey made up to $100 \mathrm{cc}$. at $20^{\circ}$, and $A=$ the direct polarization of the same solution at $87^{\circ} \mathrm{C} ., 2.3919=$ the variation in polarization of $\mathrm{I}$ gram of levulose in $100 \mathrm{cc}$. of solution between 20 and $87^{\circ} \mathrm{C}$., and $\mathrm{r} .03 \mathrm{I}_{5}=$ the factor for converting the volume of the solution at $20^{\circ}$ into that at $87^{\circ} \mathrm{C}$.

Determination of Dextrose. $\uparrow-M u l t i p l y$ the percentage of levulose as obtained in the preceeding section by 0.915 , thus obtaining the equivalent dextrose, and subtract this from the per cent of reducing sugars expressed as dextrose.

Determination of Sucrose.-Owing to the inaccuracies of Clerget's method as applied to honey, Browne recommends the following: Neutralize the free acid of Io $\mathrm{cc}$. of the solution used for invert polarization with sodium carbonate, make up to $250 \mathrm{cc}$. and determine the reducing sugars by Allihn's method. Subtract from the invert sugar thus obtained the invert sugar found before inversion, and multiply the difference by 0.95 .

Determination of Dextrin.-Browne's Method. $\uparrow-W e i g h ~ 8$ grams of honey directly into a roo-cc. flask, add $4 \mathrm{cc}$. of water, and finally with

* Principles and Practice of Agricultural Analysis, 1897, III, p. 267. Browne, loc. cit., p. 17.

† Browne, loc. cit., p. 17. Jour. Am. Chem. Soc., 28, 1906, p. 446. 
continual agitation sufficient absolute alcohol to fill to the mark. Shake thoroughly and allow to stand twenty-four hours, or until the dextrin is deposited on the bottom and sides of the flask and the liquid is perfectly clear. Decant on a filter and wash the precipitate in the flask with ro cc. of cold $95 \%$ alcohol, pouring the liquid finally on the filter. Dissolve the precipitate in the flask and on the filter in a little boiling, distilled water, collecting the solution in a tared platinum dish. Evaporate the liquid, and dry to constant weight at $100^{\circ} \mathrm{C}$. If the alcohol precipitate is considerable, it should be dried at $70^{\circ} \mathrm{C}$. in vacuo. After weighing, dissolve in water and make up to a definite volume according to the weight as follows:

Residue, grams. $0-0.5 \quad 0.5^{-1} .0 \quad$ I.O-I. $5 \quad$ I. $5^{-2.0} \quad 2.0-2.5 \quad 2.5-3 . \mathrm{C}$ Volume, cc. ... $50 \quad 100 \quad 150 \quad 200 \quad 250 \quad 300$

Filter, determine invert sugar and sucrose in aliquots by copper reduction before and after inversion, and subtract the sum of these sugars from the total alcohol precipitate.

Determination of Acids.-Dissolve 10 grams of the honey in water and titrate with tenth-normal sodium hydroxide, using phenolphthalein as indicator. Express result as formic acid.

Beckman's Test for Glucose.*-Treat a mixture of equal parts of honey and water with a solution of iodine in potassium iodide. If glucose is present, a red or violet color (due to erythro- or amylo-dextrin) appears, the shade and intensity depending on the nature and amount of the glucose present.

Determination of Commercial Glucose in Honey.-Except for rough work, the method described on page 622 for calculating the per cent of commercial glucose from the sucrose and from the direct polarization is not recommended for use with honey and other products wherein the invert sugar is so large as to considerably affect its accuracy. In this case, it is best after inversion to polarize the sample at $87^{\circ} \mathrm{C}$., the temperature at which the reading due to invert sugar would theoretically be o. At this temperature, any considerable right-handed polarization can be accounted as due to commercial glucose. (See page 639.)

As in the case of molasses, the writer advocates assuming $175^{\circ}$ as the direct polarization of the glucose used, this being about the maximum reading for a normal solution of $42^{\circ}$-Bé. glucose. Lythgoe has shown

*Zeits. Anal. Chem., 35, 1896, p. 267. 
that in polarizing at high temperatures samples of saccharine products containing commercial glucose, certain precautions have to be observed not necessary when cane or invert sugar are the only sugars present. Thus, a normal solution of glucose, when polarized at $87^{\circ} \mathrm{C}$., has a lower reading than in the cold, the difference being doubtless due partly at least to the expansion of the liquid. Again, on subjecting a normal solution of glucose to inversion with acid, as in Clerget's process, and heating to $87^{\circ} \mathrm{C}$., it will be found impossible to get a constant reading, but the reading will drop rapidly, due to a partial hydrolysis of the maltose or dextrin.

In honey and other preparations containing much invert sugar and commercial glucose, it is best to proceed as follows: Divide the polarization at $87^{\circ}$ by $163_{3}^{*}$ and multiply the result by 100 for the percentage of commercial glucose in terms of glucose polarizing at $175^{\circ}$. It should be borne in mind that the results by even this method are only approximate, as genuine honey is more or less dextrorotatory at $87^{\circ} \mathrm{C}$.

The following formula is used by European chemists: $G=\frac{b+17 \cdot 5}{\mathrm{I} .93}$ in which $G=$ the per cent of commercial glucose, and $b=$ the polarization after inversion at $20^{\circ} \mathrm{C}$.

Browne's Test for Commercial Invert Sugar. + -Reagent.-This should be freshly prepared each time before using. Shake $5 \mathrm{cc}$. of c. p. anilin with $5 \mathrm{cc}$. of water, and add sufficient glacial acetic acid ( $2 \mathrm{cc}$.) to just clear the emulsion.

Process.-Treat $5 \mathrm{cc}$. of a $\mathrm{I}: \mathrm{I}$ solution of the honey in a test tube with I to $2 \mathrm{cc}$. of the anilin reagent, allowing the latter to flow down the walls of the tube so as to form a layer upon the honey solution. If, when the tube is gently agitated, a red ring forms beneath the anilin solution, this color becoming gradually imparted to the whole layer, artificial invert sugar is present. This reaction is due to furfural formed during the high temperature employed in the commercial processes of inversion. Boiling genuine honey also causes the formation of furfural, but this treatment impairs the flavor and is probably never practiced.

Distinction of Honeydew and Glucose Honeys.-Method of König and Karsch. $\ddagger-D i s s o l v e ~ 40$ grams of honey in a cylinder in water, and

* The true polarization at $87^{\circ} \mathrm{C}$. of a normal solution of glucose subjected to inversion and neutralization as above (but without the use of the clarifier), will be about $93 \%$ that of the direct polarization of the sample in the cold. Hence $175 \times 0.93=162.7$.

† U. S. Dept. of Agric., Bur. of Chem., Bul. x10, p. 68.

$\ddagger$ Zeits. anal. Chem., 34, 1895, p. r. U. S. Dept. of Agric., Bur. of Chem., Bul. 110, p. 63 . 
make up to $40 \mathrm{cc}$. Transfer $20 \mathrm{cc}$. of the homogeneous solution to a $250-\mathrm{cc}$. flask and fill to mark with absolute alcohol with slow addition and constant shaking, and then allow to stand two or three days, with occasional agitation. At the end of this time all the dextrin has settled out. After shaking the solution, filter and evaporate $100 \mathrm{cc}$. of the filtrate until free from alcohol. To the liquid residue add a little subacetate of lead and sodium sulphate, make up to $20 \mathrm{cc}$. with water, and polarize the filtered solution. Dextrorotatory natural honeys show by this method a levorotation; honeys adulterated with dextrose or glucose to the extent of $25 \%$ or more, a dextrorotation. In case the honey contains a large amount of sucrose, the solution should be inverted with hydrochloric acid before polarizing.

BEESWAX.-The purity of beeswax is best established by determining its melting-point, its specific gravity, its saponification equivalent, and its refractometric reading. The melting-point of pure wax is about $64^{\circ} \mathrm{C}$., while that of paraffin, its chief adulterant, is from $5^{2}$ to $55^{\circ} \mathrm{C}$. Its saponification equivalent should be from 87.8 to 107 , while that of paraffin is 0 .

Method of Determining Specific Gravity of Beeswax.*-Place a weighed rod of the wax, about I to $1.5 \mathrm{~cm}$. long by $0.5 \mathrm{~cm}$. diameter, in an accurately marked 5o-cc. flask, and run in water from a burette till the water level reaches the mark. $50 \mathrm{cc}$. minus the burette reading represent the volume occupied by the wax. The rod should be made to lie flat on the bottom of the flask, so that the incoming water will force its end against the sides and prevent the end from rising above the mark. The weight of the rod, divided by its volume gives its specific gravity. The specific gravity of various mixtures of wax of 0.969 specific gravity and paraffin of $0.87 \mathrm{I}$ are given in the following table, prepared by Wagner, so that from the specific gravity of the mixture the percentage of paraffin can be calculated:

\begin{tabular}{c|c|c||c|c|c}
\hline $\begin{array}{c}\text { Wax } \\
\text { (Percentage). }\end{array}$ & $\begin{array}{c}\text { Paraffin } \\
\text { (Percentage). }\end{array}$ & $\begin{array}{c}\text { Specific } \\
\text { Gravity. }\end{array}$ & $\begin{array}{c}\text { Wax } \\
\text { (Percentage). }\end{array}$ & $\begin{array}{c}\text { Paraffin } \\
\text { (Percentage). }\end{array}$ & $\begin{array}{c}\text { Specific } \\
\text { Gravity. }\end{array}$ \\
\cline { 2 - 3 } & 100 & .871 & 75 & 25 & .942 \\
25 & 75 & .893 & 80 & 20 & .948 \\
50 & 50 & .920 & 100 & -969 \\
\hline
\end{tabular}

The Refractometer Reading is most useful in establishing the purity of wax. Observations with this instrument are best made at $65^{\circ}$ and

*U. S. Dept. of Agric., Div. of Chem., Bul. 13, p. 842 . 
great care should be taken in the case of the Zeiss butyro-refractometer not to exceed this temperature, or injury to the instrument may result.

The Abbé refractometer may be used with perfect safety and, when available, is to be preferred for the examination of beeswax. Many food laboratories are, however, not equipped with the Abbé, but nearly all find the butyro-refractometer indispensable. The latter instrument was primarily designed for such substances as butter and lard, so that the

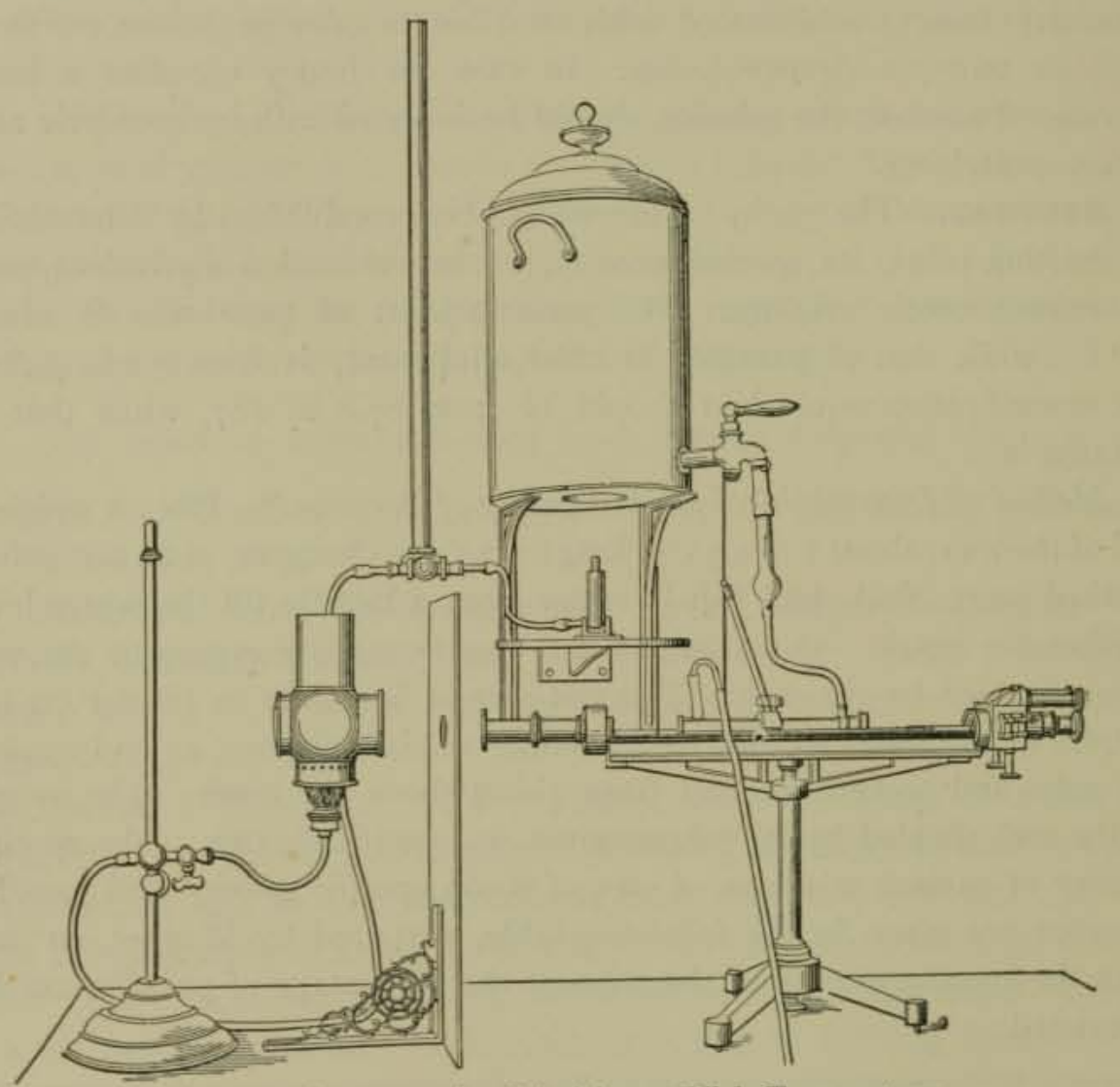

Fig. I11.-Apparatus for Polarizing at High Temperatures.

manufacturers did not intend it to be subjected to as high a temperature as $65^{\circ}$. They have, however, assured the author that if care be taken to bring the temperature very slowly and gradually to the required degree, $65^{\circ}$, and to avoid also sudden cooling, the cement that secures the prisms in place will not be appreciably affected; otherwise cracking or loosening of the cement would be liable to occur after a time. 
At $65^{\circ} \mathrm{C}$. pure beeswax should have a reading on the butyro-refractometer of 30 to 3 I. $5{ }^{*}$ while that of paraffin is from II to $14.5 . \dagger$

\section{CONFECTIONERY.}

The composition of confectionery is more complex than that of the saccharine products hitherto considered. As a rule, cane sugar, or one of its products, as molasses, forms the basis of most of the confections. Commercial glucose is also a common ingredient, while a large variety of such materials as eggs, butter, chocolate, various flavoring extracts, spices, nuts, and fruits, enter into the composition of confectionery.

U. S. Standard Candy is candy containing no terra alba, barytes, talc, chrome yellow, or other mineral substances or poisonous colors or flavors, or other ingredients injurious to health.

Adulteration.- Of late the adulteration of confectionery has been held largely in check by the National Confectioners' Association of the United States, which has fixed high standards of purity, and has been very zealous in restricting the use of harmful adulterants.

Commercial glucose is not regarded as an adulterant of confectionery by the above-named association and by but few of the state laws. On the contrary, any ingredient, other than color, that has no food value, may logically be considered as an adulterant. Under this head are included such substances as paraffin, as well as make-weight mineral matters, such as terra alba, talc, or calcium sulphate.

B. $\mathrm{H}$. Smith $\ddagger$ has called attention to the presence of arsenic in shellac used to coat certain kinds of confectionery.

Colors in Confectionery.-A very wide range of colors is necessarily employed in the manufacture of confectionery, and the almost endless variety of coal-tar dyes now available lend themselves most readily to the confectioner's needs. Elsewhere, under "colors," lists of injurious and non-injurious dyes are given as compiled by the National Confectioners' Association, though it is not always readily apparent how the lines are drawn.

The tinctorial power of these dyes is so high that the actual amount of substance contained in a thin coating of the color on the outside of the candy is exceedingly small, so that it is doubtful whether serious cases of injury have ever arisen from their use.

* $n_{D}, \mathrm{~T} .445^{2}$ to $\mathrm{I} .4463$.

$\dagger n_{D}, \mathrm{I} .4310$ to $\mathrm{I} .4335$

‡ U. S. Dept. of Agric., Bur. of Chem., Circ. 91, 1912 . 
Such was not the case formerly, before the prevalence of the coal-tar dyes, when such poisonous mineral pigments as chromate of lead were frequently used. Only one or two instances of the use of lead chromate in candy have come to the author's attention within ten years, since more satisfactory and harmless yêllow colors among the azo-dyes are now obtainable.

ANALYSIS OF CONFECTIONERY.-The following have been submitted by the author as provisional methods of procedure for the A. O. A. C.:*

(I) Products of Practically Uniform Composition Throughout.(a) Lozenges and Other Pulverizable Products.-Grind in a mortar or mill to a fine powder. For total solids, weigh from 2 to 5 grams of the powdered sample in a tared platinum dish, and dry in a MçGill oven to constant weight.

For $A s h$, ignite the residue from total solids in the original dish, observing the precautions given under sugar (p. 586), and molasses (p. 624).

(b) Semi-plastic, Syrupy, or Pasty Products.-Weigh 50 grams of the sample into a 250 -cc. graduated flask, mix thoroughly or dissolve, if soluble, in water, and fill to the mark. Be sure that the solution is uniform, or, if insoluble material is present, that it is evenly mixed by shaking before taking aliquot parts for the various determinations. For total solids and ash, measure $25 \mathrm{cc}$. of the above solution or mixture into a tared platinum dish, and proceed as directed under $(a)$.

(2) Confectionery in Layers or Sections of Different Composition.When it is desired to examine the different portions separately, they should be separated mechanically with a knife, when possible, and treated as directed under ( 1 ).

(3) Sugar-coated Fruit, Nuts, etc.-In case of a saccharine coating inclosing fruit, nuts, or any less readily soluble material, dissolve or wash off the exterior coating in water, which may, if desired, be evaporated to dryness for weighing, and proceed as in (I).

(4) Candied or Sugared Fruits.-Proceed as in the examination of fruits (Chapter XXI).

Detection of Mineral Adulterants.-As in the case of molasses, a considerable quantity, say 100 grams, should be reduced to an ash for examination for mineral adulterants, such as talc, calcium sulphate, and iron oxide, which are detected by regular qualitative tests.

* U. S. Dept. of Agric., Bur. of Chem., Bul. 65, p. 44. 
Detection of Lead Chromate.-Fuse the ash in a porcelain crucible with a mixture of sodium carbonate and potassium chlorate, boil the fused residue with water, neutralize with acetic acid, filter, and treat the filtrate with barium chloride or lead acetate solution. A yellow precipitate indicates a chromate. "Treat the insoluble part of the fusion with nitric acid, and test for lead in the usual manner.

If a drop of ammonium sulphide be applied to a piece of confectionery colored with lead chromate, it will produce a black coloration.

Determination of Ether Extract.-The ether extract includes the fat derived from chocolate, eggs, or butter, as well as any paraffin present. Measure $25 \mathrm{cc}$. of the $20 \%$ solution (I) (b) (p. 6+6) into a very thin, readily frangible glass evaporating-shell (Hoffmeister's Schälchen), containing 5 to 7 grams of freshly ignited asbestos fiber; or, if impossible to thus obtain a uniform sample, weigh out 5 grams of the mixed, finely divided sample into a dish, and wash with water into the asbestos in the evaporating-shell, using, if necessary, a small portion of the asbestos fiber on a stirring-rod to transfer the last traces of the sample from dish to shell. Dry to constant weight at $100^{\circ}$, after which cool, wrap loosely in smooth paper, and crush into rather small fragments between the fingers, carefully transferring the pieces with the aid of a camel's-hair brush to an extraction-tube, or to a Schleicher and Schull cartridge for fat extraction. Extract with anhydrous ether or with petroleum ether in a continuous extraction apparatus for at least twenty-five hours. Transfer the solution to a tared flask, evaporate the ether, dry in an oven at $100^{\circ} \mathrm{C}$. to constant weight, and weigh.

Determination of Paraffin.-Add to the ether extract in the flask, as above obtained, Io cc. of $95 \%$ alcohol, and 2 cc. of $\mathrm{I}$ : $\mathrm{r}$ sodium hydroxide solution, connect the flask with a reflux condenser, and heat for an hour on the water-bath or until saponification is complete. Remove the condenser, and allow the flask to remain on the bath till the alcohol is evaporated off, and a dry residue is left. Treat the residue with about $40 \mathrm{cc}$. of water, and heat on the bath, with frequent shaking, till everything soluble is in solution. Wash into a separatory funnel, cool, and extract with four successive portions of petroleum ether, which are collected in a tared flask or capsule. Remove the petroleum ether by evaporation, and dry in the oven to constant weight.

It should be noted that any phytosterol or cholesterol present in the fat would come down with the paraffin, but the amount would be so insignificant that, except in the most exacting work, it may be disregarded. 
The character of the final residue should, however, be confirmed by determining is melting-point and specific gravi.y, and by subjecting it to examination in the butyro-refractometer. The melting-point of paraffin is about $54.5^{\circ} \mathrm{C}$; its specific gravity at $15.5^{\circ} \mathrm{C}$. is from 0.868 to 0.915 , and on the butyro-refractometer the reading at $65^{\circ} \mathrm{C}$. is from II to 14.5 .

Determination of Starch.-Measure gradually $25 \mathrm{cc}$. of a $20 \%$ aqueous solution or uniform mixture of the sample into a hardened filter or Gooch crucible, or transfer by washing 5 grams of the finely powdered substance to the filter or Gooch, and allow the residue on the filter to become airdried. Extract with five successive portions of 10 cc. of ether, then wash with $150 \mathrm{cc}$. of $10 \%$ alcohol, and finally with $20 \mathrm{cc}$. of strong alcohol. Transfer the residue to a large flask and boil gently for four hours with $200 \mathrm{cc}$. of water and $20 \mathrm{cc}$. of hydrochloric acid (specific gravity I.125), the flask being provided with a reflux condenser. Cool, neutralize with sodium hydroxide, add $5 \mathrm{cc}$. of alumina cream, and make up the volume to $250 \mathrm{cc}$. with water. Filter and determine the dextrose in an aliquot part of the filtrate by any of the various Fehling methods. The weight of the dextrose multiplied by 0.9 gives the weight of the starch.

Polarization of Confectionery.-As a clarificr use either alumina cream or subacetate of lead, according to the nature and opacity of the sample. Ordinarily alumina cream is best, but in dark-colored samples, or those in which molasses has been used, it is sometimes necessary to employ the subacetate. When starch is absent, and the sample is practically soluble, polarize and invert in the usual manner (p. $5^{88}$ ). Where considerable starch or insoluble matter is present, use the double-dilution method of Wiley and Ewell (p. 620), thus making due allowance for the volume of the precipitate.

Cane sugar, invert sugar, and dextrin, are determined as directed for honey.

Commercial glucose is roughly determined by polarizing the sample at $87^{\circ} \mathrm{C}$., as in the case of honey (p. 639).

Confectionery is made in such a wide variety of forms, and these differ in consistency to such an extent that commercial glucose of all available degrees of density can be utilized to advantage in one product or another. In this respect confectionery is unlike honey and molasses, wherein a fairly uniform grade of commercial glucose is necessarily used for mixing, this grade being naturally selected with reference to its similarity in density to the molasses. On this account the glucose factor used 
for honey and molasses (175) may in some varieties of confectionery be too high.

Determination of Alcohol in Syrups Used in Confectionery.-(Brandydrops.) - Open each drop by cutting off a section with a sharp knife, and collect in a beaker the syrup "of from ${ }_{5} 5$ to 25 of the drops, which will usually yield from 30 to 50 grams of syrup. Strain the syrup into a tared beaker through a perforated porcelain filter-plate in a funnel to separate from particles of the inclosing shell, and ascertain the weight of the syrup. Wash into a distilling-flask, dilute with half its volume of water, and distil off into a tared receiving-flask a volume equal to the original volume of syrup taken. Ascertain the weight of the distillate and its specific gravity by means of a pycnometer. Multiply the percentage by weight of alcohol corresponding to the specific gravity, as found in the tables on page 66r et seq., by the weight of the distillate, and divide this by the weight of syrup taken. The result is the per cent by weight of alcohol in the syrup.

Detection of Colors.-It is sometimes necessary to macerate a considerable mass of the material to remove the color, which is, however, in the majority of cases readily soluble. The insoluble colors are nearly all mineral pigments to be looked for in the ash, as in the case of chromate of lead (p. 647). Frequently the coloring matter is confined to a thin outer layer, which is readily washed off.

The solution of the dyestuff is examined as directed under colors.

Detection of Arsenic.-Arsenic may be present through impure coloring-matter. If the color is confined to an exterior coating, this should be washed off and examined. If distributed through the mass, a solution of the whole should be taken. Examine for arsenic by the Gutzeit or Marsh method, as directed on pp. 74 to 77 . 


\section{REFERENCES ON SUGARS.}

Babington, F. W. Sugars, Syrups, and Molasses. Can. Inl. Rev. Dept., Bul. 25.

- Maple Syrup. Can. Inl. Rev. Dept., Bul. 45 .

BARTLEy, E. H., and MAYER, J. L. Identification of Carbohydrates. Merck's Report, 12, 1903 , p. 100.

Brown, H. T., Morris, G. H., and Minlar, J. H. Experimental Methods Employed in the Examination of the Products of Starch Hydrolysis by Diastase. Jour. Chem. Soc. Trans., 71 (1897), p. 72.

Browne, C. A. The Analysis of Sugar Mixtures. Jour. Am. Chem. Soc., 28, rgo6, p. 439 .

- Chemical Analysis and Composition of American Honeys. U. S. Dept. of Agric., Bur. of Chem., Bul. rio.

- The Unification of Saccharimetric Observations. A.O.A.C. Proc. 1908, p. 22 r.

- A Handbook of Sugar Analysis. New York, I912.

Bryan, A. H. The Estimation of Dry Substance by the Refractometer in Liquid Saccharine Food Products, Jour. Am. Chem. Soc., 30, 1908, p. 1443 .

- Methods for the Analysis of Maple Products and the Detection of Adulterants, together with the Interpretation of Results Obtained. U. S. Dept. of Agric, Bur. of Chem., Circ. No. 40.

— Maple Sap Sirup. U. S. Dept. of Agric., Bur. of Chem., Bul. I34.

- Chemical Analyses and Composition of Imported Honey from Cuba, Mexico, and Haiti. U. S. Dept. of Agric., Bur. of Chem., Bul. 154, 1912.

Doolittle, R. E., and Seeker, A. F. The Possibilities of Muscovado Sugar as an Adulterant for Maple Products. A. O. A. C. Proc, 1908. U. S. Dept. of Agric., Bur. of Chem., Bul. 122, p, 196.

Notes on the Winton Lead Number of Mixtures of Cane and Maple Syrup. Ibid., p. 198 .

Fresenius, W. Der Stärkesirup bei Zubereitung von Nahrungs- und Genussmitteln. Zeits. Unters. Nahr. Genuss., 2, I899, pp. 35 u. 279.

FRÜHLING, R. Anleitung zur Untersuchung der für die Zuckerindustrie, 6th ed. Braunsweig, 1903 .

Hintner, R. S., and Thatcher, R. W. An Improved Method for the Rapid Estimation of Sugar in Beets. Jour. Am. Chem. Soc., 23, I90r, p. 299.

Horne, W. D. The Chemical Determination of Sulphites in Sugar Products. U. S. Dept. of Agric., Bur. of Chem., Bul. 105, 1906, p. 125.

Hortver, J. The Chemical Composition of Maple-Syrup and Maple-Sugar, Methods of Analysis, and Detection of Adulteration. Jour. Am. Chem. Soc., 26, 1904, p. 1523 . 
Jones, C. H. Detection of Adulteration in Maple Sugar and Maple Syrup. Vt. Agric. Exp. Sta. Rep., 1903 , p. 446 .

-Maple Syrup and Maple Sugar Investigations with Particular Reference to the Detection of Adulteration. Vt. Agric. Exp. Sta. Rep., 1904, p. 315.

Landolt, H. Handbook of the Polariscope and its Practical Applications, I882.

- Trans. by Long, J. H. Optical Rotation of Organic Substances. Easton, I902.

LEACH, A. E. The Determination of Commercial Glucose in Molasses, Syrups and Honey. Jour. Am. Chem. Soc., 25, 1903, p. $9^{82}$.

Saccharine Products. U. S. Dept. of Agric., Bur. of Chem., Bul. 65, p. 43 .

Lock and Newlands. A Handbook for Planters and Refiners. London, 1888.

Macfarlane, T. Honey. Can. Inl. Rev. Dept., Bul. 45.

Munson, L. S., and Walker, P. H. The Unification of Reducing Sugar Methods. Jour. Am. Chem. Soc., 28, 1906, p. $66_{3}$.

Robin, L. Sucrés. Analyse des Matières Alimentaires (Girard et Dupré), p. 525. Paris, 1894 .

Ross, S. H. Suggested Modification of the Winton Lead Number, Especially as Applied to Mixtures of Maple and Cane Sugar Sirups. U. S. Dept. of Agric., Bur. of Chem., Circ. 53.

Roth, H. L. A Guide to the Literature of Sugar. London, I8go.

SACHSSE, R. Die Chemie der Kohlenhydrate. Leipzig, 1877.

SAwYer, H. E. The Commercial Analysis of Molasses. Jour. Am. Chem. Soc., 27, 1905, p. $69 \mathrm{r}$.

Shutt, F. F., and Charron, A. T. Determination of Moisture in Horey. Trans. Royal Soc. Canada, 2d Series, rgo2-3, 7, Section 3 .

Siderskx, D. Traité d'Analyse des Matières Sucrées. Paris, I8go.

Spencer, G. L. Handbook for Sugar Manufacturers and their Chemists. New York, I905.

StEydn, E. Die Untersuchung des Zuckers und Zuckerhaltiger Stoffe. Leipzig, I893.

Sy, A. P. Note on the Examination of Maple Products-The Lead Value. J. Frank. Inst., 162 , p. 71 .

- Three New Preliminary Tests for Maple Products, Jour. Am. Chem. Soc., 30, I908, p. I429.

Tollens, B. Handbuch der Kohlenhydrate. Breslau, 1888.

Tolman, L. M., and Smith, W. B. Estimation of Sugars by Means of the Refractometer. Jour. Am. Chem. Soc., 28, 1906, p. 1476.

Tucker, J. H. Manual of Sugar Chemistry. New York, 1890.

Walker, P. H. The Unification of Reducing Sugar Methods. Jour. Am. Chem. Soc., 29 , 1907 , p. 541 .

Weichmann, F. S. Sugar Analysis. New York, I89o.

WeIN, E. Tabellen zur quantitativen Bestimmung der Zuckerarten.

- Trans. by FREW, W. Tables for the Quantitative Estimation of the Sugars. London, 1896 . 
Wriey, H. W. Sugar, Molasses and Syrup, Confections, Hraey and Beeswax. U. S. Depth of Agric., Div. of Chem., Bul. 13. part 6.

- The Influence of Temperature on the Specific Rotation of Sucrose and Method of Correcting Readings of Compensating Polariscopes Therefor. Jour. Am. Chem. Soc., 21, 1899, p. 568.

Winton, A. L., and Kreider, J. LeHn. A Method for the Determination of Lead Number in Maple Syrup and Maple Sugar. Jour. Am. Chem. Soc., 28, 1906, p. 1204.

YoDER, P. A. Ueber das Vorkommen von Formaldehyd in Zuckerfabriks-erzeugnissen. Zeits. Unters. Nahr. Genuss., 20, 19ro, p. 208.

- A Polariscopic Method for the Determination of Malic Acid and its Application in Cane and Maple Products. Jour. Ind. Eng. Chem., 3, I9II, p. 563.

- Notes on the Determination of Acids in Cane Juice. Jour. Ind. Eng. Chem., 3, I0I1, p. 640.

Young, W. J. A Miscroscopic Study of Pollen. U. S. Dept. of Agric., Bur. of Chem., Bul. iro. 


\section{CHAPTER XV.}

\section{ALCOHOLIC BEVERAGES.}

Alcoholic Fermentation.-In a broad sense all alcoholic liquors are saccharine products, in that they are essentially the result of the fermentation of sugar. In the case of fruits, the sugar already exists as such in their juices, which, when expressed, almost immediately on exposure to the air begin to undergo spontaneously the process of alcoholic fermentation, in accordance with the reaction:

$$
\text { (I) } \underset{\substack{\text { Dextrose or } \\ \text { grape sugar }}}{\mathrm{C}_{2} \mathrm{H}_{12} \mathrm{O}_{6}={ }_{2} \mathrm{C}_{2} \mathrm{H}_{6} \mathrm{O}+{ }_{\text {Aloohol }} \mathrm{CO}_{2}} \text {. }
$$

In the case of grains the process is more complex, involving a preliminary saccharous fermentation, whereby the starch is first transformed into suggar.

Thus

$$
\begin{aligned}
& \text { (2) }{ }_{2} \mathrm{C}_{6} \mathrm{H}_{10} \mathrm{O}_{5}+\mathrm{H}_{2} \mathrm{O}=\underset{\text { Starch }}{\mathrm{C}_{6} \mathrm{H}_{10} \mathrm{O}_{5}}+\underset{\text { Dextrin }}{\mathrm{C}_{6} \mathrm{H}_{12} \mathrm{O}_{6^{*}}} \text {. } \\
& \text { (3) } \mathrm{C}_{6} \mathrm{H}_{10} \mathrm{O}_{5}+\mathrm{H}_{2} \mathrm{O}=\underset{\text { Dextrose }}{\mathrm{C}_{6} \mathrm{H}_{12} \mathrm{O}_{6} \text {. }}
\end{aligned}
$$

The process of alcoholic or vinous fermentation is largely dependent upon the presence of various species of yeasts, which either exist from the first in the expressed juices themselves, as in the case of wines, being derived from the skins of the grapes and from the air, or are introduced with some degree of selection, as in the case of beer.

In the juices of most fruits the sugar exists in the form of sucrose, mixed with variable amounts of invert sugar resulting from the inversion of the sucrose due to the action of ferments, such as invertase, a soluble ferment of yeast. The invert sugar nearly always predominates, and in some juices, as for instance the grape, nearly all the sugar has been inverted. 
The above reaction, No. I, illustrating the splitting up of grape sugar into alcohol and carbon dioxide, does not represent the practical yield of alcohol under ordinary conditions that occur in vinous fermentation, for, as a matter of fact, instead of $5^{\text {I.II }}$ parts of alcohol and 48.89 parts carbon dioxide, which would theoretically result as above from the fermentation of 100 parts of dextrose, only about $95 \%$ of the theoretical yield can be obtained, so that in practice it is possible to form but about $48.5 \%$ alcohol and $46.5 \%$ carbon dioxide. The balance, amounting to some $5 \%$, consists chiefly of glycerin, succinic acid, and traces of various compounds, including some of the higher-boiling alcohols (propyl, butyl, and amyl) and their ethers, which form the fusel oil of the distilled liquors.

Vinous fermentation takes place most readily in slightly acid liquids, at a temperature ranging from $25^{\circ}$ to $30^{\circ} \mathrm{C}$.

It is convenient to divide alcoholic beverages into two main groups, first the fermented and second the distilled liquors. The fermented liquors naturally subdivide themselves into $(a)$ the products of the direct spontaneous fermentation of saccharine fruit juices, such, for example, as those of the apple and the grape, to form cider and wine respectively, and $(b)$ the malted and brewed liquors, such as beer and ale, produced by the conversion of the starch of grain into sugar, and the final alcoholic fermentation of the latter.

The distilled liquors include such products as whiskey, brandy, rum, and gin, wherein alcoholic infusions prepared by previous fermentation. in various ways are further subjected to distillation.

Alcoholic Liquors and State (or Municipal) Control.-The mere adulteration of liquors does not constitute the only feature which brings them within the scope of the public analyst's work and renders them especially amenable to stringent laws. Indeed, it is often a far more important question for the analyst to decide by his results whether or not the samples submitted to him, by police seizure or otherwise, are sold in violation of the regulations in force in his particular locality governing the liquor traffic.

A common regulation in no-license localities fixes the maximum per cent of alcohol which shall decide whether or not a liquor is legally a temperance drink, and can be sold as such with impunity. From its low content in alcohol, an analyst's findings regarding a certain sample may exonerate the dealer suspected of violating this law, while yet by the very reason of its being low in alcohol the same sample would be placed 
in the adulterated list as regards non-conformance to a standard of purity. While the raising of revenue is one purpose for the existence of these laws bearing on liquor license, an equally important object sought to be gained is doubtless the repression of intemperance.

Toxic Effects.-A popular impression seems to exist that the toxic effects of an adulterated liquor are far worse from a temperance standpoint than those of a sample of good standard quality, and it is a common experience of the public analyst to have submitted to him by well-meaning temperance advocates samples which are alleged to have caused the worst forms of intoxication, and are thus suspected of being impure. As a matter of fact the chief adulterants of liquors are water, sugar, and, in the case of beer, various bitter principles and vegetable extractives, none of which are on record as being in themselves actively toxic.*

Alcohol is the one ingredient of liquor which, more than any other, produces a marked physiological effect. Many liquors, especially those of the distilled variety classed as adulterated, are so considered by reasor: of their low alcoholic content through watering or otherwise, hence this commonest form of adulteration, far from being detrimental in itself, is actually helpful to the temperance cause.

Details of Liquor Inspection.-The same precautions should be carefully observed by officers making seizures of liquors for analysis, as by food inspectors, regarding safe delivery of the samples to the analyst. The following instructions are circulated by the State Board of Health of Massachusetts, which has in charge the inspection of liquors, concerning the taking of samples in that state and the transmission to the analyst:

\section{DIRECTIONS FOR TAKING SAMPLES FOR ANALYSES.}

The officer making a seizure, or taking samples of beer, should note at the time of such seizure the general appearance of the liquor,- as to whether it is clear or cloudy, whether it is still or has a strong head.

If the liquor is in bottles, take at least one pint bottle; if in barrels, draw a pint bottle from each. Request the owner to seal each sample taken. If the bottles have cork stoppers, cut the stoppers off level with the top of the bottle and cover with wax; if with patent stoppers, a little wax placed upon the wire at the point where it lays against the neck of the bottle is sufficient. If the owner refuses to seal it, then the officer

* The writer refers to substances intentionally added, and not to accidental impurities, such as arsenic, etc, that are occasionally found. 
should seal it in his presence, calling his attention to the fact. Before leaving the premises, place upon the bottle a label or tag, with the date, the name of the owner, and the name of the officer upon it, and also the name of the town or city. Then place in a box, with the certificate required by law, and forward without delay to the analyst.

\section{FORM OF LABEL.}

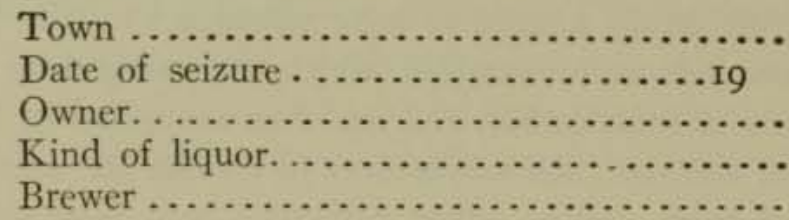

Accompanying each sample is a certificate like the following, the first part of which is filled out and signed by the officer, while the second part, containing the data of analysis, is filled out and signed by the analyst and returned by him to the officer. Such a certificate is nearly always accepted as evidence in court without the personal appearance of the analyst.

To the State Board of Health:

I send herewith a sample of taken from liquors seized by me.

Ascertain the percentage of alcohol it contains, by volume, at sixty degrees Fahrenheit, and return to me a certificate herewith upon the annexed form.

Seized from

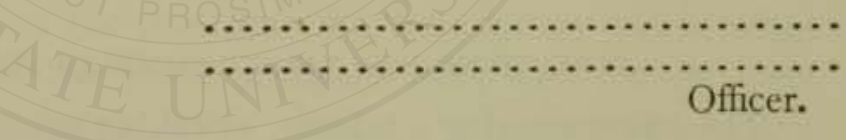

\section{COMMONWEALTH OF MASSACHUSETTS.}

No.

\section{Office of the State Board of Health.}

Boston, ................. I9

This is to certify that the $\ldots \ldots \ldots \ldots \ldots \ldots \ldots$. received by me with the above statement contains................. per cent of alcohol, by volume, at sixty degrees Fahrenheit.

Received.................... I9

Analysis made. ............... I9

[SEAL.]

Analyst State Board of Health. 
A convenient method for recording analyses is by the empleyment of numbered library cards, which bear the same number as the certificates and are kept by the analyst.

The following is a convenient form:

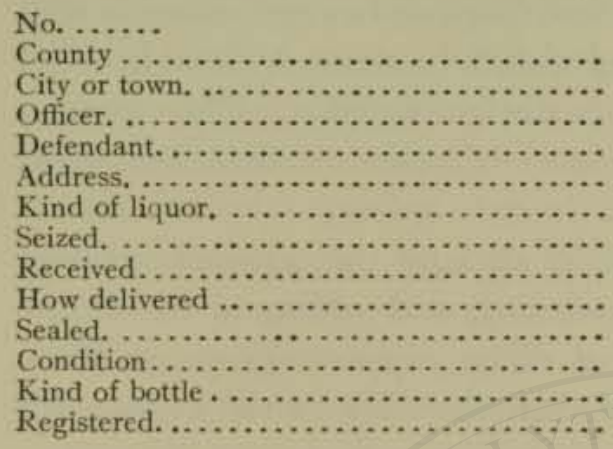

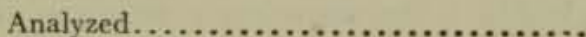

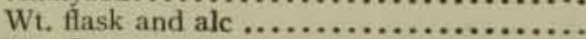

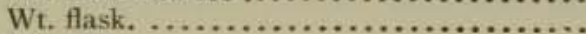

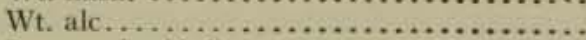

Sp. gr. alc. $\left(60^{\circ}\right), \ldots \ldots \ldots \ldots \ldots \ldots \ldots \ldots$

Per cent alcohol. . . . . . . . . . . . . . . .

Reported.

METHODS OF ANALYSIS COMMON TO ALL LIQUORS.

Specific Gravity.-This should be taken at $15.6^{\circ}$ or calculated to that temperature. The most convenient mode of procedure is to bring the temperature of the sample somewhat below that point by allowing the flask containing it to stand in cold water, and to have everything in readiness to make the determination when $15.6^{\circ}$ temperature has been reached, either by the hydrometer spindle in a glass cylinder, by the Westphal balance, or by the pycnometer. The latter is by far the most accurate, especially if it is of the form which is fitted with a thermometerstopper.

Detection of Alcohol.-It is rarely necessary to make a qualitative test for alcohol in liquors, since it is almost invariably present even in many of the so-called temperance drinks, at least in small amount. Indeed in many localities a beverage is legally a temperance drink that contains not more than $1 \%$ alcohol by volume.

The Iodojorm Test.-Alcohol, when present in aqueous solution to the extent of $0.1 \%$ or more, may be detected by the iodoform test. The solution is warmed in a test-tube with a few drops of a strong solution of iodine in potassium iodide, after which enough sodium hydroxice solution is added to nearly decolorize. On standing for some time a yellow precipitate of iodoform will appear if alcohol be present, or at once if there is a considerable amount, and the characteristic odor of iodoform will be rendered apparent, even when the precipitate is so slight as to be almost imperceptible. This iodoform precipitate is crystalline, showing under the microscope as star-shaped groups or hexagonal tablets. 
It should not be forgotten that other substances than alcohol give the reaction, as lactic acid, acetone, and various aldehydes and ketones.

Pure methyl or amyl alcohol or acetic acid do not thus react.

Berthelot recommends benzoyl chloride as a reagent for detecting alcohol. By warming a mixture of a few drops of benzoyl chloride with the solution to be tested, and adding a little sodium hydroxide, ethyl benzoate is formed, recognizable by its distinctive odor. This reaction is delicate to $0.1 \%$ alcohol. The presence of other alcohols than ethyl produces ethers of characteristic odor.

Hardy's Test for Alcohol consists in shaking the aqueous solution with some powdered guaiacum resin, filtering, and adding to the filtrate a little hydrocyanic acid and a drop of dilute copper sulphate solution. A blue coloration considerably deeper than that due to the copper salt is indicative of alcohol.

Methyl Alcohol in spirits is tested for as described on pp. 749-752.

Determination of Alcohol.-In the case of carbonated liquids it is necessary to first expel the free carbon dioxide, which is readily accomplished by pouring the liquor back and forth from one beaker to another, from time to time removing the excess of froth from the top of the vessel by the aid of the hand. Or, the sample may be shaken vigorously in a large separatory funnel, and the still liquor drawn off from below the froth, repeating the operation several times if necessary. In either case the mechanical treatment should be continued till the liquor is comparatively quiet and free from foam.

(I) By Distillation.-This is by far the most accurate method of determining alcohol, and should be carried out in all cases where any legal controversy is apt to be involved. Into a flask of $25^{\circ}$ to $400 \mathrm{cc}$. capacity introduce a convenient quantity of the liquor, which should be accurately weighed or measured, according to whether the percentage by weight or measure is desired. The following are suitable quantities: Distilled liquors, 25 grams or cc.; cordials, 25 to 50 grams or cc.; wines, ciders, and malt liquors, too grams or cc. In the case of wines or ciders which have undergone acetic fermentation, add 0.1 to 0.2 gram of precipitated calcium carbonate or neutralize with standard alkali.

Dilute the liquid to $150 \mathrm{cc}$. and distil into a Ioo-cc. flask. Nearly all alcoholic liquors, if comparatively free from carbon dioxide, will boil without undue frothing or foaming. New wine will occasionally give trouble in this regard, but foaming may usually be prevented in this 
case by the addition of tannic acid. In case of wine, cider, and beer all the alcohol will have passed over in the first $75 \mathrm{cc}$. of the distillate, or three-fourths the original measured volume, but with distilled liquors high in alcohol the process had better be continued till nearly 100 cc. or the original volume taken have passed over. If the condenser is of glass, one can observe when all the alcohol has been distilled over, for the reason that the mixed alcohol and water vapors in the upper portion of the condenser present a striated or wavy appearance, readily apparent so long as the alcohol is passing over, while after all the alcohol has been distilled, the condenser-tube appears perfectly clear. The distillation is thus continued for some time after this striated appearance has ceased. The distillate in the receiving glass is finally made up to the mark or to the original volume of the liquor taken. Strictly speaking, the measurements before and after distillation should be made at $\mathrm{I}_{5} .6^{\circ} \mathrm{C}$., but, excepting in case of distilled liquors, no appreciable error results from making both measurements at the same or room temperature. Another precaution formerly thought necessary was to have the delivery-tube from the condenser pass below the level of a little water in the receiving-flask from the start, but equally accurate results have been obtained by simply allowing the end of the condenser-tube to enter the narrow-necked flask.

Fig. II 2 shows a bank of six stills of the kind used in the author's laboratory for alcohol determination in liquors. In each still the vertical glass worm-condenser, the round-bottomed distilling-flask, and the lamp, are supported by rings held by a single upright rod. The receivingflask is readily connected with the condenser by means of a single bent tube provided with a rubber stopper. 5 The cold-water pipe supplying the condensers is shown at the top, and the gas-supply pipe at the bottom.

The distillate, made up to $100 \mathrm{cc}$., is thoroughly shaken and its specific gravity taken at exactly $15.6^{\circ}$ in a pycnometer, or by the Westphal balance. From the specific gravity the corresponding percentage of alcohol by weight or volume, or the grams per $100 \mathrm{cc}$. in the distillate, is ascertained by reference to the accompanying tables.

To obtain percentage of alcohol by weight in the sample, multiply the per cent by weight in the distillate by the weight of the distillate, and divide by the weight of the sample taken; to obtain per cent by volume, multiply the per cent by volume in the distillate by 100 , and divide by the volume of the sample used.

(2) From the Specific Gravity of the Sample.-In the case of distilled liquors having very little residue, an approximation to the true 
percentage of alcohol may be obtained by using the alcohol table in connection with the specific gravity of the liquor itself. The accuracy of this method depends largely on the freedom from residue, being absolutely correct for mixtures of alcohol and water only.

(3) By Evaporation.-Determine the specific gravity of the sample, evaporate a measured portion of the liquor $(50$ or $100 \mathrm{cc}$.) in a porcelain

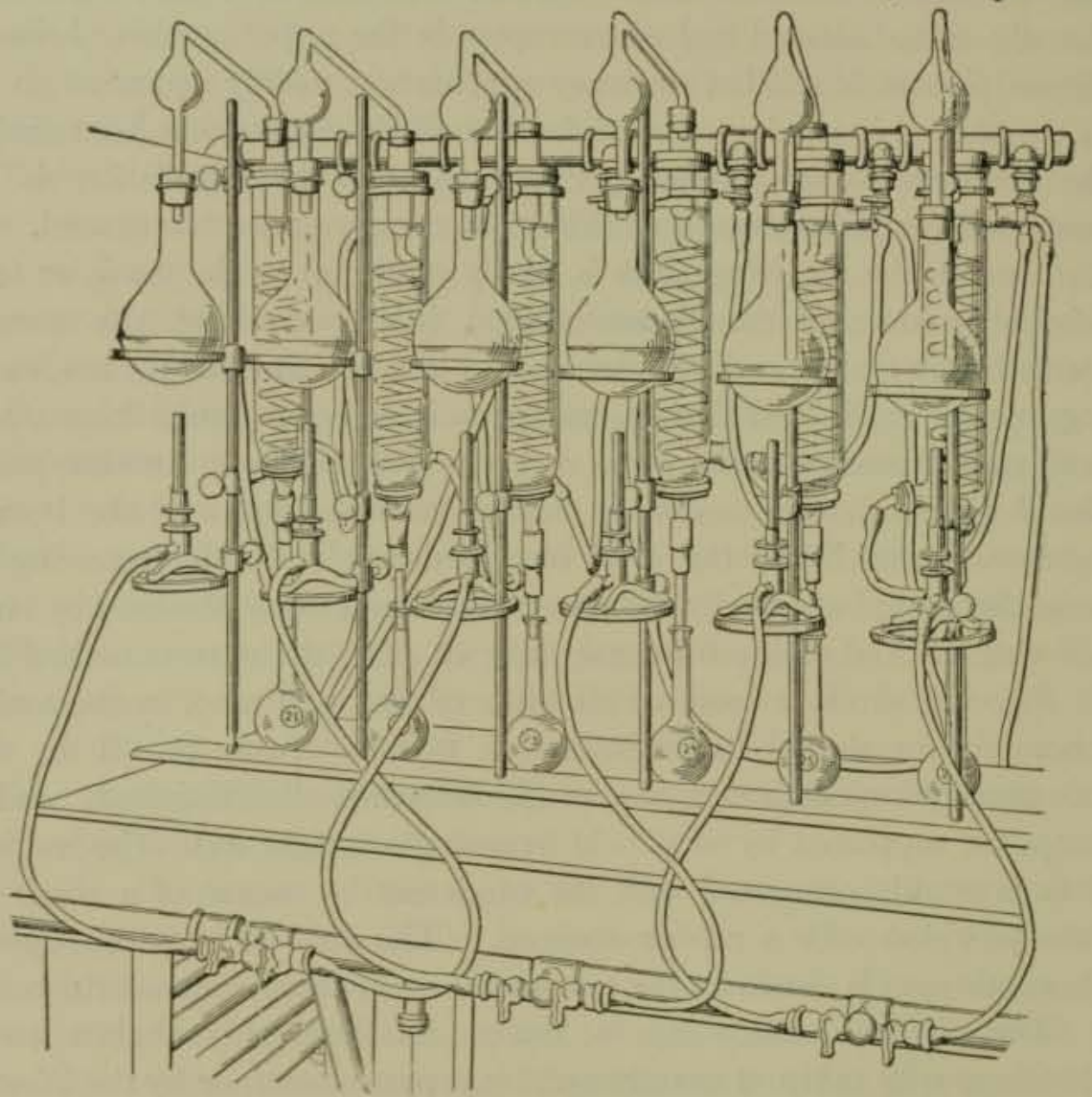

Fic. 11 2,-Bank of Stills for Alcohol Determination.

dish over the water-bath to one-fourth its bulk, make up to its original volume with distilled water, and determine the specific gravity of this second or dealcoholized portion. Add I to the original specific gravity, and from this subtract the second specific gravity. The difference is the specific gravity corresponding to the alcohol in the liquor, the per cent of which is found from the table.

Example.-Suppose the specific gravity of the original sample to be 0.9900 while that of the dealcoholized sample is 1.0009 . Then $1.9900-$ I. $00 \mathrm{c} 9=0.989$ r. $\therefore$ Per Cent by volume of alcohol $=8.10$. 
SPECIFIC GRAVITY AND PERCENTAGE OF ALCOHOL.

(ACCORDING TO HeHNER.)

\begin{tabular}{|c|c|c|c|c|c|c|c|c|c|c|c|}
\hline \multirow{2}{*}{$\begin{array}{c}\text { Spec. } \\
\text { Grav. } \\
\text { at } \\
15.6^{\circ} \mathrm{C} \text {. }\end{array}$} & \multicolumn{3}{|c|}{ Absolute Alcohol. } & \multirow{2}{*}{$\begin{array}{c}\text { Spec. } \\
\text { Grav. } \\
\text { at } \\
15.6^{\circ} \mathrm{C} \text {. }\end{array}$} & \multicolumn{3}{|c|}{ Absolute Alcohol. } & \multirow{2}{*}{$\begin{array}{c}\text { Spec. } \\
\text { Grav. } \\
\text { at } \\
15.6^{\circ} \mathrm{C} \text {. }\end{array}$} & \multicolumn{3}{|c|}{ Absolute Alcohol. } \\
\hline & $\begin{array}{c}\text { Per } \\
\text { Cent } \\
\text { by } \\
\text { Weight }\end{array}$ & $\begin{array}{c}\text { Per } \\
\text { Cent } \\
\text { by Vol- } \\
\text { ume. }\end{array}$ & $\begin{array}{c}\text { Grams } \\
\text { per } \\
\text { too ce. }\end{array}$ & & $\begin{array}{c}\text { Per } \\
\text { Cent } \\
\text { by } \\
\text { Weight }\end{array}$ & $\begin{array}{c}\text { Pet } \\
\text { Cent } \\
\text { by Vol- } \\
\text { ume. }\end{array}$ & $\begin{array}{c}\text { Grams } \\
\text { per } \\
100 \mathrm{cc} \text {. }\end{array}$ & & $\begin{array}{c}\text { Per } \\
\text { Cent } \\
\text { by } \\
\text { Weight }\end{array}$ & $\begin{array}{l}\text { Per } \\
\text { Cent } \\
\text { by Vol- } \\
\text { ume. }\end{array}$ & $\begin{array}{l}\text { Grams } \\
\text { per } \\
\text { too cc. }\end{array}$ \\
\hline I. .0000 & .00 & 0.00 & 0.00 & & & & & & & & \\
\hline .9999 & .05 & 0.07 & 0.05 & 0.9959 & 2.33 & 2.93 & 2.32 & 0.9919 & 4.69 & 5.86 & 4.65 \\
\hline & 11 & 0.13 & 0.11 & & -39 & & $2 \cdot 3^{8}$ & 8 & $4 \cdot 75$ & 5.94 & $4 \cdot 7^{I}$ \\
\hline 7 & 16 & 0.20 & 0.16 & 7 & -44 & 97 & .43 & 7 & & & -77 \\
\hline 6 & & .26 & 0.21 & 6 & 50 & & & 6 & 4.87 & & \\
\hline 5 & 26 & .33 & 0.26 & 5 & 2.56 & & .55 & 5 & 4.94 & 6. & 1.90 \\
\hline 4 & 32 & $\circ$ & $0.3^{2}$ & 4 & .61 & .28 & & 4 & & & -95 \\
\hline 3 & & 0 & 0.3 & 3 & 67 & & .65 & 3 & & & \\
\hline 2 & & 0 & 0.42 & $\frac{3}{2}$ & 72 & & .70 & 2 & & & \\
\hline I & .47 & 0.60 & 0.47 & $\mathrm{I}$ & 2.78 & 49 & 2.76 & I & 9 & 6. & 5.1 \\
\hline 0 & 0.53 & 0.66 & 0.53 & 0 & $2.8_{3}$ & 3.55 & 2.81 & 0 & 5.25 & 6.55 & 5.20 \\
\hline 0.9989 & .58 & 0 & $0.5^{8}$ & & 2.80 & .62 & 2.87 & & & 6.63 & 5.26 \\
\hline & 63 & & 0.63 & 8 & & & & 8 & & & \\
\hline 7 & 68 & 0.86 & 0.68 & 7 & & .76 & & 7 & 5. & & 5.39 \\
\hline 6 & .74 & 0.93 & 0.74 & 6 & & & & 6 & & 6.8 & $5 \cdot 45$ \\
\hline 5 & .79 & 0.99 & 0.79 & 5 & 3.12 & 3.90 & 10 & 5 & .56 & 6. & $5 \cdot 5^{1}$ \\
\hline 4 & .84 & 1.06 & 0.84 & 4 & 3.18 & 3.98 & 3.16 & 4 & 62 & 7. & 5.57 \\
\hline 3 & 0.89 & I. I 3 & 0.89 & 3 & 3.24 & 4.05 & 3.22 & 3 & 5.69 & & 5.64 \\
\hline 2 & 0.95 & 19 & 0.95 & 2 & 3.29 & $4 \cdot 1$ & 3.27 & 2 & $5 \cdot 75$ & 7 & $5 \cdot 70$ \\
\hline I & 1.00 & 1.26 & 1.00 & I & 3.35 & $4 \cdot 20$ & $3 \cdot 33$ & I & 5.8 & 5 & $5 \cdot 7^{6}$ \\
\hline 0 & 1.06 & I. 34 & 1.06 & 0 & 3.41 & 4.27 & $3 \cdot 39$ & o & 5.87 & $7 \cdot 32$ & $5.8 \mathrm{I}$ \\
\hline & 1.12 & & 1.12 & & 3.47 & $4 \cdot 34$ & 3.45 & 0.9899 & 5.94 & $7 \cdot 40$ & 5.88 \\
\hline 8 & I. 19 & 1.49 & I. 19 & 8 & $3 \cdot 53$ & $4 \cdot 42$ & $3 \cdot 5 x$ & 8 & 6.00 & $7 \cdot 4^{8}$ & 5.94 \\
\hline 7 & .25 & .57 & I. 25 & 7 & 3.59 & 4.49 & 3 & 7 & 6.07 & $7 \cdot 57$ & 6.01 \\
\hline 6 & $\cdot 3 I$ & .65 & $-3 I$ & 6 & 3.65 & $4 \cdot 56$ & $3.6_{3}$ & 6 & 6. & & 6.07 \\
\hline 5 & -37 & .73 & I. 37 & 5 & 3.71 & 4.63 & 3.69 & 5 & 6.21 & & 6.14 \\
\hline 4 & .44 & $.8 \pi$ & I. 44 & 4 & $3 \cdot 7^{6}$ & 4.71 & $3 \cdot 74$ & 4 & 6.28 & & 6.21 \\
\hline 3 & 50 & & 1.50 & 3 & 3.82 & $4 \cdot 78$ & & 3 & $6.3^{6}$ & & 6.29 \\
\hline 2 & .56 & I. 96 & I. 56 & 2 & 3.88 & 4.85 & 3.85 & 2 & 6.4 & & $6.3^{6}$ \\
\hline I & I. 62 & 2.04 & 1. $6 \mathrm{I}$ & I & 3.94 & 4.93 & 3. & $I$ & 6.50 & & 6.43 \\
\hline 0 & 1.69 & 2.12 & 1.68 & 0 & 4.00 & 5.00 & 3.97 & 0 & 6.57 & 8.18 & 6.50 \\
\hline 0.9969 & & & .74 & 0.9929 & .06 & & & 0.9889 & 6.64 & & \\
\hline 8 & I. 81 & 2.27 & I. 80 & 8 & $4 \cdot 12$ & 5.16 & 4. & 8 & 6.71 & & 6.63 \\
\hline 7 & I. 87 & 2.35 & 1. 86 & 7 & 4.1 & & & 7 & 6. & & 6.70 \\
\hline 6 & .94 & .43 & 1.93 & 6 & & & & 6 & 6.8 & & 6.78 \\
\hline 5 & 2.00 & 2.51 & 1.99 & 5 & & & & 5 & 6. & & 6.85 \\
\hline 4 & .06 & 2.58 & 2.05 & 4 & -37 & & & 4 & $7 . c$ & 8. & $6.9^{2}$ \\
\hline 3 & 2.11 & 2.62 & 2.1 & 3 & & & & 3 & 7.07 & 8.80 & 6.99 \\
\hline 2 & 2 & 2.72 & 2.16 & 2 & & 5.63 & $4 \cdot 46$ & 2 & $7 \cdot 13$ & 8.88 & 7.05 \\
\hline I & 2.22 & 2.79 & 2.21 & I & $4 \cdot 56$ & & & $\mathrm{I}$ & 720 & 8.96 & 7.12 \\
\hline o & 2.28 & 2.86 & 2.27 & 0 & 4.62 & 5.78 & $4 \cdot 5^{8}$ & o & 7.27 & 9.04 & 7.19 \\
\hline
\end{tabular}


SPECIFIC GRAVITY AND PERCENTAGE OF ALCOHOL-(Continued).

\begin{tabular}{|c|c|c|c|c|c|c|c|c|c|c|c|}
\hline \multirow{2}{*}{$\begin{array}{c}\text { Spec, } \\
\text { Grav. } \\
\text { at } \\
\times 5.6^{\circ} \mathrm{C} \text {. }\end{array}$} & \multicolumn{3}{|c|}{ Absolute Alcohol. } & \multirow[b]{2}{*}{$\begin{array}{c}\text { Spec, } \\
\text { Grav. } \\
\text { at } \\
15.6^{\circ} \mathrm{C} \text {. }\end{array}$} & \multicolumn{3}{|c|}{ Absolute Alcohol. } & \multirow{2}{*}{$\begin{array}{c}\text { Spec. } \\
\text { Grav. } \\
\text { at } \\
25.6^{\circ} \mathrm{C} \text {. }\end{array}$} & \multicolumn{3}{|c|}{ Absolute Alcohol. } \\
\hline & $\begin{array}{c}\text { Per } \\
\text { Cent } \\
\text { by } \\
\text { Weight }\end{array}$ & $\begin{array}{c}\text { Per } \\
\text { Cent } \\
\text { by Vol- } \\
\text { ume. }\end{array}$ & $\begin{array}{c}\text { Grams } \\
\text { per } \\
100 \mathrm{cc} .\end{array}$ & & $\begin{array}{c}\text { Per } \\
\text { Cent } \\
\text { by } \\
\text { Weight }\end{array}$ & $\begin{array}{c}\text { Per } \\
\text { Cent } \\
\text { by Vol- } \\
\text { ume. }\end{array}$ & $\begin{array}{c}\text { Grams } \\
\text { per } \\
100 \mathrm{cc} \text {. }\end{array}$ & & $\begin{array}{c}\text { Per } \\
\text { Cent } \\
\text { by } \\
\text { Weight }\end{array}$ & $\begin{array}{c}\text { Per } \\
\text { Cent } \\
\text { by Vol- } \\
\text { ume. }\end{array}$ & $\begin{array}{c}\text { Grame } \\
\text { per } \\
\text { roo ce. }\end{array}$ \\
\hline 0.9879 & $7 \cdot 33$ & 9.13 & $7 \cdot 24$ & 0.9829 & 10.92 & $1_{3} \cdot 5^{2}$ & 10.73 & 0.9779 & $\mathrm{I} 4.9 \mathrm{I}$ & $18 \cdot 3^{6}$ & $14.5^{8}$ \\
\hline 8 & $7 \cdot 40$ & $9.2 \mathrm{I}$ & $7 \cdot 31$ & 8 & 11.00 & 13.62 & $10.8 \mathrm{r}$ & 8 & 15.00 & 18.48 & 14.66 \\
\hline 7 & $7 \cdot 47$ & 9.29 & $7 \cdot 37$ & 7 & 11.08 & $13.7 \mathrm{I}$ & 10.89 & 7 & 15.08 & 18.58 & $14 \cdot 74$ \\
\hline 6 & $7 \cdot 53$ & $9 \cdot 37$ & $7 \cdot 43$ & 6 & $1 x \cdot I_{5}$ & 13.81 & 10.95 & 6 & 15.17 & 18.68 & 14.83 \\
\hline 5 & 7.60 & 9.45 & $7 \cdot 5^{\circ}$ & 5 & II.23 & 13.90 & I 1.03 & 5 & 15.25 & 18.78 & $\mathrm{I} 4.90$ \\
\hline 4 & 7.67 & 9.54 & $7 \cdot 57$ & 4 & 11.31 & 13.99 & 11.11 & 4 & $15 \cdot 33$ & I8.88 & 14.98 \\
\hline 3 & $7 \cdot 73$ & 9.62 & 7.63 & 3 & II. 38 & 14.09 & 11.18 & 3 & 15.42 & 18.98 & 15.07 \\
\hline 2 & 7.80 & 9.70 & $7 \cdot 70$ & 2 & 11.46 & 14.18 & II. 26 & 2 & 15.50 & 19.08 & $\mathrm{I} 5 \cdot \mathrm{I} 4$ \\
\hline I & 7.87 & $9 \cdot 7^{8}$ & $7 \cdot 77$ & I & II. 54 & 14.27 & II. 33 & I & $15.5^{8}$ & 19.18 & 15.21 \\
\hline o & 7.93 & 9.86 & $7 \cdot 83$ & o & 11.62 & $14 \cdot 37$ & II. $4 \mathrm{I}$ & 0 & 15.67 & 19.28 & 15.30 \\
\hline 0.9860 & 8.00 & 9.95 & 7.89 & 0.9819 & 11.69 & 14.46 & It. 48 & 0.9769 & I5.75 & 19.39 & $15.3^{8}$ \\
\hline & 8.07 & 10.03 & 7.96 & 8 & 11.77 & $14 \cdot 5^{6}$ & II. 56 & 8 & ${ }^{1} 5.83$ & 19.49 & 15.46 \\
\hline 7 & 8. $\mathrm{I}_{4}$ & 10.12 & 8.04 & 7 & II. 85 & 14.65 & 11.64 & 7 & 15.92 & 19.59 & 15.54 \\
\hline 6 & 8.21 & 10.21 & 8.10 & 6 & II. 92 & 14.74 & 11.70 & 6 & 16.00 & 19.68 & 15.62 \\
\hline 5 & 8.29 & 10.30 & 8.17 & 5 & 12.00 & 14.84 & 11.78 & 5 & 16.08 & 19.78 & 15.70 \\
\hline 4 & 8.36 & $10.3^{8}$ & 8.24 & 4 & 12.08 & 14.93 & 11.85 & 4 & 16.15 & 19.87 & $15 \cdot 76$ \\
\hline 3 & 8.43 & 10,47 & 8. 31 & 3 & 12.15 & 15.02 & 11.92 & 3 & 16.23 & r9. 96 & 15.84 \\
\hline 2 & $8.5^{\circ}$ & $10.5^{6}$ & $8 \cdot 3^{8}$ & 2 & 12.23 & 15.12 & 12.00 & 2 & $16.3 \mathrm{I}$ & 20.06 & 15.90 \\
\hline I & 8.57 & 10.65 & 8.45 & $\mathrm{I}$ & $12.3 \mathrm{I}$ & 15.21 & 12.08 & I & เ6. $3^{8}$ & 20.15 & 15.99 \\
\hline o & 8.64 & 10.73 & $8.5^{2}$ & o & $12.3^{8}$ & $15.3^{\circ}$ & 12.14 & 0 & 16.46 & 20.24 & 16.06 \\
\hline & 8.71 & 10.82 & $8 \cdot 5^{8}$ & 0.9809 & 12.46 & 15.40 & 12.22 & 0.9759 & I 6.54 & 20.33 & I6. 13 \\
\hline 8 & 8.79 & 10.91 & 8.66 & 8 & 12.54 & 15.49 & 12.30 & 8 & 16.62 & 20.43 & I6.21 \\
\hline 7 & 8.86 & 11.00 & 8.73 & 7 & 12.62 & r $5.5^{8}$ & 12.37 & 7 & I 6.69 & 20.52 & 16. 28 \\
\hline 6 & 8.93 & 11.08 & 8.80 & 6 & 12.69 & $I_{5} .68$ & 12.44 & 6 & 16.77 & $20.6 \mathrm{r}$ & I6. 35 \\
\hline 5 & 9.00 & II.I7 & 8.87 & 5 & 12.77 & 15.77 & 12.51 & 5 & $x 6.85$ & 20.71 & I6. 63 \\
\hline 4 & 9.07 & 11.26 & 8.93 & 4. & 12.85 & $1_{5} .86$ & 12.59 & 4 & 16.92 & 20.80 & I $6.5^{\circ}$ \\
\hline 3 & 9.14 & II. 35 & 9.00 & 3 & 12.92 & 15.96 & 12.66 & 3 & $\mathrm{I1} 7.00$ & 20.89 & I 6.57 \\
\hline 2 & 9.21 & $1 \mathrm{r} .44$ & 9.07 & 2 & 13.00 & 16.05 & 12.74 & 2 & 17.08 & 20.99 & 16.65 \\
\hline I & 9.29 & II. 52 & $9 . \mathrm{r}_{4}$ & $\mathbf{I}$ & 13.08 & 16.15 & $12.8 \mathrm{I}$ & I & 17.17 & 21.09 & 16.74 \\
\hline 0 & $9 \cdot 3^{6}$ & $11.6 \mathrm{I}$ & 9.22 & o. & 13.15 & 16.24 & 12.89 & 0 & 17.25 & 21.19 & $\mathrm{I} 6.8 \mathrm{I}$ \\
\hline $0.9^{8} 49$ & 9.43 & 11.70 & 9.29 & & 13.23 & 16.33 & 12.96 & & I $7 \cdot 33$ & 21.29 & I6.89 \\
\hline 8 & $9.5^{\circ}$ & 11.79 & $9 \cdot 35$ & 8 & $13.3 \mathrm{r}$ & I6. 43 & 13.03 & 8 & 17.42 & $21 \cdot 39$ & 16.97 \\
\hline 7 & $9 \cdot 57$ & 11.87 & 9.42 & 7 & $13 \cdot 3^{8}$ & 16.52 & 13.10 & 7 & 17.50 & $2 \mathrm{I} .49$ & 17.05 \\
\hline 6 & 9.64 & II. 96 & 9.49 & 6 & $13.4^{6}$ & $16.6 \mathrm{r}$ & 13.18 & 6 & $17.5^{8}$ & 21.59 & 17.13 \\
\hline 5 & 9.71 & 12.05 & $9 \cdot 5^{6}$ & 5 & 13.54 & 16.70 & 13.26 & 5 & 17.67 & 21.69 & 17.20 \\
\hline 4 & 9.79 & 12.13 & 9.64 & 4 & 13.62 & 16.80 & 13.33 & 4 & 17.75 & 21.79 & 17.29 \\
\hline 3 & 9.86 & 12.22 & $9.7 \mathrm{I}$ & 3 & 13.69 & 16.89 & 13.40 & 3 & 17.83 & $2 \mathrm{r} .89$ & $17 \cdot 37$ \\
\hline 2 & 9.93 & $12 \cdot 3 \mathrm{I}$ & 9.77 & 2. & 13.77 & 16.98 & 13.48 & 2 & 17.92 & 21.99 & 17.46 \\
\hline I & 10.00 & 12.40 & 9.84 & I & $\mathrm{I} 3.85$ & I 7.08 & 13.56 & 1 & 18.00 & 22.09 & 17.54 \\
\hline o & 10.03 & 12.49 & $9 \cdot 92$ & - & 13.92 & 17.17 & 13.63 & 0 & 18.08 & 22.18 & 17.61 \\
\hline 0.9839 & 10.15 & $12.5^{8}$ & 9.99 & $0.97^{89}$ & 14.00 & 17.26 & 13.71 & 0.9739 & 18.15 & 22.27 & 17.68 \\
\hline & 10.23 & 12.68 & 10.06 & 8 & 14.09 & $17 \cdot 37$ & 13.79 & 8 & I 8.23 & $22 \cdot 36$ & $17 \cdot 76$ \\
\hline 7 & 10.31 & 12.77 & 10.13 & 7 & 14.18 & 17.48 & I 3.88 & 7 & I8. $3 \mathrm{I}$ & 22.46 & 17.82 \\
\hline 6 & $10.3^{8}$ & 12.87 & 10.20 & 6 & 14.27 & $17 \cdot 59$ & 13.96 & 6 & $18.3^{8}$ & 22.55 & 17.90 \\
\hline 5 & $10.4^{6}$ & $12.9^{6}$ & 10.28 & 5 & $14 \cdot 3^{6}$ & 17.70 & 14.04 & 5 & 18.46 & 22.64 & 17.97 \\
\hline 4 & 10.54 & 13.05 & $10.3^{6}$ & 4 & 14.45 & 17.81 & 14.13 & 4 & 18.54 & 22.73 & 18.05 \\
\hline 3 & 10.62 & 13.15 & 10.44 & 3 & 14.55 & 17.92 & $\mathrm{I} 4.23$ & 3 & 18.62 & 22.82 & 18.13 \\
\hline 2 & 10.69 & 13.24 & 10.51 & 2 & 14.64 & 18.03 & $14 \cdot 32$ & 2 & I8. 69 & $22.9^{2}$ & 18. 19 \\
\hline I & 10.77 & $13 \cdot 34$ & 10.59 & I. & 14.73 & 18.14 & 14.39 & I & 18.77 & 23.01 & 18.27 \\
\hline o & 10.85 & 13.43 & 10.67 & 0 & $14-82$ & 18.25 & $14.4^{8}$ & 0 & 18.85 & 21.10 & 18.34 \\
\hline
\end{tabular}


SPECIFIC GRAVITY AND PERCENTAGE OF ALCOHOL-(Continued).

\begin{tabular}{|c|c|c|c|c|c|c|c|c|c|c|c|}
\hline \multirow[b]{2}{*}{ 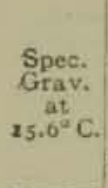 } & \multicolumn{3}{|c|}{ Absolute Alcohol. } & \multirow[b]{2}{*}{$\begin{array}{c}\text { Spec. } \\
\text { Grav. } \\
\text { at } \\
15.6^{\circ} \mathrm{C} .\end{array}$} & \multicolumn{3}{|c|}{ Absolute Alcohol. } & \multirow[b]{2}{*}{$\begin{array}{c}\text { Spec. } \\
\text { Grav. } \\
\text { at } \\
15.6^{\circ} \mathrm{C} \text {. }\end{array}$} & \multicolumn{3}{|c|}{ Absolute Aleohol. } \\
\hline & $\begin{array}{c}\text { Per } \\
\text { Cent } \\
\text { by } \\
\text { Weight }\end{array}$ & $\begin{array}{l}\text { Per } \\
\text { Cent } \\
\text { by Vol- } \\
\text { ume. }\end{array}$ & $\begin{array}{c}\text { Grams } \\
\text { per } \\
\text { too ce. }\end{array}$ & & $\begin{array}{c}\text { Per } \\
\text { Cent } \\
\text { by } \\
\text { Weight }\end{array}$ & $\begin{array}{c}\text { Per } \\
\text { Cent } \\
\text { by Vol- } \\
\text { ume. }\end{array}$ & $\begin{array}{c}\text { Grams } \\
\text { per } \\
\text { 100 cc. }\end{array}$ & & $\begin{array}{c}\text { Per } \\
\text { Cent } \\
\text { by } \\
\text { Weight }\end{array}$ & $\begin{array}{c}\text { Per } \\
\text { Cent } \\
\text { by Vol- } \\
\text { ume. }\end{array}$ & $\begin{array}{c}\text { Grams } \\
\text { per } \\
\text { too ce. }\end{array}$ \\
\hline & 18.92 & & 18.41 & & .92 & & 22.18 & 0.9629 & 26.60 & 32.27 & \\
\hline & 19.00 & & & 8 & & & & 8 & 26.67 & & \\
\hline 7 & 19.08 & $23 \cdot 3^{8}$ & 18.5 & 7 & 23.08 & 13 & $\cdot 33$ & 7 & 26.73 & & \\
\hline 6 & 19.17 & 3.48 & 1 & 6 & 23.15 & & & 6 & & & \\
\hline 5 & 19. & .58 & I & 5 & & & & 5 & & & \\
\hline 4 & & & & 4 & & & & 4 & & & \\
\hline 3 & & 8 & 18. & 3 & & & & 3 & 27.00 & & \\
\hline 2 & 19. & & 18. & 2 & & & & 2 & 27.07 & & \\
\hline I & 19.58 & 98 & $19 . C$ & $\mathbf{I}$ & .54 & 28.68 & .76 & 1 & $27 \cdot 14$ & & 26.1 \\
\hline 0 & 19.67 & & $19 \cdot 12$ & ○ & 23.62 & 28.77 & 22.83 & o & 27.21 & & 26.1 \\
\hline .9719 & 19.75 & & 19.19 & 0.9669 & 23.69 & & 22.90 & & 27.29 & 33.06 & \\
\hline & 19.83 & 28 & 19.27 & 8 & .77 & & 22.97 & 8 & $27 \cdot 36$ & & 6.3 \\
\hline 7 & 19.92 & & 19.3 & 7 & & & & 7 & 27.43 & & \\
\hline 6 & 20.00 & & 19.44 & 6 & & & & 6 & $27 \cdot 50$ & & .4 \\
\hline 5 & 20.08 & & 19.5 & 5 & & & 23.19 & 5 & & & 6.5 \\
\hline 4 & 20.17 & & 1 & 4 & & & 23.27 & 4 & 64 & & 6.5 \\
\hline 3 & 20.25 & & 19.66 & 3 & & & .33 & 3. & & & 6.6 \\
\hline 2 & 20.33 & & 19.74 & 2 & & & & 2 & & & 6.7 \\
\hline I & 20.42 & $4 \cdot 9^{8}$ & 19.83 & I & $24 \cdot 31$ & $.5^{8}$ & $23 \cdot 4^{8}$ & I & 27.86 & .73 & 26.7 \\
\hline 0 & 20.50 & 25.07 & 19.90 & o & $24 \cdot 3^{8}$ & 29.67 & $23 \cdot 55$ & 0 & 27.93 & $33.8 \mathrm{I}$ & 26.8 \\
\hline & $20.5^{8}$ & 25.17 & $19.9^{8}$ & 0.9659 & $24 \cdot 4^{6}$ & 29.76 & 23.62 & 0.9609 & 28.00 & & 9.9 \\
\hline & 20.67 & 25.27 & 20.07 & 8 & & .86 & 23.70 & 8 & 28.06 & & 5.9 \\
\hline 7 & 20.75 & $25 \cdot 37$ & 20.14 & 7 & & & .77 & 7 & 12 & & 7.0 \\
\hline 6 & $20.8_{3}$ & $5 \cdot 47$ & 20.22 & 6 & 24.69 & & 23.84 & 6 & 28.19 & & 7.0 \\
\hline 5 & 20.92 & .57 & 20.30 & 5 & & & & 5 & 28.25 & & -1 \\
\hline 4. & 21.00 & .67 & & 4 & .85 & & & 4 & $28.3^{1}$ & & 27.1 \\
\hline 3 & 21.08 & .76 & $20.4^{6}$ & 3 & & & & 3 & 28.37 & & 27.2 \\
\hline 2 & $21 \cdot 15$ & 5.86 & $20.5^{2}$ & 2 & 25. & .40 & 24.12 & 2 & 28.44 & & 3 \\
\hline I & 21.23 & 5.95 & 20.59 & I & 25.07 & 0.48 & 9 & $\mathbf{1}$ & 28.50 & & $27 \cdot 3^{6}$ \\
\hline of & $2 \mathrm{I} \cdot 3 \mathrm{I}$ & 26.04 & 20.67 & o & 25,14 & $3^{\circ} \cdot 57$ & 24.26 & 0 & 28.56 & 34.54 & 27.42 \\
\hline & $21 \cdot 3^{8}$ & .13 & 20.73 & & 21 & & $24 \cdot 3^{2}$ & & 28.62 & & .47 \\
\hline & $21 \cdot 46$ & 5.22 & .81 & 8 & & & -39 & 8 & 28.60 & & 27.53 \\
\hline 7 & $21 \cdot 54$ & $6.3 \mathrm{I}$ & .89 & 7 & $25 \cdot 3^{6}$ & & .46 & 7 & 28.75 & & $7 \cdot 5$ \\
\hline 6 & .62 & 5.40 & .96 & 6 & & & & 6 & & & 7.6 \\
\hline 5 & 21.69 & 5.49 & 07 & 5 & & $3^{0.98}$ & & 5 & 87 & & $27 \cdot 7$ \\
\hline 4. & .77 & 58 & & 4 & & 31.07 & 24.66 & 4 & & & 27.7 \\
\hline 3 & 85 & .67 & 2 & 3 & & $3^{\mathrm{I}} \cdot \mathrm{I} 3$ & $24 \cdot 7^{2}$ & 3 & 29.00 & & 27.8 \\
\hline 2 & $9^{2}$ & .77 & 21.25 & 2 & & 1.23 & 24.79 & 2 & 29.07 & & 27.8 \\
\hline 1 & $\infty$ & & & I & & $3^{x} \cdot 3^{2}$ & 24.86 & 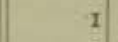 & 29.13 & & 27.9 \\
\hline 0 & 22.08 & 26.95 & 21.40 & o & 25.86 & $3 \mathrm{~T} \cdot 40$ & 24.93 & 0 & 29.20 & & 28.0 \\
\hline .9689 & & & & & & $3 \mathrm{I} \cdot 4^{8}$ & & & 29.27 & & 28.07 \\
\hline & 22.23 & & & 8 & 26.00 & $3^{x} \cdot 57$ & 25.06 & 8 & 29.33 & & 28.12 \\
\hline 7 & $22 \cdot 31$ & .22 & $6 \pi$ & 7 & 26.07 & 31.65 & & 7 & 29. & & 28.1 \\
\hline & $22.3^{8}$ & $27 \cdot 31$ & & 6 & 26.13 & $3^{x} \cdot 7^{2}$ & 25.18 & 6 & $29 \cdot 47$ & & 28.2 \\
\hline 5 & 22.46 & 27.40 & 1.76 & 5 & 26.20 & & 25.23 & 5 & $29 \cdot 53$ & & 28.3 \\
\hline 4 & 22.54 & $27 \cdot 49$ & I. 83 & 4 & 26.27 & 31.88 & & 4 & 29.60 & & 28.3 \\
\hline 3 & 22.62 & $27 \cdot 59$ & 21.90 & 3 & 26.3 & $3^{x} \cdot 9^{6}$ & $25 \cdot 3^{6}$ & 3 & 29.67 & & 28.4 \\
\hline 2 & 22.69 & 27.68 & $2 x .96$ & 2 & 26.4 & 32.03 & 25.43 & 2 & & & 28.4 \\
\hline I & 22.77 & & 22.01 & 3 & 26.47 & 32.11 & & I & 29.80 & & 28.54 \\
\hline 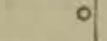 & 22.85 & 27.86 & 22.12 & 이 & 26.53 & 32.19 & 25.55 & 0 & 29.87 & 36.04 & 28.6 \\
\hline
\end{tabular}


SPECIFIC GRAVITY AND PERCENTAGE OF ALCOHOL-(Continued).

\begin{tabular}{|c|c|c|c|c|c|c|c|c|c|c|c|}
\hline \multirow{2}{*}{$\begin{array}{c}\text { Spec. } \\
\text { Grav. } \\
\text { at } \\
25.6^{\circ} \mathrm{C} .\end{array}$} & \multicolumn{3}{|c|}{ Absolute Alcohol. } & \multirow{2}{*}{$\begin{array}{c}\text { Spec, } \\
\text { Grav. } \\
\text { at } \\
15.6^{\circ} \mathrm{C} \text {. }\end{array}$} & \multicolumn{3}{|c|}{ Absolute Aicohol. } & \multirow[b]{2}{*}{$\begin{array}{c}\text { Spec. } \\
\text { Grav. } \\
\text { at. } \\
15.6^{\circ} \mathrm{C} .\end{array}$} & \multicolumn{3}{|c|}{ Absolute Alcohol. } \\
\hline & $\begin{array}{c}\text { Per } \\
\text { Cent } \\
\text { by } \\
\text { Weight }\end{array}$ & $\begin{array}{c}\text { Per } \\
\text { Cent } \\
\text { by Vol- } \\
\text { ume. }\end{array}$ & $\begin{array}{c}\text { Grams } \\
\text { per } \\
\text { 100 cc. }\end{array}$ & & $\begin{array}{c}\text { Per } \\
\text { Cent } \\
\text { by } \\
\text { Weight }\end{array}$ & $\begin{array}{c}\text { Per } \\
\text { Cent } \\
\text { by Vol- } \\
\text { ume. }\end{array}$ & $\begin{array}{c}\text { Grams } \\
\text { per } \\
\text { too ce. }\end{array}$ & & $\begin{array}{c}\text { Per } \\
\text { Cent } \\
\text { by } \\
\text { Weight }\end{array}$ & $\begin{array}{c}\text { Per } \\
\text { Cent } \\
\text { by Vol- } \\
\text { ume. }\end{array}$ & $\begin{array}{l}\text { Grams } \\
\text { per } \\
\text { 100 cc. }\end{array}$ \\
\hline 0.9579 & 29.93 & $3^{6.12}$ & 28.67 & 0.9529 & $3^{2} .94$ & $39 \cdot 54$ & $3^{1} \cdot 3^{8}$ & 0.9479 & $35 \cdot 55$ & 42.45 & $33.7 \circ$ \\
\hline & 30.00 & 36.20 & 28.73 & 8 & 33.00 & 39.61 & $3 \mathrm{I} \cdot 43$ & 8 & 35.60 & $4^{2} \cdot 51$ & 33.75 \\
\hline 7 & 30.06 & 36.26 & $28 \cdot 7^{8}$ & 7 & 33.06 & 39.68 & $3 I-4^{8}$ & 7 & 35.65 & $4^{2} \cdot 5^{6}$ & 33.79 \\
\hline 6 & 30.11 & $3^{6} \cdot 3^{2}$ & 28.82 & 6 & 33.12 & $39 \cdot 74$ & $31 \cdot 53$ & 6 & $35 \cdot 70$ & $4^{2.62}$ & $33.8_{3}$ \\
\hline 5 & $30 \cdot 17$ & 36.39 & 28.88 & 5 & $33 \cdot 18$ & $39.8 \mathrm{I}$ & $3 x \cdot 59$ & 5 & $35 \cdot 75$ & 42.67 & 33. \\
\hline 4 & 30.22 & 36.45 & 28.92 & 4 & 33.24 & 39.87 & 31.63 & 4 & 35.80 & $42 \cdot 73$ & $33-9^{2}$ \\
\hline 3 & 30.28 & $3^{6} \cdot 5^{1}$ & $28.9^{8}$ & 3 & 33.29 & $39 \cdot 94$ & $3^{1} .69$ & 3 & 35.85 & $4^{2} \cdot 78$ & 33.97 \\
\hline 2 & $3^{\circ} \cdot 33$ & 36.57 & 29.03 & 2 & $33 \cdot 35$ & 40.01 & $31 \cdot 74$ & 2 & 35.90 & $4^{2} .84$ & 34.01 \\
\hline I & $3^{0} .39$ & 36.64 & 29.08 & I & 33.41 & 40.07 & 31.80 & $I$ & 35.95 & $4^{2} .89$ & 34.05 \\
\hline 이 & 30.44 & $3^{6.70}$ & 29.13 & o & 33.47 & 40.14 & 3 I -86 & o & $3^{6.00}$ & $4^{2} .95$ & 34.09 \\
\hline 0.9569 & 30.50 & $3^{6.7} \cdot 6$ & 29.18 & 0.9519 & 33.53 & 40.20 & 31.91 & 0.9469 & 36.06 & 43.01 & 34.14 \\
\hline & $3^{0} \cdot 5^{6}$ & $3^{6.83}$ & 29.23 & 8 & 33.59 & 40.27 & $3^{\text {x. }} \cdot 96$ & 8 & $3^{6.11}$ & 07 & \\
\hline 7 & $30.6 \mathrm{I}$ & $3^{6.89}$ & 29.27 & 7 & 33.65 & 40.34 & 32.01 & 7 & 36.17 & 43.13 & 34. \\
\hline 6 & $3^{0.67}$ & 36.95 & $29 \cdot 33$ & 6 & $33 \cdot 7^{1}$ & 40.40 & 32.07 & 6 & $3^{6.22}$ & 43.19 & .28 \\
\hline 5. & $30.7^{2}$ & 37.02 & $29 \cdot 3^{8}$ & 5 & 33.76 & 40.47 & $3^{2.12}$ & 5 & 36.28 & $43 \cdot 26$ & $34 \cdot 34$ \\
\hline 4 & 30.78 & 37.08 & $29 \cdot 43$ & 4 & 33.82 & 40.53 & 32.17 & 4 & 36.33 & $43 \cdot 32$ & $3^{8}$ \\
\hline 3 & $3^{\circ} .83$ & 37.14 & $29 \cdot 4^{8}$ & 3 & 33.88 & 40.60 & 32.22 & 3 & 36.39 & $43 \cdot 3^{8}$ & 34.44 \\
\hline 2 & 30.89 & 37.20 & 29.53 & 2 & 33.94 & 40.67 & 32.27 & 2 & $3^{6} .44$ & 43.44 & $4 \cdot 4^{8}$ \\
\hline I & 30.94 & 37.27 & $29.5^{8}$ & I & 34.00 & 40.74 & $3^{2} \cdot 3^{2}$ & I & $3^{6} .50$ & $43-50$ & $34-54$ \\
\hline o & $3^{1} .00$ & $37-34$ & 29.63 & 0 & 34.05 & 40.79 & $3^{2} \cdot 37$ & o) & $3^{6.56}$ & 43.56 & $34 \cdot 5^{8}$ \\
\hline 0.9559 & 31.06 & $37 \cdot 4 \mathrm{I}$ & 29.69 & 0.9509 & $34-10$ & .40 .84 & $3^{2} \cdot 4^{1}$ & 0.9459 & $36.6 x$ & 43.63 & 34.63 \\
\hline & $3^{1}+12$ & $37 \cdot 4^{8}$ & 29.74 & 8 & 34,14 & 40.90 & $3^{2} \cdot 45$ & 8 & $3^{6} 6.67$ & 43.69 & 34 \\
\hline 7 & $3+19$ & $37-55$ & 29.81 & 7 & 34.19 & 40.95 & 32.49 & 7 & $3^{6.72}$ & 43.75 & $34 \cdot 73$ \\
\hline & $3^{\mathrm{I}}-25$ & 37.62 & 29.86 & 6 & 34.24 & 41.00 & $3^{2} \cdot 54$ & 6 & $3^{6.7^{8}}$ & 43.81 & 34 \\
\hline 5 & $3^{\mathrm{I}} \cdot 3^{\mathrm{I}}$ & 37.69 & 29.91 & 5 & 34.29 & 41.05 & 32.59 & 5 & $3^{6.83}$ & 43.87 & 34 . \\
\hline 4 & $3^{\mathrm{x}}-37$ & $37-7^{6}$ & 29.97 & 4 & $34 \cdot 33$ & $4 \mathrm{I} \cdot \mathrm{II}$ & $3^{2} .63$ & 4 & $3^{6.89}$ & 43.93 & 34. \\
\hline 3 & $3 \mathrm{r} .44$ & 37.83 & 30.03 & 3 & $34-3^{8}$ & 41,16 & 32.67 & 3 & $3^{6.94}$ & 44.00 & 34. \\
\hline 2 & $31 \cdot 50$ & 37.90 & 30.09 & 2 & $34 \cdot 43$ & 41.21 & 32.71 & 2 & 37.00 & 44.06 & 34 \\
\hline I & $3^{I} \cdot 5^{6}$ & $37 \cdot 97$ & 30.14 & 1 & $34 \cdot 4^{8}$ & 47.26 & 32.75 & 1 & 37.06 & 44.12 & 35,02 \\
\hline 0 & $3 \mathrm{x} .62$ & $3^{8} .04$ & 30.20 & 0 & $34-5^{2}$ & $4 \mathrm{~T} \cdot 3^{2}$ & 32.79 & o & $37 . \mathrm{II}$ & $44 \cdot 18$ & 35.07 \\
\hline & 31.69 & $3^{8.11}$ & 30.26 & & & 41.37 & 32.84 & & 37.17 & 44.24 & \\
\hline & $3 \mathrm{I}-75$ & $3^{8.18}$ & $3^{0} \cdot 3^{\mathrm{T}}$ & & 34.62 & $41 \cdot 42$ & 32.88 & 8 & 37.22 & $44 \cdot 30$ & \\
\hline 7 & $31.8 \mathrm{I}$ & 38.25 & $30.3^{6}$ & 7 & 34.67 & 41.48 & 32.92 & 7 & 37.28 & $44 \cdot 3^{6}$ & 35.21 \\
\hline 6 & 35.87 & $3^{8} \cdot 33$ & $3^{\circ} \cdot 4^{2}$ & 6 & $34 \cdot 71$ & 41.53 & 32.96 & 6 & $37-33$ & 44.43 & 35.26 \\
\hline 5 & $3 x .94$ & $3^{8} .40$ & $30.4^{8}$ & 5 & 34.76 & $41-5^{8}$ & 33.00 & 5 & $37-39$ & $44 \cdot 49$ & $35-31$ \\
\hline 4 & 32.00 & $3^{8} \cdot 4 i$ & $3^{0} \cdot 53$ & 4 & $34.8 \mathrm{I}$ & 41.63 & $33 . c$ & 4 & $37-44$ & 44.55 & $35-35$ \\
\hline 3 & 32.06 & $3^{8} .53$ & 30.59 & 3 & 34.86 & $4 x-69$ & 33.0 & 3 & $37 \cdot 50$ & 44.61 & $35 \cdot 41$ \\
\hline 2 & $3^{2}, 12$ & $3^{8.60}$ & 30.64 & 2 & 34.90 & $4 \mathrm{~T}-74$ & 33.1 & 2 & $37 \cdot 5^{6}$ & 44.67 & $35-46$ \\
\hline I & 32.19 & $3^{8} .68$ & 30.71 & $\mathrm{I}$ & 34.95 & $41 \cdot 79$ & $33 \cdot 17$ & 1 & 37.61 & $44 \cdot 73$ & 35.51 \\
\hline o & $3^{2.25}$ & $3^{8} .75$ & 30.77 & o. & 35.00 & $4^{1} .84$ & 33.21 & o & $37 \cdot 67$ & $44 \cdot 79$ & $35 \cdot 5^{6}$ \\
\hline 0.9539 & $3^{2} \cdot 3^{\mathrm{I}}$ & 38.82 & $30.8 \mathrm{I}$ & $0.94^{89}$ & 35.05 & 41.90 & 32.26 & & $37 \cdot 73$ & 44.86 & 35.60 \\
\hline & $3^{2}-37$ & 38.89 & 30.87 & 8 & 35.10 & 41.95 & $33 \cdot 30$ & & $37 \cdot 78$ & 44.02 & 35.65 \\
\hline 7 & $3^{2} .44$ & $3^{8.96}$ & 30.93 & 7 & 35.15 & $4^{2} .0 \mathrm{I}$ & $33 \cdot 34$ & 7 & $37 \cdot 83$ & $44.9^{8}$ & $35.7^{c}$ \\
\hline & $3^{2} \cdot 50$ & $39 \cdot 04$ & 30.99 & 6 & 35.20 & 42.06 & $33 \cdot 39$ & 6 & 37.89 & 45. & 35 . \\
\hline 5 & $3^{2} \cdot 5^{6}$ & $39.1 \mathrm{I}$ & $3 x .05$ & 5 & 35.25 & 42.12 & 33.43 & 5 & $37 \cdot 49$ & 45.10 & \\
\hline 4 & 32.62 & 39.18 & $3^{1} \cdot 10$ & 4 & $35 \cdot 30$ & 42.17 & $33 \cdot 4^{8}$ & 4 & 38.00 & $45 \cdot 16$ & 35.85 \\
\hline 3 & 32.69 & 39.25 & $3^{1} \cdot 15$ & 3 & $35 \cdot 35$ & 42.23 & $33 \cdot 53$ & 3 & 38.06 & 45.22 & 35.90 \\
\hline 2 & $3^{2} \cdot 75$ & $39 \cdot 32$ & 31.20 & 2 & 35.40 & 42.29 & $33 \cdot 57$ & 2 & 38,11 & 45.28 & 35.95 \\
\hline $\mathrm{I}$ & $3^{2} .8 \mathrm{r}$ & 39.4 잉 & 31.26 & 1 & 35.45 & $4^{2}-34$ & 33.61 & $\mathbf{I}$ & 38.17 & $45-34$ & 36.00 \\
\hline ㅇ & $3^{2} \cdot 87$ & $39 \cdot 47$ & $3^{1} \cdot 3^{2}$ & o & $35.5^{\circ}$ & 42.40 & 33.65 & 0 & $3^{8} .22$ & $45 \cdot 4^{1}$ & $3^{6.04}$ \\
\hline
\end{tabular}


SPECIFIC GRAVITY AND PERCENTAGE OF ALCOHOL-(Continued).

\begin{tabular}{|c|c|c|c|c|c|c|c|c|c|c|c|}
\hline \multirow[b]{2}{*}{$\begin{array}{c}\text { Spec. } \\
\text { Grav. } \\
\text { at } \\
\mathbf{5} 5.6^{\circ} \mathrm{C} \text {. }\end{array}$} & \multicolumn{3}{|c|}{ Absolute Alcohol. } & \multirow[b]{2}{*}{$\begin{array}{c}\text { Spec. } \\
\text { Grav. } \\
\text { at } \\
\text { rs. } 6^{\circ} \mathrm{C} \text {. }\end{array}$} & \multicolumn{3}{|c|}{ Absolute Alcohol. } & \multirow[b]{2}{*}{$\begin{array}{c}\text { Spec. } \\
\text { Grav. } \\
\text { at } \\
15.6^{\circ} \mathrm{C} \text {. }\end{array}$} & \multicolumn{3}{|c|}{ Absolute Alcohol. } \\
\hline & $\begin{array}{c}\text { Per } \\
\text { Cent } \\
\text { by } \\
\text { Weight }\end{array}$ & $\begin{array}{c}\text { Per } \\
\text { Cent } \\
\text { by Vol- } \\
\text { ume. }\end{array}$ & $\begin{array}{l}\text { Grams } \\
\text { per } \\
\text { roo cc. }\end{array}$ & & $\begin{array}{c}\text { Per } \\
\text { Cent } \\
\text { by } \\
\text { Weight }\end{array}$ & $\begin{array}{l}\text { Per } \\
\text { Cent } \\
\text { by Vol- } \\
\text { ume. }\end{array}$ & $\begin{array}{c}\text { Grams } \\
\text { per } \\
\text { roo cc. }\end{array}$ & & $\begin{array}{c}\text { Per } \\
\text { Cent } \\
\text { by } \\
\text { Weight }\end{array}$ & $\begin{array}{c}\text { Per } \\
\text { Cent } \\
\text { by Vol- } \\
\text { ume. }\end{array}$ & $\begin{array}{c}\text { Grams } \\
\text { per } \\
\text { too cc. }\end{array}$ \\
\hline 0.9429 & $3^{8} .28$ & $45 \cdot 47$ & 36.08 & 0.9379 & 40.85 & 48.26 & $38.3 \mathrm{I}$ & 0.9329 & 43.29 & 50.87 & $40 \cdot 38$ \\
\hline 8 & $3^{8} \cdot 33$ & $45 \cdot 53$ & $36 .+3$ & 8 & 40.90 & $4^{8} \cdot 3^{2}$ & $3^{8} .35$ & 8 & 43.33 & $50.9^{2}$ & 40.42 \\
\hline 7 & 38.39 & 45.59 & $3^{6} .18$ & 7 & 40.95 & 48.37 & 38.39 & 7 & $43 \cdot 39$ & 50.97 & 40.46 \\
\hline 6 & 38.44 & 45.65 & 36.23 & 6 & 41.00 & 48.43 & 38.44 & 6 & 4343 & 51.02 & 40.50 \\
\hline 5 & 38.50 & $45 \cdot 71$ & $3^{6} .28$ & 5 & 41.05 & $48 \cdot 4^{8}$ & $3^{8} \cdot 4^{8}$ & 5 & $43 \cdot 48$ & 51.07 & 40.54 \\
\hline 4 & $38.5^{6}$ & $45-77$ & $3^{6} .33$ & 4 & $4 \mathrm{r} \cdot \mathrm{ro}$ & 48.54 & $3^{8} .52$ & 4 & $43 \cdot 52$ & $5 I . I 2$ & $40 \cdot 5^{8}$ \\
\hline 3 & $38.6 r$ & $45 \cdot 83$ & $3^{6} \cdot 3^{8}$ & 3 & 41.15 & 48.59 & $3^{8} \cdot 5^{8}$ & 3 & $3 \cdot 57$ & $5 \mathrm{I} \cdot \mathrm{I} 7$ & 40.62 \\
\hline 2 & 38.67 & 45.89 & 36.43 & 2 & 41.20 & 48.64 & $3^{8} .62$ & 2 & 43.62 & $5 \mathrm{I} .22$ & 40.66 \\
\hline I & 38.72 & 45.95 & $3^{6} \cdot 4^{8}$ & I & 41.25 & 48.70 & 38.66 & $I$ & 43.67 & 51.27 & 40.70 \\
\hline o & $3^{8} \cdot 7^{8}$ & 46.02 & $3^{6} \cdot 53$ & 0 & $41 \cdot 30$ & 48.75 & $3^{8} \cdot 7^{\circ}$ & 0 & $43-7 \mathrm{I}$ & $51 \cdot 3^{2}$ & 40.74 \\
\hline 0.9419 & $3^{8} .83$ & 46.08 & $3^{6} .57$ & $0.93^{69}$ & $41 \cdot 35$ & 48.80 & $38 \cdot 74$ & & $43 \cdot 7^{6}$ & $51 \cdot 3^{8}$ & 40.78 \\
\hline 8 & 38.89 & 46.14 & 36.62 & 8 & $4 \mathrm{I} .40$ & 48.86 & 38.78 & 8 & $43.8 \mathrm{I}$ & $5 \mathrm{I}$. & $40.8 \mathrm{I}$ \\
\hline 7 & $3^{8} .94$ & 46.20 & 36.67 & 7 & $4 \mathrm{r} .45$ & 48.91 & 38.82 & 7 & .86 & .48 & 40.85 \\
\hline 6 & 39.00 & 6.26 & 36.72 & 6 & 41.50 & 48.97 & $3^{8.87}$ & 6 & .90 & $5 \mathrm{I} .53$ & 40.89 \\
\hline 5 & 39.05 & $46 \cdot 32$ & $36.7^{6}$ & 5 & $4 I-55$ & 49.02 & & 5 & .95 & $5 \mathrm{r} \cdot 5^{8}$ & 40.93 \\
\hline 4 & 39.10 & 46.37 & 80 & 4 & 41.60 & 49.07 & 38. & 4 & .00 & 51.62 & 40.97 \\
\hline 3 & 39.15 & 46.42 & 66.85 & 3 & 41.65 & 49.13 & $3^{8}$. & 3 & .05 & $5 \mathrm{r} .68$ & $4 \mathrm{I} .0 \mathrm{r}$ \\
\hline 2 & 39 & $46.4^{8}$ & $3^{6} .89$ & 2 & $4 \pi \cdot 70$ & 49.18 & 39 & 2 & 44.09 & $51 \cdot 72$ & $4 I .05$ \\
\hline $\mathbf{I}$ & 39.25 & 46.53 & 36.94 & I & 41.75 & 49.23 & 39.08 & 1 & $4 \cdot 14$ & 51.77 & 41.09 \\
\hline 0 & $39 \cdot 30$ & $46 \cdot 59$ & $3^{6} \cdot 9^{8}$ & 0 & 41.80 & $49 \cdot 29$ & $39 . \mathrm{I}_{3}$ & 0 & 44.18 & $5 \mathrm{I} .82$ & $4 \mathrm{I} \quad \mathrm{I} 3$ \\
\hline ○ 9409 & 39.35 & & 37.02 & 0.9359 & 41.85 & $49 \cdot 34$ & 39.17 & .9309 & 44.23 & $5 \mathrm{r} .87$ & $4 \mathrm{I} \cdot \mathrm{I} 7$ \\
\hline 8 & $39 \cdot 40$ & 46.70 & 37.07 & 8 & 41.90 & $49+0$ & 39.21 & 8 & 44.27 & .91 & 41.20 \\
\hline 7 & 39.45 & 46.75 & $37 \cdot \mathrm{II}$ & 7 & 41.95 & $49 \cdot 45$ & 39.25 & 7 & $44 \cdot 32$ & 51.96 & $4 I \cdot 24$ \\
\hline 6 & 39.50 & 46.80 & $37 \cdot \mathrm{I}_{5}$ & 6 & 42.00 & 4950 & & 6 & $44 \cdot 36$ & 52.01 & $4 I \cdot 28$ \\
\hline 5 & $39 \cdot 55$ & 46.86 & 37 & 5 & 42.05 & 49.55 & 34 & 5 & $.4 \mathrm{I}$ & .06 & $4 I \cdot 3 I$ \\
\hline 4 & 39.60 & $46.9 t$ & $37 \cdot 23$ & 4 & 42.10 & 49.61 & $39 \cdot 3^{8}$ & 4 & $44 \cdot 4^{6}$ & 52.10 & $41 \cdot 35$ \\
\hline 3 & 39.65 & 46.97 & $37 \cdot 27$ & 3 & $42 \cdot 14$ & 49.66 & 39.42 & 3 & $44 \cdot 50$ & 52.15 & $4 I .49$ \\
\hline 2 & 39.70 & 47.02 & $37 \cdot 32$ & 2 & 42.19 & 49.71 & $39 \cdot 46$ & 2 & 44.55 & 52.20 & $4 \mathrm{I} \cdot 43$ \\
\hline $\mathbf{I}$ & 39.75 & 47.08 & $37 \cdot 36$ & $\mathrm{I}$ & $42 \cdot 24$ & $49 \cdot 7^{6}$ & 39.50 & $I$ & $44 \cdot 59$ & 52.25 & $41 \cdot 47$ \\
\hline o & 39.80 & $47 \cdot 13$ & $37 \cdot 4 \mathrm{r}$ & 0 & 42.29 & $49.8 \mathrm{I}$ & 39.54 & 0 & $44 \cdot 64$ & 52.29 & $4 I \cdot 5 \mathrm{I}$ \\
\hline 0.9399 & 39.85 & $47 \cdot 18$ & $37 \cdot 45$ & 0.9349 & $42 \cdot 33$ & 49.86 & 39.58 & 0.9299 & 44.68 & $52 \cdot 34$ & $4 \mathrm{I} \cdot 55$ \\
\hline 8 & 39.90 & $47 \cdot 24$ & $37 \cdot 49$ & 8 & $42 \cdot 3^{8}$ & 49.91 & 39.62 & 8 & $44 \cdot 73$ & $52 \cdot 39$ & $4 I .59$ \\
\hline 7 & 39.95 & 7.29 & & 7 & $42 \cdot 43$ & $49 \cdot 96$ & & 7 & $44 \cdot 77$ & $52 \cdot 44$ & 41.63 \\
\hline 6 & 40.00 & $47 \cdot 35$ & $37 \cdot 5^{8}$ & 6 & $42 \cdot 48$ & 50.01 & 39.70 & 6 & 44.82 & 52.48 & $4 \mathrm{I} .67$ \\
\hline 5 & 40.05 & $47 \cdot 40$ & 37.62 & 5 & $42 \cdot 52$ & 50.06 & 39.74 & 5 & 44.86 & $5^{2} \cdot 53$ & $4 I .70$ \\
\hline 4 & 40.10 & $47 \cdot 45$ & 37.67 & 4 & $42 \cdot 57$ & 50.11 & 39.78 & 4 & $44.9 \mathrm{I}$ & $5^{2} \cdot 5^{8}$ & $41 \cdot 74$ \\
\hline 3 & 40.15 & $47 \cdot 5 x$ & $37.7 \mathrm{I}$ & 3 & 42.62 & 50.16 & 39.82 & 3 & $44 \cdot 96$ & 52.63 & $4 \mathrm{I} \cdot 77$ \\
\hline 2 & 40.20 & $47 \cdot 56$ & $37 \cdot 75$ & 2 & 42.67 & 50.21 & 39.86 & 2 & 45.00 & & $4 \mathrm{I} .8 \mathrm{I}$ \\
\hline $\mathbf{I}$ & 40.25 & 47.62 & 37.80 & $\mathrm{I}$ & $42 \cdot 7 \mathrm{I}$ & 50.26 & 39.90 & I & 45.05 & 52.72 & 41.85 \\
\hline 이 & $40 \cdot 30$ & 47.67 & $37 \cdot 84$ & 0 & $42 \cdot 76$ & $50.3 \mathrm{I}$ & 39.94 & 0 & 45.09 & $52 \cdot 77$ & 41.89 \\
\hline $0.93^{8} 9$ & 40.35 & $47 \cdot 72$ & & 0.9339 & $42.8 \mathrm{I}$ & 50.37 & & 0.9289 & & 52.82 & $4 \mathrm{r} .93$ \\
\hline 8 & 40.40 & $47 \cdot 78$ & $37 \cdot 92$ & 8 & 42.86 & 50.42 & 40.02 & 8 & 45.18 & 52.87 & 41.97 \\
\hline 7 & 40.45 & $47 \cdot 83$ & $37 \cdot 96$ & 7 & 42.90 & 50.47 & 40.06 & 7 & 45.23 & 52.91 & 42.00 \\
\hline 6 & 40.50 & 47.89 & $3^{8} .00$ & 6 & 42.95 & 50.52 & 40.10 & 6 & 45.27 & 52.96 & 42.04 \\
\hline 5 & 40.55 & $47 \cdot 94$ & 38.05 & 5 & 43.00 & 50.57 & 40.14 & 5 & $45 \cdot 3^{2}$ & 53.01 & 42.08 \\
\hline 4 & 40.60 & $47 \cdot 99$ & 38.09 & 4 & 43.05 & 50.62 & 40.18 & 4 & $45 \cdot 36$ & 53.06 & 42.12 \\
\hline 3 & 40.65 & 48.05 & & 3 & 43 . IO & 50.67 & 40.22 & 3 & $45 \cdot 41$ & 53.10 & 42.16 \\
\hline 2 & 40.70 & 48.10 & $3^{8} .18$ & 2 & $43 \cdot I_{3}$ & 50.72 & 40.26 & 2 & $45 \cdot 46$ & 53.15 & 42.19 \\
\hline $\mathbf{I}$ & 40.75 & 48.16 & 38.22 & 1 & 43.19 & 50.77 & 40.30 & $\mathrm{I}$ & $45 \cdot 50$ & 53.20 & $42 \cdot 23$ \\
\hline 0 & 40.80 & $48.2 \mathrm{r}$ & $3^{8} .27$ & 0 & $43 \cdot 24$ & 50.82 & 40.34 & o & $45 \cdot 55$ & $53 \cdot 24$ & 42.27 \\
\hline
\end{tabular}


SPECIFIC GRAVITY AND PERCENTAGE OF ALCOHOL-(Continued).

\begin{tabular}{|c|c|c|c|c|c|c|c|c|}
\hline \multirow{2}{*}{$\begin{array}{c}\text { Spec. } \\
\text { Grav. } \\
\text { at } \\
15.6^{2} \mathrm{C} .\end{array}$} & \multicolumn{2}{|c|}{ Absolute Alcohol. } & \multirow{2}{*}{$\begin{array}{l}\text { Spec. } \\
\text { Grav. } \\
\text { at. } \\
15.6^{\circ} \mathrm{C} \text {. }\end{array}$} & \multicolumn{2}{|c|}{ Absolute Alcohol. } & \multirow{2}{*}{$\begin{array}{c}\text { Spec. } \\
\text { Grav. } \\
\text { at } \\
15.6^{\circ} \mathrm{C} \text {. }\end{array}$} & \multicolumn{2}{|c|}{ Absolute Alcohol. } \\
\hline & $\begin{array}{c}\text { Per } \\
\text { Cent } \\
\text { by } \\
\text { Weight. }\end{array}$ & $\begin{array}{c}\text { Per } \\
\text { Cent } \\
\text { by Voi- } \\
\text { ume. }\end{array}$ & & $\begin{array}{c}\text { Per } \\
\text { Cent } \\
\text { by } \\
\text { weight. }\end{array}$ & $\begin{array}{l}\text { Per } \\
\text { Cent } \\
\text { by Vol- } \\
\text { tume. }\end{array}$ & & $\begin{array}{c}\text { Per } \\
\text { Cent } \\
\text { by } \\
\text { weight. }\end{array}$ & $\begin{array}{l}\text { Per } \\
\text { Cent } \\
\text { by Vol- } \\
\text { ume, }\end{array}$ \\
\hline 0.9279 & $45 \cdot 59$ & 53.29 & 0.9229 & 47.86 & 55.65 & 0.9179 & 50.13 & 57.97 \\
\hline 8 & 45.64 & $53-34$ & 8 & $47 \cdot 91$ & 55.69 & 8 & 50.17 & $58.0 \tau$ \\
\hline 7 & 45.68 & $53 \cdot 39$ & 7 & 47.96 & $55 \cdot 74$ & 7 & 50.22 & $5^{8} .06$ \\
\hline 6 & $45 \cdot 73$ & 53.43 & 6 & 48.00 & $55 \cdot 79$ & 6 & 50.26 & $5^{8.10}$ \\
\hline 5 & $45 \cdot 77$ & 53.48 & 5 & 48.05 & 55.83 & 5 & 50.30 & 58.14 \\
\hline 4 & 45.83 & $53 \cdot 53$ & 4 & 48.09 & 55.88 & 4 & $50 \cdot 35$ & $5^{8} .19$ \\
\hline 3 & 45.86 & $53.5^{8}$ & 3 & 48.14 & 55.93 & 3 & 50.39 & $5^{8} .23$ \\
\hline 2 & $45-91$ & 53.62 & 2 & 48.18 & 55.97 & 2 & 50.43 & 58.28 \\
\hline$I$ & $45-96$ & 53.67 & I & 48.23 & 56.02 & 1 & 50.48 & 58.32 \\
\hline$\circ$ & 46.00 & $53 \cdot 7^{2}$ & ० & 48.27 & 56.07 & $\circ$ & 50.52 & $5^{8} \cdot 3^{6}$ \\
\hline 0.9269 & 46.05 & $53 \cdot 77$ & 0.9219 & 48.32 & 56.11 & 0.9169 & 50.57 & 58.41 \\
\hline 8 & 46.09 & $53.8 \mathrm{I}$ & 8 & 48.36 & 56.16 & 8 & 50.61 & 58.45 \\
\hline 7 & 46.14 & 53.86 & 7 & $48.4 I$ & $5^{6.21}$ & 7 & 50.65 & $5^{8} \cdot 5^{\circ}$ \\
\hline 6 & 46.18 & 53.91 & 6 & 48.46 & $5^{6.25}$ & 6 & 50.70 & $5^{8} \cdot 54$ \\
\hline 5 & 46.23 & $53 \cdot 35$ & 5 & 48.50 & 56.30 & 5 & 50.74 & $5^{8}-5^{8}$ \\
\hline 4 & 46.27 & 54.00 & 4 & 48.55 & 56.35 & 4 & $5^{0.78}$ & 58.63 \\
\hline 3 & 46.32 & 54.05 & 3 & 48.59 & 56.40 & 3 & 50.83 & 58.67 \\
\hline 2 & $4^{6} \cdot 3^{6}$ & 54.10 & 2 & 48.64 & 56.44 & $=$ & 50.87 & $5^{8.72}$ \\
\hline 1 & 46.41 & $54 \cdot 14$ & I & 48.68 & 56.49 & 1 & $50.9 \mathrm{I}$ & $5^{8} .7^{6}$ \\
\hline ○ & 46.46 & 54.19 & - & 48.73 & $5^{6} \cdot 54$ & o & $50.9^{6}$ & 58.80 \\
\hline 0.9259 & 46.50 & 54.24 & 0.9200 & 48.77 & $5^{6} \cdot 5^{8}$ & 0.9159 & 51.00 & 58.85 \\
\hline & 46.55 & 54.29 & & 48.82 & 56.63 & 8 & 51.04 & 58.89 \\
\hline 7 & 46.59 & $54 \cdot 33$ & 7 & 48.86 & 56.68 & 7 & 51.08 & 58.93 \\
\hline 6 & 46.64 & $54-38$ & 6 & 48.91 & 56.72 & 6 & $51-13$ & 58.97 \\
\hline 5 & 46.68 & $54 \cdot 43$ & 5 & $4^{8} .96$ & 56.77 & 5 & $51-17$ & $59-0 \mathrm{I}$ \\
\hline 4 & 46.73 & $54 \cdot 47$ & 4 & 49.00 & 56.82 & 4 & $5^{1.21}$ & 59.05 \\
\hline 3 & 46.77 & $54 \cdot 5^{2}$ & 3 & 49.04 & 56.86 & 3 & 51.25 & 59.09 \\
\hline 2 & $46.8_{2}$ & 54.57 & 2 & 49.08 & 56.90 & $=2$ & 51.29 & $59 \cdot 14$ \\
\hline I & 46.86 & 54.62 & 1 & 49.12 & 56.94 & 1 & $5 \pm-33$ & $59 \cdot 18$ \\
\hline$\circ$ & 46.91 & 54.66 & $\circ$ & $49 \cdot 16$ & 56.98 & 0 & $5^{1} \cdot 3^{8}$ & $59 \cdot 22$ \\
\hline 0.9249 & 46.96 & $54-7 \mathrm{r}$ & 0.9199 & $49 \cdot 20$ & 57.02 & & 51.42 & 59.26 \\
\hline 8 & 47.00 & 54.76 & Proof 8 & 49.24 & 57.06 & 8 & $5^{1.46}$ & $59 \cdot 3^{\circ}$ \\
\hline 7 & 47.05 & 54.80 & $1 \quad 7$ & 49.29 & $57 \cdot 10$ & 7 & $51-50$ & $59 \cdot 34$ \\
\hline 6 & 47.09 & 54.85 & 6 & $49 \cdot 34$ & 57.15 & 6 & 51.54 & $59 \cdot 39$ \\
\hline 5 & $47 \cdot 14$ & 54.90 & 5 & 49.39 & 57.20 & 5 & $5^{1} \cdot 5^{8}$ & $59 \cdot 43$ \\
\hline 4 & 47.18 & 54.95 & 4 & 49.44 & 57.25 & 4 & 51.63 & $59 \cdot 47$ \\
\hline 3 & 47.23 & 54.99 & 3 & 49.49 & $57.3^{\circ}$ & 3 & 51.67 & $59 \cdot 5^{I}$ \\
\hline 2 & 47.27 & 55.04 & 2 & 49.54 & $57 \cdot 35$ & 2 & 51.71 & $59 \cdot 55$ \\
\hline I & $47 \cdot 32$ & 55.09 & I & $49 \cdot 59$ & 57.40 & I & 51.75 & $59 \cdot 59$ \\
\hline$\circ$ & $47 \cdot 36$ & $55 \cdot 13$ & $\circ$ & 49.64 & $57 \cdot 45$ & 0 & $51 \cdot 79$ & 59.63 \\
\hline 0.9239 & $47.4 \mathrm{I}$ & 55.18 & 0.9189 & 49.68 & $57 \cdot 49$ & 0.9139 & $51-83$ & 59.68 \\
\hline & $47 \cdot 46$ & 55.23 & 8 & $49 \cdot 73$ & $57 \cdot 54$ & 8 & 5 I. 88 & $59 \cdot 72$ \\
\hline 7 & 47.50 & 55.27 & 7 & $49 \cdot 77$ & $57 \cdot 59$ & 7 & 51.92 & $59 \cdot 76$ \\
\hline 6 & $47-55$ & $55-32$ & 6 & 49.82 & 57.64 & 6 & 51.96 & 59.80 \\
\hline 5 & 47.59 & $55 \cdot 37$ & 5 & 49.86 & 57.69 & 5 & $5^{2.00}$ & $59.8_{4}$ \\
\hline 4 & 47.64 & $55.4 \mathrm{I}$ & 4 & 49.91 & 57.74 & 4 & $5^{2} .05$ & 59.89 \\
\hline 3 & 47.68 & 55.46 & 3 & 49.95 & 57.79 & 3 & $5^{2} .09$ & 59.93 \\
\hline 2 & $47 \cdot 73$ & $55 \cdot 5 \mathrm{I}$ & 2 & 50.00 & 57.84 & 2 & 52.14 & $59.9^{8}$ \\
\hline I & 47.77 & 55.55 & 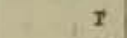 & 50.04 & 57.88 & I & 52.18 & 60.02 \\
\hline 0 & 47.82 & 55.60 & 0 & 50.09 & $5^{8} .9^{2}$ & 。 & $5^{2}, 23$ & 60.07 \\
\hline
\end{tabular}


SPECIFIC GRAVITY AND PERCENTAGE OF ALCOHOL-(Continued).

\begin{tabular}{|c|c|c|c|c|c|c|c|c|}
\hline \multirow[b]{2}{*}{$\begin{array}{c}\text { Spec. } \\
\text { Grav. } \\
\text { at } \\
15.6^{\circ} \mathrm{C} \text {. }\end{array}$} & \multicolumn{2}{|c|}{ Absolute Alcohol. } & \multirow[b]{2}{*}{$\begin{array}{c}\text { Spec. } \\
\text { Grav. } \\
\text { at } \\
15.6^{\circ} \mathrm{C} \text {. }\end{array}$} & \multicolumn{2}{|c|}{ Absolute Alcohol. } & \multirow[b]{2}{*}{$\begin{array}{c}\text { Spec. } \\
\text { Grav. } \\
\text { at } \\
15.6^{\circ} \mathrm{C} .\end{array}$} & \multicolumn{2}{|c|}{ Absolute Alcohol. } \\
\hline & $\begin{array}{c}\text { Per } \\
\text { Cent } \\
\text { by } \\
\text { Weight. }\end{array}$ & $\begin{array}{l}\text { Per } \\
\text { Cent } \\
\text { by Vol- } \\
\text { ume. }\end{array}$ & & $\begin{array}{c}\text { Per } \\
\text { Cent } \\
\text { by } \\
\text { Weight. }\end{array}$ & $\begin{array}{c}\text { Per } \\
\text { Cent } \\
\text { by Vol- } \\
\text { ume. }\end{array}$ & & $\begin{array}{c}\text { Per } \\
\text { Cent } \\
\text { by } \\
\text { Weight. }\end{array}$ & $\begin{array}{l}\text { Per } \\
\text { Cent } \\
\text { by Vol- } \\
\text { ume, }\end{array}$ \\
\hline $0.9 \times 29$ & $5^{2} .27$ & 60.12 & 0.9079 & $54 \cdot 52$ & $62 \cdot 36$ & 0.9029 & 56.82 & 64.63 \\
\hline & $5^{2} \cdot 32$ & 60.16 & & $54 \cdot 57$ & 62.41 & $\begin{array}{r}.9029 \\
8\end{array}$ & 56.86 & 64.67 \\
\hline 7 & $5^{2} \cdot 3^{6}$ & 60.21 & 7 & 54.62 & 62.45 & 7 & $56.9 \mathrm{I}$ & $64 \cdot 7 x$ \\
\hline 6 & $5^{2}-4^{1}$ & 60.25 & 6 & 54.67 & 62.50 & 6 & 56.95 & 64.76 \\
\hline 5 & $5^{2} \cdot 45$ & $60 \cdot 30$ & 5 & $54.7 \mathrm{x}$ & 62.55 & 5 & 57.00 & 64.80 \\
\hline 4 & $5^{2} \cdot 5^{\circ}$ & 60.34 & 4 & 54.76 & 62.60 & 4 & 57.04 & 64.85 \\
\hline 3 & $5^{2} .55$ & 60.39 & 3 & 54.81 & 62.65 & 3 & 57.08 & 64.89 \\
\hline 2 & 52.59 & 60.44 & 2 & 54.86 & 62.69 & 2 & 57.13 & 64.93 \\
\hline I & $5^{2} .64$ & 60.47 & I & 54.90 & 62.74 & I & $57 \cdot 17$ & 64.97 \\
\hline 0 & $5^{2} .68$ & $60 \cdot 5^{2}$ & 0 & 54.95 & 62.79 & 0 & 57.21 & 65.01 \\
\hline 0.9119 & $5^{2} \cdot 73$ & 60.56 & 0.9069 & 55.00 & 62.84 & 0.9019 & 57.25 & 65.05 \\
\hline 8 & $5^{2} .77$ & $60.6 \pi$ & 8 & 55.05 & 62.88 & 8 & 57.29 & 65.09 \\
\hline 7 & $5^{2.82}$ & 60.65 & 7 & 55.09 & $6 z .93$ & 7 & $57 \cdot 33$ & $65 . r_{3}$ \\
\hline 6 & $5^{2} .86$ & 60.70 & 6 & $55 \cdot 14$ & 62.97 & 6 & $57 \cdot 3^{8}$ & 65.17 \\
\hline 5 & $5^{2} .91$ & 60.74 & 5 & $55 \cdot 18$ & 63.02 & 5 & 57.42 & $65.2 \mathrm{I}$ \\
\hline 4 & 52.95 & 60.79 & 4 & 55.23 & 63.06 & 4 & $57 \cdot 4^{6}$ & 65.25 \\
\hline 3 & 53.00 & 60.85 & 3 & 55.27 & 63.11 & 3 & $57 \cdot 50$ & 65.29 \\
\hline 2 & 53.04 & 60.80 & 2 & 55.32 & 63.15 & 2 & $57 \cdot 54$ & $65 \cdot 33$ \\
\hline$I$ & 53.09 & 60.93 & $I$ & $55 \cdot 36$ & 63.20 & $I$ & 57.58 & $65 \cdot 37$ \\
\hline 0 & 53.13 & 60.97 & 0 & $55.4 \mathrm{I}$ & 63.24 & 0 & 57.63 & $65.4 \mathrm{I}$ \\
\hline 0.9109 & 53.17 & 61.02 & 0.9059 & 55.45 & 63.28 & 0.9009 & 57.67 & 65.45 \\
\hline 8 & 53.22 & 61.06 & 8 & $55.5^{\circ}$ & 63.33 & 8 & $57-71$ & 65.49 \\
\hline 7 & 53.26 & 61.10 & 7 & 55.55 & 63.37 & 7 & $57 \cdot 75$ & $65 \cdot 53$ \\
\hline 6 & 53.30 & $6 \mathrm{x} \cdot 15$ & 6 & 55.59 & 63.42 & 6 & $57 \cdot 79$ & 65.57 \\
\hline 5 & 53.35 & 61.19 & 5 & 55.64 & $63 \cdot 46$ & 5 & 57.83 & $65.6 \mathrm{x}$ \\
\hline 4 & 53.39 & 61.23 & 4 & 55.68 & 63.51 & 4 & 57.88 & 65.65 \\
\hline 3 & 53.43 & 61.28 & 3 & 55.73 & $63-55$ & 3 & 57.92 & 65.69 \\
\hline 2 & 53.48 & $61 \cdot 3^{2}$ & 2 & $55 \cdot 77$ & 63.60 & 2 & 57.96 & $65 \cdot 73$ \\
\hline I & $53 \cdot 5^{2}$ & 61.36 & I & 55.82 & 63.64 & $I$ & 58.00 & 65.77 \\
\hline 0 & 53.57 & 61.40 & 0 & 55.86 & 63.69 & 0 & 58.05 & $65.8 \mathrm{I}$ \\
\hline 0.9099 & $53.6 \mathrm{r}$ & $6 \mathrm{r} \cdot 45$ & 0.9049 & $55.9 \mathrm{t}$ & 63.73 & 0.8999 & 58.09 & 65.85 \\
\hline 8 & 53.65 & 61.49 & 8 & 55.95 & 63.78 & 8 & 58.14 & $65 \cdot 90$ \\
\hline 7 & 53.70 & 61.53 & 7 & 56.00 & 63.82 & 7 & 58.18 & 65.94 \\
\hline 6 & 53.74 & 61.58 & 6 & 56.05 & 63.87 & 6 & 58.23 & 65.99 \\
\hline 5 & 53.78 & 61.62 & 5 & 56.09 & $63.9 \mathrm{x}$ & 5 & 58.27 & 66.03 \\
\hline 4 & 53.83 & 61.66 & 4 & 56.14 & 63.96 & 4 & 58.32 & 66.07 \\
\hline 3 & 53.87 & $61 \cdot 71$ & 3 & 56.18 & 64.00 & 3 & 58.36 & 66.12 \\
\hline 2 & 53.91 & $6 x \cdot 75$ & 2 & 56.23 & 64.05 & 2 & 58.41 & 66.16 \\
\hline I & $53.9^{6}$ & 61,79 & I & 56.27 & 64.09 & $I$ & 58.45 & $66.2 \mathrm{I}$ \\
\hline 0 & 54.00 & 61.84 & 0 & $5^{6} \cdot 3^{2}$ & 64.14 & 0 & $5^{8} .5^{\circ}$ & 66.25 \\
\hline 0.9089 & 54.05 & 61.88 & 0.9039 & 56.36 & 64.18 & 0.8989 & $5^{8} .55$ & 66.29 \\
\hline 8 & 54.10 & 61.93 & 8 & $56.4 \mathrm{I}$ & 64.22 & 8 & 58.59 & $66 \cdot 34$ \\
\hline 7 & 54.14 & 61.98 & 7 & 56.45 & 64.27 & 7 & 58.64 & $66 \cdot 3^{8}$ \\
\hline 6 & 54.19 & 62.03 & 6 & 56.30 & $64 \cdot 31$ & 6 & 58.68 & 66.43 \\
\hline 5 & 54.24 & 62.07 & 5 & 56.55 & $64 \cdot 36$ & 5 & 58.73 & 66.47 \\
\hline 4 & 54.29 & 62.12 & 4 & 56.59 & 64.40 & 4 & 58.77 & $66.5 !$ \\
\hline 3 & $54 \cdot 33$ & 62.17 & 3 & 56.64 & 64.45 & 3 & 58.82 & 66.56 \\
\hline 2 & $.54 \cdot 3^{8}$ & 62.22 & 2 & 56.68 & 64.49 & 2 & 58.86 & $66.6 n$ \\
\hline I & 54.43 & 62.26 & I & 56.73 & $64-54$ & $I$ & 58.91 & 66.65 \\
\hline 0 & $54 \cdot 4^{8}$ & $62 \cdot 3 x$ & 0 & 56.77 & $64 \cdot 5^{8}$ & 0 & $5^{8} .95$ & 66.0 ; \\
\hline
\end{tabular}


SPECIFIC GRAVITY AND PERCENTAGE OF ALCOHOL-(Continued).

\begin{tabular}{|c|c|c|c|c|c|c|c|c|}
\hline \multirow{2}{*}{$\begin{array}{c}\text { Spec. } \\
\text { Grav. } \\
\text { at } \\
15.6^{\circ} \mathrm{C} \text {. }\end{array}$} & \multicolumn{2}{|c|}{ Absolute Alcohol. } & \multirow{2}{*}{$\begin{array}{c}\text { Spec. } \\
\text { Grav. } \\
\text { at } \\
15.6^{\circ} \mathrm{C} \text {. }\end{array}$} & \multicolumn{2}{|c|}{ Absolute Alcohol. } & \multirow{2}{*}{$\begin{array}{c}\text { Spec. } \\
\text { Grav. } \\
\text { at } \\
+5.0^{\circ} \mathrm{C} \text {. }\end{array}$} & \multicolumn{2}{|c|}{ Absolute Alcohol, } \\
\hline & $\begin{array}{c}\text { Per } \\
\text { Cent } \\
\text { by } \\
\text { Weight. }\end{array}$ & $\begin{array}{l}\text { Per } \\
\text { Cent } \\
\text { by Vol- } \\
\text { ume. }\end{array}$ & & $\begin{array}{c}\text { Per } \\
\text { Cent } \\
\text { by } \\
\text { Weight. }\end{array}$ & $\begin{array}{l}\text { Per } \\
\text { Cent } \\
\text { by Vol- } \\
\text { ume. }\end{array}$ & & $\begin{array}{c}\text { Per } \\
\text { Cent } \\
\text { by } \\
\text { Weight. }\end{array}$ & $\begin{array}{c}\text { Per } \\
\text { Cent } \\
\text { by Vol- } \\
\text { ume. }\end{array}$ \\
\hline 0.8979 & 59.00 & 66.74 & 0.8929 & 61.13 & $68 \cdot 7^{6}$ & 0.8879 & $63 \cdot 30$ & 70.81 \\
\hline & 59.04 & 66.78 & 8 & 61.17 & 68.80 & 8 & 63.35 & 70.85 \\
\hline 7 & 59.09 & 66.82 & 7 & $6 \mathrm{r} .2 \mathrm{I}$ & 68.83 & 7 & 63.39 & 70.89 \\
\hline 6 & 59.13 & 66.86 & 6 & 61.25 & 68.87 & 6 & 63.43 & 70.93 \\
\hline 5 & 59.17 & 66.90 & 5 & 61.29 & $68.9 \mathrm{i}$ & 5 & 63.48 & 70.97 \\
\hline 4 & 59.22 & 66.94 & 4 & 61.33 & 68.95 & 4 & 63.52 & 71.01 \\
\hline 3 & 59.26 & 66.99 & 3 & $61 \cdot 3^{8}$ & 68.99 & 3 & 63.57 & 71.05 \\
\hline 2 & $59 \cdot 30$ & 67.03 & 2 & 61.42 & 69.03 & 2 & $63.6 \mathrm{r}$ & 71.09 \\
\hline $\mathbf{I}$ & $59 \cdot 35$ & 67.07 & I & 61.46 & 69.07 & I & 63.65 & $71-13$ \\
\hline o & 59.39 & 67.11 & 0 & $61 \cdot 50$ & 69.11 & 0 & 63.70 & 71.17 \\
\hline 0.8969 & $59 \cdot 43$ & $67 \cdot 15$ & 0.8910 & $6 \pi \cdot 54$ & 69.15 & 0.8869 & $63-74$ & 71.22 \\
\hline 8 & $59 \cdot 4^{8}$ & $67 \cdot 19$ & 8 & $6 \pi \cdot 5^{8}$ & 69.19 & 8 & $63.7^{8}$ & 71.26 \\
\hline 7 & $59 \cdot 52$ & 67,24 & 7 & $6 \mathrm{r} .63$ & 69.22 & 7 & $6_{3} .8_{3}$ & $71-30$ \\
\hline 6 & $59 \cdot 57$ & 67.28 & 6 & 61.67 & 69.26 & 6 & 63.87 & $71-34$ \\
\hline 5 & $59.6 \mathrm{I}$ & $67 \cdot 32$ & 5 & $6 r \cdot 7 x$ & 69.30 & 5 & 63.91 & $71 \cdot 3^{8}$ \\
\hline 4 & 59.65 & $67 \cdot 36$ & 4 & 61.75 & $69 \cdot 34$ & 4 & 63.96 & 71.42 \\
\hline 3 & 59.70 & 67.40 & 3 & 61.79 & $69 \cdot 3^{8}$ & 3 & 64.00 & 71.46 \\
\hline 2 & 59.74 & 67.44 & 2 & 61.83 & 69.42 & 2 & 64.04 & $71 \cdot 5^{\circ}$ \\
\hline I & 59.78 & 67.49 & I & 61.88 & 69.46 & I & 64.09 & 71.54 \\
\hline o & $59-8_{3}$ & 67.53 & 0 & $61-92$ & 69.50 & 0 & 64.13 & $71.5^{8}$ \\
\hline 0.8959 & 59.87 & $67 \cdot 57$ & 0.8909 & $6 r .96$ & 69.54 & 0.8859 & $64 \cdot 17$ & 71.62 \\
\hline 8 & $59.9 \mathrm{I}$ & $67.6 \mathrm{r}$ & 8 & 62.00 & $69 \cdot 5^{8}$ & 8 & 64.22 & 71.66 \\
\hline 7 & 59.96 & 67.65 & 7 & 62.05 & 69.62 & 7 & 64.26 & 71.70 \\
\hline 6 & 60.00 & 67.69 & 6 & 62.09 & 69.66 & 6 & $64 \cdot 30$ & $7 x \cdot 74$ \\
\hline 5 & 60.04 & 67.73 & 5 & 62.14 & $69 \cdot 71$ & 5 & $64 \cdot 35$ & $71 \cdot 7^{8}$ \\
\hline 4 & 60.08 & 67.77 & 4 & 62.18 & 69.75 & 4 & $64 \cdot 39$ & 71.82 \\
\hline 3 & 60.13 & $67.8 \mathrm{I}$ & 3 & 62.23 & 69.79 & 3 & 64.43 & 71.86 \\
\hline 2 & 60.17 & 67.85 & 2 & 62.27 & $69.8_{4}$ & 2 & 64.48 & 71.90 \\
\hline I & 60,21 & 67.80 & I & $62 \cdot 32$ & 69.88 & $\mathrm{I}$ & $64-5^{2}$ & 71.94 \\
\hline 0 & 60.26 & 67.93 & 0 & $62 \cdot 3^{6}$ & 69.92 & 0 & $64 \cdot 57$ & 71.98 \\
\hline 0.8949 & 60.29 & 67.97 & 0.8899 & $62.4 \mathrm{I}$ & 69.96 & 0.8849 & $64.6 \mathrm{r}$ & 72.02 \\
\hline 8 & 60.33 & 68.01 & 8 & 62.45 & 70.01 & 8 & 64.65 & 72.06 \\
\hline 7 & $60 \cdot 3^{8}$ & 68.05 & 7 & 62.50 & 70.05 & 7 & 64.70 & 72.10 \\
\hline 6 & 60.42 & 68.09 & 6 & 62.55 & 70.00 & 6 & $64 \cdot 74$ & 72.14 \\
\hline 5 & 60.46 & $68.1_{3}$ & 5 & 62.59 & 70.14 & 5 & $64 \cdot 7^{8}$ & 72.18 \\
\hline 4 & 60.50 & 68.17 & 4 & 62.64 & 70.18 & 4 & $64.8_{3}$ & 72.22 \\
\hline 3 & 60.54 & $68.2 \mathrm{r}$ & 3 & 62.68 & 70.22 & 3 & 64.87 & 72.26 \\
\hline 2 & $60.5^{8}$ & 68.25 & 2 & 62.73 & 70.27 & 2 & 64.91 & $7^{2} \cdot 30$ \\
\hline I & 60.63 & $68=29$ & I & 62.77 & 70.31 & I & 64.96 & $7^{2} \cdot 34$ \\
\hline 0 & 60.67 & 68.33 & 0 & 62.82 & 70.35 & 0 & 65.00 & $7^{2} \cdot 3^{8}$ \\
\hline 0.8039 & 60.71 & 68.36 & 0.8889 & 62.86 & 70.40 & 0.8839 & 65.04 & $72.4^{2}$ \\
\hline & 60.76 & 68.40 & 8 & 62.91 & 70.44 & 8 & 65.08 & $72.4^{6}$ \\
\hline 7 & 60.79 & 68.44 & 7 & 62.95 & 70.48 & 7 & 65.13 & $7^{2} \cdot 5^{0}$ \\
\hline 6 & $60.8_{3}$ & 68.48 & 6 & 63.00 & $70.5^{2}$ & 6 & 65.17 & 72.54 \\
\hline 5 & 60.88 & 68.52 & 5 & 63.04 & 70.57 & 5 & 65.21 & $7^{2} \cdot 5^{8}$ \\
\hline 4 & 60.92 & 68.56 & 4 & 63.09 & $70.6 \mathrm{r}$ & 4 & 65.25 & 72.61 \\
\hline 3 & 60.96 & 68.60 & 3 & 63.13 & 70.65 & 3 & 65.29 & 72.65 \\
\hline 2 & 61.00 & 68.64 & 2 & $63 \cdot 17$ & 70.60 & 2 & 65.33 & 72.69 \\
\hline I & $6 \mathrm{r} .04$ & 68.68 & I & 63.22 & 70.73 & I & $65 \cdot 3^{8}$ & $7^{2} \cdot 73$ \\
\hline 0 & $6 \mathrm{r} .08$ & $68.7^{2}$ & 0 & 63.26 & 70.77 & 0 & 65.42 & 72.77 \\
\hline
\end{tabular}


ALCOHOLIC BEVERAGES.

SPECIFIC GRAVITY AND PERCENTAGE OF ALCOHOL-(Continued).

\begin{tabular}{|c|c|c|c|c|c|c|c|c|}
\hline \multirow{2}{*}{$\begin{array}{c}\text { Spec. } \\
\text { Grav. } \\
\text { at } \\
\text { x } 5.6^{\circ} \mathrm{C} .\end{array}$} & \multicolumn{2}{|c|}{ Absolute Alcohol. } & \multirow{2}{*}{$\begin{array}{c}\text { Spec. } \\
\text { Grav. } \\
\text { at } \\
15.6^{\circ} \mathrm{C} .\end{array}$} & \multicolumn{2}{|c|}{ Absolute Alcohol. } & \multirow{2}{*}{$\begin{array}{c}\text { Spec. } \\
\text { Grav. } \\
\text { at } \\
15.6^{\circ} \mathrm{C} \text {. }\end{array}$} & \multicolumn{2}{|c|}{ Absolute Alcohol. } \\
\hline & $\begin{array}{c}\text { Per } \\
\text { Cent } \\
\text { by } \\
\text { Weight. }\end{array}$ & $\begin{array}{l}\text { Per } \\
\text { Cent } \\
\text { by Vol- } \\
\text { ume. }\end{array}$ & & $\begin{array}{c}\text { Per } \\
\text { Cent } \\
\text { by } \\
\text { Weight. }\end{array}$ & $\begin{array}{l}\text { Per } \\
\text { Cent } \\
\text { by Vol- } \\
\text { ume. }\end{array}$ & & $\begin{array}{c}\text { Per } \\
\text { Cent } \\
\text { by } \\
\text { Weight. }\end{array}$ & $\begin{array}{l}\text { Per } \\
\text { Cent } \\
\text { by Vol- } \\
\text { ume. }\end{array}$ \\
\hline 0.8829 & 65.46 & 72.80 & 0.8779 & $67 \cdot 5^{8}$ & $74 \cdot 74$ & 0.8729 & 69.67 & $76.6 \mathrm{r}$ \\
\hline 8 & $65.5^{\circ}$ & 72.84 & 8 & 67.63 & $74.7^{8}$ & 8 & 69.71 & 76.65 \\
\hline 7 & $65-54$ & 72.88 & 7 & 67.67 & 74.82 & 7 & $69 \cdot 75$ & 76.68 \\
\hline 6 & $65 \cdot 5^{8}$ & 72.92 & 6 & $67 \cdot 7 x$ & 74.86 & 6 & 69.79 & 76.72 \\
\hline 5 & 65.63 & 72.96 & 5 & $67 \cdot 75$ & 74.89 & 5 & 69.83 & $7^{6} \cdot 7^{6}$ \\
\hline 4 & 65.67 & 72.99 & 4 & 67.79 & 74.93 & 4 & 69.88 & 76.80 \\
\hline 3 & 65.71 & 73.03 & 3 & $67.8_{3}$ & 74.97 & 3 & 69.92 & 76.83 \\
\hline 2 & 65.75 & 73.07 & 2 & 67.88 & 75.01 & 2 & 69.96 & 76.87 \\
\hline I & 65.79 & $73 . \mathrm{II}$ & I & 67.92 & 75.04 & I & 70.00 & 76.91 \\
\hline ○ & 65.83 & $73 \cdot 15$ & 0 & $67 \cdot 96$ & 75.08 & 0 & 70.04 & 76.94 \\
\hline 0.8819 & 65.88 & 73.19 & 0.8769 & 68.00 & $75 \cdot 12$ & 0.8719 & 70.08 & 76.98 \\
\hline 8 & 65.92 & 73.22 & 8 & 68.04 & $75 \cdot 16$ & 8 & 70.12 & 77.01 \\
\hline 7 & 65.96 & 73.26 & 7 & 68.08 & $75 \cdot 19$ & 7 & $70 \cdot 16$ & 77.05 \\
\hline 6 & 66.00 & $73 \cdot 3^{\circ}$ & 6 & 68.13 & 75.23 & 6 & 70.20 & 77.08 \\
\hline 5 & 66.04 & $73 \cdot 34$ & 5 & 68.17 & $75 \cdot 27$ & 5 & 70.24 & $77 \cdot 12$ \\
\hline 4 & 66.09 & $73 \cdot 3^{8}$ & 4 & $68.2 \mathrm{I}$ & $75 \cdot 30$ & 4 & 70.28 & $77 \cdot 15$ \\
\hline 3 & 66.13 & $73 \cdot 42$ & 3 & 68.25 & $75 \cdot 34$ & 3 & 70.32 & 77.19 \\
\hline 2 & 66.17 & $73.4^{6}$ & 2 & 68.29 & $75-3^{8}$ & 2 & $70.3^{6}$ & 77.22 \\
\hline I & 66.22 & $73 \cdot 5 \circ$ & I & 68.33 & $75 \cdot 42$ & I & 70.40 & 77.25 \\
\hline 0 & 66.26 & $73 \cdot 54$ & 0 & $68.3^{8}$ & $75 \cdot 45$ & 0 & 70.44 & 77.29 \\
\hline 0.8809 & 66.30 & $73 \cdot 57$ & 0.8759 & 68.42 & $75 \cdot 49$ & 0.8709 & $70.4^{8}$ & $77 \cdot 3^{2}$ \\
\hline 8 & 66.35 & 73.61 & 8 & $68.4^{6}$ & 75.53 & 8 & 70.52 & $77 \cdot 36$ \\
\hline 7 & 66.39 & 73.65 & 7 & 68.50 & $75 \cdot 57$ & 7 & 70.56 & $77 \cdot 39$ \\
\hline 6 & 66.43 & 73.69 & 6 & 68.54 & 75.60 & 6 & 70.60 & $77 \cdot 43$ \\
\hline 5 & 66.48 & $73 \cdot 73$ & 5 & $68 \cdot 5^{8}$ & $75 \cdot 64$ & 5 & 70.64 & $77-46$ \\
\hline 4 & 66.52 & $73 \cdot 77$ & 4 & 68.63 & 75.68 & 4 & 70.68 & $77 \cdot 50$ \\
\hline 3 & 66.57 & 73.81 & 3 & 68.67 & $75 \cdot 72$ & 3 & 70.72 & $77 \cdot 53$ \\
\hline 2 & 66.61 & 73.85 & 2 & 68.71 & $75 \cdot 75$ & 2 & $70.7^{6}$ & $77 \cdot 57$ \\
\hline I & 66.65 & 73.89 & I & 68.75 & $75 \cdot 79$ & I & 70.80 & 77.60 \\
\hline 0 & 66.70 & $73 \cdot 93$ & 0 & 68.79 & $75.8_{3}$ & 0 & 70.84 & 77.64 \\
\hline 0.8799 & 66.74 & 73.97 & 0.8749 & $68.8_{3}$ & 75.87 & 0.8699 & 70.88 & 77.67 \\
\hline 8 & 66.78 & 74.01 & 8 & 68.88 & 75.90 & 8 & 70.92 & $77 \cdot 71$ \\
\hline 7 & 66.83 & 74.05 & 7 & 68.92 & 75.94 & 7 & $70.9^{6}$ & $77 \cdot 74$ \\
\hline 6 & 66.87 & 74.09 & 6 & 68.96 & $75-98$ & 6 & 71.00 & $77 \cdot 7^{8}$ \\
\hline 5 & 66.91 & 74.13 & 5 & 69.00 & 76.01 & 5 & 71.04 & 77.82 \\
\hline 4 & 66.96 & 74.17 & 4 & 69.04 & 76.05 & 4 & 71.08 & 77.85 \\
\hline 3 & 67.00 & 74.22 & 3 & 69.08 & 76.09 & 3 & 71.13 & 77.89 \\
\hline 2 & 67.04 & 74.25 & 2 & 69.13 & 76.13 & 2 & 71.17 & 77.93 \\
\hline I & 67.08 & 74.29 & I & $69 \cdot 17$ & 76.16 & $I$ & 71.21 & 77.96 \\
\hline 0 & $67 \cdot I_{3}$ & $74-33$ & 0 & 69.21 & 76.20 & 0 & 71.25 & 78.00 \\
\hline 0.8789 & 67.17 & $74 \cdot 37$ & 0.8739 & 69.25 & 76.24 & 0.8689 & 71.29 & $7^{8.04}$ \\
\hline 8 & 67.21 & 74.40 & 8 & 69.29 & 76.27 & 8 & $7 x-33$ & 78.07 \\
\hline 7 & 67.25 & $74 \cdot 44$ & 7 & $69 \cdot 33$ & 76.31 & 7 & $71 \cdot 3^{8}$ & 78.11 \\
\hline 6 & 67.29 & $74 \cdot 48$ & 6 & $69-3^{8}$ & 76.35 & 6 & $71 \cdot 42$ & 78.14 \\
\hline 5 & 67.33 & $74 \cdot 52$ & 5 & $69 \cdot 42$ & 76.39 & 5 & $7 x \cdot 4^{6}$ & 78.18 \\
\hline 4 & $67 \cdot 3^{8}$ & 74.55 & 4 & 69.46 & 76.42 & 4 & $71-50$ & 78.22 \\
\hline 3 & $67 \cdot 42$ & $74 \cdot 59$ & 3 & 69.50 & $7^{6}-4^{6}$ & 3 & $71 \cdot 54$ & 78.25 \\
\hline 2 & $67 \cdot 46$ & 74.63 & 2 & $69 \cdot 54$ & $7^{6} .50$ & 2 & 71.58 & 78.29 \\
\hline I & $67 \cdot 50$ & 74.67 & 1 & 60.58 & 76.53 & $\mathrm{I}$ & 71.63 & 78.33 \\
\hline 0 & $67-54$ & $74-70$ & 0 & 69.63 & 76.57 & 0 & 71.67 & 78.36 \\
\hline
\end{tabular}


SPECIFIC GRAVITY AND PERCENTAGE OF ALCOHOL-(Continued).

\begin{tabular}{|c|c|c|c|c|c|c|c|c|}
\hline \multirow{2}{*}{$\begin{array}{l}\text { Spec. } \\
\text { Grav. } \\
\text { at. } \\
\times 5.6^{\circ} \mathrm{C} \text {. }\end{array}$} & \multicolumn{2}{|c|}{ Absolute Alcohol. } & \multirow{2}{*}{$\begin{array}{c}\text { Spec. } \\
\text { Grav. } \\
\text { at } \\
15.6^{\circ} \mathrm{C} \text {. }\end{array}$} & \multicolumn{2}{|c|}{ Absolute Alcohol, } & \multirow{2}{*}{$\begin{array}{l}\text { Spec, } \\
\text { Grav. } \\
\text { at. } \\
15.6^{\circ} \mathrm{C} \text {. }\end{array}$} & \multicolumn{2}{|c|}{ Absolute Alcohol. } \\
\hline & $\begin{array}{c}\text { Per } \\
\text { Cent } \\
\text { by } \\
\text { Weight. }\end{array}$ & $\begin{array}{c}\text { Per } \\
\text { Cent } \\
\text { by Vol- } \\
\text { ume. }\end{array}$ & & $\begin{array}{c}\text { Per } \\
\text { Cent } \\
\text { by } \\
\text { Weight. }\end{array}$ & $\begin{array}{c}\text { Per } \\
\text { Cent } \\
\text { by Vol- } \\
\text { ume. }\end{array}$ & & $\begin{array}{c}\text { Per } \\
\text { Cent } \\
\text { by } \\
\text { Weight. }\end{array}$ & $\begin{array}{c}\text { Per } \\
\text { Cent } \\
\text { by Vol- } \\
\text { ume. }\end{array}$ \\
\hline 0.8679 & 71.71 & $7^{8} .40$ & 0.8629 & 73.83 & 80.26 & 0.8579 & 76.08 & 82.23 \\
\hline & 71.75 & 78.44 & 8 & 73.88 & 80.30 & 8 & 76.13 & 82.26 \\
\hline 7 & 71.79 & 78.47 & 7 & 73.92 & 80.33 & 7 & 76.17 & 82.30 \\
\hline 6 & 71.83 & $7^{8} \cdot 5^{1}$ & 6 & 73.96 & 80.37 & 6 & 76.21 & 82.33 \\
\hline 5 & 71.88 & 78.55 & 5 & 74.00 & 80.40 & 5 & 76.25 & 82.37 \\
\hline 4 & 71.92 & $78.5^{8}$ & 4 & 74.05 & 80.44 & 4 & 76.29 & 82.40 \\
\hline 3 & 71.96 & 78.62 & 3 & 74.09 & 80.48 & 3 & 76.33 & 82.44 \\
\hline 2 & 72.00 & 78.66 & 2 & $74 \cdot 14$ & $80.5^{2}$ & 2 & $76 \cdot 3^{8}$ & 82.47 \\
\hline I & $7^{2} .04$ & 78.70 & I & 74.18 & 80.56 & 1 & 76.42 & 82.51 \\
\hline 0 & 72.09 & $7^{8} \cdot 73$ & 0 & 74.23 & 80.60 & 。 & 76.46 & 82.54 \\
\hline 0.8669 & 72.13 & 78.77 & 0.8619 & 74.27 & 80.64 & 0.8569 & 76.50 & $82.5^{8}$ \\
\hline & 72.17 & $78.8 \mathrm{r}$ & 8 & $74 \cdot 32$ & 80.68 & 8 & 76.54 & $82.6 \mathrm{r}$ \\
\hline 7 & 72.22 & 78.85 & 7 & $74 \cdot 3^{6}$ & 80.72 & 7 & $76.5^{8}$ & 82.65 \\
\hline 6 & 72.26 & 78.89 & 6 & $74 \cdot 41$ & 80.76 & 6 & 76.63 & 82.69 \\
\hline 5 & $7^{2} \cdot 3^{\circ}$ & 78.93 & 5 & $74 \cdot 45$ & 80.80 & 5 & 76.67 & 82.72 \\
\hline 4 & 72.35 & 78.96 & 4 & $74 \cdot 50$ & $80.8_{4}$ & 4 & 76.71 & 82.76 \\
\hline 3 & $7^{2} \cdot 39$ & 79.00 & 3 & $74 \cdot 55$ & 80.88 & 3 & 76.75 & 82.79 \\
\hline 2 & 72.43 & 79.04 & 2 & 74.59 & 80.92 & 2 & 76.79 & 82.83 \\
\hline I & $7^{2} .4^{8}$ & 79.08 & I & 74.64 & 80.96 & I & 76.83 & 82.86 \\
\hline 0 & $7^{2} \cdot 5^{2}$ & $79 \cdot 12$ & 0 & 74.68 & 81.00 & - & 76.88 & 82.90 \\
\hline 0.8659 & 72.57 & 79.16 & 0.8609 & $74 \cdot 73$ & 81.04 & 0.8559 & 76.92 & 82.93 \\
\hline 8 & 72.61 & $79 \cdot 19$ & 8 & $74 \cdot 77$ & 81.08 & 8 & 76.96 & 82.97 \\
\hline 7 & 72.65 & 79.23 & 7 & 74.82 & 81,12 & 7 & 77.00 & 83.00 \\
\hline 6 & 72.70 & 79.27 & 6 & 74.86 & 81.16 & 6 & 77.04 & 83.04 \\
\hline 5 & 72.74 & $79 \cdot 3^{\mathrm{I}}$ & 5 & $74.9 \mathrm{I}$ & 81.20 & 5 & 77.08 & 83.07 \\
\hline 4 & 72.78 & $79 \cdot 35$ & 4 & 74.95 & $8 \mathrm{I}, 24$ & 4 & $77 \cdot 13$ & $83.1 \mathrm{II}$ \\
\hline 3 & 72.83 & $79 \cdot 39$ & 3 & 75.00 & $8 \mathrm{r} .28$ & 3 & 77.17 & 83.14 \\
\hline 2 & 72.87 & $79 \cdot 42$ & 2 & 75.05 & $81 \cdot 32$ & 2 & 77.21 & 83.18 \\
\hline I & 72.91 & 79.46 & I & 75.09 & 81.3 & I & 77.25 & $83.2 \mathrm{I}$ \\
\hline$\circ$ & 72.96 & 79.50 & 0 & $75 \cdot 14$ & 81.40 & 。 & 77.29 & 83.25 \\
\hline 0.8649 & 73.00 & $79 \cdot 54$ & 0.8599 & 75.18 & 81.44 & 0.8549 & $77-33$ & 83.28 \\
\hline 8 & 73.04 & $79 \cdot 57$ & 8 & $75 \cdot 23$ & 81.48 & 8 & & $83 \cdot 32$ \\
\hline 7 & 73.08 & 79.61 & 7 & 75.27 & $81 \cdot 52$ & 7 & 77.42 & $83 \cdot 3^{6}$ \\
\hline 6 & 73.13 & 79.65 & 6 & $75-33$ & 81.56 & 6 & $77 \cdot 46$ & 83.39 \\
\hline 5 & 73.17 & 79.68 & 5 & $75 \cdot 3^{6}$ & 81.60 & 5 & $77 \cdot 50$ & 83.43 \\
\hline 4 & $73.2 \mathrm{I}$ & 79.72 & 4 & 75. & 81.64 & 4 & $77 \cdot 54$ & 83.46 \\
\hline 3 & 73.25 & 79.75 & 3 & 75.45 & 81.68 & 3 & $77.5^{8}$ & 83.50 \\
\hline 2 & 73.29 & & 2 & $75 \cdot 5^{\circ}$ & $8 \mathrm{r}$. & 2 & 77.63 & 83.53 \\
\hline I & $73 \cdot 33$ & 79.83 & I & $75 \cdot 55$ & $8 \mathrm{x} .7$ & I & 77.67 & 83.57 \\
\hline 。 & $73 \cdot 3^{8}$ & 79.86 & 0 & $75 \cdot 59$ & $8 \mathrm{r} .80$ & o & $77 \cdot 7^{1}$ & 83.60 \\
\hline 0.8639 & 73.42 & 79.90 & 0.8589 & $75 \cdot 64$ & $8 \pi .84$ & 0.8539 & $77 \cdot 75$ & 83.64 \\
\hline & 73.46 & 79.94 & 8 & & 81.88 & & $77 \cdot 79$ & 83.67 \\
\hline & $73.5^{\circ}$ & 79.97 & 7 & 75.73 & 81.92 & 7 & & $83.7 \mathrm{r}$ \\
\hline 6 & 73.54 & 80.01 & 6 & 75.77 & 81.96 & 6 & 77.88 & 83.74 \\
\hline 5 & $73 \cdot 5^{8}$ & 80.04 & 5 & 75.82 & 82.00 & 5 & 77.92 & 83.78 \\
\hline 4 & 73.63 & 80.08 & 4 & 75.86 & 82.04 & 4 & 77.96 & $83.8 \mathrm{I}$ \\
\hline 3 & 73.67 & 80.12 & 3 & 75.91 & 82.08 & 3 & 78.00 & 83.85 \\
\hline 2 & 73.71 & 80.15 & 2 & 75.95 & 82.12 & 2 & 78.04 & 83.88 \\
\hline I & 73.75 & 80.19 & I & 76.00 & 82.16 & I & 78.08 & $83.9 \mathrm{r}$ \\
\hline 。 & 73.79 & 80.22 & $\circ$ & 76.04 & 82.19 & 。 & 78.12 & 83.94 \\
\hline
\end{tabular}


SPECIFIC GRAVITY AND PERCENTAGE OF ALCOHOL-(Continued).

\begin{tabular}{|c|c|c|c|c|c|c|c|c|}
\hline \multirow{2}{*}{$\begin{array}{c}\text { Spec. } \\
\text { Grav. } \\
\text { at } \\
15.6^{\circ} \mathrm{C} \text {. }\end{array}$} & \multicolumn{2}{|c|}{ Absolute Alcohol. } & \multirow{2}{*}{$\begin{array}{c}\text { Spec. } \\
\text { Grav. } \\
\text { at } \\
\text { r } 5.6^{\circ} \mathrm{C} \text {. }\end{array}$} & \multicolumn{2}{|c|}{ Absolute Alcohol. } & \multirow{2}{*}{$\begin{array}{c}\text { Spec. } \\
\text { Grav. } \\
\text { at } \\
15.6^{\circ} \mathrm{C} \text {. }\end{array}$} & \multicolumn{2}{|c|}{ Absolute Alcohol. } \\
\hline & $\begin{array}{c}\text { Per } \\
\text { Cent } \\
\text { by } \\
\text { Weight. }\end{array}$ & $\begin{array}{l}\text { Per } \\
\text { Cent. } \\
\text { by Vol- } \\
\text { ume. }\end{array}$ & & $\begin{array}{c}\text { Per } \\
\text { Cent } \\
\text { by } \\
\text { Weight. }\end{array}$ & $\begin{array}{c}\text { Per } \\
\text { Cent } \\
\text { by Vol- } \\
\text { ume. }\end{array}$ & & $\begin{array}{c}\text { Per } \\
\text { Cent } \\
\text { by } \\
\text { Weight. }\end{array}$ & $\begin{array}{c}\text { Per } \\
\text { Cent } \\
\text { by Vol- } \\
\text { ume. }\end{array}$ \\
\hline 0.8529 & 78.16 & $8_{3.98}$ & 0.8479 & 80,17 & 85.63 & 0.8429 & 82.19 & 87.27 \\
\hline 8 & 78.20 & 84.01 & 8 & 80.21 & 85.66 & 8 & 82.23 & $87 \cdot 30$ \\
\hline 7 & 78.24 & 84.04 & 7 & 80.25 & $85 \cdot 70$ & 7 & 82.27 & $87 \cdot 34$ \\
\hline 6 & 78.28 & 84.08 & 6 & 80.29 & 85.73 & 6 & $82 \cdot 31$ & $87 \cdot 37$ \\
\hline 5 & 78.32 & 84.11 & 5 & So. 33 & 85.77 & 5 & 82.35 & 87.40 \\
\hline 4 & $78 \cdot 3^{6}$ & $84 \cdot 14$ & 4 & 80.38 & 85.80 & 4 & $82 \cdot 3^{8}$ & 87.43 \\
\hline 3 & 78.40 & $84 \cdot 18$ & 3 & 80.42 & 85.84 & 3 & 82.42 & $87 \cdot 46$ \\
\hline 2 & 78.44 & 84.21 & 2 & 80.46 & 85.87 & 2 & $82 \cdot 46$ & 87.49 \\
\hline $\mathbf{I}$ & 78.48 & $84 \cdot 24$ & 1 & 80.50 & 85.90 & I & 82.50 & 87.52 \\
\hline$\circ$ & $78.5^{2}$ & 84.27 & 0 & 80.54 & 85.94 & 0 & 82.54 & 87.55 \\
\hline 0.8519 & 78.56 & $84 \cdot 3 I$ & 0.8469 & $80.5^{8}$ & 85.97 & 0.8419 & $82 \cdot 58$ & $87 \cdot 5^{8}$ \\
\hline 8 & 78.60 & $84 \cdot 34$ & 8 & $80.6_{3}$ & 86.01 & 8 & 82.62 & $87.6 x$ \\
\hline 7 & 78.64 & $84 \cdot 37$ & 7 & 80.67 & 86.04 & 7 & 82.65 & 87.64 \\
\hline 6 & 78.68 & 84.41 & 6 & 80.71 & 86.08 & 6 & 82.69 & 87.67 \\
\hline 5 & $78 \cdot 72$ & 84.44 & 5 & 80.75 & 86.11 & 5 & 82.73 & 87.70 \\
\hline 4 & 78.76 & 84.47 & 4 & 80.79 & 86.15 & 4 & 82.77 & $87 \cdot 73$ \\
\hline 3 & 78.80 & 84.51 & 3 & $80.8_{3}$ & 86.18 & 3 & 82.81 & 87.76 \\
\hline 2 & 78.84 & 84.54 & 2 & 80.88 & 86.22 & 2 & 82.85 & 87.79 \\
\hline$I$ & 78.88 & 84.57 & 1 & 80.92 & 86.25 & $\mathrm{I}$ & 82.88 & 87.82 \\
\hline 0 & 78.92 & 84.60 & 0 & 80.96 & 86.28 & 0 & 82.92 & 87.85 \\
\hline 0.8509 & 78.96 & 84.64 & 0.8459 & 81.00 & 86.32 & 0.8400 & 82.96 & 87.88 \\
\hline 8 & 79.00 & 84.67 & 8 & 81.04 & 86.35 & 8 & 83.00 & 87.91 \\
\hline 7 & 79.04 & 84.70 & 7 & 81.08 & $86.3^{8}$ & 7 & 83.04 & 87.94 \\
\hline 6 & 79.08 & $84 \cdot 74$ & 6 & $81 \cdot 12$ & 86.42 & 6 & $8_{3} .08$ & 87.97 \\
\hline 5 & 79.12 & $84 \cdot 77$ & 5 & $81 \cdot 16$ & 86.45 & 5 & 83.12 & 88.00 \\
\hline 4 & $79 \cdot 16$ & 84.80 & 4 & 81.20 & 86.48 & 4 & 83.15 & 88.03 \\
\hline 3 & 79.20 & $8_{4} \cdot 8_{3}$ & 3 & $8 \mathrm{I} \cdot 24$ & 86.51 & 3 & 83.19 & 88.06 \\
\hline 2 & 79.24 & 84.87 & 2 & 81.28 & 86.54 & 2 & 83.23 & 88.09 \\
\hline I & 79.28 & 84.90 & I & $81 \cdot 32$ & $86.5^{8}$ & I & 83.27 & 88.13 \\
\hline 0 & $79 \cdot 32$ & $84 \cdot 93$ & 0 & $8 \mathrm{I} \cdot 3^{6}$ & $86.6 \mathrm{I}$ & 0 & $83 \cdot 3 \mathrm{I}$ & 88.16 \\
\hline c. 8499 & $79 \cdot 36$ & 84.97 & 0.8449 & $8 \mathrm{r} \cdot 40$ & 86.64 & 0.8399 & 83.35 & 88.19 \\
\hline 8 & 79.40 & 85.00 & 8 & $8 \mathrm{I} \cdot 44$ & 86.67 & 8 & $83 \cdot 38$ & 88.22 \\
\hline 7 & $79 \cdot 44$ & 85.03 & 7 & $8 x \cdot 48$ & $86.7 \mathrm{I}$ & 7 & $83 \cdot 42$ & 88.25 \\
\hline 6 & $79 \cdot 4^{8}$ & 85.06 & 6 & $8 \mathrm{r} \cdot 52$ & 86.74 & 6 & 83.46 & 88.28 \\
\hline 5 & $79 \cdot 52$ & 85.10 & 5 & $8 x \cdot 56$ & 86.77 & 5 & 83.50 & $88 \cdot 31$ \\
\hline 4 & $79 \cdot 56$ & 85.13 & 4 & 81.60 & 86.80 & 4 & $83 \cdot 54$ & 88.34 \\
\hline 3 & 79.60 & 85.16 & 3 & 81.64 & 86.83 & 3 & $8_{3} \cdot 5^{8}$ & 88.37 \\
\hline 2 & 79.64 & 85.19 & 2 & 8 I. 68 & 86.87 & 2 & $8_{3} .62$ & 88.40 \\
\hline I & 79.68 & 85.23 & I & $8 \pi \cdot 72$ & 86.90 & I & 83.65 & 88.43 \\
\hline 0 & $79 \cdot 72$ & 85.26 & 0 & $81 \cdot 76$ & 86.93 & 0 & 83.69 & 88.46 \\
\hline 0.8489 & $79 \cdot 76$ & 85.29 & 0.8439 & $8 \mathrm{r} \cdot 80$ & 86.96 & $0.83^{89}$ & $83 \cdot 73$ & 88.49 \\
\hline 8 & 79.80 & 85.33 & 8 & $8 \mathrm{r} \cdot 84$ & 86.99 & 8 & 83.77 & $88.5^{2}$ \\
\hline 7 & 79.84 & $85 \cdot 36$ & 7 & 81.88 & 87.03 & 7 & $83.8 \mathrm{r}$ & 88.55 \\
\hline 6 & 79.88 & 85.39 & 6 & 81.92 & 87.06 & 6 & 83.85 & 88.58 \\
\hline 5 & 79.92 & 85.42 & 5 & $8 \mathrm{r} \cdot 96$ & 87.09 & 5 & 83.88 & 88.61 \\
\hline 4 & 79.96 & 85.46 & 4 & 82.00 & 87.12 & 4 & $8_{3-92}$ & 88.64 \\
\hline 3 & 80.00 & 85.49 & 3 & 82.04 & $87 \cdot 15$ & 3 & 83.96 & 88.67 \\
\hline 2 & 80.04 & 85.53 & 2 & 82.08 & 87.18 & 2 & 84.00 & 88.70 \\
\hline I & 80.08 & 85.56 & I & 82.12 & 87.21 & I & 84.04 & 88.73 \\
\hline 0 & 80.13 & 85.59 & 0 & 82.15 & 87.24 & 0 & 84.08 & 88.76 \\
\hline
\end{tabular}


SPECIFIC GRAVITY AND PERCENTAGE OF ALCOHOL-(Continued).

\begin{tabular}{|c|c|c|c|c|c|c|c|c|}
\hline \multirow{2}{*}{$\begin{array}{c}\text { Spec. } \\
\text { Grav. } \\
\text { at } \\
15.6^{\circ} \mathrm{C} .\end{array}$} & \multicolumn{2}{|c|}{ Absolute Alcohol. } & \multirow{2}{*}{$\begin{array}{c}\text { Spec, } \\
\text { Grav, } \\
\text { at } \\
15.6^{\circ} \mathrm{C} \text {. }\end{array}$} & \multicolumn{2}{|c|}{ Absolute Aleohol. } & \multirow{2}{*}{$\begin{array}{c}\text { Spec. } \\
\text { Grav. } \\
\text { at } \\
15.6^{\circ} \mathrm{C} \text {. }\end{array}$} & \multicolumn{2}{|c|}{ Absolute Alcohol. } \\
\hline & $\begin{array}{c}\text { Per } \\
\text { Cent } \\
\text { by } \\
\text { Weight. }\end{array}$ & $\begin{array}{l}\text { Per } \\
\text { Cent } \\
\text { by Vol- } \\
\text { ume. }\end{array}$ & & $\begin{array}{c}\text { Per } \\
\text { Cent } \\
\text { by } \\
\text { Weight. }\end{array}$ & $\begin{array}{l}\text { Per } \\
\text { Cent } \\
\text { by Vol- } \\
\text { ume. }\end{array}$ & & $\begin{array}{c}\text { Per } \\
\text { Cent } \\
\text { by } \\
\text { Weight. }\end{array}$ & $\begin{array}{l}\text { Per } \\
\text { Cent. } \\
\text { by Vol- } \\
\text { ume. }\end{array}$ \\
\hline 0.8379 & 84.12 & 88.79 & 0.8329 & 86.08 & $90 \cdot 3^{2}$ & 0.8279 & 88.00 & $91 \cdot 78$ \\
\hline & $84 \cdot 16$ & 88.83 & 8 & 86.12 & 90.35 & 8 & 88.04 & 91.81 \\
\hline 7 & 84.20 & 88.86 & 7 & 86.15 & $90 \cdot 3^{8}$ & 7 & 88.08 & 91.84 \\
\hline 6 & 84.24 & 88.89 & 6 & 86.19 & 90.40 & 6 & 88.12 & 91.87 \\
\hline 5 & $84 \cdot 28$ & 88.92 & 5 & 86.23 & $90-43$ & 5 & 88.16 & 91.90 \\
\hline 4 & $84-32$ & 88.95 & 4 & 86.27 & 90.46 & 4 & 88.20 & 91.93 \\
\hline 3 & $84 \cdot 36$ & 88.98 & 3 & $86.3 \mathrm{i}$ & 90.49 & 3 & 88.24 & 91.96 \\
\hline 2 & 84.40 & 89.01 & 2 & 86.35 & $90.5^{2}$ & 2 & 88.28 & 91.99 \\
\hline I & 84.44 & 89.05 & I & $86.3^{8}$ & 90.55 & $\mathrm{r}$ & $88 \cdot 32$ & 92.02 \\
\hline 0 & $84 \cdot 4^{8}$ & 89.08 & 0 & 86.42 & $90.5^{8}$ & 0 & $88 \cdot 36$ & 92.05 \\
\hline 0.8369 & $84 \cdot 5^{2}$ & 89.11 & 0.8319 & 86.46 & $90.6 \mathrm{r}$ & 0.8269 & 88.40 & 92.08 \\
\hline 8 & $84 \cdot 5^{6}$ & 89.14 & 8 & 86.50 & 90.64 & 8 & 88.44 & 92.12 \\
\hline 7 & 84.60 & 89.17 & 7 & $86-54$ & 90.67 & 7 & 88.48 & 92.15 \\
\hline 6 & $8_{4} .64$ & 89.20 & 6 & $86.5^{8}$ & 90.70 & 6 & 88.52 & 92.18 \\
\hline 5 & 84.68 & 89.24 & 5 & 86.62 & 90.73 & 5 & 88.56 & $92.2 \mathrm{I}$ \\
\hline 4 & $84-72$ & 89.27 & 4 & 86.65 & 90.76 & 4 & 88.60 & 92,24 \\
\hline 3 & $84 \cdot 76$ & 89.30 & 3 & 86.69 & 90.79 & 3 & 88.64 & 92.27 \\
\hline 2 & 84.80 & 89.33 & 2 & 86.73 & 90.82 & 2 & 88.68 & $92.3^{\circ}$ \\
\hline I & 84.84 & $89 \cdot 36$ & I & 86.77 & 90.85 & I & $88 \cdot 7^{2}$ & $92 \cdot 33$ \\
\hline 0 & 84.88 & 89.39 & 0 & $86.8 \mathrm{i}$ & 90.88 & 0 & 88.76 & $9^{2} \cdot 3^{6}$ \\
\hline 0.8359 & 84.92 & 89.42 & 0.8309 & 86.85 & 90.90 & 0.8259 & 88.80 & $92 \cdot 39$ \\
\hline & 84.96 & 89.46 & 8 & 86.88 & 90.93 & 8 & 88.84 & 92.42 \\
\hline 7 & 85.00 & 89.49 & 7 & 86.92 & 90.96 & 7 & 88.88 & $9^{2}-45$ \\
\hline 6 & 85.04 & $89-52$ & 6 & 86.96 & 90.99 & 6 & 88.92 & $92-48$ \\
\hline 5 & 85.08 & 89.55 & 5 & 87.00 & 91.02 & 5 & 88.96 & $9^{2} \cdot 5^{1}$ \\
\hline 4 & 85.12 & $89.5^{8}$ & 4 & 87.04 & 91.05 & 4 & 89.00 & 92.54 \\
\hline 3 & 85.15 & $89.6 \mathrm{r}$ & 3 & 87.08 & 91.08 & 3 & 89.04 & 92.57 \\
\hline 2 & 85.19 & 89.64 & 2 & $87 \cdot 12$ & 91.11 & 2 & 89.08 & 92.60 \\
\hline I & 85.23 & 89.67 & I & $87 \cdot \mathrm{I}_{5}$ & 91.14 & 1 & 89.12 & 92.63 \\
\hline 0 & 85.27 & 89.70 & 0 & 87.19 & 91.17 & 0 & 89.16 & 92.66 \\
\hline 0.8349 & $8_{5} \cdot 31$ & $89.7^{2}$ & 0.8299 & 87.23 & $91 \cdot 20$ & 0.8249 & 89.19 & 92.68 \\
\hline 8 & $85 \cdot 35$ & 89.75 & 8 & 87.27 & 91.23 & 8 & 80.23 & $92.7 \mathrm{I}$ \\
\hline 7 & $85-3^{8}$ & 89.78 & 7 & $87 \cdot 3 x$ & 91.25 & 7 & 89.27 & 92.74 \\
\hline 6 & 85.42 & $89.8 \mathrm{t}$ & 6 & 87.35 & 91.28 & 6 & 89.31 & 92.77 \\
\hline 5 & 85.46 & 89.84 & 5 & $87 \cdot 3^{8}$ & $9 \mathrm{I} \cdot 3 \mathrm{I}$ & 5 & 89.35 & 92.80 \\
\hline 4 & 85.50 & 89.87 & 4 & $87 \cdot 42$ & 9 I. 34 & 4 & $89 \cdot 3^{8}$ & 92.83 \\
\hline 3 & 85.54 & 89.90 & 3 & $87.4^{6}$ & 91.37 & 3 & 89.42 & 92.86 \\
\hline 2 & $85 \cdot 5^{8}$ & 89.93 & 2 & $87 \cdot 50$ & 91.40 & 2 & $89-46$ & 92.89 \\
\hline I & 85.62 & 89.96 & I & 87.54 & $9 \mathrm{r} \cdot 43$ & I & 89.50 & $92.9 \mathrm{r}$ \\
\hline 0 & $8_{5} .6_{5}$ & 89.99 & 0 & 87.58 & $9 \mathrm{r} \cdot 46$ & 0 & 89.54 & 92.94 \\
\hline 0.8339 & 85.69 & 90.02 & 0.8289 & 87.62 & 91.49 & 0.8239 & $89.5^{8}$ & $9^{2} .97$ \\
\hline 8 & 85.73 & 90.05 & 8 & 87.65 & $91 \cdot 5^{2}$ & 8 & 89.62 & 93.00 \\
\hline 7 & 85.77 & 90.08 & 7 & 87.69 & 91.55 & 7 & 89.65 & 93.03 \\
\hline 6 & 85.81 & 90.11 & 6 & $87 \cdot 73$ & 91.57 & 6 & 89.69 & 93.06 \\
\hline 5 & 85.85 & 90.14 & 5 & 87.77 & 91.60 & 5 & 89.73 & 93.09 \\
\hline 4 & 85.88 & 90.17 & 4 & $87.8 \mathrm{r}$ & 91.63 & 4 & 89.77 & $93.1 \mathrm{I}$ \\
\hline 3 & 85.92 & 90.20 & 3 & 87.85 & 91.66 & 3 & $89.8 \mathrm{I}$ & 93.14 \\
\hline 2 & 85.96 & 90.23 & 2 & 87.88 & 91.69 & 2 & 89.85 & $93 \cdot 17$ \\
\hline I & 86.00 & 90.26 & I & 87.92 & $9 x \cdot 72$ & I & 89.88 & 93.20 \\
\hline 0 & 86.04 & 90.29 & 0 & 87.96 & 91.75 & 0 & 89.92 & 93.23 \\
\hline
\end{tabular}


SPECIFIC GRAVITY AND PERCENTAGE OF ALCOHOL-(Continued).

\begin{tabular}{|c|c|c|c|c|c|c|c|c|}
\hline \multirow{2}{*}{$\begin{array}{c}\text { Spec. } \\
\text { Grav. } \\
\text { at } \\
15.0^{\circ} \mathrm{C} \text {. }\end{array}$} & \multicolumn{2}{|c|}{ Absolute Alcohol. } & \multirow{2}{*}{$\begin{array}{c}\text { Spec. } \\
\text { Grav. } \\
\text { at } \\
15.6^{\circ} \mathrm{C} \text {. }\end{array}$} & \multicolumn{2}{|c|}{ Absolute Alcohol. } & \multirow{2}{*}{$\begin{array}{c}\text { Spec. } \\
\text { Grav. } \\
\text { at } \\
15.6^{\circ} \mathrm{C} \text {. }\end{array}$} & \multicolumn{2}{|c|}{ Absolute Alcohol. } \\
\hline & $\begin{array}{c}\text { Per } \\
\text { Cent } \\
\text { by } \\
\text { Weight. }\end{array}$ & $\begin{array}{l}\text { Per } \\
\text { Cent } \\
\text { by Vol- } \\
\text { ume. }\end{array}$ & & $\begin{array}{c}\text { Per } \\
\text { Cent } \\
\text { by } \\
\text { Weight. }\end{array}$ & $\begin{array}{l}\text { Per } \\
\text { Cent } \\
\text { by Vol- } \\
\text { ume. }\end{array}$ & & $\begin{array}{c}\text { Per } \\
\text { Cent } \\
\text { by } \\
\text { Weight. }\end{array}$ & $\begin{array}{l}\text { Per } \\
\text { Cent } \\
\text { by Vol- } \\
\text { ume. }\end{array}$ \\
\hline 0.8229 & 89.96 & 93.26 & 0.8179 & $9 \pi \cdot 75$ & 94.53 & 0.8129 & 93.59 & 95.84 \\
\hline 8 & 90.00 & 93.29 & 8 & 91.79 & $94 \cdot 5^{6}$ & 8 & 93.63 & 95.87 \\
\hline 7 & 90.04 & $93 \cdot 31$ & 7 & 91.82 & 94.59 & 7 & 93.67 & 95.90 \\
\hline 6 & 90.07 & $93 \cdot 34$ & 6 & 91.86 & 94.61 & 6 & 93.70 & 95.92 \\
\hline 5 & 90.11 & $93 \cdot 36$ & 5 & 9 r. 89 & 94.64 & 5 & 93.74 & $95-95$ \\
\hline 4 & $90 \cdot \mathrm{r} 4$ & 93.39 & 4 & 91.93 & 94.66 & 4 & $93.7^{8}$ & 95.97 \\
\hline 3 & 90.18 & $93.4 \mathrm{I}$ & 3 & 91.96 & 94.69 & 3 & 93.81 & 96.00 \\
\hline 2 & 90.21 & 93.44 & 2 & 92.00 & 94.71 & 2 & 93.85 & 96.03 \\
\hline I & 90.25 & 93.47 & I & 92.04 & $94 \cdot 74$ & I & 93.89 & 96.05 \\
\hline 0 & 90.29 & 93.49 & o & 92.07 & 94.76 & 0 & 93.92 & 96.08 \\
\hline 0.8219 & 90.32 & $93 \cdot 5^{2}$ & 0.8169 & $9^{2}, \mathrm{II}$ & 94.79 & $0.8 \times 19$ & 93.96 & 96.11 \\
\hline 8 & $90 \cdot 3^{6}$ & $93 \cdot 74$ & 8 & 92.15 & 94.82 & 8 & 94.00 & 96.13 \\
\hline 7 & 90.39. & $93 \cdot 57$ & 7 & 92.18 & 94.84 & 7 & 94.03 & 96.16 \\
\hline 6 & $90 .+3$ & 93.59 & 6 & 92.22 & 94.87 & 6 & 94.07 & 96.18 \\
\hline 5 & 90.46 & 93.62 & 5 & 92.26 & 94.90 & 5 & 94.10 & 96.20 \\
\hline 4 & $90.5^{\circ}$ & 93.64 & 4 & $92 \cdot 30$ & 94.92 & 4 & $94 \cdot 14$ & 96.22 \\
\hline 3 & 90.54 & 93.67 & 3 & $92 \cdot 33$ & 94.95 & 3 & 94.17 & 96.25 \\
\hline 2 & 90.57 & 93.70 & 2 & $9^{2} \cdot 37$ & $94.9^{8}$ & 2. & 94.21 & 96.27 \\
\hline I & 90.61 & $93 \cdot 72$ & 1 & 92.41 & 95.00 & $\mathrm{I}$ & 94.24 & 96.29 \\
\hline 0 & 90.64 & $93 \cdot 75$ & 0 & 92.44 & 95.03 & 0 & 94.28 & $9^{6.32}$ \\
\hline 0.8209 & 90.68 & 93.77 & 0.8159 & 92.48 & 95.06 & 0.8109 & $94 \cdot 3^{\mathrm{I}}$ & 96.34 \\
\hline 8 & 90.71 & 93.80 & 8 & $92 \cdot 5^{2}$ & 95.08 & 8 & 94.34 & $96.3^{6}$ \\
\hline 7 & 90.75 & 93.82 & 7 & 92.55 & 95.11 & 7 & $94 \cdot 3^{8}$ & 96.39 \\
\hline 6 & 90.79 & 93.85 & 6 & 92.59 & $95 \cdot 13$ & 6 & 94.41 & $96.4 \mathrm{I}$ \\
\hline 5 & 90.82 & 93.87 & 5 & 92.63 & 95.16 & 5 & $94 \cdot 45$ & 96.43 \\
\hline 4 & $9 c .86$ & 93.90 & 4 & 92.67 & 95.19 & 4 & $94 \cdot 4^{8}$ & 96.46 \\
\hline 3 & 90.89 & 93.93 & 3 & 92.70 & $95-2 \mathrm{I}$ & 3 & $94.5^{2}$ & $96.4^{8}$ \\
\hline 2 & 90.93 & 93.95 & 2 & $92 \cdot 74$ & 95.24 & 2 & $94 \cdot 55$ & 96.50 \\
\hline I & $90.9^{6}$ & 93.98 & I & $92.7^{8}$ & 95.27 & I & 94.59 & 96.53 \\
\hline 0 & 91.00 & 94.00 & 0 & $9^{2} .8 \mathrm{I}$ & 95.29 & 0 & 94.62 & 96.55 \\
\hline 0.8199 & 91.04 & 94.03 & 0.8149 & 92.85 & $95 \cdot 32$ & 0.8099 & 94.65 & 96.57 \\
\hline 8 & 91.07 & 94.05 & 8 & 92.89 & 95.35 & 8 & 94.69 & 96.60 \\
\hline 7 & 91.11 & 94.08 & 7 & 92.92 & $95 \cdot 37$ & 7 & $94 \cdot 73$ & 96.62 \\
\hline 6 & $9 \mathrm{I} \cdot \mathrm{r} 4$ & 94 . 10 & 6 & 92.96 & 95.40 & 6 & $94 \cdot 7^{6}$ & 96.64 \\
\hline 5 & $91 \cdot 18$ & 94,13 & 5 & 93.00 & 95.42 & 5 & 94.80 & 96.67 \\
\hline 4 & 91.21 & 94.15 & 4 & 93.04 & 95.45 & 4 & 94.83 & 96.69 \\
\hline 3 & 91.25 & 94.18 & 3 & 93.07 & $95 \cdot 48$ & 3 & 94.86 & 96.71 \\
\hline 2 & 91.29 & 94.21 & 2 & $93 \cdot \mathrm{II}$ & $95 \cdot 50$ & 2 & 94.90 & 96.74 \\
\hline I & $91 \cdot 32$ & 94.23 & $I$ & 93.15 & 95.53 & I & 94.93 & 96.76 \\
\hline - & $91 \cdot 36$ & 94.26 & 0 & 93.18 & 95.55 & 0 & 94.97 & 96.78 \\
\hline $0.8 \mathrm{r} 89$ & $91 \cdot 39$ & 94.28 & $0.8 x_{39}$ & 93.22 & $95 \cdot 5^{8}$ & 0.8089 & 95.00 & 96.80 \\
\hline 8 & $9 x-43$ & $94.3^{I}$ & 8 & 93.26 & $95.6 \mathrm{r}$ & 8 & 95.04 & 96.83 \\
\hline 7 & 91.46 & $94 \cdot 33$ & 7 & $93.3^{\circ}$ & 95.63 & 7 & 95.07 & 06.85 \\
\hline 6 & 91.50 & $94 \cdot 36$ & 6 & $93 \cdot 33$ & 95.66 & 6 & 95.II & 96.88 \\
\hline 5 & $9 \mathrm{r} \cdot 54$ & $94 \cdot 3^{8}$ & 5 & $93 \cdot 37$ & 95.69 & 5 & 95.14 & 96.90 \\
\hline 4 & $91 \cdot 57$ & 94.41 & 4 & $93.4 I$ & 95.71 & 4 & 95.18 & 96.93 \\
\hline 3 & $91.6 \mathrm{I}$ & 94.43 & 3 & 93.44 & $95 \cdot 74$ & 3 & 95.21 & 96.95 \\
\hline 2 & 91.64 & 94.46 & 2 & $93 \cdot 48$ & $95 \cdot 7^{6}$ & 2 & 95.25 & 96.98 \\
\hline I & 91.68 & 94.48 & $I$ & $93 \cdot 5^{2}$ & 95.79 & I & 95.29 & 97.00 \\
\hline 0 & 91.71 & $94 \cdot 51$ & 0 & $93 \cdot 55$ & 95.82 & 0 & $95 \cdot 3^{2}$ & 97.02 \\
\hline
\end{tabular}


SPECIFIC GRAVITY AND PERCENTAGE OF ALCOHOL-(Continued).

\begin{tabular}{|c|c|c|c|c|c|c|c|c|}
\hline \multirow{2}{*}{$\begin{array}{c}\text { Spec, } \\
\text { Orav } \\
\text { at } \\
\times 5.6^{\circ} \mathrm{C} \text {. }\end{array}$} & \multicolumn{2}{|c|}{ Absolute Alcohol. } & \multirow{2}{*}{$\begin{array}{c}\text { Spec. } \\
\text { Grav. } \\
\text { at } \\
15.6^{\circ} \mathrm{C} \text {. }\end{array}$} & \multicolumn{2}{|c|}{ Absolute Alcohol. } & \multirow{2}{*}{$\begin{array}{l}\text { Spec. } \\
\text { Grav. } \\
\text { at } \\
15.6^{\circ} \mathrm{C} \text {. }\end{array}$} & \multicolumn{2}{|c|}{ Absolute Alcohol. } \\
\hline & $\begin{array}{c}\text { Per } \\
\text { Cent } \\
\text { by } \\
\text { Weight. }\end{array}$ & $\begin{array}{c}\text { Per } \\
\text { Cent } \\
\text { by Vol- } \\
\text { ume. }\end{array}$ & & $\begin{array}{c}\text { Per } \\
\text { Cent } \\
\text { by } \\
\text { Weight. }\end{array}$ & $\begin{array}{c}\text { Per } \\
\text { Cent } \\
\text { by Vol- } \\
\text { ume. }\end{array}$ & & $\begin{array}{c}\text { Per } \\
\text { Cent } \\
\text { by } \\
\text { Weight. }\end{array}$ & $\begin{array}{c}\text { Per } \\
\text { Cent } \\
\text { by Vol- } \\
\text { ume. }\end{array}$ \\
\hline 0.8079 & $95 \cdot 3^{6}$ & 97.05 & 0.8029 & 97.07 & 98.18 & 0.7979 & 98.69 & 99.18 \\
\hline & $95 \cdot 39$ & 97.07 & 8 & $97 \cdot 10$ & 98.20 & & $98 \cdot 7^{2}$ & 99.20 \\
\hline 7 & 95.43 & $97 \cdot 10$ & 7 & 97,13 & 98.22 & 7 & $98 \cdot 75$ & 99.22 \\
\hline 6 & $95,4^{6}$ & $97 \cdot 12$ & 6 & $97 \cdot 16$ & 98.24 & 6 & $98 \cdot 7^{8}$ & 99.24 \\
\hline 5 & $95 \cdot 50$ & $97 \cdot 15$ & 5 & 97.20 & 98.27 & 5 & $98.8 \mathrm{I}$ & 99.26 \\
\hline 4 & $95 \cdot 54$ & $97 \cdot 17$ & 4 & 97.23 & 98.29 & 4 & 98.84 & 99.27 \\
\hline 3 & $95 \cdot 57$ & 97.20 & 3 & 97.26 & $98 \cdot 31$ & 3 & 98.87 & 99.29 \\
\hline 2 & 95.61 & 97.22 & 2 & $97 \cdot 30$ & $98 \cdot 33$ & 2 & 98.91 & $99 \cdot 3 \mathrm{I}$ \\
\hline 1 & 95.64 & 97.24 & I & $97 \cdot 33$ & $98 \cdot 35$ & I & 98.94 & $99-33$ \\
\hline - & 95.68 & 97.27 & o & $97 \cdot 37$ & $9^{8} \cdot 37$ & $\circ$ & 98.97 & $99 \cdot 35$ \\
\hline 0.8069 & $95 \cdot 71$ & 97.29 & 0.8019 & 97.40 & $98 \cdot 39$ & 0.7969 & 99.00 & $99 \cdot 37$ \\
\hline 8 & $95 \cdot 75$ & $97 \cdot 32$ & 8 & $97 \cdot 43$ & 98.42 & 8 & 99.03 & $99 \cdot 39$ \\
\hline 7 & 95.79 & $97 \cdot 34$ & 7 & $97 \cdot 46$ & 98.44 & 7 & 99.06 & $99.4 \mathrm{I}$ \\
\hline 6 & 95.82 & $97 \cdot 37$ & 6 & 97.50 & 98.46 & 6 & 99.10 & 99.43 \\
\hline 5 & 95.86 & $97 \cdot 39$ & 5 & $97 \cdot 53$ & 98.48 & 5 & $99 \cdot 13$ & $99-45$ \\
\hline 4 & 95.89 & 97.41 & 4 & 97.57 & $98 \cdot 5^{\circ}$ & 4 & 99.16 & $99 \cdot 47$ \\
\hline 3 & 95.93 & $97 \cdot 44$ & 3 & 97.60 & $9^{8} \cdot 5^{2}$ & 3 & 99.19 & 99.49 \\
\hline 2 & 95.96 & $97 \cdot 4^{6}$ & 2 & 97.63 & 98.54 & 2 & 99.23 & $99 \cdot 51$ \\
\hline I & 96.00 & 97.49 & I & 97.66 & $98 \cdot 5^{6}$ & I & 99.26 & $99 \cdot 53$ \\
\hline 0 & 96.03 & $97 \cdot 5^{I}$ & - & $97 \cdot 70$ & 98.59 & 0 & 99.29 & $99 \cdot 55$ \\
\hline 0.8059 & 96.07 & $97 \cdot 53$ & 0.8009 & $97 \cdot 73$ & $98.6 \mathrm{r}$ & 0.7959 & $99 \cdot 3^{2}$ & $99 \cdot 57$ \\
\hline 8 & 96.10 & $97 \cdot 55$ & 8 & $97 \cdot 76$ & 98.63 & 8 & $99 \cdot 3^{6}$ & 99.59 \\
\hline 7 & 96.13 & 97.57 & 7 & 97.80 & 98.65 & 7 & $99 \cdot 39$ & 99.61 \\
\hline 6 & 96.16 & 97.60 & 6 & 97.83 & 98.67 & 6 & 99.42 & 99.63 \\
\hline 5 & 96.20 & 97.62 & 5 & 97.87 & 98.69 & 5 & 99.45 & 99.65 \\
\hline 4 & $9^{6.23}$ & 97.64 & 4 & 97.90 & $9^{8} \cdot 7^{1}$ & 4 & $99.4^{8}$ & 99.67 \\
\hline 3 & 96.26 & 97.66 & 3 & 97.93 & 98.74 & 3 & $99.5^{2}$ & 99.69 \\
\hline 2 & $96.3^{\circ}$ & 97.68 & 2 & 97.96 & $98.7^{6}$ & 2 & 99.55 & 99.71 \\
\hline I & $96 \cdot 33$ & 97.70 & I & 98.00 & 98.78 & I & $99.5^{8}$ & 99.73 \\
\hline 0 & $96 \cdot 37$ & $97 \cdot 73$ & 0 & 98.03 & 98.80 & 0 & $99.6 \mathrm{I}$ & $99 \cdot 75$ \\
\hline 0.8049 & 96.40 & $97 \cdot 75$ & 0.7999 & 98.06 & 98.82 & 0.7949 & 99.65 & $99 \cdot 77$ \\
\hline 8 & 96.43 & 97.77 & 8 & 98.09 & 98.83 & 8 & 99.68 & 99.80 \\
\hline 7 & 96.46 & $97 \cdot 79$ & 7 & $9^{8.12}$ & $9^{8.85}$ & 7 & 99.71 & 99.82 \\
\hline 6 & 96.50 & $97.8 \mathrm{r}$ & 6 & 98.16 & 98.87 & 6 & 99.74 & 99.84 \\
\hline 5 & 96.53 & 97.83 & 5 & $9^{8.19}$ & 98.89 & 5 & $99.7^{8}$ & 99.86 \\
\hline 4 & 96.57 & 97.86 & 4 & 98.22 & 98.91 & 4 & $99.8 \mathrm{I}$ & 99.88 \\
\hline 3 & 96.60 & 97.88 & 3 & 98.25 & 98.93 & 3 & 99.84 & 99.90 \\
\hline 2 & 96.63 & 97.90 & 2 & 98.28 & 98.94 & 2 & 99.87 & 99.92 \\
\hline I & 96.66 & 97.92 & I & $9^{8} \cdot 3^{1}$ & 98.96 & I & 99.90 & 99.94 \\
\hline 0 & 96.70 & 97.94 & 0 & $9^{8} \cdot 34$ & 98.98 & o & 99.94 & 99.96 \\
\hline 0.8039 & 96.73 & 97.96 & 0.7989 & 98.37 & 99.00 & 0.7939 & 99.97 & 99.98 \\
\hline & 96.76 & 97.98 & & $9^{8} \cdot 4^{\mathrm{I}}$ & 99.02 & & & \\
\hline & 96.80 & 98.01 & 7 & $9^{8.44}$ & 99.04 & & Abs. & Alc. \\
\hline 6 & $96.8_{3}$ & 98.03 & 6 & $9^{8} .47$ & 99.05 & $0.793^{8}$ & 100.00 & 100.00 \\
\hline 5 & 96.87 & 98.05 & 5 & $98.5^{\circ}$ & 99.07 & & & \\
\hline 4 & 96.90 & 98.07 & 4 & 98.53 & 99.09 & & & \\
\hline 3 & 96.93 & 98.09 & 3 & 98.56 & 99.11 & & & \\
\hline 2 & 96.96 & 98.11 & 2 & $9^{8} .59$ & 99.13 & & & \\
\hline I & 97.00 & 98.14 & I & 98.62 & 99.15 & & & \\
\hline 0 & 97.03 & 98.16 & 0 & $9^{8} .66$ & $99 \cdot \mathrm{I} 6$ & & & \\
\hline
\end{tabular}


(4) Determination of Alcohol by the Ebullioscope or Vaporimeter is based on the variation in boiling-point of mixtures of alcohol and water, in accordance with the amount of alcohol present. There are various forms of this instrument, one of the simplest and most convenient being that of Salleron, Fig. 113, the apparatus being known in France as an
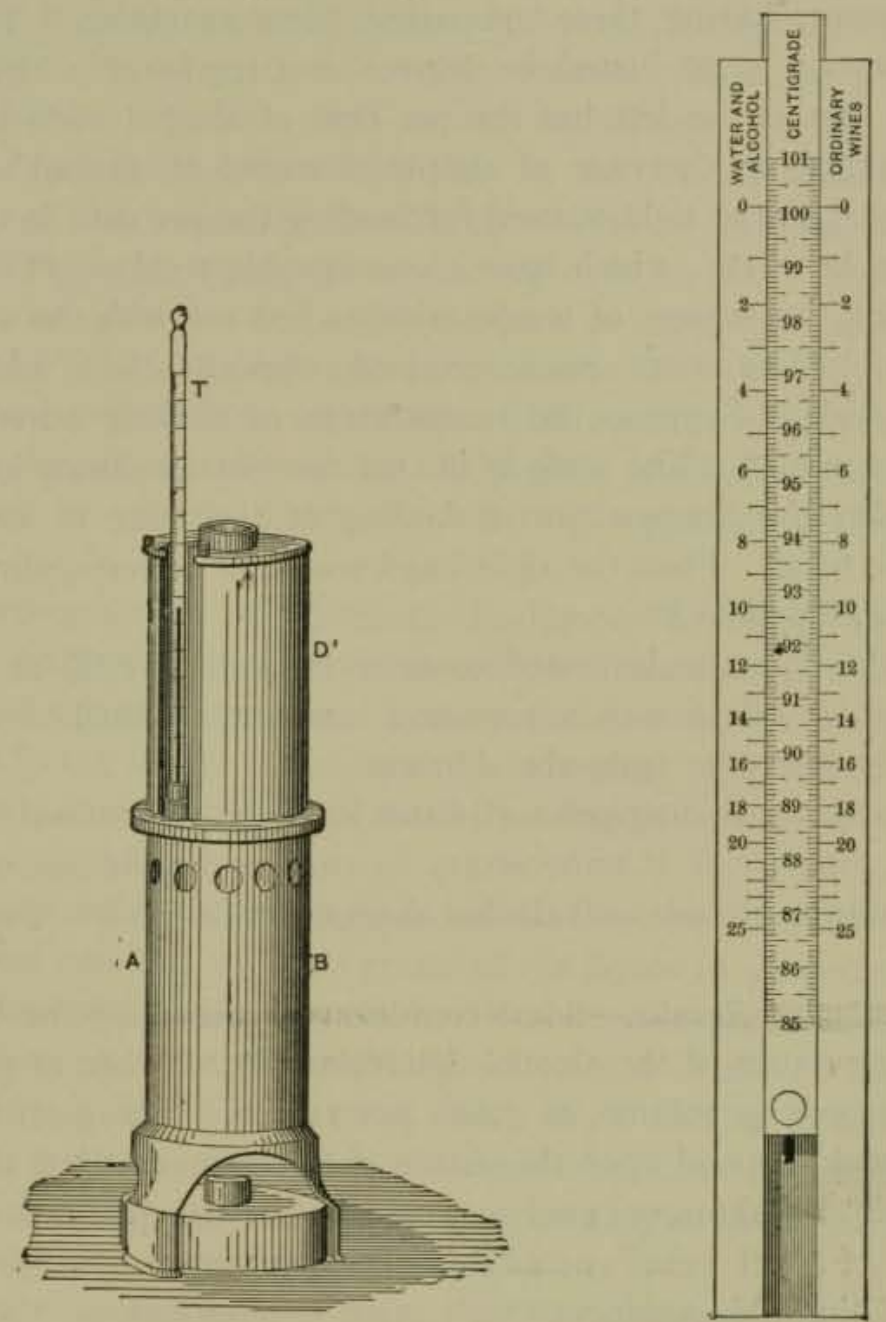

Fic. 1r3.-Salleron's Ebullioscope and Scale for Calculation of Results.

ebulliometer. This consists of a jacketed metallic reservoir, heated by a lamp placed beneath, and fitted with a return-flow condenser at the top and with a delicate thermometer graduated in tenths of a degree.

As the beiling-point of water varies with the atmospheric pressure, it is necessary to determine the actual boiling-point corresponding with the barometric conditions each time a series of determinations are made. 
This is done by boiling a measured portion of distilled water in the reservoir, and carefully noting the temperature when it becomes constant.

The reservoir is then rinsed out with a little of the liquor to be tested, after which a measured amount of this liquor is boiled in the reservoir and the temperature again noted. A sliding scale (Fig. II3) accompanies the instrument, having three graduated parts as shown. The central movable portion is graduated in degrees and tenths of a degree centigrade, the part at the left has the per cent of alcohol corresponding to the temperature in the case of simple mixtures of alcohol and water, while the part at the right is used for reading the per cent in the case of wine, cider, beer, etc., which have a considerable residue. The movable scale bearing the degrees of temperature is first set with the actual temperature of boiling water (as ascertained) opposite the o mark on the stationary scale. Suppose the temperature of boiling water has been found to be $100 \cdot 1^{\circ}$. The seale is in this case set as shown in Fig. II3. Suppose also the temperature of boiling of the wine to be tested is found to be $89 \cdot 3^{\circ}$. From the right-hand scale the corresponding per cent of alcohol is found to be 17.2 .

When the liquor to be tested contains more than $25 \%$ of alcohol, it is necessary to dilute with a measured amount of distilled water and calculate the per cent from the dilution.

When once the boiling-point of water has been determined for a given barometric pressure, it is unnecessary to change the position of the sliding scale during a series of alcohol determinations unless that pressure changes.

Expression of Results. - Some confusion is caused by the three ways of expressing results of the alcohol determination, whether as per cent by weight, per cent by volume, or grams per $100 \mathrm{cc}$. The particular mode adopted should depend upon the nature of the case and upon the prevailing custom. In laboratory analyses, unless otherwise qualified, the simple expression of "per cent" usually implies per cent by weight, and for the reason that this conforms with other determinations, the adoption of the weight-percentage plan is perhaps most natural to the chemist on the grounds of uniformity.

In enforcing the laws regulating the liquor traffic, the custom leans to volume percentage, and many of the laws are based on the "volume of alcohol at $60^{\circ} \mathrm{F}$," (see p. 656 ).

In recent years many European analysts have adopted the custom of expressing results of analyses of wines and other liquors in grams per 
$100 \mathrm{cc}$. and, in order to have a common basis of comparison between the composition of American and of European wines, this manner of expression has to some extent been adopted in the United States.

Proof-spirit in the United States is an alcoholic liquor containing $50 \%$ of absolute alcohol by volume at $15: 6^{\circ} \mathrm{C}$. A common method of expressing alcohol is in "degree proof" or simply "proof," which in the United States is twice the per cent of alcohol by volume. Thus, 91.3 proof or degree proof is the same as $45.65 \%$ alcohol by volume.

English Proof-spirit differs from that in the United States in that it contains $49.24 \%$ by weight, or $57.06 \%$ by volume of absolute alcohol at $15.6^{\circ} \mathrm{C}$. Strength is expressed in degrees over or under proof. Thus liquor $20^{\circ}$ under proof has 80 parts by volume of proof-spirit and 20 parts of water at $15.6^{\circ} \mathrm{C}$., while $20^{\circ}$ over-proof means that 100 volumes of the liquor have to be diluted to 120 volumes with water to yield proof-spirit. To calculate the per cent by volume of English proof-spirit from the per cent of alcohol by volume, divide the latter by 0.5706 , or multiply it by I. 7525 .

Direct Determination of Extract.-In liquors having a high sugar content, the extract or total solids cannot be determined accurately by evaporation at the temperature of boiling water, owing to the dehydration of the reducing sugars at temperatures exceeding $75^{\circ}$. When extreme accuracy is required, such liquors should be dried in vacuo at $75^{\circ}$, or in a McGill oven (p. 586).

Approximate results satisfactory in most cases are obtained by heating for two and one-half hours 10 grams of the liquor in a tared platinum dish at the temperature of boiling water. If the results are to be expressed in grams per soo cc., instead of weighing out ro grams, Io cc. of the liquor are measured by a pipette into a tared dish. With distilled liquors having low residues, accurate results are obtainable by direct evaporation at $100^{\circ}$, using preferably 25 grams or $25 \mathrm{cc}$. according as the result is to be expressed in per cent by weight or grams per Ioo cc.

Extract in wine and beer is more readily calculated indirectly from their specific gravity as noted elsewhere.

Determination of Ash.-The residue from the determination of the exuract is incinerated to a white ash in the original dish at a low red heat, either over a Bunsen flame or in a muffle. The dish is finally cooled in a desiccator and weighed.

Preservatives and Artificial Sweeteners in liquors are identified as. described in Chapters XVIII and XIX. 


\section{FERMENTED LIQUORS.}

The fermented juices of many varieties of fruits and berries furnish beverages more or less popular in various localities, especially for home consumption, though, with the exception of the products of the apple and the grape, few of them are found on the market. The following table shows the average percentage of sugar and free acid in the expressed juice or must of fruits, according to Fresenius, arranged in the order of their sugar content:

\begin{tabular}{|c|c|c|}
\hline & Per Cent Sugar. & $\begin{array}{l}\text { Per Cent Free } \\
\text { Acid as Malic. }\end{array}$ \\
\hline Peaches. ............... & I.99 & 0.85 \\
\hline Apricots. ............ & 2.13 & 1.29 \\
\hline Plums................ & 2.80 & 1.72 \\
\hline Green gages ............. & $4 \cdot 18$ & 0.67 \\
\hline Raspberries. ............ & 4.84 & 1.80 \\
\hline Blackberries. ........... & $5 \cdot 32$ & 1.42 \\
\hline Strawberries. ............ & 6.89 & I. 57 \\
\hline Currants, $\ldots \ldots \ldots \ldots \ldots$ & $7 \cdot 30$ & 2.43 \\
\hline German prunes .......... & $7 \cdot 56$ & 1.08 \\
\hline Gooseberries............ & 8.00 & I. 63 \\
\hline Pears. . . . . . . . . . . . . & 8.43 & 0.09 \\
\hline Apples. ............... & 9.14 & 0.82 \\
\hline Mulberries.............. & 10.00 & 2.02 \\
\hline Sour cherries. ........... & 10.44 & 1.52 \\
\hline Sweet cherries............ & $15 \cdot 3^{\circ}$ & 0.88 \\
\hline Grapes................... & 16.15 & 0.80 \\
\hline
\end{tabular}

Cider is the expressed juice of the apple. When fresh and before fermentation has set in, it is known as sweet cider, but it does not long remain in this condition, developing after a good fermentation from 3 to 6 per cent of alcohol by volume.

The predominating yeast under the influence of which the fermentation of cider takes place is Saccharomyces apiculatus, found in considerable quantity on the outside of the apples as well as in the soil in which the trees grow.

Process of Manufacture.-The best cider is made from ripe fruit, taking care to avoid the green and the rotten apples, both of which impair the quality of the product. After gathering, the apples are best allowed to stand in piles until perfectly ripe, being kept under cover. If exposed to the weather, certain of the yeast organisms found on the skins of the apples that are useful in promoting subsequent fermentation would be 
washed off. As a rule the apples commonly used by farmers for cidermaking are those that are unsalable or unfit for other purposes, being chiefly windfalls or bruised and imperfect fruit. The apples are usually first crushed in a mill to a coarse pulp, which is afterward subjected to pressure in a suitable press and the juice thus extracted.

In this country but little attention is paid to the after processes, the juice being usually transferred directly to barrels, which are not always particularly clean, and allowed to ferment spontaneously in a convenient place, subject to changes in temperature. There is little wonder that cider so made will keep but a short time and quickly goes over into vinegar, unless salicylic acid or other antiseptic is added.

In France more care is taken to regulate the temperature of fermentation, to insure absolute cleanness of all receptacles, and to separate out contaminating impurities. A preliminary fermentation is usually given to the juice in open vats, during which the yeast spores are developed, while impurities separate out both by rising to the surface and by settling to the bottom, care being taken to avoid the development of acetic fermentation. At the proper time the juice is "racked off" or drawn from the clear portion between the top and bottom, transferred to scrupulously clean barrels, and allowed to undergo a second fermentation at a lower temperature than before.

Sometimes the "racking off" is repeated, and the juice is further clarified by "fining" or treating with isinglass, which carries down certain albuminous substances.

Cider thus made is capable of keeping a very long time.

In England cider is sometimes "fined" by treatment with milk, one quart of the latter being added to eighteen gallons of cider.

The apple pomace, left as a residue, is generally steeped in water and repressed. The juice from the second pressing is occasionally added to the first for cider manufacture, but more often is concentrated and made into apple jelly, or used as a fortifier for vinegar to make up deficiency in solids.

Composition of Cider.-The following tables, due to Browne,* show the chemical composition of the freshly expressed juice of several American varieties of apple, as well as that of a few fermented samples of cider of known purity.

* Penn. Dept. of Agric., Bul. 58. 
APPLE JUICES.

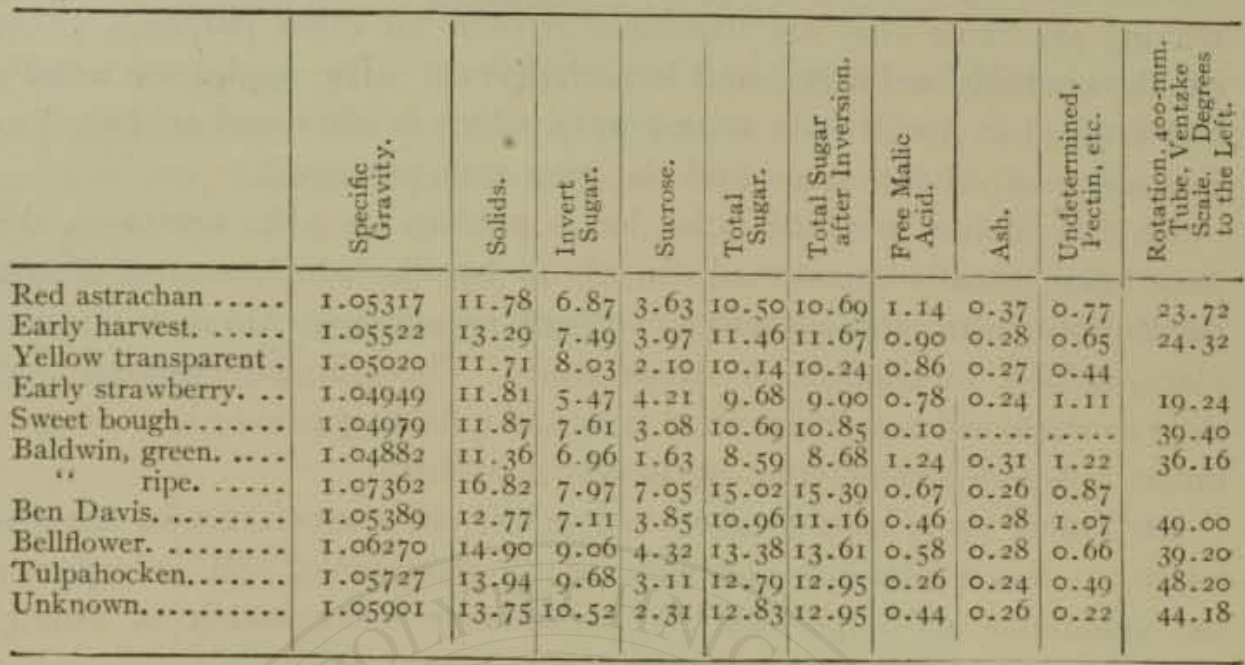

FERMENTED CIDER (MIXED APPLES).

\begin{tabular}{|c|c|c|c|c|c|c|c|c|c|}
\hline & $\begin{array}{l}\text { Specifie } \\
\text { Gravity. }\end{array}$ & Solids. & $\begin{array}{l}\text { Invert } \\
\text { Sugar. }\end{array}$ & $\begin{array}{l}\text { Malic } \\
\text { Acid. }\end{array}$ & $\begin{array}{l}\text { Acetic } \\
\text { Acid. }\end{array}$ & Alcohol. & Pectin. & Ash. & $\begin{array}{l}\text { Rotation, } \\
\text { 4oo-mm. } \\
\text { Tube, } \\
\text { Ventake } \\
\text { Scale. } \\
\text { Degrees } \\
\text { to the } \\
\text { Left. }\end{array}$ \\
\hline A... & 1. 99805 & I. 94 & 0.19 & $0.2 \mathrm{I}$ & 0.24 & 6.85 & 0.03 & 0.25 & $2 \cdot 30$ \\
\hline B... & 1.00122 & 2.71 & 0.19 & 0.24 & 0.42 & 5.13 & 0.03 & 0.32 & 2.49 \\
\hline C... & I. 00525 & 3.26 & 0.89 & 0.30 & 0.48 & 4.67 & 0.05 & 0.20 & 5.28 \\
\hline D. . & 1.00071 & $\mathrm{I} .93$ & 0.34 & 0.27 & $0.2 \mathrm{r}$ & 4.95 & 0.05 & 0.23 & 2.00 \\
\hline E... & 1.00512 & 2.71 & 0.24 & 0.29 & 1.96 & 4.26 & 0.06 & 0.36 & 1.76 \\
\hline
\end{tabular}

The following are summaries of the results of a large number of analyses of European apple juices made by Truelle, the quantities being expressed in grams per liter:

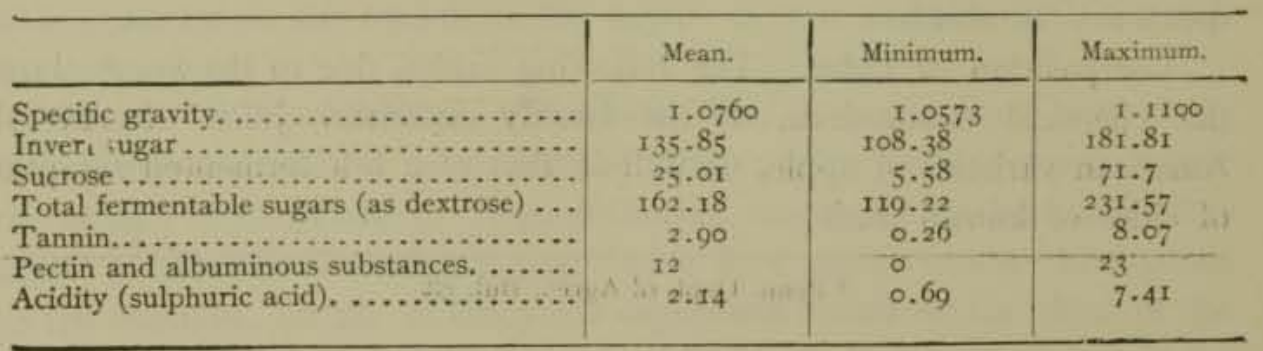


In the municipal laboratory of Paris, Sanglé Ferrière has analyzed eleven samples of known-purity cider with the following results:

\begin{tabular}{|c|c|c|c|c|c|c|c|c|c|c|}
\hline & \multirow[b]{2}{*}{ 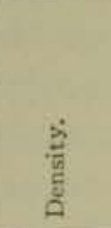 } & \multirow[b]{2}{*}{ 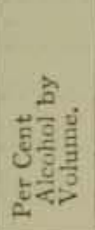 } & \multirow[b]{2}{*}{ 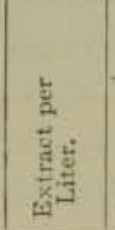 } & \multicolumn{2}{|c|}{$\begin{array}{l}\text { Sugar per } \\
\text { Liter. }\end{array}$} & \multirow[b]{2}{*}{ 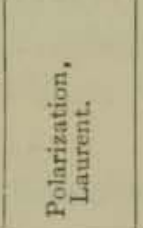 } & \multirow[b]{2}{*}{ 岁乌 } & \multirow{2}{*}{ 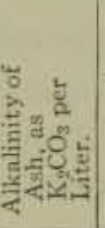 } & \multicolumn{2}{|c|}{$\begin{array}{c}\text { Acidity as } \\
\mathrm{H}_{2} \mathrm{SO}_{4} .\end{array}$} \\
\hline & & & & 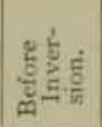 & 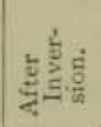 & & & & & $\begin{array}{l}\frac{d}{\vec{d}} \\
\frac{\vec{x}}{0}\end{array}$ \\
\hline $\begin{array}{l}\text { Mean ........ } \\
\text { Maximum. }\end{array}$ & $\begin{array}{l}1.0159 \\
1.0410\end{array}$ & $\begin{array}{l}3.9 \\
6.2\end{array}$ & $\begin{array}{r}52.67 \\
114.00\end{array}$ & $\begin{array}{l}21 .-31 \\
59-40\end{array}$ & $\begin{array}{l}21.62 \\
60.80\end{array}$ & $\begin{array}{l}-4^{\circ} \cdot 26 \\
-11^{\circ} \cdot 20\end{array}$ & $\begin{array}{l}3.26 \\
4.32\end{array}$ & $\begin{array}{l}2.56 \\
3.68\end{array}$ & $\begin{array}{l}5.27 \\
6.50\end{array}$ & $\begin{array}{l}2.55 \\
2.04\end{array}$ \\
\hline Minimum. .. & 1.0012 & $\mathrm{I}, \mathrm{I}$ & 22.62 & Trace & Trace & 0 & $\begin{array}{l}4.32 \\
2.48\end{array}$ & 2.04 & 4.20 & $\begin{array}{l}1.94 \\
1.47\end{array}$ \\
\hline
\end{tabular}

Six samples of bottled "sweet" cider purchased in Massachusetts were analyzed in the Food and Drug Laboratory of the Board of Health with the following results:

\begin{tabular}{|c|c|c|c|}
\hline & $\begin{array}{l}\text { Per Cent } \\
\text { Alcohnl by } \\
\text { Weight. }\end{array}$ & $\begin{array}{l}\text { Per Cent } \\
\text { Acid as } \\
\text { Malic. }\end{array}$ & $\begin{array}{l}\text { Per Cent } \\
\text { Extract. }\end{array}$ \\
\hline 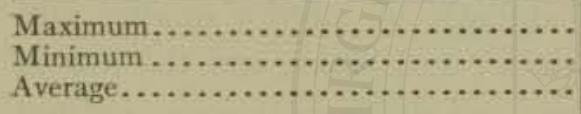 & $\begin{array}{l}8.00 \\
3 \cdot 55 \\
5 \cdot 7 x\end{array}$ & $\begin{array}{l}0.72 \\
0.48 \\
0.58\end{array}$ & $\begin{array}{l}7.82 \\
2.42 \\
4.19\end{array}$ \\
\hline
\end{tabular}

Browne gives the following as the composition of the mixed ash of several varieties of apple:

\begin{tabular}{|c|c|c|c|c|}
\hline Ingredient. & $\begin{array}{l}\text { Per- } \\
\text { cent- } \\
\text { age. }\end{array}$ & & Ingredient. & $\begin{array}{l}\text { Per- } \\
\text { cent- } \\
\text { age- }\end{array}$ \\
\hline 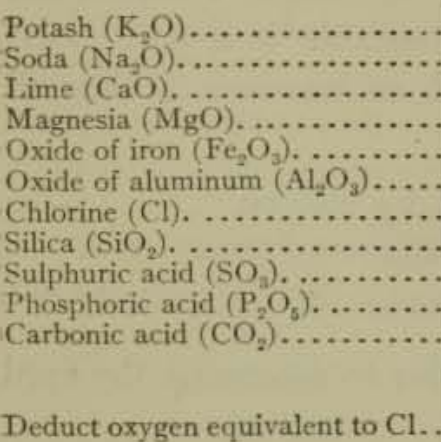 & $\begin{array}{r}55.94 \\
0.31 \\
4.43 \\
3.78 \\
0.95 \\
0.80 \\
0.39 \\
0.40 \\
2.66 \\
8.64 \\
21.60 \\
99.90 \\
.00\end{array}$ & $\frac{\stackrel{2}{E}}{\frac{\mathrm{E}}{\mathrm{E}}}$ & 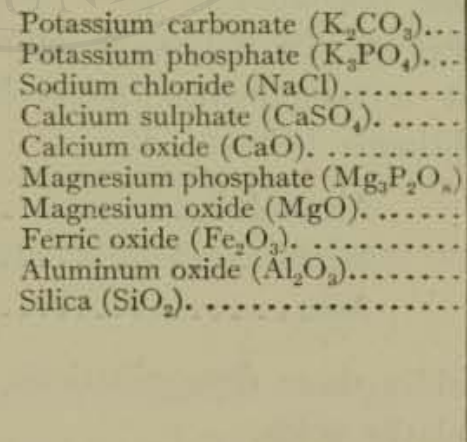 & $\begin{array}{r}6.85 \\
14.55 \\
0.60 \\
4.5^{2} \\
2.57 \\
6.97 \\
0.59 \\
0.95 \\
0.80 \\
0.40\end{array}$ \\
\hline Total. & $99.8 \mathrm{I}$ & & Total. .... & 99.80 \\
\hline
\end{tabular}


Burcker* gives the following composition of the ash of cider:

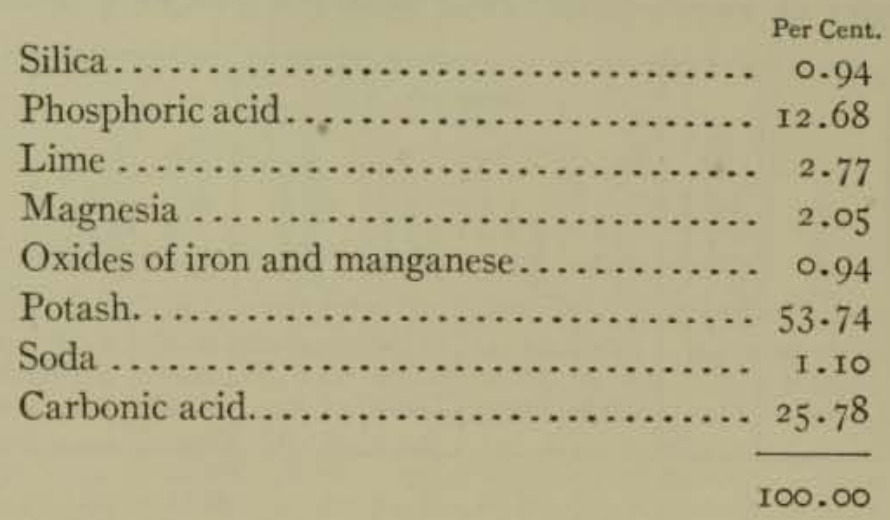

Adulteration of Cider.-The Committee on Standards of the A. O. A. C. have submitted for adoption the following standards for cider: Alcohol not more than $8 \%$, extract not less than $1.8 \%$ determined by evaporation in an open vessel at ordinary atmospheric pressure and at the temperasure of boiling water; ash not less than $0.2 \%$.

Entirely factitious cider made from other than apple stock is rarely found, though the product as sold is frequently of inferior quality and adulterated. The chief adulterants are water and sugar, and the use of antiseptics is common, especially of salicylic and sulphurous acids, sodium benzoate, and occasionally beta-naphthol.

Sodium carbonate is sometimes added to cider to neutralize the acid and thus prevent acetic fermentation. An abnormally high ash (say in excess of $0.35 \%$ ) would point toward the presence of added alkali.

Watering is apparent when the content of alcohol, solids, and ash of the suspected sample are found to be considerably below the corresponding constants of pure cider. According to Sanglé Ferrière, the following are the minimum figures for these constants in a pure cider, so that a sample may safely be pronounced as watered if they all run distinctly below:

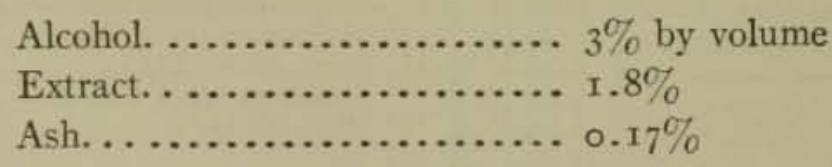

Besides these determinations, it is useful also to determine the fixed and volatile acids.

Caramel is to be looked for, especially in watered samples. Other

* Les Falsifications des Substances Alimentaires, p. 176. 
adulterants alleged to be of frequent occurrence in French cider, but not commonly found in this country are commercial glucose, tartaric acid (to increase the acidity of a watered product), and coal-tar colors.

Absence or deficiency of malates is conclusive evidence of fraud, indicating the admixture of notable quantities of the juice of the second pressing of pomace.

Sugar is rendered apparent by the right-handed polarization of the sample, pure cider always polarizing well to the left. If after inversion of a dextro-rotary cider the polarization is still to the right, commercial glucose is indicated; if the reading after inversion is to the left, cane sugar has undoubtedly been added.

Frequently the analyst has only to determine the alcohol, especially in cases of seizure, to ascertain whether or not there has been violation of the liquor laws.

\section{PERRY OR PEAR CIDER.}

This is a common French product, but is rarely if ever found on sale in this country, though sometimes made for home consumption. In composition and in method of manufacture it much resembles apple cider. It is also subject to the same forms of adulteration.

The following table summarizes a number of analyses made by Truelle on pear juice, or must, amounts being expressed in parts per thousand:

\begin{tabular}{|c|c|c|c|}
\hline & Mean. & Maximum. & Minimum. \\
\hline 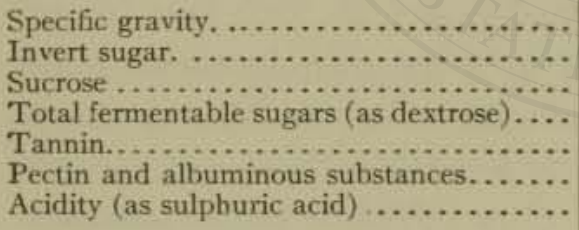 & $\begin{array}{l}1.0845 \\
145.64 \\
36.74 \\
184.14 \\
1.78 \\
13.08 \\
1.47\end{array}$ & $\begin{array}{r}1.0675 \\
108.10 \\
16.69 \\
143.78 \\
1.01 \\
3.76 \\
0.76\end{array}$ & $\begin{array}{l}1.0980 \\
200 \\
61.41 \\
220 \\
3.20 \\
18 \\
2.40\end{array}$ \\
\hline
\end{tabular}

The following analysis of champagne perry is taken from the Lancet of October I, 1892 :

Alcohol by weight. .................. 1.45

Alcohol by volume. ................. I.80

Solids............................ 11.00

Ash. ............................ 0.35 
WINE.

Wine in its broadest sense is the fermented expressed juice of any fruit, though the term, unless otherwise restricted, is generally understood to apply to the juice of the grape.

The organism present in grape juice that plays the chief part in its alcoholic fermentation is the Saccharomyces ellipsoideus, a yeast which exists on the skins of the grape.

Process of Manufacture.-The grapes, which should be fully ripe, are picked and sometimes sorted, according to the care that is taken in grading the product. They are also sometimes freed from the stems, which contain considerable tannic acid, and which when crushed with the grapes impart a certain astringency to the final product. The grapes are crushed either by machinery or by the bare feet, and the juice is pressed out from the pulp in various ways, by screw or hydraulic press, or by the centrifugal process.

A certain amount of juice runs off from the preliminary crushing known as the first run, and makes the choicest wine. The product from the pressure constitutes the second run, after which the pomace, by steeping in water and repressing, is made to yield an inferior juice used in vinegar-making.

Red wines are made from dark grapes by fermenting the pulp, before pressing, with the skins, which by this treatment yield up their rich color (anocyanin) to the juice. Besides the color, the skins contain also tannin. White wine is made from the pressed pulp, freed from the skins at once, or from the pulp of white grapes. The unfermented must constitutes from 60 to 80 per cent of the weight of the grape.

Fermentation progresses most rapidly at a temperature between $25^{\circ}$ and $30^{\circ} \mathrm{C}$., but wine having a much finer bouquet is produced by slower fermentation, hence the must is allowed to ferment in open vats or tubs in cool cellars, at a temperature of from $5^{\circ}$ to $15^{\circ}$ till it settles out comparatively clear, special care being taken to avoid development of acetic fermentation. At the end of the first or active fermentation, the wine is drawn off and allowed to undergo a second or slow fermentation in casks, during which most of the lees or crude argols, composed of potassium bitartrate, settle out, being insoluble in alcohol, and the characteristic bouquet or flavor of the wine is developed. Occasionally during this process the wine is racked or drawn off.

Undesirable fermentations and vegetable fungus growth, which are 
liable to occur at this time, are avoided as much as possible by using especially clean casks, which are frequently "sulphured" (or burnt out with sulphur) before being used. The wine is also sometimes clarified, or "fined," by treatment with gelatin, which mechanically removes many impurities by precipitation, or is subjected to pasteurization before finally being bottled or stored in casks.

Classification of Wines.-Wines are either natural or fortified. Natural wines are those which contain no added sugar or alcohol, but which are exclusively the product of the simple juice, fermented under the best conditions, either till the sugar has been used up, or till the yeast food is exhausted, or until the yeast growth has been checked by the strength of the alcohol developed. When the alcohol content amounts to $14 \%$ by weight there can be no further fermentation due to yeast, so that this is the highest limit for natural wine. Examples of natural wines are hock and claret and many California wines.

Fortified wines are those to which alcohol has been added, usually before the natural fermentation has been allowed to proceed to a finish. For this reason considerable sugar is usually left, and such wines are more often sweet. Examples of fortified wines are Madeira, sherry, and port.

Volatile ethers (products of volatile acids) predominate as a rule in natural wines, while fixed ethers (from the fixed acids as tartaric) are most characteristic in fortified wines.

Wines are also variously classified according to characteristic properties possessed by them, as still or sparkling, red or white, "dry" or sweet, etc.

Still wines are those in which there is but little carbon dioxide remaining, so that they do not effervesce. Sparkling wines are more or less heavily charged with carbon dioxide, either naturally, as in the case of champagne, wherein the gas is formed by after-fermentation of added sugar in the corked bottle, or artificially, by carbonating them in a similar manner to "soda-water."

Among the best-known red wines are those of Burgundy and the Bordeaux wines or clarets, while the Rhenish and Moselle wines and the Sauternes are examples of white wine.

"Dry" wines are those in which the sugar has been exhausted by fermentation, while sweet wines possess a considerable amount of unfermented sugar. Whether or not an excess of sugar is left after fermentation has stopped depends upon the amount of yeast food or nitrogenous substance present in the wine. When the proteins are exhausted by the 
yeast, fermentation ceases, and for this reason gelatin and other nitrogenous bodies are sometimes added to extend the period of fermentation. Sweet wines are often reinforced by the addition of sugar. Madeira, both red and white, are samples of dry wine, while port wine is one of the sweet variety.

While most of our finer wines still come from France and Germany, large quantities of California wines are now being produced of an extremely high grade and of many varieties.

Composition of Grape Must and of Wine.-König's analyses of a large number of grape musts from different sources are thus summarized:

\begin{tabular}{|c|c|c|c|c|c|c|c|}
\hline & $\begin{array}{l}\text { Specific } \\
\text { Gravity. }\end{array}$ & $\begin{array}{l}\text { Water. } \\
\text { Per Cent. }\end{array}$ & $\begin{array}{l}\text { Nitroge- } \\
\text { nous Ma- } \\
\text { terial. }\end{array}$ & Sugar. & Acid. & $\begin{array}{l}\text { Other } \\
\text { Non-ni- } \\
\text { trogenous } \\
\text { Material. }\end{array}$ & Ash. \\
\hline 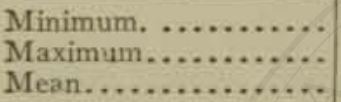 & $\begin{array}{l}1.0690 \\
1.2075 \\
1.1024\end{array}$ & $\begin{array}{l}51 \cdot 53 \\
82,10 \\
74 \cdot-49\end{array}$ & $\begin{array}{l}0.11 \\
0.57 \\
0.28\end{array}$ & $\begin{array}{l}12.89 \\
35.45 \\
19.71\end{array}$ & $\begin{array}{l}0.20 \\
1.18 \\
0.64\end{array}$ & $\begin{array}{r}1.68 \\
11.62 \\
4.48\end{array}$ & $\begin{array}{l}0.20 \\
0.6: \\
0.40\end{array}$ \\
\hline
\end{tabular}

Typical analyses of German, French, Austrian, Russian, Italian, and Spanish wines are shown in the following table, also due to König:

\begin{tabular}{|c|c|c|c|c|c|c|c|c|c|}
\hline & 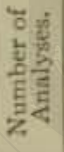 & 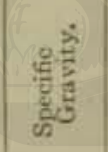 & $\frac{\hat{a}}{\frac{a}{8}} \frac{a}{8}$ & 递 & 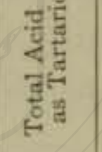 & 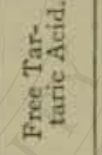 & 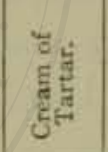 & 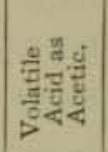 & 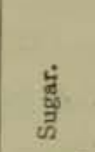 \\
\hline \multicolumn{10}{|l|}{$\begin{array}{l}\text { Germany: } \\
\text { Moselle. }\end{array}$} \\
\hline Moselle. ........ & 14 & 0.9964 & 7.99 & 2.24 & 0.79 & & & & 0.031 \\
\hline 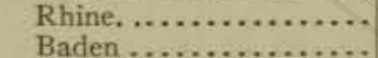 & 23 & 1.0005 & $\begin{array}{l}8.00 \\
6.6\end{array}$ & $\begin{array}{l}2.60 \\
2.16\end{array}$ & 0.81 & & 0.20 & & \\
\hline $\begin{array}{l}\text { Baden } \\
\text { Wurtemburg, white wine. }\end{array}$ & $\begin{array}{l}40 \\
\text { I5 }\end{array}$ & 0.000 & $\begin{array}{l}0.05 \\
6.10\end{array}$ & $\begin{array}{l}2.10 \\
2.27\end{array}$ & $\begin{array}{l}0.91 \\
0.95\end{array}$ & $\begin{array}{l}0.018 \\
0.095\end{array}$ & $\begin{array}{l}0.35^{\circ} \\
0.262\end{array}$ & & 0.095 \\
\hline " red wine.. & 6 & & $4 \cdot 73$ & 2.64 & 1.14 & 0.091 & 0.026 & & \\
\hline Alsace $\ldots \ldots \ldots \ldots \ldots \ldots$ & 15 & & 6.59 & 2.07 & 0.696 & 0.018 & 0.168 & 0.052 & \\
\hline Lorraine, red wine..... & 10 & 0.9967 & 8.08 & 2.27 & 0.56 & 0.032 & & 0.155 & 0.088 \\
\hline \multicolumn{8}{|l|}{ France: } & & 0.30 \\
\hline White wine. . & 5 & 0.9963 & $8.3^{\circ}$ & 3.03 & 0.66 & & & & \\
\hline \multicolumn{10}{|l|}{ Austria: } \\
\hline $\begin{array}{l}\text { Tyrol, red wine... } \\
\text { white wine. }\end{array}$ & $\begin{array}{l}60 \\
17\end{array}$ & 0.9940 & $\begin{array}{l}9.08 \\
8.8_{4}\end{array}$ & $\begin{array}{l}2.34 \\
1.87\end{array}$ & 0.62 & & & & \\
\hline \multicolumn{10}{|l|}{ Russia: } \\
\hline $\begin{array}{l}\text { Red wine. } \\
\text { White win }\end{array}$ & 10 & 0.9939 & 10.76 & 2.76 & $0.5^{6}$ & & & 0.142 & \\
\hline $\begin{array}{l}\text { White wine, ................................ } \\
\text { Italy. }\end{array}$ & 12 & $\mid 0.993 \mathrm{t}$ & 11.96 & 2.568 & 0.49 & …. & & 0.100 & $0.45^{8}$ \\
\hline \multirow{3}{*}{$\begin{array}{l}\text { Spain: } \\
\text { Ordinary red wine. ...... } \\
\text { Sweer wine. ........... }\end{array}$} & 20 & & 10.01 & 3.44 & $0.5^{2}$ & & & $\cdots \cdots$ & 1.44 \\
\hline & $\begin{array}{l}7 \\
4\end{array}$ & r....... & $\begin{array}{l}12.30 \\
12.78\end{array}$ & $\begin{array}{l}3.53 \\
9.69\end{array}$ & $\begin{array}{l}0.49 \\
0.59\end{array}$ & & & & $\begin{array}{l}0.38 \\
6.55\end{array}$ \\
\hline & & & & & & & & & \\
\hline
\end{tabular}




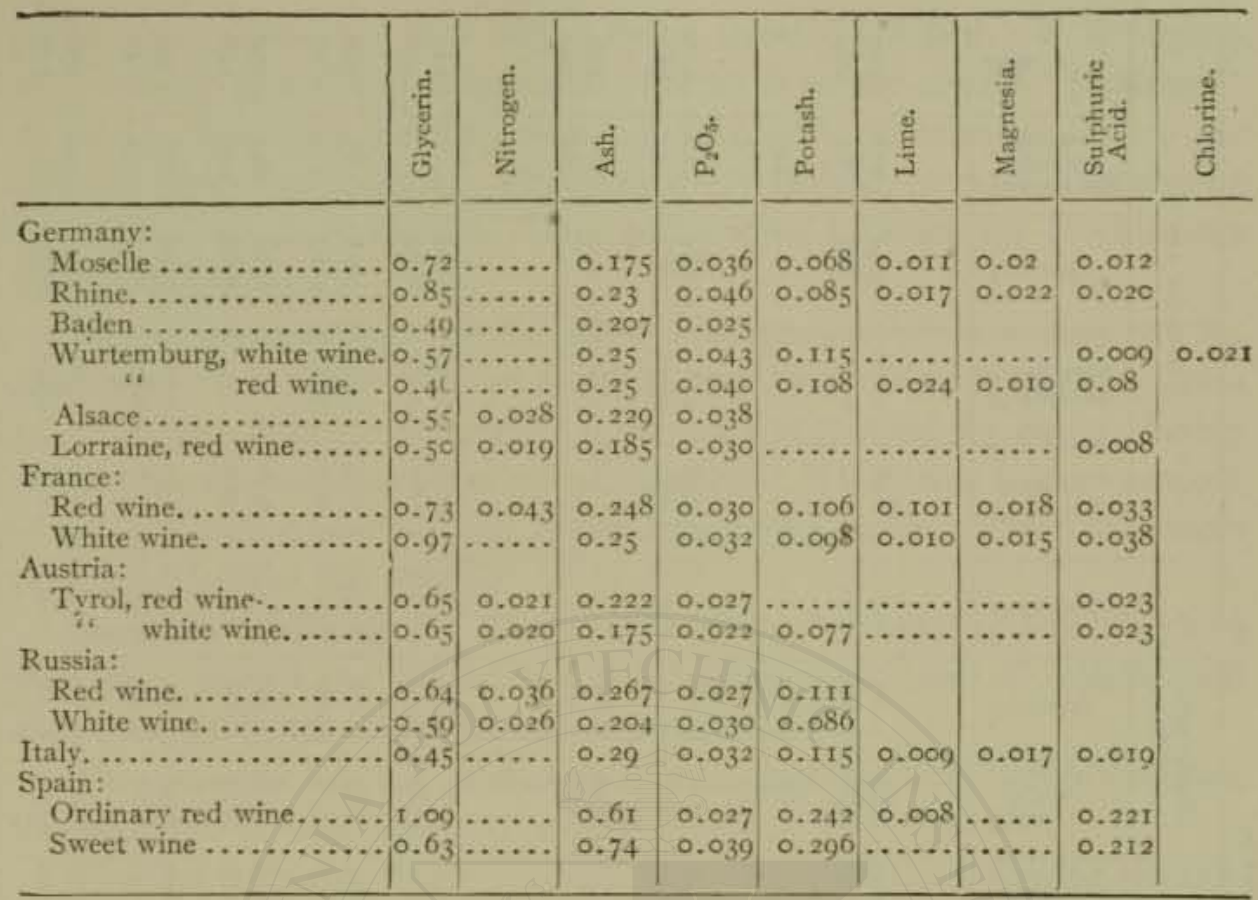

On page 688 are given summaries of analyses of American wines compiled from tables of analyses made by Bigelow.*

Varieties of Wine.-Champagne is a selected, sweet, white wine, clarified with gelatin, bottled with the addition of cane sugar, mixed with a little brandy, and tightly corked. Sometimes a small amount of yeast is also introduced. Fermentation is allowed to go on at a temperature of about $24^{\circ} \mathrm{C}$., during which the wine is highly charged with carbon dioxide. The bottles are set on their side for some months, after which they are inverted till the sediment gathers above the cork, which by careful manipulation is quickly removed so as to throw out the sediment, and is afterward replaced and secured. Champagne contains from 8 to ro per cent of alcohol and is high in sugar.

Claret is a light, red wine of a deep color, and is somewhat acid and astringent. In alcohol it varies from 8 to $I 3$ per cent by volume. It has very little sugar and is high in volatile ethers.

Madeira is a strong, white wine, possessing a refined, nutty, aromatic flavor when fully aged. It is generally forified, containing from 17 to 20 per cent of alcohol. It is named from the island which produces it. Sherry is a deep, amber-colored, sweet, Spanish wine, high in alcohol

* U. S. Dept. of Agric., Bur. of Chem., Bul. 59. 


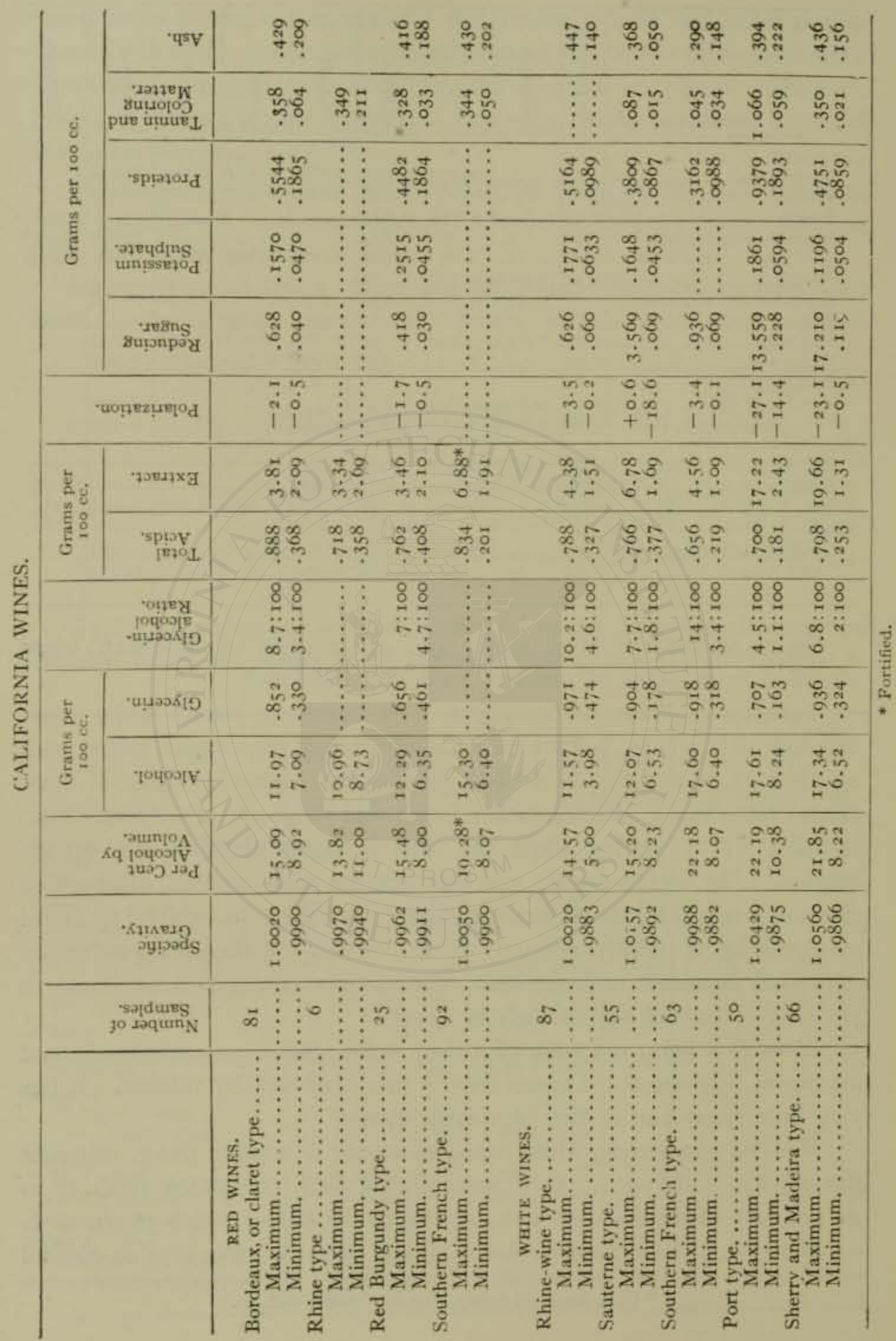


(sometimes containing over $20 \%$ ), being usually fortified. It is slightly acid and possesses much fragrance. Sherry is nearly always "plastered."

Hocks are white German wines, mildly acid, containing 9 to 12 per cent of alcohol by volume. They have very little sugar, and rank among the highest of natural wines. The best-known varieties are Hockheimer and Johanisberger.

Port (Vinum portense of the 1870 Pharmacopoia) is a dark-purple, astringent wine, almost always fortified, and hence high in alcohol (from I5 to 18 per cent by volume). It is much improved by aging, during which it looses considerable of its astringency. It contains a large amount of extract, from 2 to 6 per cent of the wine being sugar. The fixed ethers predominate over the volatile.

Standards of Purity for Wine.-The ratio of volatile to fixed acids in pure wine should not exceed $1: 3$. A higher proportion of volatile acid shows the fact that acetic fermentation has set in.

The presence of any considerable free tartaric acid would indicate the addition of this substance to the wine.

The Unied States Pharmacopoia has prescribed the following requirements in the case of wines: For white wine (Vinum album) the specific gravity at $15.6^{\circ}$ should not be less than 0.990 nor more than 1.010 ; the extract or residue at $100^{\circ}$ should not be less than 1.5 nor more than $3 \%$; as indicating the amount of free acid, not less than 3 nor more than $5.2 \mathrm{cs}$. normal potassium hydroxide should be required to neutralize $50 \mathrm{cc}$. of the wine, using phenolphthalein as an indicator; it should contain not less than 7 nor more than 12 per cent by weight of absolute alcohol; it should contain only traces of tannin.

For red wine (Vinum rubum) the specific gravity at $15.6^{\circ}$ should not be less than 0.989 nor more than I.oro; the extract should not be less than $\mathrm{r} .6 \%$ nor more than $3.5 \%$; its limits as to acidity are the same as with white wine, eosin or fluorescin, however, being used as an indicator; in alcoholic strength, it should, like white wine, come within the limits of 7 and 12 per cent alcohol by weight. It should not be artificially colored, but should show the presence of tannic acid.

The following are U. S. standards for wines: Wine is the product made by the normal alcoholic fermentation of the juice of sound, ripe grapes, and the usual cellar treatment, and contains not less than 7 nor more than i6 per cent of alcohol, by volume, and, in $100 \mathrm{cc} .\left(20^{\circ} \mathrm{C}\right.$.), 
not more than 0.1 gram of sodium chloride nor more than 0.2 gram of potassium sulphate; and for red wine not more than o.r 4 gram, and for white wine not more than 0.12 gram of volatile acids produced by fermentation and calculated as acetic acid. Red wine is wine containing the red coloring matter of the skins of grape. White wine is wine made from white grapes or the expressed fresh juice of other grapes.

Dry wine is wine in which the fermentation of the sugars is practically complete, and which contains, in $100 \mathrm{cc}$. $\left(20^{\circ} \mathrm{C}\right.$. $)$, less than I gram of sugars, and for dry red wine not less than 0.16 gram of grape ash and not less than I.6 grams of sugar-free grape solids, and for dry white wine not less than 0.13 gram of grape ash and not less than I.4 grams of sugar-free grape solids.

Fortified dry wine is dry wine to which brandy has been added, but which conforms in all other particulars to the standard of dry wine.

Sweet wine is wine in which the alcoholic fermentation has been arrested, and which contains, in $100 \mathrm{cc}$. $\left(20^{\circ} \mathrm{C}\right.$.), not less than I gram of sugars, and for sweet red wine not less than 0.16 gram of grape ash, and for sweet white wine not less than 0.13 gram of grape ash.

Fortified sweet wine is sweet wine to which wine spirits have been added. By act of Congress, "sweet wine" used for making fortified sweet wine and "wine spirits" used for such fortification are defined as follows (sec. 43 , Act. of October $1,1890,26$ Stat. 567 , as amended by section 68, Act of August 27, 1894, 28 Stat. 509, and further amended by Act of Congress, approved June 7, 1906): "That the wine spirits mentioned in section 42 of this act is the product resulting from the distillation of fermented grape juice to which water may have been added, prior to, during, or after fermentation, for the sole purpose of facilitating the fermentation, and economical distillation thereof, and shall be held to include the products from grapes or their residues, commonly known as grape brandy; and the pure sweet wine, which may be fortified free of tax, as provided in said section, is fermented grape juice only, and shall contain no other substance whatever introduced before, at the time of, or after fermentation, except as herein expressly provided; and such sweet wine shall contain not less than 4 per cent of saccharine matter, which saccharine strength may be determined 
by testing with Balling's saccharometer or must scale, such sweet wine, after the evaporation of the spirits contained therein, and restoring the sample tested to original volume by addition of water: Provided, That the addition of pure boiled or condensed grape must, or pure crystallized cane or beet sugar, or pure anhydrous sugar to the pure grape juice aforesaid, or the fermented product of such grape juice prior to the fortification provided by this act, for the sole purpose of perfecting swest wine according to commercial standard, or the addition of water in such quantities only as may be necessary in the mechanical operation of grape conveyors, crushers, and pipes leading to fermenting tanks, shall not be excluded by the definition of pure sweet wine aforesaid: Provided, however, That the cane or beet sugar, or pure anhydrous sugar, or water, so used shall not in either case be in excess of $10 \%$ of the weight of the wine to be fortified under this act: And provided further, That the addition of water herein authorized shall be under such regulations and limitations as the Commissioner of Internal Revenue, with the approval of the Secretary of the Treasury, may from time to time prescribe; but in no case shall such wines to which water has been added be eligible for fortification under the provisions of this act where the same, after fermentation and before fortification, have an alcoholic strength of less than $5 \%$ of their volume."

Sparkling wine is wine in which the after part of the fermentation is completed in the bottle, the sediment being disgorged and its place supplied by wine or sugar liquor, and which contains in $100 \mathrm{cc} .\left(20^{\circ} \mathrm{C}\right.$.), not less than 0.12 gram of grape ash.

Modified wine, ameliorated wine, corrected wine, is the product made by the alcoholic fermentation, with the usual cellar treatment, of a mixture of the juice of sound, ripe grapes with sugar (sucrose), or a syrup containing not less than $65 \%$ of sugar (sucrose), and in quantity not more than enough to raise the alcoholic strength after fermentation, to $11 \%$ by volume.

Raisin wine is the product made by the alcoholic fermentation of an infusion of dried or evaporated grapes, or of a mixture of such infusion, or of raisins with grape juice.

Adulteration of Wine. - Beverages purporting to be wine are sometimes found on sale that are entirely spurious, in that they contain little if any fermented grape juice. Apple cider is not infrequently the basis of such artificial products, and the following recipes given 
by Brannt may be taken as typical of the composition of these wine substitutes:

Burgundy.-Bring into a barrel 40 quarts of apple juice, 5 pounds of bruised raisins, $\frac{1}{4}$ pound of tartar, I quart of bilberry juice, and 3 pounds sugar. Allow the whole to ferment, filling constantly up with cider. Then clarify with isinglass, add about $\mathrm{x}$ ounce of essence of bitter almonds, and after a few weeks draw off into bottles.

Malaga Wine.-Apple juice, 40 quarts; crushed raisins, to pounds; rectified alcohol, 2 quarts; sugar solution, 2 quarts; elderberry flowers, I quart; acetic ether, I ounce and 2 drachms. The desired coloration is effected by the addition of bilberry or elderberry juice; otherwise the process is the same as given for Burgundy.

Sherry Wine.-Apple juice, 50 quarts; orange-flower water, about 2 drachms; tartar, 2 ounces and 4 drachms; rectified alcohol, 3 quarts; crushed raisins, Io pounds; acetic ether, $I$ ounce and 2 drachms. The process is the same as for Burgundy.

Claret Wine.-Apple juice, 50 quarts; rectified alcohol, 4 quarts; black currant juice, 2 quarts; tartar, 2 ounces and 4 drachms. Color with bilberry juice. The further process is the same as for Burgundy.

Artificial products similar in nature to the above are also mixed in varying proportions with pure wine.

Presence of malates, as well as absence or diminution of total tartaric acid, is also indicative of cider.

If the ash of the wine be submitted to the flame test, the sodium light will predominate in the case of pure wine, while if the basis of the sample be largely or wholly apple stock, the potash flame will be readily apparent.

Wines are most frequently adulterated by "plastering," by watering: by the addition of excessive amounts of sugar or glucose, by various flavoring principles, by coal-tar and vegetable colors, by antiseptics, and by added alcohol.

Plastering. - By this term is understood the addition of gypsum or plaster of Paris to the must before fermentation, a practice in vogue in parts of France, Italy, and Spain. The reaction which takes place with the potassium bitartrate present in the wine is, according to Chancel, as follows: 


$$
\underset{\substack{\text { Potassium } \\ \text { bitartrate }}}{2 \mathrm{KHC}_{4} \mathrm{H}_{4} \mathrm{O}_{6}}+\underset{\substack{\text { Calcium } \\ \text { sulphate }}}{\mathrm{CaSO}_{4}}=\underset{\substack{\text { Calcium } \\ \text { tartrate }}}{\mathrm{CaC}_{4} \mathrm{H}_{4} \mathrm{O}_{6}}+\underset{\substack{\text { Tartaric } \\ \text { acid }}}{\mathrm{H}_{2} \mathrm{C}_{4} \mathrm{H}_{4} \mathrm{O}_{6}}+\underset{\substack{\text { Potassium } \\ \text { sulphate }}}{\mathrm{K}_{2}} \mathrm{SO}_{4}
$$

Various advantages are said to result from this practice. The wine is clarified by the precipitation of the calcium tartrate, which mechanically carries down with it many impurities, the color of the wine is improved, since the solubility of the coloring principle present in the skins is increased, the fermentation is rendered more rapid and complete, and the keeping qualities of the wine are enhanced. The practice is, however, considered objectionable on account of the potassium sulphate which is left in solution in the wine, and in some countries plastering is forbidden, or the amount of potassium sulphate limited by statute.

The following are analyses of two Spanish wines made from the same grape juice, one of which was plastered. The results are expressed in grams per liter.

\begin{tabular}{|c|c|c|}
\hline & Not Plastered. & Plastered. \\
\hline 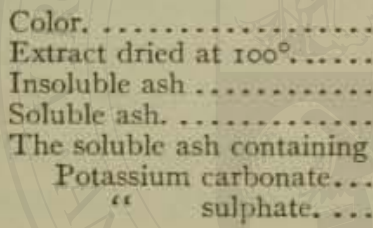 & $\begin{array}{c}\text { Yellow } \\
23.3 \\
0.66 \\
2.06 \\
\\
1.29 \\
0.4 \mathrm{r}\end{array}$ & $\begin{array}{l}\text { Bright red } \\
27 \cdot 3 \\
0.6 \mathrm{x} \\
5 \cdot 3^{8} \\
0.17 \\
5\end{array}$ \\
\hline
\end{tabular}

The effect of plastering is thus seen to distinctly increase the extract and the soluble ash. Any considerable amount of potassium sulphate is an indication of plastering.

Addition of Cane Sugar.-The term "chaptalizing" is applied in France to the addition to the must of cane sugar for the purpose of increasing the yield in alcohol. The addition of 1,700 grams of sugar to $\mathrm{I}, 000$ liters of must is said to increase the alcoholic streng h by $\mathrm{I} \%$. It was formerly customary to add with the sugar calcium carbonate, to partially neutralize the acidity, but this is rarely practiced at present.

The European wine-raising countries are not disposed to regard the reinforcement of wine by added cane sugar in the must as in itself a fraud, unless water is also added. or unless some other form of adulteration is practiced at the same time. In France, however, the addition of cane 
sugar is permitted only in wine for local consumption, and is restricted in amount.

The use of commercial glucose in wine instead of cane sugar is not regarded with as much favor, in view of the fact that glucose contains more or less unfermentable matier, and introduces dexirin and various mineral salts into the wine.

To ascertain the nature and extent of the sugars present in wine is frequently of great importance. Much information may be gained from the direct and invert polarization of the sample, as well as from the determination of reducing sugars.

Invert sugar is the only legitimate sugar that should be present in genuine wine. In normal fermentation the dextrose is more quickly destroyed than the levulose, hence the polarization of pure wine is always left-handed, unless all the sugar has been fermented, in which case the reading should be zero.

Seventy-five samples of California red wines, chiefly claret, Burgundy, Rhine, and southern France types, analyzed in the Bureau of Chemistry* of the U. S. Department of Agriculture, polarized from -0.5 to -2.I. Upwards of eighty samples of California white wine (of the types of Burgundy, Sauterne, and southern France) were submitted to polarization and all but four were left-handed. These four (evidently abnormal) polarized from o, to $+\mathrm{I}$. Most of them varied from -0.1 to -3.5 .

Thirteen of the port wine types (California) had a left-handed polarization of from -14.7 to $-27 . \mathrm{I}$. These apparently contained large quantities of unfermented, inverted cane sugar.

A sharp, right-handed polarization would indicate the presence of either commercial glucose or cane sugar. After inversion, if the reading is still right-handed, glucose is apparent, while if inversion changes the reading from right to left, cane sugar has undoubtedly been added. By application of Clerget's formula the amount of cane sugar can be estimated.

The Watering of Wine, unless excessive in degree, is not always easy to prove, by reason of the varying composition of pure wine, and because the practice is usually accompanied by other forms of sophistication intended to cover up evidences of watering. Considerable quantities of added water alone would usually be rendered apparent by a proportionate and abnormal lowering of the alcohol, extract, ash, acidity, and, indeed, nearly all the constants.

Gautier in his Traité sur la Sophistication et l'Analyse des Vins claims 
that the sum of the weight in grams of alcohol in ros cc. and the total acidity, expressed in grams of sulphuric acid per liter, varies within very narrow limits in pure wines, rarely being below $\mathrm{I}_{3}$ or above 17 . A large number of analyses made by Gautier and others would seem to confirm this, so that in the majority of cases, added water would be strongly indicated if the sum of these two constants was materially reduced below 13. It is more conservative to adopt $\mathbf{2} 2.5$ as a minimum limit for the sum of the alcohol and total acid expressed as above.

Detection of Added Alcohol.-As a result of the findings of a committee appointed in France to determine the matter of added alcohol, it was submitted that a relation existed between the weight of the extract and that of the alcohol in pure wine. In the case of red wines, if the weight of the alcohol, divided by the weight of the extract (both expressed in grams per $100 \mathrm{cc}$.) exceeds 4.6 , the addition of alcohol is strongly indicated. With white wines, the quotient obtained by dividing the weight of alcohol by weight of extract should not exceed 6.6. If it does, adder alcohol is to be suspected.

In the case of plastered wines containing sulphate of potassium, or wines having added sugar, it is necessary to deduct from the total extract the weight of the reducing sugar and of the potassium sulphate as found (less 0.1 gram for each of these substances), the difference, or reduced extract as it is called, being used in this case in obtaining the ratio.

Fruit Wines other than Grape.-Wines mostly of domestic manufacture are sometimes made from small fruits, such as raspberries, strawberries, blackberries, gooseberries, elderberries, and currants, as well as from cherries, plums, and apricots. Wines made from most of these fruits readily undergo acetic fermentation unless antiseptics are added, or unless extreme care is taken in their manufacture and keeping. Frequently mixtures of various fruit juices are made to yield excellent wine. Most of the sour fruits require a liberal admixture of sugar to produce an acceptable wine.

The following analysis of currant wine is due to Fresenius:

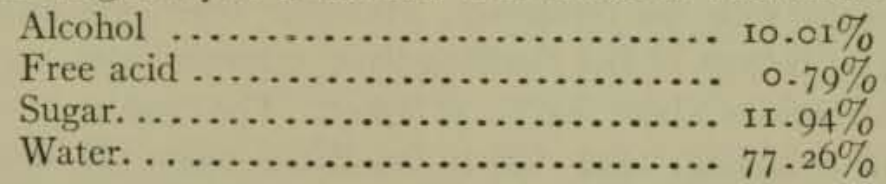

The alcoholic content of other fruit wines is thus shown by Brannt:

Gooseberry wine.............. II $84 \%$ alcohol

Elderberry wine............... $8.79 \%$ "

Orange wine................ II. $26 \%$ " 


\section{METHODS OF ANALYSIS OF WINE AND CIDER.}

For determination of specific gravity, alcohol, extract (by direct method), and ash, see pp. $657-676$.

Calculation of the Extract in Wine.-Attention has already been called to the difficulty in accurately determining the extract of sweet wines gravimetrically by evaporation. An approximate determination of the extract may be obtained by calculation from the specific gravity of the dealcoholized liquor, or one may use for this purpose the tables compiled by Windisch, and based on experiments made on drying wine in vacuo at $75^{\circ} \mathrm{C}$. In wines high in sugar, with more than $6 \%$ of extract, this method is far more accurate than drying at $100^{\circ}$, and is to be recommended.

Evaporate a measured portion of the wine on the water-bath to one-fourth its volume, and dilute with water to exactly the volume measured. Determine the specific gravity of this dealcoholized liquid at $15.6^{\circ}$, and from the following table ascertain the extract corresponding.

Determination of Total Acidity.-Carbonated beverages are first freed from carbon dioxide by agitation as described on page $65^{8}$, after which $25 \mathrm{cc}$. of the sample are heated just to the boiling-point and titrated with tenth-normal sodium hydroxide, using in the case of white wine or cider phenolphthalein as an indicator. With red wine delicate litmus paper should be used. Total acidity is usually expressed, in the case of cider as malic, and of wine as tartaric acid. Each cubic centimeter of tenth-normal alkali corresponds to 0.0067 gram malic, or 0.0075 gram tartaric acid. Some chemists express total acidity in terms of sulphuric acid, each cubic centimeter of tenth-normal alkali being equivalent to0.0049 gram of sulphuric acid.

Volatile Acids in all liquors are usually expressed as acetic, although traces of propionic and other volatile acids may be present. $5^{\circ} \mathrm{cc}$. of the cider or wine and a little tannic acid are transferred to a distillingflask, Fig. II5, the stopper of which is provided with two tubes, one of which connects with the condenser, while the other, arranged to reach nearly to the bottom of the distilling-flask, communicates with a second flask which contains about $300 \mathrm{cc}$. of water. The contents of both flasks are brought to boiling, after which the flame under the distilling-flask is lowered, and steam from the water-flask is passed through the wine till about $200 \mathrm{cc}$. of distillate have collected in the receiving-flask. Titrate this with tenth-normal sodium hydroxide, using phenolphthalein 
EXTRACT IN WINE.

[ACCORDING TO WINDISCH.]

\begin{tabular}{|c|c|c|c|c|c|c|c|c|c|c|c|}
\hline $\begin{array}{l}\text { Specific } \\
\text { Gravity. }\end{array}$ & $\begin{array}{l}\text { Ex- } \\
\text { tract. }\end{array}$ & $\begin{array}{l}\text { Specific } \\
\text { Gravity. }\end{array}$ & $\begin{array}{l}\text { Ex- } \\
\text { tract. }\end{array}$ & $\begin{array}{r}\text { Specific } \\
\text { Gravity. }\end{array}$ & $\begin{array}{l}\text { Ex- } \\
\text { tract. }\end{array}$ & $\begin{array}{l}\text { Specific } \\
\text { Gravity. }\end{array}$ & $\begin{array}{l}\text { Ex- } \\
\text { tract. }\end{array}$ & $\begin{array}{l}\text { Specific } \\
\text { Gravity. }\end{array}$ & $\begin{array}{l}\text { Ex- } \\
\text { tract. }\end{array}$ & $\begin{array}{l}\text { Specific } \\
\text { Gravity. }\end{array}$ & $\begin{array}{l}\text { Ex- } \\
\text { tract. }\end{array}$ \\
\hline 1,0000 & 0.00 & I. 0065 & t. 68 & 1.0130 & 3.36 & t, org5 & 5.04 & r. .0260 & 6.72 & 1,0325 & 8.40 \\
\hline I. 0001 & 0.03 & r. 0066 & 1.70 & 1.0131 & $3-38$ & 1.0106 & 5,06 & 1.0261 & 6.75 & 1.0326 & 8.43 \\
\hline 1.0002 & 0.05 & 1.0067 & 1.73 & 5.0132 & $3.4 x$ & 1.0197 & 5.09 & 1. 0262 & 6.77 & 1.0527 & 8.46 \\
\hline 1,0003 & 0.08 & 1. 0068 & 1.76 & 1.0133 & 3.43 & 1.0198 & 5.11 & $x .0263$ & 6.80 & 1.0328 & 8.48 \\
\hline 1.0004 & 0,10 & t.0069 & 1.78 & 1.0134 & 3.46 & 1.0190 & 5,14 & t. .0264 & 6.82 & 1.0320 & $8,5 x$ \\
\hline I.0005 & 0.13 & 1.0070 & 1.81 & t. 0135 & 3.49 & 1.0200 & 5.17 & I. 0265 & 6.85 & $\mathrm{I} .0330$ & 8.53 \\
\hline 1. 0006 & 0.15 & $\mathrm{t} .007 \mathrm{t}$ & $x \cdot 83$ & 1.0136 & 3. $5 \mathrm{t}$ & r. $.010 \mathrm{t}$ & 5,10 & t. 0266 & 6.88 & 1.0331 & 8.56 \\
\hline 1.0007 & 0.18 & 1.0072 & x. 86 & t. .0137 & 3.54 & 1.0202 & 5.22 & 1.0267 & 6.00 & 1.0332 & 8.59 \\
\hline r. .0008 & 0.20 & 1.0073 & I. 83 & r. 0538 & $3: 56$ & f.0203 & 5,25 & $t .0268$ & 6.93 & $t .0333$ & $8.6 \mathrm{r}$ \\
\hline 1.0000 & 0.23 & 1.0074 & 1.91 & 1. 0139 & 3.50 & I., 0204 & 5.27 & 1.0269 & 6.95 & 1. .0334 & 8.64 \\
\hline I.0010 & 0.26 & 1,0075 & I. 94 & 1.0140 & 3.62 & 1. 0205 & 5.30 & 1.0270 & 6.98 & $\mathrm{t} .0535$ & 8.66 \\
\hline 1.0011 & 0,28 & $x, 0076$ & $x=96$ & $1.014 \mathrm{t}$ & 3.64 & I. 0206 & $5 \cdot 32$ & 1.0271 & 7.01 & $t .0336$ & 8.60 \\
\hline I. 0012 & $0.3 \mathrm{I}$ & I. 0077 & 1.99 & I. 0142 & 3.07 & 1.0207 & $5-35$ & 1.0272 & 7.03 & 1.0337 & $8: 72$ \\
\hline 1. 0013 & 0.34 & 1,0078 & 2.01 & 1.0143 & 3.69 & 1.0208 & 5.38 & 1.0273 & 7.06 & 1.0338 & 8.74 \\
\hline 1.0014 & 0.36 & 1.0079 & 2,04 & 1.0144 & $3-72$ & 1.0200 & 5.40 & 1.0274 & 7.08 & 1.0339 & 8.77 \\
\hline 1.0015 & 0.39 & $t .0080$ & 2.07 & $t .0145$ & 3. 75 & I. 0210 & 5.43 & 1.0275 & 7.71 & 1.0340 & 8.79 \\
\hline 1.0016 & 0.41 & $1.008 \mathrm{x}$ & 2.09 & 1.0146 & $3-77$ & $\mathrm{I} .02 \mathrm{II}$ & 5.45 & 1.0276 & 7.13 & $t .034 t$ & 8.82 \\
\hline I. 0017 & 0.44 & I. 0082 & 2.12 & 1.0147 & 3.80 & I. 0212 & 5.48 & 1. 0277 & 7,16 & 1.0342 & 8.85 \\
\hline 1.0018 & 0,46 & I. 0083 & 2,14 & 1.0148 & 3,82 & 1.0213 & 5.51 & 1.0278 & $7 \cdot 10$ & 1.0343 & 8.87 \\
\hline 1.0019 & 0.40 & I. .0084 & 2.17 & I. 0149 & 3.85 & 1.0214 & $5 \cdot 53$ & 1.0279 & 7.21 & 1,0344 & 8.90 \\
\hline 1.0020 & 0.52 & $t .0085$ & $2, \pi 9$ & I. 9150 & 3.87 & 1,0215 & 5.56 & r. 0280 & 7.24 & I. 0.345 & 8.02 \\
\hline 1.0021 & 0.54 & 1.0086 & 2.22 & t.ors & 3.90 & 1.0216 & 5.58 & $1.028 x$ & 7.26 & $x: 0346$ & 8.95 \\
\hline 1,0022 & 0.37 & I., 0087 & 2.25 & 1.0152 & 3.93 & 1.0217 & $5.6 \mathrm{r}$ & 1.0282 & 7220 & 1,0347 & 8.97 \\
\hline 1.0023 & 0.59 & 1.0088 & 2,27 & 1.0153 & 3.95 & 1.0218 & 5.64 & 1.0283 & 7.32 & t. 0348 & 0.00 \\
\hline 1.0024 & 0.62 & 1.0089 & 2,30 & I. 0154 & $3: 98$ & $1.02 \times 9$ & 5.66 & 1.0284 & 7,34 & 1.0549 & 9.03 \\
\hline 1.0025 & 0.64 & 1.0000 & 2.32 & 1.0155 & 4.00 & I. .0223 & 5.60 & 1.0285 & $7-37$ & $1.055^{\circ}$ & 9.05 \\
\hline 1.0026 & 0.67 & I.0091 & 2.35 & 1.0156 & $4=03$ & 1,0220 & $5-7 x$ & 1.0286 & $7-39$ & 1. $.035 \mathrm{I}$ & 9.08 \\
\hline 1,0027 & 0.60 & 1.0002 & 2.38 & t.ors7 & 4.06 & 1. 0222 & 5.74 & $x .0287$ & $7 \cdot 42$ & r. 0352 & 9.10 \\
\hline 1. 0028 & 0.72 & 1.0093 & 2.40 & t. 0158 & 4.08 & 1,0223 & 5.77 & 1. .0288 & $7 \cdot 45$ & 1.0353 & 9.13 \\
\hline 1.0029 & 0.75 & 1.0094 & 2.43 & 1.0159 & 4.11 & 1.0224 & $5 \cdot 79$ & 1.0280 & $7 \cdot 47$ & 1.0354 & 9.16 \\
\hline 1. .0030 & 0.77 & t. .0095 & $2-45$ & $1.0: 60$ & $4 \cdot 13$ & 1.0225 & 5.82 & 1.0290 & 7,50 & 1.0355 & 9,18 \\
\hline I. $003 \mathrm{r}$ & 0.80 & 1.0006 & 2.48 & r.oror & 4.16 & 1.0226 & 5.84 & $1.029 x$ & 7.52 & $x, 0356$ & $9.2 \mathrm{r}$ \\
\hline 1,0032 & 0.82 & 1. 0007 & 2.50 & t. $-0+62$ & 4.10 & t. 0227 & 5.87 & 1.0292 & $7 \cdot 55$ & 1.0357 & 9.23 \\
\hline 1.0033 & 0.85 & 1.0008 & $2 \cdot 53$ & t.or63 & 4.21 & I. .0228 & 5.80 & 5.0293 & 7.58 & 1.0358 & 9.26 \\
\hline 1,0034 & 0.87 & 1.0009 & 2.56 & 2. 0164 & 4.24 & I.0229 & 5.92 & 1.0294 & 7,60 & $x .0359$ & 9.29 \\
\hline x. 0035 & 0.90 & 1.0100 & 2,58 & 1.0165 & 4.26 & I. .0250 & 5,94 & 1.0295 & 7.63 & 1.0360 & $0.3 t$ \\
\hline 1.0036 & 0.95 & t.0101: & $2.6 \mathrm{x}$ & 1.0166 & 4.29 & 1.0231 & 5.97 & 1.0296 & 7.65 & $x .036 t$ & $9-34$ \\
\hline 1.0037 & 0.05 & 1.0102 & 2,63 & 1. 0167 & 4.31 & 1.0232 & 6.00 & 1.0207 & 7.08 & 2.0362 & 0.36 \\
\hline 1.0038 & 0.08 & 1.0103 & 2.66 & 1.0168 & 4.34 & I. .0233 & 6.02 & 1.0208 & 7.70 & 1.0363 & 9.30 \\
\hline 1.0039 & 1.00 & 1.0104 & 2.69 & 1.0169 & 4.37 & $t .0234$ & $6 / 05$ & 1.0299 & 7.73 & 1.0364 & 9.42 \\
\hline I 9040 & 1.03 & I. 0105 & $2.7 \mathrm{r}$ & r.0170 & 4.30 & 1.0235 & 6.07 & 1.0300 & $7 \cdot 76$ & I. .0365 & $9 \cdot 44$ \\
\hline I. 0041 & 1.05 & 1.0106 & 2,74 & 1.0171 & 4.42 & I. 0230 & 6.0 & 1.0301 & $7 \cdot 78$ & $x .0366$ & $9 \cdot 47$ \\
\hline I. 0042 & 1.08 & 1,0107 & 2.76 & 1.0172 & 4.44 & 1.0237 & 6. 12 & 1.0302 & $7.8 t$ & 1.0367 & 9.49 \\
\hline I. 0043 & $x . x$ & 1.0108 & 2.79 & 1.0173 & 4.47 & 1.0238 & 6.15 & 1.0303 & 7.83 & 1,0368 & 9.52 \\
\hline I. 0044 & 1,13 & 1.0109 & 2.82 & 1.0174 & $4 \cdot 50$ & 1.0239 & 6.18 & $x .0304$ & 7.80 & $=0360$ & 9.55 \\
\hline$t .0045$ & 1. 16 & r.orto & 2,84 & $x .0175$ & 4.52 & 1.0240 & 6.20 & 1.0305 & 7.80 & 1.0370 & 0.57 \\
\hline I. .0046 & 1,18 & 1.0111 & 2.87 & 1.0176 & 4.55 & 1,0241 & 0.23 & $x .0306$ & $7+9 t$ & I. 0371 & 9.00 \\
\hline I. 0047 & 1.21 & 1.0112 & 2.80 & 1.0177 & 4.57 & $t .0242$ & 6. 25 & $x .0307$ & 7.94 & 1.0372 & $9.6 z$ \\
\hline I. .0048 & 1.24 & 2.0113 & 2.92 & 1.0178 & 4.60 & 1.0243 & 6.28 & $x .0308$ & 7.07 & I. .0373 & 9.65 \\
\hline I. .0049 & 1,26 & 1.0114 & 2.94 & 1.0179 & 4.63 & t. 0244 & 6.32 & 1.0309 & 7.99 & 1.0374 & 9.68 \\
\hline r. 0050 & 1,20 & 1.0115 & 2.07 & 1.0180 & 4.65 & I. 0245 & 6.33 & $1.03 t 0$ & 8,02 & t.0375 & 9.70 \\
\hline 1.0051 & 1,32 & 1.0116 & 3.00 & $t .028 \mathrm{t}$ & 4. 68 & 1.0246 & 6.36 & $t, 031 t$ & 8.04 & 1.0376 & 9.73 \\
\hline I. 0052 & I. 34 & 1.0117 & 3.02 & $t, 0182$ & 4.70 & 1.0247 & $6 \cdot 38$ & 1.0312 & 8.07 & r. .0377 & 9.75 \\
\hline 1.0053 & 1.37 & 1,0118 & 3.05 & 1.0183 & $4 \cdot 73$ & 1.0248 & $6.4 \mathrm{t}$ & $x .03 x 3$ & 8.09 & 1.0378 & 9.78 \\
\hline I. 0054 & ${ }^{1} .39$ & 1.0119 & 3.07 & 1.0184 & 4.75 & $x .0249$ & 6.44 & 1.0314 & 8.12 & 1.0379 & 0.80 \\
\hline t. 0055 & I. 42 & 1.0120 & 3,10 & 1.0185 & 4. 78 & 1.0250 & 6.46 & 1.0315 & $8 \cdot 14$ & 1.0380 & 9.83 \\
\hline 1.0056 & $x .45$ & 1.0121 & 3,12 & $1.0 \times 86$ & 4.81 & 1.0251 & 6.49 & 1.0316 & 8. 17 & $1.038 \mathrm{r}$ & 0.86 \\
\hline 1.0057 & $x .47$ & 1.0122 & 3.15 & 1.0187 & 4.83 & I. .0252 & 6.51 & 1.0317 & 8,20 & 1.0382 & 9.88 \\
\hline 1.0058 & 1.50 & 1.0123 & 3.18 & 1.0188 & 4.86 & $t .0253$ & 6.54 & I. 0318 & 8.22 & 1.0383 & $9.9 x$ \\
\hline $1: 0059$ & I. 52 & 1.0124 & $3: 20$ & r. 0189 & 4.88 & 1.0254 & 6.56 & 1.0319 & 8.25 & 1.0384 & 0.93 \\
\hline I. 0060 & I. 55 & 1,0125 & 3.23 & I. 0190 & 4.91 & 1.0255 & 6.59 & 1.0320 & 8,27 & $x .0385$ & 9.96 \\
\hline x. $006 x$ & 1.57 & 1.0126 & 3.26 & 1.0191 & 4.94 & 1.0256 & 6,62 & 1.0321 & 8.30 & 1.0386 & 0.90 \\
\hline 1. 0062 & 1.60 & 1,0127 & 3.28 & 1.0192 & 4.96 & 1.0237 & 6.64 & 1.0322 & 8.33 & 1.0387 & $\mathrm{xo} .0 \mathrm{x}$ \\
\hline 1.0063 & 1.63 & 1,0128 & 3.31 & 1.0193 & 4.09 & 1.0258 & 0.67 & 1.0323 & 8.35 & 1.0388 & 10.04 \\
\hline 1,0064 & 1.65 & 1.0129 & 3.33 & 1.0194 & 5.01 & 1.0259 & 6.70 & 1.0324 & 8.38 & 1.0380 & 10.06 \\
\hline
\end{tabular}


EXTRACT IN. WINE-(Continued).

\begin{tabular}{|c|c|c|c|c|c|c|c|c|c|c|c|}
\hline $\begin{array}{l}\text { Specific } \\
\text { Gravity. }\end{array}$ & $\begin{array}{l}\text { Ex- } \\
\text { tract. }\end{array}$ & $\begin{array}{l}\text { Specific } \\
\text { Gravity. }\end{array}$ & $\begin{array}{c}\text { Ex- } \\
\text { tract. }\end{array}$ & $\begin{array}{l}\text { Specifie } \\
\text { Gravity. }\end{array}$ & $\begin{array}{l}\text { Ex- } \\
\text { tract: }\end{array}$ & $\begin{array}{l}\text { Specifie } \\
\text { Gravity. }\end{array}$ & $\begin{array}{l}\text { Ex- } \\
\text { tract. }\end{array}$ & $\begin{array}{l}\text { Specific } \\
\text { Gravity. }\end{array}$ & $\underset{\text { tract. }}{\text { Ex- }}$ & $\begin{array}{l}\text { Specific } \\
\text { Gravity. }\end{array}$ & $\begin{array}{l}\text { Ex- } \\
\text { tract. }\end{array}$ \\
\hline I. 0390 & 10.09 & I. 0455 & 11.78 & 1.0520 & 13.47 & 1.0583 & 15,16 & $x, 0650$ & 16,86 & $1.07+5$ & 18.56 \\
\hline 1.0391 & 10,11 & .0456 & $11.8 \mathrm{r}$ & I. $052 \mathrm{I}=$ & 13.49 & I. 0586 & 15.19 & $I .0651$ & 16.88 & 1.0716 & 18.58 \\
\hline 1.0392 & 10.14 & I. 0457 & Ix. 83 & 1.0522 & 13.52 & I. 0587 & 15,22 & 1.0652 & $x 6.9 x$ & $x, 07 x ?$ & $18.6 x$ \\
\hline I. 0303 & 10.17 & 1.0458 & 11.86 & 1.0523 & 13.55 & 1.0588 & 15.24 & 1.0653 & $x 6,94$ & $1.07+8$ & 18.63 \\
\hline$x .0394$ & 10.19 & I. 0459 & 11.88 & I. 0524 & 13.57 & $\mathrm{I}, 0580$ & 15.27 & 1.0654 & 26.96 & 1.0719 & 18.66 \\
\hline 1. 0305 & 10.22 & I. 0460 & 11,91 & 1.0525 & 13.60 & $\mathrm{I} .0500$ & 15.20 & 1.0655 & 16.99 & $t .0720$ & 18,60 \\
\hline 1.0306 & 10.25 & 1.0461 & $x x=94$ & 1.0526 & $x 3.62$ & 1.0591 & $15 \cdot 32$ & 1. .0656 & 17.01 & 1.0721 & 18,71 \\
\hline 1.0397 & 10.27 & $x .0462$ & 11.06 & 1.0527 & 13.65 & 1.0502 & 15.35 & 1.0657 & $17 \cdot 04$ & 1,0722 & 18.74 \\
\hline 1.0398 & 10,30 & 1.0463 & 11.90 & I. 0528 & 13.68 & I. .0593 & 15.37 & 1.0658 & 17.07 & 1,0723 & 18.76 \\
\hline 1.0399 & 10.32 & 1.0404 & 12.01 & $x .0529$ & 13.70 & I. 0594 & 15.40 & I. 0659 & 17.00 & 1.0724 & 18.79 \\
\hline 1.0400 & 10.35 & 1.0465 & 12.04 & 1.0530 & 13.73 & 1.0505 & 75.42 & t. .0660 & 17.12 & 1.0725 & 18.82 \\
\hline 1.0401 & 10.37 & I. 0.466 & $x 2,06$ & $1.053 \mathrm{x}$ & 13.75 & I. 0596 & 15.45 & I. o6bu & 17.14 & 1.0726 & $\tau 8.84$ \\
\hline 1.0402 & 10,40 & 1.0 .467 & 12.00 & 1.0532 & $13 .-8$ & 1.0597 & 75.48 & 5.0662 & 17.17 & 1.0727 & 18.87 \\
\hline $\mathrm{I} .040 .3$ & $10 \cdot 43$ & $x .0468$ & 12.12 & 1.0533 & 13.81 & 1.0598 & I 5.50 & $x, 0663$ & 17.20 & $x, 0728$ & 18.00 \\
\hline x. 0404 & 10.45 & $x .0469$ & 12.14 & I. 05.34 & 13.83 & $x .0599$ & 15.53 & 1,0664 & 17.22 & 1.0729 & 18.92 \\
\hline$x .0405$ & 10.48 & I. 0420 & 12.17 & 1.0535 & $x 3.86$ & I, 0600. & 15.55 & 1.0665 & $17 \cdot 25$ & 1.07 .30 & 38.05 \\
\hline$x .0406$ & $10.5 \mathrm{I}$ & $1.047 x$ & 12,19 & I. 0536 & 13.89 & 1.0001 & 13.58 & 1.0666 & 17.27 & $x .0731$ & 18,07 \\
\hline 1.0407 & 10.53 & 1.0472 & 12,22 & 1.0537 & 13.91 & 1.0602 & $15,6 x$ & 1.0667 & $17 \cdot 30$ & 1.0732 & 10.00 \\
\hline$t .0408$ & 10.56 & 1.0473 & 12.25 & 1.0538 & 13.94 & 1.0603 & 15.03 & I. 0.008 & $17 \cdot 33$ & 1.0733 & 10.03 \\
\hline I. 0409 & 10.58 & I. 0474 & 12.27 & $x .0539$ & 13.96 & 1.0604 & 15.60 & 1.0600 & $17 \cdot 35$ & 3.0734 & 19.05 \\
\hline 1.0410 & $10.6 \mathrm{r}$ & 1.0475 & 12.30 & I. 0540 & I.3.99 & 1.0605 & 15,68 & 1,0670 & $17 \cdot 38$ & 1.0735 & 10.08 \\
\hline 1.0411 & 10.03 & 1.0476 & 12.32 & 1.0541 & 14.07 & 1.0006 & 15.71 & 1.0671 & $37 \cdot 4^{1}$ & 1.0736 & 10.10 \\
\hline 1.0412 & 10.66 & 1.0477 & 12,35 & 1.0542 & 14.04 & 1.0607 & 15,74 & 1.0672 & $17 \cdot 43$ & 1.0737 & 10.13 \\
\hline 1.0413 & 10. 69 & $1.047^{8}$ & 12.38 & $1.05+3$ & 14.07 & 1.0008 & 15.76 & I. 0673 & 17.46 & 1.0738 & 19.16 \\
\hline I. 0414 & 10.71 & I. 0479 & 12.40 & I. 0544 & 14.09 & 1.0609 & 15,79 & 1.0674 & $17.4^{8}$ & 1.0739 & 10,18 \\
\hline 1,0415 & 10,74 & 1.0480 & 12.43 & I. .0545 & 14.12 & I. 0610 & $I 5 \times 8 I$ & $x .0675$ & 17.51 & 1.0740 & $10.2 \mathrm{I}$ \\
\hline $1.04 \times 6$ & 10.76 & I. $048 \mathrm{I}$ & 12.45 & 1.0546 & 14.14 & $1.06 \mathrm{TI}^{1}$ & 15.84 & 1.0676 & 17.54 & 1.0741 & 10,23 \\
\hline 1.0417 & 10.70 & I. 0482 & 12.48 & 1.0547 & 14.17 & $1.06+2$ & 15.87 & I. .0077 & $17-56$ & $x .0742$ & 19.26 \\
\hline 1.0418 & 10.82 & I. .0483 & 12.51 & 1.0548 & $14 \cdot 20$ & 1.0613 & 15.80 & 1.0678 & 17.59 & 1.0743 & 10.29 \\
\hline 1.0470 & 10,84 & I. 0484 & 12.53 & 1.0549 & 1.4 .22 & 1,0614 & 15.92 & 1.0679 & 17,62 & I. 0744 & $19-31$ \\
\hline 1.0420 & 10.87 & I. 0485 & 12.56 & $1.055^{\circ}$ & 14.25 & 1.0675 & 15.94 & 1.0680 & 17.64 & 1.0745 & 50.34 \\
\hline 1.0421 & 10,90 & $1,0,486$ & 12.58 & 1.0551 & 14,28 & 1.0616 & 15,97 & $x, 0681$ & 17.67 & 1.0746 & 19.37 \\
\hline 1.0422 & 10,92 & 1.0487 & 12.61 & 1.0552 & 14.30 & 1.0617 & 16.00 & 1.9682 & 17.60 & 1.0747 & 10.39 \\
\hline 1.0423 & 10.93 & $I .0488$ & 12.64 & I. 0553 & $x+33$ & 1. .0618 & +6.02 & $x .0683$ & 17.72 & 1.0748 & 10.42 \\
\hline 1.0424 & 10.97 & $I .0489$ & 12.66 & 1.0554 & 14.35 & 1.0619 & 16.05 & I. .0684 & 17.75 & I. .0749 & 10.44 \\
\hline 1.0425 & 11.00 & I. 0400 & 12.69 & 1.0355 & 14,38 & $x .0626$ & 16.07 & t. .0685 & 17.77 & 1.0750 & 19.47 \\
\hline I. .0426 & 11.03 & 1.0491 & 12.71 & 1.0556 & $14.4 x$ & $x .0621$ & 16.10 & 1.0686 & 17.80 & $x=075 x$ & 19.50 \\
\hline I. .0427 & 11.05 & $1,0.492$ & 12.74 & 1.0557 & $14: 43$ & 1,0622 & 16.13 & $x .0687$ & 17.83 & 1.0752 & 19.52 \\
\hline 1.0428 & $1 x .08$ & 1. 0403 & +2.77 & 1.0558 & 14.46 & $x .0623$ & 16.15 & 1.0688 & 17.85 & 1.0753 & 19.55 \\
\hline I. 0429 & 11.10 & I. 0494 & 12.79 & 1.0559 & 14.48 & 1.0624 & 16.18 & 1.0689 & 17.88 & $x=0754$ & $\times 9.58$ \\
\hline 1.0430 & $I x \cdot 13$ & I. 0405 & 12.82 & 1.0560 & $\mathrm{r}+4.5 \mathrm{t}$ & 1,0623 & 16.21 & 1.0690 & 17.90 & 1.0755 & 10.60 \\
\hline 1.0431 & 11.15 & 1.0496 & 12.84 & 1.0561 & $14 \cdot 54$ & 1.0626 & 16.23 & $x, 0601$ & 17.93 & 1.0756 & 19.63 \\
\hline I. .0432 & 11,18 & 1.0407 & 12.87 & 1.0562 & 14.56 & $x .0627$ & 16.20 & 1.0692 & 17.95 & 1.0757 & 10.65 \\
\hline 1.0433 & 11.21 & I. 0498 & 12,90 & 1.0563 & 14.50 & I. .0628 & 16.28 & 1.0603 & $17-98$ & $x .0758$ & 19.68 \\
\hline 1.0434 & 11.25 & 1,0499 & 12.92 & $x, 0564$ & 14.61 & 1,0629 & 16.31 & I.0094 & 18.01 & I. 0759 & $x 9.71$ \\
\hline 1.0435 & 11.26 & I. 0500 & 12.95 & 1.0565 & 14.64 & 1.0630 & 16.33 & I. .0695 & 18.03 & 1.0760 & 10.73 \\
\hline 1.0436 & II. 28 & 1.0501 & 12.97 & I. 0566 & 14.67 & $x .0631$ & 16.36 & x. .0696 & 18.06 & $x .076 \mathrm{r}$ & 19.76 \\
\hline 1,0437 & $1 \mathrm{I}-3 \mathrm{I}$ & 1.0502 & 13.00 & 1.0567 & 14.60 & 1.0632 & $16-59$ & 1.0697 & 18.08 & 1.0762 & 19.79 \\
\hline 1.0438 & II $: 34$ & 1.0503 & 13,03 & 1.0568 & 14.72 & 1.0633 & 16.41 & 1. .0608 & 18.11 & $x .0763$ & 19.81 \\
\hline 1.0439 & 11.36 & 1.0504 & 13.05 & 1.0569 & $14 \cdot 74$ & I. .0034 & 16.44 & 1.0099 & 18,14 & 1.0764 & 19.84 \\
\hline $1.044^{\circ}$ & II. 30 & i. .0505 & 13,08 & 1.0570 & 8.77 & 1,0635 & 16.47 & 1.0700 & 18.16 & I. 0765 & 19.86 \\
\hline 1. 044 I & II. 42 & 1.0506 & 13.10 & 1.0571 & 14.80 & I. .0636 & 16.49 & 1.0701 & 18, & 1.0766 & 10.89 \\
\hline 1.0442 & $11: 44$ & 1.0507 & 13,13 & 1.0572 & 14.82 & 1.0637 & 16.52 & 1.0702 & 18.22 & 1.0767 & 19.92 \\
\hline I. .0443 & $\mathrm{xx} \cdot 47$ & 1,0508 & 13.16 & 1.0573 & 14.85 & I. .0638 & 16.54 & 1.0703 & 18,24 & 1.0768 & 10.94 \\
\hline 1. 0444 & 11,49 & I. 0500 & 13.18 & 1.0574 & 14.87 & 1.0639 & 16.57 & 1.0704 & 18.27 & 1.0769 & 19.97 \\
\hline 1.0445 & $x x \cdot 52$ & 1.0510 & 13.21 & 1.0575 & 14.90 & I. .0640 & 16.60 & 1.0705 & I8. 30 & 1.0770 & 20,00 \\
\hline 1.0446 & $1 x, 55$ & 1.0511 & 13.23 & 1.0576 & 14.93 & $x .0641$ & 16,62 & 1.0706 & 18.32 & 1.0771 & 20.02 \\
\hline 1.0447 & $1 \mathrm{I} \cdot 57$ & 1.0512 & 13.26 & 1,0577 & $14 \cdot 95$ & 3.0642 & 16.65 & $1.070 ?$ & 18.35 & 1.0772 & 20.05 \\
\hline I. .0448 & 11.60 & I. .0513 & 13.29 & 1,0578 & 14.98 & 1.0643 & 10.68 & 1.0708 & 18.37 & 1.0773 & 20.07 \\
\hline 1.0449 & 11.62 & I. 0514 & 13.31 & $x .0579$ & 15.00 & 1.0644 & $16.7^{\circ}$ & 1.0709 & 18.40 & 1,0774 & 20.10 \\
\hline I. $045^{\circ}$ & 11.65 & 1.0515 & 13.34 & 1,0580 & 15.03 & 1.0645 & 16.73 & 1.0710 & I 8.43 & 1.0773 & 20,12 \\
\hline $1.045 t$ & $1 \mathrm{x}, 68$ & 1.0516 & 13.36 & $\mathrm{I} .058 \mathrm{I}$ & 15.06 & i. .0646 & 16.75 & 1.0711 & 18,45 & 1.0776 & 20,15 \\
\hline 1.0452 & 11.70 & $1.05 \times ?$ & 13.39 & $x+058 z$ & 15.08 & 1,0647 & $16-78$ & 1.0712 & $18 \cdot 48$ & 1.0777 & 20.18 \\
\hline$x+0453$ & 11.73 & t. .0518 & $13.4^{2}$ & 1.0583 & $15-11$ & $x .0648$ & T 6.80 & $x .0713$ & 18,50 & 1,0778 & 20.20 \\
\hline 1.0454 & $11-75$ & 1. 0519 & $x \cdot 3.44$ & I. .0584 & $x 5 \cdot 14$ & 1.0649 & 16.83 & 1,0714 & 18.53 & 1.0770 & 20.23 \\
\hline
\end{tabular}


EXTRACT IN WINE-(Continued $)$.

\begin{tabular}{|c|c|c|c|c|c|c|c|c|c|c|c|}
\hline $\begin{array}{l}\text { Specific } \\
\text { Gravity. }\end{array}$ & $\begin{array}{l}\text { Ex- } \\
\text { tract. }\end{array}$ & $\begin{array}{l}\text { Specific } \\
\text { Gravity. }\end{array}$ & $\begin{array}{l}\text { Ex- } \\
\text { tract: }\end{array}$ & $\begin{array}{l}\text { Specific } \\
\text { Gravity. }\end{array}$ & $\begin{array}{l}\text { Ex- } \\
\text { tract: }\end{array}$ & $\begin{array}{l}\text { Specific } \\
\text { Gravity. }\end{array}$ & $\begin{array}{l}\text { Ex- } \\
\text { tract. }\end{array}$ & $\begin{array}{l}\text { Specific } \\
\text { Gravity. }\end{array}$ & $\frac{\mathrm{Ex}-}{\text { tract. }}$ & $\begin{array}{l}\text { Specific } \\
\text { Gravity. }\end{array}$ & $\begin{array}{l}\text { Ex- } \\
\text { tract. }\end{array}$ \\
\hline 1.0780 & 20,26 & $x: 0845$ & 27.96 & I. 0970 & 23.67 & 1.0075 & 25.38 & I. 1040 & 27.00 & 1. 1105 & $28,8 \mathrm{r}$ \\
\hline $1.078 \mathrm{t}$ & 20.28 & & $2 x \cdot .99$ & & 23.70 & & 25.41 & & & & \\
\hline$x .0782$ & $20 \cdot 3 x$ & I. 0847 & 22.02 & I. 0012 & 23.72 & I. .0077 & $25 \cdot 43$ & 1.1042 & 27.15 & T.1107 & \\
\hline 1.0783 & 20,34 & 1.0848 & 22.04 & $1.09 \times 3$ & 23.75 & I. .0078 & $25 \cdot 4^{6}$ & 1.1043 & 27.17 & 1.1108 & \\
\hline$x .0784$ & 20.36 & 1.0849 & 22.07 & I. 0914 & 23.77 & I. 0979 & 25.49 & I. 1044 & 27.20 & 1.1100 & 28.91 \\
\hline$t .0785$ & 20,39 & 1.0850 & 23.00 & 1.0913 & 23.80 & 1.0080 & $25.5 x$ & I. 1045 & 27.22 & I. Ttro & 28.04 \\
\hline I. 0786 & $20 \cdot 4 x$ & 1.0 & 22.12 & 1. 0916 & 23.83 & $I .098 x$ & $25 \cdot 54$ & 1. 1046 & 27.25 & 1.1111 & \\
\hline$x .0787$ & 20,44 & 1.0852 & 22.15 & 1.0017 & 23.85 & 1,0082 & 25,56 & 1.1047 & 27.27 & $x+1+12$ & 28.90 \\
\hline$x .0788$ & 20.47 & $x, 0853$ & 22.17 & 1.0018 & 23.88 & 1.0083 & 25.50 & 1.1048 & 27,30 & 1,1213 & 29.02 \\
\hline$x .0789$ & 20.49 & 1.0854 & 22.20 & 1.0919 & $23.9 \mathrm{I}$ & $x .0984$ & 25.62 & 1. 1049 & $27 \cdot 33$ & $x, x 154$ & 29.04 \\
\hline 1.0700 & 20,52 & 1.0855 & 22.22 & $t .0020$ & 23.03 & 1.0085 & 25.64 & 1. 1050 & $27 \cdot 35$ & $1.1+15$ & 29.07 \\
\hline $1: 0701$ & $20 \cdot 55$ & 56 & 22.25 & 1.0021 & 23.90 & 86 & 25.67 & & & & 29.09 \\
\hline 1.0702 & $20 \cdot 57$ & 857 & 22.28 & 922 & 23.09 & 887 & 25.70 & 52 & $27 \cdot 41$ & 17 & 20,12 \\
\hline I. 0793 & 20.60 & 1. .0858 & 22.30 & 1,0023 & 24.01 & I.0088 & 25.72 & 1. 1053 & 27.43 & $1,1+18$ & 20.15 \\
\hline 1.0794 & 20.62 & r. 0859 & 22.33 & 1.0924 & 24.04 & 1.0089 & 25.75 & I. 1054 & $27 \cdot 46$ & $x+1+1=0$ & 29.17 \\
\hline 1.0705 & 20.65 & I. 0860 & 22.36 & 1.0025 & 24.07 & 1,0990 & 25.78 & 1. 1055 & $27 \times 49$ & $I, I 120$ & 20.20 \\
\hline 1.0 & 20 & 1.0 & 22.2 & 26 & 24.09 & I. & 25.80 & 56 & 51 & & $29-23$ \\
\hline 1.07 & $20 \cdot 70$ & I. .0862 & 22.45 & 1.0027 & 24.1 .2 & 1.9 & 25.83 & & .54 & & 20.25 \\
\hline 1.0708 & 20.73 & I. .0863 & 22.43 & 1.0028 & $24 \cdot 14$ & 1.00 & 25.85 & I. 1058 & $27 \cdot 57$ & $1.1 \times 23$ & 29.28 \\
\hline$x .0790$ & 20.75 & I. 0864 & 22.46 & 1.0929 & 24.17 & I. 0994 & 25.88 & 1. 1059 & $27 \cdot 59$ & I. 1124 & 29.31 \\
\hline i. .0800 & 20.78 & & 22.49 & I. 0930 & 24.20 & 1.0995 & & 1.1060 & 27.62 & & 20,33 \\
\hline 1. $080 t$ & $20.8 \pi$ & x. .0866 & $22.5 \mathrm{r}$ & 1.0031 & 24.22 & $1,0 r$ & .93 & I. $x 06 x$ & $27 \cdot 65$ & 1.1 & 20.36 \\
\hline 1. .0802 & 20,83 & 1.0867 & 22,54 & 1,0032 & 24,25 & 1.0097 & 25.96 & 1. 1062 & 27.67 & 1. 1127 & 20.39 \\
\hline 1.0803 & 20.86 & 1.0868 & 22.57 & 1. .0933 & 24.27 & $x .0008$ & 25.00 & I. 1063 & $27 \cdot 70$ & $1.11 \geq 8$ & 20.41 \\
\hline 1,0804 & 20,89 & 1.0869 & 22.50 & 1.0934 & 24.30 & 1.0999 & $26.0 \mathrm{r}$ & x. $x p 64$ & $27 \cdot 72$ & 1.1129 & 29.44 \\
\hline 1.0805 & $20.9 \mathrm{r}$ & $1.0870^{\circ}$ & 22.62 & & 24.33 & $I .1$ & .04 & 1065 & & 1,1130 & \\
\hline 1.0806 & 20.94 & $1.087 x$ & 22.65 & 1.0036 & 24.35 & I. 1 & 26.06 & 1. 1066 & 27.78 & 1.1131 & 29.40 \\
\hline 1,0807 & 20.96 & 1,0872 & 22.67 & I. 0037 & 24.38 & 1,1002 & 26.00 & I. 1067 & 27.80 & 1.1132 & 20.52 \\
\hline x. 0808 & 20.90 & I. 0873 & 22.70 & 1.0038 & 24.41 & 1. 1003 & 26.12 & 1. 1068 & 27.83 & $x, x+33$ & 29.54 \\
\hline 1.0809 & 21.02 & $x, 0874$ & 22.72 & 1.0939 & 24.43 & I. 1004 & 26.14 & I. 1069 & 27.86 & 1.1134 & 29.57 \\
\hline I. 0810 & $21 \cdot 04$ & I. .0875 & 22.75 & $x, 0040$ & $24 \cdot 46$ & 1.1005 & 26.17 & I. 1 C & 27.88 & 1.1135 & 29.60 \\
\hline $\mathrm{x}, 08 \mathrm{xt}$ & 21.07 & 1.0876 & 22.78 & $\mathrm{x} .094 \mathrm{r}$ & 24.49 & 1. 1006 & 20,20 & I. & 27.96 & 1. 1536 & 29,62 \\
\hline 1.0812 & 21.10 & 1.0877 & 22.80 & 1.0942 & $24 \cdot 51$ & 1.1007 & 26,22 & 1,1072 & 27.93 & 1,1137 & 29.65 \\
\hline $1.08 x_{3}$ & $2 x-12$ & 1.0878 & 22.83 & I. 0943 & 24.54 & $x+1008$ & 26.25 & I. 1073 & 27.96 & 1.1138 & 39.68 \\
\hline 1,0814 & 21,15 & 1.0879 & 22.86 & t. .0944 & $24 \cdot 57$ & 1.1009 & 26.27 & I. 1074 & 27.90 & 1,1139 & 29.70 \\
\hline 1.08 & $21, x 7$ & t. 0880 & 22.88 & I. .0945 & $24 \cdot 59$ & I.toro & 26.30 & I. 1075 & 28.01 & 1.1140 & \\
\hline 1,0 & 21.20 & 81 & 22.01 & t. 0046 & 24.62 & 1.10 & 26.33 & 76 & .04 & I. $114 \mathrm{t}$ & 29.76 \\
\hline $1.08 \times 7$ & $21 \cdot 23$ & 1.0882 & 22.93 & $x .0947$ & 24.64 & 1.1012 & 26.35 & I. 1077 & 28.07 & I. 1142 & $29: 78$ \\
\hline I. .0818 & 21,25 & 1. 0883 & 22,06 & I. .0048 & 24.67 & t. $x 013$ & 26.38 & I. 1078 & 28.09 & t. I 443 & $29.8 \mathrm{I}$ \\
\hline t. $08 \mathrm{rg}$ & 21.28 & I. 0884 & 22,99 & I. 0949 & 24.70 & 1,1014 & 26.41 & 1,1079 & 28.12 & I. 1144 & 29.83 \\
\hline I. O & & 85 & & & 24,72 & $x, x$ & & & & & 86 \\
\hline $.082 \mathrm{r}$ & $2 x+3,3$ & 86 & 23.04 & $1.005 \mathrm{x}$ & 24.75 & I. 10 & .46 & 1. $108 \mathrm{i}$ & 37 & $1,11,46$ & 29.89 \\
\hline 1.0822 & 21.36 & .0887 & 23.07 & 1.0952 & $24 \cdot 78$ & I. IO & $26 \cdot 49$ & $x+1082$ & 28.20 & 1.1147 & 20.01 \\
\hline$x .0823$ & $21 \cdot 38$ & .0888 & 23.00 & i. .0053 & 24.80 & I. 1018 & 26.51 & 1. 1083 & 28.22 & 1.1148 & 29.94 \\
\hline 1.0824 & $21 \cdot 41$ & 1.0889 & 23.12 & 1.0054 & 24.83 & x. 1019 & 26.54 & I. 1084 & 28.25 & $x, 1 \times 49$ & 29.96 \\
\hline 1.08 & 44 & -08 & & & 24.85 & 1.10 & & I. & .28 & I. .17 & \\
\hline 1.0826 & $2 x \cdot 4^{6}$ & .0891 & 23.17 & 1.0 & 24.88 & 1.10 & 26.59 & 086 & 28.30 & t. $1 \times 5 t$ & 30.02 \\
\hline 1.0827 & 21.49 & .0892 & 23.20 & I. 0057 & 24.91 & 1,1022 & 26.62 & $1, \operatorname{ros} 7$ & $28 \cdot 33$ & I. $1 \times 52$ & 30.04 \\
\hline 1.0828 & $21 \cdot 52$ & z. 0893 & 23.22 & 1.0058 & 24.03 & 1.1023 & 26.64 & $x, 1088$ & 28.36 & 5.1153 & 30.07 \\
\hline 1.0820 & $21 \cdot 54$ & 1.0894 & 23.25 & 1.0959 & 24.96 & 1.1024 & 26.67 & 1. 1089 & $28 \cdot 38$ & 1. 1154 & 30,10 \\
\hline 1.0 & 1.57 & .08 & 3.28 & .0060 & & & & & & & \\
\hline$I .0831$ & $21 \cdot 59$ & .0896 & $23 \cdot 30$ & $x .096 \mathrm{x}$ & 25.01 & I. 10 & 72 & & 28.43 & 56 & 30.15 \\
\hline 7.0832 & 21.62 & .08 & 23.33 & x.096a & 25.04 & $x . x 027$ & 26.75 & $I, x c$ & $28 \cdot 40$ & $1 \cdot x$ & 30.18 \\
\hline 1.0833 & 21.65 & 1.08 & 23,35 & I. .0963 & 25.07 & 1. 1028 & 26.78 & 1. 1003 & 28.49 & 1.1158 & 30,21 \\
\hline$x .0834$ & 21.67 & 2.0899 & 23.38 & 1.0964 & 25.09 & 1.1029 & 26.80 & I. 1094 & 28.51 & 1.1159 & 30.23 \\
\hline 1.0 & 1.70 & 1 & & & & 1030 & & & & & \\
\hline 1. $=0$ & $1-73$ & $\mathrm{x} .090 \mathrm{r}$ & 3.43 & 1. 0966 & $25 \cdot 14$ & & & I. IO & & & \\
\hline & $2 t \cdot 75$ & I. 0902 & 23.46 & 1.0067 & 25,17 & 1.1032 & 26.88 & x. 1097 & & & \\
\hline 1.0 & 21.78 & 1.090 & 23.49 & I. .0968 & 25.20 & 1.1033 & 26.91 & $1 . x \circ 98$ & & & \\
\hline 1.0839 & 21.80 & 1. 0904 & 23.51 & 1. 0969 & $25 \cdot 22$ & I. 1034 & 26.93 & 1.1099 & 28.65 & & \\
\hline & .83 & & & & & & & & & & \\
\hline & 1.86 & .0906 & 23.57 & 1.0971 & 25.28 & 1.1036 & 26.99 & I. IIOI & $28 \cdot 70$ & & \\
\hline 1.0 & 1.88 & .0907 & -23.50 & 1.0972 & 25.30 & 1.1037 & 27.01 & 1.1102 & & & \\
\hline 1.0843 & & & 23.62 & 1.0673 & 25.33 & 1,1038 & 27.04 & 1.1103 & & & \\
\hline 1.0844 & $2 x \cdot 94$ & 1.0909 & 23.65 & 1.0024 & $25: 36$ & 1. 1039 & 27.07 & I. 1104 & & & \\
\hline
\end{tabular}




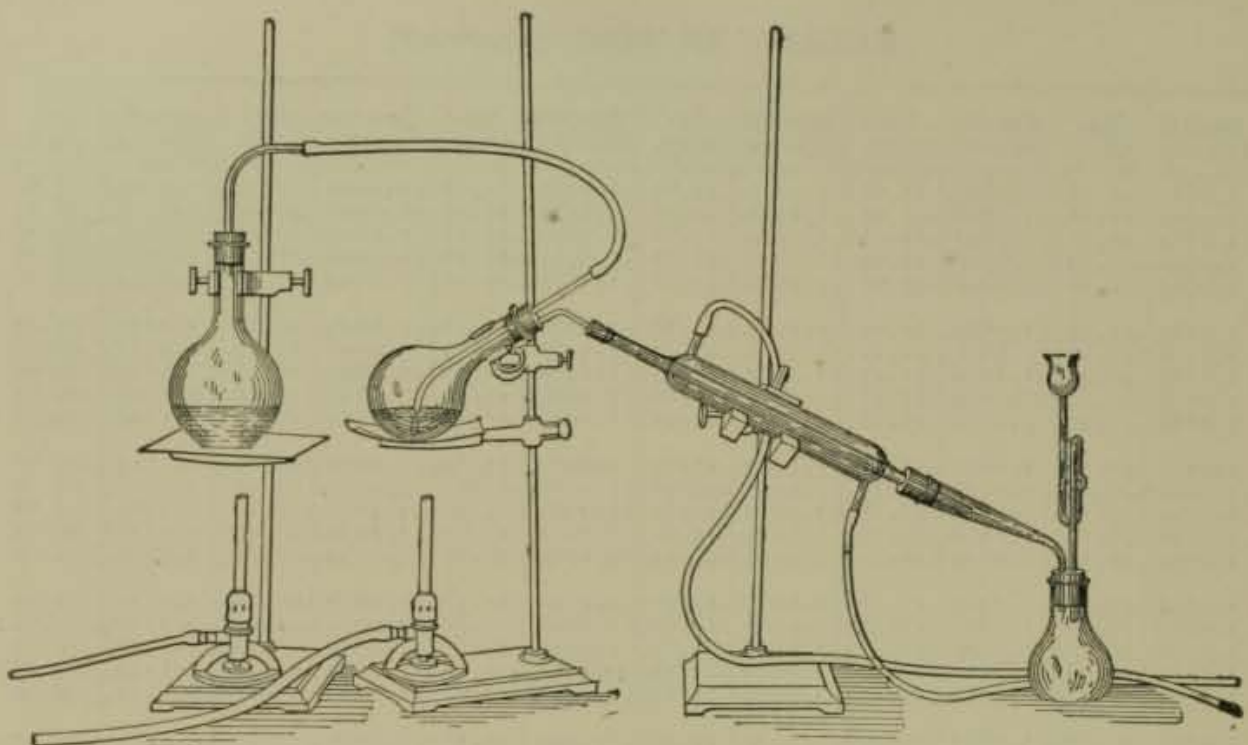

Fig. 114-Apparatus for Determining Volatile Acids in Wine.

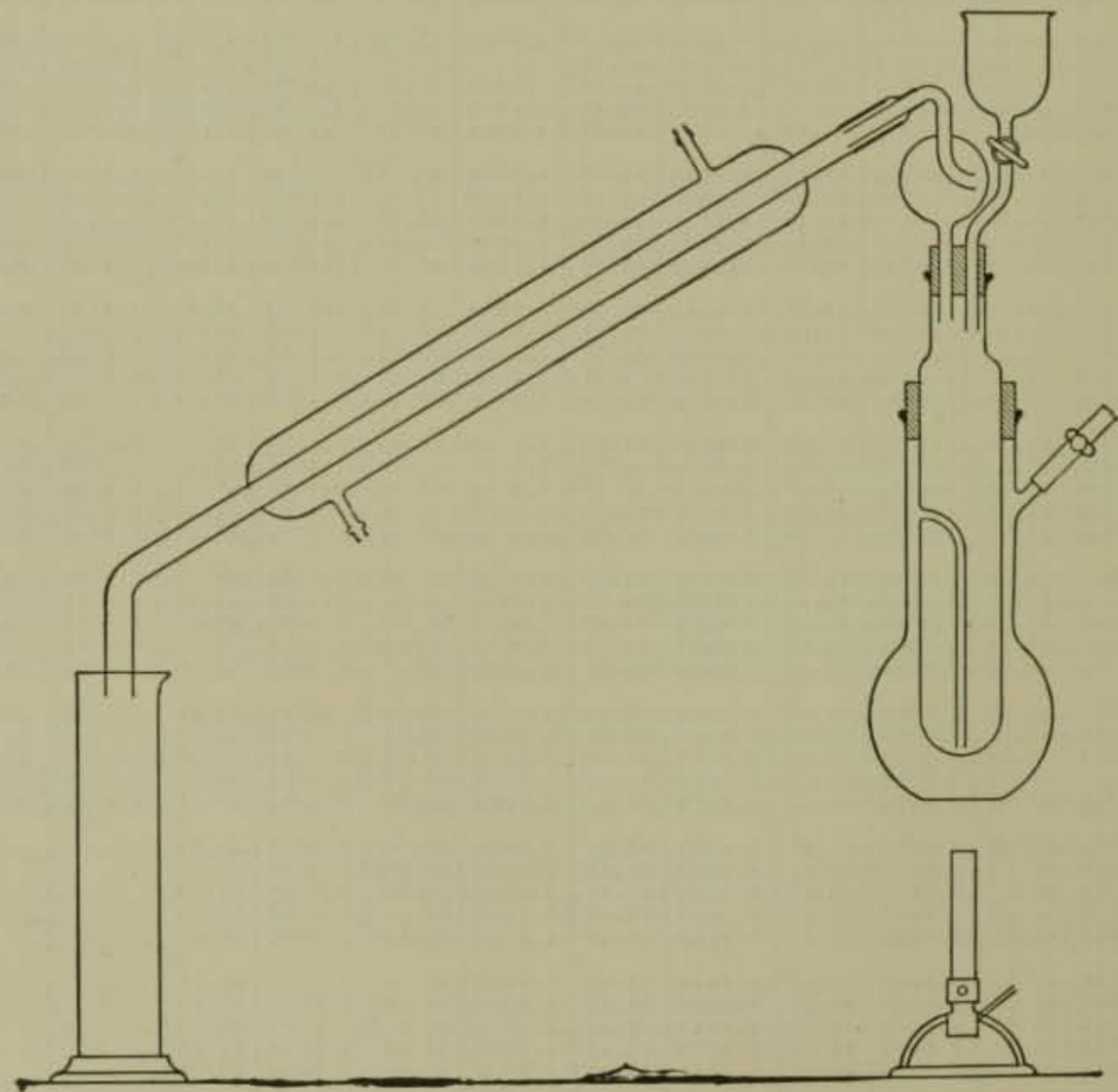

Fig. II 5.-Hortvet's Apparatus for Determining the Volatile Acids in Wine. 
as an indicator. Each cubic centimeter of tenth-normal alkali is equivalent to 0.006 gram acetic acid.

Hortvet Method.*-The apparatus (Fig. II5) consists of a $300 \mathrm{cc}$. flask into the neck of which is fitted a $200-\mathrm{cc}$. cylindrical flask, with a steam tube, a bulb-trap leading to a condenser, and a stop-cock funnel. The procedure is as follows: Pour $150 \mathrm{cc}$. of recently boiled water into the larger flask, attach the smaller flask by means of a section of rubber tubing, run in to cc. of wine (previously freed from carbonic acid), close the stop-cock and boil. In extreme cases add to the wine a small piece of paraffin to prevent foaming. When the water has boiled a moment, close the tube at the side of the larger flask and distil until $70 \mathrm{cc}$. of distillate have passed over. Transfer to a beaker, without discontinuing the distillation, and titrate, using phenolphthalein as indicator. Continue the distillation until the last ro cc. portion requires not more than one drop of tenth-normal alkali for neutralization. Usually 80 or $90 \mathrm{cc}$. of distillate includes practically all of the volatile acids. Cool the apparatus, thus allowing the wine residue to be drawn back into the lower flask, rinse with boiled water, and reserve the total liquid for determination of non-volatile acids.

Non-volatile Acids. - These may be determined by difference, calculating the voli ile acids for purposes of subtraction in terms of tartaric or other acid in which the total acidity is expressed. Non-volatile acid may be directly determined by evaporating to dryness a measured portion of the liquor, boiling the residue with water, and titrating the solution with the standard alkali.

Detection of Free Tartaric Acid.-Nessler's Method.-Some powdered cream of tartar is added to a portion of the wine in a corked flask, which is shaken from time to time, and the liquid finally filtered. To the filtrate is added a little $20 \%$ potassium acetate solution. If free tartaric acid is present, on stirring and especially after standing for some time, there will be a precipitate of cream of tartar.

Determination of Tartaric Acid, Total, Free, and Combined.-Provisional methods A. O. A. C. $†$

Total Tartaric Acid.-To too cc. of wine add $2 \mathrm{cc}$. of glacial acetic acid, 3 drops of a $20 \%$ solution of potassium acetate, and $I_{5}$ grams of powdered potassium chloride, and stir to hasten solution. Add $\mathrm{I}_{5} \mathrm{cc}$. of $95 \%$ alcohol (specific gravity $0.8 \mathrm{I}$ ) and rub the side of the beaker vigorously wi h a glass rod for about one minute to start crystallization.

* Jour. Ind. Eng. Chem., r, rgog, p. 3r

$\dagger$ U. S. Dept. of Agric,, Bur. of Chem., Bul. 65, F. 87 . 
Let stand at least fifteen hours at room iemperature; decant the liquid from the separated acid potassium tartrate as rapidly as possible (using vacuum) through a Gooch crucible prepared with a very thin film of asbestos, transferring no more of the precipitate to the crucible than necessary. Wash the precipitate and filter tree times with a small amount of a mixture of $1_{5}$ grams potassium chloride, $20 \mathrm{cc}$. of $95 \%$ alco hol (specific gravity $0.8 \mathrm{I}$ ), and $100 \mathrm{cc}$. water, using not more than $20 \mathrm{cc}$. of the wash solution in all. Transfer the asbestos film and precipitate to the beaker in which the precipitation took place, wash out the Gooch crucible with hot water, add about $50 \mathrm{cc}$. of hot water, heat to boiling, and titrate the hot solution with decinormal sodium hydroxide, using delicate litmus tincture or litmus paper as indicator. Increase the number of cubic centimeters of decinormal alkali employed by $\mathrm{I} .5$ on account of the solubility of the precipitate. This figure multiplied by 0.015 gives the amount of total tartaric acid in grams per $100 \mathrm{cc}$.

Cream of Tartar.-Ignite the residue obtained from the evaporation of $50 \mathrm{cc}$. of wine as directed under the determination of ash. Exhaust the ash with hot water, add to the filtrate $25 \mathrm{cc}$. of decinormal hydrochloric acid, heat to incipient boiling, and titrate with decinormal alkali solution, using litmus as indicator. Deduct from $25 \mathrm{cc}$. the number of cubic centimeters of decinormal alkali employed, and multiply the remainder by 0.0188 for potassium bitartrate expressed in grams.

Free Tartaric Acid.-Add $25 \mathrm{cc}$. of decinormal hydrochloric acid to the ash of $50 \mathrm{cc}$. of wine, heat to incipient boiling, and titrate with decinormal sodium hydroxide, using litmus as indicator. Deduct the number of cubic centimeters of alkali employed from 25, and multiply the remainder by 0.0075 to obtain the amount of tartaric acid necessary to combine with all the ash (considering it to consist entirely of potash). Deduct the figure so obtained from the total tartaric acid for the free tartaric acid.

Determination of Free and Combined Malic Acid in Cider and Wine. -Evaporate $100 \mathrm{cc}$. of the sample on the water-bath to half its volume, cool, and treat first with 10 cc. of $10 \%$ calcium chloride solution, and then with ammonia to strong alkaline reaction. Let stand for an hour and filter. This removes the tartaric acid. Concentrate the filtrate by evaporation on the water-bath to $25 \mathrm{cc}$., add $75 \mathrm{cc}$. of $95 \%$ alcohol, heat to the boiling-point, and filter. Wash the residue with a mixture of 3 parts $95 \%$ alcohol and I part water, dry, and burn to an ash. Add $25 \mathrm{cc}$. of tenth-normal hydrochloric acid to the ash, dilute with water, 
heat to boiling, and titrate with tenth-normal sodium hydroxide, using phenolphthalein as an indicator. Multiply the difference between 25 and the number of cubic centimeters required to neutralize by 0.0067 for the grams of malic acid.

Polarization.-Treat a measured amount of wine or cider with onetenth of its volume of lead subacetate, filter and polarize the filtrate in the $200 \mathrm{~mm}$. tube. The reading is increased by $10 \%$ for the true direct polarization.

If the reducing sugars are also to be determined, the same solutions may be used for both the polarization and the reducing sugars as follows:

Exactly neutralize with sodium hydroxide solution $200 \mathrm{cc}$. of the wine, using litmus paper as an indicator, and evaporate on the water-bath to about one-fourth its original volume. Wash with water into a $200 \mathrm{cc}$. flask, add enough normal lead acetate solution to clarify, and make up with water to the mark. Filter and to the filtrate add powdered sodium sulphate or carbonate sufficient to precipitate the lead, again filter and polarize before and after inversion (p. $5^{88}$ ).

Determination of Reducing Sugars.-Determine reducing sugars in portions of the wine treated as described in the preceding section, after dilution so as not to contain above $0.5 \%$ of sugar for the Defren and the Munson and Walker methods or above $I \%$ of sugar for the Allihn method. One may assume $2 \%$ as the sugar-free extract of wine, the number of volumes of water to be added to the filtrate being determined by the difference between 2 and the total extract as determined.

Determination of Glycerin.-In Dry Wines.-Evaporate Ioo cc. of the wine in a porcelain dish on the water-bath to about ro cc., add about 5 grams of fine sand and from 3 to $4 \mathrm{cc}$. of milk of lime (containing about I5\% of calcium oxide) for each gram of extract present and evaporate nearly to dryness. Treat the moist residue with $50 \mathrm{cc}$. of $95 \%$ (by vol.) alcohol, remove the substance adhering to the sides of the dish with a spatula, and rub the whole mass to a paste. Heat on a water-bath, with constant stirring, to incipient boiling and decant through a filter into a small flask. Wash by decantation with to cc. portions of hot $95 \%$ alcohol until the filtrate amounts to about $150 \mathrm{cc}$. Evaporate the filtrate to a sirup on a hot, but not boiling, water-bath, transfer to a small glassstoppered graduated cylinder with $20 \mathrm{cc}$. of absolute alcohol, and add 3 portions of to cc. each of absolute ether, shaking throughly after each addition. Let stand until clear, then pour off through a filter and wash 
the cylinder with a mixture of absolute alcohol and absolute ether ( 1 : I.5), pouring the wash liquor also through the filter. Evaporate the filtrate to a sirup, dry for one hour in a boiling water oven, weigh, ignite, and weigh again. The loss on ignition gives the weight of glycerin.

In Sweet Wines.-If the extract exceeds $5 \%$ heat $100 \mathrm{cc}$. to boiling in a flask and treat with successive small portions of milk of lime until the color becomes at first darker and then lighter. When cool add $200 \mathrm{cc}$. of $95 \%$ alcohol, allow the precipitate to subside, filter, and wash with $95 \%$ alcohol. With the filtrate thus obtained proceed as directed for dry wines

Determination of Potassium Sulphate.-Acidify roo cc. of the sample with hydrochloric acid, heat to boiling, and add an excess of barium chloride solution. Filter, wash, dry, ignite, and weigh as barium sulphate, calculating the equivalent of potassium sulphate. The presence of the latter in excess of 0.06 gram per $100 \mathrm{cc}$. indicates plastering.

Determination of Tannin.-An approximate method of determining tannin is that of Nessler and Barth. I2 cc. of wine are treated with 30 cc. of alcohol and filtered. $35 \mathrm{cc}$. of the filtrate, which corresponds to Io cc. of the wine, is evaporated to about $6 \mathrm{cc}$. and transferred to a ro-cc. graduated centrifuge tube. A few drops of $40 \%$ sodium acetate are then added, and a slight excess of $10 \%$ ferric chloride. The tube is corked, gently agitated, and allowed to stand for twenty-four hours. The volume of the precipitate is then measured, each cubic centimeter being equivalent to $0.033 \%$ of tannin in the wine.

Foreign Coloring Matters in Wine.-A wide variety of artificial colors have been found in red wine. Those most commonly employed at present are cochineal, fuchsin, and acid fuchsin.

The Pharmacopœia prescribes the following color tests:

If $2 \mathrm{cc}$. of red wine be mixed in a test-tube with 2 drops of chloroform and $4 \mathrm{cc}$. of normal potassium hydroxide, and the mixture carefully heated, the disagreeable odor of isonitril should not become preceptible (absence of various anilin colors).

If $50 \mathrm{cc}$. of red wine be treated with a slight excess of ammonia water, the liquid should acquire a green or brownish-green color; if it be then well shaken with $25 \mathrm{cc}$. of ether, the greater portion of the ethereal layer removed and evaporated in a porcelain capsule with an excess of acetic acid and a few fibers of uncolored silk, the latter should not acquire a crimson or violet color (absence of fuchsin).

If $25 \mathrm{cc}$. of red wine heated to about $45^{\circ} \mathrm{C}$. be well agitated with 25 grams of manganese dioxide, the liquid filtered off and acidulated with 
hydrochloric acid, it should not acquire a red color (absence of sulphofuchsin).

Duprê's Method of Detection.*-Small cubes of jelly measuring about $2 \mathrm{~cm}$. in thickness are prepared as follows: Dissolve I part of pure gelatin in to parts boiling water and pour upon a plate to harden. This is then cut into cubes of the above size by a sharp knife. When a wine is suspected of containing foreign color, one of the cubes is immersed therein and allowed to remain for twenty-four hours, after which it is removed, washed slightly in cold water, and cut through with a knife. If the color is a natural one, it will lightly tinge the outer surface of the ct:be, but will not permeate far below the surface, so that the inner portion of the cross-section will be largely free from color. Nearly all foreign coloring matters used in wine, such as most coal-tar dyes, cochineal, Brazil wood, logwood, etc., will be found to deeply permeate the jelly cube often to the center. Information as to the nature of the color may sometimes be gained by immersing the dyed jelly cube in weak ammonia. If the color be rosanilin, the cube is decolorized, if cochineal, a purple coloration will result, and if logwood, a brown tinge.

Cazeneuve's Methods for Detecting Colors in Wine.-While by no means complete, the following method of Cazeneuve as condensed and arranged by Gautier (La Sophistication des Vins) will often be found helpful. If other colors than these are evidently present, tests should be made as indicated in Chapter XVII. Cazeneuve employs the following reagents:

(1) Yellow oxide of mercury, finely pulverized.

(2) Lead hydrate, freshly precipitated, well washed, suspended in about twice its volume of water; to be kept in a stoppered bottle; should be renewed after several days' use.

(3) Gelatinous ferric hydrate, well washed from ammonia, suspended in about twice its volume of water.

(4) Manganese dioxide, pulverized.

(5) Concentrated, chemically pure sulphuric acid.

(6) White wool.

(7) Stannous hydrate, freshly precipitated, well washed, suspended in water, and kept from exposure to light and air.

(8) Collodion silk, the artificial silk produced from nitro-cellulose. This fiber has a special affinity for basic dyes.

* Jour. Chem. Soc., 37, p. 572. 
To ro cc. of the wine are added 0.2 gram finely powdered yellow oxide of mercury. Boil and pour upon a double filter.

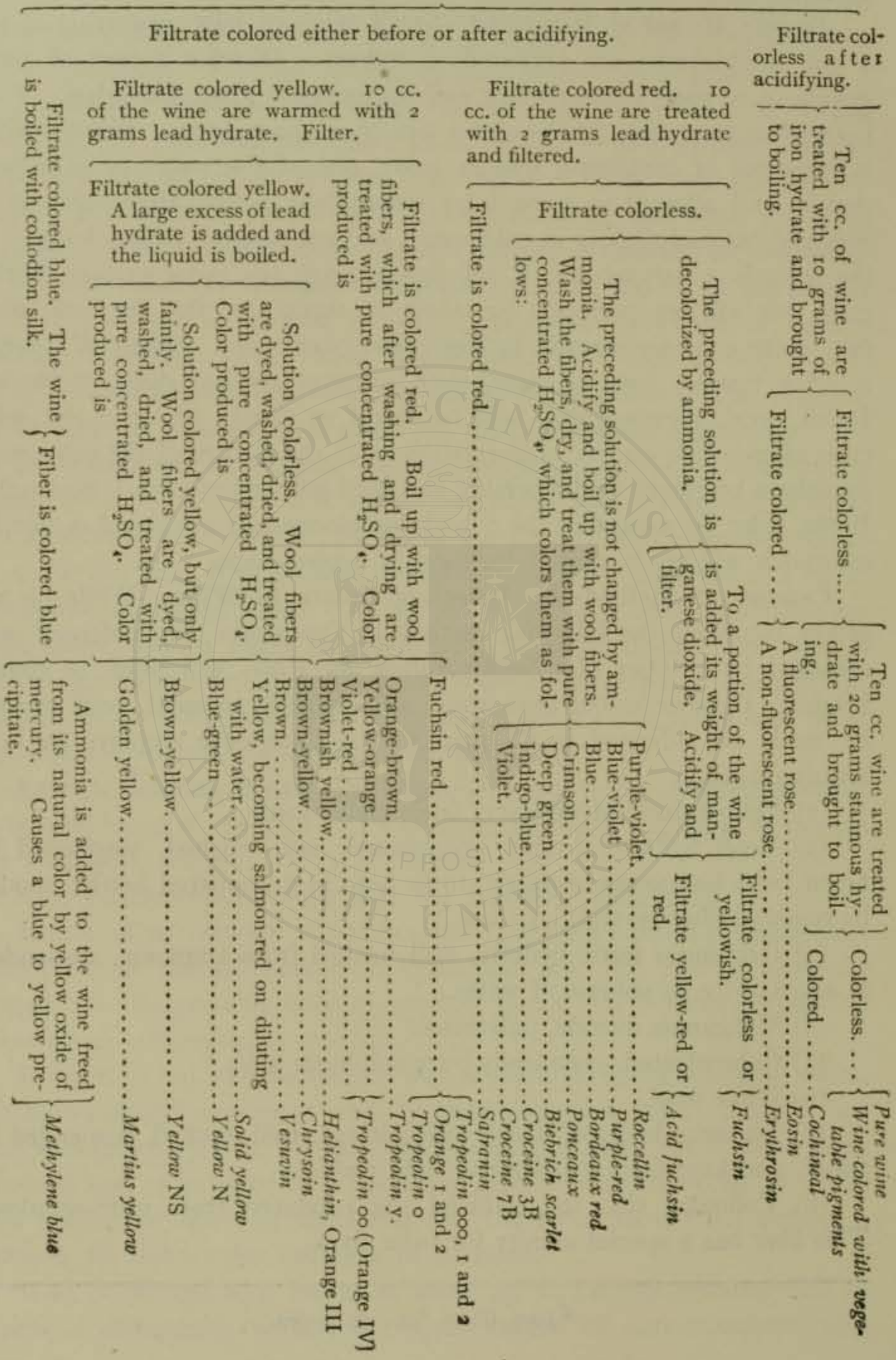




\section{MALT LIQUORS. BEER.}

In its widest sense beer may be defined as the product of fermentation of an infusion of almost any farinaceous grain with various bitter extractives, but unless otherwise qualified it should be strictly applied to the beverage resulting from the fermentation of malted barley and hops. In the manufacture of beer two distinct processes are employed, viz., malting or sprouting the grain, and brewing. Many brewers do nothing but the latter, buying their malt already prepared.

Malting.-For the preparation of malt, the barley is steeped in water for several days, after which the water is drained off and the moist grain is "couched," or piled in heaps, on a cement floor, where it undergoes a spontaneous heating process, during which it germinates, forming the ferment diastase. When the maximum amount of diastase has been produced, indicated by the length of growth of the sprout, or " acrospire" within the grain, the germination is checked by spreading the grain in layers over a perforated iron floor, and finally subjecting it to artificial heat. The character of the malt and of the beer produced from it depends largely on the heat at which the "green" malt is kiln dried. If dried between $32^{\circ}$ and $37^{\circ} \mathrm{C}$. it forms pale malt, which produces the lightest grades of beer. Most beer is made from malt dried at higher temperatures, say from $38^{\circ}$ to $50^{\circ}$, the depth of color of the liquor varying with the heat to which the malt has been subjected, while the color of the malt varies from the "pale" through the "amber" to "brown," or even black. The darkest grades are sometimes dried at temperatures over $100^{\circ} \mathrm{C}$., even to the point where the starch becomes caramelized.

A more modern method consists in the so-called pneumatic malting, wherein the whole operation is conducted in a large rotating drum, which holds the grain, and in which the temperature and moisture at different stages is carefully controlled by the admission to the interior of the drum of moisture-laden or dry air, heated to the required degree.

The chief object of malting is the production of diastase, which by its subsequent action on the starch converts it into the fermentable sugars maltose and dextrin. Malt contains much more diastase than is necessary to convert the starch simply contained therein to maltose, and is capable of acting on the starch of a considerable quantity of raw grain, such as corn or rice, when mixed with it. This practice of using other grains than malt is prohibited in some localities, such as Bavaria. 
Brewing.-The malt, or mixture of malt and raw grain, is first crushed and "mashed" by stirring with water in tubs at $50^{\circ}$ to $60^{\circ} \mathrm{C}$, finally heating to $70^{\circ}$. During this process the conversion of the starch to maltose and dextrin takes place. The resulting liquor is known as "wort," containing, besides maltose and dextrin, peptones and amides. The clear wort is then drawn off from the residue, and boiled to concentrate the product and to sterilize it, after which hops (the female flower of the Humulus lupulus) are added and the boiling continued. Hops contain resins, bitter principles, tannic acid, and a peculiar essential oil, all of which are to some extent imparted to the wort. After cooling and settling, the clear wort is run into fermenting-vats, where selected yeast, usually saccharomyces cerevisia, is added, and the alcoholic fermentation allowed to proceed. The temperature greatly affects the character of the fermentation. If kept between $5^{\circ}$ and $8^{\circ} \mathrm{C}$., a slow fermentation proceeds, known as bottom fermentation, during which the yeast settles out at the bottom. This is much more easily controlled than the quick or top fermentation, which takes place at from $15^{\circ}$ to $18^{\circ}$, much of the yeast in the latter case being carried to the surface, from which it is finally removed by skimming. In either case the yeast feeds upon the albuminous matter present. At the proper stage the beer is drawn off from the larger portion of the yeast, and run into casks, or tuns, in which an after-fermentation proceeds. The beer is finally clarified by treatment with gelatin or beech shavings or chips, to which the floating yeast-cells and other impurities attach themselves. It is finally stored in barrels coated with brewers' pitch, or pasteurized at $60^{\circ} \mathrm{C}$. and bottled.

Varieties of Beer.-Formerly the division of beers into "lager," "schenk," and "bock" was made by reason of the fact that beer had to be brewed under certain climatic conditions and at certain seasons only. Now, with improved means for artificial refrigeration, and with better methods controlling all stages of the process, these distinctions are less marked.

Lager Beer (from lager, a storehouse) is a term originally applied to Bavarian beer, but is now given to any beer that has been stored several months. Formerly lager beer was made early in the winter, and stored in cool cellars till the following spring or summer, during nearly all of which time a slow after-fermentation took place. The best lager beers contain a low proportion of hops, and are high in extract and alcohol.

Schenk Beer is a quickly fermented beer made in winter for immedi- 
ate use. It is brewed in from four to six weeks and will not keep long without souring.

Bock Beer, according to older systems of nomenclature, occupied a middle place between lager and schenk, being an extra strong beer brewed for spring use and made in limited quantities, not being intended for storage.

Berlin Weiss Bier is prepared by the quick or top fermentation of a wort consisting of a mixture of malted barley and wheat with hops. It is high in carbon dioxide, being usually bottled before the second fermen. tation has ended.

Ale is virtually the English name for beer. It is usually lighter colored than lager beer, being made from pale malt by quick or top fermentation, and containing rather more hops than beer. It has a high content of sugar, due to checking fermentation at an earlier stage than in ordinary beer.

Porter is a dark ale, the deep color of which should be due to the use of brown malt dried at a high temperature, but which is sometimes colored by the admixture of caramel. It has a large extract, chiefly sugar.

Stout is an extra-strong porter, being high both in alcohol and extract.

Composition of Beer.-Beer is a somewhat complex liquor. Besides water, alcohol, and sugar, it contains carbon dioxide, succinic acid, dextrin, glycerin, tannic acid, the resinots bitter principles of hops, nitrogenous bodies (chiefly peptones and amides), alkaline and lime salts (chiefly phosphates), fat (traces), aceic acid and lactic acid. The latter acid constitutes the chief fixed acid of beer.

The following analyses of different varieties of beer are due to König:

\begin{tabular}{|c|c|c|c|c|c|c|c|c|c|c|c|c|c|}
\hline Variety. & 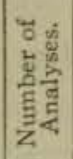 & 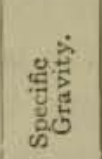 & 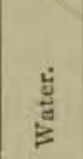 & 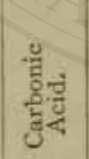 & $\frac{\hat{a}}{3}=$ & 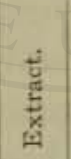 & 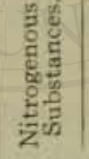 & 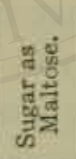 & 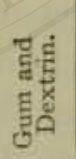 & 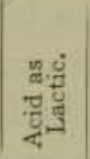 & $\begin{array}{l}\frac{E}{5} \\
\frac{\delta}{0} \\
\text { है }\end{array}$ & $\frac{d}{4}$ & 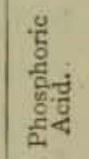 \\
\hline Schenk. & 205 & 1.0114 & 91.11 & 0.197 & $3 \cdot 36$ & $5 \cdot 34$ & 0.74 & 0.95 & 3.11 & 0.156 & 0.12 & 0.20 & \\
\hline & $25^{8}$ & 1.0162 & 90.08 & 0.196 & 3.93 & $5 \cdot 79$ & 0.71 & 0.88 & 3.73 & 0.151 & 0.165 & 0.228 & 0.0 \\
\hline Export beer & 109 & 1.0176 & 89.01 & 0.209 & 4.40 & $6.3^{8}$ & 0.74 & 1.20 & 3.47 & 0.1 & 0.1 & 0.247 & \\
\hline Bock. & 84 & 1.0213 & $87.8 \%$ & 0.234 & 4.69 & $7.2 \mathrm{I}$ & 0.73 & I. $8 \mathrm{I}$ & 3.97 & 0.165 & 0.17 & 0.263 & \\
\hline Weiss bier. & 26 & I. 0137 & 91.63 & 0.297 & 2.73 & $5 \cdot 34$ & $0.5^{8}$ & 1.62 & 2.42 & $0.39^{2}$ & 0.092 & 0.149 & \\
\hline & 40 & 1.0191 & 88.49 & 0.215 & 4.70 & 6.59 & 0.65 & 2.62 & 3.08 & $0.28 \mathrm{I}$ & & 0.363 & 0.0 \\
\hline Ale. & 38 & I. 0141 & $80.4^{2}$ & 0.201 & 4.75 & 5.65 & $0.6 \mathrm{x}$ & I. .07 & 1. 81 & 0.278 & & 0.31 & \\
\hline
\end{tabular}

Fifteen samples of lager beer and seven samples of pale ale, bou hat in Massachusetts bar-rooms, representing as nearly as possible the quali; $y$ 
of liquor sold every day to patrons by the bottle or glass, were analyzed by the Board of Health with the following results:

\begin{tabular}{|c|c|c|c|c|}
\hline & * & $\begin{array}{l}\text { Per Cent of } \\
\text { Original Wort } \\
\text { Extract. }\end{array}$ & $\begin{array}{l}\text { Per Cent of } \\
\text { Alcohol by } \\
\text { Weight. }\end{array}$ & $\begin{array}{l}\text { Per Cent of } \\
\text { Extract. }\end{array}$ \\
\hline $\begin{array}{l}\text { Beer- } \\
\text { Pale ale- }\end{array}$ & 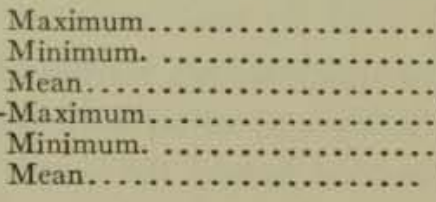 & $\begin{array}{r}18.91 \\
7.33 \\
15.04 \\
15.99 \\
10.95 \\
13.56\end{array}$ & $\begin{array}{l}7.07 \\
1.10 \\
4 \cdot 45 \\
5 \cdot 37 \\
3 \cdot 53 \\
4 \cdot 49\end{array}$ & $\begin{array}{l}7 \cdot 76 \\
3 \cdot 67 \\
5 \cdot 9^{2} \\
5 \cdot 47 \\
3 \cdot 3^{8} \\
4 \cdot 54\end{array}$ \\
\hline
\end{tabular}

Five out of the 15 beer samples and 3 out of the 7 ale samples contained salicylic acid.

The percentage composition of the ash of German beer is thus given by König as the mean of ig analyses:

\begin{tabular}{|c|c|c|c|c|c|c|c|c|c|}
\hline $\begin{array}{l}\text { Ash in } \\
\text { roo Parts } \\
\text { Beer. }\end{array}$ & Potash. & Soda. & Lime. & Magnesia. & $\begin{array}{c}\text { Iron } \\
\text { Oxide. }\end{array}$ & $\begin{array}{l}\text { Phos- } \\
\text { phoric } \\
\text { Acid. }\end{array}$ & $\begin{array}{l}\text { Sul- } \\
\text { phuric } \\
\text { Acid. }\end{array}$ & Silica. & Chlorin \\
\hline 0.306 & 33.67 & 8.94 & $2 \cdot 7^{8}$ & 6.24 & $0.4^{8}$ & $3 r \cdot 35$ & $3 \cdot 47$ & 9.29 & 2.93 \\
\hline
\end{tabular}

Malt and Hop Substitutes.-By reason of the fluctuation in market price of these two chief constituents of beer, it sometimes becomes a question of economy to employ cheaper substitutes wholly or in part for one or the other. There are two classes of malt substitutes, (I) those which, like corn, rice, and wheat, are mixed directly with the malt before "mashing," and, like the malt, have to undergo a saccharous fermentation before being acted on by yeast, and (2) such substances as cane sugar, invert sugar, commercial glucose, and dextrin, which are added to the wort at a later stage in the brewing, just before the addition of the yeast, being in condition to be readily acted on by the latter.

Glucose is by far the most common malt substitute, by reason of the fact that its sugars much resemble those of malt, and are in readily fermentable form. Diastase forms from the malt dextrin and maltose, while commercial glucose contains dextrin, maltose, and dextrose.

When the price of malt is abnormally high, the addition of glucose is decidedly economical, but when ordinary conditions prevail, the cost of the two, figured with reference to their yield in alcohol and extract, is about the same. Aside from the question of economy, however, there are advantages in the use of glucose, such as diminishing the nitrogenous content of the wort without lessening the alcohol or extract yielded. 
The nitrogenous matter left after fermentation is one of the chief causes of cloudiness or turbidity in the finished product, and is sometimes difficult to remove. By the use of glucose, especially in brewing clear bottled ales and sparkling pale beers, the appearance of the product is much enhanced. The temptation at times to add more glucose than is necessary to accomplish this is great. A high-grade malt may have as much as $40 \%$ of glucose added to its wort and still produce an acceptable beer. With a low-grade malt, glucose yields a very poor quality of beer. Hence the use of glucose may or may not be desirable, though it can hardly be considered unqualifiedly as an adulterant.

As to the employment of hop substitutes, the question of relative price again enters in. Only when the price of hops is high is there any special inducement to use substitutes. Quassia wood, chiretta, gentian, and calumba, all of which yield bitter principles, have been used in beer, and cannot be considered detrimental to health. Allen and Chattaway have found the first two in beer examined by them.* Such poisonous substances as cocculus indicus, picric acid, and strychnine are alleged to have been used as hop substitutes, but there is no authentic record of any of them having been found in recent years, if at all.

Adulteration of Malt Liquors and Standards of Purity.-The Joint Committee on Standards of the A. O. A. C. and the A. S. N. F. D. D. has adopted the following standards:

Malt Liquor is a beverage made by the alcoholic fermentation of an infusion, in potable water, of barley malt and hops, with or without unmalted grains or decorticated and degerminated grains.

Beer is a malt liquor produced by bottom fermentation, and contains in $100 \mathrm{cc}$., at $20^{\circ} \mathrm{C}$, not less than 5 grams of extractive matter and 0.16 gram of ash, chiefly potassium phosphate, and not less than 2.25 grams of alcohol.

Lager Beer, Stored Beer, is beer which has been stored in casks for a period of at least three months, and contains, in roo cc., at $20^{\circ} \mathrm{C}$., not less than 5 grams of extractive matters, and 0.16 gram of ash, chiefly potassium phosphate, and not less than 2.50 grams of alcohol.

Malted Beer is beer made of an infusion, in potable water, of barley, malt, and hops, and contains, in $100 \mathrm{cc}$., at $20^{\circ} \mathrm{C}$., not less than 5 grams of extractive matter, nor less than 0.2 gram of ash, chiefly potassium phosphate, not less than 2.25 grams of alcohol, nor less than 0.4 gram of crude protein (nitrogen $\times 6.25$ ).

\footnotetext{
* Analyst, I2, II 2 .
} 
Ale is a malt liquor produced by top fermentation, and contains, in $100 \mathrm{cc}$., at $20^{\circ} \mathrm{C}$., not less than 2.75 grams of alcohol, nor less than 5 grams of extract, and not less than 0.16 gram of ash, chiefly potassium phosphate.

Porter and Stout are varieties of malt liquors made in part from highly roasted malt.

Non-injurious bitter principles are no doubt employed in place of hops, and unless the liquor is sold for a pure malt beer, they cannot be regarded as adulterants.

The tendency to shorten the time of storage of beer, or to sell it without storing at all, lessens or does away with the after-fermentation, resulting in a lack of "life" or effervescence in the product. This is sometimes made up by the addition of sodium bicarbonate.

Distinction between Malted and Non-malted Liquors. - In some states where strict prohibitory liquor laws are in force, it is illegal to sell "'malt liquors," so that when convictions are obtained, it is necessary for the analyst to distinguish between liquors brewed wholly or in part from malt and those in which no malt has been used, but which were brewed entirely from malt substitutes. This distinction is not always easy to make with precision. In the absence of malt, glucose is usually the sole source of alcohol in these beverages. Parsons* has shown that the most striking points of difference between malted and non-malted liquors are in their per cent of phosphoric acid and albuminoids, and that pure malt beer or ale should contain at least $0.04 \% \mathrm{P}_{2} \mathrm{O}_{5}$, and $0.25 \%$ albuminoids $(\mathrm{N} \times 6.25)$. A low ash and high content of sulphates in the ash are also indicative of glucose. The following analyses made by Parsons clearly show these distinctions:

COMPOSITION OF SEVENTY-SIX SAMPLES OF AMERICAN MALT LIQUORS.

\begin{tabular}{|c|c|c|c|c|c|c|c|c|}
\hline & $\begin{array}{l}\text { Specific } \\
\text { Gravity. }\end{array}$ & $\begin{array}{l}\text { Alcohol } \\
\text { by Vol- } \\
\text { ume. }\end{array}$ & Extract. & $\begin{array}{l}\text { Albumin- } \\
\text { oids } \\
(\mathrm{N} \times 6.25)\end{array}$ & $\begin{array}{l}\text { Phos- } \\
\text { phoric } \\
\text { Acid. }\end{array}$ & Ash. & $\begin{array}{l}\text { Sul- } \\
\text { phates in } \\
\text { Ash. }\end{array}$ & $\begin{array}{l}\text { Free } \\
\text { Acid. }\end{array}$ \\
\hline Average...... & 1.0100 & $5.6 \mathrm{r}$ & $4.6 \mathrm{r}$ & 0.470 & $0.06:$ & 0.209 & 6.34 & 0.26 \\
\hline Maximum.... & 1.0210 & $7 \cdot 85$ & 7.64 & 0.614 & 0.095 & 0.296 & 12.67 & 0.87 \\
\hline Minimum. ... & 1.0047 & 0.35 & 3.15 & 0.290 & 0.045 & 0.147 & 2.44 & 0.10 \\
\hline
\end{tabular}

* Jour. Am. Chem. Soc., 24, 1902, p. 1170. 
TYPICAL ANALYSES OF BEERS APPARENTLY NOT BREWED FROM MALT.

\begin{tabular}{|c|c|c|c|c|c|c|c|c|}
\hline Number. & $\begin{array}{l}\text { Specific } \\
\text { Gravity. }\end{array}$ & $\begin{array}{l}\text { Alcohol } \\
\text { by Vol- } \\
\text { ume. }\end{array}$ & Extract. & $\begin{array}{l}\text { Albumin- } \\
\text { oids } \\
(\mathrm{N} \times 6.25)\end{array}$ & $\begin{array}{l}\text { Phos- } \\
\text { phoric } \\
\text { Acid. }\end{array}$ & Ash. & $\begin{array}{c}\text { Sul- } \\
\text { phates. }\end{array}$ & $\begin{array}{l}\text { Free } \\
\text { Acid. }\end{array}$ \\
\hline $1 \ldots \ldots \ldots$ & I. .0074 & 1. 68 & $2.5^{2}$ & 0.114 & 0.010 & 0.19 & 21.22 & Normal \\
\hline $2 \ldots \ldots \ldots \ldots$ & I. 0008 & 2.63 & 3.40 & 0.215 & 0.023 & 0.180 & $\ldots . . .$. & it \\
\hline $3 \ldots \ldots \ldots \ldots$ & 1.0062 & 2.27 & 2.25 & 0.150 & 0.015 & 0.124 & $11-30$ & “ \\
\hline $4 \ldots \ldots \ldots$ & 1.0112 & 2.11 & 3.53 & 0.133 & 0.015 & 0.140 & $10.8 \mathrm{I}$ & 4 \\
\hline $5 \ldots \ldots \ldots \ldots$ & 1.0041 & I. 85 & 1.73 & 0.031 & 0.010 & 0.088 & 12.50 & $c$ \\
\hline
\end{tabular}

The ash of the fifth sample is thus compared with that of the average beer as given by Blyth:

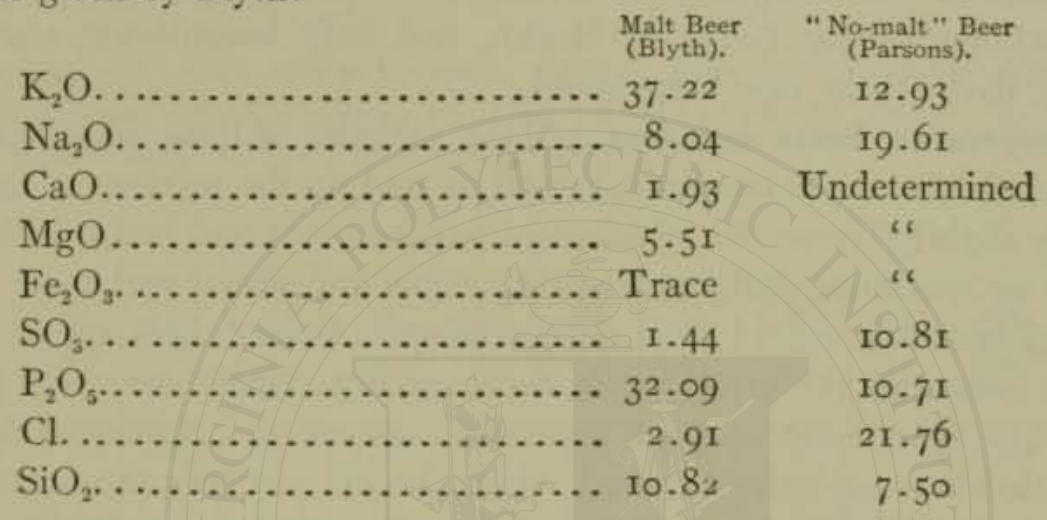

Praservatives in Beer.-Antiseptics are frequently added to malt liquors, salicylic acid being most commonly used. Fluorides of ammonium and sodium have been found in American beer. Other preservatives to be looked for are benzoic acid and sulphites. Beer casks are frequently "sulphured" or fumed with a solution of calcium bisulphite, so that the beer may derive its content of sulphites from this source.

In cases of police seizure of beer sold in bulk or in opened bottles for the purpose of ascertaining whether or not their alcoholic content exceeds certain limits fixed by law, a little formalin had best be added as soon as possible after the seizure to prevent further fermentation. This is especially desirable in cases where there is likely to be some delay in making the analysis, so as to forestall any claim on the part of the defendant of additional alcohol being formed after the seizure. From 6 to 8 drops of a $40 \%$ solution of formaldehyde to a quart of beer is sufficient, and this quantity will not appreciably affect the analysis.

Arsenic in Beer.-In I900 a very disastrous epidemic of arsenical poisoning occurred in Manchester, England, involving several thousand cases, many of which were fatal. The arsenic was traced to sulphuric 
acid which entered into the manufacture of commercial glucose uset in the beer, the acid found so highly arsenical being made from a certain variety of Swedish pyrites, which was found to be abnormally high in arsenic. There appeared to be no doubt whatever that the beer was the sole cause of the trouble. While the presence of arsenic was in this case accidental, carelessness was shown on the part of those having to do with the purity of the materials entering into the composition of the bee:Fortunately no other instances are on record of arsenical poisoning from malted liquors. A large number of samples of American beer have been examined in the laboratory of the Food and Drug Department of the Massachusetts State Board of Health, and only insignificant traces of arsenic have in any case been found.

Temperance Beers and Ales.-Many varieties of these so-called temperance drinks are home-made, as well as sold on the market. They are usually slightly fermented infusions of various roots and herbs, including ginger or sassafras, with molasses or sugar and yeast, and more often contain less than $\mathrm{x} \%$ of alcohol by volume. Among them are included spruce beer, and the various root beers, such as ginger beer and ginger ale. The latter beverages are generally carbonated. Numerous brands of bottled beer are manufactured, which contain virtually the same body and characteristic flavor as lager beer, but not the alcohol. Indeed the composition of many of these beverages is identical with that of lager beer, excepting in alcoholic content, being made by substantially the same process and out of the same ingredients, but with the alcohol finally removed by steaming, so that the liquor comes within the limits of a temperance beverage. Of this class is Uno beer, which ranges from 0.6 to 0.9 per cent in alcohol.

\section{METHODS OF ANALYSIS OF MALT LIQUORS.*}

Preparation of Sample.-Transfer the contents of the bottle or bottles to a large flask and shake vigorously to hasten the escape of carbon dioxide, care being taken that the liquor is not below $15^{\circ} \mathrm{C}$., since below this temperature the carbon dioxide is retained by the beer and is liable to form bubbles in the pycnometer.

Specific Gravity.-See page 657 .

Ash.-Determine in $25 \mathrm{cc}$. by evaporation and ignition at dull redness.

* Barnard, U. S. Dept. of Agric., Bur. of Chem., Circ. 33. A. O. A. C. Methods, ibid., Bul. 107 (rev.), p. 90 . 
Determination of Alcohol.-From the Specific Gravity of the Distillate--Proceed as described on p. 658 , employing $100 \mathrm{cc}$. of the liquor, and determining the specific gravity at $15.5^{\circ} \mathrm{C}$. If the licuor is markedly acid, add 0.1 to 0.2 gram of precipitated calcium carbonate previous to distillation.

From the Refraction of the Distillate.-Prepare the distillate as described on p. $65^{8}$, except that it is made up to the mark at $17.5^{\circ} \mathrm{C}$. Determine the refraction at $17.5^{\circ} \mathrm{C}$. Jy Leans of the immersion refractometer, and calculate the alcohol by the table of Ackermann and Steinmann below.

ACKERMANN AND STEINMANN'S TABLE FOR OBTAINING THE PERCENTAGE OF ALCOHOL IN THE DISTILLATE OF BEER FROM THE IMMERSION REFRACTOMETER READINGS.*

\begin{tabular}{|c|c|c|c|c|c|c|c|c|c|c|c|}
\hline 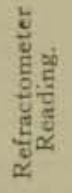 & 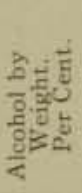 & 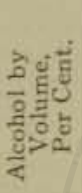 & 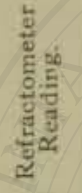 & 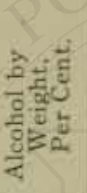 & 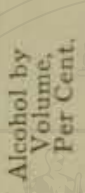 & 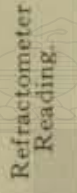 & 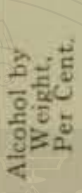 & 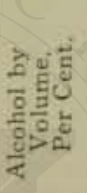 & 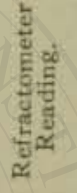 & 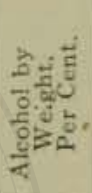 & 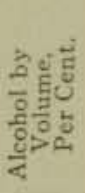 \\
\hline 15.0 & 0.00 & 0.00 & 17.2 & 1.38 & 1.74 & 19.4 & 2.74 & $3 \cdot 4^{6}$ & 21.6 & 4.02 & 5.06 \\
\hline 15.1 & 0.06 & 0.08 & 17.3 & I. 44 & 1.82 & 19.5 & 2.80 & 3.53 & 21.7 & 4.07 & 5.13 \\
\hline 15.2 & 0.13 & 0.16 & 17.4 & I. 5 I & 1.90 & 19.6 & 2.86 & 3.61 & 21.8 & 4.13 & 5.20 \\
\hline 15.3 & 0.19 & 0.24 & \pm 7.5 & I. 57 & 1.98 & 19.7 & $2.9 \mathrm{I}$ & 3.68 & $2 \mathrm{I} \cdot 9$ & 4.18 & 5.26 \\
\hline 15.4 & 0.25 & $0.3^{2}$ & 17.6 & I. 63 & 2.05 & 19.8 & 2.97 & 3.75 & 22,0 & 4.22 & $5 \cdot 3^{2}$ \\
\hline 15.5 & 0.32 & 0.40 & 17.7 & I. 68 & 2.12 & 19.9 & 3.04 & $3.8_{3}$ & 22. I & 4.28 & 5.39 \\
\hline 15.6 & $0.3^{8}$ & 0.48 & 17.8 & 1.74 & 2.20 & 20.0 & 3.10 & 3.90 & 22.2 & $4 \cdot 33$ & $5.4^{6}$ \\
\hline 15.7 & 0.44 & 0.56 & 17.9 & $1.8 \mathrm{I}$ & 2.28 & 20.1 & 3.15 & 3.97 & 22.3 & 4.39 & 5.53 \\
\hline 15.8 & 0.50 & 0.64 & 18.0 & I. 87 & 2.36 & 20.2 & 3.20 & 4.04 & 22.4 & $4 \cdot 44$ & 5.59 \\
\hline I5.9 & 0.57 & $0.7^{2}$ & 18,1 & 1.93 & 2.44 & 20.3 & 3.26 & $4.1 I$ & 22.5 & 4.49 & 5.65 \\
\hline 16.0 & 0.64 & 0.80 & 18.2 & 2.00 & 2.52 & 20.4 & $3 \cdot 33$ & 4.19 & 22.6 & 4.54 & 5.72 \\
\hline 16.1 & 0.70 & 0.88 & 18.3 & 2.06 & 2.60 & 20.5 & $3 \cdot 38$ & 4.26 & 22.7 & 4.59 & 5.78 \\
\hline 16.2 & 0.77 & 0.96 & 18.4 & 2.13 & 2.68 & 20.6 & 3.43 & $4 \cdot 33$ & 22.8 & 4.64 & 5.85 \\
\hline 16.3 & 0.83 & I. 04 & 18.5 & 2.19 & 2.76 & 20.7 & 3.50 & 4.41 & 22.9 & 4.70 & 5.92 \\
\hline I6. 4 & 0.88 & $I . I 2$ & 18.6 & 2.25 & 2.84 & 20.8 & 3.56 & 4.48 & 23.0 & $4 \cdot 76$ & 6.00 \\
\hline 10.5 & 0.95 & 1.19 & I8. 7 & $2 \cdot 3 I$ & 2.92 & 20.9 & 3.61 & 4.55 & 23.1 & 4.81 & 6.07 \\
\hline 16.6 & 1.01 & 1.27 & 18.8 & 2.37 & 2.99 & 21.0 & 3.67 & 4.63 & 23.2 & 4.86 & 6.13 \\
\hline 16.7 & 1.05 & 1.33 & 18.9 & 2.43 & 3.07 & 21,1 & 3.73 & 4.71 & $23 \cdot 3$ & 4.92 & 6.20 \\
\hline 16.8 & I. I3 & I. 43 & I9.0 & 2.49 & 3.14 & 21.2 & $3 \cdot 7^{8}$ & 4.77 & 23.4 & $4 \cdot 97$ & 6.27 \\
\hline 16.9 & I. I9 & I. $5^{1}$ & 19.1 & 2.55 & 3.22 & $2 \mathrm{r}-3$ & 3.84 & 4.84 & 23.5 & 5.02 & 6.33 \\
\hline 17.0 & 1.25 & 1. 58 & 19.2 & 2.61 & 3.29 & 21.4 & 3.90 & 4.92 & & & \\
\hline 17.1 & $1.3^{2}$ & 1.66 & 19.3 & 2.68 & $3 \cdot 37$ & 21.5 & 3.96 & 4.99 & & & \\
\hline
\end{tabular}

* Zeits. gesamte Brauwesen, 28, I905, p. 259.

Determination of Extract. - In cases where extreme accuracy is desired, the result obtained by evaporating at $100^{\circ}$ a weighed amount of the beer cannot be accepted, on account of the dehydration of the maltose at a temperature exceeding $75^{\circ} \mathrm{C}$. Unless the evaporation is conducted at that temperature (a difficult operation), a closer approxi- 
EXTRACT IN BEER WORT.*

[ACCORDING to SCHULtz AND OSTERMAanN.]

\begin{tabular}{|c|c|c|c|c|c|c|c|c|c|c|c|}
\hline \multirow[b]{2}{*}{$\begin{array}{l}\text { Specific } \\
\text { Gravity } \\
\text { at } x 5^{\circ} \mathrm{C} \text {. }\end{array}$} & \multicolumn{2}{|c|}{ Extract. } & \multirow[b]{2}{*}{$\begin{array}{l}\text { Specific } \\
\text { Gravity } \\
\text { at } t 5^{\circ} \mathrm{C} \text {. }\end{array}$} & \multicolumn{2}{|c|}{ Extract. } & \multirow[b]{2}{*}{$\begin{array}{l}\text { Specific } \\
\text { Gravity } \\
\text { at } 15^{\circ} \mathrm{C} \text {. }\end{array}$} & \multicolumn{2}{|c|}{ Extract. } & \multirow[b]{2}{*}{$\begin{array}{l}\text { Specific } \\
\text { Gravity. } \\
\text { at } 15^{\circ} \mathrm{C} \text {. }\end{array}$} & \multicolumn{2}{|c|}{ Extract. } \\
\hline & \begin{tabular}{|c|} 
Per \\
Cent \\
by \\
Weight
\end{tabular} & $\begin{array}{c}\text { Grams } \\
\text { per } \\
\text { too ce. }\end{array}$ & & $\begin{array}{c}\text { Per } \\
\text { Cent } \\
\text { by } \\
\text { Weight }\end{array}$ & $\begin{array}{c}\text { Grams } \\
\text { per } \\
\text { roo cc. }\end{array}$ & & $\begin{array}{c}\text { Per } \\
\text { Cent } \\
\text { by } \\
\text { Weight }\end{array}$ & $\begin{array}{c}\text { Grams } \\
\text { per } \\
100 \mathrm{cc} \text {. }\end{array}$ & & $\begin{array}{c}\text { Per } \\
\text { Cent } \\
\text { by } \\
\text { Weight }\end{array}$ & $\begin{array}{l}\text { Grams } \\
\text { per } \\
\text { roo cc. }\end{array}$ \\
\hline 1.0000 & 0.00 & 0.00 & I. .0065 & $x, 60$ & 1.70 & 1.0130 & 3.35 & 3.30 & I. 0105 & 5.06 & 5.16 \\
\hline$x .000 x$ & 0.03 & 0.03 & ז.0066 & 1.72 & 1.73 & 1.0131 & $3 \cdot 38$ & 3.42 & 1.0196 & 5.09 & 5,19 \\
\hline$x, 0002$ & 0.05 & 0.05 & 1.00677 & I. 7.4 & 1.75 & 1.0132 & $3 \cdot 41$ & 3.46 & 1.0107 & 5.12 & 5.22 \\
\hline 1.0003 & 0.08 & 0.08 & 1. 0068 & 1.77 & 1. 78 & 1.0133 & $3 \cdot 43$ & $3 \cdot 48$ & 1.0198 & 5,15 & 5.25 \\
\hline I. .0004 & 0.10 & 0.10 & 1.0069 & 1.79 & $x .80$ & 1.0134 & $3 \cdot 46$ & 3.51 & 1.0100 & 5.17 & 5.27 \\
\hline 1.0005 & 0.13 & 0.13 & I.0070 & 7.83 & I. 83 & r. 0135 & 3.48 & 3.53 & 1.0200 & 5.20 & 5.30 \\
\hline 1.0006 & 0.16 & 0.16 & 1.0071 & 1.84 & 1.85 & I. 0136 & 3.51 & 3.56 & 1.0201 & 5.23 & 5.34 \\
\hline 1.0007 & 0.18 & 0.18 & 1.0072 & I. 87 & $x .88$ & 1.0137 & 3.54 & 3.50 & 1.0202 & 5.25 & 5.36 \\
\hline 1. 0008 & 0.21 & 0.21 & 1.0073 & 3.00 & $1.0 \mathrm{r}$ & 1. or 38 & $3 \cdot 56$ & $36 r$ & 1.0203 & 5.28 & 5.39 \\
\hline I. 0000 & 0.24 & 0.24 & $x .0074$ & 1.92 & 1.93 & 1.0139 & 3.59 & 3.64 & I. 0204 & 5.30 & 5.41 \\
\hline I.0010 & 0.26 & 0.26 & $x .0075$ & $x, 05$ & 1.06 & I.0140 & $3.6 \mathrm{x}$ & 3.66 & I.0205 & $5 \cdot 33$ & $5 \cdot 44$ \\
\hline I. oort & 0.20 & 0.29 & 1.0076 & 1. .97 & $x+08$ & 1.0141 & 3.64 & 3.60 & 1.0206 & 5.35 & $5 \cdot 46$ \\
\hline 1.0012 & 0.31 & 0.31 & t. 0077 & 2.00 & 2,02 & 1.0142 & 3.66 & 3.71 & 1.0207 & 5.38 & 5,40 \\
\hline 1.0013 & 0.34 & 0.34 & $x .0078$ & 2.02 & 2.04 & 5.0143 & 3.60 & $3-74$ & 1.0208 & 5.40 & $5.5 \mathrm{I}$ \\
\hline 1.0014 & 0.37 & 0.37 & 1.0079 & 2.05 & 2.07 & 1.0144 & 3.22 & 3.77 & 1,0209 & 5.43 & 5.54 \\
\hline I. 0015 & 0,39 & 0.30 & 1.0080 & $2: 07$ & 2.09 & 1.0145 & 3,74 & $3 \times 79$ & 1.0210 & $5 \cdot 45$ & 5.56 \\
\hline 1.0016 & 0.42 & 0.42 & $1.008 x$ & 2.10 & 2,12 & 1. 0146 & 3.77 & 3.83 & 1.0211 & $5 \cdot 48$ & 5.60 \\
\hline 1.0017 & 0.45 & 0.45 & $t, 0082$ & 2.12 & 2.14 & $x .0147$ & 3.79 & 3.85 & 1.0212 & 5.50 & 5.62 \\
\hline 1.0018 & 0.47 & 0.47 & $1.008_{3}$ & 2.13 & 2.17 & 1.0148 & 3.82 & 3.88 & 1.0213 & 5.53 & 5.65 \\
\hline 1.0010 & 0.50 & 0.50 & $x .0084$ & 2.17 & 2.19 & $x .0149$ & 3.85 & $3.9 t$ & 1.0214 & 5.55 & 5.67 \\
\hline $1.00 \geq 0$ & 0.52 & 0.32 & 1.0085 & 2.20 & 2.22 & $1,0 \times 50$ & $3 \cdot 87$ & 3.93 & I. 0215 & $5 \cdot 57$ & 5.60 \\
\hline 1.0021 & 0.55 & 0.35 & 1.0086 & 2.23 & 2.25 & $1.015 \mathrm{r}$ & 3.90 & 3.96 & $x .0216$ & 5.60 & 5.72 \\
\hline 1.0022 & 0.58 & 0.38 & 1.0087 & 2.25 & 2.27 & 1.0152 & 3.02 & 3.98 & 1.0217 & 3.62 & $5 \cdot 74$ \\
\hline 1,0023 & 0.60 & 0,60 & t. 0088 & 2,28 & 2.30 & 1,0153 & 3.05 & $4.0 x$ & $\mathrm{I} .0218$ & 5.65 & 5.77 \\
\hline I. .0024 & 0.63. & 0.03 & 1.0080 & 2.30 & 2.32 & $x .0154$ & 3.97 & 4.03 & 1.0216 & 5,67 & 5.79 \\
\hline 1.0025 & 0.66 & 0.66 & 1.0090 & 2.33 & 2.35 & 1.0155 & 4.00 & 4.06 & $\mathrm{I}, 0220$ & 5.70 & 5.83 \\
\hline x.0026 & 0.68 & 0.68 & $t .000 t$ & 2.35 & 2.37 & 1.0156 & 4.03 & 4.00 & 1.0221 & 5.72 & 5.85 \\
\hline 1.0027 & 0.71 & 0,71 & I. .0002 & 2,38 & 2.40 & 1.0157 & 4.05 & 4.11 & 1.0222 & $5-75$ & 5.88 \\
\hline 1.0028 & 0.73 & 0.73 & I., 0093 & $2 \times 41$ & 2.43 & $1.0 \times 58$ & 4.08 & $4 \cdot 14$ & 1.0223 & 5.77 & 5.90 \\
\hline 1.0029 & 0.76 & 0.76 & 1.0094 & 2.43 & 2.45 & 1.0159 & 4,10 & 4.17 & 1.0224 & 5.80 & 5,93 \\
\hline I. .0030 & 0.79 & 0.79 & I. .0095 & 2.49 & 2.48 & $1.0 \times 60$ & 4.13 & 4.20 & 1.0225 & 5.82 & 5.95 \\
\hline I. $003 \mathrm{I}$ & 6,83 & $0.0 \mathrm{r}$ & t. 0006 & -2.48 & 2.50 & 1.0161 & 4.16 & 4.23 & 1.0226 & 5.84 & $5 \cdot 97$ \\
\hline 1,0032 & 0.84 & 0.84 & 1.,0007 & 2.51 & 2.53 & 1.0162 & 4.18 & 4.25 & I.0227 & 5.87 & 6.00 \\
\hline I. .0033 & 0.87 & 0.87 & 1.0008 & 2,53 & 2.53 & $\mathrm{x} .0163$ & $\therefore 21$ & $4 \cdot 28$ & 1.0228 & 5.80 & 6,02 \\
\hline 1.0034 & 0.89 & 0.89 & 1.0090 & 2.56 & 2.59 & 1.0164 & 4.23 & 4.30 & 1.0229 & 5.92 & 6.06 \\
\hline I. 0035 & 0.92 & 0.92 & $x .0100$ & 2.58 & $2.6 \mathrm{r}$ & $x .0165$ & 4,26 & $4 \cdot 33$ & 1.0230 & 5.94 & 6.08 \\
\hline$t .0036$ & 0.94 & 0,04 & 1.0101 & $2.6 t$ & 2.64 & 1.0166 & 4.28 & 4.35 & I. $023 x$ & 3.97 & $6.1 \mathrm{r}$ \\
\hline$x .0037$ & 0.97 & 0.97 & I:OrOZ & 2.64 & 2.67 & I.0167 & 4.31 & 4.38 & 1.0232 & 5.99 & 6.13 \\
\hline 1.0038 & $x, 00$ & 1. 00 & 1.0ro 3 & 2,66 & 2.60 & 1.0168 & $4 \cdot 34$ & $4 \cdot 4 t$ & 8.0233 & 6.02 & 6.16 \\
\hline 1.0039 & 1.02 & $x, 02$ & 1.0104 & 2.69 & 2.72 & t. 0169 & 4.36 & $4 \cdot 43$ & I. 0234 & 6.04 & 6.78 \\
\hline$x, 0040$ & 1.05 & 1.05 & r.,0105 & 2.71 & 2.74 & I. 0170 & 4.39 & $4 \cdot 46$ & 1.0235 & 6.07 & 6,21 \\
\hline 1.0041 & $x .08$ & $\pi .08$ & r.0106 & 2,74 & 2.77 & 1.0171 & 4.42 & 4.50 & 1.0236 & 6.09 & 6.23 \\
\hline 1,0042 & 1.10 & $x, x 0$ & 1.0107 & 2.76 & 2.79 & 1.0172 & 4.44 & 4.52 & $T .0237$ & 6.11 & 6.25 \\
\hline I. .0043 & $x+x 3$ & 1,13 & I.0108 & 2.79 & 2.32 & 1.9173 & $4 \cdot 47$ & $4 \cdot 55$ & 1.0238 & 6.14 & 6.29 \\
\hline$x .0044$ & 2.15 & 1.16 & 1.0100 & 2.82 & 2.85 & 2.0174 & 4.50 & $4 \cdot 5^{8}$ & 1.0230 & $6 . x 6$ & $6.3 \mathrm{I}$ \\
\hline I. 0045 & I. 18 & I. IO & I. Otro & 2.84 & 2.87 & 1.0175 & 4.53 & 4.61 & $x, 0240$ & 6.19 & \\
\hline I. 0046 & $t, 2 t$ & 1.22 & t.ortit & 2.87 & 2.90 & 1.0176 & 4.55 & 4.63 & $\mathrm{I}, 024 \mathrm{I}$ & 6.21 & 6.36 \\
\hline 1.0047 & 1. 23 & 1.24 & I.0112 & 2.89 & 2.97 & 1.0177 & 4.58 & 4.60 & 1.0242 & 6.24 & 6.39 \\
\hline 1.0048 & I. 26 & 1.27 & 1.0113 & 2.92 & 2.95 & 10178 & 4.61 & 469 & 1.0243 & 6.26 & $6.4 \mathrm{r}$ \\
\hline 1.0049 & 1.29 & 1.30 & 1.0114 & 2.94 & 2.97 & 1.0179 & 4.63 & 4.71 & 1.0244 & 6,29 & 6.44 \\
\hline I. 0050 & 1.31 & I. -32 & I.0115 & 2.97 & 3.00 & 1.0180 & 4.66 & 4.74 & $x .0245$ & $3.3 \mathrm{t}$ & 6.46 \\
\hline I. $005 \mathrm{t}$ & 1. 34 & 1. 35 & $x .0116$ & $z, 99$ & 3.02 & $x .018 x$ & 4.69 & 4.77 & $1.024^{6}$ & 6.34 & 6.50 \\
\hline 1.0052 & 1.36 & I. 37 & I.oxx & 3.03 & 3.06 & 1.0582 & 4.71 & 4.80 & $x, 024 i$ & 6.36 & 6.52 \\
\hline 1.0053 & 1. 39 & 2.40 & 1.0218 & 3.95 & 3.09 & 1.0183 & 4.74 & 4.83 & 1.0248 & 6.39 & 6.55 \\
\hline I. 0054 & 1.41 & $1 \cdot 42$ & 1.0119 & 3.07 & 3.11 & 1.0184 & 4.77 & 4.86 & 1,0249 & 6.41 & 6.57 \\
\hline $\mathbf{1}, 0055$ & I. 44 & 1. 45 & 2.0120 & 3,10 & 3.14 & x. 0185 & 4.7 & 4.88 & 1.0250 & 6.44 & 6.60 \\
\hline 1.0056 & 1.46 & $x .47$ & 1.0121 & 3.12 & 3.10 & 1.0186 & +.82 & 4.5. & I. 0251 & 6.47 & \\
\hline 1.0057 & I. 49 & $x .50$ & $x, 0122$ & 3.15 & 3.19 & 1.0187 & 4.85 & 4.94 & 1.0252 & 6.50 & \\
\hline 1.0058 & $x \cdot 5 x$ & 1.52 & 1.0123 & 3.17 & 3.21 & 1.0188 & 4.88 & 4.97 & I. .0253 & 6.52 & 6.68 \\
\hline x. 0059 & I. 54 & 1.55 & 1.0124 & 3.20 & 3.24 & 1.0180 & 4.90 & 4.99 & 3.0254 & 6.55 & 6.72 \\
\hline $\mathbf{t} .0060$ & t. 56 & I. 57 & 1,0125 & 3.23 & 3.27 & 1.0100 & 4.9 & 5.02 & 1.0255 & 6.58 & 6.75 \\
\hline I.006x & I. 59 & 1.60 & 1.0126 & 3.25 & 3.20 & 1.0191 & 4.96 & 5.05 & 1.0256 & 6.61 & \\
\hline 1.0062 & I. 62 & 1.63 & 1.0127 & 3.28 & $3 \cdot 32$ & 1.0102 & 4.98 & 3.08 & I. 0257 & 6.63 & 6.80 \\
\hline $\mathbf{2} .0063$ & I. 64 & $x, 65$ & 1.0128 & 3,30 & $3 \cdot 34$ & I.0ro3 & 5.01 & 5.11 & I. 0258 & 6.66 & 6.83 \\
\hline$x .0064$ & 1.67 & $x .68$ & 1.0129 & 3.33 & $3 \cdot 37$ & I. 0194 & 5.04 & $5 \cdot 14$ & 1.0259 & 6,60 & 6.86 \\
\hline
\end{tabular}

- Calculated from results obtained by drying below $75^{\circ} \mathrm{C}$. 
EXTRACT IN BEER WORT-(Continued).

\begin{tabular}{|c|c|c|c|c|c|c|c|c|c|c|c|}
\hline \multirow[b]{2}{*}{$\begin{array}{l}\text { Specific } \\
\text { Gravity } \\
\text { at } 15^{\circ} \mathrm{C} \text {. }\end{array}$} & \multicolumn{2}{|c|}{ Extract. } & \multirow[b]{2}{*}{$\begin{array}{l}\text { Specific } \\
\text { Gravity } \\
\text { at } 15^{\circ} \mathrm{C} \text {, }\end{array}$} & \multicolumn{2}{|c|}{ Extract. } & \multirow[b]{2}{*}{$\begin{array}{l}\text { Specific } \\
\text { Gravity } \\
\text { at } 15^{\circ} \mathrm{C} \text {. }\end{array}$} & \multicolumn{2}{|c|}{ Extract. } & \multirow[b]{2}{*}{$\begin{array}{l}\text { Specific } \\
\text { Gravity } \\
\text { at } \times 5^{\circ} \mathrm{C} \text {. }\end{array}$} & \multicolumn{2}{|c|}{ Extract. } \\
\hline & $\begin{array}{c}\text { Per } \\
\text { Cent } \\
\text { by } \\
\text { Weight }\end{array}$ & $\begin{array}{c}\text { Grams } \\
\text { per } \\
\text { roo cc. }\end{array}$ & & $\begin{array}{c}\text { Per } \\
\text { Cent } \\
\text { by } \\
\text { Weight }\end{array}$ & $\begin{array}{l}\text { Grams } \\
\text { per } \\
\text { roo cc; }\end{array}$ & & $\begin{array}{c}\text { Per } \\
\text { Cent } \\
\text { by } \\
\text { Weight }\end{array}$ & $\begin{array}{c}\text { Grams } \\
\text { per } \\
\text { roo cc. }\end{array}$ & & $\begin{array}{c}\text { Per } \\
\text { Cent } \\
\text { by } \\
\text { Weight }\end{array}$ & $\begin{array}{l}\text { Grams } \\
\text { per } \\
\text { roo cc. }\end{array}$ \\
\hline 1.0260 & 6.71 & 6.88 & 1.0325 & .27 & 8. 54 & 1.0390 & 9.92 & 10.31 & 1.0455 & 11.53 & 12.05 \\
\hline $1.026 \mathrm{t}$ & 6.74 & & & & & & 9.95 & & 456 & 11.55 & 12.08 \\
\hline 1.0262 & 6.77 & .95 & 327 & $8 \cdot 32$ & 8.59 & 1.0392 & 9.97 & 6 & 457 & 1.57 & 2.10 \\
\hline 1.0263 & 6.80 & & 1.0328 & $8 \cdot 34$ & & 1.0393 & 0.99 & 10.38 & 1,0458 & 11.60 & 12.13 \\
\hline I. 0264 & 6.82 & 7.00 & I. 0329 & 8.37 & 8.65 & I. 0394 & 10.02 & 10.41 & 1.0459 & 11.62 & 12.15 \\
\hline 1. 0265 & 6.85 & .03 & 1.0330 & 8.40 & 8.68 & I. 0395 & 10.04 & 10.44 & 1.0460 & 11.65 & 12.19 \\
\hline 1.0 & 6.88 & & 0331 & & 1 & & & & & 11.67 & 2.21 \\
\hline 1.0267 & 6.0 & & & & 3 & & 10,09 & 9 & 1.0462 & 11.70 & 2.24 \\
\hline 1.0268 & 6.9 & & & & & & II & & I. 0463 & 11.72 & .26 \\
\hline I, & 6.96 & $7 \cdot 15$ & .0334 & 8. & & 1.03 & 10.13 & 10.3 & I. 0464 & 11.75 & 12.30 \\
\hline 1.0270 & 6.99 & $7+18$ & 1,0335 & 8,53 & 8.82 & I. 0400 & 10,16 & 10.57 & I. 0465 & 17.77 & 12.32 \\
\hline 1.0271 & 7.0 & & & & & & 10.18 & 9 & I. .0466 & II. 79 & 2.34 \\
\hline 1.0272 & & & 337 & .59 & & 2 & 10.20 & 10.61 & I. 0467 & 11.82 & 12.37 \\
\hline 1.0273 & & & & .61 & & 33 & 10.23 & 10.64 & 1.0468 & 11.84 & .39 \\
\hline 1.0274 & 7 & & & 8.64 & & 1.0 & 10.25 & 10.66 & $x .0409$ & 11.87 & 12.43 \\
\hline 1.0 & 7.12 & $7 \cdot 32$ & 1.0340 & 8.67 & 8.06 & I. 0.405 & 10,27 & 10.69 & 470 & 11.89 & 12.45 \\
\hline 1.0276 & 15 & 35 & .0341 & 8.7 & & I. 0.406 & 10.30 & 10.72 & $\mathrm{I}, 047 \mathrm{I}$ & 11.92 & $x 2.48$ \\
\hline 1.0277 & $7+18$ & 38 & .0342 & 8.72 & 9.02 & 1.0407 & 10.32 & 10.74 & 1.0472 & 11.94 & 12.50 \\
\hline 1.0278 & 7.21 & $7 \cdot 41$ & $x .0343$ & 8.75 & 9.05 & 1.0408 & 10.35 & 10.77 & 1.0473 & 11.97 & 12.54 \\
\hline$x, 0279$ & 7.23 & $7 \cdot 43$ & 1.0344 & 8.78 & 9.08 & I. 0409 & 10.37 & 10.79 & I. .0474 & 11.99 & 12.56 \\
\hline 1.0280 & 26 & 46 & .0345 & .80 & 10 & 1.0410 & 10.40 & 10.83 & I. 0475 & 12.01 & 12.58 \\
\hline$x .028 \mathrm{x}$ & .28 & & .0346 & .83 & & 1.0411 & 10.42 & 10.85 & 1.0476 & 12.04 & 12.01 \\
\hline 1.0282 & $7 \cdot 30$ & $.3 t$ & $1.034 ?$ & .86 & 9.17 & 1.0412 & 10.45 & 10.88 & 1.0477 & 12.06 & 12.64 \\
\hline I. 0283 & $7 \cdot 33$ & $7 \cdot 54$ & I. 0348 & .88 & 0.10 & 1.0413 & 10.47 & 10.90 & I. 0478 & 12.09 & 12.67 \\
\hline 1.0284 & $7 \cdot 35$ & $7 \cdot 56$ & 1.0349 & $8.9 t$ & 9.22 & $t, 0414$ & 10.50 & 10.93 & 1.0479 & 12.11 & 12.69 \\
\hline I. 0285 & $\cdot 37$ & 58 & 1. 0350 & .94 & .25 & 1.0415 & 10.52 & 10.96 & I. .0480 & 12.14 & 12.72 \\
\hline 1.0286 & .39 & 60 & .0351 & 7 & 9.28 & 1.0416 & 10.55 & 10.99 & $I .048 \mathrm{I}$ & 12.16 & 12.74 \\
\hline I. 0287 & .42 & .63 & 1.0352 & .00 & 9.31 & 1.0417 & 10.57 & 11,01 & 1.0482 & 12.19 & 12.78 \\
\hline 1. .0288 & $7 \cdot 44$ & 7.65 & 1.0353 & 9.02 & $9 \cdot 34$ & 1.0418 & 10.60 & I I.04 & $I .0483$ & $12.2 \mathrm{I}$ & 12.80 \\
\hline 1.0289 & 7.46 & 7.68 & $x, 0354$ & 9.05 & $9 \cdot 37$ & 1.0419 & 10.62 & 11.06 & I. .0484 & 12.23 & 12.82 \\
\hline 8.0290 & $7 \cdot 4^{8}$ & 70 & I. 0355 & .07 & 9.39 & I. 0420 & 10.65 & 11.10 & I. .0485 & 12.25 & 12.85 \\
\hline I, 0291 & .51 & .73 & $x .0356$ & & .42 & 1.04 & 10.67 & 11.12 & I. 0486 & $12 \cdot 28$ & 12.88 \\
\hline I. 0202 & .53 & 75 & 1.0357 & 13 & 9.46 & .0422 & 10.70 & $1 \mathrm{x}, \mathrm{T} 5$ & I. .0487 & 12.31 & $12.9 \mathrm{t}$ \\
\hline t. .0293 & $7 \cdot 55$ & +77 & I 9358 & 9.15 & $9.4^{8}$ & I. 0423 & 10.72 & $x+17$ & I. .0488 & 12.33 & 12.93 \\
\hline I. 0294 & 7.57 & $7+79$ & 1.0359 & 9.18 & 9.51 & I. 0424 & 10.75 & 11.21 & I. 0489 & 12.36 & 72.96 \\
\hline t. 0295 & .60 & 82 & I. 0360 & $2 \pi$ & .54 & I. 0425 & 10.77 & 11.23 & 1.0490 & $\mathrm{I} 2.38$ & 12.99 \\
\hline I, o & .62 & 85 & $x .0361$ & 24 & .57 & 1,0426 & 10.80 & 11,26 & $x, 0491$ & 12.41 & 13.02 \\
\hline I. 0207 & .04 & 87 & 1.0362 & 26 & 9.60 & $\mathrm{t} .0427$ & 10.82 & I I. 28 & I. 0492 & 12.43 & 13.04 \\
\hline I. 0298 & 7.60 & 89 & 1.0363 & .29 & 9.03 & 1.0428 & 10.85 & $I x+3 i$ & 1.0493 & 12.45 & 13.06 \\
\hline 1.0299 & 7.69 & 7.92 & 1.0364 & 9.31 & 9.65 & 1.0429 & 10.88 & II. 35 & I. 0494 & 12.48 & 13.10 \\
\hline 2300 & .71 & & .0365 & & 68 & .0430 & 10.90 & 1.37 & 1.04 & 12.50 & 13.12 \\
\hline 1.0301 & .73 & 96 & .0360 & 36 & .70 & 1.0431 & 10.93 & 11.40 & 1,0496 & 12.53 & 13.15 \\
\hline 1.0302 & .75 & 98 & 7 & 38 & .72 & 1.0432 & 10.95 & I. 42 & 1.04 & 12.55 & $1 / 3.17$ \\
\hline 1,0303 & $7 \cdot 77$ & & & .41 & 9.76 & 1.0433 & 10.08 & 11.46 & 1.0498 & 12.58 & 13.21 \\
\hline 1.0304 & 7.80 & 8.04 & 1.0369 & 9.43 & 9.78 & 1.0434 & 11.00 & $11 \cdot 48$ & 1,0499 & 12.60 & 13.23 \\
\hline .0305 & .82 & & & & So & 43 & & & & 12.63 & 13.26 \\
\hline 1.0306 & .84 & .06 & .03 & & 9.83 & 1.0436 & 11.05 & $x+53$ & 1.0501 & 12.65 & 13.28 \\
\hline 1.0307 & 7.86 & .10 & .0372 & 50 & 9.85 & 1.0437 & 11.08 & $x 1.56$ & 1.0502 & 12.67 & $13 \cdot 3 x$ \\
\hline 1.0308 & 7,89 & & 1.0373 & .52 & 9.88 & 1.0438 & 11.10 & 11.59 & 1.0503 & 12.70 & 13.34 \\
\hline$t .0309$ & 7.91 & 8.15 & 1.0374 & 9.55 & $9.9 x$ & I. 0439 & 11.13 & 11.62 & 1.0504 & 12.72 & $13-36$ \\
\hline 1.0310 & .93 & & & & & & & 11,64 & & .75 & 39 \\
\hline 1.0311 & 7.95 & & .0376 & .59 & 9.95 & 1.0441 & 11.18 & $1 x .67$ & 1.0500 & 12.77 & 13.42 \\
\hline 1.0312 & $7 \cdot 98$ & .23 & 1.0377 & .62 & 9.98 & 1.0444 & 11.20 & $11+70$ & 1.0507 & 12,80 & $13 \times 45$ \\
\hline 1.0313 & 8.00 & & 1.0378 & 9.64 & 0.00 & I. .0443 & 11.23 & 11,73 & 1.0508 & 12.82 & 13.47 \\
\hline 1.0314 & 8.02 & 8.27 & 1.0379 & & 10.03 & I. .0444 & $x x .25$ & 11.75 & 1.0509 & 12,85 & $13.5^{\circ}$ \\
\hline & 8.04 & & & & & I. 0445 & 11,28 & 11.78 & & $\mathrm{r} 2.87$ & 13.53 \\
\hline 1.0316 & 8.07 & & & .71 & 0.08 & I. . 0446 & $11 \cdot 30$ & II, 80 & 1.0511 & 12.90 & 13.56 \\
\hline 1.0317 & 8.09 & & 82 & .73 & 0.10 & 1.0447 & $x+33$ & 11,84 & 1.0512 & 12.92 & $13 \cdot 5^{8}$ \\
\hline I. .0318 & 8.11 & & & & & 1.0448 & 11.35 & 11.86 & 1.0513 & 12.94 & 13,60 \\
\hline $1.03 \times 9$ & 8.13 & 8.39 & I. .0384 & 9.78 & $10, x 6$ & I. 0449 & Ix $>8$ & Ix. 89 & I. 0514 & 12.97 & $x 3.04$ \\
\hline$x, 0320$ & & & & & & 1.0450 & 11.40 & $11 \cdot 91$ & & & 13.66 \\
\hline 1.0321 & 8.18 & & 36 & 9.83 & 10.21 & I. $045 \mathrm{I}$ & $x+43$ & II. 05 & 1.0516 & 13.02 & I3.69 \\
\hline 1.0322 & 8.20 & & .0387 & & & I. 0452 & 11.45 & & $x .0517$ & 13.04 & 13.71 \\
\hline 1.0323 & 8.22 & & 1.0388 & 9.88 & 10.26 & I. .0453 & $1 x \cdot 4^{8}$ & 12,00 & 1.0518 & 13.07 & 13.75 \\
\hline 1.0324 & 8.25 & 8.52 & 1.0389 & 9.90 & 10.29 & 1.0454 & 11.50 & 12,02 & $7.05 \times 9$ & 13.09 & 53.77 \\
\hline
\end{tabular}


EXTRACT IN BEER WORT-(Continted).

\begin{tabular}{|c|c|c|c|c|c|c|c|c|c|c|c|}
\hline \multirow[b]{2}{*}{$\begin{array}{l}\text { Specific } \\
\text { Gravity } \\
\text { nt }+5^{\circ} \mathrm{C} \text {. }\end{array}$} & \multicolumn{2}{|c|}{ Extract. } & \multirow[b]{2}{*}{$\begin{array}{l}\text { Specific } \\
\text { Gravity } \\
\text { at } 15^{\circ} \mathrm{C} \text {. }\end{array}$} & \multicolumn{2}{|c|}{ Extract. } & \multirow[b]{2}{*}{$\begin{array}{l}\text { Specific } \\
\text { Gravity } \\
\text { at } 15^{\circ} \mathrm{C} \text {. }\end{array}$} & \multicolumn{2}{|c|}{ Extract. } & \multirow[b]{2}{*}{$\begin{array}{l}\text { Specific } \\
\text { Gravity } \\
\text { at } 15^{\circ} \text { C. }\end{array}$} & \multicolumn{2}{|c|}{ Extract. } \\
\hline & $\begin{array}{c}\text { Per } \\
\text { Cent } \\
\text { by } \\
\text { Weight }\end{array}$ & $\begin{array}{c}\text { Grams } \\
\text { per } \\
\text { roo cc. }\end{array}$ & & $\begin{array}{c}\text { Per } \\
\text { Cent } \\
\text { by } \\
\text { Weight }\end{array}$ & $\begin{array}{l}\text { Grams } \\
\text { t per } \\
\text { too cc. }\end{array}$ & & $\begin{array}{c}\text { Per } \\
\text { Cent } \\
\text { by } \\
\text { Weight }\end{array}$ & $\begin{array}{l}\text { Grams } \\
\text { per } \\
\text { too cc. }\end{array}$ & & $\begin{array}{c}\text { Per } \\
\text { Cent } \\
\text { by } \\
\text { Weight }\end{array}$ & $\begin{array}{l}\text { Grams } \\
\text { per } \\
\text { 100 cc. }\end{array}$ \\
\hline 1.0520 & 13.12 & 13.80 & 1.0585 & 74.75 & $75.6 x$ & $\mathrm{x}: 0650$ & 16.25 & $\mathrm{I} 7 \cdot 3 \mathrm{~T}$ & $1.07 \times 5$ & $17,8 x$ & 70.08 \\
\hline $\mathrm{x}, 052 \mathrm{I}$ & $13 . x$ & .82 & 1. 0586 & 1.78 & 5.65 & 1.0651 & 16,27 & $17,3.3$ & 1.0716 & 17.84 & 10.12 \\
\hline 1.0522 & 13,16 & 3.85 & 1.0587 & $x .4 .81$ & 15.68 & 1.0652 & 16.30 & 17.36 & 1.0717 & 7.7 .86 & 10.14 \\
\hline 1.0523 & 13.19 & 13.88 & 1. 0588 & 14.83 & 15.70 & 1.0653 & 16.32 & 17.39 & 1.0718 & 17.88 & 19.16 \\
\hline 1.0524 & 13.21 & 13.90 & t. 0589 & 14.86 & 15.74 & 1.0654 & 16.35 & 17.42 & 1.0719 & 17.90 & 19.19 \\
\hline 1.0525 & 13.24 & 13.94 & 1.0500 & 14.80 & 15.77 & I. 0655 & 16.37 & 17.44 & $\mathrm{x} .0720$ & 17.93 & 10.22 \\
\hline$x .0526$ & 13.26 & 13.96 & I. 0591 & 14.91 & $15-70$ & 1.0656 & 16.40 & $17-48$ & 1.0721 & 77.05 & 10.24 \\
\hline$x .0527$ & 13.29 & 13.99 & 1.0592 & 14.94 & 15.82 & 1.0657 & 16.42 & $17 \cdot 50$ & $\mathrm{r} .0722$ & 17.97 & 70.27 \\
\hline 1.0528 & 13.31 & $14.0 \mathrm{I}$ & 1.0593 & 1.4 .96 & 13.85 & I, 0658 & 16,45 & $17 \cdot 53$ & 1.0723 & 17,00 & 20.29 \\
\hline 1.0529 & 13.34 & 14.05 & 1.0594 & $14=99$ & 15,88 & 1.0659 & 16.47 & 17.56 & 1.0724 & 18,02 & $x 9 \cdot 32$ \\
\hline$x .0530$ & 1 3.36 & 14.07 & $x, 0505$ & 15.02 & $15.9 \mathrm{t}$ & 1. 0660 & 16.50 & 17.59 & 1.0725 & 18.04 & 10.35 \\
\hline 1.0531 & 13.38 & 14.09 & 1.0596 & 15,04 & 15.94 & $\mathrm{I} .066 \mathrm{t}$ & 16.52 & 17.61 & 1.0726 & 18.06 & 10.37 \\
\hline I. 0532 & $13.4 \mathrm{t}$ & 14.12 & x.0597 & 15.07 & Is -07 & 1,0002 & $x 6.54$ & 17.63 & 1.0727 & 18.08 & 19.39 \\
\hline 1.0533 & 73,43 & 14,15 & $x .0508$ & 15.00 & 15,99 & t. .0663 & 16.57 & 17.67 & 1.0728 & 18,11 & 19.43 \\
\hline$x .0534$ & $13 \cdot 4^{6}$ & 14.18 & 1,0599 & 15.11 & 16.02 & 1.0664 & 16.59 & 17.69 & 1.0729 & 18.13 & 10.45 \\
\hline 1.0535 & 1. 3.48 & 14,20 & 1.0600 & 15,14 & 16.05 & 1.0665 & 16,62 & 17.73 & 1.0730 & 18.15 & 10.47 \\
\hline 1.0536 & 13.51 & 14.23 & 1.0601 & 15.16 & 16.07 & 1.0666 & 16.04 & 17.75 & $1.073 \mathrm{t}$ & 18.17 & 10.50 \\
\hline $\begin{array}{l}1.0537 \\
1.0538\end{array}$ & 13.53 & 14.26 & 1. .0602 & 15.18 & 16.00 & 1.0667 & 16.67 & $17 \cdot 78$ & 1.0732 & 18.20 & 10.53 \\
\hline $\begin{array}{l}1.0538 \\
1.0539\end{array}$ & $\begin{array}{r}13.56 \\
13.58\end{array}$ & 34.29 & 1.0603 & 15.20 & 16.12 & 7,0608 & 76,60 & 17.80 & 1.0733 & 18.22 & 10.55 \\
\hline 1.0539 & 23.58 & 14.31 & I. 0604 & $15 \quad 23$ & 16.15 & I 0669 & 10.72 & 17.84 & I. .0734 & 18.24 & 19.58 \\
\hline $\begin{array}{l}\text { I. } 0540 \\
1.0547\end{array}$ & $13.6 x$ & $24 \cdot 34$ & 1. 0605 & 35.25 & 16.17 & 1.0670 & 16.74 & 17.86 & 1.0735 & 78.26 & 19.60 \\
\hline $\begin{array}{l}1,0541 \\
1,0542\end{array}$ & $\begin{array}{l}13.63 \\
13.66\end{array}$ & 14.37 & $\begin{array}{l}1.0606 \\
1.0607\end{array}$ & 15.27 & 16.20 & 1.0671 & 16.76 & 17.88 & 1.0736 & 18.29 & 10.64 \\
\hline $\mathrm{I} .0543$ & $\begin{array}{l}13.66 \\
13.68\end{array}$ & $\begin{array}{l}14 \cdot 40 \\
14: 42\end{array}$ & $\begin{array}{l}1.0607 \\
1.0608\end{array}$ & $\begin{array}{l}15.29 \\
15.37\end{array}$ & $\begin{array}{l}16.22 \\
16.24\end{array}$ & $\begin{array}{r}1.0672 \\
.0673\end{array}$ & 16.79 & $17-92$ & 1.0757 & 18.31 & 10.66 \\
\hline 1.0544 & $\begin{array}{l}13.68 \\
13.71\end{array}$ & $\begin{array}{l}1.4 \cdot 4^{2} \\
14 \cdot 4^{6}\end{array}$ & 1.0609 & 15.34 & 16.27 & $\begin{array}{l}1,0673 \\
1.0674\end{array}$ & $\begin{array}{l}16.81 \\
16.84\end{array}$ & $\begin{array}{l}17.94 \\
17.98\end{array}$ & $\begin{array}{l}1.0738 \\
1.0739\end{array}$ & $\begin{array}{l}18.33 \\
18.35\end{array}$ & $\begin{array}{l}10.68 \\
10.71\end{array}$ \\
\hline I. 0545 & & 74.48 & 1.0610 & 15.36 & 16.30 & & $x 6.86$ & 18.00 & & & \\
\hline 1.0546 & 13.76 & 74.51 & 1.0611 & 25.38 & 16.32 & $\begin{array}{l}1.0075 \\
1.0076\end{array}$ & $\begin{array}{r}10,80 \\
16,80\end{array}$ & $\begin{array}{l}18.00 \\
18.03\end{array}$ & $\begin{array}{l}1.0740 \\
1.0741\end{array}$ & $\begin{array}{l}x 8.38 \\
18.40\end{array}$ & 19.74 \\
\hline 1.0547 & 13.78 & $1.4 \cdot 53$ & x.0612 & 15.40 & 76.34 & 1.0677 & 36.93 & $\begin{array}{r}18.05 \\
\end{array}$ & $\begin{array}{l}1.0741 \\
1.0742\end{array}$ & $\begin{array}{l}18.40 \\
18.42\end{array}$ & $50-76$ \\
\hline 1.0548 & $13.8 \mathrm{I}$ & 14.57 & 1.0613 & 15.43 & 16.38 & 1.0678 & 16.94 & 18.09 & $\begin{array}{l}1.0742 \\
1.0743\end{array}$ & $\begin{array}{l}18.42 \\
18.44\end{array}$ & 10.79 \\
\hline x. 0549 & 13.83 & $x 4-59$ & 1.0614 & 15.45 & 10.40 & 1.0679 & $\times 6.96$ & $x 8 . x x$ & 1.0744 & $\begin{array}{l}18.44 \\
18.47\end{array}$ & $\begin{array}{l}10.81 \\
10.84\end{array}$ \\
\hline $\mathbf{x}, 0550$ & 13.86 & 14.62 & 7.0615 & 15.47 & 16.42 & 1.0680 & 16.90 & 18.15 & $\mathrm{I} .0745$ & 8.49 & \\
\hline$x, 055 x$ & 13.88 & 14.64 & 1.0610 & 15.49 & 16.44 & I. $608 \mathrm{I}$ & $17.0 \mathrm{r}$ & 18.17 & 1.0746 & $\begin{array}{l}18.49 \\
+8.51\end{array}$ & $\begin{array}{l}19.87 \\
10.89\end{array}$ \\
\hline 1.0552 & 13.91 & 14.68 & 1.0617 & 25.52 & 16.48 & 1.0682 & 17.03 & 18.19 & 3.0747 & $\begin{array}{l}18.51 \\
18.53\end{array}$ & $\begin{array}{l}19.89 \\
19.91\end{array}$ \\
\hline 1.0553 & 13.93 & 14.70 & 1.0618 & 15.54 & 16.50 & I. .0683 & 17,06 & 18.23 & 1.0748 & $\begin{array}{r}1.35 \\
8.35\end{array}$ & $\begin{array}{r}19.91 \\
10.04\end{array}$ \\
\hline$x .0554$ & 13.96 & $14 \cdot 73$ & 1.0619 & 15.56 & 26.52 & $x .0684$ & 17.08 & 78.25 & 1.0749 & 18.57 & $\begin{array}{r}19.94 \\
+9.96\end{array}$ \\
\hline 1. 0555 & 13.98 & 14.76 & 1.0620 & 15.58 & 16.55 & 685 & $17 \times x$ & $x 8,28$ & x. 0750 & 18.59 & to. 0.08 \\
\hline x.0556 & 14.01 & 14.79 & $x .062 x$ & 15.60 & 16.57 & T. 0686 & 17,13 & 38. 31 & 1. 0751 & 18.62 & $\begin{array}{l}19.00 \\
20.02\end{array}$ \\
\hline 1.0557 & 14.03 & $14.8 \mathrm{I}$ & 1.0622 & 15.63 & 16.60 & 3.0687 & $17 \cdot 16$ & 18.34 & 1.0752 & $r 8.64$ & 20.04 \\
\hline 1.0558 & 14.06 & 34.84 & 1.0623 & $\times 5.65$ & 16.02 & 3.0688 & 17.18 & 18.36 & 1.0753 & 38.66 & 20.07 \\
\hline 1.0559 & 14.08 & 14.87 & $x .0024$ & 15.67 & 16.64 & 1.0689 & 17.21 & 18.40 & 1.0754 & 18.68 & 20.09 \\
\hline x. 0560 & 14.11 & 14.90 & 1.0625 & 15.69 & 16.66 & 1,0600 & 17,23 & 18.42 & 1.0755 & 18.70 & 20,11 \\
\hline I. $056 t$ & 14.13 & 14.02 & 1.0626 & 15.72 & 16.70 & 1.0691 & 17.25 & 18.44 & 1.0750 & 18.72 & $20 \cdot 14$ \\
\hline 1.0562 & 14.16 & 14.96 & 1.0627 & 15.74 & 16.73 & 1.0602 & 17,28 & 18.48 & 1.0757 & $x 8,74$ & 20,16 \\
\hline I. 0563 & 14.18 & $\begin{array}{r}1.98 \\
15,91\end{array}$ & 1.0628 & 15.76 & 16.75 & I. 0603 & 17.30 & 18.50 & 1.0758 & 18.76 & 20.18 \\
\hline$x .0564$ & 14.21 & 15.01 & 1.0629 & 15.78 & 10.77 & I. 0004 & 17.33 & 18.53 & 1.0759 & 18.78 & 20.21 \\
\hline $\begin{array}{l}x .0565 \\
x .0566\end{array}$ & $\begin{array}{l}14.23 \\
14.26\end{array}$ & $\begin{array}{l}15.03 \\
15.07\end{array}$ & $x .0630$ & 15.80 & 16.80 & x. .0695 & 17.35 & $x 8.5^{6}$ & $x, 0760$ & $\mathrm{t} 8.8 \mathrm{t}$ & 20.24 \\
\hline $\begin{array}{l}x, 0566 \\
1.0567\end{array}$ & $\begin{array}{l}14.26 \\
14.28\end{array}$ & $\begin{array}{l}15.07 \\
15.00\end{array}$ & $\mathrm{I} .063 \mathrm{I}$ & 15.83 & 16.83 & 1.0606 & 17.38 & 18,50 & $1.076 x$ & 18.83 & 20.26 \\
\hline $\begin{array}{r}1.0567 \\
1.0568\end{array}$ & $\begin{array}{l}14 \cdot 28 \\
14.37\end{array}$ & $\begin{array}{l}15,00 \\
15,12\end{array}$ & 1.0632 & 15.85 & 16.85 & 1.0697 & 17.40 & $18.6 \mathrm{r}$ & 1.0762 & 18.85 & 20.29 \\
\hline $\begin{array}{l}1.0568 \\
1.0569\end{array}$ & $\begin{array}{l}14 \cdot 31 \\
14 \cdot 33\end{array}$ & $\begin{array}{l}15.12 \\
15.15\end{array}$ & 1.0033 & 15.87 & 10.87 & 1. .0698 & $17 \cdot 43$ & 18.65 & $\mathrm{x} .0763$ & 18.87 & 20.31 \\
\hline 1.0569 & $14 \cdot 33$ & 15.15 & 1.0634 & 15.89 & 16.90 & x. 0699 & 17.45 & 18.67 & 1.0704 & 18.80 & 20.33 \\
\hline $1.057^{\circ}$ & $\begin{array}{l}14 \cdot 36 \\
14 \cdot 35\end{array}$ & 15.18 & 1.0635 & 15.92 & .93 & $\infty$ & $17 \cdot 4^{8}$ & $18.7^{\circ}$ & 1.0765 & 18.01 & 20.36 \\
\hline$x: 0571$ & $\begin{array}{l}14.38 \\
\times 4.47\end{array}$ & $\begin{array}{l}15=20 \\
15.23\end{array}$ & I. 0630 & 15.94 & 0.95 & 1.0701 & $17-50$ & 18.73 & 1.0766 & 8.93 & 20.38 \\
\hline 1.0572 & $\begin{array}{l}x .4 .41 \\
1.4 .44\end{array}$ & $\begin{array}{l}15,23 \\
15,27\end{array}$ & 1.0637 & 15.96 & 16.08 & 1.0702 & 17.52 & 18.75 & $x .0767$ & $x 8.95$ & 20.40 \\
\hline 1,0573 & $\begin{array}{l}1.4 \cdot 44 \\
1.4 \cdot 46\end{array}$ & 15.27 & 1.0038 & 15.98 & 17.00 & 1.0703 & $17 \cdot 54$ & +8.77 & 1. 0768 & 18.97 & 20.43 \\
\hline 1.0574 & $14 \cdot 46$ & 15.29 & 1.0039 & 20.01 & 17.03 & 1.0704 & 17.57 & $18.8 t$ & 1.0769 & 19.00 & $20 \cdot 4^{6}$ \\
\hline$x, 0575$ & 14.49 & 15.32 & 1.0640 & 16,03 & 17.06 & 0705 & 17.59 & 18.83 & $1+0770$ & 10.02 & 20.48 \\
\hline $1,05,6$ & 14.52 & $\times 5.36$ & $x .0641$ & 16.05 & 17.08 & 1.0706 & 17.61 & & 1.0771 & 10.04 & 20.51 \\
\hline r. .0577 & $x .4 \cdot 54$ & 15.38 & 1.0642 & 16.07 & 17.10 & 1.0707 & 17.03 & 8.88 & $1.077^{2}$ & 10.06 & 20.53 \\
\hline$x, 0578$ & 1.4 .57 & 15.41 & 1. 0643 & 16.09 & 17.12 & 1.0708 & $\times 7.66$ & 18.91 & 1,0773 & 19.08 & 20,55 \\
\hline I.057? & 74.59 & $x 5 \cdot 43$ & 1.0644 & 16.12 & 17,16 & 1.0709 & 17.68 & 18.93 & 1.0774 & 19,10 & 20.58 \\
\hline 1.0580 & 14.62 & 15.47 & $x .0645$ & $x 6.14$ & 27.18 & 1.0710 & 17.70 & 18. 96 & 1.0775 & $19: 12$ & 20.60 \\
\hline $\mathbf{1} .0581$ & 74.65 & $15.50^{\circ}$ & I. $.064^{6}$ & 16.16 & 17.20 & 1.0711 & 17.72 & 18.98 & 1.0776 & 19.14 & 20.63 \\
\hline 1.0584 & 1.4 .67 & 15.52 & 1.0647 & 26,38 & $17+23$ & 1.0712 & 17.75 & $19.0 \mathrm{r}$ & 1.0777 & 19.17 & 20.66 \\
\hline 1.0583 & 14.70 & 15.56 & $x .0648$ & 36.21 & 17.26 & 1.0713 & $17 \cdot 77$ & 19.04 & I. 0778 & 70.19 & 20.68 \\
\hline 1,0584 & 14.73 & 15.59 & 5.0649 & 10.23 & 17.28 & 1.0714 & 17.79 & 19.06 & $x .0779$ & 90.21 & 20.71 \\
\hline
\end{tabular}


EXTRACT IN BEER WORT-(Continued).

\begin{tabular}{|c|c|c|c|c|c|c|c|c|c|c|c|}
\hline \multirow[b]{2}{*}{$\begin{array}{l}\text { Specific } \\
\text { Gravity } \\
\text { at } 15^{\circ} \mathrm{C} \text {. }\end{array}$} & \multicolumn{2}{|c|}{ Extract. } & \multirow[b]{2}{*}{$\begin{array}{l}\text { Specific } \\
\text { Gravity } \\
\text { at } 15^{\circ} \mathrm{C} .\end{array}$} & \multicolumn{2}{|c|}{ Extract. } & \multirow[b]{2}{*}{$\begin{array}{l}\text { Specific } \\
\text { Gravity } \\
\text { at } 15^{\circ} \mathrm{C} \text {. }\end{array}$} & \multicolumn{2}{|c|}{ Extract. } & \multirow[b]{2}{*}{$\begin{array}{l}\text { Specific } \\
\text { Gravity } \\
\text { at } 15^{\circ} \mathrm{C} \text {. }\end{array}$} & \multicolumn{2}{|c|}{ Extract. } \\
\hline & $\begin{array}{c}\text { Per } \\
\text { Cent } \\
\text { by } \\
\text { Weight }\end{array}$ & $\begin{array}{c}\text { Grams } \\
\text { per } \\
\text { too ec. }\end{array}$ & & $\begin{array}{c}\text { Per } \\
\text { Cent } \\
\text { by } \\
\text { Weight }\end{array}$ & $\begin{array}{c}\text { Grams } \\
\text { per } \\
\text { too ce. }\end{array}$ & & $\begin{array}{c}\text { Per } \\
\text { Cent } \\
\text { by } \\
\text { Weight }\end{array}$ & $\begin{array}{l}\text { Grams } \\
\text { per } \\
\text { too cc. }\end{array}$ & & $\begin{array}{c}\text { Per } \\
\text { Cent } \\
\text { by } \\
\text { Weight }\end{array}$ & $\begin{array}{l}\text { Grams } \\
\text { per } \\
\text { roo ce. }\end{array}$ \\
\hline 1.0780 & 19.23 & 20.73 & 3.0845 & $20 \cdot 70$ & $22: 45$ & $2.09 \times 0$ & 22.10 & $24.2 x$ & 1.0075 & 23.50 & 25.80 \\
\hline$t .078 t$ & 10.25 & 20.75 & 1.0846 & 20.73 & 22.48 & 1,0011 & 22.21 & 24.24 & $x .0076$ & $23.6 \pi$ & 25.92 \\
\hline$x .078 z$ & 19.27 & 20.78 & 1.0847 & 20.75 & 22.50 & $1.09 t_{2}$ & 22,23 & 24.26 & 1.0977 & 23.63 & $25 \cdot 04$ \\
\hline 1.0783 & 10.29 & 20.80 & 1.0848 & 20.77 & 22.53 & 1.0913 & 22.26 & 24.29 & I.0978 & 23.65 & 25.07 \\
\hline 1.0784 & 10.31 & 20.82 & 2.0849 & 20.70 & 22.55 & 1.0914 & 22,28 & $24 \cdot 31$ & 1. 0979 & 23.67 & 25.99 \\
\hline I. 0785 & 19.33 & 20.85 & 1.0850 & $20.8 \mathrm{r}$ & 22.58 & x. 0015 & 22.30 & 24.34 & 1.0980 & 23.60 & $26.0 \mathrm{r}$ \\
\hline$x .0786$ & $\times 9.36$ & 20.88 & $2.085 x$ & 20.83 & 22.61 & 1.0916 & 22,32 & 24.37 & t. $098 x$ & 23.71 & 26.04 \\
\hline x.0787 & $10-38$ & 20.90 & 1.0852 & 20.86 & 22.64 & 1.0017 & $22-34$ & 24.30 & 1.0082 & 23.73 & 26.06 \\
\hline x. 0788 & 19.40 & 20.93 & 1.0853 & 20.88 & 22.66 & $1.09 \times 8$ & 22.37 & $24 \cdot 42$ & I. 0983 & 23.76 & 26.09 \\
\hline x. 0789 & 19.42 & 20.95 & t. .0854 & 20.90 & 22.68 & $1.00 \times 9$ & 22,39 & $24 \cdot 44$ & I. .0984 & 23.78 & 26,11 \\
\hline$\tau .0700$ & to. 44 & 20.08 & $\mathrm{x} .0855$ & 20.03 & 22.72 & 1.0920 & $2.2 .4 \mathrm{~T}$ & $24 \cdot 47$ & 1.0085 & 23.80 & 26,74 \\
\hline $1.070 \pi$ & 19.46 & 21.00 & 1.0856 & 20,95 & 22.75 & $x .0921$ & 22.43 & 24.49 & I. 0986 & 23.82 & 26,17 \\
\hline 1.0792 & 10.40 & 21.03 & 1.0857 & $20.9^{8}$ & 22.78 & 1.0922 & 22.45 & 24.51 & 1.0087 & 23,84 & 26,10 \\
\hline 1.0703 & $10.5 \mathrm{t}$ & 21.06 & $x .0858$ & 21 - 91 & 22.81 & 1. 0023 & 2248 & 24.54 & $x .0988$ & 23.86 & 26.22 \\
\hline 1.0794 & 19.53 & 21.08 & 1.0850 & 21,04 & 22.84 & 1.0924 & 22.50 & 24.56 & 1.0989 & 23.88 & 26.24 \\
\hline I. 0705 & 10.56 & $2 \pi \cdot t I$ & $t .0860$ & 28.06 & 22.87 & 1.0023 & 22.52 & 24,60 & x. 0990 & 23.00 & 26.27 \\
\hline 1.0706 & 19.58 & 28,14 & $t .086 t$ & $21: 09$ & 22,00 & 1.0926 & 22.54 & 24.62 & $1.009 x$ & 23.92 & 26,30 \\
\hline I. 0707 & 19.60 & $21+16$ & 1.0862 & $2 x \cdot X$ & 22.93 & 1.0027 & 22.56 & 24.64 & 1.0092 & 23.04 & 26.32 \\
\hline 1.0708 & 10.63 & 21,20 & t. 0863 & 21,13 & 22,96 & 1.0028 & 22.59 & 24,67 & 1.0003 & 23.07 & 26.35 \\
\hline 1.0799 & 19.65 & 21,22 & 1.0864 & $21 \cdot 16$ & 22.99 & 1.0929 & $22.6 t$ & 24.70 & 1.0994 & 23.99 & 26.37 \\
\hline$x .0800$ & xo.67 & 21.24 & r. 0865 & $21+19$ & 23.02 & 1.0030 & 22.63 & 24,73 & 1.0095 & $24.0 \mathrm{t}$ & 26.40 \\
\hline 1. $080 \mathrm{r}$ & 19.70 & $2 x-28$ & $1-0866$ & $2 x \cdot 22$ & 23.06 & $1.093 \mathrm{t}$ & 22.65 & 24.76 & 1.0006 & 24.03 & 26.42 \\
\hline 1.0802 & 19.72 & $21 \cdot 30$ & x. .0867 & 21,25 & 23.09 & 1. 0032 & 22.67 & $24 \cdot 78$ & 1. .0997 & 24.05 & 26.44 \\
\hline 1.0803 & 19.74 & 21.33 & 1.0868 & 21,28 & 23.12 & 1.0033 & 22.69 & 24,81 & 1. 0008 & 24.07 & 26.47 \\
\hline I. 0804 & 19.77 & 21,36 & I. .0869 & $2 x \cdot 30$ & 23.15 & 1.0934 & 22.71 & 24.83 & I. 0999 & 24.09 & 26.49 \\
\hline 1.0805 & 19.79 & $21 \cdot 38$ & 1.0870 & $2 \mathrm{r} \cdot 33$ & 23.18 & 1.0035 & 22.73 & 24,86 & 1. 1000 & 24.11 & 26.52 \\
\hline 1.0806 & 19.81 & 21.41 & $1.087 \mathrm{I}$ & 21.35 & 23.21 & 1.0936 & 22.75 & 24.89 & I. root & 24.13 & 26.55 \\
\hline 1.0807 & To. 84 & $2 x .43$ & $x .0872$ & $21 \cdot 37$ & 93.23 & 1.0037 & 22.77 & $24 \cdot 9 x$ & 1. 1002 & 24,15 & 26.57 \\
\hline I. 0808 & 19.86 & $2 x, 46$ & 1.0873 & $21-39$ & 23.26 & 1.0038 & 22.80 & 24.93 & 1.1003 & $24 \times 7$ & 26,60 \\
\hline 1.0800 & 19.88 & 21.49 & I. .0874 & $2 x \cdot 4 x$ & 23.28 & 1.0939 & 22.82 & 24.96 & I. 1004 & 24,19 & 26.62 \\
\hline 1. 08 ro & 19.01 & 21,52 & x. .0875 & $2 \mathrm{r} .43$ & 23.31 & 1.0940 & $22: 84$ & 24.90 & 1. 1005 & 24.21 & 26,65 \\
\hline $\mathrm{I} .08 \mathrm{xI}$ & 10.93 & 21.55 & $x .0876$ & $2 x \cdot 45$ & 23.33 & 1. $.094 x$ & 22,86 & 25.01 & 1. 1006 & 24.23 & 26.68 \\
\hline 1.0812 & 10.96 & $21 \cdot 58$ & 1.0877 & 22.47 & 23.36 & 1.0042 & 22.88 & 25.03 & I. 1007 & 24.25 & 26.70 \\
\hline 1.0813 & 19.98 & 21.60 & 1.0878 & $21 \cdot 49$ & $23 \cdot 38$ & I. 0043 & 22.90 & 25.06 & 1. 1008 & $24 \cdot 28$ & 26.73 \\
\hline $\mathrm{I} .08 \mathrm{r} 4$ & 20,00 & 21.63 & 1. .0879 & $2 x \cdot 51$ & 23.40 & I. .0944 & 22.92 & 25.08 & I. 1009 & $24 \cdot 30$ & 26.75 \\
\hline 1.0815 & 20.03 & 21.66 & $1.088 a$ & $2 \mathrm{I} \cdot 54$ & $2.3 \cdot 43$ & I. .0945 & 22,94 & 25.11 & I. toro & $24 \cdot 32$ & 26.78 \\
\hline$x .0816$ & 20.05 & 25.69 & $x .088 x$ & 21.56 & 23.45 & I. 0946 & 22.96 & $25 \cdot \mathrm{I} 4$ & 1.1011 & $24 \cdot 34$ & $26.8 \mathrm{r}$ \\
\hline$x .0817$ & 20,07 & $21 \cdot 71$ & 1.0882 & 21.58 & 23.48 & i. .0047 & 22.98 & $25 \times 16$ & 1.1012 & $24 \cdot 36$ & 20.83 \\
\hline $1.08 \times 8$ & 20.10 & 21.74 & 1.0883 & 21.60 & 23,50 & I. 0048 & 23.00 & $25 \cdot 18$ & 1.1013 & 24.39 & 26.86 \\
\hline I. $08 \times 9$ & 20.12 & 21.77 & 1.0884 & 21.62 & $23 \cdot 52$ & I. 0949 & 23.03 & 25.21 & I. $1 \circ 14$ & $24 \cdot 41$ & 26.88 \\
\hline 1.0820 & 20.14 & 21.79 & $\mathbf{x} .0885$ & 21.64 & 23.55 & 1.0050 & 23.05 & 25.24 & $1.10 x 5$ & 24.45 & 26.91 \\
\hline $\mathrm{I}, 082 \mathrm{I}$ & 30.17 & 21.83 & t. 0886 & 21.66 & $23 \cdot 58$ & I. $005 x$ & 23.07 & 25.26 & 1.1016 & $24 \cdot 45$ & 26.93 \\
\hline 1.0822 & 20.10 & 21.85 & I. 0887 & 21.68 & 23.60 & 1.0952 & 23.10 & 25.29 & t. $\operatorname{ror} 7$ & $24 \cdot 47$ & 26.95 \\
\hline 1.0823 & 20.21 & 21.87 & $x .0888$ & 21.71 & 23.63 & I. 0953 & 23.12 & 25.31 & 1.1018 & 24.49 & 26,08 \\
\hline$x .0824$ & $20 \cdot 24$ & $2 \pi \cdot 9 \pi$ & 1.0889 & 21.73 & 23.66 & 1.0054 & $23 \cdot 14$ & $25 \cdot 34$ & 1. 1019 & $24 \cdot 51$ & 27.00 \\
\hline 1.0825 & 20.26 & 21,93 & 1.0890 & $2 \pi .75$ & 23.69 & $x .0955$ & 23.16 & $25 \cdot 37$ & 1.1020 & $24 \cdot 53$ & 27.03 \\
\hline 1.0826 & 20.28 & $2 x .06$ & $x .0801$ & $2 x, 77$ & 23.72 & 1.0956 & 23.18 & $25 \cdot 39$ & 1.1021 & 24.55 & 27.06 \\
\hline 1.0827 & $20 \cdot 31$ & 21.99 & 1.0892 & 21.79 & $23 \cdot 74$ & 1.0957 & 23,20 & 25.42 & 1.1022 & 24.57 & 27.08 \\
\hline 1.0828 & 20.33 & $22.0 \mathrm{I}$ & I. 0893 & 21.82 & 23.77 & I.0958 & 23.23 & 25.45 & 1.1023 & 24,60 & 27.11 \\
\hline 1.0829 & 20.35 & 22.04 & 1.0894 & 21.84 & 23.79 & 1.0059 & 23.25 & 25.47 & I. 1024 & 24.62 & 27.14 \\
\hline$x .0830$ & 20.37 & 22.06 & 1.0805 & 21.86 & 23.82 & I. 0960 & 23.27 & 25.50 & I. 1025 & 24.64 & $27-17$ \\
\hline 1.0831 & 20.39 & 22.08 & 1.0806 & 21.89 & 23.85 & I. $096 x$ & 23.29 & 25.33 & 1.1026 & 24,06 & $27 \times 19$ \\
\hline 1.0832 & 20.41 & 22.11 & 1.0897 & $2 \mathrm{x}, 9 \mathrm{r}$ & 23.87 & 1.0062 & $23 \cdot 31$ & 25.55 & 1,1027 & 24,68 & 27.21 \\
\hline 1.0833 & 20.43 & 22.13 & 1.0808 & 21.93 & 23.90 & I. 0963 & 23.33 & 25.58 & I. 1028 & 24.70 & 27.24 \\
\hline 1.0834 & 20.46 & 22,16 & 1.0899 & 21.96 & 23.93 & 1. .0964 & $23 \cdot 35$ & 25.60 & 1.1029 & 24.72 & $27 \cdot 26$ \\
\hline I. 0835 & 20.48 & 22.19 & 1.0900 & 21,08 & 23.96 & I. 0065 & 23.37 & 25.63 & $\mathrm{I} \cdot \mathrm{IO} 30$ & $24 \cdot 74$ & 27.29 \\
\hline 1.0836 & 20.50 & 22.21 & I. 0001 & 22.00 & 23.98 & 1.0966 & 23.39 & 25.66 & $2.103 \mathrm{~T}$ & 24.76 & $27 \cdot 32$ \\
\hline 1. .0837 & 20.52 & 22,24 & I. 0902 & 22.02 & 24.01 & 1.0967 & 23.41 & 25.68 & $\mathrm{I} \cdot \mathrm{10} 32$ & 24,78 & 27.34 \\
\hline$x .0838$ & 20.54 & 22.26 & t. 0003 & 22.04 & 24.03 & 1,9068 & 23,44 & $25 \cdot 71$ & I. 1033 & $24.8 \mathrm{I}$ & 27.37 \\
\hline 1.0839 & 20.56 & 22.20 & 1.0004 & 22.06 & 24.05 & 1.0969 & 23.46 & 25.73 & I. 1034 & 24.83 & 27.39 \\
\hline 1.0840 & 20.50 & 22.32 & 1.00 & 22.08 & 24.08 & 1.0970 & 23,48 & $23 \cdot 76$ & I. 1035 & 24.85 & 27.42 \\
\hline$x .084 x$ & 20.62 & 22.35 & 1.0006 & 22.10 & 24.11 & 1.0971 & 23.50 & 25,70 & 1.1036 & 24.87 & 27.45 \\
\hline$x .0842$ & 20.64 & $22 \cdot 38$ & $x .0007$ & 22.12 & 24.13 & 1.0072 & 23.52 & $25.8 x$ & 1.1037 & 24.89 & 27.47 \\
\hline 1.0843 & 20.66 & 22.40 & 1.0908 & 22,15 & 24,16 & 1. 0073 & 23.55 & 25.84 & I. 1038 & 24.92 & 27.50 \\
\hline$x .0844$ & 20.68 & 22.42 & $x .0009$ & 22.17 & 24.18 & 1.0974 & 23,57 & 25.86 & I 1039 & 24.94 & 27.53 \\
\hline
\end{tabular}


EXTRACT IN BEER WORT-(Concluded).

\begin{tabular}{|c|c|c|c|c|c|c|c|c|c|c|c|}
\hline \multirow[b]{2}{*}{$\begin{array}{l}\text { Specific } \\
\text { Gravity } \\
\text { at } 15^{\circ} \mathrm{C} \text {. }\end{array}$} & \multicolumn{2}{|c|}{ Extract. } & \multirow[b]{2}{*}{$\begin{array}{l}\text { Specific } \\
\text { Gravity } \\
\text { at } 15^{\circ} \mathrm{C} .\end{array}$} & \multicolumn{2}{|c|}{ Extract. } & \multirow[b]{2}{*}{$\begin{array}{l}\text { Specific } \\
\text { Gravity } \\
\text { at is }{ }^{\circ} \mathrm{C} .\end{array}$} & \multicolumn{2}{|c|}{ Extract. } & \multirow[b]{2}{*}{$\begin{array}{l}\text { Specific } \\
\text { Gravity } \\
\text { at } 15^{\circ} \mathrm{C} \text {. }\end{array}$} & \multicolumn{2}{|c|}{ Extract. } \\
\hline & $\begin{array}{c}\text { Per } \\
\text { Cent } \\
\text { by } \\
\text { Weight }\end{array}$ & $\begin{array}{c}\text { Grams } \\
\text { per } \\
\text { too ce. }\end{array}$ & & $\begin{array}{c}\text { Per } \\
\text { Cent } \\
\text { by } \\
\text { Weight }\end{array}$ & $\begin{array}{c}\text { Grams } \\
\text { per } \\
\text { roo cc. }\end{array}$ & & $\begin{array}{c}\text { Per } \\
\text { Cent } \\
\text { by } \\
\text { Weight }\end{array}$ & $\begin{array}{c}\text { Grams } \\
\text { per } \\
\text { too ce. }\end{array}$ & & $\begin{array}{c}\text { Per } \\
\text { Cent } \\
\text { by } \\
\text { Weight }\end{array}$ & $\begin{array}{l}\text { Grams } \\
\text { per } \\
\text { too ce. }\end{array}$ \\
\hline 1,1010 & 24.96 & $27 \cdot 56$ & 1. 1005 & 26,16 & 29.03 & 1.1150 & 27.29 & 30,43 & 1,1205 & 28,38 & $3 t .8 t$ \\
\hline 1.1041 & 24.98 & 27.58 & f. 1006 & $26 .+8$ & 20.06 & I. $115 \mathrm{x}$ & 27.31 & 30.45 & 1.1206 & 28.40 & 31.83 \\
\hline$x+1042$ & 25.00 & 27.60 & I. 1097 & 26.20 & 20.08 & $1,1+52$ & $27-33$ & $30 \cdot 47$ & 1.1207 & 28.42 & 31.86 \\
\hline$x+1043$ & 25.03 & 27.63 & $x \cdot 1098$ & 26.23 & 20.11 & 1.1153 & $27 \cdot 35$ & 30.50 & 1,1208 & $28 \cdot 44$ & 31,88 \\
\hline $1+1044$ & 25.05 & 27.66 & 1. 1009 & 26.25 & 29.13 & 1.1154 & $27-37$ & 30.52 & $x=1209$ & 28.46 & 31.02 \\
\hline$x, 1045$ & 25.07 & 27.60 & 1. 1100 & 26.27 & $29 \cdot 16$ & $t+7+55$ & $27-38$ & 30.55 & 1.1210 & $28 \cdot 48$ & 31.93 \\
\hline 1.1045 & 25.00 & 27.72 & 1. $110 \mathrm{x}$ & 26.20 & $29 \cdot 10$ & $x+156$ & 27.42 & 30.57 & 1.1211 & 28.50 & $31=95$ \\
\hline 1.1047 & 25.11 & $27 \cdot 74$ & $x, 1102$ & 26.31 & 20.21 & $2.1+57$ & 27.42 & 30.59 & 1.1212 & 28.52 & $3 t \cdot 98$ \\
\hline$x, 1048$ & 25.74 & 27.77 & 1.1103 & 26.33 & 29.24 & $1+1258$ & $27 \cdot 44$ & 30.62 & 1.1213 & 28.54 & 32.30 \\
\hline I. 1049 & 25.16 & $27+79$ & $x .1104$ & 26.35 & 29.26 & 2.1159 & $27 \cdot 46$ & 30.64 & I. 1214 & 28.56 & 32.03 \\
\hline 2.1050 & 25,18 & 27.82 & T. $x 105$ & 26.37 & 20.29 & $1=1160$ & $27 \cdot 48$ & 30.67 & 1.1215 & 28.58 & 32.05 \\
\hline 1.1051 & 25.20 & 27.85 & 1.1106 & 26.30 & $29 \cdot 32$ & $1.116 x$ & 27.50 & 30.60 & 1.1216 & 28.60 & 32.08 \\
\hline 1.7052 & 25.22 & 27.87 & I. 1107 & $26,4 r$ & $20-34$ & $x+1+62$ & 27.52 & 30.72 & $1 .+217$ & 28.62 & 32,11 \\
\hline t. 1053 & 25,24 & 27.00 & $x, 1+08$ & 26.44 & 20037 & I. 1163 & $27 \cdot 54$ & 30.75 & $1 . \tan 8$ & 28,64 & $32, I_{3}$ \\
\hline 1. 1054 & $25 \cdot 27$ & 27.93 & I. Tro9 & $20 \cdot 4^{6}$ & $29 \cdot 39$ & $1.1 \times 64$ & 27.56 & 30.77 & 1.1219 & 28.66 & 32.15 \\
\hline t. 1055 & 25,29 & 27.06 & $x, x+0$ & 26.48 & $20 \cdot 42$ & I. $.1+65$ & 27.58 & 30.80 & 1,1220 & 28.68 & 32,18 \\
\hline 1,1056 & $25-31$ & 27.98 & I. $x \mathrm{xPt}$ & 36.50 & $20 \cdot 44$ & 1. $1 \times 56$ & 27.60 & 30.82 & $1.122 t$ & 28,70 & 32.20 \\
\hline 1. 1057 & 25.33 & 28.00 & $1 \cdot \operatorname{tin} z$ & 26,52 & $20 \cdot 46$ & 5. 5167 & 27.62 & 30.85 & 1,1222 & 28.72 & 32.23 \\
\hline 1.1058 & 25.35 & 28.03 & $1,+113$ & 26.54 & 29,49 & 1.1168 & 27.64 & 30.87 & $t, 2223$ & 28,74 & 32.25 \\
\hline 1,1059 & $25 \cdot 38$ & 28,06 & $1.11 \times 4$ & 26.56 & $29.5 \mathrm{I}$ & X.x+69 & 27.66 & 30,80 & $x, 1 \geq 24$ & 28,76 & 32.27 \\
\hline 1.1060 & 25.40 & 28.69 & $1, x 115$ & 26.58 & 20.54 & $t-1170$ & 27.68 & 30,97 & 7.1225 & $28 \cdot 78$ & 32.30 \\
\hline I. $106 \mathrm{t}$ & 25,42 & 28.12 & $1 . x+16$ & 26.60 & 20.57 & $\mathrm{x}, \mathrm{xI} 7 \mathrm{r}$ & 27.72 & 30.01 & $1 .+226$ & 28.80 & 32.32 \\
\hline 1.1062 & 25.44 & 28.74 & 1.1117 & 26.62 & 20.50 & 5.8172 & 27.72 & 30.07 & 1.1227 & 28.82 & 32.35 \\
\hline$t=1063$ & 25.46 & 28.17 & $1+1+18$ & 26.64 & 20.61 & $x+1 \times 7 ;$ & $27-74$ & 31.80 & 1.1228 & 28.84 & 32.37 \\
\hline 1. 1064 & $25 \cdot 48$ & 28.19 & I. IIIO & 26.06 & 29.64 & $x \cdot 1+74$ & 27.75 & 31.02 & 1.2229 & 28.86 & 32.40 \\
\hline t. 1065 & 25.50 & 28,22 & 1,1120 & 26.68 & 20.67 & 1.1175 & $27-78$ & 31.03 & 1.1230 & 28.88 & 32.43 \\
\hline 1. 1066 & 25.52 & 23.25 & I. IIZI & $26 \cdot 70$ & 20.00 & $x .1176$ & 27.80 & 31.07 & 1.2251 & 28,90 & $32: 45$ \\
\hline ז. 1067 & 25.54 & 28,27 & I. 1122 & 26.72 & 20,71 & 1,1177 & 27.82 & 31.00 & 1.1232 & 28.92 & 32.43 \\
\hline 1. 1068 & 25,57 & 28.30 & 1.1123 & 26.75 & $29 \cdot 74$ & $1 .+178$ & 27.84 & $3 x+12$ & $T, 1233$ & 28,94 & 32.50 \\
\hline 1. 1069 & 25.59 & 28.32 & x. $\operatorname{Tr} 24$ & 26.77 & 29.77 & 1.1179 & 27.86 & 31,15 & 1. 1234 & 28.96 & 32.53 \\
\hline 1,1070 & $25,6 x$ & 28.35 & $1 .+1 \times 25$ & 26.79 & 29.80 & 1.r+80 & 27.88 & $3 t .+8$ & 1.1235 & 28.98 & 32.56 \\
\hline 1.1071 & 25.63 & 28.38 & $r \cdot x+26$ & $26.8 \mathrm{i}$ & 29.83 & $1.138 \mathrm{r}$ & 27.00 & $31-20$ & 1.1236 & 20,00 & 32,58 \\
\hline 1.1072 & 25.65 & 28.40 & $1 .+127$ & 26.83 & 20.85 & $1.1 \times 82$ & 27.02 & $3 x=23$ & $1 . \times 237$ & 29.02 & 32,60 \\
\hline I. 1073 & 25.67 & 28,43 & $1 . \operatorname{Tr} 23$ & 26.85 & 20.88 & I. 1183 & $27 \cdot 94$ & 31.25 & 1,1238 & $20 \cdot 04$ & 32.63 \\
\hline I. $x 074$ & 25.69 & 28.45 & $x \cdot \operatorname{tr} 29$ & 26.87 & 29.92 & I. 1184 & 27,96 & $3 x \cdot 27$ & 1.1239 & 29.06 & 32.65 \\
\hline 1,1075 & 25.71 & 28.48 & 1.1130 & 26.86 & 20.03 & $\mathrm{I}, \mathrm{T}+85$ & 27.98 & 31.30 & 1. 1240 & 20.08 & 32.68 \\
\hline 1.1076 & 25.73 & 28.51 & 1. $113 \mathrm{t}$ & 26.01 & 20.05 & $x .1 \pm 86$ & 28.00 & 31.32 & 1.1241 & 20.10 & $32.7 \mathrm{r}$ \\
\hline I. 1077 & 25.75 & $28 \cdot 53$ & $x .1232$ & 26.93 & 29.07 & $1+1+87$ & 28.02 & $31 \cdot 35$ & 1.1242 & 29.12 & $32 \cdot 73$ \\
\hline 1.1078 & $25 .-8$ & 28.56 & 1,1133 & 26.05 & 30.00 & T. 1188 & 28,94 & $38 \cdot 37$ & $1 .+243$ & 20.14 & 32.76 \\
\hline 1.1079 & 25.80 & 28.58 & 1.1134 & 26.97 & 30.02 & 1.1189 & 28.07 & 31.40 & 1.1244 & 29.16 & 32.78 \\
\hline 1. 1080 & 25.82 & $28.6 r$ & $1.1 \times 35$ & 26.99 & 30.06 & $x \cdot 1200$ & 28.00 & $3 i \cdot 43$ & 1.1245 & 20,18 & $32.8 t$ \\
\hline I. 1081 & 25,84 & 28.64 & 1. $7+36$ & 27.01 & 30,08 & $t, t+0 t$ & 28.11 & $3 t \cdot 45$ & 1.1246 & 20.20 & 32.83 \\
\hline 1.2082 & 25.86 & 28.66 & 1.1137 & 27.03 & 30.10 & 1.1192 & 28.13 & $3 I=48$ & I. 1247 & 20,22 & 32.86 \\
\hline 1. 1083 & 25.89 & 28.69 & $x .1138$ & 27.05 & 30.13 & 1,1103 & $28.1=$ & 31.51 & I. 1248 & $29 \cdot 24$ & 32.80 \\
\hline 1,1084 & 25.91 & 28.72 & $1.1+39$ & 27.07 & 30.15 & 1.1194 & 28.17 & 31.53 & I. $x=249$ & 29.26 & 32.91 \\
\hline 1,1085 & 25.93 & 28.75 & $\mathrm{I} . \mathrm{II}_{4} \mathrm{O}$ & 27.09 & 30.18 & 1. 1795 & 28.19 & $3 x .56$ & 1.1250 & 29.28 & $32 \cdot 94$ \\
\hline I. 7086 & 25.06 & 28.78 & $t .1+4 t$ & $27+11$ & 30.20 & 1.11906 & 28.21 & $3 r .59$ & I. 1251 & 29.30 & 32.96 \\
\hline 1. 1087 & 25.98 & 28.80 & 1.1142 & 27.13 & 30.22 & 1.1197 & 28.23 & $3 x .61$ & 1.1252 & $29-32$ & 32.99 \\
\hline 1. 1088 & 26.01 & 28.83 & $\mathrm{I}, \mathrm{II} 43$ & 27.15 & 30.25 & 1. $1+03$ & 28.25 & 37.63 & $\mathrm{I} .1253$ & 20.34 & 33.02 \\
\hline 1.1080 & 26.03 & 28.86 & $x+1144$ & 27.17 & 30.27 & I.1199 & 28.27 & 31,65 & I. 1254 & $29 \cdot 36$ & 33.04 \\
\hline 1. 1000 & 26.05 & 28.89 & I. 1145 & 27.19 & $30 \cdot 31$ & 1.1200 & 28.28 & $3 \mathrm{x} \cdot 68$ & $\mathrm{I}, \mathrm{I} 255$ & $29 \cdot 38$ & 33.27 \\
\hline 1. 1001 & 26.07 & 28.92 & 1.1146 & $27 \cdot 21$ & 30.33 & I. 1201 & 28.30 & 31.70 & 1.1250 & 29.40 & 33.09 \\
\hline $\begin{array}{l}\text { t. } 1002 \\
1,1003\end{array}$ & 26.09 & 28.94 & I. 1147 & 27.23 & 30.35 & 1.1202 & 28.32 & $3 t \cdot 73$ & 1.1257 & $29 \cdot 42$ & $33 \cdot 12$ \\
\hline $\begin{array}{l}\text { 1. } 1003 \\
\text { 1. } 1094\end{array}$ & 26.12 & 28.97 & I. 1148 & $27 \cdot 25$ & 30.37 & 1.1203 & 28.34 & $3 x .75$ & 1.1258 & $29 \cdot 45$ & $\begin{array}{r}33.14 \\
3,17\end{array}$ \\
\hline I. 1094 & 26.14 & 29.00 & 1.1149 & 27.27 & 30.40 & I. I 204 & $28 \cdot 36$ & 35.73 & 1.1259 & $29 \cdot 47$ & 33.17 \\
\hline
\end{tabular}




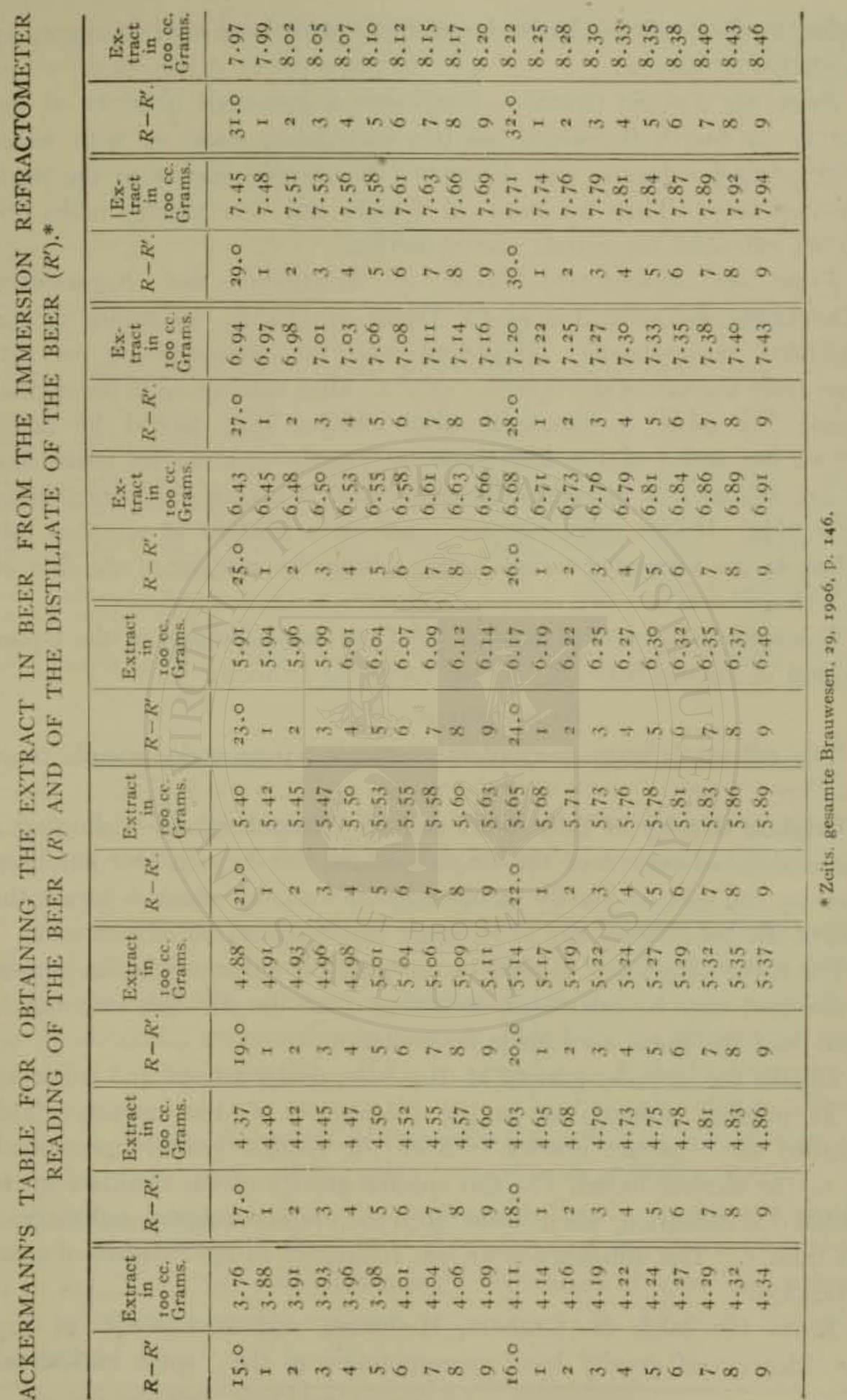


mation to the truth is obtained, especially with beer high in sugar, by calculation as follows:

From the Specific Gravity.-Evaporate a measured quantity of the beer to one-fourth its volume on the water-bath, make up with water to its original measure, and determine the specific gravity of the dealcoholized beer. Then by means of Schultz and Ostermann's table, pp. 716-20, calculate the extract corresponding.

From the Refraction.-Method of Ackermann and Foggenburg. -Determine the refraction of the liquor at $17.5^{\circ} \mathrm{C}$. by means of the immersion refractometer. Determine also the refraction of the distillate from $100 \mathrm{cc}$. of the liquor at $17.5^{\circ} \mathrm{C}$. after making up to its original volume. In order to secure accurate results, care should be taken to cool the prism of the instrument to exactly $17.5^{\circ} \mathrm{C}$. by immersing for five minutes in the water-bath previous to taking the refraction of the liquids. If determinations are made on a number of samples, this cooling is not necessary except before taking the reading of the first of the series.

Calculate the grams of extract $(E)$ from the refraction of the liquor $(R)$ and of the distillate $\left(R^{\prime}\right)$ by the following formula:

$$
E=0.25705\left(R-R^{\prime}\right) \text {. }
$$

The extract is more conveniently obtained from Ackermann's table given on p. 721 .

Original Gravity of Beer Wort and its Determination.-Following a long-established custom of the English excise, the duty on beer has been based on the specific gravity of the original wort, by which is meant the wort of the beer before any of its sugar has been lost by fermentation.

From the content of alcohol in the beer the sugar originally present in the wort may be calculated, assuming that the alcohol amounts to about half the sugar used up in fermentation.

Obtain the specific gravity of the beer, dealcoholized and made up to its original volume, as in the calculation of the extract. This is called the "extract gravity." Note the specific gravity corresponding to the alcohol found, i.e., the specific gravity of the distillate in the alcohol determination, when made up to the original volume, and subtract this from $\mathrm{I}$. The difference is known arbitrarily as the "degree of spirit indication."

From the table of Graham, Hofmann, and Redwood,* p. 723, the "degrees of gravity lost". corresponding to the "spirit indication"

\footnotetext{
* Report on Original Gravities, $185^{2}$; Allen's Com. Org Anal., 4 Ed., Vol. I, p, 151.
} 
are ascertained. This figure is added to the "extract gravity" to find the "original gravity of the wort."

SUGAR USED UP IN FERMENTATION.

\begin{tabular}{|c|c|c|c|c|c|c|c|c|c|c|}
\hline $\begin{array}{l}0.0 \\
505 \\
0: 0\end{array}$ & 11.0000 & 0.0001 & 0,0002 & 0.0003 & 0.0004 & 0,0005 & 0.0006 & 0.0007 & 0.0008 & 0.0009 \\
\hline 0.000 & $\ldots \ldots \ldots$ & 0.0003 & 0.0006 & 0.0009 & 0.0012 & 0.0015 & 0.0018 & 0.0021 & 0.0024 & 0.0027 \\
\hline .001 & .0030 & .0033 & .0037 & $.004 \mathrm{r}$ & .0044 & .0048 & $.005 \mathrm{I}$ & .0055 & .0059 & .0062 \\
\hline .002 & .0066 & .0070 & .0074 & $.007^{8}$ & .0082 & .0086 & .0090 & .0094 & .9098 & .0102 \\
\hline .003 & .0107 & . Orix & .0115 & .0120 & .0124 & .0129 & .0133 & $.013^{8}$ & .0142 & .0147 \\
\hline .004 & $.015 \mathrm{I}$ & .0155 & .0160 & .0164 & .0168 & .0173 & .0177 & .0182 & .0186 & .0191 \\
\hline .005 & .0195 & .0199 & .0204 & .0209 & .0213 & .0218 & .0222 & .0227 & .0231 & $.023^{6}$ \\
\hline .006 & .0241 & .0245 & .0250 & .0255 & .0260 & .0264 & .0269 & .0274 & .0278 & $.028_{3}$ \\
\hline .007 & .0288 & .0292 & .0297 & .0302 & .0307 & .0312 & .0317 & .0322 & .0327 & .0332 \\
\hline .008 & .0337 & .0343 & .0348 & .0354 & .0359 & .0365 & .0370 & .0375 & .0380 & .0386 \\
\hline.$\infty 09$ & $.039 \mathrm{r}$ & .0397 & .0402 & .0407 & .0412 & .0417 & .0422 & .0427 & .0432 & .0437 \\
\hline -oro & .0442 & .0447 & .0451 & .0456 & .0460 & .0465 & .0476 & .0475 & .0480 & .0485 \\
\hline -O11 & .0490 & .0496 & .0501 & .0506 & .0512 & .0517 & .0522 & .0527 & .0533 & $.053^{8}$ \\
\hline .012 & .0543 & .0549 & .0554 & .0559 & .0564 & .0569 & .0574 & .0579 & .0584 & .0589 \\
\hline .013 & .0594 & .0600 & .0605 & .0611 & .0616 & .0622 & .0627 & .0633 & .0638 & .0643 \\
\hline .014 & .0648 & .0654 & .0659 & .0665 & $.047^{1}$ & .0676 & .0682 & .0687 & .0693 & .0609 \\
\hline .015 & .0705 & .0711 & .0717 & .0723 & .0729 & .0735 & $.074 \mathrm{I}$ & .0747 & .0753 & .0759 \\
\hline
\end{tabular}

Example.-Suppose the "extract gravity" is $\mathrm{I.0389}$ and the specific gravity of the alcoholic distillate is 0.9902 , both at $\mathrm{r}_{5} .6$. Then $\mathrm{I}-0.9902=$ 0.0098 , the "degree of spirit indication." From the above table the corresponding "degree of gravity lost" is found to be 0.0432 .

$0.043^{2}+\mathrm{r} .0389=\mathrm{x} .082 \mathrm{I}$, the original gravity of the wort.

The calculation in the above simplified form is accurate for normal beer wherein the free acid present, expressed as acetic, does not exceed $0.1 \%$. In case of beer that has developed free acid much in excess of the above limit, a correction should be added to the degrees of spirit indication. This correction, which in practice it is rarely necessary to apply except in extreme cases of old or sour beer, is calculated as follows:

If $a$ represents the grams of free acid (as acetic) in 100 cc., then the correction to be added to the spirit indication $=0.0013 a-0.00014$.

Example.-Supposing the "extract gravity" to be $\mathrm{I}^{\circ} .4^{1} 3$, the specific gravity of the alcoholic distillate to be 0.9890 , and the free acid as acetic to be $0.35 \%$. Then $\mathrm{I}-0.989=0.0 \mathrm{I} \mathrm{r}$, the degree of spirit indication.

$0.35 \times 0.0013-0.00014=0.0003$, correction to be added to the spirit indication.

$0.0110+0.0003=0.0113$, corrected spirit indication. 
From the above table the corresponding degrees of gravity lost are 0.0506 :

$0.0506+1.0413=1.0919$, the original gravity of the wort.

Determination of Degree of Fermentation.-This is calculated by the formula $D=\frac{200 A}{B}$, in which $D=$ degree of fermentation, $A=$ per cent of alcohol by weight, and $B=$ the original extract.

Determination of Reducing Sugars.-Dealcoholize $25 \mathrm{cc}$. of the beer and make up to roo cc. Determine reducing sugars by the DefrenO'Sullivan or Munson-Walker method, and calculate as maltose.

Determination of Dextrin.-Dilute $50 \mathrm{cc}$. of the beer to $200 \mathrm{cc}$., hydrolize by heating in a boiling water-bath for $2 \frac{1}{2}$ hours with $20 \mathrm{cc}$. of hydrochloric acid (specific gravity 1.125), nearly neutralize the free acid with sodium hydroxide, make up to $300 \mathrm{cc}$., filter, and determine the dextrose by copper reduction. Multiply the amount of reducing sugars as maltose by 0.95 , subtract this from the dextrose, and multiply the difference by 0.9 , thus obtaining the dextrin in the bser

Determination of Glycerin.-Proceed as directed on page 703 under wine. The milk of lime is added during evaporation after the carbon dioxide has been expelled. It is advisable that the filtrate, after being evaporated to a syrupy consistency, be treated again with $5 \mathrm{cc}$. of absolute alcohol and two portions of $7.5 \mathrm{cc}$. each of absolute ether. If clear, continue as directed. If not clear, it is necessary to repeat the treatment with lime.

Determination of Total, Fixed, and Volatile Acids. - A measured volume of the beer, say to cc., is freed from carbon dioxide by bringing to boiling. It is then cooled and titrated with tenth-normal sodium hydroxide, using neutral litmus solution as an indicator. Each cubic centimeter of tenth-normal alkali is equivalent to 0.009 gram of lactic acid, in which the total acidity is usually expressed.

Fixed acid, also expressed as lactic, though small quantities of succinic, tannic, and malic acids are usually also present, is determined as follows: Dealcoholize a measured amount of the beer, say ro cc., by evaporation to one-fourth its volume, dilute with water to the original volume, and titrate with tenth-normal alkali, as before.

Volatile acid is expressed as acetic, and is usually calculated by difference between total and fixed acid. Each cubic centimeter of tenthnormal alkali is the equivalent of 0.006 gram acetic acid. 
Detarmination of Proteins.-Fifty cc. of the beer are first treated with 5 cc. of dilute sulphuric acid, and concentrated by boiling to syrupy consistency. Then proceed by the Gunning method, p. $69 . \mathrm{N} \times 6.25=$ proteins.

Determination of Phosphoric Acid.-Unless the sample is very darkcolored, sufficiently close results can usually be obtained by direct titration of the beer itself with uranium acetate solution. For very accurate results the ash should be used. Prepare a solution of uranium acetate of such strength that $20 \mathrm{cc}$. will correspond to o.I gram $\mathrm{P}_{2} \mathrm{O}_{5}$. This solution is best standardized against pure, crystallized, uneffloresced, powdered hydrogen sodium phosphate, 10.085 grams of which are dissolved in water and made up to a liter. $50 \mathrm{cc}$. of this solution contains 0.1 gram phosphoric anhydride, if the salt is pure. If the solution is of proper strength, $50 \mathrm{cc}$. evaporated to dryness and ignited in a tared platinum dish should have an ash weighing 0.1874 gram. For preliminary trial about 35 grams of uranium acetate are dissolved in water, $25 \mathrm{cc}$, of glacial acetic acid, or its equivalent in weaker acid added, and the solution made up to a liter with water.

To standardize, $50 \mathrm{cc}$. of the standard phosphate solution prepared as above are heated to $90^{\circ}$ or $100^{\circ} \mathrm{C}$., and the uranium solution run in from a burette till the resulting precipitate of hydrogen uranium phosphate is complete. The end-point is determined by transferring a few drops of the solution to a porcelain plate, and touching with a drop of freshly prepared potassium ferrocyanide solution. When the slightest excess of uranium acetate has been added, a reddish-brown color is produced by the ferrocyanide. The uranium acetate solution is purposely made rather stronger than necessary at first, and by repeated trials is brought by dilution with water to the required strength (20 cc. equivalent to $50 \mathrm{cc}$. of the phosphate solution).

Fifty cc. of the beer are heated to $90^{\circ}$ or $100^{\circ} \mathrm{C}$. and titrated wi.h the uranium acetate solution under the same conditions and in precisely the same mannor as when standardizing that solution. Each cubic centimeter of the uranium acetate corresponds to $0.01 \%$ of $\mathrm{P}_{2} \mathrm{O}_{5}$.

For the phosphoric acid determination in the ash, $50 \mathrm{cc}$. of the beer are incinerated in the regular manner, and the ash moistened with concentrated hydrochloric acid. The acid is then evaporated off on the water-bath, after which the ash is boiled with $50 \mathrm{cc}$. of distilled water, and titrated with the standard uranium solution. 
Determination of Carbon Dioxide.*-In the case of beer and other carbonated drinks put up in corked bottles, the carbon dioxide may be readily determined by piercing the cork with a metal champagne tap, which is connected by a flexible tube, first with a safety flask and then with an absorption apparatus somewhat after the style of that used in the determination of carbon dioxide in baking powder, the liberated carbon dioxide being absorbed for weighing in a concentrated solution of potassium hydroxide contained in a tared Liebig bulb. The beerbottle thus connected is immersed in a vessel of water, which is heated over a gas-flame, after all the carbon dioxide that will escape spontaneously has been allowed to do so. Before weighing the absorbed carbon dioxide, the beer-bottle is replaced by a soda-lime tube, and a current of air drawn through the tubes.

Beer and ale put up in bottles having patent metallic or rubber stoppers cannot thus be treated. In this case a measured quantity, say $200 \mathrm{cc}$, of the sample is transferred as quickly as possible to a large flask provided with an outlet-tube having a glass stopper, this being connected up with the safety-flask and absorption-tubes. In this case heat may be directly, though cautiously, applied to the flask containing the beer by means of a gas-flame, after all the carbon dioxide has gone over that will do so spontaneously. Exactly the same apparatus as that shown in Fig. 7 I may be used to advantage for determination of carbon dioxide in beer, except that a larger distilling-flask should be used in the case of beer.

Detection of Bitter Principles.-Elaborate schemes have been worked out for the systematic treatment of beer and ale for bitter principles. Nearly all of these are complicated and somewhat unsatisfactory. The presence of alkaloids in malt liquors, deliberately introduced during the process of manufacture, is now so rare that the analyst need seldom look for them, except in cases of suspected poisoning, when the scheme of Dragendorf $\dagger$ or of Otto-Stas should be employed. While it is somewhat difficult to positively identify the various alkaloids, it is usually easy to prove their absence in clear solutions, if on treatment with either of the general alkaloidal reagents, Mayer's solution (Reagent No. I 70), or iodine in potassium iodide (Reagent No. 143 ), no precipitate is formed.

It is comparatively easy to prove the mere presence or absence of hop substitutes. The bitter principle of hops is readily soluble in ether, when a sample of the beer evaporated to syrupy consistency is extracted

* U. S. Dept. of Agric., Bur. of Chem., Bul. 65, p. 95; Bul. ro7 (rev.), p. $9^{2}$.

$\dagger$ Gerichtlich-Chemische Ermittelung von Giften, St. Petersburg, 1876. 
therewith, while the bitters of quassia and aloes, common hop substitutes, are insoluble in ether. Though many varieties of bitters might be employed that are soluble in ether, the absence of a bitter taste from the ether extract is evidence of the absence of hops.

The most marked differencê analytically between hops and their substitutes in malt liquors lies in the fact that the bitter principle of hops is completely precipitated therefrom by treatment of the beer with lead acetate (either basic or neutral), leaving no bitter taste in the filtrate after concentration, while if any of the hop substitutes are present, the concentrated filtrate from the lead acetate treatment will have a bitter taste. The excess of lead should be removed from the filtrate, before concentration and tasting, by treatment with hydrogen sulphide. If the residue from the ether or chloroform extraction of the concentrated filtrate from a beer after treatment with lead acetate be found to be bitter, there is positive evidence that a foreign substitute has been employed.

The following are characteristic reactions that may help to identify some of the common hop substitutes.*

Quassiin is readily soluble by chloroform from acid solution. If a residue containing quassin be moistened with a weak alcoholic solution of ferric chioride and gently heated, a marked mahogany-brown coloration is produced.

On treatment of quassiin with bromine and sodium hydroxide or ammonia, a bright-yellow color is shown.

Chiretta is readily dissolved by ether from its"aqueous solution. Its ether residue, when treated with bromine and ammonia, gives a straw color, slowly changing to a dull purple-brown. This is not true of its chloroform residue, so that it is not to be mistaken for quassia (Allen).

Gentian Bitter may be extracted by treatment of the acid liquor with chloroform. When the residue containing gentian bitter is treated with concentrated sulphuric acid in the cold, no color is produced, but on warming gently a carmine-red color is shown; if further treated with ferric chloride solution, a green-brown color is formed.

Aloes.-This substance is separated from beer by treating the dried resilue from $200 \mathrm{cc}$. of the beer with warm ammonia, filtering, cooling, and treating the filtrate with hydrochloric acid. The resin of aloes is precipitated and collected on a filter. It is insoluble in cold water, ether,

* Allen, Analyst, 12, 1887, p. 107. 
chloroform, or petroleum ether, but is soluble in alcohol. It has a very characteristic odor, which serves to identify it. The hot-water solution gives a curdy precipitate on treatment with lead acetate.

Capsicin is extracted by treatment of the acid liquor with chloroform. It is recognizable by its sharp; pungent taste.

Detection of Arsenic.-By the Marsh Method.-Measure roo cc. of the beer (freed from carbon dioxide by agitation) into a seven-inch porcelain evaporating-dish, add $20 \mathrm{cc}$. pure concentrated nitric acid, and $3 \mathrm{cc}$. pure concentrated sulphuric acid, and cautiously heat till vigorous chemical action sets in, accompanied by frothing and swelling of the beer. Turn the flame low or remove it altogether, and stir vigorously till the frothing ceases, after which the liquid may be boiled freely. At this stage transfer to a large casserole, and continue the boiling till nearly all the nitric acid is driven off. Then, holding the casserole by the handle, continue the heating till the mass chars and the fumes of sulphuric acid are given off, giving the casserole a rotary motion to prevent sputtering. The residue should be reduced to a dry, black, pulverulent char soon after the sulphuric acid fumes begin to come off freely. If still liquid, pieces of filter-paper should be stirred in while still heating, till the residue is dry, avoiding an excess of paper.

Cool, add $50 \mathrm{cc}$. of water, and remove the masses of char from the sides of the dish by the stirring-rod. Heat to boiling and filter. Use the filtrate for the Marsh apparatus, adding it gradually.

The arsenic mirror may be weighed in the usual manner, if of suffi. cient size.

Reinsch's Test,*-Two hundred cc. of the beer are acidified with I cc. of pure, concentrated, arsenic-free hydrochloric acid, and evaporated to half its volume. $15 \mathrm{cc}$. more of hydrochloric acid are then added, and a piece of pure burnished copper foil half an inch long and a quarter of an inch wide is immersed in the liquid and kept in it for an hour while simmering, replacing from time to time the water lost by evaporation. If after the lapse of an hour the copper still remains bright, no arsenic is present.

If the copper shows a deposit, remove, wash with water, alcohol, and ether, and dry. Then place the copper in a subliming-tube, and heat over a low flame. Tetrahedral crystals, apparent under the microscope, show the presence of arsenic. Blackening of the copper does not in itself prove arsenic.

\footnotetext{
* Jour. Soc., Chem Ind., 20, p. 646.
} 
Detection and Determination of Preservatives. - See Chapter XVIII. Sulphurous acid may be determined by direct titration, as in the case of wine.

\section{MALT EXTRACT.}

True malt extract is a syrupy fluid having a specific gravity of from I. 3 to I.6, and made up in accordance with the following directions of the 1880 Pharmacopœia: Upon 100 parts of coarsely powdered malt contained in a suitable vessel, pour 100 parts of water, and macerate for six hours. Then add 400 parts of water, heated to about $30^{\circ} \mathrm{C}$. and digest for an hour at a temperature not exceeding $55^{\circ} \mathrm{C}$. Strain the mixture with strong pressure. Finally, by means of a water-bath or vacuum apparatus, at a temperature not exceeding $55^{\circ} \mathrm{C}$., evaporate the strained liquid rapidly to the consistence of thick honey.

Keep the product in well-closed vessels in a cool place.

Such an extract has a residue of at least $70 \%$, consisting chiefly of maltose, and contains about $2 \%$ of diastase. It should furthermore be capable of converting its own weight of starch at $55^{\circ} \mathrm{C}$. in less than ten minutes.

The following are analyses of three samples of pure malt extract:*

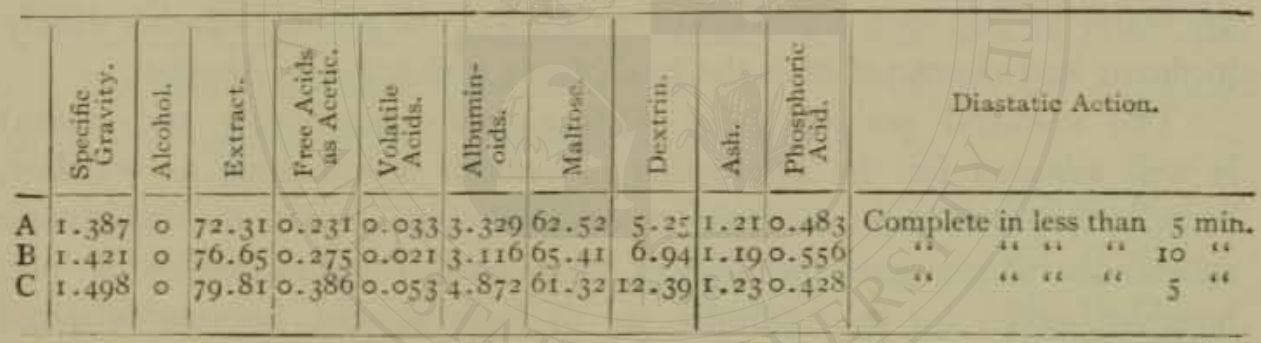

There are on the market many so-called malt extracts widely advertised for their tonic and medicinal virtues, having the taste and consistency of beer or ale. In fact they are virtually beer, differing therefrom mainly in respect to price. Such "malt extracts" have no diastase, and their value as nutrients depends almost entirely on their sugar content.

Harrington $\dagger$ has analyzed twenty-one of the best known of these alleged malt extracts, the maximum, minimum, and mean results of his analyses being as follows:

* Penn. Dept. of Agric. An. Rep., i8g8, p. 85.

$\dagger$ Boston Medical and Surgical Journal, Dec. 31, 1896 . 


\begin{tabular}{|c|c|c|c|c|}
\hline & $\begin{array}{l}\text { Specific } \\
\text { Gravity. }\end{array}$ & Alcohol. & $\begin{array}{c}\text { Total } \\
\text { Residue. }\end{array}$ & Ash, \\
\hline 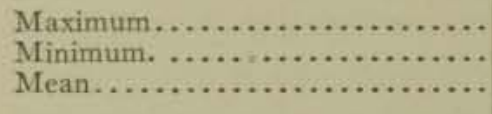 & $\begin{array}{l}1.0555 \\
1.0149 \\
\cdots \cdots\end{array}$ & $\begin{array}{l}7.13 \\
0.74 \\
3.94\end{array}$ & $\begin{array}{r}13.63 \\
5.13 \\
8.78\end{array}$ & $\begin{array}{l}0.53 \\
0.20\end{array}$ \\
\hline
\end{tabular}

None of them contained any diastase, and several were preserved with salicylic acid.

\section{DISTILLED LIQUORS.}

These beverages differ from those hitherto considered, by reason of their high alcoholic content and low extract or residue. Indeed, when first distilled they are entirely without residue, but from long storage in casks, they absorb certain extractives from the wood, that impart more or less flavor as well as color.

When any fermented alcoholic infusion is subjected to distillation under ordinary circumstances, a distillate results which consists of a mixture with water of a large number of alcohols, chief among which is ethyl alcohol. The high boiling alcohols-amyl, butyl, propyl, etc., with their esters-exist in the distillate in small amount, constituting what is known as fusel oil. The various distilled liquors. of commerce are made by just such a process of distillation, the product varying widely in flavor and character with the basis from which it was distilled.

The so-called pot-still (the old-fashioned copper still and worm) is well adapted for the production of potable spirits such as whiskey, brandy, gin, and rum, as these products should contain the congeneric substances which give the liquors their special character; it is not, however, suited for the manufacture of pure alcohol, because repeated distillation would be required for purification.

Now, however, by the use of improved apparatus, such as the Coffey still, involving the principle of fractional condensation, it is possible to obtain what is known as "silent spirit," or ethyl alcohol, free from fusel oil. With proper appurtenances for rectifying, one can now obtain $95 \%$ alcohol by two distillations.

Standards for Spirits.-The following are the standards adopted by the Joint Committee of the Association of Official Agricultural Chemists and the Association of State and National Food and Dairy Departments:

Distilled Spirit is the distillate obtained from a fermented mash of cereals, molasses, sugars, fruits, or other fermentable substance, and 
contains all the volatile flavors, essential oils, and other substances derived directly from the material used, and the higher alcohols, ethers, acids, and other volatile bodies congeneric with ethyl alcohol produced during fermentation, which are carried over at the ordinary temperature of distillation, and the principal part of which are higher alcohols estimated as amylic.

Alcohol, Cologne Spirit, Neutral Spirit, Velvet Spirit, or Silent Spirit, is distilled spirit from which all, or practically all, of its constituents except ethyl alcohol and water, are separated, and contains not less than $94.9 \%$ (189.8 proof) by volume of ethyl alcohol.

Composition of Fusel Oil.-Fusel oil varies considerably in composition with the source from which it is derived. Amyl alcohol, being in all cases its chief constituent, is frequently known commercially as fusel oil. The alcohols found in fusel oil with their formulas, specific gravity, and boiling-points are as follows:

\begin{tabular}{|c|c|c|c|}
\hline & Formula. & $\begin{array}{l}\text { Specific } \\
\text { Gravity. }\end{array}$ & Boiling-point. \\
\hline 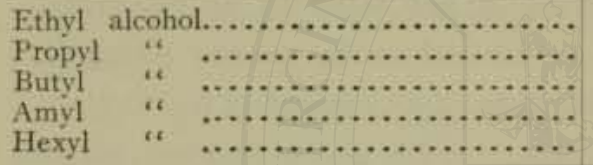 & $\begin{array}{l}\mathrm{C}_{2} \mathrm{H}_{5} \mathrm{OH} \\
\mathrm{C}_{3} \mathrm{H}_{7} \mathrm{OH} \\
\mathrm{C}_{4} \mathrm{H}_{5} \mathrm{OH} \\
\mathrm{C}_{3} \mathrm{H}_{11} \mathrm{OH} \\
\mathrm{C}_{0} \mathrm{H}_{13} \mathrm{OH}\end{array}$ & $\begin{array}{l}.794 \\
.820 \\
.803 \\
.8 \mathrm{nI}\end{array}$ & $\begin{array}{l}78 \cdot 4^{\circ} \mathrm{C} \\
97^{\circ} \mathrm{C} \\
115^{\circ} \mathrm{C} \\
130^{\circ} \mathrm{C}\end{array}$ \\
\hline
\end{tabular}

The following acids have been found in fusel oil, usually combined with the alcohols to form compound ethers:
Acetic.......... $\mathrm{HC}_{2} \mathrm{H}_{3} \mathrm{O}_{2}$
Caproic. $\mathrm{HC}_{6} \mathrm{H}_{11} \mathrm{O}_{2}$
Propionic........ $\mathrm{HC}_{3} \mathrm{H}_{5} \mathrm{O}_{2}$
Enanthylic. ...... $\mathrm{HC}_{7} \mathrm{H}_{13} \mathrm{O}_{2}$
Butyric. .......... $\mathrm{HC}_{4} \mathrm{H}_{7} \mathrm{O}_{2}$
Caprylic.......... $\mathrm{HC}_{8} \mathrm{H}_{15} \mathrm{O}_{2}$
Valerianic........ $\mathrm{HC}_{5} \mathrm{H}_{9} \mathrm{O}_{2}$
Pelargonic........ $\mathrm{HC}_{3} \mathrm{H}_{17} \mathrm{O}_{2}$

Aging.-Freshly distilled liquors all contain notable quantities of fusel oil, which renders them harsh and unfit for use, but by the process. of aging, they become in several years mellow and palatable. The chemical changes which take place during aging are discussed under whiskey.

\section{WHISKEY.}

Process of Manufacture.-Whiskey is the liquor resulting from the distillation of a fermented infusion of grain, the process being carried out in a pot-still, or some other form of still, constructed so that the resulting liquor contains not only the alcohol, but also the greater part 
of the congeneric substances which are vaporized with the alcohol. The fermented infusion known as the "mash" is obtained by steeping in water the starch-containing material, usually barley, rye, corn (maize), or oats mixed with malt, and subjecting the mixture to the action of the diastase contained in the malt, in much the same manner as the mashing process in the brewing of beer, except that for whiskey the process of saccharous fermentation is carried further, with a view to obtaining a maximum yield of maltose and a minimum of dextrin. Yeast is afterwards added, and alcoholic fermentation allowed to proceed with proper precautions.

The fermented wort from whatever source obtained is subjected to distillation, purposely avoiding rectification or separation of the fusel oil and other congeneric substances which are valuable as flavors. The product of the first distillation is called "low wines," and is redistilled; the product of the second distillation is commonly divided into three fractions, of which the middle portion, or "clean spirit" is retained for the whiskey, while the first ("foreshots") and the last fraction ("faints") are mixed with the next portion of low wine to be redistilled. If the whiskey is too high in alcohol, it is diluted to the proper strength.

As new whiskey is crude and harsh in taste, it is subjected to "aging," or storing in casks for a number of years. The aging process softens and refines the flavor, but recent investigations have proved that this is not due, as formerly believed, to transformation of fusel oil into esters, although the esters increase in amount during aging, as do also the acids -especially the volatile acids - the aldehydes, and the furfural. As a matter of fact, the percentage of fusel oil increases instead of diminishes on aging, due to the evaporation of water and, in a lesser degree, of alcohol through the wood; the actual amount, however, remains practically the same as at the start (see table, p. 737). When first distilled, whiskey is perfectly colorless, but during the aging it extracts more or less color and some flavor from the casks in which it is stored. This color is especially pronounced in American whiskies, owing to the prevailing custom of charring the inside of the cask. Its flavor varies considerably with the nature of the grain used in its preparation.

U. S. Rulings.-The following decision of President Roosevelt, based on an opinion of Attorney-General Bonaparte, was promulgated by Secretary Wilson, April II, I907:*

* This decision has been overruled by President Taft, whose opinion is the basis of Food Inspection Decision No. $\mathrm{II}_{3}$ (Feb, 16, 1910), signed by the secretaries of the Treasury, Agriculture, and Commerce and Labor. The chief points of this decision follow: 
"Straight whiskey will be labeled as such.

"A mixture of two or more straight whiskies will be labeled 'blended whiskey,' or ' whiskies.'

"A mixture of straight whiskey and ethyl alcohol, provided that there is a sufficient amount of straight whiskey to make it genuinely a ' mixture,' will be labeled as compound of, or compounded with, pure grain distillate.

"Imitation whiskey will be labeled as such."

Joint Standards. - The following are the standards of the Joint Committee of the A. O. A. C. and the A. S. N. F. D. D.:

New Whiskey is the properly distilled spirit from the properly prepared and properly fermented mash of malted grain, or of grain the starch of which has been hydrolyzed by malt; it has an alcoholic strength corresponding to the excise laws of the various countries in which it is produced, and contains in roo liters of proof spirit not less than 100 grams of the various substances other than ethyl alcohol derived from the grain from which it is made, and of those produced during fermentation, the principal part of which consists of higher alcohols estimated as amylic.

Whiskey (Potable Whiskey) is new whiskey which has been stored in wood not less than four years without any artificial heat save that which may be imparted by warming the storehouse to the usual temperature, and contains in 100 liters of proof spirit not less than 200 grams of the substances found in new whiskey, save as they arz changed or eliminated by storage, and of those produced as secondary bodies during aging; and, in addition thereto, the substances extracted from the casks in which it has been stored. It contains, when prepared for consumption

All unmixed distilled spirits from grain, colored and flavored with harmless color and flavor, in the customary ways, either by the charred barrel process, or by the addition of caramel and harmless flavor, if of potable strength and not less than $80^{\circ}$ proof, are entitled to the name whiskey without qualification.

Whiskies of the same or different kinds (i.e., straight, rectified, redistilled, and neutral spirits whiskies) are like substances and mixtures of such, with or withotit harmless color or flavor used for purposes of coloring and flavoring only, are blends.

Potable alcoholic distillates from sources other than grain (e.g., cane, fruit, or vegetables), colored and flavored, are imitations and mixtures of such with grain distillate are compounds.

A distillate of grain (e.g., corn) flavored to simulate a whiskey of another kind (e.g., rye) is an imitation of that whiskey.

Attorney-General Wickersham (F. I. D. No. 127) has further decided that the name "Canadian Club whiskey" is distinctive and it is therefore unnecessary to place the word "blend" on the label. 
as permitted by the regulations of the Bureau of Internal Revenue, not less than $45 \%$ by volume of ethyl alcohol, and, if no statement is made concerning its alcoholic strength, it contains not less than $50 \%$ of ethyl alcohol by volume, as prescribed by law.

Rye Whiskey is a whiskey in the manufacture of which rye, either in a malted condition or with sufficient barley or rye malt to hydrolyze the starch, is the only grain used.

Bourbon Whiskey is a whiskey made in Kentucky from a mash of Indian corn and rye, and barley malt, of which Indian corn forms more than $50 \%$.

Corn Whiskey is whiskey made from malted Indian corn or of Indian corn the starch of which has been hydrolyzed by barley malt.

Blended Whiskey is a mixture of two or more whiskies.

Scotch Whiskey is whiskey made in Scotland solely from barley malt, in the drying of which peat has been used. It contains in 100 liters of proof spirit not less than 150 grams of the various substances prescribed for whiskey exclusive of those extracted from the cask.

Irish Whiskey is whiskey made in Ireland, and conforms in the proportions of its various ingredients to Scotch whiskey, save that it may be made of the same materials as prescribed for whiskey, and the malt used is not dried over peat.

U. S. P. Standards.-The requirements for whiskey are as follows: It should be at least two years old; in specific gravity it should lie between the limits of 0.945 and 0.924 ; its alcoholic content should be not less than $37 \%$ nor more than $47.5 \%$ by weight; the residue from Ioo cc. should be not more than 0.5 gram, which should be neither sweet nor spicy, should dissolve in $10 \mathrm{cc}$. of cold water, and this solution should be colored only a pale green when treated with a drop of very dilute ferric chloride solution (a deeper color would indicate more than traces of tannin). In evaporating the liquor on the water-bath for the residue, the last traces volatilized should have an agreeable odor free from harshness, indicative of the absence of fusel oil. Its reaction should be slightly acid, but not more than I.2 cc. of normal alkali should be required to neutralize $100 \mathrm{cc}$. of the liquor, using litmus as an indicator. If $50 \mathrm{cc}$. are shaken vigorously with 25 grams of kaolin, allowed to stand an hour and filtered, the color of the filtrate should not be much lighter than before treatment.

Composition.-Whiskey consists chiefly of alcohol and water, with relatively small amounts of fusel oil, acids, esters, aldehydes, and fur- 
fural. Its extract, derived mainly from the casks in which it is stored, should consist only of small amounts of tannin, sugar, and coloring matter.

British Whiskies.--Scotch and Irish whiskies are aged in uncharred barrels, hence they are of a lighter color than the American product. Scotch whiskey is further characterized by its smoky taste, due to the peat over which it is dried. The following analyses by Vasey * illustrate the composition of Scotch and Irish whiskey of different ages, of neutral spirits used in compounding ("blending") and adulterating, and of the compounded liquors:

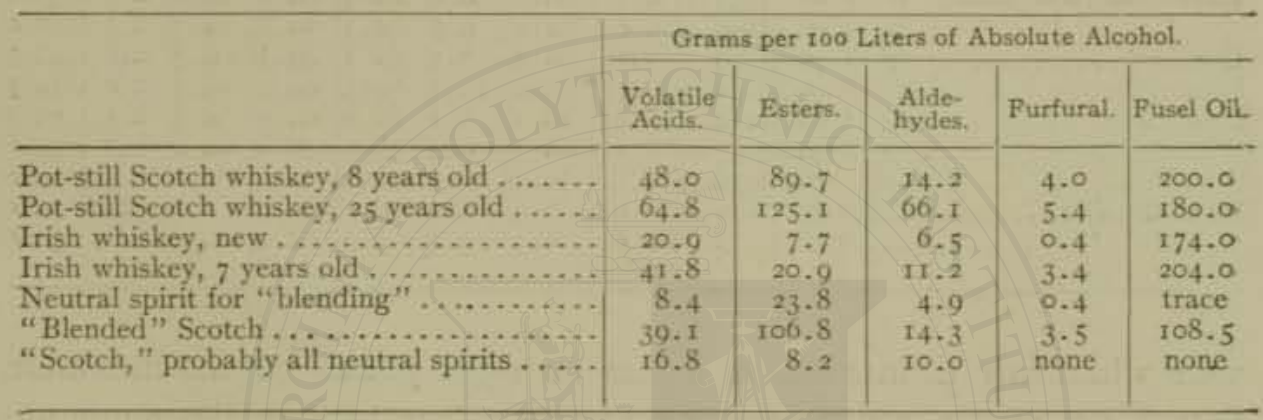

It will be noted that the congeneric substances in whiskey increase on aging, although in the case of fusel oil this apparent increase is doubtless due merely to concentration dependent on evaporation. The sample of neutral spirits contained only small amounts of the congeneric substances, while the "blended" whiskies were deficient in most of these substances.

American Whiskies.-These have a deeper color than the British whiskies (due to the charred barrel) and a rich fruity flavor without the suggestion of smoke.

In the table on p. 736 are given analyses by Shepard $\dagger$ of fourteen leading brands, including both rye and bourbon, varying in age from four to eight years; also of two samples of neutral spirits used for compounding and adulterating.

A summary of the results obtained by Crampton and Tolman $\ddagger$ in the analysis of fourteen brands of rye and seventeen brands of bourbon whiskey at differing stages of aging appear in the table on page 737. The barrels were kept in U.S. bonded warehouses during aging, and samples:

* Potable Spirits, pp. 82,83 , and 87 .

† The Constants of Whiskey, S. Dak. Food and Dairy Commission, March, I9o6.

$\ddagger$ Jour, Am. Chem. Soc., 30, 1908, p. 98. 


\begin{tabular}{|c|c|c|c|c|c|c|c|c|c|c|c|}
\hline & & & & & rams & eer 100 & Liters & of the & Liquor & & \\
\hline & & ปย & & & & Acids. & & & & & \\
\hline & 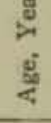 & 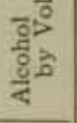 & 芯 & $\frac{5}{4}$ & हूँّ & 总 & 营 & 总 & 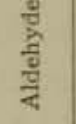 & 咅 & $\begin{array}{l}\overrightarrow{0} \\
\vec{B} \\
\text { 惫 }\end{array}$ \\
\hline & 8 & 50.1 & $\mathrm{I} 89.8$ & & 92.0 & 12.8 & 79.2 & $8 \mathrm{r} .8$ & & 3.0 & 84.9 \\
\hline & 8 & 50,1 & $18 \mathrm{r} .5$ & $7 \cdot 3$ & 68.4 & $9 \cdot 3$ & 59.1 & 60.7 & 17.5 & 3.2 & 102.6 \\
\hline Stan & 5 & 50.0 & 160.4 & 7.2 & 66.8 & 10.2 & 56.6 & $55 \cdot 9$ & 10.0 & 2.4 & 160.4 \\
\hline Hand-made sour $n$ & $4 \frac{1}{2}$ & 49.8 & 162.1 & 7.4 & 67.1 & 10.2 & 56.9 & 74.8 & 12.0 & 2.6 & 130.9 \\
\hline & 4 & 50.2 & $\mathbf{x} 48.5$ & $7 \cdot 3$ & 62.4 & $7 \cdot 5$ & $54 \cdot 9$ & 55.9 & 15.0 & 2.6 & 152.0 \\
\hline Hand-made sour mash. & 4 & $49 \cdot 9$ & 132.7 & 5.8 & 49.2 & $7 \cdot 5$ & $4 \mathrm{I} \cdot 7$ & 39.6 & 8.0 & 1.0 & 107.4 \\
\hline & 6 & 50.4 & ${ }^{3} 38$. & 6.4 & & 8.6 & 66.2 & 61.6 & 10.5 & I. 3 & 192.7 \\
\hline Hand-made sour mash. & 6 & $5 \circ$ & 153.7 & 9.7 & $5^{8} .8$ & 9.9 & 48.9 & 69.6 & 14.0 & 0.7 & 137.1 \\
\hline " & 7. & 50 & 180.0 & 10.0 & 74.4 & 9.9 & 64.5 & 70.8 & 12.5 & 2.5 & 117.0 \\
\hline Bour & $5 t$ & 49.9 & $129 \cdot 3$ & $5 \cdot 1$ & 60.9 & 7.2 & $53 \cdot 7$ & $49 \cdot 3$ & $9 \cdot 5$ & 0.8 & 141.7 \\
\hline Spec & 7 & 49.8 & $2+2.0$ & 8.0 & 93.0 & 13.5 & $79 \cdot 5$ & 94.0 & 22.5 & 5.0 & II 9.5 \\
\hline Sour mash & & 50.1 & 124.5 & 7.2 & 58.2 & 7.2 & 51.0 & 64.0 & $9 \cdot 5$ & 0.5 & $95 \cdot 3$ \\
\hline & $7 \frac{1}{2}$ & 49.8 & 177.2 & $7 \cdot 3$ & 66.5 & 9.0 & $57 \cdot 5$ & 76.6 & 10.0 & x.7 & 193.6 \\
\hline & & & 139.4 & 6.5 & 50.3 & $6 \cdot 3$ & 44.0 & 54.6 & $7 \cdot 5$ & I. 5 & 152.0 \\
\hline Neutral spirits. . & & 95.6 & 10.3 & 0.9 & $7 \cdot 5$ & 1.2 & 6.3 & $15 \cdot 4$ & 2.5 & & 30.0 \\
\hline & & $94 \cdot 4$ & $3 \cdot 2$ & 0.0 & 6.3 & I. 4 & 4.9 & 64.2 & II.O & & 39.6 \\
\hline
\end{tabular}

were withdrawn at intervals of a year for eight years. As the minimum figures for certain constituents are abnormal, the next to the minimum figures are also given. It will be noted that during the first few years there was a marked increase in actual amounts of all the constituents determined, except fusel oil, over and above that due to concentration, but after three or four years the acids and esters do not materially change. The rye whiskies contained larger amounts of solids, acids, esters, etc., than the bourbons, but this was attributed to the fact that heated warehouses are used for rye, and unheated for bourbon whiskey. The authors state that the characteristic aroma of American whiskey, also the oily appearance and the "body" (solids), are due to the charred barrels.

Thirty-seven samples of whiskey, purchased by the glass from various Massachusetts saloons, were examined by the Massachusetts State Board of Health in 1894 , with the following results:

\begin{tabular}{|c|c|c|}
\hline & $\begin{array}{l}\text { Per Cent } \\
\text { Aloohol by } \\
\text { Weight. }\end{array}$ & $\begin{array}{l}\text { Per Cent } \\
\text { Extract. }\end{array}$ \\
\hline $\begin{array}{l}\text { Maximum............... } \\
\text { Minimum. } \\
\text { Mean...................... }\end{array}$ & $\begin{array}{l}45 \cdot 9^{6} \\
30.70 \\
36.51\end{array}$ & $\begin{array}{l}1.68 \\
0.08 \\
0.50\end{array}$ \\
\hline
\end{tabular}




\begin{tabular}{|c|c|c|c|c|c|c|c|c|c|}
\hline & & \multirow{2}{*}{ Proof: } & \multicolumn{7}{|c|}{ Grams per $x 00$ Liters of 100 Proof Spirits. } \\
\hline & & & Color & Extract. & Acids. & Esters. & $\begin{array}{l}\text { Alde- } \\
\text { hydes. }\end{array}$ & $\begin{array}{l}\text { Fur- } \\
\text { fural. }\end{array}$ & $\begin{array}{l}\text { Fusel } \\
\text { Oil. }\end{array}$ \\
\hline \multicolumn{2}{|c|}{ RYE WHISKEY. } & & & & & & & & \\
\hline \multirow[t]{2}{*}{ New: } & $\begin{array}{l}\text { Average.... } \\
\text { Maximum. }\end{array}$ & $\begin{array}{l}101.2 \\
102.0\end{array}$ & $\begin{array}{l}0.0 \\
0.0\end{array}$ & $\begin{array}{l}18.3 \\
30.0\end{array}$ & $\begin{array}{r}4.4 \\
72.0\end{array}$ & $\begin{array}{l}16.3 \\
21.8\end{array}$ & $\begin{array}{r}5.4 \\
15.0\end{array}$ & $\begin{array}{l}1.0 \\
1.9\end{array}$ & $\begin{array}{r}90.4 \\
161.8\end{array}$ \\
\hline & Minimum * & 100.0 & 0.0 & 5.0 & 12.0 & $4 \cdot 3$ & 0.7 & trace & \\
\hline \multirow[t]{3}{*}{ One year old: } & Average... & 102.5 & 8.8 & 119.7 & 46.6 & 37.0 & $\gamma .0$ & 1.8 & 111.5 \\
\hline & Maximum . & 104.0 & 13.8 & 171.0 & 60.5 & 64.8 & $15 \cdot 5$ & $3 \cdot 3$ & 194.0 \\
\hline & Minimum * & 101.0 & $\begin{array}{l}7.2 \\
6.6\end{array}$ & $\begin{array}{l}93.0 \\
92.0\end{array}$ & $\begin{array}{r}3 \mathrm{r} . \mathrm{I} \\
5.8\end{array}$ & $\begin{array}{l}6.8 \\
6.8\end{array}$ & 2.8 & 0.4 & $\begin{array}{l}80.4 \\
66.4\end{array}$ \\
\hline \multirow[t]{3}{*}{ Two years old: } & Average... & 104.9 & 11.6 & 144.7 & 51.9 & 54.0 & 10.5 & 2.8 & 112.4 \\
\hline & $\mathrm{Ma}$ & 109.0 & 16.7 & 199.0 & 75.6 & 75.1 & 18.7 & $5 \cdot 7$ & 214.0 \\
\hline & Minimum * & 100.0 & & $\begin{array}{r}121.0 \\
04.0\end{array}$ & $44 \cdot 3$ & $4 \mathrm{I} \cdot 5$ & $5 \cdot 4$ & 0.7 & 83.4 \\
\hline \multirow{3}{*}{ Three years old: } & Average.... & 107.7 & 13.2 & 171.4 & 68.7 & 61.5 & 12.5 & 1.5 & 112.7 \\
\hline & Maxi & 112.0 & 18.3 & 224.0 & 81.8 & 79.8 & 20.8 & 6.1 & 202.0 \\
\hline & Minimum * & 104.0 & $\begin{array}{l}11.4 \\
10.1\end{array}$ & \begin{tabular}{|l|}
145.0 \\
119.0
\end{tabular} & $\begin{array}{l}52.3 \\
16.4\end{array}$ & 47.6 & 6.5 & 0.7 & $\begin{array}{l}79.0 \\
60.0\end{array}$ \\
\hline \multirow[t]{3}{*}{ Four years old: } & Average .... & 111.2 & 14.0 & 185.0 & 65.9 & 69.3 & 13.9 & 2.8 & 125.1 \\
\hline & Maximum . & 118.0 & 18.9 & 238.0 & 83.8 & 89.1 & 22.1 & 6.7 & 203.5 \\
\hline & Minimum * & 105.0 & $\begin{array}{l}11.6 \\
11.3\end{array}$ & $\begin{array}{l}156.0 \\
153.0\end{array}$ & $\begin{array}{l}58.6 \\
17.3\end{array}$ & $\begin{array}{l}57 \cdot 7 \\
36.3\end{array}$ & 6.4 & 0.7 & $\begin{array}{l}83.8 \\
67.8\end{array}$ \\
\hline \multirow[t]{3}{*}{ Eight years old: } & Avera & 128.8 & 18.6 & 256.0 & 82.9 & 89.1 & 16.0 & 3.4 & 154.2 \\
\hline & Ma & 132.0 & 24.2 & 339.0 & 112.0 & 126.6 & 26.5 & 9.2 & 280.3 \\
\hline & Minimum * & 112.0 & $\begin{array}{l}13.8 \\
13.7\end{array}$ & $\begin{array}{l}214.0 \\
200.0\end{array}$ & $\begin{array}{l}73 \cdot 7 \\
31 \cdot 7\end{array}$ & $\begin{array}{l}68.4 \\
40.9\end{array}$ & 7.9 & 0.8 & $\begin{array}{l}109.0 \\
107.1\end{array}$ \\
\hline \multirow{4}{*}{$\begin{array}{l}{ }^{B} \\
\text { New; }\end{array}$} & WHISKI & & & & & & & & \\
\hline & & 101.0 & 0.0 & 26.5 & 10.0 & 18.4 & 3.2 & 0.7 & 100.9 \\
\hline & & 104.0 & 0.0 & 161.0 & 29.1 & 53.2 & 7.9 & 2.0 & $171 \cdot 3$ \\
\hline & Minimum * & 100.0 & 0.0 & 4.0 & 12.0 & $t_{3} .0$ & 1.0 & trace & $\begin{array}{l}71.3 \\
42.0\end{array}$ \\
\hline \multirow[t]{3}{*}{ One year old: } & Average... & 101.8 & $\gamma .1$ & 99.6 & 41.1 & 28.6 & 5.8 & 1.6 & 110.1 \\
\hline & Maximur & $\mathrm{IO}_{3.0}$ & 10.9 & 193.0 & $55 \cdot 3$ & $55-9$ & 8.6 & 7.9 & 173.4 \\
\hline & Minimum * & 100.0 & $\begin{array}{l}5.4 \\
4.6\end{array}$ & $\begin{array}{r}61.0 \\
54.0\end{array}$ & $\begin{array}{r}24.7 \\
7.2\end{array}$ & $\begin{array}{l}17.2 \\
10.4\end{array}$ & 2.7 & trace & 58.0 \\
\hline \multirow[t]{3}{*}{ Two years old: } & Average... & 102.2 & 8.6 & 126.8 & 45.6 & 40.0 & 8.4 & 1.6 & 108.9 \\
\hline & $\mathrm{Ma}$ & 104.0 & 11.8 & 214.0 & 61.7 & 59.8 & 12.0 & 9.1 & 197.1 \\
\hline & Minimum * & 100.0 & 6.9 & $\begin{array}{l}81.0 \\
78.0\end{array}$ & $\begin{array}{l}25 \cdot 5 \\
23.3\end{array}$ & $\begin{array}{l}24.4 \\
11.2\end{array}$ & 5.9 & 0.4 & $\begin{array}{l}86.2 \\
42.8\end{array}$ \\
\hline \multirow{3}{*}{ Three years old: } & Average.... & 103.0 & 10.0 & 149.3 & 54.3 & 48.1 & 10.5 & 1.7 & 112.4 \\
\hline & Maximum . & 106.0 & ${ }_{3} 3.8$ & 245.0 & 64.8 & 73.0 & 22.1 & $9 \cdot 5$ & 221.8 \\
\hline & Minimum.* & 100.0 & & & $\begin{array}{l}38.4 \\
32.1\end{array}$ & $\begin{array}{l}27.2 \\
12.1\end{array}$ & 5.9 & 0.6 & 88.0 \\
\hline \multirow[t]{3}{*}{ Four years old: } & Aver: & $104 \cdot 3$ & 10.8 & 151.9 & 58.4 & 53.5 & 11.0 & 1.9 & 123.9 \\
\hline & Maximum . & 108.0 & 14.8 & 249.0 & 73.0 & 8o. 6 & 22.0 & 9.6 & $237 \cdot I$ \\
\hline & Minimum * & 100.0 & 8.6 & 101.0 & 40.4 & 28.2 & 6.9 & 0.8 & 95.0 \\
\hline \multirow[t]{3}{*}{ Eight years old: } & Average ... & 111.1 & 14.2 & 210.3 & 76.4 & 65.6 & 12.9 & 2.1 & 143.5 \\
\hline & & 124.0 & 20.9 & 326.0 & 91.4 & 93.6 & 28.8 & 10.0 & 241.8 \\
\hline & Minimum * & 102.0 & $\begin{array}{l}12.3 \\
10.5\end{array}$ & $\begin{array}{l}152.0 \\
141.0\end{array}$ & $\begin{array}{l}64.1 \\
53.7\end{array}$ & $\left.\begin{array}{l}37.7 \\
22.1\end{array}\right\}$ & 8.7 & 1.0 & $\begin{array}{r}110.0 \\
47.6\end{array}$ \\
\hline
\end{tabular}

* Minimum and next to the minimum. 
Seven of these samples had an excess of tannic acid, three had no tannic acid at all, and two or three had insoluble residues.

Adulteration of Whiskey.-Imitation whiskey is often concocted by diluting alcohol or neutral spirit to the proper strength, coloring with caramel, sometimes adding for body prune juice, and adding for flavor certain essential oils, such as oil of wintergreen, and artificial fruit essences, such as œnanthic and pelargonic ethers. As a rule, a small amount of pure whiskey is mixed with the artificial to give it flavor.

What has long been known as "blended whiskey" is either an imitation pure and simple, or a compound of whiskey and neutral spirits, artificially colored and flavored. According to the U. S. decisions, the term "blended whiskey" is restricted to a mixture of different kinds of genuine whiskey, colored and flavored.

Among Fleischman's recipes for "blended" whiskey is the following, which he claims to be the very lowest grade:

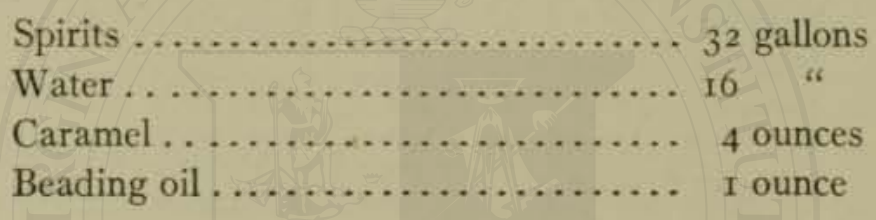

"Beading oil" is prepared by mixing 48 ounces oil of sweet almonds with 12 ounces C. P. sulphuric acid, neutralizing with ammonia, adding double the volume of proof spirits, and distilling. This preparation is so called because it is largely used for putting an artificial bead on cheap liquors.

A little creosote is sometimes added to give a burnt taste in semblance of Scotch whiskey. Pungent materials such as cayenne pepper are said to be used as adulterants, but no record is known of any substance being used more injurious than the alcohols. Sugar is a frequent adulterant.

Some doubt exists as to the injurious effects of fusel oil on the system.

The following analyses by Ladd* show the composition of neutral spirits, and imitation whiskey consisting of neutral spirits diluted with water, colored with caramel and flavored:

*N. Dak. Agric, Exp. Sta. Rep., 19o6, Part II, p. 145. 


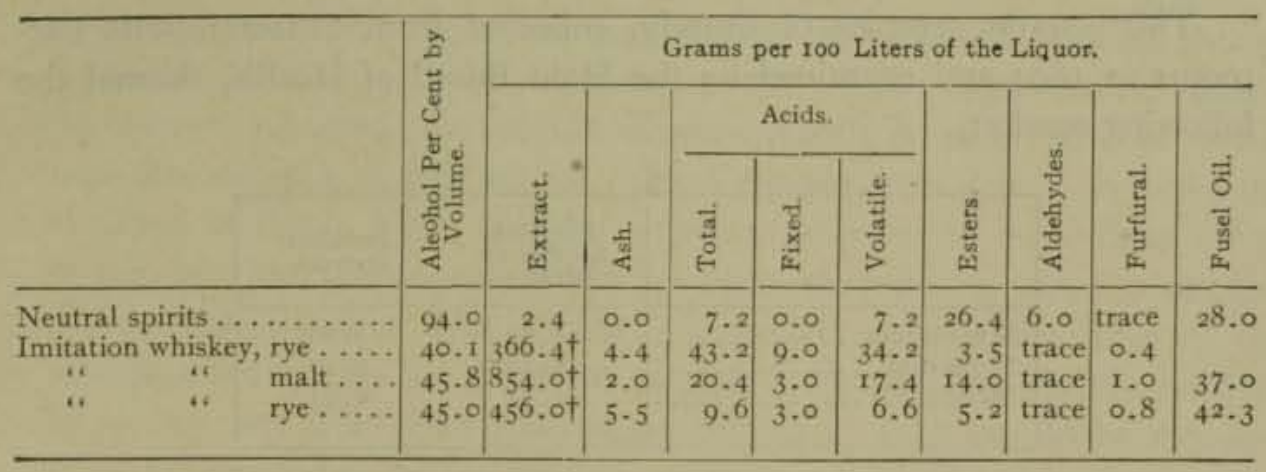

† Includes caramel color.

\section{BRANDY AND COGNAC.}

Brandy is the product of the distillation of fermented grape juice or wine. In a broader sense the term brandy is sometimes applied to liquor distilled from the juices of other fruits, such as apples, peaches, cherries; etc. The finest grades of brandy, such as pure cognac and armagnac (named from towns in France in which they were originally distilled), are made from choice white wine by the use of pot stills, and naturally command a high price. Brandy of a lower grade is distilled from the cheaper wines, and sometimes from the fermented marc, or refuse, of the grape, as well as from the lees and "scrapings" of the casks. The best brandies are sometimes rectified by a second distillation. Like whiskey, the fresh brandy is colorless, and would so remain if stored in glass or stone. The color is due to the wooden casks in which it is stored. Brandy consists of nearly pure alcohol and water, having a characteristic flavor, differing somewhat according to the nature and quality of the wine from which it was prepared. The chief flavor of pure cognac is due to œenanthic ether.

Composition.-Vasey gives the following analyses of cognac and of brandy adulterated with neutral spirits:

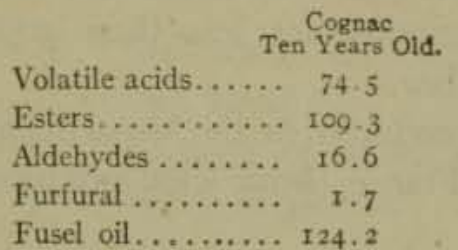

Volatile acids...... 74.5

Esters........... 109.3

Fusel oil........ 124.2
Brandy Mixed with Neutral Spirits. 79. 4 grams per 100 liters of absolute alcohol

$\begin{array}{rrrr}32.4 & \text { u } & \text { " } & \text { " } \\ 7.4 & \text { u } & \text { u } & \text { u } \\ 0.6 & \text { u } & \text { u } & \text { " } \\ 49.0 & \text { u } & \text { u }\end{array}$

* Analysis of Potable Spirits, p. 20. 
Thirty-seven samples of brandy, collected from Massachusetts barrooms in 1894 and examined by the State Board of Health, showed the: following results:

\begin{tabular}{|c|c|c|}
\hline & $\begin{array}{l}\text { Per Cent } \\
\text { Alcohol by } \\
\text { Weight. }\end{array}$ & $\begin{array}{l}\text { Per Cent } \\
\text { Extract. }\end{array}$ \\
\hline Maximum.............. & 50.70 & 3.00 \\
\hline Minimum. ............. & $21 \cdot 30$ & 0.10 \\
\hline Mean................... & 40.54 & 0.93 \\
\hline
\end{tabular}

Three of these samples were artificially prepared mixtures of alcohol and water, one showed the presence of cloves, five contained tannin in excess, nine were excessively acid, and two had insoluble residues.

Joint Standards. - The following are the standards of the A. O. A. C. and the A.S. N. F. D. D.:

New Brandy is a properly distilled spirit made from wine, and contains in 100 liters of proof spirit not less than 100 grams of the volatile flavors, oils, and other substances, derived from the material from which it is made, and of the substances congeneric with ethyl alcohol produced during fermentation and carried over at the ordinary temperatures of distillation, the principal part of which consists of the higher alcohols, estimated as amylic.

Brandy (Potable Brandy) is new brandy stored in wood for not less than four years without any artificial heat save that which may be imparted by warming the storehouse to the usual temperature, and contains in 100 liters of proof spirit not less than 150 grams of the substances found in new brandy, save as they are changed or eliminated by storage, and of those produced as secondary bodies during aging; and, in addition thereto, the substances extracted from the casks in which it has been stored. It contains, when prepared for consumption, as permitted by the regulations of the Bureau of Internal Revenue, not less than $45 \%$ by volume of ethyl alcohol, and, if no statement is made concerning its alcoholic strength, it contains not less than $50 \%$ by volume of ethyl alcohol as prescribed by law.

Cognac, Cognac Brandy, is brandy produced in the departments of the Charente and Charente Inferieure, France, from wine produced in those departments.

U. S. Pharmaco pœia Standards.-According to the U. S. Pharmacopœia, brandy should be at least four years old; its specific gravity should be 
not more than $0.94 \mathrm{I}$ nor less than 0.925 ; its alcoholic content should be from 39 to 47 per cent by weight; the residue from $100 \mathrm{cc}$. should not exceed 0.5 gram, and should dissolve readily in ro cc. of cold water, and this solution should not be colored deeper than a pale green by the addition of dilute ferric chloride solution (absence of more than traces of tannin); the residue should not be sweet nor spicy in taste; a marked disagreeable pungent odor of fusel oil should not manifest itself on the volatilization of the last traces of alcohol in evaporating for the residue; in acidity, not more than I cc. of tenth-normal alkali should be required to neutralize $100 \mathrm{cc}$. of the brandy, using litmus as an indicator.

Adulteration of Brandy.-Much of the brandy sold on the market is a compound or imitation, having for its basis alcohol reduced to the requisite strength, flavored either by the admixture of real brandy, or by various preparations such, for example, as syrup of raisins, prune juice, rum, acetic ether, œnanthic ether, infusion of green walnut-hulls, infusion of bitter almond shells, catechu, balsam of Tolu, etc.

Fleischmann gives the following recipe for artificial brandy of the cheapest grade:

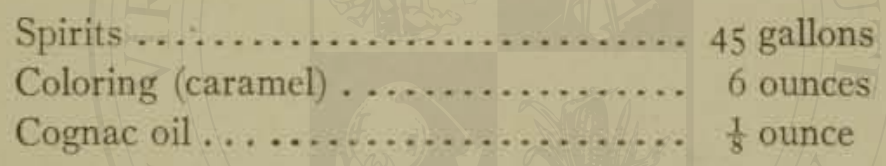

"Cognac oil " is made up of melted cocoanut oil r6 ounces, sulphuric acid 8 ounces, alcohol 16 ounces, mixed and distilled.

While commercial brandy often fails to meet the pharmacopœial requirements, and may contain any of the above flavoring materials, not one sample has been found among the many examined by the Massachusetts Board of Health during upwards of twenty years containing a more injurious ingredient than alcohol.

Genuine new brandy may be "aged" or "improved" for immediate use, according to Duplais, by adding to roo liters the following:

Old rum..................... 2.00 liters

Old kirsch *................... 1.75 "

Infusion of walnut-hulls ........... .75 liter

Syrup of raisins............... 2.00 liters

The addition of sugar and caramel to brandy is very common. The

* Brandy distilled from cherry wine. 
lack of flavor resulting from the employment of "silent spirit," or from watering the product, may be compensated for by the employment of so-called cognac essences sold for the purpose, containing mixtures of the aromatic compounds named above.

\section{RUM.}

Rum is the liquor distilled from fermented molasses or cane juice, or from the scum and other waste juices from the manufacture of raw sugar. The molasses wort is mixed with the residue from a previous fermentation and allowed to ferment for a number of days, after which it is distilled twice and stored in wood for a long time, to remove the disagreeable odor, which in the new product is especially marked. The characteristic flavor of old rum is due to a mixture of butyric and acetic ether, principally the former. Pineapples and guavas are often put in the still to impart a fruity flavor. The best varieties of rum come from Jamaica and Vera Cruz.

Composition.-The following analysis of rum is by Vasey:*

Volatile acids........... 28.0 grams per too liters of absolute alcohol

Esters................ 399.0

Aldehydes............. 8.4

Furfural.............. 2.8

Fusel oil............. 90.6

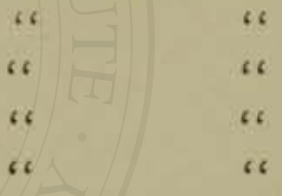

66

Thirty-nine samples of rum, sold at retail in Massachusetts in 1894 , were examined by the State Board of Health with the following results:

\begin{tabular}{|c|c|c|}
\hline & $\begin{array}{l}\text { Per Cent } \\
\text { Alcohol by } \\
\text { Weight. }\end{array}$ & $\begin{array}{l}\text { Per Cent } \\
\text { Extract. }\end{array}$ \\
\hline Maximum.......... & 42.9 & 3.93 \\
\hline Minimum. .................. & $24 \cdot 7$ & 0.04 \\
\hline Mean...$\ldots \ldots \ldots \ldots \ldots \ldots$ & $37 \cdot \mathrm{I}$ & $0.5 \mathrm{I}$ \\
\hline
\end{tabular}

Of these, two samples were new rum, and several were entirely artificial,

Joint Standards.-The following are the joint standards of the A. O. A. C. and the A. S. N. F. D. D.: 
New Rum is properly distilled spirit made from the properly fermented clean, sound juice of the sugar cane, the clean, sound massacuite made therefrom, clean, sound molasses from the massecuite, or any sound clean intermediate product save sugar, and contains in 100 liters of proof spirit not less than Ioo grams of the volatile flavors, oils, and other substances derived from the materials of which it is made, and of the substances congeneric with the ethyl alcohol produced during fermentation, which are carried over at the ordinary temperatures of distillation, the principal part of which is higher alcohols estimated as amylic.

Rum (Potable Rum) is new rum stored not less than four years in wood without any artificial heat save that which may be imparted by warming the storehouse to the usual temperature, and contains in 100 liters of proof spirit not less than 175 grams of the substances found in new rum, save as they are changed or eliminated by storage, and of those produced as secondary bodies, during aging; and, in addition thereto, the substances extracted from the casks. It contains, when prepared for consumption as permitted by the regulations of the Bureau of Internal Revenue, not less than $45 \%$ by volume of ethyl alcohol, and if no statement is made concerning its alcoholic strength, it contains not less than $50 \%$ by volume of ethyl alcohol as prescribed by law.

More or less factitious rum is sold on the market, made up of alcohoi diluted to the right strength, colored with caramel, and flavored by the addition of "rum essence." Prune juice is sometimes added.

Fleischman gives the following recipe for low-grade artificial rum:

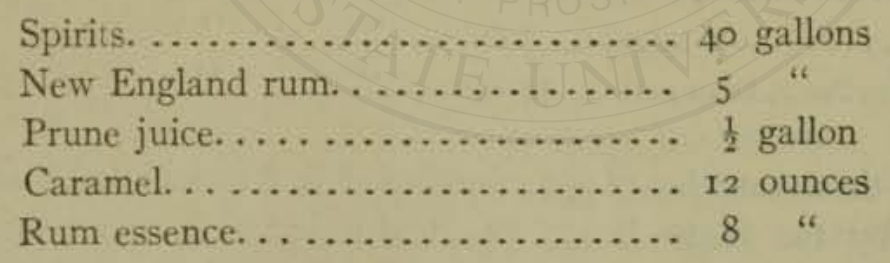

The "rum essence" is made up by distilling 32 ounces of a mixture of 2 ounces black oxide of manganese, 4 ounces pyroligneous acid, 32 ounces alcohol, and 4 ounces sulphuric acid. To this is added 32 ounces of acetic ether, 8 ounces of butyric ether, 16 ounces saffron extract, and $\frac{1}{8}$ ounce oil of birch. 


\section{GIN.}

Gin is an alcoholic liquor, flavored with the volatile oil of juniper and sometimes with other aromatic substances, such as coriander, grains of paradise, anise, cardamom, orange-peel, and fennel. The choicest variety is known as Schiedam schnapps, named from the town of Schiedam in Holland, where there are upwards of 200 distilleries devoted to the manufacture of gin. The mash used for this variety is fermented by yeast from malted barley and rye, after which it is distilled and redistilled in pot stills with juniper berries and sometimes hops.

Juniper berries, to which the most characteristic flavor of gin is due, are dark blue in color, and possess a pungent taste. They grow on the slender evergreen shrub Juniperus communis. Gin differs from the other distilled liquors by being water-white. To this end it is kept in glass and not in wood.

Much of the gin of commerce is made by redistilling corn or grain whiskey with oil of juniper, and frequently one or several of the abovenamed flavoring materials. Sugar is often added, and sometimes in the cheaper productions oil of turpentine is substituted for juniper oil.

Composition.-The following analysis of unsweetened gin is by Vasey: *

Volatile acids........... 0.0 grams per roo liters of absolute alcohol

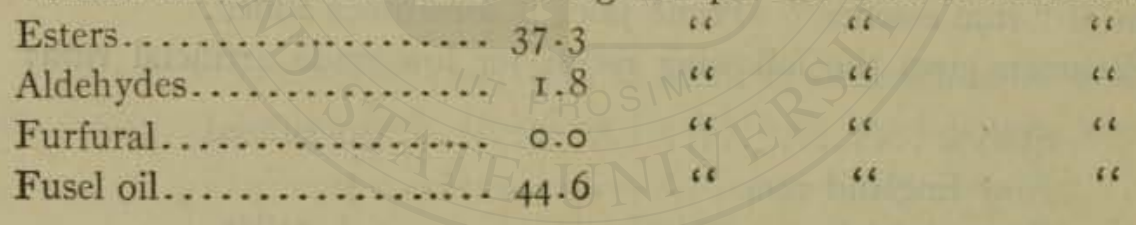

Thirty-three samples of gin, purchased in Massachusetts saloons and analyzed by the State Board of Health in 1894 , gave the following results in per cent of alcohol by weight: Maximum 42.5 , minimum 29.5, mean 38.2 .

* Analysis of Potable Spirits, p. 85 . 
METHODS OF ANALYSIS OF DISTILLED LIQUORS.

Specific gravity and alcohol are determined as described on pp. 657677. The following methods with the exception of the qualitative test for fusel oil, Mitchell's method, and McGill's opalescence test are those of the A. O. A. C.*

Determination of Extract.-Weigh or measure (at $15.6^{\circ} \mathrm{C}$.) $100 \mathrm{cc}$. of the sample, evaporate nearly to dryness on the water-bath, then transfer to a water-oven, and dry at the temperature of boiling water for $2 \frac{1}{2}$ hours.

Determination of Acids.-Titrate $100 \mathrm{cc}$. (or $50 \mathrm{cc}$. diluted to $100 \mathrm{cc}$. if the sample is dark in color) with tenth-normal alkali, using phenolphthalein as indicator. I cc. of tenth-normal alkali is equal to 0.006 of acetic acid.

Determination of Esters.-Dilute $200 \mathrm{cc}$. of the sample with $25 \mathrm{cc}$. of water and distil slowly into a graduated 200-cc. flask until nearly filled to the mark. Complete the volume, shake, and use aliquot portions for the determination of esters, aldehydes, and furfural.

Exactly neutralize $50 \mathrm{cc}$. of the distillate with tenth-normal alkali, using phenolphthalein as indicator, and add from 25 to $50 \mathrm{cc}$. of the tenth-normal alkali in excess of that required for neutralization. Either boil for one hour with a reflux condenser, or allow to stand overnight in a stoppered flask, and heat with a tube condenser for one-half hour below the boiling-point. Cool, and titrate with tenth-normal acid, using phenolphthalein as indicator. Multiply the number of $\mathrm{cc}$. of tenthnormal alkali used in the saponification by 0.0088 , thus obtaining the grams of esters calculated as ethyl acetate.

Determination of Aldehydes.-1. Reagents.-(a) Alcohol Free from Aldehydes.-Prepare by first redistilling the ordinary $95 \%$ alcohol over caustic soda or potash, then add from 2 to 3 grams per liter of $m$-phenylenediamine hydrochloride, digest at ordinary temperature for several days (or reflux on the steam-bath for several hours), and then distil slowly, rejecting the first $100 \mathrm{cc}$. and the last $200 \mathrm{cc}$.

(b) Sulphite-fuchsin Solution.-Dissolve 0.50 gram of pure fuchsin in $5 \mathrm{co} \mathrm{cc}$. of water, then add 5 grams of $\mathrm{SO}_{2}$ dissolved in water, make up to a liter, and allow to stand until colorless. Prepare this solution in small quantities, as it retains its strength for only a very few days.

*U. S. Dept. of Agric., Bur. of Chem., Bul, ro7 (rev.), pp. 95 to ror; Circular 43 . 
(c) Standard Acetic Aldehyde Solution.-Prepare according to the directions of Vasey* as follows: Grind aldehyde ammonia in a mortar with ether, and decant the ether. Repeat this operation several times, then dry the purified salt in a current of air and finally in a vacuum over sulphuric acid. Dissolve I.386 grams of this purified ammonium aldehyde in $50 \mathrm{cc}$. of $95 \%$ alcohol, to this add $22.7 \mathrm{cc}$. of normal alcoholic sulphuric acid, then make up to $100 \mathrm{cc}$. and add $0.8 \mathrm{cc}$. to compensate for the volume of the ammonium sulphate precipitate. Allow this to stand over night and filter. This solution contains I gram of acetic aldehyde in $100 \mathrm{cc}$, and will retain its strength.

The standard found most convenient for use is $2 \mathrm{cc}$. of this strong aldehyde solution diluted to $100 \mathrm{cc}$. with $50 \%$ alcohol by volume. One cc. of this solution is equal to 0.0002 gram of acetic aldehyde. This solution should be made up fresh every day or so, as it loses its strength.

2. Process.-Determine the aldehyde in thè distillate prepared for esters. Dilute from 5 to $10 \mathrm{cc}$. of the distillate to $50 \mathrm{cc}$. with aldehydefree alcohol ( $50 \%$ by volume), add $25 \mathrm{cc}$. of the fuchsin solution, and allow to stand for fifteen minutes at $15^{\circ} \mathrm{C}$. The solutions and the reagents should be at $15^{\circ} \mathrm{C}$. before they are mixed. Prepare standards of known strength in the same way.

Determination of Furfural.-Standard Furfural Solution,-Dissolve I gram of redistilled furfural in Ioc cc. of $95 \%$ alcohol. This strong solution will keep. Standards are made by diluting $\mathrm{I}$ cc. of this solution to $100 \mathrm{cc}$. with $50 \%$ by volume alcohol. One cc. of this solution contains $0.000 \mathrm{r}$ gram furfural.

Process.-Dilute from 1o to $20 \mathrm{cc}$. of the distillate, prepared as described under esters, to $50 \mathrm{cc}$. with furfural-free alcohol $(50 \%$ by volume). To this add 2 cc. of colorless anilin and $0.5 \mathrm{cc}$. of hydrochloric acid (specific gravity 1.125), and keep for fifteen minutes in a water-bath at about $15^{\circ} \mathrm{C}$. Prepare standards of known strength in the same way.

Detection of Fusel Oil.-In the process of dealcoholizing a liquor by evaporation in an open dish over the water-bath, one may readily detect fusel oil, if present, by its harsh and nauseating odor, if the nose is applied just at the moment when the last traces of alcohol are going off. At this stage any considerable trace of fusel oil will be especially apparent by the effect on the throat of the one who smells it, causing

\footnotetext{
* Analysis of Potable Spirits, p. 30.
} 
an uncontrollable desire to cough. Other ways of applying the odor test consist in pouring a small portion of the spirit into the hand, and allowing it to evaporate slowly therefrom, or in rinsing out a warm glass with the liquor, observing the odor in each case.

Goebel suggests the following test, based on the detection of the volatile acids: Agitate about $30 \mathrm{cc}$. of the liquor with 2 or $3 \mathrm{cc}$. of a dilute solution of potassium hydroxide; evaporate over the waterbath to the volume of 2 or $3 \mathrm{cc}$., cool, and to the residue add 5 or $6 \mathrm{cc}$. of concentrated sulphuric acid. If fusel oil be present, the characteristic odors of valerianic and butyric acids will be apparent.

Determination of Fusel Oil.-Allen-Marquardt Method.-Add to Ioo cc. of whiskey $20 \mathrm{cc}$. of half-normal sodium hydroxide, and saponify the mixture by boiling for one hour under a reflux condenser.* Connect the flasks with a distilling apparatus, distil $90 \mathrm{cc}$, add $25 \mathrm{cc}$. of water, and continue the distillation until an additional $25 \mathrm{cc}$. is collected.

Approximately saturate the distillate with finely ground sodium chloride, and add a saturated solution of sodium chloride until the specific gravity is I.IO.

Extract this salt solution four times with carbon tetrachloride, $\dagger$ using $40,30,20$, and $10 \mathrm{cc}$. respectively, and wash the carbon tetrachloride three times with $5 \mathrm{a}$-cc. portions of a saturated solution of sodium chloride, and twice with saturated solution of sodium sulphate. Then transfer the carbon tetrachloride to a flask containing $5 \mathrm{cc}$. of concentrated sulphuric acid, $45 \mathrm{cc}$. of water, and 5 grams of potassium bichromate, and boil for eight hours under a reflux condenser.

Add $30 \mathrm{cc}$. of water, and distil until only about $20 \mathrm{cc}$. remain; add $80 \mathrm{cc}$. of water, and distil until but $5 \mathrm{cc}$. are left. Neutralize the distillate to methyl orange, and titrate with sodium hydroxide, using phenolphthalein as indicator. One cc. of tenth-normal sodium hydroxide is equivalent to 0.0088 gram of amyl alcohol.

Rubber stoppers can be used in the saponification and first distillation, but corks covered with tinfoil must be used in the oxidation and second distillation. Corks and tinfoil must be renewed frequently.

* Or $100 \mathrm{cc}$. of the liquor may be mixed with $20 \mathrm{cc}$. of half-normal sodium hydroxide, allowed to stand overnight at room temperature, and distilled directly.

† Purify 5 liters of carbon tetrachloride by boiling for several hours under a reflux condenser with $200 \mathrm{cc}$. of sulphuric acid and 25 grams of potassium bichromate in $200 \mathrm{cc}$. of water; separate from the oxidizing mixture by distillation, and redistil over barium carbonate. 
Tolman and Hillyer's Modification of the Allen-Marquardt Method.Proceed with the Allen-Marquardt method to the point where the carbon tetrachloride solution of the higher alcohols is ready to be oxidized. Add $50 \mathrm{cc}$. of a solution of 200 grams of pulverized potassium bichromate in I800 cc. of water and $200 \mathrm{cc}$. of concentrated sulphuric acid, very carefully measured with pipette or burette, and start the eight-hour oxidation. Take great care to prevent any isolation of spots of bichromate on the flask during the oxidation. Decomposition of the bichromate from overheating can best be prevented by slow boiling over several thicknesses of asbestos board. After the oxidation is complete, separate the bichromate solution from the carbon tetrachloride in a separatory funnel, care being taken to wash the carbon tetrachloride free from bichromate. Make up the bichromate solution to $500 \mathrm{cc}$. Place $200 \mathrm{cc}$. of this solution in a liter flask, add $20 \mathrm{cc}$. of concentrated hydrochloric acid, $100 \mathrm{cc}$. of potassium iodide solution (I:I), and $50 \mathrm{cc}$. of approximately three-fourths normal thiosulphate not standardized. Make this last addition by means of a burette. (If a high content of fusel oil is present, $50 \mathrm{cc}$. of thiosulphate may be excessive and a smaller amount should be used, the same quantity being added to the sample and to the blank.) Run blanks containing exactly the same amount of reagents with each series, and treat them in the same way, starting them at the point where the carbon tetrachloride is washed with sodium chloride. The titration of this blank, to which has been added exactly the same amount of three-fourths normal thiosulphate, gives the value of the bichromate solution. The difference in cubic centimeters of tenthnormal thiosulphate used in titrating the blank and the samples gives the amount of bichromate reduced by the higher alcohols. This difference in cubic centimeters of tenth-normal thiosulphate multiplied by the factor 0.001773 gives grams of higher alcohols present.

Mitchell and Smith Method.*-This is more rapid than the AllenMarquardt method and gives more nearly the true amount of fusel oil.

Saponify, distil, shake with sodium chloride, and extract with carbon tetrachloride, as in the Allen-Marquardt method. To the carbon tetrachloride extract, contained in the separatory funnel, add $10 \mathrm{cc}$. of potassium hydroxide solution (I:I). Cool the mixture in ice-water to approximately $0^{\circ} \mathrm{C}$. Similarly cool $100 \mathrm{cc}$. of a solution of potassium permanganate solution (20 grams to the liter), accurately measured in

* A. O. A. C. Proc, rgos, U. S. Dept. of Agric., Bur. of Chem., Bul. 122, p. 199. 
a flask. To the contents of the separatory funnel add the bulk of the permanganate solution, but without rinsing, retaining the residue to be added at a later stage. Remove the mixture from the bath, and shake vigorously for five minutes; set aside for thirty minutes, with occasional shaking, permitting the liquid to warm to room temperature ( 20 to $25^{\circ} \mathrm{C}$.)

Accurately measure into a liter Erlenmeyer flask $100 \mathrm{cc}$. of a solution of hydrogen peroxide slightly (about $2 \%$ ) stronger than the permanganate solution, acidulate with $100 \mathrm{cc}$. of an approximately $25 \%$ sulphuric acid solution, and slowly add the contents of the separatory funnel with constant shaking, keeping the acid solution constantly in excess. Rinse the separatory funnel and the flask containing the residue of permanganate with water and add to the peroxide solution. Finally titrate the excess of hydrogen peroxide with standard potassium permanganate solution (Io grams to the liter).

Run a blank determination, using the same amounts of the stronger permanganate, potassium hydroxide, hydrogen peroxide, and sulphuric acid solutions, and titrating the residual peroxide with the standard potassium permanganate as before.

The difference in the amounts of permanganate consumed, in grams, times 0.696 , gives the amount of amyl alcohol.

Detection of Methyl Alcohol.-Leach and Lythgoe Immersion Refractometer Method.*-Determine at $20^{\circ} \mathrm{C}$, the refraction of the distillate obtained in the determination of alcohol by the immersion refractometer. If on reference to the table the refraction shows the percentage of alcohol agreeing with that obtained from the specific gravity, it may be safely assumed that no methyl alcohol is present. If, hewever, there is an appreciable amount of methyl alcohol, the low refractometer reading will at once indicate the fact. If the absence from the solution of other refractive substances than water and the alcohols is assured, this qualitative test by difference in refraction is conclusive.

The addition of methyl to ethyl alcohol decreases the refraction in direct proportion to the amount present; hence the quantitative calculation is readily made by interpolation in the table, using the figures for pure ethyl and methyl alcohol of the same alcoholic strength as the sample.

Example.-Suppose the distillate made up to the original volume of the measured portion taken for the alcohol determirlation has a

\footnotetext{
* Jour. Am. Chem. Soc., 27, 1905, p. 964.
} 
specific gravity of 0.9736 , corresponding to $18.38 \%$ alcohol by weight, and has a refraction of 35.8 at $20^{\circ} \mathrm{C}$. by the immersion refractometer; by interpolation in the refractometer table the readings of ethyl and methyl alcohol corresponding to $18.38 \%$ alcohol are 47.2 and 25.4 , respectively, the difference being $21.8 ; 47.2-35.8=11.4 ;$ (II. $4 \div 2 \mathrm{I} .8$ ) $I 00=5^{2} \cdot 3$, showing that $5^{2} \cdot 3$ of the alcohol present is methyl alcohol.

SCALE READINGS ON ZEISS IMMERSION REFRACTOMETER AT $20^{\circ} \mathrm{C}$, CORRESPONDING TO EACH PER CENT BY WEIGHT OF METHYL AND ETHYL ALCOHOLS.

\begin{tabular}{|c|c|c|c|c|c|c|c|c|c|c|c|}
\hline \multirow{2}{*}{$\begin{array}{c}\text { Per Cent } \\
\text { Alcoho! } \\
\text { by } \\
\text { Weight. }\end{array}$} & \multicolumn{2}{|c|}{$\begin{array}{c}\text { Scale } \\
\text { Readings. }\end{array}$} & \multirow{2}{*}{$\begin{array}{c}\text { Per Cent } \\
\text { Alcohol } \\
\text { by } \\
\text { Weight: }\end{array}$} & \multicolumn{2}{|c|}{$\begin{array}{c}\text { Scale } \\
\text { Reactings. }\end{array}$} & \multirow{2}{*}{$\begin{array}{c}\text { Per Cent } \\
\text { Alcohol } \\
\text { by } \\
\text { Weight. }\end{array}$} & \multicolumn{2}{|c|}{$\begin{array}{l}\text { Scale } \\
\text { Readings. }\end{array}$} & \multirow{2}{*}{$\begin{array}{c}\text { Per Cent } \\
\text { Alcohol } \\
\text { by } \\
\text { Weight. }\end{array}$} & \multicolumn{2}{|c|}{$\begin{array}{c}\text { Scale } \\
\text { Readings. }\end{array}$} \\
\hline & $\begin{array}{c}\text { Methyl } \\
\text { Al- } \\
\text { cohol. }\end{array}$ & $\begin{array}{l}\text { Ethyl } \\
\text { Al- } \\
\text { cohol. }\end{array}$ & & $\begin{array}{c}\text { Methyl } \\
\text { Al- } \\
\text { cohol. }\end{array}$ & $\begin{array}{l}\text { Ethyl } \\
\text { A!- } \\
\text { cohol. }\end{array}$ & & $\begin{array}{c}\text { Methyl } \\
\text { Al- } \\
\text { cohol. }\end{array}$ & $\begin{array}{l}\text { Ethyl } \\
\text { Al- } \\
\text { cohol. }\end{array}$ & & $\begin{array}{c}\text { Methyl } \\
\text { Al- } \\
\text { cohol }\end{array}$ & $\begin{array}{l}\text { Ethyl } \\
\text { Al- } \\
\text { cohol. }\end{array}$ \\
\hline 0 & 14.5 & 14.5 & 26 & 30.3 & 61.9 & $5^{1}$ & 39.7 & 91.1 & 76 & 29.0 & IOI.O \\
\hline I & $\mathrm{I} 4.8$ & 16.0 & 27 & 30.9 & 63.7 & 52 & 39.6 & 91.8 & 77 & 28.3 & 100.9 \\
\hline 2 & I 5.4 & 17,6 & 28 & 31.6 & 65.5 & 53 & 39.6 & $9^{2} \cdot 4$ & 78 & 27.6 & 100.9 \\
\hline 3 & 16.0 & 19.1 & 29 & $3^{2} \cdot 2$ & 67.2 & 54 & $39 \cdot 5$ & 93.0 & 79 & 26.8 & 100.8 \\
\hline 4 & 16.6 & 20.7 & 30 & 32.8 & 69.0 & 55 & 39.4 & 93.6 & 80 & 26.0 & 100.7 \\
\hline 5 & 17.2 & 22.3 & $3 I$ & 33.5 & 70.4 & $5^{6}$ & 39.2 & $94 . \mathrm{I}$ & $8 \mathrm{r}$ & $25 . \mathrm{I}$ & 100.6 \\
\hline 6 & 17.8 & 24.1 & $3^{2}$ & 34.1 & $71-7$ & 57 & 39.0 & 94.7 & 82 & 24.3 & 100.5 \\
\hline 7 & 18.4 & 25.9 & 33 & 34.7 & 73.1 & $5^{8}$ & $3^{8} .6$ & 95.2 & 83 & 23.6 & 100.4 \\
\hline 8 & 19.0 & 27.8 & 34 & 35.2 & 74.4 & 59 & $3^{8} .3$ & 95.7 & 84 & 22.8 & 100.3 \\
\hline 9 & 19.6 & 29.6 & 35 & 35.8 & 75.8 & 60 & 37.9 & 96.2 & 85 & 21.8 & 100.1 \\
\hline 10 & 20.2 & 31.4 & $3^{6}$ & $3^{6} \cdot 3$ & 76.9 & $6 r$ & 37.5 & 96.7 & 86 & 20.8 & 99.8 \\
\hline II & 20.8 & 33.2 & 37 & 36.8 & 78.0 & 62 & 37.0 & $97 \cdot 1$ & 87 & 19.7 & $99 \cdot 5$ \\
\hline 12 & 21.4 & 35.0 & 38 & $37 \cdot 3$ & 79.1 & 63 & 36.5 & 97.5 & 88 & 18.6 & 99.2 \\
\hline 13 & 22.0 & $3^{6} .9$ & 39 & 37.7 & 80.2 & 64 & 36.0 & 98.0 & 89 & 17.3 & 98.9 \\
\hline 14 & 22.6 & $3^{8} \cdot 7$ & 40 & $3^{8} .1$ & $8 \mathrm{r} \cdot 3$ & 65 & $35 \cdot 5$ & 98.3 & $9 \circ$ & 16.1 & 98.6 \\
\hline I5 & 23.2 & 40.5 & $4 \mathrm{I}$ & $3^{8} \cdot 4$ & 82.3 & 66 & 35.0 & 98.7 & 91 & 14.9 & 98.3 \\
\hline 16 & 23.9 & 42.5 & 42 & 38.8 & $83 \cdot 3$ & 67 & 34.5 & 99.1 & 92 & 13.7 & 97.8 \\
\hline I 7 & $24 \cdot 5$ & 44.5 & 43 & 39.2 & 84.2 & 68 & 34.0 & 99.4 & 93 & 12.4 & 97.2 \\
\hline 18 & 25.2 & 46.5 & 44 & $39 \cdot 3$ & 85.2 & 69 & $33 \cdot 5$ & 99.7 & 94 & 11.0 & 96.4 \\
\hline 19 & 25.8 & 48.5 & 45 & 39.4 & 86,2 & 70 & 33.0 & 100.0 & 95 & 9.6 & 95.7 \\
\hline 20 & 26.5 & 50.5 & $4^{6}$ & 39.5 & 87.0 & 71 & $32 \cdot 3$ & 100.2 & 96 & 8. 2 & 94.9 \\
\hline 21 & 27.1 & 52.4 & 47 & 39.6 & 87.8 & 72 & $3 \mathrm{x} .7$ & 100.4 & 97 & 6.7 & 94.0 \\
\hline 22 & 27.8 & $54 \cdot 3$ & 48 & 39.7 & 88.7 & 73 & $3 \mathrm{I}=\mathrm{I}$ & 100.6 & 98 & $5 \cdot 1$ & 93.0 \\
\hline 23 & 28.4 & $5^{6} \cdot 3$ & 49 & 39.8 & $89 \cdot 5$ & 74 & 30.4 & 100.8 & 99 & 3.5 & 92.0 \\
\hline 24 & 29.1 & 58.2 & 50 & 39.8 & 90.3 & 75 & 29.7 & 101.0 & 100 & 2.0 & 91.0 \\
\hline 25 & 29.7 & 60.1 & & & & & & & & & \\
\hline
\end{tabular}

Trillat Method.*-To $50 \mathrm{cc}$. add $50 \mathrm{cc}$. of water and 8 grams of lime, and fractionally distil by the aid of Glinksy bulb tubes. Dilute the

*A. Trillat, Analyst; 24,1899, pp. $13,211-212$. 
first ${ }_{15} \mathrm{cc}$. of the distillate to $\mathrm{I}_{5} \mathrm{O} \mathrm{cc}$., mix with $\mathrm{I}_{5}$ grams of potassium bichromate and $70 \mathrm{cc}$. of sulphuric acid $(\mathrm{r}: 5)$, and allow to stand for one hour with occasional shaking.

Distil, reject the first $25 \mathrm{cc}$., and collect $100 \mathrm{cc}$. Mix $50 \mathrm{cc}$. of the distillate with $\mathrm{I} \mathrm{cc}$ of rectified dimethyl-anilin, transfer to a stout, tightly-stoppered flask, and keep on bath at 70 to $80^{\circ} \mathrm{C}$. for three hours with occasional shaking. Make distinctly alkaline with sodium hydroxide, and distil the excess of dimethyl-anilin, stopping the distillation when $25 \mathrm{cc}$. have passed over.

Acidify the residue in the flask with acetic acid, shake, and test a few cc. by adding four or five drops of water with lead dioxide in suspension ( $\mathrm{I}$ gram in $100 \mathrm{cc}$.). If methyl alcohol be present, a blue coloration occurs which is increased by boiling.

Note.-Ethyl alcohol thus treated yields a blue coloration, changing immediately to green, afterwards to yellow, and becoming colorless when boiled.

Riche and Bardy Methoa.*-The following method for the detection of methyl alcohol in commercial spirit of wine depends on the formation of methyl-anilin violet:

Place $10 \mathrm{cc}$. of the sample, previously rectified over potassium carbonate if necessary, in a small flask with 15 grams of iodine and 2 grams of red phosphorus. Keep in ice-water for from ten to fifteen minutes until action has ceased. Distil on a water-bath the methyl and ethyl iodides formed into about $30 \mathrm{cc}$. of water. Wash with dilute alkali to eliminate free iodine. Separate the heavy oily liquid which settles, and transfer to a flask containing $5 \mathrm{cc}$. of anilin. The flask should be placed in cold water, in case the action should be violent, or, if necessary, the reaction may be stimulated by gently warming the flask. After one hour boil the product with water, and add about $20 \mathrm{cc}$. of a ${ }_{15} \%$ solution of soda; when the bases rise to the top as an oily layer, fill the flask up to the neck with water, and draw them off with a pipette. Oxidize I cc. of the oily liquid by adding to grams of a mixture of 100 parts of clean sand, 2 of common salt, and 3 of cupric nitrate; mix thoroughly, introduce into a glass tube, and heat to $90^{\circ} \mathrm{C}$. for eight or ten hours. Exhaust the product with warm alcohol, filter, and make up with alcohol to $100 \mathrm{cc}$. If the sample of spirits be pure, the liquid is of a red tint, but in the presence of ${ }_{1} \%$ of methyl alcohol, it has a distinct violet shade; with

* Allen's Commercial Organic Analysis, 3d ed., I, p. 8o. 
$2.5 \%$ the shade is very distinct, and still more so with $5 \%$. To detect more minute quantities of methyl alcohol, dilute $5 \mathrm{cc}$. of the colored liquid to $100 \mathrm{cc}$. with water, and dilute $5 \mathrm{cc}$. of this again to $400 \mathrm{cc}$. Heat the liquid thus obtained in porcelain, and immerse a fragment of white merino (free from sulphur) in it for half an hour. If the alcohol be pure. the wool will remain white, but if methylated, the fiber will become

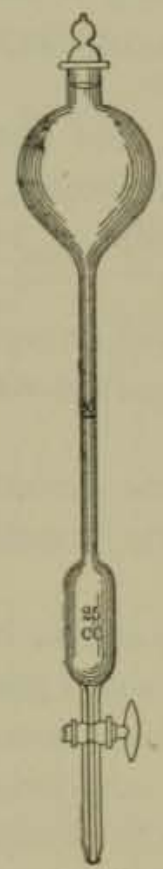
violet, the depth of tint giving a fair approximate indication of the proportion of methyl alcohol present.

Detection of Caramel.-Crampton and Simon's Method.*-Evaporate $50 \mathrm{cc}$. of the liquor nearly but not quite to dryness in an evaporating-dish on the water-bath. Wash with water into a 50 -cc. graduated glass-stoppered flask, add $25 \mathrm{cc}$. of absolute alcohol, and fill to the mark with water. Shake, and transfer $25 \mathrm{cc}$. of the solution to a separatory funnel of the type presented in Fig. Ir6, the stem of which terminates in a 25 -cc. graduated bulb pipette, provided with a stop-cock as shown.

Add $50 \mathrm{cc}$. of ether, and shake carefully at intervals during half an hour. After complete separation, make up the lower aqueous layer with water to the $25-\mathrm{cc}$. mark, which may be done by siphoning it in through a rubber tube from an elevated flask, controlling the supply by the stop-cock. Shake the separatory funnel, and again allow the layers to separate, draw off the

Fic, 116.-Separa- aqueous layer, and compare with the color of the origtory Funnel for Detecton of Caramel. inal liquor. Express the amount of color removed as per cent of the total amount. Ether will readily dissolve the natural color due to oakwood (mainly flavescin), while caramel is insoluble in ether; hence uncolored liquors are partially decolorized by this treatment, while those colored with caramel show little change.

A mishor Test, Modified by Lasché.†-Add 1o cc. of paraldehyde to $5 \mathrm{cc}$. of the sample contained in a test tube and shake. Add absolute alcohol, a few drops at a time, shaking after each addition until the mixture becomes clear. Allow to stand. Turbidity after ten minutes is an indication of caramel.

* Jour. Am. Chem. Soc., 22 r 900 , p. 8 ro.

$\dagger$ The Brewer Distiller, May, 1903 . 
Determination of Water-insoluble Color in Whiskies.-Evaporate $50 \mathrm{cc}$. of the sample just to dryness. Take up with cold water, using approximately $\mathrm{I}_{5} \mathrm{cc}$, filter, and wash until the filtrate amounts to nearly $25 \mathrm{cc}$. To this filtrate add $25 \mathrm{cc}$. of absolute alcohol or $26.3 \mathrm{cc}$. of $95 \%$ by volume alcohol, and make up to $50 \mathrm{cc}$. by the addition of water. Mix thoroughly and compare in a colorimeter with the original material. Calculate the per cent of color insoluble in water from these readings.

Determination of Color Insoluble in Amyl Alcohol.-Modified Marsh Test.-Evaporate $50 \mathrm{cc}$. of the whiskey just to dryness on the steambath. Add $26.3 \mathrm{cc}$. of $95 \%$ alcohol to dissolve the residue. Transfer to a 5o-cc. flask and make up to volume with water to obtain a uniform alcohol concentration. Place $25 \mathrm{cc}$. of this solution in a separatory funnel, and add $20 \mathrm{cc}$. of the Marsh reagent, shaking lightly so as not to form an emulsion. (This reagent consists of $100 \mathrm{cc}$. of pure amyl alcohol, 3 cc. of syrupy phosphoric acid, and $3 \mathrm{cc}$. of water; shake before using.) Allow the layers to separate, and repeat this shaking and standing twice again. After the layers have clearly separated, draw off the lower or watery layer which contains the caramel into a 25 -cc. cylinder, and make up to volume with $50 \%$ by volume alcohol. Com. pare this solution in a colorimeter with the untreated $25 \mathrm{cc}$. Calculate the result of this reading to the per cent of color insoluble in amyl alcohol.

Opalescence in Diluted Alcohol Distillate--McGill * has shown that in the case of liquors made from thoroughly rectified grain spirit, there is little or no opalescence produced when the alcoholic distillate (i.e., that used in determining the alcohol) is diluted with an equal volume of water, while in the case of liquors distilled from alcoholic infusions without rectification, the opalescence is marked. He ascribes the opalescence to the presence of minute amounts of volatile oils present in wine matc, grains, and other sources of these liquors, soluble in strong, but insoluble in dilute alcohol. Whether due to this or to the separation of minute traces of fusel oil on dilution, the presence or absence of turbidity certainly furnishes a rough distinguishing test, indicating in some cases the exclusive use of rectified spirit.

* Bul. 27, Canadian Inland Rev. Dept. 


\section{LIQUEURS AND CORDIALS.}

These are manufactured beverages, usually high in alcohol and sugar, flavored with a wide variety of aromatic herbs or essences, and often strongly colored. Red colors most frequently used for this purpose are cochineal, cudbear, and red sandal and Brazil woods; for yellow colors, caramel and saffron-yellow are employed; for blue, indigo; and for green, chlorophyll and malachite green.

Some of the oldest of the liqueurs, such as chartreuse and bénédictine, derive their names from certain monasteries of Europe, in which they have been made for many years.

Absinthe is one of the best-known cordials, made by redistilling $40 \%$ alcohol in which wormwood, anise, sweet flag, and marjoram leaves have been macerated. Sometimes coriander and fennel are also used. It is highly intoxicating.

Curaçao is made by distilling dilute spirits in which Curaçao orangepeel,* cinnamon and often other spices have been soaked, and by adding sugar to the resulting liqueur.

De Brevans gives the following recipe for curaçoa:

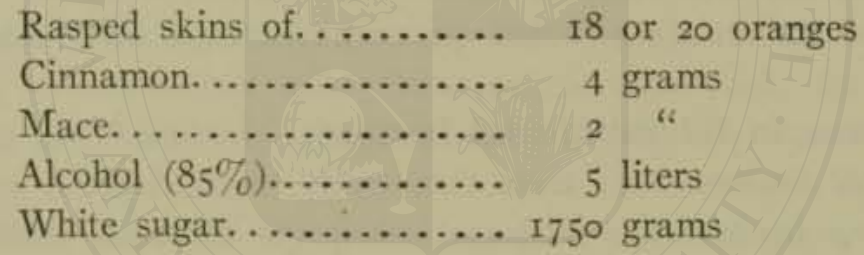

Macerate for fourteen days, distill without rectification, and color with caramel.

Angostura owes its flavor to Angostura bark and various spices.

Maraschino had originally for its basis the fermented juice of the sour Italian cherry, to which honey was added. It is more commonly made by distilling a mixture in alcohol of ripe wild cherries, raspberries, cherry leaves, peach nuts, and orris. Finally sugar is added.

Chartreuse and Benédictine contain much sugar, and are flavored with the volatile oils of angelica, hyssop, nutmeg, and peppermint.

Noyau, or Crême de Noyau, is a preparation distilled from brandy, bitter almonds, mace and nutmeg. Sugar and coloring matter, usually pink, are added to the final product.

\footnotetext{
* This is a very rare and highly prized orange, growing in the island of Curaçao.
} 
Crême de Menthe, according to De Brevans, is made by distilling a mixture of

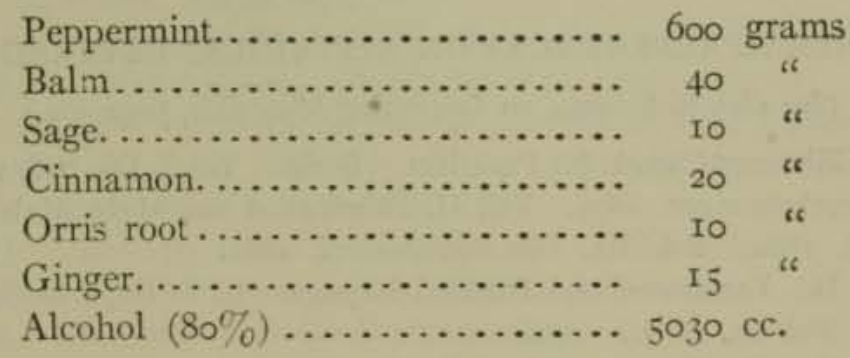

producing finally to liters of the liquor, after $375^{\circ}$ grams of white sugar have been introduced.

The better grades of creme de menthe were formerly colored with an alcoholic solution of chlorophyll, derived by macerating bruised green leaves of various plants with alcohol, but at present, coal-tar dyes are used. Frequently the desired shade is secured by mixing a green (e.g., Light Green S.F.), a blue-green (e.g., Malachite Green), or a blue (e.g., Indigo Carmine) with a yellow color.

The following analyses, due to König, show the chemical composition of the best-known cordials:

\begin{tabular}{|c|c|c|c|c|c|c|c|}
\hline & $\begin{array}{l}\text { Specific } \\
\text { Gravity. }\end{array}$ & $\begin{array}{l}\text { Alcohol } \\
\text { by Vol- } \\
\text { ume. }\end{array}$ & $\begin{array}{l}\text { Aleohol } \\
\text { by } \\
\text { Weight. }\end{array}$ & Extract. & $\begin{array}{l}\text { Cane } \\
\text { Sugar. }\end{array}$ & $\begin{array}{l}\text { Other } \\
\text { Extrac- } \\
\text { tives. }\end{array}$ & Ash. \\
\hline Absinthe... & 0.9116 & $5^{8.93}$ & & 0.18 & $\ldots \ldots$ & $0.3^{2}$ & \\
\hline Bénédictine. & 1.0709 & 52 & $3^{8} \cdot-5$ & 36.00 & 32.57 & 3.43 & 0.043 \\
\hline Ginger............ & 1. $048 \mathrm{I}$ & $47 \cdot 5$ & 36.0 & 27.79 & 25.92 & 1.87 & 0.141 \\
\hline Crême de menthe. & I. 0447 & 48.0 & 36.5 & 28.28 & 27.63 & 0.65 & 0.068 \\
\hline Anisette de Bordeaux. & 1.0847 & 42.0 & 30.7 & 34.82 & $37 \cdot 44$ & $0.3^{8}$ & 0.040 \\
\hline Curayoa.............. & 1.0300 & 55.0 & $42-5$ & 28.60 & 28.50 & 0.10 & 0,040 \\
\hline Kümmel ............. & 1.0830 & 33.9 & 24.8 & 32.02 & $3 \mathrm{r}, \mathrm{is}$ & 0.84 & $0.05^{8}$ \\
\hline Angostera. ............ & 0.9540 & $49 \cdot 7$ & $\ldots \ldots$ & 5,85 & 4.16 & 1.69 & \\
\hline Chartreuse,........... & I. 0799 & $43 \cdot 18$ & $\ldots \ldots$ & $3^{6} .11$ & $34 \cdot 35$ & 1. -76 & \\
\hline
\end{tabular}

Analysis of Cordials and Liqueurs.-The character of the essences and flavoring principles used in these beverages is so widely varied that no regular systematic plan for identifying them can be made applicable to all cases. The senses of smell and taste are most useful, both when applied directly to the liqueur itself and to the dry extract, for suggestions as to the main ingredients employed. Coloring-matters, sugars, acids, and alcohol are determined as with other liquors, except that in the case of alcohol all volatile oils must first be separated out by treatment with magnesia, as directed for alcohol in lemon extract. Presence of volatile 
oils is shown, if on treatment of a few cubic centimeters of the sample in a test-tube with water a precipitate is formed.

\section{GENERAL REFERENCES ON ALCOHOLIC BEVERAGES.}

(See also References on Leavening Materials, page $3^{64}$.)

BERSCH, J. Gährungs-Chemie für Praktiker. Berlin. Vol. I, Die Hefe und die Gährungs Erscheinungen, 1879. Vol. II, Fabrikation von Malz, Malz Extract und Dextrin, r88o. Vol. III, Die Bierbrauerei, r88ı.

Bigelow, W. D. Fermented and Distilled Liquors. U. S. Dept. of Agric., Bur. of Chem., Bul. 65 , p. 8 r. 1902 .

Botrguelot, E. Des Fermentations. Paris, 1889.

Brevans, J. DE. The Manufacture of Liquors and Preserves. New York, 1893 .

Crampton, C. A. Fermented Alcoholic Beverages. U. S. Dept. of Agric., Div. of Chem., Bul. 13, part 3. 1887 .

Duplats, P. (Translated by McKennie, M.) A Treatise on the Manufacture and Distillation of Alcoholic Liquors. Philadelphia.

Fleischmax, J. The Art of Blending and Compounding Liquors and Wines. New York, 1885 .

GIRARD, C. La Fabrication des Liqueurs et des Conserves. Paris, 189o.

Hansen, E. CH. Untersuchungen aus der Praxis der Gäłrungs-Industrie. München, 1889.

LeAch, A. E., and Lythgoe, H. C. The Detection and Determination of Ethyl and Methyl Alcohols in Mixtures by the Immersion Refractometer. Jour. Am. Chem. Soc., 27,1905 , p. 964 .

Mew, J., and Ashton, J. Drinks of the World. London, 1892.

PAsteur, M. Studies in Fermentation. London, 1879.

PREscotr, A. B. Critical Examination of Alcoholic Liquors. New York, 1880.

SPENCER, E, The Flowing Bowl. A Treatise on Drinks of all Kinds and of all Periods. London, 1899 .

Stevenson, T. A Treatise on Alcohol with Tables of Spirit Gravities. London, 1888.

- A Treatise on the Manufacture, Imitation, Adulteration and Reduction of Foreign Wines, Brandies, Rums and Gins, based upon the "French System," by a Practical Chemist and Experienced Liquor Dealer.

\section{REFERENCES ON BEER.}

Allen, A. H., and Chatraway, W. Detection of Hop Substitutes in Beer. Analyst, 12, 1887 , p. 107; also Analyst 15,1890 , p. 181 .

Barnard, H. E. Report on Beer. U. S. Dept. of Agric., Bur. of Chem., Bul. go, p. 64 .

Brevans, J. DE. Analyse des Matières Alimentaires (Girard et Dupré), p. $18_{3}$. Paris, 1894 .

Euron, H. Detection of Antiseptics in Beer. Analyst, 16, 1891, p. 116.

FAULKNER, F. Theory and Practice of Modern Brewing. London, 1888.

Hefelmann, R., and Mann, P. Detection of Fluorine in Beer. Pharm. Centralh., 16, 1895 , p. 249; Abs. Analyst, 20, 1895 , p. 185. 
Kelynack, T. N., and Kirby, W. Arsenical Poisoning in Beer Drinkers. London, IgOI.

Lindet, L. La Bierre. Paris, r892.

Lindner, C. Lehrbuch der Bierbrauerei. Braunschweig, 1878 .

Macfart.ane, T. Malt Liquors. Canada Inl. Rev. Dept., Bul., 52.

Parsons, C. L. The Identification and Composition of Malt Liquors. Jour. Am. Chem. Soc., 24, 1902, p. 1170.

Pasteur, M. Etudes sur la Bierre. Paris, 1876 .

Presse, C. H. Chemistry in the Brewing Room. London, I89r.

Prior, E. Chemie und Physiologie des Malzes und des Bieres. Leipzig, 1896 .

Stierlern, R. Das Bier und seine Verfälschungen. Berlin, 1878 .

\section{REFERENCES ON CIDER AND WINE.}

Alwoon, W. B. A Study of Cider Making. U. S. Dept. of Agric., Bur. of Chem., Bul. 71 .

Alwoop, W. B., Davimson, R. J., and Moncure, W. A. P. The Chemical Composition of Apples and Cider. U. S. Dept. of Agric., Bur. Chem., Bul. 88.

Arauner, P. Der Wein und seine Chemie. Kitzingen, a. M., 1906.

BarLllot, E. Manuel de l'Analyse des Vins. Paris, 1889.

BARTH, M. Die Weinanalyse. Leipzig, I884.

Bastide, E. Les Vins Sophistiques. Paris, 1889.

Browne, C. A. The Chemical Analysis of the Apple, and some of Its Products. Jour.

Am. Chem. Soc., 23 , I90r, p. 869.

- The Effects of Fermentation upon the Composition of Cider and Vinegar. Jour.

Am. Chem. Soc., 25, 1903 , p. 16.

Borgmann, E. Anleitung zur chemischen Analyse des Weines. Wiesbaden, 1898.

Cazeneuve, P. La Coloration des Vins par les Couleurs de la Houille. Paris, 1886.

Снасе, E. M. Qualitative Detection of Saccharine in Wine. Jour. Am. Chem. Soc., 26,1904, p. 1627.

Earbrex, G. A Comparison of English and American Cider, yith Suggestions for

Estimating the Amount of Added Water. Analyst, IG, r8gr, p. $4 \mathrm{I}$.

Gautier, A. La Sophistication des Vins. Paris, 1384.

Macfarlane, T. Wines. Canada Inl. Rev, Dept., Bul. $3^{8 .}$

Nessler, J. Die Bereitung, Pflege und Untersuchung des Weins. Stuttgart, r889.

Niviere, G., and Hubert, A. Detection of Fluorine in Wine. Monit. Scient., 9, 1895, p. 324; Abs. Analyst, 20, 1895, p. 185 .

Pasteur, M. Etudes sur le Vin, Paris, 1873 .

Robinet, E. Manuel Pratique d'Analyse des Vins. Paris, 1888.

Ross, S. H. Determination of Glycerine in Wine. A. O. A. C. Proc. rgog. U. S. Dept. of Agric., Bur. of Chem., Bul. 132, p. 85.

SAngle-Ferriere. Analyse des Matières Alimentaires (Girard et Dupré), Paris, 1894. Vin., p. 65. Cidre, p. 217.

Smith, A. W., and PARKs, N. Composition of Ohio Wines. Jour. Am. Chem. Soc., 20, 1908, p. 878 .

Windisch, K. Die chemische Untersuchung und Beurtheilung des Weines. Berlin, 1896 . 


\section{REFERENCES ON DISTILLED LIQUORS.}

Adams, A. B. The Detection of Substitution of Spirits for Aged Whiskey. Jour. Ind. Eng. Chem., 3, I9II, p. 647 .

Alten, A. H. The Chemistry of Whiskey and Allied Products. Jour. Soc. Chem. Ind, Io, IS9I, p. 312.

Brannt, W. T. Practical Treatise on the Distillation of Alcohol. Phila., 1885.

Crampton, C. A. Detection of Foreign Coloring Matter in Spirits. Jour. Am. Chem. Soc., 22, rooo, p. 8ro.

Crampton, C. A., and Tolman, L. M. A Study of the Changes Taking Place in

Whiskey Stored in Wood. Jour. Am. Chem. Soc., 30, I908, p. 98.

GABER, A. Die Fabrikation von Rum, Arrak, Cugnac, etc. Leipzig, I886.

Macfariane, T., and McGill, A. Distilled Liquors. Canada Inl. Rev Dept, Bul. 27.

Mrtchels, A. S., and Smrth, C. R. The Determination of Fusel Oil by Alakaline Permanganate. A. O. A. C. Proc. 1908. U. S. Dept. of Agric., Bur. of Chem., Bul. 122, p. 199.

Mouzert. The Practical Distiller. 1890.

LADD, E. F. Whiskey. N. Dak. Agric. Exp. Sta. Bulletins 57,63 and 69. Reports 1906 and 1907.

Rrchter, H. Analyse des Rums. Zeits. landw. Gerwerbe, 9, 1889, p. Ir.

SAngliter, A. Alcohols et Spiritueux. Analyse des Matières Alimentaires (Girard et Dupré), P. 253. Paris, 1894.

ScAl.A, A. Rum and Its Adulteration. Gazetta Chem. Ital., I89I, 396; Abs. Analyst, 17,1892 , p. 79 .

Sert, E. Ueber Cognac, Rum, Arrak, etc. Berlin, 1890 .

Sh:PARD, J. H. The Constants of Whiskey. Report of the Chemist of the South Dakota Food and Dairy Commission, March, rgo6.

Stamings, R. E. An Examination of Whiskeys. N. Dak. Agric. Exp. Sta. Rep. 19o6, p. 138 .

Tolman, L. M., and HirLyer, W. E. Methods of Analysis of Distilled Spirits. A. O. A. C. Proc. Igo8. U. S. Dept. Agric. Bur. of Chem., Bul. 122, p. 206.

Tolman, L. M., and Trescot, T. C. A Study of the Methods for the Determination of Esters, Aldehydes and Furfural in Whiskey. Jour. Am. Chem. Soc., 28, I906, p. I6r9.

Vasey, S. A. Guide to the Analysis of Potable Spirits. London, I904.

U. S. DEPt, OF AGRIC, Food Inspection Decisions, 45, 65, 95, 98, I1 3 , and 127. 


\section{CHAPTER XVI.}

\section{VINEGAR.}

VINEGAR is the product formed by the acetic fermentation of an alco holic liquid under the influence of the organism mycoderma aceli, existing in the "mother-of-vinegar." While vinegar may be made directly from a dilute solution of pure alcohol, it is more often obtained from fruit juice, wine, or other saccharine liquid that has first undergone alcoholic fermentation.

Of the following equations, (I) and (2) illustrate the processes of inversion and alcoholic fermentation respectively, while (3) and (4) show the double process of acetic fermentation, wherein the alcohol is oxidized, first to acetaldehyde and finally to acetic acid:

$$
\begin{aligned}
& \underset{\text { Cane sugar }}{\mathrm{C}_{12} \mathrm{H}_{22} \mathrm{O}_{11}}+\mathrm{H}_{2} \mathrm{O}=\underset{\text { Invert sugar }}{2} \mathrm{C}_{6} \mathrm{H}_{12} \mathrm{O}_{6} ; \cdot \cdots \cdot(\cdot) \text { (I) }
\end{aligned}
$$

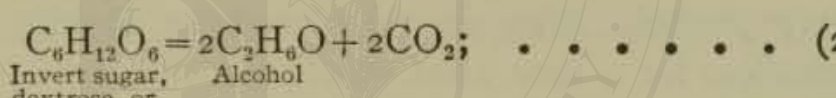

$$
\begin{aligned}
& \text { maltose } \\
& \underset{\text { Alcohol }}{\mathrm{C}_{2} \mathrm{H}_{6} \mathrm{O}}+\mathrm{O}=\underset{\text { Aldehyde }}{\mathrm{C}_{2} \mathrm{H}_{4} \mathrm{O}}+\mathrm{H}_{2} \mathrm{O} ; \cdots \cdots(3) \\
& \underset{\text { Aldehyde }}{\mathrm{C}_{2} \mathrm{H}_{4} \mathrm{O}}+\mathrm{O}=\underset{\text { Acetic acid }}{\mathrm{C}_{2} \mathrm{H}_{4} \mathrm{O}_{2}} \text {. }
\end{aligned}
$$

In addition to the acetic acid, its chief active principle, vinegar usually contains traces of other organic acids free or combined, small amounts of alcohol, aldehyde, sugar, glycerin, coloring matter, aromatic ethers, and mineral salts, its extract varying considerably with the source from which the vinegar was obtained.

Varieties.-The principal varieties of vinegar are the following: Cider vinegar, wine vinegar, malt or beer vinegar, spiril vinegar, glucose vinegar, molasses vinegar, and wood vinegar, the three last being more frequently used as adulterants of the others. 
Manufacture of Vinegar.-Cider vinegar, the principal variety used in the United States and Canada, was formerly made almost entirely by the slow process of cask fermentation, the fresh cider being allowed to undergo both alcoholic and acetic fermentation in barrels with open bung-holes in a warm cellar, or exposed to the sun. Two or three years are required for this process. Sometimes fresh cider is added to the barrels at regular intervals of two or three weeks, thus causing a series of progressive fermentations. The acetic fermentation is hastened by adding old vinegar, or mother-of-vinegar to the cider. While farmers and some manufacturers still continue to make cider vinegar by the slow process, the quick or "generator" vinegar process is now much used for cider vinegar, though originally intended and almost exclusively used in the manufacture of malt, beer, and spirit vinegar. This process requires only two or three days for complete acetification. In the quick process, the cider or other alcoholic liquor is allowed to percolate slowly through beechwood shavings or birch twigs, held in a cask known as a generator, provided with a perforated, false bottom, the shavings or twigs being previously saturated with old vinegar, and a current of air being passed up through them.

The alcoholic liquid from which genuine malt vinegar is made is derived from the wort obtained by mashing malt, or a mixture of malt and barley. Spirit vinegar is derived from diluted whiskey, brandy, or grain alcohol. Wine vinegar is made by allowing the wine to stand over wine lees for a time, after which it is clarified by passing through beech shavings, and subjected to progressive acetification in large open oak casks, to which the wine is added, the vinegar being drawn off in much the same manner as the slow-process cider vinegar.

CHARACTERISTICS AND COMPOSITION OF THE VARIOUS VINEgARS. - Cider Vinegar is brownish yellow in color, and possesses an odor of apples. It is chiefly distinguished from other vinegar by the large amount of malic acid normally present, by the character of its sugars, and by the predominance of potash in the ash. Its specific gravity varies from 1.0I3 to I.OI5. Its acidity varies from 3 to 6 per cent, and its solids from $I \frac{1}{2}$ to 3 per cent. Cider vinegar under polarized light is always lævo-rotary.

The following are summarized data of analyses made by H. C. Lythgoe in the writer's laboratory of twenty-two samples of cider vinegar of known purity: 


\begin{tabular}{|c|c|c|c|c|c|c|c|c|}
\hline & & & \multirow{2}{*}{$\begin{array}{l}\text { Acetic } \\
\text { Acid. }\end{array}$} & \multirow{2}{*}{$\begin{array}{l}\text { Total } \\
\text { Solids. }\end{array}$} & \multirow{2}{*}{ Ash. } & \multirow{2}{*}{$\begin{array}{l}\text { Alkalin- } \\
\text { ity of } \\
\text { Ash. }\end{array}$} & \multicolumn{2}{|c|}{$\begin{array}{l}\mathrm{P}_{2} \mathrm{O}_{5} \text { in Ash of } 100 \\
\text { Grams Vinegar. }\end{array}$} \\
\hline & & & & & & & $\begin{array}{l}\text { Soluble } \\
\text { (mgr.) }\end{array}$ & $\begin{array}{l}\text { Insoluble } \\
\text { (mgr.). }\end{array}$ \\
\hline \multicolumn{3}{|c|}{$\begin{array}{l}\text { Maximum } \ldots \ldots \ldots \ldots \ldots \ldots \ldots \ldots \\
\text { Minimum. } \ldots \ldots \ldots \ldots \ldots \ldots \ldots \ldots \\
\text { Average } \ldots \ldots \ldots \ldots \ldots \ldots\end{array}$} & $\begin{array}{l}5.86 \\
3.92 \\
4.84\end{array}$ & $\begin{array}{l}3.20 \\
1.84 \\
2.49\end{array}$ & $\begin{array}{l}0.42 \\
0.20 \\
0.34\end{array}$ & $\begin{array}{l}36.1 \\
22.2 \\
29 \cdot 7\end{array}$ & $\begin{array}{l}31 \cdot 7 \\
12.1 \\
19.2\end{array}$ & $\begin{array}{r}31.5 \\
6.5 \\
15.6\end{array}$ \\
\hline & \multicolumn{2}{|c|}{ Reducing Sugars. } & \multirow{2}{*}{$\begin{array}{c}\text { Polariza- } \\
\text { tion, } \\
\text { Degrees } \\
\text { Ventzke } \\
200-m m . \\
\text { Tube. }\end{array}$} & \multirow{2}{*}{$\begin{array}{l}\text { Malic } \\
\text { Acid. }\end{array}$} & \multirow{2}{*}{$\begin{array}{l}\text { Per Cent } \\
\text { Ash in } \\
\text { Total } \\
\text { Solids. }\end{array}$} & \multirow{2}{*}{$\begin{array}{l}\text { Per Cent } \\
\text { Reducing } \\
\text { Sugars } \\
\text { in Total } \\
\text { Solids. }\end{array}$} & \multirow{2}{*}{$\begin{array}{l}\text { Ratio of } \\
\text { Soluble } \\
\text { to Total } \\
\mathrm{P}_{2} \mathrm{O}_{5}\end{array}$} & \multirow{2}{*}{$\begin{array}{l}\text { Alkalin- } \\
\text { ity of } \\
\text { I Gram of } \\
\text { Ash, cc. } \\
\frac{\mathrm{N}}{10} \text { Acid. }\end{array}$} \\
\hline & $\begin{array}{c}\text { Before } \\
\text { Inversion. }\end{array}$ & $\begin{array}{c}\text { After } \\
\text { Inversion. }\end{array}$ & & & & & & \\
\hline Maximum.... & 0.51 & 0.53 & -3.6 & 0.16 & 19.0 & 16.6 & 66.9 & 125.0 \\
\hline Minimum.... & 0.15 & 0.15 & -0.3 & 0.08 & 10.0 & $7 \cdot 3$ & 50.0 & 69.0 \\
\hline Average...... & 0.25 & 0.25 & $-1 \cdot 3$ & 0.11 & 13.8 & 10.7 & 56.3 & 90.0 \\
\hline
\end{tabular}

${ }^{1}$ Number of cubic centimeters of tenth-normal acid to neutralize the ash of too grams of vinegar.

Twenty-two samples of pure cider vinegar were analyzed by A. W. Smith * with the following results:

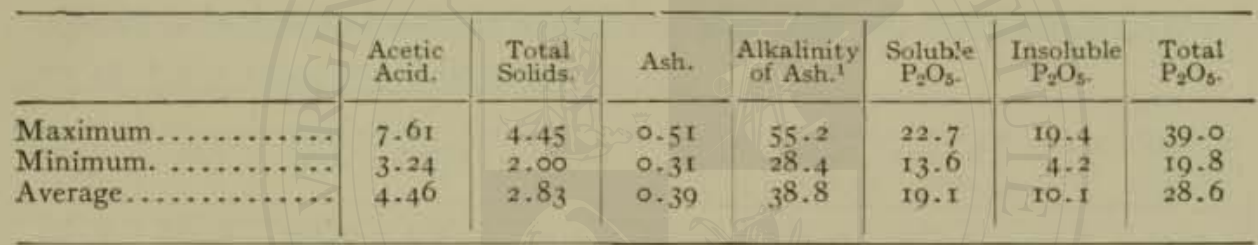

1 Number of cubic centimeters of tenth-normal acid required to neutralize the ash from roo grams of vinegar.

The composition of cider vinegar ash is found by Doolittle and Hess $\dagger$ to be as follows:

Calcium oxide........ $\mathrm{CaO} \ldots \ldots \ldots . .3 .4$ to $8.2 \mathrm{I}$

Magnesium oxide...... MgO.......... 1.88 " 3.44

Potassium oxide....... $\mathrm{K}_{2} \mathrm{O} \ldots \ldots \ldots \ldots .46 .33$ " 65.64

Sodium oxide.......... $\mathrm{Na}_{2} \mathrm{O} \ldots \ldots \ldots \ldots$ None

Sulphuric anhydride..... $\mathrm{SO}_{3} \ldots \ldots \ldots \ldots . .6 .66$ to 16.29

Phosphoric anhydride. . $\mathrm{P}_{2} \mathrm{O}_{5} \ldots \ldots \ldots \ldots .3 .29$ " 6.66

Iron oxide........... $\mathrm{Fe}_{2} \mathrm{O}_{3} \ldots \ldots \ldots$. None " trace $\mathrm{CO}_{2}$ and loss.... 0.00 " 40.44

Wine Vinegar is light yellow if made from white wine, and red if from red wine. The former is the highest prized. Wine vinegar varies in specific

* Jour. Am. Chem. Soc., 20 (1898), p. 6.

† Ibid., 22 (1900), p. 220. 
gravity from 1.0129 to 1.0213 , and contains from 6 to 9 per cent of acetic acid. It is characterized chiefly by the bitartrate of potassium (cream of tartar) which true wine vinegar always possesses. Free tartaric acid is also usually present. Wine vinegar is the principal vinegar of France and Germany. In the United States the term white wine vinegar is usually applied to distilled or spirit vinegar, which is much cheaper than the real wine vinegar and altogether inferior to it.

Wine vinegar is slightly lævo-rotary with polarized light.

The composition of genuine white wine vinegar is shown by the following summary of the analyses of twenty-two samples, made in the Municipal Laboratory of Paris:

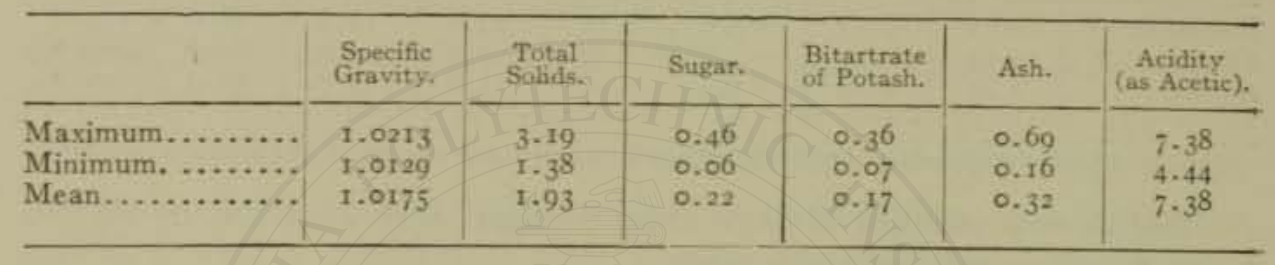

Weigmann gives the following average of analyses of red wine vinegar:

\begin{tabular}{|c|c|c|c|c|c|c|c|c|c|}
\hline $\begin{array}{l}\text { Specific } \\
\text { Gravity. }\end{array}$ & $\begin{array}{l}\text { Ace tic } \\
\text { Acid. }\end{array}$ & $\begin{array}{c}\text { Total } \\
\text { Tartaric } \\
\text { Acid. }\end{array}$ & $\begin{array}{l}\text { Free } \\
\text { Tartaric } \\
\text { Acid. }\end{array}$ & $\begin{array}{l}\text { Cream of } \\
\text { Tartar. }\end{array}$ & Alcohol. & Extract. & $\begin{array}{l}\text { Gly- } \\
\text { cerin. }\end{array}$ & Ash. & $\begin{array}{l}\text { Phos- } \\
\text { phoric } \\
\text { Acid. }\end{array}$ \\
\hline 1.0143 & $7 \cdot 79$ & 0.216 & 0.006 & 0.057 & 1.19 & $0.86_{3}$ & $0.14 \mathrm{I}$ & 0.118 & 0.012 \\
\hline
\end{tabular}

Malt or Beer Vinegar is of a brown color, and its odor is suggestive of sour beer. It varies in specific gravity from 1.015 to 1.025 ; its acidity. is about the same as cider vinegar, but the extract is much larger, varying from 4 to 6 per cent. Malt vinegar contains considerable nitrogenous. matter, and notable quantities of phosphates, dextrin, and maltose. It contains no cream of tartar. Malt vinegar is largely used in Great Britian.

Hehner gives the following data of the analyses of seven samples of vinegar undoubtedly made from malt only.*

\begin{tabular}{|c|c|c|c|c|c|}
\hline & Acidity. & $\begin{array}{l}\text { Total } \\
\text { Solids. }\end{array}$ & Ash. & $\begin{array}{l}\text { Phosphoric } \\
\text { Anhydride. }\end{array}$ & $\begin{array}{l}\text { Alkalinity } \\
\left(\mathrm{Nag}_{\mathrm{C}} \mathrm{O}_{3}\right) \text {. }\end{array}$ \\
\hline 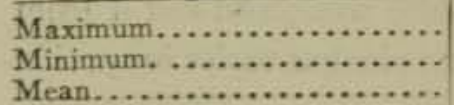 & $\begin{array}{l}6.48 \\
2.88 \\
4.23\end{array}$ & $\begin{array}{l}4.23 \\
1.68 \\
2.70\end{array}$ & $\begin{array}{l}0.47 \\
0.22 \\
0.34\end{array}$ & $\begin{array}{l}.13 \\
.067 \\
.105\end{array}$ & $\begin{array}{l}.089 \\
.017 \\
.024\end{array}$ \\
\hline
\end{tabular}

*Analyst, 16, p. 82. See also Analyst, 18, p. 240. 
Allen gives the results of the analyses of three samples of genuine vinegar brewed from a mixture of malted and unmalted barley as follows:*

\begin{tabular}{|c|c|c|c|c|c|c|c|c|}
\hline & $\begin{array}{l}\text { Specific } \\
\text { Gravity. }\end{array}$ & $\begin{array}{l}\text { Acetic } \\
\text { Acid. }\end{array}$ & $\begin{array}{l}\text { Total } \\
\text { Solids. }\end{array}$ & Ash. & $\begin{array}{c}\text { Alkalinity } \\
\text { as } \mathrm{K}_{2} \mathrm{O} \text {. }\end{array}$ & $\begin{array}{l}\text { Phos- } \\
\text { phoric } \\
\text { Acid. }\end{array}$ & Nitrogen. & $\begin{array}{l}\text { Albumin- } \\
\text { oids. }\end{array}$ \\
\hline${ }_{2} \ldots \ldots \ldots \ldots \ldots \ldots$ & $\begin{array}{l}\mathrm{r} .0170 \\
\mathrm{I} .0228\end{array}$ & $\begin{array}{l}6.39 \\
5.26\end{array}$ & $\begin{array}{l}2.67 \\
3.96\end{array}$ & $\begin{array}{l}0.34 \\
0.40\end{array}$ & $\begin{array}{l}0.091 \\
0.118\end{array}$ & $\begin{array}{l}0.077 \\
0.093\end{array}$ & $\begin{array}{l}.099 \\
.095\end{array}$ & $\begin{array}{l}.624 \\
.598\end{array}$ \\
\hline $3 \ldots \ldots \ldots \ldots$ & 1.0160 & 4.86 & 2.31 & 0.47 & $\ldots \ldots$ & 0.057 & .099 & .624 \\
\hline
\end{tabular}

Distilled, Spirit, or Alcohol Vinegar.-This vinegar, being made from diluted alcohol, is nearly colorless, unless artificially colored, as it often is, with caramel. As stated on page 762 , the "white wine" vinegar (incorrectly so-called) commonly sold in the United States is of this class. Its specific gravity ranges from 1.008 to 1.013 . Spirit vinegar contains from 3 to ro per cent of acetic acid. Its content of total solids is insignificant, and it contains only traces of ash. It always contains nonacetified alcohol and aldehyde. It has no optical activity with polarized light.

Twelve samples of alcohol vinegar analyzed in the Municipal Laboratory of Paris gave the following results:

\begin{tabular}{|c|c|c|c|c|c|}
\hline & $\begin{array}{l}\text { Specific } \\
\text { Gravity. }\end{array}$ & $\begin{array}{l}\text { Total } \\
\text { Solids. }\end{array}$ & Sugar. & Ash. & Acidity. \\
\hline $\begin{array}{l}\text { Maximum............ } \\
\text { Minimum. } \\
\text { Mean................. }\end{array}$ & $\begin{array}{l}1.0131 \\
1.0082 \\
1.0100\end{array}$ & $\begin{array}{l}0.16 \\
0.07 \\
0.35\end{array}$ & $\begin{array}{c}\text { Trace } \\
. \\
.6\end{array}$ & $\begin{array}{l}.09 \\
.04 \\
\text { Trace }\end{array}$ & $\begin{array}{l}7.98 \\
4.98 \\
6.34\end{array}$ \\
\hline
\end{tabular}

Glucose Vinegar is made from the acetification of alcohol, obtained from the fermentation of commercial glucose. This vinegar usually possesses the odor and taste of fermented starch. It is low in total solids, the extract consisting almost entirely of untransformed glucose, and the vinegar therefrom contains all the ingredients of the product from which it was made, viz., dextrin, maltose, and dextrose, as well as chloride of sodium. It is decidedly dextro-rotatory with polarized light both before and after inversion.

Molasses Vinegar.-This is largely the product of the acetic fermentation of sugar-house wastes, and sometimes of the accidental acetic fermentation of molasses itself, after it has undergone alcoholic fermentation for the manufacture of rum. This variety of vinegar is sometimes

* Analyst, I9, p. I5. 
used as an adulterant of cider vinegar. With polarized light molasses vinegar is dextro-rotary before, and lævo-rotary after inversion.

Wood Vinegar is prepared by the purification of pyroligneous acid, which may be accomplished by saturating the crude acid with lime or soda, adding hydrochloric or sulphuric acid, and distilling. It is further purified by redistillation with potassium bichromate, and filtration through boneblack. Acetic acid is sometimes added to impart flavor.

The extract and ash of wood vinegar are very small. Its specific gravity averages 1.007 according to Blyth. Empyreumatic or tarry products are nearly always present in vinegar of this class.

\section{ANALYSIS OF VINEGAR.}

Specific Gravity.-This is obtained either with the hydrometer, pyc. nometer, or Westphal balance.

Determination of Total Solids.-Weigh to grams of the sample in a tared platinum dish $50 \mathrm{~mm}$. in diameter, evaporate to dryness on a boilingwater bath and dry for two and one-half hours in a water oven at the temperature of boiling water. Cool in a desiccator and weigh.

Determination of Ash.-Transfer the dish containing the last residue or extract to a muffle, and burn at a low red heat to an ash, or the ignition may be accomplished with care over a direct flame turned low. Cool the dish and weigh.

Determination of Solubility and Alkalinity of the Ash.-Smith's Method.*-Twenty-five cc. of the vinegar are evaporated to dryness in a tared platinum dish, ignited, cooled, and the ash weighed. The ash is then repeatedly extracted with hot water by washing into a Gooch crucible provided with a layer of asbestos (previously ignited in the crucible, cooled, and weighed) or upon an ash-free filter. Dry the Gooch or filter, ignite, cool, and weigh the insoluble ash. The aqueous extract is titrated directly with tenth-normal acid, using methyl orange as an indicator, or treated by adding an excess of tenth-normal hydrochloric acid, boiling and titrating back with tenth-normal sodium hydroxide, using phenolphthalein. Express the alkalinity in terms of 100 grams of the vinegar, by multiplying by 4 the number of cubic centimeters of acid required to neutralize.

Determination of Phosphoric Acid. $†$-Extract repeatedly the insoluble ash as obtained in the preceding section with hot water acidulated with nitric acid, and acidify with nitric acid the neutralized solution of the

* Jour. Am. Chem. Soc., 20, p. 5 .

†U. S. Dept. of Agric., Bur. of Chem., Bul. 46, p. 12. 
soluble ash. Add to each solution 15 grams of ammonium nitrate, heat to boiling, and precipitate the phosphoric acid with $50 \mathrm{cc}$. of ammonium molybdate (reagent No. 53). Digest for an hour at a temperature of about $65^{\circ}$, filter, and wash with cold water. Dissolve the precipitate on the filter with ammonia and hot water, and wash into a beaker to a bulk of not more than $100 \mathrm{cc}$. Nearly neutralize with hydrochloric acid, cool, and add slowly magnesia mixture (reagent No. 164) drop by drop while stirring vigorously. After fifteen minutes add $30 \mathrm{cc}$. of ammonia (specific gravity 0.96 ), let stand for at least two hours, filter on a Gooch crucible, wash with $2.5 \%$ ammonia till practically free from chlorides, ignite, and weigh as $\mathrm{Mg}_{2} \mathrm{P}_{2} \mathrm{O}_{7}$. Express results in terms of milligrams of phosphoric anhydride in the soluble and insoluble vinegar ash from too grams of vinegar.

Phosphoric acid in the soluble and insoluble ash may be conveniently determined also by the uranium acetate method, page 725 .

Determination of Nitrogen,-Concentrate from 50 to $100 \mathrm{cc}$. of vinegar to a syrupy consistency, and proceed as directed under the Kjeldahl or Gunning method, page 69 .

Determination of Total Acidity. - Six cc. of vinegar are carefully measured from a pipette into a white porcelain dish and diluted with water. Using phenolphthalein as an indicator, titrate with tenth-normal sodium hydroxide. The number of cubic centimeters of the latter required to neutralize, divided by 10 , expresses the acidity in terms of percentage of acetic acid.

Approximate Determination of Vinegar Acidity by Lime Water. - It has generally been considered difficult for vinegar dealers and others who desire to estimate the acidity of their vinegar to do this themselves, in that it has been necessary to obtain for the purpose a carefully standardized alkaline solution, the exact strength of which it is impossible for them to determine.

It has been found that very satisfactory, though of course not absolutely accurate, results may be obtained by the use of ordinary lime water, which any one may easily prepare by making a saturated solution of ordinary air-slaked lime. The strength of such a solution is very nearly constant, and has been found to be about $\frac{1}{21.4}$ of the normal. If, therefore, it is not easy to obtain exactly normal or tenth-normal alkali, approximate figures may be obtained by employing such a saturated lime water. If $2.75 \mathrm{cc}$. of vinegar are titrated with lime water contained in a burette, using phenolphthalein as an indicator, the number of cubic centimeters 
of the lime water necessary to neutralize the vinegar, divided by ז0, gives the percentage of acetic acid in the vinegar. To make sure that the lime water is saturated, an excess of lime should always be present in the reagent bottle.

Determination of Volatile and Fixed Acids.-Thirty cc. of the vinegar are transferred to a distilling-flask and subjected to distillation, using a current of steam. Receive the distillate in a 25 -cc. graduated cylinder. After ${ }_{15} \mathrm{cc}$. have passed over, test from time to time the drops of distillate as they fall into the receiving vessel with litmus-paper, and when free from acid discontinue the distillation. Note the volume of the distillate, mix by shaking, and transfer one-fifth to a white porcelain dish. Titrate as in the case of total acidity, expressing the volatile acids as acetic.

Calculate the fixed acid, expressed in the case of cider vinegar as malic, by subtracting the percentage of volatile acid from the percentage of total acid, and multiplying the result by the factor I.II7. In the case of wine vinegar, express as tartaric acid by using the factor 1.25. To express acidity in terms of sulphuric acid, multiply the percentage of acetic acid by 0.817 .

Determination of Alcohol.-Alcohol is present in very small amounts in fruit vinegar that has not been completely acetified. Frear recommends concentrating the distillates as follows: Neutralize roo cc. of the sample and distill off $40 \mathrm{cc}$. Then redistill the distillate till $20 \mathrm{cc}$. have gone over. $\mathrm{Cool}$ to $15.6^{\circ} \mathrm{C}$. and make up to $20 \mathrm{cc}$. with distilled water. Determine the specific gravity with a ro-cc. pycnometer, and ascertain from the table on page 66I the per cent by weight of alcohol corresponding to the specific gravity. The percentage in the last distillate, divided by 5 , expresses the amount of alcohol in the vinegar.

Detection of Free Mineral Acids. - The ash of genuine cider vinegar is always alkaline. If the ash is neutral, free mineral acids are doubtless present. For their detection the following is a modification of Brannt's method of procedure:

Add to $50 \mathrm{cc}$. of the vinegar in an Erlenmeyer flask a small bit of starch the size of a wheat-grain, and shake to disseminate it through the fluid. Boil for some minutes, cool, and add a drop of iodine solution. If a blue coloration occurs, no mineral acid is present. In the presence of an appreciable amount of mineral acid, the starch will be converted to dextrin and sugar, and no coloration will be produced by the iodine.

Frear's Method.-Add 5 or 1o cc. of water to $5 \mathrm{cc}$. of the vinegar, and 
to the mixture add a few drops of a solution of methyl violet (one part of methyl violet $2 \mathrm{~B}$ in 100,000 parts of water). In the presence of mineral acids, a blue or green coloration will be produced.

Determination of Free Mineral Acids.-Hehner's Method.*-To a weighe 1 quantity of the sample add an excess of decinormal alkali, evapcrate to dryness, incinerate, and titrate the ash with decinormal acid. The difference between the number of cubic centimeters of alkali added in the first place, and the number of cubic centimeters needed to titrate the ash, represents the equivalent of the free acid present.

Detection and Determination of Sulphuric Acid.-This is determined as bariam sulphate by the addition of barium chloride solution. A slight cloudiness on the addition of the reagent indicates the presence of small quantities of sulphate as an impurity, rather than free sulphuric acid. If a minute quantity of free sulphuric acid be present, a rather heavy white cloud on the addition of the barium chloride will be formed, which slowly settles out. According to Brannt, if the quantity of sulphuric acid is more than one part in a thousand, the sulphate of barium formed by addition of the reagent produces a copious precipitate that rapidly falls to the bottom of the receptacle. This may be filtered, washed, ignited, and weighed in the usual manner.

Detection of Free Hydrochloric Acid.-Distill off half of a measured volume of vinegar into the receiving-flask of a distillation apparatus, and to the distillate add a few drops of nitrate of silver reagent. A precipitate indicates hydrochloric acid.

Detection of Malic Acid (Frec or Combined).-Absence of malic acid may be assured, if no precipitate occurs with neutral acetate of lead, when a few drops of a solution of this reagent are added to the vinegar. In the presence of malic acid, as in the case of a pure cider vinegar, the precipitate which is formed with lead acetate is flocculent, forms at once, and is of considerable amount. In pure cider vinegar the precipitate will settle to the bottom of the test-tube, leaving a clear supernatant liqui-1 within ten minutes. Unfortunately the acetate of lead test is a negative one, in that several organic acids other than malic will cause a precipitate, as, for instance, tartaric and saccharic acids, the former being found in wine and the latter in molasses vinegar. Malt vinegar also gives a copious precipitate with lead acetate, due to phosphoric acid.

The writer employs the following test $\dagger$ for detecting malic acid in

* Analyst, $\mathrm{I}, \mathrm{1877}$, p. 105.

† An. Rep. Mass. State Board of Health, 1902, p. 485 . Food and Drug Reprint, p. 33. 
vinegar: Add a few drops of a $10 \%$ solution of calcium chloride to some of the vinegar in a test-tube, and make the mixture slightly alkaline with ammonia. Filter off the precipitate that occurs at this point, to the filtrate add two or three volumes of $95 \%$ alcohol, and heat to boiling. A copious, flocculent precipitate of calcium malate will form, if malic acid be present, settling to the bottom of the tube in a few minutes. A precipitate will occur in malt and glucose vinegar, due to dextrin.

To confirm the presence of malic acid, filter, wash the precipitate with a little alcohol, dry, dissolve it in strong nitric acid in a porcelain evaporating-dish, and evaporate to dryness over the water-bath, forming calcium oxalate. Boil the residue with sodium carbonate, filter, acidify the filtrate with acetic acid, boil to expel the carbon dioxide, and add a solution of calcium sulphate. A precipitate of calci $\mathrm{m}$ oxalate confirms the presence of malic acis.

For the determination of malic acid proceed as directed on page 702 .

Lead Precipitate.-Hortvet Number.-The quantitative measurement of the precipitate formed with lead acetate, or subacetate, is of considerable importance. Even though the precipitate formed may not be due as was long thought to malic acid, but may be due to phosphoric acid (though this has not been fully proved), it nevertheless remains a fact that the qualitative lead acetate test is one of the most important of all in judging the purity of cider vinegar.

The lead precipitate is best measured as follows: To $25 \mathrm{cc}$. of the vinegar add $2.5 \mathrm{ce}$. of U. S. P. subacetate of lead solution. Shake and whirl in a graduated Hortvet tube in the centrifugal machine, and read the volume of the precipitate in the bottom of the tube. The results expressed in cc. on thirty samples of pure cider vinegar are summarized as follows: Highest, 1.4 ; lowest, 0.5 ; average, 0.84 . The lead number of adulterated cider vinegar runs from a mere trace to 0.5 and sometimes higher.

Winton's Lead Number.-This is determined by the method described for maple products, page 628 .

Bailey* obtained by this method the following results:

Cider vinegar (8 samples) $\ldots \ldots \ldots \ldots . . .075$ to 0.290

Malt vinegar (3 samples) ............ 0.158 to 0.548

Distilled vinegar (I sample) ......... 0.018

* A. O. A. C. Proc., 1908. U. S. Dept. of Agric., Bur. of Chem., Bul. 122, p. 27. 
Hickey * follows the same method, except that he employs only $5 \mathrm{cc}$. of standard lead subacetate solution and determines the lead in $50 \mathrm{cc}$. of the filtrate. The lead number found by him in twenty samples of cider vinegar varied from 0.076 to 0.166 .

Determination of Acid Tartrate of Potassium.-Berthelot and Flewrien's Method.†-Twenty-five cc. of the vinegar are evaporated on the water-bath to syrupy consistency, and the residue is dissolved in water and made up to its original volume. It is then transferred to a $250-\mathrm{cc}$. Erlenmeyer flask, and $100 \mathrm{cc}$. of a mixture of equal parts of strong alcohol and ether are added, the flask is corked, shaken, and set on ice or in a cold place for forty-eight hours. At the end of this time, if a crystalline precipitate has gathered, the supernatant liquid is decanted upon a filter, and finally the precipitate is washed upon it by a fresh quantity of the ether-alcohol mixture, and the washing continued with this reagent till practically free from acid. The filter and its contents are then transferred to the original flask, and the tartrate is dissolved in boiling water, after which the solution is titrated in the same flask with tenth-normal sodium hydroxide, using phenolphthalein as an indicator. Multiply the number of cubic centimeters of alkali required to neutralize by the factor 0.0188 , and the quotient expresses the grams of bitartrate of potash in the sample. Multiply this by 4 to obtain the percentage present.

Polarization and Determination of Sugar.-If the vinegar is lightcolored and quite free from turbidity, it may sometimes be polarized undiluted in the $100-\mathrm{mm}$. tube. Vinegar may often be sufficiently clarified for polarization by filtering twice through the same filter. It is, however, best to add 10\% of basic lead acetate solution, and to filter before polarizing, thus removing the malic or tartaric acids which may have a slight effect on the polarization. In case of dark-colored or turbid samples, add to $50 \mathrm{cc}$. of the sample $5 \mathrm{cc}$. of about equal quantities of lead subacetate and alumina cream, shake, filter, and polarize in a 200$\mathrm{mm}$. tube, adding $10 \%$ to the reading on account of the dilution. The polarization value of the vinegar is conveniently expressed in terms of actual direct reading obtained by the undiluted sample in a $200-$ or 400-mm. tube.

If the invert reading is desired for calculation of sucrose or commercial glucose, subject the sample to inversion with hydrochloric acid and heat, as in the case of sugars.

* Tbid.

† Girard et Dupré, Analyse des Matières Alimentaires, p. I2 8 
For the determination of sucrose, use Clerget's formula (p. 588), calculating the true direct and invert readings from the direct and invert readings of the undiluted vinegar on the basis of the normal weight of the sample, by multiplying the obtained readings by 0.26 in the case of the Soleil-Ventzke instrument.

Determination of Reducing Matter before and after Inversion.Measure two portions of $25 \mathrm{cc}$. each into Ioo cc. flasks. Dilute one portion with $25 \mathrm{cc}$. of water, add $5 \mathrm{cc}$. of concentrated hydrochloric acid and invert in the usual manner. Neutralize both portions with sodium hydroxide, clear with normal lead acetate, remove the excess of lead with potassium sulphate or carbonate, and make up to the mark. Determine reducing sugars in each portion by the Munson and Walker method (p. 598) and calculate as invert sugar.

Determination of Pentosans. - Place roo cc. of the vinegar in a flask, add $43 \mathrm{cc}$. of concentrated hydrochloric acid (sp.gr. I.I9) and proceed as described on page 286 .

Determination of Glycerin.-The glycerin is extracted by essentially the same process as is used for dry wines (p. 703) and determined by the Hehner method modified by Richardson and Jaffe * and Low. These processes have been adapted to vinegar analysis by Ross $\uparrow$ as follows:

Standard Solutions.-1. Strong Bichromate.-Dissolve 74.56 grams of dry, recrystallized potassium bichromate in water, add ${ }_{5} 5^{\circ} \mathrm{cc}$. concentrated sulphuric acid, cool, make up to $1000 \mathrm{cc}$. at $20^{\circ} \mathrm{C}$., and determine the specific gravity at $20^{\circ} / 20^{\circ} \mathrm{C}$.; I cc. $=0.01$ gram glycerin. Accurate measurements being difficult owing to changes in room-temperature it is well to use weighed amounts of the solution from a weight burette, dividing by the specific gravity to obtain the volume used. The solution has an apparent expansion in glass of 0.0005 (or $0.05 \%$ ) for each degree centigrade. The solution may be measured if this correction is made.

2. Dilute Bichromate.-Introduce a weighed amount (I2.5 times the specific gravity) of the strong bichromate from a weight burette into a $250 \mathrm{cc}$. glass-stoppered volumetric flask, make up to the mark with water at room temperature; $20 \mathrm{cc} .=\mathrm{I} \mathrm{cc}$. of the strong solution. If slightly more than $12.5 \mathrm{cc}$. equivalent is used, make up to the mark and then add the required amount of water to make one-twentieth dilution.

3. Ferrous Ammonium Sulphate.-Dissolve 30 grams of the crystallized salt in water, add $50 \mathrm{cc}$. of concentrated sulphuric acid, cool, and dilute

* Jour. Soc. Chem. Ind. 17 , 1898, p. 330.

$\dagger$ Proc. A.O.A.C. rgro. U. S. Dept. of Agric., Bur. of Chem., Bul. 137, p. 61. 
to $\mathrm{I} 000 \mathrm{cc}$. at room temperature; I cc. =approximately I cc. of the dilute bichromate. Owing to daily changes in strength it should be standardized against the bichromate whenever used.

Extraction of Glycerin.-Make all evaporations on a water-bath kept at $85^{\circ}$ to $90^{\circ} \mathrm{C}$. Evaporate Ioo cc. of the vinegar to about $5 \mathrm{cc}$., add $20 \mathrm{cc}$. of water and again evaporate to about $5 \mathrm{cc}$. to expel acetic acid. Add about 5 grams of fine sand and I $_{5}$ cc. of milk of lime (freshly prepared and containing about $\mathrm{I}_{5} \%$ of calcium oxide), and evaporate nearly, but not quite, to dryness, with frequent stirring, avoiding formation of dry crust. Rub into a homogeneous paste with $5 \mathrm{cc}$. of hot water, add 45 cc. of absolute alcohol, washing down paste adhering to the sides of the dish, and stir thoroughly. Heat the mixture on a water-bath with constant stirring to incipient boiling, decant onto a $12.5 \mathrm{~cm}$. fluted filter, wash twice by decantation and finally on the filter with $90 \%$ alcohol up to about $\mathrm{r}_{5} \mathrm{O} \mathrm{cc}$., or, instead of filtering, centrifuge and wash three times. Evaporate to a sirup, dissolve in $\mathrm{so} \mathrm{cc}$. of absolute alcohol, and wash into a $50 \mathrm{cc}$. glass-stoppered cylinder with two $5 \mathrm{cc}$. portions of absolute alcohol. Add three portions of ro cc. each of absolute ether, thoroughly shaking after each addition. Let stand until clear, then pour off through a filter, and wash the cylinder and filter with mixed absolute alcohol and absolute ether (I:I.5). If a heavy precipitate is observed in the cylinder, it is well to centrifuge at low speed and decant the clear liquid through a filter. Add $20 \mathrm{cc}$. of the mixture of absolute alcohol and absolute ether to the precipitate in the cylinder, shake thoroughly, centrifuge and decant, repeating three times. Evaporate filtrate and washings at $85^{\circ}-90^{\circ} \mathrm{C}$., to about $5 \mathrm{cc}$; dilute and evaporate to $5 \mathrm{cc}$. three times, using respectively 20,20 and Io cc. of water. Wash residue with hot water into a $50 \mathrm{cc}$. volumetric flask, cool, add silver carbonate freshly precipitated from o.I gram of silver sulphate, shake occasionally, and allow to stand Io minutes; then add 0.5 cc. of lead subacetate solution, shake occasionally, and allow to stand ro minutes. Make up to the mark, shake well, filter, rejecting the first portion of the filtrate, and pipette off $25 \mathrm{cc}$. of the clear filtrate into a $250 \mathrm{cc}$. glass-stoppered volumetric flask. Precipitate the excess of lead with I cc. of concentrated sulphuric acid, and determine the glycerin by the following method:

Determination.-From a weight burette introduce into the $250 \mathrm{cc}$. flask, containing the $25 \mathrm{cc}$. of purified glycerin solution, a weighed amount of the strong bichromate solution (with ordinary vinegar $30-35 \mathrm{cc}$.) sufficient to leave about $\mathrm{r} 2.5 \mathrm{cc}$. in excess, carefully add $24 \mathrm{cc}$. of concentrated 
sul, shuric acid, rotating gently to mix and avoid ebullition, then heat in boiling-water bath for exactly 20 minutes. Dilute at once, cool, and make up to mark at room temperature. The oxidation is a trifle more complete if only $\mathrm{I}_{5} \mathrm{cc}$. of concentrated sulphuric acid are added and the digestion is continued for at least 2 hours.

Standardize the ferrous ammonium sulphate solution against the dilute bichromate by introducing from burettes approximately $20 \mathrm{cc}$. of each into a beaker containing $100 \mathrm{cc}$. of water. Complete the titration, using potassium ferricyanide solution $(0.5$ to $I \%)$ as indicator on a porcelain spot plate. Calculate the volume $(F)$ of ferrous ammonium sulphate equivalent to $20 \mathrm{cc}$. of the dilute and, consequently, to I cc. of the strong bichromate solution.

Substitute for the dilute bichromate a burette containing the oxidized glycerin with excess of bichromate solution, and ascertain how many cubic centimeters of it are equivalent to $F \mathrm{cc}$, of the ferrous ammonium sulphate solution, and therefore to I cc. of the strong bichromate. Then 250 divided by this last equivalent equals the number of cubic centimeters excess of the strong bichromate present in the $250 \mathrm{cc}$. flask after oxidation of the glycerin.

The number of cubic centimeters of strong bichromate added, minus the excess found after cxidation, multiplied by $0.0 \mathrm{r}$ equals the weight of glycerin in the $25 \mathrm{cc}$. of purified solution used in the determination; this result, multiplied by 2 , gives the weight of glycerin in grams per $100 \mathrm{cc}$. of the vinegar.

\section{ADULTERATION OF VINEGAR,}

Standards of Purity.-In England, where the principal vinegar is malt vinegar, the legal standards are considerably different from those in force in France and Germany, where wine vinegar is prevalent. These differ again from the requirements found in the United States and Canada, where cider vinegar is the chief product.

Most of the state food laws fix a standard for the acidity of cider vinegar varying from 3.5 to 4.5 per cent of acetic acid, and in most cases also a minimum standard for total solids or residue of from 1.5 to 2 per cent. Special laws stipulate furthermore in some states that cider vinegar, sold as such, must be exclusively the product of pure apple cider. In such cases cider vinegar may be adulterated by non-conformance to the standard in either acidity or solids or both, while yet it may be exclusively made from pure apple cider. This may be due either to actual watering or to incomplete acetification. On the other hand, 
so-calied cider vinegar may be of legal standard as to solids and acidity, and yet be entirely spurious.

Following are the U. S. standards for the various vinegars:

Vinegar, Cider Vinegar, Apple Vinegar, is the product made by the alcoholic and subsequent acetous fermentations of the juice of apples, is lævo-rotatory, and contains not less than 4 grams of acetic acid, not less than I.6 grams of apple solids, of which not more than $50 \%$ are reducing sugars, and not less than 0.25 gram of apple ash in $100 \mathrm{cc} .\left(20^{\circ} \mathrm{C}\right.$. $)$; and the water-soluble ash from $100 \mathrm{cc}$. $\left(20^{\circ} \mathrm{C}\right.$. $)$ of the vinegar contains not less than Io milligrams of phosphoric acid $\left(\mathrm{P}_{2} \mathrm{O}_{5}\right)$, and requires not less than $30 \mathrm{cc}$. of decinormal acid to neutralize its alkalinity.

Wine Vinegar, Grape Vinegar, is the product made by the alcoholic and subsequent acetous fermentations of the juice of grapes, and contains in $100 \mathrm{cc} .\left(20^{\circ} \mathrm{C}\right.$.), not less than 4 grams of acetic acid, not less. than 1.0 gram of grape solids, and not less than 0.13 gram of grape ash.

Male Vinegar is the product made by the alcoholic and subsequent acetous fermentations, without distillation, of an infusion of barley malt, or cereals whose starch has been converted by malt, is dextro-rotatory, and contains, in fuo cc. $\left(20^{\circ} \mathrm{C}\right)$, not less than 4 grams of acetic acid, not less than 2 grams of solids, and not less than 0.2 gram of ash; and the water-soluble ash from $100 \mathrm{cc}$. $\left(20^{\circ} \mathrm{C}\right)$, of the vinegar contains not less than 9 milligrams of phosphoric acid $\left(\mathrm{P}_{2} \mathrm{O}_{5}\right)$, and requires not less. than 4 cc. of decinormal acid to neutralize its alkalinity.

Sugar Vinegar is the product made by the alcoholic and subsequent acetous fermentations of solutions of sugar, syrup, molasses, or refiners' syrup, and contains, in $100 \mathrm{cc}$. $\left(20^{\circ} \mathrm{C}\right)$, not less than 4 grams of acetic acid.

Glucose Vinegar is the product made by the alcoholic and subsequent acetous fermentations of solutions of starch sugar or glucose, is dextrorotatery, and contains, in $100 \mathrm{cc}\left(20^{\circ} \mathrm{C}\right.$.), not less than 4 grams of acetic acid.

Spirit Vinegar, Distilled Vinegar, Grain Vinegar, is the product made by the acetous fermentation of dilute distilled alcohol, and contains, in Ioo r.c. $\left(20^{\circ} \mathrm{C}\right.$.), not less than 4 grams of acetic acid.

Accidental Adulteration of vinegar may result in the presence of injurious metallic salts, such as of copper, lead, or zinc, derived from vessels or utensils used in the manufacture of vinegar, or even minute traces of arsenic may be found, when glucose has been employed as an ingredient. 
or source of the vinegar, the arsenic being in this case probably due to impure sulphuric acid used in the manufacture of the glucose.

Willful or Fraudulent Adulteration is, however, common, in which misbranded vinegar is sold under names suggesting a class other than that to which it really belongs, or wherein entirely artificial substitutes are made up for pure cider, malt, or wine vinegar, in which the color, residue, and acid principle may be either or all of spurious origin.

Artificial Cider Vinegar is in most cases readily detected, though very ingenious imitations are on the market, involving not a little skill and chemical knowledge in their manufacture.

Entirely artificial substitutes for cider vinegar are frequently made up of spirit vinegar, colored with caramel, and having the solids reinforced by apple jelly, made for the most part out of exhausted apple pomace, which is the residue left after the apple-stock has been subjected to one and sometimes two pressings. The jelly used for this purpose is not infrequently made up with commercial glucose. All grades of adulterated vinegar are to be found, from the wholly spurious substitute above described, to the varieties in which cider vinegar is itself present, but is pieced out or reinforced by the admixture of coloring matter, mineral acid, wood vinegar, or of molasses or glucose vinegar. Acetic ether is sometimes omployed to impart flavor to the product. All the characteristics of a pure cider vinegar are difficult to duplicate artificially, though some of them may be.

Character of the Residue.-The residue of pure cider vinegar should be thick, light brown in color, of a viscid or mucilaginous consistency, somewhat foamy, having an astringent acid though pleasant taste very suggestive of baked apples, which it also resembles in odor. The odor of molasses is very apparent in the residue of vinegar having sugar-house wastes, and the smell of a malt-vinegar residue is also very characteristic. If pyroligneous or wood vinegar has been introduced, the dried residue will have a tarry or smoky taste and smell.

The residue of cider vinegar is very soluble in alcohol, while that of malt vinegar is only slightly soluble. Wine vinegar residues dissolve readily in alcohol, except for the granular residue of cream of tartar. If the loop of a clean platinum wire be rubbed in the vinegar residue and ignited in a colorless Bunsen flame, the color imparted will, if the vinegar has been made from pure cider exclusively, consist altogether of the palelilac color of a potash salt without any of the yellow sodium flame being 
visible. In all vinegars other than of pure cider, the sodium flame will predominate, when the residue is burnt as above. Again, the ignited residue left in the loop of wire in the case of a pure cider vinegar will form a fusible bead, having a strong alkaline reaction upon moistened test-paper, and effervescing briskly when immersed in acid. The presence in vinegar of even a slight trace of added mineral acid will prevent the ignited residue from having the alkaline reaction, or effervescing with acid.*

The residue of malt or beer vinegar is brown and gummy, containing a considerable quantity of dextrin. Not only are the appearance and odor of the dried vinegar residue to be particularly noted, but also the odor given off in the first stages of burning this residue to an ash. With cider vinegar the apple odor is very marked while burning. In vinegar wherein molasses products have been employed, the smell of charred sugar is usually apparent, while with glucose vinegar the smell of burnt corn predominates.

On burning the residue of malt vinegar, the odor produced at first is not unlike that of toasted bread. At a later stage in the burning the vapors evolved are very pungent.

The Character of the Ash is of considerable importance in determining the source of a sample of vinegar. The ash of pure cider and malt vinegar is quite strongly alkaline, while that of distilled and wood vinegar is only slightly alkaline. The ash of cider vinegar is high in alkaline carbonates.

In cider and malt vinegar the quantity of phosphoric acid present in the ash is considerable, while only traces are present in distilled or spirit vinegar. Considerably more than half the phosphoric acid in the ash of cider vinegar is soluble, while no soluble phosphoric acid is present in the ash of spirit vinegar.

The percentage of ash in total solids is of some value in judging the purity of cider vinegar. According to Frear. $\dagger$ if the ash of the vinegar is less than $10 \%$ of the total solids, the vinegar may be suspected of having added unfermented material, while a percentage of ash less than 6 is absolute evidence that the vinegar is not genuine cider vinegar.

The alkalinity of $I$ gram of the ash of pure cider vinegar should be

* Davenport, 18 th An. Rep., Mass. Board of Health, 1887 , p. 159.

† Report of Penn. Dept. of Agric., r898, p 38. 
equivalent to at least $65 \mathrm{cc}$. of tenth-normal acid. At least $50 \%$ of the phosphates in the ash should be so'uble in water.

Character of the Sugars.-One of the most important steps in establishing the source of a sinegar consists in subjecting it to polarization (p. 769 ). From the nature of the sugar-content of the apple juice, not only when freshly expressed, but also when allowed to undergo alcoholic fermentation, and, furthermore, after it has gone over into vinegar, the polarization through all three stages is always left-handed.

Browne* has shown that the optical rotation of the freshly expressed juice of eleven varieties of apple varies from $19.24^{\circ}$ to $49^{\circ}$ to the left on the Ventzke scale, in a $400-\mathrm{mm}$. tube. Also that in the case of five samples of completely fermented cider, examined five or six months after pressing, the left-handed rotation in a $400-\mathrm{mm}$. tube varied from i. $76^{\circ}$ to $5.28^{\circ}$. He showed, furthermore, that a sample of pure cider jelly made up of concentrated apple juice had a left-handed rotation amounting to $2 \mathrm{I} .35^{\circ}$ in a $200-\mathrm{mm}$. tube ( 20 grams made up $100 \mathrm{cc}$.), and finally that four cider vinegar samples of known purity showed lefthanded readings of from $0.96^{\circ}$ to $2.94^{\circ}$ Ventzke in a $400-\mathrm{mm}$. tube.

The left-handed rotation of pure cider vinegar is a characteristic so fixed and unalterable that a right-handed polarization of more than $0.5^{\circ}$ may safely be assumed as evidence of adulteration. The polarization of cider vinegar, expressed in terms of $200 \mathrm{~mm}$. of the undiluted sample should lie between $-0 . \mathrm{I}^{\circ}$ and $-4.0^{\circ}$ Ventzke. If the direct polarization of a sample of vinegar is right-handed, while the invert is left-handed, sugar-house wastes or molasses may be suspected as an adulterant.

If both direct and invert readings are right-handed, commercial glucose is undoubtedly present. If the polarization of the vinegar is far to the left, unfermented cider jelly has probably been used to reinforce the solids.

Frear regards the ratio of reducing sugars after inversion to total solids as a useful factor in discriminating between pure cider vinegar and the common artificial substitutes in which the solids of distilled vinegar are reinforced by apple jelly, or in which commercial glucose or molasses vinegars are used. When the reducing sugars after inversion form more than $25 \%$ of the entire solids, the alleged cider vinegar is undoubtedly ducts."

* Bull. $5^{8}$, Penn. Dept. of Agric., "A Chemical Study of the Apple and Its Pro- 
spurious. In pure cider vinegar the per cent of reducing sugar is the same after inversion as before. The same is true of glucose vinegar

Vinegar containing added molasses or cane sugar will, however, naturally show an increase in reducing sugar after inversion.

A large content of alcohol in cider vinegar, otherwise showing the constants of pure vinegar except for the low acidity, would indicate incomplete acetification. A high content of nitrogen is characteristic of malt vinegar.

Data of analyses of samples of vinegar examined in the Food and Drug Department of the Massachusetts State Board of Health are given in the tables on this page and the next. The table below shows in summarized form the results obtained from the examination of eighty-four samples of undoubtedly pure cider vinegar examined in 1901.*

CIDER VINEGAR FOUND PURE.

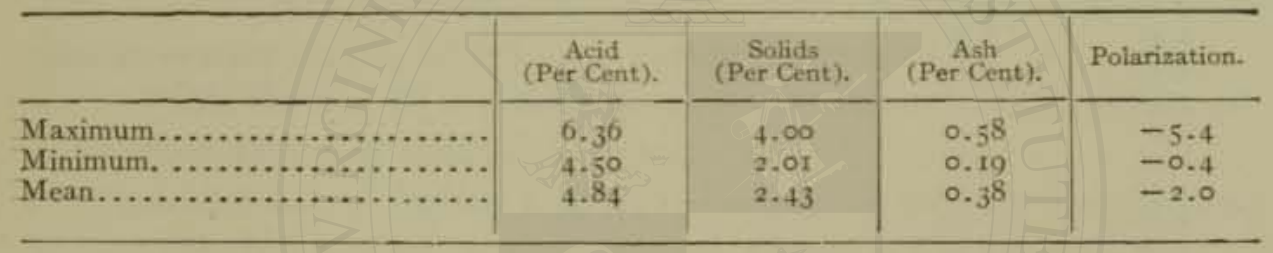

The second table includes samples of adulterated vinegar, sold for cider vinegar, none of which were probably made from cider. It will be noticed that in several of the samples the amount of glucose was abnormally large, as is shown by the very high right-handed polarization, in one case amounting to over $12^{\circ}$.

Direct Tests Made on the Vinegar.-The genuine or spurious natur: of cider vinegar may usually be established by direct tests with reagents on the vinegar itself. The appearance, taste, and odor of the vinegar should be noted. Brannt $\dagger$ applies the test of odor in vinegar as determining its character, by rising out a large beaker with the sample, and'

* 32d An. Rep. (1900), p. 66r, Food and Drug Reprint, p. 44; 33d An. Rep. (190r), p. 467, Food and Drug Reprint, p. 47; 34th An. Rep. (1902), p. 483, Food and Drug Reprint, p. 3 I.

+ A Practical Treatise on the Manufacture of Vinegar, p. 219. 
VINEGAR NOT THE EXCLUSIVE PRODUCT OF PURE APPLE CIDER.

\begin{tabular}{|c|c|c|c|c|c|}
\hline $\begin{array}{l}\text { Per Cent } \\
\text { Acetic Acid. }\end{array}$ & $\begin{array}{l}\text { Per Cent } \\
\text { Total Solids. }\end{array}$ & $\begin{array}{l}\text { Per'Cent } \\
\text { Ash. }\end{array}$ & $\begin{array}{c}\text { Per Cent } \\
\text { Ash in Total } \\
\text { Solids. }\end{array}$ & $\begin{array}{l}\text { Polarization } \\
\text { in 20o-mm. } \\
\text { Tube. }\end{array}$ & Lead Acetate. \\
\hline $\begin{array}{l}5 \cdot 90 \\
5 \cdot 14 \\
5 \cdot 12 \\
4 \cdot 83 \\
4 \cdot 82 \\
4 \cdot 80 \\
4 \cdot 80 \\
4 \cdot 66 \\
4 \cdot 60 \\
4 \cdot 56 \\
4 \cdot 54 \\
4 \cdot 54 \\
4 \cdot 54 \\
4 \cdot 54 \\
4 \cdot 50 \\
4 \cdot 50 \\
4 \cdot 50 \\
4 \cdot 48 \\
4 \cdot 46 \\
4 \cdot 42 \\
4 \cdot 42 \\
4 \cdot 40 \\
4 \cdot 40 \\
4 \cdot 38 \\
4 \cdot 32 \\
4 \cdot 08 \\
3 \cdot 98\end{array}$ & $\begin{array}{r}.40 \\
.36 \\
.53 \\
3.70 \\
2.71 \\
1.97 \\
1.03 \\
2.92 \\
2.57 \\
2.60 \\
3.97 \\
3.90 \\
2.94 \\
2.70 \\
3.05 \\
2.92 \\
2.60 \\
3.80 \\
2.80 \\
2.75 \\
2.70 \\
2.51 \\
.97 \\
.29 \\
.70 \\
3.35 \\
.55\end{array}$ & $\begin{array}{l}\ldots \ldots \\
\ldots \ldots \\
\ldots 32 \\
.32 \\
.13 \\
.20 \\
.27 \\
.20 \\
\ldots \\
\ldots \\
.19 \\
.32 \\
.23 \\
.23 \\
\ldots . \\
.22 \\
\ldots . \\
\ldots \\
\ldots \\
\ldots \\
\ldots . \\
.20 \\
\ldots . \\
\ldots \ldots \\
.09 \\
\ldots \ldots \\
\ldots \ldots\end{array}$ & $\begin{array}{r}\ldots . \\
\ldots . \\
\ldots .65 \\
4.80 \\
10.15 \\
14.75 \\
6.49 \\
\ldots . \\
\ldots \ldots \\
4.78 \\
9.72 \\
7.82 \\
8.52 \\
\ldots \ldots \\
7.52 \\
\ldots \ldots \\
\ldots . . \\
\ldots \ldots \\
\ldots . . \\
\ldots \ldots \\
11.15 \\
\ldots \ldots \\
\ldots .36 \\
12.86 \\
\ldots . \\
\ldots \ldots\end{array}$ & $\begin{array}{l}+. .0 \\
+. .6 \\
+8.0+ \\
+9.6 \neq \\
+. .9 \\
+1.1 \\
+2.2 \\
+2.6 \\
+7.0+ \\
+5.6 \\
+5.0 \\
+5.0 \\
+.4 \\
+2.2 \\
+.9 \\
+2.8 \\
+12.0 \neq \\
+2.6 \\
+3.2 \\
+9.2 \\
+1.1 \\
+.4 \\
+1.6 \\
+\ldots . .2 \\
+1.2 \\
+1.8\end{array}$ & 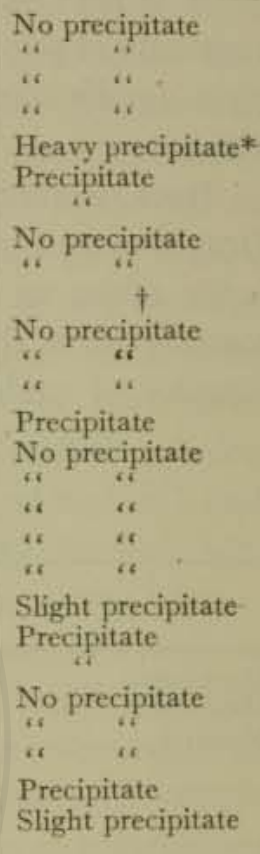 \\
\hline
\end{tabular}

* Cider vinegar to which apple jelly containing glucose had been added for the purpose of increasing the solids after watering.

+ This sample contained a large amount of phosphate, and consequently the test for malates is obscured.

‡These samples polarized practically the same after as before inversion, indicating much glucose.

after allowing it to stand for some hours, examining the few drops remaining in the beaker. The acetic acid having for the most part become volatilized, the characteristic vinous odor of pure wine vinegar would at this stage be very prominent, while that of cider vinegar would be entirely different. The odor of the two vinegars is very similar in their ordinary state. The peculiar fruity flavor of pure cider vinegar is very characteristic and not readily imitated by cheaper substitutes. Only a very slight turbidity should be produced in pure cider vinegar by the addition of either ammonium oxalate (absence of lime), barium chloride (absence of sulphuric acid or sulphate), and nitrate of silver (absence of hydrochloric acid or chlorides). 
The character of the precipitate produced by neutral lead acetate should be particularly noted. Unless it is flocculent and copious, settling out after a few minutes, cider vinegar is not pure, even if a marked turbidity is produced. Added apple jelly from exhausted apple pomace gives such a turbidity, and is to be suspected when not more than a cloudiness is produced on addition of the lead acetate reagent. Pure cider vinegar should respond in a perfectly normal manner to both the lead acetate and the calcium chloride tests for malic acid.

Wood Vinegar or Pyroligneous Acid is sometimes rendered apparent by the empyreumatic or tarry taste and odor imparted to the product. When, however, the added acetic acid has been so purified that the tarry taste and odor are lacking, its presence may often be proved by the traces of furfurol which always accompany it.

Test for Furfural.-A little of the vinegar is subjected to distillation, and to the first few drops of the distillate is added a little colorless anilin solution. A fading crimson color will be produced in presence of furfurol. This reaction may sometimes be obtained upon the vinegar itself without distillation, if sufficient added wood vinegar be present.

The first portion of the distillate of wood vinegar reduces permanganate of potassium to a marked degree.

The Addition of Spices to vinegar in order to increase the pungency is best detected by first neutralizing the vinegar with sodium carbonate and then tasting. Under these conditions, the admixture of spices is rendered very apparent.

Detection of Caramel.-Considerable added caramel in vinegar is apparent from the unnaturally dark color and extremely bitter taste of the residue after evaporation.

Tests for caramel made on the vinegar residue, if long dried at the temperature of the water-bath, are not to be depended on as establishing the presence of added caramel, since at that temperature the decomposition of the sugar may in any event cause a positive test.

Caramel is detected by Crampton and Simon's and Amthor's tests (p. 752). A further indication of caramel is the reducing power of the water solution of the precipitate obtained in Amthor's test.

Examination for Metallic Impurities.-Lead and Zinc are best looked for in the ash of the vinegar in cases where, like cider vinegar, the percentage of extract is high. A large volume of the vinegar is evaporated to substantial dryness over the water-bath. This may most readily be done in a roo-cc. platinum wine-shell, adding the vinegar in successive 
portions. To the residue add a small amount of sodium hydroxide, and burn to an ash in a muffle, or over a low flame, using potassium nitrate if necessary, a little at a time. Take up the ash in dilute hydrochloric acid, and examine for lead and zinc as in the case of canned goods.

In the case of vinegar low in extract, as in spirit vinegar, the sample may be evaporated to dryness, the residue dissolved directly in dilute hydrochloric acid without ignition, and the acid solution subjected to dircet examination for lead and zinc.

Copper is best determined by electrolysis. 100 cc. of the vinegar are evaporated to a volume of about ro cc. with a little sulphuric acid, filtered into a platinum dish, and subjected to electrolysis, using conveniently the apparatus described on page 608 .

Arsenic.-Boil down a portion of the vinegar, to which concentrated nitric acid has been added, to a small volume, then add a few cubic centimeters of concentrated sulphuric acid, and continue the heating till fumes of sulphuric acid show the nitric to have been driven off. Cool, dilute with water, and test in the Marsh apparatus.

\section{REFERENCES ON VINEGAR.}

Allen, A. H. White Wine Vinegar. Analyst, 21, 1896, p. 253.

Allen, A. H., and Moor, C. G. Vinegar. Analyst, 18, 1893, pp. 180 and 240.

BERSCH, J. Die Essigfabrikation. Vienna, 1895.

Brannt, W. Vinegar, Acetates, Cider, Fruit Wines and Preservation of Fruits. London, 1900 .

Browne, C. A. A Chemical Study of the Apple and Its Products. Penn. Dept. of Agric. Bul. 58,1899 .

- The Effects of Fermentation upon the Composition of Cider and Vinegar. Jour.

Am. Chem. Soc., 25, 1903 , p. 16.

Crampton, C. A., and Stmons, F. D. Detection of Caramel in Spirits and Vinegar. Jour. Am. Chem. Soc., 21, 1899, p. 355.

DavenPort, B. F. Analysis of Vinegar. Chem. News, 1887,3 and 66.

Doolitrle, R. E., and Hess, W. H. Cider Vinegar, Its Solids and Ash. Jour. Am. Chem. Soc., 22, 1900, p. 218.

Dusors, W. L. The Fuller's Earth Test for Caramel in Vinegar. Jour. Am. Chem. Soc., 29, 1907 , p. 75 .

Frear, W. Apple Juice, Fermented Cider and Vinegar. Penn. Dept. of Agric. Rep., 1898 , p. 138.

- Cider Vinegars of Pennsylvania. Penn. Dept. of Agric., Bul. 22, 1897.

- Vinegar. U. S. Dept. of Agric., Bur. of Chem., Bul. 65, p. 62. Washington, 1902.

Gardner, J. Acetic Acid and Vinegar. Philadelphia., 1885.

LeAch, A. E., and Lythgoe, H. C. Cider Vinegar and Suggested Standards of Purity. Jour. Am. Chem. Soc., 26, 1904, p. 375. 
Leens, A. R. Acetic Acid in Vinegar. Jour. Am. Chem. Soc., 17, 1895, p. $74 \mathrm{I}$. Macfarlane, T. Vinegar. Canada Inl. Rev. Dept., Bul. 35. Ottawa, r893. PASteur, M. Études sur la Vinaigre. Paris, r868.

SANGLÉ-FERRIÈrE. Vinaigre. Analyse des Matières Alimentaires (Girard), p. 263. Sмттн, A. W. Vinegar Analysis and Characteristics of Pure Cider Vinegar. Jour. Am. Chem. Soc., 20,1898 , p. 3 .

SykEs, W. J. Detection of Adulteration in Vinegar. Analyst, 16, 1891, p. 83 .

Connecticut Exp. Sta. An. Reports, 1897, 1898, r899.

Massachusetts State Board of Health, An. Reports, 1900, 1901, 1902, and 1903. North Carolina Exp. Station Bul, 15.3. 


\section{CHAPTER XVII.}

\section{ARTIFICIAL FOOD COLORS.}

THE use of artificial dyestuffs in food products has greatly increasec during recent years, both in degree and in variety of colors employed. Where formerly but a few well-known coloring matters, chiefly so-called vegetable colors and occasionally mineral pigments were used for this purpose, a vast array of dyes, chosen largely from the coal-tar colors, are now found in food, so that at present the exact identification of the particular dyestuff employed in all cases presents a somewhat formidable problem to the analyst. The problem may consist in determining the class to which a commercial food color or combination of colors belongs, or it may consist in isolating the color itself, and afterwards identifying it as far as possible, for the purpose of determining whether or not it is harmless within the meaning of the law.

The effect of imparting to the cheaper varieties of jellies, jams, and. ketchups which flood the market such intense and striking colors that these products in no wise resemble their pure uncolored prototypes, has a tendency in many cases to mislead the public into the idea that the genuine products are inferior by contrast, and to create a craving for unnaturally colored varieties. Indeed, the adherents to the free use of coloring matters in food assert that these brilliant hues please the eye and are hence legitimate.

Objectionable Features.-With the exception of confectionery and certain dessert preparations, in which dyes may be employed purely for æsthetic considerations only (a fact which is well understood by the consumer), the use of coloring matters in food is mainly for the purpose of deceiving as to their true character. The use of dyestuffs in food is objectionable on two accounts, first as introducing in some cases materials injurious to health, and second, in nearly all cases as deceiving the purchaser by concealing inferiority, or by making the goods 
appear of greater value than they really are. In most states the food laws regarding employment of colors are so framed, that the presence of such colors constitutes an offense under one or the other of the above heads, mainly, however, because, by reason of their use, cheaper or inferior materials are made to masquerade for the higher or genuine grades, as, for instance, when alleged currant jelly is found to consist chiefly of apple-stock and commercial glucose, colored with an artificial red dye.

In such cases the analyst has merely to prove conclusively that an artificial color is present, even if he does not identify the dye itself. It is of course more satisfactory to at least show in addition whether the dye present is of vegetable origin, or is of the coal-tar variety, and in most cases this can readily be done, even if it is not easy to identify the exact color.

In localities where laws prevail stipulating that what are commonly known as "mixtures" or "compounds" to be legally sold, must be labeled with the names and percentages of ingredients, the law applies to coloring matters as well as other ingredients, and the exact dye or dyes employed should appear on the label. Otherwise the product must be classed as adulterated.

Toxic Effects of Colors.-Formerly the use of such pigments as chromate of lead was common in coloring confectionery, but lead chromate is rarely used at present. Other mineral pigments obviously unfit for use in food by reason of their well-known poisonous effects are those which contain salts of arsenic, mercury, lead, and copper. While most of the coal-tar colors are considered harmless in themselves, some are decidedly objectionable, and should not be used in foods. Under the latter class are included, first, those in connection with the manufacture of which arsenic, mercury, or other poisonous mineral ingredients have been used, such for example as arsenical fuchsin, and, second, those which are themselves inherently poisonous, as for instance picric acid. Fuchsin is now largely made without the aid of arsenic acid, and this variety is, perhaps, harmless. The toxic effects of many of the coal-tar colors have not been thoroughly established excepting in a negative way. Weyl has made many experiments on dogs and rabbits in which these animals have been fed with varying amounts of coloring material. In nearly all cases the doses far exceeded the amounts ordinarily taken in food, and the experiments are of value mainly in so far as they show harmless results of certain colors on the animal. It is to be regretted 
that physiological experiments cannot more readily be tried on human beings, so as to study the effects of administering to them such amounts as are used in food.

More conclusive results (though still of a negative character) tending to establish the harmlessness of most of the coal-tar colors are given by Grandhomme * in statistics showing the condition of health of laborers in factories where these dyestuffs are made, in comparison with those engaged in other industries where poisonous materials are handled. From these it appears that the proportion of illness among the anilinmakers is remarkably small.

In the case of coloring confectionery by the use of mineral pigments, a considerable amount of the coloring material must be used, forming without doubt a source of danger in some cases. With coal-tar dyes, on the contrary, the case is different. One ounce of auramine, for instance, has been found sufficient to give a deep-yellow color to 2,000 pounds of confectionery, so that almost an infinitesimal amount of the actual dyestuff is taken into the system. Hence it is that very little danger need be apprehended from the use of most coal-tar colors in food, objectionable as they certainly are as a commercial fraud.

Injurious and Non-injurious Colors.-Various countries have enacted specific laws regulating the use of coloring matters in foods, especially England, France, Germany, Austria, and Italy. In some cases attempts have been made to specify harmful and harmless colors. The National Confectioners' Association of the United States has compiled a useful classified list of injurious and harmless colors, $\dagger$ the classification being based largely on the results of experiments by Weyl and König, as well as upon the Resolutions of the Association of Swiss Chemists, and on the French Ordinances regarding food colors. The list is as follows:

HARMFUL MINERAL COLORS.

Compounds of Copper.-Blue ashes, mountain blue, etc.

Compounds of Lead.-Massicot, red lead, white lead, Cassel yellow, Paris yellow, Turner yellow, Naples yellow, sulphates of lead, chrome yellow, Cologne yellow, etc.

Compounds of Barium.-Ultramarine yellow, etc.

* Weyl, Sanitary Relations of the Coal-tar Colors, pp. 28-30.

+ Colors in Confectionery. An Official Circular from the Executive Committee of the National Confectioners' Association of the U. S., 1899. 
Compounds of Mercury.-Vermilion, etc.

Compounds of Arsenic.-Scheele's green, Schweinfurth green, etc.

In Other Words colors in whose preparation mercury, lead, copper, arsenic, antimony, tin, zinc, chromium, and barium compounds are used.

\section{HARMFUL ORGANIC COLORS.}

Red Colors.-Ponceau $3 R B$.-Ponceau B extra, fast ponceau B, new red L, scarlet EC, imperial scarlet, old scarlet, Biebrich scarlet.

Crocein Scarlet ${ }_{3} B$.-Ponceau ${ }_{4} \mathrm{RB}$.

Cochenille Red A.-Crocein scarlet $4 \mathrm{~B}$ and $\mathrm{G}$, brilliant scarlet, brilliant ponceau ${ }_{4} R$, ponceau ${ }_{4} R$, ponceau brilliant ${ }_{4} R$, new coccin, scarlet.

Crocein Scarlet 7 B.-Crocein scarlet 8B, ponceau 6RB.

Crocein scarlet $O$ extra.

Safranin.-Safranin T, safranin extra G, safranin G extra GGSS, safranin GOOO, safranin FF extra No. O, safranin conc., safranin AG extra, safranin AGT extra, anilin pink.

Yellow Colors.-Gum gutta.

Picric acid.

Martius Yellow.-Naphthylamin yellow, jaune d'or, Manchester yellow, naphthalin yellow, naphthol yellow, jaune naphthol.

Acme Yellow.-Chrysoin, chryseolin yellow T, gold yellow, resorcin yellow, acid yellow RS, tropæolin O, jaune II.

Victoria Yellow.-Victoria orange, anilin orange, dinitrocresol, saffron substitute, golden yellow.

Orange II.-Orange No. 2 , orange $\mathrm{P}$, orange extra, orange $\mathrm{A}$, orange $\mathrm{G}$, acid orange, gold orange, mandarin G extra, beta-naphtholorange, tropæolin OOO No. 2, mandarin, chrysaurin.

Metanil Yellow.-Orange MN, tropæolin G, Victoria yellow (O double conc.), jaune G (metanil extra).

Sudan I.-Carminaph.

Orange IV.-Orange No. 4, orange N, orange GS, new yellow, acid yellow D, tropæolin OO, fast yellow, diphenylorange, diphenylamine orange, jaune d'anilin, anilin yellow.

Green Colors. -Naphthol green $B$.

Blue Colors. - Methylene blue BBG.-Methylene blue BB, in powder extra, methylene blue DBB extra, methylene blue BB (crystalline) ethylene blue. 
Brown Colors.-Bismarck Brown.-Bismarck brown G, Manchester brown, phenylen brown, vesuvin, anilin brown, leather brown, cinnamon brown, canelle, English brown, gold brown.

Vesuvin B.-Manchester brown EE, Manchester brown PS, Bismarck brown, Bismarck brown $\mathrm{T}$, brun Bismarck EE.

Fast Brown G.-Acid brown.

Chrysoidin.-Chrysoidin G, chrysoidin R, chrysoidin J, chrysoidin Y.

HARMLESS MINERAL COLORS.

Blue Colors.-Ultramarine blue.

Violet Colors.-Ultramarine violet.

Brown Colors.-Manganese brown.

Chocolate-brown and colors of a similar nature have as their basis natural or precipitated oxide of iron, which in an impure condition may have small quantities of arsenic in its composition. It is possible with proper care to secure a raw material entirely free from this objectionable element, and no oxide of iron containing any traces of arsenic should be used in the preparation of color.

Green Colors.-Ultramarine green.

\section{HARMLESS ORGANIC COLORS.}

Red Colors.-Cochineal carmine.

Carthamic acid (from saffron).

Redwood.

Artificial alizarin and purpurin.

Cherry and beet juices.

Eosin.-Eosin A, eosin G extra, eosin GGF, eosin water soluble, eosin $3 \mathrm{~J}$, eosin $4 \mathrm{~J}$ extra, eosin extra, eosin KS ord., eosin DH, eosin JJF.

Erythrosin.-Erythrosin D, erythrosin B, pyrosin B, primrose soluble, eosin bluish, eosin J, dianthin B.

Rose Bengale.--Rose bengale N, Rose bengale AT, rose bengale $\mathrm{G}$, bengalrosa.

Phloxin.-Phloxin TA, eosin blue, cyanosin, eosin roB.

Bordeaux and Ponceau reds, resulting from the action of naphtholsulphonic acids on diazoxylene:

Ponceau $2 R$.-Ponceau G, ponceau GR.

Ponceau R.-Brilliant ponceau G, ponceau J. 
Bordeaux B.-Fast red B, Bordeaux R extra.

Cerasin.-Rouge B.

Ponceau 2 G.-Brilliant ponceau GG, ponceau JJ.

Fuchsin S.-Acid magenta, rubin S, fuchsin acide (free from arsenic). Archil Substitute.-Naphthion red.

Orange I.-Orange No. I, naphtholorange, alpha-naphtholorange, tropæolin OOO No. I.

Congo red.

Azorubin S.-Azorubin, azorubin A, azoacidrubin, fast red C, carmoisin, brilliant carmoisin $\mathrm{O}$, rouge rubin $\mathrm{A}$.

Fast Red D.-Fast red EB, fast red NS, amaranth, azoacidrubin ${ }_{2} \mathrm{~B}$, Bordeaux DH, Bordeaux S, naphthol red S, naphthol red O, Victoria ruby, wool red (extra), cenanthinin.

Fast Red.-Fast red E, fast red S, acid carmoisin S.

Ponceau 4 GB.-Crocein orange, brilliant orange G, orange GRX, pyrotin orange, orange ENL.

Fuchsin.

Metanitrazotin.

Yellow and Orange Colors.-Annatto.

Saffron.

Saflower.

Turmeric.

Naphthol Yellow S.-Citronin A, sulphur yellow S, jaune acide, jaune acide C, anilin yellow, succinine, saftron-yellow, solid yellow, acid yellow $\mathrm{S}$.

Brilliant Yellow.-(Sch.)

Ponceau ${ }_{4} G B$. - Crocein orange, brilliant orange G, orange GRX, pyrotin orange, orange ENL.

Fast Yellow.-Fast yellow G, fast yellow (greenish), fast yellow S, acid yellow, new yellow $\mathrm{L}$.

Fast Yellow R.-Fast yellow, yellow W.

Azarin $S$.

Orange I.-Orange No. I, naphtholorange, alpha-naphtholorange, tropxolin OOO No. I.

Orange--Orange GT, orange $\mathrm{RN}$, brilliant orange $\mathrm{O}$, orange $\mathrm{N}$.

Mixtures of harmless red and yellow colors.

Green Colors.-Spinach green.

Chinese green.

Malachite Green.-Malachite green B, benzaldehyde green, new Vic- 
toria green, new green, solid green crystals, solid green $\mathrm{O}$, diamond green, bitter amond oil green, fast green.

Dinitrosoresorcin.-Solid green $\mathrm{O}$ in paste, dark green, chlorine green, Russia green, Alsace green, fast green, resorcinol green.

Mixtures of harmless blue and yellow colors.

Blue Colors.-Indigo.

Litmus.

Archil blue.

Gentian Blue 6B.-Spirit blue, spirit blue FCS, opal blue, blue lumière, Hessian blue, light blue.

Coupiers Blue.-Fast blue R and B, solid blue RR and B, indigin DF, indulin (soluble in alcohol), indophenin extra, blue CB (soluble in alcohol), nigrosin (soluble in alcohol), noir CNN.

In General such blues as are derived from triphenylrosanilin or from diphenylamin.

Violet Colors. - Paris Violet. - Methyl violet B and ${ }_{2} \mathrm{~B}$, methyl violet $\mathrm{V}_{3}$, pyoktanin cœruleum, malbery blue.

Wool black.

Naphthol black $P$.

Azoblue.

Mauvein.-Rosolan, violet paste, chrome violet, anilin violet, anilin purple, Perkins violet, indisin, phenamin, purpurin, tyralin, tyrian purple, lydin.

\section{Brown Colors.-Caramel.}

Licorice.

Chrysamin $R$.

Use of Colors in Confectionery.--Regarding the choice of colors for use in confectionery and precautions to be observed in their use, the Confectioners' Association has offered the following considerations:

First. That coal-tar colors are specially adapted to the wants of confectioners on account of their brilliancy, permanency, and high coloring power, by reason of which last-named quality only infinitesimal amounts of color need be or can be used to give the desired effects.

Second. That there is no evidence to show that any poisonous or hurtful colorings have in recent years been found in confeciionery. Reports of deaths from poisoned candy are only too frequently made, but no autopsy has ever been published confirming them.

Third. That while the exceedingly small proportions of color used in confectionery constitute a practical safeguard to the public health, con- 
fectioners are in duty bound to provide against all possible contingencies of harm, by using the utmost care in obtaining absolutely non-poisonous colors, buying only from color-dealers of established reputation and unquestioned responsibility, whose colors are tested at frequent intervals, and are vouched for by competent chemists.

Confectioners should require that a guarantee be put upon each package of color, stating not only that the contents are non-poisonous, but also that they will not in any way interfere with digestion or injure health.

Fourth. Any illegitimate use of coloring matter in contestionery as a substitute for chocolate or any other material or ingredient, or for the purpose of adding bulk or increasing the weight of the confectionery in which it is incorporated, should not be permitted or countenanced. Both the letter and the spirit of these laws should clearly prevent the illegitimate use of coal-tar colors or of earth colors, such, for example, as chocolate-brown, coconole brown, or chocolatina.

Fifth. That color-dealers furnishing colors to confectioners should publish printed lists of their colors under the various names and titles by which they are known and offered for sale, accompanying such lists with ample certifications by competent chemists to their purity and suitableness for coloring confectionery and other articles of food. They should also attach to each package or other container of color a guarantee that it does not contain anything injurious to health.

\section{VEGETABLE COLORS.}

These with a few mineral pigments and cochineal were formerly almost exclusively used for coloring food products, and are still used to some extent.

Most of the vegetable colors, according to L. Robin,* react with ammonia to form a coloration, usually passing from violet to blue, then to a brownish green, when the ammonia is added little by little in excess to the color in solution. If by the addition of ammonia to a solution of an unknown color the green coloration does not result, the presence may be suspected of orchil or cudbear, logwood, cochineal, or a coal-tar dye.

The following vegetable colors are occasionally found in food, with some of the reactions in aqueous solution, as given by Robin:

* Girard, Analyse des Matières Alimentaires, pp. 678, 679 . 
RED COLORS.

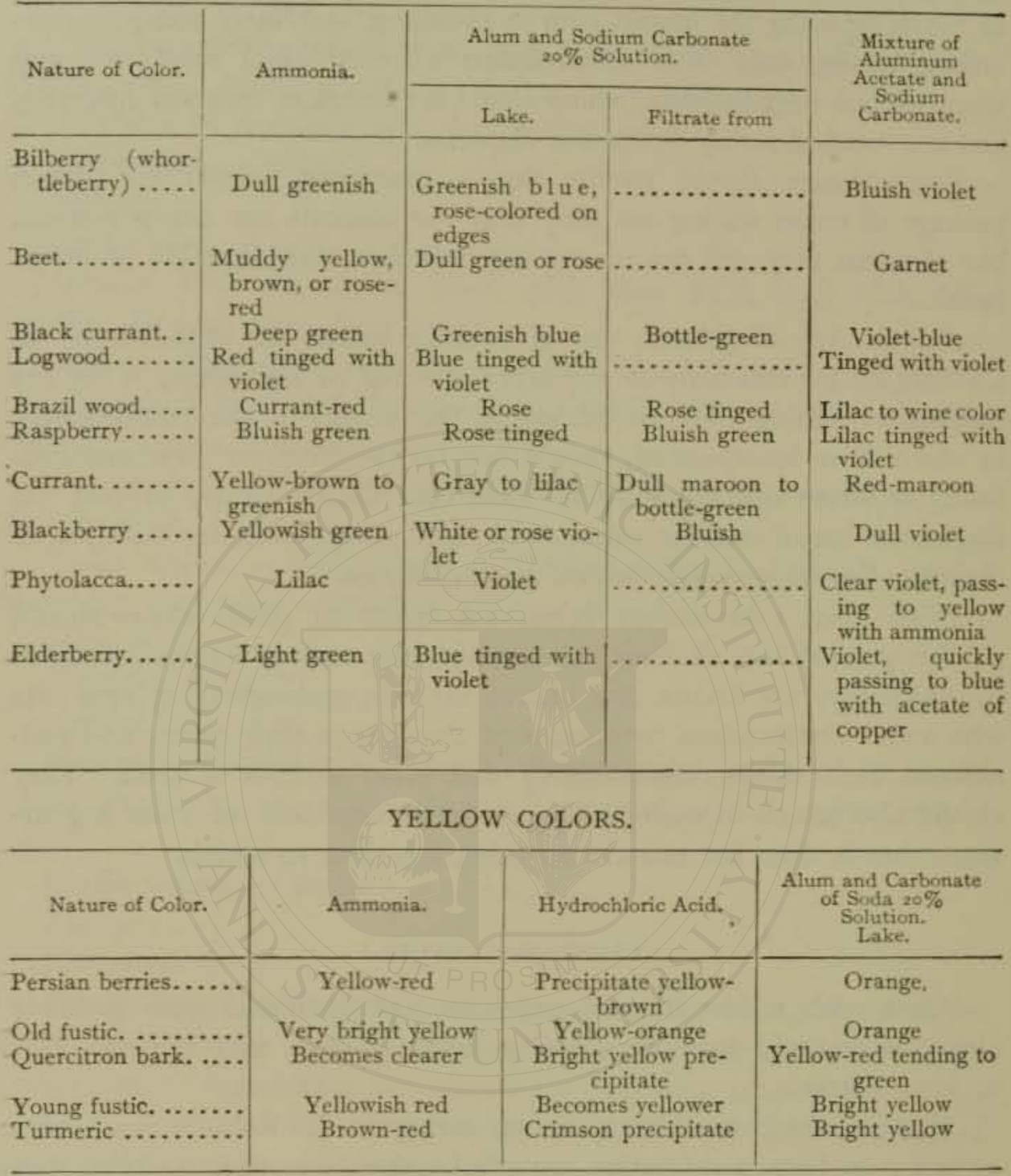

Additional yellow vegetable colors sometimes used in foods are the following, taken from a table of Leed's,* showing reactions given by treating a few drops of an alcoholic solution of the color with an equal volume of the reagent.

Most of these vegetable colors do not directly dye wool or silk a fast color, but as a rule require the use of a mordant. Many of these colors may be fixed on cotton (previously mordanted by boiling in a solution

\section{* Analyst, I2, 150 .}


REACTIONS OF COLORING MATTERS.

\begin{tabular}{|c|c|c|c|c|}
\hline $\begin{array}{l}\text { Coloring } \\
\text { Matter. }\end{array}$ & $\begin{array}{c}\text { Concentrated } \\
\mathrm{H}_{2} \mathrm{SO}_{4} \text {. }\end{array}$ & $\begin{array}{c}\text { Concentrated } \\
\mathrm{HNO}_{3}\end{array}$ & $\mathrm{H}_{2} \mathrm{SO}_{4}+\mathrm{HNO}_{3}$ & $\begin{array}{c}\text { Concentrated } \\
\text { HCl. }\end{array}$ \\
\hline Turmeric,... & $\begin{array}{l}\text { Indigo-blue, chang- } \\
\text { ing to violet } \\
\text { Pure violet }\end{array}$ & $\begin{array}{l}\text { Blue, becoming } \\
\text { colorless on } \\
\text { standing } \\
\text { Violet }\end{array}$ & $\begin{array}{l}\text { Same } \\
\text { Violet }\end{array}$ & $\begin{array}{l}\text { No change, or only } \\
\text { slight dirty yel- } \\
\text { low and brown } \\
\text { Violet, changing to } \\
\text { original color on } \\
\text { evaporation of } \\
\text { HCl }\end{array}$ \\
\hline Saffron..... & $\begin{array}{l}\text { Violet to cobalt } \\
\text { blue, changing to } \\
\text { reddish brown }\end{array}$ & $\begin{array}{l}\text { Light blue, chang- } \\
\text { ing to light red- } \\
\text { dish brown }\end{array}$ & $\begin{array}{c}\text { Same } \\
t\end{array}$ & $\begin{array}{l}\text { Yellow, changing } \\
\text { to dirty yellow }\end{array}$ \\
\hline Carrot...... & Umber brown & Decolorized & $\begin{array}{l}\text { Same with } \mathrm{NO}_{2} \\
\text { fumes and odor } \\
\text { of burnt sugar }\end{array}$ & No change \\
\hline Marigold... & $\begin{array}{l}\text { Dark olive-green, } \\
\text { permanent }\end{array}$ & $\begin{array}{l}\text { Blue, changing in- } \\
\text { stantly to dirty } \\
\text { yellow-green }\end{array}$ & Green & $\begin{array}{l}\text { Green to yellowish } \\
\text { green }\end{array}$ \\
\hline Safflower.... & Light brown & $\begin{array}{l}\text { Partially decolor- } \\
\text { ized }\end{array}$ & Decolorized & No change \\
\hline
\end{tabular}

of aluminum acetate or potassium bichromate) by boiling the mordanted fibers in a bath of the colored solution, rendered acid by acetic acid. The dyed fibers are then examined by reagents, as in tables given on pages $806-\mathrm{I}_{3}$.

SPECIAL TESTS FOR Vegetable Colors.-Orchil and Cudbear, both derived from lichens, dye wool red in acid bath. The colored fiber, in the case of cudbear, is turned blue by treatment with ammonia. For reactions of orchil on the fiber see table, page 809. Robin's test for orchil in aqueous solution consists in shaking it with ether, which, if orchil is present, is colored yellow. On treatment of the ether with ammonia, the yellow color is changed to blue, and, by adding acetic acid, goes over to a reddish violet.

Logwood, according to Robin, in aqueous solution colors ether yellow, and on treating the ether with ammonia the color becomes red or faintly violet. Potassium bichromate gives a violet coloration, mingled with greenish yellow. If cotton is first mordanted by boiling with aluminum acetate, it is dyed violet when boiled in a solution of logwood.

Turmeric is best extracted from a dry residue with alcohol, which it colors yellow. The color is transferred to a piece of filter-paper by soaking the paper in the alcoholic tincture, the paper is dried and dipped in a dilute solution of boric acid or borax slightly acidulated with hydrochloric acid. On again drying the paper, it will be of a cherry-red color if turmeric is present, and when touched with a drop of dilute alkali will turn dark olive. 
Caramel.-Care should be taken in testing for caramel not to subject the sample to long-continued heating, even on the water-bath. Indeed caramel is sometimes developed spontaneously in saccharine food products during their process of manufacture when heat is used, by the charring of the sugar. If solutions are to be concentrated or brought to dryness before testing for caramel, this should be done in a vacuum desiccator over sulphuric acid, or at a temperature not exceeding $70^{\circ}$. For detection of caramel in milk, vinegar, and liquors, special tests are given elsewhere.

Fradiss Test.*-The dried residue of the sample to be tested is extracted wi.h warm, pure methyl alcohol, which, if caramel be present, is colored brown. Filter, and to the filtrate add amyl alcohol or chloroform. In presence of caramel, a brown flocculent precipitate is formed, which slowly settles to the bottom of the tube.

Indigo in aqueous solution turns green with ammonia. On boiling, the solution becomes bright blue. Indigo in neutral or acid solution dyes wool or silk.

\section{ANIMAL COLORS.}

Cochineal.-This dyestuff is used in ketchups, cordials, confections, and other food products. Robin's test for cochineal is as follows: The aqueous solution is acidulated with hydrochloric acid, and shaken out in a separatory funnel with amyl alcohol. Cochineal imparts to this solvent a yellowish color, the depth depending on the amount present. The separated amyl alcohol is washed with water till neutral, and divided into two portions. To one of these a little water is added, and then drop by drop a solution of uranium acetate, shaking each time a drop is added. In presence of cochineal the water is colored a very characteristic emerald-green color. To the other portion ammonia is added. If cochineal has been used, a violet coloration is produced.

\section{MINERAL PIGMENTS.}

Evidence of the presence of these pigments is usually best looked for in the ash of the suspected sample. In some cases the color may be extracted from the dried residue by water, alkali, or alcohol.

Prussian Blue.-This pigment is insoluble in water. It is decomposed and decolorized by treatment with potassium hydroxide. If the

* Oestr. ungar. Zeits. Zucker. Ind., 1899, 28, 229-231; Abs. Zeits. f. Unters. Nahr. u. Genuss., 2, 1899 , p. 88 r. 
filtered alkaline solution of the coloring matter be treated with hydrochloric acid and ferric chloride, a precipitate of the original Prussian blue will be produced. For reactions on the fiber see table, page 812 .

Ultramarine Blue is decolorized by hydrochloric acid with evolution of hydrogen sulphide, which blackens filter-paper moistened with lead acetate. For the recognition of ultramarine in sugar see page 590. For its detection on the fiber see table, page $8 r_{3}$.

Chromate of Lead has never been used to any extent in food products with the exception of confectionery. For its detection, see page 647 .

\section{COAL-TAR COLORS.}

So many of the coal-tar dyes are adapted for use in food that it would be impossible to even name them all, especially in view of the fact that new coors are from time to time being added to the list. No attempt will be made in the present work to give the nature and composition of the dyes named, as such descriptions would lead beyond its scope. For detailed information along this line the reader is directed to the references on pp. $8 \mathrm{I} 9$ and 820 , especially to the works of Schultz and Julius, Benedict and Knecht, Weyl, etc.

About 2000 separate coal-tar dyes are at present on the market. Various classifications of these colors are attempted, based on (r), their origin, as anilin dyes, naphthalin dyes, anthracene dyes, etc.; (2), their chemical composition, as nitro, nitroso, azo, diazo, and other compounds; (3), their solubility in water and other solvents; and (4), their mode of application to the fiber, as basic dyes, acid dyes, direct cotton dyes, mordant dyes, etc.

These dyes are sold in the form of powder, and are readily made into solutions for food colors in the case of the water-soluble varieties, and into pastes in the case of the insoluble forms. Most of the coal-tar colors employed in foods are naturally of the soluble variety, especially such as are found in jellies, jams, fruit products, canned foods, ketchups, beverages, and milk. Pastes made from insoluble dyes are adapted mainly for exterior coatings of hard substances such as candies. Colors in the dry form are to be looked for in such spices as cayenne, mustard, and mace, but a commoner method of coloring these spices high in oil is to mix with them a solution of the color in oil (usually cottonseed). Oil solutions of coal-tar dyes are also employed for coloring butter and oleomargarine.

The chief concern of the food analyst, as regards artificial color is 
its recognition in food products. Coal-tar dyes may usualiy be identified as such, but it is not always possible to name the particular individual dye or combination of dyes employed, even though the class to which they belong may be determined. One reason for this is that not infrequently mixtures of two or more colors are employed.

Coal-tar Colors Allowed under the Federal Law.-The use of any dye, harmless or otherwise, to color food in a manner whereby damage or inferiority is concealed is in violation of Sec. 7 of the Food and Drugs Act of June 30, 1906. The addition of all mineral or metallic dyes, and of all coal-tar dyes, other than those specially provided for, is also prohibited. Pending further investigation the following coal-tar colors are permitted in foods, provided they are certified to be true to name and to be free from mineral and metallic poisons, harmful organic constituents, and contaminations due to improper or incomplete manufacture: *

Red Shades.-I07. Amaranth [M.] [C.]. Synonyms: Fast red D. [B.] Bordeaux S. [A.], azoacidrubine 2 B. [D.], fast red EB. [B.].

56. Ponceau ${ }_{3}$ R. $[A].[B].[M$.$] . Synonyms: Ponceau { }_{4}$ R. [A.], cumidin red, cumidin ponceau.

517. Erythrosin [B.] [M.] [B.S.S.]. Synonyms: Erythrosin D. [C.], erythrosin B. [A.], pyrosin B. [Mo.], iodeosin B., eosin bluish, eosin J. [B.].

Orange Shade. -85 . Orange I. Synonyms: Alphanaphthol orange, naphthol orange $[A$.$] , tropæolin 000$ No. I, orange B. $[L$.$] .$

Yellow Shade.-4. Naphthol yellow. S. [B.]. Synonyms: Naphthol yellow, acid yellow S., citronin A. [L.].

Green Shade.-435. Light green S. F. yellowish [B.]. Synonyms: Acid green $[B y].[M].[T . M].[O$.$] , acid green extra conc. [C$.$] .$

Blue Shade.-692. Indigo disulphoacid. Synonyms: Indigo carmine, indigo extract, indigotine $[B$.$] , sulphonated indigo.$

None of these seven colors is patented, hence their manufacture is not likely to become a monopoly. They may be used in combinations, thus securing any desired shade. For example, violet may be obtained by mixing indigo disulphoacid and one of the red colors, a blue-green by mixing indigo-disulphoacid with naphthol yellow S. or light-green S.F. and so on.

* The numbers preceding the dyes are those given in Green: A Systematic Survey of the Organic Colouring Matters founded on the German of Schultz and Julius, Londons 1904; the letters in brackets represent the manufacturers who originated the names. 
DETECTION OF COAL-TAR COLORS IN FOODS.-There are various methods for the separation of coloring matters from food products, and these may be divided into three general classes: First, dying silk or wool with the color by boiling the fiber in a solution of the sample to be examined; second, extracting the color from a solution of the sample by the use of an immiscible solvent; and third, extracting the color from the dried residue of the sample by means of a suitable solvent. Of these the method of dying wool lends itself most readily to the analyst's use, by reason of its simplicity, and from the fact that almost without exception coal-tar dyes adaptable for food colors are substantive dyes, being readily taken up by wool.

Basic and Acid Dyes. - The soluble coal-tar dyes are either basic or acid. Basic dyes are precipitated from their aqueous solution by tannin. Acid dyes are not so precipitated. Theoretically, all the basic colors are taken up by wool from a faintly alkaline or neutral bath, while the acid colors are left in solution. Thus if a dilute solution of the color be made faintly alkaline with ammonia and boiled with the wool, only basic colors will be taken up. If both acid and basic dyes are present in the same solution, the basic color should first be exhausted by the use of fresh pieces of wool in the ammoniacal solution, till they no longer take out color, after which the solution should be made slightly acid with hydrochloric acid and again boiled with wool, which under these conditions takes out any acid colors. Comparatively few basic colors are employed in foods. Basic colors can be removed from the fiber by boiling with $5 \%$ acetic acid. Acid colors are removed therefrom by boiling with $5 \%$ ammonia. Having dissolved the dye from the fiber by the appropriate solvent as above, the decolorized fiber may be removed, and the solution evaporated to dryness on the water-bath. The residue consists chiefly of the dyestuff, and may be put through various reactions for identification according to Rota's scheme, page 799 .

Methods of Dyeing Wool from Food Products.-The wool employed should be white worsted, or strips of white cloth, such as nun's veiling or albatross cloth. Care should be taken that the color is pure white and not the more common cream white. The woolen material should be freed from grease by boiling first in very dilute soda solution and finally in water. Strips of the woolen cloth, or pieces of the worsted this previously cleansed, are boiled in diluted filtered solutions of jams, jellies, ketchup, fruit and vegetable products, and similar food preparations, or 
in solutions of candy colors, or in wines, the clear solution of the sample to be tested being slightly acidified with hydrochloric acid.

Arata* recommends boiling the wool in a dilute solution of the food material to which potassium bisulphate has been added, using io cc. of a 10\% solution of the bisulphate to roo cc. of the solution to be tested. If the color solution is neutral, the wool should first be boiled in this before acidifying, to separate out any basic dyes. The dyed wool, after removal from the solution, is boiled first in water, and afterwards preferably in an alkali-free soap solution. It is then washed and dried. The dried fiber may then be subjected to the various reactions given in the table, pp. 806-81 3 ; for recognition of the dye, this method of identifying colors by means of reactions on the dyed fiber being one of the most con. venient.

Some of the vegetable dyes (including lichen colors), also cochineal, dye wool directly, and these may be identified by reactions given in the table with the coal-tar dyes. Other vegetable colors, and the natural colors of fruits nearly always give a slight dull coloration or stain to the wool, but this is not, as a rule, to be mistaken for the vivid hues of the coaltar dyes. Moreover most of the vegetable colors on the fiber turn green when treated with ammonia. Care should be taken to thoroughly wash the wool after the dyeing, so that colored particles simply held thereon mechanically may be removed.

Sostegni and Carpentierit recommend a method of double dyeing, applicable when acid dyes are employed. The method consists in first boiling the wool in a dilute acid solution of the food sample as above described, after which the fiber is removed and boiled, first in very dilute hydrochloric acid solution, and then in water, till free from acid. The color is then dissolved from the fiber by boiling the latter in a weak ammoniacal solution, some of the colors being more readily dissolved than others. The fiber is then removed from the solution, the latter is acidified with hydrochloric acid, and the color fixed on a fresh piece of wool by boiling therein. The second dyeing fixes coaltar and lichen colors on the fiber, but fruit colors and most others of vegetable origin remain in solution after this treatment. Any color left on the first fiber, after treatment with ammonia, is probably due to the

*Ztsch. anal. Chem., 28 (r889), 639 .

† Ibid., 35 (1896), 397 . 
natural vegetable color of the sample, and is usually no more than a dull stain.

Vegetable Colors on Wool.-In case no color is directly fixed on the fiber by boiling wool in a solution of the sample, either neutral or acid, absence of coal-tar colors may be assumed. In this case it is sometimes advisable to boil strips of previously mordanted white cotton in an acid solution of the sample, to remove certain vegetable colors for purposes of testing on the fiber. The cotton is mordanted by boiling in a dilute $(5 \%)$ solution of potassium bichromate.

Extraction of Colors from their Solution by Immiscible Solvents.Methods based on this principle are in use in the municipal laboratory at Paris.* Sangle-Ferrière uses the following method: $50 \mathrm{cc}$. of the wine or solution to be tested for color are rendered slightly alkaline by ammonia, and cautiously shaken with about ${ }_{5} \mathrm{cc}$. of amyl alcohol. If acid dyes are present, they will be dissolved, and will impart to the amyl alcohol a distinct color. $\dagger$ Basic dyes also are dissolved, but when they are present the amyl alcohol solution is colorless. Remove the amyl alcohol by means of a separatory funnel, wash with water, and finally, if the alcohol is colored, dilute with about an equal volume of distilled water and evaporate on the water-bath with a piece of white wool. The wool should be kept in the solution till the odor of the amyl alcohol has disappeared, and, if not then colored, for a short time longer, as with some colors the wool will dye more readily in the aqueous solution than in the amyl alcohol. Remove the wool, and evaporate the solution to dryness. Test for color in the dried residue, and on the fiber also.

Orchil, like the acid colors, is extracted by, and imparts a coloration to the amyl alcohol under the above conditions, the color being a light violet.

If the amyl alcohol extract after separation, washing, and filtering is colorless, acidify with acetic acid; if a basic color is present, it will be indicated by a coloration at this stage; if there is no coloration on the addition of acetic acid, no basic color is present excepting fuchsin, which is separately tested for. In case a basic dye is indicated, add distilled water and evaporate with wool as before. Test the dried residue with pure concentrated sulphuric acid.

* Girard, Analyse des Matières Alimentaires, pp. $188_{3}, 68 \mathrm{r}$.

$\dagger$ Acid fuchsin forms an exception to this rule by dissolving colorless like basic dyes. A special test is, however, given for it, p. 799 . 
Fuchsin is indicated by a yellow-brown color with sulphuric acid, which by dilution with water becomes rose; safranin, by a green color becoming first blue, then red, when diluted with water, and magdala red by a dark blue color, turning red on the addition of water.

Basic colors are also extracted readily, according to Robin, by making the solution to be tested alkaline with sodium hydroxide, and shaking with acetic ether. The solvent is removed, washed, and evaporated with wool (on which the tests are to be made), or the evaporation is carried to dryness and the tests made on the residue.

Many coal-tar colors are extracted by amyl alcohol in acid solution, but some of the natural fruit colors are also dissolved under these conditions. The coal-tar dyes thus dissolved will, however, dye wool and the fruit colors will not. Fruit colors are not extracted from acid or alkaline solution by ether, nor from alkaline solution by amyl alcohol.

Robin's method for ascertaining whether acid colors are present consists in adding to the liquid to be tested an excess of calcined magnesia, and a little $20 \%$ mercuric acetate solution, the mixture being boiled and filtered. If the filtrate is colored, or if by the addition of acetic acid to the colorless filtrate a color is developed, a coal-tar dye is indicated.

Separation of Acid and Basic Colors with Ether.*-Acid and basic colors may be separated from their dilute aqueous solution, according to Rota, by means of ether as follows: To $100 \mathrm{cc}$. of the solution add I cc. of $20 \%$ potassium hydroxide and shake in a separatory funnel with several portions of ether. Basic dyes are dissolved by the ether, leaving behind as a rule the acid colors. $\dagger$ Wash the ether extract with faintly alkaline water, and shake out with $5 \%$ acetic acid. Some colors remain in the ether, others are dissolved in the acid. Separate the two solvents, and evaporate each to dryness on the water-bath.

The acid colors left in the slightly alkaline, aqueous solution after removal of the basic colors by ether as above, may, if desired, be separated into several groups by successive extraction, as follows: first slightly acidulate with acetic acid and extract with ether, then acidify with hydrochloric acid and again extract, and finally examine the residual solution for colors that are insoluble in ether. Thus erythrosin and eosin are soluble in ether when shaken with their aqueous solution made acid with hydrochloric acid, while acid fuchsin is insoluble.

*Analyst, 24, p. 45 .

† A few acid dyes are exceptional in being soluble in ether with alkali, as for example, quinolin yellow and the sudans. 
Separation of Colors from Dried Food Residues by Solvents.-This me.hod is rarely employed, excepting in the case of colors insoluble in water, but soluble in e.her or alcohol. The dried pulp of canned vegetables, ketchups, etc., may be acidified wi.h hydrochloric acid, and the color extracted therefrom direcily" wi.h alcohol. In this case however, there is no obvious advantage over the previous methods of dyeing the fiber directly in the acid solution of the sample.

Girard's Tests for Acid Fuchsin.*-Add 2 cc. of $5 \%$ potassium hydroxide to $10 \mathrm{cc}$. of the wine or other solution to be tested, or enough of the alkali to neutralize the acid. Then add $4 \mathrm{cc}$. of $10 \%$ acetate of mercury and filter. The filtrate should be alkaline and colorless. If the solution remains uncolored after acidifying with dilute sulphuric acid, no acid fuchsin is present. If, however, there is produced a red to violet coloration, and no other coal-tar colors have been found by the amyl alcohol extraction, the presence of acid fuchsin is shown.

Beilier's Test for Acid Fuchsin.-Presence of acid fuchsin is indicated by adding to $20 \mathrm{cc}$. of wine or other solution to be tes ed aboul 4 grams of freshly precipitated yellow oxide of mercury, boiling and filtering. The filtrate, if acid fuchsin is present, is colored red, tinged with violet.

According to Blarez, all red coal-tar colors, with the exception of acid fuchsin, and all red vegetable colors are completely decolorized by acidulating their aqueous solution with tartaric acid, and digesting with dioxide of lead. $\dagger$

Schemes for Idéntification.-These serve for identifying unknown colors by their characteristic reactions, first grouping them into classes, and finally ascertaining the particular color itself. Of these may be mentioned the tabular schemes of Witt, $\ddagger$ Weingartner, \& Green,\| Martinon, $\uparrow$ and Rota.**

Rota's Scheme is one of the latest, and on some accounts the best, being based on the relation between the color and the composition of

* Analyse der Substances Alimentaires, p. 185 .

† Allen, Commercial Org. Analysis, 4 Ed., Vol. V, p. 250.

‡ Zeits, anal. Chem., 1887, 26, p. 100; Analyst, II, p. III.

$\$$ Jour. Soc. Dyers, etc., III, p. 67.

II Jour. Soc. Chem. Ind., 12, No. I.

โ Jour. Soc. Dyers, 3, p. 124.

** Chem. Zeit., 1898, pp. 437-442; Anal., 24, p. 4r. 
the dyes. The colors are divided into two main groups, according to whether or not they are reducible by stannous chloride. These two groups are each further subdivided into two classes, the reducible colors being classed according to whether the color remains unchanged, or is restored by treatment with ferric chloride, and the non-reducible colors according to their action with potassium hydroxide.

The tests are carried out on a dilute aqueous or alcoholic solution of the coloring matter, the strength being about $\mathrm{I}$ in 10,000 . Treat about $5 \mathrm{cc}$. of this solution with 4 or 5 drops of concentrated hydrochloric acid and about as much stannous chloride in a test-tube, shake the mixture, and heat if necessary to boiling. With some colors the process of decolorization is a slow one, especially if the solution is too concentrated, and it is well to repeat the experiment, if in doubt, diluting the original sample still further with water. Tin in solution in concentrated hydrochloric acid may be employed instead of stannous chloride, if desired.

Here, as in all cases of color testing, it is well to make comparative tests with known colors.

\section{CLASSIFICATION OF ORGANIC COLORING MATTERS.}

[A portion of the aqueous or alcoholic solution is treated with $\mathrm{HCl}$ and $\mathrm{SnCl}_{2}$.]

Complete decolorization. Reducible coloring matters. Colorless solution is treated with $\mathrm{Fe}_{2} \mathrm{Cl}_{6}$, or shaken with exposure to air.
The color changed no further than with $\mathrm{HCl}$ alone. Nonreducible colors. A part of original solution is mixed with $20 \% \mathrm{KOH}$ and warmed.

The liquid remains unchanged. Coloring matters not reoxidizable.

\section{Cr.Ass I.}

Nitro, nitroso, and azo colors, including oxyazo and hydrazo colors.

Picric acid, naphthol yellow, ponceau, Bordeaux, and Congo red.
The original color restored. Reoxidizable coloring matters.

\section{Class II.}

Indogenide and imidoquinone coloring matters, methylene blue, safranin, indigo carmine.
Decolorization, or a precipitate. Imidocarbo-quinone coloring matters.

\section{Cr.ass III.}

Amido-derivatives of $\mathrm{di}$ and triphenyl methane, auramins, acridins, quinolins, and color derivatives of thio benzenil. zuramin.
Fuchsin, rosanilin,
No precipitation. Liquid becomes more colored. Oxycarbo-quinone coloring matters.

Class IV.

Nonamide diphenyl methane, oxy-ketone, and most of natural organic coloring matters.

Eosins, aurin, alizarin. 


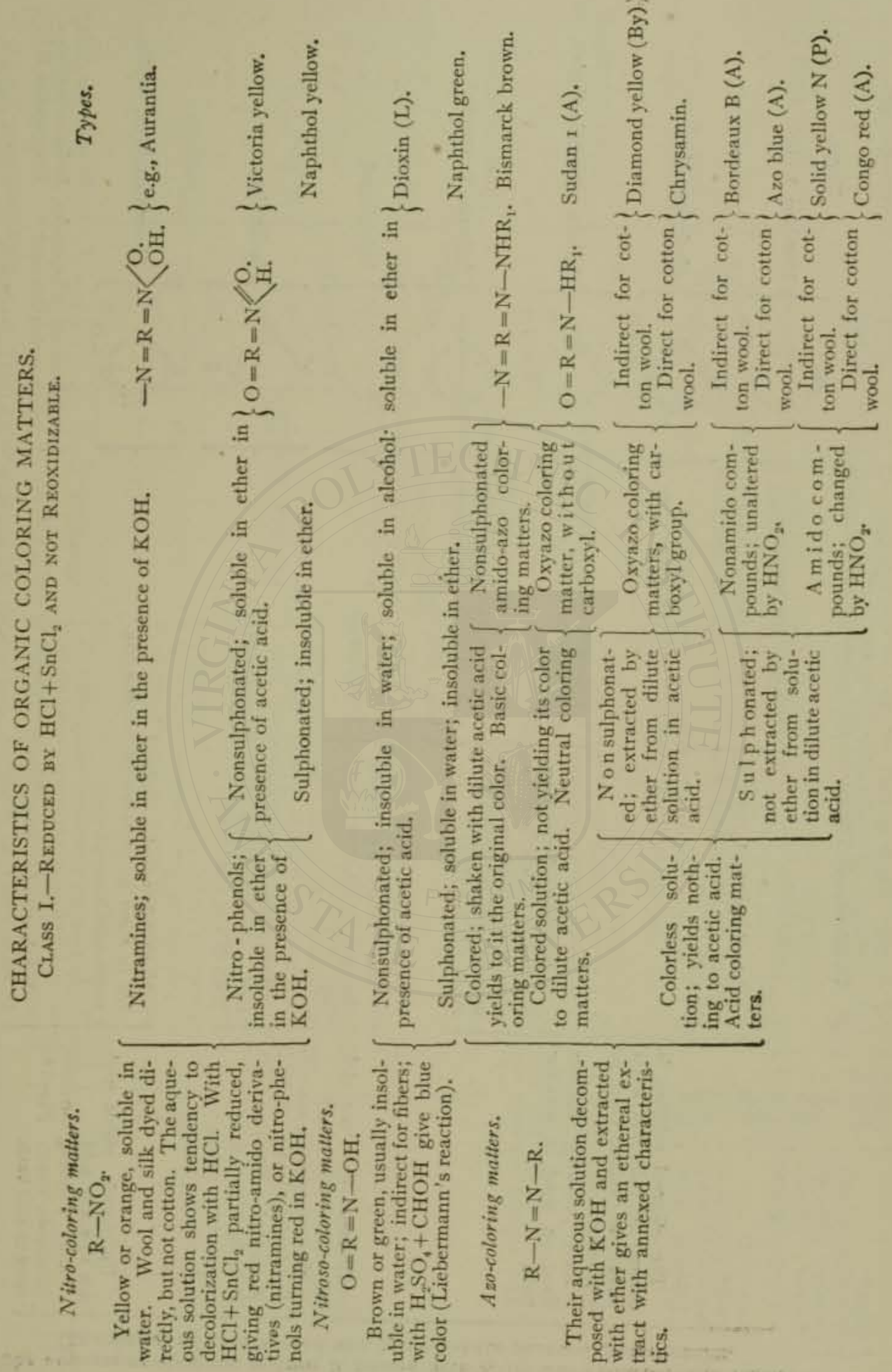




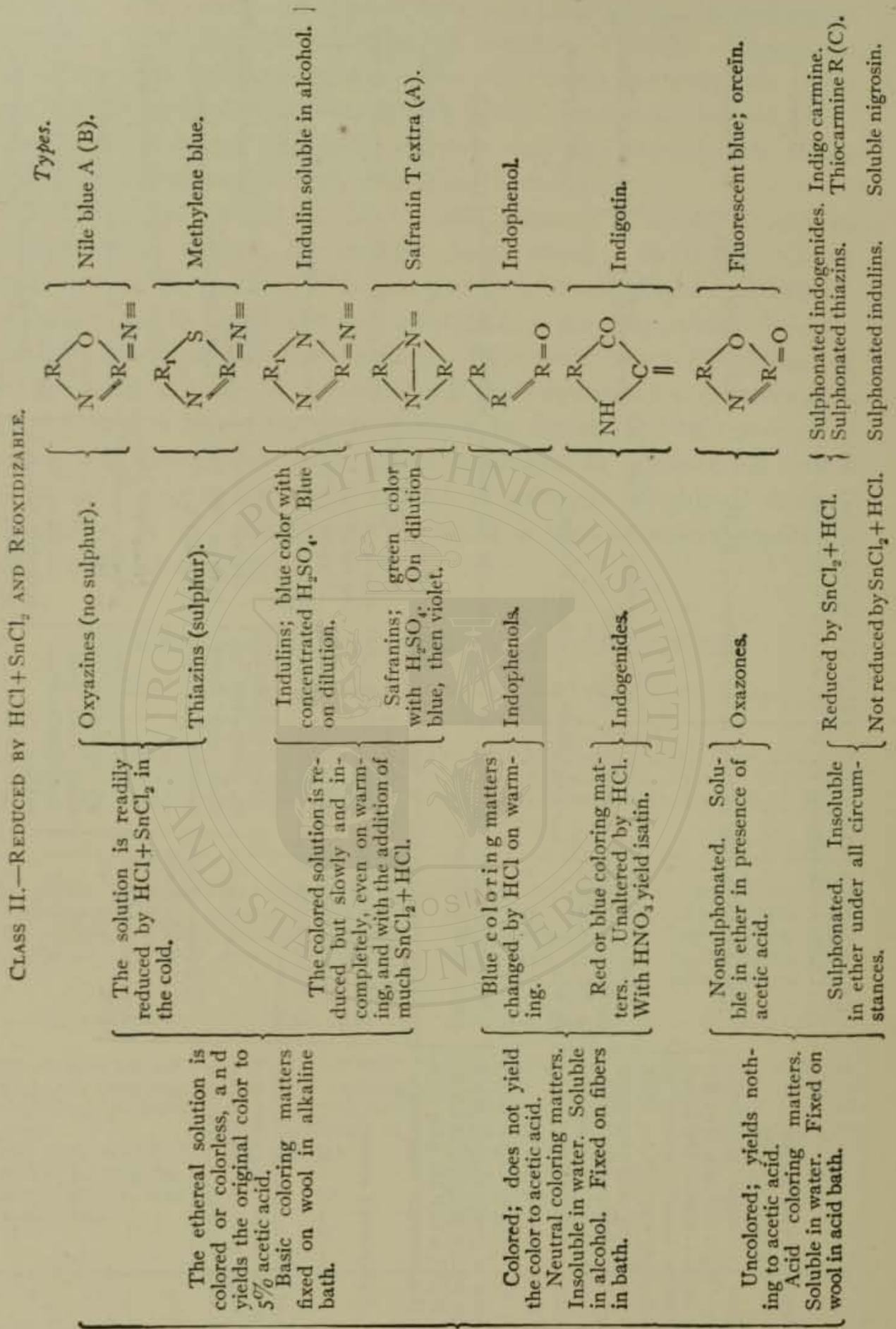

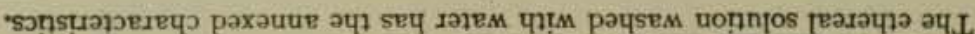

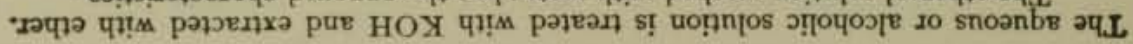




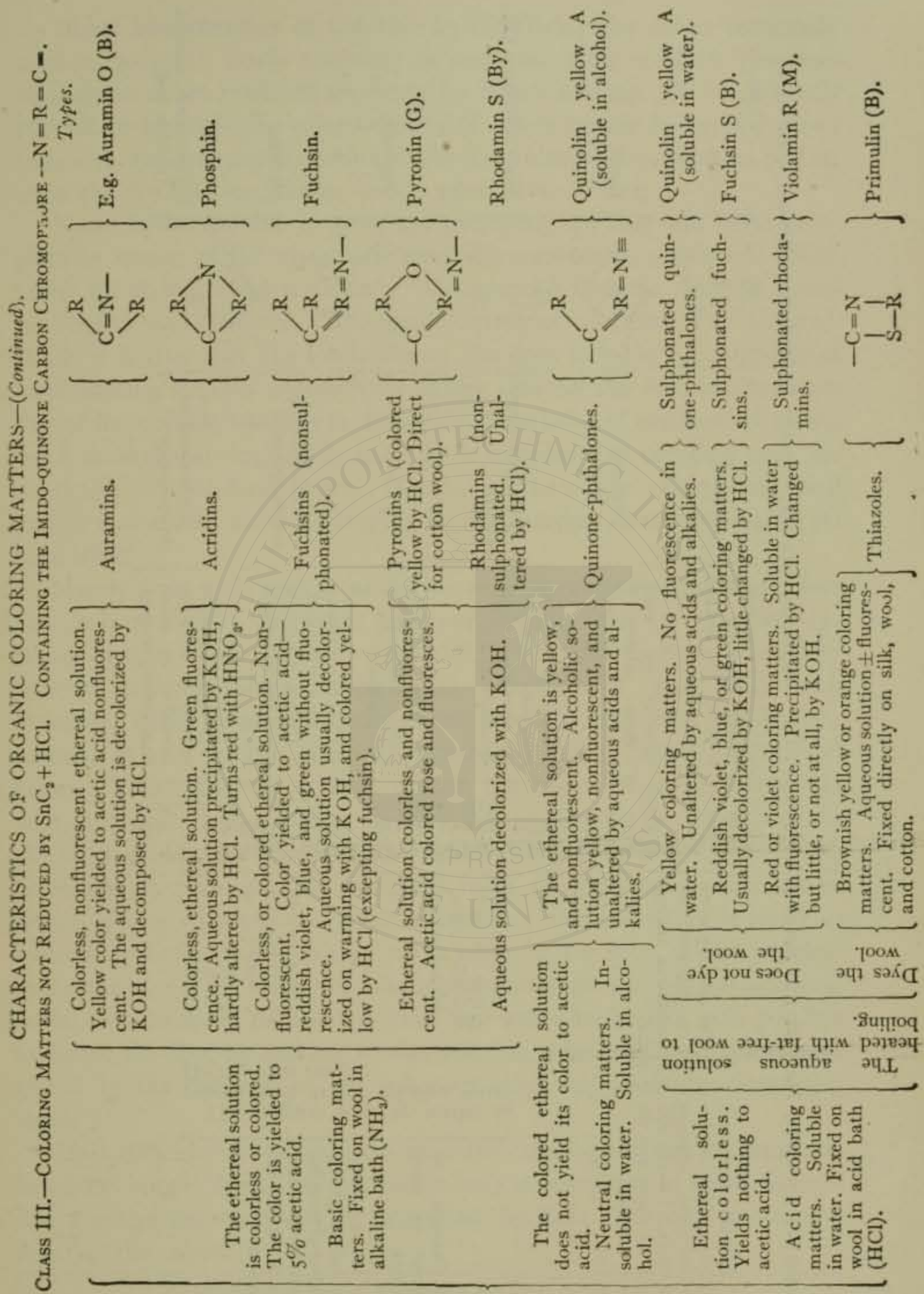

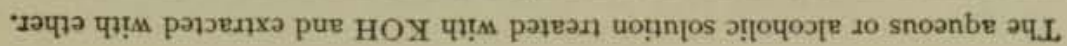




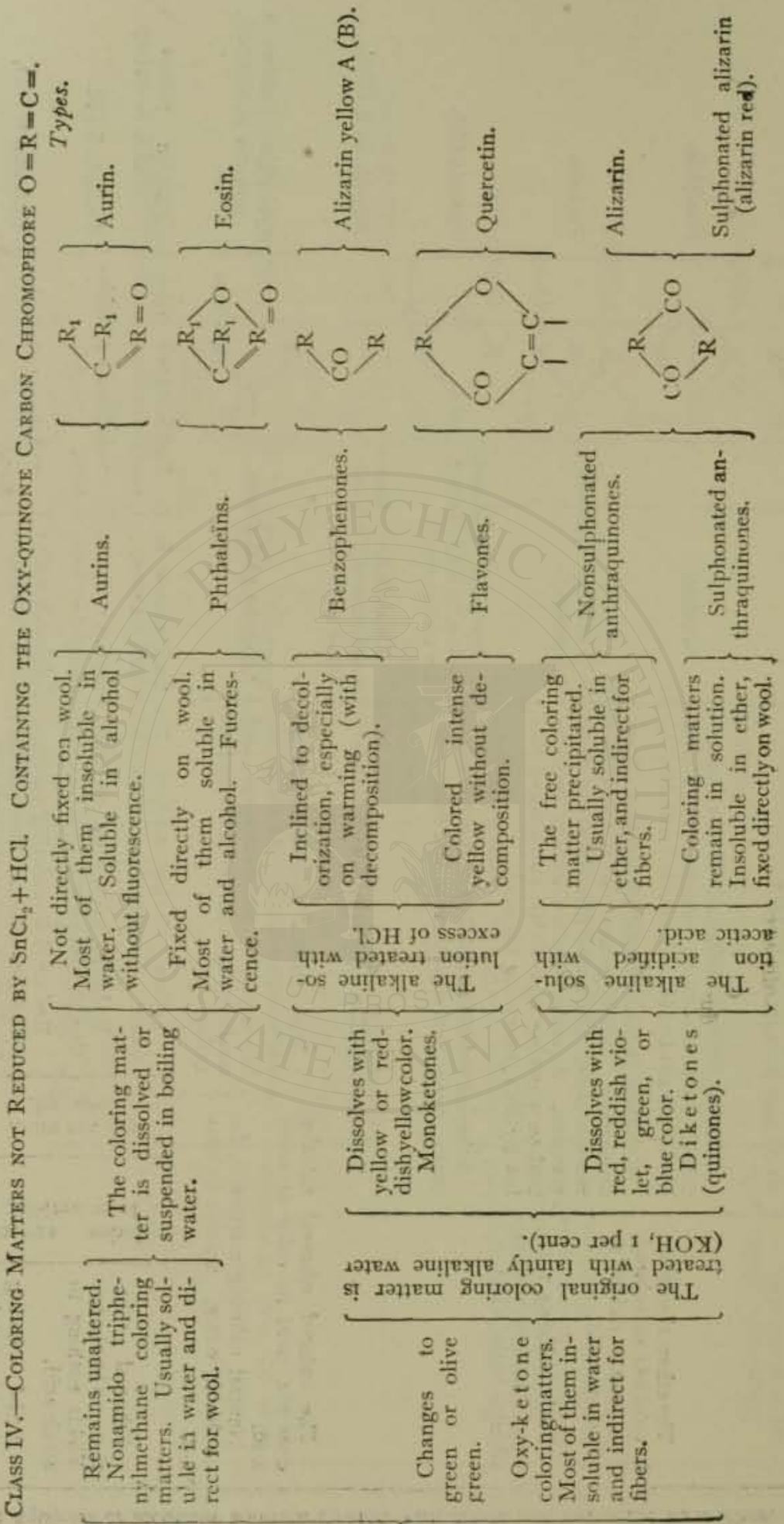

(ooor:1) ${ }^{n} 12^{3}$ ə.

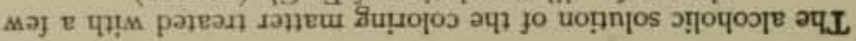


Direct Identification of Colors.-In identifying the colors commonly used in food, it is rarely necessary to carry out such involved processes of analyses as are rendered necessary by Rota's scheme. It is frequently possible to ascertain the color or group of colors present by making direct tests with various reagents, either on the dyed fiber as described on p. 8I4; or on the dry coloring matter, or in a solution containing it.

Many tables for this purpose are prepared, but they are never complete by reason of the many new dyestuffs constantly introduced. Such tables are to be found in Allen's "Commercial Organic Analysis," and in Schuiltz and Julius's "Systematic Survey of the Coal Tar Colors." While it is true that the limitation of the dyes suitable for purposes of food coloring imposes a somewhat lighter task on the food analyst than that of the chemist who has to deal with all varieties of commercial colors, yet it is obviously impossible to make a complete list covering even the restricted field of food colors. Doubtless there are colors long well known that would serve admirably for this purpose, but have never yet been tried.

Mainly from such sources as the above comprehensive tables of colors and their reactions, the writer has compiled the table on pp. 806-13, taking as a basis the scheme of Allen.* This table includes over fifty selected coloring matters, which are adapted for, and have been found in, foods by various analysts, as listed in state and government reporls, as well as in laws of various countries dealing with food. colors. This table will at least contain the colors most commonly met with, and will nearly always serve, if not to identify the exact dye, to aid in classifying it. In case the analyst wishes to identify the color, he should be provided, for standards, with as complete a collection of known purity dyestuffs as possible covering the colors he is likely to meet with in foods, and should make comparative tests, if the slightest doubt exists. If the unknown color is apparently not found in the following table, and the more exhaustive tables are unavailable, it is still possible to locate the dye, by making similar tests on other standard colors suggested by the behavior of the unknown color, and carefully comparing them.

Most difficulty is encountered when the coloring matters are mixtures instead of simple dyes. In this case it may be necessary to resort to fractional extraction by ether, as suggested by Rota (p. 799), in order to. separate the colors.

\footnotetext{
- Commercial Organic Analysis, 4 Ed., Vol. V, p. 540 et seq.
} 


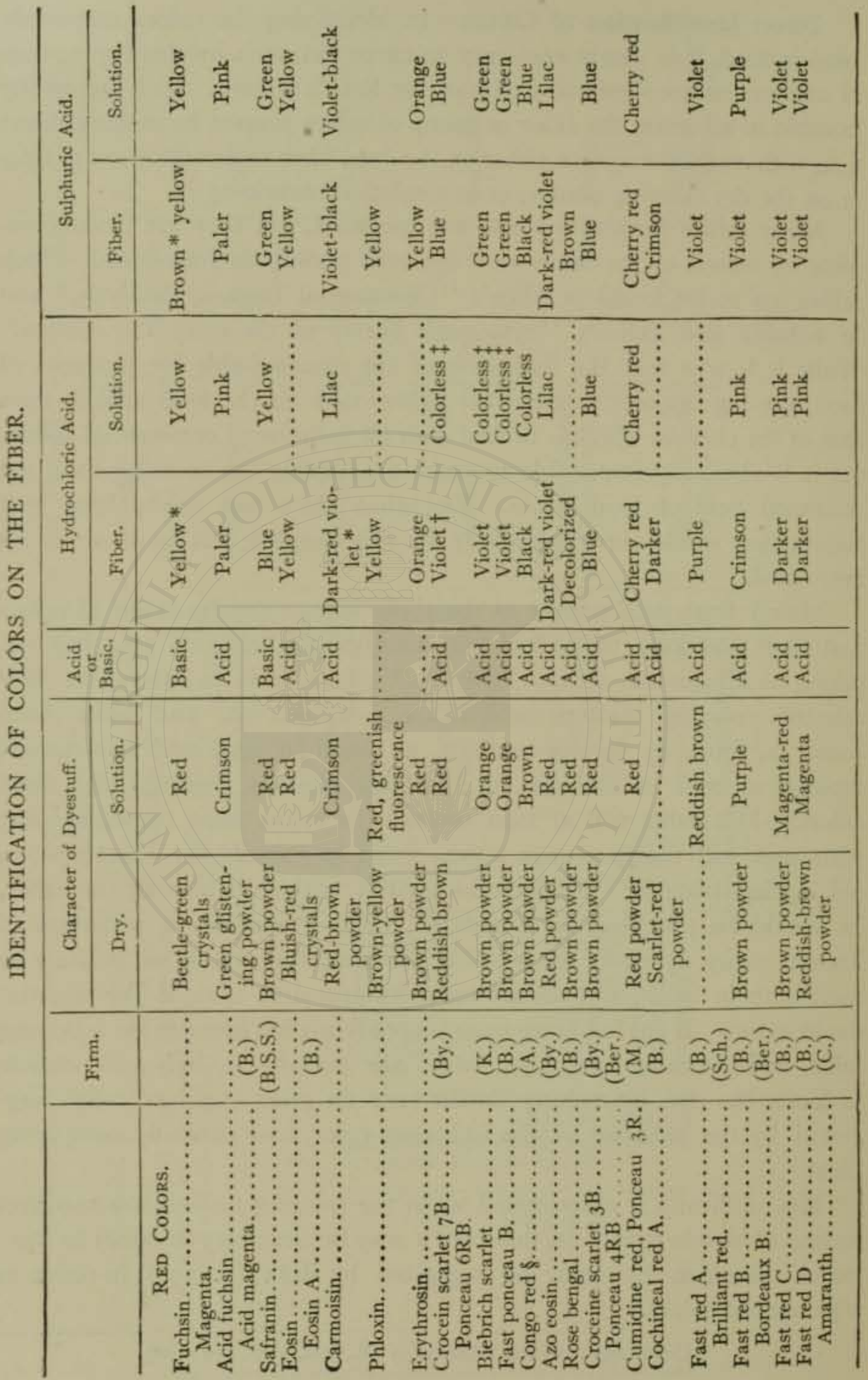




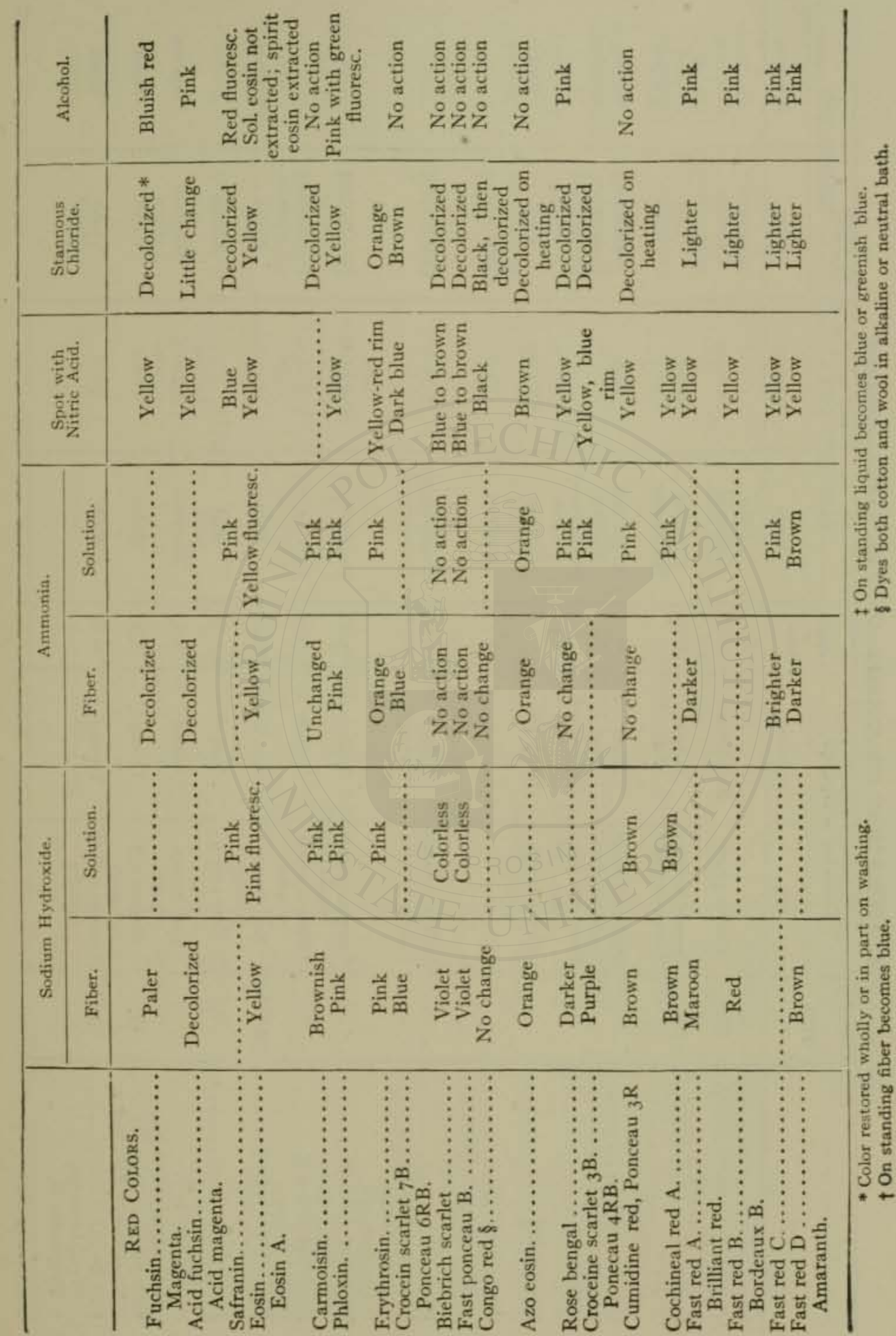




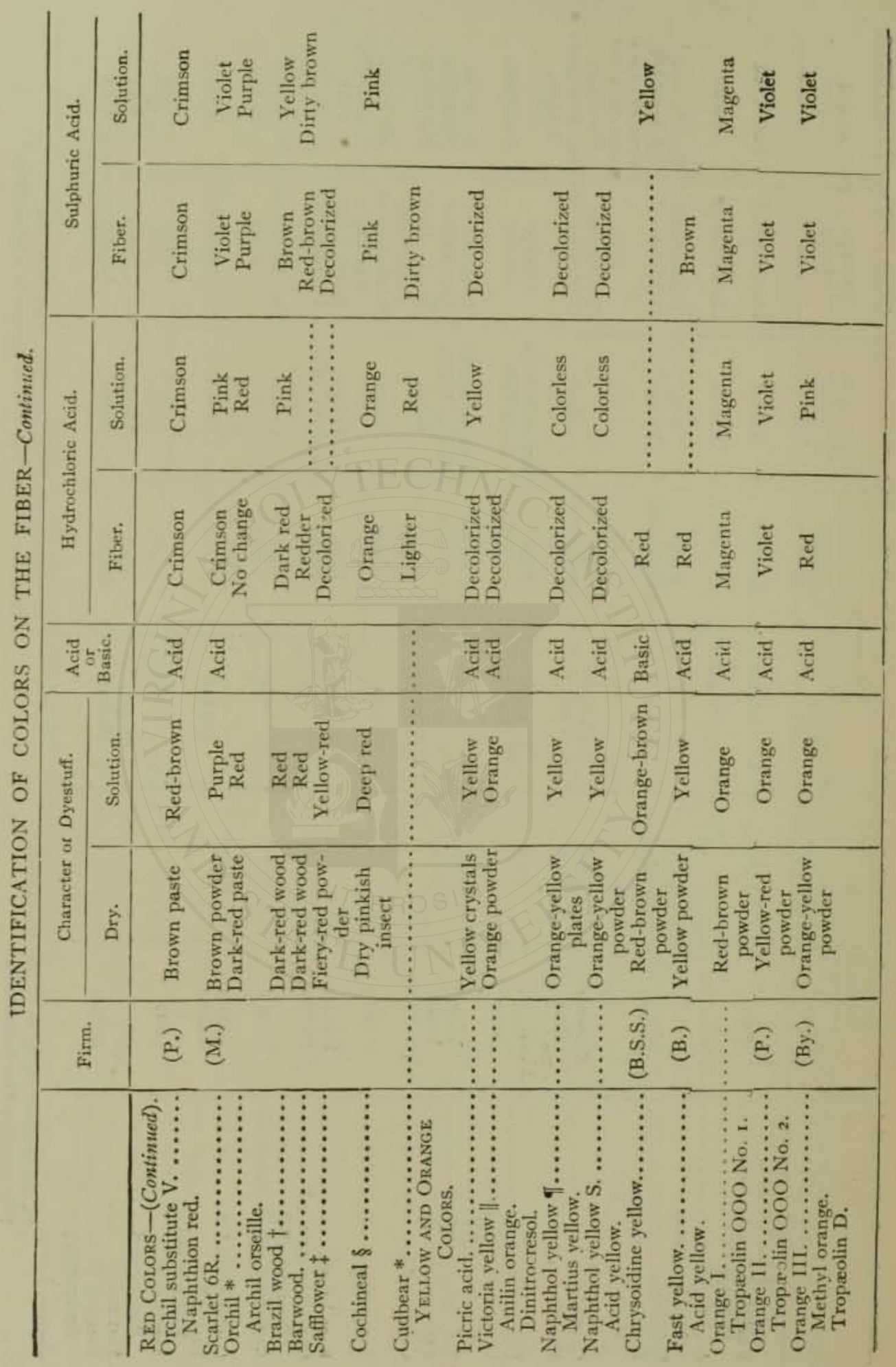




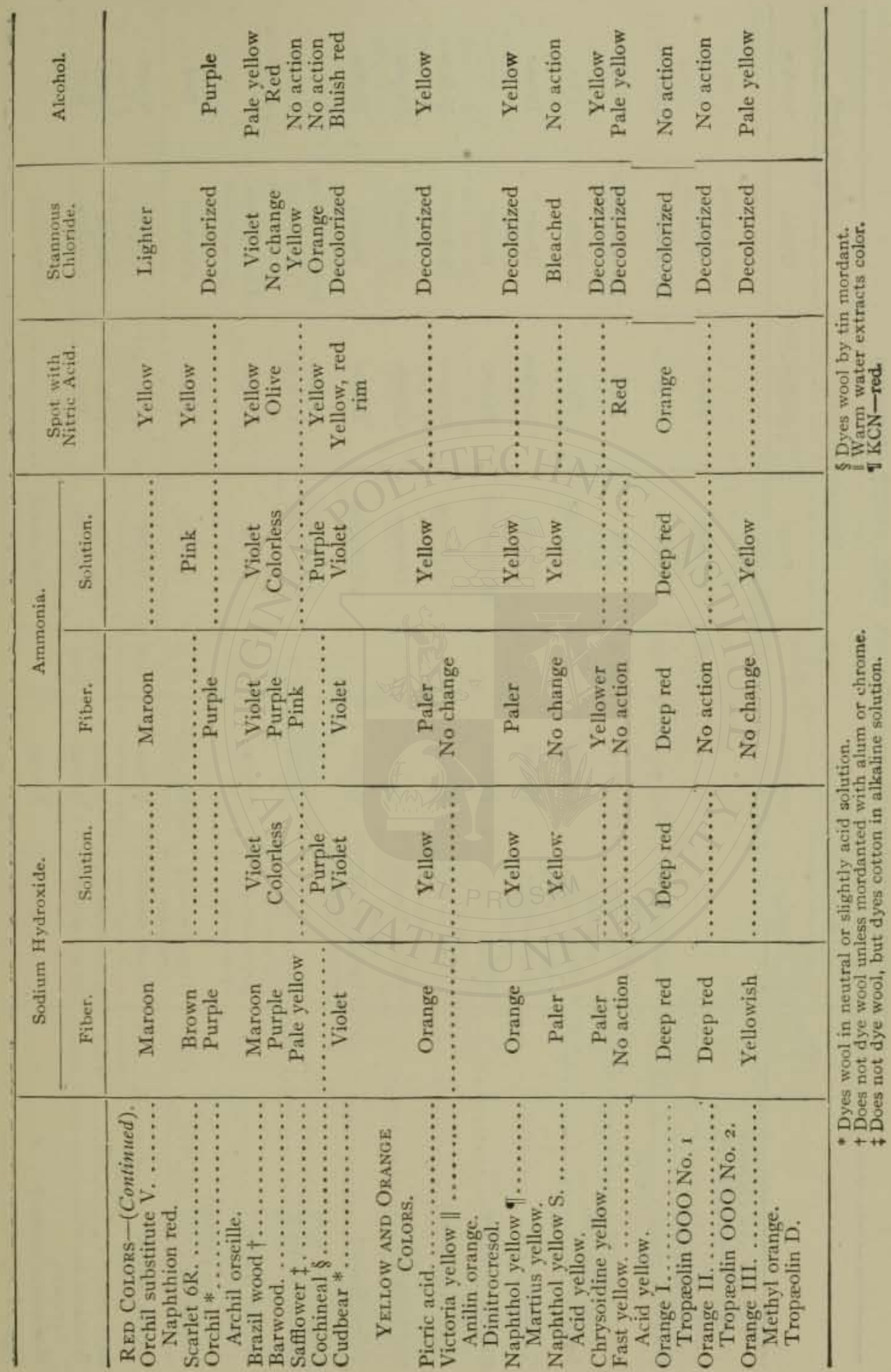




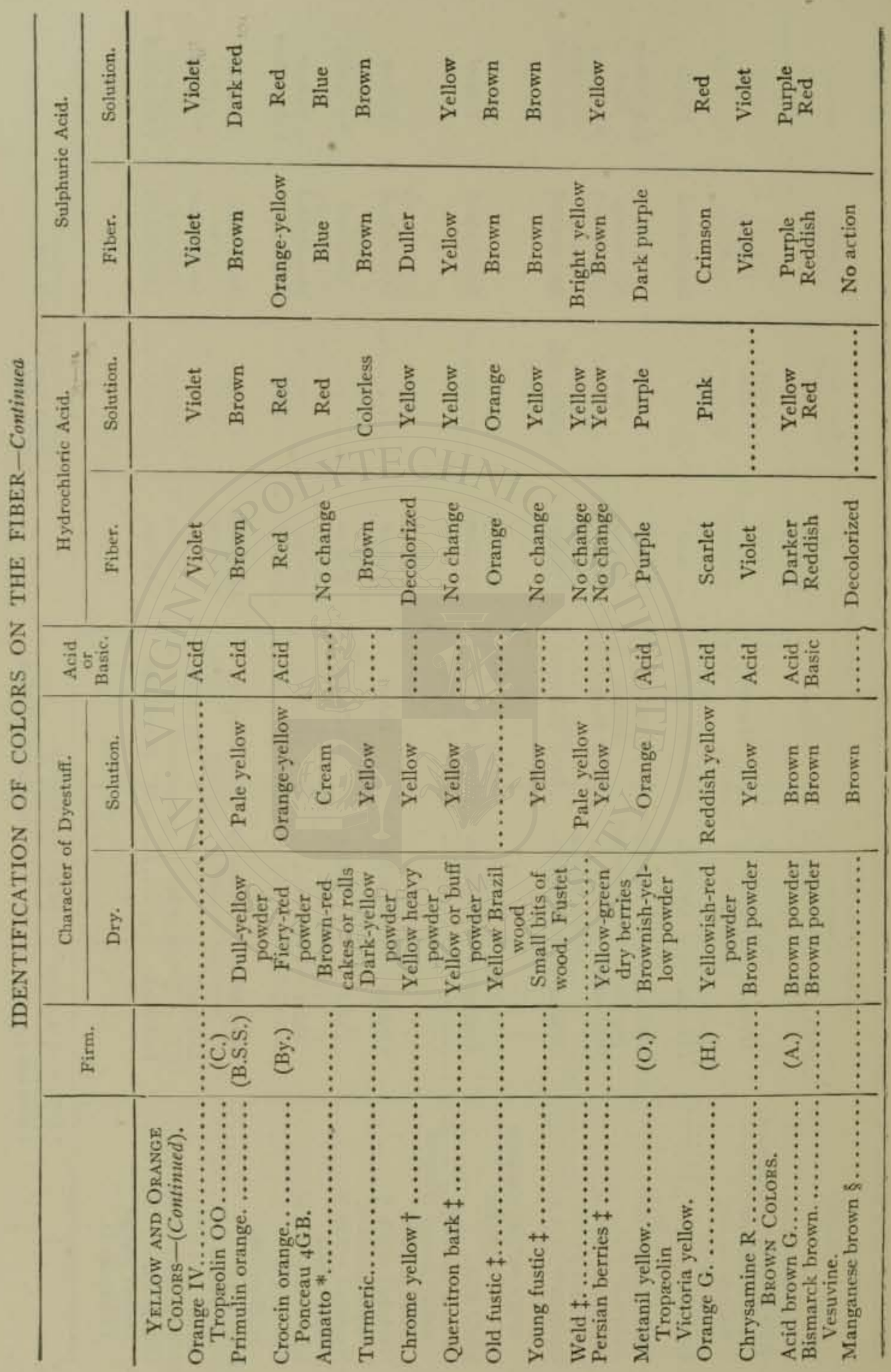


ARTIFICIAL FOOD COLORS.

811

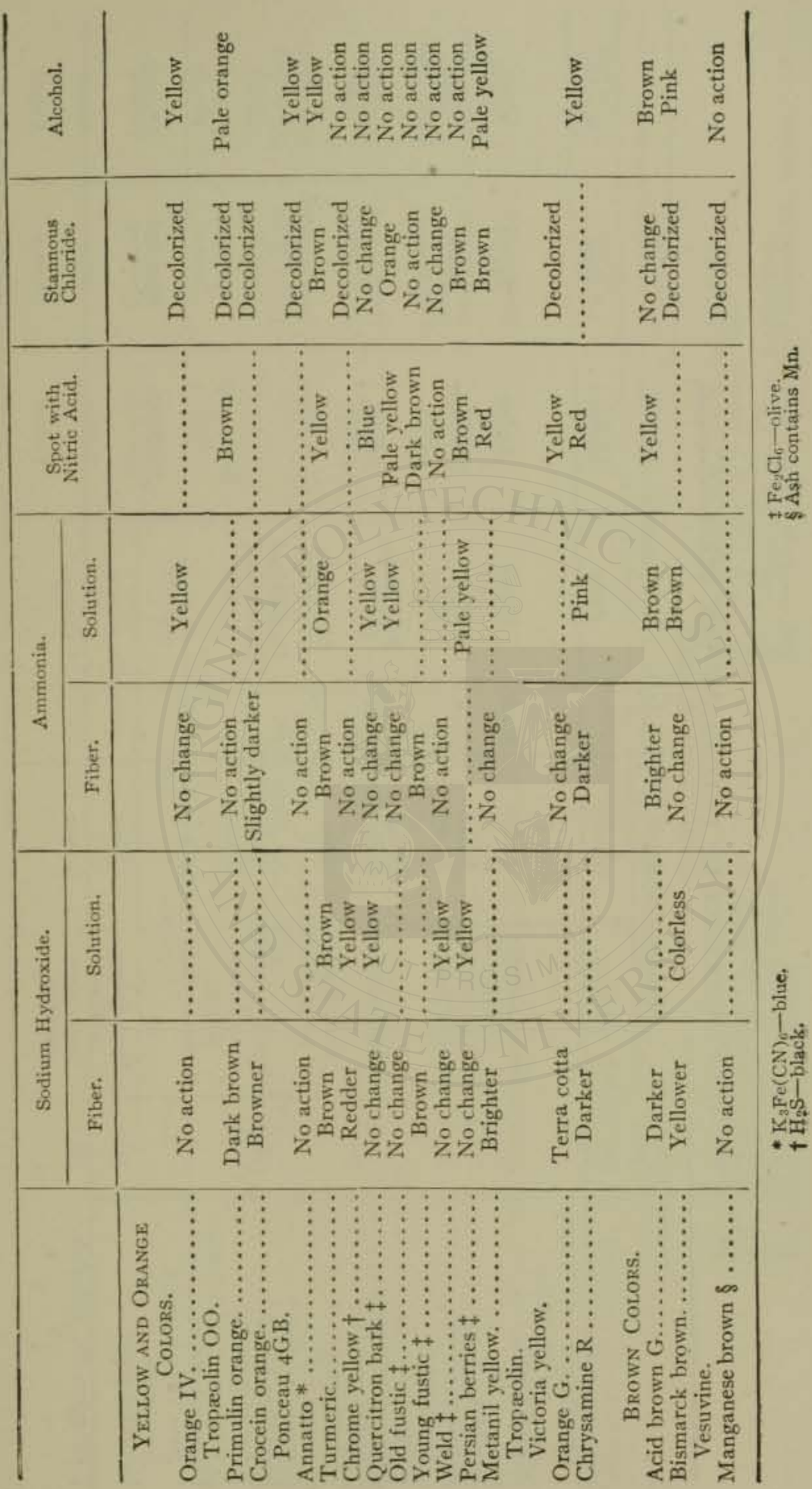




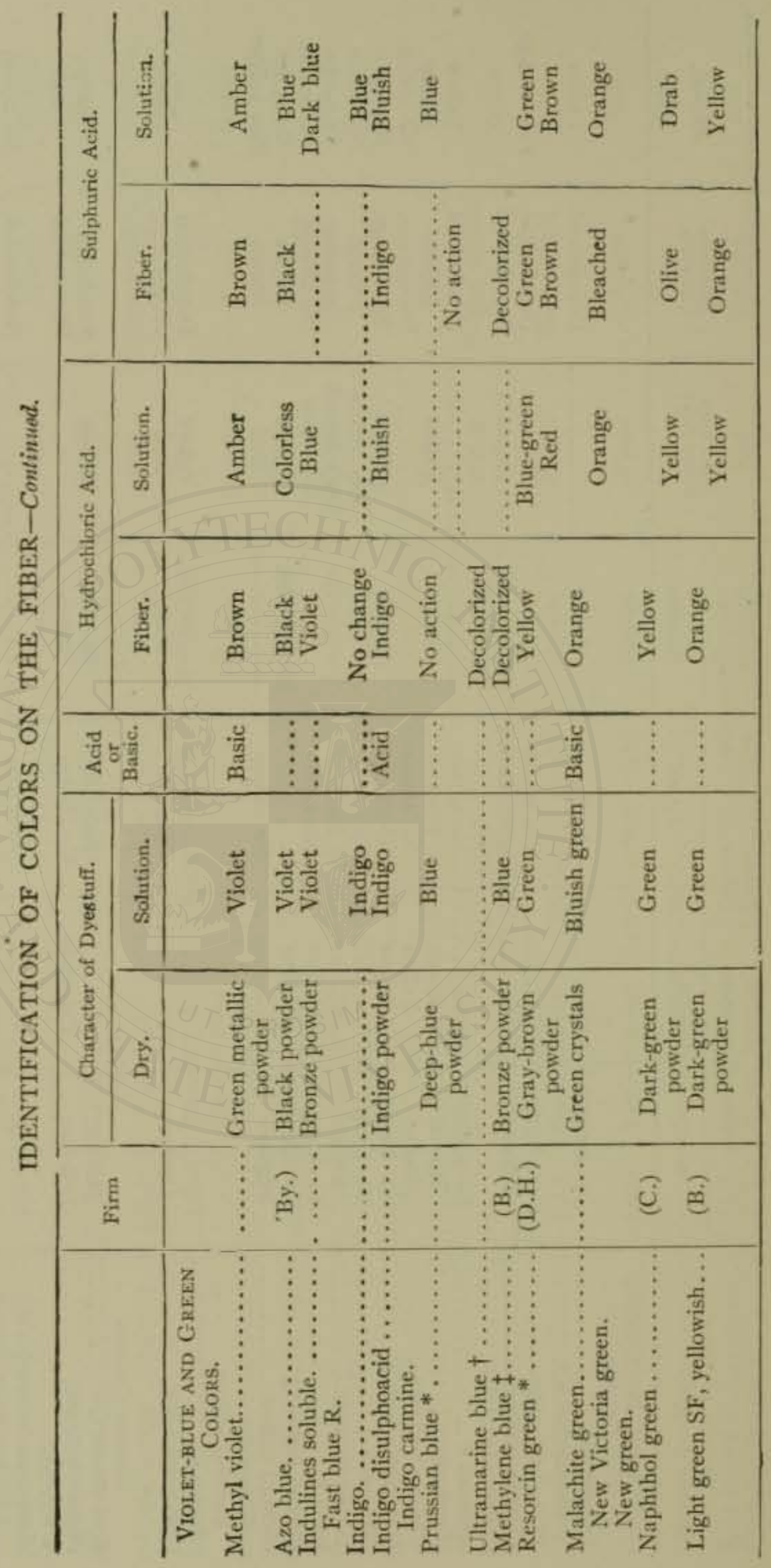


ARTIFICIAL FOOD COLORS.

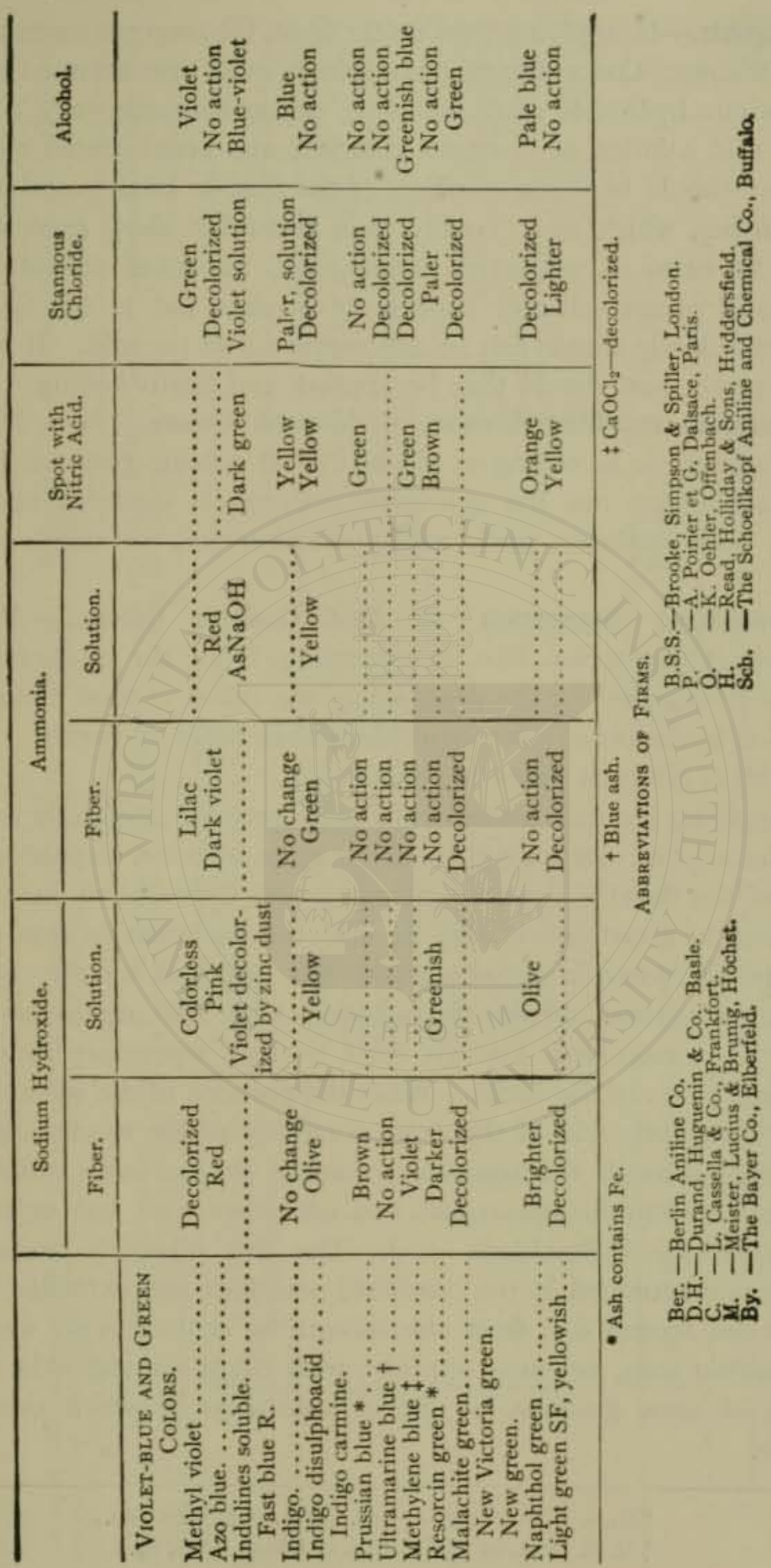


Reagents.-In applying tests on the fiber, the reagents commonly used are as follows: Concentrated hydrochloric acid, concentrated sulphuric acid, sodium hydroxide (10\% solution), strong ammonia ( $28 \%$ ), a hydrochloric acid solution of stannous chloride, and concentrated nitric acid. The tests should be made on pieces of the fiber in small porcelain evaporating-dishes, which more readily than test-tubes show exact shades of color. In cases of suspected fluorescence, test-tubes should be used. Nitric acid is conveniently applied by a glass rod to the fiber. The stannous chloride should first be allowed to act in the cold. If no change occurs, gentle heat should then be applied, and finally boiling.

Separation and Identification of Allowed Colors.-Price Method.*The procedure is according to the analytical scheme given on page $81_{5}$. As a preliminary test the powdered material is scattered upon water. alcohol, and sulphuric acid, noting whether one or more colors are present.

Quantitative Separation of Acid Coal-tar Colors. - Mathewson Method. $\uparrow-T h i s$ process, like the preceding, is for the colors themselves, but may be adapted for the detection of the colors in food products after separation by means of solvents or less satisfactorily by dyeing. Mathewson's table is given on pages 816 and 817 .

In applying the data given in the table proceed essentially as follows: Treat the solution containing 0.2 to 0.4 gram of color (depending on the nature of the dyes) with sufficient water and hydrochloric acid to bring its volume to about $5 \circ \mathrm{cc}$. and its acid concentration to that point for which the difference in percentage of color extracted for the two dyes is near its maximum. Shake the solution with the immiscible solvent, passing it in succession through three or four separatory funnels each containing $50 \mathrm{cc}$. of the latter. Wash the portions of the solvent with 50 cc. of hydrochloric acid of the same normality as the solution, passing it successively through the separatory funnels in the same order as was the original solution, and repeat this operation with one or two fresh amounts of the hydrochloric acid. The dye relatively more soluble in water is determined in the combined washings and extracted solution. Remove the second dye from the solvent by shaking with water, very dilute caustic soda, or, more quickly, with dilute caustic soda after the addition of some gasoline, or similar substance in which the color is insoluble.

*U. S. Dept. of Agric,, Bur. of An. Ind., Circ. 180.

$\dagger$ U. S. Dept. of Agric., Bur. of Chem., Circ. 89. 
ARTIFICIAL FOOD COLORS.

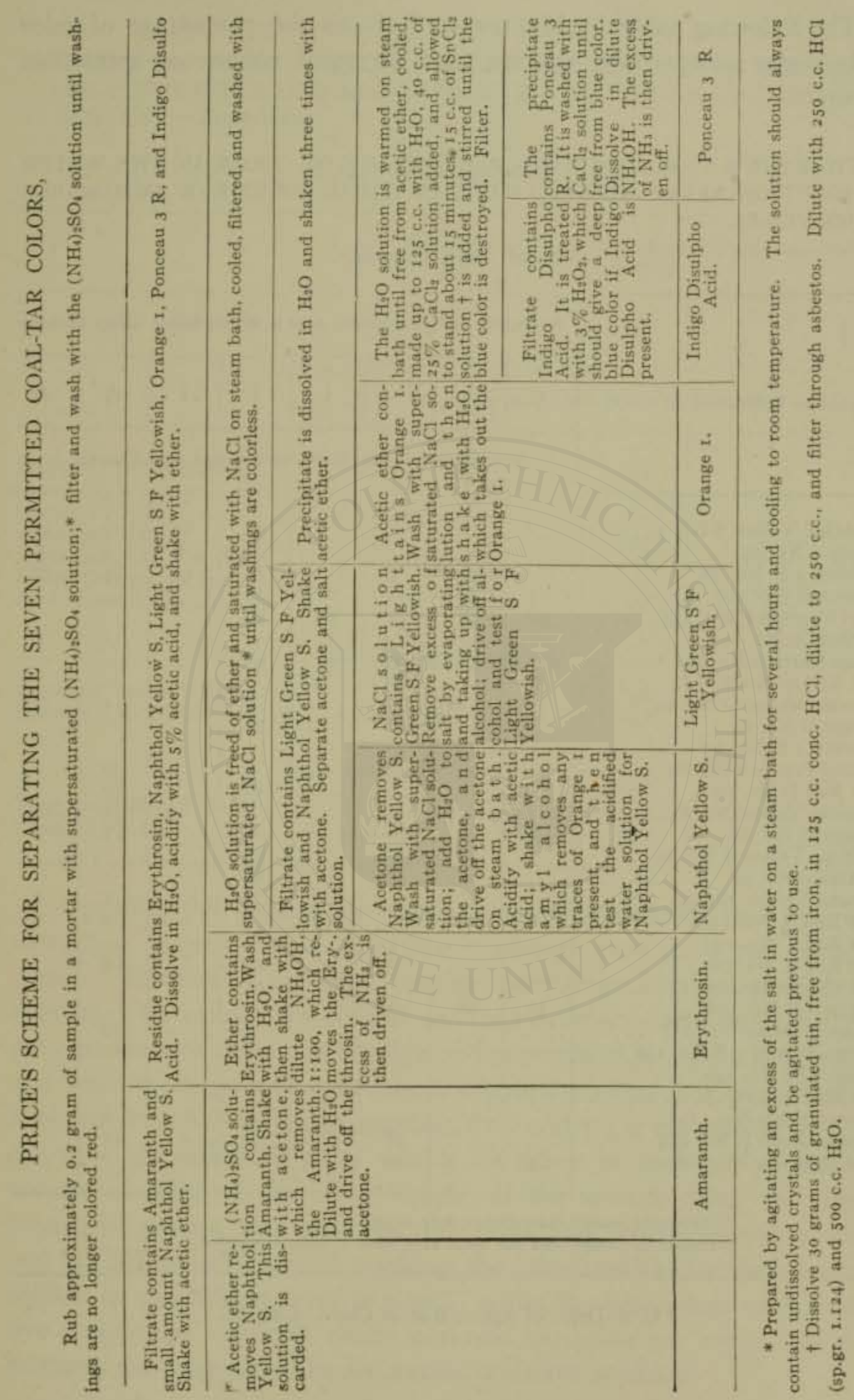


The following table by Mathewson * shows the percentage of color in the water solution after shaking with an equal volume of immiscible solvent.

\section{MATHEWSON'S TABLE SHOWING PERCENTAGE OF COLOR IN THE WATER SOLUTION AFTER SHAKING WITH AN EQUAL VOLUME OF IMMISCIBLE SOLVENT.}

SOLVENT: AMYL ALCOHOL.

\begin{tabular}{|c|c|c|c|c|c|c|c|c|}
\hline \multirow{3}{*}{ Colors. } & \multicolumn{8}{|c|}{ Normality of Hydrochloric Acid in Water Layer before Shaking } \\
\hline & 4 & 2 & I & $\frac{1}{2}$ & $\frac{1}{4}$ & $\frac{1}{8}$ & $\frac{1}{16}$ & $\frac{1}{32}$ \\
\hline & \multicolumn{8}{|c|}{ Percentage of Color in Water Solution after Shaking. } \\
\hline Naphthol Yellow S No. 4... & , & $\ldots \ldots$ & 4 & 7 & II & $x_{7}$ & 27 & 43 \\
\hline Orange I No. $85, \ldots \ldots \ldots$. & s. & $\ldots \ldots$ & $\ldots$ & $f$ & 0.5 & $\mathrm{I}$ & 2 & $\begin{array}{r}43 \\
4\end{array}$ \\
\hline Ponceau $3 \mathrm{R}$ No. 56 ............ & ....... & $\mathbf{I}$ & 3 & 7 & 21 & 43 & 64 & 78 \\
\hline Amaranth No. $107 \ldots . . . . . . .$. & 5 & 15 & 52 & 82 & 93 & & & \\
\hline Light Green S F No. $435 \ldots \ldots$. . & 90 & 95 & 97 & 99 & 99 & & & \\
\hline Erythrosin No. $517 \ldots \ldots \ldots \ldots$ & $\ldots \ldots$ & ....... & & & & & & $\circ$ \\
\hline Indigo Carmin No. $692 \ldots . .$. & 34 & $5^{1}$ & 89 & 96 & 99 & & & \\
\hline Fast Yellow No. 8 ............ & 36 & & 61 & & & & & \\
\hline Crocein Orange G No. $13 \ldots . .$. & $\ldots \ldots$ & & & & & & I & 2 \\
\hline Orange G No. $14 \ldots . . . . . . .$. & $\ldots \ldots$ & $\ldots . .$. & 18 & & 58 & & & \\
\hline Ponceau 2 R No. $55 \ldots . . . .$. & $\ldots$ & 2 & ...... & I 4 & & & & \\
\hline Crystal Ponceau No. $64 \ldots \ldots$. & $\ldots$ & $\ldots$ & & & 8 & & 39 & \\
\hline Fast Red B No. $65 \ldots \ldots \ldots \ldots$ & & $\cdots$ & 2 & & 16 & & 62 & \\
\hline Resorcin Yellow No. $84 \ldots . .$. & $\ldots \ldots$ & ...... & $\ldots \ldots$ & & 5 & & II & 17 \\
\hline Orange II No. 86 & $\ldots$. & $\ldots$ & $\ldots$ & & 6.2 & & 2 & 3 \\
\hline Brilliant Yellow S No. $80, \ldots \ldots$. & $\ldots$. & ..... & 73 & & 90 & & & \\
\hline Tartrazin No. $94 \ldots \ldots \ldots \ldots$ & $\cdots \cdots$ & .... & 47 & & 88 & & & \\
\hline Metanil Yellow No. $95 \ldots \ldots$. & 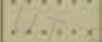 & & W. & & & & I & $\mathrm{I}$ \\
\hline Fast Red A No, ro2 ............. & $5 \%$ & $\ldots$ & & & & & 0.2 & I \\
\hline Fast Red C No, $\mathrm{IO}_{3} \ldots . . . . . .$. & . & . & I & & 4 & & & \\
\hline Fast Red E No. ro $5 . . .$. & $6 . .6$ & $\ldots . .$. & $\mathrm{I}$ & & $1 \frac{4}{7}$ & & 68 & \\
\hline New Coccin No. 106.............. & $\ldots$ & I5 & & & 75 & & & \\
\hline Scarlet $6 \mathrm{R}$ No. 108 ............... & $4 t$ & 80 & 95 & & & & & \\
\hline Resorcin Brown No. $137 \ldots \ldots$. . & ...... & …..... & ...... & & 8 & & 20 & \\
\hline Cotton Scarlet 3 B No. $146 \ldots$. & $\ldots \ldots$ & $\ldots \ldots$ & $\ldots \ldots$ & & 2 & & Io & \\
\hline Congo Red No. $240^{\circ} \ldots \ldots \ldots$ & & & & & & & & \\
\hline Azo Blue No. $287 \dagger \ldots \ldots \ldots \ldots$ & & & & & & & & \\
\hline Chrysophenin No. $329 . . . . . . .$. & $\ldots \ldots$ & & & & & & $\mathrm{I}$ & 2 \\
\hline Guinea Green B No. 433 . . . . . . . & $\ldots \ldots$ & …. & 48 & & 32 & & 25 & \\
\hline Acid Magenta No. $462 . . . . . .$. & 75 & & 93 & & & & & \\
\hline
\end{tabular}

* Color acid nearly insoluble in both layers.

† Similar to Congo Red but color acid more soluble in alcohol.

* U. S. Dept. of Agric., Bur, of Chem., Circ. 89 . 
MATHEWSON'S TABLE SHOWING PERCENTAGE OF COLOR IN THE WATER SOLUTION AFTER SHAKING WITH AN EQUAL VOLUME OF IMMISCIBLE SOLVENT-(Continued).

SOLVENT: DICHLORHYDRIN.

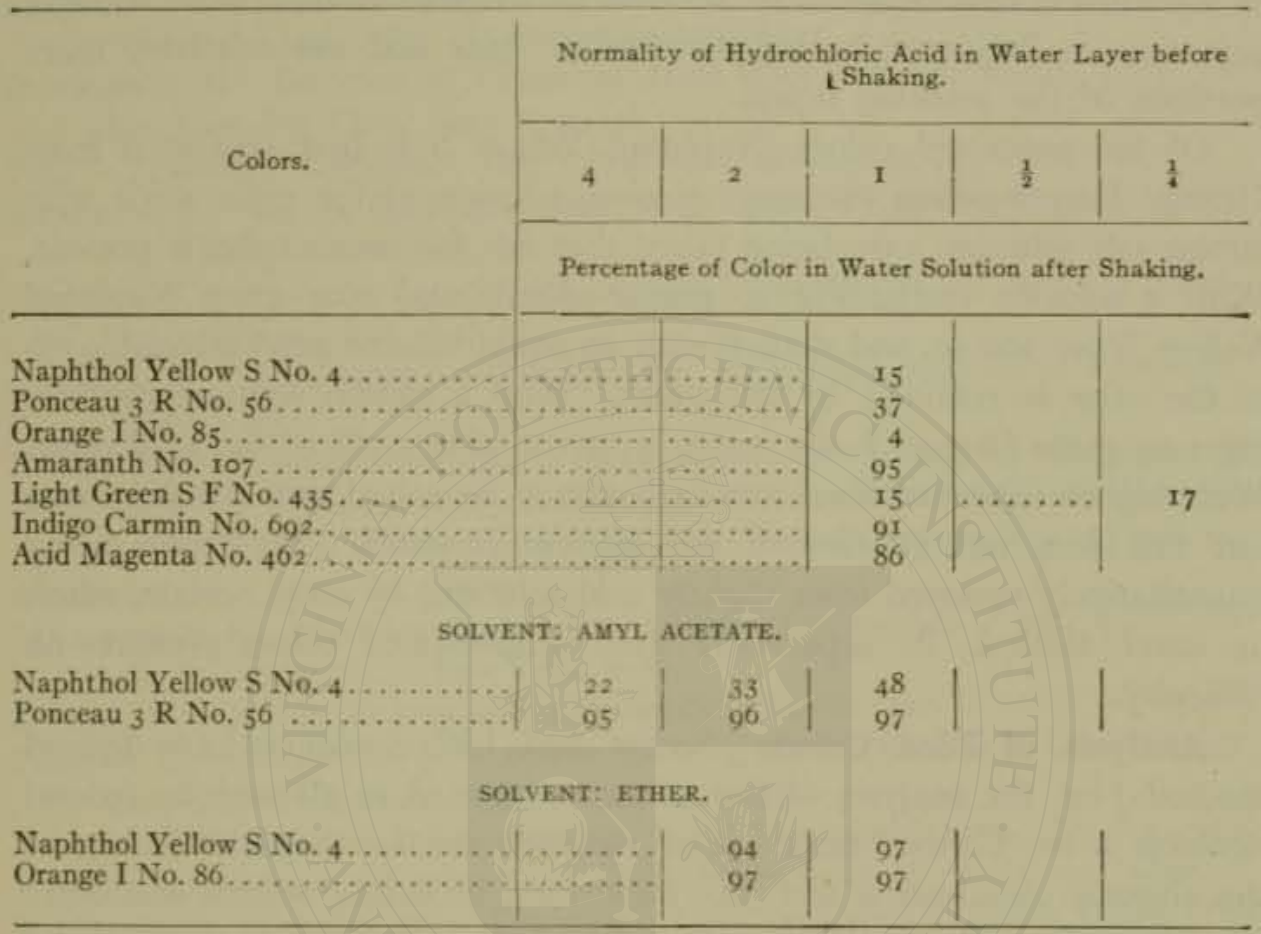

Assuming the distribution ratios to remain constant, this procedure using four funnels and making three washings gives for a pair of colors whose "distribution numbers" (as the percentage numbers given in the table may be called) are 80 and 20 , respectively, a separation of 98.30 per cent for each color. With distribution numbers 90 and to four funnels and three washings give a calculated separation of $99.73 \%$, and the same is obtained with distribution numbers $8 \mathrm{r} .8$ and 5.3 if the solvent in which the dyes are relatively more soluble be taken in portions one-half the volume of those of the other liquid. If the second, third, and fourth funnels be given a fifth washing, the third and fourth funnels a sixth, and the last funnel a seventh washing, the calculated loss for the color more soluble in the solvent layer is $0.76 \%$, while the percentage of the other dye removed is relatively much increased (to 99.99 per cent). In most mixtures the progress of the separation is always apparent. 
In practice, because of incomplete extraction and separation, and especially on acccount of uncertainty due to small amounts of subsidiary dyes always present, it is necessary to increase the number of successive extractions. The formation - of esters of the color acids is a possible source of difficulty, but is not believed to take place. With amyl alcohol as solvent it is usually desirable to make the original solution more strongly acid than is indicated by the distribution data and use relatively more portions of the washing liquid.

Of the permitted colors, Naphthol Yellow $\mathrm{S}$ is best separated from Orange I by washing the amyi alcohol solution of the color acids with strong salt solution, care being taken that not too much color is present. With a solution containing 20 grams of salt and 0.04 gram Naphthol Yellow S per $100 \mathrm{cc}$. and shaken with an equal volume amyl alcohol, $97 \%$ of the color is retained by the water. With a similar solution containing 0.07 gram Orange I, the water layer contains $x .5 \%$ of the total color. With higher concentrations some color may be salted out in solid form, but this does not interfere if the amount is small. Erythrosin being quantitatively removed from slightly acid solutions by amyl acetate, ether, or amyl alcohol, its separation from sulphonated colors presents no difficulty.

Analysis of Food Colors.-Seeker and his co-workers have devised methods for the analysis of the seven coal-tar colors allowed by federal decision in the United States. The methods are for the detemination of the ultimate constituents and for impurities, including arsenic and other heavy metals. The reader is referred to Hesse's report (see reference, page 8 (19) for details of these processes.

Solubility Tables.-Robin* has published tables showing the reactions of the coal-tar dyes used in confectionery, classified as basic, acid, and water-insoluble colors, the distinction of basic and acid colors being based on their extraction by amyl alcohol or ethyl acetate from alkaline and acid solutions. Rota (page 798) employs ether for the separation of basic colors.

Loomis (see reference, page 819 ) has prepared a table giving the solubility of food colors in various solvents, including those named above, and another table showing the relative amounts extracted from neutral, alkaline, and acid solutions, shaking with amyl alcohol, ethyl acetate and acetone, the aqueous solution in the latter case being saturated with salt.

* Girard: Analyse des Matières alimentaires, 2 Ed., pp. 679-69r. 


\section{REFERENCES ON COLORS.}

Arata, P. N. (Specielle analytische Methoden.) Zeits. anal. Chem., 28, p.639.

Bellier, J. Detection of Artificial Coloring Matters in Wine. Ann. de Chim. Anal, 5 , 1900, p. 407; Abs. Analyst, 26, 1901, p. 42.

BENEDIKr, R., and KNECHr, E. The Chemistry of the Coal-tar Colours, London, 1889. Berry, W. G. Coloring Matters for Foodstuffs and Methods for their Detection.

U. S. Dept. of Agric., Bur. of Chem., Circular No. 25.

Dommergue, G. Detection of Colors on Dyed Wool. Monit. Scient., 33, p. 25; Abs. Jour. Soc. Chem. Ind, 8, p. 216.

For, F. Testing of Dyestuffs. Jour. Chem. Soc., 28, 1875, p. 193.

Green, A. G. On the Qualitative Analysis of Coal Tar Coloring Matters. Jour.

Soc. Chem. Ind, 12,1893, p. 3.

Hesse, B. C. Coal Tar Colors Used in Food Products, U. S, Dept. of Agric., Bur. of Chem., Bul, Ia7, igr2.

LEEDS, A. R. Tabellarische Uebersicht der künstlichen organischen Farbstoffe. Berlin, 1894 .

Looms, H. M. Report on Colors: The Solubility and Extraction of Colors and the Color Reactions of Dyed Fiber and of Aqueous and Sulphuric-Acid Solutions. U. S. Dept. of Agric., Bur. of Chem., Circulars Nus. 35 and 63 . Martinon, B. Jour. Soc. Dyers, 3, p. 174.

Mriliken, S. P. Identification of Pure Organic Compounds, Vol. III. Commercial Dyestuffs. New York, 1910.

NiEtzki, R. Chemie der organischen Farbstoffe. Berlin, rgor.

Posetro, G. Composition of Vegetable Coloring Matters for Use in Confectionery. Zeits. Nahr. Unters. u. Hygiene, 9, 1895, p. 150.

Rawson, C., KNecht, E., and Lowenthat, R. A Manual of Dyeing. London, r893. Rawson, Gardner, and Laycock. A Dictionary of Dyes, Mordants, etc. 1890.

Reichel.mann and Leuscher. Detection of Coal Tar Colors in Pastry, Cakes, Fruit Products, etc. Zeit. für öffentl. Chem., 8. 1902, p, 204; Abs. Analyst, 27, 1902, p. 276 .

Rota. A. R. A Method of Analyzing Natural and Artificial Organic Coloring Matters Analyst, 24, 1899, p. 4I. From Chem. Zeit., 1898, p. 437.

Schultz, G., u. Julrus, P. Taballarische Uebersicht der künstlichen organischen Farbstoffe. 1897 .

— Translated by Green, A. G. A Systematic Survey of the Organic Coloring Matters. Ist ed., 1894; 2d ed., r904.

SeEker, A. F. Coloring Matters in Foods. Allen's Commercial Organic Analysis. $4_{\text {th Ed., Vol. V, p. } 625 .}$

Sostegni, L., and Carpentieri, F. (Specielle analytische Methoden.) Zeits. anal. Chem., 35, r896, F. 397 .

Spaeth, E. Foreign Coloring Matters in Fruit Juices. Zeits. Unters. Nahr. Genuss., 2, 1899 , p. 633 .

Tolman, L. M. Coloring Matter in Food. U. S. Dept. of Agric., Bur. of Chem., Bul. 65 , p. $11 \mathrm{r}$.

य. S. Food Inspection Decisions: No. 76. Dyes, Chemicals and Preservatives in 
Foods. No. 77. Certificate and Control of Dyes Permissible for Use in Coloring Foods and Foodstuffs. No, 106. Amendment to No. 77. No. 117. The Use of Certified Colors. No, 129. Amendment to No, 77 .

Weber, H. A. Effect of Coal Tar Colors on Digestion. Am. Chem. Jour., 8, r896, p. 1092 .

WEINGÄRTNER. Eine Anleitung zur Untersuchung der im Handel vorkommenden künstlichen Farbstoffe. Zeits. anal. Chem., 27, 1888, p. 232.

WEYL, T. Translated by Leffmann, H. The Sanitary Relations of the Coal Tar Colors. Philadelphia, 1892 .

Winton, A. L. The Use of Coal Tar Dyes in Food. Conn. Agric. Exp. Sta. Rep., I901, p. 179.

Wrrt, O. N. Versuch. einer qualitativen Analyse der im Handel vorkommenden Farbstoffe. Zeits. anal. Chem., 26, 1887, p. 100.

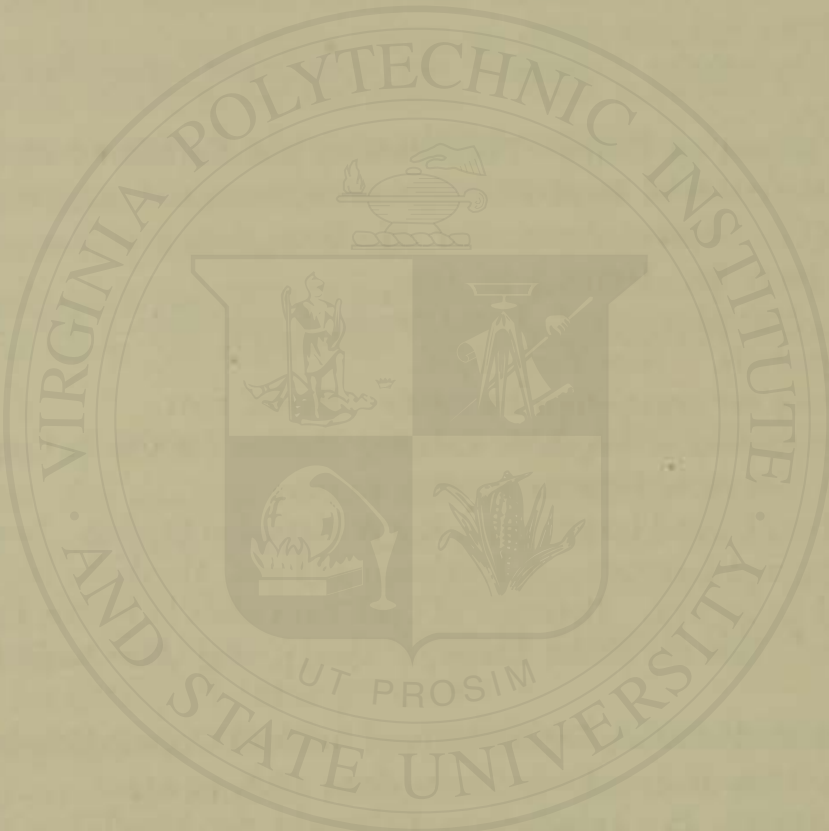




\section{CHAPTER XVIII.}

\section{FOOD PRESERVATTVES.}

Preservation of Food.-Various processes have from ancient times been known and used for arresting the fermentative changes which food products in their natural state undergo on long standing. These processes include pickling with vinegar, drying, smoking, salting, preserving with sugar, and finally in the employment of heat in sterilizing and pasteurizing, and of low temperature as in cold storage. All of them are still in use, and are universally regarded as unobjectionable. In addition to these old and well-known methods of food preservation is the comparatively modern practice of arresting fermentation by the use of such antiseptic chemical agents as formaldehyde, beta-naphthol, boric, salicylic, benzoic, and sulphurous acids or salts of these acids, etc., in regard to the wholesomeness of which there is considerable difference of opinion. These substances depend for their efficiency on the more or less complete inhibition of bacterial growth. Nearly all exert a powerful antiseptic influence, to such an extent that to accomplish their object only small quantities need be used in food.

Apart from their toxic effects, a marked difference naturally exists between the employment of such substances as salt, sugar, and vinegar for food preservation, all of which are in themselves foods, and in the use of chemical agents that have no food value. The advocates of the use of chemical antiseptics claim that there are no authentic instances on record of injury from the use of such small quantities of these substances as are necessary to arrest decay, while there are many cases of injury arising from the use of foods which, while apparently wholesome, have undergone such fermentation as to develop ptomaines or other harmful toxins, and that because antiseptics prevent such spoiling of the food, their use is decidedly beneficial; that there is, besides, no more reason why a prejudice should exist against the employment of these 
newer chemicals than against saltpeter, which has long been used in the corning of meat, or against the cresols and phenols left as a product of smoking.

The opponents to their use assert, that the addition to food of such antiseptic substances as prevent its decay also serves to retard the digestive processes when the food is eaten; that many of these substances are drugs, and as such cannot fail even in small quantities to exercise a toxic effect of some sort on the system; that finally their use is objectionable, as allowing the employment in certain foods of old materials that have in some cases already undergone incipient decomposition before the addition of the antiseptic, and are thus unwholesome.

Regulation of Antiseptics in Food.-In the absence of legislation directly prohibiting the use of any of the above-named antiseptics, and in view of the difference of opinion regarding their toxic effects when present in small quantities, it is difficult to maintain a complaint under the general food laws as they exist in most states, basing the complaint solely on their harmfulness. In some localities certain antiseptics are specifically allowed and others are prohibited. Some of the states, as, for example, Massachusetts, have special laws under which it is required that in the case of all foods thus treated, the name and percentage of such antiseptics as are used must appear plainly on labels of the packages or containers thereof, such a provision being based on the assumption that the general public should be informed of what they are buying, where any doubt exists as to the wholesomeness of any ingredient present. Where such laws as these are in force, the chemist's task is comparatively easy, in that conviction in court is not dependent on his individual opinion regarding the toxic effects of the antiseptic employed.

Physiological experiments for testing the toxicity of these chemical preservatives were formerly confined to the lower animals, but no satisfactory results could be thus obtained. Later, metabolism experiments were made on human beings treated with varying amounts of the preservatives under carefully controlled conditions, but the results of these, though made by experts of unquestioned ability, do not agree. Even if any of these substances as used in food appear to have little or no effect on people in good health, they cannot be assumed to be equally harmless to those who are inclined to be delicate or sickly. Even though pronounced harmless in themselves, there is still the objection that the chemical preservatives may readily conceal unclean methods or materials. If perishable foods are free from preservatives and are sweet and 
untainted, the consumer has reason to believe that clean and wholesome materials and sanitary processes were employed throughout in their manufacture.

Commercial Food Preservatives.-A large number of commercial preparations are sold for purposes of preserving specific articles of food and are put out under trade names that usually convey no suggestion of their true character. Some of these consist of a single antiseptic substance, such as salicylic acid, ammonium fluoride, calcium sulphate, borax, or benzoic acid, while others are mixtures of several antiseptics, of which the following are typical examples, showing their composition as found, together with the amount of the mixture to be employed.

$A$. For preserving sausage meat, using 8 ounces per 100 pounds of meat:

Borax......................... $36 \%$

Salt. ........................... $46 \%$

Saltpeter. ......................... $18 \%$

(Colored with an anilin dye.)

$B$. For preserving cider and ketchup.

A $34 \%$ solution of beta-naphthol in alcohol, using 2 fluid ounces to 45 gallons of cider, or $1 \frac{1}{2}$ ounces to 10 gallons of ketchup.

C. For preserving beer, using $I_{3}^{\frac{1}{3}}$ ounces per barrel of beer:

Salt. ............................ $45 \%$

Salicylic acid. . .................... $27 \%$

Sodium carbonate and salicylate......... $28 \%$

$D$. Fcr preserving chepped meats, using I ounce to 50 pounds of meat.

Sodium sulphite...................... $65 \%$

Borax........................... $35 \%$

E. Effective for curing beef, hams, tongues, bacon, pig's feet, etc.:

Borax.......................... $28 \%$

Boric acid......................... I2 $\%$

Sodium chloride..................... $35 \%$

Potassium nitrate................... $25 \%$

F. For preserving milk and cream:

Boric acid.......................... $75 \%$

Borax............................ $25 \%$ 
G. For preserving jellies, jams, preserves, mince-meat, and syrups, using from $I$ to 2 ounces of preservative to roo pounds of product:

Sodium benzoate. .................. $50 \%$

Boric acid.......................... $40 \%$

Sodium chloride.................... $5 \%$

Sodium bicarbonate. . ................ $5 \%$

H. For preserving ketchup and tomato pulp, using from 6 to 8 ounces to 45 gallons of the product:

Sodium benzoate. .................... $50 \%$

Sodium chloride.................... $40 \%$

Sodium sulphite..................... $10 \%$

I. Effective for keeping butter from becoming tainted or rancid, also for salt codfish, using 8 to 12 ounces per 100 pounds butter:

Boric acid........................ $25 \%$

Borax........................... $50 \%$

Sodium chloride................... $25 \%$

$J$. For preserving eggs (surface application). A saturated solution of salicylic acid in 3 quarts of water, I quart strong alcohol and 7 eunces of glycerin.

\section{FORMALDEHYDE.}

Formaldehyde ( $\mathrm{HCHO})$ is a gas formed by the action of a red-hot spiral of platinum wire on vaporized methyl alcohol. It is also produced by the dry distillation of calcium formate. In the market it commonly appears in the form of a $40 \%$ solution of the gas in water under the name of formalin, and for use as a food preservative dilute solutions of from 2 to 5 per cent strength are usually employed. Its use as a food preservative is comparatively modern. Formaldehyde, while not confined exclusively to milk products, is, as a matter of fact, more commonly used in these than in other foods. Its prompt and direct action in checking or preventing the growth of lactic acid bacteria renders it especially desirable for use as a milk and cream preservative, from the standpoint of the dairy man who does not concern himself as to whether or not its use is injurious or illegal.

When present in milk to the extent of I part formaldehyde to 20,000 parts milk (a proportion quite commonly employed), the sample is kept 
sweet for four days in summer weather, when under ordinary conditions, the milk untreated would curdle in less than forty-eight hours.

Determination of Formaldehyde in the Commercial Preservative.(I) Iodometric Method.*-Mix ro cc. of the aldehyde solution (diluted if necessary to a strength not exceeding $3 \%$ of formaldehyde) with $25 \mathrm{cc}$. of tenth-normal iodine solution, and add drop by drop a solution of sodium hydroxide, till the color of the liquid becomes clear yellow. The solution is set aside for at least ten minutes, after which hydrochloric acid is added to set free the uncombined iodine, and the latter is titrated back with tenth-normal thiosulphate. Two atoms of iodine are equivalent to one molecule of formaldehyde, in accordance with the following reactions:

$$
\begin{array}{ll}
6 \mathrm{NaOH}+6 \mathrm{I} & =\mathrm{NaIO}_{3}+5 \mathrm{NaI}+3 \mathrm{H}_{2} \mathrm{O} . \\
{ }_{3} \mathrm{CH}_{2} \mathrm{O}+\mathrm{NaIO}_{3} & ={ }_{3} \mathrm{CH}_{2} \mathrm{O}_{2}+\mathrm{NaI} . \\
5 \mathrm{NaI}+\mathrm{NaIO}_{3}+6 \mathrm{HCl}=6 \mathrm{NaCl}+\mathrm{I}_{6}+{ }_{3} \mathrm{H}_{2} \mathrm{O} .
\end{array}
$$

(2) Method of Blank and Finkenbeiner.†-Three grams of the solution are weighed into a tall Erlenmeyer flask, to which is then added from 25 to $30 \mathrm{cc}$. of twice-normal sodium hydroxide. $50 \mathrm{cc}$. of pure 2.5 to 3 per cent hydrogen peroxide solution are next gradually run in during a space of from three to ten minutes, through a funnel placed in the neck of the flask to prevent spurting, and the solution is allowed to stand for two or three minutes, after which the funnel is washed with water.

Finally the unused sodium hydroxide is titrated with twice-normal sulphuric acid, using litmus as an indicator. The less formaldehyde in the sample, the longer the mixture should stand after addition of the hydrogen peroxide, to complete the reaction. When less than $30 \%$ is present, it should stand at least ten minutes.

Ascertain the percentage of formaldehyde, by multiplying by 2 the number of cubic centimeters of soda solution used, when 3 grams of the sample are taken.

(3) Ammonia Method. $\ddagger-W e i g h$ Io grams of the formaldehyde solution into a flask, and treat with an excess of ammonia. Cork the flask and shake frequently during scveral days. The formaldehyde is by this process converted into hexamethylamine.

Transfer the solution to a tared platinum dish, and evaporate nearly

* Zeits, anal. Chem., 1897, 36, pp, 18-24; abs. Analyst, 22, p. 22 r.

$\dagger$ Ber., 3r (17), 2979.

$\ddagger$ Conn. Exp. Sta., Annual Report, 1899, p. 143 . 
to dryness on the top of a closed water-bath. Finally the dish is transferred to a desiccator, and the drying continued over sulphuric acid to constant weight. The per cent of formaldehyde is calculated from the weight of the hexamethylamine, making a correction for the residue left by the formaldehyde itself by direct evaporation:

$$
6 \mathrm{CH}_{2} \mathrm{O}+4 \mathrm{NH}_{4} \mathrm{OH}=\left(\mathrm{CH}_{2}\right)_{6} \mathrm{~N}_{4}+10 \mathrm{H}_{2} \mathrm{O} .
$$

Or an excess of a standardized ammonia solution may be added in the first place, the excess of ammonia being distilled off and titrated with standard acid, calculating the per cent of formaldehyde by the amount of ammonia absorbed.

Detection of Formaldehyde.-Methods have previously been given for the detection of formaldehyde in milk. Pure milk furnishes a convenient reagent for the detection of formaldehyde in various preparations. A solution of the sample to be tested is acidified with phosphoric acid, subjected to distillation, and the first few cubic centimeters of the distillate are tested for formaldehyde as follows:

(1) Hydrochloric Acid and Ferric Chloride Test.-Add a few drops of the suspected distillate to about Io cc. of pure milk (previously proved free from formaldehyde) in a porcelain casserole, and carry out the test as described on page 180 .

(2) Hehner's Sulphuric Acid Test.-Apply the test as described on page 180 to ro cc. of pure milk to which a few drops of the suspected distillate have been added.

(3) Resorcin or Carbolic Acid Test.-To about to cc. of the distillate to be tested, add a few drops of a $1 \%$ solution of carbolic acid or resorcin, mix thorbughly, and carefully pour the liquid down the side of a test-tube containing concentrated sulphuric acid. In the presence of formaldehyde, a rose-red zone is formed at the junction of the two liquids, sensitive to I part in 200,000 . If formaldehyde be present to an extent exceeding I part in 100,000 , a white turbidity or precipitate is formed above the colored zone.

(4) Phenylhydrazine Hydrochloride Test.*-One gram of phenylhydrazine hydrochloride and $I_{\frac{1}{2}}$ grams sodium acetate are dissolved in Io cc. of water. Add 2 to 4 drops of this reagent, and an equal amount of sulphuric acid, to I or $2 \mathrm{cc}$. of the distillate to be tested in a test-tube. A green coloration is produced in the presence of formaldehyde.

* Jour. Am. Chem. Soc., 22, p. 135 . 
If present in a very small amount (say I part formaldehyde in 200,000), heat is necessary to bring out the color.

Determination of Formaldehyde.-The exact quantitative determination of formaldehyde in food products is difficult, owing to its extreme volatility as well as the uncertainty of the compounds which it forms with proteins. A rough idea of the amount present may often be gained by the intensity of the colorations produced in carrying out the various qualitative tests.

Formaldehyde in the small amount present in food products may be roughly determined by the potassium cyanide method (p. 18I), on separate portions of the distillate of about $20 \mathrm{cc}$. each, collecting the distillate as long as an appreciable amount of formaldehyde is shown therein.

\section{BORIC ACID.}

Boric or boracic acid is commonly obtained in impure form from lagoons or fumaroles of volcanic origin in Tuscany. It is afterwards purified by recrystallization. It is weakly acid, and readily soluble in water and in alcohol. Its alcoholic solution, even when the acid is present in small quantity, burns with a characteristic green flame. The acid is quite volatile with steam.

Borax, the most commonly known salt of boric acid, is found native in Italy, California, and elsewhere, and is also made from boric acid. It is mildly alkaline, and readily soluble in water.

Boric acid and borax, either used separately or mixed, have long been used as preservatives, especially in animal foods. A mixture of 3 parts boric acid and I part borax has been found very effective as a milk and butter preservative, as well as for meat products.

Determination of Boric Anhydride in Commercial Preservatives.Gladding Method**A I50-cc. flask, Fig. II 7 , is arranged with a doubly perforated stopper having two tubes, one of which, the inlet-tube reaching nearly to the bottom, connects it with a larger flask, while the other or outlet-tube communicates with a Liebig condenser, which in turn delivers into a receiving-flask. In the 150 -cc. flask, I gram of the powdered sample is placed, with about $20 \mathrm{cc}$. of $95 \%$ methyl alcohol and $5 \mathrm{cc}$. of $85 \%$ phosphoric acid. The larger flask is then filled two-thirds full of methyl alcohol, and heated on the water-bath after the apparatus has been connected up. Heat is also applied to the 150 -cc. flask, the:

* Jour. Am. Chem. Soc., 20, 1898, p. 288. 
whole arrangement being such that a continuous current of methyl alcohol vapor bubbles through the liquid in the smaller flask, the heat being so regulated that from 15 to $25 \mathrm{cc}$. of methyl alcohol remains in the 150 cc. flask, while about roo cc. of distillate passes into the receiving-flask in half an hour. Continue the distillation till all the acid has passed over, which is usually accomplished by distilling roo cc. By a gentle aspiration upon the receiving-flask, loss by leaking may be avoided.

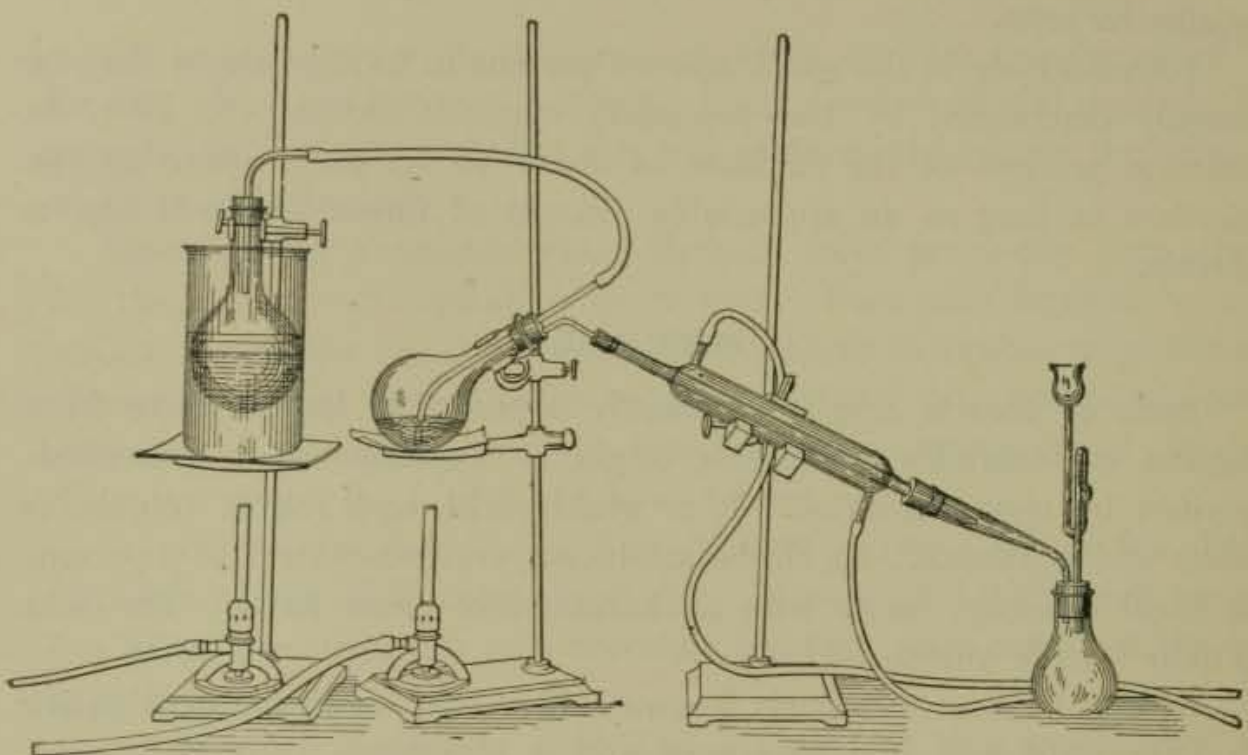

FIG. I17.-Apparatus for Determining Boric Acid According to Gladding.

Prepare a mixture of $40 \mathrm{cc}$. of glycerin and $100 \mathrm{cc}$. of water, and carefully neutralize, using phenolphthalein as an indicator. Add this mixture to the distillate, and titrate the whole with tenth-normal sodium hydroxide. Run a blank with the reagents alone, deducting any acidity. For the factors for calculation see page 830 .

Detection of Boric Acid and Borates.-These are best tested for in most cases in a solution of the ash of the sample, the quantity to be used for the test depencing largely on the case in hand. With meat products and canned goods, about 25 grams are taken for the test, being first made distinctly alkaline with lime water, dried over the water-bath, and burned. The ash is boiled with from Io to I5 cc. of water, and tests made on the solution. With such products as salt codfish, which is preserved by brushing or coating with boric mixture, portions of the coating may be scraped off and boiled in water, the tests being made on the aqueous solucions. 
(I) The Turmeric-paper Tet.-The most delicate test for boric acid, free or combined, is made by the aid of turmeric-paper, prepared by soaking a smooth, thin grade of filter-paper in an alcoholic tincture of powdered turmeric. The paper is afterwards dried and cut into strips, which are kept for convenience in a wide-mouthed bottle in a dark place.

Slightly acidulate the ash of the sample to be tested with a few drops of dilute hydrochloric acid, avoiding an excess of acid. Then dissolve the ash in a few drops of water and thoroughly saturate a strip of the turmeric-paper in the solution. On drying the paper, if boric acid either free or combined be present, a cherry-red coloration will be imparted to the paper, the depth of color depending on the amount present. As a confirmatory test, apply a drop of dilute alkali to the reddened paper, and a dark-olive color will be due to boric acid, sharply to be distinguished from the deep-red color produced when an alkaline solution is applied to ordinary turmeric-paper. The turmeric-paper reaction is delicate to I part in 8,000 .

(2) Tincture of Turmeric Test.-To the solution to be tested, slightly acidified with hydrochloric acid, add an equal volume of saturated tincture of turmeric in an evaporating-dish, and heat for a minute or two. A red color, light or dark, depending on the amotint of the preservative, is produced if boric acid be present, changed to an olive color by the addition of dilute alkali, after cooling.

(3) The Flame Test.-A few cubic centimeters of alcohol are added to the dish containing the slightly acidulated ash of the sample to be tested, or to the acidulated dried residue from the evaporation of the aqueous solution of the suspected preservative, and after mixing by the aid of a stirringrod, the alcohol is ignited. In the presence of any considerable portion of free or combined boric acid, a greenish tinge will be observed in the flame of the burning alcohol, especially at the first flash, due to the boric ether formed. This test is by no means as delicate at the paper test.

Determination of Boric Acid in Foods.-(1) Thompson's Method.*Add I or 2 grams of sodium hydroxide to Ioo grams of the sample, and evaporate to dryness in a platinum dish. Char the residue thoroughly, and boil with $20 \mathrm{cc}$. of water, adding hydrochloric acid drop by drop till all but the carbon is dissolved. In burning, avoid too high a heat, simply charring sufficiently to insure a clear solution with water. Transfer by washing to a 100 -cc. graduated flask, taking care that the volume does not exceed 50 or $60 \mathrm{cc}$. Add half a gram of dry calcium chloride, then a few drops

* Analyst, 18, p. 184 . 
of phenolphthalein solution, and next a 10\% solution of sodium hydroxide, till a permanent pink color persists. Finally add 25 cc. of lime-water. By this means all phosphoric acid is precipitated in the form of calcium phosphate. Make up to the roo-cc. mark with water, shake, and pour upon a dry filter. To $50 \mathrm{cc}$. of the filtrate add sufficient normal sulphuric acid to remove the pink color. Then add a few drops of methyl orange, and continue the addition of sulphuric acid till the yellow is just turned to pink. Tenth-normal sodium hydroxide is then added * till the liquid takes on a faint yellow, excess of alkali being avoided. The salts of the acids present at this time are all neutral to phenolphthalein except boric acid and carbon dioxide. Boil the solution to expel the carbon dioxide, cool, add a little more phenolphthalein, and a quantity of glycerin equal in volume to the solution. Finally titrate with tenth-normal sodium hydroxide to a permanent pink color. Each cubic centimeter of tenthnormal sodium hydroxide equals 0.0062 gram crystallized boric acid, $\mathrm{H}_{3} \mathrm{BO}_{3}$, or 0.0035 gram boric anhydride, $\mathrm{B}_{2} \mathrm{O}_{3}$, or 0.00955 gram crystallized borax, $\mathrm{Na}_{2} \mathrm{~B}_{4} \mathrm{O}_{7}, \mathrm{IoH}_{2} \mathrm{O}$.

(2) Gooch's Method.-Mix 400 to 500 grams of the substance with Io grams of calcium hydrate, evaporate to dryness over a water-bath in a platinum dish, and burn cautiously to an ash. Dissolve the residue in cold nitric acid, and add an excess of silver nitrate to precipitate the chlorine. Filter, make up to $500 \mathrm{cc}$. with water, shake, and measure out $25 \mathrm{cc}$. into a 200-cc. flask fitted with a stopper provided with an outlet-tube, and with a separatory funnel forming virtually a thistle-tube, capable of being closed with a glass stop-cock. Through the outlet-tube connect the flask with a Liebig condenser provided with an adapter which can dip below the liquid in the receiver. As a receiver, use a $150-c c$. tared platinum dish, which contains a weighed quantity of ignited lime in water.

Add through the thistle-tibe $10 \mathrm{cc}$. of methyl alcohol to the contents of the flask, close the stop-cock therein, and distill the contents in a paraffin-bath at a temperature of $140^{\circ} \mathrm{C}$., constantly stirring the liquid in the receiver to keep it alkaline during the distillation. Add five successive portions of methyl alcohol of 12 cc. each to the distilling-flask, and continue the distillation till all the alcohol has passed over. Finally evaporate to dryness the contents of the platinum dish, and ignite over a blast-lamp to constant weight. Multiply the increased weight due to boric oxide by 2.728 to give the equivalent in borax.

* If the value of the standard alkali solution is not absolutely certain, it had best be restandardized against pure crystallized boric acid, 0.31 gram of which should neutralize $50 \mathrm{cc}$, of tenth-normal alkali. 


\section{SALICYLIC ACID.}

Salicylic acid $\left(\mathrm{HC}_{7} \mathrm{H}_{5} \mathrm{O}_{3}\right)$ is a white, crystalline, strongly acid powder, made synthetically by treatment of carbolic acid with sodium hydroxide and carbon dioxide, or naturally from methyl salicylate (which occurs in oil of wintergreen to the extent of about $9 \circ \%$ ), by treatment of the wintergreen oil with strong potash lye. Most of the commercial salicylic acid is of the synthetic variety. Pure salicylic acid crystallizes from alcoholic solutions in 4 -sided prisms, and from aqueous solution in long, slender needles. It melts at $155^{\circ}$ to $156^{\circ} \mathrm{C}$. It is slightly soluble in cold water (I part in 450), and much more so in hot water. It is readily soluble in ether, alcohol, and chloroform.

It is frequently found on the market as a food preservative in the form of the much more soluble sodium salt, sodium salicylate, $\left(\mathrm{NaC}_{7} \mathrm{H}_{5} \mathrm{O}_{3}\right)$, which is, however, converted into salicylic acid when added to acidfruit preparations, condiments, and liquors.

Sodium salicylate is a white, amorphous powder, soluble in 0.9 parts water and in 6 parts alcohol. It is prepared by treating salicylic acid with a strong, aqueous solution of sodium carbonate, and afterwards purifying. If a known weight of the powdered preservative be ignited, and a solution of the ash titrated with tenth-normal sulphuric acid, using cochineal as an indicator, each cubic centimeter of the acid is equivalent to 0.0160 gram of sodium salicylate.

Salicylic acid is largely used as a preservative of jellies, jams, and fruit preparations, canned vegetables, ketchups, table sauces, wines, beer, and cider. It is rarely used in milk and milk products, or in meats.

Bucholz has shown that $0.15 \%$ of salicylic acid is sufficient to prevent bacteria from developing in ordinary organic substances, while as small a quantity as $0.04 \%$ produces a marked restraining influence.

Small amounts of salicylic acid occur naturally in grapes, strawberries, and other fruits, but the amounts are too small to give distinct color reactions when only 50 grams of the fruit products are used for tests.

Detection of Salicylic Acid.-If the sample to be tested is of a similar nature to jelly, jam, ketchup, cider, etc., or capable of getting into aqueous solution, slightly acidify the liquid or pasty material, diluted, if necessary, with weak sulphuric (if not already acid), and shake directly with an equal bulk of ether, petroleum ether, or chloroform, in a corked flask, or in a separatory funnel. If the sample be too thick in consistency to 
shake directly, macerate in a mortar with alkaline water, and strain through cloth. Acidify the filtrate with dilute sulphuric acid, and then proceed to shake with the immiscible solvent as above. Separate by decantation or otherwise the immiscible solvent containing the preservative, if present, and allow it to evaporate in an open sialiow dish, either at room temperature or at a low heat. In case an emulsion forms on shaking, which is quite apt to happen, especially with ether for a solvent, divide the whole mixture between two tubes of a centrifuge of the form shown in Fig. Ir, and whirl for three minutes at a high rate of speed. This usually serves to break up the most obstinate emulsion, so that it is easy to separate by decantation. If a considerable amount of salicylic acid be present, it will sometimes appear in the residue in the form of fibrous crystals.

(I) To a portion of the dry residue add a drop of ferric chloride solution. A deep purple or violet color indicates salicylic acid.* If doubt exists as to the color, dilute with water, which often serves to bring out a distinctive purple coloration otherwise unobservable.

(2) Another portion of the residue may be heated with methyl alcohol and sulphuric acid. If salicylic acid be present, the well-known odor of methyl salicylate will be produced.

(3) A portion of the dry ether extract is warmed gently with a drop of concentrated nitric acid, and two or three drops of ammonia are added. Yellow ammonium picrate will be formed if a considerable quantity of salicylic acid be present, and a thread of wool free from fat may be dyed by soaking therein. This test is by no means as delicate as the ferric chloride color test.

Instead of evaporating the ether solution of the salicylic acid to dryness, the author prefers to shake out the salicylic acid from the ether with dilute ammonia, evaporate the solution of ammonium salicylate nearly to dryness, and apply the tests given above to the concentrated solution. In this case the ether may be recovered.

Determination of Salicylic Acid.-Dubois Method.†-In the case of ketchups and similar pulped materials place 50 grams in a graduated 200-cc. flask, make slightly alkaline with ammonia, add ${ }_{5}$ cc. of milk

* Peters (U. S. Dept. of Agric., Bur. of Chem., Bul. 65, p. 160) advises the use of chloroform as more convenient for extraction when testing for salicylic acid, and recommends that the chloroform extract without evaporation be shaken in a test-tube with a drop of ferric chloride reagent and a little water. In the presence of salicylic acid, the violet color will be apparent in the supernatant aqueous layer.

† Jour. Am. Chem. Soc., 28, 1906, p. 1616, U. S. Dept. of Agric., Bur. of Chem., Bul. ro7 (rev.), p. 179 . 
of lime ( 200 grams of quicklime in $2000 \mathrm{cc}$. water), complete the volume, shake and filter. Transfer $150 \mathrm{cc}$. of the filtrate to a separatory funnel, acidify with hydrochloric acid, and extract with four portions of 75 to Ioo cc. of ether. Wash the combined extract twice with $25 \mathrm{cc}$. of water, and distil off the ether slowly, allowing the last 20 to $25 \mathrm{cc}$. to evaporate spontaneously. Dissolve the residue in a small amount of hot water, make up to a definite volume with water, and add to an aliquot portion a few drops of a $2 \%$ solution of ferric alum to develop the color. Estimate the amount of salicylic acid by matching the color thus obtained with that produced in a solution containing I mg. of salicylic in $50 \mathrm{cc}$., using either a colorimeter or Nessler tubes for making the comparison.

In the case of semisolid materials, such as mince meat, jams, etc., macerate 50 grams with water in a mortar previous to treatment as above described.

Liquids and solutions of jellies and other materials free from pulp may be extracted with ether directly after acidifying.

\section{BENZOIC ACID}

Benzoic Acid $\left(\mathrm{HC}_{7} \mathrm{H}_{5} \mathrm{O}_{2}\right)$ is produced by the oxidation of a large number of organic substances, particularly toluene. It is also extracted by sublimation from gum benzoin, which exudes from the bark of the Styrax benzoin, a tree growing in Java, Sumatra, Borneo, and Siam. Most of the commercial benzoic acid is made from toluene by treatment with chlorine and subsequent oxidation.

Benzoic acid crystallizes in leaflets, having a silky luster. It is odorless when cold, is soluble in 200 parts of cold, and 25 parts of boiling water, and readily dissolves in alcohol, ether, and chloroform. Its melting-point is $120^{\circ}$, and it sublimes at a slightly higher temperature. It occurs naturally in the cranberry and other berries of the Ericacex.

Sodium Benzoate $\left(\mathrm{NaC}_{7} \mathrm{H}_{5} \mathrm{O}_{2}\right)$ is the salt most largely used in commercial preservatives, being much more soluble than the acid itself, into which, however, it is converted when put into acid fruit preparations. Sodium benzoate is prepared by adding benzoic acid to a concentrated hot solution of sodium carbonate till there is no longer effervescence, and then cooling, and allowing the sodium benzoate to crystallize out. In titrating solutions of ignited sodium benzoate with tenth-normal sulphuric acid, each cubic centimeter of the standard acid is equivalent to 0.0144 gram of the benzoate.

Sodium benzoate is a white amorphous powder, having a sweetish, 
astringent taste, and is soluble in 1.8 parts of cold water, and in 45 parts of alcohol. It is used as a preservative of ketchups, fruit products, soft drinks, codfish, and less often of wines.

Long, Herter, and Chittenden of the Referee Board of Consulting Scientific Experts, after independent experiments, conclude that sodium benzoate in small doses (less than 0.5 gram per day) is not injurious to health and in large doses (up to 4 grams per day) has not been found to exert any deleterious effects on the general health nor to act as a poison in the general acceptance of the term. Accordingly this preservative is allowed under the federal law provided the presence and amount are declared on the label.*

Detection of Benzoic Acid.-Extract with ether or chloroform as directed for salicylic acid. If it is desired to test for both preservatives divide the extract into two parts and evaporate in separate dishes. A considerable amount of benzoic acid is apparent in the residue as shining crystalline scales or needles.

In the author's experience a better procedure than evaporating the ether solution is to extract the benzoic acid from the ether by shaking with dilute ammonia, evaporate the solution of ammonium benzoate nearly to dryness, and apply tests to the concentrated solution.

(1) Ferric Chloride Test.-A portion of the residue from the ether extract is dissloved in ammonia, and evaporated over the water-bath until neutral to test paper. The residue is stirred in a few drops of warm water, and filtered through a small filter into a narrow test tube. A drop of neutral ferric chloride (prepared by precipitating a portion of the iron from a solution of the salt by ammonia and filtering) is added, and in the presence of benzoic acid a flesh-colored precipitate of ferric benzoate is produced, very characteristic and unmistakable, because of its peculiar color, when the solution in which the test is made is colorless. It occasionally happens, however, in the case of jellies, jams, and ketchups, that these preparations are artificially colored with a dyestuff that persists by its depth of color in obscuring that of the ferric benzoate, especially when only a small amount of benzoic acid is present. Again, in such products as sweet pickles, a precipitate of basic ferric acetate might also come down with the ferric benzoate, and thus confuse. In such cases one of the following methods should be carried out.

(2) Sublimation Method. $\dagger$-Evaporate an ammoniacal solution of the

* Food Inspection Decision 104.

$\dagger$ Annual Report, Mass. State Board of Health, 1902, p. 486. 
ether extract till neutral in a large watch-glass, by the aid of a gentle heat. Fasten with clips or otherwise a second watch-glass to the first, edge to edge, so as to form a double convex chamber, with a cut filterpaper between. Place upon a small sand-bath and heat. Benzoic acid, if present, will sublime upon the surface of the upper glass in minute needles, recognizable under the microscope. It may further be tested by determining the melting-point, or by treating with ammonia, evaporating, and applying the ferric chloride test as above.

(3) Mohler Method Modified by Heide and Jakob.*-Evaporate the ether extract to dryness, take up the residue in I to $3 \mathrm{cc}$. of third-normal sodium hydroxide, and evaporate to dryness. To the residue add 5 to to drops of concentrated sulphuric acid and a small crystal of potassium nitrate. Heat for ten minutes in a glycerol bath at $120^{\circ}$ to $130^{\circ} \mathrm{C}$. (never higher), or for twenty minutes in a boiling water-bath, thus forming metadi-nitro benzoic acid. After cooling add I cc, of water and make decidedly ammoniacal; boil the solution, to break up any ammonium nitrite which may have been formed. Cool and add a drop of fresh colorless ammonium sulphide, without allowing the layers to mix. A red-brown ring (ammonium meta-di-amido benzoic acid) indicates benzoic acid. On mixing, the color diffuses through the whole liquid; on heating it finally changes to greenish yellow, owing to the decomposition of the amido acid, thus distinguishing benzoic from salicylic or cinnamic acids. Both the latter form amido compounds, which are not destroyed by heating. The presence of phenolphthalein interferes with this test.

(4) Peter Oxidation Method. $\dagger$ - This method, depending on the formation of salicylic acid, is not applicable in the presence of this acid or saccharin which also oxidizes to salicylic acid.

Transfer a portion of the residue, say o.r gram, from the ether or chloroform extraction to a large test-tube, and dissolve in from 5 to $8 \mathrm{cc}$. of concentrated sulphuric acid. Add from 0.5 to 0.8 gram of barium peroxide in successive small portions, shaking the tube in cold water. This should produce a permanent froth on the sulphuric acid solution. After standing for half an hour, fill the test-tube three-quarters full of water, shake, cool quickly, and filter. Extract the filtrate with ether or chloroform, and test the extract for salicylic acid.

Determination of Benzoic Acid.-La Wall and Bradshaw Method. Modified.-This process is based on principles brought to notice by

*Zeits. Unters. Nahr. Genuss., I9, I9ro, p. 137. A. O. A. C. Method.

$\dagger$ U. S. Dept. of Agric., Bur. of Chem., Bul. 65, p. 160. 
Moerck.* Although originally devised for catsup, $\dagger$ it has been modified by Bigelow $\ddagger$ and Dunbar, $\S$ so as to be applicable to various classes of foods. The details which follow are those elaborated by Dunbar and adopted by the A. O. A. C.

I. Preparation of Solution.-(a) General.-Grind in a sausage-machine, if solid or semi-solid, and thoroughly mix. Transfer about 150 grams to a 500-cc. flask, add enough pulverized sodium chloride to saturate the water in the sample, make alkaline with sodium hydroxide or milk of lime, and dilute to the mark with saturated salt solution. Allow to stand at least two hours with frequent shaking and filter. If the sample contains large amounts of matter precipitable by salt solution follow a method similar to that given under $(e)$; if large amounts of fats are present it is well to make an alkaline extraction of the filtrate before proceeding as directed under "Extraction and Titration."

(b) Catsup.-To 150 grams of the sample add ${ }_{15}$ grams of pulverized sodium chloride. Transfer the mixture to a $500-\mathrm{cc}$. graduated flask, using about $150 \mathrm{cc}$. of saturated salt solution for rinsing. Make slightly alkaline to litmus paper with strong sodium hydroxide and complete the dilution to $500 \mathrm{cc}$. with saturated salt solution. Allow to stand at least two hours with frequent shaking and then filter through a large folded filter. If difficulty is experienced, centrifuge or squeeze the mixture through a muslin bag before filtering.

(c) Jellies, Jams, Preserves, and Marmalades.-Dissolve 150 grams of the sample in about $300 \mathrm{cc}$. of saturated salt solution. Add I5 grams of pulverized sodium chloride. Make alkaline to litmus-paper with milk of lime. Transfer to a 500-cc. graduated flask, and dilute to the mark with saturated salt solution. Allow to stand at least two hours with frequent shaking, centrifuge, if necessary, and filter through a large folded filter.

(d) Cider and Similar Products Containing Alcohol.-Make $250 \mathrm{cc}$. of the sample alkaline to litmus-paper with sodium hydroxide and evaporate on the steam-bath to about $100 \mathrm{cc}$. Transfer to a 250 -cc. flask, add 30 grams of pulverized sodium chloride and shake until dissolved. Dilute to the mark with saturated salt solution, allow to stand at least two hours with frequent shaking, and filter through a folded filter.

* Proc. Penn. Pharm. Assn., 1905, p. r8r.

† Am. Jour. Pharm., 80, 1908, p. I7 I.

士 A. O. A. C. Proc. 1908, U. S. Dept. of Agric., Bur. of Chem., Bul. I22, p. 68.

\$ Ibid., Proc, rgog, Bul. 132, p. 138; Cire. 66, p. 14. 
(e) Salt or Dried Fish.-Transfer 50 grams of the ground sample to a 500 -cc. flask with water. Make slightly alkaline to litmus-paper with strong sodium hydroxide and dilute to the mark with water. Allow to stand at least two hours with frequent shaking and filter through a folded filter. Pipette at least $300 \mathrm{cc}$. of the filtrate into a second $500-\mathrm{cc}$. flask. add 30 grams of pulverized sodium chloride for each roo cc., shake until dissolved, and dilute to the mark with saturated salt solution. Mix thoroughly and filter off the precipitated protein matter on a folded filter.

2. Extraction and Titration.-Pipette a convenient portion of the filtrate ( 100 to $200 \mathrm{cc}$.), obtained as above, into a separatory funnel. Neutralize to litmus-paper with hydrochloric acid $(1: 3)$ and add an excess of $5 \mathrm{cc}$. In the case of salt fish, protein matter usually precipitates on acidifying, but this does not interfere with the extraction. Extract carefully with chloroform, using, for 200-cc. aliquots, successive portions of $70,50,40$, and $30 \mathrm{cc}$., and proportional quantities for smaller aliquots. To avoid emulsion, shake each time cautiously. The chloroform layer usually separates readily after standing a few minutes. If an emulsion forms, stir the chloroform layer with a glass rod. If this does not break up the emulsion, draw it off into a second funnel and shake sharply once or twice. If this also fails, centrifuge the emulsion for a few moments. Draw off with great care as much of the clear chloroform solution as possible after each extraction. If not contaminated with the emulsion, it is unnecessary to wash the chloroform extract.

Transfer the combined chloroform extract to a dish, rinsing with chloroform, evaporate to dryness at room temperature, either spontaneously or in a current of dry air, and dry over night (or, in case of catsup, until no odor of acetic acid can be detected) in a sulphuric acid desiccator. Dissolve the residue of benzoic acid in 30 to $50 \mathrm{cc}$. of neutral alcohol, add about one-fourth this volume of water, a drop or two of phenolphthalein solution and titrate with twentieth-normal sodium hydroxide. One cc. of the standard solution is equivalent to $0.007^{2}$ gram anhydrous sodium benzoate.

In the absence of a blast an electric fan may be used for evaporating the extract. If it is impracticable to evaporate the chloroform spontaneously or by means of a blast it may be transferred from the separatory funnel to a $300-\mathrm{cc}$. Erlenmeyer flask, rinsing the separatory funnel three times with 5 or ro cc. of chloroform. Distil very carefully to about onefifth the original volume, keeping the temperature down so that the: 
chloroform comes over in drops, not in a steady stream. Then transfer the extract to a porcelain evaporating dish, rinsing the flask three times with 5 or to cc. portions of chloroform, and evaporate to dryness spontaneously.

The evaporation of the chloroform is best effected by delivering to the dish a blast of air dried by means of a calcium chloride bottle.

Hilyer Method.*-This method is valuable as a check on the $\mathrm{La}$ Wall and Bradshaw method. After titrating the benzoic acid obtained as described in the preceding section, proceed as follows:

Evaporate to dryness the accurately neutralized solution (which should not have even a slight alkaline reaction), and redissolve in a few cc. of alcohol saturated with silver.benzoate. Filter if not clear, wash with a few drops of alcohol, and treat with 10 to $15 \mathrm{cc}$. of a saturated solution of silver nitrate in alcohol. Collect the precipitate in a Gooch crucible, care being taken that the asbestos filter is so prepared as to afford as rapid a filtration as possible, wash with alcohol, and finally with a little ether, heat in a water-oven until the ether is removed, cool, and weigh. Care must be taken to perform all the operations as quickly as possible to avoid separation of silver oxide.

West's Distillation Method.†-1. Apparatus.-The special form of double flask for distillation in a current of steam is the same as that employed by Hortvet $\ddagger$ in determining the volatile acids of wine (Fig. Ir5). The steam tube leading from the outer to the inner flask, being introduced half-way up the side of the inner flask, makes it possible to connect the apparatus in such a way that at the beginning of the operation the water in the outer flask will reach to the height of the contents of the inner flask. The side tube leading from the neck of the outer flask is provided with a rubber tube and pinch-cock for use in relieving the steam pressure and avoiding the danger of drawing the contents of the inner flask over into the outer flask.

2. Process.-Weigh into the inner flask of the apparatus to grams, add I.5 to 2.0 grams of paraffin free from volatile matter, and connect with the condenser. Add ro cc. of concentrated sulphuric acid, drop by drop, through the funnel tube at such a rate as to complete the addition

* A. O. A. C. Proc. 1908, U. S. Dept. of Agric., Bur, of Chem., Bul. r22, p. 74; Circ. 66 , p. 15 .

† Jour. Ind. Eng. Chem., I, 1909, p. rgo.

$\ddagger$ Ibid., I, 1909, p. 3 r. 
in two to three minutes, mix thoroughly by gentle agitation, and allow to stand five to ten minutes after all apparent action of the sulphuric acid has stopped. Measure $150 \mathrm{cc}$. of distilled water into the outer flask, heat the water slowly to boiling, and continue the boiling until roo cc. of distillate have been collected, the rate of distillation being such as to yield this amount in 25 to 30 minutes.

Filter the distillate into a separatory funnel, and rinse receiver and filter with two ro-cc. portions of water. Shake with three portions of ether, using $50 \mathrm{cc}$., $30 \mathrm{cc}$., and $20 \mathrm{cc}$., and wash the combined ether extracts by shaking with four 50-cc. portions of water and a last portion of $25 \mathrm{cc}$., which portion should not require more than a drop of tenth-normal alkali for neutralization, indicating the complete removal of volatile acids. Transfer the ether extract to a tared, wide-mouthed flask, and distil off the ether on the water-bath as quickly as possible. At just the point where ebullition of the ether ceases, remove the flask from the bath, blow air into it to remove the last traces of ether, and dry in a desiccator over night, or until constant weight is secured.

The benzoic acid may also be determined by titration, in which case the filtration of the distillate, also the drying and weighing of the acid, may be omitted. The crystals of benzoic acid are dissolved in alcohol carefully neutralized immediately before each analysis, and the solution titrated with tenth-normal alkali.

\section{SULPHUROUS ACID AND THE SULPHITES.}

Free sulphurous acid in the form of sulphur fumes is extensively employed to bleach molasses, to disinfect wine casks, and to bleach and preserve dried fruits. This process is known as "sulphuring." It is stated that the sulphur dioxide combines with the acetaldehyde of wines forming aldehyde-sulphurous acid, which is comparatively harmless. In the case of dried fruits it is believed to form compounds witb the sugars.

The sulphurous acid salts most commonly employed as food preservatives are the bisulphites of sodium and calcium, $\mathrm{NaHSO}_{3}$ and $\mathrm{Ca}\left(\mathrm{HSO}_{3}\right)_{2}$. Others used to some extent are the normal sodium sulphite, and also potassium and ammonium sulphite. The sulphites are usually commercially prepared by passing sulphurous acid gas through strong solutions of the carbonates. Acid sulphites are formed by an excess of the sulphurous acid in the solution of the sulphite. The acid sulphites are distinguishable from the sulphites by their reaction with 
litmus paper, the former being acid, while the latter are neutral or feebly alkaline. All of these salts have a bitter, salty, and highly sulphurous taste, and possess a very pungent, irritating odor. With the exception of normal calcium sulphite, all of the above are readily soluble in water.

The sulphites are most commonly used as preservatives of fruit juices, ketchups, fruit and vegetable pulps, wines, malt liquors and meat prcducts. They are frequently mixed with other antiseptics, as with the salts of salicylic and benzoic acids.

Detection and Determination of Sulphurous Acid. - The same methods are used for the detection of sulphurous acid as for its quantitative determination, except that in the former case weighed quantities need not be employed, and the precipitate obtained by the barium sulphate method need not be weighed.

Distillation Method.-This method is adapted to all food products whether solid or liquid.

- Place 50 to 200 grams of the material in a 500 -cc. flask, add water, if necessary, and $5 \mathrm{cc}$. of a $20 \%$ solution of phosphoric acid, and distil in a current of carbonic acid into water containing a few drops of bromine, until $150 \mathrm{cc}$. have passed over. If sulphides are present, as is true of decomposed meat products and possibly other foods, the steam from the distilling-flask before entering the condenser should be passed through a flask containing $40 \mathrm{cc}$. of a $2 \%$ neutral solution of cadmium chloride* or a ${ }_{1} \%$ solution of copper sulphate. $\dagger$ These solutions effectually remove the hydrogen sulphide, without retaining any appreciable amount of sulphurous acid. To avoid escape of sulphurous acid the condenser tube should dip below the surface of the bromine solution.

The method and apparatus may be simplified without material loss in accuracy by omitting the current of carbon dioxide, adding to cc. of phosphoric acid instead of $5 \mathrm{cc}$., and dropping into the distilling-flask a piece of sodium bicarbonate weighing not more than a gram, immediately before attaching the condenser.

When the distillation is finished, boil off the excess of bromine, dilute to about $250 \mathrm{cc}$, add I cc. of concentrated hydrochloric acid, heat to boiling, and add, drop by drop while boiling, an excess of barium chloride solution. Allow to stand over night in a warm place, filter (preferably on a Gooch crucible with a compact mat of woolly as-

* Horne, U. S. Dept, of Agric,, Bur. Chem., Bul. 105, p. I25.

$\uparrow W$ inton and Bailey, Jour. Am. Chem. Soc., 29, 1907, p. I 499. 
bestos), wash with hot water, ignite at a dull red heat, and weigh as barium sulphate.

Direct Titration Method.*-This method is applicable to sauternes and other white wines and to beer, but should not be used for other materials, unless found by experiment to yield accurate results.

To 25 grams of the sample, finely divided in water if solid or semisolid, add $25 \mathrm{cc}$. of a normal solution of potassium hydroxide in a $200-\mathrm{cc}$. flask. Shake thoroughly, and set aside for at least fifteen minutes with occasional shaking. Io cc. of sulphuric acid $(\mathrm{I}: 3)$ are then added with a little starch solution, and the mixture is titrated with $\mathrm{N} / 5^{\circ}$ iodine solution, introducing the iodine solution quite rapidly, and adding it till a distinct fixed blue color is produced. I cc. of the iodine solution is the equivalent of 0.00064 gram $\mathrm{SO}_{2}$.

FORMIC ACID.

Formic acid $(\mathrm{HCOOH})$ is a colorless liquid at temperatures above $8.3^{\circ} \mathrm{C}$. It boils at $10 \mathrm{I}^{\circ} \mathrm{C}$, has a pungent odor and strong caustic action when applied to the skin, causing great pain and ulceration. It occurs naturally in the bodies of certain ants (hence the name) and in small quantities in various regetable and animal substances.

On a commercial scale formic acid is usually prepared by heating glycerol with oxalic acid, the glycerol ester first formed being saponified by a fresh portion of the oxalic acid and the formic acid separated by distillation.

Formerly this acid was considered to be less active as a preservative than acetic acid, but more recently it has been shown to be very powerful, a water solution containing less than $0.1 \%$ entirely preventing the growth of yeasts and certain bacteria. Recently a $60 \%$ solution has come into use as a preservative for fruit products.

Detection 'of Formic Acid.-Bacon Method. $\uparrow-$ Strongly acidify the solution (which must not contain formaldehyde) with phosphoric acid and distil about one-third of it. To the distillate add dilute sulphuric acid and magnesium filings in sufficient quantities to cause a vigorous but not a violent evolution of hydrogen. In case quite a large quantity of

* U. S. Dept. of Agric., Bur, of Chem., Bul. 65, p. 90.

$\dagger$ U. S. Dept. of Agric., Bur. of Chem., Circ. 74. 
acid is present in the distillate it is not necessary to add any sulphuric acid. If the amount of formic acid is small (about ๑.I\%) continue the action for one hour; if larger quantities are present the reaction will be complete in a few minutes. Test the solution for formaldehyde by the methods given on page 826 .

Shannon Method*-Distil in a current of steam about $1000 \mathrm{cc}$. of the solution, collecting $2500 \mathrm{cc}$. of distillate in a receiver containing $5 \mathrm{cc}$. of lead cream. (The latter is prepared by adding sodium hydroxide to a solution of lead nitrate until a faint pink color appears with phenolphthalein and washing the precipitate 8 to ro times by decantation.) Shake and as the lead dissolves add a few cc. more of the cream until all the formic acid is combined. Evaporate to about $50 \mathrm{cc}$., filter and allow to crystallize in a desiccator. Wash the needle-like crystals of lead formate with absolute alcohol and dry on filter-paper.

An aqueous solution of the crystals should reduce silver nitrate, mercuric or platinum chloride solution on warming and should yield with sulphuric acid on warming in a test tube, carbon monoxide, which burns in the tube. Distilled with concentrated phosphoric acid, the crystals yield formic acid, identified by the acid reaction, the reducing action on the metallic salts as given above, and the formation of formaldehyde when treated according to the Bacon test.

Determination of Formic Acid. - Fincke Method.†-Dilute 25 to 50 grams of the material to $100 \mathrm{cc}$., add I gram of tartaric acid and distil in a current of steam until the distillate amounts to I000-I500 cc. Render slightly alkaline with sodium hydroxide and evaporate to $300 \mathrm{cc}$.

To the neutral or slightly acid solution add $3-5$ grams of sodium acetate and sufficient mercuric chloride solution (100 grams of mercuric chloride and 30 grams of sodium chloride per liter) so that the amount of mercuric chloride added is at least ${ }_{1} 5$ times the amount of formic acid present Heat on a steam bath under a reflux condenser for two hours. Collect the mercurous chloride on a Gooch crucible, wash with water and finally with alcohol and ether, dry at $100^{\circ} \mathrm{C}$. for one hour and weigh. Calculate the formic acid, using the factor 0.0977 .

If sulphurous acid is contained in the material, oxidize in an alkaline solution with hydrogen peroxide and remove the excess of peroxide with freshly precipitated mercuric oxide. In case salicylic acid is present add I gram of sodium chloride for each $50 \mathrm{cc}$. of the distillate.

* Jour. Ind. Eng. Chem., 4, 1912, p. 526.

† Zeits. Unters. Nahr. Genussm., 21, 1911, p. I. 
To separate from formaldehyde or other aldehydes pass the vapor from the distilling flask through a boiling suspension of $\mathrm{I}$ gram of calcium carbonate in $100 \mathrm{cc}$. of water before condensing. Separate the suspended calcium carbonate by filtering and treat the filtrate as described.

Bacon Method:*-Distil the solution containing the formic acid with a small quantity of phosphoric acid until the distillate is no longer acid. If the volume of the distillate is too large to be conveniently handled, neutralize it with sodium hydroxide and evaporate to a convenient volume. Add an excess of platinic chloride and sufficient acetic acid to make the solution strongly acid (usually about $\mathrm{I}$ or $2 \mathrm{cc}$. of glacial acetic acid for less than I gram of formic acid), and boil the solution for one hour, using a reflux condenser. Collect the reduced platinum in the usual manner and weigh. The weight of the platinum multiplied by 0.472 equals the formic acid present.

\section{FLUORIDES, FLUOSILICATES, AND FLUOBORATES.}

These substances all possess strong antiseptic qualities, and while no instances are recorded of the use of the last two classes of compounds in this country, the use of fluorides as a preservative of beer is practiced to some extent. The salt most commonly used is ammonium fluoride $\left(\mathrm{NH}_{4} \mathrm{~F}\right)$, preparations of this salt being sold commercially under various trade names as beer preservatives. Ammonium fluoride exists as small, deliquescent, hexagonal, flat crystals. Its taste is strongly saline. It is soluble in water, and slightly soluble in alcohol. Sodium fluoride $(\mathrm{NaF})$ occurs as clear, lustrous crystals, soluble in water.

Detection of Fluorides.-Modification of Blares' Method.†-Thoroughly mix the sample and heat $\mathrm{r}_{50} \mathrm{cc}$. to boiling. Add to the boiling liquid $5 \mathrm{cc}$. of a $10 \%$ solution of barium acetate. Collect the precipitate in a compact mass, using to advantage a centrifuge, wash upon a small filter, and dry in the oven. Transfer to a platinum crucible, first breaking up the dry precipitate and then adding the filter ash to the crucible. Prepare a glass plate (preferably of the thin variety commonly used for lantern-slide covers) as follows: First thoroughly clean and polish, and coat on one side by carefully dipping while hot in a mixture of equal parts of Canauba wax and paraffin. Near the middle of the plate make a small cross or other distinctive mark through the wax with a sharp.

*U. S. Dept. of Agric., Bur. of Chem., Circ. 74.

$\dagger$ Mass. State Board of Health An. Rep., 1905. p. 498. Chem. News, 91, 1905, p. 39. 
instrument, such as a pointed piece of wood or ivory, which will remove the wax and expose the glass without scratching the latter. Add a few drops of concentrated sulphuric acid to the residue in the crucible, and cover with the waxed plate, having the mark nearly over the center, and making sure that the crucible is firmly imbedded in the wax. Place in close contact with the top or unwaxed surface of the plate a cooling device, consisting of a glass cylinder the bottom of which is closed with a thin sheet of pure rubber. Keep the cylinder filled with ice water, so that the wax does not melt. Heat the bottom of the crucible gently over a low flame or on an electric stove for an hour. Remove the glass plate and indicate the location of the distinguishing mark on the unwaxed surface of the plate by means of gummed strips of paper, melt off the wax by heat or a jet of steam, and thoroughly clean the glass with a soft cloth. A distinct etching will be apparent on the glass where it was exposed, if fluoride be present.

Detection of Fluoborates and Fluosilicates.*-Two hundred cc. of the wine or other sample are made alkaline with lime water, evaporated to dryness, and ignited. The crude ash is first extracted with water acidified with acetic acid, and the solution filtered. The insoluble residue is again ignited and extracted with dilute acetic acid, which is filtered off and added to the first extract. The filtrate contains the boric acid, if present, and this is tested for as directed on page 829. Calcium silicate or fluoride, if present, is in the insoluble portion.

Incinerate the filter with the insoluble portion, transfer the ash to a test-tube, mix with some silica, and add a little concentrated sulphuric acid. A small U-tube should be attached to the test-tube, containing a very little water. The test-tube is immersed for half an hour in a beaker of water kept hot on a steam-bath. In the presence of fluoride, silicon fluoride will be generated, and will be decomposed by the water, forming a gelatinous deposit on the walls of the tube.

If both boric and hydrofluoric acids are found, the compound present is undoubtedly a borofluoride. If no boric acid is found, but silicon fluoride is detected, repeat the operation, but without the added silica. If the silicon skeleton is then formed, fluosilicate is probably present.

* U. S. Dept. of Agric., Bur. of Chem., Bul. 59, p. 63. 


\section{BETA-NAPHTHOL.}

Beta-naphthol $\left(\mathrm{C}_{10} \mathrm{H}_{7} \mathrm{OH}\right)$ is a phenol, occurring naturally in coaltar, but the commercial product is more commonly prepared artificially from naphthalene by digesting the latter with sulphuric acid, and fusing the product with alkali. It is a colorless, or pale buff-colored powder, with a faint phenolic odor and a sharp taste. It is slightly soluble in water, and readily soluble in alcohol, ether, and chloroform. Its melting-point is $122^{\circ} \mathrm{C}$. In alcoholic solution it is neutral to litmus.

It is used to some extent in alcoholic solution as a preservative of cider.

Detection of Beta-Naphthol.-Bube * states that if an ethereal extract of beta-naphthol is evaporated to dryness, and the residue dissolved in hot water made first faintly alkaline with ammonia, and then faintly acid with very dilute nitric acid, a beautiful rose color will be developed on the addition of a drop of fuming nitric acid or of a nitrite. He declares the test to be a delicate one, but it is apparently sometimes obscured by interfering substances, which the ether may dissolve. It should also be carried out in a faint light, as strong sunlight affects the color.

Ferric chloride, when applied to an aqueous solution of beta-naphthol, produces a greenish coloration.

Shake about 50 grams of the sample to be tested with chloroform in a separatory funnel, evaporate the chloroform extract to a small volume (say $\mathrm{I}$ or $2 \mathrm{cc}$.), transfer to a test-tube, add $5 \mathrm{cc}$. of an aqueous solution of potassium hydroxide $(I: 4)$, and warm gently. If beta-naphthol is present, a deep-blue color will appear in the aqueous layer, turning through green to light brown.

\section{ASAPROL, OR ABRASTOL.}

These are trade names for calcium $\alpha$-mono-sulphonate of betanaphthol, $\mathrm{Ca}\left(\mathrm{C}_{10} \mathrm{H}_{6} \mathrm{SO}_{3} \mathrm{OH}\right)_{2}$, a white, odorless, scaly powder, sometimes slightly reddish, obtained by the action of heated sulphuric acid on betanaphthol, the resulting compound being afterwards treated with a calcium salt. It is readily soluble in water and alcohol, and is neutral in reaction. Its taste is at first slightly bitter, but rapidly changes to sweet. It decomposes at about $50^{\circ} \mathrm{C}$.

\footnotetext{
* Analyst, 13 (1888), p. 52.
} 
The writer is unaware of any instance of the presence of this substancein foods, but its character is such as to adapt it for use as a preservative of wines and possibly other food products. It has long been regarded as a possible preservative, and the analyst should be prepared to encounter it at any time.

Detection of Asaprol.-Sinabaldi's Method.*-The portion of the solution to be tested (say $50 \mathrm{cc}$.) is made slightly alkaline with ammonia, and shaken with ro cc. of amyl alcohol in a separatory funnel. Alcohol is often useful in breaking up an emulsion if there is one. Separate the amyl alcohol extract, which if turbid is filtered, and evaporate to dryness. Wet the residue with about 2 cc. of nitric acid ( $\mathrm{I}: \mathrm{I})$, heat on the water-bath till the volume is about I cc., and wash with a few drops of water into a narrow test-tube. Next add about 0.2 gram of ferrous sulphate and ammonia in excess, a drop at a time, constantly shaking the solution. If a reddish-colored precipitate is formed, it is dissolved by the addition of a little sulphuric acid, and further additions of ferrous sulphate and ammonia are made as before. When a dark-colored or green precipitate appears, add $5 \mathrm{cc}$. of alcohol, dissolve in sulphuric acid, shake, and filter. If abrastrol be present to the extent of $0.0 \mathrm{I}$ gram or more, a red coloration is observed, while in its absence, the filtrate is colorless or faintly yellow.

If the solution to be tested is a fat, it should be melted and extracted with hot $20 \%$ alcohol, which is evaporated to dryness, and the above test carried out on the dry residue.

\section{REFERENCES ON PRESERVATIVES AND THEIR USE IN FOOD.}

ABEL, R. Zum Kampfe gegen die Konservierung von Nahrungsmitteln durch Antiseptica. Hyg. Runds., I90I, 265-28r.

AnNetr, H. E. Boric Acid and Formaline as Milk Preservatives. Thompson Yates Lab. Reports, Liverpool, Vol. II, I900, pp. 57-67.

Baldwin, H. B. Toxic Action of Sodium Fluoride. Jour. Am. Chem. Soc., 21, 1899, p. 517 .

Benedicenti. Action of Formaldehyde on Various Proteid Substances. Archw. f. Anat. u. Physiolog., 1897, p. 219 .

BeHRE, A., u. SEgIN, A. Ueber die Wirkung der Konservierungsmittel. Zeits. Unters. Nahr. Genuss., 12, 1906, p. 461 .

\footnotetext{
* Mon. Sэi., 1703 , (4), 7, p. 842 ; U. S. Dept. Agric., Bur. of Chem., Bul. 59, p. 9 r.
} 
Bischoff, H., and Wintgen, U. Beiträge zur Konservenfabrikation. Ztsch. für Hyg., Bd. 34 , 1900, Heft $3,49^{-} 5^{1} 3^{\text {. }}$.

Burss and Novy. Action of Formaldehyde on Enzymes. Jour. Exp. Med., 4, 47.

Chitrenden, R. H. Influence of Borax and Boracic Acid on Digestion. Diet. and Hyg. Gazette, 9, $1893,25$.

Chirtenden and Gres. Effects of Borax and Boric Acid on Nutrition. New York Med. Jour., Feb., 1898 .

- Experiments with Borax and Boric Acid on the Lower Animals. Am. Jour. of Phys., Vol. I, No. I, 1898 .

Dighr, C. F. Effect of Boric Acid and Borax on the Human Body. Minneapolis, 1902.

Folin and Flanders. Determination of Benzoic Acid. J. Am. Chem. Soc., 33, I9II, p. I6r.

Gourn, R. Le Beurre et l'Acide Borique. Jour. d'Agricult prat, I900, p. $14^{-16}$.

Gruber. Ueber die Zulässigkeit der Verwendung der Fluoride zur Konservierung von Lebensmittel. Das Oesterr. Sanitätsw., 1900, 4.

_ Ueber die Zulässigkeit der Verwendung von Chemikalien zur Konservierung von Lebensmittel. Das Oesterr. Sanitätsw., 1900.

GrüNBaud, A. S. Note on the Value of Experiments in the Question of Food Preservatives. Brit. Med. Jour., Igor, p. 1337 .

Halliburton, W. D. Remarks on the Use of Borax and Formaldehyde as Preserva tives of Food. Brit. Med. Jour., I900, Pp. I-2.

Heffter, A. Ueber den Einfluss der Borsäure auf die Ausnutzung der Nahrung. Arbeiten aus dem kaiserlichen Gesundheitsamte, Bd. I9, Part 1, 1902, p. 97.

HiLl, A. Antiseptics in Food. Pub. Health Jour., London, II (I90I), 527.

Hope, E. W. Preservatives and Coloring Matters in Foods. Thompson Yates Lab. Reports, Vol. III (1900), pp. $75-78$.

JACOBJ, C., u. WALBAUM, H. Zur Bestimmung der Grenze der Gesundheitsschädlichkeit der Schwefligen Säure in Nahrungsmitteln. Arch. Exp. Path. Pharm., 54 , I906, p. 421.

Kickion, A. Ueber die Wirkung einiger sogenannter Konservierungsmittel auf Hackfleisch. Zeits. Unters, Nahr. Genuss., I3, 1907 , p. 534.

KISTER, J. Ueber Gesundheitschädlichkeit der Borsäuer als Konservierungsmittel für Nahrungsmittel. Zeit. f. Hygiene, Bd. 37, I9or, Heft 2, p. 225.

Lauge, L. Beiträge zur Frage der Fleischkonservierungmittel. Borsäure, Borax und Schwefeligsauren Natronzusätzen. Mit einem Anhang. Milchkonservierung. betr. Arch. f. Hygiene, Bd. 40, 1901, 2, pp. 143-186.

Lebenn, G. Die Konservierung und Färbung von Fleischwaaren. Hyg. Rund., II, No. 23 .

Lebbin u. Kallmann. Ueber die Zulässigkeit Schwefeligsauer Salze in Nahrungsmitteln. Zeits. für öffentl. Chem., 7, 17, 324-334.

Lebbin, G. Preservation and Coloring of Meat Produce. Translated from the German.

- Should the Use of Boric Acid as a Food Preservative be Permitted? Translated from the German of Die medicinische Woche, Sept., rgor.

Lefrmann, H. Food Preservatives. Penn. Board of Agric., An. Rep., r897, 535. 
Leffmann, H. Influence of Preservatives on Digestive Enzymes. Diet, and Hyg. Gazette, I4, 7 I8.

- Hygienic Relations of Boric Acid and Borax. Diet. and Hyg. Gazette, 14, I7 I. Digestive Ferments and Preservatives. Jour. Frankl. Inst., I47 (I899), 97.

LepIERre. Action of Formaldehyde on Proteids. Bul. Soc. Chem., 21 (1899),p. 729.

Liebreich, O. Effects of Borax and Boric Acidon the Human System. London, 19o2.

- The So-called Danger from the Use of Boric Acid in Preserved Foods. Lancet, I900, pp. 13-15.

- Die Verwendung von Formalin zur Konservierung von Nahrungsmitteln. Therap. Monatsh., 18, 1904, p. 59.

- Zur Frage der Bor-Wirkungen. Berlin, Igo6.

LoEw. Action of Formaldehyde on Pepsin and Diastase. Jour. f. prakt. Chem., 37, 1888, p. 10r.

Low, W. H. Boric Acid: its Deflection and Determination in Small and Large Amounts. Jour. Am. Chem. Soc., 28, 1906, p. 807 .

Neumann, R, O. Ueber den Einfluss des Borax auf dem Stoffwechsel des Menschen. Arbeiten aus dem kaiserlichen Gesundheitsamte, Bd. I9, Pt. I, 1902, p. 89.

FoLFNSKE. Ueber den Borsăuregehalt von frischen und geräucherten Schweineschinken. Loc. cit., 167 .

PrICE, J. M. Die Einwirkung einiger Konservierungsmittel auf die Wirksamkeit der Verdauungsenzyme. Centralb. Bakt. II Abt., 14, 1905, p. 65.

Rideat, S. Formalin as a Milk Preservative. Analyst, 20, p. 157.

- Disinfection and the Preservation of Food. London and New York, rgo3.

On the Use of Boric Acid and Formic Aldehyde as Milk Preservatives. Public Health Jour., London, 11, 1901, p. 554.

RонARDT, W. Ueber Konservierung von frischem Fleisch und über Fleischkonserven von Hygienischen- und Sanitäts-polizeilichem Standpunkt aus. Vierteljahresschrift f. gerichtl. Med, 1901, Heft 2, p. 321.

Rost, E. Ueber die Wirkungen der Borsäure und des Borax auf den thierischen und menschlichen Körper, mit besonderer Berücksichtigung ihrer Verwendung zum Konservieren von Nahrungsmitteln. Arbeiten aus dem kaiserlichen Gesundheitsamte, Bd. 19, Part 1, 1902, P. I.

Z Zur Kenntnis der Ausscheidung der Borsäure. Arch. internat. Pharm. Thér., 15, 1905 , p. 291 .

Rost, E., u. Franz, F. Pharmakologische Wirkungen der Schwefligen Säure. Arb. Kaiserl-Gesundsheitsamt,' 2I, 1904, p. $3^{\text {I2 }}$.

Rubner. Ueber die Wirkung der Borsäure auf den Stoffwechsel des Menschen. Loc. cit, Bd. 19, Part I, 1902, p. 70.

Sonntag, G. Ueber die Quantitative Untersuchung des Ablaufs der Borsäureausscheidung aus dem menschlichen Körper. Loc. cit., Iro.

Stroscher, A. Konservierung u. Keimzahlen des Hackfleishes. Arch. f. Hyg., 40, 190I, pp. $29 \mathrm{I}-3 \mathrm{I} 9$.

Tunincliffe, F. W., and Rosenheim, O. On the Influence of Formaldehyde upon the Metabolism of Children. Jour. of Hygiene (London), Vol. I, 3, rgor.

- On the Influence of Boric Acid and Borax upon the General Metabolism of Children. Loc. cit., supra, rgor, Vol. I, No. 2, pp. 168-202. 
Vaughan, V. C., and Veenboer, W. H. The Use of Boric Acid and Borax as Food Preservatives. Am. Medicine, March, 1902.

VAILlaRd, L. Les Conserves alimentaires de Viande. Rev. d'Hyg., I900, pp. $789-792$.

Walbaum, H. Die Gesundheitsschädlichkeit der Schwefligen Säure und ihrer Verbindungen unter besonderer Bẻrücksichtigung der freien Schwefligen Säure. Arch. Hyg., 57, 1906, p. 87 .

Wertzet, A. Ueber die Labgerinnung der Kuhmilch unter dem Einfluss von Borpräparaten und anderen chemischen Stoffen. Arbeiten aus dem kaiserlichen Gesundheitsamte, Bd. 19, Part I, 1902, p. I26.

WrLeY, H. W. Influence of Food Preservatives and Artificial Colors on Digestion and Health. U. S. Dept. of Agric., Bur. of Chem., Bul. 84. Part I, Boric Acid and Borax; Part II, Salicylic Acid and Salicylates; Part III, Sulphurous Acid and Sulphites; Part IV, Benzoic Acid and Benzoates; Part V, Formaldehyde.

Report of the Departmental Commttee appointed to Inquire into the Use of Preservatives and Coloring Matters in the Preserving and Coloring of Food. 497 pp. London.

Report of REFERfe BOARD of CONSUlting EXPERTS appointed by the Secretary of Agriculture, on the Influence of Sodium Benzoate on the Nutrition and Health of Man. Chittenden, R. H., Long, J. H., and Herter, C. A. U. S. Dept. of Agric., Report No. 88, Washington, 1909 .

U. S. Food Inspection Decisions: No. 76, Dyes, Chemicals, and Preservatives in Foods. No. 89, Amendment to No. 76. No. 101, Benzoate of Soda. No. 104, Amendment to Nos. 76 and 89 . 


\section{CHAPTER XIX.}

\section{ARTIFICIAL SWEETENERS.}

UNDER this head are included the intensely sweet coal-tar derivatives, such as saccharin, dulcin, and glucin, that possess no food value whatever in themselves. From their high sweetening power, in some cases several hundred times that of cane sugar, they are capable, when used in minute quantity, of imparting an appropriate degree of sweetness to food products, which, on account of the use of inferior materials, or by reason of the presence of inert or less sweet adulterants, would otherwise be lacking in this property.

Such canned vegetables as sweet corn and peas are subject to treatment with saccharin, especially if by their age and condition before canning they are wanting in the sweet, succulent taste inherent in the fresh product.

The sweetening power of commercial glucose is considerably less than that of cane sugar, so that when large admixtures of the glucose are used in such products as jellies, jams, honey, molasses, maple syrup, etc., to the exclusion of cane sugar, the presence of the glucose might in some cases be suggested by the bland taste of the food, unless reinforced by one of the artificial sweeteners.

The analyst should therefore be on the outlook for one or another of these concentrated sweetening agents in all of the above classes of foods, especially in saccharine products wherein glucose is found to predominate largely over the cane sugar, while the taste is not lacking in sweetness. The use of saccharin in foods other than those specially designed for invalids is not allowed under the federal law.

\section{SACCHARIN.}

Saccharin or Gluside, Benzoyl sulphimide $\left(\mathrm{C}_{6} \mathrm{H}_{4} \cdot \mathrm{CO} \cdot \mathrm{SO}_{2} \mathrm{NH}\right)$, is a white powder, composed of irregular crystals, whose melting-point, when 
pure, is about $224^{\circ} \mathrm{C}$. It is prepared from toluene, which by treatment with concentrated sulphuric acid is first converted into a mixture of ortho- and para-toluene sulphonic acids. These are further converted into corresponding chlorides, and from the orthochloride, by treatment with ammonia, the imide is formed. It is soluble in 230 parts of cold water, 30 parts of alcohol, and 3 parts of ether. It is sparingly soluble in chloroform, but readily soluble in dilute ammonia. It is from 300 to 500 times as sweet as cane sugar, and, unlike cane sugar, it is not, when pure, charred by the action of concentrated sulphuric acid even on heating. Its aqueous solution is distinctly acid in reaction. Pure saccharin, when heated under diminished pressure, can be sublimed without decomposition.

The addition of I part of saccharin to I, 000 parts of commercial glucose renders the latter as sweet as cane sugar.

A sodium salt of saccharin is found on the market, prepared by neutralizing a solution of saccharin with sodium hydroxide or carbonate. The sodium salt crystallizes in the form of rhombic plates, forming a white powder readily soluble in water, and possessing nearly the same sweetening power as saccharin. It is sometimes put up in the form of tablets for the use of diabetic patients as a substitute for sugar.

Saccharin, aside from its sweet taste possesses, according to Fahlberg and List,* antiseptic properties, and on this account it is sometimes used in beer and other liquors. Squibb states that saccharin has about the same power as boric acid as an antiferment

Detection of Saccharin in Foods. - If the sample to be tested is a solution or syrup, render it acid, if not already such, with phosphoric acid, and extract with ether. In case of canned vegetables and similar goods, finely divide the material by pulping or maceration in a mortar, dilute with water, and strain through muslin. Acidify the filtrate, and extract with ether. $\dagger$ If an emulsion forms, use a centrifugal machine (p. 25), Separate the extract, evaporate off the ether, and test the residue for saccharin as follows:

(I) Add to the residue, if it tastes sweet, a few cubic centimeters of hot water, or preferably a very dilute solution of sodium carbonate, in which saccharin is more soluble. An intensely sweet taste is indicative of its presence. This test, if applied directly, will sometimes fail, especially in the case of beer, by reason of the extraction by the ether of various

* Jour, Soc. Chem, Ind., IV, p. 608.

† Allen states that a purer residue is obtained if the sample of beer be treated with lead acetate, and filtered before extraction with ether. 
bitter principles, such as hop resins, which by their strong, bitter taste mask the sweet taste of saccharin in the residue. Spaeth* recommends that such bitter substances be removed before extraction, which is done by treatment of $500 \mathrm{cc}$. of the beer with a few crystals of copper nitrate, or with a solution of copper sulphate. The flocculent precipitate formed need not be filtered off, but the liquid is preferably concentrated by evaporation to syrupy consistency, acidified with phosphoric acid, and extracted with three successive portions of a mixture of ether and petroleum ether. After extraction, separation, and evaporation of the solvent, dissolve the residue in weak sodium carbonate. As small a quantity as $0.001 \%$ of saccharin can be detected in the final alkaline solution by its sweet taste.

(2) Bornstein's Test. $†$-Heat the residue from the ether extraction of the acidified sample with resorcin and a few drops of sulphuric acid in a test-tube till it begins to swell up. Remove from the flame, and, after cooling till the action quiets down, again heat, repeating the heating and cooling several times. Finally cool, dilute with water, and neutralize with sodium hydroxide. A red-green fluorescence indicates saccharin. Ganter $\ddagger$ states that it is useless to apply this test to beer, in view of the fact that ordinary hop resin gives the same fluorescence.

(3) Schmidt's Test. $\$$-The residue is heated in a porcelain dish with x bout a gram of sodium hydroxide $\|$ for half an hour at a temperature of $250^{\circ} \mathrm{C}$, e either in an air-oven or in a linseed oil bath. This converts the saccharin if present into sodium salicylate. Dissolve the fused mass in water, acidify, and extract the solution with ether. Test the ether residue in the regular manner for salicylic acid with ferric chloride (p. 83I). This test can obviously be applied only in the absence of salicylic acid, which should first be directly tested for.

It is recommended that a mixture of equal parts of ether and petroleumether is preferable to the use of ether alone as a solvent of saccharin, as such a mixture, while readily dissolving saccharin, does not, like ether, dissolve other substances, which might form salicylic acid when fused with sodium hydroxide.

Determination of Saccharin.-When saccharin is fused with an alkali and potassium nitrate, the sulphur is oxidized to sulphuric acid. On

* Zeits, angewandte Chem., 1893 , p. 579 .

† Zeits. anal. Chem., 27 , p. 165 .

$\ddagger$ Ibid., 32, 309 .

\$ Rep. Anal. Chem., 30; Abs. Analyst, 12, p. 200.

\| Potassium hydroxide cannot be used instead of sodium hydroxide for the fusion. 
this principle depends the following method of Reischauer:* A known quantity of the beer or other liquid to be tested is concentrated by evaporation to about one-third its original volume, acidified with phosphoric acid, and extracted by repeated portions of ether. The combined ether extract is evaporated to small volume, and transferred to a platinum crucible, in which it is further brought to dryness. It is then cautiously ignited with a mixture of about 6 parts sodium carbonate and I part potassium nitrate. Dissolve the ftsion in water, acidulate with hydrochloric acid, and determine the sulphuric acid in the usual manner with barium chloride. The weight of the precipitated barium sulphate, multiplied by 0.785 , gives the weight of saccharin. In view of the fact that only small quantities of saccharin are used in beer and other foods, it is best to employ a large portion of the sample for analysis.

\section{DULCIN.}

Dulcin or sucrol, para-phenetol carbamide $\left(\mathrm{C}_{2} \mathrm{H}_{5} \mathrm{O} . \mathrm{C}_{6} \mathrm{H}_{4} \cdot \mathrm{NH} . \mathrm{CO} \cdot \mathrm{NH}_{2}\right)$ is a white powder, composed of needle-like crystals, sparingly sol-tble in cold water, ether, petroleum ether, and chloroform. It dissolves in 800 parts of cold water, 50 parts of boiling water, and 25 parts of $95 \%$ alcohol. It is readily soluble in acetic ether. Its melting-point is about $173^{\circ} \mathrm{C}$. It is not readily sublimed without decomposition. Dulcin is about four hundred times sweeter than cane sugar.

When a mixture of dulcin and dilute sodium hydroxide is subjected to distillation, phenetidin goes over with the steam into the distillate. When this is heated with glacial acetic acid, phenacetin is formed, which may be tested for as follows: Boil with hydrochloric acid, dilute with water, cool, filter if turbid, and add a few drops of a solution of chromic acid. A deep-red color indicates phenacetin.

Detection of Dulcin in Foods.-In view of the comparatively slight solubility of dulcin in ether and chloroform, acetic ether is the best solvent for purposes of removing it from foods, first making it alkaline.

(I) Bellier's Method. $\uparrow-A$ portion of the sample to be tested is mac'e alkaline and extracted with acetic ether. In the case of certain prodictis it is best to subject them to varied preliminary treatment, depending oב the case in hand. With such products as thin fruit syrups, simply make alkaline and shake out with acetic ether. In the case of thick fruit syrups, confectionery, and preserves, dilute with water, add an excess of basic

* Abst. Analyst, 11, p. 234 .

† Ann. de Chim. Anal., 1900, V, pp. 333-337; Abs. Analyst, 26, p. 43 . 
lead acetate, remove the lead by precipitation with sodium sulphate, filter, and make the filtrate alkaline.

With wines, add 2 grams of mercuric acetate and a slight excess of ammonia, shake, and filter.

With beer, add to $200 \mathrm{cc} .2$ or 3 grams of powdered sodium phosphotungstate, and a few drops of sulphuric acid, shake, allow to stand for a few minutes, and filter. Make the filtrate alkaline with ammonia.

Having thus obtained a clarified solution, use from 50 to $200 \mathrm{cc}$. of neutral acetic ether to say $500 \mathrm{cc}$. of the alkaline solution, and shake in a separatory funnel. Separate the extract, filter, and evaporate to dryness. If the dulcin exceeds 0.04 gram per liter, crystals will be apparent in the residue. If fats and resins are present in the residue, make repeated extractions with hot water, and evaporate to dryness. The purified residue is finally brought to dryness in a porcelain dish, and treated with I or $2 \mathrm{cc}$. of sulphuric acid and a few drops of a solution of formaldehyde. Let it stand for fifteen minutes, and afterwards dilute with $5 \mathrm{cc}$. of water. A turbidity or precipitate indicates dulcin.

(2) Jorissen's Test.*-The residue from the acetic ether extract of an alkaline solution of the sample is treated with 2 or $3 \mathrm{cc}$. of boiling water in a test-tube, and a few drops of mercuric nitrate $\dagger$ are added. Heat the tube and its contents for five minutes in a boiling water-bath, withdraw, and disregarding any precipitate, add a small quantity of lead peroxide. On the subsidence of the precipitate, which quickly occurs, a fine violet color appears for a short time in the clear upper layer in presence of 0.001 gram of dulcin.

(3) Morpurgo's Method: $\ddagger$-To the acetic ether residue, evaporated to dryness in a porcelain dish, add a few drops of phenol and concentrated sulphuric acid, and heat a few minutes on the water-bath. After cooling, transfer to a test-tube, and with the least possible mixing pour ammonia or sodium hydroxide over the surface. A blue zone at the plane of contact between the two layers indicates dulcin.

Determination of Dulcin.-For a quantitative determination, Bellier's method is carried out on a weighed or measured portion of the sample, as follows: In the case of alcoholic beverages first expel the alcohol by

* Chem. Zeit. Rep., I896, p. Ir4.

$\dagger$ The mercuric nitrate is prepared by dissolving 2 grams of mercuric oxide in dilute nitric acid, adding sodium hydroxide solution till a slight permanent precipitate is formed, diluting to $15 \mathrm{cc}$, and decanting the clear liquid. p. 89 .

‡ Zeits. anal. Chem., 1896,35 , p. ro4; U. S. Dept. of Agric., Bur. of Chem., Bul. 65 
evaporation, and make up to the original volume with water. Treat the various food preparations with the appropriate clarifying reagents. as in Bellier's qualitative test (p. 853), and, after filtering and making alkaline, extract twice with $50 \mathrm{cc}$. each of acetic ether. The residue is purified if necessary by extraction with hot water as above described, and the final residue is dissolved in I to $5 \mathrm{cc}$. of concentrated sulphuric acid. A few drops of formaldehyde are added. The solution is allowed to stand for fifteen minutes, and then diluted to ten times its volume with distilled water. After twenty-four hours, collect the precipitate on a tared filter, wash with water, dry, and weigh.

\section{GLUCIN.}

This comparatively new sweetening agent is the sodium salt of a mixture of the mono- and di-sulphonic acids of a substance having the composition $\mathrm{C}_{10} \mathrm{H}_{10} \mathrm{~N}_{4}$. In the market it appears as a light-brown powder, readily soluble in water. It is insoluble in ether and chloroform. It decomposes without melting at about $250^{\circ} \mathrm{C}$. It is three hundred times sweeter than cane sugar.

A color reaction with glucin is obtained by dissolving it in dilute hydrochloric acid, cooling by immersing the test-tube in water, and to the cold solution adding a little sodium nitrite solution. Finally, to the liquid is added a few drops of an alkaline solution of beta-naphthol, and a red coloration is produced. With resorcin or salicylic acid in alkaline solution, the color will be yellow.

\section{REFERENCES ON ARTIFICIAL SWEETENERS.}

Alren, A. H. The Detection of Saccharin in Beer. Analyst, ${ }_{3}$ (1888), p. ro5.

Belliter, J. The Detection and Estimacion of Dulcin in Beverages. Ann. de Chem. Anal., 1900, 5, 333; Abs. Analyst, 26, 1901, p. 43.

BerLIOz, F. Influence of Saccharin upon Digestion. Chem. Zeit., 1900, p. 416; Abs. Analyst, 25, 1900, p. 233.

Bucнка, K. V. Künstliche Süssstoffe. Vereinbarung. v. Nahr. u. Genuss. f, d. deuts. Reich., Heft II, p. I34. Berlin, I899.

CоHN, G. Ueber künstliche Süssstoffe. Apoth. Ztg., I898, I3, Pp. 796 and 804 .

Defourner, H. Determination of Saccharin in Food Products. Abs. Analyst, 26, 190r, p. 268.

DennHardt. Versuche zum Nachweise des Duicins. Ber. d. deutsch. pharm. Ges., 1898,6 , p. 287 .

Folin, O. Effect of Saccharin on the Health, Nutrition, and General Metabolism of Man. U. S, Dept. of Agric., Rep. 94. 
Gantter, F. The Detection of Saccharin in Beer. Abs. Analyst, 18, 1893, p. 184.

Gravil., E. D. Notes on Saccharin. Pharm. Jour., 8, 1887 (3), pp. 18, 337.

Herter, C. A. Influence of Saccharin on Digestion, Metabolism, Nutrition, and General Health. U. S. Dept. of Agric., Rep. 94.

Herzfeld, A., and Wolfr, F. Ueber die Bestimmung der künstlichen Süssstoffe in Nahrungsmitteln. Zeits. f. Unters. d. Nahr. u. Genussm., 1898, 1, p. 839 .

Jorissen, A. Neue Methode zum Nachweise von Dulcin in Getränken. Jour. de Pharm. de Liège; Chem. Centr., 1896, I, p. 1084; Abs. Analyst, 21, 1896, p. 164.

Leys, A. A New Test for Saccharin. Ann. de. Chim. anal., 1901, 6, p. 201; Abs. Analyst, 26, 190I, p. 321 .

ReID, E. E. Valuation of Saccharin. Am. Chem. Jour., I899, 2I, p. 461.

Ruger, C. Ueber das Fahlberg'sche Saccharin. Gesundheit, I888, 13, p. $24 \mathrm{I}$.

Scнитrт, C. Ueber den Nachweis der o. Sulfamin-benzoesäuer, genannt "Fahlberg'sches Saccharin." Rep. für anal. Ch., 1887,7 , p. 437 .

SPÄтH, E. Ueber den Nachweis des Saccharins im Bier. Zeits. f. angew. Chemie, IS93, p. 579.

Sutherland, D. A. Saccharin. Jour. Soc. Chem. Ind., 6, 1887, p. 808 .

TAYLoR, W. A. H. Commercial Saccharine. Pharm. Jour., 1887,88 (3), 18, 377.

Tном, H. Ueber Dulcin. Ber. d. deutsch. pharm. Gesellsch., 1893, 3, I33.

Wanters, J. Nachweis des Saccharins im Bier. Moniteur scientifique, 4, t896, 10, I46.

Rep, of the Referee Board of Consulting Scifntific Experts. U. S. Dept. of Agric., Rep. 94, Washington, 1911.

U. S. Food Inspection Decisions: Nos. $\mathrm{r}_{35}, \mathrm{I}_{4}$, and 146 , Saccharin in Food. 


\section{CHAPTER XX.}

FLAVORING EXTRACTS AND THEIR SUBSTITUTES.

OF the three great groups of organic compounds essential for nutrition, the fats and proteins in a state of purity are almost tasteless, as is also true of starch, dextrin, and cellulose of the carbohydrate group. Only the sugars have a pronounced taste. The flavor of food products, aside from their sweetness, is largely due to minor constituents, such as organic acids, ethers, essential oils, etc., which serve chiefly to render the products acceptable to the palate, thereby contributing to their digestibility. Many culinary preparations lacking in flavor, but not in nutritive value, are commonly mixed with substances which supply this deficiency. Spices and flavoring extracts belong to the class of materials added mainly if not entirely for their zest-giving properties.

By far the most extensively used flavoring extracts are those of vanilla and lemon, and in comparison with these the sale of all other varieties is comparatively insignificant. These two favorite extracts are employed in nearly every household, and form a necessary adjunct to almost all forms of desserts, cakes, and confections, as well as to a wide variety of commercial preparations. Others of some importance are extracts of orange, almond, wintergreen, peppermint, rose, and certain spices. Imitation fruit flavors are used in cheap confectionery, ice cream, etc., and are of questionable wholesomeness.

\section{VANILLA EXTRACT.}

The Vanilla Bean is the source of pure vanilla extract, besides being used in chopped form directly as a flavoring agent. It is the fruit of the plant of the Vanilla planifolia, or flat-leaved vanilla. This climbing, perennial plant belongs to the orchid family, and is indigenous to Central and South America and the West Indies, but by far the highest prized beans are cultivated in Mexico. While different varieties differ in some details, the best cured beans of commerce, as a rule, are from 20 to $25 \mathrm{~cm}$. in length and from 4 to $8 \mathrm{~mm}$. thick, drawn out at their ends and curved 
at the base. They are rich dark brown in color, of a soapy or waxy nature to the touch, deeply rifted lengthwise, and covered with fine frostlike crystals of vanillin. When cut cross-wise, the bean exudes a thick, odorless juice, containing calcium oxalate crystals.

The cross-section of the bean is ellipsoidal in shape. The thick brown walls inclose a triangular cavity, in which are the lobed placentas. Between these are papillæ, secreting a finely granular, yellow, balsamlike substance that contributes much to the flavor of the extract, and helps to give the cut bean its delicious odor.

When first gathered, the beans are yellowish green, fleshy, and without odor, developing their peculiar consistency, color, and smell by the process of fermentation or "sweating," which differs in various countries. According to the best methods the beans are sun-dried for nearly a month, being alternately pressed lightly between the folds of blankets, and exposed to the air. After the curing, they are packed in bundles.

Quicker methods of curing consist of the use of artificial heat and calcium chloride for drying, but the products so prepared are considered inferior in quality.

The Mexican vanilla beans are of the choicest grade, and command a high price, sometimes reaching fifteen dollars per pound. The Bourbon beans, grown in the Isle of Réunion, are next in grade. These beans are shorter than the Mexican and much less expensive. They resemble the Tonka bean in odor. Beans from Seychelles and Mauritius are even shorter than the Bourbon beans, and are largely exported to England. Cheaper varieties are those from South America, which do not bring half the price of the Mexican beans, and the cheapest are the Tahiti beans and so-called "vanillons," or beans of the wild vanilla (Vanilla pompona). These latter are used more in sachet powders and perfumes, possessing an odor not unlike heliotrope.

Composition of the Vanilla Bean.-The following are results of the analyses of two varieties of vanilla beans, according to König:

A.

Water.................... 25.85

Nitrogen bodies........... 4.87

Fat and wax............. 6.74

Reducing sugar........... 7.07

Non-nitrogen substances. ..... 30.50

Cellulose................. 19.60

Ash $\ldots \ldots \ldots \ldots \ldots \ldots \ldots \ldots, 4.73$
B.

30.94

$2 \cdot 5^{6}$

4.68

$9 \cdot 12$

32.90

15.27

$4 \cdot 53$ 
Vanillin.-Under "non-nitrogen substances" in the above table is included vanillin, the principle to which vanilla owes its peculiar odor. This body $\left(\mathrm{C}_{8} \mathrm{H}_{8} \mathrm{O}_{3}\right)$ is the methyl ether of protocatechuic aldehyde, and is found on the surface of the bean in fine crystalline needles. It has a sharp but pleasant flavor, is soluble with difficulty in cold water, but readily soluble in hot water, ether, alcohol, and chloroform. Its meltingpoint is $80^{\circ}$ to $8 \mathrm{I}^{\circ} \mathrm{C}$. and it sublimes at $280^{\circ}$. It is present in vanilla beans to an amount varying from I to $2 \frac{3}{4}$ per cent, and it is a curious fact that varieties of bean most highly prized possess the least vanillin. This is shown by Tiemann and Harmann as follows:

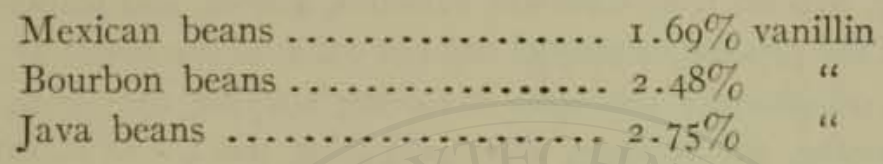

While vanillin may be readily extracted by alcohol and other solvents from the beans, such a product would be far too expensive to compete with the commercial synthetic vanillin, an artificial product, chemically identical with the vanillin from the bean. Synthetic vanillin was formerly made from the glucoside coniferin by oxidation with chromic acid. It is now largely obtained by oxidizing the eugenol of clove oil with alkalinepotassium permanganate.

If ferric chloride be added to an aqueous solution containing vanillin, a dark-blue coloration will be produced.

Besides vanillin, the bean contains notable quantities of wax, fat, sugar, tannin, gum, and resin.

Exhausted Vanilla Beans are sometimes found on sale, which have been deprived of their vanillin by being soaked in alcohol, after which they are coated with some artificial substitute, presenting the same frosty appearance as the natural vanillin crystals. This may be accomplished by rolling the beans in benzoic acid. Benzoic acid crystals are readily distinguished from those of vanillin under the microscope.

Composition of Vanilla Extract.-Vanilla extract is a dilute alcoholic tincture of the vanilla bean, sweetened by cane sugar. To be perfectly pure it should contain no other added substances, with the possible exception of glycerin, and many of the best brands are free from this. In practice i. is variously prepared, but the following method of the U. S. Pharmacopœia is a typical one:

"Vanilla, cut into small pieces and bruised, 100 grams. "Sugar, in coarse powder, 200 grams. 
"Alcohol and water, each, a sufficient quantity to make $\mathrm{I}, 000 \mathrm{cc}$.

"Mix alcohol and water in the proportion of $6_{50} \mathrm{cc}$. of alcohol to $350 \mathrm{cc}$. of water. Macerate the vanilla in $500 \mathrm{cc}$. of this mixture for twelve hours, then drain off the liquid and set it aside. Transfer the vanilla to a mortar, beat it with the sugar into a uniform powder, then pack it in a percolator, and pour upon it the reserved liquid. When this has disappeared from the surface, gradually pour on the menstruum, and continue the percolation, until $1,000 \mathrm{cc}$. of tincture are obtained."

U.S. P. Extracts.- The table on page 861, gives a summary of analyses by Winton and Berry* of vanilla extracts prepared in the laboratory by the U. S. P. process from different varieties, grades, and lengths of vanilla beans. As the process employed did not exhaust the beans as thoroughly as certain commercial processes involving soaking the beans for weeks or even months, the residues after preparing the U. S. P. extracts were further exhausted by soaking for five months in $60 \%$ alcohol and the extracts thus obtained analyzed with the results summarized at the bottom of the table.

A study of the average figures for the different grades and different lengths, irrespective of variety, showed an increase of vanillin but a decrease in normal lead number and color value from the lowest to the highest grade and also from the shortest to the longest bean.

Extracts Prepared with Different Menstrua.-Winton and Berry further found that the composition of the extract was not affected by omission of the sugar entirely, and also that when glycerin was substituted for sugar the only constant affected was the color value, which was somewhat increased. When $35 \%$ alcohol was substituted for the $62 \%$ alcohol of the U. S. P. process the percentage of vanillin was not altered, but the normal lead number, the percentages of color in the lead filtrate and insoluble in amyl alcohol were increased while the color value of the extract itself was decreased. In the preparation of a pure extract the use of alcohol weaker than $45 \%$ is not commercially practicable owing to difficulties in percolation.

Use of Alkali.-Some manufacturers employ dilute alkali, generally potassium bicarbonate, to aid in dissolving out the resinous matter from the bean, and to enable them to use a more dilute alcohol. The resulting product made by this process is distinctly inferior, both in taste and odor.

The Tonka Bean forms the basis of many of the cheaper so-called vanilla extracts on the market. It is the seed of the large tree, native to

* A. O. A. C. Proc., I9r1, U. S. Dept. of Agric., Bur. of Chem., Bul. 152, p. 146. 
U. S. P. VANILLA EXTRACTS MADE IN THE LABORATORY

\begin{tabular}{|c|c|c|c|c|c|c|c|c|c|c|c|c|c|}
\hline \multirow{3}{*}{$\begin{array}{c}\text { Variety } \\
\text { of } \\
\text { Bean. }\end{array}$} & \multirow{3}{*}{ 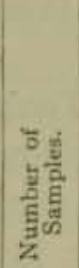 } & \multirow{3}{*}{ 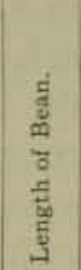 } & \multirow[b]{3}{*}{$\stackrel{\text { ह }}{\text { है }}$} & \multirow{3}{*}{ 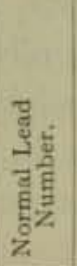 } & \multicolumn{4}{|c|}{ Color Value. } & \multirow{2}{*}{\multicolumn{2}{|c|}{$\begin{array}{l}\text { Per Cent } \\
\text { of Total } \\
\text { Color in } \\
\text { Lead } \\
\text { Filtrate. }\end{array}$}} & \multicolumn{2}{|c|}{$\begin{array}{l}\text { Ratio of } \\
\text { Red to } \\
\text { Yellow. }\end{array}$} & \multirow{3}{*}{ 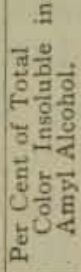 } \\
\hline & & & & & \multicolumn{2}{|c|}{$\begin{array}{l}\text { Extract } \\
\text { (Total } \\
\text { Color). }\end{array}$} & \multicolumn{2}{|c|}{$\begin{array}{l}\text { Lead } \\
\text { Filtrate.* }\end{array}$} & & & & & \\
\hline & & & & & בृ & $\frac{\frac{8}{3}}{3}$ & $\ddot{d}$ & $\frac{6}{3}$ & $\stackrel{d}{\mathscr{u}}$ & $\frac{5}{\frac{6}{3}}$ & 总 & खृ & \\
\hline Mexican ......... & 13 & cm. & $\%$ & $\%$ & & & & & $\%$ & $\%$ & $1:$ & I: & \\
\hline Maximum .......... & $\ldots \ldots$ & 23 & 0.20 & 0.68 & 56 & 154 & 2.0 & 8.0 & 6 & 9 & 3.8 & 5,6 & 24.4 \\
\hline Minimum...... & $\cdots \cdots$ & 15 & 0.15 & 0.47 & 19 & 55 & 1. 0 & 4.8 & 4 & 5 & 2.6 & 4. 0 & 19.0 \\
\hline $\begin{array}{l}\text { Average ........ } \\
\text { Bourbon ........ }\end{array}$ & $\cdots \ddot{16}$ & 19 & 0.17 & 0.58 & 32 & 97 & I. 5 & 6.5 & 5 & 7 & 3.1 & $4 \cdot 5$ & 21.3 \\
\hline Maximum....... & $\ldots \ldots$ & 22 & 0,22 & 0.63 & 55 & 127 & 2.4 & 8.2 & 8 & 10 & 3.9 & 5.0 & 30. \\
\hline Minimum...... & $\ldots .$. & 10 & 0.13 & 0.44 & 22 & 65 & I. 4 & 5. 8 & 4 & 5 & 2.3 & 2.8 & 21.3 \\
\hline Average........ & $\cdots \cdots$ & 16 & 0.18 & 0.52 & 30 & 94 & 1.9 & 7.0 & 6 & 8 & 3.2 & 3.8 & 26.6 \\
\hline 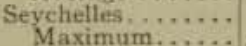 & $\begin{array}{c}9 \\
\ldots . .\end{array}$ & & & & & & & & & & & & \\
\hline $\begin{array}{l}\text { Maximum........ } \\
\text { Minimum ...... }\end{array}$ & $\mid \begin{array}{ll}\ldots \ldots \\
\ldots \ldots\end{array}$ & $\begin{array}{l}22 \\
10\end{array}$ & 0.21 & 0.60 & 50 & 162 & 3. 4 & 14.6 & 7 & 9 & 3.6 & 5.0 & $29 \cdot 4$ \\
\hline $\begin{array}{l}\text { Minimum ....... } \\
\text { Average. ........ }\end{array}$ & & $\begin{array}{l}\text { Io } \\
\text { 16 }\end{array}$ & 0.16 & 0.45 & 22 & 77 & 1. 0 & 5.0 & 4 & 6 & 2,5 & 4.0 & 22,7 \\
\hline $\begin{array}{c}\text { Average. ........ } \\
\text { Madagascar........ }\end{array}$ & $\cdots$ & 16 & 0.10 & 0.51 & 33 & 107 & 1,8 & 7.9 & 5 & 8 & 3.2 & $4 \cdot 5$ & 25.6 \\
\hline Maximum...... & $\ldots \ldots$ & 23 & 0.30 & 0.63 & 47 & 148 & 2.6 & $\mathrm{It} \cdot 5$ & 7 & 9 & 3.5 & 5.1 & 30.3 \\
\hline Minimum...... & $\cdots \cdots$ & II & 0.16 & 0.40 & 25 & 85 & 1. 4 & 6.2 & 4 & 6 & 2.7 & 3.5 & 23,2 \\
\hline Average............. & $\because \cdots$ & 16 & 0.22 & 0.50 & 34 & III & 2.0 & $8 \cdot 7$ & 6. & 8 & 3.2 & $4 \cdot 5$ & 26.8 \\
\hline $\begin{array}{l}\text { Comares.......... } \\
\text { Maximum ....... }\end{array}$ & $\begin{array}{c}\mathbf{x} 6 \\
\cdots\end{array}$ & 21 & $0.3 \mathrm{I}$ & 0.74 & 40 & 1.40 & 2.6 & 12,6 & 8 & 9 & 3.8 & 5.3 & 30,3 \\
\hline Minimum ....... & $\ldots \ldots$ & 10 & 0.12 & 0.40 & 22 & 70 & I. 4 & 6.0 & 5 & 6 & 2,8 & $\begin{array}{l}3.3 \\
3.4\end{array}$ & $\begin{array}{l}30.3 \\
20.4\end{array}$ \\
\hline Average.......... & $\ldots .$. & 15 & 0,18 & 0.59 & 31 & 99 & 1.9 & $7 \cdot 7$ & 6 & 8 & 3.2 & 4.1 & 26.7 \\
\hline South American... & 3 & & & & & & & & & & & & \\
\hline & $\ldots \ldots$ & $\ldots$ & 0.23 & 0.58 & 50 & 155 & 2.6 & 10.4 & 6. & 6 & 3.1 & 4.0 & 29.4 \\
\hline $\begin{array}{l}\text { Minimum........ } \\
\text { Average......... }\end{array}$ & $\cdots \cdots$ & $\ldots$ & 0,19 & 0.49 & 42 & 117 & 1. 8 & 6.8 & 4 & 6 & 2.5 & $3 \cdot 5$ & 20.0 \\
\hline $\begin{array}{l}\text { Average. . . . . . . } \\
\text { Ceylon. . . . . . . . }\end{array}$ & $\begin{array}{c}\cdots+\cdots \\
3\end{array}$ & $\cdots$ & 0.21 & 0.52 & 46 & 134 & 2.3 & 8.5 & 5 & 6 & 2.9 & 3.8 & $23 \cdot 3$ \\
\hline Maximum..... & $\cdots+\cdots$ & 20 & 0.08 & 0.67 & $6 t$ & 195 & 7.6 & 32,6 & 12 & 17 & 3.6 & 4.6 & 50.0 \\
\hline Minimum ....... & $\ldots+\infty$ & 12 & 0.07 & 0.57 & 40 & 145 & $\mathrm{i}, 4$ & 6.4 & 4 & 4 & $3 \cdot 2$ & 4. I & 22.7 \\
\hline $\begin{array}{l}\text { Average,......... } \\
\text { Java }\end{array}$ & $\cdots$ & 16 & 0.08 & 0.62 & 48 & 162 & $4+3$ & 18.2 & 8 & II & $3 \cdot 4$ & $4 \cdot 3$ & 36.1 \\
\hline Muximum....... & $\ldots .$. & 20 & 0.24 & $0.6 \mathrm{t}$ & 45 & 177 & 3.2 & I3. 4 & 7 & to & $3 \cdot 9$ & 4.3 & 35.7 \\
\hline Minimum...... & $\ldots \ldots$ & 10 & 0.22 & 0.44 & 44 & 130 & 2.4 & 10.4 & 5 & 6 & 3.0 & 4. 1 & 32.2 \\
\hline Average......... & $\ldots \ldots$ & 15 & 0.23 & 0.50 & 44 & I5o & 2.9 & $1+1$ & 6 & 8 & 3.4 & 4.2 & 34,5 \\
\hline 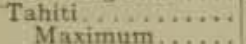 & 2 & & & & & & & & & & & & \\
\hline $\begin{array}{l}\text { Maximum...... } \\
\text { Minimum...... }\end{array}$ & $\cdots \cdots$ & W. & $0 . \mathrm{Ir}$ & 0.50 & 17. & 50 & 0.6 & 3.5 & 4 & 8 & 3,0 & 5.8 & 18.8 \\
\hline Average.............. & $\cdots \cdots$ & $\cdots \cdots$ & o. II & 0.44 & I5 & 40 & 0.6 & 3.1 & 4 & 7 & 2,7 & 5.2 & 16,0 \\
\hline Vanillons.............. & $\cdots+\frac{1}{t}$ & $\cdots \cdots$ & $0.1 \mathrm{r}$ & 0.47 & 16 & 45 & 0.6 & 3.3 & 4 & 8 & 2.9 & 5.5 & 17.4 \\
\hline Tonka Beans t.... & 2 & $\because \ldots$ & 0.06 & 0.52 & 42 & 107 & I. 4 & 6.6 & 3 & 6 & 2,5 & $4 \cdot 7$ & 22,2 \\
\hline Maximum ...... & $\cdots \ldots$ & $\ldots \ldots$ & $\ldots .$. & $0 . \mathrm{rr}$ & 5 & to & 0.5 & $2: 4$ & 10 & 13 & 3.8 & 4.8 & $3 I, 2$ \\
\hline Minimum...... & $\cdots \cdots$ & $\ldots \ldots$ & $2 \ldots \times 6$ & $0 . \mathrm{IT}$ & 5 & 18 & 0.5 & 2.4 & Io & 13 & 3.6 & 4.8 & 30,3 \\
\hline $\begin{array}{c}\text { Average. . . . . . } \\
\text { All Analyses } f \ldots . . .\end{array}$ & $\cdots \cdots$ & & & 0.11 & 5 & 19 & 0.5 & 2.4 & Io & 13 & 3.7 & 4.8 & 30,8 \\
\hline Maximum,..... & $\ldots \ldots$ & 23 & 0.31 & 0.74 & 56 & 1.77 & 3.4 & 14.6 & 8 & 10 & 3.9 & 5. 8 & 35,7 \\
\hline Minimum...... & 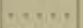 & I0 & 0.11 & 0.40 & 15 & 40 & 0.6 & 3.1 & 4 & 5 & $2-3$ & 2,8 & 16.0 \\
\hline A1I Analyses t...... & $\because 7 \mathrm{t}$ & 16 & 0.19 & 0.54 & 32 & 102 & t. 8 & 76 & 6 & 8 & $3 \cdot 2$ & 4.2 & 25.5 \\
\hline (2d Extraction) & & & & & & & & & & & & & \\
\hline Maximum,...... & & 23 & 0.07 & $0 . \mathrm{tr}$ & I7 & 62 & $0: 5$ & 2,2 & 7 & 6 & $5 \cdot 7$ & 6.5 & \\
\hline Minimum ....... & & Io & 0,01 & 0.03 & 4 & $2 t$ & $0-1$ & 0.8 & 2 & 2 & 2.5 & 3. 0 & \\
\hline Average. ....... & $\ldots \ldots$ & 16 & 0.03 & 9,05 & 9 & 32 & 0.3 & $1: 2$ & 3 & 4 & 3.4 & 4.6 & \\
\hline
\end{tabular}

* Caleulated to volume of extract.

+ Coumarin: Maximum, $0.27 \%$; minimum, $0.22 \%$; average, $0.25 \%$.

‡ Excluding Ceylon, Vanillons, and Tonka Beans. 
Guiana, known as Dipterix (or Coumarouna) odorata. The pods are almond-shaped, and contain a single seed, from 3 to $4 \mathrm{~cm}$. long, shaped like a kidney bean, of a dark-brown color, having a thin, shiny, rough, brittle skin, and containing a two-lobed oily kernel.

Coumarin $\left(\mathrm{C}_{9} \mathrm{H}_{6} \mathrm{O}_{2}\right)$, the active principle of the Tonka bean, is the anhydride of coumaric acid. It occurs in the crystalline state between the lobes of the seed kernel. Coumarin occurs also in many other plants. It may be extracted from the beans by treatment with alcohol. It crystallizes in slender, colorless, needles, melting at $67^{\circ} \mathrm{C}$. It has a fragrant odor and burning taste. It is very slightly soluble in cold water, but readily soluble in hot water, ether, chloroform, and alcohol. One pound of cut beans yields by alcoholic extraction about 108 grains of coumarin. The latter may be synthetically prepared by heating salicylic aldehyde with sodium acetate and acetic anhydride, forming aceto-coumaric acid, which decomposes into acetic acid and coumarin.

The author has found that an aqueous solution of coumarin, unlike vanillin, forms a precipitate when iodine in potassium iodide is added in excess, the precipitate being at first brown and flocculent, afterwards, on shaking, clotting together to form a dark-green, curdy mass, leaving the liquid perfectly clear.

U. S. Standards.-Vanilla extract is the flavoring extract prepared from the vanilla bean, with or without sugar or glycerin, and contains in roo cc. the soluble matters from not less than 10 grams of the vanilla bean.

Vanilla bean is the dried, cured fruit of Vanilla planifolia Andrews.

Tonka extract is the flavoring extract prepared from tonka bean, with or without sugar or glycerin, and contains not less than $0.1 \%$ by weight of coumarin extracted from the tonka bean, together with a corresponding proportion of the other soluble matters thereof.

Tonka bean is the seed of Coumarouna odorata Aublet (Dipteryx odorata (Aubl.) Willd.).

The Adulteration of Vanilla Extract consists chiefly in the use of coumarin or extract of the Tonka bean, and in the substitution of artificial vanillin, either alone or with coumarin, for the true extractives of the vanilla bean. Imitation vanilla flavors more often consist of a mixture of either tincture of Tonka or coumarin with vanillin in weak alcohol, colored with caramel, or occasionally with coal-tar colors. Or the exhausted marc from high-grade vanilla extract is macerated with hot water and extracted, the extract being reinforced with artificial vanillin or coumarin, or both. A pure vanilla extract possesses 
certain peculiarities with regard to its resins and gums that distinguish it from the artificial, or indicate whether or not it has been tampered with. While it is possible to introduce artificial resinous matter in the adulterated brands with a view to deceiving the analyst, it is almost impossible to do this without detection, since different reactions are readily apparent in this case from those of the pure extracts.

Prune juice is said to be used to give body and flavor to vanilla extract. The writer has found spirit of myrcia or bay rum in a sampl. of alleged vanilla extract, containing also vanillin and coumarin. The adulterant in this sample was present to such an extent as to be unmistakable by reason of the odor.

Factitious Vanilla Extracts are ordinarily indicated (I) by the presence of coumarin, (2) by the peculiar reactions of the resinous matter, or by the entire absence of these resins, (3) by the scanty precipitate with lead acetate, and (4) by the abnormally low or high content of vanillin.

The following figures show the content of vanillin and coumarin in a few typical cheap "vanilla" extracts, selected from a large number examined by the author. All of these were entirely artificial, and ranged from 5 to 20 per cent by weight of alcohol.
7. $\begin{array}{cc}\text { Vanillin, } \\ \text { Per Cent. }\end{array}$ Poumarin,
A ...................... $0.040 \quad 0.074$
B . ................... None 0.172
C . .................... None $\quad 0.33^{\circ}$
D ........................ 0.250 None
E.................... $0.025 \quad 0.144$

As a rule these cheap artificial preparations possess considerable body and flavor, but the latter is of a much grosser nature than the genuine vanilla extract, with the delicate and refined flavor of which they are not to be mistaken by any one at all familiar with both varieties.

Winton and Bailey* have found as high as $2.55 \%$ of vanillin in imitation extracts. They also have detected the presence of acetanilide in amounts varying up to $0.15 \%$. This substance at one time was extensively employed as an adulterant of vanillin, hence its presence in imitation extracts prepared from such vanillin. It is not only worthless as a flavor, but is a menace to health.

*Conn. Agric, Exp. Sta., Rep. 1905, p. 13 r. 
.n the limits of composition for standard vanilla extract given on page 870 , the range in vanillin content is from 0.10 to $0.35 \%$.

\section{METHODS OF ANALYSIS OF VANILLA EXTRACT}

Detection of Artificial Extracts.-The presence of coumarin or Tonka tincture to any appreciable extent in vanilla extract is usually recognizable by the odor, to one skilled in examining these flavors. The odor of coumarin is more pungent and penetrating than that of vanillin, and in mixtures is apt to predominate over the milder and more delicate odor of vanillin.

Add normal acetate of lead solution to a suspected extract. The absence of a precipitate is conclusive evidence that it is artificial. If a precipitate is formed, much information may be gained by its character. A pure vanilla extract should yield with lead acetate a heavy precipitate, due to the various extractives. The precipitate should settle in a few minutes, leaving a clear, supernatant, partially decolorized liquid. If only a mere cloudiness is formed, this may be due to the caramel present, and in any event is suspicious.

Examination of the Resins.-Resin is present in vanilla beans to the extent of from 4 to II per cent, and the manufacturer of high-grade essences endeavors to extract as much as possible of this in his product. This he can do by the use of $50 \%$ alcohol, in which all the resin is readily soluble, or by employing less alcohol and relying on the use of alkali to dissolve it. A pure extract free from alkali should produce a precipitate, when a portion of the original sample is diluted with twice its volume of water and shaken in a test-tube.

When, moreover, the alcohol is removed from such an extract, the excess of resin is naturally precipitated.

The character of the resins extracted from the vanilla bean is so different from that of other resins as to furnish conclusive tests, worked out by Hess * as follows: 25 to $50 \mathrm{cc}$. of the extract are de-alcoholized by heating in an evaporating-dish on the water-bath to about one-ihird its volume. Make up to the original volume with water, and, if no alkali has been used in the manufacture of the preparation, the resin will be in the form of a brown, flocculent precipitate. To entirely set free the resin, acidify, after cooling, with dilute hydrochloric acid, and allow to stand till all the resin has settled out, leaving a clear supernatant liquid. The resin may be quantitatively determined, if desired, by filtering, wash-

* Jour. Am. Chem. Soc., 21 (1899), p. 721. 
ing, drying, and weighing, but in this case should stand for a long time before filtering.

The resin is collected on a filter, washed, and subjected to various tests. A piece of the filter with the attached resin is placed in a beaker, containing dilute potassium hydroxide. Pure vanilla resin dissolves to a deep-red color, and is reprecipitated on acidifying with hydrochloric acid. Dissolve another portion of the precipitate in alcohol, and divida the alcoholic solution into two portions, to one of which add a few drops of ferric chloride, and to the other hydrochloric acid. Pure vanilla resin shows no marked coloration in either case, but foreign resins nearly all give color reactions under these conditions.

Tannin.-Test a portion of the filtrate from the resin for tannin by the addition of a few drops of a solution of gelatin. A small quantity of tannin only should be indicated, if the extract is pure, a large excess tending to show added tannin.

Determination of Vanillin and Coumarin.-Modified Hess and Prescott Method.-This process, in its original form devised by Hess and Prescott,* has been modified by Winton, collaborating with Silverman, $\dagger$ Bailey, $\ddagger$ Lott, $\&$, and Berry, $\mid$ in order to prevent loss of coumarin, detect the presence of acetanilide, and permit the determination of normal lead number in the same weighed portion. It depends on the principle that ammonia water, acting on the ether solution of vanillin and coumarin, forms with' the aldehyde vanillin a compound soluble in water, but does not affect the coumarin, which remains in solution in the ether.

Weigh 50 grams of the extract directly into a tared $250-\mathrm{cc}$. beaker with marks showing volumes of 80 and $50 \mathrm{cc}$., dilute to $80 \mathrm{cc}$., and evaporate to $50 \mathrm{cc}$. in a water-bath kept at $70^{\circ} \mathrm{C}$. Dilute again to $80 \mathrm{cc}$. with water and evaporate to $50 \mathrm{cc}$. Transfer to a Ioo-cc. flask, rinsing the beaker with hot water, add $25 \mathrm{cc}$. of standard lead acetate solution ( 80 grams of C. P. crystallized lead acetate, made up to one liter), make up to the mark with water, shake, and allow to stand eighteen hours at a temperature of from $37^{\circ}$ to $40^{\circ} \mathrm{C}$, , in a bacteriological incubator, in a water-bath provided with a thermostat, or in any other suitable apparatus.

\footnotetext{
* Jour. Am. Chem. Soc., 21, 1899, p. 256.

$\dagger$ Ibid., 24, 1902, p. 1128.

† Ibid., 27, 1905, p. 719 .

8 A. O. A. C. Proc. 1909, U. S. Dept, of Agric., Bur. of Chem., Bul. 132, p. rog.

II U. S. Dept. of Agric, Bur, of Chem., Circ, 66.
} 
Filter through a small dry filter and pipette off $50 \mathrm{cc}$. of the filtrate into a separatory funnel.

If a determination of normal lead number is desired, pipette off ro cc. of the filtrate into a beaker, and proceed as described on page 867. In the latter case, the water used throughout the process should be boiled until free from carbon dioxide. If coloring with caramel is suspected determine the color value of the original extract and the filtrate (p. 869).

To the $50 \mathrm{cc}$. of the filtrate in the separatory funnel, add $20 \mathrm{cc}$. of ether and shake. Draw off carefully the aqueous liquid, together with any ether emulsion and then remove the clear ether solution to another separatory funnel. Repeat the shaking of the aqueous liquid with ether three times, using $15 \mathrm{cc}$. each time.

Shake the combined ether solutions four or five times with $2 \%$ ammonium hydroxide, using $10 \mathrm{cc}$. for the first shaking and $3 \mathrm{cc}$. for each subsequent shaking. In drawing off the ammoniacal solution, care should be taken not to allow any of the ether solution to pass through with it. Reserve the ammoniacal solution for the determination of vanillin.

Transfer the ether solution to a weighed dish and allow the ether to evaporate at room temperature. Dry in a sulphuric acid desiccator and weigh. If the residue is pure coumarin, it should have a meltingpoint of $67^{\circ} \mathrm{C}$., respond to the Leach test, and be completely soluble in three or four portions of petroleum ether (boiling-point $30^{\circ}$ to $40^{\circ} \mathrm{C}$.), stirring with each portion fifteen minutes.

If a residue remains in the dish after decanting off the last portion of the petroleum ether solution, acetanilide should be looked for (p. 868).

Add to the ammoniacal solution $10 \%$ hydrochloric acid to slightly acid reaction. This should be done without delay, as the ammoniacal solution on standing grows slowly darker with a loss of vanillin. Cool, and shake out in a separatory funnel with four portions of ether, as described for the first ether extraction. Evaporate the ether solution at room temperature in a weighed dish, dry over sulphuric acid, and weigh. The residue should be pure vanillin free from any appreciable amount of color and with a melting-point of $80^{\circ} \mathrm{C}$.

If the percentage of vanillin is not desired, and coumarin only is to be separated for gravimetric determination, the author has found that good results are usually obtained by simply treating the dealcoholized original sample with ammonia, extracting it with 3 or 4 portions of chloroform in a separatory funnel, and evaporating the combined chloroform extract in a tared dish at a temperature not exceeding $60^{\circ}$ in the oven. 
Many of the precautions employed in carrying out the above processes for vanillin and coumarin determination may be dispensed with if these substances are simply to be tested for qualitatively.

Leach Test for Coumarin. - The residue, believed to be coumarin, obtained as described in the preceding section, is identified by the following test: Add a few drops of water, warm gently, and add to the solution a little iodine in potassium iodide, reagent No. 143. In presence of coumarin a brown precipitate will form, which, on stirring with the rod, will soon gather in dark-green flecks. The reaction is especially marked if done on a white plate or tile.

Wichmann Test for Coumarin.-Dilute 25 cc. of the extract with 25 cc. of water, slightly acidify, if alkaline, with sulphuric acid, and distil to dryness. To the distillate, containing the vanillin and coumarin, add 15 to 20 drops of $\mathrm{I}$ : I potassium hydroxide, hastily evaporate to $5 \mathrm{cc}$., transfer to a test tube and heat over a free flame until the water completely evaporates and the residue fuses to a colorless, or nearly colorless mass. Cool the melt and dissolve in a few cubic centimeters of water, transfer to a $50 \mathrm{cc}$. Erlenmeyer flask and acidify slightly with $25 \%$ sulphuric acid. Finally distil the solution (which should not exceed to cc.) into a test tube containing four or five drops of neutral $0.5 \%$ ferric chloride. If coumarin is present in the original extract, a purple color will develop, the intensity being proportional to the amount of coumarin.

Vanillin and Coumarin Crystals under the Microscope.-These substances are best examined when crystallized from ether solution, and several crystallizations may be found necessary, before the best results are obtained. For examination, pour a few drops of the ether solution of the purified vanillin or coumarin directly on a slide, and allow to evaporate spontaneously. Under best conditions vanillin crystallizes from ether in long, slender needles, often radiating from central points, or forming star-shaped bundles.

Coumarin crystals are shorter and thicker than vanillin.

With polarized light pure vanillin crystals give a brilliant play of colors between crossed nicols, even without the selenite plate, while pure coumarin crystals without the selenite are almost lacking in varying colors, and show very little play, even when the selenite is employed. This sharp distinction is not true when crystallized from chloroform.

Determination of Normal Lead Number.-Winton and Lott Method.Mix the ro-cc. aliquot of the filtrate from the lead acetate precipitate, obtained in the determination of vanillin and coumarin (p. 866), with 
-

25 cc. of water, boiled until free from carbon dioxide, and a moderate excess of sulphuric acid. Add $100 \mathrm{cc}$. of $95 \%$ alcohol, and mix again. Let stand over night, filter on a Gooch crucible, wash with $95 \%$ alcohol, dry at a moderate heat, ignite at low redness for three minutes, taking care to avoid the reducing flame, and weigh. The normal lead number is calculated by the following formula:

$$
P=\frac{100 \times 0.68{ }_{3}(S-W)}{5}={ }_{3} .662(S-W),
$$

in which $P=$ normal lead number, $S=$ grams of lead sulphate corresponding to $2.5 \mathrm{cc}$. of the standard lead acetate solution as determined in blank analyses, and $W=$ grams of lead sulphate obtained in $10 \mathrm{cc}$. of the filtrate from the lead acetate precipitate, as above described.

The standard of the lead acetate solution as determined by blank analyses does not change appreciably on standing; it should, however, be checked from time to time, especially if the bottle is opened frequently, thus permitting absorption of carbon dioxide. In all steps of the process only water free from carbon dioxide should be used.

Pure vanilla extract of standard strength should have a normal lead number not less than 0.40 . Dilution diminishes the number proportionately. For example, a mixture containing $50 \%$ of vanilla extract should have a normal lead number not less than 0.20 and so on.

Determination of Acetanilide.-Winton and Bailey Method.-If in the determination of vanillin and coumarin (p. 865) a residue is found after thoroughly stirring the coumarin with three or four ${ }_{15}$-cc. portions of petroleum ether and decanting off the liquid; allow this residue to stand at room temperature until apparently dry and finish the drying in a sulphuric acid desiccator. Weigh and deduct the weight from that previously obtained, thus obtaining the true amount of coumarin.

The residue, if acetanilide, should melt at $112^{\circ} \mathrm{C}$. and respond to Ritsert's tests as given below.

If acetanilide is found in the coumarin it will also be present in the vanillin, although in smaller amount. Dissolve the weighed residue of impure vanillin in $15 \mathrm{cc}$. of $10 \%$ ammonium hydroxide solution, shake twice with ether, evaporate the ether solution at room temperature, dry in a sulphuric acid desiccator, and weigh. Deduct this weight from the 
weight of impure vanillin, thus correcting for the amount of acetanilide present.

The total weight of acetanilide is found by adding the weight of the portion separated from the coumarin to that separated from the vanillin.

Ritsert's Tests for Acetanilide.*-Boil the acetanilide, obtained as described above, in a small beaker for two or three minutes with 2 to $3 \mathrm{cc}$. of concentrated hydrochloric acid, cool, divide into three portions, and test in small tubes ( 4 to $5 \mathrm{~mm}$. inside diameter), or by spotting on a porcelain plate, as follows:

(I) To one portion add carefully $\mathrm{I}$ to 3 drops of a solution of chlorinated lime $(\mathrm{I}: 200)$ in such a manner that the two solutions do not mix. A Seautiful biue color formed at the juncture of the two liquids indicates acetanilide.

(2) To another portion add a small drop of potassium permanganate solution. A clear green color is formed if any appreciable amount of acetanilide is present.

(3) Mix the third portion with a small drop of $3 \%$ chromic acid solution. Acetanilide gives a yellow-green solution, changing to dark green on standing five minutes, and forming a dark blue precipitate on addition of a drop of caustic potash solution.

These tests are conclusive only when taken in conjunction with the melting-point.

Determination of Glycerin.-The presence of any considerable quantity of glycerin is apparent by the character of the residue obtained on evaporating 5 grams to dryness, in the determination of total solids. The residue, if glycerin is present in notable amount, appears of a moist consistency, even when a practically constant weight has been attained at $100^{\circ} \mathrm{C}$.

To determine glycerin, proceed as with wines (p. 703).

Determination of Alcohol.-Measure out $25 \mathrm{cc}$. of the sample, dilute to $50 \mathrm{cc}$. with water, and distil off about $20 \mathrm{cc}$. in a $25-\mathrm{cc}$. graduated receiver. Make up to the mark with water, determine the specific gravity at $15.6^{\circ}$, and find from the alcohol table the per cent corresponding.

Cane Sugar and Glucose are determined as in the case of preserves and jellies.

Detection of Caramel.--Lead Acetate Method.-Dealcoholize, precipitate with lead acetate, and filter, as described for the determination of vanillin and coumarin (page 865). If the extract is pure, the filtratz

*Pharm. Ztg. 33, 1888, p. 383 ; Abs. Zeits, anal. Chem., 27, 1888, p. 667. 
will be light yellow; if colored with caramel, the filtrate will be yellow brown or deep brown, according to the amount present.

More definite conclusions may be reached by determining the color values of the original extract and the lead acetate filtrate in terms of yellow and red of the Lovibond scale and calculating the ratio of the two colors, also the percentage of each color remaining in the filtrate. The reading of the extract is made in the $\mathrm{I}$-inch cell after diluting $2 \mathrm{cc}$. to $50 \mathrm{cc}$. with $50 \%$ alcohol, while that of the filtrate is made directly in a $\mathrm{I}-$ inch cell or, if very dark, in a half or quarter inch cell.

Color Insoluble in Amyl Alcohol.-Evaporate $25 \mathrm{cc}$. of the extract on a water-bath until no odor of alcohol is apparent and the liquid is reduced to a thick sirup, then proceed as described on page 753 .

Limits of Composition for Standard Vanilla Extract.-The following are suggested by Winton and Berry:

Vanillin, 0.10 to $0.35 \%$.

Normal lead number, 0.40 to $0.80 \%$.

Percent of total color in lead filtrate, not more than 10\% red or 12\% yellow.

Ratio of red to yellow in the extract, not less than $1: 2.2$.

Color insoluble in amyl alcohol, not more than $40 \%$.

Coal-tar Colors are detected by the usual tests (pp. 795 to $8 \mathrm{r} 8$ ).

\section{LEMON EXTRACT.}

Spirit or essence of lemon of the National Formulary and former editions of the Pharmacopœia, is a $5 \%$ solution (by volume) of lemon oil in deodorized alcohol, colored with lemon peel.

This preparation was dropped from the 8 th revision of the Pharmacopœia, and Tinctura limonis corticis or tincture of lemon peel added. The following are the directions for the preparation of the latter:

"Lemon peel, from the fresh fruit, in thin shavings and cut in narrow shreds .................... 500 grams

"Alcohol, a sufficient quantity to make............ rooo cc.

"Macerate the lemon peel in a stoppered, wide-mouthed container, in a moderately warm place, with $1000 \mathrm{cc}$. of alcohol during forty-eight hours, with frequent agitation; then filter through purified cotton, and, when the liquor has drained off completely, gradually pour on enough alcohol to make $1000 \mathrm{cc}$. of tincture, and filter."

U. S. Standards.-Lemon Extract is the flavoring extract prepared from oil of lemon, or from lemon peel, or both, and contains not less than $5 \%$ by volume of oil of lemon. 
Oil of Lemon is the volatile oil obtained, by expression or alcoholic solution, from the fresh peel of the lemon (Citrus limonum L.), has an optical rotation $\left(25^{\circ} \mathrm{C}\right.$.) of not less than $+60^{\circ}$ in a 100-mm. tube, and contains not less than $4 \%$ by weight of citral.

Terpencless Extract of Lemon is the flavoring extract prepared by shaking oil of lemon with dilute alcohol, or by dissolving terpeneless oil of lemon in dilute alcohol, and contains not less than $0.2 \%$ by weight of citrai derived from oil of lemon.

Terpeneless Oil of Lemon is oil of lemon from which all or nearly all of the terpenes have been removed.

The U. S. standard for lemon extract ( $5 \%$ of lemon oil by volume) is a fair one. In fact there are commercial extracts on the market containing as high as ${ }_{12} \%$. An extract of lemon to contain $5 \%$ of lemon oil must contain at least $80 \%$ by volume of alcohol, lemon oil being insoluble in dilute alcohol. Deodorized, or purified alcohol, commonly known as cologne spirits or perfumers' alcohol, is used in the highest-grade preparations, since the odor of ordinary commercial alcohol produces a slightiy deleterious effect.

Adulteration of Lemon Extracts.-For making a cheap extract the cost of the lemon oil is not so important an item as that of the alcohol, and as little as possible of the latter is employed, though pure oil is doubtless used. These terpeneless extracts are made by rubbing the oil in carbonate of magnesia in a mortar, stirring in slowly a little strong alcohol, and allowing the mixture to soak for some time. A varying amount of water is added a little at a time, and the whole is shaken and again allowed to stand, sometimes for a week, before filtering. Finally the extract is filtered, and the coloring matter added, consisting sometimes of turmeric tincture and sometimes of coaltar dyes. In these cheap extracts the per cent of alcohol often runs below 40 , and as little as $4.5 \%$ by volume of alcohol has been found by the author in a commercial extract. With less than $45 \%$ of alcohol by volume, no appreciable amount of oil is dissolved, only a portion of citral, though such preparations are sometimes bottled as "pure extract of lemon." Time and again manufacturers have protested to the author that the purest oil was used by them, when notified that their brand contained no oil, or when prosecuted in court, and were with difficulty convinced that the trouble with their goods was that, on account of weak alcohol employed, the lemon oil used failed to get into the final product. It is true that a certain taste or odor of the lemon is present, even in cheap varieties wherein no oil is found, due to the fact that 
even dilute alcohol, when slowly percolating through the magnesia in which the oil is finely distributed, does abstract therefrom a certain amount of citral, which is, however, but a mere shadow of the substance and body possessed by a strong alcoholic solution of oil of lemon.

In many instances, where formulas appear stating the name and per cent of ingredients, these formulas are entirely deceptive and misleading, in that the statements are not borne out on analysis.

The flavor of the cheap extracts is sometimes reinforced by the addition of such substances as citral, oil of citronella, and oil of lemongrass, but minute quantities only of these pungent materials can be used, not exceeding $0.33 \%$ in the case of citral, and $0.1 \%$ in the case of the two last mentioned oils. Cane sugar and glycerin are sometimes found.

U. S. P. tincture of lemon peel owes its color to natural substances extracted by the alcohol. This color, however, readily fades on exposure to light. Other coloring matters employed are largely coal-tar dyes, and occasionally tincture of turmeric or saffron.

During I IOI practically all the brands of lemon extract sold in Massachusetts were collected and analyzed. ${ }^{6} 67$ samples were examined, representing ahout 100 brands, and $I_{39}$ samples were classed as adulterated, based on $5 \%$ lemon oil as a standard, and depending on whether or not the contents conformed to the labels on the bottles.

The typical analyses, given in tables on p. 873 , are selected from the tabulated results of the above examination.*

Forty-two samples contained no lemon oil, ranging in content of alcohol from 4 to 45 per cent.

\section{METHODS OF ANALYSIS OF LEMON EXTRACT.}

A. S. Mitchell was the earliest among food chemists to systematically examine lemon extract, and to him are due the methods for determining oil of lemon, as well as various other tests now adopted provisionally by the A. O. A. C. $†$

Detection of Oil of Lemon.-If on adding a large excess of water to a little of the extract in a test-tube no cloudiness occurs, the oil may

* An. Rep. Mass. State Board of Health, I901, p. 459; Food and Drug Reprint, p. 41.

$\dagger$ Jour. Am. Chem. Soc., 21, 1899, p. 1132; U. S. Dept. of Agric., Bur. of Chem., Bul. 65 , p. 73 ; Bul. 107 (rev.), p. 159 . 
LEMON EXTRACTS OF STANDARD QUALITY.

\begin{tabular}{|c|c|c|c|c|}
\hline $\begin{array}{l}\text { Polarization } \\
\text { in zoo-mm. } \\
\text { Tube. }\end{array}$ & $\begin{array}{l}\text { Lemon Oil, } \\
\text { Per Cent by } \\
\text { Volume. }\end{array}$ & $\begin{array}{l}\text { Specific } \\
\text { Gravity at } \\
15.6^{\circ} \text { C. }\end{array}$ & $\begin{array}{l}\text { Alcohol, } \\
\text { Per Cent by } \\
\text { Volume. }\end{array}$ & Foreign Ingredients. \\
\hline $\begin{array}{l}30.8 \\
26.0\end{array}$ & $\begin{array}{l}9 . r \\
7.6\end{array}$ & $\begin{array}{l}0.8280 \\
0.8402\end{array}$ & $84 \cdot 39$ & Turmeric \\
\hline 23.5 & 6.9 & 0.8352 & $\begin{array}{l}80.49 \\
8 \mathrm{x} .74\end{array}$ & Dinitrocresol \\
\hline 21.8 & 6.4 & 0.8396 & 82.88 & \\
\hline 20.0 & 5.9 & 0.8335 & $84 \cdot 24$ & \\
\hline 18.0 & $5 \cdot 3$ & 0.8268 & 86.82 & \\
\hline 17.0 & 5.0 & 0.8496 & 80.06 & \\
\hline
\end{tabular}

INFERIOR OR ADULTERATED LEMON EXTRACTS.

\begin{tabular}{|c|c|c|c|c|}
\hline $\begin{array}{l}\text { Polarization } \\
\text { in 2oo-mm. } \\
\text { Tube. }\end{array}$ & $\begin{array}{l}\text { Lemon Oil, } \\
\text { Fer Cent by } \\
\text { Volume. }\end{array}$ & $\begin{array}{l}\text { Specific } \\
\text { Gravity at } \\
\\
55.0^{\circ} \mathrm{C} \text {. }\end{array}$ & $\begin{array}{l}\text { Alcohol, } \\
\text { Per Cent by } \\
\text { Volume. }\end{array}$ & Foreign Ingredients. \\
\hline 14.0 & $4 \cdot \pi$ & 0.8592 & 77.62 & Dinitrocresol \\
\hline 12.2 & 3.6 & 0,8644 & 76.08 & \\
\hline II,O & 3.1 & 0.8620 & $77 \cdot 5^{\circ}$ & A coal-tar dye \\
\hline $9 \cdot 9$ & 2.9 & $0.86 \mathrm{r}_{5}$ & 77.90 & \\
\hline 8.0 & 2.3 & $0.853 \mathrm{r}$ & $8 \mathrm{r} .6 \mathrm{r}$ & Dinitrocresol \\
\hline 6.8 & 2.0 & 0.8416 & 87.55 & Tropacolin \\
\hline 5.0 & $\pi .5$ & 0.8832 & 71.10 & \\
\hline 3.5 & T. 0 & 0.8939 & 67.68 & \\
\hline 2.8 & 0.8 & 0.8995 & 65.23 & Dinitrocresol \\
\hline 2.2 & 0.6 & 0.8941 & 67.69 & \\
\hline 1.4 & 0.4 & 0.9136 & $59 \cdot 40$ & A nitro dye \\
\hline 0.3 & 0.1 & 0.9408 & 46.40 & Dinitrocresol \\
\hline 0.0 & 0.0 & 0.9937 & 4.49 & Tropaeolin \\
\hline-8.0 & 0.0 & ....... & $\ldots \ldots$ & Invert sugar \\
\hline 27.0 & 0.0 & ....... & 27.49 & Cane sugar \\
\hline 0.0 & 0.0 & $\ldots \ldots$ & $47 \cdot 35$ & Oil other than lemon \\
\hline
\end{tabular}

fairly be inferred to be absent. The degree of cloudiness produced is proportional to the amount of lemon oil present.

Determination of Lemon Oil.-Mitchell's Methods.-(I) By Polarization.-Polarize the undiluted extract in a $200-\mathrm{mm}$. tube at $20^{\circ} \mathrm{C}$. Divide the reading on the Ventzke cane sugar scale by 3.4 , and if cane sugar or other optically active substances are absent, the quotient expresses the per cent of lemon oil by volume. With instruments reading in circular degrees, divide the rotation in minutes at $20^{\circ} \mathrm{C}$. by 62.5 . If the Laurent instrument with sugar-scale is used, divide the sugar-scale reading by 4.8 .

Cane sugar, though rarely found in lemon extract, is occasionally used in small amount. It is seid to aid in the solution of the oil. If it is present, wash the solid residue from ro cc. of the sample (dried on a water-bath) with three portions of $5 \mathrm{cc}$. each of ether, to remove waxy 
and fatty matters, dry and weigh the residue of cane sugar, deducting 0.38 from the reading for each $0.1 \%$ of sugar so found.

(2) By Precipitation.-Transfer by a pipette $20 \mathrm{cc}$. of the extract to a Babcock milk-flask, add $\mathrm{x}$ cc. of dilute hydrochloric acid ( $\mathrm{I}: \mathrm{I})$; add 25 to $28 \mathrm{cc}$. of water previously warmed to $60^{\circ} \mathrm{C}$; ; mix, and stand in water at $60^{\circ}$ for five minutes; whirl in a centrifuge for five minutes; fill with warm water to bring the oil into the graduated neck of the flask, and repeat the whirling for two minutes; stand in water at $60^{\circ}$ for a few minutes, and read the per cent of oil by volume. Where the oil of lemon is present in amounts over $2 \%$, add to the percentage of oil found $0.4 \%$ to correct for the oil retained in solution. Where less than $2 \%$ and more than $1 \%$ is present, add $0.3 \%$ for correction.

Save the precipitated oil for the determination of refraction.

When the extract is made in accordance with the U. S. Pharmacopœia, the results by the two methods just given should agree within $0.2 \%$.

To obtain per cent by weight from per cent by volume, as found by either of the above methods, multiply the volume percentage by 0.86 , and divide the result by the specific gravity of the original extract.

Howard's Modification of Mitchell's Precipitation Method.*-Pipette ro cc. of the extract in a Babcock milk bottle, and add in the following order, 25 cc. of cold water, I cc. hydrochloric acid (specific gravity 1.2), and $0.5 \mathrm{cc}$. chloroform. Close the mouth of the bottle with the thumb, and shake vigorously for not less than one minute. Whirl the bottle in a centrifuge for one and one-half to two minutes, thus forcing the chloroform and oil to the bottom of the bottle, and remove all but 3 or $4 \mathrm{cc}$. of the clear supernatant liquid by means of a glass tube of small bore connected with an aspirator.

To the residue add I cc. of ether, agitate thoroughly, plunge the bottle to the neck in a boiling-water bath, holding at slight angle, and rotate in the bath for exactly one minute. This step is best carried out by removing one of the small rings from a water- or steam-bath and holding the bottle in the live steam. The ether serves the purpose of steadily and rapidly sweeping out every trace of chloroform without appreciable loss of oil. Finally, cool the bottle, fill nearly to

* Jour. Am. Chem. Soc., 30, rgo8, p. 608. 
the top of the neck with water at room temperature, centrifuge for one-half minute, read the column of separated oil to the top meniscus, and multiply the reading by two, thus obtaining the per cent of oil.

This method may also be used for determining the oil in extracts of orange, peppermint, clove, cinnamon, and cassia, employing in the case of the heavier oils dilute sulphuric acid $(\mathrm{I}: 2)$, instead of water, in filling the bottles before the last centrifuging.

Determination of Alcohol.-Mitchell has shown that the difference in specific gravity between oil of lemon and stronger alcohol is not so great, but that a very close approximation to the true percentage of alcohol in lemon extracts may be obtained from the specific gravity itself, assuming, of course, that foreign substances, such as sugar, glycerin, etc., are absent. In the absence of such foreign substances determine the specific gravity of the sample, ascertain from the alcohol tables on pages 66I674 , the per cent of alcohol by volume corresponding. This gross figure includes the lemon oil, the per cent of which should be deducted for the correct per cent of alcohol.

In the absence of oil of lemon, a measured portion of the original sample may be distilled, and the percentage of alcohol determined from the distillate in the usual manner, but when lemon oil is present, this should first be removed by diluting $5 \circ \mathrm{cc}$. of the extract with water to $200 \mathrm{cc}$. exclusive of the oil in the sample, and shaking the mixture with 5 grams of magnesium carbonate in a flask, filtering through a dry filter, and determining the alcohol by distillation in a portion of the filtrate. The result is multiplied by 4 to correct for the dilution.

Determination of Total Aldehydes.-Chace's Method.*-1. Reagents. -(a) Aldehyde-free Alcohol.-Allow alcohol ( $95 \%$ by vol.) containing 5 grams of metaphenylene diamine hydrochloride per liter to stand for twenty-four hours with frequent shaking. Previous treatment with potassium hydroxide is unnecessary. Boil under a reflux cooler for at least eight hours, allow to stand over night and distil, rejecting the first 10 and the last 5 per cent which come over. Store in a dark, cool place in well-filled bottles. $25 \mathrm{cc}$. of this alcohol, on standing for twenty minutes in the cooling bath with the fuchsin solution

* Jour. Am. Chem. Soc., 28, 1906, p. 1472. U. S. Dept. of Agric., Bur, of Chem., Bul. I22, p. 32 . 
(20 cc.), should develop only a faint pink coloration. If a stronger color is developed, treat again with metaphenylene diamine hydrochloride.

(b) Fuchsin Solution.-Dissolve 0.5 gram of fuchsin in $250 \mathrm{cc}$. of water, add an aqueous solution of sulphur dioxide containing 16 grams of the gas, and allow to stand until colorless, then make up to I liter with distilled water. This solution should stand twelve hours before using, and should be discarded after three days.

(c) Standard Citral Solution.-Use I mg. of c. p. citral per cc. in $50 \%$ by volume aldehyde-free alcohol. This solution deteriorates on standing, and should not be kept over three or four days.

2. Apparatus,-(a) A Cooling Bath.-Keep at from 14 to $16^{\circ} \mathrm{C}$. The aldehyde-free alcohol, fuchsin solution, and comparison tubes are to be kept in this bath.

(b) Colorimeter--Any form of colorimeter, using a large volume of solution and adapted to rapid manipulation, may be used.

The comparison may also be made in Nessler or Hehner tubes.

3. Manipulation,-Weigh in a stoppered weighing flask approximately 25 grams of extract, transfer to a 50 -cc. flask, and make up to the mark at room temperature with aldehyde-free alcohol. Measure at room temperature and transfer to a comparison tube $2 \mathrm{cc}$. of this solution. Add $25 \mathrm{cc}$. of the aldehyde-free alcohol (previously cooled in a bath), then $20 \mathrm{cc}$. of the fuchsin solution (also cooled), and finally make up to the 5o-cc. mark with more aldehyde-free alcohol. Mix thoroughly, stopper, and place in the cooling bath for fifteen minutes. Prepare a standard for comparison at the same time and in the same manner, using $2 \mathrm{cc}$. of the standard citral solution. Remove and compare the colors developed. Calculate the amount of citral present and repeat the determination, using a quantity sufficient to give the sample apprcximately the strength of the standard. From this result calculate the amount of citral in the sample. If the comparisons are made in Nessler tubes, standards containing I, I.5, 2, 2.5, 3, 3.5, and $4 \mathrm{mg}$. should be prepared, and the trial comparison made against these, the final comparison being made with standards between $\mathrm{I} .5$ and $2.5 \mathrm{mg}$., varying but $0.25 \mathrm{mg}$.

It is absolutely essential to keep the reagents and comparison tubes at the required temperature. Comparisons should be made within one minute after removing the tubes from the bath. Where the comparisons are made in the bath (this being possible only where the bath is glass), the standards should be discarded within twenty-five minutes after 
adding the fuchsin solution. Give samples and standards identical treatment.

Determination of Citral.-Hiltner's Method.*-1. Reagents.-(a) Metaphenylene Diamine Hydrochloride Solution.-Prepare a $1 \%$ solution in $50 \%$ ethyl alcohol. Decolorize by shaking with fuller's earth or animal charcoal, and filter through a double filter. The solution should be bright and clear, free from suspended matter and practically colorless. It is well to prepare only enough solution for the day's work, as it darkens on standing. The color may be removed from old solutions by shaking again with fuller's earth.

(b) Standard Citral Solution.-Dissolve 0.250 gram of c. p. citral in $50 \%$ ethyl alcohol and make up the solution to $250 \mathrm{cc}$.

(c) Alcohol.-For the analysis of lemon extracts, 90 to 95 per cent alcohol should be used, but for terpeneless extracts alcohol of 40 to 50 per cent strength is sufficient. Filter to remove any suspended matter. The alcohol need not be purified from aldehyde. If not practically colorless, render slightly alkaline with sodium hydroxide and distil.

2. Apparatus.-The Schreiner colorimeter (page 77) or Eggertz tubes may be used. With this latter apparatus, alcohol is added, small quantities at a time, to the stronger colored solution until after shaking and viewing transversely, the colors in the two tubes are exactly matched. Calculations are then made by establishing a proportion between the volumes of samples taken and the final dilutions.

3. Manipulation.-All of the operations may be carried on at room temperature. Weigh into a 50 -cc. graduated flask 25 grams of the extract, and make up to the mark with alcohol (90-95 per cent). Stopper the flask and mix the contents thoroughly. Pipette into the colorimeter tube $2 \mathrm{cc}$. of this solution, add Io cc. of metaphenylene diamine hydrochloride reagent, and complete the volume to $50 \mathrm{cc}$. (or other standard volume) with alcohol. Compare at once the color with that of the standard, which should be prepared at the same time, using $2 \mathrm{cc}$. of standard citral solution and ro cc. of the metaphenylene diamine reagent, and making up to standard volume with alcohol. From the result of this first determination, calculate the amount of standard citral solution that should be used in order to give approximately the same citral strength of the sample under examination, then repeat the determination.

* A. O. A. C. Proc., 1908, U. S. Dept. of Agric., Bur. of Chem., Bul. 122, p. 34. Jour. Ind. Eng. Chem., I, I909, p. 798. 
Methyl Alcohol has been used by unscrupulous manufacturers in lemon extracts. It is detected and determined by the refractometer method of Leach and Lythgoe (page 749).

As a confirmatory test for methyl alcohol the distillate, after testing by the Leach and Lythgoe method, may to advantage be subjected to. the method of Mulliken and Scudder,* which depends on the conversion of the methyl alcohol to formaldehyde. The latter method is also useful as a rough preliminary test on the original extract without distillation, the extract, being, however, first diluted until the liquid contains approximately $12 \%$ by weight of alcohol, shaking with magnesium carbonate, and filtering when lemon oil is present.

Oxidize Io cc. of the liquid in a test-tube as follows: Wind copper wire $1 \mathrm{~mm}$. thick upon a rod or pencil 7 to $8 \mathrm{~mm}$. thick, in such a manner as to inclose the fixed end of the wire, and to form a close coil 3 to $3.5 \mathrm{~cm}$. long. Twist the two ends of the wire into a stem $20 \mathrm{~cm}$. long, and bend the stem at right angles about $6 \mathrm{~cm}$. from the free end, or so that the coil may be plunged to the bottom of a test-tube, preferably about $16 \mathrm{~mm}$. wide and $16 \mathrm{~cm}$. long. Heat the coil in the upper or oxidizing flame of a Bunsen burner to a red heat throughout. Plunge the heated coil to the bottom of the test-tube containing the diluted alcohol. Withdraw the coil after a second's time and dip it in water. Repeat the operation from three to five times, or until the film of copper oxide ceases to be reduced. Cool the liquid in the test-tube meanwhile by immersion in cold water.

Test for Formaldehyde.-Divide the oxidized liquid in the test-tube into two parts, testing one for formaldehyde with pure milk by the hydrochloric acid and ferric chloride test. Test the other portion by Mulliken and Scudder's resorcin test for formaldehyde, page 826 , avoiding an excess of the reagent. $\dagger$

fests for Colors.-Evaporate a portion of the sample to dryness, dissolve the residue in water, and extract coal-tar colors if present by Arata's method, page 796 , or with hydrochloric acid.

Much information may often be gained by treatment of the original extract with strong hydrochloric acid. If the color employed be turmeric, no change in color will be evident on addition of the acid. If tropæolin or methyl orange is present, the solution will turn pink, while partial decoloration of the solution indicates naphthol yellow $\mathrm{S}$, and complete decoloration shows presence of dinitrocresols or naphthol yellow.

\footnotetext{
*Am. Chem. Jour., 23, 1899, p. $266 . \quad$ † Ibid, 24, 1900, p. 451.
} 
Test for turmeric by boric acid, page $79 \mathrm{I}$.

Detection of Lemon and Orange Peel Coloring Matter.-Albrech Method.*-Place a few cubic centimeters of the extract in a test-tube and add slowly 3 or 4 volumes of concentrated hydrochloric acid. Place a few cubic centimeters of the extract in a second tube and add several drops of concentrated ammonia. In the presence of lemon or orange peel color the yellow tint of the original extract will be materially deepened in both cases.

Determination of Total Solids and Ash.--Total Solids are estimated by evaporating on the water-bath Io grams of the sample in a tared dish, and drying at $100^{\circ}$ to constant weight. If glycerin be present, it is difficult if not impossible to get a constant weight. Cane sugar and glycerin, if present, will be apparent in the residue. If capsicin has been used, it will be noticed by the taste.

Burn to an ash the residue from the solids in a muffle at a low red heat, cool in a desiccator, and weigh.

Glycerin is determined as in wine, page 703 .

Detection of Tartaric or Citric Acid.-To a portion of the extract in a test-tube add an equal volume of water to precipitate the oil. Filter and add one or two drops of the filtrate to a test-tube half full of cold, clear lime water. If tartaric acid is present, a precipitate will come down, which is soluble in an excess of ammonium chloride or acetic acid.

Filter off the precipitate, or, if no precipitate is visible, heat the contents of the tube. Citric acid will precipitate in a large excess of hot lime water.

Examination of Lemon Oil.-The oil separated from the extract in the process of determining the lemon oil by precipitation (p. 874), is most readily examined for its purity, after drying with calcium chloride, by determination of its specific gravity, its index of refraction, or its refractometric reading with the Zeiss butyro-refractometer, and its polariscopic reading.

The specific gravity and refractometric readings are determined as with fixed oils, using with the butyro-refractometer a sodium flame or yellow bichromate color-screen, which gives perfectly sharp readings without dispersion.

The first table on page 880 shows readings on the Zeiss butyrorefractometer of pure lemon oil at various temperatures, using the sodium light.

*A.O.A.C. Method, Proc. for 1910, U. S. Dept. of Agric., Bur. of Chem., Bul. 137, p. 71 . 
For examination of high polarizing essential oils like oil of lemon, the author employs a $50-\mathrm{mm}$. tube, in order to get the readings on the undiluted oil well within the limits of the cane sugar scale on the polariscope. If such a tube is not available, dilute the oil with an equal volume of alcohol, and use the $100-\mathrm{mm}$. tube. The second table given below expresses constants of pure lemon oils and of various commonly employed adulterants, as determined in the laboratory of the Massachusetts State Board of Health.

READINGS ON ZEISS BUTYRO-REFRACTOMETER OF LEMON OIL.

\begin{tabular}{|c|c|c|c|c|c|c|c|}
\hline $\begin{array}{c}\text { Tempera- } \\
\text { ture, } \\
\text { Centigrade. }\end{array}$ & $\begin{array}{c}\text { Scale } \\
\text { Reading. }\end{array}$ & $\begin{array}{l}\text { Tempera- } \\
\text { ture, } \\
\text { Centigrade. }\end{array}$ & $\begin{array}{c}\text { Scale } \\
\text { Reading. }\end{array}$ & $\begin{array}{l}\text { Tempera- } \\
\text { ture, } \\
\text { Centigrade. }\end{array}$ & $\begin{array}{c}\text { Scale } \\
\text { Reading. }\end{array}$ & $\begin{array}{c}\text { Tempera- } \\
\text { ture. } \\
\text { Centigrade. }\end{array}$ & $\begin{array}{c}\text { Scale } \\
\text { Reading. }\end{array}$ \\
\hline 40.0 & $59 \cdot 4$ & 35.0 & 62.8 & 30.0 & 66.3 & 25.0 & $69 \cdot 7$ \\
\hline $39 \cdot 5$ & 59.7 & $34 \cdot 5$ & 63.1 & $29 \cdot 5$ & 66.6 & $24 \cdot 5$ & 70.0 \\
\hline 39.0 & 60.1 & 34.0 & 63.5 & 29.0 & 67.0 & 24.0 & 70.4 \\
\hline $3^{8} \cdot 5$ & 60.4 & $33 \cdot 5$ & 63.8 & 28.5 & $67-3$ & $23 \cdot 5$ & 70.7 \\
\hline $3^{8} .0$ & 60.8 & 33.0 & $64 \cdot 2$ & 28.0 & $67 \cdot 7$ & 23.0 & $71 \cdot 1$ \\
\hline $37 \cdot 5$ & 61.0 & 32.5 & $64 \cdot 5$ & $27 \cdot 5$ & 68.0 & 22.5 & 71.4 \\
\hline 37.0 & $6 \mathrm{r} \cdot 5$ & 32.0 & $64 \cdot 9$ & 27.0 & 68.4 & 22.0 & 71.8 \\
\hline $3^{6} .5$ & 61.8 & $3 x \cdot 5$ & $65 . \mathrm{I}$ & 26.5 & 68.7 & $2 \mathrm{I} \cdot 5$ & $72 . \mathrm{I}$ \\
\hline 36.0 & 62.1 & 31.0 & 65.6 & 26.0 & 69.0 & 21.0 & 13.5 \\
\hline $35 \cdot 5$ & 62.4 & $30 \cdot 5$ & 65.9 & $25 \cdot 5$ & $69 \cdot 3$ & 20.5 & 72.8 \\
\hline 35.0 & 62.8 & 30.0 & 66.3 & 25.0 & $69 \cdot 7$ & 20.0 & 73.2 \\
\hline
\end{tabular}

CONSTANTS OF SOME ESSENTIAL OILS.

\begin{tabular}{|c|c|c|c|c|}
\hline \multirow{2}{*}{ Oil. } & \multicolumn{2}{|c|}{$\begin{array}{l}\text { Butyro-refractometer } \\
\text { (Sodium Light) at - }\end{array}$} & \multirow{2}{*}{$\begin{array}{l}\text { Rotation } \\
\text { in 100- } \\
\text { Millimeter } \\
\text { Tube, } \\
\text { Ventzke } \\
\text { Scale. }\end{array}$} & \multirow{2}{*}{$\begin{array}{l}\text { Specific } \\
\text { Gravity } \\
\text { at } 15.6^{\circ} \mathrm{C} \text {. }\end{array}$} \\
\hline & Temp. & Reading. & & \\
\hline Oil of lemon (lowest)... & 25. & $69 \cdot 5$ & 173.0 & 0.8580 \\
\hline$"$ "f " (highest)........... & 25. & 71.2 & 184.5 & $0.86 \mathrm{ro}$ \\
\hline 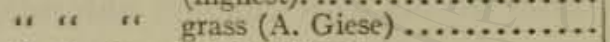 & 22.5 & 96.9 & -10.8 & 0.9309 \\
\hline "t " citronella (A. Giese)................ & 22.5 & 87.1 & -10.2 & 0.9437 \\
\hline Terpeneless oil of lemon (Hansel's)......... & 23. & 87.9 & -22.0 & 0.9463 \\
\hline "s " " " grass (Hansel's)... & 23. & 91.0 & -5.6 & 0.9232 \\
\hline 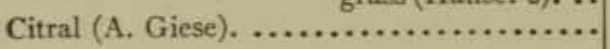 & 22.5 & 95.0 & -3.6 & 0.9296 \\
\hline
\end{tabular}

Oil of Lemon is a light-yellow liquid, having the pleasant odor of fresh lemons, and an aromatic, mild, somewhat bitter after taste. It is obtained from the grated rind of the lemon either by treatment with hot water, skimming off the oil which rises to the surface, or by pressure, or by distillation with water. It is rapidly changed by action of air and light, becoming "terpeney," and under these conditions its solubility in alcohol seems to increase. Its composition is somewhat uncertain, 
but according to Wallach * nearly $90 \%$ consists of hydrocarbons, mostly terpenes, the most important of which is the terpene limonene $\dagger$ of the dextro-gyrate variety, also known as citrene.

Another important constituent of lemon oil is the aldehyde citral, present to the extent of from 4 to* 5 per cent. To this the odor of the oil is largely due. A second aldehyde, citronellal, is also present.

A frequent adulterant of lemon oil is turpentine oil, which lowers the rotation considerably, and is thus most easily rendered apparent.

Chace $\ddagger$ detects small quantities of turpentine by the difference in crystalline form of pinene nitroso-chloride from that of limonene nitrosochloride.

Citral $\left(\mathrm{C}_{10} \mathrm{H}_{16} \mathrm{O}\right)$ is an aldehyde present in lemon oil and in oil of lemon-grass, and, while it may be separated from these oils, is prepared artificially by oxidizing geraniol with chromic acid. $\$$ It is a mobile oil, and when perfectly pure is optically inactive. The commercial citral is, however, slightly lævo-rotary, due no doubt to impurities.

Oil of Lemon-grass is distilled from lemon-grass, Andropogon citratus (D. C.), cultivated in India. It is reddish yellow in color, and has an intense lemon-like odor and taste. Very little is known of its composition, but it seems to contain several aldehydes, one of which is citronellal, and another citral. The latter, however, is its chief constituent, being present to the extent of 70 to 75 per cent.

Citronellal $\left(\mathrm{C}_{10} \mathrm{H}_{18} \mathrm{O}\right)$ is an aldehyde found in various oils, especially in citronella oil, from which it is readily separated. It is made artificially by the oxidation of the primary alcohol citronellol $\left(\mathrm{C}_{10} \mathrm{H}_{20} \mathrm{O}\right)$. It is quite strongly dextro-rotary.

Oil of Citronella is distilled from the grass Andropogon nardus (L.), growing chiefly in Ceylon, India, and tropical East Africa. It is a yellowish-brown liquid with a pleasant and lasting odor. Citronellal is present in this oil to the extent of from to to 20 per cent, and the oil contains also from to to $I_{5}$ per cent of terpenes, among which are camphene.

Tests for Citral, Citronellal, and Limonene. $\Vdash$ Shake $2 \mathrm{cc}$. of the sample to be examined in a corked test-tube with $5 \mathrm{cc}$. of a solution of

- Liebig's Annalen, 227, p. 290.

†There are two limonenes, one of which is dextro- and the other lxvo-rotary. The two are completely alike in their behavior, differing only in their optical rotation.

$\ddagger$ Jour. Am. Chem. Soc.., 30, 1908, p. 1475.

$\$$ Tiemann, Berichte, 31 , p. 3311 .

|| Burgess, Chem. and Drugg., 57, p. 732. 
ro grams of mercuric sulphate in sufficient $25 \%$ sulphuric acid to make too cc. Citral yields a bright-red color, which rapidly disappears, leaving a whitish compound, which floats on top. Citronellal forms a brightyellow color, remaining for some time. Limonene forms an evanescent, faint flesh color, and leaves a white compound.

\section{METHODS OF ANALYSIS OF LEMON OIL.}

The following are the methods of the A.O.A.C.* They apply to orange as well as lemon oil.

Determination of Specific Gravity.-Determine the specific gravity by means of a pycnometer or a Sprengel tube at $15.6^{\circ} \mathrm{C}$.

Determination of Index of Refraction.-Determine the index of refraction with any standard instrument, making the reading at $20^{\circ} \mathrm{C}$.

Determination of Rotation.-Determine the rotation at $20^{\circ} \mathrm{C}$. with any standard instrument using a $50-\mathrm{mm}$. tube and sodium light. The results should be stated in angular degrees on a $100-\mathrm{mm}$. basis. If instruments having the sugar scale are used, the reading on orange oils is above the range of the scale, but readings may be obtained by the use of standard laevo reading quartz plates.

Determination of Citral.-Kleber Method.-I. Reagents.-(a) Phenyl Hydrazin.-A $10 \%$ solution of the purified chemical in absolute alcohol. A sufficiently pure product can be obtained by rectification of the commercial article, rejecting the first portions coming over which contain ammonia.

(b) Hydrochloric Acid.-A half normal solution.

2. Manipulation.-Weigh $\mathrm{I}_{5}$ grams of the sample into a small glassstoppered flask; add ro cc. of the phenyl hydrazin solution. After allowing to stand for half an hour at room temperature, titrate with half normal hydrochloric acid, using either methyl or ethyl orange as indicator. Titrate Io $\mathrm{cc}$. of the phenyl hydrazin reagent in the same manner. The difference in cubic centimeters of half normal acids between this titration and that of the sample, multiplied by the factor 0.076 , gives the weight of citral in the sample.

If difficulty is experienced in detecting the end point of the reaction, carry out the titration until the solution is distinctly acid, transfer to a separatory funnel, and draw off the alcoholic portion. Wash the oil with water, adding the washings to the alcoholic solution, and titrate back with half normal alkali, making the necessary corrections.

\footnotetext{
*U. S. Dept. of Agric., Bul. 137, 1911, p. 72.
} 
Hiltner Method.-Proceed as under lemon extract (p. 877) weighing 2 grams of the oil, diluting to roo cc., and using $2 \mathrm{cc}$. of this solution for the comparison.

Determination of Total Aldehydes.-Proceed as under lemon extract (p. 875), using from 2 to 5 grams of the sample in 100 cc. of aldehydefree alcohol. This method should be used on orange oils the aldehydes of which are not determined by the other methods, although valuable information as to the content of added citral in the oil can be obtained by use of the Hiltner method.

Determination of Physical Constants of the Ten Per Cent Distillate. Schnmmel $\&$ Co. Method.-Place $50 \mathrm{cc}$. of the sample in a 3 -bulb Ladenburg flask in which the main bulb has a diameter of $6 \mathrm{~cm}$. and is of $200 \mathrm{cc}$. capacity and which has the condensing bulbs of the following dimensions: $5.5 \mathrm{~cm}$., $5 \mathrm{~cm} ., 2.5 \mathrm{~cm}$., and in which the distance from the bottom of the flask to the opening of the side arm is $20 \mathrm{~cm}$. Distil the oil at the rate of $2 \mathrm{cc}$. per minute until $5 \mathrm{cc}$. have been distilled. Determine the refractive index and rotation of this distillate as directed above.

Detection of Pinene.-Chace Method.-Mix the 10\% distillate as obtained above with $5 \mathrm{cc}$. of glacial acetic acid, cool the mixture thoroughly in a freezing bath, and add ro cc. of ethyl nitrite; then add slowly, with constant shaking, 2 cc. of a mixture of 2 parts concentrated hydrochloric acid and I part water which has been previously cooled. Keep this mixture in the freezing bath during this operation and allow it to remain therein for $\mathrm{I}_{5}$ minutes. Filter off the crystals formed, using vacuum and washing with strong alcohol. Return the filtrate and washings to the freezing bath and allow them to remain for I $_{5}$ minutes. Filter off the crystals formed, using the original filter-paper. Wash the two crops of crystals thoroughly with alcohol. Dry at room temperature and dissolve in the least possible amount of chloroform. Reprecipitate the nitrosochloride crystals with methyl alcohol and mount for examination under the microscope with olive oil. Pinene nitroso-chloride crystals have irregular pyramidal ends while limonene nitroso-chloride crystallizes in needle forms.

Determination of Alcohol.-The amount of alcohol present in oils which have been used for the manufacture of terpeneless extracts may be approximately determined by washing repeatedly with small portions of saturated sodium chloride solution and determining the alcohol in these washings in the usual way. 


\section{ORANGE EXTRACT.}

Orange Oil is a yellowish liquid, having the characteristic odor of orange, and a mild aromatie taste. It is prepared from orange peel in an analogous manner to that of lemon oil, which it somewhat resembles in chemical composition. At least $90 \%$ of orange oil, according to Walach, consists of dextro-limonene (citrene). It has a much higher specific rotatory power than lemon oil.

U. S. Standards. - Oil of Orange is the volatile oil obtained, by expression or alcoholic solution, from the fresh peel of the orange (Citrus auranium L.) and has an optical rotation at $25^{\circ} \mathrm{C}$. of not less than $+95^{\circ}$ in a roo-mm. tube.

Terpeneless Oil of Orange is oil of orange from which all or nearly all of the terpenes have been removed.

Orange Extract is the flavoring extract prepared from oil of orange, or from orange peel, or both, and contains not less than $5 \%$ by volume of oil of orange.

Terpeneless Extract of Orange is the flavoring extract prepared by shaking oil of orange with dilute alcohol, or by dissolving terpeneless oil of orange in dilute alcohol, and corresponds in flavoring strength to orange extract.

Method of Analysis,-Orange oil and orange extract are analyzed by the same methods as lemon oil (p. 882) and lemon extract (p. 872).

In the determination of orange oil by Mitchell's polariscopic method divide the direct reading on the Ventzke scale, calculated for the $200 \mathrm{~mm}$. tube, by 5.3 to obtain the per cent of orange oil by volume. To obtain the per cent by weight, multiply the per cent by volume by 0.85 and divide by the specific gravity of the extract.

\section{ALMOND EXTRACT.}

Oil of Bitter Almonds is obtained by distilling crushed bitter almonds, peach seeds, or apricot seeds with water. It should be remembered that both sweet and bitter almonds yield a bland fixed oil on pressure, which is not to be confounded with the volatile oil yielded on distillation of the bitter almonds after the fixed oil has been pressed out. Bitter almonds contain a glucoside, amygdalin, together with a ferment known as emulsin or synaptase, which, acting on the amygdalin in the distillation, produces benzaldehyde and hydrocyanic acid as follows: 


$$
\underset{\text { Amygdalin }}{\mathrm{C}_{20} \mathrm{H}_{27} \mathrm{NO}_{11}}+{ }_{2} \mathrm{H}_{2} \mathrm{O}=\underset{\substack{\text { Benzalde } \\ \text { hyde }}}{\mathrm{C}_{7} \mathrm{H}_{6} \mathrm{O}}+\underset{\substack{\text { Hydro } \\ \text { cyanic acid }}}{\mathrm{HCN}}+\underset{\text { Glucose }}{{ }_{2} \mathrm{C}_{6} \mathrm{H}_{12} \mathrm{O}_{6}} \text {. }
$$

The unpurified oil of bitter almonds consists largely of benzaldehyde, with a small amount of the poisonous hydrocyanic acid. Nearly all of the commerical oil is made from the cheaper apricot and peach seeds rather than those of the bitter almond, but the product is practically identical. The oil is freed from hydrocyanic acid by agitating with calcium hydrate and a solution of ferrous chloride, distilling the mixture, and drying the oil which comes over with calcium chloride.

Benzaldehyde constitutes 90 to 95 per cent of oil of bitter almonds, having a bitter, acrid, burning taste, and a marked almond odor. The specific gravity of the crude oil varies from $1.05^{2}$ to 1.082 , while that of the purified oil (benzaldehyde) at $20^{\circ}$ is 1.0455 . Its boiling-point is $180^{\circ} \mathrm{C}$. On standing it becomes readily oxidizable to benzoic acid. It is readily soluble in alcohol and ether. Its solubility in water is slight, $\mathrm{I}: 300$. Its index of refraction at $20^{\circ} \mathrm{C}$. is I.5446. It should be noted that the refractive indices of almond oil, whether with or without hydrocyanic acid, and of artificial benzaldehyde are nearly the same.

Benzaldehyde is produced artificially in a variety of ways, but is chiefly prepared by the action of chlorine on hot toluene. The resulting benzyl chloride is distilled with lead nitrate and water in an atmosphere of carbon dioxide, which forms benzoic aldehyde. Synthetic benzaldehyde has the same properties as the purified oil of bitter almonds, and has largely displaced it in the market, not the least of its advantages being its freedom from hydrocyanic acid.

Almond Extract.-Essence of bitter almonds, or Spiritus amygdala amare, is thus prepared according to the U. S. Pharmacopœia:

Oil of bitter almonds . . . . . . .

Alcohol ......................... $800 \mathrm{cc}$.

Distilled water sufficient to make........ rooo cc.

Thus $1 \%$ of almond oil is present in the product.

U. S. Standards.-Oil of Bitler Almonds, commercial, is the volatile oil obtained from the seed of the bitter almond (Amygdalus communis L.), the apricot (Prunus armeniaca L.), or the peach (Amygdalus persica L.).

Almond Extract is the flavoring extract prepared from oil of bitter 
almonds, free from hydrocyanic acid, and contains not less than $1 \%$ by volume of oil of bitter almonds.

Adulteration of Almond Oil.-The official essence of the Pharmacopoia does not specify that the almond oil used be perfectly free from hydrocyanic acid, in spite of the fact that its highly poisonous nature is well known, and that it exists in the crude oil to the extent of from 4 to 6 per cent. True, but little of it is found in the extract, but in these days, when the unannounced presence in foods of such substances as antiseptics and coloring matters is regarded as questionable from a sanitary standpoint, in spite of the fact that their toxic effects on man are still matters of controversy, there thould be little hesitancy in pronouncing the presence of prussic acid objectionable, especially when a pure almond oil entirely free from it is readily obtainable.

The presence of nitrobenzol or oil of mirbane as a substitute of almond oil is to be looked for. This substance is sometimes, though incorrectly, called artificial oil of bitter almonds. It is a heavy, yellow liquid of the composition $\mathrm{C}_{6} \mathrm{H}_{5} \mathrm{NO}_{2}$, readily soluble in water. Its specific gravity at $20^{\circ} \mathrm{C}$. is 1.2039 . Its boiling-point is $205^{\circ} \mathrm{C}$. It is formed by the action of nitric acid on benzol. It possesses a highly pungent odor, somewhat like that of oil of bitter almonds, though more penetrating and less refined. Its index of refraction at $20^{\circ} \mathrm{C}$. is $1.55^{1} 7$.

\section{METHODS OF ANALYSIS OF ALMOND EXTRACT.}

Determination of Benzaldehyde.-Denis and Dunbar Method.*-I. Reagent.-Mix $30 \mathrm{cc}$. of glacial acetic acid with $40 \mathrm{cc}$. of water, then pour in $2 \mathrm{cc}$. of phenyl hydrazine. The reagent should be made up immediately before use and discarded when more than an hour old.

2. Method.-Measure out two portions of $10 \mathrm{cc}$. each of the extract into $300-\mathrm{cc}$. Erlenmeyer flasks and add Io cc. of the reagent to one flask and $\mathrm{I}_{5} \mathrm{cc}$. to the other. Shake, stopper tightly, and allow to stand in a dark place over night. Add 200 cc. of distilled water and filter the precipitate of hydrazone on a tared Gooch crucible provided with a thin coat of asbestos. Wash first with cold water, finally with 10 cc. of $10 \%$ alcohol, and dry for three hours in a vacuum-oven at $70^{\circ} \mathrm{C}$., or to constant weight over sulphuric acid. The weight of the precipitate multiplied by the factor 5.408 , will give the weight of benzaldehyde in $100 \mathrm{cc}$. of the sample. If duplicate determinations do not agree, repeat the operations, using a larger quantity of the reagent.

\footnotetext{
* Jour. Ind. Eng. Chem., 1, r909, p. 256, A. O. A. C. Method.
} 
Hortvet and West Method.*-Measure ro cc. of the extract into a roo-cc. flask, add 10 cc. of a 10\% sodium hydroxide solution, and $20 \mathrm{cc}$. of a $3 \%$ hydrogen peroxide solution, cover with a watch-glass and place on a water-oven. Oxidation of the aldehyde to benzoic acid begins almost immediately and should be continued from five to ten minutes after all odor of benzaldehyde has disappeared, which usually requires from twenty to thirty minutes. If nitrobenzol be present, it will be indicated at this point by its odor. When the oxidation of the aldehyde is complete, remove the flask from the water-oven, transfer the contents to a separatory funnel, rinsing off the watch-glass, add to cc. of a $20 \%$ sulphuric acid solution, and cool the contents of the funnel to room temperature under the water tap. Extract the benzoic acid with three portions of 50,30 , and $20 \mathrm{cc}$. of ether, respectively, wash the combined extracts in another separatory funnel with two portions of from 25 to $30 \mathrm{cc}$. of distilled water, or until all the sulphuric acid is removed. Filter into a tared dish, wash with ether, allow to evaporate at room temperature, and finally dry over night in a desiccator, and weigh. The per cent of benzaldehyde $(B)$ is obtained from the weight of the acid $(W)$ by the following formula:

$$
B=\frac{0.869 \times 10 \times W}{1.045} .
$$

If desired the benzoic acid may be titrated, and the benzaldehyde calculated from the amount of standard alkali required for neutralization. The process is as follows: Dissolve the benzoic acid obtained as above described, except that it need not be dried in a desiccator, in $95 \%$ alcohol made neutral to phenolphthalein with tenth-normal sodium hydroxide, dilute with an equal volume of water, and titrate with tenthnormal sodium hydroxide, using phenolphthalein as indicator. The per cent of benzaldehyde $(B)$ is calculated from the cc. of tenth-normal alkali $(V)$ by the following formula:

$$
B=\frac{V \times 0.0106 \mathrm{I} \times 10}{1.045} \text {. }
$$

Detection of Nitrobenzol. $\uparrow-$ Boil ${ }_{15} \mathrm{cc}$. of the extract in a test-tube with a few drops of a strong solution of potassium hydroxide. Nitrobenzol produces a blood-red coloration.

* Jour. Ind. Eng. Chem., 1, 1909, p. 86.

† Holde, Jour. Soc. Chem. Ind., 13, 1893, p. 906. 
Distinction between Benzaldehyde and Nitrobenzol.-Treat $20 \mathrm{cc}$. of the extract with 5 to $10 \mathrm{cc}$. of a cold, saturated aqueous solution of sodium bisulphite in a test-tube, and shake vigorously. Transfer to an evaporating-dish, and heat on the water-bath till the alcohol is driven off. At this stage benzaldehyde remains in the hot solution as a crystalline salt, and the solution gives off no almond odor.

Nitrobenzol, on the contrary, does not combine wi.h the bisulphi:e and is insoluble, forming globules of oil on the surface of the hot liquid, and in addition giving off the pungent odor so characteristic of the subs ance.

Separation of Nitrobenzol and Benzaldehyde.-If by the qualitative test nitrobenzol is found, shake vigorously as before $50 \mathrm{cc}$. of the extract with $10 \mathrm{cc}$. of the saturated sodium bisulphite solution in a corked flask, and transfer with $100 \mathrm{cc}$. of water to a large separatory funnel. Shake out the nitrobenzol from the solution with four successive portions of petroleum ether of 15 to $20 \mathrm{cc}$. each, and after washing with water the combined petroleum ether, transfer it to a tared dish, in which it is allowed to evaporate spontaneously.

It is extremely difficult to avoid loss of some of the nitrobenzol by this process, but even if the weighed residue fails to shew the full amount originally used, enough will usually be extracted to admit of testing on the refractometer, and of otherwise verifying its character.

After removal of the nitrobenzol, make the residual solution in the separatory funnel strongly alkaline with sodium hydroxide, and shake out the benzaldehyde, if present, with petroleum ether as previotsly described. If after making the solution alkaline no odor of benzaldehyde is apparent, the absence of benzaldehyde may be inferred.

Distinction between Artificial Benzaldehyde and Pure Almond Oil.Test the final residue from the ether extract by shaking with an equal volume of concentrated sulphuric acid in a test-tube. Wi.h natural oil of almonds a clear, brilliant, but dark currant-red color is produced, while with artificial benzaldehyde, the acid produces a dirty brown color with the formation of a precipitate.

Determination of Alcohol,-In the absence of other flavoring substances than nitrobenzol and benzaldehyde, which are rarely present to an extent exceeding $\mathrm{r} \%$, a sufficiently close approximation for most purposes can be gained by estimating the alcohol from the direct specific gravity of the extract.

Detection of Hydrocyanic Acid.-To a few cubic centimeters of 
extract in a test-tube add a few drops of a mixture of solutions of ferrous sulphate and ferric chloride, the ferrous salt being in excess. Make alkaline with sodium hydroxide, and add enough dilute hydrochloric acid to dissolve the precipitate formed by the alkali. Presence of a blue coloration or precipitate, duc to the formation of Prussian blue, indicates hydrocyanic acid. The reaction is very delicate.

Determination of Hydrocyanic Acid.*-Hydrocyanic acid may be determined by titration with tenth-normal silver nitrate solution. $25 \mathrm{cc}$. of the extract are measured into a flask, and $5 \mathrm{cc}$. of freshly prepared magnesium hydroxide suspended in water are added, or enough to make the reaction alkaline.

A few drops of a solution of potassium chromate are then introduced, and the tenth-normal silver nitrate solution added till, with shaking, the formation of the red silver chromate indicates the end-point. I cc. of silver solution equals 0.0027 gram of hydrocyanic acid.

\section{WINTERGREEN EXTRACT.}

Wintergreen Oil.-True oil of wintergreen is obtained by distillation from the leaves of the wintergreen plant (Gaultheria procumbens L.). Gildermeister and Hoffman $\dagger$ state that the specific gravity at $15^{\circ}$ is I.I80 to 1.187 , the boiling-point 218 to $221^{\circ} \mathrm{C}$. It is slightly lævorotatory $\left(a_{D}=-0.0^{\circ} 25^{\prime}\right.$ to $\left.-1^{\circ}\right)$.

Oil of betula or sweet birch is distilled from the bark of the black birch (Betula lenta L.). It has the same specific gravity and boilingpoint as oil of wintergreen, but unlike the latter is optically inactive. It differs somewhat from oil of wintergreen in taste and odor, but is hardly distinguishable in these respects from synthetic methyl salicylate.

Both oil of wintergreen and oil of sweet birch consist almost entirely of methyl salicylate, the former containing, according to Power and Kleber, $\ddagger$ as much as $99.8 \%$ of this substance.

U. S. Standards.-Oil of Wintergreen is the volatile oil distilled from the leaves of the Gaultheria procumbens $\mathrm{L}$.

Wintergreen Extract is the flavoring extract prepared from oil of wintergreen, and contains not less than $3 \%$ by volume of oil of wintergreen.

* Vielhaber, Arch. Pharm. (3), 13, 408.

† The Volatile Oils. Translated by Kremers, Milwaukee, 1900, p. 588 .

‡ Pharm. Rund, 13, p. 228 . 
Spirit of Gautheria of the U. S. P. is a mixture of $50 \mathrm{cc}$. of oil of wintergreen and $950 \mathrm{cc}$. of alcohol. It accordingly contains $5 \%$ by volume of the essential oil.

Adulteration of Wintergreen Extract.-Synthetic methyl salicylate is very commonly substituted for both wintergreen and sweet birch oil, and sweet birch oil in turn for wintergreen oil. The production of true wintergreen oil is small, the so-called natural wintergreen oil of commerce being usually sweet birch oil. The sense of smell is the best means of distinguishing the two oils; polarization is of rather uncertain value, owing to low rotatory power of the wintergreen oil.

Determination of Wintergreen Oil.-Hortvet and West's Method." -Measure 10 cc. of the extract into a I00-cc. beaker, add 10 cc. of $10 \%$ potassium hydroxide solution, and heat the mixture over a boiling waterbath until the odor of oil of wintergreen has disappeared and the liquid is reduced to about one-half its original volume. By this treatment the methyl salicylate is converted into the potassium salt. Liberate the salicylic acid by the addition of an excess of $10 \%$ hydrochloric acid, $\mathrm{cool}$, and extract in a separatory funnel with three portions of 40,30 , and $20 \mathrm{cc}$. of ether respectively. Pour the combined ether extracts through a dry filter into a weighed dish, wash the filter with $10 \mathrm{cc}$. of ether, evaporate filtrate and washings slowly at $50^{\circ} \mathrm{C}$., dry one hour in a desiccator, and weigh. The per cent of wintergreen oil by volume $(M)$ is obtained from the weight of salicylic acid $(S)$ by the following formula:

$$
M=\frac{\text { I.10I } \times 10 \times S}{1.18} .
$$

Howard's Method.-Proceed as described on page 874, except that the heavy oil is brought into the graduated portion of the Babcock bottle by addition of dilute sulphuric acid ( $1: 2)$, taking care that the acid is not over $25^{\circ} \mathrm{C}$. and avoiding agitation.

\section{PEPPERMINT EXTRACT}

Peppermint Oil is obtained from various plants of the genus Mentha, which are commonly classed as sub-species or varieties of $M$. piperita. Owing in large part to the botanical differences in the plants from which 
it is made, peppermint oil from different regions differs greatly in its chemical and physical constants as shown by the following table compiled from figures given by Gildermeister and Hoffmann: *

\begin{tabular}{|c|c|c|c|}
\hline & Specific Gravity. & Rotation, $a_{D}$ & $\begin{array}{l}\text { Total Menthol, } \\
\text { Per Cent. }\end{array}$ \\
\hline 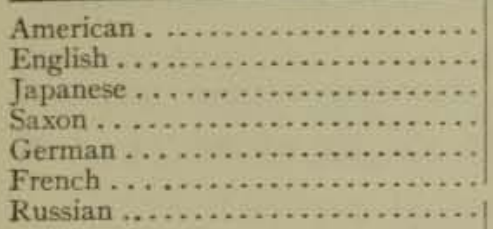 & $\begin{array}{l}0.905 \text { to } 0.920 \\
0.900 \text { to } 0.910 \\
0.895 \text { to } 0.900 \\
0.900 \text { to } 0.915 \\
0.899 \text { to } 0.930 \\
0.918 \text { to } 0.920 \\
0.905 \text { to } 0.910\end{array}$ & $\begin{array}{l}-18^{\circ} \text { to }-33^{\circ} \\
-22^{\circ} \text { to }-33^{\circ} \\
-30^{\circ} \text { to }-42^{\circ} \\
-25^{\circ} \text { to }-33^{\circ} \\
-27^{\circ} \text { to }-33^{\circ} \\
-5^{\circ} \text { to }-9^{\circ} \\
-17^{\circ} \text { to }-22^{\circ}\end{array}$ & $\begin{array}{l}48 \text { to } 60 \\
56 \text { to } 66 \\
70 \text { to } 91 \\
54 \text { to } 68 \\
43 \text { to } 46 \\
50.2\end{array}$ \\
\hline
\end{tabular}

U. S. Standards.-Peppermint is the leaves and flowering tops of Mentha piperita L.

Oil of Peppermint is the volatile oil obtained from peppermint, and contains not less than $50 \%$ by weight of menthol.

Peppermint Extract is the flavoring extract prepared from oil of peppermint, or from peppermint, or both, and contains not less than $3 \%$ by volume of oil of peppermint.

Analysis of Peppermint Extract.-Owing to the wide variation in the rotatory power of peppermint oil, only a roughly approximate idea of the oil content of peppermint extract can be gained by polarization. The variation in the percentage of menthol in the oil is also too great to permit of a method based on the amount of this constituent. Mitchell's precipitation method, as originally described (page 873), does not effect a complete separation of the oil, but Howard's modification (page 874) gives satisfactory results, and is well adapted for purposes of inspection.

\section{SPEARMINT EXTRACT.}

U. S. Standards. - Spearmint is the leaves and flowering tops of Mentha spicata L.

Oil of Spearmint is the volatile oil obtained from spearmint.

Spearmint Extract is the flavoring extract prepared from oil of spearmint, or from spearmint, or both, and contains not less than $3 \%$ by volume of oil of spearmint.

* The Volatile Oils. Translated by Edward Kremers, Milwaukee, tgoo. 


\section{SPICE EXTRACTS.}

Alcoholic solutions of the essential oils of spices are used to some extent instead of the spices themselves. The following are the definitions of these extracts and the oils from which they are prepared, as adopted by the joint committee on standards and the U. S. Secretary of Agriculture:

U. S. Standards.-Anise Extract is the flavoring extract prepared from oil of anise, and contains not less than $3 \%$ by volume of oil of anise.

Oil of Anise is the volatile oil obtained from the anise seed.

Celery Seed Extract is the flavoring extract prepared from celery seed or the oil of celery seed, or both, and contains not less than $0.3 \%$ by volume of oil of celery seed.

Oil of Celery Seed is the volatile oil obtained from celery seed.

Cassia Extract is the flavoring extract prepared from oil of cassia, and contains not less than $2 \%$ by volume of oil of cassia.

Oil of Cassia is the lead-free volatile oil obtained from the leaves or bark of Cinnamomum cassia Bl., and contains not less than $75 \%$ by weight of cinnamic aldehyde.

Cinnamon Extract is the flavoring extract prepared from oil of cinnamon, and contains not less than $2 \%$ by volume of oil of cinnamon.

Oil of Cinnamon is the lead-free volatile oil obtained from the bark of the Ceylon cinnamon (Cinnamomum zeylanicum Breyne), and contains not less than $65 \%$ by weight of cinnamic aldehyde and not more than 10\% by weight of eugenol.

Clove Extract is the flavoring extract prepared from oil of cloves, and contains not less than $2 \%$ by volume of oil of cloves.

Oil of Cloves is the lead-free, volatile oil obtained from cloves.

Ginger Extract is the flavoring extract prepared from ginger, and contains in each roo cc. the alcohol-soluble matters from not less than 20 grams of ginger.

Nutmeg Extract is the flavoring extract prepared from oil of nutmeg, and contains not less than $2 \%$ by volume of oil of nutmeg.

Oil of Nutmeg is the volatile oil obtained from nutmegs.

Savory Extract is the flavoring extract prepared from oil of savory, or from savory, or both, and contains not less than $0.35 \%$ by volume of oil of savory.

Oil of Savory is the volatile oil obtained from savory. 
Star Anise Extract is the flavoring extract prepared from oil of star anise, and contains not less than $3 \%$ by volume of oil of star anise.

Oil of Star Anise is the volatile oil distilled from the fruit of the star anise (Illicium verum Hook).

Sweet Basil Extract is the flavoring extract prepared from oil of sweet basil, or from sweet basil, or both, and contains not less than $0.1 \%$ by volume of oil of sweet basil.

Sweet Basil, Basil, is the leaves and tops of Ocymum basilicum L.

Oil of Sweet Basil is the volatile oil obtained from basil.

Sweet Marjoram Extract, Marjoram Extract, is the flavoring extract prepared from the oil of marjoram, or from marjoram, or both, and contains not less than $1 \%$ by volume of oil of marjoram.

Oil of Marjoram is the volatile oil obtained from marjoram.

Thyme Extract is the flavoring extract prepared from oil of thyme, or from thyme, or both, and contains not less than $0.2 \%$ by volume of oil of thyme.

Oil of Thyme is the volatile oil obtained from thyme.

Determination of Essential Oil in Cinnamon, Cassia, and Clove Extracts.-Howard's Method.-Proceed as with wintergreen extract, page 890 .

Hortvet and West's Method.*-Place 1o cc. of the extract and $50 \mathrm{cc}$. of water in a separatory funnel, and extract with three portions of ether measuring respectively 50,30 , and $20 \mathrm{cc}$. Wash the combined extracts successively with 25 and $30 \mathrm{cc}$. of distilled water, and filter through a dry funnel into a wide-mouth flask, washing out the funnel and filter with a little ether. In the case of cinnamon extract, transfer the ether extract before filtering to a I5o-cc. flask, shake for a few minutes with some granulated calcium chloride, then filter in the manner described. Evaporate off the ether as rapidly as possible on a boiling water-bath until only a few drops remain. At this point remove the flask from the bath, and rotate rapidly for a few minutes, spreading the residue over the sidez of the flask. The rapid evaporation of the remaining ether cools the flask to near room temperature. When the odor of ether has disappeared, stopper the flask and weigh.

In the case of cassia and clove oils, where the ether extract is not first dried with calcium chloride, a slight cloudiness gathers on the flask as the last traces of ether disappear, due to the presence of a little moisture. In such case allow the flask to stand on the balance-pan

\footnotetext{
* Jour. Ind. Eng. Chem., I, 1909, p. 88.
} 
until the film disappears, requiring not longer than two to three minutes, then stopper, and weigh.

The per cent of oil by volume $(V)$ is calculated from the weight of oil $(W)$ by the following formula:

$$
V=\frac{100 \times W}{10 \times 1.05^{\circ}} .
$$

The oil thus extracted may be used for determination of the refractive index. After dissolving in a little alcohol it may be tested with ferric chloride solution. By this test cinnamon oil gives a green, cassia oil a brown, and clove oil a deep blue, coloration.

Determination of Essential Oil in Nutmeg Extract.-Follow Mitchell's precipitation method, page 873 .

Determination of Solids in Ginger Extract.*-Evaporate Io cc. on a boiling water-bath to dryness, dry for 2 hours in a boiling water oven and weigh.

Determination of Alcohol in Ginger Extract.*-Proceed as with vanilla extract (p. 869).

Detection of Ginger in Ginger Extract.*-Seeker Method.-Dilute Io cc. of the extract to $30 \mathrm{cc}$., evaporate off $20 \mathrm{cc}$., decant into a separatory funnel and extract with an equal volume of ether. Evaporate the ether spontaneously in a porcelain dish and to the residue add $5 \mathrm{cc}$. of $75 \%$ sulphuric acid and $5 \mathrm{mg}$. of vanillin. Allow to stand for $\mathrm{I}_{5}$ minutes and add an equal volume of water. In the presence of ginger extract an azure blue color develops.

Detection of Capsicum in Ginger Extract.-La Wall Method Modified by Doyle. $\uparrow-$ To ro cc. of the extract cautiously add dilute sodium hydroxide until the solution reacts very slightly alkaline with litmus paper. Evaporate at about $70^{\circ} \mathrm{C}$. to about one-quarter of the original volume, render slightly acid with dilute sulphuric acid, testing with litmus paper. Transfer to a separatory funnel, rinsing the evaporating dish with water, and extract with an equal volume of ether, avoiding emulsification by shaking the funnel gently for a minute or two. Draw off the lower layer and wash the ether extract once with about ro cc. of water. Transfer the washed ether extract to a small evaporating dish, render decidedly alkaline with alcoholic potassium hydroxide, and evaporate at about $70^{\circ}$ until

*A.O.A.C. Method, Proc, for 19ro, U. S. Dept. of Agric., Bur. of Chem., Bul. 137, p. 75 .

† A.O.A.C. Method, Proc. for I9II, Bul. I52, p. I45. 
the residue is pasty; then add about $20 \mathrm{cc}$. more of half-normal alcoholic patash and allow to stand on a steam bath until the gingerol is completely saponified, which usually requires about one-half hour. Dissolve the residue in a little water and transfer with water to a small separatory funnel. The volume should not exceed $50 \mathrm{cc}$. Extract the alkaline solution with an equal volume of ether. Wash the ether extract repeatedly with small amounts of water until no longer alkaline to litmus. Transfer the washed extract to a small evaporating dish, allow the ether to evaporate spontaneously, and finally, last the residue for capsicum by moistening the tip of the finger, rubbing it around on the bottom and sides of the dish, and then applying the finger to the end of the tongue. A hot, stinging, or prickly sensation, which persists for several minutes, indicates capsicum or other foreign pungent substances.

\section{ROSE EXTRACT.}

U. S. Standards.-Rose Extract is the flavoring extract prepared from otto of roses, with or without red rose petals, and contains not less than $0.4 \%$ by volume of otto of roses.

Otto of Roses is the volatile oil obtained from the petals of Rosa damascena Mill., $R$. centifolia L., or $R$. moschata L.

Determination of Rose Oil.-Hortvet and West's Method.*-Measure $25 \mathrm{cc}$. of the extract into a separatory funnel, add $50 \mathrm{cc}$. of water, mix thoroughly, acidify with I cc. of hydrochloric acid (I:I), and extract with three portions of $20 \mathrm{cc}$. each of ether. Transfer the combined

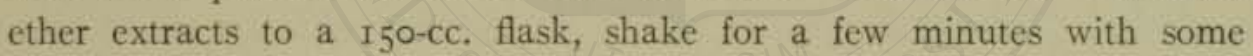
granulated calcium chloride, allow to settle until clear, then decant through a dry filter into a flat bottom glass dish previously weighed together with a cover-glass. Wash the calcium chloride and filter twice with Io cc. of ether, and add the washings to the glass dish. Cover the dish, place in a vacuum desiccator over sulphuric acid, allow to remain until all traces of ether and alcohol are removed, and weigh. Repeat the drying in the desiccator, for one hour periods, until the weight is practically constant. The final weight, divided by 0.86 and multiplied by 5 , gives the per cent of oil of rose by volume.

IMITATION FRUIT FLAVORS.

Nearly all the fruits possess distinctive flavors, which are desirable in food [preparations, and which may be made to impart their flavor to

* Jour. Ind. Eng. Chem., I, rgog, p. 89 . 
such substances as confections, ice cream, dessert mixtures, jellies, etc., by simply mixing with these foods the fresh or preserved fruit or fruit juice in sufficient quantity. In many cases, however, it is not found possible or practicable to prepare from the fruits themselves an extract sufficiently concentrated to give the distinctive fruit flavor, when used in moderate quantity, and hence the use of artificial fruit essences made up of compound ethers, mixed in varying combinations and proportions to imitate more or less closely various fruit flavors.

These ethers are usually much more pungent and penetrating than the fruits which they imitate, and, while lacking the delicacy and refinement of the original fruits, serve to impart a certain semblance of the genuine flavor in a convenient and highly concentrated form.

Some of the single compound ethers possess a remarkable resemblance to particular fruits, while to imitate other fruits a mixture of various ethers and flavoring materials, such as lemon and other volatile oils, vanilla, organic acids, chloroform, etc., is necessary. These arificial essences should in all cases be sold as such, and not as "pure fruit flavors."

Imitation Pineapple Essence is made up by dissolving in alcohol butyric ether, $\mathrm{C}_{4} \mathrm{H}_{7}\left(\mathrm{C}_{2} \mathrm{H}_{5}\right) \mathrm{O}_{2}$, which possesses a dis.inct pineapple flavor, and is prepared by mixing 100 paris of butyric acid $\left(\mathrm{C}_{4} \mathrm{H}_{8} \mathrm{O}_{2}\right)$, Ioo paris of alcohol, and $5 \circ$ parts of sulphuric acid, and shaking. Butyric ether is sparingly soluble in water, and boils at $121^{\circ} \mathrm{C}$.

Imitation Quince Essence depends as a basis on ethyl pelargonate, sometimes called pelargonic or œnanthic ether, $\mathrm{C}_{2} \mathrm{H}_{5}, \mathrm{C}_{4} \mathrm{H}_{17} \mathrm{O}_{2}$, dissolved in alcohol. Pelargonic ether is formed by digestion with the aid of heat of pelargonic acid and alcohol. Pelargonic acid, $\mathrm{C}_{4} \mathrm{H}_{18} \mathrm{O}_{2}$, is first obtained by the action of nitric acid on oil of rue. Pelargonic ether is a colorless liquid, having a specific gravity of 0.8635 at $17.5^{\circ} \mathrm{C}$. Its boiling-point is $227^{\circ}$ to $228^{\circ} \mathrm{C}$. It is insoluble in water.

Imitation Jargonelle Pear Essence consists of an alcoholic solution of amyl or pentyl acetate, $\mathrm{C}_{5} \mathrm{H}_{11}, \mathrm{C}_{2} \mathrm{H}_{3} \mathrm{O}_{2}$. This is prepared by distilling a mixture of one part of amyl alcohol, two parts of potassium acetate, and one part of concentrated sulphuric acid. It is a colorless liquid, insoluble in water, and having a boiling-point of $137^{\circ} \mathrm{C}$.

Imitation Banana Essence is made up of a mixture of amyl acetate and butyric ether.

Imitation Apple Essence is composed of an alcoholic solution of amyl valerianate, sometimes called apple oil, $\mathrm{C}_{5} \mathrm{H}_{11}, \mathrm{C}_{5} \mathrm{H}_{9} \mathrm{O}_{2}$, prepared by mixing four parts of amyl alcohol with four of sulphuric acid, and adding 
COMPOSITION OF IMITATION ESSENCES.

\begin{tabular}{|c|c|c|c|c|c|c|c|c|c|c|c|c|}
\hline & $\frac{\text { E⿱ 口) }}{\frac{0}{8}}$ & 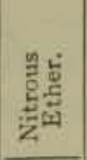 & 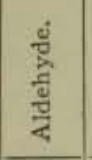 & 选范 & E. & 辞 & 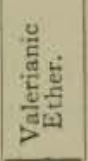 & 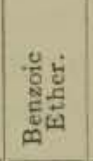 & 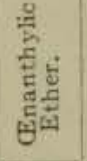 & $\begin{array}{l}\frac{1}{5} \\
\frac{c}{5} \\
\frac{0}{0}\end{array}$ & 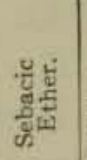 & 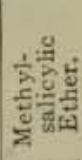 \\
\hline 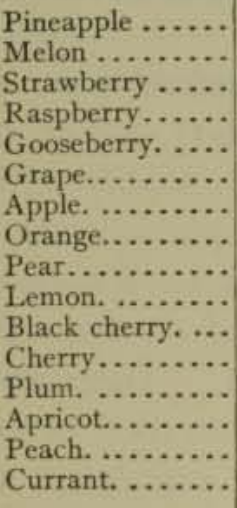 & 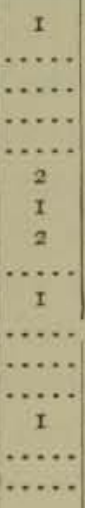 & 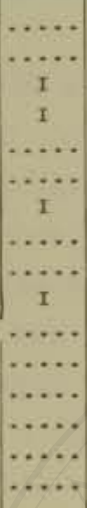 & $\begin{array}{c}1 \\
2 \\
\cdots \\
1 \\
1 \\
2 \\
2 \\
2 \\
\cdots \\
2 \\
\cdots \\
\cdots \\
5 \\
\cdots \\
2 \\
1\end{array}$ & $\begin{array}{c}\cdots \cdots \\
\cdots \\
5 \\
5 \\
5 \\
\cdots \cdots \\
1 \\
5 \\
5 \\
10 \\
10 \\
5 \\
5 \\
\cdots \\
5 \\
5\end{array}$ & $\begin{array}{c}\mathrm{I} \\
\\
\cdots \ldots \\
\cdots \cdots \\
\mathrm{I} \\
\cdots \\
5 \\
\cdots \\
\cdots\end{array}$ & $\begin{array}{c}5 \\
4 \\
5 \\
I \\
\cdots \cdots \\
\cdots \cdots \\
I\end{array}$ & $\begin{array}{c}5 \\
\cdots \cdots \\
\cdots \cdots \\
\cdots \cdots \\
\cdots \\
\cdots\end{array}$ & $\begin{array}{c}1 \\
\\
5 \\
5 \\
\cdots \cdots \\
\cdots \cdots \\
\cdots \\
1\end{array}$ & $\begin{array}{c}\ldots \ldots \\
\ldots \\
I \\
\text { IO }\end{array}$ & $\begin{array}{l}\ldots \ldots \\
\ldots \ldots \\
\ldots \ldots \\
\ldots \ldots\end{array}$ & $\begin{array}{c}10 \\
\ldots \ldots \\
I \\
\ldots \ldots \\
\ldots \ldots\end{array}$ & $\begin{array}{l}\text { I } \\
\text { I } \\
\text { I } \\
\text { I }\end{array}$ \\
\hline & & $\begin{array}{l}\frac{3}{0} \\
\frac{0}{8} \\
\frac{8}{4} \\
\frac{5}{3}\end{array}$ & $\frac{y}{\frac{y}{g}}$ & 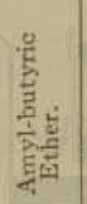 & $\frac{\frac{U}{2}}{\frac{d}{2}}$ & 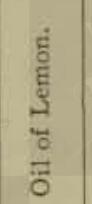 & $\begin{array}{l}\frac{5}{6} \\
\frac{5}{6} \\
\frac{5}{6} \\
\frac{0}{0}\end{array}$ & 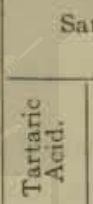 & 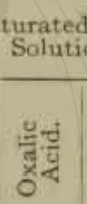 & 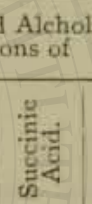 & 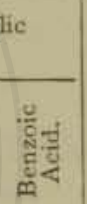 & $\frac{E}{\frac{E}{4}}$ \\
\hline 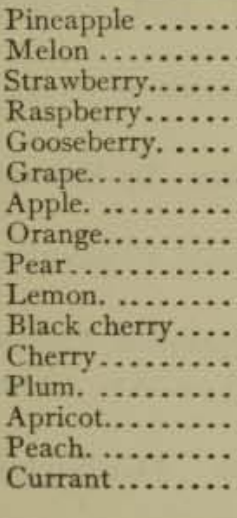 & $\begin{array}{l}\ldots \ldots \\
\ldots \ldots \\
\ldots \ldots \\
\ldots \ldots \\
\ldots \ldots \\
\ldots \ldots \\
\ldots \ldots \\
\ldots \ldots \\
\ldots \ldots \\
\ldots \ldots \\
\ldots \ldots \\
\ldots \ldots \\
\ldots \ldots \\
\ldots \ldots\end{array}$ & $\begin{array}{l}\ldots \ldots \\
\ldots \ldots \\
\ldots \ldots \\
\ldots \ldots \\
\ldots \ldots \\
\ldots \ldots \\
\ldots \ldots \\
\ldots \ldots \\
\ldots \ldots \\
\ldots \ldots \\
\ldots \ldots \\
\ldots \ldots \\
\ldots \\
2 \\
2 \\
\ldots \ldots\end{array}$ & $\begin{array}{c}\ldots \ldots \\
\ldots \ldots \\
3 \\
1 \\
\ldots \ldots \\
\ldots \ldots \\
\ldots \ldots \\
I \\
2 \\
\ldots \ldots \\
\ldots \ldots \\
\ldots \ldots \\
\ldots \ldots \\
\ldots \ldots \\
\ldots \ldots \\
\ldots .\end{array}$ & $\begin{array}{c}10 \\
\ldots \ldots . \\
2 \\
1 \\
\ldots \ldots \\
\ldots \ldots \\
\ldots \ldots \\
\ldots \ldots \\
\ldots \ldots \\
\ldots \ldots \\
\ldots \ldots \\
\ldots \ldots \\
\ldots \ldots \\
1 \\
\ldots \ldots \\
\ldots \ldots\end{array}$ & 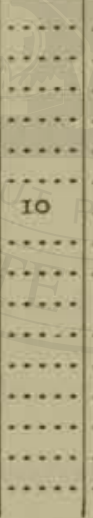 & 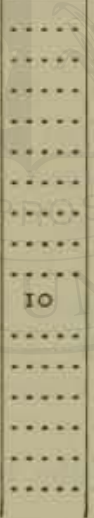 & 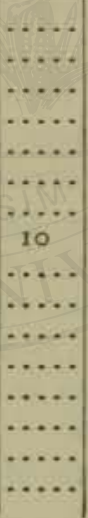 & $\begin{array}{c}\cdots \cdots \\
\cdots \\
\cdots \\
5 \\
5 \\
5 \\
\cdots \\
\mathrm{I} \\
\cdots \cdots \\
\mathrm{IO} \\
\cdots \cdots \\
\cdots \\
\cdots \\
\mathrm{I} \\
\cdots \\
5\end{array}$ & 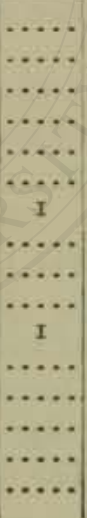 & 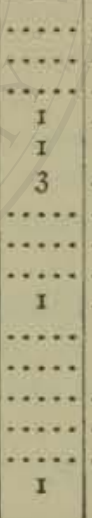 & $\begin{array}{c}\cdots \\
\cdots \\
\cdots \cdots \\
\cdots \\
I \\
\cdots \\
\cdots \cdots \\
\cdots \cdots \\
\cdots \cdots \\
\cdots \cdots \\
2 \\
I \\
\cdots \cdots \\
\cdots \cdots \\
\cdots \cdots \\
I\end{array}$ & $\begin{array}{r}3 \\
3 \\
2 \\
4 \\
\\
10 \\
4 \\
10 \\
2 \\
5 \\
3 \\
8 \\
4 \\
5\end{array}$ \\
\hline
\end{tabular}


the mixture when cold to five parts of valerianic acid. The specific gravity of amyl valerianate is 0.879 at $\circ^{\circ} \mathrm{C}$. and its boiling-point is $188^{\circ} \mathrm{C}$.

The table on p. 897 , prepared by Kletzinsky, shows the composition of a large variety of these artificial essences. The numerals in the various columns indicate the parts by volume to be added to 100 parts of deodorized alcohol.

Determination of Esters.-Add to 25 grams of the extract $2 \mathrm{cc}$. of sodium hydroxide solution ( 100 grams in $100 \mathrm{cc}$. of water), 100 cc. of water and heat under a reflux condenser one half-hour. Acidify with 5 cc. of dilute sulphuric acid $(\mathrm{r}: 4)$, add a few pieces of pumice stone, distil in a current of steam and titrate the distillate with tenth-normal alkali, using phenophthalein as indicator. The number of $\mathrm{cc}$. required represents the total volatile acids free and combined. Determine free volatile acids, if present by direct distillation and titration of the distillate. The difference between the two titrations is calculated as ethyl acetate.

\section{REFERENCES ON FLAVORING EXTRACTS.}

ChACE, E. M. A Method for the Determination of Citral in Lemon Oils and Extracts. Jour. Am. Chem. Soc., 28, 1906, p. 1472.

- The Detection of Small Quantities of Turpentine in Lemon Oil. Ibid., 3०, rgo8, p. 1475 .

Denis, W., and Dunbar, P. B. The Determination of Benzaldehyde in Almond Flavoring Extract. Jour. Ind. Eng. Chem., I, 1909, p. 256.

Gilldemeister, E., and Hofrmann, F. The Volatile Oils. Trans. by Edward Kremer. Milwaukee, 1900.

Hess, W. H. The Distinction of True Extract of Vanilla from Liquid Preparations of Vanillin. Jour. Am. Chem. Soc., 21, 1899, p. 719.

Hess, W. H., and Prescotr, A. B. Coumarin and Vanillin, their Separation, Estimation and Identification in Commercial Flavoring Extracts. Jour. Am. Chem. Soc., 2I, 1899 , p. 256 .

Heusler, F. The Chemistry of the Terpenes. Trans. by F. J. Pond. Philadelphia, 1902.

Hrltner, R. S. The Determination of Citral in Lemon Extract. A. O. A. C. Proc., I908, U. S. Dept. of Agric., Bur. of Chem., Bul. 122, p. 34. Jour. Ind. Eng. Chem., I, I909, p. 798 .

Hortver, J., and West, R. M. The Determination of Essential Oils and Alcohol in Flavoring Extracts. Jour. Ind. Eng. Chem., I, I909, p. 84.

Howard, C. D. The Precipitation Method for the Estimation of Oils in Flavoring Extracts and Pharmaceutical Preparations. Jour. Am. Chem. Soc., 30, 1908, p. 608 . 
Mrtchell, A. S. Lemon Flavoring Extract and its Substitutes. Jour. Am. Chem. Soc., 21, 1899, p. 1132 .

- Flavoring Extracts. U. S. Dept. of Agric., Bur. of Chem., Bul. 65, p. 69.

Winton, A. L., and Silverman, M. The Analysis of Vanilla Extract. Jour. Am. Chem. Soc., 24, 1902, p. I1 29.

Winton, A. L., and BArtex, E. M. The Determination of Vanillin, Coumarin, and Acetanilide in Vanilla Extract. Jour. Am. Chem. Soc., 27, 1905, p. 7 19.

Wrnton, A. L., and Lotr, C. I. Distinction of Vanilla Extract and its Imitations. U. S. Dept. of Agric., Bur. of Chem., Bul. 132, p. rog. 


\section{CHAPTER XXI. \\ VEGETABLE AND FRUIT PRODUCTS.}

\section{CANNED VEGETABLES AND FRUITS.}

STRICTLY speaking all varieties of canned foods found in the market,

- whether meats, fruits, or vegetables, in order to be entirely beyond criticism, should not differ from the corresponding freshly cooked varieties which they are intended to replace, excepting that they are free from bacteria. Such a degree of perfection is, however, difficult, even if possible, to attain, and nearly all commercial canned products, even if made from the best materials, are liable to contain either antiseptic substances or coloring-matter intentionally added by the manufacturer, or metallic impurities accidentally derived from the vessels in which they are prepared, or from the containers in which they are sealed. In spite of these objections, canned foods form a convenient, and in some cases indispensable means of furnishing both necessities and luxuries for the table. The canning of foods is especially useful for preserving them during long periods of time, for enabling certain fruits and vegetables to be enjoyed out of season, and for furnishing supplies in a convenient manner to inaccessible places where fresh foods are not readily obtainable, as in the case of armies in the field, of vessels at sea, of campers in the woods, etc. Canned goods in great variety are used in nearly every household.

When it is considered that in the United States alone something like one hundred million cans of corn are packed in a single year, about the same quantity of peas, and one hundred and fifty million cans of tomatoes, to say nothing of an ever-increasing variety of other foods, some idea may be gained of the enormous proportions to which the canning industry has grown. It is comforting to know that, in view of their wide-spread consumption, the greater portion of such foods found on the market are comparatively harmless, as is evidenced by the fact that few cases of injury to health have been directly traceable to their use.

Method of Canning Food.-Various modifications as to details exist with different products and in different localities, but in general the prin- 
ciple of canning in tin is the same in all cases. The fresh product is cleaned carefully, and packed in cans with the requisite amount of water. The cans are then sealed, and subjected to the effect of steam or boiling water till the contents are thoroughly cooked. Each can is then tapped or punctured at one end to expel the air, and again heated, after which the hole is closed by a lump of solder, thus forming a vacuum in the can, which is afterwards heated for a sufficient time to destroy the bacteria, usually for several hours.

The above mode of procedure is the time-honored one, and is still in vogue in most localities, but a more modern method, much in use at present, consists in first cooking the food at a temperature of $82^{\circ}$ to $88^{\circ} \mathrm{C}$. before transferring to the cans, and afterwards subjecting the cans when sealed to a high heat of about $125^{\circ} \mathrm{C}$. in dry air in so-called retorts, this heating or " processing," as it is termed, being carried on for a sufficient length of time to completely sterilize the contents of the can. Obviously a much shorter time is required for this than when the temperature of boiling water is employed, and the sterilization is much more effective.

Cooked vegetables and fruit products put up in glass jars or bottles are tightly sealed when hot, either with screw-caps or with some form of cover held by a clamp, or with metal or hard-rubber caps fitting over a flanged mouth. Frequently a soft-rubber ring is inserted between the cover and the mouth of the jar or bottle. The material of the cover is generally either glass, porcelain, or metal. Cork stoppers are, however, sometimes pressed into the mouths of the bottles, and made extra tight therein with sealing-wax. These stoppers are occasionally soaked in paraffin. Thus the contents of the jar may be exposed to porcelain, glass, metal, rubber, or cork, according to the material of the cover and the method of sealing.

The preservation of food by canning was long thought to be due to the perfect exclusion of air, but is now known to depend on the perfect sterilization, or destruction of bacteria, and it has been proved that as far as keeping qualities are concerned, it makes no difference whether or not air is present in the can, if the contents are sterile, though for purposes of inspection the vacuum, in the case of tin cans, is of great use, in that as a natural consequence of the vacuum, when the goods are sound, the ends of the cans are usually concave. The highest aim of the canner should be to retain in his product as far as possible the appearance, palatability, and nutritive value of the freshly cooked food. 
PROXIMATE COMPOSITION OF CANNED VEGETABLES AND FRUITS.*

\begin{tabular}{|c|c|c|c|c|c|c|c|c|}
\hline & - & है & $\frac{\dot{E}}{2}$ & 压 & 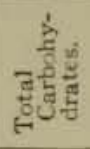 & 雚 & 趗 & 造 \\
\hline & & & & & & & & \\
\hline $\begin{array}{l}\text { Artichokes, ................. } \\
\text { Asparagas . . . . . . }\end{array}$ & $\begin{array}{r}3 \\
14\end{array}$ & $\begin{array}{l}92 \cdot 5 \\
94 \cdot 4\end{array}$ & $\therefore .8$ & $\cdots^{-1}$ & $\begin{array}{l}5.0 \\
2.8\end{array}$ & $\begin{array}{l}.6 \\
.5\end{array}$ & $\begin{array}{l}7.7 \\
1.2\end{array}$ & $\begin{array}{r}110 \\
85\end{array}$ \\
\hline Beans, baked. .......... & $2 \mathrm{I}$ & 68.9 & 6.9 & 2.5 & 19.6 & 2.5 & 2.1 & 600 \\
\hline$" \quad$ string. .......... & 29 & 93.7 & I. $\mathrm{I}$ & . I & 3.8 & $\cdot 5$ & 1.3 & 95 \\
\hline$" \quad$ Lima........... & 16 & $79 \cdot 5$ & 4.0 & $\cdot 3$ & 14.6 & 1.2 & 1.6 & $3^{60}$ \\
\hline Brussels sprouts........ & I & 93.7 & I. 5 & $-\mathrm{I}$ & 3.4 & $\cdot 5$ & 1.3 & 95 \\
\hline Corn, green ........... & 52 & 76.1 & 2.8 & 1.2 & 19.0 & .8 & .9 & 455 \\
\hline Peas, green. . .......... & 88 & $85 \cdot 3$ & 3.6 & .2 & 9.8 & I.2 & I. 1 & 255 \\
\hline Pumpkin............. & 7 & 91.6 & .8 & .2 & 6.7 & I. I & .7 & $15^{\circ}$ \\
\hline Squash. ............... & 5 & 87.6 & $\cdot 9$ & $\cdot 5$ & 10. 5 & $\cdot 7$ & .5 & 235 \\
\hline Succotash ............ & 12 & $75 \cdot 9$ & 3.6 & 1.0 & I8.6 & $\cdot 9$ & .9 & 455 \\
\hline Tomatoes .............. & I9 & 94.0 & 1.2 & .2 & 4.0 & $\cdot 5$ & .6 & 105 \\
\hline $\begin{array}{l}\text { CANNED FRUITs. } \\
\text { Apples, crab. . . . . . }\end{array}$ & & & & & & & & \\
\hline Apples, crab. ........... & I & $\begin{array}{l}42.7 \\
6 \mathrm{r} . \mathrm{I}\end{array}$ & $\begin{array}{r}.3 \\
.2\end{array}$ & $\begin{array}{r}2.4 \\
.8\end{array}$ & $\begin{array}{l}54.4 \\
37.2\end{array}$ & & -5 & $\begin{array}{r}1,120 \\
730\end{array}$ \\
\hline Apricots. .............. & $\mathrm{I}$ & $8 \mathrm{I} \cdot 4$ & .9 & ...... & $17 \cdot 3$ & $\cdots \cdots \cdot$ & .4 & $\begin{array}{l}730 \\
340\end{array}$ \\
\hline Blackberries. ......... & I & 40.0 & .8 & 2.1 & 56.4 & & .7 & $1,15^{\circ}$ \\
\hline Blueberries. ........... & 3 & 85.6 & .6 & .6 & 12.8 & $\ldots$. & .4 & 275 \\
\hline Cherries .............. & I & $77 \cdot 2$ & I. I & . I & $2 \mathrm{I} \cdot \mathrm{T}$ & ..... & .5 & $4 \mathrm{I} 5$ \\
\hline Peaches. .............. & 3 & 88.1 & $\cdot 7$ & .1 & 10.8 & & $\cdot 3$ & 220 \\
\hline Pears. ................ & 4 & $8 x-r$ & $\cdot 3$ & -3 & 18.0 & $\cdots$ & -3 & 355 \\
\hline Pineapples, ........... & I & $6 \mathrm{r} .8$ & .4 & .7 & 36.4 & $\cdots \cdots$ & .7 & 715 \\
\hline Strawberries. . ......... & I & 74.8 & .7 & & 24.0 & & .5 & 460 \\
\hline
\end{tabular}

-U. S. Dept, of Agric,, Exp. Sta. Bul. 28, p. 70.

\section{DECOMPOSITION.}

Nature and Detection of Spoilage.-In the case of canned vegetables and fruit products, decomposition rarely results in the formation of ptomaines even after the can has long been open, though these toxins are sometimes formed in canned meat and fish. Decomposition is readily apparent after opening a can, from a cursory examination of its contents. The appearance, taste, and odor will not fail to indicate the unfitness of the contents for food, if decomposition is at all advanced. It is, however, often of great advantage to detect spoiled cans without opening. As a rule, when a can is spoiled, it is usually in the condition termed " blown," i.e., with its ends convex, instead of normal or concave.

According to Prescott and Underwood, $\dagger$ although nearly all forms of bacterial decomposition are accompanied by bulging of the ends of the cans, there are some exceptions. In the souring of canned sweet corn, $\ddagger$

† Tech. Quart., II, I898, pp. 6-30; also 10 , 1897 , p. 183 .

$\ddagger$ These experimenters found at least twelve varieites of bacteria to which the souring of corn is apparently due. 
for instance, it is exceptional that swelling occurs. Ordinarily, in the factory inspection of canned goods before shipping, not only are the bulged cans or "swells," as they are termed, sifted out, but the condition of the cans is tested by sounding or striking the cans. If the contents are sweet, a peculiar note is produced when the can is struck, readily distinguishable from the dull tone of the unsound can by anyone familiar with the work.

As stated above, concavity in the ends of the can indicates that the contents are in good condition.

Prescott and Underwood further state that sound cans may be distinguished from unsound in a lot of suspicious goods, when the swelling of the ends is not apparent, by the following method:

Boil the cans for an hour, causing the ends of all to swell, then cool, and set aside for eight hours, during which the sound cans will snap back, while the unsound will continue convex, by reason of the fact that the swelling in this case is due to the generation of gas by the bacteria present.

Examination of Gases from Spoiled Cans.-When the tops of blown cans are punctured in the process of opening, an outflow of gas is usually to be noted. Doremus * has studied the character of these gases and

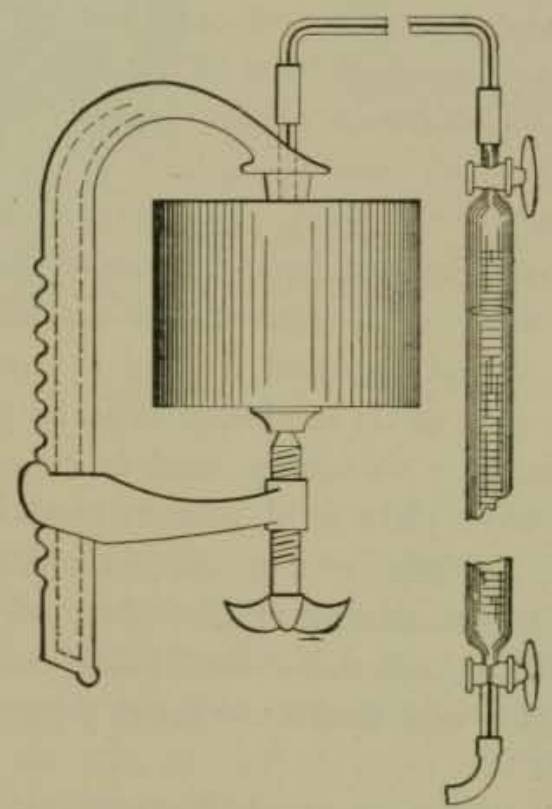

Fic. 118.-Apparatus for Collecting Gases from Spoiled Cans. (After Doremus.)

found that when the contents have become putrid, carbon dioxide and hydrogen are the chief gases to be found. Often 60 to $80 \mathrm{cc}$. of gas may be coilected from a can. For the collection of the gases, Doremus

* Jour. Am. Chem. Soc., 19, 1897, p. 733. 
uses the device shown in Fig. II8. An adjustable clamp has attached to its upper arm a beveled, hollow, steel needle. A perforated rubber stopper covers the needle and serves as a cushion. A fine tube connects the needle with the receiver of a eudiometer, both tube and receiver being filled with water or mercury. Either the stop-cock form of eudiometer, as here shown, or the kind with attached leveling-tube may be used. The can is adjusted between the arms of the clamp, and by turning the screw the needle is brought into contact with the top of the can and caused to puncture it, the rubber stopper serving to make a gas-tight joint. The gas passes through the tube into the eudiometer, and its constituents are determined in the usual manner, either by introducing the reagents directly into the eudiometer-tube in the proper order, or by transferring the gases to pipettes.* Hydrogen sulphide is tested for by subjecting a filter-paper moistened with lead acetate solution to the gas. If it turns black, the presence of hydrogen sulphide is indicated.

\section{METALLIC IMPURITIES.}

Salts of Lead and Tin are commonly met with in varying amounts in nearly all classes of products put up in tin. The quantity dissolved depends largely on the character of the tin plate used in the manufacture of the can, as well as on how the solder is applied. Much depends also on the nature of the food product and its acidity. Formerly much danger was apprehended from the use of the so-called terne plate as a material for cans. This consists of an alloy of lead and tin, coated on iron plate and intended for use as roofing. Sometimes two parts of lead to one part of tin are found in terne plate. Only the better grades of bright tin plate should be used in canning. There is reason to believe that no terne plate is at present used in cans. In 1892 the plating alloy of 47 samples of tin cans in which peas had been put up were examined in the Bureau of Chemistry of the U. S. Department of Agriculture, $\dagger$ and the amount of lead found varied from $\circ$ to ${ }_{3} 3$ per cent. Only 4 samples were found to exceed 5 per cent, and 24 contained less than I per cent.

The construction of the can should be such that practically no soldered surface is exposed to the contents, the joints being lapped and soldered on the outside. In spite of this, however, it is not unusual to find cans

* See Thorpe's Dictionary of App'd Chem., Vol. I, Pp. 232-243.

† Bul. 13 , p. 1036 . 
soldered on the inside, or lumps of solder in the can from the sealing of the tapped hole. From $5 \mathrm{x}$ to $65 \%$ of lead was found in the solder taken from the interior of twenty-four of the cans mentioned in the preceding paragraph.*

Cans lacquered on the inside to prevent contact of the metal with the foot are coming into use but as yet are not an unqualified success. Some of the lacquers which have proved most efficient are objectionable because of their lead content.

Action of Fruits and Vegetables on Tin Plate.-A large variety of canned products have been examined in the laboratory of the Massachu-

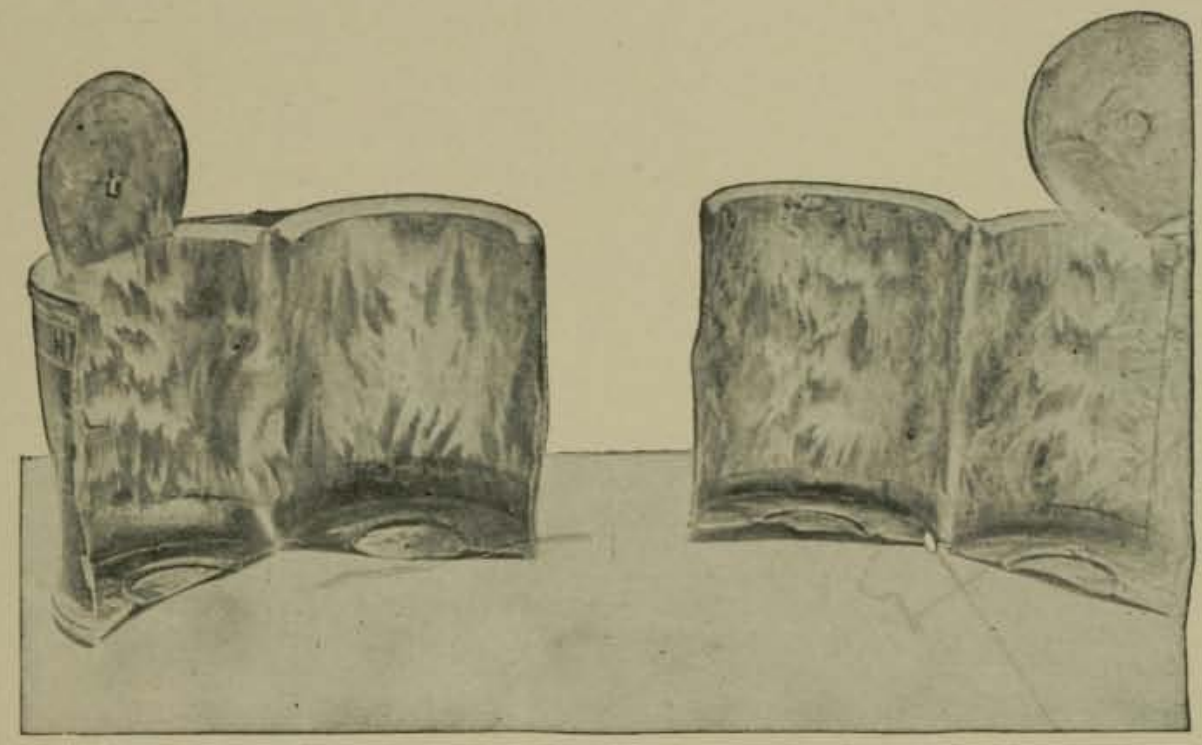

Fic. 119. - Interior of Blueberry Cans, Cut Open to Show the Corrosion by Acid of the Fruit Juice.

setts State Board of Health, with a view to determining the quantity of tin contained in solution. The results have shown that though notable traces of tin were found in acid fruits and rhubarb, and large traces in some green vegetables, canned blueberries were found to contain, as a rule, much more tin in solution than any other canned goods examined. It is assumed that the tin was, at least in considerable part, still held in solution by the fruit acids, inasmuch as the metal was found in the filtered juice. In every instance the inner tin lining was found to be extensively corroded, and in some cases it had been almost entirely dissolved off, leaving the underlying iron bare. Fig. II shows the appear-

* Bul. ${ }_{13}$, P. 1038 . 
ance of two of these cans, split open to show the inner surfaces. The corrosion is apparent. Eleven samples of canned blueberries, representing seven brands, were examined in 1894 by Worcester, showing an amount of tin in solution (calculated as $\mathrm{SnO}_{2}$ ) varying from 0.066 to 0.27 gram per can of $6 \mathrm{I}_{5} \mathrm{cc}$. capacity.

In 1899 samples of various canned products were examined for lead and tin in the author's laboratory, the results of which are thus summarized: *

\begin{tabular}{|c|c|c|c|}
\hline & Tin, Grams. & Lead, Grams. & $\begin{array}{c}\text { Capacity of } \\
\text { Can, cc. }\end{array}$ \\
\hline 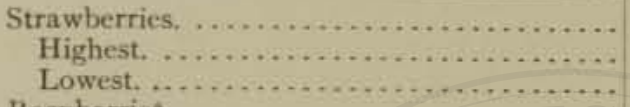 & $\begin{array}{l}.0393 \\
.0124\end{array}$ & $\begin{array}{l}.0004 \\
.0000\end{array}$ & 615 \\
\hline 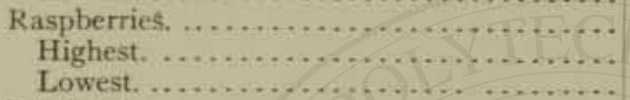 & $08_{48}$ & .0002 & 615 \\
\hline 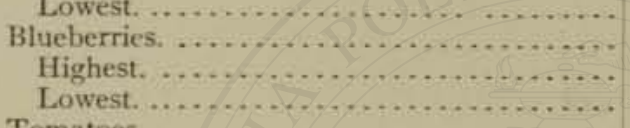 & $\begin{array}{l}.0725 \\
.2226 \\
.0056\end{array}$ & $\begin{array}{l}.0001 \\
.0021 \\
.0004\end{array}$ & 615 \\
\hline 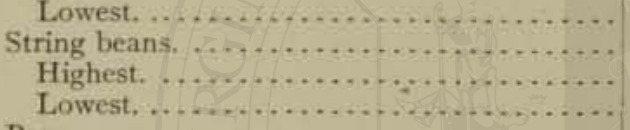 & $\begin{array}{l}.0146 \\
.0499 \\
.0065\end{array}$ & $\begin{array}{l}.0001 \\
.0003 \\
.0008\end{array}$ & 650 \\
\hline $\begin{array}{l}\text { Peas } \\
\text { Highest. } \quad \ldots \ldots \ldots \\
\text { Lowest. } \ldots \ldots \ldots \\
\end{array}$ & $\begin{array}{l}.0046 \\
.0024\end{array}$ & .0000 & 615 \\
\hline 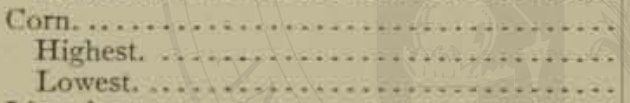 & $\begin{array}{l}\ldots . . \\
.0101 \\
.0045\end{array}$ & $\begin{array}{l}\ldots \ldots \text {. } \\
.0011 \\
.0001\end{array}$ & 615 \\
\hline 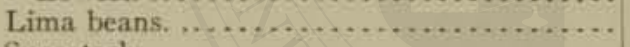 & .0064 & .0004 & 650 \\
\hline 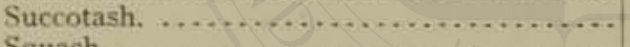 & .0039 & $.000 \mathrm{x}$ & 650 \\
\hline $\begin{array}{l}\text { Squash. } \ldots \ldots \ldots \ldots \ldots \ldots \\
\text { Highest. } \ldots \ldots \ldots \ldots \ldots \ldots\end{array}$ & 1793 & .0087 & $95^{\circ}$ \\
\hline Lowest. . . . . . . . . . . . . . . . . . . . & .1577 & .0003 & \\
\hline 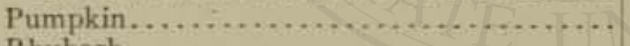 & .1844 & .0019 & 950 \\
\hline 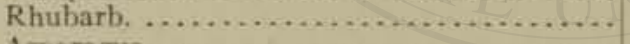 & .3506 & .0002 & 615 \\
\hline 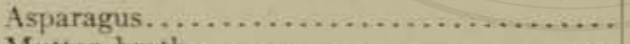 & .1249 & .0001 & $93^{\circ}$ \\
\hline Mutton broth. . . . . . . . . . . . . . . . . . & .0114 & .0001 & 950 \\
\hline 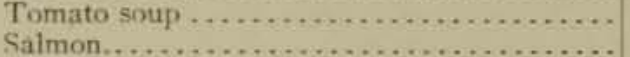 & .0023 & .0002 & $377^{\circ}$ \\
\hline 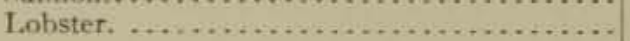 & $\begin{array}{r}0519 \\
.0411\end{array}$ & .0001 & $\begin{array}{l}470 \\
430\end{array}$ \\
\hline
\end{tabular}

A wide range of variation exists in the amount of tin dissolved. Pumpkin and squash, for example, dissolve surprisingly large quantities. considering the supposed inert nature of these vegetables.

In samples of canned sardines put up in mustard, vinegar, and oil, the Massachusetts Board has found as high as 0.376 gram of tin in a

* An. Rep. Mass. State Board of Health, r899, p. 623. 
half-pound can. In these cases the corrosion of the interior of the cans was very marked.*

Effect of Time on Amount of Tin Dissolved.-A series of experiments was conducted by the author in $1899+$ on the action of various fruit acids on tin, with a view to ascertaining, among other facts, whether or not the element of time exerts an appreciable difference in the results.

Samples of various canned fruits and vegetables were titrated for their acidity. It was found that certain samples of canned blueberries, for instance, had an scidity of about one-twentieth normal. In the case of strawberries, the acidity was about one-sixth normal. Canned raspberries were found to be about one-tenth normal in acidity, while the acidity of canned tomatoes varied from one-tenth to one-fourteenth normal. Solutions of one-fifth, one-tenth, and one-fifteenth-normal malic acid, one-tenth and one-fifteenth-normal tartaric acid, one-tenth and onefifteenth-normal citric acid, and one-tenth-normal acetic acid were prepared and sealed in pint glass jars, having about the same capacity as the ordinary-sized tin fruit cans, each jar containing an amount of tin plate equivalent to the interior exposed surface of a can. Solutions thus sealed were kept for three months, six months, and a year, and examined at the end of these respective periods for tin. The results showing the amount of tin found at the end of three months in each case are given in the following list:

ACTION OF FRUIT ACIDS ON TIN IN THREE MONTHS.

\begin{tabular}{|c|c|c|c|}
\hline Acid. & $\begin{array}{l}\text { Grams of Tin } \\
\text { in One Pint of } \\
\text { Solution. }\end{array}$ & Acid. & $\begin{array}{l}\text { Grams of Tin } \\
\text { in One Pint of } \\
\text { Solution. }\end{array}$ \\
\hline 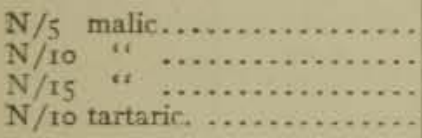 & $\begin{array}{l}0.0578 \\
0.0201 \\
0.0197 \\
0.0382\end{array}$ & 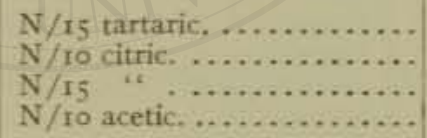 & $\begin{array}{l}0.0246 \\
0.0374 \\
0.0236 \\
0.0019\end{array}$ \\
\hline
\end{tabular}

It was found that, as a rule, the amount dissolved in three months was the same as in six months or even a year.

Tenth-normal acetic acid sealed in jars with tin plate, as in the case of the fruit acids, dissolved in three months 0.0019 gram, and in six months

* The U. S. Government, pending further investigation, permits 300 mg. of tin per kilo in canned goods. F. I. D. No, 126.

$\dagger$ Ann. Rep. Mass. State Board of Health, 1899, p. 624. 
$0.008_{3}$ gram of tin, which is much less than was obtained with fruit acids of the same strength, and with the samples of sardines referred to on page 906 .

Bigelow and Bacon find that shrimps contain monomethylamin, which corrodes the cans in which they are packed. Their experiments with volatile alkalis and amino acids present in vegetables of low acidity indicate that the corrosive action of certain vegetables is due to substances of this group.

Salts of Lead.-While it is a fact that the material of the tin plating usually found in cans is comparatively low in lead, the same is not always true of the metal caps used to cover some of the bottled goods. The French "haricots verts" are usually sold in wide-mouthed bottles, closed by a disk of very soft metal. In one instance this metal cap, which came in contact with the liquid contents of the bottle, was found to contain $93 \frac{1}{2} \%$ of lead. Of the various kinds of bottles in which are sold cheap carbonated drinks known as "pop," one style has a stopper consisting of a metallic button surrounded by a rubber ring. These metallic buttons consist of tin and lead in varying proportions. Inasmuch as the inclosed liquor was usually found to be quite acid in reaction, the danger of prolonged contact with the metallic portion of the stopper is evident.

The following table gives the percentage of lead found in the stoppers of this character, together with the amount of lead contained in the liquor:*

\begin{tabular}{|c|c|c|}
\hline Character of Sample. & $\begin{array}{c}\text { Per Cent of } \\
\text { Lead in Stopper. }\end{array}$ & $\begin{array}{c}\text { Amount of Lead } \\
\text { in Contents of } \\
\text { Bottle in Milli- } \\
\text { grams.t }\end{array}$ \\
\hline Blood orange $\ldots \ldots \ldots \ldots$. & 50.7 & 0.31 \\
\hline Birch beer. .............. & 35.0 & Large trace \\
\hline Ginger................ & 32.2 & 0.40 \\
\hline Strawberry A. ........... & 8.8 & 0.20 \\
\hline Strawberry B, .......... & 6.5 & 0.30 \\
\hline Sarsaparilla A........... & 8.5 & 0.19 \\
\hline Sarsaparilla B............ & $3 \cdot 5$ & 0.17 \\
\hline Lemon. ................ & $7 \cdot 5$ & 0.27 \\
\hline \multicolumn{3}{|l|}{ Miscellaneous ( 20 samples) } \\
\hline Maximum............ & 50.3 & 1.05 \\
\hline Minimum. ........... & 3.8 & 0.01 \\
\hline
\end{tabular}

+ Capacity of bottle about $\frac{f}{f}$ pint.

Besides the above tabulated samples, twenty were found with stoppers containing less than $3 \%$ of lead. While the amount of lead found in the

* An. Rep. Mass. State Board of Health, 1897, p. 571 . 
contents of the bottles was in no case very large, it was enough to condemn the use of lead in the manufacture of such stoppers. That the amounts of lead found in the contents of the bottles vary quite irre spective of the percentage of lead in their stoppers, may be ascribed to various causes, such as the difference in the acidity of the liquors, and the length of time that the liquor has been in contact with the stopper. Furthermore, the more soluble metal of an alloy is attacked by an acid with an energy which is not proportional to the percentage of that metal in the alloy.

Salts of Zinc.-The presence of zinc salts in canned foods is largely accidental, and is generally due either to the contact of the acid fruits and vegetables with galvanized iron in the canneries, to the occasional use of brass vessels, or to the zinc chloride used as a soldering fluid. Hilgard and Colby * have examined empty tin cans fresh from the manufacturer, and found zinc chloride in notable quantity in the seams, and especially in the empty space of the lap at the bottom of the can, where it could easily be acted on by the contents. The average amount of soluble zinc chloride found in the "lap" alone amounted to three-fourths of a grain per can. It was furthermore ascertained that it was not the practice of canners to wash the cans before packing, so that zinc present in canned goods may thus readily be accounted for.

Zinc chloride is commonly used in machine soldering, but should be displaced by rosin.

Hilgard and Colby found in some spoiled cans of asparagus, where the acidity was unusually high, an average of 6.3 grains of zinc chloride per large can.

Zinc salts are said to have been used for greening peas, but their use for this purpose is not common. Zinc chloride is the salt used, and a natural yellowish-green tint is imparted when properly applied. The process has been kept secret.

Salts of Copper.-While copper in canned goods is sometimes accidental, its presence being due to the use of copper or brass vessels in the canneries, its chief interest to the food analyst lies in the use of copper sulphate for greening peas and other vegetables. The artificial greening of vegetables is much more commonly practiced in France than in the United States.

French canners of peas, beans, Brussels sprouts, etc., are frequently so lavish in the use of sulphate of copper that the goods as found on our

* Rep. Cal. Agric. Exp. Sta., 1897-8, p. 159. 
markets can in some cases hardly be said to resemble the freshly cooked products in color. Oftentimes, indeed, they possess such a deep green as to be positively distasteful to the average American palate, though evidently this unnatural hue is craved in Europe. The use of copper in such foods is often rendered apparent by the most cursory examination.

In this country, when copper is used, smaller quantities are usually employed, with an attempt to imitate more closely the color of the natural product.

Complaint in court for this form of adulteration under the general food law as it exists in most states would naturally be brought under one of two clauses:

1st. As being colored, whereby the product appears of greatei value than it really is, or

2d. As containing an ingredient injurious to health.

An ingenious claim is sometimes advanced by the defendant in opposition to clause $r$, to the effect that copper sulphate is added, not to give an artificial green color, but to preserve the original green of the chlorophyl or natural color of the fresh peas,* so that it will not be destroyed by subsequent boiling.

This point was argued in a strongly contested court case brought in Massachusetts for copper in French peas. 1

As Worcester $\ddagger$ has shown, the fallacy of this argument can be easily demonstrated. If it were true that the copper acts as a preservative of the chlorophyl, a pure extract of chlorophyl should, by the addition of copper sulphate, be prevented from destruction on boiling, and again, on once destroying the color of the chlorophyl by boiling, it would be impossible to restore it by further boiling it with copper sulphate.

As a matter of fact, if an extract of chlorophyl is boiled with a dilute solution of copper sulphate, its color is at once destroyed, and a brown precipitate is thrown down. On the other hand, if yellow or white peas or beans devoid of chlorophyl are boiled with copper sulphate, they are colored green, the depth of color depending on the strength of the copper solution. When peas or other vegetables are thus colored, very little copper is found, as a rule, in the liquid contents of the can, but the copper is chiefly confined to the solid portions. Green compounds are produced

* The term used by the French to describe this process is reverdissage or "regreening." $\dagger$ An. Rep. Mass. State Board of Health, 1892, p. 605.

\pm Loc cit., supra, p. 64I. 
by boiling albumins with copper salts, due to the formation of albuminate, or in the case of peas, leguminate of copper. Harrington* states that it is possible to color eggs an intense green by boiling with copper sulphate.

Examination of a large number of brands of canned vegetables greened by copper, as bought in Massachusetts, showed that the amount used varied from a trace to 2.75 grams per can, calculated as copper sulphate. In justice to the consumer, who may be cautious about taking into his system copper salts, as well as to those who are indifferent to their use, it is no more than fair that all cans should have a label, plainly stating the quantity present. In the Massachusetts market, labels like the following are not uncommon: "This package of French Vegetables contains an equivalent of Metallic Copper not exceeding three-quarters of a grain."

Copper as a coloring matter has been most commonly found in peas, beans, and Brussels sprouts. Copper salts in minute quantity have been found in Massachusetts in canned tomatoes, clams, and squash, as well as in pickles.

Salts of Nickel.-Sulphate of nickel has been employed instead of sulphate of copper for greening vegetables. According to Harrington $\dagger$ 0.25 gram of nickelous sulphate per kilogram of peas is used. The peas or other vegetables are boiled in a solution of the salt, made slightly alkaline with ammonia.

Toxic Effects of Metallic Salts.-Divergence of opinion is so great as to the toxic effects of salts of the heavy metals on the human system, when present in the small amounts commonly found in food products, that it is extremely difficult to maintain a complaint in court based entirely on the harmful effects of these salts. Since the question is one for the toxicologist or physiological chemist rather than the analyst to settle, it will not be discussed here at length; suffice it to say that experiments made by the Referee Board indicate that while as much as $150 \mathrm{mg}$. of copper may be contained in the coppered beans or peas eaten in a day as little as ro $\mathrm{mg}$. under certain conditions may have a deleterious action and must be considered injurious to health. Accordingly foods greened with copper are considered adulterated by the federal authorities. $\ddagger$

* Practical Hygiene, p. 203.

$\dagger$ Ibid., p. 205.

$\ddagger$ Food Inspection Decision r48. ) 


\section{PRESERVATIVES.}

No class of food products stands so little in need of these added substances to arrest fermentation as canned foods, if properly prepared and, as a matter of fact, thê use of antiseptics has been almost entirely discontinued.

The Bleaching of Corn by artificial means before canning is usually accomplished by boiling the corn with sulphite of soda, thus giving to the product an unnaturally white color. The practice seems to have been more in vogue some years ago than at present, the popular taste now apparently preferring the natural rich yellow of fresh corn.

Saccharin is claimed to possess antiseptic powers and is used in canned goods, but its primary purpose is as a sweetener.

Salicyle acid, sodium benzoate, and beta-naphthol, although formerly used, are now seldom found in canned goods.

\section{"SOAKED GOODS."}

It has become quite common, especially in the case of peas, beans, and corn, to utilize for canning purposes those that have grown old and dried, after soaking them for a long time. The presence of soaked peas in the market is generally more common in years when there is a scarcity in the pea crop. By the process of soaking, dried and matured field corn may be softened to such an extent as to be substituted for green or swect corn in the canned product. These goods, frequently sold at a very low price, under some such tempting name as "Choice Early June Peas," are entirely devoid of that succulent property so highly prized in the fresh goods, and are altogether so inferior in quality that their sale may justly be considered as fraudulent, unless their character is specified. In some states the law provides that such a product, to be legally sold, shall have plainly marked on the label of the can the words "Soaked Goods" in letters of prescribed size.

Detection.-Methods of detecting soaked goods are distinctly physi$\mathrm{cal}$ rather than chemical. The appearance and taste of the goods furnish in most cases an unmistakable clue to their nature. Soaked goods are entirely lacking in juiciness, and in the flavors so characteristic of the various vegetables, when gathered and canned before becoming dry, The process of soaking is also said to develop the growth of the rudimentary stem of the embryo in the dried pea and bean. Peas and beans of the soaked variety are almost entirely lacking in the green color of 
the fresh vegetables, unless the color has been artificially supplied. The liquid is commonly milky.

In all cases it will be found that the solid grains or kernels of the peas, beans, and corn that have once been dried, though softened by the process of soaking, have much less water than the grains of the corresponding vegetables that were gathered while still soft and succulent.

\section{METHODS OF ANALYSIS.}

Methods of Proximate Analysis.-As a rule, the contents of canned goods are intended to be entirely edible throughout, and contain little or no refuse or portions to be rejected. An exception to this is the occasionat canning of certain fruits with stones or pits, which are, of course, to be removed. The can or package is first weighed before opening, and later the cleaned receptacle is weighed after its contents have been removed. The weight of the contents is thus ascertained by difference.

For the analytical determinations, the contents of the can or bottle are intimately mixed to form a homogeneous pulp, so that parts taken for analysis are fairly representative of the whole. If considerable liquid is present, with some solid masses held in suspension therein, the liquid is best drained off, and the solid portions pulped separately in any convenient manner, as by the use of a mortar, or by means of a household food-chopper. The whole is then thoroughly ingled together. If desired, the weight of the liquid and solid portions may be separately ascertained before mixing.

The analyst should use judgment and discrimination as to how various portions of the mass are to be best measured out for the determinations. Much depends on the consistency of the pulpy mass. It is often convenient to make a $20 \%$ solution or mixture of the material with water, using say 50 grams of the pulped sample in $250 \mathrm{cc}$. of water, such of the sample as is insoluble being disintegrated by shaking.

Methods for determining water, ether extract, crude fiber, protein, and ash do not differ materially from those employed in the case of corresponding fresh fruits and vegetables.

These determinations, in the case of canned products, while useful in showing their food value, give little information as to their adulteration by the substitution of foreign vegetable or fruit pulp.

Determination of Lead in Tin Alloy.-Method of Paris Municipal Laboratory.*-The material, if soft, is hammered into a thin plate, and

*Analyse des Matières Alimentaires et Recherche de leurs Falsifications, 1894, p. 695. 
$2 \frac{1}{2}$ grams are weighed out, transferred to a 250 -cc. flask, and dissolved in 7 to $8 \mathrm{cc}$. of concentrated nitric acid. Evaporate to dryness on the sand-bath, add to drops of nitric acid and $50 \mathrm{cc}$. of boiling water, cool, and make up to $250 \mathrm{cc}$. with water. Let the residue settle and pour off through a filter roo cc. of the clear, supernatant liquid, corresponding to I gram of the material. This contains the lead, while the tin is left behind in the residue, together with antimony if present.

Add ro cc. of a standard solution of potassium bichromate ( 7.13 grams to the liter) and shake. Each cubic centimeter of this standard solution is sufficient to precipitate 0.01 gram of lead. Allow the lead chromate formed to settle, and, if the solution is colorless, add ro cc. more of the bichromate, or sufficient to be present in excess, as indicated by the yellow color. Filter, wash, and titrate the excess of bichromate with a standard iron solution, containing 57 grams of the double sulphate of iron and ammonia and 125 grams of sulphuric acid per liter. This iron solution should be kept under a layer of petroleum, and standardized against the potassium bichromate before use.

Add, drop by drop, the iron solution to that containing the excess of bichromate. The color of the latter passes from pale green to bright green, when the chromate is completely reduced. Determine the endpoint with a freshly prepared dilute solution of potassium ferricyanide, a drop of which is placed on a porcelain plate or tile in contact with a little of the solution titrated. A blue color is prociuced when the iron is present in excess. If the standard iron and bichromate solutions exactly correspond, I cc. of the iron solution is equivalent to $\mathrm{I} \%$ of lead, but the latter solution is usually a little weak.

If $n=$ number of cubic centimeters of iron solution necessary to reduce to cc. of the standard bichromate,

$$
\text { I cc. of the iron solution }=\frac{\text { IO }}{n} \text {. }
$$

If, now, $r=$ number of cubic centimeters of iron solution necessary to reduce the excess of bichromate in the determination, and $s=$ number of cubic centimeters of bichromate used,

$$
s-\frac{10}{n} r=\text { per cent of lead in the alloy. }
$$

Separation and Determination of Tin, Copper, Lead, and Zinc in Canned Goods. - Munson's Method.*-The contents of the can are

-U. S. Dept, of Agric,, Bur. of Chem., Bul. ro7 rev., p. 62. 
first evaporated to dryness, and from 10 to 15 cc. of concentrated sulphuric acid or enough to carbonize are added to the dry residue contained in a porcelain evaporating-dish, which is very gently heated over the flame till foaming ceases. Then ignite to an ash in a muffle; or carefully over the free flame, using a little nitric acid, if necessary, for oxidation of the organic matter. Add $20 \mathrm{cc}$. of dilute hydrochloric acid, and evaporate over the water-bath to dryness. Wash the residue into a beaker, slightly acidify with hydrochloric acid, and saturate with hydrogen sulphide without previous filtration. Heat the beaker on the water-bath, and pass the contents through a filter. Wash the precipitate, which contains sulphides of tin, lead, and copper, if these metals are present, while if there is zinc, it is contained in the filtrate. The precipitate is fused with sodium hydroxide in a silver crucible for half an hour, to increase the solubility of the tin, which would otherwise be dissolved with difficulty. The fusion is boiled up with hot water, acidulated with hydrochloric acid, and transferred without filtering to a beaker, in which hydrogen sulphide is added to saturation. This precipitates the sulphides of tin, lead, and copper (if these metals are present). The sulphide precipitate is collected on a filter, and thoroughly washed with hot water, the washings being rejected. Pass through the filter several portions of boiling ammonium sulphide, using about $50 \mathrm{cc}$. in all, or till all the tin is dissolved. Precipitate the tin from the combined filtrate with hydrochloric acid, filter, wash, ignite, and weigh as stannic oxide.

The residue left on the filter, after dissolving out the tin sulphide, is then dissolved by treatment with nitric acid, which is filtered, and to the filtrate and washings ammonia is added nearly to the point of neutralization. Then add ammonium acetate. Filter off any precipitate of iron that may be formed. The filtrate is divided into two portions for determination of copper and lead. If lead is absent, determine the copper by titration with potassium cyanide* or electrolytically (p. 608). Copper is rarely present in sufficient amount to be determined, unless used for greening the vegetables. If notable quantities of lead are present, the solution is made acid with acetic, and the lead precipitated therefrom with potassium chromate, collected on a tared filter, washed with water acidified with acetic acid, dried at $100^{\circ} \mathrm{C}$., and weighed as lead chromate. Or determine the lead by color-tests, as on page $9 \mathrm{I} 8$.

For the determination of zinc, the filtrate from the first hydrogensulphide residue is evaporated to a volume of about $60 \mathrm{cc}$., and treated

* Sutton, Volumetric Anahysis, 8th ed., p. 204. 
with bromine water to oxidize the iron, as well as any excess of hydrogen sulphide remaining, the excess of bromine is then boiled off, and a few drops of concentrated ferric chloride added, to make the solution distinctly yellow, if not already so. Nearly neutralize with ammonia, and precipitate the iron with ammonium acetate. Filter, wash, acidify the filtrate with acetic acid, and precipitate the zinc with hydrogen sulphide. Filter, wash, ignite, and weigh as zinc oxide.

The metals may be determined separately, as follows:

Determination of Tin.*-Evaporate the contents of the can to dryness, and ignite in porcelain. Fuse the ash with sodium hydroxide in a silver crucible, boil the fusion with several portions of water acidulated with hydrochloric acid, filter, and precipitate the tin from the acid solution with hydrogen sulphide. Dissolve the washed precipitate in ammo. nium sulphide, filter, and deposit the tin directly from this solution by electrolysis in the platinum dish which contains it, using a current of 0.5 ampere and the electrolytic apparatus described on page 608 .

Smith and Bartlett + employ the following method of solution: Weigh 50 grams of fish or 100 grams of vegetables in a porcelain dish and dry over night. Heat from 75 to $100 \mathrm{cc}$. of concentrated sulphuric acid in a Kjeldahl flask until acid fumes are visible, then add gradually small portions of the food product, heating the acid between additions until frothing ceases. Allow to cool, then add gradually to the charred mixture $25 \mathrm{cc}$. of concentrated nitric acid, which causes the evolution of red fumes and the generation of heat. Cool, add $25 \mathrm{cc}$. of nitric acid and heat gently until all nitric fumes are expelled and the charred material is dissolved to a homogeneous solution. Boil this solution about 45 minutes, then add from Io to 15 grams of potassium sulphate and continue boiling from three to five hours until decolorized. Wash the digest into an $800 \mathrm{cc}$. beaker, dilute to about $600 \mathrm{cc}$. and bring to a boil. Almost all of the tin separates as stannic oxide, partially hydrated, some of which adheres to the sides of the flask, and cannot be removed by washing. Filter the contents of the beaker, thus separating the hydrated stannous oxide from all other compounds. Place the filter in the flask, to which $20 \mathrm{cc}$. of saturated sodium hydroxide and an equal volume of water have been added, boil for several minutes, then wash the sodium stannate into a beaker. Acidify with

*Hilger u. Laband, Zeits. Unters. Nahr. Genussm., 2, 1899, p. 795; An. Rep. Mass. State Board of Health, 1899 , p. 625 .

† A.O.A.C. Proc. 1910, U. S. Dept. of Agric., Bur. of Chem., Bul. 137, p. 134. 
hydrochloric acid, precipitate with hydrogen sulphide and proceed as above described.

Hansen and Johnson* heat a quantity of the material, containing about 25 grams of solids, with a mixture of $200 \mathrm{cc}$. of water, $100 \mathrm{cc}$. of concentrated nitric acid and $50 \mathrm{cc}$. of concentrated sulphuric acid, adding additional nitric acid from time to time and finally 25 grams of potassium sulphate.

Baker Method. $\uparrow-$ Treat 100 grams of the material with nitric and sulphuric acid as described in the preceding sections. Dilute the sulphuric acid residue, neutralize with ammonia, add hydrochloric acid until the solution contains about $2 \%$, and thoroughly saturate with hydrogen sulphide gas. Filter the impure lead sulphide on a Gooch crucible with a false bottom, wash three or four times with water, then transfer precipitate and asbestos to a $300-\mathrm{cc}$. Erlenmeyer flask, washing with a little water, and boil with strong hydrochloric acid, adding potassium chlorate from time to time to insure complete solution of the tin sulphide as well as the elimination of the sulphur. Add a few strips of pure aluminum foil, free from tin, until all the chlorine is eliminated, then dilute to from 30 to $40 \%$ acid strength and attach to a carbon dioxide generator provided with a scrubber and charged with pure marble and hydrochloric acid.

A bulb tube passing through one opening of a double-bore stopper serves to deliver the gas near the surface of the liquid and another bulb tube provides an exit, the latter being connected with a glass tube immersed in water to the depth of $20 \mathrm{~cm}$,, forming a water seal. When the flask is first attached to the carbon dioxide apparatus, lift the exit tube out of the water so as to reduce the pressure and thus force a large amount of gas through the system, expelling all air. Then raise the stopper of the flask and introduce about I gram of aluminum foil, which quickly reduces the tin to the metallic form with evolution of hydrogen.

Heat to boiling on a hot plate and boil for a few minutes, which causes the aluminum to disappear and changes the tin into stannous chloride, then cool in ice-water, still passing carbon dioxide through the system. Remove the stopper together with the tubes, washing the same and the sides of the flask with air-free water, prepared by boiling distilled water, adding a small amount of sodium bicarbonate and then a slight excess of hydrochloric acid.

* A.O.A.C. Proc. 1911, U. S. Dept, of Agric., Bur. of Chem., Bul. 152, p. 117.

† 8th Intern. Cong. Appl. Chem., 18, p. 35. 
Add starch paste and titrate directly and quickly with hundredthnormal iodine solution until a faint blue color is obtained. The iodine solution is standardized against pure tin solution or a food mixture, such as apple butter, containing an added amount of tin salt.

An alternate procedure is to add an excess of iodine solution to the flask after lifting the stopper, but while the carbon dioxide is still issuing from the neck, and titrate the excess with standard sodium thiosulphate solution.

By means of a $\mathrm{Y}$ tube the current from one generator may be divided for two flasks so that duplicates may be conducted at the same time.

Determination of Lead, especially applicable if lead is present in small amounts only. Boil the sulphated ash of the contents of the can (obtained as on page 915) with a solution of ammonium acetate, having an excess of ammonia. The tin, zinc, and iron remain insoluble, while the copper and lead are dissolved. Filter, wash, and add a few drops of potassium cyanide to the filtrate, to prevent precipitation of copper when hydrogen sulphide is subsequently added. If the solution exceeds $40 \mathrm{cc}$., concentrate to that amount by evaporation, and transfer to a 50 -cc. Nessler tube. Add hydrogen sulphide water, and make up to the mark. Cornpare the brown color imparted by the lead sulphide, with the colors obtained by treating with hydrogen sulphide water in Nessler tubes various measured amounts of a standard solution of lead acetate, made alkaline with ammonia.

Determination of Copper.-(I) Electrolytically.-Ash the contents of the can as on page 915 . Wet the ash with concentrated nitric acid, add water, and boil. Then make strongly alkaline with ammonia and filter. Unless the filtrate is colored blue, copper is absent. Transfer the filtrate to a bright tared platinum dish of roo-cc. capacity, neutralize with concentrated nitric acid, and add about $2 \mathrm{cc}$. in excess. Nearly fill the dish with water, and electrolyze with the apparatus described on page 608 , using a current of about 0.3 of an ampere.

(2) Colorimetrically, - This method is especially applicable for - nall amounts of copper. The blue-colored ammoniacal solution of the ash, filtered as in (I), is transferred to a Nessler tube, and its color matched against the colors of a series of measured amounts of an ammoniacal standard solution of copper sulphate.

Determination of Nickel.-Boil the ash with water slightly acidified with hydrochloric acid, and without filtering, saturate with hydrogen 
sulphide, thus precipitating out any copper, tin, or lead. Filter and wash. Zinc and nickel, if present, are in the filtrate. Boil the filtrate to expel the hydrogen sulphide, and add sodium carbonate till slightly alkaline. Add acetic acid without filtering till the precipitate produced by the alkaline carbonate is dissolved, and then add a considerable excess of acetic acid. The zinc is precipitated by passing hydrogen sulphide through the cold dilute solution, while the nickel is held in solution by the large excess of acetic acid. Filter, and wash with hydrogen sulphide water, to which a little ammonium acetate has been added.

Make the filtrate alkaline with ammonia, precipitate the nickel with ammonium sulphide, filter, wash, ignite, and weigh as nickelous oxide.

\section{KETCHUP,}

Standards. - The following are the standards of the A.O.A.C. and the Assn. of State and Nat. Food and Dairy Depts.:

Catchup (Ketchup, Catsup) is the clean, sound product made from the properly prepared pulp of clean, sound, fresh, ripe tomatoes, with spices and with or without sugar and vinegar; Mushroom Catchup, Walnut Catchup, etc., are catchups made as above described, and conform in name to the substances used in their preparation.

No standard is given for Chili Sauce, a product made from tomatoes, peppers, onions, vinegar, sugar, and spices differing from ketchup in that it is not strained.

Process of Manufacture.-When made in the household ripe tomatoes, with or without paring and coring, are cut in pieces and boiled down to a thick pulp, strained to remove seeds and other coarse tissues and finally heated for a time with vinegar, spices and sugar. The product is bottled while hot.

Factory-made ketchup, of good quality, is prepared by practically the same process, using special apparatus for washing, pulping and concentrating. In many factories considerable time elapses before the finishing processes are carried out, the pulp being stored in barrels or better in lacquered tin receptacles until needed. Manufacturers of ketchup often purchase the barrelled or canned pulp from canning factories, confining their attention to the final processes and bottling.

In the so-called gravity process the pulped material is allowed to stand until fermentation sets in and the cellular matter rises to the surface. The clear liquid is then removed from below. In Italy it is a common 
CHEMICAL COMPOSITION OF KETCHUP, PICKLES, AND RELISHES.*

\begin{tabular}{|c|c|c|c|c|c|c|c|c|}
\hline & $\begin{array}{c}\text { Number } \\
\text { of } \\
\text { Analyses }\end{array}$ & Refuse. & Water. & Protein. & Fat. & $\begin{array}{c}\text { Total } \\
\text { Carbo- } \\
\text { hydrates }\end{array}$ & Ash. & $\begin{array}{c}\text { Fuel } \\
\text { Value } \\
\text { per } \\
\text { Pound. }\end{array}$ \\
\hline Tomato ketchup....... & 2 & $\cdots \cdots$ & 82.8 & $x \cdot 5$ & .2 & $12 \cdot 3$ & 3.2 & 265 \\
\hline $\begin{array}{l}\text { Horseradish.............. } \\
\text { Olives, green: }\end{array}$ & 2 & & 86.4 & I. 4 & .2 & 10.5 & 1.5 & 230 \\
\hline Ertible portion....... & I & & $5^{8.0}$ & I. I & 27.6 & Ir. 6 & 1. 7 & $x, 400$ \\
\hline $\begin{array}{l}\text { As purchased......... } \\
\text { Olives, rine: }\end{array}$ & $\mathrm{I}$ & 27.0 & $42 \cdot 3$ & .8 & 20.2 & 8.5 & 1.2 & 1,025 \\
\hline Edible portion....... & I & $\cdots \cdots \cdots$ & 64.7 & I. 7 & 25.9 & $4 \cdot 3$ & $3 \cdot 4$ & I,205 \\
\hline As purchased ........ & I & 19.0 & 52.4 & $\mathrm{I} .4$ & 21.0 & 3.5 & 2.7 & 975 \\
\hline Cucumber pickles ...... & 3 & ........ & 92.9 & .5 & $\cdot 3$ & 2.7 & 3.6 & 70 \\
\hline Mixed pickles. .......... & I & & 93.8 & I. 1 & .4 & 4.0 & .7 & rio \\
\hline Spiced pickles. ........ & I & $\cdots \cdot$ & $77 \cdot 1$ & .4 & - I & 20.7 & I. 7 & 395 \\
\hline
\end{tabular}

practice in the manufacture of tomato paste to allow the pulp to ferment for a time, after which the fermentation is checked by the addition of salt. $\dagger$

Decayed Material.-According to Bacon and Dunbar $\ddagger$ fresh tomatoes contain on the average $6.5 \%$.total solids, of which $3.5 \%$ is invert sugar, $0.5 \%$ citric acid, $0.6 \%$ ash, $0.9 \%$ protein $(\mathrm{N} \times 6.25), 0.85 \%$ crude fiber and $0.05 \%$ fat. During spoilage the sugars rapidly disappear, forming alcohol, carbon dioxide, acetic and lactic acids, the amounts of each formed depending on the organisms present. Usually the citric acid is also decomposed. A good ketchup is accordingly characterized by a high citric acid content and little lactic acid, while one made from decomposed material will usually contain little or no citric acid, but a high per cent of lactic acid.

Tomato Refuse. - The skins, cores and other refuse from tomato canneries are used for the preparation of pulp which in turn is made into ketchup. Even with the use of such materials, when properly prepared, and before advanced fermentation has set in, with clean methods of handling, the product may not be unwholesome. It is, however, sometimes the practice to allow the refuse and skins to accumulate through a whole tomato-canning season, storing them all in large vats, and working them up, after they have become badly fermented, for "fresh tomato ketchup."

$\dagger$ Daily Consular and Trade Reports, I4, I9ri, p. 74.

$\ddagger$ U. S. Dept. of Agric., Bur. of Chem., Circ. 78 . 
It is largely for this reason that antiseptics and coloring matters are so commonly employed in ketchup.*

Foreign Pulp.-Pumpkin pulp and apple sauce, the latter made often from unsound material or even pomace, have been extensively used in cheap ketchups. At the present time such compound sauces are usually labelled to show the constituents present.

Preservatives.-Salicylic acid, formerly used in most commercial ketchups, more recently has given place to benzoate of soda. Bitting $\dagger$ has shown that by using sound tomatoes and exercising proper care in the process of manufacture, ketchup can be kept without a preservative. Manufacturers are themselves corroborating this, many of the standard brands being entirely free from any antiseptic material other than spices and vinegar.

Artificial Colors.-Of ninety-five samples of ketchup examined in Igor in Connecticut all but fifteen contained coal-tar colors. $\ddagger$ This practice, however, is now decreasing and is indeed quite unnecessary if fresh ripe tomatoes are used, dark-colored spices are avoided, and sugar is not added until the end of the process.

\section{METHODS OF ANALYSIS.}

Ash, Alkalinity of Ash, and Sodium Chloride are determined by the methods described for jams and jellies (p. 936). Total Acidity is calculated as citric acid from the number of cc. of $\mathrm{N} /$ ro alkali used in titration ( $\mathrm{c} \mathrm{cc} .=0.0064$ gram citric acid), Volatile Acids, as acetic, following the method for vinegar (p. 766). Tests for Preservatives and Colors are carried out as described in Chapters XVII and XVIII.

Determination of Solids. - Weigh Io grams of the sample into a flatbottomed metal dish $6 \mathrm{~cm}$. in diameter, add water to distribute the mate-

* The writer has in his possession a circular from an Indiana commission merchant, advertising for sale tomato pulp of some twelve different grades for ketchup. Among them are listed the following: "100 bbls. of old goods, made partly from whole stock and partly waste, boiled down nearly to ketchup thickness; has preservaline in it; fine goods, but some of it is fermented; packed in good oak whiskey and wine barrels. Price \$2.00 per bbl." " 225 bbls. new goods, made from waste; has bensoate of soda in it, packed in uncharred whiskey and wine barrels at $\$ 3.00$ per bbl. net cash." " 300 bbls. old goods, partly whole stock, partly waste, has salicylic acid in it; nice goods, etc. Price \$2.00 per bbl." " 400 bbls. new goods, Jersey style; solid and good red color, fine quality. Price $\$_{3} .00$ per bbl."

† U. S. Dept. of Agric., Bur. of Chem., Bul. Irg.

‡Ann. Rep. Conn. Exp. Sta., roor. 
rial, evaporate to dryness, dry 4 hours at the temperature of boiling water, and weigh.

Determination of Insoluble Solids.*-Shake 20 grams of the material with hot water in a narrow cylinder, centrifuge and decant the clear liquid on a tared filter-paper and filter with the aid of suction. Repeat the operation several times, finally transferring the material to the paper. Finish the washing on the paper and dry at $100^{\circ} \mathrm{C}$. to constant weight.

The filtering may be carried on to advantage on a Buchner funnel, using two or more tared filters, as the suction is liable to break a single layer.

Determination of Sand.*-Weigh 100 grams of the well-mixed sample into a 2 - or 3 -liter beaker, nearly fill the beaker with water, and mix the contents thoroughly. Allow to stand 5 minutes and decant the supernatant liquid into a second beaker. Refill the first with water and again mix the contents. After 5 minutes more decant the second beaker into a third, the first into the second, refill and again mix the first. Continue this operation, decanting from the third beaker into the sink until the lighter material is washed out from the ketchup. Then collect the sand from the three beakers into a tared Gooch crucible, dry, ignite, and weigh.

The method for the determination of ash insoluble in hydrochloric acid is not applicable to the determination of sand in tomato products, because the percentage present is so small and the sand is so unevenly distributed that reliable results can only be obtained by taking a larger sample than is possible in the determination of ash.

Determination of Soluble Solids. - Subtract the percentage of insoluble solids from the percentage of total solids.

Determination of Reducing Sugars.-Direct.-Place Io grams of the ketchup in a roo-cc. flask, add an excess of normal lead acetate, make up to the mark and filter. To the filtrate add powdered sodium sulphate or carbonate sufficient to precipitate the excess of lead and again filter. Determine the reducing power of the filtrate by the Munson and Walker method (p. 598) and calculate as invert sugar.

After Inversion.-Mix $50 \mathrm{cc}$. of the solution, after clarifying and removal of the lead, as described in last paragraph, with $5 \mathrm{cc}$. of concentrated hydrochloric acid, invert in the usual manner (p. 588), nearly neutralize with sodium hydroxide and determine the reducing power as before inversion.

*A.O.A.C. Proc. I911, U. S. Dept. of Agric., Bur. of Chem., Bul. 152, p. 118. 
Determination of Citric Acid.-Bacon and Dunbar Method.*-Weigh 25 grams into a $250 \mathrm{cc}$. beaker, make up to approximately $200 \mathrm{cc}$. with 95 per cent alcohol, allow to stand with frequent stirring for four hours, filter through a folded filter and wash with $50 \mathrm{cc}$. of 80 per cent alcohol. To the filtrate add sufficient water to dilute the alcohol to 50 or 60 per cent and then add Io cc. of 20 per cent barium acetate solution, stir well with a glass rod, and allow to stand over night. In the morning filter on a Gooch crucible, washing with 50 per cent alcohol, dry for from 3 to 4 hours in an oven at $100^{\circ} \mathrm{C}$. and weigh. Weight of precipitate times $0.5 \mathrm{I}$ equals anhydrous citric acid. This method is not applicable in the presence of malic acid, hence if apple pulp is a constituent of the ketchup, the Pratt method (p. 95r), should be employed.

Determination of Lactic Acid.-Bacon and Dunbar Method.*-To Ioo grams of ketchup add Io cc. of 20 per cent normal lead acetate solution, make up to $500 \mathrm{cc}$., shake well and centrifuge. To $400 \mathrm{cc}$. of the clear portion add a moderate excess of sulphuric acid, filter, wash the precipitate with a small amount of water, and evaporate the filtrate on the steam bath to about $100 \mathrm{cc}$. Extract for from 18 to 20 hours in a liquid extractor (Fig. I20) with washed ether. In case the quantity of lactic acid present is greater than 0.5 gram it is usually necessary to extract for a longer period. In any case it is well to re-extract for from 8 to to hours to make sure that the extraction is complete. (Ether sufficiently pure for this purpose may be prepared by shaking out ordinary ether once with a sodium hydrate solution and then ten times with small quantities of water.) Evaporate on the steam bath until the ether is no longer evident, and take up the residue at once in water and filter, thus removing a small amount of coloring matter and substances other than lactic acid, which may be

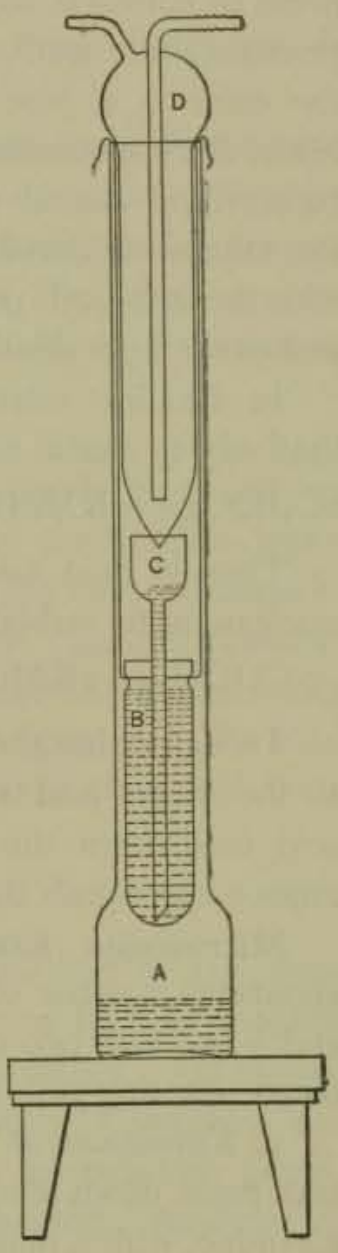

Fig. 120.-Bacon and Dunbar Extractor for Liquids. $A$, jacketflash; $B$, extract-tube; $C$, funnel-tube, $D$, condenser.

${ }^{*}$ U. S. Dept. of Agric., Bur. of Chem., Circ. 78, Igrr. 
extracted from ketchup by ether, but which are insoluble in water. Heat the filtrate on the steam bath for some time to remove all traces of ether or alcohol. Add approximately 3 grams of sodium hydroxide and 50 cc. of a $1.5 \%$ solution of potassium permanganate from a pipette. Heat on a water-bath at $100^{\circ} \mathrm{C}$. for one-half hour. At the end of that time, or before, if the color is not a decided blue-black or purple, but is green or colorless above the layer of brown precipitate, add more standard permanganate until, after heating one-half hour on a boiling water-bath, the color is a blue-black or purple. The oxidation is then complete, Make the hot solution strongly acid with ro per cent sulphuric acid (about $50 \mathrm{cc}$.) and run in 5 per cent standard oxalic acid from a burette until the solution is decolorized. Titrate back any slight excess of oxalic acid with the standard permanganate solution. (Any standard permanganate and oxalic acid solution may be used within reasonable limits of strength.)

In alkaline solution the permanganate oxidizes the lactic acid quantitatively to oxalic acid according to the equation:

${ }_{2} \mathrm{C}_{3} \mathrm{H}_{6} \mathrm{O}_{3}+\mathrm{IOKMnO}_{4}=2(\mathrm{COOH})_{2}+{ }_{4} \mathrm{H}_{2} \mathrm{O}+{ }_{2} \mathrm{CO}_{2}+{ }_{5} \mathrm{MnO}_{2}+{ }_{5} \mathrm{~K}_{2} \mathrm{MnO}_{4}$.

Then in acid solution, the oxalic acid is further oxidized by the permanganate to carbon dioxide and water according to the equation:

$$
5(\mathrm{COOH})_{2}+{ }_{2} \mathrm{KMnO}_{4}+{ }_{3} \mathrm{H}_{2} \mathrm{SO}_{4}=\mathrm{IOCO}_{2}+8 \mathrm{H}_{2} \mathrm{O}+\mathrm{K}_{2} \mathrm{SO}_{4}+2 \mathrm{MnSO}_{4} \text {. }
$$

To determine the total weight of permanganate used in the oxidation of the lactic acid subtract the permanganate equivalent of the oxalic acid used from the total amount used. The weight of permanganate times 0.237 equals the weight of lactic acid.

Microscopic Examination for Spoilage.-Howard Method*.-The apparatus consists of a compound microscope with two objectives $\left(\frac{2}{3} \mathrm{in}\right.$. and $\frac{1}{3}$ in.) and two compensating oculars $(\times 6$ and $\times 18)$, a Thoma-Zeiss blood-counting cell, slides and cover glasses.

I. Estimation of Molds.-Mount a drop of the material on a slide and press down the cover glass until the film is about $0.1 \mathrm{~mm}$. thick. Examine, with a magnification of 90 , approximately 50 fields and calculate the percentage of fields showing presence of mold filaments.

This percentage for home-made ketchups is practically zero, and for factory-made ketchups should be kept below 25 .

2. Estimation of Yeasts and Spores.-These are counted together because of the difficulty in differentiation without making cultures, which is impossible with a sterilized product. Thoroughly shake to cc. of

*U. S. Dept, of Agric., Bur of Chem., Circ. 68, 1911. 
the material with $20 \mathrm{cc}$. of water, and after standing one minute for the coarsest particles to settle, mount a drop in the Thoma-Zeiss cell. The material should not overrun the moat and Newton's rings must appear from the perfect contact of the glass surfaces to insure correct depth of liquid. With a magnification of 180 , -count the number of yeasts and spores in one-half of the ruled squares, which gives the number present in I 60 of a cubic millimeter of the original material.

The number in home-made and best factory-made ketchups, is practically none; the allowed limit is 25 .

3. Estimation of Bacteria.-Only rod-shaped forms are considered, as micrococci are easily confused with particles of clay, etc. Employing the mount used for yeasts and spores, and a magnification of 500 , count the rod-shaped organisms in several areas of five small squares each and multiply the average by $2,400,000$ which gives the number per cc.

The limit for bacteria is $25,000,000$ per cc.

Microscopic Examination for Foreign Pulp.-Apple is identified by the window-like cells of the skin, the pitted vessels of the bundles, quite unlike the vessels of the tomato, and the tissues of the core. Pumpkin may be detected by the yellow skin of the fruit with colorless stomata, somewhat obscure latex tubes and the peculiar cactus-like parenchyma of the seeds. Although only the fruit pulp is used, fragments of the skin and seeds of sufficient size to be of diagnostic importance often find their way into the product.

\section{PICKLES.}

A large variety of vegetables and fruits are preserved in the form of pickles in vinegar, either with or without spices, and kept in wooden pails, stoneware pots, kegs, or sealed wide-mouthed bottles. The containers are not of necessity air-tight. The commoner vegetables are usually pickled without cooking, while fruits such as peaches, pears, gooseberries, etc., are usually cooked, or at least heated. Analyses of pickles and relishes appear in the table, page 920.

Cucumber Pickles are the most common, and are prepared by soaking the fresh cucumbers in strong salt brine. They are then dried on frames, and afterwards treated with boiling vinegar, to which spices may or may not be added. Other vegetables pickled in similar manner, either separately or in mixture with cucumbers or "gherkins" to form " mixed pickles," are cauliflower, bean pods, white cabbage, young walnuts, and. onions. 
Such soft vegetables as young podded beans and beets are not treated with brine, but, after soaking in water, are directly treated with vinegar. The vinegar used for the finest pickling is of the cider, wine, or malt variety. Cheaper varieties of pickles are put up in "white wine" or spirit vinegar.

Mustard Pickles.-These differ from plain vinegar pickles in the character of the preserving medium, which in this case consists of a mixture of mustard and spices with the vinegar to form a thin paste.

Piccalilli consists of a mixture in vinegar of various chopped vegetables, such as cucumbers, cauliflower, green pickles, onions, green tomatoes, and various spices.

Olives for pickling are picked before they have fully ripened, and the inherent bitter taste is removed by soaking in a solution of potash and lime. This is replaced by cold water, and finally the olives are transferred to the medium in which they are bottled, which consists of salt brine, either with or without flavoring. The flavoring materials employed consist of such substances as fennel, coriander, laurel leaves, and occasionally vinegar. Ripe olives in brine are also highly esteemed.

Capers.-These are the flower buds of the shrub Capparis spinosa, which are pickled in vinegar. Nasturtium seeds, when similarly pickled, possess a flavor much resembling capers, but their substiution for capers could readily be detected by their distinctive appearance, even if colored.

Adulteration of Pickles.-Green pickles, such as cucumbers, are not uncommonly colored artificially by copper salts, either through the addition of copper sulphate, as in the greening of peas, or by the use of copper vessels. This artificial greening is to be looked for also in such products as capers and olives.

For methods of detection and estimation of copper, see page 902 . Pickles may be greened by boiling with much less harmful substances than copper salts, such, for example, as grape leaves, spinach, or parsley:

Free Sulphuric Acid has been found in a number of cases in the vinegar of pickles bought on the Massachusetts market. A pronounced test for chloride with nitrate of silver should not be attributed to free hydrochloric acid, since it may be and probably is due to the salt from the brine in which the pickles have been treated.

Alum is sometimes added to the salt solution to produce hardness and crispness in pickles. A number of samples of cucumber pickles have been found by the author to contain alum. For its detection, fuse the ash of the pickles, if free from copper, in a platinum dish with sodium 
c.rbonate, extract with boiling water, filter, and add ammonium chloride. A flocculent precipitate shows alum.

Sodium Benzoate and Saccharine are frequently used in sweet pickles.

Horseradish.-This condiment is prepared by grating the root of the perennial herb Nasturtium armoricia, and preserving in vinegar. It is very pungent and aromatic when first prepared, but by exposure to light and air quickly loses strength. Turnip, an occasional adulterant of gr ${ }^{\prime}$ ed horseradish, is best detected by the microscope.

\section{PRESERVES.}

Under this head are included various fruit products prepared with sugar syrup and often also with spices and vinegar. Some of these products differ little from canned fruits white others are really sweet pickles. Mince meat, although not strictly a fruit product, and fruits in cordials are classified for convenience as preserves. Jams are considered with jellies in the next section, as are also methods of analysis.

Fruit Butter.-According to the U. S. Standard, "fruit butter is the sound product made from fruit juice and clean, sound, properly matured and prepared fruit, evaporated to a semi-solid mass of homogeneous consistence, with or without the addition of sugar and spices or vinegar, and conforms in name to the fruit used in its preparation."

Apple Butter is the best-known product of this class. Unfortunately it is sometimes made from decayed fruit or even from apple pomace. Glucose is frequently substituted wholly or in part for sugar, in which case its presence should be declared on the label.

Mince Meat.-As prepared in the household, mince meat, the filling for mince pies, contains from to to $20 \%$ of lean meat and about twice as much apple. Other constituents appear in the following typical formula with statement of quantities in parts by weight: 2 parts each of meat, raisins, dried currants, and sugar, 4 parts of apples, I part each of suet and candied citron, 2 parts of sweet cider, wine or brandy, $I$ to 2 parts of seasoning including salt, spices, and lemons or oranges.

Standard Mince Meat of the A.O.A.C. and the Association of State and National Dairy and Food Departments, "is a mixture of not less than 10\% of cooked comminuted meat, with chopped suet, apple and other fruit, salt, and spices, and with sugar, syrup, or molasses, and with or without vinegar, fresh, concentrated, or fermented fruit juices, or spirituous liquors."

Adulteration.-There has been some conflict between food officials and certain manufacturers as to the proportion of meat in commercial 
mince meat, the manufacturers claiming that $10 \%$ is too much for the proper keeping of the product, the food officials, on the other hand, contending that the manufacturer has no right to lower the recognized standard of the housewife.

As a matter of fact the greater part of the mince meat on the market contains considerably less than $10 \%$ of meat and much of it none whatever. Glucose is a common substitute for part of the sugar, wormy or other inferior fruit is sometimes used, and benzoate of soda is added as a preservative.

Condensed Mince Meat is made in a commercial way from dried apples and other desiccated materials and is sold in compressed cakes with instructions for preparing from the cakes moist pie filling. As in the case of wet mince meat, glucose, wormy fruit and benzoate of soda are frequent admixtures and true meat is often omitted entirely. Wheat or rye flour is a common adulterant.

Examination of Mince Meat.-Meat and cereal flour may be identified by microscopic examination. Care should be taken not to confuse apple starch, which is always present in the immature fruit, with cereal starches. Meat fibers are recognized by their yellow brown color, the delicate transverse striations and their occurrence in bundles.

Determinations of nitrogen are of service in estimating the amount of meat present. Glucose and sugar are calculated from the polarization readings.

Pie Filling. Bakers and hotel cooks are supplied by manufacturers with filling prepared ready for use in pies. This material is shipped in pails or tubs preserved with benzoate of soda, and may contain fruit of questionable quality as well as admixtures such as starch, glucose, and artificial colors.

Maraschino Cherries. - This name has been applied indiscriminately to the vivid red preserved cherries used in cocktails, punches, ice cream and confectionery. Investigation by the Board of Food and Drug Inspection has led to the decision * that only marasca cherries, preserved in true maraschino cordial prepared by fermentation and distillation from marasca cherries, are entitled to the name maraschino cherries, although cherries of other types preserved in pure maraschino cordial may be labelled: "Cherries in Maraschino." Ordinary cherries preserved in syrup flavored with maraschino may be so labelled, but if the flavoring is oil of bitter almonds or benzaldehyde the product should be labelled as an imitation if the word maraschino is used.

\footnotetext{
* Food Inspection, Decision I4I.
} 
Enormous quantities of white cherries of the Bigarreau or Royal Anne type, preserved in a mixture of sulphurous acid and brine, are brought into the United States from Europe and transformed into red "Maraschino cherries" or green "Crème de menthe cherries." After removal of the sulphurous acid and brine the cherries are put through a dye bath and then, being quite without taste, are flavored with oil of bitter almond or benzaldehyde, or else peppermint, and packed in syrup. Scarcely more than the cellular structure of the original cherry remains, the fruit juice with its sugars, acids, and true cherry flavor being replaced by the syrup with its sickening flavor and aroma. Even if flavored with true maraschino the metamorphoses through which the fruit passes leave it a sorry substitute for the natural cherry.

Woodman and Davis * have shown that true maraschino contains very little benzaldehyde and that cherries flavored with maraschino should not contain more than two or three times as many milligrams of benzaldehyde per 100 cc. as there are grams of alcohol in that volume, and those containing over $20 \mathrm{mg}$. of benzaldehyde but no alcohol are evidently entirely artificial.

Artificial colors, sulphurous acid and other preservatives are detected by the methods given in the chapters on colors and perservatives, benzaldehyde by the following method:

Determination of Benzaldehyde in Maraschino Cherries.-Woodman and Davis Method.*-Reagent.-Mix $3 \mathrm{cc}$. of glacial acetic acid with $40 \mathrm{cc}$. of water, add 2 cc. of C.P. phenylhydrazine, as near colorless as possible, shake thoroughly, and filter the emulsion through several thicknesses of filter-paper. The clear filtrate should be used immediately as a turbidity appears on standing longer than five minutes.

Process.-Dilute $100 \mathrm{cc}$. of the liquor from maraschino cherries (or $50 \mathrm{cc}$. of maraschino liqueur) to $140 \mathrm{cc}$. and distill off $\mathrm{IrO} \mathrm{cc}$. Determine approximately the alcohol in the distillate by the pycnometer or immersion refractometer, then without delay transfer $100 \mathrm{cc}$. to a $300 \mathrm{cc}$. Erlenmeyer flask and add alcohol or water so that the solution shall contain approximately $10 \%$ of alcohol. Add $100 \mathrm{cc}$. of the reagent, stopper tightly with a rubber stopper, and shake vigorously for ten minutes. Collect the precipitate in a tared Gooch crucible, wash with cold water and finally with about Io cc. of $10 \%$ alcohol. Dry in a vacuum desiccator for $20-24$ hours at about $20 \mathrm{~cm}$. pressure, or in a vacuum oven at $70-80^{\circ} \mathrm{C}$. for 3 hours. Throughout the process avoid exposure of the precipitate to strong light.

* Jour. Ind. Eng. Chem., 4, 1912, p. 588. 
Run a blank determination at the same time and deduct the weight obtained from that found in the actual analysis. Multiply the corrected weight of the precipitate by $0.54 \mathrm{Ir}$, thus obtaining the weight of benzaldehyde.

\section{JAMS AND JELLIES.}

Jams or marmalades are prepared from the pulp of fruits, and jellies from the fruit juices. Both jams and jellies, to be considered of the highest degree of purity, should contain nothing but the fruit pulp or juice named on the label, mixed with pure cane sugar, and, in the case of jams, the further addition of spices and flavoring materials is permissible.

For the manufacture of jam, apples, quinces, and pears are peeled, freed from cores, and sliced; berries are simply stemmed; and stone fruits, such as peaches and apricots, are peeled, and freed from stones. The material, properly prepared, is cooked with as much water as is necessary for boiling, and with the addition of an amount of sugar varying with different manufacturers. Some prefer to use equal parts of sugar and fruit, others one part sugar to two parts fruit.

In the case of jelly, the fruit is cooked in a small amount of water till soft, transferred to a bag or press, and the juice allowed to flow out spontaneously, or is squeezed out under pressure, according to the grade of jelly desired, the clearest and finest varieties being made from the juice that flows out naturally. This juice is then evaporated down with the addition of sugar to a density of from $30^{\circ}$ to $32^{\circ}$ Bé., which is of the proper consistency to form a perfect jelly product after cooling, and, while still hot, is poured into the tumblers in which it is to be kept. Here, as in the case of jams, the amount of sugar varies, some using pound for pound, and others only half as much sugar as fruit. Some manufacturers clarify their jellies by mixing with the juice, while boiling, elutriated chalk, using a teaspoonful to each quart of juice. The impurities come to the surface with the chalk as a scum, and are skimmed off. This clarifying process is somewhat analogous to the defecation of sugar juices with lime, and is commonly carried out with apple jelly.

The " jellying" or gelatinizing of the final product is due to the presence in the fruit juice of pectin, or so-called vegetable jelly $\left(\mathrm{C}_{32} \mathrm{H}_{40} \mathrm{O}_{28} \mathrm{H}_{2} \mathrm{O}\right)$; see page 276 .

The high content of added sugar in jelly, once thought to be essential for keeping it, is now no longer considered necessary, and much less sugar 
is at present added than formerly. The finest grade of apple jelly, for instance, is made without any added sugar whatever.

In making the better grades of apple jelly, apple juice fresh from the press is run directly into the boiler or evaporator before any fermentation has ensued, and gelatinized by concentration. If boiled cider is wanted instead of jelly, it is drawn off at an earlier stage than in the case of apple jelly.

Composition of Known-purity Jellies and Jams.-In the tables on pp. 932 and 933, due to Tolman, Munson, and Bigelow,* are given results reached on the examination of the pure finished products, as well as on pure fruit juices and pulp used in their manufacture.

Adulteration of Jams and Jellies. - As a matter of fact, a small percentage of these products sold in the United States are honest prototypes of the home-made jams and jellies, which consist exclusively of the fruit specified on the label, in mixture with pure cane sugar. If we accept as a standard the product of the housewife, fully $90 \%$ of the commercial brands of these preparations would be found wanting. So great is the demand for cheap sweets of this variety, that the market is flooded with them at eight and ten cents per half-pound jar, when in reality absolutely pure goods cannot be produced at much less than twice that amount.

The cheap substitutes are made up largely of apple juice and commercial glucose, sometimes containing no fruit whatever of the kind specified on the label. Sometimes an attempt is made to imitate the flavor by the addition of artificial fruit essences, but more often the same apple-glucose stock mixture of jelly, put out under a particular brand, serves to masquerade as damson, strawberry, raspberry, current, grape, etc., differing from each other only in color, but not as a rule in flavor. A variety of artificial colors are employed, mostly coal-tar cyes. To compensate for the lack of sweetness of the gl:cose, a minute quantity of one of the concentrated sweetener, s:ch as saccharin or dulcin, is sometimes added. Besides artificial colors, antiseptic substances are occasionally used, especially sodium benzoate.

All grades of apple stock are found in these preparations. A large source of supply is furnished by the parings and cores of canning establishments, to say nothing of the refuse of these factories, such materials being boiled with water, and the extract, variously colored to imitate the different fruits, being evaborated with commercial glucose.

* Jour. Am, Chem. Soc. (1901), pp. 349-351. 


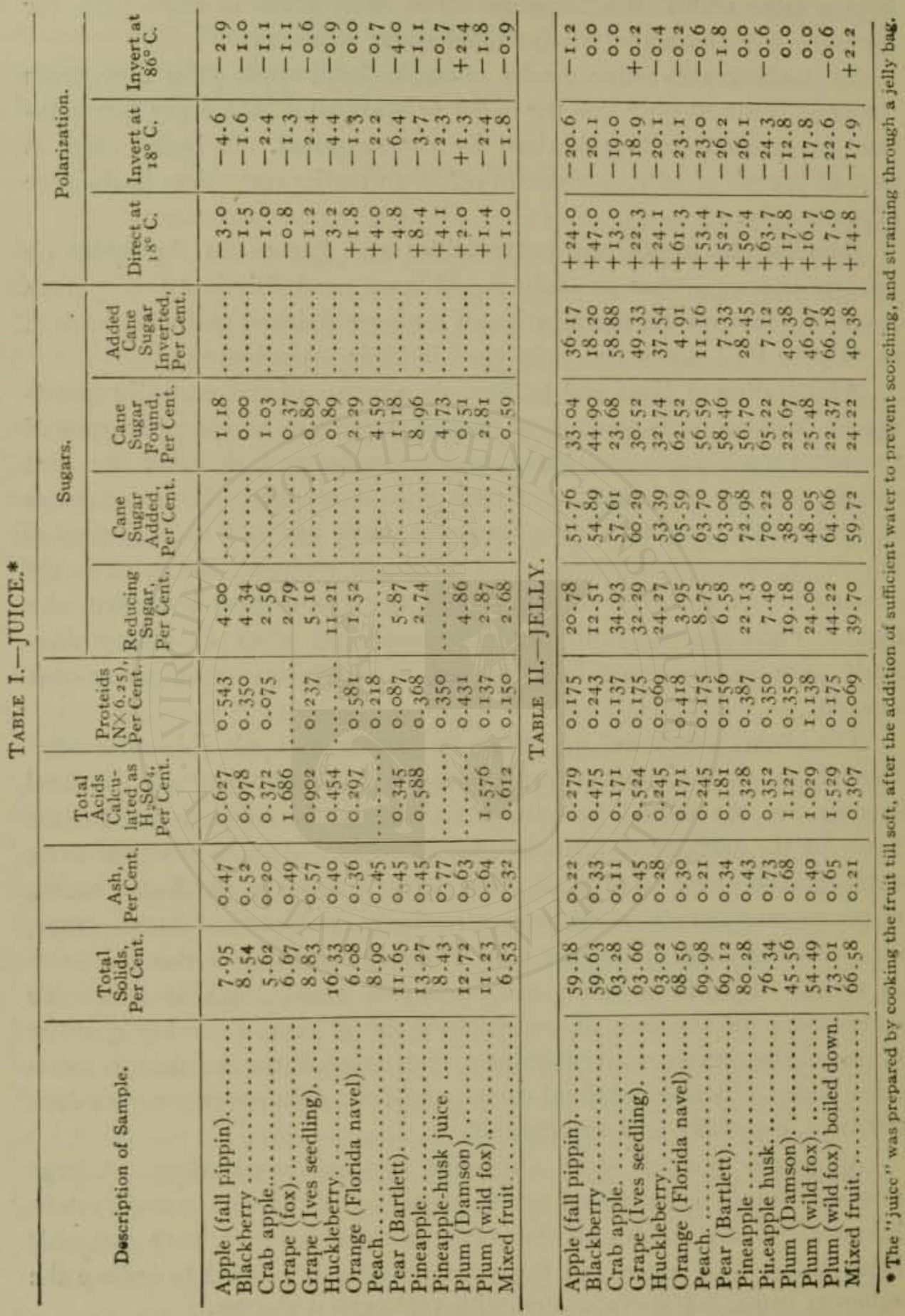




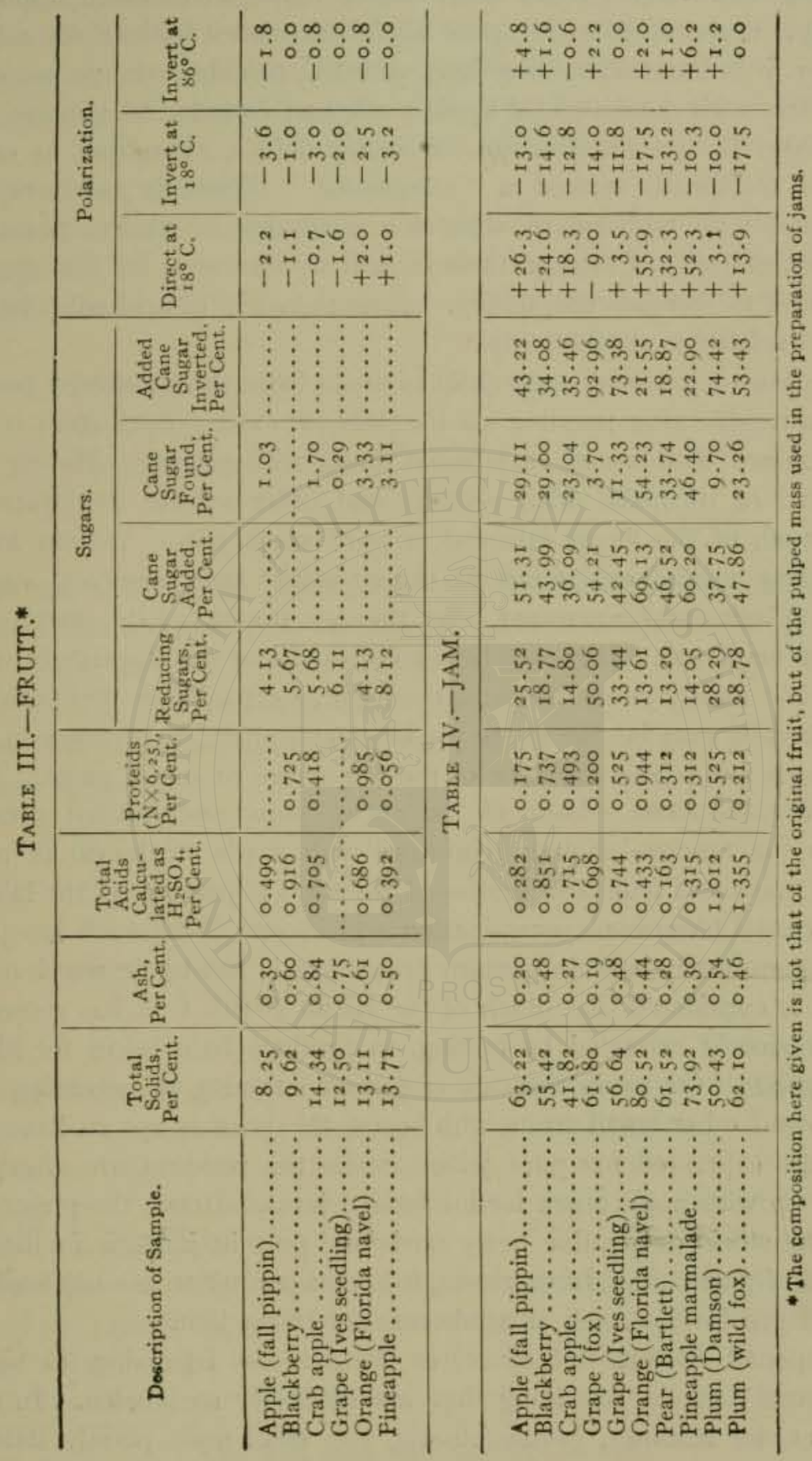


Adulterated Jelly.-While it is easy to make an excellent apple jelly by simple evaporation of the pure apple juice, even without the addition of sugar, it is impossible, or at least difficult, to obtain the proper degree of stiffness with a mixture of apple stock and commercial glucose. It is customary, in the manufaciure of cheap jellies, therefore, to employ what is technically termed a "coagulator." Formerly sulphuric acid, sometimes with addition of alum was used, but at present phosphoric acid is preferred. Citric or tartaric acid is also used for this purpose, as well as to increase the acidity. Less than ${ }_{1} \%$ of acid will cause the mass to gelatınize satisfactorily.

The lowest grade of apple jelly is made from the exhausted pomace, left as a residue after pressing out the juice for cider. Such stock is commonly mixed with water, and boiled down with glucose. Having been exhausted of its malic acid, pectose, and other soluble constituents, it lacks much of the flavor inherent in pure apple jelly. Various foreign gelatinizing agents are found in cheap jellies and preserves, such as starch, gelatin, and agar-agar. In the low-priced goods, starch paste has been employed. It should be remembered that starch exists in unripe apples, but hardly at all in the mature fruit, so that while mere traces of starch in jelly may be due to the use of green apples, its presence in large amounts is undoubted evidence of the admixture of starch paste.

Adulterated Jams.-Most of the cheap jams and bottled preserves. sold on the market, though reinforced with apple stock, do in reality contain masses of fruit and berries of the kind stipulated on the label, as. even a casual megascopic examination will show. That such low-priced preparations really contain genuine fruit pulp is not to be wondered at; when it is considered that much of the virtue of this fruit has sometimes been previously extracted by boiling, to produce fruit juices for higherpriced goods. Or, as in the case of jams containing strawberries, raspberries, and other small fruits with seeds, the juice is apt to have been previously expressed for pure jellies, while the residues are afterwards worked up with apple stock for low-priced jams. Hence the presence of pure fruit stock, or genuine berry seeds and pulp in jams, is in itself no criterion of purity, and, furthermore, it is unnecessary to use hay seed and other alleged foreign seeds as adulterants of cheap jam.

Compound Goods.-Many states have a law legalizing the sale of "compound" goods, providing they are distinctly so labeled. In other states, as, for instance, Massachusetts, the label must plainly state the name and percentage of the ingredients. In either case the analyst must 
discriminate, in classifying the inferior or low-grade preparations, between those that are labeled in accordance with the law, and those that are not. Only those not properly labeled can in such cases be classed as adulterated within the meaning of the law. Where such a law prevails, probably no class of food-products is so extensively affected by it as the low-grade jams, preserves, and jellies.

The restrictions as to labeling do not in all cases eliminate the element of deception. It is hardly justifiable, for example, to boldly label an alleged "currant jelly" which contains no currant, in the following manner:

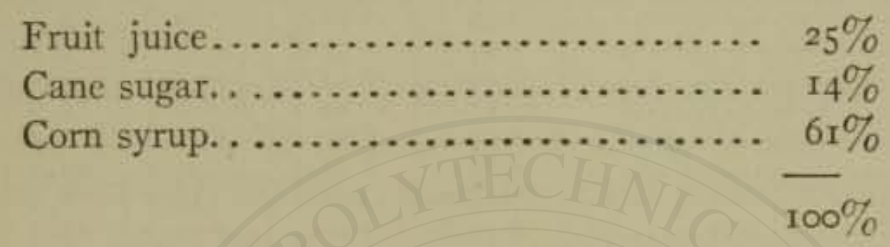

The use of the term "fruit juice" surely implies to the unsuspecting purchaser that so much pure currant juice has entered into the jelly, elsewhere labeled in large letters "Currant," whereas all the juice is apple, and no currant juice has been used.

The following label is a type of those which discriminate between pure fruit and apple juice:

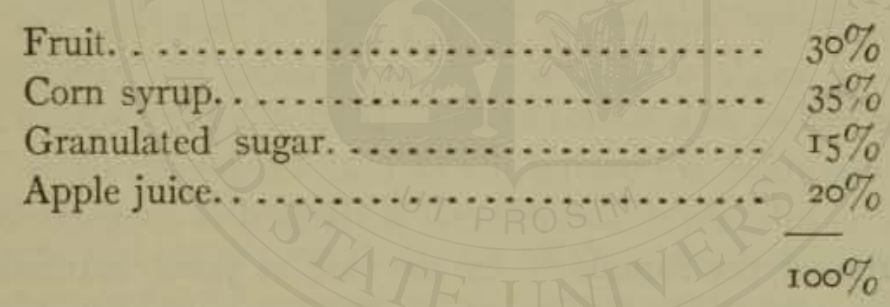

Composition of Cheaper Grades.-Out of 66 samples of jellies, jams, and preserves analyzed by Winton, Langley, and Ogden in Connecticut, the samples being purchased in that state, ${ }^{*}{ }{ }_{7}$ samples contained starch paste, 35 were artificially colored with coal-tar dyes, and ig contained salicylic or benzoic acid.

The following table has been compiled, showing the sugar content of some of the typical commercial jellies and jams analyzed in the laboratory of the Massachusetts State Board of Health. Nearly all of these were artificially colored, and found to contain little if any frui, other than apple.

- An, Rep. Conn. Exp. Sta., rgor, p. rzo. 


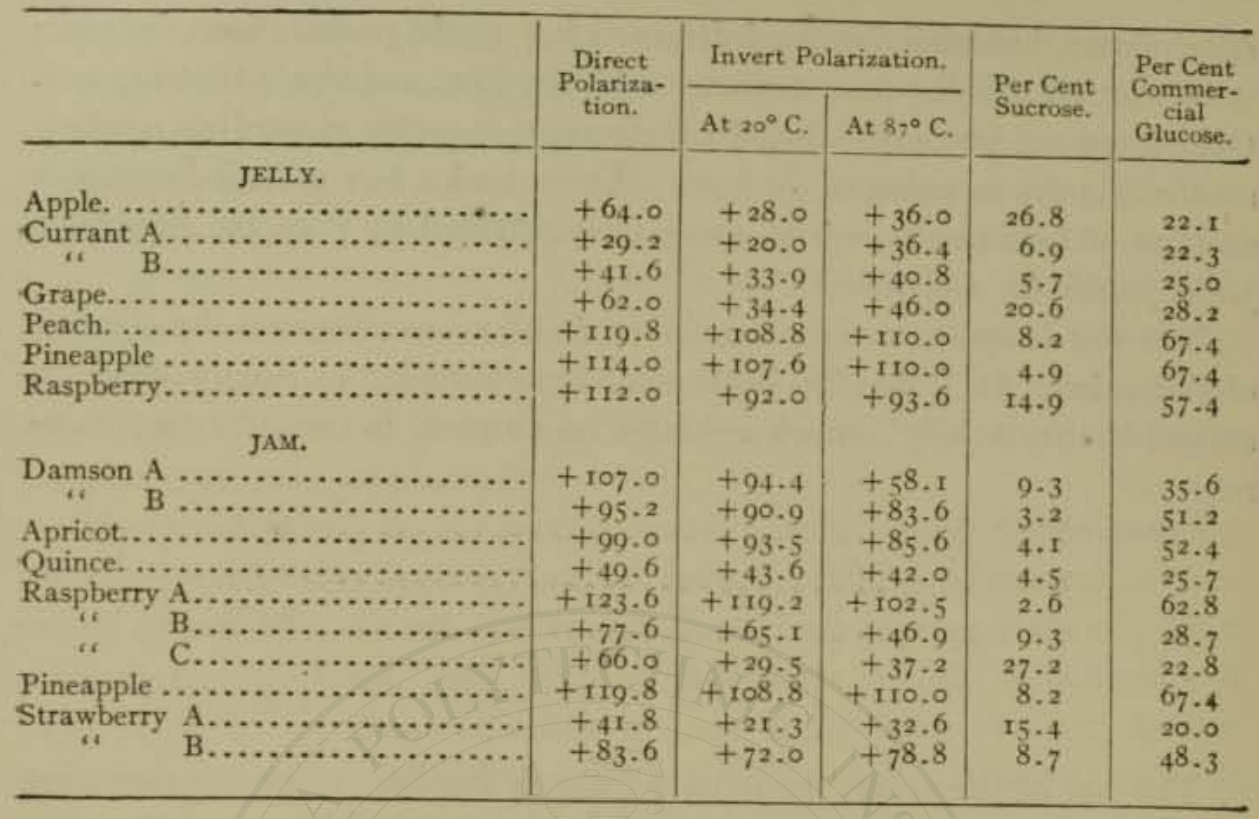

METHODS OF ANALYSIS.

As in the case of canned goods, but little information is to be derived as to adulteration of jams, jellies, and preserves by the ordinary determinations of moisture, ash, and nitrogen, and these are rarely made by the public analyst.

Of considerable importance in this regard, however, are the sugar determinations, made with a view to ascertaining the varieties of sugar employed, as well as their approximate proportion in the products examined. Still more important are the results of tests for preservatives, dyes, foreign gelatinous substances, and mineral acids used as coagulators.

Preparation of the Sample.*-In the case of jams, marmalades, and preserves, separate and weigh the stones, if present, then thoroughly pulp the sample. Stir well before weighing out the portions for analysis.

In the case of jellies prepare a solution of the thoroughly mixed sample containing 60 grams in $300 \mathrm{cc}$. and make the determinations, so far as practicable, on aliquots of this " $20 \%$ solution." If starch or insoluble matter is present shake thoroughly before aliquoting.

Determination of Total Solids.*-Weigh 4 to 5 grams of jam, or measure 25 cc. of $20 \%$ jelly solution, into a large flat-bottomed dish (preferably of platinum) containing from 4 to 5 grams of ignited asbestos and add in the case of jam enough water to uniformly distribute the material. Evap-

* A. O. A. C. Method, U. S. Dept. of Agric., Bur. of Chem., Bul. 107, rev., p. 77. 
orate to dryness and dry for from 20 to 24 hours in a boiling wateroven.

The results by this method are not strictly accurate owing to the dehydration of levulose, but for practical purposes they are sufficiently close. If extreme accuracy is required dry in vacuo at $75^{\circ}$ or in a McGill oven (p. 586).

The solids in a jelly may also be calculated from the specific gravity.

Determination of Ash.-Burn the residue from the determination of solids, or else a new portion, in a platinum dish at dull redness.

Alkalinity of ash is determined as described for insoluble ash in maple products (p. 627).

Chlorides and Sulphates are detected in the ash by the usual tests. If the portion used for determination of alkalinity is also to be used for the chlorine test the titration must be made with fifth-normal nitric acid.

The presence of chlorides is an indication of glucose, as pure fruit products do not contain appreciable amounts of chlorine compounds.

Determination of Insoluble Solids.*-Modified Kremla Method.Thoroughly macerate 50 grams of the sample in a mortar with warm water, then transfer to a muslin filter and wash thoroughly with warm water, stirring well after each addition. Wash up to $500 \mathrm{cc}$., or in extreme cases up to Iooo cc, remove the insoluble solids to a dish, dry in a boiling water-oven, and weigh.

Reserve the filtrate for determinations of soluble constituents.

German Official Method.-Transfer a weighed portion of the sample to a graduated flask, add water, shake thoroughly and make up to volume. Allow to settle and either filter or decant off the supernatant liquid. Determine the soluble solids by evaporating and drying an aliquot. The insoluble solids are obtained by subtracting the soluble from the total solids.

Determination of Acidity.-Dilute an aliquot of the solution from the insoluble solids of a jam or of the $20 \%$ solution of a jelly and titrate with standard alkali. Use phenolphthalein as indicator if the color of the solution will permit, otherwise use litmus paper. Calculate the result as sulphuric acid or as the organic acid known to predominate (see page 949).

Determination of Protein.-Determine nitrogen in 5 grams of the material by the Kjeldahl or Gunning method and calculate protein, using the factor 6.25 .

- A. O. A. C. Method, U. S. Dept. of Agric., Bur. of Chem., Bul. to7, rev., p. 78. 
Determination of Sugars.-In products of the highest grade, wherein only cane sugar is employed, a large portion of the cane sugar is inverted in the process of boiling the jam or jelly, so that when the analyst examines it, he finds, as a rule, only a small amount of sucrose, and considerable invert sugar. It is possible, however, to calculate the amount of cane sugar originally employed, if such information is desirable. It is further of interest to calculate, at least approximately, the percentage of commercial glucose, when present, especially in cases where the package contains a formula setting forth the amount of the various ingredients used. In such cases the analyst is naturally called upon to verify the formula, since a wide variation in percentage composition from the statement on the label would constitute an offense under some state laws.

Polarization.-Use half the normal weight of the preserve or jelly for the Schmidt and Haensch instrument, viz., I 3.024 grams in $100 \mathrm{cc}$. If fresh fruit or fruit juice is to be examined, use the full normal weight, 26.048 grams. Clarify, before making up to the mark, with subacetate of lead and alumina cream (using 2 to $3 \mathrm{cc}$. of each clarifier), filter, and obtain the direct reading; then invert in the usual manner, and obtain the invert readings at $20^{\circ} \mathrm{C}$, , and in the water-jacketed tube at $87^{\circ} \mathrm{C}$., proceeding in detail as directed under honey, p. 64I.

Calculation of Sugars.-Sucrose is determined by using Clerget's formula:

$$
S=\frac{(a-b) \mathrm{I} 00}{\mathrm{I} 42.66-\frac{t}{2}}, \cdots . . .
$$

This represents the sucrose actually present as such in the preserve or jelly, and not the amount originally used. If the latter is desired, it may be calculated from the formula,

$$
S^{\prime}=\frac{100 b}{42.66-\frac{t}{2}}, \quad \ldots . . . . .
$$

where $S^{\prime}$ is the per cent of cane sugar originally used, and $b$ is the invert reading at $t^{\circ}$ of the normal solution.

If, after inversion, the correct reading at $20^{\circ}$ is found to be 12 or more to the left of the zero, it can be safely inferred that no appreciable amount of commercial glucose is present, and it is unnecessary to make a third 
stading at $87^{\circ}$, unless to confirm the fact. In such a case, with cane sugar alone present, the reading at $87^{\circ}$ will not, of course, vary much from 0 .

Invert Sugar.-In the absence of commercial glucose, the invert sugar is calculated as follows:

$$
\text { Invert sugar }=\frac{(\text { Sucrose }- \text { direct reading })_{105.3}}{42.66-\frac{t}{2}}, \ldots \text { (3) }
$$

or it may be determined directly from the copper reducing power.

Any decided reading above zero at $87^{\circ}$ is due to the presence of commercial glucose, and when the latter is present, it is impossible to determine the invert sugar from the copper reduction or by formula No. 3 . The following formula is proposed for calculating approximately the invert sugar from the polarization, in the presence of commercial glucose. While theoretically correct, the method is subject to practical limitations, which admit of only roughly approximate results in such mixtures as jelly or jam. It is perfectly accurate only in mixtures of sucrose, glucose, and invert sugar.

Invert sugar $=\frac{\left(\begin{array}{c}\text { Reading due to glucose and } \\ \text { inverted sucrose at } \rho\end{array}\right)-\left(\begin{array}{c}\text { Invert reading } \\ \text { at } t^{\circ}\end{array}\right)}{ \pm\left(42.66-\frac{t}{2}\right)}$ 105.3

These formulas, (3) and (4), serve at best to indicate the approximate amount of invert sugar present in the sample, resulting from the inversion of a portion of the original sucrose in the natural process of manufacture of the jam or jelly, and not the total invert sugar resulting from the inversion by the analyst of all the sucrose.

The factor 105.3 is used, since, in the natural process of inversion, I $\infty$ parts of sucrose become 105.3 parts of invert sugar.

Example.-The invert sugar in the sample of apple jelly first on the list in the table on page 936 is calculated as follows:

Invert reading at $t^{\circ}\left(20^{\circ}\right)=28.0$.

Reading due to glucose at $20^{\circ}=.221 \times 175=38.68$.

$$
\begin{aligned}
& \text { " " " inverted sucrose at } 20^{\circ}=.268 \times-34=-9.1 \text {. } \\
& \text { Invert sugar }=\frac{(38.68-9 \cdot \mathrm{II})-28}{28,66} \text { I05.3 } \\
& =5.76 \% \text {. }
\end{aligned}
$$




\section{Determination of Reducing Sugar.-Proceed as described on page 621 .}

Commercial Glucose. - While it is impossible to determine the exact percentage of this substance in preserves and jellies, by reason of the varying composition of its component parts, it is quite feasible to approximate very closely to the amount present. Indeed, this approximate method of calculation, wherein glucose is treated as a chemical entity, has been found in practice to be much more close to the actual truth than results gained by methods wherein the copper reducing power enters as a factor, or methods for determining separately dextrin, maltose, and dextrose. Calculate the commercial glucose in jellies and jams exactly as in the case of honey, p. 641 .

Dextrin.*-If alcohol be added to a somewhat thick solution of the fruit product, a white turbidity is at once apparent, followed by the formation of a thick gummy precipitate, if dextrin is present. In the absence of dextrin there is no turbidity, but a light flocculent precipitate.

To determine the dextrin, dissolve $\dagger$ 1o grams of the sample in a roo-cc. flask; add $22 \mathrm{mg}$. of potassium fluoride, and then about one-quarter of a cake of compressed yeast. Allow the fermentation to proceed below $25^{\circ} \mathrm{C}$. for two or three hours to prevent excessive foaming, and then place in an incubator at a temperature of from $27^{\circ}$ to $30^{\circ} \mathrm{C}$. for five days. At the end of that time clarify with lead subacetate and alumina cream; make up to $100 \mathrm{cc}$. and polarize in a $200-\mathrm{mm}$. tube. A pure fruit jelly will show a rotation of not more than a few tenths of a degree either to the right or to the left. If a Schmidt and Haensch polariscope be used, and a $10 \%$ solution be polarized in a $200-\mathrm{mm}$. tube, the number of degrees read on the sugar scale of the instrument, multiplied by 0.8755 , will give the percentage of dextrin, or the following formula may be used:

in which

$$
\text { Percentage of dextrin }=\frac{C \times 1000 \times V}{198 \times L \times W},
$$

$C=$ degrees of circular rotation,

$V=$ volume in cubic centimeters of solution polarized,

$L=$ length of tube in centimeters,

$W=$ weight of sample in solution in grams.

* Bur. of Chem., Bul, 65, p. 78 ; Bul. 107 rev., p. 80.

$\dagger$ Bigelow and McElroy, Jour. Am. Chem. Soc, r893, 15, 668 . 
Determination of Alcohol Precipitate.*-Evaporate 100 cc. of a $20 \%$ solution of jelly, or of the washings from the determination of insoluble solids of a jam, to $20 \mathrm{cc}$; ; add slowly and with constant stirring $200 \mathrm{cc}$. of $95 \%$ alcohol and allow the mixture to stand over night. Filter and wash with $80 \%$ alcohol by volume. Wash this precipitate off the filter paper with hot water into a platinum dish; evaporate to dryness; dry at $100^{\circ}$ C. for several hours and weigh; then burn off the organic matter and weigh the residue as ash. The loss in weight upon ignition is called alcohol precipitate.

The ash should be largely lime and not more than $5 \%$ of the total weight of the alcohol precipitate. If it is larger than this some of the salts of the organic acids have been brought down. Titrate the watersoluble portion of this ash with tenth-normal acid, as any potassium bitartrate precipitated by the alcohol can thus be estimated.

The general appearance of the alcohol precipitate is one of the best indications as to the presence of glucose and dextrin. Upon the addition of alcohol to a pure fruit product a flocculent precipitate is formed with no turbidity while in the presence of glucose a white turbidity appears at once upon adding the alcohol, and a thick, gummy precipitate fornis.

Determination of Tartaric, Citric, and Malic Acids.-Modified SchmidtHiepe Method.*-Use the filtrate from the alcohol precipitate in this determination. After evaporating the alcohol and taking up the acids with water add lead subacetate until the solution is alkaline, then filter and wash the precipitate until only a slight amount of lead remains in the washings. Wash the precipitate off the filter-paper into a beaker with hot water, precipitate the lead by hydrogen sulphide, and filter off the lead sulphide while hot, washing with hot water. Evaporate the filtrate which contains the free organic acids to about $50 \mathrm{cc}$., neutralize with potassium hydroxide, add an excess of strong solution of neutral calcium acetate with constant stirring, and allow to stand from six to twelve hours. Throw the precipitate of calcium tartrate on a filter-paper and wash until filtrate and washings make exactly roo cc.; ignite the filter-paper and precipitate, and determine the lime by titration. A correction of 0.0286 gram of tartaric acid, which is held in solution in the roo cc. of washings as calcium tartrate, must be added. Evaporate the filtrate down to about $20 \mathrm{cc}$, and if a precipitate of calcium citrate is formed collect it on a filter while hot, wash with hot water, ignite, and titrate. From

*A. O. A!'C. Method, U. S. Dept. of Agric., Bur. of Chem,, Bul. 107, rev., p. 80. 
this result calculate the citric acid. Evaporate the filtrate and washings from the calcium citrate to about $20 \mathrm{cc}$. and add three volumes of $95 \%$ alcohol, which will throw down the calcium salt of tartaric acid held in solution, the remaining citrate, and the malate and succinate. Filter, ignite the precipitate, titrate, and calculate as malic acid after subtracting the tartaric acid present. (The amount of citric and succinic acid present is very small.)

Determination of Citric Acid.*-Fifty cubic centimeters of the fruit solution is evaporated on the water-bath to a syrupy condition. 'To the residue add, very slowly at first, stirring constantly, $95 \%$ alcohol until no further precipitate is formed; 70 to $80 \mathrm{cc}$. are generally enough. Filter, and wash the residue with $95 \%$ alcohol. Evaporate the filtrate to eliminate the alcohol, take up the residue with a little water, and transfer to a graduated cylinder, making up to ro $\mathrm{cc}$. To $5 \mathrm{cc}$. of this solution, add half a cubic centimeter of glacial acetic acid, and to this add, drop by drop, a saturated solution of lead acetate. The presence of citric acid is shown by the appearance of a precipitate, which possesses the property of disappearing on being heated, and reappearing on cooling. In order to separate the citric acid from other acids, heat to boiling, filter, and wash with boiling water; then allow to cool, and the precipitate of lead citrate will re-form. This lead precipitate may be filtered off, washed with weak alcohol, dried, weighed, and the citric acid calculated. It is necessary that there shall be no tartaric acid present. If the tartaric acid has been estimated, any error on this account may be avoided by adding enough decinormal potash to neutralize the tartaric acid before the alcohol is added.

Detection of Coloring Matter.-Boil white woolen cloth or worsted in a solution of the jelly or jam, acidified with hydrochloric acid, or with acid sulphate of potassium, according to Arata's method and test for the color on the dyed fabric by methods given in detail in Chapter XVII.

Detection of Preservatives and Concentrated Sweeteners.-Extract an acid aqueous solution of the fruit product with ether or chloroform in a separatory funnel, and test for benzoic and salicylic acids and for saccharin in the ether extract. If dulcin is suspected, extract with acetic ether.

* Moslinger, Zeits. Unter. Nahr. Genussm., 2, 1899, p. 93; U. S. Dept. of Agric., Bur。 of Chem., Bul, 65, p. 8o. 
Detection of Starch.*-Heat an aqueous solution of the preserve or jelly nearly to the boiling point, and decolorize by the addition of several cubic centimeters of dilute sulphuric acid and afterwards permanganate of potassium. This treatment does not affect the starch, which is tested for with iodine in the ordinary manner in the solution after cooling. In the clear filtrate from a boiled apple pulp solution, free from added starch, little or no darkening should occur on the addition of the iodine reagent. If, however, the reagent is added to the residue of the previously boiled pulp, the presence of starch inherent in the apple is usually recognized by the blue color produced thereon.

The presence of any considerable added starch paste in a fruit preparation is thus readily indicated by an intense blue color obtained by adding the iodine reagent to the filtrate (free from fruit pulp).

Detection of Gelatin.-Robin's Method $\uparrow$-Add to a thick aqueous solution of the preserve or jelly sufficient strong alcohol to precipitate the gelatin. Decant the supernatant liquid after settling, set aside part of the precipitate, and dissolve the remainder in water. Divide the latter solution in two parts, to one of which add, drop by drop, a fresh solution of tannin, which precipitates gelatin if present. To the remainder add picric acid solution, which in presence of gelatin forms a yellow precipitate. The portion of the yellow precipitate set aside is transferred to a test tube, and heated over the flame with a little quicklime. If gelatin is present, ammonia will be given off, apparent by the odor, and by fumes of ammonium chloride when a drop of hydrochloric acid on a glass rod is held at the mouth of the bottle.

Leffmann and Beam's Method. $\ddagger$-Boil the sample with water, filter, and boil the filtrate with an excess of potassium bichromate. Cool, and add a few drops of sulphuric acid. A flocculent precipitate indicates gelatin.

Detection of Agar Agar. $\$$-The jelly is heated with 5\% sulphuric acid, a little potassium permanganate is added, and, after settling, the sediment is examined by the microscope for diatoms, which will be found in large numbers if agar agar has been used.

Detection of Apple Pulp.-A distinct clue to the presence of apple pulp in fruit preparations is often furnished by the characteristic apple

* U. S, Dept, of Agric,, Bur, of Chem., Bul. 65, p. 8r.

$\dagger$ Girard, Analyse des Matières Alimentaires, p. 676.

$\ddagger$ Select Methods of Food Analysis, p. 324.

$\$$ Marpmann, Zeit. f. angew. Mikrosk, 1896 , p. 260; U. S. Dept. of Agric., Bur. of Chem., Bul, 65, p. 81 . 
odor given off when a small amount of the sample is heated to boiling with water in a test tube. Under such conditions, the apple odor is quite apparent, as distinguished from that of other fruits, especially if the apple is the chief fruit present, or predominates in the mixture.

Apple pulp in fruit preserves, free from added starch, may usually be recognized by a microscopical examination, using iodine reagent. The cell contents of the pulp will show the characteristic blue color, undoubtedly due to portions of unconverted starch still remaining in them.

Detection of Fruit Tissues under the Microscope. Certain of the common fruits are readily identified in jams by their microscopic characters. This is especially true of most of the small fruits, the skins, styles and seeds being more or less characteristic in structure.

The apple differs from the quince and pear in that stone cells are lacking; the starch of the green fruit is noteworthy. Peaches, plums and apricots, while possessing skins and stone peculiar to each, when pared and freed from stones are much alike in structure. Pineapples have peculiar needle-shaped crystals. Figs are identified by the "seeds" and hairs. Citrus fruits are remarkable because of the oil cavities and spongy parenchyma. Fragments of elements of the skins and cores of fruits, although pared and cored before preparation, find their way into the finished products, furnishing evidence to the microscopist. The seeds of berries are highly characteristic.

\section{DRIED FRUITS.}

Desiccation is the oldest and in some respects the most satisfactory method of preserving fruits. It is an economical method, as the apparatus and the process are simple, especially if the sun's heat is utilized for the evaporation; furthermore, the cost of the containers is small and the compact form of the product reduces the cost for transportation and storage to the minimum. From the sanitary standpoint dried fruit has certain advantages, notably the freedom from metallic impurities from the containers; on the other hand, great care is required to protect the material during drying and handling from surface contamination.

Xanti currants as well as raisins are dried grapes of certain European varieties. These, together with figs and dates, although produced in California and the Southern States, are imported into the United States in enormous quantities from the regions adjoining the Mediterranean. Apples, prunes, apricots, peaches, and cherries, on the other hand, are 
produced in the United States in quantities not only sufficient for domestic needs but also for export.

California fruits, such as raisins, prunes, apricots, peaches, and pears are sun-dried, as are also raisins, figs, dates and other fruits produced about the Mediterranean. Apples are commonly dried in the United States by artificial heat, although the old process of sun drying is still practiced on a small scale in certain regions.

Treatment with Lye.-Preliminary to drying certain fruits, such as raisins and prunes, are often dipped in a hot but weak solution of potash, which removes the bloom and otherwise acts on the skin, thus facilitating drying. Oil is also used with the lye in preparing "oil-dipped "Smyrna raisins. These methods of treatment are quite distinct from the lyepeeling process employed in preparing peaches, apricots, and some other fruits for canning.

Sulphuring of Fruit. - The treatment of fruits with the fumes of burning sulphur is practiced not only to bleach and prevent discoloration, but also to ward off the attacks of insects, fungi, and bacteria. It is allowed with restrictions in most European countries and also, pending further investigation, in the United States, provided the amount of sulphur dioxide remaining in the fruit does not exceed $350 \mathrm{mg}$. per kilo, of which not more than $70 \mathrm{mg}$. is free sulphurous acid.*

There is reason to believe that the sulphur dioxide exists in dried fruits in combination largely, if not wholly, with sugar, although possibly to some extent, as in wines, with acetaldehyde, or even with protein and cellulose.

Sulphuring when used for purposes of deception, as for example in rejuvenating old or damaged stock or when used in excessive amount, is obviously improper. Analyses by government chemists show that when no restrictions were placed on sulphuring as high as $3072 \mathrm{mg}$. per kilo were present in dried peaches, $2842 \mathrm{mg}$. in California apricots and ${ }^{1} 73^{8} \mathrm{mg}$. in evaporated apples.

Moisture Content of Dried Fruits, - An excessive amount of moisture in dried fruit is not only a worthless make-weight, but also facilitates the growth of molds and bacteria, causing rapid deterioration. In I904 a law was passed in New York State requiring that dried apples contain not above $27 \%$ of moisture, determined by drying 4 hours at the temperature of boiling water.

Wormy and Decomposed Dried Fruits.-Figs, dates, and currants from Europe, also dried apples, cherries, and other fruits of domestic

\footnotetext{
* U. S. Dept. of Agric., Off. of Sec., Food Inspection Decision 76.
} 
production often are infected with worms or are in a moldy or fermented condition due to careless drying or packing. Under the federal law such "filthy, decomposed or putrid " fruit is adulterated.

Zinc in Evaporated Fruit.-Apples dried in contact with galvanized iron trays may contain a small amount of this metal combined as malate, which usually amounts to only 0.01 to $0.02 \%$, but reaches in extreme cases, according to Loock, $0.09 \%$. This contamination can be entirely avoided by greasing the galvanized iron trays or covering them with greased cloth or else by the use of wooden trays.

\section{METHODS OF ANALYSIS.}

Preparation of the Sample. - If stones are present, separate and weigh. Reduce the edible portion to a uniform mass by grinding in a food chopper and thorough mixing.

Determine Ash, Nitrogen, Sugars, and Acids as described under Jams and Jellies, pp. 937 to 942 , and Zinc as described on p. $9^{1} 5$.

Determination of Moisture.-Dry 5 grams of the ground sample for 24 hours in a flat-bottomed dish at the temperature of boiling water, and weigh.

The New York State law with reference to dried apples makes no provision for grinding the sample, but does specify that the drying must be for 4 hours. Naturally this method yields lower results than that given above.

Sulphurous Acid.-Determine by the distillation method as described in Chapter XVIII.

\section{FRUIT JUICES.}

Sweet cider, orange juice, lime juice, grape juice, raspberry shrub, and the juices of various other fruits and berries, may be so prepared and sterilized as to keep without fermentation when bottled, and are so put up in considerable variety, either with or without the addition of sugar.

Such preparations, if of the highest purity, should consist of the undiluted juices of these fruits, separated by pressure and carefully sterilized and bottled. They should contain no other fruit juice than that specified on their labels, and should be free from alcohol, added antiseptics, or coloring matter, unless the label specifies the presence of the added foreign materials. The addition of pure cane sugar to such preparations as grape juice is allowable if declared, as well as charging with carbon dioxide to form so-called carbonated drinks.

The following analyses of pure fruit juices are taken from tables 
prepared by Winton, Ogden, and Mitchell, showing results on samples purchased in the Connecticut market, as well as on some samples made in the laboratory.*

\begin{tabular}{|c|c|c|c|c|c|c|c|c|}
\hline & \multirow[b]{2}{*}{ Solids. } & \multirow{2}{*}{$\begin{array}{l}\text { Acids } \\
\text { Other } \\
\text { than } \\
\mathrm{CO}_{2} \text { as } \\
\text { Citric. }\end{array}$} & \multirow{2}{*}{$\begin{array}{c}\text { Cane } \\
\text { Bugar. }\end{array}$} & \multirow[b]{2}{*}{$\begin{array}{l}\text { Invert } \\
\text { Sugar, }\end{array}$} & \multicolumn{4}{|c|}{ Polarization. } \\
\hline & & & & & Direct. & $\begin{array}{l}\text { After } \\
\text { Inver- } \\
\text { sion. }\end{array}$ & $\begin{array}{l}\text { Temper- } \\
\text { ature } \\
\text { C. }\end{array}$ & $\begin{array}{l}\text { Invert } \\
\text { Reading } \\
\text { at } 80^{\circ} \mathrm{C} \text {. }\end{array}$ \\
\hline $\begin{array}{l}\text { COMMERCIAL FRUIT } \\
\text { JUICES. }\end{array}$ & & & & & & & & \\
\hline Blackberry .......... & $5 \cdot 3^{2}$ & 0.65 & 0.0 & 4.6 & $-1 \cdot 3$ & $-\mathrm{r} \cdot 3$ & 29.0 & \\
\hline 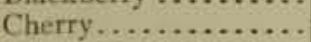 & 14.33 & 0.80 & 0.0 & 6.5 & $-\mathrm{t} .9$ & -1.9 & 26.0 & \\
\hline Black currant........ & 10.00 & 2.41 & 0.0 & 9.2 & -2.7 & $-2 \cdot 7$ & 26.0 & \\
\hline Red currant.......... & $7 \cdot 58$ & 2.09 & 0.0 & $7 \cdot 2$ & $-2 . \mathrm{I}$ & -2.1 & 27.0 & \\
\hline Grape.............. & 15.29 & 0.91 & 0.0 & $\therefore$. & -6.5 & -6.5 & 25.0 & -1.0 \\
\hline Lime fruit......... & 7.78 & 6.50 & 0.0 & 0.0 & 0.0 & 0.0 & & \\
\hline Orange............... & 12.72 & 2.44 & 0.0 & $7 \cdot 1$ & $-2,1$ & -2.1 & 26.0 & \\
\hline Pineapple ......... & 8.07 & 0.81 & I. 5 & 5.1 & 0.0 & -2.0 & 26.0 & \\
\hline Plum. ............ & $10.8 \mathrm{i}$ & 1.00 & 0.0 & 0.3 & $-0 . I$ & -0.1 & 26.0 & \\
\hline Quince.............. & 10.41 & 0.09 & 0.0 & $\ldots$ & -5.0 & -5.0 & 25.0 & -2.2 \\
\hline Bilack raspberry...... & 8.47 & $x-36$ & 0.0 & 7.8 & $-2 \cdot 3$ & -2.3 & 26.0 & \\
\hline Strawberry ........... & 5.69 & 0.99 & 0.0 & $5 \cdot 1$ & $-1 \cdot 5$ & $-1,5$ & 26.0 & \\
\hline $\begin{array}{l}\text { MADE IN I.ABORA- } \\
\text { TORY. }\end{array}$ & & & & & & & & \\
\hline Peach. ............. & 12.70 & 0.95 & 5.4 & & 4.8 & -2.2 & 28.0 & 0.0 \\
\hline Red raspberry........ & $9.4 \mathrm{I}$ & 1.19 & 0.8 & 8.6 & -1.6 & -2.8 & 26.0 & 0.0 \\
\hline Blackberry .......... & 8.94 & 1.22 & 0.0 & 8. 7 & -2.4 & -2.4 & 30.0 & 0.0 \\
\hline Huckleberry. . . . . . & 11.40 & 0.51 & 0.6 & $\ldots$ & -4.0 & -4.8 & 30.0 & -1.0 \\
\hline Pineapple ........... & 13.90 & 0.68 & $7 \cdot 4$ & $9 \cdot \mathrm{T}$ & $4-7$ & -4.8 & 28.0 & -0.8 \\
\hline
\end{tabular}

Preservatives.-Formerly salicylic and boric acids were frequent additions, now sulphurous acid and sodium benzoate are the common preservatives. Beta-naphthol, formaldehyde, formic acid and fluorides have also been used.

Unfermented Grape Juice has the following average composition :

\begin{tabular}{|c|c|c|}
\hline & $\begin{array}{l}\text { Austria. } \\
\text { Per Cent. }\end{array}$ & $\begin{array}{l}\text { California, } \\
\text { Per Cent. }\end{array}$ \\
\hline 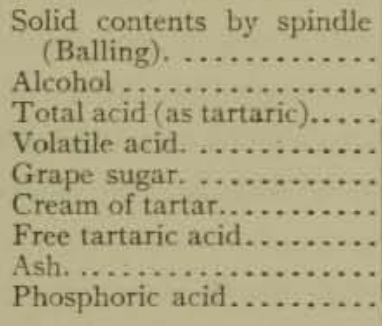 & $\begin{array}{r}21.62 \\
\text { None } \\
.78 \\
.01 \\
19.62 \\
.61 \\
.03 \\
.37 \\
.02\end{array}$ & $\begin{array}{r}20.60 \\
\text { None } \\
.53 \\
.03 \\
19.15 \\
.59 \\
.07 \\
.19 \\
.04\end{array}$ \\
\hline
\end{tabular}

*An. Rep. Conn. Exp. Sta., 1899, p. $136 . \quad$ † California Exp. Sta., Bul. I30. 
Grape juice is prepared by sterilizing at a temperature of $80^{\circ}$ the juice expressed from the crushed grapes, filtering by means of a press or otherwise, and sealing in carefully sterilized bottles. After bottling, a final sterilization is conducted at a temperature $5^{\circ}$ below the first. Bottled grape juices are rarely carbonated.

Bottled Sweet Cider.-The composition of pure, freshly expressed apple juice is shown by the following table of analyses by Browne:*

\begin{tabular}{|c|c|c|c|c|c|c|c|c|c|c|}
\hline & $\begin{array}{l}\text { Specific } \\
\text { Gravity. }\end{array}$ & Solids. & $\begin{array}{l}\text { Invert } \\
\text { Sugar. }\end{array}$ & $\begin{array}{l}\text { Su- } \\
\text { crose, }\end{array}$ & $\begin{array}{l}\text { Total } \\
\text { Sugar. }\end{array}$ & $\begin{array}{l}\text { Total } \\
\text { Sugar } \\
\text { after } \\
\text { Inver- } \\
\text { sion. }\end{array}$ & $\begin{array}{l}\text { Free } \\
\text { Malic } \\
\text { Acid. }\end{array}$ & Ash. & $\begin{array}{l}\text { Unde- } \\
\text { ter- } \\
\text { mined } \\
\text { (Pectin, } \\
\text { ete.). }\end{array}$ & $\begin{array}{c}\text { Left- } \\
\text { handed } \\
\text { Rotation } \\
\text { Deerees } \\
\text { Ventzke } \\
\text { 4oo mm, } \\
\text { Tube. }\end{array}$ \\
\hline$e d a=$ & 1.0532 & $12.7^{8}$ & 6.87 & 3.63 & 10.50 & 10.69 & & & & \\
\hline Earl & 1.0552 & 13.29 & $7 \cdot 49$ & 3.97 & II. 46 & 11.67 & 0.90 & 0.28 & 0.65 & $24 \cdot 3^{2}$ \\
\hline Yellow transparent. & 1.0502 & II. 71 & 8.03 & & & & 0.86 & 0.27 & 0.44 & \\
\hline & 1.0498 & 11.87 & 7.61 & 3.08 & 10.69 & 10.85 & 0.10 & & & 39.40 \\
\hline Baldwin, gr & 1.0488 & $11.3^{6}$ & 6.96 & 1. 63 & 8.59 & 8.68 & 1.24 & 0.31 & I. 2 & 36.16 \\
\hline & $x .073^{6}$ & 16.82 & 7.97 & 7.05 & 15.02 & $15 \cdot 39$ & 0.67 & 0.26 & 0.87 & \\
\hline Ben Davis. & 1.0539 & I 2.77 & 7.11 & 3.85 & 10.96 & 11.16 & 0.46 & 0.28 & 1.07 & 49.00 \\
\hline
\end{tabular}

Bottled sweet cider, properly sterilized, should not differ materially from the fresh juice, and should contain no alcohol.

Salicylic acid, sodium benzoate and sodium or calcium bisulphite have been extensively used as preservatives. Benzoate is still much used.

Lime or Lemon Juice.-This, according to the U. S. Pharmacopœeia, should consist of the freshly expressed juice of the ripe fruit of Citrus limonum (Risso), natural order of Rutacex. Our supply of both lemons and limes comes chiefly from the West Indies and the Mediterranean. Both varieties of the genus Citrus are used indiscriminately for furnishing commercial lime juice, though strictly speaking, only that of the lemon is recognized in the Pharmacopœia. The juice is sharply acid, and is largely composed of citric acid (about $7 \%$ ), gum, sugar ( 3 to 4 per cent), and inorganic salts from 2 to $2 \frac{1}{2}$ per cent. It also usually contains a little lemon oil from the rind. According to the pharmacopœia, lemon juice (Limonis succus) should conform to the following requirements:

"Specific gravity: not less than 1.030 at $15^{\circ} \mathrm{C}$.

"It has an acid reaction upon litmus paper, due to the presence of about $7 \%$ of citric acid. 
"On evaporating 100 grams of the juice to dryness, and igniting the residue, not more than 0.5 gram of ash should remain."

Of thirty samples of commercial lime juice examined in the Massachusetts Staie Board of Health laboratory, representing fifteen brands, all were deficient in citric acid, containing from 1.92 to $4 . \mathrm{I} 5$ per cent, thus showing that these preparations are frequently watered. Fifteen were found to contain salicylic acid, seven had sulphurous acid, while two contained both these preservatives. Several were found colored with coal-tar dyes.

One sample examined by the author, purporting to be a "pure West Indian Lime Juice, triple refined," proved to be a mixture of hydrochloric and salicylic acids, colored with a coal-tar dye, and contained no lime juice whatever.

\section{METHODS OF ANALYSIS.}

Total Solids, Total Nitrogen, Ash, and Sugars are determined by the methods employed for jams and jellies (pp. 936 to 940), Solubility and Alkalinity of the Ash and Phosphoric Acid as described in the chapter on vinegar (p. 764).

Colors and Preservatives are detected and determined as described in Chapters XVII and XVIII.

Total Acidity.-Titrate Io grams of the juice, diluted to $250 \mathrm{cc}$. with freshly boiled water, with tenth-normal alkali. Use phenolphthalein as indicator if the color of the juicewill permit,otherwise delicate litmus paper. Calculate either as sulphuric acid or as the organic acid known to predominate.

One cc. of tenth-normal alkali is equivalent to 0.0075 gram tartaric acid, 0.0067 gram malic acid and 0.0064 gram citric acid.

Determination of Tartaric Acid-Proceed as directed for total tartaric acid in wine, p. $70 \mathrm{r}$, except that $20 \mathrm{cc}$. instead of $\mathrm{I}_{5} \mathrm{cc}$. of alcohol are used for precipitation.

Determination of Malic Acid.-Dunbar and Bacon Method.*-Dilute a weighed or measured amount of the fruit juice, usually to grams, with quite a large volume of water, add phenolphthalein, and titrate with standard alkali to a decided pink color. Weigh or measure another portion of the liquid ( 75 grams or cc. is a convenient amount) into a roocc. graduated flask, and add enough standard alkali, calculated from the

*U. S. Dept, of Agric., Bur. of Chem., Circ. 76. Jour. Ind. Eng. Chem., 3, 19rr, p. 826. 
above titration, to neutralize the acidity. A slight excess of alkali is not objectionable. If the solution is dark colored, add 5 or 1o cc. of alumina cream. Dilute to the mark, mix thoroughly, and filter if necessary through a folded filter.

Treat about $25 \mathrm{cc}$. of the filtrate with enough powdered uranyl acetate so that a small amount remains undissolved after two hours, 2.5 grams usually being sufficient, except in the presence of large amounts of malic acid. In case all the uranium salt dissolves more should be added. Allow to stand for two hours, shaking frequently, filter through a folded filter until clear and polarize if possible in a $200 \mathrm{~mm}$. tube or, if too dark, in a 100 or $50 \mathrm{~mm}$. tube. Designate this solution and reading as $A$.

Treat the remainder of the original filtrate with powdered normal lead acetate until the precipitation is just complete, avoiding a large excess and consequent solution of lead malate. Cool in an ice bath and filter through a folded filter until clear. Warm the filtrate to room temperature and add a small crystal of lead acetate. If no precipitate forms, remove the excess of lead with anhydrous sodium sulphate, filter until clear, and polarize. Designate this solution and its polarization reading as $B$. Solutions which are sufficiently clear and contain less than $10 \%$ of sugar may be polarized directly without treatment with lead acetate.

If reading $B$ is negative treat a portion of solution $B$ with uranyl acetate in the manner already described and polarize. Designate this as $C$. If reading $B$ is positive, reading $C$ need not be made.

Polarize all solutions at a uniform room temperature with white light, using the average of at least six readings and calculating to the basis of a $200 \mathrm{~mm}$. tube. If reading $C$ is numerically less than reading $B$, the latter should be discarded; otherwise use reading $B$ in the subsequent calculation. Multiply the algebraic difference between this reading and reading $A$ by 0.036 , the product being the percentage of malic acid $\left(\mathrm{C}_{4} \mathrm{H}_{6} \mathrm{O}_{5}\right)$ in the solution as polarized.

Pratt's Modification.*-Place a weighed amount of juice, generally I00 grams, in a $500 \mathrm{cc}$. beaker and add, with vigorous stirring, two or three times its volume of $95 \%$ alcohol. The pectin bodies are precipitated and usually in such a form that after standing a few minutes they may be gathered into a coherent mass. Decant the liquid through a filter and wash the precipitate twice with $95 \%$ alcohol. Evaporate the filtrate in a current of air on the water-bath to about $75 \mathrm{cc}$. After cooling make up to Ioo cc. in a measured flask, using to to $15 \mathrm{cc}$. of $95 \%$ alcohol and dis-

${ }^{*}$ U. S. Dept. of Agric., Bur. of Chem., Circ. 87. 
tilled water. The temperature when the volume is finally made up to the mark should be close to that at which the polariscope readings are to be taken. Treat this solution exactly as in the original method, except that no clarification is necessary.

Determination of Citric Acid.-Pratt's Method.*-This method is applicable in the presence of malic and tartaric acids.

I. Apparatus.-This consists of a 500 cc. distilling flask provided with a small dropping funnel drawn down to a small opening and protruding one-half inch below the stopper. In the flask is placed a glass rod with a piece of small tubing one-half inch long, sealed on the lower end to insure steady ebullition. This small tube should be filled with air when the heating begins. A condenser preferably of the spiral type is connected with the flask.

2. Denigès Reagent.-Add about $500 \mathrm{cc}$. of water to 50 grams of mercuric oxide; then add $200 \mathrm{cc}$. of concentrated sulphuric acid with constant stirring, and heat the mixture, if necessary, on a steam bath until the solution is complete. After cooling make up to a liter and filter.

3. Determination. - Weigh $5^{\circ}$ grams of the fruit juice into a beaker and add 1 ro $\mathrm{cc}$. of $95 \%$ alcohol to throw out the pectin bodies. After standing fifteen minutes filter and wash with $95 \%$ alcohol. Dilute the filtrate with water to approximately $50 \%$ alcohol content and add enough $20 \%$ barium acetate solution to precipitate the citric acid. Stir, let stand until the barium citrate partially settles, and filter. Wash twice with $50 \%$ alcohol to remove the greater part of the sugar present. Remove all alcohol from the precipitate and filter either by drying in the beaker used for precipitation or else by washing with ether before removing from the funnel. Add $50 \mathrm{cc}$. of water and 3 to $5 \mathrm{cc}$. of sirupy phosphoric acid to the beaker containing the filter-paper and precipitate and warm, thus dissolving the barium citrate completely. Filter into a roo cc. measuring flask and wash up to the mark.

Measure an aliquot containing from 0.05 to 0.15 gram of citric acid, into the distilling flask, add 5 to 10 cc. of sirupy phosphoric acid and $400 \mathrm{cc}$. of hot water. Connect with the condenser, heat and when briskly boiling, add potassium permanganate solution ( 0.5 gram per liter), I to 2 drops per second, until a pink color persists throughout the solution. Distill off the acetone formed by the oxidation into a liter Erlenmeyer flask containing 30 to $40 \mathrm{cc}$. of Denigès reagent, continuing the distillation until 50 to $100 \mathrm{cc}$. remain in the flask. Boil the distillate gently

*U. S. Dept, of Agric, Bur. of Chem., Circ, 88. 
under a reflux condenser for forty-five minutes after it turns milky. Filter hot through a Gooch crucible, wash the precipitate with water, alcohol, and finally with ether, and dry in a water-oven for half an hour. The weight of the precipitate multiplied by 0.22 gives the weight of citric acid.

\section{FRUIT SYRUPS.}

Two classes of these preparations are on the market, one for use in soda-fountains, and one for "family trade," intended as a basis for sweetened drinks to be diluted with water and sugar. Some are made exclusively from pure fruit pulp and sugar, sterilized by heating and put up in tightly sealed bottles, while others of the cheaper variety are more apt to be entirely artificial both in color and in flavor, deriving the latter principally from the wide variety of artificial fruit essences now available. Commercial glucose is a frequent ingredient. The same classes of coaltar dyes and antispetics are found in these preparations as in the other fruit products. Citric or tartaric acid is frequently added to genuine fruit syrups to bring out the flavor and to imitation fruit syrups to better simulate the characters of the genuine product.

For purposes of comparison with such fruit-pulp preparations as may come to the analyst for examination, he is referred to the analysis of fruits found on page 274 .

\section{NON-ALCOHOLIC CARBONATED BEVERAGES.}

Soda Water. - Originally the beverage known as soda water was prepared by the action of an acid on sodium bicarbonate in solution and corresponded to what is now obtained by dissolving Seidlitz powders in water. Later it was found that water charged with carbon dioxide was not only more practicable commercially but also more acceptable to the palate and this product was substituted for true soda water without change of name.

As dispensed by the pharmacist and confectioner in the United States, soda water consists of a syrup, variously flavored, mixed at the "fountain " with carbonated water. The syrup is first placed in the glass, then the carbonated water is drawn into it in a large stream and finally more added in a fine stream to mix and froth the liquid. Ice cream or liquid "cream " is used with certain flavors, eggs and milk in "egg chocolate," "egg shake" and other nutritious mixtures, a solution of calcium acid phosphate in "orange phosphate" and other phosphates-in fact there appears to be 
no end to the preparations and combinations introduced by ingenious venders to quench the thirst, gratify the palate, and furnish nourishment in an easily digestible form.

Carbonated Water, the basis of all effervescent drinks, is prepared by charging ordinary water with carbon dioxide in a steel drum, known as the fountain. Formerly the gas was generated on the premises by the action of mineral acid on marble, but now it is obtained in liquid form in steel cylinders from mineral springs and the fermentation industries where it formerly went to waste.

The process of carbonating consists in allowing the gas to discharge into the water, rocking the fountain continually to aid absorption. A gauge indicates the pressure in the fountain, which should be about 170 pounds per square inch for soda water and somewhat less for ginger ale and root beer. The steel drum or fountain proper is kept in the cellar or other convenient place and the carbonated water is piped to the so-called fountain where the drinks are served, or, in the case of bottled beverages, to the machine for filling the bottles.

Needless to say both the water and the gas should be free from contamination, and the introduction of metallic salts from the lead pipes and other sources should be guarded against.

Soda Water Syrups.- Sugar and flavors are added to carbonated beverages in the form of syrups. At the soda fountain these are drawn into the glass from small reservoirs or poured from bottles, while in the bottling works measured quantities both of syrup and carbonated water are introduced into each bottle by an automatic machine.

Fruit Syrups are prepared either by the manufacturer of soda water supplies or else by the pharmacist or confectioner who serves the beverages. More commonly the manufacturer supplies the fruit juice or fruit pulp in bottles or jars, spoilage being avoided either by sterilization or the use of sodium benzoate. The vender mixes the juice or pulp with sugar syrup as needed. Orange, lemon, and lime syrups are commonly made from the oils rather than from the fresh fruit, the necessary acidity being supplied by citric acid. This acid as well as tartaric acid is also used in strawberry, raspberry and other true fruit syrups to bring out the flavor.

Imitation Fruit Syrups flavored with mixtures of ethers such as are described on pp. 895 to 897 , are frequently substituted for genuine fruit syrups at soda fountains and quite universally in the preparation of cheap bottled soda water. Aside from the deception to the consumer these mixtures are highly objectionable because of their nauseating and unwholesome properties. 
Various Syrups not belonging under the head of fruit syrups are drawn from fountains and used in bottled beverages. Among these are vanilla, coffee, chocolate (really cocoa), ginger, sarsaparilla, and mixtures sold under distinctive names.

Bottled Carbonated Beverages.-To this class belong various nonalcoholic beverages known as "soda" "soft-drinks" and "temperance drinks." Some of these are high-grade articles of national or even international reputation, so prepared as to keep indefinitely, while others are cheap preparations of local manufacture sold for immediate consumption in pleasure resorts.

Ginger Ale, by far the best-known bottled carbonated beverage, is made from ginger (or ginger extract) with the addition of lemon juice (or lemon oil and citric acid) and carbonated water. Capsicum extract, known in solid form as capsicin, is frequently substituted in part for the ginger because of its greater pungency.

Root Beer was formerly brewed from a sweetened infusion of various roots and herbs, the gas being formed by a true fermentation process. A similar beverage is now made in the household, using so-called "rootbeer extract," but the commercial product is commonly charged, like soda water, with carbon dioxide gas.

Birch Beer, formerly made by fermentation, is now merely soda water flavored with oil of birch or synthetic methyl salicylate.

Sarsaparilla, so called, is flavored with a mixture of oil of birch, natural or synthetic, and oil of sassafras. The dark color is due to caramel or other artificial colors.

Lemon Soda and Orange Soda are flavored respectively with terpeneless lemon and orange extract, the acidity being contributed by citric acid. Orangeade belongs in the same class. So-called blood-orange soda is probably never made from blood oranges, the color being artificial.

Vanilla Soda is more correctly vanillin soda or vanillin and.coumarin soda. The term cream soda applied to this colorless beverage is equally misleading.

Strawberry Soda, Raspberry Soda and other bottled beverages purporting to be made from fruits are commonly imitations flavored with ethers and colored with coal-tar dyes. So-called Cherry Soda is flavored with an extract of cherry bark or benzaldahyde.

Sweeteners in Beverages.-Sugar is the only proper sweetener for syrups or bottled beverages. Glucose because of its lower sweetening power is unsuited for the purpose, while saccharin and other chemical sweeteners are objectionable both because of their lack of nutritive prop- 
erties and their possible injury to health. The use of saccharin, which has hitherto been extensive, is now prohibited in beverages entering into interstate commerce.

Acids in Beverages. - Citric and tartaric acids are used not only in imitation but also in true fruit syrups to bring out the flavor. Lemon juice serves the same purpose, but is more expensive and does not keep so well. Calcium acid phosphate is a characteristic constituent of orange and other fruit phosphates.

Preservatives.--Sodium benzoate is the common preservative of beverages, although its use is by no means universal. Formerly salicylic, boric and sulphurous acids and even fluorides were employed.

Artificial Colors.-Cochineal, cudbear, caramel and the seven colors allowed by $\mathrm{U}$. S. decisions are most commonly met with. The use of fuchsin, acid fuchsin, rhodamine, tartrazine and other coal-tar colors has been largely discontinued.

Foam Producers. - Froth on soda water is cheaper to produce than the same bulk of liquid, furthermore it is sanctioned by custom.

Soap-bark, the bark of Quillaja Saponaria, a common foam producer, contains two saponins, sapotoxin and quillaiac acid, both of which are poisonous. In addition these principles combine with the cholesterin of the blood and if in excess dissolve the corpuscles.

Commercial saponin, prepared from Saponaria officinalis, and consisting largely of sapotoxin, is also extensively used.

Foam producers are also used in malt liquors.

Glycerrhisin, the characteristic principle of licorice, also serves as a foam producer.

Habit-forming Drugs in Beverages.-Caffein, extract of cola leaves, and cocaine are ingredients of certain proprietary syrups and beverages, contributing their well-known stimulating properties. The use of caffein is defended on the ground that it is the active principle of tea and coffee. Opponents of this drug have pointed out that tea and coffee are recognized as improper articles of diet for children and invalids, furthermore, the presence of other consituents tends to prevent the excessive use of these beverages. Again the presence of caffein in carbonated beverages is not usually known to the consumer, and he forms the habit without proper warning.

It would be difficult to find an argument in favor of the use of a drug so potent as cocaine or products containing cocaine. 


\section{METHODS OF ANALYSIS.}

Transfer the sample to a flask and shake at intervals for an hour or two, at room temperature, thus removing most of the carbon dioxide. Use the liquid thus obtained for the several determinations, measuring out the portions, if desired, and calculating the weight from the specific gravity.

Total Solids, Ash, Acidity, Sugars, and Organic Acids are determined as directed for jams and jellies (pp. 936 to 942 ) using 25 grams of the liquid except for the polarizations, which may be made on normal quantities.

Vanillin, Coumarin, Citral, and Methyl Salicylate are detected and determined by the methods described under the head of Flavoring Extracts, with such modifications as are necessitated by the absence of alcohol on the one hand and the greater dilution on the other. Methods for the detection of Ginger and Capsicum are given on page 894 .

Detection of Colors, Preservatives, and Sweeteners. - See Chapters XVII, XVIII and XIX.

Determination of Phosphoric Acid.-This determination is made in so-called "orange phosphate," "raspberry phosphate" and other beverages containing calcium acid phosphate.

Treat 25 grams of the liquid according to the method described on p. 346 , except that the entire residue, after ignition with magnesium nitrate, is used for the determination, without aliquoting.

Determination of Alcohol.-Follow the method prescribed for wines (p. 658). The amount of volatile oil present is seldom sufficient to appreciably affect the result.

Detection of Saponin.-Of the various color tests that have been proposed none has been found absolutely characteristic, especially if glycerrhizin is present, although the reactions with sulphuric acid and Fröhde reagent are of considerable value. The haemolysis test is believed to be reliable even in the presence of glycerrhizin. Whichever test is applied the saponin should be separated from interfering substances as follows:

I. Extraction of Saponin by the Rühle-Brummer Method.*-In the case of soda water and other products containing organic or mineral acids (other than carbonic), to roo cc. of the liquid add an excess of precipitated magnesium carbonate and filter. If dextrin is present, as in the case of malt liquors, evaporate $100 \mathrm{cc}$. of the liquid to $20 \mathrm{cc}$., pre-

\footnotetext{
*Zeits. Unters. Nahr, Genussm., 5, 1902, p. $1197 ; 16$, 1908, p. 165; 23, 1912, p. 566.
} 
cipitate with $\mathrm{I}_{5} \mathrm{O} \mathrm{cc}$. of $95 \%$ alcohol, let stand 30 minutes then heat to boiling, filter, dilute the filtrate with water and dealcoholize, finally making up the solution to $100 \mathrm{cc}$.

To roo cc. of the neutral, dextrin-free solution in a separatory funnel, add 20 grams of ammonium sulphate, $g \mathrm{cc}$. of phenol and shake thoroughly. Draw off the watery layer and shake the phenol solution with a mixture of $50 \mathrm{cc}$. of water, I00 cc. of ether, and (if necessary to avoid an emulsion) $4 \mathrm{cc}$. of alcohol. Allow to stand until the liquids separate, which usually requires 12 to 24 hours. Draw off the aqueous solution and evaporate nearly to dryness, finishing the drying either at $100^{\circ} \mathrm{C}$. or in a desiccator, the latter being preferable if the residue is to be purified by treatment with acetone, which is usually desirable. Employ this extract, consisting of saponin and impurities, in the following tests:

II. Tests for Saponin.-1. Sulphuric Acid Test.-Rub up a portion of the extract with a few drops of sulphuric acid. Saponin is indicated by the appearance in a few minutes of a reddish color changing in half an hour to red-violet and finally to gray.

2. Fröhde Test.-Treat another portion in like manner with a few drops of a mixture of $\mathrm{IOO} \mathrm{cc}$. of concentrated sulphuric acid and I gram of ammonium molybdate. In the presence of saponin the drops in $I_{5}$ minutes become violet, changing later to green and finally to gray.

3. Foam Test.-Shake another portion of the extract with water and note its foam-producing properties.

In the presence of glycerrhizin none of the last three tests is reliable.

4. Haemolysis Test.-This process is recommended by Rusconi,* Sormali, $\dagger$ and Rhüle. $\ddagger$ The following details are given by Rhüle and are based on the method as described by Gadamer: $\S$

(a) Reagents.-(I) Physiological Salt Solution.-Dissolve 8 grams of sodium chloride in water and make up to one liter.

(2) One per cent Defibrinated Blood.-Shake vigorously fresh ox blood in a sterilized, salt-mouth, 500-cc. bottle with 20 glass beads $5^{-7}$ $\mathrm{mm}$. in diameter. Separate from the clot of fibrin and store in a sterilized container in a refrigerator. Properly cared for it should keep for several days.

Dilute with too volumes of physiological salt solution for use.

(3) One per cent Blood Corpuscles.-Centrifuge $100 \mathrm{cc}$. of the ${ }_{1} \%$

* Bol. Soc. Med.-Chi. Pavia, rgro.

$\dagger$ Zeits. Unters. Nahr. Genussm. 23, 1912, p. 562 .

$\ddagger$ Ibid. p. 566 .

§Lehrbuch der chemischen Toxicologie. Göttingen, 1909, p. 443. 
defibrinated blood in physiological salt solution, pour off the clear solution containing the cholesterol and make up again to roo cc. with the salt solution. This preparation is more sensitive than solution (2).

(b) Process.-Dissolve about 0.1 gram of the extract in $25 \mathrm{cc}$, , of physiological salt solution, filter, and shake $\mathrm{I}, 2$, and $3 \mathrm{cc}$. of this solution in small test-tubes with I cc. portions of ${ }_{1} \%$ defribinated blood. If saponin is present the liquid becomes clear in from a minute to an hour or two, depending on the amount of saponin in the beverage and the number of $\mathrm{cc}$. of the solution used.

As a confirmatory test dissolve I mg. of cholesterol in a small amount of ether, shake with Io $\mathrm{cc}$. of the solution of the extract in salt solution, heat at $36^{\circ} \mathrm{C}$., for a few hours to remove ether, avoiding concentration, and treat portions of this solution with $\mathrm{I} \%$ defibrinated blood as above described. Cholesterol destroys the haemolytic action of the saponin, hence the liquids should not become clear in these tests. In order to exert this influence cholesterol should be present to the extent of I part to 5 parts of saponin.

If only a small amount of saponin is present the haemolytic action can best be noted under a microscope magnifying to 300 diameters. Mount a drop of the solution of the extract in salt solution and place a drop of either solution (2) or (3) in contact with it. The saponin causes the corpuscles in contact with it to swell, then become strongly refractive, and finally dissolve.

Determination of Caffein.-Fuller Method.*-Weigh 50 grams or measure an equivalent volume into a small beaker, add $5 \mathrm{cc}$. of concentrated ammonium hydroxide, allow to digest over night; then add 2 cc. more of ammonium hydroxide, heat for two hours, transfer to a large separatory funnel, dilute with 3 volumes of acid, add $5 \mathrm{cc}$. of ammonium hydroxide and shake out with four successive portions of chloroform, each of $50 \mathrm{cc}$. In case any dyestuff is removed by the chloroform, shake out with a saturated solution of sodium bisulphite, which will remove some of the color.

Distil off the bulk of the chloroform and evaporate the remainder in a porcelain dish. Dissolve the residue in $25 \mathrm{cc}$. of $2 \%$ sulphuric acid, shake out with five portions of $\mathrm{I}_{5} \mathrm{cc}$. each of chloroform, filter the combined chloroform solutions into a flask, distil off the bulk of the chloroform and evaporate in a tared dish; dry at $100^{\circ} \mathrm{C}$. and weigh.

If the caffein is not pure, dissolve in $15 \mathrm{cc}$. of $10 \%$ hydrochloric acid, add an excess of a solution of 10 grams of iodine and 20 grams of potas-

* A. O.A.C. Proc, 19ro, U.S. Dept. of Agric., Bur. of Chem., Bul. 137, p. 191. 
isium iodide in roo cc. of water, allow to stand over night, filter, and wash twice with to $\mathrm{cc}$. of the iodine solution. Transfer filter and precipitate to the original precipitation flask, add sufficient sulphurous acid to dissolve the precipitate, heating gently, filter into a separatory funnel, wash three times with water, and add ammonium hydroxide in excess, shake out four times with $\mathrm{I}_{5} \mathrm{cc}$. portions of chloroform, and filter the chloroform extracts into a flask, using a $7 \mathrm{~cm}$. filter and keeping the funnel covered with a watch glass. Wash the filter with 5 portions of $5 \mathrm{cc}$. of chloroform. If the chloroform extract is colored, concentrate, add a small amount of animal charcoal, rotate several times and filter. Distil off part of the solvent and evaporate the remainder in a tared dish, dry at $100^{\circ} \mathrm{C}$., and weigh.

Detection and Determination of Cocaine.-Fuller Method.*-To $200 \mathrm{cc}$. of the sample in a large separatory funnel, add concentrated ammonium hydroxide to alkaline reaction, and shake out with three portions of $50 \mathrm{cc}$. each of Prolius mixture (4 parts ether, I part chloroform, I part alcohol), collecting the solvent in another separatory funnel. If desired the aqueous solution may be reserved for the detection of salicylic and benzoic acids and saccharine. Filter the combined Prolius extracts into an evaporating dish, and evaporate on a steam bath, removing the dish as the last traces of solvent disappear. Dissolve the residue in normal sulphuric acid, transfer to a separatory funnel and shake out four times with I 5 cc. portions of chloroform; wash the combined chloroform solutions once with water, reject the chloroform, and add the water extract to the original acid solution. Add ro cc. of petroleum ether boiling at $40^{\circ}$ to $50^{\circ} \mathrm{C}$., and shake; draw off the acid layer, rejecting the petroleum ether, add concentrated ammonium hydroxide in excess and shake out three times with I $_{5} \mathrm{cc}$. portions of petroleum ether, collecting the ethereal solutions in another separatory funnel. To the latter add ro cc. of water and shake thoroughly; reject the water extract and filter the petroleum ether into a beaker, washing twice with ro cc. portions of the solvent. Evaporate over a steam bath, using a fan. By this method, if coca alkaloids are present, a nearly colorless residue will be obtained, which will finally crystallize on standing.

Dissolve the residue in petroleum ether and divide into four portions, one of which may be small. Evaporate the solvent and to the small portion add a few drops of normal sulphuric acid, warm gently, filter into a test-tube, and add a drop of potassium mercuric iodide test solution (Meyer's reagent). A precipitate indicates an alkaloid but does not

*A.O.A.C. Proc. 1910, U. S. Dept. of Agric., Bur. of Chem., Bul. 137, p. 192. 
identify it as cocaine; if no precipitate forms, cocaine is not present and further test is unnecessary.

To another portion add a few drops of concentrated nitric acid, and evaporate on a steam bath until the acid is all driven off, then add a few drops of half normal alcoholic potash and note the first odor that comes off, which, if cocaine is present, is that of ethyl benzoate.

The residue of the third portion should be applied to the end of the tongue by rubbing with the finger. Cocaine will cause a numbness which is not apparent immediately, but develops gradually, and persists for a longer or shorter time according to the amount present.

Remove a portion of the fourth residue to a microscopic slide, add a drop or two of gold chloride test solution, and stir vigorously, noting the character of the crystals under the microscope.

All the above tests should be checked by controls on pure cocaine.

If a quantitative determination of coca alkaloids is desired the residue after evaporating the petroleum ether should be weighed, then, as a check on the gravimetric determination, warmed in $50 \mathrm{cc}$. of fiftieth-normal sulphuric acid until dissolved, cooled, and titrated with fiftieth-normal potassium or sodium hydroxide, using cochineal as indicator. The factor for cocaine is 0.006018 .

Determination of Caffein and Detection of Cocaine and Glycerine.Fuller Method.*-Weigh 50 grams of the sample into an evaporating dish, add $5 \mathrm{cc}$. of concentrated ammonium hydroxide, cover with a watch-glass and allow to stand I2 hours. Add 2 cc. more of ammonium hydroxide and evaporate on the steam bath. Warm the residue with $25 \mathrm{cc}$. of $95 \%$ alcohol, on the steam bath, cool, and pour off the alcohol into another evaporating dish, repeating the treatment four times. Evaporate the combined alcoholic extract, dissolve the residue in $25 \mathrm{cc}$. of $2 \%$ sulphuric acid, transfer to a separatory funnel and shake out 5 times with $15 \mathrm{cc}$. portions of chloroform.

Reserve the acid liquid for subsequent tests for cocaine and glycerine.

Distil off most of the chloroform, evaporate in a dish on a steam bath, dissolve the residue in $10 \%$ hydrochloric acid and transfer to a small flask. Add to the acid solution an excess of iodine solution (Io grams iodine and 20 grams potassium iodide in $100 \mathrm{cc}$. of water), rotate flask, allow to settle over night, filter, and wash flask and precipitate twice with the iodine solution, then transfer filter and precipitate to the flask. Heat gently with sufficient sulphurous acid solution to dissolve the precipitate,

*A. O. A. C. Proc. 19ro, U. S. Dept. of Agric., Bur. of Chem., Bul. 137, p, 192. 
filter into a separatory funnel, cool, add excess of concentrated ammonium hydroxide, and shake out four times with ${ }_{15} \mathrm{cc}$. portions of chloroform. Filter the chloroform extract into a flask, using a $7 \mathrm{~cm}$. filter in a small funnel covered with a watch-glass, or filter through cotton plugged in the stem of the separatory funnel. Decolorize the chloroform, if necessary, with animal charcoal, distil off most of the chloroform, then evaporate in a tared dish over steam, dry at $100^{\circ} \mathrm{C}$. and weigh.

Add an excess of concentrated ammonium hydroxide to the solution from which the caffein was extracted, shake out three times with petroleum ether, boiling at $40^{\circ}$ to $60^{\circ} \mathrm{C}$., filter ether solution, divide into four parts, evaporate, and test for cocaine as described in the preceding method.

Evaporate the aqueous solution from the cocaine extraction with milk of lime and proceed as in the determination of glycerine in wines (p. $7 \circ 3$ ). The glycerine thus obtained will be only an approximation to the true amount.

\section{REFERENCES ON VEGETABLE AND FRUIT PRODUCTS.}

ADAMs, M. A. Composition and Adulteration of Fruit Jams. Analyst, 9, I884, p. 100 ,

Anger.., A. Microscopical Structure of Fruits, etc., to be met with in Jams and Preserves. Analyst, 1, 1877, p. 73 .

Bacon, R. F, and Dunbar, P. B. Changes taking place during Spoilage of Tomatoes, with Methods for Detection of Spoilage. U. S. Dept, of Agric., Bur. of Chem., Circ. 78 .

BarER, E., u. Hasse, P. Ueber die Zusammensetzung von rgo7-er Obst- und Beerenfrüchte. Zeits. unters. Nahr. Genuss., 15, 1908, p. 140.

BFHre, A., Grosse, F, u. Thimme, K. Beiträge zur Kenntnis der Fruchtsäfte des Jahrganges 1907. Zeits. Unters. Nahr. Genuss, 15, 1908, p. 131.

Beythis, A. Ueber Fruchtsäfte und Marmeladen. Zeits. Unters. Nahr. Genuss 6,1903, p. 1095.

- Ueber die Verwendung der Schwefligen Säure als Konservierungsmittel, insbesondere den jetzigen Stand der Beurteilung geschwefelten Dörrobstes. Zeits Unters. Nahr. Genuss., 8, 1904, p. 36.

- Einige weitere Analysen von Fruchtsaften und Beerenfrüchten. Zeits. Unters. Nahr. Genuss., 8, 1904, p. 544 .

BeythIEN, A, u. BoHrisch, P. Ueber amerikanisches getrocknetes Obst. Zeits. Unters. Nahr. Genuss., 5, 1902, p. 401.

- Beiträge zur Untersuchung und Beurteilung des Citronensaftes. Zeits. Unters. Nahr. Genuss., 9, I005, p. 449.

— Ueber geschwefeltes Dörrsobst. Zeits. Unters. Nahr. Genuss., 6, I993, p. 355. 
Beythien, A., Borisch, P., u. Hempel, H. Ueber die Zusammensetzung der 1905-eriCitronensâfte. Zeits. Unters. Nahr. Genuss., I1, 1906, p. 651.

Beythien, A., u. Smmich, P. Beiträge zur Untersuchung und Beurtheilung der Marmeladen. Zeits. Unters. Nahr. Genuss., 20, 1910, p. 241.

Bigelow, W. D., and Gore, H: C. Studies on Peaches. U. S. Dept. of Agric., Bur. of Chem., Bul. 97.

- Ripening of Oranges. Jour. Am. Chem. Soc., 29, 1907, p. 767.

Study of Apple Marc. Jour. Am. Chem. Soc., 28, r9o6, p. 200.

Bigelow, W. D., Gore, H. C., and Howard, B. J. Studies on Apples. U. S. Dept. of Agric., Bur, of Chem., Bul. 94.

Growth and Ripening of Persimmons. Jour. Am. Chem. Soc,, 28, 1906, p. 688.

Bioletit, F. T., and dal Plaz, A. M. Preservation of Unfermented Grape Must. Cal. Exp. Sta. Bul. I30.

Brrming, A. W. Experiments on the Spoilage of Tomato Ketchup. U. S. Dept. of Agric., Bur, of Chem., Bul. rig.

Bodmer, R., and Moor, C, G. On Copper in Peas. Analyst, 22, 1897, p. I4r.

Boseley, L. R. The Analysis of Marmalade. Analyst, 23, 1898, p. 123.

Browne, C. A. A Chemical Study of the Apple and Its Products. Penn. Dept. of Agriculture, Bul, 58. Jour. Am. Chem. Soc., 23, 1901, p. 869.

Buchanan, G. S., and Schryver, S. B. On the Presence of Tin in Certain Canned Foods. Local (British) Govt. Board, Rep. of Insp. of Foods, 7, 1908.

Budden, E. R., and Hardy, H. Colorimetric Estimation of Lead, Copper, Tin, and Iron. Analyst, 19, $1894,168$.

Buttenberg, P. Zur Kenntnis und Beurteilung des Himbeersaftes. Zeits. Unters. Nahr. Genuss., 9, 1905, p. 141 .

Buttenberg, Hempel, Thamm, Luhrig, Juckenack, Barer, et al. Fruchtsaftstatistik, 1906. Zeits. Unters. Nahr. Genuss., 12, 1906, p. 721.

Chace, E. M., To man, L. M., and Munson, L. S. Chemical Composition of Some Tropical Fruits and their Products. U. S. Dept. of Agric., Bur. of Chem., Bul. 87.

Doremus, C. A. Collecting and Analyzing Gases in Canned Goods. Jour. Am. Chem. Soc., 19, 1897, p. 733 .

Dubors, W. L. Analyses of Canned Peas, Showing Composition of Different Grades. U. S. Dept. of Agric., Bur. of Chem., Circ. 54.

FARNSteIneR, K. Ueber organisch gebundene Schweflige Säure in Nahrungsmitteln. Zeits. Unters. Nahr. Genuss., 7, 1904, p. 449.

Fischer, R., u. ALPERS, K. Beiträge zur Kenntnis der rgo7-er Fruchtsäfte und Marmeladen. Zeits. Unters. Nahr. Genuss., I5, 1908, p. I44.

Formenti, C., u. Aristide, S. Zusammensetzung italienischer Tomatensäfte. Zeits. Unters. Nahr. Genuss., 12, 1906, p. 283.

Gore, H. C. Studies on Apple Juice. Jour. Am. Chem. Soc., 29, 1907, p. I112.

- Unfermented Apple Juice. U. S. Dept. of Agric., Bur. of Chem., Bul. ir8.

HaL.nI, J. Ueber ungarische Fruchtsäfte, etc. Zeits. Unters, Nahr. Genuss., 15, 1908, pp. 153,277 .

HAUSNER, A. The Manufacture of Preserved Foods and Sweetmeats. London, 1902. 
Hrigard, E. W., and Colby, G. E. Investigations of Canned Products. Rep. Cal. Exp. Sta. $1897-98$, p. 159 .

HiLgar, A., u. LaBAND, L. Ueber electrolytische Abscheidung von Kupfer Zink und Zinn aus Konserven. Zeits. Unters. Nahr. Genuss., 2, 1899 , p. 795.

Hiltner, R. S. A Rapid Method for the Analysis of Tin and Terne Plate. Western Chem. and Met. 4, 1908, p. 262.

Husmann, G. C. Manufacture and Preservation of Unfermented Grape Must. U. S. Dept. of Agric., Bur. of Plant Ind., Bul. 24.

Juckenack, A., u. Pasternack, R. Ueber die Zusammensetzung der Fruchtsäfte und Fruchtsyrupe. Zeits Unters. Nahr. Genuss., 8, I904, p. 548.

Untersuchung und Beurtheilung von Fruchtsäften. Zeits. Unters. Nahr. Genuss. 8 , 1904 , p. Io.

Juckenack, A., u. Pranse, H. Untersuchung und Beurteilung der Marmeladen, Fruchtmuse, Gelees und ähnlicher Erzeugnisse der Obstverwertungs-Industrie. Zeits. Unters. Nahr. Genuss., 8, 1904, p. 26.

KıсктоN, A. Untersuchung getrockneter Aprikosen. Zeits. Unters. Nahr. Genuss., 8,1904, p. 675 .

Krzizan, R., u. PLaHL, W. I905-er Himbeersäfte und-syrupe böhmischer Herkunft. Zeits. Unters. Nahr. Genuss., I1, 1906, p. 205.

LADD, E. F. Food Products and their Adulteration. (Canned Goods, Ketchups, Jellies, Jams and Extracts.) North Dak. Exp. Sta., Buls. 53 and 57 .

Ludwig, W. Beitrag zur Untersuchung und Beurteilung von Marmeladen. Zeits, Unters. Nahr. Genuss., 13, 1907, p. 5.

— Beiträge zur Untersuchung und Beurteilung von Fruchtsäften. Zeits. Unters, Nahr. Genuss., I1, 1906, p. 212.

LüHRIG, H. Zur Kenntnis und Beurteilung des Himbeersäftes, Zeits. Unters. Nahr. Genuss., 8, 1904 , p. 657 .

_- Zur Kenntnis des Citronensaftes. Zeits. Unters. Nahr. Genuss., II, rgo6, p. 44 I. LÜHRIG, BEYTHIEN, JuCKenACK u. BaIER. Fruchtsaftstatistik, r905. Zeits. Unters. Nahr. Genuss., Io, I905, p. 713 .

Macfarlane, T. Unfermented Grape Juice. Can. Inl. Rev. Dept., Bul. 82.

McElroy, K. P., and Bigelow, W. D. Canned Vegetables. U. S. Dept. of Agric., Div. of Chem., Bul. $1_{3}$, part 8.

McGint, A. Canned Meats. Can. Inl. Rev. Dept,, Bul. 85.

- Canned Vegetables. Can. Inl. Rev. Dept., Bul. 87 .

Lime Juice and Catsup. Can. Inl. Rev. Dept., Bul. 83 .

Munson, L. S. Canned Vegetables. U. S. Dept. of Agric., Bur. of Chem., Bul. 50, p. 50 .

Munson, L. S., and Tolman, L. M. Fruits and Fruit Products. U. S. Dept. of Agric., Bur. of Chem., Bul. 65, p. 74 .

Munson, L. S., Tolman, L. M., and Howard, B. J. Fruits and Fruit Products. U. S. Dept. of Agric., Bur. of Chem., Bul. 66.

Norton, F. A. Discoloration of Fruits and Vegetables put up in Tin. Jour. Am. Chem. Soc., 28, 1906, p. 1503 .

- The Ripening of Peaches. Jour. Am. Chem. Soc., 29, rgo5, p. 915.

Prescott, S. C., and Underwoon, W. L. Micro-organisms and Sterilizing Processes in the Canning Industry. Tech. Quarterly, ro, 1897 , p. 183 ; also 11,1898 , p. 6 . 
v. RAUMER. Untersuchung und Beurteilung eingekochter Beeren und Fruchtmarmeladen. Zeits. Unters. Nahr. Genuss., 6, 1903, p. $48 \mathrm{r}$.

RöHrig, A. Konzentrierte Fruchtsäfte. Zeits. Unters. Nahr. Genuss., I5, I9a8, p. 148 .

SchWarz, F., u. Weber, O. Beitrag zur Fruchtsaftstatistik für das Jahr. 1907. Zeits. Unters. Nahr. Genuss, I5, 1908, p. 147.

SpAzth, E. Ueber Fruchtsäfte (besonders Himbeersaft) und deren Untersuchung. Zeits. Unters. Nahr. Genuss., 2, 1899, p. 633; 4, r9or, pp. $97,920$.

- Ueber die Untersuchung und Beurteilung von Himbeersyrup. Zeits. Unters. Nahr. Genuss., 8, I904, p. $53^{8}$.

Street, J. P. Canned Peas. Conn. Exp. Sta., Rep., I9ro, p. 456.

Ketchup. Ibid., p. $52 \mathrm{I}$.

StuBER, W. Zusammensetzung der Tomate. Zeits. Unters. Nahr. Genuss., II, 1906, p. 578 .

Ueber Apfelsinensaft. Zeits. Unters. Nahr. Genuss,, I5, 1908, p. 273.

Tolman, L. M. The Polarization of Fruits, Jellies, Jams and Honies. Jour. Am. Chem. Soc., 24, 1902, 515.

Tolman, L. M., Munson, L. S., and Bigelow, W. D. The Composition of Jellies and Jams. Jour. Am. Chem. Soc., 23, I901, D. $3+7$.

Trafhagen, F, W., and Burke, E. Occurrence of Salicylic Acid in Fruits. Jour. Am. Chem. Soc., 25, 1903, p. 242.

vox WEst, E. Beiträge zur Analyse des Johannisbeersäftes. Zeits. Unters. Nahr. Genuss., 15, 1908, p. 595 .

Witson, H. M. Adulteration of Tinned Peas by Copper. Pub. Health, April, r892, p. 203.

WINDIsch, K. Ueber das natürliche Vorkommen von Salicylsäure in Erdbeeren und Himbeeren. Zeits. Unters. Nahr. Genuss., 6, 1903, p. $\angle 47$.

Windisch, K., u. Schmmt, P. Beiträge zur Kenntnis der Fruchtsäfte. Zeits. Unters. Nahr. Genuss,, 17, 1009, p. 584 .

Winton, A. L. Detection of Coal Tar Dyes in Fruit Products. Jour. Am. Chem. Soc., 22, 1000 , p. $5^{82}$.

- Beiträge zur Anatomie des Beerenobstes, Zeits. Unters. Nahr. Genuss., 5, I902, p. 785 .

Writhers, W. A., and Primrose, H. W. Preservatives in Canned Foods in North Carolina. North Car. Exp. Sta., Bul. 165. 


\title{
APPENDIX.
}

THE FOOD AND DRUGS ACT, JUNE 30, 1906, AS AMENDED AUGUST 23, 1912.

\begin{abstract}
AN ACT FOR PREVENTING THE MANUFACTURE, SALE, OR TRANSPORTATION OF ADULTERATED OR MISBRANDED OR POISONOUS OR DELETERIOUS FOODS, DRUGS, MEDICINES, AND LIQUORS, AND FOR REGULATING TRAFTIC THEREIN, AND FOR OTHER PURPOSES.
\end{abstract}

Be it enacted by the Senate and House of Representatives of the United States of America in Congress assembled, That it shall be unlawful for any person to manufacture within any Territory or the District of Columbia any article of food or drug which is adulterated or misbranded, within the meaning of this Act; and any person who shall violate any of the provisions of this section shall be guilty of a misdemeanor, and for each offense shall, upon conviction thereof, be fined not to exceed five hundred dollars or shall be sentenced to one year's imprisonment, or both such fine and imprisonment, in the discretion of the court, and for each subsequent offense and conviction thereof shall be fined not less than one thousand dollars or sentenced to one year's imprisonment, or both such fine and imprisonment, in the discretion of the Court.

SEc. 2. That the introduction into any State or Territory or the District of Columbia from any other State or Territory or the District of Columbia, or from any foreign country, or shipment to any foreign country of any article of food or drugs which is adulterated or misbranded, within the meaning of this Act, is hereby prohibited; and any person who shall ship or deliver for shipment from any State or Territory or the District of Columbia to any other State or Territory or the District of Columbia, or to a foreign country, or who shall receive in any State or Territory or the District of Columbia from any other State or Territory or the District of Columbia, or foreign country, and having so received, shall deliver, in original unbroken packages, for pay or otherwise, or offer to deliver to any other person, any such article so adulterated or misbranded within the meaning of this Act, or any person who shall sell or offer for sale in the District of Columbia or the Territories of the United States any such adulterated or misbranded foods or drugs, or export or offer to export the same to any foreign country, shall be guilty of a misdemeanor, and for such offense be fined not exceeding two hundred dollars for the first offense, and upon conviction for each subsequent offense not exceeding three hundred dollars or be imprisoned not exceeding one year, or both, in the discretion of the court: Provided, That no article shall be deemed misbranded or adulterated within the provisions of this Act when intended for export to any foreign country and prepared or packed according to the specifications or directions of the foreign purchaser when no substance is used in the preparation or packing thereof in conflict with the laws of the foreign country to which said article is intended to be shipped; but if said article shall be in fact sold or offered for sale for domestic use or consumption, then this proviso shall not exempt said article from the operation of any of the other provisions of this Act. 
SEC. 3. That the Secretary of the Treasury, the Secretary of Agriculture, and the Secretary of Commerce and Labor shall make uniform rules and regulations for carrying out the provisions of this Act, including the collection and examination of specimens of foods and drugs manufactured or offered for sale in the District of Columbia, or in any Territory of the United States, or which shall be offered for sale in unbroken packages in any State other than that in which they shall have been respectively manufactured or produced, or which shall be received from any foreign country, or intended for shipment to any foreign country, or which may be submitted for examination by the chief health, food, or drug officer of any State, Territory, or the District of Columbia, or at any domestic or foreign port through which such product is offered for interstate commerce, or for export or import between the United States and any foreign port or country.

SEC. 4. That the examinations of specimens of foods and drugs shall be made in the Bureau of Chemistry of the Department of Agriculture, or under the direction and supervision of such Bureau, for the purpose of determining from such examinations whether such articles are adulterated or misbranded within the meaning of this Act; and if it shall appear from any such examination that any of such specimens is adulterated or misbranded within the meaning of this Act, the Secretary of Agriculture shall cause notice thereof to be given to the party from whom such sample was obtained. Any party so notified shall be given an opportunity to be heard, under such rules and regulations as may be prescribed as aforesaid, and if it appears that any of the provisions of this Act have been violated by such party, then the Secretary of Agriculture shall at once certify the facts to the proper United States district attorney, with a copy of the results of the analysis or the examination of such article duly authenticated by the analyst or officer making such examination, under the oath of such officer. After judgment of the court, notice shall be given by publication in such manner as may be prescribed by the rules and regulations aforesaid.

SEC. 5. That it shall be the duty of each district attorney to whom the Secretary of Agriculture shall report any violation of this Act, or to whom any health or food or drug officer or agent of any State, Territory, or the District of Columbia shall present satisfactory evidence of any such violation, to cause appropriate proceedings to be commenced and prosecuted in the proper courts of the United States, without delay, for the enforcement of the penalties as in such case herein provided.

SEC. 6. That the term "drug," as used in this Act, shall include all medicines and preparations recognized in the United States Pharmacopceia or National Formulary for internal or external use, and any substance or mixture of substances intended to be used for the cure, mitigation, or prevention of disease of either man or other animals. The term "food," as used herein, shall include all articles used for food, drink, confectionery, or condiment by man or other animals, whether simple, mixed, or compound.

SEC. 7. That for the purposes of this Act an article shall be deemed to be adulterated:

In case of drugs:

First. If, when a drug is sold under or by a name recognized in the United States Pharmacopceia or National Formulary, it differs from the standard of strength, quality, or purity, as determined by the test laid down in the United States Pharmacopœia or National Formulary official at the time of investigation: Provided, That no drug defined in the United States Pharmacopeia or National Formulary shall be deemed to be adulterated under this provision if the standard of strength, quality, or purity be plainly stated upon the bottle, box, or other container thereof although the standard may differ from that determined by the test laid down in the United States Pharmacopoia or National Formulary.

Second. If its strength or purity fall below the professed standard or quality under which it is sold. 
In the case of confectionery:

If it contain terra alba, barytes, talc, chrome yellow, or other mineral substance or poisonous color or flavor, or other ingredient deleterious or 'detrimental to health, or any vinous, malt, or spirituous liquor or compound or narcotic drug.

In the case of food:

First. If any substance has been mixed and packed with it so as to reduce or lower or injuriously affect its quality or strength.

Second. If any substance has been substituted wholly or in part for the article.

Third. If any valuable constituent of the article has been wholly or in part abstracted.

Fourth. If it be mixed, colored, powdered, coated, or stained in a manner whereby damage or inferiority is concealed.

Fifth. If it contain any added posionous or other added deleterious ingredient which may render such article injurious to health: Provided, That when in the preparation of food products for shipment they are preserved by any external application applied in such manner that the preservative is necessarily removed mechanically, or by maceration in water, or otherwise, and directions for the removal of said preservative shall be printed on the covering or the package, the provisions of this Act shall be construed as applying only when said products are ready for consumption.

Sixth. If it consists in whole or in part of a filthy, decomposed, or putrid animal or vegetable substance, or any portion of an animal unfit for food, whether manufactured or not, or if it is the product of a diseased animal, or one that has died otherwise than by slaughter.

SEC. 8. That the term "misbranded," as used herein, shall apply to all drugs, or articles of food, or articles which enter into the composition of food, the package or label of which shall bear any statement, design, or device regarding such article, or the ingredients or substances contained therein which shall be false or misleading in any particular, and to any food or drug product which is falsely branded as to the State, Territory, or country in which it is manufactured or produced.

That for the purposes of this Act an article shall also be deemed to be misbranded:

In case of drugs:

First. If it be an imitation of offered for sale under the name of another article.

Second. If the contents of the package as originally put up shall have been removed, in whole or in part, and other contents shall have been placed in such package, or if the package fail to bear a statement on the label of the quantity or proportion of any alcohol, morphine, opium, cocaine, heroin, alpha or beta eucaine, chloroform, cannabis indica, chloral hydrate, or acetanilide, or any derivative or preparation of any such substances contained therein.

Third.* If its package or label shall bear or contain any statement, design, or device regarding the curative or theraupetic effect of such article or any of the ingredients or substances contained therein, which is false and fraudulent.

In the case of food:

First. If it be an imitation of or offered for sale under the distinctive name of another article.

Second. If it be labeled or branded so as to deceive or mislead the purchaser, or purport to be a foreign product when not so, or if the contents of the package as originally put up shall have been removed in whole or in part and other contents shall have been placed in such package, or if it fail to bear a statement on the label of the quantity or proportion of any morphine, opium, cocaine, heroin, alpha or beta eucaine, chloroform, cannabis

* This paragraph constitutes the amendment. 
indica, chioral hydrate, or acetanilide, or any derivative or preparation of any of such substances contained therein.

Third. If in package form, and the contents are stated in terms of weight or measure, they are not plainly and correctly stated on the outside of the package.

Fourth. If the package containing it or its label shall bear any statement, design, or device regarding the ingredients or the substances contained therein, which statement, design, or device shall be false or misleading in any particular: Provided, That an article of food which does not contain any added poisonous or deleterious ingredients shall not be deemed to be adulterated or misbranded in the following cases:

First. In the case of mixtures or compounds which may be now or from time to time hereafter known as articles of food, under their own distinctive names, and not an imitation of or offered for sale under the distinctive name of another article, if the name be accompanied on the same label or brand with a statement of the place where said article has been manufactured or produced.

Second. In the case of articles labeled, branded, or tagged so as to plainly indicate that they are compounds, imitations, or blends, and the word "compound," "imitation," or "blend," as the case may be, is plainly stated on the package in which it is offered for sale: Provided, That the term blend as used herein shall be construed to mean a mixture of like substances, not excluding harmless coloring or flavoring ingredients used for the purpose of coloring and flavoring only: And provided futher. That nothing in this Act shall be construed as requiring or compelling proprietors or manufacturers of proprietary foods which contain no unwholesome added ingredient to disclose their trade formulas, except in so far as the provisions of this Act may require to secure freedom from adulteration or misbranding.

SEc. 9. That no dealer shall be prosecuted under the provisions of this Act when he can establish a guaranty signed by the wholesaler, jobber, manufacturer, or other party residing in the United States, from whom he purchases such articles, to the effect that the same is not adulterated or misbranded within the meaning of this Act, designating it. Said guaranty, to afford protection, shall contain the name and address of the party or parties making the sale of such articles to such dealer, and in such case said party or parties shall be amenable to the prosecutions, fines, and other penalties which would attach, in due course, to the dealer under the provisions of this Act.

SEC. 10. That any article of food, drug, or liquor that is adulterated or misbranded within the meaning of this Act, and is being transported from one State, Territory, District, or insular possession to another for sale, or, having been transported, remains unloaded, unsold, or in original unbroken packages, or if it be sold or offered for sale in the District of Columbia or the Territories, or insular possessions of the United States, or if it be imported from a foreign country for sale, or if it is intended for export to a foreign country, shall be liable to be proceeded against in any district court of the United States within the district where the same is found, and seized for confiscation by a process of libel for condemnation. And if such article is condemned as being adulterated or misbranded, or of a poisonous or deleterious character, within the meaning of this Act, the same shall be disposed of by destruction or sale, as the said court may direct, and the proceeds thereof, if sold, less the legal costs and charges, shall be paid into the Treasury of the United States, but such goods shall not be sold in any jurisdiction contrary to the provisions of this Act or the laws of that jurisdiction: Provided however, That upon the payment of the costs of such libel proceedings and the execution and delivery of a good and sufficient bond to the effect that such articles shall not be sold or otherwise disposed of contrary to the provisions of this Act, or the laws of any State, Territory, District, or insular possession, the court may by order direct that such articles be delivered to the owner thereof. The proceedings of such libel 
cases shall conform, as near as may be, to the proceedings in admiralty, except that either party may demand trial by jury of any issue of fact joined in any such case, and all such proceedings shall be at the suit of and in the name of the United States.

SEC. 11. The Secretary of the Treasury shall deliver to the Secretary of Agriculture, upon his request from time to time, samples of foods and drugs which are being imported into the United States or offered for import, giving notice thereof to the owner or consignee, who may appear before the Secretary of Agriculture, and have the right to introduce testimony, and if it appear from the examination of such samples that any article of food or drug offered to be imported into the United States is adulterated or misbranded within the meaning of this Act, or is otherwise dangerous to the health of the people of the United States, or is of a kind forbidden entry into, or forbidden to be sold or restricted in sale in the country in which it is made or from which it is exported, or is otherwise falsely labeled in any respect, the said article shall be refused admission, and the Secretary of the Treasury shall refuse delivery to the consignee and shall cause the destruction of any goods refused delivery which shall not be exported by the consignee within three months from the date of notice of such refusal under such regulations as the Secretary of the Treasury may prescribe: Provided, That the Secretary of the Treasury may deliver to the consignee such goods pending examination and decision in the matter on execution of a penal bond for the amount of the full invoice value of such goods, together with the duty thereon, and on refusal to return such goods for any cause to the custody of the Secretary of the Treasury, when demanded, for the purpose of excluding them from the country, or for any other purpose, said consignee shall forfeit the full amount of the bond: And provided furlher, That all charges for storage, cartage, and labor on goods which are refused admission or delivery shall be paid by the owner or consignee, and in default of such payment shall constitute a lien against any future importation made by such owner or consignee.

SEC. 12. That the term "Territory" as used in this Act shall include the insular possessions of the United States. The word "person" as used in this Act shall be construed to import both the plural and the singular, as the case demands, and shall include corporations, companies, societies and associations. When construing and enforcing the provisions of this Act, the act, omission, or failure of any officer, agent, or other person acting for or employed by any corporation, company, society, or association, within the scope of his employment or office, shall in every case be also deemed to be the act, omission, or failure of such corporation, company, society, or association as well as that of the person.

SEC, 13. That this Act shall be in force and effect from and after the first day of January, nineteen hundred and seven.

\section{THE MEAT-INSPECTION LAW.}

EXtract from an ACT OF CONGRESS ENTITLED "AN ACT MAKING apPropriations For THE.

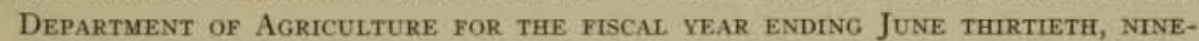
TEEN HUNDRED AND SEVEN," APPROVED JUNE 30 , I906.

That for the purpose of preventing the use in interstate or foreign commerce, as hereinafter provided, of meat and meat food products which are unsound, unhealthful, unwholesome, or otherwise unfit for human food, the Secretary of Agriculture, at his discretion, may cause to be made, by inspectors appointed for that purpose, an examination and inspection of all cattle, sheep, swine, and goats before they shall be allowed to enter into any slaughtering, packing, meat-canning, rendering, or similar establishment, in which they are to be slaughtered and the meat and meat food products thereof are to be used in interstate or foreign commerce; and all cattle, swine, sheep, and goats found on such inspection to 
show symptoms of disease shall be set apart and slaughtered separately from all other cattle, sheep, swine, or goats, and when so slaughtered the carcasses of said cattle, sheep, swine, or goats shall be subject to a careful examination and inspection, all as provided by the rules and regulations to be prescribed by the Secretary of Agriculture as herein provided for.

That for the purposes hereinbefore set forth the Secretary of Agriculture shall cause to be made by inspectors appointed for that purpose, as hereinafter provided, a post-mortem examination and inspection of the carcasses and parts thereof of all cattle, sheep, swine, and goats to be prepared for human consumption at any slaughtering, meat-canning, salting, packing, rendering, or similar establishment in any State, Territory, or the District of Columbia for transportation or sale as articles of interstate or foreign commerce, and the carcasses and parts thereof of all such animals found to be sound, healthful, wholesome, and fit for human food shall be marked, stamped, tagged, or labeled as "Inspected and Passed;" and said inspectors shall label, mark, stamp, or tag as "Inspected and Condemned," all carcasses and parts thereof of animals found to be unsound, unhealthful, unwholesome, or otherwise unfit for human food; and all carcasses and parts thereof thus inspected and condemned shall be destroyed for food purposes by the said establishment in the presence of an inspector, and the Secretary of Agriculture may remove inspectors from any such establishment which fails to so destroy any such condemned carcass or part thereof, and said inspectors, after said first inspection shall, when they deem it necessary, reinspect said carcasses or parts thereof to determine whether since the first inspection the same have become unsound, unhealthful, unwholesome, or in any way unfit for human food, and if any carcass or any part thereof shall, upon examination and inspection subsequent to the first examination and inspection, be found to be unsound, unhealthful, unwholesome, or otherwise unfit for human food, it shall be destroyed for food purposes by the said establishment in the presence of an inspector, and the Secretary of Agriculture may remove inspectors from any establishment which fails to so destroy any such condemned carcass or part thereof.

The foregoing provisions shall apply to all carcasses or parts of carcasses of cattle, sheep, swine, and goats, or the meat or meat products thereof which may be brought into any slaughtering, meat-canning, salting, packing, rendering, or similar establishment, and such examination and inspection shall be had before the said carcasses or parts thereof shall be allowed to enter into any department wherein the same are to be treated and prepared for meat food products; and the foregoing provisions shall also apply to all such products which, after having been issued from any slaughtering, meat-canning, salting, packing, rendering, or similar establishment, shall be returned to the same or to any similar establishment where such inspection is maintained.

That for the purposes hereinbefore set forth the Secretary of Agriculture shall cause to be made by inspectors appointed for that purpose an examination and inspection of all meat food products prepared for interstate or foreign commerce in any slaughtering, meat-canning, salting, packing, rendering, or similar establishment, and for the purposes of any examination and inspection said inspectors shall have access at all times, by day or night, whether the establishment be operated or not, to every part of said establishment; and said inspectors shall mark, stamp, tag, or label as "Inspected and Passed" all such products found to be sound, healthful, and wholesome, and which contain no dyes, chemicals, preservatives, or ingredients which render such meat or meat food products unsound, unhealthful, unwholesome, or unfit for human food; and said inspectors shall label, mark, stamp, or tag as "Inspected and Condemned" all such products found unsound, unhealthful, and unwholesome, or which contain dyes, chemicals, preservatives, or ingredients which render such meat or meat food products unsound, unhealthiful, unwholesome, or unfit for human food, and all such condemned meat food products shall be destroyed for food pur- 
poses, as hereinbofore provided, and the Secretary of Agriculture may remove inspectors from any establishment which fails to so destroy such condemned meat food products: Frovided, That, subject to the rules and regulations of the Secretary of Agriculture, the provisions hereof in regard to preservatives shall not apply to meat food products for export to any foreign country and whi h are prepared or packed according to the specifications or directions of the foreign purchaser, when no substance is used in the preparation or packing thereof in conflict with the laws of the foreign country to which said article is to be exported; but if said article shall be in fact sold or offered for sale for domestic use or cunsumption, then this proviso shall not exempt said article from the operation of all the other provisions of this act.

That when any meat or meat food product prepared for interstate or foreign commerce which has bzen inspected as hereinbefore provided and marked "Inspected and Passed " shall ba placed or packed in any can, pot, tin, canvas, or other receptacle or covering in any establishment where inspection under the provisions of this act is maintained, the person, firm, or corporation preparing said product shall cause a label to be attached to said can, pot, tin, canvas, or other receptacle or covering, under the supervision of an inspector, which label shall state that the contents threof have been "Inspected and Passed " under the provisions of this act; and no inspection and examination of meat or meat food products deposited or inclosed in eans, tins, pots, canvas, or other receptacle or covering in any establishment where inspection under the provisions of this act is maintained shall be deened to be complete until such meat or meat food products have been sealed or inclosed in said can, tin, pot, canvas, or other receptacle or covering under the supervision of an inspector, and no such meat or meat food products shall be sold or offered for sale by any person, firm, or corporation in interstate or foreign commerce under any false or deceptive name; but established trade name or names which are usual to such products and which are not false and deceptive and which shall be approved by the Secretary of Agriculture are permitted.

The Secretary of Agriculture shall cause to be made, by experts in sanitation or by other competent inspectors, such inspection of all slaughtering, meat-canning, salting, packing, rendering, or similar establishments in which cattle, sheep, swine, and goats are slaughtered and the meat and meat food products thereof are prepared for interstate or foreign commerce as may be necessary to inform himself concerning the sanitary conditions of the same, and to prescribe the rules and regulations of sanitation under which such establishments shall be maintained; and where the sanitary conditions of any such establishment are such that the meat or meat food products are rendered unclean, unsound, unhealthful, unwholesome, or otherwise unfit for human food, he shall refuse to allow said meat or meat food pioducts to be labeled, marked, stamped, or tagged as " Inspected and Passed."

That the Secretary of Agriculture shall cause an examination and inspection of all cattle, sheep, swine, and goats, and the food products thereof, slaughtered and prepared in the establishments hereinbefore described for the purposes of interstate or foreign commerce to be made during the nighttime as well as during the daytime when the slaughtering of said cattle, sheep, swine, and goats, or the preparation of said food products is conducted during the nighttime.

That on and after October first, nineteen hundred and six, no person, firm, or corporation shall transport or offer for transportation, and no carrier of interstate or foreign commerce. shall transport or receive for transportation from one State or Territory or the District of Columbia to any other State or Territory or the District of Columbia, or to any place under the jurisdiction of the United States, or to any foreign country, any carcasses or parts thereof, meat, or meat food products thereof which have not been inspected, examined, and marked as "Inspected and Passed," in accordance with the terms of this act and with the rules and 
regulations prescribed by the Secretary of Agriculture: Provided, That all meat and meat food products on hand on October first, nineteen hundred and six, at establishments where inspection has not been maintained, or which have been inspected under existing law, shall be examined and labeled under such rules and regulations as the Secretary of Agriculture shall prescribe, and then shall be allowed to be sold in interstate or foreign commerce.

That no person, firm, or corporation, or officer, agent, or employee thereof, shall forge, counterfeit, simulate, or falsely represent, or shall without proper authority use, fail to use, or detach, or shall knowingly or wrongfully alter, deface, or destroy, or fail to deface or destroy, any of the marks, stamps, tags, labels, or other identification devices provided for in this act, or in and as directed by the rules and regulations prescribed hereunder by the Secretary of Agriculture, on any carcasses, parts of carcasses, or the food product, or containers thereof, subject to the provisions of this act, or any certificate in relation thereto, authorized or required by this act or by the said rules and regulations of the Secretary of Agriculture.

That the Secretary of Agriculture shall cause to be made a careful inspection of all cattle, sheep, swine, and goats intended and offered for export to foreign countries at such times and places, and in such manner as he may deem proper, to ascertain whether such cattle, sheep, swine, and goats are free from disease.

And for this purpose he may appoint inspectors who shall be authorized to give an official certificate clearly stating the condition in which such cattle, sheep, swine, and goats are found.

And no clearance shall be given to any vessel having on board cattle, sheep, swine, or goats for export to a foreign country until the owner or shipper of such cattle, sheep, swine, or goats has a certificate from the inspector herein authorized to be appointed, stating that the said cattle, sheep, swine, or goats are sound and healthy, or unless the Secretary of Agriculture shall have waived the requirement of such certificate for export to the particular country to which such cattle, sheep, swine, or goats are to be exported.

That the Secretary of Agriculture shall also cause to be made a careful inspection of the carcasses and parts thereof of all cattle, sheep, swine, and goats, the meat of which, fresh, salted, canned, corned, packed, cured, or otherwise prepared, is intended and offered for export to any foreign country, at such times and places and in such manner as he may deem proper.

And for this purpose he may appoint inspectors who shall be authorized to give an official certificate stating the condition in which said cattle, sheep, swine, or goats, and the meat thereof, are found.

And no clearance shall be given to any vessel having on board any fresh, salted, canned, corned, or packed beef, mutton, pork, or goat meat, being the meat of animals killed after the passage of this act, or except as hereinbefore provided for export to and sale in a foreign country from any port in the United States, until the owner or shipper thereof shall obtain from an inspector appointed under the provisions or this act a certificate that the said cattle, sheep, swine, and goats were sound and healthy at the time of inspection, and that their meat is sound and wholesome, unless the Secretary of Agriculture shall have waived the requirements of such certificate for the country to which said cattle, sheep, swine and goats or meats are to be exported.

That the inspectors provided for herein shall be authorized to give official certificates of the sound and wholesome condition of the cattle, sheep, swine, and goats, their carcasses and products as herein described, and one copy of every certificate granted under the provisions of this act shall be filed in the Department of Agriculture, another copy shall be delivered to the owner or shipper, and when the cattle, sheep, swine, and goats or their carcasses and products are sent abroad, a third copy shall be delivered to the chief officer of the vessel on which the shipment shall be made. 
That no person, firm, or corporation engaged in the interstate commerce of meat or meat food products shall transport or offer for transportation, sell or offer to sell any such meat or meat food products in any State or Territory or in the District of Columbia or any place under the jurisdiction of the United States, other than in the State or Territory or in the District of Columbia or any place under the jurisdiction of the United States in which the slaughtering, packing, canning, rendering, or other similar establishment owned, leased, operated by said firm, person, or corporation is located unless and until said person, firm, or corporation shall have complied with all of the provisions of this act.

That any person, firm, or corporation, or any officer or agent of any such person, firm, or corporation, who shall violate any of the provisions of this act shall be deemed guilty of a misdemeanor, and shall be punished on conviction thereof by a fine of not exceeding ten thousand dollars or imprisonment for a period not more than two years, or by both such fine and imprisonment, in the discretion of the court.

That the Secretary of Agriculture shall appoint from time to time inspectors to make examination and inspection of all cattle, sheep, swine, and goats, the inspection of which is hereby provided for, and of all carcasses and parts thereof, and of all meats and meat food products thereof, and of the sanitary conditions of all establishments in which such meat and meat food products hereinbefore described are prepared; and said inspectors shall refuse to stamp, mark, tag, or label any carcass or any part thereof, or meat food product. therefrom, prepared in any establishment hereinbefore mentioned, until the same shall have actually been inspected and found to be sound, healthful, wholesome, and fit for human food, and to contain no dyes, chemicals, preservatives, or ingredients which render such meat food product unsound, unhealthful, unwholesome, or unfit for human food; and to have been prepared under proper sanitary conditions, hereinbefore provided for; and shall perform such other duties as are provided by this act and by the rules and regulations to be prescribed by said Secretary of Agriculture; and said Secretary of Agriculture shall, from time to time, make such rules and regulations as are necessary for the efficient execution of the provisions of this act, and all inspections and examinations made under this act shall be such and made in such manner as described in the rules and regulations prescribed by said Secretary of Agriculture not inconsistent with the provisions of this act.

That any person, firm, or corporation, or any agent or employee of any person, firm, or corporation, who shall give, pay, or offer, directly or indirectly, to any inspector, deputy inspector, chief inspector, or any other officer or employee of the United States authorized to perform any of the duties prescribed by this act or by the rules and regulations of the Secretary of Agriculture any money or other thing of value, with intent to influence said inspector, deputy inspector, chief inspector, or other officer or employee of the United States in the discharge of any duty herein provided for, shall be deemed guilty of a felony and, upon conviction thereof, shall be punished by a fine not less than five thousand dollars nor more than ten thousand dollars and by imprisonment not less than one year nor more than three years; and any inspector, deputy inspector, chief inspector, or other officer or employee of the United States authorized to perform any of the duties prescribed by this act who shall accept any money, gift, or other thing of value from any person, firm, or corporation, or officers, agents, or employees thereof, given with intent to influence his official action, or who shall receive or accept from any person, firm, or corporation engaged in interstate or foreign commerce any gift, money, or other thing of value given with any purpose or intent whatsoever, shall be deemed guilty of a felony and shall, upon conviction thereof, be summarily discharged from office and shall be punished by a fine not less than one thousand dollars nor more than ten thousand dollars and by imprisonment not less than one year nor more than three years.

That the provisions of this act requiring inspection to be made by the Secretary of 
Agriculture shall not apply to animals slaughtered by any farmer on the farm and sold and transported as interstate or foreign commerce, nor to retail butchers and retail dealers in meat and meat food products, supplying their customers: Provided, That if any person shall sell or offer for sale or transportation for interstate or foreign commerce any meat or meat food products which are diseased, unsound, unhealthful, unwholesome, or otherwise unfit for human food, knowing that such meat food products are intended for human consumption, he shall be guilty of a misdemeanor, and on conviction thereof shall be punished by a fine not exceeding one thousand dollars ot by imprisonment for a period of not exceeding one year, or by both such fine and imprisonment: Provided also, That the Secretary of Agriculture is authorized to maintain the inspection in this act.provided for at any slaughtering, meat canning, salting, packing, rendering, or similar establishment notwithstanding this exception, and that the persons operating the same may be retail butchers and retail dealers or farmers; and where the Secretary of Agriculture shall establish such inspection then the provisions of this act shall apply notwithstanding this exception.

That there is permanently appropriated, out of any money in the Treasury not otherwise appropriated, the sum of three million dollars, for the expenses of the inspection of cattle, sheep, swine, and goats and the meat and meat food products thereof which enter into interstate or foreign commerce and for all expenses necessary to carry into effect the provisions of this act relating to meat inspection, including rent and the employment of labor in Washington and elsewhere, for each year. And the Secretary of Agriculture shall, in his annual estimates made to Congress, submit a statement in detail, showing the number of persons employed in such inspections and the salary or per diem paid to each, together with the contingent expenses of such inspectors and where they have been and are employed.

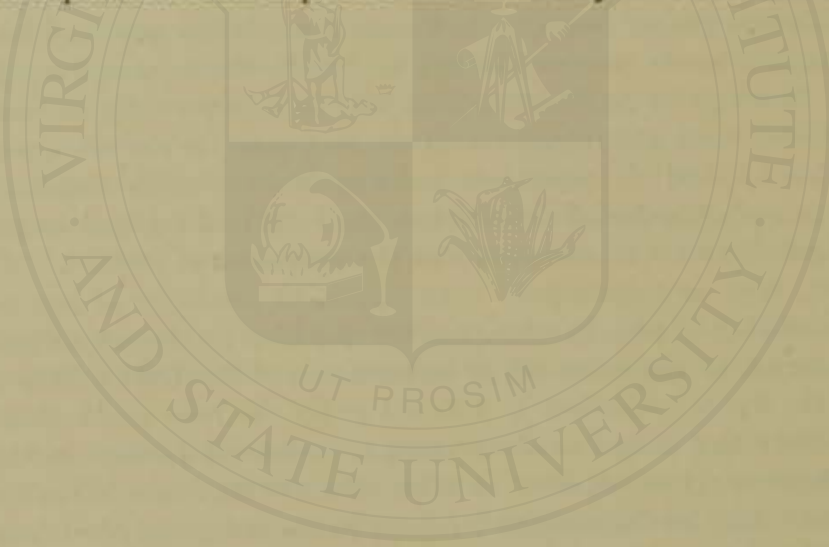




\section{IN DEX.}

Abbé refractometer, 100, 108

construction, 109

influence of temperature, 110

manipulation, 100

Abrastol, 837

Absinthe, 754

Acetanilide in vanilla extract, 858 tests for, 859

Acetyl value, 497

Achroodextrine, 575

Acid fuchsin, 799

brown, 810

green, 794

magenta, 816, 8I 7

yellow, 794,818

Acids, fatty, $481,484,499,500$

of acetic series, $47 \mathrm{I}$

of linoleic series, 472

of oleic series, 472

mineral, in vinegar, 767

organic, $47,941,949$

Ackermann and Steinmann's table for alcohol from refraction, 715

Ackermann's table for extract from refraction, 721

Adams' fat method, I34

"Aerated " butter, 540

Agar agar, in jelly, 934, 943

Aging of liquors, 731, 732

Albumin, acid, 44

alkali, 44

determination in milk, 146

of muscle, $2 \mathrm{II}$

Albuminoids, 42 preparation of, 263

Albumins, 41, 297

Albumose, 44,45

Alcohol, detection, 657

determination, $65^{8}$

by distillation, $65^{8}$

by ebulioscope, 675

by evaporation, 660

from refraction, 715

from specific gravity, 658,659
Alcohol, extract of spices, 470

in malt liquors, 715

methyl-, 749,878

preparation of, 730

stills, 659

tables, 66r-674

Alcoholic beverages, 653,654 . See also

Liquors.

references on, 756

siate control of, 654

toxic effect of, 655

fermentation, 653

Aldehydes, determination, 745

Ale, 709, 712. See also Beer. ginger, 954

Aleurone, 00

Alizarin, 804

Alkaloidal nitrogen, 40,46

Alkaloids, proof of absence of, 726

Alkanna tincture, 92

Allantoin, 299

Allen-Marquardt method for fusel oil, 747

Allihn's sugar method, 608

tables, 609

Allspice, 420

adulteration, 424

composition of, 420

microscopical structure, 422

standard, 424

tannin in, $42 \mathrm{I}$

Almond extract, 884

adulteration of, 886

alcohol in, 888

benzaldehyde in, 886,888

hydrocyanic acid in, 888 , 889

nitro benzol in, 887,888

meal, $35^{8}$ standards, 885

Almonds, bitter, oil of, $88_{4}, 88_{5}$

Alum in baking powder, 333,344

in bread, 326

in flour, 315

in pickles, 926 
Alumina, determination of, 344

Aluminum salts in baking powder, 344 in cream of tartar, 344

Amagat and Jean's refractometer, 100

Amaranth, $794,806,815,816,817$

Amides, 45

$$
\text { in milk, } 147
$$

Amido nitrogen determination, 74,147

$$
\text { in wheat, } 299
$$

Amino acids, 40,45

Ammonia, determination, 74

in baking powder, 346

in foods, 40,46

in milk, 147

Ammonium fluoride, 835

Amthor test for caramel, 752

Amylodextrin, 575

Amyloid, 91, 92

Analyst, functions of, 3, 4

Angostura, 754

Anilin orange, 808

Animal diastase, 284

$$
\text { in milk, } 177
$$

Anise extract, standards, 892

$$
\text { oil, standards, } 892
$$

Annatto in butter, 536,537

$$
\text { in milk, } 175,177
$$$$
\text { tests for, 701, 8ro }
$$

Antiseptics, see Preservatives.

Apparatus, 20

Apple butter, 927

essence, imitation, 896,897

juice, 680

pulp, detection, 943

Apples, composition of, 274,275

Araban, 285,288

Arabinose, 285,288

Arata's color test, 796

Army rations, 257

Arsenic detection and determination, 74 compounds in colors, 785

in baking chemicals, 346

in beer, 713,728

in confectionery, 649

in glucose, 633

in vinegar, 780

Johnson-Chittenden-Gautier method, 74

Marsh apparatus, 75

Sanger-Black-Gutzeit test for, 76

Artificial colors, 782

fruit essences, 895,897

sweeteners, 850

$$
\text { references on, } 855
$$

Asaprol, 845

Asbestos fiber, preparation of, 594,598
Ash analysis, scheme for, 301

determination of, 62

of food, 47

Asparagin, 45, 299

Auramin, 784,803

Aurantia, 8 or

Aurin, 804

Azo blue, $801,812,816$

Azoacidrubine, 794

Babcock asbestos milk fat method, 135 milk solids method, $\mathrm{r}_{34}$ centrifugal fat method, 136 milk formulx, 153 test bottles, 138

Bacon formic acid method, $84 \mathrm{r}, 843$

Bacon and Dunbar citric acid method, 923 lactic acid method, 923

Baier and Neuman's test for sucrose in milk 197

Baker tin method, 917

Baking powders, 332

adulteration of, 334

alum, 333,334

methods of analysis, 336

phosphate, 334

Balances, 20

tartrate, 332

Bamihl test for gluten, 322

Banana essence, artificial, 896,897

Barium compounds in colors, 784

Bark as an adulterant, 428

Bariey, 271, 272

$$
\text { ash, } 302
$$

microscopy of, 300

proteins, 300

starch, $28 \mathrm{I}$

Barwood, 808

Basic colors, 795, 798

Baudouin's sesame oil test, 519

Beading oil, 738

Beans, 272,388

Bechi's cottonseed oil test, $5 \mathrm{I} 7$

Beckman's test for glucose in honey, 641

Beef, composition of, 213

cuts of, 213

stearin, microscopical structure, $55^{8}$

tallow, 529

Beer, 707

acids in, 724

adulteration of, $7 \mathrm{II}$

alcohol in, 715

aloes in, 727

arsenic in, 713,728

ash of, 714

birch, 954 
Beer, bitter principles of, 726

bock-, 709

brewing of, 708

carbon dioxide in, 726

chiretta in, 711,727

composition of, 709

degree of fermentation of, 724

dextrin in, 724

extract gravity of, 722

extract in, 715 specific gravity method, 722

refractometer method, 722

gentian bitter in, 711,727

glucose in, 710

glycerin in, 724

lager-, 708

methods of analysis, 714

phosphoric acid in, 725

preservatives in, 713,729

proteins of, 725

quassiin in, 711,727

references on, 756

root, 954

schenk-, 708

standards, 711

temperance-, $7 \mathrm{I} 4$

varieties of, 708

uno-, 714

weiss-, 709

wort, 708

Beeswax, 643

$$
\text { gravity of, } 722
$$

$$
\text { refractometer reading of, } 645
$$

Beet (color), 790 sugar, 560

Bellier's peanut oil test, 524

Benches, 15

Bénédictine, 754

Benzaldehyde, $885,886,929$ artificial, 885

in almond extract, 886

Benzoic acid, 833 in maraschino cherries, 929

detection of, 834

determination, 835

in milk, 180

Betaine, 45, 299

toxicity of, 834

Beta-naphthol, 845

Beverages, carbonated. See Carbonated beverages.

Biebrich scarlet, 806

Bigelow and McElroy's cane-sugar method, 192

Bilberry (color), 790

Birch beer, 954
Birotation, 584,639

Biscuit, gluten, $35^{8}$ soja bean, 358

Bishop arsenic apparatus, 76

Bismarck brown, 8or, 8 ro

Bisulphites as preservatives, 839

Bitter almonds, oil of, $88_{4}, 88_{5}$

Biuret reaction, $4 \mathrm{I}$

Blackberry (color), 790

Blarez test for fluorides, 843

Blast pump, 19

Blue colors, $785,786,788,794,812$

"Blown" cans, 902

Bock-beer, 709

"Boiled " butter, $54^{\circ}$

Bombay mace, 467

Bomb calorimeter, 47

Bömer's phytosterol acetate test, 507

Borax, 827

Bordeaux B, 801, 806

S, 794

Boric acid, 827

detection, $182,184,828$

determination, 827,829

in butter, 538

in meat, 220,232

in milk, 182,184

Bourbon whiskey, $732,734,737$

Brandy, 730

adulteration of, $74 \mathrm{I}$

composition of, 739

"drops," 649

methods of analysis, 745

new, 740

potable, 740

standards, 740

Brazil wood, 790,808

Bread, 317,323

acidity of, 325

adulteration of, 326

alum in, 326

baking of, 323

composition of, 324,325

fat in, 326

Breakfast cereals, 352

Brewing beer, 708

Brie cheese, 202

Brilliant red, 806 yellow, $8 \mathrm{I} 6$

Bromination oil test, 494

Bromine absorption of oils, 492

Brown and Duvel's method for moisture in grain, 278

Brown colors, $786,788,8$ Io

sugar, 568

Browne's method for dextrin in honey, 640 
Browne's test for invert sugar in honey, 642 Brucke's glycogen method, 236

$$
\text { reagent, } 236
$$

Buckwheat, 271

ash of, 302

composition of, 271,272

flour, 313

microscepy of, $31 \mathrm{I}$

Burgundy wine, artificial, 692

Butter, 20r, 529

adulteration of, 535

annatto in, 536

apple, 927

ash in, 534

azo colors in, 536,537

boric acid in, 538

carrotin in, 536

casein in, $534,55 \mathrm{I}$

coloring in, 535

composition of, 530

distinction from oleomargarine and process butter, 546

effects of feeding. 531

fat, composition of, 530 standard, 535

fat in, 533,534

filled, 540

foam test, 549

formaldehyde in, 539

fruit, 927

glucose in, 539

methods of analysis, 53I

microscopical examination of, 552

milk test, 550

preservatives in, 538

references on, 562

renovated, 540

salicylic acid in, 539

salt in, 534

standard, 535

sulphurous acid in, 539

turmeric in, 536

water in, 5.31

Waterhouse test, 550

Butterine, 54 $\mathrm{T}$

Butterine oil, 522

Butyro-refractometer, 100, IOI

critical line of, 106

limits of butter readings, 547

manipulation, 102

oil readings on, 478,479

olive and cottonseed oil readings, 514

sliding scale for, 107

special thermometer for, 549

table of equivalent refractive indices,

104, 105
Butyro-refractometer, temperature variation testing scale, 104 of reading, 107

Caffeine, 372,955

determination of, $373,384,958,960$ in carbonated beverages, 955

Caffeol, 379

in cocoa, 400

Caffetannic acid, 379,382

Cake, 327

Calcium carbonate crystals, 90 oxalate crystals, 90 sucrate, ro 6

California wines, 688

Calorie, 47,48

Calorimeter, bomb, 47

oil, 495

respiration, 2

Camembert cheese, 202

Camera, 96

Canada balsam, 86

Candy, see Confectionery. standard, 645

Cane sugar, 566

ash of, 567

composition of, 568

detection of, $5^{8} 5$

determination of :

$$
\text { in milk, } 197
$$

by copper reduction, 590 , 612

by polarimetry, 586,614

in cereals, 295

inversion of, $588,5^{89}$

manufacture of, 567

methods of analysis, $5^{85}$

moisture in, 586

quotient of purity, 586

refining, 570

test for, $5^{8} 5$

Canned food, 900
composition of, 002
decomposition of, 902 metallic impurities in, 904 method of canning, 900 methods of analysis, 913 preservatives in, 912 references on, 961

Canned fruits, 900,902

meats, 22

vegetables, 900,902

Cans, detection of spoiled, 902

gases from spoiled, 903

Capers, 926

Capsicin, 440 
Capsicums, 439

Caramel, 792

in distilled liquors, $75^{2}$

in milk, 176,177

in vanilla extract, 869

in vinegar, 779

Carbohydrates, $46,47,74,279$

of cereals, 279,295

of eggs, 263

Carbon dioxide in baking chemicals, 336

in beer, 726

in yeast, 330

Carbonated beverages, 952

acids in, 955

bottled, 954

caffein in, $95^{8}$

cocaine in, 959

colors in, 955

foam producers in, 955

habit-forming drugs in, 955

methods of analysis, $95^{6}$

preservatives in, 955

saponin in, 956

sweeteners in, 954

water, 953

syrups for, 955

Carmosin, 806

Carnin, 211

Carrot (color), 791

Casein, 43 125, 126

determination in milk, 145

Caseose, 44

$$
\text { in cheese, } 203
$$

in milk, 146

Casoid flour, $35^{8}$

Cassia, 424

adulteration of, 428

buds, 425

composition of, 422

extract, standards, 892

microscopical structure, 426

oil, 425,892

standards, 892

standard, 428

Catsup, see Ketchup.

Cayenne, 439

adulteration of, 443

coal-tar colors in, 444

colors in, 444

composition of, $44 \mathrm{I}$

microscopical structure, 44 I

mineral adulterants in, 444

oil of, 440
Cayenne, redwood in, 444

standard, 443

Cazeneuve's color scheme, 705, 706

Celery seed extract, standards, 892

Cellulose, 47,285

oil, standards, 892

Centrifuge, milk-fat, ${ }_{3} 6,1_{37}$

Centrifuge, universal, 25

Cereal products, microscopy of, 305

Cereals, 271

ash of, 302

breakfast foods, $35^{2}$

cane sugar in, 295

carbohydrates of, 279

separation of, 295

composition of, 271

crude fiber in, 277,296

dextrin in, 205

hemicelluloses in, 206

methods of proximate analysis, 276

pentosans in, 285,296

proteins of, 296

references on, $36 \mathrm{r}$

starch determination in, 283,296

Chace total aldehyde method, 875

Champagne, 687

Chaptalizing. 693

Charlock, 459

Chartreuse, 745

Cheddar cheese, 202

Cheese, 202

adulteration of, 203

amides in, 206

ammonia in, 206

ash in, 205

composition of, 202,203

cream, 203

fat in, 205,207

filled, 204

lactic acid in, 207

methods of analysis, 204

milk sugar in, 207

nitrogen compounds of, 206

paranuclein in, 206

peptones in, 206

proteins in, 205

sampling, 204

skimmed milk, 203, 204

standards, 203

varieties of, 202

water in, 204

whole milk, $2 \mathrm{O}_{3}$

Cherries, maraschino, 928

Cherry soda, 954

Chicory, $386,388,389$ 
Chili sauce, 9 rg

Chiretta, 727

Chlor iodide of zinc, $9 \mathrm{I}$

Chloral hydrate, 93

test for charlock, 460

Chlorine in vegetable substances, 305

Chocolate, see Cocoa.

milk, 307 composition of, 397 sucrose and lactose in, 590

Cholesterol, 502

crystallizations of, 504

determination of, 503

distinction from phytosterol, 503

Cholin, 45, 299 separation of, $5 \circ 3$

Chromate of lead, 647

Chrome yellow, 8 ro

Chromogenic bacteria, 130

Chrysamin, 801, 810

Chrysoidin yellow, 808

Chrysophenin, 8 r6

Cider, 678

adulteration of, 682

ash of, 682

composition of, 679

fermented, 680

malic acid in, 683

manufacture of, 678

methods of, analysis, 696

references on, 757

sweet, 948

vinegar, 760,773

watering of, 682

yeast in, 678

Cinnamon, 424

composition of, 425,426

extract, 892

microscopical structure of, 426 oil, standards, 892

Citral, $88 \mathrm{r}$ standard, 428

determination, 877

in carbonated beverages, 955

in fruit juices, $95 \mathrm{I}$

Citric acid in fruit products, 920, 923, 941 $942,948,951$

in ketchup, 920, 923

in milk, 126,127

Citronellal, 881

Citronella oil, 880,88 I

Citronin, 794

Clams, 256

Claret wine, 687

Clarifying reagents in microscopy, 92

in sugar analysis, 586,614
Clerget's formula, $5^{88}$

Clove extract, 892

$$
\text { oil, } 892
$$

Cloves, 412

adulteration of, $4 \mathrm{~T} 8$

cocoanut shells in, 419

composition of, $4 \mathrm{r} 4$

exhausted, 418

microscopical structure, 416

oil of, $\mathrm{Sg}_{2}$

standard, 418

stems, 417

tannin in, 415

Clupein, 43

Coal-tar colors, 793

acid, 798

allowed, 704

Mathewson method of determination, 814

Arata's test, 796

Price method of identification, $8 \mathrm{I} 4$

basic, 795

classification, 793, 800

detection of, 795

double dyeing method, 796

dyeing wool by, 795

extraction by amyl alcohol, 797

identification of, 795,799 ,

801,805

in milk, 177

in sausages, 239

Rota's scheme for, 790

separation with ether, 798

Sostegni and Carpentieri's color test, 796

Cocaine, detection of, 959,960

in carbonated beverages, 955

Cochineal, 792, 808

$$
\begin{aligned}
& \text { in sausages, } 238 \\
& \text { red, } 806
\end{aligned}
$$

Cocoa, 392,393

adulteration of, 402

alkali in, 403

ash of, 396

butter, 393,529

caffeine in, 400

composition of, 393

foreign fat in, 402

manufacture of, 393

methods of, analysis, 398

microscopical structure, 403

nibs, 394,402

nitrogeneous bodies in, 396

pentosans in, 396 
Cocoa, references on, 406

shells, $394,395,405$

standards, 402

starch in, $394,395,399,405$

sugar in, 399, 405

theobromine in, 396,400

Cocoanut oil, 528

$$
\text { pulp, } 528
$$

shells, 419

Coffee, 379

adulteration of, 384

ash of, 380,382

caffeine in, $3^{80}, 3^{84}$

caffeol in, 379

caffetannic acid in, 379,382

chicory in, $388,3^{89}$

coloring of, 384

composition of, $379,380,38 \mathrm{I}$

essential oil of, 379

fat in, 379

glazing of, 385

hygienic, 390

methods of analysis, 382

microscopical structure, 386

"pellets," 384

references on, 406

standards for, 384

starch in, 386

substitutes, 392

Cognac, 739. See also Brandy. oil, 741

Collagen, 42,211

Collodion silk, 705

Colorimeter, Schreiner's, 77

Colorometric analysis, 77

Colors, acid fuchsin, 799

artificial, 782

allowed, 794

animal, 792

arsenic compounds, 785

barium compounds, 784

basic, 795

blue, $785,786,788,794,812$

brown, $786,788,8$ ro

caramel, 792

coal tar, 793, 794, 795

cochineal, 792

copper compounds, 784

cudbear, 791

extraction of, by immiscible solvents, 797

fuchsin, 800,806

green, $785,786,787,794,812$

harmless, 784,786

identification of, 795, 799, 805

in butter, 535
Colors, in carbonated beverages, 955

in cayenne, 444

in confectionery, $649,784,788$

indigo, 792,812

in jams and jellies, 942

injurious, 784,785

in ketchup, 921

in milk, $174-177$

in mustard, 460

in sugar, 590

lead chromate, $647,784,793$

lead compounds, 784

logwood, 791

mercury compounds, 785

mineral, 792

non-injurious, 784,786

orange, $785,794,808$

orchil, $79 \mathrm{I}$

Prussian blue, $375,792,812$

reagents for identifying, $8 \mathrm{I} 4$

red, $785,786,790,806$

references on, $8 \mathrm{In}$

Rota's scheme for, 799

separation by solvents, 797,814

toxic effect of, $78_{3}$

turmeric, 791

ultramarine blue, $793,8 \mathrm{r} 2$

vegetable, $789,791,797$

violet, $786,789,812$

wool dyeing, 795,796

yellow, $785,787,790,794,808$

Colostrum, 120

Commercial glucose, see Glucose.

Compressed yeast, 328

Conalbumin, 262

Concentrated foods, 257

Condensed milk, 186

as a milk adulterant, 186

ash of, 189

cane sugar in, 191, 192

composition of, 187

fat in, $189,191,192$

foreign fats in, $19 \mathrm{I}$

milk sugar in, 100

methods in analysis, 188

proteins in, 190,192

solids of, 188

Confectionery, 645 -standards for, 188

adulteration of, 645

alcohol in, 649

arsenic in, 649

cane sugar in, 648

colors in, 645,647

dextrin in, 648

glucose in, 648 
Confectionery, invert sugar in, 648

lead chromate in, 647

methods of analysis, 646

mineral adulterants, 646

paraffin in, 647

starch in, 648

Congo red, 8or, 800,8 r 6

Connective tissue, 211

Copper salts, 900

$$
\text { determination of, } 914,918
$$$$
\text { in vinegar, } 780
$$

Copra oil, 528

Cordials, 754

analysis of, 755

composition of, 755

Corky tissue, 89

Corn, 271, 272

ash of, 302

bleaching of canned, $9 \mathrm{I}_{2}$

composition of, 271,272

microscopical structure, 309

oil, 521

sitosterol in, $5^{22}$

proteins of, 300

starch, $28 \mathrm{I}$

syrup, 575

Cornelison's butter color test, 537

Corning of meat, 219

Cotton scarlet 3 B, 816

Cottonseed, 5 I 6

$$
\begin{aligned}
& \text { oil, } 516 \\
& \text { standards for, } 517 \\
& \text { tests for, } 5^{17} \\
& \text { stearin, } 5^{17}
\end{aligned}
$$

Cotton's cane sugar method, 185

Coumarin, 862

determination, 865

microscopical structure, 867

Crampton and Simon's caramel test, $75^{2}$

Cream, 193 palm oil tests, 542

adulteration of, 194

cheese, 202

evaporated, 194

fat in, 195

foreign fats in, 194,196

gelatin in, 195, 106

methods of analysis, 195

standards for, 194

sucrate of lime in, 195, 197

test scale, 194

viscogen in, 195,197

Cream of tartar, $33^{6}$

$$
\text { in wine, } 702
$$

Creatin, 46, 21I
Creatinin, 46, 211

Crême de menthe, 755

Crême de Noyau, 754

Crocein orange, 810,816 scarlet, 806

Crude fiber, 277

$$
\text { in cereals, } 296
$$

Crustaceans, 256

Crystals, plant, 90

Cucumber pickles, 925

Cudbear, 791,808

Cumidin red, 794, 806 ponceau, 794

Cuprammonia, 93

Curaçoa, 754

Curcuma, 450

Curcumin, 45I

Curd tests in butter, $55 \mathrm{I}, 55^{2}, 553$

Curing meat, 219

Currant (color), 790 black (color), 790

Curry powder, $45^{\circ}$

Custard powders, 270

Dakota mustard, 460

Date stones, 390

Decker-Kunze method for theobromine and caffeine, 400

Defren-O'Sullivan sugar method, 150,594

Defren's sugar tables, 595

Denis and Dunbar benzaldehyde method, 886

Desiccated egg, 268

Deutyro-albumose, 44,45

Dextrin, 575

determination of, in cereals, 295 in glucose, 602

in honey, 640

in jams and jellies, 940

in molasses, 624

Dextrose, 573

determination of, $591,593,594,598$

Diabetic foods, 357

Diamond yellow, 801

$$
\text { analyses, } 35^{8}
$$

Diastase, animal, 284

in malt extract, 729

starch methods, 283

Dietetics, references on, 49

Dinitrocresol, 808

Dioxin, 8 or

Distilled liquors, 730

aldehydes in, 745

analytical methods, 745

caramel in, $75^{2}$

color tests, 752,753 
Distilled liquors, esters in, 745

extract in, 745

furfural in, 746

fusel oil in, 746

methyl alcohol in, 749

opalescence test, 753

references on, 758

Doolittle and Woodruff theine method, 373

Doolittle butter color test, 537

Double dilution sugar method, 149

Dough, expansion of, 317

Drains, 17

Dried fruits, 944

decomposed, 945

lye treatment of, 945

methods of analysis, 946

moisture content of, 945

sulphuring of, 945

wormy, 945

zinc in, 946

Drugs, habit-forming, 955

Dry wines, 690

Dry yeast, 328

Dubois salicylic acid method, 832

sugar method, 390

Dubosc's saccharimeter, $5^{8}{ }_{3}$

Dulcin, 853 determination, 854

Dunbar and Bacon malic acid method, 949

Dupré's color method, 705

Dvorkovitsch theine method, 373

Ebulioscope, 675

Edam cheese, 202

Edestan, 44

Edestin, 299, 300

Eggs, 261

ash of, 264

carbohydrates of, 263

cold storage, 267

composition of, 264,265

desiccated, 268

fat of, 264

frozen, 268 .

lecithin determination, 265

methods of analysis, 265

opened, 268

physical examination of, 267

preservation of, 266

proteins of, 262

references on, 270

substitutes for, 269

waterglass as a preservative, 266

weights of, 264

white of, 262

yolk of, 263
Elaĩdin oil test, 499

Elastin, 2 II

Elderberry (color), 790

Electrolytic apparatus, 608

Elm bark, 428

Emergency rations, 257

Eosin, 794, 804, 806

Ergot, 313

Erythrodextrin, 575

Erythrosin, 794, 806, 815, 816,817

Esters, in distilled liquors, 745 in imitation flavors, 898

Ether, ethyl, preparation of absolute, 66 petroleum, preparation of, for a solvent, 66

Eucasin, ${ }_{5} 8$

Eugenol, 412

Ewe's milk, 127

Exhausted cloves, 418 ginger, $45^{\circ}$ tea leaves, 375 vanilla beans, 859

Exhaust pump, 20

Extraction with immiscible solvents, 68 volatile solvents, 63

Extractor, Johnson, 55 Soxhlet, 63

"Faints," 732

Farinaceous infants' foods, 356

Fast red, 794, 806, 816

Fat globules, $9 \circ$

Fat of food, 39 of meat, 226,227

Fats, edible, 47r. See also Oils. filtering, 473

measuring, 473

melting-point of, 480

methods of analysis, 473

microscopical examination of, 510

paraffin in, 510

references on, $56 \mathrm{r}$

weighing, 473

Fatty acids, 499

constants of, 500

insoluble, 485

solidifying point of, 500

soluble, 484

volatile, $84 \mathrm{I}$

Fehling processes, 590

Fehling's solution, $59 \mathrm{I}$ gravimetric, 150,593 volumetric, 150 , 591

equivalents of, 592

Fermentation, acetic, 759

alcoholic, 653 
Fermentation, lactic, 129

proteolytic, 158,202

Fermented liquors, 678

Feser's lactoscope, $\mathrm{I}_{3}$

Fibrin, 125

Fibro vascular tissue, 88

Fibroin, 42

Filled cheese, 204

Fincke formic acid method, 842

Fish, analyses of, 255 preservatives in, 257 colors in, 257

Flavoring extracts, 857

Flesh foods, 211 references on, 898 references on, 258

Fletcher and Allen's tannin method, $37 \mathrm{I}$

Floor, 15

Flour, 3 II

absorption test of, 317

acidity in, 320

adulteration of, $3 \mathrm{I} 4$

alcohol soluble protein in, 320

alum in, 315

baking tests of, 318

bleaching of, 315

detection, $32 \mathrm{I}$

cold water extract of, 320

color test of, 317

composition of, 312

damaged, $3+3$

dough test of, 317

fineness of, 316

gluten in, 320,322

inspection, 316

iodine number of fat of, $32 \mathrm{I}$

methods of analysis, 316

nitrites in, $32 \mathrm{I}$

proximate constituents of, 3 r9

salt soluble protein in, 320

Fluoborates, 843,844

Fluorides, 843

$$
\text { detection of, } 843
$$

Fluosilicates, 843,844

Foam producers, 955

"Foam" test for butter, 549

Folin method for ammonia in meat, 226

Food adulteration, 5

analysis, commercial, 3

from dietetic standpoint, 2

general methods, 4

references on, 70

and drugs act, 965

concentrated, 257

economy, references on, 49

inspection, 3,6
Food inspection, references on, 11 misbranding, 6

nature and composition of, 39

official control of, I

standards, 4

Fore milk, 128

Foreshots, 732

Formaldehyde, 824

detection of, 180,824

determination of, $18 \mathrm{r}, 825$, 827

in eggs, 268

in milk, 178

Formic acid, $84 \mathrm{r}$

references on, II

detection of, 841

determination of, 842

Fortified wine, 685,600

Freas drying oven, 22

Fresenius' method for colors in pastes, $35^{\circ}$

Frozen milk, test for, 129 meat, 239

Fructose, $d$-, 574

Fruit, 274

$$
1-8-, 574
$$

butter, 927

candied, 640

composition of, 274

essences, artificial, $895,896,897$

juices, 946,947

methods of analysis, 949

methods of proximate analysis, 276

organic acids in, 949

products, 900

references on, $96 \mathrm{r}$

references on, $36 \mathrm{r}$

sugar, see Levulose.

sugar-coated, 646

sugar in, $5^{66}$

syrups, 952, 953

tissues under the microscope, 944

Fruits, dried, see Dried fruits.

Fuchsin, 798, 803,806

Fuel value, 47

Fuller caffein method, 958,960 cocaine method, 959,960

Funnel, jacketed, 474 separatory, 67,68

Furfural, 285

determination, 746

in distilled liquors, 746

in vinegar, 779

Furnace, electric, 62,24 gas, 24

Fusel oil, 73 detection, 746 
Fusel oil, determination, 747

Fustic, 790,810

Game, composition of, 216

Gases, in spoiled cans, 903

Geerlig's table for dry substances in sugar. products, 615

Geissler's carbon dioxide apparatus, 337

Gelatin, 42,211

$$
\begin{aligned}
& \text { in cream, } 195 \\
& \text { in jams and jellies, } 943 \\
& \text { in meat, } 231
\end{aligned}
$$

Gerber's milk centrifuge, 136

Gill and Hatch's oil calorimeter, 495

Gin, 744

Ginger, 445

adulteration of, $45^{\circ}$

ale, 954

black, 446

cold water extract of, 448

composition of, 446,447

exhausted, 447

extract, methods of analysis, 894 standards, 892

liming of, 446

microscopical structure of, 449

oil of, 4.46

root, 445

standard, $45^{\circ}$

white, 446

Gliadin, 42, 208, 299

Globulins, 42, 297

Globulose, 44

Glucin, 855

Glucose, 575

arsenic in, 632

composition of, 576 .

$d-, 573$

determination of, in honey, 637,641 in jams and jellies, 940

in molasses, 621

dextrin in, 632

healthfulness of, 576

in beer, 710,712

in butter, 539

methods of analysis, 630

standards for, 576

test for, $632,64 \mathrm{I}$

Glucoses, 565

Glutelins, 42

Gluten, 298, 299

Bamihl's test for, 322

biscuit, $35^{8}$

determination of, 319

flour, 357,358

Glutenin, 42, 298, 300
Glycerin in carbonated beverages, 960 in vanilla extract, 869 in wine, 703

jelly, 86

Glycerrhizin, 955

Glycogen, 212

detection, 235

determination, 236

Glycoproteins, 43

Goat's milk, 127

Gooch's boric acid method, 830

Görter caffeine method, 384

Graham flour, 3 II

Grain, moisture in, 278

Grape juice, 947

Grape sugar, standard, 574

Grav's method for water in butter, 532

Green colors, $785,786,787,794,812$

Groats, 312

Gruyère cheese, 202

Guinea green, 816

Gums, 89

Gunning-Arnold nitrogen method, 432

Gunning nitrogen methods, $69,7 \mathrm{I}$

Gutzeit arsenic test, 76

Habit-forming drugs, 955

Hamoglobins, 43

Hæmolysis test for saponin, 957

Halphen cottonseed oil test, 518

Hansen and Johnson tin method, 917

Hanus' iodine absorption method, $49 \mathrm{r}$

Hefelmann's Bombay mace test, 467

Hehner and Richmond's milk formula, ${ }_{5} \mathrm{I}$

Hehner's method for insoluble fatty acids, 486

Heidenhain's tartaric acid method, 340

Hemicellulose, 285,296

Hess and Prescott vanillin and coumarin method, 865

Hetero-albumose, 44, 45

Hiltner's citral method, 877

Hilyer's benzoic acid method, 838

Histones, 43

Hock wine, 689

Hoffmeister's schälchen, 64

Holstein cows, milk from, 162

Homogenized fats, 191, 194, 199

Honey, 633

adulteration of, 636

American, 634

analysis of, 639

Canadian, 634

composition of, $633,635,636$

dextro-rotatory, 635,636

European, 633 
Honey, gelatin in, 639

glucose in, $637,64 \mathrm{I}, 642$

Hawaiian, 634

invert sugar in, 638,642 methods of analysis, 639

Honeydew, 636, 642

Hoods, I6, 21

Hops, 708

substitute, 710

Hordein, 42

Horseflesh, characteristics of, 234

composition of, 222

detection of, 235, 237

Horseradish, 920, 927 glycogen in, 235

Hortvet method for acids in wine, 701 number, of maple products, 628 of vinegar, 768

and West's benza!dehyde method, 887

rose oil method, 895 spice oil method, 893 wintergreen oil method, 890

Hoskins' electric furnace, 63

Howard microscopic ketchup method, 924 test for gums in ice cream, 201 volatile oil method, 874

Hübl's iodine absorption method, 487

Human milk, 127

Hungarian red pepper, 439, 44I, 442

Hunt's iodine reagent, 492

Hydrocyanic acid, 888, 889

Hydrometer, 55

Hypoxanthin, 211

Ice cream, 198

analytical methods, 199

colors in, 201

cones, 199

detection of thickeners, 200

fat in, 199

gelatine in, $20 \mathrm{r}$

homogenized, 199

preservatives in, 201

standards, 198

starch in, 20r

Imitation coffee, 384

Immersion refractometer, 100, III

adjustment of scale, $\mathrm{II}_{3}$

distilled water readings on, $\mathrm{II}_{3}$

investigation of small quantities of solutions by, 115

of solutions excluded from air by, 115

milk examination by, 166
Immersion refractometer, scale readings compared with $n_{D}, 116$ solutions standardized by, 120

references on, 122

temperature corrections for, 121

Incinerator, 173

Indicators, 38

Indices of refraction, 105,116

Indigo, $792,8 \mathrm{I}_{2}$

carmine, 794, 802, $812,815,816$, 817

disulphosacid, $794,8 \mathrm{r} 2$

Indigotine, 794,802

Indol, 92

Indophenol, 802

Indulin, $802,8 \mathrm{I}_{2}$

Infants' foods, 354

classification of, 355

cold water extract of, 360

composition of, 356

methods of analysis, 350

microscopical examination of, 360

preparation of, 355

Inosite, 276

Inspection of foods, $3,5,6,9$

flour, 316

liquors, 655

Inulin, 276

milk, 159

Invalids' foods, 354. See also Infants'

Foods.

Inversion, 588

Invert sugar, 589

detection of, $580,625,642$

determination of, 589,598

in honey, 638,642

Iodeosin, 794

Iodine absorption of oils, $487,491,492$

Iodine in potassium iodide, 91

Irish whiskey, 732, 734, 735

Iams, 930

acids in, 94I, 942

adulteration of, 93 I, 934

agar agar in, 943

apple stock in, 943

coagulator in, 934

coloring matter in, 942

composition of, $932,933,936$

compound, 934

dextrin in, 940

fruit tissues in, 944

gelatin in, 943

glucose in, 940

methods of analysis, 936 
Jams, organic acids in, $94 \mathrm{r}$ polarization of, 938 preservatives in, 942 starch in, 943 sugars in, 938

Jellies, see Jams.

Johnson extractor, 65

Johnson-Chittenden-Gautier arsenic method, 74

Juckenack's lecithin phosphoric acid method, 349

Kenrick's tartaric acid method, 340

Kephir, 159

Keratins, 42

Ketchup, 919

citric acid in, 920, 923

colors in, 921

decayed material in, 920

foreign pulp in, 921

lactic acid in, 920, 923

manufacture, 919

methods of analysis, 921

microscopy of, 924,925

organisms in, 924

preservatives in, $92 \mathrm{I}$

refuse in, 920

standards, 919

Kjeldahl nitrogen method, 72

Knorr's carbon dioxide apparatus, 338

Koelner's baking test, 318

Koettstorfer's saponification method, 486

König and Karach's method for distinguishing honeydew and glucose, 642

Koumis, $15^{8}$

Kröber's table for pentosans and pentoses, 288

Laboratory benches, 15

drains, 17

$$
\text { stain for, } 16
$$

equipment, 14,15 references on, 38

floor, 15

hoods, 16

lighting, 15

location, 14

sinks, 17

ventilation, 15

Lactalbumin, 125

Lactated infants' foods, 356

Lactic acid in ketchup, 923

Lactoglobulin, 125

in tomatoes, 920

Lactometer, 131

Lactoscope, 163
Lactose, 125,577

Defren's table for, 595

detection of, 625

determination of, $593,594,598,626$

in milk, $126,127,147$

Munson and Walker's table for, 599

Soxhlet's table for, 152

Lager beer, 708

Lamb, composition of, 215

cuts of, 215

Landwehr's glycogen method, 236

Lard, 554

adulteration of, 556

back, 554

composition of, 554

composition of as affected by feeding, - 560

"compound," 556

constants of, 555

iodine number, 559

kettle-rendered, 554

leaf, 554

microscopical examination of, 557

neutral, 554

oil, 555

references on, 563

standards, 556

stearin, $\mathbf{5 5 5}$

substitutes, 559

Laurent's saccharimeter, 583

Law, food and drugs, 965

meat inspection, 960

La Wall and Bradshaw benzoic acid method, 835

Leach and Lythgoe method for malic value in maple products, 627

methyl alcohol method, 749

Lead chromate, 647, 784, 793 number, maple products, 628

$$
\text { vinegar, } 768
$$

salts, of, 904,908 determination of, $91_{3}, 914,918$

Leavening materials, 327,332

Lecitalbumin, 43 references on, 364

Lecithin, 46

determination of, 265,349

nucleovitellin, 43

Lecithoproteins, 43

Leffmann and Beam's method for volatile fatty acids; 482

fat method, 49

Legumelin, $4 \mathrm{I}$

Legumes, 272

$$
\text { ash of, } 302
$$

Legumin, 42,300 
Lemon extract, 870

adulteration of, 871

alcohol in, 875

aldehydes in, 875

citral in, 877

citric acid in, $879^{\circ}$

colors in, 878

composition of, 870

lemon oil in, 871,872

methods of analysis, 872

methyl alcohol in, 878

standard for, 870

tartaric acid in, 879

terpeneless, $87 \mathrm{I}$

oil, terpeneless, $87 \mathrm{r}$

Lemongrass oil, $872,880,88 \mathrm{I}$

Lemon juice, 948

Lemon oil, $870,871,880$

alcohol in, $88_{3}$

aldehydes in, $88_{3}$

citral in, 882

determination of, 872,873

examination of, 882

pinene in, $88_{3}$

Lentils, 272

soda, 954

Leucosin, 41, 290, 300

Levallois' bromine absorption method, 493

Levulose, 574 determination of, 626,640

Liebig's meat extract, 242

Light green S. F., 794, 812, 815, 816, 81 7

Lighting, 15

Lignin, 94

Lime, determination of, 303

in baking powder, 345

in spices, 410

juice, 947.948

sucrate of, 196

water, in vinegar analysis, 765

Liming of ginger, 446

Limonene, 88I

Liqueurs, 754

analysis of, 755

Liquor inspection, 655

Liquors, alcohol in, 658,715

ash of, 677

distilled, 730

methods of analysis, 745

extract of, 677

fermented, 678

malt, 707

methods of analysis, 714

maited and non-malted, 712

methods of analysis, 657

preservatives in, 677
Liquors, specific gravity of, 657

Lobster, composition of, 256

Logwood, 790, 791

Long fermentation baking test, 319 pepper, 438

Lovibond tintometer, 77

Lowenthal's tannin method, 370

Low wines, 732

Lye treatment of fruit, 945

Lythgoe's sucrose test for milk, 197

Macaroni, 347. See also Pastes.

Macassar mace, 468

Mace, 462,465

adulteration of, 466

Bombay, 467 composition of, 465

Macassar, 468

microscopical structure of, 466

standard, 406

Madeira wine, 687

Magenta, 806, 816

Maize, see Corn.

Malachite green, $8 \mathrm{r}_{2}$

Malaga wine, artificial, 692

Malic acid in cider, 702

in fruit juices, 949

in vinegar, 767

in wine, 702

Malt, 707 value in maple products, 627

extracts, 284,729

liquors, 707. See also Beer.

substitutes, 7 ro

vinegar, 762

Malting, 707

Maltose, 574

detection of, 625

determination of, $594,598,626$

Manganese brown, 810

Maple sap, 570

sugar, 570. See also Maple syrup.

syrup, 570

adulteration of, 572

ash of, 571,572

composition of, $57 \mathrm{I}, 572$

Hortvet number of, 628

lead number of, 628

malic acid value, 627

methods of analysis, 627

moisture in, 627

standards, 572

Maraschino, 754

cherries, 928

Mare's milk, 127 
Marigold, 79r

Marpmann's color method, 239

Marsh arsenic test, 75, 728 test for caramel, 753

Martin's color scheme, 535

"Materna " milk modifier, ${ }_{57}$

Mathewson color method, 814

Maumené thermal test, 494

Mayrhofer's glycogen method, 237

McGill's drying oven, $5^{86}$

Meat, 2 II

ammoniacal nitrogen in, 226

antiseptics in, 220

ash in, 225

bases, $211,222,228,23 \mathrm{I}$

boric acid in, 232

canned, $22 \mathrm{I}$

canning of, 22I

colors in, 238

composition of, $22 \mathrm{t}$

cooking, effect of, 220

corning of, 219

curing of, 219

extracts, 240

acidity of, 253

albumoses in, 250

ash in, 249

composition of, $242,243,247$

creatin in, 244,252

creatinin in, 244,252

fat in, 249

fluid, $241,243,244$

gelatin in, 253

glycerol in, 254

meat bases in, 252

methods of analysis, 246

nitrogen compounds of, 249 , 250

peptones in, $25 \mathrm{I}$

preservatives in, 254

proteoses in, $25^{\circ}$

solid, $24 \mathrm{I}, 242,244$

standards, $24 \mathrm{I}$

xanthin bases in, 253

fat, acidity of, 226

composition of, 226

determination, 226

gelatin determination, 231

glycogen in, 236

inspection, 217

$$
\text { law, } 969
$$

juices, $24 \mathrm{I}, 245,247,248$

manufactured, 218

methods of analysis, 225

mince, 927

nitrates in, 232
Meat, nitrogen determination, 226

nitrogenous bodies, separation of, 228

peptones in, $25 \mathrm{I}$

pickled, 218

powders, 247,248

preservation of, 218

preservatives in, 232

proteins, coagulable, $23 \mathrm{r}$

proteoses in, 231

ptomaines in, 218

refrigeration of, 219

salicylic acid in, 233

salted, 210

smoked, 219

standards of, 218

sulphurous acid in, 231

unwholesome, 218

water in, 225

Melting point, 480

Mercury compounds in colors, 785

Metallic salts in canned goods, toxic effects of, 9 II

determination, $9 I_{4}$

Metanil yellow, 810,816

Metaproteins, 44

Methyl alcohol, detection of, 749,878

Methylene blue, 802,812

orange, 808

violet, $8 \mathrm{I} 2$

Micro-chemical reactions, 94

Micro-polariscope, 84

Microscope in food analysis, $8 \mathrm{I}$

references on, 98

reagents for, 90

stand, 82

Microscopical accessories, 84

analysis, $8 \mathrm{r}$

apparatus, 82

diagnosis, 86

reagents, 90

analytical, 91

clarifying, 92

Microscopy of agar agar, 943

allspice, 422

arowroot, 282

barley, 309

bean, 388

$$
\text { starch, } 28 \mathrm{I}
$$

starch, 282

buckwheat, $3 \mathrm{II}, 437$

starch, $28 \mathrm{r}$

butter, $55^{2}$

cassia, 426

cayenne, 441

cereal products, 305

charlock, 460 
Microscopy of chicory, 386

cinnamon, 426

cloves, $4 \mathrm{I} 6$

cocoa, 403

cocoanut shells, $4 \mathrm{I} 9$

coffee, 386

corn, 309

starch, 28I

date stones, 390

fats, 510

flour, 306,322

fruit tissues, 944

ginger, 449

honey, 633

jams, 944

jellies, 944

ketchup, 724, 725

lard, 557

mace, 466

milk, 124

mustard, $45^{8}$

nutmeg, 464

oats, 309

oat starch, 282

oils, 510

oleomargarine, $55^{2}$

olive stones, 436

paprika, 44I

pea, 388 starch, 282

pepper, black, 433

long, 439

red, $44 \mathrm{r}$

white, 433

potato starch, 282

rice, 3 Io starch, 282

rye, 308 starch, $28 \mathrm{I}$

sago, 283

sawdust, 444

starches, 280

tapioca starch, 282

tea, $37^{8}$

turmeric, 451

wheat, 306 starch, $28 \mathrm{r}$

Micro-technique, 82

Milk, 124

acidity of, 124,153

adulteration of, 159

alkalinity of ash, 198

anilin orange in, 175,177

annatto in, 175,176

ash of, 127,134

ashing of, 134
Milk, ass's, I27

boiled milk, detection, I55

boric acid in, $\mathrm{I} 82$

calcium oxide in, 189

calculation of proteins, $\mathrm{I}_{53}$

caramel in, 176,177

carbonate in, 180,182

chocolate, 397

citric acid in, 127

coloring matter in, $174-177$

composition of, $124^{-1} 26$

constants, 169

ewe's, 127

fat of, 127,134

fermentations of, 129

foods, prepared, 157

fore milk, 128

formaldehyde in, $178,18 \mathrm{I}$

goat's, 127

human, 127

inspection, 159

known purity, : 69

mare's, 127

methods of analysis, I $\mathrm{I}_{3} \mathrm{O}, \mathrm{I} 6_{3}, \mathrm{I} 68$

microscopical appearance, 124

modified, 155

nitrogen compounds in, 125,145

powder, 157

preservatives in, 177

proteins of, $125,145,153$

records of analysis of, $x 72$

references on, 208

ropy, 130

sampler, $13 \mathrm{I}$

serum, refraction of, 166,167 specific gravity of, 166,167

skimmed, 161

sour, analysis of, 186

souring of, 129

standards, 160

strippings, 128

sucrate of lime in, 106 detection, 197,198

sugar, 125,577

determination of, $593,594,598$

determination of, in milk, 147 . I $49, I_{5} 1$

systematic examination of, 130 , 168

total solids in, 133,134 calculation of, $151,153,154$

watering of, $16 \mathrm{I}$

Milliau's cottonseed oil test, 518

Millon's reaction, $4 \mathrm{I}, 9^{2}$

reagent, 92

Mill's bromine absorption method, 493

Mince meat, 927 
Mince meat, adulteration of, 927 condensed, 928 standards, 927

Mineral colors, 792 content of food, 47

Mirbane, oil of, 886,888

Mitchell and Smith fusel oil method, 748

Modified milk, 155

Mohler's test for benzoic acid, 835

Moisture, determination of, $6 \mathrm{I}$

Molasses, $5^{67}$ adulteration of, $62 \mathrm{I}$ ashing of, $6 \mathrm{I}_{4}, 624$ clarifying, 614 composition of, 568 glucose in, 621 invert polarization at $87^{\circ} \mathrm{C}$., 623 methods of analysis, $61_{3}$ standard for, $62 \mathrm{I}$ sucrose in, 614 tin in, 625 total solids in, $6 \mathrm{r}_{3}$

Mollusks, 256 vinegar, 763

Mucoid protein, 127

Munson method for metallic salts, 9 I4

Munson and Walker sugar method, r51, 598

Muscle albumin, 2II table, 599 fibers in meat, $2 \mathrm{II}$ sugar, 212,238

Muscovado, 567,568

Mushroom ketchup, 9 I9

Mustard, 453 adulteration of, 459 ash of, 457 black, 453 cake, 455 charlock in, 459,460 coloring matter in, 460 composition of, 455,456

Dakota, 459

flour, 454

methods of analysis, 457 microscopical structure of, 458 oil, fixed, 454,525 volatile, 453,457 pickles, 926 prepared, 460 adulteration of, 460 composition of, $460,46 \mathrm{I}$ sinalbin in, 454 methods of analysis, $46 \mathrm{I}$ sinapin sulphocyanate, 457 standard, 459
Mustard, starch in, 459 turmeric in, 460 volatile oil of, 453 wheat in, 459 white, 453

Mutton, composition of 215 cuts of, 215 tallow, 529

Myosin, 42 insoluble, 44

Naphthion red, 808

Naphthol green, 80I, 8I2 orange, 794 yellow, 794, 80r, 808

Natural wine, $68_{5}$ S., $794,808,8$ I 6,8 I 7

Neufchâtel cheese, 202

New coccin, 8 I6 green, 812

Nickel salts, $9 I 1$ determination of, 918

Niebel's glycogen method, 236

Nigrosin, 802

Nile blue, 802

Nitrates in food, 40,46 in watered milk, 168

Nitrobenzol, 886,888

Nitrogen apparatus, 72,73 compounds in milk, 145 determination of, 69,73 free extract, 54

Nitrogenous bodies, 40 classification of, 40 separation of, in cheese, 205 in meat, 228

Noodles, 347 in milk, $125,126,145$

Notification, 10

Noyau, 754

Nuclein, 43

Nucleoproteins, 43

Nutmeg, 462, 463 adulteration of, 464 composition of, 462,463 extract, standards, $88 \mathrm{I}$

Macassar, 465 microscopical structure of, 464 oil of, 463 standard, 892 standard, 464

Nutrose, $15^{8}$

Nuts, composition of, 275

Oats, $27 \mathrm{I}$

analysis of, 271,272 
Oats, ash of, 302

microscopic structure, 309

starch in, 282

Oil cakes, effects on butter of feeding, 531

anise, 802

lard of feeding, 560

basil, 893

bitter almond, $884,88_{5}, 886$

calorimeter, 495

cassia, 425,802

celery seed, 892

cinnamon, 892 .

cloves, 892

cocoanut, 528

corn, 521

cottonseed, 516

ginger, 446

lard, 555

lemon, $870,871,880$

terpeneless, 871

lemongrass, $872,880,88 \mathrm{I}$

majoram, 803

mustard, fixed, 454,525

nutmeg, 892 volatile, 453,457

oleo, 541

olive, 511

orange, 884

peanut, 522

peppermint, 890

poppyseed, 526

rape, 520

rose, 895

rosin, 527

savory, 892

sesame, 519

spearmint, $89 \mathrm{I}$

staranise, 893

sunflower, $5^{26}$

thyme, 893

wintergreen, 880

Oils, edible, 471 . See also Fats.

acetyl value, 497

bromine absorption of, 492

bromination test, 494

cholesterol in, 502, 503, 507

composition of, 471,472

constants of, 508,509

elaĩdin test, 499

fatty acids in, 484,499

iodine absorption of, 487,492

judgment as to purity of, 473

Maumené test, 494

melting point, 480

methods of analysis, 473

microscopical examination, 510
Oils, edible, phytosterol in, 502, 503, 507

Polenske number of, $48_{3}$

rancidity of, $473,53 \circ$

references on, 561

refractive index of, 477

Reichert-Meissl number, $48 \mathrm{I}$

saponification of, $472,484,486$

sitosterol in, $5^{22}$

specific gravity of, 474

factors, 475

thermal tests, 493

titer test, 500

unsaponifiable matter in, 501

Valenta test, 499

Oleomargarine, 541 viscosity of, 477

adulteration of, 543

coloring of, 542

constants of, 544

distinction from butter, 544,546

healthfulness of, 543

manufacture of, $54 \mathrm{I}$

microscopical examination, $55^{2}$

odor and taste, 545

palm oil in, 542

Zega's test for, 553

Oleo oil, 54 I

Olive, composition, $5 \mathrm{II}$

oil, $5^{12}$

adulteration of, 512,515

examination of, 515

refraction of, $5^{14}$

standard, 513

Olives, pickled, 920,926

Olive stones, 436

Orange colors, $787,794,808,810,815,816,817$

extract, 884

oil, 884

soda, 954

standards, 884

terpeneless, 884

Orchil, 791, 808 substitute, 808

O'Sullivan-Defren sugar method, 150

Ovalbumin, 262

Oven, drying, 22 McGill's, 586

Ovomucin, 262

Ovomucoid, 263

Oxygen absorbed, 4I5 equivalent, 415

Oxyhremoglobin, 43

Oysters, 257

Palas rapeseed oil test, 521

Paprika, 439 
Paprika, added oil in, 445

adulteration of, 444,445

composition of, 442

methods of analysis, 445

microscopical structure of, $44 \mathrm{I}$

Paraffin in beeswax, 643

in confectionery, 647

in fats, 510

in oleomargarine, 543

Paranuclein, 206

Parenchyma, 87

Pastes, adulteration of, 349

artificial colors in, 349

edible, 347

Italian, 347

lecithin phosphoric acid in, 349

methods of analysis, 349

noodles, 347

Patrick's method for water in butter, 531 test for thickeners in ice cream, 200

Paul method for foreign fats, $19 \mathrm{r}$

Pea, composition, 272

proteins of, 300

starch of, 282

Peanut oil, $5^{22}$

adulteration of, 523

standards for, 522

tests for, $523,5^{25}$

Pear cider, 683

essence, imitation, 896,897

Pectose. 93,276

Pekar's color test of flour, 317

Pentosans, 285,296

determination of, 285,296

in cocoa products, 396

table for, 288

Pentose, 285,296

Pepper, 428

adulteration of, 435

black, 429

buckwheat in, 437

composition of, 430,432

dust, 436

ether extract in, 410

long, 438

microscopical structure of, 433

nitrogen in, 432

in ether extract, 433

olive stones in, 436

piperin in, 429

determination of, 433

red, see Cayenne and Paprika.

shells, 435

standard, 435

varieties of, 429

white, 429
Peppermint extract, 890

composition of, $89 \mathrm{r}$

standards, 891

oil, 891

Peptides, 45

Peptones, 44

in cheese, 202

in meat, $21 \mathrm{I}, 23 \mathrm{I}$

in milk, 146

Peter's test for benzoic acid, 835

Perry, 683

Persian berries, 790,810

Petroleum ether, 60

Phloroglucide, 286

Phloroglucinol, 287

Phloxin, 806

Phosphate baking powders, 333

Phosphin, 803

Phosphoproteins, 43

Phosphoric acid in baking chemicals, 346 in beer, 725

Phosphotungstic acid reaction, 45

Photomicrography, 93

Phytolacca, 790 camera for, 96

Phytosterol, 502 acetate test, 507 crystallization of, 503

determination of, 503

distinction from cholesterol, $5 \circ 3$

Piccalilli, 926 separation of, 503

Pickled meats, 218

Pickles, 925 adulteration of, 926

Pickling pump, 219

Pirric acid, 350,808

Pie filling, 928

Pimiento, 439, 442

Pineapple essence, imitation, 896,897

Pioscope, 164

Piperin, 429 determination of, 433

Piutti an 1 Bentivoglio's method for colors in pastes, $35 \mathrm{I}$

Plant crystals, 90

Plasmon, $15^{8}$

Plastering, of wine, 629

Platinum dishes, $61,133,134,170$ counterweights for, 170

Poisoned foods, 74

Poivrette, 436

Polariscope, 578 . See also Saccharimeter. micro, 84

Polariscope tube jacketed, 639 short, for oils, 880 
Polarization at high temperature, 639 of essential oils, 880 honey, 630 jams and jellies, 938 lemon extract, 873 molasses, 614 orange extract, 884 sugar, 578 vinegar, 769

Polenske number, 483 wine, 694,703

Ponceau, 794, 806, 810, 81 5, 816, 81 7

Poppyseed, 526

$$
\text { oil, } 526
$$

Pork, composition of, 216 cuts of, 216

Porter, 709, 712 . See also Beer.

Port wine, 689

Potash determination, 304,345

Potassium myronate, 453,457

Potatoes, composition of, 273 proteins of, 3 Or
starch of, 282

Poultry, composition of, 216

Pratt citric acid method, 95I

Preparation of sample, 55

Preservatives, 821

commercial food, 823

in butter, $53^{8}$

in canned goods, 912

in carbonated beverages, 955

in fish, 257

in fruit juices, 947

in jams and jellies, 942

in ketchup, 921

in meats, 220,232

in milk, 177,183

in preserves, 928

of eggs, 266,268

references on, 846

Preserves, 927

regulation of, 822

Pressure pump, 20

Price color method, 814

Primulin, $80_{3}$ orange, 8 io

Process butter, 540

Prolamins, 42

Proof spirit, 677

Prosecution, 10

Protamins, 43

Proteans, 44

Protein grains, $9 \circ$

Proteins, 40 coagulated, 44 conjugated, 43
Proteins, derived, 44

factor for. 40

of barley, 277,300

of beer, 725

of cereals, 296

of condensed milk, 190

of eggs, 262

of milk, 125 calculation of, 153 determination of, 145

of peas, 300

of potatoes, 3 or

of rye, 277,300

of wheat, 277,298

secondary derivatives, 44

simple, $4 \mathrm{I}$

tests for, $4 \mathrm{I}$

Proteolytic fermentation, 158,202

Proteoses, 44, 297

Proto-albumose, 44, 45

Proximate analysis, extent of, 53 expression of resuits of. 53

Prussian blue, 792,812

Ptomaines, 218 in tea, 375

Publication of adulterated foods, ro

Pulfrich refractometer, 100

Pycnometer, 57

Pyroligneous acid, 764

Pyronin, 803

in meats, 219

Pyrosin, 794

Quassiin, 727

Quercetin, 804

Quercitannic acid, 4I5

Quercitron bark, 790, 8 10

Quevenne's lactometer, $\mathrm{I}_{32}$

Quince essence, imitation, 896,897

Quinolin yellow, $80_{3}$

Quotient of purity of sugar, $5^{86}$

Raffinose, 279, 577 determination of, 620

Rancidity, 473, 530

Rape oil, 520 test for, $52 \mathrm{I}$

seed, 520

Raphides, 90

Raspberry (color), 790

Reagents, $35,9 \circ$ soda, 954 references on, 38 table of, $26-34$

Red colors, $785,786,790,794,806$ 
Red ochre in sausages, 238

Red pepper, see Cayenne and Paprika.

Red wines, 684,689

Red wood, 444

References on beer, 756

butter, 562

canned goods, $96 \mathrm{I}$

cereals, $36 \mathrm{I}$

cocoa, 406

coffee, 406

colors, 8 ig

dietetics, 49

distilled liquors, $75^{8}$

eggs, 270

flavoring extracts, 808

flesh foods, 258

food economy, 49 inspection, II

fruit products, $96 \mathrm{r}$

fruits, 361

general analytical methods, 79

laboratory equipment, 38

leavening materials, 364

liquors, 756

microscope, 98

milk, 208

oils, $5^{61}$

preservatives, 846

reagents, 38

refractometer, 122

spices, 468

sugars, 650

sweeteners, 855

tea, 406

vegetable products, 961

vinegar, 780

wine, 757

Refractometer, 100

\section{Abbé, 100}

Amagat and Jean, 100

butyro, 100, 10I

heater for, 102

immersion, III

in oil analysis, 477

Pulfrich, 100

sliding scale for, 107

tables for, $104,105,113,116$, I20, 121

Wollny, 100,139

Reichert-Meissl method, $48 \mathrm{I}$

Reichert number of butter, 549

Reinsch's test for arsenic, 728

Relishes, 920 , 926

Renard's test for peanut oil, 523

for rosin oil, 527

Renovated butter, $54^{\circ}$
Renovated butter, distinction from' butter and oleomargarine, 546

Resins, 89

Resorcin brown, $8 \mathrm{I} 6$

green, 812

yellow, 8I6

Respiration calorimeter, 2

Rhodamin, 803

Rice, composition of, 272

microscopical structure of, 310

polished, 272

starch, 282

Riche and Bardy methyl alcohol method $75 \mathrm{I}$

Richmond's cane sugar method, 185

sliding milk scale, 153

Ritsert's tests for acetanilide, 869

Ritthausen's method for milk proteins, 145

Roese-Gottlieb fat method, 190,199

Roeser's mustard oil method, 457

Röhrig tube, 199

Root beer, 954

Ropy milk, ${ }_{3} 30$

Roquefort cheese, 202

Rose, attar of, 895

Bengal, 806

extract, 895

standards, 895

rose oil in, 895

Rosin oil, 527

Rota's color scheme, 799

Rubner's fuel value factors, 48

Rühle-Brummer saponin method, 956

Rum, 742

composition of, 742

essence, 743

methods of analysis, 745

new, 743

standards, 742

Rye, composition of, $27 \mathrm{I}$

microscopical structure of, 308

proteins of, 300

starch, 281

Saccharimeter, 578

double wedge, 581

forms of, $5^{8} 3$

normal weights for, $5^{8} 3$

scales compared, $5^{8} 3$

single wedge, 579

Soleil-Ventzke, 578

Saccharimetry, 578

triple field, $5^{81}$

Saccharin, 850

$$
\begin{aligned}
& \text { detection of, } 85 \mathrm{r} \\
& \text { determination of, } 852
\end{aligned}
$$

Saccharine products, 565 
Saccharoses, 565

Safflower, 791, 808

Saffron, 791

Safranin, 802, 806

Sago, $28_{3}$

Saleratus, 332

Salicylic acid, $83 \mathrm{I}$

detection of, 831

determination of, 832

in meat, 233

Salmin, 43

in milk, 180

Salted meats, 218

Sample, preparation, 55

Sanatogen, 158

Sanger arsenic method, 75

Black-Gutzeit method, 76

Sanose, 158

Saponification, $472,484,486$

Saponin, 955

detection, 956

tests for, 957

Sarcolemma, $2 \mathrm{II}$

Sarsaparilla, 954

Sausages, 223

ash of, 225

color of, 224

composition of, 223

fat in, 226

glycogen in, 234

horseflesh in, 234

method; of analysis, 225

starch in, 223

water in, 225

Sauterne wine, 185,688

Savory extract, standards, 892 oil, standards, 892

Sawdust, $45^{\circ}$

Scarlet $6 \mathrm{R}, 8 \mathrm{I} 6$

Schiedam schnapps, 744

Schenk beer, 708

Schlegel's method for colors in pastes, $35^{\circ}$

Schreiner's colorimeter, 77

Schultze's reagent, 93

Sclerenchyma, 87

Scovell sampling tube, $\mathrm{I}_{31}$

Sealed samples, 6, 159

Semolina, 347

Separatory funnel support, 68

Sericin, 42

Sesame oil, 518

aduiteration of, $5^{19}$

tests for, 510

seeds, 518

Shannon formic acid method, 842

Sherry wine, 687
Short's method for fat in cheese, 205

Shredded wheat, 352

Sieve tubes, 89

Silent spirit, 731

Sinabaldi's asaprol method 846

Sinalbin, 545

Sinigrin, 453

$$
\text { mustard oil, } 454
$$

Sinks, 17

Sitosterol, 522

Smith and Bartlett tin method, 916

Smoked meats, 218

"Soaked" goods, 912

Soap-bark, 955

Soda, cherry, 954 determination of, 304,345

lemon, 054

orange, 954

raspberry, 954

strawberry, 954

vanilla, 054

water, $95^{2}$ syrups, 953

Sodium benzoate, 833 bicarbonate, 332

bisulphite, 839

carbonate, in milk, 180,182

hydroxide, tenth-normal solution, 35

salicylate, $8_{31}$

Soja bean meal, 357

Soleil-Ventzke saccharimeter, 578

Solid yellow, 8 or

Sorghum, 573

Sostegni and Carpentieri's test, 796

Souring of milk, 129

Sour milk, I39

Soxhlet, extractor, 64

Soxhlet's milk sugar method, 150,152

Spaghetti, 347. See also Pastes.

Sparkling wine, $685,69 \mathrm{r}$

Spearmint, extract, 891

oil, 89 I

Specific gravity bottle, 57

of beeswax, 643

of liquids, 55

of liquors, 657

of milk, $13 \mathrm{I}$

of milk serum. 166

temperature correc-

tion for, 133

of oils, 474

of vinegar, 764

rotary power, 584

Spent tea leaves, 375

Spices, 408 
Spices, adulterants of, $4 \mathrm{I} 3$ alcohol extract of, 410 ash of, 400 crude fiber of, 4 II ether extract of, 4 IO lime in, 410 methods of analysis, 408 microscopical examination of, $4 \mathrm{I}^{2} 2$ nitrogen in, 410 references on, 468 starch in, $4 \mathrm{II}$ volatile oil of, $4 \mathrm{II}$

Spiral ducts, 89

Spirits, cologne, $73 \mathrm{I}$ distilled, 730 neutral, 731 silent, 731 standards, 730 velvet, $73 \mathrm{I}$

Spirit vinegar, 760,763

Spoon test for butter, 549 Sprengel tube, 60

Stahlschmidt's caffeine method, 374

Standards for allspice, 424 anise extract, 892 beer, 7 II oil, 982

brandy, 740

butter, 535

cassia, 428 extract, 892 oil, 892

cayenne, 443 celery seed extract, 892 oil, 892

cheese, 203

cinnamon, 428 extract, 892 oil, 892

clove extract, 892 oil, 892

cloves, 418

cocoa, 402

cream, 195

foods, 4

fruit butter, 927

ginger, $45 \circ$

ice cream, 198

$$
\text { extract, } 892
$$

ketchups, 9 I9

lard, 556

lemon extract, 870

mace, 460

$$
\text { oil, } 871
$$

maple products, 572

meats, 218
Standards for meat extracts, $24 \mathrm{I}$

milk, 160,162

mince meat, 927

molasses, 621

mustard, 459

nutmeg, 464 extract, 892

olive oil, $5{ }^{1} 3$ oil, 892

pepper, 435

renovated butter, $54 \mathrm{I}$

rum, 742

savory extract, 892 oil, 892

staranise extract, 893 oil, 893

starch sugar, 574

sugars, $566,574,772$

sweet basil extract, 893 oil, 893

marjoram extract, 893 oil, 893

thyme extract, 893 oil, 893

vanilla extract, 862

vinegar, 772

wine, 689

whiskey, 733

Standard solutions, equivalents of, 36 refractometric readings of, 120

Staranise extract, standards, 893 oil, standards, 893

Starch, $47,89,279$

arrowroot, 282

barley, $28 \mathrm{x}$

bean, 282

buckwheat, $28 \mathrm{I}$

classification of, 280

corn, $28 \mathrm{r}$

detection of, 279,943

determination of, 283

by acid conversion, 283

by diastase method, 283

in baking powder, 343

in cereals, 283,296

in jams and jellies, 943

in milk, 185

in sausages, 233

in spices, $4 \mathrm{II}$

oat, 282

pea, 282

potato, 282

rice, 282

rye, $28 \mathrm{r}$

sago, 283 
Starch, syrup, 575

tapioca, 282

under polarized light, 283

wheat, $28 \mathrm{I}$

Stearin, beef, 541 cottonseed, 517

lard, 555

-Sterilized butter, 540

Still, alcohol, 659

fractionating, 67

nitrogen, 73

water, 22

wine, 685

Stilton cheese, 202

Stokes' milk centrifuge, ${ }_{13} 6$

Stone's method of carbohydrate separation, 295

Storch's method for boiled milk, I 55 mucoid protein, 127

Stout, 709, 712. See also Beer.

Strawberry soda, 954

Strippings, 128

Stutzer's gelatin method, $23 \mathrm{I}$

Suberin, 89

Sucrate of lime, 195, 197

Sucrose, see Cane sugar.

Suction pump, 19

Sudan I, 80I

Suet, 529

Sugar, 561

beet, 569

brown, composition of ash, 567

cane, 566,567

classification of, 565

composition of, 568

grape, see Dextrose.

in fruits, 566

in jams, 938

maple, see Maple syrup.

methods of analysis, $5^{8} 5$

muscovado, 567

organic non-sugars in, $5^{86}$

quotient of purity, 586

raw, 568,569

references on, $65^{\circ}$

refining, 570

standards, $5^{66,}, 572,574$

ultramarine in, 570,590

Sulphur, dete mination of, 305

Sulphuric acid in baking chemicals, 346

Sulphuring, 839 in vinegar, 767

Sulphurous acid, 839

$$
\text { of fruits, } 945
$$

detection of, 840

determination of, 840
Sulphurous acid, in meat, 220,232

Sunflower oil, 526 seeds, 527

Sweet basil extract, standards, 893 oil, standards, 893

Sweeteners, artificial, $85^{\circ}$

Sweet marjoram extract, standards, 893

Sweet wine, 685,690 oil, standards, 893

Syrup, analysis of, 613 ashing of, $6 \mathrm{r} 4$ maple, see Maple syrup.

mixing, 576

starch, 576

total solids in, $6 \mathrm{r}_{3}$

Syrups, fruit, 952 soda water, 953

Sy's lead method, 630

Table sauces, $9 \mathrm{r} 9,920$

Tallow, 529 preservatives in, 921

Tannin in cloves, 415

in tea, 370

Tapoica, 282 in wine, 704

Tartaric acid in baking powder, 339,340 in fruit products, $94 \mathrm{I}, 949$

Tartrate baking powders, 332

Tartrazin, 816

Tea, 365

adulteration of, 374

ash of, 368,369

astringents in, 377

caffeine in, 372,373

composition of, 366,367

exhaustive leaves in, 375

extract of, 370

facing of, 374 .

foreign leaves in, 376

leaf, characetristics of, 376

methods of analysis, 368

microscopical examination of, 378

references on, 406

spent leaves in, 375

stems in, 376

tablets, 377

tannin in, 370

theine in, 372,373

Technique, 82

Teller's method of separating wheat proteins, 298

Theine, 372

Theobromine, 396,400

Thompson's boric acid method, 829

Thyme extract, standards, 893 
Thyme oil, standards, 893

Tin, action of fruits and vegetables on, 904 , 905,906

determination of, 914,916

salts in molasses, 625

Tintometer, Lovibond, 78

Titer test, 500

Tocher's sesame oil test, 5 Io

Tomato ketchup, see Ketchup.

Tonka bean, 860 tincture, 862

Trillat methyl alcohol test, $75^{\circ}$

Tropæolin, 794, 808, 810

Turmeric, $450,790,810$ as an adulterant, 452 microscopical structure of, $45 \mathrm{I}$ tests for, $453,79 \mathrm{r}$

Ultramarine blue, $793,8{ }_{12}$

Uno beer, 714

in sugar, 570,590

in tea, 375

Unsaponifiable matter, 501

Vacuoles in yeast cells, 330

Vanilla bean, $8_{57}, 858$ exhausted, 859

extract, 857 acetanilide in, 868 adulteration of, 862 alcohol in, 869 alkali in, 860 artificial, 863 caramel in, 869 color value of, 870 composition of, 859 coumarin in, $86_{3}, 865$

glucose in, 869 glycerin in, 869 lead number of, 867 methods of analysis, 864 prune juice in, $86_{3}$ resins in, 864

standards, 862

tannin in, 865

tonka in, $86_{3}$ vanillin in, $863,865,870$ soda, 954

Vanillin, 859

determination, 865

microscopical structure, 867

Van Slyke's protein formula, 153 method of nitrogen separation in cheese, 205,206 in milk, 146

Vaporimeter, 675
Veal, composition of, 2 I 4 cuts of, 214

Vegetable colors, 789

Vegetable colors in sausages, 239

Vegetables, 273 ash of, 302
composition of, 273
methods of proximate analy-
sis of, 276
references on, $36 \mathrm{r}$

Ventilation, $\mathrm{I}_{5}$

Vermicelli, 347. See also Pastes.

Vessels, 89

Vesuvine, 809

Victoria yellow, $352,801,808,8$ ro

Villivecchia and Fabris' sesame oil test, 520

Vinegar, 759 acidity of, 765 acids of, 766 adulterated, 778 adulteration of, 772 alcohol in, 766 apple, 773 arsenic in, 780 artificial, 774 ash of, $761,764,775$ solubility and alkalinity of, 764 beer, 762

caramel in, 779

cider, 760,773 artificial, 774 composition of, 760 copper in, 780

distilled, $76_{3}, 773$

extract of, 764

furfural in, 779

g!ucose, 763,773

glycerine in, 770

grain, 773

Hortvet number of, 768

hydrochloric acid in, 767

lead in, 779 acetate, test for, 768, 779 number of, 768

malic acid in, 767

malt, 762,773

manufacture of, 760

metallic impurities in, 779

methods of analysis, 764

mineral acids in, 766,767

molasses, $76_{3}$

nitrogen in, 765

pentosans in, 770

phosphoric acid in, 764

polarization of, 769,776

reducing sugars in, 770 
Vinegar, references on, 780

residue of, 774

specific gravity of, 764

spices in, 779

spirit, 773

standards, 772

sugar, 773

sugars in, 769,776

sulphuric acid in, 767

tartrate in, 769

tests on, 779

varieties of, 759

volatile acids of, 766

wine, 761,773

wood, 764,779

zinc in, 779

Vinous fermentation, 654

Violamin, 803

Viscogen, 196

Viscosity of cream, 195

of oils, 477

Vitellin, 43

Waage's Bombay mace test, 468

Walnut ketchup, grg

Water-bath, 2I

Water glass, 266

Waterhouse butter test, $55^{\circ}$

Weiss beer, 709

Weld, 8 ro

Werner-Schmidt method for fat in cheese,

Westphal balance, 56

West's benzoic acid method, 838

Wheat, 271,272

ash of, 302

composition of, 271,272

microscopic structure of, 306

proteins of, 277,298

shredded, 352

starch, $28 \mathrm{I}$

Whiskey, 731. See also Distilled liquors. adulteration of, 738

aging of, 732

American, 735

Bourbon, 732, 734, 736, 737

British, 735

composition of, 734

imitation, 738

Irish, 732, 734, 735

manufacture of, $73 \mathrm{I}$

methods of analysis, 745

rye, $732,734,737$

Scotch, $732,734,735$
Whiskey, standards, 733, 734

Wijs's iodine absorption method, 492

Wild's saccharimeter, 583

Wiley's bromine pipette, 495

Wiley and Ewell's double dilution sugar method, 149

Wine, 684 acidity of, 696 added alcohol in, 695 adulteration of, $69 \mathrm{r}$ ameliorated, 69I Burgundy, artificial, 692

California, 688

cane sugar in, 693

Cazeneuve's color method, 705

chaptalizing, 693

claret, 687 artificial. 602

classification of, 685

coloring matter in, 704, 705

composition of, 686

corrected, $69 \mathrm{I}$

cream of tartar in, 702

"dry," 600

Dupré's color method, 705

extract in, 696, 697

fortified, 685,600

fruit other than grape, 695

glycerin in, 703

hocks, 689

Madeira, 685,686

Malaga, artificial, 692

malic acid in, 702

manufacture of, 684

methods of analysis, 696

modified, $69 \mathrm{t}$

natural, 685

non-volatile acids in, 701

plastering, 602

polarization of, 703

port, 689

potassium sulphate in, 704

raisin, $69 \mathrm{I}$

red, 684,689

reducing sugar in, 703

references on, 757

sherry, 687 artificial, 692

sparkling, $685,69 \mathrm{I}$

standards, 689

still, 685

sweet, 690

tannin in, 704

tartaric acid in, 7or

varieties of, 687

vinegar, 761,773 
Wine, volatile acids in, 696 watering of, 694 white, 684,689 yeast of, $68_{4}$

Wintergreen extract, 889 oil of, 880 adulteration of, 890 wintergreen oil in, $89 \circ$.

Winton lead number, 628,768

moisture apparatus, 62

Wollny milk fat refractometer, 100, 139

tables for using, $14 \mathrm{I}$

table for converting Wollny degrees into $n_{D}, \mathrm{r}_{4} 3$

Woodman and Davis benzaldehyde method, 929

and Taylor's caffetannic acid method, 383

Wood vinegar, 764,777

Wool, double dyeing method with, 796 dyeing of, 795

for color tests, 795

vegetable colors on, 797

Wormy fruit, 945
Xanthin, $46,21 \mathrm{I}$

Xantho-proteic reaction, $4 \mathrm{I}$

Xylan, $285,288,296$

Xylose, $285,288,296$

Yeast, 327

adulteration of, $33 \mathrm{r}$

composition of, 329

compressed, 328

dry, 328

in cider, 678

in wine, 684

microscopical examination of, 329

starch in, 331

testing, $33^{\circ}$

vacuoles in, 330

Yeast extracts, 246

Yellow colors, $785,787,790,794,808$

Zega's test for oleomargine, 553

Zein, 42, 300

Zinc salts, 909

determination of, 914 


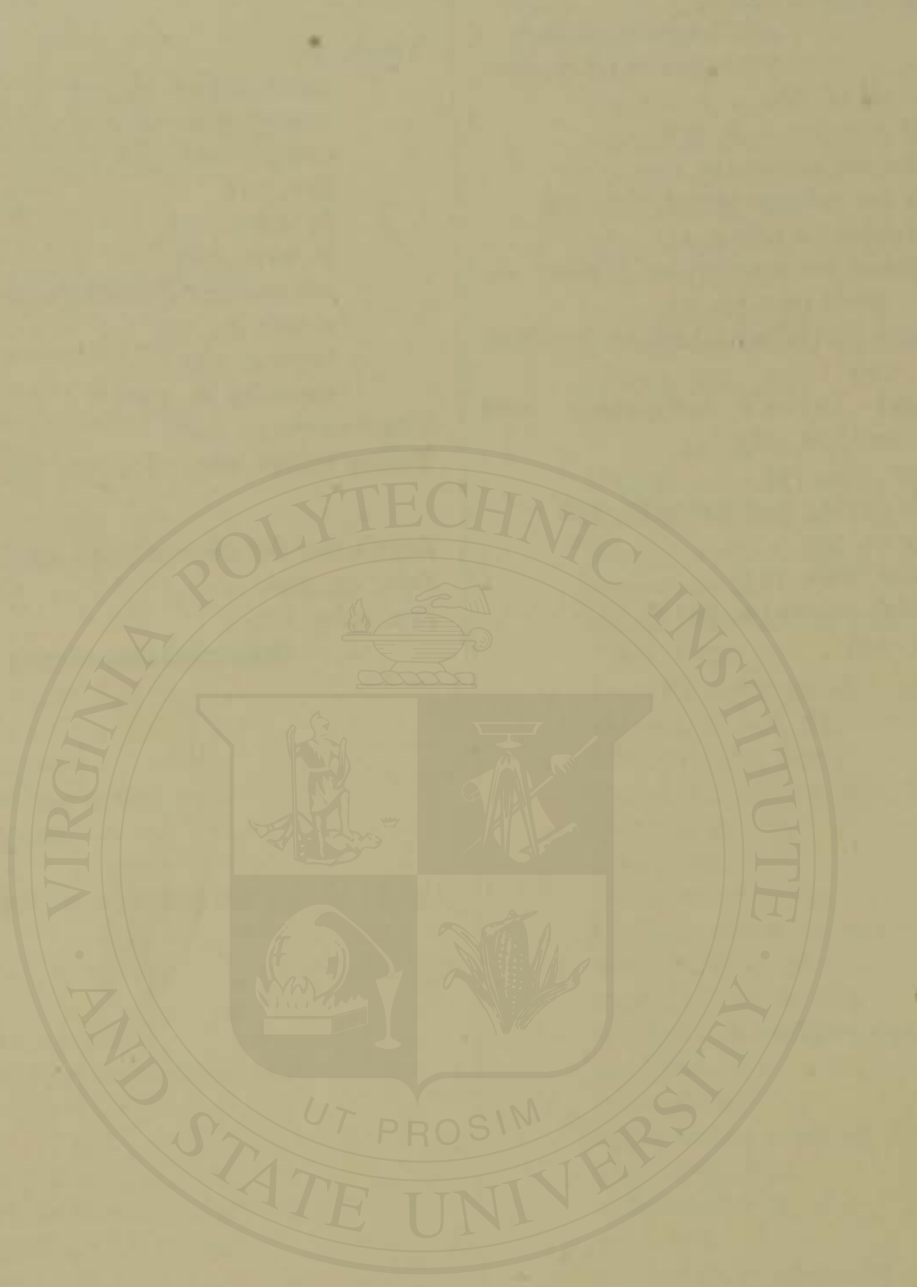


PLATE I.

\section{CEREALS.}

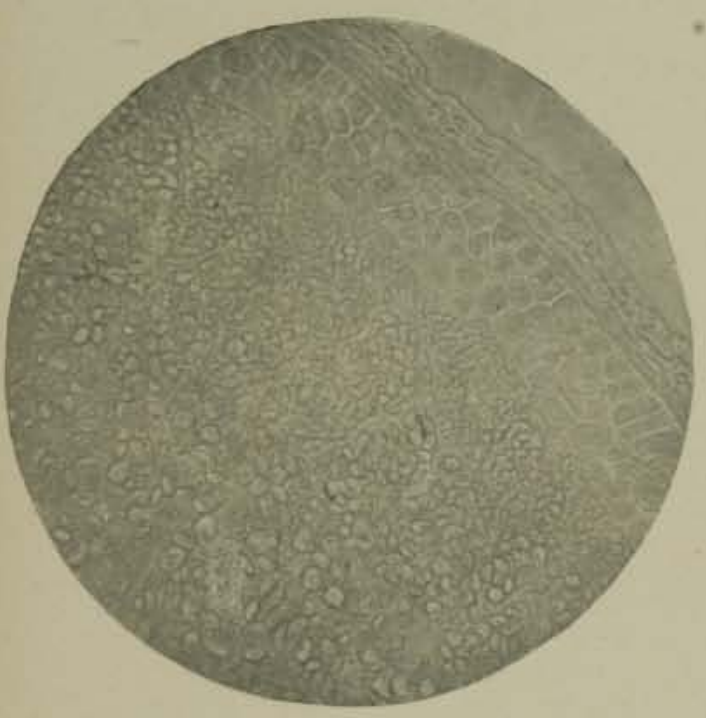

Frs, 121.-Barley, X110.

Transverse section, showing in order, pericarp, seed coats, aleurone layer, and starch cells.

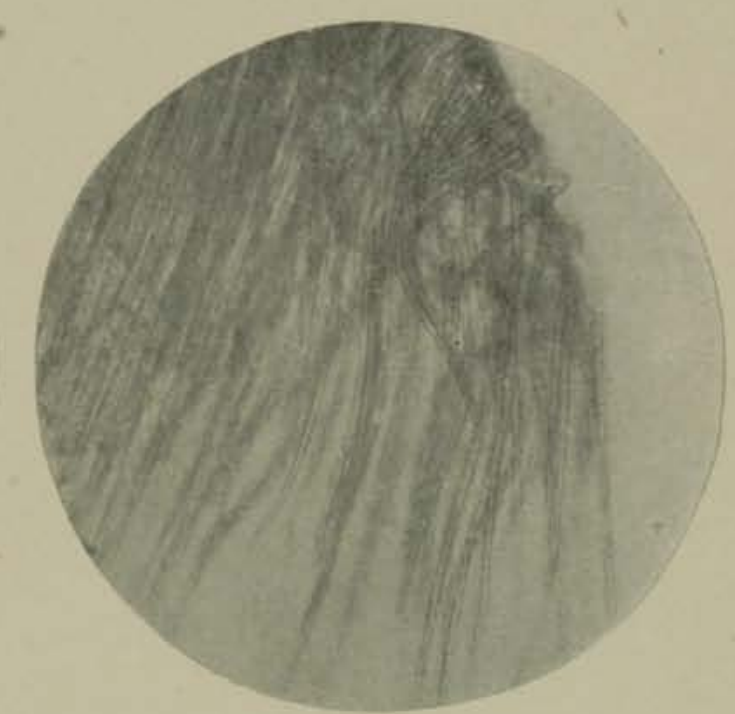

Fig. I22,-Barley, $\times 55$.

Surface view of epidermis with hairs.

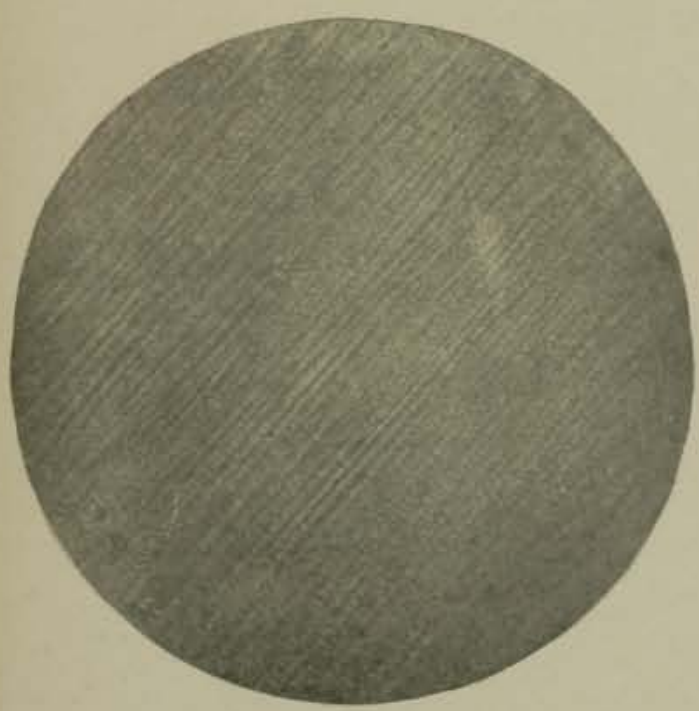

Fig, 123,-Barley, $X_{125}$.

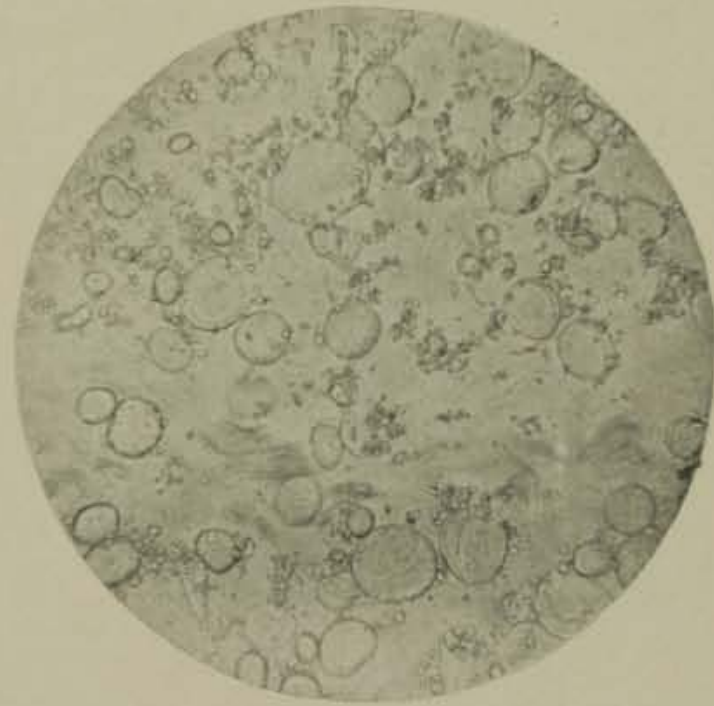

Fig. 124.-Barley Starch, $\times 220$.

Surface view of upper chaff layer. 


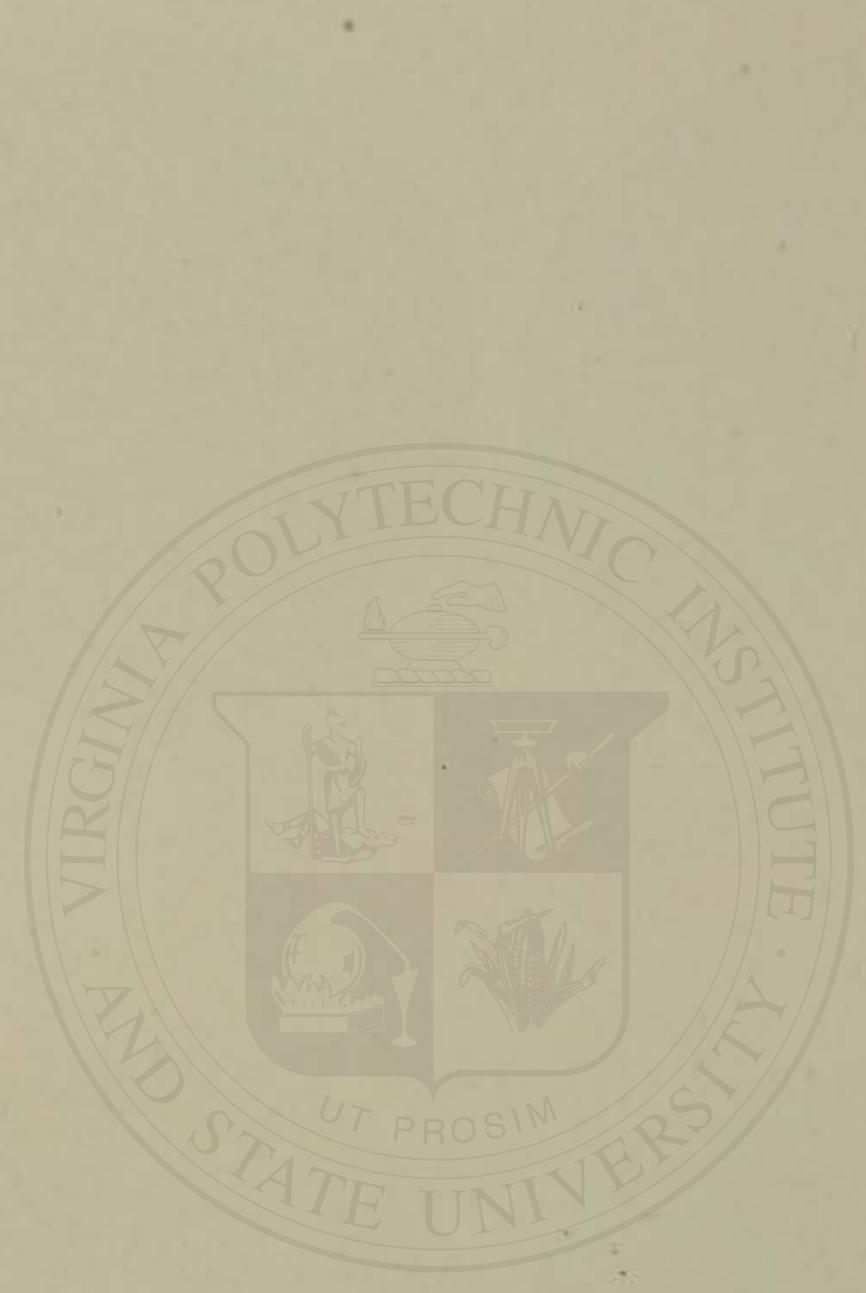


PLATE II. .

\section{CEREALS.}

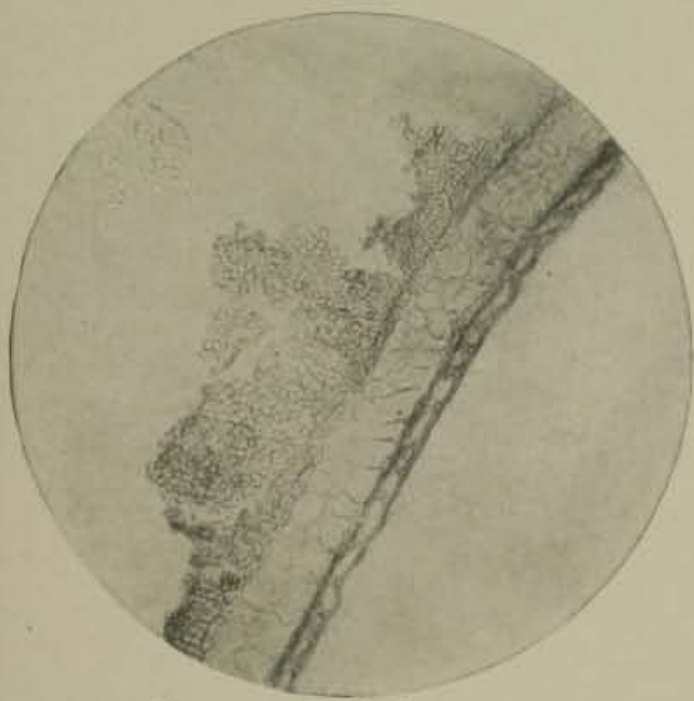

FiG. 125.-Buckwheat, $X$ rio.

Transverse section through part of pericarp, seed coat. and part of endosperm.

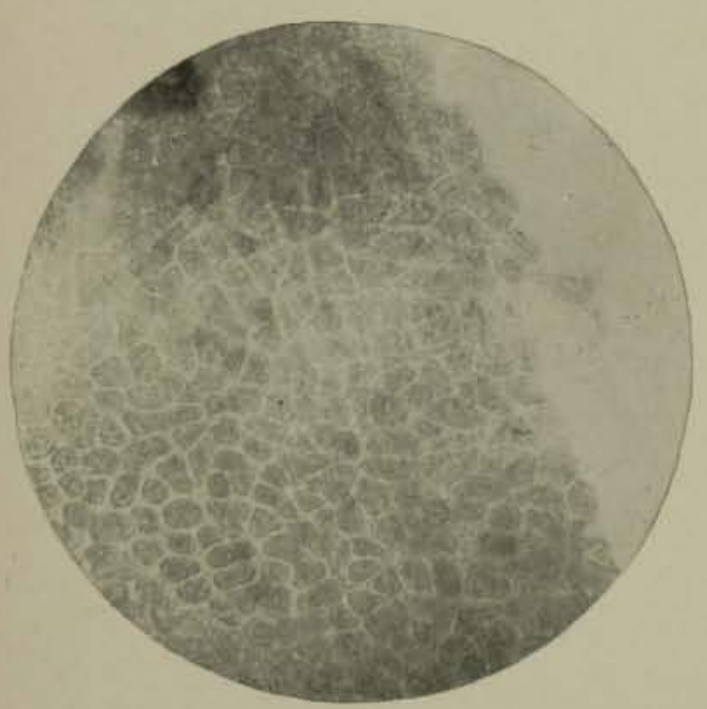

Fic. 127--Buckwheat, $X$ rro.

Surface section. Aleurone or proteid layer.

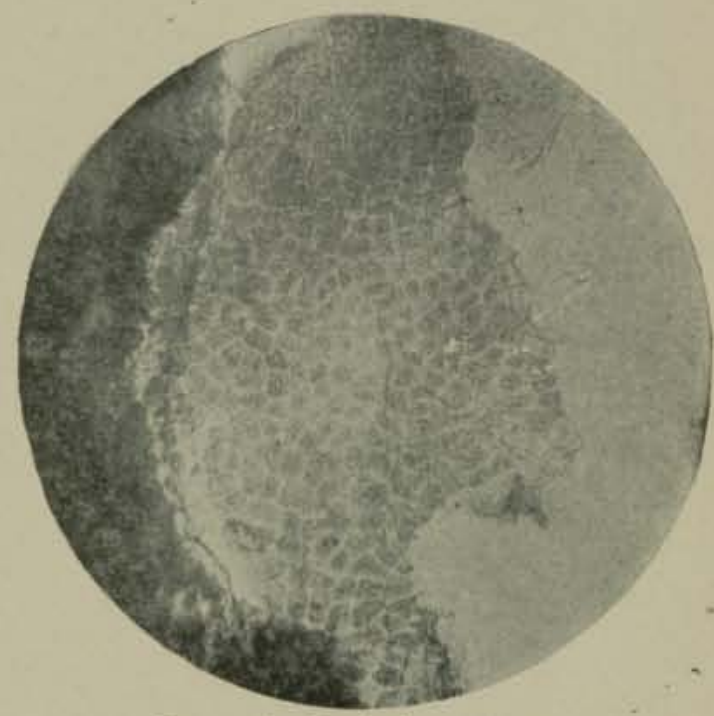

Fig. 126,-Buckwheat, $\times$ 110. Surface view of scutellum.

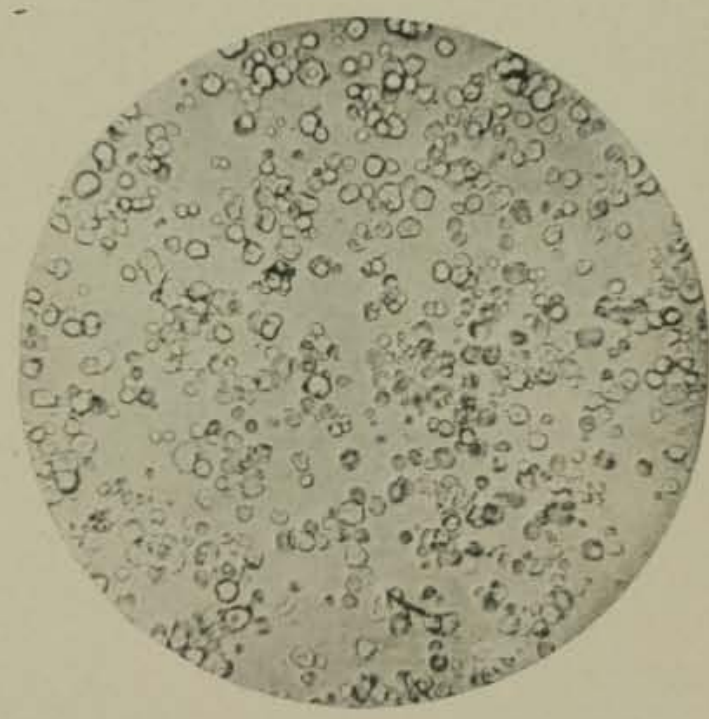

FIG. ${ }_{22} \mathrm{~S}$,-Buckwheat Starch, $\times 220$. Starch granules separated. 


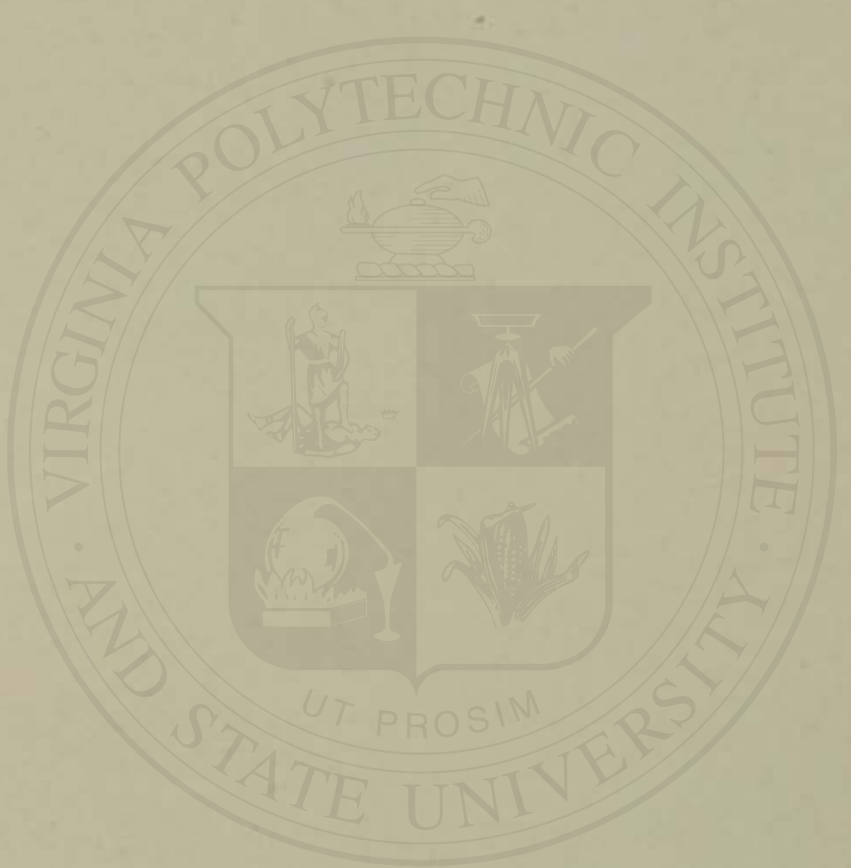




\section{PLATE III.}

\section{CEREALS.}

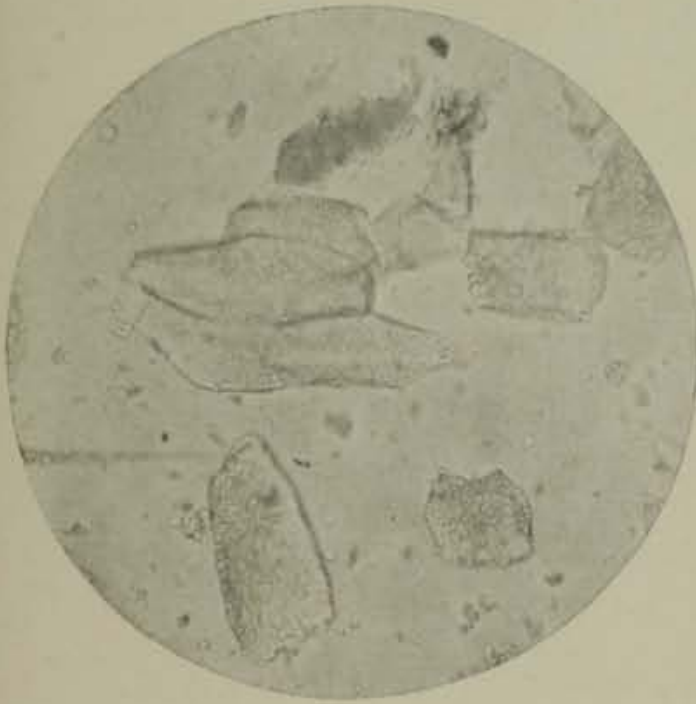

Fig. 129.-Buckwheat Starch, Xiro. Starch grains in masses.

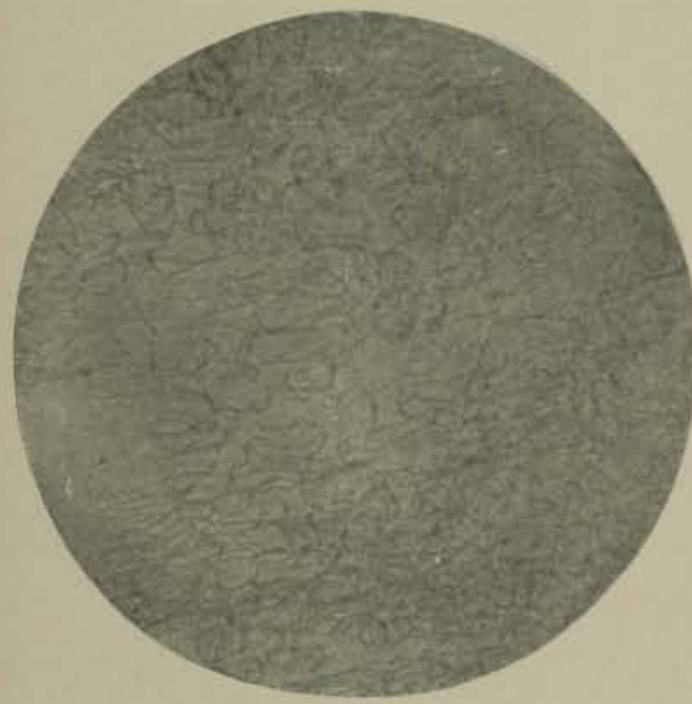

Fig. 131.-Corn, Xiro.

Surface view showing two layers of the mesocarp.

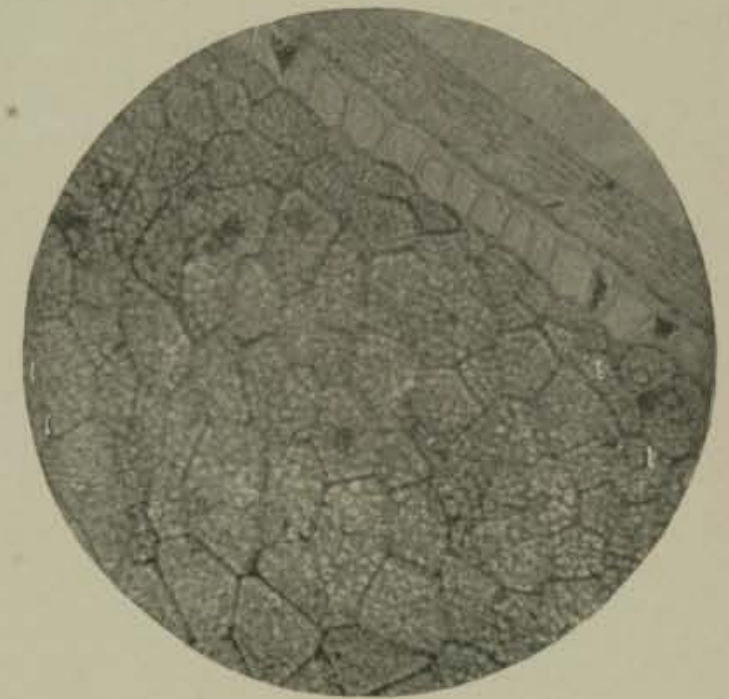

Fig. 130,-Corn, X110.

Transverse section through pericarp, seed coat, proteid layer, and part of endosperm, showing starch cells.

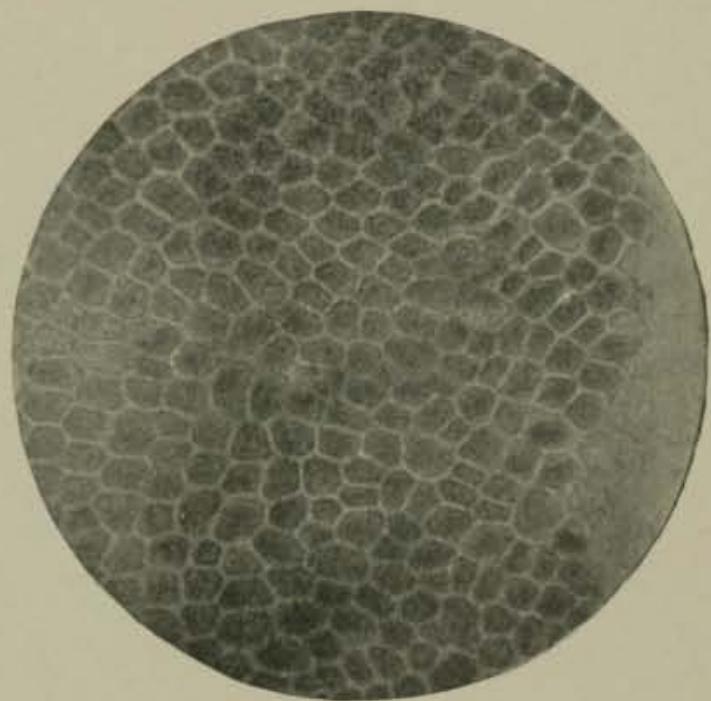

F1G. 132.-Com, Xrio.

Surface section. Proteid layer. 


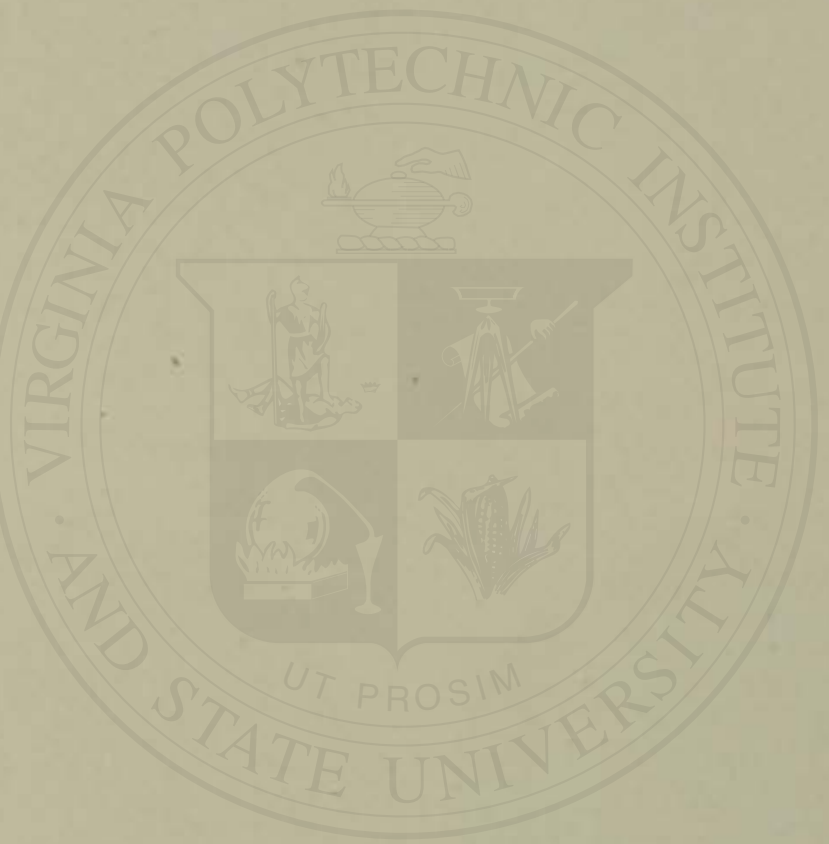




\section{CEREALS.}

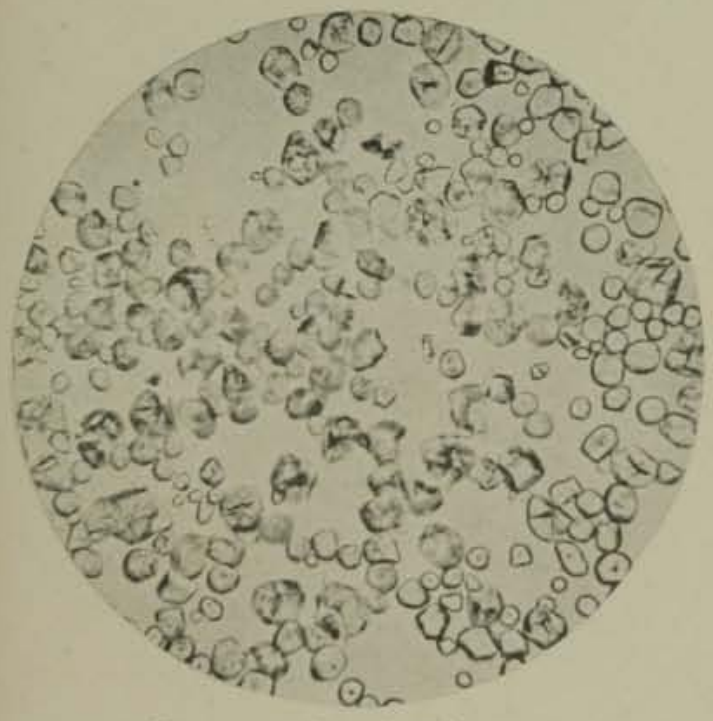

Fig. 133 -Corn Starch, $X_{220}$.

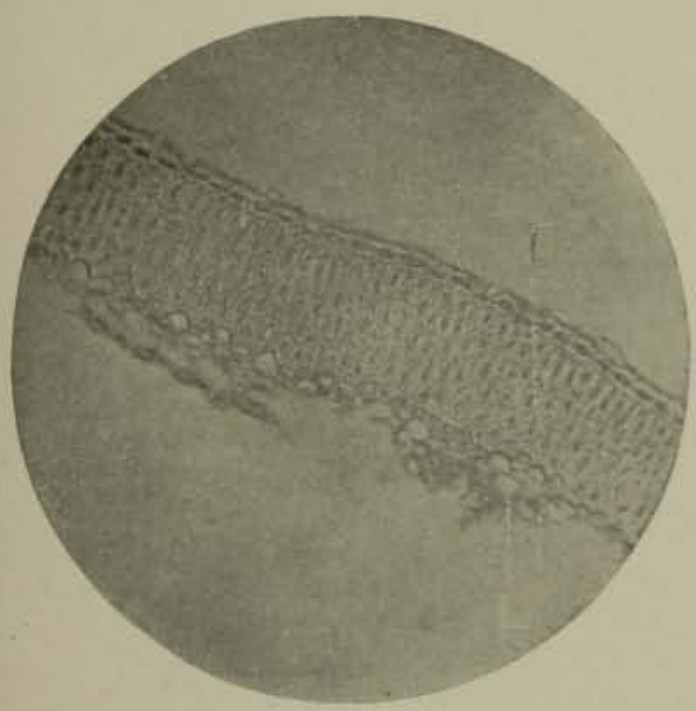

FiG. 135.-Oat, Xiro.

Transverse section through chaff.

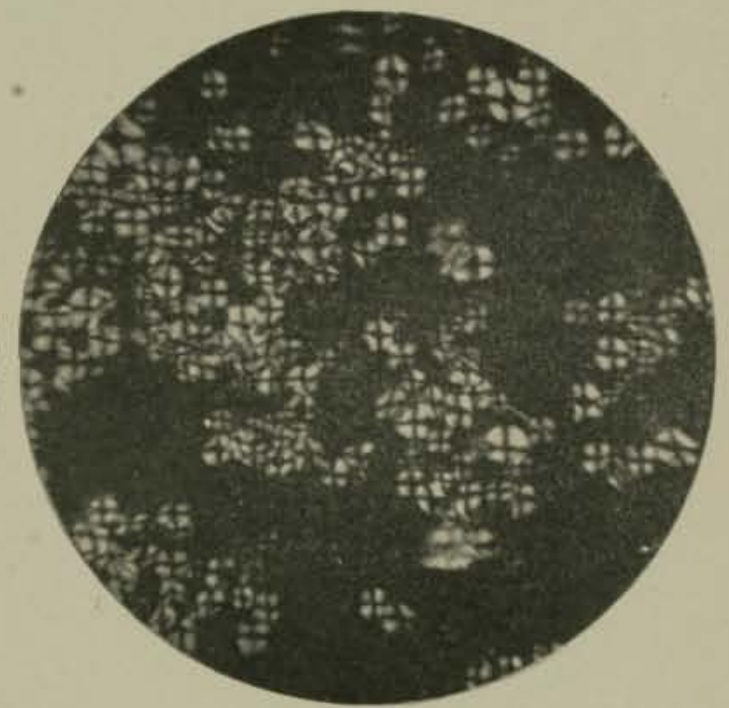

Fig. 134.-Corn Starch, $\mathrm{X}_{220}$ With polarized light.

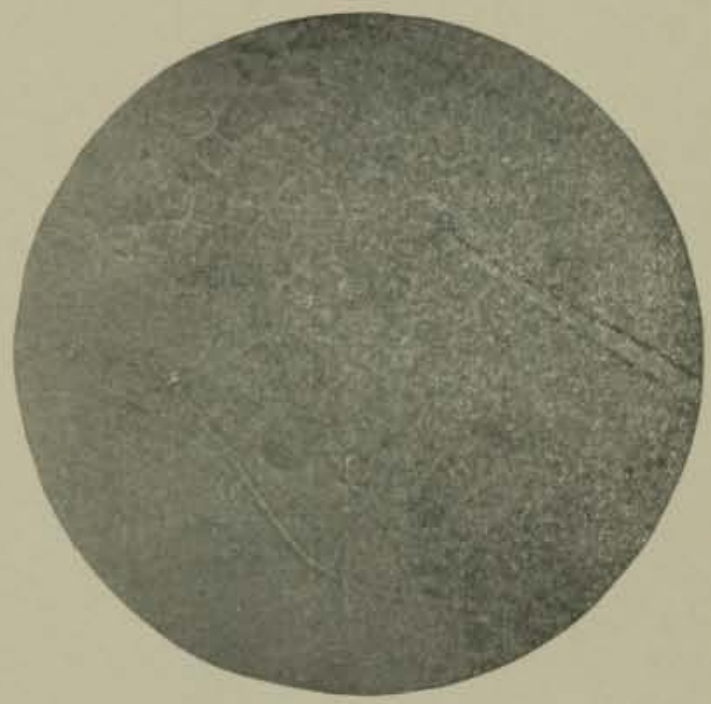

Fig. 136.-Oat, $\times$ rio.

Surface section. Proteid layer with fragments of epidermis and hairs. 


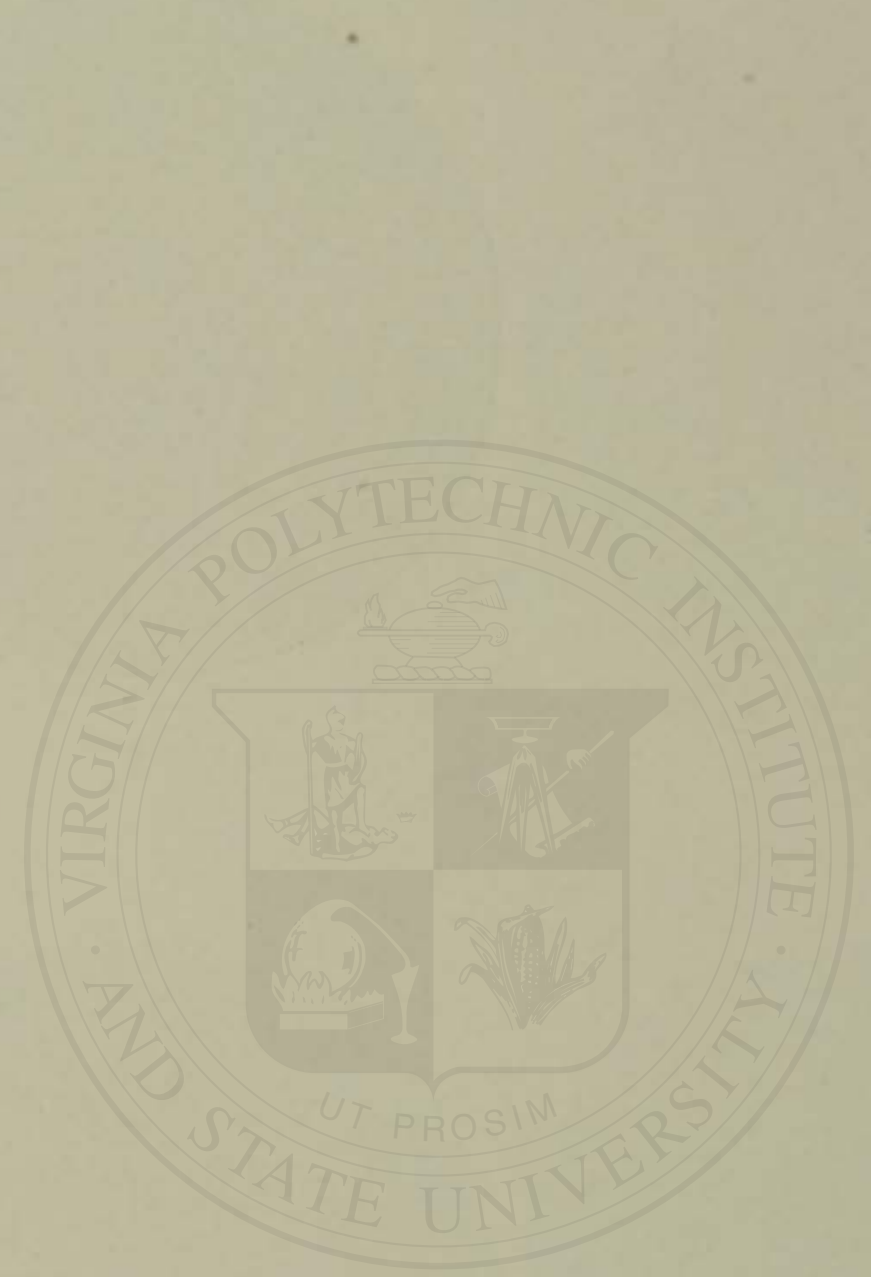


PLATE V.

\section{CEREAIS.}

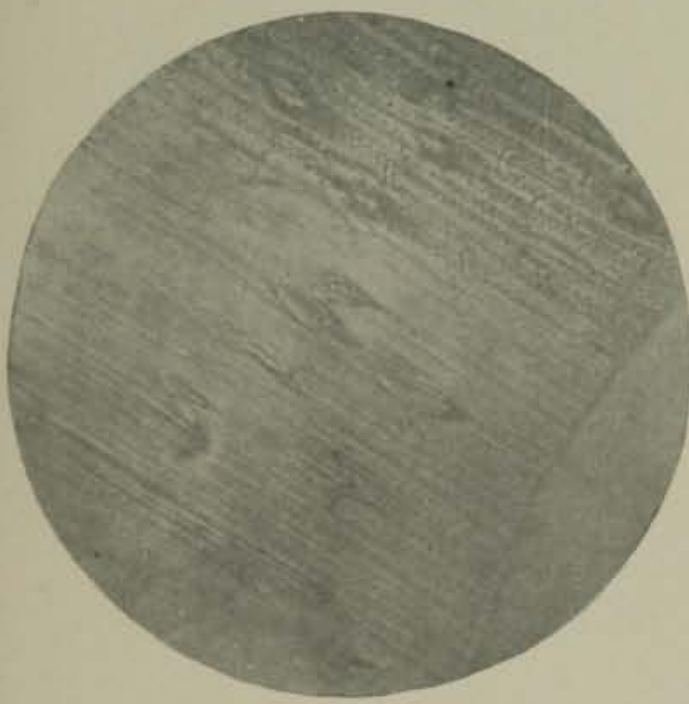

Fig. 137.-Oat, Xrio.

Surface view of upper chaff layer.

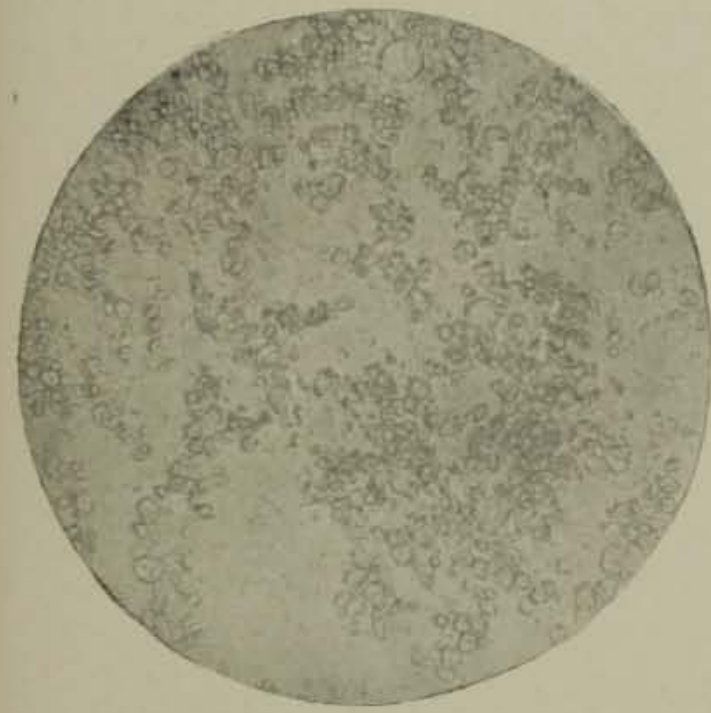

Fic. 139.-Oat Starch, $\times 220$.

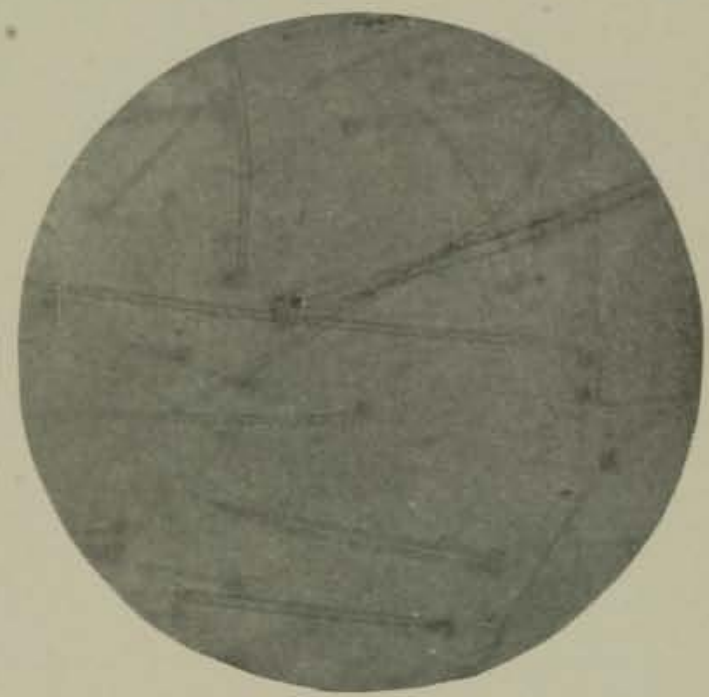

Fic, I $_{3} 8$. - Oat, $\times 55$.

Surface view of epidermis and hairs.

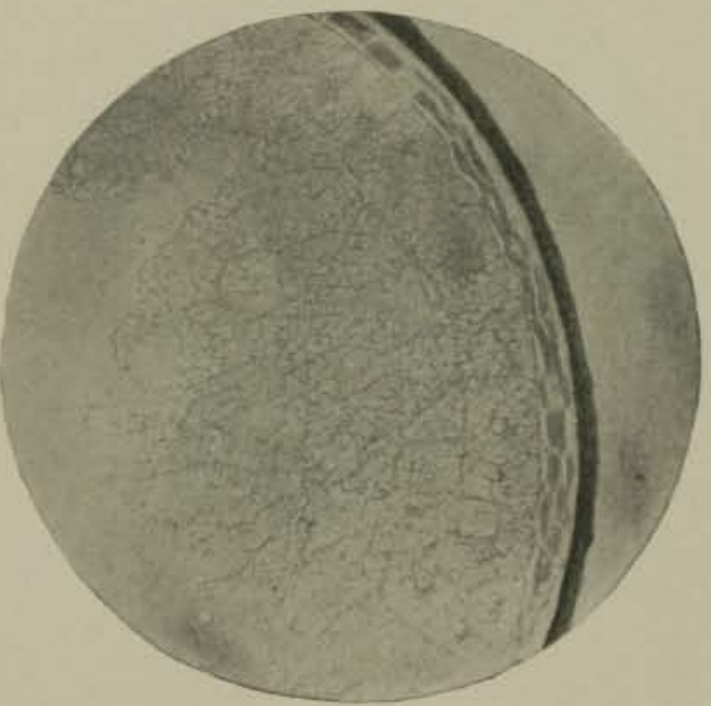

Fis. $t+0 .-$ Rice, $\times$ rio

Transverse section through seed coat and part of endosperm 


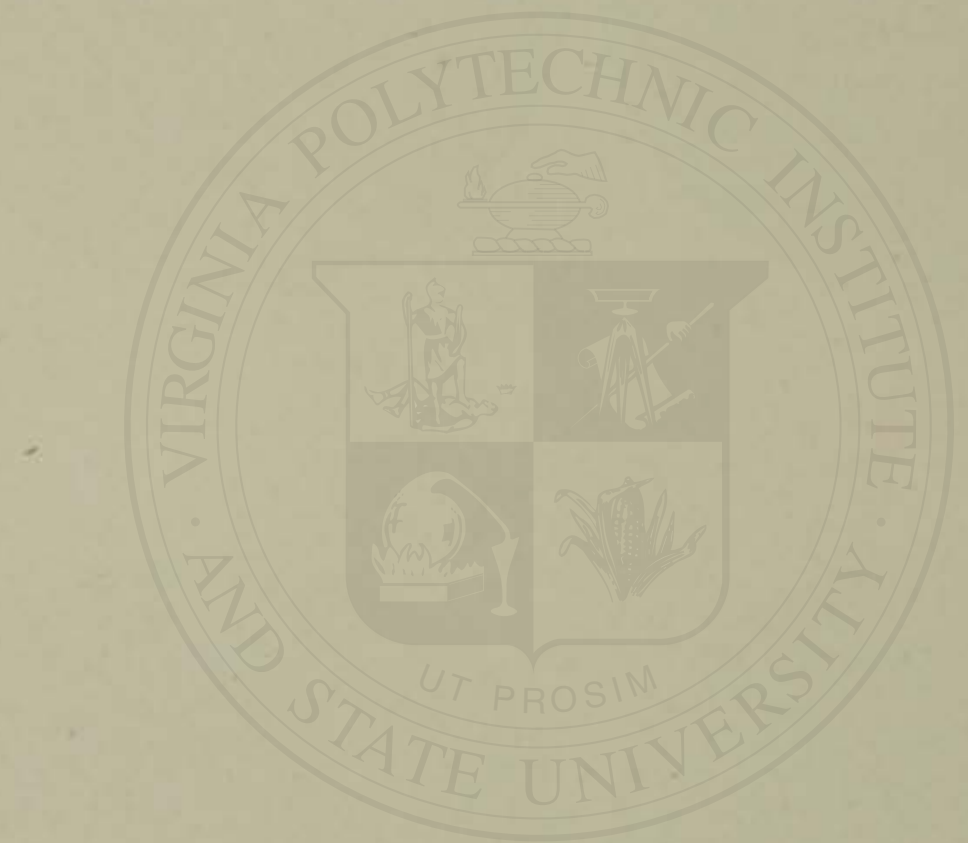


PLATE VI.

\section{CEREALS.}

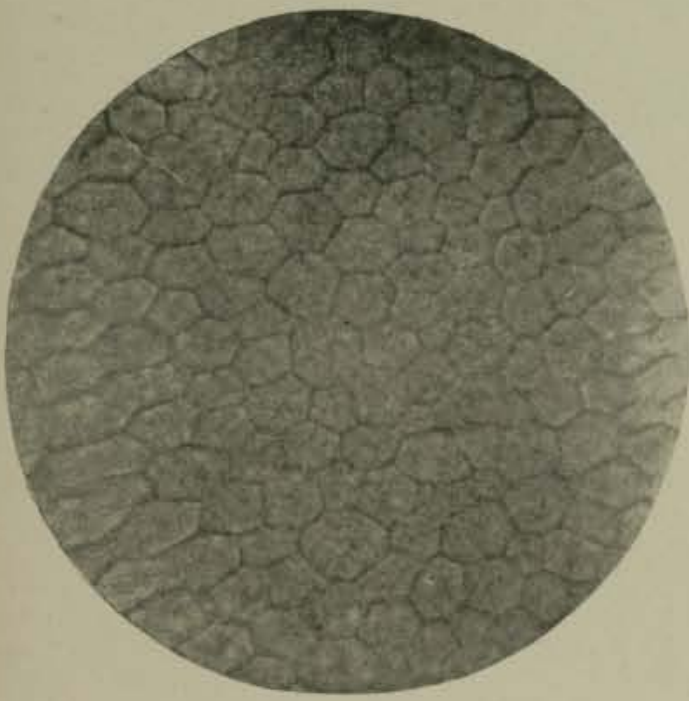

Fic. I4I,-Rice, X $x$ ro.

Surface section through starch cells.

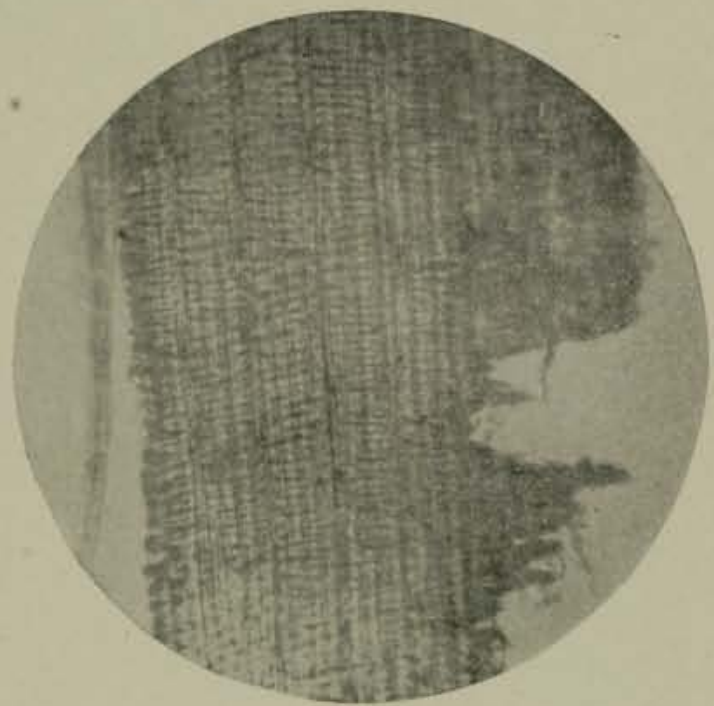

Frg. $142 .-$ Rice, $\times 110$.

Surface view of upper chaff laver

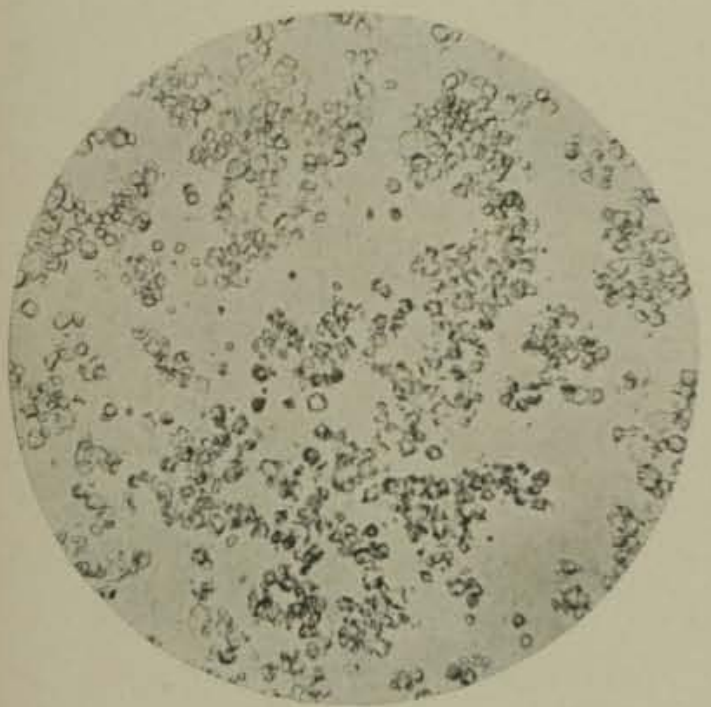

Fic. 143.-Rice Starch, $\times 220$.

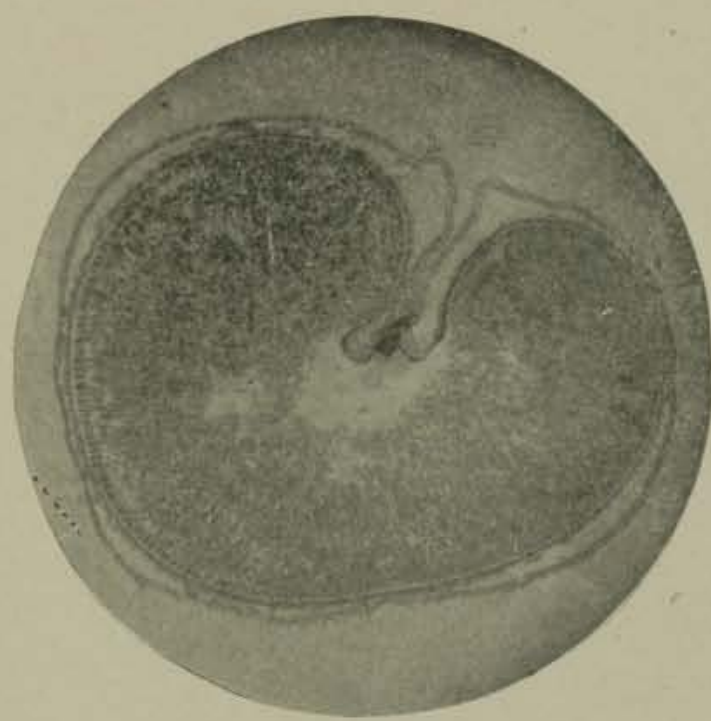

Fig. T44.-Rye, $\times 18$

Transverse section through the entire grain. 


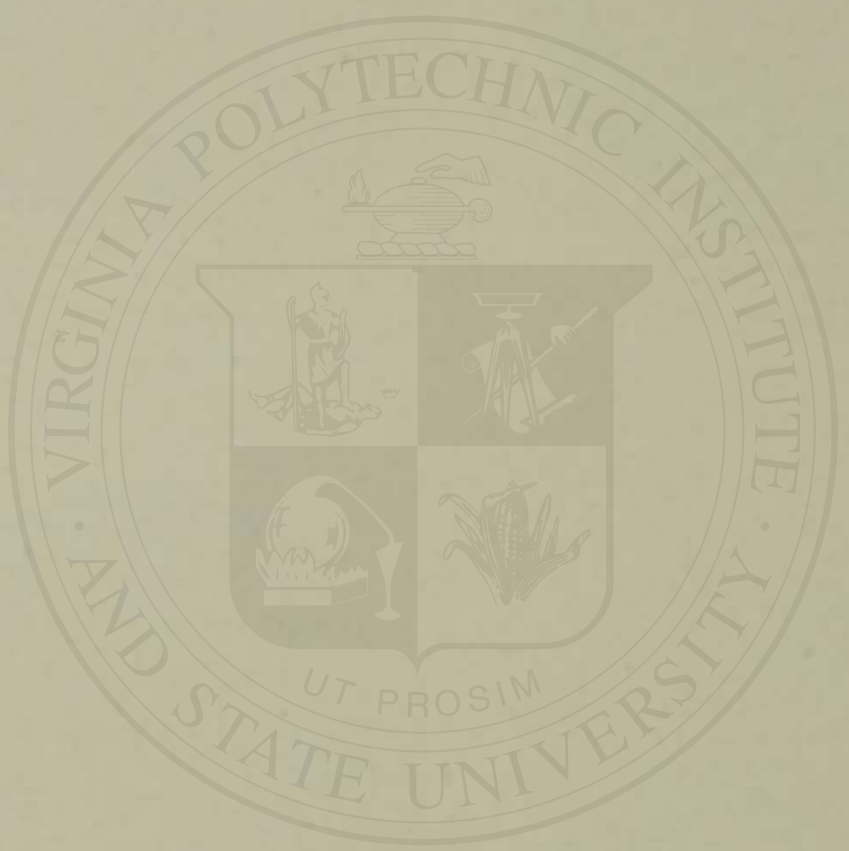


PLATE VII.

\section{CEREALS.}

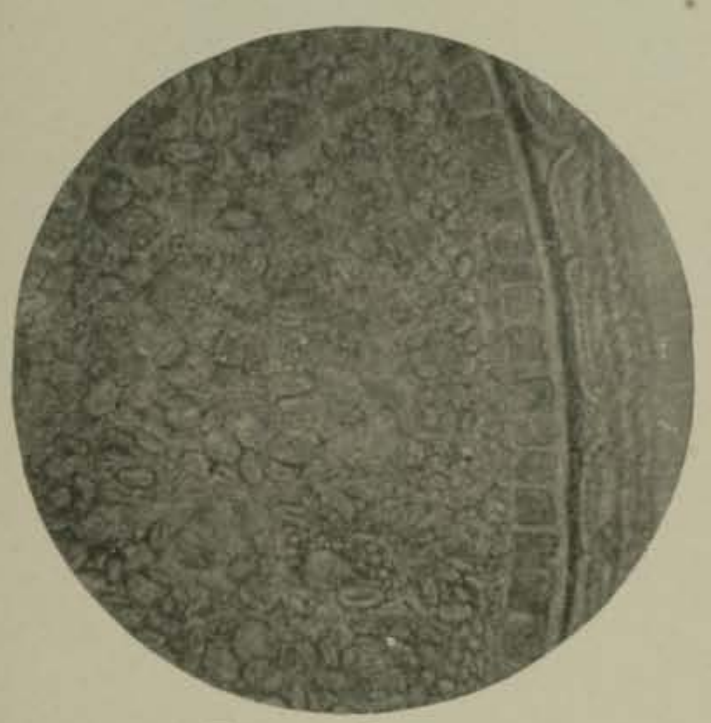

Fro. I45.-Rye, $X$ rio.

Transverse section through pericarp, seed coat, aleurone layer, and starch cells of endosperm.

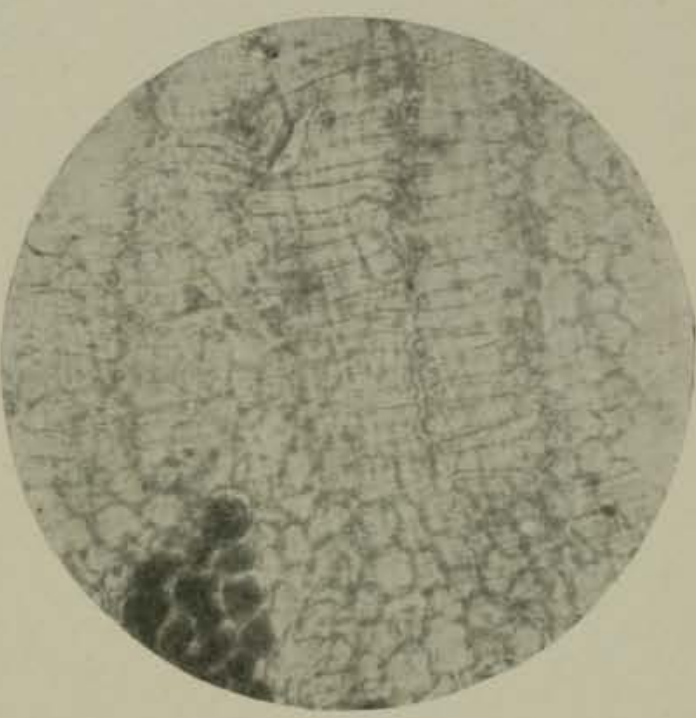

Frg. $146 .-$ Rye, $\times$ rio.

Surface view of epidermis and underlying layers.

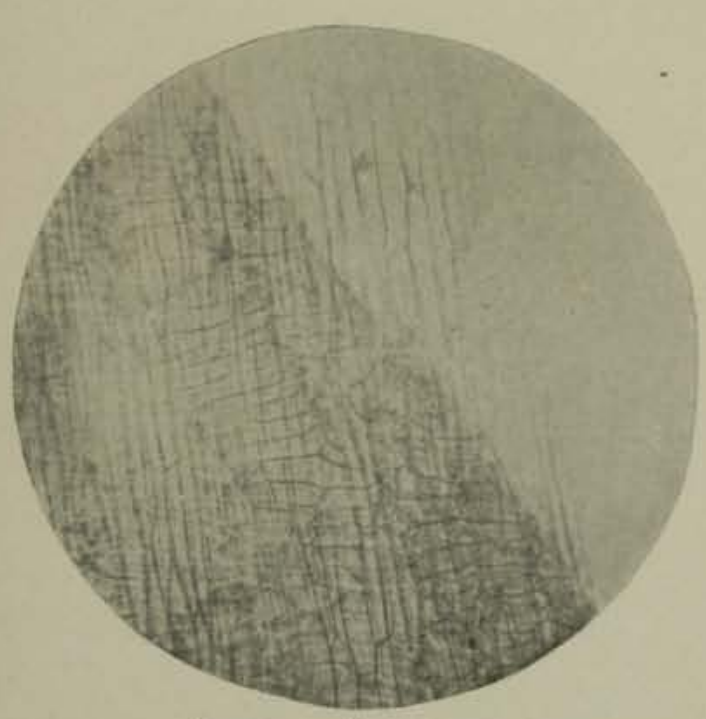

FtG, 147.-Rye, X110.

Surface view of epidermis and of seed coat.

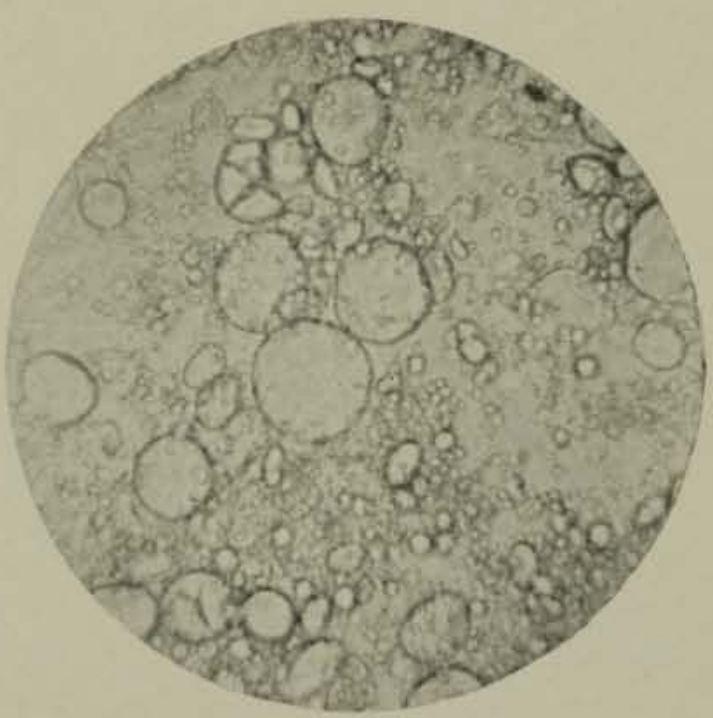

Fig. I48,-Rye Starch, $X_{220}$. 


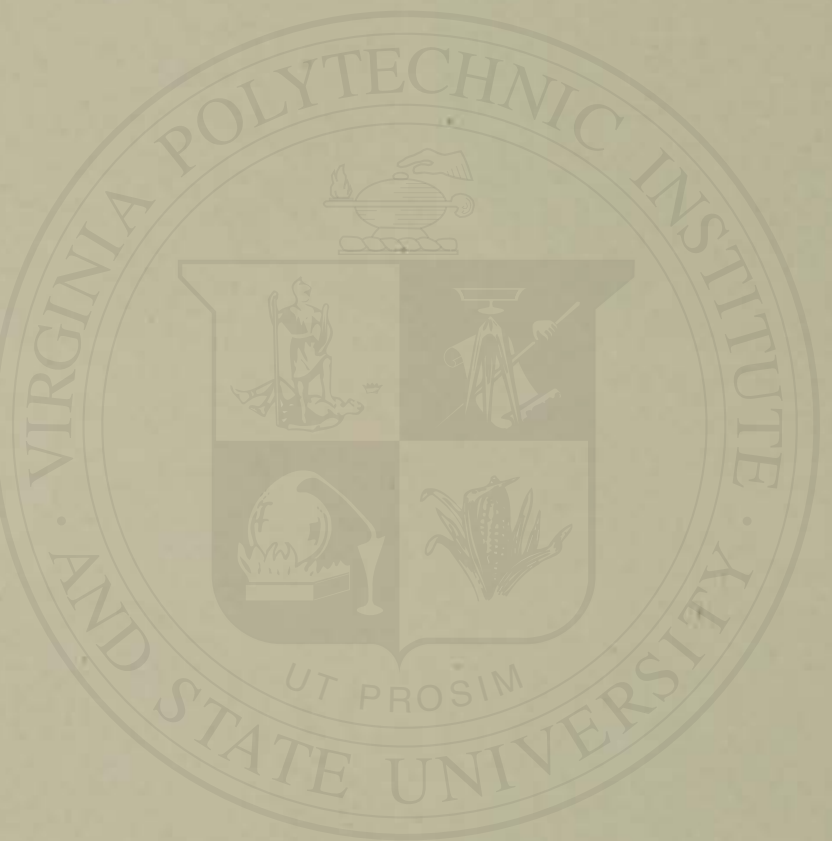




\section{CEREALS.}

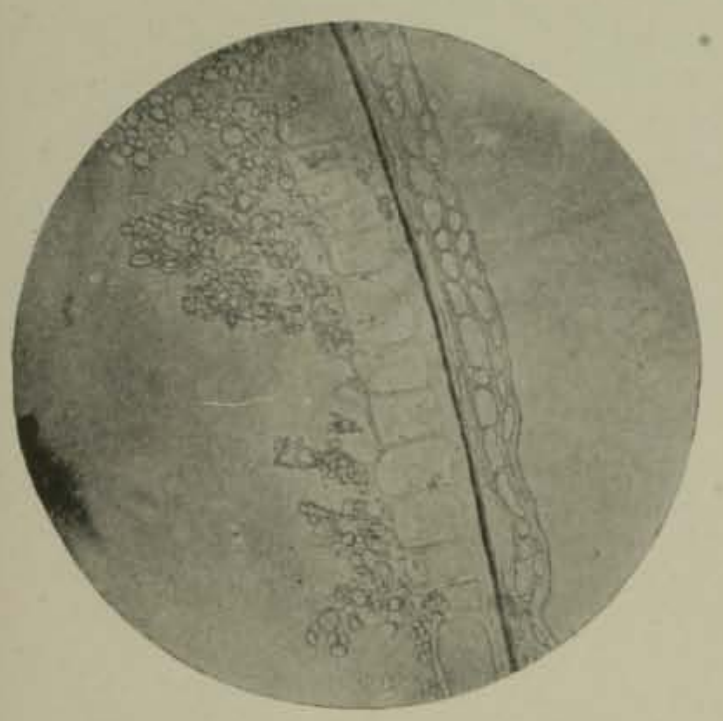

Fic. r49.-Wheat, $\times$ rro.

Transverse section through pericarp, seed coat, proteid layer, and starch cells of endosperm.

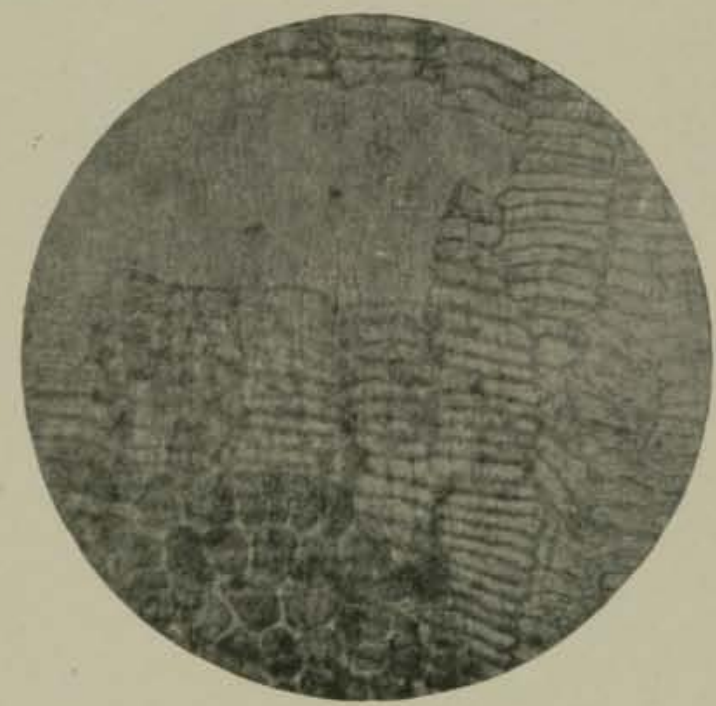

FrG. I50.-Wheat, $X$ rro.

Surface view of outer and inner epidermis Also showing proteid layer.

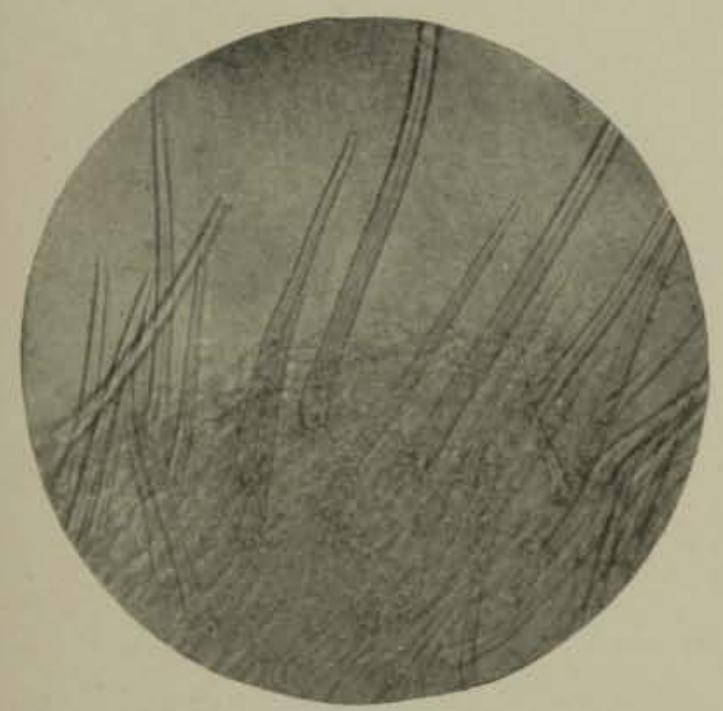

Fic. I5x.-Wheat, Xiro.

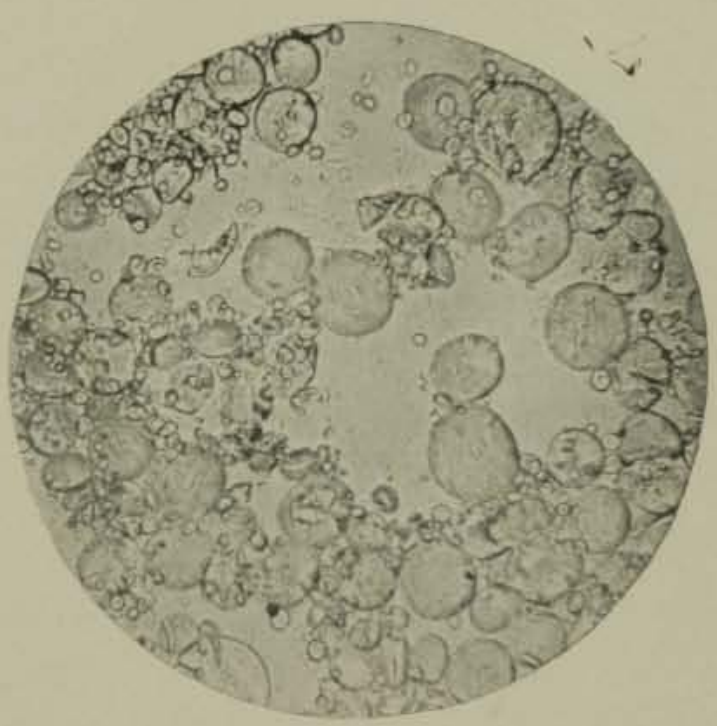

Fic. 152.-Wheat Starch, $\times 220$.

Surface view of epidermis, with hairs. 

PLATE IX.

\section{LEGUMES.}

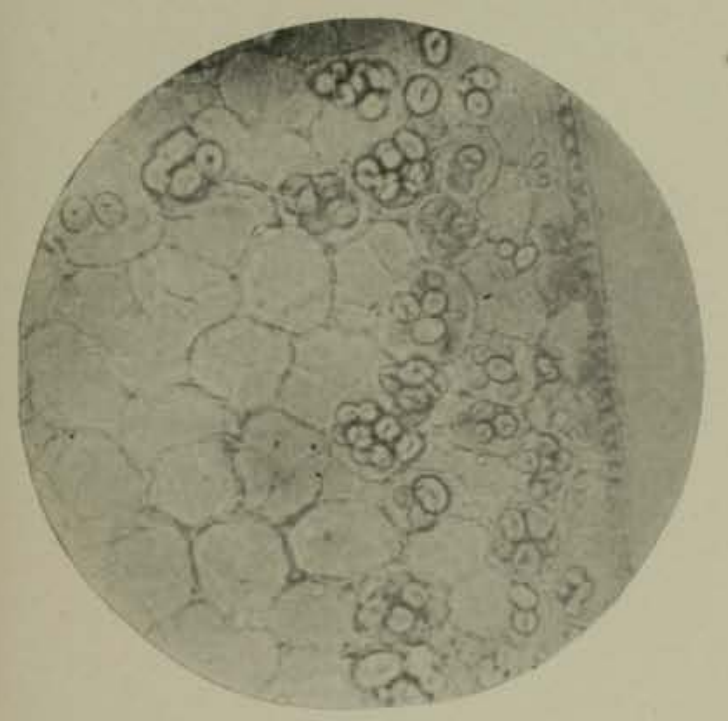

Fir. 153--Bean, Xiro.

Transverse section through starch cells.

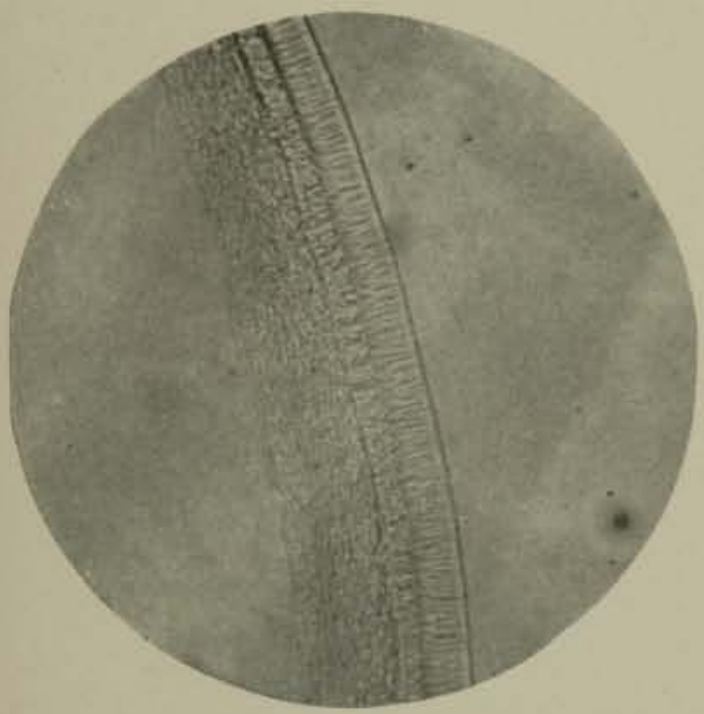

Fic. 155.-Bean, Xiro.

Transverse cection through hull, showing palisade cells of epidermis, and underlying hypoderma.

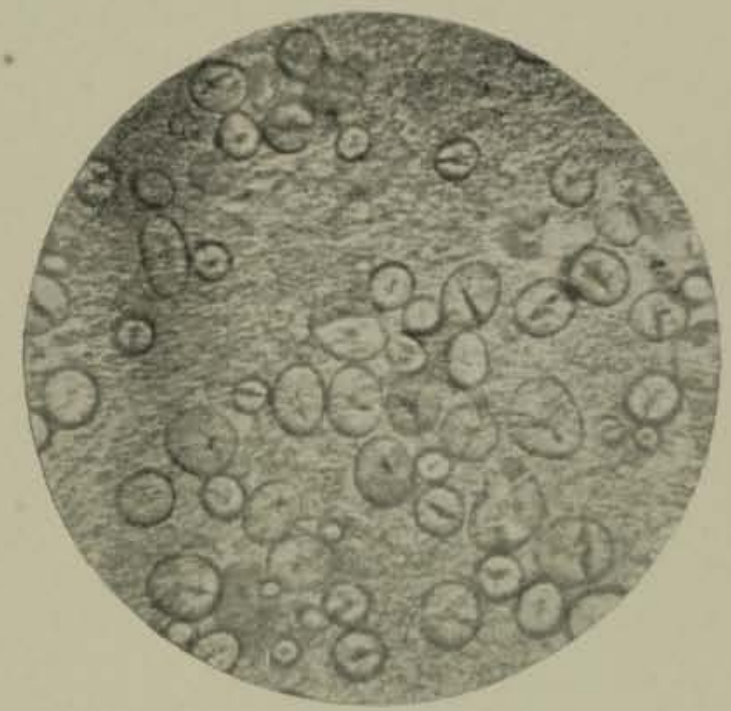

Fic. 154.-Bean Starch, $\times 220$.

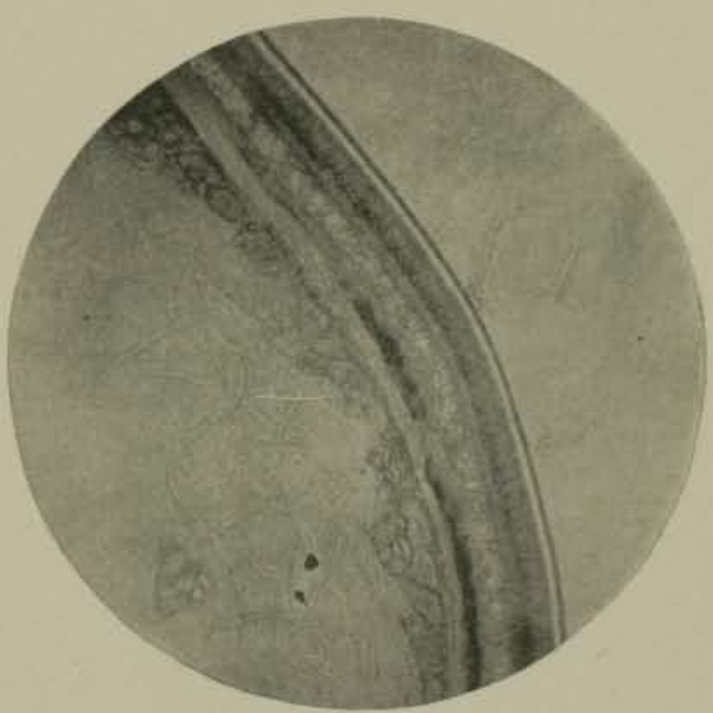

Ftc. 156 . -Lentil, $\times 110$.

Transverse section through hull and part of endosperm, showing some of the starch cells. 


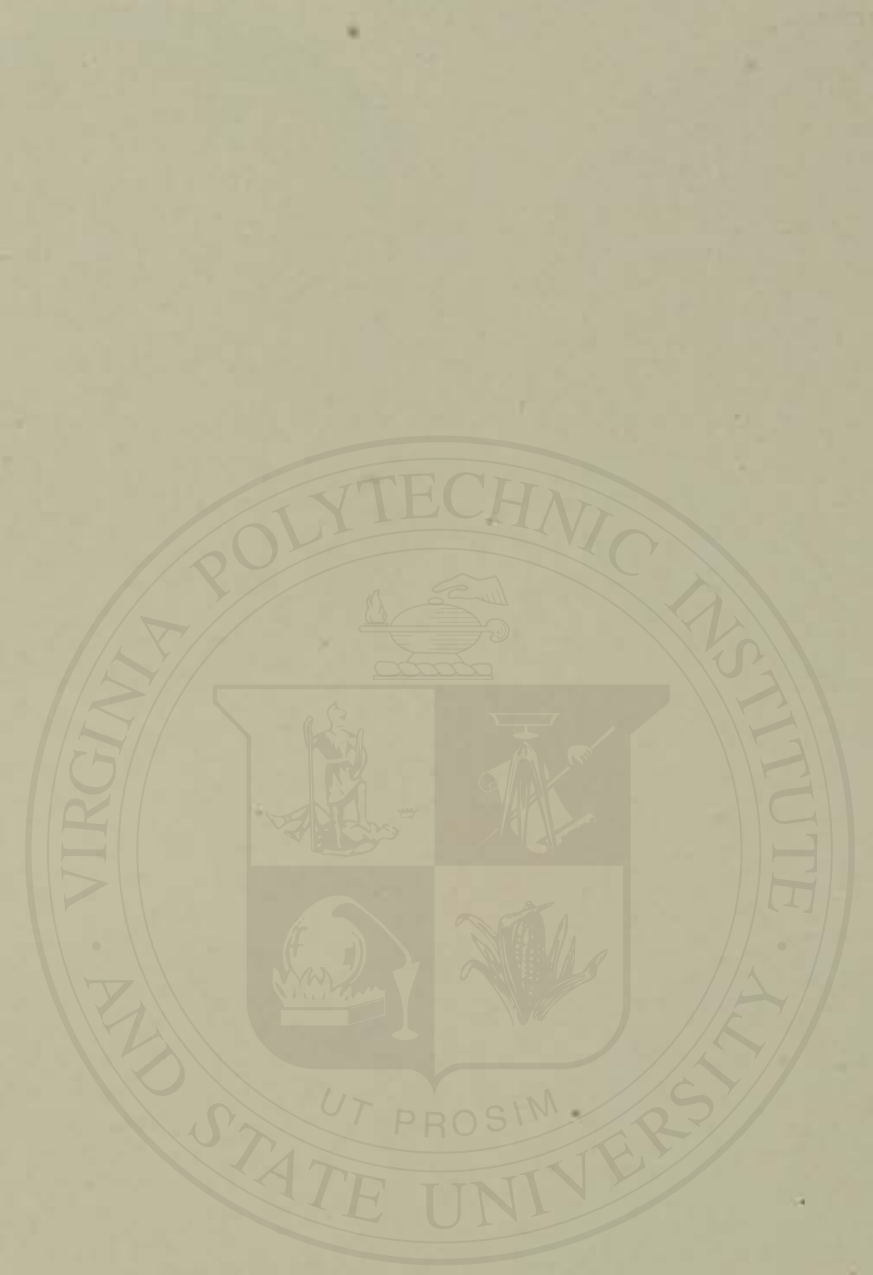




\section{LEGUMES.}

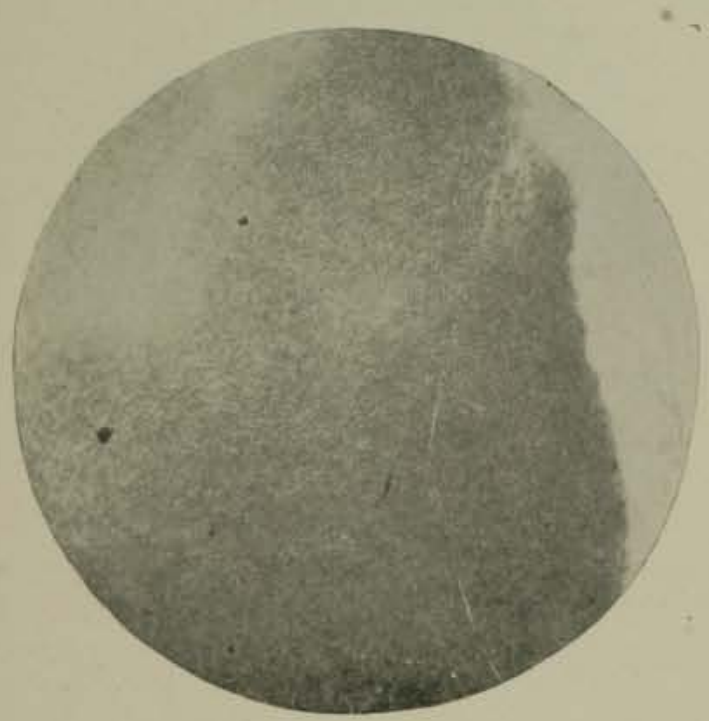

Fig. 157 -Lentil, $X$ rto. Surface view of epidermis.

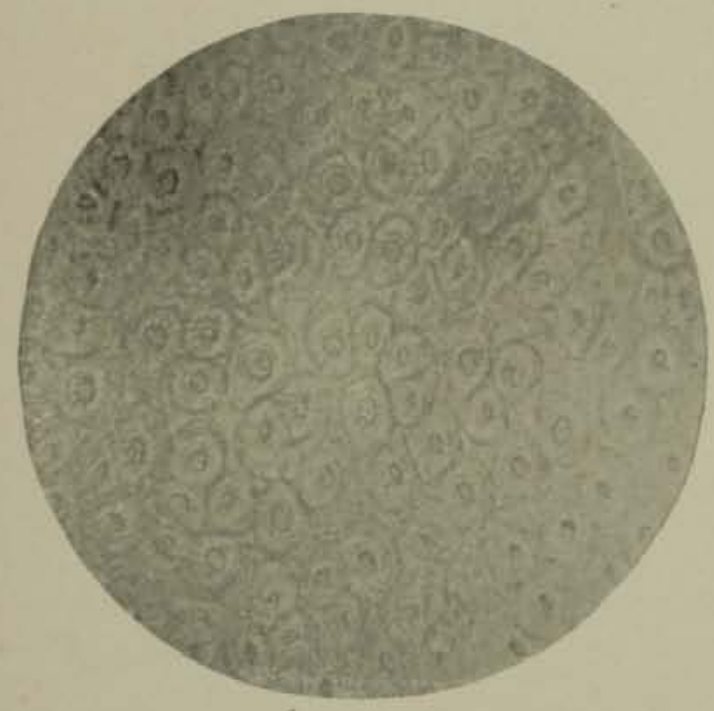

Fig. $159-$ Pea, $\times 110$.

Eurface section through base of palisade layer.

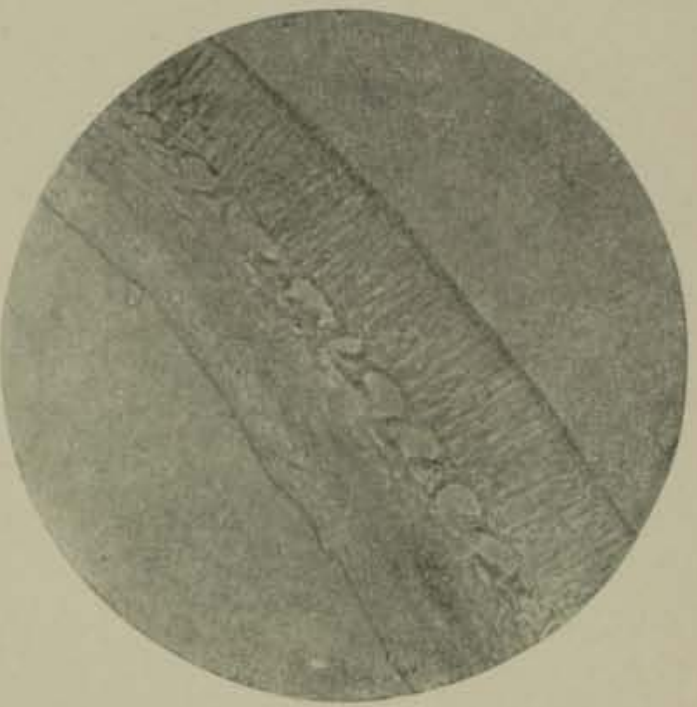

Fic. 158.-Pea, Xiro.

Transverse section through hull and seed coat, showing outer palisade cells and underlying hypoderma.

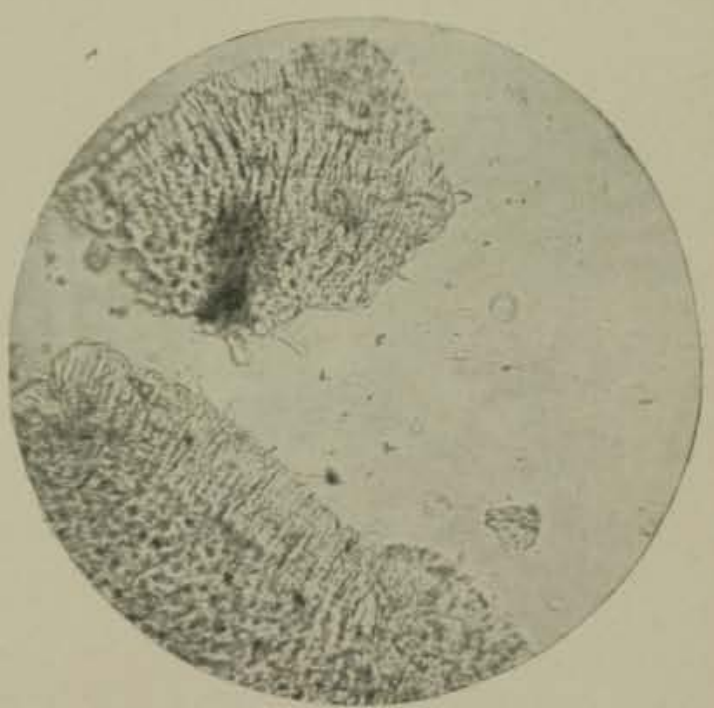

Fro, $160 .-\mathrm{Pea}, \times 110$.

Powdered pea hulls. 


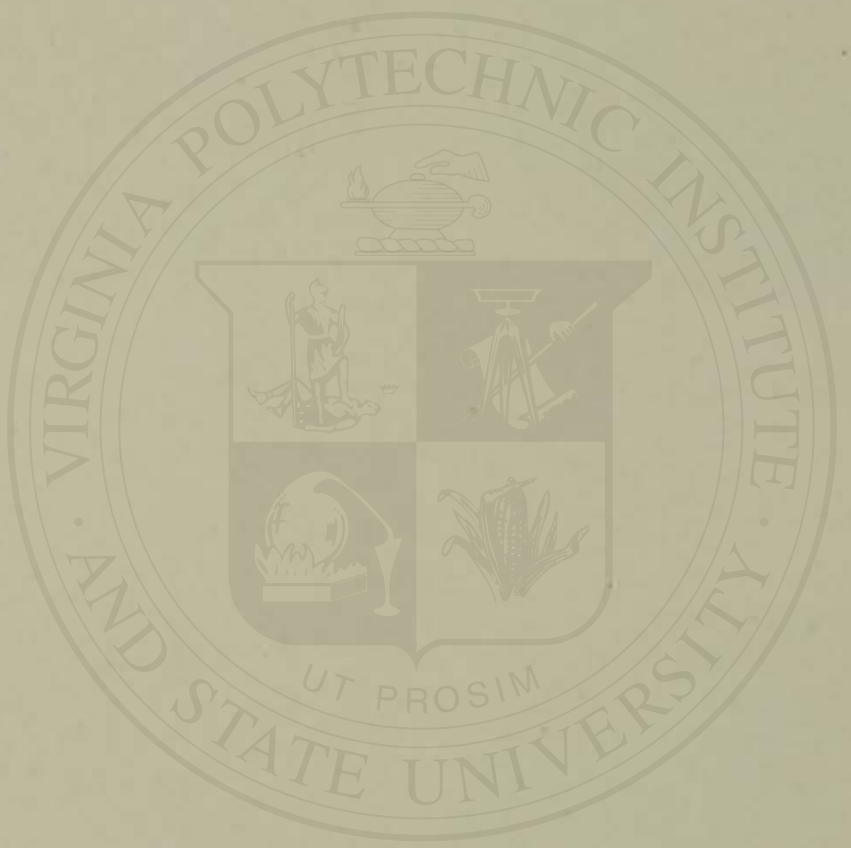


PLATE XI.

\section{LEGUMES.}

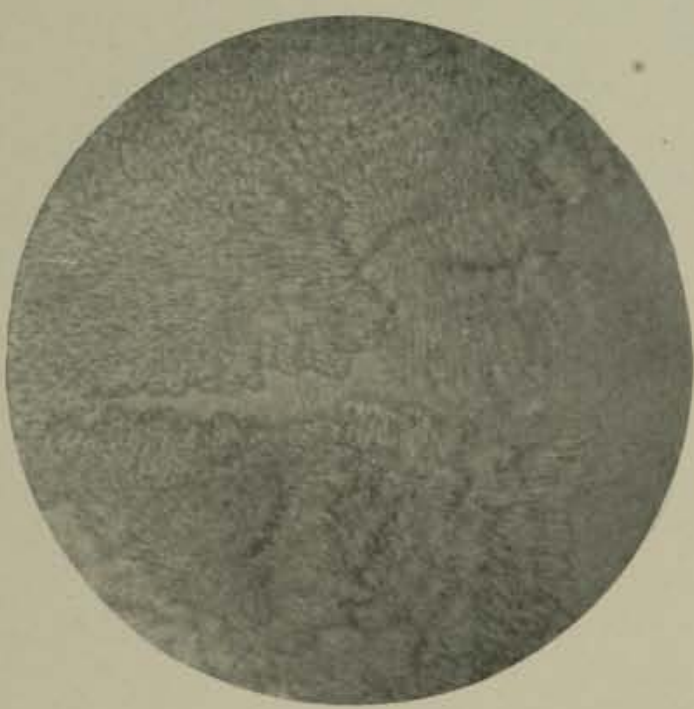

FrG. r6r.-Pea, Xrro.

Surface view of palisade cells.

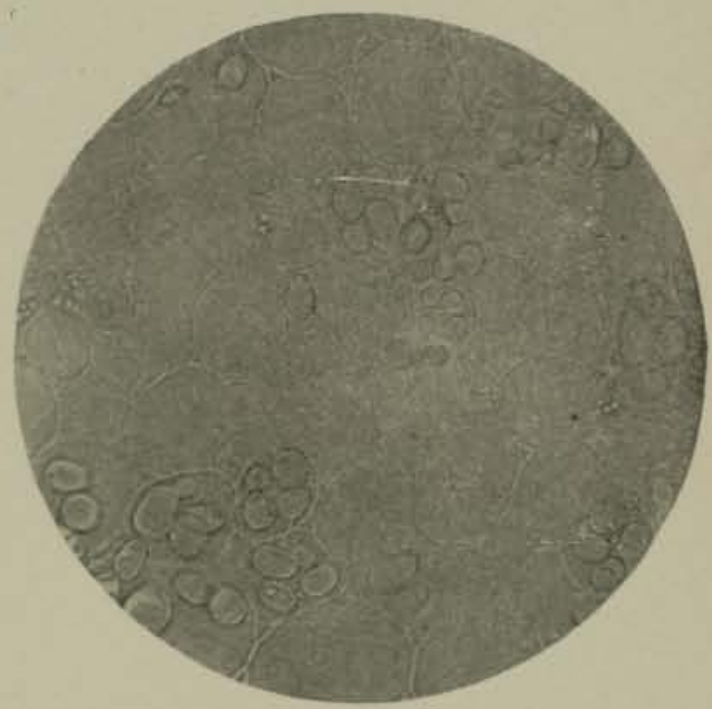

Fis, $162,-P e a, \times$ rro.

Transverse section through starch cells.

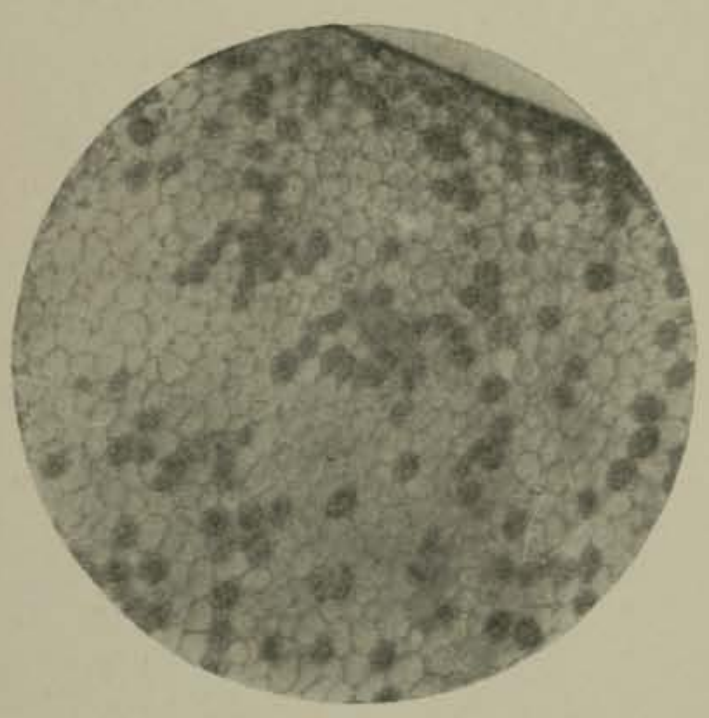

Fic. 163. - Pea, $\times 30$.

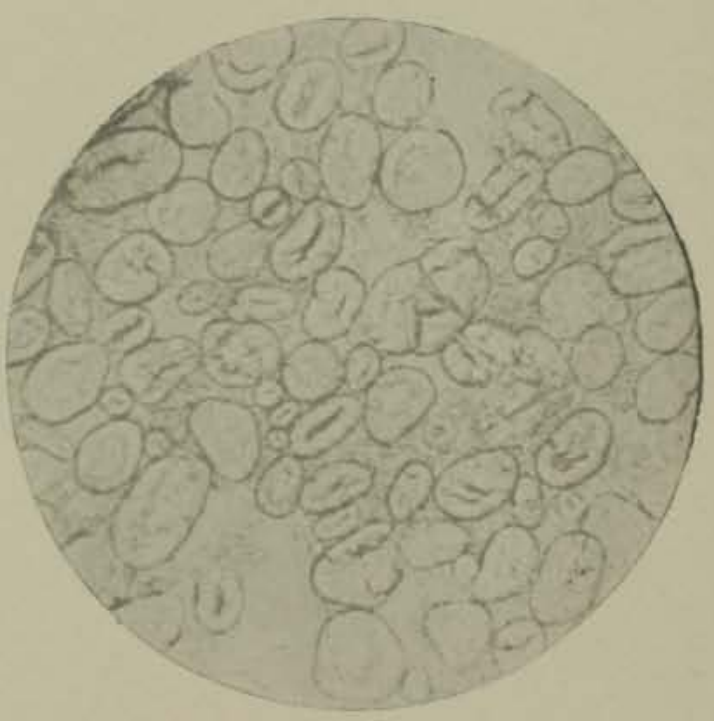

Fig. $164,-P e a$ Starch, $\times 220$.

Transverse section through starch cells. 


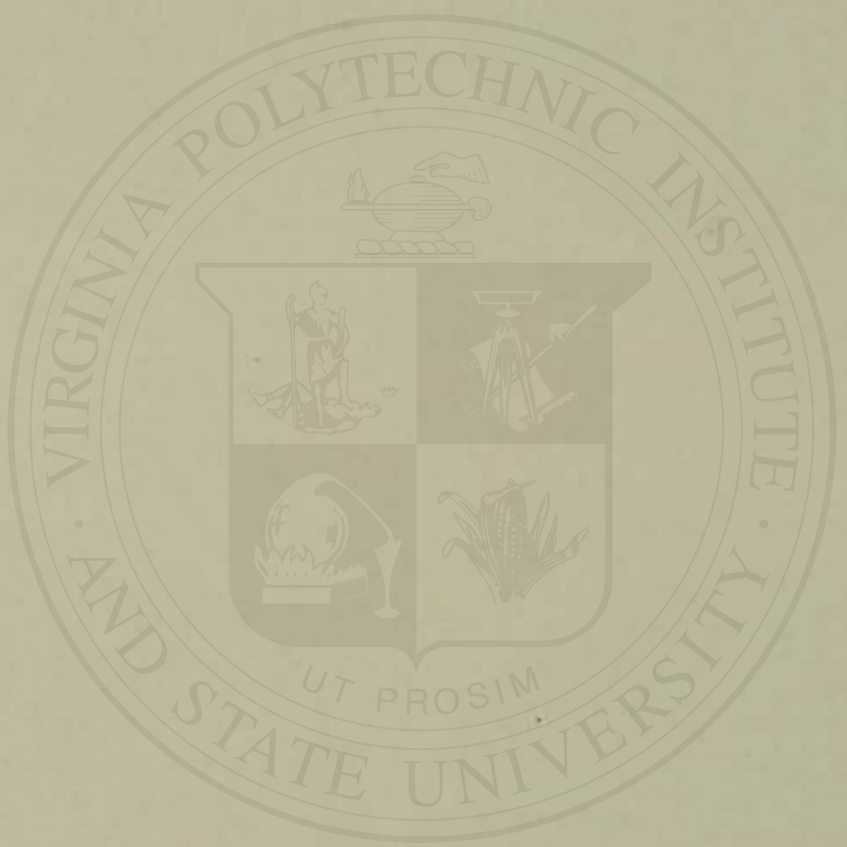


PLATE XII.

MISCELLANEOE'S STARCHES.

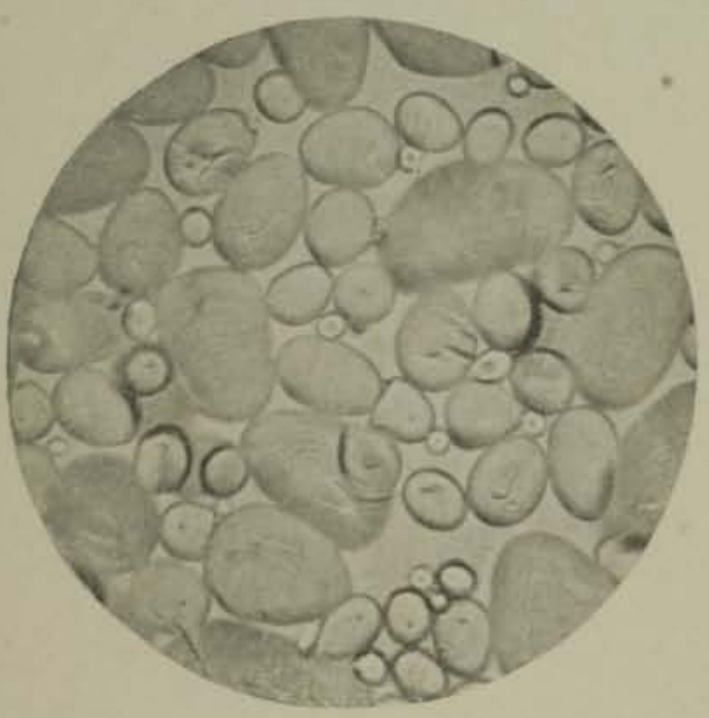

Fic. $165 .-$ Potato Starch, $\times 220$.

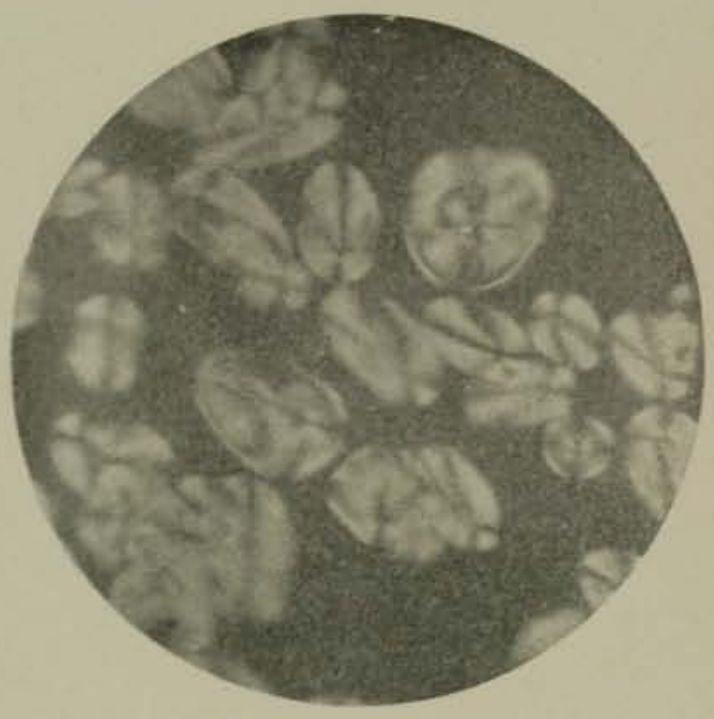

Fic. 166.-Potato Starch, X 220 . With polarized light.

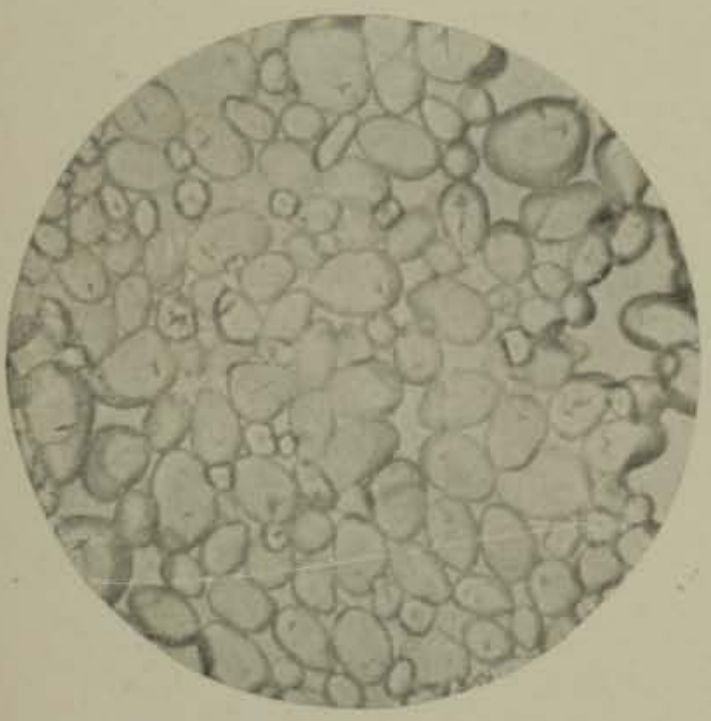

F16. 167.-Arrowroot Starch, $X_{220}$

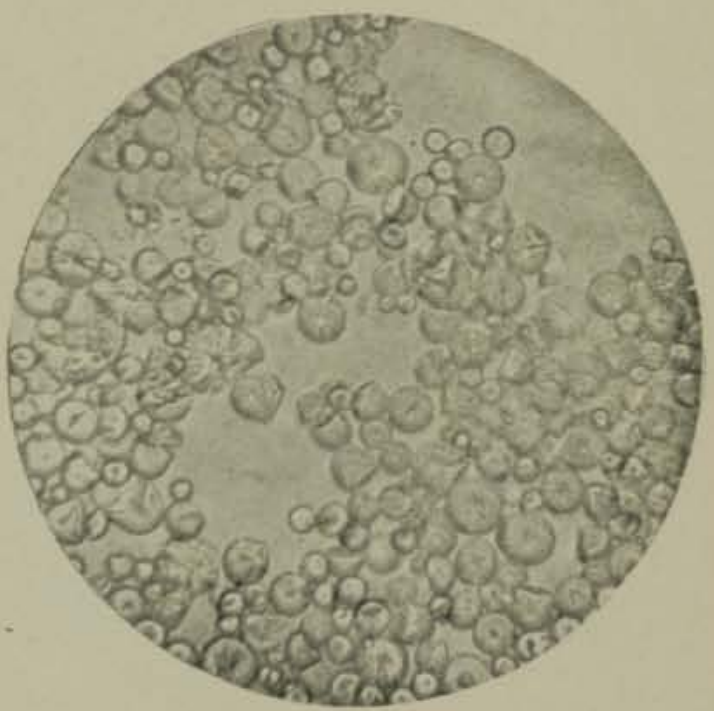

Fig. 168,-Tapioca Starch, $\times 220$. (Cassava.) 


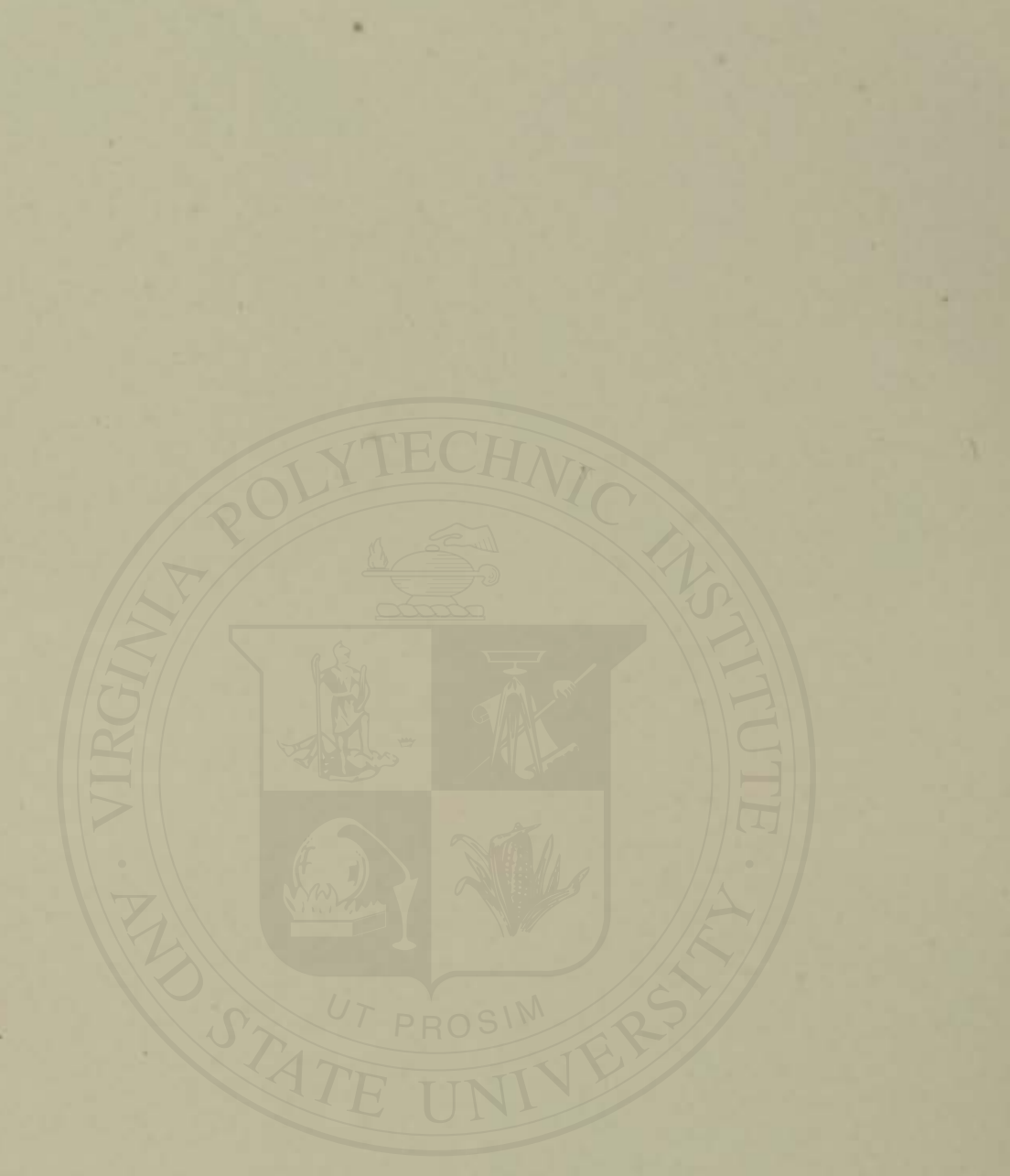


PLATE XIII.

TURMERIC. SAGO.

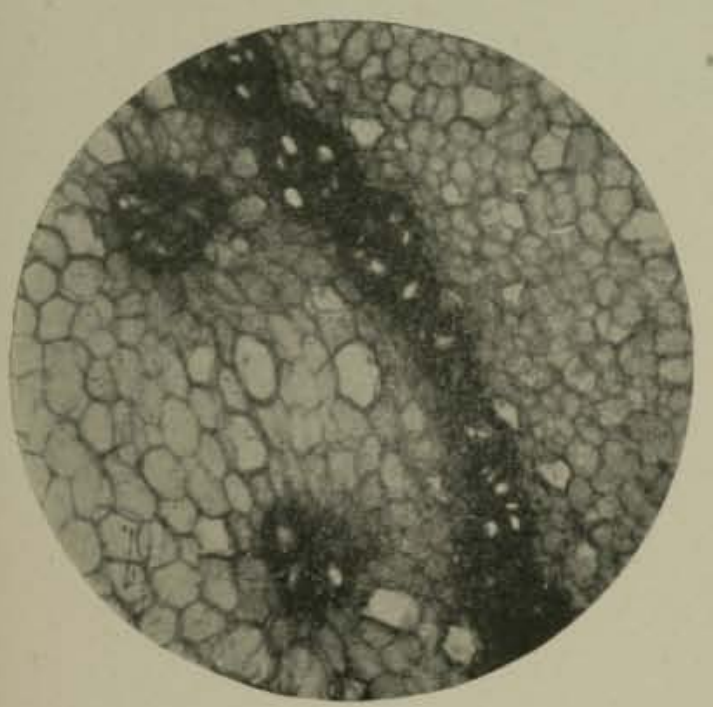

FIG. 160 -Turmeric, $\times 70$.

Transverse section through rhizome.

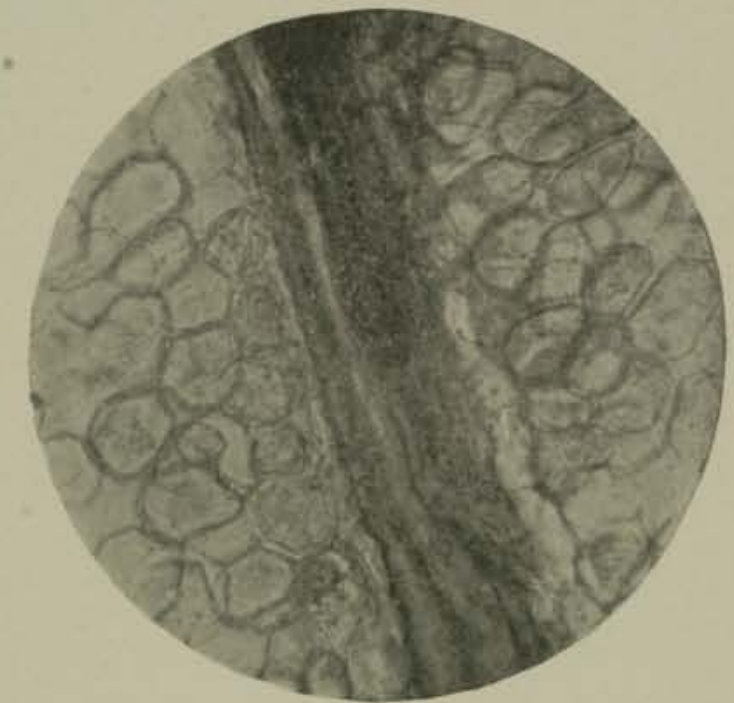

Fro. r7o.-Turmeric, $\times 1$ ro.

Longitudinal section. Note spiral ducts through the center.

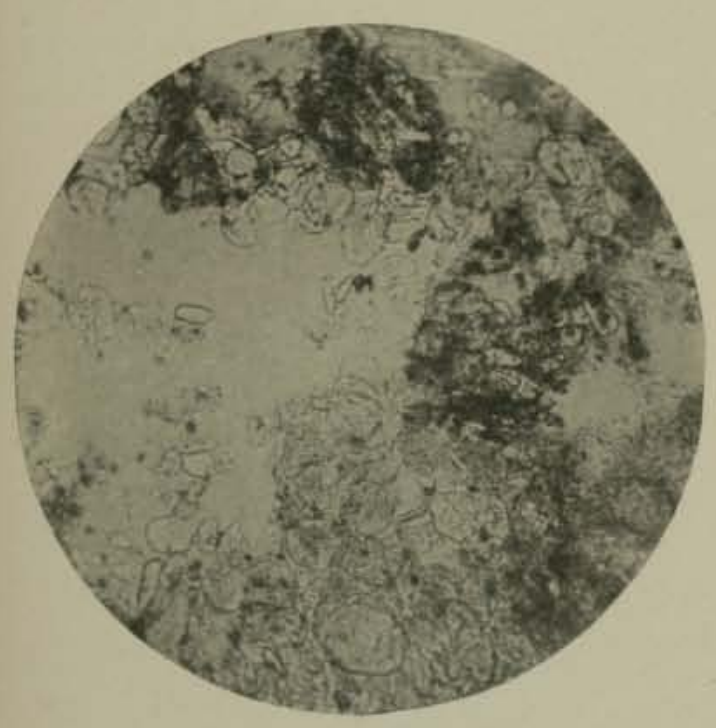

FrG. 171.-Powdered Turmeric, X ro.

Showing starch grains, fragments of cell tissue, coloring matter, etc.

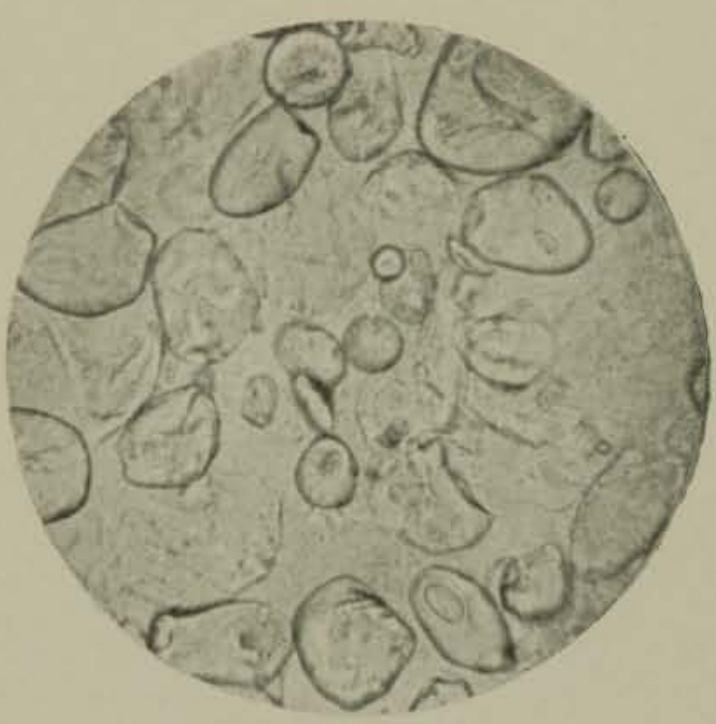

Fic. 172.-Sago Starch, X220. 


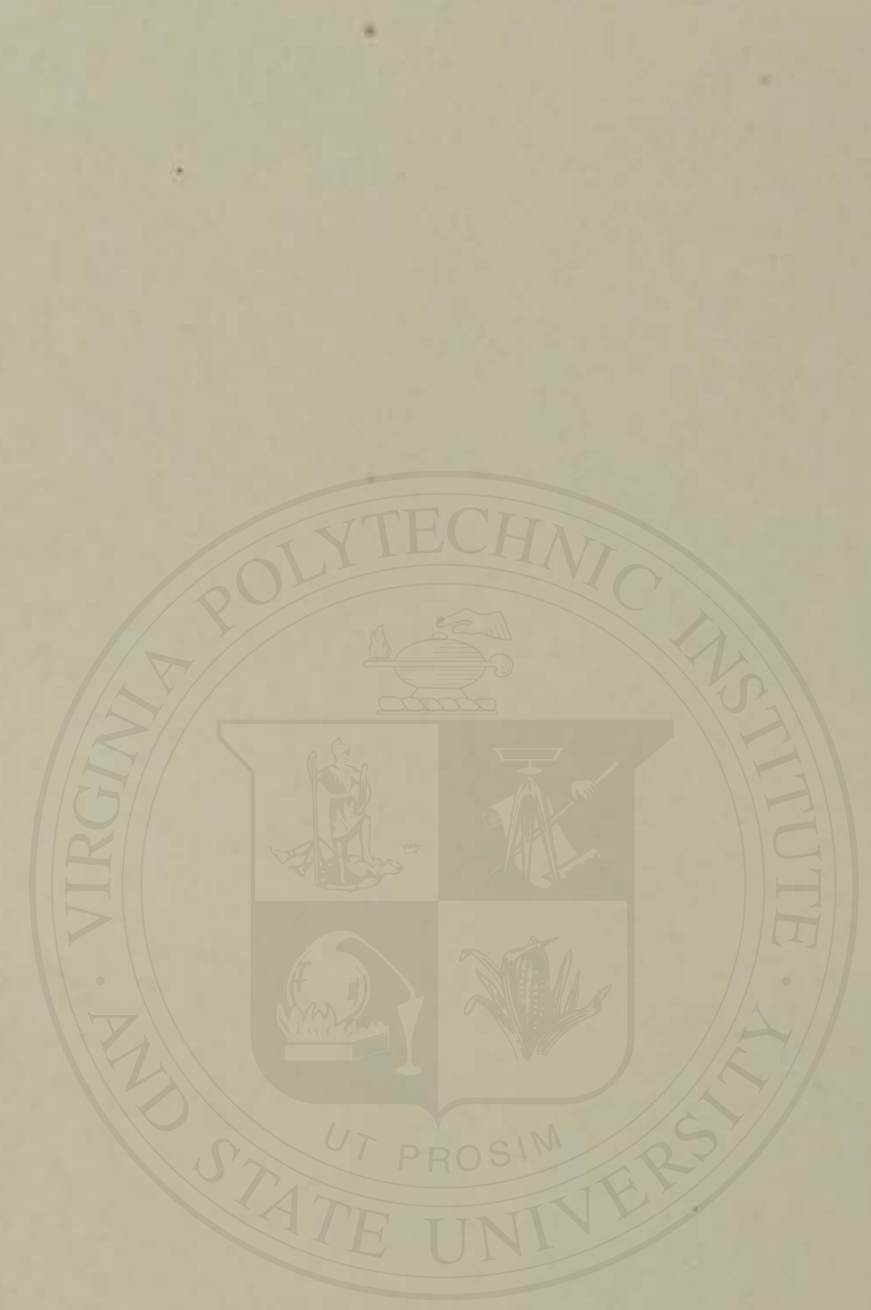


PLATE XIV.

\section{COFFEE.}

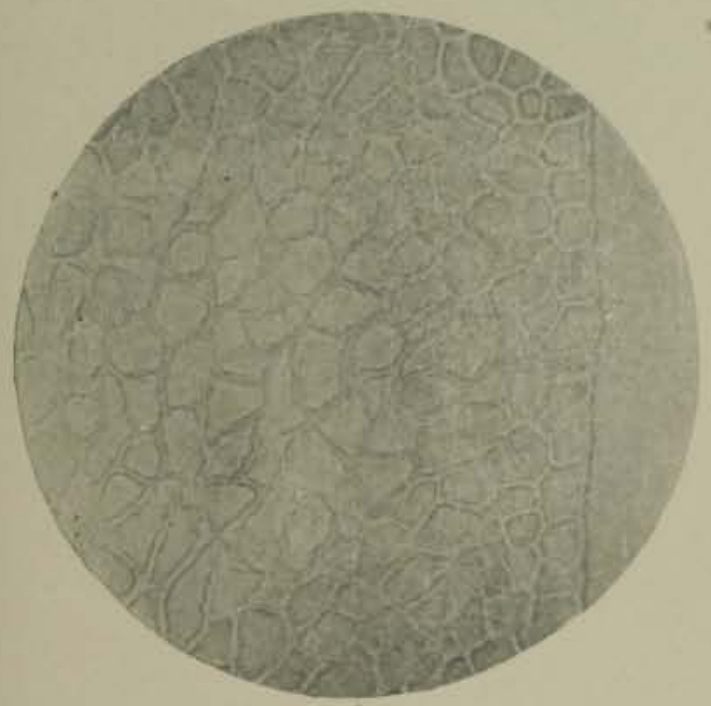

Fic. I73-Raw Coffee, $X$ iso.

Transverse section of outer portion of endosperm.

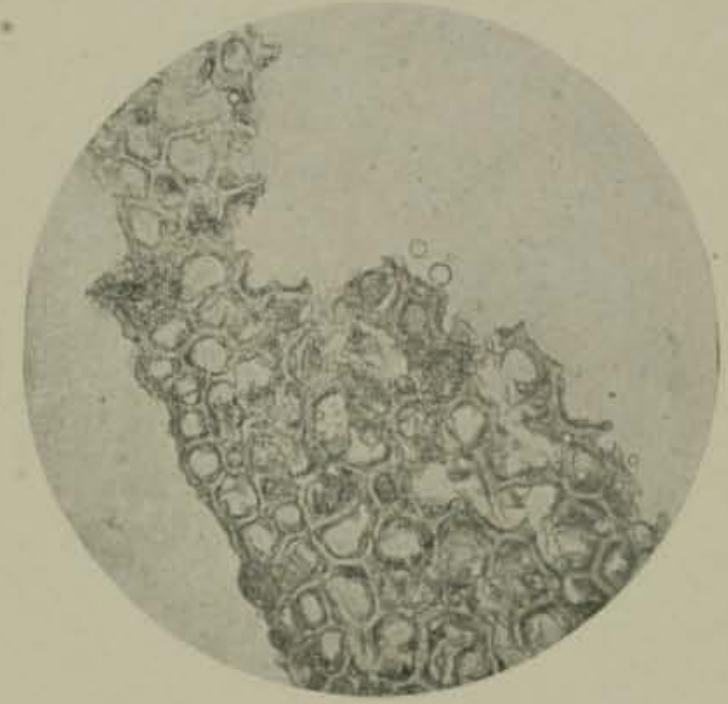

FtG. I74.-Roasted Coffee, $\times 130$.

Traasverse section through parenchyma of endosperm.

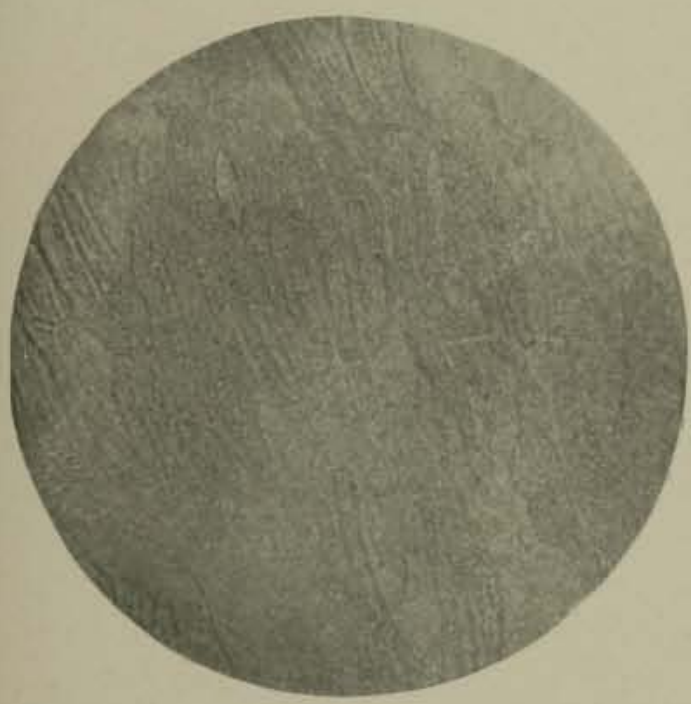

Frg. 175.-Coffee, $X$ rio.

Surface view of seed coat.

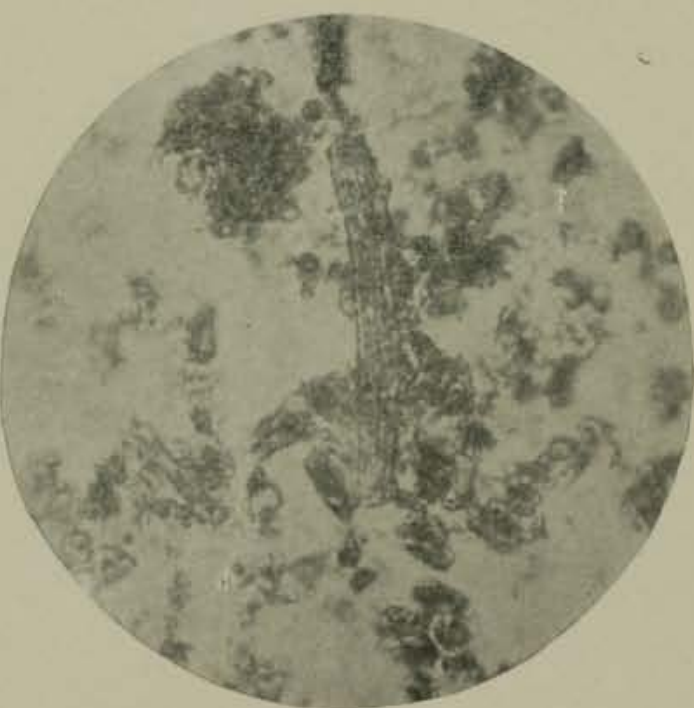

Fic. 176.-Coffee, $\times$ rro.

Roasted, ground coffee, showing fragments of endosperm parenchyma and of seed coat. 


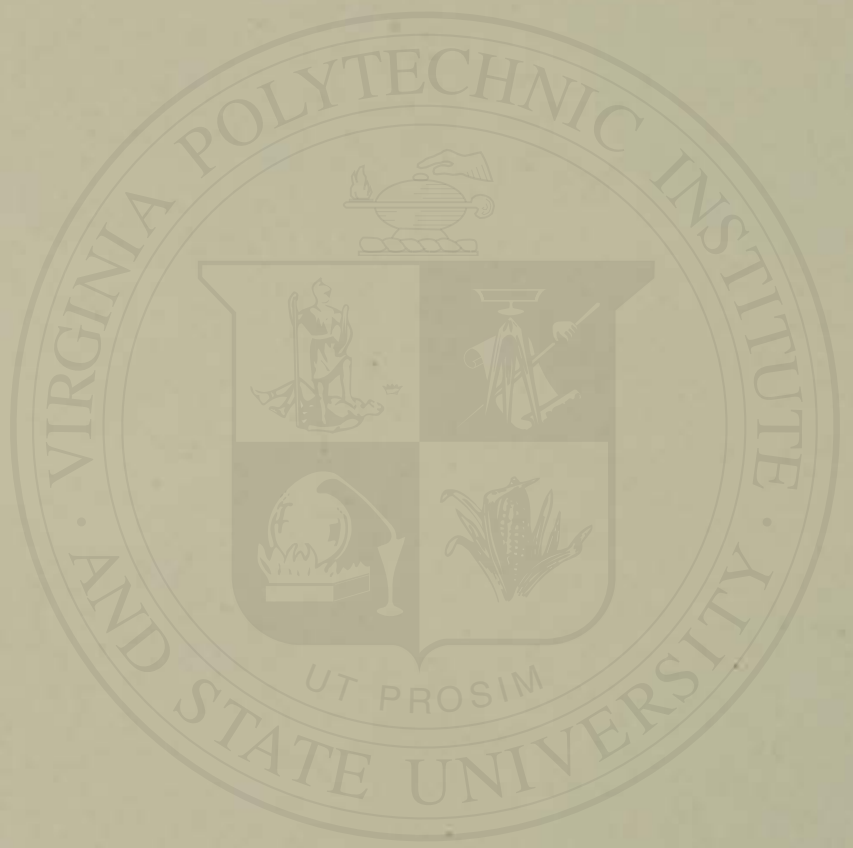


PLATE XV.

\section{COFFEE. CHICORY.}

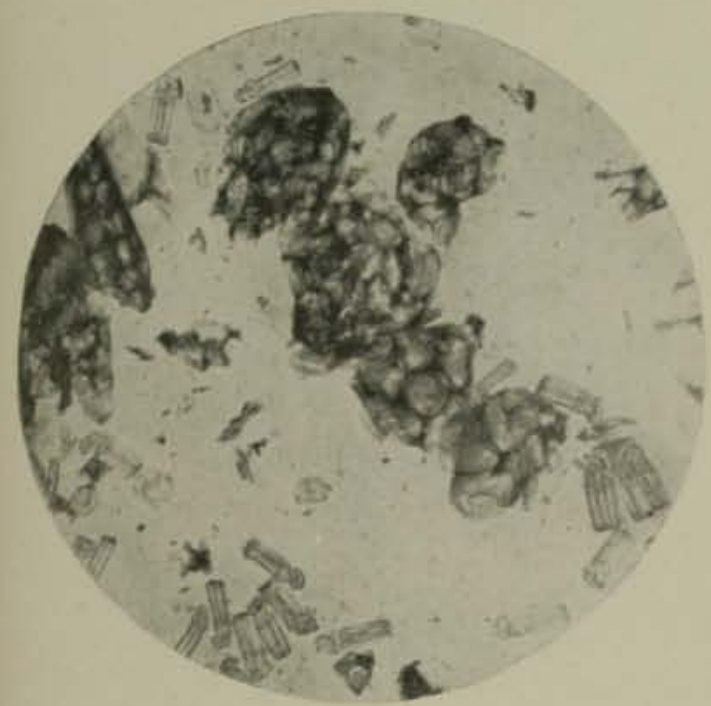

Fig, 177 ,-Adulterated Coffee, $\times 130$.

Dark masses of roasted pea starch are shown, with transparent fragments of the palisade cells of the pea-tull.

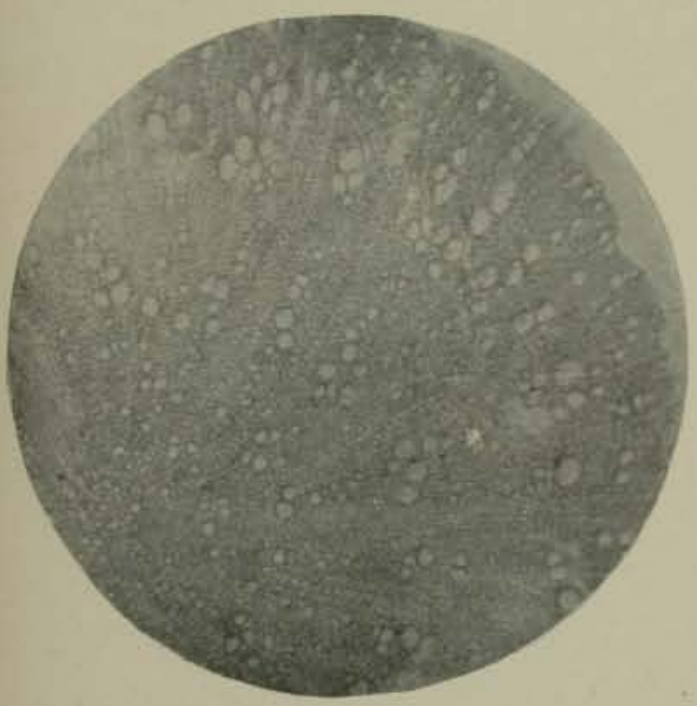

Fig. 179.-Chicory, $\times 25$.

Transverse section through the root.

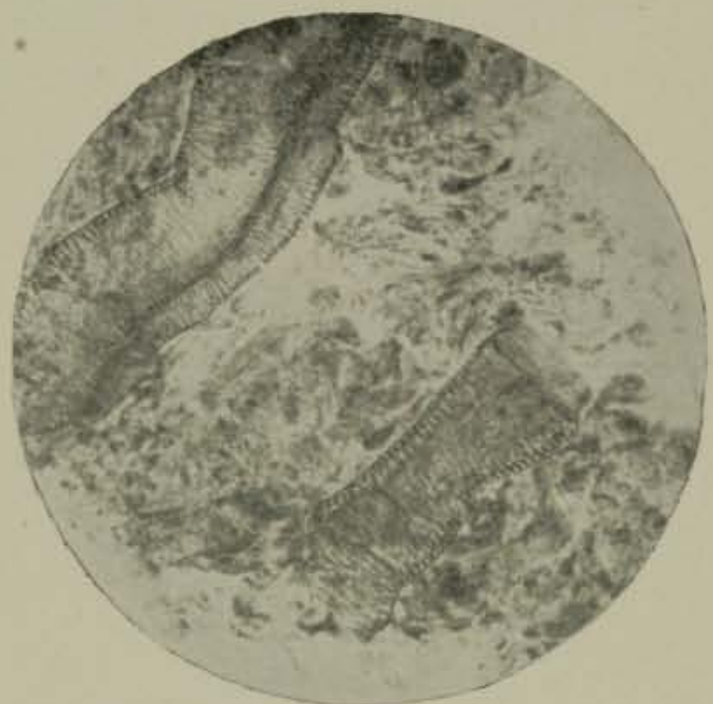

Fic. 178 . - Adulterated Coffee, $\times 130$.

The vascular ducts of chicory show most conspicuously in this field.

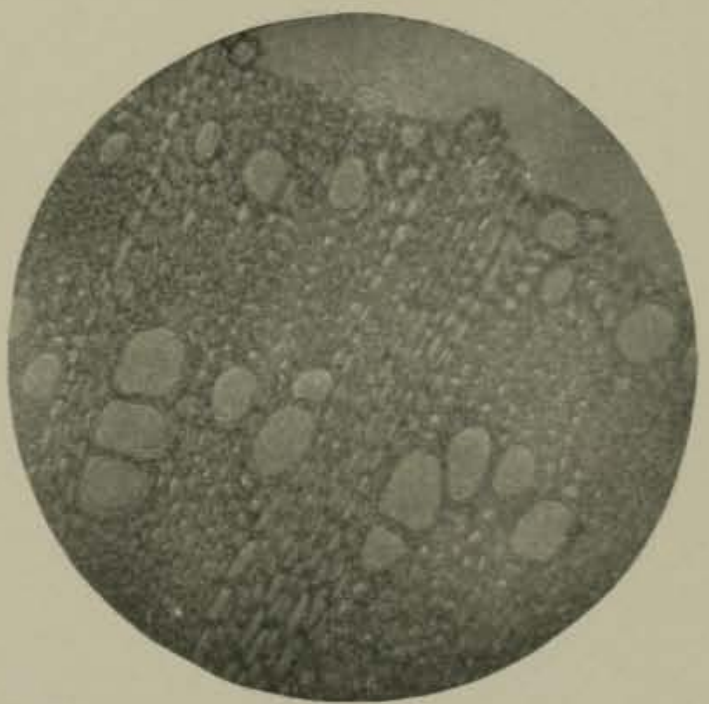

Fis. 180.-Chicory, Xi10.

Transverse section. 


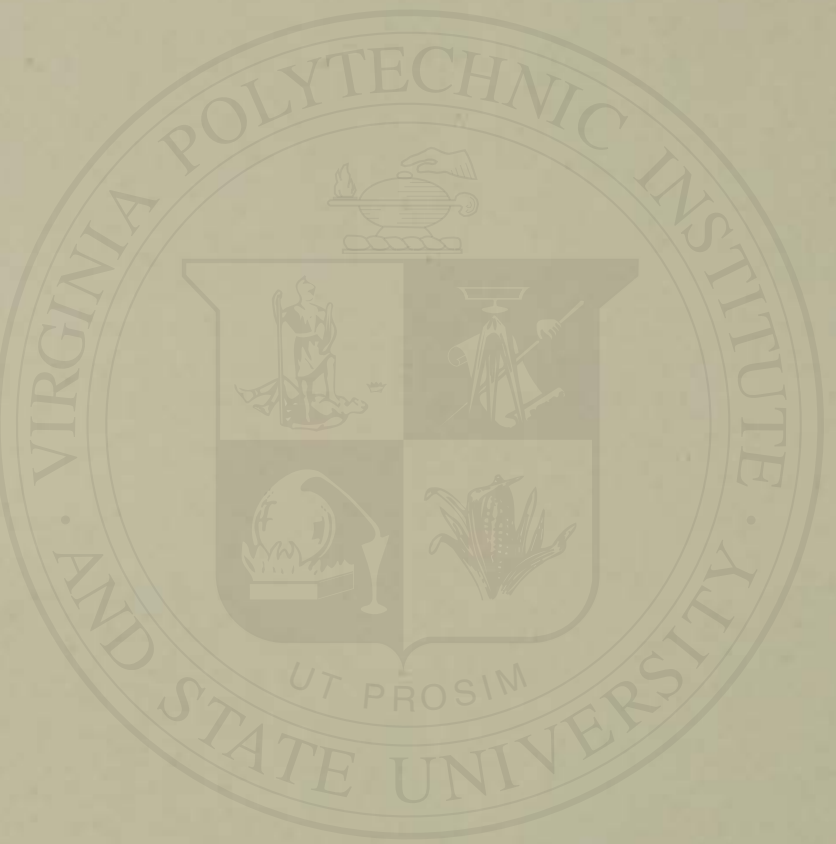




\section{CHICORY. COCOA.}

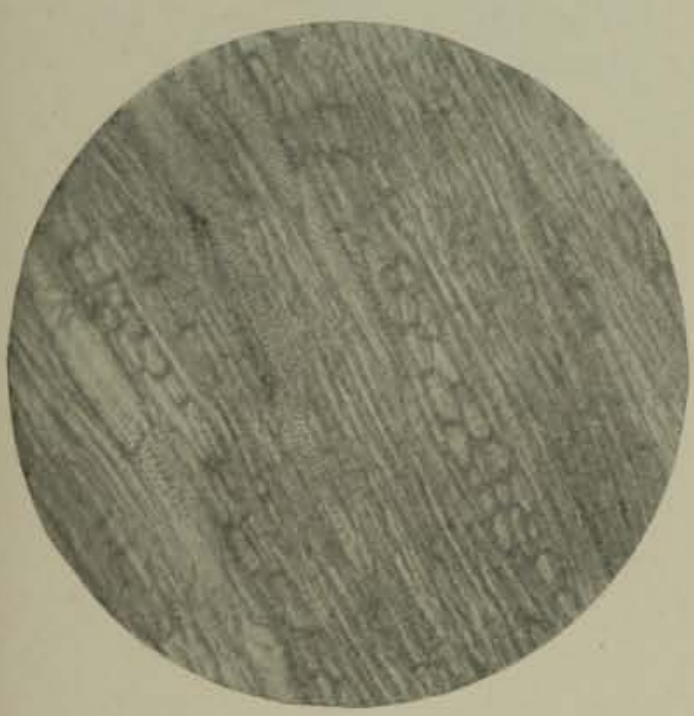

Fit. 181 . - Chicory, $X \mathrm{Ito}$.

Tangential section, showing reticulated ducts and wood parenchyma.

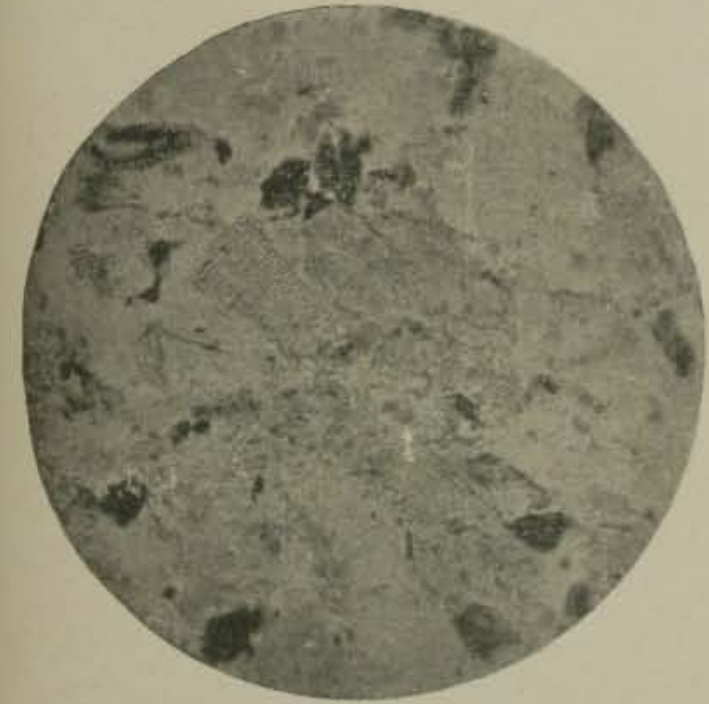

FIG. 183 .-Chicory, $\mathrm{X}_{\text {rto. }}$

Roasted and ground, showing fragments of ducts and other tissues.

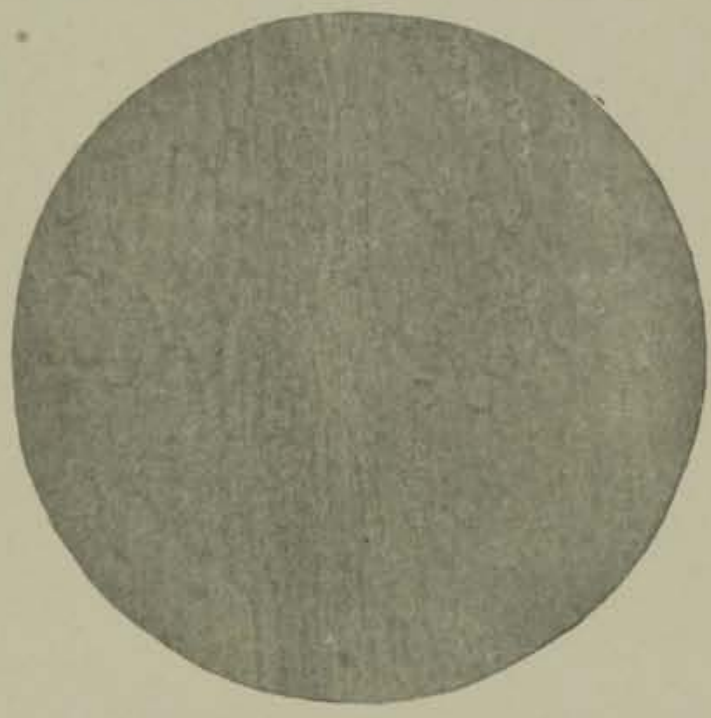

- Fis. IS2,-Chicory, $\mathrm{X}$ iro

Radial section, showing bark parenchyma and milk ducts.

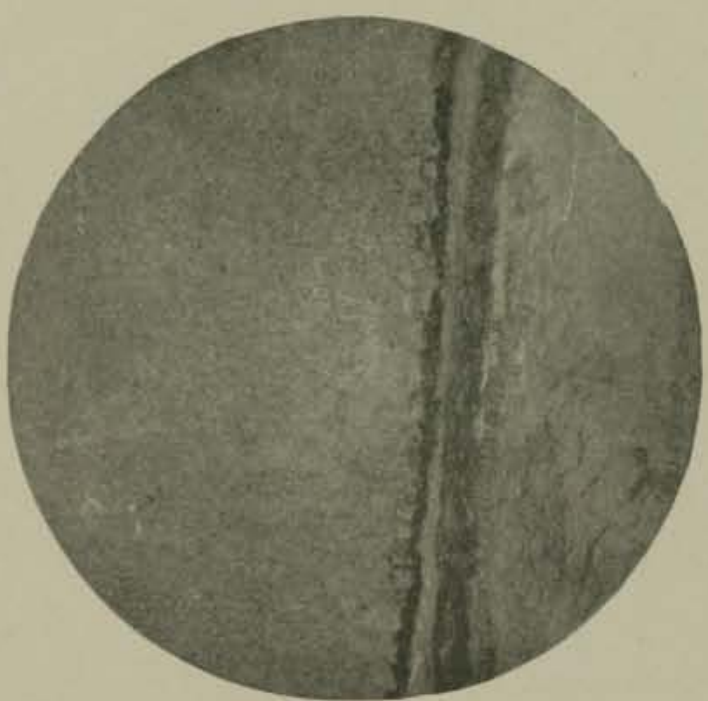

Fic. $\mathrm{IS}_{4}$-Cocoa, $\mathrm{X}_{\mathrm{rio}}$

Transverse section through periphery of seed, seed coats, and cotyledon. 


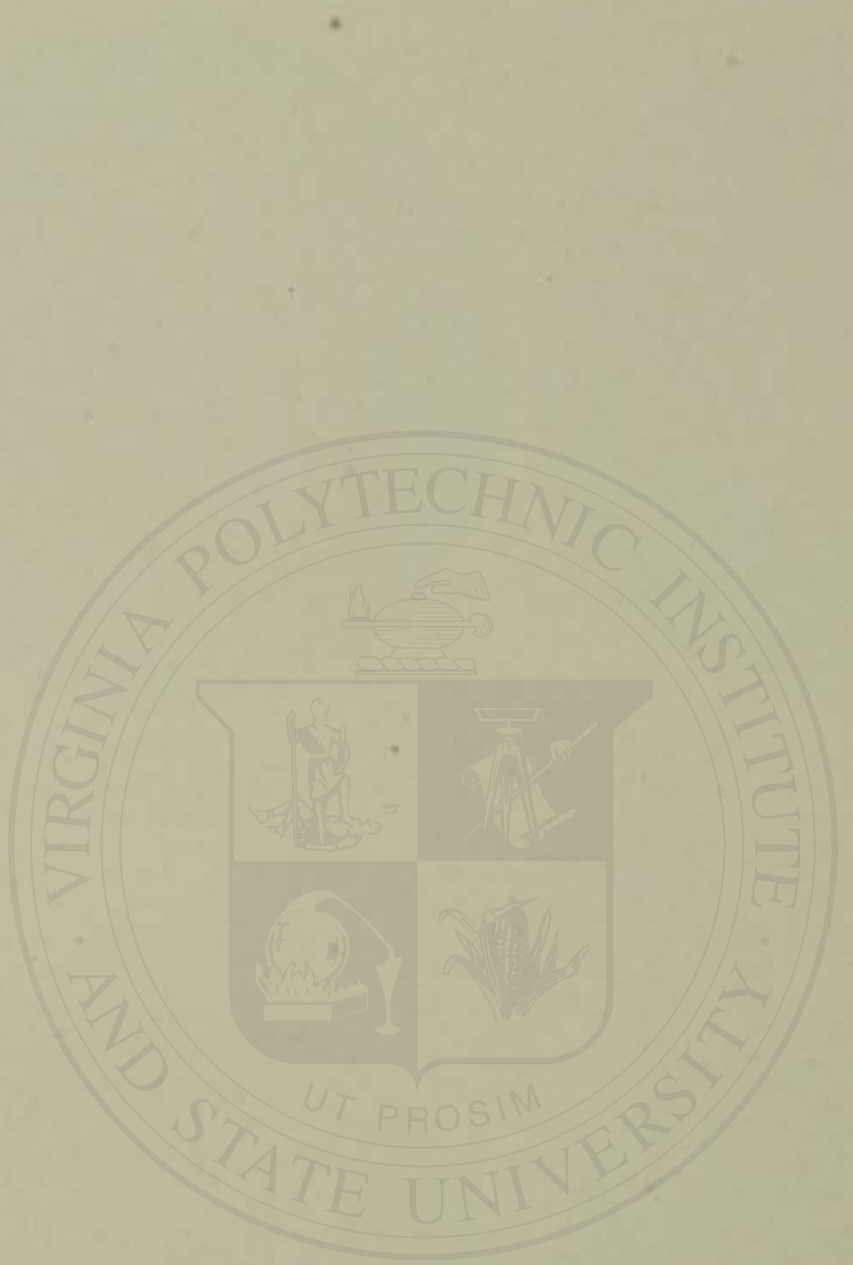




\section{COCOA.}

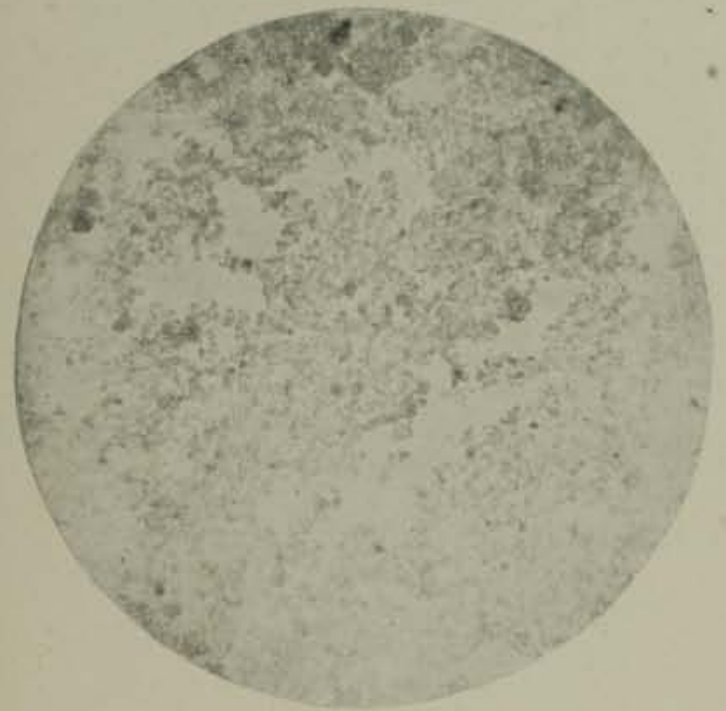

Fig. 185--Powdered Cocoa, Xiro.

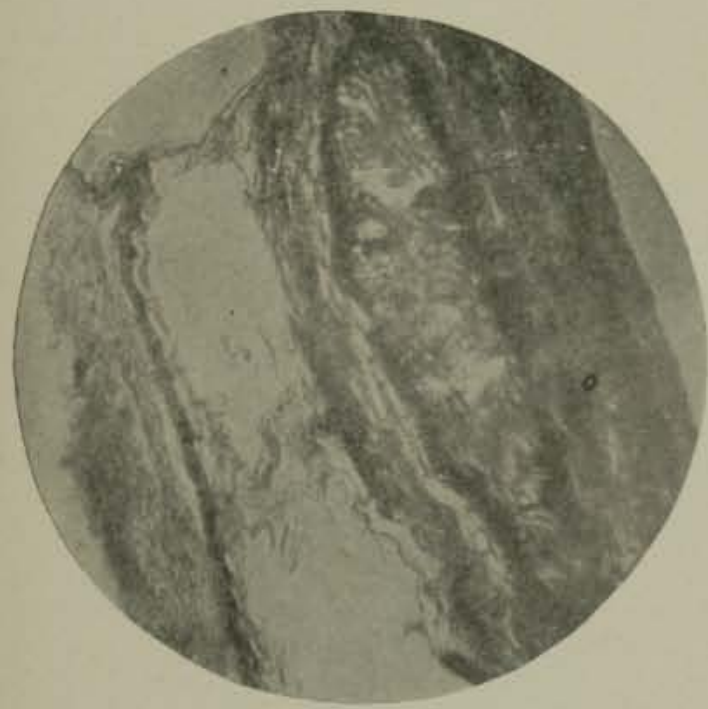

Fig 187 --Cocoa Shell, Xiro.

Transverse section through epidermis, pulp, and mucilaginous layers of the pericarp and seed coat.

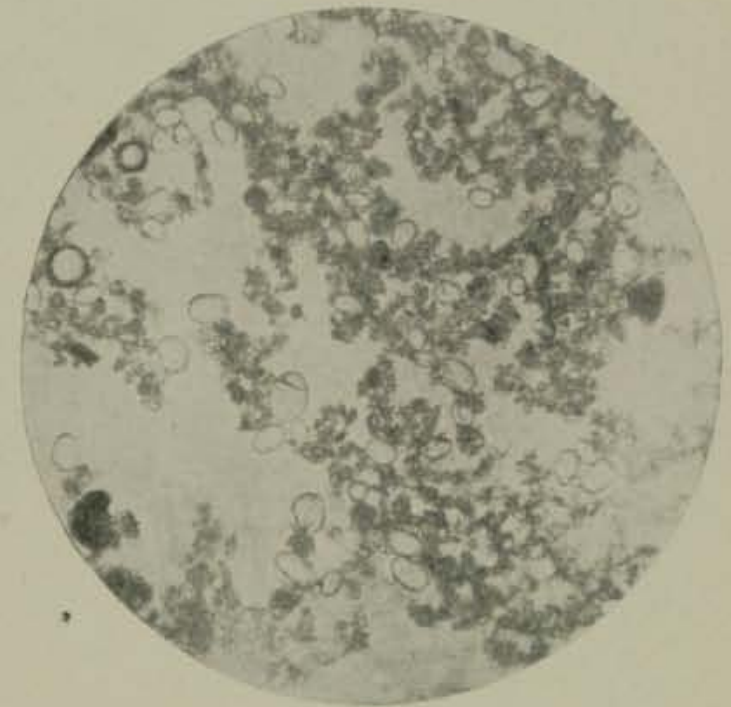

Fig, $186,-$ - Adulterated Cocoa, $X 110$

Showing admixture of arrowroot with the cocoa powder.

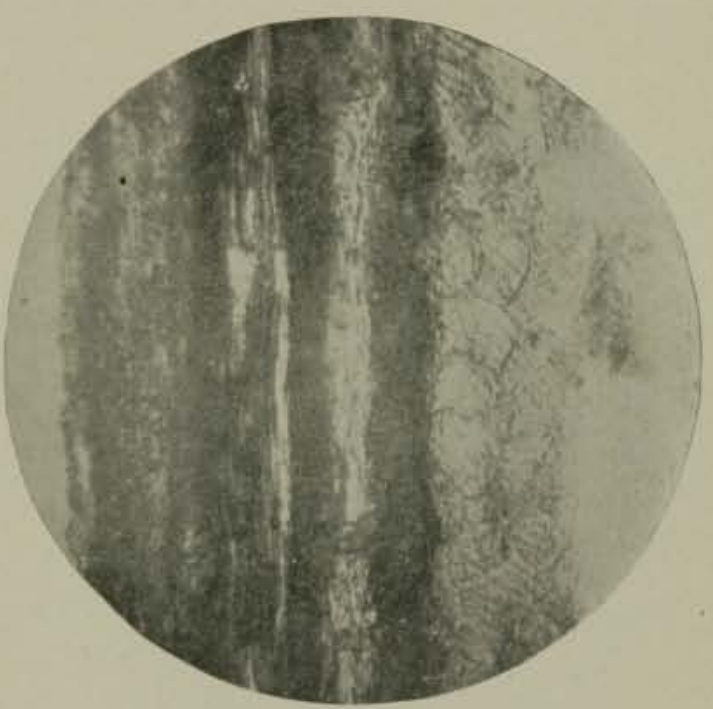

Fig, 188.-Cocoa Shell, Xirc.

Longitudinal section through shell. 


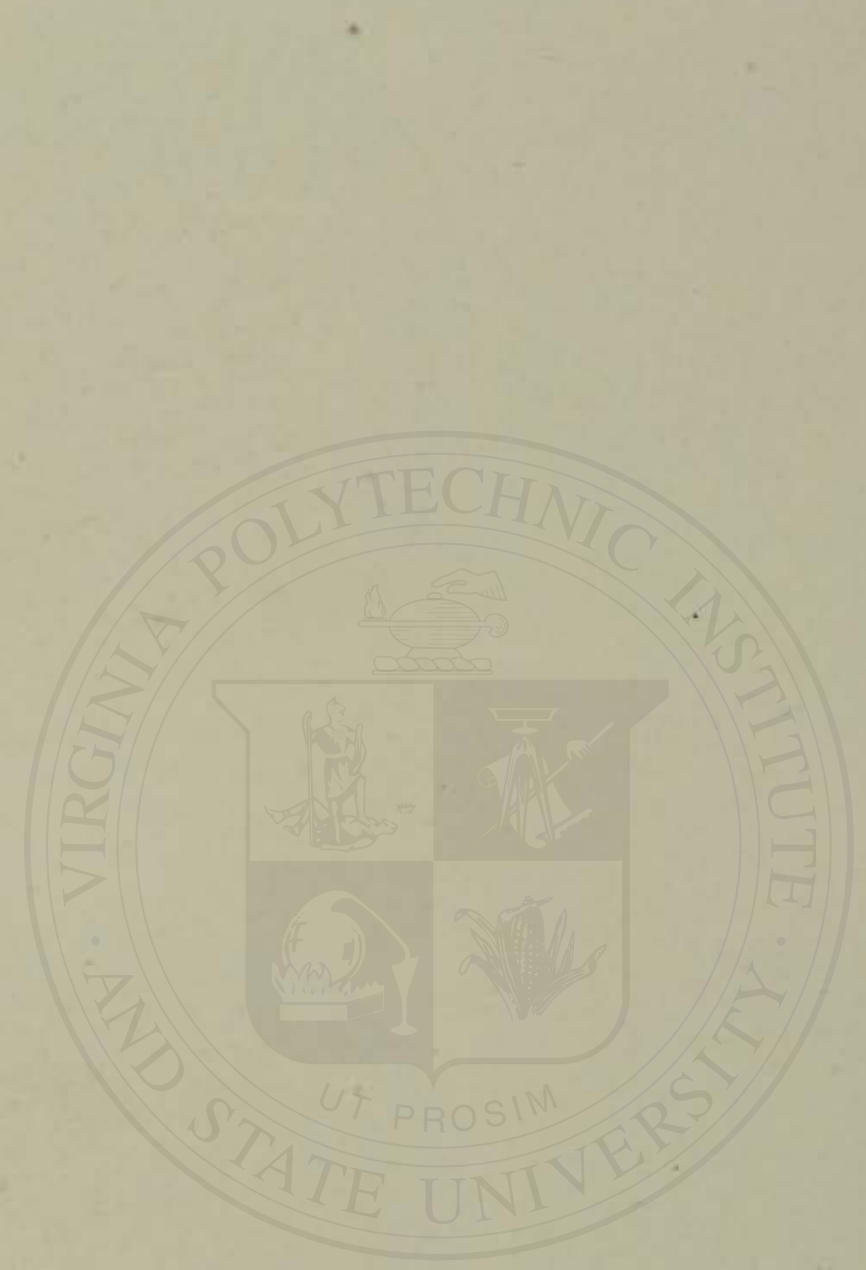


TEA. SPICES.

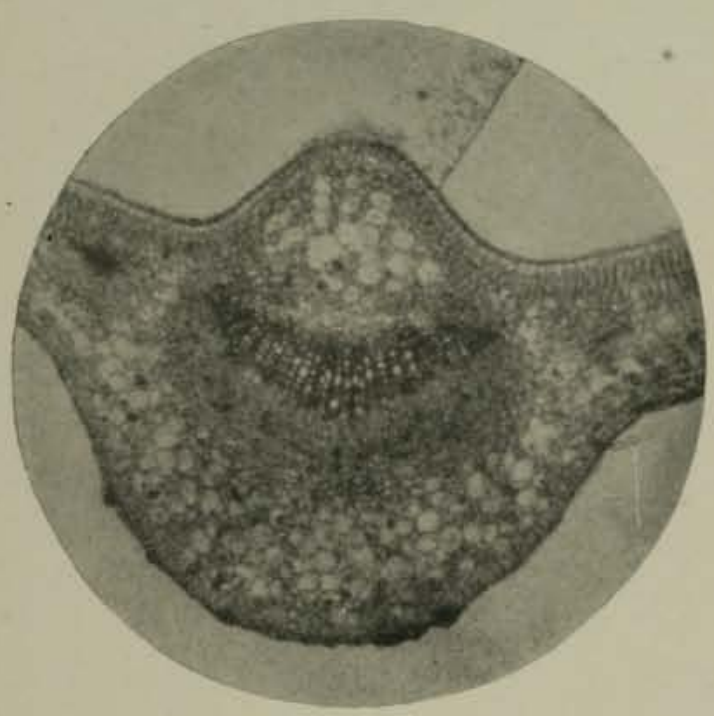

Frg. $189 .-$ Tea, $\times 55$.

Transverse section through midrib of leaf. Note the palisade layer below the upper epidermis, the inner wood vessels above the center, and the parenchyma of the pulp.

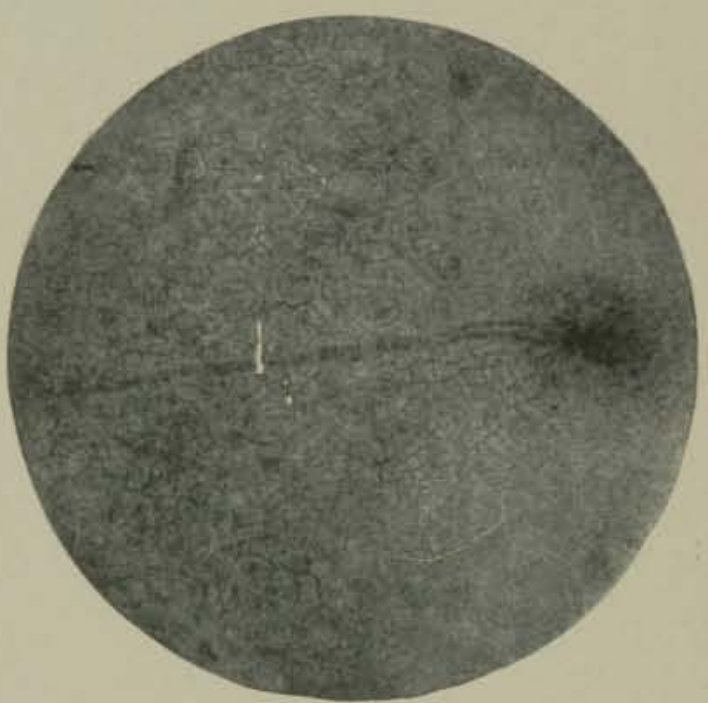

Fig. 190.-Tea, Xiro.

Surface view of lower epidermis, with stomata and one of the hairs.

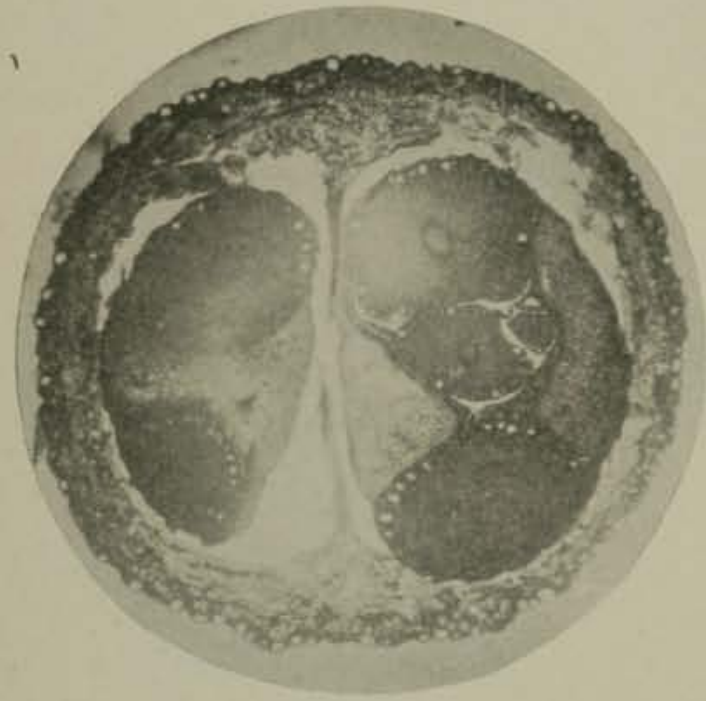

Fig. I91.-Allspice, $\times 9$.

Transverse section through the entire berry, showing the two cells, with kidney shaped seed in each.

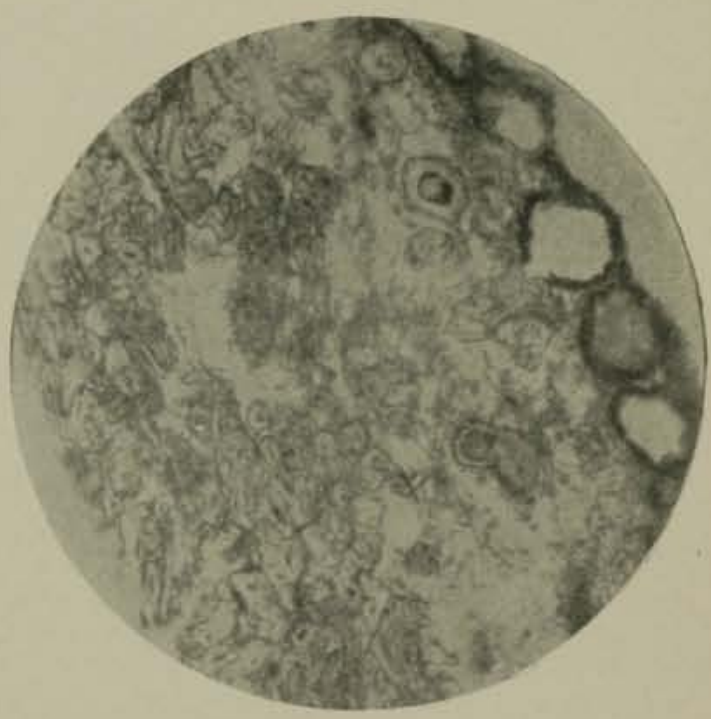

FrG. 192.-Allspice, $\times 70$.

Transverse section through pericarp, showing oil spaces and stone cells. 

PLATE XIX

\section{SPICES.}

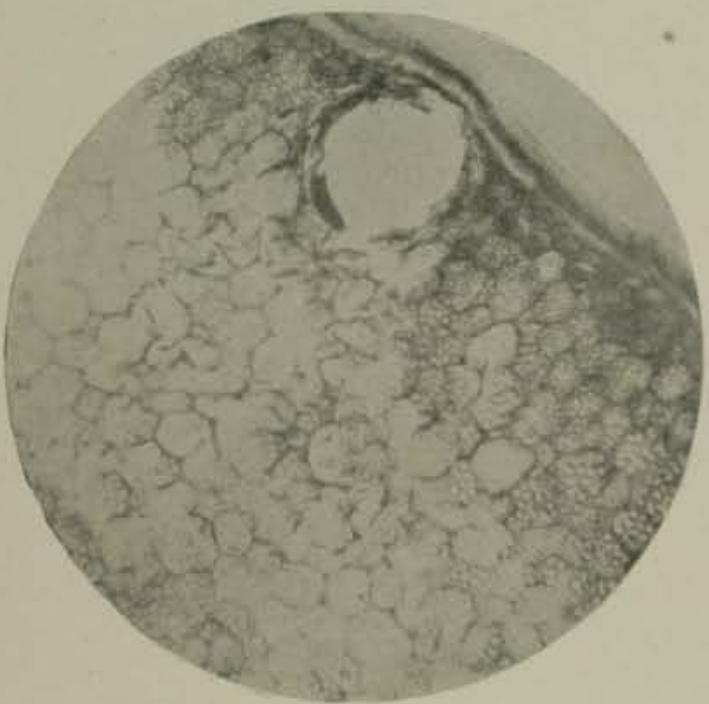

Fro. 193.-Allspice Seed $\times$ rro.

Transverse section through seed shell and part of embryo, showing starch cells.

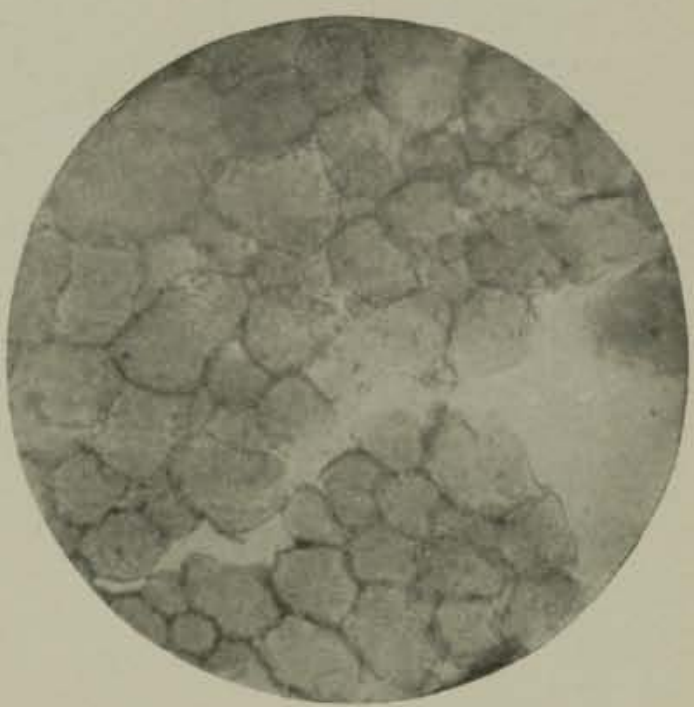

Frg. 194.-Allspice Seed, Xiro.

Transverse section through the resinous portion of the seed coat, showing port wine colored lumps of gum or resin.

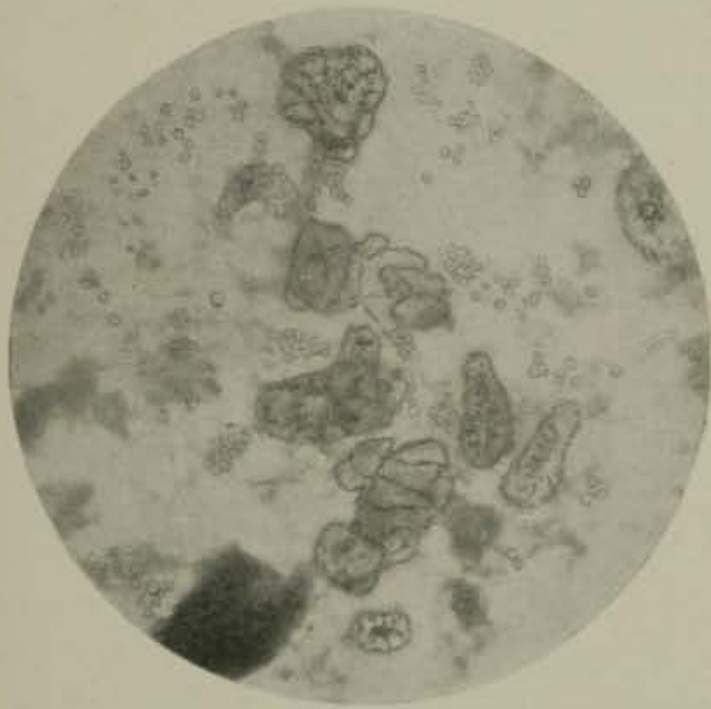

Fig. I95.-Powdered Allspice, $X$ r1o.

Showing stone cells, resinous lumps, and starch.

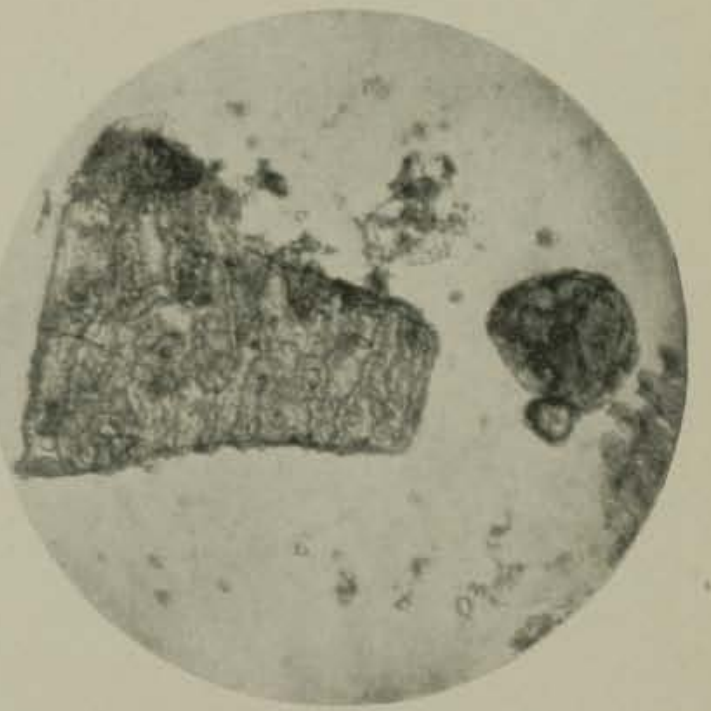

FiG. I96.-Adulterated Allspice, $X$ m10. Showing a large fragment of the seed skin of cayenne at the left. 


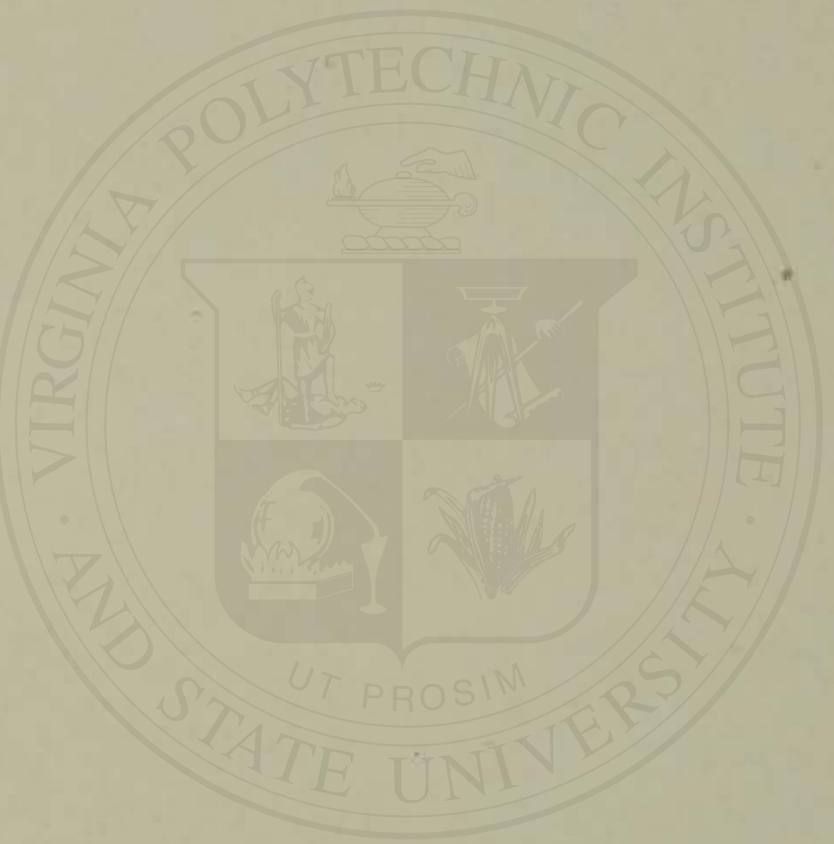


PLATE XX.

\section{SPICES.}

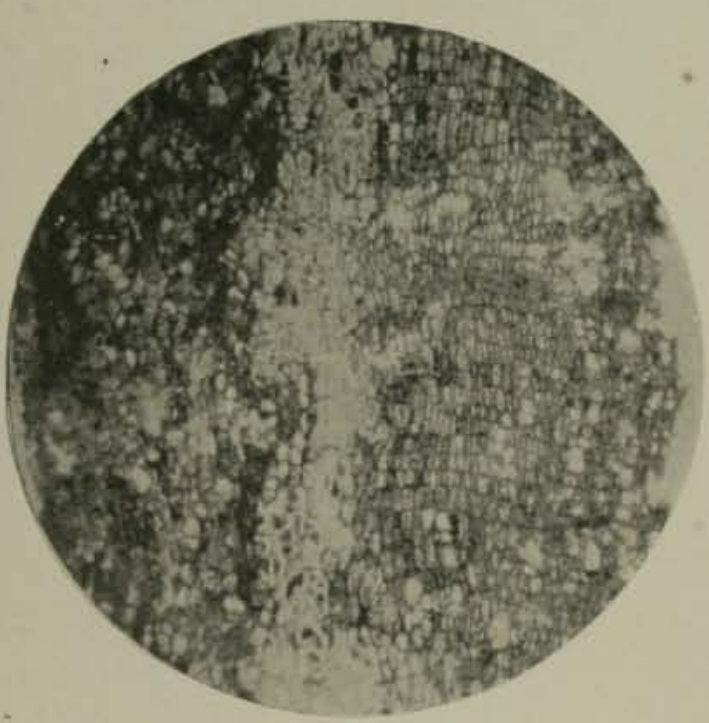

FiG. 197,-Cassia Bark, $\times 45$.

Transverse section through the bark.

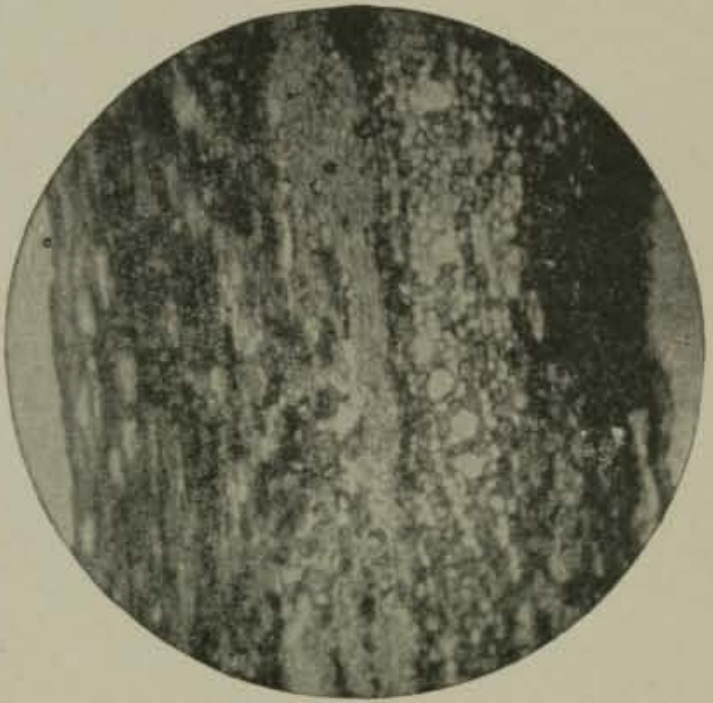

Frg. 198.-Cassia Bark, $X_{45}$. Longitudinal section.

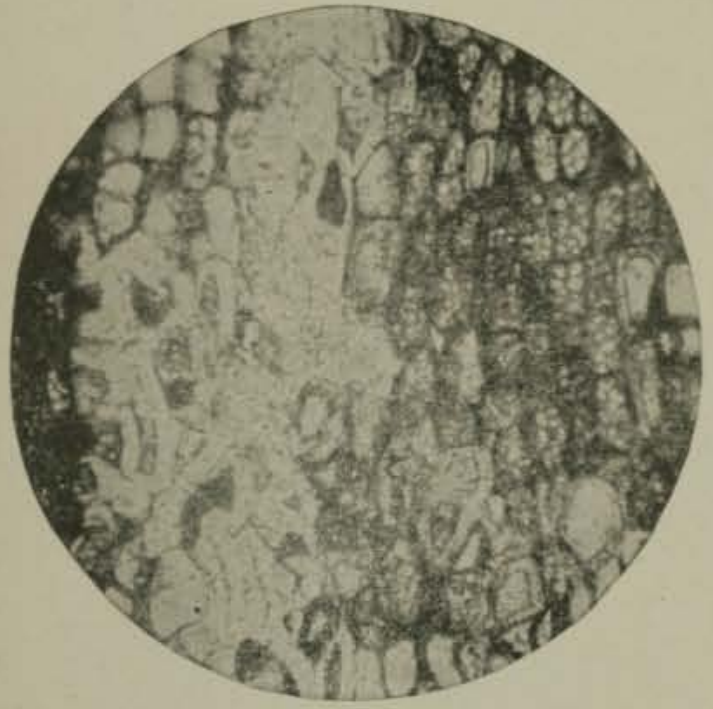

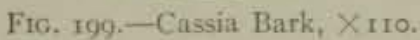

Transverse section, showing cork cells, parenchy$\mathrm{ma}$, and stone cells.

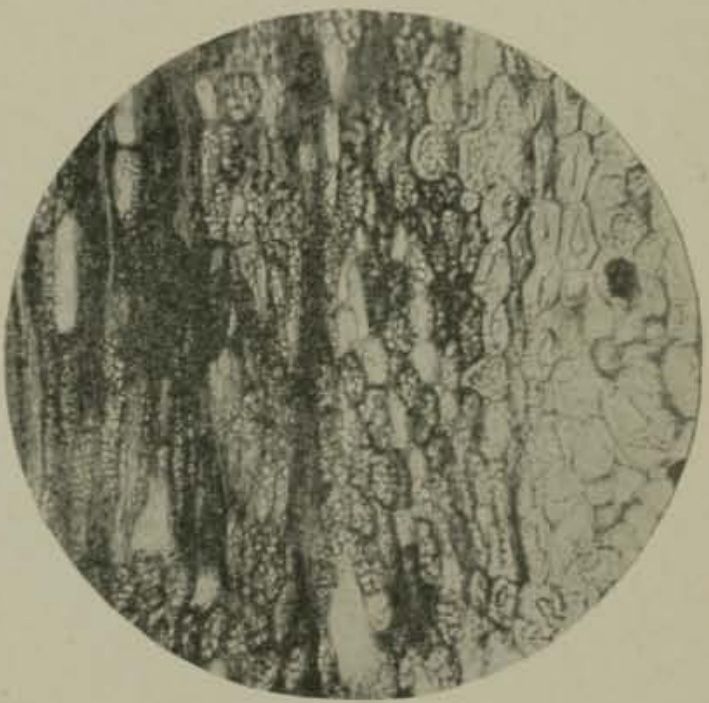

Fig. 200.-Cassia Bark, Xiro.

Longitudinal section, showing bunches of bast fibers at the left, starch cells in the center, and stone cells at the right. 


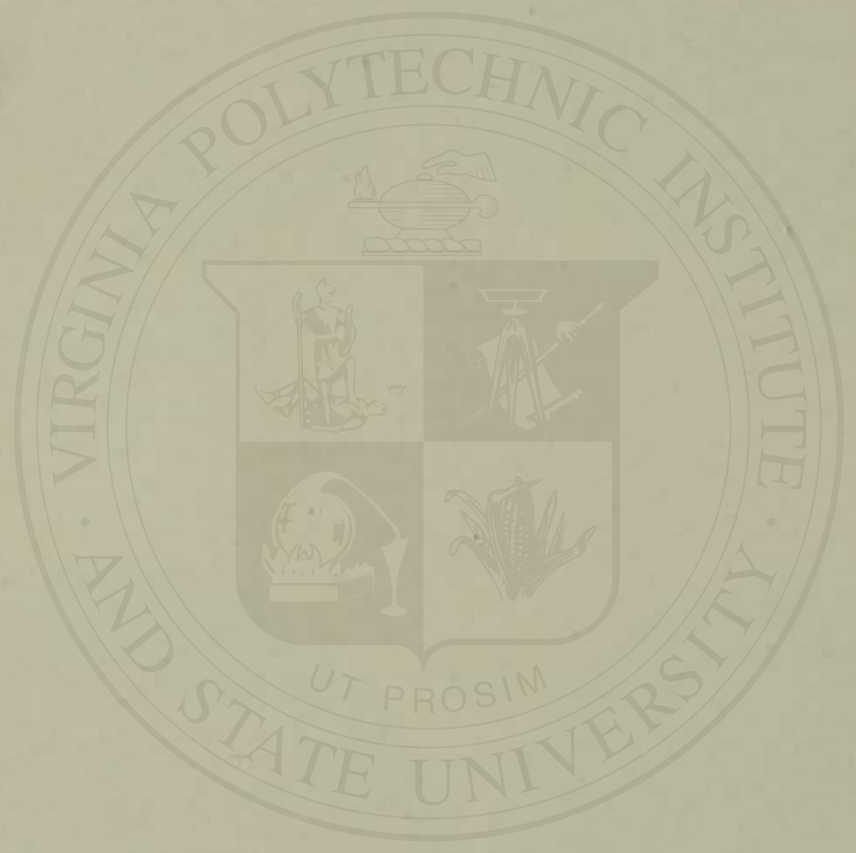




\section{SPICES.}

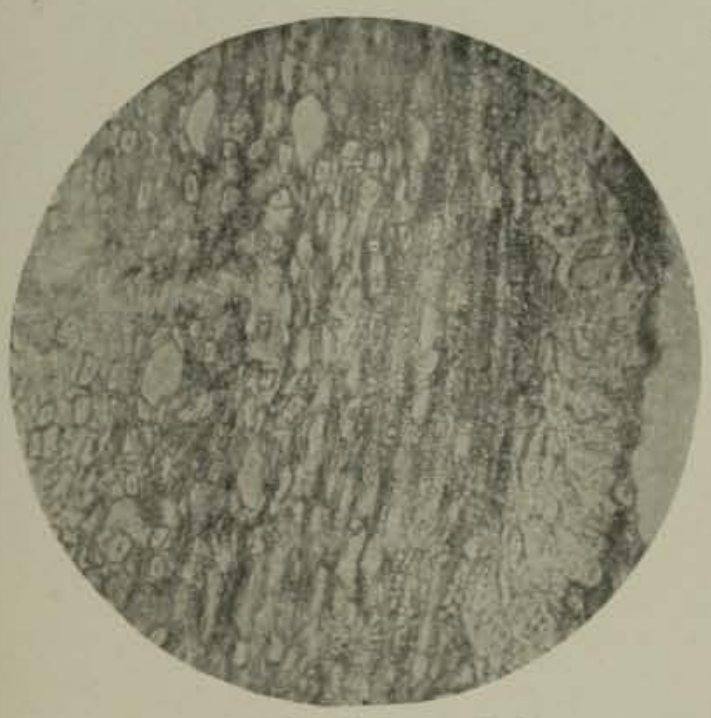

Frc. 201.-Ceylon Cinnamon Bark, $\times 110$.

Transverse section, showing many bast fibers and starch cells.

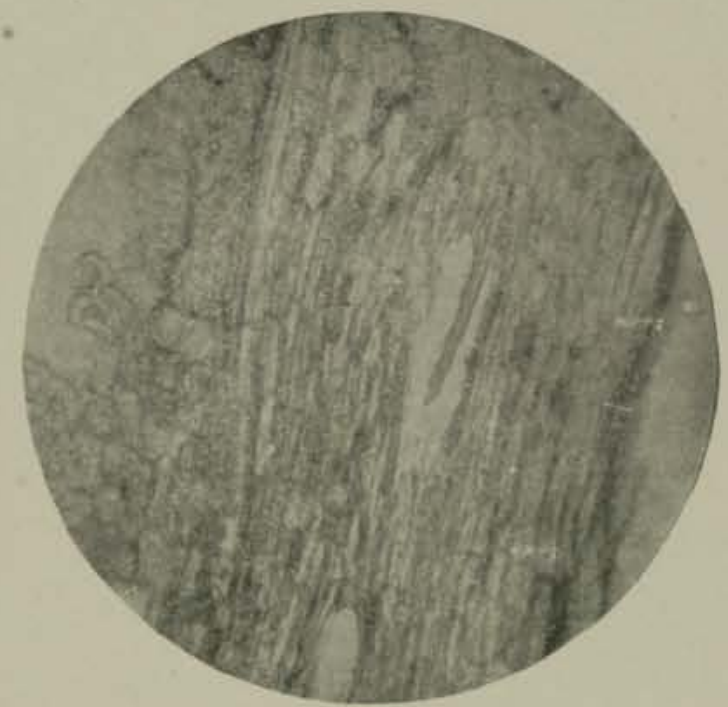

FrG. 202.-Ceylon Cinnamon Bark, Xruo.

Longitudinal section, showing bast fibers, stone cells, and parenchyma.

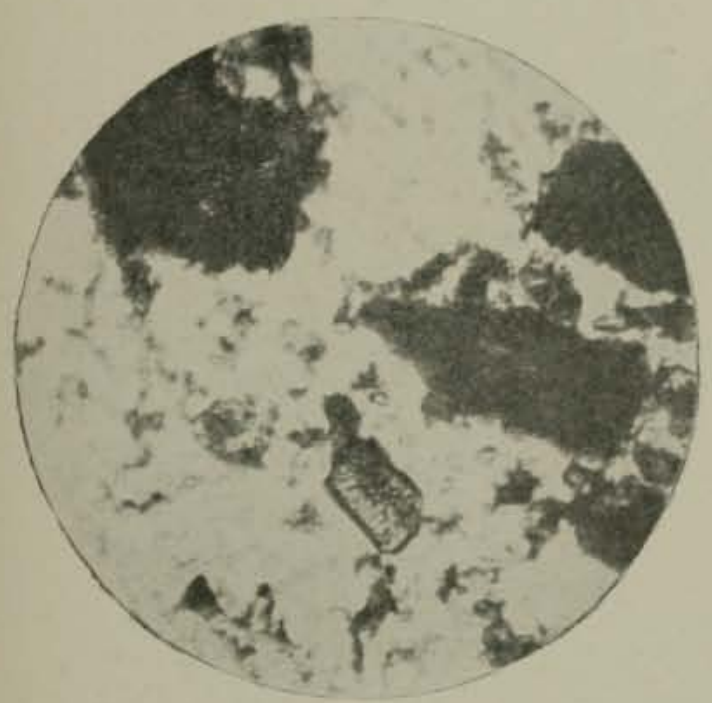

Fic. 203.-Powdered Cassia, Xro.

Showing stone cells, starch, and corky tissue.

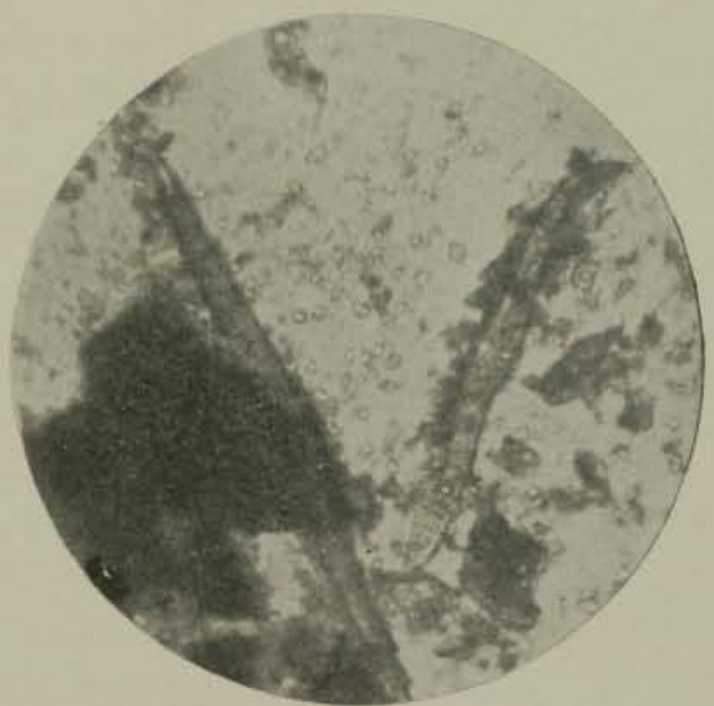

Frg. 204.-Powdered Cassia, $X_{\text {I }}$ ro.

Showing bast fibers and starch. 

PLATE XXII.

\section{SPICES.}

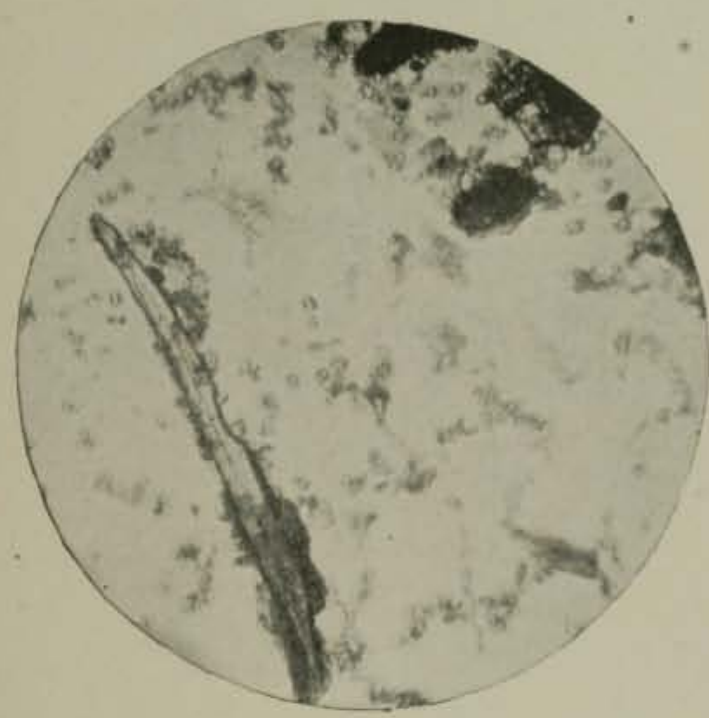

Fic. 205--Powdered Cassia, $X$ rro.

Showing large bast fiber and starch grains.

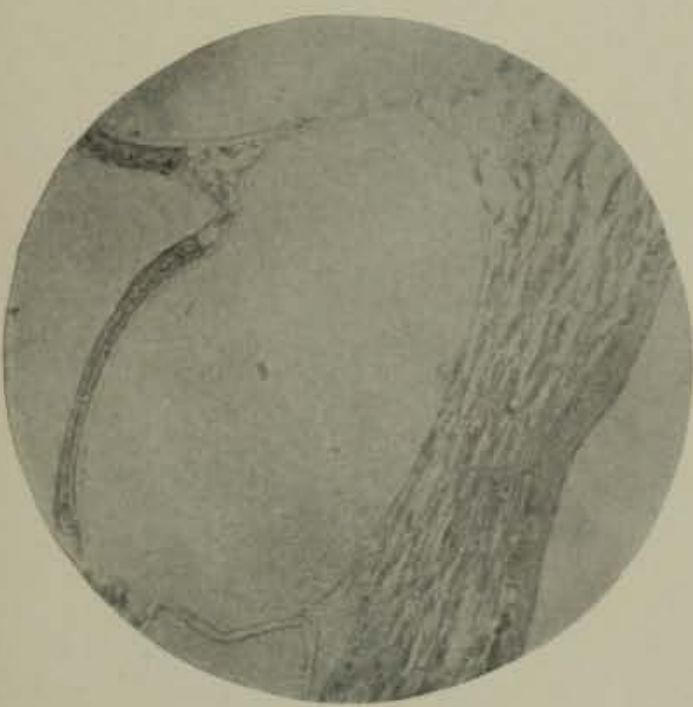

Frg. 207 -Cayenne, $X$ r1o.

Transverse section through pericarp.

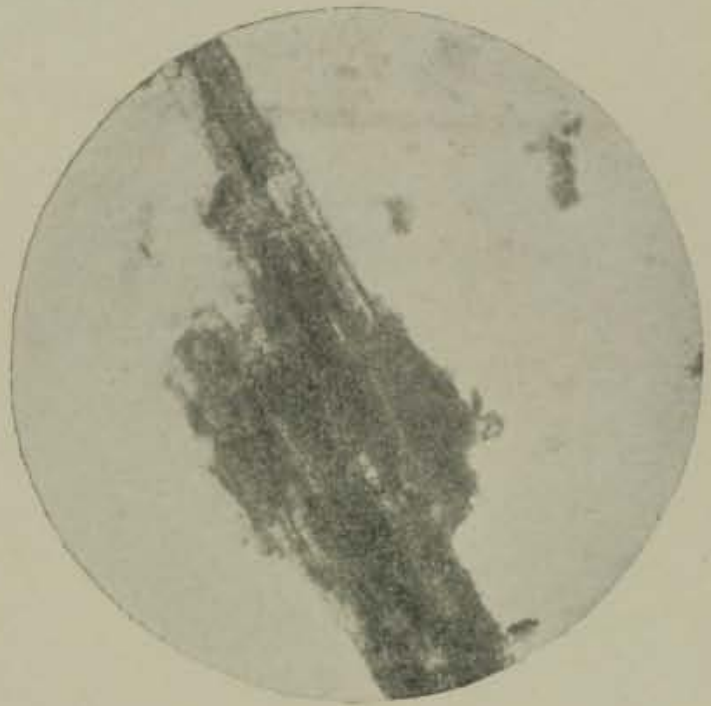

Frc. 206.-Adulterated Cassia, $\times 110$. A mass of foreign bark.

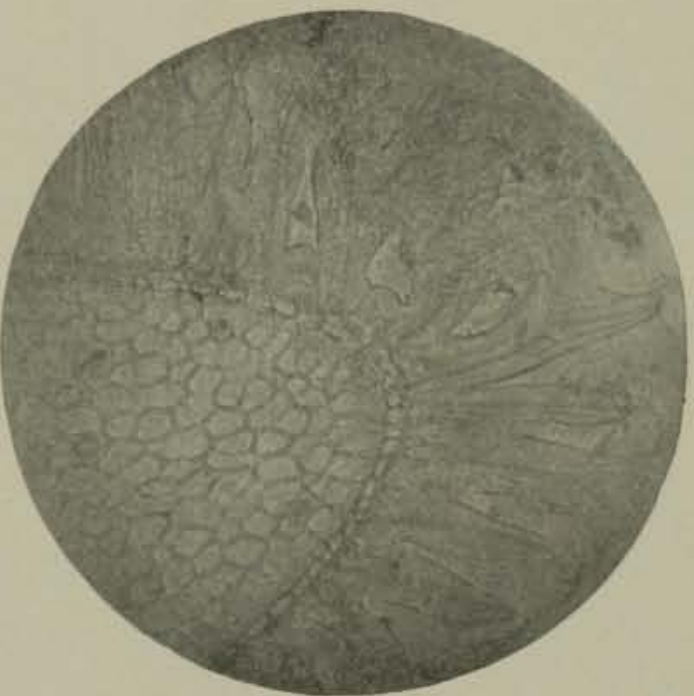

Fic. 208.-Cayenne, $X$ rio.

Transverse section through seed coat and part of endosperm. Collapsed parenchyma cells separate endosperm from long epidermal cells. 


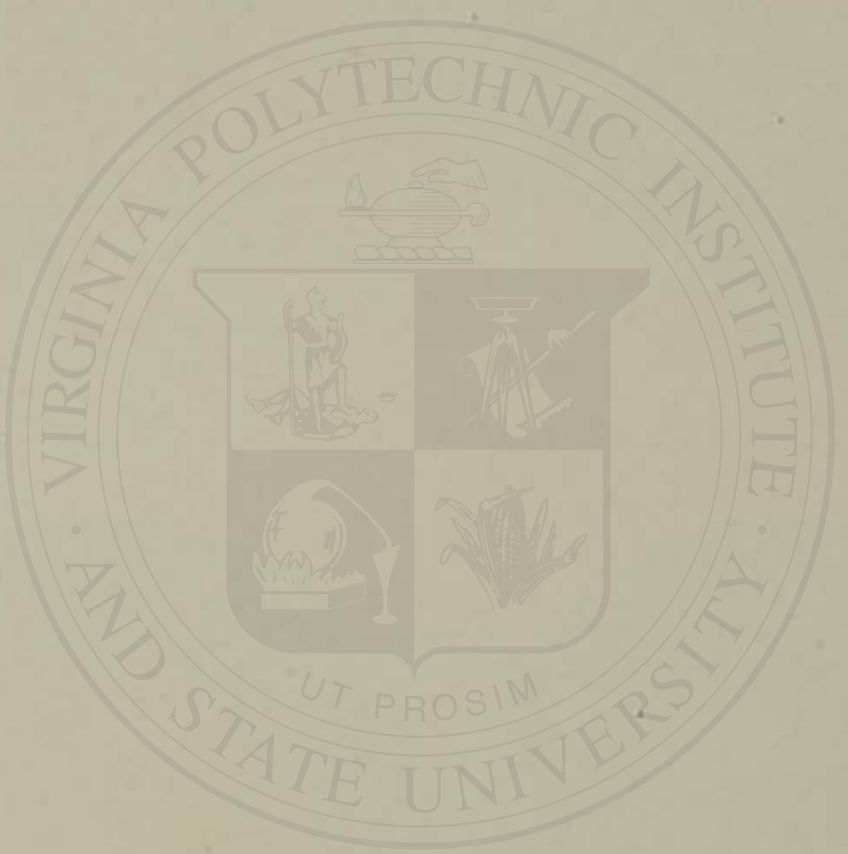


PLATE XXIII.

\section{SPICES.}

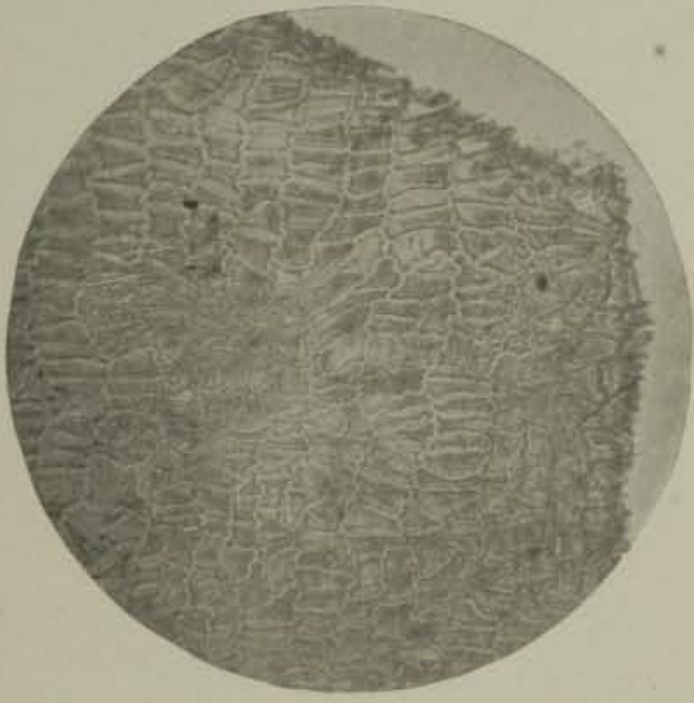

Fits. 200.-Cayenne, Xr10.

Surface view of fruit epidermis.

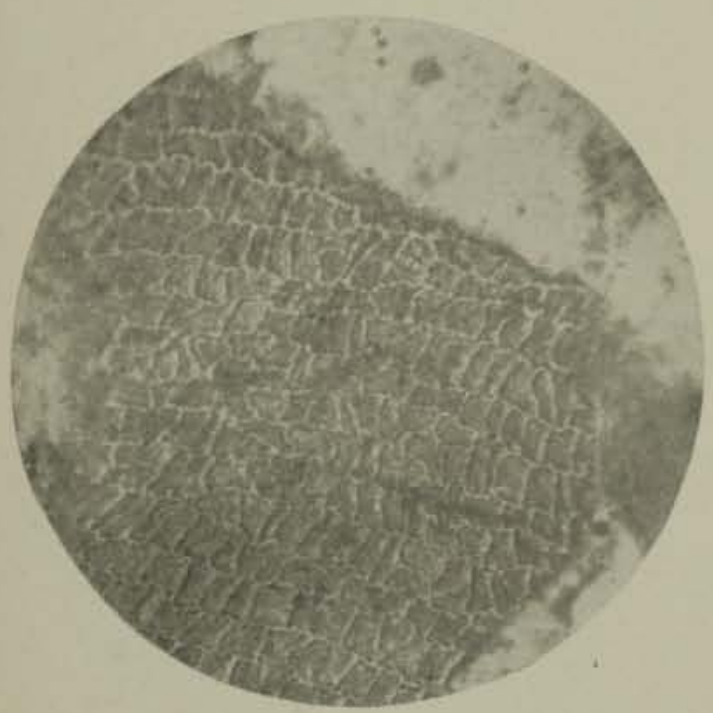

FIG. 211 .-Powdered Cayenne, Xrro. A large mass of fruit epidermis.

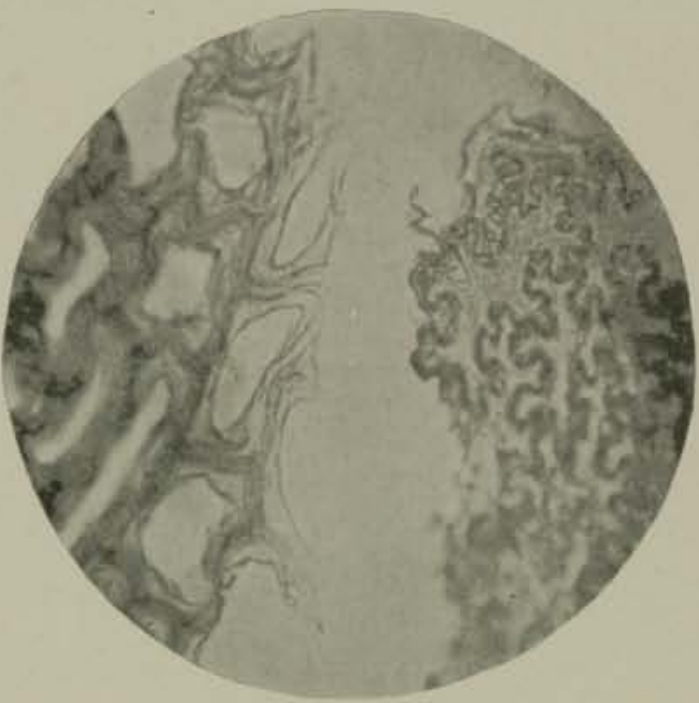

Fic. 2ro,-Cayenne, $\times$ tro.

Surface view of two layers of seed coat.

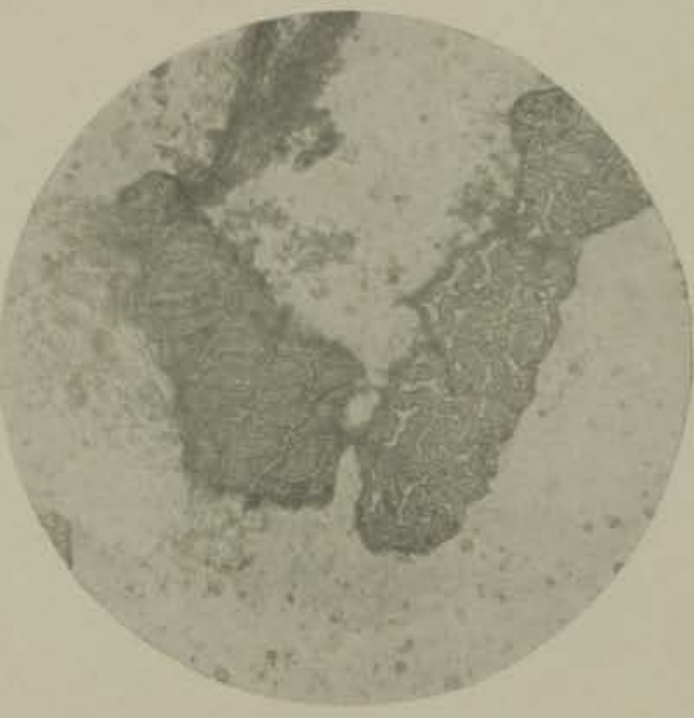

FIG. 212.-Powdered Cayenne, $\times 110$.

Showing chiefly two of the seed coat layers. 


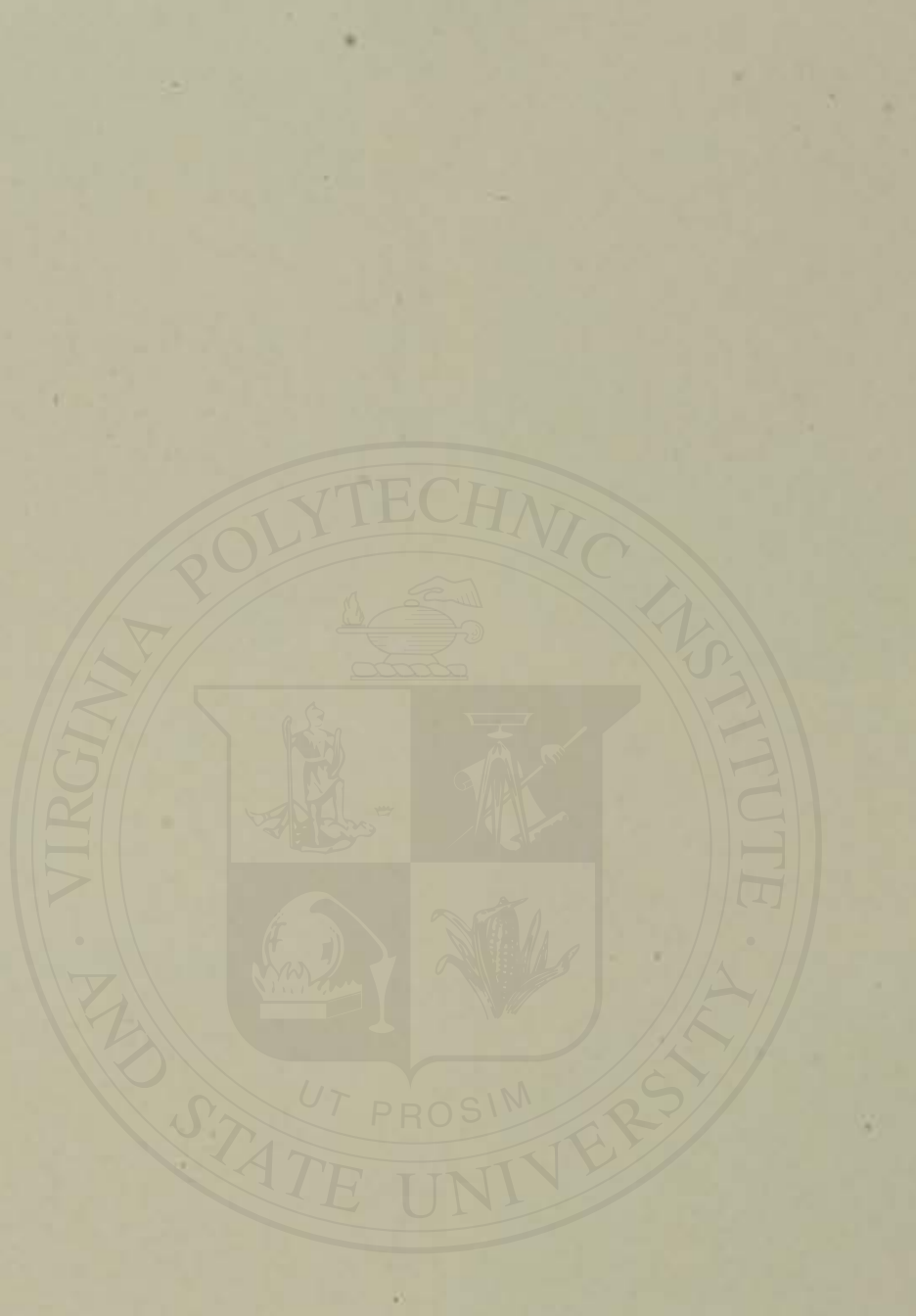




\section{PLATE XXIV.}

\section{SPICES.}

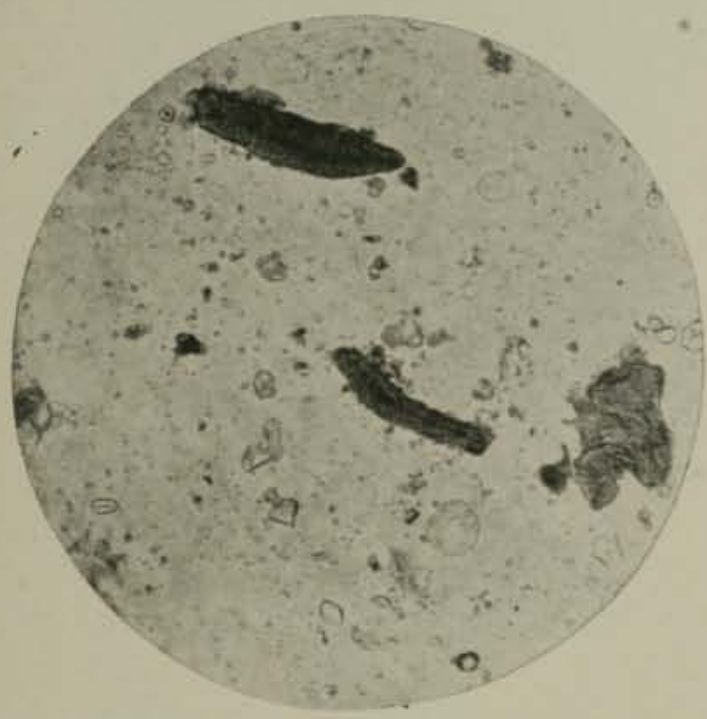

Fic. 213.-Adulterated Cayenne, $X$ I3O $_{3}$.

Corn and wheat starch and cocoanut shells appear chiefly. A bit of cayenne is shown at the right.

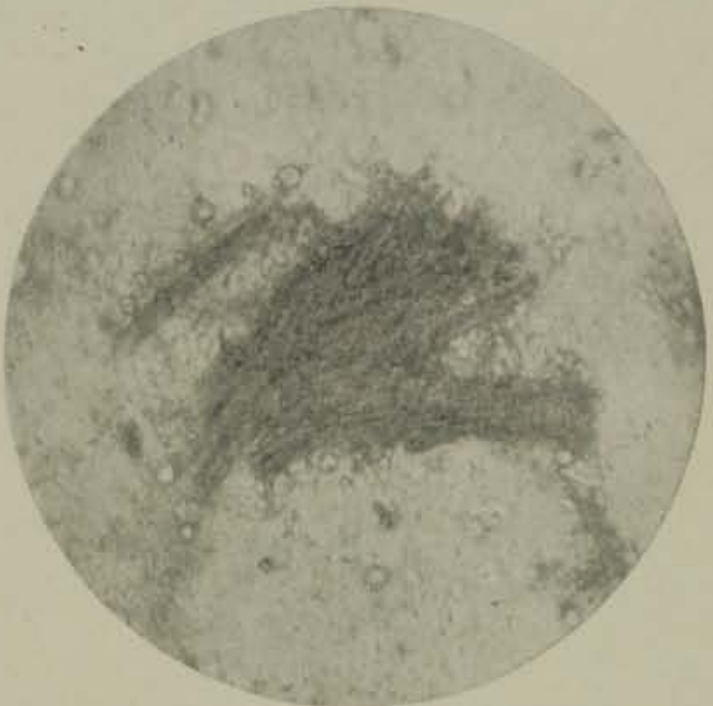

FrG. 214.-Adulterated Cayenne, $\times 214$.

The central mass is ground red wood, surrounded by corn starch grains.

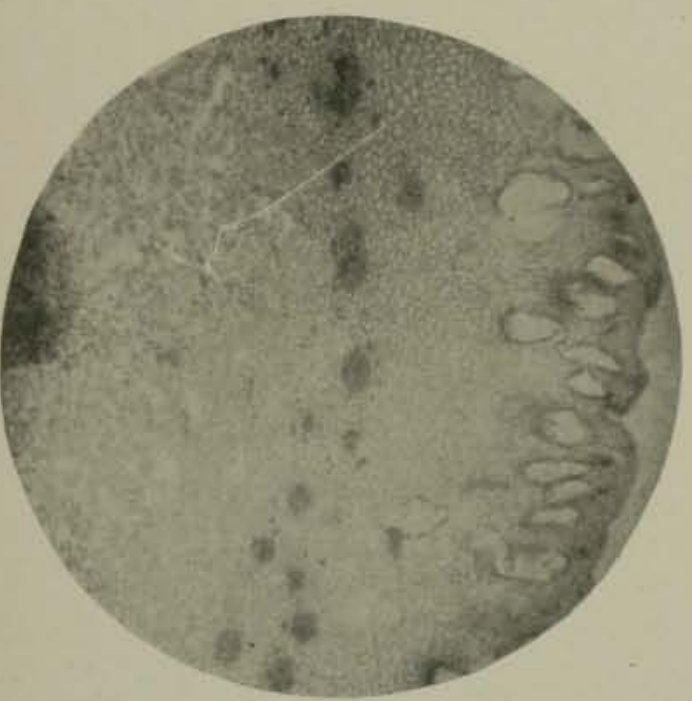

Fic. 215.-Clove, $\times 65$.

Transverse section from the center outward to epidermis, showing parenchyma.

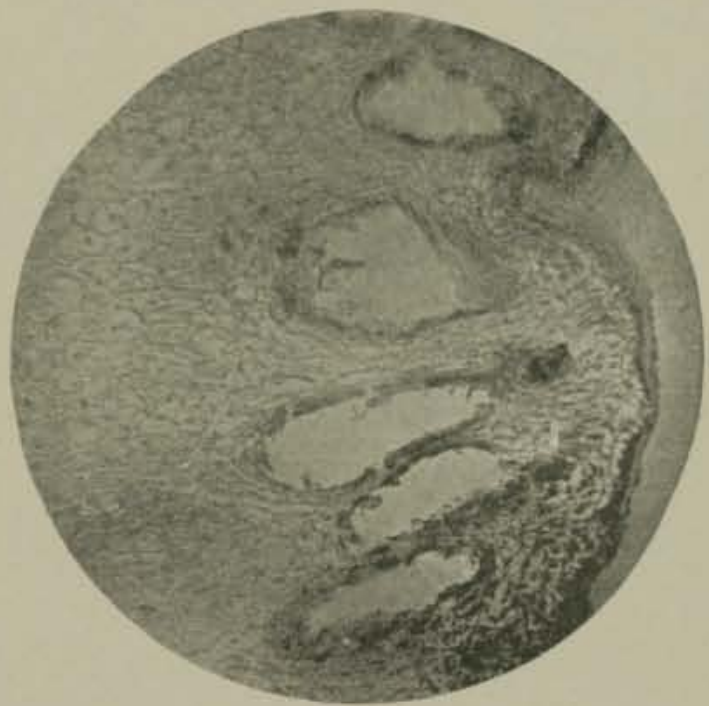

Fro. 216.-Clove, $\times$ r1o.

Transverse section near epidermis, showing large oil cavities. 


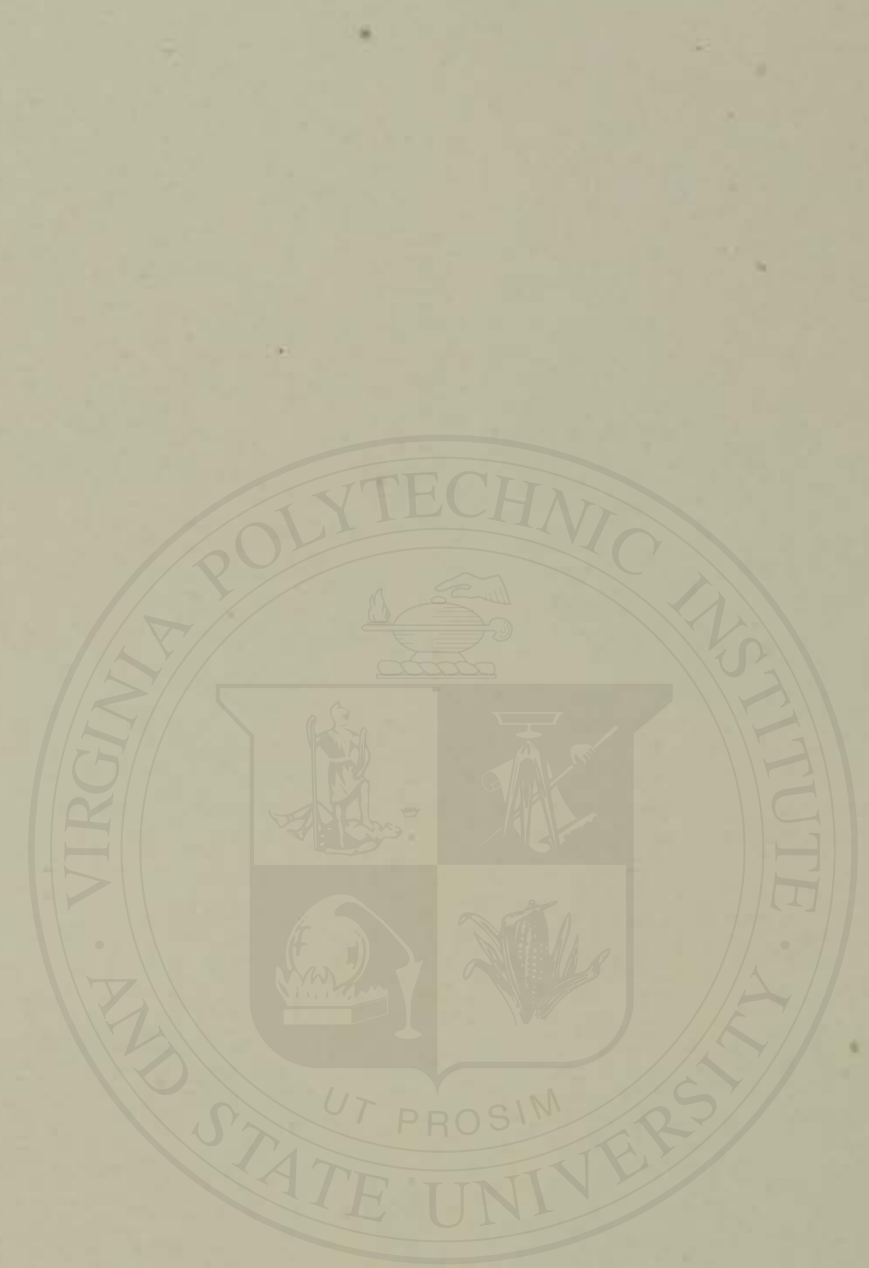




\section{PLATE XXV.}

\section{SPICES.}

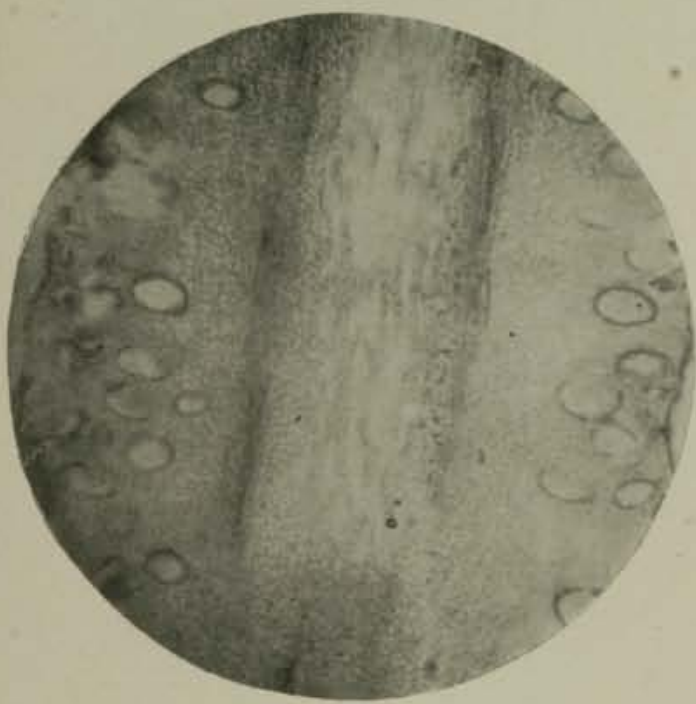

FiG. 217.-Clove, $X_{28} 8$.

Longitudinal section through entire clove.

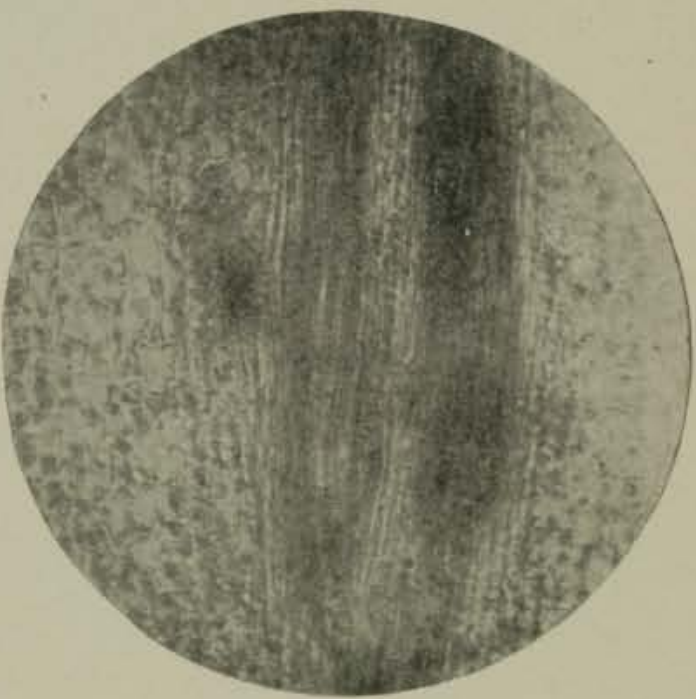

Fig. 218 -Clove, $\times 70$.

Central longitudinal section, showing duct bundles.

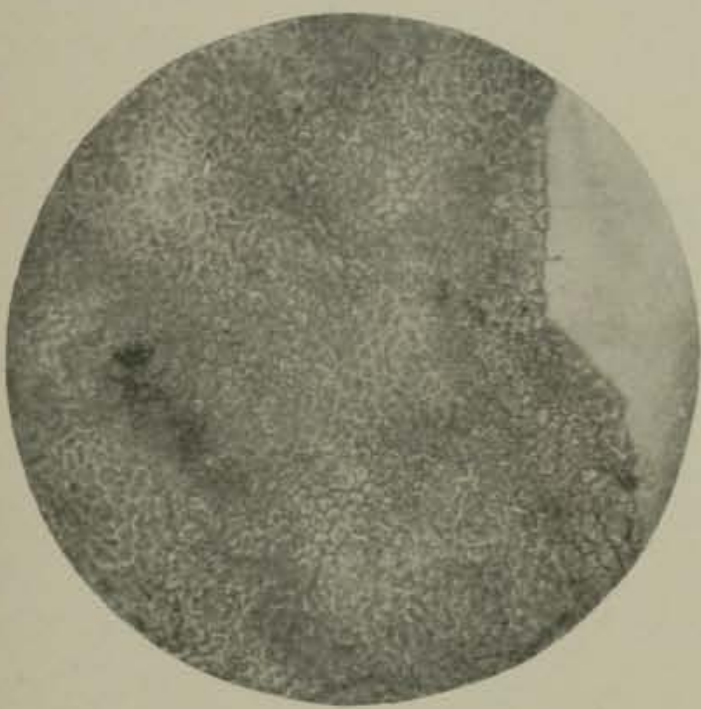

Fig. 219.-Clove, XIro.

Surface view of epidermis.

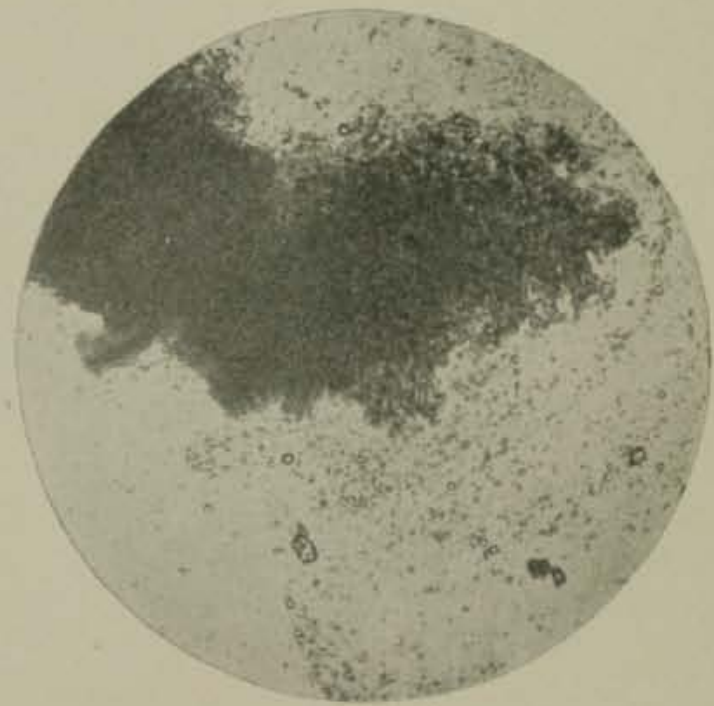

FIG. 220.-Powdered Cloves, $X_{13}$.

Dense, spongy tissue, with small oil drops. 


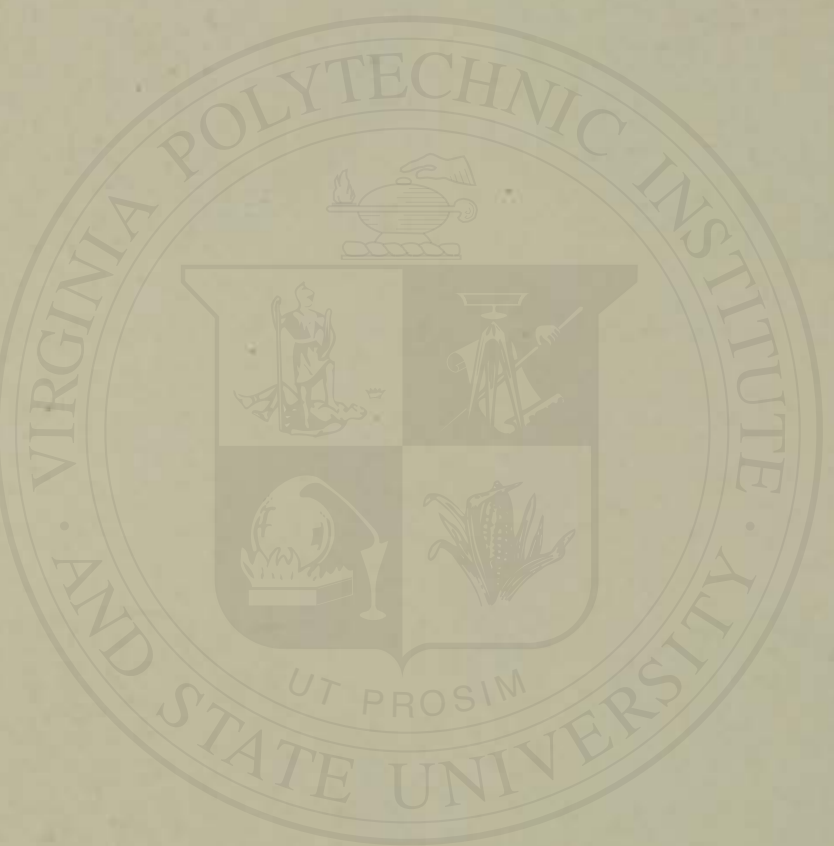




\section{PLATE XXVI.}

\section{SPICES.}

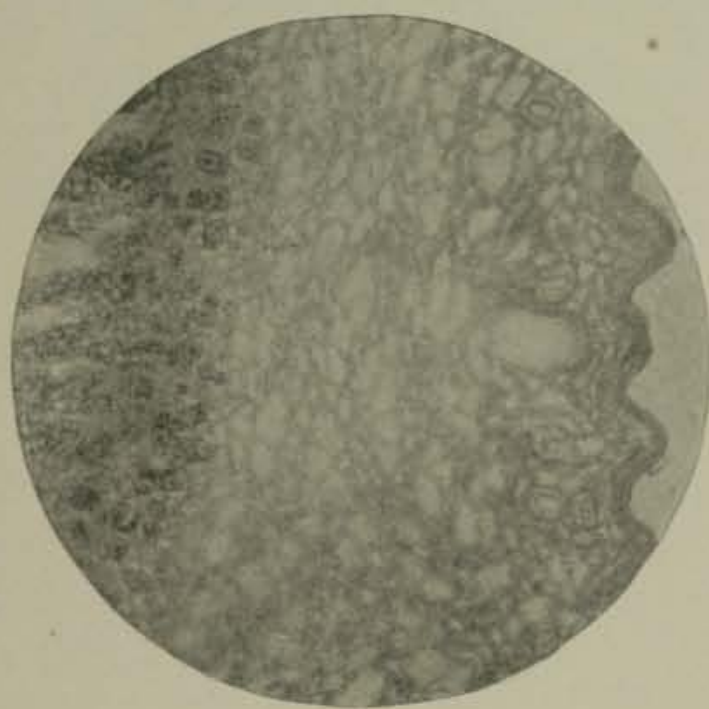

Fro, 221.-Clove Stem, $\times 70$.

Transverse section through outer part of stem, showing bast fibers at the left, parenchyma in the center, and stone cells near the epidermis.

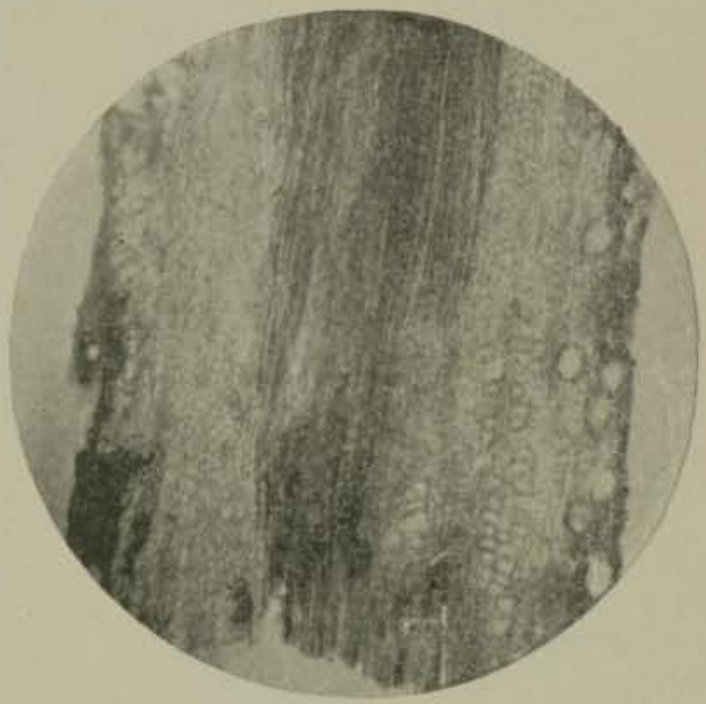

Fig. 222.-Clove Stem, $X_{25}$.

Central longitudinal section through entire stem, showing bast fibers in the center, and stone cells at the right.

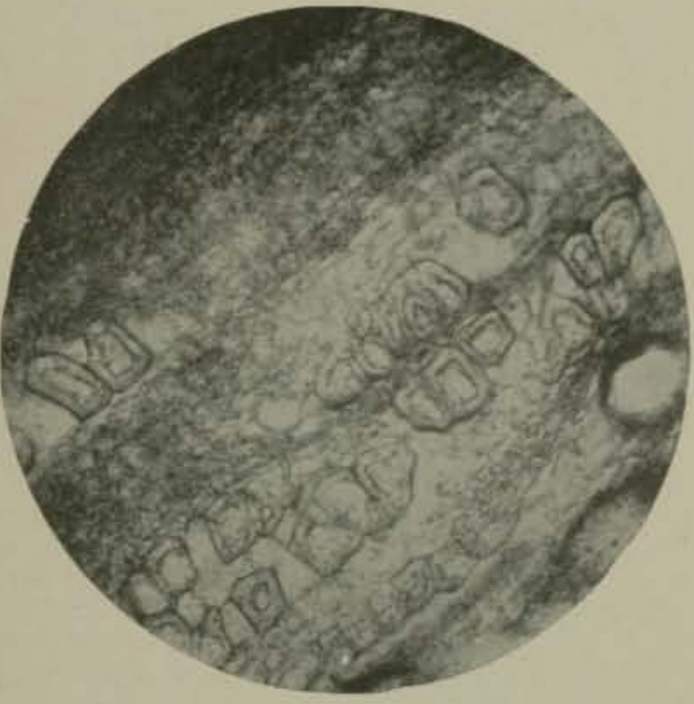

Frg. 223.-Clove Stem, $\times 7$.

Longitudinal section, showing the stone cells.

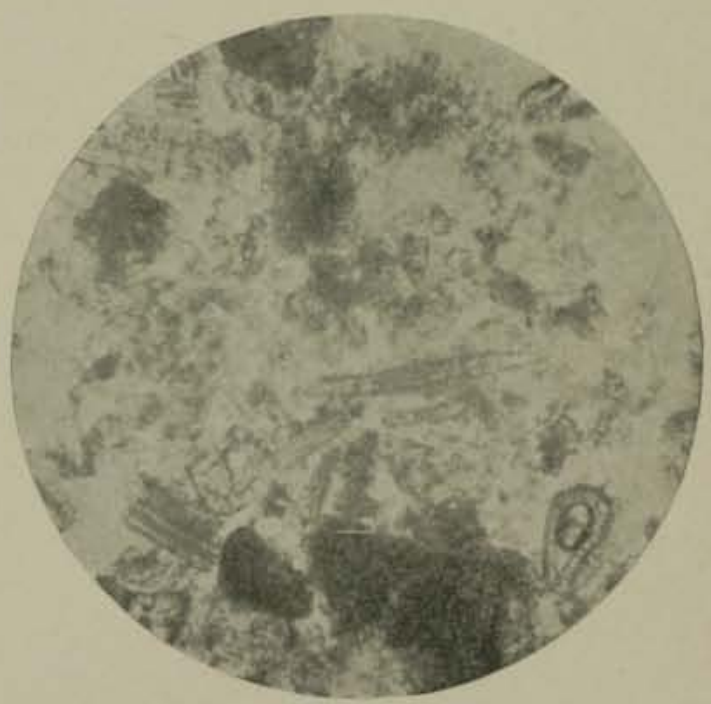

FIG. 224.-Powdered Clove Stems, XIIO.

Showing fragments of tissues, stone cells, and bast fibers. 


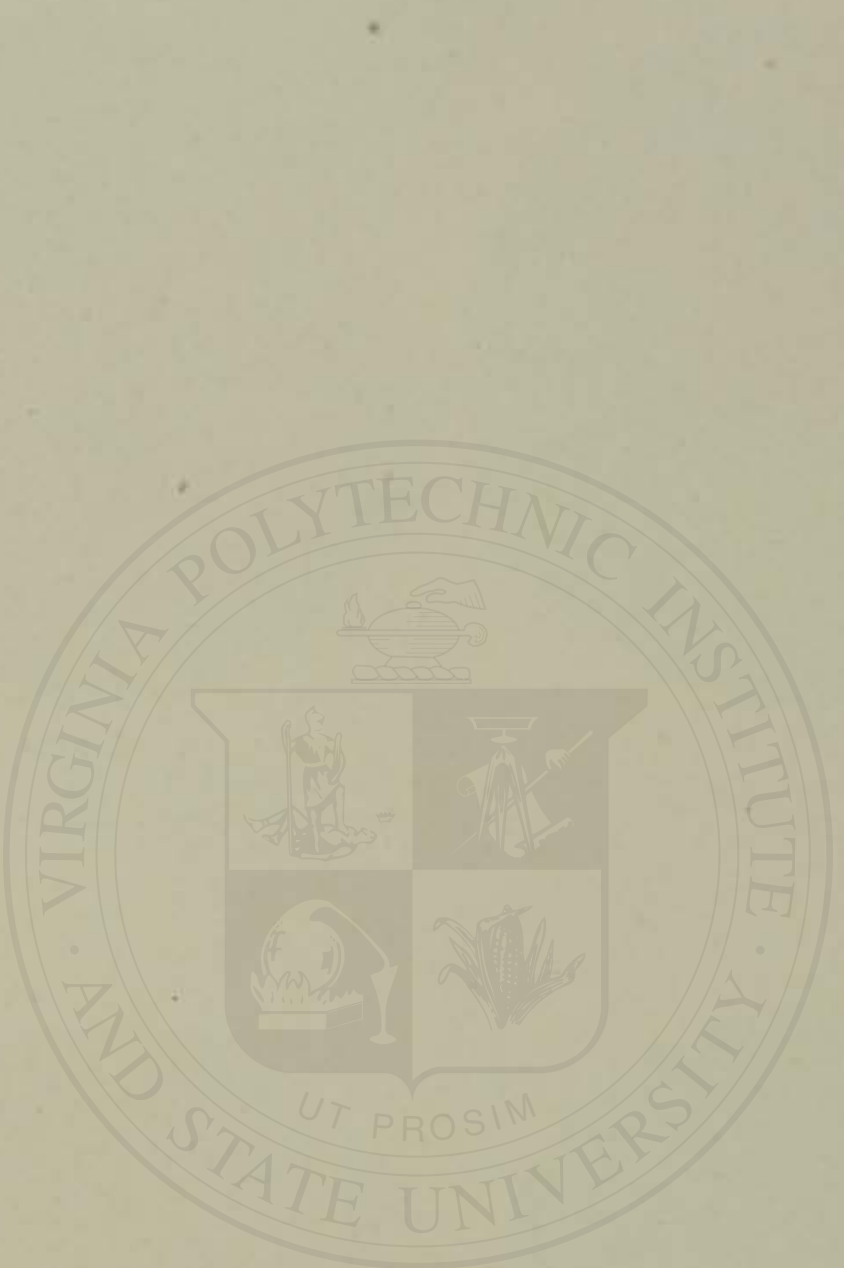




\section{SPICES.}

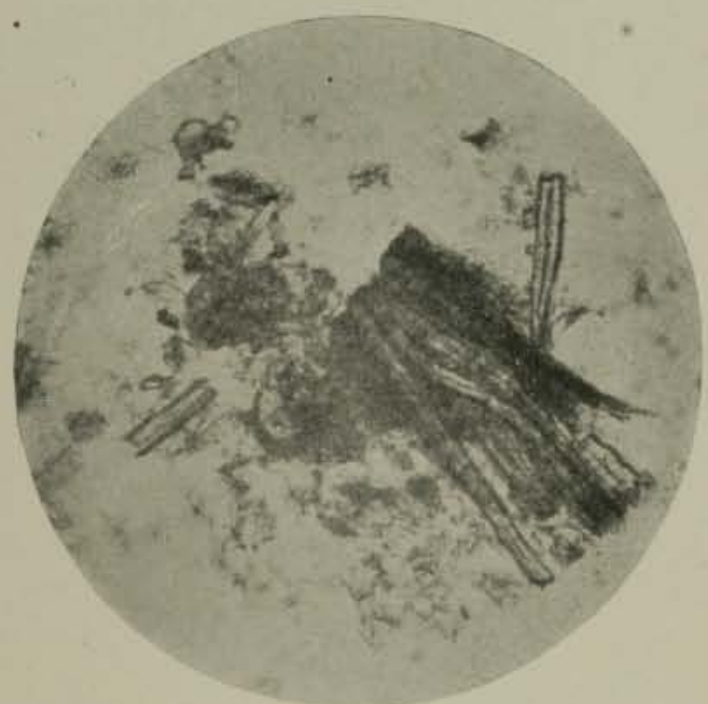

Fig. 225.-Powdered Clove Stems, Xito. Showing bundle of bast fibers.

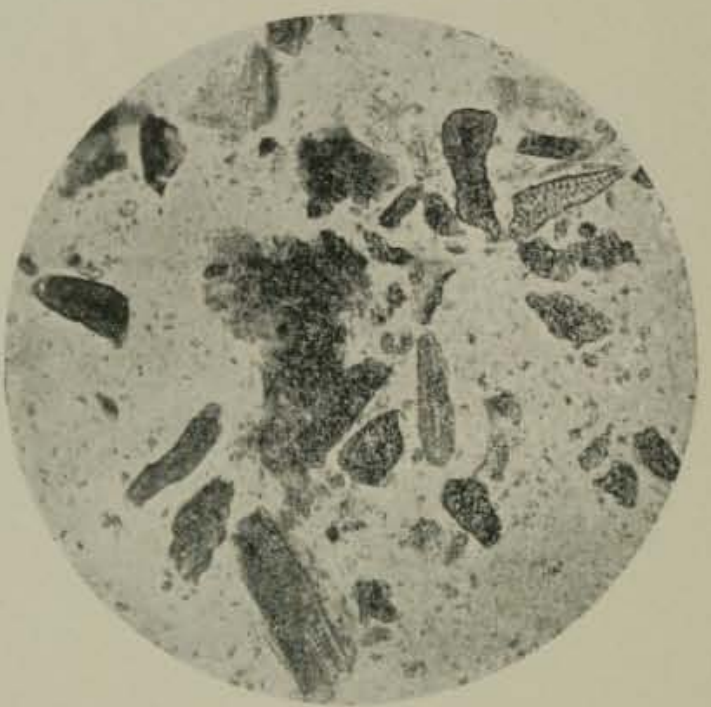

FIG. 226.-Adulterated Cloves, $X_{\text {I }} 30$.

Showing chiefly stone cells of cocoanut shells.

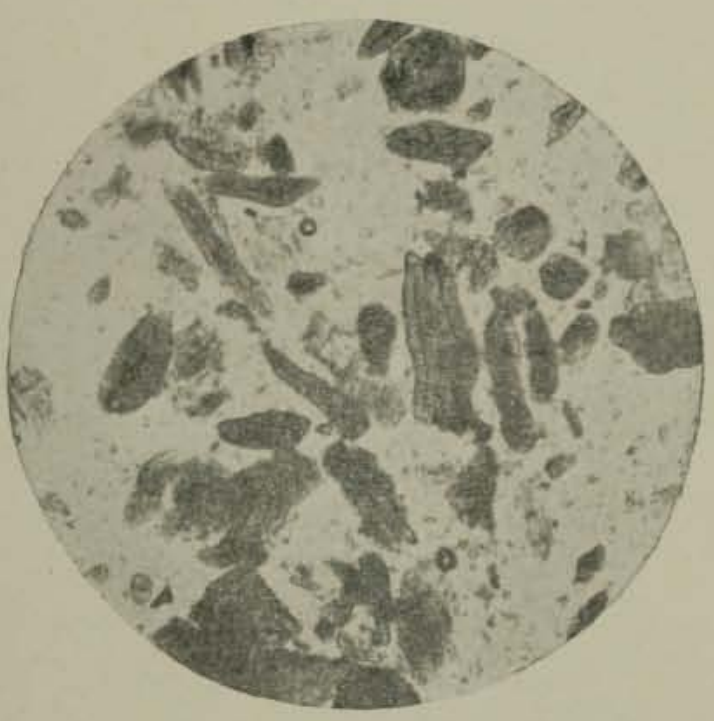

Fig. 227.-Adulterated Cloves, $X+30$. With large admixture of cocoanut shells.

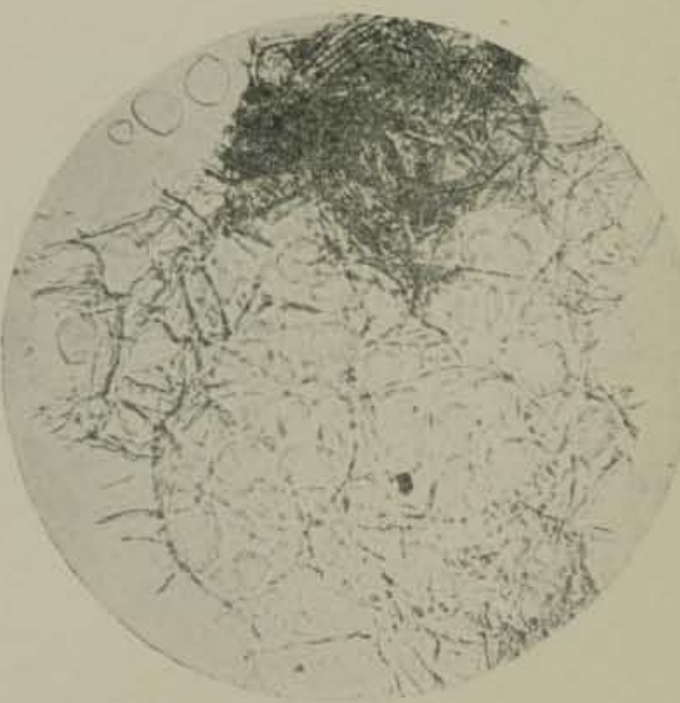

Frg. 228.-Ginger, $\times$ Iro.

Transwerse section, showing starch cells with contents. 


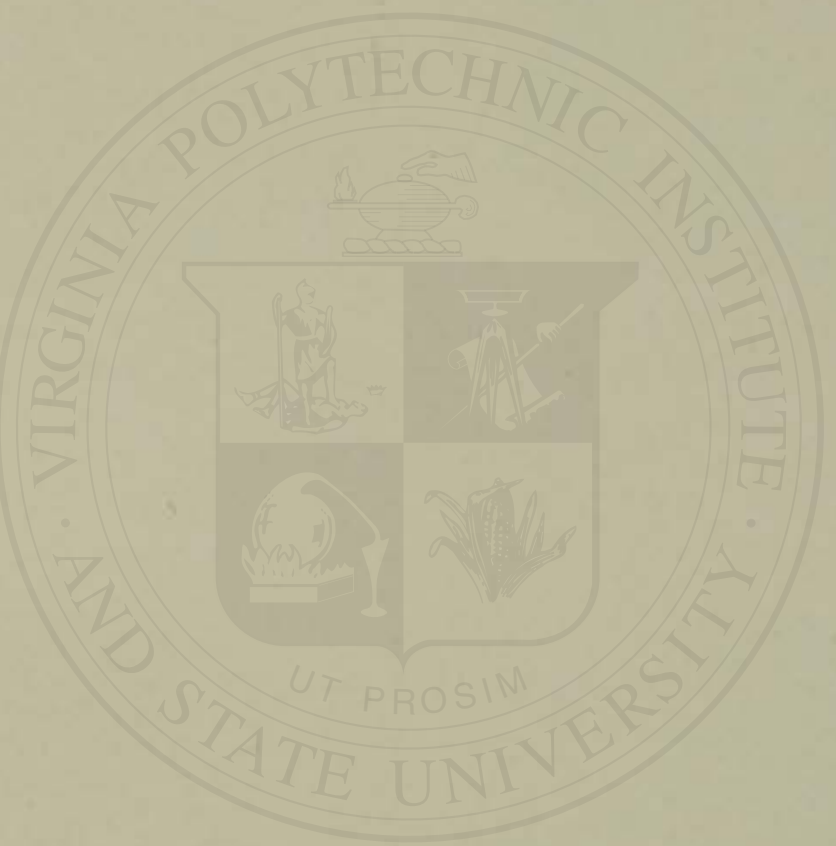




\section{SPICES.}

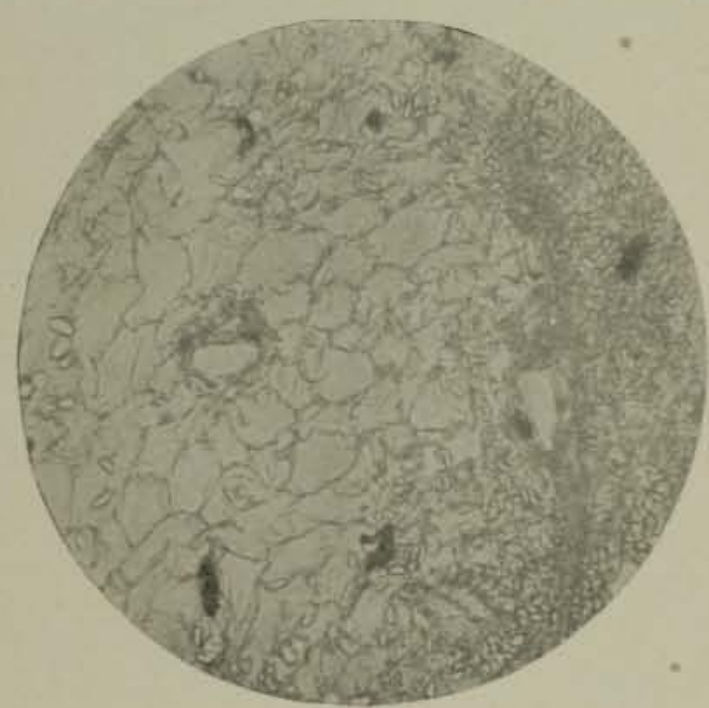

Fis. 229. - (tinger, $X 110$.

Transverse section, showing parenchyma, starch grains, and duct vessels.

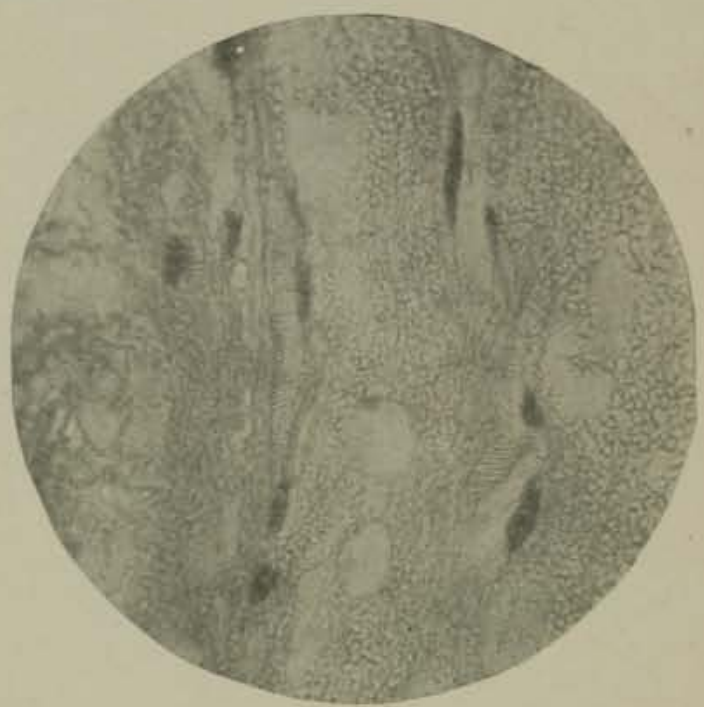

Fic. $230,-$ Ginger, $\times 110$.

Longitudinal section, showing spiral ducts and pigment cells.

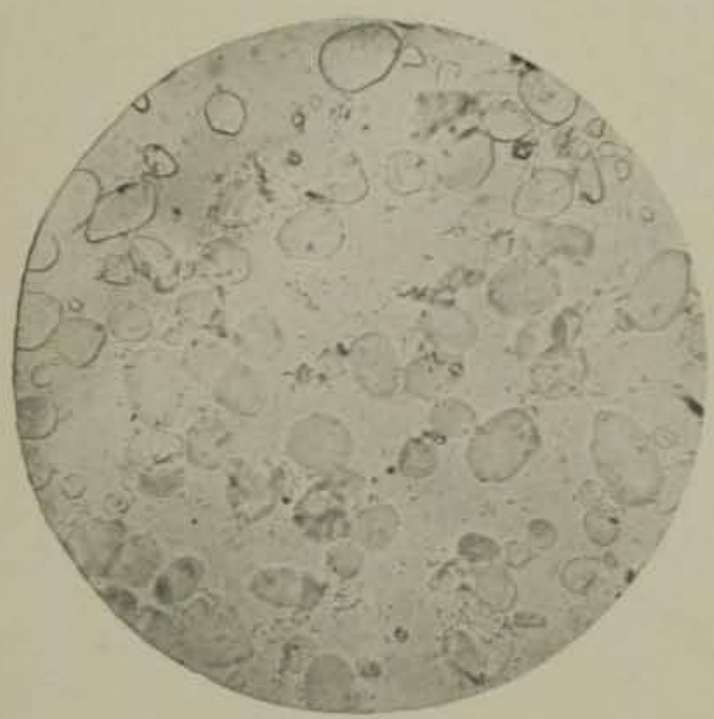

Fis, 231.-Ginger Starch, $X_{220}$

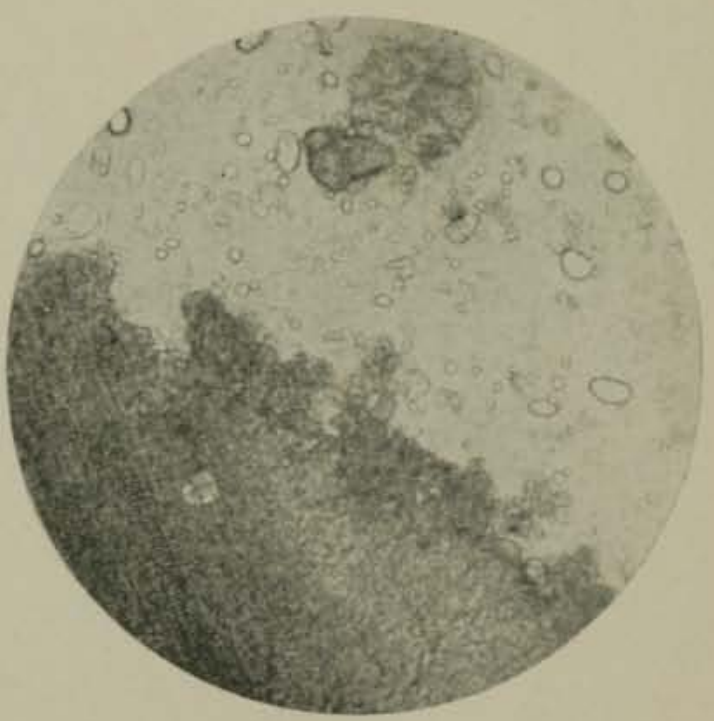

Fig. 232.-Adulterated Ginger, $\times$ i 30 .

A mass of wheat bran tissue is most conspicuous. 


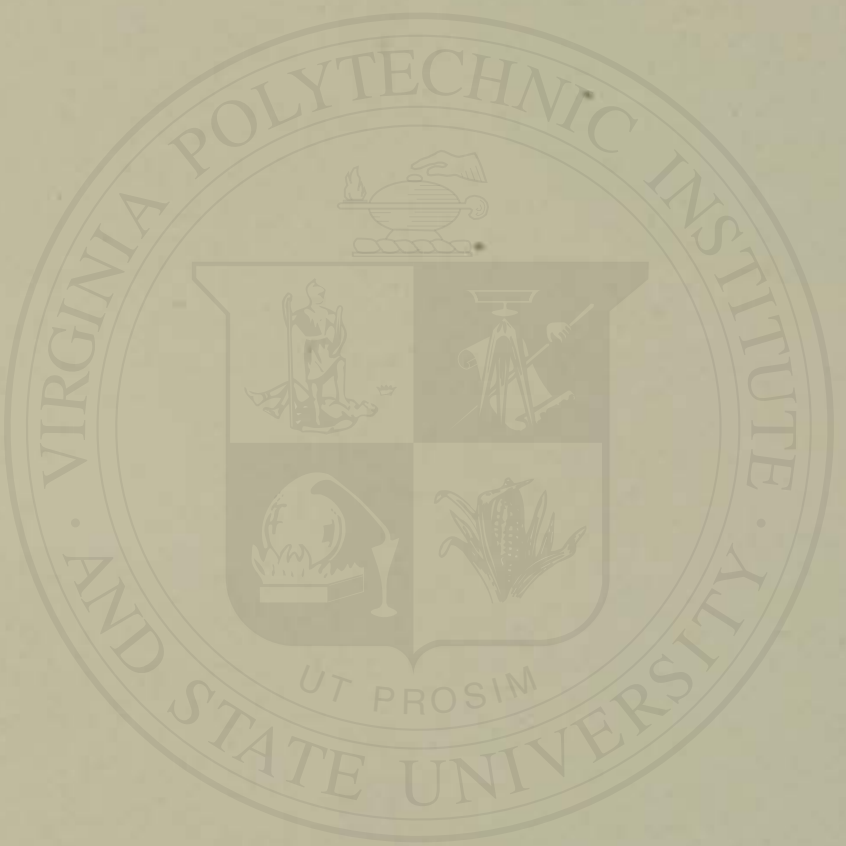




\section{SPICES.}

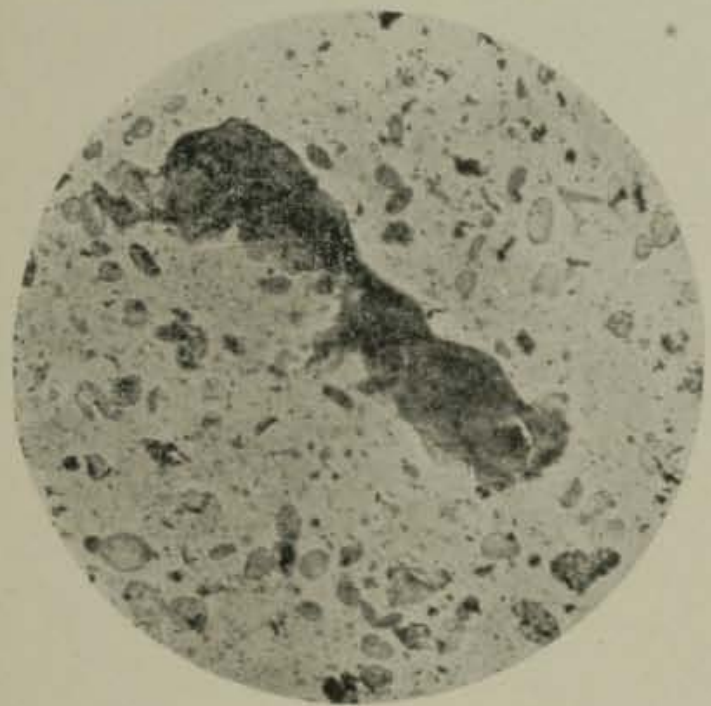

FIG. 233.-Adulterated Ginger, X 130.

The central dark mass is a yellow fragment of turmeric.

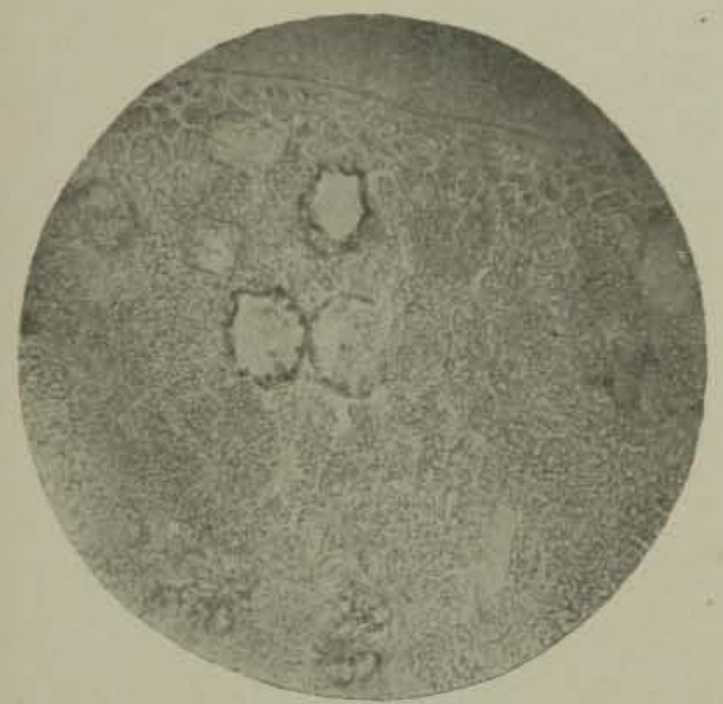

Frc. 235.-Penang Mace, Xr10.

Transverse section through epidermis and oil cells, showing also parenchyma with contents of amylodextrin.

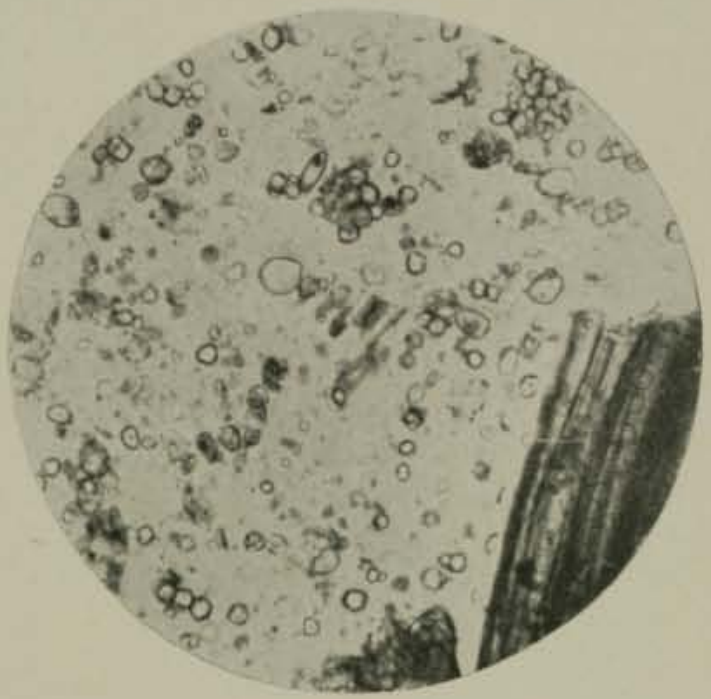

FIC. 234--Adulterated Ginger, X I 30 .

Containing a large admixture of corn and wheat starches.

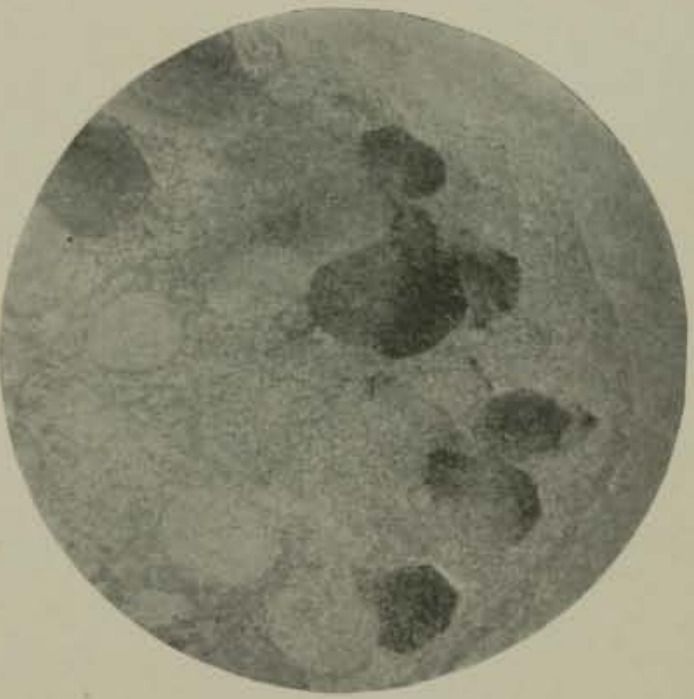

Frg, 236.-Bombay or Wild Mace, Xiro.

Transverse section through outer layers, showing yellow and red resinous lumps. 


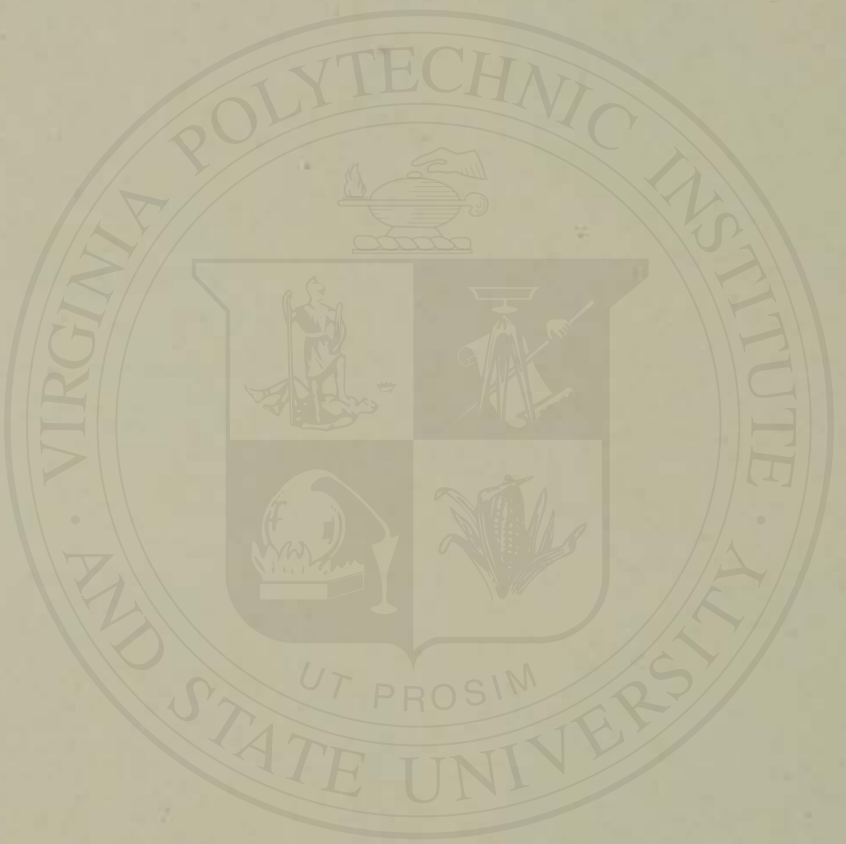




\section{PLATE XXX.}

\section{SPICES}

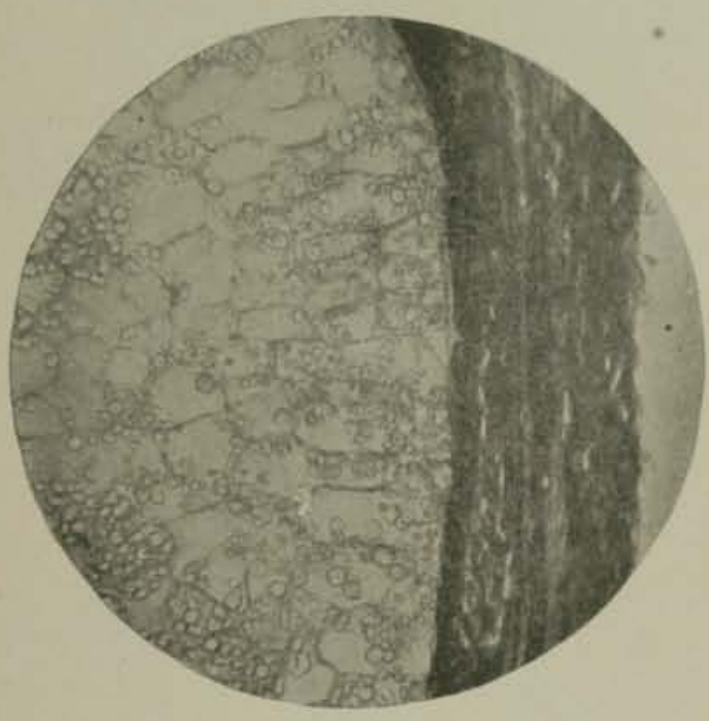

Fis. 237.-Nutmeg, $X$ rio.

Transverse section through the exterior and interior teguments of the seed and part of the endosperm, showing starch cells.

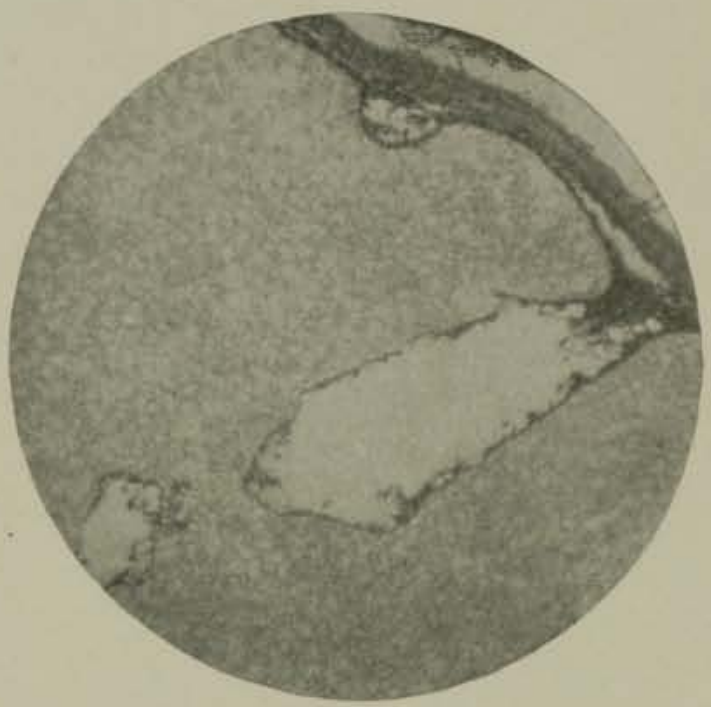

Fic. $23^{8}$. - Nutmeg, $\times 25$.

Transverse section near exterior of seed.

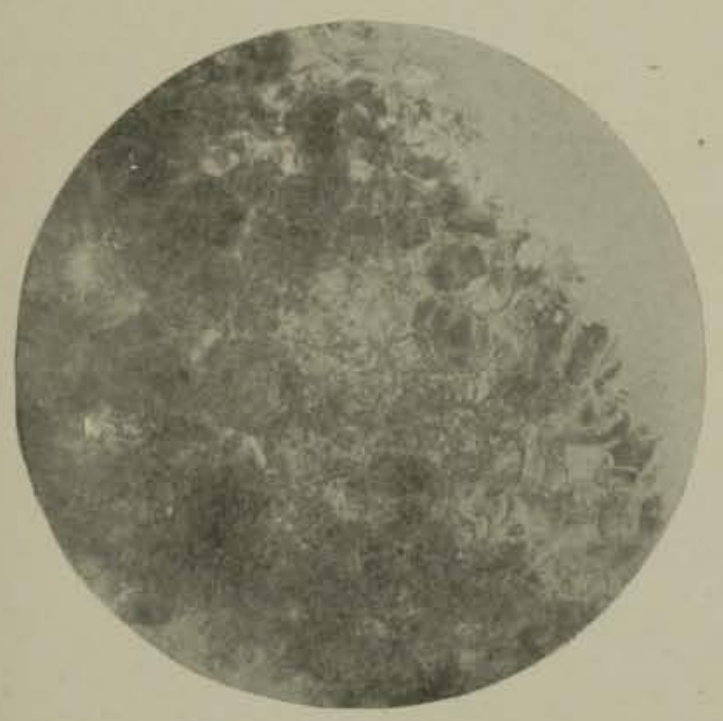

Fic 239.-Nutmeg, $\times 110$.

Surface view of seed coat, showing also portions of underlying tissues.

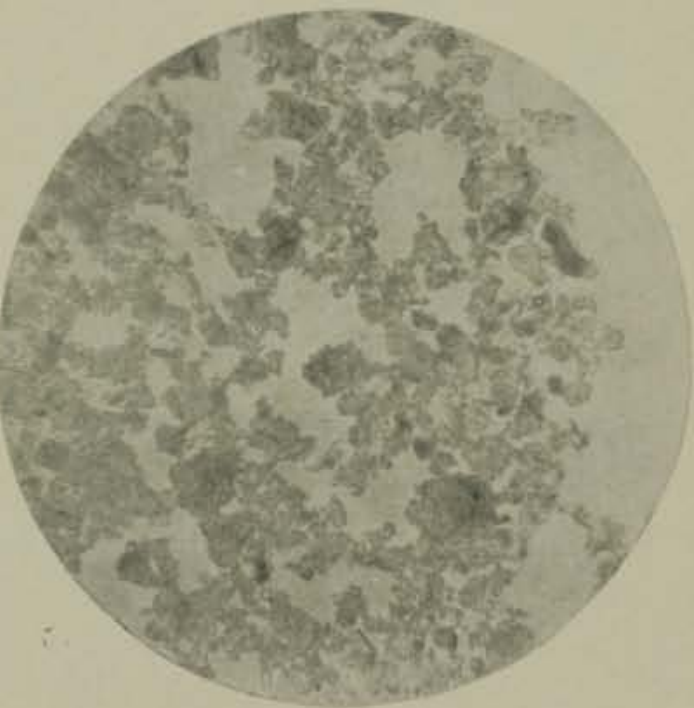

Fig, 240.-Powdered Nutmeg, $X_{110}$ 


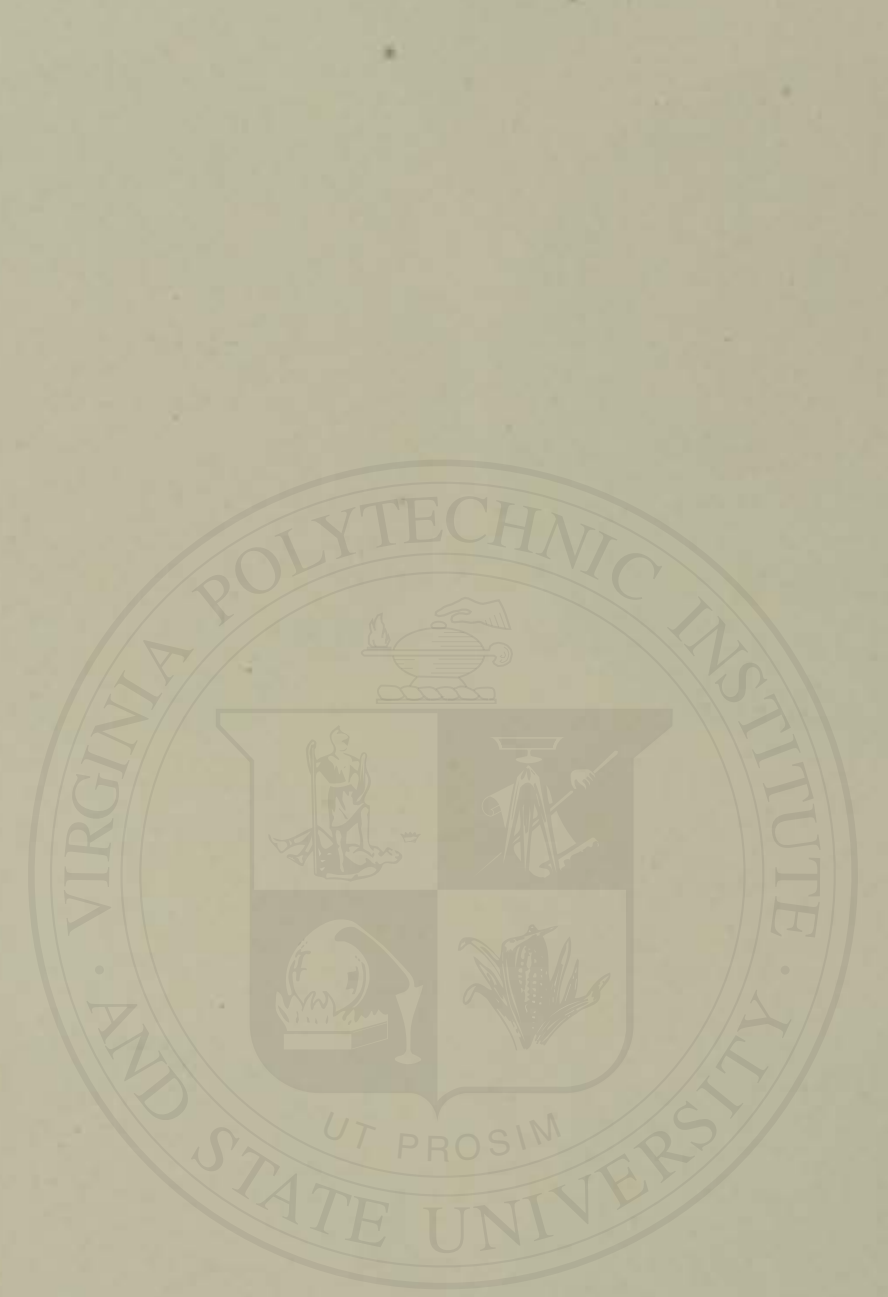




\section{PLATE XXXI.}

\section{SPICES.}

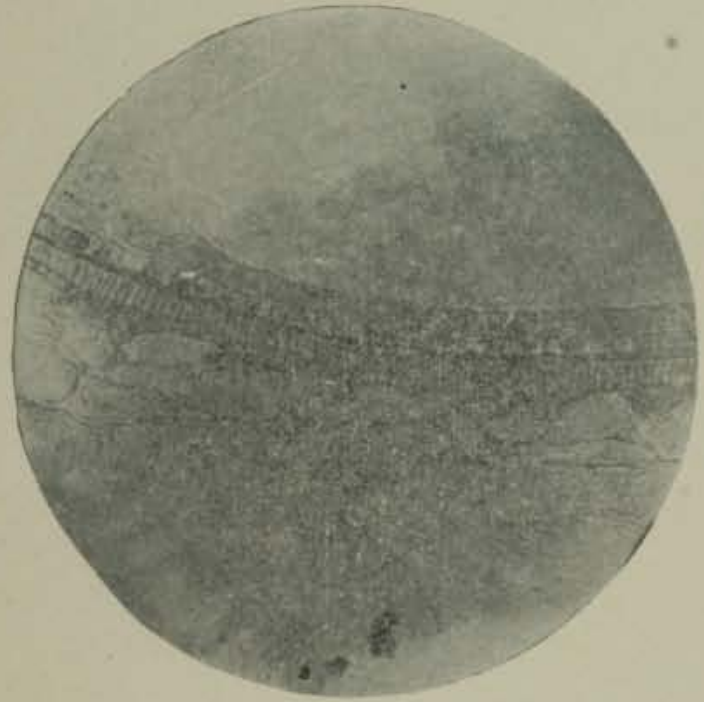

Fra. 241.-White Mustard, Xiro

Transverse section through mucilaginous epidermis, sub-epidermal parenchyma layer (square cells), palisade cells, and broken parenchyma layer of the hull.

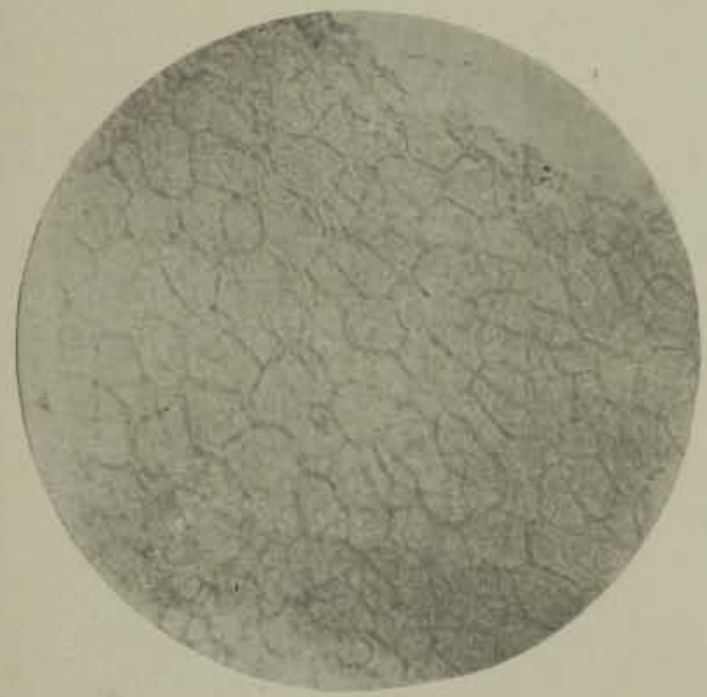

Frg. 243,-White Mustard X1ro

Surface view of two layers of the hull or seed coat.

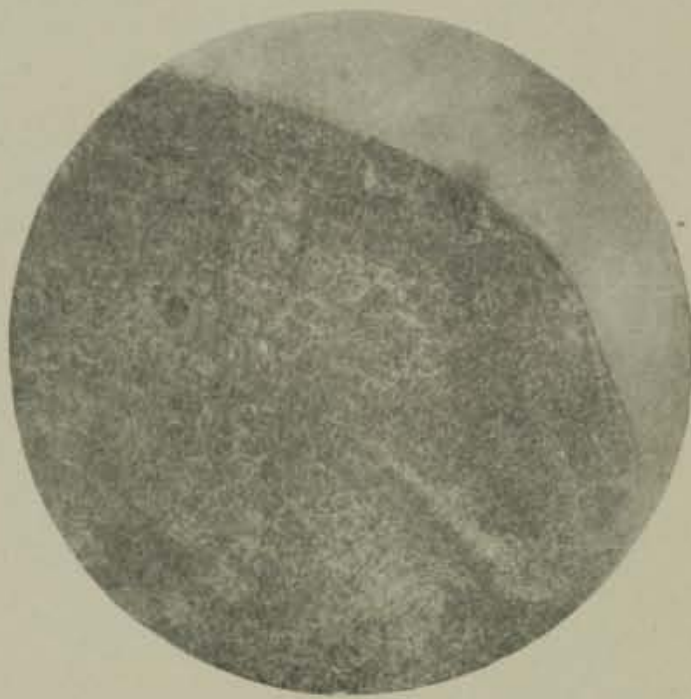

FIG. 242.-White Mustard, $X_{110}$

Transverse section through the tissue of the radicle.

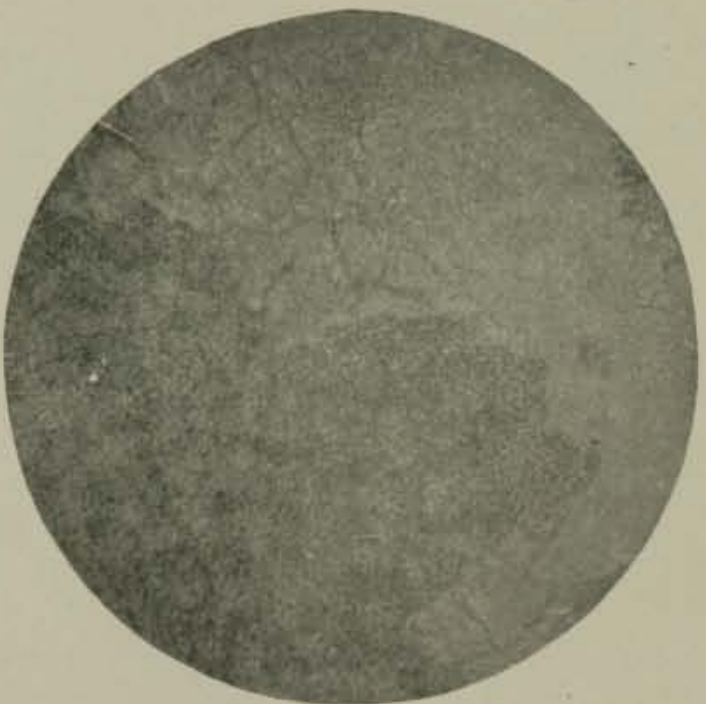

Fig. 244.-White Mustard, Xrro.

Surface section through palisade cells and underlying layer of the seed coat. 


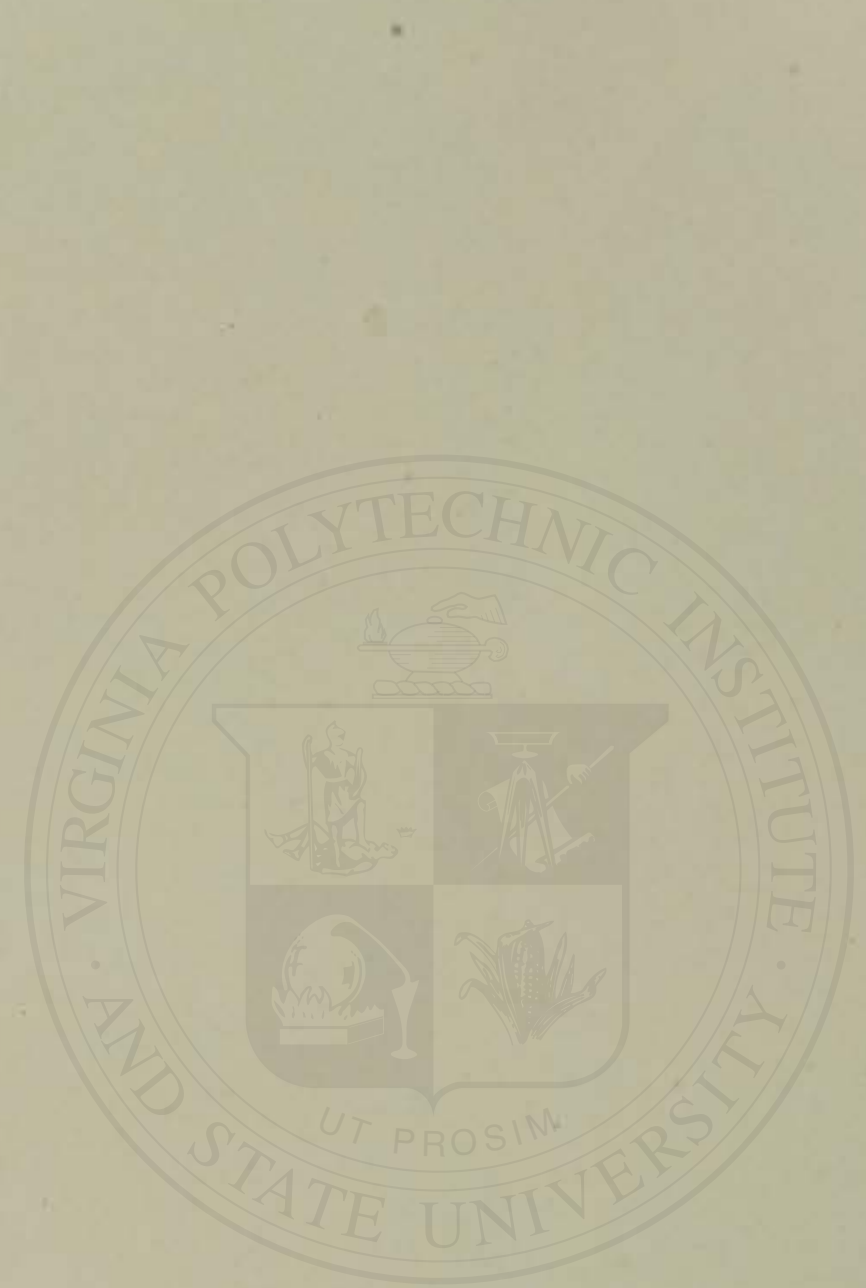




\section{PLATE XXXII.}

\section{SPICES.}

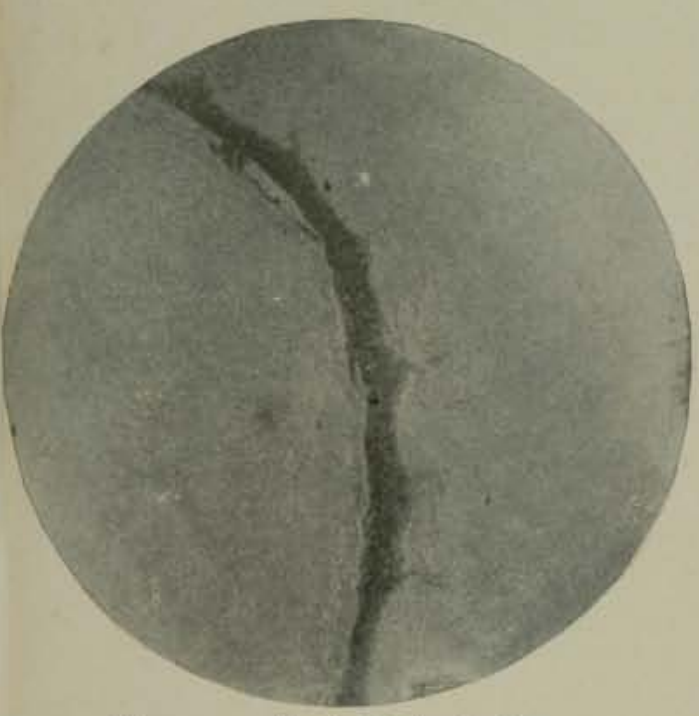

Fig. 245.-Black Mustard, $X_{110}$.

Transverse section, showing fragments of the epidermis and dark colored palisade cells of the seed coat.

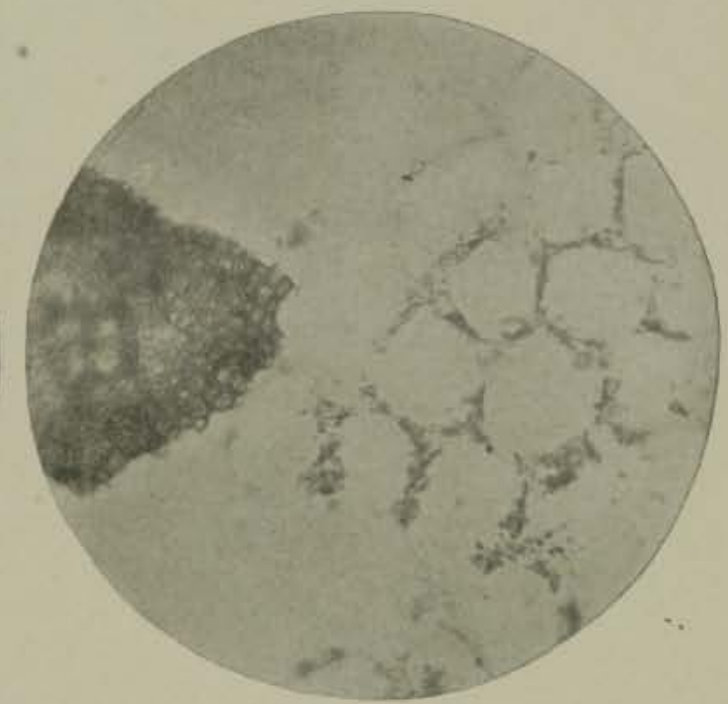

Fig. 246.-Black Mustard, Xiro.

Surface view of two of the seed coat lavers.

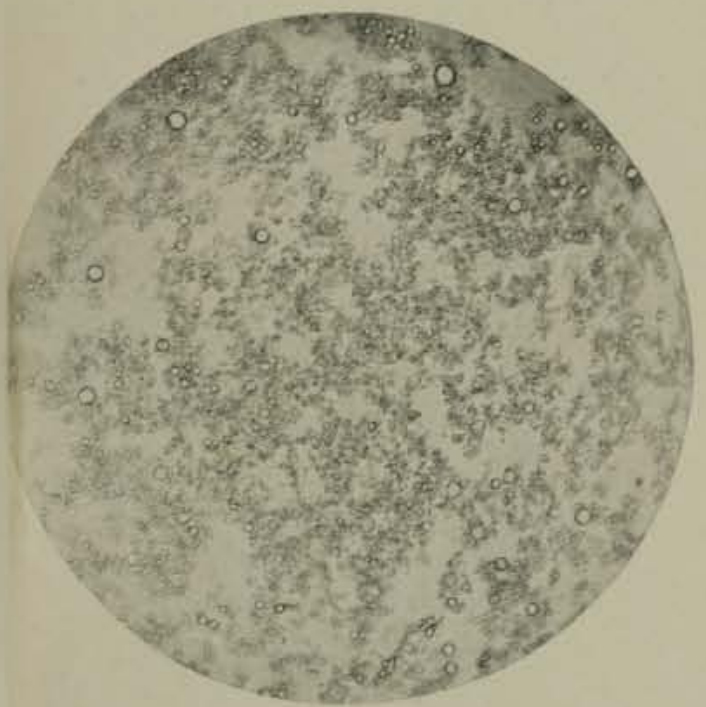

FIG, 247.-Ground Mustard, XI30.

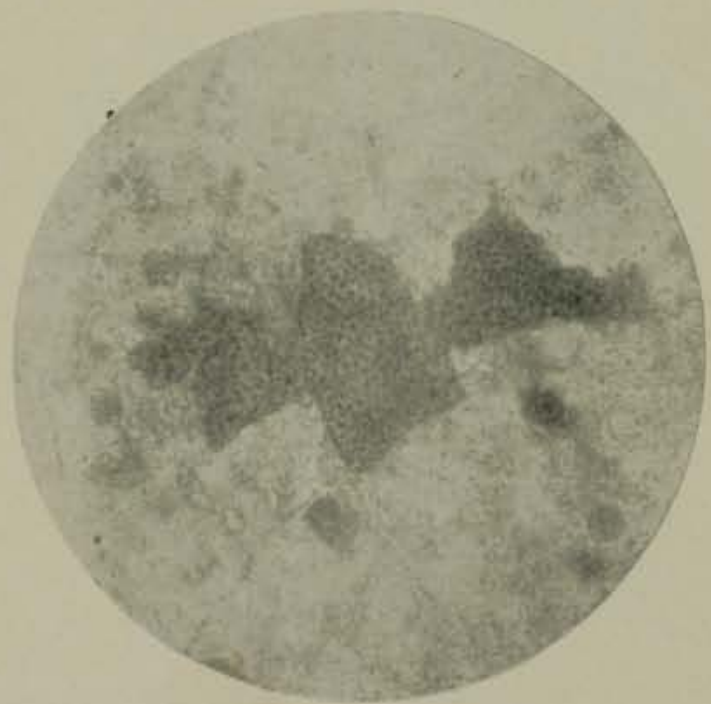

FrG. 248.- Ground Mustard Hulls, Xiro.

Ground without removal of the oil.

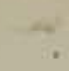




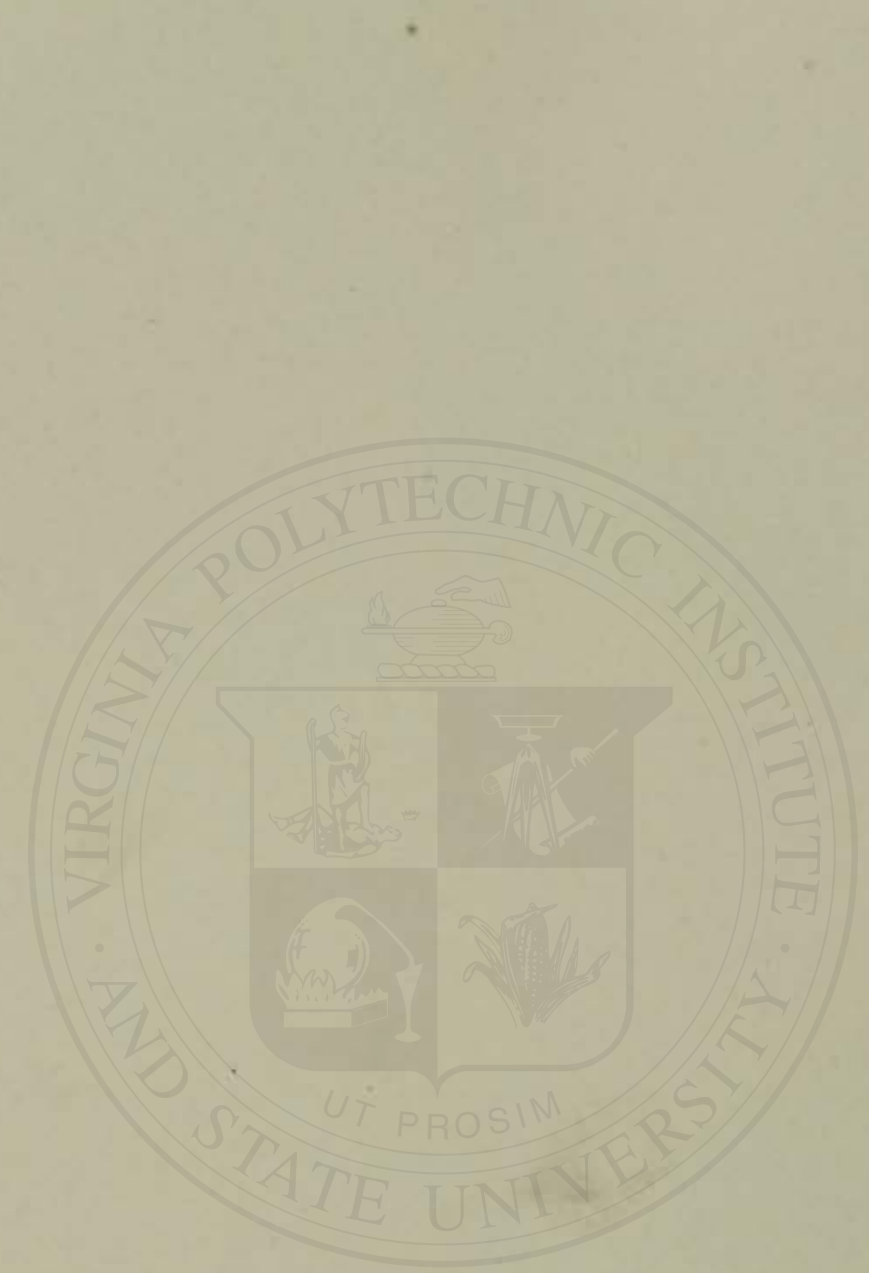




\section{SPICES.}

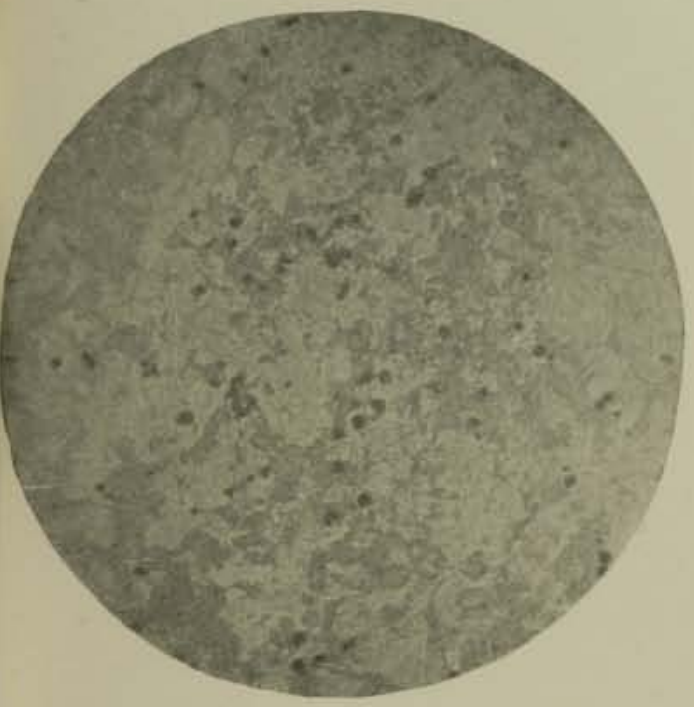

Fig. 249.-Dakota Mustard Flour, X 1 ro.

Dark spots show starch grains of foreign weed seed, stained with iodine.

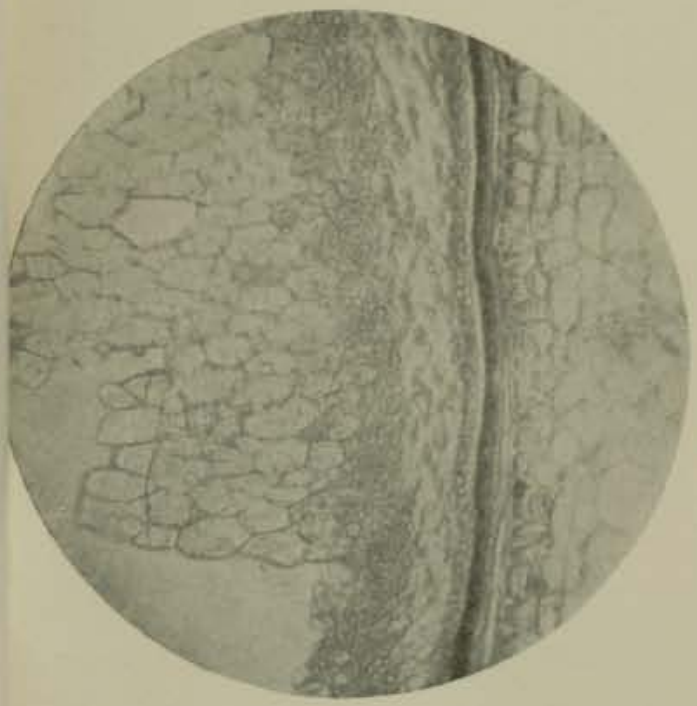

FrG. 251.-Pepper, Xrro.

Transverse section through inner part of pericarp (including parenchyma and seed coat layers) and portion of perisperm, showing starch and oil cells.

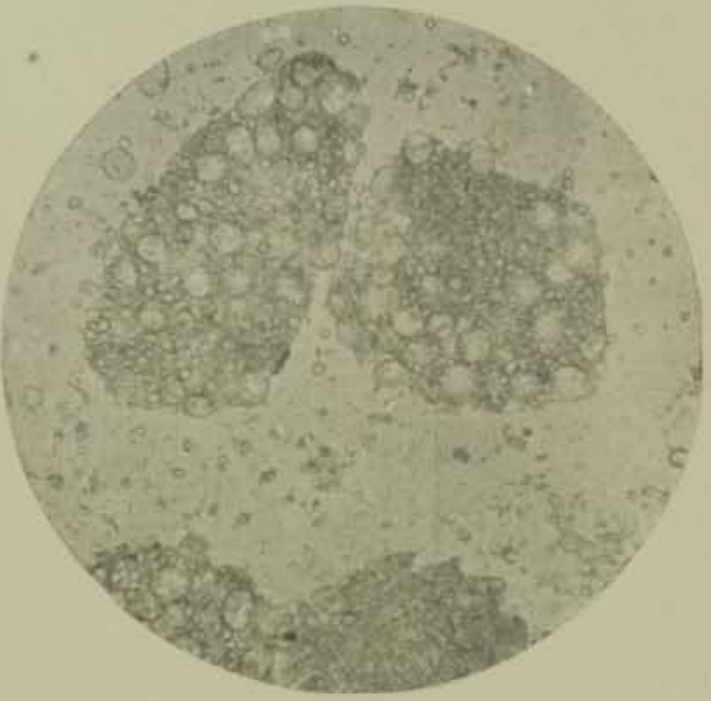

Frc, 250.-Adulterated Mustard Flour, X r 30. Showing masses of wheat starch.

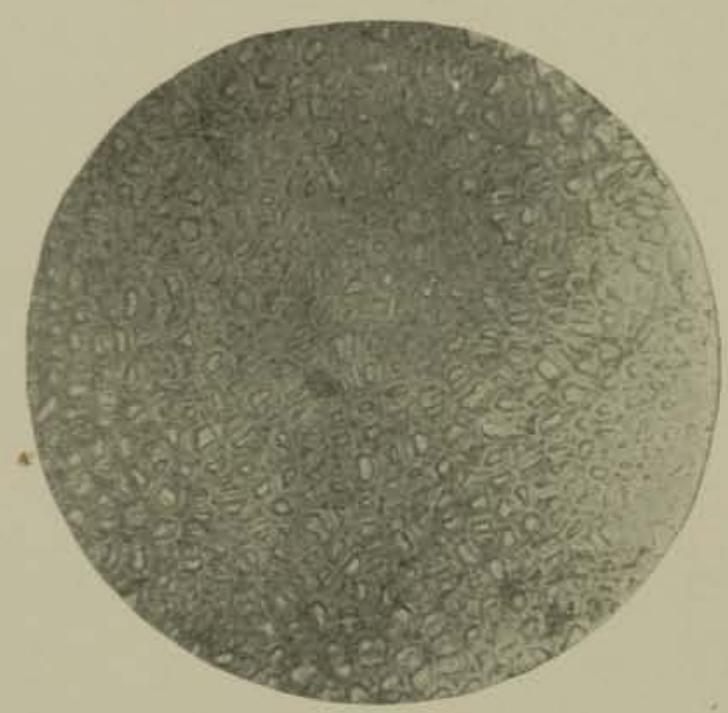

Fic. 252.-Pepper, Xiro.

Surface view of hypodermal layer. 


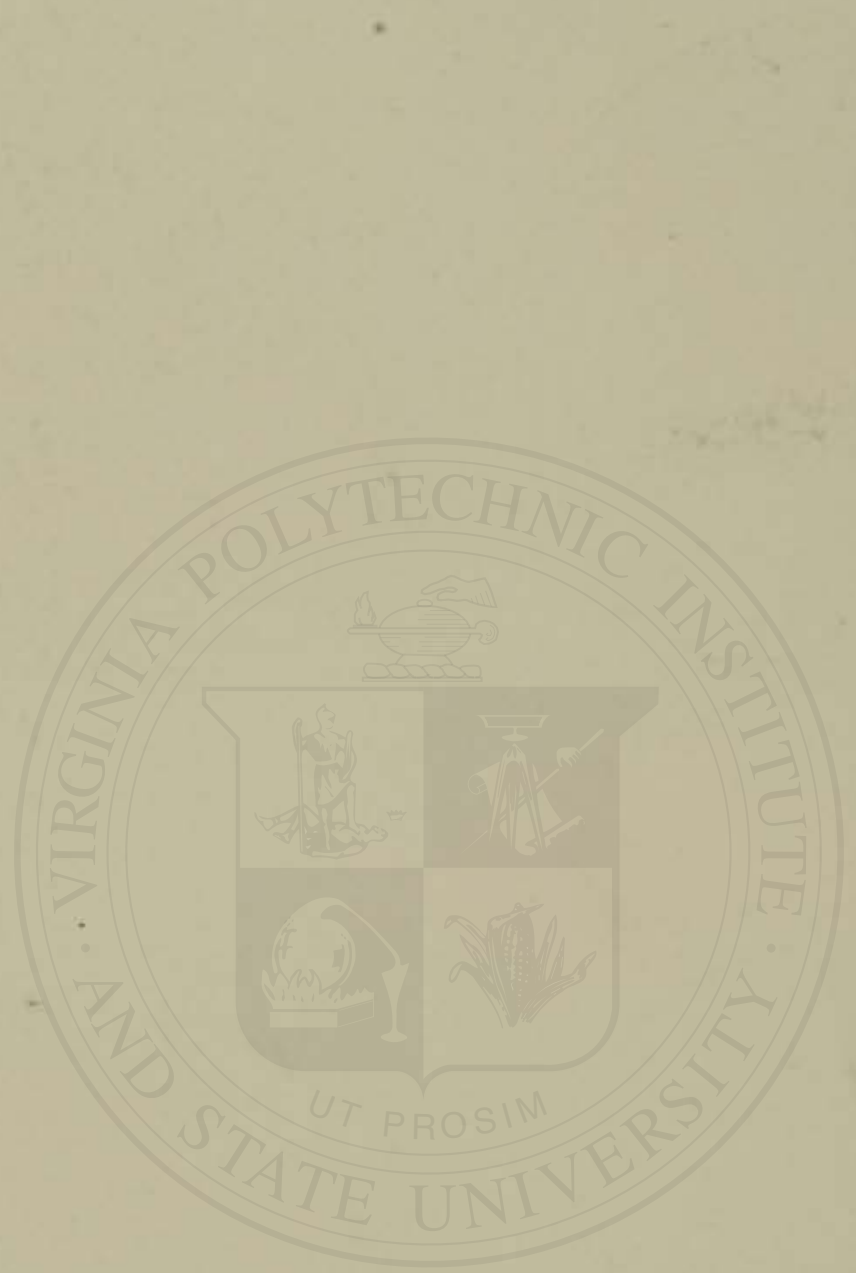


PLATE XXXIV.

\section{SPICES.}

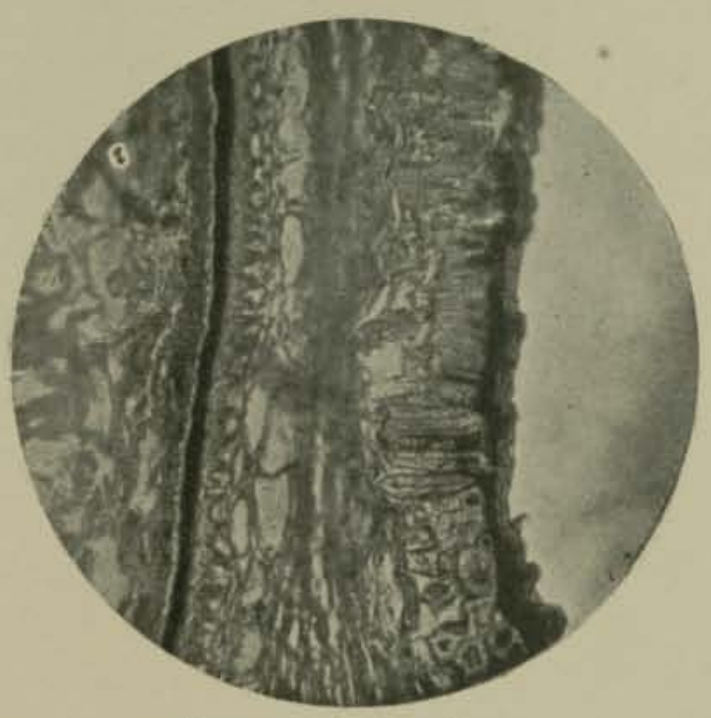

Frg. 253.-Pepper, Xrso.

Transverse section through outer part of pericarp, showing epidermis, underlying stone cell layers, parenchyma, and seed coat.

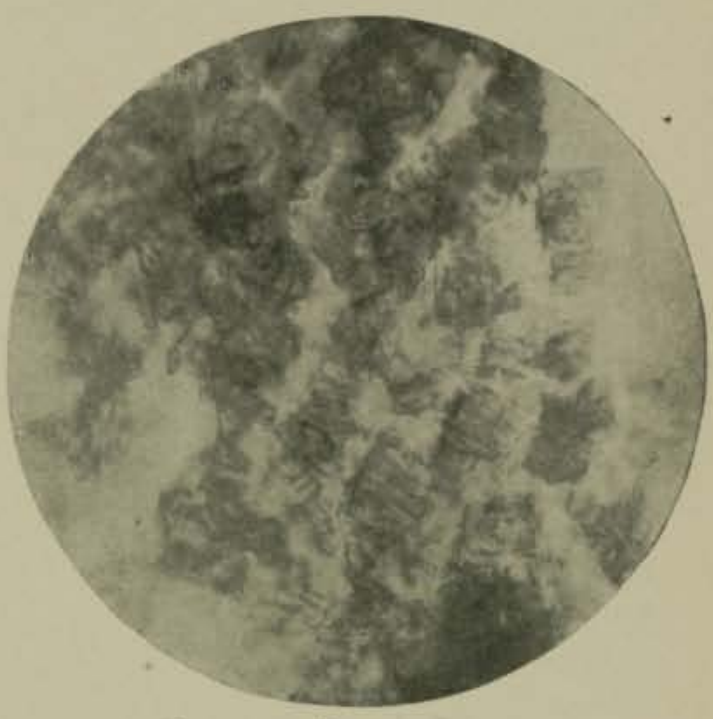

Fig. 254.-Pepper, X 110.

Surface section through stone cell layer.

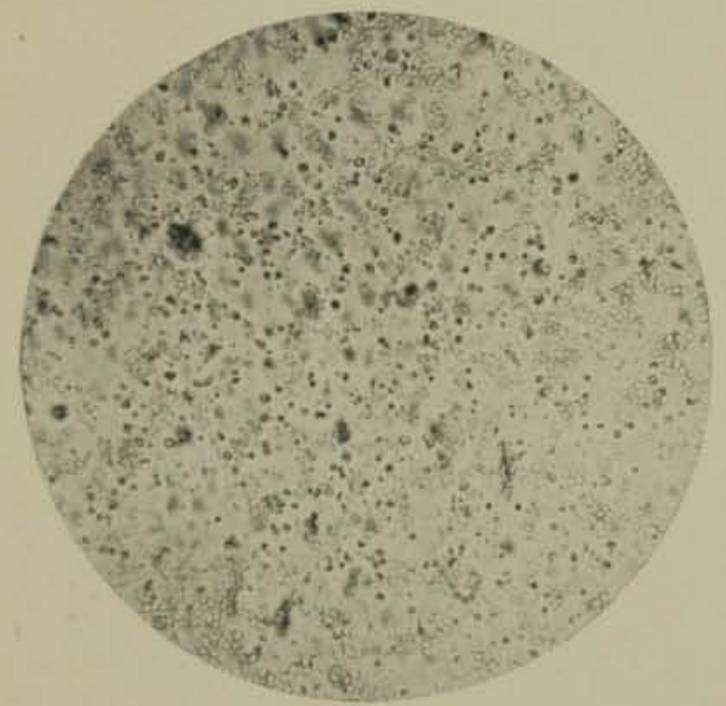

Fic. 255.-Pepper Starch, $X_{220}$ Starch granules separated.

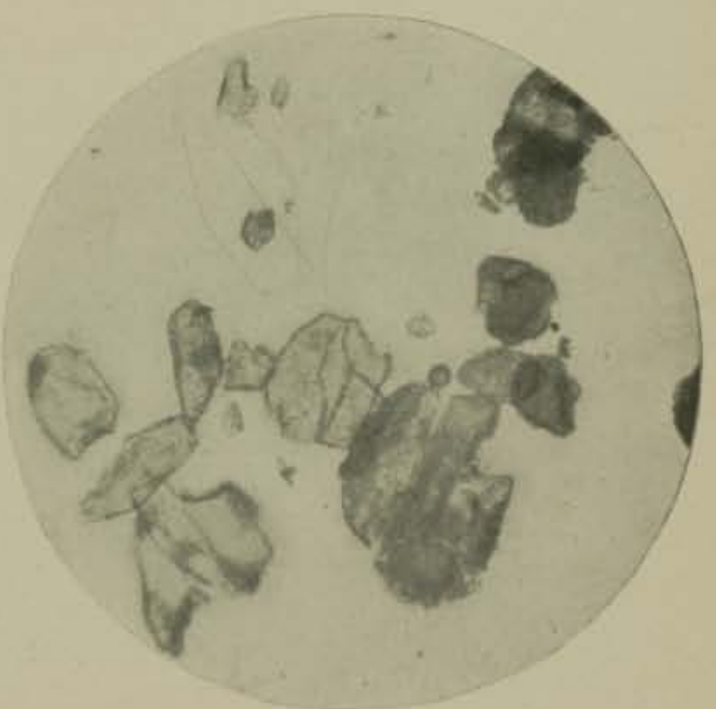

Fig. 256.-Pepper Starch, Xtro. Starch grains in masses. 


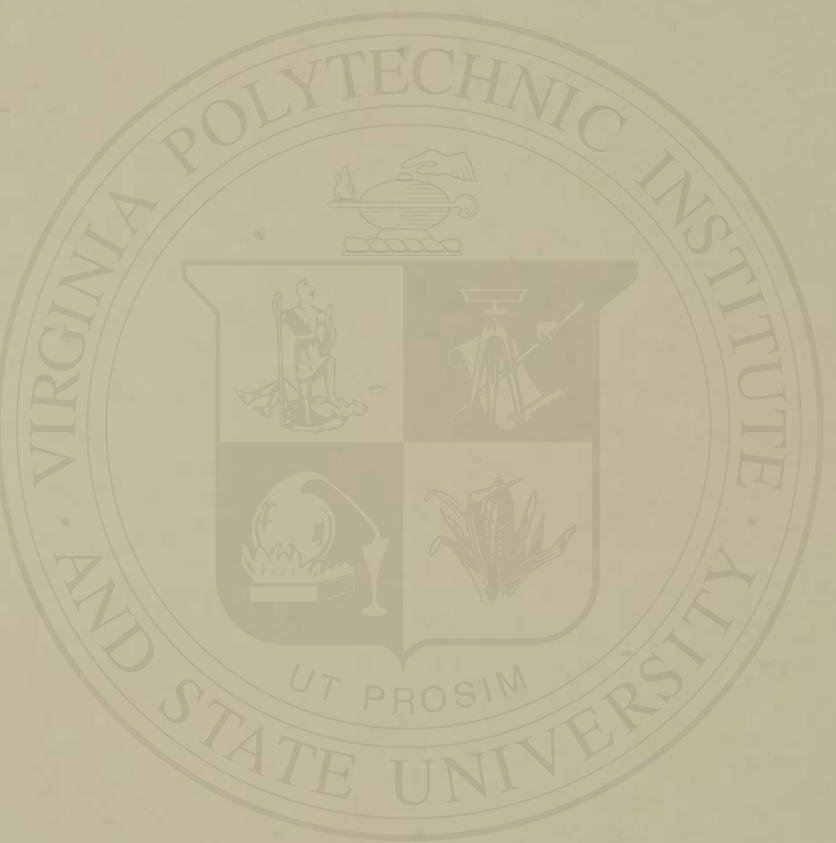


PLATE XXXV.

\section{SPICES.}

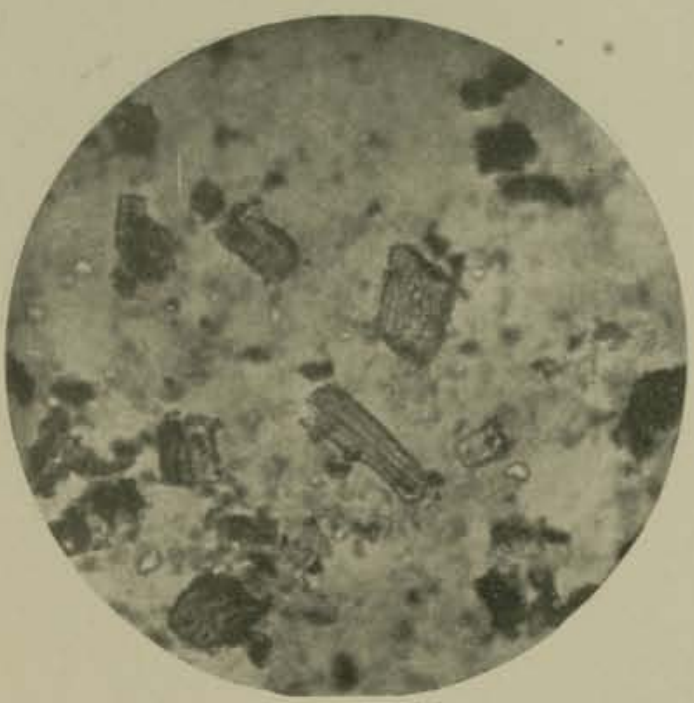

FtG. 257.-Ground Pepper Shells, X 110. Mainly showing stone cells.

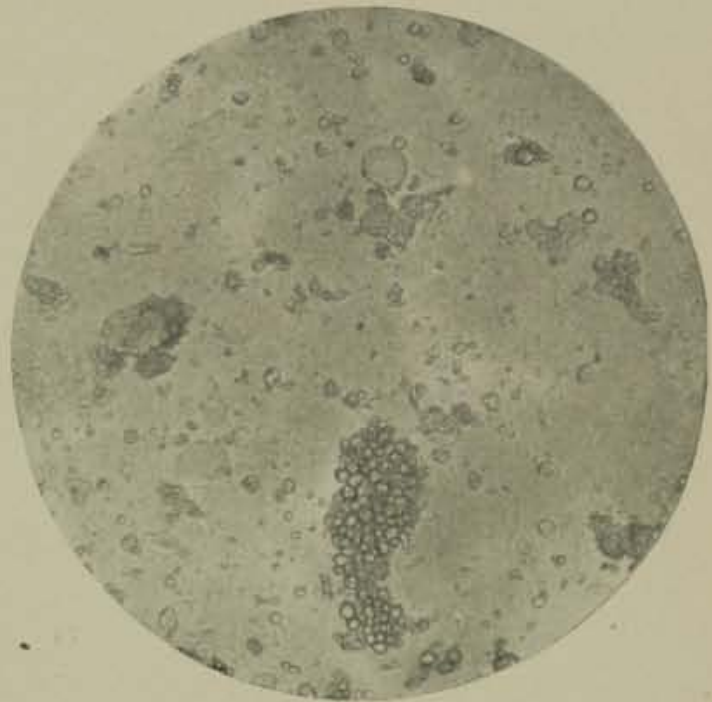

Fic. 258.-Adulterated Pepper, $X_{130}$.

Showing wheat and buckwheat starches.

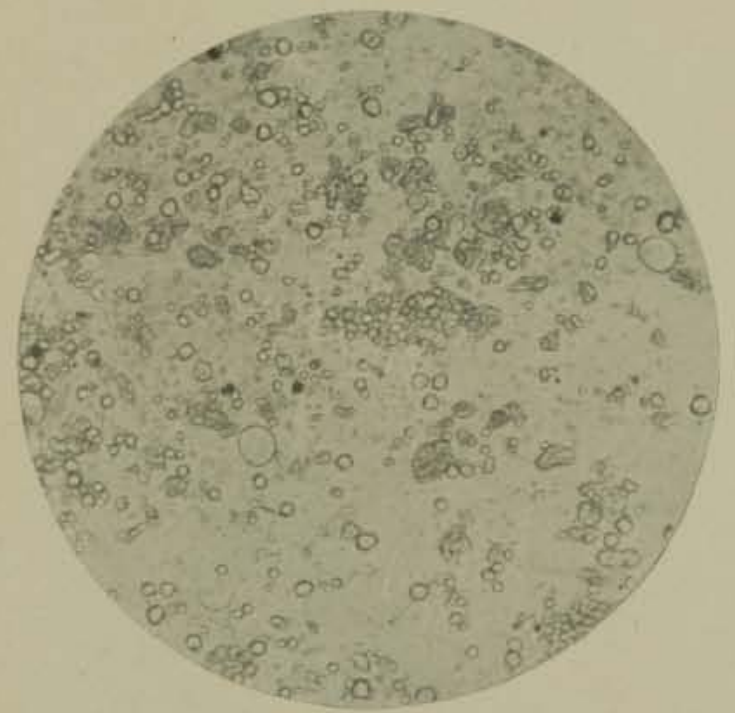

Fic. 259.-Adulterated Pepper, $\times 130$.

Showing wheat, corn, and rice starches.

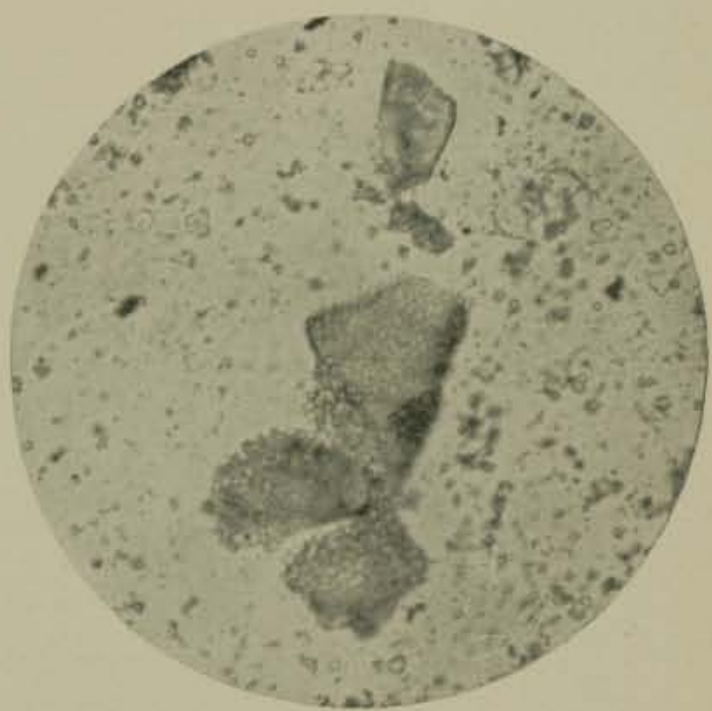

Fig. 260.-Adulterated Pepper, $X 130$.

The large, lower mass shows buckwheat starch, while the finer-grained mass near the top is of pepper. 


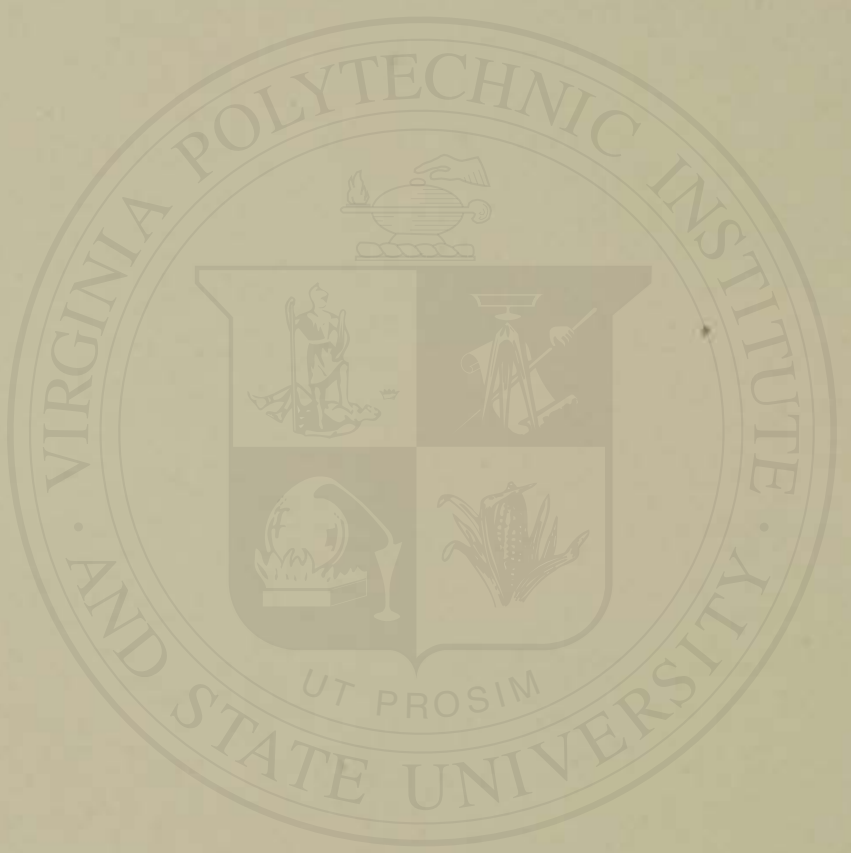


PLATE XXXVI.

\section{SPICES. SPICE ADULTERANTS.}

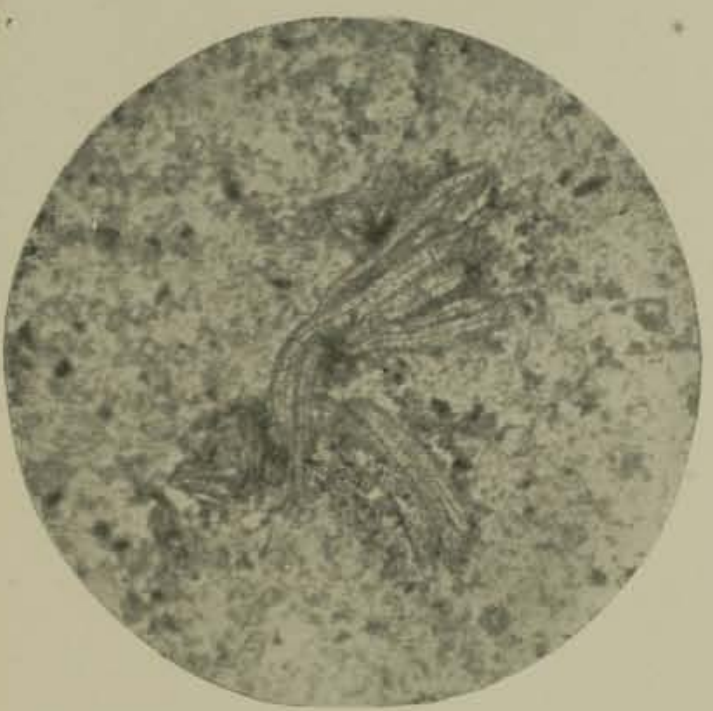

Fig. 261.-Adulterated Pepper, $X 110$.

The central mass shows the sclerenchyma cells of olive stones.

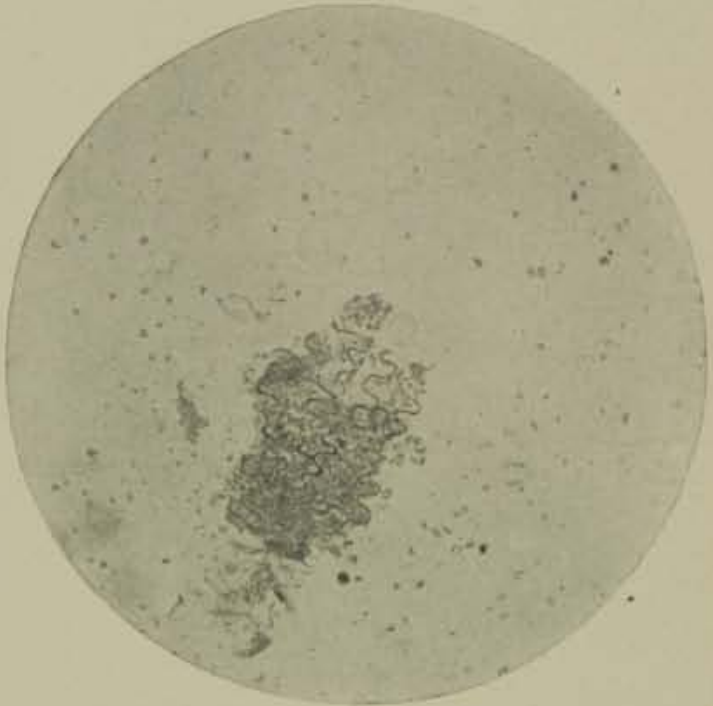

Fic. 262.-Adulterated Pepper, XI30.

Cayenne and wheat starch are the adulterants.

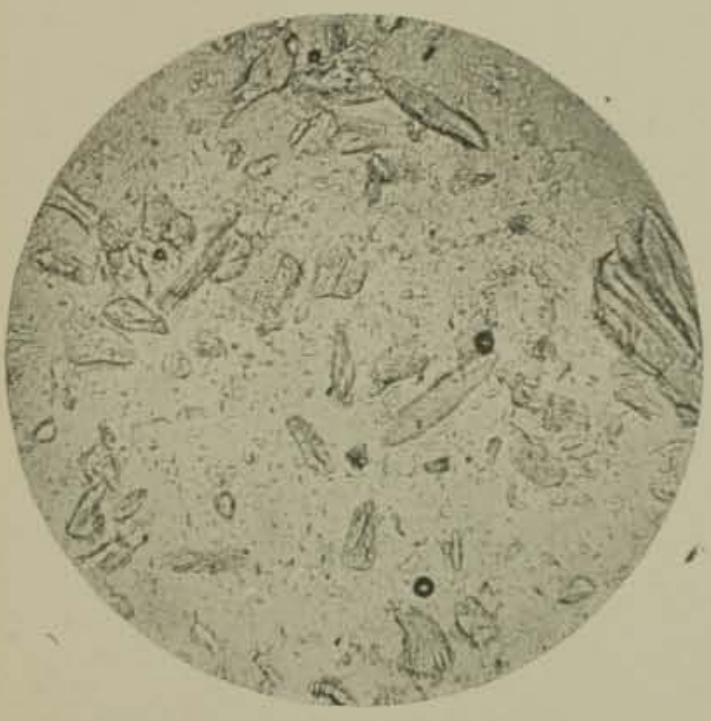

FrG. 263.-Powdered Olive Stones, Xrro.

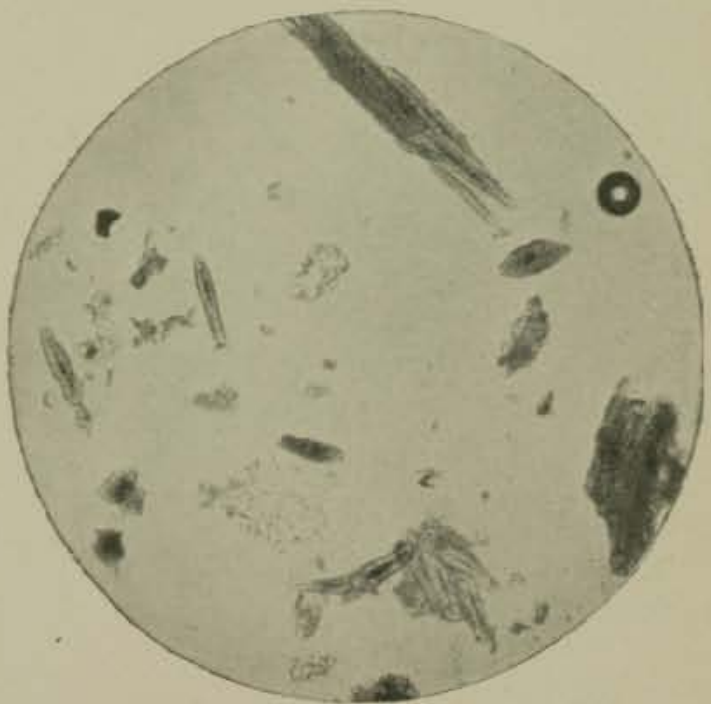

FrG. 264.--Powdered Cocoanut Shells, Xrro 


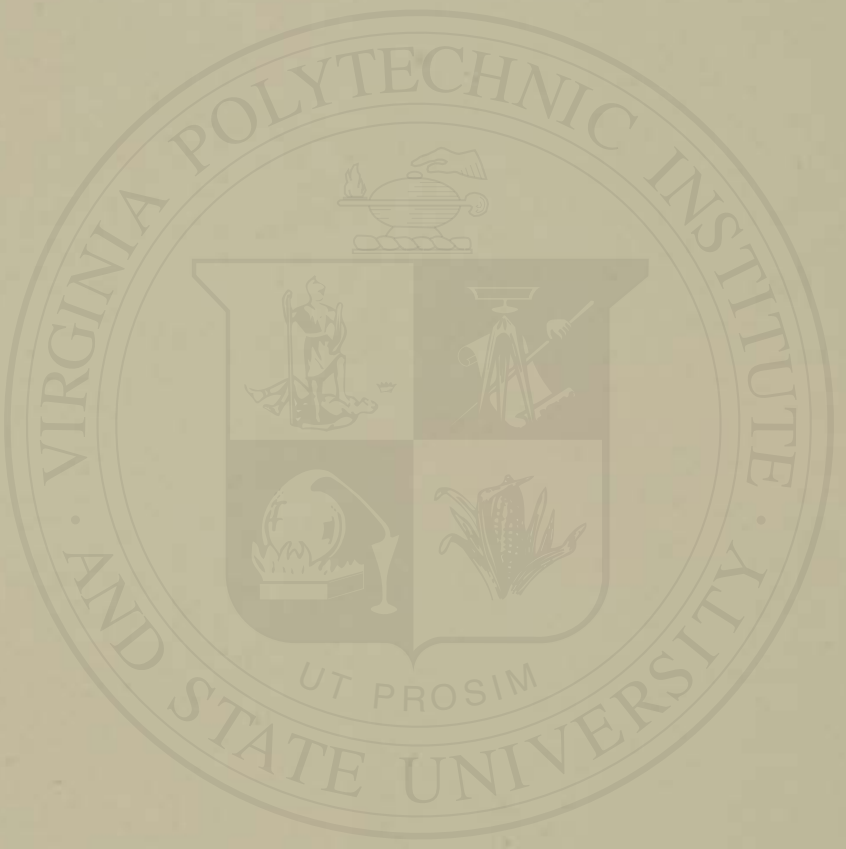




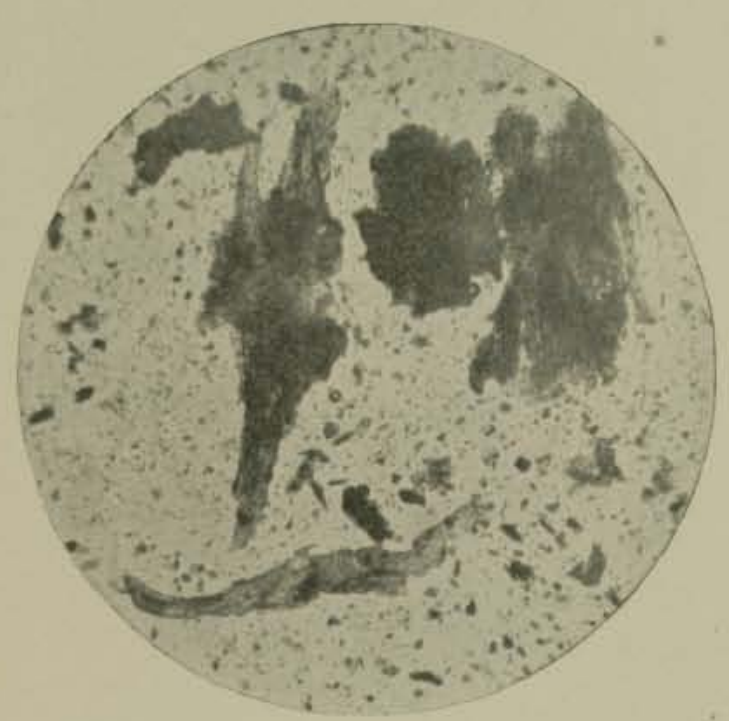

Fig. 265 .-Powdered Elm Bark, X110.

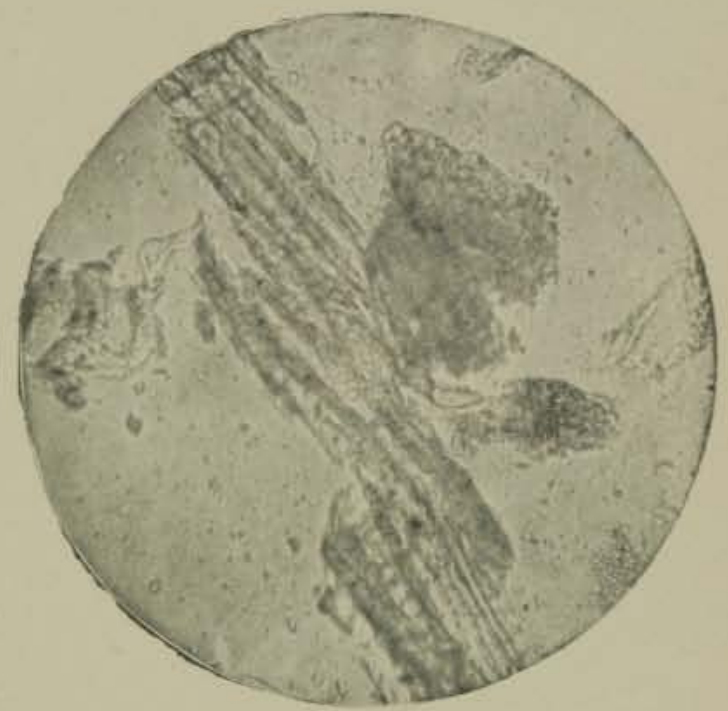

FiG. 266.-Pine Sawdust, $\times 110$. Finely ground.

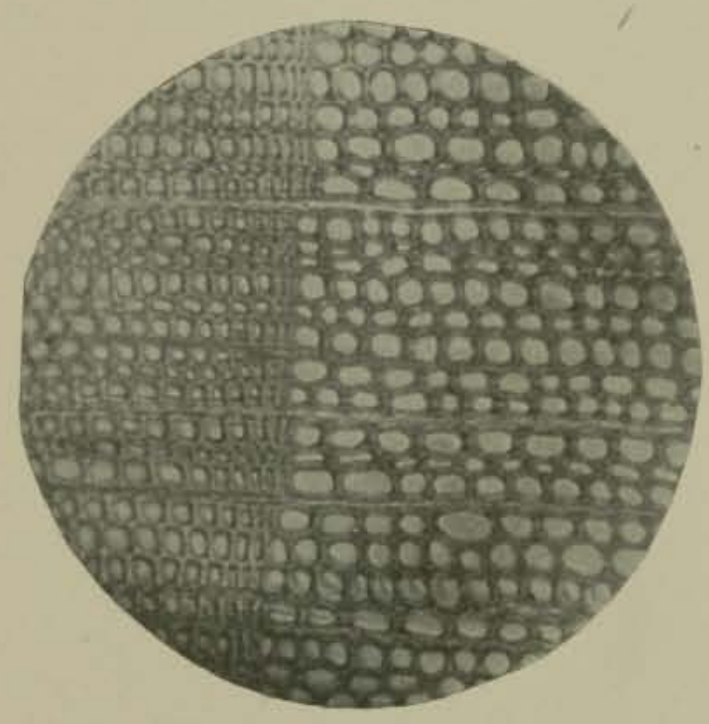

Fig. 267.-Pine Wood, X110. Transverse section.

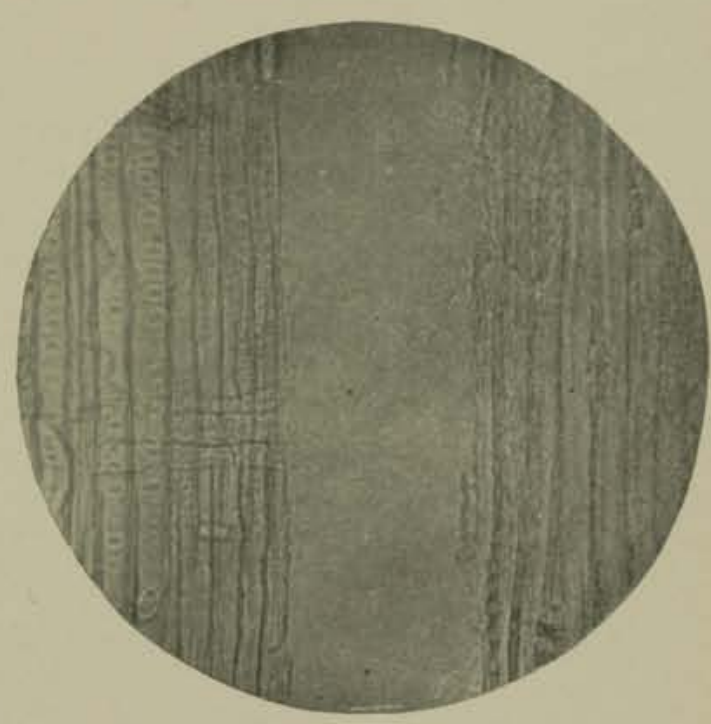

Fic. 268.-Pine Wood, Xrio. Radial and tangential sections. 


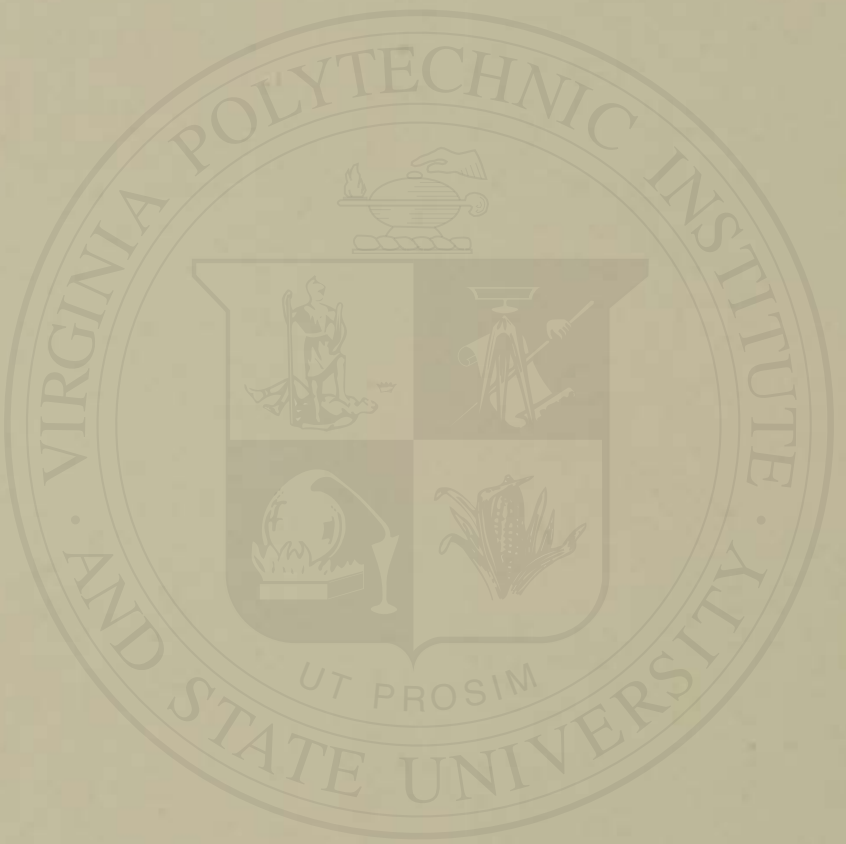


EDIBLE FATS.

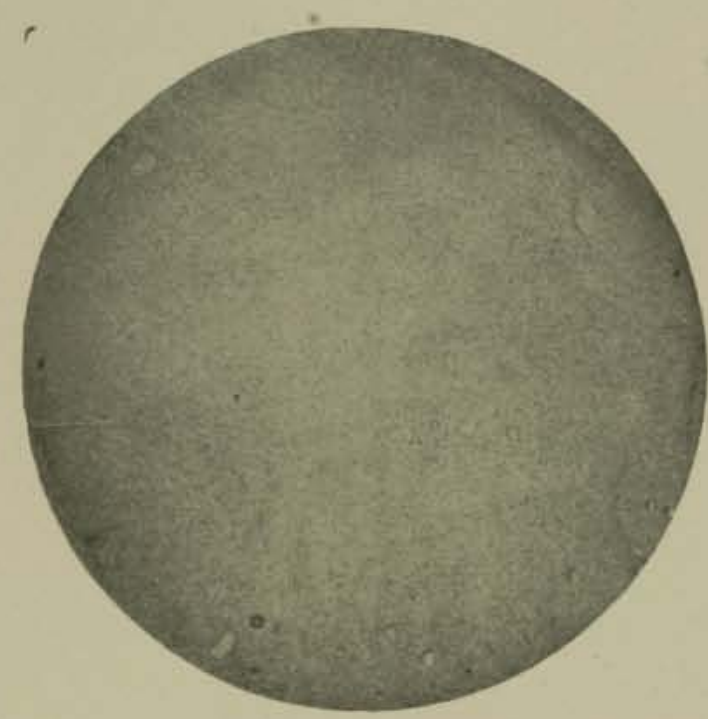

Fig. 260.-Pure Butter, $X_{25}$.

With polarized light and selenite plate.

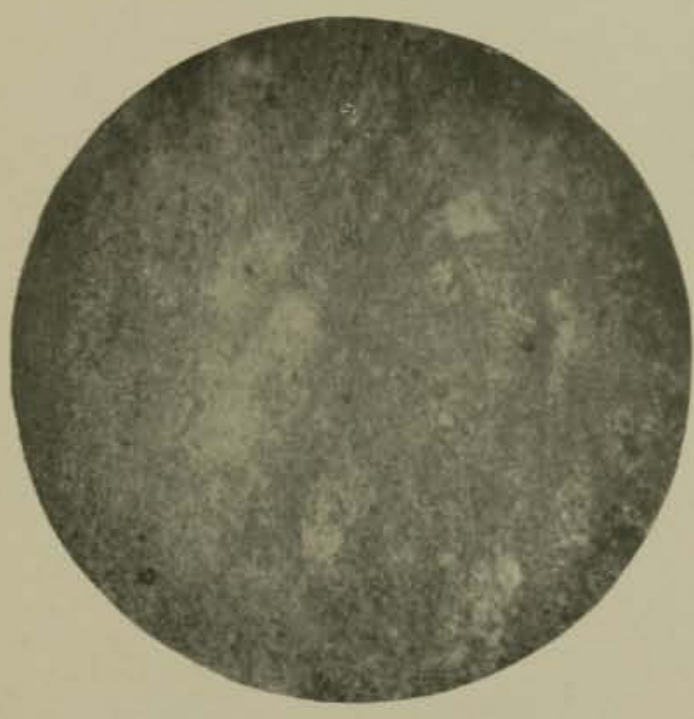

FiG. 270.-Process or Renovated Butter, X25. With polarized light and selenite plate.

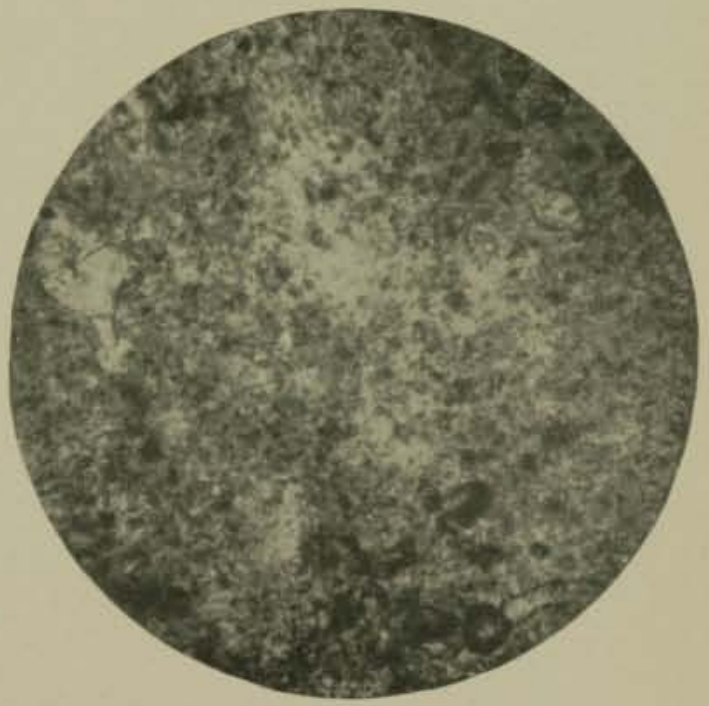

FiG. 271 ,-Oleomargarine, $X_{25}$.

With polarized light and selenite plate. 


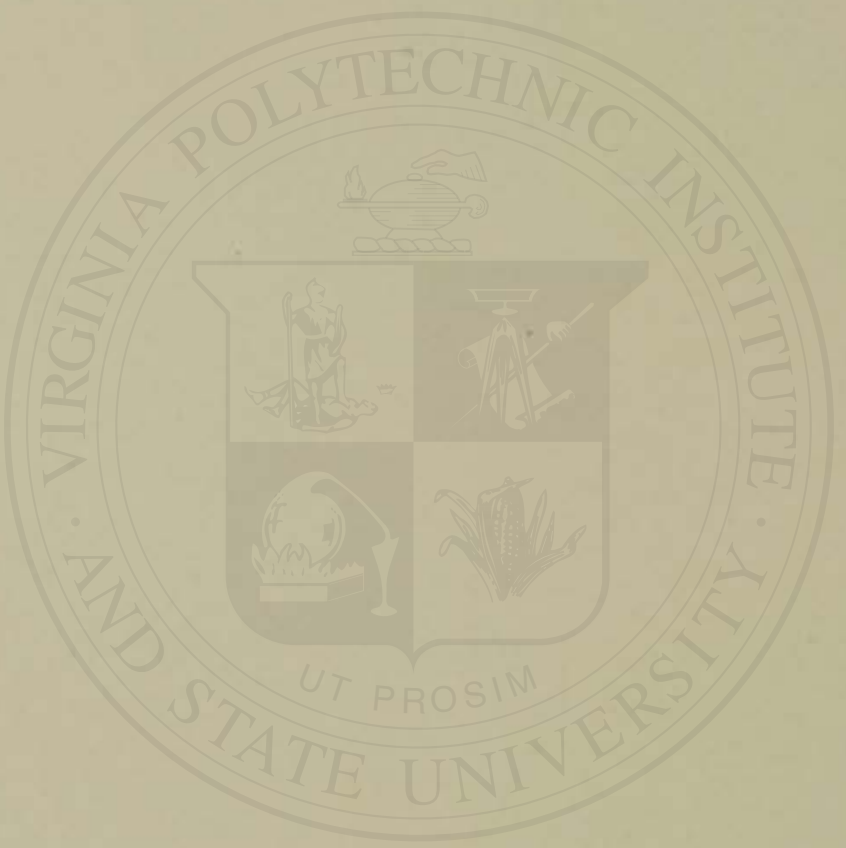


PLATE XXXIX.

\section{EDIBLE FATS.}

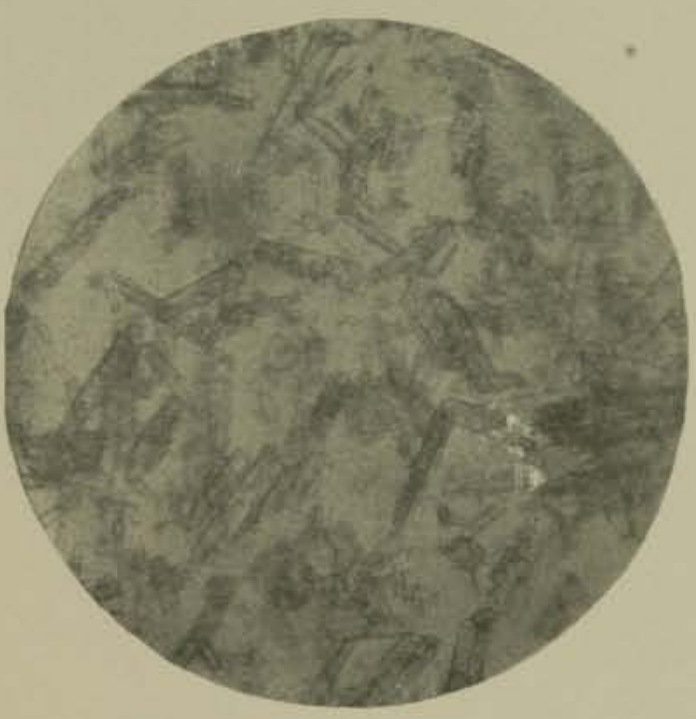

Fic. 272,-Lard Stearin, $X 110$.

I.eaf lard, crystallized from ether.

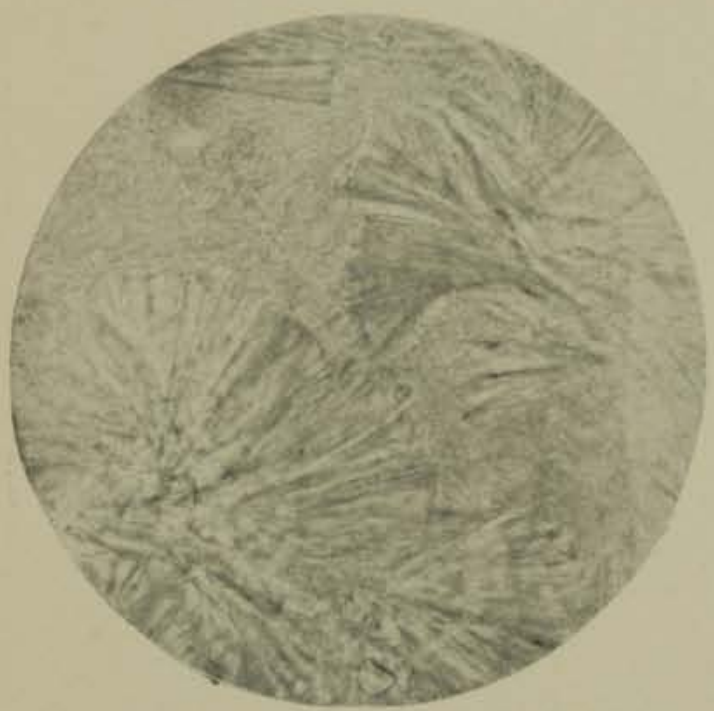

Fig, 274.-Lard Stearin, $\times 220$.

"Back" lard, crystallized from ether.

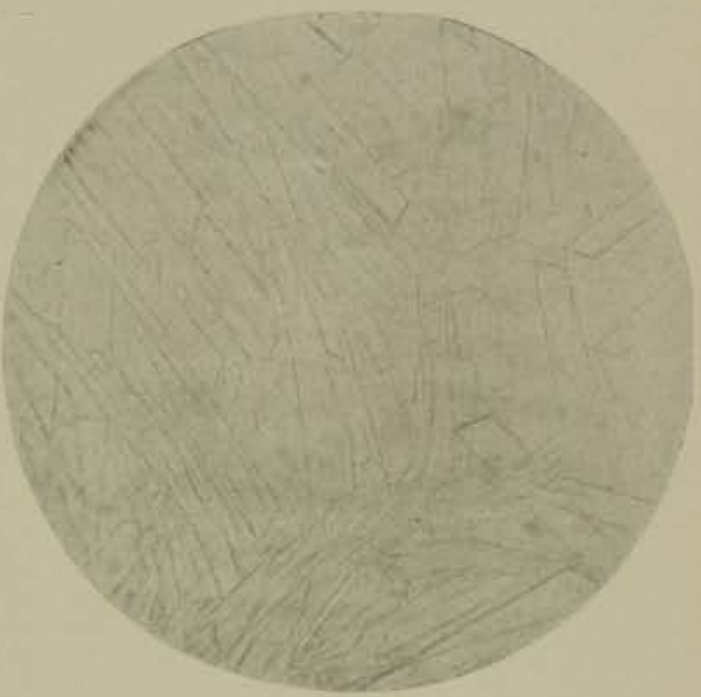

Fic. 273,-Lard Stearin, $\times 220$.

Leaf lard, crystallized from ether.

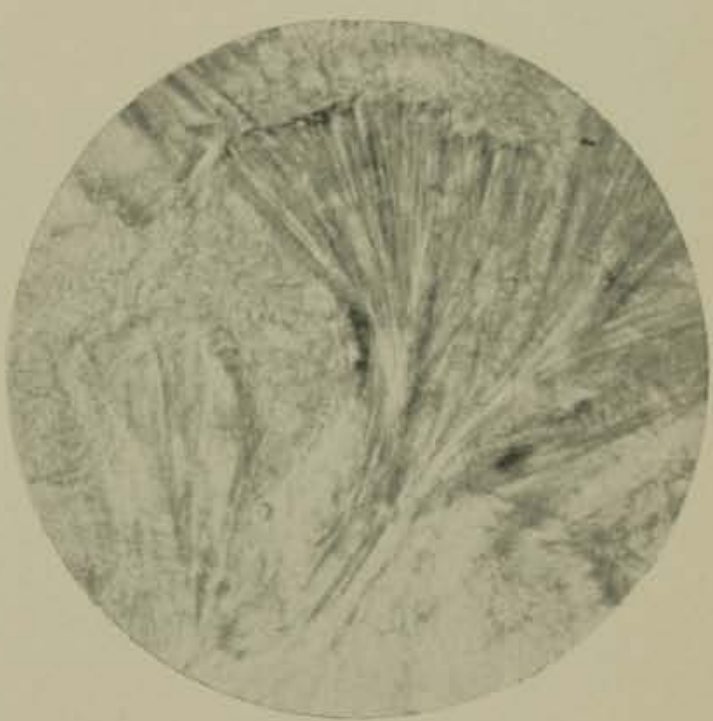

Fig, 275.-Lard Stearin, $X_{4} 80$.

"Back" lard, crystallized from ether. 


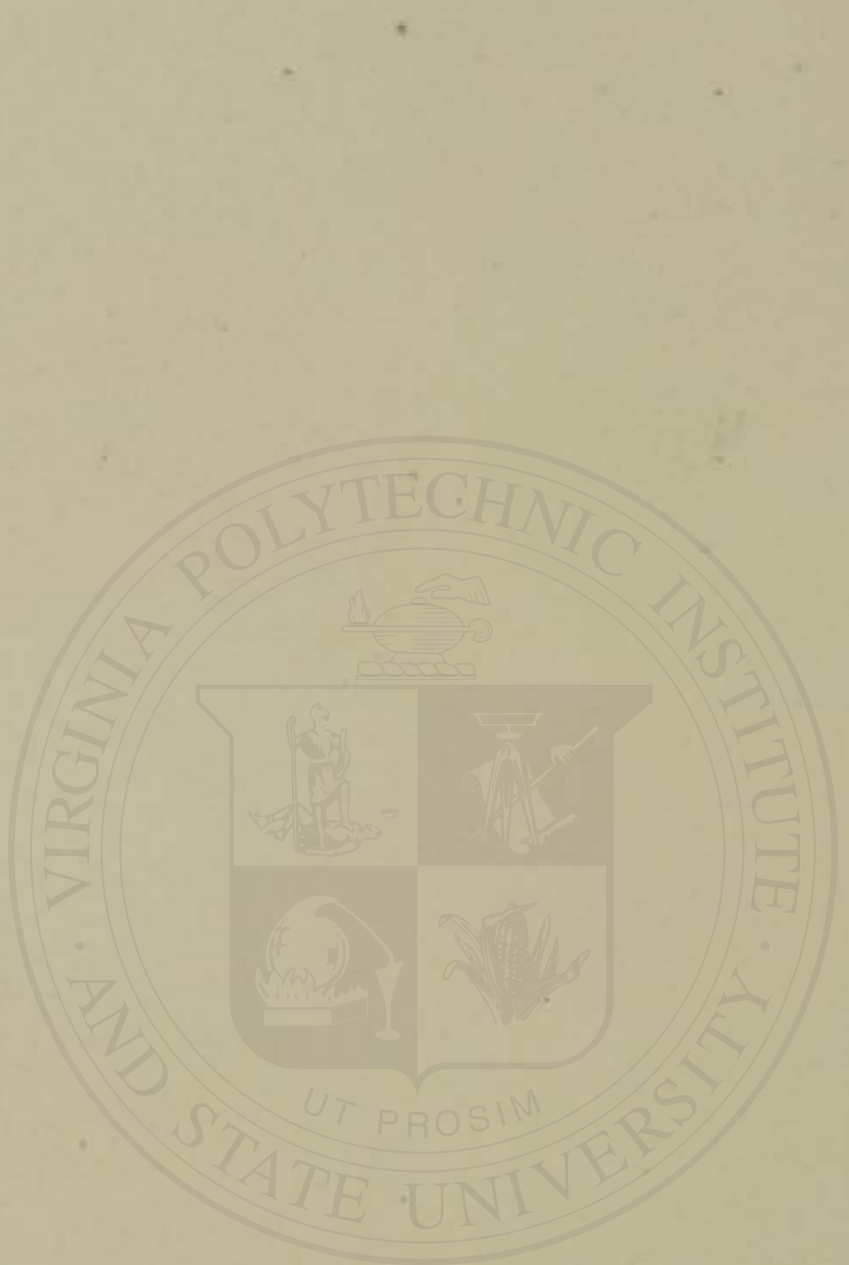


EDIBLE FATS.

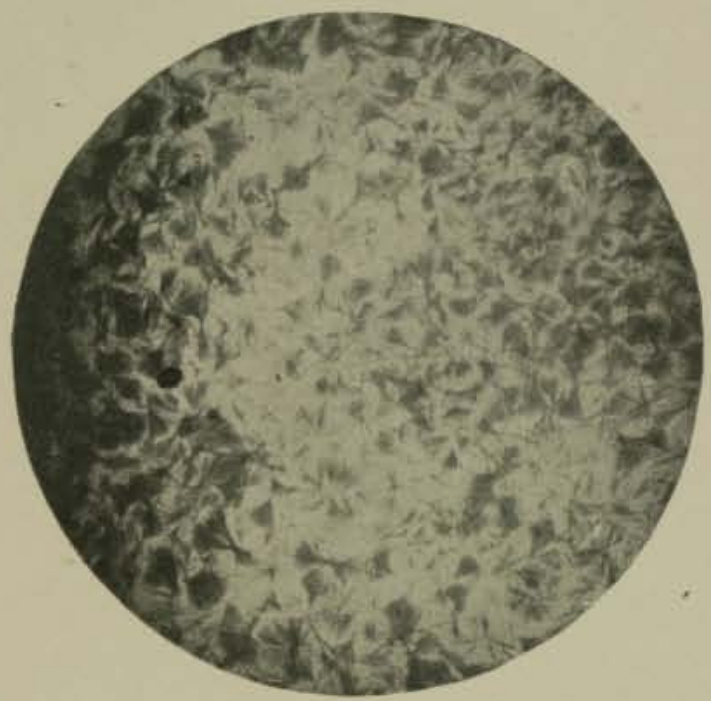

Fig. 276.-Beef Stearin, $\times 35$.

Crystallized from ether.

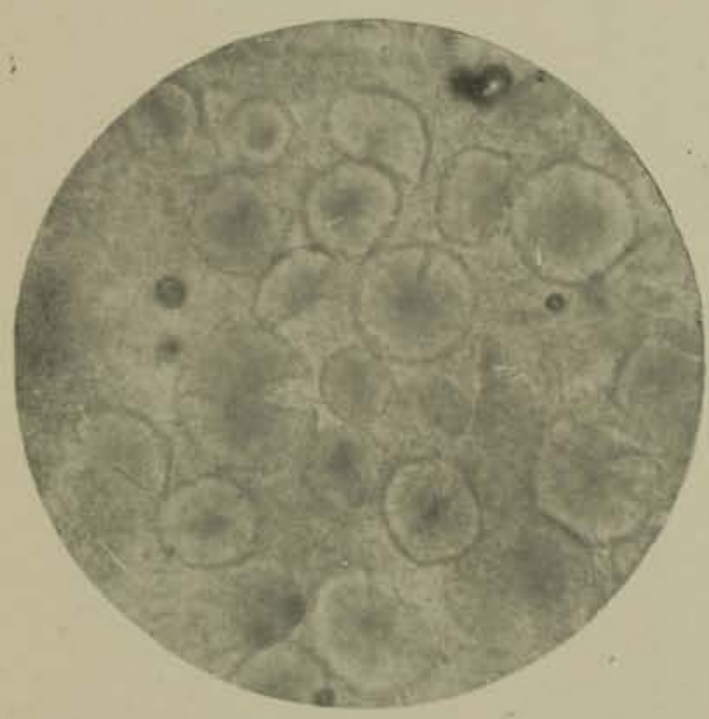

Fic. 277:-Beef Stearin, $X_{110}$ Crystallized from ether.

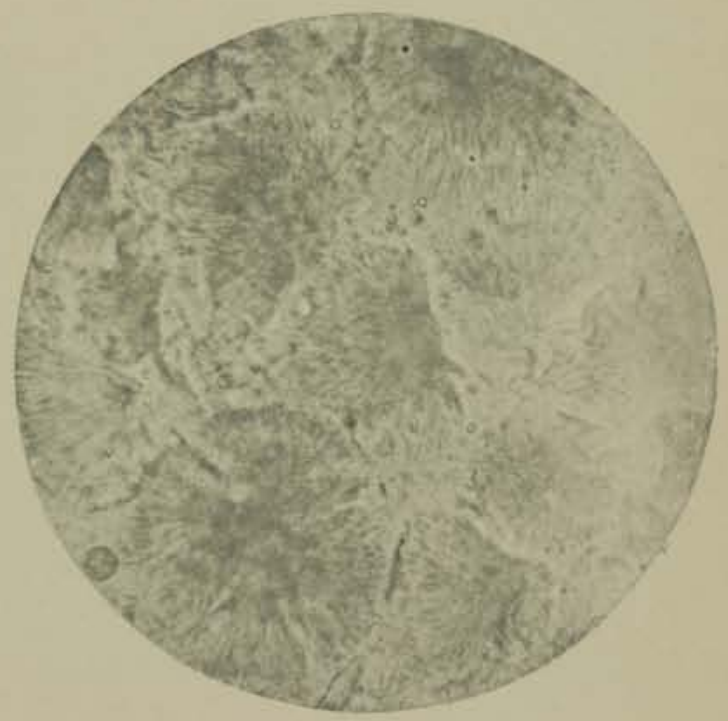

Fig. $278 .-$ Beef Stearin, $\times 220$. Crystallized from etticr. 


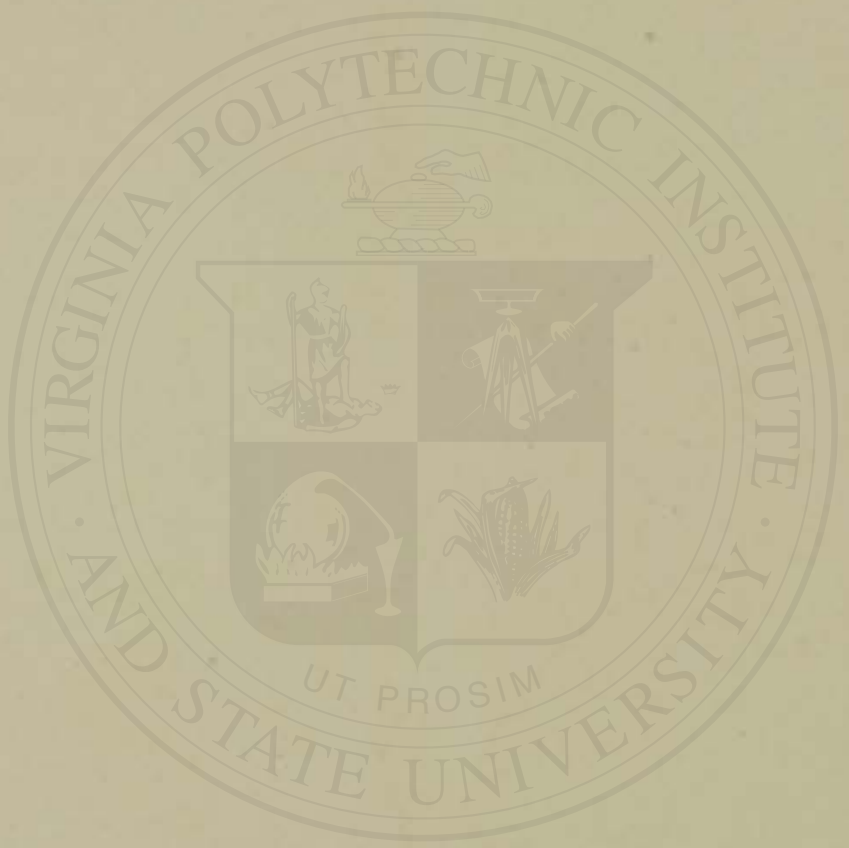




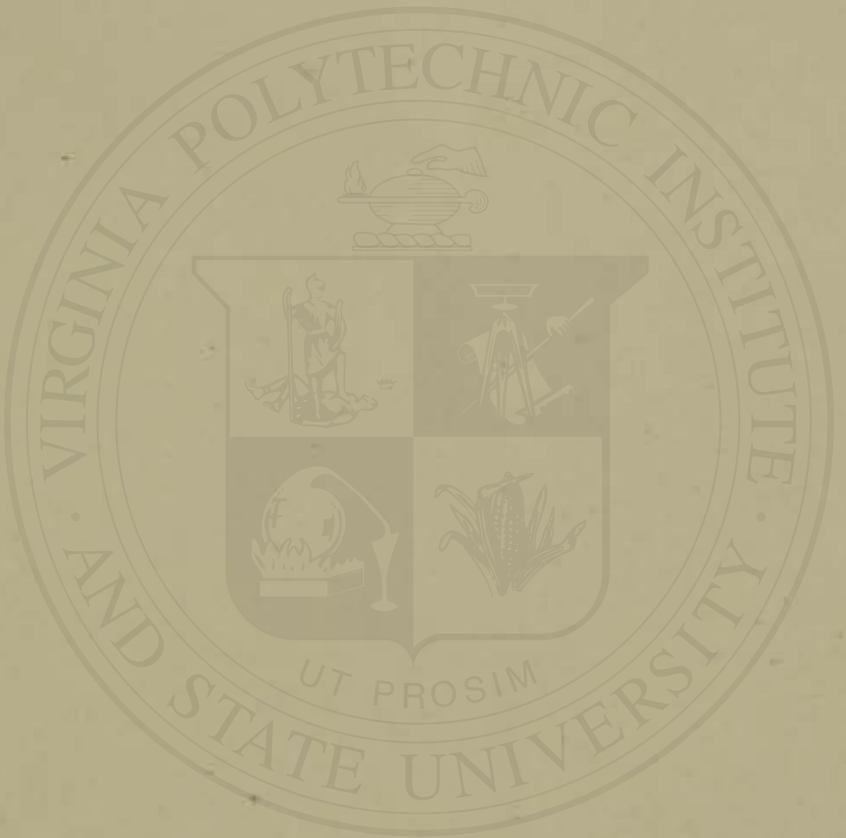






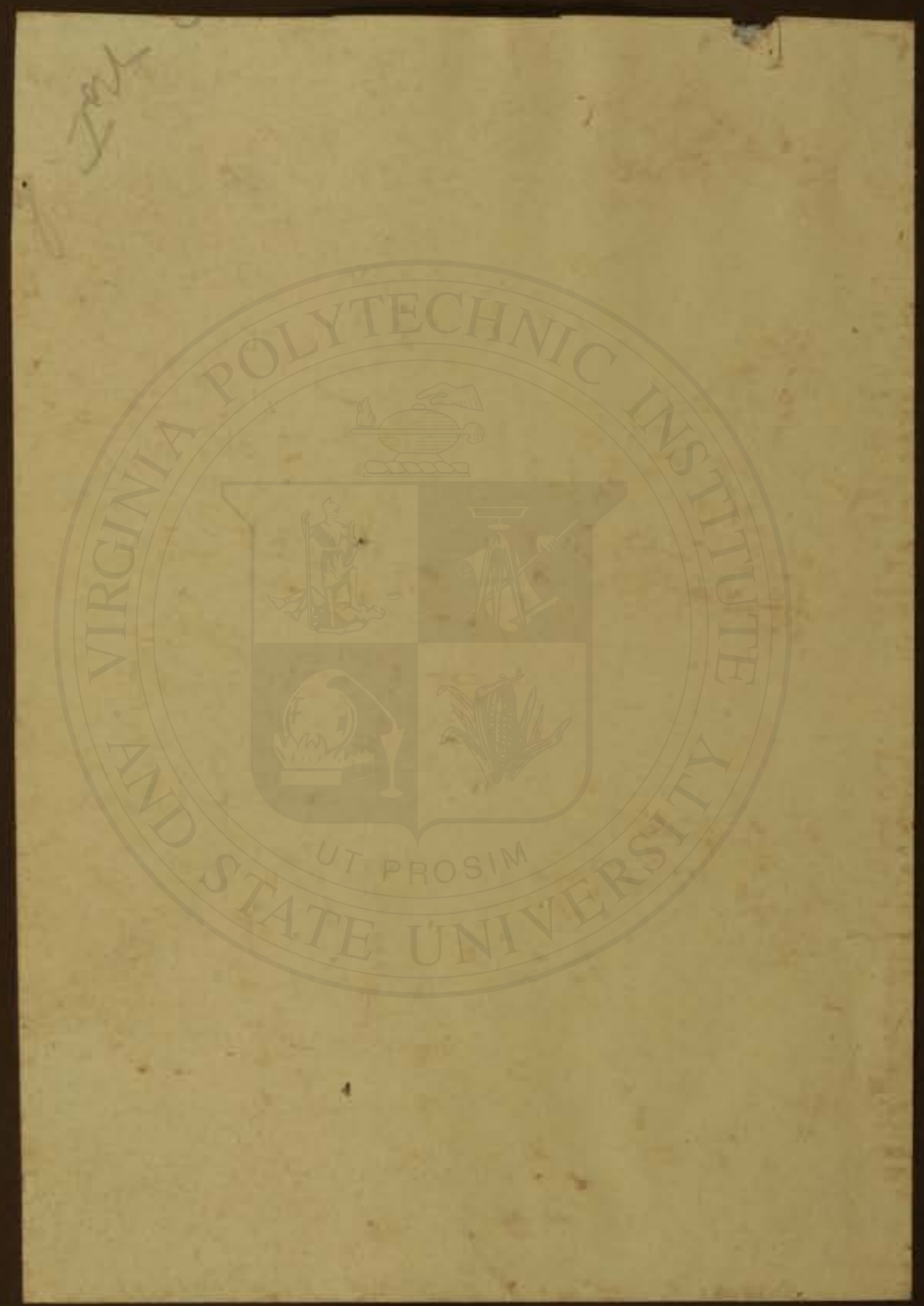



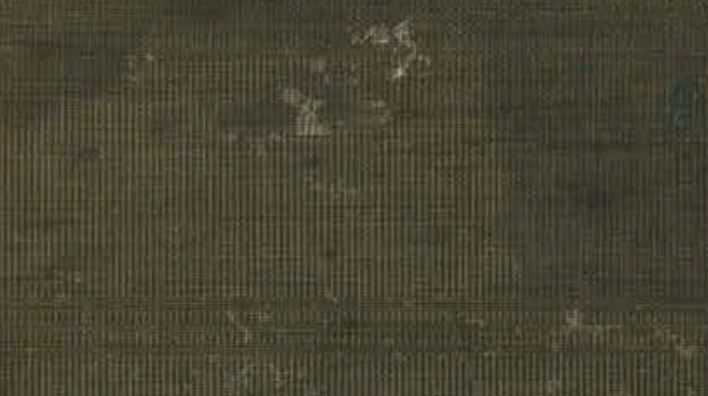

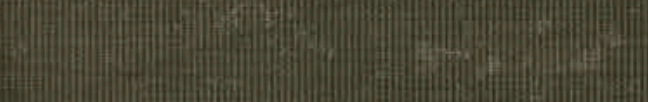

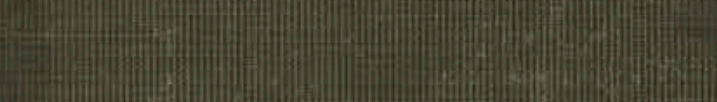

${ }^{5-\infty}$

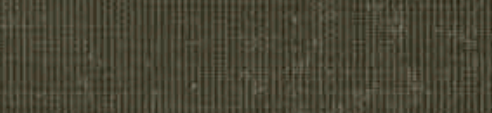

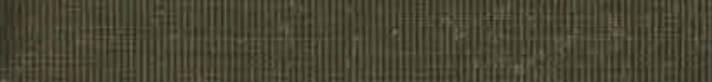

-

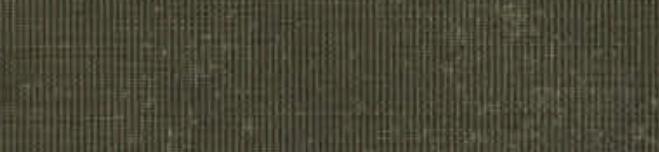

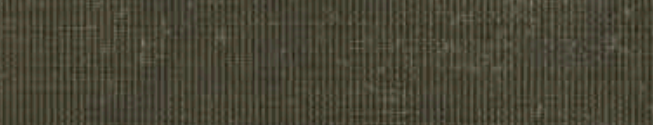

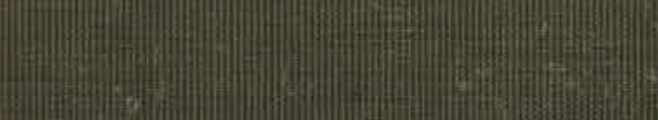

-

${ }^{2}$

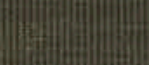

(3)

$(-)^{-(x)}$

(1)

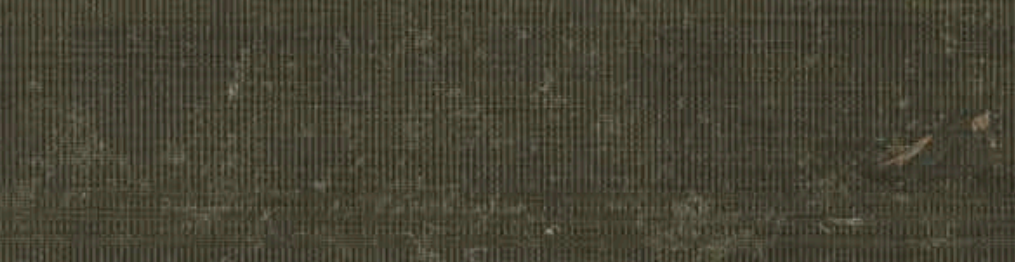

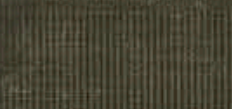

$\frac{1}{4}$

-

(i)

(y)

and 